
UNITED STATES DEPARTMENT OF COMMERCE - Luther H. Hodges, Secretary NATIONAL BUREAU OF STANDARDS - A. V. Astin, Director

\section{Tables of Electron Radial Functions and Tangents of Phase Shifts for Light Nuclei $(Z=1$ through 10$)$}

C. P. Bhalla

This publication sponsored by the National Standard Reference Data Center at the National Bureau of Standards

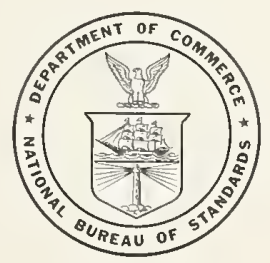

National Bureau of Standards Monograph 81 Issued August 6, 1964 


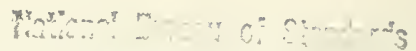

SEP $\triangle 1964$

123,294

QCIOO

- L 556

cop. 2

Library of Congress Catalog Number: 64-60017 


\section{Contents}

I. Int roduction

II. Formulation of the problem ....... vi

III. Computational procedure ............... viii

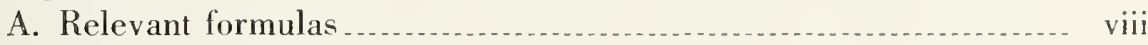

B. Numerical approximations ........... ix

IV. Explanation of the tables _...........

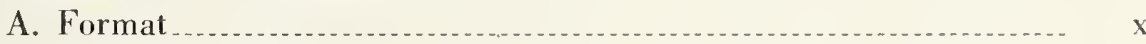

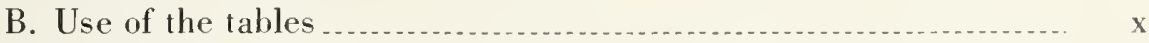

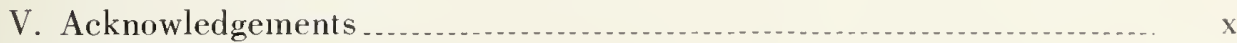

VI. References _.......

VII. Tables .......... 1

$f_{\kappa}, g_{\kappa}, \tan \delta_{\kappa}$ for $\kappa= \pm 1$ and $\kappa= \pm 2$ corresponding to a uniform charge distribution inside a nucleus of radius $\rho$, and $F_{0}$ for a point-nucleus in the following cases:
A. Electrons, $\rho=1 \cdot 2 A^{1 / 3} 10^{-13} \mathrm{~cm}$ and $Z=1$ through 10
B. Positrons, $\rho=1.2 A^{1 / 3} 10^{-13} \mathrm{~cm}$ and $Z=1$ through $10 \ldots \ldots \ldots$
C. Electrons, $\rho=1.3 A^{1 / 3} 10^{-13} \mathrm{~cm}$ and $Z=6$ and $7 \ldots \ldots \ldots 28$
D. Positrons, $\rho=1.3 A^{1 / 3} 10^{-13} \mathrm{~cm}$ and $Z=6$ and $7 \ldots \ldots \ldots \ldots$
E. Electrons, $\rho=1.1 A^{1 / 3} 10^{-13} \mathrm{~cm}$ and $Z=6$ and $7 \ldots \ldots \ldots \ldots \ldots$
F. Positrons, $\rho=1 \cdot 1 A^{1 / 3} 10^{-13} \mathrm{~cm}$ and $Z=6$ and $7 \ldots \ldots \ldots \ldots \ldots$ 



\title{
Tables of Electron Radial Functions and Tangents of Phase Shifts for Light Nuclei $(Z=1$ through 10$)$
}

\author{
C. P. Bhalla*
}

\begin{abstract}
To facilitate the theoretical analyses of beta-decay experiments in light nuclei electronic radial wave functions, evaluated at the nuclear radius, and tangents of phase-shifts are tabulated for total angular momentum equal to $1 / 2$ and $3 / 2$. Separate tables for electrons and positrons are given for ten values of $Z$, starting from $Z$ equal to one, in steps of unity and for beta momentum values from 0.1 mc to $42.0 \mathrm{mc}$ in steps of $0.1 \mathrm{mc}$. The nucleus is represented as a sphere with uniform charge distribution. The nuclear radius, $\rho$, is taken to be $1.2 A^{1 / 3} 10^{-13} \mathrm{~cm}$ in the major body of these tables. However, additional tables for $Z=6$ and $Z=7$ are given for $\rho=1.1 A^{1 / 3} 10^{-13} \mathrm{~cm}$ and $\rho=1.3 A^{1 / 3} 10^{-13} \mathrm{~cm}$.
\end{abstract}

\section{Introduction}

For the analyses of beta-decay experiments one needs to know electronic radial functions, $f_{\kappa}$ and $g_{\kappa}$, and some appropriate combinations of sines and/or cosines of the phase shift, $\delta_{\kappa}$, (the total angular momentum, $j$, being equal to $|\kappa|-1 / 2$ ). In this Monograph $f_{\kappa}, g_{\kappa}$, and $\tan \delta_{\kappa}$ for $\kappa= \pm 1$ and $\kappa= \pm 2$ are tabulated by assuming a smeared charge distribution inside a nucleus. The Fermi function. $F_{0}$. for the Coulomb field potential of a point nucleus is also tabulated. $Z$-values range from 1 through 10 with beta momentum in me units varying from 0.1 to a maximum value of 42.0 at intervals of 0.1 .

From time to time several investigators have made available tables for the analyses of beta-decay experiments. The first such extensive tables were published by the National Bureau of Standards [1 ${ }^{1}$ in 1952. A Coulomb potential of a point-charge nucleus was used. The Fermi functions were tabulated by using the nuclear radius, $\rho$, equal to $1.37 A^{1 / 3} 10^{-13} \mathrm{~cm}$. In subsequent years Rose and his collaborators $[2,3,4]$ made significant improvements over the original NBS publication. For example, Rose, Perry, and Dismuke [2] published tables to include the forbidden beta transitions. The effects arising from the finite de Broglie wavelength [3] were incorporated by considering the contribution of higher order terms in the evaluation of the confluent hypergeometric function. This confluent hypergeometric function occurs in the expression for the relativistic radial function in the case of a Coulomb potential for a point-nucleus. In the calculations of Rose et al., [2] $\rho$ was taken to be equal to $1.4 A^{1 / 3} 10^{-13} \mathrm{~cm}$. These tables, due to Rose, Perry, and Dismuke, were used for the analyses of beta-shape factors but were not adequate for the analyses of $\beta-\gamma$ correlation experiments. At about the same time, Reitz [5] was the first to report on extensive numerical calculations of Fermi functions with atomic screening potential and for a point nucleus. Significant corrections were found for low energy posi- trons. Fortunately, however, because of the smallness of the statistical factor for such low energy $\left(\sim 1.01 \mathrm{mc}^{2}\right)$ positrons the screening corrections can safely be ignored in most calculations of the Fermi functions.

As it is well known, the variety of the experiments in beta decay increased by orders of magnitude when the prediction of parity violation by Lee and Yang [6] was confirmed experimentally [7]. In the analyses of the postparity experiments, new combinations of $f_{\kappa}, g_{\kappa}$, and $\tan \delta_{\kappa}$ are required. All of the earlier tables on beta-decay functions were inadequate for this purpose. Furthermore, it turns out that some of the radial functions are very sensitive to the finite nuclear size effects which arise from the fact that the nucleus is better represented by a smeared charge distribution than by a point nucleus. This was pointed out by Rose [8]. Recognizing a need for accurate tabulated functions, Bhalla and Rose [9] prepared tables of $f_{\kappa}, g_{\kappa}$, and $\tan \delta_{\kappa}$ for $\kappa= \pm 1$ and $\kappa= \pm 2$ for a finite nuclear charge distribution. Numerical results correspond to $\rho=1.2 A^{1 / 3} 10^{-13} \mathrm{~cm}$. The extensive tables of Bhalla and Rose did not extend below $Z=10$. and to high beta momentum values. This Monograph is intended to fill this need.

This Monograph, supplemented by ORNL-3207 [9], should eliminate completely the need for use of approximate formulas (e.g. those of Kotani and Ross [10]). This is especially so because accurate beta-decay functions can be conveniently calculated in as little time as would be required by the use of approximate formulas. Furthermore. it turns out that in the empirical evaluation of nuclear matrix elements [11] the use of accurate beta-decay functions is most desirable.

The formulation of the problem has been summarized in sec. II. This is followed in sec. 1II by a discussion of the methods used in the numerical calculations. Sec. IV contains a detailed explanation to facilitate the use of the present tables.

*Present address: Westinghouse Electric Corporation, Atomic Power Division, Piltsburgh 30, Pa.

' Figures in brackets indicate the titerature references on page $\boldsymbol{X}$. 


\section{Formulation of the Problem}

The relevant theory has already been described in the literature $[12,4]$. For the sake of completeness and reference, we describe the general formalism.

Throughout this work we use the rationalized units: $\nvdash=m=c=1$. We express the solution of the Dirac equation,

$$
\begin{gathered}
{[-\alpha \cdot \mathbf{p}-\beta+V(r)] \psi_{\kappa}^{\mu}=W \psi_{\kappa}{ }^{\mu}} \\
\psi_{\kappa}^{\mu}=\left(\begin{array}{c}
-i f_{\kappa}(r) \chi_{-\kappa}^{\mu} \\
g_{\kappa}(r) \chi_{\kappa}^{\mu}
\end{array}\right)
\end{gathered}
$$

where $\chi_{\kappa}^{\mu}$ is the familiar spin angular function [13]:

$$
\chi_{\kappa}^{\mu}=\sum_{\tau} C\left(l_{\kappa} \frac{1}{2} j ; \tau, \mu-\tau\right) \chi_{\mathbf{i} / 2}^{\tau} Y_{l_{\kappa}}^{\mu-\tau}
$$

$V(r)$ is the potential energy of an electron in the field of a uniform charge distribution, i.e.:

$$
\begin{aligned}
& V(r)=-\frac{\alpha Z}{2 \rho}\left[3-(r / \rho)^{2}\right] \text { for } r<\rho \\
& V(r)=-\frac{\alpha Z}{r} \text { for } r>\rho
\end{aligned}
$$

The nuclear radius, $\rho$, is in units of $\hbar / \mathrm{mc} . W$ is energy in $\mathrm{mc}^{2}$ units. The fine structure constant, $\alpha$, is equal to $(1 / 137.037)$. The subscript $\kappa$, which can be a positive or negative integer but never equal to zero, specifies the total angular momentum, $j$, and the orbital angular momentum, $l$.

$$
\begin{aligned}
j & =|\kappa|-1 / 2 \\
l & =\kappa \text { for } \kappa>0 \\
\text { and } \quad l & =-(\kappa+1) \text { for } \kappa<0 .
\end{aligned}
$$

Normalization of $f_{\kappa}(r)$ and $g_{\kappa}(r)$ was taken to correspond to one particle in a sphere of unit radius. The asymptotic behavior of the radial functions is given by

$$
\begin{gathered}
r f_{\kappa} \rightarrow-\left(\frac{W-1}{W}\right)^{1 / 2} \sin \left(p r+\frac{\alpha Z W}{p} \ln 2 p r+\delta_{\kappa}\right) \\
r g_{\kappa} \rightarrow\left(\frac{W+1}{W}\right)^{1 / 2} \cos \left(p r+\frac{\alpha Z W}{p} \ln 2 p r+\delta_{\kappa}\right) \\
W=\left(p^{2}+1\right)^{1 / 2}
\end{gathered}
$$

In eqs $(5) \delta_{\kappa}$ represents the phase shift, exclusive of the $\log$ term. The radial functions $f_{\kappa}(r)$ and $g_{\kappa}(r)$ obey two coupled first order equations:

$$
\left.\left.\frac{d}{d r}\left[r f_{\kappa}(r)\right]=\frac{\kappa}{r}\left[r f_{\kappa}(r)\right]-\mid W-\right]-V(r)\right]\left[r g_{\kappa}(r)\right]
$$

$$
\frac{d}{d r}\left\{r g_{\kappa}(r)\right]=[W+1-V(r)]\left[r f_{\kappa}(r)\right]-\frac{\kappa}{r}\left[r g_{\kappa}(r)\right]
$$

We represent by $F_{\kappa}(r) / r$ and $G_{\kappa}(r) / r$ the (regular) radial functions for a point nucleus. The solution of eq (1) in our notation with $V(r)=-\frac{\alpha Z}{r}$ for $r \geqslant 0$ is:

$$
\Psi_{\kappa}^{\mu}=\left(\begin{array}{l}
-\frac{i F_{\kappa}(r)}{r} \chi_{-\kappa}^{\mu} \\
\frac{G_{\kappa}(r)}{r} \chi_{\kappa}^{\mu}
\end{array}\right)
$$

The asymptotic behavior of $F_{\kappa}(r)$ and $G_{\kappa}(r)$ is given by eqs (5) with $\delta_{\kappa}$ replaced by $\delta_{\kappa}^{\prime}$, where [13]

$$
\delta_{\kappa}^{\prime}=-\arg \Gamma\left(\gamma_{\kappa}+i y\right)+\eta_{\kappa}-1 / 2 \pi \gamma_{\kappa}
$$

We use the standard notation:

$$
\begin{gathered}
\gamma_{\kappa}=\left[\kappa^{2}-(\alpha Z)^{2}\right]^{1 / 2} \\
y=\alpha Z W / p \\
e^{2 i \eta_{\kappa}}=-\frac{(\kappa-i \alpha Z / p)}{\left(\gamma_{\kappa}+i y\right)}
\end{gathered}
$$

The irregular solution for the Coulomb potential of a point source is denoted by $\bar{F}_{\kappa}(r)$ and $\bar{G}_{\kappa}(r)$, which can be obtained by replacing $\gamma_{\kappa}$ by $-\gamma_{\kappa}$ in the expressions for $F_{\kappa}(r)$ and $G_{\kappa}(r)$ respectively. Following Rose [13], we have

$$
\left(\begin{array}{l}
F_{\kappa}(r) \\
G_{\kappa}(r)
\end{array}\right)=\frac{(1 \mp W)^{1 / 2}}{W^{1 / 2}}(2 p r)^{\gamma \kappa} e^{\pi y / 2} \frac{\left|\Gamma\left(\gamma_{\kappa}+i y\right)\right|}{\Gamma\left(2 \gamma_{\kappa}+1\right)}
$$

$1 / 2\left\{\begin{array}{c}e^{-i p r+i \eta}\left(\gamma_{\kappa}+i y\right) \\ { }_{1} F_{1}\left(\gamma_{\kappa}+1+i y, 2 \gamma_{\kappa}+1 ; 2 i p r\right) \mid \mp \text { c.c. }\end{array}\right\}$

where the upper and lower signs refer to $F_{\text {. }}(r)$ and $G_{\kappa}(r)$ respectively. For $F_{\kappa}(r)$ we have ! $-W)^{1 / 2}=+i(W-1)^{1 / 2}$. The confluent hypergeometric function can be represented by the following series:

$$
{ }_{1} F_{1}(a, b, z)=\frac{\Gamma(b)}{\Gamma(a)} \sum_{m=0}^{\infty} \frac{\Gamma(a+m)}{\Gamma(b+m)} \frac{z^{m}}{m !}
$$

over the entire complex plane $|z|<\infty$. We repre- 
sent by $\frac{F_{\kappa}^{i}(r)}{r}$ and $\frac{G_{\kappa}^{i}(r)}{r}$ the solution of the Dirac equation, eq (1), for $r \leqslant \rho$. The superscript $i$ has been used to denote the radial functions inside the nucleus.

Our definitions imply, then, that

and

$$
\left.\begin{array}{l}
r f_{\kappa}(r)=A_{\kappa} F_{\kappa}^{i}(r) \\
r g_{\kappa}(r)=A_{\kappa} G_{\kappa}^{i}(r)
\end{array}\right\} \text { for } r \leqslant \rho
$$

$$
\left.\begin{array}{r}
r f_{\kappa}(r)=B_{\kappa} F_{\kappa}(r)+C_{\kappa} \bar{F}_{\kappa}(r) \\
r g_{\kappa}(r)=B_{\kappa} G_{\kappa}(r)+C_{\kappa} \bar{G}_{\kappa}(r)
\end{array}\right\} \text { for } r \geqslant \rho
$$

In eqs (11) and (12), $A_{\kappa}, B_{\kappa}$, and $C_{\kappa}$ are parameters to be determined so as to satisfy the conditions of continuity of radial functions at $r=\rho$ and the normalization of $f_{\kappa}(r)$ and $g_{\kappa}(r)$. The normalization corresponding to one particle in a sphere of unit radius gives from eqs (12) and (5):

$$
B_{\kappa}^{2}+C_{\kappa}^{2}+2 B_{\kappa} C_{\kappa} \cos \left(\delta_{\kappa}^{\prime}-\bar{\delta}_{\kappa}^{\prime}\right)=1
$$

where

$$
\begin{aligned}
\delta_{\kappa}^{\prime}-\bar{\delta}_{\kappa}^{\prime}= & -\arg \Gamma\left(\gamma_{\kappa}+i y\right) \\
& +\arg \Gamma\left(-\gamma_{\kappa}+i y\right)+\eta_{\kappa}+\bar{\eta}_{\kappa}-\pi \gamma_{\kappa}
\end{aligned}
$$

Here we have made use of the relevant asymptotic behavior of the Coulomb regular and irregular radial functions.

From now onwards, when we do not give the argument of a function, it will imply that this quantity is evaluated at the nuclear radius i.e. $r=\rho$. The continuity of the radial functions gives from eqs (11) and (12):

$$
A_{\kappa}=\left(\frac{F_{\kappa} \bar{G}_{\kappa}-G_{\kappa} \bar{F}_{\kappa}}{F_{\kappa}^{i} \bar{G}_{\kappa}-G_{\kappa}^{i} \bar{F}_{\kappa}}\right) B_{\kappa}
$$

Similarly, we obtain

$$
C_{\kappa}=\left(\frac{F_{\kappa} G_{\kappa}^{i}-F_{\kappa}^{i} G_{\kappa}}{\bar{G}_{\kappa} F_{\kappa}^{i}-\bar{F}_{\kappa} G_{\kappa}^{i}}\right) B_{\kappa}
$$

For the sake of convenience, we define $H_{\kappa}$ so that

$$
C_{\kappa}=H_{\kappa} B_{\kappa}
$$

where

$$
H_{\kappa}=\left(\frac{F_{\kappa} / G_{\kappa}-F_{\kappa}^{i} / G_{\kappa}^{i}}{F_{\kappa}^{i} / G_{\kappa}^{i}-\bar{F}_{\kappa} / \bar{G}_{\kappa}}\right) G_{\kappa} / \bar{G}_{\kappa}
$$

Substituting for $C_{k}$ (from eq (16)) in eq (13a) gives

$$
B_{\kappa}=\left[1+H_{\kappa}^{2}+2 H_{\kappa} \cos \left(\delta_{\kappa}^{\prime}-\bar{\delta}_{\kappa}^{\prime}\right)\right]^{-1 / 2}
$$

To evaluate $f_{\kappa}(r)$ and $g_{\kappa}(r)$ for $r$ equal to the nuclear radius, $\rho$, one uses the following relations:

and

$$
\rho f_{\kappa}=A_{\kappa} F_{\kappa}^{i}
$$

$$
\rho g_{\kappa}=A_{\kappa} G_{\kappa}^{i}
$$

where $A_{\kappa}$ is given by eq (14). Using eq (14) in eq (20), one gets

$$
g_{\kappa}=B_{\kappa}\left(\frac{F_{\kappa} / G_{\kappa}-\bar{F}_{\kappa} / \bar{G}_{\kappa}}{F_{\kappa}^{i} / G_{\kappa}^{i}-\bar{F}_{\kappa} / \bar{G}_{\kappa}}\right) G_{\kappa} / \rho .
$$

$f_{\kappa}$ can be calculated from the following:

$$
f_{\kappa}=\left(F_{\kappa}^{i} / G_{\kappa}^{i}\right) g_{\kappa}
$$

This, then, completes the derivation for $f_{\kappa}$ and $g_{\kappa}$.

For the calculation of the tangent of the phase shift, $\delta_{\kappa}$, defined in eq (5), we proceed as follows. We make use of eq (12) and the asymptotic behavior of $f_{\kappa}(r), g_{\kappa}(r), F_{\kappa}(r)$, and $G_{\kappa}(r)$. After simplification, we obtain:

$\tan \delta_{\kappa}=\left[\frac{-a_{0}+a_{1} \tan \eta_{\kappa}+H_{\kappa} \frac{\cos \bar{\eta}_{\kappa}}{\cos \eta_{\kappa}}\left(a_{2}+a_{3} \tan \eta_{\kappa}\right)}{a_{1}+a_{0} \tan \eta_{\kappa}+H_{\kappa} \frac{\cos \bar{\eta}_{\kappa}}{\cos \eta_{\kappa}}\left(a_{3}-a_{2} \tan \bar{\eta}_{\kappa}\right)}\right]$

where

$$
\begin{aligned}
& a_{0}=a_{4} \sin \left(\pi \gamma_{\kappa} / 2\right)+a_{5} \cos \left(\pi \gamma_{\kappa} / 2\right) \\
& a_{1}=a_{4} \cos \left(\pi \gamma_{\kappa} / 2\right)-a_{5} \sin \left(\pi \gamma_{\kappa} / 2\right) \\
& a_{2}=a_{6} \sin \left(\pi \gamma_{\kappa} / 2\right)-a_{7} \cos \left(\pi \gamma_{\kappa} / 2\right) \\
& a_{3}=a_{6} \cos \left(\pi \gamma_{\kappa} / 2\right)+a_{7} \sin \left(\pi \gamma_{\kappa} / 2\right)
\end{aligned}
$$

with

$a_{4} \equiv \operatorname{Re} \frac{\Gamma\left(\gamma_{\kappa}+i y\right)}{\left|\Gamma\left(\gamma_{\kappa}+i y\right)\right|}, \quad a_{5} \equiv \operatorname{Im} \frac{\Gamma\left(\gamma_{\kappa}+i y\right)}{\left|\Gamma\left(\gamma_{\kappa}+i y\right)\right|}$

$a_{6} \equiv \operatorname{Re} \frac{\Gamma\left(\gamma_{\kappa}+i y\right)}{\left|\Gamma\left(-\gamma_{\kappa}+i y\right)\right|}, a_{7} \equiv \operatorname{Im} \frac{\Gamma\left(-\gamma_{\kappa}+i y\right)}{\left|\Gamma\left(-\gamma_{\kappa}+i y\right)\right|}$ 


\section{Computational Procedure}

\section{A. Relevant Formulas}

The functions, to be evaluated, are $f_{\kappa}, g_{\kappa}$ at the nuclear radius, $\tan \delta_{\kappa}$ for $\kappa= \pm 1$ and $\kappa= \pm 2$, and the Fermi function, $F_{0}$. We define the Fermi function as follows:

$$
F_{0}=4(2 p \rho)^{2\left(\gamma_{1}-1\right)} e^{\pi y} \frac{\left|\Gamma\left(\gamma_{1}+i y\right)\right|^{2}}{\left(\Gamma\left(1+2 \gamma_{1}\right)\right)^{2}}
$$

It may be noted that our definition of $F_{0}$ differs slightly (by a factor of $\frac{1+\gamma_{1}}{2}$ ) from the definition used by some others.

The relevant equations were derived in sec. II and are given in eqs (21), (22), and (23) with definitions in eqs (18), (13b), and (10). It should be noted that the ratios of radial functions (e.g. $F_{\kappa} / G_{\kappa}$ ) are independent of the choice of a phase convention, and such ratios are readily evaluated in a relatively short computer time. The appropriate ratios of radial functions can be obtained from the coupled first-order equations. (see for example eq (6)). As an illustration, let us consider the evaluation of $F_{\kappa} / G_{\kappa}$. The relevant equations are (for an electron):

$$
\begin{aligned}
& \frac{d F_{\kappa}(r)}{d r}=\frac{\kappa}{r} F_{\kappa}(r)-\left(W-1+\frac{\alpha Z}{r}\right) G_{\kappa}(r) \\
& \frac{d G_{\kappa}(r)}{d r}=\left(W+1+\frac{\alpha Z}{r}\right) F_{\kappa}(r)-\frac{\kappa}{r} G_{\kappa}(r)
\end{aligned}
$$

Making use of the following series expansions we obtain

$$
\begin{gathered}
F_{\kappa}(r)=C_{f} r^{\gamma} \sum_{n=0}^{\infty} S_{n}\left(\frac{r}{\rho}\right)^{n} \\
G_{\kappa}(r)=C_{g} r^{\gamma} \sum_{n=0}^{\infty} T_{n}\left(\frac{r}{\rho}\right)^{n}
\end{gathered}
$$

given the appropriate recurrence relations

$$
\begin{aligned}
S_{n}=-\frac{\alpha Z \rho}{n\left(n+2 \gamma_{\kappa}\right)}\left[\frac{(W-1)\left(\gamma_{\kappa}+n+\kappa\right)}{\kappa+\gamma_{\kappa}}\right. & T_{n-1} \\
& \left.+(W+1) S_{n-1}\right] \\
T_{n}=\frac{\alpha Z \rho}{n\left(n+2 \gamma_{\kappa}\right)}\left[\frac{(W+1)\left(\gamma_{\kappa}+n-\kappa\right)}{\kappa-\gamma_{\kappa}} S_{n-1}\right. & \\
& \left.-(W-1) T_{n-1}\right]
\end{aligned}
$$

with

$$
\frac{C_{f}}{C_{g}}=\frac{\gamma_{\kappa}+\kappa}{\alpha Z}
$$

We take $S_{0}=T_{0}=1$. This leads to

$$
\frac{F_{\kappa}}{G_{\kappa}}=\frac{\gamma_{\kappa}+\kappa}{\alpha Z} \frac{\sum_{n=0}^{\infty} S_{n}}{\sum_{n=0}^{\infty} T_{n}} .
$$

Similarly,

$$
\frac{\bar{F}_{\kappa}}{\bar{G}_{\kappa}}=\frac{-\gamma_{\kappa}+\kappa}{\alpha Z} \frac{\sum_{n=0}^{\infty} \bar{S}_{n}}{\sum_{n=0}^{\infty} \bar{T}_{n}}
$$

where $\bar{S}_{n}$ and $\bar{T}_{n}$ are obtained from eqs (27) by replacing $\gamma$ by $-\gamma$ and setting $\bar{S}_{0}=\bar{T}_{0}=1$.

For $\kappa<0, F_{\kappa}^{i} / G_{\kappa}^{i}$ can be calculated from eq (6) by the substitution:

$$
\begin{aligned}
& F_{\kappa}^{i}(r)=r^{|\kappa|+1} \sum_{n=0}^{\infty} b_{n}(r / \rho)^{2 n} \\
& G_{\kappa}^{i}(r)=r^{|\kappa|} \sum_{n=0}^{\infty} b_{n}^{\prime}(r / \rho)^{2 n}
\end{aligned}
$$

After simplifying, one obtains these recurrence relations:

$$
(2 n+2|\kappa|+1) b_{n}=-\left[W-1+\frac{3 \alpha Z}{2 \rho}\right] b_{n}^{\prime}+\frac{\alpha Z}{2 \rho} b_{n-1}^{\prime}
$$

and

$$
\frac{2 n b_{n}^{\prime}}{\rho^{2}}=\left[W+1+\frac{3 \alpha Z}{2 \rho}\right] b_{n-1}-\frac{\alpha Z}{2 \rho} b_{n-2}
$$

We take

$$
b_{0}^{\prime}=1, \text { and } b_{0}=-\frac{W-1+3 \alpha Z / 2 \rho}{2|\kappa|+1}
$$

Thus.

$$
\frac{F_{\kappa}^{i}}{G_{\kappa}^{i}}=\rho \frac{\sum_{n=0}^{\infty} b_{n}}{\sum_{n=0}^{\kappa} b_{n}^{\prime}} \text { for } \kappa=0
$$

A corresponding expression for $\kappa>0$ can immediately be obtained by interchanging $F_{\kappa}^{i}$ and $G_{\kappa}^{i}$ and by changing the signs of $W$ and $Z$ in eqs (31).

At this stage, we observe that whereas $H_{\kappa}$ and cus $\left(\delta_{\kappa}^{\prime}-\bar{\delta}_{k}^{\prime}\right)$ do depend on any particular choice of phase convention, $H_{\kappa} \cos \left(\delta_{k}^{\prime}-\overline{\delta_{k}^{\prime}}\right)$ is independent 
of these considerations. Consequently, $B_{\kappa}$ does not have any phase ambiguity. In the evaluation of $B_{\kappa}$ we made use of the following relationships:

$$
\frac{G_{\kappa}}{\bar{G}_{\kappa}}=\sigma_{\kappa} \frac{\sum_{n=0}^{\infty} T_{n}}{\sum_{n=0}^{\infty} \bar{T}_{n}}
$$

where

$\sigma_{\kappa} \equiv(2 p \rho)^{2 \gamma} \kappa_{\kappa} \frac{\left|\Gamma\left(\gamma_{\kappa}+i y\right)\right| \Gamma\left(1-2 \gamma_{\kappa}\right)}{\left|\Gamma\left(-\gamma_{\kappa}+i y\right)\right| \Gamma\left(1+2 \gamma_{\kappa}\right)}\left(\frac{W \kappa-\gamma_{\kappa}}{W \kappa+\gamma_{\kappa}}\right) \frac{\cos \eta_{\kappa}}{\cos \bar{\eta}_{\kappa}}$

and

$$
\frac{\cos \eta_{\kappa}}{\cos \bar{\eta}_{\kappa}}=\left[\frac{\left(\kappa-\gamma_{\kappa}\right)}{\left(\kappa+\gamma_{\kappa}\right)} \cdot \frac{\left(\kappa W+\gamma_{\kappa}\right)}{\left(\kappa W-\gamma_{\kappa}\right)}\right]^{1 / 2}
$$

$\cos \left(\delta_{\kappa}^{\prime}-\bar{\delta}_{\kappa}^{\prime}\right) \equiv \operatorname{Re} \exp \left[\arg \Gamma\left(-\gamma_{\kappa}+i y\right)\right.$

$$
\begin{aligned}
& \left.-\arg \Gamma\left(\gamma_{\kappa}+i y\right)+\eta_{\kappa}-\bar{\eta}_{\kappa}-\pi \gamma_{\kappa}\right] \\
& =\frac{1}{\left(\gamma_{\kappa}^{2}+y^{2}\right)^{1 / 2}}\left[y \cos \pi \gamma_{\kappa}\right. \\
& \left.+\gamma_{\kappa} \sin \pi \gamma_{\kappa}\right] \operatorname{Re} \frac{\Gamma\left(-\gamma_{\kappa}+i y\right)}{\left|\Gamma\left(-\gamma_{\kappa}+i y\right)\right|} \\
& \cdot \frac{\left|\Gamma\left(\gamma_{\kappa}+i y\right)\right|}{\Gamma\left(\gamma_{\kappa}+i y\right)}+\frac{1}{\left(\gamma_{\kappa}^{2}+y^{2}\right)^{1 / 2}}\left[\gamma_{\kappa} \cos \pi \gamma_{\kappa}\right. \\
& \left.-y \sin \pi \gamma_{\kappa}\right] \operatorname{Im} \frac{\Gamma\left(-\gamma_{\kappa}+i y\right)}{\left|\Gamma\left(-\gamma_{\kappa}+i y\right)\right|} \\
& \cdot \frac{\left|\Gamma\left(\gamma_{\kappa}+i y\right)\right|}{\Gamma\left(\gamma_{\kappa}+i y\right)} \quad(35 \mathrm{~d})
\end{aligned}
$$

In the above equation, Re and Im imply the real part and the imaginary part of the respective quantities.

From eq (10),

$$
\begin{aligned}
\frac{G_{\kappa}}{\rho}= & \left(\frac{W+1}{W}\right)^{1 / 2} \frac{(2 p \rho)^{\gamma_{\kappa}}}{\rho} \frac{\left|\Gamma\left(\gamma_{\kappa}+i y\right)\right|}{\Gamma(1+2 \gamma)} \cdot \cos \eta_{\kappa} \\
& \left\{\cos p \rho\left[\left(\gamma_{\kappa} U_{\kappa}-y V_{\kappa}\right)-\left(\gamma_{\kappa} V_{\kappa}+y U_{\kappa}\right) \tan \eta_{\kappa}\right]\right. \\
& \left.\quad+\sin p \rho\left[\left(\gamma_{\kappa} U_{\kappa}-y V_{\kappa}^{\prime}\right) \tan \eta_{\kappa}+\left(\gamma_{\kappa} V_{\kappa}+y U_{\kappa}\right)\right]\right\}
\end{aligned}
$$

where $U_{\kappa}$ and $V_{\kappa}$ are real and are defined by

$$
U_{\kappa}+i V_{\kappa}=F\left(\gamma_{\kappa}+1+i y, 2 \gamma_{\kappa}+1,2 i p \rho\right) \text {. (36b) }
$$

\section{B. Numerical Approximations}

(i) The nuclear radius, $\rho$, equal $101.2 A^{1 / 3} \mathrm{I}^{-1 / 3}$ $\mathrm{cm}\left(0.42585 \alpha A^{1 / 3} \mathrm{X}_{1} / \mathrm{mc}\right)$ was used in the major body of the present tables. However, for purposes of illustration, numerical results are also presented for $Z=6$ and $Z=7$ by taking $\rho=1.3 A^{1 / 3} 10^{-13} \mathrm{~cm}$ $\left(0.46134 \alpha A^{1 / 3} \quad \mathrm{~K} / \mathrm{mc}\right)$ and $\rho=1.1 A^{1 / 3} \quad 10^{-13} \mathrm{~cm}$ $\left(0.39036 \alpha A^{1 / 3} \mathrm{~K} / \mathrm{mc}\right)$. It may be noted that a value of $\rho=0.4276 \alpha A^{1 / 3} \mathrm{~h} / \mathrm{mc}$ was employed in ORNL3207.

(ii) In the evaluation of $S_{n}, T_{n}, \bar{S}_{n}, \bar{T}_{n}, b_{n}, b_{n}^{\prime}$ and other similar coefficients, the series were terminated when the last coefficient became less than $10^{-8}$.

(iii) The confluent hypergeometric series was terminated when both the real part and the imaginary part berame less than $10^{-8}$. from

(iv) Complex gamma functions [14] were obtained

$$
\begin{aligned}
\Gamma(Z)=e^{-Z} Z^{Z-1 / 2}(2 \pi)^{1 / 2}\left\{1+1 / 12 Z+1 / 288 Z^{2}\right. \\
\left.\quad-139 / 51840 Z^{3}-571 / 2488320 Z^{4}+0\left(1 / Z^{5}\right)\right\}
\end{aligned}
$$

The argument, $Z$, was first tested whether $\operatorname{Re} Z$ $>10$. When this argument did not satisfy this condition, the recurrence relation, $\Gamma(Z+1)=$ $Z \Gamma(Z)$, was used until $\operatorname{Re} Z>10$. 'The accuracy in this calculation was better than 1 part in $10^{6}$.

(v) The real gamma functions were evaluated by the following formula

$$
\begin{gathered}
\text { For }-1 / 2 \leqslant x<1 / 2 \\
\Gamma(1+x)=\left[\frac{(1-x)}{(1+x)} \frac{\pi x}{\sin \pi x}\right]^{1 / 2} e^{-\lambda}
\end{gathered}
$$

where

$$
\lambda=\sum_{n=1}^{10} C_{2 n-1} x^{2 n-1}
$$

with the values of the coefficients $C_{2 n-1}$ taken from reference [15]. The arguments of the real gamma functions were reduced by the following recurrence relations:

$$
\Gamma(1+x)=x \Gamma(x) \text { for } 1 / 2 \leqslant x
$$

and

$$
\Gamma(1+x)=\frac{\pi x}{\Gamma(1+|x|) \sin \pi x} \text { for } x<-1 / 2
$$

An accuracy of seven significant figures was accomplished.

The calculations were performed on the IBM 7094 of the National Bureau of Standards. 


\section{Explanation of the Tables}

\section{A. Format}

In this Monograph, $Z$ and $A$ are the atomic number and the mass number, respectively, of the daughter nucleus. The parent nucleus is, then, specified by $Z-1$ and $A$ for electron decays, and by $Z+1$ and $A$ for positron decays. The value of the nuclear radius, $\rho$, in fermis, is listed on the top of each page. The subscripts of $f_{\kappa}, g_{\kappa}$ and tan $\delta_{\kappa}$ specify the total angular momentum and the orbital angular momentum (see eq (4)). For purposes of reference only, the Fermi function, $F_{0}$, is also tabulated for a point nucleus. Another quantity labeled $\bar{F}$ is given for a convenient format.

Each entry, except for beta momentum, is given in the format of a signed mantissa followed by a signed exponent to the base 10: blank space indicates + sign. For example, a number listed as $1.23456 \mathrm{E} 07$ implies $1.23456 \times(10)^{7}$, and an entry of $1.23456 \mathrm{E}-07$ means $1.23456 \times 10^{-7}$.

\section{B. Use of the Tables}

At the very outset we wish to point out that we have not included the corrections arising from atomic screening.

The Fermi function corresponding to a smeared charge distribution should be calculated by

$$
\text { Fermi function }=\left(f_{1}{ }^{2}+g_{-1}{ }^{2}\right) / 2 p^{2} .
$$

In our calculations we have taken the normalization of $f_{\kappa}$ and $g_{\kappa}$ corresponding to a sphere of unit radius. To convert the numerical results to a nor- malization per unit energy, the radial functions should be multiplied by $(W / \pi p)^{1 / 2}$.

Since we tablulate $\tan \delta_{|\kappa|}$ and $\tan \delta_{-|\kappa|}$, the following prescription may be used in the calculation of $\sin \left(\delta_{\kappa}-\delta_{\kappa^{\prime}}\right)$ and $\cos \left(\delta_{\kappa}-\delta_{\kappa^{\prime}}\right)$ :

For $\beta^{+}$particles,

$$
\cos \left(\delta_{\kappa}-\delta_{\kappa^{\prime}}\right)=\frac{1+\tan \delta_{\kappa} \cdot \tan \delta_{\kappa^{\prime}}}{\left[\left(1+\tan ^{2} \delta_{\kappa}\right)\left(1+\tan ^{2} \delta_{K^{\prime}}\right)\right]^{1 / 2}}
$$

and

$$
\sin \left(\delta_{\kappa}-\delta_{\kappa^{\prime}}\right)=\frac{\tan \delta_{\kappa}-\tan \delta_{\kappa^{\prime}}}{\left[\left(1+\tan ^{2} \delta_{\kappa}\right)\left(1+\tan ^{2} \delta_{\kappa^{\prime}}\right)\right]^{1 / 2}} .
$$

Also, whenever $\tan \delta_{-1}$ is negative, the square root of $\left(1+\tan \delta_{-1}{ }^{2}\right)$ should be taken as negative.

For $\beta^{-}$particles,

$$
\cos \left(\delta_{\kappa}-\delta_{\kappa^{\prime}}\right)=\epsilon_{\kappa \kappa^{\prime}} \frac{1+\tan \delta_{\kappa} \tan \delta_{\kappa^{\prime}}}{\left[\left(1+\tan ^{2} \delta_{\kappa}\right)\left(1+\tan ^{2} \delta_{\kappa^{\prime}}\right)\right]^{1 / 2}}
$$

and

$$
\sin \left(\delta_{\kappa}-\delta_{\kappa^{\prime}}\right)=\epsilon_{\kappa \kappa^{\prime}} \frac{\tan \delta_{\kappa}-\tan \delta_{\kappa^{\prime}}}{\left[\left(1+\tan ^{2} \delta_{\kappa}\right)\left(1+\tan ^{2} \delta_{\kappa^{\prime}}\right)\right]^{1 / 2}}
$$

where

$$
\begin{gathered}
\epsilon_{\kappa \kappa^{\prime}}=1 \text { if }|\kappa|=\left|\kappa^{\prime}\right| \\
\epsilon_{\kappa \kappa^{\prime}}=-1 \text { if }|\kappa| \neq\left|\kappa^{\prime}\right|
\end{gathered}
$$

The appropriate theoretical formulas relevant to nuclear beta decay appear in the literature [16].

\section{Acknowledgments}

It is a pleasure to thank Dr. R. W. Hayward for suggesting this project, for encouragement throughout this work and for several stimulating discussions. The author is grateful to the administration of the National Bureau of Standards for their hospitality and for the financial support, and to the administra- tion of the Westinghouse Atomic Power Division, Pittsburgh, in particular Mr. R. J. French, for grant. ing a leave of absence. Special thanks are also due to Mr. W. Bozman and Mr. C. Drew for writing a program to convert the computer output, for suitable printing in the present format of these tables.

\section{References}

[1] Tables for the analysis of beta spectra, National Bureau of Standards, Applied Mathematics Series No. 13 (1952). Also see 1. Feister, Phys. Rev. 70, 375 (1950).

[2] M. E. Rose, C. L. Perry, and N. M. Dismuke, Oak Ridge National Laboratory Report ORNL-1459. Also see M. E. Rose, in Beta-and-gamma ray spectroscopy (ed. K. Siegbahn; North-Holland Publishing Company, Amsterdam, 1955).

[3] M. E. Rose and C. L. Perry, Phys. Rev. 90, 479 (1953). In this paper the upper limit of $P_{0}$ in figure 4 should be 1.0 (and not 10).
[4] M. E. Rose and D. K. Holmes, Phys. Rev. 83, 190 (1951). Also see M. E. Rose and D. K. Holmes, Oak Ridge National Laboratory Report ORNL-1022.

[5] J. R. Reitz, Phys. Rev. 77, 10 (1950). Also see J. R. Reitz, Relativistic electron-wave-functions for a Thomas-FermiDirac statistical atom, (Department of Physics of the University of Chicago, 1949.) For approximate treatment of this problem see M. E. Rose, Phys. Rev. 49, 727 (1937), and C. Longmire and H. Brown, Phys. Rev. 75, 102 (1949).

[6] T. D. Lee and C. N. Yang, Phys. Rev. 104, 254 (1956).

[7] C. S. Wu, E. Ambler, R. W. Hayward, D. D. Hoppes, and R. P. Hudson, Phys. Rev. 105, 1413 (1957). 
[8] M. E. Rose, Phys. Rev. 82, 389 (1951).

[9] (a) C. P. Bhalla and M. E. Rose, Oak Ridge National Laboratory Report, ORNL-3207 (1962). In this report, $f_{\kappa}, g_{\kappa}, \tan \delta_{\kappa}$ for $\kappa= \pm 1$ and $\kappa= \pm 2$, and the Fermi function are tabulated for thirty values of beta momentum in steps of $0.2 \mathrm{mc}$ up to a maximum of 6.2 for about 93 values of $Z$ separately for electrons and positrons. This' report is available from the Office of Technical Services, Department of Commerce, Washington, D. C. 20234.

[9] (b) C. P. Bhalla and M. E. Rose, Oak Ridge National Laboratory Report ORNL-2954 (1960). In ORNL-2954, entries under $\sin \Delta$ should be taken with a negative sign for positrons.

[10] T. Kot ani and M. Ross, Progr. Theoret. Phys., 20, 643 (1958).

[11] D. D. Hoppes, E. Ambler, R. W. Hayward, and R. S. Kaeser, Phys. Rev. letters 6, 115 (1961).

[12] C. P. Bhalla and M. E. Rose, Phys. Rev. 128, 774 (1962). Also see reference [4].
[13] M. E. Rose, Elementary theory of angular momentum (John Wiley and Sons Inc. New York-1957). M. E. Rose, Phys. Rev. 51, 484 (1937).

[14] C. T. Whittaker and G. N. Watson, A Course of Modern Analysis (Cambridge, 1952).

[15] E. Jahnke, F. Emde, and F. Lïsch, Tables of Higher Functions. (Mccraw-1lill, New York 1960).

[16] A. M. Bincer, Phys. Rev. 112, 244 (1958); E. Greuling, Phys. Rev. 61, 568 (1942); D. L. Pursey, Phil. Mag. 412, 1193 (1951), C. P. Bhalla and M. E. Rose, Phys. Rev. 120, 1415 (1960); M. Morita, Phys. Rev. 113, 1584 (1959); M. Gell-Mann, Phys. Rev. 111, 362 (1958); M. Morita and R. S. Morita, Phys. Rev. 109, 2048 (1958); L. C. Biedenharn and M. E. Rose, Revs. Modern Pluys. 25, 729 (1953); J. Huffaker, Ph.D. dissertation Duke University (1962). R. B. Curtis and R. R. Lewis, Phys. Rev. 107, 543 (1957). H. A. Tolhoek, Selected Topics in Nuclear Theory (ed. F. Janouch, International Atomic Energy Agency, Vienna 1963). 
VII. Tables of Electron Radial Functions and

Tangents of Phase Shifts for Light Nuclei

Corresponding to a Uniform Charge Distribution

Inside a Nucleus of Radius $\rho$ 


\section{p $F_{0}$}

0.10

0.20

0.30

0.40

0.50

0.60

0.70

0.80

0.90

1.00

1.10

1.20

1.30

1.40

1.50

1.60

1.70

1.80

1.90

2.00

2.10

2.20

2.30

2.40

2.50

2.60

2.70

2.80

2.90

3.00

3.10

3.20

3.30

3.40

3.50

3.60

3.70

3.80

3.90

4.00

4.10

4.20

4.30

4.40

4.50

4.60

4.70

4.80

4.90

5.00

5.10

5.20

5.30

5.40

5.50

5.60

5.70

5.80

5.90

6.00 $f_{1}$

$1.24867 \mathrm{E} \quad 00$

$1.12199 \mathrm{E} \quad 00$

$1.08240 \mathrm{E} \quad 00$

$1.06347 \mathrm{E} \quad 00$

$1.05260 \mathrm{E} \quad 00$

$1.04566 \mathrm{E} \quad 00$

$1.04094 \mathrm{E} \quad 00$

$1.03757 \mathrm{E} \quad 00$

$1.03508 \mathrm{E} \quad 00$

$1.03318 \mathrm{E} \quad 00$

$1.03171 \mathrm{E} \quad 00$

$1.03054 \mathrm{E} \quad 00$

$1.02960 \mathrm{E} \quad 00$

$1.02883 \mathrm{E} \quad 00$

$1.02819 \mathrm{E} \quad 00$

$1.02766 \mathrm{E} \quad 00$ $1.02721 \mathrm{E} \quad 00$ $1.02683 \mathrm{E} \quad 00$ $1.02650 \mathrm{E} \quad 00$ $1.02622 \mathrm{E} \quad 00$

$1.02597 \mathrm{E} \quad 00$ $1.02576 \mathrm{E} \quad 00$ $1.02557 \mathrm{E} \quad 00$

$1.02540 \mathrm{E} \quad 00$

$1.02525 \mathrm{E} \quad 00$

$1.02512 \mathrm{E} \quad 00$ $1.02500 \mathrm{E} \quad 00$ $1.02489 \mathrm{E} \quad 00$ $1.02480 \mathrm{E} \quad 00$ $\begin{array}{ll}1.02471 \mathrm{E} & 00\end{array}$

$1.02463 \mathrm{E} \quad 00$ $1.02455 \mathrm{E} \quad 00$ $1.02449 \mathrm{E} \quad 00$ $1.02443 \mathrm{E} \quad 00$ $1.02437 \mathrm{E} \quad 00$

$1.02432 \mathrm{E} \quad 00$ $1.02427 \mathrm{E} \quad 00$ $1.02423 \mathrm{E} \quad 00$ $1.02419 \mathrm{E} \quad 00$ $1.02415 \mathrm{E} \quad 00$

$1.02411 \mathrm{E} \quad 00$ $1.02408 \mathrm{E} \quad 00$ $1.02405 \mathrm{E} \quad 00$ $1.02402 \mathrm{E} \quad 00$

$1.02399 \mathrm{E} \quad 00$

$1.02397 \mathrm{E} \quad 00$ $1.02394 \mathrm{E} \quad 00$ $1.02392 \mathrm{E} \quad 00$ $1.02390 \mathrm{E} \quad 00$ $1.02388 \mathrm{E} \quad 00$

$1.02386 \mathrm{E} \quad 00$ $1.02384 \mathrm{E} \quad 00$ $1.02383 \mathrm{E} \quad 00$ $1.02381 \mathrm{E} \quad 00$ $1.02380 \mathrm{E} \quad 00$

$1.02378 \mathrm{E} \quad 00$ $1.02377 \mathrm{E} \quad 00$ $1.02376 \mathrm{E} \quad 00$ $1.02374 \mathrm{E} \quad 00$ $1.02373 \mathrm{E} \quad 00$ $g_{1}$

$-7.68252 \mathrm{E}-03$

$-2.93435 \mathrm{E}-02$

$-6.39199 \mathrm{E}-02$

$-1.10141 \mathrm{E}-01$

$-1.66498 \mathrm{E}-01$

$-2.31431 \mathrm{E}-01$

$-3.03462 E-01$

$-3.81270 \mathrm{E}-01$

$-4.63727 \mathrm{E}-01$

$-5.49899 \mathrm{E}-01$

$-6.39027 \mathrm{E}-01$

$-7.30507 \mathrm{E}-01$

$-8.23859 \mathrm{E}-01$

$-9.18702 \mathrm{E}-01$

$-1.01474 \mathrm{E} \quad 00$

$-1.11173 \mathrm{E} \quad 00$

$-1.20949 \mathrm{E} \quad 00$

$-1.30788 \mathrm{E} \quad 00$

$-1.40677 \mathrm{E} \quad 00$

$-1.50608 \mathrm{E} 00$

$-1.60572 \mathrm{E} \quad 00$

$-1.70565 \mathrm{E} 00$

$-1.80580 \mathrm{E} \quad 00$

$-1.90615 \mathrm{E} 00$

-2.00665 E 00

$-2.10728 \mathrm{E} \quad 00$

$-2.20803 \mathrm{E} \quad 00$

$-2.30887 \mathrm{E} \quad 00$

$-2.40978 \mathrm{E} \quad 00$

$-2.51076 \mathrm{E} \quad 00$

$-2.61179 \mathrm{E} \quad 00$

$-2.71287 \mathrm{E} \quad 00$

$-2.81399 \mathrm{E} \quad 00$

$-2.91514 \mathrm{E} \quad 00$

$-3.01632 \mathrm{E} 00$

$-3.11752 \mathrm{E} \quad 00$

$-3.21874 \mathrm{E} \quad 00$

$-3.31997 \mathrm{E} \quad 00$

$-3.42122 \mathrm{E} 00$

$-3.52248 \mathrm{E} \quad 00$

$-3.62374 \mathrm{E} \quad 00$

$-3.72502 \mathrm{E} \quad 00$

$-3.82630 \mathrm{E} \quad 00$

$-3.92758 \mathrm{E} \quad 00$

$-4.02887 \mathrm{E} \quad 00$

$-4.13016 \mathrm{E} \quad 00$

$-4.23145 \mathrm{E} \quad 00$

$-4.33274 \mathrm{E} \quad 00$

$-4.43403 \mathrm{E} 00$

$-4.53533 \mathrm{E} 00$

$-4.63662 \mathrm{E} \quad 00$

$-4.73791 \mathrm{E} \quad 00$

$-4.83920 \mathrm{E} \quad 00$

$-4.94048 \mathrm{E} 00$

$-5.04177 \mathrm{E} \quad 00$

$-5.14305 \mathrm{E} \quad 00$

$-5.24433 \mathrm{E} 00$

$-5.34561 \mathrm{E} \quad 00$

$\begin{array}{ll}-5.44689 \mathrm{E} & 00\end{array}$

$-5.54816 \mathrm{E} \quad 00$

\begin{abstract}
$-3.83806 \mathrm{E}-05$
$-1.47046 \mathrm{E}-04$

$-3.21918 \mathrm{E}-04$

$-5.58464 \mathrm{E}-04$

$-8.51293 \mathrm{E}-04$
\end{abstract}

$-1.19484 \mathrm{E}-03$

$-1.58384 \mathrm{E}-03$

$-2.01363 \mathrm{E}-03$

$-2.48021 \mathrm{E}-03$

$-2.98031 \mathrm{E}-03$

$-3.51129 \mathrm{E}-03$

$-4.07103 \mathrm{E}-03$

$-4.65791 \mathrm{E}-03$

$-5.27058 \mathrm{E}=03$

$-5.90805 \mathrm{E}-03$

$-6.56952 \mathrm{E}-03$

$-7.25437 \mathrm{E}-03$

$-7.96210 \mathrm{E}-03$

$-8.69234 \mathrm{E}-03$

$-9.44477 \mathrm{E}-03$

$-1.02192 \mathrm{E}-02$

$-1.10153 \mathrm{E}-02$

$-1.18330 \mathrm{E}-02$

$-1.26723 \mathrm{E}-02$

$-1.35329 \mathrm{E}-02$

$-1.44148 \mathrm{E}-02$

$-1.53179 \mathrm{E}-02$

$-1.62423 \mathrm{E}-02$

$-1.71877 \mathrm{E}-02$

$-1.81543 \mathrm{E}-02$

$-1.91419 \mathrm{E}-02$

$-2.01506 \mathrm{E}-02$

$-2.11803 \mathrm{E}-02$

$-2.22310 \mathrm{E}-02$

$-2.33027 \mathrm{E}-02$

$-2.43954 \mathrm{E}-02$

$-2.55090 \mathrm{E}-02$

$-2.66436 \mathrm{E}-02$

$-2.77992 \mathrm{E}-02$

$-2.89757 \mathrm{E}-02$

$-3.01732 \mathrm{E}-02$ $-3.13916 \mathrm{E}-02$ $-3.26310 \mathrm{E}-02$

$-3.38913 \mathrm{E}-02$

$-3.51725 \mathrm{E}-02$

$-3.64747 \mathrm{E}-02$

$-3.77978 \mathrm{E}-02$

$-3.91418 \mathrm{E}-02$

$-4.05068 \mathrm{E}-02$

$-4.18927 \mathrm{E}-02$

$-4.32995 \mathrm{E}-02$ $-4.47273 \mathrm{E}-02$

$-4.61760 \mathrm{E}-02$

$-4.76456 \mathrm{E}-02$

$-4.91361 \mathrm{E}-02$

$-5.06476 \mathrm{E}-02$

$-5.21800 \mathrm{E}-02$

$-5.37333 \mathrm{E}-02$

$-5.53075 \mathrm{E}-02$

$-5.69027 \mathrm{E}-02$

$\tan \delta_{1}$

$f_{-1}$

$-3.08232 \mathrm{E}-02-4.61504 \mathrm{E}-04$

$-1.53348 \mathrm{E}-02 \quad-8.76334 \mathrm{E}-04$

$-1.01616 \mathrm{E}-02-1.29462 \mathrm{E}-03$

$-7.56351 \mathrm{E}-03-1.71771 \mathrm{E}-03$

$-5.99582 \mathrm{E}-03-2.14717 \mathrm{E}-03$

$-4.94405 \mathrm{E}-03$

$-4.18772 \mathrm{E}-03$

$-3.61662 \mathrm{E}-03$

$-3.16945 \mathrm{E}-03$

$-2.80940 \mathrm{E}-03$

$-2.51300 \mathrm{E}-03$

$-2.26457 \mathrm{E}-03$

$-2.05322 \mathrm{E}-03$

$-1.87116 \mathrm{E}-03$

$-1.71263 \mathrm{E}-03$

$-1.57332 \mathrm{E}-03$

$-1.44990 \mathrm{E}-03$

$-1.33978 \mathrm{E}-03$

$-1.24092 \mathrm{E}-03$

$-1.15166 \mathrm{E}-03$

$-1.07066 \mathrm{E}-03$

$-9.96823 \mathrm{E}-04$

$-9.29232 \mathrm{E}-04$

$-8.67125 \mathrm{E}-04$

$-8.09859 \mathrm{E}-04$

$-7.56887 \mathrm{E}-04$

$-7.07740 \mathrm{E}-04$

$-6.62024 \mathrm{E}-04$

$-6.19384 \mathrm{E}-04$

$-5.79523 \mathrm{E}-04$

$-5.42177 \mathrm{E}-04$

$-5.07114 \mathrm{E}-04$

$-4.74132 \mathrm{E}-04$

$-4.43049 \mathrm{E}-04$

$-4.13706 \mathrm{E}-04$

$-3.85963 \mathrm{E}-04$

$-3.59688 \mathrm{E}-04$

$-3.34772 \mathrm{E}-04$

$-3.11111 \mathrm{E}-04$ 
ELECTRON RADIAL FUNCTIONS

$Z=1 \quad A=1 \quad \rho=1.2 A^{1 / 3} F$

Electron

$\bar{F}$

$3.36669 \mathrm{E}-09$

$1.20526 \mathrm{E}-08$

$2.61425 \mathrm{E}-08$

$4.56513 \mathrm{E}-08$

$7.05927 \mathrm{E}-08$

$1.00978 \mathrm{E}-07$

$1.36817 \mathrm{E}-07$

$1.78117 \mathrm{E}-07$

$2.24884 \mathrm{E}-07$

$2.77124 \mathrm{E}-07$

$3.34839 \mathrm{E}-07$ $3.98032 \mathrm{E}-07$ $4.66707 \mathrm{E}-07$ $5.40864 \mathrm{E}-07$ $6.20505 \mathrm{E}-07$

$7.05631 \mathrm{E}-07$

$7.96243 \mathrm{E}-07$

$8.92342 \mathrm{E}-07$

$9.93928 \mathrm{E}-07$

$1.10100 \mathrm{E}-06$

$1.21356 \mathrm{E}-06$

$1.33161 \mathrm{E}-06$

$1.45515 \mathrm{E}-06$

$1.58418 \mathrm{E}-06$

$1.71869 \mathrm{E}-06$

$1.85870 \mathrm{E}-06$

$2.00419 \mathrm{E}-06$

$2.15518 \mathrm{E}-06$

$2.31165 \mathrm{E}-06$

$2.47361 \mathrm{E}-06$

$2.64106 \mathrm{E}-06$

$2.81400 \mathrm{E}-06$

$2.99243 \mathrm{E}-06$

$3.17635 \mathrm{E}-06$

$3.36576 \mathrm{E}-06$

$3.56066 \mathrm{E}-06$

$3.76105 \mathrm{E}-06$

$3.96693 \mathrm{E}-06$

$4.17829 \mathrm{E}-06$

$4.39515 \mathrm{E}-06$

$4.61750 \mathrm{E}-06$ $4.84533 \mathrm{E}-06$

$5.07866 \mathrm{E}-06$

$5.31748 \mathrm{E}-06$

$5.56178 \mathrm{E}-06$

4.60

4.70

4.80

4.90

5.00

5.10

5.20

5.30

5.40

5.50

5.60

5.70

5.80

5.90

6.00

$5.81158 \mathrm{E}-06$ $6.06686 \mathrm{E}-06$

$6.32764 \mathrm{E}-06$

$6.59390 \mathrm{E}-06$

$6.86566 \mathrm{E}-06$

$7.14290 \mathrm{E}-06$

$7.42564 \mathrm{E}-06$

$7.71386 \mathrm{E}-06$

$8.00757 \mathrm{E}-06$

$8.30678 \mathrm{E}-06$

$8.61148 \mathrm{E}-06$ $8.92166 \mathrm{E}-06$ $9.23733 \mathrm{E}-06$

$9.55849 \mathrm{E}-06$

$9.88515 \mathrm{E}-06$ $f_{2}$

$-6.86458 \mathrm{E}-07$

$-1.95560 \mathrm{E}-05$

$-4.52266 \mathrm{E}-05$

$-8.57206 \mathrm{E}-05$

$-1.43218 \mathrm{E}-04$

$-2.19312 \mathrm{E}-04$

$-3.15113 \mathrm{E}-04$

$-4.31366 \mathrm{E}-04$

$-5.68548 \mathrm{E}-04$

$-7.26950 \mathrm{E}-04$

$-9.06737 \mathrm{E}-04$

$-1.10800 \mathrm{E}-03$

$-1.33076 \mathrm{E}-03$

$-1.57502 \mathrm{E}-03$

$-1.84077 \mathrm{E}-03$

$-2.12797 \mathrm{E}-03$

$-2.43658 \mathrm{E}-03$

$-2.76658 \mathrm{E}-03$

$-3.11792 \mathrm{E}-03$

$-3.49058 \mathrm{E}-03$

$-3.88451 \mathrm{E}-03$

$-4.29970 \mathrm{E}-03$

$-4.73612 \mathrm{E}-03$

$-5.19374 \mathrm{E}-03$

$-5.67253 \mathrm{E}-03$

$-6.17249 \mathrm{E}-03$

$-6.69359 \mathrm{E}-03$

$-7.23582 \mathrm{E}-03$

$-7.79915 \mathrm{E}-03$

$-8.38359 \mathrm{E}-03$

$-8.98912 \mathrm{E}-03$

$-9.61572 \mathrm{E}-03$

$-1.02634 \mathrm{E}-02$

$-1.09321 \mathrm{E}-02$

$-1.16219 \mathrm{E}-02$

$-1.23327 \mathrm{E}-02$

$-1.30646 \mathrm{E}-02$

$-1.38175 \mathrm{E}-02$

$-1.45914 \mathrm{E}-02$

$-1.53864 \mathrm{E}-02$

$-1.62023 \mathrm{E}-02$

$-1.70393 \mathrm{E}-02$

$-1.78973 \mathrm{E}-02$

$-1.87763 \mathrm{E}-02$

$-1.96763 \mathrm{E}-02$

$-2.05973 \mathrm{E}-02$

$-2.15393 \mathrm{E}-02$

$-2.34863 \mathrm{E}-02$

$-2.44913 \mathrm{E}-02$

$-2.55172 \mathrm{E}-02$

$-2.65642 \mathrm{E}-02$

$-2.76321 \mathrm{E}-02$

$-2.87210 \mathrm{E}-02$

$-2.98309 \mathrm{E}-02$

$-3.09618 \mathrm{E}-02$

$-3.21136 \mathrm{E}-02$

$-3.32865 \mathrm{E}-02$

$-3.44803 \mathrm{E}-02$
$-5.87149 \mathrm{E}-06$

$g_{2}$

$-2.00040 \mathrm{E}-09$

$-1.71641 \mathrm{E}-08$

$-5.74625 \mathrm{E}-08$

$-1.33819 \mathrm{E}-07$

$-2.55820 \mathrm{E}-07$

$-4.31699 \mathrm{E}-07$

$-6.68491 \mathrm{E}-07$

$-9.72251 \mathrm{E}-07$

$-1.34829 \mathrm{E}-06$

$-1.80140 \mathrm{E}-06$

$-2.33600 \mathrm{E}-06$

$-2.95625 \mathrm{E}-06$

$-3.66619 \mathrm{E}-06$

$-4.46973 \mathrm{E}-06$

$-5.37072 \mathrm{E}-06$

$-6.37302 \mathrm{E}-06$

$-7.48043 \mathrm{E}-06$

$-8.69678 \mathrm{E}-06$

$-1.00259 \mathrm{E}-05$

$-1.14715 \mathrm{E}-05$

$-1.30376 \mathrm{E}-05$

$-1.47279 \mathrm{E}-05$

$-1.65463 \mathrm{E}-05$

$-1.84965 \mathrm{E}-05$

$-2.05826 \mathrm{E}-05$

$-2.28083 \mathrm{E}-05$

$-2.51774 \mathrm{E}-05$

$-2.76940 \mathrm{E}-05$

$-3.03617 \mathrm{E}-05$

$-3.31846 \mathrm{E}-05$

$-3.61664 \mathrm{E}-05$

$-3.93112 \mathrm{E}-05$

$-4.26226 \mathrm{E}-05$

$-4.61047 \mathrm{E}-05$

$-4.97614 \mathrm{E}-05$

$-5.35965 \mathrm{E}-05$

$-5.76139 \mathrm{E}-05$

$-6.18175 \mathrm{E}-05$

$-6.62111 \mathrm{E}-05$

$-7.07989 \mathrm{E}-05$

$-7.55844 \mathrm{E}-05$

$-8.05718 \mathrm{E}-05$

$-8.57649 \mathrm{E}-05$

$-9.11676 \mathrm{E}-05$

$-9.67837 \mathrm{E}-05$

$-2.25023 \mathrm{E}-02$

$-1.02617 \mathrm{E}-04$

$-1.08672 \mathrm{E}-04$

$-1.14952 \mathrm{E}-04$

$-1.21461 \mathrm{E}-04$

$-1.28203 \mathrm{E}-04$

$-1.35183 \mathrm{E}-04$

$-1.42402 \mathrm{E}-04$

$-1.49867 \mathrm{E}-04$

$-1.57580 \mathrm{E}-04$

$-1,65546 \mathrm{E}-04$

$-1.73768 \mathrm{E}-04$

$-1.82250 \mathrm{E}-04$

$-1.90997 \mathrm{E}-04$

$-2.00012 \mathrm{E}-04$ $\tan \delta_{2}$

$f_{-2}$

$1.47717 \mathrm{E} \quad 01-2.73900 \mathrm{E}-08$

$2.92753 \mathrm{E} \quad 01 \quad-1.03841 \mathrm{E}-07$

$4.31856 \mathrm{E}$ ol $-2.30122 \mathrm{E}-07$

$5.62911 \mathrm{E} \quad 01-4.07280 \mathrm{E}-07$

$6.84495 \mathrm{E}$ ol $-6.36781 \mathrm{E}-07$

$7.95900 \mathrm{E} \quad 01$

$8.97015 \mathrm{E} \quad 01$

$9.88175 \mathrm{E} \quad 01$

$1.07000 \mathrm{E} \quad 02$

$1.14325 \mathrm{E} \quad 02$

$-9.20515 \mathrm{E}-07$

$-1.26077 \mathrm{E}-06$

$-1.66019 \mathrm{E}-06$

$-2.12173 \mathrm{E}-06$

$-2.64856 \mathrm{E}-06$

$1.20878 \mathrm{E} \quad 02$

$1.26740 \mathrm{E} \quad 02$

$1.31990 \mathrm{E} \quad 02$

$1.36700 \mathrm{E} \quad 02$

$1.40935 \mathrm{E} \quad 02$

$1.44751 \mathrm{E} \quad 02$

$1.48199 \mathrm{E} \quad 02$

$1.51324 \mathrm{E} \quad 02$

$\begin{array}{ll}1.54163 \mathrm{E} & 02\end{array}$

$1.56750 \mathrm{E} \quad 02$

$1.59113 \mathrm{E} \quad 02$

$1.61278 \mathrm{E} \quad 02$

$1.63266 \mathrm{E} \quad 02$

$\begin{array}{lll}1.65097 \mathrm{E} & 02\end{array}$

$\begin{array}{ll}1.66787 \mathrm{E} & 02\end{array}$

$-3.24407 \mathrm{E}-06$

$-3.91178 \mathrm{E}-06$

$-4.65532 \mathrm{E}-06$

$-5.47839 \mathrm{E}-06$

$-6.38477 \mathrm{E}-06$

$-7.37826 \mathrm{E}-06$

$-8.46270 \mathrm{E}-06$

$-9.64195 \mathrm{E}-06$

$-1.09199 \mathrm{E}-05$

$-1.23004 \mathrm{E}-05$

$-1.37874 \mathrm{E}-05$

$-1.53848 \mathrm{E}-05$

$-1.70964 \mathrm{E}-05$

$-1.89262 \mathrm{E}-05$

$-2.08781 \mathrm{E}-05$

$1.68350 \mathrm{E} \quad 02$

$1.69800 \mathrm{E} \quad 02$

$\begin{array}{ll}1.71147 \mathrm{E} & 02\end{array}$

$1.72401 \mathrm{E} \quad 02$

$1.73572 \mathrm{E} \quad 02$

$-2.29561 \mathrm{E}-05$

$-2.51639 \mathrm{E}-05$

$-2.75056 \mathrm{E}-05$

$-2.99850 \mathrm{E}-05$

$-3.26060 \mathrm{E}-05$

$1.74666 \mathrm{E} \quad 02$

$1.75690 \mathrm{E} \quad 02$

$1.76651 \mathrm{E} \quad 02$

$1.77554 \mathrm{E} \quad 02$

$1.78404 \mathrm{E} \quad 02$

$-3.53726 \mathrm{E}-05$

$-3.82887 \mathrm{E}-05$

$-4.13582 \mathrm{E}-05$

$-4.45850 \mathrm{E}-05$

$1.79205 \mathrm{E} \quad 02$

$1.79962 \mathrm{E} \quad 02$

$1.80676 \mathrm{E} \quad 02$

$1.81353 \mathrm{E} \quad 02$

$1.81994 \mathrm{E} \quad 02$

$1.82603 \mathrm{E} \quad 02$

$\begin{array}{ll}1.83181 \mathrm{E} & 02\end{array}$

$1.83731 \mathrm{E} \quad 02$

$\begin{array}{ll}1.84254 \mathrm{E} & 02\end{array}$

$1.84753 \mathrm{E} \quad 02$

$-2.09299 \mathrm{E}-04$

$1.85229 \mathrm{E} \quad 02$

$1.85684 \mathrm{E} \quad 02$

$1.86119 \mathrm{E} \quad 02$

$1.86535 \mathrm{E} \quad 02$

$1.86933 \mathrm{E} \quad 02$

\begin{tabular}{ll}
$1.87315 \mathrm{E} \quad 02$ \\
\hline
\end{tabular}

$\begin{array}{lll}1.87681 \mathrm{E} & 02\end{array}$

$\begin{array}{ll}1.88032 \mathrm{E} & 02\end{array}$

$\begin{array}{ll}1.88370 \mathrm{E} & 02\end{array}$

$\begin{array}{ll}1.88694 \mathrm{E} & 02\end{array}$

$1.89007 \mathrm{E} \quad 02$

$\begin{array}{lll}1.89307 \mathrm{E} & 02\end{array}$

$\begin{array}{lll}1.89597 \mathrm{E} & 02\end{array}$ 
ELECTRON RADIAL FUNCTIONS

Electron

\begin{tabular}{|c|c|c|c|c|c|}
\hline$p$ & $F_{0}$ & & $f_{1}$ & & $g_{2}$ \\
\hline 6.10 & $1.02372 \mathrm{E}$ & 00 & $-5.64943 \mathrm{E}$ & 00 & $-5.85188 \mathrm{E}-02$ \\
\hline 6.20 & $1.02371 \mathrm{E}$ & 00 & $-5.75070 \mathrm{E}$ & 00 & $-6.01559 \mathrm{E}-02$ \\
\hline 6.30 & $1.02370 \mathrm{E}$ & 00 & $-5.85197 \mathrm{E}$ & 00 & $6.18138 \mathrm{E}-02$ \\
\hline 6.40 & $.02369 \mathrm{E}$ & 00 & $-5.95323 \mathrm{E}$ & 00 & $6.34927 \mathrm{E}-02$ \\
\hline 6.50 & $1.02368 \mathrm{E}$ & 00 & $-6.05448 \mathrm{E}$ & 00 & $-6.51925 E-02$ \\
\hline 6.60 & $1.02367 \mathrm{E}$ & 00 & $-6.15574 \mathrm{E}$ & 00 & $-6.69133 \mathrm{E}-02$ \\
\hline .70 & $1.02366 \mathrm{E}$ & 00 & $-6.25699 \mathrm{E}$ & 00 & $-6.86549 \mathrm{E}-02$ \\
\hline 6.80 & $.02365 \mathrm{E}$ & 00 & $-6.35824 \mathrm{E}$ & 00 & $-7.04175 \mathrm{E}-02$ \\
\hline 6.90 & $.02365 \mathrm{E}$ & 00 & $-6.45949 \mathrm{E}$ & 00 & $-7.22010 \mathrm{E}-02$ \\
\hline 7.00 & $1.02364 \mathrm{E}$ & 00 & $-6.56073 \mathrm{E}$ & 00 & $-7.40055 \mathrm{E}-02$ \\
\hline 7.10 & $1.02363 \mathrm{E}$ & 00 & $-6.66197 \mathrm{E}$ & 00 & $-7.58308 \mathrm{E}-02$ \\
\hline 20 & $1.02362 \mathrm{E}$ & 00 & $-6.76321 \mathrm{E}$ & 00 & $-7.76772 \mathrm{E}-02$ \\
\hline 30 & $1.02362 \mathrm{E}$ & 00 & $-6.86444 \mathrm{E}$ & 00 & $-7.95444 \mathrm{E}-02$ \\
\hline & $61 \mathrm{E}$ & 00 & $-6.96567 \mathrm{E}$ & 00 & $-8.14325 \mathrm{E}-02$ \\
\hline 7.50 & $1.02360 \mathrm{E}$ & 00 & $-7.06690 \mathrm{E}$ & 00 & $3416 E-02$ \\
\hline 7.60 & $1.02360 \mathrm{E}$ & 00 & $-7.16812 \mathrm{E}$ & 00 & $-8.52716 \mathrm{E}-02$ \\
\hline 7.70 & $1.02359 \mathrm{E}$ & 00 & $-7.26935 \mathrm{E}$ & 00 & $-8.72226 \mathrm{E}-02$ \\
\hline .80 & $.02359 \mathrm{E}$ & 00 & $-7.37056 \mathrm{E}$ & 00 & $-8.91945 \mathrm{E}-02$ \\
\hline 90 & $58 \mathrm{E}$ & 00 & $7178 \mathrm{E}$ & 00 & $-9.11873 \mathrm{E}-02$ \\
\hline 8.00 & $1.02358 \mathrm{E}$ & 00 & $7299 \mathrm{E}$ & 00 & $32010 \mathrm{E}-02$ \\
\hline 8.10 & $1.02357 \mathrm{E}$ & 00 & $-7.67420 \mathrm{E}$ & 00 & $-9.52356 \mathrm{E}-02$ \\
\hline 20 & $1.02356 \mathrm{E}$ & 00 & $-7.77541 \mathrm{E}$ & 00 & $-9.72912 \mathrm{E}-02$ \\
\hline 8.30 & $1.02356 \mathrm{E}$ & 00 & $-7.87661 \mathrm{E}$ & 00 & $-9.93677 \mathrm{E}-02$ \\
\hline 40 & $56 \mathrm{E}$ & 00 & $781 \mathrm{E}$ & 00 & $-1.01465 \mathrm{E}-01$ \\
\hline 8.50 & $355 \mathrm{E}$ & 00 & $7901 \mathrm{E}$ & 00 & $3584 \mathrm{E}-01$ \\
\hline 8.60 & $.02355 \mathrm{E}$ & 00 & $-8.18021 \mathrm{E}$ & 00 & $-1.05723 \mathrm{E}-01$ \\
\hline 70 & $.02354 \mathrm{E}$ & 00 & $-8.28140 E$ & 00 & $-1.07883 \mathrm{E}-01$ \\
\hline 8.80 & $54 \mathrm{E}$ & 00 & $-8.38259 \mathrm{E}$ & 00 & $-1.10064 \mathrm{E}-$ \\
\hline 8.90 & $53 \mathrm{E}$ & 00 & $377 \mathrm{E}$ & 00 & $2266 \mathrm{E}-01$ \\
\hline 9.00 & $1.02353 \mathrm{E}$ & 00 & $-8.58496 \mathrm{E}$ & 00 & $.14489 \mathrm{E}-01$ \\
\hline 9.10 & $.02353 \mathrm{E}$ & 00 & $8614 \mathrm{E}$ & 00 & $-1.16733 \mathrm{E}-01$ \\
\hline 20 & $1.02352 \mathrm{E}$ & 00 & $3732 \mathrm{E}$ & 00 & $-1.18998 \mathrm{E}-01$ \\
\hline 9.30 & & 00 & -8.8 & 00 & 01 \\
\hline 9.40 & & 00 & -8 & 00 & $1 \mathrm{E}-01$ \\
\hline 9.50 & $1.02351 \mathrm{E}$ & 00 & $-9.09084 \mathrm{E}$ & 00 & $-1.25918 \mathrm{E}-01$ \\
\hline 9.60 & $51 \mathrm{E}$ & 00 & $-9.19200 \mathrm{E}$ & 00 & $8267 \mathrm{E}-01$ \\
\hline 9.70 & $1 \mathrm{E}$ & 00 & $17 \mathrm{E}$ & 00 & $36 E-01$ \\
\hline 9.8 & & 00 & -9 & 00 & $27 \mathrm{E}-$ \\
\hline 9.90 & $2350 \mathrm{E}$ & 00 & $-9.49549 \mathrm{E}$ & 00 & $-1.35438 \mathrm{E}-01$ \\
\hline 10.00 & $1.02350 \mathrm{E}$ & 00 & $-9.59665 \mathrm{E}$ & 00 & $-1.37870 \mathrm{E}-01$ \\
\hline 10 & $49 \mathrm{E}$ & 00 & $-9.69780 \mathrm{E}$ & 00 & $0323 E-01$ \\
\hline & $49 \mathrm{E}$ & 00 & -9 & 0 & \\
\hline & & 0 & & 00 & $5293 \mathrm{E}-$ \\
\hline & $1.02349 \mathrm{E}$ & 00 & $-1.00012 \mathrm{E}$ & 01 & $-1.47809 \mathrm{E}-01$ \\
\hline & $1.02348 \mathrm{E}$ & 00 & $-1.01024 \mathrm{E}$ & 01 & $-1.50345 \mathrm{E}-01$ \\
\hline 60 & $1.02348 \mathrm{E}$ & 00 & $-1.02035 \mathrm{E}$ & 01 & $2903 E-01$ \\
\hline & & 00 & & 01 & $32 E-01$ \\
\hline & $1.02348 \mathrm{E}$ & 00 & $-1.04058 \mathrm{E}$ & 01 & $-1.58082 \mathrm{E}-01$ \\
\hline & $1.02347 \mathrm{E}$ & 00 & $-1.05069 \mathrm{E}$ & 01 & $-1.60702 \mathrm{E}-01$ \\
\hline & $1.02347 \mathrm{E}$ & 00 & $-1.06081 \mathrm{E}$ & 01 & $-1.63344 \mathrm{E}-0 \mathrm{I}$ \\
\hline 1 & $1.02347 \mathrm{E}$ & 00 & $-1.07092 \mathrm{E}$ & 01 & $-1.66006 \mathrm{E}-01$ \\
\hline & & 00 & $-1.08103 \mathrm{E}$ & 01 & $-1.68689 \mathrm{E}-01$ \\
\hline & $1.02347 \mathrm{E}$ & 00 & $-1.09115 \mathrm{E}$ & 01 & $-1.71394 \mathrm{E}-01$ \\
\hline & $1.02346 \mathrm{E}$ & 0 & $-1.10126 \mathrm{E}$ & 01 & $-1.74119 \mathrm{E}-01$ \\
\hline & $1.02346 \mathrm{E}$ & 00 & $-1.11137 \mathrm{E}$ & 01 & $-1.76865 E-01$ \\
\hline & $1.02346 \mathrm{E}$ & 00 & $-1.12148 \mathrm{E}$ & 01 & $-1.79632 \mathrm{E}-01$ \\
\hline & $1.02346 \mathrm{E}$ & 00 & $-1.13159 \mathrm{E}$ & 01 & $-1.82420 \mathrm{E}-01$ \\
\hline & $1.02346 \mathrm{E}$ & 0 & $-1.14170 \mathrm{E}$ & 01 & $-1.85229 \mathrm{E}-01$ \\
\hline & $1.02345 \mathrm{E}$ & 00 & $-1.15181 \mathrm{E}$ & 01 & $-1.88059 \mathrm{E}-01$ \\
\hline & & 00 & $-1.16193 \mathrm{E}$ & 01 & $-1.90909 \mathrm{E}-01$ \\
\hline
\end{tabular}

$\tan \delta_{1}$ $f_{-1}$

$1.54946 \mathrm{E}-05$ $2.48951 \mathrm{E}-05$ $3.40009 \mathrm{E}-05$ $4.28265 \mathrm{E}-05$ 5.13820 E- 05

$5.96808 \mathrm{E}-05$ $6.77346 \mathrm{E}-05$ $7.55544 \mathrm{E}-05$ $8.31487 \mathrm{E}-05$ $9.05268 \mathrm{E}-05$

$9.77018 \mathrm{E}-05$ $1.04678 \mathrm{E}-04$

$1.11464 \mathrm{E}-04$ $1.18069 \mathrm{E}-04$

$1.24499 \mathrm{E}-04$

$1.30761 \mathrm{E}-04$ $1.36862 \mathrm{E}-04$

$1.42806 \mathrm{E}-04$

$1.48603 \mathrm{E}-04$

$1.54254 \mathrm{E}-04$

$1.59767 \mathrm{E}-04$

$1.65147 \mathrm{E}-04$

$1.70397 \mathrm{E}-04$

$1.75524 \mathrm{E}-04$

$1.80531 \mathrm{E}-04$

$1.85422 \mathrm{E}-04$ $1.90201 \mathrm{E}-04$ $1.94871 \mathrm{E}-04$ $1.99438 \mathrm{E}-04$ $2.03902 \mathrm{E}-04$

$2.08271 \mathrm{E}-04$

$2.12543 \mathrm{E}-04$

$2.16726 \mathrm{E}-04$

$2.20818 \mathrm{E}-04$.

$2.24825 \mathrm{E}-04$

$2.28749 \mathrm{E}-04$ $2.32592 \mathrm{E}-04$

$2.36357 \mathrm{E}-04$

$2.40046 \mathrm{E}-04$

2.43662 E-04

$2.47205 \mathrm{E}-04$

$2.50680 \mathrm{E}-04$

$2.54087 \mathrm{E}-04$

$2.57428 \mathrm{E}-04$

$2.60707 \mathrm{E}-04$

$2.63923 \mathrm{E}-04$

$2.67079 \mathrm{E}-04$

$2.70177 \mathrm{E}-04$

$2.73219 \mathrm{E}-04$

$2.76203 \mathrm{E}-04$.

$2.79135 \mathrm{E}-04$ $2.82015 \mathrm{E}-04$

$2.84844 \mathrm{E}-04$

$2.87621 \mathrm{E}-04$

$2.90353 \mathrm{E}-04$

$2.93035 \mathrm{E}-04$ $2.95672 \mathrm{E}-04$.

$2.98265 \mathrm{E}-04$

$3.00813 \mathrm{E}-04$

$3.03320 \mathrm{E}-04$

\section{$-5.51144 \mathrm{E}-02$ $-5.66465 \mathrm{E}-02$ $-5.81996 \mathrm{E}-02$ $-5.97736 \mathrm{E}-02$ $-6.13685 \mathrm{E}-02$}

$-6.29844 \mathrm{E}-02$

$-6.46211 \mathrm{E}-02$

$-6.62788 \mathrm{E}-02$

$-6.79574 \mathrm{E}-02$

$-6.96569 \mathrm{E}-02$

$-7.13773 \mathrm{E}-02$

$-7.31187 \mathrm{E}-02$

$-7.48810 \mathrm{E}-02$

$-7.66642 \mathrm{E}-02$

$-7.84683 \mathrm{E}-02$

$-8.02933 \mathrm{E}-02$

$-8.21393 \mathrm{E}-02$

$-8.40062 \mathrm{E}-02$

$-8.58941 \mathrm{E}-02$

$-8.78028 \mathrm{E}-02$

$-8.97325 \mathrm{E}-02$

$-9.16831 \mathrm{E}-02$

$-9.36546 \mathrm{E}-02$

$-9.56471 \mathrm{E}-02$

$-9.76605 \mathrm{E}-02$

$-9.96948 \mathrm{E}-02$

$-1.01750 \mathrm{E}-01$

$-1.03826 \mathrm{E}-01$

$-1.05923 \mathrm{E}-01$

$-1.08041 \mathrm{E}-01$

$-1.10180 \mathrm{E}-01$

$-1.12340 \mathrm{E}-01$

$-1.14521 \mathrm{E}-01$

$-1.16723 \mathrm{E}-01$

$-1.18945 \mathrm{E}-01$

$-1.21189 \mathrm{E}-01$

$-1.23453 \mathrm{E}-01$

$-1.25739 \mathrm{E}-01$

$-1.28045 \mathrm{E}-01$

$-1.30372 \mathrm{E}-01$

$-1.32721 \mathrm{E}-01$

$-1.35090 \mathrm{E}-01$

$-1.37480 \mathrm{E}-01$

$-1.39891 \mathrm{E}-01$

$-1.42323 \mathrm{E}-01$

$-1.44776 \mathrm{E}-01$

$-1.47249 \mathrm{E}-01$

$-1.49744 \mathrm{E}-01$

$-1.52260 \mathrm{E}-01$

$-1.54796 \mathrm{E}-01$

$-1.57354 \mathrm{E}-01$

$-1.59932 \mathrm{E}-01$

$-1.62531 \mathrm{E}-01$

$-1.65152 \mathrm{E}-01$

$-1.67793 \mathrm{E}-01$

$-1.70455 \mathrm{E}-01$

$-1.73138 \mathrm{E}-01$

$-1.75842 \mathrm{E}-01$

$-1.78566 \mathrm{E}-01$

$-1.81312 \mathrm{E}-01$

\section{$g_{-1}$}

$6.65106 \mathrm{E} \quad 00$ $6.75263 \mathrm{E} \quad 00$ $6.85419 \mathrm{E} \quad 00$ $6.95572 \mathrm{E} \quad 00$ $7.05724 \mathrm{E} \quad 00$

$7.15875 \mathrm{E} \quad 00$ $7.26024 \mathrm{E} \quad 00$ $7.36171 \mathrm{E} \quad 00$

$7.46318 \mathrm{E} \quad 00$

$7.56463 \mathrm{E} \quad 00$

$7.66607 \mathrm{E} \quad 00$ $7.76749 \mathrm{E} \quad 00$ $7.86891 \mathrm{E} \quad 00$ $7.97031 \mathrm{E} \quad 00$ $8.07171 \mathrm{E} \quad 00$

$8.17309 \mathrm{E} \quad 00$ $8.27447 \mathrm{E} \quad 00$ $8.37584 \mathrm{E} \quad 00$ $8.47719 \mathrm{E} \quad 00$ $8.57854 \mathrm{E} \quad 00$

$8.67988 \mathrm{E} \quad 00$ $8.78121 \mathrm{E} \quad 00$ $8.88254 \mathrm{E} \quad 00$ $8.98386 \mathrm{E} \quad 00$ $9.08517 \mathrm{E} \quad 00$

$9.18647 \mathrm{E} \quad 00$ $9.28776 \mathrm{E} \quad 00$ $9.38905 \mathrm{E} \quad 00$ $9.49034 \mathrm{E} \quad 00$ $9.59161 \mathrm{E} \quad 00$

$9.69288 \mathrm{E} \quad 00$ $9.79415 \mathrm{E} \quad 00$ $9.89540 \mathrm{E} \quad 00$ $9.99666 \mathrm{E} \quad 00$ $1.00979 \mathrm{E} \quad 01$

$1.01991 \mathrm{E} \quad 01$ $1.03004 \mathrm{E} \quad 01$ $1.04016 \mathrm{E} \quad 01$ $1.05028 \mathrm{E} \quad 01$ $1.06041 \mathrm{E} \quad 01$

$1.07053 \mathrm{E} \quad 01$ $1.08065 \mathrm{E} \quad 01$ $1.09077 \mathrm{E} 01$ $1.10089 \mathrm{E} \quad 0 \mathrm{I}$ $1.11101 \mathrm{E} \quad 01$

$1.12113 \mathrm{E} \quad 01$ $1.13125 \mathrm{E} \quad 01$ $1.14137 \mathrm{E} \quad 01$

$1.15148 \mathrm{E} \quad 01$

$1.16160 \mathrm{E} 01$

$1.17172 \mathrm{E} \quad 01$ $1.18184 \mathrm{E} 01$ $1.19195 \mathrm{E} 01$ $1.20207 \mathrm{E} \quad 01$ $1.21218 \mathrm{E} 01$

$1.22230 \mathrm{E} \quad 01$ $1.23241 \mathrm{E} 01$ $1.24253 \mathrm{E} 01$ $1.25264 \mathrm{E} \quad 01$ $1.26276 \mathrm{E} 01$ $\tan \delta_{-1}$

$-8.24997 \mathrm{E} \quad 02$ $-8.31788 \mathrm{E} \quad 02$ $-8.38469 \mathrm{E} \quad 02$ $\begin{array}{ll}-8.45041 \mathrm{E} & 02\end{array}$ -8.51509 E 02

$\begin{array}{ll}-8.57874 \mathrm{E} & 02\end{array}$ $-8.64139 \mathrm{E} \quad 02$ $-8.70305 \mathrm{E} \quad 02$ $-8.76377 \mathrm{E} \quad 02$ $-8.82357 \mathrm{E} 02$

$-8.88243 \mathrm{E} \quad 02$ $-8.94040 \mathrm{E} \quad 02$ $-8.99752 \mathrm{E} \quad 02$ $-9.05377 \mathrm{E} \quad 02$ $-9.10920 \mathrm{E} 02$

$-9.16381 \mathrm{E} \quad 02$ $-9.21763 \mathrm{E} \quad 02$ $-9.27067 \mathrm{E} \quad 02$ $-9.32295 \mathrm{E} \quad 02$ $-9.37449 \mathrm{E} \quad 02$

$-9.42530 \mathrm{E} \quad 02$ $-9.47539 \mathrm{E} \quad 02$ $-9.52479 \mathrm{E} \quad 02$ $\begin{array}{ll}-9.57350 \mathrm{E} & 02\end{array}$ $-9.62154 \mathrm{E} \quad 02$

$-9.66892 \mathrm{E} \quad 02$ $-9.71567 \mathrm{E} \quad 02$ $-9.76180 \mathrm{E} \quad 02$ $-9.80730 \mathrm{E} \quad 02$ $-9.85220 \mathrm{E} \quad 02$

$-9.89651 \mathrm{E} \quad 02$ $-9.94024 \mathrm{E} \quad 02$ $-9.98339 \mathrm{E} 02$ $-1.00260 \mathrm{E} \quad 03$ $-1.00681 \mathrm{E} 03$

$-1.01096 \mathrm{E} \quad 03$ $-1.01506 \mathrm{E} \quad 03$ $-1.01911 \mathrm{E} \quad 03$ $-1.02310 \mathrm{E} \quad 03$ $-1.02705 \mathrm{E} \quad 03$

$-1.03095 \mathrm{E} 03$ $-1.03480 \mathrm{E} 03$ $-1.03861 \mathrm{E} 03$ $-1.04237 \mathrm{E} 03$ $-1.04608 \mathrm{E} \quad 03$

$\begin{array}{rl}-1.04975 \mathrm{E} & 03\end{array}$ $-1.05338 \mathrm{E} \quad 03$ $-1.05696 \mathrm{E} \quad 03$ $-1.06050 \mathrm{E} \quad 03$ $-1.06401 \mathrm{E} 03$

$-1.06747 \mathrm{E} \quad 03$ $-1.07089 \mathrm{E} 03$ $-1.07427 \mathrm{E} 03$ $-1.07761 \mathrm{E} \quad 03$ $-1.08092 \mathrm{E} \quad 03$

$-1.08418 \mathrm{E} 03$ $-1.08742 \mathrm{E} 03$ $-1.09061 \mathrm{E} 03$ $-1.09377 \mathrm{E} 03$ $-1.09690 \mathrm{E} \quad 03$ 
$\bar{F}$

$6.10 \quad 1.02173 \mathrm{E}-05$

$6.20 \quad 1.05549 \mathrm{E}-05$

$6.30 \quad 1.08980 \mathrm{E}-05$

$6.40 \quad 1.12467 \mathrm{E}-05$

$6.50 \quad 1.16008 \mathrm{E}-05$

$6.60 \quad 1.19603 \mathrm{E}-05$

$6.70 \quad 1.23254 \mathrm{E}-05$

$6.80 \quad 1.26960 \mathrm{E}-05$

$6.90 \quad 1.30721 \mathrm{E}-05$

$7.00 \quad 1.34536 \mathrm{E}-05$

$7.10 \quad 1.38406 \mathrm{E}-05$

$7.20 \quad 1.42332 \mathrm{E}-05$

$7.30 \quad 1.46312 \mathrm{E}-05$

$7.40 \quad 1.50347 \mathrm{E}-05$

$7.50 \quad-1.54437 \mathrm{E}-05$

$7.60 \quad 1.58582 \mathrm{E}-05$

$7.70 \quad 1.62782 \mathrm{E}-05$

$7.80 \quad 1.67036 \mathrm{E}-05$

$7.90 \quad 1.71346 \mathrm{E}-05$

$8.00 \quad 1.75710 \mathrm{E}-05$

$8.10 \quad 1.80130 \mathrm{E}-05$

$8.20 \quad 1.84604 \mathrm{E}-05$

$8.30 \quad 1.89133 \mathrm{E}-05$

$8.40 \quad 1.93717 \mathrm{E}-05$

$8.50 \quad 1.98356 \mathrm{E}-05$

$8.60 \quad 2.03050 \mathrm{E}-05$

$8.70 \quad 2.07799 \mathrm{E}-05$

$8.80 \quad 2.12603 \mathrm{E}-05$

$8.90 \quad 2.17461 \mathrm{E}-05$

$9.00 \quad 2.22374 \mathrm{E}-05$

$9.10 \quad 2.27343 \mathrm{E}-05$

$9.20 \quad 2.32366 \mathrm{E}-05$

$9.30 \quad 2.37444 \mathrm{E}-05$

$9.40 \quad 2.42577 \mathrm{E}-05$

$9.50 \quad 2.47765 \mathrm{E}-05$

$9.60 \quad 2.53008 \mathrm{E}-05$

$9.70 \quad 2.58306 \mathrm{E}-05$

$9.80 \quad 2.63658 \mathrm{E}-05$

$9.90 \quad 2.69066 \mathrm{E}-05$

$10.00 \quad 2.74528 \mathrm{E}-05$

10.10

10.20

10.30

10.40

10.50

$2.80046 \mathrm{E}-05$

$2.85618 \mathrm{E}-05$

$2.91245 \mathrm{E}-05$

$2.96927 \mathrm{E}-05$

$3.02664 \mathrm{E}-05$

10.60

10.70

10.80

10.90

11.00

11.10

11.20

11.30

11.40

11.50

11.60

11.70

11.80

11.90

12.00

$3.08456 \mathrm{E}-05$

$3.14302 \mathrm{E}-05$

$3.20204 \mathrm{E}-05$

$3.26160 \mathrm{E}-05$

$3.32172 \mathrm{E}-05$

$3.38238 \mathrm{E}-05$ $3.44359 \mathrm{E}-05$

$3.50535 \mathrm{E}-05$

$3.56766 \mathrm{E}-05$

$3.63052 \mathrm{E}-05$

$3.69393 \mathrm{E}-05$

$3.75789 \mathrm{E}-05$

$3.82239 \mathrm{E}-05$

$3.88745 \mathrm{E}-05$

$3.95305 \mathrm{E}-05$ $f_{2}$

$-3.56951 \mathrm{E}-02$

$-3.69308 \mathrm{E}-02$

$-3.81876 \mathrm{E}-02$

$-3.94653 \mathrm{E}-02$

$-4.07639 \mathrm{E}-02$

$-4.20836 \mathrm{E}-02$

$-4.34242 \mathrm{E}-02$

$-4.47857 \mathrm{E}-02$

$-4.61683 \mathrm{E}-02$

$-4.75718 \mathrm{E}-02$

$-4.89963 \mathrm{E}-02$

$-5.04417 \mathrm{E}-02$

$-5.19081 \mathrm{E}-02$

$-5.33955 \mathrm{E}-02$

$-5.49038 \mathrm{E}-02$

$-5.64331 \mathrm{E}-02$

$-5.79833 \mathrm{E}-02$

$-5.95545 \mathrm{E}-02$

$-6.11467 \mathrm{E}-02$

$-6.27598 \mathrm{E}-02$

$-6.43939 \mathrm{E}-02$

$-6.60490 \mathrm{E}-02$

$-6.77250 \mathrm{E}-02$

$-6.94219 \mathrm{E}-02$

$-7.11398 \mathrm{E}-02$

$-7.28787 \mathrm{E}-02$

$-7.46385 \mathrm{E}-02$

$-7.64193 \mathrm{E}-02$

$-7.82210 \mathrm{E}-02$

$-8.00437 \mathrm{E}-02$

$-8.18874 \mathrm{E}-02$

$-8.37520 \mathrm{E}-02$

$-8.56375 \mathrm{E}-02$

$-8.75440 \mathrm{E}-02$

$-8.94715 \mathrm{E}-02$

$-9.14199 \mathrm{E}-02$

$-9.33892 \mathrm{E}-02$

$-9.53795 \mathrm{E}-02$

$-9.73908 \mathrm{E}-02$

$-9.94230 \mathrm{E}-02$

$-1.01476 \mathrm{E}-01$

$-1.03550 \mathrm{E}-01$

$-1.05645 \mathrm{E}-01$

$-1.07761 \mathrm{E}-01$

$-1.09898 \mathrm{E}-01$

$-1.12056 \mathrm{E}-01$

$-1.14235 \mathrm{E}-01$

$-1.16435 \mathrm{E}-01$

$-1.18655 \mathrm{E}-01$

$-1.20897 \mathrm{E}-01$

$-1.23160 \mathrm{E}-01$

$-1.25443 \mathrm{E}-01$

$-1.27748 \mathrm{E}-01$

$-1.30073 \mathrm{E}-01$

$-1.32420 \mathrm{E}-01$

$-1.34787 \mathrm{E}-01$

$-1.37175 \mathrm{E}-01$

$-1.39585 \mathrm{E}-01$

$-1.42015 \mathrm{E}-01$

$-1.44466 \mathrm{E}-01$ $g_{2}$

$-2.18862 \mathrm{E}-04$

$-2.28704 \mathrm{E}-04$

$-2.38831 \mathrm{E}-04$

$-2.49245 \mathrm{E}-04$

$-2.59950 \mathrm{E}-04$

$-2.70951 \mathrm{E}-04$

$-2.82252 \mathrm{E}-04$

$-2.93855 \mathrm{E}-04$

$-3.05766 \mathrm{E}-04$

$-3.17988 \mathrm{E}-04$

$-3.30525 \mathrm{E}-04$

$-3.43381 \mathrm{E}-04$

$-3.56560 \mathrm{E}-04$

$-3.83901 \mathrm{E}-04$

$-3.98072 \mathrm{E}-04$

$-4.12581 \mathrm{E}-04$

$-4.27432 \mathrm{E}-04$

$-4.42629 \mathrm{E}-04$

$-4.58177 \mathrm{E}-04$

$-4.74079 \mathrm{E}-04$

$-4.90338 \mathrm{E}-04$

$-5.06960 \mathrm{E}-04$

$-5.23947 \mathrm{E}-04$

$-5.41304 \mathrm{E}-04$

$-5.59034 \mathrm{E}-04$ $-5.77142 \mathrm{E}-04$

$-5.95631 \mathrm{E}-04$

$-6.14506 \mathrm{E}-04$

$-6.33769 \mathrm{E}-04$

$-6.53426 \mathrm{E}-04$

$-6.73480 \mathrm{E}-04$

$-6.93935 \mathrm{E}-04$

$-7.14794 \mathrm{E}-04$

$-7.36063 \mathrm{E}-04$

$-7.57743 \mathrm{E}-04$

$-7.79841 \mathrm{E}-04$

$-8.02358 \mathrm{E}-04$

$-8.25300 \mathrm{E}-04$

$-8.48670 \mathrm{E}-04$

$-8.72473 \mathrm{E}-04$

$-8.96711 \mathrm{E}-04$

$-9.21390 \mathrm{E}-04$

$-9.46512 \mathrm{E}-04$

$-9.72082 \mathrm{E}-04$

$-9.98104 \mathrm{E}-04$

$-1.02458 \mathrm{E}-03$

$-1.05152 \mathrm{E}-03$

$-1.07892 \mathrm{E}-03$

$-1.10678 \mathrm{E}-03$

$-1.13512 \mathrm{E}-03$ $-1.16394 \mathrm{E}-03$ $-1.19323 \mathrm{E}-03$ $-1.22300 \mathrm{E}-03$ $-1.25327 \mathrm{E}-03$

$-1.28402 \mathrm{E}-\mathrm{-0} 3$

$-1.34701 \mathrm{E}-03$

$-1.37926 \mathrm{E}-03$

$-1.41202 \mathrm{E}-03$
$-3.70065 \mathrm{E}-04$

$\tan \delta_{2}$

$1.90405 \mathrm{E} \quad 02$

$1.90656 \mathrm{E} \quad 02$

$1.90898 \mathrm{E} \quad 02$

$1.91132 \mathrm{E} \quad 02$

$1.91359 \mathrm{E} \quad 02$

$1.91579 \mathrm{E} \quad 02$

$1.91791 \mathrm{E} \quad 02$

$1.91997 \mathrm{E} \quad 02$

$1.92196 \mathrm{E} \quad 02$

$1.92389 \mathrm{E} \quad 02$

$1.92577 \mathrm{E} \quad 02$

$1.92759 \mathrm{E} \quad 02$

$1.92935 \mathrm{E} \quad 02$

$1.93107 \mathrm{E} \quad 02$

$1.93274 \mathrm{E} \quad 02$

$1.93436 \mathrm{E} \quad 02$

$1.93593 \mathrm{E} \quad 02$

$1.93747 \mathrm{E} \quad 02$

$1.93896 \mathrm{E} \quad 02$

$1.94041 \mathrm{E} \quad 02$

$1.94182 \mathrm{E} \quad 02$

$1.94320 \mathrm{E} \quad 02$

$1.94454 \mathrm{E} \quad 02$

$1.94585 \mathrm{E} \quad 02$

$1.94712 \mathrm{E} \quad 02$

$1.94837 \mathrm{E} \quad 02$

$1.94958 \mathrm{E} \quad 02$

$1.95076 \mathrm{E} \quad 02$

$1.95192 \mathrm{E} \quad 02$

$1.95305 \mathrm{E} \quad 02$

$1.95415 \mathrm{E} \quad 02$

$1.95523 \mathrm{E} \quad 02$

$1.95628 \mathrm{E} \quad 02$

$1.95731 \mathrm{E} \quad 02$

$1.95831 \mathrm{E} \quad 02$

$1.95930 \mathrm{E} \quad 02$

$1.96026 \mathrm{E} \quad 02$

$1.96120 \mathrm{E} \quad 02$

$1.96212 \mathrm{E} \quad 02$

$1.96302 \mathrm{E} \quad 02$

$1.96390 \mathrm{E} \quad 02$

$1.96477 \mathrm{E} \quad 02$

$1.96561 \mathrm{E} \quad 02$

$1.96644 \mathrm{E} \quad 02$

$1.96726 \mathrm{E} \quad 02$

$-1.31527 \mathrm{E}-03$

$f_{-2}$

$-2.05451 \mathrm{E}-04$

$-2.14669 \mathrm{E}-04$

$-2.24158 \mathrm{E}-04$

-2.33921 E-04

$-2.43963 \mathrm{E}-04$

$-2.54287 \mathrm{E}-04$

$-2.64897 \mathrm{E}-04$

$-2.75798 \mathrm{E}-04$

$-2.86992 \mathrm{E}-04$

$-2.98485 \mathrm{E}-04$

$-3.10279 \mathrm{E}-04$

$-3.22379 \mathrm{E}-04$

$-3.34789 \mathrm{E}-04$

$-3.47512 \mathrm{E}-04$

$-3.60553 \mathrm{E}-04$

$-3.73916 \mathrm{E}-04$

$-3.87603 \mathrm{E}-04$

$-4.01620 \mathrm{E}-04$

$-4.15970 \mathrm{E}-04$

$-4.30657 \mathrm{E}-04$

$-4.45685 \mathrm{E}-04$

$-4.61058 \mathrm{E}-04$

$-4.76779 \mathrm{E}-04$

$-4.92853 \mathrm{E}-04$

$-5.09284 \mathrm{E}-04$

$-5.26075 \mathrm{E}-04$

$-5.43231 \mathrm{E}-04$

$-5.60754 \mathrm{E}-04$

$-5.78650 \mathrm{E}-04$

$-5.96922 \mathrm{E}-04$

$-6.15574 \mathrm{E}-04$

$-6.34610 \mathrm{E}-04$

$-6.54033 \mathrm{E}-04$

$-6.73849 \mathrm{E}-04$

$-6.94060 \mathrm{E}-04$

$-7.14670 \mathrm{E}-04$

$-7.35684 \mathrm{E}-04$ 


\section{ELECTRON RADIAL FUNCTIONS}

D

$F_{0}$

\section{$f_{1}$}

12.10

12.20

12.30

12.40

12.50

12.60

12.70

12.80

12.90

13.00

13.10

13.20

13.30

13.40

13.50

13.60

13.70

13.80

13.90

14.00

14.10

14.20

14.30

14.40

14.50

14.60

14.70

14.80

14.90

15.00

15.10

15.20

15.30

15.40

15.50

15.60

15.70

15.80

15.90

16.00

16.10

16.20

16.30

16.40

16.50

16.60

16.70

16.80

16.90

17.00

17.10

17.20

17.30

17.40

17.50

17.60

17.70

17.80

17.90

18.00
$1.02345 \mathrm{E} \quad 00$

$1.02345 \mathrm{E} 00$

$1.02345 \mathrm{E} \quad 00$

$1.02345 \mathrm{E} \quad 00$

$1.02344 \mathrm{E} \quad 00$

$1.02344 \mathrm{E} \quad 00$

$1.02344 \mathrm{E} \quad 00$

$1.02344 \mathrm{E} \quad 00$

$1.02344 \mathrm{E} \quad 00$

$1.02344 \mathrm{E} \quad 00$

$1.02343 \mathrm{E} \quad 00$

$1.02343 \mathrm{E} \quad 00$

$1.02343 \mathbf{E} 00$

$1.02343 \mathrm{E} \quad 00$

$1.02343 \mathrm{E} 00$

$1.02343 \mathrm{E} \quad 00$

$1.02343 \mathrm{E} \quad 00$

$\begin{array}{ll}1.02343 \mathrm{E} & 00\end{array}$

$1.02342 \mathrm{E} \quad 00$

$1.02342 \mathrm{E} \quad 00$

$1.02342 \mathrm{E} \quad 00$

$1.02342 \mathrm{E} \quad 00$

$1.02342 \mathrm{E} \quad 00$

$1.02342 \mathrm{E} \quad 00$

$1.02342 \mathrm{E} \quad 00$

$1.02342 \mathrm{E} \quad 00$ $1.02341 \mathrm{E} \quad 00$ $1.02341 \mathrm{E} \quad 00$ $1.02341 \mathrm{E} \quad 00$

$1.02341 \mathrm{E} \quad 00$

$1.02341 \mathrm{E} \quad 00$

$1.02341 \mathrm{E} \quad 00$

$1.02341 \mathrm{E} \quad 00$

$1.02341 \mathrm{E} \quad 00$

$1.02341 \mathrm{E} \quad 00$

$1.02341 \mathrm{E} \quad 00$ $1.02340 \mathrm{E} \quad 00$ $1.02340 \mathrm{E} \quad 00$ $1.02340 \mathrm{E} \quad 00$ $1.02340 \mathrm{E} \quad 00$

$1.02340 \mathrm{E} \quad 00$ $1.02340 \mathrm{E} \quad 00$ $1.02340 \mathrm{E} \cdot 00$ $1.02340 \mathrm{E} \quad 00$ $1.02340 \mathrm{E} \quad 00$

$1.02340 \mathrm{E} \quad 00$ $1.02340 \mathrm{E} \quad 00$ $1.02339 \mathrm{E} \quad 00$ $1.02339 \mathrm{E} \quad 00$ $1.02339 \mathrm{E} \quad 00$

$1.02339 \mathrm{E} \quad 00$ $1.02339 \mathrm{E} \quad 00$ $1.02339 \mathrm{E} \quad 00$ $1.02339 \mathrm{E} \quad 00$ $1.02339 \mathrm{E} \quad 00$

$1.02339 \mathrm{E} \quad 00$ $1.02339 \mathrm{E} \quad 00$ $1.02339 \mathrm{E} \quad 00$ $1.02339 \mathrm{E} \quad 00$ $1.02339 \mathrm{E} \quad 00$
$-1.17204 \mathrm{E} \quad 01 \quad-1.93781 \mathrm{E}-01$ $-1.18215 \mathrm{E}$ 01 $-1.96674 \mathrm{E}-01$ $-1.19226 \mathrm{E}$ 01 $-1.99587 \mathrm{E}-01$ $-1.20237 \mathrm{E}$ 01 $-2.02521 \mathrm{E}-01$ $-1.21248 \mathrm{E} 01$

$-1.22258 \mathrm{E} \quad 01$ $-1.23269 \mathrm{E} 01$ $-1.24280 \mathrm{E} 01$ $-1.25291 \mathrm{E} 01$ $-1.26302 \mathrm{E} 01$

$-1.27313 \mathrm{E} \quad 01$ $-1.28323 \mathrm{E} 01$ $-1.29334 \mathrm{E} 01$ $-1.30345 \mathrm{E} 01$ $-1.31356 \mathrm{E} 01$

$-1.32366 \mathrm{E} 01$ $-1.33377 \mathrm{E} \quad 01$ $-1.34388 \mathrm{E} 01$ $-1.35398 \mathrm{E} 01$ $-1.36409 \mathrm{E} 01$

$-1.37419 \mathrm{E} 01$ $-1.38430 \mathrm{E} 01$ $-1.40451 \mathrm{E} 01$ $-1.41461 \mathrm{E} 01$

$-1.42472 \mathrm{E} 01$ $-1.43482 \mathrm{E} 01$ $-1.44493 \mathbf{E} 01$ $-1.46513 \mathrm{E} 01$

$\begin{array}{ll}-1.47524 \mathrm{E} & 01\end{array}$ $-1.48534 \mathrm{E} 01$ $-1.49544 \mathrm{E} 01$ $-1.50555 \mathrm{E} 01$ $-1.51565 \mathrm{E} 01$

$-1.52575 \mathrm{E} 01$ $-1.53585 \mathrm{E} 01$ $-1.54595 \mathrm{E} 01$ $-1.55606 \mathrm{E} 01$ $-1.56616 \mathrm{E} 01$

$-1.57626 \mathrm{E} 01$ $-1.58636 \mathrm{E} 01$ $-1.59646 \mathrm{E} 01$ $-1.60656 \mathrm{E} 01$ $-1.61666 \mathrm{E} 01$

$-1.62676 \mathrm{E} 01$ $-1.63686 \mathrm{E} 01$ $-1.64696 \mathrm{E} 01$ $-1.65706 \mathrm{E} 01$ $-1.66716 \mathrm{E} 01$

$\begin{array}{ll}-1.67725 \mathrm{E} & 01\end{array}$ $-1.68735 \mathrm{E} 01$ $-1.69745 \mathrm{E} 01$ $-1.70755 \mathrm{E} 01$ -1.71765 E 01

$-1.72774 \mathrm{E} \quad 01$ $-1.73784 \mathrm{E} 01$ $-1.74794 \mathrm{E} 01$ $-1.75803 \mathbf{E} 01$ $-1.76813 \mathrm{E} 01$ $-3.00278 \mathrm{E}-01$ -3.92031 E-01 $-1.39441 \mathrm{E} 01$ $-1.45503 \mathrm{E} 01$ $g_{1}$

$\tan \delta_{1}$

$3.05784 \mathrm{E}-04$

$3.08207 \mathrm{E}-04$

$3.10592 \mathrm{E}-04$

$3.12938 \mathrm{E}-04$

$3.15246 \mathrm{E}-04$

$-2.08453 \mathrm{E}-01$

$-2.11450 \mathrm{E}-01$

$-2.14468 \mathrm{E}-01$

$-2.17507 \mathrm{E}-01$

$-2.20567 \mathrm{E}-01$

$-2.23648 \mathrm{E}-01$ $-2.26750 \mathrm{E}-01$

$-2.29872 \mathrm{E}-01$

$-2.33016 \mathrm{E}-01$

$-2,36180 \mathrm{E}-01$

$-2.39366 \mathrm{E}-01$

$-2.42572 \mathrm{E}-01$

$-2.45799 \mathrm{E}-01$

$-2.49047 \mathrm{E}-01$

$-2.52316 \mathrm{E}-01$

$-2.55606 \mathrm{E}-01$

$-2.58917 \mathrm{E}-01$

$-2.62249 \mathrm{E}-01$

$-2.65602 \mathrm{E}-01$

$-2.68975 \mathrm{E}-01$

$-2.72370 \mathrm{E}-01$ $-2.75785 \mathrm{E}-01$ $-2.79221 \mathrm{E}-01$ $-2.82679 \mathrm{E}-01$ $-2.86157 \mathrm{E}-01$

$-2.89656 \mathrm{E}-01$ $-2.93176 \mathrm{E}-01$ $-2.96716 \mathrm{E}-01$ -3.03861 E-01

$-3.07464 \mathrm{E}-01$ $-3.11089 \mathrm{E}-01$ $-3.14734 \mathrm{E}-01$ $-3.18401 \mathrm{E}-01$ $-3.22088 \mathrm{E}-01$

$-3.25796 \mathrm{E}-01$ $-3.29525 \mathrm{E}-01$ $-3.33275 \mathrm{E}-01$ $-3.37046 \mathrm{E}-01$ $-3.40837 \mathrm{E}-01$

$-3,44650 \mathrm{E}-01$ $-3.48483 \mathrm{E}-01$ $-3.52338 \mathrm{E}-01$ -3.56213 E-01 $-3.60109 \mathrm{E}-01$

$-3.64026 \mathrm{E}-01$ $-3.67964 \mathrm{E}-01$ $-3.71923 \mathrm{E}-01$ $-3.75903 \mathrm{E}-01$ -3.79904 E-01

$-3.83925 \mathrm{E}-01$ $-3.87968 \mathrm{E}-01$ $-3.96115 \mathrm{E}-01$ $-4.00220 \mathrm{E}-01$

$3.17517 \mathrm{E}-04$

3.19752 E-04 $3.21952 \mathrm{E}-04$

$3.24118 \mathrm{E}-04$

$3.26249 \mathrm{E}-04$

$3.28350 \mathrm{E}-04$

$3.30417 \mathrm{E}-04$

$3.32452 \mathrm{E}-04$

$3.34458 \mathrm{E}-04$

$3.36434 \mathrm{E}-04$

3.38379 E- 04 $3.40297 \mathrm{E}-04$

$3.42187 \mathrm{E}-04$

3.44048 E-04

$3.45884 \mathrm{E}-04$

$3.47692 \mathrm{E}-04$

$3.49476 \mathrm{E}-04$

$3.51233 \mathrm{E}-04$

$3.52967 \mathrm{E}-04$

3.54675 E- 04

3.56361 E-04 $3.58023 \mathrm{E}-04$ $3.59662 \mathrm{E}-04$ $3.61278 \mathrm{E}-04$

$3.62874 \mathrm{E}-04$

$3.64448 \mathrm{E}-04$

$3.66000 \mathrm{E}-04$

$3.67533 \mathrm{E}-04$

3.69044 E-04

$3.70537 \mathrm{E}-04$

$3.72009 \mathrm{E}-04$

$3.73463 \mathrm{E}-04$

$3.74897 \mathrm{E}-04$

$3.76313 \mathrm{E}-04$

3.77711 E-04

$3.79093 \mathrm{E}-04$

$3.80455 \mathrm{E}-04$

3.81802 E-04 
ELECTRON RADIAL FUNCTIONS

$Z=1 \quad A=1 \quad \rho=1.2 A^{1 / 3} F$

Electron

$\bar{F}$

$4.01921 \mathrm{E}-05$ $4.08591 \mathrm{E}-05$ $4.15316 \mathrm{E}-05$ $4.22096 \mathrm{E}-05$ $4.28931 \mathrm{E}-05$

$4.35820 \mathrm{E}-05$ $4.42765 \mathrm{E}-05$ $4.49764 \mathrm{E}-05$ $4.56819 \mathrm{E}-05$ $4.63928 \mathrm{E}-05$

$4.71092 \mathrm{E}-05$ $4.78312 \mathrm{E}-05$ $4.85586 \mathrm{E}-05$ $4.92914 \mathrm{E}-05$ $5.00298 \mathrm{E}-05$

$5.07737 \mathrm{E}-05$ $5.15231 \mathrm{E}-05$ $5.22779 \mathrm{E}-05$ $5.30382 \mathrm{E}-05$ $5.38041 \mathrm{E}-05$

$5.45754 \mathrm{E}-05$ $5.53522 \mathrm{E}-05$ $5.61345 \mathrm{E}-05$ $5.69223 \mathrm{E}-05$ $5.77156 \mathrm{E}-05$

$5.85143 \mathrm{E}-05$ $5.93186 \mathrm{E}-05$ $6.01283 \mathrm{E}-05$ $6.09436 \mathrm{E}-05$ $6.17643 \mathrm{E}-05$

$6.25905 \mathrm{E}-05$ 6.34222 E-05 $6.42594 \mathrm{E}-05$ $6.51021 \mathrm{E}-05$ $6.59503 \mathrm{E}-05$

$6.68039 \mathrm{E}-05$ $6.76631 \mathrm{E}-05$ $6.85277 \mathrm{E}-05$ $6.93979 \mathrm{E}-05$ $7.02735 \mathrm{E}-05$

$7.11546 \mathrm{E}-05$ $7.20412 \mathrm{E}-05$ $7.29333 \mathrm{E}-05$ $7.38309 \mathrm{E}-05$ 7.47339 E- 05

$7.56425 \mathrm{E}-05$ $7.65565 \mathrm{E}-05$ $7.74761 \mathrm{E}-05$ $7.84011 \mathrm{E}-05$ $7.93316 \mathrm{E}-05$

$8.02676 \mathrm{E}-05$ $8.12091 \mathrm{E}-05$ $8.21561 \mathrm{E}-05$ $8.31086 \mathrm{E}-05$ $8.40666 \mathrm{E}-05$

$8.50300 \mathrm{E}-05$ $8.59990 \mathrm{E}-05$ $8.69734 \mathrm{E}-05$ $8.79533 \mathrm{E}-05$ $8.89387 \mathrm{E}-05$ $f_{2}$

$-1.46938 \mathrm{E}-01$ $-1.49431 \mathrm{E}-01$ $-1.51945 \mathrm{E}-01$ $-1.54480 \mathrm{E}-01$ $-1.57036 \mathrm{E}-01$

$-1.59612 \mathrm{E}-01$ $-1.62210 \mathrm{E}-01$ $-1.64829 \mathrm{E}-01$ $-1.67468 \mathrm{E}-01$ $-1.70129 \mathrm{E}-01$

$-1.72810 \mathrm{E}-01$

$-1.75513 \mathrm{E}-01$

$-1.78236 \mathrm{E}-01$

$-1.80980 \mathrm{E}-01$

$-1.83745 \mathrm{E}-01$

$-1.86531 \mathrm{E}-01$ $-1.89338 \mathrm{E}-01$ $-1.92166 \mathrm{E}-01$ $-1.95015 \mathrm{E}-01$ $-1.97885 \mathrm{E}-01$

$-2.00776 \mathrm{E}-01$ $-2.03688 \mathrm{E}-01$ $-2.06620 \mathrm{E}-01$ $-2.09574 \mathrm{E}-01$ $-2.12548 \mathrm{E}-01$

$-2.15544 \mathrm{E}-01$ $-2.18560 \mathrm{E}-01$ $-2.21598 \mathrm{E}-01$ $-2.24656 \mathrm{E}-01$ $-2.27735 \mathrm{E}-01$

$-2.30835 \mathrm{E}-01$ $-2.33956 \mathrm{E}-01$ $-2.37098 \mathrm{E}-01$ $-2.40261 \mathrm{E}-01$ $-2.43445 \mathrm{E}-01$

$-2.46649 \mathrm{E}-01$ $-2.49875 \mathrm{E}-01$ $-2.53121 \mathrm{E}-01$ $-2.56389 \mathrm{E}-01$ $-2.59677 \mathrm{E}-01$

$-2.62987 \mathrm{E}-01$ $-2.66317 \mathrm{E}-01$ $-2.69668 \mathrm{E}-01$ $-2.73040 \mathrm{E}-01$ $-2.76433 \mathrm{E}-01$

$-2.79847 \mathrm{E}-01$ $-2.83282 \mathrm{E}-01$ $-2.86738 \mathrm{E}-01$ $-2.90215 \mathrm{E}-01$ $-2.93712 \mathrm{E}-01$

$-2.97231 \mathrm{E}-01$ $-3.00770 \mathrm{E}-01$ $-3.04331 \mathrm{E}-01$ $-3.07912 \mathrm{E}-01$ $-3.11514 \mathrm{E}-01$

$-3.15137 \mathrm{E}-01$ $-3.18781 \mathrm{E}-01$ $-3.22446 \mathrm{E}-01$ $-3.26132 \mathrm{E}-01$ $-3.29839 \mathrm{E}-01$ $g_{2}$

$-1.44528 \mathrm{E}-03$

$-1.47906 \mathrm{E}-03$

$-1.51336 \mathrm{E}-03$

$-1.54818 \mathrm{E}-03$

$-1.58352 \mathrm{E}-03$

$-1.61939 \mathrm{E}-03$

$-1.65580 \mathrm{E}-03$

$-1.69275 \mathrm{E}-03$

$-1.73023 \mathrm{E}-03$

$-1.76827 \mathrm{E}-03$

$-1.80685 \mathrm{E}-03$

$-1.84598 \mathrm{E}-03$

$-1.88567 \mathrm{E}-03$

$-1.92593 \mathrm{E}-03$

$-1.96674 \mathrm{E}-03$

$-2.00813 \mathrm{E}-03$

$-2.05009 \mathrm{E}-03$

$-2.09262 \mathrm{E}-03$

$-2.13574 \mathrm{E}-03$

$-2.17944 \mathrm{E}-03$

$-2.22373 \mathrm{E}-03$

$-2.26861 \mathrm{E}-03$

$-2.31408 \mathrm{E}-03$

$-2.36016 \mathrm{E}-03$

$-2.40684 \mathrm{E}-03$

$-2.45413 \mathrm{E}-03$

$-2.50203 \mathrm{E}-03$

$-2.55054 \mathrm{E}-03$

$-2.59967 \mathrm{E}-03$

$-2.64943 \mathrm{E}-03$

$-2.69982 \mathrm{E}-03$

$-2.75083 \mathrm{E}-03$

$-2.80248 \mathrm{E}-03$

$-2.85477 \mathrm{E}-03$

$-2.90771 \mathrm{E}-03$

$-2.96129 \mathrm{E}-03$

$-3.01552 \mathrm{E}-03$

$-3.07040 \mathrm{E}-03$

$-3.12594 \mathrm{E}-03$

$-3.18215 \mathrm{E}-03$

$-3.23902 \mathrm{E}-03$

$-3.29656 \mathrm{E}-03$

$-3.35478 \mathrm{E}-03$

$-3.41367 \mathrm{E}-03$

$-3.47325 \mathrm{E}-03$

$-3.53351 \mathrm{E}-03$

$-3.59446 \mathrm{E}-03$

$-3.65610 \mathrm{E}-03$

$-3.71844 \mathrm{E}-03$

$-3.78149 \mathrm{E}-03$

$-3.84524 \mathrm{E}-03$ $-3.90969 \mathrm{E}-03$ $-3.97486 \mathrm{E}-03$ $-4.04075 \mathrm{E}-03$ $-4.10736 \mathrm{E}-03$

$-4.17469 \mathrm{E}-03$ $-4.24275 \mathrm{E}-03$ $-4.31155 \mathrm{E}-03$ $-4.38108 \mathrm{E}-03$ $-4.45135 \mathrm{E}-03$ $\tan \delta_{2}$

$1.97833 \mathrm{E} \quad 02$
$0.97893 \mathrm{E}$ $1.97893 \mathrm{E} \quad 02$ $\begin{array}{ll}1.97951 \mathrm{E} & 02\end{array}$ $1.98008 \mathrm{E} \quad 02$ $1.98064 \mathrm{E} \quad 02$

$1.98119 \mathrm{E} \quad 02$ $1.98173 \mathrm{E} \quad 02$ $1.98227 \mathrm{E} \quad 02$ $1.98279 \mathrm{E} \quad 02$ $1.98331 \mathrm{E} \quad 02$

$1.98382 \mathrm{E} \quad 02$ $1.98432 \mathrm{E} \quad 02$ $1.98481 \mathrm{E} \quad 02$ $1.98530 \mathrm{E} \quad 02$ $1.98577 \mathrm{E} \quad 02$

$1.98624 \mathrm{E} \quad 02$ $1.98671 \mathrm{E} \quad 02$ $1.98716 \mathrm{E} \quad 02$ $1.98761 \mathrm{E} \quad 02$ $1.98805 \mathrm{E} \quad 02$

$1.98849 \mathrm{E} \quad 02$ $1.98892 \mathrm{E} \quad 02$ $1.98934 \mathrm{E} \quad 02$ $1.98976 \mathrm{E} \quad 02$ $1.99017 \mathrm{E} \quad 02$

$1.99057 \mathrm{E} \quad 02$ $1.99097 \mathrm{E} \quad 02$ $1.99136 \mathrm{E} \quad 02$ $1.99175 \mathrm{E} \quad 02$ $1.99213 \mathrm{E} \quad 02$

$1.99251 \mathrm{E} \quad 02$ $1.99288 \mathrm{E} \quad 02$ $1.99325 \mathrm{E} \quad 02$ $1.99361 \mathrm{E} \quad 02$ $1.99397 \mathrm{E} \quad 02$

$1.99432 \mathrm{E} \quad 02$ $1.99467 \mathrm{E} \quad 02$ $1.99501 \mathrm{E} \quad 02$ $1.99535 \mathrm{E} \quad 02$ $1.99568 \mathrm{E} \quad 02$

$1.99601 \mathrm{E} \quad 02$ $1.99634 \mathrm{E} \quad 02$ $1.99666 \mathrm{E} \quad 02$ $1.99697 \mathrm{E} \quad 02$ $1.99729 \mathrm{E} \quad 02$

$1.99760 \mathrm{E} \quad 02$ $1.99790 \mathrm{E} \quad 02$ $1.99820 \mathrm{E} \quad 02$ $1.99850 \mathrm{E} \quad 02$ $1.99880 \mathrm{E} \quad 02$

$1.99909 \mathrm{E} \quad 02$ $1.99937 \mathrm{E} \quad 02$ $1.99966 \mathrm{E} \quad 02$ $1.99994 \mathrm{E} \quad 02$ $2.00021 \mathrm{E} \quad 02$

$2.00049 \mathrm{E} \quad 02$ $2.00076 \mathrm{E} \quad 02$ $2.00102 \mathrm{E} \quad 02$ $2.00129 \mathrm{E} \quad 02$ $2.00155 \mathrm{E} \quad 02$

$f_{-2}$

$-1.37118 \mathrm{E}-03$

$-1.40355 \mathrm{E}-03$

$-1.43642 \mathrm{E}-03$

$-1.46980 \mathrm{E}-03$

$-1.50369 \mathrm{E}-03$

$-1.53810 \mathrm{E}-03$

$-1.57303 \mathrm{E}-03$

$-1.60848 \mathrm{E}-03$

$-1.64447 \mathrm{E}-03$

$-1.68098 \mathrm{E}-03$

$-1.71803 \mathrm{E}-03$

$-1.75562 \mathrm{E}-03$

$-1.79376 \mathrm{E}-03$

$-1.83244 \mathrm{E}-03$

$-1.87168 \mathrm{E}-03$

$-1.91147 \mathrm{E}-03$

$-1.95182 \mathrm{E}-03$

$-1.99273 \mathrm{E}-03$

$-2.03421 \mathrm{E}-03$

$-2.07626 \mathrm{E}-03$

$-2.11889 \mathrm{E}-03$

$-2.16209 \mathrm{E}-03$

$-2.20588 \mathrm{E}-03$

$-2.25025 \mathrm{E}-03$

$-2.29522 \mathrm{E}-03$

$-2.34078 \mathrm{E}-03$

$-2.38694 \mathrm{E}-03$

$-2.43370 \mathrm{E}-03$

$-2.48106 \mathrm{E}-03$

$-2.52904 \mathrm{E}-03$

$-2.57763 \mathrm{E}-03$

$-2.62684 \mathrm{E}-03$

$-2.67667 \mathrm{E}-03$

$-2.72713 \mathrm{E}-03$

$-2.77822 \mathrm{E}-03$

$-2.82994 \mathrm{E}-03$

$-2.88229 \mathrm{E}-03$

$-2.93529 \mathrm{E}-03$

$-2.98894 \mathrm{E}-03$

$-3.04323 \mathrm{E}-03$

$-3.09818 \mathrm{E}-03$

$-3.15378 \mathrm{E}-03$ 
$F_{0}$

18.10

18.20

18.30

18.40

18.50

18.60

18.70

18.80

18.90

19.00

19.10

19.20

19.00

19.10

19.20

19.40

19.50

19.60

19.70

19.80

19.90

20.00

20.10

20.20

20.30

20.40

20.50

20.60

20.70

20.80

20.90

21.00

21.10

21.20

21.30

21.40

21.50

21.60

21.70

21.80

21.90

22.00

22.10

22.20

22.30

22.40

22.50

22.60

22.70

22.80

22.90

23.00

23.10

23.20

23.30

23.40

23.50

23.60

23.70

23.80

23.90

24.00 $f_{1}$

$1.02338 \mathrm{E} \quad 00$ $1.02338 \mathrm{E} \quad 00$ $1.02338 \mathrm{E} \quad 00$ $1.02338 \mathrm{E} \quad 00$ $1.02338 \mathrm{E} \quad 00$

$1.02338 \mathrm{E} \quad 00$ $1.02338 \mathrm{E} \quad 00$ $1.02338 \mathrm{E} \quad 00$ $1.02338 \mathrm{E} \quad 00$ $1.02338 \mathrm{E} \quad 00$

$1.02338 \mathrm{E} \quad 00$ $1.02338 \mathrm{E} \quad 00$

$1.02338 \mathrm{E} \quad 00$

$1.02338 \mathrm{E} \quad 00$

$1.02338 \mathrm{E} \quad 00$

$1.02338 \mathrm{E} \quad 00$

$1.02338 \mathrm{E} \quad 00$

$1.02338 \mathrm{E} \quad 00$ $1.02337 \mathrm{E} \quad 00$ $1.02337 \mathrm{E} \quad 00$ $1.02337 \mathrm{E} \quad 00$ $1.02337 \mathrm{E} \quad 00$

$1.02337 \mathrm{E} \quad 00$

$1.02337 \mathrm{E} \quad 00$

$1.02337 \mathrm{E} \quad 00$

$1.02337 \mathrm{E} \quad 00$

$1.02337 \mathrm{E} \quad 00$

$1.02337 \mathrm{E} \quad 00$ $1.02337 \mathrm{E} \quad 00$ $1.02337 \mathrm{E} \quad 00$ $1.02337 \mathrm{E} \quad 00$ $1.02337 \mathrm{E} \quad 00$

$1.02337 \mathrm{E} \quad 00$ $1.02337 \mathrm{E} \quad 00$ $1.02337 \mathrm{E} \quad 00$ $1.02337 \mathrm{E} \quad 00$ $1.02337 \mathrm{E} \quad 00$

$1.02336 \mathrm{E} \quad 00$ $1.02336 \mathrm{E} \quad 00$ $1.02336 \mathrm{E} \quad 00$ $1.02336 \mathrm{E} \quad 00$ $1.02336 \mathrm{E} \quad 00$

$1.02336 \mathrm{E} \quad 00$ $1.02336 \mathrm{E} \quad 00$ $1.02336 \mathrm{E} \quad 00$ $1.02336 \mathrm{E} \quad 00$ $1.02336 \mathrm{E} \quad 00$

$1.02336 \mathrm{E} \quad 00$ $1.02336 \mathrm{E} \quad 00$ $1.02336 \mathrm{E} \quad 00$ $1.02336 \mathrm{E} \quad 00$ $1.02336 \mathrm{E} \quad 00$

$1.02336 \mathrm{E} \quad 00$ $1.02336 \mathrm{E} \quad 00$ $1.02336 \mathrm{E} \quad 00$ $1.02336 \mathrm{E} \quad 00$ $1.02336 \mathrm{E} \quad 00$

$1.02336 \mathrm{E} \quad 00$ $1.02336 \mathrm{E} \quad 00$ $1.02335 \mathrm{E} \quad 00$ $1.02335 \mathrm{E} \quad 00$ $1.02335 \mathrm{E} \quad 00$
$-1.77823 \mathrm{E} \quad 01$ $\begin{array}{lll}-1.78832 \mathrm{E} & 01\end{array}$ $-1.79842 \mathrm{E} 01$ $\begin{array}{lll}-1.80851 \mathrm{E} & 01\end{array}$ $-1.81861 \mathrm{E} 01$

$-1.82870 \mathrm{E} \quad 01$ $-1.83880 \mathrm{E} 01$ $-1.84889 \mathrm{E} 01$ $-1.85899 \mathrm{E} \quad 01$ $-1.86908 \mathrm{E} 01$

$\begin{array}{lll}-1.87917 \mathrm{E} & 01\end{array}$ $-1.88927 \mathrm{E} 01$ $-1.86908 \mathrm{E} 01$

$-1.87917 \mathrm{E} \quad 01$ $-1.88927 \mathrm{E} 01$ $-1.90945 \mathrm{E} 01$ $-1.91954 \mathrm{E} 01$

$\begin{array}{ll}-1.92964 \mathrm{E} & 01\end{array}$ $-1.93973 \mathrm{E} 01$ $-1.94982 \mathrm{E} 01$ $-1.95991 \mathrm{E} 01$ $-1.97000 \mathrm{E} 01$

$-1.98009 \mathrm{E} \quad 01$ $-1.99019 \mathrm{E} 01$ $-2.00028 \mathrm{E} 01$ $\begin{array}{lll}-2.01037 \mathrm{E} & 01\end{array}$ $-2.02046 \mathrm{E} 01$

$-2.03055 \mathrm{E} 01$ $-2.04064 \mathrm{E} \quad 01$ $-2.05072 \mathrm{E} 01$ $-2.06081 \mathrm{E} 01$ $-2.07090 \mathrm{E} 01$

$-2.08099 \mathrm{E} \quad 01$ $-2.09108 \mathrm{E} 01$ $-2.10117 \mathrm{E} \quad 01$ $-2.11125 \mathrm{E} 01$ $-2.12134 \mathrm{E} 01$

$-2.13143 \mathrm{E} 01$

$-2.14151 \mathrm{E} 01$

$-2.15160 \mathrm{E} 01$

$-2.16169 \mathrm{E} \quad 01$

$-2.17177 \mathrm{E} 01$

$-2.18186 \mathrm{E} \quad 01$ $-2.19194 \mathrm{E} \quad 01$ $-2.20203 \mathrm{E} 01$ $-2.21211 \mathrm{E}$ 01 $-2.22220 \mathrm{E}$ 0l

$-2.23228 \mathrm{E} \quad 01$ $-2.24237 \mathrm{E} 01$ $-2.25245 \mathrm{E} \quad 01$ $-2.26253 \mathrm{E} \quad 01$ $-2.27262 \mathrm{E} 01$

$-2.28270 \mathrm{E} 01$ $-2.29278 \mathrm{E} \quad 01$ $-2.30286 \mathrm{E} 01$ $-2.31295 \mathrm{E} 01$ $-2.32303 \mathrm{E} 01$

$-2.33311 \mathrm{E} \quad 01$ $-2.34319 \mathrm{E} \quad 01$ $-2.35327 \mathrm{E} 01$ $-2.36335 \mathrm{E} 01$ $-2.37343 \mathrm{E} 01$ $g_{1}$

$-4.04347 \mathrm{E}-01$ $-4.08494 \mathrm{E}-01$ $-4.12661 \mathrm{E}-01$ $-4.16850 \mathrm{E}-01$ $-4.21060 \mathrm{E}-01$

$-4.25290 \mathrm{E}-01$ $-4.29542 \mathrm{E}-01$ $-4.33814 \mathrm{E}-01$ $-4.38107 \mathrm{E}-01$ $-4.42421 \mathrm{E}-01$

$-4.46756 \mathrm{E}-01$ $-4.51112 \mathrm{E}-01$

$-4.42421 \mathrm{E}-01$

$-4.46756 \mathrm{E}-01$

$-4.51112 \mathrm{E}-01$

$-4.59886 \mathrm{E}-01$

$-4.64305 \mathrm{E}-01$

$-4.68744 \mathrm{E}-01$ $-4.73204 \mathrm{E}-01$ $-4.77685 \mathrm{E}-01$ $-4.82187 \mathrm{E}-01$ $-4.86710 \mathrm{E}-01$

$-4.91254 \mathrm{E}-01$

$-4.95819 \mathrm{E}-01$

$-5.00404 \mathrm{E}-01$

$-5.05011 \mathrm{E}-01$

$-5.09638 \mathrm{E}-01$

$-5.14286 \mathrm{E}-01$

$-5.18955 \mathrm{E}-01$

$-5.23645 \mathrm{E}-01$

$-5.28356 \mathrm{E}-01$

$-5.33087 \mathrm{E}-01$

$-5.37840 \mathrm{E}-01$

$-5.42613 \mathrm{E}-01$

$-5.47408 \mathrm{E}-01$

$-5.52223 \mathrm{E}-01$

$-5.57059 \mathrm{E}-01$

$-5.61916 \mathrm{E}-01$

$-5.66794 \mathrm{E}-01$

$-5.71692 \mathrm{E}-01$

$-5.76612 \mathrm{E}-01$

$-5.81552 \mathrm{E}-01$

$-5.86513 \mathrm{E}-01$

$-5.91496 \mathrm{E}-01$

$-5.96499 \mathrm{E}-01$

$-6.01522 \mathrm{E}-01$

$-6.06567 \mathrm{E}-01$

$-6.11633 \mathrm{E}-01$

$-6.16719 \mathrm{E}-01$ $-6.21827 \mathrm{E}-01$

$-6.26955 \mathrm{E}-01$

$-6.32104 \mathrm{E}-01$

$-6.37274 \mathrm{E}-01$

$-6.42464 \mathrm{E}-01$

$-6.47676 \mathrm{E}-01$ $-6.52909 \mathrm{E}-01$ $-6.58162 \mathrm{E}-01$

$-6.63436 \mathrm{E}-01$ $-6.68731 \mathrm{E}-01$ $-6.74047 \mathrm{E}-01$ $-6.79384 \mathrm{E}-01$ $-6.84742 \mathrm{E}-01$

$\tan \delta_{1}$

$f_{-1}$

$4.03433 \mathrm{E}-04$

$4.04506 \mathrm{E}-04$

$4.05567 \mathrm{E}-04$

$4.06616 \mathrm{E}-04$

$4.07653 \mathrm{E}-04$

$4.08678 \mathrm{E}-04$

$4.09693 \mathrm{E}-04$

$4.10696 \mathrm{E}-04$

$4.11688 \mathrm{E}-04$

$4.12669 \mathrm{E}-04$

$4.13641 \mathrm{E}-04$

4.14601 E-04

$4.12669 \mathrm{E}-04$

$4.13641 \mathrm{E}-04$

$4.14601 \mathrm{E}-04$

$4.16491 \mathrm{E}-04$

$4.17421 \mathrm{E}-04$

$4.18341 \mathrm{E}-04$

$4.19250 \mathrm{E}-04$

$4.20152 \mathrm{E}-04$

$4.21042 \mathrm{E}-04$

$4.21924 \mathrm{E}-04$

$4.22797 \mathrm{E}-04$

$4.23661 \mathrm{E}-04$

$4.24516 \mathrm{E}-04$

$4.25361 \mathrm{E}-04$

$4.26198 \mathrm{E}-04$

$4.27027 \mathrm{E}-04$ $4.27847 \mathrm{E}-04$

$4.28660 \mathrm{E}-04$

$4.29464 \mathrm{E}-04$

$4.30260 \mathrm{E}-04$ 
18.10

18.20

18.30

18.40

18.50

18.60

18.70

18.80

18.90

19.00

19.10

19.20

19.30

19.40

19.50

19.60

19.70

19.80

19.90

20.00

20.10

20.20

20.30

20.40

20.50

20.60

20.70

20.80

20.90

21.00

21.10

21.20

21.30

21.40

21.50

21.60

21.70

21.80

21.90

22.00

22.10

22.20

22.30

22.40

22.50

22.60

22.70

22.80

22.90

23.00

23.10

23.20

23.30

23.40

23.50

23.60

23.70

23.80

23.90

24.00
8.99297E-05 $9.09261 \mathrm{E}-05$ $9.19280 \mathrm{E}-05$ $9.29354 \mathrm{E}-05$ $9.39482 \mathrm{E}-05$

$9.49666 \mathrm{E}-0.5$ $9.59904 \mathrm{E}-05$ $9.70198 \mathrm{E}-05$ $9.80546 \mathrm{E}-0$. $9.90949 \mathrm{E}-05$

$1.00141 \mathrm{E}-04$ $1.01192 \mathrm{E}-04$ $1.02249 \mathrm{E}-04$

$1.03311 \mathrm{E}-04$ $1.04379 \mathrm{E}-04$

$1.05452 \mathrm{E}-04$ $1.06531 \mathrm{E}-04$ $1.07615 \mathrm{E}-04$

$1.08705 \mathrm{E}-04$

$1.09800 \mathrm{E}-04$

$1.10901 \mathrm{E}-04$ $1.12007 \mathrm{E}-04$

$1.13119 \mathrm{E}-04$

$1.14236 \mathrm{E}-04$

$1.15358 \mathrm{E}-04$

$1.16486 \mathrm{E}-04$ $1.17620 \mathrm{E}-04$ $1.18759 \mathrm{E}-04$ $1.19904 \mathrm{E}-04$ $1.21054 \mathrm{E}-04$

$1.22210 \mathrm{E}-04$ $1.23371 \mathrm{E}-04$ $1.24537 \mathrm{E}-04$ $1.25709 \mathrm{E}-04$ $1.26887 \mathrm{E}-04$

$1.28070 \mathrm{E}-04$ $1.29258 \mathrm{E}-04$ $1.30452 \mathrm{E}-04$ $1.31652 \mathrm{E}-04$ $1.32857 \mathrm{E}-04$

$1.34067 \mathrm{E}-04$

$1.35283 \mathrm{E}-04$

$1.36505 \mathrm{E}-04$

$1.37732 \mathrm{E}-04$

$1.38964 \mathrm{E}-04$

$1.40202 \mathrm{E}-04$

$1.41446 \mathrm{E}-04$ $1.42695 \mathrm{E}-04$ $1.43949 \mathrm{E}-04$ $1.45209 \mathrm{E}-04$

$1.46474 \mathrm{E}-04$ $1.47745 \mathrm{E}-04$. $1.49021 \mathrm{E}-04$ $1.50303 \mathrm{E}-04$ $1.51591 \mathrm{E}-04$

$1.52883 \mathrm{E}-04$ $1.54182 \mathrm{E}-04$ $1.55486 \mathrm{E}-04$ $1.56795 \mathrm{E}-04$ $1.58110 \mathrm{E}-04$
$-3.33567 \mathrm{E}-01$

$-3.37315 \mathrm{E}-01$

$-3.41085 \mathrm{E}-01$

$-3.44875 \mathrm{E}-01$

$-3.48687 \mathrm{E}-01$

$-3.52519 \mathrm{E}-01$

$-3.56372 \mathrm{E}-01$

$-3.60246 \mathrm{E}-01$

$-3.64141 \mathrm{E}-01$

$-3.68057 \mathrm{E}-01$

$-3.71994 \mathrm{E}-01$

$-3.75952 \mathrm{E}-01$

$-3.79930 \mathrm{E}-01$

$-3.83930 \mathrm{E}-01$

$-3.87950 \mathrm{E}-01$

$-3.91992 \mathrm{E}-01$

$-3.96054 \mathrm{E}-01$

$-4.00137 \mathrm{E}-01$

$-4.04241 \mathrm{E}-01$

$-4.08366 \mathrm{E}-01$

$-4.12512 \mathrm{E}-01$

$-4.16679 \mathrm{E}-01$

$-4.20866 \mathrm{E}-01$

$-4.25075 \mathrm{E}-01$

$-4.29304 \mathrm{E}-01$

$-4.33555 E-01$

$-4.37826 \mathrm{E}-01$

$-4.42118 \mathrm{E}-01$

$-4.46431 \mathrm{E}-01$

$-4.50765 \mathrm{E}-01$

$-4.55120 \mathrm{E}-01$

$-4.59496 \mathrm{E}-01$

$-4.63892 \mathrm{E}-01$

$-4.68310 \mathrm{E}-01$

$-4.72748 \mathrm{E}-01$

$-4.77208 \mathrm{E}-01$

$-4.81688 \mathrm{E}-01$

$-4.86189 \mathrm{E}-01$

$-4.90711 \mathrm{E}-01$

$-4.95254 \mathrm{E}-01$

$-4.99818 \mathrm{E}-01$

$-5.04403 \mathrm{E}-01$

$-5.09008 \mathrm{E}-0]$

$-5.13635 \mathrm{E}-01$

$-5.18282 \mathrm{E}-01$

$-5.22950 \mathrm{E}-01$

$-5.27639 \mathrm{E}-01$

$-5.32350 \mathrm{E}-01$

$-5.37080 \mathrm{E}-01$

$-5.41832 \mathrm{E}-01$

$-5.46605 \mathrm{E}-01$

$-5.51398 \mathrm{E}-01$

$-5.56213 \mathrm{E}-01$

$-5.61048 \mathrm{E}-01$

$-5.65905 \mathrm{E}-01$

$-5.70782 \mathrm{E}-01$

$-5.75680 \mathrm{E}-01$

$-5.80598 \mathrm{E}-01$

$-5.85538 \mathrm{E}-01$

$-5.90499 \mathrm{E}-01$ $g_{2}$

$-4.52237 \mathrm{E}-03$

$-4.59413 \mathrm{E}-03$

$-4.66664 \mathrm{E}-03$

$-4.73992 \mathrm{E}-03$

$-4.81395 \mathrm{E}-03$

$-4.88874 \mathrm{E}-03$

$-4.96430 \mathrm{E}-03$

$-5.04064 \mathrm{E}-03$

$-5.11774 \mathrm{E}-03$

$-5.19563 \mathrm{E}-03$

$-5.27430 \mathrm{E}-03$

$-5.35376 \mathrm{E}-03$

$-5.43401 \mathrm{E}-03$

$-5.51505 \mathrm{E}-03$

$-5.59689 \mathrm{E}-03$

$-5.67954 \mathrm{E}-03$

$-5.76299 \mathrm{E}-03$

$-5.84725 \mathrm{E}-03$

$-5.93233 \mathrm{E}-03$

$-6.01822 \mathrm{E}-03$

$-6.10494 \mathrm{E}-03$

$-6.19248 \mathrm{E}-03$

$-6.28086 \mathrm{E}-03$

$-6.37006 \mathrm{E}-03$

$-6.46011 \mathrm{E}-03$

$-6.55099 \mathrm{E}-03$

$-6.64272 \mathrm{E}-03$

$-6.73530 \mathrm{E}-03$

$-6.82874 \mathrm{E}-03$

$-6.92303 \mathrm{E}-03$

$-7.01818 \mathrm{E}-03$

$-7.11420 \mathrm{E}-03$

$-7.21108 \mathrm{E}-03$

$-7.30884 \mathrm{E}-03$

$-7.40747 \mathrm{E}-03$

$-7.50699 \mathrm{E}-03$

$-7.60739 \mathrm{E}-03$

$-7.70868 \mathrm{E}-03$

$-7.81086 \mathrm{E}-03$

$-7.91393 \mathrm{E}-03$

$-8.01791 \mathrm{E}-03$

$-8.12279 \mathrm{E}-03$

$-8.22858 \mathrm{E}-03$

$-8.33528 \mathrm{E}-03$

$-8.44289 \mathrm{E}-03$

$-8.55143 \mathrm{E}-03$

$-8.66088 \mathrm{E}-03$

$-8.77127 \mathrm{E}-03$

$-8.88258 \mathrm{E}-03$

$-8.99483 \mathrm{E}-03$

$-9.10802 \mathrm{E}-03$

$-9.22216 \mathrm{E}-0.3$

$-9.33724 \mathrm{E}-03$

$-9.45327 \mathrm{E}-0.3$

$-9.57025 \mathrm{E}-03$

$-9.68819 \mathrm{E}-03$

$-9.80710 \mathrm{E}-03$

$-9.92697 \mathrm{E}-03$

$-1.00478 \mathrm{E}-02$

$-1.01696 \mathrm{E}-02$

$\tan \delta_{z}$

$f_{-2}$

$\tan \delta_{2}$

$2.00181 \mathrm{E} \quad 02-4.34025 \mathrm{E}-03$ $2.00206 \mathrm{E} \quad 02-4.40981 \mathrm{E}-03$ $2.00231 \mathrm{E} \quad 02-4.48010 \mathrm{E}-03$ $2.00256 \mathrm{E} \quad 02-4.55115 \mathrm{E}-03$ $2.00281 \mathrm{E} \quad 02-4.62294 \mathrm{E}-03$

$2.00305 \mathrm{E} \quad 02$ $2.00329 \mathrm{E} \quad 02$ $2.00353 \mathrm{E} \quad 02$ $2.00377 \mathrm{E} \quad 02$ $2.00400 \mathrm{E} \quad 02$

$2.00423 \mathrm{E} \quad 02$ $2.00446 \mathrm{E} \quad 02$ $2.00468 \mathrm{E} \quad 02$ $2.00491 \mathrm{E} \quad 02$ $2.00513 \mathrm{E} \quad 02$

$2.00535 \mathrm{E} \quad 02$ $2.00556 \mathrm{E} \quad 02$ $2.00578 \mathrm{E} \quad 02$

$2.00599 \mathrm{E} \quad 02$

$2.00620 \mathrm{E} \quad 02$

$2.00641 \mathrm{E} \quad 02$

$2.00661 \mathrm{E} \quad 02$

$2.00681 \mathrm{E} \quad 02$

$2.00701 \mathrm{E} \quad 02$

$2.00721 \mathrm{E} \quad 02$

$2.00741 \mathrm{E} \quad 02$ $2.00761 \mathrm{E} \quad 02$ $2.00780 \mathrm{E} \quad 02$ $2.00799 \mathrm{E} \quad 02$ $2.00818 \mathrm{E} \quad 02$

$2.00837 \mathrm{E} \quad 02$ $2.00855 \mathrm{E} \quad 02$ $2.00874 \mathrm{E} \quad 02$ $2.00892 \mathrm{E} \quad 02$ $2.00910 \mathrm{E} \quad 02$

$2.00928 \mathrm{E} \quad 02$

$2.00945 \mathrm{E} \quad 02$

$2.00963 \mathrm{E} \quad 02$ 
ELECTRON RADIAL FUNCTIONS

Electron

$p$

24.10

24.20

24.30

24.40

24.50

24.60

24.70

24.80

24.90

25.00

25.10

25.20

25.30

25.40

25.50

25.60

25.70

25.80

25.90

26.00

26.10

26.20

26.30

26.40

26.50

26.60

26.70

26.80

26.90

27.00

27.10

27.20

27.30

27.40

27.50

27.60

27.70

27.80

27.90

28.00

28.10

28.20

28.30

28.40

28.50

28.60

28.70

28.80

28.90

29.00

29.10

29.20

29.30

29.40

29.50

29.60

29.70

29.80

29.90

30.00

$$
Z=1 \quad A=1
$$

$f_{1}$

$1.02335 \mathrm{E} \quad 00$ $1.02335 \mathrm{E} \quad 00$ $1.02335 \mathrm{E} \quad 00$ $1.02335 \mathrm{E} \quad 00$ $1.02335 \mathrm{E} \quad 00$

$1.02335 \mathrm{E} \quad 00$ $1.02335 \mathrm{E} \quad 00$ $1.02335 \mathrm{E} \quad 00$ $1.02335 \mathrm{E} \quad 00$ $1.02335 \mathrm{E} \quad 00$

$1.02335 \mathrm{E} \quad 00$ $1.02335 \mathrm{E} \quad 00$ $1.02335 \mathrm{E} \quad 00$ $1.02335 \mathrm{E} \quad 00$ $1.02335 \mathrm{E} \quad 00$

$1.02335 \mathrm{E} \quad 00$ $1.02335 \mathrm{E} \quad 00$ $1.02335 \mathrm{E} \quad 00$ $1.02335 \mathrm{E} \quad 00$ $1.02335 \mathrm{E} \quad 00$

$1.02335 \mathrm{E} \quad 00$ $1.02335 \mathrm{E} \quad 00$ $1.02335 \mathrm{E} \quad 00$ $1.02335 \mathrm{E} \quad 00$ $1.02335 \mathrm{E} \quad 00$

$1.02334 \mathrm{E} \quad 00$ $1.02334 \mathrm{E} \quad 00$ $1.02334 \mathrm{E} \quad 00$ $1.02334 \mathrm{E} \quad 00$ $1.02334 \mathrm{E} \quad 00$

$1.02334 \mathrm{E} \quad 00$ $1.02334 \mathrm{E} \quad 00$ $1.02334 \mathrm{E} \quad 00$ $\begin{array}{ll}1.02334 \mathrm{E} & 00\end{array}$ $1.02334 \mathrm{E} \quad 00$

$1.02334 \mathrm{E} \quad 00$ $1.02334 \mathrm{E} \quad 00$ $1.02334 \mathrm{E} \quad 00$ $1.02334 \mathrm{E} \quad 00$ $1.02334 \mathrm{E} \quad 00$

$1.02334 \mathrm{E} \quad 00$ $1.02334 \mathrm{E} \quad 00$ $1.02334 \mathrm{E} \quad 00$ $1.02334 \mathrm{E}-00$ $1.02334 \mathrm{E} \quad 00$

$1.02334 \mathrm{E} \quad 00$ $1.02334 \mathrm{E} \quad 00$ $1.02334 \mathrm{E} \quad 00$ $1.02334 \mathrm{E} \quad 00$ $1.02334 \mathrm{E} \quad 00$

$1.02334 \mathrm{E} \quad 00$ $1.02334 \mathrm{E} \quad 00$ $1.02334 \mathrm{E} \quad 00$ $1.02334 \mathrm{E} \quad 00$ $1.02334 \mathrm{E} \quad 00$

$1.02334 \mathrm{E} \quad 00$ $1.02334 \mathrm{E} \quad 00$ $1.02334 \mathrm{E} \quad 00$ $1.02333 \mathrm{E} \quad 00$ $1.02333 \mathrm{E} \quad 00$ $g_{1}$ $-2.38351 \mathrm{E} \quad 01$ $-2.39359 \mathrm{E} 01$ $-2.40367 \mathrm{E} 01$ $-2.41375 \mathrm{E} 01$ -2.42383 E 01

$-2.43391 \mathrm{E} \quad 01$ $-2.44399 \mathrm{E} \quad 01$ $-2.45406 \mathrm{E} \quad 01$ $-2.46414 \mathrm{E} \quad 01$ $-2.47422 \mathrm{E} 01$

$-2.48430 \mathrm{E} 01$ $-2.49437 \mathrm{E} \quad 01$ $-2.50445 \mathrm{E} \quad 01$ $-2.51453 \mathrm{E} 01$ $-2.52460 \mathrm{E}$ ol

$-2.53468 \mathrm{E} \quad 01$ $-2.54475 \mathrm{E} 01$ $-2.55483 \mathrm{E} 01$ $-2.56490 \mathrm{E} 01$ $-2.57498 \mathrm{E} 01$

$-2.58505 \mathrm{E} \quad 0 \mathrm{I}$ $-2.59512 \mathrm{E} 01$ $-2.60520 \mathrm{E} 01$ $-2.61527 \mathrm{E} 01$ $-2.62534 \mathrm{E} \quad 01$

$-2.63542 \mathrm{E} \quad 01$ $-2.64549 \mathrm{E} 01$ $-2.65556 \mathrm{E} \quad 01$ $-2.66563 \mathrm{E}$ 0I $-2.67570 \mathrm{E} 01$

$\begin{array}{lll}-2.68577 \mathrm{E} & 01\end{array}$ $-2.69584 \mathrm{E} \quad 01$ $-2.70591 \mathrm{E} 01$ $-2.71598 \mathrm{E} 01$ -2.72605 E 01

$-2.73612 \mathrm{E} \quad 01$ $-2.74619 \mathrm{E} \quad 01$ $-2.75626 \mathrm{E} \quad 01$ $-2.76633 \mathrm{E} \quad 01$ $-2.77640 \mathrm{E} \quad 01$

$-2.78646 \mathrm{E} \quad 01$ $-2.79653 \mathrm{E} \quad 01$ $-2.80660 \mathrm{E} \quad 01$ $-2.81667 \mathrm{E} 01$ $-2.82673 \mathrm{E} 01$

$-2.83680 \mathrm{E} \quad 01$ $-2.84686 \mathrm{E} 01$ $-2.85693 \mathrm{E}$ 0l $-2.86699 \mathrm{E} \quad 01$ $-2.87706 \mathrm{E} \quad 01$

$-2.88712 \mathrm{E} \quad 01$ $-2.89719 \mathrm{E} \quad 01$ $-2.90725 \mathrm{E} \quad 01$ $-2.91731 \mathrm{E} \quad 01$ $-2.92738 \mathrm{E} 01$

$-2.93744 \mathrm{E} \quad 01$ $-2.94750 \mathrm{E} \quad 01$ $-2.95757 \mathrm{E} 01$ $-2.96763 \mathrm{E} \quad 01$ $-2.97769 \mathrm{E} \quad 01$
$-6.90120 \mathrm{E}-01$ $-6.95520 \mathrm{E}-01$ $-7.00940 \mathrm{E}-01$ $-7.06381 \mathrm{E}-01$ $-7.11843 \mathrm{E}-01$

$-7.17325 \mathrm{E}-01$ $-7.22829 \mathrm{E}-0 \mathrm{l}$ $-7.28354 \mathrm{E}-01$ $-7.33899 \mathrm{E}-01$ $-7.39465 \mathrm{E}-01$

$-7.45052 \mathrm{E}-01$ $-7.50660 \mathrm{E}-01$ $-7.56289 \mathrm{E}-01$ $-7.61938 \mathrm{E}-01$ $-7.67608 \mathrm{E}-01$

$-7.73300 \mathrm{E}-01$ $-7.79012 \mathrm{E}-01$ $-7.84745 \mathrm{E}-01$ $-7.90498 \mathrm{E}-01$ $-7.96273 \mathrm{E}-01$

$-8.02068 \mathrm{E}-01$ $-8.07885 \mathrm{E}-01$ $-8.13722 \mathrm{E}-01$ $-8.19580 \mathrm{E}-01$ $-8.25459 \mathrm{E}-01$

$-8.31358 \mathrm{E}-01$ $-8.37279 \mathrm{E}-01$ $-8.43220 \mathrm{E}-01$ $-8.49182 \mathrm{E}-01$ $-8.55165 \mathrm{E}-01$

$-8.61169 \mathrm{E}-01$ $-8.67193 \mathrm{E}-01$ $-8.73239 \mathrm{E}-01$ $-8.79305 \mathrm{E}-01$ $-8.85392 \mathrm{E}-01$

$-8.91500 \mathrm{E}-01$ $-8.97629 \mathrm{E}-01$ $-9.03779 \mathrm{E}-0 \mathrm{l}$ $-9.09949 \mathrm{E}-01$ $-9.16140 \mathrm{E}-01$

$-9.22352 \mathrm{E}-01$ $-9.28585 \mathrm{E}-01$ $-9.34839 \mathrm{E}-01$ $-9.41114 \mathrm{E}-01$ $-9.47409 \mathrm{E}-01$

$-9.53725 \mathrm{E}-01$ $-9.60062 \mathrm{E}-01$ $-9.66420 \mathrm{E}-01$ $-9.72799 \mathrm{E}-01$ $-9.79198 \mathrm{E}-01$

$-9.85618 \mathrm{E}-01$ $-9.92059 \mathrm{E}-01$ $-9.98521 \mathrm{E}-01$ $-1.00500 \mathrm{E} 00$ $-1.01151 \mathrm{E} 00$

$-1.01803 \mathrm{E} \quad 00$ $-1.02458 \mathrm{E} 00$ $-1.03114 \mathrm{E} \quad 00$ $-1.03773 \mathrm{E} 00$ $-1.04434 \mathrm{E} 00$

$\tan \delta_{1}$

$\rho=1.2 A^{1 / 3} F$

$4.51477 \mathrm{E}-04$ $4.52065 \mathrm{E}-04$ $4.52647 \mathrm{E}-04$ $4.53225 \mathrm{E}-04$ $4.53796 \mathrm{E}-04$

$4.54364 \mathrm{E}-04$ $4.54927 \mathrm{E}-04$

$4.55484 \mathrm{E}-04$

$4.56037 \mathrm{E}-04$

$4.56583 \mathrm{E}-04$

$4.57127 \mathrm{E}-04$ 4.57665 E-- 04 $4.58198 \mathrm{E}-04$

$4.58727 \mathrm{E}-04$

4.59251 E-04

4.59771 E-0 04 $4.60286 \mathrm{E}-04$ $4.60797 \mathrm{E}-04$

4.61304 E-04

$4.61806 \mathrm{E}-04$

4.62304 E- 04 $4.62797 \mathrm{E}-04$ $4.63287 \mathrm{E}-04$

$4.63773 \mathrm{E}-04$

4.64254 E- 04

$4.64732 \mathrm{E}-04$ 4.65204 E-04 $4.65674 \mathrm{E}-04$

$4.66140 \mathrm{E}-04$

$4.66601 \mathrm{E}-04$

$4.67059 \mathrm{E}-04$

$4.67513 \mathrm{E}-04$

$4.67963 \mathrm{E}-04$

$4.68410 \mathrm{E}-04$

$4.68853 \mathrm{E}-04$

$4.69292 \mathrm{E}-04$ $4.69728 \mathrm{E}-04$ $4.70160 \mathrm{E}-04$ 
$Z=1 \quad A=1 \quad \rho=1.2 A^{1 / 3} \mathrm{~F}$

24.60

24.70

24.80

24.90

25.00

25.10

25.20

25.30

25.40

25.50

25.60

25.70

25.80

25.90

26.00

26.10

26.20

26.30

26.40

26.50

26.60

26.70

26.80

26.90

27.00

27.10

27.20

27.30

27.40

27.50

27.60

27.70

27.80

27.90

28.00

28.10

28.20

28.30

28.40

28.50

28.60

28.70

28.80

28.90

29.00

29.10

29.20

29.30

29.40

29.50

29.60

29.70

29.80

29.90

30.00
$\bar{F}$

$1.59430 \mathrm{E}-04$ $1.60756 \mathrm{E}-04$ $1.62087 \mathrm{E}-04$

$1.63424 \mathrm{E}-04$

$1.64766 \mathrm{E}-04$

$1.66114 \mathrm{E}-04$

$1.67467 \mathrm{E}-04$

$1.68826 \mathrm{E}-04$

$1.70190 \mathrm{E}-04$

$1.71560 \mathrm{E}-04$

$1.72935 \mathrm{E}-04$

$1.74315 \mathrm{E}-04$

$1.75702 \mathrm{E}-04$

$1.77093 \mathrm{E}-04$

$1.78490 \mathrm{E}-04$

$1.79893 \mathrm{E}-04$ $1.81301 \mathrm{E}-04$

$1.82715 \mathrm{E}-04$

$1.84134 \mathrm{E}-04$

$1.85558 \mathrm{E}-04$

$1.86989 \mathrm{E}-04$ $1.88424 \mathrm{E}-04$ $1.89865 \mathrm{E}-04$

$1.91312 \mathrm{E}-04$

$1.92764 \mathrm{E}-04$

$1.94221 \mathrm{E}-04$

$1.95684 \mathrm{E}-04$

$1.97153 \mathrm{E}-04$

$1.98627 \mathrm{E}-04$

$2.00106 \mathrm{E}-04$

$2.01591 \mathrm{E}-04$

$2.03082 \mathrm{E}-04$

$2.04578 \mathrm{E}-04$

$2.06079 \mathrm{E}-04$

$2.07586 \mathrm{E}-04$

$2.09098 \mathrm{E}-04$

$2.10616 \mathrm{E}-04$

$2.12140 \mathrm{E}-04$

$2.13669 \mathrm{E}-04$

2.15203 E- 04

$2.16743 \mathrm{E}-04$

$2.18288 \mathrm{E}-04$

$2.19839 \mathrm{E}-04$

$2.21395 \mathrm{E}-04$

$2.22957 \mathrm{E}-04$

$2.24524 \mathrm{E}-04$

$2.26097 \mathrm{E}-04$

$2.27676 \mathrm{E}-04$

$2.29259 \mathrm{E}-04$

$2.30849 \mathrm{E}-04$

$2.32443 \mathrm{E}-04$ $2.34044 \mathrm{E}-04$ $2.35649 \mathrm{E}-04$ 2.37261 E-04 $2.38877 \mathrm{E}-04$

$2.40499 \mathrm{E}-04$ $2.42127 \mathrm{E}-04$ 2.43760 E- 04 $2.45399 \mathrm{E}-04$

$2.47043 \mathrm{E}-04$ $f_{2}$

$g_{2}$

$\tan \delta_{2}$

$f_{-2}$

$-5.95481 \mathrm{E}-01$ $-6.00483 \mathrm{E}-01$

$-6.05506 \mathrm{E}-01$

$-6.10550 \mathrm{E}-01$

$-6.15615 \mathrm{E}-01$

$-6.20701 \mathrm{E}-01$

$-6.25808 \mathrm{E}-01$

$-6.30936 \mathrm{E}-01$

$-6.36084 \mathrm{E}-01$

$-6.41254 \mathrm{E}-01$

$-6.46444 \mathrm{E}-01$

$-6.51655 \mathrm{E}-01$

$-6.56887 \mathrm{E}-0 \mathrm{l}$

$-6.62140 \mathrm{E}-01$

$-6.67413 \mathrm{E}-01$

$-6.72708 \mathrm{E}-01$

$-6.78024 \mathrm{E}-01$

$-6.83360 \mathrm{E}-01$

$-6.88717 \mathrm{E}-01$

$-6.94095 \mathrm{E}-01$

$-6.99494 \mathrm{E}-01$

$-7.04914 \mathrm{E}-01$

$-7.10355 \mathrm{E}-01$

$-7.15816 \mathrm{E}-01$

$-7.21299 \mathrm{E}-01$

$-7.26802 \mathrm{E}-01$

$-7.32326 \mathrm{E}-01$

$-7.37871 \mathrm{E}-01$

$-7.43437 \mathrm{E}-01$

$-7.49023 \mathrm{E}-01$

$-7.54631 \mathrm{E}-01$

$-7.60259 \mathrm{E}-01$

$-7.65909 \mathrm{E}-01$

$-7.71579 \mathrm{E}-01$

$-7.77270 \mathrm{E}-01$

$-7.82982 \mathrm{E}-01$

$-7.88714 \mathrm{E}-01$

$-7.94468 \mathrm{E}-01$

$-8.00242 \mathrm{E}-01$

$-8.06038 \mathrm{E}-01$

$-8.11854 \mathrm{E}-01$

$-8.17691 \mathrm{E}-01$

$-8.23548 \mathrm{E}-01$

$-8.29427 \mathrm{E}-01$

$-8.35327 \mathrm{E}-01$

$-8.41247 \mathrm{E}-0 \mathrm{l}$

$-8.47188 \mathrm{E}-01$

$-8.53150 \mathrm{E}-01$

$-8.59133 \mathrm{E}-01$

$-8.65137 \mathrm{E}-01$

$-8.71161 \mathrm{E}-01$

$-8.77207 \mathrm{E}-01$

$-8.83273 \mathrm{E}-01$

$-8.89360 \mathrm{E}-01$

$-8.95468 \mathrm{E}-01$

$-9.01597 \mathrm{E}-01$

$-9.07746 \mathrm{E}-01$

$-9.13917 \mathrm{E}-01$

$-9.20108 \mathrm{E}-01$

$-9.26320 \mathrm{E}-01$
$-1.04162 \mathrm{E}-02$

$-1.05410 \mathrm{E}-02$

$-1.06667 \mathrm{E}-02$

$-1.07934 \mathrm{E}-02$

$-1.09212 \mathrm{E}-02$

$-1.10499 \mathrm{E}-02$

$-1.11797 \mathrm{E}-02$

$-1.13104 \mathrm{E}-02$

$-1.14422 \mathrm{E}-02$

$-1.15750 \mathrm{E}-02$

$-1.17088 \mathrm{E}-02$

$-1.18436 \mathrm{E}-02$

$-1.19795 \mathrm{E}-02$

$-1.21164 \mathrm{E}-02$

$-1.22543 \mathrm{E}-02$

$-1.23932 \mathrm{E}-02$

$-1.25333 \mathrm{E}-02$

$-1.26743 \mathrm{E}-02$

$-1.28164 \mathrm{E}-02$

$-1.29596 \mathrm{E}-02$

$-1.31038 \mathrm{E}-02$

$-1.32491 \mathrm{E}-02$

$-1.33955 \mathrm{E}-02$

$-1.35429 \mathrm{E}-02$

$-1.36914 \mathrm{E}-02$

$-1.38409 \mathrm{E}-02$

$-1.39916 \mathrm{E}-02$

$-1.41434 \mathrm{E}-02$

$-1.42962 \mathrm{E}-02$

$-1.44501 \mathrm{E}-02$

$-1.46052 \mathrm{E}-02$

$-1.47613 \mathrm{E}-02$

$-1.49185 \mathrm{E}-02$

$-1.50769 \mathrm{E}-02$

$-1.52363 \mathrm{E}-02$

$-1.53969 \mathrm{E}-02$

$-1.55586 \mathrm{E}-02$

$-1.57215 \mathrm{E}-02$

$-1.58854 \mathrm{E}-02$

$-1.60505 \mathrm{E}-02$

$-1.62167 \mathrm{E}-02$

$-1.63841 \mathrm{E}-02$

$-1.65526 \mathrm{E}-02$

$-1.67223 \mathrm{E}-02$

$-1.68931 \mathrm{E}-02$

$-1.70651 \mathrm{E}-02$

$-1.72382 \mathrm{E}-02$

$-1.74125 \mathrm{E}-02$

$-1.75879 \mathrm{E}-02$

$-1.77646 \mathrm{E}-02$

$-1.79424 \mathrm{E}-02$

$-1.81214 \mathrm{E}-02$

$-1.83016 \mathrm{E}-02$

$-1.84829 \mathrm{E}-02$

$-1.86655 \mathrm{E}-02$

$-1.88492 \mathrm{E}-02$

$-1.90342 \mathrm{E}-02$

$-1.92203 \mathrm{E}-02$

$-1.94077 \mathrm{E}-02$
$-1.02924 \mathrm{E}-02$

2.01324E 02 $2.01338 \mathrm{E} \quad 02$

$2.01352 \mathrm{E} \quad 02$

$2.01366 \mathrm{E} \quad 02$

$2.01380 \mathrm{E} \quad 02$

$2.01393 \mathrm{E} \quad 02$

$2.01407 \mathrm{E} \quad 02$

$2.01420 \mathrm{E} \quad 02$

$2.01434 \mathrm{E} \quad 02$

$2.01447 \mathrm{E} \quad 02$

$2.01460 \mathrm{E} \quad 02$

$2.01473 \mathrm{E} \quad 02$

$2.01486 \mathrm{E} \quad 02$

$2.01498 \mathrm{E} \quad 02$

$2.01511 \mathrm{E} \quad 02$

$2.01524 \mathrm{E} \quad 02$

$2.01536 \mathrm{E} \quad 02$

$2.01548 \mathrm{E} \quad 02$

$2.01561 \mathrm{E} \quad 02$

$2.01573 \mathrm{E} \quad 02$

$2.01585 \mathrm{E} \quad 02$

$2.01597 \mathrm{E} \quad 02$

$2.01609 \mathrm{E} \quad 02$

$2.01621 \mathrm{E} \quad 02$

$2.01632 \mathrm{E} \quad 02$

$2.01644 \mathrm{E} \quad 02$

$2.01655 \mathrm{E} \quad 02$

$2.01667 \mathrm{E} \quad 02$

$2.01678 \mathrm{E} \quad 02$

$2.01689 \mathrm{E} \quad 02$

$2.01701 \mathrm{E} \quad 02$ $2.01712 \mathrm{E} \quad 02$

$2.01723 \mathrm{E} \quad 02$

$2.01733 \mathrm{E} \quad 02$

$2.01744 \mathrm{E} \quad 02$

$2.01755 \mathrm{E} \quad 02$

$2.01766 \mathrm{E} \quad 02$

$2.01776 \mathrm{E} \quad 02$

$2.01787 \mathrm{E} \quad 02$

$2.01797 \mathrm{E} \quad 02$

$2.01808 \mathrm{E} \quad 02$ 
ELECTRON RADIAL FUNCTIONS

Electron

$p$

30.10

30.20

30.30

30.40

30.50

30.60

30.70

30.80

30.90

31.00

31.10

31.20

31.30

31.40

31.50

31.60

31.70

31.80

31.90

32.00

32.10

32.20

32.30

32.40

32.50

32.60

32.70

32.80

32.90

33.00

33.10

33.20

33.30

33.40

33.50

33.60

33.70

33.80

33.90

34.00

34.10

34.20

34.30

34.40

34.50

34.60

34.70

34.80

34.90

35.00

35.10

35.20

35.30

35.40

35.50

35.60

35.70

35.80

35.90

36.00

$$
Z=1 \quad A=1
$$

$f_{1}$

$1.02333 \mathrm{E} \quad 00$ $1.02333 \mathrm{E} \quad 00$ $1.02333 \mathrm{E} \quad 00$ $1.02333 \mathrm{E} \quad 00$

$1.02333 \mathrm{E} \quad 00$

$1.02333 \mathrm{E} \quad 00$ $1.02333 \mathrm{E} \quad 00$ $1.02333 \mathrm{E} \quad 00$ $1.02333 \mathrm{E} \quad 00$ $1.02333 \mathrm{E} \quad 00$

$1.02333 \mathrm{E} \quad 00$ $1.02333 \mathrm{E} \quad 00$ $1.02333 \mathrm{E} \quad 00$ $1.02333 \mathrm{E} \quad 00$ $1.02333 \mathrm{E} \quad 00$

$1.02333 \mathrm{E} \quad 00$ $1.02333 \mathrm{E} \quad 00$ $\begin{array}{ll}1.02333 \mathrm{E} & 00\end{array}$ $1.02333 \mathrm{E} \quad 00$ $1.02333 \mathrm{E} \quad 00$

$1.02333 \mathrm{E} \quad 00$ $1.02333 \mathrm{E} \quad 00$ $1.02333 \mathrm{E} \quad 00$ $1.02333 \mathrm{E} \quad 00$ $1.02333 \mathrm{E} \quad 00$

$1.02333 \mathrm{E} \quad 00$ $1.02333 \mathrm{E} \quad 00$ $1.02333 \mathrm{E} \quad 00$ $1.02333 \mathrm{E} 00$ $1.02333 \mathrm{E} \quad 00$

$1.02333 \mathrm{E} \quad 00$ $1.02333 \mathrm{E} \quad 00$ $1.02333 \mathrm{E} \quad 00$ $1.02333 \mathrm{E} \quad 00$ $1.02333 \mathrm{E} \quad 00$

$1.02333 \mathrm{E} \quad 00$ $1.02333 \mathrm{E} \quad 00$ $1.02333 \mathrm{E} \quad 00$ $1.02333 \mathrm{E} \quad 00$ $1.02333 \mathrm{E} \quad 00$

$1.02332 \mathrm{E} \quad 00$ $1.02332 \mathrm{E} \quad 00$ $1.02332 \mathrm{E} \quad 00$ $1.02332 \mathrm{E}-00$ $1.02332 \mathrm{E} \quad 00$

$1.02332 \mathrm{E} \quad 00$ $\begin{array}{ll}1.02332 \mathrm{E} & 00\end{array}$ $1.02332 \mathrm{E} \quad 00$ $1.02332 \mathrm{E} \quad 00$ $1.02332 \mathrm{E} \quad 00$

\begin{tabular}{ll}
$1.02332 \mathrm{E} \quad 00$ \\
\hline
\end{tabular} $1.02332 \mathrm{E} \quad 00$ $\begin{array}{ll}1.02332 \mathrm{E} & 00\end{array}$ $\begin{array}{ll}1.02332 \mathrm{E} & 00\end{array}$ $1.02332 \mathrm{E} \quad 00$

$1.02332 \mathrm{E} \quad 00$ $\begin{array}{ll}1.02332 \mathrm{E} & 00\end{array}$ $\begin{array}{ll}1.02332 \mathrm{E} & 00\end{array}$ $1.02332 \mathrm{E} \quad 00$ $1.02332 \mathrm{E} \quad 00$ $g_{1}$ $-2.98775 \mathrm{E} \quad 01$ $\begin{array}{lll}-2.99781 \mathrm{E} & 01\end{array}$ $-3.00787 \mathrm{E} 01$ $-3.01793 \mathrm{E} 01$ $-3.02799 \mathrm{E} 01$

-3.03805 E 01 $-3.04811 \mathrm{E} 01$ $-3.05817 \mathrm{E} 01$ $-3.06822 \mathrm{E} 01$ $-3.07828 \mathrm{E} 01$

$-3.08834 \mathrm{E}$ 0l $-3.09840 \mathrm{E} 01$ $-3.10845 \mathrm{E} 01$ $-3.11851 \mathrm{E} 01$ -3.12857 E 01

-3.13862 E 01 $-3.14868 \mathrm{E} 01$ $-3.15873 \mathrm{E} 01$ $-3.16879 \mathrm{E} 01$ -3.17884 E 01

-3.18890 E 01 $-3.19895 \mathrm{E} 01$ $-3.20900 \mathrm{E} \quad 01$ $-3.21906 \mathrm{E} 01$ $-3.22911 \mathrm{E} 01$

$\begin{array}{ll}-3.23916 \mathrm{E} & 01\end{array}$ $-3.24921 \mathrm{E} \quad 01$ $-3.25926 \mathrm{E} \quad 01$ $-3.26932 \mathrm{E} 01$ $-3.27937 \mathrm{E}$ 0l

$-3.28942 \mathrm{E} 01$ $-3.29947 \mathrm{E} 01$ $-3.30952 \mathrm{E} 01$ $-3.31957 \mathrm{E} 01$ $-3.32961 \mathrm{E} 01$

$-3.33966 \mathrm{E}$ 01 $-3.34971 \mathrm{E} 01$ $-3.35976 \mathrm{E} 01$ $-3.36981 \mathrm{E} \quad 01$ $-3.37985 \mathrm{E} 01$

$-3.38990 \mathrm{E} 01$ $-3.39995 \mathrm{E} 01$ $-3.40999 \mathrm{E} 01$ $-3.42004 \mathrm{E} 01$ $-3.43008 \mathrm{E} 01$

$-3.44013 \mathrm{E}$ 0l $-3.45017 \mathrm{E}$ 0l $-3.46022 \mathrm{E} 01$ $-3.47026 \mathrm{E} 0 \mathrm{l}$ $-3.48030 \mathrm{E}$ 0l

$-3.49034 \mathrm{E} \quad 01$ $-3.50039 \mathrm{E} 01$ $-3.51043 \mathrm{E} 01$ $-3.52047 \mathrm{E}$ 01 $-3.53051 \mathrm{E} 01$

$-3.54055 \mathrm{E} 01$ $-3.55059 \mathrm{E} 01$ $-3.56063 \mathrm{E} 01$ $-3.57067 \mathrm{E} 01$ $-3.58071 \mathrm{E} 01$
$-1.05097 \mathrm{E} \quad 00$ $-1.05762 \mathrm{E} \quad 00$ $-1.06429 \mathrm{E} 00$ $-1.07098 \mathrm{E} \quad 00$ $-1.07769 \mathrm{E} \quad 00$

$-1.08442 \mathrm{E} \quad 00$ $-1.09117 \mathrm{E} 00$ $-1.09795 \mathrm{E} 00$ $-1.10474 \mathrm{E} \cdot 00$ $-1.11156 \mathrm{E} 00$

$-1.11839 \mathrm{E} \quad 00$ $-1.12525 \mathrm{E} \quad 00$ $-1.13213 \mathrm{E} 00$ $-1.13903 \mathrm{E} 00$ $-1.14595 \mathrm{E} 00$

$-1.15289 \mathrm{E} \quad 00$ $-1.15985 \mathrm{E} 00$ $-1.16683 \mathrm{E} \quad 00$ $-1.17383 \mathrm{E} \quad 00$ $-1.18086 \mathrm{E} \quad 00$

$-1.18790 \mathrm{E} \quad 00$ $-1.19497 \mathrm{E} \quad 00$ $-1.20205 \mathrm{E} \quad 00$ $-1.20916 \mathrm{E} 00$ $-1.21629 \mathrm{E} 00$

$-1.22344 \mathrm{E} \quad 00$ $-1.23061 \mathrm{E} \quad 00$ $-1.23780 \mathrm{E} 00$ $-1.24501 \mathrm{E} \quad 00$

$-1.25224 \mathrm{E} \quad 00$

$-1.25949 \mathrm{E} \quad 00$

$-1.26676 \mathrm{E} 00$

$-1.27406 \mathrm{E} \quad 00$

$-1.28137 \mathrm{E} \quad 00$

$\begin{array}{lll}-1.28871 \mathrm{E} & 00\end{array}$

$-1.29606 \mathrm{E} \quad 00$

$-1.30344 \mathrm{E} \quad 00$

$-1.31084 \mathrm{E} \quad 00$

$-1.31826 \mathrm{E} 00$

$-1.32569 \mathrm{E} 00$

$-1.33315 \mathrm{E} \quad 00$ $-1.34064 \mathrm{E} 00$

$-1.34814 \mathrm{E} 00$

$-1.35566 \mathrm{E} \quad 00$

$-1.36320 \mathrm{E} \quad 00$

$-1.37077 \mathrm{E} \quad 00$

$-1.37835 \mathrm{E} \quad 00$

$-1.38596 \mathrm{E} 00$

$-1.39358 \mathrm{E} \quad 00$

$-1.40123 \mathrm{E} \quad 00$

$-1.40890 \mathrm{E} \quad 00$

$-1.41659 \mathrm{E} \quad 00$

$-1.42429 \mathrm{E} \quad 00$

$-1.43202 \mathrm{E} \quad 00$

$-1.43978 \mathrm{E} \quad 00$

$-1.44755 \mathrm{E} \quad 00$ $-1.45534 \mathrm{E} \quad 00$

$-1.46315 \mathrm{E} 00$

$-1.47099 \mathrm{E} 00$

$-1.47884 \mathrm{E} \quad 00$

$$
\rho=1.2 A^{1 / 3} F
$$

$\tan \delta_{1}$

4.79200E-04 $4.79557 \mathrm{E}-04$ $4.79911 \mathrm{E}-04$ $4.80263 \mathrm{E}-04$

$4.80612 \mathrm{E}-04$

$4.80958 \mathrm{E}-04$

$4.81301 \mathrm{E}-04$

$4.81642 \mathrm{E}-04$

$4.81980 \mathrm{E}-04$

$4.82316 \mathrm{E}-04$

$4.82648 \mathrm{E}-04$ $4.82979 \mathrm{E}-04$

$4.83306 \mathrm{E}-04$

$4.83632 \mathrm{E}-04$

$4.83955 \mathrm{E}-04$

$4.84275 \mathrm{E}-04$

$4.84593 \mathrm{E}-04$

$4.84908 \mathrm{E}-04$

$4.85221 \mathrm{E}-04$

$4.85532 \mathrm{E}-04$

$4.85840 \mathrm{E}-04$

$4.86147 \mathrm{E}-04$

$4.86450 \mathrm{E}-04$

$4.86752 \mathrm{E}-04$

4.87051 E-04

$4.87348 \mathrm{E}-04$

$4.87643 \mathrm{E}-04$

$4.87935 \mathrm{E}-04$

$4.88226 \mathrm{E}-04$

$4.88514 \mathrm{E}-04$

$4.88799 \mathrm{E}-04$

$4.89083 \mathrm{E}-04$

$4.89365 \mathrm{E}-04$

$4.89644 \mathrm{E}-04$

$4.89922 \mathrm{E}-04$

$4.90197 \mathrm{E}-04$

4.90471 E-04

$4.90743 \mathrm{E}-04$ 
$\bar{F}$

$2.48693 \mathrm{E}-04$.

$2.50348 \mathrm{E}-04$

$2.52009 \mathrm{E}-04$

$2.53675 \mathrm{E}-04$

$2.55347 \mathrm{E}-04$

$2.57024 \mathrm{E}-04$

$2.58706 \mathrm{E}-04$

$2.60394 \mathrm{E}-04$

$2.62088 \mathrm{E}-04$

$2.63787 \mathrm{E}-04$

$2.65491 \mathrm{E}-04$

$2.67201 \mathrm{E}-04$

$2.68917 \mathrm{E}-04$

$2.70638 \mathrm{E}-04$

$2.72365 \mathrm{E}-04$

$2.74097 \mathrm{E}-04$

$2.75834 \mathrm{E}-04$

$2.77577 \mathrm{E}-04$

$2.79325 \mathrm{E}-04$

$2.81079 \mathrm{E}-04$

$2.82839 \mathrm{E}-04$

$2.84604 \mathrm{E}-04$

$2.86374 \mathrm{E}-04$

$2.88150 \mathrm{E}-04$

$2.89932 \mathrm{E}-04$

$2.91718 \mathrm{E}-04$

$2.93511 \mathrm{E}-04$

$2.95309 \mathrm{E}-04$

$2.97112 \mathrm{E}-04$

$2.98921 \mathrm{E}-04$

$3.00735 \mathrm{E}-04$

$3.02555 \mathrm{E}-04$

$3.04380 \mathrm{E}-04$

$3.06211 \mathrm{E}-04$

$3.08048 \mathrm{E}-04$

$3.09889 \mathrm{E}-04$

$3.11737 \mathrm{E}-04$

$3.13589 \mathrm{E}-04$

$3.15448 \mathrm{E}-04$

$3.17311 \mathrm{E}-04$

$3.19181 \mathrm{E}-04$

$3.21055 \mathrm{E}-04$

$3.22936 \mathrm{E}-04$

$3.24821 \mathrm{E}-04$

$3.26712 \mathrm{E}-04$

$3.28609 \mathrm{E}-04$

$3.30511 \mathrm{E}-04$

$3.32419 \mathrm{E}-04$

$3.34332 \mathrm{E}-04$

3.36251 E-04

$3.38175 \mathrm{E}-04$

$3.40105 \mathrm{E}-04$

$3.42040 \mathrm{E}-04$

$3.43980 \mathrm{E}-04$

$3.45926 \mathrm{E}-04$

$3.47878 \mathrm{E}-04$

$3.49835 \mathrm{E}-04$

$3.51798 \mathrm{E}-04$

$3.53766 \mathrm{E}-04$

$3.55739 \mathrm{E}-04$ $f_{2}$

$-9.32554 \mathrm{E}-01$

$-9.38807 \mathrm{E}-01$

$-9.45082 \mathrm{E}-01$

$-9.51377 \mathrm{E}-01$

$-9.57694 \mathrm{E}-01$

$-9.64031 \mathrm{E}-01$

$-9.70389 \mathrm{E}-01$

$-9.76768 \mathrm{E}-01$

$-9.83167 \mathrm{E}-01$

$-9.89588 \mathrm{E}-01$

$-9.96029 \mathrm{E}-01$

$-1.00249 \mathrm{E} \quad 00$

$-1.00897 \mathrm{E} \quad 00$

$-1.01548 \mathrm{E} \quad 00$

$-1.02200 \mathrm{E} 00$

$-1.02855 \mathrm{E} \quad 00$

$-1.03511 \mathrm{E} \quad 00$

$-1.04170 \mathrm{E} \quad 00$

$-1.04831 \mathrm{E} 00$

$-1.05494 \mathrm{E} \quad 00$

$-1.06159 \mathrm{E} \quad 00$

$-1.06826 \mathrm{E} \quad 00$

$-1.07495 \mathrm{E} 00$

$-1.08166 \mathrm{E} \quad 00$

$-1.08839 \mathrm{E} 00$

$-1.09515 \mathrm{E} \quad 00$

$-1.10192 \mathrm{E} 00$

$-1.10872 \mathrm{E} \quad 00$

$-1.11553 \mathrm{E} 00$

$-1.12237 \mathrm{E} \quad 00$

$-1.12923 \mathrm{E} \quad 00$

$-1.13611 \mathrm{E} 00$

$-1.14300 \mathrm{E} \quad 00$

$-1.14992 \mathrm{E} \quad 00$

$-1.15687 \mathrm{E} \quad 00$

$-1.16383 \mathrm{E} \quad 00$

$-1.17081 \mathrm{E} \quad 00$

$-1.17781 \mathrm{E} \quad 00$

$-1.18484 \mathrm{E} \quad 00$

$-1.19188 \mathrm{E} \quad 00$

$-1.19895 \mathrm{E} \quad 00$

$-1.20603 \mathrm{E} \quad 00$

$-1.21314 \mathrm{E} 00$

$-1.22027 \mathrm{E} \quad 00$

$-1.22742 \mathrm{E} 00$

$-1.23459 \mathrm{E} \quad 00$

$-1.24178 \mathrm{E} 00$

$-1.24899 \mathrm{E} 00$

$-1.25622 \mathrm{E} 00$

$-1.26348 \mathrm{E} 00$

$-1.27075 \mathrm{E} \quad 00$

$-1.27804 \mathrm{E} \quad 00$

$-1.28536 \mathrm{E} \quad 00$

$-1.29269 \mathrm{E} \quad 00$

$-1.30005 \mathrm{E} 00$

-1.30743 E 00

$-1.31483 \mathrm{E} 00$

$-1.32225 \mathrm{E} 00$

$-1.32969 \mathrm{E} 00$

$-1.33715 \mathrm{E} 00$ $g_{2}$

$-1.95963 \mathrm{E}-02$

$-1.97861 \mathrm{E}-02$

$-1.99771 \mathrm{E}-02$

$-2.01693 \mathrm{E}-02$

$-2.03627 \mathrm{E}-02$

$-2.05574 \mathrm{E}-02$

$-2.07533 \mathrm{E}-02$

$-2.09505 \mathrm{E}-02$

$-2.11489 \mathrm{E}-02$

$-2.13485 \mathrm{E}-02$

$-2.15494 \mathrm{E}-02$

$-2.17516 \mathrm{E}-02$

$-2.19549 \mathrm{E}-02$

$-2.21596 \mathrm{E}-02$

$-2.23655 \mathrm{E}-02$

$-2.25727 \mathrm{E}-02$

$-2.27812 \mathrm{E}-02$

$-2.29910 \mathrm{E}-02$

$-2.32020 \mathrm{E}-02$

$-2.34143 \mathrm{E}-02$

$-2.36279 \mathrm{E}-02$

$-2.38428 \mathrm{E}-02$

$-2.40590 \mathrm{E}-02$

$-2.42765 \mathrm{E}-02$

$-2.44952 \mathrm{E}-02$

$-2.47153 \mathrm{E}-02$

$-2.49368 \mathrm{E}-02$

$-2.51595 \mathrm{E}-02$

$-2.53835 \mathrm{E}-02$

$-2.56089 \mathrm{E}-02$

$-2.58356 \mathrm{E}-02$

$-2.60636 \mathrm{E}-02$

$-2.62930 \mathrm{E}-02$

$-2.65237 \mathrm{E}-02$

$-2.67557 \mathrm{E}-02$

$-2.69891 \mathrm{E}-02$

$-2.72239 \mathrm{E}-02$

$-2.74600 \mathrm{E}-02$

$-2.76974 \mathrm{E}-02$

$-2.79362 \mathrm{E}-02$

$-2.81764 \mathrm{E}-02$

$-2.84179 \mathrm{E}-02$

$-2.86609 \mathrm{E}-02$

$-2.89052 \mathrm{E}-02$

$-2.91509 \mathrm{E}-02$

$-2.93979 \mathrm{E}-02$

$-2.96464 \mathrm{E}-02$

$-2.98962 \mathrm{E}-02$

$-3.01475 \mathrm{E}-02$

$-3.04001 \mathrm{E}-02$

$-3.06542 \mathrm{E}-02$

$-3.09096 \mathrm{E}-02$

$-3.11665 \mathrm{E}-02$

$-3.14248 \mathrm{E}-02$

$-3.16845 \mathrm{E}-02$

$-3.19456 \mathrm{E}-02$

$-3.22082 \mathrm{E}-02$

$-3.24722 \mathrm{E}-02$

$-3.27376 \mathrm{E}-02$

$-3.30045 \mathrm{E}-02$ $\tan \delta$.

$2.02000 \mathrm{E} \quad 02$

$2.02009 \mathrm{E} \quad 02$

$2.02018 \mathrm{E} \quad 02$

$2.02026 \mathrm{E} \quad 02$

$2.02035 \mathrm{E} \quad 02$

$2.02044 \mathrm{E} \quad 02$

$2.02052 \mathrm{E} \quad 02$

$2.02061 \mathrm{E} \quad 02$

$2.02070 \mathrm{E} \quad 02$

$2.02078 \mathrm{E} \quad 02$

$2.02086 \mathrm{E} \quad 02$ $2.02095 \mathrm{E} \quad 02$

$2.02103 \mathrm{E} \quad 02$

$2.02111 \mathrm{E} \quad 02$

$2.02119 \mathrm{E} \quad 02$

$2.02128 \mathrm{E} \quad 02$

$2.02136 \mathrm{E} \quad 02$

$2.02144 \mathrm{E} \quad 02$

$2.02152 \mathrm{E} \quad 02$

$2.02160 \mathrm{E} \quad 02$

$2.02168 \mathrm{E} \quad 02$

$2.02175 \mathrm{E} \quad 02$

$2.02183 \mathbf{E} \quad 02$

$2.02191 \mathrm{E} \quad 02$

$2.02199 \mathrm{E} \quad 02$

$2.02206 \mathrm{E} \quad 02$

$2.02214 \mathrm{E} \quad 02$

$2.02221 \mathrm{E} \quad 02$

$2.02229 \mathrm{E} \quad 02$

$2.02236 \mathrm{E} \quad 02$

$2.02244 \mathrm{E} \quad 02$

$2.02251 \mathrm{E} \quad 02$

$2.02258 \mathrm{E} \quad 02$

$2.02265 \mathrm{E} \quad 02$

$2.02273 \mathrm{E} \quad 02$

$2.02280 \mathrm{E} \quad 02$

$2.02287 \mathrm{E} \quad 02$

$2.02294 \mathrm{E} \quad 02$

$2.02301 \mathrm{E} \quad 02$

$2.02308 \mathrm{E} \quad 02$

$2.02315 \mathrm{E} \quad 02$

$2.02322 \mathrm{E} \quad 02$

$2.02329 \mathrm{E} \quad 02$

$2.02336 \mathrm{E} \quad 02$

$2.02342 \mathrm{E} \quad 02$

$2.02349 \mathrm{E} \quad 02$ $2.02356 \mathrm{E} \quad 02$

$2.0236 .3 \mathrm{E} \quad 02$

$2.02369 \mathrm{E} \quad 02$

$2.02376 \mathrm{E} \quad 02$

$2.02382 \mathrm{E} \quad 02$ $2.02389 \mathrm{E} \quad 02$ $2.02395 \mathrm{E} \quad 02$

$2.02402 \mathrm{E} \quad 02$

$2.02408 \mathrm{E} \quad 02$

$2.02415 \mathrm{E} \quad 02$ $2.02421 \mathrm{E} \quad 02$ $2.02427 \mathrm{E} \quad 02$

$2.02433 \mathrm{E} \quad 02$

2.02440 E $\quad 02$

$f_{-2}$

$-1.90552 \mathrm{E}-02$

$-1.92412 \mathrm{E}-02$

$-1.94284 \mathrm{E}-02$

$-1.96168 \mathrm{E}-02$

$-1.98064 \mathrm{E}-02$

$-1.99973 \mathrm{E}-02$

$-2.01893 \mathrm{E}-02$

$-2.03826 \mathrm{E}-02$

$-2.05771 \mathrm{E}-02$

$-2.07729 \mathrm{E}-02$

$-2.09698 \mathrm{E}-02$

$-2.11680 \mathrm{E}-02$

$-2.13675 \mathrm{E}-02$

$-2.15682 \mathrm{E}-02$

$-2.17702 \mathrm{E}-02$

$-2.19734 \mathrm{E}-02$

$-2.21779 \mathrm{E}-02$

$-2.23836 \mathrm{E}-02$

$-2.25906 \mathrm{E}-02$

$-2.27989 \mathrm{E}-02$

$-2.30084 \mathrm{E}-02$

$-2.32193 \mathrm{E}-02$

$-2.34314 \mathrm{E}-02$

$-2.36448 \mathrm{E}-02$

$-2.38595 \mathrm{E}-02$

$-2.40755 \mathrm{E}-02$

$-2.42927 \mathrm{E}-02$

$-2.45113 \mathrm{E}-02$

$-2.47312 \mathrm{E}-02$

$-2.49524 \mathrm{E}-02$

$-2.51749 \mathrm{E}-02$

$-2.53988 \mathrm{E}-02$

$-2.56239 \mathrm{E}-02$

$-2.58504 \mathrm{E}-02$

$-2.60782 \mathrm{E}-02$

$-2.63073 \mathrm{E}-02$

$-2.65378 \mathrm{E}-02$

$-2.67696 \mathrm{E}-02$

$-2.70028 \mathrm{E}-02$

$-2.72373 \mathrm{E}-02$

$-2.74732 \mathrm{E}-02$

$-2.77104 \mathrm{E}-02$ 
ELECTRON RADIAL FUNCTIONS

\begin{tabular}{|c|c|c|c|c|c|c|c|c|c|c|c|c|c|}
\hline Electro & & & & & $=1$ & $A=1$ & $\rho=$ & $A^{1 / 3} F$ & & & & & \\
\hline$p$ & $F_{0}$ & & $f_{1}$ & & $g_{1}$ & & $\tan \delta_{1}$ & $f_{-1}$ & & $g_{-1}$ & & $\tan \delta_{-1}$ & \\
\hline 36.10 & $1.02332 \mathrm{E}$ & 00 & $-3.59075 \mathrm{E}$ & 01 & $-1.48672 \mathrm{E}$ & 00 & $4.96459 \mathrm{E}-04$ & $-1.45185 E$ & 00 & $3.69143 \mathrm{E}$ & 01 & $-1.43182 \mathrm{E}$ & 03 \\
\hline 36.20 & $1.02332 \mathrm{E}$ & 00 & $-3.60079 E$ & 01 & $-1.49461 \mathrm{E}$ & 00 & $4.96686 \mathrm{E}-04$ & $-1.45964 \mathrm{E}$ & 00 & $3.70146 \mathrm{E}$ & 01 & & 03 \\
\hline 36.30 & $1.02332 \mathrm{E}$ & 00 & $-3.61083 E$ & 01 & $-1.50253 \mathrm{E}$ & 00 & $4.96911 \mathrm{E}-04$ & $-1.46745 \mathrm{E}$ & 00 & $3.71150 \mathrm{E}$ & 01 & $.43318 \mathrm{E}$ & 03 \\
\hline 36.40 & $1.02332 \mathrm{E}$ & 00 & $-3.62087 \mathbf{E}$ & 01 & $-1.51047 \mathbf{E}$ & 00 & $4.97135 \mathrm{E}-04$ & $-1.47529 \mathrm{E}$ & 00 & $3.72153 \mathrm{E}$ & 01 & $1.43386 \mathrm{E}$ & 03 \\
\hline 36.50 & $1.02332 \mathrm{E}$ & 00 & $-3.63090 \mathrm{E}$ & 01 & $-1.51842 \mathrm{E}$ & 00 & $4.97357 \mathrm{E}-04$ & $-1.48314 \mathrm{E}$ & 00 & $3.73157 \mathrm{E}$ & 01 & $-1.43454 \mathrm{E}$ & 03 \\
\hline 36.60 & $1.02332 \mathrm{E}$ & 00 & $-3.64094 \mathrm{E}$ & 01 & $-1.52640 \mathrm{E}$ & 00 & $4.97577 \mathrm{E}-04$ & $-1.49101 \mathrm{E}$ & 00 & $3.74160 \mathrm{E}$ & 01 & $-1.43521 \mathrm{E}$ & 03 \\
\hline 36.70 & $1.02332 \mathrm{E}$ & 00 & $-3.65098 \mathrm{E}$ & 01 & $-1.53440 \mathrm{E}$ & 00 & $4.97797 \mathrm{E}-$ & $-1.49891 \mathrm{E}$ & 00 & $3.75164 \mathrm{E}$ & 01 & & 03 \\
\hline 36.80 & $1.02332 \mathrm{E}$ & 00 & $-3.66101 \mathrm{E}$ & 01 & $-1.54242 \mathrm{E}$ & 00 & $98014 \mathrm{E}-$ & $-1.50682 \mathrm{E}$ & 00 & $3.76167 \mathrm{E}$ & 01 & $43655 \mathbf{E}$ & 03 \\
\hline 36.90 & $1.02332 \mathrm{E}$ & 00 & $-3.67105 \bar{E}$ & 01 & $-1.55046 \mathbf{E}$ & 00 & $4.98230 \mathrm{E}-$ & $-1.51476 \mathrm{E}$ & 00 & $3.77171 \mathrm{E}$ & 01 & $-1.43722 \mathrm{E}$ & 03 \\
\hline 37.00 & $1.02332 \mathrm{E}$ & 00 & $-3.68108 \mathrm{E}$ & 01 & $-1.55853 \mathrm{E}$ & 00 & $4.98444 \mathrm{E}-04$ & $-1.52272 \mathrm{E}$ & 00 & $3.78174 \mathrm{E}$ & 01 & $-1.43789 \mathrm{E}$ & 03 \\
\hline 37.10 & $1.02332 \mathrm{E}$ & 00 & $-3.69112 \mathbf{E}$ & 01 & $-1.56661 \mathrm{E}$ & 00 & $4.98657 \mathrm{E}-04$ & $-1.53070 \mathrm{E}$ & 00 & $3.79177 \mathrm{E}$ & 01 & $-1.43855 E$ & 03 \\
\hline 37.20 & $1.02332 \mathrm{E}$ & 00 & $0115 \mathrm{E}$ & 01 & $57471 \mathrm{E}$ & 00 & $67 \mathrm{E}-04$ & $53869 \mathrm{E}$ & 00 & $3.80181 \mathrm{E}$ & 01 & $22 \bar{E}$ & 03 \\
\hline 37.30 & $1.02332 \mathrm{E}$ & 00 & $-3.71119 \mathrm{E}$ & 01 & $-1.58284 \mathrm{E}$ & 00 & $4.99077 \mathrm{E}-$ & $-1.54671 \mathrm{E}$ & 00 & $3.81184 \mathrm{E}$ & 01 & $-1.43987 \mathrm{E}$ & 03 \\
\hline 37.40 & $1.02332 \mathrm{E}$ & 00 & $-3.72122 \mathrm{E}$ & 01 & $-1.59098 \mathrm{E}$ & 00 & $4.99284 \mathrm{E}-04$ & $-1.55475 \mathrm{E}$ & 00 & $3.82187 \mathrm{E}$ & 01 & & 03 \\
\hline 37.50 & $1.02332 \mathrm{E}$ & 00 & $-3.73125 \mathrm{E}$ & 01 & $-1.59915 \mathrm{E}$ & 00 & $4.99491 \mathrm{E}-04$ & $-1.56281 \mathrm{E}$ & 00 & $3.83190 \mathrm{E}$ & 01 & $-1.44119 \mathrm{E}$ & 03 \\
\hline 37.60 & $1.02332 \mathrm{E}$ & 00 & $-3.74129 \mathrm{E}$ & 01 & $-1.60733 \mathrm{E}$ & 00 & $4.99696 \mathrm{E}-04$ & $-1.57090 \mathrm{E}$ & 00 & $3.84193 \mathrm{E}$ & 01 & $-1.44184 \mathrm{E}$ & 03 \\
\hline 37.70 & $1.02332 \mathrm{E}$ & 00 & $-3.75132 \mathrm{E}$ & 01 & $-1.61554 \mathrm{E}$ & 00 & $4.99899 \mathrm{E}-$ & $-1.57900 \mathrm{E}$ & 00 & $3.85196 \mathrm{E}$ & 01 & $49 \mathrm{E}$ & 03 \\
\hline 37.80 & $1.02332 \mathrm{E}$ & 00 & $-3.76135 \mathrm{E}$ & 01 & $2377 \mathbf{E}$ & 00 & $00 \mathrm{E}-$ & $58712 \mathrm{E}$ & 00 & 3.861 & 01 & & 03 \\
\hline 37.90 & $1.02332 \mathrm{E}$ & 00 & $-3.77138 \mathrm{E}$ & 01 & $-1.63202 \mathbf{E}$ & 00 . & $90 \mathrm{E}-$ & $-1.59527 \mathrm{E}$ & $0 c$ & $3.87202 \mathrm{E}$ & 01 & $379 \mathrm{E}$ & 03 \\
\hline 38.00 & $1.02332 \mathrm{E}$ & 00 & $-3.78141 \mathrm{E}$ & 01 & $-1.64029 \mathrm{E}$ & 00 & $5.00500 \mathrm{E}-04$ & $-1.60343 \mathrm{E}$ & 00 & $3.88205 \mathrm{E}$ & 01 & $.44444 \mathrm{E}$ & 03 \\
\hline 38.10 & $1.02332 \mathrm{E}$ & 00 & $-3.79145 \mathrm{E}$ & 01 & $-1.64858 \mathrm{E}$ & 00 & $5.00697 \mathbf{E}-04$ & $-1.61162 \mathrm{E}$ & 00 & $3.89208 \mathrm{E}$ & 01 & $508 \mathrm{E}$ & 03 \\
\hline 38.20 & $1.02332 \mathrm{E}$ & 00 & $-3.80148 E$ & 01 & $5689 \mathrm{E}$ & 00 & $F$ & $1982 \mathrm{E}$ & 00 & $211 \mathrm{E}$ & 01 & $72 \mathrm{E}$ & 03 \\
\hline 38.30 & $1.02332 \mathrm{E}$ & 00 & $-3.81151 \mathbf{E}$ & 01 & $-1.66522 \mathbf{E}$ & 00 & $88 \mathrm{E}-0$ & $-1.62805 \mathrm{E}$ & 00 & $14 \mathrm{E}$ & 01 & $636 \mathrm{E}$ & 03 \\
\hline 38.40 & $1.02332 \mathrm{E}$ & 00 & $-3.82153 \mathbf{E}$ & 01 & $-1.67357 \mathrm{E}$ & 00 & 81 E-04 & $-1.63630 \mathrm{E}$ & 00 & $3.92216 \mathrm{E}$ & 01 & $700 \mathrm{E}$ & 03 \\
\hline 38.50 & $1.02332 \mathrm{E}$ & 00 & $-3.83156 \mathrm{E}$ & 01 & $-1.68194 \mathrm{E}$ & 00 & $5.01472 \mathrm{E}-04$ & $-1.64456 \mathrm{E}$ & 00 & $3.93219 \mathrm{E}$ & 01 & $4764 \mathrm{E}$ & 03 \\
\hline 38.60 & $1.02332 \mathrm{E}$ & 00 & $4159 \mathrm{E}$ & 01 & -1 & 00 & $63 \mathrm{E}-04$ & $65285 \mathrm{E}$ & 00 & $22 \mathrm{E}$ & 01 & & 03 \\
\hline 38.70 & $1.02332 \mathrm{E}$ & 00 & $-3.85162 \mathbf{E}$ & 01 & $-1.69875 \mathrm{E}$ & 00 & $51 \mathrm{E}-0$ & $-1.66116 \mathrm{E}$ & 0( & $5224 \mathrm{E}$ & 01 & $4891 \mathrm{E}$ & 03 \\
\hline 38.80 & $1.02332 \mathrm{E}$ & 00 & $-3.86165 \mathrm{E}$ & 01 & $-1.70719 \mathrm{E}$ & 00 & $2039 E-0$ & $-1.66949 \mathrm{E}$ & 00 & $3.96227 \mathrm{E}$ & 01 & $44954 \mathrm{E}$ & 03 \\
\hline 38.90 & $1.02332 \mathrm{E}$ & 00 & $-3.87168 \mathrm{E}$ & 01 & $1564 \mathrm{E}$ & 00 & $5 \mathrm{E}-04$ & $67785 \mathrm{E}$ & 00 & $7230 \mathrm{E}$ & 01 & $5017 \mathrm{E}$ & 03 \\
\hline 39.00 & $1.02332 \mathrm{E}$ & 00 & $-3.88170 \mathrm{E}$ & 01 & $-1.72412 \mathrm{E}$ & 00 & $5.02409 \mathrm{E}-\mathrm{C}$ & $-1.68622 \mathrm{E}$ & 00 & $3.98232 \mathrm{E}$ & 01 & $45079 \mathrm{E}$ & 03 \\
\hline 39.10 & $02331 \mathrm{E}$ & 00 & $-3.89173 \mathbf{E}$ & 01 & $-1.73262 \mathrm{E}$ & 00 & $5.02594 \mathrm{E}-04$ & $-1.69461 \mathrm{E}$ & 00 & $3.99235 \mathrm{E}$ & 01 & $45142 \mathrm{E}$ & 03 \\
\hline 39.20 & $02331 \mathrm{E}$ & 00 & $90176 \mathbf{E}$ & 01 & $114 \mathrm{E}$ & 00 & $4 \mathrm{E}-$ & $70302 \mathrm{E}$ & 00 & $37 \mathrm{E}$ & 01 & $205 \mathrm{E}$ & 03 \\
\hline 39.30 & $331 \mathrm{E}$ & 00 & $178 \mathrm{E}$ & 01 & $967 \mathrm{E}$ & 00 & $6 \mathrm{~F}-0$ & $1146 \mathrm{E}$ & 00 & $39 \mathrm{E}$ & 01 & $5267 \mathrm{E}$ & 03 \\
\hline 39.40 & $1.02331 \mathrm{E}$ & 00 & $-3.92181 \mathrm{E}$ & 01 & $5823 \mathrm{E}$ & 00 & $35 \mathrm{E}-$ & $1991 \mathrm{E}$ & 00 & $4.02242 \mathrm{E}$ & 01 & $45329 E$ & 03 \\
\hline 39.50 & $1.02331 \mathrm{E}$ & 00 & $-3.93183 \bar{E}$ & 01 & $-1.76681 \mathrm{E}$ & 00 & $5.03313 \mathrm{E}-0$ & $-1.72839 \mathrm{E}$ & 00 & $4.03244 \mathrm{E}$ & 01 & $-1.45390 \mathrm{E}$ & 03 \\
\hline 39.60 & $.02331 \mathrm{E}$ & 00 & $-3.94186 \mathrm{E}$ & 1 & $-1.77542 \mathrm{E}$ & 00 & $0 \mathrm{E}-$ & $73688 \mathrm{E}$ & 00 & $46 E$ & 01 & $52 \mathrm{E}$ & 03 \\
\hline 39.70 & & 00 & & 01 & & 00 & & & $0 \mathrm{r}$ & & 01 & & 03 \\
\hline 39.80 & $31 \mathrm{E}$ & 00 & $6190 \mathrm{E}$ & 01 & $268 \mathrm{E}$ & 00 & $F_{-}$ & $5394 \mathrm{E}$ & 00 & $50 \mathrm{E}$ & 01 & $5 \mathrm{E}$ & 03 \\
\hline 39.90 & $1.02331 \mathrm{E}$ & 00 & $-3.97193 \bar{E}$ & 01 & $0134 \mathrm{E}$ & 00 & $2 \mathrm{E}-$ & $6250 \mathrm{E}$ & 00 & $4.07253 \bar{E}$ & 01 & $5636 \mathrm{E}$ & 03 \\
\hline 40.00 & $1.02331 \mathrm{E}$ & 00 & $-3.98195 \mathrm{E}$ & & $-1.81003 \mathrm{E}$ & & $5.04183 \mathrm{E}-04$ & & & $4.08255 \mathrm{E}$ & & $-1.45697 \mathrm{E}$ & 03 \\
\hline 40.10 & $02331 \mathrm{E}$ & 00 & $-3.99197 \mathrm{E}$ & 01 & $-1.81873 \mathrm{E}$ & 00 & $5.04354 \mathrm{E}-04$ & $-1.77968 \mathbf{E}$ & 00 & $4.09257 \mathrm{E}$ & 01 & $758 \mathrm{E}$ & 03 \\
\hline 40.20 & & 00 & -4 & 01 & & 00 & 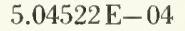 & -1 & 00 & & 01 & & 03 \\
\hline 40.30 & $2331 \mathrm{E}$ & 00 & $-4.01201 \overline{\mathbf{E}}$ & 01 & $620 \mathrm{E}$ & 00 & E- & -1 & 0 & $1 \mathrm{E}$ & 0 & $0 \mathrm{E}$ & 03 \\
\hline 40.40 & $.02331 \mathrm{E}$ & 00 & $-4.02204 \mathrm{E}$ & 01 & $4497 \mathrm{E}$ & 00 & & & 0 & & 01 & $15940 \mathrm{E}$ & 03 \\
\hline 40.50 & $1.02331 \mathrm{E}$ & 00 & & 01 & $-1.85376 \mathrm{E}$ & 00 & & $81428 \mathrm{E}$ & 00 & $4.13264 \mathrm{E}$ & 01 & $.46000 \mathrm{E}$ & \\
\hline 40.60 & $02331 \mathrm{E}$ & 00 & $-4.04208 \mathrm{E}$ & 01 & $-1.86256 \mathrm{E}$ & 00 & $36 \mathrm{E}-04$ & $-1.82299 \mathbf{E}$ & 00 & & 01 & $0 \mathrm{E}$ & 03 \\
\hline & & 00 & & 0 & & 00 & & & 0 & $8 \mathrm{E}$ & 0 & & 03 \\
\hline 40.8 & $2331 \mathrm{E}$ & 00 & $-4.06211 \mathrm{E}$ & 01 & $8024 \mathrm{E}$ & 00 & & -1 & 0 & $270 E$ & 01 & & 03 \\
\hline 40.90 & $1.02331 \mathrm{E}$ & 00 & $-4.07213 \mathrm{E}$ & 01 & $8911 \mathrm{E}$ & 00 & & & 00 & $7271 \mathrm{E}$ & 01 & $6240 \mathrm{E}$ & 03 \\
\hline 41.00 & $1.02331 \mathrm{E}$ & 00 & $-4.08215 \mathrm{E}$ & 01 & $-1.89800 \mathrm{E}$ & 00 & $5.05830 \mathrm{E}-04$ & $-1.85801 \mathrm{E}$ & 00 & $4.18273 \mathrm{E}$ & 01 & $.46299 \mathrm{E}$ & 03 \\
\hline 41.1 & $31 \mathrm{E}$ & 00 & -4.092 & 0 & $-1.90691 \mathrm{E}$ & 00 & $8 \mathrm{E}-04$ & -1 . & & & 01 & & \\
\hline & & 00 & $-4.10219 \mathrm{E}$ & 01 & $-1.91585 \mathrm{E}$ & 00 & $4 \mathrm{E}-$ & & 00 & & 01 & $418 \mathrm{E}$ & 3 \\
\hline 41.30 & & 00 & $-4.11220 \mathrm{E}$ & 01 & $92480 \mathrm{E}$ & 00 & 4 & & 00 & $1277 \mathrm{E}$ & 01 & $5477 \mathrm{E}$ & \\
\hline 41.4 & & 00 & & 01 & & 00 & & & 00 & & 01 & & 03 \\
\hline 41.50 & $1.02331 \mathrm{E}$ & 00 & $-4.13224 \mathrm{E}$ & 01 & $-1.94277 \mathrm{E}$ & 00 & $5.06608 \mathrm{E}-04$ & $-1.90225 \mathrm{E}$ & & $4.23280 \mathrm{E}$ & 01 & $-1.46594 \mathrm{E}$ & \\
\hline 41.60 & $.02331 \mathrm{E}$ & 00 & $-4.14225 \mathrm{E}$ & 01 & $-1.95178 \mathrm{E}$ & 00 & $6759 \mathrm{E}-04$ & $-1.91116 \mathrm{E}$ & 00 & & 01 & & 3 \\
\hline & $1.02331 \mathrm{E}$ & 00 & $-4.15227 \mathrm{E}$ & 01 & $-1.96082 \mathrm{E}$ & 00 & $6910 \mathrm{E}-04$ & $-1.92009 \mathrm{E}$ & 00 & $4.25283 \mathrm{E}$ & 01 & $-1.46712 \mathrm{E}$ & \\
\hline 41.80 & $1.02331 \mathrm{E}$ & 00 & $-4.16228 \mathrm{E}$ & 01 & $-1.96987 \mathrm{E}$ & 00 & $0 \mathrm{E}-04$ & & & $4.26284 \mathrm{E}$ & 01 & $46770 \mathrm{E}$ & \\
\hline & $1.02331 \mathrm{E}$ & 00 & $-4.17230 \mathrm{E}$ & 01 & $-1.97895 \mathrm{E}$ & 00 & $5.07209 \mathrm{E}-04$ & $-1.93801 \mathrm{E}$ & 00 & $4.27285 \mathrm{E}$ & 01 & $-1.46828 \mathrm{E}$ & 03 \\
\hline 42.00 & $1.02331 \mathrm{E}$ & 00 & $-4.18231 \mathrm{E}$ & 01 & $-1.98805 \mathrm{E}$ & 00 & $5.07356 \mathrm{E}-04$ & $-1.94701 \mathrm{E}$ & 00 & $4.28287 \mathrm{E}$ & 01 & $-1.46886 \mathrm{E}$ & 03 \\
\hline
\end{tabular}


ELECTRON RADIAL FUNCTIONS

$Z=1 \quad A=1 \quad \rho=1.2 A^{1 / 3} F$

Electron

$\bar{F}$

$f_{2}$

$3.57718 \mathrm{E}-04$

$3.59703 \mathrm{E}-04$

$3.61693 \mathrm{E}-04$

$3.63688 \mathrm{E}-04$

$3.65689 \mathrm{E}-04$

$3.67696 \mathrm{E}-04$

$3.69708 \mathrm{E}-04$

$3.71725 \mathrm{E}-04$

$3.73748 \mathrm{E}-04$

$3.75777 \mathrm{E}-04$

$3.77810 \mathrm{E}-04$

$3.79850 \mathrm{E}-04$

$3.81895 \mathrm{E}-04$

$3.83945 \mathrm{E}-04$

$3.86001 \mathrm{E}-04$

$3.88062 \mathrm{E}-04$

$3.90129 \mathrm{E}-04$

$3.92202 \mathrm{E}-04$

$3.94279 \mathrm{E}-04$

3.96363 E-04

3.98452 E- 04 $4.00546 \mathrm{E}-04$

$4.02646 \mathrm{E}-04$

4.04751 E-0 4

$4.06862 \mathrm{E}-04$

$4.08978 \mathrm{E}-04$

$4.11100 \mathrm{E}-04$

$4.13227 \mathrm{E}-04$

$4.15360 \mathrm{E}-04$

4.17498E-04

4.19642E-04

$4.21791 \mathrm{E}-04$

$4.23946 \mathrm{E}-04$

$4.26106 \mathrm{E}-04$

$4.28272 \mathrm{E}-04$

$4.30443 \mathrm{E}-04$

$4.32619 \mathrm{E}-04$

4.34801 E-0 4

$4.36989 \mathrm{E}-04$

$4.39182 \mathrm{E}-04$

4.41381 E-04

$4.43585 \mathrm{E}-04$

$4.45795 \mathrm{E}-04$

$4.48010 \mathrm{E}-04$

$4.50230 \mathrm{E}-04$

$4.52456 \mathrm{E}-04$

$4.54688 \mathrm{E}-04$

$4.56925 \mathrm{E}-04$

$4.59167 \mathrm{E}-04$

4.61415E-04

$4.63669 \mathrm{E}-04$

$4.65928 \mathrm{E}-04$

$4.68192 \mathrm{E}-04$

$4.70462 \mathrm{E}-04$

$4.72738 \mathrm{E}-04$

4.75019E-04

4.77305E-04

$4.79597 \mathrm{E}-04$

$4.81894 \mathrm{E}-04$

$4.84197 \mathrm{E}-04$ $g_{2}$

$-1.34463 \mathrm{E} \quad 00 \quad-3.32728 \mathrm{E}-02$

$-1.35213 \mathrm{E} \quad 00 \quad-3.35426 \mathrm{E}-02$

$\begin{array}{lll}-1.35965 \mathrm{E} & 00 & -3.38138 \mathrm{E}-02\end{array}$

$-1.36720 \mathrm{E} \quad 00 \quad-3.40864 \mathrm{E}-02$

$-1.37476 \mathrm{E} \quad 00 \quad-3.43606 \mathrm{E}-02$

$-1.38235 \mathrm{E} \quad 00$

$-1.38996 \mathrm{E} \quad 00$

$-1.39758 \mathrm{E} 00$

$-1.40523 \mathrm{E} \quad 00$

$-1.41290 \mathrm{E} \quad 00$

$-1.42059 \mathrm{E} \quad 00$

$-1.42830 \mathrm{E} 00$

$-1.43603 \mathrm{E} \quad 00$

$-1.44378 \mathrm{E} 00$

$-1.45155 \mathrm{E} \quad 00$

$-1.45935 \mathrm{E} \quad 00$

$-1.46716 \mathrm{E} \quad 00$

$-1.47500 \mathrm{E} \quad 00$

$-1.48285 \mathrm{E} 00$

$-1.49073 \mathrm{E} \quad 00$

$-1.49862 \mathrm{E} \quad 00$

$-1.50654 \mathrm{E} \quad 00$

$-1.51448 \mathrm{E} \quad 00$

$-1.52244 \mathrm{E} 00$

$-1.53042 \mathrm{E} \quad 00$

$-1.53842 \mathrm{E} \quad 00$

$-1.54644 \mathrm{E} \quad 00$

$-1.55448 \mathrm{E} 00$

$-1.56255 \mathrm{E} \quad 00$

$-1.57063 \mathrm{E} 00$

$-1.57874 \mathrm{E} \quad 00$

$-1.58686 \mathrm{E} \quad 00$

$-1.59501 \mathrm{E} 00$

$-1.60318 \mathrm{E} \quad 00$

$-1.61136 \mathrm{E} 00$

$-1.61957 \mathrm{E} \quad 00$

$-1.62780 \mathrm{E} 00$

$-1.63605 \mathrm{E} 00$

$-1.64432 \mathrm{E} \quad 00$

$-1.65261 \mathrm{E} 00$

$-1.66092 \mathrm{E} \quad 00$

$-1.66926 \mathrm{E} \quad 00$

$-1.67761 \mathrm{E} \quad 00$

$-1.68599 \mathrm{E} 00$

$-1.69438 \mathrm{E} \quad 00$

$-1.70280 \mathrm{E} \quad 00$

$-1.71123 \mathrm{E} 00$

$-1.71969 \mathrm{E} \quad 00$

$-1.72817 \mathrm{E} 00$

$-1.73667 \mathrm{E} \quad 00$

$-1.74519 \mathrm{E} \quad 00$

$-1.75373 \mathrm{E} 00$

$-1.76229 \mathrm{E} \quad 00$

$-1.77087 \mathrm{E} 00$

$-1.77947 \mathrm{E} \quad 00$

$-1.78810 \mathrm{E} \quad 00$

$-1.79674 \mathrm{E} \quad 00$

$-1.80540 \mathrm{E} 00$

$-1.81409 \mathrm{E} \quad 00$

$-1.82280 \mathrm{E} 00$

$-3.46361 \mathrm{E}-02$

$-3.49132 \mathrm{E}-02$

$-3.51917 \mathbf{E}-02$

$-3.54717 \mathrm{E}-02$

$-3.57532 \mathrm{E}-02$

$-3.60361 \mathrm{E}-02$

$-3.63206 \mathrm{E}-02$

$-3.66065 \mathrm{E}-02$

$-3.68939 \mathrm{E}-02$

$-3.71829 \mathrm{E}-02$

$-3.74733 \mathrm{E}-02$

$-3.77652 \mathrm{E}-02$

$-3.80586 \mathrm{E}-02$

$-3.86501 \mathbf{E}-02$

$-3.89480 \mathrm{E}-02$

$-3.92476 \mathrm{E}-02$

$-3.95486 \mathrm{E}-02$

$-3.98512 \mathrm{E}-02$

$-4.01553 \mathrm{E}-02$

$-4.04609 \mathrm{E}-02$

$-4.07681 \mathrm{E}-02$

$-4.10768 \mathrm{E}-02$

$-4.13871 \mathrm{E}-02$

$-4.16990 \mathrm{E}-02$

$-4.20124 \mathrm{E}-02$

$-4.23274 \mathrm{E}-02$

$-4.26439 \mathrm{E}-02$

$-4.29620 \mathrm{E}-02$

$-4.32817 \mathrm{E}-02$

$-4.36029 \mathrm{E}-02$

$-4.39258 \mathrm{E}-02$

$-4.42502 \mathrm{E}-02$

$-4.45762 \mathrm{E}-02$

$-4.49038 \mathrm{E}-02$

$-4.52330 \mathrm{E}-02$

$-4.55638 \mathrm{E}-02$

$-4.58963 \mathrm{E}-02$

$-4.62303 \mathrm{E}-02$

$-4.65659 \mathrm{E}-02$

$-4.69032 \mathrm{E}-02$

$-4.72421 \mathrm{E}-02$

$-4.75826 \mathrm{E}-02$

$-4.79247 \mathrm{E}-02$

$-4.86138 \mathrm{E}-02$

$-4.89609 \mathrm{E}-02$

$-4.93096 \mathrm{E}-02$

$-4.96599 \mathrm{E}-02$

$-5.00119 \mathrm{E}-02$

$-5.03655 \mathrm{E}-02$

$-5.07208 \mathrm{E}-02$

$-5.10778 \mathrm{E}-02$

$-5.14364 \mathrm{E}-02$

$-5.17967 \mathrm{E}-02$ $\tan \delta$.

$f_{2}$

$2.02446 \mathrm{E} \quad 02 \quad-3.24805 \mathrm{E}-02$ $2.02452 \mathrm{E} \quad 02-3.27456 \mathrm{E}-02$

$2.02458 \mathrm{E} \quad 02-3.30122 \mathrm{E}-02$

$2.02464 \mathrm{E} \quad 02-3.32803 \mathrm{E}-02$

$2.02470 \mathrm{E} \quad 02-3.35498 \mathrm{E}-02$

$-3.83536 \mathrm{E}-02$

$-4.82684 \mathrm{E}-02$
$2.02476 \mathrm{E} \quad 02-3.38207 \mathrm{E}-02$

$2.02482 \mathrm{E} \quad 02 \quad-3.40931 \mathrm{E}-02$

$2.02488 \mathrm{E} \quad 02-3.43669 \mathrm{E}-02$

$2.02494 \mathrm{E} \quad 02-3.46422 \mathrm{E}-02$

$2.02500 \mathrm{E} \quad 02-3.49190 \mathrm{E}-02$

$2.02506 \mathrm{E} \quad 02 \quad-3.51972 \mathrm{E}-02$

$2.02512 \mathrm{E} \quad 02 \quad-3.54769 \mathrm{E}-02$

$\begin{array}{lll}2.02517 \mathrm{E} & 02 & -3.57581 \mathrm{E}-02\end{array}$

$2.02523 \mathrm{E} \quad 02-3.60408 \mathrm{E}-02$

$2.02529 \mathrm{E} \quad 02 \quad-3.63249 \mathrm{E}-02$

$2.02535 \mathrm{E} \quad 02 \quad-3.66106 \mathrm{E}-02$

$2.02540 \mathrm{E} \quad 02 \quad-3.68977 \mathrm{E}-02$

$2.02546 \mathrm{E} \quad 02 \quad-3.71863 \mathrm{E}-02$

$2.02557 \mathrm{E} \quad 02 \quad-3.77681 \mathrm{E}-02$

$2.02563 \mathrm{E} \quad 02$

$2.02568 \mathrm{E} \quad 02$

$2.025 \pi 4 \mathrm{E} \quad 02$

$2.02579 \mathrm{E} \quad 02$

$2.02584 \mathrm{E} \quad 02$

$2.02590 \mathrm{E} \quad 02$

$2.02595 \mathrm{E} \quad 02$

$2.02600 \mathrm{E} \quad 02$

$2.02606 \mathrm{E} \quad 02$

$2.02611 \mathrm{E} \quad 02$

$2.02616 \mathrm{E} \quad 02$

$2.02622 \mathrm{E} \quad 02$

$2.02627 \mathrm{E} \quad 02$

$2.02632 \mathrm{E} \quad 02$

$2.02637 \mathrm{E} \quad 02$

$2.02642 \mathrm{E} \quad 02$

$2.02647 \mathrm{E} \quad 02$

$2.02652 \mathrm{E} \quad 02$

$2.02657 \mathrm{E} \quad 02$

$2.02662 \mathrm{E} \quad 02$

$2.02667 \mathrm{E} \quad 02$

$2.02672 \mathrm{E} \quad 02$

2.02677 E 02

$2.02682 \mathrm{E} \quad 02$

2.02687 E 02

$2.02692 \mathrm{E} \quad 02$

$2.02697 \mathrm{E} \quad 02$

$2.02702 \mathrm{E} \quad 02$

$2.02706 \mathrm{E} \quad 02$

$2.02711 \mathrm{E} \quad 02$

$2.02716 \mathrm{E} \quad 02$

$2.02721 \mathrm{E} \quad 02$

$2.02725 \mathrm{E} \quad 02$

$2.02730 \mathrm{E} \quad 02$

$2.02735 \mathrm{E} \quad 02$

$2.02739 \mathrm{E} \quad 02$

$2.02744 \mathrm{E} \quad 02$

$2.02748 \mathrm{E} \quad 02$

$2.02753 \mathrm{E} \quad 02$

$2.02757 \mathrm{E} \quad 02$
$-3.80612 \mathrm{E}-02$ $-3.83559 \mathrm{E}-02$ $-3.86520 \mathrm{E}-02$ $-3.89497 \mathrm{E}-02$

$-3.92489 \mathrm{E}-02$

$-3.95497 \mathrm{E}-02$

$-3.98519 \mathrm{E}-02$

$-4.01557 \mathrm{E}-02$

$-4.04610 \mathrm{E}-02$

$-4.07679 \mathrm{E}-02$

$-4.10763 \mathrm{E}-02$

$-4.13863 \mathrm{E}-02$

$-4.16978 \mathrm{E}-02$

$-4.20109 \mathrm{E}-02$

$-4.23255 \mathrm{E}-02$

$-4.26417 \mathrm{E}-02$ $-4.29595 \mathrm{E}-02$ $-4.32789 \mathrm{E}-02$ $-4.35998 \mathrm{E}-02$ $-4.39223 \mathrm{E}-02$

$-4.42464 \mathrm{E}-02$ $-4.45721 \mathrm{E}-02$ $-4.48993 \mathrm{E}-02$ $-4.52282 \mathrm{E}-02$ $-4.55587 \mathrm{E}-02$

$-4.58907 \mathrm{E}-02$ $-4.65597 \mathrm{E}-02$ $-4.72351 \mathrm{E}-02$

$-4.75753 \mathrm{E}-02$ $-4.79170 \mathrm{E}-02$ $-4.82604 \mathrm{E}-02$ $-4.86055 \mathrm{E}-02$

$-4.89521 \mathrm{E}-02$

-4.93004 E-02

$-4.96504 \mathrm{E}-02$

$-5.00020 \mathrm{E}-02$

$-5.03553 \mathrm{E}-02$

$-5.07102 \mathrm{E}-02$ $-4.68966 \mathrm{E}-02$
$2.02551 \mathrm{E} \quad 02 \quad-3.74765 \mathrm{E}-02$ $-4.62244 \mathrm{E}-02$

\section{$b^{\prime}-2$}

$\tan \delta$

$1.38206 \mathrm{E} \quad 00 \quad-4.83726 \mathrm{E}-0.3$

$1.38967 \mathrm{E} \quad 00 \quad-4.83739 \mathrm{E}-03$ 


\begin{tabular}{|c|c|c|c|c|c|c|c|c|c|}
\hline$p$ & $F_{0}$ & & $f_{1}$ & $g_{1}$ & $\tan \delta_{1}$ & $f_{-1}$ & $g_{-1}$ & $\tan \delta_{-1}$ & \\
\hline 0.10 & $1.53362 \mathrm{E}$ & 00 & $-8.66162 E-03$ & $-7.91236 \mathrm{E}-05$ & $-6.17684 \mathrm{E}-02$ & $-1.02246 \mathrm{E}-03$ & $1.74892 \mathrm{E}-01$ & $-1.19854 \mathrm{E}$ & 01 \\
\hline 0.20 & $1.25425 \mathrm{E}$ & 00 & $-3.11565 \mathrm{E}-02$ & $-2.85373 \mathrm{E}-04$ & $-3.06084 \mathrm{E}-02$ & $-1.85027 \mathrm{E}-03$ & $3.15176 \mathrm{E}-01$ & $-2.36547 \mathrm{E}$ & 01 \\
\hline 0.30 & $1.17009 \mathrm{E}$ & 00 & $-6.65830 \mathrm{E}-02$ & $-6.12509 E-04$ & $-2.02450 \mathrm{E}-02$ & $-2.68332 \mathrm{E}-03$ & $4.53995 \mathrm{E}-01$ & $-3.52376 \mathrm{E}$ & 01 \\
\hline 0.40 & $1.13044 \mathrm{E}$ & 00 & $-1.13675 \mathrm{E}-01$ & $-1.05188 \mathrm{E}-03$ & $-1.50451 \mathrm{E}-02$ & $-3.52221 \mathrm{E}-03$ & $5.90506 \mathrm{E}-01$ & $-4.66592 \mathrm{E}$ & 01 \\
\hline 0.50 & $1.10785 \mathrm{E}$ & 00 & $-1.70925 \mathrm{E}-01$ & $-1.59317 \mathrm{E}-03$ & $-1.19086 \mathrm{E}-02$ & $-4.36870 \mathrm{E}-03$ & $7.24236 \mathrm{E}-01$ & $-5.78820 \mathrm{E}$ & 01 \\
\hline 0.60 & $1.093 .52 \mathrm{E}$ & 00 & $-2.36775 \mathrm{E}-01$ & $-2.22570 \mathrm{E}-03$ & $-9.80461 E-03$ & $-5.22510 \mathrm{E}-03$ & $8.54984 \mathrm{E}-01$ & $-6.88806 \mathrm{E}$ & 01 \\
\hline 0.70 & $1.08379 \mathrm{E}$ & 00 & $-3.09744 \mathrm{E}-01$ & $-2.93936 \mathrm{E}-03$ & $-8.29181 \mathrm{E}-03$ & $-6.09394 \bar{E}-03$ & $9.82749 \mathrm{E}-01$ & $-7.96379 \bar{E}$ & 01 。 \\
\hline 0.80 & $1.07685 \mathrm{E}$ & 00 & $-3.88513 \mathrm{E}-01$ & $-3.72517 \mathrm{E}-03$ & $-7.14956 \mathrm{E}-03$ & $-6.97781 \mathrm{E}-03$ & $1.10767 \mathrm{E} \quad 00$ & $-9.01430 \mathrm{E}$ & 01 \\
\hline 0.90 & $1.07173 \bar{E}$ & 00 & $-4.71951 \mathrm{E}-01$ & $-4.57543 \mathrm{E}-03$ & $-6.25521 \mathrm{E}-03$ & $-7.87920 \mathrm{E}-03$ & $1.22997 \mathrm{E} \quad 00$ & $-1.00390 \mathrm{E}$ & 02 \\
\hline 1.00 & $1.06784 \mathrm{E}$ & 00 & $-5.59121 \mathrm{E}-01$ & $-5.48384 \mathrm{E}-03$ & $-5.53512 \mathrm{E}-03$ & $-8.80038 E-03$ & $1.34992 \mathrm{E} \quad 00$ & $-1.10378 \mathrm{E}$ & 02 \\
\hline 1.10 & $1.06482 \mathrm{E}$ & 00 & $-6.49265 \mathrm{E}-01$ & $-6.44526 \mathrm{E}-03$ & $-4.94235 \mathrm{E}-03$ & $-9.74340 \mathrm{E}-03$ & $1.46776 \mathrm{E} \quad 00$ & $-1.20107 \mathrm{E}$ & 02 \\
\hline 1.20 & $1.06242 \mathrm{E}$ & 00 & $-7.41775 \mathrm{E}-01$ & $-7.45563 \mathrm{E}-03$ & $-4.44551 \mathrm{E}-03$ & $-1.07100 \mathrm{E}-02$ & $1.58378 \mathrm{E} \quad 00$ & $-1.29582 \mathrm{E}$ & 02 \\
\hline 1.30 & $1.06049 \mathrm{E}$ & 00 & $-8.36168 \mathrm{E}-01$ & $1174 \mathrm{E}-03$ & $-4.02284 \mathrm{E}-03$ & $17017 \mathrm{E}-02$ & $1.69819 \mathrm{E} \quad 00$ & $-1.38806 \mathrm{E}$ & 02 \\
\hline 1.40 & $1.05891 \mathrm{E}$ & 00 & $-9.32063 \mathrm{E}-01$ & $-9.61106 \mathrm{E}-03$ & $-3.65873 \mathrm{E}-03$ & $-1.27199 \mathrm{E}-02$ & $1.81122 \mathrm{E} \quad 00$ & $-1.47787 \mathrm{E}$ & 02 \\
\hline 1.50 & $1.05761 \mathrm{E}$ & 00 & $-1.02916 \mathrm{E} \quad 00$ & $-1.07516 \mathrm{E}-02$ & $-3.34169 \mathrm{E}-03$ & $-1.37655 \mathrm{E}-02$ & $1.92304 \mathrm{E} \quad 00$ & $-1.56529 \mathrm{E}$ & 02 \\
\hline 1.60 & $1.05652 \mathrm{E}$ & 00 & $-1.12722 \mathrm{E} \quad 00$ & $-1.19318 \mathrm{E}-02$ & $-3.06309 \mathrm{E}-03$ & $-1.48395 \mathrm{E}-02$ & $2.03382 \mathrm{E} \quad 00$ & $-1.65042 \mathrm{E}$ & 02 \\
\hline 1.70 & $1.05560 \mathrm{E}$ & 00 & $-1.22606 \mathrm{E} \quad 00$ & $1505 \mathrm{E}-02$ & $-2.81626 \mathrm{E}-03$ & $59427 \mathrm{E}-02$ & $2.14369 \mathrm{E} \quad 00$ & $-1.73330 \mathrm{E}$ & 02 \\
\hline 1.80 & $1.05481 \mathrm{E}$ & 00 & $-1.32554 \mathrm{E} \quad 00$ & $-1.44067 \mathrm{E}-02$ & $-2.59606 \mathrm{E}-03$ & $1.70756 \mathrm{E}-02$ & $2.25279 \mathrm{E} \quad 00$ & $-1.81403 \mathrm{E}$ & 02 \\
\hline 1.90 & $1.05414 \mathrm{E}$ & 00 & $-1.42552 \mathrm{E} \quad 00$ & $-1.56996 \mathrm{E}-02$ & $-2.39835 \mathrm{E}-03$ & $-1.82389 \mathrm{E}-02$ & $2.36119 \mathrm{E} \quad 00$ & $-1.89267 \mathrm{E}$ & 02 \\
\hline 2.00 & $1.05356 \mathrm{E}$ & 00 & $-1.52593 \mathrm{E} \quad 00$ & $-1.70287 \mathrm{E}-02$ & $-2.21985 \mathrm{E}-03$ & $-1.94330 \mathrm{E}-02$ & $2.46900 \mathrm{E} \quad 00$ & $-1.96930 \mathrm{E}$ & 02 \\
\hline 2.10 & $1.05305 \mathrm{E}$ & 00 & $-1.62667 \mathrm{E} \quad 00$ & $-1.83934 \mathrm{E}-02$ & $-2.05787 \mathrm{E}-03$ & $-2.06582 \mathrm{E}-02$ & $2.57629 \mathrm{E}$ & $-2.04397 \mathrm{E}$ & 02 \\
\hline 2.20 & $1.05261 \mathrm{E}$ & 00 & $-1.72770 \mathrm{E} \quad 00$ & $934 \mathrm{E}-02$ & $1020 \mathrm{E}-03$ & $9149 \mathrm{E}-02$ & $2.68312 \mathrm{E} \quad 00$ & $-2.11676 \bar{E}$ & 02 \\
\hline 2.30 & $1.05222 \mathrm{E}$ & 00 & $-1.82896 \mathrm{E} \quad 00$ & $-2.12283 \mathrm{E}-02$ & $-1.77504 \mathrm{E}-03$ & $32033 \mathrm{E}-02$ & $2.78954 \mathrm{E} \quad 00$ & $-2.18775 \mathrm{E}$ & 02 \\
\hline 2.40 & $1.05187 \mathrm{E}$ & 00 & $-1.93042 \mathrm{E} \quad 00$ & $-2.26980 \mathrm{E}-02$ & $-1.65084 \mathrm{E}-03$ & $-2.45238 \mathrm{E}-02$ & $2.89561 \mathrm{E} \quad 00$ & $-2.25698 \mathrm{E}$ & 02 \\
\hline 2.50 & $1.05156 \mathrm{E}$ & 00 & $-2.03203 \mathrm{E} \quad 00$ & $-2.42022 \mathrm{E}-02$ & $-1.53632 \mathrm{E}-03$ & $-2.58764 \mathrm{E}-02$ & $3.00136 \mathrm{E} \quad 00$ & $-2.32452 \mathrm{E}$ & 02 \\
\hline 2.60 & $1.05129 \mathrm{E}$ & 00 & $-2.13378 \mathrm{E} \quad 00$ & $-2.57408 \mathrm{E}-02$ & $-1.43039 \mathrm{E}-03$ & $-2.72613 \mathrm{E}-02$ & $3.10682 \mathrm{E}$ & $-2.39042 \mathrm{E}$ & 02 \\
\hline 2.70 & $1.05104 \mathrm{E}$ & 00 & $-2.23564 \mathrm{E} \quad 00$ & $3136 \mathrm{E}-02$ & $211 \mathrm{E}-03$ & $6787 \mathrm{E}-02$ & $3.21204 \mathrm{E} \quad 00$ & $-2.45476 \mathrm{E}$ & 02 \\
\hline 2.80 & $1.05082 \mathrm{E}$ & 00 & $-2.33760 \mathrm{E} \quad 00$ & $-2.89205 \mathrm{E}-02$ & $-1.24069 \mathrm{E}-03$ & $-3.01288 \mathrm{E}-02$ & $3.31703 \mathrm{E} \quad 00$ & $-2.51757 \mathrm{E}$ & 02 \\
\hline 2.90 & $1.05062 \mathrm{E}$ & 00 & $-2.43963 \mathrm{E}$ & $-3.05615 \mathrm{E}-02$ & $-1.15543 \mathrm{E}-03$ & $-3.16116 \mathrm{E}-02$ & $3.42181 \mathrm{E} \quad 00$ & $-2.57892 \mathrm{E}$ & 02 \\
\hline 3.00 & $1.05044 \mathrm{E}$ & 00 & $-2.54173 \mathrm{E} \quad 00$ & $-3.22364 \mathrm{E}-02$ & $-1.07572 \mathrm{E}-03$ & $-3.31272 E-02$ & $3.52641 \mathrm{E} \quad 00$ & $-2.63885 \mathrm{E}$ & 02 \\
\hline 3.10 & $1.05027 \mathrm{E}$ & 00 & $-2.64388 \mathrm{E} \quad 00$ & $-3.39452 \mathrm{E}-02$ & $-1.00105 \mathrm{E}-03$ & $-3.46757 \mathrm{E}-02$ & $3.63085 \mathrm{E}$ & $-2.69741 \mathrm{E}$ & 02 \\
\hline 3.20 & $1.05012 \mathrm{E}$ & 00 & $-2.74608 \mathrm{E}$ & $-3.56878 \mathrm{E}-02$ & $-9.30938 \mathrm{E}-04$ & $-3.62572 \mathrm{E}-02$ & $3.73514 \mathrm{E} \quad 00$ & $-2.75464 \mathrm{E}$ & 02 \\
\hline 3.30 & $1.04998 \mathrm{E}$ & 00 & $-2.84832 \mathrm{E}$ & $-3.74642 \mathrm{E}-02$ & $-8.64988 \mathrm{E}-04$ & $-3.78717 \mathrm{E}-02$ & $3.83929 \mathrm{E}$ & $-2.81059 \mathrm{E}$ & 02 \\
\hline 3.40 & $1.04985 \mathrm{E}$ & 00 & $-2.95059 \mathrm{E} \quad 00$ & $-3.92744 \mathrm{E}-02$ & E-04 & $-3.95193 \mathrm{E}-02$ & $3.94332 \mathrm{E}$ & $-2.86530 \mathrm{E}$ & 02 \\
\hline 3.50 & $1.04973 \mathrm{E}$ & 00 & $-3.05288 \mathrm{E} \quad 00$ & $-4.11183 \mathrm{E}-02$ & $-7.44171 \mathrm{E}-04$ & $-4.12000 \mathrm{E}-02$ & $4.04723 \mathrm{E} \quad 00$ & $-2.91881 \mathrm{E}$ & 02 \\
\hline 3.60 & $1.04963 \mathrm{E}$ & 00 & $-3.15520 \mathrm{E} \quad 00$ & $-4.29959 \mathrm{E}-02$ & $-6.88696 \mathrm{E}-04$ & $-4.29139 \mathrm{E}-02$ & $4.15104 \mathrm{E} \quad 00$ & $-2.97117 \mathrm{E}$ & 02 \\
\hline 3.70 & $1.04953 \mathrm{E}$ & 00 & $-3.25754 \mathrm{E}$ & $-4.49073 \mathrm{E}-02$ & $-6.36168 \mathrm{E}-04$ & $-4.46611 \mathrm{E}-02$ & $4.25476 \mathrm{E}$ & $-3.02240 \mathrm{E}$ & 02 \\
\hline 3.80 & $1.04943 \mathrm{E}$ & 00 & $-3.35990 \mathrm{E}$ & $-4.68523 \mathrm{E}-02$ & $-5.86354 E-04$ & $-4.64415 \mathrm{E}-02$ & $4.35839 \mathrm{E}$ & $-3.07254 \mathrm{E}$ & 02 \\
\hline 3.90 & $1.04935 \mathrm{E}$ & 00 & $-3.46226 \mathrm{E} \quad 00$ & $-4.88309 \mathrm{E}-02$ & $9046 \mathrm{E}-04$ & 2551 E-02 & $4.46194 \mathrm{E}$ & $-3.12163 \mathrm{E}$ & 02 \\
\hline 4.00 & $1.04927 \mathrm{E}$ & 00 & $-3.56464 \mathrm{E} \quad 00$ & $-5.08432 \mathrm{E}-02$ & $-4.94063 \mathrm{E}-04$ & $-5.01021 \mathrm{E}-02$ & $4.56541 \mathrm{E} \quad 00$ & $-3.16970 \mathrm{E}$ & 02 \\
\hline 4.10 & $1.04919 \mathrm{E}$ & 00 & $-3.66702 \mathrm{E}$ & $-5.28891 \mathrm{E}-02$ & $-4.51236 E-04$ & $-5.19824 \mathrm{E}-02$ & $4.66881 \mathrm{E}$ & $-3.21678 \mathrm{E}$ & 02 \\
\hline 4.20 & $1.04912 \mathrm{E}$ & 00 & $-3.76941 \mathrm{E}$ & $86 \mathrm{E}-02$ & $-4.10417 \mathrm{E}-04$ & $-5.38961 \mathrm{E}-02$ & $4.77216 \mathrm{E}$ & $.26291 \mathrm{E}$ & 02 \\
\hline 4.30 & $1.04906 \mathrm{E}-$ & 00 & $-3.87181 \mathrm{E}$ & $7 \mathrm{E}-02$ & $1464 \bar{E}-($ & $-5.58432 \mathrm{E}-02$ & $4.87544 \mathrm{E}$ & $-3.30810 \mathrm{E}$ & 02 . \\
\hline 4.40 & $1.04900 \mathrm{E}$ & 00 & $-3.97421 \mathrm{E} \quad 00$ & $2285 \mathrm{E}-02$ & $4257 \mathrm{E}-04$ & $8236 \mathrm{E}-02$ & $4.97867 \mathrm{E}$ & $-3.35240 \mathrm{E}$ & 02 \\
\hline 4.50 & $1.04894 \mathrm{E}$ & 00 & $-4.07661 \mathrm{E} \quad 00$ & $-6.14088 E-02$ & $-2.98676 \mathrm{E}-04$ & $-5.98375 \mathrm{E}-02$ & $5.08184 \mathrm{E}$ & $-3.39582 \mathrm{E}$ & 02 \\
\hline 4.60 & $1.04888 \mathrm{E}$ & 00 & $-4.17901 \mathrm{E} \quad 00$ & $-6.36228 \mathrm{E}-02$ & $-2.64620 \mathrm{E}-04$ & $-6.18847 \mathrm{E}-02$ & $5.18497 \mathrm{E}$ & $-3.43839 \mathrm{E}$ & 02 \\
\hline & $1.04883 \mathrm{E}$ & 00 & $-4.28141 \mathrm{E} \quad 00$ & $-6.58703 \mathrm{E}-02$ & $-2.31991 \mathrm{E}-04$ & $39654 \bar{E}-02$ & $5.28806 \mathrm{E}$ & $-3.48013 \mathrm{E}$ & 02 \\
\hline 4.80 & $1.04879 \mathrm{E}$ & 00 & $-4.38381 \mathrm{E} \quad 00$ & $-6.81514 \mathrm{E}-02$ & $-2.00704 E-04$ & $50796 \mathrm{E}-02$ & $5.39110 \mathrm{E}$ & $-3.52108 E$ & 02 \\
\hline 4.90 & $1.04874 \mathrm{E}$ & 00 & $-4.48621 \mathrm{E} \quad 00$ & $E-02$ & $675 \mathrm{E}-04$ & $272 \mathrm{E}-02$ & 00 & $.56124 \mathrm{E}$ & 02 \\
\hline 5.00 & $1.04870 \mathrm{E}$ & 00 & $-4.58861 \mathrm{E} \quad 00$ & $-7.28144 \mathrm{E}-02$ & $-1.41832 \mathrm{E}-04$ & $-7.04082 \mathrm{E}-02$ & $5.59707 \mathrm{E} \quad 00$ & $-3.60066 \mathrm{E}$ & 02 \\
\hline 5.10 & $1.04866 \mathrm{E}$ & 00 & $-4.69101 \mathrm{E} \quad 00$ & $-7.51962 \mathrm{E}-02$ & $-1.14106 \mathrm{E}-04$ & $-7.26227 \mathrm{E}-02$ & $5.70000 \mathrm{E}$ & $-3.63933 \mathrm{E}$ & 02 \\
\hline 5.20 & $1.04862 \mathrm{E}$ & 00 & $-4.79341 \mathrm{E} \quad 00$ & $-7.76117 \bar{E}-02$ & $4324 \mathrm{E}-05$ & $708 \mathrm{E}-02$ & $5.80289 \mathrm{E}$ & $30 \mathrm{E}$ & 02 \\
\hline 5.30 & $1.04858 \mathrm{E}$ & 00 & $-4.89580 \mathrm{E} \quad 00$ & $-8.00607 \bar{E}-02$ & $7519 E-05$ & $1523 \mathrm{E}-02$ & $5.90576 \mathrm{E}$ & $-3.71457 \mathrm{E}$ & 02 \\
\hline 5.40 & $1.04855 \mathrm{E}$ & 00 & $-4.99819 \mathrm{E} \quad 00$ & $-8.25433 \mathrm{E}-02$ & $0122 \mathrm{E}-05$ & $-7.94672 \mathrm{E}-02$ & $6.00859 \mathrm{E}$ & $-3.75116 \mathrm{E}$ & 02 \\
\hline & $1.04851 \mathrm{E}$ & 00 & $-5.10058 \mathrm{E} \quad 00$ & $-8.50594 \mathrm{E}-02$ & $-1.31618 \mathrm{E}-05$ & $-8.18158 \mathrm{E}-02$ & $6.11141 \mathrm{E} \quad 00$ & $-3.78710 \mathrm{E}$ & 02 \\
\hline 5.60 & $1.04848 \mathrm{E}$ & 00 & $-5.20296 \mathrm{E} \quad 00$ & $-8.76092 \mathrm{E}-02$ & $9.84750 \mathrm{E}-06$ & $-8.41977 \mathrm{E}-02$ & $6.21419 \mathrm{E} \quad 00$ & $-3.82240 \mathrm{E}$ & 02 \\
\hline 5.70 & $1.04845 \mathrm{E}$ & 00 & $-5.30534 \mathrm{E} \quad 00$ & $-9.01924 \mathrm{E}-02$ & $3.20555 \mathrm{E}-05$ & $6132 \mathrm{E}-02$ & $6.31694 \mathrm{E}$ & $-3.85708 \mathrm{E}$ & 02 \\
\hline 5.80 & $1.04843 \mathrm{E}$ & 00 & $-5.40771 \mathrm{E} \quad 00$ & $-9.28093 \mathrm{E}-02$ & $5.35081 \mathrm{E}-05$ & $-8.90622 \mathrm{E}-02$ & $6.41968 \mathrm{E}$ & $-3.89116 \mathrm{E}$ & 02 \\
\hline 5.90 & $1.04840 \mathrm{E}$ & 00 & $-5.51008 \mathrm{E} \quad 00$ & $-9.54597 \mathrm{E}-02$ & 7.423 & $-9.15448 \mathrm{E}-02$ & $6.52239 \mathrm{E}$ & $-3.92465 \mathrm{E}$ & 02 \\
\hline 6.00 & $1.04837 \mathrm{E}$ & 00 & $-5.61245 \mathrm{E} \quad 00$ & $-9.81437 E-02$ & $9.42857 \mathrm{E}-05$ & $-9.40608 \mathrm{E}-02$ & $6.62508 \mathrm{E} \quad 00$ & $-3.95756 \mathrm{E}$ & 02 \\
\hline
\end{tabular}


$\rho$

0.10

0.20

0.30

0.40

0.50

0.60

0.70

0.80

0.90

1.00

1.10

1.20

1.30

1.40

1.50

1.60

1.70

1.80

1.90

2.00

2.10

2.20

2.30

2.40

2.50

2.60

2.70

2.80

2.90

3.00

3.10

3.20

3.30

3.40

3.50

3.60

3.70

3.80

3.90

4.00

4.10

4.20

4.30

4.40

4.50

4.60

4.70

4.80

4.90

5.00

5.10

5.20

5.30

5.40

5.50

5.60

5.70

5.80

5.90

6.00
$\vec{F}$

$1.05791 \mathrm{E}-08$
$3.40690 \mathrm{E}-08$
$7.13041 \mathrm{E}-08$
$1.22345 \mathrm{E}-07$
$1.87258 \mathrm{E}-07$
$2.66100 \mathrm{E}-07$
$3.58918 \mathrm{E}-07$
$4.65751 \mathrm{E}-07$
$5.86629 \mathrm{E}-07$
$7.21576 \mathrm{E}-07$

$7.21576 \mathrm{E}-07$

$8.70610 \mathrm{E}-07$

$1.03375 \mathrm{E}-06$

$1.21100 \mathrm{E}-06$

$1.40237 \mathrm{E}-06$

$1.60787 \mathrm{E}-06$

$1.82750 \mathrm{E}-06$

$2.06128 \mathrm{E}-06$

$2.30919 \mathrm{E}-06$

$2.57125 \mathrm{E}-06$

$2.84746 \mathrm{E}-06$

$3.13781 \mathrm{E}-06$

$3.44232 \mathrm{E}-06$

$3.76097 \mathrm{E}-06$

$4.09378 \mathrm{E}-06$

4.44074 E-06

$4.80186 \mathrm{E}-06$

$5.17713 \mathrm{E}-06$

$5.56655 \mathrm{E}-06$

$5.97013 \mathrm{E}-06$

$6.38787 \mathrm{E}-06$

$6.81976 \mathrm{E}-06$

$7.26581 \mathrm{E}-06$

$7.72601 \mathrm{E}-06$

$8.20037 \mathrm{E}-06$

$8.68889 \mathrm{E}-06$

9.19155E-06

$9.70838 \mathrm{E}-06$

$1.02394 \mathrm{E}-05$

$1.07845 \mathrm{E}-05$

$1.13438 \mathrm{E}-05$

$1.19173 \mathrm{E}-05$

$1.25049 \mathrm{E}-05$

$1.31066 \mathrm{E}-05$

$1.37226 \mathrm{E}-05$

$1.43526 \mathrm{E}-05$

$1.49969 \mathrm{E}-05$

$1.56553 \mathrm{E}-0.5$

$1.63278 \mathrm{E}-05$

$1.70145 \mathrm{E}-0.5$

$1.77154 \mathrm{E}-05$

$1.84304 \mathrm{E}-05$

$1.91596 \mathrm{E}-05$

$1.99029 \mathrm{E}-0.5$

$2.06604 \mathrm{E}-05$

$2.14321 \mathrm{E}-05$

$2.22179 \mathrm{E}-05$

$2.30179 \mathrm{E}-0.5$

$2.38320 \mathrm{E}-0.5$

$2.46603 \mathrm{E}-05$

$2.55027 \mathrm{E}-05$ $f_{2}$

$-1.41357 \mathrm{E}-06$

$-1.02251 \mathrm{E}-05$

$-3.28095 \mathrm{E}-05$

$-7.47115 \mathrm{E}-05$

$-1.40447 \mathrm{E}-04$

$-2.33488 \mathrm{E}-04$

$-3.56374 \mathrm{E}-04$

$-5.10881 \mathrm{E}-04$

$-6.98195 \mathrm{E}-04$

$-9.19084 \mathrm{E}-04$

$-1.17401 \mathrm{E}-03$

$-1.46325 \mathrm{E}-03$

$-1.78694 \mathrm{E}-03$

$-2.14512 \mathrm{E}-03$

$-2.53781 \mathrm{E}-03$

$-2.96496 \mathrm{E}-03$

$-3.42655 \mathrm{E}-03$

$-3.92250 \mathrm{E}-03$

$-4.45277 \mathrm{E}-03$

$-5.01730 \mathrm{E}-03$

$-5.61604 \mathrm{E}-03$

$-6.24894 \mathrm{E}-03$

$-6.91594 \mathrm{E}-03$

$-7.61701 \mathrm{E}-03$

$-8.35211 \mathrm{E}-03$

$-9.12121 \mathrm{E}-03$

$-9.92427 \mathrm{E}-03$

$-1.07613 \mathrm{E}-02$

$-1.16322 \mathrm{E}-02$

$-1.25370 \mathrm{E}-02$

$-1.34756 \mathrm{E}-02$

$-1.44481 \mathrm{E}-02$

$-1.54545 \mathrm{E}-02$

$-1.64946 \mathrm{E}-02$

$-1.75686 \mathrm{E}-02$

$-1.86763 \mathrm{E}-02$

$-1.98178 \mathrm{E}-02$

$-2.09931 \mathrm{E}-02$

$-2.22021 \mathrm{E}-02$

$-2.34449 \mathrm{E}-02$

$-2.47214 \mathrm{E}-02$

$-2.60316 \mathrm{E}-02$

$-2.73756 \mathrm{E}-02$

$-2.87533 \mathrm{E}-02$

$-3.01646 \mathrm{E}-02$

$-3.16097 \mathrm{E}-02$

$-3.30885 \mathrm{E}-02$

$-3.46010 \mathrm{E}-02$

$-3.61472 \mathrm{E}-02$

$-3.77271 \mathrm{E}-02$

$-3.93406 \mathrm{E}-02$

$-4.09878 \mathrm{E}-02$

$-4.26687 \mathrm{E}-02$

$-4.43833 \mathrm{E}-02$

$-4.61315 \mathrm{E}-02$

$-4.79134 \mathrm{E}-02$

$-4.97290 \mathrm{E}-02$

$-5.15782 \mathrm{E}-02$

$-5.34611 \mathrm{E}-02$

$-5.53776 \mathrm{E}-02$ g.,

$-7.51183 \mathrm{E}-09$

$-5.44867 \mathrm{E}-08$

$-1.75616 \mathrm{E}-07$

$-4.02333 \mathrm{E}-07$

$-7.62010 \mathrm{E}-07$

$-1.27791 \mathrm{E}-06$

$-1.96963 \mathrm{E}-06$

$-2.85379 \mathrm{E}-06$

$-3.94474 \mathrm{E}-06$

$-5.25517 \mathrm{E}-06$

$-6.79666 \mathrm{E}-06$

$-8.58006 \mathrm{E}-06$

$-1.06157 \mathrm{E}-05$

$-1.29136 \mathrm{E}-05$

$-1.54837 \mathrm{E}-05$

$-1.83357 \mathrm{E}-05$

$-2.14792 \mathrm{E}-05$

$-2.49241 \mathrm{E}-05$

$-2.86800 \mathrm{E}-05$

$-3.27566 \mathrm{E}-05$

$-3.71635 \mathrm{E}-05$

$-4.19107 \mathrm{E}-05$

$-4.70077 \mathrm{E}-05$

$-5.24644 \mathrm{E}-05$

$-5.82906 \mathrm{E}-05$

$-6.44960 \mathrm{E}-05$

$-7.10906 \mathrm{E}-05$

$-7.80841 \mathrm{E}-05$

$-8.54864 \mathrm{E}-05$

$-9.33074 \mathrm{E}-05$

$-1.01557 \mathrm{E}-04$

$-1.10245 \mathrm{E}-04$

$-1.19381 \mathrm{E}-04$

$-1.28975 \mathrm{E}-04$

$-1.39038 \mathrm{E}-04$

$-1.49578 \mathrm{E}-04$

$-1.60606 \mathrm{E}-04$

$-1.72132 \mathrm{E}-04$

$-1.84166 \mathrm{E}-04$

$-1.96717 \mathrm{E}-04$

$-2.09796 \mathrm{E}-04$

$-2.23413 \mathrm{E}-04$

$-2.37576 \mathrm{E}-04$

$-2.52297 \mathrm{E}-04$

$-2.67586 \mathrm{E}-04$

$-2.83451 \mathrm{E}-04$

$-2.99904 \mathrm{E}-04$

$-3.16954 \mathrm{E}-04$

$-3.34610 \mathrm{E}-04$

$-3.52884 \mathrm{E}-04$

$-3.71784 \mathrm{E}-04$

$-3.91322 \mathrm{E}-04$

$-4.11506 \mathrm{E}-04$

$-4.32346 \mathrm{E}-04$

$-4.53853 \mathrm{E}-04$

$-4.76037 \mathrm{E}-04$

$-4.98907 \mathrm{E}-04$

$-5.22474 \mathrm{E}-04$

$-5.46747 \mathrm{E}-04$

$-5.71736 \mathrm{E}-04$

$\tan \delta_{2}$

$f$

$7.35323 \mathrm{E} \quad 00$

$1.46320 \mathrm{E}$ 01 $-3.48634 \mathrm{E}-07$

$2.16053 \mathrm{E} \quad 01 \quad-7.57551 \mathrm{E}-07$

$2.81759 \mathrm{E} \quad 01-1.32578 \mathrm{E}-06$

$3.42736 \mathrm{E} \quad 01 \quad-2.05616 \mathrm{E}-06$

$3.98628 \mathrm{E} \quad 0 \mathrm{l}-2.95257 \mathrm{E}-06$

$4.49376 \mathrm{E} \quad 01-4.01994 \mathrm{E}-06$

$4.95143 \mathrm{E}$ 01 $-5.26421 \mathrm{E}-06$

$5.36234 \mathrm{E}$ 01 $-6.69222 \mathrm{E}-06$

$5.73035 \mathrm{E}$ 01 $-8.31154 \mathrm{E}-06$

$6.05960 \mathrm{E} \quad 01-1.01304 \mathrm{E}-05$

$6.35423 \mathrm{E}$ 01 $-1.21573 \mathrm{E}-05$

$6.61815 \mathrm{E} \quad 01-1.44014 \mathrm{E}-05$

$6.85496 \mathrm{E} 01-1.68719 \mathrm{E}-05$

$7.06790 \mathrm{E} \quad 01-1.95783 \mathrm{E}-05$

$7.25984 \mathrm{E} \quad 01 \quad-2.25301 \mathrm{E}-05$

$7.43330 \mathrm{E} 01 \quad-2.57370 \mathrm{E}-05$

$7.59049 \mathrm{E} \quad 01 \quad-2.92089 \mathrm{E}-0.5$

$7.73333 \mathrm{E}$ o1 $-3.29556 \mathrm{E}-05$

$7.86348 \mathrm{E}$ ol $-3.69869 \mathrm{E}-05$

$7.98240 \mathrm{E} \quad 01$ $8.09135 \mathrm{E} \quad 01$

$8.19142 \mathrm{E} \quad 01$

$8.28357 \mathrm{E} .01$

$8.36863 \mathrm{E} 01$

$8.44733 \mathrm{E} \quad 01$

$8.52030 \mathrm{E} \quad 01$

$8.58812 \mathrm{E} \quad 01$

$8.65127 \mathrm{E} \quad 01$

8.71020 E 01

$8.76528 \mathrm{E} 01$

$8.81687 \mathrm{E} 01$

$8.86526 \mathrm{E} 01$

$8.91074 \mathrm{E} \quad 01$

$8.95354 \mathrm{E} \quad 01$

$8.99388 \mathrm{E} \quad 01$ $9.03197 \mathrm{E} \quad 01$

$9.06797 \mathrm{E} \quad 01$

$9.10205 \mathrm{E} \quad 01$

$9.13434 \mathrm{E} \quad 01$

$9.16499 \mathrm{E} 01$ $9.19411 \mathrm{E} 01$ $9.22180 \mathrm{E} \quad 01$ $9.24817 \mathrm{E} \quad 01$

$9.27331 \mathrm{E} \quad 01$

$9.29729 \mathrm{E} \quad 01$ $9.32019 \mathrm{E} \quad 01$ 


\begin{tabular}{|c|c|c|c|c|c|c|c|c|c|c|c|}
\hline$p$ & $F_{0}$ & & $f_{\mathrm{l}}$ & & $g_{1}$ & $\tan \delta_{1}$ & $f_{-1}$ & $g_{-\mathbf{i}}$ & & $\tan \delta_{-1}$ & \\
\hline 6.10 & $1.04835 \mathrm{E}$ & 00 & $-5.71481 \mathrm{E}$ & 00 & $-1.00861 \mathrm{E}-01$ & $1.13682 \mathrm{E}-04$ & $-9.66104 \mathrm{E}-02$ & $6.72775 \mathrm{E}$ & 00 & $-3.98991 \mathrm{E}$ & 02 \\
\hline 6.20 & $1.04832 \mathrm{E}$ & 00 & $-5.81717 \mathrm{E}$ & 00 & $-1.03612 \mathrm{E}-01$ & $1.32458 \mathrm{E}-04$ & $-9.91935 \mathrm{E}-02$ & $6.83039 \mathrm{E}$ & 00 & $-4.02172 \mathrm{E}$ & 02 \\
\hline 6.30 & $1.04830 \mathrm{E}$ & 00 & $-5.91952 \mathrm{E}$ & 00 & $-1.06397 \mathrm{E}-01$ & $1.50643 \mathrm{E}-04$ & $-1.01810 \mathrm{E}-01$ & $6.93302 \mathrm{E}$ & 00 & $-4.05299 \mathrm{E}$ & 02 \\
\hline 6.40 & $1.04828 \mathrm{E}$ & 00 & $-6.02187 \mathrm{E}$ & 00 & $-1.09215 \mathrm{E}-01$ & $1.68266 \mathrm{E}-04$ & $-1.04460 \mathrm{E}-01$ & $7.03563 \mathrm{E}$ & 00 & $-4.08375 \mathrm{E}$ & 02 \\
\hline 6.50 & $1.04826 \mathrm{E}$ & 00 & $-6.12422 \mathrm{E}$ & 00 & $-1.12067 \bar{E}-01$ & $1.85348 \mathrm{E}-04$ & $-1.07144 \mathrm{E}-01$ & $7.13823 \mathrm{E}$ & 00 & $-4.11401 \mathrm{E}$ & 02 \\
\hline 6.60 & $1.04824 \mathrm{E}$ & 00 & $-6.22655 \mathrm{E}$ & 00 & $-1.14953 \mathrm{E}-01$ & $2.01919 \mathrm{E}-04$ & $-1.09861 \mathrm{E}-01$ & $7.24080 \mathrm{E}$ & 00 & $-4.14377 \mathrm{E}$ & 02 \\
\hline 6.70 & $1.04822 \mathrm{E}$ & 00 & $-6.32889 \mathrm{E}$ & 00 & $-1.17871 \mathrm{E}-01$ & $2.17998 \mathrm{E}-04$ & $-1.12612 \mathrm{E}-01$ & $7.34336 \mathrm{E}$ & 00 & $-4.17305 \mathrm{E}$ & 02 \\
\hline 6.80 & $1.04820 \mathrm{E}$ & 00 & $-6.43122 \mathrm{E}$ & 00 & $-1.20824 \mathrm{E}-01$ & $2.33607 \mathrm{E}-04$ & $-1.15396 \mathrm{E}-01$ & $7.44591 \mathrm{E}$ & 00 & $-4.20187 \mathrm{E}$ & 02 \\
\hline 6.90 & $1.04818 \mathrm{E}$ & 00 & $-6.53354 \mathrm{E}$ & 00 & $-1.23810 \mathrm{E}-01$ & $2.48767 \mathrm{E}-04$ & $-1.18214 \mathrm{E}-01$ & $7.54844 \mathrm{E}$ & 00 & $-4.23022 \mathrm{E}$ & 02 \\
\hline 7.00 & $1.04817 \mathrm{E}$ & 00 & $-6.63586 \mathrm{E}$ & 00 & $-1.26830 \mathrm{E}-01$ & $2.63494 \mathrm{E}-04$ & $-1.21065 \mathrm{E}-01$ & $7.65096 \mathrm{E}$ & 00 & $-4.25814 \mathrm{E}$ & 02 \\
\hline 7.10 & $1.04815 \mathrm{E}$ & 00 & $-6.73818 E$ & 00 & $-1.29883 \mathrm{E}-01$ & $2.77812 \mathrm{E}-04$ & $-1.23950 \mathrm{E}-01$ & $7.75346 \mathrm{E}$ & 00 & $-4.28562 \mathrm{E}$ & 02 \\
\hline 7.20 & $1.04813 \mathrm{E}$ & 00 & $-6.84048 \mathrm{E}$ & 00 & $-1.32969 \mathrm{E}-01$ & $2.91733 \mathrm{E}-04$ & $-1.26869 \mathrm{E}-01$ & $7.85595 \mathrm{E}$ & 00 & $-4.31267 \mathrm{E}$ & 02 \\
\hline 7.30 & $1.04812 \mathrm{E}$ & 00 & $-6.94279 \mathrm{E}$ & 00 & $-1.36090 \mathrm{E}-01$ & $3.05275 \mathrm{E}-04$ & $-1.29821 \mathrm{E}-01$ & $7.95842 \mathrm{E}$ & 00 & $-4.33931 \mathrm{E}$ & 02 \\
\hline 7.40 & $1.04810 \mathrm{E}$ & 00 & $-7.04509 \mathrm{E}$ & 00 & $-1.39243 \mathrm{E}-01$ & $3.18451 \mathrm{E}-04$ & $-1.32806 \mathrm{E}-01$ & $8.06088 \mathrm{E}$ & 00 & $-4.36554 \mathrm{E}$ & 02 \\
\hline & $1.04809 \mathrm{E}$ & 00 & $-7.14738 \mathrm{E}$ & 00 & $-1.42431 \mathrm{E}-01$ & $3.31280 \mathrm{E}-04$ & $-1.35825 \mathrm{E}-01$ & $8.16333 \mathrm{E}$ & 00 & $-4.39137 \mathrm{E}$ & 02 \\
\hline 7.60 & $1.04807 \mathrm{E}$ & 00 & $-7.24967 \mathrm{E}$ & 00 & $-1.45652 \mathrm{E}-01$ & $3.43772 \mathrm{E}-04$ & $-1.38878 \mathrm{E}-01$ & $8.26577 \mathrm{E}$ & 00 & $-4.41682 \mathrm{E}$ & 02 \\
\hline 7.70 & $1.04806 \mathrm{E}$ & 00 & $-7.35195 \mathrm{E}$ & 00 & $-1.48906 \mathrm{E}-01$ & $3.55938 \mathrm{E}-04$ & $-1.41964 \mathrm{E}-01$ & $8.36820 \mathrm{E}$ & 00 & $-4.44190 \mathrm{E}$ & 02 \\
\hline 7.80 & $1.04805 \mathrm{E}$ & 00 & $-7.45423 E$ & 00 & $-1.52194 \mathrm{E}-01$ & $3.67796 \mathrm{E}-04$ & $-1.45083 \mathrm{E}-01$ & $8.47061 \mathrm{E}$ & 00 & $-4.46660 \mathrm{E}$ & 02 \\
\hline 7.90 & $1.04804 \mathrm{E}$ & 00 & $-7.55651 \mathrm{E}$ & 00 & $-1.55516 \mathrm{E}-01$ & $3.79354 \mathrm{E}-04$ & $-1.48237 \mathrm{E}-01$ & $8.57302 \mathrm{E}$ & 00 & $-4.49095 \mathrm{E}$ & 02 \\
\hline 8.00 & $1.04802 \mathrm{E}$ & 00 & $-7.65878 \mathrm{E}$ & 00 & $-1.58871 \mathrm{E}-01$ & $3.90622 \mathrm{E}-04$ & $-1.51423 \mathrm{E}-01$ & $8.67541 \mathrm{E}$ & 00 & $-4.51494 \mathrm{E}$ & 02 \\
\hline 8.10 & $1.04801 \mathrm{E}$ & 00 & $-7.76104 \mathrm{E}$ & 00 & $-1.62259 \mathrm{E}-01$ & $4.01614 \mathrm{E}-04$ & $-1.54644 \mathrm{E}-01$ & $8.77779 \mathrm{E}$ & 00 & $-4.53858 \mathrm{E}$ & 02 \\
\hline 8.20 & $1.04800 \mathrm{E}$ & 00 & $-7.86330 \mathrm{E}$ & 00 & $-1.65682 \mathrm{E}-01$ & $4.12338 \mathrm{E}-04$ & $-1.57897 \mathrm{E}-01$ & $8.88017 \mathrm{E}$ & 00 & $-4.56189 \mathrm{E}$ & 02 \\
\hline 8.30 & $1.04799 \mathrm{E}$ & 00 & $-7.96555 \mathrm{E}$ & 00 & $-1.69137 \mathrm{E}-01$ & $4.22806 \mathrm{E}-04$ & $-1.61185 \mathrm{E}-01$ & $8.98253 \mathrm{E}$ & 00 & $-4.58487 \mathrm{E}$ & 02 \\
\hline 8.40 & $1.04798 \mathrm{E}$ & 00 & $-8.06780 \mathrm{E}$ & 00 & $-1.72627 \mathrm{E}-01$ & $4.33020 \mathrm{E}-04$ & $-1.64506 \mathrm{E}-01$ & $9.08488 \mathrm{E}$ & 00 & $-4.60752 \mathrm{E}$ & 02 \\
\hline 8.50 & $1.04797 \mathrm{E}$ & 00 & $-8.17004 \mathrm{E}$ & 00 & $-1.76149 \mathrm{E}-01$ & $4.42999 \mathrm{E}-04$ & $-1.67860 \mathrm{E}-01$ & $9.18723 \mathrm{E}$ & 00 & $-4.62986 \mathrm{E}$ & 02 \\
\hline 8.60 & $1.04796 \mathrm{E}$ & 00 & $-8.27228 \mathrm{E}$ & 00 & $-1.79706 \mathrm{E}-01$ & $4.52741 \mathrm{E}-04$ & $-1.71248 \mathrm{E}-01$ & $9.28956 \mathrm{E}$ & 00 & $-4.65190 \mathrm{E}$ & 02 \\
\hline 8.70 & $1.04795 \mathrm{E}$ & 00 & $-8.37452 \mathrm{E}$ & 00 & $-1.83296 \mathrm{E}-01$ & $4.62261 \mathrm{E}-04$ & $-1.74670 \mathrm{E}-01$ & $9.39189 \mathrm{E}$ & 00 & $-4.67363 \mathrm{E}$ & 02 \\
\hline 8.80 & $1.04794 \mathrm{E}$ & 00 & $-8.47674 \mathrm{E}$ & 00 & $-1.86919 \mathrm{E}-01$ & $4.71563 \mathrm{E}-04$ & $-1.78125 \mathrm{E}-01$ & $9.49420 \mathrm{E}$ & 00 & $-4.69506 \mathrm{E}$ & 02 \\
\hline 8.90 & $1.04793 \mathrm{E}$ & 00 & $-8.57897 \mathrm{E}$ & 00 & $-1.90576 \mathrm{E}-01$ & $4.80660 \mathrm{E}-04$ & $.81613 \mathrm{E}-01$ & $9.59651 \mathrm{E}$ & 00 & $-4.71620 \mathrm{E}$ & 02 \\
\hline 9.00 & $1.04792 \mathrm{E}$ & 00 & $-8.68118 \mathrm{E}$ & 00 & $-1.94267 \mathrm{E}-01$ & $4.89552 \mathrm{E}-04$ & $-1.85135 \mathrm{E}-01$ & $9.69881 \mathrm{E}$ & 00 & $-4.73706 \mathrm{E}$ & 02 \\
\hline 9.10 & $1.04791 \mathrm{E}$ & 00 & $-8.78340 \mathrm{E}$ & 00 & $-1.97991 \mathrm{E}-01$ & $4.98246 \mathrm{E}-04$ & $-1.88691 \mathrm{E}-01$ & $9.80110 \mathrm{E}$ & 00 & $-4.75764 \mathrm{E}$ & 02 \\
\hline 9.20 & $1.04790 \mathrm{E}$ & 00 & $-8.88561 \mathrm{E}$ & 00 & $-2.01748 \mathrm{E}-01$ & $5.06755 \mathrm{E}-04$ & $-1.92280 \mathrm{E}-01$ & $9.90338 \mathrm{E}$ & 00 & $-4.77795 \mathrm{E}$ & 02 \\
\hline 9.30 & $1.04789 \mathrm{E}$ & 00 & $-8.98781 \mathrm{E}$ & 00 & $-2.05539 \mathrm{E}-01$ & $5076 \mathrm{E}-04$ & $-1.95903 \mathrm{E}-01$ & $1.00057 \mathrm{E}$ & 01 & $-4.79799 \mathrm{E}$ & 02 \\
\hline 9.40 & $1.04789 \mathrm{E}$ & 00 & $-9.09001 \mathrm{E}$ & 00 & $-2.09364 \mathrm{E}-01$ & $5.23221 \mathrm{E}-04$ & $-1.99559 \mathrm{E}-01$ & $1.01079 \mathrm{E}$ & 01 & $-4.81778 \mathrm{E}$ & 02 \\
\hline 9.50 & $1.04788 \mathrm{E}$ & 00 & $-9.19220 \mathrm{E}$ & 00 & $-2.13222 \mathrm{E}-01$ & $5.31196 \mathrm{E}-04$ & $-2.03249 \mathrm{E}-01$ & $1.02102 \mathrm{E}$ & 01 & $-4.83730 \mathrm{E}$ & 02 \\
\hline 9.60 & $1.04787 \mathrm{E}$ & 00 & $-9.29439 \mathrm{E}$ & 00 & $-2.17114 \mathrm{E}-01$ & $5.39001 \mathrm{E}-04$ & $-2.06972 \mathrm{E}-01$ & $1.03124 \mathrm{E}$ & 01 & $-4.85657 \mathrm{E}$ & 02 \\
\hline 9.70 & $1.04786 \mathrm{E}$ & 00 & $-9.39657 \mathrm{E}$ & 00 & $-2.21039 \mathrm{E}-01$ & $5.46647 \mathrm{E}-04$ & $-2.10729 \mathrm{E}-01$ & $1.04147 \mathrm{E}$ & 01 & $-4.87560 \mathrm{E}$ & 02 \\
\hline 9.80 & $1.04786 \mathrm{E}$ & 00 & $-9.49875 \mathrm{E}$ & 00 & $-2.24998 \mathrm{E}-0 \mathrm{l}$ & $5.54134 \mathrm{E}-04$ & $-2.14519 \mathrm{E}-01$ & $1.05169 \mathrm{E}$ & 01 & $-4.89439 E$ & 02 \\
\hline 9.90 & $1.04785 \mathrm{E}$ & 00 & $-9.60092 \mathrm{E}$ & 00 & $-2.28990 \mathrm{E}-01$ & $5.61467 \mathrm{E}-04$ & $-2.18343 \mathrm{E}-01$ & $1.06191 \mathrm{E}$ & 01 & $-4.91295 E$ & 02 \\
\hline 10.00 & $1.04784 \mathrm{E}$ & 00 & $-9.70309 \mathrm{E}$ & 00 & $-2.33016 \mathrm{E}-01$ & $5.68656 \mathrm{E}-04$ & $-2.22200 \mathrm{E}-01$ & $1.07214 \mathrm{E}$ & 01 & $-4.93127 \mathrm{E}$ & 02 \\
\hline 10.10 & $1.04783 \mathrm{E}$ & 00 & $-9.80525 \mathrm{E}$ & 00 & $-2.37075 \mathrm{E}-01$ & $5.75699 \mathrm{E}-04$ & $-2.26091 \mathrm{E}-01$ & $1.08236 \mathrm{E}$ & 01 & $-4.94936 \mathrm{E}$ & 02 \\
\hline 10.20 & & 00 & $-9.90741 \mathrm{E}$ & 00 & $-2.41168 \mathrm{E}-01$ & $5.82606 \mathrm{E}-04$ & $30016 \mathrm{E}-01$ & $1.09258 \mathrm{E}$ & 01 & $-4.96723 \mathrm{E}$ & 02 \\
\hline 10.30 & $1.04782 \mathrm{E}$ & 00 & $-1.00096 \mathrm{E}$ & 01 & $-2.45294 \mathrm{E}-01$ & $5.89373 \mathrm{E}-04$ & $-2.33974 \mathrm{E}-01$ & $1.10280 \mathrm{E}$ & 01 & $-4.98489 E$ & 02 \\
\hline 10.40 & $1.04782 \mathrm{E}^{-}$ & 00 & $-1.01117 \mathrm{E}$ & 01 & $-2.49454 \mathrm{E}-0 \mathrm{l}$ & $5.96014 \mathrm{E}-04$ & $-2.37965 \mathrm{E}-01$ & $1.11302 \mathrm{E}$ & 01 & $-5.00233 \mathrm{E}$ & 02 \\
\hline 10.50 & $1.04781 \mathrm{E}$ & 00 & $-1.02139 \mathrm{E}$ & 01 & $-2.53648 \mathrm{E}-01$ & $6.02522 \mathrm{E}-04$ & $-2.41990 \mathrm{E}-01$ & $1.12324 \mathrm{E}$ & 01 & $-5.01956 \mathrm{E}$ & 02 \\
\hline 10.60 & $1.04780 \mathrm{E}$ & 00 & $-1.03160 \mathrm{E}$ & 01 & $-2.57874 \mathrm{E}-01$ & $6.08909 \mathrm{E}-04$ & $-2.46049 \mathrm{E}-01$ & $1.13345 \mathrm{E}$ & 01 & $-5.03659 \mathrm{E}$ & 02 \\
\hline 0.70 & $1.04780 \mathrm{E}$ & 00 & $-1.04181 \mathrm{E}$ & 01 & $-2.62135 \mathrm{E}-01$ & $6.15177 \mathrm{E}-04$ & $-2.50141 \mathrm{E}-01$ & $1.14367 \mathrm{E}$ & 01 & $-5.05341 \mathrm{E}$ & 02 \\
\hline 10.80 & $1.04779 \mathrm{E}$ & 00 & $-1.05202 \mathrm{E}$ & 01 & $-2.66429 \mathrm{E}-01$ & $6.21325 \mathrm{E}-04$ & $-2.54266 \mathrm{E}-01$ & $1.15389 \mathrm{E}$ & 01 & $-5.07003 \mathrm{E}$ & 02 \\
\hline 10. & $1.04779 \mathrm{E}$ & 00 & $-1.06224 \mathrm{E}$ & 01 & $-2.70756 \mathrm{E}-01$ & $6.27359 \mathrm{E}-04$ & $-2.58425 \mathrm{E}-01$ & $1.16410 \mathrm{E}$ & 01 & $-5.08646 \mathrm{E}$ & 02 \\
\hline 11.00 & $1.04778 \mathrm{E}$ & 00 & $-1.07245 \mathrm{E}$ & 01 & $-2.75117 \mathrm{E}-01$ & $6.33282 \mathrm{E}-04$ & $-2.62618 \mathrm{E}-01$ & $1.17432 \mathrm{E}$ & 01 & $-5.10270 \mathrm{E}$ & 02 \\
\hline 1.10 & $1.04777 \mathrm{E}$ & 00 & $-1.08266 \mathrm{E}$ & 01 & $-2.79512 \mathrm{E}-01$ & $6.39100 \mathrm{E}-04$ & $-2.66844 \mathrm{E}-01$ & $1.18453 \mathrm{E}$ & 01 & $-5.11875 \mathrm{E}$ & 02 \\
\hline & $1.04777 \mathrm{E}$ & 00 & $-1.09287 \mathrm{E}$ & 01 & $-2.83939 \mathrm{E}-01$ & $6.44808 \mathrm{E}-04$ & $-2.71103 \mathrm{E}-01$ & $1.19474 \mathrm{E}$ & 01 & $-5.13462 \mathrm{E}$ & 02 \\
\hline & $1.04776 \mathrm{E}$ & 00 & $-1.10308 \mathrm{E}$ & 01 & $-2.88401 \mathrm{E}-01$ & $6.50417 \mathrm{E}-04$ & $-2.75396 \mathrm{E}-01$ & $1.20496 \mathrm{E}$ & 01 & $-5.15031 \mathrm{E}$ & 02 \\
\hline 11.4 & $1.04776 \mathrm{E}$ & 00 & $-1.11329 \mathrm{E}$ & 01 & $-2.92896 \mathrm{E}-01$ & $6.55927 \mathrm{E}-04$ & $-2.79723 \mathrm{E}-01$ & $1.21517 \mathrm{E}$ & 01 & $-5.16581 \mathrm{E}$ & 02 \\
\hline 11.50 & $1.04775 \mathrm{E}$ & 00 & $-1.12350 \mathrm{E}$ & 01 & $-2.97424 \mathrm{E}-01$ & $6.61335 \mathrm{E}-04$ & $-2.84083 \mathrm{E}-01$ & $1.22538 \mathrm{E}$ & 01 & $-5.18115 \mathrm{E}$ & 02 \\
\hline 60 & $1.04775 \mathrm{E}$ & 00 & $-1.13371 \mathrm{E}$ & 01 & $-3.01986 \mathrm{E}-01$ & $6.66650 \mathrm{E}-04$ & $-2.88477 \mathrm{E}-01$ & $1.23559 \mathrm{E}$ & 01 & $-5.19632 \mathrm{E}$ & 02 \\
\hline & & 00 & $-1.14392 \mathrm{E}$ & 01 & $-3.06582 \mathrm{E}-01$ & $6.71873 \mathrm{E}-04$ & $-2.92904 \mathrm{E}-01$ & $1.24580 \mathrm{E}$ & 01 & $-5.21132 \mathrm{E}$ & 02 \\
\hline & & 00 & $-1.15412 \mathrm{E}$ & 01 & $-3.11211 \mathrm{E}-01$ & $6.77009 \mathrm{E}-04$ & $-2.97364 \mathrm{E}-01$ & $1.25601 \mathrm{E}$ & 01 & $-5.22614 \mathrm{E}$ & 02 \\
\hline & $1.04773 \mathrm{E}$ & 00 & $-1.16433 \mathrm{E}$ & 01 & $-3.15873 \mathrm{E}-01$ & $6.82052 \mathrm{E}-04$ & $-3.01858 \mathrm{E}-01$ & $1.26622 \mathrm{E}$ & 01 & $-5.24082 \mathrm{E}$ & 02 \\
\hline 12.00 & $1.04773 \mathrm{E}$ & 00 & $-1.17454 \mathrm{E}$ & 01 & $-3.20569 \mathrm{E}-01$ & $6.87011 \mathrm{E}-04$ & $-3.06386 \mathrm{E}-01$ & $1.27643 \mathrm{E}$ & 01 & $-5.25533 \mathrm{E}$ & 02 \\
\hline
\end{tabular}




\begin{tabular}{|c|c|c|c|}
\hline$p$ & $F$ & $f_{2}$ & $g_{2}$ \\
\hline $\begin{array}{l}6.10 \\
6.20 \\
6.30 \\
6.40 \\
6.50\end{array}$ & $\begin{array}{l}2.63593 \mathrm{E}-05 \\
2.72301 \mathrm{E}-05 \\
2.81150 \mathrm{E}-05 \\
2.90140 \mathrm{E}-05 \\
2.99272 \mathrm{E}-05\end{array}$ & $\begin{array}{l}-5.73278 \mathrm{E}-02 \\
-5.93116 \mathrm{E}-02 \\
-6.13290 \mathrm{E}-02 \\
-6.33801 \mathrm{E}-02 \\
-6.54649 \mathrm{E}-02\end{array}$ & $\begin{array}{l}-5.97452 \mathrm{E}-04 \\
-6.23904 \mathrm{E}-04 \\
-6.51102 \mathrm{E}-04 \\
-6.79055 \mathrm{E}-04 \\
-7.07775 \mathrm{E}-04\end{array}$ \\
\hline $\begin{array}{l}6.60 \\
6.70 \\
6.80 \\
6.90 \\
7.00\end{array}$ & $\begin{array}{l}3.08546 \mathrm{E}-05 \\
3.17962 \mathrm{E}-05 \\
3.27519 \mathrm{E}-05 \\
3.37217 \mathrm{E}-05 \\
3.47057 \mathrm{E}-05\end{array}$ & $\begin{array}{l}-6.75833 \mathrm{E}-02 \\
-6.97353 \mathrm{E}-02 \\
-7.19210 \mathrm{E}-02 \\
-7.41403 \mathrm{E}-02 \\
-7.63932 \mathrm{E}-02\end{array}$ & $\begin{array}{l}-7.37271 \mathrm{E}-04 \\
-7.67553 \mathrm{E}-04 \\
-7.98630 \mathrm{E}-04 \\
-8.30513 \mathrm{E}-04 \\
-8.63212 \mathrm{E}-04\end{array}$ \\
\hline $\begin{array}{l}7.10 \\
7.20 \\
7.30 \\
7.40 \\
7.50\end{array}$ & $\begin{array}{l}3.57039 \mathrm{E}-05 \\
3.67162 \mathrm{E}-05 \\
3.77427 \mathrm{E}-05 \\
3.87833 \mathrm{E}-05 \\
3.98381 \mathrm{E}-05\end{array}$ & $\begin{array}{l}-7.86797 \mathrm{E}-02 \\
-8.09999 \mathrm{E}-02 \\
-8.33537 \mathrm{E}-02 \\
-8.57411 \mathrm{E}-02 \\
-8.81622 \mathrm{E}-02\end{array}$ & $\begin{array}{l}-8.96737 \mathrm{E}-04 \\
-9.31097 \mathrm{E}-04 \\
-9.66302 \mathrm{E}-04 \\
-1.00236 \mathrm{E}-03 \\
-1.03929 \mathrm{E}-03\end{array}$ \\
\hline $\begin{array}{l}7.60 \\
7.70 \\
7.80 \\
7.90 \\
8.00\end{array}$ & $\begin{array}{l}4.09070 \mathrm{E}-05 \\
4.19901 \mathrm{E}-05 \\
4.30874 \mathrm{E}-05 \\
4.41988 \mathrm{E}-05 \\
4.53244 \mathrm{E}-05\end{array}$ & $\begin{array}{l}-9.06168 \mathrm{E}-02 \\
-9.31051 \mathrm{E}-02 \\
-9.56270 \mathrm{E}-02 \\
-9.81825 \mathrm{E}-02 \\
-1.00772 \mathrm{E}-01\end{array}$ & $\begin{array}{l}-1.07709 \mathrm{E}-03 \\
-1.11578 \mathrm{E}-03 \\
-1.15536 \mathrm{E}-03 \\
-1.19585 \mathrm{E}-03 \\
-1.23725 \mathrm{E}-03\end{array}$ \\
\hline $\begin{array}{l}8.10 \\
8.20 \\
8.30 \\
8.40 \\
8.50\end{array}$ & $\begin{array}{l}4.64641 \mathrm{E}-05 \\
4.76180 \mathrm{E}-05 \\
4.87860 \mathrm{E}-05 \\
4.99682 \mathrm{E}-05 \\
5.11646 \mathrm{E}-05\end{array}$ & $\begin{array}{l}-1.03394 \mathrm{E}-01 \\
-1.06051 \mathrm{E}-01 \\
-1.08741 \mathrm{E}-01 \\
-1.11464 \mathrm{E}-01 \\
-1.14221 \mathrm{E}-01\end{array}$ & $\begin{array}{l}-1.27958 \mathrm{E}-03 \\
-1.32284 \mathrm{E}-03 \\
-1.36705 \mathrm{E}-03 \\
-1.41221 \mathrm{E}-03 \\
-1.45833 \mathrm{E}-03\end{array}$ \\
\hline $\begin{array}{l}8.60 \\
8.70 \\
8.80 \\
8.90 \\
9.00\end{array}$ & $\begin{array}{l}5.23751 \mathrm{E}-05 \\
5.35997 \mathrm{E}-05 \\
5.48385 \mathrm{E}-05 \\
5.60915 \mathrm{E}-05 \\
5.73587 \mathrm{E}-05\end{array}$ & $\begin{array}{l}-1.17012 \mathrm{E}-01 \\
-1.19836 \mathrm{E}-01 \\
-1.22694 \mathrm{E}-01 \\
-1.25586 \mathrm{E}-01 \\
-1.28511 \mathrm{E}-01\end{array}$ & $\begin{array}{l}-1.50544 \mathrm{E}-03 \\
-1.55352 \mathrm{E}-03 \\
-1.60260 \mathrm{E}-03 \\
-1.65269 \mathrm{E}-03 \\
-1.70379 \mathrm{E}-03\end{array}$ \\
\hline $\begin{array}{l}9.10 \\
9.20 \\
9.30 \\
9.40 \\
9.50\end{array}$ & $\begin{array}{l}5.86399 \mathrm{E}-05 \\
5.99354 \mathrm{E}-05 \\
6.12450 \mathrm{E}-05 \\
6.25687 \mathrm{E}-05 \\
6.39066 \mathrm{E}-05\end{array}$ & $\begin{array}{l}-1.31470 \mathrm{E}-01 \\
-1.34462 \mathrm{E}-01 \\
-1.37488 \mathrm{E}-01 \\
-1.40548 \mathrm{E}-01 \\
-1.43641 \mathrm{E}-01\end{array}$ & $\begin{array}{l}-1.75591 \mathrm{E}-03 \\
-1.80907 \mathrm{E}-03 \\
-1.86327 \mathrm{E}-03 \\
-1.91852 \mathrm{E}-03 \\
-1.97484 \mathrm{E}-03\end{array}$ \\
\hline $\begin{array}{l}9.60 \\
9.70 \\
9.80 \\
9.90\end{array}$ & $\begin{array}{l}6.52587 \mathrm{E}-05 \\
6.66249 \mathrm{E}-05 \\
6.80053 \mathrm{E}-05 \\
6.93998 \mathrm{E}-05 \\
7.08085 \mathrm{E}-05\end{array}$ & $\begin{array}{l}-1.46768 \mathrm{E}-01 \\
-1.49928 \mathrm{E}-01 \\
-1.53122 \mathrm{E}-01 \\
-1.56349 \mathrm{E}-01 \\
-1.59610 \mathrm{E}-01\end{array}$ & $\begin{array}{l}-2.03224 \mathrm{E}-03 \\
-2.09072 \mathrm{E}-03 \\
-2.15029 \mathrm{E}-03 \\
-2.21096 \mathrm{E}-03 \\
-2.27275 \mathrm{E}-03\end{array}$ \\
\hline & $\begin{array}{l}7.22314 \mathrm{E}-05 \\
7.36684 \mathrm{E}-05 \\
7.51196 \mathrm{E}-05 \\
7.65849 \mathrm{E}-05 \\
7.80644 \mathrm{E}-05\end{array}$ & $\begin{array}{l}-1.62905 \mathrm{E}-01 \\
-1.66233 \mathrm{E}-01 \\
-1.69595 \mathrm{E}-01 \\
-1.72990 \mathrm{E}-01 \\
-1.76419 \mathrm{E}-01\end{array}$ & $\begin{array}{l}-2.33567 \mathrm{E}-03 \\
-2.39971 \mathrm{E}-03 \\
-2.46490 \mathrm{E}-03 \\
-2.53125 \mathrm{E}-03 \\
-2.59875 \mathrm{E}-03\end{array}$ \\
\hline & $\begin{array}{l}7.95580 \mathrm{E}-05 \\
8.10658 \mathrm{E}-05 \\
8.25878 \mathrm{E}-05 \\
8.41239 \mathrm{E}-05 \\
8.56741 \mathrm{E}-05\end{array}$ & $\begin{array}{l}-1.79882 \mathrm{E}-01 \\
-1.83378 \mathrm{E}-01 \\
-1.86907 \mathrm{E}-01 \\
-1.90471 \mathrm{E}-01 \\
-1.94067 \mathrm{E}-01\end{array}$ & $\begin{array}{l}-2.66743 \mathrm{E}-03 \\
-2.73730 \mathrm{E}-03 \\
-2.80835 \mathrm{E}-03 \\
-2.88061 \mathrm{E}-03 \\
-2.95408 \mathrm{E}-03\end{array}$ \\
\hline & $\begin{array}{l}8.72385 \mathrm{E}-05 \\
8.88171 \mathrm{E}-05 \\
9.04098 \mathrm{E}-05 \\
9.20167 \mathrm{E}-05 \\
9.36377 \mathrm{E}-05\end{array}$ & $\begin{array}{l}-1.97698 \mathrm{E}-01 \\
-2.01362 \mathrm{E}-01 \\
-2.05059 \mathrm{E}-01 \\
-2.08790 \mathrm{E}-01 \\
-2.12555 \mathrm{E}-01\end{array}$ & $\begin{array}{l}-3.02878 \mathrm{E}-03 \\
-3.10471 \mathrm{E}-03 \\
-3.18188 \mathrm{E}-03 \\
-3.26030 \mathrm{E}-03 \\
-3.33999 \mathrm{E}-03\end{array}$ \\
\hline & $\begin{array}{l}9.52729 \mathrm{E}-05 \\
9.69223 \mathrm{E}-05 \\
9.85858 \mathrm{E}-05 \\
1.00263 \mathrm{E}-04\end{array}$ & $\begin{array}{l}-2.16353 \mathrm{E}-01 \\
-2.20185 \mathrm{E}-01 \\
-2.24050 \mathrm{E}-01 \\
-2.27949 \mathrm{E}-01\end{array}$ & $\begin{array}{l}-3.42094 \mathrm{E}-03 \\
-3.50319 \mathrm{E}-03 \\
-3.58672 \mathrm{E}-03 \\
-3.67155 \mathrm{E}-03\end{array}$ \\
\hline
\end{tabular}

\begin{abstract}
$\tan \delta_{2}$ $f_{-2}$ $9.55799 \mathrm{E} \quad 01 \quad-5.70201 \mathrm{E}-04$ $9.57063 \mathrm{E} \quad 01 \quad-5.95178 \mathrm{E}-04$ $9.58284 \mathrm{E} \quad 01-6.20868 \mathrm{E}-04$ $9.59465 \mathrm{E} \quad 01-6.47280 \mathrm{E}-04$ $\begin{array}{lll}9.60607 \mathrm{E} & 01 & -6.74425 \mathrm{E}-04\end{array}$
\end{abstract}

$9.61712 \mathrm{E} \quad 01$ $9.62782 \mathrm{E} \quad 01$ $9.63818 \mathrm{E} 01$ $9.64823 \mathrm{E} \quad 01$ $9.65797 \mathrm{E} 01$

$-7.02313 \mathrm{E}-04$ $-7.30953 \mathrm{E}-04$ $-7.60355 \mathrm{E}-04$ $-7.90530 \mathrm{E}-04$ $-8.21488 \mathrm{E}-04$

$9.66741 \mathrm{E} 01$ $9.67658 \mathrm{E} 01$ $9.68548 \mathrm{E} 01$ $9.69413 \mathrm{E} 01$ $9.70253 \mathrm{E} \quad 01$

$9.71069 \mathrm{E} \quad 01$ $9.71863 \mathrm{E} \quad 01$ $9.72635 \mathrm{E} \quad 01$ $9.73386 \mathrm{E} \quad 01$ $9.74118 \mathrm{E} \quad 01$

$9.74830 \mathrm{E} \quad 01$ $9.75524 \mathrm{E} \quad 01$ $9.76200 \mathrm{E} \quad 01$ $9.76859 \mathrm{E} 01$ $9.77501 \mathrm{E} \quad 01$

$9.78128 \mathrm{E} \quad 01$ $9.78739 \mathrm{E} 01$ $9.79335 \mathrm{E} \quad 01$ $9.79918 \mathrm{E} 01$ $9.80486 \mathrm{E} \quad 01$

$9.81041 \mathrm{E} \quad 01$ $9.81584 \mathrm{E} \quad 01$ $9.82114 \mathrm{E} \quad 0 \mathrm{l}$ $9.82632 \mathrm{E} \quad 01$ $9.83139 \mathrm{E} \quad 01$

$9.83634 \mathrm{E} \quad 01$ $9.84119 \mathrm{E} 01$ $9.84593 \mathrm{E} \quad 01$ $9.85057 \mathrm{E} \quad 01$ $9.85511 \mathrm{E} \quad 01$

$9.85956 \mathrm{E} \quad 01$ $9.86391 \mathrm{E} \quad 01$ $9.86818 \mathrm{E} 01$ $9.87235 \mathrm{E} \quad 01$ $9.87645 \mathrm{E} \quad 01$

$9.88046 \mathrm{E} \quad 01$ $9.88439 \mathrm{E} \quad 01$ $9.88825 \mathrm{E} \quad 01$ $9.89203 \mathrm{E} 01$ $9.89574 \mathrm{E} \quad 01$

$9.89938 \mathrm{E} \quad 01$ $9.90295 \mathrm{E} 01$ $9.90645 \mathrm{E} \quad 01$ $9.90989 \mathrm{E} \quad 01$ $9.91326 \mathrm{E} \quad 01$

$9.91658 \mathrm{E} \quad 01$ $9.91983 \mathrm{E} 01$ $9.92303 \mathrm{E} \quad 01$ $9.92616 \mathrm{E} 01$ $9.92925 \mathrm{E} 01$
$-8.53237 \mathrm{E}-04$ $-8.85789 \mathrm{E}-04$ $-9.19153 \mathrm{E}-04$ $-9.53339 \mathrm{E}-04$ $-9.88358 \mathrm{E}-04$

$-1.02422 \mathrm{E}-03$ $-1.06093 \mathrm{E}-03$ $-1.09850 \mathrm{E}-03$ $-1.13695 \mathrm{E}-03$ $-1.17628 \mathrm{E}-03$

$-1.21650 \mathrm{E}-03$ $-1.25762 \mathrm{E}-03$ $-1.29965 \mathrm{E}-03$ $-1.34261 \mathrm{E}-03$ $-1.38649 \mathrm{E}-03$

$-1.43132 \mathrm{E}-03$ $-1.47710 \mathrm{E}-03$ $-1.52384 \mathrm{E}-03$ $-1.57155 \mathrm{E}-03$ $-1.62024 \mathrm{E}-03$

$-1.66992 \mathrm{E}-03$ $-1.72061 \mathrm{E}-03$ $-1.77230 \mathrm{E}-03$ $-1.82502 \mathrm{E}-03$ $-1.87876 \mathrm{E}-03$

$-1.93355 \mathrm{E}-03$ $-1.98939 \mathrm{E}-03$ $-2.04629 \mathrm{E}-03$ $-2.10426 \mathrm{E}-03$ $-2.16331 \mathrm{E}-03$

$-2.22345 \mathrm{E}-03$ $-2.28469 \mathrm{E}-03$ $-2.34704 \mathrm{E}-03$ $-2.41051 \mathrm{E}-03$ $-2.47511 \mathrm{E}-03$

$-2.54085 \mathrm{E}-03$ $-2.60774 \mathrm{E}-03$ $-2.67579 \mathrm{E}-03$ $-2.74501 \mathrm{E}-03$ $-2.81541 \mathrm{E}-03$

$-2.88700 \mathrm{E}-03$ $-2.95979 \mathrm{E}-03$ $-3.03379 \mathrm{E}-03$ $-3.10901 \mathrm{E}-03$ $-3.18546 \mathrm{E}-03$ $-3.26315 \mathrm{E}-03$ $-3.34208 \mathrm{E}-03$ $-3.42228 \mathrm{E}-03$ $-3.50374 \mathrm{E}-03$ $-3.58649 \mathrm{E}-03$

\section{$g_{-2}$}

$6.74923 \mathrm{E}-02$ $6.96458 \mathrm{E}-02$ $7.18329 \mathrm{E}-02$

$7.40536 \mathrm{E}-02$

$7.63078 \mathrm{E}-02$

$7.85957 \mathrm{E}-02$ $8.09172 \mathrm{E}-02$

$8.32722 \mathrm{E}-02$

$8.56609 \mathrm{E}-02$

$8.80831 \mathrm{E}-02$

$9.05390 \mathrm{E}-02$ $9.30284 \mathrm{E}-02$ $9.55514 \mathrm{E}-02$ $9.81080 \mathrm{E}-02$ $1.00698 \mathrm{E}-01$

$1.03322 \mathrm{E}-01$ $1.05979 \mathrm{E}-01$ $1.08670 \mathrm{E}-01$ $1.11395 \mathrm{E}-01$ $1.14153 \mathrm{E}-01$

$1.16945 \mathrm{E}-01$ $1.19770 \mathrm{E}-01$ $1.22629 \mathrm{E}-01$ $1.25521 \mathrm{E}-01$

$1.28447 \mathrm{E}-01$

$1.31407 \mathrm{E}-01$ $1.34400 \mathrm{E}-01$ $1.37427 \mathrm{E}-01$ $1.40487 \mathrm{E}-01$ $1.43581 \mathrm{E}-01$

$1.46708 \mathrm{E}-01$ $1.49870 \mathrm{E}-01$ $1.53064 \mathrm{E}-01$ $1.56292 \mathrm{E}-01$ $1.59554 \mathrm{E}-01$

$1.62849 \mathrm{E}-01$

$1.66178 \mathrm{E}-01$

$1.69541 \mathrm{E}-01$

$1.72937 \mathrm{E}-01$

$1.76366 \mathrm{E}-01$

$1.79830 \mathrm{E}-01$ $1.83326 \mathrm{E}-01$ $1.86857 \mathrm{E}-01$ $1.90421 \mathrm{E}-01$ $1.94018 \mathrm{E}-01$

$1.97649 \mathrm{E}-01$ $2.01313 \mathrm{E}-01$ $2.05011 \mathrm{E}-01$ $2.08743 \mathrm{E}-01$ $2.12508 \mathrm{E}-01$

$2.16307 \mathrm{E}-01$ $2.20139 \mathrm{E}-01$ $2.24005 \mathrm{E}-01$ $2.27904 \mathrm{E}-01$ $2.31837 \mathrm{E}-01$

2.35804 E-01 $2.39804 \mathrm{E}-01$ $2.43837 \mathrm{E}-01$ $2.47904 \mathrm{E}-0 \mathrm{l}$ $2.52005 \mathrm{E}-01$ $\tan \delta_{-2}$

$-9.26561 \mathrm{E}-03$ $-9.27109 \mathrm{E}-03$ $-9.27646 \mathrm{E}-03$ $-9.28172 \mathrm{E}-03$ $-9.28688 \mathrm{E}-0.3$

$-9.29193 \mathrm{E}-0.3$ $-9.29688 \mathrm{E}-0.3$ $-9.30173 \mathrm{E}-03$ $-9.30648 \mathrm{E}-0.3$ $-9.31114 \mathrm{E}-03$

$-9.31571 \mathrm{E}-03$ $-9.32019 \mathrm{E}-03$ $-9.32458 \mathrm{E}-03$ $-9.32888 \mathrm{E}-03$ $-9.33310 \mathrm{E}-03$

$-9.33724 \mathrm{E}-03$ $-9.34130 \mathrm{E}-03$ $-9.34528 \mathrm{E}-03$ $-9.34919 \mathrm{E}-03$ $-9.35302 \mathrm{E}-03$

$-9.35679 \mathrm{E}-03$ $-9.36048 \mathrm{E}-03$ $-9.36411 \mathrm{E}-03$ $-9.36766 \mathrm{E}-03$ $-9.37116 \mathrm{E}-03$

$-9.37459 \mathrm{E}-03$ $-9.37796 \mathrm{E}-03$ $-9.38127 \mathrm{E}-03$ $-9.38452 \mathrm{E}-03$ $-9.38772 \mathrm{E}-03$

$-9.39085 \mathrm{E}-03$ $-9.39394 \mathrm{E}-03$ $-9.39697 \mathrm{E}-03$ $-9.39995 \mathrm{E}-03$ $-9.40288 \mathrm{E}-03$

$-9.40576 \mathrm{E}-03$ $-9.40859 \mathrm{E}-03$ $-9.41137 \mathrm{E}-03$ $-9.41411 \mathrm{E}-03$ $-9.41680 \mathrm{E}-03$

$-9.41946 \mathrm{E}-03$ $-9.42206 \mathrm{E}-03$ $-9.42463 \mathrm{E}-03$ $-9.42715 \mathrm{E}-03$ $-9.42964 \mathrm{E}-03$

$-9.43208 \mathrm{E}-03$ $-9.43449 \mathrm{E}-03$ $-9.43686 \mathrm{E}-03$ $-9.43920 \mathrm{E}-03$ $-9.44149 \mathrm{E}-03$

$-9.44376 \mathrm{E}-03$ $-9.44599 \mathrm{E}-03$ $-9.44818 \mathrm{E}-03$ $-9.45035 \mathrm{E}-03$ $-9.45248 \mathrm{E}-03$

$-9.45458 \mathrm{E}-03$ $-9.45665 \mathrm{E}-03$ $-9.45869 \mathrm{E}-03$ $-9.46070 \mathrm{E}-03$ $-9.46268 \mathrm{E}-03$ 
$p \quad F$

12.10

12.20

12.30

12.40

12.50

12.60

12.70

12.80

12.90

13.00

13.10

13.20

13.30

13.40

13.50

13.60

13.70

13.80

13.90

14.00

14.10

14.20

14.30

14.40

14.50

14.60

14.70

14.80

14.90

15.00

15.10

15.20

15.30

15.40

15.50

15.60

15.70

15.80

15.90

16.00

16.10

16.20

16.30

16.40

16.50

16.60

16.70

16.80

16.90

17.00

17.10

17.20

17.30

17.40

17.50

17.60

17.70

17.80

17.90

18.00 $f_{1}$

$1.04772 \mathrm{E} \quad 00$ $1.04772 \mathrm{E} \quad 00$ $1.04772 \mathrm{E} \quad 00$ $1.04771 \mathrm{E} \quad 00$ $1.04771 \mathrm{E} \quad 00$

$1.04770 \mathrm{E} \quad 00$ $1.04770 \mathrm{E} \quad 00$ $1.04769 \mathrm{E} \quad 00$ $1.04769 \mathrm{E} \quad 00$ $1.04769 \mathrm{E} \quad 00$

$1.04768 \mathrm{E} \quad 00$ $1.04768 \mathrm{E} \quad 00$ $1.04768 \mathrm{E} \quad 00$ $1.04767 \mathrm{E} \quad 00$ $1.04767 \mathrm{E} \quad 00$

$1.04766 \mathrm{E} \quad 00$

$1.04766 \mathrm{E} \quad 00$

$1.04766 \mathrm{E} \quad 00$

$1.04765 \mathrm{E} \quad 00$

$1.04765 \mathrm{E} \quad 00$

$1.04765 \mathrm{E} \quad 00$

$1.04764 \mathrm{E} \quad 00$

$1.04764 \mathrm{E} \quad 00$

$1.04764 \mathrm{E} \quad 00$

$1.04764 \mathrm{E} \quad 00$

$1.04763 \mathrm{E} \quad 00$

$1.04763 \mathrm{E} \quad 00$

$1.04763 \mathrm{E} \quad 00$

$1.04762 \mathrm{E} \quad 00$

$1.04762 \mathrm{E} \quad 00$

$1.04762 \mathrm{E} \quad 00$

$1.04761 \mathrm{E} \quad 00$

$1.04761 \mathrm{E} \quad 00$

$1.04761 \mathrm{E} \quad 00$

$1.04761 \mathrm{E} \quad 00$

$1.04760 \mathrm{E} \quad 00$

$1.04760 \mathrm{E} \quad 00$

$1.04760 \mathrm{E} \quad 00$

$1.04760 \mathrm{E} \quad 00$

$1.04759 \mathrm{E} \quad 00$

$1.04759 \mathrm{E} \quad 00$

$1.04759 \mathrm{E} \quad 00$

$1.04759 \mathrm{E} \quad 00$

$1.04758 \mathrm{E} \quad 00$

$1.04758 \mathrm{E} \quad 00$

$1.04758 \mathrm{E} \quad 00$

$1.04758 \mathrm{E} \quad 00$

$1.04757 \mathrm{E} \quad 00$

$1.04757 \mathrm{E} \quad 00$

$\begin{array}{ll}1.04757 \mathrm{E} & 00\end{array}$

$1.04757 \mathrm{E} \quad 00$

$1.04756 \mathrm{E} \quad 00$

$1.04756 \mathrm{E} \quad 00$

$1.04756 \mathrm{E} \quad 00$

$1.04756 \mathrm{E} \quad 00$

$1.04756 \mathrm{E} \quad 00$ $1.04755 \mathrm{E} \quad 00$ $1.04755 \mathrm{E} \quad 00$ $1.04755 \mathrm{E} \quad 00$ $1.04755 \mathrm{E} \quad 00$ $g_{1}$

$-1.18474 \mathrm{E} \quad 01 \quad-3.25299 \mathrm{E}-01$ $-1.19495 \mathrm{E}$ 01 $-3.30062 \mathrm{E}-01$ $-1.20515 \mathrm{E} 01 \quad-3.34858 \mathrm{E}-01$ $-1.21536 \mathrm{E}$ 01 $-3.39688 \mathrm{E}-01$ $-1.22556 \mathrm{E} 01 \quad-3.44551 \mathrm{E}-01$

$-1.23577 \mathrm{E} \quad 01$ $-1.24597 \mathrm{E} \quad 01$ $-1.25617 \mathrm{E} \quad 01$ $-1.26637 \mathrm{E} 01$ $-1.27657 \mathrm{E} 01$

$-1.28678 \mathrm{E} 01$ $-1.29698 \mathrm{E} 01$ $-1.30718 \mathrm{E} \quad 01$ $-1.31738 \mathrm{E} 01$ $-1.32757 \mathrm{E} 01$

$-1.33777 \mathrm{E} \quad 01$ $-1.34797 \mathrm{E} 01$ $-1.35817 \mathrm{E}$ 01 $-1.36836 \mathrm{E} 01$ $-1.37856 \mathrm{E} 01$

-1.38875 E 01 $-1.39895 \mathrm{E} 01$ $-1.40914 \mathrm{E} \quad 01$ $-1.41934 \mathrm{E} \quad 01$ $-1.42953 \mathrm{E} 01$

$-1.43972 \mathrm{E} \quad 01$ $-1.44992 \mathrm{E} 01$ $-1.46011 \mathrm{E} \quad 01$ $-1.47030 \mathrm{E} 01$ $-1.48049 \mathrm{E} 01$

\section{$-1.49068 \mathrm{E} 01$}

$-1.50087 \mathrm{E} \quad 01$

$-1.51106 \mathrm{E} 01$

$-1.52125 \mathrm{E} \quad 01$

$-1.53143 \mathrm{E} 01$

$-1.54162 \mathrm{E} \quad 01$

$-1.55181 \mathrm{E} 01$

$-1.56199 \mathrm{E} 01$

$-1.57218 \mathrm{E} 01$

$-1.58236 \mathrm{E} 01^{\circ}$

$-1.59255 \mathrm{E} 01$ $-1.60273 \mathrm{E} 01$ $-1.61291 \mathrm{E} 01$ $-1.62310 \mathrm{E} 01$

$-1.63328 \mathrm{E} 01$

$-1.64346 \mathrm{E} \quad 01$ $-1.65364 \mathrm{E}$ 01 $-1.66382 \mathrm{E} 01$ $-1.67400 \mathrm{E} 01$ $-1.68418 \mathrm{E} 01$

$-1.69436 \mathrm{E} \quad 01$ $-1.70454 \mathrm{E} 01$ $-1.71471 \mathrm{E} 01$ $-1.72489 \mathrm{E} 01$ $-1.73507 \mathrm{E} 01$

$-1.74524 \mathrm{E} \quad 01$ $-1.75542 \mathrm{E} 01$ $-1.76559 \mathrm{E} 01$ $-1.77576 \mathrm{E} 01$ $-1.78594 \mathrm{E} \cdot 01$
$-3.49448 \mathrm{E}-01$

$-3.54378 \mathrm{E}-01$

$-3.59342 \mathrm{E}-01$

$-3.64339 \mathrm{E}-01$

$-3.69370 \mathrm{E}-01$

$-3.74434 \mathrm{E}-01$ $-3.79532 \mathrm{E}-01$ $-3.84663 \mathrm{E}-01$ $-3.89828 \mathrm{E}-01$

$-3.95026 \mathrm{E}-01$

$-4.00257 \mathrm{E}-01$

$-4.05523 \mathrm{E}-01$

$-4.10821 \mathrm{E}-01$ $-4.16153 \mathrm{E}-01$

$-4.21518 \mathrm{E}-01$

$-4.26917 \mathrm{E}-01$ $-4.32349 \mathrm{E}-01$ $-4.43314 \mathrm{E}-01$ $-4.48847 \mathrm{E}-01$

$-4.54413 \mathrm{E}-01$ $-4.60013 \mathrm{E}-01$ $-4.65646 \mathrm{E}-01$ $-4.71312 \mathrm{E}-01$ $-4.77012 \mathrm{E}-01$

$-4.82745 \mathrm{E}-01$ $-4.88512 \mathrm{E}-01$ $-4.94312 \mathrm{E}-01$ $-5.00145 \mathrm{E}-01$ $-5.06012 \mathrm{E}-01$

$-5.11913 \mathrm{E}-01$ $-5.17847 \mathrm{E}-01$ $-5.23814 \mathrm{E}-01$ $-5.29814 \mathrm{E}-01$ $-5.35849 . \mathrm{E}-01$

$-5.41916 \mathrm{E}-01$ $-5.48017 \mathrm{E}-0]$ $-5.54151 \mathrm{E}-01$ $-5.66520 \mathrm{E}-01$

$-5.72754 \mathrm{E}-01$ $-5.79022 \mathrm{E}-01$ $-5.85324 \mathrm{E}-01$ $-5.91658 \mathrm{E}-01$ $-5.98026 \mathrm{E}-01$

$-6.04428 \mathrm{E}-01$ $-6.10863 \mathrm{E}-01$ $-6.17331 \mathrm{E}-01$ $-6.23833 \mathrm{E}-01$ $-6.30368 \mathrm{E}-01$

$-6.36936 \mathrm{E}-01$ $-6.43538 \mathrm{E}-01$ $-6.50173 \mathrm{E}-01$ $-6.56842 \mathrm{E}-0]$ $-6.63543 \mathrm{E}-01$ $-4.37815 \mathrm{E}-01$ $-5.60319 \mathrm{E}-01$

$\tan \delta_{1}$

$f_{-1}$

$6.91888 \mathrm{E}-04$ $6.96685 \mathrm{E}-04$ $7.01398 \mathrm{E}-04$

$7.06035 \mathrm{E}-04$

$7.10597 \mathrm{E}-04$

$7.15083 \mathrm{E}-04$

$7.19498 \mathrm{E}-04$

$7.23843 \mathrm{E}-04$

$7.28117 \mathrm{E}-04$

$7.32326 \mathrm{E}-04$

$7.36469 \mathrm{E}-04$

$7.40546 \mathrm{E}-04$

$7.44559 \mathrm{E}-04$

$7.48511 \mathrm{E}-04$

$7.52403 \mathrm{E}-04$

$7.56236 \mathrm{E}-04$

$7.60011 \mathrm{E}-04$

$7.63729 \mathrm{E}-04$

$7.67394 \mathrm{E}-04$

$7.71003 \mathrm{E}-04$

$7.74558 \mathrm{E}-04$

$7.78061 \mathrm{E}-04$

$7.81516 \mathrm{E}-04$

$7.84919 \mathrm{E}-04$

$7.88273 \mathrm{E}-04$

$7.91579 \mathrm{E}-04$

$7.94838 \mathrm{E}-04$

$7.98053 \mathrm{E}-04$

$8.01220 \mathrm{E}-04$

$8.04344 \mathrm{E}-04$

$8.07425 \mathrm{E}-04$

$8.104 .63 \mathrm{E}-04$

$8.13461 \mathrm{E}-04$

$8.16417 \mathrm{E}-04$

$8.19333 \mathrm{E}-04$

$8.22209 \mathrm{E}-04$

$8.25048 \mathrm{E}-04$

$8.27848 \mathrm{E}-04$

$8.30611 \mathrm{E}-04$

$8.33338 \mathrm{E}-04$

$8.36029 \mathrm{E}-04$

$8.38684 \mathrm{E}-04$

$8.41305 \mathrm{E}-04$

$8.43889 \mathrm{E}-04$ 
$\bar{F}$

$1.03661 \mathrm{E}-04$ $1.05381 \mathrm{E}-04$ $1.07116 \mathrm{E}-04$ $1.08864 \mathrm{E}-04$ $1.10627 \mathrm{E}-04$

$1.12404 \mathrm{E}-04$ $1.14194 \mathrm{E}-04$ $1.16000 \mathrm{E}-04$ $1.17819 \mathrm{E}-04$ $1.19652 \mathrm{E}-04$

$1.21500 \mathrm{E}-04$ $1.23361 \mathrm{E}-04$ $1.25237 \mathrm{E}-04$ $1.27127 \mathrm{E}-04$ $1.29031 \mathrm{E}-04$

$1.30950 \mathrm{E}-04$ $1.32882 \mathrm{E}-04$ $1.34829 \mathrm{E}-04$ $1.36789 \mathrm{E}-04$ $1.38764 \mathrm{E}-04$

$1.40754 \mathrm{E}-04$ $1.42757 \mathrm{E}-04$ $1.44774 \mathrm{E}-04$ $1.46806 \mathrm{E}-04$ $1.48851 \mathrm{E}-04$

$1.50911 \mathrm{E}-04$ $1.52985 \mathrm{E}-04$ $1.55074 \mathrm{E}-04$ $1.57176 \mathrm{E}-04$ $1.59292 \mathrm{E}-04$

$1.61423 \mathrm{E}-04$ $1.63568 \mathrm{E}-04$ $1.65727 \mathrm{E}-04$ $1.67900 \mathrm{E}-04$ $1.70087 \mathrm{E}-04$

$1.72289 \mathrm{E}-04$ $1.74504 \mathrm{E}-04$ $1.76734 \mathrm{E}-04$ $1.78978 \mathrm{E}-04$ $1.81236 \mathrm{E}-04$

$1.83508 \mathrm{E}-04$ $1.85794 \mathrm{E}-04$ $1.88095 \mathrm{E}-04$ $1.90410 \mathrm{E}-04$ $1.92738 \mathrm{E}-04$

$1.95081 \mathrm{E}-04$ $1.97438 \mathrm{E}-04$ $1.99810 \mathrm{E}-04$ $2.02195 \mathrm{E}-04$ 2.04595 E- 04

$2.07009 \mathrm{E}-04$ $2.09436 \mathrm{E}-04$ $2.11879 \mathrm{E}-04$ $2.14335 \mathrm{E}-04$ $2.16805 \mathrm{E}-04$

$2.19290 \mathrm{E}-04$ $2.21788 \mathrm{E}-04$ $2.24301 \mathrm{E}-04$ $2.26828 \mathrm{E}-04$ $2.29369 \mathrm{E}-04$ $f_{2}$

$-2.35847 \mathrm{E}-01$

$-2.39847 \mathrm{E}-01$

$-2.43880 \mathrm{E}-01$

$-2.47946 \mathrm{E}-01$

$-2.52046 \mathrm{E}-01$

$-2.56180 \mathrm{E}-01$

$-2.60347 \mathrm{E}-01$

$-2.64548 \mathrm{E}-01$

$-2.68782 \mathrm{E}-01$

$-2.73050 \mathrm{E}-01$

$-2.77351 \mathrm{E}-01$

$-2.81686 \mathrm{E}-01$

$-2.86054 \mathrm{E}-01$

$-2.90456 \mathrm{E}-01$

$-2.94892 \mathrm{E}-01$

$-2.99360 \mathrm{E}-01$

$-3.03863 \mathrm{E}-0 \mathrm{I}$

$-3.08399 \mathrm{E}-01$

$-3.12968 \mathrm{E}-01$

-3.17571 E-01

$-3.22208 \mathrm{E}-01$

$-3.26878 \mathrm{E}-01$

$-3.31582 \mathrm{E}-0 \mathrm{I}$

$-3.36319 \mathrm{E}-01$

$-3.41089 \mathrm{E}-01$

$-3.45893 \mathrm{E}-01$

$-3.50731 \mathrm{E}-01$

$-3.55602 \mathrm{E}-01$

$-3.60507 \mathrm{E}-01$

$-3.65445 \mathrm{E}-01$

$-3.70416 \mathrm{E}-01$

$-3.75421 \mathrm{E}-01$

$-3.80460 \mathrm{E}-01$

$-3.85532 \mathrm{E}-01$

$-3.90638 \mathrm{E}-01$

$-3.95777 \mathrm{E}-01$

$-4.00949 \mathrm{E}-01$

$-4.06155 \mathrm{E}-01$

$-4.11395 \mathrm{E}-01$

$-4.16668 \mathrm{E}-01$

$-4.21974 \mathrm{E}-01$

$-4.27314 \mathrm{E}-01$

$-4.32688 \mathrm{E}-01$

$-4.38095 \mathrm{E}-01$

$-4.43535 \mathrm{E}-01$

$-4.49009 \mathrm{E}-01$

$-4.54516 \mathrm{E}-01$

$-4.60057 \mathrm{E}-01$

$-4.65631 \mathrm{E}-01$

$-4.71239 \mathrm{E}-01$

$-4.76880 \mathrm{E}-01$

$-4.82554 \mathrm{E}-01$

$-4.88263 \mathrm{E}-01$

$-4.94004 \mathrm{E}-01$

$-4.99779 \mathrm{E}-01$

$-5.05587 \mathrm{E}-01$

$-5.11429 \mathrm{E}-01$

$-5.17305 \mathrm{E}-01$

$-5.23213 \mathrm{E}-01$

$-5.29156 \mathrm{E}-01$ $g_{2}$

$-3.84518 \mathrm{E}-03$

$-3.93398 \mathrm{E}-03$

$-4.02412 \mathrm{E}-03$

$-4.11562 \mathrm{E}-03$

$-4.20847 \mathrm{E}-03$

$-4.30270 \mathrm{E}-03$

$-4.39831 \mathrm{E}-03$

$-4.49531 \mathrm{E}-03$

$-4.59372 \mathrm{E}-03$

$-4.69353 \mathrm{E}-03$

$-4.79477 \mathrm{E}-03$

$-4.89743 \mathrm{E}-03$

$-5.00154 \mathrm{E}-03$

$-5.10710 \mathrm{E}-03$

$-5.21412 \mathrm{E}-03$

$-5.32261 \mathrm{E}-03$

$-5.43258 \mathrm{E}-03$

$-5.54404 \mathrm{E}-03$

$-5.65701 \mathrm{E}-03$

$-5.77148 \mathrm{E}-03$

$-5.88747 \mathrm{E}-03$ $-6.00500 \mathrm{E}-03$

$-6.12406 \mathrm{E}-03$

$-6.24467 \mathrm{E}-03$

$-6.36685 \mathrm{E}-03$

$-6.49059 \mathrm{E}-03$

$-6.61591 \mathrm{E}-03$

$-6.74283 \mathrm{E}-03$

$-6.87134 \mathrm{E}-03$

$-7.00146 \mathrm{E}-03$

$-7.13320 \mathrm{E}-03$

$-7.26657 \mathrm{E}-03$

$-7.40158 \mathrm{E}-03$

$-7.53824 \mathrm{E}-03$

$-7.67656 \mathrm{E}-03$

$-7.81655 \mathrm{E}-03$

$-7.95821 \mathrm{E}-03$

$-8.10156 \mathrm{E}-03$

$-8.24662 \mathrm{E}-03$

$-8.39338 \mathrm{E}-03$

$-8.54185 \mathrm{E}-03$

$-8.69206 \mathrm{E}-03$

$-8.84401 \mathrm{E}-03$

$-8.99770 \mathrm{E}-03$

$-9.15315 \mathrm{E}-03$

$-9.31037 \mathrm{E}-03$

$-9.46937 \mathrm{E}-03$

$-9.63015 \mathrm{E}-03$

$-9.79273 \mathrm{E}-03$

$-9.95712 \mathrm{E}-03$

$-1.01233 \mathrm{E}-02$

$-1.02914 \mathrm{E}-02$

$-1.04612 \mathrm{E}-02$

$-1.06329 \mathrm{E}-02$

- $1.08065 \mathrm{E}-02$

$-1.09820 \mathrm{E}-02$

$-1.11593 \mathrm{E}-02$

$-1.13385 \mathrm{E}-02$

$-1.15196 \mathrm{E}-02$

$-1.17026 \mathrm{E}-02$ $\tan \delta_{2}$

$f_{2}$

$9.93228 \mathrm{E} \quad 01$ $9.93526 \mathrm{E} 01$ $9.93818 \mathrm{E}$ ol $9.94106 \mathrm{E} 01$ $9.94389 \mathrm{E} \quad 01$

$9.94667 \mathrm{E} \quad 01$ $9.94941 \mathrm{E} \quad 01$ $9.95210 \mathrm{E} \quad 01$ $9.95474 \mathrm{E} \quad 01$ $9.95735 \mathrm{E} \quad 01$

$9.95991 \mathrm{E} \quad 01$ $9.96243 \mathrm{E} \quad 01$ $9.96491 \mathrm{E}$ ol $9.96736 \mathrm{E} \quad 01$ $9.96976 \mathrm{E}$ ol

$9.97213 \mathrm{E} \quad 01$ $9.97446 \mathrm{E} \quad 01$ $9.97676 \mathrm{E}$ ol $9.97902 \mathrm{E} \quad 01$ $9.98125 \mathrm{E} \quad 01$

$9.98344 \mathrm{E} \quad 01$ $9.98560 \mathrm{E} \quad 01$ $9.98773 \mathrm{E} \quad 01$ $9.98983 \mathrm{E} \quad 01$ $9.99190 \mathrm{E} \quad 01$

$9.99394 \mathrm{E} \quad 01$ $9.99595 \mathrm{E} \quad 01$ $9.99793 \mathrm{E} \quad 01$ $9.99988 \mathrm{E} \quad 01$ $1.00018 \mathrm{E} \quad 02$

$1.00037 \mathrm{E} \quad 02$ $1.00056 \mathrm{E} \quad 02$ $1.00074 \mathrm{E} \quad 02$ $1.00093 \mathrm{E} \quad 02$

$1.00110 \mathrm{E} \quad 02$

$1.00128 \mathrm{E} \quad 02$ $1.00146 \mathrm{E} \quad 02$ $1.00163 \mathrm{E} \quad 02$ $1.00180 \mathrm{E} \quad 02$ $1.00197 \mathrm{E} \quad 02$

$1.00213 \mathrm{E} \quad 02$ $1.00230 \mathrm{E} \quad 02$ $1.00246 \mathrm{E} \quad 02$ $1.00262 \mathrm{E} \quad 02$ $1.00278 \mathrm{E} \quad 02$

$1.00293 \mathrm{E} \quad 02$ $1.00309 \mathrm{E} \quad 02$ $1.00324 \mathrm{E} \quad 02$ $1.00339 \mathrm{E} \quad 02$ $1.00354 \mathrm{E} \quad 02$

$1.00368 \mathrm{E} \quad 02$ $1.00383 \mathrm{E} \quad 02$ $1.00397 \mathrm{E} \quad 02$

$1.00411 \mathrm{E} \quad 02$

$1.00425 \mathrm{E} \quad 02$

$1.00439 \mathrm{E} \quad 02$ $1.00453 \mathrm{E} \quad 02$ $1.00466 \mathrm{E} \quad 02$ $1.00479 \mathrm{E} \quad 02$ $1.00493 \mathrm{E} \quad 02$

$-3.67052 \mathrm{E}-03$

$-3.75586 \mathrm{E}-03$

$-3.84250 \mathrm{E}-03$

$-3.93045 \mathrm{E}-03$

$-4.01974 \mathrm{E}-03$

$-4.11037 \mathrm{E}-03$ $-4.20234 \mathrm{E}-03$

$-4.29567 \mathrm{E}-03$

$-4.39037 \mathrm{E}-03$

$-4.48645 \mathrm{E}-03$

$-4.58392 \mathrm{E}-03$

$-4.68278 \mathrm{E}-03$

$-4.78306 \mathrm{E}-03$

$-4.88475 \mathrm{E}-03$

$-4.98786 \mathrm{E}-03$

$-5.09242 \mathrm{E}-03$

$-5.19842 \mathrm{E}-03$

$-5.30588 \mathrm{E}-03$

$-5.41481 \mathrm{E}-03$

$-5.52522 \mathrm{E}-03$

$-5.63711 \mathrm{E}-03$ $-5.75050 \mathrm{E}-03$

$-5.86539 \mathrm{E}-03$ $-5.98181 \mathrm{E}-03$ $-6.09975 \mathrm{E}-03$

$-6.21922 \mathrm{E}-03$ $-6.34025 \mathrm{E}-03$ $-6.46283 \mathrm{E}-03$ $-6.58697 \mathrm{E}-03$ $-6.71269 \mathrm{E}-03$

$-6.84000 \mathrm{E}-03$ $-6.96891 \mathrm{E}-03$ $-7.09942 \mathrm{E}-03$ $-7.23154 \mathrm{E}-03$ $-7.36529 \mathrm{E}-03$

$-7.50068 \mathrm{E}-03$ $-7.63771 \mathrm{E}-03$ $-7.77640 \mathrm{E}-03$ $-7.91676 \mathrm{E}-03$ $-8.05879 \mathrm{E}-03$

$-8.20250 \mathrm{E}-03$ $-8.34791 \mathrm{E}-03$ 
$p$

18.10

18.20

18.30

18.40

18.50

18.60

18.70

18.80

18.90

19.00

19.10

19.20

19.30

19.40

19.50

19.60

19.70

19.80

19.90

20.00

20.10

20.20

20.30

20.40

20.50

20.60

20.70

20.80

20.90

21.00

21.10

21.20

21.30

21.40

21.50

21.60

21.70

21.80

21.90

22.00

22.10

22.20

22.30

22.40

22.50

22.60

22.70

22.80

22.90

23.00

23.10

23.20

23.30

23.40

23.50

23.60

23.70

23.80

23.90

24.00
$F_{0}$

$f_{1}$

$1.04755 \mathrm{E} \quad 00$

$1.04754 \mathrm{E} \quad 00$

$1.04754 \mathrm{E} \quad 00$

$1.04754 \mathrm{E} \quad 00$

$1.04754 \mathrm{E} \quad 00$

$1.04754 \mathrm{E} \quad 00$

$1.04753 \mathrm{E} \quad 00$

$1.04753 \mathrm{E} \quad 00$

$1.04753 \mathrm{E} \quad 00$

$1.04753 \mathrm{E} \quad 00$

$1.04753 \mathrm{E} \quad 00$

$1.04752 \mathrm{E} \quad 00$

$1.04752 \mathrm{E} \quad 00$

$\begin{array}{ll}1.04752 \mathrm{E} & 00\end{array}$

$1.04752 \mathrm{E} \quad 00$

$\begin{array}{ll}1.04752 \mathrm{E} & 00\end{array}$

$1.04752 \mathrm{E} \quad 00$

$1.04751 \mathrm{E} \quad 00$

$1.04751 \mathrm{E} \quad 00$

$1.04751 \mathrm{E} \quad 00$

$1.04751 \mathrm{E} \quad 00$

$1.04751 \mathrm{E} \quad 00$

$1.04751 \mathrm{E} \quad 00$

$1.04750 \mathrm{E} \quad 00$

$1.04750 \mathrm{E} \quad 00$

$1.04750 \mathrm{E} \quad 00$

$1.04750 \mathrm{~F}, 00$

$1.04750 \mathrm{E} \quad 00$

$1.04750 \mathrm{E} \quad 00$

$1.04749 \mathrm{E} \quad 00$

$1.04749 \mathrm{E} \quad 00$

$1.04749 \mathrm{E} \quad 00$

$1.04749 \mathrm{E} \quad 00$

$1.04749 \mathrm{E} \quad 00$

$1.04749 \mathrm{E} \quad 00$

$1.04748 \mathrm{E} \quad 00$

$1.04748 \mathrm{E} \quad 00$

$1.04748 \mathrm{E} \quad 00$

$1.04748 \mathrm{E} \quad 00$

$1.04748 \mathrm{E} \quad 00$

$1.04748 \mathrm{E} \quad 00$

$1.04748 \mathrm{E} \quad 00$

$1.04747 \mathrm{E} \quad 00$

$1.04747 \mathrm{E} \quad 00$

$1.04747 \mathrm{E} \quad 00$

$1.04747 \mathrm{E} \quad 00$

$1.04747 \mathrm{E} \quad 00$

$1.04747 \mathrm{E} \quad 00$

$1.04747 \mathrm{E} \quad 00$

$1.04746 \mathrm{E} \quad 00$

$1.04746 \mathrm{E} \quad 00$

$1.04746 \mathrm{E} \quad 00$

$1.04746 \mathrm{E} \quad 00$

$1.04746 \mathrm{E} \quad 00$

$1.04746 \mathrm{E} \quad 00$

$1.04746 \mathrm{E} \quad 00$

$1.04746 \mathrm{E} \quad 00$

$1.04745 \mathrm{E} \quad 00$

$1.04745 \mathrm{E} \quad 00$

$1.04745 \mathrm{E} \quad 00$ $g_{1}$

$-1.79611 \mathrm{E} \quad 01$

$-1.80628 \mathrm{E} 01$

$-1.81645 \mathrm{E} 0 \mathrm{l}$

$-1.82662 \mathrm{E} 01$

$-1.83679 \mathrm{E} 01$

$-1.84696 \mathrm{E} \quad 01$

$-1.85713 \mathrm{E} 01$

$-1.86730 \mathrm{E}$ 01

$-1.87747 \mathrm{E} 01$

$-1.88763 \mathrm{E} 01$

$-1.89780 \mathrm{E} 01$

$-1.90796 \mathrm{E} \quad 01$

$-1.91813 \mathrm{E}$ 01

$-1.92829 \mathrm{E} 01$

$-1.93846 \mathrm{E} 01$

$-1.94862 \mathrm{E} 01$

$-1.95878 \mathrm{E} 01$

$-1.96894 \mathrm{E} 01$

$-1.97910 \mathrm{E} 01$

$-1.98926 \mathrm{E} 01$

$-1.99942 \mathrm{E} 01$

$-2.00958 \mathrm{E} \quad 0 \mathrm{l}$

$-2.01974 \mathrm{E} \quad 01$

$-2.02990 \mathrm{E} 01$

$-2.04006 \mathrm{E} 01$

$-2.05021 \mathrm{E} \quad 01$

$-2.06037 \mathrm{E} \quad 01$

$-2.07052 \mathrm{E} \quad 01$

$-2.08068 \mathrm{E} 01$

$-2.09083 \mathrm{E} 0 \mathrm{l}$

$-2.10098 \mathrm{E} \quad 01$

$-2.11114 \mathrm{E} \quad 01$

$-2.12129 \mathrm{E}$ 01

$-2.13144 \mathrm{E} \quad 01$

$-2.14159 \mathrm{E} 01$

$-2.15174 \mathrm{E} \quad 01$

$-2.16189 \mathrm{E} \quad 01$

-2.17203 E 01

$-2.18218 \mathrm{E} 01$

$-2.19233 \mathrm{E}$ ol

$-2.20247 \mathrm{E} \quad 01$ $-2.21262 \mathrm{E} 01$ $-2.22276 \mathrm{E} \quad 01$

$-2.23291 \mathrm{E}$ 01

$-2.24305 \mathrm{E} 01$

$-2.25319 \mathrm{E} \quad 01$

$-2.26334 \mathrm{E} \quad 01$

$-2.27348 \mathrm{E} 01$

$-2.28362 \mathrm{E} 01$

$-2.29376 \mathrm{E} 01$

$-2.30390 \mathrm{E} \quad 01$

$-2.31403 \mathrm{E} 01$

$-2.32417 \mathrm{E} 01$

$-2.33431 \mathrm{E} 01$

$-2.34444 \mathrm{E} \quad 01$

$-2.35458 \mathrm{E}$ ol -2.36471 E 01

$-2.37485 \mathrm{E} 01$

$-2.38498 \mathrm{E} 01$

$-2.39511 \mathrm{E} 01$
$-6.70279 \mathrm{E}-01$

$-6.77048 \mathrm{E}-01$

$-6.83850 \mathrm{E}-01$

$-6.90685 \mathrm{E}-01$

$-6.97554 \mathrm{E}-01$

$-7.04456 \mathrm{E}-01$

$-7.11391 \mathrm{E}-01$

$-7.18360 \mathrm{E}-01$

$-7.25362 \mathrm{E}-01$

$-7.32397 \mathrm{E}-01$

$-7.39466 \mathrm{E}-01$

$-7.46568 \mathrm{E}-01$

$-7.53704 \mathrm{E}-01$

$-7.60872 \mathrm{E}-01$

$-7.68075 \mathrm{E}-01$

$-7.75310 \mathrm{E}-01$

$-7.82579 \mathrm{E}-01$

$-7.89881 \mathrm{E}-01$

$-7.97216 \mathrm{E}-01$

$-8.04585 \mathrm{E}-01$

$-8.11987 \mathrm{E}-01$

$-8.19422 \mathrm{E}-01$

$-8.26891 \mathrm{E}-01$

$-8.34393 \mathrm{E}-01$

$-8.41929 \mathrm{E}-01$

$-8.49497 \mathrm{E}-01$

$-8.57099 \mathrm{E}-01$

$-8.64734 \mathrm{E}-01$

$-8.72403 \mathrm{E}-01$

$-8.80104 \mathrm{E}-01$

$-8.87840 \mathrm{E}-01$

$-8.95608 \mathrm{E}-01$

$-9.03409 \mathrm{E}-01$

$-9.11244 \mathrm{E}-01$

$-9.19113 \mathrm{E}-01$

$-9.27014 \mathrm{E}-01$

$-9.34949 \mathrm{E}-01$

$-9.42917 \mathrm{E}-01$

$-9.50918 \mathrm{E}-01$

$-9.58953 \mathrm{E}-01$

$-9.67021 \mathrm{E}-01$

$-9.75122 \mathrm{E}-01$

$-9.83256 \mathrm{E}-01$

$-9.91424 \mathrm{E}-01$

$-9.99624 \mathrm{E}-01$

$-1.00786 \mathrm{E} 00$

$-1.01613 \mathrm{E} \quad 00$

$-1.02443 \mathrm{E} 00$

$-1.03276 \mathrm{E} \quad 00$

$-1.04113 \mathrm{E} 00$

$-1.04953 \mathrm{E} \quad 00$

$-1.05796 \mathrm{E} 00$

$-1.06643 \mathrm{E} 00$

$-1.07493 \mathrm{E} 00$

$-1.08346 \mathrm{E} 00$

$-1.09203 \mathrm{E} \quad 00$

$-1.10063 \mathrm{E} 00$

$-1.10926 \mathrm{E} 00$

$-1.11793 \mathrm{E} 00$

$-1.12663 \mathrm{E} 00$

$\tan \delta_{1}$

$f_{-1}$

$8.83200 \mathrm{E}-04$

$8.85265 \mathrm{E}-04$

$8.87307 \mathrm{E}-04$

$8.89323 \mathrm{E}-04$

$8.91316 \mathrm{E}-04$

$8.93286 \mathrm{E}-04$

$8.95233 \mathrm{E}-04$

$8.97154 \mathrm{E}-04$

$8.99058 \mathrm{E}-04$

$9.00936 \mathrm{E}-04$

$9.02793 \mathrm{E}-04$

$9.04629 \mathrm{E}-04$

$9.06444 \mathrm{E}-04$

$9.08239 \mathrm{E}-04$

$9.10011 \mathrm{E}-04$

$9.11764 \mathrm{E}-04$

$9.13498 \mathrm{E}-04$

$9.15212 \mathrm{E}-04$

$9.16906 \mathrm{E}-04$

$9.18580 \mathrm{E}-04$

$9.20238 \mathrm{E}-04$

$9.21877 \mathrm{E}-04$

$9.23497 \mathrm{E}-04$

$9.25097 \mathrm{E}-04$

$9.26682 \mathrm{E}-04$

$9.28250 \mathrm{E}-04$

$9.29798 \mathrm{E}-04$

$9.31329 \mathrm{E}-04$

$9.32845 \mathrm{E}-04$

$9.34345 \mathrm{E}-04$

$9.35828 \mathrm{E}-04$

$9.37294 \mathrm{E}-04$

$9.38746 \mathrm{E}-04$

$9.40181 \mathrm{E}-04$

$9.41600 \mathrm{E}-04$

$9.43004 \mathrm{E}-04$

$9.44394 \mathrm{E}-04$

$9.45768 \mathrm{E}-04$

$9.47126 \mathrm{E}-04$ 
18.60

18.70

18.80

18.90

19.00

19.10

19.20

19.30

19.40

19.50

19.60

19.70

19.80

19.90

20.00

20.10

20.20

20.30

20.40

20.50

20.60

20.70

20.80

20.90

21.00

21.10

21.20

21.30

21.40

21.50

21.60

21.70

21.80

21.90

22.00

22.10

22.20

22.30

22.40

22.50

22.60

22.70

22.80

22.90

23.00

23.10

23.20

23.30

23.40

23.50

23.60

23.70

23.80

23.90

24.00
$\bar{F}$

$2.31925 \mathrm{E}-04$ $2.34494 \mathrm{E}-04$ $2.37078 \mathrm{E}-04$ $2.39676 \mathrm{E}-04$ $2.42288 \mathrm{E}-04$

$2.44914 \mathrm{E}-04$ $2.47554 \mathrm{E}-04$ $2.50208 \mathrm{E}-04$ $2.52877 \mathrm{E}-04$ $2.55560 \mathrm{E}-04$

$2.58256 \mathrm{E}-04$ $2.60967 \mathrm{E}-04$ $2.63693 \mathrm{E}-04$ $2.66432 \mathrm{E}-04$ $2.69185 \mathrm{E}-04$

$2.71953 \mathrm{E}-04$ $2.74735 \mathrm{E}-04$ 2.77531 E- 04 $2.80341 \mathrm{E}-04$

$2.83165 \mathrm{E}-04$

$2.86004 \mathrm{E}-04$ $2.88856 \mathrm{E}-04$ $2.91723 \mathrm{E}-04$ $2.94604 \mathrm{E}-04$ $2.97499 \mathrm{E}-04$

$3.00408 \mathrm{E}-04$ 3.03331 E-04 $3.06269 \mathrm{E}-04$ $3.09221 \mathrm{E}-04$

$3.12186 \mathrm{E}-04$

$3.15166 \mathrm{E}-04$ $3.18160 \mathrm{E}-04$ $3.21169 \mathrm{E}-04$ $3.24191 \mathrm{E}-04$ $3.27228 \mathrm{E}-04$

$3.30278 \mathrm{E}-04$ $3.33343 \mathrm{E}-04$ $3.36422 \mathrm{E}-04$ $3.39516 \mathrm{E}-04$ $3.42623 \mathrm{E}-04$

$3.45745 \mathrm{E}-04$ $3.48880 \mathrm{E}-04$ $3.52030 \mathrm{E}-04$ $3.55194 \mathrm{E}-04$ 3.58372 E- 04

$3.61564 \mathrm{E}-04$ $3.64771 \mathrm{E}-04$ $3.67991 \mathrm{E}-04$ $3.71226 \mathrm{E}-04$ $3.74475 \mathrm{E}-04$

$3.77738 \mathrm{E}-04$ $3.81015 \mathrm{E}-04$ $3.84307 \mathrm{E}-04$ $3.87612 \mathrm{E}-04$ $3.90932 \mathrm{E}-04$

$3.94266 \mathrm{E}-04$ $3.97614 \mathrm{E}-04$ $4.00976 \mathrm{E}-04$ $4.04352 \mathrm{E}-04$ $4.07743 \mathrm{E}-04$ $f_{2}$

$-5.35131 \mathrm{E}-01$ $-5.41141 \mathrm{E}-01$ $-5.47183 \mathrm{E}-01$ $-5.53259 \mathrm{E}-01$ $-5.59368 \mathrm{E}-01$

$-5.65511 \mathrm{E}-01$

$-5.71687 \mathrm{E}-01$

$-5.77897 \mathrm{E}-01$

$-5.84140 \mathrm{E}-01$

$-5.90416 \mathrm{E}-01$

$-5.96726 \mathrm{E}-01$

$-6.03070 \mathrm{E}-01$

$-6.09446 \mathrm{E}-01$

$-6.15857 \mathrm{E}-01$

$-6.22300 \mathrm{E}-01$

$-6.28777 \mathrm{E}-01$

$-6.35287 \mathrm{E}-01$

$-6.41831 \mathrm{E}-01$

$-6.48408 \mathrm{E}-01$

$-6.55019 \mathrm{E}-01$

$-6.61663 \mathrm{E}-01$

$-6.68340 \mathrm{E}-01$

$-6.75051 \mathrm{E}-01$

$-6.81795 \mathrm{E}-01$

$-6.88572 \mathrm{E}-01$

$-6.95383 \mathrm{E}-01$ $-7.02227 \mathrm{E}-01$ $-7.09105 \mathrm{E}-01$ $-7.16016 \mathrm{E}-01$ $-7.22961 \mathrm{E}-01$

$-7.29938 \mathrm{E}-01$ $-7.36949 \mathrm{E}-01$ $-7.43994 \mathrm{E}-01$ $-7.51071 \mathrm{E}-01$ $-7.58183 \mathrm{E}-01$

$-7.65327 \mathrm{E}-01$ $-7.72505 \mathrm{E}-01$ $-7.79716 \mathrm{E}-01$ $-7.86961 \mathrm{E}-01$ $-7.94239 \mathrm{E}-01$

$-8.01550 \mathrm{E}-01$ $-8.08895 \mathrm{E}-01$ $-8.16273 \mathrm{E}-01$ $-8.23684 \mathrm{E}-01$ $-8.31129 \mathrm{E}-01$

$-8.38607 \mathrm{E}-01$ $-8.46118 \mathrm{E}-01$ $-8.53663 \mathrm{E}-01$

$-8.61241 \mathrm{E}-01$ -8.68852 E-01

$-8.76497 \mathrm{E}-01$ $-8.84175 \mathrm{E}-01$ $-8.91886 \mathrm{E}-01$ $-8.99631 \mathrm{E}-01$ $-9.07409 \mathrm{E}-01$

$-9.15220 \mathrm{E}-01$ $-9.23065 \mathrm{E}-01$ $-9.30942 \mathrm{E}-01$ $-9.38853 \mathrm{E}-01$ $-9.46798 \mathrm{E}-0 \mathrm{l}$ $g_{2}$

$-1.18875 \mathrm{E}-02$

$-1.20744 \mathrm{E}-02$

$-1.22631 \mathrm{E}-02$

$-1.24539 \mathrm{E}-02$

$-1.26466 \mathrm{E}-02$

$-1.28412 \mathrm{E}-02$

$-1.30378 \mathrm{E}-02$

$-1.32365 \mathrm{E}-02$

$-1.34371 \mathrm{E}-02$

$-1.36397 \mathrm{E}-02$

$-1.38443 \mathrm{E}-02$

$-1.40509 \mathrm{E}-02$

$-1.42596 \mathrm{E}-02$

$-1.44704 \mathrm{E}-02$

$-1.46831 \mathrm{E}-02$

$-1.48980 \mathrm{E}-02$

$-1.51149 \mathrm{E}-02$

$-1.53339 \mathrm{E}-02$

$-1.55550 \mathrm{E}-02$

$-1.57782 \mathbf{E}-02$

$-1.60035 \mathrm{E}-02$ $-1.62310 \mathrm{E}-02$

$-1.64605 \mathrm{E}-02$ $-1.66923 \mathrm{E}-02$

$-1.69261 \mathrm{E}-02$

$-1.71622 \mathrm{E}-02$ $-1.74004 \mathrm{E}-02$ $-1.76407 \mathrm{E}-02$ $-1.78833 \mathrm{E}-02$

$-1.81281 \mathrm{E}-02$

$-1.83751 \mathrm{E}-02$ $-1.86243 \mathrm{E}-02$

$-1.88758 \mathrm{E}-02$

$-1.91294 \mathrm{E}-02$

$-1.93854 \mathrm{E}-02$

$-1.96436 \mathrm{E}-02$ $-1.99040 \mathrm{E}-02$

$-2.01668 \mathrm{E}-02$

$-2.04318 \mathrm{E}-02$

$-2.06992 \mathrm{E}-02$

$-2.09688 \mathrm{E}-02$

$-2.12408 \mathrm{E}-02$

$-2.15151 \mathrm{E}-02$

$-2.17918 \mathrm{E}-02$

$-2.20708 \mathrm{E}-02$

$-2.23521 \mathrm{E}-02$

$-2.26358 \mathrm{E}-02$

$-2.29219 \mathrm{E}-02$

$-2.32104 \mathrm{E}-02$

$-2.35013 \mathrm{E}-02$

$-2.37946 \mathrm{E}-02$

$-2.40903 \mathrm{E}-02$

$-2.43885 \mathrm{E}-02$

$-2.46891 \mathrm{E}-02$

$-2.49921 \mathrm{E}-02$

$-2.52976 \mathrm{E}-02$

$-2.56056 \mathrm{E}-02$

$-2.59160 \mathrm{E}-02$

$-2.62289 \mathrm{E}-02$

$-2.65444 \mathrm{E}-02$

$\tan \delta_{2}$

$1.00506 \mathrm{E} \quad 02$

$1.00518 \mathrm{E} \quad 02$

$1.00531 \mathrm{E} \quad 02$

$1.00544 \mathrm{E} \quad 02$

$1.00556 \mathrm{E} \quad 02$

$1.00568 \mathrm{E} \quad 02$

$1.00580 \mathrm{E} \quad 02$

$1.00592 \mathrm{E} \quad 02$

$1.00604 \mathrm{E} \quad 02$

$1.00616 \mathrm{E} \quad 02$

$\begin{array}{ll}1.00628 \mathrm{E} & 02\end{array}$

$1.00639 \mathrm{E} \quad 02$

$1.00651 \mathrm{E} \quad 02$

$1.00662 \mathrm{E} \quad 02$

$1.00673 \mathrm{E} \quad 02$

$\begin{array}{ll}1.00684 \mathrm{E} & 02\end{array}$

$1.00695 \mathrm{E} \quad 02$

$1.00706 \mathrm{E} \quad 02$

$1.00716 \mathrm{E} \quad 02$

$1.00727 \mathrm{E} \quad 02$

$\begin{array}{ll}1.00737 \mathrm{E} & 02\end{array}$

$1.00748 \mathrm{E} \quad 02$

$1.00758 \mathrm{E} \quad 02$

$1.00768 \mathrm{E} \quad 02$

$1.00778 \mathrm{E} \quad 02$

$1.00788 \mathrm{E} \quad 02$ $1.00798 \mathrm{E} \quad 02$ $\begin{array}{ll}1.00807 \mathrm{E} & 02\end{array}$ $1.00817 \mathrm{E} \quad 02$ $1.00827 \mathrm{E} \quad 02$

$1.00836 \mathrm{E} \quad 02$ $1.00845 \mathrm{E} \quad 02$ $1.00855 \mathrm{E} \quad 02$ $1.00864 \mathrm{E} \quad 02$ $1.00873 \mathrm{E} \quad 02$

$1.00882 \mathrm{E} \quad 02$ $1.00891 \mathrm{E} \quad 02$ $1.00900 \mathrm{E} \quad 02$ $1.00908 \mathrm{E} \quad 02$ $1.00917 \mathrm{E} \quad 02$

$1.00926 \mathrm{E} \quad 02$ $1.00934 \mathrm{E} \quad 02$ 
p

24.10

24.20

24.30

24.40

24.50

24.60

24.70

24.80

24.90

25.00

25.10

25.20

25.30

25.40

25.50

25.60

25.70

25.80

25.90

26.00

26.10

26.20

26.30

26.40

26.50

26.60

26.70

26.80

26.90

27.00

27.10

27.20

27.30

27.40

27.50

27.60

27.70

27.80

27.90

28.00

28.10

28.20

28.30

28.40

28.50

28.60

28.70

28.80

28.90

29.00

29.10

29.20

29.30

29.40

29.50

29.60

29.70

29.80

29.90

30.00

$$
F_{0}
$$

$f_{1}$

$g_{1}$

$\tan \delta_{1}$

$f_{-1}$

$-1.13536 \mathrm{E} \quad 00$ $-1.14412 \mathrm{E} \quad 00$ $-1.15292 \mathrm{E} \quad 00$ $-1.16175 \mathrm{E} 00$ $-1.17062 \mathrm{E} \quad 00$

$-1.17952 \mathrm{E} \quad 00$ $-1.18845 \mathrm{E} 00$ $-1.19742 \mathrm{E} 00$ $-1.20641 \mathrm{E} 00$ $-1.21544 \mathrm{E} \quad 00$

$-1.22451 \mathrm{E} \quad 00$ $-1.23361 \mathrm{E} \quad 00$ $-1.24274 \mathrm{E} \quad 00$ $-1.25190 \mathrm{E} \quad 00$ $-1.26110 \mathrm{E} \quad 00$

$-1.27033 \mathrm{E} \quad 00$ $-1.27959 \mathrm{E} \quad 00$ $-1.28889 \mathrm{E} 00$ $-1.29822 \mathrm{E} \quad 00$ $-1.30758 \mathrm{E} \quad 00$

$-1.31698 \mathrm{E} \quad 00$ $-1.32640 \mathrm{E} 00$ $-1.33587 \mathrm{E} \quad 00$ $-1.34536 \mathrm{E} \quad 00$ $1.04743 \mathrm{E} \quad 00 \quad-2.61783 \mathrm{E}$ 01 $\begin{array}{llll}1.04742 \mathrm{E} & 00 & -2.62795 \mathrm{E} & 01\end{array}$ $\begin{array}{llll}1.04742 \mathrm{E} & 00 & -2.63806 \mathrm{E} & 01\end{array}$ $1.04742 \mathrm{E} \quad 00$

$1.04742 \mathrm{E} \quad 00$ 1.04742 E 00 $1.04742 \mathrm{E} \quad 00$ $1.04742 \mathrm{E} \quad 00$

$1.04742 \mathrm{E} \quad 00$

$1.04742 \mathrm{E} \quad 00$ $1.04741 \mathrm{E} \quad 00$ $1.04741 \mathrm{E} \quad 00$ $1.04741 \mathrm{E} \quad 00$ $1.04741 \mathrm{E} \quad 00$

$1.04741 \mathrm{E} \quad 00$ $1.04741 \mathrm{E} \quad 00$

$1.04741 \mathrm{E} \quad 00$

$1.04741 \mathrm{E} \quad 00$

$1.04741 \mathrm{E} \quad 00$

$1.04741 \mathrm{E} \quad 00$ $1.04740 \mathrm{E} \quad 00$ $1.04740 \mathrm{E} \quad 00$ $1.04740 \mathrm{E} \quad 00$ $1.04740 \mathrm{E} \quad 00$

$1.04740 \mathrm{E} \quad 00$ $1.04740 \mathrm{E} \quad 00$ $1.04740 \mathrm{E} \quad 00$ $1.04740 \mathrm{E} \quad 00$ $1.04740 \mathrm{E} \quad 00$

$1.04740 \mathrm{E} \quad 00$ $1.04739 \mathrm{E} \quad 00$

$1.04739 \mathrm{E} \quad 00$

$1.04739 \mathrm{E} \quad 00$

$1.04739 \mathrm{E} \quad 00$

$1.04739 \mathrm{E} \quad 00$ $1.04739 \mathrm{E} \quad 00$ $1.04739 \mathrm{E} \quad 00$ $1.04739 \mathrm{E} \quad 00$ $1.04739 \mathrm{E} 00$ -1.35489 E 00

$-2.64817 \mathrm{E} 01$

$-2.65828 \mathrm{E} \quad 01$ $-2.66840 \mathrm{E} \quad 01$ $-2.67851 \mathrm{E} \quad 01$ -2.68862 E 01 -2.69873 E 01

-2.70883 E 01 $-2.71894 \mathrm{E} \quad 01$ $-2.72905 \mathrm{E} 01$ $-2.73915 \mathrm{E} \quad 01$ $-2.74926 \mathrm{E} \quad 01$

$-2.75936 \mathrm{E} \quad 01$ $-2.76947 \mathrm{E} 01$ $\begin{array}{lll}-2.77957 \mathrm{E} & 01\end{array}$ $-2.78967 \mathrm{E} 01$ $-2.79977 \mathrm{E} \quad 01$

$-1.36445 \mathrm{E} \quad 00$ $-1.37405 \mathrm{E} \quad 00$ $-1.38367 \mathrm{E} 00$ $-1.39334 \mathrm{E} \quad 00$ $-1.40303 \mathrm{E} 00$

$-1.41276 \mathrm{E} \quad 00$ $-1.42252 \mathrm{E} \quad 00$ $-1.43231 \mathrm{E} 00$ $-1.44214 \mathrm{E} \quad 00$ $-1.45200 \mathrm{E} \quad 00$

$-1.46189 \mathrm{E} \quad 00$ $-1.47182 \mathrm{E} \quad 00$ $-1.48177 \mathrm{E} \quad 00$ $-1.49177 \mathrm{E} 00$ $-1.50179 \mathrm{E} 00$

$-1.51185 \mathrm{E} \quad 00$ $-1.52194 \mathrm{E} \quad 00$ $-1.53206 \mathrm{E} 00$ $-1.54222 \mathrm{E} 00$ $-1.55241 \mathrm{E} 00$

$-1.56264 \mathrm{E} \quad 00$ $-1.57289 \mathrm{E} 00$ $-1.58318 \mathrm{E} 00$ $-1.59350 \mathrm{E} \quad 00$ $-1.60386 \mathrm{E} \quad 00$

$-1.61425 \mathrm{E} \quad 00$ $-1.62467 \mathrm{E} 00$ $-1.63512 \mathrm{E} 00$ $-1.64561 \mathrm{E} 00$

$-1.66669 \mathrm{E} \quad 00$ $-1.67727 \mathrm{E} 00$ $-1.68789 \mathrm{E} 00$ $-1.69855 \mathrm{E} \quad 00$ $-1.70923 \mathrm{E} 00$ $-1.65613 \mathrm{E} 00$
$9.73682 \mathrm{E}-04$ $9.74749 \mathrm{E}-04$ $9.75805 \mathrm{E}-04$ $9.76854 \mathrm{E}-04$ $9.77889 \mathrm{E}-04$

$9.78915 \mathrm{E}-04$ $9.79931 \mathrm{E}-04$ $9.80935 \mathrm{E}-04$ $9.81929 \mathrm{E}-04$ $9.82913 \mathrm{E}-04$

$9.83887 \mathrm{E}-04$ $9.84850 \mathrm{E}-04$ $9.85806 \mathrm{E}-04$ $9.86752 \mathrm{E}-04$ $9.87687 \mathrm{E}-04$

$9.88610 \mathrm{E}-04$ $9.89529 \mathrm{E}-04$ $9.90437 \mathrm{E}-04$ $9.91334 \mathrm{E}-04$ $9.92224 \mathrm{E}-04$

$9.93103 \mathrm{E}-04$ $9.93973 \mathrm{E}-04$ $9.94837 \mathrm{E}-04$ $9.95692 \mathrm{E}-04$ $9.96537 \mathrm{E}-04$

$9.97374 \mathrm{E}-04$ $9.98204 \mathrm{E}-04$ $9.99022 \mathrm{E}-04$ $9.99835 \mathrm{E}-04$ $1.00064 \mathrm{E}-03$

$1.00143 \mathrm{E}-03$ $1.00222 \mathrm{E}-0.3$ $1.00377 \mathrm{E}-03$ $1.00454 \mathrm{E}-03$

$1.00529 \mathrm{E}-03$ $1.00604 \mathrm{E}-03$ $1.00678 \mathrm{E}-03$ $1.00751 \mathrm{E}-03$ $1.00824 \mathrm{E}-03$

$1.00896 \mathrm{E}-03$ $1.01038 \mathrm{E}-03$ $1.01176 \mathrm{E}-03$

$1.01245 \mathrm{E}-03$ $1.01312 \mathrm{E}-03$ $1.01446 \mathrm{E}-03$ $1.01511 \mathrm{E}-03$

$1.01576 \mathrm{E}-03$ $1.01640 \mathrm{E}-03$ $1.01704 \mathrm{E}-03$

$1.01767 \mathrm{E}-03$

$1.01829 \mathrm{E}-03$

$1.01891 \mathrm{E}-03$ $1.01952 \mathrm{E}-03$ $1.02013 \mathrm{E}-03$ $1.02131 \mathrm{E}-03$ $1.00300 \mathrm{E}-03$ $1.00967 \mathrm{E}-03$ $1.01107 \mathrm{E}-03$ $1.01379 \mathrm{E}-03$ $1.02072 \mathrm{E}-0.3$

$-1.10083 \mathrm{E} \quad 00$ $-1.10943 \mathrm{E} \quad 00$ $-1.11806 \mathrm{E} \quad 00$ $-1.12673 \mathrm{E} 00$ $-1.13542 \mathrm{E} 00$

$-1.14416 \mathrm{E} \quad 00$ $-1.15292 \mathrm{E} 00$ $-1.16172 \mathrm{E} 00$ -1.17055 E 00 $-1.17941 \mathrm{E} 00$

$-1.18831 \mathrm{E} \quad 00$ $-1.19724 \mathrm{E} \quad 00$ $-1.20620 \mathrm{E} \quad 00$ $-1.21520 \mathrm{E} \quad 00$ $-1.22422 \mathrm{E} 00$

$-1.23329 \mathrm{E} \quad 00$ $-1.24238 \mathrm{E} \quad 00$ $-1.25151 \mathrm{E} 00$ $-1.26067 \mathrm{E} 00$ $-1.26987 \mathrm{E} 00$

$-1.27910 \mathrm{E} \quad 00$ $-1.28836 \mathrm{E} \quad 00$ $-1.29765 \mathrm{E} \quad 00$ $-1.30698 \mathrm{E} 00$ $-1.31634 \mathrm{E} \quad 00$

$-1.32574 \mathrm{E} \quad 00$ $-1.33516 \mathrm{E} \quad 00$ $-1.34462 \mathrm{E} 00$ $-1.35412 \mathrm{E} 00$ $-1.36364 \mathrm{E} \quad 00$

$-1.37320 \mathrm{E} \quad 00$ $-1.38279 \mathrm{E} \quad 00$ $-1.39242 \mathrm{E} 00$ $-1.40208 \mathrm{E} 00$ $-1.41177 \mathrm{E} \quad 00$

$-1.42150 \mathrm{E} \quad 00$ $-1.43125 \mathrm{E} 00$ $-1.44105 \mathrm{E} 00$ $-1.45087 \mathrm{E} \quad 00$ $-1.46073 \mathrm{E} \quad 00$

$-1.47062 \mathrm{E} \quad 00$ $-1.48054 \mathrm{E} 00$ 
ELECTRON RADIAL FUNCTIONS

$Z=2 \quad A=4 \quad \rho=1.2 A^{1 / 3} F$

Elecetron

$\bar{F}$

$4.11148 \mathrm{E}-04$ $4.14566 \mathrm{E}-04$ $4.17999 \mathrm{E}-04$

$4.21446 \mathrm{E}-04$

$4.24908 \mathrm{E}-04$

$4.28383 \mathrm{E}-04$

$4.31873 \mathrm{E}-04$

$4.35376 \mathrm{E}-04$

$4.38894 \mathrm{E}-04$

$4.42426 \mathrm{E}-04$

4. $45972 \mathrm{E}-04$

$4.49533 \mathrm{E}-04$

$4.53107 \mathrm{E}-04$

$4.56696 \mathrm{E}-04$

$4.60298 \mathrm{E}-04$

$4.63915 \mathrm{E}-04$

$4.67547 \mathrm{E}-04$

$4.71192 \mathrm{E}-04$

4.74851 E-04

$4.78525 \mathrm{E}-04$

$4.82212 \mathrm{E}-04$

$4.85914 \mathrm{E}-04$

$4.89630 \mathrm{E}-04$

$4.93360 \mathrm{E}-04$

$4.97105 \mathrm{E}-04$

$5.00863 \mathrm{E}-04$

$5.04636 \mathrm{E}-04$

$5.08423 \mathrm{E}-04$

$5.12224 \mathrm{E}-04$

$5.16039 \mathrm{E}-04$

$5.19868 \mathrm{E}-04$

$5.23711 \mathrm{E}-04$

$5.27569 \mathrm{E}-04$

$5.31441 \mathrm{E}-04$

$5.35327 \mathrm{E}-04$

$5.39227 \mathrm{E}-04$

$5.43141 \mathrm{E}-04$

$5.47069 \mathrm{E}-04$

$5.51012 \mathrm{E}-04$

$5.54968 \mathrm{E}-04$

$5.58939 \mathrm{E}-04$

$5.62924 \mathrm{E}-04$

$5.66923 \mathrm{E}-04$

$5.70937 \mathrm{E}-04$

$5.74964 \mathrm{E}-04$

$5.79006 \mathrm{E}-04$

$5.83061 \mathrm{E}-04$

$5.87131 \mathrm{E}-04$

$5.91215 \mathrm{E}-04$

$5.95313 \mathrm{E}-04$

$5.99426 \mathrm{E}-04$ $6.03552 \mathrm{E}-04$ $6.07693 \mathrm{E}-04$ $6.11848 \mathrm{E}-04$

$6.16017 \mathrm{E}-04$

$6.20200 \mathrm{E}-04$ $6.24397 \mathrm{E}-04$ $6.28609 \mathrm{E}-04$ $6.32834 \mathrm{E}-04$ $6.37074 \mathrm{E}-04$ $f_{2}$

$g_{2}$

$-9.54776 \mathrm{E}-01$

$-9.62787 \mathrm{E}-01$

$-9.70831 \mathrm{E}-01$

$-9.78909 \mathrm{E}-01$

$-9.87020 \mathrm{E}-01$

$-9.95164 \mathrm{E}-01$

$-1.00334 \mathrm{E} \quad 00$

$-1.01155 \mathrm{E} 00$

$-1.01980 \mathrm{E} \quad 00$

$-1.02807 \mathrm{E} 00$

$-1.03638 \mathrm{E} \quad 00$

$-1.04473 \mathrm{E} 00$

$-1.05310 \mathrm{E} 00$

$-1.06151 \mathrm{E} 00$

$-1.06996 \mathrm{E} 00$

$-1.07843 \mathrm{E} \quad 00$

$-1.08695 \mathrm{E} 00$

$-1.09549 \mathrm{E} 00$

$-1.10407 \mathrm{E} 00$

$-1.11267 \mathrm{E} 00$

$-1.12132 \mathrm{E} \quad 00$

$-1.12999 \mathrm{E} 00$

$-1.13870 \mathrm{E} 00$

-1.14745 E 00

$-1.15622 \mathrm{E} 00$

$-1.16503 \mathrm{E} 00$

$-1.17387 \mathrm{E} 00$

-1.18275 E 00

$-1.19166 \mathrm{E} 00$

$-1.20060 \mathrm{E} \quad 00$

$-1.20958 \mathrm{E} \quad 00$

$-1.21858 \mathrm{E} 00$

$-1.22763 \mathrm{E} 00$

$-1.23670 \mathrm{E} \quad 00$

$-1.24581 \mathrm{E} 00$

$-1.25495 \mathrm{E} \quad 00$

$-1.26412 \mathrm{E} 00$

$-1.27333 \mathrm{E} 00$

$-1.28257 \mathrm{E} \quad 00$

$-1.29185 \mathrm{E} 00$

$-1.30115 \mathrm{E} \quad 00$

$-1.31049 \mathrm{E} \quad 00$

$-1.31987 \mathrm{E} \quad 00$

$-1.32927 \mathrm{E} 00$

$-1.33871 \mathrm{E} 00$

$-1.34819 \mathrm{E} \quad 00$

$-1.35769 \mathrm{E} 00$

$-1.36723 \mathrm{E} 00$

$-1.37681 \mathrm{E} \quad 00$

$-1.38641 \mathrm{E} 00$

$-1.39605 \mathrm{E} \quad 00$

$-1.40572 \mathrm{E} 00$

$-1.41543 \mathrm{E} \quad 00$

$-1.42516 \mathrm{E} 00$

$-1.43494 \mathrm{E} \quad 00$

$-1.44474 \mathrm{E} \quad 00$

$-1.45458 \mathrm{E} 00$

$-1.46445 \mathrm{E} \quad 00$

$-1.47435 \mathrm{E} \quad 00$

$-1.48429 \mathrm{E} \quad 00$
$-2.68623 \mathrm{E}-02$ $-2.71828 \mathrm{E}-02$

$-2.75057 \mathrm{E}-02$

$-2.78312 \mathrm{E}-02$

$-2.81593 \mathrm{E}-02$

$-2.84899 \mathrm{E}-02$

$-2.88231 \mathrm{E}-02$

$-2.91589 \mathrm{E}-02$

$-2.94972 \mathrm{E}-02$

$-2.98381 \mathrm{E}-02$

$-3.01817 \mathrm{E}-02$

$-3.05278 \mathrm{E}-02$

$-3.08766 \mathrm{E}-02$

$-3.12280 \mathrm{E}-02$

$-3.15821 \mathrm{E}-02$

$-3.19388 \mathrm{E}-02$

$-3.22982 \mathrm{E}-02$

$-3.26603 \mathrm{E}-02$

$-3.30250 \mathrm{E}-02$

$-3.33924 \mathrm{E}-02$

$-3.37626 \mathrm{E}-02$

$-3.41354 \mathrm{E}-02$

$-3.45110 \mathrm{E}-02$

$-3.48893 \mathrm{E}-02$

$-3.52704 \mathrm{E}-02$

$-3.56542 \mathrm{E}-02$

$-3.60407 \mathrm{E}-02$

$-3.64301 \mathrm{E}-02$

$-3.68222 \mathrm{E}-02$

$-3.72171 \mathrm{E}-02$

$-3.76148 \mathrm{E}-02$

$-3.80153 \mathrm{E}-02$

$-3.84187 \mathrm{E}-02$

$-3.88249 \mathrm{E}-02$

$-3.92339 \mathrm{E}-02$

$-3.96458 \mathrm{E}-02$

$-4.00605 \mathrm{E}-02$

$-4.04781 \mathrm{E}-02$

$-4.08986 \mathrm{E}-02$

$-4.13219 \mathrm{E}-02$

$-4.17482 \mathrm{E}-02$

$-4.21774 \mathrm{E}-02$

$-4.26095 \mathrm{E}-02$

$-4.30445 \mathrm{E}-02$

$-4.34824 \mathrm{E}-02$

$-4.39234 \mathrm{E}-02$

$-4.43672 \mathrm{E}-02$

$-4.48141 \mathrm{E}-02$

$-4.52639 \mathrm{E}-02$

$-4.57167 \mathrm{E}-02$

$-4.61725 \mathrm{E}-02$

$-4.66313 \mathrm{E}-02$

$-4.70931 \mathrm{E}-02$

$-4.75580 \mathrm{E}-02$

$-4.80259 \mathrm{E}-02$

$-4.84968 \mathrm{E}-02$

$-4.89708 \mathrm{E}-02$

$-4.94478 \mathrm{E}-02$

$-4.99280 \mathrm{E}-02$

$-5.04112 \mathrm{E}-02$ $\tan \delta_{2}$

$f_{-2}$

$1.01082 \mathrm{E} \quad 02$ $1.01089 \mathrm{E} \quad 02$

$1.01096 \mathrm{E} \quad 02$

$1.01103 \mathrm{E} \quad 02$

$1.01110 \mathrm{E} \quad 02$

$1.01117 \mathrm{E} \quad 02$

$1.01123 \mathrm{E} \quad 02$

$1.01130 \mathrm{E} \quad 02$

$1.01137 \mathrm{E} \quad 02$

$1.01144 \mathrm{E} \quad 02$

$1.01150 \mathrm{E} \quad 02$

$1.01157 \mathrm{E} \quad 02$

$1.01163 \mathrm{E} \quad 02$

$1.01170 \mathrm{E} \quad 02$

$1.01176 \mathrm{E} \quad 02$

$1.01182 \mathrm{E} \quad 02$

$1.01189 \mathrm{E} \quad 02$

$1.01195 \mathrm{E} \quad 02$

$1.01201 \mathrm{E} \quad 02$

$1.01207 \mathrm{E} \quad 02$

$1.01213 \mathrm{E} \quad 02$

$1.01219 \mathrm{E} \quad 02$

$1.01225 \mathrm{E} \quad 02$

$1.01231 \mathrm{E} \quad 02$

$1.01237 \mathrm{E} \quad 02$

$1.01243 \mathrm{E} \quad 02$

$1.01249 \mathrm{E} \quad 02$

$1.01254 \mathrm{E} \quad 02$

$1.01260 \mathrm{E} \quad 02$

$1.01266 \mathrm{E} \quad 02$

$1.01271 \mathrm{E} \quad 02$

$1.01277 \mathrm{E} \quad 02$

$\begin{array}{ll}1.01282 \mathrm{E} & 02\end{array}$

$1.01288 \mathrm{E} \quad 02$

$1.01293 \mathrm{E} \quad 02$

$1.01299 \mathrm{E} \quad 02$

$1.01304 \mathrm{E} \quad 02$

$1.01310 \mathrm{E} \quad 02$

$1.01315 \mathrm{E} \quad 02$

$1.01320 \mathrm{E} \quad 02$

$1.01325 \mathrm{E} \quad 02$

$1.01331 \mathrm{E} \quad 02$

$1.01336 \mathrm{E} \quad 02$

$1.01341 \mathrm{E} \quad 02$

$1.01346 \mathrm{E} \quad 02$

$1.01351 \mathrm{E} \quad 02$

$1.01356 \mathrm{E} \quad 02$

$1.01361 \mathrm{E} \quad 02$

$1.01366 \mathrm{E} \quad 02$

$1.01371 \mathrm{E} \quad 02$

$1.01375 \mathrm{E} \quad 02$

$1.01380 \mathrm{E} \quad 02$

$1.01385 \mathrm{E} \quad 02$

$1.01390 \mathrm{E} \quad 02$

$1.01395 \mathrm{E} \quad 02$

$1.01399 \mathrm{E} \quad 02$

$1.01404 \mathrm{E} \quad 02$

$1.01408 \mathrm{E} \quad 02$

$1.01413 \mathrm{E} .02$

$1.01418 \mathrm{E} \quad 02$

$-2.60344 \mathrm{E}-02$ $-2.63474 \mathrm{E}-02$

$-2.66629 \mathrm{E}-02$

$-2.69809 \mathrm{E}-02$

$-2.73014 \mathrm{E}-02$

$-2.76245 \mathrm{E}-02$

$-2.79500 \mathrm{E}-02$

$-2.82781 \mathrm{E}-02$

$-2.86088 \mathrm{E}-02$

$-2.89420 \mathrm{E}-02$

$-2.92778 \mathrm{E}-02$

$-2.96162 \mathrm{E}-02$

$-2.99572 \mathrm{E}-02$

$-3.03008 \mathrm{E}-02$

$-3.06470 \mathrm{E}-02$

$-3.09958 \mathrm{E}-02$

$-3.13472 \mathrm{E}-02$

$-3.17013 \mathrm{E}-02$

$-3.20580 \mathrm{E}-02$

$-3.24174 \mathrm{E}-02$

$-3.27795 \mathrm{E}-02$

$-3.31442 \mathrm{E}-02$

$-3.35116 \mathrm{E}-02$

$-3.38818 \mathrm{E}-02$

$-3.42546 \mathrm{E}-02$

$-3.46302 \mathrm{E}-02$

$-3.50085 \mathrm{E}-02$

$-3.53895 \mathrm{E}-02$

$-3.57733 \mathrm{E}-02$

$-3.61598 \mathrm{E}-02$

$-3.65491 \mathrm{E}-02$

$-3.69412 \mathrm{E}-02$

$-3.73361 \mathrm{E}-02$

$-3.77337 \mathrm{E}-02$

$-3.81342 \mathrm{E}-02$

$-3.85375 \mathrm{E}-02$

$-3.89436 \mathrm{E}-02$

$-3.93526 \mathrm{E}-02$

$-3.97644 \mathrm{E}-02$

$-4.01791 \mathrm{E}-02$

$-4.05966 \mathrm{E}-02$

$-4.10170 \mathrm{E}-02$ 
ELECTRON RADIAL FUNCTIONS

Electron

p

30.10

30.20

30.30

30.40

30.50

30.60

30.70

30.80

30.90

31.00

31.10

31.20

31.30

31.40

31.50

31.60

31.70

31.80

31.90

32.00

32.10

32.20

32.30

32.40

32.50

32.60

32.70

32.80

32.90

33.00

33.10

33.20

33.30

33.40

33.50

33.60

33.70

33.80

33.90

34.00

34.10

34.20

34.30

34.40

34.50

34.60

34.70

34.80

34.90

35.00

35.10

35.20

35.30

35.40

35.50

35.60

35.70

35.80

35.90

36.00
$Z=2$

$f_{1}$

$\begin{array}{llll}1.04739 \mathrm{E} & 00 & -3.01169 \mathrm{E} & 01\end{array}$ $\begin{array}{llll}1.04739 \mathrm{E} & 00 & -3.02177 \mathrm{E} & 01\end{array}$ $\begin{array}{llll}1.04738 \mathrm{E} & 00 & -3.03185 \mathrm{E} & 01\end{array}$ $\begin{array}{llll}1.04738 \mathrm{E} & 00 & -3.04193 \mathrm{E} & 01\end{array}$ $1.04738 \mathrm{E} \quad 00$

$-3.05201 \mathrm{E} \quad 01$

$1.04738 \mathrm{E} \quad 00$

$1.04738 \mathrm{E} \quad 00$

$1.04738 \mathrm{E} \quad 00$

$1.04738 \mathrm{E} \quad 00$

$1.04738 \mathrm{E} \quad 00$

$1.04738 \mathrm{E} \quad 00$

$1.04738 \mathrm{E} \quad 00$

$1.04738 \mathrm{E} \quad 00$

$1.04737 \mathrm{E} \quad 00$

$1.04737 \mathrm{E} \quad 00$

$1.04737 \mathrm{E} \quad 00$

$1.04737 \mathrm{E} \quad 00$

$1.04737 \mathrm{E} \quad 00$

$1.04737 \mathrm{E} \quad 00$

$1.04737 \mathrm{E} \quad 00$

$1.04737 \mathrm{E} \quad 00$

$1.04737 \mathrm{E} \quad 00$

$1.04737 \mathrm{E} \quad 00$

$1.04737 \mathrm{E} \quad 00$

$1.04737 \mathrm{E} \quad 00$

$1.04736 \mathrm{E} \quad 00$

$1.04736 \mathrm{E} \quad 00$

$1.04736 \mathrm{E} \quad 00$

$1.04736 \mathrm{E} \quad 00$

$1.04736 \mathrm{E} \quad 00$

$1.04736 \mathrm{E} \quad 00$

$1.04736 \mathrm{E} \quad 00$

$1.04736 \mathrm{E} \quad 00$

$1.04736 \mathrm{E} \quad 00$

$1.04736 \mathrm{E} \quad 00$

$1.04736 \mathrm{E} \quad 00$ $1.04736 \mathrm{E} \quad 00$

$1.04735 \mathrm{E} \quad 00$

$1.04735 \mathrm{E} \quad 00$

$1.04735 \mathrm{E} \quad 00$

$1.04735 \mathrm{E} \quad 00$

$1.04735 \mathrm{E} \quad 00$

$1.04735 \mathrm{E} \quad 00$

$1.04735 \mathrm{E} \quad 00$

$1.04735 \mathrm{E} \quad 00$

$1.04735 \mathrm{E} \quad 00$

$1.04735 \mathrm{E} \quad 00$

$1.04735 \mathrm{E} \quad 00$

$1.04735 \mathrm{E} \quad 00$

$1.04735 \mathrm{E} \quad 00$

$1.04734 \mathrm{E} \quad 00$

$1.04734 \mathrm{E} \quad 00$

$1.04734 \mathrm{E} \quad 00$

$1.04734 \mathrm{E} \quad 00$

$1.04734 \mathrm{E} \quad 00$

$1.04734 \mathrm{E} \quad 00$

$1.04734 \mathrm{E} \quad 00$

$1.04734 \mathrm{E} \quad 00$

$1.04734 \mathrm{E} \quad 00$

$1.04734 \mathrm{E} \quad 00$
$-3.06209 \mathrm{E} \quad 01$

$-3.07216 \mathrm{E} \quad 01$

$-3.08224 \mathrm{E} \quad 01$

$-3.09232 \mathrm{E} \quad 01$

$-3.10239 \mathrm{E} 01$

$-3.11246 \mathrm{E} 01$

$-3.12254 \mathrm{E} \quad 01$

$-3.13261 \mathrm{E}$ 01

$-3.14268 \mathrm{E}$ 01

$-3.15275 \mathrm{E} 01$

$-3.16282 \mathrm{E} \quad 01$

$-3.17289 \mathrm{E} \quad 01$

$-3.18295 \mathrm{E} \quad 01$

$-3.19302 \mathrm{E} 01$

$-3.20309 \mathrm{E} 01$

$-3.21315 \mathrm{E} \quad 01$

$-3.22321 \mathrm{E} 01$

$-3.23328 \mathrm{E} 01$

$-3.24334 \mathrm{E} 01$

$-3.25340 \mathrm{E} 01$

$-3.26346 \mathrm{E} 01$

$-3.27352 \mathrm{E} \quad 01$

$-3.28357 \mathrm{E} 01$

$-3.29363 \mathrm{E} 01$

$-3.30369 \mathrm{E} 01$

$-3.31374 \mathrm{E} \quad 01$

$-3.32380 \mathrm{E}$ 01

$-3.33385 \mathrm{E}$ 01

$-3.34390 \mathrm{E}$ 01

$-3.35395 \mathrm{E} 01$

$-3.36400 \mathrm{E} \quad 01$ $-3.38410 \mathrm{E} \quad 01$ $-3.39415 \mathrm{E}$ 01

$-3.40419 \mathrm{E} 01$

$-3.41424 \mathrm{E} 01$ $-3.42428 \mathrm{E} \quad 01$

$-3.43433 \mathrm{E}$ 01

$-3.44437 \mathrm{E} \quad 01$

$-3.45441 \mathrm{E} 01$

$-3.46445 \mathrm{E}$ 0I

$-3.47449 \mathrm{E} 01$

$-3.48453 \mathrm{E} 01$

$-3.49456 \mathrm{E} 01$

$-3.50460 \mathrm{E} 01$

$-3.51463 \mathrm{E} \quad 01$ $-3.52467 \mathrm{E} \quad 01$ $-3.53470 \mathrm{E} \quad 01$ $-3.54474 \mathrm{E} \quad 01$

$-3.55477 \mathrm{E} \quad 01$

$-3.56480 \mathrm{E} 01$ $-3.57483 \mathrm{E} 01$ $-3.58485 \mathrm{E}$ 01 $-3.59488 \mathrm{E} \quad 01$ $-3.60491 \mathrm{E} 01$ $-3.37405 \mathrm{E}$ 01 $g_{1}$

$A=4$

$\rho=1.2 A^{1 / 3} F$

$-1.71995 \mathrm{E} \quad 00$ $-1.73070 \mathrm{E} \quad 00$ $-1.74149 \mathrm{E} \quad 00$ $-1.75231 \mathrm{E} \quad 00$ $-1.76316 \mathrm{E} \quad 00$

$-1.77404 \mathrm{E} 00$ $-1.78496 \mathrm{E} \quad 00$ $-1.79591 \mathrm{E} 00$ $-1.80689 \mathrm{E} \quad 00$ $-1.81791 \mathrm{E} 00$

$-1.82896 \mathrm{E} \quad 00$ $-1.84004 \mathrm{E} \quad 00$ $-1.85115 \mathrm{E} \quad 00$ $-1.86230 \mathrm{E} \quad 00$ $-1.87348 \mathrm{E} 00$

$-1.88469 \mathrm{E} 00$ $-1.89594 \mathrm{E} \quad 00$ $-1.90722 \mathrm{E} \quad 00$ $-1.91853 \mathrm{E} 00$ $-1.92988 \mathrm{E} \quad 00$

$-1.94126 \mathrm{E} \quad 00$ $-1.95267 \mathrm{E} 00$ $-1.96411 \mathrm{E} \quad 00$ $-1.97559 \mathrm{E} \quad 00$ $-1.98710 \mathrm{E} 00$

$-1.99864 \mathrm{E} \quad 00$ $-2.01022 \mathrm{E} \quad 00$ $-2.02183 \mathrm{E} 00$ $-2.03347 \mathrm{E} \quad 00$ $-2.04514 \mathrm{E} \quad 00$

$-2.05685 \mathrm{E} \quad 00$ $-2.06859 \mathrm{E} \quad 00$ $-2.08036 \mathrm{E} \quad 00$ $-2.09217 \mathrm{E} \quad 00$ $-2.10401 \mathrm{E} \quad 00$

$-2.11588 \mathrm{E} \quad 00$ $-2.12778 \mathrm{E} \quad 00$ $-2.13972 \mathrm{E} \quad 00$ $-2.15169 \mathrm{E} \quad 00$ $-2.16370 \mathrm{E} \quad 00$

$-2.17573 \mathrm{E} \quad 00$ $-2.18780 \mathrm{E} \quad 00$ $-2.19990 \mathrm{E} \quad 00$ $-2.21204 \mathrm{E} \quad 00$ $-2.22420 \mathrm{E} 00$

$-2.23640 \mathrm{E} \quad 00$ $-2.24864 \mathrm{E} \quad 00$ $-2.26090 \mathrm{E} \quad 00$ $-2.27320 \mathrm{E} \quad 00$ $-2.28553 \mathrm{E} \quad 00$

$-2.29790 \mathrm{E} \quad 00$ $-2.31030 \mathrm{E} \quad 00$ $-2.32273 \mathrm{E} \quad 00$ $-2.33519 \mathrm{E} \quad 00$ $-2.34768 \mathrm{E} \quad 00$

$-2.36021 \mathrm{E} \quad 00$ $-2.37277 \mathrm{E} \quad 00$ $-2.38537 \mathrm{E} \quad 00$ $-2.39800 \mathrm{E} \quad 00$ $-2.41066 \mathrm{E} 00$

$\tan \delta_{1}$

$1.02190 \mathrm{E}-03$

$1.02248 \mathrm{E}-03$

$1.02305 \mathrm{E}-03$

$1.02362 \mathrm{E}-03$

$1.02418 \mathrm{E}-03$

$1.02474 \mathrm{E}-03$

$1.02529 \mathrm{E}-03$

$1.02584 \mathrm{E}-03$

$1.02637 \mathrm{E}-03$

$1.02691 \mathrm{E}-03$

$1.02743 \mathrm{E}-03$

$1.02796 \mathrm{E}-03$

$1.02847 \mathrm{E}-03$

$1.02898 \mathrm{E}-03$

$1.02949 \mathrm{E}-03$

$1.02999 \mathrm{E}-03$

$1.03049 \mathrm{E}-03$

$1.03098 \mathrm{E}-03$

$1.03146 \mathrm{E}-03$

$1.03194 \mathrm{E}-03$

$1.03242 \mathrm{E}-03$

$1.03289 \mathrm{E}-03$

$1.03335 \mathrm{E}-03$

$1.03381 \mathrm{E}-03$

$1.03426 \mathrm{E}-03$

$1.03471 \mathrm{E}-03$ $1.03516 \mathrm{E}-03$

$1.03559 \mathrm{E}-03$

$1.03603 \mathrm{E}-03$

$1.03646 \mathrm{E}-03$

$1.03688 \mathrm{E}-03$

$1.03730 \mathrm{E}-03$

$1.03772 \mathrm{E}-03$

$1.03813 \mathrm{E}-03$

$1.03854 \mathrm{E}-03$

$1.03894 \mathrm{E}-03$

$1.03933 \mathrm{E}-03$

$1.03973 \mathrm{E}-03$

$1.04011 \mathrm{E}-03$

$1.04050 \mathrm{E}-03$

$1.04088 \mathrm{E}-03$

$1.04125 \mathrm{E}-03$

$1.04162 \mathrm{E}-03$

$1.04199 \mathrm{E}-03$

$f_{-1}$ 


\section{$\bar{F}$}

30.10

30.20

30.30

30.40

30.50

30.60

30.70

30.80

30.90

31.00

31.10

31.20

31.30

31.40

31.50

31.60

31.70

31.80

31.90

32.00

32.10

32.20

32.30

32.40

32.50

32.60

32.70

32.80

32.90

33.00

33.10

33.20

33.30

33.40

33.50

33.60

33.70

33.80

33.90

34.00

34.10

34.20

34.30

34.40

34.50

34.60

34.70

34.80

34.90

35.00

35.10

35.20

35.30

35.40

35.50

35.60

35.70

35.80

35.90

36.00
$6.41328 \mathrm{E}-04$ $6.45596 \mathrm{E}-04$ $6.49878 \mathrm{E}-04$ $6.54175 \mathrm{E}-04$ $6.58485 \mathrm{E}-04$

$6.62810 \mathrm{E}-04$ $6.67149 \mathrm{E}-04$ $6.71502 \mathrm{E}-04$ $6.75869 \mathrm{E}-04$ $6.80250 \mathrm{E}-04$

$6.84645 \mathrm{E}-04$ $6.89055 \mathrm{E}-04$ $6.93479 \mathrm{E}-04$ $6.97917 \mathrm{E}-04$ $7.02369 \mathrm{E}-04$

$7.06835 \mathrm{E}-04$ $7.11315 \mathrm{E}-04$ $7.15810 \mathrm{E}-04$ $7.20319 \mathrm{E}-04$ 7.2484.1 E-04

$7.29378 \mathrm{E}-04$ $7.33930 \mathrm{E}-04$ $7.38495 \mathrm{E}-04$ $7.43074 \mathrm{E}-04$ $7.47668 \mathrm{E}-04$

$7.52276 \mathrm{E}-04$ $7.56898 \mathrm{E}-04$

$7.61534 \mathrm{E}-04$

$7.66184 \mathrm{E}-04$

$7.70848 \mathrm{E}-04$

$7.75527 \mathrm{E}-04$

$7.80220 \mathrm{E}-04$

$7.84926 \mathrm{E}-04$

$7.89647 \mathrm{E}-04$

$7.94383 \mathrm{E}-04$

$7.99132 \mathrm{E}-04$ $8.03895 \mathrm{E}-04$

$8.08673 \mathrm{E}-04$

$8.13465 \mathrm{E}-04$

8.18271 E-04

$8.23091 \mathrm{E}-04$ $8.27925 \mathrm{E}-04$

$8.32773 \mathrm{E}-04$

$8.37636 \mathrm{E}-04$

$8.42513 \mathrm{E}-04$

$8.47403 \mathrm{E}-04$ $8.52308 \mathrm{E}-04$

$8.57228 \mathrm{E}-04$

$8.62161 \mathrm{E}-04$

8.67108E-04

$8.72070 \mathrm{E}-04$ $8.77046 \mathrm{E}-04$ $8.82036 \mathrm{E}-04$ $8.87040 \mathrm{E}-04$ $8.92058 \mathrm{E}-04$

$8.97090 \mathrm{E}-04$ $9.02137 \mathrm{E}-04$ $9.07198 \mathrm{E}-04$ $9.12272 \mathrm{E}-04$ $9.17362 \mathrm{E}-04$ $f_{2}$

$g_{2}$

$-1.49426 \mathrm{E} \quad 00 \quad-5.08975 \mathrm{E}-02$ $-1.50426 \mathrm{E} \quad 00 \quad-5.13869 \mathrm{E}-02$ $-1.51430 \mathrm{E} \quad 00 \quad-5.18795 \mathrm{E}-02$ $-1.52437 \mathrm{E} \quad 00 \quad-5.23751 \mathrm{E}-02$ $-1.53447 \mathrm{E} \quad 00 \quad-5.28739 \mathrm{E}-02$

$-1.54461 \mathrm{E} \quad 00$ $-1.55478 \mathrm{E} \quad 00$ $-1.56498 \mathrm{E} \quad 00$ $-1.57521 \mathrm{E} \quad 00$ $-1.58548 \mathrm{E} \quad 00$

$-1.59578 \mathrm{E} \quad 00$ $-1.60612 \mathrm{E} \quad 00$ $-1.61648 \mathrm{E} \quad 00$ $-1.62688 \mathrm{E} 00$ $-1.63732 \mathrm{E} \quad 00$

$-1.64778 \mathrm{E} \quad 00$ $-1.65828 \mathrm{E} 00$ $-1.66882 \mathrm{E} \quad 00$ $-1.67938 \mathrm{E} 00$ $-1.68998 \mathrm{E} \quad 00$

$-1.70061 \mathrm{E} \quad 00$ $-1.71128 \mathrm{E} 00$ $-1.72198 \mathrm{E} \quad 00$ $-1.73271 \mathrm{E} \quad 00$ -1.74347 E 00

$-1.75427 \mathrm{E} \quad 00$ $-1.76510 \mathrm{E} 00$ $-1.77596 \mathrm{E} \quad 00$ $-1.78686 \mathrm{E} \quad 00$

$-1.79778 \mathrm{E} \quad 00$

$-1.80875 \mathrm{E} \quad 00$

$-1.81974 \mathrm{E} \quad 00$

$-1.83077 \mathrm{E} \quad 00$

$-1.84183 \mathrm{E} \quad 00$

$-1.85293 \mathrm{E} 00$

$-1.86405 \mathrm{E} \quad 00$ $-1.87521 \mathrm{E} \quad 00$ $-1.8864 .1 \mathrm{E} 00$ $-1.89763 \mathrm{E} \quad 00$ $-1.90889 \mathrm{E} \quad 00$

$-5.33758 \mathrm{E}-02$ $-5.38809 \mathrm{E}-02$ $-5.43892 \mathrm{E}-02$ $-5.49006 \mathrm{E}-02$ $-5.54152 \mathrm{E}-02$

$-5.59330 \mathrm{E}-02$ $-5.64540 \mathrm{E}-02$ $-5.69782 \mathrm{E}-02$ $-5.75056 \mathrm{E}-02$ $-5.80363 \mathrm{E}-02$

$-5.85702 \mathrm{E}-02$ $-5.91074 \mathrm{E}-02$ $-5.96478 \mathrm{E}-02$ $-6.01915 \mathrm{E}-02$ $-6.07384 \mathrm{E}-02$

$-6.12887 \mathrm{E}-02$ $-6.18423 \mathrm{E}-02$ $-6.23991 \mathrm{E}-02$ $-6.29593 \mathrm{E}-02$ $-6.35228 \mathrm{E}-02$

$-6.40897 \mathrm{E}-02$ $-6.46599 \mathrm{E}-02$ $-6.52334 \mathrm{E}-02$ $-6.58104 \mathrm{E}-02$ $-6.63907 \mathrm{E}-02$

$-6.69744 \mathrm{E}-02$ $-6.75615 \mathrm{E}-02$ $-6.81520 \mathrm{E}-02$ $-6.87459 \mathrm{E}-02$ $-6.93432 \mathrm{E}-02$

$-6.99440 \mathrm{E}-02$ $-7.05482 \mathrm{E}-02$ $-7.11559 \mathrm{E}-02$ $-7.17670 \mathrm{E}-02$ $-7.23817 \mathrm{E}-02$

$-1.92018 \mathrm{E} \quad 00$ $-1.93151 \mathrm{E} \quad 00$ $-1.94287 \mathrm{E} \quad 00$ $-1.95426 \mathrm{E} \quad 00$ $-1.96568 \mathrm{E} 00$

$-1.97714 \mathrm{E} \quad 00$ $-1.98863 \mathrm{E} \quad 00$ $-2.00015 \mathrm{E} \quad 00$ $-2.01171 \mathrm{E} \quad 00$ $-2.02329 \mathrm{E} \quad 00$

$-2.03491 \mathrm{E} \quad 00$ $-2.04657 \mathrm{E} \quad 00$ $-2.05826 \mathrm{E} \quad 00$ $-2.06998 \mathrm{E} \quad 00$ $-2.08173 \mathrm{E} 00$

$-2.09352 \mathrm{E} \quad 00$ $-2.10534 \mathrm{E} \quad 00$ $-2.11719 \mathrm{E} \quad 00$ $-2.12907 \mathrm{E} \quad 00$ $-2.14099 \mathrm{E} \quad 00$ $\tan \delta_{2}$

$f_{2}$

$1.01422 \mathrm{E} \quad 02$ $1.01427 \mathrm{E} \quad 02$ $1.01431 \mathrm{E} \quad 02$ $1.01436 \mathrm{E} \quad 02$ $1.01440 \mathrm{E} \quad 02$

$1.01444 \mathrm{E} \quad 02$ $1.01449 \mathrm{E} \quad 02$ $\begin{array}{ll}1.01453 \mathrm{E} & 02\end{array}$

$1.01457 \mathrm{E} \quad 02$

$1.01462 \mathrm{E} \quad 02$

$1.01466 \mathrm{E} \quad 02$

$1.01470 \mathrm{E} \quad 02$

$1.01474 \mathrm{E} \quad 02$

$1.01478 \mathrm{E} \quad 02$

$1.01482 \mathrm{E} \quad 02$

$1.01487 \mathrm{E} \quad 02$

$1.01491 \mathrm{E} \quad 02$

$1.01495 \mathrm{E} \quad 02$

$1.01499 \mathrm{E} \quad 02$

$1.01503 \mathrm{E} \quad 02$

$\begin{array}{lll}1.01507 \mathrm{E} & 02\end{array}$

$\begin{array}{lll}1.01511 \mathrm{E} & 02\end{array}$

$\begin{array}{ll}1.01514 \mathrm{E} & 02\end{array}$

$1.01518 \mathrm{E} \quad 02$

$1.01522 \mathrm{E} \quad 02$

$\begin{array}{ll}1.01526 \mathrm{E} & 02\end{array}$

$1.01530 \mathrm{E} \quad 02$

$1.01534 \mathrm{E} \quad 02$

$1.01537 \mathrm{E} \quad 02$

$1.01541 \mathrm{E} \quad 02$

$1.01545 \mathrm{E} \quad 02$

$\begin{array}{ll}1.01549 \mathrm{E} & 02\end{array}$

$\begin{array}{ll}1.01552 \mathrm{E} & 02\end{array}$

$1.01556 \mathrm{E} \quad 02$

$1.01560 \mathrm{E} \quad 02$

$\begin{array}{ll}1.01563 \mathrm{E} & 02\end{array}$

$1.01567 \mathrm{E} \quad 02$

$\begin{array}{ll}1.01570 \mathrm{E} & 02\end{array}$

$1.01574 \mathrm{E} \quad 02$

$1.01577 \mathrm{E} \quad 02$

$1.01581 \mathrm{E} \quad 02$ $1.01584 \mathrm{E} \quad 02$ $\begin{array}{ll}1.01588 \mathrm{E} & 02\end{array}$ $1.01591 \mathrm{E} \quad 02$ $1.01595 \mathrm{E} \quad 02$

$1.01598 \mathrm{E} \quad 02$ $\begin{array}{ll}1.01601 \mathrm{E} & 02\end{array}$ $1.01605 \mathrm{E} \quad 02$ $\begin{array}{ll}1.01608 \mathrm{E} & 02\end{array}$

$1.01611 \mathrm{E} \quad 02$

$\begin{array}{ll}1.01615 \mathrm{E} & 02\end{array}$ $1.01618 \mathrm{E} \quad 02$ $1.01621 \mathrm{E} \quad 02$ $\begin{array}{ll}1.01625 \mathrm{E} & 02\end{array}$ $1.01628 \mathrm{E} \quad 02$

$1.01631 \mathrm{E} \quad 02$ $1.01634 \mathrm{E} \quad 02$ $\begin{array}{lll}1.01637 \mathrm{E} & 02\end{array}$ $1.01641 \mathrm{E} \quad 02$ $1.01644 \mathrm{E} \quad 02$

$-4.95643 \mathrm{E}-02$ $-5.00443 \mathrm{E}-02$ $-5.05273 \mathrm{E}-02$ $-5.10135 \mathrm{E}-02$ $-5.15028 \mathrm{E}-02$

$-5.19951 \mathrm{E}-02$ 
ELECTRON RADIAL FUNCTIONS

Electron

$F_{0}$

36.10

36.20

36.30

36.40

36.50

36.60

36.70

36.80

36.90

37.00

37.10

37.20

37.30

37.40

37.50

37.60

37.70

37.80

37.90

38.00

38.10

38.20

38.30

38.40

38.50

38.60

38.70

38.80

38.90

39.00

39.10

39.20

39.30

39.40

39.50

39.60

39.70

39.80

39.90

40.00

40.10

40.20

40.30

40.40

40.50

40.60

40.70

40.80

40.90

41.00

41.10

41.20

41.30

41.40

41.50

41.60

41.70

41.80

41.90

42.00

$$
Z=2 \quad A=4
$$

$g_{1}$

$1.04734 \mathrm{E} \quad 00$ $1.04734 \mathrm{E} \quad 00$ $1.04734 \mathrm{E} \quad 00$ $1.04734 \mathrm{E} 00$

$1.04733 \mathrm{E} \quad 00$

$1.04733 \mathrm{E} \quad 00$ $1.04733 \mathrm{E} \quad 00$ $1.04733 \mathrm{E} \quad 00$ $1.04733 \mathrm{E} 00$ $1.04733 \mathrm{E} \quad 00$

$1.04733 \mathrm{E} \quad 00$ $1.04733 \mathrm{E} \quad 00$ $1.04733 \mathrm{E} \quad 00$ $1.04733 \mathrm{E} \quad 00$ $1.04733 \mathrm{E} \quad 00$

$1.04733 \mathrm{E} \quad 00$ $1.04733 \mathrm{E} \quad 00$ $1.04733 \mathrm{E} \quad 00$ $1.04732 \mathrm{E} \quad 00$

$1.04732 \mathrm{E} \quad 00$

$1.04732 \mathrm{E} \quad 00$ $1.04732 \mathrm{E} \quad 00$ $1.04732 \mathrm{E} \quad 00$ $1.04732 \mathrm{E} \quad 00$ $1.04732 \mathrm{E} \quad 00$

$1.04732 \mathrm{E} \quad 00$ $1.04732 \mathrm{E} \quad 00$ $1.04732 \mathrm{E} \quad 00$ $1.04732 \mathrm{E} \quad 00$ $1.04732 \mathrm{E} \quad 00$

$1.04732 \mathrm{E} \quad 00$ $1.04732 \mathrm{E} \quad 00$ $1.04732 \mathrm{E} \quad 00$ $1.04732 \mathrm{E} \quad 00$ $1.04731 \mathrm{E} \quad 00$

$1.04731 \mathrm{E} \quad 00$ $1.04731 \mathrm{E} \quad 00$ $1.04731 \mathrm{E} \quad 00$ $1.04731 \mathrm{E} \quad 00$ $1.04731 \mathrm{E} \quad 00$

$1.04731 \mathrm{E} \quad 00$ $1.04731 \mathrm{E} \quad 00$ $1.04731 \mathrm{E} \quad 00$ $1.04731 \mathrm{E} \quad 00$ $1.04731 \mathrm{E} \quad 00$

$1.04731 \mathrm{E} \quad 00$ $1.04731 \mathrm{E} \quad 00$ $1.04731 \mathrm{E} \quad 00$ $1.04731 \mathrm{E} \quad 00$

$1.04730 \mathrm{E} \quad 00$

$1.04730 \mathrm{E} \quad 00$ $1.04730 \mathrm{E} \quad 00$ $1.04730 \mathrm{E} \quad 00$ $1.04730 \mathrm{E} \quad 00$ $1.04730 \mathrm{E} \quad 00$

$1.04730 \mathrm{E} \quad 00$ $1.04730 \mathrm{E} \quad 00$ $1.04730 \mathrm{E} \quad 00$ $1.04730 \mathrm{E} \quad 00$ $1.04730 \mathrm{E} \quad 00$ $f_{1}$

$-3.61493 \mathrm{E} 01$ $-3.62496 \mathrm{E} \quad 01$ $-3.63498 \mathrm{E} 0 \mathrm{I}$ $-3.64500 \mathrm{E}$ 0I $-3.65502 \mathrm{E} 01$

$-3.66504 \mathrm{E} \quad 01$ $-3.67506 \mathrm{E} 01$ $-3.68508 \mathrm{E} 01$ $-3.69510 \mathrm{E} 01$ $-3.70511 \mathrm{E} 01$

$-3.71513 \mathrm{E} 01$ $-3.72514 \mathrm{E} 01$ $-3.73515 \mathrm{E} 01$ $-3.74516 \mathrm{E} 01$ $-3.75517 \mathrm{E} \quad 01$

$-3.76518 \mathrm{E} 01$ $-3.77519 \mathrm{E} 01$ $-3.78520 \mathrm{E} \quad 01$ $-3.79521 \mathrm{E} 01$ $-3.80521 \mathrm{E} \quad 01$

$-3.81522 \mathrm{E} 01$ $-3.82522 \mathrm{E} 0 \mathrm{l}$ $-3.83522 \mathrm{E} 01$ $-3.84522 \mathrm{E} 01$ $-3.85522 \mathrm{E}$ 01

$-3.86522 \mathrm{E} 01$ $-3.87522 \mathrm{E} 01$ $-3.88521 \mathrm{E} 01$ $-3.89521 \mathrm{E} 01$ $-3.90520 \mathrm{E} 01$

$-3.91520 \mathrm{E}$ 01 $-3.92519 \mathrm{E} 01$ $-3.93518 \mathrm{E} 01$ $-3.94517 \mathrm{E} 01$ $-3.95516 \mathrm{E} \quad 01$

$-3.96514 \mathrm{E} \quad 01$ $-3.97513 \mathrm{E}$ 01 $-3.98512 \mathrm{E} 01$ $-3.99510 \mathrm{E} 01$ $-4.00508 \mathrm{E} 01$

$-4.01507 \mathrm{E} 01$ $-4.02505 \mathrm{E} 01$ $-4.03503 \mathrm{E} 01$ $-4.04501 \mathrm{E} 01$ $-4.05498 \mathrm{E} 01$

$-4.06496 \mathrm{E} \quad 01$ $-4.07494 \mathrm{E} 01$ $-4.08491 \mathrm{E} 01$ $-4.09488 \mathrm{E} \quad 01$ -4.10485 E 01

$-4.11482 \mathrm{E} \quad 01$ $-4.12479 \mathrm{E}$ 01 $-4.13476 \mathrm{E} 01$ $-4.14473 \mathrm{E} 01$ $-4.15470 \mathrm{E} 01$

$-4.16466 \mathrm{E} \quad 01$ $-4.17462 \mathrm{E} 01$ $-4.18459 \mathrm{E} 01$ $-4.19455 \mathrm{E} 01$ $-4.20451 \mathrm{E} 01$
$-2.42335 \mathrm{E} \cdot 00$ $-2.43607 \mathrm{E} \quad 00$ $-2.44883 \mathrm{E} \quad 00$

$-2.46162 \mathrm{E} \quad 00$

$-2.47445 \mathrm{E} 00$

$-2.48730 \mathrm{E} \quad 00$ $-2.50019 \mathrm{E} 00$

$-2.51311 \mathrm{E} 00$ $-2.52607 \mathrm{E} \quad 00$

$-2.53905 \mathrm{E} \quad 00$

$-2.55207 \mathrm{E} 00$

$-2.56513 \mathrm{E} 00$

$-2.57821 \mathrm{E} \quad 00$

$-2.59133 \mathrm{E} 00$

$-2.60448 \mathrm{E} \quad 00$

$-2.61766 \mathrm{E} \quad 00$ $-2.63088 \mathrm{E} \quad 00$ $-2.64413 \mathrm{E} \quad 00$ $-2.65741 \mathrm{E} \quad 00$

$-2.67073 \mathrm{E} 00$

$-2.68407 \mathrm{E} \quad 00$ $-2.69745 \mathrm{E} 00$ $-2.71086 \mathrm{E} \quad 00$ $-2.72431 \mathrm{E} \quad 00$ $-2.73779 \mathrm{E} 00$

$-2.75130 \mathrm{E} \quad 00$ $-2.76484 \mathrm{E} \quad 00$

$-2.77842 \mathrm{E} 00$

$-2.79202 \mathrm{E} \quad 00$

$-2.80567 \mathrm{E} \quad 00$

$-2.81934 \mathrm{E} \quad 00$

$-2.83305 \mathrm{E} \quad 00$

$-2.84678 \mathrm{E} \quad 00$

$-2.86056 \mathrm{E} \quad 00$

$-2.87436 \mathrm{E} \quad 00$

$-2.88820 \mathrm{E} \quad 00$

$-2.90207 \mathrm{E} \quad 00$

$-2.91597 \mathrm{E} \quad 00$

$-2.92990 \mathrm{E} \quad 00$

$-2.94387 \mathrm{E} 00$

$-2.95787 \mathrm{E} \quad 00$

$-2.97190 \mathrm{E} 00$

$-2.98597 \mathrm{E} \quad 00$

$-3.00007 \mathrm{E} 00$

$-3.01420 \mathrm{E} 00$

$-3.02836 \mathrm{E} 00$ $-3.04255 \mathrm{E} \quad 00$ $-3.05678 \mathrm{E} \quad 00$

$-3.07104 \mathrm{E} \quad 00$

$-3.08534 \mathrm{E} \quad 00$

$-3.09966 \mathrm{E} \quad 00$ $-3.11402 \mathrm{E} \quad 00$

$-3.12841 \mathrm{E} 00$ $-3.14283 \mathrm{E} \quad 00$ $-3.15729 \mathrm{E} 00$

$-3.17178 \mathrm{E} \quad 00$ $-3.18630 \mathrm{E} 00$ $-3.20085 \mathrm{E} \quad 00$

$-3.21544 \mathrm{E} \quad 00$

$-3.23006 \mathrm{E} \quad 00$

$\tan \delta_{1}$

$1.04759 \mathrm{E}-03$ $1.04788 \mathrm{E}-03$ $1.04817 \mathrm{E}-03$

$1.04845 \mathrm{E}-03$

$1.04873 \mathrm{E}-03$

$1.04901 \mathrm{E}-03$

$1.04929 \mathrm{E}-03$

$1.04956 \mathrm{E}-03$

$1.04983 \mathrm{E}-03$

$1.05009 \mathrm{E}-03$

$1.05035 \mathrm{E}-03$

$1.05061 \mathrm{E}-03$

$1.05086 \mathrm{E}-03$

$1.05111 \mathrm{E}-03$

$1.05135 \mathrm{E}-03$

$1.05160 \mathrm{E}-03$

$1.05184 \mathrm{E}-03$

$1.05207 \mathrm{E}-03$

$1.05230 \mathrm{E}-03$

$1.05253 \mathrm{E}-03$

$1.05276 \mathrm{E}-03$

$1.05298 \mathrm{E}-03$

$1.05320 \mathrm{E}-03$

$1.05342 \mathrm{E}-03$

$1.05363 \mathrm{E}-03$

$1.05384 \mathrm{E}-03$

$1.05404 \mathrm{E}-03$

$1.05425 \mathrm{E}-03$

$1.05445 \mathrm{E}-03$

$1.05464 \mathrm{E}-03$

$1.05483 \mathrm{E}-03$

$1.05503 \mathrm{E}-03$

$1.05521 \mathrm{E}-03$

$1.05540 \mathrm{E}-03$

$1.05558 \mathrm{E}-03$

$1.05576 \mathrm{E}-03$

$1.05593 \mathrm{E}-03$

$1.05610 \mathrm{E}-03$

$1.05627 \mathrm{E}-03$

$1.05643 \mathrm{E}-03$

$1.05660 \mathrm{E}-03$

$1.05675 \mathrm{E}-03$ 
ELECTRON RADIAL FUNCTIONS

$Z=2 \quad A=4 \quad \rho=1.2 A^{1 / 3} F$

Filectron

$\bar{F}$

$9.22465 \mathrm{E}-04$ $9.27582 \mathrm{E}-04$ $9.32714 \mathrm{E}-04$ $9.37859 \mathrm{E}-04$ $9.43019 \mathrm{E}-04$

$9.48193 \mathrm{E}-04$ $9.53381 \mathrm{E}-04$ $9.58583 \mathrm{E}-04$ $9.63800 \mathrm{E}-04$ $9.69030 \mathrm{E}-04$

$9.74275 \mathrm{E}-04$ $9.79534 \mathrm{E}-04$ $9.84807 \mathrm{E}-04$ $9.90094 \mathrm{E}-04$ $9.95395 \mathrm{E}-04$

37.50

37.60

37.70

37.80

37.90

38.00

38.10

38.20

38.30

38.40

38.50

38.60

38.70

38.80

38.90

39.00

39.10

39.20

39.30

39.40

39.50

39.60

39.70

39.80

39.90

40.00

40.10

40.20

40.30

40.40

40.50

40.60

40.70

40.80

40.90

41.00

41.10

41.20

41.30

41.40

41.50

41.60

41.70

41.80

41.90

42.00

$1.00071 \mathrm{E}-03$ $1.00604 \mathrm{E}-03$ $1.01138 \mathrm{E}-03$ $1.01674 \mathrm{E}-03$ $1.02211 \mathrm{E}-03$

$1.02750 \mathrm{E}-03$ $1.03290 \mathrm{E}-03$ $1.03832 \mathrm{E}-03$ $1.04374 \mathrm{E}-03$ $1.04919 \mathrm{E}-03$

$1.05464 \mathrm{E}-03$ $1.06012 \mathrm{E}-03$ $1.06560 \mathrm{E}-03$ $1.07110 \mathrm{E}-03$ $1.07661 \mathrm{E}-03$

$1.08214 \mathrm{E}-03$ $1.08768 \mathrm{E}-03$ $1.09324 \mathrm{E}-03$ $1.09881 \mathrm{E}-03$ $1.10439 \mathrm{E}-03$

$1.10999 \mathrm{E}-03$ $1.11561 \mathrm{E}-03$ $1.12123 \mathrm{E}-03$ $1.12687 \mathrm{E}-03$ $1.13253 \mathrm{E}-03$

$1.13820 \mathrm{E}-03$ $1.14388 \mathrm{E}-03$ $1.14958 \mathrm{E}-03$ $1.15529 \mathrm{E}-03$ $1.16102 \mathrm{E}-03$

$1.16676 \mathrm{E}-03$

$1.17251 \mathrm{E}-03$

$1.17828 \mathrm{E}-03$

$1.18406 \mathrm{E}-03$

$1.18986 \mathrm{E}-03$

$1.19567 \mathrm{E}-03$

$1.20150 \mathrm{E}-03$

$1.20733 \mathrm{E}-03$

$1.21319 \mathrm{E}-03$

$1.21906 \mathrm{E}-03$

$1.22494 \mathrm{E}-03$ $1.23083 \mathrm{E}-03$ $1.23674 \mathrm{E}-03$ $1.24267 \mathrm{E}-03$ $1.24860 \mathrm{E}-03$ $f_{2}$
$-2.15294 \mathrm{E} \quad 00$

$-2.16493 \mathrm{E} \quad 00$

$-2.17694 \mathrm{E} \quad 00$

$-2.18899 \mathrm{E} \quad 00$

$-2.20107 \mathrm{E} \quad 00$

$-2.21319 \mathrm{E} 00$ $-2.22534 \mathrm{E} \quad 00$ $-2.23752 \mathrm{E} \quad 00$ $-2.24973 \mathrm{E} \quad 00$ $-2.26198 \mathrm{E} 00$

$-2.27426 \mathrm{E} \quad 00$ $-2.28657 \mathrm{E} \quad 00$ $-2.29892 \mathrm{E} \quad 00$ $-2.31130 \mathrm{E} \quad 00$ $-2.32371 \mathrm{E} \quad 00$

$g_{2}$

$-8.61085 \mathrm{E}-02$

$-8.68020 \mathrm{E}-02$

$-8.74992 \mathrm{E}-02$

$-8.82002 \mathrm{E}-02$

$-8.89048 \mathrm{E}-02$

$-8.96132 \mathrm{E}-02$

$-9.03253 \mathrm{E}-02$

$-9.10411 \mathrm{E}-02$

$-9.17607 \mathrm{E}-02$

$-9.32112 \mathrm{E}-02$

$-9.39422 \mathrm{E}-02$

$-9.46769 \mathrm{E}-02$

$-9.54154 \mathrm{E}-02$

$-9.61578 \mathrm{E}-02$

$-2.33615 \mathrm{E} \quad 00$

$-2.34863 \mathrm{E} \quad 00$

$-2.36114 \mathrm{E} \quad 00$

$-2.37368 \mathrm{E} \quad 00$

$-2.38625 \mathrm{E} \quad 00$

$-2.39886 \mathbf{E} \quad 00$

$-2.41150 \mathrm{E} 00$

$-2.42418 \mathrm{E} \quad 00$

$-2.43688 \mathrm{E} \quad 00$

$-2.44962 \mathrm{E} \quad 00$

$-2.46239 \mathrm{E} \quad 00$

$-2.47520 \mathrm{E} \quad 00$

$-2.48804 \mathrm{E} \quad 00$

$\begin{array}{ll}-2.50091 \mathrm{E} & 00\end{array}$

$-2.51381 \mathrm{E} \quad 00$

$-2.52675 \mathrm{E} \quad 00$

$-2.53972 \mathrm{E} \quad 00$

$-2.55272 \mathrm{E} \quad 00$

$-2.56575 \mathrm{E} \quad 00$

$-2.57882 \mathrm{E} \quad 00$

$-2.59192 \mathrm{E} \quad 00$ $-2.60505 \mathrm{E} \quad 00$ $-2.61822 \mathrm{E} \quad 00$

$-2.63141 \mathrm{E} \quad 00$

$-2.64465 \mathrm{E} \quad 00$

$-2.65791 \mathrm{E} \quad 00$ $-2.67121 \mathrm{E} \quad 00$ $-2.68453 \mathrm{E} \quad 00$ $-2.69790 \mathrm{E} \quad 00$

$-2.71129 \mathrm{E} \quad 00$

$-2.72472 \mathrm{E} \quad 00$

$-2.73818 \mathrm{E} \quad 00$

$-2.75167 \mathrm{E} \quad 00$

$-2.76520 \mathrm{E} \quad 00$

$-2.77875 \mathrm{E} \quad 00$

$-2.79235 \mathrm{E} \quad 00$

$-2.80597 \mathrm{E} \quad 00$

$-2.81963 \mathrm{E} \quad 00$

$-2.83331 \mathrm{E} 00$

$-2.84704 \mathrm{E} \quad 00$

$-2.86079 \mathrm{E} \quad 00$ $-2.87458 \mathrm{E} \quad 00$ $-2.88840 \mathrm{E} \quad 00$ $-2.90225 \mathbf{E} \quad 00$ $-2.91613 \mathrm{E} 00$

$-1.00692 \mathrm{E}-01$

$-1.01462 \mathrm{E}-0$

$-1.03012 \mathrm{E}-01$

$-1.03793 \mathrm{E}-01$

$-1.04578 \mathrm{E}-01$

$-1.05367 \mathrm{E}-01$

$-1.06160 \mathrm{E}-01$

$-1.06956 \mathrm{E}-01$

$-1.07757 \mathrm{E}-01$

$-1.08562 \mathrm{E}-01$

$-1.09371 \mathrm{E}-01$

$-1.10183 \mathrm{E}-01$

$-1.11000 \mathrm{E}-01$

$-1.11820 \mathrm{E}-01$

$-1.12645 \mathrm{E}-01$

$-1.13474 \mathrm{E}-01$

$-1.14307 \mathrm{E}-01$

$-1.15143 \mathrm{E}-01$

$-1.15984 \mathrm{E}-01$

$-1.16829 \mathrm{E}-01$

$-1.17678 \mathrm{E}-01$

$-1.18531 \mathrm{E}-01$

$-1.19388 \mathrm{E}-01$

$-1.20249 \mathrm{E}-0 \mathrm{I}$

$-1.21115 \mathrm{E}-01$

$-1.21984 \mathrm{E}-01$

$-1.22858 \mathrm{E}-01$

$-1.23736 \mathrm{E}-01$

$-1.24618 \mathrm{E}-01$

$-1.25504 \mathrm{E}-01$

$-1.26394 \mathrm{E}-01$

$-1.27288 \mathrm{E}-01$

$-1.28187 \mathrm{E}-01$

$-1.29090 \mathrm{E}-01$

$-1.29997 \mathrm{E}-01$

$-1.30908 \mathrm{E}-01$

$-1.31823 \mathrm{E}-01$

$-1.32743 \mathrm{E}-01$

$-1.33667 \mathrm{E}-01$
$-9.24841 \mathrm{E}-02$

$\tan \delta_{2}$

$f_{-2}$

$1.01647 \mathrm{E} \quad 02 \quad-8.41512 \mathrm{E}-02$

$\begin{array}{lll}1.01650 \mathrm{E} & 02 & -8.48333 \mathrm{E}-02\end{array}$

$\begin{array}{lll}1.01653 \mathrm{E} & 02 & -8.55190 \mathrm{E}-02\end{array}$

$\begin{array}{lll}1.01656 \mathrm{E} & 02 & -8.62085 \mathrm{E}-02\end{array}$

$\begin{array}{lll}1.01659 \mathrm{E} & 02 & -8.69016 \mathrm{E}-02\end{array}$

$\begin{array}{lll}1.01662 \mathrm{E} & 02 & -8.75985 \mathrm{E}-02\end{array}$

$\begin{array}{lll}1.01665 \mathrm{E} & 02 & -8.82990 \mathrm{E}-02\end{array}$

$\begin{array}{lll}1.01668 \mathrm{E} & 02 & -8.90032 \mathrm{E}-02\end{array}$

$\begin{array}{lll}1.01671 \mathrm{E} & 02 & -8.97112 \mathrm{E}-02\end{array}$

$\begin{array}{lll}1.01674 \mathrm{E} & 02 & -9.04229 \mathrm{E}-02\end{array}$

$\begin{array}{ll}1.01677 \mathrm{E} & 02\end{array}$

$1.01680 \mathrm{E} \quad 02$

$\begin{array}{ll}1.01683 \mathrm{E} & 02\end{array}$

$1.01686 \mathrm{E} \quad 02$

\begin{tabular}{ll}
$1.01689 \mathrm{E} \quad 02$ \\
\hline
\end{tabular}

$1.01691 \mathrm{E} \quad 02$

$1.01694 \mathrm{E} \quad 02$

$\begin{array}{ll}1.01697 \mathrm{E} & 02\end{array}$

$1.01700 \mathrm{E} \quad 02$

$\begin{array}{ll}1.01703 \mathrm{E} & 02\end{array}$

$1.01705 \mathrm{E} \quad 02$

$1.01708 \mathrm{E} \quad 02$

$1.01711 \mathrm{E} \quad 02$

$1.01714 \mathrm{E} \quad 02$

$1.01717 \mathrm{E} \quad 02$

$-9.11384 \mathrm{E}-02$

$-9.18576 \mathrm{E}-02$

$-9.25805 \mathrm{E}-02$

$-9.33072 \mathrm{E}-02$

$-9.40378 \mathrm{E}-02$

-9.47721 E-02 $-9.55102 \mathrm{E}-02$

$-9.62521 \mathrm{E}-02$

$-9.69978 \mathrm{E}-02$

$-9.77474 \mathrm{E}-02$

$1.01719 \mathrm{E} \quad 02$

$1.01722 \mathrm{E} \quad 02$

$1.01725 \mathrm{E} \quad 02$

$1.01727 \mathrm{E} \quad 02$

$1.01730 \mathrm{E} \quad 02$

$-9.85008 \mathrm{E}-02$

$-9.92580 \mathrm{E}-02$

$-1.00019 \mathrm{E}-01$

$-1.00784 \mathrm{E}-01$

$-1.01553 \mathrm{E}-01$

$\begin{array}{ll}1.01733 \mathbf{E} & 02\end{array}$

$1.01735 \mathrm{E} \quad 02$

$1.01738 \mathrm{E} \quad 02$

$1.01740 \mathrm{E} \quad 02$

$1.01743 \mathrm{E} \quad 02$

$-1.02326 \mathrm{E}-01$

$-1.03102 \mathrm{E}-0 \mathrm{I}$

$-1.03883 \mathbf{E}-01$

$-1.04667 \mathrm{E}-0 \mathrm{I}$

$-1.05456 \mathrm{E}-01$

$1.01746 \mathrm{E} \quad 02$

$1.01748 \mathrm{E} \quad 02$

$1.01751 \mathrm{E} \quad 02$

$\begin{array}{ll}1.01753 \mathrm{E} & 02\end{array}$

$1.01756 \mathrm{E} \quad 02$

$-1.06248 \mathrm{E}-01$

$-1.07044 \mathrm{E}-01$ 


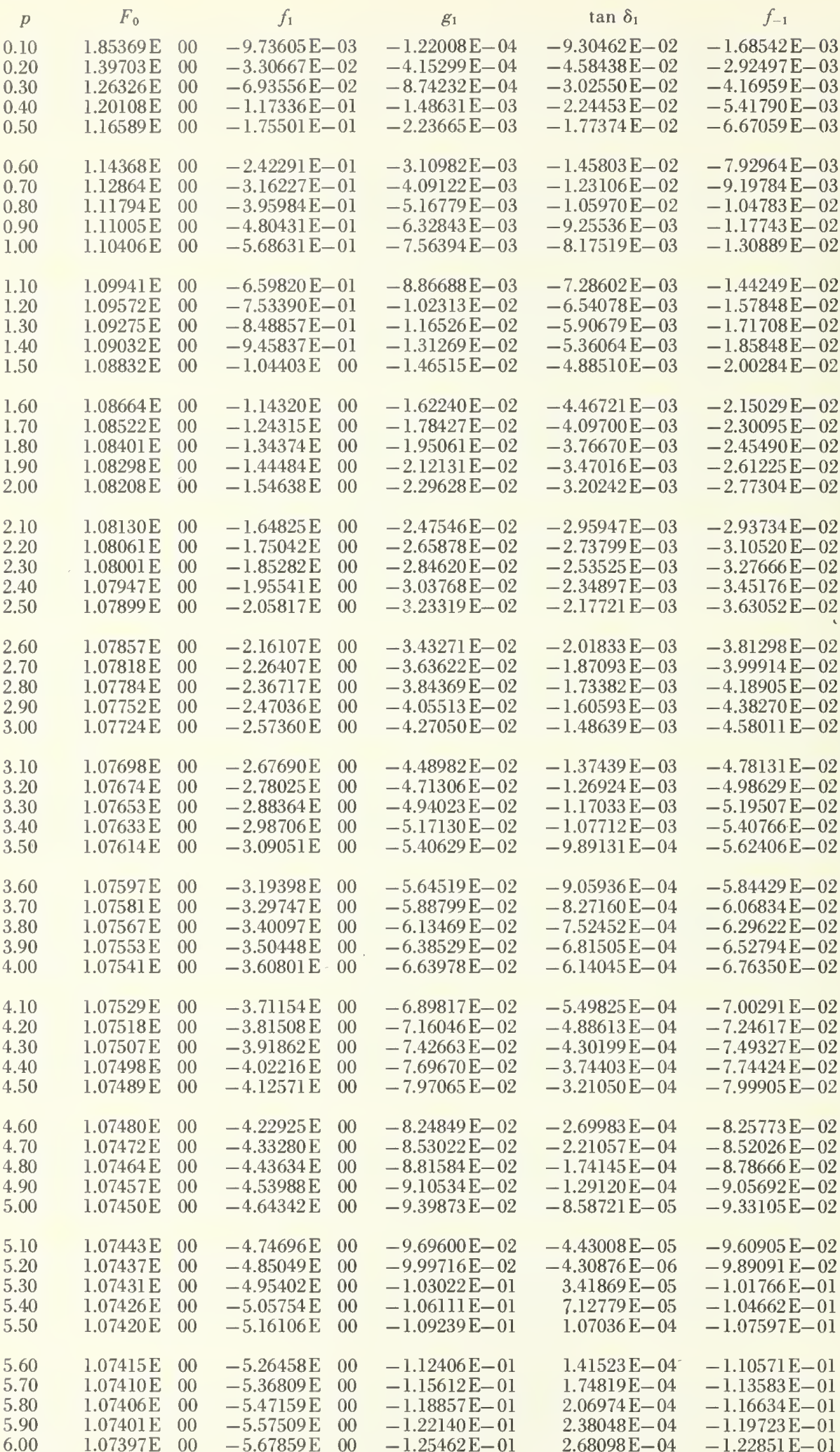

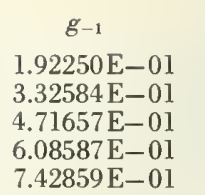

$8.74245 \mathrm{E}-0 \mathrm{l}$ $1.00273 \mathrm{E} \quad 00$ $1.12843 \mathrm{E} \quad 00$ $1.25158 \mathrm{E} \quad 00$ $1.37240 \mathrm{E} \quad 00$

$1.49117 \mathrm{E} \quad 00$ $1.60814 \mathrm{E} \quad 00$ $1.72354 \mathrm{E} \quad 00$ $\begin{array}{lll}1.83757 \mathrm{E} & 00\end{array}$ $1.95042 \mathrm{E} \quad 00$

$2.06224 \mathrm{E} \quad 00$ $2.17317 \mathrm{E} \quad 00$ $2.28333 \mathrm{E} \quad 00$ $2.39282 \mathrm{E} \quad 00$ $2.50171 \mathrm{E} \quad 00$

$2.61010 \mathrm{E} \quad 00$ $2.71802 \mathrm{E} \quad 00$ $2.82555 \mathrm{E} \quad 00$ $2.93273 \mathrm{E} \quad 00$ $3.03959 \mathrm{E} \quad 00$

$3.14618 \mathrm{E} \quad 00$ $3.25251 \mathrm{E} \quad 00$ $3.35862 \mathrm{E} \quad 00$ $3.46453 \mathrm{E} \quad 00$ $3.57026 \mathrm{E} \quad 00$

$3.67583 \mathrm{E} \quad 00$ $3.78125 \mathrm{E} \quad 00$ $3.88653 \mathrm{E} \quad 00$ $3.99169 \mathrm{E} \quad 00$ $4.09673 \mathrm{E} \quad 00$

$4.20168 \mathrm{E} \quad 00$ $4.30652 \mathrm{E} \quad 00$ $4.41129 \mathrm{E} \quad 00$ $4.51597 \mathrm{E} \quad 00$ $4.62057 \mathrm{E} \quad 00$

$4.72511 \mathrm{E} \quad 00$ $4.82959 \mathrm{E} \quad 00$ $4.93400 \mathrm{E} 00$ $5.03836 \mathrm{E} \quad 00$ $5.14267 \mathrm{E} \quad 00$

$5.24692 \mathrm{E} \quad 00$ $5.35113 \mathrm{E} \quad 00$ $5.45530 \mathrm{E} \quad 00$ $5.55943 \mathrm{E} \quad 00$ $\begin{array}{lll}5.66352 \mathrm{E} & 00\end{array}$

$5.76758 \mathrm{E} \quad 00$ $5.87160 \mathrm{E} \quad 00$ $5.97559 \mathrm{E} \quad 00$ $6.07955 \mathrm{E} \quad 00$ $6.18348 \mathrm{E} \quad 00$

$6.28738 \mathrm{E} \quad 00$ $6.39126 \mathrm{E} \quad 00$ $6.49511 \mathrm{E} \quad 00$ $6.59893 \mathrm{E} \quad 00$ $6.70274 \mathrm{E} \quad 00$ $\tan \delta_{-1}$

$-8.10595 \mathrm{E} \quad 00$ $-1.57975 \mathrm{E} 01$ $-2.34599 \mathrm{E} 01$ $-3.10113 \mathrm{E} 01$ $-3.84211 \mathrm{E} 01$

$-4.56714 \mathrm{E} 01$ $-5.27507 \mathrm{E} \quad 01$ $-5.96525 \mathrm{E} \quad 01$ $-6.63737 \mathrm{E} 01$ $-7.29138 \mathrm{E} 01$

$-7.92746 \mathrm{E} \quad 01$ $-8.54589 \mathrm{E}$ 01 $-9.14708 \mathrm{E} 01$ $-9.73147 \mathrm{E} \quad 01$ $-1.02996 \mathrm{E} 02$

$-1.08519 \mathrm{E} \quad 02$ $-1.13890 \mathrm{E} 02$ $-1.19114 \mathrm{E} \quad 02$ $-1.24196 \mathrm{E} 02$ $-1.29142 \mathrm{E} 02$ $-1.33955 \mathrm{E} \quad 02$ -1.38642 E 02 $-1.43207 \mathrm{E} \quad 02$ $-1.47654 \mathrm{E} \quad 02$ -1.51987 E 02 $-1.56212 \mathrm{E} \quad 02$ $-1.60331 \mathrm{E} 02$ $-1.64348 \mathrm{E} 02$ $-1.68268 \mathrm{E} 02$ $\begin{array}{ll}-1.72093 \mathrm{E} & 02\end{array}$

$-1.75827 \mathrm{E} \quad 02$ -1.79473 E 02 $-1.83035 \mathrm{E} 02$ $\begin{array}{ll}-1.86514 \mathrm{E} & 02\end{array}$ $-1.89914 \mathrm{E} \quad 02$ $-1.93238 \mathrm{E} \quad 02$ $-1.96488 \mathrm{E} \quad 02$ $-1.99666 \mathrm{E} 02$ $-2.02775 \mathrm{E} \quad 02$ $-2.05817 \mathrm{E} \quad 02$ $\begin{array}{ll}-2.08794 \mathrm{E} & 02\end{array}$ $-2.11708 \mathrm{E} \quad 02$ $\begin{array}{ll}-2.14562 \mathrm{E} & 02\end{array}$ $-2.17357 \mathrm{E} \quad 02$ $-2.20094 \mathrm{E} \quad 02$ $-2.22777 \mathrm{E} \quad 02$ $-2.25405 \mathrm{E} \quad 02$ $-2.27982 \mathrm{E} \quad 02$ $-2.30508 \mathrm{E} 02$ $-2.32985 \mathrm{E} \quad 02$ $-2.35415 \mathrm{E} \quad 02$ $-2.37798 \mathrm{E} \quad 02$ $-2.40136 \mathrm{E} \quad 02$ $-2.42431 \mathrm{E} 02$ $-2.44683 \mathrm{E} \quad 02$

$-2.46895 \mathrm{E} \quad 02$ $-2.49066 \mathrm{E} \quad 02$ $-2.51198 \mathrm{E} 02$ $-2.53292 \mathrm{E} 02$ $\begin{array}{ll}-2.55349 \mathrm{E} & 02\end{array}$ 
ELECTRON RADIAL FUNCTIONS

$Z=3 \quad A=6 \quad \rho=1.2 A^{1 / 3} F$

Electron

$\bar{F}$

$1.71766 \mathrm{E}-08$ $5.00126 \mathrm{E}-08$ $1.01094 \mathrm{E}-07$ $1.70492 \mathrm{E}-07$ $2.58326 \mathrm{E}-07$

$3.64704 \mathrm{E}-07$

$4.89720 \mathrm{E}-07$

$6.33447 \mathrm{E}-07$

$7.95945 \mathrm{E}-07$

$9.77260 \mathrm{E}-07$

$1.17743 \mathrm{E}-06$

$1.39648 \mathrm{E}-06$

$1.63443 \mathrm{E}-06$

$1.89131 \mathrm{E}-06$

$2.16712 \mathrm{E}-06$

$2.46187 \mathrm{E}-06$

$2.77558 \mathrm{E}-06$

$3.10824 \mathrm{E}-06$

$3.45987 \mathrm{E}-06$

$3.83047 \mathrm{E}-06$

$4.22004 \mathrm{E}-06$ $4.62859 \mathrm{E}-06$

$5.05611 \mathrm{E}-06$

$5.50262 \mathrm{E}-06$

$5.96811 \mathrm{E}-06$

$6.45257 \mathrm{E}-06$ $6.95602 \mathrm{E}-06$

$7.47845 \mathrm{E}-06$

$8.01987 \mathrm{E}-06$

$8.58027 \mathrm{E}-06$

$9.15967 \mathrm{E}-06$

$9.75804 \mathrm{E}-06$

$1.03754 \mathrm{E}-05$

$1.10118 \mathrm{E}-05$

$1.16671 \mathrm{E}-05$

$1.23414 \mathrm{E}-05$ $1.30347 \mathrm{E}-05$

$1.37470 \mathrm{E}-05$

$1.44783 \mathrm{E}-05$

$1.52286 \mathrm{E}-05$

$1.59978 \mathrm{E}-05$

$1.67861 \mathrm{E}-05$

$1.75933 \mathrm{E}-05$

$1.84195 \mathrm{E}-05$

$1.92647 \mathrm{E}-05$

$2.01289 \mathrm{E}-05$

$2.10121 \mathrm{E}-05$

$2.19143 \mathrm{E}-05$

$2.28354 \mathrm{E}-05$

$2.37756 \mathrm{E}-05$

$2.47347 \mathrm{E}-05$

$2.57128 \mathrm{E}-05$

$2.67099 \mathrm{E}-05$

$2.77260 \mathrm{E}-0.5$

$2.87611 \mathrm{E}-05$

$2.98152 \mathrm{E}-05$ $3.08882 \mathrm{E}-05$

$3.19803 \mathrm{E}-05$

$3.30913 \mathrm{E}-05$

$3.42213 \mathrm{E}-05$ $f_{2}$

$-1.77913 \mathrm{E}-06$

$-1.23503 \mathrm{E}-05$

$-3.90103 \mathrm{E}-05$

$-8.81199 \mathrm{E}-05$

$-1.64862 \mathrm{E}-04$

$-2.73225 \mathrm{E}-04$

$-4.16131 \mathrm{E}-04$

$-5.95620 \mathrm{E}-04$

$-8.13065 \mathrm{E}-04$

$-1.06935 \mathrm{E}-03$

$-1.36501 \mathrm{E}-03$

$-1.70037 \mathrm{E}-03$

$-2.07559 \mathrm{E}-03$

$-2.49073 \mathrm{E}-03$

$-2.94579 \mathrm{E}-03$

$-3.44075 \mathrm{E}-03$

$-3.97555 \mathrm{E}-03$

$-4.55013 \mathrm{E}-03$

$-5.16443 \mathrm{E}-03$

$-5.81839 \mathrm{E}-03$

$-6.51195 \mathrm{E}-03$

$-7.24504 \mathrm{E}-03$

$-8.01762 \mathrm{E}-03$

$-8.82964 \mathrm{E}-03$

$-9.68106 \mathrm{E}-03$

$-1.05718 \mathrm{E}-02$

$-1.15019 \mathrm{E}-02$

$-1.24713 \mathrm{E}-02$

$-1.34799 \mathrm{E}-02$

$-1.45278 \mathrm{E}-02$

$-1.56149 \mathrm{E}-02$

$-1.67411 \mathrm{E}-02$

$-1.79066 \mathrm{E}-02$

$-1.91111 \mathrm{E}-02$

$-2.03548 \mathrm{E}-02$

$-2.16376 \mathrm{E}-02$

$-2.29595 \mathrm{E}-02$

$-2.43206 \mathrm{E}-02$

$-2.57206 \mathrm{E}-02$

$-2.71598 \mathrm{E}-02$

$-2.86380 \mathrm{E}-02$

$-3.01552 \mathrm{E}-02$

$-3.17115 \mathrm{E}-02$

$-3.33069 \mathrm{E}-02$

$-3.49412 \mathrm{E}-02$

$-3.66146 \mathrm{E}-02$

$-3.83269 \mathrm{E}-02$

$-4.00783 \mathrm{E}-02$

$-4.18687 \mathrm{E}-02$

$-4.36980 \mathrm{E}-02$

$-4.55664 \mathrm{E}-02$

$-4.74737 \mathrm{E}-02$

$-4.94201 \mathrm{E}-02$

$-5.14053 \mathrm{E}-02$

$-5.34296 \mathrm{E}-02$

$-5.54929 \mathrm{E}-02$

$-5.75950 \mathrm{E}-02$

$-5.97362 \mathrm{E}-02$

$-6.19163 \mathrm{E}-02$

$-6.41353 \mathrm{E}-02$ $g_{2}$

$-1.29314 \mathrm{E}-08$

$-8.99737 \mathrm{E}-08$

$-2.85263 \mathrm{E}-07$

$-6.47663 \mathrm{E}-07$

$-1.21934 \mathrm{E}-06$

$-2.03566 \mathrm{E}-06$

$-3.12598 \mathrm{E}-06$

$-4.51465 \mathrm{E}-06$

$-6.22227 \mathrm{E}-06$

$-8.26674 \mathrm{E}-06$

$-1.06640 \mathrm{E}-05$

$-1.34289 \mathrm{E}-05$

- $1.65752 \mathrm{E}-05$

$-2.01165 \mathrm{E}-05$

$-2.40657 \mathrm{E}-05$

$-2.84357 \mathrm{E}-05$

$-3.32395 \mathrm{E}-05$

$-3.84898 \mathrm{E}-05$

$-4.41993 \mathrm{E}-05$

$-5.03809 \mathrm{E}-05$

$-5.70474 \mathrm{E}-05$

$-6.42116 \mathrm{E}-05$

$-7.18864 \mathrm{E}-05$

$-8.00847 \mathrm{E}-05$

$-8.88195 \mathrm{E}-05$

$-9.81035 \mathrm{E}-05$

$-1.07950 \mathrm{E}-04$

$-1.18372 \mathrm{E}-04$

$-1.29382 \mathrm{E}-04$

$-1.40994 \mathrm{E}-04$

$-1.53220 \mathrm{E}-04$

$-1.66074 \mathrm{E}-04$

$-1.79568 \mathrm{E}-04$

$-1.93716 \mathrm{E}-04$

$-2.08531 \mathrm{E}-04$

$-2.24026 \mathrm{E}-04$

$-2.40213 \mathrm{E}-04$

$-2.57107 \mathrm{E}-04$

$-2.74720 \mathrm{E}-04$

$-2.93066 \mathrm{E}-04$

$-3.12157 \mathrm{E}-04$

$-3.32007 \mathrm{E}-04$

$-3.52629 \mathrm{E}-04$

$-3.74035 \mathrm{E}-04$

$-3.96240 \mathrm{E}-04$

$-4.19256 \mathrm{E}-04$

$-4.43096 \mathrm{E}-04$

$-4.67774 \mathrm{E}-04$

$-4.93303 \mathrm{E}-04$

$-5.19695 \mathrm{E}-04$

$-5.46965 \mathrm{E}-04$

$-5.75125 \mathrm{E}-04$

$-6.04188 \mathrm{E}-04$

$-6.34167 \mathrm{E}-04$

$-6.65077 \mathrm{E}-04$

$-6.96929 \mathrm{E}-04$

$-7.29737 \mathrm{E}-04$

$-7.63514 \mathrm{E}-04$

$-7.98274 \mathrm{E}-04$

$-8.34029 \mathrm{E}-04$ $\tan \delta_{2}$

$4.86226 \mathrm{E} \quad 00$ $9.73975 \mathrm{E} \quad 00$

$1.44022 \mathrm{E} \quad 01$

$1.87949 \mathrm{E} \quad 01$

2.28723 E 01

$2.66111 \mathrm{E} \quad 01$

$3.00070 \mathrm{E} \quad 01$

$3.30706 \mathrm{E} \quad 01$

$3.58221 \mathrm{E} 01$

$3.82871 \mathrm{E}$ 01

$4.04931 \mathrm{E} \quad 01$

$4.24675 \mathrm{E} \quad 01$

$4.42366 \mathrm{E} \quad 01$

$4.58243 \mathrm{E} \quad 01$

$\begin{array}{lll}4.72522 \mathrm{E} & 01\end{array}$

$4.85395 \mathrm{E} \quad 01$ $4.97030 \mathrm{E} 01$

5.07575 E 01

$5.17159 \mathrm{E} \quad 01$

$5.25892 \mathrm{E} \quad 01$

$5.33873 \mathrm{E} \quad 01$

$5.41185 \mathrm{E} \quad 01$

$5.47902 \mathrm{E} \quad 01$

$5.54087 \mathrm{E} \quad 01$

$5.59797 \mathrm{E} 01$

$5.65081 \mathrm{E} \quad 01$

$5.69980 \mathrm{E}$ 01

$5.74534 \mathrm{E} \quad 01$

$5.78774 \mathrm{E}$ 0l

5.82731 E 01

$5.86430 \mathrm{E} \quad 01$

$5.89895 \mathrm{E} 01$

$5.93145 \mathrm{E} \quad 01$

$5.96199 \mathrm{E} \quad 01$

$5.99074 \mathrm{E} \quad 01$

$6.01783 \mathrm{E} \quad 01$ $6.04342 \mathrm{E} \quad 01$ $6.06760 \mathrm{E} 01$ $6.09049 \mathrm{E} \quad 01$

$6.11218 \mathrm{E} 01$

$6.13277 \mathrm{E} \quad 01$

$6.15233 \mathrm{E} \quad 01$

$6.17094 \mathrm{E} \quad 01$

6.18865 E 01

$6.20554 \mathrm{E} \quad 01$

$6.22165 \mathrm{E} \quad 01$

$6.23704 \mathrm{E} \quad 01$

$6.25174 \mathrm{E} \quad 01$

$6.26582 \mathrm{E} \quad 01$

$6.27930 \mathrm{E} 01$

$6.29222 \mathrm{E} \quad 01$ $6.30461 \mathrm{E} \quad 01$ $6.31650 \mathrm{E} 01$ $6.32793 \mathrm{E} 01$ $6.33892 \mathrm{E} 01$

$6.34948 \mathrm{E} \quad 01$ $6.35966 \mathrm{E} \quad 01$ $6.36946 \mathrm{E} \quad 01$ $6.37891 \mathrm{E} 01$ $6.38802 \mathrm{E} \quad 01$

\section{$f_{-2}$}

$-1.85455 \mathrm{E}-07$ $-6.32716 \mathrm{E}-07$ $-1.34888 \mathrm{E}-06$ $-2.33512 \mathrm{E}-06$

$-3.59348 \mathrm{E}-06$

$-5.12720 \mathrm{E}-06$ $-6.94105 \mathrm{E}-06$ $-9.04134 \mathrm{E}-06$ $-1.14358 \mathrm{E}-05$

$-1.41334 \mathrm{E}-05$

$-1.71442 \mathrm{E}-05$ $-2.04789 \mathrm{E}-05$ $-2.41490 \mathrm{E}-05$ $-2.81664 \mathrm{E}-05$ $-3.25434 \mathrm{E}-05$

$-3.72924 \mathrm{E}-05$ $-4.24261 \mathrm{E}-05$ $-4.79575 \mathrm{E}-05$ $-5.38994 \mathrm{E}-05$ $-6.02649 \mathrm{E}-05$

$-6.70670 \mathrm{E}-05$ $-7.43190 \mathrm{E}-05$ $-8.20338 \mathrm{E}-05$ $-9.02248 \mathrm{E}-05$ $-9.89050 \mathrm{E}-05$

$-1.08088 \mathrm{E}-04$ $-1.17786 \mathrm{E}-04$ $-1.28013 \mathrm{E}-04$ $-1.38783 \mathrm{E}-04$ $-1.50107 \mathrm{E}-04$

$-1.62001 \mathrm{E}-04$ $-1.74476 \mathrm{E}-04$ $-1.87545 \mathrm{E}-04$ $-2.01223 \mathrm{E}-04$ $-2.15523 \mathrm{E}-04$

$-2.30457 \mathrm{E}-04$ $-2.46038 \mathrm{E}-04$ $-2.62281 \mathrm{E}-04$ $-2.79198 \mathrm{E}-04$ $-2.96802 \mathrm{E}-04$

$-3.15107 \mathrm{E}-04$ 
ELECTRON RADIAL FUNCTIONS

Electron

\begin{tabular}{|c|c|c|c|c|c|}
\hline$p$ & $F_{0}$ & & $f_{1}$ & & $g_{1}$ \\
\hline 6.10 & $1.07393 \mathrm{E}$ & 00 & $-5.78207 \mathrm{E}$ & 00 & $-1.28824 \mathrm{E}-01$ \\
\hline 6.20 & $1.07389 \mathrm{E}$ & 00 & $-5.88555 \mathrm{E}$ & 00 & $-1.32223 \mathrm{E}-01$ \\
\hline 6.30 & $1.07385 \mathrm{E}$ & 00 & $-5.98903 \mathrm{E}$ & 00 & $.35662 \mathrm{H}$ \\
\hline 6.40 & $1.07382 \mathrm{E}$ & 00 & $-6.09250 \mathrm{E}$ & 00 & $.39140 \mathrm{E}$ \\
\hline 6.50 & $1.07378 \mathrm{E}$ & 00 & $-6.19596 \mathrm{E}$ & 00 & $42656 \mathrm{E}-01$ \\
\hline 6.60 & $1.07375 \mathrm{E}$ & 00 & $-6.29942 \mathrm{E}$ & 00 & $1.46211 \mathrm{E}-01$ \\
\hline 6.70 & $1.07372 \mathrm{E}$ & 00 & $-6.40287 \mathrm{E}$ & 00 & 49805 \\
\hline 6.80 & $1.07369 \mathrm{E}$ & 00 & $-6.50631 \mathrm{E}$ & 00 & $.53438 \mathrm{E}-$ \\
\hline 6.90 & $1.07366 \mathrm{E}$ & 00 & $-6.60975 \mathrm{E}$ & 00 & $-1.57110 \mathrm{E}-01$ \\
\hline 7.00 & $1.07363 \mathrm{E}$ & 00 & $-6.71318 \mathrm{E}$ & 00 & $-1.60820 \mathrm{E}-01$ \\
\hline 7.10 & $1.07360 \mathrm{E}$ & 00 & $-6.81660 \mathrm{E}$ & 00 & $.64569 \mathrm{E}-01$ \\
\hline 7.20 & $1.07358 \mathrm{E}$ & 00 & $-6.92002 \mathrm{E}$ & 00 & $6835 / \mathrm{E}-$ \\
\hline 7.30 & $1.07355 \mathrm{E}$ & 00 & $-7.02343 E$ & 00 & $1.72184 \mathrm{E}-$ \\
\hline 7.40 & $1.07352 \mathrm{E}$ & 00 & $-7.12684 \mathrm{E}$ & 00 & $.76049 \mathrm{E}-$ \\
\hline 7.50 & $1.07350 \mathrm{E}$ & 00 & $-7.23024 \mathrm{E}$ & 00 & $-1.79954 \mathrm{E}-01$ \\
\hline 7.60 & $1.07348 \mathrm{E}$ & 00 & $-7.33363 \mathrm{E}$ & 00 & $83897 \mathrm{~F}$ \\
\hline 7.70 & $07345 \mathrm{E}$ & 00 & $-7.43701 \mathrm{E}$ & 00 & $.87879 \mathrm{E}-$ \\
\hline 7.80 & $.07343 \mathrm{E}$ & 00 & $-7.54039 \mathrm{E}$ & 00 & $-1.91900 \mathrm{E}-01$ \\
\hline 7.90 & $07341 \mathrm{E}$ & 00 & $-7.64376 \mathrm{E}$ & 00 & $.95959 \mathrm{E}-01$ \\
\hline 8.00 & $1.07339 \mathrm{E}$ & 00 & $-7.74713 \mathrm{E}$ & 00 & $.00057 \mathrm{E}-01$ \\
\hline 8.10 & $1.07337 \mathrm{E}$ & 00 & $-7.85048 \mathrm{E}$ & 00 & $04194]$ \\
\hline 8.20 & $07335 \mathrm{E}$ & 00 & $-7.95383 E$ & 00 & $.08370 \mathrm{E}-01$ \\
\hline .30 & $333 \mathrm{E}$ & 00 & $-8.05718 \mathrm{E}$ & 00 & $12585 \mathrm{E}-01$ \\
\hline 8.40 & $31 \mathrm{E}$ & 00 & $6051 \mathrm{E}$ & 00 & $6838 \mathrm{E}-01$ \\
\hline 8.50 & $1.07329 \mathrm{E}$ & 00 & $-8.26384 \mathrm{E}$ & 00 & -01 \\
\hline 8.60 & $.07328 \mathrm{E}$ & 00 & $6717 \mathrm{E}$ & 00 & $25462 \mathrm{E}-01$ \\
\hline 8.70 & $26 \mathrm{E}$ & 00 & $7049 \mathrm{E}$ & 00 & $9831 \mathrm{E}-$ \\
\hline 8.80 & $324 \mathrm{E}$ & 00 & $380 \mathrm{E}$ & 00 & 01 \\
\hline 8.90 & $23 \mathrm{E}$ & 00 & $7710 \mathrm{E}$ & 00 & $-2.38687 \mathrm{E}-01$ \\
\hline 9.00 & $1.07321 \mathrm{E}$ & 00 & $-8.78040 \mathrm{E}$ & 00 & $43173 \mathrm{E}-01$ \\
\hline 9.10 & $20 \mathrm{E}$ & 00 & $8369 \mathrm{E}$ & 00 & E-01 \\
\hline 9.20 & & 00 & $697 \mathrm{E}$ & 00 & \\
\hline 9.30 & $17 \mathrm{E}$ & 00 & $-9.09025 \mathrm{E}$ & 00 & $56864 \mathrm{E}-$ \\
\hline 9.40 & $1.07315 \mathrm{E}$ & 00 & $-9.19351 \mathrm{E}$ & 00 & $-2.61505 \mathrm{E}-01$ \\
\hline 9.50 & $1.07314 \mathrm{E}$ & 00 & $-9.29678 \mathrm{E}$ & 00 & $66185 \mathrm{E}-01$ \\
\hline 9.60 & $.07313 \mathrm{E}$ & 00 & $-9.40003 \mathrm{E}$ & 00 & $\mathrm{E}-01$ \\
\hline 9.70 & $11 \mathrm{E}$ & 00 & $0328 \mathrm{E}$ & 00 & -2 \\
\hline 9.80 & $.07310 \mathrm{E}$ & 00 & $-9.60652 \mathrm{E}$ & 00 & $-2.80456 \mathrm{E}-01$ \\
\hline 9.90 & $.07309 \mathrm{E}$ & 00 & $0976 \mathrm{E}$ & 00 & $85291 \mathrm{E}-$ \\
\hline 10.00 & $1.07307 \mathrm{E}$ & 00 & $-9.81298 E$ & 00 & $5 \mathrm{E}-01$ \\
\hline 0.10 & $1.07306 \mathrm{E}$ & 00 & $-9.91620 \mathrm{E}$ & 00 & E-01 \\
\hline 20 & $07305 \mathrm{E}$ & 00 & $-1.00194 \mathrm{E}$ & 01 & 00028 \\
\hline 30 & $304 \mathrm{E}$ & 00 & $-1.01226 \mathrm{E}$ & 01 & $05018 \mathrm{E}-01$ \\
\hline .40 & & 00 & $258 \mathrm{E}$ & 01 & 01 \\
\hline 10.50 & $1.07302 \mathrm{E}$ & 00 & $-1.03290 \mathrm{E}$ & 01 & $15113 \mathrm{E}-01$ \\
\hline 0.60 & $0 \mathrm{E}$ & 00 & $-1.04322 \mathrm{E}$ & 01 & $E-01$ \\
\hline 70 & & 00 & $354 \mathrm{E}$ & 01 & 01 \\
\hline & & 00 & $386 \mathrm{E}$ & 01 & \\
\hline & & 00 & & 01 & $9 \mathrm{E}-01$ \\
\hline 11.00 & $1.07296 \mathrm{E}$ & 00 & $-1.08449 \mathrm{E}$ & 01 & $0 \mathrm{~F} \quad 0$ \\
\hline 11.10 & & 00 & $-1.09480 \mathrm{E}$ & 01 & $-3.46329 \mathrm{E}-01$ \\
\hline 20 & & 00 & & 01 & \\
\hline 11.30 & $293 \mathrm{E}$ & 00 & $1543 \mathrm{E}$ & 01 & $7044 \mathrm{E}-01$ \\
\hline & $1.07292 \mathrm{E}$ & 00 & $2574 \mathrm{E}$ & 01 & $-3.62460 \mathrm{E}-01$ \\
\hline & $1.07291 \mathrm{E}$ & 00 & $-1.13606 \mathrm{E}$ & 01 & $-3.67914 \mathrm{E}-01$ \\
\hline 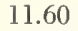 & $7291 \mathrm{E}$ & 00 & & 01 & $7 \mathrm{E}-01$ \\
\hline & $.07290 \mathrm{E}$ & 00 & & 01 & \\
\hline & $289 \mathrm{E}$ & 0( & $-1.16699 \mathrm{E}$ & 0 & $-3.84509 \mathrm{E}-01$ \\
\hline & $1.07288 \mathrm{E}$ & 00 & $-1.17730 \mathrm{E}$ & 01 & $-3.90118 \mathrm{E}-01$ \\
\hline & $1.07287 \mathrm{E}$ & 00 & $-1.18761 \mathrm{E}$ & 01 & $-3.95766 \mathrm{E}-01$ \\
\hline
\end{tabular}
$\rho=1.2 A^{1 / 3} F$ $\begin{array}{cc}\tan \delta_{1} & f_{-1} \\ 2.97170 \mathrm{E}-04 & -1.26019 \mathrm{E}-\end{array}$

$3.25311 \mathrm{E}-04$

$3.52566 \mathrm{E}-04$

$3.78973 \mathrm{E}-04$

$4.04578 \mathrm{E}-04$

$4.29410 \mathrm{E}-04$

$4.53502 \mathrm{E}-04$

$4.76889 \mathrm{E}-04$

$4.99607 \mathrm{E}-04$

$5.21673 \mathrm{E}-04$

$5.43121 \mathrm{E}-04$

$5.63978 \mathrm{E}-04$

$5.84266 \mathrm{E}-04$

$6.04002 \mathrm{E}-04$

$6.23217 \mathrm{E}-04$

$6.41926 \mathrm{E}-04$

$6.60150 \mathrm{E}-04$

$6.77908 \mathrm{E}-04$

$6.95214 \mathrm{E}-04$

$7.12093 \mathrm{E}-04$

$7.28552 \mathrm{E}-04$

$7.44607 \mathrm{E}-04$

$7.60275 \mathrm{E}-04$

$7.75570 \mathrm{E}-04$

$7.90503 \mathrm{E}-04$

$8.05088 \mathrm{E}-04$

$8.19338 \mathrm{E}-04$

$8.33261 \mathrm{E}-04$

$8.46874 \mathrm{E}-04$

$8.60176 \mathrm{E}-04$

$8.73184 \mathrm{E}-04$ $8.85913 \mathrm{E}-04$

$8.98364 \mathrm{E}-04$

$9.10549 \mathrm{E}-04$

$9.22473 \mathrm{E}-04$

$9.34146 \mathrm{E}-04$

$9.45578 \mathrm{E}-04$

$9.56770 \mathrm{E}-04$

$9.67739 \mathrm{E}-04$

9.78485 E-04

$9.89014 \mathrm{E}-04$ $9.99337 \mathrm{E}-04$

$1.00945 \mathrm{E}-03$

$1.01937 \mathrm{E}-03$

$1.02910 \mathrm{E}-03$

$1.03864 \mathrm{E}-03$ $1.04800 \mathrm{E}-03$ $1.05719 \mathrm{E}-03$ $1.06620 \mathrm{E}-03$

$1.07504 \mathrm{E}-03$

$1.08372 \mathrm{E}-03$ $1.09225 \mathrm{E}-03$ $1.10062 \mathrm{E}-03$ $1.10884 \mathrm{E}-03$ $1.11692 \mathrm{E}-03$

$1.12485 \mathrm{E}-03$ $1.13264 \mathrm{E}-03$ $1.14029 \mathrm{E}-03$ $1.14782 \mathrm{E}-03$ $1.15521 \mathrm{E}-03$
$-1.26019 \mathrm{E}-01$

$-1.29224 \mathrm{E}-01$

$-1.32469 \mathrm{E}-01$

$-1.35752 \mathrm{E}-01$

$-1.39074 \mathrm{E}-01$

$-1.42435 \mathrm{E}-01$

$-1.45835 \mathrm{E}-01$

$-1.49273 \mathrm{E}-01$

$-1.52750 \mathrm{E}-01$

$-1.56266 \mathrm{E}-01$

$-1.59821 \mathrm{E}-01$

$-1.63414 \mathrm{E}-01$

$-1.67046 \mathrm{E}-01$

$-1.70717 \mathrm{E}-01$

$-1.74427 \mathrm{E}-01$

$-1.78176 \mathrm{E}-01$

$-1.81963 \mathrm{E}-01$

$-1.85789 \mathrm{E}-01$

$-1.89654 \mathrm{E}-01$

$-1.93557 \mathrm{E}-01$

$-1.97500 \mathrm{E}-01$

$-2.01481 \mathrm{E}-01$

$-2.05500 \mathrm{E}-01$

$-2.09559 \mathrm{E}-01$

$-2.13656 \mathrm{E}-0 \mathrm{I}$

$-2.17793 \mathrm{E}-01$

$-2.21967 \mathrm{E}-01$

$-2.26181 \mathrm{E}-01$

$-2.30433 \mathrm{E}-01$

$-2.34725 \mathrm{E}-01$

$-2.39055 \mathrm{E}-01$

$-2.43423 \mathrm{E}-01$

$-2.47831 \mathrm{E}-01$

$-2.52277 \mathrm{E}-01$

$-2.56762 \mathrm{E}-01$

$-2.61285 \mathrm{E}-01$

$-2.65848 \mathrm{E}-01$

$-2.70449 \mathrm{E}-01$

$-2.75089 \mathrm{E}-01$

$-2.79767 \mathrm{E}-01$

$-2.84485 \mathrm{E}-01$

$-2.89241 . \mathrm{E}-01$

$-2.94036 \mathrm{E}-01$

$-2.98869 \mathrm{E}-01$

$-3.03741 \mathrm{E}-01$

$-3.08652 \mathrm{E}-01$

$-3.13602 \mathrm{E}-01$

$-3.18590 \mathrm{E}-01$

$-3.23618 \mathrm{E}-01$

$-3.28683 \mathrm{E}-01$

$-3.33788 \mathrm{E}-01$ $-3.38931 \mathrm{E}-01$

$-3.44113 \mathrm{E}-01$

$-3.49334 \mathrm{E}-01$

$-3.54593 \mathrm{E}-01$

$-3.59891 \mathrm{E}-01$

$-3.65228 \mathrm{E}-01$

$-3.70604 \mathrm{E}-01$

$-3.76018 \mathrm{E}-01$

$-3.81471 \mathrm{E}-01$ g-1

$6.80652 \mathrm{E} \quad 00$ $6.91028 \mathrm{E} \quad 00$

$7.01402 \mathrm{E} \quad 00$

$7.11774 \mathrm{E} \quad 00$

$7.22144 \mathrm{E} \quad 00$

$7.32512 \mathrm{E} \quad 00$

$7.42879 \mathrm{E} \quad 00$

$7.53244 \mathrm{E} \quad 00$

$7.63607 \mathrm{E} \quad 00$

$7.73968 \mathrm{E} \quad 00$

$7.84328 \mathrm{E} \quad 00$

$7.94687 \mathrm{E} \quad 00$

$8.05044 \mathrm{E} \quad 00$

$8.15399 \mathrm{E} 00$

$8.25754 \mathrm{E} \quad 00$

$8.36107 \mathrm{E} \quad 00$

$8.46458 \mathrm{E} \quad 00$

$8.56809 \mathrm{E} \quad 00$

$8.67158 \mathrm{E} \quad 00$

$8.77505 \mathrm{E} \quad 00$

$8.87852 \mathrm{E} \quad 00$

$8.98197 \mathrm{E} \quad 00$

$9.08542 \mathrm{E} \quad 00$

$9.18885 \mathrm{E} \quad 00$

$9.29227 \mathrm{E} \quad 00$

$9.39568 \mathrm{E} \quad 00$

$9.49907 \mathrm{E} \quad 00$

$9.60246 \mathrm{E} \quad 00$

$9.70584 \mathrm{E} \quad 00$

$9.80920 \mathrm{E} \quad 00$

$9.91256 \mathrm{E} \quad 00$ $1.00159 \mathrm{E} \quad 01$

$1.01192 \mathrm{E} \quad 01$

$1.02226 \mathrm{E} \quad 01$

$1.03259 \mathrm{E} 01$

$1.04292 \mathrm{E} \quad 01$

$1.05325 \mathrm{E} 0$

$1.06358 \mathrm{E} 01$

$1.07391 \mathrm{E} \quad 01$

$1.08423 \mathrm{E} 01$

$1.09456 \mathrm{E} \quad 01$

$1.10488 \mathrm{E} \quad 01$

$1.11521 \mathrm{E} \quad 01$

$1.12553 \mathrm{E}$ 01

$1.13585 \mathrm{E} \quad 01$

$1.14617 \mathrm{E} \quad 01$ $1.15650 \mathrm{E} 01$ $1.16682 \mathrm{E} 01$ $1.17713 \mathrm{E} 01$

$1.18745 \mathrm{E} 01$

$\begin{array}{lll}1.19777 \mathrm{E} & 01\end{array}$ $120809 \mathrm{E} 01$ $1.21840 \mathrm{E} 01$ $1.22872 \mathrm{E} \quad 01$ $1.23903 \mathrm{E} \quad 01$

$\begin{array}{ll}1.24934 \mathrm{E} & 01\end{array}$ $1.25965 \mathrm{E} \quad 01$ $\begin{array}{lll}1.26996 \mathrm{E} & 01\end{array}$ $1.28028 \mathrm{E} 01$ $1.29058 \mathrm{E} \quad 01$ $\tan \delta_{-1}$

$-2.57371 \mathrm{E} \quad 02$ $-2.59357 \mathrm{E} \quad 02$ $-2.61310 \mathrm{E} \quad 02$ $-2.63229 \mathrm{E} \quad 02$ $-2.65116 \mathrm{E} 02$

$-2.66971 \mathrm{E} \quad 02$ $-2.68796 \mathrm{E} \quad 02$ $-2.70591 \mathrm{E} \quad 02$ $-2.72356 \mathrm{E} \quad 02$ $-2.74094 \mathrm{E} \quad 02$ $-2.75803 \mathrm{E} \quad 02$ $-2.77486 \mathrm{E} \quad 02$ $-2.79142 \mathrm{E} \quad 02$ $\begin{array}{ll}-2.80772 \mathrm{E} & 02\end{array}$ $-2.82377 \mathrm{E} 02$

$-2.83958 \mathrm{E} 02$ $-2.85515 \mathrm{E} \quad 02$ $-2.87048 \mathrm{E} 02$ $-2.88559 \mathrm{E} \quad 02$ $-2.90046 \mathrm{E} \quad 02$

$\begin{array}{lll}-2.91512 \mathrm{E} & 02\end{array}$ $-2.92957 \mathrm{E} \quad 02$ $\begin{array}{lll}-2.94382 \mathrm{E} & 02\end{array}$ $-2.95785 \mathrm{E} 02$ $-2.97169 \mathrm{E} 02$

$-2.98533 \mathrm{E} \quad 02$ $\begin{array}{ll}-2.99878 \mathrm{E} & 02\end{array}$ $-3.01205 \mathrm{E} 02$ $-3.02513 \mathrm{E} 02$ $-3.03804 \mathrm{E} 02$

$-3.05077 \mathrm{E} \quad 02$ $-3.06332 \mathrm{E} 02$ $-3.07571 \mathrm{E} \quad 02$ $-3.08794 \mathrm{E} \quad 02$ $-3.10001 \mathrm{E} 02$

$-3.11192 \mathrm{E} \quad 02$ $\begin{array}{lll}-3.12367 \mathrm{E} & 02\end{array}$ $-3.13528 \mathrm{E} 02$ $-3.14674 \mathrm{E} \quad 02$ -3.15805 E 02

$-3.16922 \mathrm{E} \quad 02$ $-3.18025 \mathrm{E} \quad 02$ $-3.19114 \mathrm{E} \quad 02$ $-3.20191 \mathrm{E} 02$ $-3.21254 \mathrm{E} 02$

$-3.22304 \mathrm{E} \quad 02$ $-3.23342 \mathrm{E} 02$ $-3.24367 \mathrm{E} \quad 02$ $-3.25380 \mathrm{E} \quad 02$ $\begin{array}{lll}-3.26381 \mathrm{E} & 02\end{array}$

$-3.27371 \mathrm{E} \quad 02$ $\begin{array}{lll}-3.28349 \mathrm{E} & 02\end{array}$ $-3.29316 \mathrm{E} \quad 02$ $\begin{array}{lll}-3.30272 \mathrm{E} & 02\end{array}$ $-3.31217 \mathrm{E} \quad 02$

$-3.32152 \mathrm{E} \quad 02$ $-3.33076 \mathrm{E} 02$ $-3.33990 \mathrm{E} \quad 02$ $\begin{array}{ll}-3.34894 \mathrm{E} & 02\end{array}$ $-3.35788 \mathrm{E} 02$ 
ELECTRON RADIAL FUNCTIONS

$Z=3 \quad A=6 \quad \rho=1.2 A^{1 / 3} F$

Electron

$\bar{F}$

$3.53703 \mathrm{E}-05$

$3.65383 \mathrm{E}-05$

$3.77253 \mathrm{E}-05$

$3.89312 \mathrm{E}-05$

$4.01562 \mathrm{E}-05$

$4.14001 \mathrm{E}-05$

$4.26630 \mathrm{E}-05$

$4.39450 \mathrm{E}-05$

$4.52458 \mathrm{E}-05$

$4.65657 \mathrm{E}-05$

$4.79046 \mathrm{E}-05$ $4.92624 \mathrm{E}-05$

$5.06393 \mathrm{E}-05$

$5.20351 \mathrm{E}-05$

$5.34499 \mathrm{E}-05$

$5.48837 \mathrm{E}-05$

$5.63365 \mathrm{E}-05$

$5.78083 \mathrm{E}-05$

$5.92990 \mathrm{E}-05$

$6.08088 \mathrm{E}-05$

$6.23375 \mathrm{E}-05$

$6.38852 \mathrm{E}-05$

$6.54519 \mathrm{E}-05$

$6.70376 \mathrm{E}-05$

$6.86422 \mathrm{E}-05$

$7.02659 \mathrm{E}-05$

$7.19085 \mathrm{E}-05$

$7.35701 \mathrm{E}-05$

$7.52507 \mathrm{E}-05$

$7.69504 \mathrm{E}-05$

$7.86689 \mathrm{E}-05$ $8.04065 \mathrm{E}-05$ $8.21631 \mathrm{E}-05$ $8.39386 \mathrm{E}-05$

$8.57331 \mathrm{E}-05$

$8.75466 \mathrm{E}-05$ $8.93791 \mathrm{E}-05$ $9.12306 \mathrm{E}-05$ $9.31010 \mathrm{E}-05$

$9.49905 \mathrm{E}-05$

$9.68989 \mathrm{E}-05$

$9.88263 \mathrm{E}-05$

$1.00773 \mathrm{E}-04$

$1.02738 \mathrm{E}-04$

$1.04722 \mathrm{E}-04$

$1.06726 \mathrm{E}-04$

$1.08748 \mathrm{E}-04$

$1.10789 \mathrm{E}-04$

$1.12850 \mathrm{E}-04$

$1.14929 \mathrm{E}-04$

$1.17027 \mathrm{E}-04$

$1.19145 \mathrm{E}-04$

$1.21281 \mathrm{E}-04$

$1.23436 \mathrm{E}-04$

$1.25610 \mathrm{E}-04$

$1.27803 \mathrm{E}-04$ $1.30016 \mathrm{E}-04$ $1.32247 \mathrm{E}-04$.

$1.34497 \mathrm{E}-04$

$1.36766 \mathrm{E}-04$ $f_{2}$

$-6.63933 \mathrm{E}-02$

$-6.86903 \mathrm{E}-02$

$-7.10262 \mathrm{E}-02$

$-7.34010 \mathrm{E}-02$

$-7.58147 \mathrm{E}-02$

$-7.82674 \mathrm{E}-02$

$-8.07590 \mathrm{E}-02$

$-8.32896 \mathrm{E}-02$

$-8.58590 \mathrm{E}-02$

$-8.84674 \mathrm{E}-02$

$-9.11147 \mathrm{E}-02$ $-9.38009 \mathrm{E}-02$

$-9.65260 \mathrm{E}-02$

$-9.92901 \mathrm{E}-02$

$-1.02093 \mathrm{E}-01$

$-1.04935 \mathrm{E}-01$

$-1.07816 \mathrm{E}-0$

$-1.10735 \mathrm{E}-01$

$-1.13694 \mathrm{E}-01$

$-1.16691 \mathrm{E}-01$

$-1.19728 \mathrm{E}-01$

$-1.22803 \mathrm{E}-01$

$-1.25917 \mathrm{E}-01$

$-1.29070 \mathrm{E}-01$

$-1.32262 \mathrm{E}-01$

$-1.35492 \mathrm{E}-01$

$-1.38762 \mathrm{E}-01$

$-1.42071 \mathrm{E}-01$

$-1.45418 \mathrm{E}-01$

$-1.48804 \mathrm{E}-01$

$-1.52229 \mathrm{E}-01$

$-1.55693 \mathrm{E}-01$

$-1.59196 \mathrm{E}-01$

$-1.62738 \mathrm{E}-01$

$-1.66318 \mathrm{E}-01$

$-1.69938 \mathrm{E}-01$

$-1.73596 \mathrm{E}-01$

$-1.77293 \mathrm{E}-01$

$-1.81029 \mathrm{E}-01$

$-1.84804 \mathrm{E}-01$

$-1.88618 \mathrm{E}-01$

$-1.92470 \mathrm{E}-01$

$-1.96361 \mathrm{E}-01$

$-2.00292 \mathrm{E}-01$

$-2.04261 \mathrm{E}-01$

$-2.08268 \mathrm{E}-01$

$-2.12315 \mathrm{E}-01$

$-2.16401 \mathrm{E}-01$

$-2.20525 \mathrm{E}-01$

$-2.24688 \mathrm{E}-01$

$-2.28890 \mathrm{E}-01$

$-2.33131 \mathrm{E}-01$

$-2.37410 \mathrm{E}-01$

$-2.41729 \mathrm{E}-01$

$-2.46086 \mathrm{E}-01$

$-2.50482 \mathrm{E}-01$

$-2.54917 \mathrm{E}-01$

$-2.59390 \mathrm{E}-01$

$-2.63903 \mathrm{E}-01$

$-2.68454 \mathrm{E}-01$ $g_{2}$

$-8.70793 \mathrm{E}-04$

$-9.08579 \mathrm{E}-04$

$-9.47399 \mathrm{E}-04$

$-9.87268 \mathrm{E}-04$

$-1.02820 \mathrm{E}-03$

$-1.07020 \mathrm{E}-03$

$-1.11329 \mathrm{E}-03$

$-1.15749 \mathrm{E}-03$

$-1.20279 \mathrm{E}-03$

$-1.24923 \mathrm{E}-03$

$-1.29680 \mathrm{E}-03$

$-1.34553 \mathrm{E}-03$

$-1.39542 \mathrm{E}-03$

$-1.44650 \mathrm{E}-03$

$-1.49876 \mathrm{E}-03$

$-1.55224 \mathrm{E}-03$

$-1.60693 \mathrm{E}-03$

$-1.66285 \mathrm{E}-03$

$-1.72002 \mathrm{E}-03$

$-1.77845 \mathrm{E}-03$

$-1.83815 \mathrm{E}-03$

$-1.89914 \mathrm{E}-03$

$-1.96142 \mathrm{E}-03$

$-2.02501 \mathrm{E}-03$

$-2.08993 \mathrm{E}-03$

$-2.15619 \mathrm{E}-03$

$-2.22380 \mathrm{E}-03$

$-2.29277 \mathrm{E}-03$

$-2.36311 \mathrm{E}-03$

$-2.43485 \mathrm{E}-03$

$-2.50800 \mathrm{E}-03$

$-2.58255 \mathrm{E}-03$

$-2.65854 \mathrm{E}-03$

$-2.73597 \mathrm{E}-03$

$-2.81486 \mathrm{E}-03$

$-2.89521 \mathrm{E}-03$

$-2.97705 \mathrm{E}-03$

$-3.06038 \mathrm{E}-03$

$-3.14522 \mathrm{E}-03$

$-3.23159 \mathrm{E}-03$

$-3.31949 \mathrm{E}-03$

$-3.40893 \mathrm{E}-03$

$-3.49994 \mathrm{E}-03$

$-3.59252 \mathrm{E}-03$

$-3.68669 \mathrm{E}-03$

$-3.78246 \mathrm{E}-03$

$-3.87984 \mathrm{E}-03$

$-3.97885 \mathrm{E}-03$

$-4.07950 \mathrm{E}-03$

$-4.18180 \mathrm{E}-03$

$-4.28576 \mathrm{E}-03$

$-4.39141 \mathrm{E}-03$

$-4.49875 \mathrm{E}-03$

$-4.60780 \mathrm{E}-03$

$-4.71856 \mathrm{E}-03$

$-4.83105 \mathrm{E}-03$

$-4.94529 \mathrm{E}-03$

$-5.06129 \mathrm{E}-03$

$-5.17906 \mathrm{E}-03$

$-5.29861 \mathrm{E}-03$ $\tan \delta_{2}$

$f_{-2}$

$6.39682 \mathrm{E} \quad 01 \quad-8.48640 \mathrm{E}-04$ $6.40531 \mathrm{E} \quad 01 \quad-8.84703 \mathrm{E}-04$ $6.41352 \mathrm{E} \quad 01 \quad-9.21758 \mathrm{E}-04$ $6.42145 \mathrm{E} \quad 01 \quad-9.59816 \mathrm{E}-04$ $6.42913 \mathrm{E} \quad 01 \quad-9.98892 \mathrm{E}-04$

$6.43655 \mathrm{E} \quad 01-1.03900 \mathrm{E}-03$ $6.44374 \mathrm{E}$ ol $-1.08015 \mathrm{E}-03$ $6.45071 \mathrm{E} \quad 01-1.12235 \mathrm{E}-03$ $6.45746 \mathrm{E} \quad 01-1.16563 \mathrm{E}-03$ $6.46400 \mathrm{E} \quad 01-1.20999 \mathrm{E}-03$

$6.47035 \mathrm{E} \quad 01$ $6.47651 \mathrm{E} \quad 01$ $6.48250 \mathrm{E}$ 01 $6.48830 \mathrm{E} \quad 01$ $6.49395 \mathrm{E} \quad 01$

$6.49944 \mathrm{E} \quad 01$ $6.50477 \mathrm{E} \quad 01$ $6.50996 \mathrm{E} \quad 01$ $6.51501 \mathrm{E} \quad 01$ $6.51993 \mathrm{E} \quad 01$

$6.52471 \mathrm{E} \quad 01$ $6.52938 \mathrm{E} \quad 01$ $6.53392 \mathrm{E} \quad 01$ $6.53835 \mathrm{E} \quad 01$ $6.54266 \mathrm{E} \quad 01$

$6.54688 \mathrm{E} \quad 01$ $6.55098 \mathrm{E} \quad 01$ $6.55499 \mathrm{E} \quad 01$ $6.55891 \mathrm{E} \quad 01$ $6.56273 \mathrm{E} \quad 01$

$6.56646 \mathrm{E} \quad 01$ $6.57011 \mathrm{E} \quad 01$ $6.57367 \mathrm{E} \quad 01$ $6.57715 \mathrm{E} \quad 01$ $6.58056 \mathrm{E} \quad 01$

$6.58389 \mathrm{E} \quad 01$ $6.58715 \mathrm{E} 01$ $6.59033 \mathrm{E} \quad 01$ $6.59345 \mathrm{E} \quad 01$ $6.59650 \mathrm{E} \quad 01$

$6.59949 \mathrm{E} \quad 01$ $6.60242 \mathrm{E} \quad 01$ $6.60528 \mathrm{E} 01$ $6.60809 \mathrm{E} \quad 01$ $6.61084 \mathrm{E} \quad 0$

$6.61354 \mathrm{E} \quad 01$ $6.61619 \mathrm{E} \quad 01$ $6.61878 \mathrm{E} \quad 01$ $6.62132 \mathrm{E} \quad 01$ $6.62381 \mathrm{E} 01$

$6.62626 \mathrm{E} \quad 01$ $6.62866 \mathrm{E} \quad 01$ $6.63101 \mathrm{E} \quad 01$ $6.63332 \mathrm{E} \quad 01$ $6.63559 \mathrm{E} \quad 01$

$6.63782 \mathrm{E} \quad 01$ $6.64000 \mathrm{E} \quad 01$ $6.64215 \mathrm{E} 01$ $6.64426 \mathrm{E} \quad 01$ $6.64634 \mathrm{E} \quad 01$

$-1.25544 \mathrm{E}-03$ $-1.30200 \mathrm{E}-03$ $-1.34969 \mathrm{E}-03$ $-1.39851 \mathrm{E}-03$ $-1.44848 \mathrm{E}-03$

$-1.49961 \mathrm{E}-03$ $-1.55192 \mathrm{E}-03$ $-1.60541 \mathrm{E}-03$ $-1.66011 \mathrm{E}-03$ $-1.71602 \mathrm{E}-03$

$-1.77316 \mathrm{E}-03$ $-1.83154 \mathrm{E}-03$ $-1.89117 \mathrm{E}-03$ $-1.95207 \mathrm{E}-03$ $-2.01426 \mathrm{E}-03$

$-2.07773 \mathrm{E}-03$ $-2.14251 \mathrm{E}-03$ $-2.20861 \mathrm{E}-03$ $-2.27605 \mathrm{E}-03$ $-2.34483 \mathrm{E}-03$

$-2.41497 \mathrm{E}-03$ $-2.48648 \mathrm{E}-03$ $-2.55937 \mathrm{E}-03$ $-2.63367 \mathrm{E}-03$

$-2.70938 \mathrm{E}-03$

$-2.78651 \mathrm{E}-03$ $-2.86508 \mathrm{E}-03$ $-2.94510 \mathrm{E}-03$ $-3.02659 \mathrm{E}-03$

$-3.10955 \mathrm{E}-03$

$-3.19401 \mathrm{E}-03$

$-3.27997 \mathrm{E}-03$

$-3.36744 \mathrm{E}-03$

$-3.45645 \mathrm{E}-03$

$-3.54700 \mathrm{E}-03$

$-3.63910 \mathrm{E}-03$

$-3.73278 \mathrm{E}-03$

$-3.82803 \mathrm{E}-03$

$-3.92489 \mathrm{E}-03$

$-4.02335 \mathrm{E}-03$

$-4.12343 \mathrm{E}-03$ $-4.22515 \mathrm{E}-03$ 


\begin{tabular}{|c|c|c|c|c|c|}
\hline$p$ & $F_{0}$ & & $f_{1}$ & & $g_{1}$ \\
\hline 12.10 & $1.07286 \mathrm{E}$ & 00 & $-1.19791 \mathrm{E}$ & 01 & $-4.01452 \mathrm{E}-01$ \\
\hline 12.20 & $1.07285 \mathrm{E}$ & 00 & $-1.20822 \mathrm{E}$ & 01 & $-4.07177 \mathrm{E}-01$ \\
\hline 12.30 & $1.07285 \mathrm{E}$ & 00 & $-1.21853 \mathrm{E}$ & 01 & $-4.12941 \mathrm{E}-01$ \\
\hline 12.40 & $1.07284 \mathrm{E}$ & 00 & $-1.22883 \mathrm{E}$ & 01 & $-4.18744 \mathrm{E}-01$ \\
\hline 2.50 & $1.07283 \mathrm{E}$ & 00 & $-1.23914 \mathrm{E}$ & 01 & $-4.24585 \mathrm{E}-01$ \\
\hline 2.60 & $1.07282 \mathrm{E}$ & 00 & $-1.24944 \mathrm{E}$ & 01 & $-4.30465 \mathrm{E}-01$ \\
\hline 2.70 & $1.07281 \mathrm{E}$ & 00 & $-1.25975 \mathrm{E}$ & 01 & $.36383 \mathrm{E}-01$ \\
\hline .80 & $1.07281 \mathrm{E}$ & 00 & $-1.27005 \mathrm{E}$ & 01 & $-4.42340 \mathrm{E}-01$ \\
\hline .90 & $1.07280 \mathrm{E}$ & 00 & $-1.28035 \mathrm{E}$ & 01 & $-4.48336 \mathrm{E}-01$ \\
\hline .00 & $1.07279 \mathrm{E}$ & 00 & $-1.29065 \mathrm{E}$ & 01 & $-4.54370 \mathrm{E}-01$ \\
\hline .10 & $1.07278 \mathrm{E}$ & 00 & $-1.30095 \mathrm{E}$ & 01 & $-4.60443 \mathrm{E}-01$ \\
\hline .20 & $1.07278 \mathrm{E}$ & 00 & $31125 \mathrm{E}$ & 01 & $-4.66555 \mathrm{E}-01$ \\
\hline .30 & $1.07277 \mathrm{E}$ & 00 & $-1.32155 \mathrm{E}$ & 01 & $-4.72705 \mathrm{E}-01$ \\
\hline .40 & $.07276 \mathrm{E}$ & 00 & $.33185 \mathrm{E}$ & 01 & $-4.78894 \mathrm{E}-01$ \\
\hline 50 & $1.07276 \mathrm{E}$ & 00 & $.34215 \mathrm{E}$ & 01 & $-4.85122 \mathrm{E}-01$ \\
\hline 3.60 & $1.07275 \mathrm{E}$ & 00 & $-1.35245 \mathrm{E}$ & 01 & $-4.91388 \mathrm{E}-01$ \\
\hline .70 & $1.07274 \mathrm{E}$ & 00 & $-1.36274 \mathbf{E}$ & 01 & $-4.97693 \mathrm{E}-01$ \\
\hline .80 & $1.07274 \mathrm{E}$ & 00 & $-1.37304 \mathrm{E}$ & 01 & $-5.04036 \mathrm{E}-01$ \\
\hline & $1.07273 \mathrm{E}$ & 00 & $38333 \mathrm{E}$ & 01 & $10418 \mathrm{E}-01$ \\
\hline .00 & $1.07272 \mathrm{E}$ & 00 & $9362 \mathrm{E}$ & 01 & $6839 \mathrm{~F}$ \\
\hline .10 & $1.07272 \mathrm{E}$ & 00 & $-1.40392 \mathrm{E}$ & 01 & $-5.23299 \mathrm{E}-01$ \\
\hline & $07271 \mathrm{E}$ & 00 & $-1.41421 \mathrm{E}$ & 01 & $.29797 \mathrm{E}-01$ \\
\hline & $1.07271 \mathrm{E}$ & 00 & $2450 \mathrm{E}$ & 01 & $-5.36333 \mathrm{E}-01$ \\
\hline & $270 \mathrm{E}$ & 00 & $479 \mathrm{E}$ & 01 & 429081 \\
\hline J & $69 \mathrm{E}$ & 00 & $14508 \mathrm{E}$ & 01 & $9522 \mathrm{E}-$ \\
\hline .60 & $1.07269 \mathrm{E}$ & 00 & $45537 \mathrm{E}$ & 01 & -5.56175 \\
\hline & $268 \mathrm{E}$ & 00 & $5666 \mathrm{E}$ & 01 & .028001 \\
\hline & $268 \mathrm{E}$ & 00 & $595 \mathrm{E}$ & 01 & - \\
\hline & $267 \mathrm{E}$ & 00 & $18623 \mathrm{E}$ & 01 & 63631 \\
\hline 15.00 & $1.07266 \mathrm{E}$ & 00 & $49652 \mathrm{E}$ & 01 & $-5.83170 \mathrm{E}-$ \\
\hline 10 & $1.07266 \mathrm{E}$ & 00 & $-1.50681 \mathrm{E}$ & 01 & $0016 \mathrm{E}-01$ \\
\hline & $265 \mathrm{E}$ & 00 & $709 \mathrm{E}$ & 01 & \\
\hline J & $5 \mathrm{E}$ & 00 & $-1.52737 \mathrm{E}$ & 01 & $03822 \mathrm{~F}$ \\
\hline .40 & $1.07264 \mathrm{E}$ & 00 & $-1.53766 \mathrm{E}$ & 01 & $-6.10783 \mathrm{E}-01$ \\
\hline .50 & $1.07264 \mathrm{E}$ & 00 & $4794 \mathrm{E}$ & 01 & $17783 \mathrm{E}-01$ \\
\hline .60 & $1.07263 \mathrm{E}$ & 00 & $5822 \mathrm{E}$ & 01 & $1 E-01$ \\
\hline & $63 \mathrm{E}$ & 00 & $850 \mathrm{E}$ & 01 & \\
\hline 80 & $1.07262 \mathrm{E}$ & 00 & $57878 \mathrm{E}$ & 01 & $-6.39013 \mathrm{E}-01$ \\
\hline .90 & $1.07262 \mathrm{E}$ & 00 & $-1.58906 \mathrm{E}$ & 01 & $-6.46167 \mathrm{E}-$ \\
\hline .00 & $1.07261 \mathrm{E}$ & 00 & $9934 \mathrm{E}$ & 01 & $-6.53360 \mathrm{E}-01$ \\
\hline .10 & $1.07261 \mathrm{E}$ & 00 & $-1.60962 \mathrm{E}$ & 01 & $1 \mathrm{E}-01$ \\
\hline & & 00 & $1989 \mathrm{E}$ & 01 & -6 \\
\hline 30 & $1.07260 \mathrm{E}$ & 00 & $-1.63017 \mathrm{E}$ & 01 & $-6.75168 \mathrm{E}-$ \\
\hline 4 & $.07259 \mathrm{E}$ & 00 & $-1.64044 \mathrm{E}$ & 01 & $-6.82515 \mathrm{E}$ \\
\hline 55 & $59 \mathrm{E}$ & 00 & $5072 \mathrm{E}$ & 01 & $9900 \mathrm{~F}$ \\
\hline 16.60 & $1.07258 \mathrm{E}$ & 00 & $-1.66099 \mathrm{E}$ & 01 & $E-01$ \\
\hline 70 & $258 \mathrm{E}$ & 00 & $57126 \mathrm{E}$ & 01 & $-7.04786 \mathrm{E}-$ \\
\hline 8 & $1.07257 \mathrm{E}$ & 00 & $68153 \mathrm{E}$ & $0]$ & $-7.12287 \mathrm{E}$ \\
\hline 6.9 & $257 \mathrm{E}$ & 00 & $59180 \mathrm{E}$ & 01 & -7.19826 \\
\hline 8 & $56 \mathrm{E}$ & 00 & $207 \mathrm{E}$ & 01 & 2 \\
\hline .1 & $1.07256 \mathrm{E}$ & 00 & $-1.71234 \mathrm{E}$ & 01 & -7.3502 \\
\hline & $256 \mathrm{E}$ & 00 & $2261 \mathrm{E}$ & 01 & -7.4267 \\
\hline & $1.07255 \mathrm{E}$ & 00 & $73288 \mathrm{E}$ & 0 & $-7.50368 \mathrm{H}$ \\
\hline & $255 \mathrm{E}$ & 00 & & 01. & $-7.58100 \mathrm{E}-01$ \\
\hline 17.50 & $1.07254 \mathrm{E}$ & 00 & $-1.75341 \mathrm{E}$ & 01 & $-7.65870 \mathrm{E}-01$ \\
\hline & $\mathbf{E}$ & 00 & $368 \mathrm{E}$ & 01 & -01 \\
\hline & $253 \mathrm{E}$ & 0 & $1.77394 \mathrm{E}$ & 0 & -7.815261 \\
\hline & $253 \mathrm{E}$ & 0 & $1.78420 \mathrm{E}$ & & -7.894121 \\
\hline & $53 \mathrm{E}$ & 00 & $-1.79447 \mathrm{E}$ & & $-7.97337 \mathrm{E}-01$ \\
\hline
\end{tabular}

$\tan \delta_{1} \quad f_{-1}$

$1.16248 \mathrm{E}-03$

$1.16963 \mathrm{E}-03$

$1.17666 \mathrm{E}-03$

$1.18357 \mathrm{E}-03$

$1.19037 \mathrm{E}-03$

$1.19705 \mathrm{E}-03$

$1.20363 \mathrm{E}-03$

$1.21010 \mathrm{E}-03$

$1.21646 \mathrm{E}-03$

$1.22273 \mathrm{E}-03$

$1.22889 \mathrm{E}-03$

$1.23496 \mathrm{E}-03$

$1.24093 \mathrm{E}-03$

$1.24681 \mathrm{E}-03$

$1.25260 \mathrm{E}-03$

$1.25830 \mathrm{E}-03$

$1.26391 \mathrm{E}-03$

$1.26944 \mathrm{E}-03$

$1.27488 \mathrm{E}-03$

$1.28024 \mathrm{E}-03$

$1.28552 \mathrm{E}-03$

$1.29073 \mathrm{E}-03$

$1.29586 \mathrm{E}-03$

$1.30091 \mathrm{E}-03$

$1.30588 \mathrm{E}-03$

$1.31079 \mathrm{E}-03$

$1.31563 \mathrm{E}-03$

$1.32040 \mathrm{E}-03$

$1.32509 \mathrm{E}-03$

$1.32973 \mathrm{E}-03$

$1.33429 \mathrm{E}-03$

$1.33879 \mathrm{E}-03$

$1.34323 \mathrm{E}-03$

$1.34761 \mathrm{E}-03$

$1.35193 \mathrm{E}-03$

$1.35618 \mathrm{E}-03$ $1.36038 \mathrm{E}-03$

$1.36452 \mathrm{E}-03$

$1.36861 \mathrm{E}-03$

$1.37264 \mathrm{E}-03$

$1.37662 \mathrm{E}-03$

$1.38054 \mathrm{E}-03$

$1.38441 \mathrm{E}-03$

$1.38823 \mathrm{E}-03$

$1.39200 \mathrm{E}-03$

$1.39572 \mathrm{E}-03$

$1.39939 \mathrm{E}-03$

$1.40301 \mathrm{E}-03$

$1.40659 \mathrm{E}-03$

$1.41012 \mathrm{E}-03$

$1.41361 \mathrm{E}-03$ $1.41705 \mathrm{E}-03$ $1.42044 \mathrm{E}-03$ $1.42380 \mathrm{E}-03$ $1.42711 \mathrm{E}-03$

$1.43038 \mathrm{E}-03$

$1.43360 \mathrm{E}-03$

$1.43679 \mathrm{E}-03$

$1.43994 \mathrm{E}-03$

$1.44305 \mathrm{E}-03$
$-3.86962 \mathrm{E}-01$ $-3.92493 \mathrm{E}-01$ $-3.98061 \mathrm{E}-01$ $-4.03669 \mathrm{E}-01$ $-4.09315 \mathrm{E}-01$

$-4.15000 \mathrm{E}-01$ $-4.20724 \mathrm{E}-01$ $-4.26486 \mathrm{E}-01$ $-4.32287 \mathrm{E}-01$ $-4.38126 \mathrm{E}-01$

$-4.44005 \mathrm{E}-01$ $-4.49922 \mathrm{E}-01$ $-4.55877 \mathrm{E}-01$ $-4.61871 \mathrm{E}-01$ $-4.67904 \mathrm{E}-01$

$-4.73975 \mathrm{E}-01$ $-4.80086 \mathrm{E}-01$ $-4.86234 \mathrm{E}-01$ $-4.92422 \mathrm{E}-01$ $-4.98648 \mathrm{E}-01$

$-5.04912 \mathrm{E}-01$ $-5.11215 \mathrm{E}-01$ $-5.17557 \mathrm{E}-01$ $-5.23938 \mathrm{E}-01$ $-5.30357 \mathrm{E}-01$

$-5.36815 \mathrm{E}-01$ $-5.43311 \mathrm{E}-01$ $-5.49846 \mathrm{E}-01$ $-5.56419 \mathrm{E}-01$ $-5.63031 \mathrm{E}-01$

$-5.69682 \mathrm{E}-01$ $-5.76371 \mathrm{E}-01$ $-5.83099 \mathrm{E}-01$ $-5.89865 \mathrm{E}-01$ $-5.96670 \mathrm{E}-01$

$-6.03514 \mathrm{E}-01$ $-6.10396 \mathrm{E}-01$ $-6.17317 \mathrm{E}-01$ $-6.24276 \mathrm{E}-01$ $-6.31274 \mathrm{E}-01$

$-6.38310 \mathrm{E}-01$ $-6.45385 \mathrm{E}-01$ $-6.52499 \mathrm{E}-01$ $-6.59651 \mathrm{E}-01$ $-6.66841 \mathrm{E}-01$

$-6.74070 \mathrm{E}-01$ $-6.81338 \mathrm{E}-01$ $-6.88644 \mathrm{E}-01$ $-6.95989 \mathrm{E}-01$ $-7.03372 \mathrm{E}-01$

$-7.10794 \mathrm{E}-01$ $-7.18254 \mathrm{E}-01$ $-7.25753 \mathrm{E}-01$ $-7.33290 \mathrm{E}-01$ $-7.40866 \mathrm{E}-01$

$-7.48480 \mathrm{E}-01$ $-7.56133 \mathrm{E}-01$ $-7.63824 \mathrm{E}-01$ $-7.71554 \mathrm{E}-01$ $-7.79322 \mathrm{E}-01$ $g_{-1}$ $\tan \delta_{-1}$

$\begin{array}{llll}1.30089 \mathrm{E} & 01 & -3.36672 \mathrm{E} & 02\end{array}$ $\begin{array}{lllll}1.31120 \mathrm{E} & 01 & -3.37547 \mathrm{E} & 02\end{array}$ $\begin{array}{llll}1.32151 \mathrm{E} & 01 & -3.38413 \mathrm{E} & 02\end{array}$ $\begin{array}{llll}1.33181 \mathrm{E} & 01 & -3.39268 \mathrm{E} & 02\end{array}$ $\begin{array}{llll}1.34212 \mathrm{E} & 01 & -3.40116 \mathrm{E} & 02\end{array}$

$1.35242 \mathrm{E} \quad 01$ $1.36273 \mathrm{E} \quad 01$ $1.37303 \mathrm{E} \quad 01$ $1.38333 \mathrm{E} \quad 01$ $1.39363 \mathrm{E} \quad 01$

$1.40393 \mathrm{E} \quad 01$

$1.41423 \mathrm{E} \quad 01$

$1.42453 \mathrm{E} \quad 01$

$1.43483 \mathrm{E} \quad 01$

$1.44513 \mathrm{E} 01$

$1.45542 \mathrm{E} \quad 01$ $1.46572 \mathrm{E} \quad 01$

$1.47601 \mathrm{E}$ 01

$1.48631 \mathrm{E} \quad 01$

$1.49660 \mathrm{E} \quad 01$

$1.50689 \mathrm{E} \quad 01$

$1.51718 \mathrm{E} 01$

$1.52747 \mathrm{E} \quad 01$

$1.53776 \mathrm{E} \quad 01$

$1.54805 \mathrm{E} \quad 01$

$1.55834 \mathrm{E} \quad 01$ $1.56863 \mathrm{E} \quad 01$ $1.57891 \mathrm{E} \quad 01$

$1.58920 \mathrm{E} \quad 01$

$1.59948 \mathrm{E} \quad 01$

$1.60977 \mathrm{E} \quad 01$ $1.62005 \mathrm{E} \quad 01$ $1.63033 \mathrm{E} \quad 01$ $1.64061 \mathrm{E} 01$ $1.65089 \mathrm{E} 01$

$1.66117 \mathrm{E} \quad 01$ $1.67145 \mathrm{E} \quad 01$ $1.68173 \mathrm{E} 01$ $1.69201 \mathrm{E} 01$ $1.70228 \mathrm{E} \quad 01$

$1.71256 \mathrm{E} \quad 01$ $1.72283 \mathrm{E} \quad 01$ $1.73310 \mathrm{E} \quad 01$ $1.74338 \mathrm{E} \quad 01$ $1.75365 \mathrm{E} 01$

$1.76392 \mathrm{E} \quad 01$ $1.77419 \mathrm{E} \quad 01$ $1.78446 \mathrm{E} \quad 01$ $1.79473 \mathrm{E} \quad 01$ $1.80499 \mathrm{E} 01$

$1.81526 \mathrm{E} \quad 01$ $1.82553 \mathrm{E} \quad 01$ $1.83579 \mathrm{E} \quad 01$ $1.84606 \mathrm{E} \quad 01$ $1.85632 \mathrm{E} \quad 01$

$1.86658 \mathrm{E} \quad 01$ $1.87684 \mathrm{E} \quad 01$ $1.88710 \mathrm{E} \quad 01$ $1.89736 \mathrm{E} \quad 01$ $1.90762 \mathrm{E} \quad 01$
$-3.40954 \mathrm{E} \quad 02$

$-3.41784 \mathrm{E} \quad 02$ $-3.42605 \mathrm{E} \quad 02$

$-3.43418 \mathrm{E} \quad 02$

$-3.44222 \mathrm{E} \quad 02$

$-3.45019 \mathrm{E} \quad 02$ $-3.45807 \mathrm{E} \quad 02$ $-3.46588 \mathrm{E} 02$ $-3.47361 \mathrm{E} 02$ $-3.48126 \overline{\mathrm{E}} 02$

$-3.48884 \mathrm{E} \quad 02$ $-3.49634 \mathrm{E} \quad 02$ $-3.50377 \mathrm{E} \quad 02$ $-3.51114 \mathrm{E} \quad 02$ $-3.51843 \mathrm{E} 02$ $-3.52565 \mathrm{E} \quad 02$ $-3.53281 \mathrm{E} 02$ $-3.53990 \mathrm{E} 02$ $-3.54692 \mathrm{E} \quad 02$ $-3.55388 \mathrm{E} 02$

$-3.56078 \mathrm{E} \quad 02$ $-3.56761 \mathrm{E} 02$ $\begin{array}{lll}-3.57438 \mathrm{E} & 02\end{array}$ $-3.58110 \mathrm{E} 02$ $-3.58775 \mathrm{E} 02$

$-3.59435 \mathrm{E} \quad 02$ $-3.60089 \mathrm{E} \quad 02$ $-3.60736 \mathrm{E} 02$ $-3.61379 \mathrm{E} \quad 02$ $-3.62016 \mathrm{E} 02$

$-3.62648 \mathrm{E} \quad 02$ $-3.63274 \mathrm{E} \quad 02$ $-3.63895 \mathrm{E} \quad 02$ $-3.64511 \mathrm{E} 02$ $-3.65122 \mathrm{E} \quad 02$

$-3.65728 \mathrm{E} \quad 02$ $-3.66329 \mathrm{E} 02$ $\begin{array}{ll}-3.66925 \mathrm{E} & 02\end{array}$ $-3.67516 \mathrm{E} 02$ $-3.68103 \mathrm{E} 02$

$-3.68685 \mathrm{E} \quad 02$ $-3.69262 \mathrm{E} 02$ $-3.69835 \mathrm{E} \quad 02$ $-3.70403 \mathrm{E} 02$ \begin{tabular}{ll}
$-3.70968 \mathrm{E} \quad 02$ \\
\hline
\end{tabular} $-3.71527 \mathrm{E} \quad 02$ $-3.72083 \mathrm{E} 02$ $-3.72634 \mathrm{E} \quad 02$ $-3.73181 \mathrm{E} 02$ $-3.73724 \mathrm{E} \quad 02$

$-3.74263 \mathrm{E} 02$ $-3.74799 \mathrm{E} 02$ $-3.75330 \mathrm{E} 02$ $-3.75857 \mathrm{E} 02$ $-3.76381 \mathrm{E} \quad 02$ 
$\bar{F}$

$1.39054 \mathrm{E}-04$ $1.41361 \mathrm{E}-04$ $1.43688 \mathrm{E}-04$ $1.46033 \mathrm{E}-04$ $1.48397 \mathrm{E}-04$

$1.50780 \mathrm{E}-04$ $1.53182 \mathrm{E}-04$ $1.55603 \mathrm{E}-04$ $1.58043 \mathrm{E}-04$ $1.60502 \mathrm{E}-04$

$1.62980 \mathrm{E}-04$ $1.65477 \mathrm{E}-04$ $1.67992 \mathrm{E}-04$ $1.70527 \mathrm{E}-04$ $1.73081 \mathrm{E}-04$

$1.75654 \mathrm{E}-04$ $1.78246 \mathrm{E}-04$ $1.80857 \mathrm{E}-04$ $1.83487 \mathrm{E}-04$ $1.86135 \mathrm{E}-04$

$1.88803 \mathrm{E}-04$ $1.91490 \mathrm{E}-04$ $1.94196 \mathrm{E}-04$ $1.96921 \mathrm{E}-04$ $1.99664 \mathrm{E}-04$

$2.02427 \mathrm{E}-04$ $2.05209 \mathrm{E}-04$ $2.08009 \mathrm{E}-04$ $2.10829 \mathrm{E}-04$ $2.13668 \mathrm{E}-04$

$2.16525 \mathrm{E}-04$ $2.19402 \mathrm{E}-04$ $2.22297 \mathrm{E}-04$ $2.25212 \mathrm{E}-04$ $2.28145 \mathrm{E}-04$

$2.31098 \mathrm{E}-04$ $2.34070 \mathrm{E}-04$ $2.37060 \mathrm{E}-04$ $2.40070 \mathrm{E}-04$ $2.43098 \mathrm{E}-04$

$2.46145 \mathrm{E}-04$ $2.49212 \mathrm{E}-04$ $2.52297 \mathrm{E}-04$ $2.55402 \mathrm{E}-04$ $2.58525 \mathrm{E}-04$

$2.61667 \mathrm{E}-04$ $2.64829 \mathrm{E}-04$ $2.68009 \mathrm{E}-04$ $2.71208 \mathrm{E}-04$ 2.74427 E-04

$2.77664 \mathrm{E}-04$ $2.80920 \mathrm{E}-04$ $2.84195 \mathrm{E}-04$ $2.87490 \mathrm{E}-04$ $2.90803 \mathrm{E}-04$

$2.94135 \mathrm{E}-04$ $2.97486 \mathrm{E}-04$ $3.00856 \mathrm{E}-04$ $3.04245 \mathrm{E}-04$ $3.07654 \mathrm{E}-04$ $f_{2}$

$g_{2}$

$-2.73044 \mathrm{E}-01$ $-2.77673 \mathrm{E}-01$ $-2.82341 \mathrm{E}-01$ $-2.87047 \mathrm{E}-01$ $-2.91792 \mathrm{E}-01$

$-2.96576 \mathrm{E}-01$ $-3.01399 \mathrm{E}-01$ $-3.06260 \mathrm{E}-01$ $-3.11161 \mathrm{E}-01$ $-3.16100 \mathrm{E}-01$

$-3.21078 \mathrm{E}-01$ $-3.26094 \mathrm{E}-01$ $-3.31149 \mathrm{E}-01$ $-3.36244 \mathrm{E}-01$ $-3.41376 \mathrm{E}-01$

$-3.46548 \mathrm{E}-01$ $-3.51758 \mathrm{E}-01$ $-3.57007 \mathrm{E}-01$ $-3.62295 \mathrm{E}-01$ $-3.67622 \mathrm{E}-01$

$-3.72987 \mathrm{E}-01$ $-3.78391 \mathrm{E}-01$ $-3.83834 \mathrm{E}-01$ $-3.89316 \mathrm{E}-01$

$-3.94836 \mathrm{E}-01$

$-4.00395 \mathrm{E}-01$ $-4.05993 \mathrm{E}-01$ $-4.11629 \mathrm{E}-01$ $-4.17304 \mathrm{E}-01$ $-4.23018 \mathrm{E}-01$

$-4.28771 \mathrm{E}-01$

$-4.34562 \mathrm{E}-01$ $-4.40392 \mathrm{E}-01$ $-4.46261 \mathrm{E}-01$ $-4.52168 \mathrm{E}-01$

$-4.58114 \mathrm{E}-01$ $-4.64099 \mathrm{E}-01$ $-4.70123 \mathrm{E}-01$ $-4.76185 \mathrm{E}-01$ $-4.82286 \mathrm{E}-01$

$-4.88425 \mathrm{E}-01$ $-4.94603 \mathrm{E}-01$ $-5.00820 \mathrm{E}-01$ $-5.07076 \mathrm{E}-01$ $-5.13370 \mathrm{E}-01$

$-5.19703 \mathrm{E}-01$ $-5.26074 \mathrm{E}-01$ $-5.32485 \mathrm{E}-01$ $-5.38934 \mathrm{E}-01$ $-5.45421 \mathrm{E}-01$

$-5.51947 \mathrm{E}-01$ $-5.58512 \mathrm{E}-01$ $-5.65116 \mathrm{E}-01$ $-5.71758 \mathrm{E}-01$ $-5.78438 \mathrm{E}-01$

$-5.85158 \mathrm{E}-01$ $-5.91916 \mathrm{E}-01$ $-5.98713 \mathrm{E}-01$ $-6.05548 \mathrm{E}-01$ $-6.12422 \mathrm{E}-01$ $\tan \delta_{2}$ $f_{-2}$

$-5.41997 \mathrm{E}-03$ $-5.54313 \mathrm{E}-03$ $-5.66812 \mathrm{E}-03$ $-5.79494 \mathrm{E}-03$ $-5.92361 \mathrm{E}-03$

$-6.05414 \mathrm{E}-03$ $-6.18655 \mathrm{E}-03$ $-6.32085 \mathrm{E}-03$ $-6.45706 \mathrm{E}-03$ $-6.59517 \mathrm{E}-03$

$-6.73522 \mathrm{E}-03$ $-6.87720 \mathrm{E}-03$ $-7.02115 \mathrm{E}-03$ $-7.16706 \mathrm{E}-03$ $-7.31495 \mathrm{E}-03$

$-7.46483 \mathrm{E}-03$ $-7.61672 \mathrm{E}-03$ $-7.77063 \mathrm{E}-03$ $-7.92658 \mathrm{E}-03$ $-8.08457 \mathrm{E}-03$

$-8.24462 \mathrm{E}-03$ $-8.40674 \mathrm{E}-03$ $-8.57095 \mathrm{E}-03$ $-8.73726 \mathrm{E}-03$ $-8.90568 \mathrm{E}-03$

$-9.07623 \mathrm{E}-03$ $-9.24891 \mathrm{E}-03$ $-9.42374 \mathrm{E}-03$ $-9.60074 \mathrm{E}-03$ $-9.77992 \mathrm{E}-03$

$-9.96128 \mathrm{E}-03$ $-1.01449 \mathrm{E}-02$ $-1.03306 \mathrm{E}-02$ $-1.05187 \mathrm{E}-02$ $-1.07089 \mathrm{E}-02$

$-1.09014 \mathrm{E}-02$ $-1.10962 \mathrm{E}-02$ $-1.12933 \mathrm{E}-02$ $-1.14926 \mathrm{E}-02$ $-1.16943 \mathrm{E}-02$

$-1.18983 \mathrm{E}-02$ $-1.21046 \mathrm{E}-02$ $-1.23133 \mathrm{E}-02$ $-1.25243 \mathrm{E}-02$ $-1.27377 \mathrm{E}-02$

$-1.29535 \mathrm{E}-02$ $-1.31717 \mathrm{E}-02$ $-1.33923 \mathrm{E}-02$ $-1.36154 \mathrm{E}-02$ $-1.38408 \mathrm{E}-02$

$-1.40687 \mathrm{E}-02$ $-1.42991 \mathrm{E}-02$ $-1.45320 \mathrm{E}-02$ $-1.47673 \mathrm{E}-02$ $-1.50052 \mathrm{E}-02$

$-1.52456 \mathrm{E}-02$ $-1.54885 \mathrm{E}-02$ $-1.57339 \mathrm{E}-02$ $-1.59819 \mathrm{E}-02$ $-1.62325 \mathrm{E}-02$

$6.64837 \mathrm{E}$ 0l $\quad-5.21638 \mathrm{E}-03$ $6.65037 \mathrm{E} 01 \quad-5.33517 \mathrm{E}-03$ $6.65234 \mathrm{E}$ 01 $-5.45575 \mathrm{E}-03$ $6.65428 \mathrm{E} \quad 01 \quad-5.57811 \mathrm{E}-03$ $6.65618 \mathrm{E} 01-5.70228 \mathrm{E}-03$

$6.65805 \mathrm{E} \quad 01$ $6.65989 \mathrm{E} 01$ $6.66170 \mathrm{E} 01$ $6.66347 \mathrm{E} \quad 01$ $6.66522 \mathrm{E} \quad 01$

$6.66695 \mathrm{E} \quad 01$ $6.66864 \mathrm{E} 01$ $6.67031 \mathrm{E} \quad 01$ $6.67195 \mathrm{E} \quad 01$ $6.67357 \mathrm{E} \quad 01$

$6.67516 \mathrm{E} \quad 01$ $6.67673 \mathrm{E} 01$ $6.67827 \mathrm{E} \quad 01$ $6.67979 \mathrm{E} \quad 01$ $6.68129 \mathrm{E} \quad 01$

$6.68276 \mathrm{E} \quad 01$ $6.68422 \mathrm{E}$ 0l $6.68565 \mathrm{E} \quad 01$ $6.68706 \mathrm{E}$ 01 $6.68845 \mathrm{E} \quad 01$

$6.68982 \mathrm{E} \quad 01$ $6.69117 \mathrm{E} \quad 01$ $6.69250 \mathrm{E} \quad 01$ $6.69382 \mathrm{E} \quad 01$ $6.69511 \mathrm{E} \quad 01$

$6.69639 \mathrm{E} \quad 01$ $6.69765 \mathrm{E} \quad 01$ $6.69889 \mathrm{E} \quad 01$ $6.70011 \mathrm{E} \quad 01$ $6.70132 \mathrm{E}$ ol

$6.70252 \mathrm{E} \quad 01$ $6.70369 \mathrm{E} \quad 01$ $6.70485 \mathrm{E}$ ol $6.70600 \mathrm{E} \quad 01$ $6.70713 \mathrm{E} \quad 01$

$6.70825 \mathrm{E} \quad 01$ $6.70935 \mathrm{E} \quad 01$ $6.71044 \mathrm{E}$ 01 $6.71151 \mathrm{E} \quad 01$ $6.71257 \mathrm{E} \quad 01$

$6.71362 \mathrm{E} \quad 01$ $6.71465 \mathrm{E} \quad 01$ $6.71567 \mathrm{E} \quad 01$ $6.71668 \mathrm{E} \quad 01$ $6.71768 \mathrm{E} \quad 01$

$6.71866 \mathrm{E}$ 01 $-1.35929 \mathrm{E}-02$ $6.71963 \mathrm{E}$ 01 $-1.38167 \mathrm{E}-02$ $6.72059 \mathrm{E} 01-1.40430 \mathrm{E}-02$ $6.72154 \mathrm{E} \quad 01-1.42717 \mathrm{E}-02$ $6.72248 \mathrm{E}$ 01 $-1.45028 \mathrm{E}-02$

$6.72341 \mathrm{E} \quad 01 \quad-1.47364 \mathrm{E}-02$ $6.72432 \mathrm{E}$ 01 $-1.49726 \mathrm{E}-02$ $6.72523 \mathrm{E}$ 01 $-1.52112 \mathrm{E}-02$ $6.72612 \mathrm{E}$ 01 $-1.54523 \mathrm{E}-02$ $6.72700 \mathrm{E} 01 \quad-1.56960 \mathrm{E}-02$

$-5.82828 \mathrm{E}-03$ $-5.95610 \mathrm{E}-03$ $-6.08576 \mathrm{E}-03$ $-6.21729 \mathrm{E}-03$ $-6.35069 \mathrm{E}-03$

$-6.48597 \mathrm{E}-03$ $-6.62315 \mathrm{E}-03$ $-6.76224 \mathrm{E}-03$ $-6.90325 \mathrm{E}-03$ $-7.04620 \mathrm{E}-03$

$-7.19110 \mathrm{E}-03$ $-7.33797 \mathrm{E}-03$ $-7.48681 \mathrm{E}-03$ $-7.63763 \mathrm{E}-03$ $-7.79047 \mathrm{E}-03$

$-7.94531 \mathrm{E}-03$

$-8.10219 \mathrm{E}-03$

$-8.26111 \mathrm{E}-03$

$-8.42208 \mathrm{E}-03$

$-8.58512 \mathrm{E}-03$

$-8.75024 \mathrm{E}-03$ $-8.91746 \mathrm{E}-03$ $-9.08678 \mathrm{E}-03$ $-9.25822 \mathrm{E}-03$

$-9.43180 \mathrm{E}-03$

$-9.60752 \mathrm{E}-03$

$-9.78540 \mathrm{E}-03$

$-9.96546 \mathrm{E}-03$

$-1.01477 \mathrm{E}-02$

$-1.03321 \mathrm{E}-02$

$-1.05188 \mathrm{E}-02$

$-1.07077 \mathrm{E}-02$

$-1.08988 \mathrm{E}-02$ $-1.10921 \mathrm{E}-02$ $-1.12878 \mathrm{E}-02$

$-1.14857 \mathrm{E}-02$ $-1.16859 \mathrm{E}-02$

$-1.18884 \mathrm{E}-02$ $-1.20932 \mathrm{E}-02$ $-1.23003 \mathrm{E}-02$

$-1.25098 \mathrm{E}-02$ $-1.27217 \mathrm{E}-02$

$-1.29359 \mathrm{E}-02$

$-1.31525 \mathrm{E}-02$ 
ELECTRON RADIAL FUNCTIONS

Electron

$Z=3 \quad A=6$

$\rho=1.2 A^{1 / 3} F$

$P$

18.10

18.20

18.30

18.40

18.50

18.60

18.70

18.80

18.90

19.00

19.10

19.20

19.30

19.40

19.50

19.60

19.70

19.80

19.90

20.00

20.10

20.20

20.30

20.40

20.50

20.60

20.70

20.80

20.90

21.00

21.10

21.20

21.30

21.40

21.50

21.60

21.70

21.80

21.90

22.00

22.10

22.20

22.30

22.40

22.50

22.60

22.70

22.80

22.90

23.00

23.10

23.20

23.30

23.40

23.50

23.60

23.70

23.80

23.90

24.00
$F_{0}$

$f_{1}$

$g_{1}$

$\begin{array}{lllll}1.07252 \mathrm{E} & 00 & -1.81499 \mathrm{E} & 01\end{array}$

$1.07251 \mathrm{E} \quad 00$

$1.07251 \mathrm{E} \quad 00$

$\begin{array}{ll}1.07251 \mathrm{E} & 00\end{array}$

$1.07250 \mathrm{E} \quad 00$

$1.07250 \mathrm{E} \quad 00$ $1.07249 \mathrm{E} \quad 00$ $1.07249 \mathrm{E} \quad 00$ $1.07249 \mathrm{E} \quad 00$

$1.07248 \mathrm{E} \quad 00$

$1.07248 \mathrm{E} \quad 00$

$1.07248 \mathrm{E} \quad 00$

$\begin{array}{lll}1.07247 \mathrm{E} & 00\end{array}$

$1.07247 \mathrm{E} \quad 00$

$1.07246 \mathrm{E} \quad 00$

$1.07246 \mathrm{E} \quad 00$

$1.07246 \mathrm{E} \quad 00$

$1.07245 \mathrm{E} \quad 00$

$1.07245 \mathrm{E} \quad 00$

$1.07245 \mathrm{E} \quad 00$

$1.07244 \mathrm{E} \quad 00$

$1.07244 \mathrm{E} \quad 00$

$1.07244 \mathrm{E} \quad 00$

$1.07243 \mathrm{E} \quad 00$

$1.07243 \mathrm{E} \quad 00$

$1.07243 \mathrm{E} \quad 00$

$1.07242 \mathrm{E} \quad 00$

$1.07242 \mathrm{E} \quad 00$

$1.07242 \mathrm{E} \quad 00$

$1.07241 \mathrm{E} \quad 00$

$1.07241 \mathrm{E} \quad 00$

$1.07241 \mathrm{E} \quad 00$

$1.07240 \mathrm{E} \quad 00$

$1.07240 \mathrm{E} \quad 00$

$1.07240 \mathrm{E} \quad 00$

$1.07239 \mathrm{E} \quad 00$

$1.07239 \mathrm{E} \quad 00$

$1.07239 \mathrm{E} \quad 00$

$1.07239 \mathrm{E} \quad 00$

$1.07238 \mathrm{E} \quad 00$

$1.07238 \mathrm{E} \quad 00$

$1.07238 \mathrm{E} \quad 00$

$\begin{array}{lll}1.07237 \mathrm{E} & 00\end{array}$

$1.07237 \mathrm{E} \quad 00$

$1.07237 \mathrm{E} \quad 00$

$1.07236 \mathrm{E} \quad 00$

$1.07236 \mathrm{E} \quad 00$

$1.07236 \mathrm{E} \quad 00$

$1.07236 \mathrm{E} \quad 00$

$1.07235 \mathrm{E} \quad 00$

$1.07235 \mathrm{E} \quad 00$

$1.07235 \mathrm{E} \quad 00$

$1.07234 \mathrm{E} \quad 00$

$1.07234 \mathrm{E} \quad 00$

$1.07234 \mathrm{E} \quad 00$

$1.07234 \mathrm{E} 00$

$1.07233 \mathrm{E} \quad 00$

$1.07233 \mathrm{E} \quad 00$

$1.07233 \mathrm{E} \quad 00$

$1.07233 \mathrm{E} 00$
$-1.82525 \mathrm{E} \quad 01$

$-1.83551 \mathrm{E} 01$

$-1.84576 \mathrm{E} 01$

$-1.85602 \mathrm{E} \quad 01$

$-1.86628 \mathrm{E} \quad 01$

$-1.87653 \mathrm{E} \quad 01$

$-1.88679 \mathrm{E} \quad 01$

$-1.89704 \mathrm{E} \quad 01$

$-1.90729 \mathrm{E} 01$

$-1.91754 \mathrm{E} \quad 01$

$-1.92780 \mathrm{E}$ 0l

$-1.93804 \mathrm{E}$ 01

$-1.94829 \mathrm{E}$ 0I

$-1.95854 \mathrm{E} 01$

$-1.96879 \mathrm{E} \quad 01$

$-1.97904 \mathrm{E} \quad 01$

$-1.98928 \mathrm{E} \quad 01$

$-1.99953 \mathrm{E} 01$

$-2.00977 \mathrm{E} \quad 01$

$-2.02001 \mathrm{E} \quad 01$

$-2.03025 \mathrm{E} \quad 01$

$-2.04049 \mathrm{E} 01$

$-2.05073 \mathrm{E} 01$

$-2.06097 \mathrm{E} \quad 01$

$-2.07121 \mathrm{E} \quad 01$

$-2.08145 \mathrm{E} \quad 01$

$-2.09168 \mathrm{E} \quad 01$

$-2.10192 \mathrm{E} 01$

$-2.11215 \mathrm{E} 01$

$-2.12239 \mathrm{E} \quad 01$ $-2.13262 \mathrm{E} 0 \mathrm{O}$

$-2.14285 \mathrm{E} \quad 01$

$-2.15308 \mathrm{E} 01$

$-2.16331 \mathrm{E} \quad 01$

$-2.17354 \mathrm{E} \quad 01$

$-2.18377 \mathrm{E} 01$

$-2.19399 \mathrm{E} 01$

$-2.20422 \mathrm{E} 01$

$-2.21444 \mathrm{E} \quad 01$

$\begin{array}{ll}-2.22467 \mathrm{E} & 01\end{array}$

$-2.23489 \mathrm{E} \quad 01$

$-2.24511 \mathrm{E} 01$

$-2.25533 \mathrm{E} 01$

$-2.26555 \mathrm{E} 01$

$-2.27577 \mathrm{E} \quad 01$

$-2.28599 \mathrm{E} \quad 01$

$-2.29620 \mathrm{E} 01$

$-2.30642 \mathrm{E} 01$

$-2.31663 \mathrm{E} 01$

$-2.32685 \mathrm{E} 01$

$-2.33706 \mathrm{E} 01$

$-2.34727 \mathrm{E} 01$

$-2.35748 \mathrm{E} 01$

$-2.36769 \mathrm{E} 01$

$-2.37790 \mathrm{E} \quad 01$

$-2.38811 \mathrm{E} 01$

$-2.39831 \mathrm{E} 01$

$-2.40852 \mathrm{E} 01$

$-2.41872 \mathrm{E} 01$
$-8.13301 \mathrm{E}-01$

$-8.21341 \mathrm{E}-01$

$-8.29419 \mathrm{E}-01$

$-8.37535 \mathrm{E}-01$

$-8.45690 \mathrm{E}-01$

$-8.53884 \mathrm{E}-01$

$-8.62116 \mathrm{E}-01$

$-8.70386 \mathrm{E}-01$

$-8.78695 \mathrm{E}-01$

$-8.87042 \mathrm{E}-01$

$-8.95428 \mathrm{E}-01$

$-9.03852 \mathrm{E}-01$

$-9.12315 \mathrm{E}-01$

$-9.20816 \mathrm{E}-01$

$-9.29355 \mathrm{E}-01$

$-9.37933 \mathrm{E}-01$

$-9.46549 \mathrm{E}-01$

$-9.55204 \mathrm{E}-01$

$-9.63897 \mathrm{E}-01$

$-9.72628 \mathrm{E}-01$

$-9.81398 \mathrm{E}-01$

$-9.90206 \mathrm{E}-01$

$-9.99053 \mathrm{E}-01$

$-1.00794 \mathrm{E} \quad 00$

$-] .01686 \mathrm{E} \quad 00$

$-1.02582 \mathrm{E} \quad 00$

$-1.03482 \mathrm{E} \quad 00$

$-1.04386 \mathrm{E} 00$

$-1.05294 \mathrm{E} 00$

$-1.06205 \mathrm{E} 00$

$-1.07121 \mathrm{E} \quad 00$

$-1.08040 \mathrm{E} 00$

$-1.08963 \mathrm{E} \quad 00$

$-1.09890 \mathrm{E} \quad 00$

$-1.10820 \mathrm{E} \quad 00$

$-1.11755 \mathrm{E} \quad 00$

$-1.12693 \mathrm{E} \quad 00$

$-1.13635 \mathrm{E} \quad 00$

$-1.14581 \mathrm{E} \quad 00$

$-1.15531 \mathrm{E} 00$

$-1.16485 \mathrm{E} \quad 00$

$-1.17442 \mathrm{E} \quad 00$

$-1.18404 \mathrm{E} \quad 00$

$-1.19369 \mathrm{E} \quad 00$

$-1.20338 \mathrm{E} 00$

$-1.21311 \mathrm{E} \quad 00$

$-1.22287 \mathrm{E} 00$

$-1.23268 \mathrm{E} 00$

$-1.24252 \mathrm{E} \quad 00$

$-1.25240 \mathrm{E} 00$

$-1.26232 \mathrm{E} \quad 00$

$-1.27228 \mathrm{E} \quad 00$

$-1.28228 \mathrm{E} 00$

$-1.29231 \mathrm{E} 00$

$-1.30238 \mathrm{E} \quad 00$

$-1.31249 \mathrm{E} 00$

$-1.32264 \mathrm{E} \quad 00$

$-1.33283 \mathrm{E} 00$

$-1.34306 \mathrm{E} \quad 00$

$-1.35332 \mathrm{E} 00$

$\tan \delta_{1}$

$f_{-1}$

$1.44612 \mathrm{E}-03$

$1.44914 \mathrm{E}-03$

$1.45214 \mathrm{E}-03$

$1.45510 \mathrm{E}-03$

$1.45802 \mathrm{E}-03$

$1.46090 \mathrm{E}-03$

$1.46376 \mathrm{E}-03$

$1.46657 \mathrm{E}-03$

$1.46935 \mathrm{E}-03$

$1.47210 \mathrm{E}-03$

$1.47482 \mathrm{E}-03$

$1.47750 \mathrm{E}-03$

$1.48015 \mathrm{E}-03$

$1.48277 \mathrm{E}-03$

$1.48536 \mathrm{E}-03$

$1.48791 \mathrm{E}-03$

$1.49044 \mathrm{E}-03$

$1.49294 \mathrm{E}-03$

$1.49541 \mathrm{E}-03$

$1.49785 \mathrm{E}-03$

$1.50026 \mathrm{E}-03$

$1.50264 \mathrm{E}-03$

$1.50500 \mathrm{E}-03$

$1.50733 \mathrm{E}-03$

$1.50962 \mathrm{E}-03$

$1.51190 \mathrm{E}-03$

$1.51414 \mathrm{E}-03$

$1.51636 \mathrm{E}-03$

$1.51856 \mathrm{E}-03$

$1.52073 \mathrm{E}-03$

$1.52288 \mathrm{E}-03$

$1.52500 \mathrm{E}-03$

$1.52710 \mathrm{E}-03$

$1.52917 \mathrm{E}-03$

$1.53122 \mathrm{E}-03$

$1.53325 \mathrm{E}-03$

$1.53525 \mathrm{E}-03$

$1.53723 \mathrm{E}-03$

$1.53919 \mathrm{E}-03$

$1.54113 \mathrm{E}-03$

$1.54304 \mathrm{E}-03$ 
ELECTRON RAIDIAL FUNCTIONS

$Z=3 \quad A=6 \quad \rho=1.2 A^{1 / 3} F$

Elecetron

$\bar{F}$

$3.11081 \mathrm{E}-04$ $3.14527 \mathrm{E}-04$ $3.17992 \mathrm{E}-04$ $3.21476 \mathrm{E}-04$ $3.24979 \mathrm{E}-04$

$3.28501 \mathrm{E}-04$ $3.32042 \mathrm{E}-04$ $3.35602 \mathrm{E}-04$ $3.39181 \mathrm{E}-04$ $3.42779 \mathrm{E}-04$

$3.46396 \mathrm{E}-04$ $3.50032 \mathrm{E}-04$ $3.53687 \mathrm{E}-04$ $3.57360 \mathrm{E}-04$ $3.61053 \mathrm{E}-04$

$3.64765 \mathrm{E}-04$ $3.68496 \mathrm{E}-04$ $3.72246 \mathrm{E}-04$ $3.76014 \mathrm{E}-04$ $3.79802 \mathrm{E}-04$

$3.83609 \mathrm{E}-04$ $3.87435 \mathrm{E}-04$ $3.91279 \mathrm{E}-04$ $3.95143 \mathrm{E}-04$ $3.99026 \mathrm{E}-04$

$4.02927 \mathrm{E}-04$ $4.06848 \mathrm{E}-04$ $4.10788 \mathrm{E}-04$ $4.14746 \mathrm{E}-04$ $4.1872+\mathrm{E}-04$

$4.22720 \mathrm{E}-04$ $4.26736 \mathrm{E}-04$ 4. $30770 \mathrm{E}-04$ $4.34824 \mathrm{E}-04$ $4.38896 \mathrm{E}-04$

$4.42988 \mathrm{E}-04$ $4.47098 \mathrm{E}-04$ $4.51228 \mathrm{E}-04$ $4.55376 \mathrm{E}-04$ $4.59 .543 \mathrm{E}-04$

$4.63730 \mathrm{E}-04$ $4.67935 \mathrm{E}-04$ $4.72159 \mathrm{E}-04$ 4.76403 E-04 $4.80665 \mathrm{E}-04$

$4.84946 \mathrm{E}-04$ $4.89246 \mathrm{E}-04$ $4.93566 \mathrm{E}-04$ $4.97904 \mathrm{E}-04$ 5.02261 E-04

$5.06637 \mathrm{E}-04$ $5.11032 \mathrm{E}-04$ $5.15446 \mathrm{E}-04$ $5.19880 \mathrm{E}-04$ $5.24332 \mathrm{E}-04$

$5.28803 \mathrm{E}-04$ $5.33293 \mathrm{E}-04$ $5.37802 \mathrm{E}-04$ $5.42330 \mathrm{E}-04$ $5.46877 \mathrm{E}-04$ $f_{2}$

$-6.193 .34 \mathrm{E}-01$ $-6.26286 \mathrm{E}-0 \mathrm{I}$ $-6.33275 \mathrm{E}-0 \mathrm{l}$ $-6.40304 \mathrm{E}-01$ $-6.47371 \mathrm{E}-01$

$-6.54476 \mathrm{E}-01$

$-6.61620 \mathrm{E}-01$

$-6.68803 \mathrm{E}-01$

$-6.76024 \mathrm{E}-01$

$-6.83284 \mathrm{E}-0]$

$-6.90583 \mathrm{E}-01$

$-6.97920 \mathrm{E}-01$

$-7.05295 \mathrm{E}-01$

$-7.12710 \mathrm{E}-01$

$-7.20162 \mathrm{E}-01$

$-7.276 .54 \mathrm{E}-01$

$-7.35183 \mathrm{E}-01$

$-7.42752 \mathrm{E}-01$

$-7.50359 \mathrm{E}-01$

$-7.5800 .5 \mathrm{E}-01$

$-7.65689 \mathrm{E}-01$

$-7.73+11 \mathrm{E}-01$

$-7.81172 \mathrm{E}-01$

$-7.88972 \mathrm{E}-01$

$-7.96810 \mathrm{E}-01$

$-8.04687 \mathrm{E}-01$

$-8.12603 \mathrm{E}-01$

$-8.20556 \mathrm{E}-01$

$-8.28549 \mathrm{E}-01$

$-8.36580 \mathrm{E}-01$

$-8.44649 \mathrm{E}-01$

$-8.52757 \mathrm{E}-01$

$-8.60904 \mathrm{E}-01$

$-8.69088 \mathrm{E}-01$

$-8.77312 \mathrm{E}-01$

$-8.85574 \mathrm{E}-01$

$-8.93874 \mathrm{E}-01$

$-9.02213 \mathrm{E}-01$

$-9.10590 \mathrm{E}-01$

$-9.19006 \mathrm{E}-01$

$-9.27460 \mathrm{E}-01$

$-9.35953 \mathrm{E}-01$

$-9.44484 \mathrm{E}-01$

$-9.53054 \mathrm{E}-01$

$-9.61662 \mathrm{E}-01$

$-9.70308 \mathrm{E}-01$

$-9.78994 \mathrm{E}-01$

$-9.87717 \mathrm{E}-01$

$-9.96479 \mathrm{E}-01$

$-1.00528 \mathrm{E} 00$

$-1.01412 \mathrm{E} \quad 00$

$-1.02300 \mathrm{E} \quad 00$

$-1.03191 \mathrm{E} \quad 00$

$-1.04086 \mathrm{E} \quad 00$

$-1.04986 \mathrm{E} 00$

$-1.05889 \mathrm{E} \quad 00$

$-1.06796 \mathrm{E} 00$

$-1.07706 \mathrm{E} \quad 00$

$-1.08621 \mathrm{E} \quad 00$

$-1.09540 \mathrm{E} \quad 00$ $g_{2}$

$-1.64857 \mathrm{E}-02$

$-1.67414 \mathrm{E}-02$

$-1.69998 \mathrm{E}-02$

$-1.72608 \mathrm{E}-02$

$-1.75244 \mathrm{E}-02$

$-1.77907 \mathrm{E}-02$

$-1.80596 \mathrm{E}-02$

$-1.83312 \mathrm{E}-02$

$-1.86055 \mathrm{E}-02$

$-1.88825 \mathrm{E}-02$

$-1.91622 \mathrm{E}-02$

$-1.94446 \mathrm{E}-02$

$-1.97298 \mathrm{E}-02$

$-2.00177 \mathrm{E}-02$

$-2.0308+\mathrm{E}-02$

$-2,06018 \mathrm{E}-02$

$-2.08981 \mathrm{E}-02$

$-2.11971 \mathrm{E}-02$

$-2.14990 \mathrm{E}-02$

$-2.18037 \mathrm{E}-02$

$-2.21112 \mathrm{E}-02$

$-2.24216 \mathrm{E}-02$

$-2.27349 \mathrm{E}-02$

$-2.30511 \mathrm{E}-02$

$-2.33701 \mathrm{E}-02$

$-2.36921 \mathrm{E}-02$

$-2.40169 \mathrm{E}-02$

$-2.43448 \mathrm{E}-02$

$-2.46755 \mathrm{E}-02$

$-2.50092 \mathrm{E}-02$

$-2.53459 \mathrm{E}-02$

$-2.56856 \mathrm{E}-02$

$-2.60283 \mathrm{E}-02$

$-2.63740 \mathrm{E}-02$

$-2.67227 \mathrm{E}-02$

$-2.70744 \mathrm{E}-02$

$-2.74292 \mathrm{E}-02$

$-2.77871 \mathrm{E}-02$

$-2.81480 \mathrm{E}-02$

$-2.85121 \mathrm{E}-02$

$-2.88792 \mathrm{E}-02$

$-2.92495 \mathrm{E}-02$

$-2.96228 \mathrm{E}-02$

$-2.99994 \mathrm{E}-02$

$-3.03791 \mathrm{E}-02$

$-3.07619 \mathrm{E}-02$

$-3.11479 \mathrm{E}-02$

$-3.15371 \mathrm{E}-02$

$-3.19296 \mathrm{E}-02$

$-3.23252 \mathrm{E}-02$

$-3.27241 \mathrm{E}-02$

$-3.31262 \mathrm{E}-02$

$-3.35316 \mathrm{E}-02$

$-3.39403 \mathrm{E}-02$

$-3.43522 \mathrm{E}-02$

$-3.47675 \mathrm{E}-02$

$-3.51860 \mathrm{E}-02$

$-3.56079 \mathrm{E}-02$

$-3.60331 \mathrm{E}-02$

$-3.64617 \mathrm{E}-02$ $\tan \delta_{2}$

$6.72788 \mathrm{E}$ (0) $-1.59422 \mathrm{E}-02$ $6.72874 \mathrm{E}$ 01 $-1.61909 \mathrm{E}-02$ $6.72960 \mathrm{E} \quad 01-1.64423 \mathrm{E}-02$ $6.73044 \mathrm{E}$ 01 $-1.66961 \%-02$ $6.73127 \mathrm{E} \quad 01-1.69526 \mathrm{E}-02$

$6.73210 \mathrm{E}$ 01 $-1.72117 \mathrm{E}-02$ $6.73292 \mathrm{E}$ 01 $-1.74734 \mathrm{E}-02$ $6.73372 \mathrm{E}$ 01 $-1.77377 \mathrm{E}-02$ $6.73452 \mathrm{E}$ 01 $-1.80047 \mathrm{E}-02$ $6.73531 \mathrm{E} \quad 01-1.82743 \mathrm{E}-02$

$6.73609 \mathrm{E} \quad 01-1.85466 \mathrm{E}-02$ $6.73686 \mathrm{E} \quad 01-1.88216 \mathrm{E}-02$ $6.73763 \mathrm{E} \quad 01-1.90993 \mathrm{E}-02$ $6.73839 \mathrm{E}$ 0l $-1.93797 \mathrm{E}-02$ $6.73913 \mathrm{E} \quad 01-1.96628 \mathrm{E}-02$

$6.73987 \mathrm{E} \quad 01 \quad-1.99486 \mathrm{E}-02$ $6.7+061 \mathrm{E} \quad 01 \quad-2.02372 \mathrm{E}-02$ $6.74133 \mathrm{E} \quad 01 \quad-2.05286 \mathrm{E}-02$ $6.74205 \mathrm{E} \quad 0 \mathrm{l}-2.08227 \mathrm{E}-02$ $6.74276 \mathrm{E}$ ol $-2.11196 \mathrm{E}-02$

$6.74346 \mathrm{E}$ 0l $-2.14193 \mathrm{E}-02$ $6.74415 \mathrm{E}$ 01 $-2.17218 \mathrm{E}-02$ $6.74484 \mathrm{E} 01-2.20272 \mathrm{E}-02$ $6.74552 \mathrm{E}$ ol $-2.23353 \mathrm{E}-02$ $6.74620 \mathrm{E} \quad 01 \quad-2.26464 \mathrm{E}-02$

$6.74686 \mathrm{E} \quad 01 \quad-2.29603 \mathrm{E}-02$ $6.74752 \mathrm{E} \quad 01 \quad-2.32770 \mathrm{E}-02$ $6.74818 \mathrm{E}$ 01 $-2.35967 \mathrm{E}-02$ $6.74882 \mathrm{E}$ 01 $-2.39193 \mathrm{E}-02$ $6.74947 \mathrm{E}$ 0l $-2.42448 \mathrm{E}-02$

$6.75010 \mathrm{E}$ 0l $-2.45732 \mathrm{E}-02$ $6.75073 \mathrm{E}$ 01 $-2.4904 .5 \mathrm{E}-02$ $6.75135 \mathrm{E}$ 01 $-2.52388 \mathrm{E}-02$ $6.75197 \mathrm{E} \quad 01 \quad-2.55761 \mathrm{E}-02$ $6.75258 \mathrm{E}$ ol $-2.59164 \mathrm{E}-02$

$6.75318 \mathrm{E} \quad 01$ $6.75438 \mathrm{E} \quad 01 \quad-2.69552 \mathrm{E}-02$ $6.75496 \mathrm{E}$ 01 $-2.73075 \mathrm{E}-02$ $6.75554 \mathrm{E}$ ol $-2.76628 \mathrm{E}-02$

$6.75612 \mathrm{E}$ 01 $-2.80213 \mathrm{E}-02$ $6.75669 \mathrm{E}$ 01 $-2.83828 \mathrm{E}-02$ $6.75726 \mathrm{E}$ 01 $-2.87473 \mathrm{E}-02$ $6.75782 \mathrm{E}$ 01 $-2.91150 \mathrm{E}-02$ $6.75837 \mathrm{E} \quad 01 \quad-2.94858 \mathrm{E}-02$

$6.75892 \mathrm{E} \quad 01 \quad-2.98597 \mathrm{E}-02$ $6.75947 \mathrm{E}$ 01 $-3.02368 \mathrm{E}-02$ $6.76001 \mathrm{E} \quad 01-3.06170 \mathrm{E}-02$ $6.76055 \mathrm{E}$ 01 $-3.10003 \mathrm{E}-02$ $6.76108 \mathrm{E}$ 0l $-3.13869 \mathrm{E}-02$

$6.76160 \mathrm{E} \quad 01$ $6.76212 \mathrm{E} \quad 01$ $6.76264 \mathrm{E} \quad 01$ $6.76315 \mathrm{E} \quad 01$ $6.76366 \mathrm{E} \quad 01$

$6.76416 \mathrm{E} \quad 01$ $6.76466 \mathrm{E} \quad 01$ $6.76516 \mathrm{E}$ 01 $6.76565 \mathrm{E} \quad 01$ $6.76613 \mathrm{E}$ ol
$-3.17766 \mathrm{E}-02$ $-3.21695 \mathrm{E}-02$ $-3.25657 \mathrm{E}-02$ $-3.29650 \mathrm{E}-02$ $-3.33677 \mathrm{E}-02$

$-3.37735 \mathrm{E}-02$ $-3.41827 \mathrm{E}-02$ $-3.45951 \mathrm{E}-02$ $-3.50108 \mathrm{E}-02$ $-3.54298 \mathrm{E}-02$

$6.54478 \mathrm{~F}-0]$ $6.61623 \mathrm{E}-01$ $6.68806 \mathrm{E}-0) 1$ 6. $76028 \mathrm{E}-0]$ $6.83289 \mathrm{E}-0 \mathrm{l}$

$6.90588 \mathrm{E}-0]$ $6.97926 \mathrm{l}-01$ 7.05302E-0)

$7.12717 \mathrm{E}-01$

$7.20170 \mathrm{E}-01$

7.27662E-0]

$7.35193 \mathrm{E}-01$

$7.42762 \mathrm{E}-01$

$7.50370 \mathrm{E}-0]$

$7.58016 \mathrm{E}-01$

$7.65701 \mathrm{E}-01$ $7.73424 \mathrm{E}-01$

$7.81186 \mathrm{E}-01$

$7.88987 \mathrm{E}-0]$

$7.96826 \mathrm{E}-01$

$8.04703 \mathrm{E}-01$

$8.12619 \mathrm{E}-01$

$8.20574 \mathrm{E}-01$

$8.28567 \mathrm{E}-0]$

$8.36598 \mathrm{E}-01$

$8.44669 \mathrm{E}-01$ $8.52777 \mathrm{E}-01$

$8.60924 \mathrm{E}-01$

$8.69110 \mathrm{E}-01$

$8.77334 \mathrm{E}-01$ 
ELECTRON RADIAL FUNCTIONS

Electron

\begin{tabular}{|c|c|c|c|c|c|c|}
\hline$p$ & $F_{0}$ & & $f_{1}$ & & $g_{1}$ & \\
\hline 30.10 & $1.07219 \mathrm{E}$ & 00 & $-3.03910 \mathrm{E}$ & 01 & $-2.05146 \mathrm{E}$ & 00 \\
\hline 30.20 & $1.07218 \mathrm{E}$ & 00 & $-3.04923 \bar{E}$ & 01 & $-2.06408 \mathrm{E}$ & 00 \\
\hline 30.30 & $1.07218 \mathrm{E}$ & 00 & $-3.05937 \mathbf{E}$ & 01 & $-2.07674 \mathrm{E}$ & 00 \\
\hline 30.40 & $1.07218 \mathrm{E}$ & 00 & $-3.06950 \mathrm{E}$ & 01 & $-2.08944 \mathrm{E}$ & 00 \\
\hline 30.50 & $1.07218 \mathrm{E}$ & 00 & $-3.07963 \mathrm{E}$ & 01 & $-2.10218 \mathrm{E}$ & 09 \\
\hline 30.60 & $1.07218 \mathrm{E}$ & 00 & $-3.08976 \mathrm{E}$ & 01 & $-2.11495 \mathrm{E}$ & 00 \\
\hline 30.70 & $1.07218 \mathrm{E}$ & 00 & $-3.09989 \mathrm{E}$ & 01 & $-2.12776 \mathrm{E}$ & 00 \\
\hline 30.80 & $1.07217 \mathrm{E}$ & 00 & $-3.11001 \mathrm{E}$ & 01 & $-2.14061 \mathrm{E}$ & 00 \\
\hline 30.90 & $1.07217 \mathrm{E}$ & 00 & $-3.12014 \mathrm{E}$ & 01 & $-2.15350 \mathrm{E}$ & 00 \\
\hline 31.00 & $1.07217 \mathrm{E}$ & 00 & $-3.13026 \mathrm{E}$ & 01 & $-2.16643 \mathrm{E}$ & 00 \\
\hline 31.10 & $1.07217 \mathrm{E}$ & 00 & $-3.14038 \mathrm{E}$ & 01 & $-2.17939 \mathrm{E}$ & 00 \\
\hline 31.20 & $1.07217 \mathrm{E}$ & 00 & $-3.15050 \mathrm{E}$ & $0 \mathrm{I}$ & $-2.19239 \mathrm{E}$ & 00 \\
\hline 31.30 & $1.07216 \mathrm{E}$ & 00 & $-3.16062 \mathrm{E}$ & 01 & $-2.20543 \mathrm{E}$ & 00 \\
\hline 31.40 & $1.07216 \mathrm{E}$ & 00 & $-3.17074 \mathrm{E}$ & $0 \mathrm{I}$ & $-2.21851 \mathrm{E}$ & 00 \\
\hline 31.50 & $1.07216 \mathrm{E}_{2}$ & & $-3.18086 \mathrm{E}$ & $0 \mathrm{I}$ & $-2.23162 \mathrm{E}$ & 00 \\
\hline 31.60 & $1.07216 \mathrm{E}$ & 00 & $-3.19098 \mathrm{E}$ & 01 & $-2.24477 \mathrm{E}$ & 00 \\
\hline 31.70 & $1.07216 \mathrm{E}$ & 00 & $-3.20109 \mathrm{E}$ & 01 & $-2.25797 \mathrm{E}$ & 00 \\
\hline 31.80 & $1.07215 \mathrm{E}$ & 00 & $-3.21121 \mathrm{E}$ & 01 & $-2.27119 \mathrm{E}$ & 00 \\
\hline 31.90 & $1.07215 \mathrm{E}$ & 00 & $-3.22132 \mathrm{E}$ & 01 & $-2.28446 \mathrm{E}$ & 00 \\
\hline 32.00 & $1.07215 \mathrm{E}$ & & $-3.23143 \mathrm{E}$ & 01 & $-2.29776 \mathrm{E}$ & 00 \\
\hline 32.10 & $1.07215 \mathrm{E}$ & 00 & $-3.24154 \mathrm{E}$ & 01 & $-2.31110 \mathrm{E}$ & 00 \\
\hline 32.20 & $1.07215 \mathrm{E}$ & 00 & $-3.25165 \mathrm{E}$ & 01 & $-2.32448 \mathrm{E}$ & 00 \\
\hline 32.30 & $1.07215 \mathrm{E}$ & 00 & $-3.26176 \mathrm{E}$ & 01 & $-2.33790 \mathrm{E}$ & 00 \\
\hline 32.40 & $1.07214 \mathrm{E}$ & 00 & $-3.27186 \mathrm{E}$ & 01 & $-2.35136 \mathrm{E}$ & 00 \\
\hline 32.50 & $1.07214 \mathrm{E}$ & 00 & $-3.28197 \mathrm{E}$ & 01 & $-2.36485 \mathrm{E}$ & 00 \\
\hline 32.60 & $1.07214 \mathrm{E}$ & 00 & $-3.29207 \mathrm{E}$ & 01 & $-2.37838 \mathrm{E}$ & 00 \\
\hline 32.70 & $1.07214 \mathrm{E}$ & 00 & $-3.30217 \mathrm{E}$ & 01 & $-2.39195 \mathrm{E}$ & 00 \\
\hline 32.80 & $1.07214 \mathrm{E}$ & 00 & $-3.31227 \mathrm{E}$ & 01 & $-2.40555 \mathrm{E}$ & 00 \\
\hline 32.90 & $1.07213 \mathrm{E}$ & 00 & $-3.32237 \mathrm{E}$ & 01 & $-2.41919 \mathrm{E}$ & 00 \\
\hline 33.00 & $1.07213 \mathrm{E}$ & 00 & $-3.33247 \mathrm{E}$ & 01 & $-2.43288 \mathrm{E}$ & 00 \\
\hline 33.10 & $1.07213 \mathrm{E}$ & 00 & $-3.34257 \mathrm{E}$ & 01 & $-2.44659 \mathrm{E}$ & 00 \\
\hline 33.20 & $1.07213 \mathrm{E}$ & 00 & $-3.35266 \mathrm{E}$ & 01 & $46035 \mathrm{E}$ & 00 \\
\hline 33.30 & $1.07213 \mathrm{E}$ & 00 & $-3.36276 \mathrm{E}$ & 01 & $-2.47415 \mathrm{E}$ & 00 \\
\hline 33.40 & $1.07213 \mathrm{E}$ & 00 & $-3.37285 \mathrm{E}$ & 01 & $-2.48798 \mathrm{E}$ & 00 \\
\hline 33.50 & $1.07212 \mathrm{E}$ & 00 & $-3.38294 \mathrm{E}$ & 01 & $-2.50185 \mathrm{E}$ & 00 \\
\hline 33.60 & $1.07212 \mathrm{E}$ & 00 & $-3.39303 E$ & 01 & $-2.51575 \mathrm{E}$ & 00 \\
\hline 33.70 & $1.07212 \mathrm{E}$ & 00 & $-3.40312 \mathrm{E}$ & 01 & $-2.52970 \mathrm{E}$ & 00 \\
\hline 33.80 & $1.07212 \mathrm{E}$ & 00 & $-3.41321 \mathrm{E}$ & 01 & $-2.54368 \mathrm{E}$ & 00 \\
\hline 33.90 & $1.07212 \mathrm{E}$ & 00 & $-3.42329 \mathrm{E}$ & 01 & $-2.55770 \mathrm{E}$ & 00 \\
\hline 34.00 & $1.07212 \mathrm{E}$ & 00 & $-3.43338 \mathrm{E}$ & 01 & $-2.57176 \mathrm{E}$ & 00 \\
\hline 34.10 & $1.07211 \mathrm{E}$ & 00 & $-3.44346 \mathrm{E}$ & 01 & $-2.58586 \mathrm{E}$ & 00 \\
\hline 34.20 & $1.07211 \mathrm{E}$ & 00 & $-3.45354 \mathrm{E}$ & 01 & $-2.59999 \mathrm{E}$ & 00 \\
\hline 34.30 & $1.07211 \mathrm{E}$ & 00 & $-3.46363 E$ & 01 & $-2.61+16 \mathrm{E}$ & 00 \\
\hline 34.40 & $1.07211 \mathrm{E}-$ & 00 & $-3.47370 \mathrm{E}$ & 01 & $-2.62837 \mathrm{E}$ & 00 \\
\hline 34.50 & $1.07211 \mathrm{E}$ & 00 & $-3.48378 \mathbf{E}$ & 01 & $-2.64261 \mathrm{E}$ & 00 \\
\hline 34.60 & $1.07211 \mathrm{E}$ & 00 & $-3.49386 \mathrm{E}$ & 01 & $-2.65690 \mathrm{E}$ & 00 \\
\hline 34.70 & $1.07210 \mathrm{E}$ & 00 & $-3.50394 \mathrm{E}$ & 01 & $-2.67122 \mathrm{E}$ & 00 \\
\hline 34.80 & $1.07210 \mathrm{E}$ & 00 & $-3.51401 \mathrm{E}$ & 01 & $-2.68558 \mathrm{E}$ & 00 \\
\hline 34.90 & $1.07210 \mathrm{E}$ & 00 & $-3.52408 E$ & 01 & $-2.69998 \mathrm{E}$ & 00 \\
\hline 35.00 & $1.07210 \mathrm{E}$ & 00 & $-3.53415 \mathbf{E}$ & 01 & $-2.71441 \mathrm{E}$ & 00 \\
\hline 35.10 & $1.07210 \mathrm{E}$ & 00 & $-3.54422 \mathrm{E}$ & 01 & $-2.72888 \mathrm{E}$ & 00 \\
\hline 35.20 & $1.07210 \mathrm{E}$ & 00 & $-3.55429 E$ & 01 & $-2.74339 \mathrm{E}$ & 00 \\
\hline 35.30 & $1.07209 \mathrm{E}$ & 00 & $-3.56436 \mathrm{E}$ & 01 & $-2.75794 \mathrm{E}$ & 00 \\
\hline 35.40 & $1.07209 \mathrm{E}$ & 00 & $-3.57442 \mathbf{E}$ & 01 & $-2.77252 \mathrm{E}$ & 00 \\
\hline 35.50 & $1.07209 \mathrm{E}$ & 00 & $-3.58449 \mathrm{E}$ & 01 & $-2.78714 \mathrm{E}$ & 00 \\
\hline 35.60 & $1.07209 \mathrm{E}$ & 00 & $-3.59455 \mathrm{E}$ & 01 & $-2.80180 E$ & 00 \\
\hline 35.70 & $1.07209 \mathrm{E}$ & 00 & $-3.60461 \mathrm{E}$ & 01 & $-2.81650 \mathrm{E}$ & 00 \\
\hline 35.80 & $1.07209 \mathrm{E}$ & 00 & $-3.61467 \mathrm{E}$ & 01 & $-2.83124 \mathrm{E}$ & 00 \\
\hline 35.90 & $1.07208 \mathrm{E}$ & 00 & $-3.62473 \mathrm{E}$ & 01 & $-2.84601 \mathrm{E}$ & 00 \\
\hline 36.00 & $1.07208 \mathrm{E}$ & 00 & $-3.63479 \mathrm{E}$ & 01 & $-2.86082 \mathrm{E}$ & 00 \\
\hline
\end{tabular}

$\tan \delta_{1}$

$$
\rho=1.2 \mathrm{~A}^{1 / 3} \mathrm{~F}
$$

$f_{-1}$

$1.64344 \mathrm{E}-03$

$1.64420 \mathrm{E}-03$

$1.64495 \mathrm{E}-03$

$1.64569 \mathrm{E}-03$

$1.64642 \mathrm{E}-03$

$1.64714 \mathrm{E}-03$

$1.64785 \mathrm{E}-03$

$1.64855 \mathrm{E}-03$

$1.64925 \mathrm{E}-03$

$1.64993 \mathrm{E}-03$

$1.65061 \mathrm{E}-03$

$1.65127 \mathrm{E}-03$

$1.65193 \mathrm{E}-03$

$1.65258 \mathrm{E}-03$

$1.65323 \mathrm{E}-03$

$1.65386 \mathrm{E}-03$

$1.65448 \mathrm{E}-03$

$1.65510 \mathrm{E}-03$

$1.65571 \mathrm{E}-03$

$1.65631 \mathrm{E}-03$

$1.65690 \mathrm{E}-03$

$1.65749 \mathrm{E}-03$

$1.65806 \mathrm{E}-03$

$1.65863 \mathrm{E}-03$

$1.65919 \mathrm{E}-03$

$1.65975 \mathrm{E}-03$

$1.66029 \mathrm{E}-03$

$1.66083 \mathrm{E}-03$

$1.66136 \mathrm{E}-03$

$1.66188 \mathrm{E}-03$

$1.66240 \mathrm{E}-03$ $1.66290 \mathrm{E}-03$ $1.66340 \mathrm{E}-03$

$1.66389 \mathrm{E}-03$

$1.66438 \mathrm{E}-03$

$1.66486 \mathrm{E}-03$

$1.66533 \mathrm{E}-03$

$1.66579 \mathrm{E}-03$

$1.66625 \mathrm{E}-03$

$1.66670 \mathrm{E}-03$

$1.66714 \mathrm{E}-03$

$1.66757 \mathrm{E}-03$

$1.66801 \mathrm{E}-03$

$1.66843 \mathrm{E}-03$

$1.66884 \mathrm{E}-03$

$1.66925 \mathrm{E}-03$

$1.66965 \mathrm{E}-03$

$1.67005 \mathrm{E}-03$

$1.67044 \mathrm{E}-03$

$1.67082 \mathbf{E}-03$

$1.67119 \mathrm{E}-03$

$1.67156 \mathrm{E}-03$

$1.67192 \mathrm{E}-03$

$1.67228 \mathrm{E}-03$

$1.67263 \mathrm{E}-03$

$1.67297 \mathrm{E}-03$

$1.67331 \mathrm{E}-03$

$1.67364 \mathrm{E}-03$

$1.67396 \mathrm{E}-03$

$1.67428 \mathrm{E}-03$

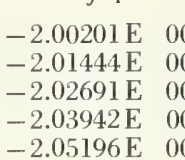

$-2.06454 \mathrm{E} \quad 00$

$-2.07716 \mathrm{E} \quad 00$

$-2.08982 \mathrm{E} \quad 00$

$-2.10251 \mathrm{E} \quad 00$

$-2.11524 \mathrm{E} \quad 00$

$-2.12801 \mathrm{E} \quad 00$

$-2.14082 \mathrm{E} 00$

$-2.15367 \mathrm{E} \quad 00$

$-2.16655 \mathrm{E} 00$

$-2.17947 \mathrm{E} 00$

$-2.19243 \mathrm{E} \quad 00$

$-2.20543 \mathrm{E} \quad 00$

$-2.21846 \mathrm{E} 00$

$-2.23154 \mathrm{E} \quad 00$

$-2.24465 \mathrm{E} 00$

$-2.25780 \mathrm{E} \quad 00$

$-2.27098 \mathrm{E} 00$

$-2.28421 \mathrm{E} 00$

$-2.29747 \mathrm{E} \quad 00$

$-2.31077 \mathrm{E} \quad 00$

$-2.32410 \mathrm{E} \quad 00$

$-2.33748 \mathrm{E} 00$

$-2.35089 \mathrm{E} \quad 00$

$-2.36434 \mathrm{E} 00$

$-2.37783 \mathrm{E} 00$

$-2.39136 \mathrm{E} \quad 00$ $-2.40492 \mathrm{E} \quad 00$ $-2.41852 \mathrm{E} \quad 00$ $-2.43216 \mathrm{E} \quad 00$

$-2.44584 \mathrm{E} \quad 00$

$-2.45955 \mathrm{E} \quad 00$ $-2.47330 \mathrm{E} \quad 00$ $-2.48709 \mathrm{E} 00$ $-2.50092 \mathrm{E} \quad 00$ $-2.51479 \mathrm{E} 00$

-2.52869 E 00 $-2.54263 \mathrm{E} \quad 00$ $-2.55661 \mathrm{E} \quad 00$ $-2.57062 \mathrm{E} \quad 00$ -2.58468 E 00

$-2.59877 \mathrm{E} \quad 00$ $-2.61290 \mathrm{E} 00$ $-2.62706 \mathrm{E} \quad 00$ $-2.64127 \mathrm{E} 00$ $-2.65551 \mathrm{E} \quad 00$

$-2.66979 \mathrm{E} \quad 00$ $-2.68410 \mathrm{E} \quad 00$ $-2.69846 \mathrm{E} \quad 00$ $-2.71285 \mathrm{E} \quad 00$ $-2.72728 \mathrm{E} 00$

$-2.74175 \mathrm{E} 00$ $-2.75625 \mathrm{E} 00$ $-2.77079 \mathrm{E} \quad 00$ $-2.78537 \mathrm{E} \quad 00$ $-2.79999 \mathrm{E} \quad 00$ $g_{-1}$

$3.14150 \mathrm{E} \quad 01$

$3.15162 \mathrm{E} \quad 01$

$3.16175 \mathrm{E} \quad 01$

$3.17188 \mathrm{E} 01$

$3.18200 \mathrm{E} \quad 01$

$3.19213 \mathrm{E} 01$ $3.20225 \mathrm{E} \quad 01$ $3.21237 \mathrm{E}$ 01 $3.22249 \mathrm{E} \quad 01$ 3.23261 E 01

$3.24273 \mathrm{E} \quad 01$ $3.25284 \mathrm{E} \quad 01$ $3.26296 \mathrm{E} \quad 01$ $3.27307 \mathrm{E} \quad 01$ $3.28318 \mathrm{E} 01$

$3.29330 \mathrm{E} \quad 01$ $3.30341 \mathrm{E} \quad 01$ $3.31351 \mathrm{E} \quad 01$ $3.32362 \mathrm{E} 01$ $3.33373 \mathrm{E} 01$

$3.34383 \mathrm{E} \quad 01$ $3.35393 \mathrm{E}$ 01 $3.36404 \mathrm{E} \quad 01$ $3.37414 \mathrm{E} \quad 01$ $3.38424 \mathrm{E} \quad 01$

$3.39433 \mathrm{E} \quad 01$ $3.40443 \mathrm{E} \quad 01$

$3.41453 \mathrm{E} \quad 01$

$3.42462 \mathrm{E} 01$

$3.43471 \mathrm{E} \quad 01$

$3.44480 \mathrm{E} \quad 01$ $3.45489 \mathrm{E} \quad 01$ $3.46498 \mathrm{E} \quad 01$

$3.47507 \mathrm{E} \quad 01$

$3.48516 \mathrm{E} 01$

$3.49524 \mathrm{E} \quad 01$ $3.50532 \mathrm{E}$ 0I

$3.51541 \mathrm{E} \quad 01$ $3.52549 \mathrm{E} 01$

$3.53557 \mathrm{E} \quad 01$

$3.54564 \mathrm{E} \quad 01$ $3.55572 \mathrm{E} \quad 01$ $3.56580 \mathrm{E} \quad 01$ $3.57587 \mathrm{E}, 01$ $3.58594 \mathrm{E} \quad 01$

$3.59601 \mathrm{E} 01$ $3.60608 \mathrm{E} \quad 01$ $3.61615 \mathrm{E} \quad 01$ $3.62622 \mathrm{E} \quad 01$ $3.63628 \mathrm{E} \quad 01$

$3.64635 \mathrm{E} \quad 01$

$3.65641 \mathrm{E} \quad 01$ $3.66647 \mathrm{E} \quad 01$ $3.67653 \mathrm{E} \quad 01$

$3.68659 \mathrm{E} 01$

$3.69665 \mathrm{E} \quad 01$ $3.70670 \mathrm{E} \quad 01$ $3.71676 \mathrm{E} \quad 01$ $3.72681 \mathrm{E} \quad 01$ $3.73686 \mathrm{E} \quad 01$ $\tan \delta_{-1}$

$-4.22559 \mathrm{E} \quad 02$ $-4.22857 \mathrm{E} \quad 02$ $-4.23154 \mathrm{E} \quad 02$ $-4.23450 \mathrm{E} \quad 02$ $-4.23745 \mathrm{E} \quad 02$

$\begin{array}{lll}-4.24040 \mathrm{E} & 02\end{array}$ $-4.24334 \mathrm{E} \quad 02$ $-4.24628 \mathrm{E} \quad 02$ $-4.24920 \mathrm{E} \quad 02$ $-4.25212 \mathrm{E} \quad 02$

$-4.25503 \mathrm{E} \quad 02$ $\begin{array}{lll}-4.25794 \mathrm{E} & 02\end{array}$ $-4.26084 \mathrm{E} \quad 02$ $-4.26373 \mathrm{E} \quad 02$ $-4.26661 \mathrm{E} 02$

$-4.26950 \mathrm{E} \quad 02$ $-4.27237 \mathrm{E} \quad 02$ $-4.27524 \mathrm{E} \quad 02$ $-4.27810 \mathrm{E} \quad 02$ $-4.28095 \mathrm{E} \quad 02$

$-4.28380 \mathrm{E} \quad 02$ $-4.28665 \mathrm{E} \quad 02$ -4.28949 E 02 $-4.29232 \mathrm{E} \quad 02$ $\begin{array}{ll}-4.29515 \mathrm{E} & 02\end{array}$

$\begin{array}{ll}-4.29797 \mathrm{E} & 02\end{array}$ $-4.30079 \mathrm{E} \quad 02$ $-4.30360 \mathrm{E} \quad 02$ $-4.30641 \mathrm{E} \quad 02$ $-4.30921 \mathrm{E} \quad 02$

$-4.31200 \mathrm{E} \quad 02$ $-4.31480 \mathrm{E} \quad 02$ $-4.31758 \mathrm{E} \quad 02$ $-4.32036 \mathrm{E} \quad 02$ $-4.32314 \mathrm{E} \quad 02$

$-4.32591 \mathrm{E} \quad 02$ $-4.32867 \mathrm{E} \quad 02$ $-4.33144 \mathrm{E} \quad 02$ $-4.33420 \mathrm{E} 02$ -4.33695 E 02

$-4.33970 \mathrm{E} \quad 02$ $-4.34245 \mathrm{E} \quad 02$ $-4.34519 \mathrm{E} \quad 02$ $-4.34793 \mathrm{E} \quad 02$ $-4.35066 \mathrm{E} 02$

$-4.35339 \mathrm{E} \quad 02$ $-4.35611 \mathrm{E} \quad 02$ $-4.35883 \mathrm{E} 02$ $-4.36155 \mathrm{E} \quad 02$ $-4.36426 \mathrm{E} \quad 02$

-4.36698 E 02 $-4.36968 \mathrm{E} \quad 02$ $-4.37239 \mathrm{E} 02$ $-4.37509 \mathrm{E} \quad 02$ -4.37778 E 02

$-4.38048 \mathrm{E} \quad 02$ $-4.38317 \mathrm{E} \quad 02$ $-4.38585 \mathrm{E} \quad 02$ $-4.38854 \mathrm{E} \quad 02$ $-4.39122 \mathrm{E} \quad 02$ 
$\bar{F}$

30.10

30.20

30.30

30.40

30.50

30.60

30.70

30.80

30.90

31.00

31.10

31.20

31.30

31.40

31.50

31.60

31.70

31.80

31.90

32.00

32.10

32.20

32.30

32.40

32.50

32.60

32.70

32.80

32.90

33.00

33.10

33.20

33.30

33.40

33.50

33.60

33.70

33.80

33.90

34.00

34.10

34.20

34.30

34.40

34.50

34.60

34.70

34.80

34.90

35.00

35.10

35.20

35.30

35.40

35.50

35.60

35.70

35.80

35.90

36.00

\section{$f_{2}$}

$8.60137 \mathrm{E}-04$ $8.65861 \mathrm{E}-04$ $8.71603 \mathrm{E}-04$ $8.77365 \mathrm{E}-04$ $8.83146 \mathrm{E}-04$

$8.88945 \mathrm{E}-04$ $8.94764 \mathrm{E}-04$ $9.00602 \mathrm{E}-04$ $9.064 .59 \mathrm{E}-04$ $9.12334 \mathrm{E}-04$

$9.18229 \mathrm{E}-04$ $9.24142 \mathrm{E}-04$ $9.30075 \mathrm{E}-04$ $9.36026 \mathrm{E}-04$ $9.41997 \mathrm{E}-04$

$9.47986 \mathrm{E}-04$ $9.53995 \mathrm{E}-04$ $9.60022 \mathrm{E}-04$ $9.66069 \mathrm{E}-04$ $9.72134 \mathrm{E}-04$

$9.78218 \mathrm{E}-04$ $9.84322 \mathrm{E}-04$ $9.90444 \mathrm{E}-04$ $9.96586 \mathrm{E}-04$ $1.00275 \mathrm{E}-03$

$1.00893 \mathrm{E}-03$ $1.01512 \mathrm{E}-03$ $1.02134 \mathrm{E}-03$ $1.02758 \mathrm{E}-03$ $1.03383 \mathrm{E}-03$

$1.01011 \mathrm{E}-03$ $1.04640 \mathrm{E}-03$ $1.05271 \mathrm{E}-03$ $1.05904 \mathrm{E}-03$ $1.06539 \mathrm{E}-03$

$1.07176 \mathrm{E}-03$ $1.07815 \mathrm{E}-03$ $1.08456 \mathrm{E}-03$ $1.09098 \mathrm{E}-03$ $1.09743 \mathrm{E}-03$

$1.10389 \mathrm{E}-03$ $1.11038 \mathrm{E}-03$ $1.11688 \mathrm{E}-03$ $1.12340 \mathrm{E}-03$ $1.12994 \mathrm{E}-03$

$1.13650 \mathrm{E}-03$ $1.14307 \mathrm{E}-03$ $1.14967 \mathrm{E}-03$ $1.15629 \mathrm{E}-03$ $1.16292 \mathrm{E}-03$

$1.16958 \mathrm{E}-03$ $1.17625 \mathrm{E}-03$ $1.18294 \mathrm{E}-03$ $1.18965 \mathrm{E}-03$ $1.19638 \mathrm{E}-03$

$1.20313 \mathrm{E}-03$ $1.20990 \mathrm{E}-03$ $1.21668 \mathrm{E}-03$ $1.22349 \mathrm{E}-03$ $1.23031 \mathrm{E}-03$ $g_{2}$

$-1.72808 \mathrm{E} \quad 00$ $-1.73963 \mathrm{E} 00$ $-1.75123 \mathrm{E} \quad 00$ $-1.76286 \mathrm{E} \quad 00$ $-1.77453 \mathrm{E} \quad 00$

$-1.78624 \mathrm{E} \quad 00$ $-1.79798 \mathrm{E} \quad 00$ $-1.80977 \mathrm{E} \quad 00$ $-1.82159 \mathrm{E} \quad 00$

-1.83345 E 00

$-1.84535 \mathrm{E} 00$

$-1.85729 \mathrm{E} 00$

$-1.86926 \mathrm{E} \quad 00$

$-1.88127 \mathrm{E} \quad 00$

$-1.89333 \mathrm{E} 00$

$-1.90541 \mathrm{E} \quad 00$

$-1.91754 \mathrm{E} \quad 00$

$-1.92971 \mathrm{E} \quad 00$

$-1.94191 \mathrm{E} \quad 00$

$-1.95415 \mathrm{E} \quad 00$

$-1.96643 \mathrm{E} \quad 00$ $-1.97875 \mathrm{E} \quad 00$ $-1.99110 \mathrm{E} \quad 00$ $-2.00349 \mathrm{E} \quad 00$ $-2.01593 \mathrm{E} 00$

$-2.02839 \mathrm{E} \quad 00$ $-2.04090 \mathrm{E} \quad 00$ $-2.05345 \mathrm{E} \quad 00$ $-2.06603 \mathrm{E} \quad 00$ $-2.07865 \mathrm{E} 00$

$-2.09131 \mathrm{E} \quad 00$ $-2.10401 \mathrm{E} \quad 00$ $-2.11674 \mathrm{E} 00$ $-2.12951 \mathrm{E} \quad 00$ $-2.14232 \mathrm{E} \quad 00$

$-2.15517 \mathrm{E} \quad 00$ $-2.16806 \mathrm{E} \quad 00$ $-2.18098 \mathrm{E} \quad 00$ $-2.19395 \mathrm{E} \quad 00$ $-2.20695 \mathrm{E} \quad 00$

$-2.21998 \mathrm{E} \quad 00$ $-2.23306 \mathrm{E} \quad 00$ $-2.24617 \mathrm{E} \quad 00$ $-2.25933 \mathrm{E} \quad 00$ $-2.27251 \mathrm{E} 00$

$-2.28574 \mathrm{E} \quad 00$ $-2.29901 \mathrm{E} \quad 00$ $-2.31231 \mathrm{E} \quad 00$ $-2.32565 \mathrm{E} 00$ $-2.33903 \mathrm{E} \quad 00$

$-2.35245 \mathrm{E} \quad 00$ $-2.36590 \mathrm{E} \quad 00$ $-2.37939 \mathrm{E} 00$ $-2.39292 \mathrm{E} \quad 00$ $-2.40649 \mathrm{E} \quad 00$

$-2.42010 \mathrm{E} \quad 00$ $-2.43374 \mathrm{E} \quad 00$ -2.44742 E 00 $-2.46114 \mathrm{E} \quad 00$ $-2.47490 \mathrm{E} 00$ $\tan \delta_{2}$

$f_{-2}$

$-6.94514 \mathrm{E}-02$ $-7.01128 \mathrm{E}-02$ $-7.07785 \mathrm{E}-02$ $-7.14482 \mathrm{E}-02$ $-7.21222 \mathrm{E}-02$

$-7.28004 \mathrm{E}-02$ $-7.34828 \mathrm{E}-02$ $-7.41694 \mathrm{E}-02$ $-7.48603 \mathrm{E}-02$ $-7.55554 \mathrm{E}-02$

$-7.62548 \mathrm{E}-02$ $-7.69585 \mathrm{E}-02$ $-7.76664 \mathrm{E}-02$ $-7.83786 \mathrm{E}-02$ $-7.90952 \mathrm{E}-02$

$-7.98161 \mathrm{E}-02$ $-8.05414 \mathrm{E}-02$ $-8.12709 \mathrm{E}-02$ $-8.20049 \mathrm{E}-02$ $-8.27432 \mathrm{E}-02$

$-8.34860 \mathrm{E}-02$ $-8.42331 \mathrm{E}-02$ $-8.49846 \mathrm{E}-02$ $-8.57406 \mathrm{E}-02$ $-8.65011 \mathrm{E}-02$

$-8.726 .59 \mathrm{E}-02$ $-8.803 .53 \mathrm{E}-02$ $-8.88091 \mathrm{E}-02$ $-8.95875 \mathrm{E}-02$ $-9.03703 \mathrm{E}-02$

$-9.11577 \mathrm{E}-02$ $-9.19496 \mathrm{E}-02$ $-9.27460 \mathrm{E}-02$ $-9.35470 \mathrm{E}-02$ $-9.43526 \mathrm{E}-02$

$-9.51627 \mathrm{E}-02$ $-9.59775 \mathrm{E}-02$ $-9.67968 \mathrm{E}-02$ $-9.76208 \mathrm{E}-02$ $-9.84494 \mathrm{E}-02$

$-9.92827 \mathrm{E}-02$ $-1.00121 \mathrm{E}-01$ $-1.00963 \mathrm{E}-01$ $-1.01810 \mathrm{E}-01$ $-1.02662 \mathrm{E}-01$

$-1.03519 \mathrm{E}-01$ $-1.04381 \mathrm{E}-01$ $-1.05247 \mathrm{E}-01$ $-1.06118 \mathrm{E}-01$ $-1.06993 \mathrm{E}-01$

$-1.07874 \mathrm{E}-01$

$-1.08759 \mathrm{E}-01$

$-1.09649 \mathrm{E}-01$

$-1.10544 \mathrm{E}-01$

$-1.11444 \mathrm{E}-01$

$-1.12348 \mathrm{E}-01$

$-1.13257 \mathrm{E}-01$

$-1.14172 \mathrm{E}-01$

$-1.15091 \mathrm{E}-01$

$-1.16015 \mathrm{E}-01$

$6.78950 \mathrm{E}$ 0l $\quad-6.77543 \mathrm{~L}-02$ $6.78980 \mathrm{E}$ 01 $-6.84035 \mathrm{~L}-02$ $6.79010 \mathrm{E}$ 01 $\quad-6.90568 \mathrm{l}-02$ $6.79040 \mathrm{E}$ ol $-6.97142 \mathrm{E}-02$ $6.79069 \mathrm{E} \quad 01-7.03758 \mathrm{E}-02$

$6.79099 \mathrm{E} \quad 01 \quad-7.10416 \mathrm{E}-02$ $6.79128 \mathrm{E} 01-7.17115 \mathrm{E}-02$ $6.79157 \mathrm{E}$ 0l $-7.23856 \mathrm{E}-02$ $6.79186 \mathrm{E}$ 01 $-7.30639 \mathrm{E}-02$ $6.79214 \mathrm{E} \quad 01 \quad-7.37464 \mathrm{E}-02$

6.79243E $01 \quad-7.44331 \mathrm{E}-02$ $6.79271 \mathrm{E} 01 \quad-7.51241 \mathrm{E}-02$ $6.79299 \mathrm{E} \quad 01 \quad-7.58193 \mathrm{E}-02$ $6.79327 \mathrm{E} \quad 01 \quad-7.65188 \mathrm{E}-02$ $6.79355 \mathrm{E} \quad 01 \quad-7.72225 \mathrm{E}-02$

$6.79382 \mathrm{E}$ 01 $-7.79305 \mathrm{E}-02$ $6.79409 \mathrm{E} \quad 01 \quad-7.86429 \mathrm{E}-02$ $6.79437 \mathrm{E} 01 \quad-7.93595 \mathrm{E}-02$ $6.79464 \mathrm{E} \quad 01 \quad-8.00804 \mathrm{E}-02$ $6.79490 \mathrm{E} \quad 01 \quad-8.08057 \mathrm{E}-02$

$6.79517 \mathrm{E} \quad 01$ $6.79544 \mathrm{E} \quad 01$ $6.79570 \mathrm{E} \quad 01$ $6.79596 \mathrm{E} \quad 0]$ $6.79622 \mathrm{E} 01$

$6.79648 \mathrm{E} \quad 01$ $6.79673 \mathrm{E} \quad 0$ $6.79699 \mathrm{E} \quad 01$ $6.79724 \mathrm{E} \quad 01$ $6.79749 \mathrm{E} \quad 01$

$6.79774 \mathrm{E} \quad 01$ $6.79799 \mathrm{E} \quad 01$ $6.79824 \mathrm{E} \quad 01$ $6.79848 \mathrm{E} \quad 01$ $6.79873 \mathrm{E} \quad 01$

$-8.15354 \mathrm{E}-02$ $-8.22694 \mathrm{E}-02$ $-8.30078 \mathrm{E}-02$ $-8.37505 \mathrm{E}-02$ $-8.44977 \mathrm{E}-02$

$-8.52493 E-02$ 
$p$

36.10

36.20

36.30

36.40

36.50

36.60

36.70

36.80

36.90

37.00

37.10

37.20

37.30

37.40

37.50

37.60

37.70

37.80

37.90

38.00

38.10

38.20

38.30

38.40

38.50

38.60

38.70

38.80

38.90

39.00

39.10

39.20

39.30

39.40

39.50

39.60

39.70

39.80

39.90

40.00

40.10

40.20

40.30

40.40

40.50

40.60

40.70

40.80

40.90

41.00

41.10

41.20

41.30

41.40

41.50

41.60

41.70

41.80

41.90

42.00
$F_{0}$

$1.07208 \mathrm{E} \quad 00$ $1.07208 \mathrm{E} \quad 00$ $1.07208 \mathrm{E} \quad 00$ $1.07208 \mathrm{E} \quad 00$ $1.07208 \mathrm{E} \quad 00$

$1.07207 \mathrm{E} \quad 00$ $1.07207 \mathrm{E} \quad 00$ $1.07207 \mathrm{E} \quad 00$ $1.07207 \mathrm{E} \quad 00$ $1.07207 \mathrm{E} \quad 00$

$1.07207 \mathrm{E} \quad 00$ $1.07206 \mathrm{E} \quad 00$ $1.07206 \mathrm{E} \quad 00$ $1.07206 \mathrm{E} \quad 00$ $1.07206 \mathrm{E} \quad 00$

$1.07206 \mathrm{E} \quad 00$ $1.07206 \mathrm{E} \quad 00$ $1.07206 \mathrm{E} \quad 00$ $1.07205 \mathrm{E} \quad 00$ $1.07205 \mathrm{E} \quad 00$

$1.07205 \mathrm{E} \quad 00$ $1.07205 \mathrm{E} \quad 00$ $1.07205 \mathrm{E} \quad 00$ $1.07205 \mathrm{E} \quad 00$ $1.07205 \mathrm{E} \quad 00$

$1.07204 \mathrm{E} \quad 00$ $\begin{array}{ll}1.07204 \mathrm{E} & 00\end{array}$ $1.07204 \mathrm{E} \quad 00$ $1.07204 \mathrm{E} \quad 00$ $1.07204 \mathrm{E} \quad 00$

$1.07204 \mathrm{E} \quad 00$ $1.07204 \mathrm{E} \quad 00$ $1.07203 \mathrm{E} \quad 00$ $1.07203 \mathrm{E} \quad 00$ $1.07203 \mathrm{E} \quad 00$

$1.07203 \mathrm{E} \quad 00$ $1.07203 \mathrm{E} \quad 00$ $1.07203 \mathrm{E} \quad 00$ $1.07203 \mathrm{E} \quad 00$ $1.07202 \mathrm{E} \quad 00$

$1.07202 \mathrm{E} \quad 00$ $1.07202 \mathrm{E} \quad 00$ $1.07202 \mathrm{E} \quad 00$ $1.07202 \mathrm{E}-00$ $1.07202 \mathrm{E} \quad 00$

$1.07202 \mathrm{E} \quad 00$ $1.07201 \mathrm{E} \quad 00$ $1.07201 \mathrm{E} \quad 00$ $1.07201 \mathrm{E} \quad 00$ $1.07201 \mathrm{E} \quad 00$

$1.07201 \mathrm{E} \quad 00$ $1.07201 \mathrm{E} \quad 00$ $1.07201 \mathrm{E} \quad 00$ $1.07200 \mathrm{E} \quad 00$ $1.07200 \mathrm{E} \quad 00$

$1.07200 \mathrm{E} \quad 00$ $1.07200 \mathrm{E} \quad 00$ $1.07200 \mathrm{E} \quad 00$ $1.07200 \mathrm{E} \quad 00$ $1.07200 \mathrm{E} \quad 00$

\section{$f_{\mathrm{i}}$}

$g_{1}$

$\begin{array}{llll}-3.64484 \mathrm{E} & 01 & -2.87567 \mathrm{E} & 00\end{array}$ $\begin{array}{llll}-3.65490 \mathrm{E} & 01 & -2.89055 \mathrm{E} & 00\end{array}$ $\begin{array}{llll}-3.66495 \mathrm{E} & 01 & -2.90547 \mathrm{E} & 00\end{array}$ $\begin{array}{llll}-3.67500 \mathrm{E} & 01 & -2.92043 \mathrm{E} & 00\end{array}$ $-3.68505 \mathrm{E} \quad 01$ $-2.93543 \mathrm{E} 00$

$\begin{array}{llll}-3.69510 \mathrm{E} & 01 & -2.95047 \mathrm{E} & 00\end{array}$ $\begin{array}{llll}-3.70515 \mathrm{E} & 01 & -2.96554 \mathrm{E} & 00\end{array}$ $\begin{array}{llll}-3.71519 \mathrm{E} & 01 & -2.98065 \mathrm{E} & 00\end{array}$ $\begin{array}{llll}-3.72524 \mathrm{E} & 01 & -2.99579 \mathrm{E} & 00\end{array}$ $-3.73528 \mathrm{E} \quad 01$ $-3.01098 \mathrm{E} 00$

$-3.74532 \mathrm{E} \quad 01$ $-3.75536 \mathrm{E}$ 01 $-3.76540 \mathrm{E} \quad 01$ $-3.77544 \mathrm{E} \quad 01$ $-3.78547 \mathrm{E} \quad 01$

$-3.79551 \mathrm{E} \quad 01$ $-3.80554 \mathrm{E}$ 01 $-3.81557 \mathrm{E} 01$ $-3.82560 \mathrm{E} 01$ $-3.83563 \mathrm{E} 01$

$-3.84566 \mathrm{E} \quad 01$ $-3.85568 \mathrm{E} 01$ $-3.86571 \mathrm{E} \quad 01$ $-3.87573 \mathrm{E} 01$ -3.88575 E 01

$\begin{array}{ll}-3.89577 \mathrm{E} & 01\end{array}$ $-3.90579 \mathrm{E} 01$ $-3.91580 \mathrm{E} \quad 01$ $-3.92582 \mathrm{E} \quad 01$ -3.93583 E 01

$-3.94584 \mathrm{E}$ 0l $-3.95585 \mathrm{E} \quad 01$ $-3.96586 \mathrm{E} 01$ $-3.97587 \mathrm{E}$ 01 -3.98588 E 01

$-3.99588 \mathrm{E} \quad 01$ $-4.00588 \mathrm{E} \quad 01$ $-4.01588 \mathrm{E} \quad 01$ $-4.02588 \mathrm{E} \quad 01$ $-4.03588 \mathrm{E} 01$

$-4.04588 \mathrm{E} \quad 01$ $-4.05587 \mathrm{E} 01$ $-4.06587 \mathrm{E} \quad 01$ $-4.07586 \mathrm{E} 01$ -4.08585 E 01

$-4.09584 \mathrm{E} \quad 0$ $-4.10583 \mathrm{E} 01$ $-4.11581 \mathrm{E} 01$ $-4.12580 \mathrm{E} 01$ $-4.13578 \mathrm{E} 01$

$-4.14576 \mathrm{E} \quad 01$ $-4.15574 \mathrm{E} 01$ $-4.16572 \mathrm{E} 01$ $-4.17569 \mathrm{E} 01$ $-4.18567 \mathrm{E} \quad 01$

$-4.19564 \mathrm{E} \quad 01$ $-4.20561 \mathrm{E} 01$ $-4.21558 \mathrm{E} \quad 01$ $-4.22555 \mathrm{E} 01$ $-4.23552 \mathrm{E} \quad 01$
$-3.02620 \mathrm{E} \quad 00$ $-3.05676 \mathrm{E} \quad 00$ $-3.08747 \mathrm{E} \quad 00$

\section{$-3.10287 \mathrm{E} \quad 00$} $-3.11832 \mathrm{E} \quad 00$ $-3.14933 \mathrm{E} 00$ $-3.16489 \mathrm{E} \quad 00$ $-3.19612 \mathrm{E} 00$ $-3.21179 \mathrm{E} 00$ $-3.22750 \mathrm{E} \quad 00$ $-3.24324 \mathrm{E} 00$

$-3.25902 \mathrm{E} \quad 00$ $-3.27485 \mathrm{E} 00$ $-3.29070 \mathrm{E} \quad 00$ $-3.30660 \mathrm{E} 00$ $-3.32253 \mathrm{E} \quad 00$

-3.33850 E 00 -3.35451 E 00 $-3.37055 \mathrm{E} 00$ $-3.38663 \mathrm{E} \quad 00$ $-3.40275 \mathrm{E} 00$ $-3.43510 \mathrm{E} \quad 00$ $-3.45133 \mathrm{E} 00$ $-3.46760 \mathrm{E} 00$ -3.48391 E 00

$-3.50025 \mathrm{E} \quad 00$ $-3.51663 \mathrm{E} 00$ $-3.53305 \mathrm{E} 00$ $-3.54950 \mathrm{E} 00$ $-3.56599 \mathrm{E} \quad 00$

$-3.58252 \mathrm{E} \quad 00$ $-3.59909 \mathrm{E} \quad 00$ $-3.61569 \mathrm{E} \quad 00$ $-3.63233 \mathrm{E} \quad 00$ $-3.64901 \mathrm{E} 00$

$-3.66572 \mathrm{E} 00$ $-3.68248 \mathrm{E} 00$ $-3.69926 \mathrm{E} \quad 00$ $-3.71609 \mathrm{E} \quad 00$ $-3.73295 \mathrm{E} \quad 00$

$-3.74985 \mathrm{E} \quad 00$ $-3.78377 \mathrm{E} \quad 00$ $-3.80078 \mathrm{E} \quad 00$ $-3.81783 \mathrm{E} 00$ $-3.04146 \mathrm{E} 00$ $-3.07209 \mathrm{E} 00$ $-3.13381 \mathrm{E} 00$

$-3.18048 \mathrm{E} 00$

$-3.41891 \mathrm{E} 00$ $-3.76679 \mathrm{E} 00$

$\tan \delta_{1}$

$f_{-1}$

$1.67460 \mathrm{E}-03$ $1.67490 \mathrm{E}-03$ $1.67520 \mathrm{E}-03$ $1.67549 \mathrm{E}-03$ $1.67578 \mathrm{E}-03$

$1.67606 \mathrm{E}-03$ $1.67634 \mathrm{E}-03$

$1.67661 \mathrm{E}-03$ $1.67688 \mathrm{E}-03$ $1.67713 \mathrm{E}-03$

$1.67739 \mathrm{E}-03$ $1.67763 \mathrm{E}-03$ $1.67787 \mathrm{E}-03$ $1.67811 \mathrm{E}-03$ $1.67834 \mathrm{E}-03$

$1.67857 \mathrm{E}-03$ $1.67879 \mathrm{E}-03$ $1.67900 \mathrm{E}-03$ $1.67921 \mathrm{E}-03$ $1.67941 \mathrm{E}-03$

$1.67961 \mathrm{E}-03$ $1.67980 \mathrm{E}-03$ $1.67999 \mathrm{E}-03$ $1.68017 \mathrm{E}-03$

$1.68034 \mathrm{E}-03$

$1.68051 \mathrm{E}-03$ $1.68068 \mathrm{E}-03$ $1.68084 \mathrm{E}-03$ $1.68099 \mathrm{E}-03$

$1.68115 \mathrm{E}-03$

$1.68129 \mathrm{E}-03$

$1.68143 \mathrm{E}-03$

$1.68156 \mathrm{E}-03$

$1.68169 \mathrm{E}-03$

$1.68182 \mathrm{E}-03$

$1.68194 \mathrm{E}-03$ $1.68205 \mathrm{E}-03$

$1.68216 \mathrm{E}-03$

$1.68226 \mathrm{E}-03$

$1.68237 \mathrm{E}-03$

$1.68246 \mathrm{E}-03$ $1.68255 \mathrm{E}-03$ $1.68264 \mathrm{E}-03$

$1.68271 \mathrm{E}-03$

$1.68279 \mathrm{E}-03$

$1.68286 \mathrm{E}-03$ 
$\bar{F}$

36.10

36.20

36.30

36.40

36.50

36.60

36.70

36.80

36.90

37.00

37.10

37.20

37.30

37.40

37.50

37.60

37.70

37.80

37.90

38.00

38.10

38.20

38.30

38.40

38.50

38.60

38.70

38.80

38.90

39.00

39.10

39.20

39.30

39.40

39.50

39.60

39.70

39.80

39.90

40.00

40.10

40.20

40.30

40.40

40.50

40.60

40.70

40.80

40.90

41.00

41.10

41.20

41.30

41.40

41.50

41.60

4.70

41.80

41.90

42.00 $f_{2}$

$1.23716 \mathrm{E}-03$

$1.24402 \mathrm{E}-03$

$1.25090 \mathrm{E}-03$

$1.25780 \mathrm{E}-03$

$1.26472 \mathrm{E}-03$

$1.27166 \mathrm{E}-03$

$1.27862 \mathrm{E}-03$

$1.28559 \mathrm{E}-03$

$1.29259 \mathrm{E}-03$

$1.29960 \mathrm{E}-03$

$1.30664 \mathrm{E}-03$

$1.31369 \mathrm{E}-03$

$1.32076 \mathrm{E}-03$

$1.32785 \mathrm{E}-03$

$1.33496 \mathrm{E}-03$

$1.34209 \mathrm{E}-03$

$1.34924 \mathrm{E}-03$

$1.35640 \mathrm{E}-03$

$1.36359 \mathrm{E}-03$

$1.37079 \mathrm{E}-03$

$1.37801 \mathrm{E}-03$

$1.38526 \mathrm{E}-03$

$1.39252 \mathrm{E}-03$

$1.39980 \mathrm{E}-03$

$1.40710 \mathrm{E}-03$

$1.41441 \mathrm{E}-03$

$1.42175 \mathrm{E}-03$

$1.42911 \mathrm{E}-03$

$1.43648 \mathrm{E}-03$

$1.44388 \mathrm{E}-03$

$1.45129 \mathrm{E}-03$ $1.45872 \mathrm{E}-03$

$1.46617 \mathrm{E}-03$

$1.47364 \mathrm{E}-03$

$1.48113 \mathrm{E}-03$

$1.48864 \mathrm{E}-03$

$1.49617 \mathrm{E}-03$

$1.50371 \mathrm{E}-03$

$1.51128 \mathrm{E}-03$

$1.51886 \mathrm{E}-03$

$1.52646 \mathrm{E}-03$

$1.53408 \mathrm{E}-03$

$1.54173 \mathrm{E}-03$

$1.54938 \mathrm{E}-03$

$1.55706 \mathrm{E}-03$

$1.56476 \mathrm{E}-03$

$1.57248 \mathrm{E}-03$

$1.58021 \mathrm{E}-03$

$1.58797 \mathrm{E}-03$

$1.59574 \mathrm{E}-03$

$1.60353 \mathrm{E}-03$

$1.61135 \mathrm{E}-03$

$1.61918 \mathrm{E}-03$

$1.62703 \mathrm{E}-03$

$1.63489 \mathrm{E}-03$

$1.64278 \mathrm{E}-03$ $1.65069 \mathrm{E}-03$ $1.65861 \mathrm{E}-03$

$1.66656 \mathrm{E}-03$

$1.67452 \mathrm{E}-03$
$-2.48869 \mathrm{E} \quad 00$ $-2.50253 \mathrm{E} \quad 00$ $-2.51640 \mathrm{E} \quad 00$ $-2.53030 \mathrm{E} \quad 00$

$-2.54425 \mathrm{E} 00$

$-2.55823 \mathrm{E} \quad 00$ $-2.57225 \mathrm{E} \quad 00$

$-2.58631 \mathrm{E} \quad 00$

$-2.6004 \mathrm{lE} \quad 00$

$-2.61454 \mathrm{E} \quad 00$

$-2.62872 \mathrm{E} \quad 00$

$-2.64293 \mathrm{E} 00$

$-2.65717 \mathrm{E} \quad 00$

$-2.67146 \mathrm{E} \quad 00$

$-2.68578 \mathrm{E} 00$

$-2.70014 \mathrm{E} \quad 00$

$-2.71454 \mathrm{E} \quad 00$

$-2.72898 \mathrm{E} \quad 00$

$-2.74345 \mathrm{E} \quad 00$

$-2.75796 \mathrm{E} \quad 00$

$-2.77251 \mathrm{E} \quad 00$

$-2.78710 \mathrm{E} \quad 00$

$-2.80172 \mathrm{E} \quad 00$

$-2.81638 \mathrm{E} \quad 00$

$-2.83108 \mathrm{E} \quad 00$

$-2.84582 \mathrm{E} \quad 00$ $-2.86060 \mathrm{E} \quad 00$ $-2.87541 \mathrm{E} \quad 00$ $-2.89026 \mathrm{E} 00$

$-2.90515 \mathrm{E} 00$

$-2.92007 \mathrm{E} \quad 00$ $-2.93503 \mathrm{E} 00$ $-2.95003 \mathrm{E} \quad 00$ $-2.96507 \mathrm{E} \quad 00$

$-2.98015 \mathrm{E} \quad 00$

$-2.99526 \mathrm{E} \quad 00$ $-3.01041 \mathrm{E} \quad 00$ $-3.02560 \mathrm{E} \quad 00$ $-3.04082 \mathrm{E} \quad 00$

$-3.05609 \mathrm{E} \quad 00$

$-3.07139 \mathrm{E} \quad 00$

$-3.08673 \mathrm{E} \quad 00$

$-3.10210 \mathrm{E} \quad 00$

$-3.11752 \mathrm{E} 00$

$-3.13297 \mathrm{E} 00$

$g_{2}$

$\tan \delta_{z}$

$f_{-2}$

$\tan \delta$

$-1.16944 \mathrm{E}-01$

$-1.17878 \mathrm{E}-01$

$-1.18816 \mathrm{E}-01$

$-1.19760 \mathrm{E}-01$

$-1.20709 \mathrm{E}-01$

$-1.21662 \mathrm{E}-01$

$-1.22621 \mathrm{E}-0 \mathrm{~J}$

$-1.23584 \mathrm{E}-01$

$-1.24553 \mathrm{E}-01$

$-1.25526 \mathrm{E}-01$

$-1.26505 \mathrm{E}-01$

$-1.27489 \mathrm{E}-0 \mathrm{]}$

$-1.28477 \mathrm{E}-01$

$-1.29471 \mathrm{E}-01$

$-1.30470 \mathrm{E}-01$

$-1.31474 \mathrm{E}-01$

$-1.32483 \mathrm{E}-01$

$-1.33497 \mathrm{E}-01$

$-1.34517 \mathrm{E}-01$

$-1.35541 \mathrm{E}-01$

$-1.36571 \mathrm{E}-01$

$-1.37605 \mathrm{E}-01$

$-1.38645 \mathrm{E}-01$

$-1.39691 \mathrm{E}-01$

$-1.40741 \mathrm{E}-01$

$-1.41797 \mathrm{E}-01$

$-1.42857 \mathrm{E}-01$

$-1.43923 \mathrm{E}-01$

$-1.44995 \mathrm{E}-01$

$-1.46071 \mathrm{E}-01$

$-1.47153 \mathrm{E}-01$ $-1.48240 \mathrm{E}-01$

$-1.49333 \mathrm{E}-01$

$-1.50431 \mathrm{E}-01$

$-1.51534 \mathrm{E}-01$

$-1.52642 \mathrm{E}-01$

$-1.53756 \mathrm{E}-01$

$-1.54875 \mathrm{E}-01$

$-1.56000 \mathrm{E}-01$

$-1.57130 \mathrm{E}-01$

$-1.58265 \mathrm{E}-01$

$-1.59406 \mathrm{E}-01$

$-1.60552 \mathrm{E}-01$

$-1.61704 \mathrm{E}-01$

$-1.62861 \mathrm{E}-01$

$-3.14846 \mathrm{E} \quad 00$ $-3.16398 \mathrm{E} \quad 00$

$-3.17954 \mathrm{E} \quad 00$

$-3.19515 \mathrm{E} \quad 00$

$-3.21078 \mathrm{E} 00$

$-1.64024 \mathrm{E}-01$

$-1.65192 \mathrm{E}-01$

$-1.66365 \mathrm{E}-01$

$-1.6754+\mathrm{E}-01$

$-1.68729 \mathrm{E}-01$

$-3.22646 \mathrm{E} \quad 00$

$-3.24217 \mathrm{E} \quad 00$

$-3.25792 \mathrm{E} \quad 00$

$-3.27371 \mathrm{E} \quad 00$

$-3.28954 \mathrm{E} \quad 00$

$-3.30540 \mathrm{E} \quad 00$

$-3.32130 \mathrm{E} \quad 00$

$-3.33724 \mathrm{E} \quad 00$

$-3.35321 \mathrm{E} 00$

$-3.36923 \mathrm{E} 00$

$6.80458 \mathrm{E} \quad 01$ $6.80479 \mathrm{E} \quad 01$ $6.80500 \mathrm{E} \quad 01$ $6.80521 \mathrm{E}$ 0l $6.80541 \mathrm{E} \quad 01$

$6.80561 \mathrm{E} \quad 01$ $6.80582 \mathrm{E} \quad 01$ $6.80602 \mathrm{E} 01$ $6.80622 \mathrm{E} \quad 01$ $6.80642 \mathrm{E} \quad 01$

$6.80661 \mathrm{E} \quad 01$ $6.80681 \mathrm{E} \quad 01$ $6.80700 \mathrm{E} \quad 01$ $6.80720 \mathrm{E} \quad 01$ $6.80739 \mathrm{E} \quad 01$

$6.80758 \mathrm{E} \quad 01$ $6.80778 \mathrm{E} \quad 01$ $6.80797 \mathrm{E} \quad 01$ $6.80815 \mathrm{E} \quad 01$

$6.80834 \mathrm{E} \quad 01$

$6.80853 \mathrm{E} \quad 01$ $6.80872 \mathrm{E} \quad 01$ $6.80890 \mathrm{E} \quad 01$ $6.80909 \mathrm{E}$ 0l $6.80927 \mathrm{E} \quad 01$

$6.80945 \mathrm{E} \quad 01$ $6.80963 \mathrm{E} \quad 01$ $6.80981 \mathrm{E} \quad 01$ $6.80999 \mathrm{E} \quad 01$ $6.81017 \mathrm{E} \quad 01$

$6.81035 \mathrm{E} \quad 01$ $6.81052 \mathrm{E} \quad 01$ $6.81070 \mathrm{E} \quad 01$ $6.81087 \mathrm{E} \quad 01$ $6.81105 \mathrm{E} \quad 01$

$6.81122 \mathrm{E} \quad 01$ $6.81139 \mathrm{E} \quad 01$ $6.81156 \mathrm{E} \quad 01$ $6.81173 \mathrm{E} \quad 01$ $6.81190 \mathrm{E} \quad 01$

$6.81207 \mathrm{E} \quad 01$ $6.81224 \mathrm{E} \quad 01$ $6.81241 \mathrm{E} \quad 01$ $6.81257 \mathrm{E} \quad 01$ $6.81274 \mathrm{E} 01$

$6.81290 \mathrm{E} \quad 01$ $6.81306 \mathrm{E} 01$ $6.81323 \mathrm{E} \quad 01$ $6.81339 \mathrm{E} 01$ $6.81355 \mathrm{E} \quad 01$

$-1.69919 \mathrm{E}-01$ $-1.71115 \mathrm{E}-01$ $-1.72316 \mathrm{E}-01$ 


$$
Z=4 \quad A=8
$$

$$
\rho=1.2 A^{1 / 3} F
$$

p $F_{0}$

0.10

0.20

0.30

0.40

0.50

0.60

0.70

0.80

0.90

1.00

1.10

1.20
1.30

1.30
1.40

1.50

1.60

1.70

1.80

1.90

2.00

2.10

2.20

2.30

2.40

2.50

2.60

2.70

2.80

2.90

3.00

3.10

3.20

3.30

3.40

3.50

3.60

3.70

3.80

3.90

4.00

4.10

4.20

4.30

4.40

4.50

4.60

4.70

4.80

4.90

5.00

5.10

5.20

5.30

5.40

5.50

5.60
5.70

5.80

5.90

6.00 $f_{1}$

$2.20673 \mathrm{E} \quad 00$

$1.55047 \mathrm{E} \quad 00$

$1.36210 \mathrm{E} \quad 00$

$1.27551 \mathrm{E} \quad 00$

$1.22684 \mathrm{E} 00$

$1.19624 \mathrm{E} \quad 00$

$1.17557 \mathrm{E} \quad 00$

$1.16090 \mathrm{E} \quad 00$

$1.15009 \mathrm{E} \quad 00$

$1.14189 \mathrm{E} \quad 00$

$1.13552 \mathrm{E} \quad 00$ $1.13048 \mathrm{E} \quad 00$ $1.12642 \mathrm{E} \quad 00$ $1.12310 \mathrm{E} \quad 00$ $1.12035 \mathrm{E} \quad 00$

$1.11805 \mathrm{E} \quad 00$ $1.11611 \mathrm{E} \quad 00$ $1.11446 \mathrm{E} \quad 00$ $1.11304 \mathrm{E} \quad 00$ $1.11180 \mathrm{E} \quad 00$

$1.11073 \mathrm{E} \quad 00$ $1.10979 \mathrm{E} \quad 00$ $1.10896 \mathrm{E} \quad 00$ $1.10822 \mathrm{E} \quad 00$ $1.10756 \mathrm{E} \quad 00$

$1.10697 \mathrm{E} \quad 00$ $1.10644 \mathrm{E} \quad 00$ $1.10596 \mathrm{E} \quad 00$ $1.10553 \mathrm{E} \quad 00$ $1.10514 \mathrm{E} \quad 00$

$1.10478 \mathrm{E} \quad 00$ $1.10445 \mathrm{E} \quad 00$ $1.10415 \mathrm{E} \quad 00$ $1.10387 \mathrm{E} \quad 00$ $1.10361 \mathrm{E} \quad 00$

$1.10338 \mathrm{E} \quad 00$ $1.10316 \mathrm{E} \quad 00$ $1.10295 \mathrm{E} \quad 00$ $1.10276 \mathrm{E} \quad 00$ $1.10258 \mathrm{E} \quad 00$

$1.10242 \mathrm{E} \quad 00$ $1.10226 \mathrm{E}, 00$ $1.10211 \mathrm{E}^{-} 00$ $1.10198 \mathrm{E} \quad 00$ $1.10185 \mathrm{E} \quad 00$

$1.10172 \mathrm{E} \quad 00$ $1.10161 \mathrm{E} \quad 00$ $1.10150 \mathrm{E} \quad 00$ $1.10140 \mathrm{E} \quad 00$ $1.10130 \mathrm{E} \quad 00$

$1.10120 \mathrm{E} \quad 00$ $1.10112 \mathrm{E} \quad 00$ $1.10103 \mathrm{E} \quad 00$ $1.10095 \mathrm{E} \quad 00$ $1.10088 \mathrm{E} \quad 00$

$1.10080 \mathrm{E} \quad 00$ $1.10073 \mathrm{E} \quad 00$ $1.10067 \mathrm{E} \quad 00$ $1.10060 \mathrm{E} \quad 00$ $1.10054 \mathrm{E} \quad 00$
$-1.08695 \mathrm{E}-02$ $-3.50435 \mathrm{E}-02$ $-7.22089 \mathrm{E}-02$ $-1.21096 \mathrm{E}-01$ $-1.80197 \mathrm{E}-01$

$-2.47952 \mathrm{E}-01$ $-3.22879 \mathrm{E}-01$

$-4.03652 \mathrm{E}-01$

$-4.89137 \mathrm{E}-01$

$-5.78394 \mathrm{E}-01$

$-6.70658 \mathrm{E}-01$

$-7.65318 \mathrm{E}-01$

$-8.61887 \mathrm{E}-01$

$-9.59982 \mathrm{E}-01$

$-1.05930 \mathrm{E} \quad 00$

$-1.15960 \mathrm{E} 00$

$-1.26069 \mathrm{E} \quad 00$

$-1.36242 \mathrm{E} 00$

$-1.46468 \mathrm{E} \quad 00$

$-1.56737 \mathrm{E} \quad 00$

$-1.67041 \mathrm{E} \quad 00$ $-1.77373 \mathrm{E} 00$ $-1.87730 \mathrm{E} \quad 00$ $-1.98106 \mathrm{E} 00$ $-2.08499 \mathrm{E} \quad 00$

$-2.18906 \mathrm{E} \quad 00$ $-2.29323 \mathrm{E} \quad 00$ $-2.39751 \mathrm{E} \quad 00$ $-2.50186 \mathrm{E} \quad 00$ $-2.60628 \mathrm{E} \quad 00$

$-2.71076 \mathrm{E} \quad 00$

$-2.81528 \mathrm{E} \quad 00$

$-2.91985 \mathrm{E} \quad 00$

$-3.02444 \mathrm{E} 00$

$-3.12906 \mathrm{E} \quad 00$

$-3.23370 \mathrm{E} \quad 00$ $-3.33836 \mathrm{E} 00$ $-3.44304 \mathrm{E} \quad 00$

$-3.54772 \mathrm{E} \quad 00$ $-3.65242 \mathrm{E} 00$

$-3.75712 \mathrm{E} 00$ $-3.86183 \mathrm{E} 00$ $-3.96653 \mathrm{E} 00$ $-4.07124 \mathrm{E} \quad 00$

$-4.17596 \mathrm{E} \quad 00$

$-4.28067 \mathrm{E} \quad 00$ $-4.38537 \mathrm{E} \quad 00$ $-4.49008 \mathrm{E} 00$ $-4.59478 \mathrm{E} \quad 00$ $-4.69948 \mathrm{E} 00$

$-4.80417 \mathrm{E} \quad 00$ $-4.90886 \mathrm{E} \quad 00$ $-5.01354 \mathrm{E} 00$ $-5.11822 \mathrm{E} \quad 00$ $-5.22289 \mathrm{E} 00$

$-5.32756 \mathrm{E} \quad 00$ $-5.43222 \mathrm{E} \quad 00$ $-5.53687 \mathrm{E} \quad 00$ $-5.64151 \mathrm{E} 00$ $-5.74615 \mathrm{E} 00$ $g_{1}$

$-1.72079 \mathrm{E}-04$

$-5.55861 \mathrm{E}-04$

$-1.14900 \mathrm{E}-03$

$-1.93518 \mathrm{E}-03$

$-2.89497 \mathrm{E}-03$

$-4.00824 \mathrm{E}-03$

$-5.25592 \mathrm{E}-03$

$-6.62093 \mathrm{E}-03$

$-8.08873 \mathrm{E}-03$

$-9.64729 \mathrm{E}-03$

$-1.12868 \mathrm{E}-02$

$-1.29996 \mathrm{E}-02$

$-1.47793 \mathrm{E}-02$

$-1.66213 \mathrm{E}-02$

$-1.85216 \mathrm{E}-02$

$-2.04772 \mathrm{E}-02$

$-2.24859 \mathrm{E}-02$

$-2.45456 \mathrm{E}-02$

$-2.66550 \mathrm{E}-02$

$-2.88128 \mathrm{E}-02$

$-3.10181 \mathrm{E}-02$ $-3.32701 \mathrm{E}-02$

$-3.55683 \mathrm{E}-02$

$-3.79121 \mathrm{E}-02$

$-4.03012 \mathrm{E}-02$

$-4.27351 \mathrm{E}-02$

$-4.52137 \mathrm{E}-02$

$-4.77366 \mathrm{E}-02$

$-5.03038 \mathrm{E}-02$

$-5.29151 \mathrm{E}-02$

$-5.55702 \mathrm{E}-02$

$-5.82692 \mathrm{E}-02$

$-6.10119 \mathrm{E}-02$

$-6.37983 \mathrm{E}-02$

$-6.66283 \mathrm{E}-02$

$-6.95018 \mathrm{E}-02$

$-7.24188 \mathrm{E}-02$

$-7.53792 \mathrm{E}-02$

$-7.83831 \mathrm{E}-02$

$-8.14303 \mathrm{E}-02$

$-8.45209 \mathrm{E}-02$

$-8.76548 \mathrm{E}-02$

$-9.08320 \mathrm{E}-02$

$-9.40526 \mathrm{E}-02$

$-9.73164 \mathrm{E}-02$

$-1.00623 \mathrm{E}-01$

$-1.03974 \mathrm{E}-01$

$-1.07367 \mathrm{E}-01$

$-1.10804 \mathrm{E}-01$

$-1.14284 \mathrm{E}-01$

$-1.17808 \mathrm{E}-01$

$-1.21374 \mathrm{E}-01$

$-1.24984 \mathrm{E}-01$

$-1.28637 \mathrm{E}-01$

$-1.32333 \mathrm{E}-01$

$-1.36072 \mathrm{E}-01$

$-1.39854 \mathrm{E}-01$

$-1.43680 \mathrm{E}-01$

$-1.47549 \mathrm{E}-01$

$-1.51461 \mathrm{E}-01$

$\tan \delta_{1}$

$f_{-1}$

$-1.24874 \mathrm{E}-01 \quad-2.45112 \mathrm{E}-03$

$-6.10672 \mathrm{E}-02-4.10498 \mathrm{E}-03$

$-4.01989 \mathrm{E}-02 \quad-5.76264 \mathrm{E}-03$

$-2.97670 \mathrm{E}-02-7.42220 \mathrm{E}-03$

$-2.34836 \mathrm{E}-02 \quad-9.08300 \mathrm{E}-03$

$-1.92716 \mathrm{E}-02$

$-1.62443 \mathrm{E}-02$

$-1.39589 \mathrm{E}-02$

$-1.21698 \mathrm{E}-02$

$-1.07294 \mathrm{E}-02$

$-9.54379 \mathrm{E}-03$

$-8.55009 \mathrm{E}-03$

$-7.70475 \mathrm{E}-03$

$-6.97654 \mathrm{E}-03$

$-6.34250 \mathrm{E}-03$

$-5.78531 \mathrm{E}-03$

$-5.29170 \mathrm{E}-03$

$-4.85132 \mathrm{E}-03$

$-4.45594 \mathrm{E}-03$

$-4.09897 \mathrm{E}-03$

$-3.77504 \mathrm{E}-03$

$-3.47975 \mathrm{E}-03$

$-3.20945 \mathrm{E}-03$

$-2.96109 \mathrm{E}-03$

$-2.73209 \mathrm{E}-03$

$-2.52026 \mathrm{E}-03$

$-2.32374 \mathrm{E}-03$

$-2.14093 \mathrm{E}-03$

$-1.97044 \mathrm{E}-03$

$-1.81106 \mathrm{E}-03$

$-1.66174 \mathrm{E}-03$

$-1.52156 \mathrm{E}-03$

$-1.38970 \mathrm{E}-03$

$-1.26543 \mathrm{E}-03$

$-1.14813 \mathrm{E}-03$

$-1.03722 \mathrm{E}-03$

$-9.32200 \mathrm{E}-04$

$-8.32607 \mathrm{E}-04$

$-7.38033 \mathrm{E}-04$

$-6.48108 \mathrm{E}-04$ 
$\bar{F}$

$f_{2}$

0.10

0.20

0.30

0.40

0.50

0.60

0.70

0.80

0.90

1.00

1.10

1.20

1.30

1.40

1.50

1.60

1.70

1.80

1.90

2.00

2.10

2.20

2.30

2.40

2.50

2.60

2.70

2.80

2.90

3.00

3.10

3.20

3.30

3.40

3.50

3.60

3.70

3.80

3.90

4.00

4. 10

4.20

4.30

4.40

4.50

4.60

4.70

4.80

4.90

5.00

5.10

5.20

5.30

5.40

5.50

5.60

5.70

5.80

5.90

6.00
$2.56196 \mathrm{E}-08$ $6.77821 \mathrm{E}-08$ $1.32452 \mathrm{E}-07$

$2.19623 \mathrm{E}-07$

$3.29467 \mathrm{E}-07$

$4.62154 \mathrm{E}-07$

$6.17830 \mathrm{E}-07$

$7.96611 \mathrm{E}-07$

$9.98594 \mathrm{E}-07$

$1.22385 \mathrm{E}-06$

$1.47244 \mathrm{E}-06$

$1.74442 \mathrm{E}-06$

$2.03980 \mathrm{E}-06$

$2.35863 \mathrm{E}-06$

$2.70092 \mathrm{E}-06$

$-3.06669 \mathrm{E}-06$

$3.45595 \mathrm{E}-06$

$3.86872 \mathrm{E}-06$

$4.30500 \mathrm{E}-06$

$4.76480 \mathrm{E}-06$

$5.24812 \mathrm{E}-06$

$5.75497 \mathrm{E}-06$

$6.28535 \mathrm{E}-06$

$6.83927 \mathrm{E}-06$

$7.41672 \mathrm{E}-06$

$8.01771 \mathrm{E}-06$

$8.64224 \mathrm{E}-06$

$9.29032 \mathrm{E}-06$

$9.96193 \mathrm{E}-06$

$1.06571 \mathrm{E}-05$

$1.13758 \mathrm{E}-05$ $1.21180 \mathrm{E}-05$ $1.28838 \mathrm{E}-05$ $1.36732 \mathrm{E}-05$

$1.44861 \mathrm{E}-0.5$

$1.53225 \mathrm{E}-0.5$ $1.61825 \mathrm{E}-0.5$ $1.70660 \mathrm{E}-0.5$ $1.79730 \mathrm{E}-05$ $1.89037 \mathrm{E}-05$

$1.98578 \mathrm{E}-0.5$

$2.08355 \mathrm{E}-05$

$2.18368 \mathrm{E}-05$

$2.28616 \mathrm{E}-05$

$2.39099 \mathrm{E}-0.5$

$2.49818 \mathrm{E}-05$

$2.60772 \mathrm{E}-0.5$

$2.71962 \mathrm{E}-05$

$2.83387 \mathrm{E}-05$

$2.95048 \mathrm{E}-05$

$3.06944 \mathrm{E}-05$ $3.19076 \mathrm{E}-05$ $3.31443 \mathrm{E}-05$

$3.44046 \mathrm{E}-05$

$3.56884 \mathrm{E}-0.5$

$3.69957 \mathrm{E}-05$

$3.83266 \mathrm{E}-0.5$

$3.96810 \mathrm{E}-05$

$4.10590 \mathrm{E}-05$

$4.24605 \mathrm{E}-05$
$-2.23568 \mathrm{E}-06$

$-1.44803 \mathrm{E}-05$

$-4.47951 \mathrm{E}-05$

$-1.00197 \mathrm{E}-04$

$-1.86407 \mathrm{E}-04$

$-3.07832 \mathrm{E}-04$

$-4.67702 \mathrm{E}-04$

$-6.68276 \mathrm{E}-04$

$-9.11077 \mathrm{E}-04$

$-1.19709 \mathrm{E}-03$

$-1.52691 \mathrm{E}-03$

$-1.90089 \mathrm{E}-03$

$-2.31923 \mathrm{E}-03$

$-2.78198 \mathrm{E}-03$

$-3.28916 \mathrm{E}-03$

$-3.84074 \mathrm{E}-03$

$-4.43666 \mathrm{E}-03$

$-5.07687 \mathrm{E}-03$

$-5.76128 \mathrm{E}-03$

$-6.48983 \mathrm{E}-03$

$-7.26246 \mathrm{E}-03$

$-8.07910 \mathrm{E}-03$

$-8.93969 \mathrm{E}-03$

$-9.84419 \mathrm{E}-03$

$-1.07925 \mathrm{E}-02$

$-1.17847 \mathrm{E}-02$

$-1.28206 \mathrm{E}-02$

$-1.39003 \mathrm{E}-02$

$-1.50237 \mathrm{E}-02$

$-1.61907 \mathrm{E}-02$

$-1.74014 \mathrm{E}-02$

$-1.86557 \mathrm{E}-02$

$-1.99536 \mathrm{E}-02$

$-2.12951 \mathrm{E}-02$

$-2.26801 \mathrm{E}-02$

$-2.41087 \mathrm{E}-02$

$-2.55808 \mathrm{E}-02$

$-2.70964 \mathrm{E}-02$

$-2.86555 \mathrm{E}-02$

$-3.02581 \mathrm{E}-02$

$-3.19042 \mathrm{E}-02$

$-3.35937 \mathrm{E}-02$

$-3.53268 \mathrm{E}-02$

$-3.71032 \mathrm{E}-02$

$-3.89231 \mathrm{E}-02$

$-4.07864 \mathrm{E}-02$

$-4.26931 \mathrm{E}-02$

$-4.46432 \mathrm{E}-02$

$-4.66368 \mathrm{E}-02$

$-4.86737 \mathrm{E}-02$

$-5.07540 \mathrm{E}-02$

$-5.28778 \mathrm{E}-02$

$-5.50449 \mathrm{E}-02$

$-5.72553 \mathrm{E}-02$

$-5.95092 \mathrm{E}-02$

$-6.18064 \mathrm{E}-02$

$-6.41470 \mathrm{E}-02$

$-6.65309 \mathrm{E}-02$

$-6.89582 \mathrm{E}-02$

$-7.14288 \mathrm{E}-02$ $g_{2}$

$-2.04889 \mathrm{E}-08$

$-1.32971 \mathrm{E}-07$

$-4.12699 \mathrm{E}-07$

$-9.27229 \mathrm{E}-07$

$-1.73452 \mathrm{E}-06$

$-2.88281 \mathrm{E}-06$

$-4.41165 \mathrm{E}-06$

$-6.35341 \mathrm{E}-06$

$-8.73509 \mathrm{E}-06$

$-1.15797 \mathrm{E}-05$

$-1.49076 \mathrm{E}-05$

$-1.87372 \mathrm{E}-0.5$

$-2.30858 \mathrm{E}-05$

$-2.79700 \mathrm{E}-05$

$-3.34058 \mathrm{E}-05$

$-3.94091 \mathrm{E}-05$

$-4.59954 \mathrm{E}-05$

$-5.31804 \mathrm{E}-05$

$-6.09797 \mathrm{E}-05$

$-6.94090 \mathrm{E}-05$

$-7.84837 \mathrm{E}-05$

$-8.82197 \mathrm{E}-05$

$-9.86327 \mathrm{E}-05$

$-1.09738 \mathrm{E}-04$

$-1.21552 \mathrm{E}-04$

$-1.34091 \mathrm{E}-04$

$-1.47369 \mathrm{E}-04$

$-1.61404 \mathrm{E}-04$

$-1.76211 \mathrm{E}-04$

$-1.91805 \mathrm{E}-04$

$-2.08204 \mathrm{E}-04$

$-2.25422 \mathrm{E}-04$

$-2.43476 \mathrm{E}-04$

$-2.62382 \mathrm{E}-04$

$-2.82156 \mathrm{E}-04$

$-3.02814 \mathrm{E}-04$

$-3.24372 \mathrm{E}-04$

$-3.46846 \mathrm{E}-04$

$-3.70251 \mathrm{E}-04$

$-3.94606 \mathrm{E}-04$

$-4.19924 \mathrm{E}-04$

$-4.46223 \mathrm{E}-04$

$-4.73518 \mathrm{E}-04$

$-5.01825 \mathrm{E}-04$

$-5.31162 \mathrm{E}-04$

$-5.61542 \mathrm{E}-04$

$-5.92984 \mathrm{E}-04$

$-6.25503 \mathrm{E}-04$

$-6.59114 \mathrm{E}-04$

$-6.93835 \mathrm{E}-04$

$-7.29681 \mathrm{E}-04$

$-7.66668 \mathrm{E}-04$

$-8.04813 \mathrm{E}-04$

$-8.44131 \mathrm{E}-04$

$-8.84639 \mathrm{E}-04$

$-9.26353 \mathrm{E}-04$

$-9.69288 \mathrm{E}-04$

$-1.01346 \mathrm{E}-03$

$-1.05889 \mathrm{E}-03$

$-1.10559 \mathrm{E}-03$

$\tan \delta_{2}$

$f_{-2}$

$3.60148 \mathrm{E} \quad 00$

$7.28799 \mathrm{E} \quad 00$

$1.07975 \mathrm{E} \quad 01$

$1.41025 \mathrm{E} \quad 01$

$1.71708 \mathrm{E} \quad 01$

$1.99850 \mathrm{E} \quad 01$

$2.254 .20 \mathrm{E}$ 0l

$2.48496 \mathrm{E} \quad 01$

$2.69228 \mathrm{E} \quad 01$

$2.87806 \mathrm{E} \quad 01$

$3.04436 \mathrm{E} \quad 01$ $3.19325 \mathrm{E} 01$

$3.32668 \mathrm{E} \quad 01$

$3.44646 \mathrm{E} \quad 01$

$3.55419 \mathrm{E} \quad 01$

$3.65134 \mathrm{E} \quad 01$ $3.73915 \mathrm{E} \quad 01$

$3.81875 \mathrm{E} \quad 01$

$3.89110 \mathrm{E} \quad 01$

$3.95704 \mathrm{E} \quad 01$

$4.01731 \mathrm{E} \quad 01$

$4.07252 \mathrm{E} 01$

$4.12325 \mathrm{E} \quad 01$

$4.16997 \mathrm{E} \quad 01$

$4.21310 \mathrm{E} \quad 01$

$4.25301 \mathrm{E} \quad 01$

$4.29003 \mathrm{E} 01$

$4.32443 \mathrm{E} \quad 01$

$4.35646 \mathrm{E} \quad 01$

$4.38636 \mathrm{E} \quad 01$

$4.41431 \mathrm{E} \quad 01$

$4.44049 \mathrm{E} \quad 01$

$4.46505 \mathrm{E} \quad 01$

$4.48813 \mathrm{E} 01$

$4.50985 \mathrm{E} \quad 01$

$4.53033 \mathrm{E}$ OI

$4.54967 \mathrm{E} \quad 01$

$4.56794 \mathrm{E}$ 0I

$4.58524 \mathrm{E}$ 0]

$4.60164 \mathrm{E} 01$

$4.61720 \mathrm{E} \quad 01$

$4.63199 \mathrm{E} 01$ 
ELECTRON RADIAL FUNCTIONS

Electron

\begin{tabular}{|c|c|c|c|c|c|}
\hline$p$ & $F_{0}$ & & $f_{1}$ & & $g_{1}$ \\
\hline 6.10 & $1.10048 \mathrm{E}$ & 00 & $-5.85078 \mathrm{E}$ & 00 & $-1.55416 \mathrm{E}-01$ \\
\hline 6.20 & $1.10042 \mathrm{E}$ & 00 & $-5.955+0 \mathrm{E}$ & 00 & $-1.59414 \mathrm{~F}$ \\
\hline 6.30 & $1.10037 \mathrm{E}$ & 00 & $-6.06002 \mathrm{E}$ & 00 & $-1.63456 \mathrm{E}-01$ \\
\hline 6.40 & $1.10032 \mathrm{E}$ & 00 & $-6.16462 \mathrm{E}$ & 00 & $-1.67541 \mathrm{E}-01$ \\
\hline 6.50 & $1.10027 \mathrm{E}$ & 00 & $-6.26922 \mathrm{E}$ & 00 & $-1.71668 E-01$ \\
\hline 6.60 & $1.10022 \mathrm{E}$ & 00 & $-6.37381 E$ & 00 & $-1.75839 E-01$ \\
\hline 6.70 & $1.10017 \mathrm{E}$ & 00 & $-6.47840 \mathrm{E}$ & 00 & $-1.80054 \mathrm{E}-01$ \\
\hline 6.80 & $1.10013 \mathrm{E}$ & 00 & $-6.58297 \mathrm{E}$ & 00 & $-1.84311 \mathrm{E}-01$ \\
\hline 6.90 & $1.10008 \mathrm{E}$ & 00 & $-6.68754 \mathrm{E}$ & 00 & $-1.88611 \mathrm{E}-01$ \\
\hline 7.00 & $1.10004 \mathrm{E}$ & 00 & $-6.79209 \mathrm{E}$ & 00 & $-1.92955 \mathrm{E}-01$ \\
\hline 7.10 & $1.10000 \mathrm{E}$ & 00 & $-6.89664 \mathrm{E}$ & 00 & $.97341 \mathrm{E}-01$ \\
\hline 7.20 & $1.09996 \mathrm{E}$ & 00 & $-7.00118 \mathrm{E}$ & 00 & $-2.01771 \mathrm{E}-01$ \\
\hline 7.30 & $1.09992 \mathrm{E}$ & 00 & $-7.10572 \mathrm{E}$ & 00 & $-2.06244 \mathrm{E}-01$ \\
\hline 7.40 & $1.09988 \mathrm{E}$ & 00 & $-7.21024 \mathrm{E}$ & 00 & $-2.10760 \mathrm{E}-01$ \\
\hline 7.50 & $1.09985 \mathrm{E}$ & 00 & $-7.31476 \mathrm{E}$ & 00 & $-2.15319 \mathrm{E}-01$ \\
\hline 7.60 & $1.09981 \mathrm{E}$ & 00 & $-7.41927 \mathrm{E}$ & 00 & -2.1992 \\
\hline 7.70 & $78 \mathrm{E}$ & 00 & $-7.52377 \mathrm{E}$ & 00 & $-2.24567 \mathrm{E}-01$ \\
\hline 7.80 & $74 \mathrm{E}$ & 00 & $-7.62826 \mathrm{E}$ & 00 & $-2.29255 \mathrm{E}-0 \mathrm{l}$ \\
\hline 7.90 & $1 \mathrm{E}$ & 00 & $-7.73274 \mathrm{E}$ & 00 & $-2.33987 \mathrm{E}-01$ \\
\hline 8.00 & $1.09968 \mathrm{E}$ & 00 & $-7.83722 \mathrm{E}$ & 00 & $-2.38762 \mathrm{E}-01$ \\
\hline 8.10 & $1.09965 \mathrm{E}$ & 00 & $-7.94168 \mathrm{E}$ & 00 & $-2.43580 \mathrm{E}-01$ \\
\hline 8.20 & $62 \mathrm{E}$ & 00 & $-8.04614 \mathrm{E}$ & 00 & $-2.48441 \mathrm{E}-01$ \\
\hline 30 & $59 \mathrm{E}$ & 00 & $-8.15058 \mathrm{E}$ & 00 & $-2.53345 \mathrm{E}-01$ \\
\hline 40 & $7 \mathrm{E}$ & 00 & $-8.25502 \mathrm{E}$ & 00 & -2.5829 \\
\hline 8.50 & $4 \mathrm{E}$ & 00 & -8.3 & 00 & $2 \mathrm{E}-01$ \\
\hline 8.60 & $.09951 \mathrm{E}$ & 00 & $-8.46388 E$ & 00 & -2.6831 \\
\hline 8.70 & & 00 & $6829 \mathrm{E}$ & 00 & -01 \\
\hline 80 & & 00 & $269 \mathrm{E}$ & 00 & \\
\hline 8.90 & & 00 & $-8.77709 \mathrm{E}$ & 00 & -2.8367 \\
\hline 9.00 & $1.09941 \mathrm{E}$ & 00 & $-8.88148 \mathrm{E}$ & 00 & -2.888 \\
\hline 9.10 & & 00 & $3585 \mathrm{E}$ & 00 & $3 E-01$ \\
\hline 9.20 & & 00 & & 00 & -01 \\
\hline 9.30 & & 00 & $9458 \mathrm{E}$ & 00 & $04754 \mathrm{E}-01$ \\
\hline 9.40 & $32 \mathrm{E}$ & 00 & $-9.29894 \mathrm{E}$ & 00 & $-3.10132 \mathrm{E}-01$ \\
\hline 9.50 & $1.09930 \mathrm{E}$ & 00 & $-9.40328 \mathrm{E}$ & 00 & $-3.15553 \mathrm{E}-01$ \\
\hline 9.60 & $1.09928 \mathrm{E}$ & 00 & $-9.50761 \mathrm{E}$ & 00 & $E-01$ \\
\hline 9.70 & & 00 & -9. & 00 & -01 \\
\hline 9.80 & $1.09924 \mathrm{E}$ & 00 & $-9.71625 \mathrm{E}$ & 00 & $-3.32074 \mathrm{E}-01$ \\
\hline 9.90 & $22 \mathrm{E}$ & 00 & $-9.82056 \mathrm{E}$ & 00 & $-3.37667 \mathrm{E}-01$ \\
\hline 10.00 & $1.09920 \mathrm{E}$ & 00 & $-9.92486 \mathrm{E}$ & 00 & $-3.43303 \mathrm{E}-01$ \\
\hline .10 & & 00 & $-1.00291 \mathrm{E}$ & 01 & $E-01$ \\
\hline 120 & & 00 & $1334 \mathrm{E}$ & 01 & $5 \mathrm{E}-01$ \\
\hline 30 & $4 \mathrm{E}$ & 00 & $-1.02377 \mathrm{E}$ & 01 & $-3.60470 \mathrm{E}-01$ \\
\hline & $3 E$ & 00 & $-1.03420 \mathrm{E}$ & 01 & $-3.66278 \mathrm{E}-01$ \\
\hline 10.5 & $1.09911 \mathrm{E}$ & 00 & $-1.04462 \mathrm{E}$ & 01 & $-3.72129 \mathrm{E}-01$ \\
\hline 10.60 & $1.09909 \mathrm{E}$ & 00 & $-1.05505 \mathrm{E}$ & 01 & $E-01$ \\
\hline & & 00 & -1.0 & 01 & -3.8396 \\
\hline 0.80 & $6 \mathrm{E}$ & 00 & $-1.07589 \mathrm{E}$ & 01 & $-3.89941 \mathrm{E}-01$ \\
\hline & & 00 & -1.0 & 01 & \\
\hline 11.00 & $1.09903 \mathrm{E}$ & 00 & $-1.09673 \mathrm{E}$ & 01 & $-4.02030 \mathrm{E}-01$ \\
\hline 11.10 & $1.09901 \mathrm{E}$ & 00 & -1.10 & 01 & $E-01$ \\
\hline & & 00 & $1757 \mathrm{E}$ & 01 & -01 \\
\hline & $98 \mathrm{E}$ & 00 & & 01 & \\
\hline & & 00 & & 01 & $-4.26725 \mathrm{E}-01$ \\
\hline & $1.09895 \mathrm{E}$ & 00 & $-1.14882 \mathrm{E}$ & 01 & $-4.33006 \mathrm{E}-01$ \\
\hline 11.60 & $1.09894 \mathrm{E}$ & 00 & -1.1592 & 01 & $-4.39330 \mathrm{E}-01$ \\
\hline & & 00 & $-1.16965 \mathrm{E}$ & 01 & $-4.45698 \mathrm{E}-01$ \\
\hline & & 00 & $-1.18007 \mathrm{E}$ & 01 & $-4.52107 \mathrm{E}-01$ \\
\hline & $1.09889 \mathrm{E}$ & 00 & $-1.19048 \mathrm{E}$ & 01 & $-4.58560 \mathrm{E}-01$ \\
\hline 12.00 & $1.09888 \mathrm{E}$ & 00 & $-1.20089 \mathrm{E}$ & 01 & $-4.65056 \mathrm{E}-01$ \\
\hline
\end{tabular}

$\tan \delta_{1}$ $\rho=1.2 A^{1 / 3} F$

$$
Z=4 \quad A=8
$$

$5.66373 \mathrm{E}-04$ $6.03869 \mathrm{E}-04$ $6.40180 \mathrm{E}-04$ $6.75361 \mathrm{E}-04$ $7.09468 \mathrm{E}-04$

$7.42545 \mathrm{E}-04$ $7.74643 \mathrm{E}-04$ $8.05794 \mathrm{E}-04$ $8.36050 \mathrm{E}-04$

$8.65441 \mathrm{E}-04$

$8.94011 \mathrm{E}-04$ $9.21788 \mathrm{E}-04$ $9.48804 \mathrm{E}-04$ $9.75090 \mathrm{E}-04$ $1.00067 \mathrm{E}-03$

$1.02559 \mathrm{E}-03$ $1.04985 \mathrm{E}-03$

$1.07349 \mathrm{E}-03$

$1.09653 \mathrm{E}-03$

$1.11900 \mathrm{E}-03$

$1.14090 \mathrm{E}-03$

$1.16228 \mathrm{E}-03$

$1.18313 \mathrm{E}-03$

$1.20348 \mathrm{E}-03$

$1.22336 \mathrm{E}-03$

$1.24276 \mathrm{E}-03$

$1.26172 \mathrm{E}-03$

$1.28025 \mathrm{E}-03$

$1.29836 \mathrm{E}-03$

$1.31605 \mathrm{E}-03$

$1.33336 \mathrm{E}-03$

$1.35029 \mathrm{E}-03$

$1.36685 \mathrm{E}-03$

$1.38305 \mathrm{E}-03$

$1.39891 \mathrm{E}-03$

$1.41443 \mathrm{E}-03$

$1.42963 \mathrm{E}-03$

$1.44451 \mathrm{E}-03$

$1.45909 \mathrm{E}-03$

$1.47337 \mathrm{E}-03$

$1.48736 \mathrm{E}-03$

$1.50107 \mathrm{E}-03$

$1.51452 \mathrm{E}-03$

$1.52770 \mathrm{E}-03$

$1.54062 \mathrm{E}-03$

$1.55329 \mathrm{E}-03$

$1.56572 \mathrm{E}-03$

$1.57792 \mathrm{E}-03$

$1.58989 \mathrm{E}-03$

$1.60163 \mathrm{E}-03$

$1.61316 \mathrm{E}-03$

$1.62447 \mathrm{E}-03$

$1.63558 \mathrm{E}-03$

$1.64649 \mathrm{E}-03$

$1.65720 \mathrm{E}-03$

$1.66772 \mathrm{E}-03$

$1.67806 \mathrm{E}-03$

$1.68821 \mathrm{E}-03$

$1.69819 \mathrm{E}-03$

$1.70800 \mathrm{E}-03$ $f_{-1}$

$-1.54394 \mathrm{E}-01$

$-1.58177 \mathrm{E}-01$

$-1.62002 \mathrm{E}-01$

$-1.65871 \mathrm{E}-01$

$-1.69783 \mathrm{E}-01$

$-1.73738 \mathrm{E}-01$

$-1.77736 \mathrm{E}-01$

$-1.81777 \mathrm{E}-01$

$-1.85861 \mathrm{E}-01$

$-1.89988 \mathrm{E}-01$

$-1.94158 \mathrm{E}-01$

$-1.98372 \mathrm{E}-01$

$-2.02628 \mathrm{E}-01$

$-2.06928 \mathrm{E}-01$

$-2.11270 \mathrm{E}-01$

$-2.15656 \mathrm{E}-01$

$-2.20085 \mathrm{E}-01$

$-2.24556 \mathrm{E}-01$

$-2.29071 \mathrm{E}-01$

$-2.33629 \mathrm{E}-01$

$-2.38230 \mathrm{E}-01$ $-2.42875 \mathrm{E}-01$ $-2.47562 \mathrm{E}-01$ $-2.52292 \mathrm{E}-01$ $-2.57065 \mathrm{E}-01$

$-2.61882 \mathrm{E}-01$ $-2.66741 \mathrm{E}-01$ $-2.71644 \mathrm{E}-01$ $-2.76589 \mathrm{E}-01$ $-2.81578 \mathrm{E}-01$

$-2.86610 \mathrm{E}-01$ $-2.91685 \mathrm{E}-01$ $-2.96802 \mathrm{E}-01$ $-3.01963 \mathrm{E}-01$ $-3.07167 \mathrm{E}-01$

$-3.12414 \mathrm{E}-01$ $-3.17704 \mathrm{E}-01$ $-3.23037 \mathrm{E}-01$ $-3.28413 \mathrm{E}-01$ $-3.33832 \mathrm{E}-01$

$-3.39295 \mathrm{E}-01$ $-3.44800 \mathrm{E}-01$ $-3.50348 \mathrm{E}-01$ $-3.55939 \mathrm{E}-01$ $-3.61573 \mathrm{E}-01$

$-3.67250 \mathrm{E}-01$ $-3.72971 \mathrm{E}-01$ $-3.78734 \mathrm{E}-01$ $-3.84540 \mathrm{E}-01$ $-3.90389 \mathrm{E}-01$

$-3.96281 \mathrm{E}-01$ $-4.02217 \mathrm{E}-01$ $-4.08195 \mathrm{E}-01$ $-4.14216 \mathrm{E}-01$ $-4.20280 \mathrm{E}-01$

$-4.26387 \mathrm{E}-01$ $-4.32537 \mathrm{E}-01$ $-4.38730 \mathrm{E}-01$ $-4.44966 \mathrm{E}-01$ $-4.51245 \mathrm{E}-01$ $g_{-1}$ $\tan \delta_{-1}$

$6.88690 \mathrm{E} \quad 00$ $6.99179 \mathrm{E} \quad 00$ $7.09665 \mathrm{E} \quad 00$ $7.20150 \mathrm{E} \quad 00$ $7.30632 \mathrm{E} \quad 00$

$7.41112 \mathrm{E} \quad 00$ $7.51591 \mathrm{E} \quad 00$ $7.62067 \mathrm{E} \quad 00$ $7.72542 \mathrm{E} \quad 00$ $7.83014 \mathrm{E} \quad 00$

$7.93485 \mathrm{E} \quad 00$ $8.03955 \mathrm{E} \quad 00$ $8.14423 \mathrm{E} \quad 00$ $8.24889 \mathrm{E} \quad 00$ $8.35354 \mathrm{E} \quad 00$

$8.45817 \mathrm{E} \quad 00$ $8.56279 \mathrm{E} \quad 00$ $8.66739 \mathrm{E} \quad 00$

$8.77198 \mathrm{E} \quad 00$

$8.87655 \mathrm{E} \quad 00$

$8.98111 \mathrm{E} \quad 00$ $9.08566 \mathrm{E} \quad 00$ $9.19019 \mathrm{E} \quad 00$ $9.29471 \mathrm{E} \quad 00$ $9.39922 \mathrm{E} \quad 00$

$9.50371 \mathrm{E} \quad 00$ $9.60819 \mathrm{E} \quad 00$ $9.71266 \mathrm{E} \quad 00$ $9.81712 \mathrm{E} \quad 00$ $9.92156 \mathrm{E} \quad 00$

$1.00260 \mathrm{E} \quad 01$ $1.01304 \mathrm{E} \quad 01$ $1.02348 \mathrm{E} \quad 01$ $1.03392 \mathrm{E} 01$ $1.04436 \mathrm{E} \quad 01$

$1.05480 \mathrm{E} \quad 01$ $1.06523 \mathrm{E} \quad 01$ $\begin{array}{ll}1.07567 \mathrm{E} & 01\end{array}$ $1.08610 \mathrm{E} \quad 01$ $1.09653 \mathrm{E} \quad 01$

$1.10697 \mathrm{E} \quad 01$ $1.11740 \mathrm{E} \quad 01$ $1.12783 \mathrm{E} 01$ $1.13825 \mathrm{E} \quad 01$ $1.14868 \mathrm{E} 01$

$1.15911 \mathrm{E} \quad 01$ $1.16953 \mathrm{E} \quad 01$ $1.17996 \mathrm{E} \quad 01$ $1.19038 \mathrm{E} \quad 01$ $1.20080 \mathrm{E} \quad 01$

$\begin{array}{ll}1.21122 \mathrm{E} & 01\end{array}$ $1.22164 \mathrm{E} 01$ $1.23206 \mathrm{E} 01$ $\begin{array}{lll}1.24248 \mathrm{E} & 01\end{array}$ $1.25289 \mathrm{E} 01$

$1.26331 \mathrm{E} 01$ $1.27372 \mathrm{E} \quad 01$ $1.28413 \mathrm{E} 0 \mathrm{I}$

$1.29455 \mathrm{E} \quad 01$ $1.30496 \mathrm{E} 01$
$-1.86912 \mathrm{E} \quad 02$ $-1.88309 \mathrm{E} \quad 02$ $-1.89682 \mathrm{E} \quad 02$ $-1.91032 \mathrm{E} 02$ $-1.92358 \mathrm{E} \quad 02$

$-1.93661 \mathrm{E} \quad 02$ -1.94942 E 02
$-1.96202 E$ $-1.96202 \mathrm{E} 02$ $-1.97441 \mathrm{E} 02$ $-1.98659 \mathrm{E} 02$

$-1.99858 \mathrm{E} \quad 02$ $-2.01037 \mathrm{E} \quad 02$ $-2.02197 \mathrm{E} \quad 02$ $-2.03339 \mathrm{E} \quad 02$ $-2.04463 \mathrm{E} 02$ $-2.05569 \mathrm{E} \quad 02$
$-2.06659 \mathrm{E}$ $-2.06659 \mathrm{E} \quad 02$ -2.07731 E 02 $\begin{array}{ll}-2.08787 \mathrm{E} & 02\end{array}$ $-2.09828 \mathrm{E} \quad 02$

$-2.10853 \mathrm{E} \quad 02$ $\begin{array}{ll}-2.11862 \mathrm{E} & 02\end{array}$ $-2.12857 \mathrm{E} \quad 02$ $\begin{array}{ll}-2.13837 \mathrm{E} & 02\end{array}$ $-2.14803 \mathrm{E} \quad 02$

$-2.15755 \mathrm{E} \quad 02$ $-2.16694 \mathrm{E} \quad 02$ $-2.17620 \mathrm{E} \quad 02$ $-2.18532 \mathrm{E} \quad 02$ $\begin{array}{ll}-2.19432 \mathrm{E} & 02\end{array}$ $\begin{array}{lll}-2.20320 \mathrm{E} & 02\end{array}$ $-2.21195 \mathrm{E} \quad 02$ $-2.22059 \mathrm{E} \quad 02$ $-2.22911 \mathrm{E} \quad 02$ $-2.23751 \mathrm{E} \quad 02$

$-2.24581 \mathrm{E} \quad 02$ $\begin{array}{ll}-2.25399 \mathrm{E} & 02\end{array}$ $\begin{array}{lll}-2.26207 \mathrm{E} & 02\end{array}$ $-2.27005 \mathrm{E} \quad 02$ $-2.27793 \mathrm{E} \quad 02$

$-2.28570 \mathrm{E} \quad 02$ $-2.29338 \mathrm{E} \quad 02$ $-2.30096 \mathrm{E} \quad 02$ $-2.30844 \mathrm{E} \quad 02$ $\begin{array}{ll}-2.31584 \mathrm{E} & 02\end{array}$

$-2.32314 \mathrm{E} \quad 02$ $-2.33036 \mathrm{E} \quad 02$ $-2.33749 \mathrm{E} \quad 02$ $-2.34453 \mathrm{E} \quad 02$ $-2.35149 \mathrm{E} \quad 02$ $-2.35837 \mathrm{E} \quad 02$ $-2.36517 \mathrm{E} \quad 02$ $-2.37189 \mathrm{E} \quad 02$ $-2.37854 \mathrm{E} 02$ $-2.38510 \mathrm{E} \quad 02$ $-2.39160 \mathrm{E} \quad 02$ $-2.39802 \mathrm{E} \quad 02$ $-2.40437 \mathrm{E} \quad 02$ $-2.41065 \mathrm{E} \quad 02$ $-2.41686 \mathrm{E} \quad 02$ 
ELECTRON RADIAL FUNCTIONS

$Z=4 \quad A=8 \quad \rho=1.2 A^{1 / 3} F$

Electron

$\bar{F}$

$4.38856 \mathrm{E}-05$

$4.53342 \mathrm{E}-05$

$4.68063 \mathrm{E}-05$

$4.83020 \mathrm{E}-05$

$4.98213 \mathrm{E}-05$

$5.13641 \mathrm{E}-05$ $5.29304 \mathrm{E}-05$

$5.45203 \mathrm{E}-05$

$5.61337 \mathrm{E}-05$

$5.77706 \mathrm{E}-05$

$5.94311 \mathrm{E}-05$ $6.11151 \mathrm{E}-05$ $6.28227 \mathrm{E}-05$ $6.45539 \mathrm{E}-05$

$6.63085 \mathrm{E}-05$

$6.80867 \mathrm{E}-05$ $6.98885 \mathrm{E}-05$

$7.17138 \mathrm{E}-05$

$7.35626 \mathrm{E}-05$

$7.54350 \mathrm{E}-05$

$7.73309 \mathrm{E}-05$

$7.92504 \mathrm{E}-05$

$8.11934 \mathrm{E}-05$

$8.31599 \mathrm{E}-05$

$8.51500 \mathrm{E}-05$

$8.71636 \mathrm{E}-05$ $8.92008 \mathrm{E}-05$ $9.12615 \mathrm{E}-05$ $9.33458 \mathrm{E}-05$

$9.54536 \mathrm{E}-05$

$9.75849 \mathrm{E}-05$

$9.97398 \mathrm{E}-0.5$

$1.01918 \mathrm{E}-04$

$1.04120 \mathrm{E}-04$

$1.06346 \mathrm{E}-04$

$1.08595 \mathrm{E}-04$

$1.10867 \mathrm{E}-04$

$1.13163 \mathrm{E}-04$

$1.15483 \mathrm{E}-04$

$1.17826 \mathrm{E}-04$

$1.20193 \mathrm{E}-04$

$1.22583 \mathrm{E}-04$

$1.24997 \mathrm{E}-04$

$1.27434 \mathrm{E}-04$

$1.29895 \mathrm{E}-04$

$1.32380 \mathrm{E}-04$ $1.34888 \mathrm{E}-04$ $1.37419 \mathrm{E}-04$ $1.39974 \mathrm{E}-04$

$1.42553 \mathrm{E}-04$

$1.45155 \mathrm{E}-04$ $1.47780 \mathrm{E}-04$ $1.50430 \mathrm{E}-04$ $1.53102 \mathrm{E}-04$ $1.55799 \mathrm{E}-04$

$1.58518 \mathrm{E}-04$

$1.61262 \mathrm{E}-04$

$1.64029 \mathrm{E}-04$

$1.66819 \mathrm{E}-04$

$1.69633 \mathrm{E}-04$ $f_{2}$

$-7.39428 \mathrm{E}-02$

$-7.65001 \mathrm{E}-02$

$-7.91007 \mathrm{E}-02$

$-8.17447 \mathrm{E}-02$

$-8.44320 \mathrm{E}-02$

$-8.71626 \mathrm{E}-02$

$-8.99365 \mathrm{E}-02$

$-9.27538 \mathrm{E}-02$

$-9.56143 \mathrm{E}-02$

- 9.85181 E-02

$-1.01465 \mathrm{E}-01$

$-1.04456 \mathrm{E}-01$

$-1.07490 \mathrm{E}-01$

$-1.10567 \mathrm{E}-01$

$-1.13687 \mathrm{E}-01$

$-1.16851 \mathrm{E}-01$

$-1.20057 \mathrm{E}-01$

$-1.23308 \mathrm{E}-01$

$-1.26601 \mathrm{E}-01$

$-1.29938 \mathrm{E}-01$

$-1.33318 \mathrm{E}-01$

$-1.36741 \mathrm{E}-01$

$-1.40208 \mathrm{E}-01$

$-1.43717 \mathrm{E}-01$

$-1.47270 \mathrm{E}-01$

$-1.50867 \mathrm{E}-01$

$-1.54506 \mathrm{E}-01$

$-1.58189 \mathrm{E}-01$

$-1.61915 \mathrm{E}-01$

$-1.65684 \mathrm{E}-01$

$-1.69497 \mathrm{E}-01$

$-1.73352 \mathrm{E}-01$

$-1.77251 \mathrm{E}-01$

$-1.81193 \mathrm{E}-01$

$-1.85179 \mathrm{E}-01$

$-1.89207 \mathrm{E}-01$

$-1.93279 \mathrm{E}-01$

$-1.97394 \mathrm{E}-01$

$-2.01552 \mathbf{E}-01$

$-2.05754 \mathrm{E}-01$

$-2.09998 \mathrm{E}-01$

$-2.14286 \mathrm{E}-01$

$-2.18617 \mathrm{E}-01$

$-2.22991 \mathrm{E}-01$

$-2.27409 \mathrm{E}-01$

$-2.31869 \mathrm{E}-01$

$-2.36373 \mathrm{E}-01$

$-2.40920 \mathrm{E}-01$

$-2.45510 \mathrm{E}-01$

$-2.50144 \mathrm{E}-01$

$-2.54820 \mathrm{E}-01$ $-2.59540 \mathrm{E}-0$ ? $-2.64302 \mathrm{E}-01$ $-2.69108 \mathrm{E}-01$ $-2.73957 \mathrm{E}-01$

$-2.78849 \mathrm{E}-01$

$-2.83785 \mathrm{E}-01$

$-2.88763 \mathrm{E}-01$

$-2.93785 \mathrm{E}-01$

$-2.98850 \mathrm{E}-01$ $g_{2}$

$-1.15357 \mathrm{E}-03$

$-1.20286 \mathrm{E}-03$

$-1.25346 \mathrm{E}-03$

$-1.30540 \mathrm{E}-03$

$-1.35869 \mathrm{E}-03$

$-1.41335 \mathrm{E}-03$

$-1.46939 \mathrm{E}-03$

$-1.52683 \mathbf{E}-03$

$-1.58569 \mathbf{E}-03$

$-1.64597 \mathrm{E}-03$

$-1.70770 \mathrm{E}-03$

$-1.77090 \mathrm{E}-03$

$-1.83558 \mathrm{E}-03$

$-1.90175 \mathrm{E}-03$

$-1.96943 \mathrm{E}-03$

$-2.03865 \mathrm{E}-03$

$-2.10940 \mathrm{E}-03$

$-2.18172 \mathrm{E}-03$

$-2.25561 \mathrm{E}-03$

$-2.33110 \mathrm{E}-03$

$-2.40819 \mathrm{E}-03$

$-2.48691 \mathrm{E}-03$

$-2.56727 \mathrm{E}-03$

$-2.64928 \mathrm{E}-03$

$-2.73297 \mathrm{E}-03$

$-2.81835 \mathrm{E}-03$

$-2.90543 \mathrm{E}-03$

$-2.99423 \mathrm{E}-03$

$-3.08477 \mathrm{E}-03$

$-3.17706 \mathrm{E}-03$

$-3.27113 \mathrm{E}-03$

$-3.36697 \mathrm{E}-03$

$-3.46462 \mathrm{E}-03$

$-3.56409 \mathrm{E}-03$

$-3.66539 \mathrm{E}-03$

$-3.76854 \mathrm{E}-03$

$-3.87356 \mathrm{E}-03$

$-3.98046 \mathrm{E}-03$

$-4.08926 \mathrm{E}-03$

$-4.19997 \mathrm{E}-03$

$-4.31261 \mathrm{E}-03$

$-4.42719 \mathrm{E}-03$

$-4.54374 \mathrm{E}-03$

$-4.66227 \mathrm{E}-03$

$-4.78279 \mathrm{E}-03$

$-4.90532 \mathrm{E}-03$

$-5.02988 \mathrm{E}-03$

$-5.15648 \mathrm{E}-03$

$-5.28515 \mathrm{E}-03$

$-5.41588 \mathrm{E}-03$

$-5.54871 \mathrm{E}-03$

$-5.68364 \mathrm{E}-03$

$-5.82069 \mathrm{E}-03$

$-5.95989 \mathrm{E}-03$

$-6.10124 \mathrm{E}-03$

$-6.24476 \mathrm{E}-03$

$-6.39047 \mathrm{E}-03$

$-6.53838 \mathrm{E}-03$

$-6.68851 \mathrm{E}-03$

$-6.84087 \mathrm{E}-03$ $\tan \delta_{2}$

$f_{2}$

$4.81682 \mathrm{E} \quad 01 \quad-1.14163 \mathrm{E}-03$ $4.82324 \mathrm{E}$ ol $-1.18906 \mathrm{E}-03$

$4.82945 \mathrm{E} \quad 01 \quad-1.23776 \mathrm{E}-03$

$4.83545 \mathrm{E}$ 0l $-1.28773 \mathrm{E}-03$

$4.84125 \mathrm{E}$ ol $-1.33900 \mathrm{E}-03$

$\begin{array}{ll}4.84686 \mathrm{E} & 01\end{array}$

$4.85230 \mathrm{E}$ 0l

$4.85757 \mathrm{E} \quad 01$

$4.86267 \mathrm{E} \quad 01$

$4.86762 \mathrm{E}$ ol

$-1.39159 \mathrm{E}-03$

$-1.44550 \mathrm{E}-03$

$-1.50076 \mathrm{E}-03$

$-1.55737 \mathrm{E}-03$

$-1.61537 \mathrm{E}-03$

$4.87242 \mathrm{E} \quad 01$

$4.87708 \mathrm{E} \quad 01$

$4.88160 \mathrm{E} \quad 01$

$4.88600 \mathrm{E} \quad 01$

$4.89027 \mathrm{E} \quad 0$

$4.89441 \mathrm{E} \quad 01$

$4.89845 \mathrm{E} \quad 01$

$4.90237 \mathrm{E} \quad 01$

$4.90619 \mathrm{E} \quad 01$

$4.90991 \mathrm{E} \quad 01$

$4.91353 \mathrm{E} \quad 01$

$4.91705 \mathrm{E} \quad 01$

$4.92049 \mathrm{E} \quad 01$

$4.92384 \mathrm{E} \quad 01$

$4.92710 \mathrm{E} \quad 01$

$4.93029 \mathrm{E} \quad 01$ $4.93340 \mathrm{E} 01$

$4.93643 \mathrm{E} \quad 01$

$4.93939 \mathrm{E} \quad 01$

$4.94228 \mathrm{E} 01$

$4.94510 \mathrm{E} \quad 01$ $4.94786 \mathrm{E} \quad 01$

$4.95055 \mathrm{E}$ 01

$495319 \mathrm{E}$ ol

$4.95576 \mathrm{E} \quad 01$

$4.95828 \mathrm{E} \quad 01$

$4.96074 \mathrm{E} \quad 01$

$4.96315 \mathrm{E} \quad 01$

$4.96551 \mathrm{E} \quad 01$

$4.96782 \mathrm{E} \quad 01$

$4.97008 \mathrm{E} \quad 01$

$4.97229 \mathrm{E} \quad 01$

$4.97446 \mathrm{E} \quad 01$

$4.97659 \mathrm{E}$ 01

$4.97867 \mathrm{E} \quad 01$

$4.98071 \mathrm{E} \quad 01$

$4.98271 \mathrm{E} \quad 0$

$4.98467 \mathrm{E} \quad 01$

$4.98659 \mathrm{E} \quad 0$

$4.98847 \mathrm{E} \quad 01$

$4.99032 \mathrm{E} \quad 01$ $4.99214 \mathrm{E}$ 01 $4.99392 \mathrm{E} \quad 01$

$4.99567 \mathrm{E} \quad 01$

$4.99738 \mathrm{E} \quad 01$

$4.99907 \mathrm{E} \quad 01$

$5.00072 \mathrm{E} \quad 01$

$5.00234 \mathrm{E} \quad 01$

$5.00394 \mathrm{E} \quad 01$

$5.00551 \mathrm{E} 01$

$-1.67476 \mathrm{E}-03$

$-1.73556 \mathrm{E}-03$

$-1.79778 \mathrm{E}-03$

$-1.86144 \mathrm{E}-03$

$-1.92657 \mathrm{E}-03$

$-1.99316 \mathrm{E}-03$

$-2.06125 \mathrm{E}-03$

$-2.13084 \mathrm{E}-03$

$-2.20196 \mathrm{E}-03$

$-2.27461 \mathrm{E}-03$

$-2.34882 \mathrm{E}-03$

$-2.42459 \mathrm{E}-03$

$-2.50196 \mathrm{E}-03$

$-2.58092 \mathrm{E}-03$

$-2.66151 \mathrm{E}-03$

$-2.74373 \mathrm{E}-03$

$-2.82760 \mathrm{E}-03$

$-2.91314 \mathrm{E}-03$

$-3.00036 \mathrm{E}-03$

$-3.08928 \mathrm{E}-03$

$-3.17992 \mathrm{E}-03$

$-3.27229 \mathrm{E}-03$

$-3.36640 \mathrm{E}-03$

$-3.46228 \mathrm{E}-03$

$-3.55994 \mathrm{E}-03$

$-3.65939 \mathrm{E}-03$

$-3.76066 \mathrm{E}-03$

$-3.86375 \mathrm{E}-03$

$-3.96869 \mathrm{E}-03$

$-4.07549 \mathrm{E}-03$

$-4.18417 \mathrm{E}-03$

$-4.29473 \mathrm{E}-03$

$-4.40721 \mathrm{E}-03$

$-4.52161 \mathrm{E}-03$

$-4.63795 \mathrm{E}-03$

$-4.75624 \mathrm{E}-03$

$-4.87651 \mathrm{E}-03$ 
ELECTRON RADIAL FUNCTIONS

Electron

$p$

12.10

12.20

12.30

12.40

12.50

12.60

12.70

12.80

12.90

1.3 .00

13.10

13.20

13.30

13.40

13.50

13.60

13.70

13.80

13.90

14.00

14.10

14.20

14.30

14.40

14.50

I 4.60

14.70

14.80

$1+.90$

15.00

15.10

15.20

15.30

15.40

15.50

15.60

15.70

15.80

15.90

16.00

16.10

16.20

16.30

I6. 40

16.50

16.60

16.70

16.80

16.90

17.00

17.10

17.20

17.30

17.40

17.50

17.60

17.70

17.80

17.90

18.00

$$
Z=4 \quad A=8
$$

$g_{1}$

$\begin{array}{llll}1.09887 \mathrm{E} & 00 & -1.21130 \mathrm{E} & 01\end{array}$ $\begin{array}{llll}1.09885 \mathrm{E} & 00 & -1.22171 \mathrm{E} & 01\end{array}$ $\begin{array}{lllll}1.09884 \mathrm{E} & 00 & -1.23212 \mathrm{E} & 01\end{array}$ $1.09883 \mathrm{E} \quad 00$ $1.09882 \mathrm{E} \quad 00$

$1.09880 \mathrm{E} \quad 00$ $1.09879 \mathrm{E} \quad 00$ $1.09878 \mathrm{E} \quad 00$ $1.09877 \mathrm{E} \quad 00$ $1.09876 \mathrm{E} \quad 00$

$1.09874 \mathrm{E} \quad 00$ $1.09873 \mathrm{E} \quad 00$ $1.09872 \mathrm{E} \quad 00$ $1.09871 \mathrm{E} \quad 00$ $1.09870 \mathrm{E} \quad 00$

$1.09869 \mathrm{E} \quad 00$ $1.09868 \mathrm{E} \quad 00$ $1.09867 \mathrm{E} \quad 00$ $1.09866 \mathrm{E} \quad 00$ $1.09865 \mathrm{E} \quad 00$

$1.09864 \mathrm{E} \quad 00$ $1.09863 \mathrm{E} \quad 00$ $1.09862 \mathrm{E} \quad 00$ $1.09861 \mathrm{E} \quad 00$ $1.09860 \mathrm{E} \quad 00$

$1.09859 \mathrm{E} \quad 00$ $1.09858 \mathrm{E} \quad 00$ $1.09857 \mathrm{E} \quad 00$ $1.09856 \mathrm{E} \quad 00$ $1.09855 \mathrm{E} \quad 00$

$1.09854 \mathbf{E} \quad 00$ $1.09853 \mathrm{E} \quad 00$ $1.09852 \mathrm{E} \quad 00$ $1.09851 \mathrm{E} \quad 00$

$1.09851 \mathrm{E} \quad 00$

$1.09850 \mathrm{E} \quad 00$ $1.09849 \mathrm{E} \quad 00$ $1.09848 \mathrm{E} \quad 00$ $1.09847 \mathrm{E} \quad 00$ $1.09846 \mathrm{E} \quad 00$

$1.09845 \mathrm{E} \quad 00$ $1.09845 \mathrm{E} \quad 00$ $1.09844 \mathrm{E} \quad 00$ $1.09843 \mathrm{E}-00$ $1.09842 \mathrm{E} \quad 00$

$1.09841 \mathrm{E} \quad 00$ $1.09841 \mathrm{E} \quad 00$ $1.09840 \mathrm{E} \quad 00$ $1.09839 \mathrm{E} \quad 00$ $1.09838 \mathrm{E} \quad 00$

$1.09838 \mathrm{E} \quad 00$ $1.09837 \mathrm{E} \quad 00$ $1.09836 \mathrm{E} \quad 00$ $1.09836 \mathrm{E} \quad 00$ $1.09835 \mathrm{E} \quad 00$

$1.09834 \mathrm{E} \quad 00$ $1.09833 \mathrm{E} \quad 00$ $1.09833 \mathrm{E} \quad 00$ $1.09832 \mathrm{E} \quad 00$ $1.09831 \mathrm{E} \quad 00$
$-1.24253 \mathrm{E} 01$

$-1.25294 \mathrm{E} \quad 01$

$-1.26334 \mathrm{E} \quad 01$ $-1.27375 \mathrm{E} \quad 01$ $-1.28415 \mathrm{E} \quad 01$ $-1.29455 \mathrm{E} \quad 0$ $-1.30496 \mathrm{E} \quad 01$

$-1.31536 \mathrm{E} \quad 01$ $-1.32576 \mathrm{E} 01$ $-1.33616 \mathrm{E} \quad 01$ $-1.34655 \mathrm{E} 01$ $-1.35695 \mathrm{E} \quad 01$

$-1.36735 \mathrm{E} \quad 01$ $-1.37774 \mathrm{E} \quad 01$ $-1.38814 \mathrm{E} 01$ $-1.40892 \mathrm{E} \quad 01$

$-1.41931 \mathrm{E} \quad 01$ $-1.42970 \mathrm{E} \quad 01$ $-1.44009 \mathrm{E} \quad 01$ $-1.45048 \mathrm{E} \quad 01$ $-1.46087 \mathrm{E} 01$

$-1.47126 \mathrm{E} \quad 01$ $-1.48164 \mathrm{E} \quad 01$ $-1.49203 \mathrm{E} \quad 01$ $-1.50241 \mathrm{E} \quad 01$ $-1.51279 \mathrm{E} \quad 01$

$-1.52317 \mathrm{E} \quad 01$ $-1.53355 \mathrm{E} \quad 01$ $-1.54393 \mathrm{E} \quad 01$ -1.55431 E 01 $-1.56469 \mathrm{E} \quad 01$

$-1.57506 \mathrm{E} 01$ $-1.58544 \mathrm{E} \quad 01$ $-1.59581 \mathrm{E} 01$ $-1.60618 \mathrm{E} 01$ $-1.61656 \mathrm{E} 01$

$-1.62693 \mathbf{E} 01$ $-1.63730 \mathrm{E} 01$ $-1.64766 \mathbf{E} 01$ $-\mathrm{I} .65803 \mathrm{E} 01$ $-1.66840 \mathrm{E} 01$

$\begin{array}{lll}-1.67876 \mathrm{E} & 01\end{array}$ $-1.68913 \mathrm{E} 01$ $-1.69949 \mathrm{E} \quad 01$ $-1.70985 \mathrm{E} \quad 01$ $-1.72022 \mathrm{E} \quad 01$

$-4.71596 \mathrm{E}-01$ $-4.78177 \mathrm{E}-01$ $-4.84802 \mathrm{E}-01$ $-4.91470 \mathrm{E}-01$ $-4.98181 \mathrm{E}-01$

$-5.04934 \mathrm{E}-01$ $-5.11731 \mathrm{E}-01$ $-5.18570 \mathrm{E}-01$ $-5.25452 \mathrm{E}-01$ $-5.32378 \mathrm{E}-01$

$-5.39346 \mathrm{E}-01$ $-5.46357 \mathrm{E}-01$ $-5.53411 \mathrm{E}-01$ $-5.60507 \mathrm{E}-01$

$-5.67647 \mathrm{E}-01$

$-5.74830 \mathrm{E}-01$ $-5.82055 \mathrm{E}-01$ $-5.89323 \mathrm{E}-01$ $-5.96635 \mathrm{E}-01$ $-6.03989 \mathrm{E}-01$

$-6.11386 \mathrm{E}-01$ $-6.18825 \mathrm{E}-01$ $-6.26308 \mathrm{E}-01$ $-6.33833 \mathrm{E}-01$ $-6.41401 \mathrm{E}-01$

$-6.49013 \mathrm{E}-01$ $-6.56666 \mathrm{E}-01$ $-6.64363 \mathrm{E}-01$ $-6.72103 \mathrm{E}-01$ $-6.79885 \mathrm{E}-01$

$-6.87710 \mathrm{E}-01$ $-6.95578 \mathrm{E}-01$ $-7.03489 \mathrm{E}-01$ $-7.11442 \mathrm{E}-01$ $-7.19439 \mathrm{E}-01$

$-7.27478 \mathrm{E}-01$ $-7.35560 \mathrm{E}-01$ $-7.43684 \mathrm{E}-0 \mathrm{l}$ $-7.51852 \mathrm{E}-01$ $-7.60062 \mathrm{E}-01$

$-7.68315 \mathrm{E}-01$ $-7.76610 \mathrm{E}-01$ $-7.84949 \mathrm{E}-01$ $-7.93330 \mathrm{E}-01$ $-8.01754 \mathrm{E}-01$

$-8.10220 \mathrm{E}-01$ $-8.18730 \mathrm{E}-01$ $-8.27282 \mathrm{E}-01$ $-8.35876 \mathrm{E}-0 \mathrm{I}$ $-8.44514 \mathrm{E}-01$

$-8.53194 \mathrm{E}-01$

$-8.61917 \mathrm{E}-01$

$-8.70683 \mathrm{E}-01$

$-8.79491 \mathrm{E}-01$

$-8.88342 \mathrm{E}-01$

$-8.97235 \mathrm{E}-01$ $-9.06171 \mathrm{E}-01$ $-9.15150 \mathrm{E}-01$ $-9.24172 \mathrm{E}-01$ $-9.33236 \mathrm{E}-01$ $-1.39853 \mathrm{E} \quad 01$

$\tan \delta_{1}$

$1.71764 \mathrm{E}-03$ $1.72711 \mathrm{E}-03$

$1.73643 \mathrm{E}-03$

$1.74558 \mathrm{E}-03$

$1.75459 \mathrm{E}-03$

$1.76344 \mathrm{E}-03$

$1.77215 \mathrm{E}-03$

$1.78072 \mathrm{E}-03$

$1.78915 \mathrm{E}-03$

$1.79744 \mathrm{E}-03$

$1.80560 \mathrm{E}-03$

$1.81363 \mathrm{E}-03$

$1.82153 \mathrm{E}-03$

$1.82931 \mathrm{E}-03$

$1.83696 \mathrm{E}-03$

$1.84450 \mathrm{E}-03$

$1.85192 \mathrm{E}-03$

$1.85923 \mathrm{E}-03$

$1.86642 \mathrm{E}-03$

$1.87350 \mathrm{E}-03$

$1.88048 \mathrm{E}-03$

$1.88736 \mathrm{E}-03$

$1.89413 \mathrm{E}-03$

$1.90080 \mathrm{E}-03$

$1.90737 \mathrm{E}-03$

$1.91384 \mathrm{E}-03$

$1.92022 \mathrm{E}-03$

$1.92651 \mathrm{E}-03$

$1.93271 \mathrm{E}-03$

$1.93881 \mathrm{E}-03$

$1.94483 \mathrm{E}-03$

$1.95076 \mathrm{E}-03$

$1.95661 \mathrm{E}-03$

$1.96238 \mathrm{E}-03$

$1.96806 \mathrm{E}-03$

$1.97367 \mathrm{E}-03$

$1.97919 \mathrm{E}-03$

$1.98465 \mathrm{E}-03$

$1.99002 \mathrm{E}-03$

$1.99532 \mathrm{E}-03$

$2.00055 \mathrm{E}-03$ $2.00570 \mathrm{E}-03$

$2.01079 \mathrm{E}-03$

$2.01581 \mathrm{E}-03$

$2.02076 \mathrm{E}-03$

$2.02564 \mathrm{E}-03$

$2.03046 \mathrm{E}-03$

$2.03521 \mathrm{E}-03$

$2.03990 \mathrm{E}-03$

$2.04453 \mathrm{E}-03$

$2.04910 \mathrm{E}-03$

$2.05361 \mathrm{E}-03$

$2.05806 \mathrm{E}-03$

$2.06245 \mathrm{E}-03$

$2.06678 \mathrm{E}-03$

$2.07106 \mathrm{E}-03$ $2.07528 \mathrm{E}-03$

$2.07944 \mathrm{E}-03$ 
$\bar{F}$

12.10

12.20

12.30

12.40

12.50

12.60

12.70

12.80

12.90

13.00

13.10

13.20

13.30

13.40

13.50

13.60

13.70

13.80

13.90

14.00

14.10

14.20

14.30

14.40

14.50

14.60

14.70

14.80

14.90

15.00

15.10

15.20

15.30

15.40

15.50

15.60

15.70

15.80

15.90

16.00

16.10

16.20

16.30

16.40

16.50

16.60

16.70

16.80

16.90

17.00

17.10

17.20

17.30

17.40

17.50

17.60

17.70

17.80

17.90

18.00 $f_{2}$

$1.72471 \mathrm{E}-04$

$1.75332 \mathrm{E}-04$

$1.78216 \mathrm{E}-04$

$1.81124 \mathrm{E}-04$

$1.84056 \mathrm{E}-04$

$1.87011 \mathrm{E}-04$

$1.89990 \mathrm{E}-04$

$1.92992 \mathbf{E}-04$

$1.96018 \mathrm{E}-04$

$1.99067 \mathrm{E}-04$

$2.02140 \mathrm{E}-04$

$2.05237 \mathrm{E}-04$

$2.08357 \mathrm{E}-04$

$2.11500 \mathrm{E}-04$

$2.14667 \mathrm{E}-04$

$2.17858 \mathrm{E}-04$

$2.21072 \mathrm{E}-04$

$2.24309 \mathrm{E}-04$

$2.27570 \mathrm{E}-04$

$2.30855 \mathrm{E}-04$

$2.34163 \mathrm{E}-04$ $2.37495 \mathrm{E}-04$ $2.40850 \mathrm{E}-04$

$2.44229 \mathrm{E}-04$

$2.47632 \mathrm{E}-04$

$2.51058 \mathrm{E}-04$

$2.54507 \mathrm{E}-04$

$2.57980 \mathrm{E}-04$

$2.61477 \mathrm{E}-04$

$2.64997 \mathrm{E}-04$

$2.68540 \mathrm{E}-04$

$2.72107 \mathrm{E}-04$

$2.75698 \mathrm{E}-04$

$2.79312 \mathrm{E}-04$

$2.82950 \mathrm{E}-04$

$2.86611 \mathrm{E}-04$ $2.90296 \mathrm{E}-04$ $2.94004 \mathrm{E}-04$. $2.97736 \mathrm{E}-04$ $3.01492 \mathrm{E}-04$

$3.05271 \mathrm{E}-04$ $3.09073 \mathrm{E}-04$ $3.12899 \mathrm{E}-04$ $3.16749 \mathrm{E}-04$ $3.20622 \mathrm{E}-04$

$3.24518 \mathrm{E}-04$ $3.28438 \mathrm{E}-04$ $3.32382 \mathrm{E}-04$ $3.36349 \mathrm{E}-04$ $3.40340 \mathrm{E}-04$

$3.44355 \mathrm{E}-04$ $3.48392 \mathrm{E}-04$ $3.52454 \mathrm{E}-04$ $3.56539 \mathrm{E}-04$ $3.60647 \mathrm{E}-04$.

$3.64779 \mathrm{E}-04$ $3.68934 \mathrm{E}-04$ $3.73113 \mathrm{E}-04$ $3.77316 \mathrm{E}-04$ $3.81542 \mathrm{E}-04$ $g_{2}$

$-3.03958 \mathrm{E}-01$

$-3.09109 \mathrm{E}-01$

$-3.14303 \mathrm{E}-01$

$-3.19540 \mathrm{E}-01$

$-3.24821 \mathrm{E}-01$

$-3.30144 \mathrm{E}-01$

$-3.35511 \mathrm{E}-01$

$-3.40921 \mathrm{E}-01$

$-3.46373 \mathrm{E}-01$

$-3.51869 \mathrm{E}-01$

$-3.57408 \mathrm{~F}-01$

$-3.62990 \mathrm{E}-01$

$-3.68615 \mathrm{E}-01$

$-3.74284 \mathrm{E}-01$

$-3.79995 \mathrm{E}-01$

$-3.85749 \mathrm{E}-01$

$-3.91547 \mathrm{E}-01$

$-3.97387 \mathrm{E}-01$

$-4.03271 \mathrm{E}-01$

$-4.09198 \mathrm{E}-01$

$-4.15167 \mathrm{E}-01$

$-4.21180 \mathrm{E}-01$

$-4.27236 \mathrm{E}-01$

$-4.33335 \mathrm{E}-01$

$-4.39477 \mathrm{E}-01$

$-4.45661 \mathrm{E}-01$

$-4.51889 \mathrm{E}-01$

$-4.58160 \mathrm{E}-01$

$-4.64474 \mathrm{E}-01$

$-4.70831 \mathrm{E}-01$

$-4.77231 \mathrm{E}-01$

$-4.83674 \mathrm{E}-01$

$-4.90160 \mathrm{E}-01$

$-4.96689 \mathrm{E}-01$

$-5.03261 \mathrm{E}-01$

$-5.09876 \mathrm{E}-01$

$-5.16534 \mathrm{E}-0]$

$-5.23235 \mathrm{E}-01$

$-5.29979 \mathrm{E}-01$

$-5.36766 \mathrm{E}-01$

$-5.43596 \mathrm{E}-01$

$-5.50469 \mathrm{E}-01$

$-5.57385 \mathrm{E}-01$

$-5.64344 \mathrm{E}-01$

$-5.71345 \mathrm{E}-01$

$-5.78390 \mathrm{E}-01$

$-5.85478 \mathrm{E}-01$

$-5.92608 \mathrm{E}-01$

$-5.99782 \mathrm{E}-01$

$-6.06998 \mathrm{E}-01$

$-6.14258 \mathrm{E}-01$

$-6.21560 \mathrm{E}-01$

$-6.28905 \mathrm{E}-01$

$-6.36293 \mathrm{E}-01$

$-6.43724 \mathrm{E}-01$

$-6.51198 \mathrm{E}-01$

$-6.58715 \mathrm{E}-01$

$-6.66275 \mathrm{E}-01$

$-6.73877 \mathrm{E}-01$

$-6.81523 \mathrm{E}-01$
$-6.995 .50 \mathrm{E}-03$

$-7.15238 \mathrm{E}-03$

$-7.3115 .5 \mathrm{E}-0.3$

$-7.47302 \mathrm{E}-03$

$-7.63680 \mathrm{E}-03$

$-7.80291 \mathrm{E}-03$

$-7.97137 \mathrm{E}-03$

$-8.14220 \mathrm{E}-03$

$-8.31540 \mathrm{E}-03$

$-8.49100 \mathrm{E}-03$

$-8.66901 \mathrm{E}-03$

$-8.84945 \mathrm{E}-03$

$-9.03234 \mathrm{E}-03$

$-9.21768 \mathrm{E}-03$

$-9.40550 \mathrm{E}-03$

$-9.59581 \mathrm{E}-03$

$-9.78863 \mathrm{E}-03$

$-9.98397 \mathrm{E}-03$

$-1.01818 \mathrm{E}-02$

$-1.03823 \mathrm{E}-02$

$-1.05853 \mathrm{E}-02$

$-1.07909 \mathrm{E}-02$

$-1.09991 \mathrm{E}-02$

$-1.12099 \mathrm{E}-02$

$-1.14234 \mathrm{E}-02$

$-1.16395 \mathrm{E}-02$

$-1.18583 \mathrm{E}-02$

$-1.20797 \mathrm{E}-02$

$-1.23039 \mathrm{E}-02$

$-1.25307 \mathrm{E}-02$

$-1.27604 \mathrm{E}-02$

$-1.29927 \mathrm{E}-02$

$-1.32278 \mathrm{E}-02$

$-1.34657 \mathrm{E}-02$

$-1.37064 \mathrm{E}-02$

$-1.39499 \mathrm{E}-02$

$-1.41963 \mathrm{E}-02$

$-1.44454 \mathrm{E}-02$

$-1.46975 \mathrm{E}-02$

$-1.49524 \mathrm{E}-02$

$-1.52102 \mathrm{E}-02$

$-1.54709 \mathrm{E}-02$

$-1.57345 \mathrm{E}-02$

$-1.60011 \mathrm{E}-02$

$-1.62706 \mathrm{E}-02$

$-1.65431 \mathrm{E}-02$

$-1.68186 \mathrm{E}-02$

$-1.70971 \mathrm{E}-02$

$-1.73786 \mathrm{E}-02$

$-1.76632 \mathrm{E}-02$

$-1.79508 \mathrm{E}-02$

$-1.82414 \mathrm{E}-02$

$-1.85352 \mathrm{E}-02$

$-1.88320 \mathrm{E}-02$

$-1.91320 \mathrm{E}-02$

$-1.94351 \mathrm{E}-02$

$-1.97413 \mathrm{E}-02$

$-2.00507 \mathrm{E}-02$

$-2.03633 \mathrm{E}-02$

$-2.06791 \mathrm{E}-02$

$\tan \delta_{2}$

$f_{2}$

$5.00705 \mathrm{E} \quad 01$

$-6.92819 \mathrm{E}-03$

5.01005E $01-7.08221 \mathrm{E}-03$

$5.01151 \mathrm{E}$ 01 $-7.23847 \mathrm{E}-03$

$5.01295 \mathrm{E} \quad 01 \quad-7.39699 \mathrm{~F}-0.3$

$5.01437 \mathrm{E} \quad 01 \quad-7.55779 \mathrm{E}-03$

$5.01576 \mathrm{E}$ o1 $-7.72089 \mathrm{E}-03$

$5.01713 \mathrm{E}$ 01 $-7.88629 \mathrm{E}-03$

$5.01847 \mathrm{E}$ 01 $-8.05402 \mathrm{E}-03$

$5.01979 \mathrm{E}$ 01 $-8.22409 \mathrm{E}-0.3$

$5.02110 \mathrm{E} \quad 01 \quad-8.39652 \mathrm{E}-03$

$5.02238 \mathrm{E} \quad 01 \quad-8.57132 \mathrm{E}-03$

$5.02364 \mathrm{E}$ 01 $-8.74852 \mathrm{E}-03$

$5.02488 \mathrm{E} \quad 01 \quad-8.92812 \mathrm{E}-03$

$5.02611 \mathrm{E} \quad 01 \quad-9.11014 \mathrm{E}-03$

$5.02731 \mathrm{E} \quad 01$

$5.02850 \mathrm{E} \quad 01$

$5.02966 \mathrm{E} \quad 01$

$5.03081 \mathrm{E} \quad 01$

$5.03194 \mathrm{E} 01$

$5.03306 \mathrm{E} \quad 01$

$5.03416 \mathrm{E} \quad 01$

$5.03524 \mathrm{E} 01$

$5.03631 \mathrm{E} 01$

$5.03736 \mathrm{E} \quad 01$

$5.03840 \mathrm{E} \quad 01$

$5.03942 \mathrm{E} \quad 01$

$5.04043 \mathrm{E} \quad 01$

$5.04142 \mathrm{E} 01$

$5.04240 \mathrm{E} \quad 01$

$-9.29459 \mathrm{E}-03$

$-9.48151 \mathrm{E}-03$

$-9.67089 \mathrm{E}-03$

$-9.86276 \mathrm{E}-03$

$-1.00571 \mathrm{E}-02$

$-1.02540 \mathrm{E}-02$

$-1.04534 \mathrm{E}-02$

$-1.06554 \mathrm{E}-02$

$-1.08599 \mathrm{E}-02$

$-1.10671 \mathrm{E}-02$

$-1.12768 \mathrm{E}-02$ 
ELECTRON RADIAL FUNCTIONS

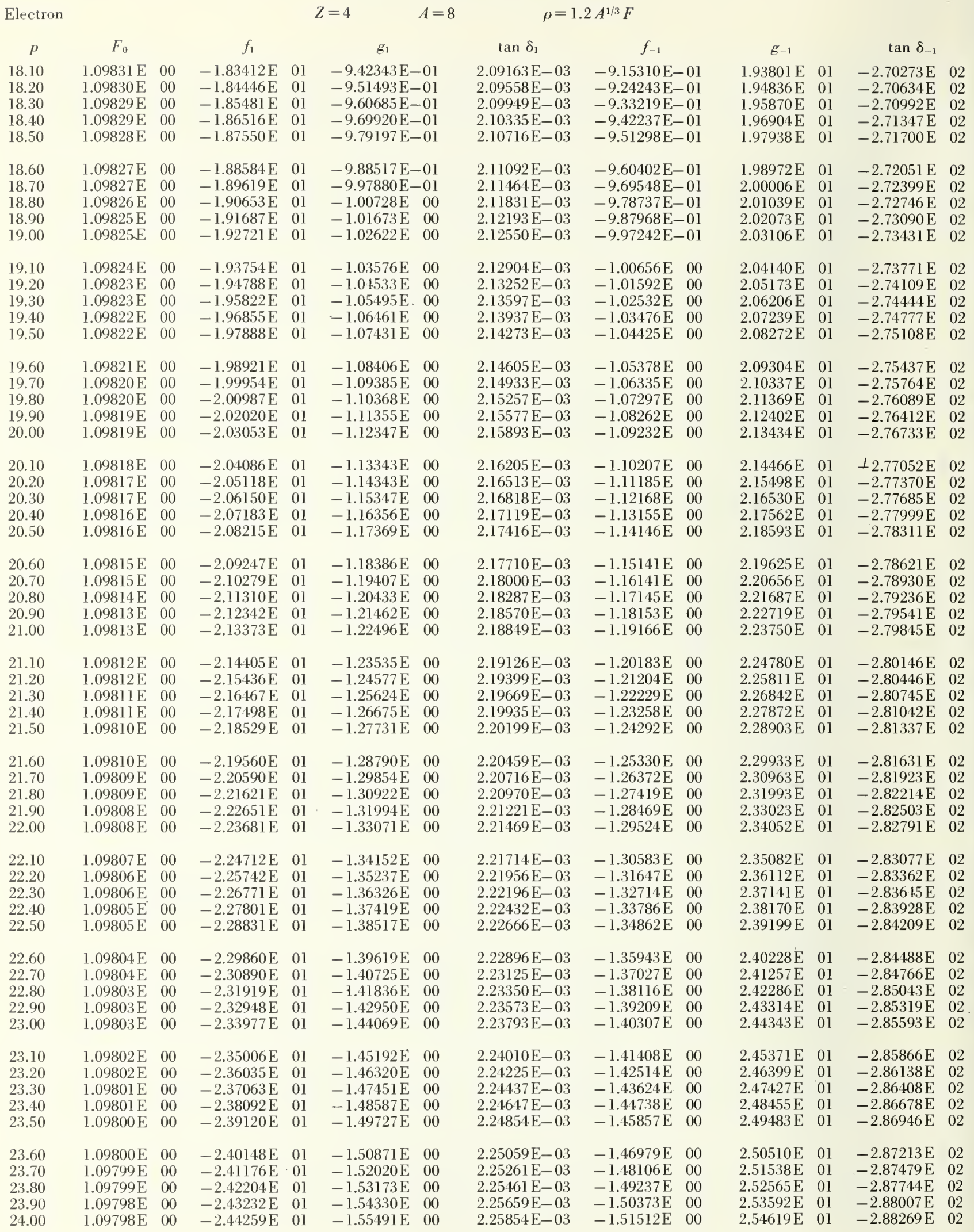


19.10

19.20

19.30

19.40

19.50

19.60

19.70

19.80

19.90

20.00

20.10

20.20

20.30

20.40

20.50

20.60

20.70

20.80

20.90

21.00

21.10

21.20

21.30

21.40

21.50

21.60

21.70

21.80

21.90

22.00

22.10

22.20

22.30

22.40

22.50

22.60

22.70

22.80

22.90

23.00

23.10

23.20

23.30

23.40

23.50

23.60

23.70

23.80

23.90

24.00
$\bar{F}$

$3.85792 \mathrm{E}-04$ $3.90065 \mathrm{E}-04$ $3.94362 \mathrm{E}-04$ $3.98682 \mathrm{E}-04$ $4.03026 \mathrm{E}-04$

$4.07393 \mathrm{E}-04$ $4.11784 \mathrm{E}-04$ $4.16198 \mathrm{E}-04$ $4.20636 \mathrm{E}-04$ $4.25097 \mathrm{E}-04$

$4.29582 \mathrm{E}-04$ $4.34091 \mathrm{E}-04$ $4.38623 \mathrm{E}-04$ $4.43178 \mathrm{E}-04$ $4.47757 \mathrm{E}-04$

$4.52360 \mathrm{E}-04$ $4.56986 \mathrm{E}-04$ $4.61636 \mathrm{E}-04$ $4.66309 \mathrm{E}-04$ $4.71006 \mathrm{E}-04$

$4.75726 \mathrm{E}-04$ $4.80470 \mathrm{E}-04$ $4.85237 \mathrm{E}-04$ $4.90028 \mathrm{E}-04$ $4.94842 \mathrm{E}-04$

$4.99680 \mathrm{E}-04$ $5.04542 \mathrm{E}-04$ $5.09427 \mathrm{E}-04$ $5.14335 \mathrm{E}-04$ $5.19268 \mathrm{E}-04$

$5.24223 \mathrm{E}-04$ $5.29202 \mathrm{E}-04$ $5.34205 \mathrm{E}-04$ $5.39231 \mathrm{E}-04$ 5.44281 E-04

$5.49354 \mathrm{E}-04$ $5.54451 \mathrm{E}-04$ 5.59571 E-04 $5.64715 \mathrm{E}-04$ $5.69883 \mathrm{E}-04$

$5.75073 \mathrm{E}-04$ $5.80288 \mathrm{E}-04$ $5.85526 \mathrm{E}-04$ $5.90787 \mathrm{E}-04$ $5.96072 \mathrm{E}-04$

$6.01381 \mathrm{E}-04$ $6.06713 \mathrm{E}-04$ $6.12069 \mathrm{E}-04$ $6.17448 \mathrm{E}-04$ $6.22851 \mathrm{E}-04$

$6.28277 \mathrm{E}-04$ $6.33727 \mathrm{E}-04$ $6.39200 \mathrm{E}-04$ $6.44697 \mathrm{E}-04$ $6.50217 \mathrm{E}-04$

$6.55761 \mathrm{E}-04$ $6.61328 \mathrm{E}-04$ $6.66919 \mathrm{E}-04$ $6.7253+\mathrm{E}-04$ $6.78172 \mathrm{E}-04$ $f_{2}$

$-6.89211 \mathrm{E}-01$ $-6.96943 \mathrm{E}-01$ $-7.04717 \mathrm{E}-01$ $-7.12534 \mathrm{E}-01$ $-7.2039 .4 \mathrm{E}-01$

$-7.28296 \mathrm{E}-01$ $-7.36241 \mathrm{E}-01$ $-7.44230 \mathrm{E}-01$ $-7.52261 \mathrm{E}-01$ $-7.60335 \mathrm{E}-01$

$-7.68+52 \mathrm{E}-01$ $-7.76612 \mathrm{E}-01$ $-7.84814 \mathrm{E}-01$ $-7.93060 \mathrm{E}-01$ $-8.01348 \mathrm{E}-01$

$-8.09679 \mathrm{E}-01$

$-8.18052 \mathrm{E}-01$

$-8.26469 \mathrm{E}-01$

$-8.34928 \mathrm{E}-01$

$-8.43430 \mathrm{E}-01$

$-8.51975 \mathrm{E}-01$

$-8.60563 \mathrm{E}-01$

$-8.69194 \mathrm{E}-01$

$-8.77867 \mathrm{E}-01$

$-8.86583 \mathrm{E}-01$

$-8.95341 \mathrm{E}-01$ $-9.04143 \mathrm{E}-01$

$-9.12987 \mathrm{E}-01$ $-9.21874 \mathrm{E}-01$

$-9.30804 \mathrm{E}-01$

$-9.39776 \mathrm{E}-01$

$-9.48791 \mathrm{E}-01$

$-9.57849 \mathrm{E}-01$

$-9.66950 \mathrm{E}-01$

$-9.76093 \mathrm{E}-01$

$-9.85279 \mathrm{E}-01$

$-9.94508 \mathrm{E}-01$

$-1.00378 \mathrm{E} \quad 00$

$-1.01309 \mathrm{E} 00$

$-1.02245 \mathrm{E} \quad 00$

$-1.03185 \mathrm{E} \quad 00$

$-1.04129 \mathrm{E} \quad 00$

$-1.05078 \mathrm{E} 00$

$-1.06030 \mathrm{E} 00$

$-1.06987 \mathrm{E} \quad 00$

$-1.07949 \mathrm{E} \quad 00$ $-1.08914 \mathrm{E} 00$ $-1.09884 \mathrm{E} \quad 00$ $-1.10858 \mathrm{E} 00$ $-1.11836 \mathrm{E} \quad 00$

$-1.12819 \mathrm{E} \quad 00$ $-1.13806 \mathrm{E} 00$ $-1.14797 \mathrm{E} \quad 00$ $-1.15792 \mathrm{E} \quad 00$ $-1.16792 \mathrm{E} \quad 00$

$-1.17796 \mathrm{E} \quad 00$ $-1.18804 \mathrm{E} \quad 00$ $-1.19816 \mathrm{E} \quad 00$ $-1.20833 \mathrm{E} \quad 00$ $-1.2185+\mathrm{E} 00$ $g_{2}$

$-2.09981 \mathrm{E}-02$

$-2.13203 \mathrm{E}-02$

$-2.16458 \mathrm{E}-02$

$-2.19745 \mathrm{E}-02$

$-2.23065 \mathrm{E}-02$

$-2.26417 \mathrm{E}-02$

$-2.29803 \mathrm{E}-02$

$-2.33222 \mathrm{E}-02$

$-2.36675 \mathrm{E}-02$

$-2.40161 \mathrm{E}-02$

$-2.43680 \mathrm{E}-02$

$-2.47234 \mathrm{E}-02$

$-2.50821 \mathrm{E}-02$

$-2.54443 \mathrm{E}-02$

$-2.58099 \mathrm{E}-02$

$-2.61790 \mathrm{E}-02$

$-2.65515 \mathrm{E}-02$

$-2.69275 \mathrm{E}-02$

$-2.73070 \mathrm{E}-02$

$-2.76900 \mathrm{E}-02$

$-2.80765 \mathrm{E}-02$

$-2.84666 \mathrm{E}-02$

$-2.88603 \mathrm{E}-02$

$-2.92575 \mathrm{E}-02$

$-2.96583 \mathrm{E}-02$

$-3.00627 \mathrm{E}-02$ $-3.04707 \mathrm{E}-02$

$-3.08824 \mathrm{E}-02$

$-3.12978 \mathrm{E}-02$

$-3.17168 \mathrm{E}-02$

$-3.21395 \mathrm{E}-02$

$-3.25659 \mathrm{E}-02$

$-3.29960 \mathrm{E}-02$

$-3.34299 \mathrm{E}-02$

$-3.38675 \mathrm{E}-02$

$-3.43088 \mathrm{E}-02$

$-3.47540 \mathrm{E}-02$

$-3.52029 \mathrm{E}-02$

$-3.56557 \mathrm{E}-02$

$-3.61123 \mathrm{E}-02$

$-3.65728 \mathrm{E}-02$

$-3.70371 \mathrm{E}-02$

$-3.75052 \mathrm{E}-02$

$-3.79773 \mathrm{E}-02$

$-3.84533 \mathrm{E}-02$

$-3.89332 \mathrm{E}-02$

$-3.94171 \mathrm{E}-02$

$-3.99049 \mathrm{E}-02$

$-4.03967 \mathrm{E}-02$

$-4.08924 \mathrm{E}-02$

$-4.13922 \mathrm{E}-02$

$-4.18960 \mathrm{E}-02$

$-4.24038 \mathrm{E}-02$

$-4.29157 \mathrm{E}-02$

$-4.34316 \mathrm{E}-02$

$-4.39517 \mathrm{E}-02$

$-4.44758 \mathrm{E}-02$

$-4.50040 \mathrm{E}-02$

$-4.5536+\mathrm{E}-02$

$-4.60729 \mathrm{E}-02$

$\tan \delta_{2}$

$f$ :

$5.06718 \mathrm{E} 01 \quad-2.03779 \mathrm{E}-02$ $5.06784 \mathrm{E} 01 \quad-2.06918 \mathrm{E}-02$

$5.06848 \mathrm{E} \quad 01-2.10089 \mathrm{E}-02$

$5.06912 \mathrm{E} \quad 01-2.13292 \mathrm{E}-02$

$5.06975 \mathrm{E} \quad 01 \quad-2.16527 \mathrm{E}-02$

$5.07038 \mathrm{E} \quad 01$

$5.07100 \mathrm{E} \quad 01$

$5.07161 \mathrm{E} 01$

$5.07221 \mathrm{E} \quad 01$

$5.07281 \mathrm{E} \quad 01$

$-2.19794 \mathrm{E}-02$

$-2.23094 \mathrm{E}-02$

$-2.26426 \mathrm{E}-02$

$-2.29792 \mathrm{E}-02$

$-2.33190 \mathrm{E}-02$

$5.07340 \mathrm{E} \quad 01$

$5.07398 \mathrm{E} 01$

$5.07456 \mathrm{E} \quad 01$

$5.07513 \mathrm{E} \quad 01$

$5.07570 \mathrm{E} \quad 01$

$-2.36622 \mathrm{E}-02$ $-2.40087 \mathrm{E}-02$ $-2.43585 \mathrm{E}-02$ $-2.47117 \mathrm{E}-02$

$-2.50683 \mathrm{E}-02$

$5.07626 \mathrm{E} \quad 01$ $5.07681 \mathrm{E} \quad 01$

$5.07736 \mathrm{E} \quad 01$

$5.07790 \mathrm{E} \quad 01$

$5.07844 \mathrm{E} \quad 01$

$5.07897 \mathrm{E} \quad 01$

$5.07950 \mathrm{E} \quad 01$

$5.08002 \mathrm{E} \quad 01$

$5.08053 \mathrm{E} \quad 01$

$5.08104 \mathrm{E} 01$

$-2.54283 \mathrm{E}-02$

$-2.57917 \mathrm{E}-02$

$-2.61585 \mathrm{E}-02$

$-2.65288 \mathrm{E}-02$

$-2.69025 \mathrm{E}-02$

$-2.72797 \mathrm{E}-02$ 
ELECTRON RADIAL FUNCTIONS

$$
Z=4 \quad A=8
$$

24.10

24.20

24.30

24.40

24.50

24.60

24.70

24.80

24.90

25.00

25.10

25.20

25.30

25.40

25.50

25.60

25.70

25.80

25.90

26.00

26.10

26.20

26.30

26.40

26.50

26.60

26.70

26.80

26.90

27.00

27.10

27.20

27.30

27.40

27.50

27.60

27.70

27.80

27.90

28.00

28.10

28.20

28.30

28.40

28.50

28.60

28.70

28.80

28.90

29.00

29.10

29.20

29.30

29.40

29.50

29.60

29.70

29.80

29.90

30.00
$F_{0}$

\section{$f_{1}$}

$1.09797 \mathrm{E} \quad 00$ $1.09797 \mathrm{E} \quad 00$ $1.09796 \mathrm{E} \quad 00$ $1.09796 \mathrm{E} \quad 00$ $1.09796 \mathrm{E} \quad 00$

$1.09795 \mathrm{E} \quad 00$ $1.09795 \mathrm{E} \quad 00$ $1.09794 \mathrm{E} \quad 00$ $1.09794 \mathrm{E} \quad 00$ $1.09793 \mathrm{E} \quad 00$

$1.09793 \mathrm{E} \quad 00$ $1.09792 \mathrm{E} \quad 00$ $1.09792 \mathrm{E} \quad 00$ $1.09792 \mathrm{E} \quad 00$ $1.09791 \mathrm{E} \quad 00$

$1.09791 \mathrm{E} \quad 00$ $1.09790 \mathrm{E} \quad 00$ $1.09790 \mathrm{E} \quad 00$ $1.09789 \mathrm{E} \quad 00$ $1.09789 \mathrm{E} \quad 00$

$1.09789 \mathrm{E} \quad 00$ $1.09788 \mathrm{E} \quad 00$ $1.09788 \mathrm{E} \quad 00$ $1.09787 \mathrm{E} \quad 00$

$1.09787 \mathrm{E} \quad 00$ $1.09786 \mathrm{E} \quad 00$ $1.09786 \mathrm{E} \quad 00$ $1.09785 \mathrm{E} \quad 00$

$1.09785 \mathrm{E} \quad 00$ $1.09784 \mathrm{E} \quad 00$ $1.09784 \mathrm{E} \quad 00$ $1.09783 \mathrm{E} \quad 00$ $1.09783 \mathrm{E} \quad 00$

$1.09783 \mathrm{E} \quad 00$ $1.09782 \mathrm{E} \quad 00$ $1.09782 \mathrm{E} \quad 00$ $1.09781 \mathrm{E} \quad 00$ $1.09781 \mathrm{E} \quad 00$

$1.09781 \mathrm{E} \quad 00$ $1.09780 \mathrm{E} \quad 00$ $1.09780 \mathrm{E}-00$ $1.09780 \mathrm{E} \quad 00$ $1.09779 \mathrm{E} \quad 00$

$1.09779 \mathrm{E} \quad 00$ $1.09778 \mathrm{E} \quad 00$ $1.09778 \mathrm{E} \quad 00$ $1.09778 \mathrm{E} \quad 00$ $1.09777 \mathrm{E} \quad 00$

$1.09777 \mathrm{E} \quad 00$ $1.09777 \mathrm{E} \quad 00$ $1.09776 \mathrm{E} \quad 00$ $1.09776 \mathrm{E} \quad 00$

$1.09775 \mathrm{E} \quad 00$ $1.09775 \mathrm{E} \quad 00$ $1.09775 \mathrm{E} \quad 00$ $1.09774 \mathrm{E} \quad 00$ $1.09774 \mathrm{E} \quad 00$ $1.09787 \mathrm{E} \quad 00$ $1.09785 \mathrm{E} \quad 00$ $1.09776 \mathrm{E} \quad 00$ $\begin{array}{ll}-2.45287 \mathrm{E} & 01\end{array}$ $-2.46314 \mathrm{E} 01$ $-2.47342 \mathrm{E} 01$ $-2.48369 \mathrm{E} \quad 01$ $-2.49396 \mathrm{E} \quad 01$

$-2.50423 \mathrm{E} \quad 01$ $-2.51449 \mathrm{E} 01$ $-2.52476 \mathrm{E} 01$ $-2.53502 \mathrm{E} \quad 01$ $-2.54529 \mathrm{E} \quad 01$

$-2.55555 \mathrm{E} 01$ $-2.56581 \mathrm{E} \quad 01$ $-2.57607 \mathrm{E} 01$ $-2.58632 \mathrm{E} 01$ $-2.59658 \mathrm{E} 01$

$\begin{array}{ll}-2.60683 \mathrm{E} & 01\end{array}$ $-2.61709 \mathrm{E} 01$ $-2.62734 \mathrm{E} 01$ $-2.63759 \mathrm{E} \quad 01$ $-2.64784 \mathrm{E} 01$

$-2.65809 \mathrm{E} \quad 01$ $-2.66833 \mathrm{E} 01$ $-2.67858 \mathrm{E} 01$ $-2.68882 \mathrm{E} 01$ $-2.69906 \mathrm{E} \quad 01$

$-2.70930 \mathrm{E} \quad 01$ $-2.71954 \mathrm{E}$ 01 $-2.72978 \mathrm{E} 01$ $-2.74002 \mathrm{E} 01$ $-2.75025 \mathrm{E} \quad 01$

$-2.76048 \mathrm{E}$ 01 $-2.77072 \mathrm{E} \quad 01$ $-2.78095 \mathrm{E} 01$ $-2.79118 \mathrm{E} \quad 01$ $-2.80140 \mathrm{E} \quad 01$

$-2.81163 \mathrm{E} \quad 01$ $-2.82185 \mathrm{E} 01$ $-2.83208 \mathrm{E} 01$ $-2.84230 \mathrm{E} 01$ $-2.85252 \mathrm{E} 01$

$-2.86274 \mathrm{E} \quad 01$ $-2.87295 \mathrm{E} 01$ $-2.88317 \mathrm{E} 01$ $-2.89338 \mathrm{E} \quad 01$ $-2.90359 \mathrm{E} \quad 01$

$-2.91381 \mathrm{E} \quad 01$ $-2.92402 \mathrm{E} \quad 01$ $-2.93422 \mathrm{E} 01$ $-2.94443 \mathrm{E} 01$ $-2.95464 \mathrm{E} \quad 01$

$\begin{array}{lll}-2.96484 \mathrm{E} & 01\end{array}$ $-2.97504 \mathrm{E} 01$ $-2.98524 \mathrm{E} \quad 01$ $-2.99544 \mathrm{E} \quad 01$ $-3.00564 \mathrm{E} 01$

$-3.01583 \mathrm{E} 01$ $-3.02603 \mathrm{E} 01$ $-3.03622 \mathrm{E} 01$ $-3.05660 \mathrm{E} 01$ $-3.04641 \mathrm{E} 01$ $g_{1}$

$-1.56656 \mathrm{E} \quad 00$ $-1.57826 \mathrm{E} \quad 00$ $-1.59000 \mathrm{E}$ 00 $-1.60178 \mathrm{E} \quad 00$ $-1.61360 \mathrm{E} 00$

$-1.62547 \mathrm{E} \quad 00$ $-1.63737 \mathrm{E} \quad 00$ $-1.64932 \mathrm{E} \quad 00$ $-1.66132 \mathrm{E} \quad 00$ $-1.67335 \mathrm{E} \quad 00$

$-1.68543 \mathrm{E} \quad 00$ $-1.69755 \mathrm{E} \quad 00$ $-1.70971 \mathrm{E} \quad 00$ $-1.72191 \mathrm{E} \quad 00$ $-1.73415 \mathrm{E} \quad 00$

$-1.74644 \mathrm{E} \quad 00$ $-1.75877 \mathrm{E} \quad 00$ $-1.77114 \mathrm{E} \quad 00$ $-1.78356 \mathrm{E} \quad 00$ -1.79601 E 00

$-1.80851 \mathrm{E} \quad 00$ -1.82105 E 00 $-1.83363 \mathrm{E} \quad 00$ $-1.84626 \mathrm{E} \quad 00$ $-1.85892 \mathrm{E} \quad 00$

$-1.87163 \mathrm{E} \quad 00$ $-1.88438 \mathrm{E} 00$ $-1.89717 \mathrm{E} \quad 00$ $-1.91001 \mathrm{E} 00$ $-1.92289 \mathrm{E} \quad 00$

$-1.93581 \mathrm{E} \quad 00$ $-1.94877 \mathrm{E} 00$ $-1.96177 \mathrm{E} \quad 00$ $-1.97481 \mathrm{E} 00$ -1.98790 E 00

\section{$-2.00103 \mathrm{E} \quad 00$}

$-2.01420 \mathrm{E} \quad 00$ $-2.02741 \mathrm{E} 00$ $-2.04067 \mathrm{E} \quad 00$ $-2.05396 \mathrm{E} \quad 00$

$-2.06730 \mathrm{E} \quad 00$ $-2.08068 \mathrm{E} \quad 00$ $-2.09411 \mathrm{E} \quad 00$ $-2.10757 \mathrm{E} \quad 00$ $-2.12108 \mathrm{E} \quad 00$

$-2.13463 \mathrm{E} \quad 00$ $-2.14822 \mathrm{E} \quad 00$ $-2.16185 \mathrm{E} \quad 00$ $-2.17552 \mathrm{E} \quad 00$ $-2.18924 \mathrm{E} \quad 00$

$-2.20300 \mathrm{E} \quad 00$ $-2.21680 \mathrm{E} \quad 00$ $-2.23064 \mathrm{E} \quad 00$ $-2.24452 \mathrm{E} \quad 00$ $-2.25845 \mathrm{E} \quad 00$

$-2.27242 \mathrm{E} \quad 00$ $-2.28643 \mathrm{E} \quad 00$ $-2.30048 \mathrm{E} 00$ $-2.31457 \mathrm{E} \quad 00$ $-2.32871 \mathrm{E} \quad 00$

$\tan \delta_{1}$

$2.26047 \mathrm{E}-03$ $2.26237 \mathrm{E}-03$

$2.26426 \mathrm{E}-03$

$2.26612 \mathrm{E}-03$

$2.26796 \mathrm{E}-03$

$2.26977 \mathrm{E}-03$ $2.27157 \mathrm{E}-03$

$2.27334 \mathrm{E}-03$

$2.27509 \mathrm{E}-03$

$2.27682 \mathbf{E}-03$

$2.27853 \mathrm{E}-03$

$2.28021 \mathrm{E}-03$

$2.28188 \mathrm{E}-03$

$2.28352 \mathrm{E}-03$

$2.28516 \mathrm{E}-03$

$2.28676 \mathrm{E}-03$

$2.28835 \mathrm{E}-03$

$2.28992 \mathbf{E}-03$

$2.29147 \mathrm{E}-03$

$2.29300 \mathrm{E}-03$

$2.29451 \mathrm{E}-03$

$2.29600 \mathrm{E}-03$

$2.29747 \mathrm{E}-03$

$2.29893 \mathrm{E}-03$

$2.30036 \mathrm{E}-03$

$2.30178 \mathrm{E}-03$

$2.30318 \mathrm{E}-03$

$2.30457 \mathrm{E}-03$

2.30594 E-03

$2.30728 \mathrm{E}-03$

$2.30862 \mathrm{E}-03$

$2.30993 \mathrm{E}-03$

$2.31123 \mathrm{E}-03$

2.31251 E-03

$2.31377 \mathbf{E}-03$

$2.31502 \mathrm{E}-03$

$2.31625 \mathrm{E}-03$

$2.31747 \mathrm{E}-03$

$2.31867 \mathrm{E}-03$

$2.31986 \mathrm{E}-03$ 
$Z=4 \quad A=8 \quad \rho=1.2 A^{1 / 3} F$

$\bar{F}$

$6.83834 \mathrm{E}-04$ $6.89519 \mathrm{E}-04$ $6.95227 \mathrm{E}-04$ $7.00960 \mathrm{E}-04$ $7.06715 \mathrm{E}-04$

$7.12495 \mathrm{E}-04$ $7.18297 \mathrm{E}-04$ $7.24124 \mathrm{E}-04$ $7.29973 \mathrm{E}-04$ $7.35847 \mathrm{E}-04$

$7.41743 \mathrm{E}-04$ $7.47664 \mathrm{E}-04$ $7.53608 \mathrm{E}-04$

$7.59575 \mathrm{E}-04$

$7.65566 \mathrm{E}-04$

$7.71581 \mathrm{E}-04$ $7.77619 \mathrm{E}-04$ $7.83680 \mathrm{E}-04$ $7.89765 \mathrm{E}-04$ $7.95874 \mathrm{E}-04$

$8.02006 \mathrm{E}-04$ $8.08162 \mathrm{E}-04$ $8.1434 .1 \mathrm{E}-04$ $8.20544 \mathrm{E}-04$ $8.26770 \mathrm{E}-04$

$8.33020 \mathrm{E}-04$ $8.39293 \mathrm{E}-04$ $8.45590 \mathrm{E}-04$ $8.51910 \mathrm{E}-04$ $8.58254 \mathrm{E}-04$

$8.64622 \mathrm{E}-04$ $8.71013 \mathrm{E}-04$ $8.77427 \mathrm{E}-04$ $8.83865 \mathrm{E}-04$ $8.90327 \mathrm{E}-04$

$8.96812 \mathrm{E}-04$ $9.03321 \mathrm{E}-04$ $9.09853 \mathrm{E}-04$ $9.16408 \mathrm{E}-04$ $9.22988 \mathrm{E}-04$

$9.29590 \mathrm{E}-04$ $9.36217 \mathrm{E}-04$ $9.42866 \mathrm{E}-04$ $9.49540 \mathrm{E}-04$ $9.56237 \mathrm{E}-04$

$9.62957 \mathrm{E}-04$ $9.69701 \mathrm{E}-04$ $9.76468 \mathrm{E}-04$ $9.83259 \mathrm{E}-04$ $9.90074 \mathrm{E}-04$

$9.96912 \mathrm{E}-04$ $1.00377 \mathrm{E}-03$ $1.01066 \mathrm{E}-03$ $1.01757 \mathrm{E}-03$ $1.02450 \mathrm{E}-03$

$29.60 \quad 1.03146 \mathrm{E}-03$ $29.70 \quad 1.03843 \mathrm{E}-03$ $29.80 \quad 1.04544 \mathrm{E}-03$ $29.90 \quad 1.05246 \mathrm{E}-03$ $30.00 \quad 1.05951 \mathrm{E}-03$ $f_{2}$

$-1.22879 \mathrm{E} \quad 00$ $-1.23909 \mathrm{E} \quad 00$ $-1.24942 \mathrm{E} \quad 00$ $-1.25980 \mathrm{E} 00$ $-1.27022 \mathrm{E} 00$

$-1.28069 \mathrm{E} \quad 00$ $-1.29120 \mathrm{E} 00$ $-1.30175 \mathrm{E} \quad 00$ $-1.31234 \mathrm{E} 00$ $-1.32297 \mathrm{E} \quad 00$

$-1.33365 \mathrm{E} \quad 00$ $-1.34437 \mathrm{E} \quad 00$ $-1.35513 \mathrm{E} 00$ $-1.36593 \mathrm{E} \quad 00$

$-1.37678 \mathrm{E} 00$

$-1.38767 \mathrm{E} \quad 00$

$-1.39860 \mathrm{E} 00$

$-1.40958 \mathrm{E} 00$

$-1.42059 \mathrm{E} \quad 00$

$-1.43165 \mathrm{E} 00$

$-1.44275 \mathrm{E} 00$ $-1.45390 \mathrm{E} 00$

$-1.46509 \mathrm{E} \quad 00$ $-1.47631 \mathrm{E} \quad 00$ $-1.48759 \mathrm{E} 00$

$-1.49890 \mathrm{E} \quad 00$ $-1.51026 \mathrm{E} 00$ $-1.52165 \mathrm{E} \quad 00$ $-1.53310 \mathrm{E} \quad 00$ $-1.54458 \mathrm{E} \quad 00$

$-1.55610 \mathrm{E} \quad 00$ $-1.56767 \mathrm{E} 00$ $-1.57928 \mathrm{E} 00$ $-1.59094 \mathrm{E} \quad 00$ $-1.60263 \mathrm{E} 00$

$-1.61437 \mathrm{E} \quad 00$ $-1.62615 \mathrm{E} 00$ $-1.63797 \mathrm{E} 00$ $-1.64983 \mathrm{E} 00$ $-1.66174 \mathrm{E} \quad 00$

$-1.67369 \mathrm{E} \quad 00$ $-1.68568 \mathrm{E} 00$ $-1.69772 \mathrm{E} \quad 00$ $-1.70979 \mathrm{E} \quad 00$ $-1.72191 \mathrm{E} \quad 00$

$-1.73407 \mathrm{E} \quad 00$ $-1.74627 \mathrm{E} 00$ $-1.75852 \mathrm{E} \quad 00$ $-1.77081 \mathrm{E} \quad 00$ $-1.78314 \mathrm{E} 00$

-1.79551 E 00 $-1.80792 \mathrm{E} \quad 00$ $-1.82038 \mathrm{E} \quad 00$ $-1.83288 \mathrm{E} 00$ $-1.84542 \mathrm{E} \quad 00$

$-1.85800 \mathrm{E} \quad 00$ $-1.87063 \mathrm{E} 00$ $-1.88329 \mathrm{E} \quad 00$ $-1.89600 \mathrm{E} \quad 00$ -1.90875 E 00 $g_{2}$

$-4.66136 \mathrm{E}-02$ $-4.71585 \mathrm{E}-02$ $-4.77076 \mathrm{E}-02$ $-4.82608 \mathrm{E}-02$ $-4.88183 \mathrm{E}-02$

$-4.93800 \mathrm{E}-02$ $-4.99460 \mathrm{E}-02$ $-5.05163 \mathrm{E}-02$ $-5.10908 \mathrm{E}-02$ $-5.16697 \mathrm{E}-02$

$-5.22528 \mathrm{E}-02$ $-5.28403 \mathrm{E}-02$ $-5.34322 \mathrm{E}-02$ $-5.40284 \mathrm{E}-02$ $-5.46290 \mathrm{E}-02$

$-5.52340 \mathrm{E}-02$ $-5.58435 \mathrm{E}-02$ $-5.64573 \mathrm{E}-02$ $-5.70756 \mathrm{E}-02$ $-5.76984 \mathrm{E}-02$

$-5.83256 \mathrm{E}-02$ $-5.89573 \mathrm{E}-02$ $-5.95935 \mathrm{E}-02$ $-6.02343 \mathrm{E}-02$ $-6.08796 \mathrm{E}-02$

$-6.15295 \mathrm{E}-02$ $-6.21839 \mathrm{E}-02$ $-6.28429 \mathrm{E}-02$ $-6.35065 \mathrm{E}-02$ $-6.41747 \mathrm{E}-02$

$-6.48476 \mathrm{E}-02$ $-6.55251 \mathrm{E}-02$ $-6.62073 \mathrm{E}-02$ $-6.68942 \mathrm{E}-02$ $-6.75857 \mathrm{E}-02$

$-6.82820 \mathrm{E}-02$ $-6.89830 \mathrm{E}-02$ $-6.96887 \mathrm{E}-02$ $-7.03993 \mathrm{E}-02$ $-7.11145 \mathrm{E}-02$

$-7.18346 \mathrm{E}-02$ $-7.25595 \mathrm{E}-02$ $-7.32892 \mathrm{E}-02$ $-7.40237 \mathrm{E}-02$ $-7.47631 \mathrm{E}-02$

$-7.55074 \mathrm{E}-02$ $-7.62565 \mathrm{E}-02$ $-7.70106 \mathrm{E}-02$ $-7.77696 \mathrm{E}-02$ $-7.85334 \mathrm{E}-02$

$-7.93023 \mathrm{E}-02$ $-8.00761 \mathrm{E}-02$ $-8.08549 \mathrm{E}-02$ $-8.16387 \mathrm{E}-02$ $-8.24274 \mathrm{E}-02$

$-8.32213 \mathrm{E}-02$ $-8.40201 \mathrm{E}-02$ $-8.48240 \mathrm{E}-02$ $-8.56330 \mathrm{E}-02$ $-8.64471 \mathrm{E}-02$ $\tan \delta_{2}$

$5.09648 \mathrm{E} \quad 01$ $5.09684 \mathrm{E} \quad 01$ $5.09720 \mathrm{E} \quad 01$

$5.09756 \mathrm{E} \quad 01$

5.09791 E 01

$5.09826 \mathrm{E} \quad 01$

$5.09860 \mathrm{E} \quad 01$

$5.09895 \mathrm{E} \quad 01$

$5.09929 \mathrm{E} \quad 01$

$5.09963 \mathrm{E} \quad 0$

$\begin{array}{lll}5.09996 \mathrm{E} & 01\end{array}$

$5.10029 \mathrm{E} \quad 01$

$5.10062 \mathrm{E} 01$

$5.10095 \mathrm{E} 01$

$5.10127 \mathrm{E} \quad 01$

$5.10159 \mathrm{E} \quad 01$

$5.10191 \mathrm{E} 01$

$5.10223 \mathrm{E} 01$

$5.10254 \mathrm{E} \quad 01$

$5.10286 \mathrm{E} 01$

$5.10316 \mathrm{E} 01$ $5.10347 \mathrm{E} \quad 01$

$5.10377 \mathrm{E} \quad 01$

$5.10408 \mathrm{E} \quad 01$

5.10438 E 01

$5.10467 \mathrm{E} \quad 01$ $5.10497 \mathrm{E} \quad 01$

$5.10526 \mathrm{E} 01$

$5.10555 \mathrm{E} \quad 01$

$5.10584 \mathrm{E} \quad 01$

$5.10612 \mathrm{E} \quad 01$

$5.10641 \mathrm{E} \quad 01$

$5.10669 \mathrm{E} \quad 01$

$5.10697 \mathrm{E} 01$

$5.10724 \mathrm{E} 01$

$5.10752 \mathrm{E} 01$

$5.10779 \mathrm{E} \quad 01$

$5.10806 \mathrm{E} \quad 01$

$5.10833 \mathrm{E} \quad 01$

$5.10860 \mathrm{E} \quad 01$

$5.10887 \mathrm{E} \quad 01$

$5.10913 \mathrm{E} \quad 01$

$5.10939 \mathrm{E} 01$

$5.10965 \mathrm{E} \quad 0$

$5.10991 \mathrm{E} 01$

$5.11016 \mathrm{E} \quad 01$

$5.11042 \mathrm{E} \quad 01$

$5.11067 \mathrm{E} 01$

$5.11092 \mathrm{E} \quad 01$

$5.11117 \mathrm{E} 01$

$5.11141 \mathrm{E} \quad 01$ $5.11166 \mathrm{E} \quad 01$ $5.11190 \mathrm{E} 01$ $5.11214 \mathrm{E} \quad 01$ $5.11238 \mathrm{E} 01$

$5.11262 \mathrm{E} \quad 01$ $5.11286 \mathrm{E} \quad 01$ $5.11309 \mathrm{E} 01$

$5.11333 \mathrm{E}$ 0l

$5.11356 \mathrm{E} 01$ $f_{-2}$

$f_{-2}$
$-4.53992 \mathrm{E}-02$
$-4.59326 \mathrm{E}-02$
$-4.64700 \mathrm{E}-02$
$-4.70117 \mathrm{E}-02$
$-4.75574 \mathrm{E}-02$
$-4.81074 \mathrm{E}-02$
$-4.86616 \mathrm{E}-02$
$-4.92200 \mathrm{E}-02$
$-4.97826 \mathrm{E}-02$
$-5.03495 \mathrm{E}-02$
$-5.09207 \mathrm{E}-02$
$-5.14961 \mathrm{E}-02$
$-5.20759 \mathrm{E}-02$
$-5.26599 \mathrm{E}-02$
$-5.32483 \mathrm{E}-02$
$-5.38410 \mathrm{E}-02$
$-5.44381 \mathrm{E}-02$
$-5.50395 \mathrm{E}-02$
$-5.56454 \mathrm{E}-02$
$-5.62557 \mathrm{E}-02$
$-5.68703 \mathrm{E}-02$
$-5.74895 \mathrm{E}-02$
$-5.81130 \mathrm{E}-02$
$-5.87411 \mathrm{E}-02$
$-5.93736 \mathrm{E}-02$

$-6.00107 \mathrm{E}-02$ $-6.06522 \mathrm{E}-02$ $-6.12983 \mathrm{E}-02$ $-6.19490 \mathrm{E}-02$ $-6.260+2 \mathrm{E}-02$

$-6.32639 \mathrm{E}-02$ $-6.39283 \mathrm{E}-02$ $-6.45973 \mathrm{E}-02$ $-6.52709 \mathrm{E}-02$ $-6.59492 \mathrm{E}-02$

$-6.66321 \mathrm{E}-02$ $-6.73197 \mathrm{E}-02$ $-6.80120 \mathrm{E}-02$ $-6.87090 \mathrm{E}-02$ $-6.94107 \mathrm{E}-02$

$-7.01172 \mathrm{E}-02$ $-7.08284 \mathrm{E}-02$ $-7.154+4 \mathrm{E}-02$ $-7.22652 \mathrm{E}-02$ $-7.29907 \mathrm{E}-02$

$-7.37211 \mathrm{E}-02$ $-7.44563 \mathrm{E}-02$ $-7.51964 \mathrm{E}-02$ $-7.59413 \mathrm{E}-02$ $-7.66911 \mathrm{E}-02$

$-7.74458 \mathrm{E}-02$ $-7.82054 \mathrm{E}-02$ $-7.89699 \mathrm{E}-02$ $-7.97394 \mathrm{E}-02$ $-8.05139 \mathrm{E}-02$

$-8.12933 \mathrm{E}-02$ $-8.20776 \mathrm{E}-02$ $-8.28670 \mathrm{E}-02$ $-8.36615 \mathrm{E}-02$ $-8.44609 \mathrm{E}-02$

$b_{2} \quad \tan \delta_{2}$

$1.28080 \mathrm{E} 00-1.90151 \mathrm{E}-02$ 
Electron

\begin{tabular}{|c|c|c|c|c|c|c|}
\hline$p$ & $F_{0}$ & & $f_{1}$ & & $g_{1}$ & \\
\hline 30.10 & $1.09773 \mathrm{E}$ & 00 & $-3.06679 \mathrm{E}$ & 01 & $-2.34288 \mathrm{E}$ & 00 \\
\hline 30.20 & $1.09773 \mathrm{E}$ & 00 & $-3.07698 \mathrm{E}$ & 01 & $-2.35710 \mathrm{E}$ & 00 \\
\hline 30.30 & $1.09773 \mathrm{E}$ & 00 & $-3.08716 \mathrm{E}$ & 01 & $-2.37136 \mathrm{E}$ & 00 \\
\hline 30.40 & $1.09772 \mathrm{E}$ & 00 & $-3.09734 \mathrm{E}$ & 01 & $-2.38566 \mathrm{E}$ & 00 \\
\hline 30.50 & $1.09772 \mathrm{E}$ & 00 & $-3.10753 \mathrm{E}$ & 01 & $-2.40001 \mathrm{E}$ & 00 \\
\hline 30.60 & $1.09772 \mathrm{E}$ & 00 & $-3.11771 \mathrm{E}$ & 01 & $-2.41439 \mathrm{E}$ & 00 \\
\hline 30.70 & $1.09771 \mathrm{E}$ & 00 & $-3.12788 \mathrm{E}$ & 01 & $-2.42882 \mathrm{E}$ & 00 \\
\hline 30.80 & $1.09771 \mathrm{E}$ & 00 & $-3.13806 \mathrm{E}$ & 01 & $-2.44329 \mathrm{E}$ & 00 \\
\hline 30.90 & $1.09771 \mathrm{E}$ & 00 & $-3.14824 \mathrm{E}$ & 01 & $-2.45780 \mathrm{E}$ & 00 \\
\hline 31.00 & $1.09770 \mathrm{E}$ & 00 & $-3.15841 \mathrm{E}$ & 01 & $-2.47235 \mathrm{E}$ & 00 \\
\hline 31.10 & $1.09770 \mathrm{E}$ & 00 & $-3.16858 \mathrm{E}$ & 01 & $-2.48695 \mathrm{E}$ & 00 \\
\hline 31.20 & $1.09770 \mathrm{E}$ & 00 & $-3.17875 \mathrm{E}$ & 01 & $-2.50158 \mathrm{E}$ & 00 \\
\hline 31.30 & $1.09769 \mathrm{E}$ & 00 & $-3.18892 \mathrm{E}$ & 01 & $-2.51626 \mathrm{E}$ & 00 \\
\hline 31.40 & $1.09769 \mathrm{E}$ & 00 & $-3.19909 \mathrm{E}$ & 01 & $-2.53098 \mathrm{E}$ & 00 \\
\hline 31.50 & $1.09769 \mathrm{E}$ & 00 & $-3.20925 \mathrm{E}$ & 01 & $-2.54574 \mathrm{E}$ & 00 \\
\hline 31.60 & $1.09768 \mathrm{E}$ & 00 & $-3.21942 \mathrm{E}$ & 01 & $-2.56054 \mathrm{E}$ & 00 \\
\hline 31.70 & $1.09768 \mathrm{E}$ & 00 & $-3.22958 \mathrm{E}$ & 01 & $-2.57539 \mathrm{E}$ & 00 \\
\hline 31.80 & $1.09768 \mathrm{E}$ & 00 & $-3.23974 \mathrm{E}$ & 01 & $-2.59028 \mathrm{E}$ & 00 \\
\hline 31.90 & $1.09767 \mathrm{E}$ & 00 & $-3.24990 \mathrm{E}$ & 01 & $-2.60520 \mathrm{E}$ & 00 \\
\hline 32.00 & $1.09767 \mathrm{E}$ & 00 & $-3.26006 \mathrm{E}$ & 01 & $-2.62017 \mathrm{E}$ & 00 \\
\hline 32.10 & $1.09767 \mathrm{E}$ & 00 & $-3.27021 \mathrm{E}$ & 01 & $-2.63518 E$ & 00 \\
\hline 32.20 & $1.09766 \mathrm{E}$ & 00 & $-3.28037 \mathrm{E}$ & 01 & $-2.65024 \mathrm{E}$ & 00 \\
\hline 32.30 & $1.09766 \mathrm{E}$ & 00 & $-3.29052 \mathrm{E}$ & 01 & $-2.66533 \mathrm{E}$ & 00 \\
\hline 32.40 & $1.09766 \mathrm{E}$ & 00 & $-3.30067 \mathrm{E}$ & 01 & $-2.68047 \mathrm{E}$ & 00 \\
\hline 32.50 & $1.09766 \mathrm{E}$ & 00 & $-3.31082 \mathrm{E}$ & 01 & $-2.69564 \mathrm{E}$ & 00 \\
\hline 32.60 & $1.09765 \mathrm{E}$ & 60 & $-3.32097 \mathrm{E}$ & 01 & $-2.71086 \mathrm{E}$ & 00 \\
\hline 32.70 & $1.09765 \mathrm{E}$ & 00 & $-3.33112 \mathrm{E}$ & 01 & $-2.72612 \mathrm{E}$ & 00 \\
\hline 32.80 & $1.09765 \mathrm{E}$ & 00 & $-3.34126 \mathrm{E}$ & 01 & $-2.74143 \mathrm{E}$ & 00 \\
\hline 32.90 & $1.09764 \mathrm{E}$ & 00 & $-3.35140 \mathrm{E}$ & 01 & $-2.75677 \mathrm{E}$ & 00 \\
\hline 33.00 & $1.09764 \mathrm{E}$ & 00 & $-3.36154 \mathrm{E}$ & 01 & $-2.77216 \mathrm{E}$ & 00 \\
\hline 33.10 & $1.09764 \mathrm{E}$ & 00 & $-3.37168 \mathrm{E}$ & 01 & $-2.78758 \mathrm{E}$ & 00 \\
\hline 33.20 & $1.09763 \mathrm{E}$ & 00 & $-3.38182 \mathrm{E}$ & 01 & $-2.80305 \mathrm{E}$ & 00 \\
\hline 33.30 & $1.09763 \mathrm{E}$ & 00 & $-3.39196 \mathrm{E}$ & 01 & $-2.81856 \mathrm{E}$ & 00 \\
\hline 33.40 & $1.09763 \mathrm{E}$ & 00 & $-3.40209 \mathrm{E}$ & 01 & $-2.83411 \mathrm{E}$ & 00 \\
\hline 33.50 & $1.09762 \mathrm{E}$ & 00 & $-3.41222 \mathrm{E}$ & 01 & $-2.84971 \mathrm{E}$ & 00 \\
\hline 33.60 & $1.09762 \mathrm{E}$ & 00 & $-3.42235 \mathrm{E}$ & 01 & $-2.86534 \mathrm{E}$ & 00 \\
\hline 33.70 & $1.09762 \mathrm{E}$ & 00 & $-3.43248 \mathrm{E}$ & 01 & $-2.88102 \mathrm{E}$ & 00 \\
\hline 33.80 & $1.09762 \mathrm{E}$ & 00 & $-3.44261 \mathrm{E}$ & 01 & $-2.89674 \mathrm{E}$ & 00 \\
\hline 33.90 & $1.09761 \mathrm{E}$ & 00 & $-3.45274 \mathrm{E}$ & 01 & $-2.91250 \mathrm{E}$ & 00 \\
\hline 34.00 & $1.09761 \mathrm{E}$ & 00 & $-3.46286 \mathrm{E}$ & 01 & $-2.92830 \mathrm{E}$ & 00 \\
\hline 34.10 & $1.09761 \mathrm{E}$ & 00 & $-3.47298 \mathrm{E}$ & 01 & $-2.94414 \mathrm{E}$ & 00 \\
\hline 34.20 & $1.09760 \mathrm{E}$ & 00 & $-3.48310 \mathrm{E}$ & 01 & $-2.96002 \mathrm{E}$ & 00 \\
\hline 34.30 & $1.09760 \mathrm{E}$ & 00 & $-3.49322 \mathrm{E}$ & 01 & $-2.97595 \mathrm{E}$ & 00 \\
\hline 34.40 & $1.09760 \mathrm{E}$ & 00 & $-3.50334 \mathrm{E}$ & 01 & $-2.99192 \mathrm{E}$ & 00 \\
\hline 34.50 & $1.09759 \mathrm{E}$ & 00 & $-3.51346 \mathrm{E}$ & 01 & $-3.00792 \mathrm{E}$ & 00 \\
\hline 34.60 & $1.09759 \mathrm{E}$ & 00 & $-3.52357 \mathrm{E}$ & 01 & $-3.02397 \mathrm{E}$ & 00 \\
\hline 34.70 & $1.09759 \mathrm{E}$ & 00 & $-3.53368 \mathrm{E}$ & 01 & $-3.04006 \mathrm{E}$ & 00 \\
\hline 34.80 & $1.09759 \mathrm{E}$ & 00 & $-3.54379 \mathrm{E}$ & 01 & $-3.05620 \mathrm{E}$ & 00 \\
\hline 34.90 & $1.09758 \mathrm{E}$ & 00 & $-3.55390 \mathrm{E}$ & 01 & $-3.07237 \mathrm{E}$ & 00 \\
\hline 35.00 & $1.09758 \mathrm{E}$ & 00 & $-3.56401 \mathrm{E}$ & 01 & $-3.08859 \mathrm{E}$ & 00 \\
\hline 35.10 & $1.09758 \mathrm{E}$ & 00 & $-3.57411 \mathrm{E}$ & 01 & $-3.10484 \mathrm{E}$ & 00 \\
\hline 35.20 & $1.09757 \mathrm{E}$ & 00 & $-3.58421 \mathrm{E}$ & 01 & $-3.12114 \mathrm{E}$ & 00 \\
\hline 35.30 & $1.09757 \mathrm{E}$ & 00 & $-3.59432 \mathrm{E}$ & 01 & $-3.13748 \mathrm{E}$ & 00 \\
\hline 35.40 & $1.09757 \mathrm{E}$ & 00 & $-3.60442 \mathrm{E}$ & 01 & $-3.15386 \mathrm{E}$ & 00 \\
\hline 35.50 & $1.09757 \mathrm{E}$ & 00 & $-3.61451 \mathrm{E}$ & 01 & $-3.17028 \mathrm{E}$ & 00 \\
\hline 35.60 & $1.09756 \mathrm{E}$ & 00 & $-3.62461 \mathrm{E}$ & 01 & $-3.18675 \mathrm{E}$ & 00 \\
\hline 35.70 & $1.09756 \mathrm{E}$ & 00 & $-3.63470 \mathrm{E}$ & 01 & $-3.20325 \mathrm{E}$ & 00 \\
\hline 35.80 & $1.09756 \mathrm{E}$ & 00 & $-3.64480 \mathrm{E}$ & 01 & $-3.21980 \mathrm{E}$ & 00 \\
\hline 35.90 & $1.09755 \mathrm{E}$ & 00 & $-3.65489 \mathrm{E}$ & 01 & $-3.23638 \mathrm{E}$ & 00 \\
\hline
\end{tabular}

$\tan \delta_{1}$

$$
\rho=1.2 A^{1 / 3} \mathrm{~F}
$$

$2.34133 \mathrm{E}-03 \quad-2.28997 \mathrm{E} \quad 00$ $2.34220 \mathrm{E}-03 \quad-2.30397 \mathrm{E} \quad 00$ $2.34306 \mathrm{E}-03 \quad-2.31802 \mathrm{E} \quad 00$ $2.34390 \mathrm{E}-03 \quad-2.33210 \mathrm{E} \quad 00$ $2.34474 \mathrm{E}-03 \quad-2.34623 \mathrm{E} \quad 00$

$2.34555 \mathrm{E}-03$

$2.34636 \mathrm{E}-03 \quad-2.37462 \mathrm{E} \quad 00$

$2.34716 \mathrm{E}-03 \quad-2.38887 \mathrm{E} \quad 00$

$2.34794 \mathrm{E}-03 \quad-2.40317 \mathrm{E} \quad 00$

$2.34871 \mathrm{E}-03 \quad-2.41750 \mathrm{E} \quad 00$

$2.34947 \mathrm{E}-03$ $2.35022 \mathrm{E}-03$

$2.35095 \mathrm{E}-03$

$2.35167 \mathrm{E}-03$

$2.35238 \mathrm{E}-03$

$2.35308 \mathrm{E}-03$

$2.35377 \mathrm{E}-03$

$2.35444 \mathrm{E}-03$

$2.35511 \mathrm{E}-03$

$2.35576 \mathrm{E}-03$

$2.35640 \mathrm{E}-03$

$2.35703 \mathrm{E}-03$

$2.35765 \mathrm{E}-03$

$2.35826 \mathrm{E}-03$

$2.35886 \mathrm{E}-03$

$2.35945 \mathrm{E}-03$ $2.36002 \mathrm{E}-03$

$2.36058 \mathrm{E}-03$

$2.36114 \mathrm{E}-03$

$2.36168 \mathrm{E}-03$

$2.36221 \mathrm{E}-03$

$2.36274 \mathrm{E}-03$

$2.36325 \mathrm{E}-03$

$2.36375 \mathrm{E}-03$

$2.36425 \mathrm{E}-03$

$2.36473 \mathrm{E}-03$

$2.36520 \mathrm{E}-03$

$2.36566 \mathrm{E}-03$

$2.36612 \mathrm{E}-03$

$2.36656 \mathrm{E}-03$

$2.36699 \mathrm{E}-03$ $2.36742 \mathrm{E}-03$

$2.36783 \mathrm{E}-03$

$2.36823 \mathrm{E}-03$

$2.36862 \mathrm{E}-03$

$2.36901 \mathrm{E}-03$

$2.36938 \mathrm{E}-03$

$2.36975 \mathrm{E}-03$

$2.37011 \mathrm{E}-03$

$2.37046 \mathrm{E}-03$

$2.37079 \mathrm{E}-03$

$2.37112 \mathrm{E}-03$

$2.37144 \mathrm{E}-03$

$2.37176 \mathrm{E}-03$

$2.37206 \mathrm{E}-03$

2.37235 E-03 $2.37264 \mathrm{E}-03$ $2.37291 \mathrm{E}-03$

$2.37318 \mathrm{E}-03$

$2.37344 \mathrm{E}-03$
$-2.43188 \mathrm{E} \quad 00$ $-2.44631 \mathrm{E} 00$ $-2.46077 \mathrm{E} \quad 00$ $-2.47527 \mathrm{E} \quad 00$ $-2.48982 \mathrm{E} \quad 00$

$-2.50441 \mathrm{E} \quad 00$ $-2.51904 \mathrm{E} \quad 00$ $-2.53371 \mathrm{E} \quad 00$ $-2.54842 \mathrm{E} \quad 00$ $-2.56318 \mathrm{E} 00$

$-2.57797 \mathrm{E} \quad 00$ $-2.59281 \mathrm{E} \quad 00$ $-2.60769 \mathrm{E} \quad 00$ $-2.62261 \mathrm{E} \quad 00$ $-2.63758 \mathrm{E} 00$

$-2.65258 \mathrm{E} \quad 00$ $-2.66763 \mathrm{E} 00$ $-2.68272 \mathrm{E} 00$ $-2.69785 \mathrm{E} \quad 00$ $-2.71302 \mathrm{E} \quad 00$

$-2.72823 \mathrm{E} \quad 00$ $-2.74349 \mathrm{E} \quad 00$ $-2.75878 \mathrm{E} \quad 00$ $-2.77412 \mathrm{E} \quad 00$ $-2.78950 \mathrm{E} \quad 00$

$-2.80492 \mathrm{E} \quad 00$ $-2.82038 \mathrm{E} \quad 00$ $-2.83589 \mathrm{E} \quad 00$ $-2.85143 \mathrm{E} \quad 00$ $-2.86702 \mathrm{E} \quad 00$

$-2.88265 \mathrm{E} \quad 00$ $-2.89832 \mathrm{E} \quad 00$ $-2.91403 \mathrm{E} 00$ $-2.92978 \mathrm{E} \quad 00$ $-2.94557 \mathrm{E} 00$

$-2.96141 \mathrm{E} \quad 00$ $-2.97729 \mathrm{E} 00$ $-2.99320 \mathrm{E} \quad 00$ $-3.00916 \mathrm{E} \quad 00$ $-3.02517 \mathrm{E} 00$ $-3.05729 \mathrm{E} 00$ $-3.07342 \mathrm{E} 00$ $-3.08958 \mathrm{E} 00$ $-3.10579 \mathrm{E} 00$

$-3.12204 \mathrm{E} \quad 00$ $-3.13833 \mathrm{E} \quad 00$ $-3.15467 \mathrm{E} 00$ $-3.17104 \mathrm{E} 00$ -3.18745 E 00
$-3.04121 \mathrm{E} \quad 00$ $g_{-1}$ $\tan \delta_{-1}$

$\begin{array}{llll}3.17001 \mathrm{E} & 01 & -3.02682 \mathrm{E} & 02\end{array}$ $\begin{array}{lllll}3.18019 \mathrm{E} & 01 & -3.02899 \mathrm{E} & 02\end{array}$ $3.19037 \mathrm{E} \quad 01 \quad-3.03115 \mathrm{E} \quad 02$ $3.20055 \mathrm{E} \quad 01 \quad-3.03331 \mathrm{E} \quad 02$ $3.21072 \mathrm{E} \quad 01 \quad-3.03546 \mathrm{E} \quad 02$

$3.22089 \mathrm{E} \quad 01$ $3.23107 \mathrm{E} \quad 01$ $3.24124 \mathrm{E} \quad 01$ $3.25141 \mathrm{E} \quad 01$

$3.26157 \mathrm{E} \quad 01$

$3.27174 \mathrm{E} \quad 01$ $3.28190 \mathrm{E} 01$ $3.29206 \mathrm{E} \quad 01$ $3.30222 \mathrm{E} \quad 01$ $3.31238 \mathrm{E} \quad 01$

$3.32254 \mathrm{E} \quad 01$ $3.33270 \mathrm{E} \quad 01$ $3.34285 \mathrm{E} 01$ $3.35300 \mathrm{E} \quad 01$ $3.36315 \mathrm{E} 01$

$3.37330 \mathrm{E} \quad 01$ $3.38345 \mathrm{E} \quad 01$ $3.39359 \mathrm{E} \quad 01$ $3.40374 \mathrm{E}$ 01

$3.41388 \mathrm{E} \quad 01$

$3.42402 \mathrm{E} \quad 01$ $3.43416 \mathrm{E} \quad 01$ $3.44430 \mathrm{E} 01$ $3.45443 \mathrm{E} \quad 01$ $3.46457 \mathrm{E} \quad 01$

$3.47470 \mathrm{E} \quad 01$ $3.48483 \mathrm{E}$ 01 $3.49496 \mathrm{E} \quad 01$ $3.50509 \mathrm{E} \quad 01$ $3.51521 \mathrm{E} 01$

$3.52533 \mathrm{E} \quad 01$ $3.53546 \mathrm{E} \quad 01$ $3.54558 \mathrm{E} \quad 01$ $3.55569 \mathrm{E} 01$ $3.56581 \mathrm{E} \quad 01$

$3.57593 \mathrm{E} \quad 01$ $3.58604 \mathrm{E} \quad 01$ $3.59615 \mathrm{E} \quad 01$ $3.60626 \mathrm{E} \quad 01$ $3.61637 \mathrm{E} \quad 01$

$3.62648 \mathrm{E} \quad 01$ $3.63658 \mathrm{E} 01$ $3.64668 \mathrm{E} 01$ $3.65678 \mathrm{E} 01$ $3.66688 \mathrm{E} 01$

$3.67698 \mathrm{E} \quad 01$ $3.68708 \mathrm{E} \quad 01$ $3.69717 \mathrm{E} 01$ $3.70726 \mathrm{E} \quad 01$ $3.71735 \mathrm{E} \quad 01$

$3.72744 \mathrm{E} \quad 01$ $3.73753 \mathrm{E} \quad 01$ $3.74762 \mathrm{E} \quad 01$ $3.75770 \mathrm{E} \quad 01$ $3.76778 \mathrm{E} \quad 01$
$-3.03762 \mathrm{E} \quad 02$ $-3.03976 \mathrm{E} 02$ $-3.04190 \mathrm{E} 02$ $-3.04404 \mathrm{E} \quad 02$ $-3.04617 \mathrm{E} \quad 02$ $-3.04831 \mathrm{E} \quad 02$ $-3.05043 \mathrm{E} 02$ $-3.05255 \mathrm{E} \quad 02$ $-3.05467 \mathrm{E} 02$ $-3.05678 \mathrm{E} 02$ $-3.05890 \mathrm{E} \quad 02$ $-3.06101 \mathrm{E} 02$ $-3.06311 \mathrm{E} \quad 02$ $-3.06521 \mathrm{E} \quad 02$ $-3.06731 \mathrm{E} \quad 02$ $-3.06940 \mathrm{E} \quad 02$ $-3.07149 \mathrm{E} \quad 02$ $-3.07358 \mathrm{E} \quad 02$ $-3.07567 \mathrm{E} 02$ -3.07775 E 02 -3.07982 E 02 $-3.08190 \mathrm{E} 02$ $-3.08398 \mathrm{E} \quad 02$ $-3.08604 \mathrm{E} \quad 02$ $-3.08811 \mathrm{E} \quad 02$ $-3.09018 \mathrm{E} \quad 02$ $-3.09224 \mathrm{E} \quad 02$ $-3.09430 \mathrm{E} 02$ $-3.09635 \mathrm{E} \quad 02$ $-3.09840 \mathrm{E} \quad 02$

$-3.10045 \mathrm{E} \quad 02$ $-3.10250 \mathrm{E} 02$ $-3.10455 \mathrm{E} 02$ $-3.10659 \mathrm{E} 02$ $\begin{array}{ll}-3.10864 \mathrm{E} & 02\end{array}$ $-3.11271 \mathrm{E} \quad 02$ $-3.11475 \mathrm{E} 02$ $-3.11678 \mathrm{E} 02$ $-3.11882 \mathrm{E} \quad 02$ $\begin{array}{ll}-3.12085 \mathrm{E} & 02\end{array}$ $-3.12287 \mathrm{E} 02$ $-3.12490 \mathrm{E} 02$ $-3.12692 \mathrm{E} 02$ $-3.12894 \mathrm{E} \quad 02$

$\begin{array}{ll}-3.13096 \mathrm{E} & 02\end{array}$ $-3.13298 \mathrm{E} 02$ $-3.13500 \mathrm{E} \quad 02$ $-3.13701 \mathrm{E} \quad 02$ $-3.13903 \mathrm{E} 02$ $-3.14104 \mathrm{E} \quad 02$ $-3.14305 \mathrm{E} \quad 02$ $-3.14506 \mathrm{E} \quad 02$ $-3.14706 \mathrm{E} 02$ --3.14907 E 02 $-3.11067 \mathrm{E} \quad 02$ 
ELECTRON RADIAL FUNCTIONS

$Z=4 \quad A=8 \quad \rho=1.2 A^{1 / 3} F$

Electron

$\bar{F}$

30.10

30.20

30.30

30.40

30.50

30.60

30.70

30.80

30.90

31.00

31.10

31.20

31.30

31.40

31.50

31.60

31.70

31.80

31.90

32.00

32.10

32.20

32.30

32.40

32.50

32.60

32.70

32.80

32.90

33.00

33.10

33.20

33.30

33.40

33.50

33.60

33.70

33.80

33.90

34.00

34.10

34.20

34.30

34.40

34.50

34.60

34.70

34.80

34.90

35.00

35.10

35.20

35.30

35.40

35.50

35.60

35.70

35.80

35.90

36.00 $f_{2}$

$1.06659 \mathrm{E}-03$ $1.07368 \mathrm{E}-03$ $1.08080 \mathrm{E}-03$ $1.08795 \mathrm{E}-03$ $1.09512 \mathrm{E}-03$

$1.10231 \mathrm{E}-03$ $1.10952 \mathrm{E}-03$ $1.11676 \mathrm{E}-03$ $1.12402 \mathrm{E}-03$ $1.13131 \mathrm{E}-03$

$1.13861 \mathrm{E}-03$

$1.14595 \mathrm{E}-03$

$1.15330 \mathrm{E}-03$

$1.16068 \mathrm{E}-03$

$1.16808 \mathrm{E}-03$

$1.17551 \mathrm{E}-03$

$1.18296 \mathrm{E}-03$

$1.19043 \mathrm{E}-03$

$1.19793 \mathrm{E}-03$

$1.20545 \mathrm{E}-03$

$1.21299 \mathrm{E}-03$

$1.22056 \mathrm{E}-03$

$1.22815 \mathrm{E}-03$

$1.23577 \mathrm{E}-03$

$1.24340 \mathrm{E}-03$

$1.25107 \mathrm{E}-03$

$1.25875 \mathrm{E}-03$

$1.26646 \mathrm{E}-03$

$1.27419 \mathrm{E}-03$

$1.28195 \mathrm{E}-03$

$1.28973 \mathrm{E}-03$

$1.29753 \mathrm{E}-03$

$1.30536 \mathrm{E}-03$

$1.31321 \mathrm{E}-03$

$1.32108 \mathrm{E}-03$

$1.32898 \mathrm{E}-03$

$1.33690 \mathrm{E}-03$

$1.34484 \mathrm{E}-03$

$1.35281 \mathrm{E}-03$

$1.36080 \mathrm{E}-03$

$1.36881 \mathrm{E}-03$ $1.37685 \mathrm{E}-03$

$1.38491 \mathrm{E}-03$

$1.39300 \mathrm{E}-03$

$1.40111 \mathrm{E}-03$

$1.40924 \mathrm{E}-03$

$1.41739 \mathrm{E}-03$

$1.42557 \mathrm{E}-03$

$1.43377 \mathrm{E}-03$

$1.44200 \mathrm{E}-03$

$1.45025 \mathrm{E}-03$

$1.45852 \mathrm{E}-03$

$1.46682 \mathrm{E}-03$

$1.47514 \mathrm{E}-03$

$1.48348 \mathrm{E}-03$

$1.49185 \mathrm{E}-03$

$1.50024 \mathrm{E}-03$

$1.50866 \mathrm{E}-03$

$1.51710 \mathrm{E}-03$

$1.52556 \mathrm{E}-03$ $g_{2}$

$-1.92155 \mathrm{E} \quad 00$

$-1.93438 \mathrm{E} 00$

$-1.94726 \mathrm{E} \quad 00$

$-1.96018 \mathrm{E} 00$

$-1.97315 \mathrm{E} \quad 00$

$-1.98615 \mathrm{E} \quad 00$

$-1.99920 \mathrm{E} \quad 00$

$-2.01229 \mathrm{E} \quad 00$

$-2.02542 \mathrm{E} \quad 00$

$-2.03859 \mathrm{E} \quad 00$

$-2.05181 \mathrm{E} \quad 00$

$-2.06506 \mathrm{E} \quad 00$

$-2.07836 \mathrm{E} 00$

$-2.09170 \mathrm{E} 00$

$-2.10509 \mathrm{E} \quad 00$

$-2.11851 \mathrm{E} \quad 00$

$-2.13198 \mathrm{E} \quad 00$

$-2.14549 \mathrm{E} 00$

$-2.15904 \mathrm{E} \quad 00$

$-2.17264 \mathrm{E} \quad 00$

$-2.18627 \mathrm{E} \quad 00$

$-2.19995 \mathrm{E} 00$

$-2.21367 \mathrm{E} \quad 00$

$-2.22743 \mathrm{E} \quad 00$

$-2.24123 \mathrm{E} 00$

$-2.25508 \mathrm{E} \quad 00$

$-2.26897 \mathrm{E} \quad 00$

$-2.28290 \mathrm{E} 00$

$-2.29687 \mathrm{E} 00$

$-2.31088 \mathrm{E} 00$

$-2.32494 \mathrm{E} \quad 00$

$-2.33904 \mathrm{E} \quad 00$

$-2.35318 \mathrm{E} 00$

$-2.36736 \mathrm{E} \quad 00$

$-2.38158 \mathrm{E} 00$

$-2.39585 \mathrm{E} \quad 00$

$-2.41015 \mathrm{E} 00$

$-2.42450 \mathrm{E} \quad 00$

$-2.43889 \mathrm{E} 00$

$-2.45333 \mathrm{E} \quad 00$

$-2.46780 \mathrm{E} \quad 00$

$-2.48232 \mathrm{E} \quad 00$

$-2.49688 \mathrm{E} \quad 00$

$-2.51148 \mathrm{E} 00$

$-2.52612 \mathrm{E} \quad 00$

$-2.54080 \mathrm{E} \quad 00$

$-2.55553 \mathrm{E} \quad 00$

$-2.57030 \mathrm{E} \quad 00$

$-2.58511 \mathrm{E} \quad 00$

$-2.59996 \mathrm{E} \quad 00$

$-2.61485 \mathrm{E} \quad 00$

$-2.62978 \mathrm{E} 00$

$-2.64476 \mathrm{E} 00$

$-2.65978 \mathrm{E} 00$

$-2.67484 \mathrm{E} \quad 00$

$-2.68994 \mathrm{E} \quad 00$

$-2.70509 \mathrm{E} 00$

$-2.72027 \mathrm{E} 00$

$-2.73550 \mathrm{E} \quad 00$

$-2.75077 \mathrm{E} \quad 00$ $\tan \delta_{2}$

$f_{-2}$

$-8.72663 \mathrm{E}-02$

$-8.80906 \mathrm{E}-02$

$-8.89200 \mathrm{E}-02$

$-8.97546 \mathrm{E}-02$

$-9.05944 \mathrm{E}-02$

$-9.14393 \mathrm{E}-02$

$-9.22894 \mathrm{E}-02$

$-9.31448 \mathrm{E}-02$

$-9.40054 \mathrm{E}-02$

$-9.48712 \mathrm{E}-02$

$-9.57422 \mathrm{E}-02$

$-9.66186 \mathrm{E}-02$

$-9.75002 \mathrm{E}-02$

$-9.83872 \mathrm{E}-02$

$-9.92794 \mathrm{E}-02$

$-1.00177 \mathrm{E}-01$

$-1.01080 \mathrm{E}-01$

$-1.01988 \mathrm{E}-01$

$-1.02902 \mathrm{E}-01$

$-1.03821 \mathrm{E}-01$

$-1.04746 \mathrm{E}-01$

$-1.05676 \mathrm{E}-01$

$-1.06611 \mathrm{E}-01$

$-1.07552 \mathrm{E}-01$

$-1.08498 \mathrm{E}-01$

$-1.09450 \mathrm{E}-01$

$-1.10407 \mathrm{E}-01$

$-1.11370 \mathrm{E}-01$

$-1.12339 \mathrm{E}-01$

$-1.13313 \mathrm{E}-01$

$-1.14292 \mathrm{E}-01$

$-1.15277 \mathrm{E}-01$

$-1.16268 \mathrm{E}-01$

$-1.17264 \mathrm{E}-01$

$-1.18266 \mathrm{E}-01$

$-1.19274 \mathrm{E}-01$

$-1.20287 \mathrm{E}-01$

$-1.21306 \mathrm{E}-01$

$-1.22331 \mathrm{E}-01$

$-1.23361 \mathrm{E}-01$

$-1.24397 \mathrm{E}-01$

$-1.25439 \mathrm{E}-01$

$-1.26487 \mathrm{E}-01$

$-1.27540 \mathrm{E}-01$

$-1.28599 \mathrm{E}-01$

$-1.29664 \mathrm{E}-01$

$-1.30735 \mathrm{E}-01$

$-1.31811 \mathrm{E}-01$

$-1.32894 \mathrm{E}-01$

$-1.33982 \mathrm{E}-01$

$-1.35076 \mathrm{E}-01$

$-1.36176 \mathrm{E}-01$

$-1.37282 \mathrm{E}-01$

$-1.38394 \mathrm{E}-01$

$-1.39512 \mathrm{E}-01$

$-1.40636 \mathrm{E}-01$

$-1.41766 \mathrm{E}-01$

$-1.42902 \mathrm{E}-01$

$-1.44043 \mathrm{E}-01$

$-1.45191 \mathrm{E}-01$

$5.11379 \mathrm{E} \quad 01 \quad-8.52655 \mathrm{E}-02$

$5.11402 \mathrm{E}$ 01 $-8.60750 \mathrm{E}-02$

$5.11424 \mathrm{E} \quad 01 \quad-8.68897 \mathrm{E}-02$

$5.11447 \mathrm{E}$ 01 $-8.77094 \mathrm{E}-02$

$5.11469 \mathrm{E} \quad 01 \quad-8.85343 \mathrm{E}-02$

$5.11491 \mathrm{E} \quad 01 \quad-8.93643 \mathrm{E}-02$

$5.11513 \mathrm{E} \quad 01 \quad-9.01994 \mathrm{E}-02$

$5.11535 \mathrm{E} \quad 01 \quad-9.10397 \mathrm{E}-02$

$5.11557 \mathrm{E}$ 01 $-9.18852 \mathrm{E}-02$

$5.11579 \mathrm{E}$ 01 $-9.27358 \mathrm{E}-02$

$5.11600 \mathrm{E} \quad 01$

$5.11622 \mathrm{E} \quad 01$

$5.11643 \mathrm{E} 01$

$5.11664 \mathrm{E} \quad 01$

$5.11685 \mathrm{E} \quad 01$

$5.11706 \mathrm{E} \quad 01$

$5.11726 \mathrm{E} \quad 01$

$5.11747 \mathrm{E} 01$

$5.11767 \mathrm{E} \quad 01$

$5.11788 \mathrm{E} 01$

$5.11808 \mathrm{E} \quad 01$

$5.11828 \mathrm{E} \quad 01$

$5.11848 \mathrm{E} \quad 01$

$5.11867 \mathrm{E} \quad 01$

$5.11887 \mathrm{E} \quad 01$

$5.11907 \mathrm{E} \quad 01$

$5.11926 \mathrm{E} \quad 01$

$5.11945 \mathrm{E} \quad 01$

$5.11964 \mathrm{E} \quad 01$

$5.11983 \mathrm{E} \quad 0$

$-9.35917 \mathrm{E}-02$

$-9.44528 \mathrm{E}-02$

$-9.53191 \mathrm{E}-02$

$-9.61907 \mathrm{E}-02$

$-9.70675 \mathrm{E}-02$

$-9.79496 \mathrm{E}-02$

$-9.88371 \mathrm{E}-02$

$-9.97298 \mathrm{E}-02$

$-1.00628 \mathrm{E}-01$

$-1.01531 \mathrm{E}-01$

$-1.02440 \mathrm{E}-01$

$-1.03354 \mathrm{E}-01$ 


\begin{tabular}{|c|c|c|c|c|c|c|c|c|}
\hline$p$ & $F_{0}$ & & $f_{1}$ & & $g_{1}$ & & $\tan \delta_{1}$ & $f_{-1}$ \\
\hline 36.10 & $1.09755 \mathrm{E}$ & 00 & $-3.67506 \mathrm{E}$ & 01 & $-3.26968 \mathrm{E}$ & 00 & $2.37368 \mathrm{E}-03$ & $-3.20391 \mathrm{E}$ \\
\hline 36.20 & $1.09755 \mathrm{E}$ & 00 & $-3.68515 E$ & 01 & $-3.28640 \mathrm{E}$ & 00 & $2.37392 \mathrm{E}-03$ & $-3.22041 \mathrm{E}$ \\
\hline .30 & $1.09754 \mathrm{E}$ & 00 & $-3.69523 \mathrm{E}$ & 01 & $-3.30315 \mathrm{E}$ & 00 & $2.37416 \mathrm{E}-03$ & $-3.23695 \mathrm{E}$ \\
\hline .40 & $1.09754 \mathrm{E}$ & 00 & $-3.70532 \mathrm{E}$ & 01 & $-3.31994 \mathrm{E}$ & 00 & $2.37438 \mathrm{E}-03$ & $-3.25353 \mathrm{E}$ \\
\hline .50 & $1.09754 \mathrm{E}$ & 00 & $-3.71539 \mathrm{E}$ & 01 & $-3.33678 \mathrm{E}$ & 00 & $2.37460 \mathrm{E}-03$ & $-3.27015 \mathrm{E}$ \\
\hline 5.60 & $1.09753 \mathrm{E}$ & 00 & $-3.72547 \mathrm{E}$ & 01 & $-3.35365 \mathrm{E}$ & 00 & $2.37481 \mathrm{E}-03$ & $-3.28681 \mathrm{E}$ \\
\hline .70 & $1.09753 \mathrm{E}$ & 00 & $-3.73555 \mathrm{E}$ & 01 & $-3.37057 \mathrm{E}$ & 00 & $2.37500 \mathrm{E}-03$ & $-3.30352 \mathrm{E}$ \\
\hline .80 & $1.09753 \mathrm{E}$ & 00 & $-3.74562 \mathrm{E}$ & 01 & $-3.38753 \mathrm{E}$ & 00 & $2.37520 \mathrm{E}-03$ & $-3.32026 \mathrm{E}$ \\
\hline .90 & $1.09753 \mathrm{E}$ & 00 & $-3.75570 \mathrm{E}$ & 01 & $-3.40453 \mathrm{E}$ & 00 & $2.37538 \mathrm{E}-03$ & $-3.33705 \mathrm{E}$ \\
\hline .00 & $1.09752 \mathrm{E}$ & 00 & $-3.76577 \mathrm{E}$ & 01 & $-3.42157 \mathrm{E}$ & 00 & $2.37555 \mathrm{E}-03$ & $-3.35388 \mathrm{E}$ \\
\hline .10 & $1.09752 \mathrm{E}$ & 00 & $-3.77584 \mathrm{E}$ & 01 & $-3.43865 \mathrm{E}$ & 00 & $2.37572 \mathrm{E}-03$ & $-3.37074 \mathrm{E}$ \\
\hline .20 & $1.09752 \mathrm{E}$ & 00 & $-3.78590 \mathrm{E}$ & 01 & $-3.45578 \mathrm{E}$ & 00 & $2.37588 \mathrm{E}-03$ & $3.38765 \mathrm{E}$ \\
\hline .30 & $1.09752 \mathrm{E}$ & 00 & $-3.79597 \mathrm{E}$ & 01 & $-3.47294 \mathrm{E}$ & 00 & $2.37603 \mathrm{E}-03$ & $-3.40461 \mathrm{E}$ \\
\hline .40 & $1.09751 \mathrm{E}$ & 00 & $-3.80603 \mathrm{E}$ & 01 & $-3.49015 \mathrm{E}$ & 00 & $2.37617 \mathrm{E}-03$ & $-3.42160 \mathrm{E}$ \\
\hline 7.50 & $1.09751 \mathrm{E}$ & 00 & $-3.81610 \mathrm{E}$ & 01 & $-3.50739 \mathrm{E}$ & 00 & $2.37630 \mathrm{E}-03$ & $-3.43863 \mathrm{E}$ \\
\hline 60 & $1.09751 \mathrm{E}$ & 00 & $-3.82616 \mathrm{E}$ & 01 & $-3.52468 \mathrm{E}$ & 00 & $2.37643 \mathrm{E}-03$ & $-3.45571 \mathrm{E}$ \\
\hline .70 & $1.09750 \mathrm{E}$ & 00 & $-3.83621 E$ & 01 & $-3.54201 \mathrm{E}$ & 00 & $2.37655 \mathrm{E}-03$ & $-3.47282 \mathrm{E}$ \\
\hline .80 & $1.09750 \mathrm{E}$ & 00 & $-3.84627 \mathrm{E}$ & 01 & $-3.55938 \mathrm{E}$ & 00 & $2.37666 \mathrm{E}-$ & $-3.48998 \mathrm{E}$ \\
\hline .90 & $1.09750 \mathrm{E}$ & 00 & $-3.85632 \mathrm{E}$ & 01 & $-3.57679 \mathrm{E}$ & 00 & $2.37676 \mathrm{E}-03$ & $.50718 \mathrm{E}$ \\
\hline 38.00 & $1.09750 \mathrm{E}$ & 00 & $-3.86638 E$ & 01 & $-3.59425 \mathrm{E}$ & 00 & $2.37686 \mathrm{E}-03$ & $-3.52442 \mathrm{E}$ \\
\hline .10 & $1.09749 \mathrm{E}$ & 00 & $-3.87643 E$ & 01 & $-3.61174 \mathrm{E}$ & 00 & $2.37694 \mathrm{E}-03$ & $-3.54170 \mathrm{E}$ \\
\hline .20 & $1.09749 \mathrm{E}$ & 00 & $-3.88648 \mathrm{E}$ & 01 & $3.62928 \mathrm{E}$ & 00 & 2.377 & $.55902 \mathrm{E}$ \\
\hline .30 & $1.09749 \mathrm{E}$ & 00 & $-3.89652 \mathrm{E}$ & 01 & $.64685 \mathrm{E}$ & 00 & $10 \mathrm{E}-03$ & $3.57638 \mathrm{E}$ \\
\hline 38.40 & $1.09749 \mathrm{E}$ & 00 & $-3.90657 \mathrm{E}$ & 01 & $-3.66447 \mathrm{E}$ & 00 & $2.37716 \mathrm{E}-03$ & $-3.59379 \mathrm{E}$ \\
\hline .50 & $1.09748 \mathrm{E}$ & 00 & $-3.91661 \mathrm{E}$ & 01 & $-3.68213 E$ & 00 & $2.37722 \mathrm{E}-03$ & $-3.61123 \mathrm{E}$ \\
\hline .60 & $1.09748 \mathrm{E}$ & 00 & $-3.92665 \mathrm{E}$ & 01 & $-3.69983 E$ & 00 & $2.37727 \mathrm{E}-03$ & $-3.62872 \mathrm{E}$ \\
\hline .70 & $1.09748 \mathrm{E}$ & 00 & $-3.93669 \mathrm{E}$ & 01 & $1757 \mathrm{E}$ & 00 & $1 \mathrm{E}-03$ & $54625 \mathrm{E}$ \\
\hline 38.80 & $1.09748 \mathrm{E}$ & 00 & $-3.94673 \mathrm{E}$ & 01 & $-3.73535 \mathrm{E}$ & 00 & $5 E-03$ & $3.66381 \mathrm{E}$ \\
\hline 3.90 & $1.09747 \mathrm{E}$ & 00 & $-3.95677 \mathrm{E}$ & 01 & $-3.75317 \mathrm{E}$ & 00 & $2.37737 \mathrm{E}-03$ & $-3.68142 \mathrm{E}$ \\
\hline .00 & $1.09747 \mathrm{E}$ & 00 & $-3.96680 \mathrm{E}$ & 01 & $-3.77104 \mathrm{E}$ & 00 & $2.37739 \mathrm{E}-03$ & $-3.69907 \mathrm{E}$ \\
\hline 9.10 & $1.09747 \mathrm{E}$ & 00 & $-3.97683 E$ & 01 & $-3.78894 E$ & 00 & $2.37741 \mathrm{E}-03$ & $-3.71676 \mathrm{E}$ \\
\hline .20 & $1.09747 \mathrm{E}$ & 00 & $-3.98686 \mathrm{E}$ & 01 & $-3.80688 \mathrm{E}$ & 00 & $\mathrm{E}-03$ & $73450 \mathrm{E}$ \\
\hline 9.30 & $1.09746 \mathrm{E}$ & 00 & $-3.99689 \mathrm{E}$ & 01 & $-3.82487 \mathrm{E}$ & 00 & $2.37741 \mathrm{E}-03$ & $-3.75227 \mathrm{E}$ \\
\hline 39.40 & $1.09746 \mathrm{E}$ & 00 & $-4.00691 \mathrm{E}$ & 01 & $-3.84290 \mathrm{E}$ & 00 & $2.37740 \mathrm{E}-03$ & $-3.77008 \mathrm{E}$ \\
\hline 39.50 & $1.09746 \mathrm{E}$ & 00 & $-4.01694 \mathrm{E}$ & 01 & $-3.86097 \mathrm{E}$ & 00 & $2.37739 \mathrm{E}-03$ & $-3.78794 \mathrm{E}$ \\
\hline 9.60 & $1.09746 \mathrm{E}$ & 00 & $-4.02696 \mathrm{E}$ & 01 & $-3.87908 \mathrm{E}$ & 00 & $2.37737 \mathrm{E}-03$ & $-3.80584 \mathrm{E}$ \\
\hline .70 & $1.09745 \mathrm{E}$ & 00 & $-4.03698 \mathrm{E}$ & 01 & $-3.89723 \mathrm{E}$ & 00 & $2.37733 \mathrm{E}-03$ & $-3.82377 \mathrm{E}$ \\
\hline 9.80 & $1.09745 \mathrm{E}$ & 00 & $-4.04700 \mathrm{E}$ & 01 & $-3.91542 \mathrm{E}$ & 00 & $2.37730 \mathrm{E}-03$ & $-3.84175 \mathrm{E}$ \\
\hline 9.90 & $1.09745 \mathrm{E}$ & 00 & $-4.05702 \mathrm{E}$ & 01 & $-3.93365 \mathrm{E}$ & 00 & $2.37726 \mathrm{E}$ & $-3.85977 \mathrm{E}$ \\
\hline 40.00 & $1.09745 \mathrm{E}$ & 00 & $-4.06703 \mathrm{E}$ & 01 & $-3.95192 \mathrm{E}$ & 00 & $2.37720 \mathrm{E}-03$ & $-3.87783 \mathrm{E}$ \\
\hline 40.10 & $1.09744 \mathrm{E}$ & 00 & $-4.07704 \mathrm{E}$ & 01 & $-3.97024 \mathrm{E}$ & 00 & $2.37715 \mathrm{E}-03$ & $-3.89593 \mathrm{E}$ \\
\hline .20 & $1.09744 \mathrm{E}$ & 00 & $-4.08705 \mathrm{E}$ & 0 & $-3.98859 \mathrm{E}$ & 00 & $2.37708 \mathrm{E}-03$ & $-3.91407 \mathrm{E}$ \\
\hline 10.3 & $1.09744 \mathrm{E}$ & 00 & $-4.09706 \mathrm{E}$ & 0 & $-4.00699 \mathrm{E}$ & 0 & 3 & $93226 \mathrm{E}$ \\
\hline .40 & $9744 \mathrm{E}$ & 00 & $0707 \mathrm{E}$ & 01 & $542 \mathrm{E}$ & 00 & 93 & $5048 \mathrm{E}$ \\
\hline 40.50 & $1.09743 \mathrm{E}$ & 00 & $-4.11707 \mathrm{E}$ & 01 & $-4.04390 \mathrm{E}$ & 00 & $2.37685 \mathrm{E}-03$ & $-3.96875 \mathrm{E}$ \\
\hline 40.60 & $.09743 \mathrm{E}$ & 00 & $-4.12708 \mathrm{E}$ & 01 & $-4.06242 \mathrm{E}$ & 00 & $2.37675 \mathrm{E}-03$ & $-3.98705 \mathrm{E}$ \\
\hline & $1.09743 \mathrm{E}$ & 00 & $-4.13708 \mathrm{E}$ & 01 & $.08098 \mathrm{E}$ & 00 & 03 & $0540 \mathrm{E}$ \\
\hline 0.80 & $9743 \mathrm{E}$ & 00 & $-4.14708 \mathrm{E}$ & 01 & & 00 & 03 & $2379 \mathrm{E}$ \\
\hline 90 & $1.09742 \mathrm{E}$ & 00 & $-4.15707 \mathrm{E}$ & 01 & $-4.11822 \mathrm{E}$ & 00 & $2.37644 \mathrm{E}-03$ & $-4.04221 \mathrm{E}$ \\
\hline 41.00 & $1.09742 \mathrm{E}$ & 00 & $-4.16707 \mathrm{E}$ & 01 & $-4.13690 \mathrm{E}$ & 00 & $2.37632 \mathrm{E}-03$ & $-4.06068 \mathrm{E}$ \\
\hline 41.10 & $1.09742 \mathrm{E}$ & 00 & $-4.17706 \mathrm{E}$ & 01 & $-4.15563 \mathrm{E}$ & 00 & $2.37620 \mathrm{E}-03$ & $-4.07919 \mathrm{E}$ \\
\hline & $1.09742 \mathrm{E}$ & 00 & $-4.18705 \mathrm{E}$ & 01 & & 0 & & $-4.09774 \mathrm{E}$ \\
\hline 4. 30 & $1.09741 \mathrm{E}$ & 00 & $-4.19704 \mathrm{E}$ & 01 & $-4.19319 \mathrm{E}$ & 00 & $2.37593 \mathrm{E}-$ & $-4.11633 \mathrm{E}$ \\
\hline 41.40 & $1.09741 \mathrm{E}$ & 00 & $-4.20703 \mathrm{E}$ & 01 & $-4.21204 \mathrm{E}$ & 00 & $2.37578 \mathrm{E}-03$ & $-4.13497 \mathrm{E}$ \\
\hline 41.50 & $1.09741 \mathrm{E}$ & 00 & $-4.21701 \mathrm{E}$ & 01 & $-4.23092 \mathrm{E}$ & 00 & $2.37563 \mathrm{E}-03$ & $-4.15364 \mathrm{E}$ \\
\hline 41.60 & $1.09741 \mathrm{E}$ & 00 & $-4.22700 \mathrm{E}$ & 01 & $-4.24985 \mathrm{E}$ & 00 & $7 \mathrm{E}-03$ & $-4.17235 \mathrm{E}$ \\
\hline & $.09740 \mathrm{E}$ & 00 & $-4.23698 \mathrm{E}$ & 01 & $-4.26882 \mathrm{E}$ & 06 & $2.37531 \mathrm{E}$ & $-4.19111 \mathrm{E}$ \\
\hline & $1.09740 \mathrm{E}$ & 00 & $-4.24696 \mathrm{E}$ & 0 & $-4.28783 \mathrm{E}$ & 00 & $2.37514 \mathrm{E}-03$ & $-4.20990 \mathrm{E}$ \\
\hline . & $1.09740 \mathrm{E}$ & 00 & $-4.25693 \mathrm{E}$ & 01 & $-4.30687 \mathrm{E}$ & 00 & $2.37496 \mathrm{E}-03$ & $-4.22874 \mathrm{E}$ \\
\hline 12.00 & $1.09740 \mathrm{E}$ & 00 & $-4.26691 \mathrm{E}$ & 01 & $-4.32596 \mathrm{E}$ & 00 & $2.37478 \mathrm{E}-03$ & $-4.24762 \mathrm{E}$ \\
\hline
\end{tabular}

$g_{-1}$ $\tan \delta_{-1}$

$3.77786 \mathrm{E} \quad 01$ $3.78794 \mathrm{E}$ 01 $3.79801 \mathrm{E} \quad 01$ $3.80809 \mathrm{E} \quad 01$ $3.81816 \mathrm{E} \quad 01$

$3.82823 \mathrm{E} \quad 01$ $3.83830 \mathrm{E} \quad 01$ $3.84837 \mathrm{E}$ 0l $3.85843 \mathrm{E} 01$ $3.86850 \mathrm{E} \quad 01$

$3.87856 \mathrm{E} \quad 01$ $3.88862 \mathrm{E} \quad 01$ $3.89867 \mathrm{E} \quad 01$ $3.90873 \mathrm{E} \quad 01$ $3.91878 \mathrm{E} \quad 01$

$3.92884 \mathrm{E} \quad 01$ $3.93889 \mathrm{E}$ 0l $3.94894 \mathrm{E}$ 0l $3.95898 \mathrm{E}$ 0l $3.96903 \mathrm{E} 01$

$3.97907 \mathrm{E} \quad 01$ $3.98911 \mathrm{E} \quad 01$ $3.99915 \mathrm{E} 01$ $4.00919 \mathrm{E} \quad 01$ $4.01922 \mathrm{E} \quad 01$

$4.02926 \mathrm{E} \quad 01$ $4.03929 \mathrm{E} \quad 01$ $4.04932 \mathrm{E} 01$ $4.05934 \mathrm{E} \quad 01$ $4.06937 \mathrm{E} \quad 01$

$4.07939 \mathrm{E} \quad 01$ $4.08942 \mathrm{E} \quad 01$ $4.09944 \mathrm{E} \quad 01$ $4.10945 \mathrm{E} \quad 01$ $4.11947 \mathrm{E} \quad 01$

$4.12948 \mathrm{E} \quad 01$ $4.13950 \mathrm{E} \quad 01$ $4.14951 \mathrm{E} \quad 01$ $4.15952 \mathrm{E} \quad 01$ $4.16952 \mathrm{E} \quad 01$

4.17953 E 01 $4.18953 \mathrm{E} \quad 01$ $4.19953 \mathrm{E} \quad 01$ $4.20953 \mathrm{E} \quad 01$ $4.21952 \mathrm{E} 01$

$4.22952 \mathrm{E} \quad 01$ $4.23951 \mathrm{E} \quad 01$ $4.24950 \mathrm{E} 01$ $4.25949 \mathrm{E} \quad 01$ $4.26948 \mathrm{E} \quad 01$

$4.27946 \mathrm{E} \quad 01$ $4.28945 \mathrm{E} 01$ $4.29943 \mathrm{E} \quad 01$ $4.30940 \mathrm{E} 01$ $4.31938 \mathrm{E} \quad 01$

$4.32936 \mathrm{E} \quad 01$ $4.33933 \mathrm{E} \quad 01$ $4.34930 \mathrm{E} 01$ $4.35927 \mathrm{E} 01$ $4.36923 \mathrm{E} 01$
$-3.15108 \mathrm{E} \quad 02$ $-3.15308 \mathrm{E} \quad 02$ $-3.15508 \mathrm{E} \quad 02$ $-3.15708 \mathrm{E} \quad 02$ $-3.15908 \mathrm{E} \quad 02$

$-3.16108 \mathrm{E} \quad 02$ $-3.16308 \mathrm{E} 02$ $-3.16508 \mathrm{E} 02$ $-3.16707 \mathrm{E} 02$ $-3.16907 \mathrm{E} \quad 02$

$-3.17107 \mathrm{E} \quad 02$ $-3.17306 \mathrm{E} \quad 02$ $-3.17505 \mathrm{E} \quad 02$ $-3.17704 \mathrm{E} \quad 02$ $-3.17903 \mathrm{E} \quad 02$

$-3.18102 \mathrm{E} 02$ $-3.18301 \mathrm{E} \quad 02$ $-3.18500 \mathrm{E} 02$ $-3.18699 \mathrm{E} \quad 02$ $-3.18898 \mathrm{E} 02$

$-3.19097 \mathrm{E} \quad 02$ $-3.19295 \mathrm{E} 02$ $-3.19494 \mathrm{E} \quad 02$ $-3.19692 \mathrm{E} 02$ -3.19891 E 02

$-3.20089 \mathrm{E} \quad 02$ $-3.20288 \mathrm{E} \quad 02$ $\begin{array}{ll}-3.20486 \mathrm{E} & 02\end{array}$ $-3.20685 \mathrm{E} \quad 02$ -3.20883 E 02

$-3.21081 \mathrm{E} \quad 02$ $-3.21280 \mathrm{E} \quad 02$ $-3.21478 \mathrm{E} \quad 02$ $-3.21676 \mathrm{E} \quad 02$ $\begin{array}{lll}-3.21874 \mathrm{E} & 02\end{array}$

$-3.22073 \mathrm{E} \quad 02$ $-3.22272 \mathrm{E} 02$ $-3.22469 \mathrm{E} \quad 02$ $-3.22668 \mathrm{E} 02$ -3.22867 E 02

$-3.23064 \mathrm{E} \quad 02$ -3.23263 E 02 $-3.23461 \mathrm{E} 02$ $-3.23660 \mathrm{E} \quad 02$ $-3.23858 \mathrm{E} 02$

$-3.24057 \mathrm{E} \quad 02$ $-3.24255 \mathrm{E} 02$ $-3.24454 \mathrm{E} \quad 02$ $-3.24652 \mathrm{E} \quad 02$ -3.24851 E 02

$-3.25050 \mathrm{E} \quad 02$ $-3.25248 \mathrm{E} \quad 02$ $-3.25447 \mathrm{E} 02$ $-3.25646 \mathrm{E} \quad 02$ -3.25845 E 02

$-3.26044 \mathrm{E} \quad 02$ $-3.26243 \mathrm{E} 02$ $-3.26442 \mathrm{E} \quad 02$ $-3.26641 \mathrm{E} \quad 02$ $-3.26840 \mathrm{E} 02$ 
ELECTRON RADIAL FUNCTIONS

$Z=4 \quad A=8 \quad \rho=1.2 A^{1 / 3} F$

Electron

$\bar{F}$

$1.53404 \mathrm{E}-03$

$1.54255 \mathrm{E}-03$

$1.55108 \mathrm{E}-03$

$1.55964 \mathrm{E}-03$

$1.56822 \mathrm{E}-03$

$1.57682 \mathrm{E}-03$

$1.58545 \mathrm{E}-03$

$1.59410 \mathrm{E}-03$

$1.60277 \mathrm{E}-03$

$1.61147 \mathrm{E}-03$

$1.62019 \mathrm{E}-03$

$1.62893 \mathrm{E}-03$

$1.63770 \mathrm{E}-03$

$1.64649 \mathrm{E}-03$

$1.65530 \mathrm{E}-03$

$1.66414 \mathrm{E}-03$

$1.67300 \mathrm{E}-03$

$1.68189 \mathrm{E}-03$

$1.69079 \mathrm{E}-03$

$1.69973 \mathrm{E}-03$

$1.70868 \mathrm{E}-03$

$1.71766 \mathrm{E}-03$

$1.72666 \mathrm{E}-03$

$1.73569 \mathrm{E}-03$

$1.74474 \mathrm{E}-03$

38.60

38.70

38.80

38.90

39.00

39.10

39.20

39.30

39.40

39.50

39.60

39.70

39.80

39.90

40.00

40.10

40.20

40.30

40.40

40.50

40.60

40.70

40.80

40.90

41.00

41.10

41.20

41.30

41.40

41.50

41.60

41.70

41.80

41.90

42.00

$1.75381 \mathrm{E}-03$ $1.77203 \mathrm{E}-03$ $1.78117 \mathrm{E}-03$

$1.79034 \mathrm{E}-03$

$1.79953 \mathrm{E}-03$

$1.80875 \mathrm{E}-03$

$1.81798 \mathrm{E}-03$

$1.82724 \mathrm{E}-03$

$1.83653 \mathrm{E}-03$

$1.84584 \mathrm{E}-03$

$1.85517 \mathrm{E}-03$

$1.86453 \mathrm{E}-03$

$1.87390 \mathrm{E}-03$

$1.88331 \mathrm{E}-03$

$1.89273 \mathrm{E}-03$

$1.90218 \mathrm{E}-03$

$1.91166 \mathrm{E}-03$

$1.92115 \mathrm{E}-03$

$1.93067 \mathrm{E}-03$.

$1.94022 \mathrm{E}-03$

$1.94978 \mathrm{E}-03$

$1.95937 \mathrm{E}-03$

$1.96899 \mathrm{E}-03$

$1.97863 \mathrm{E}-03$

$1.98829 \mathrm{E}-03$

$1.99797 \mathrm{E}-03$

$2.00768 \mathrm{E}-03$

$2.01741 \mathrm{E}-03$

$2.02717 \mathrm{E}-03$

$2.03695 \mathrm{E}-03$ $2.04675 \mathrm{E}-03$ $2.05657 \mathrm{E}-03$

$2.06642 \mathrm{E}-03$

$2.07630 \mathrm{E}-03$ $f_{2}$

$g_{2}$

$-2.76608 \mathrm{E} \quad 00 \quad-1.46345 \mathrm{E}-01$ -2.78143 E 00 $-2.79682 \mathrm{E} 00$ $-2.81226 \mathrm{E} \quad 00$ $-2.82774 \mathrm{E} \quad 00$

$-2.84326 \mathrm{E} \quad 00$ $-2.85882 \mathrm{E} \quad 00$ $-2.87442 \mathrm{E} \quad 00$ $-2.89006 \mathrm{E} \quad 00$ $-2.90575 \mathrm{E} \quad 00$

$-2.92148 \mathrm{E} \quad 00$ $-2.93725 \mathrm{E} \quad 00$ $-2.95306 \mathrm{E} \quad 00$ $-2.96891 \mathrm{E} \quad 00$ $-2.98480 \mathrm{E} \quad 00$

$-3.00074 \mathrm{E} \quad 00$ $-3.01671 \mathrm{E} \quad 00$ $-3.03273 \mathrm{E} \quad 00$ $-3.04879 \mathrm{E} 00$ $-3.06489 \mathrm{E} 00$

$-3.08104 \mathrm{E} \quad 00$ $-3.09722 \mathrm{E} \quad 00$ $-3.11345 \mathrm{E} \quad 00$ $-3.12972 \mathrm{E} 00$ $-3.14603 \mathrm{E} \quad 00$ $1.76291 \mathrm{E}-03$
$-3.16238 \mathrm{E} \quad 00$

$-3.17877 \mathrm{E} \quad 00$

$-3.19520 \mathrm{E} \quad 00$

$-3.21168 \mathrm{E} \quad 00$

$-3.22819 \mathrm{E} 00$

$-3.24475 \mathrm{E} \quad 00$

$-3.26135 \mathrm{E} \quad 00$

$-3.27799 \mathrm{E} 00$

$-3.29467 \mathrm{E} \quad 00$

$-3.31140 \mathrm{E} 00$

$-3.32816 \mathrm{E} \quad 00$

$-3.34497 \mathrm{E} \quad 00$

$-3.36182 \mathrm{E} \quad 00$

$-3.37871 \mathrm{E} 00$

$-3.41261 \mathrm{E} \quad 00$

$-3.42962 \mathrm{E} 00$

$-3.44668 \mathrm{E} 00$

$-3.46378 \mathrm{E} 00$

-3.48091 E 00

$-3.49809 \mathrm{E} \quad 00$

$-3.51531 \mathrm{E} \quad 00$

$-3.53257 \mathrm{E} 00$

$-3.54988 \mathrm{E} \quad 00$

-3.56722 E 00

-3.58461 E 00

$-3.60203 \mathrm{E} \quad 00$

$-3.61950 \mathrm{E} 00$

$-3.63701 \mathrm{E} \quad 00$

$-3.65456 \mathrm{E} \quad 00$

$-3.67215 \mathrm{E} \quad 00$

$-3.70746 \mathrm{E} 00$

$-3.72517 \mathrm{E} \quad 00$

$-3.74293 \mathrm{E} 00$
$-3.39564 \mathrm{E} 00$

$-3.68979 \mathrm{E} 00$
$-1.47505 \mathrm{E}-01$

$-1.48671 \mathrm{E}-01$

$-1.49843 \mathrm{E}-01$

$-1.51021 \mathrm{E}-01$

$-1.52205 \mathrm{E}-01$

$-1.53396 \mathrm{E}-01$

$-1.54592 \mathrm{E}-01$

$-1.55795 \mathrm{E}-01$

$-1.57004 \mathrm{E}-01$

$-1.58219 \mathrm{E}-01$

$-1.59440 \mathrm{E}-01$

$-1.60667 \mathrm{E}-01$

$-1.61901 \mathrm{E}-01$

$-1.63141 \mathrm{E}-01$

$-1.64387 \mathrm{E}-01$

$-1.65640 \mathrm{E}-01$

$-1.66898 \mathrm{E}-01$

$-1.68163 \mathrm{E}-01$

$-1.69435 \mathrm{E}-01$

$-1.70713 \mathrm{E}-01$

$-1.71997 \mathrm{E}-01$

$-1.73287 \mathrm{E}-01$

$-1.74584 \mathrm{E}-01$

$-1.75887 \mathrm{E}-01$

$-1.77197 \mathrm{E}-01$

$-1.78513 \mathrm{E}-01$

$-1.79836 \mathrm{E}-01$

$-1.81165 \mathrm{E}-01$

$-1.82500 \mathrm{E}-01$

$-1.83842 \mathrm{E}-01$

$-1.85191 \mathrm{E}-01$

$-1.86546 \mathrm{E}-01$

$-1.87907 \mathrm{E}-01$

$-1.89275 \mathrm{E}-01$

$-1.90650 \mathrm{E}-01$

$-1.92031 \mathrm{E}-01$

$-1.93419 \mathrm{E}-01$

$-1.94814 \mathrm{E}-01$

$-1.96215 \mathrm{E}-01$

$-1.97623 \mathrm{E}-01$

$-1.99037 \mathrm{E}-01$

$-2.00458 \mathrm{E}-01$

$-2.01886 \mathrm{E}-01$

$-2.03321 \mathrm{E}-01$

$-2.04762 \mathrm{E}-01$

$-2.06210 \mathrm{E}-01$

$-2.07664 \mathrm{E}-01$

$-2.09126 \mathrm{E}-01$

$-2.10594 \mathrm{E}-01$

$-2.12069 \mathrm{E}-01$

$-2.13551 \mathrm{E}-01$

$-2.15040 \mathrm{E}-01$

$-2.16535 \mathrm{E}-01$

$-2.18038 \mathrm{E}-01$

$-2.19547 \mathrm{E}-01$

$-2.21063 \mathrm{E}-01$

$-2.22586 \mathrm{E}-01$

$-2.24117 \mathrm{E}-01$

$-2.25653 \mathrm{E}-01$ $\tan \delta_{2}$

$f_{-2}$

$5.12519 \mathrm{E} \quad 01$

$5.12535 \mathrm{E} 01$

5.12551 E 01

$5.12566 \mathrm{E} 01$

$5.12582 \mathrm{E} 01$

$5.12597 \mathrm{E} \quad 01$

$5.12612 \mathrm{E} 01$

$5.12628 \mathrm{E} \quad 01$

$5.12643 \mathrm{E} \quad 01$

$5.12658 \mathrm{E} \quad 01$

$5.12673 \mathrm{E} \quad 01$

$5.12687 \mathrm{E}$ 01

$5.12702 \mathrm{E} 01$

$5.12717 \mathrm{E}$ 01

$5.12731 \mathrm{E} \quad 01$

$5.12746 \mathrm{E} \quad 01$

$5.12760 \mathrm{E} \quad 01$

$5.12775 \mathrm{E}$ 01

$5.12789 \mathrm{E} \quad 01$

$5.12803 \mathrm{E} 01$

$5.12817 \mathrm{E} \quad 01$

$5.12831 \mathrm{E} \quad 01$

$5.12845 \mathrm{E} \quad 01$

$5.12859 \mathrm{E} \quad 01$

$5.12873 \mathrm{E} \quad 01$

$5.12887 \mathrm{E} \quad 01$

$5.12901 \mathrm{E} \quad 01$

$5.12914 \mathrm{E} \quad 01$

$5.12928 \mathrm{E} \quad 01$

$5.12941 \mathrm{E} \quad 01$

5.12955 E 01

$5.12968 \mathrm{E} \quad 01$

$5.12981 \mathrm{E} \quad 01$

$5.12994 \mathrm{E} \quad 01$

$5.13008 \mathrm{E} \quad 01$

5.13021 E 01 $5.13034 \mathrm{E} \quad 01$

$5.13046 \mathrm{E} \quad 01$

$5.13059 \mathrm{E}$ ol

$5.13072 \mathrm{E} 01$

$\begin{array}{lll}5.13085 \text { E } & 01\end{array}$ $5.13097 \mathrm{E} 01$

$5.13110 \mathrm{E} \quad 01$

$5.13122 \mathrm{E} 01$

$5.13135 \mathrm{E} 01$

$5.13147 \mathrm{E} \quad 01$

$5.13160 \mathrm{E} 01$

$5.13172 \mathrm{E} \quad 01$

$5.13184 \mathrm{E} \quad 01$

$5.13196 \mathrm{E} 01$

$5.13208 \mathrm{E} 01$

$5.13220 \mathrm{E} \quad 01$

$5.13232 \mathrm{E} 01$

$5.13244 \mathrm{E} \quad 01$

$5.13256 \mathrm{E} \quad 01$

$5.13268 \mathrm{E} \quad 01$

$5.13280 \mathrm{E} \quad 01$

$5.13291 \mathrm{E} 01$

$5.13303 \mathrm{E} 01$

$5.13314 \mathrm{E} \quad 01$

$-1.43367 \mathrm{E}-01$ $-1.44509 \mathrm{E}-01$ $-1.45657 \mathrm{E}-01$ $-1.46811 \mathrm{E}-01$ $-1.47971 \mathrm{E}-01$ 
ELECTRON RADIAL FUNCTIONS

Electron

\begin{tabular}{|c|c|c|c|}
\hline$F_{0}$ & & $f_{1}$ & $g_{1}$ \\
\hline $2.58993 \mathrm{E}$ & 00 & $-1.20359 \mathrm{E}-02$ & $-2.31293 \mathrm{E}-04$ \\
\hline $1.71462 \mathrm{E}$ & 00 & $-3.70674 \mathrm{E}-02$ & $-7.13589 \mathrm{E}-04$ \\
\hline $1.46672 \mathrm{E}$ & 00 & $-7.51248 \mathbf{E}-02$ & $-1.45043 \mathrm{E}-03$ \\
\hline $1.35384 \mathrm{E}$ & 00 & $-1.24936 \mathbf{E}-01$ & $-2.42165 \mathrm{E}-03$ \\
\hline $1.29075 \mathrm{E}$ & 00 & $-1.84995 \mathrm{E}-01$ & $-3.60324 \mathrm{E}-03$ \\
\hline $1.25124 \mathrm{E}$ & 00 & $-2.53737 \mathrm{E}-01$ & $-4.97034 \mathrm{E}-03$ \\
\hline $1.22462 \mathrm{E}$ & 00 & $-3.29679 \mathrm{E}-01$ & $-6.49932 \mathrm{E}-03$ \\
\hline $1.20575 \mathrm{E}$ & 00 & $-4.11492 \mathbf{E}-01$ & $-8.16907 \mathrm{E}-03$ \\
\hline $1.19187 \mathrm{E}$ & 00 & $-4.98040 \mathrm{E}-01$ & $-9.96158 \mathrm{E}-03$ \\
\hline $1.18134 \mathrm{E}$ & 00 & $-5.88379 E-01$ & $-1.18619 \mathrm{E}-02$ \\
\hline $1.17317 \mathrm{E}$ & 00 & $-6.81743 \mathbf{E}-01$ & $-1.38579 \mathrm{E}-02$ \\
\hline $16670 \mathrm{E}$ & 00 & $-7.77518 \mathrm{E}-01$ & $-1.59400 \mathrm{E}-02$ \\
\hline $16149 \mathrm{E}$ & 00 & $-8.75215 \mathrm{E}-01$ & $-1.81004 \mathrm{E}-02$ \\
\hline $15724 \mathrm{E}$ & 00 & $-9.74449 \mathrm{E}-0 \mathrm{l}$ & $-2.03331 \mathrm{E}-02$ \\
\hline $1.15371 \mathrm{E}$ & 00 & $-1.07491 \mathrm{E} \quad 00$ & $-2.26334 \mathrm{E}-02$ \\
\hline $1.15076 \mathrm{E}$ & 60 & $-1.17637 \mathrm{E} \quad 00$ & $-2.49976 \mathrm{E}-02$ \\
\hline $14827 \mathrm{E}$ & 00 & $-1.27862 \overline{\mathbf{E}} \quad 00$ & $-2.74225 \mathrm{E}-02$ \\
\hline $.14614 \mathrm{E}$ & 00 & $-1.38153 \mathbf{E} \quad 00$ & $-2.99060 \mathrm{E}-02$ \\
\hline $.14431 \mathrm{E}$ & 00 & $-1.48496 \mathrm{E} \quad 00$ & $-3.24462 \mathbf{E}-02$ \\
\hline $1.14273 \mathrm{E}$ & 00 & $-1.58883 \mathrm{E} \quad 00$ & $-3.50415 \mathrm{E}-02$ \\
\hline $1.14135 \mathrm{E}$ & 00 & $-1.69305 \mathrm{E} \quad 00$ & $-3.76909 \mathrm{E}-02$ \\
\hline $1.14013 \mathrm{E}$ & 00 & $-1.79756 \mathrm{E} \quad 00$ & $-4.03933 \mathrm{E}-02$ \\
\hline $1.13906 \mathrm{E}$ & 00 & $-1.90231 \mathrm{E} \quad 00$ & $-4.31479 \mathrm{E}-02$ \\
\hline $1.13811 \mathrm{E}$ & 00 & $-2.00726 \mathrm{E} \quad 00$ & $-4.59543 \mathrm{E}-02$ \\
\hline $1.13726 \mathrm{E}$ & 00 & $-2.11238 \mathrm{E} \quad 00$ & $-4.88117 \mathrm{E}-02$ \\
\hline $1.13650 \mathrm{E}$ & 00 & $-2.21763 \mathrm{E}$ & $-5.17199 \mathrm{~b}$ \\
\hline $31 \mathrm{E}$ & 00 & $-2.32300 \mathrm{E} \quad 00$ & $-5.46785 E-02$ \\
\hline $13519 \mathrm{E}$ & 00 & $-2.42847 \mathrm{E} \quad 00$ & $-5.76872 \mathrm{E}-02$ \\
\hline $1.13463 \mathrm{E}$ & 00 & $-2.53401 \mathrm{E} \quad 00$ & $-6.07457 \mathrm{E}-02$ \\
\hline $1.13412 \mathrm{E}$ & 00 & $-2.63962 \mathrm{E} \quad 00$ & $-6.38539 \mathrm{E}-02$ \\
\hline $1.13365 \mathrm{E}$ & 00 & $-2.74529 \mathrm{E} \quad 00$ & $-6.70116 \mathrm{E}-02$ \\
\hline $13322 \mathrm{E}$ & 00 & $-2.85100 \mathrm{E} \quad 00$ & $-7.02186 \mathrm{E}-02$ \\
\hline $.13283 \mathrm{E}$ & 00 & $-2.95675 \mathrm{E} \quad 00$ & $-7.34749 \mathrm{E}-02$ \\
\hline $1.13246 \mathrm{E}$ & 00 & $-3.06254 \mathrm{E} \quad 00$ & $-7.67803 \mathrm{E}-02$ \\
\hline $1.13213 \mathrm{E}$ & 00 & $-3.16835 \mathrm{E}$ & $-8.01348 \mathrm{E}-02$ \\
\hline $1.13182 \mathrm{E}$ & 00 & $-3.27418 \mathrm{E} \quad 00$ & $-8.35382 \mathrm{E}-02$ \\
\hline $53 \mathrm{E}$ & 00 & $-3.38002 \mathrm{E} \quad 00$ & $-8.69906 \mathrm{E}-02$ \\
\hline $1.13126 \mathrm{E}$ & 00 & $-3.48588 \mathrm{E}$ & $-9.04919 \mathrm{E}-02$ \\
\hline $1.13101 \mathrm{E}$ & 00 & $-3.59175 \mathrm{E}$ & $-9.40419 \mathrm{E}-02$ \\
\hline $1.13078 \mathrm{E}$ & 00 & $-3.69763 \mathrm{E}$ & $-9.76408 \mathrm{E}-02$ \\
\hline $1.13056 \mathrm{E}$ & 00 & $-3.80351 \mathrm{E} \quad 00$ & $-1.01288 \mathrm{E}-01$ \\
\hline $13035 \mathrm{E}$ & 00 & $-3.90939 \mathrm{E}$ & $-1.04985 \mathrm{E}-01$ \\
\hline $1.13016 \mathrm{E}$ & 00 & $-4.01528 \mathrm{E}$ & $-1.08730 \mathrm{E}-01$ \\
\hline $.12998 \mathrm{E}$ & 00 & $-4.12117 \mathrm{E} \quad 00$ & $-1.12523 \mathrm{E}-01$ \\
\hline $1.12981 \mathrm{E}$ & 00 & $-4.22705 \mathrm{E} \quad 00$ & $-1.16366 \mathrm{E}-01$ \\
\hline $1.12964 \mathrm{E}$ & 00 & $-4.33293 \mathrm{E} \quad 00$ & $-1.20257 \mathrm{E}-01$ \\
\hline $2949 \mathrm{E}$ & 00 & $-4.43881 \mathrm{E}$ & $-1.24196 \mathrm{E}-01$ \\
\hline $1.12934 \mathrm{E}$ & 00 & $-4.54469 \mathrm{E}$ & $-1.28184 \mathrm{E}-01$ \\
\hline $12921 \mathrm{E}$ & 00 & $-4.65056 \mathrm{E} \quad 00$ & $-1.32221 \mathrm{E}-01$ \\
\hline $1.12908 \mathrm{E}$ & 00 & $-4.75642 \mathrm{E} \quad 00$ & $-1.36307 \mathrm{E}-01$ \\
\hline $1.12895 \mathrm{E}$ & 00 & $-4.86228 \mathrm{E} \quad 00$ & $-1.40440 \mathrm{E}-01$ \\
\hline $2883 \mathrm{E}$ & 00 & $-4.96813 \mathrm{E}$ & $-1.44623 \mathrm{E}-01$ \\
\hline $2872 \mathrm{E}$ & 00 & $-5.07397 \mathbf{E}$ & $-1.48854 \mathrm{E}-01$ \\
\hline & 00 & $-5.17981 \mathrm{E} \quad 00$ & $-1.53133 \mathrm{E}-01$ \\
\hline $1.12851 \mathrm{E}$ & 00 & $-5.28563 \mathrm{E} \quad 00$ & $-1.57461 \mathrm{E}-01$ \\
\hline $1.12841 \mathrm{E}$ & 00 & $-5.39145 \mathrm{E} \quad 00$ & $-1.61838 \mathrm{E}-01$ \\
\hline & 00 & $-5.49726 \mathrm{E} \quad 00$ & $-1.66263 \mathrm{E}-01$ \\
\hline $1.12823 \mathrm{E}$ & 00 & $-5.60306 \mathrm{E} \quad 00$ & $-1.70737 \mathrm{E}-01$ \\
\hline $1.12814 \mathrm{E}$ & 00 & $-5.70885 \mathrm{E} \quad 00$ & $-1.75259 \mathrm{E}-01$ \\
\hline $1.12805 \mathrm{E}$ & 00 & $-5.81463 \mathrm{E} \quad 00$ & $-1.79829 \mathrm{E}-01$ \\
\hline
\end{tabular}

$$
Z=5 \quad A=11 \quad \rho=1.2 A^{1 / 3} F
$$

$$
\begin{gathered}
\tan \delta_{1} \\
-1.57474 \mathrm{E}-01 \\
-7.63041 \mathrm{E}-02 \\
-5.00840 \mathrm{E}-02 \\
-3.70130 \mathrm{E}-02 \\
-2.91484 \mathrm{E}-02 \\
-2.38792 \mathrm{E}-02 \\
-2.00930 \mathrm{E}-02 \\
-1.72353 \mathrm{E}-02 \\
-1.49983 \mathrm{E}-02 \\
-1.31976 \mathrm{E}-02 \\
-1.17153 \mathrm{E}-02 \\
-1.04731 \mathrm{E}-02 \\
-9.41633 \mathrm{E}-03 \\
-8.50603 \mathrm{E}-03 \\
-7.71343 \mathrm{E}-03
\end{gathered}
$$

$-7.01693 \mathrm{E}-03$ $-6.39992 \mathrm{E}-03$ $-5.84944 \mathrm{E}-03$

$-5.35522 \mathrm{E}-03$

$-4.90901 \mathrm{E}-03$

$-4.50410 \mathrm{E}-03$ $-4.13500 \mathrm{E}-03$ $-3.79713 \mathrm{E}-03$ $-3.48669 \mathrm{E}-03$ $-3.20044 \mathrm{E}-03$

$-2.93567 \mathrm{E}-03$ $-2.69005 \mathrm{E}-03$ $-2.46156 \mathrm{E}-03$ $-2.24846 \mathrm{E}-03$ $-2.04925 \mathrm{E}-03$

$-1.86262 \mathrm{E}-03$ $-1.68741 \mathrm{E}-03$ $-1.52260 \mathrm{E}-03$ $-1.36729 \mathrm{E}-03$ $-1.22069 \mathrm{E}-03$

$-1.08208 \mathrm{E}-03$ $-9.50829 \mathrm{E}-04$ $-8.26363 \mathrm{E}-04$ $-7.08172 \mathrm{E}-04$ $-5.95788 \mathrm{E}-04$

$-4.88806 \mathrm{E}-04$ $-3.86838 \mathrm{E}-04$ $-2.89540 \mathrm{E}-04$ $-1.96597 \mathrm{E}-04$ $-1.07735 \mathrm{E}-04$

\section{$-2.26840 \mathrm{E}-05$} $5.88034 \mathbf{E}-05$ $1.36928 \mathrm{E}-04$ $2.11910 \mathrm{E}-04$ $2.83920 \mathrm{E}-04$

$3.53137 \mathrm{E}-04$ 4.19728E-04 $4.83826 \mathrm{E}-04$

$5.45570 \mathrm{E}-04$

$6.05093 \mathrm{E}-04$

$6.62502 \mathrm{E}-04$ $7.17924 \mathrm{E}-04$ $7.71439 \mathrm{E}-04$ $8.23150 \mathrm{E}-04$ $8.73156 \mathrm{E}-04$ $f_{-1}$

$-3.31841 \mathrm{E}-03$ $-5.39304 \mathrm{E}-03$ $-7.46718 \mathrm{E}-03$ $-9.54254 \mathrm{E}-03$

$-1.16174 \mathrm{E}-02$

$-1.36927 \mathrm{E}-02$ $-1.57713 \mathrm{E}-02$ $-1.78571 \mathrm{E}-02$ $-1.99546 \mathrm{E}-02$ $-2.20682 \mathrm{E}-02$

$-2.42023 \mathrm{E}-02$ $-2.63607 \mathrm{E}-02$ $-2.85468 \mathrm{E}-02$ $-3.07637 \mathrm{E}-02$ $-3.30137 \mathrm{E}-02$

$-3.52993 \mathrm{E}-02$ $-3.76221 \mathrm{E}-02$ $-3.99839 \mathrm{E}-02$ $-4.23858 \mathrm{E}-02$ $-4.48291 \mathrm{E}-02$

$-4.73146 \mathrm{E}-02$ $-4.98432 \mathrm{E}-02$

$-5.24156 \mathrm{E}-02$ $-5.50324 \mathrm{E}-02$ $-5.76941 \mathrm{E}-02$

$-6.04010 \mathrm{E}-02$ $-6.31536 \mathrm{E}-02$ $-6.59523 \mathrm{E}-02$ $-6.87972 \mathrm{E}-02$ $-7.16886 \mathrm{E}-02$

$-7.46268 \mathrm{E}-02$ $-7.76119 \mathrm{E}-02$ $-8.0644 \mathrm{~J} \mathrm{E}-02$ $-8.37235 \mathrm{E}-02$ $-8.68503 \mathrm{E}-02$

$-9.00246 \mathrm{E}-02$

$-9.32464 \mathrm{E}-02$

$-9.65159 \mathrm{E}-02$

$-9.98331 \mathrm{E}-02$

$-1.03198 \mathrm{E}-01$

$-1.06611 \mathrm{E}-01$ $-1.10072 \mathrm{E}-01$

$-1.13580 \mathrm{E}-01$

$-1.17137 \mathrm{E}-01$

$-1.20742 \mathrm{E}-01$

$-1.24395 \mathrm{E}-01$

$-1.28096 \mathrm{E}-01$

$-1.31845 \mathrm{E}-01$

$-1.35643 \mathrm{E}-01$

$-1.39488 \mathrm{E}-01$

$-1.43382 \mathrm{E}-01$

$-1.47324 \mathrm{E}-01$

$-1.51314 \mathrm{E}-01$

$-1.55353 \mathrm{E}-01$

$-1.59439 \mathrm{E}-01$

$-1.63574 \mathrm{E}-01$ $-1.67758 \mathrm{E}-01$ $-1.71989 \mathrm{E}-01$ $-1.76269 \mathrm{E}-01$ $-1.80598 \mathrm{E}-01$ $g_{-1}$

$2.27151 \mathrm{E}-01$

$3.68302 \mathrm{E}-0]$

$5.08012 \mathrm{E}-01$

$6.45862 \mathrm{E}-01$

$7.81297 \mathrm{E}-01$

$9.14043 \mathrm{E}-01$ $1.04405 \mathrm{E} \quad 00$

$1.17141 \mathrm{E} \quad 00$

$1.29631 \mathrm{E} \quad 00$

$1.41899 \mathrm{E} \quad 00$

$1.53969 \mathrm{E} \quad 00$ $1.65865 \mathrm{E} \quad 00$

$1.77610 \mathrm{E} \quad 00$

$1.89222 \mathrm{E} \quad 00$

$2.00719 \mathrm{E} \quad 00$

$2.12117 \mathrm{E} \quad 00$

$2.23429 \mathrm{E} \quad 00$

$2.34666 \mathrm{E} \quad 00$

$2.45837 \mathrm{E} \quad 00$

$2.56951 \mathrm{E} 00$

$2.68015 \mathrm{E} \quad 00$ $2.79034 \mathrm{E} \cdot 00$

$2.90015 \mathrm{E} \quad 00$

$3.00961 \mathrm{E} \quad 00$

$3.11877 \mathrm{E} \quad 00$

$3.22765 \mathrm{E} \quad 00$

$3.33629 \mathrm{E} \quad 00$

$3.44470 \mathrm{E} \quad 00$

$3.55292 \mathrm{E} 00$

$3.66096 \mathrm{E} 00$

$3.76884 \mathrm{E} \quad 00$ $3.87658 \mathrm{E} 00$ $3.98417 \mathrm{E} \quad 00$ $4.09165 \mathrm{E} \quad 00$

$4.19901 \mathrm{E} \quad 00$

$4.30627 \mathrm{E} \quad 00$ $4.41343 \mathrm{E} \quad 00$ $4.52051 \mathrm{E} \quad 00$ $4.62750 \mathrm{E} \quad 00$ $4.73442 \mathrm{E} \quad 00$

$4.84127 \mathrm{E} \quad 00$ $4.94805 \mathrm{E} \quad 00$ $5.05477 \mathrm{E} \quad 00$

$5.16143 \mathrm{E} \quad 00$

$5.26804 \mathrm{E} \quad 00$

$5.37459 \mathrm{E} \quad 00$ $5.48110 \mathrm{E} \quad 00$ $5.58756 \mathrm{E} \quad 00$ $5.69399 \mathrm{E} 00$ 5.80036 E. 00

$5.90670 \mathrm{E} \quad 00$ $6.01301 \mathrm{E} 00$ $6.11928 \mathrm{E} \quad 00$ $6.22551 \mathrm{E} \quad 00$ $6.33171 \mathrm{E} 00$

$6.43788 \mathrm{E} \quad 00$ $6.54403 \mathrm{E} \quad 00$ $6.65014 \mathrm{E} \quad 00$ $6.75622 \mathrm{E} \quad 00$ $6.86228 \mathrm{E} \quad 00$ $\tan \delta_{-1}$

$-5.09879 \mathrm{E} \quad 00$ $-9.55361 \mathrm{E} \quad 00$ $-1.40637 \mathrm{E} \quad 01$ $-1.85112 \mathrm{E} 01$ $-2.28666 \mathrm{E} \quad 01$

$-2.71162 \mathrm{E} \quad 01$ $-3.12528 \mathrm{E} \quad 01$ $-3.52727 \mathrm{E} \quad 01$ $-3.91748 \mathrm{E} \quad 01$ $-4.29597 \mathrm{E} 01$

$-4.66293 \mathrm{E} \quad 01$ $-5.01863 \mathrm{E} \quad 01$ $-5.36337 \mathrm{E} \quad 01$ $-5.69751 \mathrm{E} 01$ $-6.02141 \mathrm{E} 01$

$-6.33546 \mathrm{E} \quad 01$ $-6.64002 \mathrm{E} 01$ $-6.93548 \mathrm{E} \quad 01$ $-7.22218 \mathrm{E} 01$ $-7.50050 \mathrm{E} 01$

$-7.77074 \mathrm{E} 01$ $-8.03327 \mathrm{E} \quad 01$ $-8.28836 \mathrm{E} 01$ $-8.53633 \mathrm{E} 0 \mathrm{0l}$ $-8.77746 \mathrm{E} 01$

$-9.01202 \mathrm{E} \quad 01$ $-9.24028 \mathrm{E} \quad 01$ $-9.46247 \mathrm{E} \quad 01$ $-9.67883 \mathrm{E} \quad 01$ $-9.88958 \mathrm{E} 01$

$-1.00949 \mathrm{E} \quad 02$ $-1.02951 \mathrm{E} \quad 02$ $-1.04903 \mathrm{E} 02$ $-1.06807 \mathrm{E} \quad 02$ $-1.08664 \mathrm{E} \quad 02$

$-1.10476 \mathrm{E} \quad 02$ $-1.12246 \mathrm{E} \quad 02$ $-1.13974 \mathrm{E} \quad 02$ $-1.15662 \mathrm{E} 02$ $-1.17311 \mathrm{E} \quad 02$

$-1.18922 \mathrm{E} \quad 02$ $-1.20498 \mathrm{E} \quad 02$ $-1.22038 \mathrm{E} \quad 02$ $-1.23545 \mathrm{E} \quad 02$ $-1.25019 \mathrm{E} \quad 02$

$-1.26462 \mathrm{E} \quad 02$ $-1.27874 \mathrm{E} \quad 02$ $-1.29256 \mathrm{E} \quad 02$ $-1.30610 \mathrm{E} \quad 02$ $-1.31936 \mathrm{E} 02$

$-1.33235 \mathrm{E} \quad 02$ $-1.34508 \mathrm{E} \quad 02$ $-1.35756 \mathrm{E} 02$ $-1.36979 \mathrm{E} \quad 02$ $-1.38178 \mathrm{E} \quad 02$

$-1.39355 \mathrm{E} \quad 02$ $-1.40509 \mathrm{E} \quad 02$ $-1.41641 \mathrm{E} \quad 02$ $\begin{array}{ll}-1.42752 \mathrm{E} & 02\end{array}$ $\begin{array}{lll}-1.43842 \text { E } & 02\end{array}$ 


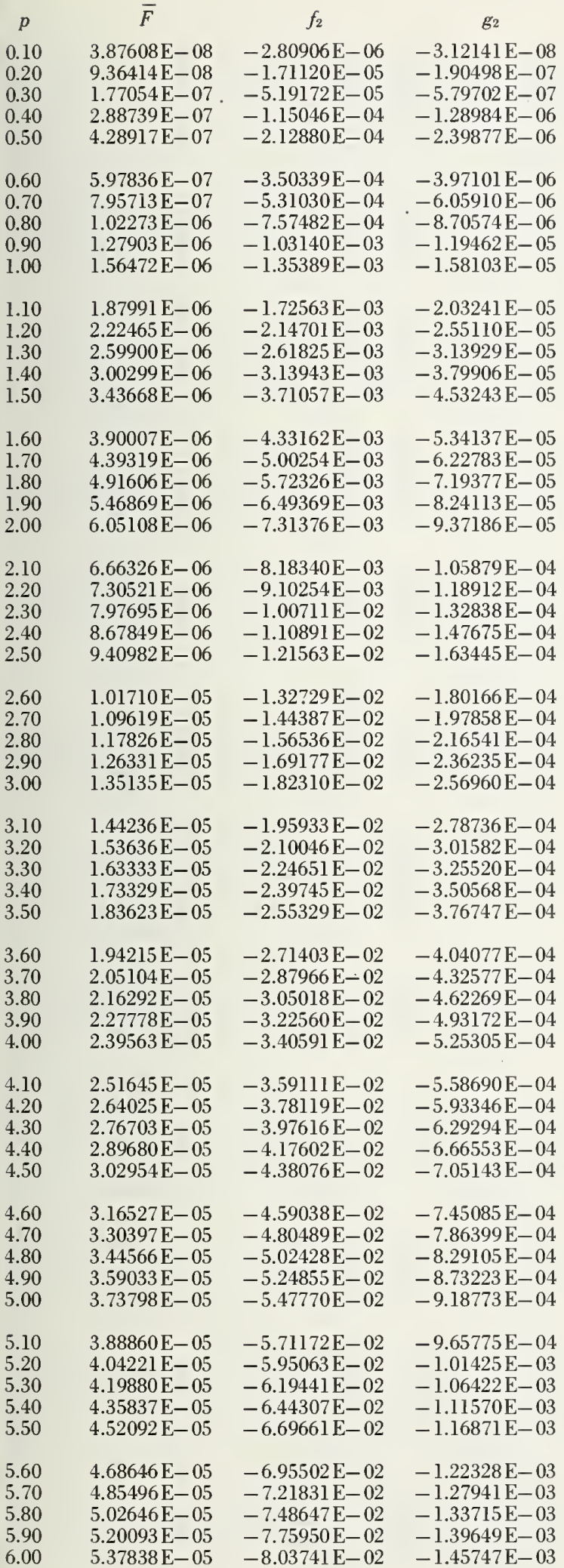

$\tan \delta_{2} \quad f_{-2}$

$2.83957 \mathrm{E} \quad 00 \quad-4.64143 \mathrm{E}-07$ $5.81189 \mathrm{E} \quad 00-1.44113 \mathrm{E}-06$ $\begin{array}{lll}8.63122 \mathrm{E} & 00 & -2.96714 \mathrm{E}-06\end{array}$ $\begin{array}{lll}1.12845 \mathrm{E} & 01 & -5.04136 \mathrm{E}-06\end{array}$ $\begin{array}{lll}1.37478 \mathrm{E} & 01-7.66348 \mathrm{E}-06\end{array}$

$1.60077 \mathrm{E} \quad 01-1.08348 \mathrm{E}-05$ $1.80616 \mathrm{E} \quad 01-1.45590 \mathrm{E}-05$ $1.99158 \mathrm{E}$ 01 $-1.88426 \mathrm{E}-05$ $2.15822 \mathrm{E} \quad 01 \quad-2.36946 \mathrm{E}-05$ $\begin{array}{lll}2.30758 \mathrm{E} & 01 & -2.91266 \mathrm{E}-05\end{array}$

$2.44133 \mathrm{E}$ 0l $-3.51523 \mathrm{E}-05$ $2.56110 \mathrm{E}$ 01 $-4.17867 \mathrm{E}-05$ $2.66845 \mathrm{E} \quad 01 \quad-4.90464 \mathrm{E}-05$ $2.76484 \mathrm{E} \quad 01-\quad-5.69489 \mathrm{E}-05$ $2.85155 \mathrm{E}$ ol $-6.55122 \mathrm{E}-05$

$2.92975 \mathrm{E}$ 01 $-7.47552 \mathrm{E}-05$ $3.00046 \mathrm{E}$ 01 $-8.46970 \mathrm{E}-05$ $3.06456 \mathrm{E}$ ol $-9.53570 \mathrm{E}-05$ $3.12282 \mathrm{E}$ 01 $-1.06755 \mathrm{E}-04$ $3.17593 \mathrm{E}$ 01 $-1.18911 \mathrm{E}-04$

$3.22447 \mathrm{E} \quad 01$ $3.26896 \mathrm{E}$ 01 $3.30983 \mathrm{E} \quad 01$ $3.34747 \mathrm{E} \quad 01$ $3.38222 \mathrm{E} 01$

$3.41438 \mathrm{E} \quad 01$

$3.44421 \mathrm{E} \quad 01$

$3.47194 \mathrm{E} \quad 01$

$3.49776 \mathrm{E} \quad 01$

$3.52185 \mathrm{E} 01$

$3.54438 \mathrm{E} \quad 01$ $3.56549 \mathrm{E} \quad 01$ $3.58528 \mathrm{E} 01$ $3.60389 \mathrm{E}$ ol $3.62140 \mathrm{E}$ 0l

$3.63792 \mathrm{E} \quad 01$ $3.65350 \mathrm{E} \quad 01$ $3.66824 \mathrm{E} 01$ $3.68219 \mathrm{E} 01$ $3.69541 \mathrm{E} 01$

$3.70796 \mathrm{E} \quad 01$ $3.71988 \mathrm{E}$ 01 $3.73122 \mathrm{E} \quad 01$ $3.74202 \mathrm{E} 01$ $3.75231 \mathrm{E} 01$

$3.76213 \mathrm{E} \quad 01$ $3.77151 \mathrm{E} \quad 01$ $3.78048 \mathrm{E} \quad 01$ $3.78906 \mathrm{E} \quad 01$ $3.79728 \mathrm{E} \quad 01$

$3.80516 \mathrm{E} \quad 01$ $3.81271 \mathrm{E} \quad 01$ $3.81996 \mathrm{E}$ ol $3.82693 \mathrm{E} \quad 01$ $3.83363 \mathrm{E}$ ol

$3.84008 \mathrm{E} \quad 01$ $3.84628 \mathrm{E} \quad 0 \mathrm{l}$ $3.85226 \mathrm{E} \quad 01$ $3.85802 \mathrm{E} 01$ $3.86358 \mathrm{E} \quad 01$ $-1.34161 \mathrm{E}-03$ $-1.39906 \mathrm{E}-03$ $-1.45807 \mathrm{E}-03$

\section{$g_{-2}$}

$5.56040 \mathrm{E}-0.5$ $1.72224 \mathrm{E}-04$ $3.53178 \mathrm{E}-04$ $5.96830 \mathrm{E}-04$ $9.01207 \mathrm{E}-04$

$1.26425 \mathrm{E}-03$ $1.68401 \mathrm{E}-03$

$2.15878 \mathrm{E}-03$

$2.68711 \mathrm{E}-03$

$3.90012 \mathrm{E}-03$ $4.58316 \mathrm{E}-03$ $5.31647 \mathrm{E}-03$ $6.09962 \mathrm{E}-03$ $6.93232 \mathrm{E}-03$

$7.81434 \mathrm{E}-03$ $8.74549 \mathrm{E}-03$ $9.72567 \mathrm{E}-03$ $1.07548 \mathrm{E}-02$ $1.18327 \mathrm{E}-02$

$1.29594 \mathrm{E}-02$ $1.41350 \mathrm{E}-02$ $1.53592 \mathrm{E}-02$ $1.66321 \mathrm{E}-02$ $1.79538 \mathrm{E}-02$

$1.93241 \mathrm{E}-02$ $2.07431 \mathrm{E}-02$ $2.22108 \mathrm{E}-02$ 2.37272 E- 02 $2.52922 \mathrm{E}-02$

$2.69059 \mathrm{E}-02$ $2.85683 \mathrm{E}-02$ $3.02794 \mathrm{E}-02$ $3.20391 \mathrm{E}-02$ $3.38474 \mathrm{E}-02$

$3.57045 \mathrm{E}-02$ $3.76102 \mathrm{E}-02$ $3.95646 \mathrm{E}-02$ $4.15677 \mathrm{E}-02$ $4.36194 \mathrm{E}-02$

$4.57198 \mathrm{E}-02$ $4.78689 \mathrm{E}-02$ $5.00666 \mathrm{E}-02$

$5.23130 \mathrm{E}-02$

$5.46081 \mathrm{E}-02$

$5.69519 \mathrm{E}-02$ $5.93443 \mathrm{E}-02$ $6.17853 \mathrm{E}-02$ $6.42751 \mathrm{E}-02$ $6.68135 \mathrm{E}-02$

$6.94006 \mathrm{E}-02$ $7.20363 \mathrm{E}-02$ $7.47207 \mathrm{E}-02$ $7.74538 \mathrm{E}-02$ $8.02355 \mathrm{E}-02$

$8.30658 \mathrm{E}-02$ $8.59448 \mathrm{E}-02$ $8.88725 \mathrm{E}-02$ $9.18488 \mathrm{E}-02$ $9.48737 \mathrm{E}-02$
$3.26786 \mathrm{E}-03$

$\tan \delta_{-2}$

$-1.59485 \mathrm{E}-01$ $-7.95946 \mathrm{E}-02$ $-5.46619 \mathrm{E}-02$ $-4.28357 \mathrm{E}-02$ $-3.61562 \mathrm{E}-02$

$-3.20035 \mathrm{E}-02$ $-2.92617 \mathrm{E}-02$ $-2.73757 \mathrm{E}-02$ $-2.60396 \mathrm{E}-02$ $-2.50721 \mathrm{E}-02$

$-2.43598 \mathrm{E}-02$ $-2.38287 \mathrm{E}-02$ $-2.34291 \mathrm{E}-02$ $-2.31265 \mathrm{E}-02$ $-2.28965 \mathrm{E}-02$

$-2.27215 \mathrm{E}-02$ $-2.25887 \mathrm{E}-02$ $-2.24884 \mathrm{E}-02$ $-2.24136 \mathrm{E}-02$ $-2.23586 \mathrm{E}-02$

$-2.23194 \mathrm{E}-02$ $-2.22926 \mathrm{E}-02$ $-2.22757 \mathrm{E}-02$ $-2.22668 \mathrm{E}-02$ $-2.22641 \mathrm{E}-02$

$-2.22665 \mathrm{E}-02$ $-2.22730 \mathrm{E}-02$ $-2.22826 \mathrm{E}-02$ $-2.22948 \mathrm{E}-02$ $-2.23091 \mathrm{E}-02$

$-2.23249 \mathrm{E}-02$ $-2.23420 \mathrm{E}-02$ $-2.23600 \mathrm{E}-02$ $-2.23787 \mathrm{E}-02$ $-2.23979 \mathrm{E}-02$

$-2.24175 \mathrm{E}-02$ $-2.24372 \mathrm{E}-02$ $-2.24571 \mathrm{E}-02$ $-2.24770 \mathrm{E}-02$ $-2.24969 \mathrm{E}-02$

$-2.25166 \mathrm{E}-02$ $-2.25362 \mathrm{E}-02$ $-2.25556 \mathrm{E}-02$ $-2.25747 \mathrm{E}-02$ $-2.25936 \mathrm{E}-02$

$-2.26122 \mathrm{E}-02$ $-2.26306 \mathrm{E}-02$ $-2.26486 \mathrm{E}-02$ $-2.26663 \mathrm{E}-02$ $-2.26837 \mathrm{E}-02$

$-2.27008 \mathrm{E}-02$ $-2.27175 \mathrm{E}-02$ $-2.27340 \mathrm{E}-02$ $-2.27501 \mathrm{E}-02$ $-2.27659 \mathrm{E}-02$

$-2.27814 \mathrm{E}-02$
$-2.27966 \mathrm{E}-02$ $-2.2811+\mathrm{E}-02$ $-2.28260 \mathrm{E}-02$ $-2.28403 \mathrm{E}-02$ 
ELECTRON RADIAL FUNCTIONS

\begin{tabular}{|c|c|c|c|c|c|c|c|c|c|c|c|}
\hline Electror & & & & & $=5$ & $\rho=1$ & $A^{1 / 3} F$ & & & & \\
\hline$p$ & $F_{0}$ & & $f_{1}$ & & $g_{\mathrm{I}}$ & $\tan \delta_{1}$ & $f_{-1}$ & $g_{-1}$ & & $\tan \delta_{-1}$ & \\
\hline $\begin{array}{l}6.10 \\
6.20 \\
6.30 \\
6.40 \\
6.50\end{array}$ & $\begin{array}{l}1.12797 \mathrm{E} \\
1.12790 \mathrm{E} \\
1.12782 \mathrm{E} \\
1.12775 \mathrm{E} \\
1.12768 \mathrm{E}\end{array}$ & $\begin{array}{l}00 \\
00 \\
00 \\
00 \\
00\end{array}$ & $\begin{array}{l}-5.92041 \mathrm{E} \\
-6.02617 \mathrm{E} \\
-6.13192 \mathrm{E} \\
-6.23767 \mathrm{E} \\
-6.34340 \mathrm{E}\end{array}$ & $\begin{array}{l}00 \\
00 \\
00 \\
00 \\
00\end{array}$ & $\begin{array}{l}-1.84448 \mathrm{E}-01 \\
-1.89116 \mathrm{E}-01 \\
-1.93832 \mathrm{E}-01 \\
-1.98596 \mathrm{E}-01 \\
-2.03409 \mathrm{E}-01\end{array}$ & $\begin{array}{l}9.21537 \mathrm{E}-04 \\
9.68352 \mathrm{E}-04 \\
1.01370 \mathrm{E}-03 \\
1.05763 \mathrm{E}-03 \\
1.10022 \mathrm{E}-03\end{array}$ & $\begin{array}{l}-1.84974 \mathrm{E}-01 \\
-1.89399 \mathrm{E}-01 \\
-1.93873 \mathrm{E}-01 \\
-1.98394 \mathrm{E}-01 \\
-2.02964 \mathrm{E}-01\end{array}$ & $\begin{array}{l}6.96832 \mathrm{E} \\
7.07433 \mathrm{E} \\
7.18031 \mathrm{E} \\
7.28627 \mathrm{E} \\
7.39221 \mathrm{E}\end{array}$ & $\begin{array}{l}00 \\
00 \\
00 \\
00 \\
00\end{array}$ & $\begin{array}{l}-1.44912 \mathrm{E} \\
-1.45964 \mathrm{E} \\
-1.46996 \mathrm{E} \\
-1.48010 \mathrm{E} \\
-1.49006 \mathrm{E}\end{array}$ & $\begin{array}{l}02 \\
02 \\
02 \\
02 \\
02\end{array}$ \\
\hline $\begin{array}{l}6.60 \\
6.70 \\
6.80 \\
6.90 \\
7.00\end{array}$ & $\begin{array}{l}1.12762 \mathrm{E} \\
1.12755 \mathrm{E} \\
1.12749 \mathrm{E} \\
1.12743 \mathrm{E} \\
1.12737 \mathrm{E}\end{array}$ & $\begin{array}{l}00 \\
00 \\
00 \\
00 \\
00\end{array}$ & $\begin{array}{l}-6.44912 \mathrm{E} \\
-6.55484 \mathrm{E} \\
-6.66054 \mathrm{E} \\
-6.76623 \mathrm{E} \\
-6.87191 \mathrm{E}\end{array}$ & $\begin{array}{l}00 \\
00 \\
00 \\
00 \\
00\end{array}$ & $\begin{array}{l}-2.08270 \mathrm{E}-01 \\
-2.13180 \mathrm{E}-01 \\
-2.18139 \mathrm{E}-01 \\
-2.23145 \mathrm{E}-01 \\
-2.28200 \mathrm{E}-01\end{array}$ & $\begin{array}{l}1.14151 \mathrm{E}-03 \\
1.18158 \mathrm{E}-03 \\
1.22047 \mathrm{E}-03 \\
1.25824 \mathrm{E}-03 \\
1.29493 \mathrm{E}-03\end{array}$ & $\begin{array}{l}-2.07583 \mathrm{E}-01 \\
-2.12249 \mathrm{E}-01 \\
-2.16964 \mathrm{E}-01 \\
-2.21728 \mathrm{E}-01 \\
-2.26540 \mathrm{E}-01\end{array}$ & $\begin{array}{l}7.49813 \mathrm{E} \\
7.60402 \mathrm{E} \\
7.70990 \mathrm{E} \\
7.81575 \mathrm{E} \\
7.92158 \mathrm{E}\end{array}$ & $\begin{array}{l}00 \\
00 \\
00 \\
00 \\
00\end{array}$ & $\begin{array}{l}-1.49984 \mathrm{E} \\
-1.50946 \mathrm{E} \\
-1.51891 \mathrm{E} \\
-1.52821 \mathrm{E} \\
-1.53734 \mathrm{E}\end{array}$ & $\begin{array}{l}02 \\
02 \\
02 \\
02 \\
02\end{array}$ \\
\hline $\begin{array}{l}7.10 \\
7.20 \\
7.30 \\
7.40 \\
7.50\end{array}$ & $\begin{array}{l}1.12731 \mathrm{E} \\
1.12726 \mathrm{E} \\
1.12721 \mathrm{E} \\
1.12716 \mathrm{E} \\
1.12711 \mathrm{E}\end{array}$ & $\begin{array}{l}00 \\
00 \\
00 \\
00 \\
00\end{array}$ & $\begin{array}{l}-6.97758 \mathrm{E} \\
-7.08324 \mathrm{E} \\
-7.18889 \mathrm{E} \\
-7.29453 \mathrm{E} \\
-7.40016 \mathrm{E}\end{array}$ & $\begin{array}{l}00 \\
00 \\
00 \\
00 \\
00\end{array}$ & $\begin{array}{l}-2.33304 \mathrm{E}-01 \\
-2.38456 \mathrm{E}-01 \\
-2.43656 \mathrm{E}-01 \\
-2.48905 \mathrm{E}-01 \\
-2.54202 \mathrm{E}-01\end{array}$ & $\begin{array}{l}1.33059 \mathrm{E}-03 \\
1.36525 \mathrm{E}-03 \\
1.39896 \mathrm{E}-03 \\
1.43176 \mathrm{E}-03 \\
1.46369 \mathrm{E}-03\end{array}$ & $\begin{array}{l}-2.31400 E-01 \\
-2.36308 E-01 \\
-2.41265 E-01 \\
-2.46270 E-01 \\
-2.51324 E-01\end{array}$ & $\begin{array}{l}8.02740 \mathrm{E} \\
8.13319 \mathrm{E} \\
8.23897 \mathrm{E} \\
8.34473 \mathrm{E} \\
8.45047 \mathrm{E}\end{array}$ & $\begin{array}{l}00 \\
00 \\
00 \\
00 \\
00\end{array}$ & $\begin{array}{l}-1.54633 \mathrm{E} \\
-1.55517 \mathrm{E} \\
-1.56386 \mathrm{E} \\
-1.57241 \mathrm{E} \\
-1.58083 \mathrm{E}\end{array}$ & $\begin{array}{l}02 \\
02 \\
02 \\
02 \\
02\end{array}$ \\
\hline $\begin{array}{l}7.60 \\
7.70 \\
7.80 \\
7.90 \\
8.00\end{array}$ & $\begin{array}{l}1.12706 \mathrm{E} \\
1.12701 \mathrm{E} \\
1.12696 \mathrm{E} \\
1.12692 \mathrm{E} \\
1.12688 \mathrm{E}\end{array}$ & $\begin{array}{l}00 \\
00 \\
00 \\
00 \\
00\end{array}$ & $\begin{array}{l}-7.50577 \mathrm{E} \\
-7.61138 \mathrm{E} \\
-7.71698 \mathrm{E} \\
-7.82256 \mathrm{E} \\
-7.92813 \mathrm{E}\end{array}$ & $\begin{array}{l}00 \\
00 \\
00 \\
00 \\
00\end{array}$ & $\begin{array}{l}-2.59548 \mathrm{E}-01 \\
-2.64942 \mathrm{E}-01 \\
-2.70384 \mathrm{E}-01 \\
-2.75875 \mathrm{E}-01 \\
-2.81414 \mathrm{E}-01\end{array}$ & $\begin{array}{l}1.49477 \mathrm{E}-03 \\
1.52504 \mathrm{E}-03 \\
1.55453 \mathrm{E}-03 \\
1.58327 \mathrm{E}-03 \\
1.61129 \mathrm{E}-03\end{array}$ & $\begin{array}{l}-2.56426 \mathrm{E}-01 \\
-2.61576 \mathrm{E}-01 \\
-2.66775 \mathrm{E}-01 \\
-2.72021 \mathrm{E}-01 \\
-2.77317 \mathrm{E}-01\end{array}$ & $\begin{array}{l}8.55619 \mathrm{E} \\
8.66190 \mathrm{E} \\
8.76759 \mathrm{E} \\
8.87326 \mathrm{E} \\
8.97892 \mathrm{E}\end{array}$ & $\begin{array}{l}00 \\
00 \\
00 \\
00 \\
00\end{array}$ & $\begin{array}{l}-1.58911 \mathrm{E} \\
-1.59726 \mathrm{E} \\
-1.60529 \mathrm{E} \\
-1.61319 \mathrm{E} \\
-1.62097 \mathrm{E}\end{array}$ & $\begin{array}{l}02 \\
02 \\
02 \\
02 \\
02\end{array}$ \\
\hline $\begin{array}{l}8.10 \\
8.20 \\
8.30 \\
8.40 \\
8.50\end{array}$ & $\begin{array}{l}1.12683 \mathrm{E} \\
1.12679 \mathrm{E} \\
1.12675 \mathrm{E} \\
1.12671 \mathrm{E} \\
1.12668 \mathrm{E}\end{array}$ & $\begin{array}{l}00 \\
00 \\
00 \\
00 \\
00\end{array}$ & $\begin{array}{l}-8.03370 \mathrm{E} \\
-8.13925 \mathrm{E} \\
-8.24479 \mathrm{E} \\
-8.35032 \mathrm{E} \\
-8.45583 \mathrm{E}\end{array}$ & $\begin{array}{l}00 \\
00 \\
00 \\
00 \\
00\end{array}$ & $\begin{array}{l}-2.87002 \mathrm{E}-01 \\
-2.92638 \mathrm{E}-01 \\
-2.98322 \mathrm{E}-01 \\
-3.04055 \mathrm{E}-01 \\
-3.09836 \mathrm{E}-01\end{array}$ & $\begin{array}{l}1.63861 \mathrm{E}-03 \\
1.66527 \mathrm{E}-03 \\
1.69127 \mathrm{E}-03 \\
1.71665 \mathrm{E}-03 \\
1.74143 \mathrm{E}-03\end{array}$ & $\begin{array}{l}-2.82660 \mathrm{E}-01 \\
-2.88052 \mathrm{E}-01 \\
-2.93493 \mathrm{E}-01 \\
-2.98981 \mathrm{E}-01 \\
-3.04518 \mathrm{E}-01\end{array}$ & $\begin{array}{l}9.08456 \mathrm{E} \\
9.19018 \mathrm{E} \\
9.29579 \mathrm{E} \\
9.40138 \mathrm{E} \\
9.50695 \mathrm{E}\end{array}$ & $\begin{array}{l}00 \\
00 \\
00 \\
00 \\
00\end{array}$ & $\begin{array}{l}-1.62863 \mathrm{E} \\
-1.63618 \mathrm{E} \\
-1.64361 \mathrm{E} \\
-1.65093 \mathrm{E} \\
-1.65815 \mathrm{E}\end{array}$ & $\begin{array}{l}02 \\
02 \\
02 \\
02 \\
02\end{array}$ \\
\hline $\begin{array}{l}8.60 \\
8.70 \\
8.80 \\
8.90 \\
9.00\end{array}$ & $\begin{array}{l}1.12664 \mathrm{E} \\
1.12660 \mathrm{E} \\
1.12657 \mathrm{E} \\
1.12653 \mathrm{E} \\
1.12650 \mathrm{E}\end{array}$ & $\begin{array}{l}00 \\
00 \\
00 \\
00 \\
00\end{array}$ & $\begin{array}{l}-8.56134 \mathrm{E} \\
-8.66684 \mathrm{E} \\
-8.77232 \mathrm{E} \\
-8.87779 \mathrm{E} \\
-8.98326 \mathrm{E}\end{array}$ & $\begin{array}{l}00 \\
00 \\
00 \\
00 \\
00\end{array}$ & $\begin{array}{l}-3.15665 \mathrm{E}-01 \\
-3.21543 \mathrm{E}-01 \\
-3.27469 \mathrm{E}-01 \\
-3.33443 \mathrm{E}-01 \\
-3.39466 \mathrm{E}-01\end{array}$ & $\begin{array}{l}1.76562 \mathrm{E}-03 \\
1.78926 \mathrm{E}-03 \\
1.81234 \mathrm{E}-03 \\
1.83491 \mathrm{E}-03 \\
1.85696 \mathrm{E}-03\end{array}$ & $\begin{array}{l}-3.10104 \mathrm{E}-01 \\
-3.15737 \mathrm{E}-01 \\
-3.21419 \mathrm{E}-01 \\
-3.27149 \mathrm{E}-01 \\
-3.32928 \mathrm{E}-01\end{array}$ & $\begin{array}{l}9.61252 \mathrm{E} \\
9.71806 \mathrm{E} \\
9.82359 \mathrm{E} \\
9.92911 \mathrm{E} \\
1.00346 \mathrm{E}\end{array}$ & $\begin{array}{l}00 \\
00 \\
00 \\
00\end{array}$ & $\begin{array}{l}-1.66526 \mathrm{E} \\
-1.67227 \mathrm{E} \\
-1.67918 \mathrm{E} \\
-1.68600 \mathrm{E} \\
-1.69271 \mathrm{E}\end{array}$ & $\begin{array}{l}02 \\
02 \\
02 \\
02 \\
02\end{array}$ \\
\hline $\begin{array}{l}9.10 \\
9.20 \\
9.30 \\
9.40 \\
9.50\end{array}$ & $\begin{array}{l}1.12646 \mathrm{E} \\
1.12643 \mathrm{E} \\
1.12640 \mathrm{E} \\
1.12637 \mathrm{E} \\
1.12634 \mathrm{E}\end{array}$ & $\begin{array}{l}00 \\
00 \\
00 \\
00 \\
00\end{array}$ & $\begin{array}{l}-9.08871 \mathrm{E} \\
-9.19415 \mathrm{E} \\
-9.29957 \mathrm{E} \\
-9.40499 \mathrm{E} \\
-9.51039 \mathrm{E}\end{array}$ & $\begin{array}{l}00 \\
00 \\
00 \\
00 \\
00\end{array}$ & $\begin{array}{l}-3.45537 \mathrm{E}-01 \\
-3.51656 \mathrm{E}-01 \\
-3.57824 \mathrm{E}-01 \\
-3.64040 \mathrm{E}-01 \\
-3.70304 \mathrm{E}-01\end{array}$ & $\begin{array}{l}1.87853 \mathrm{E}-03 \\
1.89961 \mathrm{E}-03 \\
1.92024 \mathrm{E}-03 \\
1.94042 \mathrm{E}-03 \\
1.96017 \mathrm{E}-03\end{array}$ & $\begin{array}{l}-3.38755 \mathrm{E}-01 \\
-3.44630 \mathrm{E}-01 \\
-3.50554 \mathrm{E}-01 \\
-3.56525 \mathrm{E}-01 \\
-3.62545 \mathrm{E}-01\end{array}$ & $\begin{array}{l}1.01401 \mathrm{E} \\
1.02456 \mathrm{E} \\
1.03510 \mathrm{E} \\
1.04565 \mathrm{E} \\
1.05619 \mathrm{E}\end{array}$ & $\begin{array}{l}01 \\
01 \\
01 \\
01 \\
01\end{array}$ & $\begin{array}{l}-1.69934 \mathrm{E} \\
-1.70587 \mathrm{E} \\
-1.71231 \mathrm{E} \\
-1.71867 \mathrm{E} \\
-1.72494 \mathrm{E}\end{array}$ & $\begin{array}{l}02 \\
02 \\
02 \\
02 \\
02\end{array}$ \\
\hline $\begin{array}{r}9.60 \\
9.70 \\
9.80 \\
9.90 \\
10.00\end{array}$ & $\begin{array}{l}1.12631 \mathrm{E} \\
1.12628 \mathrm{E} \\
1.12625 \mathrm{E} \\
1.12622 \mathrm{E} \\
1.12619 \mathrm{E}\end{array}$ & $\begin{array}{l}00 \\
00 \\
00 \\
00 \\
00\end{array}$ & $\begin{array}{l}-9.61579 \mathrm{E} \\
-9.72117 \mathrm{E} \\
-9.82654 \mathrm{E} \\
-9.93190 \mathrm{E} \\
-1.00372 \mathrm{E}\end{array}$ & $\begin{array}{l}00 \\
00 \\
00 \\
00 \\
01\end{array}$ & $\begin{array}{l}-3.76617 \mathrm{E}-01 \\
-3.82977 \mathrm{E}-01 \\
-3.89386 \mathrm{E}-01 \\
-3.95844 \mathrm{E}-01 \\
-4.02350 \mathrm{E}-01\end{array}$ & $\begin{array}{l}1.97950 \mathrm{E}-03 \\
1.99842 \mathrm{E}-03 \\
2.01695 \mathrm{E}-03 \\
2.03509 \mathrm{E}-03 \\
2.05287 \mathrm{E}-03\end{array}$ & $\begin{array}{l}-3.68614 \mathrm{E}-01 \\
-3.74730 \mathrm{E}-01 \\
-3.80895 \mathrm{E}-01 \\
-3.87108 \mathrm{E}-01 \\
-3.93370 \mathrm{E}-01\end{array}$ & $\begin{array}{l}1.06673 \mathrm{E} \\
1.07727 \mathrm{E} \\
1.08781 \mathrm{E} \\
1.09835 \mathrm{E} \\
1.10888 \mathrm{E}\end{array}$ & $\begin{array}{l}01 \\
01 \\
01 \\
01 \\
01\end{array}$ & $\begin{array}{l}-1.73112 \mathrm{E} \\
-1.73723 \mathrm{E} \\
-1.74325 \mathrm{E} \\
-1.74920 \mathrm{E} \\
-1.75507 \mathrm{E}\end{array}$ & $\begin{array}{l}02 \\
02 \\
02 \\
02 \\
02\end{array}$ \\
\hline $\begin{array}{l}10.10 \\
10.20 \\
10.30 \\
10.40 \\
10.50\end{array}$ & $\begin{array}{l}1.12617 \mathrm{E} \\
1.12614 \mathrm{E} \\
1.12611 \mathrm{E} \\
1.12609 \mathrm{E} \\
1.12606 \mathrm{E}\end{array}$ & $\begin{array}{r}00 \\
00 \\
00 \\
00 \\
00\end{array}$ & $\begin{array}{l}-1.01426 \mathrm{E} \\
-1.02479 \mathrm{E} \\
-1.03532 \mathrm{E} \\
-1.04585 \mathrm{E} \\
-1.05638 \mathrm{E}\end{array}$ & $\begin{array}{l}01 \\
01 \\
01 \\
01 \\
01\end{array}$ & $\begin{array}{l}-4.08903 \mathrm{E}-01 \\
-4.15506 \mathrm{E}-01 \\
-4.22156 \mathrm{E}-01 \\
-4.28855 \mathrm{E}-01 \\
-4.35602 \mathrm{E}-01\end{array}$ & $\begin{array}{l}2.07028 \mathrm{E}-03 \\
2.08734 \mathrm{E}-03 \\
2.10406 \mathrm{E}-03 \\
2.12046 \mathrm{E}-03 \\
2.13653 \mathrm{E}-03\end{array}$ & $\begin{array}{l}-3.99679 \mathrm{E}-01 \\
-4.06037 \mathrm{E}-01 \\
-4.12444 \mathrm{E}-01 \\
-4.18898 \mathrm{E}-01 \\
-4.25401 \mathrm{E}-01\end{array}$ & $\begin{array}{l}1.11942 \mathrm{E} \\
1.12995 \mathrm{E} \\
1.14048 \mathrm{E} \\
1.15101 \mathrm{E} \\
1.16154 \mathrm{E}\end{array}$ & $\begin{array}{l}01 \\
01 \\
01 \\
01 \\
01\end{array}$ & $\begin{array}{l}-1.76086 \mathrm{E} \\
-1.76659 \mathrm{E} \\
-1.77224 \mathrm{E} \\
-1.77781 \mathrm{E} \\
-1.78332 \mathrm{E}\end{array}$ & $\begin{array}{l}02 \\
02 \\
02 \\
02 \\
02\end{array}$ \\
\hline $\begin{array}{l}10.60 \\
10.70 \\
10.80 \\
10.90 \\
11.00\end{array}$ & $\begin{array}{l}1.12604 \mathrm{E} \\
1.12601 \mathrm{E} \\
1.12599 \mathrm{E} \\
1.12597 \mathrm{E} \\
1.12594 \mathrm{E}\end{array}$ & $\begin{array}{l}00 \\
00 \\
00 \\
00 \\
00\end{array}$ & $\begin{array}{l}-1.06691 \mathrm{E} \\
-1.07744 \mathrm{E} \\
-1.08796 \mathrm{E} \\
-1.09848 \mathrm{E} \\
-1.10901 \mathrm{E}\end{array}$ & $\begin{array}{l}01 \\
01 \\
01 \\
01 \\
01\end{array}$ & $\begin{array}{l}-4.42397 \mathrm{E}-01 \\
-4.49240 \mathrm{E}-01 \\
-4.56132 \mathrm{E}-01 \\
-4.63072 \mathrm{E}-01 \\
-4.70060 \mathrm{E}-01\end{array}$ & $\begin{array}{l}2.15229 \mathrm{E}-03 \\
2.16774 \mathrm{E}-03 \\
2.18291 \mathrm{E}-03 \\
2.19779 \mathrm{E}-03 \\
2.21237 \mathrm{E}-03\end{array}$ & $\begin{array}{l}-4.31952 \mathrm{E}-01 \\
-4.38551 \mathrm{E}-01 \\
-4.45198 \mathrm{E}-01 \\
-4.51894 \mathrm{E}-01 \\
-4.58638 \mathrm{E}-01\end{array}$ & $\begin{array}{l}1.17207 \mathrm{E} \\
1.18259 \mathrm{E} \\
1.19312 \mathrm{E} \\
1.20364 \mathrm{E} \\
1.21417 \mathrm{E}\end{array}$ & $\begin{array}{l}01 \\
01 \\
01 \\
01 \\
01\end{array}$ & $\begin{array}{l}-1.78877 \mathrm{E} \\
-1.79414 \mathrm{E} \\
-1.79945 \mathrm{E} \\
-1.80470 \mathrm{E} \\
-1.80989 \mathrm{E}\end{array}$ & $\begin{array}{l}02 \\
02 \\
02 \\
02 \\
02\end{array}$ \\
\hline $\begin{array}{l}11.10 \\
11.20 \\
11.30 \\
11.40 \\
11.50\end{array}$ & $\begin{array}{l}1.12592 \mathrm{E} \\
1.12590 \mathrm{E} \\
1.12588 \mathrm{E} \\
1.12586 \mathrm{E} \\
1.12583 \mathrm{E}\end{array}$ & $\begin{array}{l}00 \\
00 \\
00 \\
00 \\
00\end{array}$ & $\begin{array}{l}-1.11953 \mathrm{E} \\
-1.13005 \mathrm{E} \\
-1.14057 \mathrm{E} \\
-1.15109 \mathrm{E} \\
-1.16161 \mathrm{E}\end{array}$ & $\begin{array}{l}01 \\
01 \\
01 \\
01 \\
01\end{array}$ & $\begin{array}{l}-4.77096 \mathrm{E}-01 \\
-4.84180 \mathrm{E}-01 \\
-4.91313 \mathrm{E}-01 \\
-4.98494 \mathrm{E}-01 \\
-5.05723 \mathrm{E}-01\end{array}$ & $\begin{array}{l}2.22669 \mathrm{E}-03 \\
2.24076 \mathrm{E}-03 \\
2.25456 \mathrm{E}-03 \\
2.26810 \mathrm{E}-03 \\
2.28140 \mathrm{E}-03\end{array}$ & $\begin{array}{l}-4.65430 \mathrm{E}-01 \\
-4.72270 \mathrm{E}-01 \\
-4.79158 \mathrm{E}-01 \\
-4.86095 \mathrm{E}-01 \\
-4.93080 \mathrm{E}-01\end{array}$ & $\begin{array}{l}1.22469 \mathrm{E} \\
1.23521 \mathrm{E} \\
1.24572 \mathrm{E} \\
1.25624 \mathrm{E} \\
1.26676 \mathrm{E}\end{array}$ & $\begin{array}{l}01 \\
01 \\
01 \\
01 \\
01\end{array}$ & $\begin{array}{l}-1.81501 \mathrm{E} \\
-1.82008 \mathrm{E} \\
-1.82508 \mathrm{E} \\
-1.83003 \mathrm{E} \\
-1.83492 \mathrm{E}\end{array}$ & $\begin{array}{l}02 \\
02 \\
02 \\
02 \\
02\end{array}$ \\
\hline $\begin{array}{l}11.60 \\
11.70 \\
11.80 \\
11.90 \\
12.00\end{array}$ & $\begin{array}{l}1.12581 \mathrm{E} \\
1.12579 \mathrm{E} \\
1.12577 \mathrm{E} \\
1.12575 \mathrm{E} \\
1.12573 \mathrm{E}\end{array}$ & $\begin{array}{l}00 \\
00 \\
00 \\
00 \\
00\end{array}$ & $\begin{array}{l}-1.17212 \mathrm{E} \\
-1.18264 \mathrm{E} \\
-1.19315 \mathrm{E} \\
-1.20366 \mathrm{E} \\
-1.21417 \mathrm{E}\end{array}$ & $\begin{array}{l}01 \\
01 \\
01 \\
01 \\
01\end{array}$ & $\begin{array}{l}-5.13000 \mathrm{E}-01 \\
-5.20325 \mathrm{E}-01 \\
-5.27699 \mathrm{E}-01 \\
-5.35120 \mathrm{E}-01 \\
-5.42590 \mathrm{E}-01\end{array}$ & $\begin{array}{l}2.29446 \mathrm{E}-03 \\
2.30729 \mathrm{E}-03 \\
2.31989 \mathrm{E}-03 \\
2.33227 \mathrm{E}-03 \\
2.34444 \mathrm{E}-03\end{array}$ & $\begin{array}{l}-5.00113 \mathrm{E}-01 \\
-5.07194 \mathrm{E}-01 \\
-5.14323 \mathrm{E}-01 \\
-5.21500 \mathrm{E}-01 \\
-5.28726 \mathrm{E}-01\end{array}$ & $\begin{array}{l}1.27727 \mathrm{E} \\
1.28778 \mathrm{E} \\
1.29829 \mathrm{E} \\
1.30880 \mathrm{E} \\
1.31931 \mathrm{E}\end{array}$ & $\begin{array}{l}01 \\
01 \\
01 \\
01 \\
01\end{array}$ & $\begin{array}{l}-1.83976 \mathrm{E} \\
-1.84454 \mathrm{E} \\
-1.84927 \mathrm{E} \\
-1.85395 \mathrm{E} \\
-1.85858 \mathrm{E}\end{array}$ & $\begin{array}{l}02 \\
02 \\
02 \\
02 \\
02\end{array}$ \\
\hline
\end{tabular}


$\bar{F}$

5.55881 E-0 05

$5.74223 \mathrm{E}-05$ $5.92862 \mathrm{E}-05$

$6.11799 \mathrm{E}-05$

$6.31035 \mathrm{E}-05$

$6.50568 \mathrm{E}-05$ $6.70400 \mathrm{E}-05$ $6.90529 \mathrm{E}-05$ $7.10956 \mathrm{E}-05$

$7.31682 \mathrm{E}-05$

7.00

7.10

7.20

7.30

7.40

7.50

7.60

7.70

7.80

7.90

8.00

8.10

8.20

8.30

8.40

8.50

8.60

8.70

8.80

8.90

9.00

9.10

9.20

9.30

9.40

9.50

9.60

9.70

9.80

9.90

10.00

10.10

10.20

10.30

10.40

10.50

10.60

10.70

10.80

10.90

11.00

11.10

11.20

11.30

11.40

11.50

11.60

11.70

11.80

11.90

12.00
$7.52705 \mathrm{E}-05$

$7.74026 \mathrm{E}-05$

$7.95646 \mathrm{E}-05$

$8.17563 \mathrm{E}-05$

$8.39779 \mathrm{E}-05$

$8.62292 \mathrm{E}-05$

$8.85103 \mathrm{E}-05$

$9.08213 \mathrm{E}-05$

$9.31620 \mathrm{E}-05$

$9.55325 \mathrm{E}-05$

$9.79329 \mathrm{E}-05$

$1.00363 \mathrm{E}-04$

$1.02823 \mathrm{E}-04$

$1.05313 \mathrm{E}-04$

$1.07832 \mathrm{E}-04$

$1.10381 \mathrm{E}-04$

$1.12961 \mathrm{E}-04$

$1.15569 \mathrm{E}-04$

$1.18208 \mathrm{E}-04$

$1.20877 \mathrm{E}-04$

$1.23575 \mathrm{E}-04$

$1.26303 \mathrm{E}-04$

$1.29061 \mathrm{E}-04$

$1.31849 \mathrm{E}-04$

$1.34666 \mathrm{E}-04$

$1.37513 \mathrm{E}-04$

$1.40390 \mathrm{E}-04$

$1.43297 \mathrm{E}-04$

$1.46234 \mathrm{E}-04$

$1.49200 \mathrm{E}-04$

$1.52197 \mathrm{E}-04$

$1.55223 \mathrm{E}-04$

$1.58278 \mathrm{E}-04$

$1.61364 \mathrm{E}-04$

$1.64479 \mathrm{E}-04$

$1.67624 \mathrm{E}-04$ $1.70799 \mathrm{E}-04$

$1.74004 \mathrm{E}-04$

$1.77239 \mathrm{E}-04$

$1.80503 \mathrm{E}-04$

$1.83797 \mathrm{E}-04$ $1.87121 \mathrm{E}-04$

$1.90475 \mathrm{E}-04$

$1.93859 \mathrm{E}-04$

$1.97272 \mathrm{E}-04$

$2.00715 \mathrm{E}-04$

$2.04188 \mathrm{E}-04$

$2.07691 \mathrm{E}-04$

$2.11223 \mathrm{E}-04$

$2.14785 \mathrm{E}-04$ $f_{2}$

$-8.32020 \mathrm{E}-02$

$-8.60785 \mathrm{E}-02$

$-8.90037 \mathrm{E}-02$

$-9.19777 \mathrm{E}-02$

$-9.50003 \mathrm{E}-02$

$-9.80717 \mathrm{E}-02$

$-1.01192 \mathrm{E}-01$

$-1.04360 \mathrm{E}-01$

$-1.07578 \mathrm{E}-01$

$-1.10844 \mathrm{E}-01$

$-1.14159 \mathrm{E}-01$

$-1.17522 \mathrm{E}-01$

$-1.20934 \mathrm{E}-01$

$-1.24395 \mathrm{E}-01$

$-1.27904 \mathrm{E}-01$

$-1.31462 \mathrm{E}-01$

$-1.35069 \mathrm{E}-01$

$-1.38724 \mathrm{E}-01$

$-1.42428 \mathrm{E}-01$

$-1.46181 \mathrm{E}-01$

$-1.49982 \mathrm{E}-01$

$-1.53832 \mathrm{E}-01$

$-1.57730 \mathrm{E}-01$

$-1.61677 \mathrm{E}-01$

$-1.65673 \mathrm{E}-01$

$-1.69717 \mathrm{E}-01$

$-1.73810 \mathrm{E}-01$

$-1.77951 \mathrm{E}-01$

$-1.82141 \mathrm{E}-01$

$-1.86380 \mathrm{E}-01$

$-1.90667 \mathrm{E}-01$

$-1.95003 \mathrm{E}-01$

$-1.99387 \mathrm{E}-01$

$-2.03820 \mathrm{E}-01$

$-2.08302 \mathrm{E}-01$

$-2.12831 \mathrm{E}-01$

$-2.17410 \mathrm{E}-01$

$-2.22037 \mathrm{E}-01$

$-2.26713 \mathrm{E}-01$

$-2.31437 \mathrm{E}-01$

$-2.36209 \mathrm{E}-01$

$-2.41031 \mathrm{E}-01$

$-2.45900 \mathrm{E}-01$

$-2.50818 \mathrm{E}-01$

$-2.55785 \mathrm{E}-01$

$-2.60800 \mathrm{E}-01$

$-2.65864 \mathrm{E}-01$

$-2.70976 \mathrm{E}-01$

$-2.76137 \mathrm{E}-01$

$-2.81346 \mathrm{E}-01$

$-2.86604 \mathrm{E}-01$

$-2.91910 \mathrm{E}-01$

$-2.97264 \mathrm{E}-01$

$-3.02667 \mathrm{E}-01$

$-3.08119 \mathrm{E}-01$

$-3.13619 \mathrm{E}-01$

$-3.19167 \mathrm{E}-01$

$-3.24764 \mathrm{E}-01$

$-3.30409 \mathrm{E}-01$

$-3.36103 \mathrm{E}-01$ $g_{2}$

$-1.52010 \mathrm{E}-03$

$-1.58441 \mathrm{E}-03$

$-1.65041 \mathrm{E}-03$

$-1.71812 \mathrm{E}-03$

$-1.78757 \mathrm{E}-03$

$-1.85877 \mathrm{E}-03$

$-1.93175 \mathrm{E}-03$

$-2.00652 \mathrm{E}-03$

$-2.08310 \mathrm{E}-03$

$-2.16152 \mathrm{E}-03$

$-2.24180 \mathrm{E}-03$

$-2.32395 \mathbf{E}-03$

$-2.40799 \mathrm{E}-03$

$-2.49395 \mathrm{E}-03$

$-2.58184 \mathrm{E}-03$

$-2.67169 \mathrm{E}-03$

$-2.76352 \mathrm{E}-03$

$-2.85734 \mathrm{E}-03$

$-2.95318 \mathrm{E}-03$

$-3.05105 \mathrm{E}-03$

$-3.15098 \mathrm{E}-03$

$-3.25298 \mathrm{E}-03$

$-3.35709 \mathrm{E}-03$

$-3.46330 \mathrm{E}-03$

$-3.57166 \mathrm{E}-03$

$-3.68217 \mathbf{E}-03$

$-3.79485 \mathrm{E}-03$

$-3.90973 \mathrm{E}-03$

$-4.02683 \mathrm{E}-03$

$-4.14617 \mathrm{E}-03$

$-4.26776 \mathrm{E}-03$

$-4.39163 \mathrm{E}-03$

$-4.51780 \mathrm{E}-03$

$-4.64628 \mathrm{E}-03$

$-4.77710 \mathrm{E}-03$

$-4.91028 \mathrm{E}-03$

$-5.04583 \mathrm{E}-03$

$-5.18379 \mathrm{E}-03$

$-5.32416 \mathrm{E}-03$

$-5.46697 \mathrm{E}-03$

$-5.61223 \mathrm{E}-03$

$-5.75997 \mathrm{E}-03$

$-5.91021 \mathrm{E}-03$

$-6.06297 \mathrm{E}-03$

$-6.21826 \mathrm{E}-03$

$-6.37612 \mathrm{E}-03$

$-6.53655 \mathrm{E}-03$

$-6.69958 \mathrm{E}-03$

$-6.86522 \mathrm{E}-03$

$-7.03351 \mathrm{E}-03$

$-7.20445 \mathrm{E}-03$

$-7.37807 \mathrm{E}-03$

$-7.55439 \mathbf{E}-03$

$-7.73343 \mathrm{E}-03$

$-7.91520 \mathrm{E}-03$

$-8.09974 \mathrm{E}-03$

$-8.28705 \mathrm{E}-03$

$-8.47716 \mathrm{E}-03$

$-8.67008 \mathrm{E}-03$

$-8.86585 \mathrm{E}-03$

$\tan \delta_{2}$

$f_{2}$

$3.86894 \mathrm{E} \quad 01$

$3.87412 \mathrm{E} 01$

$3.87913 \mathrm{E} \quad 01$

$3.88397 \mathrm{E} \quad 01$

$3.88865 \mathrm{E} \quad 01$

$3.89318 \mathrm{E} \quad 01$

$3.89756 \mathrm{E} \quad 01$

$3.90181 \mathrm{E} \quad 01$

$3.90593 \mathrm{E} \quad 01$

$3.90992 \mathrm{E} \quad 0$

$3.91379 \mathrm{E} \quad 01$

$3.91755 \mathrm{E} \quad 01$

$3.92120 \mathrm{E} \quad 01$

$3.92474 \mathrm{E} \quad 01$

$3.92819 \mathrm{E} 01$

$3.93153 \mathrm{E} \quad 01$

$3.93479 \mathrm{E} \quad 01$

$3.93795 \mathrm{E}$ ol

$3.94103 \mathrm{E} \quad 01$

$3.94403 \mathrm{E} \quad 01$

$3.94695 \mathrm{E} \quad 01$

$3.94980 \mathrm{E} \quad 01$

$3.95257 \mathrm{E} 01$

$3.95527 \mathrm{E} \quad 01$

$3.95790 \mathrm{E} 01$

$3.96047 \mathrm{E} \quad 01$

$3.96298 \mathrm{E} \quad 01$

$3.96543 \mathrm{E} 01$

$3.96781 \mathrm{E} \quad 01$

$3.97015 \mathrm{E} 01$

$3.97242 \mathrm{E} \quad 01$

$3.97465 \mathrm{E} \quad 01$

$3.97682 \mathrm{E} \quad 01$

$3.97895 \mathrm{E} \quad 0$

$3.98102 \mathrm{E} \quad 01$

$3.98306 \mathrm{E} \quad 01$

$3.98504 \mathrm{E} \quad 01$

$3.98699 \mathrm{E} \quad 01$

$3.98889 \mathrm{E} \quad 01$

$3.99075 \mathrm{E} \quad 01$

$3.99258 \mathrm{E} \quad 01$

$3.99436 \mathrm{E} 01$

$3.99611 \mathrm{E} \quad 01$

$3.99783 \mathrm{E} 01$

$3.99950 \mathrm{E} \quad 01$

$4.00115 \mathrm{E} \quad 01$

$4.00276 \mathrm{E} 01$

4.00434 E 01

$4.00590 \mathrm{E} \quad 01$

$4.00742 \mathrm{E} \quad 01$

$4.00891 \mathrm{E} \quad 01$ 
ELECTRON RADIAL FUNCTIONS

Electron

\begin{tabular}{|c|c|c|c|c|c|}
\hline$p$ & $F_{0}$ & & $f_{1}$ & & $g_{1}$ \\
\hline 12.10 & $1.12571 \mathrm{E}$ & 00 & $-1.22468 \mathrm{E}$ & 01 & $-5.50108 \mathrm{E}-$ \\
\hline 12.20 & $.12569 \mathrm{E}$ & 00 & $-1.23519 \mathrm{E}$ & 01 & -5.5767 \\
\hline 12.30 & $1.12567 \mathrm{E}$ & 00 & $-1.24570 \mathrm{E}$ & 01 & $5.65289 \mathrm{I}$ \\
\hline 12.40 & $.12566 \mathrm{E}$ & 00 & $-1.25620 \mathrm{E}$ & 01 & $-5.72951 \mathrm{~F}$ \\
\hline 2.50 & $.12564 \mathrm{E}$ & 00 & $-1.26671 \mathrm{E}$ & 01 & $-5.80662 \mathrm{E}$ \\
\hline 12.60 & $1.12562 \mathrm{E}$ & 00 & $-1.27721 \mathrm{E}$ & 01 & $-5.88420 \mathrm{E}-01$ \\
\hline 12.70 & $1.12560 \mathrm{E}$ & 00 & $28772 \mathrm{E}$ & 01 & $-5.96227 \mathrm{E}$ \\
\hline 2.80 & $558 \mathrm{E}$ & 00 & $-1.29822 \mathrm{E}$ & 01 & $-6.04081 \mathrm{E}$ \\
\hline 2.90 & $557 \mathrm{E}$ & 00 & $-1.30872 \mathrm{E}$ & 01 & $-6.11984 \mathrm{~F}$ \\
\hline 3.00 & $555 \mathrm{E}$ & 00 & $-1.31922 \mathrm{E}$ & 01 & $-6.19935 \mathrm{E}-01$ \\
\hline 13.10 & $.12553 \mathrm{E}$ & 00 & $-1.32971 \mathrm{E}$ & 01 & $-6.27934 \mathrm{E}-01$ \\
\hline 3.20 & $51 \mathrm{E}$ & 00 & $34021 \mathrm{E}$ & 01 & $-6.35981 \mathrm{E}-01$ \\
\hline 30 & $550 \mathrm{E}$ & 00 & $-1.35070 \mathrm{E}$ & 01 & $-6.44076 \mathrm{E}-$ \\
\hline .40 & $548 \mathrm{E}$ & 00 & $36120 \mathrm{E}$ & 01 & $-6.52220 \mathrm{E}-$ \\
\hline 13.50 & $64 \mathrm{E}$ & 00 & $7169 \mathrm{E}$ & 01 & $-6.60411 \mathrm{E}-01$ \\
\hline 3.60 & $12545 \mathrm{E}$ & 00 & $-1.38218 \mathrm{E}$ & 01 & -6.686501 \\
\hline 3.70 & $543 \mathrm{E}$ & 00 & $39267 \mathrm{E}$ & 01 & $-6.76937 \mathrm{E}$ \\
\hline .80 & $542 \mathrm{E}$ & 00 & $40316 \mathrm{E}$ & 01 & $-6.85272 \mathrm{E}-01$ \\
\hline 90 & $40 \mathrm{E}$ & 00 & $1365 \mathrm{E}$ & 01 & -6.93656 \\
\hline 4.00 & $39 \mathrm{E}$ & 00 & $2413 \mathrm{E}$ & 01 & -01 \\
\hline 1.10 & $12537 \mathrm{E}$ & 00 & $-1.43462 \mathrm{E}$ & 01 & -7 \\
\hline 1.20 & $36 \mathrm{E}$ & 00 & $4510 \mathrm{E}$ & $0]$ & -7.190941 \\
\hline .30 & $34 \mathrm{E}$ & 00 & $5558 \mathrm{E}$ & 01 & -7 \\
\hline 4.40 & $3 \mathrm{E}$ & 00 & $6606 \mathrm{E}$ & 01 & -7 \\
\hline 14.50 & $31 \mathrm{E}$ & 00 & $7654 \mathrm{E}$ & 01 & -7 \\
\hline 1.60 & $30 \mathrm{E}$ & 00 & $8702 \mathrm{E}$ & 01 & $E-01$ \\
\hline & $28 \mathrm{E}$ & 00 & $750 \mathrm{E}$ & 01 & -7 \\
\hline & & 00 & & 01 & -7 \\
\hline 4.90 & $526 \mathrm{E}$ & 00 & $1845 \mathrm{E}$ & 01 & $-7.80129 \mathrm{E}$ \\
\hline 15.00 & $524 \mathrm{E}$ & 00 & $892 \mathrm{E}$ & 01 & -7.890401 \\
\hline 15.10 & $23 \mathrm{E}$ & 00 & $9 \mathrm{E}$ & 01 & -7 \\
\hline 5.20 & & 00 & & 01 & -8 \\
\hline 15.30 & $520 E$ & 00 & $6033 \mathrm{E}$ & 01 & $-8.16060 \mathrm{E}$ \\
\hline 15.40 & $519 E$ & 00 & $57080 \mathrm{E}$ & 01 & $-8.25163 \mathrm{E}$ \\
\hline 15.50 & $18 \mathrm{E}$ & 00 & $8127 \mathrm{E}$ & 01 & -8.34313 \\
\hline 15.60 & $16 \mathrm{E}$ & 00 & $73 \mathrm{E}$ & 01 & -8 \\
\hline 15.70 & $15 \mathrm{E}$ & 00 & $0219 \mathrm{E}$ & 01 & -8.52758 \\
\hline 15.80 & $514 E$ & 00 & $61266 \mathrm{E}$ & 01 & $-8.62053 \mathrm{E}-$ \\
\hline 15.90 & $13 \mathrm{E}$ & 00 & $2312 \mathrm{E}$ & $0]$ & -8.71395 \\
\hline 16.00 & & 00 & & 01 & 800705 \\
\hline 6.10 & $12510 \mathrm{E}$ & 00 & $03 \mathrm{E}$ & 01 & -8 . \\
\hline & $609 \mathrm{E}$ & 00 & $65449 \mathrm{E}$ & 0] & $-8.99708 \mathrm{E}$ \\
\hline 16. & $608 \mathrm{E}$ & 00 & $66495 \mathrm{E}$ & 01 & $-9.09242 \mathrm{I}$ \\
\hline & & 00 & & 01 & \\
\hline 16.50 & $605 \mathrm{E}$ & 00 & $8585 \mathrm{E}$ & 01 & $-9.28452 \mathrm{E}-01$ \\
\hline 6.60 & & 00 & -1 & 01 & -9 \\
\hline & $3 \mathrm{E}$ & 00 & $0675 \mathrm{E}$ & 0 & -9 \\
\hline & & 00 & & 01 & \\
\hline & & 00 & & 01 & -9. \\
\hline 17.00 & & 00 & $.73809 \mathrm{E}$ & 01 & $-9.77315 \mathrm{H}$ \\
\hline 17.10 & & 00 & & 01 & $1 \mathrm{E}-01$ \\
\hline & & 00 & & 01 & $-9.97195 \mathrm{E}-01$ \\
\hline & $196 \mathrm{E}$ & 00 & & $0]$ & $-1.00721 \mathrm{E} \quad 0$ \\
\hline & $495 \mathrm{E}$ & 00 & $7986 \mathrm{E}$ & $0]$ & $-1.01727 \mathrm{E} \quad 0$ \\
\hline & $1.12494 \mathrm{E}$ & 00 & $79030 \mathrm{E}$ & 01 & $-1.02737 \mathrm{E} \quad 0$ \\
\hline 17.60 & $2493 \mathrm{E}$ & 00 & & 01 & $-1.03753 \mathrm{E} \quad 00$ \\
\hline & $12492 \mathrm{E}$ & 00 & $-1.81117 \mathrm{E}$ & 01 & $-1.04773 \mathrm{E}$ \\
\hline & $491 \mathrm{E}$ & 00 & $-1.82161 \mathrm{E}$ & $0]$ & $-1.05798 \mathrm{E}$ \\
\hline & $490 \mathrm{E}$ & $0 \mathrm{r}$ & $83204 \mathrm{E}$ & $0]$ & $-1.06828 \mathrm{E}$ \\
\hline 18.00 & $2489 \mathrm{E}$ & 00 & $-1.84247 \mathrm{E}$ & 01 & $-1.07862 \mathrm{E}$ \\
\hline
\end{tabular}

\begin{abstract}
$\tan \delta_{1}$
$2.35639 \mathrm{E}-03$

$2.36814 \mathrm{E}-03$

$2.37969 \mathrm{E}-03$

$2.39103 \mathrm{E}-03$

$2.40219 \mathrm{E}-03$

$2.41316 \mathrm{E}-03$

$2.42395 \mathrm{E}-03$

$2.43456 \mathrm{E}-03$

$2.44499 \mathrm{E}-03$

$2.45526 \mathrm{E}-03$

$2.46536 \mathrm{E}-03$

$2.47529 \mathrm{E}-03$

$2.48506 \mathrm{E}-03$

$2.49468 \mathrm{E}-03$

$2.50414 \mathrm{E}-03$

$2.51346 \mathrm{E}-03$

$2.52262 \mathrm{E}-03$

$2.53165 \mathrm{E}-03$

$2.54053 \mathrm{E}-03$

$2.54928 \mathrm{E}-03$

$f_{-1}$

$-5.36000 \mathrm{E}-01$

$-5.43322 \mathrm{E}-01$

$-5.50692 \mathrm{E}-01$

$-5.58110 \mathrm{E}-01$

$-5.65577 \mathrm{E}-01$

$-5.73091 \mathrm{E}-01$

$-5.80653 \mathrm{E}-01$

$-5.88264 \mathrm{E}-01$

$-5.95923 \mathrm{E}-01$

$-6.03630 \mathrm{E}-01$

$-6.11384 \mathrm{E}-01$

$-6.19187 \mathrm{E}-01$

$-6.27038 \mathrm{E}-01$

$-6.34938 \mathrm{E}-01$

$-6.42885 \mathrm{E}-01$

$-6.50880 \mathrm{E}-01$

$-6.58923 \mathrm{E}-01$

$-6.67014 \mathrm{E}-01$

$-6.75154 \mathrm{E}-01$

$-6.83341 \mathrm{E}-01$
\end{abstract}

$\rho=1.2 A^{1 / 3} F$
$2.55789 \mathrm{E}-03$

$2.56637 \mathrm{E}-03$

$2.57472 \mathrm{E}-03$

$2.58295 \mathrm{E}-03$

$2.59104 \mathrm{E}-03$

$2.59902 \mathrm{E}-03$

$2.60689 \mathrm{E}-03$

$2.61463 \mathrm{E}-03$

$2.62226 \mathrm{E}-03$

$2.62977 \mathrm{E}-03$

$2.63717 \mathrm{E}-03$

$2.64447 \mathrm{E}-03$

$2.65166 \mathrm{E}-03$

$2.65875 \mathrm{E}-03$

$2.66573 \mathrm{E}-03$

$2.67261 \mathrm{E}-03$

$2.67940 \mathrm{E}-03$

$2.68609 \mathrm{E}-03$

$2.69268 \mathrm{E}-03$

$2.69918 \mathrm{E}-03$

$2.70559 \mathrm{E}-03$

$2.71191 \mathrm{E}-03$

$2.71814 \mathrm{E}-03$

$2.72428 \mathrm{E}-03$

$2.73033 \mathrm{E}-03$

$2.73631 \mathrm{E}-03$ $2.74220 \mathrm{E}-03$

$2.74801 \mathrm{E}-03$

$2.75374 \mathrm{E}-03$

$2.75939 \mathrm{E}-03$

$2.76497 \mathrm{E}-03$ $2.77046 \mathrm{E}-03$ $2.77589 \mathrm{E}-03$ $2.78124 \mathrm{E}-03$ $2.78652 \mathrm{E}-03$

$2.79173 \mathrm{E}-03$ $2.79687 \mathrm{E}-03$ $2.80193 \mathrm{E}-03$ $2.80694 \mathrm{E}-03$ $2.81186 \mathrm{E}-03$
$-6.91576 \mathrm{E}-01$

$-6.99860 \mathrm{E}-01$

$-7.08191 \mathrm{E}-01$

$-7.16570 \mathrm{E}-01$

$-7.24998 \mathrm{E}-01$

$-7.33473 \mathrm{E}-01$

$-7.41996 \mathrm{E}-01$

$-7.50568 \mathrm{E}-01$

$-7.59187 \mathrm{E}-01$

$-7.67854 \mathrm{E}-01$

$-7.76569 \mathrm{E}-01$

$-7.85332 \mathrm{E}-01$

$-7.94143 \mathrm{E}-01$

$-8.03002 \mathrm{E}-01$

$-8.11909 \mathrm{E}-01$

$-8.20863 \mathrm{E}-01$

$-8.29866 \mathrm{E}-01$

$-8.38916 \mathrm{E}-01$

$-8.48015 \mathrm{E}-01$

$-8.57161 \mathrm{E}-01$

$-8.66355 \mathrm{E}-01$

$-8.75597 \mathrm{E}-01$

$-8.84887 \mathrm{E}-01$

$-8.94224 \mathrm{E}-01$

$-9.03610 \mathrm{E}-01$

$-9.13043 \mathrm{E}-01$

$-9.22524 \mathrm{E}-01$

$-9.32053 \mathrm{E}-01$

$-9.41630 \mathrm{E}-01$

$-9.51254 \mathrm{E}-01$

$-9.60927 \mathrm{E}-01$ $-9.70647 \mathrm{E}-01$ $-9.80415 \mathrm{E}-01$ $-9.90230 \mathrm{E}-01$ $-1.00009 \mathrm{E} 00$

$-1.01000 \mathrm{E} \quad 00$ $-1.01996 \mathrm{E} 00$ $-1.02997 \mathrm{E} 00$ $-1.04002 \mathrm{E} 00$ $-1.05013 \mathrm{E} 00$ g-1

$\tan \delta_{-1}$

$1.32982 \mathrm{E} \quad 01$ $1.34033 \mathrm{E} \quad 01$ $1.35083 \mathrm{E} \quad 01$

$1.36133 \mathrm{E} \quad 01$

$1.37184 \mathrm{E} \quad 01$

$1.38234 \mathrm{E} \quad 01$ $1.39284 \mathrm{E} \quad 01$ $1.40334 \mathrm{E} \quad 01$

$1.41383 \mathrm{E} \quad 01$

$1.42433 \mathrm{E} 01$

$1.43482 \mathrm{E} \quad 01$ $1.44531 \mathrm{E} \quad 01$ $1.45581 \mathrm{E} \quad 01$ $1.46630 \mathrm{E} 01$ $1.47678 \mathrm{E} \quad 01$

$\begin{array}{ll}1.48727 \mathrm{E} & 01\end{array}$ $1.49776 \mathrm{E} \quad 01$ $1.50824 \mathrm{E} \quad 01$

$1.51873 \mathrm{E} \quad 01$

$1.52921 \mathrm{E} \quad 01$

$1.53969 \mathrm{E} \quad 01$ $1.55017 \mathrm{E} \quad 01$ $1.56064 \mathrm{E} \quad 01$

$1.57112 \mathrm{E} \quad 01$

$1.58160 \mathrm{E} \quad 01$

$\begin{array}{ll}1.59207 \mathrm{E} & 01\end{array}$ $1.60254 \mathrm{E} \quad 01$

$1.61301 \mathrm{E} 01$ $1.62348 \mathrm{E} \quad 01$

$1.63395 \mathrm{E} 01$

$\begin{array}{ll}1.64442 \mathrm{E} & 01\end{array}$ $1.65488 \mathrm{E} \quad 01$

$1.66535 \mathrm{E} \quad 01$

$1.67581 \mathrm{E} 01$

$1.68627 \mathrm{E} \quad 01$

$1.69673 \mathrm{E} \quad 01$ $1.70719 \mathrm{E} \quad 01$ $1.71765 \mathrm{E} \quad 01$ $1.72810 \mathrm{E} \quad 01$ $1.73856 \mathrm{E} \quad 01$

$1.74901 \mathrm{E} \quad 01$ $1.75946 \mathrm{E} \quad 01$ $1.76991 \mathrm{E} 01$ $1.78036 \mathrm{E} \quad 01$ $1.79081 \mathrm{E} 01$

$1.80125 \mathrm{E} \quad 01$ $1.81170 \mathrm{E} \quad 01$ $1.82214 \mathrm{E} \quad 01$ $1.83258 \mathrm{E} \quad 01$ $1.84302 \mathrm{E} \quad 01$

$1.85346 \mathrm{E} \quad 01$ $1.86389 \mathrm{E} \quad 01$ $1.87433 \mathrm{E} \quad 01$ $1.88476 \mathrm{E} \quad 01$ $1.89520 \mathrm{E} \quad 01$

$1.90563 \mathrm{E} \quad 01$ $1.91606 \mathrm{E} \quad 01$ $1.92649 \mathrm{E} \quad 01$ $1.93691 \mathrm{E} 01$ $1.94734 \mathrm{E} \quad 01$
$-1.86315 \mathrm{E} \quad 02$ $-1.86768 \mathrm{E} 02$ $-1.87216 \mathrm{E} 02$ $-1.87659 \mathrm{E} \quad 02$ $-1.88097 \mathrm{E} 02$

$-1.88531 \mathrm{E} \quad 02$ $-1.88960 \mathrm{E} \quad 02$ $-1.89385 \mathrm{E} \quad 02$ $-1.89806 \mathrm{E} \quad 02$ $-1.90223 \mathrm{E} \quad 02$

$-1.90635 \mathrm{E} \quad 02$ $-1.91043 \mathrm{E} \quad 02$ $-1.91447 \mathrm{E} \quad 02$ $-1.91847 \mathrm{E} 02$ -1.92243 E 02

$\begin{array}{ll}-1.92636 \mathrm{E} & 02\end{array}$ $-1.93025 \mathrm{E} 02$ $-1.93410 \mathrm{E} 02$ $-1.93791 \mathrm{E} 02$ $-1.94169 \mathrm{E} 02$

$-1.94543 \mathrm{E} \quad 02$ $-1.94914 \mathrm{E} \quad 02$ $-1.95282 \mathrm{E} \quad 02$ $-1.95646 \mathrm{E} 02$ $-1.96007 \mathrm{E} \quad 02$

$-1.96365 \mathrm{E} 02$ $-1.96719 \mathrm{E} 02$ $-1.97071 \mathrm{E} 02$ $-1.97419 \mathrm{E} \quad 02$ $\begin{array}{ll}-1.97765 \mathrm{E} & 02\end{array}$

$-1.98107 \mathrm{E} \quad 02$ $-1.98447 \mathrm{E} \quad 02$ $-1.98784 \mathrm{E} 02$ $-1.99117 \mathrm{E} \quad 02$ $-1.99449 \mathrm{E} \quad 02$

$-1.99778 \mathrm{E} 02$ $-2.00103 \mathrm{E} \quad 02$ $-2.00427 \mathrm{E} \quad 02$ $-2.00747 \mathrm{E} \quad 02$ $-2.01065 \mathrm{E} \quad 02$

$-2.01381 \mathrm{E} \quad 02$ $-2.01694 \mathrm{E} \quad 02$ $-2.02005 \mathrm{E} \quad 02$ $-2.02313 \mathrm{E} 02$ $-2.02619 \mathrm{E} 02$

$-2.02923 \mathrm{E} \quad 02$ $-2.03224 \mathrm{E} \quad 02$ $-2.03523 \mathrm{E} \quad 02$ $-2.03821 \mathrm{E} \quad 02$ $-2.04115 \mathrm{E} \quad 02$

$\begin{array}{ll}-2.04408 \mathrm{E} & 02\end{array}$ $\begin{array}{ll}-2.04699 \mathrm{E} & 02\end{array}$ $\begin{array}{lll}-2.04987 \mathrm{E} & 02\end{array}$ $-2.05274 \mathrm{E} \quad 02$ $-2.05558 \mathrm{E} \quad 02$

$-2.05841 \mathrm{E} \cdot 02$ $-2.06121 \mathrm{E} \quad 02$ $-2.06400 \mathrm{E} \quad 02$ $-2.06677 \mathrm{E} \quad 02$ $\begin{array}{ll}-2.06952 \mathrm{E} & 02\end{array}$ 
$\bar{F}$

$2.18377 \mathrm{E}-04$ $2.21999 \mathrm{E}-04$ $2.25651 \mathrm{E}-04$ $2.29332 \mathrm{E}-04$ $2.33044 \mathrm{E}-04$

$2.36785 \mathrm{E}-04$ $2.40555 \mathrm{E}-04$ $2.44356 \mathrm{E}-04$ $2.48186 \mathrm{E}-04$ $2.52047 \mathrm{E}-04$

$2.55937 \mathrm{E}-04$ $2.59856 \mathrm{E}-04$ $2.63806 \mathrm{E}-04$ $2.67785 \mathrm{E}-04$ $2.71794 \mathrm{E}-04$

$2.75833 \mathrm{E}-04$ $2.79902 \mathrm{E}-04$ $2.84000 \mathrm{E}-04$ $2.88128 \mathrm{E}-04$ $2.92286 \mathrm{E}-04$

2.96474E-04 $3.00692 \mathrm{E}-04$ $3.04939 \mathrm{E}-04$ $3.09217 \mathrm{E}-04$ 3.13523 E-04

$3.17860 \mathrm{E}-04$ $3.22227 \mathrm{E}-04$ $3.26623 \mathrm{E}-04$ $3.31049 \mathrm{E}-04$ $3.35505 \mathrm{E}-04$

$3.39991 \mathrm{E}-04$ $3.44506 \mathrm{E}-04$ $3.49052 \mathrm{E}-04$ $3.53627 \mathrm{E}-04$ $3.58231 \mathrm{E}-04$

$3.62866 \mathrm{E}-04$ $3.67530 \mathrm{E}-04$ $3.72225 \mathrm{E}-04$ $3.76949 \mathrm{E}-04$ 3.81702 E-04

$3.86486 \mathrm{E}-04$ $3.91299 \mathrm{E}-04$ $3.96142 \mathrm{E}-04$ $4.01015 \mathrm{E}-04$ $4.05918 \mathrm{E}-04$

$4.10850 \mathrm{E}-04$ $4.15813 \mathrm{E}-04$ $4.20805 \mathrm{E}-04$ $4.25826 \mathrm{E}-04$ $4.30878 \mathrm{E}-04$

17.10

17.20

17.30

17.40

17.50

17.60

17.70

17.80

17.90

18.00

$4.35959 \mathrm{E}-04$ $4.41070 \mathrm{E}-04$ $4.46211 \mathrm{E}-04$ $4.51382 \mathrm{E}-04$

$4.61813 \mathrm{E}-04$ $4.67073 \mathrm{E}-04$ $4.72363 \mathrm{E}-04$ $4.77682 \mathrm{E}-04$ $4.83032 \mathrm{E}-04$ $f_{2}$

$-3.41846 \mathrm{E}-01$ $-3.47636 \mathrm{E}-01$ $-3.53475 \mathrm{E}-01$ $-3.59362 \mathrm{E}-01$ $-3.65298 \mathrm{E}-01$

$-3.71282 \mathrm{E}-01$ $-3.77315 \mathrm{E}-01$ $-3.83396 \mathrm{E}-01$ $-3.89525 \mathrm{E}-01$ $-3.95703 \mathrm{E}-01$

$-4.01929 \mathrm{E}-01$ $-4.08204 \mathrm{E}-01$ $-4.14526 \mathrm{E}-01$ $-4.20898 \mathrm{E}-01$ $-4.27317 \mathrm{E}-01$

$-4.33785 \mathrm{E}-01$ $-4.40301 \mathrm{E}-01$ $-4.46866 \mathrm{E}-01$ $-4.53479 \mathrm{E}-01$ $-4.60140 \mathrm{E}-01$

$-4.66849 \mathrm{E}-01$ $-4.73607 \mathrm{E}-01$ $-4.80413 \mathrm{E}-01$ $-4.87268 \mathrm{E}-01$ $-4.94170 \mathrm{E}-01$

$-5.01121 \mathrm{E}-01$ $-5.08121 \mathrm{E}-01$ $-5.15168 \mathrm{E}-01$ $-5.22264 \mathrm{E}-01$ $-5.29408 \mathrm{E}-01$

$-5.36600 \mathrm{E}-01$ $-5.43841 \mathrm{E}-01$ $-5.51130 \mathrm{E}-01$ $-5.58467 \mathrm{E}-01$ $-5.65852 \mathrm{E}-01$

$-5.73286 \mathrm{E}-01$ $-5.80767 \mathrm{E}-01$ $-5.88297 \mathrm{E}-01$ $-5.95876 \mathrm{E}-01$ $-6.03502 \mathrm{E}-01$

$-6.11177 \mathrm{E}-01$ $-6.18899 \mathrm{E}-01$ $-6.26670 \mathrm{E}-01$ $-6.34489 \mathrm{E}-01$ $-6.42357 \mathrm{E}-01$

$-6.50272 \mathrm{E}-01$ $-6.58236 \mathrm{E}-0 \mathrm{l}$ $-6.66247 \mathrm{E}-01$ $-6.74307 \mathrm{E}-01$ $-6.82415 \mathrm{E}-01$ $4.56583 \mathrm{E}-04$

$-6.90572 \mathrm{E}-01$ $-6.98776 \mathrm{E}-01$ $-7.07028 \mathrm{E}-01$ $-7.15329 \mathrm{E}-01$ $-7.23678 \mathrm{E}-01$

$-7.32074 \mathrm{E}-01$

$-7.40519 \mathrm{E}-01$

$-7.49012 \mathrm{E}-01$

$-7.57553 \mathrm{E}-01$

$-7.66142 \mathrm{E}-01$ $g_{2}$

$-9.06448 \mathrm{E}-03$ $-9.26599 \mathrm{E}-03$ $-9.47039 \mathrm{E}-03$ $-9.67771 \mathrm{E}-03$ $-9.88797 \mathrm{E}-03$

$-1.01012 \mathrm{E}-02$ $-1.03174 \mathrm{E}-02$ $-1.05366 \mathrm{E}-02$ $-1.07588 \mathrm{E}-02$ $-1.09840 \mathrm{E}-02$

$-1.12123 \mathrm{E}-02$ -1.14437 E-02 $-1.16782 \mathrm{E}-02$ $-1.19158 \mathrm{E}-02$ $-1.21565 \mathrm{E}-02$

$-1.24004 \mathrm{E}-02$ $-1.26475 \mathrm{E}-02$ $-1.28978 \mathrm{E}-02$ $-1.31513 \mathrm{E}-02$ $-1.34080 \mathrm{E}-02$

$-1.36680 \mathrm{E}-02$ $-1.39313 \mathrm{E}-02$ $-1.41978 \mathrm{E}-02$ $-1.44677 \mathrm{E}-02$ $-1.47409 \mathrm{E}-02$

$-1.50175 \mathrm{E}-02$ $-1.52974 \mathrm{E}-02$ $-1.55808 \mathrm{E}-02$ $-1.58676 \mathrm{E}-02$ $-1.61578 \mathrm{E}-02$

$-1.64514 \mathrm{E}-02$ $-1.67486 \mathrm{E}-02$ $-1.73534 \mathrm{E}-02$ $-1.76611 \mathrm{E}-02$

$-1.79723 \mathrm{E}-02$ $-1.82871 \mathrm{E}-02$ $-1.86055 \mathrm{E}-02$ $-1.89276 \mathrm{E}-02$ $-1.92533 \mathrm{E}-02$

$-1.95826 \mathrm{E}-02$ $-1.99156 \mathrm{E}-02$ $-2.02523 \mathrm{E}-02$ $-2.05927 \mathrm{E}-02$ $-2.09369 \mathrm{E}-02$

$-2.12848 \mathrm{E}-02$ $-2.16365 \mathrm{E}-02$ $-2.19920 \mathrm{E}-02$ $-2.23513 \mathrm{E}-02$ $-2.27144 \mathrm{E}-02$

$-2.30814 \mathrm{E}-02$ $-2.34522 \mathrm{E}-02$ $-2.38270 \mathrm{E}-02$ $-2.45883 \mathrm{E}-02$

$-2.49748 \mathrm{E}-02$ $-2.53654 \mathrm{E}-02$ $-2.57599 \mathrm{E}-02$ $-2.61585 \mathrm{E}-02$ $-2.65610 \mathrm{E}-02$ $-1.70492 \mathrm{E}-02$

$\tan \delta_{2}$

$f_{-2}$

g-2

$4.02240 \mathrm{E}$ 01 $-8.81737 \mathrm{E}-03$ $4.02363 \mathrm{E} \quad 01 \quad-9.01272 \mathrm{E}-03$ $4.02483 \mathrm{E} 01 \quad-9.21090 \mathrm{E}-03$ $4.02601 \mathrm{E} \quad 01 \quad-9.41193 \mathrm{E}-03$ $4.02717 \mathrm{E} \quad 01 \quad-9.61584 \mathrm{E}-03$

$4.02831 \mathrm{E} \quad 01$ $4.02943 \mathrm{E} \quad 01$ $4.03054 \mathrm{E} \quad 01$ $4.03162 \mathrm{E} 01$ $4.03269 \mathrm{E} \quad 01$

$4.03374 \mathrm{E} \quad 01$ $4.03477 \mathrm{E} \quad 01$ $4.03579 \mathrm{E} \quad 01$ $4.03679 \mathrm{E} 01$ $4.03778 \mathrm{E} \quad 01$

$4.03875 \mathrm{E} \quad 01$ $4.03971 \mathrm{E} \quad 01$ $4.04065 \mathrm{E} \quad 01$ $4.04158 \mathrm{E} 01$ $4.04249 \mathrm{E} \quad 01$

$4.04339 \mathrm{E} \quad 01$

$4.04428 \mathrm{E} 01$

$4.04515 \mathrm{E} \quad 01$

$4.04601 \mathrm{E} \quad 01$

$4.04686 \mathrm{E} \quad 01$

$4.04770 \mathrm{E} \quad 01$ $4.04852 \mathrm{E} 01$ $4.04934 \mathrm{E} 01$ $4.05014 \mathrm{E} \quad 01$

$4.05093 \mathrm{E} 01$

$4.05171 \mathrm{E} \quad 01$ $4.05248 \mathrm{E} \quad 01$ $4.05323 \mathrm{E} 01$ $4.05398 \mathrm{E} 01$ $4.05472 \mathrm{E} \quad 01$

$4.05545 \mathrm{E} \quad 01$ $4.05616 \mathrm{E} \quad 01$ $4.05687 \mathrm{E} \quad 01$ $4.05757 \mathrm{E} \quad 01$ $4.05826 \mathrm{E} \quad 01$

$4.05894 \mathrm{E} \quad 01$ $4.05962 \mathrm{E} 01$ $4.06028 \mathrm{E} 01$ $4.06094 \mathrm{E} \quad 01$ $4.06158 \mathrm{E} \quad 01$

$4.06222 \mathrm{E} \quad 01$ $4.06285 \mathrm{E} \quad 01$ $4.06348 \mathrm{E} \quad 01$ $4.06409 \mathrm{E} \quad 01$ $4.06470 \mathrm{E} 01$ $-2.42057 \mathrm{E}-02$

$-9.82263 \mathrm{E}-03$ $-1.00323 \mathrm{E}-02$ $-1.02450 \mathrm{E}-02$ $-1.04606 \mathrm{E}-02$ $-1.06791 \mathrm{E}-02$

$-1.09007 \mathrm{E}-02$ $-1.11252 \mathrm{E}-02$ $-1.13528 \mathrm{E}-02$ $-1.15835 \mathrm{E}-02$ $-1.18172 \mathrm{E}-02$

$-1.20540 \mathrm{E}-02$ $-1.22939 \mathrm{E}-02$ $-1.25369 \mathrm{E}-02$ $-1.27831 \mathrm{E}-02$ $-1.30325 \mathrm{E}-02$

$-1.32851 \mathrm{E}-02$ $-1.35408 \mathrm{E}-02$ $-1.37998 \mathrm{E}-02$ $-1.40621 \mathrm{E}-02$ $-1.43276 \mathrm{E}-02$

$-1.45964 \mathrm{E}-02$ $-1.48685 \mathrm{E}-02$ $-1.51440 \mathrm{E}-02$ $-1.54228 \mathrm{E}-02$ $-1.57049 \mathrm{E}-02$

$-1.59905 \mathrm{E}-02$ $-1.62795 \mathrm{E}-02$ $-1.65719 \mathrm{E}-02$ $-1.68677 \mathrm{E}-02$ $-1.71671 \mathrm{E}-02$

$-1.74699 \mathrm{E}-02$ $-1.77762 \mathrm{E}-02$ $-1.80860 \mathrm{E}-02$ $-1.83994 \mathrm{E}-02$ $-1.87164 \mathrm{E}-02$

$-1.90370 \mathrm{E}-02$ $-1.93611 \mathrm{E}-02$ $-1.96889 \mathrm{E}-02$ $-2.00204 \mathrm{E}-02$ $-2.03555 \mathrm{E}-02$

$-2.06943 \mathrm{E}-02$ 


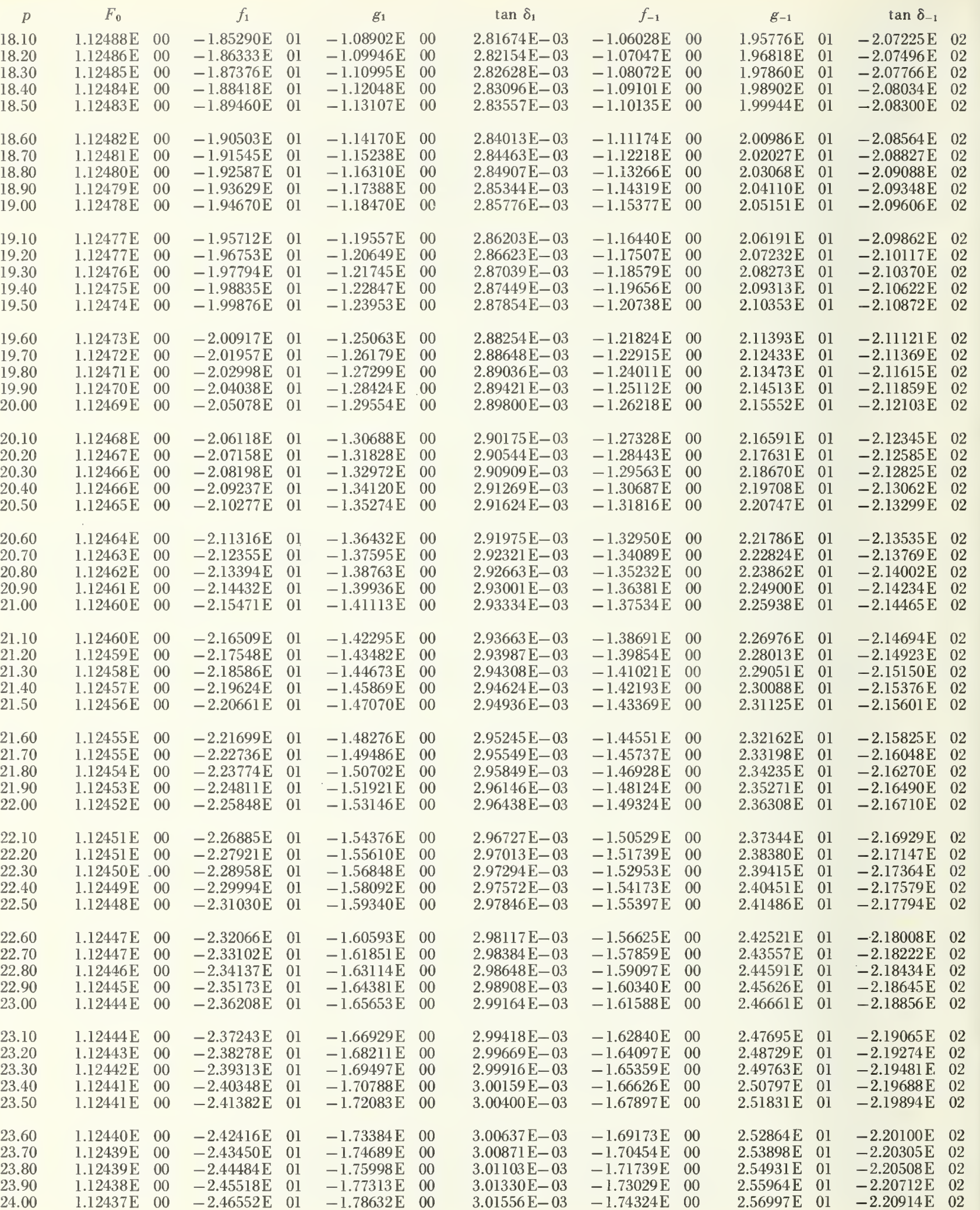


ELECTRON RADIAL FUNCTIONS

$Z=5 \quad A=11 \quad \rho=1.2 A^{1 / 3} F$

Electron

$\bar{F}$

$4.88411 \mathrm{E}-04$

$4.93820 \mathrm{E}-04$

$4.99259 \mathrm{E}-04$

$5.04727 \mathrm{E}-04$

$5.10226 \mathrm{E}-04$

5.15754 E- 04

$5.21312 \mathrm{E}-04$

$5.26899 \mathrm{E}-04$

$5.32517 \mathrm{E}-04$

$5.38164 \mathrm{E}-04$

$5.43841 \mathrm{E}-04$

$5.49548 \mathrm{E}-04$

$5.55285 \mathrm{E}-04$

$5.61051 \mathrm{E}-04$

$5.66847 \mathrm{E}-04$

$5.72673 \mathrm{E}-04$

$5.78529 \mathrm{E}-04$

$5.84414 \mathrm{E}-04$

$5.90329 \mathrm{E}-04$

$5.96274 \mathrm{E}-04$

$6.02249 \mathrm{E}-04$

$6.08254 \mathrm{E}-04$

$6.14288 \mathrm{E}-04$

$6.20352 \mathrm{E}-04$

$6.26446 \mathrm{E}-04$

$6.32570 \mathrm{E}-04$ $6.38723 \mathrm{E}-04$ $6.44907 \mathrm{E}-04$ $6.51120 \mathrm{E}-04$

$6.57363 \mathrm{E}-04$

$6.63635 \mathrm{E}-04$

$6.69937 \mathrm{E}-04$

$6.76270 \mathrm{E}-04$

$6.82632 \mathrm{E}-04$

$6.89023 \mathrm{E}-04$

$6.95445 \mathrm{E}-04$

$7.01896 \mathrm{E}-04$

$7.08377 \mathrm{E}-04$

$7.14888 \mathrm{E}-04$

$7.21428 \mathrm{E}-04$

$7.27999 \mathrm{E}-04$

$7.34599 \mathrm{E}-04$

$7.41229 \mathrm{E}-04$

$7.47889 \mathrm{E}-04$

$7.54578 \mathrm{E}-04$

$7.61297 \mathrm{E}-04$

$7.68046 \mathrm{E}-04$

$7.74825 \mathrm{E}-04$

$7.81634 \mathrm{E}-04$

$7.884 .72 \mathrm{E}-04$

$7.95340 \mathrm{E}-04$ $8.02238 \mathrm{E}-04$

$8.09166 \mathrm{E}-04$

$8.16124 \mathrm{E}-04$

8.23111 E-04

$8.30128 \mathrm{E}-04$. $8.37175 \mathrm{E}-04$ 8.44251 E-04 $8.51357 \mathrm{E}-04$

$8.58494 \mathrm{E}-04$ $f_{2}$

$-7.74780 \mathrm{E}-01$

$-7.83465 \mathrm{E}-01$

$-7.92198 \mathrm{E}-01$

$-8.00979 \mathrm{E}-01$

$-8.09809 \mathrm{E}-01$

$-8.18686 \mathrm{E}-01$

$-8.27611 \mathrm{E}-01$

$-8.36585 \mathrm{E}-01$

$-8.45606 \mathrm{E}-01$

$-8.54676 \mathrm{E}-01$

$-8.63793 \mathrm{E}-01$

$-8.72959 \mathrm{E}-01$

$-8.82172 \mathrm{E}-01$

$-8.91434 \mathrm{E}-01$

$-9.00743 \mathrm{E}-01$

$-9.10100 \mathrm{E}-01$

$-9.19505 \mathrm{E}-01$

$-9.28959 \mathrm{E}-01$

$-9.38460 \mathrm{E}-01$

$-9.48009 \mathrm{E}-01$

$-9.57606 \mathrm{E}-01$

$-9.67251 \mathrm{E}-01$

$-9.76944 \mathrm{E}-01$

$-9.86684 \mathrm{E}-01$

$-9.96473 \mathrm{E}-01$

$-1.00631 \mathrm{E} \quad 00$

$-1.01619 \mathrm{E} 00$

$-1.02613 \mathrm{E} \quad 00$

-1.03611 E 00

$-1.04613 \mathrm{E} 00$

$-1.05621 \mathrm{E} \quad 00$

$-1.06633 \mathrm{E} 00$

$-1.07651 \mathrm{E} 00$

$-1.08672 \mathrm{E} 00$

$-1.09699 \mathrm{E} 00$

$-1.10731 \mathrm{E} 00$

$-1.11767 \mathrm{E} 00$

$-1.12808 \mathrm{E} 00$

-1.13854 E 00

$-1.14904 \mathrm{E} 00$

$-1.15960 \mathrm{E} 00$

$-1.17020 \mathrm{E} \quad 00$

-1.18085 E 00

$-1.19155 \mathrm{E} 00$

$-1.20229 \mathrm{E} 00$

$-1.21308 \mathrm{E} 00$

$-1.22392 \mathrm{E} 00$

-1.23481 E 00

$-1.24575 \mathrm{E} 00$

$-1.25673 \mathrm{E} 00$

$-1.26776 \mathrm{E} 00$

$-1.27884 \mathrm{E} 00$

$-1.28997 \mathrm{E} \quad 00$

$-1.30114 \mathrm{E} 00$

$-1.31237 \mathrm{E} 00$

$-1.32363 \mathrm{E} 00$

$-1.33495 \mathrm{E} 00$

$-1.34632 \mathrm{E} 00$

$-1.35773 \mathrm{E} \quad 00$

-1.36919 E 00 $g_{2}$

$-2.69677 \mathrm{E}-02$

$-2.73784 \mathrm{E}-02$

$-2.77932 \mathrm{E}-02$

$-2.82121 \mathrm{E}-02$

$-2.86351 \mathrm{E}-02$

$-2.90624 \mathrm{E}-02$

$-2.94937 \mathrm{E}-02$

$-2.99293 \mathrm{E}-02$

$-3.03691 \mathrm{E}-02$

$-3.08131 \mathrm{E}-02$

$-3.12613 \mathrm{E}-02$

$-3.17138 \mathrm{E}-02$

$-3.21707 \mathrm{E}-02$

$-3.26318 \mathrm{E}-02$

$-3.30973 \mathrm{E}-02$

-3.35671 E-- 02

$-3.404 .13 \mathrm{E}-02$

$-3.45198 \mathrm{E}-02$

$-3.50028 \mathrm{E}-02$

$-3.54902 \mathrm{E}-02$

$-3.59821 \mathrm{E}-02$

$-3.64784 \mathrm{E}-02$

$-3.69793 \mathrm{E}-02$

$-3.74846 \mathrm{E}-02$

$-3.79945 \mathrm{E}-02$

$-3.85089 \mathrm{E}-02$

$-3.90278 \mathrm{E}-02$

$-3.95514 \mathrm{E}-02$

$-4.00796 \mathrm{E}-02$

$-4.06123 \mathrm{E}-02$

$-4.114 .98 \mathrm{E}-02$

$-4.16919 \mathrm{E}-02$

$-4.22387 \mathrm{E}-02$

$-4.27902 \mathrm{E}-02$

$-4.33464 \mathrm{E}-02$

$-4.39074 \mathrm{E}-02$

$-4.44731 \mathrm{E}-02$

$-4.50437 \mathrm{E}-02$

$-4.56190 \mathrm{E}-02$

$-4.61991 \mathrm{E}-02$

$-4.67841 \mathrm{E}-02$

$-4.73740 \mathrm{E}-02$

$-4.79687 \mathrm{E}-02$

$-4.85684 \mathrm{E}-02$

$-4.91729 \mathrm{E}-02$

$-4.97825 \mathrm{E}-02$

$-5.03969 \mathrm{E}-02$

$-5.10164 \mathrm{E}-02$

$-5.16408 \mathrm{E}-02$

$-5.22703 \mathrm{E}-02$

$-5.29048 \mathrm{E}-02$

$-5.35444 \mathrm{E}-02$

$-5.41890 \mathrm{E}-02$

$-5.48388 \mathrm{E}-02$

$-5.54937 \mathrm{E}-02$

$-5.61537 \mathrm{E}-02$

$-5.68188 \mathrm{E}-02$

$-5.74892 \mathrm{E}-02$

$-5.81647 \mathrm{E}-02$

$-5.88455 \mathrm{E}-02$ $\tan \delta_{2}$

$f_{-2}$

$4.07093 \mathrm{E} \quad 01 \quad-2.62325 \mathrm{E}-02$

$4.07145 \mathrm{E} \quad 01 \quad-2.66331 \mathrm{E}-02$

$4.07197 \mathrm{E} \quad 01 \quad-2.70376 \mathrm{E}-02$

$4.07249 \mathrm{E} \quad 01 \quad-2.74462 \mathrm{E}-02$

$\begin{array}{lll}4.07300 \mathrm{E} & 01 & -2.78589 \mathrm{E}-02\end{array}$

$4.07350 \mathrm{E} \quad 01 \quad-2.82756 \mathrm{E}-02$

$4.07400 \mathrm{E} \quad 01 \quad-2.86965 \mathrm{E}-02$

$\begin{array}{lll}4.07449 \mathrm{E} & 01 & -2.91215 \mathrm{E}-02\end{array}$

$4.07498 \mathrm{E} \quad 01 \quad-2.95506 \mathrm{E}-02$

$\begin{array}{lll}4.07546 \mathrm{E} & 01 & -2.99839 \mathrm{E}-02\end{array}$

$4.07594 \mathrm{E} \quad 01$

$4.07641 \mathrm{E} \quad 01$

$4.07688 \mathrm{E} \quad 01$

$4.07734 \mathrm{E} \quad 01$

$4.07780 \mathrm{E} \quad 01$

$4.07825 \mathrm{E} \quad 01$

$4.07869 \mathrm{E} \quad 01$

$4.07914 \mathrm{E} \quad 01$

$4.07957 \mathrm{E} \quad 01$

$4.08001 \mathrm{E} \quad 01$

$4.08043 \mathrm{E} \quad 01$

$4.08086 \mathrm{E} \quad 01$

$4.08128 \mathrm{E} \quad 01$

$4.08169 \mathrm{E} \quad 01$

$4.08211 \mathrm{E} \quad 01$

$4.08251 \mathrm{E} \quad 01$ $4.08292 \mathrm{E} 01$

$4.08331 \mathrm{E} \quad 01$

$4.08371 \mathrm{E} 01$

$4.084 .10 \mathrm{E} 01$

$4.08449 \mathrm{E} 01$

$4.08487 \mathrm{E} \quad 01$

$4.08525 \mathrm{E} 01$

$4.08563 \mathrm{E} \quad 01$

$4.08600 \mathrm{E} \quad 01$

$4.08637 \mathrm{E} \quad 01$

$4.08674 \mathrm{E} \quad 01$

$4.08710 \mathrm{E} \quad 01$

$4.08746 \mathrm{E} \quad 01$

$4.08781 \mathrm{E} \quad 01$

$4.08816 \mathrm{E} \quad 01$

$4.08851 \mathrm{E} 01$

$4.08886 \mathrm{E} \quad 01$

$4.08920 \mathrm{E} \quad 01$

$4.08954 \mathrm{E} \quad 01$

$4.08987 \mathrm{E} \quad 01$

$4.09021 \mathrm{E} \quad 01$

$4.09054 \mathrm{E} \quad 01$

$4.09086 \mathrm{E}$ ol

$4.09119 \mathrm{E} 01$

$4.09151 \mathrm{E} \quad 01$

$4.09182 \mathrm{E} \quad 01$

$4.09214 \mathrm{E} \quad 01$

$4.09245 \mathrm{E} \quad 01$

$4.09276 \mathrm{E} 01$

$4.09307 \mathrm{E} \quad 01$

$4.09337 \mathrm{E} \quad 01$

$4.09368 \mathrm{E} \quad 01$

$4.09398 \mathrm{E} \quad 01$

$4.09427 \mathrm{E} \quad 01$

$-3.04214 \mathrm{E}-02$

$-3.08630 \mathrm{E}-02$

$-3.13090 \mathrm{E}-02$

$-3.17591 \mathrm{E}-02$

$-3.22135 \mathrm{E}-02$

$-3.26722 \mathrm{E}-02$

$-3.31352 \mathrm{E}-02$

$-3.36025 \mathrm{E}-02$

$-3.40742 \mathrm{E}-02$

$-3.45502 \mathrm{E}-02$

$-3.50307 \mathrm{E}-02$

$-3.55155 \mathrm{E}-02$

$-3.60047 \mathrm{E}-02$

$-3.64984 \mathrm{E}-02$

$-3.69965 \mathrm{E}-02$

$-3.74992 \mathrm{E}-02$ $-3.80063 \mathrm{E}-02$ $-3.85179 \mathrm{E}-02$

$-3.90341 \mathrm{E}-02$

$-3.95549 \mathrm{E}-02$

$-4.00802 \mathrm{E}-02$

$-4.06101 \mathrm{E}-02$

$-4.11446 \mathrm{E}-02$

$-4.16838 \mathrm{E}-02$

$-4.22277 \mathrm{E}-02$

$-4.27762 \mathrm{E}-02$

$-4.33294 \mathrm{E}-02$

$-4.38874 \mathrm{E}-02$

$-4.44500 \mathrm{E}-02$

$-4.50175 \mathrm{E}-02$

$-4.55897 \mathrm{E}-02$

$-4.61667 \mathrm{E}-02$

$-4.67485 \mathrm{E}-02$

$-4.73352 \mathrm{E}-02$

$-4.79267 \mathrm{E}-02$

$-4.85231 \mathrm{E}-02$

$-4.91244 \mathrm{E}-02$

$-4.97306 \mathrm{E}-02$

$-5.03417 \mathrm{E}-02$

$-5.09578 \mathrm{E}-02$

$-5.15789 \mathrm{E}-02$

$-5.22049 \mathrm{E}-02$ 
ELECTRON RADIAL FUNCTIONS

Electron

\begin{tabular}{|c|c|c|c|c|c|c|}
\hline$p$ & $F_{0}$ & & $f_{1}$ & & $g_{1}$ & \\
\hline 24.10 & $1.12436 \mathrm{E}$ & 00 & $-2.47585 \mathrm{E}$ & 01 & $-1.79956 \mathrm{E}$ & 00 \\
\hline 24.20 & $1.12436 \mathrm{E}$ & 00 & $-2.48618 \mathrm{E}$ & 01 & $-1.81284 \mathrm{E}$ & 00 \\
\hline 24.30 & $1.12435 \mathrm{E}$ & 00 & $-2.49652 \mathrm{E}$ & 01 & $-1.82618 \mathrm{E}$ & 00 \\
\hline 24.40 & $1.12434 \mathrm{E}$ & 00 & $-2.50684 \mathrm{E}$ & 01 & $-1.83956 \mathrm{E}$ & 00 \\
\hline 24.50 & $1.12434 \mathrm{E}$ & 00 & $-2.51717 \mathrm{E}$ & 01 & $-1.85298 \mathrm{E}$ & 00 \\
\hline 24.60 & $1.12433 \mathrm{E}$ & 00 & $-2.52750 \mathrm{E}$ & 01 & $-1.86646 \mathrm{E}$ & 00 \\
\hline 24.70 & $1.12432 \mathrm{E}$ & 00 & $-2.53782 \mathrm{E}$ & 01 & $-1.87998 \mathrm{E}$ & 00 \\
\hline 24.80 & $1.12432 \mathrm{E}$ & 00 & $-2.54814 \bar{E}$ & 01 & $-1.89355 \mathrm{E}$ & 00 \\
\hline 24.90 & $1.12431 \mathrm{E}$ & 00 & $-2.55846 \mathrm{E}$ & 01 & $-1.90716 \mathrm{E}$ & 00 \\
\hline 25.00 & $1.12430 \mathrm{E}$ & 00 & $-2.56878 E$ & 01 & $-1.92082 \mathbf{E}$ & 00 \\
\hline 25.10 & $1.12429 \mathrm{E}$ & 00 & $-2.57910 \mathrm{E}$ & 01 & $-1.93453 \mathrm{E}$ & 00 \\
\hline 25.20 & $1.12429 \mathrm{E}$ & 00 & $-2.58941 \mathrm{E}$ & 01 & $-1.94829 \mathrm{E}$ & 00 \\
\hline 25.30 & $1.12428 \mathrm{E}$ & 00 & $-2.59973 \bar{E}$ & 01 & $-1.96209 \mathrm{E}$ & 00 \\
\hline 25.40 & $1.12427 \mathrm{E}$ & 00 & $-2.61004 \mathrm{E}$ & 01 & $-1.97594 \mathrm{E}$ & 00 \\
\hline 25.50 & $1.12427 \mathrm{E}$ & 00 & $-2.62035 \mathrm{E}$ & 01 & $-1.98984 \mathrm{E}$ & 00 \\
\hline 25.60 & $1.12426 \mathrm{E}$ & 00 & $-2.63065 E$ & 01 & $-2.00379 \mathrm{E}$ & 00 \\
\hline 25.70 & $1.12425 \mathrm{E}$ & 00 & $-2.64096 \mathrm{E}$ & 01 & $-2.01778 \mathrm{E}$ & 00 \\
\hline 25.80 & $1.12425 \mathrm{E}$ & 00 & $-2.65126 \mathrm{E}$ & 01 & $-2.03181 \mathrm{E}$ & 00 \\
\hline 25.90 & $1.12424 \mathrm{E}$ & 00 & $-2.66157 \mathrm{E}$ & 01 & $-2.04590 \mathrm{E}$ & 00 \\
\hline 26.00 & $1.12424 \mathrm{E}$ & 00 & $-2.67187 \mathrm{E}$ & 01 & $-2.06003 \mathrm{E}$ & 00 \\
\hline 26.10 & $1.12423 \mathrm{E}$ & 00 & $-2.68217 \mathrm{E}$ & 01 & $-2.07421 \mathrm{E}$ & 00 \\
\hline 26.20 & $1.12422 \mathrm{E}$ & 00 & $-2.69246 \mathrm{E}$ & 01 & $-2.08844 \mathrm{E}$ & 00 \\
\hline 26.30 & $1.12422 \mathrm{E}$ & 00 & $-2.70276 \mathrm{E}$ & 01 & $-2.10271 \mathrm{E}$ & 00 \\
\hline 26.40 & $1.12421 \mathrm{E}$ & 00 & $-2.71305 \mathrm{E}$ & 01 & $-2 . J 1703 \mathrm{E}$ & 00 \\
\hline 26.50 & $1.12420 \mathrm{E}$ & 00 & $-2.72334 \mathrm{E}$ & 01 & $-2.13140 \mathrm{E}$ & 00 \\
\hline 26.60 & $1.12420 \mathrm{E}$ & 00 & $-2.73363 \mathrm{E}$ & 01 & $-2.14581 \mathrm{E}$ & 00 \\
\hline 26.70 & $1.12419 \mathrm{E}$ & 00 & $-2.74392 \mathrm{E}$ & 01 & $-2.16027 \mathrm{E}$ & 00 \\
\hline 26.80 & $1.12418 \mathrm{E}$ & 00 & $-2.75420 \mathrm{E}$ & 01 & $-2.17478 \mathrm{E}$ & 00 \\
\hline 26.90 & $1.12418 \mathrm{E}$ & 00 & $-2.76449 \mathrm{E}$ & 01 & $-2.18933 \mathrm{E}$ & 00 \\
\hline 27.00 & $1.12417 \mathrm{E}$ & 00 & $-2.77477 \mathrm{E}$ & 01 & $-2.20394 \mathrm{E}$ & 00 \\
\hline 27.10 & $1.12417 \mathrm{E}$ & 00 & $-2.78505 \mathrm{E}$ & 01 & $-2.21858 \mathrm{E}$ & 00 \\
\hline 27.20 & $1.12416 \mathrm{E}$ & 00 & $-2.79533 \mathrm{E}$ & 01 & $-2.23328 \mathrm{E}$ & 00 \\
\hline 27.30 & $1.12415 \mathrm{E}$ & 00 & $-2.80561 \mathrm{E}$ & 01 & $-2.24802 \mathrm{E}$ & 00 \\
\hline 27.40 & $1.12415 \mathrm{E}$ & 00 & $-2.81588 \mathrm{E}$ & 01 & $-2.26281 \mathrm{E}$ & 00 \\
\hline 27.50 & $1.12414 \mathrm{E}$ & 00 & $-2.82615 \mathrm{E}$ & 01 & $-2.27764 \mathrm{E}$ & 00 \\
\hline 27.60 & $1.12414 \mathrm{E}$ & 00 & $-2.83642 \mathrm{E}$ & 01 & $-2.29253 \mathrm{E}$ & 00 \\
\hline 27.70 & $1.12413 \mathrm{E}$ & 00 & $-2.84669 \mathrm{E}$ & 01 & $-2.30745 \mathrm{E}$ & 00 \\
\hline 27.80 & $1.12412 \mathrm{E}$ & 00 & $-2.85696 \mathrm{E}$ & 01 & $-2.32243 \mathrm{E}$ & 00 \\
\hline 27.90 & $1.12412 \mathrm{E}$ & 00 & $-2.86722 \mathrm{E}$ & 01 & $-2.33745 \mathrm{E}$ & 00 \\
\hline 28.00 & $1.12411 \mathrm{E}$ & 00 & $-2.87749 \mathrm{E}$ & 01 & $-2.35252 \mathbf{E}$ & 00 \\
\hline 28.10 & $1.12411 \mathrm{E}$ & 00 & $-2.88775 E$ & 01 & $-2.36764 \mathrm{E}$ & 00 \\
\hline 28.20 & $1.12410 \mathrm{E}$ & 00 & $-2.89801 \mathrm{E}$ & 01 & $-2.38280 \mathrm{E}$ & 00 \\
\hline 28.30 & $1.12409 \mathrm{E}$ - & 00 & $-2.90827 \mathrm{E}$ & 01 & $-2.39801 \mathrm{E}$ & 00 \\
\hline 28.40 & $1.12409 \mathrm{E}$ & 00 & $-2.91852 \mathrm{E}$ & 01 & $-2.41326 \mathrm{E}$ & 00 \\
\hline 28.50 & $1.12408 \mathrm{E}$ & 00 & $-2.92878 \mathrm{E}$ & 01 & $-2.42857 \mathrm{E}$ & 00 \\
\hline 28.60 & $1.12408 \mathrm{E}$ & 00 & $-2.93903 \mathrm{E}$ & 01 & $-2.44392 \mathrm{E}$ & 00 \\
\hline 28.70 & $1.12407 \mathrm{E}$ & 00 & $-2.94928 \mathrm{E}$ & 01 & $-2.45931 \mathrm{E}$ & 00 \\
\hline 28.80 & $1.12407 \mathrm{E}$ & 00 & $-2.95952 \mathrm{E}$ & 01 & $-2.47475 \mathrm{E}$ & 00 \\
\hline 28.90 & $1.12406 \mathrm{E}$ & 00 & $-2.96977 \mathrm{E}$ & 01 & $-2.49024 \mathrm{E}$ & 00 \\
\hline 29.00 & $1.12405 \mathrm{E}$ & 00 & $-2.98001 \mathrm{E}$ & 01 & $-2.50578 \mathrm{E}$ & 00 \\
\hline 29.10 & $1.12405 \mathrm{E}$ & 00 & $-2.99026 \mathrm{E}$ & 01 & $-2.52136 \mathrm{E}$ & 00 \\
\hline 29.20 & $1.12404 \mathrm{E}$ & 00 & $-3.00050 \mathrm{E}$ & 01 & $-2.53699 \mathrm{E}$ & 00 \\
\hline 29.30 & $1.12404 \mathrm{E}$ & 00 & $-3.01073 \mathrm{E}$ & 01 & $-2.55267 \mathrm{E}$ & 00 \\
\hline 29.40 & $1.12403 \mathrm{E}$ & 00 & $-3.02097 \mathrm{E}$ & 01 & $-2.56839 E$ & 00 \\
\hline 29.50 & $1.12403 \mathrm{E}$ & 00 & $-3.03120 \mathrm{E}$ & 01 & $-2.58416 \mathrm{E}$ & 00 \\
\hline 29.60 & $1.12402 \mathrm{E}$ & 00 & $-3.04144 \mathrm{E}$ & 01 & $-2.59997 \mathrm{E}$ & 00 \\
\hline 29.70 & $1.12401 \mathrm{E}$ & 00 & $-3.05167 \mathrm{E}$ & 01 & $-2.61583 \mathrm{E}$ & 00 \\
\hline 29.80 & $1.12401 \mathrm{E}$ & 00 & $-3.06189 E$ & 01 & $-2.63174 \mathrm{E}$ & 00 \\
\hline 29.90 & $1.12400 \mathrm{E}$ & 00 & $-3.07212 \mathrm{E}$ & 01 & $-2.64770 \mathrm{E}$ & 00 \\
\hline 30.00 & $1.12400 \mathrm{E}$ & 00 & $-3.08234 \mathrm{E}$ & 01 & $-2.66370 \mathrm{E}$ & 00 \\
\hline
\end{tabular}

$Z=5 \quad A=11 \quad \rho=1.2 A^{1 / 3} F$

$\tan \delta_{1} \quad f_{-1}$

$3.01778 \mathrm{E}-03 \quad-1.75624 \mathrm{E} \quad 00$

$3.01997 \mathrm{E}-03-1.76928 \mathrm{E} \quad 00$

$3.02213 \mathrm{E}-03-1.78237 \mathrm{E} \quad 00$

$3.02426 \mathrm{E}-03-1.79551 \mathrm{E} \quad 00$

$3.02636 \mathrm{E}-03-1.80870 \mathrm{E} \quad 00$

$3.02844 \mathrm{E}-03 \quad-1.82193 \mathrm{E} \quad 00$

$3.03049 \mathrm{E}-03-1.83521 \mathrm{E} \quad 00$

$\begin{array}{lll}3.03251 \mathrm{E}-03 & -1.84853 \mathrm{E} & 00\end{array}$

$3.03450 \mathrm{E}-03-1.86191 \mathrm{E} \quad 00$

$3.03646 \mathrm{E}-03-1.87533 \mathrm{E} \quad 00$

$3.03840 \mathrm{E}-03$

$3.04031 \mathrm{E}-03$

$3.04220 \mathrm{E}-03$

$3.04406 \mathrm{E}-03$

$3.04589 \mathrm{E}-03$

$-1.88879 \mathrm{E} \quad 00$ $-1.90231 \mathrm{E} 00$ $-1.91587 \mathrm{E} \quad 00$ $-1.92948 \mathrm{E} 00$ $-1.94313 \mathrm{E} 00$

$3.04770 \mathrm{E}-03 \quad-1.95683 \mathrm{E} \quad 00$ $3.04948 \mathrm{E}-03-1.97058 \mathrm{E} \quad 00$ $3.05123 \mathrm{E}-03 \quad-1.98438 \mathrm{E} \quad 00$ $3.05297 \mathrm{E}-03 \quad-1.99822 \mathrm{E} \quad 00$ $3.05467 \mathrm{E}-03 \quad-2.01211 \mathrm{E} \quad 00$

$3.05635 \mathrm{E}-03 \quad-2.02605 \mathrm{E} \quad 00$

$3.05801 \mathrm{E}-03 \quad-2.04003 \mathrm{E} \quad 00$

$\begin{array}{lll}3.05965 \mathrm{E}-03 & -2.05407 \mathrm{E} & 00\end{array}$

$3.06126 \mathrm{E}-03 \quad-2.06814 \mathrm{E} \quad 00$

$3.06285 \mathrm{E}-03 \quad-2.08227 \mathrm{E} \quad 00$

$3.06441 \mathrm{E}-03 \quad-2.09644 \mathrm{E} \quad 00$

$3.06595 \mathrm{E}-03 \quad-2.11066 \mathrm{E} \quad 00$

$3.06747 \mathrm{E}-03 \quad-2.12493 \mathrm{E} \quad 00$

$3.06897 \mathrm{E}-03 \quad-2.13924 \mathrm{E} \quad 00$

$3.07044 \mathrm{E}-03 \quad-2.15360 \mathrm{E} \quad 00$

$3.07188 \mathrm{E}-03 \quad-2.16800 \mathrm{E} \quad 00$

$3.07332 \mathrm{E}-03 \quad-2.18246 \mathrm{E} \quad 00$

$3.07472 \mathrm{E}-03 \quad-2.19696 \mathrm{E} \quad 00$

$3.07611 \mathrm{E}-03 \quad-2.21150 \mathrm{E} \quad 00$

$3.07748 \mathrm{E}-03 \quad-2.22610 \mathrm{E} \quad 00$

$3.07882 \mathrm{E}-03 \quad-2.24074 \mathrm{E} \quad 00$

$3.08014 \mathrm{E}-03 \quad-2.25542 \mathrm{E} \quad 00$

$3.08144 \mathrm{E}-03 \quad-2.27016 \mathrm{E} \quad 00$

$3.08272 \mathrm{E}-03 \quad-2.28494 \mathrm{E} \quad 00$

$3.08398 \mathrm{E}-03 \quad-2.29977 \mathrm{E} \quad 00$

$3.08522 \mathrm{E}-03 \quad-2.31464 \mathrm{E} \quad 00$

$3.08644 \mathrm{E}-03 \quad-2.32956 \mathrm{E} \quad 09$

$3.08764 \mathrm{E}-03 \quad-2.34453 \mathrm{E} \quad 00$

$3.08882 \mathrm{E}-03 \quad-2.35954 \mathrm{E} \quad 00$

$3.08998 \mathrm{E}-03 \quad-2.37460 \mathrm{E} \quad 00$

$3.09112 \mathrm{E}-03 \quad-2.38971 \mathrm{E} \quad 00$

$3.09224 \mathrm{E}-03 \quad-2.40487 \mathrm{E} \quad 00$

$3.09335 \mathrm{E}-03 \quad-2.42007 \mathrm{E} \quad 00$

$3.09443 \mathrm{E}-03 \quad-2.43531 \mathrm{E} \quad 00$

$3.09550 \mathrm{E}-03 \quad-2.45061 \mathrm{E} \quad 00$

$3.09654 \mathrm{E}-03 \quad-2.46595 \mathrm{E} \quad 00$

$3.09757 \mathrm{E}-03 \quad-2.48134 \mathrm{E} \quad 00$

$3.09858 \mathrm{E}-03 \quad-2.49677 \mathrm{E} \quad 00$

$3.09957 \mathrm{E}-03 \quad-2.51225 \mathrm{E} \quad 00$

$3.10054 \mathrm{E}-03 \quad-2.52778 \mathrm{E} \quad 00$

$3.10149 \mathrm{E}-03 \quad-2.54335 \mathrm{E} \quad 00$

$3.10243 \mathrm{E}-03 \quad-2.55897 \mathrm{E} \quad 00$

$3.10335 \mathrm{E}-03 \quad-2.57464 \mathrm{E} \quad 00$

$3.10426 \mathrm{E}-03 \quad-2.59036 \mathrm{E} \quad 00$

$3.10514 \mathrm{E}-03 \quad-2.60612 \mathrm{E} \quad 00$ $g_{-1}$

$\tan \delta_{-1}$

$\begin{array}{lllll}2.58029 \mathrm{E} & 01 & -2.21115 \mathrm{E} & 02\end{array}$ $\begin{array}{lllll}2.59062 \mathrm{E} & 01 & -2.21316 \mathrm{E} & 02\end{array}$ $2.60094 \mathrm{E} \quad 01 \quad-2.21516 \mathrm{E} \quad 02$ $\begin{array}{lllll}2.61126 \mathrm{E} & 01 & -2.21716 \mathrm{E} & 02\end{array}$ $\begin{array}{llll}2.62158 \mathrm{E} & 01 & -2.21914 \mathrm{E} & 02\end{array}$

$\begin{array}{llll}2.63190 \mathrm{E} & 01 & -2.22112 \mathrm{E} & 02\end{array}$ $\begin{array}{llll}2.64222 \mathrm{E} & 01 & -2.22310 \mathrm{E} & 02\end{array}$ $\begin{array}{llll}2.65253 \mathrm{E} & 01 & -2.22506 \mathrm{E} & 02\end{array}$ $\begin{array}{llll}2.66284 \mathrm{E} & 01 & -2.22702 \mathrm{E} & 02\end{array}$ $\begin{array}{llll}2.67315 \mathrm{E} & 01 & -2.22898 \mathrm{E} & 02\end{array}$

$\begin{array}{llll}2.68346 \mathrm{E} & 01 & -2.23092 \mathrm{E} & 02\end{array}$ $\begin{array}{llll}2.69377 \mathrm{E} & 01 & -2.23286 \mathrm{E} & 02\end{array}$ $\begin{array}{lllll}2.70407 \mathrm{E} & 01 & -2.23480 \mathrm{E} & 02\end{array}$ $\begin{array}{llll}2.71438 \mathrm{E} & 01 & -2.23673 \mathrm{E} & 02\end{array}$ $\begin{array}{llll}2.72468 \mathrm{E} & 01 & -2.23865 \mathrm{E} & 02\end{array}$

$\begin{array}{llll}2.73498 \mathrm{E} & 01 & -2.24056 \mathrm{E} & 02\end{array}$ $\begin{array}{llll}2.74528 \mathrm{E} & 01 & -2.24248 \mathrm{E} & 02\end{array}$ $\begin{array}{lllll}2.75557 \mathrm{E} & 01 & -2.24438 \mathrm{E} & 02\end{array}$ $\begin{array}{lllll}2.76587 \mathrm{E} & 01 & -2.24628 \mathrm{E} & 02\end{array}$ $\begin{array}{llll}2.77616 \mathrm{E} & 01 & -2.24817 \mathrm{E} & 02\end{array}$

$\begin{array}{llll}2.78645 \mathrm{E} & 01 & -2.25006 \mathrm{E} & 02\end{array}$ $2.79674 \mathrm{E} \quad 01 \quad-2.25195 \mathrm{E} \quad 02$ $\begin{array}{llll}2.80703 \mathrm{E} & 01 & -2.25382 \mathrm{E} & 02\end{array}$ $\begin{array}{lllll}2.81731 \mathrm{E} & 01 & -2.25570 \mathrm{E} & 02\end{array}$ $\begin{array}{llll}2.82759 \mathrm{E} & 01 & -2.25756 \mathrm{E} & 02\end{array}$

$\begin{array}{llll}2.83787 \mathrm{E} & 01 & -2.25942 \mathrm{E} & 02\end{array}$ $\begin{array}{llll}2.84815 \mathrm{E} & 01 & -2.26128 \mathrm{E} & 02\end{array}$ $\begin{array}{lllll}2.85843 \mathrm{E} & 01 & -2.26313 \mathrm{E} & 02\end{array}$ $\begin{array}{lllll}2.86871 \mathrm{E} & 01 & -2.26498 \mathrm{E} & 02\end{array}$ $\begin{array}{llll}2.87898 \mathrm{E} & 01 & -2.26682 \mathrm{E} & 02\end{array}$

$\begin{array}{llll}2.88925 \mathrm{E} & 01 & -2.26866 \mathrm{E} & 02\end{array}$ $\begin{array}{lllll}2.89952 \mathrm{E} & 01 & -2.27049 \mathrm{E} & 02\end{array}$ $\begin{array}{lllll}2.90979 \mathrm{E} & 01 & -2.27232 \mathrm{E} & 02\end{array}$ $\begin{array}{llll}2.92006 \mathrm{E} & 01 & -2.27415 \mathrm{E} & 02\end{array}$ $\begin{array}{llll}2.93032 \mathrm{E} & 01 & -2.27597 \mathrm{E} & 02\end{array}$

$2.94058 \mathrm{E} \quad 01$ $2.95084 \mathrm{E} \quad 01$ $2.96110 \mathrm{E} 01$ $2.97136 \mathrm{E} \quad 01$ $2.98161 \mathrm{E} \quad 01$

$2.99187 \mathrm{E} \quad 01$ $3.00212 \mathrm{E} \quad 01$ $3.01237 \mathrm{E} \quad 01$ $3.02261 \mathrm{E} 01$ $3.03286 \mathrm{E} \quad 01$

$3.04310 \mathrm{E} \quad 01$ $3.05334 \mathrm{E} \quad 01$ $3.06358 \mathrm{E} \quad 01$ $3.07382 \mathrm{E} 01$ $3.08406 \mathrm{E} \quad 01$

$3.09429 \mathrm{E} 01$

$3.10452 \mathrm{E} \quad 01$

$3.11475 \mathrm{E} 01$

$3.12498 \mathrm{E} \quad 01$

$3.13520 \mathrm{E} 01$

$3.14542 \mathrm{E} \quad 01$ $3.15565 \mathrm{E} \quad 01$ $3.16587 \mathrm{E} 01$

$3.17608 \mathrm{E} 01$

$3.18630 \mathrm{E} \quad 01$
$-2.27778 \mathrm{E} \quad 02$ $-2.27959 \mathrm{E} \quad 02$ $-2.28140 \mathrm{E} \quad 02$ $-2.28320 \mathrm{E} \quad 02$

$-2.28680 \mathrm{E} \quad 02$ $-2.28859 \mathrm{E} \quad 02$ $-2.29038 \mathrm{E} \quad 02$ $-2.29216 \mathrm{E} \quad 02$ $-2.29394 \mathrm{E} \quad 02$ $\begin{array}{ll}-2.29749 \mathrm{E} & 02\end{array}$ $-2.29926 \mathrm{E} \quad 02$ $-2.30103 \mathrm{E} 02$ $-2.30279 \mathrm{E} \quad 02$

$-2.30455 \mathrm{E} \quad 02$ $-2.30631 \mathrm{E} \quad 02$ $-2.30806 \mathrm{E} \quad 02$ $\begin{array}{lll}-2.30981 \mathrm{E} & 02\end{array}$ $-2.31156 \mathrm{E} 02$ $-2.31330 \mathrm{E} \quad 02$ $-2.31504 \mathrm{E} \quad 02$ $-2.31678 \mathrm{E} 02$ $-2.31852 \mathrm{E} \quad 02$ $-2.32025 \mathrm{E} \quad 02$ $-2.28500 \mathrm{E} 02$

$\begin{array}{lll}-2.29572 \mathrm{E} & 02\end{array}$ 


$$
Z=5 \quad A=11 \quad \rho=1.2 A^{1 / 3} F
$$

$\bar{F}$

$f_{2}$

24.10

24.20

24.30

24.40

24.50

24.60

24.70

24.80

24.90

25.00

25.10

25.20

25.30

25.40

25.50

25.60

25.70

25.80

25.90

26.00

26.10

26.20

26.30

26.40

26.50

26.60

26.70

26.80

26.90

27.00

27.10

27.20

27.30

27.40

27.50

27.60

27.70

27.80

27.90

28.00

28.10

28.20

28.30

28.40

28.50

28.60

28.70

28.80

28.90

29.00

29.10

29.20

29.30

29.40

29.50

29.60

29.70

29.80

29.90

30.00
$8.65660 \mathrm{E}-04$ 8.80081 E- 04 $8.87336 \mathrm{E}-04$ 8.94621 E-04.

$9.01936 \mathrm{E}-04$ 9.09281 E-04 $9.16655 \mathrm{E}-04$ $9.24059 \mathrm{E}-04$

$9.31493 \mathrm{E}-04$

$9.38957 \mathrm{E}-04$ $9.46450 \mathrm{E}-04$ $9.53974 \mathrm{E}-04$ $9.61526 \mathrm{E}-04$ $9.69109 \mathrm{E}-04$

$9.76722 \mathrm{E}-04$ $9.84364 \mathrm{E}-04$ $9.92036 \mathrm{E}-04$ $9.99738 \mathrm{E}-04$ $1.00747 \mathrm{E}-03$

$1.01523 \mathrm{E}-03$ $1.02302 \mathrm{E}-03$

$1.03084 \mathrm{E}-03$

$1.03869 \mathrm{E}-03$

$1.04657 \mathrm{E}-03$

$1.05448 \mathrm{E}-03$ $1.06242 \mathrm{E}-03$ $1.07039 \mathrm{E}-03$ $1.07839 \mathrm{E}-03$

$1.08642 \mathrm{E}-03$

$1.09448 \mathrm{E}-03$

$1.10257 \mathrm{E}-03$

$1.11069 \mathrm{E}-03$

$1.11884 \mathrm{E}-03$

$1.12702 \mathrm{E}-03$

$1.13522 \mathrm{E}-03$

$1.14346 \mathrm{E}-03$

$1.15173 \mathrm{E}-03$

$1.16003 \mathrm{E}-03$

$1.16835 \mathrm{E}-03$

$1.17671 \mathrm{E}-03$

$1.18510 \mathrm{E}-03$

$1.19351 \mathrm{E}-03$

$1.20196 \mathrm{E}-03$

$1.21044 \mathrm{E}-03$ $1.22748 \mathrm{E}-03$ $1.23604 \mathrm{E}-03$

$1.24464 \mathrm{E}-03$

$1.25326 \mathrm{E}-03$

$1.26192 \mathrm{E}-03$ $1.27060 \mathrm{E}-03$ $1.27932 \mathrm{E}-03$ $1.28806 \mathrm{E}-03$ $1.29683 \mathrm{E}-03$

$1.30564 \mathrm{E}-03$ $1.31447 \mathrm{E}-03$ $1.32333 \mathrm{E}-03$ $1.33223 \mathrm{E}-03$

$1.34115 \mathrm{E}-03$ $8.72856 \mathrm{E}-04$

$1.21894 \mathrm{E}-03$
$-1.38070 \mathrm{E} \quad 00$ $-1.39225 \mathrm{E} 00$ $-1.40386 \mathrm{E} 00$ $-1.41551 \mathrm{E} 00$ $-1.42720 \mathrm{E} 00$

$-1.43895 \mathrm{E} \quad 00$ $-1.45074 \mathrm{E} \quad 00$ $-1.46258 \mathrm{E} \quad 00$ $-1.47447 \mathrm{E} \quad 00$ $-1.48641 \mathrm{E} 00$

$-1.49839 \mathrm{E} \quad 00$ $-1.51042 \mathrm{E} 00$ $-1.52250 \mathrm{E} 00$ $-1.53463 \mathrm{E} 00$ $-1.54680 \mathrm{E} 00$

$-1.55902 \mathrm{E} \quad 00$ $-1.57129 \mathrm{E} 00$ $-1.58360 \mathrm{E} 00$ $-1.59597 \mathrm{E} 00$ $-1.60838 \mathrm{E} \quad 00$

$-1.62084 \mathrm{E} \quad 00$ $-1.63334 \mathrm{E} 00$ $-1.64590 \mathrm{E} 00$ $-1.65850 \mathrm{E} 00$ $-1.67114 \mathrm{E} \quad 00$

$-1.68384 \mathrm{E} \quad 00$ $-1.69658 \mathrm{E} 00$ $-1.70937 \mathrm{E} \quad 00$ $-1.72221 \mathrm{E} 00$ $-1.73509 \mathrm{E} 00$

$-1.74802 \mathrm{E} \quad 00$ $-1.77403 \mathrm{E} 00$ $-1.78711 \mathrm{E} \quad 00$ $-1.80023 \mathrm{E} 00$

$-1.81340 \mathrm{E} \quad 00$ $-1.82661 \mathrm{E} \quad 00$ $-1.83987 \mathrm{E} 00$ $-1.85318 \mathrm{E} 00$

-1.86654 E 00

$-1.87995 \mathrm{E} 00$ $-1.89340 \mathrm{E} 00$ $-1.90690 \mathrm{E} 00$ $-1.92045 \mathrm{E} 00$ $-1.93404 \mathrm{E} 00$

$-1.94768 \mathrm{E} \quad 00$ $-1.96137 \mathrm{E} 00$ $-1.97510 \mathrm{E} 00$ $-1.98889 \mathrm{E} 00$ $-2.00272 \mathrm{E} \quad 00$

$-2.01659 \mathrm{E} \quad 00$ $-2.03052 \mathrm{E} \quad 00$ $-2.04449 \mathrm{E} \quad 00$ $-2.05851 \mathrm{E} 00$ $-2.07257 \mathrm{E} \quad 00$

$-2.08669 \mathrm{E} \quad 00$ $-2.11505 \mathrm{E} \quad 00$ $-2.12931 \mathrm{E} \quad 00$ $-2.14361 \mathrm{E} 00$ $-1.76100 \mathrm{E} 00$ $-2.10085 \mathrm{E} 00$ $g_{2}$

$\tan \delta_{2}$

$-5.95315 \mathrm{E}-02$ $-6.02228 \mathrm{E}-02$ $-6.09193 \mathrm{E}-02$ $-6.16211 \mathrm{E}-02$ $-6.23283 \mathrm{E}-02$

$-6.30408 \mathrm{E}-02$ $-6.37586 \mathrm{E}-02$ $-6.44818 \mathrm{E}-02$ $-6.52104 \mathrm{E}-02$ $-6.59444 \mathrm{E}-02$

$-6.66838 \mathrm{E}-02$ $-6.74287 \mathrm{E}-02$ $-6.81791 \mathrm{E}-02$ $-6.89349 \mathrm{E}-02$ $-6.96963 \mathrm{E}-02$

$-7.04632 \mathrm{E}-02$ $-7.12356 \mathrm{E}-02$ $-7.20136 \mathrm{E}-02$ $-7.27972 \mathrm{E}-02$ $-7.35864 \mathrm{E}-02$

$-7.43813 \mathrm{E}-02$ $-7.51818 \mathrm{E}-02$ $-7.59879 \mathrm{E}-02$ $-7.67997 \mathrm{E}-02$ $-7.76173 \mathrm{E}-02$

$-7.84405 \mathrm{E}-02$ $-7.92695 \mathrm{E}-02$ $-8.01043 \mathrm{E}-02$ $-8.09449 \mathrm{E}-02$ $-8.17912 \mathrm{E}-02$

$-8.26434 \mathrm{E}-02$ $-8.35015 \mathrm{E}-02$ $-8.43653 \mathrm{E}-02$ $-8.52351 \mathrm{E}-02$ $-8.61108 \mathrm{E}-02$

$-8.69924 \mathrm{E}-02$ $-8.78799 \mathrm{E}-02$ $-8.87734 \mathrm{E}-02$ $-8.96728 \mathrm{E}-02$ $-9.05783 \mathrm{E}-02$

$-9.14898 \mathrm{E}-02$ $-9.24073 \mathrm{E}-02$ $-9.33309 \mathrm{E}-02$ $-9.42605 \mathrm{E}-02$ $-9.51962 \mathrm{E}-02$

$-9.61381 \mathrm{E}-02$ $-9.70861 \mathrm{E}-02$ $-9.80402 \mathrm{E}-02$ $-9.90005 \mathrm{E}-02$ $-9.99670 \mathrm{E}-02$

$-1.00940 \mathrm{E}-01$ $-1.01919 \mathrm{E}-01$ $-1.02904 \mathrm{E}-01$ $-1.03895 \mathrm{E}-01$ $-1.04893 \mathrm{E}-01$

$-1.05897 \mathrm{E}-01$ $-1.06907 \mathrm{E}-01$ $-1.07924 \mathrm{E}-01$ $-1.08947 \mathrm{E}-01$ $-1.09977 \mathrm{E}-0$ $4.09486 \mathrm{E} \quad 01$ $4.09515 \mathrm{E} \quad 01$ $4.09543 \mathrm{E} \quad 01$ $4.09655 \mathrm{E} \quad 01$ $4.09683 \mathrm{E} \quad 01$ $4.09710 \mathrm{E} 01$

$4.09737 \mathrm{E} \quad 01$ $4.09764 \mathrm{E} \quad 01$ $4.09817 \mathrm{E} \quad 01$ $4.09895 \mathrm{E} \quad 01$ $4.09946 \mathrm{E} \quad 01$ $4.09971 \mathrm{E} \quad 01$ $4.10020 \mathrm{E} \quad 01$ $4.10045 \mathrm{E} \quad 01$ $4.10093 \mathrm{E} \quad 01$

$4.10117 \mathrm{E}$ 01 $4.10141 \mathrm{E} 01$ $4.10164 \mathrm{E} \quad 01$ $4.10211 \mathrm{E} \quad 01$

$4.10234 \mathrm{E}$ 0] $4.10257 \mathrm{E} 01$ $4.10280 \mathrm{E}$ 01 $4.10302 \mathrm{E} \quad 01$

$4.10347 \mathrm{E} \quad 01$ $4.10369 \mathrm{E} \quad 01$ $4.10412 \mathrm{E} \quad 01$ 4.10434. E 01

4.10455E 01 $4.10477 \mathrm{E} \quad 01$

$4.09456 \mathrm{E} \quad 01$ $4.09572 \mathrm{E} \quad 01$

$4.09600 \mathrm{E} \quad 01$ $4.09628 \mathrm{E} \quad 01$ $4.09790 \mathrm{E} \quad 01$ $4.09843 \mathrm{E} \quad 01$

$4.09869 \mathrm{E} \quad 01$ $4.09920 \mathrm{E} 01$

$4.09996 \mathrm{E} \quad 01$ $4.10069 \mathrm{E} 01$ $4.10188 \mathrm{E} \quad 01$ $4.10325 \mathrm{E} \quad 01$ $4.10391 \mathrm{E} 01$ 
ELECTRON RADIAL FUNCTIONS

\begin{tabular}{|c|c|c|c|c|c|c|c|c|c|c|c|c|c|}
\hline Electro & & & & & $=5$ & $A=1$ & $\rho=1$ & $A^{1 / 3} F$ & & & & & \\
\hline$p$ & $F_{0}$ & & $f_{1}$ & & $g_{1}$ & & $\tan \delta_{1}$ & $f_{-1}$ & & $g_{-1}$ & & $\tan \delta_{-1}$ & \\
\hline $\begin{array}{l}30.10 \\
30.20 \\
30.30 \\
30.40 \\
30.50\end{array}$ & $\begin{array}{l}1.12399 \mathrm{E} \\
1.12399 \mathrm{E} \\
1.12398 \mathrm{E} \\
1.12398 \mathrm{E} \\
1.12397 \mathrm{E}\end{array}$ & $\begin{array}{l}00 \\
00 \\
00 \\
00 \\
00\end{array}$ & $\begin{array}{l}-3.09257 \mathrm{E} \\
-3.10279 \mathrm{E} \\
-3.11300 \mathrm{E} \\
-3.12322 \mathrm{E} \\
-3.13343 \mathrm{E}\end{array}$ & $\begin{array}{l}01 \\
01 \\
01 \\
01 \\
01\end{array}$ & $\begin{array}{l}-2.67975 \mathrm{E} \\
-2.69584 \mathrm{E} \\
-2.71198 \mathrm{E} \\
-2.72817 \mathrm{E} \\
-2.74440 \mathrm{E}\end{array}$ & $\begin{array}{l}00 \\
00 \\
00 \\
00 \\
00\end{array}$ & $\begin{array}{l}3.10601 \mathrm{E}-03 \\
3.10686 \mathrm{E}-03 \\
3.10769 \mathrm{E}-03 \\
3.10851 \mathrm{E}-03 \\
3.10931 \mathrm{E}-03\end{array}$ & $\begin{array}{l}-2.62192 \mathrm{E} \\
-2.63778 \mathrm{E} \\
-2.65368 \mathrm{E} \\
-2.66962 \mathrm{E} \\
-2.68562 \mathrm{E}\end{array}$ & $\begin{array}{l}00 \\
00 \\
00 \\
00 \\
00\end{array}$ & $\begin{array}{l}3.19651 \mathrm{E} \\
3.20672 \mathrm{E} \\
3.21693 \mathrm{E} \\
3.22714 \mathrm{E} \\
3.23734 \mathrm{E}\end{array}$ & $\begin{array}{l}01 \\
01 \\
01 \\
01 \\
01\end{array}$ & $\begin{array}{l}-2.32198 \mathrm{E} \\
-2.32371 \mathrm{E} \\
-2.32543 \mathrm{E} \\
-2.32715 \mathrm{E} \\
-2.32887 \mathrm{E}\end{array}$ & $\begin{array}{l}02 \\
02 \\
02 \\
02 \\
02\end{array}$ \\
\hline $\begin{array}{l}30.60 \\
30.70 \\
30.80 \\
30.90 \\
31.00\end{array}$ & $\begin{array}{l}1.12397 \mathrm{E} \\
1.12396 \mathrm{E} \\
1.12396 \mathrm{E} \\
1.12395 \mathrm{E} \\
1.12394 \mathrm{E}\end{array}$ & $\begin{array}{l}00 \\
00 \\
00 \\
00 \\
00\end{array}$ & $\begin{array}{l}-3.14365 \mathrm{E} \\
-3.15386 \mathrm{E} \\
-3.16406 \mathrm{E} \\
-3.17427 \mathrm{E} \\
-3.18447 \mathrm{E}\end{array}$ & $\begin{array}{l}01 \\
01 \\
01 \\
01 \\
01\end{array}$ & $\begin{array}{l}-2.76068 \mathrm{E} \\
-2.77701 \mathrm{E} \\
-2.79338 \mathrm{E} \\
-2.80980 \mathrm{E} \\
-2.82627 \mathrm{E}\end{array}$ & $\begin{array}{l}00 \\
00 \\
00 \\
00 \\
00\end{array}$ & $\begin{array}{l}3.11009 \mathrm{E}-03 \\
3.11086 \mathrm{E}-03 \\
3.11161 \mathrm{E}-03 \\
3.11235 \mathrm{E}-03 \\
3.11307 \mathrm{E}-03\end{array}$ & $\begin{array}{l}-2.70165 \mathrm{E} \\
-2.71774 \mathrm{E} \\
-2.73387 \mathrm{E} \\
-2.75005 \mathrm{E} \\
-2.76628 \mathrm{E}\end{array}$ & $\begin{array}{l}00 \\
00 \\
00 \\
00 \\
00\end{array}$ & $\begin{array}{l}3.24755 \mathrm{E} \\
3.25775 \mathrm{E} \\
3.26795 \mathrm{E} \\
3.27814 \mathrm{E} \\
3.28834 \mathrm{E}\end{array}$ & $\begin{array}{l}01 \\
01 \\
01 \\
01 \\
01\end{array}$ & $\begin{array}{l}-2.33059 \mathrm{E} \\
-2.33230 \mathrm{E} \\
-2.33402 \mathrm{E} \\
-2.33573 \mathrm{E} \\
-2.33744 \mathrm{E}\end{array}$ & $\begin{array}{l}02 \\
02 \\
02 \\
02 \\
02\end{array}$ \\
\hline $\begin{array}{l}31.10 \\
31.20 \\
31.30 \\
31.40 \\
31.50\end{array}$ & $\begin{array}{l}1.12394 \mathrm{E} \\
1.12393 \mathrm{E} \\
1.12393 \mathrm{E} \\
1.12392 \mathrm{E} \\
1.12392 \mathrm{E}\end{array}$ & $\begin{array}{l}00 \\
00 \\
00 \\
00 \\
00\end{array}$ & $\begin{array}{l}-3.19468 \mathrm{E} \\
-3.20488 \mathrm{E} \\
-3.21507 \mathrm{E} \\
-3.22527 \mathrm{E} \\
-3.23546 \mathrm{E}\end{array}$ & $\begin{array}{l}01 \\
01 \\
01 \\
01 \\
01\end{array}$ & $\begin{array}{l}-2.84278 \mathrm{E} \\
-2.85934 \mathrm{E} \\
-2.87594 \mathrm{E} \\
-2.89259 \mathrm{E} \\
-2.90929 \mathrm{E}\end{array}$ & $\begin{array}{l}00 \\
00 \\
00 \\
00 \\
00\end{array}$ & $\begin{array}{l}3.11377 \mathrm{E}-03 \\
3.11446 \mathrm{E}-03 \\
3.11513 \mathrm{E}-03 \\
3.11579 \mathrm{E}-03 \\
3.11642 \mathrm{E}-03\end{array}$ & $\begin{array}{l}-2.78255 \mathrm{E} \\
-2.79886 \mathrm{E} \\
-2.81523 \mathrm{E} \\
-2.83164 \mathrm{E} \\
-2.84810 \mathrm{E}\end{array}$ & $\begin{array}{l}00 \\
00 \\
00 \\
00 \\
00\end{array}$ & $\begin{array}{l}3.29853 \mathrm{E} \\
3.30872 \mathrm{E} \\
3.31891 \mathrm{E} \\
3.32910 \mathrm{E} \\
3.33928 \mathrm{E}\end{array}$ & $\begin{array}{l}01 \\
01 \\
01 \\
01 \\
01\end{array}$ & $\begin{array}{l}-2.33914 \mathrm{E} \\
-2.34085 \mathrm{E} \\
-2.34254 \mathrm{E} \\
-2.34424 \mathrm{E} \\
-2.34594 \mathrm{E}\end{array}$ & $\begin{array}{l}02 \\
02 \\
02 \\
02 \\
02\end{array}$ \\
\hline $\begin{array}{l}31.60 \\
31.70 \\
31.80 \\
31.90 \\
32.00\end{array}$ & $\begin{array}{l}1.12391 \mathrm{E} \\
1.12391 \mathrm{E} \\
1.12390 \mathrm{E} \\
1.12390 \mathrm{E} \\
1.12389 \mathrm{E}\end{array}$ & $\begin{array}{l}00 \\
00 \\
00 \\
00 \\
00\end{array}$ & $\begin{array}{l}-3.24565 \mathrm{E} \\
-3.25584 \mathrm{E} \\
-3.26603 \mathrm{E} \\
-3.27622 \mathrm{E} \\
-3.28640 \mathrm{E}\end{array}$ & $\begin{array}{l}01 \\
01 \\
01 \\
01 \\
01\end{array}$ & $\begin{array}{l}-2.92604 \mathrm{E} \\
-2.94283 \mathrm{E} \\
-2.95966 \mathrm{E} \\
-2.97655 \mathrm{E} \\
-2.99348 \mathrm{E}\end{array}$ & $\begin{array}{l}00 \\
00 \\
00 \\
00 \\
00\end{array}$ & $\begin{array}{l}3.11705 \mathrm{E}-03 \\
3.11766 \mathrm{E}-03 \\
3.11826 \mathrm{E}-03 \\
3.11883 \mathrm{E}-03 \\
3.11940 \mathrm{E}-03\end{array}$ & $\begin{array}{l}-2.86460 \mathrm{E} \\
-2.88115 \mathrm{E} \\
-2.89774 \mathrm{E} \\
-2.91439 \mathrm{E} \\
-2.93108 \mathrm{E}\end{array}$ & $\begin{array}{l}00 \\
00 \\
00 \\
00 \\
00\end{array}$ & $\begin{array}{l}3.34946 \mathrm{E} \\
3.35964 \mathrm{E} \\
3.36982 \mathrm{E} \\
3.38000 \mathrm{E} \\
3.39017 \mathrm{E}\end{array}$ & $\begin{array}{l}01 \\
01 \\
01 \\
01 \\
01\end{array}$ & $\begin{array}{l}-2.34764 \mathrm{E} \\
-2.34933 \mathrm{E} \\
-2.35102 \mathrm{E} \\
-2.35271 \mathrm{E} \\
-2.35440 \mathrm{E}\end{array}$ & $\begin{array}{l}02 \\
02 \\
02 \\
02 \\
02\end{array}$ \\
\hline $\begin{array}{l}32.10 \\
32.20 \\
32.30 \\
32.40 \\
32.50\end{array}$ & $\begin{array}{l}1.12389 \mathrm{E} \\
1.12388 \mathrm{E} \\
1.12388 \mathrm{E} \\
1.12387 \mathrm{E} \\
1.12387 \mathrm{E}\end{array}$ & $\begin{array}{l}00 \\
00 \\
00 \\
00 \\
00\end{array}$ & $\begin{array}{l}-3.29658 \mathrm{E} \\
-3.30676 \mathrm{E} \\
-3.31694 \mathrm{E} \\
-3.32711 \mathrm{E} \\
-3.33728 \mathrm{E}\end{array}$ & $\begin{array}{l}01 \\
01 \\
01 \\
01 \\
01\end{array}$ & $\begin{array}{l}-3.01045 \mathrm{E} \\
-3.02747 \mathrm{E} \\
-3.04454 \mathrm{E} \\
-3.06165 \mathrm{E} \\
-3.07881 \mathrm{E}\end{array}$ & $\begin{array}{l}00 \\
00 \\
00 \\
00 \\
00\end{array}$ & $\begin{array}{l}3.11995 \mathrm{E}-03 \\
3.12048 \mathrm{E}-03 \\
3.12101 \mathrm{E}-03 \\
3.12151 \mathrm{E}-03 \\
3.12200 \mathrm{E}-03\end{array}$ & $\begin{array}{l}-2.94781 \mathrm{E} \\
-2.96459 \mathrm{E} \\
-2.98142 \mathrm{E} \\
-2.99829 \mathrm{E} \\
-3.01521 \mathrm{E}\end{array}$ & $\begin{array}{l}00 \\
00 \\
00 \\
00 \\
00\end{array}$ & $\begin{array}{l}3.40034 \mathrm{E} \\
3.41051 \mathrm{E} \\
3.42068 \mathrm{E} \\
3.43085 \mathrm{E} \\
3.44101 \mathrm{E}\end{array}$ & $\begin{array}{l}01 \\
01 \\
01 \\
01 \\
01\end{array}$ & $\begin{array}{l}-2.35609 \mathrm{E} \\
-2.35777 \mathrm{E} \\
-2.35946 \mathrm{E} \\
-2.36114 \mathrm{E} \\
-2.36282 \mathrm{E}\end{array}$ & $\begin{array}{l}02 \\
02 \\
02 \\
02 \\
02\end{array}$ \\
\hline $\begin{array}{l}32.60 \\
32.70 \\
32.80 \\
32.90 \\
33.00\end{array}$ & $\begin{array}{l}1.12386 \mathrm{E} \\
1.12386 \mathrm{E} \\
1.12385 \mathrm{E} \\
1.12385 \mathrm{E} \\
1.12384 \mathrm{E}\end{array}$ & $\begin{array}{l}00 \\
00 \\
00 \\
00 \\
00\end{array}$ & $\begin{array}{l}-3.34745 \mathrm{E} \\
-3.35762 \mathrm{E} \\
-3.36779 \mathrm{E} \\
-3.37795 \mathrm{E} \\
-3.38811 \mathrm{E}\end{array}$ & $\begin{array}{l}01 \\
01 \\
01 \\
01 \\
01\end{array}$ & $\begin{array}{l}-3.09602 \mathrm{E} \\
-3.11327 \mathrm{E} \\
-3.13057 \mathrm{E} \\
-3.14792 \mathrm{E} \\
-3.16531 \mathrm{E}\end{array}$ & $\begin{array}{l}00 \\
00 \\
00 \\
00 \\
00\end{array}$ & $\begin{array}{l}3.12248 \mathrm{E}-03 \\
3.12294 \mathrm{E}-03 \\
3.12339 \mathrm{E}-03 \\
3.12382 \mathrm{E}-03 \\
3.12425 \mathrm{E}-03\end{array}$ & $\begin{array}{l}-3.03218 \mathrm{E} \\
-3.04919 \mathrm{E} \\
-3.06625 \mathrm{E} \\
-3.08335 \mathrm{E} \\
-3.10050 \mathrm{E}\end{array}$ & $\begin{array}{l}00 \\
00 \\
00 \\
00 \\
00\end{array}$ & $\begin{array}{l}3.45117 \mathrm{E} \\
3.46133 \mathrm{E} \\
3.47149 \mathrm{E} \\
3.48164 \mathrm{E} \\
3.49179 \mathrm{E}\end{array}$ & $\begin{array}{l}01 \\
01 \\
01 \\
01 \\
01\end{array}$ & $\begin{array}{l}-2.36450 \mathrm{E} \\
-2.36617 \mathrm{E} \\
-2.36785 \mathrm{E} \\
-2.36953 \mathrm{E} \\
-2.37120 \mathrm{E}\end{array}$ & $\begin{array}{l}02 \\
02 \\
02 \\
02 \\
02\end{array}$ \\
\hline $\begin{array}{l}33.10 \\
33.20 \\
33.30 \\
33.40 \\
33.50\end{array}$ & $\begin{array}{l}1.12384 \mathrm{E} \\
1.12383 \mathrm{E} \\
1.12383 \mathrm{E} \\
1.12382 \mathrm{E} \\
1.12382 \mathrm{E}\end{array}$ & $\begin{array}{l}00 \\
00 \\
00 \\
00 \\
00\end{array}$ & $\begin{array}{l}-3.39827 \mathrm{E} \\
-3.40843 \mathrm{E} \\
-3.41859 \mathrm{E} \\
-3.42874 \mathrm{E} \\
-3.43889 \mathrm{E}\end{array}$ & $\begin{array}{l}01 \\
01 \\
01 \\
01 \\
01\end{array}$ & $\begin{array}{l}-3.18275 \mathrm{E} \\
-3.20023 \mathrm{E} \\
-3.21776 \mathrm{E} \\
-3.23533 \mathrm{E} \\
-3.25296 \mathrm{E}\end{array}$ & $\begin{array}{l}00 \\
00 \\
00 \\
00 \\
00\end{array}$ & $\begin{array}{l}3.12465 \mathrm{E}-03 \\
3.12504 \mathrm{E}-03 \\
3.12543 \mathrm{E}-03 \\
3.12579 \mathrm{E}-03 \\
3.12615 \mathrm{E}-03\end{array}$ & $\begin{array}{l}-3.11770 \mathrm{E} \\
-3.13494 \mathrm{E} \\
-3.15223 \mathrm{E} \\
-3.16957 \mathrm{E} \\
-3.18695 \mathrm{E}\end{array}$ & $\begin{array}{l}00 \\
00 \\
00 \\
00 \\
00\end{array}$ & $\begin{array}{l}3.50194 \mathrm{E} \\
3.51209 \mathrm{E} \\
3.52224 \mathrm{E} \\
3.53238 \mathrm{E} \\
3.54252 \mathrm{E}\end{array}$ & $\begin{array}{l}01 \\
01 \\
01 \\
01 \\
01\end{array}$ & $\begin{array}{l}-2.37287 \mathrm{E} \\
-2.37454 \mathrm{E} \\
-2.37621 \mathrm{E} \\
-2.37788 \mathrm{E} \\
-2.37954 \mathrm{E}\end{array}$ & $\begin{array}{l}02 \\
02 \\
02 \\
02 \\
02\end{array}$ \\
\hline $\begin{array}{l}33.60 \\
33.70 \\
33.80 \\
33.90 \\
34.00\end{array}$ & $\begin{array}{l}1.12381 \mathrm{E} \\
1.12381 \mathrm{E} \\
1.12381 \mathrm{E} \\
1.12380 \mathrm{E} \\
1.12380 \mathrm{E}\end{array}$ & $\begin{array}{l}00 \\
00 \\
00 \\
00 \\
00\end{array}$ & $\begin{array}{l}-3.44904 \mathrm{E} \\
-3.45919 \mathrm{E} \\
-3.46933 \mathrm{E} \\
-3.47947 \mathrm{E} \\
-3.48961 \mathrm{E}\end{array}$ & $\begin{array}{l}01 \\
01 \\
01 \\
01 \\
01\end{array}$ & $\begin{array}{l}-3.27062 \mathrm{E} \\
-3.28834 \mathrm{E} \\
-3.30610 \mathrm{E} \\
-3.32390 \mathrm{E} \\
-3.34176 \mathrm{E}\end{array}$ & $\begin{array}{l}00 \\
00 \\
00 \\
00 \\
00\end{array}$ & $\begin{array}{l}3.12648 \mathrm{E}-03 \\
3.12681 \mathrm{E}-03 \\
3.12712 \mathrm{E}-03 \\
3.12742 \mathrm{E}-03 \\
3.12771 \mathrm{E}-03\end{array}$ & $\begin{array}{l}-3.20438 \mathrm{E} \\
-3.22185 \mathrm{E} \\
-3.23937 \mathrm{E} \\
-3.25694 \mathrm{E} \\
-3.27455 \mathrm{E}\end{array}$ & $\begin{array}{l}00 \\
00 \\
00 \\
00 \\
00\end{array}$ & $\begin{array}{l}3.55266 \mathrm{E} \\
3.56280 \mathrm{E} \\
3.57294 \mathrm{E} \\
3.58307 \mathrm{E} \\
3.59320 \mathrm{E}\end{array}$ & $\begin{array}{l}01 \\
01 \\
01 \\
01 \\
01\end{array}$ & $\begin{array}{l}-2.38121 \mathrm{E} \\
-2.38288 \mathrm{E} \\
-2.38454 \mathrm{E} \\
-2.38621 \mathrm{E} \\
-2.38787 \mathrm{E}\end{array}$ & $\begin{array}{l}02 \\
02 \\
02 \\
02 \\
02\end{array}$ \\
\hline $\begin{array}{l}34.10 \\
34.20 \\
34.30 \\
34.40 \\
34.50\end{array}$ & $\begin{array}{l}1.12379 \mathrm{E} \\
1.12379 \mathrm{E} \\
1.12378 \mathrm{E} \\
1.12378 \mathrm{E} \\
1.12377 \mathrm{E}\end{array}$ & $\begin{array}{l}00 \\
00 \\
00 \\
00 \\
00\end{array}$ & $\begin{array}{l}-3.49975 \mathrm{E} \\
-3.50989 \mathrm{E} \\
-3.52002 \mathrm{E} \\
-3.53015 \mathrm{E} \\
-3.54028 \mathrm{E}\end{array}$ & $\begin{array}{l}01 \\
01 \\
01 \\
01 \\
01\end{array}$ & $\begin{array}{l}-3.35965 \mathrm{E} \\
-3.37760 \mathrm{E} \\
-3.39559 \mathrm{E} \\
-3.41362 \mathrm{E} \\
-3.43171 \mathrm{E}\end{array}$ & $\begin{array}{l}00 \\
00 \\
00 \\
00 \\
00\end{array}$ & $\begin{array}{l}3.12798 \mathrm{E}-03 \\
3.12823 \mathrm{E}-03 \\
3.12848 \mathrm{E}-03 \\
3.12872 \mathrm{E}-03 \\
3.12894 \mathrm{E}-03\end{array}$ & $\begin{array}{l}-3.29221 \mathrm{E} \\
-3.30991 \mathrm{E} \\
-3.32766 \mathrm{E} \\
-3.34546 \mathrm{E} \\
-3.36330 \mathrm{E}\end{array}$ & $\begin{array}{l}00 \\
00 \\
00 \\
00 \\
00\end{array}$ & $\begin{array}{l}3.60333 \mathrm{E} \\
3.61345 \mathrm{E} \\
3.62358 \mathrm{E} \\
3.63370 \mathrm{E} \\
3.64382 \mathrm{E}\end{array}$ & $\begin{array}{l}01 \\
01 \\
01 \\
01 \\
01\end{array}$ & $\begin{array}{l}-2.38953 \mathrm{E} \\
-2.39119 \mathrm{E} \\
-2.39285 \mathrm{E} \\
-2.39451 \mathrm{E} \\
-2.39617 \mathrm{E}\end{array}$ & $\begin{array}{l}02 \\
02 \\
02 \\
02 \\
02\end{array}$ \\
\hline $\begin{array}{l}34.60 \\
34.70 \\
34.80 \\
34.90 \\
35.00\end{array}$ & $\begin{array}{l}1.12377 \mathrm{E} \\
1.12376 \mathrm{E} \\
1.12376 \mathrm{E} \\
1.12375 \mathrm{E} \\
1.12375 \mathrm{E}\end{array}$ & $\begin{array}{l}00 \\
00 \\
00 \\
00 \\
00\end{array}$ & $\begin{array}{l}-3.55041 \mathrm{E} \\
-3.56053 \mathrm{E} \\
-3.57066 \mathrm{E} \\
-3.58078 \mathrm{E} \\
-3.59089 \mathrm{E}\end{array}$ & $\begin{array}{l}01 \\
01 \\
01 \\
01 \\
01\end{array}$ & $\begin{array}{l}-3.44983 \mathrm{E} \\
-3.46801 \mathrm{E} \\
-3.48623 \mathrm{E} \\
-3.50449 \mathrm{E} \\
-3.52280 \mathrm{E}\end{array}$ & $\begin{array}{l}00 \\
00 \\
00 \\
00 \\
00\end{array}$ & $\begin{array}{l}3.12915 \mathrm{E}-03 \\
3.12934 \mathrm{E}-03 \\
3.12953 \mathrm{E}-03 \\
3.12970 \mathrm{E}-03 \\
3.12986 \mathrm{E}-03\end{array}$ & $\begin{array}{l}-3.38118 \mathrm{E} \\
-3.39912 \mathrm{E} \\
-3.41710 \mathrm{E} \\
-3.43512 \mathrm{E} \\
-3.45320 \mathrm{E}\end{array}$ & $\begin{array}{l}00 \\
00 \\
00 \\
00 \\
00\end{array}$ & $\begin{array}{l}3.65394 \mathrm{E} \\
3.66405 \mathrm{E} \\
3.67417 \mathrm{E} \\
3.68428 \mathrm{E} \\
3.69438 \mathrm{E}\end{array}$ & $\begin{array}{l}01 \\
01 \\
01 \\
01 \\
01\end{array}$ & $\begin{array}{l}-2.39782 \mathrm{E} \\
-2.39949 \mathrm{E} \\
-2.40114 \mathrm{E} \\
-2.40280 \mathrm{E} \\
-2.40445 \mathrm{E}\end{array}$ & $\begin{array}{l}02 \\
02 \\
02 \\
02 \\
02\end{array}$ \\
\hline $\begin{array}{l}35.10 \\
35.20 \\
35.30 \\
35.40 \\
35.50\end{array}$ & $\begin{array}{l}1.12374 \mathrm{E} \\
1.12374 \mathrm{E} \\
1.12374 \mathrm{E} \\
1.12373 \mathrm{E} \\
1.12373 \mathrm{E}\end{array}$ & $\begin{array}{l}00 \\
00 \\
00 \\
00 \\
00\end{array}$ & $\begin{array}{l}-3.60101 \mathrm{E} \\
-3.61112 \mathrm{E} \\
-3.62123 \mathrm{E} \\
-3.63134 \mathrm{E} \\
-3.64145 \mathrm{E}\end{array}$ & $\begin{array}{l}01 \\
01 \\
01 \\
01 \\
01\end{array}$ & $\begin{array}{l}-3.54116 \mathrm{E} \\
-3.55956 \mathrm{E} \\
-3.57801 \mathrm{E} \\
-3.59651 \mathrm{E} \\
-3.61505 \mathrm{E}\end{array}$ & $\begin{array}{l}00 \\
00 \\
00 \\
00 \\
00\end{array}$ & $\begin{array}{l}3.13001 \mathrm{E}-03 \\
3.13015 \mathrm{E}-03 \\
3.13027 \mathrm{E}-03 \\
3.13038 \mathrm{E}-03 \\
3.13048 \mathrm{E}-03\end{array}$ & $\begin{array}{l}-3.47131 \mathrm{E} \\
-3.48948 \mathrm{E} \\
-3.50769 \mathrm{E} \\
-3.52594 \mathrm{E} \\
-3.54424 \mathrm{E}\end{array}$ & $\begin{array}{l}00 \\
00 \\
00 \\
00\end{array}$ & $\begin{array}{l}3.70449 \mathrm{E} \\
3.71459 \mathrm{E} \\
3.72470 \mathrm{E} \\
3.73480 \mathrm{E} \\
3.74489 \mathrm{E}\end{array}$ & $\begin{array}{l}01 \\
01 \\
01 \\
01 \\
01\end{array}$ & $\begin{array}{l}-2.40611 \mathrm{E} \\
-2.40776 \mathrm{E} \\
-2.40941 \mathrm{E} \\
-2.41107 \mathrm{E} \\
-2.41272 \mathrm{E}\end{array}$ & $\begin{array}{l}02 \\
02 \\
02 \\
02 \\
02\end{array}$ \\
\hline $\begin{array}{l}35.60 \\
35.70 \\
35.80 \\
35.90 \\
36.00\end{array}$ & $\begin{array}{l}1.12372 \mathrm{E} \\
1.12372 \mathrm{E} \\
1.12371 \mathrm{E} \\
1.12371 \mathrm{E} \\
1.12370 \mathrm{E}\end{array}$ & $\begin{array}{l}00 \\
00 \\
00 \\
00 \\
00\end{array}$ & $\begin{array}{l}-3.65156 \mathrm{E} \\
-3.66166 \mathrm{E} \\
-3.67176 \mathrm{E} \\
-3.68186 \mathrm{E} \\
-3.69195 \mathrm{E}\end{array}$ & $\begin{array}{l}01 \\
01 \\
01 \\
01 \\
01\end{array}$ & $\begin{array}{l}-3.63364 \mathrm{E} \\
-3.65227 \mathrm{E} \\
-3.67095 \mathrm{E} \\
-3.68967 \mathrm{E} \\
-3.70844 \mathrm{E}\end{array}$ & $\begin{array}{l}00 \\
00 \\
00 \\
00 \\
00\end{array}$ & $\begin{array}{l}3.13057 \mathrm{E}-03 \\
3.13064 \mathrm{E}-03 \\
3.13071 \mathrm{E}-03 \\
3.13076 \mathrm{E}-03 \\
3.13080 \mathrm{E}-03\end{array}$ & $\begin{array}{l}-3.56259 \mathrm{E} \\
-3.58098 \mathrm{E} \\
-3.59942 \mathrm{E} \\
-3.61791 \mathrm{E} \\
-3.63644 \mathrm{E}\end{array}$ & $\begin{array}{l}00 \\
00 \\
00 \\
00\end{array}$ & $\begin{array}{l}3.75499 \mathrm{E} \\
3.76508 \mathrm{E} \\
3.77517 \mathrm{E} \\
3.78526 \mathrm{E} \\
3.79534 \mathrm{E}\end{array}$ & $\begin{array}{l}01 \\
01 \\
01 \\
01 \\
01\end{array}$ & $\begin{array}{l}-2.41437 \mathrm{E} \\
-2.41603 \mathrm{E} \\
-2.41768 \mathrm{E} \\
-2.41933 \mathrm{E} \\
-2.42098 \mathrm{E}\end{array}$ & $\begin{array}{l}02 \\
02 \\
02 \\
02 \\
02\end{array}$ \\
\hline
\end{tabular}


$Z=5 \quad A=11 \quad \rho=1.2 A^{1 / 3} F$

$\bar{F}$

$1.35010 \mathrm{E}-03$

$1.35908 \mathrm{E}-03$

$1.36810 \mathrm{E}-03$

$1.37714 \mathrm{E}-03$

$1.38621 \mathrm{E}-03$

$1.39531 \mathrm{E}-03$

$1.40444 \mathrm{E}-03$

$1.41360 \mathrm{E}-03$

$1.42279 \mathrm{E}-03$

$1.43201 \mathrm{E}-03$

$1.44126 \mathrm{E}-03$

$1.45054 \mathrm{E}-03$

$1.45985 \mathrm{E}-03$

$1.46919 \mathrm{E}-03$

$1.47856 \mathrm{E}-03$

$1.48796 \mathrm{E}-03$

$1.49739 \mathrm{E}-03$

$1.50685 \mathrm{E}-03$

$1.51633 \mathrm{E}-03$

$1.52585 \mathrm{E}-03$

$1.53540 \mathrm{E}-03$

$1.54498 \mathrm{E}-03$

$1.55459 \mathrm{E}-03$

$1.56422 \mathrm{E}-03$

$1.57389 \mathrm{E}-03$

$1.58359 \mathrm{E}-03$

$1.59331 \mathrm{E}-03$

$1.60307 \mathrm{E}-03$

$1.61285 \mathrm{E}-03$

$1.62267 \mathrm{E}-03$

$1.63252 \mathrm{E}-03$

$1.64239 \mathrm{E}-03$

$1.65230 \mathrm{E}-03$

$1.66223 \mathrm{E}-03$

$1.67220 \mathrm{E}-03$

$1.68219 \mathrm{E}-03$

$1.69221 \mathrm{E}-03$

$1.70227 \mathrm{E}-03$

$1.71235 \mathrm{E}-03$

$1.72246 \mathrm{E}-03$

$1.73261 \mathrm{E}-03$

$1.74278 \mathrm{E}-03$

$1.75298 \mathrm{E}-03$

$1.76322 \mathrm{E}-03$

$1.77348 \mathrm{E}-03$

$1.78377 \mathrm{E}-03$

$1.79409 \mathrm{E}-03$

$1.80444 \mathrm{E}-03$

$1.81482 \mathrm{E}-03$

$1.82524 \mathrm{E}-03$

$1.83568 \mathrm{E}-03$

$1.84615 \mathrm{E}-03$

$1.85665 \mathrm{E}-03$

$1.86718 \mathrm{E}-03$

$1.87774 \mathrm{E}-03$

$1.88833 \mathrm{E}-03$

$1.89895 \mathrm{E}-03$

$1.90960 \mathrm{E}-03$

$1.92027 \mathrm{E}-03$

$1.93098 \mathrm{E}-03$ $f_{2}$

$-2.15796 \mathrm{E} \quad 00$ $-2.17235 \mathrm{E} \quad 00$ $-2.18679 \mathrm{E} \quad 00$ $-2.20128 \mathrm{E} \quad 00$

$-2.21582 \mathrm{E} \quad 00$

$-2.23040 \mathrm{E} \quad 00$

$-2.24503 \mathrm{E} \quad 00$

$-2.25971 \mathrm{E} \quad 00$

$-2.27443 \mathrm{E} \quad 00$

$-2.28920 \mathrm{E} \quad 00$

$-2.30402 \mathrm{E} \quad 00$

$-2.31889 \mathrm{E} \quad 00$

$-2.33380 \mathrm{E} \quad 00$

$-2.34876 \mathrm{E} \quad 00$

$-2.36376 \mathrm{E} 00$

$-2.37882 \mathrm{E} \quad 00$

$-2.39392 \mathrm{E} \quad 00$

$-2.40906 \mathrm{E} \quad 00$

$-2.42426 \mathrm{E} \quad 00$

$-2.43950 \mathrm{E} 00$

$-2.45478 \mathrm{E} \quad 00$

$-2.47012 \mathrm{E} \quad 00$

$-2.48550 \mathrm{E} \quad 00$

$-2.50093 \mathrm{E} \quad 00$

$-2.51640 \mathrm{E} 00$

$-2.53192 \mathrm{E} \quad 00$

$-2.54749 \mathrm{E} \quad 00$

$-2.56311 \mathrm{E} \quad 00$

$-2.57877 \mathrm{E} \quad 00$

$-2.59448 \mathrm{E} \quad 00$

$-2.61023 \mathrm{E} \quad 00$

$-2.62603 \mathrm{E} \quad 00$

$-2.64188 \mathrm{E} \quad 00$

$-2.65778 \mathrm{E} \quad 00$

$-2.67372 \mathrm{E} \quad 00$

$-2.68971 \mathrm{E} \quad 00$

$-2.70574 \mathrm{E} \quad 00$

$-2.72182 \mathrm{E} \quad 00$

$-2.73795 \mathrm{E} \quad 00$

$-2.75413 \mathrm{E} \quad 00$

$-2.77035 \mathrm{E} \quad 00$

$-2.78662 \mathrm{E} \quad 00$

$-2.80294 \mathrm{E} \quad 00$

$-2.81930 \mathrm{E} \quad 00$

$-2.83571 \mathrm{E} 00$

$-2.85216 \mathrm{E} \quad 00$

$-2.86866 \mathrm{E} \quad 00$

$-2.88521 \mathrm{E} \quad 00$

$-2.90181 \mathrm{E} \quad 00$

$-2.91845 \mathrm{E} \quad 00$

$-2.93513 \mathrm{E} \quad 00$

$-2.95187 \mathrm{E} \quad 00$

$-2.96865 \mathrm{E} \quad 00$

$-2.98548 \mathrm{E} \quad 00$

$-3.00235 \mathrm{E} 00$

$-3.01927 \mathrm{E} \quad 00$

$-3.03624 \mathrm{E} \quad 00$

$-3.05325 \mathrm{E} 00$

$-3.07031 \mathrm{E} \quad 00$

$-3.08742 \mathrm{E} \quad 00$ $g_{2}$

$-1.11013 \mathrm{E}-01$

$-1.12055 \mathrm{E}-01$

$-1.13104 \mathrm{E}-01$

$-1.14159 \mathrm{E}-01$

$-1.15221 \mathrm{E}-01$

$-1.16289 \mathrm{E}-01$

$-1.17364 \mathrm{E}-01$

$-1.18445 \mathrm{E}-01$

$-1.19533 \mathrm{E}-01$

$-1.20627 \mathrm{E}-01$

$-1.21728 \mathrm{E}-01$

$-1.22836 \mathrm{E}-01$

$-1.23950 \mathrm{E}-01$

$-1.25071 \mathrm{E}-01$

$-1.26198 \mathrm{E}-01$

$-1.27333 \mathrm{E}-01$

$-1.28473 \mathrm{E}-01$

$-1.29621 \mathrm{E}-01$

$-1.30776 \mathrm{E}-01$

$-1.31937 \mathrm{E}-01$

$-1.33105 \mathrm{E}-01$

$-1.34280 \mathrm{E}-01$

$-1.35461 \mathrm{E}-01$

$-1.36650 \mathrm{E}-01$

$-1.37845 \mathrm{E}-01$

$-1.39047 \mathrm{E}-01$

$-1.40257 \mathrm{E}-01$

$-1.41473 \mathrm{E}-01$

$-1.42696 \mathrm{E}-01$

$-1.43926 \mathrm{E}-01$

$-1.45163 \mathrm{E}-01$

$-1.46407 \mathrm{E}-01$

$-1.47658 \mathrm{E}-01$

$-1.48916 \mathrm{E}-01$

$-1.50181 \mathrm{E}-01$

$-1.51453 \mathrm{E}-01$

$-1.52732 \mathrm{E}-01$

$-1.54018 \mathrm{E}-01$

$-1.55312 \mathrm{E}-01$

$-1.56613 \mathrm{E}-01$

$-1.57920 \mathrm{E}-01$

$-1.59235 \mathrm{E}-01$

$-1.60558 \mathrm{E}-01$

$-1.61887 \mathrm{E}-01$

$-1.63224 \mathrm{E}-01$

$-1.64568 \mathrm{E}-01$

$-1.65919 \mathrm{E}-01$

$-1.67278 \mathrm{E}-01$

$-1.68643 \mathrm{E}-01$

$-1.70017 \mathrm{E}-01$

$-1.71397 \mathrm{E}-01$

$-1.72785 \mathrm{E}-01$

$-1.74181 \mathrm{E}-01$

$-1.75583 \mathrm{E}-01$

$-1.76994 \mathrm{E}-01$

$-1.78411 \mathrm{E}-01$

$-1.79837 \mathrm{E}-01$

$-1.81269 \mathrm{E}-01$

$-1.82709 \mathrm{E}-01$

$-1.84157 \mathrm{E}-01$ $\tan \delta_{2}$

$f_{-2}$

$4.10852 \mathrm{E} \quad 01 \quad-1.08580 \mathrm{E}-01$

$4.10871 \mathrm{E}$ 0l $-1.09604 \mathrm{E}-0$

$4.10889 \mathrm{E} 01-1.10635 \mathrm{E}-01$

$4.10907 \mathrm{E}$ 01 $-1.11672 \mathrm{E}-01$

$4.10925 \mathrm{E} \quad 01 \quad-1.12715 \mathrm{E}-01$

$4.10943 \mathrm{E}$ 01 $-1.13765 \mathrm{E}-01$

$4.10961 \mathrm{E} 01-1.14821 \mathrm{E}-01$

$4.10979 \mathrm{E} \quad 01-1.15884 \mathrm{E}-01$

$4.10996 \mathrm{E}$ 01 $-1.16953 \mathrm{E}-01$

$4.11014 \mathrm{E} \quad 01-1.18029 \mathrm{E}-01$

$4.11031 \mathrm{E} \quad 01$

$4.11048 \mathrm{E} \quad 01$

$4.11065 \mathrm{E} \quad 01$

$4.11082 \mathrm{E} \quad 01$

$4.11099 \mathrm{E} 01$

$4.11116 \mathrm{E} \quad 01$

$4.11133 \mathrm{E} \quad 01$

$4.11149 \mathrm{E} \quad 01$

$4.11166 \mathrm{E} \quad 01$

$4.11182 \mathrm{E} \quad 01$

$4.11198 \mathrm{E} \quad 01$ $4.11214 \mathrm{E} \quad 01$ $4.11230 \mathrm{E} \quad 01$

$4.11246 \mathrm{E} \quad 01$

$4.11262 \mathrm{E} 01$

$4.11278 \mathrm{E} \quad 01$

$4.11294 \mathrm{E} \quad 01$

$4.11309 \mathrm{E} \quad 01$

$4.11325 \mathrm{E} \quad 01$

$4.11340 \mathrm{E} \quad 01$

$4.11355 \mathrm{E} \quad 01$

$4.11370 \mathrm{E} \quad 01$

$4.11385 \mathrm{E} \quad 01$

$4.11400 \mathrm{E} \quad 01$

$4.11415 \mathrm{E} \quad 01$

$4.11430 \mathrm{E} \quad 01$ $4.11444 \mathrm{E} \quad 01$ $4.11459 \mathrm{E} \quad 01$ $4.11474 \mathrm{E} \quad 01$ $4.11488 \mathrm{E} \quad 01$

$4.11502 \mathrm{E} \quad 01$ $4.11517 \mathrm{E} \quad 01$ $4.11531 \mathrm{E} 01$

$4.11545 \mathrm{E} \quad 01$

$4.11559 \mathrm{E} 01$

$4.11573 \mathrm{E} \quad 01$

$4.11586 \mathrm{E} \quad 01$

$4.11600 \mathrm{E} \quad 01$

$4.11614 \mathrm{E} 01$

$4.11627 \mathrm{E}$ 01

$4.11641 \mathrm{E} \quad 01$ $4.11654 \mathrm{E}$ 0l $4.11668 \mathrm{E} \quad 01$ $4.11681 \mathrm{E} \quad 01$ $4.11694 \mathrm{E} 01$

$4.11707 \mathrm{E} \quad 01$ $4.11720 \mathrm{E}$ 0I $4.11733 \mathrm{E} \quad 01$

$4.11746 \mathrm{E} \quad 01$ $4.11759 \mathrm{E} 01$

$-1.19111 \mathrm{E}-01$

$-1.20200 \mathrm{E}-01$

$-1.21295 \mathrm{E}-01$

$-1.22397 \mathrm{E}-01$

$-1.23506 \mathrm{E}-01$

$-1.24621 \mathrm{E}-01$ $-1.25743 \mathrm{E}-01$

$-1.26871 \mathrm{E}-01$

$-1.28006 \mathrm{E}-01$

$-1.29148 \mathrm{E}-01$

$-1.30297 \mathrm{E}-01$ $-1.31452 \mathrm{E}-01$

$-1.32614 \mathrm{E}-01$

$-1.33783 \mathrm{E}-01$

$-1.34959 \mathrm{E}-01$

$-1.36141 \mathrm{E}-01$

$-1.37331 \mathrm{E}-01$

$-1.38527 \mathrm{E}-01$

$-1.39730 \mathrm{E}-01$

$-1.40940 \mathrm{E}-01$

$-1.42157 \mathrm{E}-01$

$-1.43381 \mathrm{E}-01$

$-1.44611 \mathrm{E}-01$

$-1.45849 \mathrm{E}-01$

$-1.47094 \mathrm{E}-01$

$-1.48346 \mathrm{E}-01$

$-1.49604 \mathrm{E}-01$

$-1.50870 \mathrm{E}-01$

$-1.52143 \mathrm{E}-01$

$-1.53423 \mathrm{E}-01$

$-1.54710 \mathrm{E}-01$

$-1.56004 \mathrm{E}-01$

$-1.57306 \mathrm{E}-01$

$-1.58614 \mathrm{E}-0 \mathrm{l}$

$-1.59930 \mathrm{E}-01$

$-1.61253 \mathrm{E}-01$

$-1.62583 \mathrm{E}-01$

$-1.63921 \mathrm{E}-01$

$-1.65265 \mathrm{E}-01$

$-1.66617 \mathrm{E}-01$

$-1.67976 \mathrm{E}-01$ $-1.69343 \mathrm{E}-01$ 
ELECTRON RADIAL FUNCTIONS

Electron

(n)

36.10

36.20

36.30

36.40

36.50

36.60

36.70

36.80

36.90

37.00

37.10

37.20

37.30

37.40

37.50

37.60

37.70

37.80

37.90

38.00

38.10

38.20

38.30

38.40

38.50

38.60

38.70

38.80

38.90

39.00

39.10

39.20

39.30

39.40

39.50

39.60

39.70

39.80

39.90

40.00

40.10

40.20

40.30

40.40

40.50

40.60

40.70

40.80

40.90

41.00

41.10

41.20

41.30

41.40

41.50

41.60

41.70

41.80

41.90

42.00

$Z=5$

$$
A=11
$$

$\rho=1.2 A^{1 / 3} F$

$f_{1}$

$\begin{array}{llll}1.12370 \mathrm{E} & 00 & -3.70204 \mathrm{E} & 01\end{array}$

$1.12370 \mathrm{E} \quad 00$

$1.12369 \mathrm{E} \quad 00$

$1.12369 \mathrm{E} \quad 00$

$1.12368 \mathrm{E} \quad 00$

$1.12368 \mathrm{E} \quad 00$

$1.12367 \mathrm{E} \quad 00$

$1.12367 \mathrm{E} \quad 00$

$1.12366 \mathrm{E} \quad 00$

$1.12366 \mathrm{E} \quad 00$

$1.12366 \mathrm{E} \quad 00$

$1.12365 \mathrm{E} \quad 00$

$1.12365 \mathrm{E} \quad 00$

$1.12364 \mathrm{E} \quad 00$
0

$1.12364 \mathrm{E} \quad 00$

$1.12364 \mathrm{E} \quad 00$

$1.12363 \mathrm{E} \quad 00$

$1.12363 \mathrm{E} \quad 00$

$\begin{array}{ll}1.12362 \mathrm{E} & 00\end{array}$

$1.12362 \mathrm{E} \quad 00$

$1.12361 \mathrm{E} \quad 00$

$1.12361 \mathrm{E} \quad 00$

$1.12361 \mathrm{E} \quad 00$

$1.12360 \mathrm{E} \quad 00$

$1.12360 \mathrm{E} \quad 00$

$1.12359 \mathrm{E} \quad 00$

$1.12359 \mathrm{E} \quad 00$

$1.12359 \mathrm{E} \quad 00$

$1.12358 \mathrm{E} \quad 00$

$1.12358 \mathrm{E} \quad 00$

$1.12357 \mathrm{E} \quad 00$

$1.12357 \mathrm{E} \quad 00$

$1.12357 \mathrm{E} \quad 00$

$1.12356 \mathrm{E} \quad 00$

$1.12356 \mathrm{E} \quad 00$

$1.12355 \mathrm{E} \quad 00$

$1.12355 \mathrm{E} \quad 00$

$1.12355 \mathrm{E} \quad 00$

$1.12354 \mathrm{E} \quad 00$

$1.12354 \mathrm{E} 00$

$1.12353 \mathrm{E} \quad 00$

$1.12353 \mathrm{E} \quad 00$

$1.12353 \mathrm{E} \quad 00$

$1.12352 \mathrm{E} \quad 00$

$1.12352 \mathrm{E} \quad 00$

$1.12351 \mathrm{E} \quad 00$

$1.12351 \mathrm{E} \quad 00$

$1.12351 \mathrm{E} \quad 00$

$1.12350 \mathrm{E} \quad 00$

$1.12350 \mathrm{E} \quad 00$

$1.12350 \mathrm{E} \quad 00$

$1.12349 \mathrm{E} \quad 00$

$1.12349 \mathrm{E} \quad 00$

$1.12348 \mathrm{E} \quad 00$

$1.12348 \mathrm{E} \quad 00$

$1.12348 \mathrm{E} \quad 00$

$1.12347 \mathrm{E} \quad 00$

$1.12347 \mathrm{E} \quad 00$

$1.12347 \mathrm{E} \quad 00$

$1.12346 \mathrm{E} \quad 00$ $g_{1}$

$-3.72726 \mathrm{E} \quad 00$

$-3.74612 \mathrm{E} 00$

$-3.76503 \mathrm{E} 00$

$-3.78398 \mathrm{E} \quad 00$

$-3.80298 \mathrm{E} 00$

$-3.82202 \mathrm{E} \quad 00$ $-3.84111 \mathrm{E} \quad 00$

$-3.86025 \mathrm{E} \quad 00$

$-3.87943 \mathrm{E} \quad 00$

$-3.89866 \mathbf{E} 00$

$-3.91793 \mathrm{E} 00$

-3.93725 E 00

$-3.95661 \mathrm{E} 00$

$-3.97602 \mathrm{E} \quad 00$

$-3.99547 \mathrm{E} \quad 00$

$-4.01498 \mathrm{E} \quad 00$

$-4.03452 \mathrm{E} \quad 00$

$-4.05411 \mathrm{E} \quad 00$

$-4.07375 \mathrm{E} \quad 00$

$-4.09343 \mathrm{E} \quad 00$

$-4.11316 \mathrm{E} \quad 00$

$-4.13293 \mathrm{E} \quad 00$

$-4.15275 \mathrm{E} \quad 00$

$-4.17262 \mathrm{E} \quad 00$

$-4.19253 \mathrm{E} \quad 00$

$-4.21248 \mathrm{E} \quad 00$

$-4.23248 \mathrm{E} \quad 00$

$-4.25253 \mathrm{E} \quad 00$

$-4.27262 \mathrm{E} \quad 00$

$-4.29276 \mathrm{E} \quad 00$

$-4.31294 \mathrm{E} \quad 00$

$-4.33317 \mathrm{E} \quad 00$

$-4.35344 \mathrm{E} \quad 00$

$-4.37376 \mathrm{E} \quad 00$

$-4.39412 \mathrm{E} \quad 00$

$-4.41453 \mathrm{E} \quad 00$

$-4.43498 \mathrm{E} \quad 00$

$-4.45548 \mathrm{E} \quad 00$

$-4.47603 \mathrm{E} \quad 00$

$-4.49662 \mathrm{E} \quad 00$

$-4.51725 \mathrm{E} \quad 00$

$-4.53793 \mathrm{E} \quad 00$

$-4.55866 \mathrm{E} 00$

$-4.57943 \mathrm{E} \quad 00$

$-4.60024 \mathrm{E} \quad 00$

$-4.62111 \mathrm{E} \quad 00$

$-4.64201 \mathrm{E} \quad 00$

$-4.66296 \mathrm{E} \quad 00$

$-4.68396 \mathrm{E} \quad 00$

$-4.70500 \mathrm{E} \quad 00$

$-4.72609 \mathrm{E} \quad 00$

$-4.74722 \mathrm{E} \quad 00$

$-4.76839 \mathrm{E} \quad 00$

$-4.78962 \mathrm{E} \quad 00$

$-4.81088 \mathrm{E} 00$

$-4.83219 \mathrm{E} \quad 00$

$-4.85355 \mathrm{E} \quad 00$

$-4.87495 \mathrm{E} \quad 00$

$-4.89640 \mathrm{E} 00$

$-4.91789 \mathrm{E} \quad 00$ $\tan \delta_{1}$

$3.13083 \mathrm{E}-03$

$3.13085 \mathrm{E}-03$

$3.13085 \mathrm{E}-03$

$3.13085 \mathrm{E}-03$

$3.13083 \mathrm{E}-03$

$3.13081 \mathrm{E}-03$

$3.13077 \mathrm{E}-03$

$3.13072 \mathrm{E}-03$

$3.13066 \mathrm{E}-03$

$3.13059 \mathrm{E}-03$

$3.13050 \mathrm{E}-03$

$3.13041 \mathrm{E}-03$

$3.13030 \mathrm{E}-03$

$3.13020 \mathrm{E}-03$

$3.13007 \mathrm{E}-03$

$3.12993 \mathrm{E}-03$

$3.12978 \mathrm{E}-03$

$3.12963 \mathrm{E}-03$

$3.12946 \mathrm{E}-03$

$3.12928 \mathrm{E}-03$

$3.12910 \mathrm{E}-03$

$3.12889 \mathrm{E}-03$

$3.12869 \mathrm{E}-03$

$3.12846 \mathrm{E}-03$

$3.12824 \mathrm{E}-03$

$3.12800 \mathrm{E}-03$

$3.12774 \mathrm{E}-03$

$3.12748 \mathrm{E}-03$

3.12721 E-03

$3.12694 \mathrm{E}-03$

$3.12665 \mathrm{E}-03$

$3.12635 \mathrm{E}-03$

$3.12604 \mathrm{E}-03$

$3.12572 \mathrm{E}-03$

$3.12539 \mathrm{E}-03$

$3.12505 \mathrm{E}-03$

$3.12471 \mathrm{E}-03$

$3.12435 \mathrm{E}-03$

$3.12398 \mathrm{E}-03$

$3.12361 \mathrm{E}-03$

$3.12322 \mathrm{E}-03$

$3.12283 \mathrm{E}-03$

$3.12242 \mathrm{E}-03$

$3.12201 \mathrm{E}-03$

$3.12159 \mathrm{E}-03$

$3.12116 \mathrm{E}-03$

$3.12071 \mathrm{E}-03$

$3.12026 \mathrm{E}-03$

$3.11981 \mathrm{E}-03$

$3.11934 \mathrm{E}-03$

$3.11886 \mathrm{E}-03$

$3.11838 \mathrm{E}-03$

$3.11789 \mathrm{E}-03$

$3.11738 \mathrm{E}-03$

$3.11687 \mathrm{E}-03$

$3.11634 \mathrm{E}-03$

$3.11581 \mathrm{E}-03$

$3.11527 \mathrm{E}-03$

$3.11473 \mathrm{E}-03$

$3.11417 \mathrm{E}-03$

$f_{-1}$

$-3.65501 \mathrm{E} \quad 00$ $-3.67364 \mathrm{E} \quad 00$

$-3.69230 \mathrm{E} \quad 00$

$-3.71102 \mathrm{E} \quad 00$

$-3.72977 \mathrm{E} \quad 00$

$-3.74858 \mathrm{E} \quad 00$

$-3.76743 \mathrm{E} \quad 00$

$-3.78633 \mathrm{E} 00$

$-3.80527 \mathrm{E} \quad 00$

$-3.82426 \mathrm{E} 00$

$-3.84329 \mathrm{E} \quad 00$

$-3.86237 \mathrm{E} \quad 00$

$-3.88149 \mathrm{E} \quad 00$

$-3.90066 \mathrm{E} \quad 00$

$-3.91988 \mathrm{E} \quad 00$

$-3.93914 \mathrm{E} \quad 00$

$-3.95845 \mathrm{E} \quad 00$

$-3.97780 \mathrm{E} \quad 00$

$-3.99720 \mathrm{E} \quad 00$

$-4.01664 \mathrm{E} \quad 00$

$-4.03613 \mathrm{E} \quad 00$

$-4.05566 \mathrm{E} \quad 00$

$-4.07524 \mathrm{E} 00$

$-4.09487 \mathrm{E} \quad 00$

$-4.11454 \mathrm{E} \quad 00$

$-4.13426 \mathrm{E} \quad 00$

$-4.15402 \mathrm{E} \quad 00$

$-4.17383 \mathrm{E} \quad 00$

$-4.19368 \mathrm{E} 00$

$-4.21358 \mathrm{E} 00$

$-4.23352 \mathrm{E} \quad 00$

$-4.25351 \mathrm{E} \quad 00$

$-4.27354 \mathrm{E} \quad 00$

$-4.29362 \mathrm{E} \quad 00$

$-4.31375 \mathrm{E} \quad 00$

$-4.33392 \mathrm{E} \quad 00$

$-4.35413 \mathrm{E} \quad 00$

$-4.37439 \mathrm{E} \quad 00$ 
$Z=5 \quad A=11 \quad \rho=1.2 A^{1 / 3} F$

\section{$\bar{F}$}

$1.94172 \mathrm{E}-03$

$1.95249 \mathrm{E}-03$

$1.96329 \mathrm{E}-03$

$1.97412 \mathrm{E}-03$

$1.98497 \mathrm{E}-03$

$1.99586 \mathrm{E}-03$

$2.00678 \mathrm{E}-03$

$2.01773 \mathrm{E}-03$

$2.02870 \mathrm{E}-03$

$2.03971 \mathrm{E}-03$

$2.05075 \mathrm{E}-03$

$2.06181 \mathrm{E}-03$

$2.07291 \mathrm{E}-03$

$2.08403 \mathrm{E}-03$

$2.09519 \mathrm{E}-03$

$2.10637 \mathrm{E}-03$

$2.11759 \mathrm{E}-03$

$2.12883 \mathrm{E}-03$

$2.14011 \mathrm{E}-03$

$2.15141 \mathrm{E}-03$

$2.16275 \mathrm{E}-03$

$2.17411 \mathrm{E}-03$

$2.18550 \mathrm{E}-03$

$2.19693 \mathrm{E}-03$

$2.20838 \mathrm{E}-03$

$2.21986 \mathrm{E}-03$

$2.23137 \mathrm{E}-03$

$2.24292 \mathrm{E}-03$

$2.25449 \mathrm{E}-03$

$2.26609 \mathrm{E}-03$

$2.27772 \mathrm{E}-03$ $2.28938 \mathrm{E}-03$

$2.30107 \mathrm{E}-03$

$2.31279 \mathrm{E}-03$

$2.32455 \mathrm{E}-03$

$2.33633 \mathrm{E}-03$ $2.34814 \mathrm{E}-03$

2.35998E-03

$2.37185 \mathrm{E}-03$

$2.38374 \mathrm{E}-03$

$2.39567 \mathrm{E}-03$

$2.40763 E-03$

$2.41962 \mathrm{E}-03$

$2.43164 \mathrm{E}-03$

$2.44369 \mathrm{E}-03$

$2.45577 \mathrm{E}-03$

$2.46787 \mathrm{E}-03$

$2.48001 \mathrm{E}-03$

$2.49218 \mathrm{E}-03$

$2.50438 \mathrm{E}-03$

$2.51660 \mathrm{E}-03$

$2.52886 \mathrm{E}-03$

$2.54115 \mathrm{E}-03$

$2.55346 \mathrm{E}-03$

2.56581 E- 03

$2.57818 \mathrm{E}-03$ $2.59059 \mathrm{E}-03$ $2.60302 \mathrm{E}-03$

$2.61549 \mathrm{E}-03$

$2.62798 \mathrm{E}-03$ $f_{2} \quad g_{2}$

$-3.10457 \mathrm{E} \quad 00 \quad-1.85613 \mathrm{E}-01$

$-3.12177 \mathrm{E} \quad 00 \quad-1.87075 \mathrm{E}-01$

$-3.13901 \mathrm{E} \quad 00-1.88546 \mathrm{E}-01$

$-3.15631 \mathrm{E} \quad 00 \quad-1.90024 \mathrm{E}-01$

$-3.17364 \mathrm{E} \quad 00$

$-1.91510 \mathrm{E}-01$

$-3.19103 \mathrm{E} \quad 00$

$-1.93003 \mathrm{E}-01$

$-3.20846 \mathrm{E} \quad 00$

$-3.22594 \mathrm{E} \quad 00$

$-3.24346 \mathrm{E} \quad 00$

$-3.26103 \mathrm{E} \quad 00$

$-1.94504 \mathrm{E}-0 \mathrm{l}$

$-1.96013 \mathrm{E}-01$

$-1.97529 \mathrm{E}-01$

$-1.99053 \mathrm{E}-01$

$-3.27864 \mathrm{E} \quad 00$

$-2.00585 \mathrm{E}-01$

$-3.29631 \mathrm{E} 00$

$-3.31402 \mathrm{E} \quad 00$

$-3.33177 \mathrm{E} \quad 00$

$-3.34957 \mathrm{E} \quad 00$

$25 \mathrm{E}-01$

$-2.03672 \mathrm{E}-01$

$-2.05227 \mathrm{E}-01$

$-2.06790 \mathrm{E}-01$

$-3.36742 \mathrm{E} \quad 00$

$-3.38531 \mathrm{E} 00$

$-3.40325 \mathrm{E} \quad 00$

$-3.42124 \mathrm{E} \quad 00$

$-3.43927 \mathrm{E} \quad 00$

$-3.45735 \mathrm{E} \quad 00$

$-3.47547 \mathrm{E} \quad 00$

$-3.49364 \mathrm{E} \quad 00$

$-3.51186 \mathrm{E} 00$

$-3.53012 \mathrm{E} 00$

$-3.54843 \mathrm{E} \quad 00$

$-3.56679 \mathrm{E} \quad 00$

$-3.58519 \mathrm{E} \quad 00$

$-3.60364 \mathrm{E} \quad 00$

$-3.62213 \mathrm{E} 00$

$-3.64067 \mathrm{E} \quad 00$

$-2.08361 \mathrm{E}-01$

$-2.09940 \mathrm{E}-01$

$-2.11526 \mathrm{E}-01$

$-2.13121 \mathrm{E}-01$

$-2.14723 \mathrm{E}-01$

$-2.16333 \mathrm{E}-01$

$-2.17952 \mathrm{E}-01$

$-2.19578 \mathrm{E}-01$

$-2.21212 \mathrm{E}-01$

$-2.22854 \mathrm{E}-01$

$-3.65925 \mathrm{E} \quad 00$

$-3.67789 \mathrm{E} 00$

$-3.69656 \mathrm{E} 00$

$-3.71529 \mathrm{E} 00$

$-3.73406 \mathrm{E} \quad 00$

$-2.24505 \mathrm{E}-01$

$-2.26163 \mathrm{E}-01$

$-2.27829 \mathrm{E}-01$

$-2.29504 \mathrm{E}-01$

$-2.31186 \mathrm{E}-01$

$-3.75287 \mathrm{E} \quad 00$

$-3.77173 \mathrm{E} 00$

$-3.79064 \mathrm{E} \quad 00$

$-3.80959 \mathrm{E} \quad 00$

$-2.32877 \mathrm{E}-01$

$-2.34576 \mathrm{E}-01$

$-2.36283 \mathrm{E}-01$

$-2.37998 \mathrm{E}-01$

$-2.39721 \mathrm{E}-01$

$-2.41453 \mathrm{E}-01$

$-2.43193 \mathrm{E}-01$

$-2.44941 \mathrm{E}-01$

$-2.46697 \mathrm{E}-01$

$-2.48462 \mathrm{E}-01$

$-3.82859 \mathrm{E} \quad 00$

$-2.50235 \mathrm{E}-01$

$-3.84764 \mathrm{E} \quad 00$

$-3.86673 \mathrm{E} 00$

$-3.88587 \mathrm{E} \quad 00$

$-3.90505 \mathrm{E} \quad 00$

$-2.52016 \mathrm{E}-01$

$-2.53806 \mathrm{E}-01$

$-2.55603 \mathrm{E}-01$

$-3.92428 \mathrm{E} \quad 00$

$-2.57410 \mathrm{E}-01$

$-3.94355 \mathrm{E} \quad 00$

$-3.96287 \mathrm{E} \quad 00$

$-3.98224 \mathrm{E} \quad 00$

$-4.00165 \mathrm{E} \quad 00$

$-2.59225 \mathrm{E}-01$

$-2.61048 \mathrm{E}-01$

$-2.62879 \mathrm{E}-01$

$-2.64719 \mathrm{E}-01$

$-4.02111 \mathrm{E} \quad 00$

$-2.66568 \mathrm{E}-01$

$-4.04061 \mathrm{E} \quad 00$

$-4.06016 \mathrm{E} \quad 00$

$-4.07976 \mathrm{E} \quad 00$

$-2.68425 \mathrm{E}-01$

$-2.70290 \mathrm{E}-01$

$-2.72164 \mathrm{E}-01$

$-4.09940 \mathrm{E} \quad 00$

$-2.74047 \mathrm{E}-01$

$-4.11908 \mathrm{E} \quad 00$ $-4.13882 \mathrm{E} \quad 00$ $-4.15859 \mathrm{E} \quad 00$

$-4.17842 \mathrm{E} \quad 00$

$-4.19829 \mathrm{E} \quad 00$ $\tan \delta_{2}$

$f_{-2}$

$4.11772 \mathrm{E} \quad 01 \quad-1.81974 \mathrm{E}-01$

$4.11784 \mathrm{E}$ 0l $-1.83415 \mathrm{E}-01$

$4.11797 \mathrm{E}$ 01 $-1.84863 \mathrm{E}-01$

$4.11809 \mathrm{E}$ 01 $-1.86319 \mathrm{E}-01$

$4.11822 \mathrm{E}$ ol $-1.87782 \mathrm{E}-01$

$4.11834 \mathrm{E}$ ol $-1.89253 \mathrm{E}-01$

$4.11846 \mathrm{E}$ 0l $-1.90732 \mathrm{E}-01$

$4.11859 \mathrm{E}$ 01 $-1.92218 \mathrm{E}-01$

$4.11871 \mathrm{E}$ 01 $-1.93712 \mathrm{E}-01$

$4.11883 \mathrm{E}$ 0l $-1.95213 \mathrm{E}-01$

$4.11895 \mathrm{E} \quad 01 \quad-1.96723 \mathrm{E}-01$

$4.11907 \mathrm{E} \quad 01 \quad-1.98239 \mathrm{E}-01$

$4.11919 \mathrm{E} \quad 01 \quad-1.99764 \mathrm{E}-01$

$4.11931 \mathrm{E}$ 01 $-2.01296 \mathrm{E}-01$

$4.11942 \mathrm{E} \quad 01 \quad-2.02836 \mathrm{E}-01$

$4.11954 \mathrm{E} \quad 01 \quad-2.04384 \mathrm{E}-01$

$4.11966 \mathrm{E}$ 0l $-2.05940 \mathrm{E}-01$

$4.11977 \mathrm{E}$ 01 $-2.07503 \mathrm{E}-01$

$4.11989 \mathrm{E}$ 01 $-2.09075 \mathrm{E}-01$

$4.12000 \mathrm{E} \quad 01 \quad-2.10654 \mathrm{E}-01$

$4.12012 \mathrm{E} \quad 01 \quad-2.12241 \mathrm{E}-01$

$4.12023 \mathrm{E}$ 01 $-2.13836 \mathrm{E}-01$

$4.12034 \mathrm{E}$ 01 $-2.15438 \mathrm{E}-01$

$4.12045 \mathrm{E}$ 0l $-2.17049 \mathrm{E}-01$

$4.12056 \mathrm{E} \quad 01 \quad-2.18668 \mathrm{E}-01$

$4.12068 \mathrm{E}$ 0l $-2.20294 \mathrm{E}-01$

$4.12079 \mathrm{E}$ ol $-2.21929 \mathrm{E}-01$

$4.12090 \mathrm{E} \quad 01 \quad-2.23571 \mathrm{E}-01$

$4.12100 \mathrm{E}$ 01 $-2.25222 \mathrm{E}-01$

$4.12111 \mathrm{E} \quad 01 \quad-2.26881 \mathrm{E}-01$

$4.12122 \mathrm{E} \quad 01 \quad-2.28547 \mathrm{E}-01$

$4.12133 \mathrm{E} \quad 01 \quad-2.30222 \mathrm{E}-01$

$4.12143 \mathrm{E} \quad 01 \quad-2.31905 \mathrm{E}-01$

$4.12154 \mathrm{E}$ 0l $-2.33596 \mathrm{E}-01$

$4.12165 \mathrm{E} \quad 01 \quad-2.35295 \mathrm{E}-01$

$4.12175 \mathrm{E} \quad 01 \quad-2.37002 \mathrm{E}-01$ $4.12186 \mathrm{E} \quad 01$

$4.12196 \mathrm{E} 01$

$4.12206 \mathrm{E} \quad 01$

$4.12216 \mathrm{E} \quad 01$

$4.12227 \mathrm{E} \quad 01$

$4.12237 \mathrm{E} \quad 01$

$4.12247 \mathrm{E} \quad 01$

$4.12257 \mathrm{E} \quad 0$

$4.12267 \mathrm{E} \quad 01$

$4.12277 \mathrm{E} \quad 01$

$4.12287 \mathrm{E}$ 0]

$4.12297 \mathrm{E}$ 0]

$4.12307 \mathrm{E} 01$

$4.12316 \mathrm{E} 01$

$4.12326 \mathrm{E} \quad 01$

$4.12336 \mathrm{E}$ ol

$4.12345 \mathrm{E} \quad 01$

$4.12355 \mathrm{E} \quad 01$

$4.12364 \mathrm{E} \quad 01$

$4.12374 \mathrm{E} \quad 01$

$4.12383 \mathrm{E} 01$

$4.12393 \mathrm{E} \quad 01$

$4.12402 \mathrm{E} 01$

$4.12411 \mathrm{E} 01$

g-2

$3.19165 \mathrm{E} \quad 00$

$3.20908 \mathrm{E} \quad 00$

$3.22656 \mathrm{E} \quad 00$

$3.24409 \mathrm{E} \quad 00$

$3.26167 \mathrm{E} \quad 00$

$3.27929 \mathrm{E} \quad 00$

$3.29696 \mathrm{E} \quad 00$

$3.31467 \mathrm{E} \quad 00$

$3.33243 \mathrm{E} \quad 00$

$3.35024 \mathrm{E} \quad 00$

$3.36809 \mathrm{E} \quad 00$

$3.38599 \mathrm{E} \quad 00$

$3.40393 \mathrm{E} \quad 00$

$3.42192 \mathrm{E} \quad 00$

$3.43996 \mathrm{E} \quad 00$

$3.45805 \mathrm{E} \quad 00$

$3.47618 \mathrm{E} \quad 00$

$3.49435 \mathrm{E} \quad 00$

$3.51257 \mathrm{E} \quad 00$

$3.53084 \mathrm{E} \quad 00$

$3.54916 \mathrm{E} \quad 00$

$3.56752 \mathrm{E} \quad 00$

$3.58592 \mathrm{E} \quad 00$

$3.60438 \mathrm{E} \quad 00$

$3.62288 \mathrm{E} \quad 00$

$3.64142 \mathrm{E} \quad 00$

$3.66001 \mathrm{E} \quad 00$

$3.67865 \mathrm{E} \quad 00$

$3.69733 \mathrm{E} \quad 00$

$3.71606 \mathrm{E} \quad 00$

$3.73484 \mathrm{E} \quad 00$

$3.75366 \mathrm{E} \quad 00$

$3.77253 \mathrm{E} \quad 00$

$3.79144 \mathrm{E} \quad 00$

$3.81040 \mathrm{E} \quad 00$

$3.82940 \mathrm{E} \quad 00$

$3.84846 \mathrm{E} \quad 00$

$3.86755 \mathrm{E} \quad 00$

$3.88670 \mathrm{E} \quad 00$

$3.90589 \mathrm{E} \quad 00$

$3.92512 \mathrm{E} \quad 00$

$3.94440 \mathrm{E} \quad 00$

$3.96373 \mathrm{E} \quad 00$

$3.98310 \mathrm{E} \quad 00$

$4.00252 \mathrm{E} \quad 00$

$4.02198 \mathrm{E} \quad 00$

$4.04149 \mathrm{E} \quad 00$

$4.06105 \mathrm{E} \quad 00$

$4.08065 \mathrm{E} \quad 00$

$4.10030 \mathrm{E} \quad 00$

$4.11999 \mathrm{E} \quad 00$

$4.13973 \mathrm{E} \quad 00$

$4.15951 \mathrm{E} \quad 00$ 
ELECTRON RADIAL FUNCTIONS

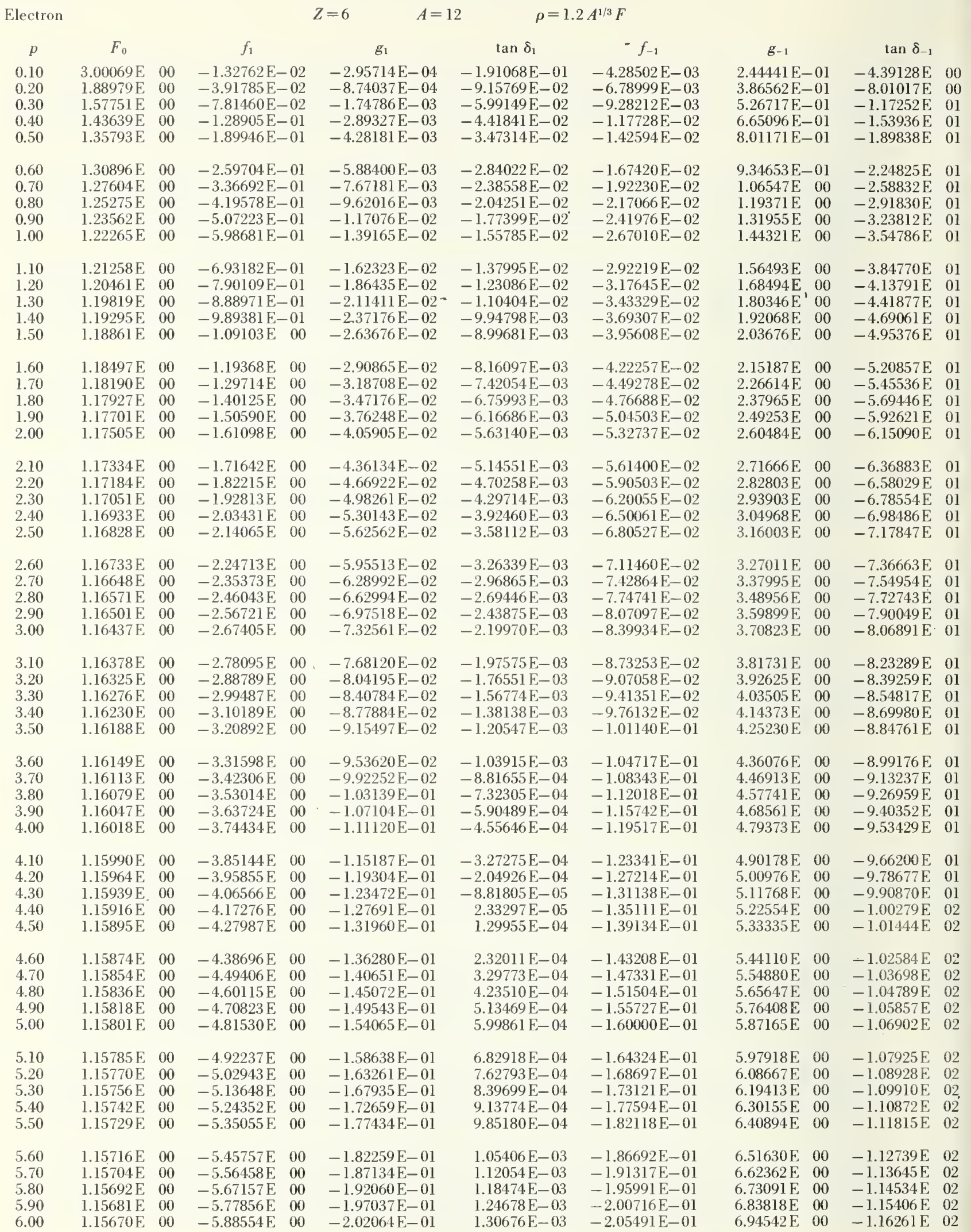


ELECTRON RADIAL FUNCTIONS

$Z=6 \quad A=12 \quad \rho=1.2 A^{1 / 3} F$

Electron

$\bar{F}$

$4.99525 \mathrm{E}-08$

$1.10726 \mathrm{E}-07$

$2.02758 \mathrm{E}-07$

$3.25310 \mathrm{E}-07$

$4.78586 \mathrm{E}-07$

$6.62878 \mathrm{E}-07$

$8.78455 \mathrm{E}-07$

$1.12554 \mathrm{E}-06$

$1.40432 \mathrm{E}-06$

$1.71493 \mathrm{E}-06$

$2.05748 \mathrm{E}-06$

$2.43206 \mathrm{E}-06$

$2.83875 \mathrm{E}-06$

$3.27759 \mathrm{E}-06$

$3.74862 \mathrm{E}-06$

$4.25188 \mathrm{E}-06$ $4.78738 \mathrm{E}-06$ $5.35516 \mathrm{E}-06$

$5.95522 \mathrm{E}-06$

$6.58758 \mathrm{E}-06$

$7.25226 \mathrm{E}-06$

$7.94924 \mathrm{E}-06$

$8.67855 \mathrm{E}-06$

$9.44020 \mathrm{E}-06$

$1.02342 \mathrm{E}-05$

$1.10605 \mathrm{E}-05$

$1.19191 \mathrm{E}-05$

$1.28101 \mathrm{E}-05$

$1.37334 \mathrm{E}-05$

$1.46891 \mathrm{E}-05$

$1.56771 \mathrm{E}-05$

$1.66975 \mathrm{E}-05$

$1.77501 \mathrm{E}-05$

$1.88352 \mathrm{E}-05$

$1.99526 \mathrm{E}-05$

2.11023 E-05 $2.22844 \mathrm{E}-05$ $2.34988 \mathrm{E}-05$ $2.47456 \mathrm{E}-05$ $2.60247 \mathrm{E}-05$

$2.73362 \mathrm{E}-05$ $2.86800 \mathrm{E}-05$

$3.00561 \mathrm{E}-05$

$3.14646 \mathrm{E}-05$

$3.29054 \mathrm{E}-05$

$3.43786 \mathrm{E}-05$

$3.58841 \mathrm{E}-05$

$3.74220 \mathrm{E}-05$

$3.89922 \mathrm{E}-05$

$4.05947 \mathrm{E}-05$

$4.22296 \mathrm{E}-05$ $4.38968 \mathrm{E}-05$ $4.55964 \mathrm{E}-05$

$4.73283 \mathrm{E}-05$

$4.90925 \mathrm{E}-05$

$5.08891 \mathrm{E}-05$

$5.27180 \mathrm{E}-05$

$5.45792 \mathrm{E}-05$

$5.64728 \mathrm{E}-05$

$5.83987 \mathrm{E}-05$ $f_{2}$

$g_{2}$

$-3.19460 \mathrm{E}-06$ $-1.86153 \mathrm{E}-05$ $-5.55665 \mathrm{E}-05$ $-1.22122 \mathrm{E}-04$

$-2.24871 \mathrm{E}-04$

$-3.68901 \mathrm{E}-04$

$-5.57943 \mathrm{E}-04$

$-7.94616 \mathrm{E}-04$

$-1.08069 \mathrm{E}-03$

$-1.41731 \mathrm{E}-03$

$-1.80519 \mathrm{E}-03$

$-2.24474 \mathrm{E}-03$

$-2.73618 \mathrm{E}-03$

$-3.27961 \mathrm{E}-03$

$-3.87503 \mathrm{E}-03$

$-4.52244 \mathrm{E}-03$

$-5.22175 \mathrm{E}-03$

$-5.97291 \mathrm{E}-03$

$-6.77583 \mathrm{E}-03$

$-7.63044 \mathrm{E}-03$

$-8.53667 \mathrm{E}-03$

$-9.49443 \mathrm{E}-03$

$-1.05037 \mathrm{E}-02$

$-1.15643 \mathrm{E}-02$

$-1.26764 \mathrm{E}-02$

$-1.38397 \mathrm{E}-02$

$-1.50543 \mathrm{E}-02$

$-1.63201 \mathrm{E}-02$

$-1.76371 \mathrm{E}-02$

$-1.90053 \mathrm{E}-02$

$-2.04245 \mathrm{E}-02$

$-2.18949 \mathrm{E}-02$

$-2.34163 \mathrm{E}-02$

$-2.49887 \mathrm{E}-02$

$-2.66122 \mathrm{E}-02$

$-2.82866 \mathrm{E}-02$

$-3.00121 \mathrm{E}-02$

$-3.17884 \mathrm{E}-02$

$-3.36157 \mathrm{E}-02$

$-3.54940 \mathrm{E}-02$

$-3.74231 \mathrm{E}-02$

$-3.94032 \mathrm{E}-02$

$-4.14341 \mathrm{E}-02$

$-4.35159 \mathrm{E}-02$

$-4.56485 \mathrm{E}-02$

$-4.78320 \mathrm{E}-02$

$-5.00663 \mathrm{E}-02$

$-5.23515 \mathrm{E}-02$

$-5.46875 \mathrm{E}-02$

$-5.70743 \mathrm{E}-02$

$-5.95118 \mathrm{E}-02$

$-6.20002 \mathrm{E}-02$

$-6.45394 \mathrm{E}-02$

$-6.71293 \mathrm{E}-02$

$-6.97700 \mathrm{E}-02$

$-7.24614 \mathrm{E}-02$

$-7.52037 \mathrm{E}-02$

$-7.79966 \mathrm{E}-02$

$-8.08403 \mathrm{E}-02$

$-8.37347 \mathrm{E}-02$ $\tan \delta_{2}$

$-4.10887 \mathrm{E}-08$

$-2.39821 \mathrm{E}-07$

$-7.17780 \mathrm{E}-07$

$-1.58324 \mathrm{E}-06$

$-2.92846 \mathrm{E}-06$

$-4.82942 \mathrm{E}-06$

$-7.34749 \mathrm{E}-06$

$-1.05320 \mathrm{E}-05$

$-1.44233 \mathrm{E}-05$

$-1.90549 \mathrm{E}-05$

$-2.44557 \mathrm{E}-05$

$-3.06515 \mathrm{E}-05$

$-3.76661 \mathrm{E}-05$

$-4.55220 \mathrm{E}-05$

$-5.42408 \mathrm{E}-05$

$-6.38437 \mathrm{E}-05$

$-7.43516 \mathrm{E}-05$

$-8.57853 \mathrm{E}-05$

$-9.81656 \mathrm{E}-05$

$-1.11513 \mathrm{E}-04$

$-1.25849 \mathrm{E}-04$

$-1.41194 \mathrm{E}-04$

$-1.57569 \mathrm{E}-04$

$-1.74996 \mathrm{E}-04$

$-1.93494 \mathrm{E}-04$

$-2.13085 \mathrm{E}-04$

$-2.33791 \mathrm{E}-04$

$-2.55632 \mathrm{E}-04$

$-2.78631 \mathrm{E}-04$

-3.02807 E-04

$-3.28182 \mathrm{E}-04$

$-3.54779 \mathrm{E}-04$

$-3.82618 \mathrm{E}-04$

$-4.11720 \mathrm{E}-04$

$-4.42108 \mathrm{E}-04$

$-4.73801 \mathrm{E}-04$

$-5.06823 \mathrm{E}-04$

$-5.41194 \mathrm{E}-04$

$-5.76936 \mathrm{E}-04$

$-6.14071 \mathrm{E}-04$

$-6.52620 \mathrm{E}-04$

$-6.92603 \mathrm{E}-04$

$-7.34044 \mathrm{E}-04$

$-7.76963 \mathrm{E}-04$

$-8.21382 \mathrm{E}-04$

$-8.67323 \mathrm{E}-04$

$-9.14806 \mathrm{E}-04$

$-9.63855 \mathrm{E}-04$

$-1.01449 \mathrm{E}-03$

$-1.06673 \mathrm{E}-03$

$-1.12060 \mathrm{E}-03$

$-1.17613 \mathrm{E}-03$

$-1.23332 \mathrm{E}-03$

$-1.29221 \mathrm{E}-03$

$-1.35281 \mathrm{E}-03$

$-1.41515 \mathrm{E}-03$

$-1.47925 \mathrm{E}-03$

$-1.54513 \mathrm{E}-03$

$-1.61282 \mathrm{E}-03$

$-1.68232 \mathrm{E}-03$
$2.32085 \mathrm{E} \quad 00$

$\begin{array}{ll}4.82351 \mathrm{E} & 00\end{array}$

$7.18406 \mathrm{E} \quad 00$

$9.40344 \mathrm{E} \quad 00$

$1.14638 \mathrm{E} \quad 01$

$1.33544 \mathrm{E} \quad 01$

$1.50732 \mathrm{E} \quad 01$

$1.66252 \mathrm{E} \quad 01$

$1.80205 \mathrm{E} \quad 01$

$1.92715 \mathrm{E} \quad 01$

$2.03920 \mathrm{E} \quad 01$

$2.13956 \mathrm{E} \quad 01$

$2.22954 \mathrm{E}$ 01

$2.31034 \mathrm{E} \quad 01$

$2.38305 \mathrm{E} \quad 01$

$2.44863 \mathrm{E} \quad 01$

$2.50793 \mathrm{E} \quad 01$

$2.56170 \mathrm{E} \quad 01$

$2.61059 \mathrm{E} \quad 01$

$2.65515 \mathrm{E} \quad 01$

$2.69588 \mathrm{E} \quad 01$

$2.73321 \mathrm{E} \quad 01$

$2.76751 \mathrm{E} 01$

$2.79911 \mathrm{E} \quad 01$

$2.82828 \mathrm{E} 01$

$2.85528 \mathrm{E} \quad 01$

$2.88032 \mathrm{E} \quad 01$

$2.90360 \mathrm{E} \quad 01$

$2.92528 \mathrm{E} \quad 01$

2.94551 E 01

$2.96443 \mathrm{E} \quad 01$

$2.98215 \mathrm{E} \quad 01$

2.99877 E 01

$3.01440 \mathrm{E} \quad 01$

$3.02911 \mathrm{E} \quad 01$

$3.04298 \mathrm{E} \quad 01$

$3.05607 \mathrm{E} \quad 01$

$3.06844 \mathrm{E} \quad 01$

$3.08016 \mathrm{E}$ 0l

$3.09127 \mathrm{E} \quad 01$

$3.10181 \mathrm{E} \quad 01$

$3.11182 \mathrm{E}$ ol

$3.12135 \mathrm{E} \quad 01$

$3.13042 \mathrm{E} \quad 01$

$3.13907 \mathrm{E} \quad 01$

$3.14732 \mathrm{E} \quad 01$

$3.15520 \mathrm{E}$ ol

$3.16273 \mathrm{E} 01$

$3.16994 \mathrm{E} \quad 01$

$3.17684 \mathrm{E} \quad 01$

$3.18346 \mathrm{E} \quad 01$ $3.18981 \mathrm{E} \quad 01$ $3.19590 \mathrm{E} \quad 01$ $3.20176 \mathrm{E} \quad 01$ $3.20739 \mathrm{E} \quad 01$

$3.21280 \mathrm{E} \quad 01$ $3.21802 \mathrm{E} \quad 01$ $3.22304 \mathrm{E} \quad 01$ $3.22788 \mathrm{E}$ 0l

$3.23255 \mathrm{E} \quad 01$

$f_{-2}$

$-6.32188 \mathrm{E}-07$

$-1.87956 \mathrm{E}-06$

$-3.80619 \mathrm{E}-06$

$-6.40955 \mathrm{E}-06$

$-9.68715 \mathrm{E}-06$

$-1.36380 \mathrm{E}-05$

$-1.82638 \mathrm{E}-05$

$-2.35695 \mathrm{E}-05$

$-2.95629 \mathrm{E}-05$

$-3.62552 \mathrm{E}-05$

$-4.36597 \mathrm{E}-05$

$-5.17919 \mathrm{E}-05$

$-6.06687 \mathrm{E}-05$

$-7.03085 \mathrm{E}-05$

$-8.07301 \mathrm{E}-05$

$-9.19536 \mathrm{E}-05$

$-1.03999 \mathrm{E}-04$

$-1.16887 \mathrm{E}-04$

$-1.30640 \mathrm{E}-04$

$-1.45277 \mathrm{E}-04$

$-1.60820 \mathrm{E}-04$

$-1.77291 \mathrm{E}-04$

$-1.94711 \mathrm{E}-04$

$-2.13102 \mathrm{E}-04$

$-2.32485 \mathrm{E}-04$

$-2.52882 \mathrm{E}-04$

$-2.74315 \mathrm{E}-04$

$-2.96805 \mathrm{E}-04$

$-3.20374 \mathrm{E}-04$

$-3.45044 \mathrm{E}-04$

$-3.70836 \mathrm{E}-04$

$-3.97772 \mathrm{E}-04$

$-4.25874 \mathrm{E}-04$

$-4.55164 \mathrm{E}-04$

$-4.85662 \mathrm{E}-04$

$-5.17391 \mathrm{E}-04$

$-5.50373 \mathrm{E}-04$

$-5.84629 \mathrm{E}-04$

$-6.20181 \mathrm{E}-04$

$-6.57051 \mathrm{E}-04$

$-6.95260 \mathrm{E}-04$

$-7.34829 \mathrm{E}-04$ 
ELECTRON RADIAL FUNCTIONS

\begin{tabular}{|c|c|c|c|c|c|c|c|}
\hline$p$ & $F_{0}$ & & $f_{1}$ & & $g_{1}$ & $\tan \delta_{1}$ & $f_{-1}$ \\
\hline 6.10 & $1.15659 \mathrm{E}$ & 00 & $-5.99251 \mathrm{E}$ & 00 & $-2.07141 \mathrm{E}-01$ & $1.36479 \mathrm{E}-03$ & $-2.10316 \mathrm{E}-01$ \\
\hline 6.20 & $1.15649 \mathrm{E}$ & 00 & $-6.09946 \mathrm{E}$ & 00 & $-2.12269 \mathrm{E}-01$ & $1.42095 \mathrm{E}-03$ & $-2.15191 \mathrm{E}-01$ \\
\hline 6.30 & $1.15640 \mathrm{E}$ & 00 & $-6.20640 \mathrm{E}$ & 00 & $-2.17447 \mathrm{E}-01$ & $1.47534 \mathrm{E}-03$ & $-2.20117 \mathrm{E}-01$ \\
\hline 6.40 & $1.15630 \mathrm{E}$ & 00 & $-6.31334 \mathrm{E}$ & 00 & $-2.22675 E-01$ & $1.52803 \mathrm{E}-03$ & $-2.25093 \mathrm{E}-01$ \\
\hline 6.50 & $1.15621 \mathrm{E}$ & 00 & $-6.42026 \mathrm{E}$ & 00 & $-2.27954 \mathrm{E}-01$ & $1.57911 \mathrm{E}-03$ & $-2.30119 \mathrm{E}-01$ \\
\hline 6.60 & $1.15613 \mathrm{E}$ & 00 & $-6.52716 \mathrm{E}$ & 00 & $-2.33284 \mathrm{E}-01$ & $1.62865 \mathrm{E}-03$ & $-2.35195 \mathrm{E}-01$ \\
\hline 6.70 & $1.15604 \mathrm{E}$ & 00 & $-6.63406 \mathrm{E}$ & 00 & $-2.38663 \mathrm{E}-01$ & $70 \mathrm{E}-03$ & $-2.40321 \mathrm{E}-01$ \\
\hline 6.80 & $1.15596 \mathrm{E}$ & 00 & $-6.74094 \mathrm{E}$ & 00 & $-2.44093 \mathrm{E}-01$ & $336 \mathrm{E}-03$ & $-2.45498 \mathrm{E}-01$ \\
\hline 6.90 & $1.15588 \mathrm{E}$ & 00 & $-6.84781 \mathrm{E}$ & 00 & $-2.49574 \mathrm{E}-01$ & $1.76864 \mathrm{E}-03$ & $-2.50725 \mathrm{E}-01$ \\
\hline 7.00 & $1.15580 \mathrm{E}$ & 00 & $-6.95467 \mathrm{E}$ & 00 & $-2.55105 E-01$ & $1.81265 \mathrm{E}-03$ & $-2.56003 \mathrm{E}-01$ \\
\hline 7.10 & $1.15573 \mathrm{E}$ & 00 & $-7.06152 \mathrm{E}$ & 00 & $-2.60686 \mathrm{E}-01$ & $1.85541 \mathrm{E}-03$ & $-2.61330 \mathrm{E}-01$ \\
\hline 7.20 & $1.15566 \mathrm{E}$ & 00 & $-7.16835 \mathrm{E}$ & 00 & $-2.66317 \mathrm{E}-01$ & $1.89698 \mathrm{E}-03$ & $-2.66708 \mathrm{E}-01$ \\
\hline 7.30 & $1.15559 \mathrm{E}$ & 00 & $-7.27517 \mathrm{E}$ & 00 & $-2.71999 \mathrm{E}-01$ & $1.93741 \mathrm{E}-$ & $-2.72136 \mathrm{E}-01$ \\
\hline 7.40 & $15552 \mathrm{E}$ & 00 & $-7.38198 \mathrm{E}$ & 00 & $-2.77731 \mathrm{E}-01$ & $1.97675 \mathrm{E}-03$ & $-2.77615 \mathrm{E}-01$ \\
\hline 7.50 & $1.15545 \mathrm{E}$ & 00 & $-7.48878 E$ & 00 & $-2.83513 \mathrm{E}$ & $2.01503 \mathrm{E}-03$ & $-2.83143 \mathrm{E}-01$ \\
\hline 7.60 & $.15539 \mathrm{E}$ & 00 & $-7.59556 \mathrm{E}$ & 00 & $-2.89346 \mathrm{E}-01$ & $2.05229 \mathrm{E}-03$ & $-2.88722 \mathrm{E}-01$ \\
\hline 7.70 & $1.15533 \mathrm{E}$ & 00 & $-7.70234 \mathrm{E}$ & 00 & $-2.95229 \mathrm{E}-01$ & $359 \mathrm{E}-$ & $-2.94351 \mathrm{E}-01$ \\
\hline 7.80 & $15526 \mathrm{E}$ & 00 & $-7.80909 \mathrm{E}$ & 00 & $-3.01162 \mathrm{E}-01$ & $2.12396 \mathrm{E}-$ & $-3.00031 \mathrm{E}-01$ \\
\hline 7.90 & $521 \mathrm{E}$ & 00 & $-7.91584 \mathrm{E}$ & 00 & $.07146 \mathrm{E}-01$ & 2.15 & $-3.05760 \mathrm{E}-01$ \\
\hline 8.00 & $1.15515 \mathrm{E}$ & 00 & $-8.02257 \mathrm{E}$ & 00 & $13180 \mathrm{E}$ & $2.19202 \mathrm{E}$ & $-3.11540 \mathrm{E}-01$ \\
\hline .10 & $.15509 \mathrm{E}$ & 00 & $-8.12929 \mathrm{E}$ & 00 & $-3.19264 \mathrm{E}-01$ & $2.22477 \mathrm{E}-03$ & $-3.17370 \mathrm{E}-01$ \\
\hline 20 & $.15504 \mathrm{E}$ & 00 & $-8.23600 \mathrm{E}$ & 00 & $-3.25399 \mathrm{E}-01$ & $2.25672 \mathrm{E}-$ & $-3.23251 \mathrm{E}-01$ \\
\hline 30 & $1.15498 \mathrm{E}$ & 00 & $-8.34270 \mathrm{E}$ & 00 & $31584 \mathrm{E}-$ & $2.28790 \mathrm{E}$ & $-3.29181 \mathrm{E}-01$ \\
\hline & $3 \mathrm{E}$ & 00 & $-8.44938 \mathrm{E}$ & 00 & 01 & 2.31 & $-3.35162 \mathrm{E}-$ \\
\hline 8.50 & $5488 \mathrm{E}$ & 00 & $-8.55605 \mathrm{E}$ & 00 & -01 & 2.348 & 41193 \\
\hline 3.60 & $.15483 \mathrm{E}$ & 00 & $-8.66270 \mathrm{E}$ & 00 & $-3.50440 \mathrm{E}-01$ & $2.37702 \mathrm{E}-03$ & $-3.47275 \mathrm{E}-01$ \\
\hline 70 & $1.15478 \mathrm{E}$ & 00 & $-8.76935 \mathrm{E}$ & 00 & $-3.56825 \mathrm{E}$ & 2.40 & $-3.53406 \mathrm{E}-01$ \\
\hline 8.80 & & 00 & $-8.87598 \mathrm{E}$ & 00 & - & 03 & 59588 \\
\hline 8.90 & $59 \mathrm{E}$ & 00 & $-8.98259 \mathrm{E}$ & 00 & $48 \mathrm{E}-01$ & 2.46 & $-3.65820 \mathrm{E}-01$ \\
\hline 9.00 & $1.15464 \mathrm{E}$ & 00 & $-9.08919 \mathrm{E}$ & 00 & $-3.76284 \mathrm{E}-01$ & $650 \mathrm{E}-03$ & $-3.72102 \mathrm{E}-01$ \\
\hline 9.10 & $1.15460 \mathrm{E}$ & 00 & $-9.19578 E$ & 00 & $1 \mathrm{E}-01$ & $34 \mathrm{E}-03$ & $-3.78434 \mathrm{E}-01$ \\
\hline & & 00 & $236 \mathrm{E}$ & 00 & & 03 & 01 \\
\hline 9.30 & $1.15451 \mathrm{E}$ & 00 & $-9.40893 \mathrm{E}$ & 00 & $6195 \mathrm{E}-01$ & 2.56 & $-3.91249 \mathrm{E}-01$ \\
\hline 9.40 & $1.15447 \mathrm{E}$ & 00 & $-9.51548 E$ & 00 & $-4.02932 \mathrm{E}-01$ & 2.586 & $-3.97732 \mathrm{E}-01$ \\
\hline 9.50 & $1.15443 \mathrm{E}$ & 00 & $-9.62201 \mathrm{E}$ & 00 & $-4.09720 \mathrm{E}-$ & 2.61 & $-4.04265 \mathrm{E}-01$ \\
\hline 9.60 & $1.15439 \mathrm{E}$ & 00 & $-9.72854 \mathrm{E}$ & 00 & $8 \mathrm{E}-01$ & $33 \mathrm{E}-03$ & $-4.10848 \mathrm{E}-01$ \\
\hline 9.7 & & 00 & $505 \mathrm{E}$ & 00 & - & 03 & $82 \mathrm{E}-01$ \\
\hline 9.80 & $1.15431 \mathrm{E}$ & 00 & $-9.94155 \mathrm{E}$ & 00 & $-4.30383 \mathrm{E}-01$ & $19 \mathrm{E}-$ & $-4.24165 \mathrm{E}-01$ \\
\hline & $427 \mathrm{E}$ & 00 & $-1.00480 \mathrm{E}$ & 01 & $-4.37372 \mathrm{E}$ & 2.69 & $-4.30899 \mathrm{E}-01$ \\
\hline & $423 \mathrm{E}$ & 00 & $-1.01545 \mathrm{E}$ & 01 & $44410 \mathrm{E}$ & & $-4.37683 \mathrm{E}-01$ \\
\hline 10 & $1.15419 \mathrm{E}$ & 00 & $-1.02610 \mathrm{E}$ & 01 & $9 E-01$ & $08 E-03$ & $-4.44516 \mathrm{E}-01$ \\
\hline & $16 \mathrm{E}$ & 00 & $-1.03674 \mathrm{E}$ & 01 & - & 2.76 & $51401 \mathrm{E}-01$ \\
\hline & $412 \mathrm{E}$ & 00 & $-1.04738 \mathrm{E}$ & 01 & $65826 \mathrm{~F}-$ & 2.78 & $-4.58335 \mathrm{E}-01$ \\
\hline & $409 \mathrm{E}$ & 00 & $-1.05803 \mathrm{E}$ & 01 & $73065 \mathrm{~F}$ & 2.80 & $-4.65319 \mathrm{E}-01$ \\
\hline & & 00 & $5867 \mathrm{E}$ & 01 & -4 & $43 \mathrm{E}-03$ & $72354 \mathrm{E}-01$ \\
\hline 60 & $1.15402 \mathrm{E}$ & 00 & $-1.07930 \mathrm{E}$ & 01 & $7693 \mathrm{E}-01$ & $2.84030 \mathrm{E}-03$ & $-4.79438 \mathrm{E}-01$ \\
\hline & & 00 & $3994 \mathrm{E}$ & 01 & & 2.85 & $-4.86573 \mathrm{E}-01$ \\
\hline & $395 \mathrm{E}$ & 00 & $-1.10058 \mathrm{E}$ & 01 & -5 & 37696 & $-4.93758 \mathrm{E}-01$ \\
\hline & & 00 & & 01 & & & 00993E-01 \\
\hline & & 00 & & 01 & $-5.17551 \mathrm{E}-01$ & & $-5.08277 \mathrm{E}-01$ \\
\hline 10 & $1.15386 \mathrm{E}$ & 00 & $-1.13248 \mathrm{E}$ & 01 & $E-01$ & $8 \mathrm{E}-03$ & $.15613 \mathrm{E}-01$ \\
\hline & & 00 & $4311 \mathrm{E}$ & 01 & -5 & 2.94 & $-5.22998 \mathrm{E}-01$ \\
\hline & & 00 & & 01 & -5 & & $-5.30433 \mathrm{E}-01$ \\
\hline & & 00 & $-1.16437 \mathrm{E}$ & 01 & -5 . & 2.978 & $-5.37918 \mathrm{E}-01$ \\
\hline & $1.15374 \mathrm{E}$ & 00 & $-1.17499 \mathrm{E}$ & 01 & $-5.55999 \mathrm{E}-01$ & 2.99 & $545453 \mathrm{~F}$ \\
\hline 6 & $1.15371 \mathrm{E}$ & 00 & $-1.18562 \mathrm{E}$ & 01 & $-5.63839 \mathrm{E}-01$ & $3.01049 \mathrm{E}-03$ & $-5.53039 \mathrm{E}-01$ \\
\hline & & 00 & $-1.19624 \mathrm{E}$ & 01 & $-5.71729 \mathrm{E}-01$ & & $-5.60674 \mathrm{E}-01$ \\
\hline & & 00 & $-1.20687 \mathrm{E}$ & 01 & $-5.79669 \mathrm{E}-01$ & $3.04092 \mathrm{E}-$ & $-5.68359 \mathrm{E}-01$ \\
\hline & $5362 \mathrm{E}$ & 00 & $-1.21749 \mathrm{E}$ & 01 & $-5.87659 \mathrm{E}-$ & $3.05573 \mathrm{E}-$ & $-5.76095 \mathrm{E}-01$ \\
\hline 2.00 & & 00 & -1 & 01 & $-5.95699 E-01$ & $3.07028 \mathrm{E}-03$ & $-5.83880 \mathrm{E}-01$ \\
\hline
\end{tabular}

$g-1$

$7.05263 \mathrm{E} \quad 00$ $7.15981 \mathrm{E} \quad 00$ $7.26697 \mathrm{E} \quad 00$ $7.37410 \mathrm{E} \quad 00$ $7.48121 \mathrm{E} \quad 00$

$7.58830 \mathrm{E} \quad 00$ $7.69536 \mathrm{E} \quad 00$ $7.80240 \mathrm{E} \quad 00$ $7.90942 \mathrm{E} \quad 00$ 8.0164.1E 00

$8.12339 \mathrm{E} \quad 00$ $8.23034 \mathrm{E} \quad 00$ $8.33727 \mathrm{E} \quad 00$ $8.44419 \mathrm{E} \quad 00$ $8.55108 \mathrm{E} \quad 00$

$8.65796 \mathrm{E} \quad 00$ $8.76481 \mathrm{E} \quad 00$ $8.87165 \mathrm{E} \quad 00$ $8.97847 \mathrm{E} \quad 00$ $9.08527 \mathrm{E} \quad 00$

$9.19205 \mathrm{E} \quad 00$ $9.29882 \mathrm{E} \quad 00$ $9.40556 \mathrm{E} \quad 00$ $9.51229 \mathrm{E} \quad 00$ $9.61900 \mathrm{E} \quad 00$

$9.72570 \mathrm{E} \quad 00$ $9.83238 \mathrm{E} \quad 00$ $9.93904 \mathrm{E} \quad 00$ $1.00457 \mathrm{E} \quad 01$ $1.01523 \mathrm{E} 01$

$1.02589 \mathrm{E}$ $1.03655 \mathrm{E}$ 01 $1.04721 \mathrm{E} \quad 01$ $1.05787 \mathrm{E} \quad 01$ $1.06852 \mathrm{E} \quad 01$

$1.07917 \mathrm{E} \quad 01$ $1.08982 \mathrm{E} \quad 01$ $1.10047 \mathrm{E} \quad 01$ $1.11112 \mathrm{E} 01$ $1.12177 \mathrm{E} \quad 01$

$1.13241 \mathrm{E} \quad 01$ $1.14306 \mathrm{E} \quad 01$ $1.15370 \mathrm{E}$ 01 $1.16434 \mathrm{E} \quad 01$ $1.17498 \mathrm{E}$ 0l

$\begin{array}{ll}1.18562 \mathrm{E} & 01\end{array}$ $1.19625 \mathrm{E} \quad 01$ $1.20689 \mathrm{E} 01$ $1.21752 \mathrm{E} \quad 01$ $1.22815 \mathrm{E} \quad 01$

$1.23878 \mathrm{E} \quad 01$ $1.24941 \mathrm{E} 01$ $1.26004 \mathrm{E} \quad 01$ $1.27066 \mathrm{E} \quad 01$ $1.28128 \mathrm{E} \quad 01$

$1.29191 \mathrm{E} \quad 01$ $1.30253 \mathrm{E} \quad 01$ $1.31315 \mathrm{E}$ 01 $1.32376 \mathrm{E} \quad 01$ $1.33438 \mathrm{E} \quad 01$ $\tan \delta_{-1}$

$-1.17101 \mathrm{E} \quad 02$ $-1.17924 \mathrm{E} \quad 02$ $-1.18733 \mathrm{E} \quad 02$ $-1.19527 \mathrm{E} \quad 02$ $-1.20307 \mathrm{E} \quad 02$

$-1.21073 \mathrm{E} \quad 02$ $-1.21825 \mathrm{E} \quad 02$ $-1.22564 \mathrm{E} \quad 02$ $-1.23291 \mathrm{E} 02$ $-1.24004 \mathrm{E} \quad 02$

$-1.24706 \mathrm{E} \quad 02$ $-1.25396 \mathrm{E} \quad 02$ $-1.26075 \mathrm{E} \quad 02$ $-1.26742 \mathrm{E} \quad 02$ $-1.27399 \mathrm{E} 02$

$-1.28045 \mathrm{E} \quad 02$ $-1.28680 \mathrm{E} \quad 02$ $-1.29306 \mathrm{E} \quad 02$ $-1.29921 \mathrm{E} \quad 02$ $-1.30527 \mathrm{E} \quad 02$

$-1.31124 \mathrm{E} \quad 02$ $-1.31712 \mathrm{E} \quad 02$ $-1.32290 \mathrm{E} \quad 02$ $-1.32860 \mathrm{E} \quad 02$ $-1.33422 \mathrm{E} \quad 02$

$-1.33975 \mathrm{E} \quad 02$ $-1.34520 \mathrm{E} \quad 02$ $-1.35057 \mathrm{E} \quad 02$ $-1.35586 \mathrm{E} \quad 02$ $-1.36108 \mathrm{E} 02$

$-1.36623 \mathrm{E} \quad 02$ $-1.37130 \mathrm{E} \quad 02$ $-1.37630 \mathrm{E} \quad 02$ $-1.38124 \mathrm{E} \quad 02$ $-1.38610 \mathrm{E} \quad 02$

$-1.39090 \mathrm{E} \quad 02$ $-1.39564 \mathrm{E} \quad 02$ $-1.40032 \mathrm{E} \quad 02$ $-1.40493 \mathrm{E} 02$ $\begin{array}{ll}-1.40948 \mathrm{E} & 02\end{array}$

$-1.41397 \mathrm{E} \quad 02$ $-1.41841 \mathrm{E} \quad 02$ $-1.42279 \mathrm{E} \quad 02$ $-1.42711 \mathrm{E} 02$ $-1.43138 \mathrm{E} \quad 02$

$-1.43559 \mathrm{E} \quad 02$ $-1.43976 \mathrm{E} 02$ $-1.44387 \mathrm{E} \quad 02$ $-1.44793 \mathrm{E} \quad 02$ $-1.45195 \mathrm{E} \quad 02$

$-1.45592 \mathrm{E} \quad 02$ $-1.45984 \mathrm{E} 02$ $-1.46371 \mathrm{E} 02$ $-1.46754 \mathrm{E} \quad 02$ $-1.47133 \mathrm{E} 02$

$\begin{array}{lll}-1.47507 \mathrm{E} & 02\end{array}$ $-1.47877 \mathrm{E} \quad 02$ $-1.48243 \mathrm{E} 02$ $-1.48604 \mathrm{E} 02$ $-1.48962 \mathrm{E} 02$ 
ELECTRON RADIAL FUNCTIONS

$Z=6 \quad A=12 \quad \rho=1.2 A^{1 / 3} F$

Electron

$\bar{F}$

$6.03570 \mathrm{E}-05$ $6.23476 \mathrm{E}-05$ $6.43706 \mathrm{E}-05$ $6.64258 \mathrm{E}-05$ $6.85134 \mathrm{E}-05$

$7.06333 \mathrm{E}-05$ $7.27856 \mathrm{E}-05$ $7.49702 \mathrm{E}-05$ $7.71871 \mathrm{E}-05$ $7.94364 \mathrm{E}-05$

$8.17180 \mathrm{E}-05$ $8.40319 \mathrm{E}-05$ $8.63782 \mathrm{E}-05$ $8.87567 \mathrm{E}-05$ $9.11677 \mathrm{E}-05$

$9.36109 \mathrm{E}-05$ $9.60865 \mathrm{E}-05$ $9.85944 \mathrm{E}-05$ $1.01135 \mathrm{E}-04$ $1.03707 \mathrm{E}-04$

$1.06312 \mathrm{E}-04$ $1.08949 \mathrm{E}-04$ $1.11619 \mathrm{E}-04$ $1.14321 \mathrm{E}-04$ $1.17055 \mathrm{E}-04$

$1.19821 \mathrm{E}-04$ $1.22620 \mathrm{E}-04$ $1.25451 \mathrm{E}-04$ $1.28315 \mathrm{E}-04$ $1.31211 \mathrm{E}-04$

$1.34139 \mathrm{E}-04$ $1.37099 \mathrm{E}-04$ $1.40092 \mathrm{E}-04$ $1.43117 \mathrm{E}-04$ $1.46174 \mathrm{E}-04$

$1.49264 \mathrm{E}-04$ $1.52386 \mathrm{E}-04$ $1.55541 \mathrm{E}-04$ $1.58727 \mathrm{E}-04$ $1.61947 \mathrm{E}-04$

$1.65198 \mathrm{E}-04$ $1.68482 \mathrm{E}-04$ $1.71798 \mathrm{E}-04$ $1.75146 \mathrm{E}-04$ $1.78527 \mathrm{E}-04$

$1.81939 \mathrm{E}-04$ $1.85385 \mathrm{E}-04$ $1.88862 \mathrm{E}-04$ $1.92372 \mathrm{E}-04$ $1.95915 \mathrm{E}-04$

$1.99489 \mathrm{E}-04$ $2.03096 \mathrm{E}-04$ $2.06735 \mathrm{E}-04$ $2.10407 \mathrm{E}-04$ 2.14111 E-04

$2.17847 \mathrm{E}-04$ $2.21615 \mathrm{E}-04$ $2.25416 \mathrm{E}-04$ $2.29249 \mathrm{E}-04$ $2.33114 \mathrm{E}-04$ $f_{2}$

$-8.66799 \mathrm{E}-02$

$-8.96758 \mathrm{E}-02$

$-9.27224 \mathrm{E}-02$

$-9.58196 \mathrm{E}-02$

$-9.89676 \mathrm{E}-02$

$-1.02166 \mathrm{E}-01$

$-1.05416 \mathrm{E}-01$

$-1.08716 \mathrm{E}-01$

$-1.12066 \mathrm{E}-01$

$-1.15468 \mathrm{E}-01$

$-1.18920 \mathrm{E}-01$

$-1.22423 \mathrm{E}-01$

$-1.25976 \mathrm{E}-01$

$-1.29580 \mathrm{E}-01$

$-1.33235 \mathrm{E}-01$

$-1.36940 \mathrm{E}-01$

$-1.40696 \mathrm{E}-01$

$-1.44502 \mathrm{E}-01$

$-1.48359 \mathrm{E}-01$

$-1.52267 \mathrm{E}-01$

$-1.56226 \mathrm{E}-01$

$-1.60235 \mathrm{E}-01$

$-1.64294 \mathrm{E}-01$

$-1.68404 \mathrm{E}-01$

$-1.72565 \mathrm{E}-01$

$-1.76776 \mathrm{E}-01$

$-1.81038 \mathrm{E}-01$

$-1.85351 \mathrm{E}-01$

$-1.89714 \mathrm{E}-01$

$-1.94127 \mathrm{E}-01$

$-1.98591 \mathrm{E}-01$

$-2.03106 \mathrm{E}-01$

$-2.07671 \mathrm{E}-01$

$-2.12287 \mathrm{E}-01$

$-2.16953 \mathrm{E}-01$

$-2.21670 \mathrm{E}-01$

$-2.26437 \mathrm{E}-01$

$-2.31255 \mathrm{E}-01$

$-2.36123 \mathrm{E}-01$

$-2.41042 \mathrm{E}-01$

$-2.46011 \mathrm{E}-01$

$-2.51031 \mathrm{E}-01$

$-2.56102 \mathrm{E}-01$

$-2.61222 \mathrm{E}-01$

$-2.66394 \mathrm{E}-01$

$-2.71615 \mathrm{E}-01$

$-2.76887 \mathrm{E}-01$

$-2.82210 \mathrm{E}-01$

$-2.87583 \mathrm{E}-01$

$-2.93007 \mathrm{E}-01$

$-2.98480 \mathrm{E}-01$

$-3.04005 \mathrm{E}-01$

$-3.09580 \mathrm{E}-01$

$-3.15205 \mathrm{E}-01$

$-3.20880 \mathrm{E}-01$

$-3.26606 \mathrm{E}-01$

$-3.32383 \mathrm{E}-01$

$-3.38210 \mathrm{E}-01$

$-3.44087 \mathrm{E}-01$

$-3.50014 \mathrm{E}-01$ $g_{2}$

$-1.75367 \mathrm{E}-03$

$-1.82689 \mathrm{E}-03$

$-1.90199 \mathrm{E}-03$

$-1.97900 \mathrm{E}-03$

$-2.05795 \mathrm{E}-03$

$-2.13885 \mathrm{E}-03$

$-2.22172 \mathrm{E}-03$

$-2.30658 \mathrm{E}-03$

$-2.39347 \mathrm{E}-03$

$-2.48239 \mathrm{E}-03$

$-2.57337 \mathrm{E}-03$

$-2.66644 \mathrm{E}-03$

$-2.76161 \mathrm{E}-03$

$-2.85890 \mathrm{E}-03$

$-2.95834 \mathrm{E}-03$

$-3.05995 \mathrm{E}-03$

$-3.16375 \mathrm{E}-03$

$-3.26976 \mathrm{E}-03$

$-3.37800 \mathrm{E}-03$

$-3.48849 \mathrm{E}-03$

$-3.60126 \mathrm{E}-03$ $-3.71633 \mathrm{E}-03$

$-3.83372 \mathrm{E}-03$

$-3.95345 \mathrm{E}-03$

$-4.07553 \mathrm{E}-03$

$-4.20001 \mathrm{E}-03$ $-4.32688 \mathrm{E}-03$

$-4.45619 \mathrm{E}-03$

$-4.58794 \mathrm{E}-03$

$-4.72216 \mathrm{E}-03$

$-4.85887 \mathrm{E}-03$

$-4.99809 \mathrm{E}-03$

$-5.13985 \mathrm{E}-03$

$-5.28416 \mathrm{E}-03$

$-5.43105 \mathrm{E}-03$

$-5.58053 \mathrm{E}-03$

$-5.73264 \mathrm{E}-03$

$-5.88739 \mathrm{E}-03$

$-6.04480 \mathrm{E}-03$

$-6.20489 \mathrm{E}-03$

$-6.36769 \mathrm{E}-03$

$-6.53322 \mathrm{E}-03$

$-6.70150 \mathrm{E}-03$

$-6.87254 \mathrm{E}-03$

$-7.04638 \mathrm{E}-03$

$-7.22303 \mathrm{E}-03$

$-7.40252 \mathrm{E}-03$

$-7.58486 \mathrm{E}-03$

$-7.77007 \mathrm{E}-03$

$-7.95819 \mathrm{E}-03$

$-8.14923 \mathrm{E}-03$

$-8.34321 \mathrm{E}-03$

$-8.54015 \mathrm{E}-03$

$-8.74007 \mathrm{E}-03$

$-8.94300 \mathrm{E}-03$

$-9.14896 \mathrm{E}-03$

$-9.35797 \mathrm{E}-03$

$-9.57005 \mathrm{E}-03$

$-9.78522 \mathrm{E}-03$

$-1.00035 \mathrm{E}-02$ $\tan \delta_{2}$

$f_{-2}$

$3.23706 \mathrm{E}$ 01 $-1.77399 \mathrm{E}-03$

$3.24141 \mathrm{E} \quad 01-1.84532 \mathrm{E}-03$

$3.24562 \mathrm{E} \quad 01-1.91847 \mathrm{E}-03$

$3.24968 \mathrm{E} \quad 01-1.99345 \mathrm{E}-03$

$3.25362 \mathrm{E} \quad 01-2.07028 \mathrm{E}-03$

$3.25742 \mathrm{E} \quad 01 \quad-2.14900 \mathrm{E}-03$

$3.26111 \mathrm{E}$ 01 $-2.22962 \mathrm{E}-03$

$3.26468 \mathrm{E} \quad 01-2.31217 \mathrm{E}-03$

$3.26814 \mathrm{E}$ 01 $-2.39665 \mathrm{E}-03$

$3.27149 \mathrm{E} \quad 01 \quad-2.48311 \mathrm{E}-03$

$3.27475 \mathrm{E} \quad 01$

$3.27790 \mathrm{E} \quad 01$

$3.28097 \mathrm{E} \quad 01$

$3.28395 \mathrm{E} \quad 01$

$3.28684 \mathrm{E} \quad 01$

$3.28966 \mathrm{E} \quad 01$ $3.29239 \mathrm{E} \quad 01$ $3.29505 \mathrm{E} \quad 01$ $3.29764 \mathrm{E} \quad 01$ $3.30016 \mathrm{E} \quad 01$

$-2.57155 \mathrm{E}-03$ $-2.66200 \mathrm{E}-03$ $-2.75448 \mathrm{E}-03$ $-2.84901 \mathrm{E}-03$ $-2.94562 \mathrm{E}-03$

$-3.04432 \mathrm{E}-03$ $-3.14514 \mathrm{E}-03$ $-3.24809 \mathrm{E}-03$ $-3.35321 \mathrm{E}-03$ $-3.46051 \mathrm{E}-03$

$3.30261 \mathrm{E} \quad 01$ $3.30500 \mathrm{E} \quad 01$ $3.30733 \mathrm{E} \quad 01$ $3.30961 \mathrm{E} \quad 01$ $3.31182 \mathrm{E} \quad 01$

$-3.57001 \mathrm{E}-03$ $-3.68174 \mathrm{E}-03$ $-3.79571 \mathrm{E}-03$ $-3.91195 \mathrm{E}-03$

$3.31398 \mathrm{E} \quad 01$ $3.31609 \mathrm{E} \quad 01$

$3.31814 \mathrm{E} \quad 01$

$3.32015 \mathrm{E} 01$

$3.32211 \mathrm{E} \quad 01$

$3.32402 \mathrm{E} \quad 01$ $3.32589 \mathrm{E} \quad 01$ $3.32772 \mathrm{E} \quad 01$ $3.32950 \mathrm{E} \quad 01$ $3.33125 \mathrm{E} \quad 01$

$3.33296 \mathrm{E} \quad 01$ $3.33463 \mathrm{E} \quad 01$ $3.33626 \mathrm{E} 01$

$3.33786 \mathrm{E} \quad 0$

$3.33943 \mathrm{E} \quad 01$

$3.34096 \mathrm{E} \quad 01$

$3.34246 \mathrm{E} \quad 01$

$3.34393 \mathrm{E}$ 0l

$3.34537 \mathrm{E} \quad 01$

$3.34678 \mathrm{E} 01$

$-4.03048 \mathrm{E}-03$

$-4.15132 \mathrm{E}-03$ $-4.27449 \mathrm{E}-03$ $-4.40002 \mathrm{E}-03$ $-4.52792 \mathrm{E}-03$ $-4.65821 \mathrm{E}-03$

$3.34817 \mathrm{E} \quad 01$ $3.34952 \mathrm{E} \quad 01$ $3.35085 \mathrm{E} \quad 01$ $3.35215 \mathrm{E} \quad 01$ $3.35343 \mathrm{E} 01$

$-4.79093 \mathrm{E}-03$ $-4.92609 \mathrm{E}-03$ $-5.06370 \mathrm{E}-03$ $-5.20380 \mathrm{E}-03$ $-5.34641 \mathrm{E}-03$

$-5.49154 \mathrm{E}-03$ $-5.63922 \mathrm{E}-03$ $-5.78946 \mathrm{E}-03$ $-5.94230 \mathrm{E}-03$ $-6.09775 \mathrm{E}-03$

$3.35469 \mathrm{E} 01$ $3.35592 \mathrm{E} \quad 01$ $3.35713 \mathrm{E} \quad 01$

$3.35831 \mathrm{E} \quad 0$ $3.35947 \mathrm{E} 01$

$-6.25583 \mathrm{E}-03$ $-6.41656 \mathrm{E}-03$ $-6.57998 \mathrm{E}-03$ $-6.74609 \mathrm{E}-03$ $-6.91492 \mathrm{E}-03$

$3.36062 \mathrm{E} \quad 01$ $3.36174 \mathrm{E} \quad 01$ $3.36284 \mathrm{E} \quad 01$ $3.36392 \mathrm{E} 01$ $3.36499 \mathrm{E} 01$

$-7.08649 \mathrm{E}-03$ $-7.26082 \mathrm{E}-03$ $-7.43794 \mathrm{E}-03$ $-7.61785 \mathrm{E}-03$ $-7.80060 \mathrm{E}-03$

$-7.98620 \mathrm{E}-03$ $-8.17466 \mathrm{E}-03$ 
ELECTRON RADIAL FUNCTIONS

Electron

\begin{tabular}{|c|c|c|c|c|c|}
\hline$p$ & $F_{0}$ & & $f_{1}$ & & $g_{1}$ \\
\hline 12.10 & $1.15357 \mathrm{E}$ & 00 & $-1.23872 \mathrm{E}$ & 01 & $-6.03789 \mathrm{E}-01$ \\
\hline 12.20 & $1.15354 \mathrm{E}$ & 00 & $-1.24934 \mathrm{E}$ & 01 & $-6.11929 \mathrm{E}-01$ \\
\hline 12.30 & $1.15352 \mathrm{E}$ & 00 & $25996 \mathrm{E}$ & 01 & -6.201191 \\
\hline 12.40 & $1.15349 \mathrm{E}$ & 00 & $-1.27057 \mathrm{E}$ & 01 & $-6.28359 \mathrm{E}-$ \\
\hline 12.50 & $1.15347 \mathrm{E}$ & 00 & $-1.28118 \mathrm{E}$ & 01 & $-6.36648 E-$ \\
\hline 2.60 & $1.15344 \mathrm{E}$ & 00 & $-1.29179 \mathrm{E}$ & 01 & $-6.44988 \mathrm{E}-01$ \\
\hline 12.70 & $1.15341 \mathrm{E}$ & 00 & $30240 \mathrm{E}$ & 01 & $-6.53378 E-01$ \\
\hline 12.80 & $1.15339 \mathrm{E}$ & 00 & $-1.31301 \mathrm{E}$ & 01 & $-6.61818 \mathrm{E}-$ \\
\hline 12.90 & $1.15337 \mathrm{E}$ & 00 & $-1.32362 \mathrm{E}$ & 01 & $-6.70307 \mathrm{E}-$ \\
\hline 13.00 & $1.15334 \mathrm{E}$ & 00 & $-1.33422 \mathrm{E}$ & 01 & $-6.78847 \mathrm{E}-$ \\
\hline 13.10 & $1.15332 \mathrm{E}$ & 00 & $-1.34483 \mathrm{E}$ & 01 & $-6.87436 \mathrm{E}-$ \\
\hline 13.20 & $1.15329 \mathrm{E}$ & 00 & $5543 \mathrm{E}$ & 01 & $-6.96075 \mathrm{E}-$ \\
\hline 30 & $1.15327 \mathrm{E}$ & 00 & $-1.36603 \mathrm{E}$ & 01 & $-7.04764 \mathrm{E}-$ \\
\hline .40 & $1.15325 \mathrm{E}$ & 00 & $-1.37663 \mathrm{E}$ & 01 & $-7.13504 \mathrm{E}-$ \\
\hline 3.50 & $1.15323 \mathrm{E}$ & 00 & $-1.38723 \mathrm{E}$ & 01 & $-7.22293 \mathrm{E}-$ \\
\hline 13.60 & $1.15320 \mathrm{E}$ & 00 & $-1.39783 E$ & 01 & $-7.31132 \mathrm{E}$ \\
\hline 13.70 & $18 \mathrm{E}$ & 00 & $0842 \mathrm{E}$ & 01 & $-7.40021 \mathrm{E}-$ \\
\hline .80 & $1.15316 \mathrm{E}$ & 00 & $1901 \mathrm{E}$ & 01 & $-7.48959 \mathrm{E}-$ \\
\hline .90 & $14 \mathrm{E}$ & 00 & $2961 \mathrm{E}$ & 01 & $-7.57948 \mathrm{E}-$ \\
\hline 14.00 & $312 \mathrm{E}$ & 00 & $4020 \mathrm{E}$ & 01 & $-7.66986 \mathrm{E}$ \\
\hline 14.10 & $1.15310 \mathrm{E}$ & 00 & $45079 \mathrm{E}$ & 01 & $-7.76074 \mathrm{E}-$ \\
\hline .20 & $1.15307 \mathrm{E}$ & 00 & $6137 \mathrm{E}$ & 01 & $-7.85212 \mathrm{E}-$ \\
\hline 30 & $05 \mathrm{E}$ & 00 & $7196 \mathrm{E}$ & 01 & $-7.94400 \mathrm{E}-$ \\
\hline 0 & $3 \mathrm{E}$ & 00 & $255 \mathrm{E}$ & 01 & -8 \\
\hline 14.50 & $01 \mathrm{E}$ & 00 & $313 \mathrm{E}$ & 01 & -8 \\
\hline 14.60 & $99 \mathrm{E}$ & 00 & $0371 \mathrm{E}$ & 01 & $-8.22262 \mathrm{E}-$ \\
\hline .70 & $97 \mathrm{E}$ & 00 & $1429 \mathrm{E}$ & 01 & -8.3 \\
\hline & & 00 & $187 \mathrm{E}$ & 01 & -8 \\
\hline 14.9 & & 00 & $545 \mathrm{E}$ & 01 & -8 \\
\hline 15.00 & $91 \mathrm{E}$ & 00 & $602 \mathrm{E}$ & 01 & $-8.60108 \mathrm{E}$ \\
\hline 15.10 & $39 \mathrm{E}$ & 00 & $5660 \mathrm{E}$ & 01 & -8 \\
\hline & & 00 & $17 \mathrm{E}$ & 01 & \\
\hline 15 & $86 \mathrm{E}$ & 00 & $774 \mathrm{E}$ & 01 & $-8.89015 \mathrm{E}-$ \\
\hline 15.4 & $1.15284 \mathrm{E}$ & 00 & $8831 \mathrm{E}$ & 01 & $-8.98750 \mathrm{E}-$ \\
\hline 15.50 & $282 \mathrm{E}$ & 00 & $9888 \mathrm{E}$ & 01 & $-9.08535 \mathrm{E}$ \\
\hline 15.60 & $80 \mathrm{E}$ & 00 & $-1.60944 \mathrm{E}$ & 01 & -9 \\
\hline 15 & & 00 & $001 \mathrm{E}$ & 01 & -9 \\
\hline 5.80 & $77 \mathrm{E}$ & 00 & $3057 \mathrm{E}$ & 01 & $-9.38188 \mathrm{E}-$ \\
\hline & $75 \mathrm{E}$ & 00 & $1113 \mathrm{E}$ & 01 & $-9.48172 \mathrm{E}$ \\
\hline 16.0 & & 00 & & 01 & -9 . \\
\hline 16.10 & $1 \mathrm{E}$ & 00 & $6225 \mathrm{E}$ & 01 & -9 \\
\hline 16 & & 00 & $7281 \mathrm{E}$ & 01 & $-9.78421 \mathrm{E}-$ \\
\hline & $68 \mathrm{E}$ & 00 & $337 \mathrm{E}$ & 0 & $-9.88603 \mathrm{E}$ \\
\hline & & 00 & & 01 & -9 \\
\hline 16.5 & & 00 & $447 \mathrm{E}$ & 01 & $-1.00912 \mathrm{E} \quad 0$ \\
\hline 16.60 & & 00 & & 01 & -1. \\
\hline & & 00 & $57 \mathrm{E}$ & 0 & -1.0 \\
\hline & & 00 & & 01 & \\
\hline & & 00 & & 01 & $-1.05074 \mathrm{E}$ \\
\hline 17.00 & & 00 & $75721 \mathrm{E}$ & 01 & $-1.06127 \mathrm{E}$ \\
\hline 17.10 & & 00 & & 01 & $-1.07185 \mathrm{E} \quad 00$ \\
\hline & & 00 & & 01 & $-1.08247 \mathrm{E}$ \\
\hline & & 00 & $883 \mathrm{E}$ & 01 & $-1.09315 \mathrm{E}$ \\
\hline & & 00 & $9937 \mathrm{E}$ & 01 & $-1.10388 \mathrm{E}$ \\
\hline & $1.15248 \mathrm{E}$ & 00 & $0991 \mathrm{E}$ & 01 & $-1.11466 \mathrm{E}$ \\
\hline 17.60 & & 00 & $044 \mathrm{E}$ & 01 & $-1.12548 \mathrm{E}$ \\
\hline & & 00 & $3097 \mathrm{E}$ & 01 & $-1.13636 \mathrm{E}$ \\
\hline & $44 \mathrm{E}$ & 00 & $4151 \mathrm{E}$ & 01 & $-1.14729 \mathrm{E}$ \\
\hline & & 00 & $-1.85203 \mathrm{E}$ & 01 & $-1.15826 \mathrm{E}$ \\
\hline 18.00 & $1.15241 \mathrm{E}$ & 00 & $-1.86256 \mathrm{E}$ & 01 & $-1.16929 \mathrm{E} \quad 00$ \\
\hline
\end{tabular}

$\tan \delta_{1}$

$3.08458 \mathrm{E}-03$ $3.09863 \mathrm{E}-03$ 3.11244 E-03 $3.12601 \mathrm{E}-03$ $3.13936 \mathrm{E}-03$

$3.15247 \mathrm{E}-03$

$3.16537 \mathrm{E}-03$

$3.17804 \mathrm{E}-03$

$3.19053 \mathrm{E}-03$

$3.20279 \mathrm{E}-03$

$3.21485 \mathrm{E}-03$

$3.22672 \mathrm{E}-03$

$3.23841 \mathrm{E}-03$

$3.24 .990 \mathrm{E}-03$

$3.26120 \mathrm{E}-03$

$3.27232 \mathrm{E}-03$

$3.28327 \mathrm{E}-03$

$3.29406 \mathrm{E}-03$

$3.30466 \mathrm{E}-03$

$3.31510 \mathrm{E}-03$

$3.32538 \mathrm{E}-03$

$3.33550 \mathrm{E}-03$

$3.34548 \mathrm{E}-03$

$3.35529 \mathrm{E}-03$

$3.36495 \mathrm{E}-03$

$3.37447 \mathrm{E}-03$ $3.38385 \mathrm{E}-03$

$3.39308 \mathrm{E}-03$

$3.40217 \mathrm{E}-03$

$3.41114 \mathrm{E}-03$

$3.41996 \mathrm{E}-03$

$3.42867 \mathrm{E}-03$

$3.43724 \mathrm{E}-03$

$3.44568 \mathrm{E}-03$

$3.45400 \mathrm{E}-03$

$3.46221 \mathrm{E}-03$

$3.47028 \mathrm{E}-03$

$3.47826 \mathrm{E}-03$

$3.48610 \mathrm{E}-03$

$3.49384 \mathrm{E}-03$

$3.50147 \mathrm{E}-03$ $3.50899 \mathrm{E}-03$

3.51641 E-03

$3.52371 \mathrm{E}-03$

$3.53092 \mathrm{E}-03$

$3.53802 \mathrm{E}-03$

$3.54503 \mathrm{E}-03$

$3.55194 \mathrm{E}-03$

$3.55875 \mathrm{E}-03$

$3.56547 \mathrm{E}-03$

$3.57209 \mathrm{E}-03$

$3.57862 \mathrm{E}-03$

$3.58506 \mathrm{E}-03$

$3.59142 \mathrm{E}-03$

$3.59769 \mathrm{E}-03$

$3.60387 \mathrm{E}-03$ $3.60997 \mathrm{E}-03$ $3.61599 \mathrm{E}-03$ $3.62191 \mathrm{E}-03$ $3.62777 \mathrm{E}-03$ $f_{-1}$

$-5.91715 \mathrm{E}-01$ $-5.99601 \mathrm{E}-01$ $-6.07536 \mathrm{E}-01$ $-6.15522 \mathrm{E}-01$ $-6.23557 \mathrm{E}-01$

$-6.31642 \mathrm{E}-01$ $-6.39777 \mathrm{E}-01$ $-6.47963 \mathrm{E}-01$ $-6.56198 \mathrm{E}-01$ $-6.64483 \mathrm{E}-01$

$-6.72818 \mathrm{E}-01$ $-6.81203 \mathrm{E}-01$ $-6.89637 \mathrm{E}-01$ $-6.98122 \mathrm{E}-01$ $-7.06657 \mathrm{E}-01$

$-7.15241 \mathrm{E}-01$ $-7.23876 \mathrm{E}-01$ $-7.32560 \mathrm{E}-01$ $-7.41294 \mathrm{E}-01$ $-7.50078 \mathrm{E}-01$

$-7.58912 \mathrm{E}-01$

$-7.67796 \mathrm{E}-01$

$-7.76729 \mathrm{E}-01$

$-7.85712 \mathrm{E}-01$

$-7.94745 \mathrm{E}-01$

$-8.03828 \mathrm{E}-01$ $-8.12961 \mathrm{E}-01$ $-8.22143 \mathrm{E}-01$ $-8.31376 \mathrm{E}-01$ $-8.40658 \mathrm{E}-01$

$-8.49989 \mathrm{E}-01$ $-8.59371 \mathrm{E}-01$ $-8.68802 \mathrm{E}-01$ $-8.78283 \mathrm{E}-01$ $-8.87814 \mathrm{E}-01$

$-8.97394 \mathrm{E}-01$ $-9.07024 \mathrm{E}-01$ $-9.16704 \mathrm{E}-01$ $-9.26434 \mathrm{E}-01$ $-9.36213 \mathrm{E}-01$

$-9.46042 \mathrm{E}-01$ $-9.55920 \mathrm{E}-01$ $-9.65848 \mathrm{E}-01$ $-9.75826 \mathrm{E}-01$ $-9.85854 \mathrm{E}-01$

$-9.95931 \mathrm{E}-01$ $-1.00606 \mathrm{E} \quad 00$ $-1.01623 \mathrm{E} 00$ $-1.02646 \mathrm{E} 00$ -1.03674 E 00

$-1.04706 \mathrm{E} \quad 00$ $-1.05743 \mathrm{E} \quad 00$ $-1.06786 \mathrm{E} 00$ $-1.07833 \mathrm{E} 00$ $-1.08886 \mathrm{E} 00$

$-1.09943 \mathrm{E} \quad 00$ $-1.11005 \mathrm{E} 00$ $-1.12072 \mathrm{E} 00$ $-1.13144 \mathrm{E} 00$ $-1.14221 \mathrm{E} \quad 00$ $g_{-1}$

$1.34499 \mathrm{E} \quad 01$

$1.35561 \mathrm{E} \quad 01$

$1.36622 \mathrm{E} \quad 01$

$1.37683 \mathrm{E} \quad 01$

$1.38744 \mathrm{E}$ 0l

$1.39804 \mathrm{E} \quad 01$ $1.40865 \mathrm{E} 01$

$1.41925 \mathrm{E} \quad 01$

$1.42985 \mathrm{E} \quad 01$

$1.44046 \mathrm{E} \quad 01$

$1.45105 \mathrm{E} \quad 01$

$1.46165 \mathrm{E} \quad 01$

$1.47225 \mathrm{E} 01$

$1.48284 \mathrm{E} \quad 01$

$1.49344 \mathrm{E} \quad 01$

$1.50403 \mathrm{E} \quad 01$

$1.51462 \mathrm{E} 01$

$1.52521 \mathrm{E} \quad 01$

$1.53579 \mathrm{E} \quad 01$

$1.54638 \mathrm{E} \quad 01$

$\begin{array}{lll}1.55696 \mathrm{E} & 01\end{array}$

$1.56754 \mathrm{E} \quad 01$

$1.57812 \mathrm{E} \quad 01$

$\begin{array}{ll}1.58870 \mathrm{E} & 01\end{array}$

$1.59928 \mathrm{E} 01$

$1.60986 \mathrm{E} \quad 01$ $1.62043 \mathrm{E} \quad 01$

$1.63100 \mathrm{E} \quad 01$

$1.64157 \mathrm{E} \quad 01$

$1.65214 \mathrm{E} \quad 01$

$1.66271 \mathrm{E} \quad 01$

$1.67328 \mathrm{E}-01$

$1.68384 \mathrm{E} \quad 01$

$1.69441 \mathrm{E} \quad 01$

$1.70497 \mathrm{E} \quad 01$

$1.71553 \mathrm{E} \quad 01$

$1.72609 \mathrm{E} \quad 01$

$1.73664 \mathrm{E} \quad 01$

$1.74720 \mathrm{E} 01$

$1.75775 \mathrm{E} 01$

$1.76830 \mathrm{E} \quad 01$ $1.77885 \mathrm{E} \quad 01$

$1.78940 \mathrm{E} 01$

$1.79995 \mathrm{E} 01$

$1.81050 \mathrm{E} \quad 01$

$1.82104 \mathrm{E} \quad 01$ $1.83158 \mathrm{E} \quad 01$ $1.84212 \mathrm{E} \quad 01$

$1.85266 \mathrm{E} \quad 01$

$1.86320 \mathrm{E} \quad 0]$

$1.87373 \mathrm{E} \quad 01$

$1.88427 \mathrm{E} \quad 01$

$1.89480 \mathrm{E} 01$

$1.90533 \mathrm{E} \quad 01$

$1.91586 \mathrm{E} 01$

$1.92639 \mathrm{E} \quad 01$ $1.93691 \mathrm{E} \quad 01$ $1.94744 \mathrm{E} \quad 01$

$1.95796 \mathrm{E}$ 01 $1.96848 \mathrm{E} 01$ $\tan \delta_{-1}$

$-1.49316 \mathrm{E} \quad 02$ $-1.49666 \mathrm{E} 02$ $-1.50012 \mathrm{E} \quad 02$ $-1.50354 \mathrm{E} \quad 02$ -1.50693 E 02

$-1.51029 \mathrm{E} \quad 02$ $-1.51360 \mathrm{E} 02$ $-1.51689 \mathrm{E} \quad 02$ $-1.52014 \mathrm{E} 02$ $-1.52336 \mathrm{E} 02$

$-1.52654 \mathrm{E} \quad 02$ $-1.52969 \mathrm{E} 02$ $-1.53281 \mathrm{E} 02$ $-1.53590 \mathrm{E} 02$ $-1.53897 \mathrm{E} 02$

$-1.54200 \mathrm{E} \quad 02$ $-1.54500 \mathrm{E} 02$ $-1.54797 \mathrm{E} \quad 02$ $-1.55092 \mathrm{E} \quad 02$ $-1.55384 \mathrm{E} \quad 02$

$-1.55673 \mathrm{E} \quad 02$ $-1.55959 \mathrm{E} 02$ $-1.56243 \mathrm{E} 02$ $-1.56524 \mathrm{E} 02$ $-1.56803 \mathrm{E} 02$ $-1.57079 \mathrm{E} \quad 02$ $-1.57353 \mathrm{E} \quad 02$ $-1.57624 \mathrm{E} \quad 02$ $-1.57893 \mathrm{E} 02$ $-1.58160 \mathrm{E} \quad 02$

$-1.58424 \mathrm{E} \quad 02$ $-1.58686 \mathrm{E} 02$ $-1.58946 \mathrm{E} 02$ $-1.59204 \mathrm{E} \quad 02$ -1.59460 E 02

$-1.59713 \mathrm{E} 02$ $-1.59965 \mathrm{E} 02$ $-1.60214 \mathrm{E} \quad 02$ $-1.60462 \mathrm{E} 02$ $-1.60708 \mathrm{E} \quad 02$ $-1.60951 \mathrm{E} \quad 02$ $-1.61193 \mathrm{E} \quad 02$ $-1.61433 \mathrm{E} \quad 02$ $-1.61671 \mathrm{E} \quad 02$ $-1.61907 \mathrm{E} 02$ $-1.62141 \mathrm{E} \quad 02$ $-1.62374 \mathrm{E} \quad 02$ $-1.62605 \mathrm{E} \quad 02$ $-1.62835 \mathrm{E} \quad 02$ -1.63062 E 02

$-1.63288 \mathrm{E} \quad 02$ $-1.63512 \mathrm{E} 02$ $-1.63735 \mathrm{E} 02$ $\begin{array}{lll}-1.63957 \mathrm{E} & 02\end{array}$ $\begin{array}{ll}-1.64176 \mathrm{E} & 02\end{array}$

$-1.64394 \mathrm{E} \quad 02$ $-1.64611 \mathrm{E} 02$ $-1.64826 \mathrm{E} 02$ $-1.65040 \mathrm{E} \quad 02$ $-1.65252 \mathrm{E} 02$ 
$\bar{F}$

12.10

12.20

12.30

12.40

12.50

12.60

12.70

12.80

12.90

13.00

13.10

13.20

13.30

13.40

13.50

13.60

13.70

13.80

13.90

14.00

14.10

14.20

14.30

14.40

14.50

14.60

14.70

14.80

14.90

15.00

15.10

15.20

15.30

15.40

15.50

15.60

15.70

15.80

15.90

16.00

16.10

16.20

16.30

16.40

16.50

16.60

16.70

16.80

16.90

17.00

17.10

17.20

17.30

17.40

17.50

17.60

17.70

17.80

17.90

18.00 $f_{2}$

$2.37012 \mathrm{E}-04$ $2.40942 \mathrm{E}-04$ $2.44905 \mathrm{E}-04$ $2.48899 \mathrm{E}-04$ $2.52926 \mathrm{E}-04$

$2.56986 \mathrm{E}-04$ $2.61077 \mathrm{E}-04$ $2.65201 \mathrm{E}-04$ $2.69357 \mathrm{E}-04$

$2.73546 \mathrm{E}-04$

$2.77767 \mathrm{E}-04$ $2.82020 \mathrm{E}-04$ $2.86305 \mathrm{E}-04$ $2.90623 \mathrm{E}-04$ 2.94973 E-04

$2.99356 \mathrm{E}-04$ $3.03770 \mathrm{E}-04$ $3.08218 \mathrm{E}-04$ $3.12697 \mathrm{E}-04$ $3.17208 \mathrm{E}-04$

$3.21752 \mathrm{E}-04$ $3.26329 \mathrm{E}-04$

3.30937E-04

$3.35578 \mathrm{E}-04$

$3.40251 \mathrm{E}-04$

$3.44957 \mathrm{E}-04$ $3.49695 \mathrm{E}-04$

$3.54465 \mathrm{E}-04$

$3.59267 \mathrm{E}-04$

3.64102E-04

$3.68969 \mathrm{E}-04$

$3.73869 \mathrm{E}-04$

$3.78800 \mathrm{E}-04$

$3.83764 \mathrm{E}-04$

$3.88760 \mathrm{E}-04$

$3.93789 \mathrm{E}-04$

$3.98850 \mathrm{E}-04$

$4.03943 \mathrm{E}-04$

$4.09069 \mathrm{E}-04$

4.14227E-04

4.19417E-04

$4.24639 \mathrm{E}-04$

$4.29894 \mathrm{E}-04$

$4.35181 \mathrm{E}-04$

$4.40500 \mathrm{E}-04$

$4.45852 \mathrm{E}-04$ $4.51236 \mathrm{E}-04$ $4.56652 \mathrm{E}-04$ $4.62101 \mathrm{E}-04$ $4.67582 \mathrm{E}-04$

4.73095E-04 $4.78640 \mathrm{E}-04$ $4.84218 \mathrm{E}-04$

$4.89828 \mathrm{E}-04$ 4.95471 E-04

$5.01145 \mathrm{E}-04$ $5.06852 \mathrm{E}-04$

$5.12592 \mathrm{E}-04$

$5.18363 \mathrm{E}-04$

$5.24167 \mathrm{E}-04$ $g_{2}$

$-3.55993 \mathrm{E}-01$ $-3.62021 \mathrm{E}-01$

$-3.68100 \mathrm{E}-01$

$-3.74229 \mathrm{E}-01$

$-3.80408 \mathrm{E}-01$

$-3.86638 \mathrm{E}-01$

$-3.92918 \mathrm{E}-01$

$-3.99248 \mathrm{E}-01$

$-4.05629 \mathrm{E}-01$

$-4.12060 \mathrm{E}-01$

$-4.18541 \mathrm{E}-01$

$-4.25073 \mathrm{E}-01$

$-4.31655 \mathrm{E}-01$

$-4.38287 \mathrm{E}-01$

$-4.44970 \mathrm{E}-01$

$-4.51703 \mathrm{E}-01$

$-4.58486 \mathrm{E}-01$

$-4.65319 \mathrm{E}-01$

$-4.72203 \mathrm{E}-01$

$-4.79137 \mathrm{E}-01$

-4.86121 E-01

$-4.93155 \mathrm{E}-01$

$-5.00240 \mathrm{E}-01$

$-5.07374 \mathrm{E}-01$

$-5.14559 \mathrm{E}-01$

$-5.21795 \mathrm{E}-01$

$-5.29080 \mathrm{E}-01$

$-5.36416 \mathrm{E}-01$

$-5.43801 \mathrm{E}-01$

$-5.51238 \mathrm{E}-01$

$-5.58724 \mathrm{E}-01$ $-5.66260 \mathrm{E}-01$ $-5.73847 \mathrm{E}-01$

$-5.81483 \mathrm{E}-01$

$-5.89170 \mathrm{E}-01$

$-5.96907 \mathrm{E}-01$

$-6.04694 \mathrm{E}-01$

$-6.12532 \mathrm{E}-01$

$-6.20419 \mathrm{E}-01$

$-6.28356 \mathrm{E}-01$

$-6.36344 \mathrm{E}-01$

$-6.44382 \mathrm{E}-01$

$-6.52470 \mathrm{E}-01$

$-6.60607 \mathrm{E}-01$

$-6.68795 \mathrm{E}-01$

$-6.77033 \mathrm{E}-01$

$-6.85321 \mathrm{E}-01$

$-6.93660 \mathrm{E}-01$

$-7.02048 \mathrm{E}-01$

$-7.10486 \mathrm{E}-01$

$-7.18974 \mathrm{E}-01$

$-7.27513 \mathrm{E}-01$

$-7.36101 \mathrm{E}-01$

$-7.44739 \mathrm{E}-01$

$-7.53427 \mathrm{E}-01$

$-7.62166 \mathrm{E}-01$

$-7.70954 \mathrm{E}-01$

$-7.79792 \mathrm{E}-01$

$-7.88681 \mathrm{E}-01$

$-7.97619 \mathrm{E}-01$ $\tan \delta_{2}$

$f_{-2}$

$-1.02249 \mathrm{E}-02$

$-1.04495 \mathrm{E}-02$

$-1.06773 \mathrm{E}-02$

$-1.09082 \mathrm{E}-02$

$-1.11424 \mathrm{E}-02$

$-1.13798 \mathrm{E}-02$

$-1.16205 \mathrm{E}-02$

$-1.18644 \mathrm{E}-02$

$-1.21117 \mathrm{E}-02$

$-1.23623 \mathrm{E}-02$

$-1.26162 \mathrm{E}-02$ $-1.28735 \mathrm{E}-02$

$-1.31342 \mathrm{E}-02$

$-1.33983 \mathrm{E}-02$

$-1.36659 \mathrm{E}-02$

$-1.39369 \mathrm{E}-02$ $-1.42114 \mathrm{E}-02$

$-1.44893 \mathrm{E}-02$

$-1.47708 \mathrm{E}-02$

$-1.50559 \mathrm{E}-02$

$-1.53445 \mathrm{E}-02$

$-1.56366 \mathrm{E}-02$

$-1.59324 \mathrm{E}-02$

$-1.62318 \mathrm{E}-02$

$-1.65349 \mathrm{E}-02$

$-1.68416 \mathrm{E}-02$

$-1.71520 \mathrm{E}-02$

$-1.74662 \mathrm{E}-02$

$-1.77840 \mathrm{E}-02$

$-1.81056 \mathrm{E}-02$

$-1.84310 \mathrm{E}-02$

- $1.87602 \mathrm{E}-02$

$-1.90932 \mathrm{E}-02$

$-1.94301 \mathrm{E}-02$

$-1.97708 \mathrm{E}-02$

$-2.01154 \mathrm{E}-02$

$-2.04639 \mathrm{E}-02$

$-2.08163 \mathrm{E}-02$

$-2.11726 \mathrm{E}-02$

$-2.15330 \mathrm{E}-02$

$-2.18973 \mathrm{E}-02$

$-2.22656 \mathrm{E}-02$

$-2.26380 \mathrm{E}-02$

$-2.30144 \mathrm{E}-02$

$-2.33949 \mathrm{E}-02$

$-2.37794 \mathrm{E}-02$

$-2.41681 \mathrm{E}-02$

$-2.45609 \mathrm{E}-02$

$-2.49579 \mathrm{E}-02$

$-2.53591 \mathrm{E}-02$

$-2.57645 \mathrm{E}-02$

$-2.61741 \mathrm{E}-02$

$-2.65879 \mathrm{E}-02$

$-2.70060 \mathrm{E}-02$

$-2.74284 \mathrm{E}-02$

$-2.78551 \mathrm{E}-02$

$-2.82861 \mathrm{E}-02$

$-2.87214 \mathrm{E}-02$

$-2.91612 \mathrm{E}-02$

$-2.96053 \mathrm{E}-02$

$3.36603 \mathrm{E} \quad 01-1.00035 \mathrm{E}-02$

$3.36706 \mathrm{E} \quad 01-1.02219 \mathrm{E}-02$

$3.36807 \mathrm{E} \quad 01-1.04433 \mathrm{E}-02$

$3.36906 \mathrm{E} \quad 01-1.06679 \mathrm{E}-02$

$3.37003 \mathrm{E} 01-1.08956 \mathrm{E}-02$

$3.37099 \mathrm{E}$ 01 $-1.11265 \mathrm{E}-02$

$3.37194 \mathrm{E} \quad 01-1.13605 \mathrm{E}-02$

$3.37286 \mathrm{E}$ 01 $-1.15978 \mathrm{E}-02$

$3.37378 \mathrm{E}$ 01 $-1.18384 \mathrm{E}-02$

$3.37468 \mathrm{E}$ 01 $-1.20821 \mathrm{E}-02$

$3.37556 \mathrm{E} \quad 01$

$3.37643 \mathrm{E} \quad 01$

$3.37728 \mathrm{E} \quad 01$

$3.37813 \mathrm{E} \quad 01$

$3.37896 \mathrm{E} 01$

$3.37977 \mathrm{E} \quad 01$

$3.38058 \mathrm{E} \quad 01$

$3.38137 \mathrm{E} \quad 01$

$3.38215 \mathrm{E} 01$

$3.38292 \mathrm{E} \quad 01$

$3.38367 \mathrm{E} \quad 01$

$3.38442 \mathrm{E} \quad 01$

$3.38515 \mathrm{E} \quad 01$

$3.38588 \mathrm{E} \quad 01$

$3.38659 \mathrm{E} 01$

$3.38729 \mathrm{E} \quad 01$

$3.38799 \mathrm{E}$ 0]

$3.38867 \mathrm{E} \quad 01$

$3.38934 \mathrm{E} \quad 01$

$3.39001 \mathrm{E} 01$

$3.39066 \mathrm{E} \quad 01$ $3.39131 \mathrm{E} \quad 01$ $3.39195 \mathrm{E} \quad 01$ $3.39257 \mathrm{E} \quad 01$

$3.39319 \mathrm{E} \quad 01$

$3.39381 \mathrm{E} \quad 01$

$3.39441 \mathrm{E} \quad 01$

$3.39501 \mathrm{E} \quad 01$

$3.39559 \mathrm{E} \quad 01$

$3.39617 \mathrm{E} \quad 01$

$3.39675 \mathrm{E} \quad 01$

$3.39731 \mathrm{E} 01$ 
p

18.10

18.20

18.30

18.40

18.50

18.60

18.70

18.80

18.90

19.00

19.10

19.20

19.30

19.40

19.50

19.60

19.70

19.80

19.90

20.00

20.10

20.20

20.30

20.40

20.50

20.60

20.70

20.80

20.90

21.00

21.10

21.20

21.30

21.40

21.50

21.60

21.70

21.80

21.90

22.00

22.10

22.20

22.30

22.40

22.50

22.60

22.70

22.80

22.90

23.00

23.10

23.20

23.30

23.40

23.50

23.60

23.70

23.80

23.90

24.00
$F_{0}$

$f_{1}$

$\begin{array}{llll}1.15239 \mathrm{E} & 00 & -1.87309 \mathrm{E} & 01\end{array}$

$\begin{array}{llll}1.15238 \mathrm{E} & 00 & -1.88361 \mathrm{E} & 01\end{array}$

$\begin{array}{llll}1.15236 \mathrm{E} & 00 & -1.89414 \mathrm{E} & 01\end{array}$

$\begin{array}{llll}1.15235 \mathrm{E} & 00 & -1.90466 \mathrm{E} & 01\end{array}$

$1.15234 \mathrm{E} \quad 00$

$1.15232 \mathrm{E} \quad 00$

$1.15231 \mathrm{E} \quad 00$

$1.15229 \mathrm{E} \quad 00$

$1.15228 \mathrm{E} \quad 00$

$1.15227 \mathrm{E} \quad 00$

$1.15225 \mathrm{E} \quad 00$

$1.15224 \mathrm{E} \quad 00$

$1.15222 \mathrm{E} \quad 00$

$1.15221 \mathrm{E} \quad 00$

$1.15220 \mathrm{E} \quad 00$

$1.15218 \mathrm{E} \quad 00$ $1.15217 \mathrm{E} \quad 00$

$1.15216 \mathrm{E} \quad 00$

$1.15214 \mathrm{E} \quad 00$

$1.15213 \mathrm{E} \quad 00$

$1.15212 \mathrm{E} \quad 00$

$1.15211 \mathrm{E} \quad 00$

$1.15209 \mathrm{E} \quad 00$

$1.15208 \mathrm{E} \quad 00$

$1.15207 \mathrm{E} \quad 00$

$1.15206 \mathrm{E} \quad 00$

$1.15204 \mathrm{E} \quad 00$

$1.15203 \mathrm{E} \quad 00$

$1.15202 \mathrm{E} \quad 00$

$1.15201 \mathrm{E} \quad 00$

$1.15199 \mathrm{E} \quad 00$

$1.15198 \mathrm{E} \quad 00$

$1.15197 \mathrm{E} \quad 00$

$1.15196 \mathrm{E} \quad 00$

$1.15195 \mathrm{E} \quad 00$

$1.15193 \mathrm{E} \quad 00$

$1.15192 \mathrm{E} \quad 00$

$1.15191 \mathrm{E} \quad 00$

$1.15190 \mathrm{E} \quad 00$

$1.15189 \mathrm{E} \quad 00$

$1.15188 \mathrm{E} \quad 00$

$1.15187 \mathrm{E} \quad 00$

$1.15185 \mathrm{E} \quad 00$

$1.15184 \mathrm{E} \quad 00$

$1.15183 \mathrm{E} \quad 00$

$1.15182 \mathrm{E} \quad 00$

$1.15181 \mathrm{E} \quad 00$

$1.15180 \mathrm{E} \quad 00$

$1.15179 \mathrm{E} \quad 00$

$1.15178 \mathrm{E} \quad 00$

$1.15177 \mathrm{E} \quad 00$

$1.15176 \mathrm{E} \quad 00$

$1.15174 \mathrm{E} \quad 00$

$1.15173 \mathrm{E} \quad 00$

$1.15172 \mathrm{E} \quad 00$

$1.15171 \mathrm{E} \quad 00$

$1.15170 \mathrm{E} \quad 00$

$1.15169 \mathrm{E} \quad 00$

$1.15168 \mathrm{E} \quad 00$

$1.15167 \mathrm{E} \quad 00$ $g_{1}$

$-1.18036 \mathrm{E} \quad 00$ $-1.19148 \mathrm{E} \quad 00$ $-1.20266 \mathrm{E} 00$ $-1.21388 \mathrm{E} \quad 00$ $-1.22515 \mathrm{E} 00$

$-1.23647 \mathrm{E} \quad 00$ $-1.24784 \mathrm{E} 00$ $-1.25926 \mathrm{E} 00$ $-1.27073 \mathrm{E} \quad 00$ $-1.28225 \mathrm{E} \quad 00$

$-1.29382 \mathrm{E} \quad 00$ $-1.30544 \mathrm{E} 00$ $-1.31710 \mathrm{E} 00$ $-1.32882 \mathrm{E} 00$ $-1.34059 \mathrm{E} \quad 00$

$-1.35240 \mathrm{E} 00$ $-1.36426 \mathrm{E} \quad 00$ $-1.37618 \mathrm{E} \quad 00$ $-1.38814 \mathrm{E} \quad 00$ $-1.40015 \mathrm{E} 00$

$-1.41221 \mathrm{E} \quad 00$ $-1.42432 \mathrm{E} \quad 00$ $-1.43648 \mathrm{E} \quad 00$ $-1.44869 \mathrm{E} \quad 00$ $-1.46095 \mathrm{E} \quad 00$

$-1.47326 \mathrm{E} \quad 00$ $-1.48561 \mathrm{E} 00$ $-1.49802 \mathrm{E} \quad 00$ $-1.51047 \mathrm{E} \quad 00$ $-1.52298 \mathrm{E} 00$

$-1.53553 \mathrm{E} 00$ $-1.54813 \mathrm{E} \quad 00$ $-1.56079 \mathrm{E} 00$ $-1.57349 \mathrm{E} 00$ $-1.58624 \mathrm{E} \quad 00$

$-1.59904 \mathrm{E} 00$ $-1.61188 \mathrm{E} 00$ $-1.62478 \mathrm{E} 00$ $-1.63773 \mathrm{E} 00$ $-1.65072 \mathrm{E} \quad 00$

$-1.66377 \mathrm{E} \quad 00$ $-1.67686 \mathrm{E} \quad 00$ $-1.69000 \mathrm{E} 00$ $-1.70319 \mathrm{E} \quad 00$ $-1.71643 \mathrm{E} \quad 00$

$-1.72972 \mathrm{E} \quad 00$ $-1.74306 \mathrm{E} \quad 00$ $-1.75645 \mathrm{E} \quad 00$ $-1.76988 \mathrm{E} \quad 00$ $-1.78337 \mathrm{E} \quad 00$

$-1.79690 \mathrm{E} 00$ $-1.81049 \mathrm{E} \quad 00$ $-1.82412 \mathrm{E} 00$ $-1.83780 \mathrm{E} 00$ $-1.85153 \mathrm{E} 00$

$-1.86531 \mathrm{E} \quad 00$ $-1.87913 \mathrm{E} 00$ $-1.89301 \mathrm{E} \quad 00$ $-1.90693 \mathrm{E} 00$ $-1.92091 \mathrm{E} 00$ $\tan \delta_{1}$

$3.63354 \mathrm{E}-03$ $3.63924 \mathrm{E}-03$ $3.64486 \mathrm{E}-03$ $3.65040 \mathrm{E}-03$

$3.65587 \mathrm{E}-03$

$3.66126 \mathrm{E}-03$ $3.66659 \mathrm{E}-03$

$3.67184 \mathrm{E}-03$

$3.67702 \mathrm{E}-03$

$3.68213 \mathrm{E}-03$

$3.68718 \mathrm{E}-03$

$3.69216 \mathrm{E}-03$

$3.69707 \mathrm{E}-03$

$3.70192 \mathrm{E}-03$

$3.70670 \mathrm{E}-03$

$3.71143 \mathrm{E}-03$

$3.71608 \mathrm{E}-03$

$3.72067 \mathrm{E}-03$

$3.72521 \mathrm{E}-03$

$3.72968 \mathrm{E}-03$

$3.73410 \mathrm{E}-03$

$3.73846 \mathrm{E}-03$

$3.74275 \mathrm{E}-03$

$3.74700 \mathrm{E}-03$

$3.75118 \mathrm{E}-03$

$3.75531 \mathrm{E}-03$ $3.75940 \mathrm{E}-03$

$3.76342 \mathrm{E}-03$

$3.76739 \mathrm{E}-03$

3.77131 E-03

$3.77517 \mathrm{E}-03$

$3.77898 \mathrm{E}-03$

$3.78275 \mathrm{E}-03$

$3.78646 \mathrm{E}-03$

$3.79013 \mathrm{E}-03$

$3.79375 \mathrm{E}-03$

$3.79731 \mathrm{E}-03$

$3.80084 \mathrm{E}-03$

$3.80431 \mathrm{E}-03$

$3.80775 \mathrm{E}-03$

$3.81113 \mathrm{E}-03$ $3.81447 \mathrm{E}-03$

$3.81775 \mathrm{E}-03$

$3.82101 \mathrm{E}-03$

3.82421 E-03

$3.82737 \mathrm{E}-03$

$3.83050 \mathrm{E}-03$

$3.83357 \mathrm{E}-03$

$3.83661 \mathrm{E}-03$

$3.83961 \mathrm{E}-03$

$3.84256 \mathrm{E}-03$

$3.84547 \mathrm{E}-03$

$3.84835 \mathrm{E}-03$

$3.85119 \mathrm{E}-03$

$3.85398 \mathrm{E}-03$

$3.85674 \mathrm{E}-03$ $3.85946 \mathrm{E}-03$

$3.86214 \mathrm{E}-03$

$3.86479 \mathrm{E}-03$

$3.86740 \mathrm{E}-03$

$f_{-1}$

$-1.15304 \mathrm{E} 00$ $-1.16391 \mathrm{E} 00$ $-1.17482 \mathrm{E} \quad 00$ $-1.18579 \mathrm{E} \quad 00$ $-1.19681 \mathrm{E} 00$

$-1.20788 \mathrm{E} \quad 00$ $-1.21900 \mathrm{E} \quad 00$ $-1.23016 \mathrm{E} \quad 00$ $-1.24138 \mathrm{E} \quad 00$ $-1.25264 \mathrm{E} 00$

$-1.26396 \mathrm{E} \quad 00$ $-1.27532 \mathrm{E} \quad 00$ $-1.28674 \mathrm{E} \quad 00$ $-1.29820 \mathrm{E} \quad 00$ $-1.30971 \mathrm{E} 00$

$-1.32127 \mathrm{E} \quad 00$ $-1.33288 \mathrm{E} \quad 00$ $-1.34454 \mathrm{E} \quad 00$ $-1.35625 \mathrm{E} \quad 00$ $-1.36801 \mathrm{E} \quad 00$

$-1.37982 \mathrm{E} \quad 00$ $-1.39167 \mathrm{E} \quad 00$ $-1.40358 \mathrm{E} \quad 00$ $-1.41554 \mathrm{E} \quad 00$ $-1.42754 \mathrm{E} \quad 00$

$-1.43960 \mathrm{E} \quad 00$ $-1.45170 \mathrm{E} \quad 00$ $-1.46385 \mathrm{E} \quad 00$ $-1.47605 \mathrm{E} 00$ $-1.48830 \mathrm{E} 00$

$-1.50060 \mathrm{E} \quad 00$ $-1.51295 \mathrm{E} \quad 00$ $-1.52535 \mathrm{E} \quad 00$ $-1.53780 \mathrm{E} \quad 00$ $-1.55030 \mathrm{E} \quad 00$

$-1.56284 \mathrm{E} \quad 00$ $-1.57544 \mathrm{E} \quad 00$ $-1.58808 \mathrm{E} 00$ $-1.60077 \mathrm{E} \quad 00$ $-1.61351 \mathrm{E} 00$ 
ELECTRON RADIAL FUNCTIONS

$Z=6 \quad A=12 \quad \rho=1.2 A^{1 / 3} F$

Electron

18.60

18.70

18.80

18.90

19.00

19.10

19.20

19.30

19.40

19.50

19.60

19.70

19.80

19.90

20.00

20.10

20.20

20.30

20.40

20.50

20.60

20.70

20.80

20.90

21.00

21.10

21.20

21.30

21.40

21.50

21.60

21.70

21.80

21.90

22.00

22.10

22.20

22.30

22.40

22.50

22.60

22.70

22.80

22.90

23.00

23.10

23.20

23.30

23.40

23.50

23.60

23.70

23.80

23.90

24.00
$\bar{F}$

$5.30004 \mathrm{E}-04$ $5.35872 \mathrm{E}-04$

$5.41773 \mathrm{E}-04$

$5.47706 \mathrm{E}-04$

5.53672 E-04

$5.59669 \mathrm{E}-04$

$5.65699 \mathrm{E}-04$

$5.71762 \mathrm{E}-04$

$5.77856 \mathrm{E}-04$

$5.83983 \mathrm{E}-04$

$5.90143 \mathrm{E}-04$ $5.96334 \mathrm{E}-04$ $6.02558 \mathrm{E}-04$ $6.08814 \mathrm{E}-04$

$6.15103 \mathrm{E}-04$

$6.21424 \mathrm{E}-04$ $6.27777 \mathrm{E}-04$ $6.34162 \mathrm{E}-04$ $6.40580 \mathrm{E}-04$ $6.47029 \mathrm{E}-04$

$6.53512 \mathrm{E}-04$ $6.60026 \mathrm{E}-04$ $6.66573 \mathrm{E}-04$ $6.73152 \mathrm{E}-04$ $6.79764 \mathrm{E}-04$

$6.86407 \mathrm{E}-04$ $6.93083 \mathrm{E}-04$ $6.99792 \mathrm{E}-04$ $7.06532 \mathrm{E}-04$ 7.13305 E- 04

7.20111 E- 04 $7.26948 \mathrm{E}-04$ $7.33818 \mathrm{E}-04$ $7.40720 \mathrm{E}-04$ $7.47655 \mathrm{E}-04$

7.54621 E- 04 $7.61620 \mathrm{E}-04$ $7.68652 \mathrm{E}-04$ 7.75715 E-04 7.82811 E- 04

$7.89939 \mathrm{E}-04$ $7.97100 \mathrm{E}-04$ $8.04292 \mathrm{E}-04$ 8.11518 E-04 8.18775 E- 04

$8.26065 \mathrm{E}-04$ 8.33387E-04 $8.40741 \mathrm{E}-04$ $8.48128 \mathrm{E}-04$ $8.55546 \mathrm{E}-04$

$8.62998 \mathrm{E}-04$ $8.70481 \mathrm{E}-04$ $8.77997 \mathrm{E}-04$ $8.85545 \mathrm{E}-04$ $8.93125 \mathrm{E}-04$

$9.00738 \mathrm{E}-04$ $9.08383 \mathrm{E}-04$ $9.16060 \mathrm{E}-04$ $9.23769 \mathrm{E}-04$ 9.31511 E-04 $f_{2}$

$g_{2}$

$\tan \delta_{2}$

$f_{-2}$

$-8.06608 \mathrm{E}-01$

$-8.15646 \mathrm{E}-01$

$-8.24734 \mathrm{E}-01$

$-8.33872 \mathrm{E}-01$

$-8.43060 \mathrm{E}-01$

$-8.52298 \mathrm{E}-01$

$-8.61585 \mathrm{E}-01$

$-8.70923 \mathrm{E}-01$

$-8.80311 \mathrm{E}-01$

$-8.89748 \mathrm{E}-01$

$-8.99236 \mathrm{E}-01$ $-9.08773 \mathrm{E}-01$ $-9.18360 \mathrm{E}-01$ $-9.27997 \mathrm{E}-01$

$-9.37684 \mathrm{E}-01$

$-9.47420 \mathrm{E}-01$

$-9.57207 \mathrm{E}-01$

$-9.67043 \mathrm{E}-01$

$-9.76929 \mathrm{E}-01$

$-9.86865 \mathrm{E}-0 \mathrm{l}$

$-9.96850 \mathrm{E}-01$

$-1.00689 \mathrm{E} 00$

$-1.01697 \mathrm{E} 00$

$-1.02711 \mathrm{E} 00$

$-1.03729 \mathrm{E} 00$

$-1.04753 \mathrm{E} \quad 00$

$-1.05781 \mathrm{E} \quad 00$

$-1.06814 \mathrm{E} 00$

$-1.07853 \mathrm{E} \quad 00$

$-1.08896 \mathrm{E} 00$

$-1.09944 \mathrm{E} \quad 00$

$-1.10998 \mathrm{E} \quad 00$

$-1.12056 \mathrm{E} \quad 00$

$-1.13119 \mathrm{E} 00$

$-1.14187 \mathrm{E} \quad 00$

$-1.15260 \mathrm{E} \quad 00$

$-1.16339 \mathrm{E} 00$

$-1.17422 \mathrm{E} \quad 00$

$-1.18510 \mathrm{E} 00$

$-1.19603 \mathrm{E} 00$

$-1.20701 \mathrm{E} \quad 00$ $-1.21804 \mathrm{E} 00$ $-1.22912 \mathrm{E} 00$ $-1.24024 \mathrm{E} 00$ $-1.25142 \mathrm{E} 00$

$-1.26265 \mathrm{E} \quad 00$ $-1.27393 \mathrm{E} 00$ $-1.28525 \mathrm{E} \quad 00$ $-1.29663 \mathrm{E} \quad 00$ $-1.30806 \mathrm{E} 00$

$-1.31953 \mathrm{E} 00$ $-1.33106 \mathrm{E} 00$ $-1.34263 \mathrm{E} 00$ $-1.35425 \mathrm{E} 00$ $-1.36593 \mathrm{E} 00$

$-1.37765 \mathrm{E} \quad 00$ $-1.38942 \mathrm{E} \quad 00$ $-1.40125 \mathrm{E} 00$ $-1.41312 \mathrm{E} \quad 00$ $-1.42504 \mathrm{E} 00$
$-3.00539 \mathrm{E}-02$ $-3.05068 \mathrm{E}-02$

$-3.09643 \mathrm{E}-02$

$-3.14262 \mathrm{E}-02$

$-3.18926 \mathrm{E}-02$

$-3.23635 \mathrm{E}-02$

$-3.28390 \mathrm{E}-02$

$-3.33190 \mathrm{E}-02$

$-3.38036 \mathrm{E}-02$

$-3.42928 \mathrm{E}-02$

$-3.47867 \mathrm{E}-02$

-3.52851 E-02

$-3.57883 \mathrm{E}-02$

-3.62961 E-02

$-3.68087 \mathrm{E}-02$

$-3.73259 \mathrm{E}-02$

$-3.78480 \mathrm{E}-02$

$-3.83748 \mathrm{E}-02$

$-3.89064 \mathrm{E}-02$

$-3.94428 \mathrm{E}-02$

$-3.99840 \mathrm{E}-02$

$-4.05301 \mathrm{E}-02$

-4.10811 E-02

$-4.16370 \mathrm{E}-02$

$-4.21978 \mathrm{E}-02$

$-4.27635 \mathrm{E}-02$

$-4.33342 \mathrm{E}-02$

$-4.39099 \mathrm{E}-02$

$-4.44906 \mathrm{E}-02$

$-4.50763 \mathrm{E}-02$

$-4.56670 \mathrm{E}-02$

$-4.62629 \mathrm{E}-02$

$-4.68638 \mathrm{E}-02$

$-4.74698 \mathrm{E}-02$

$-4.80810 \mathrm{E}-02$

$-4.86973 \mathrm{E}-02$

$-4.93188 \mathrm{E}-02$

$-4.99454 \mathrm{E}-02$

$-5.05773 \mathrm{E}-02$

$-5.12145 \mathrm{E}-02$

$-5.18568 \mathrm{E}-02$

$-5.25045 \mathrm{E}-02$

$-5.31575 \mathrm{E}-02$

$-5.38158 \mathrm{E}-02$

$-5.44794 \mathrm{E}-02$

$-5.51484 \mathrm{E}-02$

$-5.58227 \mathrm{E}-02$

$-5.65025 \mathrm{E}-02$

$-5.71877 \mathrm{E}-02$

$-5.78783 \mathrm{E}-02$

$-5.85745 \mathrm{E}-02$

$-5.92761 \mathrm{E}-02$

$-5.99832 \mathrm{E}-02$

$-6.06958 \mathrm{E}-02$

$-6.14140 \mathrm{E}-02$

$-6.21378 \mathrm{E}-02$

$-6.28671 \mathrm{E}-02$

$-6.36021 \mathrm{E}-02$

$-6.43427 \mathrm{E}-02$

$-6.50889 \mathrm{E}-02$
$3.40682 \mathrm{E} \quad 01$

$3.40726 \mathrm{E} \quad 01$

$3.40770 \mathrm{E}$ 01

$3.40813 \mathrm{E} 01$

$3.40856 \mathrm{E} \quad 01$

$3.40898 \mathrm{E} \quad 01$

$3.40940 \mathrm{E} \quad 01$

$3.40982 \mathrm{E} 01$

$3.41023 \mathrm{E} \quad 01$

$3.41063 \mathrm{E} 01$

$3.41103 \mathrm{E} \quad 01$

$3.41143 \mathrm{E} \quad 01$

$3.41182 \mathrm{E} 01$

$3.41221 \mathrm{E} 01$

$3.41259 \mathrm{E} \quad 01$

$3.41297 \mathrm{E} \quad 01$

$3.41335 \mathrm{E} \quad 01$

$3.41372 \mathrm{E}$ 01

$3.41409 \mathrm{E} \quad 01$

$3.41445 \mathrm{E} \quad 01$

$3.41481 \mathrm{E} \quad 01$

$3.41517 \mathrm{E}$ 01

$3.41552 \mathrm{E}$ 01

$3.41587 \mathrm{E}$ 01

$3.41622 \mathrm{E}$ 01

$3.41656 \mathrm{E} \quad 01$ $3.41690 \mathrm{E}$ 01

$3.41723 \mathrm{E} 01$

$3.41756 \mathrm{E}$ 01

$3.41789 \mathrm{E}$ ol

$3.41822 \mathrm{E} \quad 01$

$3.41854 \mathrm{E}$ 01

$3.41886 \mathrm{E} \quad 01$

$3.41918 \mathrm{E} \quad 01$

$3.41949 \mathrm{E}$ ol

$3.41980 \mathrm{E} 01$

$3.42011 \mathrm{E}$ 01

$3.42041 \mathrm{E} \quad 01$

$3.42071 \mathrm{E}$ 01

$3.42101 \mathrm{E} \quad 01$

$3.42131 \mathrm{E} \quad 01$ 
ELECTRON RADIAL FUNCTIONS

Electron

$Z=6 \quad A=12 \quad \rho=1.2 A^{1 / 3} F$

24.
24.
24.
24.
24.

24.
24.
24.
24.
25.
25.
25.
25.
25.
25.

$F_{0}$

$f_{1}$

$\begin{array}{llll}1.15166 \mathrm{E} & 00 & -2.50145 \mathrm{E} & 01\end{array}$ $1.15165 \mathrm{E} \quad 00$

$1.15164 \mathrm{E} \quad 00$

$1.15163 \mathrm{E} \quad 00$

$1.15162 \mathrm{E} \quad 00$

$1.15161 \mathrm{E} \quad 00$

$1.15160 \mathrm{E} \quad 00$

$1.15159 \mathrm{E} \quad 00$

$1.15158 \mathrm{E} \quad 00$

$1.15157 \mathrm{E} \quad 00$

$1.15156 \mathrm{E} \quad 00$

$1.15155 \mathrm{E} \quad 00$

$1.15154 \mathrm{E} \quad 00$

$1.15153 \mathrm{E} \quad 00$

$1.15152 \mathrm{E} \quad 00$

$1.15151 \mathrm{E} \quad 00$

$1.15150 \mathrm{E} \quad 00$

25.80

25.90

26.00

26.10

26.20

26.30

26.40

26.50

26.60

26.70

26.80

26.90

27.00

27.10

27.20

27.30

27.40

27.50

27.60

27.70

27.80

27.90

28.00

28.10

28.20

28.30

28.40

28.50

28.60

28.70

28.80

28.90

29.00

29.10

29.20

29.30

29.40

29.50

29.60

29.70

29.80

29.90

30.00
$1.15149 \mathrm{E} \quad 00$

$1.15148 \mathrm{E} \quad 00$

$1.15147 \mathrm{E} \quad 00$

$1.15147 \mathrm{E} \quad 00$

$1.15146 \mathrm{E} \quad 00$

$1.15145 \mathrm{E} \quad 00$

$1.15144 \mathrm{E} \quad 00$

$1.15143 \mathrm{E} \quad 00$

$1.15142 \mathrm{E} \quad 00$

$1.15141 \mathrm{E} \quad 00$

$1.15140 \mathrm{E} \quad 00$

$1.15139 \mathrm{E} \quad 00$

$1.15138 \mathrm{E} \quad 00$

$1.15137 \mathrm{E} \quad 00$

$1.15137 \mathrm{E} \quad 00$

$1.15136 \mathrm{E} \quad 00$

$1.15135 \mathrm{E} \quad 00$

$1.15134 \mathrm{E} \quad 00$

$1.15133 \mathrm{E} \quad 00$

$1.15132 \mathrm{E} \quad 00$

$1.15131 \mathrm{E} \quad 00$

$1.15130 \mathrm{E} \quad 00$

$1.15130 \mathrm{E} \quad 00$

$1.15129 \mathrm{E} \quad 00$

$1.15128 \mathrm{E} \quad 00$

$1.15127 \mathrm{E} \quad 00$

$1.15126 \mathrm{E} \quad 00$

$1.15125 \mathrm{E} \quad 00$

$1.15125 \mathrm{E} \quad 00$

$1.15124 \mathrm{E} \quad 00$

$1.15123 \mathrm{E} \quad 00$

$1.15122 \mathrm{E} \quad 00$

$1.15121 \mathrm{E} \quad 00$

$1.15120 \mathrm{E} \quad 00$

$1.15120 \mathrm{E} \quad 00$

$1.15119 \mathrm{E} \quad 00$

$1.15118 \mathrm{E} \quad 00$

$1.15117 \mathrm{E} \quad 00$

$1.15116 \mathrm{E} \quad 00$

$1.15116 \mathrm{E} \quad 00$

$1.15115 \mathrm{E} \quad 00$

$1.15114 \mathrm{E} \quad 00$

$1.15113 \mathrm{E} \quad 00$
$-2.51187 \mathrm{E} \quad 01$

$-2.52228 \mathrm{E} 01$

$-2.53270 \mathrm{E} \quad 0 \mathrm{l}$

$-2.54311 \mathrm{E} 01$

$-2.55351 \mathrm{E} \quad 01$

$-2.56392 \mathrm{E} 01$

$-2.57432 \mathrm{E} 01$

$-2.58473 \mathrm{E} 01$

$-2.59513 \mathrm{E} 01$

$-2.60553 \mathrm{E} \quad 01$

$-2.61592 \mathrm{E} 01$

$-2.62632 \mathrm{E} 0 \mathrm{l}$

$-2.64710 \mathrm{E} 0 \mathrm{l}$

$\begin{array}{lll}-2.65749 \mathrm{E} & 01\end{array}$

-2.66787 E 01

$-2.67826 \mathrm{E} 01$

$-2.68864 \mathrm{E} \quad 01$

-2.69902 E 01

$-2.70940 \mathrm{E} \quad 01$

$-2.71977 \mathrm{E} \quad 01$

$-2.73015 \mathrm{E} \quad 01$

$-2.74052 \mathrm{E} 01$

-2.75089 E 01

$-2.76126 \mathrm{E} \quad 01$

$-2.77162 \mathrm{E} 01$

$-2.78199 \mathrm{E} 01$

$-2.79235 \mathrm{E} \quad 01$

$-2.80271 \mathrm{E} 01$

$-2.81306 \mathrm{E} \quad 01$

$-2.82342 \mathrm{E} 01$

$-2.83377 \mathrm{E} 01$

$-2.84412 \mathrm{E} 01$

$-2.85447 \mathrm{E} \quad 01$

$-2.86482 \mathrm{E} \quad 01$

$-2.87516 \mathrm{E} 01$

$-2.88551 \mathrm{E}$ 01

$-2.89585 \mathrm{E} 01$

$-2.90619 \mathrm{E}$ 0l

$-2.91652 \mathrm{E} \quad 01$

$-2.92686 \mathrm{E} 01$

$-2.93719 \mathrm{E}$ 0l

$-2.94752 \mathrm{E} 01$

$-2.95785 \mathrm{E} 01$

$-2.96817 \mathrm{E} \quad 01$

$-2.97849 \mathrm{E} \quad 01$

$-2.98882 \mathrm{E} \quad 01$

$-2.99913 \mathrm{E} 01$

$-3.00945 \mathrm{E} 01$

$-3.01977 \mathrm{E} \quad 01$

$-3.03008 \mathrm{E} 01$

$-3.04039 \mathrm{E}$ al

$-3.06100 \mathrm{E} 01$

$-3.07131 \mathrm{E} \quad 01$

$-3.08161 \mathrm{E} 01$

$-3.09191 \mathrm{E} 01$

$-3.10220 \mathrm{E}$ 01
$-2.63671 \mathrm{E} \quad 01$

$-3.05070 \mathrm{E} 01$

$-3.11250 \mathrm{E} 01$ $g_{1}$

$\tan \delta_{1}$

$f_{-1}$

$-1.93493 \mathrm{E} 00$

$-1.94900 \mathrm{E} 00$

$-1.96312 \mathrm{E} 00$

$-1.97729 \mathrm{E} 00$

$-1.99151 \mathrm{E} 00$

$-2.00578 \mathrm{E} \quad 00$

$-2.02009 \mathrm{E} \quad 00$

$-2.03446 \mathrm{E} \quad 00$

$-2.04887 \mathrm{E} \quad 00$

$-2.06333 \mathrm{E} \quad 00$

$-2.07784 \mathrm{E} \quad 00$

$-2.09240 \mathrm{E} \quad 00$

$-2.10701 \mathrm{E} \quad 00$

$-2.12167 \mathrm{E} \quad 00$

$-2.13637 \mathrm{E} 00$

$-2.15112 \mathrm{E} \quad 00$

$-2.16593 \mathrm{E} \quad 00$

$-2.18078 \mathrm{E} \quad 00$

$-2.19568 \mathrm{E} \quad 00$

$-2.21062 \mathrm{E} \quad 00$

$-2.22562 \mathrm{E} \quad 00$

$-2.24067 \mathrm{E} \quad 00$

$-2.25576 \mathrm{E} 00$

$-2.27090 \mathrm{E} \quad 00$

$-2.28609 \mathrm{E} \quad 00$

$-2.30133 \mathrm{E} \quad 00$

$-2.31662 \mathrm{E} \quad 00$

$-2.33196 \mathrm{E} \quad 00$

$-2.34734 \mathrm{E} 00$

$-2.36277 \mathrm{E} 00$

$-2.37826 \mathrm{E} \quad 00$ $-2.39379 \mathrm{E} \quad 00$

$-2.40937 \mathrm{E} \quad 00$

$-2.42499 \mathrm{E} \quad 00$

$-2.44067 \mathrm{E} \quad 00$

$-2.45639 \mathrm{E} \quad 00$

$-2.47216 \mathrm{E} \quad 00$

$-2.48799 \mathrm{E} 00$

$-2.50385 \mathrm{E} \quad 00$

$-2.51977 \mathrm{E} 00$

$-2.53574 \mathrm{E} \quad 00$

$-2.55175 \mathrm{E} \quad 00$

$-2.56781 \mathrm{E} 00$

$-2.58392 \mathrm{E} \quad 00$

$-2.60008 \mathrm{E} 00$

$-2.61629 \mathrm{E} \quad 00$

$-2.63255 \mathrm{E} \quad 00$

$-2.64885 \mathrm{E} \quad 00$

$-2.66520 \mathrm{E} \quad 00$

$-2.68160 \mathrm{E} \quad 00$

$-2.69805 \mathrm{E} \quad 00$

$-2.71455 \mathrm{E} \quad 00$

$-2.73109 \mathrm{E} \quad 00$

$-2.74768 \mathrm{E} 00$

$-2.76433 \mathrm{E} 00$

$-2.78101 \mathrm{E} \quad 00$

-2.79775 E 00

$-2.81454 \mathrm{E} \quad 00$

$-2.83137 \mathrm{E} \quad 00$

$-2.84825 \mathrm{E} 00$

$3.86997 \mathrm{E}-03$

$3.87251 \mathrm{E}-03$

$3.87501 \mathrm{E}-03$

$3.87748 \mathrm{E}-03$

$3.87991 \mathrm{E}-03$

$3.88230 \mathrm{E}-03$

$3.88467 \mathrm{E}-03$

$3.88700 \mathrm{E}-03$

$3.88930 \mathrm{E}-03$

$3.89156 \mathrm{E}-03$

$3.89378 \mathrm{E}-03$

$3.89599 \mathrm{E}-03$

$3.89815 \mathrm{E}-03$

$3.90029 \mathrm{E}-03$

$3.90239 \mathrm{E}-03$

$3.90446 \mathrm{E}-03$

3.90651 E-03

$3.90852 \mathrm{E}-03$

$3.91050 \mathrm{E}-03$

$3.91245 \mathrm{E}-03$

$3.91436 \mathrm{E}-03$

$3.91626 \mathrm{E}-03$

$3.91811 \mathrm{E}-03$

$3.91995 \mathrm{E}-03$

$3.92175 \mathrm{E}-03$

$3.92353 \mathrm{E}-03$

$3.92528 \mathrm{E}-03$

$3.92700 \mathrm{E}-03$

$3.92869 \mathrm{E}-03$

$3.93036 \mathrm{E}-03$

$3.93200 \mathrm{E}-03$

$3.93361 \mathrm{E}-03$

$3.93520 \mathrm{E}-03$

$3.93676 \mathrm{E}-03$

$3.93829 \mathrm{E}-03$

$3.93980 \mathrm{E}-03$

$3.94128 \mathrm{E}-03$

$3.94274 \mathrm{E}-03$

$3.94417 \mathrm{E}-03$

$3.94558 \mathrm{E}-03$

$3.94695 \mathrm{E}-03$

$3.94832 \mathrm{E}-03$

$3.94965 \mathrm{E}-03$

$3.95095 \mathrm{E}-03$

$3.95224 \mathrm{E}-03$

$3.95350 \mathrm{E}-03$

$3.95474 \mathrm{E}-03$

$3.95595 \mathrm{E}-03$

$3.95715 \mathrm{E}-03$

$3.95832 \mathrm{E}-03$

$3.95946 \mathrm{E}-03$

$3.96058 \mathrm{E}-03$

$3.96168 \mathrm{E}-03$

$3.96276 \mathrm{E}-03$

$3.96382 \mathrm{E}-03$

$3.96486 \mathrm{E}-03$

$3.96587 \mathrm{E}-03$

$3.96686 \mathrm{E}-03$ 
ELECTRON RADIAL FUNCTIONS

$Z=6 \quad A=12 \quad \rho=1.2 A^{1 / 3} F$

Electron

$\bar{F}$

24.10

24.20

24.30

24.40

24.50

24.60

24.70

24.80

24.90

25.00

25.10

25.20

25.30

25.40

25.50

25.60

25.70

25.80

25.90

26.00

26.10

26.20

26.30

26.40

26.50

26.60

26.70

26.80

26.90

27.00

27.10

27.20

27.30

27.40

27.50

27.60

27.70

27.80

27.90

28.00

28.10

28.20

28.30

28.40

28.50

28.60

28.70

28.80

28.90

29.00

29.10

29.20

29.30

29.40

29.50

29.60

29.70

29.80

29.90

30.00
$9.39286 \mathrm{E}-04$ $9.47092 \mathrm{E}-04$ $9.54931 \mathrm{E}-04$ $9.62802 \mathrm{E}-04$ $9.70705 \mathrm{E}-04$

$9.78641 \mathrm{E}-04$ $9.86609 \mathrm{E}-04$ $9.94609 \mathrm{E}-04$ $1.00264 \mathrm{E}-03$

$1.01071 \mathrm{E}-03$

$1.01880 \mathrm{E}-03$

$1.02693 \mathrm{E}-03$

$1.03509 \mathrm{E}-03$

$1.04329 \mathrm{E}-03$

$1.05151 \mathrm{E}-03$

$1.05977 \mathrm{E}-03$

$1.06806 \mathrm{E}-03$

$1.07639 \mathrm{E}-03$

$1.08474 \mathrm{E}-03$

$1.09313 \mathrm{E}-03$

$1.10155 \mathrm{E}-03$

$1.11000 \mathrm{E}-03$

$1.11849 \mathrm{E}-03$

$1.12700 \mathrm{E}-03$

$1.13555 \mathrm{E}-03$

$1.14413 \mathrm{E}-03$

$1.15275 \mathrm{E}-03$

$1.16139 \mathrm{E}-03$

$1.17007 \mathrm{E}-03$

$1.17878 \mathrm{E}-03$

$1.18753 \mathrm{E}-03$

$1.19630 \mathrm{E}-03$

$1.20511 \mathrm{E}-03$

$1.21395 \mathrm{E}-03$

$1.22282 \mathrm{E}-03$

$1.23172 \mathrm{E}-03$ $1.24066 \mathrm{E}-03$ $1.24963 \mathrm{E}-03$

$1.25863 \mathrm{E}-03$

$1.26766 \mathrm{E}-03$

$1.27673 \mathrm{E}-03$

$1.28583 \mathrm{E}-03$

$1.29496 \mathrm{E}-03$

$1.30412 \mathrm{E}-03$

$1.31332 \mathrm{E}-03$

$1.32254 \mathrm{E}-03$

$1.33180 \mathrm{E}-03$

$1.34109 \mathrm{E}-03$

$1.35042 \mathrm{E}-03$

$1.35977 \mathrm{E}-03$

$1.36916 \mathrm{E}-03$

$1.37858 \mathrm{E}-03$

$1.38804 \mathrm{E}-03$

$1.39752 \mathrm{E}-03$

$1.40704 \mathrm{E}-03$

$1.41659 \mathrm{E}-03$

$1.42617 \mathrm{E}-03$

$1.43579 \mathrm{E}-03$

$1.44543 \mathrm{E}-03$

$1.45511 \mathrm{E}-03$ $f_{2}$

$-1.43701 \mathrm{E} \quad 00$ $-1.44903 \mathrm{E} 00$ $-1.46110 \mathrm{E} \quad 00$ $-1.47322 \mathrm{E} \quad 00$ $-1.48538 \mathrm{E} 00$

$-1.49760 \mathrm{E} \quad 00$ $-1.50987 \mathrm{E} \quad 00$ $-1.52218 \mathrm{E} \quad 00$ $-1.53455 \mathrm{E} 00$

$-1.54696 \mathrm{E} \quad 00$

$-1.55943 \mathrm{E} \quad 00$

$-1.57194 \mathrm{E} \quad 00$

$-1.58450 \mathrm{E} \quad 00$

$-1.59712 \mathrm{E} \quad 00$

$-1.60978 \mathrm{E} 00$

$-1.62249 \mathrm{E} \quad 00$

$-1.63525 \mathrm{E} \quad 00$

$-1.64806 \mathrm{E} \quad 00$

$-1.66092 \mathrm{E} \quad 00$

$-1.67382 \mathrm{E} \quad 00$

$-1.68678 \mathrm{E} \quad 00$

$-1.69979 \mathrm{E} \quad 00$

$-1.71284 \mathrm{E} \quad 00$

$-1.72595 \mathrm{E} \quad 00$

$-1.73910 \mathrm{E} 00$

$-1.75230 \mathrm{E} \quad 00$

$-1.76556 \mathrm{E} \quad 00$

$-1.77886 \mathrm{E} \quad 00$

$-1.79221 \mathrm{E} \quad 00$

$-1.80561 \mathrm{E} \quad 00$

$-1.81906 \mathrm{E} \quad 00$

$-1.83255 \mathrm{E} 00$

$-1.84610 \mathrm{E} \quad 00$

$-1.85970 \mathrm{E} \quad 00$

$-1.87334 \mathrm{E} \quad 00$

$-1.88704 \mathrm{E} \quad 00$

$-1.90078 \mathrm{E} \quad 00$

$-1.91457 \mathrm{E} \quad 00$

$-1.92841 \mathrm{E} 00$

$-1.94230 \mathrm{E} \quad 00$

$-1.95624 \mathrm{E} \quad 00$

$-1.97023 \mathrm{E} \quad 00$

$-1.98427 \mathrm{E} \quad 00$

$-1.99836 \mathrm{E} \quad 00$

$-2.01249 \mathrm{E} \quad 00$

$-2.02668 \mathrm{E} \quad 00$

$-2.04091 \mathrm{E} \quad 00$

$-2.05519 \mathrm{E} 00$

$-2.06952 \mathrm{E} \quad 00$

$-2.08390 \mathrm{E} 00$

$-2.09833 \mathrm{E} \quad 00$

$-2.11281 \mathrm{E} \quad 00$

$-2.12734 \mathrm{E} 00$

$-2.14191 \mathrm{E} \quad 00$

$-2.15654 \mathrm{E} \quad 00$

$-2.17121 \mathrm{E} \quad 00$

$-2.18594 \mathrm{E} \quad 00$

$-2.20071 \mathrm{E} \quad 00$

$-2.21553 \mathrm{E} \quad 00$

$-2.23040 \mathrm{E} 00$ $\tan \delta_{2}$

$f_{-2}$

$-6.58409 \mathrm{E}-02$

$-6.65985 \mathrm{E}-02$

$-6.73619 \mathrm{E}-02$

$-6.81310 \mathrm{E}-02$

$-6.89058 \mathrm{E}-02$

$-6.96865 \mathrm{E}-02$

$-7.04729 \mathrm{E}-02$

$-7.12651 \mathrm{E}-02$

$-7.20632 \mathrm{E}-02$

$-7.28672 \mathrm{E}-02$

$-7.36770 \mathrm{E}-02$

$-7.44928 \mathrm{E}-02$

$-7.53145 \mathrm{E}-02$

$-7.61421 \mathrm{E}-02$

$-7.69757 \mathrm{E}-02$

$-7.78153 \mathrm{E}-02$

$-7.86609 \mathrm{E}-02$

$-7.95125 \mathrm{E}-02$

$-8.03702 \mathrm{E}-02$

$-8.12339 \mathrm{E}-02$

$-8.21038 \mathrm{E}-02$

$-8.29797 \mathrm{E}-02$

$-8.38619 \mathrm{E}-02$

$-8.47501 \mathrm{E}-02$

$-8.56445 \mathrm{E}-02$

$-8.65452 \mathrm{E}-02$

$-8.74520 \mathrm{E}-02$

$-8.83651 \mathrm{E}-02$

$-8.92845 \mathrm{E}-02$

$-9.02101 \mathrm{E}-02$

$-9.11420 \mathrm{E}-02$

$-9.20803 \mathrm{E}-02$

$-9.30248 \mathrm{E}-02$

$-9.39758 \mathrm{E}-02$

$-9.49331 \mathrm{E}-02$

$-9.58969 \mathrm{E}-02$

$-9.68671 \mathrm{E}-02$

$-9.78437 \mathrm{E}-02$

$-9.88268 \mathrm{E}-02$

$-9.98164 \mathrm{E}-02$

$-1.00812 \mathrm{E}-01$

$-1.01815 \mathrm{E}-01$

$-1.02824 \mathrm{E}-01$

$-1.03840 \mathrm{E}-01$

$-1.04862 \mathrm{E}-01$

$-1.05891 \mathrm{E}-01$

$-1.06927 \mathrm{E}-01$

$-1.07969 \mathrm{E}-01$

$-1.09018 \mathrm{E}-01$

$-1.10073 \mathrm{E}-01$

$-1.11136 \mathrm{E}-01$ $-1.12205 \mathrm{E}-01$

$-1.13281 \mathrm{E}-01$

$-1.14363 \mathrm{E}-01$

$-1.15453 \mathrm{E}-01$

$-1.16549 \mathrm{E}-01$

$-1.17652 \mathrm{E}-01$

$-1.18762 \mathrm{E}-01$

$-1.19879 \mathrm{E}-01$

$-1.21002 \mathrm{E}-01$

$3.42669 \mathrm{E} \quad 01$

$3.42693 \mathrm{E} 01$

$3.42718 \mathrm{E} \quad 01$

$3.42742 \mathrm{E}$ 01

$3.42766 \mathrm{E} \quad 01$

$3.42789 \mathrm{E} \quad 01$

$3.42813 \mathrm{E} 01$

$3.42836 \mathrm{E} 01$

$3.42859 \mathrm{E}$ ol

$3.42882 \mathrm{E} \quad 01$

$3.42905 \mathrm{E}$ 0l

$3.42927 \mathrm{E} \quad 01$

$3.42950 \mathrm{E}$ 01

$3.42972 \mathrm{E} \quad 01$

$3.42994 \mathrm{E} 01$

$3.43016 \mathrm{E} \quad 01$

$3.43037 \mathrm{E} \quad 01$

$3.43059 \mathrm{E} 01$

$3.43080 \mathrm{E} \quad 01$

$3.43101 \mathrm{E} \quad 01$

$3.43122 \mathrm{E} \quad 01$

$3.43143 \mathrm{E}$ 01

$3.43163 \mathrm{E} 01$

$3.43184 \mathrm{E} \quad 01$

$3.43204 \mathrm{E} 01$

$3.43224 \mathrm{E} \quad 01$

$3.43244 \mathrm{E} \quad 01$

$3.43264 \mathrm{E}$ 01

$3.43284 \mathrm{E} \quad 01$

$3.43303 \mathrm{E} \quad 01$

$3.43323 \mathrm{E} \quad 01$

$3.43342 \mathrm{E} 01$

$3.43361 \mathrm{E} 01$

$3.43380 \mathrm{E}$ 01

$3.43399 \mathrm{E} 01$

$3.43417 \mathrm{E} \quad 01$

$3.43436 \mathrm{E} 01$

$3.43454 \mathrm{E} \quad 01$

$3.43472 \mathrm{E} 01$

$3.43491 \mathrm{E} 01$

$3.43509 \mathrm{E} \quad 01$

$3.43526 \mathrm{E} \quad 01$

$3.43544 \mathrm{E}$ 0l 
ELECTRON RADIAL FUNCTIONS

Electron

\begin{tabular}{|c|c|c|c|c|c|c|}
\hline$p$ & $F_{0}$ & & $f_{1}$ & & $g_{1}$ & \\
\hline 30.10 & $1.15112 \mathrm{E}$ & 00 & $-3.12279 \mathrm{E}$ & 01 & $-2.86518 E$ & 00 \\
\hline 30.20 & $1.15112 \mathrm{E}$ & 00 & $-3.13308 \mathrm{E}$ & 01 & $-2.88216 \mathrm{E}$ & 00 \\
\hline 30.30 & $1.15111 \mathrm{E}$ & 00 & $-3.14337 \mathrm{E}$ & 01 & $-2.89919 \mathrm{E}$ & 00 \\
\hline 30.40 & $1.15110 \mathrm{E}$ & 00 & $-3.15365 \mathrm{E}$ & 01 & $-2.91626 \mathrm{E}$ & 00 \\
\hline 30.50 & $1.15109 \mathrm{E}$ & 00 & $-3.16394 \mathrm{E}$ & 01 & $-2.93338 \mathrm{E}$ & 00 \\
\hline 30.60 & $1.15108 \mathrm{E}$ & 00 & $-3.17422 \mathrm{E}$ & 01 & $-2.95055 \mathrm{E}$ & 00 \\
\hline 30.70 & $1.15108 \mathrm{E}$ & 00 & $-3.18450 \mathrm{E}$ & 01 & $-2.96777 \mathrm{E}$ & 00 \\
\hline 30.80 & $1.15107 \mathrm{E}$ & 00 & $-3.19477 \mathrm{E}$ & 01 & $-2.98504 \mathrm{E}$ & 00 \\
\hline 30.90 & $1.15106 \mathrm{E}$ & 00 & $-3.20505 \mathrm{E}$ & 01 & $-3.00235 \mathrm{E}$ & 00 \\
\hline 31.00 & $1.15105 \mathrm{E}$ & 00 & $-3.21532 \mathrm{E}$ & 01 & $-3.01971 \mathrm{E}$ & 00 \\
\hline 31.10 & $1.15105 \mathrm{E}$ & 00 & $-3.22559 \mathrm{E}$ & 01 & $-3.03712 \mathrm{E}$ & 00 \\
\hline 31.20 & $1.15104 \mathrm{E}$ & 00 & $-3.23586 \mathrm{E}$ & 01 & $-3.05458 \mathrm{E}$ & 00 \\
\hline 31.30 & $1.15103 \mathrm{E}$ & 00 & $-3.24612 \mathrm{E}$ & 01 & $-3.07208 \mathrm{E}$ & 00 \\
\hline 31.40 & $1.15102 \mathrm{E}$ & 00 & $-3.25638 \mathrm{E}$ & 01 & $-3.08964 \mathrm{E}$ & 00 \\
\hline 31.50 & $1.15102 \mathrm{E}$ & 00 & $-3.26664 \mathrm{E}$ & 01 & $-3.10724 \mathrm{E}$ & 00 \\
\hline 31.60 & $1.15101 \mathrm{E}$ & 00 & $-3.27690 \mathrm{E}$ & 01 & $-3.12489 \mathrm{E}$ & 00 \\
\hline 31.70 & $1.15100 \mathrm{E}$ & 00 & $-3.28716 \mathrm{E}$ & 01 & $-3.14258 \mathrm{E}$ & 00 \\
\hline 31.80 & $1.15099 \mathrm{E}$ & 00 & $-3.29741 \mathrm{E}$ & 01 & $-3.16033 E$ & 00 \\
\hline 31.90 & $1.15099 \mathrm{E}$ & 00 & $-3.30766 \mathrm{E}$ & 01 & $-3.17812 \mathrm{E}$ & 00 \\
\hline 32.00 & $1.15098 \mathrm{E}$ & 00 & $-3.31791 \mathrm{E}$ & 01 & $-3.19596 \mathrm{E}$ & 00 \\
\hline 32.10 & $1.15097 \mathrm{E}$ & 00 & $-3.32816 \mathrm{E}$ & 01 & $-3.21385 E$ & 00 \\
\hline 32.20 & $1.15096 \mathrm{E}$ & 00 & $-3.33840 \mathrm{E}$ & 01 & $-3.23179 \mathrm{E}$ & 00 \\
\hline 32.30 & $1.15096 \mathrm{E}$ & 00 & $-3.34864 \mathrm{E}$ & 01 & $-3.24977 \mathrm{E}$ & 00 \\
\hline 32.40 & $1.15095 \mathrm{E}$ & 00 & $-3.35888 \mathrm{E}$ & 01 & $-3.26780 \mathrm{E}$ & 00 \\
\hline 32.50 & $1.15094 \mathrm{E}$ & 00 & $-3.36912 \mathrm{E}$ & 01 & $-3.28588 \mathrm{E}$ & 00 \\
\hline 32.60 & $1.15093 \mathrm{E}$ & 00 & $-3.37935 \mathrm{E}$ & 01 & $-3.30401 \mathrm{E}$ & 00 \\
\hline 32.70 & $1.15093 \mathrm{E}$ & 00 & $-3.38959 \mathrm{E}$ & 01 & $-3.32218 \mathrm{E}$ & 00 \\
\hline 32.80 & $1.15092 \mathrm{E}$ & 00 & $-3.39982 \mathrm{E}$ & 01 & $-3.34041 \mathrm{E}$ & 00 \\
\hline 32.90 & $1.15091 \mathrm{E}$ & 00 & $-3.41005 \mathrm{E}$ & 01 & $-3.35868 \mathrm{E}$ & 00 \\
\hline 33.00 & $1.15091 \mathrm{E}$ & 00 & $-3.42027 \mathrm{E}$ & 01 & $-3.37699 \mathrm{E}$ & 00 \\
\hline 33.10 & $1.15090 \mathrm{E}$ & 00 & $-3.43049 \mathrm{E}$ & 01 & $-3.39536 \mathrm{E}$ & 00 \\
\hline 33.20 & $1.15089 \mathrm{E}$ & 00 & $-3.44071 \mathrm{E}$ & 01 & $-3.41377 \mathrm{E}$ & 00 \\
\hline 33.30 & $1.15089 \mathrm{E}$ & 00 & $-3.45093 \mathrm{E}$ & 01 & $-3.43223 \mathrm{E}$ & 00 \\
\hline 33.40 & $1.15088 \mathrm{E}$ & 00 & $-3.46115 \mathrm{E}$ & 01 & $-3.45074 \mathrm{E}$ & 00 \\
\hline 33.50 & $1.15087 \mathrm{E}$ & 00 & $-3.47136 \mathrm{E}$ & 01 & $-3.46930 \mathrm{E}$ & 00 \\
\hline 33.60 & $1.15086 \mathrm{E}$ & 00 & $-3.48157 \mathrm{E}$ & 01 & $-3.48790 \mathrm{E}$ & 00 \\
\hline 33.70 & $1.15086 \mathrm{E}$ & 00 & $-3.49178 \mathrm{E}$ & 01 & $-3.50655 \mathrm{E}$ & 00 \\
\hline 33.80 & $1.15085 \mathrm{E}$ & 00 & $-3.50199 \mathrm{E}$ & 01 & $-3.52525 \mathrm{E}$ & 00 \\
\hline 33.90 & $1.15084 \mathrm{E}$ & 00 & $-3.51219 E$ & 01 & $-3.54400 \mathrm{E}$ & 00 \\
\hline 34.00 & $1.15084 \mathrm{E}$ & 00 & $-3.52239 \mathrm{E}$ & 01 & $-3.56279 \mathrm{E}$ & 00 \\
\hline 34.10 & $1.15083 \mathrm{E}$ & 00 & $-3.53259 E$ & 01 & $-3.58163 \mathrm{E}$ & 00 \\
\hline 34.20 & $1.15082 \mathrm{E}$ & 00 & $-3.54279 E$ & 01 & $-3.60052 \mathrm{E}$ & 00 \\
\hline 34.30 & $1.15082 \mathrm{E}$ & 00 & $-3.55298 \mathrm{E}$ & 01 & $-3.61946 \mathrm{E}$ & 00 \\
\hline 34.40 & $1.15081 \mathrm{E}$ & 00 & $-3.56317 \mathrm{E}$ & 01 & $-3.63844 \mathrm{E}$ & 00 \\
\hline 34.50 & $1.15080 \mathrm{E}$ & 00 & $-3.57336 \mathrm{E}$ & 01 & $-3.65747 \mathrm{E}$ & 00 \\
\hline 34.60 & $1.15080 \mathrm{E}$ & 00 & $-3.58355 E$ & 01 & $-3.67655 \mathrm{E}$ & 00 \\
\hline 34.70 & $1.15079 \mathrm{E}$ & 00 & $-3.59373 E$ & 01 & $-3.69568 E$ & 00 \\
\hline 34.80 & $1.15078 \mathrm{E}$ & 00 & $-3.60391 \mathrm{E}$ & 01 & $-3.71485 \mathrm{E}$ & 00 \\
\hline 34.90 & $1.15078 \mathrm{E}$ & 00 & $-3.61409 \mathrm{E}$ & 01 & $-3.73407 E$ & 00 \\
\hline 35.00 & $1.15077 \mathrm{E}$ & 00 & $-3.62427 \mathrm{E}$ & 01 & $-3.75334 \mathrm{E}$ & 00 \\
\hline 35.10 & $1.15076 \mathrm{E}$ & 00 & $-3.63444 \mathrm{E}$ & 01 & $-3.77266 \mathrm{E}$ & 00 \\
\hline 35.20 & $1.15076 \mathrm{E}$ & 00 & $-3.64461 \mathrm{E}$ & 01 & $-3.79202 \mathrm{E}$ & 00 \\
\hline 35.30 & $1.15075 \mathrm{E}$ & 00 & $-3.65478 \mathrm{E}$ & 01 & $-3.81143 \mathrm{E}$ & 00 \\
\hline 35.40 & $1.15074 \mathrm{E}$ & 00 & $-3.66495 \mathrm{E}$ & 01 & $-3.83089 \mathrm{E}$ & 00 \\
\hline & $1.15074 \mathrm{E}$ & 00 & $-3.67511 \mathrm{E}$ & 01 & $-3.85039 \mathrm{E}$ & 00 \\
\hline 35.60 & $1.15073 \mathrm{E}$ & 00 & $-3.68527 \mathrm{E}$ & 01 & $-3.86995 \mathrm{E}$ & 00 \\
\hline 35.70 & $1.15072 \mathrm{E}$ & 00 & $-3.69543 \mathrm{E}$ & 01 & $-3.88954 \mathrm{E}$ & 00 \\
\hline 35.80 & $1.15072 \mathrm{E}$ & 00 & $-3.70559 \mathrm{E}$ & 01 & $-3.90919 E$ & 00 \\
\hline 35.90 & $1.15071 \mathrm{E}$ & 00 & $-3.71574 \mathrm{E}$ & 01 & $-3.92889 E$ & 00 \\
\hline 36.00 & $1.15070 \mathrm{E}$ & 00 & $-3.72589 \mathrm{E}$ & 01 & $-3.94863 \mathrm{E}$ & 00 \\
\hline
\end{tabular}

$\tan \delta_{1} \quad f_{-1}$

$3.96970 \mathrm{E}-03 \quad-2.80756 \mathrm{E} \quad 00$ $3.97062 \mathrm{E}-03 \quad-2.82429 \mathrm{E} \quad 00$ $3.97150 \mathrm{E}-03 \quad-2.84106 \mathrm{E} \quad 00$ $3.97237 \mathrm{E}-03 \quad-2.85788 \mathrm{E} \quad 00$ $3.97321 \mathrm{E}-03 \quad-2.87475 \mathrm{E} \quad 00$

$3.97404 \mathrm{E}-03 \quad-2.89167 \mathrm{E} \quad 00$ $3.97485 \mathrm{E}-03 \quad-2.90864 \mathrm{E} \quad 00$ $3.97563 \mathrm{E}-03 \quad-2.92565 \mathrm{E} \quad 00$ $3.97639 \mathrm{E}-03 \quad-2.94272 \mathrm{E} \quad 00$ $3.97714 \mathrm{E}-03 \quad-2.95983 \mathrm{E} \quad 00$

$3.97787 \mathrm{E}-03 \quad-2.97699 \mathrm{E} \quad 00$ $3.97858 \mathrm{E}-03 \quad-2.99419 \mathrm{E} \quad 00$ $3.97926 \mathrm{E}-03-3.01145 \mathrm{E} \quad 00$ $3.97994 \mathrm{E}-03 \quad-3.02875 \mathrm{E} \quad 00$ $3.98058 \mathrm{E}-03-3.04610 \mathrm{E} \quad 00$

$3.98121 \mathrm{E}-03 \quad-3.06350 \mathrm{E} \quad 00$ $3.98182 \mathrm{E}-03 \quad-3.08095 \mathrm{E} \quad 00$ $3.98243 \mathrm{E}-03-3.09844 \mathrm{E} \quad 00$ $3.98300 \mathrm{E}-03-3.11598 \mathrm{E} \quad 00$ $3.98355 \mathrm{E}-03 \quad-3.13357 \mathrm{E} \quad 00$

$3.98410 \mathrm{E}-03 \quad-3.15121 \mathrm{E} \quad 00$ $3.98462 \mathrm{E}-03-3.16890 \mathrm{E} \quad 00$ $3.98512 \mathrm{E}-03-3.18663 \mathrm{E} \quad 00$ $3.98561 \mathrm{E}-03 \quad-3.20441 \mathrm{E} \quad 00$ $3.98608 \mathrm{E}-03-3.22224 \mathrm{E} \quad 00$

$3.98652 \mathrm{E}-03$ $3.98696 \mathrm{E}-03$ $3.98737 \mathrm{E}-03$

$3.98777 \mathrm{E}-03$

$3.98815 \mathrm{E}-03$

$3.98852 \mathrm{E}-03$ $3.98886 \mathrm{E}-03$ $3.98919 \mathrm{E}-03$ $3.98951 \mathrm{E}-03$

$3.98980 \mathrm{E}-03$

$3.99009 \mathrm{E}-03$

$3.99035 \mathrm{E}-03$

$3.99060 \mathrm{E}-03$

$3.99083 \mathrm{E}-03$

$3.99105 \mathrm{E}-03$

$3.99125 \mathrm{E}-03$

$3.99144 \mathrm{E}-03$

$3.99160 \mathrm{E}-03$

$3.99176 \mathrm{E}-03$

$3.99189 \mathrm{E}-03$

$3.99202 \mathrm{E}-03$

$3.99212 \mathrm{E}-03$

$3.99221 \mathrm{E}-03$

$3.99229 \mathrm{E}-03$

$3.99235 \mathrm{E}-03$

$3.99240 \mathrm{E}-03$

$3.99243 \mathrm{E}-03$

$3.99245 \mathrm{E}-03$

$3.99245 \mathrm{E}-03$

$3.99243 \mathrm{E}-03$

$3.99241 \mathrm{E}-03$

$3.99237 \mathrm{E}-03$

$3.99231 \mathrm{E}-03$

$3.99224 \mathrm{E}-03$

$3.99216 \mathrm{E}-03$

$-3.24012 \mathrm{E} \quad 00$ $-3.25804 \mathrm{E} 00$ $-3.27601 \mathrm{E} \quad 00$ $-3.29403 \mathrm{E} 00$ $-3.31210 \mathrm{E} \quad 00$

$-3.33021 \mathrm{E} \quad 00$ $-3.34838 \mathrm{E} 00$ $-3.36659 \mathrm{E} 00$ $-3.38485 \mathrm{E} 00$ $-3.40315 \mathrm{E} 00$

$-3.42151 \mathrm{E} \quad 00$ $-3.43991 \mathrm{E} 00$ $-3.45836 \mathrm{E} \quad 00$ $-3.47685 \mathrm{E} \quad 00$ $-3.49540 \mathrm{E} 00$

$-3.51399 \mathrm{E} \quad 00$ $-3.53263 \mathrm{E} 00$ $-3.55131 \mathrm{E} 00$ $-3.57005 \mathrm{E} \quad 00$ $-3.58883 \mathrm{E} 00$

$-3.60766 \mathrm{E} \quad 00$ $-3.62653 \mathrm{E} \quad 00$ $-3.64546 \mathrm{E} 00$ $-3.66443 \mathrm{E} 00$ $-3.68345 \mathrm{E} 00$

$-3.70251 \mathrm{E} \quad 00$ $-3.72163 \mathrm{E} 00$ $-3.74079 \mathrm{E} 00$ $-3.75999 \mathrm{E} 00$ $-3.77925 \mathrm{E} 00$

$-3.79855 \mathrm{E} 00$ $-3.81790 \mathrm{E} \quad 00$ $-3.83730 \mathrm{E} 00$ $-3.85675 \mathrm{E} \quad 00$ $-3.87624 \mathrm{E} \quad 00$ g-1

$\tan \delta_{-1}$

$\begin{array}{llll}3.22762 \mathrm{E} & 01 & -1.84863 \mathrm{E} & 02\end{array}$ $\begin{array}{llll}3.23790 \mathrm{E} & 01 & -1.84998 \mathrm{E} & 02\end{array}$ $\begin{array}{lllll}3.24817 \mathrm{E} & 01 & -1.85134 \mathrm{E} & 02\end{array}$ $\begin{array}{llll}3.25845 \mathrm{E} & 01 & -1.85268 \mathrm{E} & 02\end{array}$ $\begin{array}{llll}3.26872 \mathrm{E} & 01 & -1.85404 \mathrm{E} & 02\end{array}$

$3.27899 \mathrm{E} \quad 01$ $3.28926 \mathrm{E} \quad 01$ $3.29953 \mathrm{E} \quad 01$ $3.30979 \mathrm{E} \quad 01$

$3.32005 \mathrm{E} 01$

$3.33031 \mathrm{E} \quad 01$ $3.34057 \mathrm{E} 01$ $3.35082 \mathrm{E} \quad 01$ $3.36107 \mathrm{E} \quad 01$ $3.37132 \mathrm{E} \quad 01$

$3.38157 \mathrm{E} \quad 01$ $3.39182 \mathrm{E} \quad 01$ $3.40206 \mathrm{E} \quad 01$ $3.41230 \mathrm{E} \quad 01$ $3.42254 \mathrm{E} \quad 01$

$3.43278 \mathrm{E} \quad 01$ $3.44301 \mathrm{E} \quad 01$ $3.45324 \mathrm{E} \quad 01$

$3.46347 \mathrm{E} \quad 01$

$3.47369 \mathrm{E} \quad 01$
$3.48392 \mathrm{E} \quad 01$ $3.49414 \mathrm{E} \quad 01$ $3.50436 \mathrm{E} \quad 01$ $3.51458 \mathrm{E} \quad 01$ $3.52479 \mathrm{E} 01$

$3.53500 \mathrm{E} \quad 01$ $\begin{array}{lll}3.54521 \mathrm{E} & 01\end{array}$ $3.55542 \mathrm{E} \quad 01$ $3.56562 \mathrm{E} 01$ $3.57583 \mathrm{E} \quad 01$

$3.58603 \mathrm{E} \quad 01$ $3.59622 \mathrm{E} \quad 01$ $3.60642 \mathrm{E} \quad 01$

$3.61661 \mathrm{E} \quad 01$

$3.62680 \mathrm{E}$ ol

$3.63699 \mathrm{E} \quad 01$ $3.64717 \mathrm{E} \quad 01$ $3.65736 \mathrm{E}$ ol $3.66754 \mathrm{E} \quad 01$ $3.67772 \mathrm{E} \quad 01$

$3.68789 \mathrm{E} \quad 01$ $3.69806 \mathrm{E} \quad 01$ $3.70823 \mathrm{E} 01$ $3.71840 \mathrm{E} \quad 01$ $3.72857 \mathrm{E} \quad 01$

$3.73873 \mathrm{E} \quad 01$ $3.74889 \mathrm{E} \quad 01$ $3.75905 \mathrm{E} \quad 01$ $3.76920 \mathrm{E} \quad 01$ $3.77936 \mathrm{E} 01$

$3.78951 \mathrm{E} \quad 01$ $3.79965 \mathrm{E} \quad 01$ $3.80980 \mathrm{E} \quad 01$ $3.81994 \mathrm{E} \quad 01$ $3.83008 \mathrm{E} \quad 01$
$-1.85538 \mathrm{E} \quad 02$ $-1.85673 \mathrm{E} \quad 02$ $-1.85807 \mathrm{E} \quad 02$ $-1.85941 \mathrm{E} \quad 02$ $-1.86075 \mathrm{E} \quad 02$

$-1.86209 \mathrm{E} \quad 02$ $-1.86343 \mathrm{E} \quad 02$ $-1.86477 \mathrm{E} \quad 02$ $-1.86610 \mathrm{E} \quad 02$ $-1.86743 \mathrm{E} \quad 02$

$-1.86877 \mathrm{E} \quad 02$ $-1.87010 \mathrm{E} \quad 02$ $-1.87142 \mathrm{E} \quad 02$ $-1.87275 \mathrm{E} \quad 02$ $-1.87408 \mathrm{E} \quad 02$

$-1.87541 \mathrm{E} \quad 02$ $-1.87673 \mathrm{E} \quad 02$ $-1.87806 \mathrm{E} \quad 02$ $-1.87938 \mathrm{E} \quad 02$ $-1.88070 \mathrm{E} \quad 02$

$-1.88202 \mathrm{E} \quad 02$ $-1.88334 \mathrm{E} \quad 02$ $-1.88598 \mathrm{E} \quad 02$ $-1.88729 \mathrm{E} \quad 02$

$-1.88861 \mathrm{E} \quad 02$ $-1.88993 \mathrm{E} \quad 02$ $-1.89124 \mathrm{E} \quad 02$ $-1.89256 \mathrm{E} \quad 02$ $-1.89387 \mathrm{E} \quad 02$

$-1.89518 \mathrm{E} \quad 02$ $-1.89650 \mathrm{E} 02$ $-1.89781 \mathrm{E} \quad 02$ $-1.89912 \mathrm{E} \quad 02$ $-1.90043 \mathrm{E} 02$

$-1.90174 \mathrm{E} \quad 02$ $-1.90305 \mathrm{E} \quad 02$ $-1.90436 \mathrm{E} 02$ $-1.90567 \mathrm{E} \quad 02$ $-1.90698 \mathrm{E} \quad 02$ $-1.90828 \mathrm{E} \quad 02$ $-1.90959 \mathrm{E} \quad 02$ $-1.91090 \mathrm{E} 02$ $-1.91221 \mathrm{E} 02$ $-1.91351 \mathrm{E} \quad 02$

$-1.91482 \mathrm{E} \quad 02$ $-1.91613 \mathrm{E} 02$ $-1.91743 \mathrm{E} \quad 02$ $-1.91874 \mathrm{E} \quad 02$ $-1.92005 \mathrm{E} 02$ $-1.92135 \mathrm{E} \quad 02$ $-1.92266 \mathrm{E} \quad 02$ $\begin{array}{lll}-1.92397 \mathrm{E} & 02\end{array}$ $-1.92527 \mathrm{E} \quad 02$ $-1.92658 \mathrm{E} \quad 02$ $-1.88466 \mathrm{E} 02$ 
$Z=6 \quad A=12 \quad \rho=1.2 A^{1 / 3} F$

$\bar{F}$

$1.46483 \mathrm{E}-03$ $1.47457 \mathrm{E}-03$ $1.48435 \mathrm{E}-03$ $1.49415 \mathrm{E}-03$ $1.50400 \mathrm{E}-03$

$1.51387 \mathrm{E}-03$ $1.52377 \mathrm{E}-03$ $1.53371 \mathrm{E}-03$ $1.55368 \mathrm{E}-03$

$1.56372 \mathrm{E}-03$ $1.57378 \mathrm{E}-03$ $1.58388 \mathrm{E}-03$ $1.59401 \mathrm{E}-03$ $1.60418 \mathrm{E}-03$

$1.61437 \mathrm{E}-03$ $1.62460 \mathrm{E}-03$ $1.63486 \mathrm{E}-03$ $1.65548 \mathrm{E}-03$

$1.66584 \mathrm{E}-03$ $1.67623 \mathrm{E}-03$ $1.68665 \mathrm{E}-03$ $1.69710 \mathrm{E}-03$ $1.70759 \mathrm{E}-03$

$1.71811 \mathrm{E}-03$ $1.72866 \mathrm{E}-03$ $1.73924 \mathrm{E}-03$ $1.74986 \mathrm{E}-03$ $1.76050 \mathrm{E}-03$

$1.77118 \mathrm{E}-03$ $1.78190 \mathrm{E}-03$ $1.79264 \mathrm{E}-03$ $1.80342 \mathrm{E}-03$ $1.81423 \mathrm{E}-03$

33.60

33.70

33.80

33.90

34.00

34.10

34.20

34.30

34.40

34.50

34.60

34.70

34.80

34.90

35.00

35.10

35.20

35.30

35.40

35.50

35.60

35.70

35.80

35.90

36.00

$1.82507 \mathrm{E}-03$ $1.83594 \mathrm{E}-03$ $1.84685 \mathrm{E}-03$ $1.85779 \mathrm{E}-03$ $1.86876 \mathrm{E}-03$

$1.87976 \mathrm{E}-03$ $1.89080 \mathrm{E}-03$ $1.90186 \mathrm{E}-03$ $1.91296 \mathrm{E}-03$ $1.92410 \mathrm{E}-03$

$1.93526 \mathrm{E}-03$ $1.94646 \mathrm{E}-03$ $1.95769 \mathrm{E}-03$ $1.96895 \mathrm{E}-03$ $1.98024 \mathrm{E}-03$

$1.99157 \mathrm{E}-03$ $2.00292 \mathrm{E}-03$ 2.01431 E-03 $2.02574 \mathrm{E}-03$ $2.03719 \mathrm{E}-03$

$2.04868 \mathrm{E}-03$ $2.06020 \mathrm{E}-03$ $2.08333 \mathrm{E}-03$ $2.09495 \mathrm{E}-03$ $1.54368 \mathrm{E}-03$ $1.64515 \mathrm{E}-03$

\section{$f_{2}$ \\ $g_{2}$}

$\tan \delta_{2}$

$f_{-2}$

$-2.24531 \mathrm{E} \quad 00 \quad-1.22133 \mathrm{E}-01$ $-2.26028 \mathrm{E} \quad 00-1.23270 \mathrm{E}-01$ $-2.27530 \mathrm{E} \quad 00-1.24415 \mathrm{E}-01$ $-2.29036 \mathrm{E}-00 \quad-1.25566 \mathrm{E}-01$ $-2.30547 \mathrm{E} \quad 00 \quad-1.26725 \mathrm{E}-01$

$-2.32064 \mathrm{E} \quad 00$ $-2.33585 \mathrm{E} \quad 00$ $-2.35110 \mathrm{E} \quad 00$ $-2.36641 \mathrm{E} \quad 00$ $-2.38177 \mathrm{E} \quad 00$

$-2.39717 \mathrm{E} \quad 00$ $-2.41263 \mathrm{E} 00$ $-2.42813 \mathrm{E} \quad 00$ $-2.44368 \mathrm{E} \quad 00$ $-2.45928 \mathrm{E} \quad 00$

$-2.47493 \mathrm{E} \quad 00$ $-2.49063 \mathrm{E} \quad 00$ $-2.50637 \mathrm{E} 00$ $-2.52217 \mathrm{E} \quad 00$ $-2.53801 \mathrm{E} \quad 00$

\section{$-2.55390 \mathrm{E} \quad 00$} $-2.56984 \mathrm{E} \quad 00$ $-2.58583 \mathrm{E} \quad 00$ $-2.60187 \mathrm{E} \quad 00$ $-2.61796 \mathrm{E} \quad 00$

$-2.63409 \mathrm{E} \quad 00$ $-2.65027 \mathrm{E} \quad 00$ $-2.66650 \mathrm{E} \quad 00$ $-2.68278 \mathrm{E} \quad 00$ $-2.69911 \mathrm{E} \quad 00$

$-2.71549 \mathrm{E} \quad 00$ $-2.73191 \mathrm{E} 00$ $-2.74839 \mathrm{E} \quad 00$ $-2.76491 \mathrm{E} \quad 00$ $-2.78148 \mathrm{E} \quad 00$

$-2.79810 \mathrm{E} \quad 00$ $-2.81477 \mathrm{E} \quad 00$ $-2.83148 \mathrm{E} 00$ $-2.84825 \mathrm{E} \quad 00$ $-2.86506 \mathrm{E} \quad 00$

$-2.88192 \mathrm{E} \quad 00$ $-2.89883 \mathrm{E} \quad 00$ $-2.91578 \mathrm{E} 00$ $-2.93279 \mathrm{E} \quad 00$ $-2.94984 \mathrm{E} \quad 00$ $2.07175 \mathrm{E}-03$
$-2.96695 \mathrm{E} \quad 00$

$-2.98410 \mathrm{E} \quad 00$ $-3.00129 \mathrm{E} 00$ $-3.03584 \mathrm{E} \quad 00$

$-3.05318 \mathrm{E} 00$ $-3.07057 \mathrm{E} 00$ $-3.08801 \mathrm{E} 00$ $-3.10550 \mathrm{E} 00$ $-3.15825 \mathrm{E} \quad 00$ $-3.17593 \mathrm{E} 00$ $-3.19366 \mathrm{E} 00$ $-3.21144 \mathrm{E} \quad 00$ $-3.01854 \mathrm{E} \quad 00$ $-3.12303 \mathrm{E} 00$

$-3.14062 \mathrm{E} 00$
$-1.27890 \mathrm{E}-01$ $-1.29063 \mathrm{E}-01$ $-1.30243 \mathrm{E}-01$ $-1.31429 \mathrm{E}-01$ $-1.32623 \mathrm{E}-01$

$-1.33824 \mathrm{E}-01$ $-1.35032 \mathrm{E}-01$ $-1.36247 \mathrm{E}-01$ $-1.37470 \mathrm{E}-01$ $-1.38700 \mathrm{E}-01$

$3.43842 \mathrm{E} \quad 01$

$3.43858 \mathrm{E} 01$

$3.43873 \mathrm{E} 01$

$3.43888 \mathrm{E} \quad 01$

$3.43903 \mathrm{E} \quad 01$

$3.43919 \mathrm{E} \quad 01$

$3.43934 \mathrm{E} \quad 01$

$3.43948 \mathrm{E} 01$

$3.43963 \mathrm{E} \quad 01$

$3.43978 \mathrm{E} 01$

$3.43992 \mathrm{E} \quad 01$

$3.44007 \mathrm{E} \quad 01$

$3.44021 \mathrm{E} \quad 01$

$3.44036 \mathrm{E} \quad 01$

$3.44050 \mathrm{E} \quad 01$

$-1.39937 \mathrm{E}-01$

$-1.41181 \mathrm{E}-01$

$-1.42432 \mathrm{E}-01$

$-1.43691 \mathrm{E}-01$

$-1.44957 \mathrm{E}-01$

$-1.46230 \mathrm{E}-01$

$-1.47511 \mathrm{E}-01$

$-1.48799 \mathrm{E}-01$

$-1.50094 \mathrm{E}-01$

$-1.51397 \mathrm{E}-01$

$-1.52708 \mathrm{E}-01$ $-1.54025 \mathrm{E}-0$

- $1.55351 \mathrm{E}-01$

$-1.56683 \mathrm{E}-01$

$-1.58024 \mathrm{E}-01$

$-1.59371 \mathrm{E}-01$ $-1.60727 \mathrm{E}-01$

$-1.63460 \mathrm{E}-01$

$-1.64838 \mathrm{E}-01$

$-1.66224 \mathrm{E}-01$

$-1.67618 \mathrm{E}-01$

$-1.69019 \mathrm{E}-01$

$-1.70428 \mathrm{E}-01$

$-1.71844 \mathrm{E}-01$

$-1.73268 \mathrm{E}-01$ $-1.74701 \mathrm{E}-01$ $-1.76140 \mathrm{E}-01$ $-1.77588 \mathrm{E}-01$ $-1.79043 \mathrm{E}-01$

$-1.80507 \mathrm{E}-01$ $-1.81978 \mathrm{E}-01$ $-1.83457 \mathrm{E}-01$ $-1.84944 \mathrm{E}-01$ $-1.86439 \mathrm{E}-01$

$-1.87942 \mathrm{E}-01$ $-1.89452 \mathrm{E}-01$ $-1.90971 \mathrm{E}-01$ $-1.92498 \mathrm{E}-01$ $-1.94033 \mathrm{E}-01$

$-1.95576 \mathrm{E}-01$ $-1.97126 \mathrm{E}-01$ $-2.00252 \mathrm{E}-01$ $-2.01828 \mathrm{E}-01$

$3.44604 \mathrm{E}$ 0l
$-1.62090 \mathrm{E}-01$ $-1.98685 \mathrm{E}-01$
$3.44064 \mathrm{E} \quad 01$ $3.44078 \mathrm{E} 01$ $3.44092 \mathrm{E} \quad 01$

$3.44106 \mathrm{E} \quad 01$

$3.44119 \mathrm{E} \quad 01$

$3.44133 \mathrm{E} \quad 01$

$3.44146 \mathrm{E} \quad 01$

$3.44160 \mathrm{E} \quad 01$

$3.44173 \mathrm{E} \quad 01$

$3.44187 \mathrm{E} 01$

$3.44200 \mathrm{E} \quad 01$ $3.44213 \mathrm{E} \quad 01$

$3.44226 \mathrm{E} 01$

$3.44239 \mathrm{E} 01$

$3.44252 \mathrm{E} 01$

$3.44265 \mathrm{E} \quad 01$

$3.44277 \mathrm{E}$ 01

$3.44290 \mathrm{E} \quad 01$

$3.44303 \mathrm{E} \quad 01$

$3.44315 \mathrm{E} \quad 01$

$3.44327 \mathrm{E} \quad 01$

$3.44340 \mathrm{E} 01$

$3.44352 \mathrm{E} \quad 01$

$3.44364 \mathrm{E} \quad 01$

$3.44376 \mathrm{E} \quad 01$

$3.44388 \mathrm{E} \quad 01$

$3.44400 \mathrm{E}$ ol

$3.44412 \mathrm{E} 01$

$3.44424 \mathrm{E}$ ol

$3.44436 \mathrm{E} \quad 01$

$3.44447 \mathrm{E} \quad 01$

$3.44459 \mathrm{E} \quad 01$

$3.44471 \mathrm{E} \quad 01$

$3.44482 \mathrm{E} \quad 01$

$3.44493 \mathrm{E} \quad 01$

$3.44505 \mathrm{E} \quad 01$

$3.44516 \mathrm{E} \quad 01$

$3.44527 \mathrm{E} 01$

$3.44538 \mathrm{E} \quad 01$

$3.44550 \mathrm{E} \quad 01$

$3.44561 \mathrm{E} \quad 01$

$3.44571 \mathrm{E} \quad 01$

$3.44582 \mathrm{E}$ ol

$3.44593 \mathrm{E}$ ol

$-1.19634 \mathrm{E}-01$ $-1.20752 \mathrm{E}-0 \mathrm{l}$ $-1.21877 \mathrm{E}-01$ $-1.23010 \mathrm{E}-01$ $-1.24149 \mathrm{E}-01$

$-1.25295 \mathrm{E}-01$ $-1.26448 \mathrm{E}-01$ $-1.27608 \mathrm{E}-01$ $-1.28775 \mathrm{E}-01$ $-1.29950 \mathrm{E}-01$

$-1.31131 \mathrm{E}-01$ $-1.32319 \mathrm{E}-01$ $-1.33514 \mathrm{E}-01$ $-1.34717 \mathrm{E}-01$ $-1.35927 \mathrm{E}-01$

$-1.37143 \mathrm{E}-01$ 
ELECTRON RADIAL FUNCTIONS

Electron

\begin{tabular}{|c|c|c|c|c|c|}
\hline$p$ & $F_{0}$ & & $f_{1}$ & & $g_{1}$ \\
\hline 36.10 & $1.15070 \mathrm{E}$ & 00 & $-3.73604 \mathrm{E}$ & 01 & $-3.96842 \mathrm{E}$ \\
\hline & $1.15069 \mathrm{E}$ & 00 & $-3.74619 \bar{E}$ & 01 & $-3.98825 \mathrm{E}$ \\
\hline 36.30 & $1.15068 \mathrm{E}$ & 00 & $-3.75633 \mathrm{E}$ & 01 & $-4.00814 \mathrm{E}$ \\
\hline 36.40 & $1.15068 \mathrm{E}$ & 00 & $-3.76647 \mathrm{E}$ & 01 & $-4.02807 \mathrm{E}$ \\
\hline 36.50 & $1.15067 \mathrm{E}$ & 00 & $-3.77661 \mathrm{E}$ & 01 & $-4.04804 \mathrm{E}$ \\
\hline 36.60 & $1.15066 \mathrm{E}$ & 00 & $-3.78675 E$ & 01 & $-4.06807 \mathrm{E}$ \\
\hline 36.70 & $066 \mathrm{E}$ & 00 & $-3.79688 E$ & 01 & $08814 \mathrm{E}$ \\
\hline 36.80 & $1.15065 \mathrm{E}$ & 00 & $-3.80701 \mathrm{E}$ & 01 & $-4.10826 \mathrm{E}$ \\
\hline 36.90 & $1.15065 \mathrm{E}$ & 00 & $-3.81714 \mathrm{E}$ & 01 & $-4.12842 \mathrm{E}$ \\
\hline 37.00 & $1.15064 \mathrm{E}$ & 00 & $-3.82727 \mathbf{E}$ & 01 & $-4.14864 \mathrm{E}$ \\
\hline 37.10 & $1.15063 \mathrm{E}$ & 00 & $-3.83739 E$ & 01 & $-4.16890 \mathrm{E}$ \\
\hline 37.20 & $1.15063 \mathrm{E}$ & 00 & $-3.84751 \mathrm{E}$ & 01 & $-4.18920 \mathrm{E}$ \\
\hline .30 & $1.15062 \mathrm{E}$ & 00 & $-3.85763 \mathrm{E}$ & 01 & $-4.20956 \mathrm{E}$ \\
\hline .40 & $1.15061 \mathrm{E}$ & 00 & $-3.86775 E$ & 01 & $-4.22996 \mathrm{E}$ \\
\hline 37.50 & $061 \mathrm{E}$ & 00 & $-3.87786 \mathrm{E}$ & 01 & $-4.25041 \mathrm{E}$ \\
\hline 37.60 & $1.15060 \mathrm{E}$ & 00 & $-3.88797 \mathrm{E}$ & 01 & $-4.27090 \mathrm{E}$ \\
\hline 37.70 & $1.15060 \mathrm{E}$ & 00 & $-3.89808 E$ & 01 & $-4.29144 \mathrm{E}$ \\
\hline 0 & $059 \mathrm{E}$ & 00 & $-3.90818 \mathrm{E}$ & 01 & $-4.31203 \mathrm{E}$ \\
\hline 37.90 & $058 \mathrm{E}$ & 00 & $-3.91828 E$ & 01 & $.33267 \mathrm{E}$ \\
\hline 38.00 & $1.15058 \mathrm{E}$ & 00 & $-3.92838 E$ & 01 & $-4.35335 \mathrm{E}$ \\
\hline 38.10 & $15057 \mathrm{E}$ & 00 & $-3.93848 \mathrm{E}$ & 01 & $-4.37408 \mathrm{E}$ \\
\hline 20 & $67 \mathrm{E}$ & 00 & $3.94858 \mathrm{E}$ & 01 & $39486 \mathrm{E}$ \\
\hline 38. & $56 \mathrm{E}$ & 00 & $5867 \mathrm{E}$ & 01 & $1568 \mathrm{E}$ \\
\hline 38.4 & $55 \mathrm{E}$ & 00 & $6876 \mathrm{E}$ & 01 & $43655 \mathrm{E}$ \\
\hline 38.50 & $1.15055 \mathrm{E}$ & 00 & $-3.97884 \mathrm{E}$ & 01 & $45747 \mathrm{E}$ \\
\hline 38.60 & $15054 \mathrm{E}$ & 00 & $-3.98893 E$ & 01 & $44 \mathrm{E}$ \\
\hline 38.70 & & 00 & $01 \mathrm{E}$ & 01 & \\
\hline 38.80 & $53 \mathrm{E}$ & 00 & $-4.00909 \mathrm{E}$ & 01 & $2050 \mathrm{E}$ \\
\hline 38.90 & $1.15052 \mathrm{E}$ & 00 & $-4.01916 \mathrm{E}$ & 01 & $-4.54161 \mathrm{E}$ \\
\hline 39.00 & $1.15052 \mathrm{E}$ & 00 & $-4.02924 \mathrm{E}$ & 01 & $-4.56276 \mathrm{E}$ \\
\hline 39.10 & $1.15051 \mathrm{E}$ & 00 & $-4.03931 \mathrm{E}$ & 01 & \\
\hline 39.20 & $51 \mathrm{E}$ & 00 & $-4.04937 \mathrm{E}$ & 01 & $60520 \mathrm{E}$ \\
\hline 39.30 & $5050 \mathrm{E}$ & 00 & $-4.05944 \mathrm{E}$ & 01 & $-4.62650 \mathrm{E}$ \\
\hline 39.40 & $49 \mathrm{E}$ & 00 & $-4.06950 \mathrm{E}$ & 01 & $-4.64783 \mathrm{E}$ \\
\hline 39.50 & $1.15049 \mathrm{E}$ & 00 & $956 \mathrm{E}$ & 01 & $6922 \mathrm{E}$ \\
\hline 39.60 & $15048 \mathrm{E}$ & 00 & $-4.08962 \mathrm{E}$ & 01 & $5 \mathrm{E}$ \\
\hline 39.70 & $5048 \mathrm{E}$ & 00 & $-4.09967 \mathrm{E}$ & 01 & $.71213 \mathrm{E}$ \\
\hline 39.80 & $47 \mathrm{E}$ & 00 & $-4.10973 \mathrm{E}$ & 01 & $-4.73365 \mathrm{E}$ \\
\hline 39.90 & & 00 & $77 \mathrm{E}$ & 01 & -4 \\
\hline 40.00 & & 00 & $-4.12982 \mathrm{E}$ & 01 & $77684 \mathrm{E}$ \\
\hline 40.10 & $5 \mathrm{E}$ & 00 & $-4.13986 \mathrm{E}$ & 01 & -4 \\
\hline 40.20 & $5 \mathrm{E}$ & 00 & $91 \mathrm{E}$ & 01 & $82022 \mathrm{E}$ \\
\hline & & 00 & $4 \mathrm{E}$ & 01 & \\
\hline & & 00 & $98 \mathrm{E}$ & 01 & -4 \\
\hline 40.50 & $1.15043 \mathrm{E}$ & 00 & $-4.18001 \mathrm{E}$ & 01 & -4 \\
\hline 40.60 & & 00 & -4 & 01 & \\
\hline & & 00 & & 01 & \\
\hline 40.8 & & 00 & -4.2 & 01 & $5147 \mathrm{E}$ \\
\hline 40.9 & & 00 & -4 & 01 & $7350 \mathrm{E}$ \\
\hline 41.00 & $1.15040 \mathrm{E}$ & 00 & $-4.23013 \mathrm{E}$ & 01 & $9559 \mathrm{E}$ \\
\hline 41.10 & & 00 & -4.2 & 01 & \\
\hline & & 00 & -4.2 & 0 & $3989 \mathrm{E}$ \\
\hline & $9 \mathrm{E}$ & 00 & $6017 \mathrm{E}$ & 0 & J6212E \\
\hline & & 00 & $7018 \mathrm{E}$ & 01 & $08439 \mathrm{E}$ \\
\hline 41.50 & $1.15037 \mathrm{E}$ & 00 & $-4.28018 \mathrm{E}$ & 01 & $-5.10670 \mathrm{E}$ \\
\hline & $037 \mathrm{E}$ & 00 & $-4.29019 \mathrm{E}$ & 01 & -5 \\
\hline & & 00 & $0018 \mathrm{E}$ & 0 & \\
\hline & & 00 & $1018 \mathrm{E}$ & 01 & $-5.17393 \mathrm{E}$ \\
\hline & $1.15035 \mathrm{E}$ & 00 & $-4.32017 \mathrm{E}$ & 01 & $-5.19643 \mathrm{E}$ \\
\hline & $1.15035 \mathrm{E}$ & 00 & $-4.33017 \mathrm{E}$ & 01 & $-5.21898 \mathrm{E}$ \\
\hline
\end{tabular}

$\tan \delta_{1} \quad f_{-1}$

$3.99206 \mathrm{E}-03$ $3.99194 \mathrm{E}-03$

$3.99182 \mathrm{E}-03$

$3.99167 \mathrm{E}-03$

$3.99152 \mathrm{E}-03$

$3.99134 \mathrm{E}-03$

$3.99117 \mathrm{E}-03$

$3.99097 \mathrm{E}-03$

$3.99076 \mathrm{E}-03$

$3.99054 \mathrm{E}-03$

$3.99030 \mathrm{E}-03$

$3.99004 \mathrm{E}-03$

$3.98978 \mathrm{E}-03$

$3.98950 \mathrm{E}-03$

$3.98921 \mathrm{E}-03$

$3.98891 \mathrm{E}-03$

$3.98860 \mathrm{E}-03$

$3.98827 \mathrm{E}-03$

$3.98793 \mathrm{E}-03$

$3.98757 \mathrm{E}-03$

$3.98720 \mathrm{E}-03$

$3.98683 \mathrm{E}-03$

$3.98643 \mathrm{E}-03$

$3.98602 \mathrm{E}-03$

3.98561 E-03

$3.98518 \mathrm{E}-03$

$3.98473 \mathrm{E}-03$

$3.98428 \mathrm{E}-03$

$3.98381 \mathrm{E}-03$

$3.98333 \mathrm{E}-03$

$3.98284 \mathrm{E}-03$

$3.98234 \mathrm{E}-03$

$3.98182 \mathrm{E}-03$

$3.98129 \mathrm{E}-03$

$3.98075 \mathrm{E}-03$

$3.98020 \mathrm{E}-03$

$3.97963 \mathrm{E}-03$

$3.97906 \mathrm{E}-03$

$3.97848 \mathrm{E}-03$

$3.97788 \mathrm{E}-03$

$3.97726 \mathrm{E}-03$

$3.97664 \mathrm{E}-03$

$3.97601 \mathrm{E}-03$

$3.97536 \mathrm{E}-03$

$3.97471 \mathrm{E}-03$

$3.97404 \mathrm{E}-03$

$3.97337 \mathrm{E}-03$

$3.97267 \mathrm{E}-03$

$3.97197 \mathrm{E}-03$

$3.97126 \mathrm{E}-03$

$3.97053 \mathrm{E}-03$

$3.96980 \mathrm{E}-03$

$3.96905 \mathrm{E}-03$

$3.96829 \mathrm{E}-03$

$3.96753 \mathrm{E}-03$

$3.96675 \mathrm{E}-03$ $3.96596 \mathrm{E}-03$

$3.96516 \mathrm{E}-03$

$3.96435 \mathrm{E}-03$

$3.96352 \mathrm{E}-03$
$-3.89578 \mathrm{E} \quad 00$ $-3.91536 \mathrm{E} \quad 00$ $-3.93500 \mathrm{E} 00$

$-3.95468 \mathrm{E} \quad 00$

$-3.97441 \mathrm{E} \quad 00$

$-3.994 .18 \mathrm{E} \quad 00$ $-4.01400 \mathrm{E} 00$ $-4.03387 \mathrm{E} \quad 00$ $-4.05379 \mathrm{E} \quad 00$

$-4.07375 \mathrm{E} \quad 00$

$-4.09377 \mathrm{E} \quad 00$

$-4.11382 \mathrm{E} \quad 00$

$-4.13393 \mathrm{E} 00$

$-4.15408 \mathrm{E} \quad 00$

$-4.17428 \mathrm{E} 00$

$-4.19453 \mathrm{E} \quad 00$

$-4.21482 \mathrm{E} \quad 00$

$-4.23516 \mathrm{E} \quad 00$

$-4.25555 \mathrm{E} \quad 00$

$-4.27598 \mathrm{E} \quad 00$

$-4.29646 \mathrm{E} \quad 00$

$-4.31699 \mathrm{E} 00$

$-4.33757 \mathrm{E} \quad 00$

$-4.35819 \mathrm{E} \quad 00$

$-4.37886 \mathrm{E} \quad 00$

$-4.39957 \mathrm{E} \quad 00$

$-4.42033 \mathrm{E} \quad 00$

$-4.44114 \mathrm{E} \quad 00$

$-4.46200 \mathrm{E} \quad 00$

$-4.48290 \mathrm{E} 00$

$-4.50385 \mathrm{E} \quad 00$

$-4.52485 \mathrm{E} \quad 00$

$-4.54589 \mathrm{E} \quad 00$

$-4.56698 \mathrm{E} 00$

$-4.58812 \mathrm{E} \quad 00$

$-4.60930 \mathrm{E} \quad 00$

$-4.63053 \mathrm{E} \quad 00$

$-4.65181 \mathrm{E} \quad 00$

$-4.67313 \mathrm{E} \quad 00$

$-4.69450 \mathrm{E} \quad 00$

$-4.71592 \mathrm{E} \quad 00$

$-4.73738 \mathrm{E} \quad 00$

$-4.75889 \mathrm{E} 00$

$-4.78045 \mathrm{E} \quad 00$

$-4.80205 \mathrm{E} 00$

$-4.82370 \mathrm{E} 00$

$-4.84540 \mathrm{E} \quad 00$

$-4.86714 \mathrm{E} \quad 00$

$-4.88893 \mathrm{E} 00$

$-4.91077 \mathrm{E} \quad 00$

$-4.93265 \mathrm{E} \quad 00$

$-4.95458 \mathrm{E} 00$

$-4.97655 \mathrm{E} \quad 00$

$-4.99858 \mathrm{E} \quad 00$

$-5.02064 \mathrm{E} \quad 00$

$-5.04276 \mathrm{E} \quad 00$ $-5.06492 \mathrm{E} \quad 00$ $-5.08713 \mathrm{E} \quad 00$ $-5.10938 \mathrm{E} \quad 00$ $-5.13168 \mathrm{E} \quad 00$ $g_{-1}$

$\tan \delta_{-1}$

$\begin{array}{llll}3.84022 \mathrm{E} & 01 & -1.92788 \mathrm{E} & 02\end{array}$ $3.85036 \mathrm{E}$ 01 $-1.92919 \mathrm{E} \quad 02$ $3.86049 \mathrm{E}$ ol $-1.93050 \mathrm{E} \quad 02$ $3.87062 \mathrm{E}$ 01 $-1.93180 \mathrm{E} \quad 02$ $3.88075 \mathrm{E}$ ol $\quad-1.93311 \mathrm{E} \quad 02$

$3.89087 \mathrm{E}$ 01 $\quad-1.93442 \mathrm{E} \quad 02$ $3.90099 \mathrm{E} \quad 01 \quad-1.93573 \mathrm{E} \quad 02$ $3.91111 \mathrm{E} \quad 01 \quad-1.93703 \mathrm{E} \quad 02$ $3.92123 \mathrm{E} \quad 01 \quad-1.93834 \mathrm{E} \quad 02$ $\begin{array}{llll}3.93134 \mathrm{E} & 01 & -1.93965 \mathrm{E} & 02\end{array}$

$3.94146 \mathrm{E} \quad 01$ $3.95156 \mathrm{E} \quad 01$ $3.96167 \mathrm{E} \quad 01$ $3.97177 \mathrm{E} \quad 01$ $3.98188 \mathrm{E} \quad 01$

$3.99197 \mathrm{E} \quad 01$ $4.00207 \mathrm{E} \quad 01$ $4.01216 \mathrm{E} 01$ $4.02226 \mathrm{E} \quad 01$ $4.03234 \mathrm{E} \quad 01$

$4.04243 \mathrm{E} \quad 01$ $4.05251 \mathrm{E} \quad 01$ $4.06259 \mathrm{E} \quad 01$ $\begin{array}{lll}4.07267 \mathrm{E} & 01\end{array}$

$4.08274 \mathrm{E} \quad 01$

$4.09282 \mathrm{E} \quad 01$ $4.10289 \mathrm{E} \quad 01$ $4.11295 \mathrm{E} \quad 01$ $4.12302 \mathrm{E} \quad 01$ $4.13308 \mathrm{E} \quad 01$

$4.14314 \mathrm{E} \quad 01$ $4.15319 \mathrm{E} \quad 01$

$4.16325 \mathrm{E} \quad 01$

$4.17330 \mathrm{E}$ 01

$4.18334 \mathrm{E} \quad 01$

$4.19339 \mathrm{E} \quad 01$ $4.20343 \mathrm{E} \quad 01$ $4.21347 \mathrm{E} \quad 01$ $4.22351 \mathrm{E} \quad 01$

$4.23354 \mathrm{E} \quad 01$

$4.24358 \mathrm{E} \quad 01$ $4.25360 \mathrm{E}$ 01 $4.26363 \mathrm{E} 01$ $4.27365 \mathrm{E} \quad 01$ $4.28367 \mathrm{E} \quad 01$

$4.29369 \mathrm{E} \quad 01$ $4.30371 \mathrm{E} \quad 01$

$4.31372 \mathrm{E} 01$

$4.32373 \mathrm{E} 01$

$4.33373 \mathrm{E} \quad 01$

$4.34374 \mathrm{E} \quad 01$ $4.35374 \mathrm{E} \quad 01$ $4.36374 \mathrm{E} \quad 01$ $4.37373 \mathrm{E} \quad 01$ $4.38372 \mathrm{E} 01$

$4.39371 \mathrm{E} \quad 01$ $4.40370 \mathrm{E} \quad 01$ $4.41369 \mathrm{E} \quad 01$ $4.42367 \mathrm{E} \quad 01$ $4.43364 \mathrm{E} \quad 01$
$-1.94096 \mathrm{E} \quad 02$ $-1.94227 \mathrm{E} \quad 02$ $-1.94358 \mathrm{E} \quad 02$ $-1.94489 \mathrm{E} 02$ $-1.94620 \mathrm{E} 02$

$-1.94751 \mathrm{E} \quad 02$ $-1.94882 \mathrm{E} \quad 02$ $-1.95013 \mathrm{E} \quad 02$ $-1.95144 \mathrm{E} 02$ $-1.95276 \mathrm{E} \quad 02$

$-1.95407 \mathrm{E} \quad 02$ $-1.95538 \mathrm{E} \quad 02$ $-1.95669 \mathrm{E} \quad 02$ $-1.95801 \mathrm{E} \quad 02$ $-1.95932 \mathrm{E} \quad 02$

$-1.96064 \mathrm{E} \quad 02$ $-1.96196 \mathrm{E} \quad 02$ $-1.96327 \mathrm{E} 02$ $-1.96459 \mathrm{E} \quad 02$ $-1.96591 \mathrm{E} 02$

$-1.96723 \mathrm{E} \quad 02$ $-1.96855 \mathrm{E} \quad 02$ $-1.96987 \mathrm{E} \quad 02$ $-1.97119 \mathrm{E} 02$ $-1.97252 \mathrm{E} 02$

$-1.97384 \mathrm{E} \quad 02$ $-1.97517 \mathrm{E} \quad 02$ $-1.97649 \mathrm{E} 02$ $-1.97782 \mathrm{E} \quad 02$ $-1.97914 \mathrm{E} \quad 02$

$-1.98047 \mathrm{E} \quad 02$ $-1.98180 \mathrm{E} \quad 02$ $-1.98313 \mathrm{E} 02$ $-1.98446 \mathrm{E} \quad 02$ $-1.98580 \mathrm{E} 02$

$-1.98713 \mathrm{E} \quad 02$ $-1.98846 \mathrm{E} \quad 02$ $\begin{array}{lll}-1.98980 \mathrm{E} & 02\end{array}$ $-1.99114 \mathrm{E} \quad 02$ $-1.99247 \mathrm{E} \quad 02$

$-1.99381 \mathrm{E} \quad 02$ $-1.99515 \mathrm{E} \quad 02$ $-1.99650 \mathrm{E} \quad 02$ $-1.99784 \mathrm{E} \quad 02$ $-1.99918 \mathrm{E} \quad 02$

$-2.00053 \mathrm{E} \quad 02$ $\begin{array}{lll}-2.00187 \mathrm{E} & 02\end{array}$ $-2.00322 \mathrm{E} \quad 02$ $-2.00457 \mathrm{E} \quad 02$ $-2.00592 \mathrm{E} \quad 02$ 
$\bar{F}$

$2.10660 \mathrm{E}-03$ $2.11828 \mathrm{E}-03$ $2.12999 \mathrm{E}-03$ $2.14174 \mathrm{E}-03$ $2.15352 \mathrm{E}-03$

$2.16533 \mathrm{E}-03$ $2.17717 \mathrm{E}-03$ $2.18904 \mathrm{E}-03$ $2.20095 \mathrm{E}-03$ $2.21289 \mathrm{E}-03$

$2.22486 \mathrm{E}-03$ $2.23686 \mathrm{E}-03$ $2.24 .890 \mathrm{E}-03$ $2.26097 \mathrm{E}-03$ $2.27307 \mathrm{E}-03$

$2.28520 \mathrm{E}-03$ $2.29737 \mathrm{E}-03$ $2.30956 \mathrm{E}-03$ $2.32179 \mathrm{E}-03$ $2.33406 \mathrm{E}-03$

$2.34635 \mathrm{E}-03$ $2.35868 \mathrm{E}-03$ $2.37103 \mathrm{E}-03$ $2.38343 \mathrm{E}-03$ $2.39585 \mathrm{E}-03$

$2.40830 \mathrm{E}-03$ $2.42079 \mathrm{E}-03$ $2.43331 \mathrm{E}-03$ $2.44586 \mathrm{E}-03$ $2.45845 \mathrm{E}-03$

$2.47107 \mathrm{E}-03$ $2.48372 \mathrm{E}-03$ $2.49640 \mathrm{E}-03$ $2.50911 \mathrm{E}-03$ $2.52186 \mathrm{E}-03$

2.53464 E-03 $2.54745 \mathrm{E}-03$ $2.56029 \mathrm{E}-03$ $2.57316 \mathrm{E}-03$ $2.58607 \mathrm{E}-03$

$2.59901 \mathrm{E}-03$ $2.61198 \mathrm{E}-03$ $2.62499 \mathrm{E}-03$ $2.63802 \mathrm{E}-03$ 2.65109E-03

2.66419E-03 $2.67733 \mathrm{E}-03$ $2.69049 \mathrm{E}-03$ $2.70369 \mathrm{E}-03$ $2.71692 \mathrm{E}-03$

$2.73018 \mathrm{E}-03$ $2.74348 \mathrm{E}-03$ $2.75681 \mathrm{E}-03$ $2.77017 \mathrm{E}-03$ $2.78356 \mathrm{E}-03$

$2.79698 \mathrm{E}-03$ $2.81044 \mathrm{E}-03$ $2.82393 \mathrm{E}-03$ $2.83745 \mathrm{E}-03$ $2.85100 \mathrm{E}-03$ $f_{2}$

$g_{2}$

$\tan \delta_{2}$

$f_{-2}$

$-3.22926 \mathrm{E} \quad 00$ $-3.24713 \mathrm{E} 00$ $-3.26506 \mathrm{E} 00$ $-3.28302 \mathrm{E} 00$ $-3.30104 \mathrm{E} 00$

$-3.31911 \mathrm{E} 00$ $-3.33722 \mathrm{E} \quad 00$ $-3.35538 \mathrm{E} 00$ $-3.37359 \mathrm{E}$ 00 $-3.39184 \mathrm{E} \quad 00$

$-3.41015 \mathrm{E} \quad 00$ $-3.42850 \mathrm{E} \quad 00$ $-3.44690 \mathrm{E} \quad 00$ $-3.46535 \mathrm{E} 00$ -3.48385 E 00

$-3.50239 \mathrm{E} \quad 00$ $-3.52098 \mathrm{E} \quad 00$ $-3.53962 \mathrm{E} \quad 00$ $-3.55831 \mathrm{E} 00$ $-3.57705 \mathrm{E} \quad 00$

$-3.59583 \mathrm{E} \quad 00$ $-3.61466 \mathrm{E} \quad 00$ $-3.63354 \mathrm{E} \quad 00$ $-3.65247 \mathrm{E} \quad 00$ $-3.67144 \mathrm{E} \quad 00$

$-3.69046 \mathrm{E} \quad 00$ $-3.70953 \mathrm{E} 00$ $-3.72865 \mathrm{E} \quad 00$ $-3.74782 \mathrm{E} \quad 00$ $-3.76703 \mathrm{E} 00$

$-3.78629 \mathrm{E} \quad 00$ $-3.80560 \mathrm{E} 00$ $-3.82495 \mathrm{E} 00$ $-3.84436 \mathrm{E} \quad 00$ -3.86381 E 00

$-3.88331 \mathrm{E} \quad 00$ $-3.90285 \mathrm{E} \quad 00$ $-3.92245 \mathrm{E} 00$ $-3.94209 \mathrm{E} 00$ $-3.96178 \mathrm{E} 00$

$-3.98152 \mathrm{E} \quad 00$ $-4.00130 \mathrm{E} 00$ $-4.02113 \mathrm{E} \quad 00$ $-4.04101 \mathrm{E} \quad 00$ $-4.06094 \mathrm{E} \quad 00$

$-4.08091 \mathrm{E} \quad 00$ $-4.10093 \mathrm{E} 00$ $-4.12100 \mathrm{E} 00$ $-4.14112 \mathrm{E} 00$ $-4.16128 \mathrm{E} \quad 00$

$-4.18149 \mathrm{E} \quad 00$ $-4.20175 \mathrm{E} \quad 00$ $-4.22206 \mathrm{E} \quad 00$ $-4.24241 \mathrm{E} 00$ $-4.26281 \mathrm{E} 00$

$-4.28326 \mathrm{E} \quad 00$ $-4.30375 \mathrm{E} 00$ $-4.32430 \mathrm{E} 00$ $-4.34489 \mathrm{E} \quad 00$ $-4.36552 \mathrm{E} \quad 00$
$-2.03411 \mathrm{E}-01$ $-2.05003 \mathrm{E}-01$ $-2.06602 \mathrm{E}-01$ $-2.08210 \mathrm{E}-01$ $-2.09826 \mathrm{E}-01$

$-2.11451 \mathrm{E}-01$ $-2.13083 \mathrm{E}-01$ $-2.14724 \mathrm{E}-01$ $-2.16374 \mathrm{E}-01$ $-2.18031 \mathrm{E}-01$

$-2.19697 \mathrm{E}-01$ $-2.21372 \mathrm{E}-01$ $-2.23054 \mathrm{E}-01$ $-2.26445 \mathrm{E}-01$

$-2.28153 \mathrm{E}-01$ $-2.29869 \mathrm{E}-01$ $-2.31594 \mathrm{E}-01$ $-2.35070 \mathrm{E}-01$

$-2.36820 \mathrm{E}-01$ $-2.38579 \mathrm{E}-01$ $-2.40347 \mathrm{E}-01$ $-2.42123 \mathrm{E}-01$ $-2.43908 \mathrm{E}-01$

$-2.45702 \mathrm{E}-01$ $-2.47504 \mathrm{E}-01$ $-2.49315 \mathrm{E}-01$ $-2.51134 \mathrm{E}-01$ $-2.52963 \mathrm{E}-01$

$-2.54800 \mathrm{E}-01$ $-2.56646 \mathrm{E}-01$ $-2.58500 \mathrm{E}-01$ $-2.60364 \mathrm{E}-01$ $-2.62236 \mathrm{E}-01$

$-2.64117 \mathrm{E}-01$ $-2.66007 \mathrm{E}-01$ $-2.67906 \mathrm{E}-01$ $-2.69814 \mathrm{E}-01$ $-2.71731 \mathrm{E}-01$

$-2.73656 \mathrm{E}-01$ $-2.75591 \mathrm{E}-01$ $-2.77535 \mathrm{E}-01$ $-2.79487 \mathrm{E}-01$ $-2.81449 \mathrm{E}-01$

$-2.83420 \mathrm{E}-01$ $-2.85400 \mathrm{E}-01$ $-2.87388 \mathrm{E}-01$ $-2.89386 \mathrm{E}-01$ $-2.91393 \mathrm{E}-01$

$-2.93410 \mathrm{E}-01$ $-2.95435 \mathrm{E}-01$ $-2.97470 \mathrm{E}-01$ $-2.99514 \mathrm{E}-01$ $-3.01567 \mathrm{E}-01$

$-3.03629 \mathrm{E}-01$ $-3.05701 \mathrm{E}-01$ $-3.07781 \mathrm{E}-01$ $-3.09872 \mathrm{E}-01$ $-3.11971 \mathrm{E}-01$ $-2.24745 \mathrm{E}-01$ $-2.33328 \mathrm{E}-01$
$3.44615 \mathrm{E} \quad 01 \quad-1.99641 \mathrm{E}-01$ $3.44625 \mathrm{E}$ 01 $-2.01209 \mathrm{E}-0$ $3.44636 \mathrm{E}$ 01 $-2.02786 \mathrm{E}-01$ $3.44646 \mathrm{E}$ 01 $-2.04370 \mathrm{E}-01$ $3.44657 \mathrm{E} 01 \quad-2.05963 \mathrm{E}-01$

$3.44667 \mathrm{E} \quad 01 \quad-2.07564 \mathrm{E}-01$ $3.44677 \mathrm{E}$ 01 $-2.09173 \mathrm{E}-01$ $3.44688 \mathrm{E}$ 01 $-2.10790 \mathrm{E}-01$ $3.44698 \mathrm{E}$ 01 $-2.12415 \mathrm{E}-01$ $3.44708 \mathrm{E}$ ol $-2.14049 \mathrm{E}-01$

$3.44718 \mathrm{E}$ 01 $-2.15691 \mathrm{E}-01$ $3.44728 \mathrm{E}$ 01 $-2.17341 \mathrm{E}-01$ $3.44738 \mathrm{E} \quad 01-2.19000 \mathrm{E}-01$ $3.44748 \mathrm{E}$ 0l $-2.20667 \mathrm{E}-01$ $3.44758 \mathrm{E} \quad 01 \quad-2.22342 \mathrm{E}-01$

$3.44768 \mathrm{E} \quad 01 \quad-2.24026 \mathrm{E}-01$ $3.44778 \mathrm{E}$ 01 $-2.25718 \mathrm{E}-01$ $3.44787 \mathrm{E}$ 01 $-2.27418 \mathrm{E}-01$ $3.44797 \mathrm{E}$ 01 $-2.29127 \mathrm{E}-01$ $3.44807 \mathrm{E}$ ol $-2.30845 \mathrm{E}-01$

$3.44816 \mathrm{E} \quad 01$

$3.44826 \mathrm{E} \quad 01$

$3.44835 \mathrm{E} \quad 01$

$3.44845 \mathrm{E} \quad 01$

$3.44854 \mathrm{E} \quad 01$

$-2.32571 \mathrm{E}-0$ $-2.34305 \mathrm{E}-01$ $-2.36048 \mathrm{E}-01$ $-2.37799 \mathrm{E}-01$

$3.44863 \mathrm{E} \quad 01$ $3.44872 \mathrm{E} \quad 01$ $3.44882 \mathrm{E} \quad 01$ $3.44891 \mathrm{E} 01$ $3.44900 \mathrm{E} 01$

$3.44909 \mathrm{E} \quad 01$ $3.44918 \mathrm{E} \quad 01$ $3.44927 \mathrm{E} \quad 01$ $3.44936 \mathrm{E} \quad 01$ $3.44945 \mathrm{E} \quad 01$

$3.44953 \mathrm{E} \quad 01$ $3.44962 \mathrm{E} \quad 01$ $3.44971 \mathrm{E} 01$ $3.44980 \mathrm{E} \quad 01$ $3.44988 \mathrm{E} 01$

$3.44997 \mathrm{E} \quad 01$ $3.45005 \mathrm{E} \quad 01$ $3.45014 \mathrm{E} \quad 01$ $3.45022 \mathrm{E} \quad 01$ $3.45031 \mathrm{E} 01$

$3.45039 \mathrm{E} \quad 01$

$3.45047 \mathrm{E} \quad 01$

$3.45056 \mathrm{E} \quad 01$

$3.45064 \mathrm{E} \quad 01$

$3.45072 \mathrm{E} \quad 01$

3.45080 E 01

$3.45088 \mathrm{E} \quad 01$

$3.45097 \mathrm{E}$ 01

$3.45104 \mathrm{E} \quad 01$

$3.45113 \mathrm{E} 01$

$3.45120 \mathrm{E} \quad 01$

$3.45128 \mathrm{E} 01$

$3.45136 \mathrm{E} \quad 01$

$3.45144 \mathrm{E} \quad 01$

$3.45152 \mathrm{E} \quad 01$

g-2

$\tan \delta_{-2}$

$3.31980 \mathrm{E} \quad 00$ $3.33792 \mathrm{E} \quad 00$ $3.35608 \mathrm{E} \quad 00$ $3.37430 \mathrm{E} \quad 00$ $3.39256 \mathrm{E} \quad 00$

$3.41087 \mathrm{E} \quad 00$ $3.42923 \mathrm{E} \quad 00$

$3.44764 \mathrm{E} \quad 00$

$3.46609 \mathrm{E} \quad 00$

$3.48459 \mathrm{E} \quad 00$

$3.50314 \mathrm{E} \quad 00$ $3.52174 \mathrm{E} \quad 00$ $3.54039 \mathrm{E} \quad 00$ $3.55908 \mathrm{E} \quad 00$ $3.57782 \mathrm{E} \quad 00$

$3.59661 \mathrm{E} \quad 00$ $3.61545 \mathrm{E} \quad 00$ $3.63433 \mathrm{E} 00$

$3.65326 \mathrm{E} \quad 00$

$3.67225 \mathrm{E} \quad 00$

$3.69127 \mathrm{E} \quad 00$ $3.71035 \mathrm{E} \quad 00$ $3.72947 \mathrm{E} \quad 00$ $3.74865 \mathrm{E} \quad 00$

$3.76787 \mathrm{E} \quad 00$

$3.78713 \mathrm{E} \quad 00$ $3.80645 \mathrm{E} \quad 00$ $3.82581 \mathrm{E} \quad 00$ $3.84522 \mathrm{E} \quad 00$ $3.86468 \mathrm{E} \quad 00$

$3.88418 \mathrm{E} \quad 00$ $3.90374 \mathrm{E} \quad 00$ $3.92334 \mathrm{E}$ 00 $3.94298 \mathrm{E} \quad 00$ $3.96268 \mathrm{E} \quad 00$

$3.98242 \mathrm{E} \quad 00$ 


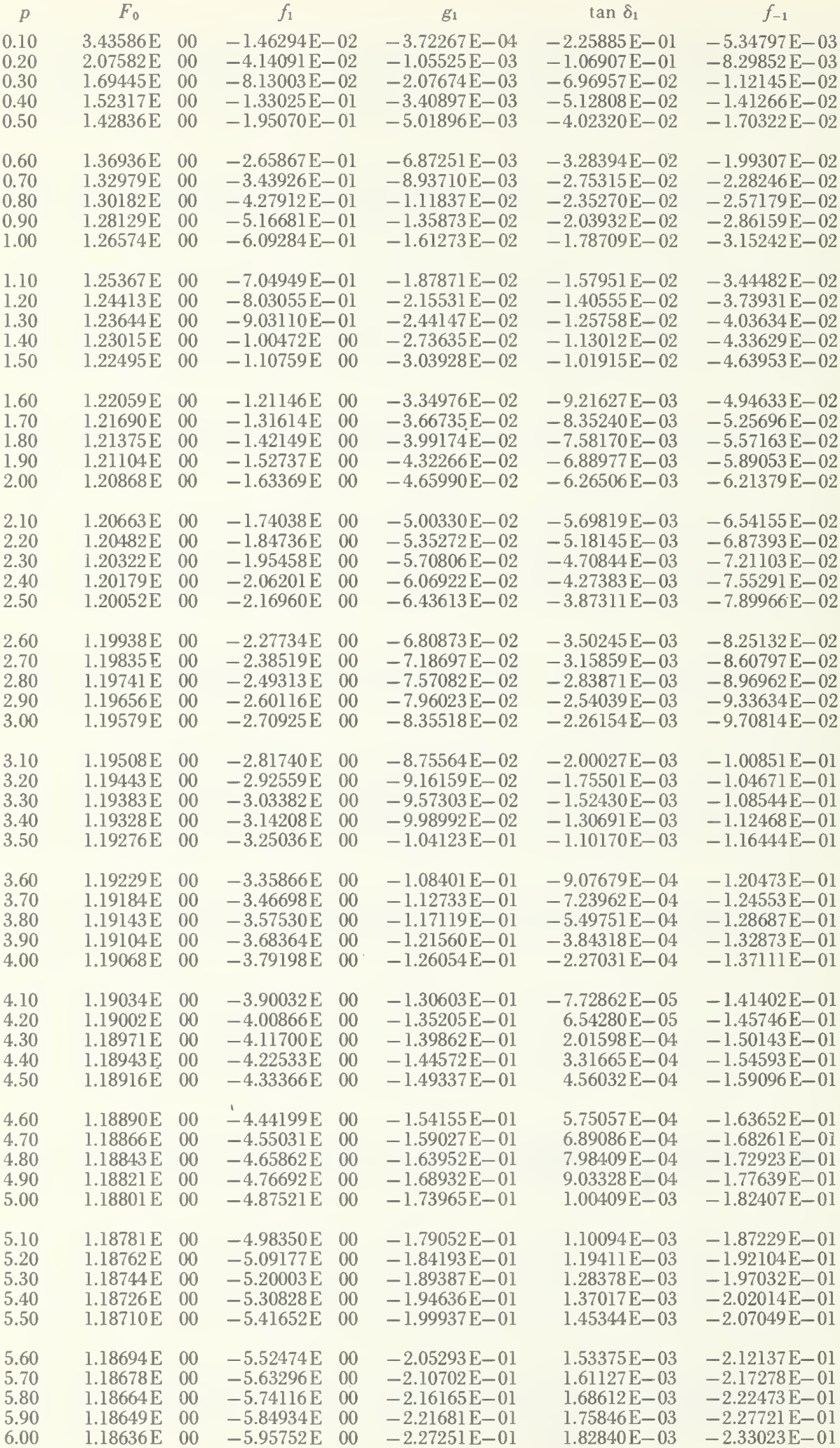

$g_{-1}$

$2.61489 \mathrm{E}-01$ $4.05023 \mathrm{E}-01$ $5.45731 \mathrm{E}-01$ $6.84690 \mathrm{E}-01$ $8.21440 \mathrm{E}-01$

$9.55689 \mathrm{E}-01$ $1.08735 \mathrm{E} \quad 00$ $1.21650 \mathrm{E} \quad 00$ $1.34330 \mathrm{E} \quad 00$ $1.46797 \mathrm{E} \quad 00$

$1.59073 \mathrm{E} \quad 00$ $1.71182 \mathrm{E} \quad 00$ $1.83143 \mathrm{E} \quad 00$ $1.94977 \mathrm{E} \quad 00$ $2.06700 \mathrm{E} \quad 00$

$2.18326 \mathrm{E} \quad 00$ $2.29868 \mathrm{E} \quad 00$ $2.41338 \mathrm{E} \quad 00$ $2.52744 \mathrm{E} \quad 00$ $2.64094 \mathrm{E} \quad 00$

$2.75395 \mathrm{E} \quad 00$ $2.86653 \mathrm{E} \quad 00$ $2.97873 \mathrm{E} \quad 00$ $3.09059 \mathrm{E} \quad 00$ $3.20215 \mathrm{E} \quad 00$

$3.31344 \mathrm{E} \quad 00$ $3.42449 \mathrm{E} \quad 00$ $3.53532 \mathrm{E} \quad 00$ $3.64596 \mathrm{E} \quad 00$ $3.75642 \mathrm{E} \quad 00$

$3.86672 \mathrm{E} \quad 00$ $3.97688 \mathrm{E} \quad 00$ $4.08690 \mathrm{E} \quad 00$ $4.19679 \mathrm{E} \quad 00$ $4.30658 \mathrm{E} \quad 00$

$4.41626 \mathrm{E} \quad 00$ $4.52584 \mathrm{E} \quad 00$ $4.63533 \mathrm{E} \quad 00$ $4.74474 \mathrm{E} \quad 00$ $4.85408 \mathrm{E} \quad 00$

$4.96334 \mathrm{E} \quad 00$ $5.07253 \mathrm{E} \quad 00$ $5.18165 \mathrm{E} \quad 00$ $5.29072 \mathrm{E} \quad 00$ $5.39973 \mathrm{E} \quad 00$

$5.50869 \mathrm{E} \quad 00$ $5.61760 \mathrm{E} \quad 00$ $5.72645 \mathrm{E} \quad 00$ $5.83527 \mathrm{E} \quad 00$ $5.94403 \mathrm{E} \quad 00$

$6.05276 \mathrm{E} \quad 00$ $6.16144 \mathrm{E} \quad 00$ $6.27009 \mathrm{E} \quad 00$ $6.37870 \mathrm{E} \quad 00$ $6.48727 \mathrm{E} \quad 00$

$6.59581 \mathrm{E} \quad 00$ $6.70432 \mathrm{E} \quad 00$ $6.81280 \mathrm{E} \quad 00$ $6.92124 \mathrm{E} \quad 00$ $7.02965 \mathrm{E} \quad 00$ $\tan \delta_{-1}$

$-3.91461 \mathrm{E} 00$ $-6.91789 \mathrm{E} \quad 00$ $-1.00608 \mathrm{E} 01$ $-1.31709 \mathrm{E} 01$ $-1.62135 \mathrm{E} 01$

$-1.91755 \mathrm{E} \quad 01$ $-2.20506 \mathrm{E} 01$ $-2.48363 \mathrm{E} 01$ $-2.75321 \mathrm{E} 01$ $-3.01391 \mathrm{E} 01$

$-3.26591 \mathrm{E} \quad 01$ $-3.50946 \mathrm{E}$ 01 $-3.74483 \mathrm{E}$ 01 $-3.97232 \mathrm{E} 01$ $-4.19225 \mathrm{E} 01$

$-4.40493 \mathrm{E} 01$ $-4.61065 \mathrm{E} \quad 01$ $-4.80973 \mathrm{E} 01$ $-5.00245 \mathrm{E} \quad 01$ $-5.18908 \mathrm{E} 01$

$-5.36990 \mathrm{E} \quad 01$ $-5.54516 \mathrm{E} 01$ $-5.71510 \mathrm{E} 01$ $-5.87994 \mathrm{E} 01$ $-6.03992 \mathrm{E} \quad 01$

$-6.19524 \mathrm{E} \quad 01$ $-6.34609 \mathrm{E} \quad 01$ $-6.49266 \mathrm{E} 01$ $-6.63512 \mathrm{E} 01$ $-6.77366 \mathrm{E} 01$

$-6.90842 \mathrm{E} 01$ $-7.03956 \mathrm{E} 01$ $-7.16722 \mathrm{E} 01$ $-7.29153 \mathrm{E} 01$ $-7.41264 \mathrm{E} \quad 01$

$-7.53065 \mathrm{E} \quad 01$ $-7.64569 \mathrm{E} 01$ $-7.75787 \mathrm{E}$ 0l $-7.86729 \mathrm{E} 01$ $-7.97406 \mathrm{E} 01$

$-8.07827 \mathrm{E} 01$ $-8.18001 \mathrm{E} \quad 01$ $-8.27938 \mathrm{E} 01$ $-8.37645 \mathrm{E} \quad 01$ $-8.47131 \mathrm{E} 01$

$-8.56402 \mathrm{E} 01$ $-8.65466 \mathrm{E}$ 01 $-8.74332 \mathrm{E}$ 0l $-8.83003 \mathrm{E} \quad 01$ $-8.91489 \mathrm{E} 01$

$-8.99793 \mathrm{E} 01$ $-9.07922 \mathrm{E} 01$ $-9.15883 \mathrm{E}$ 0l $-9.23679 \mathrm{E} 01$ $-9.31317 \mathrm{E} 01$ $-9.38801 \mathrm{E} 01$ $-9.46135 \mathrm{E} 01$ $-9.53326 \mathrm{E} 01$ $-9.60375 \mathrm{E} \quad 01$ $-9.67289 \mathrm{E} 01$ 
$Z=7 \quad A=14 \quad \rho=1.2 A^{1 / 3} \mathrm{~F}$

$f_{2}$

$-3.74751 \mathrm{E}-06$ $-2.07584 \mathrm{E}-05$ $-6.08898 \mathrm{E}-05$ $-1.32665 \mathrm{E}-04$ $-2.43046 \mathrm{E}-04$

$-3.97409 \mathrm{E}-04$ $-5.99702 \mathrm{E}-04$ $-8.52702 \mathrm{E}-04$ $-1.15828 \mathrm{E}-03$ $-1.51767 \mathrm{E}-03$

$-1.93161 \mathrm{E}-03$ $-2.40055 \mathrm{E}-03$ $-2.92474 \mathrm{E}-03$ $-3.50426 \mathrm{E}-03$ $-4.13916 \mathrm{E}-03$

$-4.82938 \mathrm{E}-03$ $-5.57488 \mathrm{E}-03$ $-6.37558 \mathrm{E}-03$ $-7.23141 \mathrm{E}-03$ $-8.14227 \mathrm{E}-03$

$-9.10810 \mathrm{E}-03$ $-1.01288 \mathrm{E}-02$ $-1.12043 \mathrm{E}-02$ $-1.23346 \mathrm{E}-02$ $-1.35196 \mathrm{E}-02$

$-1.47592 \mathrm{E}-02$ $-1.60535 \mathrm{E}-02$ $-1.74022 \mathrm{E}-02$ $-1.88055 \mathrm{E}-02$ $-2.02632 \mathrm{E}-02$

$-2.17754 \mathrm{E}-02$ $-2.33420 \mathrm{E}-02$ $-2.49629 \mathrm{E}-02$ $-2.66382 \mathrm{E}-02$ $-2.83678 \mathrm{E}-02$

$-3.01518 \mathrm{E}-02$ $-3.19899 \mathrm{E}-02$ $-3.38824 \mathrm{E}-02$ $-3.58291 \mathrm{E}-02$ $-3.78300 \mathrm{E}-02$

$-3.98851 \mathrm{E}-02$ $-4.19944 \mathrm{E}-02$ $-4.41579 \mathrm{E}-02$ $-4.63756 \mathrm{E}-02$ $-4.86474 \mathrm{E}-02$

$-5.09733 \mathrm{E}-02$ $-5.33534 \mathrm{E}-02$ $-5.57876 \mathrm{E}-02$ $-5.82759 \mathrm{E}-02$ $-6.08183 \mathrm{E}-02$

$-6.34147 \mathrm{E}-02$ $-6.60653 \mathrm{E}-02$ $-6.87699 \mathrm{E}-02$ $-7.15285 \mathrm{E}-02$ $-7.43412 \mathrm{E}-02$

$-7.72079 \mathrm{E}-02$

$-8.01287 \mathrm{E}-02$

$-8.31035 \mathrm{E}-02$

$-8.61323 \mathrm{E}-02$

$-8.92151 \mathrm{E}-02$ $g_{2}$

$-5.50171 \mathrm{E}-08$ $-3.05214 \mathrm{E}-07$ $-8.97484 \mathrm{E}-07$ $-1.96197 \mathrm{E}-06$ $-3.60932 \mathrm{E}-06$

$-5.93035 \mathrm{E}-06$ $-8.99803 \mathrm{E}-06$ $-1.28707 \mathrm{E}-05$ $-1.75956 \mathrm{E}-05$ $-2.32116 \mathrm{E}-05$

$-2.97520 \mathrm{E}-05$ $-3.72465 \mathrm{E}-05$ $-4.57218 \mathrm{E}-05$ $-5.52034 \mathrm{E}-05$ $-6.57155 \mathrm{E}-05$

$-7.72821 \mathrm{E}-05$ $-8.99264 \mathrm{E}-05$ $-1.03672 \mathrm{E}-04$ $-1.18541 \mathrm{E}-04$ $-1.34559 \mathrm{E}-04$

$-1.51747 \mathrm{E}-04$ $-1.70129 \mathrm{E}-04$ $-1.89729 \mathrm{E}-04$ $-2.10569 \mathrm{E}-04$ $-2.32675 \mathrm{E}-04$

$-2.56068 \mathrm{E}-04$ $-2.80774 \mathrm{E}-04$ $-3.06815 \mathrm{E}-04$ $-3.34215 \mathrm{E}-04$ $-3.62999 \mathrm{E}-04$.

$-3.93189 \mathrm{E}-04$ $-4.24811 \mathrm{E}-04$ $-4.57888 \mathrm{E}-04$ $-4.92443 \mathrm{E}-04$ -5.28501 E-04

$-5.66087 \mathrm{E}-04$ $-6.05223 \mathrm{E}-04$ $-6.45934 \mathrm{E}-04$ $-6.88244 \mathrm{E}-04$ $-7.32177 \mathrm{E}-04$

$-7.77758 \mathrm{E}-04$ $-8.25009 \mathrm{E}-04$ $-8.73956 \mathrm{E}-04$ $-9.24623 \mathrm{E}-04$ $-9.77033 \mathrm{E}-04$

$-1.03121 \mathrm{E}-03$ $-1.08718 \mathrm{E}-03$ $-1.14497 \mathrm{E}-03$ $-1.20459 \mathrm{E}-03$ $-1.26608 \mathrm{E}-03$

$-1.32946 \mathrm{E}-03$ $-1.39475 \mathrm{E}-03$ $-1.46198 \mathrm{E}-03$ $-1.53116 \mathrm{E}-03$ $-1.60234 \mathrm{E}-03$

$-1.67552 \mathrm{E}-03$ $-1.75073 \mathrm{E}-03$ $-1.82801 \mathrm{E}-03$ $-1.90736 \mathrm{E}-03$ $-1.98882 \mathrm{E}-03$ $\tan \delta_{2}$

$f_{-2}$

$1.94277 \mathrm{E} \quad 00 \quad-8.53559 \mathrm{E}-07$ $4.11403 \mathrm{E} \quad 00 \quad-2.43600 \mathrm{E}-06$ $6.14817 \mathrm{E} \quad 00 \quad-4.85524 \mathrm{E}-06$ $8.05829 \mathrm{E} \quad 00 \quad-8.10734 \mathrm{E}-06$ $9.83124 \mathrm{E} \quad 00 \quad-1.21880 \mathrm{E}-05$

$1.14583 \mathrm{E} \quad 01$ $1.29379 \mathrm{E} \quad 01$ $1.42744 \mathrm{E} \quad 01$ $1.54762 \mathrm{E} \quad 01$ $1.65540 \mathrm{E} 01$

$1.75196 \mathrm{E} \quad 01$ $1.83847 \mathrm{E} \quad 01$ $1.91605 \mathrm{E} \quad 01$ $1.98573 \mathrm{E} \quad 01$ $2.04844 \mathrm{E} \quad 01$

$2.10502 \mathrm{E} \quad 01$ $2.15618 \mathrm{E} 01$ $2.20258 \mathrm{E} \quad 01$ $2.24476 \mathrm{E} \quad 01$ $2.28322 \mathrm{E} \quad 01$

$2.31838 \mathrm{E} \quad 01$ $2.35061 \mathrm{E} 01$ $2.38022 \mathrm{E} \quad 01$ $2.40750 \mathrm{E} \quad 01$ $2.43269 \mathrm{E} \quad 01$

$2.45600 \mathrm{E} \quad 01$ $2.47763 \mathrm{E} \quad 01$ $2.49773 \mathrm{E} \quad 01$ $2.51646 \mathrm{E} \quad 01$ $2.53393 \mathrm{E} 01$

$2.55027 \mathrm{E} \quad 01$ $2.56558 \mathrm{E} \quad 01$ $2.57994 \mathrm{E} \quad 01$ $2.59344 \mathrm{E} \quad 01$ $2.60615 \mathrm{E} 01$

$2.61813 \mathrm{E} \quad 01$ $2.62944 \mathrm{E} \quad 01$ $2.64013 \mathrm{E} \quad 01$ $2.65026 \mathrm{E} \quad 01$ $2.65985 \mathrm{E} \quad 01$

$2.66896 \mathrm{E} \quad 01$ $2.67761 \mathrm{E} \quad 01$ $2.68585 \mathrm{E} \quad 01$ $2.69368 \mathrm{E} \quad 01$

$2.70116 \mathrm{E} \quad 01$

$2.70829 \mathrm{E} \quad 01$ $2.71510 \mathrm{E} \quad 01$ $2.72161 \mathrm{E} \quad 01$ $2.72784 \mathrm{E} \quad 01$ $2.73381 \mathrm{E} \quad 01$

$2.73952 \mathrm{E} \quad 01$ $2.74501 \mathrm{E} \quad 01$ $2.75028 \mathrm{E} \quad 01$

$2.75534 \mathrm{E} \quad 01$

$2.76020 \mathrm{E} \quad 01$

$2.76488 \mathrm{E} \quad 01$ $2.76939 \mathrm{E} \quad 01$ $2.77373 \mathrm{E} \quad 01$ $2.77792 \mathrm{E} \quad 01$ $2.78195 \mathrm{E} \quad 01$
$-1.70946 \mathrm{E}-05$ $-2.28271 \mathrm{E}-05$ $-2.93897 \mathrm{E}-05$ $-3.67899 \mathrm{E}-05$ $-4.50390 \mathrm{E}-05$

$-5.41511 \mathrm{E}-05$ $-6.41430 \mathrm{E}-05$ $-7.50332 \mathrm{E}-05$ $-8.68417 \mathrm{E}-05$ $-9.95896 \mathrm{E}-05$

$-1.13299 \mathrm{E}-04$ $-1.27992 \mathrm{E}-04$ $-1.43693 \mathrm{E}-04$ $-1.60424 \mathrm{E}-04$ $-1.78209 \mathrm{E}-04$

$-1.97072 \mathrm{E}-04$ $-2.17038 \mathrm{E}-04$ $-2.38129 \mathrm{E}-04$ $-2.60371 \mathrm{E}-04$ $-2.83787 \mathrm{E}-04$

$-3.084 .01 \mathrm{E}-04$ $-3.34239 \mathrm{E}-04$ $-3.61324 \mathrm{E}-04$ $-3.89681 \mathrm{E}-04$ $-4.19333 \mathrm{E}-04$

$-4.50306 \mathrm{E}-04$ $-4.82623 \mathrm{E}-04$ $-5.16309 \mathrm{E}-04$ $-5.51388 \mathrm{E}-04$ $-5.87884 \mathrm{E}-04$

$-6.25822 \mathrm{E}-04$ $-6.65226 \mathrm{E}-04$ $-7.06121 \mathrm{E}-04$ $-7.48529 \mathrm{E}-04$ $-7.92477 \mathrm{E}-04$

$-8.37988 \mathrm{E}-04$ $-8.85086 \mathrm{E}-04$ $-9.33796 \mathrm{E}-04$ $-9.84142 \mathrm{E}-04$ $-1.03615 \mathrm{E}-03$

$-1.08984 \mathrm{E}-03$ $-1.14524 \mathrm{E}-03$ $-1.20237 \mathrm{E}-03$ $-1.26126 \mathrm{E}-03$ $-1.32193 \mathrm{E}-03$

$-1.38440 \mathrm{E}-03$ $-1.44871 \mathrm{E}-03$ $-1.51487 \mathrm{E}-03$ $-1.58291 \mathrm{E}-03$ $-1.65285 \mathrm{E}-03$

$-1.72472 \mathrm{E}-03$ $-1.79853 \mathrm{E}-03$ $-1.87433 \mathrm{E}-03$ $-1.95212 \mathrm{E}-03$ $-2.03194 \mathrm{E}-03$

E]cetron

$\tan \delta_{2}$

$7.30489 \mathrm{E}-0.5$ $2.08082 \mathrm{E}-04$ $4.13449 \mathrm{E}-04$ $6.87487 \mathrm{E}-04$ $1.02817 \mathrm{E}-03$

$1.43335 \mathrm{E}-03$ $1.90100 \mathrm{E}-03$ $2.42934 \mathrm{E}-0.3$ $3.01684 \mathrm{E}-03$

$3.66230 \mathrm{E}-03$

$4.36475 \mathrm{E}-03$ $5.12344 \mathrm{E}-03$ $5.93780 \mathrm{E}-03$ $6.80741 \mathrm{E}-03$ $7.73195 \mathrm{E}-03$

$8.71116 \mathrm{E}-03$ $9.74487 \mathrm{E}-03$

$1.08329 \mathrm{E}-02$

$1.19753 \mathrm{E}-02$

$1.31718 \mathrm{E}-02$

$1.44224 \mathrm{E}-02$ $1.57271 \mathrm{E}-02$ $1.70859 \mathrm{E}-02$ $1.84987 \mathrm{E}-02$ $1.99656 \mathrm{E}-02$

$2.14864 \mathrm{E}-02$ $2.30613 \mathrm{E}-02$ $2.46901 \mathrm{E}-02$ $2.63729 \mathrm{E}-02$ $2.81097 \mathrm{E}-02$

$2.99005 \mathrm{E}-02$ $3.17452 \mathrm{E}-02$ $3.36439 \mathrm{E}-02$ $3.55966 \mathrm{E}-02$ $3.76033 \mathrm{E}-02$

$3.96639 \mathrm{E}-02$ $4.17785 \mathrm{E}-02$ $4.39471 \mathrm{E}-02$ $4.61696 \mathrm{E}-02$ $4.84461 \mathrm{E}-02$

$5.07766 \mathrm{E}-02$ $5.31610 \mathrm{E}-02$ $5.55994 \mathrm{E}-02$ $5.80918 \mathrm{E}-02$ $6.06381 \mathrm{E}-02$

$6.32384 \mathrm{E}-02$ 
ELECTRON RADIAL FUNCTIONS

Electron

\begin{tabular}{|c|c|c|c|c|c|}
\hline$p$ & $F_{0}$ & & $f_{1}$ & & $g_{1}$ \\
\hline 6.10 & $1.18623 \mathrm{E}$ & 00 & $-6.06568 E$ & 00 & $-2.32875 \mathrm{E}-01$ \\
\hline & $1.18610 \mathrm{E}$ & 00 & $-6.17383 \mathrm{E}$ & 00 & $-2.38552 \mathrm{E}-01$ \\
\hline 6.30 & $18598 \mathrm{E}$ & 00 & $-6.28196 \mathrm{E}$ & 00 & $44283 \mathrm{E}-01$ \\
\hline 6.40 & $586 \mathrm{E}$ & 00 & $-6.39008 E$ & 00 & $.50067 \mathrm{E}-01$ \\
\hline 6.50 & $1.18574 \mathrm{E}$ & 00 & $-6.49818 \mathrm{E}$ & 00 & $.55905 \mathrm{E}-01$ \\
\hline 6.60 & $1.18563 \mathrm{E}$ & 00 & $-6.60627 \mathrm{E}$ & 00 & $-2.61796 \mathrm{E}-01$ \\
\hline 6.70 & $53 \mathrm{E}$ & 00 & $1435 \mathrm{E}$ & 00 & $67741 \mathrm{E}-01$ \\
\hline 6.80 & $12 \mathrm{E}$ & 00 & $-6.82241 \mathrm{E}$ & 00 & $.73740 \mathrm{E}-01$ \\
\hline 6.90 & $32 \mathrm{E}$ & 00 & $-6.93046 \mathrm{E}$ & 00 & $-2.79792 \mathrm{E}-01$ \\
\hline 7.00 & $23 \mathrm{E}$ & 00 & $-7.03849 \mathrm{E}$ & 00 & $-2.85898 \mathrm{E}-01$ \\
\hline 7.10 & $1.18513 \mathrm{E}$ & 00 & $-7.14651 \mathrm{E}$ & 00 & $-2.92057 \mathrm{E}-01$ \\
\hline 7.20 & $04 \mathrm{E}$ & 00 & $5451 \mathrm{E}$ & 00 & $.98269 \mathrm{E}-01$ \\
\hline 7.30 & $95 \mathrm{E}$ & 00 & $-7.36250 \mathrm{E}$ & 00 & $-3.04535 \mathrm{E}-01$ \\
\hline 7.40 & $36 \mathrm{E}$ & 00 & $-7.47048 \mathrm{E}$ & 00 & $-3.10855 \mathrm{E}-01$ \\
\hline 7.50 & $78 \mathrm{E}$ & 00 & $57843 \mathrm{E}$ & 00 & $-3.17228 \mathrm{E}-01$ \\
\hline 7.60 & $1.18469 \mathrm{E}$ & 00 & $-7.68638 \mathrm{E}$ & 00 & $-3.23654 \mathrm{E}-01$ \\
\hline 7.70 & $461 \mathrm{E}$ & 00 & $9431 \mathrm{E}$ & 00 & - \\
\hline 7.80 & $54 \mathrm{E}$ & 00 & $0222 \mathrm{E}$ & 00 & $.36668 \mathrm{E}-01$ \\
\hline 7.90 & $446 \mathrm{E}$ & 00 & $1012 \mathrm{E}$ & 00 & $-3.43255 \mathrm{E}-01$ \\
\hline 8.00 & $1.18439 \mathrm{E}$ & 00 & $1800 \mathrm{E}$ & 00 & $-3.49895 \mathrm{E}-01$ \\
\hline 8.10 & $1.18431 \mathrm{E}$ & 00 & $-8.22587 \mathrm{E}$ & 00 & $-3.56589 \mathrm{E}-01$ \\
\hline 8.20 & $18424 \mathrm{E}$ & 00 & $3373 \mathrm{E}$ & 00 & $-3.63336 \mathrm{E}-$ \\
\hline 8.30 & $417 \mathrm{E}$ & 00 & $156 \mathrm{E}$ & 00 & $-3.70136 \mathrm{E}-$ \\
\hline 8.40 & $11 \mathrm{E}$ & 00 & $4939 \mathrm{E}$ & 00 & $76990 \mathrm{E}-01$ \\
\hline 8.50 & $1.18404 \mathrm{E}$ & 00 & $5719 \mathrm{E}$ & 00 & $-3.83898 \mathrm{E}$ \\
\hline 8.60 & $97 \mathrm{E}$ & 00 & $198 \mathrm{E}$ & 00 & $858 E-01$ \\
\hline 8.70 & $91 \mathrm{E}$ & 00 & $276 \mathrm{E}$ & 00 & $7873 \mathrm{E}-$ \\
\hline 8.80 & $85 \mathrm{E}$ & 00 & $052 \mathrm{E}$ & 00 & $940 \mathrm{E}-$ \\
\hline 8.90 & $379 \mathrm{E}$ & 00 & $-9.08827 \mathrm{E}$ & 00 & $-4.12061 \mathrm{E}-$ \\
\hline 9.00 & $1.18373 \mathrm{E}$ & 00 & $600 \mathrm{E}$ & 00 & $9235 \mathrm{E}-$ \\
\hline 9.10 & $67 \mathrm{E}$ & 00 & $0371 \mathrm{E}$ & 00 & $-4.26463 \mathrm{E}-01$ \\
\hline 9.20 & $1 \mathrm{E}$ & 00 & & 00 & $3 \mathrm{E}-$ \\
\hline 9.30 & $356 \mathrm{E}$ & 00 & $1909 \mathrm{E}$ & 00 & $1078 \mathrm{E}-$ \\
\hline 9.40 & $50 \mathrm{E}$ & 00 & $676 \mathrm{E}$ & 00 & $3465 \mathrm{E}-$ \\
\hline 9.50 & $1.18345 \mathrm{E}$ & 00 & $-9.73441 \mathrm{E}$ & 00 & $-4.55906 \mathrm{E}-01$ \\
\hline 9.60 & $1.18340 \mathrm{E}$ & 00 & -9 & 00 & $0 E-01$ \\
\hline 9.70 & $34 \mathrm{E}$ & 00 & $67 \mathrm{E}$ & 00 & - \\
\hline 9.80 & $329 \mathrm{E}$ & 00 & $-1.00573 \mathrm{E}$ & 01 & $-4.78548 \mathrm{E}-01$ \\
\hline 9.90 & $24 E$ & 00 & $649 \mathrm{E}$ & 01 & $1.86202 \mathrm{E}$ \\
\hline 10.00 & $19 \mathrm{E}$ & 00 & $724 \mathrm{E}$ & 01 & $-4.93909 \mathrm{E}-01$ \\
\hline .10 & $15 \mathrm{E}$ & 00 & $-1 .(\mathrm{r}-1)$ & 01 & $69 \mathrm{E}-01$ \\
\hline 20 & $10 \mathrm{E}$ & 00 & $4875 \mathrm{E}$ & 01 & $-5.09483 \mathrm{E}-$ \\
\hline & $05 \mathrm{E}$ & 00 & -1 . & 01 & - \\
\hline & & 00 & & 01 & \\
\hline 10.50 & $1.18296 \mathrm{E}$ & 00 & & 01 & $532943 \mathrm{~F}$ \\
\hline .60 & $92 \mathrm{E}$ & 00 & $-1.09175 \mathrm{E}$ & 01 & $-5.41270 \mathrm{E}-01$ \\
\hline & $37 \mathrm{E}$ & 00 & & 01 & \\
\hline & & 00 & & 01 & -5 . \\
\hline & & 00 & -1 & 01 & -5 \\
\hline & $274 \mathrm{E}$ & 00 & -1 & 01 & $3008 \mathrm{~F} \quad 01$ \\
\hline 11.10 & $1.18270 \mathrm{E}$ & 00 & $-1.14547 \mathrm{E}$ & 01 & $-5.82201 \mathrm{E}-01$ \\
\hline & & 00 & & 01 & \\
\hline & $1.18262 \mathrm{E}$ & 00 & $-1.16694 \mathrm{E}$ & 01 & -5.989 \\
\hline & $258 \mathrm{E}$ & 00 & $7768 \mathrm{E}$ & 01 & $7397 \mathrm{E}-01$ \\
\hline 5 & $254 \mathrm{E}$ & 00 & $18841 \mathrm{E}$ & 01 & $-6.15902 \mathrm{E}-01$ \\
\hline & $1.18251 \mathrm{E}$ & 00 & -1.199 & 01 & $-6.24460 \mathrm{E}-01$ \\
\hline & $247 \mathrm{E}$ & 00 & $-1.20987 \mathrm{E}$ & 0 & $-6.33071 \mathrm{E}-$ \\
\hline & $243 E$ & 00 & $-1.22060 \mathrm{E}$ & 01 & $-6.41735 \mathrm{E}-01$ \\
\hline & $1.18239 \mathrm{E}$ & 00 & $-1.23132 \mathrm{E}$ & 01 & $-6.50453 \mathrm{E}-01$ \\
\hline 2.00 & $1.18236 \mathrm{E}$ & 00 & $-1.24205 \mathrm{E}$ & 01 & $-6.59223 \mathrm{E}-01$ \\
\hline
\end{tabular}

$\tan \delta_{1}$

$1.89605 \mathrm{E}-03$

$1.96154 \mathrm{E}-03$

$2.02494 \mathrm{E}-03$

$2.08638 \mathrm{E}-03$

$2.14592 \mathrm{E}-03$

$2.20367 \mathrm{E}-03$

$2.25968 \mathrm{E}-03$

$2.31406 \mathrm{E}-03$

$2.36684 \mathrm{E}-03$

$2.41813 \mathrm{E}-03$

$2.46796 \mathrm{E}-03$

$2.51641 \mathrm{E}-03$

$2.56353 \mathrm{E}-03$

$2.60936 \mathrm{E}-03$

$2.65397 \mathrm{E}-03$

$2.69740 \mathrm{E}-03$

$2.73969 \mathrm{E}-03$

$2.78088 \mathrm{E}-03$

$2.82103 \mathrm{E}-03$

$2.86016 \mathrm{E}-03$

$2.89832 \mathrm{E}-03$

$2.93554 \mathrm{E}-03$

$2.97184 \mathrm{E}-03$

$3.00727 \mathrm{E}-03$

$3.04186 \mathrm{E}-03$

$3.07563 \mathrm{E}-03$

$3.10861 \mathrm{E}-03$

$3.14083 \mathrm{E}-03$

$3.17231 \mathrm{E}-03$

$3.20309 \mathrm{E}-03$

$3.23317 \mathrm{E}-03$

$3.26259 \mathrm{E}-03$

$3.29135 \mathrm{E}-03$

$3.31949 \mathrm{E}-03$

$3.34702 \mathrm{E}-03$

$3.37398 \mathrm{E}-03$

$3.40035 \mathrm{E}-03$

$3.42617 \mathrm{E}-03$

$3.45146 \mathrm{E}-03$

$3.47622 \mathrm{E}-03$

$3.50048 \mathrm{E}-03$

$3.52425 \mathrm{E}-03$

$3.54753 \mathrm{E}-03$

$3.57037 \mathrm{E}-03$

$3.59274 \mathrm{E}-03$

$3.61468 \mathrm{E}-03$

$3.63619 \mathrm{E}-03$

$3.65728 \mathrm{E}-03$

$3.67797 \mathrm{E}-03$

$3.69827 \mathrm{E}-03$

$3.71819 \mathrm{E}-03$

$3.73774 \mathrm{E}-03$

$3.75691 \mathrm{E}-03$

$3.77574 \mathrm{E}-03$

3.79423 E-03

$3.81238 \mathrm{E}-03$

$3.83019 \mathrm{E}-03$

$3.84770 \mathrm{E}-03$

$3.86488 \mathrm{E}-03$

$3.88178 \mathrm{E}-03$ $f_{-1}$

$-2.38378 \mathrm{E}-01$

$-2.43786 \mathrm{E}-01$

$-2.49248 \mathrm{E}-01$

$-2.54763 \mathrm{E}-01$

$-2.60332 \mathrm{E}-01$

$-2.65954 \mathrm{E}-01$

$-2.71629 \mathrm{E}-01$

$-2.77358 \mathrm{E}-01$

$-2.83140 \mathrm{E}-01$

$-2.88975 \mathrm{E}-01$

$-2.94864 \mathrm{E}-01$

$-3.00807 \mathrm{E}-01$

$-3.06802 \mathrm{E}-01$

$-3.12851 \mathrm{E}-01$

$-3.18954 \mathrm{E}-01$

$-3.25110 \mathrm{E}-01$

$-3.31319 \mathrm{E}-01$

$-3.37582 \mathrm{E}-01$

$-3.43898 \mathrm{E}-01$

$-3.50267 \mathrm{E}-01$

$-3.56690 \mathrm{E}-01$

$-3.63166 \mathrm{E}-01$

$-3.69696 \mathrm{E}-01$

$-3.76279 \mathrm{E}-01$

-3.82915 E-01

$-3.89604 \mathrm{E}-01$

$-3.96347 \mathrm{E}-01$

$-4.03144 \mathrm{E}-01$

$-4.09993 \mathrm{E}-01$

$-4.16896 \mathrm{E}-01$

$-4.23852 \mathrm{E}-01$

$-4.30862 \mathrm{E}-01$

$-4.37925 \mathrm{E}-01$

$-4.45041 \mathrm{E}-01$

$-4.52210 \mathrm{E}-01$

$-4.59433 \mathrm{E}-01$

$-4.66709 \mathrm{E}-01$

$-4.74038 \mathrm{E}-01$

$-4.81421 \mathrm{E}-01$

$-4.88856 \mathrm{E}-01$

$-4.96345 \mathrm{E}-01$

$-5.03888 \mathrm{E}-01$

$-5.11483 \mathrm{E}-01$

$-5.19132 \mathrm{E}-01$

$-5.26834 \mathrm{E}-01$

$-5.34589 \mathrm{E}-01$

$-5.42397 \mathrm{E}-01$

$-5.50259 \mathrm{E}-01$

$-5.58173 \mathrm{E}-01$

$-5.66141 \mathrm{E}-01$

$-5.74162 \mathrm{E}-01$

$-5.82236 \mathrm{E}-01$

$-5.90364 \mathrm{E}-01$

$-5.98544 \mathrm{E}-01$

$-6.06777 \mathrm{E}-01$

$-6.15064 \mathrm{E}-01$

$-6.23404 \mathrm{E}-01$

$-6.31797 \mathrm{E}-01$

$-6.40243 \mathrm{E}-01$

$-6.48742 \mathrm{E}-01$ g-1

$7.13804 \mathrm{E} \quad 00$

$7.24639 \mathrm{E} \quad 00$

$7.35472 \mathrm{E} \quad 00$

$7.46302 \mathrm{E} \quad 00$

$7.57129 \mathrm{E} \quad 00$

$7.67954 \mathrm{E} \quad 00$

$7.78776 \mathrm{E} \quad 00$

$7.89596 \mathrm{E} \quad 00$

$8.00414 \mathrm{E} \quad 00$

$8.11229 \mathrm{E} \quad 00$

$8.22042 \mathrm{E} \quad 00$

$8.32852 \mathrm{E} \quad 00$

$8.43660 \mathrm{E} \quad 00$

$8.54466 \mathrm{E} \quad 00$

$8.65269 \mathrm{E} \quad 00$

$8.76071 \mathrm{E} \quad 00$

$8.86870 \mathrm{E} \quad 00$

$8.97668 \mathrm{E} 00$

$9.08463 \mathrm{E} \quad 00$

$9.19256 \mathrm{E} \quad 00$

$9.30047 \mathrm{E} \quad 00$

$9.40837 \mathrm{E} \quad 00$

$9.51624 \mathrm{E} \quad 00$

$9.62409 \mathrm{E} \quad 00$

$9.73192 \mathrm{E} \quad 00$

$9.83973 \mathrm{E} \quad 00$

$9.94752 \mathrm{E} \quad 00$

$1.00553 \mathrm{E} \quad 01$

$1.01631 \mathrm{E} 01$

$1.02708 \mathrm{E} \quad 01$

$1.03785 \mathrm{E} \quad 01$

$1.04862 \mathrm{E} \quad 01$

$1.05939 \mathrm{E} \quad 01$

$1.07015 \mathrm{E} \quad 01$

$1.08092 \mathrm{E} 01$

$1.09168 \mathrm{E} \quad 01$

$1.10244 \mathrm{E} \quad 01$

$1.11320 \mathrm{E} 01$

$1.12396 \mathrm{E} \quad 01$

$1.13471 \mathrm{E} 01$

$1.14546 \mathrm{E} \quad 01$

$1.15622 \mathrm{E} \quad 01$

$1.16697 \mathrm{E} \quad 01$

$1.17771 \mathrm{E} 01$

$1.18846 \mathrm{E} \quad 01$

$1.19920 \mathrm{E} \quad 0$

$1.20995 \mathrm{E} \quad 01$

$1.22069 \mathrm{E} 01$

$1.23143 \mathrm{E} \quad 01$

$1.24216 \mathrm{E} \quad 01$

$1.25290 \mathrm{E} \quad 01$

$1.26363 \mathrm{E} 01$

$1.27436 \mathrm{E} \quad 01$

$1.28509 \mathrm{E} 01$

$1.29582 \mathrm{E} 01$

$1.30655 \mathrm{E} \quad 01$

$1.31727 \mathrm{E} \quad 0$

$1.32800 \mathrm{E} \quad 01$

$1.33872 \mathrm{E} 01$

$1.34944 \mathrm{E} \quad 01$ $\tan \delta_{-1}$

$-9.74071 \mathrm{E} \quad 01$

$-9.80724 \mathrm{E} 01$

$-9.87253 \mathrm{E} 01$

$-9.93662 \mathrm{E} 01$

$-9.99953 \mathrm{E} 01$

$-1.00613 \mathrm{E} \quad 02$

$-1.01220 \mathrm{E} \quad 02$

-1.01815 E 02

$-1.02401 \mathrm{E} 02$

$-1.02976 \mathrm{E} 02$

$-1.03541 \mathrm{E} \quad 02$

$-1.04097 \mathrm{E} \quad 02$

$-1.04643 \mathrm{E} 02$

-1.05180 E 02

$-1.05708 \mathrm{E} 02$

$-1.06227 \mathrm{E} \quad 02$ $-1.06738 \mathrm{E} 02$ $-1.07241 \mathrm{E} 02$ $-1.07736 \mathrm{E} \quad 02$ $-1.08223 \mathrm{E} 02$

-1.08702 E 02 $-1.09174 \mathrm{E} \quad 02$ $-1.09638 \mathrm{E} 02$ $-1.10096 \mathrm{E} 02$ $\begin{array}{ll}-1.10546 \mathrm{E} & 02\end{array}$

$-1.10990 \mathrm{E} \quad 02$ $-1.11428 \mathrm{E} \quad 02$ $-1.11858 \mathrm{E} 02$ $-1.12283 \mathrm{E} \quad 02$ -1.12701 E 02

$-1.13114 \mathrm{E} 02$ $-1.13521 \mathrm{E} \quad 02$ $-1.13921 \mathrm{E} 02$ $-1.14317 \mathrm{E} \quad 02$ -1.14707 E 02

$-1.15091 \mathrm{E} \quad 02$ $-1.15471 \mathrm{E} \quad 02$ $-1.15845 \mathrm{E} \quad 02$ $-1.16214 \mathrm{E} 02$ $-1.16579 \mathrm{E} 02$

$-1.16939 \mathrm{E} \quad 02$ $-1.17294 \mathrm{E} 02$ $-1.17644 \mathrm{E} 02$ $-1.17990 \mathrm{E} 02$ $-1.18332 \mathrm{E} 02$

$-1.18669 \mathrm{E} 02$ $-1.19002 \mathrm{E} \quad 02$ $-1.19331 \mathrm{E} 02$ $-1.19656 \mathrm{E} \quad 02$ -1.19979 E 02

$-1.20295 \mathrm{E} \quad 02$ $-1.20608 \mathrm{E} \quad 02$ $-1.20918 \mathrm{E} 02$ $-1.21225 \mathrm{E} 02$ $-1.21527 \mathrm{E} \quad 02$

$-1.21826 \mathrm{E} \quad 02$ $-1.22122 \mathrm{E} 02$ $-1.22415 \mathrm{E} \quad 02$ $-1.22704 \mathrm{E} 02$ $-1.22990 \mathrm{E} \quad 02$ 
ELECTRON RADIAL FUNCTIONS

$Z=7 \quad A=14 \quad \rho=1.2 A^{1 / 3} F$

Electron

$\bar{F}$

6.10

6.20

6.30

6.40

6.50

6.60

6.70

6.80

6.90

7.00

7.10

7.20

7.30

7.40

7.50

7.60

7.70

7.80

7.90

8.00

8.10

8.20

8.30

8.40

8.50

8.60

8.70

8.80

8.90

9.00

9.10

9.20

9.30

9.40

9.50

9.60

9.70

9.80

9.90

10.00

10.10

10.20

10.30

10.40

10.50

10.60

10.70

10.80

10.90

11.00

11.10

11.20

11.30

11.40

11.50

11.60

11.70

11.80

11.90

12.00 $f_{2}$

$6.85451 \mathrm{E}-05$ $7.08047 \mathrm{E}-05$ $7.31009 \mathrm{E}-05$ $7.54338 \mathrm{E}-05$ $7.78035 \mathrm{E}-05$

$8.02098 \mathrm{E}-05$ $8.26528 \mathrm{E}-05$ $8.51325 \mathrm{E}-05$ $8.76490 \mathrm{E}-05$ $9.02020 \mathrm{E}-05$

$9.27918 \mathrm{E}-05$ $9.54183 \mathrm{E}-05$ $9.80814 \mathrm{E}-05$ $1.00781 \mathrm{E}-04$ $1.03518 \mathrm{E}-04$

$1.06291 \mathrm{E}-04$. $1.09101 \mathrm{E}-04$ $1.11947 \mathrm{E}-04$. $1.14831 \mathrm{E}-04$ 1.17751 E-04

$1.20707 \mathrm{E}-04$ $1.23700 \mathrm{E}-04$

$1.26730 \mathrm{E}-04$

$1.29797 \mathrm{E}-04$

$1.32900 \mathrm{E}-04$

$1.36040 \mathrm{E}-04$ $1.39217 \mathrm{E}-04$ $1.42430 \mathrm{E}-04$ $1.45680 \mathrm{E}-04$ $1.48967 \mathrm{E}-04$

$1.52290 \mathrm{E}-04$ $1.55650 \mathrm{E}-04$ $1.59047 \mathrm{E}-04$ $1.62480 \mathrm{E}-04$

$1.65951 \mathrm{E}-04$

$1.69457 \mathrm{E}-04$

$1.73001 \mathrm{E}-04$

$1.76581 \mathrm{E}-04$

$1.80197 \mathrm{E}-04$

$1.83851 \mathrm{E}-04$

$1.87541 \mathrm{E}-04$ $1.91268 \mathrm{E}-04$ $1.95031 \mathrm{E}-04$

$1.98831 \mathrm{E}-04$

$2.02668 \mathrm{E}-04$

$2.06541 \mathrm{E}-04$

$2.10451 \mathrm{E}-04$

$2.14398 \mathrm{E}-04$

$2.18382 \mathrm{E}-04$

$2.22402 \mathrm{E}-04$

$2.26458 \mathrm{E}-04$ $2.30552 \mathrm{E}-04$ $2.34682 \mathrm{E}-04$ $2.38849 \mathrm{E}-04$ 2.43052 E- 04

$2.47292 \mathrm{E}-04$ $2.51569 \mathrm{E}-04$ $2.55882 \mathrm{E}-04$ $2.60232 \mathrm{E}-04$

$2.64619 \mathrm{E}-04$ $g_{2}$

$-9.23519 \mathrm{E}-02$ $-9.55427 \mathrm{E}-02$ $-9.87875 \mathrm{E}-02$ $-1.02086 \mathrm{E}-01$ $-1.05439 \mathrm{E}-01$

$-1.08846 \mathrm{E}-01$ $-1.12306 \mathrm{E}-01$ $-1.15821 \mathrm{E}-01$ $-1.19389 \mathrm{E}-01$ $-1.23012 \mathrm{E}-01$

$-1.26688 \mathrm{E}-01$ $-1.30418 \mathrm{E}-01$ $-1.34202 \mathrm{E}-01$ $-1.38040 \mathrm{E}-01$ $-1.41932 \mathrm{E}-01$

$-1.45878 \mathrm{E}-01$ $-1.49878 \mathrm{E}-01$ $-1.53931 \mathrm{E}-01$ $-1.58039 \mathrm{E}-01$ $-1.62200 \mathrm{E}-01$

$-1.66415 \mathrm{E}-01$ $-1: 70684 \mathrm{E}-01$ $-1.75007 \mathrm{E}-01$

$-1.79384 \mathrm{E}-01$ $-1.83814 \mathrm{E}-01$

$-1.88299 \mathrm{E}-01$ $-1.92837 \mathrm{E}-01$ $-1.97429 \mathrm{E}-01$ $-2.02074 \mathrm{E}-01$ $-2.06774 \mathrm{E}-01$

$-2.11527 \mathrm{E}-01$ $-2.16334 \mathrm{E}-01$ $-2.21195 \mathrm{E}-01$ $-2.26110 \mathrm{E}-01$ $-2.31078 \mathrm{E}-01$

$-2.36100 \mathrm{E}-01$

$-2.41176 \mathrm{E}-01$

$-2.46306 \mathrm{E}-0 \mathrm{l}$

$-2.51489 \mathrm{E}-01$

$-2.56726 \mathrm{E}-01$

$-2.62017 \mathrm{E}-01$

$-2.67361 \mathrm{E}-01$

$-2.72759 \mathrm{E}-01$

$-2.78211 \mathrm{E}-01$

$-2.83717 \mathrm{E}-01$

$-2.89276 \mathrm{E}-01$ $-2.94889 \mathrm{E}-01$ $-3.00555 \mathrm{E}-01$ $-3.06275 \mathrm{E}-01$ $-3.12049 \mathrm{E}-01$

$-3.17877 \mathrm{E}-01$ $-3.23758 \mathrm{E}-01$ $-3.29693 \mathrm{E}-01$ $-3.35681 \mathrm{E}-01$ $-3.41723 \mathrm{E}-01$

$-3.47818 \mathrm{E}-01$ $-3.53968 \mathrm{E}-01$ $-3.60170 \mathrm{E}-01$ $-3.66427 \mathrm{E}-\mathrm{Cl}$ $-3.72736 \mathrm{E}-01$ $\tan \delta_{2}$ $f_{-2}$

$-2.07241 \mathrm{E}-03$ $-2.15815 \mathrm{E}-03$ $-2.24607 \mathrm{E}-03$ $-2.33620 \mathrm{E}-03$ $-2.42855 \mathrm{E}-03$

$-2.52315 \mathrm{E}-03$

$-2.62002 \mathrm{E}-03$

$-2.71919 \mathrm{E}-03$

$-2.82069 \mathrm{E}-03$

$-2.92453 \mathrm{E}-03$

$-3.03074 \mathrm{E}-03$

$-3.13935 \mathrm{E}-03$

$-3.25038 \mathrm{E}-03$

$-3.36385 \mathrm{E}-03$

$-3.47979 \mathrm{E}-03$

$-3.59822 \mathrm{E}-03$ $-3.71917 \mathrm{E}-03$

$-3.84265 \mathrm{E}-03$

$-3.96870 \mathrm{E}-03$

$-4.09734 \mathrm{E}-03$

$-4.22859 \mathrm{E}-03$

$-4.36247 \mathrm{E}-03$

$-4.49902 \mathrm{E}-03$

$-4.63825 \mathrm{E}-03$

$-4.78019 \mathrm{E}-03$

$-4.92485 \mathrm{E}-03$

$-5.07228 \mathrm{E}-03$

$-5.22249 \mathrm{E}-03$

$-5.37550 \mathrm{E}-03$

$-5.53134 \mathrm{E}-03$

$-5.69004 \mathrm{E}-03$

$-5.85161 \mathrm{E}-03$

$-6.01608 \mathrm{E}-03$

$-6.18348 \mathrm{E}-03$

$-6.35382 \mathrm{E}-03$

$-6.52714 \mathrm{E}-03$

$-6.70346 \mathrm{E}-03$

$-6.88279 \mathrm{E}-03$

$-7.06517 \mathrm{E}-03$

$-7.25063 \mathrm{E}-03$

$-7.43917 \mathrm{E}-03$

$-7.63083 \mathrm{E}-03$

$-7.82563 \mathrm{E}-03$

$-8.02360 \mathrm{E}-03$

$-8.22476 \mathrm{E}-03$

$-8.42913 \mathrm{E}-03$

$-8.63674 \mathrm{E}-03$

$-8.84761 \mathrm{E}-03$

$-9.06176 \mathrm{E}-03$

$-9.27922 \mathrm{E}-03$

$-9.50002 \mathrm{E}-03$

$-9.72417 \mathrm{E}-03$

$-9.95171 \mathrm{E}-03$

$-1.01826 \mathrm{E}-02$

$-1.04170 \mathrm{E}-02$

$-1.06548 \mathrm{E}-02$

$-1.08961 \mathrm{E}-02$

$-1.11409 \mathrm{E}-02$

$-1.13893 \mathrm{E}-02$

$-1.16411 \mathrm{E}-02$

$2.78585 \mathrm{E} \quad 01$

$2.78961 \mathrm{E} \quad 01$ $2.79325 \mathrm{E} \quad 01$

$2.79676 \mathrm{E} 01$

$2.80016 \mathrm{E} \quad 01$

$2.80345 \mathrm{E} \quad 01$ $2.80664 \mathrm{E} \quad 01$ $2.80972 \mathrm{E} \quad 01$

$2.81271 \mathrm{E} \quad 01$

$2.81561 \mathrm{E} 01$

$2.81843 \mathrm{E} \quad 01$ $2.82116 \mathrm{E} \quad 01$

$2.82381 \mathrm{E} 01$

$2.82638 \mathrm{E} \quad 01$

$2.82888 \mathrm{E} 01$

$2.83132 \mathrm{E} \quad 01$ $2.83368 \mathrm{E} \quad 01$ $2.83598 \mathrm{E} \quad 01$ $2.83822 \mathrm{E} \quad 01$ $2.84040 \mathrm{E} 01$

$2.84252 \mathrm{E} \quad 01$

$2.84458 \mathrm{E} \quad 01$

$2.84660 \mathrm{E} \quad 01$

$2.84856 \mathrm{E} \quad 01$

$2.85048 \mathrm{E} \quad 01$

$2.85234 \mathrm{E} \quad 01$

$2.85416 \mathrm{E} 01$

$2.85594 \mathrm{E} 01$

$2.85768 \mathrm{E} 01$

$2.85937 \mathrm{E}$ 0]

$2.86102 \mathrm{E} \quad 01$

$2.86264 \mathrm{E} \quad 01$

$2.86422 \mathrm{E} \quad 01$

$2.86576 \mathrm{E}$ 0l

$2.86727 \mathrm{E} \quad 01$

$2.86875 \mathrm{E} \quad 01$

$2.87020 \mathrm{E} \quad 01$

$2.87161 \mathrm{E} 01$

$2.87299 \mathrm{E} \quad 0 \mathrm{l}$

$2.87434 \mathrm{E} \quad 01$

$2.87567 \mathrm{E} \quad 01$

$2.87697 \mathrm{E} \quad 01$ 


\begin{tabular}{|c|c|c|c|c|c|}
\hline$p$ & $F_{0}$ & & $f_{1}$ & & $g_{1}$ \\
\hline 12.10 & $1.18232 \mathrm{E}$ & 00 & $-1.25277 \mathrm{E}$ & 01 & $-6.68047 \mathrm{E}-01$ \\
\hline & $1.18229 \mathrm{E}$ & 00 & $-1.26349 E$ & 01 & $-6.76923 \mathrm{E}-01$ \\
\hline & $.18225 \mathrm{E}$ & 00 & $-1.27421 \mathrm{E}$ & 01 & $-6.85853 \mathrm{E}-$ \\
\hline & $1.18222 \mathrm{E}$ & 00 & $-1.28493 E$ & 01 & $-6.94836 \mathrm{E}-$ \\
\hline & $218 \mathrm{E}$ & 00 & $-1.29565 \mathrm{E}$ & 01 & $-7.03871 \mathrm{E}-01$ \\
\hline & $1.18215 \mathrm{E}$ & 00 & $-1.30636 \mathrm{E}$ & 01 & $-7.12960 \mathrm{E}-$ \\
\hline & & 00 & $-1.31708 \mathrm{E}$ & 01 & $-7.22101 \mathrm{E}-$ \\
\hline & $08 \mathrm{E}$ & 00 & $32779 E$ & 01 & $-7.31296 \mathrm{E}-$ \\
\hline & $05 \mathrm{E}$ & 00 & $33850 \mathrm{E}$ & 01 & $-7.40543 \mathrm{E}-$ \\
\hline .00 & $1.18202 \mathrm{E}$ & 00 & $34921 \mathrm{E}$ & 01 & $-7.49844 \mathrm{E}-01$ \\
\hline & $1.18199 \mathrm{E}$ & 00 & $-1.35991 \mathrm{E}$ & 01 & $-7.59198 \mathrm{E}-$ \\
\hline & & 00 & $7062 \mathrm{E}$ & 01 & $-7.68604 \mathrm{E}-$ \\
\hline & $2 \mathrm{E}$ & 00 & $8132 \mathrm{E}$ & 01 & 78064. E- 01 \\
\hline & $39 \mathrm{E}$ & 00 & 39202E & 01 & $-7.87576 \mathrm{E}-01$ \\
\hline .50 & $86 \mathrm{E}$ & 00 & $40272 \mathrm{E}$ & 01 & $-7.97141 \mathrm{E}$ \\
\hline .60 & & 00 & $1342 \mathrm{E}$ & 01 & $-8.06759 \mathrm{E}-0]$ \\
\hline & $80 \mathrm{E}$ & 00 & $411 \mathrm{E}$ & 01 & -8 \\
\hline & $77 \mathrm{E}$ & 00 & $-1.43481 \mathrm{E}$ & 01 & $-8.26154 \mathrm{E}-0]$ \\
\hline & $75 \mathrm{E}$ & 00 & $-1.44550 \mathrm{E}$ & 01 & $-8.35931 \mathrm{E}-$ \\
\hline ( & $72 E$ & 00 & $5619 \mathrm{E}$ & 01 & $-8.45761 \mathrm{E}$ \\
\hline 10 & $1.18169 \mathrm{E}$ & 00 & $46688 \mathrm{E}$ & 01 & $-8.55643 \mathrm{E}-$ \\
\hline & $18166 \mathrm{E}$ & 00 & $757 \mathrm{E}$ & 01 & $5579 \mathrm{E}-$ \\
\hline 30 & $1.18163 \mathrm{E}$ & 00 & $-1.48825 \mathrm{E}$ & 01 & $-8.75567 \mathrm{E}-$ \\
\hline & $161 \mathrm{E}$ & 00 & $-1.49893 \mathrm{E}$ & 01 & $-8.85608 \mathrm{E}-$ \\
\hline . & $58 \mathrm{E}$ & 00 & $.50962 \mathrm{E}$ & 01 & $-8.95702 \mathrm{E}-$ \\
\hline 60 & $1.18155 \mathrm{E}$ & 00 & $-1.52030 \mathrm{E}$ & 01 & $-9.05848 \mathrm{E}-$ \\
\hline & $1.18152 \mathrm{E}$ & 00 & $-1.53097 \mathrm{E}$ & 01 & $-9.16048 \mathrm{E}-$ \\
\hline & $50 \mathrm{E}$ & 00 & $-1.54165 \mathrm{E}$ & 01 & $-9.26300 \mathrm{E}-$ \\
\hline & $7 \mathrm{E}$ & 00 & $5233 \mathrm{E}$ & 01 & $-9.36605 \mathrm{E}$ \\
\hline & $44 \mathrm{E}$ & 00 & $.56300 \mathrm{E}$ & 01 & $6962 \mathrm{E}$ \\
\hline 10 & $1.18142 \mathrm{E}$ & 00 & $-1.57367 \mathrm{E}$ & 01 & $-9.57373 \mathrm{E}-01$ \\
\hline & $9 \mathrm{E}$ & 00 & $8434 \mathrm{E}$ & 01 & $-9.67836 \mathrm{E}-$ \\
\hline & & 00 & $1 \mathrm{E}$ & 01 & $352 \mathrm{E}-$ \\
\hline & $4 \mathrm{E}$ & 00 & $0567 \mathrm{E}$ & 01 & $8921 \mathrm{E}-0$ \\
\hline 50 & 1.18 & 00 & $-1.61634 \mathrm{E}$ & 01 & $9542 \mathrm{E}-0$ \\
\hline .60 & $29 \mathrm{E}$ & 00 & $700 \mathrm{E}$ & 01 & $1022 \mathrm{E} \quad 0 \mathrm{C}$ \\
\hline & & 00 & $66 \mathrm{E}$ & 01 & 2094E $\quad 00$ \\
\hline & & 00 & $32 \mathrm{E}$ & 01 & $3172 \mathrm{E} \quad 00$ \\
\hline & $122 \mathrm{E}$ & 00 & $-1.65897 \mathrm{E}$ & 01 & $-1.04255 \mathrm{E}$ \\
\hline 0 & $120 E$ & 00 & $.66963 \mathrm{E}$ & 01 & $-1.05344 \mathrm{E}$ \\
\hline 10 & $117 \mathrm{E}$ & 00 & $-1.68028 \mathrm{E}$ & 01 & $6438 \mathrm{E} \quad 00$ \\
\hline & & 00 & $93 \mathrm{E}$ & 01 & $-1.07537 \mathrm{E}$ \\
\hline 30 & $113 \mathrm{E}$ & 00 & $0158 \mathrm{E}$ & 01 & $-1.08641 \mathrm{E}$ \\
\hline & & 00 & $-1.71223 \mathrm{E}$ & 01 & $-1.09750 \mathrm{E}$ \\
\hline & & 00 & & 01 & $0865 \mathrm{E}$ \\
\hline 5.60 & $1.18106 \mathrm{E}$ & 00 & $-1.73352 \mathrm{E}$ & 01 & $1985 \mathrm{E}$ \\
\hline & & 00 & $16 \mathrm{E}$ & 01 & $-1.13110 \mathrm{E}$ \\
\hline & & 00 & $80 \mathrm{E}$ & 01 & $-1.14241 \mathrm{E}$ \\
\hline & & 00 & & 01 & $-1.15377 \mathrm{E} \quad 00$ \\
\hline & 1.1 & 00 & $7607 \mathrm{E}$ & 01 & $-1.16518 \mathrm{E} \quad 00$ \\
\hline & 1.18 & 00 & -1 . & 01 & -1.1 \\
\hline & & 00 & & 01 & $-1.18816 \mathrm{E}$ \\
\hline & & 00 & & 01 & $-1.19972 \mathrm{E}$ \\
\hline & & 00 & $-1.81860 \mathrm{E}$ & 01 & $-1.21134 \mathrm{E} \quad 00$ \\
\hline & $1.18086 \mathrm{E}$ & 00 & $-1.82922 \mathrm{E}$ & 01 & $-1.22302 \mathrm{E} \quad 0$ \\
\hline 6.6 & $1.18084 \mathrm{E}$ & 00 & $-1.83985 \mathrm{E}$ & 01 & $-1.23474 \mathrm{E} \quad 00$ \\
\hline & & 00 & & 01 & $-1.24652 \mathrm{E} \quad 00$ \\
\hline & & 00 & $-1.86109 \mathrm{E}$ & 01 & $-1.25835 \mathrm{E}$ \\
\hline & $78 \mathrm{E}$ & 00 & $-1.87171 \mathrm{E}$ & 01 & $-1.27023 \mathrm{E}$ \\
\hline & & 00 & $-1.88233 \mathrm{E}$ & 01 & $-1.28217 \mathrm{E} \quad 00$ \\
\hline
\end{tabular}

$\tan \delta_{1} \quad f_{-1}$

$3.89836 \mathrm{E}-03$ $3.91466 \mathrm{E}-03$

$3.93067 \mathrm{E}-03$

$3.94642 \mathrm{E}-03$

$3.96189 \mathrm{E}-03$

$3.97710 \mathrm{E}-03$

$3.99205 \mathrm{E}-03$

$4.00675 \mathrm{E}-03$

$4.02120 \mathrm{E}-03$

$4.03541 \mathrm{E}-03$

$4.04939 \mathrm{E}-03$

$4.06314 \mathrm{E}-03$

$4.07666 \mathrm{E}-03$

$4.08996 \mathrm{E}-03$

$4.10305 \mathrm{E}-03$

4.11592 E-03

$4.12859 \mathrm{E}-03$

$4.14106 \mathrm{E}-03$

4.15334. E-03

4.16541 E-03

$4.17730 \mathrm{E}-03$

$4.18900 \mathrm{E}-03$

4.20051 E-03

$4.21186 \mathrm{E}-03$

$4.22303 \mathrm{E}-03$

$4.23401 \mathrm{E}-03$

$4.24484 \mathrm{E}-03$

$4.25550 \mathrm{E}-03$

$4.26600 \mathrm{E}-03$

4.27634. E-03

$4.28653 \mathrm{E}-03$ $4.29656 \mathrm{E}-03$

4.30644 E-03

$4.31617 \mathrm{E}-03$

4. $32576 \mathrm{E}-03$

$4.33521 \mathrm{E}-03$ 4.34452 E-03 $4.35369 \mathrm{E}-03$ 4.36274 E-03

4.37164. E-03

4. $38042 \mathrm{E}-03$ $4.38907 \mathrm{E}-03$ $4.39760 \mathrm{E}-03$ 4.40599 E-03 $4.41428 \mathrm{E}-03$

$4.42243 \mathrm{E}-03$ $4.43048 \mathrm{E}-03$ $4.43841 \mathrm{E}-03$ $4.44623 \mathrm{E}-03$ 4.45394 E-03

$4.46153 \mathrm{E}-03$ $4.46903 \mathrm{E}-03$ $4.47641 \mathrm{E}-03$ $4.48369 \mathrm{E}-03$ $4.49087 \mathrm{E}-03$

$4.49795 \mathrm{E}-03$ $4.50493 \mathrm{E}-03$ $4.51180 \mathrm{E}-03$ $4.51859 \mathrm{E}-03$ $4.52528 \mathrm{E}-03$
$-6.57294 \mathrm{E}-01$ $-6.65899 \mathrm{E}-01$ $-6.74557 \mathrm{E}-01$ $-6.83268 \mathrm{E}-01$ $-6.92033 \mathrm{E}-01$

$-7.00850 \mathrm{E}-01$ $-7.09720 \mathrm{E}-01$ $-7.18643 \mathrm{E}-01$ $-7.27620 \mathrm{E}-01$ $-7.36649 \mathrm{E}-01$

$-7.45731 \mathrm{E}-01$ $-7.54867 \mathrm{E}-01$ $-7.64055 \mathrm{E}-01$ $-7.73296 \mathrm{E}-01$ $-7.82590 \mathrm{E}-01$

$-7.91937 \mathrm{E}-01$ $-8.01336 \mathrm{E}-01$ $-8.10789 \mathrm{E}-01$ $-8.20295 \mathrm{E}-01$ $-8.29853 \mathrm{E}-01$

$-8.39464 \mathrm{E}-01$ $-8.49129 \mathrm{E}-01$ $-8.58846 \mathrm{E}-01$ $-8.68616 \mathrm{E}-01$ $-8.78439 \mathrm{E}-01$

$-8.88314 \mathrm{E}-01$ $-8.98242 \mathrm{E}-01$ $-9.08223 \mathrm{E}-01$ $-9.18257 \mathrm{E}-01$ $-9.28344 \mathrm{E}-01$

$-9.38483 \mathrm{E}-01$ $-9.48676 \mathrm{E}-01$ $-9.58921 \mathrm{E}-01$ $-9.69218 \mathrm{E}-01$ $-9.79569 \mathrm{E}-01$

$-9.89972 \mathrm{E}-01$ $-1.00043 \mathrm{E} \quad 00$ $-1.01094 \mathrm{E} 00$ $-1.02150 \mathrm{E} 00$ $-1.03211 \mathrm{E} 00$

$-1.04278 \mathrm{E} \quad 00$ $-1.05350 \mathrm{E} 00$ $-1.06427 \mathrm{E} 00$ $-1.07509 \mathrm{E} 00$ $-1.08597 \mathrm{E} 00$

$-1.09690 \mathrm{E} \quad 00$ $-1.10788 \mathrm{E} 00$ $-1.11892 \mathrm{E} 00$ $-1.13000 \mathrm{E} 00$ $-1.14114 \mathrm{E} \quad 00$

$-1.15234 \mathrm{E} \quad 00$ $-1.16358 \mathrm{E} \quad 00$ $-1.17488 \mathrm{E} 00$ $-1.18623 \mathrm{E} \quad 00$ $-1.19763 \mathrm{E} 00$

$-1.20908 \mathrm{E} 00$ $-1.22059 \mathrm{E} \quad 00$ $-1.23215 \mathrm{E} 00$ $-1.24376 \mathrm{E} 00$ $-1.25543 \mathrm{E} 00$ g-1 $\tan \delta_{-1}$

$\begin{array}{llll}1.36016 \mathrm{E} & 01 & -1.23273 \mathrm{E} & 02\end{array}$ $\begin{array}{llll}1.37087 \mathrm{E} & 01 & -1.23553 \mathrm{E} & 02\end{array}$ $1.38158 \mathrm{E} 01$ $1.39230 \mathrm{E}$ 01 $1.40301 \mathrm{E} 01$

$-1.23830 \mathrm{E} \quad 02$ $-1.24104 \mathrm{E} 02$ $-1.24374 \mathrm{E} \quad 02$

$1.41372 \mathrm{E} \quad 01$ $1.42442 \mathrm{E} \quad 01$ $1.43513 \mathrm{E} \quad 01$ $1.44583 \mathrm{E} \quad 01$ $1.45653 \mathrm{E} \quad 01$

$1.46723 \mathrm{E} \quad 01$ $1.47793 \mathrm{E} 01$ $1.48863 \mathrm{E} \quad 01$ $1.49932 \mathrm{E} \quad 01$ $1.51001 \mathrm{E} 01$

$-1.24642 \mathrm{E} \quad 02$ $-1.24908 \mathrm{E} 02$ $-1.25170 \mathrm{E} 02$ $-1.25430 \mathrm{E} \quad 02$ $-1.25687 \mathrm{E} \quad 02$

$1.52070 \mathrm{E} \quad 01$ $1.53139 \mathrm{E}$ 0I $1.54208 \mathrm{E} \quad 01$ $1.55276 \mathrm{E} \quad 01$ $1.56345 \mathrm{E} \quad 01$

$-1.25942 \mathrm{E} \quad 02$ $-1.26194 \mathrm{E} \quad 02$ $-1.26444 \mathrm{E} 02$ $-1.26691 \mathrm{E} 02$ $\begin{array}{ll}-1.26935 \mathrm{E} & 02\end{array}$

$1.57413 \mathrm{E} \quad 01$ $1.58481 \mathrm{E} 01$ $1.59549 \mathrm{E} \quad 01$ $1.60616 \mathrm{E} \quad 01$ $1.61684 \mathrm{E} \quad 01$

$1.62751 \mathrm{E} \quad 01$ $1.63818 \mathrm{E} \quad 01$ $1.64885 \mathrm{E} \quad 01$ $1.65952 \mathrm{E}$ 01 $1.67018 \mathrm{E} 01$

$1.68084 \mathrm{E}-01$ $1.69151 \mathrm{E} \quad 01$ $1.70216 \mathrm{E} \quad 01$ $1.71282 \mathrm{E}$ 0l $1.72348 \mathrm{E} 01$

$1.73413 \mathrm{E} \quad 01$ $1.74478 \mathrm{E} \quad 01$ $1.75543 \mathrm{E} \quad 01$

$1.76608 \mathrm{E} 01$

$1.77673 \mathrm{E} \quad 01$

$1.78737 \mathrm{E} \quad 01$ $1.79802 \mathrm{E} \quad 01$ $1.80866 \mathrm{E}$ 01 $1.81930 \mathrm{E} \quad 01$ $1.82993 \mathrm{E} \quad 01$

$1.84057 \mathrm{E} \quad 01$ $1.85120 \mathrm{E} 01$ $1.86183 \mathrm{E} \quad 01$ $1.87246 \mathrm{E} \quad 01$ $1.88309 \mathrm{E} 01$

$1.89371 \mathrm{E} \quad 01$ $1.90434 \mathrm{E} \quad 01$ $1.91496 \mathrm{E}$ 01 $1.92558 \mathrm{E} \quad 01$ $1.93619 \mathrm{E} 01$

$1.94681 \mathrm{E} \quad 01$ $1.95742 \mathrm{E} \quad 01$ $1.96804 \mathrm{E} \quad 01$ $1.97864 \mathrm{E} \quad 01$ $1.98925 \mathrm{E} \quad 01$
$-1.27178 \mathrm{E} \quad 02$ $-1.27418 \mathrm{E} 02$ $-1.27656 \mathrm{E} 02$ $-1.27891 \mathrm{E} 02$ $-1.28124 \mathrm{E} 02$ $-1.28585 \mathrm{E} 02$ $-1.28812 \mathrm{E} 02$ $-1.29037 \mathrm{E} \quad 02$ $-1.29259 \mathrm{E} 02$

$-1.29481 \mathrm{E} \quad 02$ $-1.29700 \mathrm{E} \quad 02$ $-1.29917 \mathrm{E} 02$ $-1.30132 \mathrm{E} \quad 02$ $-1.30345 \mathrm{E} \quad 02$

$-1.30557 \mathrm{E} \quad 02$ $-1.30767 \mathrm{E} 02$ $-1.30975 \mathrm{E} \quad 02$ $-1.31181 \mathrm{E} 02$ -1.31386 E 02 $-1.31589 \mathrm{E} \quad 02$ $-1.31790 \mathrm{E} 02$ $-1.31990 \mathrm{E} 02$ $-1.32188 \mathrm{E} 02$ $-1.32385 \mathrm{E} \quad 02$

$-1.32580 \mathrm{E} \quad 02$ $-1.32774 \mathrm{E} 02$ $-1.32966 \mathrm{E} 02$ $-1.33157 \mathrm{E} 02$ $-1.33346 \mathrm{E} \quad 02$

$-1.33534 \mathrm{E} \quad 02$ $-1.33721 \mathrm{E} \quad 02$ $-1.33906 \mathrm{E} 02$ $-1.34090 \mathrm{E} 02$ $-1.34272 \mathrm{E} 02$

$-1.34454 \mathrm{E} \quad 02$ $-1.34633 \mathrm{E} 02$ $-1.34812 \mathrm{E} 02$ $-1.34990 \mathrm{E} 02$ $-1.35166 \mathrm{E} 02$

$-1.35341 \mathrm{E} \quad 02$ $-1.35515 \mathrm{E} 02$ $-1.35688 \mathrm{E} 02$ $-1.35860 \mathrm{E} 02$ -1.36030 E 02
$-1.28355 \mathrm{E} 02$ 
$\bar{F}$

12.10

12.20

12.30

12.40

12.50

12.60

12.70

12.80

12.90

13.00

13.10

13.20

13.30

13.40

13.50

13.60

13.70

13.80

13.90

14.00

14.10

14.20

14.30

14.40

14.50

14.60

14.70

14.80

14.90

15.00

15.10

15.20

15.30

15.40

15.50

15.60

15.70

15.80

15.90

16.00

16.10

16.20

16.30

16.40

16.50

16.60

16.70

16.80

16.90

17.00

17.10

17.20

17.30

17.40

17.50

17.60

17.70

17.80

17.90

18.00 $f_{2}$

$2.69042 \mathrm{E}-04$

$2.73503 \mathrm{E}-04$

$2.77999 \mathrm{E}-04$

$2.82533 \mathrm{E}-04$

$2.87103 \mathrm{E}-04$

$2.91709 \mathrm{E}-04$

$2.96353 \mathrm{E}-04$

$3.01033 \mathrm{E}-04$

$3.05749 \mathrm{E}-04$

$3.10503 \mathrm{E}-04$

$3.15293 \mathrm{E}-04$

$3.20119 \mathrm{E}-04$

$3.24982 \mathrm{E}-04$

3.29882 E- 04

$3.34819 \mathrm{E}-04$

$3.39792 \mathrm{E}-04$

$3.44802 \mathrm{E}-04$

$3.49848 \mathrm{E}-04$

$3.54932 \mathrm{E}-04$

$3.60051 \mathrm{E}-04$

$3.65208 \mathrm{E}-04$

$3.70401 \mathrm{E}-04$

$3.75631 \mathrm{E}-04$

$3.80897 \mathrm{E}-04$

$3.86201 \mathrm{E}-04$

$3.91540 \mathrm{E}-04$

$3.96917 \mathrm{E}-04$

$4.02330 \mathrm{E}-04$

$4.07779 \mathrm{E}-04$

$4.13266 \mathrm{E}-04$

4.18789E-04

$4.24349 \mathrm{E}-04$

$4.29945 \mathrm{E}-04$

$4.35578 \mathrm{E}-04$

$4.41247 \mathrm{E}-04$

4.46954E-04 $4.52697 \mathrm{E}-04$

$4.58476 \mathrm{E}-04$

$4.64293 \mathrm{E}-04$

$4.70145 \mathrm{E}-04$

$4.76035 \mathrm{E}-04$

$4.81961 \mathrm{E}-04$

$4.87924 \mathrm{E}-04$

$4.93923 \mathrm{E}-04$

$4.99959 \mathrm{E}-04$

$5.06032 \mathrm{E}-04$

$5.12141 \mathrm{E}-04$

$5.18287 \mathrm{E}-04$

$5.24470 \mathrm{E}-04$

$5.30689 \mathrm{E}-04$

$5.36945 \mathrm{E}-04$

$5.43238 \mathrm{E}-04$

$5.49567 \mathrm{E}-04$

$5.55933 \mathrm{E}-04$

$5.62336 \mathrm{E}-04$

$5.68775 \mathrm{E}-04$

$5.75251 \mathrm{E}-04$

$5.81763 \mathrm{E}-04$

$5.88312 \mathrm{E} \longrightarrow 04$

$5.94898 \mathrm{E}-04$ $g_{2}$

$\tan \delta_{2}$

$f_{-2}$

$-3.79100 \mathrm{E}-01 \quad \rightarrow 1.18966 \mathrm{E}-02$

$-3.85517 \mathrm{E}-01 \quad-1.21556 \mathrm{E}-02$

$-3.91988 \mathrm{E}-01 \quad-1.24183 \mathrm{E}-02$

$-3.98512 \mathrm{E}-01 \quad-1.26846 \mathrm{E}-02$

$-4.05090 \mathrm{E}-01-1.29546 \mathrm{E}-02$

$-4.11721 \mathrm{E}-01$

$-4.18406 \mathrm{E}-01$

$-4.25144 \mathrm{E}-01$

$-4.31936 \mathrm{E}-01$

$-4.38781 \mathrm{E}-01$

$-4.45679 \mathrm{E}-01$

$-4.52632 \mathrm{E}-01$

$-4.59637 \mathrm{E}-01$

$-4.66696 \mathrm{E}-01$

$-4.73809 \mathrm{E}-01$

$-4.80975 \mathrm{E}-01$

$-4.88194 \mathrm{E}-01$

$-4.95467 \mathrm{E}-01$

$-5.02794 \mathrm{E}-01$

$-5.10173 \mathrm{E}-01$

$-5.17606 \mathrm{E}-01$

$-5.25093 \mathrm{E}-01$

$-5.32633 \mathrm{E}-01$

$-5.40226 \mathrm{E}-01$

$-5.47873 \mathrm{E}-01$

$-5.55573 \mathrm{E}-01$

$-5.63327 \mathrm{E}-01$

$-5.71133 \mathrm{E}-0 \mathrm{l}$

$-5.78993 \mathrm{E}-01$

$-5.86907 \mathrm{E}-01$

$-5.94874 \mathrm{E}-01$

$-6.02894 \mathrm{E}-01$

$-6.10967 \mathrm{E}-01$

$-6.19094 \mathrm{E}-01$

$-6.27274 \mathrm{E}-01$

$-6.35507 \mathrm{E}-01$

$-6.43794 \mathrm{E}-01$

$-6.52134 \mathrm{E}-01$

$-6.60527 \mathrm{E}-01$

$-6.68973 \mathrm{E}-01$

$-6.77473 \mathrm{E}-01$

$-6.86025 \mathrm{E}-01$

$-6.94631 \mathrm{E}-01$

$-7.03290 \mathrm{E}-01$

$-7.12003 \mathrm{E}-01$

$-7.20769 \mathrm{E}-01$

$-7.29587 \mathrm{E}-01$

$-7.38459 \mathrm{E}-01$

$-7.47385 \mathrm{E}-01$

$-7.56363 \mathrm{E}-01$

$-7.65394 \mathrm{E}-01$

$-7.74479 \mathrm{E}-01$

$-7.83616 \mathrm{E}-0 \mathrm{l}$

$-7.92807 \mathrm{E}-01$

$-8.02051 \mathrm{E}-01$

$-8.11348 \mathrm{E}-01$

$-8.20698 \mathrm{E}-01$

$-8.30102 \mathrm{E}-01$

$-8.39558 \mathrm{E}-01$

$-8.49067 \mathrm{E}-01$
$-1.32283 \mathrm{E}-02$

$-1.35057 \mathrm{E}-02$

$-1.37868 \mathrm{E}-02$

$-1.40717 \mathrm{E}-02$

$-1.43604 \mathrm{E}-02$

$-1.46528 \mathrm{E}-02$

$-1.49492 \mathrm{E}-02$

$-1.52494 \mathrm{E}-02$

$-1.55534 \mathrm{E}-02$

$-1.58614 \mathrm{E}-02$

$-1.61733 \mathrm{E}-02$

$-1.64892 \mathrm{E}-02$

$-1.68090 \mathrm{E}-02$

$-1.71329 \mathrm{E}-02$

$-1.74608 \mathrm{E}-02$

$-1.77927 \mathrm{E}-02$

$-1.81287 \mathrm{E}-02$

$-1.84688 \mathrm{E}-02$

$-1.88130 \mathrm{E}-02$

$-1.91614 \mathrm{E}-02$

$-1.95139 \mathrm{E}-02$

$-1.98707 \mathrm{E}-02$

$-2.02316 \mathrm{E}-02$

$-2.05968 \mathrm{E}-02$

$-2.09663 \mathrm{E}-02$

$-2.13400 \mathrm{E}-02$

$-2.17181 \mathrm{E}-02$

$-2.21005 \mathrm{E}-02$

$-2.24872 \mathrm{E}-02$

$-2.28784 \mathrm{E}-02$

$-2.32739 \mathrm{E}-02$

$-2.36739 \mathrm{E}-02$

$-2.40784 \mathrm{E}-02$

$-2.44873 \mathrm{E}-02$

$-2.53186 \mathrm{E}-02$

$-2.57411 \mathrm{E}-02$

$-2.61682 \mathrm{E}-02$

$-2.65999 \mathrm{E}-02$

$-2.70362 \mathrm{E}-02$

$-2.74771 \mathrm{E}-02$

$-2.79227 \mathrm{E}-02$

$-2.83730 \mathrm{E}-02$

-2.88281 E- 02

$-2.92878 \mathrm{E}-02$

$-2.97524 \mathrm{E}-02$

$-3.02217 \mathrm{E}-02$

$-3.06958 \mathrm{E}-02$

$-3.11748 \mathrm{E}-02$

$-3.16586 \mathrm{E}-02$

$-3.21473 \mathrm{E}-02$

$-3.26409 \mathrm{E}-02$

$-3.31394 \mathrm{E}-02$

$-3.36429 \mathrm{E}-02$

$-3.41514 \mathrm{E}-02$
$-2.49007 \mathrm{E}-02$

$2.89735 \mathrm{E} \quad 0 \mathrm{l}-1.16853 \mathrm{E}-02$

$2.89823 \mathrm{E} \quad 01-1.19377 \mathrm{E}-02$

$2.89911 \mathrm{E}$ 01 $-1.21936 \mathrm{E}-02$

$2.89997 \mathrm{E} \quad 01 \quad-1.24530 \mathrm{E}-02$

$2.90081 \mathrm{E} \quad 01 \quad-1.27161 \mathrm{E}-02$

$2.90164 \mathrm{E} \quad 01$

$2.90245 \mathrm{E} \quad 01$

$2.90326 \mathrm{E} \quad 01$

$2.90404 \mathrm{E} \quad 01$

$2.90482 \mathrm{E} 01$

$2.90558 \mathrm{E} \quad 01$

$2.90634 \mathrm{E} \quad 01$

$2.90708 \mathrm{E} \quad 01$

$2.90780 \mathrm{E} \quad 01$

$2.90852 \mathrm{E} \quad 01$

$2.90923 \mathrm{E} \quad 01$

$2.90992 \mathrm{E} 01$

$2.91061 \mathrm{E} \quad 01$

$2.91128 \mathrm{E} 01$

$2.91195 \mathrm{E} \quad 01$

$2.91260 \mathrm{E} \quad 01$

$2.91324 \mathrm{E} \quad 01$

$2.91388 \mathrm{E} \quad 01$

$2.91451 \mathrm{E} 01$

$2.91512 \mathrm{E} \quad 01$

$2.91573 \mathrm{E} \quad 01$

$2.91633 \mathrm{E} \quad 01$

$2.91692 \mathrm{E} 01$

$2.91750 \mathrm{E} \quad 01$

$2.91808 \mathrm{E} \quad 01$

$-1.29828 \mathrm{E}-02$

$-1.32531 \mathrm{E}-02$

$-1.35270 \mathrm{E}-02$

$-1.38047 \mathrm{E}-02$

$-1.40860 \mathrm{E}-02$

$-1.43711 \mathrm{E}-02$

$-1.46599 \mathrm{E}-02$

$-1.49525 \mathrm{E}-02$

$-1.52489 \mathrm{E}-02$

$-1.55492 \mathrm{E}-02$

$-1.58533 \mathrm{E}-02$

$-1.61612 \mathrm{E}-02$

$-1.64731 \mathrm{E}-02$

$-1.67889 \mathrm{E}-02$

$-1.71086 \mathrm{E}-02$

$-1.74323 \mathrm{E}-02$

$-1.77600 \mathrm{E}-02$ 


\begin{tabular}{|c|c|c|c|c|c|c|c|c|}
\hline$p$ & $F_{0}$ & & $f_{1}$ & & $g_{1}$ & & $\tan \delta_{1}$ & $f_{-1}$ \\
\hline 18.10 & $1.18074 \mathrm{E}$ & 00 & $-1.89294 \mathrm{E}$ & 01 & $-1.29416 \mathrm{E}$ & 00 & $4.53188 \mathrm{E}-03$ & $-1.26715 \mathrm{E}$ \\
\hline 18.20 & $1.18072 \mathrm{E}$ & 00 & $-1.90356 \mathrm{E}$ & 01 & $-1.30620 \mathrm{E}$ & 00 & $4.53838 \mathrm{E}-03$ & $-1.27892 \mathrm{E}$ \\
\hline 18.30 & $1.18070 \mathrm{E}$ & 00 & $-1.91417 \mathrm{E}$ & 01 & $-1.31829 \mathrm{E}$ & 00 & $4.54481 \mathrm{E}-$ & $.29074 \mathrm{E}$ \\
\hline 18.40 & $1.18068 \mathrm{E}$ & 00 & $-1.92478 \mathrm{E}$ & 01 & $-1.33043 \mathrm{E}$ & 00 & $4.55113 \mathrm{E}-$ & $1.30261 \mathrm{E}$ \\
\hline 8.50 & $1.18066 \mathrm{E}$ & 00 & $-1.93538 \mathrm{E}$ & 01 & $-1.34263 \mathrm{E}$ & 00 & $4.55736 \mathrm{E}-03$ & $-1.31454 \mathrm{E}$ \\
\hline 3.60 & $1.18064 \mathrm{E}$ & 00 & $-1.94599 \mathrm{E}$ & 01 & $-1.35488 \mathrm{E}$ & 00 & $4.56352 \mathrm{E}-03$ & $-1.32652 \mathrm{E}$ \\
\hline 3.70 & $1.18062 \mathrm{E}$ & 00 & $-1.95659 \mathrm{E}$ & 01 & $-1.36718 \mathrm{E}$ & 00 & $4.56958 \mathrm{E}-03$ & $-1.33855 \mathrm{E}$ \\
\hline 3.80 & $1.18060 \mathrm{E}$ & 00 & $-1.96719 \mathrm{E}$ & 01 & $-1.37954 \mathrm{E}$ & 00 & $4.57557 \mathrm{E}-$ & $.35063 \mathrm{E}$ \\
\hline 18.90 & $1.18059 \mathrm{E}$ & 00 & $-1.97779 \mathrm{E}$ & 01 & $-1.39194 \mathrm{E}$ & 00 & $4.58147 \mathrm{E}-03$ & $-1.36277 \mathrm{E}$ \\
\hline 19.00 & $1.18057 \mathrm{E}$ & 00 & $-1.98839 \mathrm{E}$ & 01 & $-1.40440 \mathrm{E}$ & 00 & $4.58729 \mathrm{E}-03$ & $-1.37496 \mathrm{E}$ \\
\hline 19.10 & $1.18055 \mathrm{E}$ & 00 & $-1.99899 \mathrm{E}$ & 01 & $-1.41691 \mathrm{E}$ & 00 & $4.59303 \mathrm{E}-03$ & $-1.38720 \mathrm{E}$ \\
\hline 19.20 & $1.18053 \mathrm{E}$ & 00 & $-2.00958 \mathrm{E}$ & 01 & $-1.42947 \mathrm{E}$ & 00 & $4.59869 \mathrm{E}-03$ & $-1.39949 \mathrm{E}$ \\
\hline 19.30 & $1.18051 \mathrm{E}$ & 00 & $-2.02017 \mathrm{E}$ & 01 & $-1.44209 \mathrm{E}$ & 00 & $4.60428 \mathrm{E}-03$ & $-1.41183 \mathrm{E}$ \\
\hline 19.40 & $1.18049 \mathrm{E}$ & 00 & $-2.03076 \mathrm{E}$ & 01 & $.45476 \mathrm{E}$ & 00 & $4.60978 \mathrm{E}-03$ & $1.42423 \mathrm{E}$ \\
\hline 19.50 & $1.18047 \mathrm{E}$ & 00 & $-2.04135 \mathrm{E}$ & 01 & $-1.46748 \mathrm{E}$ & 00 & $4.61521 \mathrm{E}-03$ & $-1.43668 \mathrm{E}$ \\
\hline 19.60 & $1.18046 \mathrm{E}$ & 00 & $-2.05193 \mathrm{E}$ & 01 & $-1.48025 \mathrm{E}$ & 00 & $4.62057 \mathrm{E}-03$ & $-1.44918 \mathrm{E}$ \\
\hline 19.70 & $1.18044 \mathrm{E}$ & 00 & $-2.06251 \mathrm{E}$ & 01 & $49307 \mathrm{E}$ & 00 & $4.62585 \mathrm{E}-03$ & $-1.46174 \mathrm{E}$ \\
\hline 19.80 & $1.18042 \mathrm{E}$ & 00 & $-2.07310 \mathrm{E}$ & 01 & $-1.50595 \mathrm{E}$ & 00 & $4.63106 \mathrm{E}-03$ & $-1.47434 \mathrm{E}$ \\
\hline 19.90 & $1.18040 \mathrm{E}$ & 00 & $-2.08367 \mathrm{E}$ & 01 & $-1.51888 \mathrm{E}$ & 00 & $4.63621 \mathrm{E}-03$ & $-1.48700 \mathrm{~F}$ \\
\hline 20.00 & $1.18038 \mathrm{E}$ & 00 & $-2.09425 \mathrm{E}$ & 01 & $-1.53186 \mathrm{E}$ & 00 & $4.64126 \mathrm{E}-03$ & $-1.49971 \mathrm{E}$ \\
\hline 20.10 & $1.18037 \mathrm{E}$ & 00 & $-2.10483 \mathrm{E}$ & 01 & $-1.54489 \mathrm{E}$ & 00 & $4.64626 \mathrm{E}-03$ & $-1.51247 \mathrm{E}$ \\
\hline 20.20 & $1.18035 \mathrm{E}$ & 00 & $-2.11540 \mathrm{E}$ & 01 & $-1.55797 \mathrm{E}$ & 00 & $4.65119 \mathrm{E}-03$ & $-1.52529 \mathrm{E}$ \\
\hline 20.30 & $1.18033 \mathrm{E}$ & 00 & $-2.12597 \mathrm{E}$ & 01 & $-1.57111 \mathrm{E}$ & 00 & $4.65605 \mathrm{E}-03$ & $-1.53816 \mathrm{E}$ \\
\hline 20.40 & $1.18031 \mathrm{E}$ & 00 & $-2.13654 \mathrm{E}$ & 01 & $-1.58430 \mathrm{E}$ & 00 & $4.66085 \mathrm{E}-03$ & $-1.55108 \mathrm{E}$ \\
\hline 20.50 & $1.18030 \mathrm{E}$ & 00 & $-2.14711 \mathrm{E}$ & 01 & $-1.59754 \mathrm{E}$ & 00 & $4.66557 \mathrm{E}-03$ & $-1.56405 \mathrm{E}$ \\
\hline 20.60 & $1.18028 \mathrm{E}$ & 00 & $-2.15767 \mathrm{E}$ & 01 & $-1.61084 \mathrm{E}$ & 00 & $4.67024 \mathrm{E}-03$ & $-1.57707 \mathrm{E}$ \\
\hline 20.70 & $1.18026 \mathrm{E}$ & 00 & $-2.16823 \mathrm{E}$ & 01 & $-1.62418 \mathrm{E}$ & 00 & $4.67484 \mathrm{E}$ & $-1.59015 \mathrm{E}$ \\
\hline 20.80 & $1.18025 \mathrm{E}$ & 00 & $-2.17879 \mathrm{E}$ & 01 & $-1.63758 \mathrm{E}$ & 00 & $4.67938 \mathrm{E}-$ & $-1.60327 \mathrm{E}$ \\
\hline 20.90 & $023 \mathrm{E}$ & 00 & $-2.18935 \mathrm{E}$ & 01 & $55103 \mathrm{E}$ & 00 & $84 \mathrm{E}-03$ & $-1.61645 \mathrm{E}$ \\
\hline 21.00 & $1.18021 \mathrm{E}$ & 00 & $-2.19991 \mathrm{E}$ & 01 & $66453 \mathrm{E}$ & 00 & $4.68825 \mathrm{E}-03$ & $-1.62969 \mathrm{E}$ \\
\hline 21.10 & $.18020 \mathrm{E}$ & 00 & $-2.21046 \mathrm{E}$ & 01 & $-1.67808 \mathrm{E}$ & 00 & $4.69261 \mathrm{E}-03$ & $-1.64297 \mathrm{E}$ \\
\hline 21.20 & $18 \mathrm{E}$ & 00 & $-2.22101 \mathrm{E}$ & 01 & $9169 \mathrm{E}$ & 00 & -03 & $1.65630 \mathrm{E}$ \\
\hline 21.3 & & 00 & -2.2 & 01 & $535 \mathrm{E}$ & 00 & $12 \mathrm{E}-03$ & $1.66969 \mathrm{E}$ \\
\hline 21.40 & $1.18015 \mathrm{E}$ & 00 & $-2.24211 \mathrm{E}$ & 01 & $.71905 \mathrm{E}$ & 00 & $4.70529 \mathrm{E}$ & $-1.68313 \mathrm{E}$ \\
\hline 21.50 & $1.18013 \mathrm{E}$ & 00 & $-2.25266 \mathrm{E}$ & 01 & $73282 \mathrm{E}$ & 00 & $4.70940 \mathrm{E}-03$ & $-1.69662 \mathrm{E}$ \\
\hline 21.60 & $1.18012 \mathrm{E}$ & 00 & $-2.26320 \mathrm{E}$ & 01 & $-1.74663 \mathrm{E}$ & 00 & $16 \mathrm{E}-03$ & $-1.71017 \mathrm{E}$ \\
\hline .70 & & 00 & $374 \mathrm{E}$ & 01 & $9 \mathrm{E}$ & 00 & -03 & $376 \mathrm{E}$ \\
\hline 21.80 & $1.18008 \mathrm{E}$ & 00 & $-2.28428 \mathrm{E}$ & 01 & $7441 \mathrm{E}$ & 00 & $4.72140 \mathrm{E}-03$ & $-1.73741 \mathrm{E}$ \\
\hline 21.90 & $1.18007 \mathrm{E}$ & 00 & $-2.29482 \mathrm{E}$ & 01 & $-1.78838 \mathrm{E}$ & 00 & $4.72529 \mathrm{E}$ & $-1.75111 \mathrm{E}$ \\
\hline 22.00 & $1.18005 \mathrm{E}$ & 00 & $-2.30535 \mathrm{E}$ & 01 & $.80240 \mathrm{E}$ & 00 & $4.72912 \mathrm{E}$ & $-1.76486 \mathrm{E}$ \\
\hline 22.10 & $1.18004 \mathrm{E}$ & 00 & $-2.31589 \mathrm{E}$ & 01 & $-1.81648 \mathrm{E}$ & 00 & $4.73290 \mathrm{E}-03$ & $-1.77867 \mathrm{E}$ \\
\hline 22.20 & $1.18002 \mathrm{E}$ & 00 & $-2.32642 \mathrm{E}$ & 01 & $33060 \mathrm{E}$ & 00 & $62 \mathrm{E}-03$ & $-1.79252 \mathrm{E}$ \\
\hline 22.30 & $1.18000 \mathrm{E}$ & 00 & $-2.33695 \mathrm{E}$ & 01 & $-1.84478 \mathrm{E}$ & 00 & $4.74029 \mathrm{~F}$ & $-1.80643 \mathrm{E}$ \\
\hline 22.40 & $99 \mathrm{E}$ & 00 & $-2.34747 \mathrm{E}$ & 01 & $5901 \mathrm{E}$ & 00 & $1 \mathrm{E}-03$ & $-1.82039 \mathrm{E}$ \\
\hline 22.50 & $1.17997 \mathrm{E}$ & 00 & $-2.35800 \mathrm{E}$ & 01 & $87329 \mathrm{E}$ & 00 & $4.74748 \mathrm{E}-03$ & $-1.83440 \mathrm{E}$ \\
\hline 22.60 & $1.17996 \mathrm{E}$ & 00 & $-2.36852 \mathrm{E}$ & 01 & $-1.88762 \mathrm{E}$ & 00 & $4.75100 \mathrm{E}-03$ & $-1.84846 \mathrm{E}$ \\
\hline 22.70 & $994 \mathrm{E}$ & 00 & $-2.37904 \mathrm{E}$ & 01 & $-1.90200 \mathrm{E}$ & 00 & & $-1.86258 \mathrm{E}$ \\
\hline 22.80 & & 00 & $956 \mathrm{E}$ & 01 & 544.E & 00 & 4. & $-1.87674 \mathrm{E}$ \\
\hline 22.90 & & 00 & $007 \mathrm{E}$ & 01 & $092 \mathrm{E}$ & 00 & & $-1.89096 \mathrm{E}$ \\
\hline 23.00 & $1.17990 \mathrm{E}$ & 00 & $-2.41059 \mathrm{E}$ & 01 & $94546 \mathrm{E}$ & 00 & $4.76458 \mathrm{E}-03$ & $-1.90523 \mathrm{E}$ \\
\hline 23.10 & $1.17988 \mathrm{E}$ & 00 & $-2.42110 \mathrm{E}$ & 01 & $-1.96005 \mathrm{E}$ & 00 & $4.76784 \mathrm{E}-03$ & $-1.91955 \mathrm{E}$ \\
\hline 23.20 & & 00 & $-2.43161 \mathrm{E}$ & 01 & & 00 & & $93392 \mathrm{E}$ \\
\hline 23.30 & $1.17986 \mathrm{E}$ & 00 & $-2.44211 \mathrm{E}$ & 01 & $98939 \mathrm{E}$ & 00 & 4.7 & $-1.94835 \mathrm{E}$ \\
\hline 23.40 & $1.17984 \mathrm{E}$ & 00 & $-2.45262 \mathrm{E}$ & 01 & $-2.00414 \mathrm{E}$ & 00 & $4.77737 \mathrm{E}-$ & $-1.96283 \mathrm{E}$ \\
\hline 23.50 & $1.17983 \mathrm{E}$ & 00 & $-2.46312 \mathrm{E}$ & 01 & $-2.01894 \mathrm{E}$ & 00 & $4.78046 \mathrm{E}-03$ & $-1.97736 \mathrm{E}$ \\
\hline 23.60 & $1.17981 \mathrm{E}$ & 00 & $-2.47362 \mathrm{E}$ & 01 & $-2.03379 \mathrm{E}$ & 00 & $30 \mathrm{E}-03$ & $-1.99194 \mathrm{E}$ \\
\hline 23.70 & $1.17980 \mathrm{E}$ & 00 & $-2.48412 \mathrm{E}$ & 01 & $-2.04869 \mathrm{E}$ & 00 & $49 E-03$ & $-2.00657 \mathrm{E}$ \\
\hline 23.80 & $17978 \mathrm{E}$ & 00 & $-2.49461 \mathrm{E}$ & 01 & $-2.06364 \mathrm{E}$ & 00 & $4.78944 \mathrm{E}-03$ & $-2.02125 \mathrm{E}$ \\
\hline 23.90 & $1.17977 \mathrm{E}$ & 00 & $-2.50510 \mathrm{E}$ & 01 & $-2.07864 \mathrm{E}$ & 00 & $4.79235 \mathrm{E}-03$ & $-2.03599 \mathrm{E}$ \\
\hline
\end{tabular}

$g_{-1}$

$1.99986 \mathrm{E} \quad 01$ $2.01046 \mathrm{E} \quad 01$ $2.02106 \mathrm{E} \quad 01$

$2.03166 \mathrm{E} 01$

$2.04226 \mathrm{E} \quad 01$

$2.05286 \mathrm{E} \quad 01$

$2.06345 \mathrm{E} \quad 01$

$2.07404 \mathrm{E} \quad 01$

$2.08463 \mathrm{E} \quad 0$

$2.09522 \mathrm{E} 01$

$2.10581 \mathrm{E} \quad 01$ $2.11639 \mathrm{E} \quad 01$ $2.12697 \mathrm{E} \quad 01$ $2.13755 \mathrm{E} \quad 0$ $2.14813 \mathrm{E} 01$

$2.15870 \mathrm{E} \quad 01$ $2.16928 \mathrm{E} \quad 01$

$2.17985 \mathrm{E} \quad 01$

$2.19042 \mathrm{E} \quad 01$

$2.20098 \mathrm{E} 01$

$2.21155 \mathrm{E} \quad 01$ $2.22211 \mathrm{E} \quad 01$ $2.23267 \mathrm{E} \quad 01$ $2.24323 \mathrm{E} \quad 01$ $2.25379 \mathrm{E} 01$

$2.26434 \mathrm{E} \quad 01$ $2.27490 \mathrm{E} \quad 01$ $2.28545 \mathrm{E} \quad 01$ $2.29599 \mathrm{E} 01$ 2.30654E 01

$2.31709 \mathrm{E} \quad 01$ $2.32763 \mathrm{E}$ ol $2.33817 \mathrm{E} \quad 01$ $2.34870 \mathrm{E} \quad 01$ $2.35924 \mathrm{E} \quad 01$

$2.36977 \mathrm{E} \quad 01$ $2.38030 \mathrm{E} \quad 01$ $2.39083 \mathrm{E} \quad 01$ $2.40136 \mathrm{E} \quad 01$

$2.41188 \mathrm{E} 01$

$2.42241 \mathrm{E} \quad 01$ $2.43293 \mathrm{E} 0$ $2.44345 \mathrm{E}$ 0l $2.45396 \mathrm{E} \quad 0$ $2.46448 \mathrm{E} \quad 01$

$2.474 .99 \mathrm{E} \quad 01$ $2.48550 \mathrm{E} \quad 01$ $2.49600 \mathrm{E} \quad 01$ $2.50651 \mathrm{E} 01$ $2.51701 \mathrm{E} \quad 01$

$2.52751 \mathrm{E} \quad 01$ $2.53801 \mathrm{E} 01$ $2.54851 \mathrm{E} 01$ $2.55900 \mathrm{E} \quad 01$ $2.56949 \mathrm{E} 01$ $2.57998 \mathrm{E} \quad 01$ $2.59047 \mathrm{E} \quad 01$ $2.60095 \mathrm{E} \quad 01$ $2.61143 \mathrm{E} 01$ $2.62191 \mathrm{E} 01$ $\tan \delta_{-1}$

$-1.36200 \mathrm{E} 02$ $-1.36368 \mathrm{E} 02$ $-1.36536 \mathrm{E} \quad 02$ $-1.36702 \mathrm{E} 02$ $-1.36867 \mathrm{E} \quad 02$

$-1.37032 \mathrm{E} 02$ $-1.37195 \mathrm{E} \quad 02$ $-1.37357 \mathrm{E} 02$ $-1.37518 \mathrm{E} 02$ $-1.37679 \mathrm{E} 02$

$-1.37838 \mathrm{E} \quad 02$ $\begin{array}{lll}-1.37997 \mathrm{E} & 02\end{array}$ $-1.38154 \mathrm{E} 02$ $-1.38311 \mathrm{E} 02$ $-1.38467 \mathrm{E} \quad 02$ $-1.38622 \mathrm{E} \quad 02$ $-1.38776 \mathrm{E} \quad 02$ $-1.38929 \mathrm{E} \quad 02$ $-1.39082 \mathrm{E} 02$ $-1.39234 \mathrm{E} 02$

$-1.39385 \mathrm{E} \quad 02$ $-1.39535 \mathrm{E} \quad 02$ $-1.39684 \mathrm{E} 02$ $-1.39833 \mathrm{E} 02$ $-1.39981 \mathrm{E} 02$

$-1.40128 \mathrm{E} \quad 02$ $-1.40274 \mathrm{E} \quad 02$ $-1.40420 \mathrm{E} 02$ $-1.40565 \mathrm{E} 02$ $-1.40709 \mathrm{E} 02$

$-1.40852 \mathrm{E} 02$ $-1.40995 \mathrm{E} \quad 02$ $-1.41138 \mathrm{E} \quad 02$ $-1.41279 \mathrm{E} 02$ $-1.41420 \mathrm{E} 02$

$-1.41560 \mathrm{E} \quad 02$ $-1.41700 \mathrm{E} \quad 02$ $-1.41839 \mathrm{E} 02$ $-1.41978 \mathrm{E} \quad 02$ $-1.42116 \mathrm{E} \quad 02$

$\begin{array}{ll}-1.42253 \mathrm{E} & 02\end{array}$ $-1.42390 \mathrm{E} 02$ $-1.42526 \mathrm{E} 02$ $-1.42662 \mathrm{E} 02$ $-1.42797 \mathrm{E} \quad 02$

$-1.42932 \mathrm{E} \quad 02$ $-1.43065 \mathrm{E} 02$ $-1.43199 \mathrm{E} 02$ $-1.43332 \mathrm{E} \quad 02$ $-1.43465 \mathrm{E} \quad 02$

$-1.43597 \mathrm{E} \quad 02$ $-1.43728 \mathrm{E} 02$ $-1.43859 \mathrm{E} \quad 02$ $-1.43990 \mathrm{E} \quad 02$ $-1.44120 \mathrm{E} \quad 02$

$-1.44250 \mathrm{E} \quad 02$ $-1.44379 \mathrm{E} \quad 02$ $-1.44508 \mathrm{E} 02$ $-1.44636 \mathrm{E} 02$ $-1.44764 \mathrm{E} \quad 02$ 
$\bar{F}$

18.10

18.20

18.30

18.40

18.50

18.60

18.70

18.80

18.90

19.00

19.10

19.20

19.30

19.40

19.50

19.60

19.70

19.80

19.90

20.00

20.10

20.20

20.30

20.40

20.50

20.60

20.70

20.80

20.90

21.00

21.10

21.20

21.30

21.40

21.50

21.60

21.70

21.80

21.90

22.00

22.10

22.20

22.30

22.40

22.50

22.60

22.70

22.80

22.90

23.00

23.10

23.20

23.30

23.40

23.50

23.60

23.70

23.80

23.90

24.00 $f_{2}$

$6.01521 \mathrm{E}-04$

$6.08180 \mathrm{E}-04$

$6.14876 \mathrm{E}-04$

$6.21608 \mathrm{E}-04$

$6.28377 \mathrm{E}-04$

$6.35182 \mathrm{E}-04$

$6.42025 \mathrm{E}-04$

$6.48904 \mathrm{E}-04$

$6.55819 \mathrm{E}-04$

$6.62771 \mathrm{E}-04$

$6.69760 \mathrm{E}-04$

$6.76785 \mathrm{E}-04$

$6.83847 \mathrm{E}-04$

$6.90946 \mathrm{E}-04$

6.98081 E-04

$7.05253 \mathrm{E}-04$

$7.12462 \mathrm{E}-04$

$7.19707 \mathrm{E}-04$

$7.26989 \mathrm{E}-04$

7.34308 E- 04

$7.41663 \mathrm{E}-04$

$7.49055 \mathrm{E}-04$

$7.56483 \mathrm{E}-04$

$7.63948 \mathrm{E}-04$

7.71450 E-04

$7.78988 \mathrm{E}-04$

$7.86563 \mathrm{E}-04$

$7.94175 \mathrm{E}-04$

$8.01823 \mathrm{E}-04$

$8.09508 \mathrm{E}-04$

$8.17229 \mathrm{E}-04$ $8.24987 \mathrm{E}-04$ $8.32782 \mathrm{E}-04$ $8.40613 \mathrm{E}-04$

8.48482 E-04

$8.56386 \mathrm{E}-04$

$8.64328 \mathrm{E}-04$

$8.72305 \mathrm{E}-04$

$8.80320 \mathrm{E}-04$

$8.88371 \mathrm{E}-04$

$8.96459 \mathrm{E}-04$

$9.04584 \mathrm{E}-04$

$9.12745 \mathrm{E}-04$

$9.20943 \mathrm{E}-04$

$9.29177 \mathrm{E}-04$

$9.37448 \mathrm{E}-04$

$9.45756 \mathrm{E}-04$

$9.54100 \mathrm{E}-04$

$9.62481 \mathrm{E}-04$

$9.70898 \mathrm{E}-04$

$9.79352 \mathrm{E}-04$ $9.87843 \mathrm{E}-04$ 9.96371 E-04

$1.00493 \mathrm{E}-03$

$1.01354 \mathrm{E}-03$

$1.02217 \mathrm{E}-03$

$1.03085 \mathrm{E}-03$

$1.03956 \mathrm{E}-03$

$1.04830 \mathrm{E}-03$

$1.05709 \mathrm{E}-03$
$-8.58630 \mathrm{E}-01$

$-8.68246 \mathrm{E}-01$

$-8.77914 \mathrm{E}-01$

$-8.87636 \mathrm{E}-01$

$-8.97411 \mathrm{E}-01$

$-9.07238 \mathrm{E}-01$

$-9.17119 \mathrm{E}-01$

$-9.27052 \mathrm{E}-01$

$-9.37039 \mathrm{E}-01$

$-9.47078 \mathrm{E}-01$

$-9.57170 \mathrm{E}-01$

$-9.67316 \mathrm{E}-01$

$-9.77514 \mathrm{E}-01$

$-9.87766 \mathrm{E}-01$

$-9.98070 \mathrm{E}-01$

$-1.00843 \mathrm{E} \quad 00$

$-1.01884 \mathrm{E} \quad 00$

$-1.02930 \mathrm{E} \quad 00$

$-1.03982 \mathrm{E} \quad 00$

$-1.05038 \mathrm{E} 00$

$-1.06101 \mathrm{E} \quad 00$

$-1.07168 \mathrm{E} \quad 00$

$-1.08241 \mathrm{E} 00$

$-1.09319 \mathrm{E} 00$

$-1.10402 \mathrm{E} 00$

$-1.11491 \mathrm{E} \quad 00$

$-1.12584 \mathrm{E} \quad 00$

$-1.13683 \mathrm{E} \quad 00$

$-1.14788 \mathrm{E} 00$

$-1.15898 \mathrm{E} 00$

$-1.17012 \mathrm{E} \quad 00$

$-1.18133 \mathrm{E} \quad 00$

$-1.19258 \mathrm{E} \quad 00$

$-1.20389 \mathrm{E} 00$

$-1.21525 \mathrm{E} 00$

$-1.22666 \mathrm{E} 00$

$-1.23813 \mathrm{E} \quad 00$

$-1.24965 \mathrm{E} \quad 00$

$-1.26122 \mathrm{E} \quad 00$

$-1.27284 \mathrm{E} 00$

$-1.28452 \mathrm{E} \quad 00$

$-1.29625 \mathrm{E} \quad 00$

$-1.30803 \mathrm{E} \quad 00$

$-1.31986 \mathrm{E} 00$

$-1.33175 \mathrm{E} 00$

$-1.34369 \mathrm{E} \quad 00$

$-1.35568 \mathrm{E} 00$

$-1.36773 \mathrm{E} 00$

$-1.37982 \mathrm{E} 00$

$-1.39197 \mathrm{E} 00$

$-1.40418 \mathrm{E} \quad 00$

$-1.41643 \mathrm{E} \quad 00$

$-1.42874 \mathrm{E} \quad 00$

$-1.44110 \mathrm{E} \quad 00$

$-1.45351 \mathrm{E} 00$

$-1.46598 \mathrm{E} \quad 00$

$-1.47849 \mathrm{E} 00$

$-1.49106 \mathrm{E} 00$

$-1.50368 \mathrm{E} 00$

$-1.51636 \mathrm{E} \quad 00$ $g_{2}$

$-3.46649 \mathrm{E}-02$

$-3.51834 \mathrm{E}-02$

$-3.57070 \mathrm{E}-02$

$-3.62356 \mathrm{E}-02$

$-3.67693 \mathrm{E}-02$

$-3.73082 \mathrm{E}-02$

$-3.78521 \mathrm{E}-02$

$-3.84013 \mathrm{E}-02$

$-3.89556 \mathrm{E}-02$

$-3.95152 \mathrm{E}-02$

$-4.00800 \mathrm{E}-02$

$-4.06500 \mathrm{E}-02$

$-4.12254 \mathrm{E}-02$

$-4.18060 \mathrm{E}-02$

$-4.23920 \mathrm{E}-02$

$-4.29833 \mathrm{E}-02$

$-4.35800 \mathrm{E}-02$

- 4.41821 E-02

$-4.47897 \mathrm{E}-02$

$-4.54027 \mathrm{E}-02$

$-4.60211 \mathrm{E}-02$

$-4.66451 \mathrm{E}-02$

$-4.72746 \mathrm{E}-02$

$-4.79096 \mathrm{E}-02$

$-4.85502 \mathrm{E}-02$

$-4.91964 \mathrm{E}-02$

$-4.98482 \mathrm{E}-02$

$-5.05056 \mathrm{E}-02$

$-5.11687 \mathrm{E}-02$

$-5.18375 \mathrm{E}-02$

$-5.25120 \mathrm{E}-02$

$-5.31923 \mathrm{E}-02$

$-5.38782 \mathrm{E}-02$

$-5.45700 \mathrm{E}-02$

$-5.52676 \mathrm{E}-02$

$-5.59710 \mathrm{E}-02$

-5.66802E-02

$-5.73953 \mathrm{E}-02$

$-5.81163 \mathrm{E}-02$

$-5.88433 \mathrm{E}-02$

$-5.95761 \mathrm{E}-02$

$-6.03150 \mathrm{E}-02$

$-6.10598 \mathrm{E}-02$

$-6.18107 \mathrm{E}-02$

$-6.25675 \mathrm{E}-02$

$-6.33305 \mathrm{E}-02$

$-6.40995 \mathrm{E}-02$

$-6.48747 \mathrm{E}-02$

$-6.56559 \mathrm{E}-02$

$-6.64433 \mathrm{E}-02$

$-6.72369 \mathrm{E}-02$

$-6.80367 \mathrm{E}-02$

$-6.88427 \mathrm{E}-02$

$-6.96550 \mathrm{E}-02$

$-7.04736 \mathrm{E}-02$

$-7.12984 \mathrm{E}-02$

$-7.21296 \mathrm{E}-02$

$-7.29671 \mathrm{E}-02$

$-7.38109 \mathrm{E}-02$

$-7.46611 \mathrm{E}-02$

$\tan \delta_{2}$

$f_{-2}$

$2.93261 \mathrm{E} \quad 01 \quad-3.39097 \mathrm{E}-02$ $2.93300 \mathrm{E}$ 01 $-3.44167 \mathrm{E}-02$ $2.93338 \mathrm{E} \quad 01 \quad-3.49286 \mathrm{E}-02$

$2.93375 \mathrm{E} \quad 01 \quad-3.54455 \mathrm{E}-02$

$2.93412 \mathrm{E}$ 01 $-3.59675 \mathrm{E}-02$

$2.93449 \mathrm{E} \quad 01$

$2.93485 \mathrm{E} \quad 01$

$2.93521 \mathrm{E} \quad 01$

$2.93556 \mathrm{E} \quad 01$

$2.93591 \mathrm{E} \quad 01$

$2.93626 \mathrm{E} \quad 01$

$2.93660 \mathrm{E} \quad 01$

$2.93694 \mathrm{E}$ 01

$2.93727 \mathrm{E} \quad 01$

$2.93761 \mathrm{E} \quad 01$

$2.93794 \mathrm{E} \quad 01$

$2.93826 \mathrm{E} \quad 01$

$2.93858 \mathrm{E} \quad 01$

$2.93890 \mathrm{E} \quad 01$

$2.93921 \mathrm{E} \quad 01$

$2.93953 \mathrm{E} \quad 01$

$2.93983 \mathrm{E} \quad 01$

$2.94014 \mathrm{E} \quad 01$

$2.94044 \mathrm{E} \quad 01$

$2.94074 \mathrm{E} \quad 01$

2.94104E 01

$2.94133 \mathrm{E}$ 01

$2.94162 \mathrm{E} \quad 01$

$2.94191 \mathrm{E} 01$

$2.94219 \mathrm{E} 01$

$2.94247 \mathrm{E} \quad 01$

$2.94275 \mathrm{E} \quad 01$

$2.94303 \mathrm{E} \quad 01$

$2.94330 \mathrm{E} 01$

$2.94357 \mathrm{E} \cdot 01$

2.94384.E 01

$2.94411 \mathrm{E} \quad 01$

$2.94437 \mathrm{E} \quad 01$

$2.94463 \mathrm{E} \quad 01$

$2.94489 \mathrm{E} 01$

$2.94514 \mathrm{E} \quad 01$

$2.94540 \mathrm{E} \quad 01$ 
ELECTRON RADIAL FUNCTIONS

Electron

$Z=7 \quad A=14$

$\rho=1.2 A^{1 / 3} F$

$p$

24.10

24.20

24.30

24.40

24.50

24.60

24. 70

24.80

24.90

25.00

25.10

25.20

25.30

25.40

25.50

25.60

25.70

25.80

25.90

26.00

26.10

26.20

26.30

26.40

26.50

26.60

26.70

26.80

26.90

27.00

27.10

27.20

27.30

27.40

27.50

27.60

27.70

27.80

27.90

28.00

28.10

28.20

28.30

28.40

28.50

28.60

28.70

28.80

28.90

29.00

29.10

29.20

29.30

29.40

29.50

29.60

29.70

29.80

29.90

30.00

$$
F_{0}
$$

$1.17974 \mathrm{E} \quad 00$ $1.17973 \mathrm{E} \quad 00$ $1.17971 \mathrm{E} \quad 00$ $1.17970 \mathrm{E} \quad 00$ $1.17968 \mathrm{E} \quad 00$

$1.17967 \mathrm{E} \quad 00$ $1.17966 \mathrm{E} \quad 00$ $1.17964 \mathrm{E} \quad 00$ $1.17963 \mathrm{E} \quad 00$ $1.17962 \mathrm{E} \quad 00$

$1.17960 \mathrm{E} \quad 00$ $1.17959 \mathrm{E} \quad 00$ $1.17958 \mathrm{E} \quad 00$ $1.17956 \mathrm{E} \quad 00$ $1.17955 \mathrm{E} \quad 00$

$1.17954 \mathrm{E} \quad 00$ $1.17952 \mathrm{E} \quad 00$ $1.17951 \mathrm{E} \quad 00$ $1.17950 \mathrm{E} \quad 00$ $1.17948 \mathrm{E} \quad 00$

$1.17947 \mathrm{E} \quad 00$ $1.17946 \mathrm{E} \quad 00$ $1.17945 \mathrm{E} \quad 00$ $1.17943 \mathrm{E} \quad 00$ $\begin{array}{ll}1.17942 \mathrm{E} & 00\end{array}$

$1.17941 \mathrm{E} \quad 00$ $1.17940 \mathrm{E} \quad 00$ $1.17938 \mathrm{E} \quad 00$ $1.17937 \mathrm{E} \quad 00$ $1.17936 \mathrm{E} \quad 00$

$1.17935 \mathrm{E} \quad 00$ $1.17933 \mathrm{E} \quad 00$ $\begin{array}{ll}1.17932 \mathrm{E} & 00\end{array}$ $1.17931 \mathrm{E} \quad 00$ $1.17930 \mathrm{E} \quad 00$

$1.17929 \mathrm{E} \quad 00$ $1.17927 \mathrm{E} \quad 00$ $\begin{array}{ll}1.17926 \mathrm{E} & 00\end{array}$ $\begin{array}{lll}1.17925 \mathrm{E} & 00\end{array}$ $1.17924 \mathrm{E} \quad 00$
0

$\begin{array}{lll}1.17923 \mathrm{E} & 00\end{array}$ $1.17921 \mathrm{E} \quad 00$ $1.17920 \mathrm{E} \quad 00$ $1.17919 \mathrm{E}^{-} 00$ $1.17918 \mathrm{E} \quad 00$

$1.17917 \mathrm{E} \quad 00$ $\begin{array}{ll}1.17916 \mathrm{E} & 00\end{array}$ $1.17915 \mathrm{E} \quad 00$ $1.17913 \mathrm{E} \quad 00$ $1.17912 \mathrm{E} \quad 00$

$1.17911 \mathrm{E} \quad 00$ $1.17910 \mathrm{E} \quad 00$ $1.17909 \mathrm{E} \quad 00$ $1.17908 \mathrm{E} \quad 00$ $1.17907 \mathrm{E} \quad 00$

$1.17906 \mathrm{E} \quad 00$ $1.17904 \mathrm{E} \quad 00$ $1.17903 \mathrm{E} \quad 00$ $1.17902 \mathrm{E} \quad 00$ $1.17901 \mathrm{E} \quad 00$ $f_{1}$

$g_{1}$

$\begin{array}{lll}-2.52609 \mathrm{E} & 01\end{array}$ $-2.53657 \mathrm{E}$ 0l $-2.54706 \mathrm{E} \quad 01$ $-2.55754 \mathrm{E}$ ol -2.56802 E 01

$-2.57849 \mathrm{E} \quad 0 \mathrm{l}$ $-2.58897 \mathrm{E} \quad 01$ $-2.59944 \mathrm{E} \quad 01$ $-2.60991 \mathrm{E} 01$

$-2.62038 \mathrm{E}$ ol

$-2.63085 \mathrm{E} \quad 01$

$-2.64131 \mathrm{E} \quad 01$

$-2.65177 \mathrm{E} 01$

$-2.66223 \mathrm{E} \quad 01$

$-2.67269 \mathrm{E} 01$

$-2.68314 \mathrm{E} \quad 01$ $-2.69359 \mathrm{E}$ 0l $-2.70404 \mathrm{E} \quad 01$ $-2.71449 \mathrm{E}$ ol $-2.72493 \mathrm{E} \quad 01$

$-2.73538 \mathrm{E}$ ol $-2.74582 \mathrm{E} \quad 01$ $-2.75625 \mathrm{E} 01$ $-2.76669 \mathrm{E} 01$ $-2.77712 \mathrm{E} 0 \mathrm{l}$

$-2.78755 \mathrm{E} 01$ $-2.79798 \mathrm{E} \quad 01$ $-2.80840 \mathrm{E}$ 0l $-2.81883 \mathrm{E} \quad 01$ $-2.82925 \mathrm{E} 01$

$-2.83967 \mathrm{E} \quad 01$ $-2.85008 \mathrm{E} 01$ $-2.86050 \mathrm{E}$ 0l $-2.87091 \mathrm{E} \quad 01$ $-2.88132 \mathrm{E}$ 0l

$-2.89172 \mathrm{E} \quad 01$ $-2.90213 \mathrm{E} 01$ $-2.91253 \mathrm{E} \quad 01$ $-2.92293 \mathrm{E}$ ol $-2.93332 \mathrm{E} 01$

$-2.94372 \mathrm{E} 01$ $-2.95411 \mathrm{E} 01$ $-2.96450 \mathrm{E} \quad 01$ $-2.97488 \mathrm{E} 01$ $-2.98527 \mathrm{E}$ 0l

$-2.99565 \mathrm{E} \quad 01$ $-3.00603 \mathrm{E} 01$ $-3.01640 \mathrm{E} 01$ $-3.02678 \mathrm{E}$ ol $-3.03715 \mathrm{E} 01$

$-3.04752 \mathrm{E} 01$ $-3.05788 \mathrm{E} 01$ $-3.06825 \mathrm{E} 01$ $-3.07861 \mathrm{E} 01$ $-3.08897 \mathrm{E} 01$

$-3.09932 \mathrm{E} 01$ $-3.10968 \mathrm{E} 01$ $-3.12003 \mathrm{E} 01$ $-3.13037 \mathrm{E} 01$ $-3.14072 \mathrm{E}$ 0l
$-2.10881 \mathrm{E} \quad 00$ $-2.12397 \mathrm{E} \quad 00$

$-2.13918 \mathrm{E} \quad 00$

$-2.15444 \mathrm{E} \quad 00$

-2.16975 E 00

$-2.18512 \mathrm{E} \quad 00$

$-2.20054 \mathrm{E} \quad 00$

$-2.21600 \mathrm{E} 00$

$-2.23152 \mathrm{E} \quad 00$

$-2.24710 \mathrm{E} \quad 00$

$-2.26272 \mathrm{E} \quad 00$

$-2.27839 \mathrm{E} \quad 00$

$-2.29412 \mathrm{E} \quad 00$

$-2.30990 \mathrm{E} 00$

$-2.32572 \mathrm{E} \quad 00$

$-2.34160 \mathrm{E} \quad 00$

$-2.35754 \mathrm{E} \quad 00$

$-2.37352 \mathrm{E} \quad 00$

$-2.38955 \mathrm{E} \quad 00$

$-2.40564 \mathrm{E} \quad 00$

$-2.42177 \mathrm{E} \quad 00$

$-2.43796 \mathrm{E} \quad 00$

$-2.45420 \mathrm{E} \quad 00$

$-2.47049 \mathrm{E} \quad 00$

-2.48683 E 00

$\begin{array}{lll}-2.50323 \mathrm{E} & 00\end{array}$

$-2.51967 \mathrm{E} \quad 00$

$-2.53617 \mathrm{E} \quad 00$

$-2.55271 \mathrm{E} 00$

$-2.56931 \mathrm{E} \quad 00$

$-2.58596 \mathrm{E} \quad 00$

$\begin{array}{lll}-2.60266 \mathrm{E} & 00\end{array}$

$-2.61941 \mathrm{E} 00$

$-2.63621 \mathrm{E} \quad 00$

$-2.65307 \mathrm{E} \quad 00$

$-2.66997 \mathrm{E} \quad 00$

$-2.68693 \mathrm{E} 00$

$-2.70393 \mathrm{E} 00$

$-2.72099 \mathrm{E} 00$

$-2.73810 \mathrm{E} 00$

$-2.75526 \mathrm{E} \quad 00$ $-2.77247 \mathrm{E} \quad 00$ $-2.78973 \mathrm{E} \quad 00$ $-2.80704 \mathrm{E} \quad 00$ $-2.82441 \mathrm{E} \quad 00$

$-2.84182 \mathrm{E} \quad 00$ $-2.85929 \mathrm{E} \quad 00$ $-2.87680 \mathrm{E} \quad 00$ $-2.89437 \mathrm{E} \quad 00$ $-2.91199 \mathrm{E} \quad 00$

$-2.92966 \mathrm{E} \quad 00$ $-2.94738 \mathrm{E} \quad 00$ $-2.96515 \mathrm{E} 00$ $-2.98297 \mathrm{E} 00$ $-3.00084 \mathrm{E} 00$

$-3.01876 \mathrm{E} \quad 00$ $-3.03674 \mathrm{E} 00$ $-3.05476 \mathrm{E} \quad 00$ $-3.07284 \mathrm{E} \quad 00$ $-3.09096 \mathrm{E} 00$

$\tan \delta_{1}$

$f_{-1}$

$4.79802 \mathrm{E}-03$ $4.80080 \mathrm{E}-03$ $4.80354 \mathrm{E}-03$

$4.80623 \mathrm{E}-03$

$4.80887 \mathrm{E}-03$

$4.81149 \mathrm{E}-03$

$4.81406 \mathrm{E}-03$

$4.81659 \mathrm{E}-03$

$4.81908 \mathrm{E}-03$

$4.82153 \mathrm{E}-03$

$4.82393 \mathrm{E}-03$

$4.82630 \mathrm{E}-03$

$4.82864 \mathrm{E}-03$

$4.83094 \mathrm{E}-03$

$4.83320 \mathrm{E}-03$

$4.83541 \mathrm{E}-03$

$4.83761 \mathrm{E}-03$

$4.83976 \mathrm{E}-03$

$4.84187 \mathrm{E}-03$

4.84394 E-03

$4.84598 \mathrm{E}-03$

$4.84800 \mathrm{E}-03$

$4.84996 \mathrm{E}-03$

$4.85190 \mathrm{E}-03$

$4.85380 \mathrm{E}-03$

$4.85568 \mathrm{E}-03$

$4.85751 \mathrm{E}-03$

$4.85931 \mathrm{E}-03$

$4.86108 \mathrm{E}-03$

$4.86282 \mathrm{E}-03$

$4.86453 \mathrm{E}-03$

$4.86620 \mathrm{E}-03$

$4.86785 \mathrm{E}-03$

$4.86946 \mathrm{E}-03$

4.87104 E-03

$4.87259 \mathrm{E}-03$

$4.87411 \mathrm{E}-03$

$4.87560 \mathrm{E}-03$

$4.87705 \mathrm{E}-03$ 


\section{$\bar{F}$}

24.10

24.20

24.30

24.40

24.50

24.60

24.70

24.80

24.90

25.00

25,10

25.20

25.30

25.40

25.50

25.60

25.70

25.80

25.90

26.00

26.10

26.20

26.30

26.40

26.50

26.60

26.70

26.80

26.90

27.00

27.10

27.20

27.30

27.40

27.50

27.60

27.70

27.80

27.90

28.00

28.10

28.20

28.30

28.40

28.50

28.60

28.70

28.80

28.90

29.00

29.10

29.20

29.30

29.40

29.50

29.60

29.70

29.80

29.90

30.00 $f_{2}$

$1.06591 \mathrm{E}-03$

$1.07477 \mathrm{E}-03$

$1.08366 \mathrm{E}-03$

$1.09259 \mathrm{E}-03$

$1.10156 \mathrm{E}-03$

$1.11056 \mathrm{E}-03$

$1.11960 \mathrm{E}-03$

$1.12868 \mathrm{E}-03$

$1.13779 \mathrm{E}-03$

$1.14694 \mathrm{E}-03$

$1.15613 \mathrm{E}-03$

$1.16535 \mathrm{E}-03$

$1.17461 \mathrm{E}-03$

$1.18391 \mathrm{E}-03$

$1.19324 \mathrm{E}-03$

$1.20261 \mathrm{E}-03$

$1.21202 \mathrm{E}-03$

$1.22146 \mathrm{E}-03$

$1.23094 \mathrm{E}-03$

$1.24046 \mathrm{E}-03$

$1.25001 \mathrm{E}-03$

$1.25960 \mathrm{E}-03$

1.26922 E- 03

$1.27889 \mathrm{E}-03$

$1.28859 \mathrm{E}-03$

$1.29832 \mathrm{E}-03$

$1.30810 \mathrm{E}-03$

$1.31790 \mathrm{E}-03$

$1.32775 \mathrm{E}-03$

$1.33763 \mathrm{E}-03$

$1.34755 \mathrm{E}-03$

$1.35751 \mathrm{E}-03$

$1.36750 \mathrm{E}-03$

$1.37753 \mathrm{E}-03$

$1.38759 \mathrm{E}-03$

$1.39770 \mathrm{E}-03$

$1.40784 \mathrm{E}-03$

$1.41801 \mathrm{E}-03$

$1.42822 \mathrm{E}-03$

$1.43847 \mathrm{E}-03$

$1.44876 \mathrm{E}-03$

$1.45908 \mathrm{E}-03$

$1.46944 \mathrm{E}-03$

$1.47983 \mathrm{E}-03$

$1.49027 \mathrm{E}-03$

$1.50073 \mathrm{E}-03$

$1.51124 \mathrm{E}-03$

$1.52178 \mathrm{E}-03$

$1.53236 \mathrm{E}-03$

$1.54297 \mathrm{E}-03$

$1.55363 \mathrm{E}-03$

$1.56431 \mathrm{E}-03$

$1.57504 \mathrm{E}-03$

$1.58580 \mathrm{E}-03$

$1.59660 \mathrm{E}-03$

$1.60743 \mathrm{E}-03$

$1.61830 \mathrm{E}-03$

$1.62921 \mathrm{E}-03$

$1.64016 \mathrm{E}-03$

$1.65114 \mathrm{E}-03$
$-1.52909 \mathrm{E} \quad 00$

$-1.54187 \mathrm{E} \quad 00$

$-1.55470 \mathrm{E} \quad 00$

$-1.56758 \mathrm{E} \quad 00$

$-1.58052 \mathrm{E} \quad 00$

$-1.59351 \mathrm{E} \quad 00$

$-1.60655 \mathrm{E} \quad 00$

$-1.61964 \mathrm{E} \quad 00$

$-1.63279 \mathrm{E} \quad 00$

$-1.64599 \mathrm{E} 00$

$-1.65924 \mathrm{E} \quad 00$

$-1.67254 \mathrm{E} \quad 00$

$-1.68590 \mathrm{E} 00$

$-1.69930 \mathrm{E} 00$

$-1.71276 \mathrm{E} 00$

$-1.72627 \mathrm{E} \quad 00$

$-1.73984 \mathrm{E} \quad 00$

$-1.75346 \mathrm{E} \quad 00$

$-1.76712 \mathrm{E} \quad 00$

-1.78085 E 00

$-1.79462 \mathrm{E} \quad 00$

$-1.80844 \mathrm{E} \quad 00$

$-1.82232 \mathrm{E} 00$

$-1.83625 \mathrm{E} \cdot 00$

$-1.85023 \mathrm{E} \quad 00$

$-1.86427 \mathrm{E} \quad 00$

$-1.87835 \mathrm{E} \quad 00$

$-1.89249 \mathrm{E} 00$

$-1.90668 \mathrm{E} 00$

$-1.92092 \mathrm{E} \quad 00$

$-1.93521 \mathrm{E} \quad 00$ $-1.94956 \mathrm{E} \quad 00$

$-1.96396 \mathrm{E} 00$

-1.97841 E 00

$-1.99291 \mathrm{E} \quad 00$

$-2.00747 \mathrm{E} \quad 00$

$-2.02207 \mathrm{E} \quad 00$

$-2.03673 \mathrm{E} \quad 00$

$-2.05144 \mathrm{E} \quad 00$

$-2.06620 \mathrm{E} \quad 00$

$-2.08102 \mathrm{E} \quad 00$

$-2.09588 \mathrm{E} \quad 00$

$-2.11080 \mathrm{E} \quad 00$

$-2.12577 \mathrm{E} \quad 00$

$-2.14079 \mathrm{E} \quad 00$

$-2.15587 \mathrm{E} \quad 00$

$-2.17099 \mathrm{E} \quad 00$

$-2.18617 \mathrm{E} \quad 00$

$-2.20140 \mathrm{E} \quad 00$

$-2.21668 \mathrm{E} \quad 00$

$-2.23201 \mathrm{E} \quad 00$

$-2.24739 \mathrm{E} \quad 00$

$-2.26283 \mathrm{E} \quad 00$

$-2.27832 \mathrm{E} \quad 00$

$-2.29386 \mathrm{E} 00$

$-2.30945 \mathrm{E} \quad 00$

$-2.32509 \mathrm{E} \quad 00$

$-2.34079 \mathrm{E} \quad 00$

$-2.35653 \mathrm{E} \quad 00$

$-2.37233 \mathrm{E} 00$ $g_{2}$

$-7.55178 \mathrm{E}-02$

$-7.63809 \mathrm{E}-02$

$-7.72505 \mathrm{E}-02$

$-7.81265 \mathrm{E}-02$

$-7.90091 \mathrm{E}-02$

$-7.98981 \mathrm{E}-02$

$-8.07937 \mathrm{E}-02$

$-8.16959 \mathrm{E}-02$

$-8.26047 \mathrm{E}-02$

$-8.35201 \mathrm{E}-02$

$-8.44422 \mathrm{E}-02$

$-8.53709 \mathrm{E}-02$

$-8.63063 \mathrm{E}-02$

$-8.72484 \mathrm{E}-02$

$-8.81972 \mathrm{E}-02$

$-8.91528 \mathrm{E}-02$

$-9.01152 \mathrm{E}-02$

$-9.10844 \mathrm{E}-02$

$-9.20605 \mathrm{E}-02$

$-9.30434 \mathrm{E}-02$

$-9.40331 \mathrm{E}-02$

$-9.50298 \mathrm{E}-02$

$-9.60333 \mathrm{E}-02$

$-9.70439 \mathrm{E}-02$

$-9.80613 \mathrm{E}-02$

$-9.90859 \mathrm{E}-02$

$-1.00117 \mathrm{E}-01$

$-1.01156 \mathrm{E}-01$

$-1.02201 \mathrm{E}-01$

$-1.03254 \mathrm{E}-01$

$-1.04314 \mathrm{E}-01$

- $1.05381 \mathrm{E}-01$

$-1.06455 \mathrm{E}-01$

$-1.07536 \mathrm{E}-01$

$-1.08625 \mathrm{E}-01$

$-1.09720 \mathrm{E}-01$

$-1.10823 \mathrm{E}-01$

$-1.11933 \mathrm{E}-01$

-1.13051 E-01

$-1.14176 \mathrm{E}-01$

$-1.15308 \mathrm{E}-01$

$-1.16447 \mathrm{E}-01$

$-1.17594 \mathrm{E}-01$

$-1.18748 \mathrm{E}-01$

$-1.19910 \mathrm{E}-01$

$-1.21079 \mathrm{E}-01$

$-1.22256 \mathrm{E}-01$

$-1.23440 \mathrm{E}-01$

$-1.24632 \mathrm{E}-01$

$-1.25831 \mathrm{E}-01$

$-1.27038 \mathrm{E}-01$

$-1.28252 \mathrm{E}-01$

$-1.29474 \mathrm{E}-01$

$-1.30704 \mathrm{E}-01$

- 1.31941 E-01

$-1.33186 \mathrm{E}-01$

$-1.34439 \mathrm{E}-01$

$-1.35699 \mathrm{E}-01$

$-1.36967 \mathrm{E}-01$

$-1.38243 \mathrm{E}-01$

$\tan \delta_{2}$

$f_{-2}$

$2.94980 \mathrm{E} \quad 01 \quad-7.3929 .5 \mathrm{E}-02$

$2.95001 \mathrm{E}$ ol $-7.47762 \mathrm{E}-02$

$2.95022 \mathrm{E} \quad 01 \quad-7.56294 \mathrm{E}-02$

$2.95043 \mathrm{E}$ 01 $-7.64889 \mathrm{E}-02$

$2.95063 \mathrm{E} \quad 01 \quad-7.73549 \mathrm{E}-02$

$2.95084 \mathrm{E} \quad 01 \quad-7.82273 \mathrm{E}-02$

$2.95104 \mathrm{E}$ 01 $-7.91062 \mathrm{E}-02$

$2.95124 \mathrm{E}$ 01 $-7.99916 \mathrm{E}-02$

$2.95144 \mathrm{E}$ 01 $-8.088 .35 \mathrm{E}-02$

$2.95164 \mathrm{E} \quad 01 \quad-8.17819 \mathrm{E}-02$

$2.95184 \mathrm{E} \quad 01 \quad-8.26870 \mathrm{E}-02$

$2.95203 \mathrm{E}$ 01 $-8.35985 \mathrm{E}-02$

$2.95222 \mathrm{E}$ ol $-8.45168 \mathrm{E}-02$

$2.95241 \mathrm{E}$ 01 $-8.54416 \mathrm{E}-02$

$2.95260 \mathrm{E}$ ol $-8.63731 \mathrm{E}-02$

$2.95279 \mathrm{E} \quad 01$

$2.95298 \mathrm{E} \quad 01$

$2.95317 \mathrm{E} \quad 01$

$2.95335 \mathrm{E} \quad 01$

$2.95353 \mathrm{E} \quad 01$

$2.95371 \mathrm{E} \quad 01$

$2.95389 \mathrm{E} \quad 01$

$2.95407 \mathrm{E} \quad 01$

$2.95425 \mathrm{E} \quad 01$

$2.95442 \mathrm{E}$ 0I

$2.95460 \mathrm{E} \quad 0 \mathrm{I}$

$2.95477 \mathrm{E} \quad 01$

$2.95494 \mathrm{E} \quad 01$

$2.95511 \mathrm{E} 01$

$2.95528 \mathrm{E} \quad 01$

$2.95545 \mathrm{E} \quad 01$

$2.95561 \mathrm{E} 01$

$2.95578 \mathrm{E} \quad 01$

$2.95594 \mathrm{E} \quad 01$

2.95611 E 01

$2.95627 \mathrm{E} \quad 01$

$2.95643 \mathrm{E} \quad 01$

$2.95659 \mathrm{E} 01$

$2.95674 \mathrm{E} \quad 01$

$2.95690 \mathrm{E}$

$-8.73113 E-02$ 
$p$

$F_{0}$

30.10

30.20

30.30

30.40

30.50

30.60

30.70

30.80

30.90

31.00

31.10

31.20

31.30

31.40

31.50

31.60

31.70

31.80

31.90

32.00

32.10

32.20

32.30

32.40

32.50

32.60

32.70

32.80

32.90

33.00

33.10

33.20

33.30

33.40

33.50

33.60

33.70

33.80

33.90

34.00

34.10

34.20

34.30

34.40

34.50

34.60

34.70

34.80

34.90

35.00

35.10

35.20

35.30

35.40

35.50

35.60

35.70

35.80

35.90

36.00

\section{$f_{1}$}

$1.17900 \mathrm{E} \quad 00$ $1.17899 \mathrm{E} \quad 00$ $1.17898 \mathrm{E} \quad 00$ $1.17897 \mathrm{E} \quad 00$

$1.17896 \mathrm{E} \quad 00$

$1.17895 \mathrm{E} \quad 00$

$1.17894 \mathrm{E} \quad 00$

$1.17893 \mathrm{E} \quad 00$

$1.17891 \mathrm{E} \quad 00$

$1.17890 \mathrm{E} 00$

$1.17889 \mathrm{E} \quad 00$

$1.17888 \mathrm{E} \quad 00$

$1.17887 \mathrm{E} \quad 00$

$1.17886 \mathrm{E} \quad 00$

$1.17885 \mathrm{E} \quad 00$

$1.17884 \mathrm{E} \quad 00$

$1.17883 \mathrm{E} \quad 00$

$1.17882 \mathrm{E} \quad 00$

$1.17881 \mathrm{E} \quad 00$

$1.17880 \mathrm{E} \quad 00$

$1.17879 \mathrm{E} \quad 00$

$1.17878 \mathrm{E} \quad 00$

$1.17877 \mathrm{E} \quad 00$

$1.17876 \mathrm{E} \quad 00$

$1.17875 \mathrm{E} \quad 00$

$1.17874 \mathrm{E} \quad 00$ $1.17873 \mathrm{E} \quad 00$ $1.17872 \mathrm{E} \quad 00$ $\begin{array}{ll}1.17871 \mathrm{E} & 00\end{array}$ $1.17870 \mathrm{E} \quad 00$

$1.17869 \mathrm{E} \quad 00$ $1.17868 \mathrm{E} \quad 00$ $1.17867 \mathrm{E} \quad 00$ $1.17866 \mathrm{E} \quad 00$ $1.17865 \mathrm{E} \quad 00$

$1.17864 \mathrm{E} \quad 00$ $1.17863 \mathrm{E} \quad 00$ $1.17862 \mathrm{E} \quad 00$ $1.17861 \mathrm{E} \quad 00$ $1.17860 \mathrm{E} \quad 00$

$1.17859 \mathrm{E} \quad 00$ $1.17859 \mathrm{E} \quad 00$ $1.17858 \mathrm{E} .00$ $1.17857 \mathrm{E} \quad 00$ $1.17856 \mathrm{E} \quad 00$

$1.17855 \mathrm{E} \quad 00$ $1.17854 \mathrm{E} \quad 00$ $1.17853 \mathrm{E} \quad 00$ $1.17852 \mathrm{E} \quad 00$ $1.17851 \mathrm{E} \quad 00$

$1.17850 \mathrm{E} \quad 00$ $1.17849 \mathrm{E} \quad 00$ $\begin{array}{ll}1.17848 \mathrm{E} & 00\end{array}$ $1.17847 \mathrm{E} \quad 00$ $1.17847 \mathrm{E} \quad 00$

$1.17846 \mathrm{E} \quad 00$ 1.17845 E 00 $1.17844 \mathrm{E} \quad 00$ $1.17843 \mathrm{E} \quad 00$ $1.17842 \mathrm{E} \quad 00$ $g_{1}$

$-3.15106 \mathrm{E} 01$ $-3.16140 \mathrm{E} 01$ $-3.17174 \mathrm{E} \quad 01$ $-3.18208 \mathrm{E} 01$ $-3.19241 \mathrm{E} 01$

$-3.20274 \mathrm{E} \quad 01$ $-3.21307 \mathrm{E} 01$ $-3.22339 \mathrm{E} 01$ $-3.23372 \mathrm{E} 01$ $-3.24404 \mathrm{E} 01$

$-3.25435 \mathrm{E} \quad 01$ $-3.26467 \mathrm{E} 01$ $-3.27498 \mathrm{E} \quad 01$ $-3.28529 \mathrm{E} 01$ $-3.29559 \mathrm{E} 01$

$-3.30590 \mathrm{E} \quad 01$ $-3.31620 \mathrm{E} 01$ $-3.32650 \mathrm{E} 01$ $-3.33679 \mathrm{E} 01$ $-3.34709 \mathrm{E} 01$

$-3.35738 \mathrm{E} 01$ $-3.36766 \mathrm{E} 01$ $-3.37795 \mathrm{E} 01$ $-3.38823 \mathrm{E} 01$ $-3.39851 \mathrm{E} 01$

$-3.40879 \mathrm{E} \quad 01$ $-3.41906 \mathrm{E} 01$ $-3.42933 \mathrm{E} 01$ $-3.43960 \mathrm{E} 01$ $-3.44987 \mathrm{E} 01$

$-3.46013 \mathrm{E} \quad 01$ $-3.47039 \mathrm{E} 01$ $-3.48065 \mathrm{E} 01$ $-3.49090 \mathrm{E} 01$ $-3.50115 \mathrm{E} 01$

$-3.51140 \mathrm{E} 01$ $-3.52165 \mathrm{E} 01$ $-3.53189 \mathrm{E} 01$ $-3.54213 \mathrm{E} 01$ $-3.55237 \mathrm{E} 01$

$-3.56261 \mathrm{E} 01$ $-3.57284 \mathrm{E} 01$ $-3.58307 \mathrm{E} 01$ $-3.59330 \mathrm{E} 01$ $-3.60352 \mathrm{E} 01$

$-3.61374 \mathrm{E} 01$ $-3.62396 \mathrm{E} 01$ $-3.63418 \mathrm{E} 01$ $-3.64439 \mathrm{E} 01$ $-3.65460 \mathrm{E} 01$

$-3.66480 \mathrm{E} 01$ $-3.67501 \mathrm{E} 01$ $-3.68521 \mathrm{E} 01$ $-3.69541 \mathrm{E} 01$ $-3.70560 \mathrm{E} 01$

$-3.71580 \mathrm{E} 01$ $-3.72599 \mathrm{E} 01$ $-3.73617 \mathrm{E} 01$ $-3.74636 \mathrm{E}$ 0l $-3.75654 \mathrm{E}$ 0l $\tan \delta_{1}$

$-3.10914 \mathrm{E} \quad 00$ $-3.12737 \mathrm{E} 00$ $-3.14565 \mathrm{E} \quad 00$ $-3.16398 \mathrm{E} \quad 00$ $-3.18235 \mathrm{E} \quad 00$

$-3.20078 \mathrm{E} \quad 00$ $-3.21926 \mathrm{E} 00$ $-3.23779 \mathrm{E} 00$ $-3.25638 \mathrm{E} \quad 00$ $-3.27501 \mathrm{E} \quad 00$

$-3.29369 \mathrm{E} \quad 00$ $-3.31242 \mathrm{E} 00$ $-3.33121 \mathrm{E} 00$ $-3.35004 \mathrm{E} \quad 00$ $-3.36893 \mathrm{E} 00$

$-3.38786 \mathrm{E} \quad 00$ $-3.40685 \mathrm{E} \quad 00$ $-3.42588 \mathrm{E} \quad 00$ $-3.44497 \mathrm{E} \quad 00$ $-3.46410 \mathrm{E} \quad 00$

$-3.48329 \mathrm{E} \quad 00$ $-3.50253 \mathrm{E} 00$ $-3.52182 \mathrm{E} 00$ $-3.54115 \mathrm{E} \quad 00$ $-3.56054 \mathrm{E} \quad 00$

$-3.57998 \mathrm{E} \quad 00$ $-3.59947 \mathrm{E} \quad 00$ $-3.61901 \mathrm{E} \quad 00$ $-3.63860 \mathrm{E} \quad 00$ $-3.65824 \mathrm{E} \quad 00$

$-3.67793 \mathrm{E} \quad 00$ $-3.69767 \mathrm{E} \quad 00$ $-3.71746 \mathrm{E} \quad 00$ $-3.73730 \mathrm{E} 00$ $-3.75719 \mathrm{E} \quad 00$

$-3.77713 \mathrm{E} \quad 00$ $-3.79712 \mathrm{E} \quad 00$ $-3.81716 \mathrm{E} \quad 00$ $-3.83725 \mathrm{E} \quad 00$ $-3.85739 \mathrm{E} \quad 00$

$-3.87758 \mathrm{E} \quad 00$ $-3.89782 \mathrm{E} \quad 00$ $-3.91811 \mathrm{E} \quad 00$ $-3.93845 \mathrm{E} \quad 00$ $-3.95884 \mathrm{E} \quad 00$

$-3.97928 \mathrm{E} \quad 00$ $-3.99977 \mathrm{E} \quad 00$ $-4.02031 \mathrm{E} 00$ $-4.04090 \mathrm{E} \quad 00$ $-4.06154 \mathrm{E} \quad 00$

$-4.08223 \mathrm{E} \quad 00$ $-4.10297 \mathrm{E} \quad 00$ $-4.12376 \mathrm{E} \quad 00$ $-4.14460 \mathrm{E} \quad 00$ $-4.16549 \mathrm{E} \quad 00$

$-4.18643 \mathrm{E} \quad 00$ $-4.20742 \mathrm{E} \quad 00$ $-4.22845 \mathrm{E} \quad 00$ $-4.24954 \mathrm{E} \quad 00$ $-4.27068 \mathrm{E} \quad 00$ $4.90201 \mathrm{E}-03 \quad-3.04986 \mathrm{E} \quad 00$ $4.90284 \mathrm{E}-03 \quad-3.06782 \mathrm{E} \quad 00$ $4.90364 \mathrm{E}-03 \quad-3.08583 \mathrm{E} \quad 00$ $4.90441 \mathrm{E}-03 \quad-3.10389 \mathrm{E} \quad 00$ $4.90517 \mathrm{E}-03 \quad-3.12200 \mathrm{E} \quad 00$

$4.90591 \mathrm{E}-03 \quad-3.14017 \mathrm{E} \quad 00$ $4.90661 \mathrm{E}-03 \quad-3.15838 \mathrm{E} \quad 00$ $4.90730 \mathrm{E}-03 \quad-3.17664 \mathrm{E} \quad 00$ $4.90796 \mathrm{E}-03 \quad-3.19496 \mathrm{E} \quad 00$ $4.90860 \mathrm{E}-03 \quad-3.21332 \mathrm{E} \quad 00$

$4.90921 \mathrm{E}-03 \quad-3.23174 \mathrm{E} \quad 00$ $4.90980 \mathrm{E}-03 \quad-3.25021 \mathrm{E} \quad 00$ $4.91036 \mathrm{E}-03 \quad-3.26872 \mathrm{E} \quad 00$ $4.91091 \mathrm{E}-03 \quad-3.28729 \mathrm{E} \quad 00$ $4.91143 \mathrm{E}-03 \quad-3.30591 \mathrm{E} \quad 00$

$4.91192 \mathrm{E}-03 \quad-3.32458 \mathrm{E} \quad 00$ $4.91240 \mathrm{E}-03 \quad-3.34329 \mathrm{E} \quad 00$ $4.91286 \mathrm{E}-03 \quad-3.36206 \mathrm{E} \quad 00$ $4.91329 \mathrm{E}-03-3.38088 \mathrm{E} \quad 00$ $4.91369 \mathrm{E}-03-3.39975 \mathrm{E} \quad 00$

$4.91408 \mathrm{E}-03 \quad-3.41867 \mathrm{E} \quad 00$ $4.91445 \mathrm{E}-03 \quad-3.43764 \mathrm{E} \quad 00$ $4.91479 \mathrm{E}-03-3.45667 \mathrm{E} \quad 00$ $4.91511 \mathrm{E}-03 \quad-3.47574 \mathrm{E} \quad 00$ $4.91542 \mathrm{E}-03 \quad-3.49486 \mathrm{E} \quad 00$

$4.91570 \mathrm{E}-03$ $4.91595 \mathrm{E}-03$ $4.91620 \mathrm{E}-03$ $4.91641 \mathrm{E}-03$ $4.91660 \mathrm{E}-03$

$4.91678 \mathrm{E}-03$ $4.91693 \mathrm{E}-03$ $4.91707 \mathrm{E}-03$ $4.91718 \mathrm{E}-03$ $4.91728 \mathrm{E}-03$

$4.91735 \mathrm{E}-03$ 
ELECTRON RADIAL FUNCTIONS

$Z=7 \quad A=14 \quad \rho=1.2 A^{1 / 3} F$

Electron

$\bar{F}$

$f_{2}$

$1.66216 \mathrm{E}-03$

$1.67321 \mathrm{E}-03$

$1.68430 \mathrm{E}-03$

$1.69543 \mathrm{E}-03$

$1.70659 \mathrm{E}-03$

$1.71 .779 \mathrm{E}-03$

$1.72903 \mathrm{E}-03$

$1.74031 \mathrm{E}-03$

$1.75162 \mathrm{E}-03$

$1.76296 \mathrm{E}-03$

$1.77435 \mathrm{E}-03$

$1.78577 \mathrm{E}-03$

$1.79723 \mathrm{E}-03$

$1.80872 \mathrm{E}-03$

$1.82025 \mathrm{E}-03$

$1.83182 \mathrm{E}-03$

$1.84342 \mathrm{E}-03$

$1.85506 \mathrm{E}-03$

$1.86674 \mathrm{E}-03$

$1.87845 \mathrm{E}-03$

$1.89020 \mathrm{E}-03$

$1.90199 \mathrm{E}-03$

$1.91381 \mathrm{E}-03$

$1.92567 \mathrm{E}-03$

$1.93757 \mathrm{E}-03$

$1.94950 \mathrm{E}-03$

$1.96147 \mathrm{E}-03$

$1.97348 \mathrm{E}-03$

$1.98552 \mathrm{E}-03$

$1.99760 \mathrm{E}-03$

$2.00971 \mathrm{E}-03$

$2.02187 \mathrm{E}-03$

$2.03406 \mathrm{E}-03$

$2.04628 \mathrm{E}-03$

$2.05855 \mathrm{E}-03$

$2.07085 \mathrm{E}-03$

$2.08318 \mathrm{E}-03$

$2.09555 \mathrm{E}-03$

$2.10796 \mathrm{E}-03$

$2.12041 \mathrm{E}-03$

$2.13289 \mathrm{E}-03$

$2.14541 \mathrm{E}-03$

$2.15796 \mathrm{E}-03$

$2.17056 \mathrm{E}-03$

$2.18319 \mathrm{E}-03$

$2.19585 \mathrm{E}-03$

$2.20855 \mathrm{E}-03$

$2.22129 \mathrm{E}-03$

$2.23407 \mathrm{E}-03$

$2.24688 \mathrm{E}-03$

$2.25973 \mathrm{E}-03$ $2.27261 \mathrm{E}-03$

$2.28553 \mathrm{E}-03$

$2.29849 \mathrm{E}-03$

$2.31149 \mathrm{E}-03$

$2.32452 \mathrm{E}-03$

$2.33759 \mathrm{E}-03$

$2.35069 \mathrm{E}-03$

$2.36383 \mathrm{E}-03$

$2.37701 \mathrm{E}-03$ $g_{2}$

$-2.38818 \mathrm{E} \quad 00 \quad-1.39527 \mathrm{E}-01$

$-2.40409 \mathrm{E} \quad 00 \quad-1.40818 \mathrm{E}-01$

$-2.42004 \mathrm{E} \quad 00 \quad-1.42118 \mathrm{E}-01$

$-2.43604 \mathrm{E} \quad 00 \quad-1.43425 \mathrm{E}-01$

$-2.45210 \mathrm{E} \quad 00 \quad-1.44740 \mathrm{E}-01$

$-2.46821 \mathrm{E} \quad 00$

$-2.48437 \mathrm{E} \quad 00$

$-2.50058 \mathrm{E} \quad 00$

$-2.51684 \mathrm{E} \quad 00$

$-2.53316 \mathrm{E} \quad 00$

$-2.54952 \mathrm{E} \quad 00$

$-2.56594 \mathrm{E} \quad 00$

$-2.58241 \mathrm{E} 00$

$-2.59893 \mathrm{E} \quad 00$

$-2.61550 \mathrm{E} \quad 00$

$-2.63212 \mathrm{E} \quad 00$

$-2.64880 \mathrm{E} \quad 00$

$-2.66552 \mathrm{E} \quad 00$

$-2.68230 \mathrm{E} \quad 00$

$-2.69913 \mathrm{E} \quad 00$

$-2.71601 \mathrm{E} \quad 00$

$-2.73294 \mathrm{E} \quad 00$

$-2.74993 \mathrm{E} \quad 00$

$-2.76696 \mathrm{E} \quad 00$

$-2.78405 \mathrm{E} \quad 00$

$-1.46063 \mathrm{E}-01$

$-1.47394 \mathrm{E}-01$

$-1.48733 \mathrm{E}-01$

$-1.50080 \mathrm{E}-01$

$-1.51435 \mathrm{E}-01$

$-1.52798 \mathrm{E}-01$

$-1.54169 \mathrm{E}-01$

$-1.55548 \mathrm{E}-01$

$-1.56935 \mathrm{E}-01$

$-1.58331 \mathrm{E}-01$

$-1.59734 \mathrm{E}-01$

$-1.61146 \mathrm{E}-01$

$-1.62565 \mathrm{E}-01$

$-1.63993 \mathrm{E}-01$

$-1.65430 \mathrm{E}-01$

$-1.66874 \mathrm{E}-01$

$-1.68327 \mathrm{E}-01$

$-1.69788 \mathrm{E}-01$

$-1.71257 \mathrm{E}-01$

$-1.72735 \mathrm{E}-01$

$-2.80118 \mathrm{E} \quad 00$ $-2.81837 \mathrm{E} \quad 00$ $-2.83561 \mathrm{E} \quad 00$ $-2.85290 \mathrm{E} \quad 00$

$-2.87024 \mathrm{E} \quad 00$

$-2.88764 \mathrm{E} \quad 00$

$-2.90508 \mathrm{E} \quad 00$

$-2.92258 \mathrm{E} \quad 00$

$-2.94013 \mathrm{E} \quad 00$

$-2.95772 \mathrm{E} 00$

$-2.97537 \mathrm{E} \quad 00$

$-2.99307 \mathrm{E} \quad 00$

$-3.01083 \mathrm{E} 00$

$-3.02863 \mathrm{E} 00$

$-3.04648 \mathrm{E} 00$

$-3.06439 \mathrm{E} \quad 00$

$-3.08234 \mathrm{E} 00$

$-3.10035 \mathrm{E} 00$

$-3.11841 \mathrm{E} 00$

$-3.13652 \mathrm{E} 00$

$-3.15468 \mathrm{E} \quad 00$

$-3.17289 \mathrm{E} 00$

$-3.19115 \mathrm{E} \quad 00$

$-3.20946 \mathrm{E} 00$

$-3.22783 \mathrm{E} \quad 00$

$-3.24624 \mathrm{E} \quad 00$

$-3.26471 \mathrm{E} 00$

$-3.28323 \mathrm{E} \quad 00$

$-3.30179 \mathrm{E} 00$

$-3.32041 \mathrm{E} 00$

$-3.33908 \mathrm{E} \quad 00$

$-3.35780 \mathrm{E} \quad 00$

$-3.37658 \mathrm{E} \quad 00$

$-3.39540 \mathrm{E} 00$

$-3.41427 \mathrm{E} 00$ $\tan \delta_{2}$

$2.95994 \mathrm{E} \quad 01$

$2.96007 \mathrm{E} \quad 01$

$2.96021 \mathrm{E}$ ol

$2.96034 \mathrm{E} \quad 01$

$2.96047 \mathrm{E} \quad 01$

$2.96060 \mathrm{E} \quad 01$

$2.96073 \mathrm{E} \quad 01$

$2.96086 \mathrm{E} \quad 01$

$2.96098 \mathrm{E} \quad 01$

$2.96111 \mathrm{E} \quad 01$

$2.96124 \mathrm{E} \quad 01$

$2.96136 \mathrm{E} \quad 01$

$2.96149 \mathrm{E} 01$

$2.96161 \mathrm{E} \quad 01$

$2.96173 \mathrm{E} \quad 01$

$2.96185 \mathrm{E} \quad 01$

$2.96197 \mathrm{E} \quad 01$

$2.96209 \mathrm{E} \quad 01$

$2.96221 \mathrm{E} \quad 01$

2.96233 E 01

$2.96245 \mathrm{E} \quad 01$

$2.96257 \mathrm{E} 01$

$2.96268 \mathrm{E} \quad 01$

$2.96280 \mathrm{E} \quad 01$

$2.96291 \mathrm{E} \quad 01$

$2.96303 \mathrm{E} \quad 01$

$2.96314 \mathrm{E} \quad 01$

$2.96325 \mathrm{E} \quad 01$

$2.96337 \mathrm{E} \quad 01$

$2.96348 \mathrm{E} \quad 01$

$2.96359 \mathrm{E} \quad 01$

$2.96370 \mathrm{E} \quad 01$

$2.96381 \mathrm{E} 01$

$2.96392 \mathrm{E} \quad 01$

$2.96402 \mathrm{E} 01$

$2.96413 \mathrm{E} \quad 01$

$2.96424 \mathrm{E} \quad 01$

$2.96434 \mathrm{E}$ ol

$2.96445 \mathrm{E} \quad 01$

$2.96455 \mathrm{E} 01$

$2.96466 \mathrm{E} \quad 01$

$2.96476 \mathrm{E} \quad 01$

$2.96486 \mathrm{E} \quad 01$

$2.96497 \mathrm{E} 01$

$2.96507 \mathrm{E} \quad 01$

$2.96517 \mathrm{E} \quad 01$

$2.96527 \mathrm{E} 01$

$2.96537 \mathrm{E} \quad 01$

$2.96547 \mathrm{E} 01$

$2.96557 \mathrm{E} \quad 01$

$2.96566 \mathrm{E} \quad 01$

$2.96576 \mathrm{E} \quad 01$

$2.96586 \mathrm{E} \quad 01$

$2.96595 \mathrm{E} \quad 01$

$2.96605 \mathrm{E} \quad 01$

$2.96614 \mathrm{E} \quad 01$

$2.96624 \mathrm{E} \quad 01$

$2.96633 \mathrm{E} \quad 01$

$2.96643 \mathrm{E} \quad 01$

$2.96652 \mathrm{E} \quad 01$ $f-2$

$-1.36819 \mathrm{E}-01$ $-1.38089 \mathrm{E}-01$ $-1.39367 \mathrm{E}-01$ $-1.40653 \mathrm{E}-01$

$-1.41947 \mathrm{E}-01$

$-1.43249 \mathrm{E}-01$

$-1.44558 \mathrm{E}-01$

$-1.45876 \mathrm{E}-01$

$-1.47201 \mathrm{E}-01$

$-1.48534 \mathrm{E}-01$

$-1.49875 \mathrm{E}-01$

$-1.51224 \mathrm{E}-01$

$-1.52582 \mathrm{E}-01$

$-1.53947 \mathrm{E}-01$

$-1.55320 \mathrm{E}-01$

$-1.56701 \mathrm{E}-01$

$-1.58090 \mathrm{E}-01$

$-1.59488 \mathrm{E}-01$

$-1.60893 \mathrm{E}-01$

$-1.62307 \mathrm{E}-01$

$-1.63729 \mathrm{E}-01$

$-1.65159 \mathrm{E}-01$

$-1.66597 \mathrm{E}-01$

$-1.68044 \mathrm{E}-01$

$-1.69499 \mathrm{E}-01$

$-1.70962 \mathrm{E}-01$

$-1.72433 \mathrm{E}-01$

$-1.73913 \mathrm{E}-01$

$-1.75401 \mathrm{E}-01$

$-1.76898 \mathrm{E}-01$

$-1.78402 \mathrm{E}-01$

$-1.79916 \mathrm{E}-01$

$-1.81437 \mathrm{E}-01$

$-1.82968 \mathrm{E}-01$

$-1.84506 \mathrm{E}-01$

$-1.86054 \mathrm{E}-01$

$-1.87609 \mathrm{E}-01$

$-1.89174 \mathrm{E}-01$

$-1.90747 \mathrm{E}-01$

$-1.92328 \mathrm{E}-01$

$-1.93918 \mathrm{E}-01$

$-1.95517 \mathrm{E}-01$

$-1.97125 \mathrm{E}-01$

-1.98741 E-01

$-2.00366 \mathrm{E}-01$

$-2.01999 \mathrm{E}-01$

$-2.03642 \mathrm{E}-01$

$-2.05293 \mathrm{E}-01$

$-2.06953 \mathrm{E}-01$

$-2.08622 \mathrm{E}-01$

$-2.10299 \mathrm{E}-01$

$-2.11986 \mathrm{E}-01$

$-2.13681 \mathrm{E}-01$

$-2.15386 \mathrm{E}-01$

$-2.17099 \mathrm{E}-01$

$-2.18821 \mathrm{E}-01$

$-2.20552 \mathrm{E}-01$

$-2.22293 \mathrm{E}-01$

$-2.24042 \mathrm{E}-01$

$-2.25800 \mathrm{E}-01$

g-2

$\tan \delta_{-2}$

$2.46871 \mathrm{E} \quad 00 \quad-3.29349 \mathrm{E}-02$

$2.48487 \mathrm{E} \quad 00 \quad-3.29362 \mathrm{E}-02$

$2.50109 \mathrm{E} \quad 00 \quad-3.29375 \mathrm{E}-02$

$2.51736 \mathrm{E} \quad 00 \quad-3.29387 \mathrm{E}-02$

$2.53367 \mathrm{E} \quad 00 \quad-3.29400 \mathrm{E}-02$

$2.55005 \mathrm{E} \quad 00$

$2.56647 \mathrm{E} \quad 00$

$2.58294 \mathrm{E} \quad 00$

$2.59947 \mathrm{E} \quad 00$

$2.61604 \mathrm{E} \quad 00$

$2.63267 \mathrm{E} \quad 00$

$2.64935 \mathrm{E} \quad 00$

$2.66608 \mathrm{E} \quad 00$

$2.68287 \mathrm{E} \quad 00$

$2.69970 \mathrm{E} \quad 00$

$2.71659 \mathrm{E} \quad 00$

$2.73352 \mathrm{E} \quad 00$

$2.75051 \mathrm{E} \quad 00$

$2.76755 \mathrm{E} \quad 00$

$2.78464 \mathrm{E} \quad 00$ 
ELECTRON RADIAL FUNCTIONS

Electron

p

36.10

36.20
36.30

36.40

36.50

36.60

36.70

36.80

36.90

37.00

37.10

37.20

37.30

37.40

37.50

37.60

37.70

37.80

37.90

38.00

38.10

38.20

38.30

38.40

38.50

38.60

38.70

38.80

38.90

39.00

39.10

39.20

39.30

39.40

39.50

39.60

39.70

39.80

39.90

40.00

40.10

40.20

40.30

40.40

40.50

40.60

40.70

40.80

40.90

41.00

41.10

41.20

41.30

41.40

41.50

41.60

41.70

41.80

41.90

42.00
$Z=7 \quad A=14$

$F_{0}$

$f_{1}$

$g_{1}$

$-4.29187 \mathrm{E} \quad 00$

$1.17841 \mathrm{E} \quad 00$

$1.17840 \mathrm{E} \quad 00$

$1.17839 \mathrm{E} \quad 00$

$\begin{array}{ll}1.17838 \mathrm{E} & 00\end{array}$

$1.17838 \mathrm{E} \quad 00$

$1.17837 \mathrm{E} \quad 00$

$1.17836 \mathrm{E} \quad 00$

$1.17835 \mathrm{E} \quad 00$

$1.17834 \mathrm{E} \quad 00$

$1.17833 \mathrm{E} \quad 00$

$1.17832 \mathrm{E} \quad 00$ $1.17831 \mathrm{E} \quad 00$

$1.17831 \mathrm{E} \quad 00$

$1.17830 \mathrm{E} \quad 00$

$1.17829 \mathrm{E} \quad 00$

$1.17828 \mathrm{E} \quad 00$

$1.17827 \mathrm{E} \quad 00$

$1.17826 \mathrm{E} \quad 00$

$1.17825 \mathrm{E} \quad 00$

$1.17825 \mathrm{E} \quad 00$

$1.17824 \mathrm{E} \quad 00$

$1.17823 \mathrm{E} \quad 00$

$1.17822 \mathrm{E} \quad 00$

$1.17821 \mathrm{E} \quad 00$

$1.17820 \mathrm{E} \quad 00$

$1.17820 \mathrm{E} \quad 00$ $1.17819 \mathrm{E} \quad 00$ $1.17818 \mathrm{E} \quad 00$

$1.17817 \mathrm{E} \quad 00$

$1.17816 \mathrm{E} \quad 00$

$1.17815 \mathrm{E} \quad 00$

$1.17815 \mathrm{E} \quad 00$

$1.17814 \mathrm{E} \quad 00$

$\begin{array}{ll}1.17813 \mathrm{E} & 00\end{array}$

$1.17812 \mathrm{E} \quad 00$

$1.17811 \mathrm{E} \quad 00$

$1.17811 \mathrm{E} \quad 00$

$1.17810 \mathrm{E} \quad 00$

$1.17809 \mathrm{E} \quad 00$

$1.17808 \mathrm{E} \quad 00$

$\begin{array}{ll}1.17807 \mathrm{E} & 00\end{array}$ $1.17807 \mathrm{E} \quad 00$

$1.17806 \mathrm{E}-00$

$1.17805 \mathrm{E} \quad 00$

$1.17804 \mathrm{E} \quad 00$

$1.17803 \mathrm{E} \quad 00$

$1.17803 \mathrm{E} \quad 00$

$1.17802 \mathrm{E} \quad 00$

$1.17801 \mathrm{E} \quad 00$

$1.17800 \mathrm{E} \quad 00$

$1.17800 \mathrm{E} \quad 00$

$1.17799 \mathrm{E} \quad 00$

$1.17798 \mathrm{E} \quad 00$

$1.17797 \mathrm{E} \quad 00$

$1.17796 \mathrm{E} \quad 00$

$1.17796 \mathrm{E} \quad 00$

$1.17795 \mathrm{E} \quad 00$

$1.17794 \mathrm{E} \quad 00$

$1.17793 \mathrm{E} \quad 00$

$1.17793 \mathrm{E} \quad 00$
$-3.76672 \mathrm{E} 01$ $-3.77689 \mathrm{E}$ 0I $-3.78706 \mathrm{E} 01$ $-3.79723 \mathrm{E} \quad 01$ $-3.80740 \mathrm{E} 01$

$-3.81756 \mathrm{E} 01$ $-3.82772 \mathrm{E} \quad 01$ $-3.83788 \mathrm{E} \quad 01$ $-3.84804 \mathrm{E} \quad 01$ $-3.85819 \mathrm{E} 01$

$-3.86834 \mathrm{E} \quad 01$ $-3.87848 \mathrm{E}$ 01 $-3.88862 \mathrm{E} \quad 01$ $-3.89876 \mathrm{E} \quad 01$ $-3.90890 \mathrm{E}$ 0I

$-3.91903 \mathrm{E} 01$ $-3.92916 \mathrm{E} 01$ $-3.93929 \mathrm{E} 01$ $-3.94942 \mathrm{E}$ 0l $-3.95954 \mathrm{E} 01$

$-3.96966 \mathrm{E} 01$ $-3.97977 \mathrm{E} \quad 01$ -3.98989 E 01 $-4.00000 \mathrm{E} \quad 01$ $-4.01010 \mathrm{E} 01$

$-4.02021 \mathrm{E} 01$ $-4.03031 \mathrm{E} 01$ $-4.04040 \mathrm{E} \quad 01$ $-4.05050 \mathrm{E} 01$ $-4.06059 \mathrm{E} 01$

$-4.07068 \mathrm{E} 01$ $-4.08076 \mathrm{E} 01$ $-4.09084 \mathrm{E} 01$ $-4.10092 \mathrm{E} 01$ $-4.11100 \mathrm{E} 01$

$-4.12107 \mathrm{E} 01$ $-4.13114 \mathrm{E} \quad 01$ $-4.14121 \mathrm{E} 01$ $-4.15127 \mathrm{E}$ 01 -4.16133 E 01

$-4.17139 \mathrm{E} \quad 01$ $-4.18144 \mathrm{E} 01$ $-4.19150 \mathrm{E} 01$ $-4.20154 \mathrm{E} \quad 01$ $-4.21159 \mathrm{E} 01$

$-4.22163 \mathrm{E} 01$ $-4.23167 \mathrm{E} 01$ $-4.24170 \mathrm{E} 01$ $-4.25173 \mathrm{E} 01$ $-4.26176 \mathrm{E} 01$

$-4.27179 \mathrm{E} 01$ $-4.28181 \mathrm{E} 01$ $-4.29183 \mathrm{E}$ 0I $-4.30185 \mathrm{E} 01$ $-4.31186 \mathrm{E} 01$

$-4.32187 \mathrm{E} 01$ -4.33188 E 01 $-4.34188 \mathrm{E} 01$ $-4.35188 \mathrm{E}$ 01 $-4.36188 \mathrm{E} 01$
$-4.31310 \mathrm{E} \quad 00$ $-4.33439 \mathrm{E} 00$ $-4.35573 \mathrm{E} \quad 00$ $-4.37711 \mathrm{E} 00$

$-4.39855 \mathrm{E} \quad 00$ $-4.42003 \mathrm{E} \quad 00$ $-4.44157 \mathrm{E} \quad 00$ $-4.46315 \mathrm{E} 00$ $-4.48478 \mathrm{E} \quad 00$

$-4.50646 \mathrm{E} \quad 00$ $-4.52820 \mathrm{E} \quad 00$ $-4.54998 \mathrm{E} \quad 00$ $-4.57181 \mathrm{E} \quad 00$ $-4.59369 \mathrm{E} 00$

$-4.61562 \mathrm{E} \quad 00$ $-4.63760 \mathrm{E} \quad 00$ $-4.65963 \mathrm{E} 00$ $-4.68170 \mathrm{E} \quad 00$ $-4.70383 \mathrm{E} \quad 00$

$-4.72601 \mathrm{E} \quad 00$ $-4.74823 \mathrm{E} \quad 00$ $-4.77051 \mathrm{E} 00$ $-4.79283 \mathrm{E} \quad 00$ $-4.81521 \mathrm{E} \quad 00$

$-4.83763 \mathrm{E} \quad 00$ $-4.88262 \mathrm{E} \quad 00$ $-4.90519 \mathrm{E} 00$ $-4.92781 \mathrm{E} 00$

$-4.95048 \mathrm{E} \quad 00$ $-4.97320 \mathrm{E} \quad 00$ $-4.99596 \mathrm{E} \quad 00$ $-5.01878 \mathrm{E} \quad 00$

$-5.06456 \mathrm{E} \quad 00$ $-5.08752 \mathrm{E} 00$ $-5.11053 \mathrm{E} \quad 00$ $-5.13359 \mathrm{E} \quad 00$ $-5.15670 \mathrm{E} \quad 00$

$-5.17986 \mathrm{E} \quad 00$ $-5.20307 \mathrm{E} \quad 00$ $-5.22632 \mathrm{E} \quad 00$ $-5.24963 \mathrm{E} 00$ $-5.27298 \mathrm{E} \quad 00$

$-5.29638 \mathrm{E} \quad 00$ $-5.31983 \mathrm{E} \quad 00$ $-5.36688 \mathrm{E} \quad 00$ $-5.39048 \mathrm{E} 00$

$-5.41413 \mathrm{E} \quad 00$ $-5.43782 \mathrm{E} 00$ $-5.46156 \mathrm{E} \quad 00$ $-5.48536 \mathrm{E} \quad 00$ $-5.50920 \mathrm{E} \quad 00$

$-5.53309 \mathrm{E} \quad 00$ $-5.58101 \mathrm{E} \quad 00$ $-5.60505 \mathrm{E} \quad 00$ $-5.62913 \mathrm{E} 00$ $-4.86010 \mathrm{E} \quad 00$ $-5.04164 \mathrm{E} \quad 00$ $-5.34333 \mathrm{E} 00$ $-5.55703 \mathrm{E} \quad 00$

$\tan \delta_{1}$

$f_{-1}$

$4.91309 \mathrm{E}-03$

$4.91269 \mathrm{E}-03$

$4.91226 \mathrm{E}-03$

$4.91183 \mathrm{E}-03$

$4.91137 \mathrm{E}-03$

$4.91091 \mathrm{E}-03$

$4.91041 \mathrm{E}-03$

$4.90991 \mathrm{E}-03$

$4.90938 \mathrm{E}-03$

$4.90885 \mathrm{E}-03$

$4.90829 \mathrm{E}-03$

$4.90772 \mathrm{E}-03$

$4.90714 \mathrm{E}-03$

$4.90654 \mathrm{E}-03$

$4.90592 \mathrm{E}-03$

$4.90528 \mathrm{E}-03$

$4.90463 \mathrm{E}-03$

$4.90397 \mathrm{E}-03$

$4.90328 \mathrm{E}-03$

$4.90258 \mathrm{E}-03$

$4.90187 \mathrm{E}-03$

$4.90114 \mathrm{E}-03$

$4.90040 \mathrm{E}-03$

$4.89964 \mathrm{E}-03$

$4.89885 \mathrm{E}-03$

$4.89807 \mathrm{E}-03$

$4.89726 \mathrm{E}-03$

$4.89645 \mathrm{E}-03$

4.89561 E- 03

$4.89476 \mathrm{E}-03$

$4.89389 \mathrm{E}-03$

$4.89302 \mathrm{E}-03$

$4.89212 \mathrm{E}-03$

$4.89120 \mathrm{E}-03$

$4.89028 \mathrm{E}-03$

$4.88934 \mathrm{E}-03$

$4.88839 \mathrm{E}-03$

$4.88743 \mathrm{E}-03$

$4.88643 \mathrm{E}-03$

$4.88544 \mathrm{E}-03$

$4.88443 \mathrm{E}-03$

$4.88340 \mathrm{E}-03$ 


\section{$\bar{F}$}

36.10

36.20

36.30

36.40

36.50

36.60

36.70

36.80

36.90

37.00

37.10

37.20

37.30

37.40

37.50

37.60

37.70

37.80

37.90

38.00

38.10

38.20

38.30

38.40

38.50

38.60

38.70

38.80

38.90

39.00

39.10

39.20

39.30

39.40

39.50

39.60

39.70

39.80

39.90

40.00

40.10

40.20

40.30

40.40

40.50

40.60

40.70

40.80

40.90

41.00

41.10

41.20

41.30

41.40

41.50

41.60

41.70

41.80

41.90

42.00 $f_{2}$

$g_{2}$

$2.39022 \mathrm{E}-03$ $2.40348 \mathrm{E}-03$ $2.41676 \mathrm{E}-03$ $2.43009 \mathrm{E}-03$ $2.44345 \mathrm{E}-03$

$2.45685 \mathrm{E}-03$ $2.47028 \mathrm{E}-03$ $2.48375 \mathrm{E}-03$ $2.49726 \mathrm{E}-03$ $2.51080 \mathrm{E}-03$

$2.52438 \mathrm{E}-03$ $2.53800 \mathrm{E}-03$ $2.55165 \mathrm{E}-03$ $2.56535 \mathrm{E}-03$ $2.57907 \mathrm{E}-03$

$2.59284 \mathrm{E}-03$ $2.60664 \mathrm{E}-03$ $2.62047 \mathrm{E}-03$ $2.63435 \mathrm{E}-03$ $2.64826 \mathrm{E}-03$

$2.66220 \mathrm{E}-03$ $2.67619 \mathrm{E}-03$ $2.69021 \mathrm{E}-03$ $2.70426 \mathrm{E}-03$ $2.71836 \mathrm{E}-03$

$2.73248 \mathrm{E}-03$ $2.74665 \mathrm{E}-03$ $2.76085 \mathrm{E}-03$ $2.77509 \mathrm{E}-03$ $2.78937 \mathrm{E}-03$

$2.80368 \mathrm{E}-03$ $2.81803 \mathrm{E}-03$ $2.83242 \mathrm{E}-03$ $2.84684 \mathrm{E}-03$ $2.86130 \mathrm{E}-03$

$2.87579 \mathrm{E}-03$ $2.89033 \mathrm{E}-03$ $2.90490 \mathrm{E}-03$ $2.91950 \mathrm{E}-03$ $2.93414 \mathrm{E}-03$

$2.94882 \mathrm{E}-03$ $2.96354 \mathrm{E}-03$ $2.97829 E-03$ $2.99308 \mathrm{E}-03$ $3.00790 \mathrm{E}-03$

$3.02276 \mathrm{E}-03$ $3.03766 \mathrm{E}-03$ $3.05260 \mathrm{E}-03$ $3.06757 \mathrm{E}-03$ $3.08258 \mathrm{E}-03$

$3.09762 \mathrm{E}-03$ $3.11270 \mathrm{E}-03$ $3.12782 \mathrm{E}-03$ $3.14298 \mathrm{E}-03$ $3.15817 \mathrm{E}-03$

$3.17339 \mathrm{E}-03$ $3.18866 \mathrm{E}-03$ $3.20396 \mathrm{E}-03$ $3.21930 \mathrm{E}-03$ $3.23467 \mathrm{E}-03$
$-3.43319 \mathrm{E} \quad 00$ $-3.45217 \mathrm{E} \quad 00$ $-3.47120 \mathrm{E} \quad 00$ $-3.49027 \mathrm{E} \quad 00$ $-3.50940 \mathrm{E} 00$

$-3.52857 \mathrm{E} \quad 00$ $-3.54780 \mathrm{E} 00$ $-3.56708 \mathrm{E} 00$ $-3.58641 \mathrm{E} 00$ -3.60579 E 00

$-3.62522 \mathrm{E} \quad 00$ $-3.64470 \mathrm{E} \quad 00$ $-3.66424 \mathrm{E} \quad 00$ $-3.68382 \mathrm{E} \quad 00$ $-3.70345 \mathrm{E} 00$

$-3.72313 \mathrm{E} \quad 00$ $-3.74287 \mathrm{E} \quad 00$ $-3.76265 \mathrm{E} 00$ $-3.78249 \mathrm{E} \quad 00$ $-3.80237 \mathrm{E} \quad 00$

$-3.82231 \mathrm{E} \quad 00$ $-3.84230 \mathrm{E} 00$ $-3.86233 \mathrm{E} \quad 00$ $-3.88242 \mathrm{E} \quad 00$ $-3.90256 \mathrm{E} 00$

$-3.92275 \mathrm{E} \quad 00$ $-3.94299 \mathrm{E} 00$ $-3.96327 \mathrm{E} 00$ $-3.98361 \mathrm{E} \quad 00$ $-4.00400 \mathrm{E} \quad 00$

$-2.31680 \mathrm{E}-01$ $-2.33483 \mathrm{E}-01$ $-2.35294 \mathrm{E}-01$ $-2.37115 \mathrm{E}-01$ $-2.38945 \mathrm{E}-01$

$-2.40784 \mathrm{E}-01$ $-2.42633 \mathrm{E}-01$ $-2.44491 \mathrm{E}-01$ $-2.46358 \mathrm{E}-01$ $-2.48235 \mathrm{E}-01$

$-2.50120 \mathrm{E}-01$ $-2.52016 \mathrm{E}-01$ $-2.53921 \mathrm{E}-01$ $-2.55835 \mathrm{E}-01$ $-2.57759 \mathrm{E}-01$

$-2.59692 \mathrm{E}-01$ $-2.61634 \mathrm{E}-01$ $-2.63587 \mathrm{E}-01$ $-2.65548 \mathrm{E}-01$ $-2.67520 \mathrm{E}-01$

$-2.69501 \mathrm{E}-01$ $-2.71491 \mathrm{E}-01$ $-2.73492 \mathrm{E}-01$ $-2.75502 \mathrm{E}-01$ $-2.77521 \mathrm{E}-01$

$-2.79551 \mathrm{E}-01$ $-2.81590 \mathrm{E}-01$ $-2.83638 \mathrm{E}-01$ $-2.85697 \mathrm{E}-01$ $-2.87765 \mathrm{E}-01$

$-2.89844 \mathrm{E}-01$ $-2.91932 \mathrm{E}-01$ $-2.94030 \mathrm{E}-01$ $-2.96138 \mathrm{E}-01$ $-2.98255 \mathrm{E}-01$

$-3.00383 \mathrm{E}-01$ $-3.02521 \mathrm{E}-01$ $-3.04668 \mathrm{E}-01$ $-3.06826 \mathrm{E}-01$ $-3.08993 \mathrm{E}-01$

$-3.11171 \mathrm{E}-01$ $-3.13359 \mathrm{E}-01$ $-3.15557 \mathrm{E}-01$ $-3.17765 \mathrm{E}-01$ $-3.19983 \mathrm{E}-01$

$-3.22211 \mathrm{E}-01$ $-3.24449 \mathrm{E}-01$ $-3.26698 \mathrm{E}-01$ $-3.28957 \mathrm{E}-01$ $-3.31226 \mathrm{E}-01$

$-4.44378 \mathrm{E} \quad 00$ $-4.46527 \mathrm{E} \quad 00$ $-4.48681 \mathrm{E} \quad 00$ $-4.50840 \mathrm{E} \quad 00$ $-4.53004 \mathrm{E} 00$

$-3.33505 \mathrm{E}-01$ $-3.35795 \mathrm{E}-01$ $-3.38095 \mathrm{E}-01$ $-3.40405 \mathrm{E}-01$ $-3.42726 \mathrm{E}-01$

$-4.55174 \mathrm{E} \quad 00$

$-4.57348 \mathrm{E} 00$

$-4.59527 \mathrm{E} \quad 00$

$-4.61711 \mathrm{E} 00$

$-4.63900 \mathrm{E} 00$

$-3.45057 \mathrm{E}-01$

$-3.47398 \mathrm{E}-01$

$-3.49750 \mathrm{E}-01$

$-3.52112 \mathrm{E}-01$

$-3.54485 \mathrm{E}-01$ $\tan \delta_{2}$

$f_{-2}$

$2.96661 \mathrm{E} \quad 01 \quad-2.27568 \mathrm{E}-01$ $2.96670 \mathrm{E} \quad 01 \quad-2.29344 \mathrm{E}-01$

$2.96679 \mathrm{E}$ 01 $-2.31130 \mathrm{E}-01$

$2.96689 \mathrm{E} 01 \quad-2.32925 \mathrm{E}-01$

$2.96698 \mathrm{E} \quad 01-2.34729 \mathrm{E}-0 \mathrm{l}$

$2.96707 \mathrm{E} \quad 01 \quad-2.36542 \mathrm{E}-01$

$2.96715 \mathrm{E}$ 0l $-2.38364 \mathrm{E}-01$

$2.96724 \mathrm{E}$ 01 $-2.40196 \mathrm{E}-01$

$2.96733 \mathrm{E} 01 \quad-2.42037 \mathrm{E}-01$

$2.96742 \mathrm{E} \quad 01 \quad-2.43887 \mathrm{E}-01$

$2.96751 \mathrm{E} \quad 01 \quad-2.45747 \mathrm{E}-01$

$2.96759 \mathrm{E} 01-2.47615 \mathrm{E}-01$

$2.96768 \mathrm{E} \quad 01 \quad-2.49494 \mathrm{E}-01$

$2.96777 \mathrm{E} \quad 01 \quad-2.51381 \mathrm{E}-01$

$2.96785 \mathrm{E} \quad 01-2.53278 \mathrm{E}-01$

$2.96793 \mathrm{E} \quad 01 \quad-2.55184 \mathrm{E}-01$

$2.96802 \mathrm{E}$ 01 $-2.57100 \mathrm{E}-01$

$2.96810 \mathrm{E} \quad 01-2.59025 \mathrm{E}-01$

$2.96819 \mathrm{E} 01 \quad-2.60960 \mathrm{E}-01$

$2.96827 \mathrm{E} \quad 01 \quad-2.62904 \mathrm{E}-01$

$2.96835 \mathrm{E} \quad 01 \quad-2.64858 \mathrm{E}-01$

$2.96843 \mathrm{E}$ 01 $-2.66821 \mathrm{E}-01$

$2.96852 \mathrm{E} \quad 01 \quad-2.68794 \mathrm{E}-01$

$2.96860 \mathrm{E} \quad 01-2.70776 \mathrm{E}-01$

$2.96868 \mathrm{E} \quad 01-2.72768 \mathrm{E}-01$

$2.96876 \mathrm{E}$ ol $-2.74770 \mathrm{E}-01$

$2.96884 \mathrm{E} \quad 01 \quad-2.76781 \mathrm{E}-01$

$2.96892 \mathrm{E}$ 01 $-2.78802 \mathrm{E}-01$

$2.96900 \mathrm{E} \quad 01 \quad-2.80833 \mathrm{E}-01$

$2.96907 \mathrm{E} \quad 01 \quad-2.82873 \mathrm{E}-01$

$2.96915 \mathrm{E} \quad 01 \quad-2.84924 \mathrm{E}-01$

$2.96923 \mathrm{E} \quad 01 \quad-2.86983 \mathrm{E}-01$

$2.96931 \mathrm{E}$ 01 $-2.89053 \mathrm{E}-01$

$2.96938 \mathrm{E} 0 \mathrm{~J} \quad-2.91133 \mathrm{E}-01$

$2.96946 \mathrm{E} \quad 01-2.93222 \mathrm{E}-01$

$2.96954 \mathrm{E} \quad 01 \quad-2.95321 \mathrm{E}-01$

$2.96961 \mathrm{E} 01 \quad-2.97431 \mathrm{E}-01$

$2.96969 \mathrm{E} \quad 01-2.99550 \mathrm{E}-01$

$2.96976 \mathrm{E} \quad 01-3.01679 \mathrm{E}-01$

$2.96984 \mathrm{E}$ ol $-3.03817 \mathrm{E}-01$

$2.96991 \mathrm{E}$ ol $-3.05966 \mathrm{E}-01$

$2.96998 \mathrm{E}$ 01 $-3.08125 \mathrm{E}-01$

$2.97006 \mathrm{E}$ 01 $-3.10294 \mathrm{E}-01$

$2.97013 \mathrm{E} 01 \quad-3.12473 \mathrm{E}-01$

$2.97020 \mathrm{E} \quad 01-3.14662 \mathrm{E}-01$

$2.97027 \mathrm{E} \quad 01 \quad-3.16861 \mathrm{E}-01$ $2.97035 \mathrm{E}$ 01 $-3.19070 \mathrm{E}-01$ $2.97042 \mathrm{E} \quad 01-3.21289 \mathrm{E}-01$ $2.97049 \mathrm{E} \quad 01-3.23519 \mathrm{E}-01$ $2.97056 \mathrm{E}$ 0l $-3.25758 \mathrm{E}-0 \mathrm{l}$

$2.97063 \mathrm{E} \quad 01 \quad-3.28008 \mathrm{E}-01$ $2.97070 \mathrm{E} \quad 01-3.30268 \mathrm{E}-01$ $2.97077 \mathrm{E} \quad 01-3.32538 \mathrm{E}-01$ $2.97084 \mathrm{E}$ 01 $-3.34819 \mathrm{E}-01$ $2.97091 \mathrm{E}$ 01 $-3.37109 \mathrm{E}-01$

$2.97098 \mathrm{E} \quad 01 \quad-3.39410 \mathrm{E}-01$ $2.97104 \mathrm{E} \quad 01 \quad-3.41722 \mathrm{E}-01$ $2.97111 \mathrm{E} 01-3.44043 \mathrm{E}-01$ $2.97118 \mathrm{E} \quad 01-3.46376 \mathrm{E}-01$ $2.97125 \mathrm{E} 01-3.48718 \mathrm{E}-01$ 
ELECTRON RADIAL FUNCTIONS

$Z=8 \quad A=17 \quad \rho=1.2 A^{1 / 3} F$

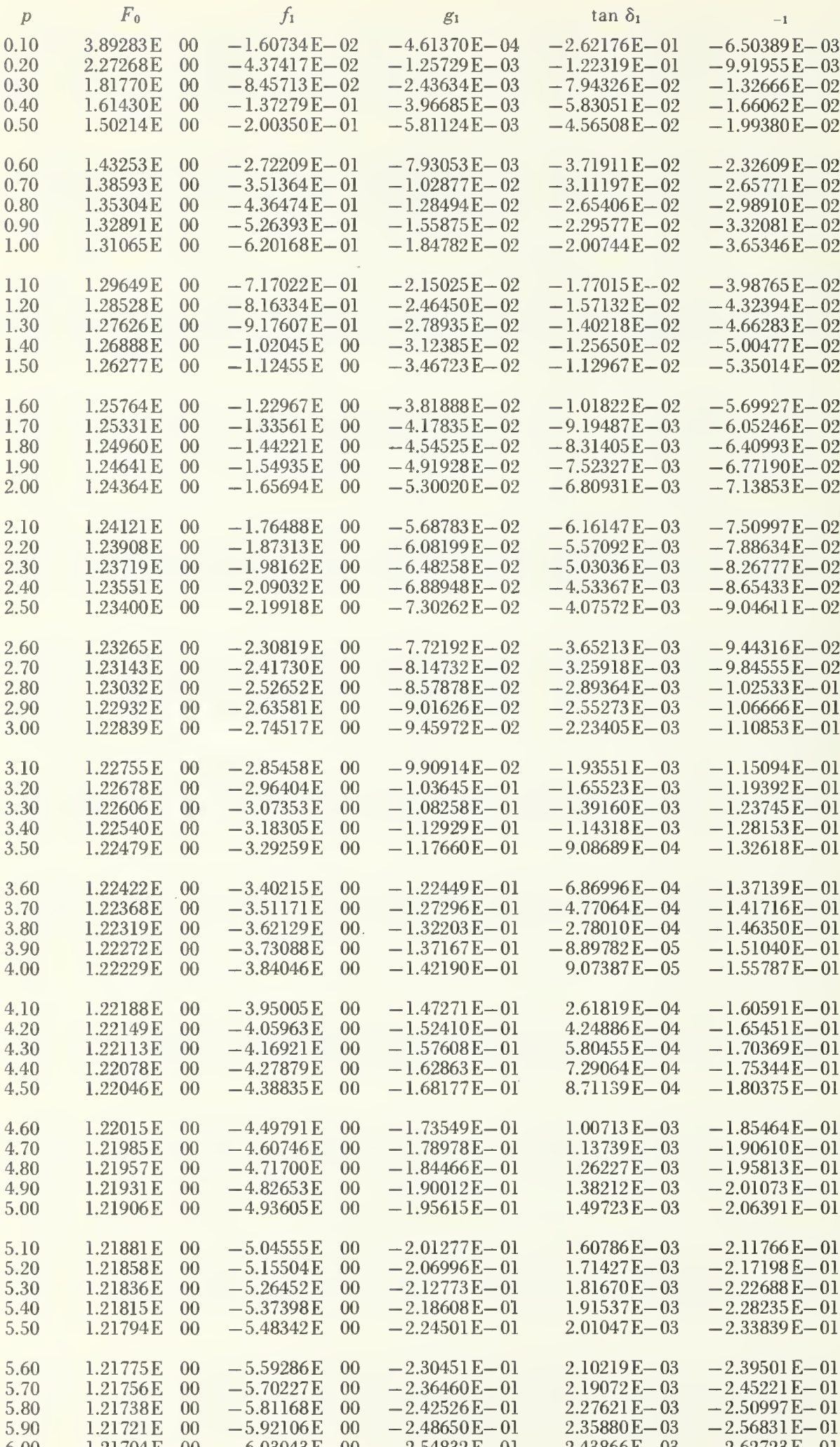

$g_{-1}$

$2.78240 \mathrm{E}-01$

$4.23647 \mathrm{E}-01$

$5.65036 \mathrm{E}-01$

$7.04631 \mathrm{E}-01$

$8.42096 \mathrm{E}-01$

$9.77144 \mathrm{E}-01$

$1.10968 \mathrm{E} \quad 00$

$1.23977 \mathrm{E} \quad 00$

$1.36756 \mathrm{E} \quad 00$

$1.49325 \mathrm{E} \quad 00$

$1.61708 \mathrm{E} \quad 00$

$1.73926 \mathrm{E} \quad 00$

$1.86000 \mathrm{E} \quad 00$

$1.97948 \mathrm{E} \quad 00$

$2.09786 \mathrm{E} \quad 00$

$2.21530 \mathrm{E} \quad 00$

$2.33191 \mathrm{E} \quad 00$

$2.44779 \mathrm{E} \quad 00$

$2.56305 \mathrm{E} \quad 00$

$2.67776 \mathrm{E} \quad 00$

$2.79199 \mathrm{E} \quad 00$ $2.90578 \mathrm{E} \quad 00$ $3.01920 \mathrm{E} \quad 00$

$3.13229 \mathrm{E} \quad 00$

$3.24508 \mathrm{E} \quad 00$

$3.35759 \mathrm{E} \quad 00$ $3.46987 \mathrm{E} \quad 00$ $3.58194 \mathrm{E} \quad 00$

$3.69380 \mathrm{E} \quad 00$

$3.80549 \mathrm{E} \quad 00$

$3.91702 \mathrm{E} \quad 00$ $4.02841 \mathrm{E} \quad 00$ $4.13965 \mathrm{E} \quad 00$ $4.25078 \mathrm{E} \quad 00$

$4.36179 \mathrm{E} \quad 00$

$4.47269 \mathrm{E} \quad 00$ $4.58349 \mathrm{E} \quad 00$ $4.69421 \mathrm{E} \quad 00$ $4.80484 \mathrm{E} \quad 00$ $4.91539 \mathrm{E} \quad 00$

$5.02587 \mathrm{E} \quad 00$ $\begin{array}{ll}5.13627 \mathrm{E} & 00\end{array}$ $5.24661 \mathrm{E} \quad 00$ $5.35689 \mathrm{E} \quad 00$ $5.46711 \mathrm{E} \quad 00$

$5.57727 \mathrm{E} \quad 00$ $5.68738 \mathrm{E} \quad 00$ $5.79744 \mathrm{E} \quad 00$ $5.90745 \mathrm{E} \quad 00$ $6.01741 \mathrm{E} \quad 00$

$6.12733 \mathrm{E} \quad 00$ $6.23721 \mathrm{E} \quad 00$ $6.34705 \mathrm{E} \quad 00$ $6.45684 \mathrm{E} \quad 00$ $6.56660 \mathrm{E} \quad 00$

$6.67632 \mathrm{E} \quad 00$ $6.78601 \mathrm{E} \quad 00$ $6.89566 \mathrm{E} \quad 00$ $\begin{array}{ll}7.00527 \mathrm{E} & 00\end{array}$ $7.11486 \mathrm{E} \quad 00$ $\tan \delta_{-1}$

$-3.58527 \mathrm{E} 00$ $-6.10771 \mathrm{E} \quad 00$ $-8.81765 \mathrm{E} \quad 00$ $-1.15073 \mathrm{E} \quad 01$ $-1.41385 \mathrm{E} 01$

$-1.66976 \mathrm{E} 01$ $-1.91784 \mathrm{E} \quad 01$ $-2.15787 \mathrm{E} \quad 01$ $-2.38983 \mathrm{E} \quad 01$ $-2.61381 \mathrm{E} \quad 01$

$-2.83001 \mathrm{E} \quad 01$ $-3.03866 \mathrm{E} 01$ $-3.24002 \mathrm{E} 01$ $-3.43438 \mathrm{E} 01$ $-3.62204 \mathrm{E} \quad 0 \mathrm{l}$

$-3.80327 \mathrm{E} \quad 01$ $-3.97837 \mathrm{E} 01$ $-4.14761 \mathrm{E} 01$ $-4.31125 \mathrm{E} 01$ $-4.46954 \mathrm{E} 01$

$-4.62274 \mathrm{E} \quad 01$ $-4.77107 \mathrm{E} \quad 01$ $-4.91475 \mathrm{E} \quad 01$ $-5.05399 \mathrm{E} \quad 01$ $-5.18899 \mathrm{E} 01$

$-5.31993 \mathrm{E} \quad 01$ $-5.44700 \mathrm{E}$ 01 $-5.57035 \mathrm{E} \quad 01$ $-5.69014 \mathrm{E} \quad 01$ $-5.80653 \mathrm{E} \quad 01$

$-5.91967 \mathrm{E} \quad 01$ $-6.02967 \mathrm{E} 01$ $-6.13667 \mathrm{E} 01$ $-6.24081 \mathrm{E} 01$ $-6.34217 \mathrm{E} 01$

$-6.44088 \mathrm{E} \quad 01$ $-6.53704 \mathrm{E} \quad 01$ $-6.63075 \mathrm{E} \quad 01$ $-6.72209 \mathrm{E} \quad 01$ $-6.81117 \mathrm{E} 01$

$-6.89806 \mathrm{E} \quad 01$ $-6.98284 \mathrm{E} 01$ $-7.06560 \mathrm{E} \quad 01$ $-7.14639 \mathrm{E} 01$ $-7.22531 \mathrm{E} 01$

$-7.30240 \mathrm{E} \quad 01$ $-7.37773 \mathrm{E} 01$ $-7.45137 \mathrm{E} 01$ $-7.52337 \mathrm{E} \quad 01$ $\begin{array}{lll}-7.59379 \mathrm{E} & 01\end{array}$ $-7.66267 \mathrm{E} \quad 01$ $-7.73008 \mathrm{E} \quad 01$ $-7.79606 \mathrm{E} 01$ $-7.86064 \mathrm{E} \quad 01$ $-7.92389 \mathrm{E} 01$

$\begin{array}{ll}-7.98584 \mathrm{E} & 01\end{array}$ $-8.04653 \mathrm{E} 01$ $-8.10600 \mathrm{E} 0 \mathrm{l}$ $-8.16430 \mathrm{E} 01$ $-8.22145 \mathrm{E} 01$ 
ELECTRON RADIAL FUNC'TIONS

$Z=8 \quad A=17 \quad \rho=1.2 A^{1 / 3} F$

Electron

$\bar{F}$

$9.15275 \mathrm{E}-08$

$1.73184 \mathrm{E}-07$

$2.98263 \mathrm{E}-07$

$4.63619 \mathrm{E}-07$

$6.69264 \mathrm{E}-07$

$9.15587 \mathrm{E}-07$

$1.20299 \mathrm{E}-06$

$1.53184 \mathrm{E}-06$

$1.90242 \mathrm{E}-06$

$2.31497 \mathrm{E}-06$

$2.76966 \mathrm{E}-06$ $3.26665 \mathrm{E}-06$ $3.80604 \mathrm{E}-06$ $4.38793 \mathrm{E}-06$ $5.01236 \mathrm{E}-06$

$5.67941 \mathrm{E}-06$ $6.38910 \mathrm{E}-06$ $7.14149 \mathrm{E}-06$ $7.93657 \mathrm{E}-06$ $8.77438 \mathrm{E}-06$

$9.65494 \mathrm{E}-06$ $1.05782 \mathrm{E}-05$ $1.15443 \mathrm{E}-05$ $1.25532 \mathrm{E}-05$ $1.36048 \mathrm{E}-05$

2.50

2.60

2.70

2.80

2.90

3.00

3.10

3.20

3.30

3.40

3.50

3.60

3.70

3.80

3.90

4.00

4.10

4.20

4.30

4.40

4.50

4.60

4.70

4.80

4.90

5.00

5.10

5.20

5.30

5.40

5.50

5.60

5.70

5.80

5.90

6.00 $1.58364 \mathrm{E}-05$ $1.70163 \mathrm{E}-05$ $1.82391 \mathrm{E}-05$ $1.95047 \mathrm{E}-05$

$2.08130 \mathrm{E}-05$ $2.21642 \mathrm{E}-05$ $2.35581 \mathrm{E}-05$ $2.49948 \mathrm{E}-05$ $2.64743 \mathrm{E}-05$

$2.79967 \mathrm{E}-05$ $2.95617 \mathrm{E}-05$ $3.11696 \mathrm{E}-05$ $3.28203 \mathrm{E}-05$ $3.45138 \mathrm{E}-05$

$3.62500 \mathrm{E}-05$ $3.80291 \mathrm{E}-05$ $3.98509 \mathrm{E}-05$ $4.17155 \mathrm{E}-05$ $4.36229 \mathrm{E}-05$

$4.55731 \mathrm{E}-05$ $4.75660 \mathrm{E}-05$ $4.96018 \mathrm{E}-05$ $5.16803 \mathrm{E}-05$ $5.38015 \mathrm{E}-05$

$5.59656 \mathrm{E}-05$ $5.81725 \mathrm{E}-05$ $6.04221 \mathrm{E}-05$ $6.27144 \mathrm{E}-05$ $6.50496 \mathrm{E}-05$

$6.74275 \mathrm{E}-05$ $6.98482 \mathrm{E}-05$ $7.23117 \mathrm{E}-05$ $7.48180 \mathrm{E}-05$ $7.73669 \mathrm{E}-05$ $f_{2}$

$-4.42647 \mathrm{E}-06$

$-2.34222 \mathrm{E}-05$

$-6.75755 \mathrm{E}-05$

$-1.46007 \mathrm{E}-04$

$-2.66171 \mathrm{E}-04$

$-4.33827 \mathrm{E}-04$

$-6.53211 \mathrm{E}-04$

$-9.27304 \mathrm{E}-04$

$-1.25812 \mathrm{E}-03$

$-1.64698 \mathrm{E}-03$

$-2.09469 \mathrm{E}-03$

$-2.60173 \mathrm{E}-03$

$-3.16838 \mathrm{E}-03$

$-3.79473 \mathrm{E}-03$

$-4.48083 \mathrm{E}-03$

$-5.22663 \mathrm{E}-03$

$-6.03207 \mathrm{E}-03$

$-6.89709 \mathrm{E}-03$

$-7.82159 \mathrm{E}-03$

$-8.80549 \mathrm{E}-03$

$-9.84870 \mathrm{E}-03$

$-1.09512 \mathrm{E}-02$

$-1.21128 \mathrm{E}-02$

$-1.33335 \mathrm{E}-02$

$-1.46132 \mathrm{E}-02$

$1.46992 \mathrm{E}-05$

$-1.59519 \mathrm{E}-02$ $-1.73495 \mathrm{E}-02$ $-1.88060 \mathrm{E}-02$ $-2.03213 \mathrm{E}-02$ $-2.18954 \mathrm{E}-02$

$-2.35283 \mathrm{E}-02$ $-2.52198 \mathrm{E}-02$ $-2.69700 \mathrm{E}-02$ $-2.87789 \mathrm{E}-02$ $-3.06464 \mathrm{E}-02$

$-3.25725 \mathrm{E}-02$ $-3.45572 \mathrm{E}-02$ $-3.66004 \mathrm{E}-02$ $-3.87021 \mathrm{E}-02$ $-4.08624 \mathrm{E}-02$

$-4.30811 \mathrm{E}-02$ $-4.53584 \mathrm{E}-02$ $-4.76940 \mathrm{E}-02$ $-5.25407 \mathrm{E}-02$

$-5.50517 \mathrm{E}-02$ $-5.76210 \mathrm{E}-02$ $-6.02488 \mathrm{E}-02$ $-6.29349 \mathrm{E}-02$ $-6.56793 \mathrm{E}-02$

$-6.84821 \mathrm{E}-02$ $-7.13433 \mathrm{E}-02$ $-7.42628 \mathrm{E}-02$ $-7.72406 \mathrm{E}-02$ $-8.02767 \mathrm{E}-02$

$-8.33710 \mathrm{E}-02$ $-8.65237 \mathrm{E}-02$ $-8.97346 \mathrm{E}-02$ $-9.30039 \mathrm{E}-02$ $-9.63313 \mathrm{E}-02$ $g_{2}$

$-7.32608 \mathrm{E}-08$ $-3.88206 \mathrm{E}-07$ $-1.12264 \mathrm{E}-06$ $-2.43332 \mathrm{E}-06$ $-4.45340 \mathrm{E}-06$

$-7.29192 \mathrm{E}-06$ $-1.10363 \mathrm{E}-05$ $-1.57561 \mathrm{E}-05$ $-2.15074 \mathrm{E}-05$ $-2.83362 \mathrm{E}-05$

$-3.62815 \mathrm{E}-05$ $-4.53778 \mathrm{E}-05$ $-5.56564 \mathrm{E}-05$ $-6.71466 \mathrm{E}-05$ $-7.98765 \mathrm{E}-05$

$-9.38737 \mathrm{E}-05$ $-1.09165 \mathrm{E}-04$ $-1.25777 \mathrm{E}-04$ $-1.43737 \mathrm{E}-04$ $-1.63071 \mathrm{E}-04$

$-1.83806 \mathrm{E}-04$ $-2.05968 \mathrm{E}-04$ $-2.29586 \mathrm{E}-04$ $-2.54685 \mathrm{E}-04$ $-2.81293 \mathrm{E}-04$ $-5.00882 \mathrm{E}-02$

$-3.09437 \mathrm{E}-04$ $-3.39145 \mathrm{E}-04$ $-3.70442 \mathrm{E}-04$ $-4.03358 \mathrm{E}-04$ $-4.37918 \mathrm{E}-04$

$-4.74152 \mathrm{E}-04$

$-5.12085 \mathrm{E}-04$

$-5.51746 \mathrm{E}-04$

$-5.93162 \mathrm{E}-04$

$-6.36361 \mathrm{E}-04$

$-6.81371 \mathrm{E}-04$ $-7.28218 \mathrm{E}-04$ $-7.76931 \mathrm{E}-04$ $-8.27537 \mathrm{E}-04$ $-8.80065 \mathrm{E}-04$

$-9.34540 \mathrm{E}-04$ $-9.90993 \mathrm{E}-04$ $-1.04945 \mathrm{E}-03$ $-1.10994 \mathrm{E}-03$ $-1.17249 \mathrm{E}-03$

$-1.23712 \mathrm{E}-03$ $-1.30387 \mathrm{E}-03$ $-1.37276 \mathrm{E}-03$ $-1.44383 \mathrm{E}-03$ $-1.51709 \mathrm{E}-03$

$-1.59258 \mathrm{E}-03$ $-1.67032 \mathrm{E}-03$ $-1.75034 \mathrm{E}-03$ $-1.83267 \mathrm{E}-03$ $-1.91735 \mathrm{E}-03$

$-2.00438 \mathrm{E}-03$ $-2.09381 \mathrm{E}-03$ $-2.18565 \mathrm{E}-03$ $-2.27995 \mathrm{E}-03$ $-2.37672 \mathrm{E}-03$ $\tan \delta_{2}$

$f_{-2}$

$1.65232 \mathrm{E} \quad 00 \quad-1.14074 \mathrm{E}-06$ $3.57875 \mathrm{E} \quad 00 \quad-3.13245 \mathrm{E}-06$ $5.36916 \mathrm{E} \quad 00-6.14846 \mathrm{E}-06$ $7.04787 \mathrm{E} \quad 00-1.01838 \mathrm{E}-05$ $\begin{array}{lll}8.60558 \mathrm{E} & 00 & -1.52326 \mathrm{E}-05\end{array}$

$1.00352 \mathrm{E} \quad 01$ $1.13356 \mathrm{E} \quad 01$ $1.25105 \mathrm{E} 01$ $1.35673 \mathrm{E} \quad 01$

$1.45154 \mathrm{E} \quad 01$

$1.53649 \mathrm{E} \quad 01$ $1.61262 \mathrm{E} \quad 01$ $1.68091 \mathrm{E} \quad 01$ $1.74225 \mathrm{E} \quad 01$ $1.79747 \mathrm{E} 01$

$1.84730 \mathrm{E} \quad 01$ $1.89236 \mathrm{E} \quad 01$ $1.93323 \mathrm{E} \quad 01$ $1.97040 \mathrm{E} \quad 01$ $2.00429 \mathrm{E} \quad 01$

$2.03527 \mathrm{E} \quad 01$ $2.06367 \mathrm{E} \quad 01$ $2.08977 \mathrm{E} \quad 01$ $2.11382 \mathrm{E} \quad 01$ $2.13602 \mathrm{E} \quad 01$

$-2.12907 \mathrm{E}-05$

$-2.83573 \mathrm{E}-05$

$-3.64357 \mathrm{E}-05$ $-4.55342 \mathrm{E}-05$ $-5.56647 \mathrm{E}-05$

$-6.68431 \mathrm{E}-05$ $-7.90880 \mathrm{E}-05$ $-9.24205 \mathrm{E}-05$ $-1.06863 \mathrm{E}-04$ $-1.22441 \mathrm{E}-04$

$-1.39178 \mathrm{E}-04$ $-1.57100 \mathrm{E}-04$ $-1.76234 \mathrm{E}-04$ $-1.96608 \mathrm{E}-04$ $-2.18247 \mathrm{E}-04$

$-2.41180 \mathrm{E}-04$ $-2.65435 \mathrm{E}-04$ $-2.91038 \mathrm{E}-04$ $-3.18017 \mathrm{E}-04$ $-3.46401 \mathrm{E}-04$

$2.15658 \mathrm{E} \quad 01$ $2.17564 \mathrm{E} \quad 01$ 2.19337 E 01 $2.20988 \mathrm{E} \quad 01$ $2.22529 \mathrm{E} \quad 01$

$2.23970 \mathrm{E} \quad 01$ $2.25320 \mathrm{E} \quad 01$ $2.26586 \mathrm{E} 01$ $2.27777 \mathrm{E} \quad 01$

$2.28898 \mathrm{E} \quad 01$

$2.29954 \mathrm{E} \quad 01$ $2.30952 \mathrm{E} \quad 01$ $2.31895 \mathrm{E} \quad 01$ $2.32788 \mathrm{E} \quad 01$ $2.33635 \mathrm{E} \quad 01$

$2.34438 \mathrm{E} \quad 01$ $2.35201 \mathrm{E} \quad 01$ 2.35928 E 01 $2.36619 \mathrm{E} \quad 01$ $2.37278 \mathrm{E} \quad 01$

$2.37907 \mathrm{E} \quad 01$ 


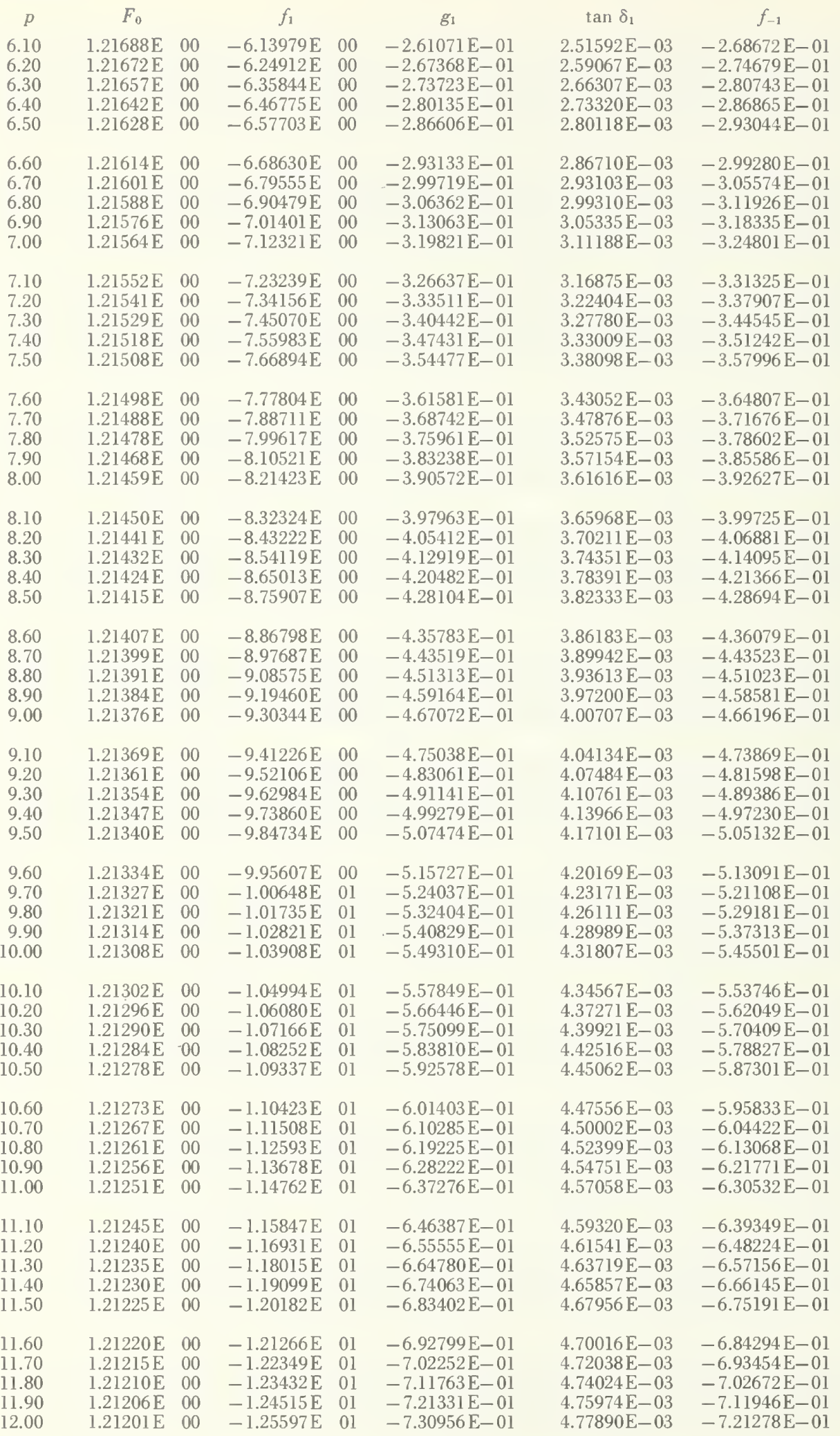

\begin{tabular}{|c|c|c|c|}
\hline$g_{-1}$ & & $\tan \delta_{-1}$ & \\
\hline $7.22441 \mathrm{E}$ & 00 & $-8.27748 E$ & \\
\hline & 00 & & \\
\hline $2 \mathrm{E}$ & 00 & $8636 \mathrm{E}$ & \\
\hline $88 \mathrm{E}$ & 00 & $3926 \mathrm{E}$ & \\
\hline $31 \mathrm{E}$ & 00 & $017 \mathrm{~F}$ & \\
\hline $172 \mathrm{E}$ & 00 & -8 . & \\
\hline & 00 & & \\
\hline & 00 & $64128 \mathrm{E}$ & \\
\hline & 00 & & \\
\hline 05 & 00 & -8 . & \\
\hline $32 \mathrm{E}$ & 00 & -8.78 & \\
\hline & 00 & & \\
\hline & 00 & -8.87 & \\
\hline & 00 & -8 & \\
\hline $75514 \mathrm{E}$ & 00 & -8 & \\
\hline & 00 & -9.00 & \\
\hline & 00 & & \\
\hline & 00 & & \\
\hline & 00 & $373 \mathrm{E}$ & \\
\hline $30061 \mathrm{E}$ & 00 & -9.1 & \\
\hline & 00 & & \\
\hline & 00 & & \\
\hline $1 \mathrm{E}$ & 00 & $13 \mathrm{E}$ & \\
\hline & 00 & -9.32 & \\
\hline$F$ & 00 & -9.3 & \\
\hline $41 \mathrm{E}$ & 00 & - & \\
\hline E & 01 & - & \\
\hline & 01 & $-9.46751 \mathrm{E}$ & \\
\hline & 01 & & \\
\hline$E$ & 01 & -9. & \\
\hline E & 01 & -9. & \\
\hline E & 01 & -9. & \\
\hline & 01 & & \\
\hline & 01 & -9 & \\
\hline 8 & 01 & -9.7 & \\
\hline E & 01 & -9 & \\
\hline & 01 & & \\
\hline & 01 & & \\
\hline & 01 & -9. & \\
\hline & 01 & & \\
\hline & 01 & & \\
\hline & 01 & -9. & \\
\hline & 01 & -9 . & \\
\hline & 01 . & & \\
\hline & 01 & & \\
\hline & 01 & -1 & \\
\hline & 01 & - & \\
\hline & 01 & & \\
\hline & 01 & & \\
\hline & 01 & & \\
\hline$E$ & 01 & & \\
\hline & 01 & & \\
\hline & 01 & -1 & \\
\hline & 01 & -1 . & 0 \\
\hline & 01 & 1 & \\
\hline & 01 & & 0 \\
\hline & 01 & & \\
\hline & 01 & $-1 .($ & \\
\hline & 0 & & \\
\hline & & & \\
\hline
\end{tabular}


$\bar{F}$

$7.99587 \mathrm{E}-05$ $8.25932 \mathrm{E}-05$ $8.52705 \mathrm{E}-05$ $8.79906 \mathrm{E}-05$ $9.07533 \mathrm{E}-05$

$9.35589 \mathrm{E}-05$ $9.64073 \mathrm{E}-05$ $9.92983 \mathrm{E}-05$ $1.02232 \mathrm{E}-04$ $1.05209 \mathrm{E}-04$

7.10

7.20

7.30

7.40

7.50

7.60

7.70

7.80

7.90

8.00

8.10

8.20

8.30

8.40

8.50

8.60

8.70

8.80

8.90

9.00

9.10

9.20

9.30

9.40

9.50

9.60

9.70

9.80

9.90

10.00

10.10

10.20

10.30

10.40

10.50

10.60

10.70

10.80

10.90

11.00

11.10

11.20

11.30

11.40

11.50

11.60

11.70

11.80

11.90

12.00

$1.08228 \mathrm{E}-04$

$1.11290 \mathrm{E}-04$

$1.14395 \mathrm{E}-04$

$1.17543 \mathrm{E}-04$

$1.20733 \mathrm{E}-04$

$1.23966 \mathrm{E}-04$

$1.27242 \mathrm{E}-04$

1.30561 E-04

$1.33922 \mathrm{E}-04$

$1.37326 \mathrm{E}-04$

$1.40773 \mathrm{E}-04$

$1.44262 \mathrm{E}-04$

$1.47795 \mathrm{E}-04$

$1.51370 \mathrm{E}-04$

$1.54988 \mathrm{E}-04$

$1.58648 \mathrm{E}-04$

$1.62351 \mathrm{E}-04$

$1.66097 \mathrm{E}-04$

$1.69886 \mathrm{E}-04$

$1.73718 \mathrm{E}-04$

$1.77592 \mathrm{E}-04$ $1.81509 \mathrm{E}-04$

$1.85468 \mathrm{E}-04$

$1.89471 \mathrm{E}-04$

$1.93516 \mathrm{E}-04$

$1.97604 \mathrm{E}-04$

$2.01735 \mathrm{E}-04$

$2.05908 \mathrm{E}-04$

$2.10124 \mathrm{E}-04$

2.14383 E- 04

$2.18684 \mathrm{E}-04$

$2.23029 \mathrm{E}-04$

$2.27416 \mathrm{E}-04$

$2.31846 E-04$

$2.36318 \mathrm{E}-04$

$2.40833 \mathrm{E}-04$

$2.45391 \mathrm{E}-04$

$2.49992 \mathrm{E}-04$

$2.54635 \mathrm{E}-04$

$2.59321 \mathrm{E}-04$

$2.64050 \mathrm{E}-04$

$2.68821 \mathrm{E}-04$

$2.73636 \mathrm{E}-04$

$2.78493 \mathrm{E}-04$

$2.83392 \mathrm{E}-04$

$2.88334 \mathrm{E}-04$

$2.93320 \mathrm{E}-04$

$2.98347 \mathrm{E}-04$

$3.03418 \mathrm{E}-04$

$3.08531 \mathrm{E}-04$ $f_{2}$

$-9.97170 \mathrm{E}-02$

-1.03161 E-01

$-1.06663 \mathrm{E}-01$

$-1.10223 \mathrm{E}-01$

-1.13842 E-01

$-1.17519 \mathrm{E}-01$

$-1.21254 \mathrm{E}-01$

$-1.25047 \mathrm{E}-01$

$-1.28898 \mathrm{E}-01$

$-1.32808 \mathrm{E}-01$
$-1.36775 \mathrm{E}-01$

-1.40801 E-01

$-1.44885 \mathrm{E}-01$

$-1.49027 \mathrm{E}-01$

$-1.53227 \mathrm{E}-01$

$-1.57485 \mathrm{E}-01$

$-1.61801 \mathrm{E}-01$

$-1.66176 \mathrm{E}-01$

$-1.70608 \mathrm{E}-01$

$-1.75099 \mathrm{E}-01$

$-1.79647 \mathrm{E}-01$

$-1.84254 \mathrm{E}-01$

$-1.88919 \mathrm{E}-01$

$-1.93642 \mathrm{E}-01$

$-1.98422 \mathrm{E}-01$

$-2.03261 \mathrm{E}-01$

$-2.08158 \mathrm{E}-01$

$-2.13113 \mathrm{E}-01$

$-2.23196 \mathrm{E}-01$

$-2.28325 \mathrm{E}-01$

$-2.33512 \mathrm{E}-01$

$-2.38756 \mathrm{E}-01$

$-2.44059 \mathrm{E}-01$

$-2.49420 \mathrm{E}-01$

$-2.54838 \mathrm{E}-01$

$-2.60315 \mathrm{E}-01$

$-2.65849 \mathrm{E}-01$

$-2.71441 \mathrm{E}-01$

-2.77091 E-01

$-2.82799 E-01$

$-2.88565 \mathrm{E}-01$

-2.94389 E-01

$-3.00271 \mathrm{E}-01$

$-3.06210 \mathrm{E}-01$

$-3.12207 \mathrm{E}-01$

$-3.18263 \mathrm{E}-01$

$-3.24376 \mathrm{E}-01$

$-3.30546 \mathrm{E}-01$

$-3.36775 \mathrm{E}-01$

$-3.43061 \mathrm{E}-01$

$-3.49406 \mathrm{E}-01$

$-3.55808 \mathrm{E}-01$

$-3.62267 \mathrm{E}-01$

$-3.68785 \mathrm{E}-01$

$-3.75360 \mathrm{E}-01$

$-3.81993 \mathrm{E}-01$

$-3.88684 \mathrm{E}-01$

$-4.02238 \mathrm{E}-01$
$-2.18126 \mathrm{E}-01$

$-3.95432 \mathrm{E}-01$ $g_{2}$

$-2.47600 \mathrm{E}-03$
$-2.57780 \mathrm{E}-03$
$-2.68217 \mathrm{E}-03$
$-2.78912 \mathrm{E}-03$
$-2.89869 \mathrm{E}-03$

$-3.01090 \mathrm{E}-03$

$-3.12577 \mathrm{E}-03$

$-3.24335 \mathrm{E}-03$

$-3.36365 \mathrm{E}-03$

$-3.48671 \mathrm{E}-03$

$-3.61254 \mathrm{E}-03$

$-3.74119 \mathrm{E}-03$

$-3.87267 \mathrm{E}-03$

$-4.00702 \mathrm{E}-03$

$-4.14426 \mathrm{E}-03$

$-4.28441 \mathrm{E}-03$

$-4.42752 \mathrm{E}-03$

$-4.57360 \mathrm{E}-03$

$-4.72268 \mathrm{E}-03$

$-4.87479 \mathrm{E}-03$

$-5.02996 \mathrm{E}-03$

$-5.18822 \mathrm{E}-03$

$-5.34958 \mathrm{E}-03$

$-5.51409 \mathrm{E}-03$

$-5.68177 \mathrm{E}-03$

$-5.85265 \mathrm{E}-03$

$-6.02675 \mathrm{E}-03$

$-6.20410 \mathrm{E}-03$

$-6.38473 \mathrm{E}-03$

$-6.56866 \mathrm{E}-03$

$-6.75593 \mathrm{E}-03$

$-6.94657 \mathrm{E}-03$

$-7.14059 \mathrm{E}-03$

$-7.33803 \mathrm{E}-03$

$-7.53892 \mathrm{E}-03$

$-7.74328 \mathrm{E}-03$

$-7.95114 \mathrm{E}-03$

$-8.16253 \mathrm{E}-03$

$-8.37747 \mathrm{E}-03$

$-8.59600 \mathrm{E}-03$

$-8.81813 \mathrm{E}-03$

$-9.04391 \mathrm{E}-03$

$-9.27335 \mathrm{E}-03$

$-9.50648 \mathrm{E}-03$

$-9.74334 \mathrm{E}-03$

$-9.98394 \mathrm{E}-03$

$-1.02283 \mathrm{E}-02$

$-1.04765 \mathrm{E}-02$

$-1.07285 \mathrm{E}-02$

$-1.09844 \mathrm{E}-02$

$-1.12442 \mathrm{E}-02$

$-1.15078 \mathrm{E}-02$

$-1.17755 \mathrm{E}-02$

$-1.20470 \mathrm{E}-02$

$-1.23226 \mathrm{E}-02$

$-1.26022 \mathrm{E}-02$

$-1.28859 \mathrm{E}-02$

$-1.31736 \mathrm{E}-02$

$-1.34655 \mathrm{E}-02$

$-1.37614 \mathrm{E}-02$

$\tan \delta_{2}$

$f_{-2}$

$2.44751 \mathrm{E}$ 01 $-2.53930 \mathrm{E}-03$

$2.45083 \mathrm{E} \quad 01 \quad-2.63928 \mathrm{E}-03$

$2.45404 \mathrm{E}$ 01 $-2.74172 \mathrm{E}-03$

$2.45714 \mathrm{E}$ 01 $-2.84666 \mathrm{E}-03$

$2.46014 \mathrm{E}$ 01 $-2.95412 \mathrm{E}-03$

$2.46304 \mathrm{E} \quad 01$

$2.46586 \mathrm{E} \quad 01$

$2.46858 \mathrm{E}$ 01

$2.47122 \mathrm{E} \quad 01$

$2.47378 \mathrm{E} \quad 0$

$2.47626 \mathrm{E} \quad 01$

$2.47867 \mathrm{E} 01$

$2.48101 \mathrm{E} \quad 01$

$2.48328 \mathrm{E} 01$

$2.48549 \mathrm{E} \quad 01$

$2.48764 \mathrm{E} \quad 01$

$2.48972 \mathrm{E} \quad 01$

$2.49175 \mathrm{E} \quad 01$

$2.49373 \mathrm{E} \quad 01$

2.49565 E 01

$2.49752 \mathrm{E} \quad 01$

$2.49935 \mathrm{E}$ 01

$2.50112 \mathrm{E} 01$

$2.50286 \mathrm{E}$ 0I

2.50455 E 01

$2.50619 \mathrm{E}$ 01

$2.50780 \mathrm{E}$ ol

$2.50937 \mathrm{E} \quad 01$

$2.51090 \mathrm{E}$ 0]

$2.51240 \mathrm{E} \quad 01$

$2.51386 \mathrm{E} \quad 01$

$2.51528 \mathrm{E} \quad 01$

$2.51668 \mathrm{E} 01$

$2.51804 \mathrm{E} \quad 01$

$2.51937 \mathrm{E} \quad 01$

$2.52068 \mathrm{E} 01$

$2.52195 \mathrm{E} \quad 01$

$2.52320 \mathrm{E} 01$

$2.52442 \mathrm{E} 01$

$2.52561 \mathrm{E} 01$

$2.52678 \mathrm{E} \quad 01$

$2.52793 \mathrm{E} 01$

$2.52905 \mathrm{E} 01$

$2.53015 \mathrm{E} \quad 01$

$2.53123 \mathrm{E}$ 01

$2.53228 \mathrm{E} \quad 01$

$2.53332 \mathrm{E} 01$

$2.53+33 \mathrm{E} \quad 01$

$2.53533 \mathrm{E} \quad 01$

$2.53630 \mathrm{E} \quad 01$

$2.53726 \mathrm{E} \quad 01$

$2.53820 \mathrm{E}$ 01

$2.53912 \mathrm{E} 01$

$2.54003 \mathrm{E} \quad 01$

2.54091 E 01

$2.54179 \mathrm{E} \quad 01$ 
ELECTRON RADIAL FUNCTIONS

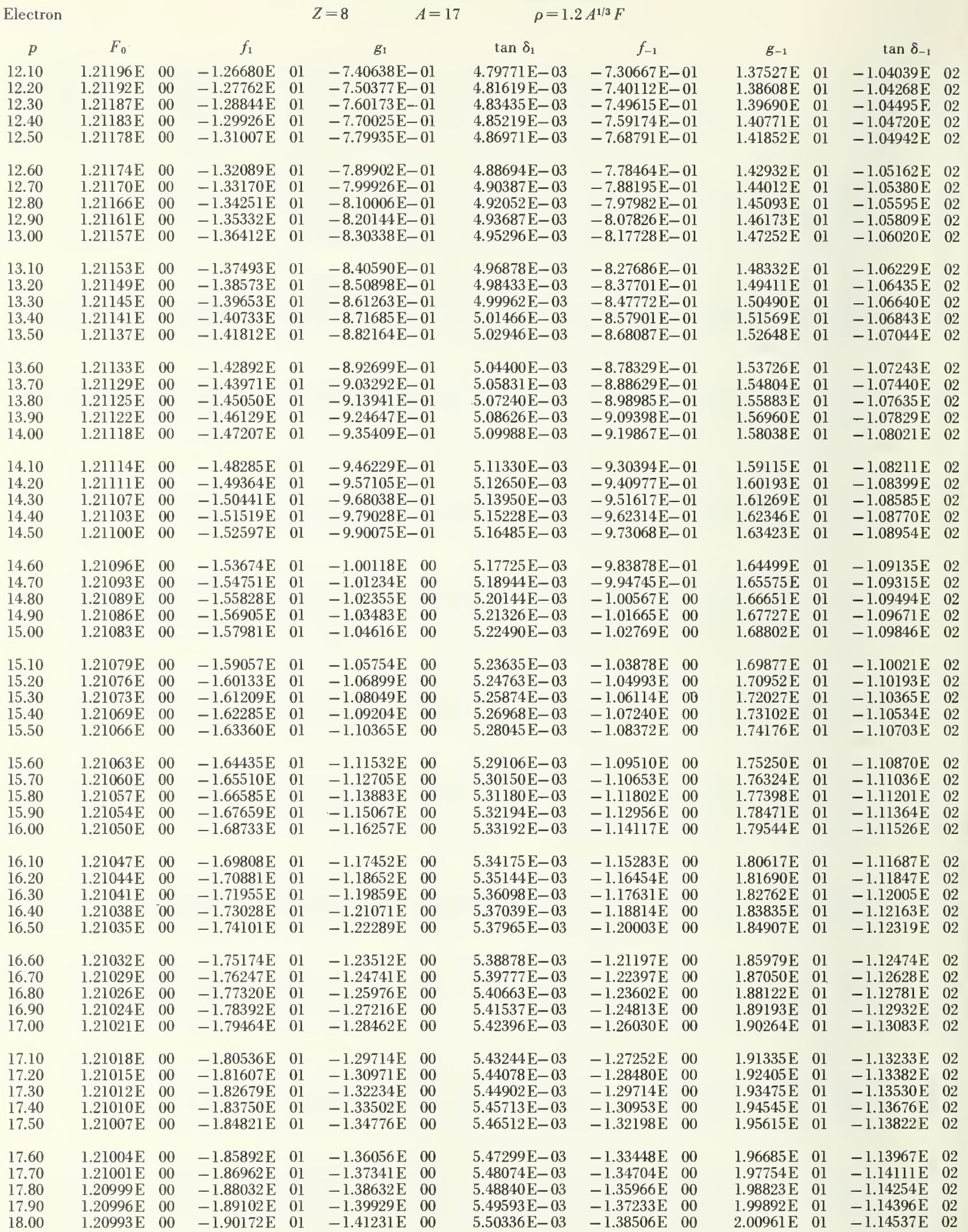


ELECTRON RADIAL FUNCTIONS

$Z=8 \quad A=17 \quad \rho=1.2 A^{1 / 3} F$

Electron

$\bar{F}$

$3.13687 \mathrm{E}-04$ $3.18886 \mathrm{E}-04$ $3.24128 \mathrm{E}-04$ $3.29412 \mathrm{E}-04$ $3.34739 \mathrm{E}-04$

$3.40108 \mathrm{E}-04$ $3.45520 \mathrm{E}-04$ $3.50975 \mathrm{E}-04$ $3.56473 \mathrm{E}-04$ $3.62013 \mathrm{E}-04$

$3.67596 \mathrm{E}-04$ $3.73222 \mathrm{E}-04$ $3.78891 \mathrm{E}-04$ $3.84602 \mathrm{E}-04$ $3.90356 \mathrm{E}-04$

$3.96153 \mathrm{E}-04$ $4.01992 \mathrm{E}-04$ $4.07874 \mathrm{E}-04$ $4.13799 \mathrm{E}-04$ $4.19766 \mathrm{E}-04$

14.10

14.20

14.30

14.40

14.50

14.60

14.70

14.80

14.90

15.00

15.10

15.20

15.30

15.40

15.50

15.60

15.70

15.80

15.90

16.00

16.10

16.20

16.30

16.40

16.50

16.60

16.70

16.80

16.90

17.00

17.10

17.20

17.30

17.40

17.50

17.60

17.70

17.80

17.90

18.00
$4.25776 \mathrm{E}-04$ $4.31829 \mathrm{E}-04$ $4.37925 \mathrm{E}-04$ $4.44063 \mathrm{E}-04$ $4.50244 \mathrm{E}-04$

$4.56468 \mathrm{E}-04$ $4.62734 \mathrm{E}-04$ $4.69043 \mathrm{E}-04$ $4.75395 \mathrm{E}-04$ $4.81790 \mathrm{E}-04$

$4.88227 \mathrm{E}-04$ $4.94706 \mathrm{E}-04$ $5.01229 \mathrm{E}-04$ $5.07794 \mathrm{E}-04$ $5.14403 \mathrm{E}-04$

$5.21053 \mathrm{E}-04$ $5.27747 \mathrm{E}-04$ $5.34483 \mathrm{E}-04$ $5.41261 \mathrm{E}-04$ $5.48083 \mathrm{E}-04$

$5.54947 \mathrm{E}-04$ $5.61854 \mathrm{E}-04$ $5.68803 \mathrm{E}-04$ $5.75796 \mathrm{E}-04$ $5.82830 \mathrm{E}-04$

$5.89908 \mathrm{E}-04$ $5.97028 \mathrm{E}-04$ $6.04191 \mathrm{E}-04$ $6.11397 \mathrm{E}-04$ $6.18645 \mathrm{E}-04$

$6.25937 \mathrm{E}-04$ $6.33271 \mathrm{E}-04$ $6.40647 \mathrm{E}-04$ $6.48066 \mathrm{E}-04$ $6.55528 \mathrm{E}-04$

$6.63032 \mathrm{E}-04$ $6.70579 \mathrm{E}-04$ $6.78169 \mathrm{E}-04$ $6.85802 \mathrm{E}-04$ $6.93477 \mathrm{E}-04$ $f_{2}$

$g_{2}$

$-4.09102 \mathrm{E}-01$ $-4.16024 \mathrm{E}-01$ $-4.23003 \mathrm{E}-01$ $-4.30040 \mathrm{E}-01$ $-4.37134 \mathrm{E}-01$

$-4.44287 \mathrm{E}-01$

$-4.51496 \mathrm{E}-01$

$-4.58764 \mathrm{E}-01$

$-4.66089 \mathrm{E}-01$

$-4.73472 \mathrm{E}-01$

$-4.80912 \mathrm{E}-01$

$-4.88410 \mathrm{E}-01$

$-4.95966 \mathrm{E}-01$

$-5.03579 \mathrm{E}-01$

$-5.11249 \mathrm{E}-01$

$-5.18977 \mathrm{E}-01$

$-5.26763 \mathrm{E}-01$

$-5.34606 \mathrm{E}-01$

$-5.42507 \mathrm{E}-01$

$-5.50465 \mathrm{E}-01$

$-5.58481 \mathrm{E}-01$

$-5.66555 \mathrm{E}-01$

$-5.74685 \mathrm{E}-01$

$-5.82874 \mathrm{E}-01$

$-5.91119 \mathrm{E}-01$

$-5.99423 \mathrm{E}-01$

$-6.07783 \mathrm{E}-01$

$-6.16201 \mathrm{E}-01$

$-6.24677 \mathrm{E}-01$

$-6.33209 \mathrm{E}-01$

$-6.41800 \mathrm{E}-01$

$-6.50447 \mathrm{E}-01$

$-6.59152 \mathrm{E}-01$

$-6.67915 \mathrm{E}-01$

$-6.76735 \mathrm{E}-01$

$-6.85612 \mathrm{E}-01$

$-6.94546 \mathrm{E}-01$

$-7.03538 \mathrm{E}-01$

$-7.12587 \mathrm{E}-01$

$-7.21693 \mathrm{E}-01$

$-7.30857 \mathrm{E}-01$

$-7.40078 \mathrm{E}-01$

$-7.49356 \mathrm{E}-01$

$-7.58691 \mathrm{E}-01$

$-7.68084 \mathrm{E}-01$

$-7.77534 \mathrm{E}-01$

$-7.87041 \mathrm{E}-01$

$-7.96605 \mathrm{E}-01$

$-8.06227 \mathrm{E}-01$

$-8.15906 \mathrm{E}-01$

$-8.25641 \mathrm{E}-01$

$-8.35435 \mathrm{E}-01$

$-8.45285 \mathrm{E}-01$

$-8.55192 \mathrm{E}-01$

$-8.65157 \mathrm{E}-01$

$-8.75178 \mathrm{E}-01$

$-8.85257 \mathrm{E}-01$

$-8.95393 \mathrm{E}-01$

$-9.05585 \mathrm{E}-01$

$-9.15835 \mathrm{E}-01$ $\tan \delta_{2}$

$f_{-2}$

$-1.40616 \mathrm{E}-02$ $-1.43659 \mathrm{E}-02$

$-1.46745 \mathrm{E}-02$

$-1.49873 \mathrm{E}-02$

$-1.53043 \mathrm{E}-02$

$-1.56257 \mathrm{E}-02$

$-1.59514 \mathrm{E}-02$

$-1.62814 \mathrm{E}-02$

$-1.66158 \mathrm{E}-02$

$-1.69547 \mathrm{E}-02$

$-1.72979 \mathrm{E}-02$

$-1.76456 \mathrm{E}-02$

$-1.79979 \mathrm{E}-02$

$-1.83546 \mathrm{E}-02$

$-1.87159 \mathrm{E}-02$

$-1.90817 \mathrm{E}-02$

$-1.94522 \mathrm{E}-02$

$-1.98273 \mathrm{E}-02$

$-2.02070 \mathrm{E}-02$

$-2.05915 \mathrm{E}-02$

$-2.09806 \mathrm{E}-02$

$-2.13745 \mathrm{E}-02$

$-2.17731 \mathrm{E}-02$

$-2.21765 \mathrm{E}-02$

$-2.25848 \mathrm{E}-02$

$-2.29979 \mathrm{E}-02$

$-2.34158 \mathrm{E}-02$

$-2.38387 \mathrm{E}-02$

$-2.42665 \mathrm{E}-02$

$-2.46993 \mathrm{E}-02$

$-2.51370 \mathrm{E}-02$ $-2.55798 \mathrm{E}-02$

$-2.60275 \mathrm{E}-02$

$-2.64804 \mathrm{E}-02$

$-2.69384 \mathrm{E}-02$

$-2.74014 \mathrm{E}-02$

$-2.78696 \mathrm{E}-02$

$-2.83430 \mathrm{E}-02$

$-2.88216 \mathrm{E}-02$

$-2.93054 \mathrm{E}-02$

$-2.97945 \mathrm{E}-02$

$-3.02889 \mathrm{E}-02$

$-3.07885 \mathrm{E}-02$

$-3.12935 \mathrm{E}-02$

$-3.18039 \mathrm{E}-02$

$-3.23197 \mathrm{E}-02$

$-3.28409 \mathrm{E}-02$

$-3.33675 \mathrm{E}-02$

$-3.38996 \mathrm{E}-02$

$-3.44372 \mathrm{E}-02$

$-3.49803 \mathrm{E}-02$

$-3.55290 \mathrm{E}-02$

$-3.60833 \mathrm{E}-02$

$-3.66432 \mathrm{E}-02$

$-3.72087 \mathrm{E}-02$

$-3.77799 \mathrm{E}-02$

$-3.83568 \mathrm{E}-02$

$-3.89394 \mathrm{E}-02$

$-3.95277 \mathrm{E}-02$

$-4.01218 \mathrm{E}-02$

$2.54592 \mathrm{E} \quad 01 \quad-1.38493 \mathrm{E}-02$ $2.54670 \mathrm{E}$ 01 $-1.41462 \mathrm{E}-02$ $2.54747 \mathrm{E} \quad 01-1.44472 \mathrm{E}-02$ $2.54823 \mathrm{E} 01-1.47524 \mathrm{E}-02$ $2.54897 \mathrm{E}$ 01 $-1.50617 \mathrm{E}-02$

$2.54971 \mathrm{E} \quad 01 \quad-1.53753 \mathrm{E}-02$ $2.55043 \mathrm{E} \quad 01-1.56930 \mathrm{E}-02$

$2.55113 \mathrm{E} \quad 01 \quad-1.60151 \mathrm{E}-02$

$2.55183 \mathrm{E} \quad 01-1.63414 \mathrm{E}-02$

$2.55252 \mathrm{E} \quad 01-1.66720 \mathrm{E}-02$

$2.55319 \mathrm{E} \quad 01-1.70070 \mathrm{E}-02$

$2.55385 \mathrm{E} \quad 01 \quad-1.73464 \mathrm{E}-02$

$2.55451 \mathrm{E} \quad 01 \quad-1.76901 \mathrm{E}-02$

$2.55515 \mathrm{E} \quad 01-1.80383 \mathrm{E}-02$

$\begin{array}{lll}2.55578 \mathrm{E} & 01-1.83910 \mathrm{E}-02\end{array}$

$2.55641 \mathrm{E} \quad 01$

$2.55702 \mathrm{E} \quad 01$

$2.55762 \mathrm{E} \quad 01$

$2.55822 \mathrm{E} \quad 01$

$2.55881 \mathrm{E} \quad 01$

$2.55938 \mathrm{E} \quad 01$

$2.55995 \mathrm{E} \quad 01$

$2.56051 \mathrm{E} 01$

$2.56106 \mathrm{E} \quad 01$

$2.56161 \mathrm{E} \quad 01$

$2.56215 \mathrm{E} \quad 01$

$2.56268 \mathrm{E} \quad 01$

$2.56320 \mathrm{E} \quad 01$

$2.56371 \mathrm{E} \quad 01$

$2.56422 \mathrm{E} \quad 01$

$2.56472 \mathrm{E} \quad 01$

$2.56521 \mathrm{E} \quad 01$

$2.56570 \mathrm{E} \quad 01$

$2.56618 \mathrm{E} \quad 01$

$2.56665 \mathrm{E} \quad 01$

$2.56712 \mathrm{E} \quad 01$

$2.56758 \mathrm{E} \quad 01$

$2.56803 \mathrm{E} \quad 01$

$2.56848 \mathrm{E}$ 0l

$2.56893 \mathrm{E} \quad 01$

$2.56936 \mathrm{E} \quad 01$

$2.56979 \mathrm{E}$ 0l

$2.57022 \mathrm{E}$ 0I

$2.57064 \mathrm{E} 01$

$2.57106 \mathrm{E} \quad 01$

$-1.87481 \mathrm{E}-02$

$-1.91097 \mathrm{E}-02$

$-1.94758 \mathrm{E}-02$

$-1.98466 \mathrm{E}-02$

$-2.02219 \mathrm{E}-02$

$-2.06018 \mathrm{E}-02$ $-2.09864 \mathrm{E}-02$ $-2.13756 \mathrm{E}-02$ $-2.17696 \mathrm{E}-02$

$-2.21682 \mathrm{E}-02$

$-2.25716 \mathrm{E}-02$

$-2.29798 \mathrm{E}-02$

$-2.33929 \mathrm{E}-02$

$-2.38107 \mathrm{E}-02$

$-2.42334 \mathrm{E}-02$

$-2.46610 \mathrm{E}-02$

$-2.50935 \mathrm{E}-02$ 


$$
Z=8 \quad A=17 \quad \rho=1.2 A^{1 / 3} F
$$

\begin{tabular}{|c|c|c|c|c|c|c|c|c|c|c|c|c|c|}
\hline$p$ & $F_{0}$ & & $f_{1}$ & & $g_{1}$ & & $\tan \delta_{1}$ & $f_{-1}$ & & $g_{-1}$ & & $\tan \delta_{-1}$ & \\
\hline 18.10 & $1.20991 \mathrm{E}$ & 00 & $-1.91242 \mathrm{E}$ & 01 & $-1.42539 \mathrm{E}$ & 00 & $5.51069 \mathrm{E}-03$ & $-1.39785 \mathrm{E}$ & 00 & $2.02029 \mathrm{E}$ & 01 & $-1.14678 \mathrm{E}$ & 02 \\
\hline 18.20 & $1.20988 \mathrm{E}$ & 00 & $-1.92311 \mathrm{E}$ & 01 & $-1.43852 \bar{E}$ & 00 & $5.51790 \mathrm{E}-03$ & $-1.41069 E$ & 00 & $2.03097 \mathrm{E}$ & 01 & $-1.14817 \mathrm{E}$ & 02 \\
\hline 18.30 & $1.20985 \mathrm{E}$ & 00 & $-1.93380 \mathrm{E}$ & 01 & $-1.45171 \mathrm{E}$ & 00 & $5.52501 \mathrm{E}-03$ & $-1.42359 \mathrm{E}$ & 00 & $2.04165 \mathrm{E}$ & 01 & $-1.14956 \mathrm{E}$ & 02 \\
\hline 18.40 & $1.20983 \mathrm{E}$ & 00 & $-1.94449 \mathrm{E}$ & 01 & $-1.46496 \mathrm{E}$ & 00 & $5.53200 \mathrm{E}-03$ & $-1.43655 \mathrm{E}$ & 00 & $2.05233 \mathrm{E}$ & 01 & $-1.15094 \mathrm{E}$ & 02 \\
\hline 18.50 & $1.20980 \mathrm{E}$ & 00 & $-1.95517 \mathrm{E}$ & 01 & $-1.47826 \mathrm{E}$ & 00 & $5.53891 \mathrm{E}-03$ & $-1.44956 \mathrm{E}$ & 00 & $2.06300 \mathrm{E}$ & 01 & $-1.15231 \mathrm{E}$ & 02 \\
\hline 18.60 & $1.20978 \mathrm{E}$ & 00 & $-1.96586 \mathrm{E}$ & 01 & $-1.49162 \mathrm{E}$ & 00 & $5.54573 \mathrm{E}-03$ & $-1.46262 \mathrm{E}$ & 00 & $2.07368 \mathrm{E}$ & 01 & $-1.15367 \mathrm{E}$ & 02 \\
\hline 18.70 & $1.20975 \mathrm{E}$ & 00 & $-1.97654 \mathrm{E}$ & 01 & $-1.50504 \mathrm{E}$ & 00 & $243 E-03$ & $-1.47575 \mathrm{E}$ & 00 & $2.08435 \mathrm{E}$ & 01 & $-1.15503 \mathrm{E}$ & 02 \\
\hline 18.80 & $1.20973 \mathrm{E}$ & 00 & $-1.98722 \mathrm{E}$ & 01 & $-1.51851 \mathrm{E}$ & 00 & $5.55904 \mathrm{E}-03$ & $-1.48893 \mathrm{E}$ & 00 & $2.09501 \mathrm{E}$ & 01 & $-1.15637 \mathrm{E}$ & 02 \\
\hline 18.90 & $1.20970 \mathrm{E}$ & 00 & $-1.99790 \mathrm{E}$ & 01 & $-1.53203 \mathrm{E}$ & 00 & $5.56555 \mathrm{E}-03$ & $-1.50216 \mathrm{E}$ & 00 & $2.10568 \mathrm{E}$ & 01 & $-1.15771 \mathrm{E}$ & 02 \\
\hline 19.00 & $1.20968 \mathrm{E}$ & 00 & $-2.00857 \mathrm{E}$ & 01 & $-1.54562 \mathrm{E}$ & 00 & $5.57197 \mathrm{E}-03$ & $-1.51545 \mathrm{E}$ & 00 & $2.11634 \mathrm{E}$ & 01 & $-1.15904 \mathrm{E}$ & 02 \\
\hline 19.10 & $1.20965 \mathrm{E}$ & 00 & $-2.01924 \mathrm{E}$ & 01 & $-1.55926 \mathrm{E}$ & 00 & $5.57830 \mathrm{E}-03$ & $-1.52880 \mathrm{E}$ & 00 & $2.12700 \mathrm{E}$ & 01 & $-1.16037 \mathrm{E}$ & 02 \\
\hline 19.20 & $1.20963 \mathrm{E}$ & 00 & $-2.02991 \mathrm{E}$ & 01 & $-1.57295 \mathrm{E}$ & 00 & $5.58455 \mathrm{E}-03$ & $-1.54220 \mathrm{E}$ & 00 & $2.13766 \mathrm{E}$ & 01 & $-1.16168 \mathrm{E}$ & 02 \\
\hline 9.30 & $1.20960 \mathrm{E}$ & 00 & $-2.04058 \mathrm{E}$ & 01 & $-1.58670 \mathrm{E}$ & 00 & $5.59068 \mathrm{E}-03$ & $-1.55566 \mathrm{E}$ & 00 & $2.14831 \mathrm{E}$ & 01 & $-1.16299 \mathrm{E}$ & 02 \\
\hline .40 & $1.20958 \mathrm{E}$ & 00 & $-2.05124 \mathrm{E}$ & 01 & $-1.60051 \mathrm{E}$ & 00 & $5.59674 \mathrm{E}-03$ & $-1.56918 E$ & 00 & $2.15897 \mathrm{E}$ & 01 & $-1.16430 \mathrm{E}$ & 02 \\
\hline 19.50 & $1.20956 \mathrm{E}$ & 00 & $-2.06191 \mathrm{E}$ & 01 & $-1.61437 \mathrm{E}$ & 00 & $5.60272 \mathrm{E}-03$ & $-1.58275 \mathrm{E}$ & 00 & $2.16962 \mathrm{E}$ & 01 & $-1.16559 \mathrm{E}$ & 02 \\
\hline 19.60 & $1.20953 \mathrm{E}$ & 00 & $-2.07257 \mathrm{E}$ & 01 & $-1.62829 \mathrm{E}$ & 00 & $5.60860 \mathrm{E}-03$ & $-1.59637 \mathrm{E}$ & 00 & $2.18027 \mathrm{E}$ & 01 & $-1.16688 \mathrm{E}$ & 02 \\
\hline 19.70 & $1.20951 \mathrm{E}$ & 00 & $-2.08322 \mathrm{E}$ & 01 & $-1.64226 \mathrm{E}$ & 00 & $5.61440 \mathrm{E}-03$ & $-1.61006 \mathrm{E}$ & 00 & $2.19091 \mathrm{E}$ & 01 & $-1.16817 \mathrm{E}$ & 02 \\
\hline .80 & $1.20948 \mathrm{E}$ & 00 & $-2.09388 \mathrm{E}$ & 01 & $-1.65630 \mathrm{E}$ & 00 & $5.62011 \mathrm{E}-03$ & $-1.62380 \mathrm{E}$ & 00 & $2.20156 \mathrm{E}$ & 01 & $-1.16944 \mathrm{E}$ & 02 \\
\hline .90 & $1.20946 \mathrm{E}$ & 00 & $0453 \mathrm{E}$ & 01 & $7038 \mathrm{E}$ & 00 & $2574 \mathrm{E}-03$ & $-1.63759 \mathrm{E}$ & 00 & $2.21220 \mathrm{E}$ & 01 & $17071 \mathrm{E}$ & 02 \\
\hline 20.00 & $1.20944 \mathrm{E}$ & 00 & $-2.11518 \mathrm{E}$ & 01 & $-1.68452 \mathrm{E}$ & 00 & $5.63129 \mathrm{E}-03$ & $-1.65144 \mathrm{E}$ & 00 & $2.22283 \mathrm{E}$ & 01 & $-1.17197 \mathrm{E}$ & 02 \\
\hline 0.10 & $1.20941 \mathrm{E}$ & 00 & $-2.12583 \mathrm{E}$ & 01 & $-1.69872 \mathrm{E}$ & 00 & $5.63676 \mathrm{E}-03$ & $-1.66535 \mathrm{E}$ & 00 & $2.23347 \mathrm{E}$ & 01 & $-1.17323 \mathrm{E}$ & 02 \\
\hline .20 & $1.20939 \mathrm{E}$ & 00 & $-2.13648 \mathrm{E}$ & 01 & $-1.71298 E$ & 00 & $5.64215 \mathrm{E}-03$ & $-1.67931 \mathrm{E}$ & 00 & $2.24410 \mathrm{E}$ & 01 & $-1.17448 \mathrm{E}$ & 02 \\
\hline .30 & $1.20937 \mathrm{E}$ & 00 & $-2.14712 \mathrm{E}$ & 01 & $-1.72729 \mathrm{E}$ & 00 & $5.64745 \mathrm{E}-03$ & $-1.69333 \mathrm{E}$ & 00 & $2.25474 \mathrm{E}$ & 01 & $-1.17573 \mathrm{E}$ & 02 \\
\hline 0.40 & 1.20 & 00 & $-2.15776 \mathrm{E}$ & 01 & $4165 \mathrm{E}$ & 00 & $268 \mathrm{E}-03$ & $70740 \mathrm{E}$ & 00 & $2.26536 \mathrm{E}$ & 01 & $17697 \mathrm{E}$ & 02 \\
\hline 20.50 & $1.20932 \mathrm{E}$ & 00 & $-2.16840 \mathrm{E}$ & 01 & $-1.75607 \mathrm{E}$ & 00 & $5.65784 \mathrm{E}-03$ & $-1.72153 \mathrm{E}$ & 00 & $2.27599 \mathrm{E}$ & 01 & $-1.17820 \mathrm{E}$ & 02 \\
\hline 20.60 & $1.20930 \mathrm{E}$ & 00 & $-2.17903 E$ & 01 & $-1.77055 \mathrm{E}$ & 00 & $5.66292 \mathrm{E}-03$ & $-1.73572 \mathrm{E}$ & 00 & $2.28661 \mathrm{E}$ & 01 & $-1.17943 \mathrm{E}$ & 02 \\
\hline .70 & $1.20928 \mathrm{E}$ & 00 & $-2.18967 \mathrm{E}$ & 01 & $8508 \mathrm{E}$ & 00 & $5.66793 \mathrm{E}-03$ & $-1.74996 \mathrm{E}$ & 00 & $2.29723 \mathrm{E}$ & 01 & $-1.18065 \mathrm{E}$ & 02 \\
\hline 2 & 1.20 & 00 & $030 \mathrm{E}$ & 01 & $67 \mathrm{E}$ & 00 & -03 & $426 \mathrm{E}$ & 00 & $785 \mathrm{E}$ & 01 & $3187 \mathrm{E}$ & 02 \\
\hline 20.90 & $1.20923 \mathrm{E}$ & 00 & $-2.21093 \mathrm{E}$ & 01 & $-1.81432 \mathrm{E}$ & 00 & $5.67771 \mathrm{E}-03$ & $1.77861 \mathrm{E}$ & 00 & $2.31847 \mathrm{E}$ & 01 & $18308 \mathrm{E}$ & 02 \\
\hline 21.00 & $1.20921 \mathrm{E}$ & 00 & $-2.22155 \mathrm{E}$ & 01 & $-1.82902 \mathrm{E}$ & 00 & $5.68249 \mathrm{E}-03$ & $-1.79302 \mathrm{E}$ & 00 & $2.32908 \mathrm{E}$ & 01 & $18428 \mathrm{E}$ & 02 \\
\hline 21.10 & $1.20919 \mathrm{E}$ & 00 & $-2.23218 \mathrm{E}$ & 01 & $-1.84377 \mathrm{E}$ & 00 & $5.68720 \mathrm{E}-03$ & $-1.80749 \mathrm{E}$ & 00 & $2.33969 \mathrm{E}$ & 01 & $-1.18548 \mathrm{E}$ & 02 \\
\hline .20 & 1.20 & 00 & & 01 & -1 . & 00 & $5 E-03$ & $1 \mathrm{E}$ & 00 & $030 \mathrm{E}$ & 01 & $668 \mathrm{E}$ & 02 \\
\hline 21.30 & $1.20915 \mathrm{E}$ & 00 & $-2.25342 \mathrm{E}$ & 01 & $-1.87345 \mathrm{E}$ & 00 & $5.69642 \mathrm{E}-03$ & $-1.83658 E$ & 00 & $2.36091 \mathrm{E}$ & 01 & $-1.18787 \mathrm{E}$ & 02 \\
\hline 21.40 & $1.20913 \mathrm{E}$ & 00 & $-2.26403 \mathrm{E}$ & 01 & $-1.88837 \mathrm{E}$ & 00 & $5.70093 \mathrm{E}-03$ & $-1.85121 \mathrm{E}$ & 00 & $2.37151 \mathrm{E}$ & 01 & $-1.18905 \mathrm{E}$ & 02 \\
\hline 1.50 & $1.20910 \mathrm{E}$ & 00 & $-2.27464 \mathrm{E}$ & 01 & $-1.90335 \mathrm{E}$ & 00 & $5.70537 \mathrm{E}-03$ & $-1.86590 \mathrm{E}$ & 00 & $2.38211 \mathrm{E}$ & 01 & $-1.19023 \mathrm{E}$ & 02 \\
\hline 21.60 & $1.20908 \mathrm{E}$ & 00 & $-2.28526 \mathrm{E}$ & 01 & $-1.91839 \mathrm{E}$ & 00 & $5.70975 \mathrm{E}-03$ & $-1.88064 \mathrm{E}$ & 00 & $2.39271 \mathrm{E}$ & 01 & $-1.19140 \mathrm{E}$ & 02 \\
\hline 1.7 & 1.209 & 00 & $9586 \mathrm{E}$ & 01 & $18 \mathrm{E}$ & 00 & $5 E-03$ & $544 \mathrm{E}$ & 00 & $2.40331 \mathrm{E}$ & 01 & $9258 \mathrm{E}$ & 02 \\
\hline 21.80 & $1.20904 \mathrm{E}$ & 00 & $-2.30647 \mathrm{E}$ & 01 & $-1.94862 \mathrm{E}$ & 00 & $5.71829 \mathrm{E}-03$ & $-1.91030 \mathrm{E}$ & 00 & $2.41390 \mathrm{E}$ & 01 & $-1.19374 \mathrm{E}$ & 02 \\
\hline 21.90 & $1.20902 \mathrm{E}$ & 00 & $-2.31707 \mathrm{E}$ & 01 & $-1.96382 \mathrm{E}$ & 00 & $5.72246 \mathrm{E}-03$ & $-1.92521 \mathrm{E}$ & 00 & $2.42449 \mathrm{E}$ & 01 & $-1.19490 \mathrm{E}$ & 02 \\
\hline 22.00 & $1.20900 \mathrm{E}$ & 00 & $-2.32767 \mathrm{E}$ & 01 & $-1.97908 \mathrm{E}$ & 00 & $5.72658 \mathrm{E}-03$ & $-1.94017 \mathrm{E}$ & 00 & $2.43508 \mathrm{E}$ & 01 & $-1.19606 \mathrm{E}$ & 02 \\
\hline 22.10 & $1.20898 \mathrm{E}$ & 00 & $-2.33827 \mathrm{E}$ & 01 & $-1.99439 \mathrm{E}$ & 00 & $5.73064 \mathrm{E}-03$ & $-1.95519 \mathrm{E}$ & 00 & $2.44566 \mathrm{E}$ & 01 & $-1.19721 \mathrm{E}$ & 02 \\
\hline .20 & $1.20896 \mathrm{E}$ & 00 & $-2.34887 \mathrm{E}$ & 01 & $-2.00976 \mathrm{E}$ & 00 & $5.73462 \mathrm{E}-03$ & $-1.97027 \mathrm{E}$ & 00 & $2.45625 \mathrm{E}$ & 01 & $-1.19836 \mathrm{E}$ & 02 \\
\hline 22.30 & $1.20894 \mathrm{E}$ & 00 & $-2.35946 \mathrm{E}$ & 01 & $-2.02518 \mathrm{E}$ & 00 & $5.73854 \mathrm{E}-03$ & $-1.98540 \mathrm{E}$ & 00 & $2.46683 \mathrm{E}$ & 01 & $-1.19950 \mathrm{E}$ & 02 \\
\hline 22.40 & $1.20892 \mathrm{E}$ & 00 & $-2.37005 \mathrm{E}$ & 01 & $-2.04066 \mathrm{E}$ & 00 & $5.74241 \mathrm{E}-03$ & $-2.00059 \mathrm{E}$ & 00 & $2.47741 \mathrm{E}$ & 01 & $-1.20064 \mathrm{E}$ & 02 \\
\hline 2.5 & $1.20890 \mathrm{E}$ & 00 & $-2.38064 \mathrm{E}$ & 01 & $-2.05619 \mathrm{E}$ & 00 & $5.74622 \mathrm{E}-0$ & $-2.01583 \mathrm{E}$ & 00 & $2.48798 \mathrm{E}$ & 01 & $-1.20177 \mathrm{E}$ & 02 \\
\hline 22.60 & $1.20888 \mathrm{E}$ & 00 & $-2.39122 \mathrm{E}$ & 01 & $-2.07178 \mathrm{E}$ & 00 & $5.74996 \mathrm{E}-03$ & $-2.03113 \mathrm{E}$ & 00 & $2.49855 \mathrm{E}$ & 01 & $-1.20291 \mathrm{E}$ & 02 \\
\hline 22.70 & $1.20886 \mathrm{E}$ & 00 & $-2.40181 \mathrm{E}$ & 01 & $-2.08743 \mathrm{E}$ & 00 & $5.75364 \mathrm{E}-03$ & $-2.04649 \mathrm{E}$ & 00 & $2.50912 \mathrm{E}$ & 01 & $-1.20403 \mathrm{E}$ & 02 \\
\hline 22.80 & $1.20884 \mathrm{E}$ & 00 & $-2.41239 E$ & 01 & $-2.10313 \mathrm{E}$ & 00 & $5.75728 \mathrm{E}-03$ & $-2.06190 \mathrm{E}$ & 00 & $2.51969 \mathrm{E}$ & 01 & $-1.20515 \mathrm{E}$ & 02 \\
\hline 22.90 & $1.20882 \mathrm{E}$ & 00 & $-2.42297 \mathbf{E}$ & 01 & $-2.11888 E$ & 00 & $5.76085 \mathrm{E}-$ & $-2.07736 \mathrm{E}$ & 00 & $2.53026 \mathrm{E}$ & 01 & $-1.20627 \mathrm{E}$ & 02 \\
\hline 23.00 & $1.20880 \mathrm{E}$ & 00 & $-2.43354 \mathrm{E}$ & 01 & $-2.13469 \mathrm{E}$ & 00 & $5.76437 \mathrm{E}-0$ & $-2.09288 E$ & 00 & $2.54082 \mathrm{E}$ & 01 & $-1.20739 \mathrm{E}$ & 02 \\
\hline 23.10 & $1.20878 \mathrm{E}$ & 00 & $-2.44411 \mathrm{E}$ & 01 & $-2.15056 \mathrm{E}$ & 00 & $5.76782 \mathrm{E}-03$ & $-2.10846 \mathrm{E}$ & 00 & $2.55138 \mathrm{E}$ & 01 & $-1.20850 \mathrm{E}$ & 02 \\
\hline & $1.20876 \mathrm{E}$ & 00 & $-2.45468 \mathrm{E}$ & 01 & $-2.16648 \mathrm{E}$ & 00 & $5.77122 \mathrm{E}-0$ & $-2.12409 \mathrm{E}$ & 00 & $2.56193 \mathrm{E}$ & 01 & $-1.20961 \mathrm{E}$ & 02 \\
\hline 23.30 & $1.20874 \mathrm{E}$ & 00 & $-2.46525 \mathrm{E}$ & 01 & $-2.18246 \mathrm{E}$ & 00 & $5.77458 \mathrm{E}-03$ & $-2.13977 \mathrm{E}$ & 00 & $2.57249 \mathrm{E}$ & 01 & $-1.21071 \mathrm{E}$ & 02 \\
\hline 23.40 & $1.20872 \mathrm{E}$ & 00 & $-2.47581 \mathrm{E}$ & 01 & $-2.19849 \mathrm{E}$ & 00 & $5.77787 \mathrm{E}-0$ & $-2.15551 \mathrm{E}$ & 00 & $2.58304 \mathrm{E}$ & 01 & $21181 \mathrm{E}$ & 02 \\
\hline 23.50 & $1.20870 \mathrm{E}$ & 00 & $-2.48638 \mathrm{E}$ & 01 & $-2.21458 \mathrm{E}$ & 00 & $5.78110 \mathrm{E}-03$ & $-2.17131 \mathrm{E}$ & 00 & $2.59359 \mathrm{E}$ & 01 & $-1.21291 \mathrm{E}$ & 02 \\
\hline 23.60 & $1.20868 \mathrm{E}$ & 00 & $-2.49694 \mathrm{E}$ & 01 & $-2.23072 \mathrm{E}$ & 00 & $5.78430 \mathrm{E}-03$ & $-2.18716 \mathrm{E}$ & 00 & $2.60414 \mathrm{E}$ & 01 & $-1.21400 \mathrm{E}$ & 02 \\
\hline & $1.20866 \mathrm{E}$ & 00 & $-2.50749 \mathrm{E}$ & 01 & $-2.24692 \mathrm{E}$ & 00 & $5.78743 \mathrm{E}-03$ & $-2.20307 \mathbf{E}$ & 00 & $2.61468 \mathrm{E}$ & 01 & $-1.21509 \mathrm{E}$ & 02 \\
\hline & $1.20864 \mathrm{E}$ & 00 & $-2.51805 \mathrm{E}$ & 01 & $-2.26317 \mathrm{E}$ & 00 & $5.79051 \mathrm{E}-$ & $-2.21903 \mathrm{E}$ & 00 & $2.62522 \mathrm{E}$ & 01 & $-1.21618 \mathrm{E}$ & 02 \\
\hline 23.90 & $1.20863 \mathrm{E}$ & 00 & $-2.52860 \mathrm{E}$ & 01 & $-2.27948 \mathbf{E}$ & 00 & $5.79354 \mathrm{E}-$ & $-2.23505 \mathrm{E}$ & 00 & $2.63576 \mathrm{E}$ & 01 & $-1.21726 \mathrm{E}$ & 02 \\
\hline 00 & $1.20861 \mathrm{E}$ & 00 & $-2.53915 \mathrm{E}$ & 01 & $-2.29584 \mathrm{E}$ & 00 & $5.79652 \mathrm{E}-03$ & $-2.25113 \mathrm{E}$ & 00 & $2.64629 \mathrm{E}$ & $0]$ & $-1.21834 \mathrm{E}$ & \\
\hline
\end{tabular}


$\bar{F}$

18.10

18.20

18.30

18.40

18.50

18.60

18.70

18.80

18.90

19.00

19.10

19.20

19.30

19.40

19.50

19.60

19.70

19.80

19.90

20.00

20.10

20.20

20.30

20.40

20.50

20.60

20.70

20.80

20.90

21.00

21.10

21.20

21.30

21.40

21.50

21.60

21.70

21.80

21.90

22.00

22.10

22.20

22.30

22.40

22.50

22.60

22.70

22.80

22.90

23.00

23.10

23.20

23.30

23.40

23.50

23.60

23.70

23.80

23.90

24.00 $f_{2}$

$7.01196 \mathrm{E}-04$

$7.08956 \mathrm{E}-04$

$7.16759 \mathrm{E}-04$

$7.24605 \mathrm{E}-04$

$7.32494 \mathrm{E}-04$

$7.40426 \mathrm{E}-04$ $7.48399 \mathrm{E}-04$ $7.56416 \mathrm{E}-04$

$7.64476 \mathrm{E}-04$

$7.72578 \mathrm{E}-04$

$7.80722 \mathrm{E}-04$

$7.88910 \mathrm{E}-04$

$7.97140 \mathrm{E}-04$

$8.05413 \mathrm{E}-04$

$8.13729 \mathrm{E}-04$

$8.22087 \mathrm{E}-04$

$8.30488 \mathrm{E}-04$

8.38931 E-04

$8.47418 \mathrm{E}-04$

$8.55946 \mathrm{E}-04$

8.64518E-04

$8.73132 \mathrm{E}-04$

$8.81789 \mathrm{E}-04$

$8.90489 \mathrm{E}-04$

8.99231 E-04

$9.08016 \mathrm{E}-04$

$9.16844 \mathrm{E}-04$

$9.25714 \mathrm{E}-04$

$9.34627 \mathrm{E}-04$

$9.43583 \mathrm{E}-04$

$9.52581 \mathrm{E}-04$

$9.61622 E-04$

$9.70706 \mathrm{E}-04$

$9.79832 \mathrm{E}-04$

$9.89001 \mathrm{E}-04$

$9.98213 \mathrm{E}-04$

$1.00747 \mathrm{E}-03$

$1.01676 \mathrm{E}-03$

$1.02610 \mathrm{E}-03$

$1.03549 \mathrm{E}-03$

$1.04491 \mathrm{E}-03$

$1.05438 \mathrm{E}-03$

$1.06389 \mathrm{E}-03$

$1.07344 \mathrm{E}-03$

$1.08304 \mathrm{E}-03$

$1.09268 \mathrm{E}-03$

$1.10236 \mathrm{E}-03$

$1.11208 \mathrm{E}-03$

$1.12185 \mathrm{E}-03$

$1.13166 \mathrm{E}-03$

$1.14151 \mathrm{E}-03$

$1.15140 \mathrm{E}-03$

$1.16134 \mathrm{E}-03$

$1.17132 \mathrm{E}-03$

$1.18134 \mathrm{E}-03$

$1.19141 \mathrm{E}-03$

$1.20152 \mathrm{E}-03$

$1.21167 \mathrm{E}-03$

$1.22186 \mathrm{E}-03$

$1.23210 \mathrm{E}-03$ $g_{2}$

$-9.26143 \mathrm{E}-01$

$-9.36507 \mathrm{E}-01$

$-9.46928 \mathrm{E}-01$

$-9.57406 \mathrm{E}-01$

$-9.67941 \mathrm{E}-01$

$-9.78533 \mathrm{E}-0 \mathrm{l}$

$-9.89182 E-01$

$-9.99888 \mathrm{E}-01$

-1.01065 E 00

$-1.02147 \mathrm{E} 00$

$-1.03235 \mathrm{E} \quad 00$

$-1.04328 \mathrm{E} 00$

$-1.05427 \mathrm{E} 00$

$-1.06532 \mathrm{E} 00$

$-1.07643 \mathrm{E} 00$

$-1.08759 \mathrm{E} \quad 00$

$-1.09880 \mathrm{E} 00$

$-1.11008 \mathrm{E} 00$

$-1.12141 \mathrm{E} 00$

$-1.13280 \mathrm{E} 00$

$-1.14425 \mathrm{E} \quad 00$

$-1.15575 \mathrm{E} 00$

-1.1673 I E 00

$-1.17892 \mathrm{E} 00$

-1.19060 E 00

$-1.20233 \mathrm{E} 00$

$-1.21411 \mathrm{E} 00$

$-1.22595 \mathrm{E} 00$

$-1.23785 \mathrm{E} 00$

-1.24981 E 00

$-1.26182 \mathrm{E} \quad 00$

$-1.27389 \mathrm{E} 00$

$-1.28602 \mathrm{E} \quad 00$

-1.29820 E 00

$-1.31044 \mathrm{E} 00$

$-1.32274 \mathrm{E} \quad 00$ $-1.33509 \mathrm{E} 00$ $-1.34750 \mathrm{E} 00$ $-1.35997 \mathrm{E} 00$

$-1.37249 \mathrm{E} 00$

$-1.38507 \mathrm{E} \quad 00$ $-1.39770 \mathrm{E} 00$ $-1.41040 \mathrm{E} 00$ $-1.42314 \mathrm{E} 00$

$-1.43595 \mathrm{E} 00$

$-1.44881 \mathrm{E} \quad 00$

$-1.46173 \mathrm{E} 00$

$-1.47470 \mathrm{E} \quad 00$

$-1.48773 \mathrm{E} \quad 00$

$-1.50082 \mathrm{E} 00$

$-1.51396 \mathrm{E} \quad 00$

$-1.52717 \mathrm{E} 00$

$-1.54042 \mathrm{E} 00$

$-1.55373 \mathrm{E} 00$

$-1.56710 \mathrm{E} 00$

$-1.58053 \mathrm{E} \quad 00$

$-1.59401 \mathrm{E} 00$

$-1.60755 \mathrm{E} \quad 00$

$-1.62114 \mathrm{E} \quad 00$

$-1.63479 \mathrm{E} 00$
$-4.07218 \mathrm{E}-02$

$-4.13275 E-02$

$-4.19391 \mathrm{E}-02$

$-4.25565 \mathrm{E}-02$

$-4.31799 \mathrm{E}-02$

$-4.38092 \mathrm{E}-02$

$-4.44445 \mathrm{E}-02$

$-4.50858 \mathrm{E}-02$

$-4.57331 \mathrm{E}-02$

$-4.63864 E-02$

$-4.70458 \mathrm{E}-02$

$-4.77113 \mathrm{E}-02$

$-4.83829 \mathrm{E}-02$

$-4.90607 \mathrm{E}-02$

$-4.97446 \mathrm{E}-02$

$-5.04348 \mathrm{E}-02$

$-5.11312 \mathrm{E}-02$

$-5.18338 \mathrm{E}-02$

$-5.25428 \mathrm{E}-02$

$-5.32580 \mathrm{E}-02$

$-5.39796 \mathrm{E}-02$

$-5.47076 \mathrm{E}-02$

$-5.54419 \mathrm{E}-02$

$-5.61827 \mathrm{E}-02$

$-5.69299 \mathrm{E}-02$

$-5.76836 \mathrm{E}-02$

$-5.84438 \mathrm{E}-02$

$-5.92105 \mathrm{E}-02$

$-5.99838 \mathrm{E}-02$

$-6.07636 \mathrm{E}-02$

$-6.15501 \mathrm{E}-02$

$-6.23432 \mathrm{E}-02$

$-6.31430 \mathrm{E}-02$

$-6.39494 \mathrm{E}-02$

$-6.47626 \mathrm{E}-02$

$-6.55826 \mathrm{E}-02$

$-6.64092 \mathrm{E}-02$

$-6.72427 \mathrm{E}-02$

$-6.80830 \mathrm{E}-02$

$-6.89302 \mathrm{E}-02$

$-6.97843 \mathrm{E}-02$

$-7.06452 \mathrm{E}-02$

$-7.15131 \mathrm{E}-02$

$-7.23879 \mathrm{E}-02$

$-7.32697 \mathrm{E}-02$

$-7.41586 \mathrm{E}-02$

$-7.50545 \mathrm{E}-02$

$-7.59574 \mathrm{E}-02$

$-7.68674 \mathrm{E}-02$

$-7.77846 \mathrm{E}-02$

$-7.87089 \mathrm{E}-02$

$-7.96404 \mathrm{E}-02$

$-8.05790 \mathrm{E}-02$

$-8.15249 \mathrm{E}-02$

-8.24781 E-02

$-8.34385 \mathrm{E}-02$

$-8.44062 \mathrm{E}-02$

$-8.53812 \mathrm{E}-02$

$-8.63637 \mathrm{E}-02$

$-8.73535 \mathrm{E}-02$

$\tan \delta_{2}$

$f_{-2}$

$2.57705 \mathrm{E} 01$

.57772E $01-4.10898 \mathrm{E}-02$

$2.57805 \mathrm{E}$ 01 $-4.16941 \mathrm{E}-02$

$2.57838 \mathrm{E} \quad 01 \quad-4.23042 \mathrm{E}-02$

$2.57870 \mathrm{E}$ 0l

$2.57902 \mathrm{E} 01$

$2.57934 \mathrm{E} \quad 01$

$2.57965 \mathrm{E}$ 01

$2.57996 \mathrm{E} \quad 01$

$2.58027 \mathrm{E} \quad 01$

$2.58057 \mathrm{E} \quad 01$

$2.58087 \mathrm{E}$ 0l

$2.58117 \mathrm{E} \quad 01$

$2.58146 \mathrm{E} \quad 01$

$2.58175 \mathrm{E}$ 01

$2.58203 \mathrm{E}$ 01

$2.58232 \mathrm{E}$ 01

$2.58260 \mathrm{E} \quad 01$

$2.58288 \mathrm{E} \quad 01$

$2.58315 \mathrm{E} 01$

$2.58342 \mathrm{E} 01$

$2.58369 \mathrm{E} 01$

$2.58396 \mathrm{E} \quad 01$

2.58422 E 01

$2.58449 \mathrm{E} \quad 01$

$2.584 .74 \mathrm{E} 01$

$2.58500 \mathrm{E}$ 01

$2.58525 \mathrm{E} \quad 01$

$2.58550 \mathrm{E} \quad 01$

$2.58575 \mathrm{E} \quad 01$

$2.58600 \mathrm{E}$ 0]

$2.58624 \mathrm{E} \quad 01$

$2.58648 \mathrm{E} 01$

$2.58672 \mathrm{E} \quad 01$

$2.58696 \mathrm{E} \quad 0]$

$2.58719 \mathrm{E} \quad 01$

$2.58743 \mathrm{E} 01$

$2.58766 \mathrm{E}$ ol

$2.58788 \mathrm{E} \quad 0 \mathrm{l}$

$2.58811 \mathrm{E}$ 0l 


\begin{tabular}{|c|c|c|c|c|c|c|c|c|c|c|c|c|c|}
\hline$p$ & $F_{0}$ & & $f_{1}$ & & $g_{1}$ & & $\tan \delta_{1}$ & $f_{-1}$ & & $g_{\sim 1}$ & & $\tan \delta_{-1}$ & \\
\hline 24.10 & $1.20859 \mathrm{E}$ & 00 & $-2.54969 \mathrm{E}$ & 01 & $-2.31226 \mathrm{E}$ & 00 & $5.79945 \mathrm{E}-03$ & $-2.26725 \mathrm{E}$ & 00 & $2.65683 \mathrm{E}$ & 01 & $-1.21942 \mathrm{E}$ & 02 \\
\hline 24.20 & $1.20857 \mathrm{E}$ & 00 & $-2.56024 \mathrm{E}$ & 01 & $-2.32874 \mathrm{E}$ & 00 & $5.80232 \mathrm{E}-03$ & $-2.28344 \mathrm{E}$ & 00 & $2.66736 \mathrm{E}$ & 01 & $-1.22050 \mathrm{E}$ & 02 \\
\hline 24.30 & $1.20855 \mathrm{E}$ & 00 & $-2.57078 \mathrm{E}$ & 01 & $-2.34527 \mathrm{E}$ & 00 & $5.80516 \mathrm{E}-03$ & $-2.29968 \mathrm{E}$ & 00 & $2.67789 \mathrm{E}$ & 01 & $-1.22157 \mathrm{E}$ & 02 \\
\hline 24.40 & $1.20853 \mathrm{E}$ & 00 & $-2.58132 \mathrm{E}$ & 01 & $-2.36185 E$ & 00 & $5.80793 \mathrm{E}-03$ & $-2.31597 \mathrm{E}$ & 00 & $2.68841 \mathrm{E}$ & 01 & $-1.22264 \mathrm{E}$ & 02 \\
\hline 24.50 & $1.20851 \mathrm{E}$ & 00 & $-2.59185 \mathrm{E}$ & 01 & $-2.37849 \mathrm{E}$ & 00 & $5.81067 \mathrm{E}-03$ & $-2.33232 \mathrm{E}$ & 00 & $2.69893 \mathrm{E}$ & 01 & $-1.22370 \mathrm{E}$ & 02 \\
\hline 24.60 & $1.20850 \mathrm{E}$ & 00 & $-2.60238 \mathrm{E}$ & 01 & $-2.39518 \mathrm{E}$ & 00 & $5.81335 \mathrm{E}-03$ & $-2.34872 \mathrm{E}$ & 00 & $2.70945 \mathrm{E}$ & 01 & $-1.22476 \mathrm{E}$ & 02 \\
\hline 24.70 & $1.20848 \mathrm{E}$ & 00 & $-2.61291 \mathrm{E}$ & 01 & $-2.41193 \mathrm{E}$ & 00 & -03 & $-2.36518 \mathrm{E}$ & 00 & $2.71997 \mathrm{E}$ & 01 & $-1.22582 \mathrm{E}$ & 02 \\
\hline 24.80 & $1.20846 \mathrm{E}$ & 00 & $-2.62344 \mathrm{E}$ & 01 & $-2.42874 \mathrm{E}$ & 00 & $5.81858 \mathrm{E}-03$ & $-2.38170 \mathrm{E}$ & 00 & $2.73048 \mathrm{E}$ & 01 & $-1.22688 E$ & 02 \\
\hline 4.90 & $1.20844 \mathrm{E}$ & 00 & $-2.63397 \mathrm{E}$ & 01 & $-2.44560 \mathrm{E}$ & 00 & $5.82112 \mathrm{E}-03$ & $-2.39827 \mathrm{E}$ & 00 & $2.74099 \mathrm{E}$ & 01 & $-1.22793 \mathrm{E}$ & 02 \\
\hline 25.00 & $1.20842 \mathrm{E}$ & 00 & $-2.64449 \mathrm{E}$ & 01 & $-2.46251 \mathrm{E}$ & 00 & $5.82362 \mathrm{E}-03$ & $-2.41489 \mathrm{E}$ & 00 & $2.75150 \mathrm{E}$ & 01 & $-1.22898 \mathrm{E}$ & 02 \\
\hline 25.10 & $1.20841 \mathrm{E}$ & 00 & $-2.65501 \mathrm{E}$ & 01 & $-2.47948 \mathrm{E}$ & 00 & $5.82607 \mathrm{E}-03$ & $-2.43157 \mathrm{E}$ & 00 & $2.76201 \mathrm{E}$ & 01 & $-1.23003 \mathrm{E}$ & 02 \\
\hline 25.20 & $1.20839 \mathrm{E}$ & 00 & $-2.66552 \mathrm{E}$ & 01 & $-2.49650 \mathrm{E}$ & 00 & $5.82849 \mathrm{E}-03$ & $-2.44831 \mathrm{E}$ & 00 & $2.77251 \mathrm{E}$ & 01 & $-1.23108 \mathrm{E}$ & 02 \\
\hline 25.30 & $1.20837 \mathrm{E}$ & 00 & $-2.67604 \mathrm{E}$ & 01 & $-2.51358 \mathrm{E}$ & 00 & $5.83085 \mathrm{E}-03$ & $-2.46510 \mathrm{E}$ & 00 & $2.78301 \mathrm{E}$ & 01 & $-1.23212 \mathrm{E}$ & 02 \\
\hline 25.40 & $1.20835 \mathrm{E}$ & 00 & $-2.68655 \mathrm{E}$ & 01 & $-2.53072 \mathrm{E}$ & 00 & $5.83317 \mathrm{E}-03$ & $-2.48194 \mathrm{E}$ & 00 & $2.79351 \mathrm{E}$ & 01 & $-1.23316 \mathrm{E}$ & 02 \\
\hline 25.50 & $1.20834 \mathrm{E}$ & 00 & $-2.69706 \mathrm{E}$ & 01 & $-2.54791 \mathrm{E}$ & 00 & $5.83544 \mathrm{E}-03$ & $-2.49884 \mathrm{E}$ & 00 & $2.80400 \mathrm{E}$ & 01 & $-1.23420 \mathrm{E}$ & 02 \\
\hline 5.60 & $1.20832 \mathrm{E}$ & 00 & $-2.70756 \mathrm{E}$ & 01 & $-2.56515 \mathrm{E}$ & 00 & $5.83766 \mathrm{E}-03$ & $-2.51579 \mathrm{E}$ & 00 & $2.81449 \mathrm{E}$ & 01 & $-1.23524 \mathrm{E}$ & 02 \\
\hline 25.70 & $1.20830 \mathrm{E}$ & 00 & $-2.71806 \mathrm{E}$ & 01 & $-2.58245 \mathrm{E}$ & 00 & $5.83985 \mathrm{E}-03$ & $-2.53280 \mathrm{E}$ & 00 & $2.82498 \mathrm{E}$ & 01 & $-1.23627 \mathrm{E}$ & 02 \\
\hline 25.80 & $1.20828 \mathrm{E}$ & 00 & $-2.72856 \mathrm{E}$ & 01 & $-2.59980 \mathrm{E}$ & 00 & $5.84200 \mathrm{E}-03$ & $-2.54987 \mathrm{E}$ & 00 & $2.83547 \mathrm{E}$ & 01 & $-1.23731 \mathrm{E}$ & 02 \\
\hline 25.90 & $1.20827 \mathrm{E}$ & 00 & $-2.73906 \mathrm{E}$ & 01 & $-2.61721 \mathrm{E}$ & 00 & $5.84410 \mathrm{E}-03$ & $-2.56699 \mathrm{E}$ & 00 & $2.84595 \mathrm{E}$ & 01 & $-1.23834 \mathrm{E}$ & 02 \\
\hline 26.00 & $1.20825 \mathrm{E}$ & 00 & $-2.74955 \mathrm{E}$ & 01 & $-2.63468 \mathrm{E}$ & 00 & $5.84616 \mathrm{E}-03$ & $-2.58416 \mathrm{E}$ & 00 & $2.85643 \mathrm{E}$ & 01 & $-1.23936 \mathrm{E}$ & 02 \\
\hline 26.10 & $1.20823 \mathrm{E}$ & 00 & $-2.76005 \mathrm{E}$ & 01 & $-2.65219 \mathrm{E}$ & 00 & $5.84818 \mathrm{E}-03$ & $-2.60139 \mathrm{E}$ & 00 & $2.86691 \mathrm{E}$ & 01 & $-1.24039 \mathrm{E}$ & 02 \\
\hline 26.20 & $1.20822 \mathrm{E}$ & 00 & $-2.77053 \mathrm{E}$ & 01 & $-2.66977 \mathrm{E}$ & 00 & $5.85015 \mathrm{E}-03$ & $-2.61867 \mathrm{E}$ & 00 & $2.87739 \mathrm{E}$ & 01 & $-1.24141 \mathrm{E}$ & 02 \\
\hline 26.30 & $1.20820 \mathrm{E}$ & 00 & $-2.78102 \mathrm{E}$ & 01 & $-2.68740 \mathrm{E}$ & 00 & $5.85209 \mathrm{E}-03$ & $-2.63601 \mathrm{E}$ & 00 & $2.88786 \mathrm{E}$ & 01 & $-1.24243 \mathrm{E}$ & 02 \\
\hline 26.40 & $1.20818 \mathrm{E}$ & 00 & $-2.79150 \mathrm{E}$ & 01 & $-2.70508 \mathrm{E}$ & 00 & -03 & $-2.65340 \mathrm{E}$ & 00 & $2.89833 \mathrm{E}$ & 01 & $-1.24345 \mathrm{E}$ & 02 \\
\hline 26.50 & $1.20817 \mathrm{E}$ & 00 & $-2.80198 E$ & 01 & $-2.72282 \mathrm{E}$ & 00 & $5.85584 \mathrm{E}-03$ & $-2.67085 \mathrm{E}$ & 00 & $2.90879 \mathrm{E}$ & 01 & $-1.24447 \mathrm{E}$ & 02 \\
\hline 26.60 & $1.20815 \mathrm{E}$ & 00 & $-2.81246 \mathrm{E}$ & 01 & $-2.74061 \mathrm{E}$ & 00 & $5.85766 \mathrm{E}-03$ & $-2.68835 \mathrm{E}$ & 00 & $2.91926 \mathrm{E}$ & 01 & $-1.24549 \mathrm{E}$ & 02 \\
\hline 26.70 & $1.20813 \mathrm{E}$ & 00 & $-2.82294 \mathrm{E}$ & 01 & $-2.75845 \mathrm{E}$ & 00 & $4 \mathrm{E}-03$ & $-2.70591 \mathrm{E}$ & 00 & $2.92972 \mathrm{E}$ & 01 & $-1.24650 \mathrm{E}$ & 02 \\
\hline 26.80 & $1.20812 \mathrm{E}$ & 00 & $3341 \mathrm{E}$ & 01 & $36 \mathrm{E}$ & 00 & & $2352 \mathrm{E}$ & 00 & $18 \mathrm{E}$ & 01 & -1 & 02 \\
\hline 26.90 & $1.20810 \mathrm{E}$ & 00 & $-2.84388 E$ & 01 & $-2.79431 \mathrm{E}$ & 00 & $5.86287 \mathrm{E}-03$ & $-2.74119 \mathrm{E}$ & 00 & $2.95063 \mathrm{E}$ & 01 & $-1.24852 \mathrm{E}$ & 02 \\
\hline 27.00 & $1.20808 \mathrm{E}$ & 00 & $-2.85434 \mathrm{E}$ & 01 & $-2.81232 \mathrm{E}$ & 00 & $5.86454 \mathrm{E}-03$ & $-2.75891 \mathrm{E}$ & 00 & $2.96108 \mathrm{E}$ & 01 & $-1.24953 \mathrm{E}$ & 02 \\
\hline 27.10 & $1.20807 \mathrm{E}$ & 00 & $-2.86481 \mathrm{E}$ & 01 & $-2.83039 \mathrm{E}$ & 00 & $5.86616 \mathrm{E}-03$ & $-2.77669 \mathrm{E}$ & 00 & $2.97153 \mathrm{E}$ & 01 & $-1.25054 \mathrm{E}$ & 02 \\
\hline 27.20 & $1.20805 \mathrm{E}$ & 00 & $7527 \mathrm{E}$ & 01 & & 00 & -03 & $9452 \mathrm{E}$ & 00 & $198 \mathrm{E}$ & 01 & $54 \mathrm{E}$ & 02 \\
\hline 27.30 & $1.20803 \mathrm{E}$ & 00 & $-2.88572 \mathrm{E}$ & 01 & $-2.86668 \mathrm{E}$ & 00 & $5.86930 \mathrm{E}-03$ & $-2.81240 \mathrm{E}$ & 00 & $2.99242 \mathrm{E}$ & 01 & $-1.25254 \mathrm{E}$ & 02 \\
\hline 27.40 & $1.20802 \mathrm{E}$ & 00 & $-2.89618 \mathrm{E}$ & 01 & $-2.88491 \mathrm{E}$ & 00 & $5.87080 \mathrm{E}-03$ & $-2.83034 \mathrm{E}$ & 00 & $3.00286 \mathrm{E}$ & 01 & $-1.25355 \mathrm{E}$ & 02 \\
\hline 27.50 & $1.20800 \mathrm{E}$ & 00 & $-2.90663 E$ & 01 & $-2.90319 \mathrm{E}$ & 00 & $5.87229 \mathrm{E}-03$ & $-2.84834 \mathrm{E}$ & 00 & $3.01330 \mathrm{E}$ & 01 & $-1.25454 \mathrm{E}$ & 02 \\
\hline 27.60 & $1.20799 \mathrm{E}$ & 00 & $-2.91708 \mathrm{E}$ & 01 & $-2.92153 \mathrm{E}$ & 00 & $5.87371 \mathrm{E}-03$ & $-2.86639 \mathrm{E}$ & 00 & $3.02374 \mathrm{E}$ & 01 & $-1.25554 \mathrm{E}$ & 02 \\
\hline 27.70 & $1.20797 \mathrm{E}$ & 00 & $-2.92753 \mathrm{E}$ & 01 & $-2.93993 \mathrm{E}$ & 00 & $5.87512 \mathrm{E}-03$ & $-2.88449 E$ & 00 & $3.03417 \mathrm{E}$ & 01 & $-1.25654 \mathrm{E}$ & 02 \\
\hline 27.80 & $1.20795 \mathrm{E}$ & 00 & $-2.93797 \mathrm{E}$ & 01 & $-2.95837 \mathrm{E}$ & 00 & $5.87649 \mathrm{E}-03$ & $-2.90265 \mathrm{E}$ & 00 & $3.04460 \mathrm{E}$ & 01 & $-1.25754 \mathrm{E}$ & 02 \\
\hline 27.90 & $1.20794 \mathrm{E}$ & 00 & $-2.94841 \mathrm{E}$ & 01 & $-2.97687 \mathrm{E}$ & 00 & $5.87782 \mathrm{E}-03$ & $-2.92086 \mathrm{E}$ & 00 & $3.05502 \mathrm{E}$ & 01 & $-1.25853 \mathrm{E}$ & 02 \\
\hline 28.00 & $1.20792 \mathrm{E}$ & 00 & $-2.95885 \mathrm{E}$ & 01 & $-2.99543 \mathrm{E}$ & 00 & $5.87911 \mathrm{E}-03$ & $-2.93913 \mathrm{E}$ & 00 & $3.06545 \mathrm{E}$ & 01 & $-1.25952 \mathrm{E}$ & 02 \\
\hline 28.10 & $1.20791 \mathrm{E}$ & 00 & $-2.96928 \mathrm{E}$ & 01 & $-3.01404 \mathrm{E}$ & 00 & $5.88038 \mathrm{E}-03$ & $-2.95745 \mathrm{E}$ & 00 & $3.07587 \mathrm{E}$ & 01 & $-1.26051 \mathrm{E}$ & 02 \\
\hline 28.20 & $1.20789 \mathrm{E}$ & 00 & $-2.97971 \mathrm{E}$ & 01 & $-3.03271 \mathrm{E}$ & 00 & $5.88160 \mathrm{E}-03$ & $-2.97583 E$ & 00 & $3.08629 \mathrm{E}$ & 01 & $-1.26151 \mathrm{E}$ & 02 \\
\hline 28.30 & $1.20788 \mathrm{E}$ & 00 & $-2.99014 \mathrm{E}$ & 01 & $-3.05142 \mathrm{E}$ & 00 & $5.88279 \mathrm{E}-03$ & $-2.99426 \mathrm{E}$ & 00 & $3.09670 \mathrm{E}$ & 01 & $-1.26249 \mathrm{E}$ & 02 \\
\hline 28.40 & $1.20786 \mathrm{E}$ & 00 & $-3.00057 \mathrm{E}$ & 01 & $-3.07020 \mathrm{E}$ & 00 & $5.88394 \mathrm{E}-$ & $-3.01274 \mathrm{E}$ & 00 & $3.10711 \mathrm{E}$ & 01 & $-1.26348 \mathrm{E}$ & 02 \\
\hline 28.50 & $1.20784 \mathrm{E}$ & 00 & $-3.01099 \mathrm{E}$ & 01 & $-3.08903 \mathrm{E}$ & 00 & $5.88507 \mathrm{E}-03$ & $-3.03128 \mathrm{E}$ & 00 & $3.11752 \mathrm{E}$ & 01 & $-1.26447 \mathrm{E}$ & 02 \\
\hline 28.60 & $1.20783 \mathrm{E}$ & 00 & $-3.02141 \mathrm{E}$ & 01 & $-3.10791 \mathrm{E}$ & 00 & $5.88616 \mathrm{E}-03$ & $-3.04988 \mathrm{E}$ & 00 & $3.12792 \mathrm{E}$ & 01 & $-1.26545 \mathrm{E}$ & 02 \\
\hline 28.70 & $20781 \mathrm{E}$ & 00 & $-3.03182 \mathrm{E}$ & 01 & $-3.12684 \mathrm{E}$ & 00 & $5.88722 \mathrm{E}$ & $-3.06852 \mathrm{E}$ & 00 & $3.13833 \mathrm{E}$ & 0] & $-1.26644 \mathrm{E}$ & 02 \\
\hline 28.80 & $1.20780 \mathrm{E}$ & 00 & $-3.04224 \mathrm{E}$ & 01 & $-3.14584 \mathrm{E}$ & 00 & $5.88825 \mathrm{E}-03$ & $-3.08723 \mathrm{E}$ & 00 & $3.14873 \mathrm{E}$ & 01 & $-1.26742 \mathrm{E}$ & 02 \\
\hline 28.90 & $8 \mathrm{E}$ & 00 & -3.0 & 01 & -3 & 00 & & $98 \mathrm{E}$ & 00 & & 01 & $26840 \mathrm{E}$ & 02 \\
\hline 29.00 & $1.20777 \mathrm{E}$ & 00 & $-3.06306 \mathrm{E}$ & 01 & $-3.18398 E$ & 00 & $5.89019 \mathrm{E}-03$ & $-3.12479 \mathrm{E}$ & 00 & $3.16952 \mathrm{E}$ & 01 & $-1.26938 \mathrm{E}$ & 02 \\
\hline 29.10 & $1.20775 \mathrm{E}$ & 00 & $-3.07346 \mathrm{E}$ & 01 & $-3.20313 \mathrm{E}$ & 00 & $5.89112 \mathrm{E}-03$ & $-3.14366 \mathrm{E}$ & 00 & 3.179 & 01 & -1.27 & 02 \\
\hline 29.20 & $20774 \mathrm{E}$ & 00 & $-3.08386 \mathrm{E}$ & 01 & $234 \mathrm{E}$ & 00 & & $6258 \mathrm{E}$ & 00 & 3.1903 & 01 & $-1.27134 \mathrm{E}$ & 02 \\
\hline 29.30 & $1.20772 \mathrm{E}$ & 00 & $-3.09426 \mathrm{E}$ & 01 & $-3.24160 \mathrm{E}$ & 00 & & $.18155 \mathrm{E}$ & 00 & $3.20068 \mathrm{E}$ & 01 & $-1.27232 \mathrm{E}$ & 02 \\
\hline 29.40 & $1.20771 \mathrm{E}$ & 00 & $-3.10466 \mathrm{E}$ & 01 & $-3.26092 \mathrm{E}$ & 00 & $5.89371 \mathrm{E}-03$ & $-3.20058 \mathrm{E}$ & 00 & $3.21106 \mathrm{E}$ & 01 & $-1.27330 \mathrm{E}$ & 02 \\
\hline 29.50 & $1.20769 \mathrm{E}$ & 00 & $-3.11505 \mathrm{E}$ & 01 & $-3.28029 \mathrm{E}$ & 00 & $5.89451 \mathrm{E}-03$ & $-3.21966 \mathrm{E}$ & 00 & $3.22144 \mathrm{E}$ & 01 & $-1.27427 \mathrm{E}$ & 02 \\
\hline 29.60 & $1.20768 \mathrm{E}$ & 00 & $-3.12544 \mathrm{E}$ & 01 & $-3.29971 \mathrm{E}$ & 00 & $5.89527 \mathrm{E}-03$ & $-3.23880 \mathrm{E}$ & 00 & $3.23182 \mathrm{E}$ & 01 & $-1.27525 \mathrm{E}$ & 02 \\
\hline 29.70 & $1.20766 \mathrm{E}$ & 00 & $-3.13583 E$ & 01 & $-3.31919 E$ & 00 & $5.89601 \mathrm{E}-03$ & $-3.25799 \mathrm{E}$ & 00 & $3.24219 \mathrm{E}$ & 01 & $-1.27623 \mathrm{E}$ & 02 \\
\hline 9.80 & $1.20765 \mathrm{E}$ & 00 & $-3.14621 \mathrm{E}$ & 01 & $-3.33873 E$ & 00 & $5.89671 \mathrm{E}-\mathrm{C}$ & $-3.27723 \mathrm{E}$ & 00 & $3.25256 \mathrm{E}$ & 01 & $-1.27720 \mathrm{E}$ & 02 \\
\hline 9.90 & $1.20764 \mathrm{E}$ & 00 & $-3.15659 \mathrm{E}$ & -01 & $-3.35831 \mathrm{E}$ & 00 & $5.89740 \mathrm{E}-$ & $-3.29653 \mathrm{E}$ & 00 & $3.26292 \mathrm{E}$ & $0]$ & $-1.27817 \mathrm{E}$ & 02 \\
\hline 30.00 & $1.20762 \mathrm{E}$ & 00 & $-3.16697 \mathrm{E}$ & 01 & $-3.37795 E$ & 00 & $5.89804 \mathrm{E}-03$ & $-3.31588 \mathrm{E}$ & 00 & $3.27329 \mathrm{E}$ & 01 & $-1.27915 \mathrm{E}$ & 02 \\
\hline
\end{tabular}


ELECTRON RADIAL FUNCTIONS

$Z=8 \quad A=17 \quad \rho=1.2 A^{1 / 3} F$

Electron

$p$

24.10

24.20

24.30

24.40

24.50

24.60

24.70

24.80

24.90

25.00

25.10

25.20

25.30

25.40

25.50

25.60

25.70

25.80

25.90

26.00

26.10

26.20

26.30

26.40

26.50

26.60

26.70

26.80

26.90

27.00

27.10

27.20

27.30

27.40

27.50

27.60

27.70

27.80

27.90

28.00

28.10

28.20

28.30

28.40

28.50

28.60

28.70

28.80

28.90

29.00

29.10

29.20

29.30

29.40

29.50

29.60

29.70

29.80

29.90

30.00
$\bar{F}$

$1.24237 \mathrm{E}-03$

$1.25270 \mathrm{E}-03$

$1.26306 \mathrm{E}-03$

$1.27347 \mathrm{E}-03$

$1.28391 \mathrm{E}-03$

$1.29441 \mathrm{E}-03$

$1.30494 \mathrm{E}-03$

$1.31552 \mathrm{E}-03$

$1.32614 \mathrm{E}-03$

$1.33680 \mathrm{E}-03$

$1.34751 \mathrm{E}-03$

$1.35825 \mathrm{E}-03$

$1.36904 \mathrm{E}-03$

$1.37988 \mathrm{E}-03$

$1.39075 \mathrm{E}-03$

$1.40167 \mathrm{E}-03$

$1.41263 \mathrm{E}-03$

$1.42364 \mathrm{E}-03$

$1.43468 \mathrm{E}-03$

$1.44577 \mathrm{E}-03$

$1.45690 \mathrm{E}-03$

$1.46808 \mathrm{E}-03$

$1.47929 \mathrm{E}-03$

$1.49055 \mathrm{E}-03$

$1.50186 \mathrm{E}-03$

$1.51320 \mathrm{E}-03$

$1.52459 \mathrm{E}-03$

$1.53602 \mathrm{E}-03$

$1.54749 \mathrm{E}-03$

$1.55901 \mathrm{E}-03$

$1.57057 \mathrm{E}-03$

$1.58217 \mathrm{E}-03$

$1.59381 \mathrm{E}-03$

$1.60550 \mathrm{E}-03$

$1.61722 \mathrm{E}-03$

$1.62900 \mathrm{E}-03$

$1.64081 \mathrm{E}-03$

$1.65267 \mathrm{E}-03$

$1.66457 \mathrm{E}-03$

$1.67651 \mathrm{E}-03$

$1.68849 \mathrm{E}-03$

$1.70052 \mathrm{E}-03$

$1.71259 \mathrm{E}-03$

$1.72470 \mathrm{E}-03$

$1.73686 \mathrm{E}-03$

$1.74906 \mathrm{E}-03$

$1.76130 \mathrm{E}-03$

$1.77358 \mathrm{E}-03$

$1.78591 \mathrm{E}-03$

$1.79828 \mathrm{E}-03$

$1.81069 \mathrm{E}-03$

$1.82314 \mathrm{E}-03$

$1.83564 \mathrm{E}-03$

$1.84818 \mathrm{E}-03$

$1.86076 \mathrm{E}-03$

$1.87338 \mathrm{E}-03$

$1.88605 \mathrm{E}-03$

$1.89876 \mathrm{E}-03$

$1.91151 \mathrm{E}-03$

$1.92431 \mathrm{E}-03$ $f_{2}$

$g_{2}$

$\tan \delta_{2}$

$f_{-2}$

$-1.64850 \mathrm{E} \quad 00$

$-1.66227 \mathrm{E} \quad 00$

$-1.67608 \mathrm{E} 00$

$-1.68996 \mathrm{E} \quad 00$

$-1.70389 \mathrm{E} 00$

$-1.71788 \mathrm{E} \quad 00$

$-1.73192 \mathrm{E} \quad 00$

$-1.74603 \mathrm{E} \quad 00$

$-1.76018 \mathrm{E} 00$

$-1.77439 \mathrm{E} \quad 00$

$-1.78866 \mathrm{E} \quad 00$

$-1.80299 \mathrm{E} 00$

$-1.81737 \mathrm{E} \quad 00$

$-1.83181 \mathrm{E} \quad 00$

$-1.84630 \mathrm{E} \quad 00$

$-1.86085 \mathrm{E} \quad 00$

$-1.87545 \mathrm{E} \quad 00$

$-1.89012 \mathrm{E} \quad 00$

$-1.90483 \mathrm{E} 00$

$-1.91961 \mathrm{E} 00$

$-1.93444 \mathrm{E} 00$

$-1.94932 \mathrm{E} \quad 00$

$-1.96426 \mathrm{E} 00$

$-1.97926 \mathrm{E} \quad 00$

$-1.99431 \mathrm{E} 00$

$-2.00942 \mathrm{E} \quad 00$

$-2.02459 \mathrm{E} \quad 00$

$-2.03981 \mathrm{E} 00$

$-2.05508 \mathrm{E} \quad 00$

$-2.07041 \mathrm{E} \quad 00$

$-2.08580 \mathrm{E} \quad 00$

$-2.10125 \mathrm{E} 00$

$-2.11675 \mathrm{E} \quad 00$

$-2.13230 \mathrm{E} \quad 00$

-2.14791 E 00

$-2.16358 \mathrm{E} \quad 00$

$-2.17930 \mathrm{E} \quad 00$

$-2.19508 \mathrm{E} 00$

$-2.21091 \mathrm{E} \quad 00$

$-2.22680 \mathrm{E} \quad 00$

$-2.24275 \mathrm{E} \quad 00$

$-2.25875 \mathrm{E} \quad 00$

$-2.27481 \mathrm{E} \quad 00$

$-2.29092 \mathrm{E} \quad 00$

$-2.30709 \mathrm{E} \quad 00$

$-2.32331 \mathrm{E} 00$

$-2.33959 \mathrm{E} \quad 00$

$-2.35592 \mathrm{E} \quad 00$

$-2.37231 \mathrm{E} \quad 00$

$-2.38876 \mathrm{E} \quad 00$

$-2.40526 \mathrm{E} \quad 00$

$-2.42182 \mathrm{E} \quad 00$

$-2.43843 \mathrm{E} \quad 00$

$-2.45509 \mathrm{E} \quad 00$

$-2.47182 \mathrm{E} \quad 00$

$-2.48860 \mathrm{E} \quad 00$

$-2.50543 \mathrm{E} \quad 00$

$-2.52232 \mathrm{E} 00$

$-2.53926 \mathrm{E} \quad 00$

$-2.55626 \mathrm{E} \quad 00$
$-8.83507 \mathrm{E}-02$ $-8.93554 \mathrm{E}-02$

$-9.03675 \mathrm{E}-02$

$-9.13871 \mathrm{E}-02$

$-9.24143 \mathrm{E}-02$

$-9.34490 \mathrm{E}-02$

$-9.44912 \mathrm{E}-02$

$-9.55410 \mathrm{E}-02$

$-9.65985 \mathrm{E}-02$

$-9.76636 \mathrm{E}-02$

$-9.87364 \mathrm{E}-02$

$-9.98169 \mathrm{E}-02$

$-1.00905 \mathrm{E}-01$

$-1.02001 \mathrm{E}-01$

$-1.03105 \mathrm{E}-01$

$-1.04217 \mathrm{E}-01$

$-1.05336 \mathrm{E}-01$

$-1.06463 \mathrm{E}-01$

$-1.07598 \mathrm{E}-01$

$-1.08742 \mathrm{E}-01$

$-1.09893 \mathrm{E}-01$

$-1.11052 \mathrm{E}-01$

$-1.12219 \mathrm{E}-01$

$-1.13394 \mathrm{E}-01$

$-1.14577 \mathrm{E}-01$

$-1.15768 \mathrm{E}-01$

$-1.16967 \mathrm{E}-01$

$-1.18175 \mathrm{E}-01$

$-1.19390 \mathrm{E}-01$

$-1.20614 \mathrm{E}-01$

$-1.21846 \mathrm{E}-01$

$-1.23086 \mathrm{E}-01$

$-1.24334 \mathrm{E}-01$

$-1.25591 \mathrm{E}-01$

$-1.26856 \mathrm{E}-01$

$-1.28129 \mathrm{E}-01$

$-1.29411 \mathrm{E}-01$

$-1.30701 \mathrm{E}-01$

$-1.32000 \mathrm{E}-01$

$-1.33307 \mathrm{E}-01$

$-1.34622 \mathrm{E}-01$

$-1.35946 \mathrm{E}-01$

$-1.37279 \mathrm{E}-01$

$-1.38620 \mathrm{E}-01$

$-1.39969 \mathrm{E}-01$

$-1.41327 \mathrm{E}-01$

$-1.42694 \mathrm{E}-01$

$-1.44070 \mathrm{E}-01$

$-1.46847 \mathrm{E}-01$

$-1.48248 \mathrm{E}-01$

$-1.49659 \mathrm{E}-01$

$-1.51078 \mathrm{E}-01$

$-1.52506 \mathrm{E}-01$

$-1.53943 \mathrm{E}-01$

$-1.55388 \mathrm{E}-01$

$-1.56843 \mathrm{E}-01$

$-1.58306 \mathrm{E}-01$

$-1.59779 \mathrm{E}-01$
$-1.45454 \mathrm{E}-01$

$-1.61260 \mathrm{E}-01$

$2.59222 \mathrm{E}$ 01 $-8.65842 \mathrm{E}-02$ $2.59240 \mathrm{E}$ 01 $-8.75703 \mathrm{E}-02$ $2.59259 \mathrm{E}$ ol $\quad-8.85638 \mathrm{E}-02$ $2.59277 \mathrm{E}$ 01 $-8.95647 \mathrm{E}-02$ $2.59296 \mathrm{E} \quad 01 \quad-9.05731 \mathrm{E}-02$

$2.59314 \mathrm{E}$ ol $-9.15888 \mathrm{E}-02$

$2.59332 \mathrm{E} \quad 01 \quad-9.26121 \mathrm{E}-02$

$2.59349 \mathrm{E}$ o1 $-9.36429 \mathrm{E}-02$

$2.59367 \mathrm{E} \quad 01 \quad-9.46812 \mathrm{E}-02$

$2.59384 \mathrm{E}$ o1 $-9.57271 \mathrm{E}-02$

$\begin{array}{lll}2.59402 \mathrm{E} & 01 & -9.67805 \mathrm{E}-02\end{array}$

$2.59419 \mathrm{E}$ ol $-9.784 .16 \mathrm{E}-02$

$2.59436 \mathrm{E}$ 01 $-9.89103 \mathrm{E}-02$

$2.59453 \mathrm{E} \quad 01 \quad-9.99867 \mathrm{E}-02$

$2.59470 \mathrm{E} \quad 01-1.01071 \mathrm{E}-01$

$2.59486 \mathrm{E} \quad 01-1.02163 \mathrm{E}-01$ $2.59503 \mathrm{E} \quad 01-1.03262 \mathrm{E}-01$ $2.59519 \mathrm{E} \quad 01-1.04369 \mathrm{E}-01$ $2.59535 \mathrm{E}$ 01 $-1.05485 \mathrm{E}-01$ $2.59551 \mathrm{E} 01 \quad-1.06607 \mathrm{E}-01$

$2.59567 \mathrm{E} \quad 01-1.07738 \mathrm{E}-01$ $2.59583 \mathrm{E} \quad 01 \quad-1.08877 \mathrm{E}-01$ $2.59599 \mathrm{E} \quad 01-1.10023 \mathrm{E}-01$ $2.59615 \mathrm{E} \quad 01-1.11178 \mathrm{E}-01$

$2.59630 \mathrm{E} \quad 01-1.12340 \mathrm{E}-01$

$2.59645 \mathrm{E} \quad 01 \quad-1.13511 \mathrm{E}-01$ $2.59661 \mathrm{E} 01-1.14689 \mathrm{E}-01$

$2.59676 \mathrm{E}$ 01 $-1.15876 \mathrm{E}-01$

$2.59691 \mathrm{E} 01-1.17070 \mathrm{E}-01$

$2.59706 \mathrm{E} \quad 01-1.18273 \mathrm{E}-01$

$2.59721 \mathrm{E}$ 01 $-1.19484 \mathrm{E}-01$ $2.59735 \mathrm{E}$ 01 $-1.20703 \mathrm{E}-01$ $2.59750 \mathrm{E}$ ol $-1.21930 \mathrm{E}-01$ $2.59764 \mathrm{E}$ 01 $-1.23165 \mathrm{E}-01$ $2.59779 \mathrm{E} 01-1.24408 \mathrm{E}-01$

$2.59793 \mathrm{E} \quad 01 \quad-1.25660 \mathrm{E}-01$ $2.59807 \mathrm{E}$ 01 $-1.26920 \mathrm{E}-01$ $2.59821 \mathrm{E}$ 01 $-1.28188 \mathrm{E}-01$ $2.59835 \mathrm{E}$ 01 $-1.29465 \mathrm{E}-01$ $2.59849 \mathrm{E} \quad 01 \quad-1.30750 \mathrm{E}-01$

$\begin{array}{lll}2.59862 \mathrm{E} & 01 & -1.32043 \mathrm{E}-01\end{array}$ 


$$
Z=8 \quad A=17
$$$$
\rho=1.2 A^{1 / 3} F
$$

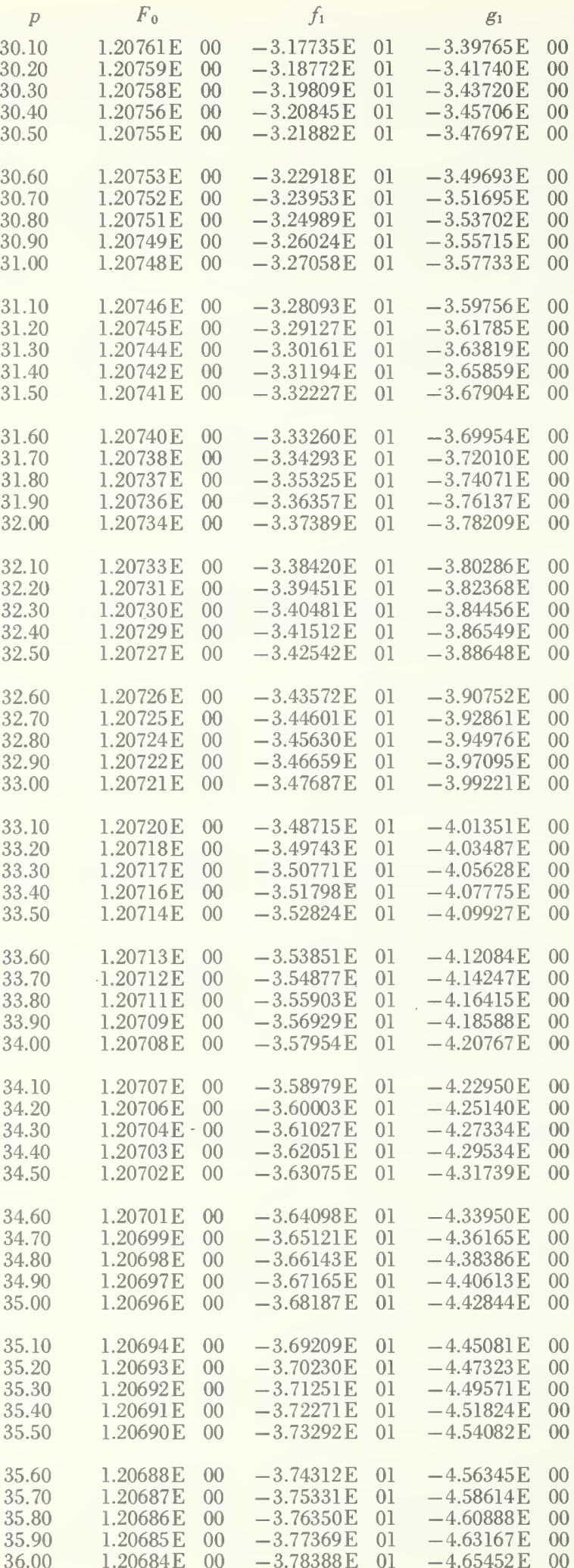

$\tan \delta_{1}$

$f_{-1}$

$5.89865 \mathrm{E}-03$

$5.89924 \mathrm{E}-03$

$5.89979 \mathrm{E}-03$

$5.90033 \mathrm{E}-03$

$5.90083 \mathrm{E}-03$

$5.90130 \mathrm{E}-03$

$5.90174 \mathrm{E}-03$

$5.90215 \mathrm{E}-03$

$5.90253 \mathrm{E}-03$

$5.90288 \mathrm{E}-03$

$5.90321 \mathrm{E}-03$

$5.90351 \mathrm{E}-03$

$5.90378 \mathrm{E}-03$

$5.90402 \mathrm{E}-03$

$5.90424 \mathrm{E}-03$

$5.90443 \mathrm{E}-03$

$5.90460 \mathrm{E}-03$

$5.90474 \mathrm{E}-03$

$5.90485 \mathrm{E}-03$

$5.90494 \mathrm{E}-03$

$5.90499 \mathrm{E}-03$

$5.90502 \mathrm{E}-03$

$5.90503 \mathrm{E}-03$

$5.90501 \mathrm{E}-03$

$5.90496 \mathrm{E}-03$

$5.90489 \mathrm{E}-03$ $5.90480 \mathrm{E}-03$ $5.90467 \mathrm{E}-03$

$5.90453 \mathrm{E}-03$

$5.90436 \mathrm{E}-03$

$5.90417 \mathrm{E}-03$

$5.90395 \mathrm{E}-03$

$5.90370 \mathrm{E}-03$

$5.90344 \mathrm{E}-03$

$5.90314 \mathrm{E}-03$

$5.90283 \mathrm{E}-03$

$5.90249 \mathrm{E}-03$

$5.90213 \mathrm{E}-03$

$5.90174 \mathrm{E}-03$

$5.90132 \mathrm{E}-03$

$5.90089 \mathrm{E}-03$ $5.90044 \mathrm{E}-03$ $5.89996 \mathrm{E}-03$

$5.89946 \mathrm{E}-03$

$5.89893 \mathrm{E}-03$

$5.89839 \mathrm{E}-03$ $5.89782 \mathrm{E}-03$

$5.89723 \mathrm{E}-03$

$5.89662 \mathrm{E}-03$

$5.89598 \mathrm{E}-03$

$5.89531 \mathrm{E}-03$

$5.89465 \mathrm{E}-03$

$5.89394 \mathrm{E}-03$

$5.89321 \mathrm{E}-03$

$5.89248 \mathrm{E}-03$

$5.89170 \mathrm{E}-03$ $5.89092 \mathrm{E}-03$

$5.89011 \mathrm{E}-03$

$5.88928 \mathrm{E}-03$

$5.88843 \mathrm{E}-03$

$g_{-1}$ $\tan \delta_{-1}$

$-3.33529 \mathrm{E} \quad 00$ $-3.35475 \mathrm{E} 00$ $-3.37427 \mathrm{E} \quad 00$ $-3.39384 \mathrm{E} \quad 00$ $-3.41346 \mathrm{E} \quad 00$

$-3.43314 \mathrm{E} \quad 00$ $-3.45287 \mathrm{E} \quad 00$ $-3.47265 \mathrm{E} 00$ $-3.49249 \mathrm{E} 00$ $-3.51238 \mathrm{E} 00$

$-3.53233 \mathrm{E} \quad 00$ $-3.55233 \mathrm{E} \quad 00$ $-3.57238 \mathrm{E} \quad 00$ $-3.59249 \mathrm{E} 00$ $-3.61265 \mathrm{E} \quad 00$

$-3.63287 \mathrm{E} \quad 00$ $-3.65314 \mathrm{E} 00$ $-3.67346 \mathrm{E} 00$ $-3.69384 \mathrm{E} \quad 00$ $-3.71427 \mathrm{E} \quad 00$

$-3.73475 \mathrm{E} \quad 00$ $-3.75529 \mathrm{E} \quad 00$ $-3.77588 \mathrm{E} \quad 00$ $-3.79652 \mathrm{E} \quad 00$ $-3.81722 \mathrm{E} \quad 00$

$-3.83797 \mathrm{E} \quad 00$ $-3.85878 \mathrm{E} \quad 00$ $-3.87964 \mathrm{E} \quad 00$ $-3.90055 \mathrm{E} 00$ $-3.92152 \mathrm{E} \quad 00$

$-3.94254 \mathrm{E} \quad 00$ $-3.96361 \mathrm{E} \quad 00$ $-3.98473 \mathrm{E} \quad 00$ $-4.00591 \mathrm{E} \quad 00$ $-4.02715 \mathrm{E} \quad 00$

$-4.04843 \mathrm{E} \quad 00$ $-4.06977 \mathrm{E} \quad 00$ $-4.09116 \mathrm{E} \quad 00$ $-4.11261 \mathrm{E} \quad 00$ $-4.13411 \mathrm{E} \quad 00$

$-4.15566 \mathrm{E} \quad 00$ $-4.17727 \mathrm{E} \quad 00$ $-4.19893 \mathrm{E} \quad 00$ $-4.22064 \mathrm{E} 00$ $-4.24240 \mathrm{E} 00$

$-4.26422 \mathrm{E} \quad 00$ $-4.28609 \mathrm{E} \quad 00$ $-4.30802 \mathrm{E} \quad 00$ $-4.32999 \mathrm{E} \quad 00$ $-4.35203 \mathrm{E} \quad 00$

$-4.37411 \mathrm{E} 00$ $-4.39625 \mathrm{E} 00$ $-4.41844 \mathrm{E} \quad 00$ $-4.44068 \mathrm{E} 00$ $-4.46297 \mathrm{E} \quad 00$

$-4.48532 \mathrm{E} \quad 00$ $-4.50772 \mathrm{E} \quad 00$ $-4.53018 \mathrm{E} \quad 00$ $-4.55268 \mathrm{E} \quad 00$ $-4.57524 \mathrm{E} \quad 00$ $\begin{array}{llll}3.28365 \mathrm{E} & 01 & -1.28012 \mathrm{E} & 02\end{array}$ $3.29401 \mathrm{E} \quad 01 \quad-1.28109 \mathrm{E} \quad 02$ $3.30436 \mathrm{E}$ 01 $-1.28206 \mathrm{E} \quad 02$ $\begin{array}{llll}3.31471 \mathrm{E} & 01 & -1.28303 \mathrm{E} & 02\end{array}$ $\begin{array}{llll}3.32506 \mathrm{E} & 01 & -1.28400 \mathrm{E} & 02\end{array}$ $3.33540 \mathrm{E} \quad 01 \quad-1.28497 \mathrm{E} \quad 02$ $\begin{array}{llll}3.34575 \mathrm{E} & 01 & -1.28593 \mathrm{E} & 02\end{array}$ $\begin{array}{llll}3.35609 \mathrm{E} & 01 & -1.28690 \mathrm{E} & 02\end{array}$ $\begin{array}{llll}3.36642 \mathrm{E} & 01 & -1.28787 \mathrm{E} & 02\end{array}$ $\begin{array}{llll}3.37675 \mathrm{E} & 01 & -1.28884 \mathrm{E} & 02\end{array}$

$3.38708 \mathrm{E} \quad 01$ $3.39741 \mathrm{E} \quad 01$ $3.40773 \mathrm{E} \quad 01$ $3.41805 \mathrm{E} \quad 01$ $3.42837 \mathrm{E}$ 01

$3.43868 \mathrm{E} \quad 01$ $3.44899 \mathrm{E} \quad 01$ $3.45930 \mathrm{E} \quad 01$ $3.46961 \mathrm{E} \quad 01$ $3.47991 \mathrm{E} 01$

$3.49021 \mathrm{E} \quad 01$ $3.50050 \mathrm{E} \quad 01$

$3.51079 \mathrm{E} \quad 01$

$3.52108 \mathrm{E} \quad 01$

$3.53137 \mathrm{E} \quad 01$

$3.54165 \mathrm{E} \quad 01$ $3.55193 \mathrm{E} 01$ $3.56220 \mathrm{E} \quad 01$

$3.57247 \mathrm{E} \quad 01$

$3.58274 \mathrm{E} \quad 01$

$3.59301 \mathrm{E} \quad 01$ $3.60327 \mathrm{E} \quad 01$ $3.61353 \mathrm{E} 01$ $3.62379 \mathrm{E} \quad 01$ $3.63404 \mathrm{E} 01$

$3.64429 \mathrm{E} \quad 01$ $3.65454 \mathrm{E} \quad 01$ $3.66478 \mathrm{E} 01$ $3.67502 \mathrm{E} 01$ $3.68526 \mathrm{E} \quad 01$

$3.69549 \mathrm{E} \quad 01$ $3.70572 \mathrm{E} \quad 01$ $3.71595 \mathrm{E} \quad 01$ $3.72617 \mathrm{E} \quad 01$

$3.73639 \mathrm{E} 01$

$3.74660 \mathrm{E} \quad 01$ $3.75682 \mathrm{E} \quad 01$ $3.76703 \mathrm{E} 01$ $3.77723 \mathrm{E} 01$ $3.78744 \mathrm{E}$ 0l

$3.79764 \mathrm{E} \quad 01$ $3.80783 \mathrm{E} 01$ $3.81803 \mathrm{E} 01$ $3.82822 \mathrm{E} \quad 01$ $3.83840 \mathrm{E} 01$

$3.84859 \mathrm{E} \quad 01$ $3.85877 \mathrm{E} \quad 01$ $3.86894 \mathrm{E} \quad 01$ $3.87911 \mathrm{E} \quad 01$ $3.88928 \mathrm{E} 01$
$-1.28980 \mathrm{E} \quad 02$ $-1.29077 \mathrm{E} \quad 02$ $-1.29173 \mathrm{E} \quad 02$ $-1.29270 \mathrm{E} \quad 02$ $-1.29367 \mathrm{E} 02$

$-1.29463 \mathrm{E} 02$ $-1.29559 \mathrm{E} \quad 02$ $-1.29656 \mathrm{E} \quad 02$ $-1.29752 \mathrm{E} \quad 02$ $-1.29848 \mathrm{E} 02$

$-1.29945 \mathrm{E} \quad 02$ $-1.30041 \mathrm{E} \quad 02$ $-1.30137 \mathrm{E} \quad 02$ $-1.30234 \mathrm{E} \quad 02$ $-1.30330 \mathrm{E} \quad 02$

$-1.30426 \mathrm{E} \quad 02$ $-1.30523 \mathrm{E} \quad 02$ $-1.30619 \mathrm{E} \quad 02$ $-1.30715 \mathrm{E} \quad 02$ $-1.30812 \mathrm{E} \quad 02$

$-1.30908 \mathrm{E} \quad 02$ $-1.31004 \mathrm{E} \quad 02$ $-1.31101 \mathrm{E} 02$ $-1.31197 \mathrm{E} 02$ $-1.31293 \mathrm{E} 02$

$-1.31390 \mathrm{E} \quad 02$ $-1.31486 \mathrm{E} \quad 02$ $-1.31582 \mathrm{E} \quad 02$ $-1.31679 \mathrm{E} \quad 02$ -1.31775 E 02

$-1.31872 \mathrm{E} \quad 02$ $-1.31968 \mathrm{E} 02$ $-1.32065 \mathrm{E} \quad 02$ $-1.32162 \mathrm{E} 02$ $-1.32258 \mathrm{E} 02$

$-1.32355 \mathrm{E} \quad 02$ $-1.32452 \mathrm{E} \quad 02$ $-1.32548 \mathrm{E} \quad 02$ $-1.32645 \mathrm{E} \quad 02$ $-1.32742 \mathrm{E} 02$

$-1.32839 \mathrm{E} 02$ $-1.32936 \mathrm{E} \quad 02$ $-1.33033 \mathrm{E} 02$ $-1.33130 \mathrm{E} 02$ $-1.33227 \mathrm{E} \quad 02$

$-1.33324 \mathrm{E} \quad 02$ $-1.33421 \mathrm{E} 02$ $-1.33518 \mathrm{E} \quad 02$ $-1.33616 \mathrm{E} 02$ $-1.33713 \mathrm{E} 02$ 
ELECTRON RADIAL FUNCTIONS

$Z=8 \quad A=17 \quad \rho=1.2 A^{1 / 3} F$

Electron

$\bar{F}$

$1.93715 \mathrm{E}-03$

$1.95003 \mathrm{E}-03$

$1.96295 \mathrm{E}-03$

$1.97592 \mathrm{E}-0.3$

$1.98893 \mathrm{E}-03$

$2.00198 \mathrm{E}-03$

$2.01507 \mathrm{E}-03$

$2.02821 \mathrm{E}-03$

$2.04139 \mathrm{E}-03$

$2.05461 \mathrm{E}-03$

$2.06787 \mathrm{E}-03$

$2.08118 \mathrm{E}-03$

$2.09453 \mathrm{E}-03$

$2.10792 \mathrm{E}-03$

$2.12136 \mathrm{E}-03$

$2.13483 \mathrm{E}-03$

$2.14835 \mathrm{E}-03$

$2.16192 \mathrm{E}-03$

$2.17552 \mathrm{E}-03$

$2.18917 \mathrm{E}-03$

$2.20286 \mathrm{E}-03$

$2.21659 \mathrm{E}-03$

$2.23037 \mathrm{E}-03$

$2.24419 \mathrm{E}-03$

$2.25805 \mathrm{E}-03$

$2.27195 \mathrm{E}-03$

$2.28590 \mathrm{E}-03$

$2.29989 \mathrm{E}-03$

$2.31392 \mathrm{E}-03$

$2.32800 \mathrm{E}-03$

$2.34211 \mathrm{E}-03$

$2.35627 \mathrm{E}-03$

$2.37048 \mathrm{E}-03$

$2.38472 \mathrm{E}-03$

$2.39901 \mathrm{E}-03$

$2.41334 \mathrm{E}-03$

$2.42771 \mathrm{E}-03$

$2.44213 \mathrm{E}-03$

$2.45659 \mathrm{E}-03$

$2.47109 \mathrm{E}-03$

$2.48563 \mathrm{E}-03$

$2.50022 \mathrm{E}-03$

$2.51485 \mathrm{E}-03$

$2.52952 \mathrm{E}-03$

$2.54423 \mathrm{E}-03$

$2.55899 \mathrm{E}-03$ $2.57379 \mathrm{E}-03$ 2.58863 E-03

$2.60352 \mathrm{E}-03$

$2.61844 \mathrm{E}-03$

$2.63341 \mathrm{E}-03$

$2.64843 \mathrm{E}-03$

$2.66348 \mathrm{E}-03$

$2.67858 \mathrm{E}-03$

$2.69372 \mathrm{E}-03$

$2.70890 \mathrm{E}-03$

$2.72413 \mathrm{E}-03$

$2.73940 \mathrm{E}-03$

$2.75471 \mathrm{E}-03$

$2.77006 \mathrm{E}-03$ $f_{2}$

$-2.57332 \mathrm{E} \quad 00$

$-2.59043 \mathrm{E} \quad 00$

$-2.60759 \mathrm{E} \quad 00$

$-2.62481 \mathrm{E} \quad 00$

$-2.64209 \mathrm{E} \quad 00$

$-2.65942 \mathrm{E} \quad 00$

$-2.67681 \mathrm{E} \quad 00$

$-2.69425 \mathrm{E} \quad 00$

$-2.71175 \mathrm{E} \quad 00$

$-2.72930 \mathrm{E} 00$

$-2.74691 \mathrm{E} \quad 00$

$-2.76457 \mathrm{E} \quad 00$

$-2.78229 \mathrm{E} \quad 00$

$-2.80006 \mathrm{E} \quad 00$

$-2.81789 \mathrm{E} 00$

$-2.83577 \mathrm{E} \quad 00$

$-2.85371 \mathrm{E} \quad 00$

$-2.87170 \mathrm{E} 00$

$-2.88975 \mathrm{E} \quad 00$

-2.90785 E 00

$-2.92601 \mathrm{E} \quad 00$

$-2.94422 \mathrm{E} \quad 00$

$-2.96249 \mathrm{E} 00$

$-2.98081 \mathrm{E} 00$

$-2.99919 \mathrm{E} \quad 00$

$-3.01762 \mathrm{E} \quad 00$

$-3.03611 \mathrm{E} 00$

$-3.05465 \mathrm{E} \quad 00$

$-3.07325 \mathrm{E} 00$

$-3.09190 \mathrm{E} 00$

$-3.11061 \mathrm{E} \quad 00$

$-3.12937 \mathrm{E} 00$

$-3.14818 \mathrm{E} \quad 00$

$-3.16705 \mathrm{E} 00$

$-3.18598 \mathrm{E} \quad 00$

$-3.20496 \mathrm{E} \quad 00$

$-3.22399 \mathrm{E} 00$

$-3.24308 \mathrm{E} 00$

$-3.26223 \mathrm{E} 00$

$-3.28143 \mathrm{E} 00$

$-3.30068 \mathrm{E} \quad 00$

$-3.31999 \mathrm{E} \quad 00$

$-3.33935 \mathrm{E} 00$

$-3.35877 \mathrm{E} 00$

$-3.37824 \mathrm{E} 00$

$-3.39777 \mathrm{E} 00$

$-3.41735 \mathrm{E} \quad 00$

$-3.43698 \mathrm{E} 00$

$-3.45667 \mathrm{E} 00$

$-3.47642 \mathrm{E} 00$

$-3.49621 \mathrm{E} \quad 00$

$-3.51607 \mathrm{E} 00$

$-3.53597 \mathrm{E} \quad 00$

$-3.55594 \mathrm{E} \quad 00$

$-3.57595 \mathrm{E} \quad 00$

$-3.59602 \mathrm{E} \quad 00$

$-3.61615 \mathrm{E} \quad 00$

$-3.63633 \mathrm{E} 00$

$-3.65656 \mathrm{E} 00$

-3.67685 E 00 $g_{2}$

$-1.62750 \mathrm{E}-01$

$-1.642 .50 \mathrm{E}-01$

$-1.65758 \mathrm{E}-01$

$-1.67276 \mathrm{E}-01$

$-1.68802 \mathrm{E}-01$

$-1.70338 \mathrm{E}-01$

$-1.71883 \mathrm{E}-0 \mathrm{I}$

$-1.73437 \mathrm{E}-01$

$-1.75000 \mathrm{E}-01$

$-1.76573 \mathrm{E}-01$

$-1.78154 \mathrm{E}-01$

$-1.79746 \mathrm{E}-01$

$-1.81346 \mathrm{E}-01$

$-1.82955 \mathrm{E}-01$

$-1.84574 \mathrm{E}-01$

$-1.86203 \mathrm{E}-01$

$-1.87841 \mathrm{E}-01$

$-1.89488 \mathrm{E}-01$

$-1.91145 \mathrm{E}-01$

$-1.92811 \mathrm{E}-01$

$-1.94486 \mathrm{E}-01$

$-1.96172 \mathrm{E}-01$

$-1.97867 \mathrm{E}-01$

$-1.99571 \mathrm{E}-01$

$-2.01285 \mathrm{E}-01$

$-2.03009 \mathrm{E}-01$

$-2.04742 \mathrm{E}-01$

$-2.06485 \mathrm{E}-01$

$-2.08238 \mathrm{E}-01$

$-2.10000 \mathrm{E}-01$

$-2.11772 \mathrm{E}-01$

$-2.13555 \mathrm{E}-01$

$-2.15346 \mathrm{E}-01$

$-2.17148 \mathrm{E}-01$

$-2.18960 \mathrm{E}-01$

$-2.20781 \mathrm{E}-01$

$-2.22613 \mathrm{E}-01$

$-2.24454 \mathrm{E}-01$

$-2.26305 \mathrm{E}-01$

$-2.28167 \mathrm{E}-01$

$-2.30038 \mathrm{E}-01$

$-2.31920 \mathrm{E}-01$

$-2.33811 \mathrm{E}-01$

$-2.35713 \mathrm{E}-01$

$-2.37624 \mathrm{E}-01$

$-2.39546 \mathrm{E}-01$

$-2.41478 \mathrm{E}-01$

$-2.43421 \mathrm{E}-01$

$-2.45373 \mathrm{E}-01$

$-2.47336 \mathrm{E}-01$

$-2.49309 \mathrm{E}-01$

$-2.51293 \mathrm{E}-01$

$-2.53286 \mathrm{E}-01$

$-2.55290 \mathrm{E}-01$

$-2.57305 \mathrm{E}-01$

$-2.59330 \mathrm{E}-01$

$-2.61365 \mathrm{E}-01$

$-2.63411 \mathrm{E}-01$

$-2.65467 \mathrm{E}-01$

$-2.67534 \mathrm{E}-01$ $\tan \delta_{2}$

$f_{-2}$

$2.60117 \mathrm{E} \quad 01 \quad-1.59711 \mathrm{E}-01$

$2.60128 \mathrm{E} 01-1.61187 \mathrm{E}-01$

$2.60140 \mathrm{E} \quad 01 \quad-1.62671 \mathrm{E}-01$

$2.60152 \mathrm{E} 01-1.64 .164 \mathrm{E}-01$

$2.60163 \mathrm{E} \quad 01-1.65667 \mathrm{E}-01$

$2.60175 \mathrm{E}$ 01 $-1.67178 \mathrm{E}-01$

$2.60186 \mathrm{E}$ 01 $-1.68698 \mathrm{E}-01$

$2.60198 \mathrm{E} \quad 01-1.70228 \mathrm{E}-01$

$2.60209 \mathrm{E} 01-1.71767 \mathrm{E}-01$

$2.60220 \mathrm{E} \quad 01-1.73314 \mathrm{E}-01$

$2.60231 \mathrm{E} \quad 01 \quad-1.74871 \mathrm{E}-01$

$2.60242 \mathrm{E} \quad 01-1.76437 \mathrm{E}-01$

$2.60253 \mathrm{E} \quad 01-1.78013 \mathrm{E}-01$

$2.60264 \mathrm{E} \quad 01-1.79597 \mathrm{E}-01$

$2.60275 \mathrm{E}$ 01 $-1.81191 \mathrm{E}-01$

$2.60286 \mathrm{E} \quad 01$

$2.60296 \mathrm{E} \quad 01$

$2.60307 \mathrm{E} \quad 01$

$2.60317 \mathrm{E} \quad 01$

$2.60328 \mathrm{E} \quad 01$

$2.60338 \mathrm{E} \quad 01$

$2.60349 \mathrm{E} \quad 01$

$2.60359 \mathrm{E} \quad 01$

$2.60369 \mathrm{E} \quad 01$

$2.60379 \mathrm{E} \quad 01$

$-1.82794 \mathrm{E}-01$

$-1.844 .07 \mathrm{E}-01$

$-1.86029 \mathrm{E}-01$

$-1.87660 \mathrm{E}-01$

- 1.89300E-01

$2.60389 \mathrm{E} \quad 01$

$2.60399 \mathrm{E} \quad 01$

$2.60409 \mathrm{E} \quad 01$

$2.60419 \mathrm{E} 01$

$2.60429 \mathrm{E} 01$

$-1.90950 \mathrm{E}-01$

$-1.92610 \mathrm{E}-01$

$-1.94279 \mathrm{E}-01$

$-1.95957 \mathrm{E}-01$

$-1.97645 \mathrm{E}-01$

$-1.99343 \mathrm{E}-01$

$-2.01050 \mathrm{E}-01$

$-2.02766 \mathrm{E}-01$

$-2.04493 \mathrm{E}-01$

$-2.06229 \mathrm{E}-01$

$2.60439 \mathrm{E} \quad 01$

$2.60448 \mathrm{E} 01$

$2.60458 \mathrm{E} \quad 01$

$2.60467 \mathrm{E} \quad 01$

$2.60477 \mathrm{E} 01$

$2.60486 \mathrm{E} \quad 01$

$2.60496 \mathrm{E} 01$

$2.60505 \mathrm{E} \quad 01$

2.60514E 01

$2.60524 \mathrm{E} \quad 01$

$2.60533 \mathrm{E} \quad 01$

$2.60542 \mathrm{E} \quad 01$

$2.60551 \mathrm{E} 01$

$2.60560 \mathrm{E} \quad 01$

$2.60569 \mathrm{E} \quad 01$

$2.60578 \mathrm{E} \quad 01$ $2.60587 \mathrm{E} \quad 01$

$2.60595 \mathrm{E}$ 01

$2.60604 \mathrm{E} \quad 01$

$2.60613 \mathrm{E} 01$

$2.60621 \mathrm{E} \quad 01$

$2.60630 \mathrm{E} \quad 01$

$2.60639 \mathrm{E} \quad 01$

$2.60647 \mathrm{E} \quad 01$

$2.60655 \mathrm{E} 01$

$2.60664 \mathrm{E} \quad 01$

$2.60672 \mathrm{E} \quad 01$

$2.60680 \mathrm{E} \quad 01$

$2.60689 \mathrm{E} \quad 01$

$2.60697 \mathrm{E} \quad 01$

$-2.07974 \mathrm{E}-01$

$-2.09730 \mathrm{E}-01$

$-2.11495 \mathrm{E}-01$

$-2.13270 \mathrm{E}-01$

$-2.15054 \mathrm{E}-01$

$-2.16849 \mathrm{E}-01$

$-2.18653 \mathrm{E}-01$

$-2.20467 \mathrm{E}-01$

$-2.22291 \mathrm{E}-01$

$-2.24125 \mathrm{E}-01$

$-2.25969 \mathrm{E}-01$

$-2.27823 \mathrm{E}-01$

$-2.29687 \mathrm{E}-01$

$-2.31561 \mathrm{E}-01$

$-2.33444 \mathrm{E}-01$

$-2.35338 \mathrm{E}-01$

$-2.37242 \mathrm{E}-01$

$-2.39157 \mathrm{E}-01$

$-2.41081 \mathrm{E}-01$

$-2.43015 \mathrm{E}-01$

$-2.44960 \mathrm{E}-01$

$-2.46915 \mathrm{E}-01$

$-2.48880 \mathrm{E}-01$

$-2.50856 \mathrm{E}-01$

-2.52841 E-01

$-2.54837 \mathrm{E}-01$

$-2.56844 \mathrm{E}-01$ 
ELECTRON RADIAL FUNCTIONS

Electron

\begin{tabular}{|c|c|c|c|c|c|c|}
\hline$p$ & $F_{0}$ & & $f_{1}$ & & $g_{1}$ & \\
\hline 36.10 & $1.20682 \mathrm{E}$ & 00 & $-3.79406 \mathrm{E}$ & 01 & $-4.67742 \mathrm{E}$ & 00 \\
\hline 36.20 & $1.20681 \mathrm{E}$ & 00 & $-3.80424 \mathrm{E}$ & 01 & $-4.70037 \mathrm{E}$ & 00 \\
\hline 36.30 & $1.20680 \mathrm{E}$ & 00 & $-3.81441 \mathrm{E}$ & 01 & $-4.72337 \mathrm{E}$ & 00 \\
\hline 36.40 & $1.20679 \mathrm{E}$ & 00 & $-3.82458 \mathrm{E}$ & 01 & $-4.74642 \mathrm{E}$ & 00 \\
\hline 36.50 & $1.20678 \mathrm{E}$ & 00 & $-3.83475 \mathrm{E}$ & 01 & $-4.76953 \mathrm{E}$ & 00 \\
\hline 36.60 & $1.20676 \mathrm{E}$ & 00 & $-3.84492 \mathrm{E}$ & 01 & $-4.79269 \mathrm{E}$ & 00 \\
\hline 36.70 & $1.20675 \mathrm{E}$ & 00 & $-3.85508 \mathrm{E}$ & 01 & $-4.81591 \mathrm{E}$ & 00 \\
\hline 36.80 & $1.20674 \mathrm{E}$ & 00 & $-3.86523 \mathrm{E}$ & 01 & $-4.83917 \mathrm{E}$ & 00 \\
\hline 36.90 & $1.20673 \mathrm{E}$ & 00 & $-3.87539 \mathrm{E}$ & 01 & $-4.86249 \mathrm{E}$ & 00 \\
\hline 37.00 & $1.20672 \mathrm{E}$ & 00 & $-3.88554 \mathrm{E}$ & 01 & $-4.88587 \mathrm{E}$ & 00 \\
\hline 37.10 & $1.20671 \mathrm{E}$ & 00 & $-3.89569 \mathrm{E}$ & 01 & $-4.90929 \mathrm{E}$ & 00 \\
\hline 37.20 & $1.20670 \mathrm{E}$ & 00 & $-3.90583 E$ & 01 & $-4.93277 \mathrm{E}$ & 00 \\
\hline 37.30 & $1.20668 \mathrm{E}$ & 00 & $-3.91597 \mathrm{E}$ & 01 & $-4.95629 \mathrm{E}$ & 00 \\
\hline 37.40 & $1.20667 \mathrm{E}$ & 00 & $-3.92611 \mathrm{E}$ & 01 & $-4.97988 \mathrm{E}$ & 00 \\
\hline 37.50 & $1.20666 \mathrm{E}$ & 00 & $-3.93624 \mathrm{E}$ & 01 & $-5.00351 \mathrm{E}$ & 00 \\
\hline 37.60 & $1.20665 \mathrm{E}$ & 00 & $-3.94637 \mathrm{E}$ & 01 & $-5.02720 \mathrm{E}$ & 00 \\
\hline 37.70 & $1.20664 \mathrm{E}$ & 00 & $-3.95650 \mathrm{E}$ & 01 & $-5.05093 \mathrm{E}$ & 00 \\
\hline 37.80 & $1.20663 \mathrm{E}$ & 00 & $-3.96662 \mathrm{E}$ & 01 & $-5.07473 \mathrm{E}$ & 00 \\
\hline 37.90 & $1.20662 \mathrm{E}$ & 00 & $-3.97674 \mathrm{E}$ & 01 & $-5.09857 \mathrm{E}$ & 00 \\
\hline 38.00 & $1.20660 \mathrm{E}$ & 00 & $-3.98686 \mathrm{E}$ & & $-5.12246 \mathrm{E}$ & 00 \\
\hline 38.10 & $1.20659 \mathrm{E}$ & 00 & $-3.99697 \mathrm{E}$ & 01 & $-5.14641 \mathrm{E}$ & 00 \\
\hline 38.20 & $1.20658 \mathrm{E}$ & 00 & $-4.00708 \mathrm{E}$ & 01 & $-5.17041 \mathrm{E}$ & 00 \\
\hline 38.30 & $1.20657 \mathrm{E}$ & 00 & $-4.01718 \mathrm{E}$ & 01 & $-5.19446 \mathrm{E}$ & 00 \\
\hline 38.40 & $1.20656 \mathrm{E}$ & 00 & $-4.02729 \mathrm{E}$ & 01 & $-5.21857 \mathrm{E}$ & 00 \\
\hline 38.50 & $1.20655 \mathrm{E}$ & 00 & $-4.03738 \mathrm{E}$ & 01 & $-5.24272 \mathrm{E}$ & 00 \\
\hline 38.60 & $1.20654 \mathrm{E}$ & 00 & $-4.04748 \mathrm{E}$ & 01 & $-5.26693 \mathrm{E}$ & 00 \\
\hline 38.70 & $1.20653 \mathrm{E}$ & 00 & $-4.05757 \mathrm{E}$ & 01 & $-5.29119 \mathrm{E}$ & 00 \\
\hline 38.80 & $1.20652 \mathrm{E}$ & 00 & $-4.06766 \mathrm{E}$ & 01 & $-5.31551 \mathrm{E}$ & 00 \\
\hline 38.90 & $51 \mathrm{E}$ & 00 & $-4.07774 \mathrm{E}$ & 01 & $-5.33987 \mathrm{E}$ & 00 \\
\hline 39.00 & $1.20649 \mathrm{E}$ & 00 & $-4.08782 \mathrm{E}$ & 01 & $-5.36429 \mathrm{E}$ & 00 \\
\hline 39.10 & $1.20648 \mathrm{E}$ & 00 & $-4.09790 \mathrm{E}$ & 01 & $-5.38876 \mathrm{E}$ & 00 \\
\hline 39.20 & $1.20647 \mathrm{E}$ & 00 & $-4.10797 \mathrm{E}$ & 01 & $-5.41328 \mathrm{E}$ & 00 \\
\hline 39.30 & $46 \mathrm{E}$ & 00 & $-4.11804 \mathrm{E}$ & 01 & $-5.43785 \mathrm{E}$ & 00 \\
\hline 39.40 & $1.20645 \mathrm{E}$ & 00 & $-4.12811 \mathrm{E}$ & 01 & $-5.46248 \mathrm{E}$ & 00 \\
\hline 39.50 & $1.20644 \mathrm{E}$ & 00 & $-4.13817 \mathrm{E}$ & 01 & $-5.48716 \mathrm{E}$ & 00 \\
\hline 39.60 & $1.20643 \mathrm{E}$ & 00 & $-4.14823 \mathrm{E}$ & 01 & $-5.51189 E$ & 00 \\
\hline 39.70 & $1.20642 \mathrm{E}$ & 00 & $-4.15828 \mathrm{E}$ & 01 & $-5.53667 \mathrm{E}$ & 00 \\
\hline 39.80 & $1.20641 \mathrm{E}$ & 00 & $-4.16834 \mathrm{E}$ & 01 & $-5.56150 \mathrm{E}$ & 00 \\
\hline 39.90 & $1.20640 \mathrm{E}$ & 00 & $-4.17838 \mathrm{E}$ & 01 & $-5.58638 \mathrm{E}$ & 00 \\
\hline 40.00 & $1.20639 \mathrm{E}$ & 00 & $-4.18843 \mathrm{E}$ & 01 & $-5.61132 \mathrm{E}$ & 00 \\
\hline 40.10 & $1.20638 \mathrm{E}$ & 00 & $-4.19847 \mathrm{E}$ & 01 & $-5.63631 \mathrm{E}$ & 00 \\
\hline 40.20 & $1.20637 \mathrm{E}$ & 00 & $-4.20851 \mathrm{E}$ & 01 & $-5.66135 \mathrm{E}$ & 00 \\
\hline 40.30 & $1.20635 \mathrm{E}$ & 00 & $-4.21854 \mathrm{E}$ & 01 & $-5.68644 \mathrm{E}$ & 00 \\
\hline 40.40 & $1.20634 \mathrm{E}$ & 00 & $-4.22857 \mathrm{E}$ & 01 & $-5.71159 \mathrm{E}$ & 00 \\
\hline 40.50 & $1.20633 \mathrm{E}$ & 00 & $-4.23860 \mathrm{E}$ & 01 & $-5.73678 \mathrm{E}$ & 00 \\
\hline 40.60 & $1.20632 \mathrm{E}$ & 00 & $-4.24862 \mathrm{E}$ & 01 & $-5.76203 \mathrm{E}$ & 00 \\
\hline 40.70 & $1.20631 \mathrm{E}$ & 00 & $-4.25864 \mathrm{E}$ & 01 & $-5.78733 \mathrm{E}$ & 00 \\
\hline 40.80 & $1.20630 \mathrm{E}$ & 00 & $-4.26865 \mathrm{E}$ & 01 & $-5.81268 \mathrm{E}$ & 00 \\
\hline 40.90 & $1.20629 \mathrm{E}$ & 00 & $-4.27866 \mathrm{E}$ & 01 & $-5.83808 \mathrm{E}$ & 00 \\
\hline 41.00 & $1.20628 \mathrm{E}$ & 00 & $-4.28867 \mathrm{E}$ & 01 & $-5.86353 \mathrm{E}$ & 00 \\
\hline 41.10 & $1.20627 \mathrm{E}$ & 00 & $-4.29867 \mathrm{E}$ & 01 & $-5.88904 \mathrm{E}$ & 00 \\
\hline 41.20 & $1.20626 \mathrm{E}$ & 00 & $-4.30867 \mathrm{E}$ & 01 & $-5.91459 \mathrm{E}$ & 00 \\
\hline 41.30 & $1.20625 \mathrm{E}$ & 00 & $-4.31867 \mathrm{E}$ & 01 & $-5.94020 \mathrm{E}$ & 00 \\
\hline 41.40 & $1.20624 \mathrm{E}$ & 00 & $-4.32866 \mathrm{E}$ & 01 & $-5.96586 \mathrm{E}$ & 00 \\
\hline 41.50 & $1.20623 \mathrm{E}$ & 00 & $-4.33865 \mathrm{E}$ & 01 & $-5.99157 \mathrm{E}$ & 00 \\
\hline 41.60 & $1.20622 \mathrm{E}$ & 00 & $-4.34864 \mathrm{E}$ & 01 & $-6.01734 \mathrm{E}$ & 00 \\
\hline 41.70 & $1.20621 \mathrm{E}$ & 00 & $-4.35862 \mathrm{E}$ & 01 & $-6.04315 \mathrm{E}$ & 00 \\
\hline 41.80 & $1.20620 \mathrm{E}$ & 00 & $-4.36860 \mathrm{E}$ & 01 & $-6.06902 \mathrm{E}$ & 00 \\
\hline 41.90 & $1.20619 \mathrm{E}$ & 00 & $-4.37857 \mathrm{E}$ & 01 & $-6.09493 \mathrm{E}$ & 00 \\
\hline 42.00 & $1.20618 \mathrm{E}$ & 00 & $-4.38854 \mathrm{E}$ & 01 & $-6.12090 \mathrm{E}$ & 00 \\
\hline
\end{tabular}

$\tan \delta_{1}$ $\rho=1.2 A^{1 / 3} F$
$5.88756 \mathrm{E}-03$

$5.88666 \mathrm{E}-03$

$5.88576 \mathrm{E}-03$

$5.88483 \mathrm{E}-03$

$5.88387 \mathrm{E}-03$

$5.88290 \mathrm{E}-03$

$5.88190 \mathrm{E}-03$

$5.88089 \mathrm{E}-03$

$5.87986 \mathrm{E}-03$

$5.87880 \mathrm{E}-03$

$5.87773 \mathrm{E}-03$

$5.87663 \mathrm{E}-03$

$5.87552 \mathrm{E}-03$

$5.87439 \mathrm{E}-03$

$5.87324 \mathrm{E}-03$

$5.87206 \mathrm{E}-03$

$5.87088 \mathrm{E}-03$

$5.86966 \mathrm{E}-03$

$5.86844 \mathrm{E}-03$

$5.86719 \mathrm{E}-03$

$5.86592 \mathrm{E}-03$ $5.86463 \mathrm{E}-03$

$5.86333 \mathrm{E}-03$

$5.86201 \mathrm{E}-03$

$5.86067 \mathrm{E}-03$

$5.85931 \mathrm{E}-03$

$5.85793 \mathrm{E}-03$

$5.85653 \mathrm{E}-03$

$5.85512 \mathrm{E}-03$

$5.85368 \mathrm{E}-03$

$5.85223 \mathrm{E}-03$

$5.85076 \mathrm{E}-03$

$5.84928 \mathrm{E}-03$

$5.84777 \mathrm{E}-03$

$5.84625 \mathrm{E}-03$

$5.84470 \mathrm{E}-03$ $5.84313 \mathrm{E}-03$

$5.84157 \mathrm{E}-03$

$5.83997 \mathrm{E}-03$

$5.83836 \mathrm{E}-03$

$5.83673 \mathrm{E}-03$

$5.83508 \mathrm{E}-03$

$5.83342 \mathrm{E}-03$

$5.83175 \mathrm{E}-03$

$5.83004 \mathrm{E}-03$

$5.82833 \mathrm{E}-03$

$5.82660 \mathrm{E}-03$

$5.82486 \mathrm{E}-03$

$5.82309 \mathrm{E}-03$

$5.82131 \mathrm{E}-03$

$5.81951 \mathrm{E}-03$ $5.81769 \mathrm{E}-03$ $5.81586 \mathrm{E}-03$ $5.81402 \mathrm{E}-03$

$5.81215 \mathrm{E}-03$

$5.81027 \mathrm{E}-03$ $5.80837 \mathrm{E}-03$ $5.80645 \mathrm{E}-03$ $5.80452 \mathrm{E}-03$ $5.80258 \mathrm{E}-03$ $f_{-1}$

$-4.59786 \mathrm{E} \quad 00$ $-4.62052 \mathrm{E} \quad 00$ $-4.64324 \mathrm{E} \quad 00$

$-4.66601 \mathrm{E} \quad 00$

$-4.68883 \mathrm{E} \quad 00$

$-4.71171 \mathrm{E} \quad 00$

$-4.73464 \mathrm{E} \quad 00$

$-4.75762 \mathrm{E} \quad 00$

$-4.78065 \mathrm{E} \quad 00$

$-4.80374 \mathrm{E} \quad 00$

$-4.82688 \mathrm{E} \quad 00$

$-4.85007 \mathrm{E} \quad 00$

$-4.87331 \mathrm{E} 00$

$-4.89661 \mathrm{E} \quad 00$

$-4.91996 \mathrm{E} \quad 00$

$-4.94336 \mathrm{E} \quad 00$

$-4.96681 \mathrm{E} \quad 00$

$-4.99032 \mathrm{E} \quad 00$

$-5.01388 \mathrm{E} 00$

$-5.03749 \mathrm{E} \quad 00$

$-5.06115 \mathrm{E} \quad 00$

$-5.08487 \mathrm{E} \quad 00$

$-5.10864 \mathrm{E} \quad 00$

$-5.13246 \mathrm{E} \quad 00$

-5.15633 E 00

$-5.18026 \mathrm{E} \quad 00$

$-5.20423 \mathrm{E} \quad 00$

$-5.22826 \mathrm{E} \quad 00$

$-5.25234 \mathrm{E} \quad 00$

$-5.27648 \mathrm{E} 00$

$-5.30066 \mathrm{E} \quad 00$

$-5.32490 \mathrm{E} 00$

$-5.34919 \mathrm{E} \quad 00$

$-5.37353 \mathrm{E} \quad 00$

$-5.39792 \mathrm{E} 00$

$-5.42237 \mathrm{E} \quad 00$

$-5.44687 \mathrm{E} \quad 00$

$-5.47141 \mathrm{E} 00$

$-5.49602 \mathrm{E} \quad 00$

$-5.52067 \mathrm{E} \quad 00$

$-5.54537 \mathrm{E} \quad 00$

$-5.57013 \mathrm{E} \quad 00$

$-5.59494 \mathrm{E} 00$

$-5.61980 \mathrm{E} \quad 00$

$-5.64471 \mathrm{E} 00$

$-5.66968 \mathrm{E} \quad 00$

$-5.69469 \mathrm{E} \quad 00$

$-5.71976 \mathrm{E} \quad 00$

$-5.74488 \mathrm{E} \quad 00$

$-5.77005 \mathrm{E} 00$

$-5.79527 \mathrm{E} \quad 00$

$-5.82054 \mathrm{E} 00$

$-5.84587 \mathrm{E} \quad 00$

$-5.87125 \mathrm{E} \quad 00$

$-5.89668 \mathrm{E} \quad 00$

$-5.92215 \mathrm{E} \quad 00$ $-5.94769 \mathrm{E} \quad 00$

$-5.97327 \mathrm{E} \quad 00$

$-5.99890 \mathrm{E} \quad 00$

$-6.02459 \mathrm{E} 00$ $g_{-1}$ $\tan \delta_{-1}$

$3.89945 \mathrm{E} \quad 01$ $3.90961 \mathrm{E} \quad 01$ $3.91977 \mathrm{E} 01$ $3.92993 \mathrm{E} 01$ $3.94008 \mathrm{E} \quad 01$

$3.95023 \mathrm{E} 01$ $3.96037 \mathrm{E} \quad 01$ $3.97052 \mathrm{E} 01$ $3.98065 \mathrm{E} \quad 01$ $3.99079 \mathrm{E} 01$

$4.00092 \mathrm{E} \quad 01$ $4.01105 \mathrm{E} \quad 01$ $4.02117 \mathrm{E} \quad 01$ $4.03129 \mathrm{E} \quad 01$ $4.04141 \mathrm{E} 01$

$4.05153 \mathrm{E} \quad 01$ $4.06164 \mathrm{E} \quad 01$ $4.07174 \mathrm{E} \quad 01$ $4.08185 \mathrm{E} 01$ $4.09195 \mathrm{E} \quad 01$

$4.10204 \mathrm{E} \quad 01$ $4.11213 \mathrm{E} \quad 01$ $4.12222 \mathrm{E} 01$ $4.13231 \mathrm{E} 01$ $4.14239 \mathrm{E} 01$

$4.15247 \mathrm{E} \quad 01$ $4.16254 \mathrm{E} \quad 01$ $4.17262 \mathrm{E} 01$ $4.18268 \mathrm{E} \quad 01$ $4.19275 \mathrm{E} 01$

$4.20281 \mathrm{E} \quad 01$ $4.21287 \mathrm{E} \quad 01$ $4.22292 \mathrm{E} \quad 01$ $4.23297 \mathrm{E} 01$ $4.24301 \mathrm{E} 01$

$4.25306 \mathrm{E} \quad 01$ $4.26310 \mathrm{E}$ 01 $4.27313 \mathrm{E} 01$ $4.28316 \mathrm{E} \quad 01$ $4.29319 \mathrm{E} \quad 01$

$4.30321 \mathrm{E} \quad 01$ $4.31323 \mathrm{E} \quad 01$ $4.32325 \mathrm{E}$ 01 $4.33326 \mathrm{E}$ 0] $4.34327 \mathrm{E} 01$

$4.35328 \mathrm{E} \quad 01$ $4.36328 \mathrm{E} \quad 01$ $4.37328 \mathrm{E} \quad 01$ $4.38328 \mathrm{E} 01$ $4.39327 \mathrm{E} \quad 01$

$4.40325 \mathrm{E} \quad 01$ $4.41324 \mathrm{E}$ 01 $4.42322 \mathrm{E}$ 01 $4.43319 \mathrm{E} \quad 01$ $4.44317 \mathrm{E} \quad 01$

$4.45313 \mathrm{E} \quad 01$ $4.46310 \mathrm{E} \quad 01$ $4.47306 \mathrm{E} 01$ $4.48302 \mathrm{E} 01$ $4.49297 \mathrm{E} \quad 01$
$-1.33811 \mathrm{E} 02$ $-1.33908 \mathrm{E} \quad 02$ $-1.34006 \mathrm{E} 02$ $-1.34103 \mathrm{E} 02$ $-1.34201 \mathrm{E} 02$

$-1.34299 \mathrm{E} \quad 02$ $-1.34397 \mathrm{E} 02$ $-1.34495 \mathrm{E} 02$ $-1.34593 \mathrm{E} 02$ $-1.34691 \mathrm{E} 02$

$-1.34789 \mathrm{E} \quad 02$ $-1.34888 \mathrm{E} \quad 02$ -1.34986 E 02 $-1.35084 \mathrm{E} \quad 02$ $-1.35183 \mathrm{E} \quad 02$

$-1.35282 \mathrm{E} \quad 02$ $-1.35380 \mathrm{E} 02$ $-1.35479 \mathrm{E} \quad 02$ $-1.35578 \mathrm{E} 02$ $-1.35677 \mathrm{E} 02$

$\begin{array}{lll}-1.35777 \mathrm{E} & 02\end{array}$ $-1.35876 \mathrm{E} \quad 02$ $-1.35975 \mathrm{E} \quad 02$ $-1.36075 \mathrm{E} 02$ $-1.36174 \mathrm{E} 02$

$-1.36274 \mathrm{E} \quad 02$ $-1.36374 \mathrm{E} 02$ $-1.36474 \mathrm{E} \quad 02$
$-1.36574 \mathrm{E}$ $-1.36574 \mathrm{E} \quad 02$ -1.36674 E 02

$-1.36774 \mathrm{E} \quad 02$ $-1.36874 \mathrm{E} \quad 02$ $-1.36975 \mathrm{E} \quad 02$ $-1.37075 \mathrm{E} \quad 02$ $-1.37176 \mathrm{E} 02$

$-1.37277 \mathrm{E} \quad 02$ $-1.37378 \mathrm{E} 02$ $-1.37479 \mathrm{E} 02$ $-1.37581 \mathrm{E} 02$ $-1.37682 \mathrm{E} 02$

$-1.37783 \mathrm{E} \quad 02$ $-1.37885 \mathrm{E} \quad 02$ $-1.37987 \mathrm{E} \quad 02$ $-1.38089 \mathrm{E} \quad 02$ $-1.38191 \mathrm{E} \quad 02$

$-1.38293 \mathrm{E} \quad 02$ $-1.38395 \mathrm{E} 02$ $-1.38498 \mathrm{E} 02$ $-1.38601 \mathrm{E} \quad 02$ $-1.38703 \mathrm{E} 02$

$-1.38806 \mathrm{E} 02$ $-1.38909 \mathrm{E} 02$ $-1.39013 \mathrm{E} \quad 02$ $-1.39116 \mathrm{E} 02$ -1.39219 E 02

$-1.39323 \mathrm{E} \quad 02$ $-1.39427 \mathrm{E} \quad 02$ $-1.39531 \mathrm{E} 02$ $-1.39635 \mathrm{E} 02$ $-1.39739 \mathrm{E} 02$ 
ELECTRON RADIAL FUNCTIONS

$$
Z=8 \quad A=17 \quad \rho=1.2 A^{1 / 3} F
$$

$\bar{F}$

36.10

36.20

36.30

36.40

36.50

36.60

36.70

36.80

36.90

37.00

37.10

37.20

37.30

37.40

37.50

37.60

37.70

37.80

37.90

38.00

38.10

38.20

38.30

38.40

38.50

38.60

38.70

38.80

38.90

39.00

39.10

39.20

39.30

39.40

39.50

39.60

39.70

39.80

39.90

40.00

40.10

40.20

40.30

40.40

40.50

40.60

40.70

40.80

40.90

41.00

41.10

41.20

41.30

41.40

41.50

41.60

41.70

41.80

41.90

42.00 $f_{2}$

$2.78546 \mathrm{E}-03$

$2.80090 \mathrm{E}-03$

$2.81638 \mathrm{E}-03$

$2.83190 \mathrm{E}-03$

$2.84747 \mathrm{E}-03$

$2.86308 \mathrm{E}-03$

$2.87873 \mathrm{E}-03$

$2.89443 \mathrm{E}-03$

$2.91016 \mathrm{E}-03$

$2.92594 \mathrm{E}-03$

$2.94177 \mathrm{E}-03$

$2.95763 \mathrm{E}-03$

$2.97354 \mathrm{E}-03$

$2.98949 \mathrm{E}-03$

$3.00548 \mathrm{E}-03$

$3.02152 \mathrm{E}-03$

$3.03760 \mathrm{E}-03$

3.05372 E-03

$3.06988 \mathrm{E}-03$

$3.08609 \mathrm{E}-03$

3.10234E-03

$3.11863 \mathrm{E}-03$

$3.13496 \mathrm{E}-03$

$3.15134 \mathrm{E}-03$

$3.16776 \mathrm{E}-03$

$3.18422 \mathrm{E}-03$

$3.20073 \mathrm{E}-03$

$3.21728 \mathrm{E}-03$

$3.23387 \mathrm{E}-03$

$3.25050 \mathrm{E}-03$

$3.26717 \mathrm{E}-03$

$3.28389 \mathrm{E}-03$

$3.30065 \mathrm{E}-03$

$3.31746 \mathrm{E}-03$

$3.33430 \mathrm{E}-03$

$3.35119 \mathrm{E}-03$

$3.36812 \mathrm{E}-03$

$3.38509 \mathrm{E}-03$

3.40211 E-03

$3.41917 \mathrm{E}-03$

$3.43627 \mathrm{E}-03$

3.45342 E-03

$3.47060 \mathrm{E}-03$

$3.48783 \mathrm{E}-03$

$3.50510 \mathrm{E}-03$

$3.52242 \mathrm{E}-03$

$3.53978 \mathrm{E}-03$

$3.55718 \mathrm{E}-03$

$3.57462 \mathrm{E}-03$

$3.59210 \mathrm{E}-03$

$3.60963 \mathrm{E}-03$

$3.62720 \mathrm{E}-03$

$3.64482 \mathrm{E}-03$

$3.66247 \mathrm{E}-03$

$3.68017 \mathrm{E}-03$

3.69791 E-03

$3.71569 \mathrm{E}-03$

$3.73352 \mathrm{E}-03$

$3.75139 \mathrm{E}-03$

$3.76930 \mathrm{E}-03$ $g_{2}$

$\tan \delta_{2}$

$f_{-2}$

$-3.69719 \mathrm{E} \quad 00 \quad-2.69611 \mathrm{E}-01$

$-3.71759 \mathrm{E} \quad 00 \quad-2.71699 \mathrm{E}-01$

$-3.73804 \mathrm{E} \quad 00 \quad-2.73798 \mathrm{E}-01$

$-3.75854 \mathrm{E} \quad 00 \quad-2.75907 \mathrm{E}-01$

$-3.77910 \mathrm{E} \quad 00 \quad-2.78027 \mathrm{E}-01$

$-3.79971 \mathrm{E} \quad 00$

$-2.80157 \mathrm{E}-01$

$-3.82038 \mathrm{E} \quad 00$

$-3.84110 \mathrm{E} \quad 00$

$-3.86187 \mathrm{E} \quad 00$

$-3.88270 \mathrm{E} \quad 00$

$-3.90359 \mathrm{E} \quad 00$

$-3.92452 \mathrm{E} \quad 00$

$-3.94551 \mathrm{E} \quad 00$

$-3.96656 \mathrm{E} \quad 00$

$-3.98766 \mathrm{E} \quad 00$

$-4.00881 \mathrm{E} \quad 00$

$-4.03002 \mathrm{E} \quad 00$

$-4.05128 \mathrm{E} \quad 00$

$-4.07259 \mathrm{E} \quad 00$

$-4.09396 \mathrm{E} \quad 00$

$-4.11538 \mathrm{E} \quad 00$

$-4.13686 \mathrm{E} \quad 00$

$-4.15839 \mathrm{E} \quad 00$

$-4.17997 \mathrm{E} \quad 00$

$-4.20161 \mathrm{E} \quad 00$

$-4.22330 \mathrm{E} \quad 00$

$-4.24504 \mathrm{E} \quad 00$

$-4.26684 \mathrm{E} \quad 00$

$-4.28869 \mathrm{E} 00$

$-4.31060 \mathrm{E} 00$

$-4.33256 \mathrm{E} \quad 00$

$-4.35457 \mathrm{E} 00$

$-4.37664 \mathrm{E} \quad 00$

$-4.39876 \mathrm{E} 00$

$-4.42093 \mathrm{E} \quad 00$

$-4.44316 \mathrm{E} \quad 00$

$-4.46544 \mathrm{E} \quad 00$

$-4.48777 \mathrm{E} \quad 00$

$-4.51016 \mathrm{E} \quad 00$

$-4.53260 \mathrm{E} 00$

$-4.55510 \mathrm{E} \quad 00$

$-4.57765 \mathrm{E} 00$

$-4.60025 \mathrm{E} 00$

$-4.62290 \mathrm{E} 00$

$-4.64561 \mathrm{E} \quad 00$

$-4.66837 \mathrm{E} \quad 00$

$-4.69119 \mathrm{E} \quad 00$

$-4.71405 \mathrm{E} \quad 00$

$-4.73697 \mathrm{E} \quad 00$

$-4.75995 \mathrm{E} 00$

$-4.78298 \mathrm{E} \quad 00$

$-4.80606 \mathrm{E} \quad 00$

$-4.82919 \mathrm{E} 00$

$-4.85238 \mathrm{E} \quad 00$

$-4.87562 \mathrm{E} \quad 00$

$-4.89891 \mathrm{E} \quad 00$

$-4.92226 \mathrm{E} \quad 00$

$-4.94566 \mathrm{E} 00$

$-4.96911 \mathrm{E} \quad 00$

$-4.99261 \mathrm{E} \quad 00$
$-2.82298 \mathrm{E}-01$

$-2.84450 \mathrm{E}-01$

$-2.86613 \mathrm{E}-01$

$-2.88786 \mathrm{E}-01$

$-2.90970 \mathrm{E}-01$

$-2.93165 \mathrm{E}-01$

$-2.95371 \mathrm{E}-01$

$-2.97587 \mathrm{E}-01$

$-2.99815 \mathrm{E}-01$

$-3.02054 \mathrm{E}-01$

$-3.04303 \mathrm{E}-01$

$-3.06563 \mathrm{E}-01$

$-3.08835 \mathrm{E}-01$

$-3.11117 \mathrm{E}-01$

$-3.13411 \mathrm{E}-01$

$-3.15715 \mathrm{E}-01$

$-3.18031 \mathrm{E}-01$

$-3.20357 \mathrm{E}-01$

$-3.22695 \mathrm{E}-01$

$-3.25044 \mathrm{E}-01$

$-3.27405 \mathrm{E}-01$

$-3.29776 \mathrm{E}-01$

$-3.32159 \mathrm{E}-01$

$-3.34553 \mathrm{E}-01$

$-3.36958 \mathrm{E}-01$

$-3.39375 \mathrm{E}-01$

$-3.41803 \mathrm{E}-01$

$-3.46693 \mathrm{E}-01$

$-3.49155 \mathrm{E}-01$

$-3.51629 \mathrm{E}-01$

$-3.54114 \mathrm{E}-01$

$-3.59118 \mathrm{E}-01$

$-3.61638 \mathrm{E}-01$

$-3.64169 \mathrm{E}-01$

$-3.66712 \mathrm{E}-01$

$-3.69266 \mathrm{E}-01$

$-3.71832 \mathrm{E}-01$

$-3.74410 \mathrm{E}-01$

$-3.77000 \mathrm{E}-01$

$-3.79601 \mathrm{E}-01$

$-3.82214 \mathrm{E}-01$

$-3.84838 \mathrm{E}-01$

$-3.87475 \mathrm{E}-01$

$-3.90123 \mathrm{E}-01$

$-3.92783 \mathrm{E}-01$

$-3.95455 \mathrm{E}-01$

$-4.00835 \mathrm{E}-01$

$-4.03543 \mathrm{E}-01$

$-4.06263 \mathrm{E}-01$

$-4.08995 \mathrm{E}-01$

$-4.11738 \mathrm{E}-01$
$-3.44242 \mathrm{E}-01$

$-3.56610 \mathrm{E}-01$

$-3.98139 \mathrm{E}-01$

$2.60705 \mathrm{E} \quad 01 \quad-2.64973 \mathrm{E}-01$

$2.60713 \mathrm{E} \quad 01-2.67032 \mathrm{E}-01$

$\begin{array}{lll}2.60721 \mathrm{E} & 01 & -2.69101 \mathrm{E}-01\end{array}$

$2.60729 \mathrm{E} \quad 01 \quad-2.71181 \mathrm{E}-01$

$2.60737 \mathrm{E} \quad 01-2.73271 \mathrm{E}-01$

$2.60745 \mathrm{E} \quad 01 \quad-2.75371 \mathrm{E}-01$

$2.60753 \mathrm{E} \quad 01 \quad-2.77483 \mathrm{E}-01$

$2.60761 \mathrm{E} \quad 01-2.79605 \mathrm{E}-01$

$2.60768 \mathrm{E} \quad 01 \quad-2.81737 \mathrm{E}-01$

$2.60776 \mathrm{E} \quad 01-2.83880 \mathrm{E}-01$

$2.60784 \mathrm{E} \quad 01$

$2.60791 \mathrm{E} 01$

$2.60799 \mathrm{E} \quad 01$

$2.60806 \mathrm{E} \quad 01$

$2.60814 \mathrm{E} \quad 01$

$2.60821 \mathrm{E} \quad 01$

$2.60829 \mathrm{E} \quad 01$

$2.60836 \mathrm{E} \quad 01$

$2.60844 \mathrm{E} \quad 01$

$2.60851 \mathrm{E} 01$

$2.60858 \mathrm{E} \quad 01$

$2.60865 \mathrm{E} \quad 01$

$2.60872 \mathrm{E} \quad 01$

$2.60880 \mathrm{E} \quad 01$

$2.60887 \mathrm{E} \quad 01$

$2.60894 \mathrm{E} \quad 01$

$2.60901 \mathrm{E} \quad 01$

$2.60908 \mathrm{E} \quad 01$

$2.60915 \mathrm{E} \quad 01$

$2.60922 \mathrm{E} \quad 01$

$2.60929 \mathrm{E} \quad 01$

$-2.86034 \mathrm{E}-01$

$-2.88199 \mathrm{E}-01$

$-2.90374 \mathrm{E}-01$

$-2.92561 \mathrm{E}-01$

$-2.94758 \mathrm{E}-01$

$-2.96965 \mathrm{E}-01$

$-2.99184 \mathrm{E}-01$

$-3.01414 \mathrm{E}-01$

$-3.03654 \mathrm{E}-01$

$-3.05906 \mathrm{E}-01$

$-3.08168 \mathrm{E}-01$

$-3.10441 \mathrm{E}-01$

$-3.12726 \mathrm{E}-01$

$-3.15021 \mathrm{E}-01$

$-3.17328 \mathrm{E}-01$ 
ELECTRON RADIAL FUNCTIONS

Electron

\begin{tabular}{|c|c|c|c|c|}
\hline$p$ & $F_{0}$ & & $f_{1}$ & $g_{1}$ \\
\hline $\begin{array}{l}0.10 \\
0.20 \\
0.30 \\
0.40 \\
0.50\end{array}$ & $\begin{array}{l}4.37042 \mathrm{E} \\
2.48087 \mathrm{E} \\
1.94783 \mathrm{E} \\
1.71030 \mathrm{E} \\
1.57973 \mathrm{E}\end{array}$ & $\begin{array}{l}00 \\
00 \\
00 \\
00 \\
00\end{array}$ & $\begin{array}{l}-1.75903 \mathrm{E}-02 \\
-4.61667 \mathrm{E}-02 \\
-8.79568 \mathrm{E}-02 \\
-1.41675 \mathrm{E}-01 \\
-2.05800 \mathrm{E}-01\end{array}$ & $\begin{array}{l}-5.58147 \mathrm{E}-04 \\
-1.46674 \mathrm{E}-03 \\
-2.80024 \mathrm{E}-03 \\
-4.52315 \mathrm{E}-03 \\
-6.59337 \mathrm{E}-03\end{array}$ \\
\hline $\begin{array}{l}.60 \\
.70 \\
.80 \\
.90 \\
.00\end{array}$ & $\begin{array}{l}1.49889 \mathrm{E} \\
1.44487 \mathrm{E} \\
1.40679 \mathrm{E} \\
1.37887 \mathrm{E} \\
1.35776 \mathrm{E}\end{array}$ & $\begin{array}{l}00 \\
00 \\
00 \\
00 \\
00\end{array}$ & $\begin{array}{l}-2.78754 \mathrm{E}-01 \\
-3.59040 \mathrm{E}-01 \\
-4.45313 \mathrm{E}-01 \\
-5.36421 \mathrm{E}-01 \\
-6.31407 \mathrm{E}-01\end{array}$ & $\begin{array}{l}-8.96717 \mathrm{E}-03 \\
-1.16030 \mathrm{E}-02 \\
-1.44637 \mathrm{E}-02 \\
-1.75173 \mathrm{E}-02 \\
-2.07374 \mathrm{E}-02\end{array}$ \\
\hline $\begin{array}{l}.10 \\
.20 \\
.30 \\
.0\end{array}$ & $\begin{array}{l}1.34138 \mathrm{E} \\
1.32843 \mathrm{E} \\
1.31799 \mathrm{E} \\
1.30946 \mathrm{E} \\
1.30239 \mathrm{E}\end{array}$ & $\begin{array}{l}00 \\
00 \\
00 \\
00 \\
00\end{array}$ & $\begin{array}{l}-7.29492 \mathrm{E}-01 \\
-8.30051 \mathrm{E}-01 \\
-9.32585 \mathrm{E}-01 \\
-1.03670 \mathrm{E} \quad 00 \\
-1.14208 \mathrm{E} \quad 00\end{array}$ & $\begin{array}{l}-2.41025 \mathrm{E}-02 \\
-2.75953 \mathrm{E}-02 \\
-3.12021 \mathrm{E}-02 \\
-3.49121 \mathrm{E}-02 \\
-3.87167 \mathrm{E}-02\end{array}$ \\
\hline 00 & $\begin{array}{l}1.29646 \mathrm{E} \\
1.29144 \mathrm{E} \\
1.28715 \mathrm{E} \\
1.28345 \mathrm{E} \\
1.28024 \mathrm{E}\end{array}$ & $\begin{array}{l}00 \\
00 \\
00 \\
00 \\
00\end{array}$ & $\begin{array}{ll}-1.24849 \mathrm{E} & 0 \\
-1.35572 \mathrm{E} & 0 \\
-1.46362 \mathrm{E} & 0 \\
-1.57207 \mathrm{E} & 0 \\
-1.68096 \mathrm{E} & 0\end{array}$ & $\begin{array}{l}-4.26092 \mathrm{E}-02 \\
-4.65841 \mathrm{E}-02 \\
-5.06374 \mathrm{E}-02 \\
-5.47655 \mathrm{E}-02 \\
-5.89658 \mathrm{E}-02\end{array}$ \\
\hline $\begin{array}{r}.20 \\
.30 \\
.50\end{array}$ & $\begin{array}{l}1.27742 \mathrm{E} \\
1.27494 \mathrm{E} \\
1.27275 \mathrm{E} \\
1.27079 \mathrm{E} \\
1.26904 \mathrm{E}\end{array}$ & $\begin{array}{l}00 \\
00 \\
00 \\
00 \\
00\end{array}$ & $\begin{array}{ll}-1.79022 \mathrm{E} & 0 \\
-1.89978 \mathrm{E} & 0 \\
-2.00958 \mathrm{E} & 0 \\
-2.11959 \mathrm{E} & 0 \\
-2.22977 \mathrm{E} & 0\end{array}$ & $\begin{array}{l}-6.32361 \mathrm{E}-02 \\
-6.75746 \mathrm{E}-02 \\
-7.19800 \mathrm{E}-02 \\
-7.64509 \mathrm{E}-02 \\
-8.09866 \mathrm{E}-02\end{array}$ \\
\hline $\begin{array}{l}2.80 \\
2.90 \\
3.00\end{array}$ & $\begin{array}{l}1.26746 \mathrm{E} \\
1.26604 \mathrm{E} \\
1.26474 \mathrm{E} \\
1.26357 \mathrm{E} \\
1.26249 \mathrm{E}\end{array}$ & $\begin{array}{l}00 \\
00 \\
00 \\
00 \\
00\end{array}$ & $\begin{array}{ll}-2.34009 \mathrm{E} & 00 \\
-2.45052 \mathrm{E} & 00 \\
-2.56105 \mathrm{E} & 00 \\
-2.67165 \mathrm{E} & 00 \\
-2.78232 \mathrm{E} & 00\end{array}$ & $\begin{array}{l}-8.55861 \mathrm{E}-02 \\
-9.02489 \mathrm{E}-02 \\
-9.49743 \mathrm{E}-02 \\
-9.97621 \mathrm{E}-02 \\
-1.04612 \mathrm{E}-01\end{array}$ \\
\hline $\begin{array}{l}3.10 \\
.20 \\
.30 \\
.40 \\
.40\end{array}$ & & $\begin{array}{l}00 \\
00 \\
00 \\
00 \\
00\end{array}$ & $\begin{array}{ll}-2.89305 \mathrm{E} & 00 \\
-3.00381 \mathrm{E} & 00 \\
-3.11461 \mathrm{E} & 00 \\
-3.22543 \mathrm{E} & 00 \\
-3.33628 \mathrm{E} & 00\end{array}$ & $\begin{array}{l}-1.09523 \mathrm{E}-01 \\
-1.14495 \mathrm{E}-01 \\
-1.19529 \mathrm{E}-01 \\
-1.24623 \mathrm{E}-01 \\
-1.29778 \mathrm{E}-01\end{array}$ \\
\hline $\begin{array}{l}3.60 \\
3.70 \\
3.80 \\
3.90 \\
4.00\end{array}$ & & $\begin{array}{l}00 \\
00 \\
00 \\
00 \\
00\end{array}$ & $\begin{array}{ll}-3.44714 \mathrm{E} & 00 \\
-3.55801 \mathrm{E} & 0 \\
-3.66889 \mathrm{E} & 00 \\
-3.77977 \mathrm{E} & 00 \\
-3.89065 \mathrm{E} & 00\end{array}$ & $\begin{array}{l}-1.34993 \mathrm{E}-01 \\
-1.40269 \mathrm{E}-01 \\
-1.45605 \mathrm{E}-01 \\
-1.51001 \mathrm{E}-01 \\
-1.56458 \mathrm{E}-01\end{array}$ \\
\hline $\begin{array}{l}4.10 \\
4.20 \\
4.30 \\
4.40 \\
4.50\end{array}$ & & $\begin{array}{l}00 \\
00 \\
00 \\
00 \\
00\end{array}$ & $\begin{array}{ll}-4.00153 \mathrm{E} & 0 \\
-4.11241 \mathrm{E} & 0 \\
-4.22328 \mathrm{E} & 0 \\
-4.33414 \mathrm{E} & 0 \\
-4.44500 \mathrm{E} & 0\end{array}$ & $\begin{array}{l}-1.61974 \mathrm{E}-01 \\
-1.67551 \mathrm{E}-01 \\
-1.73187 \mathrm{E}-01 \\
-1.78883 \mathrm{E}-01 \\
-1.84639 \mathrm{E}-01\end{array}$ \\
\hline $\begin{array}{l}4.90 \\
5.00\end{array}$ & $\begin{array}{l}5276 \mathrm{E} \\
5241 \mathrm{E} \\
5208 \mathrm{E} \\
5176 \mathrm{E} \\
5146 \mathrm{E}\end{array}$ & $\begin{array}{l}00 \\
00 \\
00 \\
00 \\
00\end{array}$ & $\begin{array}{ll}-4.55584 \mathrm{E} & 0 \\
-4.66667 \mathrm{E} & 0 \\
-4.77749 \mathrm{E} & 0 \\
-4.88829 \mathrm{E} & 0 \\
-4.99909 \mathrm{E} & 0\end{array}$ & $\begin{array}{l}-1.90455 \mathrm{E}-01 \\
-1.96331 \mathrm{E}-01 \\
-2.02266 \mathrm{E}-01 \\
-2.08261 \mathrm{E}-01 \\
-2.14315 \mathrm{E}-01\end{array}$ \\
\hline $\begin{array}{l}5.10 \\
5.20\end{array}$ & $\begin{array}{l}1.25117 \mathrm{E} \\
1.25089 \mathrm{E} \\
1.25063 \mathrm{E} \\
1.25037 \mathrm{E} \\
1.25012 \mathrm{E}\end{array}$ & $\begin{array}{l}00 \\
00 \\
00 \\
00 \\
00\end{array}$ & $\begin{array}{ll}-5.10986 \mathrm{E} & 0 \\
-5.22062 \mathrm{E} & 0 \\
-5.33137 \mathrm{E} & 0 \\
-5.44209 \mathrm{E} & 0 \\
-5.55280 \mathrm{E} & 0\end{array}$ & $\begin{array}{l}-2.20429 \mathrm{E}-01 \\
-2.26603 \mathrm{E}-01 \\
-2.32836 \mathrm{E}-01 \\
-2.39129 \mathrm{E}-01 \\
-2.45481 \mathrm{E}-01\end{array}$ \\
\hline $\begin{array}{l}5.90 \\
6.00\end{array}$ & $\begin{array}{l}1.24989 \mathrm{E} \\
1.24966 \mathrm{E} \\
1.24945 \mathrm{E} \\
1.24924 \mathrm{E} \\
1.24903 \mathrm{E}\end{array}$ & $\begin{array}{l}00 \\
00 \\
00 \\
00 \\
00\end{array}$ & $\begin{array}{ll}-5.66350 \mathrm{E} & 0 \\
-5.77417 \mathrm{E} & 0 \\
-5.88483 \mathrm{E} & 0 \\
-5.99547 \mathrm{E} & 0 \\
-6.10610 \mathrm{E} & 0\end{array}$ & $\begin{array}{l}-2.51893 \mathrm{E}-01 \\
-2.58365 \mathrm{E}-01 \\
-2.64895 \mathrm{E}-01 \\
-2.71486 \mathrm{E}-01 \\
-2.78135 \mathrm{E}-01\end{array}$ \\
\hline
\end{tabular}

\begin{abstract}
$\tan \delta_{1}$
\end{abstract}

$$
\rho=1.2 A^{1 / 3} F
$$

$$
\begin{aligned}
& -3.00210 \mathrm{E}-01 \\
& -1.37838 \mathrm{E}-01 \\
& -8.91322 \mathrm{E}-02 \\
& -6.52592 \mathrm{E}-02 \\
& -5.09885 \mathrm{E}-02 \\
& -4.14573 \mathrm{E}-02 \\
& -3.46205 \mathrm{E}-02 \\
& -2.94656 \mathrm{E}-02 \\
& -2.54332 \mathrm{E}-02 \\
& -2.21883 \mathrm{E}-02 \\
& -1.95182 \mathrm{E}-02 \\
& -1.72809 \mathrm{E}-02 \\
& -1.53780 \mathrm{E}-02 \\
& -1.37389 \mathrm{E}-02 \\
& -1.23119 \mathrm{E}-02
\end{aligned}
$$

$-1.10580 \mathrm{E}-02$

$-9.94723 \mathrm{E}-03$

$-8.95627 \mathrm{E}-03$

$-8.06662 \mathrm{E}-03$

$-7.26341 \mathrm{E}-03$

$-6.53457 \mathrm{E}-03$

$-5.87019 \mathrm{E}-03$

$-5.26205 \mathrm{E}-03$

$-4.70329 \mathrm{E}-03$

$-4.18809 \mathrm{E}-03$

$-3.71156 \mathrm{E}-03$ $-3.26950 \mathrm{E}-03$

$-2.85826 \mathrm{E}-03$

$-2.47475 \mathrm{E}-03$

$-2.11626 \mathrm{E}-03$

$-1.78041 \mathrm{E}-03$

$-1.46510 \mathrm{E}-03$

$-1.16853 \mathrm{E}-03$

$-8.89074 \mathrm{E}-04$

$-6.25284 \mathrm{E}-04$

$-3.75896 \mathrm{E}-04$

$-1.39740 \mathrm{E}-04$

$8.41860 \mathrm{E}-05$

$2.96819 \mathrm{E}-04$

$4.98991 \mathrm{E}-04$

$6.91431 \mathrm{E}-04$

$8.74845 \mathrm{E}-04$

$1.04986 \mathrm{E}-03$

$1.21703 \mathrm{E}-03$

$1.37683 \mathrm{E}-03$

$1.52979 \mathrm{E}-03$

$1.67631 \mathrm{E}-03$

$1.81678 \mathrm{E}-03$

$1.95159 \mathrm{E}-03$

$2.08105 \mathrm{E}-03$

$2.20549 \mathrm{E}-03$

$2.32517 \mathrm{E}-03$

$2.44038 \mathrm{E}-03$

$2.55134 \mathrm{E}-03$

$2.65831 \mathrm{E}-03$

$2.76146 \mathrm{E}-03$

$2.86102 \mathrm{E}-03$

$2.95716 \mathrm{E}-03$

$3.05006 \mathrm{E}-03$

$3.13987 \mathrm{E}-03$ $f_{-1}$

$-7.75040 \mathrm{E}-03$

$-1.16541 \mathrm{E}-02$

$-1.54389 \mathrm{E}-02$

$-1.92090 \mathrm{E}-02$

$-2.29678 \mathrm{E}-02$

$-2.67144 \mathrm{E}-02$

$-3.04504 \mathrm{E}-02$

$-3.41803 \mathrm{E}-02$

$-3.79099 \mathrm{E}-02$

$-4.16457 \mathrm{E}-02$

$-4.53942 \mathrm{E}-02$

$-4.91615 \mathrm{E}-02$

$-5.29532 \mathrm{E}-02$

$-5.67741 \mathrm{E}-02$

$-6.06284 \mathrm{E}=02$

$-6.45200 \mathrm{E}-02$

$-6.84517 \mathrm{E}-02$

$-7.24264 \mathrm{E}-02$

$-7.64463 \mathrm{E}-02$

$-8.05132 \mathrm{E}-02$

$-8.46290 \mathrm{E}-02$

$-8.87948 \mathrm{E}-02$

$-9.30119 \mathrm{E}-02$

$-9.72814 \mathrm{E}-02$

$-1.01604 \mathrm{E}-01$

$-1.05981 \mathrm{E}-01$ $-1.10412 \mathrm{E}-01$

$-1.14898 \mathrm{E}-01$

$-1.19440 \mathrm{E}-01$

$-1.24039 \mathrm{E}-01$

$-1.28693 \mathrm{E}-01$

$-1.33404 \mathrm{E}-01$

$-1.38173 \mathrm{E}-01$

$-1.42998 \mathrm{E}-01$

$-1.47882 \mathrm{E}-01$

$-1.52822 \mathrm{E}-01$

$-1.57821 \mathrm{E}-01$

$-1.62878 \mathrm{E}-01$

$-1.67993 \mathrm{E}-01$

$-1.73166 \mathrm{E}-01$

$-1.78397 \mathrm{E}-01$

$-1.83687 \mathrm{E}-01$

$-1.89036 \mathrm{E}-01$

$-1.94443 \mathrm{E}-01$

$-1.99908 \mathrm{E}-01$

$-2.05433 \mathrm{E}-01$

$-2.11016 \mathrm{E}-01$

$-2.16658 \mathrm{E}-01$

$-2.22359 \mathrm{E}-01$

$-2.28119 \mathrm{E}-01$

$-2.33938 \mathrm{E}-01$

$-2.39816 \mathrm{E}-01$

$-2.45753 \mathrm{E}-01$

$-2.51749 \mathrm{E}-01$

$-2.57803 \mathrm{E}-01$

$-2.63917 \mathrm{E}-01$

$-2.70091 \mathrm{E}-01$

$-2.76323 \mathrm{E}-01$

$-2.82614 \mathrm{E}-01$

$-2.88964 \mathrm{E}-01$ $g-1$

$2.94709 \mathrm{E}-01$

$4.42469 \mathrm{E}-01$

$5.84703 \mathrm{E}-01$

$7.25021 \mathrm{E}-01$

$8.63263 \mathrm{E}-01$

$9.99163 \mathrm{E}-01$

$1.13262 \mathrm{E} \quad 00$

$1.26370 \mathrm{E} \quad 00$

$1.39252 \mathrm{E} \quad 00$

$1.51930 \mathrm{E} \quad 00$

$1.64424 \mathrm{E} \quad 00$

$1.76756 \mathrm{E} \quad 00$

$1.88947 \mathrm{E} \quad 00$

$2.01014 \mathrm{E} \quad 00$

$2.12972 \mathrm{E} \quad 00$

$2.24838 \mathrm{E} \quad 00$

$2.36621 \mathrm{E} \quad 00$

$2.48334 \mathrm{E} \quad 00$

$2.59985 \mathrm{E} \quad 00$

$2.71581 \mathrm{E} \quad 00$

$2.83129 \mathrm{E} \quad 00$

$2.94635 \mathrm{E} \quad 00$

$3.06104 \mathrm{E} \quad 00$

$3.17539 \mathrm{E} \quad 00$

$3.28945 \mathrm{E} \quad 00$

$3.40324 \mathrm{E} \quad 00$ $3.51680 \mathrm{E} \quad 00$ $3.63014 \mathrm{E} \quad 00$ $3.74328 \mathrm{E} \quad 00$ $3.85625 \mathrm{E} \quad 00$

$3.96905 \mathrm{E} \quad 00$ $4.08171 \mathrm{E} \quad 00$ $4.19423 \mathrm{E} \quad 00$ $4.30663 \mathrm{E} \quad 00$ $4.41891 \mathrm{E} \quad 00$

$4.53109 \mathrm{E} \quad 00$ $4.64317 \mathrm{E} \quad 00$ $4.75516 \mathrm{E} \quad 00$ $4.86705 \mathrm{E} \quad 00$ $4.97887 \mathrm{E} \quad 00$

$5.09061 \mathrm{E} \quad 00$ $5.20228 \mathrm{E} \quad 00$ $5.31389 \mathrm{E} \quad 00$ $5.42543 \mathrm{E} \quad 00$ $5.53690 \mathrm{E} \quad 00$

$5.64832 \mathrm{E} \quad 00$ $5.75969 \mathrm{E} \quad 00$ $5.87100 \mathrm{E} \quad 00$ $5.98227 \mathrm{E} \quad 00$ $6.09348 \mathrm{E} \quad 00$

$6.20465 \mathrm{E} \quad 00$ $6.31577 \mathrm{E} \quad 00$ $6.42685 \mathrm{E} \quad 00$ $6.53789 \mathrm{E} \quad 00$ $6.64889 \mathrm{E} \quad 00$

$6.75985 \mathrm{E} \quad 00$ $6.87077 \mathrm{E} \quad 00$ $6.98166 \mathrm{E} \quad 00$ $7.09250 \mathrm{E} \quad 00$ $7.20332 \mathrm{E} \quad 00$ $\tan \delta_{-1}$

$-3.35765 \mathrm{E} \quad 00$

$-5.48576 \mathrm{E} \quad 00$ $-7.85528 \mathrm{E} \quad 00$ $-1.02165 \mathrm{E} \quad 01$ $-1.25270 \mathrm{E} 01$

$-1.47722 \mathrm{E} \quad 01$ $-1.69464 \mathrm{E} \quad 01$ $-1.90472 \mathrm{E} \quad 01$ $-2.10744 \mathrm{E} \quad 01$ $-2.30292 \mathrm{E} \quad 01$

$-2.49134 \mathrm{E} \quad 01$ $-2.67292 \mathrm{E} \quad 01$ $\begin{array}{lll}-2.84793 \mathrm{E} & 01\end{array}$ $-3.01664 \mathrm{E} \quad 01$ $-3.17931 \mathrm{E} 01$

$-3.33622 \mathrm{E} \quad 01$ $-3.48763 \mathrm{E} 01$ $-3.63381 \mathrm{E} 01$ $-3.77499 \mathrm{E} \quad 01$ $-3.91141 \mathrm{E} \quad 01$

$-4.04329 \mathrm{E} 01$ $-4.17086 \mathrm{E} \quad 01$ $-4.29430 \mathrm{E} 01$ $-4.41381 \mathrm{E} \quad 01$ $-4.52958 \mathrm{E} \quad 01$

$-4.64176 \mathrm{E} \quad 01$ $-4.75052 \mathrm{E} \quad 01$ $-4.85602 \mathrm{E} \quad 01$ $-4.95839 \mathrm{E} 01$ $\begin{array}{lll}-5.05777 \mathrm{E} & 01\end{array}$

$-5.15430 \mathrm{E} \quad 01$ $-5.24808 \mathrm{E} 01$ $-5.33924 \mathrm{E} \quad 01$ $-5.42788 \mathrm{E} \quad 01$ $-5.51412 \mathrm{E} \quad 01$

$-5.59804 \mathrm{E} \quad 01$ $-5.67973 \mathrm{E} 01$ $-5.75929 \mathrm{E} \quad 01$ $-5.83680 \mathrm{E} \quad 01$ $-5.91233 \mathrm{E} 01$

$-5.98597 \mathrm{E} \quad 01$ $-6.05779 \mathrm{E} 01$ $-6.12784 \mathrm{E} 01$ $-6.19620 \mathrm{E} 01$ $-6.26293 \mathrm{E} 01$

$-6.32809 \mathrm{E} \quad 01$ $-6.39173 \mathrm{E} \quad 01$ $-6.45391 \mathrm{E} 01$ $-6.51467 \mathrm{E} 01$ -6.5740 ? E 01

$-6.63215 \mathrm{E} \quad 01$ $-6.68896 \mathrm{E} 01$ $-6.74453 \mathrm{E} 01$ $-6.79893 \mathrm{E} 01$ $-6.85216 \mathrm{E} 01$

$-6.90428 \mathrm{E} 01$ $-6.95533 \mathrm{E} 01$ $-7.00533 \mathrm{E} 01$ $-7.05431 \mathrm{E} 01$ $-7.10232 \mathrm{E} 01$ 
ELECTRON RADIAL FUNCTIONS

$Z=9 \quad A=18 \quad \rho=1.2 A^{1 / 3} F$

Electron

$\bar{F}$

$1.13621 \mathrm{E}-07$ $1.99953 \mathrm{E}-07$ $3.34412 \mathrm{E}-07$ $5.11881 \mathrm{E}-07$ $7.32124 \mathrm{E}-07$

$9.95515 \mathrm{E}-07$ $1.30250 \mathrm{E}-06$ $1.65348 \mathrm{E}-06$ $2.04879 \mathrm{E}-06$ $2.48869 \mathrm{E}-06$

$2.97341 \mathrm{E}-06$ $3.50309 \mathrm{E}-06$ $4.07787 \mathrm{E}-06$ $4.69785 \mathrm{E}-06$ $5.36310 \mathrm{E}-06$

$6.07369 \mathrm{E}-06$ $6.82966 \mathrm{E}-06$ $7.63105 \mathrm{E}-06$ $8.47789 \mathrm{E}-06$ $9.37019 \mathrm{E}-06$

$1.03080 \mathrm{E}-05$ $1.12913 \mathrm{E}-05$ $1.23201 \mathrm{E}-05$ $1.33944 \mathrm{E}-05$ $1.45142 \mathrm{E}-05$

$1.56796 \mathrm{E}-05$ $1.68905 \mathrm{E}-05$ $1.81470 \mathrm{E}-05$ $1.94489 \mathrm{E}-0.5$ $2.07965 \mathrm{E}-05$

$2.21895 \mathrm{E}-05$ $2.36281 \mathrm{E}-05$ $2.51123 \mathrm{E}-05$ $2.66419 \mathrm{E}-05$

$2.82171 \mathrm{E}-05$

$2.98379 \mathrm{E}-05$ $3.15042 \mathrm{E}-05$ $3.32160 \mathrm{E}-05$ $3.49733 \mathrm{E}-05$ $3.67762 \mathrm{E}-05$

$3.86246 \mathrm{E}-05$ $4.05185 \mathrm{E}-05$ $4.24580 \mathrm{E}-05$ $4.44430 \mathrm{E}-05$ $4.64735 \mathrm{E}-05$

$4.85495 \mathrm{E}-05$ $5.06711 \mathrm{E}-05$ $5.28381 \mathrm{E}-05$ $5.50508 \mathrm{E}-05$ $5.73088 \mathrm{E}-05$

$5.96125 \mathrm{E}-05$ $6.19616 \mathrm{E}-05$ $6.43562 \mathrm{E}-05$ $6.67964 \mathrm{E}-05$ $6.92820 \mathrm{E}-05$

$7.18132 \mathrm{E}-05$ $7.43899 \mathrm{E}-05$ $7.70120 \mathrm{E}-05$ $7.96796 \mathrm{E}-05$ 8.23928E-05 $f_{2}$

$-4.98688 \mathrm{E}-06$ $-2.52414 \mathrm{E}-05$ $-7.16471 \mathrm{E}-05$ $-1.53529 \mathrm{E}-04$ $-2.78516 \mathrm{E}-04$

$-4.52503 \mathrm{E}-04$ $-6.79833 \mathrm{E}-04$ $-9.63562 \mathrm{E}-04$ $-1.30576 \mathrm{E}-03$ $-1.70779 \mathrm{E}-03$

$-2.17047 \mathrm{E}-03$ $-2.69433 \mathrm{E}-03$ $-3.27962 \mathrm{E}-03$ $-3.92647 \mathrm{E}-03$ $-4.63491 \mathrm{E}-03$

$-5.40491 \mathrm{E}-03$ $-6.23640 \mathrm{E}-03$ $-7.12933 \mathrm{E}-03$ $-8.08358 \mathrm{E}-03$ $-9.09909 \mathrm{E}-03$

$-1.01758 \mathrm{E}-02$ $-1.13135 \mathrm{E}-02$ $-1.25123 \mathrm{E}-02$ $-1.37720 \mathrm{E}-02$ $-1.50926 \mathrm{E}-02$

$-1.64740 \mathrm{E}-02$ -1.79161 E-02 $-1.94189 \mathrm{E}-02$ $-2.09825 \mathrm{E}-02$ $-2.26066 \mathrm{E}-02$

$-2.42913 \mathrm{E}-02$ $-2.60365 \mathrm{E}-02$ $-2.78423 \mathrm{E}-02$ $-2.97085 \mathrm{E}-02$ $-3.16352 \mathrm{E}-02$

$-3.36223 \mathrm{E}-02$ $-3.56698 \mathrm{E}-02$ $-3.77777 \mathrm{E}-02$ $-3.99459 \mathrm{E}-02$ $-4.21745 \mathrm{E}-02$

$-4.44633 \mathrm{E}-02$ $-4.68125 \mathrm{E}-02$ $-4.92219 \mathrm{E}-02$ $-5.16916 \mathrm{E}-02$ $-5.42215 \mathrm{E}-02$

$-5.68117 \mathrm{E}-02$ $-5.94620 \mathrm{E}-02$ $-6.21725 \mathrm{E}-02$ $-6.49433 \mathrm{E}-02$ $-6.77742 \mathrm{E}-02$

$-7.06652 \mathrm{E}-02$ $-7.36164 \mathrm{E}-02$ $-7.66278 \mathrm{E}-02$ $-7.96992 \mathrm{E}-02$ $-8.28307 \mathrm{E}-02$

$-8.60224 \mathrm{E}-02$ $-8.92741 \mathrm{E}-02$ $-9.25858 \mathrm{E}-02$ $-9.59577 \mathrm{E}-02$ $-9.93896 \mathrm{E}-02$ $g_{2}$

$-9.11665 \mathrm{E}-08$ $-4.62055 \mathrm{E}-07$ $-1.31436 \mathrm{E}-06$ $-2.82474 \mathrm{E}-06$ $-5.14294 \mathrm{E}-06$

$-8.39122 \mathrm{E}-06$ $-1.26672 \mathrm{E}-05$ $-1.80480 \mathrm{E}-05$ $-2.45954 \mathrm{E}-05$ $-3.23596 \mathrm{E}-05$

$-4.13829 \mathrm{E}-05$ $-5.17022 \mathrm{E}-05$ $-6.33509 \mathrm{E}-05$ $-7.63600 \mathrm{E}-05$ $-9.07593 \mathrm{E}-05$

$-1.06577 \mathrm{E}-04$ $-1.23843 \mathrm{E}-04$ $-1.42583 \mathrm{E}-04$ $-1.62827 \mathrm{E}-04$ $-1.84602 \mathrm{E}-04$

$-2.07937 \mathrm{E}-04$ $-2.32858 \mathrm{E}-04$ $-2.59396 \mathrm{E}-04$ $-2.87577 \mathrm{E}-04$ $-3.17430 \mathrm{E}-04$

$-3.48984 \mathrm{E}-04$ $-3.82267 \mathrm{E}-04$ $-4.17308 \mathrm{E}-04$ $-4.54135 \mathrm{E}-04$ $-4.92778 \mathrm{E}-04$

$-5.33264 \mathrm{E}-04$

$-5.75624 \mathrm{E}-04$

$-6.19885 \mathrm{E}-04$

$-6.66076 \mathrm{E}-04$

$-7.14227 \mathrm{E}-04$

$-7.64367 \mathrm{E}-04$

$-8.16524 \mathrm{E}-04$

$-8.70727 \mathrm{E}-04$

$-9.27006 \mathrm{E}-04$

$-9.85390 \mathrm{E}-04$

$-1.04591 \mathrm{E}-03$

$-1.10859 \mathrm{E}-03$

$-1.17346 \mathrm{E}-03$

$-1.24055 \mathrm{E}-03$

$-1.30990 \mathrm{E}-03$

$-1.38152 \mathrm{E}-03$ $-1.45545 \mathrm{E}-03$ $-1.53172 \mathrm{E}-03$ $-1.61035 \mathrm{E}-03$ $-1.69138 \mathrm{E}-03$

$-1.77483 \mathrm{E}-03$ $-1.86074 \mathrm{E}-03$ $-1.94913 \mathrm{E}-03$ $-2.04004 \mathrm{E}-03$ $-2.13348 \mathrm{E}-03$

$-2.22950 \mathrm{E}-03$

$-2.32811 \mathrm{E}-03$

$-2.42936 \mathrm{E}-03$

$-2.53326 \mathrm{E}-03$

$-2.63985 \mathrm{E}-03$ $\tan \delta_{2}$

$f_{-2}$

$1.42009 \mathrm{E} \quad 00$

$3.15962 \mathrm{E} \quad 00$

$4.76144 \mathrm{E} \quad 00$

$6.26065 \mathrm{E} \quad 00$

$7.65125 \mathrm{E} \quad 00$

$8.92759 \mathrm{E} \quad 00$

$\begin{array}{lll}1.00887 \mathrm{E} & 01\end{array}$

$1.11381 \mathrm{E} 01$

$1.20822 \mathrm{E} \quad 0$

$1.29294 \mathrm{E} 01$

$1.36888 \mathrm{E} \quad 01$

$1.43694 \mathrm{E} \quad 01$

$1.49801 \mathrm{E}$ 0]

$1.55288 \mathrm{E}$ 0I

$1.60227 \mathrm{E} 0]$

$1.64685 \mathrm{E} \quad 01$

$1.68718 \mathrm{E} \quad 01$

$1.72376 \mathrm{E}$ 0l

$1.75702 \mathrm{E} \quad 01$

$1.78736 \mathrm{E} \quad 01$

$1.81510 \mathrm{E} \quad 01$

$1.84053 \mathrm{E} \quad 01$

$1.86390 \mathrm{E} \quad 01$

$1.88543 \mathrm{E} 01$

$1.90532 \mathrm{E} \quad 01$

$1.92373 \mathrm{E} \quad 01$

$1.94081 \mathrm{E} \quad 01$

$1.95668 \mathrm{E}$ ol

$1.97147 \mathrm{E} \quad 01$

$1.98528 \mathrm{E} \quad 01$

$1.99819 \mathrm{E} \quad 01$

$2.01028 \mathrm{E} \quad 01$

$2.02163 \mathrm{E} \quad 01$

$2.03230 \mathrm{E} \quad 01$

$2.04234 \mathrm{E} 01$

$2.05181 \mathrm{E} 01$

$2.06075 \mathrm{E} \quad 01$

$2.06920 \mathrm{E}$ 01

$2.07720 \mathrm{E} \quad 01$

$2.08479 \mathrm{E} \quad 01$

2.09199 E 01 $2.09883 \mathrm{E} \quad 01$ $2.10534 \mathrm{E} \quad 01$

$2.11154 \mathrm{E} \quad 01$

$2.11745 \mathrm{E}$ 01

$2.12309 \mathrm{E} \quad 01$ $2.12847 \mathrm{E} \quad 01$ $2.13362 \mathrm{E} \quad 01$ $2.13855 \mathrm{E}$ 0l $2.14327 \mathrm{E} \quad 01$

$2.14779 \mathrm{E} \quad 01$ $2.15213 \mathrm{E} 01$ $2.15630 \mathrm{E} \quad 0 \mathrm{I}$

$2.16030 \mathrm{E} \quad 01$

$2.16415 \mathrm{E} 01$

$2.16785 \mathrm{E} \quad 01$ $2.17141 \mathrm{E} 01$ $2.17485 \mathrm{E} \quad 01$ $2.17816 \mathrm{E} \quad 01$ $2.18135 \mathrm{E} \quad 01$

$g_{2}$

$9.51673 \mathrm{E}-0.5$ $2.51577 \mathrm{E}-04$ $4.85209 \mathrm{E}-04$

$7.94380 \mathrm{E}-04$

$1.17699 \mathrm{E}-03$

$1.63083 \mathrm{E}-03$ $2.15375 \mathrm{E}-03$

$2.74388 \mathrm{E}-03$

$3.39961 \mathrm{E}-03$

$4.11967 \mathrm{E}-0.3$

$4.90303 \mathrm{E}-03$

$5.74891 \mathrm{E}-03$

$6.65669 \mathrm{E}-03$

$7.62593 \mathrm{E}-03$

8.65627 E-03

$9.74746 \mathrm{E}-03$ $1.08993 \mathrm{E}-02$

$1.21117 \mathrm{E}-02$

$1.33844 \mathrm{E}-02$

$1.47175 \mathrm{E}-02$

$1.61108 \mathrm{E}-02$

$1.75643 \mathrm{E}-02$

$1.90780 \mathrm{E}-02$

$2.06519 \mathrm{E}-02$

$2.22859 \mathrm{E}-02$

$2.39800 \mathrm{E}-02$ $2.57342 \mathrm{E}-02$ $2.75485 \mathrm{E}-02$ $2.94229 \mathrm{E}-02$

$3.13574 \mathrm{E}-02$

$3.33519 \mathrm{E}-02$

$3.54065 \mathrm{E}-02$

$3.75212 \mathrm{E}-02$

$3.96959 \mathrm{E}-02$

$4.19307 \mathrm{E}-02$

$4.42256 \mathrm{E}-02$ $4.65806 \mathrm{E}-02$ $4.89955 \mathrm{E}-02$ $5.14705 \mathrm{E}-02$

$5.40056 \mathrm{E}-02$

$5.66007 \mathrm{E}-02$ 


\begin{tabular}{|c|c|c|c|c|c|c|c|}
\hline$p$ & $F_{0}$ & & $f_{1}$ & & $g_{1}$ & $\tan \delta_{1}$ & $f_{-1}$ \\
\hline $\begin{array}{l}6.10 \\
6.20 \\
6.30 \\
6.40 \\
6.50\end{array}$ & $\begin{array}{l}1.24884 \mathrm{E} \\
1.24865 \mathrm{E} \\
1.24846 \mathrm{E} \\
1.24829 \mathrm{E} \\
1.24811 \mathrm{E}\end{array}$ & $\begin{array}{l}00 \\
00 \\
00 \\
00 \\
00\end{array}$ & $\begin{array}{l}-6.21670 \mathrm{E} \\
-6.32728 \mathrm{E} \\
-6.43785 \mathrm{E} \\
-6.54840 \mathrm{E} \\
-6.65892 \mathrm{E}\end{array}$ & $\begin{array}{l}00 \\
00 \\
00 \\
00 \\
00\end{array}$ & $\begin{array}{l}-2.84844 \mathrm{E}-01 \\
-2.91613 \mathrm{E}-01 \\
-2.98441 \mathrm{E}-01 \\
-3.05328 \mathrm{E}-01 \\
-3.12275 \mathrm{E}-01\end{array}$ & $\begin{array}{l}3.22674 \mathrm{E}-03 \\
3.31081 \mathrm{E}-03 \\
3.39221 \mathrm{E}-03 \\
3.47108 \mathrm{E}-03 \\
3.54752 \mathrm{E}-03\end{array}$ & $\begin{array}{l}-2.95374 \mathrm{E}-01 \\
-3.01842 \mathrm{E}-01 \\
-3.08370 \mathrm{E}-01 \\
-3.14957 \mathrm{E}-01 \\
-3.21603 \mathrm{E}-01\end{array}$ \\
\hline $\begin{array}{l}6.60 \\
6.70 \\
6.80 \\
6.90 \\
7.00\end{array}$ & $\begin{array}{l}1.24795 \mathrm{E} \\
1.24779 \mathrm{E} \\
1.24763 \mathrm{E} \\
1.24748 \mathrm{E} \\
1.24733 \mathrm{E}\end{array}$ & $\begin{array}{l}00 \\
00 \\
00 \\
00 \\
00\end{array}$ & $\begin{array}{l}-6.76943 \mathrm{E} \\
-6.87992 \mathrm{E} \\
-6.99039 \mathrm{E} \\
-7.10084 \mathrm{E} \\
-7.21127 \mathrm{E}\end{array}$ & $\begin{array}{l}00 \\
00 \\
00 \\
00 \\
00\end{array}$ & $\begin{array}{l}-3.19280 \mathrm{E}-01 \\
-3.26346 \mathrm{E}-01 \\
-3.33470 \mathrm{E}-01 \\
-3.40654 \mathrm{E}-01 \\
-3.47897 \mathrm{E}-01\end{array}$ & $\begin{array}{l}3.62162 \mathrm{E}-03 \\
3.69352 \mathrm{E}-03 \\
3.76330 \mathrm{E}-03 \\
3.83104 \mathrm{E}-03 \\
3.89686 \mathrm{E}-03\end{array}$ & $\begin{array}{l}-3.28308 \mathrm{E}-01 \\
-3.35072 \mathrm{E}-01 \\
-3.41895 \mathrm{E}-01 \\
-3.48778 \mathrm{E}-01 \\
-3.55720 \mathrm{E}-01\end{array}$ \\
\hline $\begin{array}{l}7.10 \\
7.20 \\
7.30 \\
7.40 \\
7.50\end{array}$ & $\begin{array}{l}1.24718 \mathrm{E} \\
1.24705 \mathrm{E} \\
1.24691 \mathrm{E} \\
1.24678 \mathrm{E} \\
1.24665 \mathrm{E}\end{array}$ & $\begin{array}{l}00 \\
00 \\
00 \\
00 \\
00\end{array}$ & $\begin{array}{l}-7.32168 \mathrm{E} \\
-7.43207 \mathrm{E} \\
-7.54243 \mathrm{E} \\
-7.65278 \mathrm{E} \\
-7.76311 \mathrm{E}\end{array}$ & $\begin{array}{l}00 \\
00 \\
00 \\
00 \\
00\end{array}$ & $\begin{array}{l}-3.55199 \mathrm{E}-01 \\
-3.62561 \mathrm{E}-01 \\
-3.69982 \mathrm{E}-01 \\
-3.77462 \mathrm{E}-01 \\
-3.85001 \mathrm{E}-01\end{array}$ & $\begin{array}{l}3.96079 \mathrm{E}-03 \\
4.02294 \mathrm{E}-03 \\
4.08338 \mathrm{E}-03 \\
4.14217 \mathrm{E}-03 \\
4.19937 \mathrm{E}-03\end{array}$ & $\begin{array}{l}-3.62720 \mathrm{E}-01 \\
-3.69780 \mathrm{E}-01 \\
-3.76899 \mathrm{E}-01 \\
-3.84077 \mathrm{E}-01 \\
-3.91315 \mathrm{E}-01\end{array}$ \\
\hline $\begin{array}{l}7.60 \\
7.70 \\
7.80 \\
7.90 \\
8.00\end{array}$ & $\begin{array}{l}1.24652 \mathrm{E} \\
1.24640 \mathrm{E} \\
1.24628 \mathrm{E} \\
1.24616 \mathrm{E} \\
1.24604 \mathrm{E}\end{array}$ & $\begin{array}{l}00 \\
00 \\
00 \\
00 \\
00\end{array}$ & $\begin{array}{l}-7.87342 \mathrm{E} \\
-7.98371 \mathrm{E} \\
-8.09398 \mathrm{E} \\
-8.20422 \mathrm{E} \\
-8.31445 \mathrm{E}\end{array}$ & $\begin{array}{l}00 \\
00 \\
00 \\
00 \\
00\end{array}$ & $\begin{array}{l}-3.92600 \mathrm{E}-01 \\
-4.00258 \mathrm{E}-01 \\
-4.07975 \mathrm{E}-01 \\
-4.15751 \mathrm{E}-01 \\
-4.23586 \mathrm{E}-01\end{array}$ & $\begin{array}{l}4.25507 \mathrm{E}-03 \\
4.30930 \mathrm{E}-03 \\
4.36210 \mathrm{E}-03 \\
4.41357 \mathrm{E}-03 \\
4.46373 \mathrm{E}-03\end{array}$ & $\begin{array}{l}-3.98611 \mathrm{E}-01 \\
-4.05967 \mathrm{E}-01 \\
-4.13381 \mathrm{E}-01 \\
-4.20855 \mathrm{E}-01 \\
-4.28387 \mathrm{E}-01\end{array}$ \\
\hline $\begin{array}{l}8.10 \\
8.20 \\
8.30 \\
8.40 \\
8.50\end{array}$ & $\begin{array}{l}1.24593 \mathrm{E} \\
1.24582 \mathrm{E} \\
1.24571 \mathrm{E} \\
1.24561 \mathrm{E} \\
1.24550 \mathrm{E}\end{array}$ & $\begin{array}{l}00 \\
00 \\
00 \\
00 \\
00\end{array}$ & $\begin{array}{l}-8.42465 \mathrm{E} \\
-8.53484 \mathrm{E} \\
-8.64501 \mathrm{E} \\
-8.75515 \mathrm{E} \\
-8.86527 \mathrm{E}\end{array}$ & $\begin{array}{l}00 \\
00 \\
00 \\
00 \\
00\end{array}$ & $\begin{array}{l}-4.31480 \mathrm{E}-01 \\
-4.39434 \mathrm{E}-01 \\
-4.47446 \mathrm{E}-01 \\
-4.55518 \mathrm{E}-01 \\
-4.63649 \mathrm{E}-01\end{array}$ & $\begin{array}{l}4.51263 \mathrm{E}-03 \\
4.56032 \mathrm{E}-03 \\
4.60684 \mathrm{E}-03 \\
4.65223 \mathrm{E}-03 \\
4.69654 \mathrm{E}-03\end{array}$ & $\begin{array}{l}-4.35979 \mathrm{E}-01 \\
-4.43630 \mathrm{E}-01 \\
-4.51340 \mathrm{E}-01 \\
-4.59109 \mathrm{E}-01 \\
-4.66937 \mathrm{E}-01\end{array}$ \\
\hline $\begin{array}{l}8.60 \\
8.70 \\
8.80 \\
8.90 \\
9.00\end{array}$ & $\begin{array}{l}1.24540 \mathrm{E} \\
1.24531 \mathrm{E} \\
1.24521 \mathrm{E} \\
1.24511 \mathrm{E} \\
1.24502 \mathrm{E}\end{array}$ & $\begin{array}{l}00 \\
00 \\
00 \\
00 \\
00\end{array}$ & $\begin{array}{l}-8.97538 \mathrm{E} \\
-9.08546 \mathrm{E} \\
-9.19552 \mathrm{E} \\
-9.30556 \mathrm{E} \\
-9.41558 \mathrm{E}\end{array}$ & $\begin{array}{l}00 \\
00 \\
00 \\
00 \\
00\end{array}$ & $\begin{array}{l}-4.71839 E-01 \\
-4.80088 E-01 \\
-4.88396 E-01 \\
-4.96763 E-01 \\
-5.05189 E-01\end{array}$ & $\begin{array}{l}4.73979 \mathrm{E}-03 \\
4.78203 \mathrm{E}-03 \\
4.82329 \mathrm{E}-03 \\
4.86359 \mathrm{E}-03 \\
4.90297 \mathrm{E}-03\end{array}$ & $\begin{array}{l}-4.74824 \mathrm{E}-01 \\
-4.82770 \mathrm{E}-01 \\
-4.90775 \mathrm{E}-01 \\
-4.98839 \mathrm{E}-01 \\
-5.06962 \mathrm{E}-01\end{array}$ \\
\hline $\begin{array}{l}9.10 \\
9.20 \\
9.30 \\
9.40 \\
9.50\end{array}$ & $\begin{array}{l}1.24493 \mathrm{E} \\
1.24484 \mathrm{E} \\
1.24475 \mathrm{E} \\
1.24466 \mathrm{E} \\
1.24458 \mathrm{E}\end{array}$ & $\begin{array}{l}00 \\
00 \\
00 \\
00 \\
00\end{array}$ & $\begin{array}{l}-9.52558 \mathrm{E} \\
-9.63556 \mathrm{E} \\
-9.74551 \mathrm{E} \\
-9.85545 \mathrm{E} \\
-9.96536 \mathrm{E}\end{array}$ & $\begin{array}{l}00 \\
00 \\
00 \\
00 \\
00\end{array}$ & $\begin{array}{l}-5.13674 \mathrm{E}-01 \\
-5.22218 \mathrm{E}-01 \\
-5.30821 \mathrm{E}-01 \\
-5.39483 \mathrm{E}-01 \\
-5.48204 \mathrm{E}-01\end{array}$ & $\begin{array}{l}4.94148 \mathrm{E}-03 \\
4.97911 \mathrm{E}-03 \\
5.01592 \mathrm{E}-03 \\
5.05192 \mathrm{E}-03 \\
5.08713 \mathrm{E}-03\end{array}$ & $\begin{array}{l}-5.15144 \mathrm{E}-01 \\
-5.23385 \mathrm{E}-01 \\
-5.31685 \mathrm{E}-01 \\
-5.40044 \mathrm{E}-01 \\
-5.48462 \mathrm{E}-01\end{array}$ \\
\hline $\begin{array}{r}9.60 \\
9.70 \\
9.80 \\
9.90 \\
10.00\end{array}$ & $\begin{array}{l}1.24449 \mathrm{E} \\
1.24441 \mathrm{E} \\
1.24433 \mathrm{E} \\
1.24425 \mathrm{E} \\
1.24417 \mathrm{E}\end{array}$ & $\begin{array}{l}00 \\
00 \\
00 \\
00 \\
00\end{array}$ & $\begin{array}{l}-1.00753 \mathrm{E} \\
-1.01851 \mathrm{E} \\
-1.02950 \mathrm{E} \\
-1.04048 \mathrm{E} \\
-1.05146 \mathrm{E}\end{array}$ & $\begin{array}{l}01 \\
01 \\
01 \\
01 \\
01\end{array}$ & $\begin{array}{l}-5.56984 \mathrm{E}-01 \\
-5.65823 \mathrm{E}-01 \\
-5.74720 \mathrm{E}-01 \\
-5.83677 \mathrm{E}-01 \\
-5.92693 \mathrm{E}-01\end{array}$ & $\begin{array}{l}5.12159 \mathrm{E}-03 \\
5.15530 \mathrm{E}-03 \\
5.18832 \mathrm{E}-03 \\
5.22064 \mathrm{E}-03 \\
5.25228 \mathrm{E}-03\end{array}$ & $\begin{array}{l}-5.56939 \mathrm{E}-01 \\
-5.65474 \mathrm{E}-01 \\
-5.74069 \mathrm{E}-01 \\
-5.82722 \mathrm{E}-01 \\
-5.91435 \mathrm{E}-01\end{array}$ \\
\hline $\begin{array}{l}0.10 \\
0.20 \\
0.30 \\
0.40 \\
0.50\end{array}$ & $\begin{array}{l}1.24410 \mathrm{E} \\
1.24402 \mathrm{E} \\
1.24395 \mathrm{E} \\
1.24387 \mathrm{E} \\
1.24380 \mathrm{E}\end{array}$ & $\begin{array}{l}00 \\
00 \\
00 \\
00 \\
00\end{array}$ & $\begin{array}{l}-1.06244 \mathrm{E} \\
-1.07342 \mathrm{E} \\
-1.08439 \mathrm{E} \\
-1.09536 \mathrm{E} \\
-1.10633 \mathrm{E}\end{array}$ & $\begin{array}{l}01 \\
01 \\
01 \\
01 \\
01\end{array}$ & $\begin{array}{l}-6.01767 \mathrm{E}-01 \\
-6.10900 \mathrm{E}-01 \\
-6.20093 \mathrm{E}-01 \\
-6.29343 \mathrm{E}-01 \\
-6.38654 \mathrm{E}-01\end{array}$ & $\begin{array}{l}5.28327 \mathrm{E}-03 \\
5.31363 \mathrm{E}-03 \\
5.34337 \mathrm{E}-03 \\
5.37251 \mathrm{E}-03 \\
5.40107 \mathrm{E}-03\end{array}$ & $\begin{array}{l}-6.00206 \mathrm{E}-01 \\
-6.09036 \mathrm{E}-01 \\
-6.17925 \mathrm{E}-01 \\
-6.26873 \mathrm{E}-01 \\
-6.35880 \mathrm{E}-01\end{array}$ \\
\hline $\begin{array}{l}0.60 \\
0.70 \\
0.80 \\
0.90 \\
1.00\end{array}$ & $\begin{array}{l}1.24373 \mathrm{E} \\
1.24366 \mathrm{E} \\
1.24359 \mathrm{E} \\
1.24352 \mathrm{E} \\
1.24346 \mathrm{E}\end{array}$ & $\begin{array}{l}00 \\
00 \\
00 \\
00 \\
00\end{array}$ & $\begin{array}{l}-1.11730 \mathrm{E} \\
-1.12827 \mathrm{E} \\
-1.13923 \mathrm{E} \\
-1.15019 \mathrm{E} \\
-1.16115 \mathrm{E}\end{array}$ & $\begin{array}{l}01 \\
01 \\
01 \\
01 \\
01\end{array}$ & $\begin{array}{l}-6.48022 \mathrm{E}-01 \\
-6.57449 \mathrm{E}-01 \\
-6.66936 \mathrm{E}-01 \\
-6.76481 \mathrm{E}-01 \\
-6.86084 \mathrm{E}-01\end{array}$ & $\begin{array}{l}5.42908 \mathrm{E}-03 \\
5.45652 \mathrm{E}-03 \\
5.48342 \mathrm{E}-03 \\
5.50982 \mathrm{E}-03 \\
5.53571 \mathrm{E}-03\end{array}$ & $\begin{array}{l}-6.44945 \mathrm{E}-01 \\
-6.54069 \mathrm{E}-01 \\
-6.63252 \mathrm{E}-01 \\
-6.72494 \mathrm{E}-01 \\
-6.81795 \mathrm{E}-01\end{array}$ \\
\hline $\begin{array}{l}1.10 \\
1.20 \\
1.30 \\
1.40 \\
1.50\end{array}$ & $\begin{array}{l}1.24339 \mathrm{E} \\
1.24333 \mathrm{E} \\
1.24326 \mathrm{E} \\
1.24320 \mathrm{E} \\
1.24313 \mathrm{E}\end{array}$ & $\begin{array}{l}00 \\
00 \\
00 \\
00 \\
00\end{array}$ & $\begin{array}{l}-1.17211 \mathrm{E} \\
-1.18306 \mathrm{E} \\
-1.19402 \mathrm{E} \\
-1.20497 \mathrm{E} \\
-1.21591 \mathrm{E}\end{array}$ & $\begin{array}{l}01 \\
01 \\
01 \\
01 \\
01\end{array}$ & $\begin{array}{l}-6.95747 \mathrm{E}-01 \\
-7.05468 \mathrm{E}-01 \\
-7.15248 \mathrm{E}-01 \\
-7.25086 \mathrm{E}-01 \\
-7.34984 \mathrm{E}-01\end{array}$ & $\begin{array}{l}5.56109 \mathrm{E}-03 \\
5.58600 \mathrm{E}-03 \\
5.61044 \mathrm{E}-03 \\
5.63442 \mathrm{E}-03 \\
5.65796 \mathrm{E}-03\end{array}$ & $\begin{array}{l}-6.91154 \mathrm{E}-01 \\
-7.00572 \mathrm{E}-01 \\
-7.10049 \mathrm{E}-01 \\
-7.19584 \mathrm{E}-01 \\
-7.29178 \mathrm{E}-01\end{array}$ \\
\hline $\begin{array}{l}.60 \\
.70 \\
.80 \\
.90\end{array}$ & $\begin{array}{l}1.24307 \mathrm{E} \\
1.24301 \mathrm{E} \\
1.24295 \mathrm{E} \\
1.24289 \mathrm{E} \\
1.24283 \mathrm{E}\end{array}$ & $\begin{array}{l}00 \\
00 \\
00 \\
00 \\
00\end{array}$ & $\begin{array}{l}-1.22686 \mathrm{E} \\
-1.23780 \mathrm{E} \\
-1.24875 \mathrm{E} \\
-1.25969 \mathrm{E} \\
-1.27062 \mathrm{E}\end{array}$ & $\begin{array}{l}01 \\
01 \\
01 \\
01 \\
01\end{array}$ & $\begin{array}{l}-7.44939 \mathrm{E}-01 \\
-7.54954 \mathrm{E}-01 \\
-7.65027 \mathrm{E}-01 \\
-7.75159 \mathrm{E}-01 \\
-7.85350 \mathrm{E}-01\end{array}$ & $\begin{array}{l}5.68107 \mathrm{E}-03 \\
5.70375 \mathrm{E}-03 \\
5.72602 \mathrm{E}-03 \\
5.74790 \mathrm{E}-03 \\
5.76936 \mathrm{E}-03\end{array}$ & $\begin{array}{l}-7.38831 \mathrm{E}-01 \\
-7.48542 \mathrm{E}-01 \\
-7.58312 \mathrm{E}-01 \\
-7.68141 \mathrm{E}-01 \\
-7.78028 \mathrm{E}-01\end{array}$ \\
\hline
\end{tabular}

$$
g-1
$$$$
\tan \delta_{-1}
$$

$7.31410 \mathrm{E} \quad 00$ $7.42484 \mathrm{E} \quad 00$ $7.53556 \mathrm{E} \quad 00$

$7.64624 \mathrm{E} \quad 00$ $7.75689 \mathrm{E} \quad 00$

$7.86751 \mathrm{E} \quad 00$ $7.97810 \mathrm{E} \quad 00$ $8.08866 \mathrm{E} \quad 00$ $8.19919 \mathrm{E} \quad 00$ $8.30969 \mathrm{E} \quad 00$

$8.42016 \mathrm{E} \quad 00$ $8.53061 \mathrm{E} \quad 00$ $8.64103 \mathrm{E} \quad 00$ $8.75142 \mathrm{E} \quad 00$ $8.86178 \mathrm{E} \quad 00$ $-7.14938 \mathrm{E} \quad 01$ $-7.19552 \mathrm{E} 01$ $-7.24077 \mathrm{E} 01$ $-7.28514 \mathrm{E} 01$ $-7.32868 \mathrm{E} 01$

$8.97212 \mathrm{E} \quad 00$ $9.08243 \mathrm{E} \quad 00$ $9.19271 \mathrm{E} \quad 00$ $9.30297 \mathrm{E} \quad 00$ $9.41320 \mathrm{E} \quad 00$

$9.52340 \mathrm{E} \quad 00$ $9.63359 \mathrm{E} \quad 00$ $9.74374 \mathrm{E} \quad 00$ $9.85387 \mathrm{E} \quad 00$ $9.96398 \mathrm{E} \quad 00$

$1.00741 \mathrm{E} \quad 01$ $1.01841 \mathrm{E} \quad 01$ $1.02941 \mathrm{E} 01$ $1.04042 \mathrm{E} \quad 01$

$1.05141 \mathrm{E} \quad 01$

$1.06241 \mathrm{E} \quad 01$ $1.07340 \mathrm{E} 01$ $1.08439 \mathrm{E} 01$ $1.09538 \mathrm{E} 01$ $1.10637 \mathrm{E} 01$

$1.11735 \mathrm{E} \quad 01$ $1.12833 \mathrm{E}$ 01 $1.13931 \mathrm{E} 01$ $1.15029 \mathrm{E} \quad 01$ $1.16127 \mathrm{E} \quad 01$

$1.17224 \mathrm{E} \quad 01$ $1.18321 \mathrm{E} \quad 01$ $1.19417 \mathrm{E} 01$

$1.20514 \mathrm{E} 01$

$\begin{array}{ll}1.21610 \mathrm{E} & 01\end{array}$

$1.22706 \mathrm{E} \quad 01$ $1.23802 \mathrm{E} 01$ $1.24898 \mathrm{E} \quad 01$ $1.25993 \mathrm{E} 01$ $1.27088 \mathrm{E} \quad 01$

$1.28183 \mathrm{E} \quad 01$ $1.29278 \mathrm{E} 01$ $1.30372 \mathrm{E} \quad 01$ $1.31466 \mathrm{E} \quad 01$ $1.32560 \mathrm{E} 01$

$1.33654 \mathrm{E} \quad 01$ $1.34748 \mathrm{E} \quad 01$ $1.35841 \mathrm{E} 01$ $1.36934 \mathrm{E} \quad 01$ $1.38027 \mathrm{E} \quad 01$
$-7.37141 \mathrm{E} 01$ $-7.41334 \mathrm{E} 01$ $-7.45450 \mathrm{E} 01$ $-7.49491 \mathrm{E} 01$ $-7.53459 \mathrm{E} 01$

$-7.57358 \mathrm{E} \quad 01$ $-7.61188 \mathrm{E} \quad 01$ $-7.64951 \mathrm{E} 01$ $-7.68649 \mathrm{E} 01$ $-7.72284 \mathrm{E} 01$ $-7.79371 \mathrm{E} \quad 01$ $-7.82827 \mathrm{E} 01$ $-7.86226 \mathrm{E} 01$ $-7.89570 \mathrm{E} 01$

$-7.92860 \mathrm{E} 01$ $-7.96099 \mathrm{E} 01$ $-7.99285 \mathrm{E} 01$ $-8.02423 \mathrm{E} 01$ $-8.05512 \mathrm{E} 01$

$-8.08553 \mathrm{E} 01$ $-8.11549 \mathrm{E} 01$ $-8.14499 \mathrm{E} 01$ $-8.17406 \mathrm{E} 01$ $-8.20270 \mathrm{E} 01$

$-8.23092 \mathrm{E} 01$ $-8.25873 \mathrm{E} 01$ $-8.28615 \mathrm{E} 01$ $-8.31318 \mathrm{E} 01$ $-8.33983 \mathrm{E} 01$

$-8.36611 \mathrm{E} 01$ $-8.39203 \mathrm{E} 01$ $-8.41760 \mathrm{E} 01$ $-8.44281 \mathrm{E} 01$ $-8.46770 \mathrm{E} 01$

$-8.49224 \mathrm{E}$ ol $-8.51648 \mathrm{E} \quad 01$ $-8.54039 \mathrm{E} 01$ $-8.56400 \mathrm{E} \quad 01$ $-8.58730 \mathrm{E} 01$

$-8.61031 \mathrm{E} 01$ $-8.63303 \mathrm{E} 01$ $-8.65548 \mathrm{E} 01$ $-8.67764 \mathrm{E} \quad 01$ $-8.69952 \mathrm{E} 01$

$-8.72116 \mathrm{E} \quad 01$ $-8.74253 \mathrm{E} 01$ $-8.76365 \mathrm{E} \quad 01$ $-8.78451 \mathrm{E} \quad 01$ $-8.80514 \mathrm{E} 01$

$-8.82552 \mathrm{E} \quad 01$ $-8.84568 \mathrm{E} 01$ $-8.86560 \mathrm{E} \quad 01$ $-8.88530 \mathrm{E} \quad 01$ $-8.90479 \mathrm{E} \quad 01$
$-7.75857 \mathrm{E} 01$ 
$Z=9 \quad A=18 \quad \rho=1.2 A^{1 / 3} F$

$f_{2}$

$8.51515 \mathrm{E}-05$

$8.79557 \mathrm{E}-05$

$9.08053 \mathrm{E}-05$

$9.37005 \mathrm{E}-05$

$9.66412 \mathrm{E}-05$

$9.96272 \mathrm{E}-05$

$1.02659 \mathrm{E}-04$

$1.05736 \mathrm{E}-04$

$1.08859 \mathrm{E}-04$

$1.12027 \mathrm{E}-04$

$1.15240 \mathrm{E}-04$

$1.18499 \mathrm{E}-04$

$1.21804 \mathrm{E}-04$

$1.25154 \mathrm{E}-04$

$1.28549 \mathrm{E}-04$

$1.31990 \mathrm{E}-04$

$1.35477 \mathrm{E}-04$

$1.39009 \mathrm{E}-04$

$1.42586 \mathrm{E}-04$

$1.46209 \mathrm{E}-04$

$1.49877 \mathrm{E}-04$

$1.53591 \mathrm{E}-04$

$1.57350 \mathrm{E}-04$

$1.61155 \mathrm{E}-04$

$1.65005 \mathrm{E}-04$

$1.68901 \mathrm{E}-04$ $1.72842 \mathrm{E}-04$

$1.76828 \mathrm{E}-04$

$1.80860 \mathrm{E}-04$

$1.84938 \mathrm{E}-04$

$1.89061 \mathrm{E}-04$

$1.93229 \mathrm{E}-04$

$1.97443 \mathrm{E}-04$

$2.01703 \mathrm{E}-04$

$2.06007 \mathrm{E}-04$

$2.10358 \mathrm{E}-04$

$2.14753 \mathrm{E}-04$

$2.19195 \mathrm{E}-04$

2.23681 E- 04

$2.28213 \mathrm{E}-04$

$2.32791 \mathrm{E}-04$

$2.37414 \mathrm{E}-04$

$2.42082 \mathrm{E}-04$

$2.46796 \mathrm{E}-04$

$2.51555 \mathrm{E}-04$

$2.56360 \mathrm{E}-04$

$2.61210 \mathrm{E}-04$

$2.66106 \mathrm{E}-04$

$2.71047 \mathrm{E}-04$

$2.76033 \mathrm{E}-04$

$2.81065 \mathrm{E}-04$

$2.86143 \mathrm{E}-04$

$2.91265 \mathrm{E}-04$

$2.96434 \mathrm{E}-04$

$3.01647 \mathrm{E}-04$

$3.06906 \mathrm{E}-04$ 3.12211 E-04 3.17561 E-04 $3.22956 \mathrm{E}-04$ $3.28397 \mathrm{E}-04$ $g_{2}$

$\tan \delta_{2}$

$f_{-2}$

$-1.02882 \mathrm{E}-01 \quad-2.74916 \mathrm{E}-03$

$-1.06434 \mathrm{E}-01$

$-1.10045 \mathrm{E}-01$

$-1.13717 \mathrm{E}-01$

$-1.17449 \mathrm{E}-01$

$-1.21241 \mathrm{E}-01$

$-1.25093 \mathrm{E}-01$

$-1.29005 \mathrm{E}-01$

$-1.32977 \mathrm{E}-01$

$-1.37009 \mathrm{E}-01$

$-1.41100 \mathrm{E}-01$

$-1.45252 \mathrm{E}-01$

$-1.49464 \mathrm{E}-01$

$-1.53735 \mathrm{E}-01$

$-1.58066 \mathrm{E}-01$

$-1.62458 \mathrm{E}-01$

$-1.66909 \mathrm{E}-01$

$-1.71420 \mathrm{E}-01$

$-1.75990 \mathrm{E}-01$

$-1.80621 \mathrm{E}-01$

$-1.85312 \mathrm{E}-01$

$-1.90062 \mathrm{E}-01$

$-1.94872 \mathrm{E}-01$

$-1.99742 \mathrm{E}-01$

$-2.04672 \mathrm{E}-01$

$-2.09661 \mathrm{E}-01$

$-2.14710 \mathrm{E}-01$

$-2.19819 \mathrm{E}-01$

$-2.24988 \mathrm{E}-01$

$-2.30217 \mathrm{E}-01$

$-2.35505 \mathrm{E}-01$

$-2.40853 \mathrm{E}-01$

$-2.46261 \mathrm{E}-01$

$-2.51728 \mathrm{E}-01$

$-2.57255 \mathrm{E}-01$

$-2.62842 \mathrm{E}-01$

$-2.68489 \mathrm{E}-01$

$-2.74195 \mathrm{E}-01$

$-2.79961 \mathrm{E}-01$

$-2.85786 \mathrm{E}-01$

$-2.91671 \mathrm{E}-01$

$-2.97616 \mathrm{E}-01$

$-3.03620 \mathrm{E}-01$

$-3.09684 \mathrm{E}-01$

$-3.15808 \mathrm{E}-01$

$-3.21991 \mathrm{E}-01$

$-3.28233 \mathrm{E}-01$

$-3.34536 \mathrm{E}-01$

$-3.40898 \mathrm{E}-01$

$-3.47319 \mathrm{E}-01$

$-3.53800 \mathrm{E}-01$

$-3.60340 \mathrm{E}-01$

$-3.66940 \mathrm{E}-01$

$-3.73599 \mathrm{E}-01$

$-3.80318 \mathrm{E}-01$

$-3.87097 \mathrm{E}-01$

$-3.93935 \mathrm{E}-01$

$-4.00832 \mathrm{E}-01$

$-4.07789 \mathrm{E}-01$

$-4.14805 \mathrm{E}-01$
$-2.86121 \mathrm{E}-03$

$-2.97603 \mathrm{E}-03$

$-3.09366 \mathrm{E}-03$

$-3.33744 \mathrm{E}-03$

$-3.46365 \mathrm{E}-03$

$-3.59279 \mathrm{E}-03$

$-3.72487 \mathrm{E}-03$

$-3.85993 \mathrm{E}-03$

$-3.99800 \mathrm{E}-03$

$-4.13910 \mathrm{E}-03$

$-4.28327 \mathrm{E}-03$

$-4.43053 \mathrm{E}-03$

$-4.58092 \mathrm{E}-03$

$-4.73446 \mathrm{E}-03$

$-4.89118 \mathrm{E}-03$

$-5.05111 \mathrm{E}-03$

$-5.21429 \mathrm{E}-03$

$-5.38073 \mathrm{E}-03$

$-5.55047 \mathrm{E}-03$

$-5.72353 \mathrm{E}-03$

$-5.89996 \mathrm{E}-03$

$-6.07976 \mathrm{E}-03$

$-6.26298 \mathrm{E}-03$

$-6.44964 \mathrm{E}-03$

$-6.63978 \mathrm{E}-03$

$-6.83342 \mathrm{E}-03$

$-7.03059 \mathrm{E}-03$

$-7.23131 \mathrm{E}-03$

$-7.43563 \mathrm{E}-03$

$-7.64356 \mathrm{E}-03$

$-7.85514 \mathrm{E}-03$

$-8.07040 \mathrm{E}-03$

$-8.28935 \mathrm{E}-03$

$-8.51205 \mathrm{E}-03$

$-8.73850 \mathrm{E}-03$

$-8.96874 \mathrm{E}-03$

$-9.20281 \mathrm{E}-03$

$-9.44072 \mathrm{E}-03$

$-9.68251 \mathrm{E}-03$ $-9.92821 \mathrm{E}-03$

$-1.01778 \mathrm{E}-02$

$-1.04314 \mathrm{E}-02$

$-1.06890 \mathrm{E}-02$

$-1.09506 \mathrm{E}-02$

$-1.12163 \mathrm{E}-02$

$-1.14861 \mathrm{E}-02$

$-1.17599 \mathrm{E}-02$

$-1.20379 \mathrm{E}-02$

$-1.23201 \mathrm{E}-02$

$-1.26064 \mathrm{E}-02$

$-1.28970 \mathrm{E}-02$

$-1.31918 \mathrm{E}-02$

$-1.34910 \mathrm{E}-02$

$-1.37944 \mathrm{E}-02$

$-1.41022 \mathrm{E}-02$

$-1.44143 \mathrm{E}-02$

$-1.47308 \mathrm{E}-02$

$-1.50518 \mathrm{E}-02$
$-3.21412 \mathrm{E}-03$
$2.18443 \mathrm{E} \quad 01$

$2.18741 \mathrm{E} 01$

$2.19029 \mathrm{E} 01$

$2.19307 \mathrm{E} 01$

$2.19576 \mathrm{E} 01$

$2.19836 \mathrm{E} \quad 01$

$2.20088 \mathrm{E} \quad 01$

$2.20332 \mathrm{E} 01$

$2.20569 \mathrm{E} \quad 01$

$2.20798 \mathrm{E} 01$

$2.21021 \mathrm{E} \quad 01$

$2.21237 \mathrm{E} \quad 01$

$2.21447 \mathrm{E} \quad 01$

$2.21651 \mathrm{E} \quad 01$

$2.21849 \mathrm{E} \quad 01$

$2.22041 \mathrm{E} \quad 01$

$2.22228 \mathrm{E} 01$

$2.22410 \mathrm{E} 01$

$2.22587 \mathrm{E}$ 01

$2.22760 \mathrm{E} \quad 01$

$2.22928 \mathrm{E} \quad 01$

$2.23091 \mathrm{E}$ 0l

$2.23251 \mathrm{E} 01$

$2.23406 \mathrm{E} 01$

$2.23557 \mathrm{E} 01$

$2.23705 \mathrm{E} \quad 01$

$2.23849 \mathrm{E}$ 0l

$2.23990 \mathrm{E} 01$

$2.24127 \mathrm{E} \quad 01$

$2.24261 \mathrm{E} \quad 01$

$2.24392 \mathrm{E} 01$

$2.24520 \mathrm{E} 01$

$2.24645 \mathrm{E} \quad 01$

$2.24767 \mathrm{E} \quad 01$

$2.24887 \mathrm{E} \quad 01$

$2.25004 \mathrm{E} \quad 01$

$2.25118 \mathrm{E} \quad 01$

$2.25230 \mathrm{E}$ 0I

$2.25339 \mathrm{E} \quad 01$

$2.25551 \mathrm{E} \quad 01$ 2.25654. E 01

$2.25755 \mathrm{E} 01$

$2.25853 \mathrm{E} 01$

$2.25950 \mathrm{E}$ 01

$2.26045 \mathrm{E} \quad 01$

$2.26137 \mathrm{E} \quad 01$

$2.26228 \mathrm{E} 01$

$2.26318 \mathrm{E} \quad 01$

$2.26405 \mathrm{E} 01$

$2.26491 \mathrm{E} 01$

$2.26575 \mathrm{E} \quad 01$

$2.26658 \mathrm{E}$ ol

$2.26739 \mathrm{E}$ ol

$2.26819 \mathrm{E}$ 0l

$2.26897 \mathrm{E} \quad 01$

$2.26974 \mathrm{E} \quad 01$

$2.27049 \mathrm{E}$ ol

$2.27123 \mathrm{E} \quad 01$

$2.27196 \mathrm{E} \quad 01$
$2.25447 \mathrm{E}$ ol

$-2.84138 \mathrm{E}-03$

$-2.95194 \mathrm{E}-03$

$-3.06517 \mathrm{E}-03$

$-3.18110 \mathrm{E}-03$

$-3.29977 \mathrm{E}-03$

$-3.42120 \mathrm{E}-03$

$-3.54543 \mathrm{E}-03$

$-3.67247 \mathrm{E}-03$

$-3.80237 \mathrm{E}-03$

$-3.93514 \mathrm{E}-03$

$-4.07082 \mathrm{E}-03$

$-4.20944 \mathrm{E}-03$

$-4.35102 \mathrm{E}-03$

$-4.49560 \mathrm{E}-03$

$-4.64321 \mathrm{E}-03$

$-4.79386 \mathrm{E}-03$

$-4.94761 \mathrm{E}-03$

$-5.10446 \mathrm{E}-03$

$-5.26445 \mathrm{E}-03$

$-5.42762 \mathrm{E}-03$

$-5.59398 \mathrm{E}-03$

$-5.76357 \mathrm{E}-03$

$-5.93643 \mathrm{E}-03$

$-6.11256 \mathrm{E}-03$

$-6.29201 \mathrm{E}-03$

$-6.47481 \mathrm{E}-03$

$-6.66098 \mathrm{E}-03$

$-6.85055 \mathrm{E}-03$

$-7.04355 \mathrm{E}-03$

$-7.24002 \mathrm{E}-03$

$-7.43997 \mathrm{E}-03$

$-7.64345 \mathrm{E}-03$

$-7.85047 \mathrm{E}-03$

$-8.06106 \mathrm{E}-03$

$-8.27527 \mathrm{E}-03$

$-8.49311 \mathrm{E}-03$

$-8.71461 \mathrm{E}-03$

$-8.93980 \mathrm{E}-03$

$-9.16872 \mathrm{E}-03$

$-9.40139 \mathrm{E}-03$

$-9.63783 \mathrm{E}-03$ $-9.87809 \mathrm{E}-03$

$-1.01222 \mathrm{E}-02$

$-1.03701 \mathrm{E}-02$ 


\begin{tabular}{|c|c|c|c|c|c|}
\hline$p$ & $F_{0}$ & & $f_{1}$ & & $g_{1}$ \\
\hline 12.10 & $1.24277 \mathrm{E}$ & 00 & $-1.28156 \mathrm{E}$ & 01 & $-7.95599 E-0$ \\
\hline 12.20 & $1.24272 \mathrm{E}$ & 00 & $-1.29249 \mathrm{E}$ & 01 & $-8.05907 \mathrm{E}-0$ \\
\hline 2.30 & $1.24266 \mathrm{E}$ & 00 & $-1.30342 \mathrm{E}$ & 01 & $-8.16273 E$ \\
\hline 2.40 & $1.24260 \mathrm{E}$ & 00 & $-1.31435 \mathrm{E}$ & 01 & $-8.26698 \mathrm{E}$ \\
\hline 2.50 & $1.24255 \mathrm{E}$ & 00 & $-1.32528 \mathrm{E}$ & 01 & $-8.37181 \mathrm{E}-$ \\
\hline 2.60 & $1.24249 \mathrm{E}$ & 00 & $-1.33620 \mathrm{E}$ & 01 & $-8.47723 \mathrm{E}-0$ \\
\hline 2.70 & $.24244 \mathrm{E}$ & 00 & $-1.34712 \mathrm{E}$ & 01 & $-8.58323 E-0$ \\
\hline .80 & $.24239 \mathrm{E}$ & 00 & $-1.35804 \mathrm{E}$ & 01 & $-8.68982 E-$ \\
\hline .90 & $.24233 \mathrm{E}$ & 00 & $-1.36896 \mathrm{E}$ & 01 & $-8.79699 \mathrm{E}$ \\
\hline 13.00 & $1.24228 \mathrm{E}$ & 00 & $-1.37987 \mathrm{E}$ & 01 & $-8.90475 \mathrm{E}-0$ \\
\hline 3.10 & $1.24223 \mathrm{E}$ & 00 & $-1.39078 \mathrm{E}$ & 01 & $-9.01309 \mathrm{E}-$ \\
\hline .20 & $.24218 \mathrm{E}$ & 00 & $-1.40169 \mathrm{E}$ & 01 & $-9.12202 \mathrm{E}-$ \\
\hline 30 & $.24213 \mathrm{E}$ & 00 & $-1.41260 \mathrm{E}$ & 01 & $-9.23153 \mathrm{E}-$ \\
\hline 40 & $08 \mathrm{E}$ & 00 & $42351 \mathrm{E}$ & 01 & $-9.34162 \mathrm{E}-$ \\
\hline 13.50 & $1.24203 \mathrm{E}$ & 00 & $-1.43441 \mathrm{E}$ & 01 & $5230 \mathrm{E}-$ \\
\hline 3.60 & $1.24198 \mathrm{E}$ & 00 & $-1.44531 \mathrm{E}$ & 01 & $-9.56356 \mathrm{E}-0$ \\
\hline & $93 \mathrm{E}$ & 00 & $1.5621 \mathrm{E}$ & 01 & $-9.67541 \mathrm{E}-0$ \\
\hline .80 & $88 \mathrm{E}$ & 00 & $6711 \mathrm{E}$ & 01 & $-9.78784 \mathrm{E}-0$ \\
\hline .90 & $83 \mathrm{E}$ & 00 & $7800 \mathrm{E}$ & 01 & $.90085 \mathrm{E}-0$ \\
\hline 14.00 & $1.24179 \mathrm{E}$ & 00 & $48889 E$ & 01 & $-1.00144 \mathrm{E} \quad 0$ \\
\hline 14.10 & $174 \mathrm{E}$ & 00 & $9978 \mathrm{E}$ & 01 & $-1.01286 \mathrm{E} \quad 0$ \\
\hline & & 00 & $067 \mathrm{E}$ & 01 & $-1.02434 \mathrm{E}$ \\
\hline 4.30 & $165 \mathrm{E}$ & 00 & $156 \mathrm{E}$ & 01 & $3587 \mathrm{E} \quad 0$ \\
\hline 1.40 & $1.24160 \mathrm{E}$ & 00 & $-1.53244 \mathrm{E}$ & 01 & $-1.04 .747 \mathrm{E}$ \\
\hline 14.50 & 1.24 & 00 & $54332 \mathrm{E}$ & 01 & $-1.05912 \mathrm{E}$ \\
\hline 1.60 & $.24151 \mathrm{E}$ & 00 & $5420 \mathrm{E}$ & 01 & $-1.07083 \mathrm{E} \quad 0$ \\
\hline .70 & $47 \mathrm{E}$ & 00 & $6507 \mathrm{E}$ & 01 & $8259 \mathrm{E}$ \\
\hline .80 & $42 \mathrm{E}$ & 00 & $57595 \mathrm{E}$ & 01 & $-1.09442 \mathrm{E}$ \\
\hline & $38 \mathrm{E}$ & 00 & $58682 \mathrm{E}$ & 01 & $-1.10630 \mathrm{E}$ \\
\hline 15.00 & $34 \mathrm{E}$ & 00 & $9768 \mathrm{E}$ & 01 & $-1.11824 \mathrm{E}$ \\
\hline 5.10 & $130 \mathrm{E}$ & 00 & $0855 \mathrm{E}$ & 01 & $-1.13024 \mathrm{E}$ \\
\hline .20 & $25 \mathrm{E}$ & 00 & $51941 \mathrm{E}$ & 01 & $-1.14230 \mathrm{E}$ \\
\hline & $21 \mathrm{E}$ & 00 & $53028 \mathrm{E}$ & 01 & $-1.1544 .2 \mathrm{E}$ \\
\hline & $7 \mathrm{E}$ & 00 & $113 \mathrm{E}$ & 01 & $-1.16659 \mathrm{E}$ \\
\hline & $3 \mathrm{E}$ & 00 & $199 \mathrm{E}$ & 01 & $7882 \mathrm{E}$ \\
\hline 60 & $.24109 \mathrm{E}$ & 00 & $6284 \mathrm{E}$ & 01 & $-1.19111 \mathrm{E}$ \\
\hline & $55 \mathrm{E}$ & 00 & $7370 \mathrm{E}$ & 01 & $-1.20346 \mathrm{E}$ \\
\hline & $\mathrm{E}$ & 00 & $154 \mathrm{E}$ & 01 & $-1.21587 \mathrm{E}$ \\
\hline & & 00 & & 01 & $.22833 \mathrm{E}$ \\
\hline 16.00 & $1.24 .093 \mathrm{E}$ & 00 & 0624E & 01 & $24.086 \mathrm{E}$ \\
\hline 16.10 & $089 \mathrm{E}$ & 00 & $1708 \mathrm{E}$ & 01 & $-1: 25344 \mathrm{E}$ \\
\hline & & 00 & & 01 & $26607 \mathrm{E}$ \\
\hline & & 00 & & 01 & $7877 \mathrm{E}$ \\
\hline & $7 \mathrm{E}$ & 00 & $959 \mathrm{E}$ & 01 & $152 \mathrm{E}$ \\
\hline 16.50 & $1.24074 \mathrm{E}$ & 00 & $042 \mathrm{E}$ & 01 & $-1.30434 \mathrm{E}$ \\
\hline 16.60 & 1.24 & 00 & $77125 \mathrm{E}$ & 01 & $-1.31721 \mathrm{E}$ \\
\hline & & 00 & & 01 & $3013 \mathrm{E}$ \\
\hline & $62 \mathrm{E}$ & 00 & $290 \mathrm{E}$ & 01 & $34312 \mathrm{E}$ \\
\hline & $\mathrm{E}$ & 00 & $372 \mathrm{E}$ & 01 & $1.35616 \mathrm{E}$ \\
\hline & $5 \mathrm{E}$ & 00 & $4 \mathrm{E}$ & 0 & $-1.36927 \mathrm{E}$ \\
\hline 7.10 & .24 & 00 & $2536 \mathrm{E}$ & 01 & $3242 \mathrm{E}$ \\
\hline & $3 \mathrm{E}$ & 00 & $3618 \mathrm{E}$ & 0 & $-1.39564 \mathrm{E}$ \\
\hline & $4 \mathrm{E}$ & 00 & $4699 \mathrm{E}$ & 0 & $-1.40892 \mathrm{E}$ \\
\hline & & 00 & & 01 & $42225 \mathrm{E}$ \\
\hline & $1.24037 \mathrm{E}$ & 00 & $86861 \mathrm{E}$ & 01 & $-1.43564 \mathrm{E} \quad 0$ \\
\hline & 2 & 00 & -1 & 01 & $-1.44909 \mathrm{E}$ \\
\hline & & 00 & & 01 & $-1.46259 \mathrm{E}$ \\
\hline & & 00 & & 01 & $-1.47616 \mathrm{E}$ \\
\hline & $1.24023 \mathrm{E}$ & 00 & $-1.91181 \mathrm{E}$ & 01 & $-1.48978 \mathrm{E}$ \\
\hline & $1.24020 \mathrm{E}$ & 00 & $-1.92260 \mathrm{E}$ & 01 & $-1.50345 \mathrm{E} \quad 0$ \\
\hline
\end{tabular}

$\tan \delta_{1} \quad f_{-1}$

$5.79045 \mathrm{E}-03$

$5.81117 \mathrm{E}-03$

$5.83151 \mathrm{E}-03$

$5.85151 \mathrm{E}-03$

$5.87115 \mathrm{E}-03$

$5.89045 \mathrm{E}-03$

$5.90943 \mathrm{E}-03$

$5.92808 \mathrm{E}-03$

$5.94639 \mathrm{E}-03$

5.9644 .1 E-03

$5.98212 \mathrm{E}-03$

$5.99954 \mathrm{E}-03$

$6.01665 \mathrm{E}-03$

$6.03350 \mathrm{E}-03$

$6.05006 \mathrm{E}-03$

$6.06635 \mathrm{E}-03$ $6.08236 \mathrm{E}-03$ $6.09813 \mathrm{E}-03$

$6.11363 \mathrm{E}-03$

$6.12887 \mathrm{E}-03$

$6.14388 \mathrm{E}-03$

$6.15864 \mathrm{E}-03$

$6.17317 \mathrm{E}-03$

$6.18746 \mathrm{E}-03$

6.20154 E-03

$6.21538 \mathrm{E}-03$ $6.22900 \mathrm{E}-03$ $6.24241 \mathrm{E}-03$

$6.25562 \mathrm{E}-03$

$6.26862 \mathrm{E}-03$

$6.28141 \mathrm{E}-03$ $6.29401 \mathrm{E}-03$ $6.30641 \mathrm{E}-03$ $6.31862 \mathrm{E}-03$ $6.33065 \mathrm{E}-03$

$6.34248 \mathrm{E}-03$ $6.35415 \mathrm{E}-03$ $6.36562 \mathrm{E}-03$ $6.37693 \mathrm{E}-03$ $6.38806 \mathrm{E}-03$

$6.39903 \mathrm{E}-03$ $6.40983 \mathrm{E}-03$ $6.42046 \mathrm{E}-03$ $6.43094 \mathrm{E}-03$ $6.44127 \mathrm{E}-03$

$6.45143 \mathrm{E}-03$ $6.46145 \mathrm{E}-03$ $6.47131 \mathrm{E}-03$ $6.48103 \mathrm{E}-03$ $6.49059 \mathrm{E}-03$

6.50004E-03 $6.50932 \mathrm{E}-03$ $6.51847 \mathrm{E}-03$ $6.52748 \mathrm{E}-03$ $6.53638 \mathrm{E}-03$

$6.54513 \mathrm{E}-03$ $6.55374 \mathrm{E}-03$ $6.56224 \mathrm{E}-03$ $6.57060 \mathrm{E}-03$ $6.57885 \mathrm{E}-03$
$-7.87975 \mathrm{E}-01$ $-7.97979 \mathrm{E}-01$ $-8.08042 \mathrm{E}-01$ $-8.18164 \mathrm{E}-01$ $-8.28344 \mathrm{E}-01$

$-8.38583 \mathrm{E}-01$ $-8.48880 \mathrm{E}-01$ $-8.59236 \mathrm{E}-01$ $-8.69651 \mathrm{E}-01$ $-8.80123 \mathrm{E}-01$

$-8.90655 \mathrm{E}-01$ $-9.01244 \mathrm{E}-01$ $-9.11892 \mathrm{E}-01$ $-9.22599 \mathrm{E}-01$ $-9.33364 \mathrm{E}-01$

$-9.44187 \mathrm{E}-01$ $-9.55069 \mathrm{E}-01$ $-9.66009 \mathrm{E}-01$ $-9.77008 \mathrm{E}-01$ $-9.88064 \mathrm{E}-01$

$-9.99179 \mathrm{E}-01$ $-1.01035 \mathrm{E} 00$ $-1.02158 \mathrm{E} 00$ $-1.03287 \mathrm{E} 00$ $-1.04422 \mathrm{E} 00$

$-1.05563 \mathrm{E} 00$ $-1.06709 \mathrm{E} 00$ $-1.07862 \mathrm{E} 00$ $-1.09020 \mathrm{E} \quad 00$ $-1.10184 \mathrm{E} 00$

$-1.11353 \mathrm{E} \quad 00$ $-1.12529 \mathrm{E} \quad 00$ $-1.13710 \mathrm{E} \quad 00$ $-1.14898 \mathrm{E} 00$ $-1.16091 \mathrm{E} 00$

$-1.17289 \mathrm{E} \quad 00$ $-1.18494 \mathrm{E} \quad 00$ $-1.19704 \mathrm{E} 00$ $-1.20921 \mathrm{E} 00$ $-1.22143 \mathrm{E} 00$

$-1.23370 \mathrm{E} \quad 00$ $-1.24604 \mathrm{E} 00$ $-1.25843 \mathrm{E} \quad 00$ $-1.27089 \mathrm{E} 00$ $-1.28340 \mathrm{E} 00$

$-1.29596 \mathrm{E} \quad 00$ $-1.30859 \mathrm{E} \quad 00$ $-1.32127 \mathrm{E} \quad 00$ $-1.33401 \mathrm{E} 00$ $-1.34681 \mathrm{E} 00$

$-1.35967 \mathrm{E} \quad 00$ $-1.37259 \mathrm{E} \quad 00$ $-1.38556 \mathrm{E} \quad 00$ $-1.39859 \mathrm{E} \quad 00$ $-1.41168 \mathrm{E} \quad 00$

$-1.42482 \mathrm{E} \quad 00$ $-1.43803 \mathrm{E} \quad 00$ $-1.45129 \mathrm{E} \quad 00$ $-1.46461 \mathrm{E} \quad 00$ $-1.47799 \mathrm{E} \quad 00$ $g_{-1}$

$\tan \delta_{-1}$

$\begin{array}{lllll}1.39119 \mathrm{E} & 01 & -8.92407 \mathrm{E} & 01\end{array}$ $1.40211 \mathrm{E}$ 01 $-8.94312 \mathrm{E} \quad 01$

$1.41304 \mathrm{E} \quad 01$

$1.42395 \mathrm{E} \quad 01$

$1.43487 \mathrm{E} \quad 01$

$1.44578 \mathrm{E} \quad 01$

$1.45669 \mathrm{E} \quad 01$

$1.46760 \mathrm{E} 01$

$1.47851 \mathrm{E} \quad 01$

$1.48942 \mathrm{E} \quad 01$

$1.50032 \mathrm{E} 01$

$1.51122 \mathrm{E} \quad 01$

$1.52211 \mathrm{E} 01$

$1.53301 \mathrm{E} 01$

$1.54390 \mathrm{E} \quad 01$

$1.554 .79 \mathrm{E} \quad 01$ $1.56568 \mathrm{E} \quad 01$

$1.57657 \mathrm{E} \quad 01$

$1.58745 \mathrm{E} \quad 01$

$1.59833 \mathrm{E} 01$

$1.60921 \mathrm{E} 01$

$1.62008 \mathrm{E} \quad 01$

$1.63096 \mathrm{E} \quad 01$

$1.64183 \mathrm{E} 01$

$1.65270 \mathrm{E} \quad 01$

$1.66356 \mathrm{E} \quad 01$

$1.67443 \mathrm{E} \quad 01$

$1.68529 \mathrm{E} \quad 01$

$1.69615 \mathrm{E} 01$

$1.70700 \mathrm{E} \quad 01$

$1.71786 \mathrm{E} \quad 01$ $1.72871 \mathrm{E} \quad 01$

$1.73956 \mathrm{E} \quad 01$

$1.75041 \mathrm{E} 01$

$1.76125 \mathrm{E} \quad 01$

$1.77209 \mathrm{E} \quad 01$ $1.78293 \mathrm{E} \quad 01$ $\begin{array}{ll}1.79377 \mathrm{E} & 01\end{array}$ $1.80460 \mathrm{E} \quad 01$ $1.81544 \mathrm{E} \quad 01$

$1.82627 \mathrm{E} \quad 01$ $1.83709 \mathrm{E} \quad 01$ $1.84792 \mathrm{E} 01$ $1.85874 \mathrm{E} \quad 01$ $1.86956 \mathrm{E} 01$

$1.88038 \mathrm{E} \quad 01$ $1.89119 \mathrm{E} 01$ $1.90200 \mathrm{E} \quad 01$ $1.91281 \mathrm{E} \quad 01$ $1.92362 \mathrm{E} \quad 01$

$1.93442 \mathrm{E} \quad 01$ $1.94523 \mathrm{E} \quad 01$ $1.95603 \mathrm{E} \quad 01$ $1.96682 \mathrm{E} \quad 01$ $1.97762 \mathrm{E} \quad 01$

$1.98841 \mathrm{E} \quad 01$ $1.99920 \mathrm{E} \quad 01$ $2.00998 \mathrm{E} 01$ $2.02077 \mathrm{E} \quad 01$ $2.03155 \mathrm{E} \quad 01$ $\begin{array}{ll}-8.96199 \mathrm{E} & 01\end{array}$ $-8.98063 \mathrm{E} \quad 01$ $-8.99909 \mathrm{E} 01$

$-9.01736 \mathrm{E} \quad 01$ $-9.03542 \mathrm{E} 01$ $-9.05330 \mathrm{E} 01$ $-9.07101 \mathrm{E} 01$ $-9.08854 \mathrm{E} 01$

$-9.10588 \mathrm{E} 01$ $-9.12305 \mathrm{E} \quad 01$ $-9.14006 \mathrm{E} 01$ $-9.15690 \mathrm{E} 01$ $-9.17357 \mathrm{E} 01$

$-9.19009 \mathrm{E} 01$ $-9.20645 \mathrm{E} \quad 01$ $-9.22266 \mathrm{E} 01$ $-9.23871 \mathrm{E} 01$ $-9.25463 \mathrm{E} 01$ $-9.28601 \mathrm{E} 01$ $-9.30149 \mathrm{E} 01$ $-9.31683 \mathrm{E} 01$ $-9.33204 \mathrm{E} 01$

$-9.34712 \mathrm{E} \quad 01$ $-9.36207 \mathrm{E} 01$ $-9.37689 \mathrm{E}$ 0l $-9.39158 \mathrm{E}$ 0l $-9.40615 \mathrm{E} 01$

$-9.42060 \mathrm{E} 01$ $-9.43494 \mathrm{E} \quad 01$ $-9.44915 \mathrm{E} \quad 01$ $-9.46325 \mathrm{E}$ 01

$-9.49112 \mathrm{E} \quad 01$ $-9.50488 \mathrm{E} 01$ $-9.51855 \mathrm{E} \quad 01$ $-9.53211 \mathrm{E} \quad 01$ $-9.54557 \mathrm{E} 01$

$-9.55892 \mathrm{E}$ ol $-9.57217 \mathrm{E} \quad 01$ $-9.58533 \mathrm{E} 01$ $-9.59840 \mathrm{E} 01$ $-9.61135 \mathrm{E} 01$

$-9.62423 \mathrm{E} \quad 01$ $-9.63701 \mathrm{E} 01$ $-9.64971 \mathrm{E} 01$ $-9.66232 \mathrm{E} \quad 01$ $-9.67484 \mathrm{E} 01$

$-9.68726 \mathrm{E} \quad 01$ $-9.69963 \mathrm{E} 01$ $-9.71190 \mathrm{E} \quad 01$ $-9.72409 \mathrm{E} 01$ $-9.73619 \mathrm{E} 01$

$-9.74822 \mathrm{E} \quad 01$ $-9.76019 \mathrm{E} \quad 01$ $-9.77207 \mathrm{E} 01$ $-9.78388 \mathrm{E} \quad 01$ $-9.79561 \mathrm{E} 01$
$-9.27039 \mathrm{E} 01$ $-9.47724 \mathrm{E} \quad 01$ 
ELECTRON RADIAL FUNCTIONS

$Z=9 \quad A=18 \quad \rho=1.2 A^{1 / 3} F$

Electron

$\bar{F}$

$3.33884 \mathrm{E}-04$

$3.39416 \mathrm{E}-04$

$3.44993 \mathrm{E}-04$

$3.50615 \mathrm{E}-04$

$3.56283 \mathrm{E}-04$

$3.61997 \mathrm{E}-04$

$3.67756 \mathrm{E}-04$

$3.73560 \mathrm{E}-04$

$3.79410 \mathrm{E}-04$

$3.85305 \mathrm{E}-04$

$3.91245 \mathrm{E}-04$

$3.97231 \mathrm{E}-04$

$4.03263 \mathrm{E}-04$

$4.09340 \mathrm{E}-04$

$4.15462 \mathrm{E}-04$

$4.21630 \mathrm{E}-04$

$4.27843 \mathrm{E}-04$

$4.34101 \mathrm{E}-04$

$4.40405 \mathrm{E}-04$

4. $46755 \mathrm{E}-04$

$4.53149 \mathrm{E}-04$

$4.59590 \mathrm{E}-04$

4.66075 E-04

$4.72606 \mathrm{E}-04$

$4.79183 \mathrm{E}-04$

$4.85805 \mathrm{E}-04$

$4.92472 \mathrm{E}-04$

$4.99184 \mathrm{E}-04$

$5.05942 \mathrm{E}-04$

$5.12746 \mathrm{E}-04$

$5.19595 \mathrm{E}-04$

$5.26489 \mathrm{E}-04$

$5.33429 \mathrm{E}-04$

$5.40414 \mathrm{E}-04$

$5.47444 \mathrm{E}-04$

$5.54520 \mathrm{E}-04$

$5.61642 \mathrm{E}-04$

$5.68808 \mathrm{E}-04$

$5.76021 \mathrm{E}-04$

$5.83278 \mathrm{E}-04$

$5.90581 \mathrm{E}-04$

$5.97929 \mathrm{E}-04$

$6.05323 \mathrm{E}-04$

$6.12763 \mathrm{E}-04$

$6.20247 \mathrm{E}-04$

$6.27777 \mathrm{E}-04$

$6.35352 \mathrm{E}-04$

$6.42973 \mathrm{E}-04$

$6.50639 \mathrm{E}-04$

$6.58350 \mathrm{E}-04$

$6.66107 \mathrm{E}-04$

$6.73910 \mathrm{E}-04$

$6.81757 \mathrm{E}-04$

$6.89651 \mathrm{E}-04$

$6.97589 \mathrm{E}-04$

$7.05573 \mathrm{E}-04$

$7.13602 \mathrm{E}-04$

$7.21677 \mathrm{E}-04$

$7.29797 \mathrm{E}-04$

$7.37963 \mathrm{E}-04$ $f_{2}$

$g_{2}$

$\tan \delta_{2}$

$f_{-2}$

$-4.21881 \mathrm{E}-01-1.53772 \mathrm{E}-02$

$-4.29016 \mathrm{E}-01-1.57071 \mathrm{E}-02$

$-4.36210 \mathrm{E}-01-1.60416 \mathrm{E}-02$

$-4.43464 \mathrm{E}-01-1.63805 \mathrm{E}-02$

$-4.50777 \mathrm{E}-01$

$-1.67240 \mathrm{E}-02$

$-4.58150 \mathrm{E}-01$

$-4.65582 \mathrm{E}-01$

$-4.73073 \mathrm{E}-01$

$-4.80624 \mathrm{E}-01$

$-4.88234 \mathrm{E}-01$

$-4.95903 \mathrm{E}-01$

$-5.03632 \mathrm{E}-01$

$-5.11420 \mathrm{E}-01$

$-5.19267 \mathrm{E}-01$

$-5.27173 \mathrm{E}-01$

$-5.35139 \mathrm{E}-01$

$-5.43164 \mathrm{E}-01$

$-5.51248 \mathrm{E}-01$

$-5.59392 \mathrm{E}-01$

$-5.67594 \mathrm{E}-01$

$-5.75856 \mathrm{E}-01$

$-5.84177 \mathrm{E}-01$

$-5.92557 \mathrm{E}-01$

$-6.00997 \mathrm{E}-01$

$-6.09495 \mathrm{E}-01$

$-6.18053 \mathrm{E}-01$

$-6.26670 \mathrm{E}-01$

$-6.35346 \mathrm{E}-01$

$-6.44081 \mathrm{E}-01$

$-6.52875 \mathrm{E}-01$

$-6.61728 \mathrm{E}-01$

$-6.70641 \mathrm{E}-01$

$-6.79612 \mathrm{E}-01$

$-6.88642 \mathrm{E}-01$

$-6.97732 \mathrm{E}-01$

$-7.06880 \mathrm{E}-01$

$-7.16088 \mathrm{E}-01$

$-7.25354 \mathrm{E}-01$

$-7.34680 \mathrm{E}-01$

$-7.44064 \mathrm{E}-01$

$-7.53508 \mathrm{E}-01$

$-7.63010 \mathrm{E}-01$

$-7.72572 \mathrm{E}-01$

$-7.82192 \mathrm{E}-01$

$-7.91871 \mathrm{E}-01$

$-8.01609 \mathrm{E}-01$

$-8.11406 \mathrm{E}-01$

$-8.21262 \mathrm{E}-01$

$-8.31176 \mathrm{E}-01$

$-8.41150 \mathrm{E}-01$

$-8.51182 \mathrm{E}-01$

$-8.61273 \mathrm{E}-01$

$-8.71423 \mathrm{E}-01$

$-8.81632 \mathrm{E}-01$

$-8.91900 \mathrm{E}-01$

$-9.02226 \mathrm{E}-01$

$-9.12611 \mathrm{E}-01$

$-9.23055 \mathrm{E}-01$

$-9.33558 \mathrm{E}-01$

$-9.44119 \mathrm{E}-01$
$-1.70722 \mathrm{E}-02$

$-1.74249 \mathrm{E}-02$

$-1.77823 \mathrm{E}-02$

$-1.81444 \mathrm{E}-02$

$-1.85113 \mathrm{E}-02$

$-1.88828 \mathrm{E}-02$

$-1.92592 \mathrm{E}-02$

$-1.96403 \mathrm{E}-02$

$-2.00262 \mathrm{E}-02$

$-2.04171 \mathrm{E}-02$

$-2.08128 \mathrm{E}-02$

$-2.12134 \mathrm{E}-02$

$-2.16190 \mathrm{E}-02$

$-2.20295 \mathrm{E}-02$

$-2.24451 \mathrm{E}-02$

$-2.28656 \mathrm{E}-02$

$-2.32913 \mathrm{E}-02$

$-2.37220 \mathrm{E}-02$

$-2.41578 \mathrm{E}-02$

$-2.45988 \mathrm{E}-02$

$-2.50450 \mathrm{E}-02$

$-2.54963 \mathrm{E}-02$

$-2.59529 \mathrm{E}-02$

$-2.64148 \mathrm{E}-02$

$-2.68819 \mathrm{E}-02$

$-2.73544 \mathrm{E}-02$

$-2.78322 \mathrm{E}-02$

$-2.83153 \mathrm{E}-02$

$-2.88039 \mathrm{E}-02$

$-2.92979 \mathrm{E}-02$

$-2.97974 \mathrm{E}-02$

$-3.03024 \mathrm{E}-02$

$-3.08129 \mathrm{E}-02$

$-3.13289 \mathrm{E}-02$

$-3.18505 \mathrm{E}-02$

$-3.23777 \mathrm{E}-02$

$-3.29105 \mathrm{E}-02$

$-3.34490 \mathrm{E}-02$

$-3.39932 \mathrm{E}-02$

$-3.45431 \mathrm{E}-02$

$-3.50987 \mathrm{E}-02$

$-3.56602 \mathrm{E}-02$

$-3.62274 \mathrm{E}-02$

$-3.68004 \mathrm{E}-02$

$-3.73793 \mathrm{E}-02$

$-3.79641 \mathrm{E}-02$

$-3.85548 \mathrm{E}-02$

$-3.91515 \mathrm{E}-02$

$-3.97542 \mathrm{E}-02$

$-4.03628 \mathrm{E}-02$

$-4.09775 \mathrm{E}-02$

$-4.15982 \mathrm{E}-02$

$-4.22250 \mathrm{E}-02$

$-4.28579 \mathrm{E}-02$

$-4.34970 \mathrm{E}-02$
$2.27267 \mathrm{E} \quad 01 \quad-1.52051 \mathrm{E}-02$

$2.27338 \mathrm{E}$ 01 $-1.55276 \mathrm{E}-02$

$2.27407 \mathrm{E}$ 01 $-1.58545 \mathrm{E}-02$

$2.27475 \mathrm{E} 01 \quad-1.61858 \mathrm{E}-02$

$2.27541 \mathrm{E} \quad 01 \quad-1.65217 \mathrm{E}-02$

$2.27607 \mathrm{E} \quad 01$

$2.27672 \mathrm{E} \quad 01$

$2.27735 \mathrm{E} \quad 01$

$2.27798 \mathrm{E} \quad 01$

$2.27859 \mathrm{E} \quad 01$

$2.27920 \mathrm{E} \quad 01$

$2.27979 \mathrm{E} \quad 01$

$2.28038 \mathrm{E} \quad 01$

$2.28095 \mathrm{E} \quad 01$

$2.28152 \mathrm{E} \quad 01$

$2.28208 \mathrm{E} \quad 01$

$2.28317 \mathrm{E} 01$

$2.28371 \mathrm{E} \quad 01$

$2.28423 \mathrm{E} \quad 01$

$-1.68620 \mathrm{E}-02$

$-1.72069 \mathrm{E}-02$

$-1.75563 \mathrm{E}-02$

$-1.79103 \mathrm{E}-02$

$-1.82689 \mathrm{E}-02$

$-1.86322 \mathrm{E}-02$

$-1.90002 \mathrm{E}-02$

$-1.93728 \mathrm{E}-02$

$-1.97502 \mathrm{E}-02$

$-2.01324 \mathrm{E}-02$

$-2.05193 \mathrm{E}-02$

$-2.09110 \mathrm{E}-02$

$-2.13076 \mathrm{E}-02$

$-2.17091 \mathrm{E}-02$

$-2.21155 \mathrm{E}-02$

$2.28475 \mathrm{E} \quad 01$

$2.28526 \mathrm{E} 01$

$2.28576 \mathrm{E} \quad 01$

$2.28626 \mathrm{E} 01$

$2.28723 \mathrm{E} \quad 01$

$2.28770 \mathrm{E}$ oI

2.28817 E 01

$2.28863 \mathrm{E} 01$

$2.28909 \mathrm{E} \quad 01$

$-2.25268 \mathrm{E}-02$

$-2.29431 \mathrm{E}-02$

$-2.33644 \mathrm{E}-02$

$-2.37906 \mathrm{E}-02$

$-2.42220 \mathrm{E}-02$

$2.28954 \mathrm{E} \quad 01$

$2.28998 \mathrm{E} \quad 01$

$2.29041 \mathrm{E} \quad 01$

$2.29084 \mathrm{E} \quad 01$

$2.29127 \mathrm{E} \quad 01$

$2.29169 \mathrm{E} \quad 01$

$2.29210 \mathrm{E} \quad 01$

$2.29251 \mathrm{E} \quad 01$

$2.29291 \mathrm{E} \quad 01$

$2.29331 \mathrm{E} \quad 01$

$2.29370 \mathrm{E} \quad 01$

$2.29409 \mathrm{E} 01$

$2.29447 \mathrm{E} 01$

$2.29485 \mathrm{E} \quad 01$

$2.29522 \mathrm{E} 01$

$2.29559 \mathrm{E} \quad 01$

$2.29595 \mathrm{E} \quad 01$

$2.29631 \mathrm{E} 01$

$2.29666 \mathrm{E} \quad 01$

$2.29701 \mathrm{E} 01$

$2.29736 \mathrm{E} \quad 01$

$2.29770 \mathrm{E} \quad 0 \mathrm{I}$

$2.29804 \mathrm{E}$ 01

$2.29837 \mathrm{E} \quad 01$

$2.29870 \mathrm{E} \quad 01$

$2.29903 \mathrm{E} \quad 01$ $2.29935 \mathrm{E} \quad 01$

$2.29966 \mathrm{E} 01$

$2.29998 \mathrm{E} \quad 01$

2.30029E 01
$2.28263 \mathrm{E} \quad 01$

$2.28675 \mathrm{E} \quad 01$

g-2

$4.58138 \mathrm{E}-01$

$4.65572 \mathrm{E}-01$

$4.73065 \mathrm{E}-01$

$4.80618 \mathrm{E}-01$

$4.88230 \mathrm{E}-01$

$4.95901 \mathrm{E}-01$

$5.03632 \mathrm{E}-01$

$5.11422 \mathrm{E}-01$

$5.19271 \mathrm{E}-01$

$5.27179 \mathrm{E}-01$

$5.35147 \mathrm{E}-01$

$5.43174 \mathrm{E}-01$

$5.51261 \mathrm{E}-01$

$5.59406 \mathrm{E}-01$

$5.67611 \mathrm{E}-01$

$5.75875 \mathrm{E}-01$

$5.84198 \mathrm{E}-01$

$5.92581 \mathrm{E}-01$

$6.01022 \mathrm{E}-01$

$6.09523 \mathrm{E}-01$

$6.18083 \mathrm{E}-01$

$6.26702 \mathrm{E}-01$

$6.35380 \mathrm{E}-01$

$6.44118 \mathrm{E}-01$

$6.52914 \mathrm{E}-01$ 
ELECTRON RADIAL FUNCTIONS

Electron

\begin{tabular}{|c|c|c|c|c|c|}
\hline$p$ & $F_{0}$ & & $f_{1}$ & & $g_{1}$ \\
\hline 18.10 & $1.24017 \mathrm{E}$ & 00 & $-1.93340 \mathrm{E}$ & 01 & $-1.51719 \mathrm{E}$ \\
\hline & $1.24013 \mathrm{E}$ & 00 & $-1.94419 \mathrm{E}$ & 01 & $-1.53099 \mathrm{E}$ \\
\hline 18.30 & $1.24010 \mathrm{E}$ & 00 & $-1.95497 \mathrm{E}$ & 01 & $.54484 \mathrm{E}$ \\
\hline 18.40 & $1.24007 \mathrm{E}$ & 00 & $-1.96576 \mathrm{E}$ & 01 & $-1.55875 \mathrm{E}$ \\
\hline 18.50 & $1.24003 \mathrm{E}$ & 00 & $-1.97654 \mathrm{E}$ & 01 & $-1.57271 \mathrm{E}$ \\
\hline 18.60 & $1.24000 \mathrm{E}$ & 00 & $-1.98732 \mathrm{E}$ & 01 & $-1.58674 \mathrm{E}$ \\
\hline 18.70 & $1.23997 \mathrm{E}$ & 00 & $99810 \mathrm{E}$ & 01 & $0082 \mathrm{E}$ \\
\hline 8.80 & $1.23994 \mathrm{E}$ & 00 & $-2.00887 \mathrm{E}$ & 01 & $1496 \mathrm{E}$ \\
\hline .90 & $1.23990 \mathrm{E}$ & 00 & $-2.01964 \mathrm{E}$ & 01 & $2916 \mathrm{E}$ \\
\hline 9.00 & $1.23987 \mathrm{E}$ & 00 & $-2.03041 \mathrm{E}$ & 01 & $341 \mathrm{E}$ \\
\hline .10 & $1.23984 \mathrm{E}$ & 00 & $-2.04118 \mathrm{E}$ & 01 & $65772 \mathrm{E}$ \\
\hline .20 & $1.23981 \mathrm{E}$ & 00 & $05194 \mathrm{E}$ & 01 & $57209 E$ \\
\hline .30 & $1.23978 \mathrm{E}$ & 00 & $-2.06270 \mathrm{E}$ & 0. & $1.68652 \mathrm{E}$ \\
\hline 19.40 & $1.23975 \mathrm{E}$ & 00 & $-2.0734 .6 \mathrm{E}$ & 01 & $.70100 \mathrm{E}$ \\
\hline 19.50 & $1.23972 \mathrm{E}$ & 00 & $-2.08421 \mathrm{E}$ & 01 & $71554 \mathrm{E}$ \\
\hline .60 & $1.23969 \mathrm{E}$ & 00 & $-2.09497 \mathrm{E}$ & 01 & $73014 \mathrm{E}$ \\
\hline .70 & $1.23966 \mathrm{E}$ & 00 & $10572 \mathrm{E}$ & 01 & $74480 \mathrm{E}$ \\
\hline .80 & $1.23963 \mathrm{E}$ & 00 & $11647 \mathrm{E}$ & 01 & $.75951 \mathrm{E}$ \\
\hline & $1.23960 \mathrm{E}$ & 00 & $12721 \mathrm{E}$ & 01 & $77428 \mathrm{E}$ \\
\hline 20.00 & $1.23957 \mathrm{E}$ & 00 & $-2.13795 \mathrm{E}$ & 01 & $3911 \mathrm{E}$ \\
\hline 0.10 & $23954 \mathrm{E}$ & 00 & $-2.14869 \mathrm{E}$ & 01 & $0399 \mathrm{E}$ \\
\hline & $3951 \mathrm{E}$ & 00 & $5943 \mathrm{E}$ & 01 & $4 \mathrm{E}$ \\
\hline 0 & $1.23948 \mathrm{E}$ & 00 & $7016 \mathrm{E}$ & 01 & $394 \mathrm{E}$ \\
\hline 20.40 & $1.23945 \mathrm{E}$ & 00 & $-2.18090 \mathrm{E}$ & 01 & $4899 \mathrm{E}$ \\
\hline 20.50 & $1.23942 \mathrm{E}$ & 00 & $-2.19163 \mathrm{E}$ & 01 & $86411 \mathrm{E}$ \\
\hline 20.60 & $1.23939 \mathrm{E}$ & 00 & $-2.20235 \mathrm{E}$ & 01 & \\
\hline 20.7 & $6 \mathrm{E}$ & 00 & $08 \mathrm{E}$ & 0. & \\
\hline 20.80 & $1.23933 \mathrm{E}$ & 00 & $-2.22380 \mathrm{E}$ & 01 & $-1.90979 \mathrm{E}$ \\
\hline 0.90 & $1.23931 \mathrm{E}$ & 00 & $-2.23451 \mathrm{E}$ & 01 & $-1.92514 \mathrm{E}$ \\
\hline 1.00 & $1.23928 \mathrm{E}$ & 00 & $-2.24523 \mathrm{E}$ & 01 & \\
\hline 21.10 & $1.23925 \mathrm{E}$ & 00 & $25594 \mathrm{E}$ & 01 & \\
\hline 21.20 & $1.23922 \mathrm{E}$ & 00 & $26665 \mathrm{E}$ & 01 & $-1.97151 \mathrm{E}$ \\
\hline 21.30 & $1.23919 \mathbf{E}$ & 00 & $-2.27736 \mathrm{E}$ & 01 & $-1.98708 \mathrm{E}$ \\
\hline 21.40 & $1.23917 \mathrm{E}$ & 00 & $-2.28806 \mathrm{E}$ & 01 & $-2.00271 \mathrm{E}$ \\
\hline 21.50 & $1.23914 \mathrm{E}$ & 00 & $-2.29877 \mathrm{E}$ & 01 & \\
\hline 1.60 & $1.23911 \mathrm{E}$ & 00 & $-2.30947 \mathrm{E}$ & 01 & \\
\hline 21.70 & $23909 \mathrm{E}$ & 00 & $-2.32016 \mathrm{E}$ & 01 & $-2.04993 \mathrm{E}$ \\
\hline 21.80 & $23906 \mathrm{E}$ & 00 & $-2.33086 \mathrm{E}$ & 01 & $.06579 \mathrm{E}$ \\
\hline & & 00 & & 0 & \\
\hline 22.00 & $1.23900 \mathrm{E}$ & 00 & $-2.35223 \mathrm{E}$ & 01 & $-2.09767 \mathrm{E}$ \\
\hline 22.10 & $8 \mathrm{E}$ & 00 & $6292 \mathrm{E}$ & 01 & \\
\hline 22.20 & $95 \mathrm{E}$ & 00 & $-2.37360 \mathrm{E}$ & 01 & $-2.12978 \mathrm{E}$ \\
\hline 22.30 & & 00 & & 01 & \\
\hline 22.40 & & 00 & $9496 \mathrm{E}$ & 01 & \\
\hline 22.50 & $1.23887 \mathrm{E}$ & 00 & $-2.40563 \mathrm{E}$ & 01 & $-2.17837 \mathrm{E}$ \\
\hline 22.60 & $.23885 \mathrm{E}$ & 00 & & 01 & \\
\hline 22.7 & & 00 & -2 & 0 & $5 \mathrm{E}$ \\
\hline 22.80 & & 00 & -2.4 & 01 & \\
\hline 22.90 & $1.23877 \mathrm{E}$ & 00 & $-2.44830 \mathrm{E}$ & 01 & $24396 \mathrm{E}$ \\
\hline 23.00 & $1.23875 \mathrm{E}$ & 00 & $-2.45896 \mathrm{E}$ & 01 & $-2.26050 \mathrm{E}$ \\
\hline 23.10 & $1.23872 \mathrm{E}$ & 00 & $-2.46962 \mathrm{E}$ & 01 & \\
\hline & & 00 & & 01 & \\
\hline 23.30 & $1.23867 \mathrm{E}$ & 00 & $-2.49092 \mathrm{E}$ & 01 & $31045 \mathrm{E}$ \\
\hline 23.40 & $1.23865 \mathrm{E}$ & 00 & $-2.50157 \mathrm{E}$ & 01 & $-2.32722 \mathrm{E}$ \\
\hline 23.50 & $1.23862 \mathrm{E}$ & 00 & $-2.51221 \mathrm{E}$ & 01 & $-2.34404 \mathrm{E}$ \\
\hline 23.60 & 1.2386 & 00 & $2286 \mathrm{E}$ & 01 & -2 \\
\hline & $23857 \mathrm{E}$ & 00 & $-2.53350 \mathrm{E}$ & 0 & $-2.37785 \mathrm{E}$ \\
\hline & $1.23855 \mathrm{E}$ & 00 & $-2.54413 \mathrm{E}$ & 0 & $-2.39484 \mathrm{E}$ \\
\hline & $1.23852 \mathrm{E}$ & 00 & $-2.55477 \mathrm{E}$ & 01 & $-2.41189 \mathrm{E}$ \\
\hline 24.00 & $1.23850 \mathrm{E}$ & 00 & $-2.56540 \mathrm{E}$ & 01 & $-2.42900 \mathrm{E}$ \\
\hline
\end{tabular}

$\tan \delta_{1}$
$Z=9 \quad A=18 \quad \rho=1.2 A^{1 / 3} F$

$6.58698 \mathrm{E}-03-1.49142 \mathrm{E} \quad 00$ $6.59498 \mathrm{E}-03-1.50491 \mathrm{E} \quad 00$ $6.60286 \mathrm{E}-03-1.51846 \mathrm{E} \quad 00$ $6.61064 \mathrm{E}-03-1.53207 \mathrm{E} \quad 00$ $6.61830 \mathrm{E}-03-1.54574 \mathrm{E} \quad 00$

$6.62583 \mathrm{E}-03$ $6.63326 \mathrm{E}-03$ $6.64058 \mathrm{E}-03$ $6.64781 \mathrm{E}-03$ $6.65491 \mathrm{E}-03$

$6.66190 \mathrm{E}-03$ $6.66881 \mathrm{E}-03$ $6.67560 \mathrm{E}-03$ $6.68230 \mathrm{E}-03$ $6.68890 \mathrm{E}-03$

$6.69540 \mathrm{E}-03$ $6.70179 \mathrm{E}-03$ $6.70811 \mathrm{E}-03$ $6.71431 \mathrm{E}-03$ $6.72044 \mathrm{E}-03$

$6.72646 \mathrm{E}-03$ $6.73240 \mathrm{E}-03$ $6.73826 \mathrm{E}-03$ $6.74401 \mathrm{E}-03$ $6.74968 \mathrm{E}-03$

$6.75528 \mathrm{E}-03$ $6.76077 \mathrm{E}-03$ $6.76621 \mathrm{E}-03$ $6.77153 \mathrm{E}-03$ $6.77679 \mathrm{E}-03$

$6.78197 \mathrm{E}-03$ $6.78706 \mathrm{E}-03$ $6.79207 \mathrm{E}-03$ $6.79702 \mathrm{E}-03$ $6.80188 \mathrm{E}-03$

$6.80667 \mathrm{E}-03$ $6.81138 \mathrm{E}-03$ $6.81602 \mathrm{E}-03$ $6.82059 \mathrm{E}-03$ $6.82508 \mathrm{E}-03$

$6.82950 \mathrm{E}-03$ $6.83386 \mathrm{E}-03$ $6.83813 \mathrm{E}-03$ $6.84234 \mathrm{E}-03$ $6.84649 \mathrm{E}-03$

$6.85056 \mathrm{E}-03$ $6.85457 \mathrm{E}-03$ $6.85852 \mathrm{E}-03$ $6.86240 \mathrm{E}-03$ $6.86622 \mathrm{E}-03$

$6.86998 \mathrm{E}-03$ $6.87366 \mathrm{E}-03$ $6.87728 \mathrm{E}-03$ $6.88084 \mathrm{E}-03$ $6.88435 \mathrm{E}-03$

$6.88779 \mathrm{E}-03$ $6.89117 \mathrm{E}-03$ $6.89450 \mathrm{E}-03$ $6.89776 \mathrm{E}-03$ $6.90098 \mathrm{E}-03$
$-1.55946 \mathrm{E} \quad 00$ $-1.57324 \mathrm{E} \quad 00$ $-1.58708 \mathrm{E} \quad 00$ $-1.60097 \mathrm{E} \quad 00$ $-1.61492 \mathrm{E} 00$

$-1.62893 \mathrm{E} \quad 00$ $-1.64300 \mathrm{E} \quad 00$ $-1.65713 \mathrm{E} \quad 00$ $-1.67131 \mathrm{E} \quad 00$ $-1.68555 \mathrm{E} \quad 00$

$-1.69985 \mathrm{E} \quad 00$ $-1.71420 \mathrm{E} 00$ $-1.72861 \mathrm{E} \quad 00$ $-1.74308 \mathrm{E} 00$ -1.75761 E 00

$-1.77220 \mathrm{E} \quad 00$ $-1.78684 \mathrm{E} \quad 00$ $-1.80154 \mathrm{E} \quad 00$ $-1.81629 \mathrm{E} \quad 00$ $-1.83111 \mathrm{E} 00$

$-1.84598 \mathrm{E} \quad 00$ $-1.86090 \mathrm{E} \quad 00$ $-1.87589 \mathrm{E} 00$ $-1.89093 \mathrm{E} 00$ $-1.90603 \mathrm{E} \quad 00$

$-1.92119 \mathrm{E} \quad 00$ $-1.93640 \mathrm{E} 00$ $-1.95167 \mathrm{E} \quad 00$ $-1.96700 \mathrm{E} 00$ $-1.98238 \mathrm{E} \quad 00$

$-1.99782 \mathrm{E} \quad 00$ $-2.01332 \mathrm{E} \quad 00$ $-2.02888 \mathrm{E} \quad 00$ $-2.06016 \mathrm{E} 00$

$-2.07589 \mathrm{E} \quad 00$ $-2.09167 \mathrm{E} \quad 00$ $-2.10751 \mathrm{E} \quad 00$ $-2.12341 \mathrm{E} \quad 00$ $-2.13936 \mathrm{E} \quad 00$

$-2.15537 \mathrm{E} \quad 00$ $-2.17144 \mathrm{E} \quad 00$ $-2.18757 \mathrm{E} \quad 00$ $-2.20375 \mathrm{E} 00$ $-2.21998 \mathrm{E} \quad 00$

$-2.23628 \mathrm{E} \quad 00$ $-2.25263 \mathrm{E} \quad 00$ $-2.26904 \mathrm{E} \quad 00$ $-2.28551 \mathrm{E} 00$ -2.30203 E 00

$-2.31861 \mathrm{E} \quad 00$ $-2.33524 \mathrm{E} \quad 00$ $-2.35193 \mathrm{E} 00$ $-2.36868 \mathrm{E} \quad 00$ $-2.38548 \mathrm{E} \quad 00$ $-2.04449 \mathrm{E} \quad 00$ $g_{-1}$ $\tan \delta_{-1}$

$\begin{array}{lllll}2.04233 \mathrm{E} & 01 & -9.80727 \mathrm{E} & 01\end{array}$ $2.05311 \mathrm{E} \quad 01$ $2.06388 \mathrm{E} \quad 01$ $2.07465 \mathrm{E} \quad 01$ $2.08542 \mathrm{E} \quad 01$

$2.09618 \mathrm{E} \quad 01$ $2.10695 \mathrm{E} \quad 01$ $2.11771 \mathrm{E} 01$ $2.12847 \mathrm{E} \quad 01$ $2.13922 \mathrm{E} \quad 01$

$2.14997 \mathrm{E} \quad 01$ $2.16072 \mathrm{E} \quad 01$ $2.17147 \mathrm{E} \quad 01$ $2.18222 \mathrm{E} \quad 01$ $2.19296 \mathrm{E} \quad 01$

2.20370 E 01 $2.21444 \mathrm{E} \quad 01$ $2.22517 \mathrm{E} \quad 01$ $2.23590 \mathrm{E} 01$ $2.24663 \mathrm{E} \quad 01$

$2.25736 \mathrm{E} \quad 01$ $2.26808 \mathrm{E} \quad 01$ $2.27880 \mathrm{E} \quad 01$ 2.28952 E 01

2.30023 E 01

$2.31095 \mathrm{E} \quad 01$ $2.32166 \mathrm{E} \quad 01$ $2.33236 \mathrm{E} \quad 01$ $2.34307 \mathrm{E} \quad 01$ $2.35377 \mathrm{E} \quad 01$

$2.36447 \mathrm{E} \quad 01$ $2.37516 \mathrm{E} \quad 01$ $2.38586 \mathrm{E} 01$ $2.39655 \mathrm{E} \quad 01$ $2.40723 \mathrm{E} \quad 01$

$2.41792 \mathrm{E} \quad 01$ $2.42860 \mathrm{E} \quad 01$ $2.43928 \mathrm{E} 01$ $2.44996 \mathrm{E} \quad 01$

2.46063 E 01

$2.47130 \mathrm{E} \quad 01$ $2.48197 \mathrm{E} \quad 01$ $2.49263 \mathrm{E} \quad 01$ $2.50330 \mathrm{E} \quad 01$ $2.51396 \mathrm{E} \quad 01$

$2.52461 \mathrm{E} \quad 01$ $2.53527 \mathrm{E} \quad 01$ $2.54592 \mathrm{E} 01$ $2.55656 \mathrm{E} \quad 01$ $2.56721 \mathrm{E} \quad 01$

2.57785 E 01 $2.58849 \mathrm{E} \quad 01$ $2.59913 \mathrm{E} \quad 01$ $2.60976 \mathrm{E} 01$ $2.62039 \mathrm{E} 01$

$2.63102 \mathrm{E} \quad 01$ $2.64165 \mathrm{E} 01$ $2.65227 \mathrm{E} \quad 01$ $2.66289 \mathrm{E} \quad 01$ $2.67350 \mathrm{E} \quad 01$
$-9.81886 \mathrm{E} \quad 01$ $-9.83039 \mathrm{E} 01$ $-9.84184 \mathrm{E} \quad 01$ $-9.85322 \mathrm{E} 01$

$-9.86456 \mathrm{E} \quad 01$ $-9.87582 \mathrm{E} \quad 01$ $-9.88701 \mathrm{E} \quad 01$ $-9.89813 \mathrm{E} \quad 01$ $-9.90921 \mathrm{E} 01$

$-9.92023 \mathrm{E} \quad 01$ $-9.93116 \mathrm{E} \quad 01$ $-9.94207 \mathrm{E} 01$ $-9.95290 \mathrm{E} \quad 01$ $-9.96368 \mathrm{E} \quad 01$

$-9.97440 \mathrm{E} 01$ $-9.98507 \mathrm{E} \quad 01$ $-9.99568 \mathrm{E} \quad 01$ $-1.00063 \mathrm{E} 02$ -1.00168 E 02

$-1.00272 \mathrm{E} \quad 02$ $-1.00376 \mathrm{E} \quad 02$ $-1.00480 \mathrm{E} \quad 02$ -1.00583 E 02 $-1.00686 \mathrm{E} \quad 02$

$-1.00788 \mathrm{E} \quad 02$ $-1.00890 \mathrm{E} \quad 02$ $-1.00991 \mathrm{E} \quad 02$ $-1.01092 \mathrm{E} \quad 02$ $-1.01192 \mathrm{E} \quad 02$

$-1.01292 \mathrm{E} 02$ $-1.01391 \mathrm{E} \quad 02$ $-1.01491 \mathrm{E} \quad 02$ $-1.01688 \mathrm{E} \quad 02$

$\begin{array}{ll}-1.01785 \mathrm{E} & 02\end{array}$ $-1.01883 \mathrm{E} 02$ $-1.01980 \mathrm{E} \quad 02$ $-1.02077 \mathrm{E} \quad 02$ $-1.02173 \mathrm{E} \quad 02$ $-1.02269 \mathrm{E} \quad 02$ $-1.02365 \mathrm{E} \quad 02$ $-1.02460 \mathrm{E} \quad 02$ $-1.02555 \mathrm{E} \quad 02$ $-1.02650 \mathrm{E} \quad 02$ $-1.02744 \mathrm{E} \quad 02$ $-1.02838 \mathrm{E} 02$ $-1.02932 \mathrm{E} 02$ $-1.03025 \mathrm{E} 02$ $-1.03118 \mathrm{E} \quad 02$ $-1.03211 \mathrm{E} \quad 02$ $-1.03304 \mathrm{E} 02$ $-1.03396 \mathrm{E} 02$ $-1.03488 \mathrm{E} 02$ $-1.03579 \mathrm{E} 02$ $-1.03670 \mathrm{E} \quad 02$ $\begin{array}{lll}-1.03762 \mathrm{E} & 02\end{array}$ $-1.03852 \mathrm{E} 02$ $-1.03943 \mathrm{E} 02$ $-1.04033 \mathrm{E} 02$ $-1.01589 \mathrm{E} 02$ 
ELECTRON RADIAL FUNCTIONS

$Z=9 \quad A=18 \quad \rho=1.2 A^{1 / 3} F$

Electron

$\bar{F}$

$f_{2}$

$g_{2}$

$7.46174 \mathrm{E}-04$

$7.54431 \mathrm{E}-04$

$7.62732 \mathrm{E}-04$

$7.71079 \mathrm{E}-04$

$7.79472 \mathrm{E}-04$

$7.87910 \mathrm{E}-04$

$7.96393 \mathrm{E}-04$

$8.04921 \mathrm{E}-04$

$8.13495 \mathrm{E}-04$

$8.22115 \mathrm{E}-04$

$8.30779 \mathrm{E}-04$

$8.39490 \mathrm{E}-04$

$8.48245 \mathrm{E}-04$

$8.57046 \mathrm{E}-04$

$8.65893 \mathrm{E}-04$

$8.74784 \mathrm{E}-04$

$8.83721 \mathrm{E}-04$

$8.92704 \mathrm{E}-04$

$9.01732 \mathrm{E}-04$

$9.10805 \mathrm{E}-04$

$9.19924 \mathrm{E}-04$ $9.29088 \mathrm{E}-04$ $9.38297 \mathrm{E}-04$

$9.47552 \mathrm{E}-04$

$9.56852 \mathrm{E}-04$

$9.66198 \mathrm{E}-04$ $9.75589 \mathrm{E}-04$ $9.85025 \mathrm{E}-04$ $9.94507 \mathrm{E}-04$

$1.00403 \mathrm{E}-03$

$1.01361 \mathrm{E}-03$ $1.02322 \mathrm{E}-03$ $1.03289 \mathrm{E}-03$ $1.04260 \mathrm{E}-03$

$1.05235 \mathrm{E}-03$

$1.06215 \mathrm{E}-03$

$1.07199 \mathrm{E}-03$

$1.08188 \mathrm{E}-03$

$1.09182 \mathrm{E}-03$

$1.10180 \mathrm{E}-03$

$1.11183 \mathrm{E}-03$

$1.12190 \mathrm{E}-03$

$1.13201 \mathrm{E}-03$

$1.14218 \mathrm{E}-03$

$1.15238 \mathrm{E}-03$

$1.16264 \mathrm{E}-03$

$1.17294 \mathrm{E}-03$

$1.18328 \mathrm{E}-03$

$1.19367 \mathrm{E}-03$

$1.20410 \mathrm{E}-03$

$1.21458 \mathrm{E}-03$

$1.22511 \mathrm{E}-03$

$1.23568 \mathrm{E}-03$

$1.24629 \mathrm{E}-03$

$1.25696 \mathrm{E}-03$

$1.26766 \mathrm{E}-03$

$1.27842 \mathrm{E}-03$

$1.28921 \mathrm{E}-03$

$1.30006 \mathrm{E}-03$

$1.31094 \mathrm{E}-03$
$-9.54740 \mathrm{E}-01$

$-9.65419 \mathrm{E}-01$

$-9.76156 \mathrm{E}-01$

$-9.86952 \mathrm{E}-01$

$-9.97807 \mathrm{E}-01$

$-1.00872 \mathrm{E} \quad 00$ $-1.01969 \mathrm{E} \quad 00$ $-1.03072 \mathrm{E} 00$ $-1.04181 \mathrm{E} 00$ $-1.05296 \mathrm{E} 00$

$-1.06417 \mathrm{E} \quad 00$ $-1.07543 \mathrm{E} \quad 00$ $-1.08675 \mathrm{E} 00$ $-1.09814 \mathrm{E} \quad 00$ $-1.10958 \mathrm{E} 00$

$-1.12108 \mathrm{E} 00$ $-1.13263 \mathrm{E} \quad 00$ $-1.14425 \mathrm{E} \quad 00$ $-1.15592 \mathrm{E} \quad 00$ $-1.16766 \mathrm{E} 00$

$-1.17945 \mathrm{E} \quad 00$ $-1.19130 \mathrm{E} 00$ $-1.20321 \mathrm{E} \quad 00$ $-1.21517 \mathrm{E} \quad 00$ $-1.22720 \mathrm{E} 00$

$-1.23928 \mathrm{E} \quad 00$ $-1.25142 \mathrm{E} 00$ $-1.26362 \mathrm{E} 00$ $-1.27588 \mathrm{E} 00$ $-1.28820 \mathrm{E} 00$

$-1.30057 \mathrm{E} \quad 00$ $-1.31301 \mathrm{E} 00$ $-1.32550 \mathrm{E} \quad 00$ $-1.33805 \mathrm{E} \quad 00$ $-1.35066 \mathrm{E} 00$

$-1.36332 \mathrm{E} \quad 00$ $-1.37605 \mathrm{E} \quad 00$ $-1.38883 \mathrm{E} 00$ $-1.40167 \mathrm{E} \quad 00$ $-1.41457 \mathrm{E} 00$

\section{$-1.42752 \mathrm{E} \quad 00$} $-1.44054 \mathrm{E} \quad 00$ $-1.45361 \mathrm{E} \quad 00$ $-1.46674 \mathrm{E} \quad 00$ $-1.47993 \mathrm{E} 00$

$-1.49318 \mathrm{E} \quad 00$ $-1.50649 \mathrm{E} 00$ $-1.51985 \mathrm{E} 00$ $-1.53327 \mathrm{E} 00$ $-1.54675 \mathrm{E} 00$

$-1.56029 \mathrm{E} \quad 00$ $-1.57388 \mathrm{E} 00$ $-1.58754 \mathrm{E} 00$ $-1.60125 \mathrm{E} \quad 00$ $-1.61502 \mathrm{E} \quad 00$

$-1.62885 \mathrm{E} 00$ $-1.64273 \mathrm{E} 00$ $-1.65667 \mathrm{E} 00$ $-1.67068 \mathrm{E} 00$ $-1.68473 \mathrm{E} 00$ $\tan \delta_{2}$

$f_{-2}$

$-4.41423 \mathrm{E}-02$

$-4.47937 \mathrm{E}-02$

$-4.54514 \mathrm{E}-02$

$-4.61153 \mathrm{E}-02$

$-4.67856 \mathrm{E}-02$

-4.74621 E-02

$-4.81450 \mathrm{E}-02$

-4.88342 E-02

$-4.95299 \mathrm{E}-02$

$-5.02319 \mathrm{E}-02$

$-5.09405 \mathrm{E}-02$ $-5.16555 \mathrm{E}-02$ $-5.23770 \mathrm{E}-02$ $-5.31051 \mathrm{E}-02$ $-5.38397 \mathrm{E}-02$

$-5.45809 \mathrm{E}-02$ $-5.53288 \mathrm{E}-02$ $-5.60833 \mathrm{E}-02$ $-5.68445 \mathrm{E}-02$ $-5.76124 \mathrm{E}-02$

$-5.83871 \mathrm{E}-02$ $-5.91685 \mathrm{E}-02$ $-5.99567 \mathrm{E}-02$ $-6.07517 \mathrm{E}-02$ $-6.15536 \mathrm{E}-02$

$-6.23623 \mathrm{E}-02$ $-6.31780 \mathrm{E}-02$ $-6.40006 \mathrm{E}-02$ $-6.48302 \mathrm{E}-02$ $-6.56667 \mathrm{E}-02$

$-6.65103 \mathrm{E}-02$ $-6.73609 \mathrm{E}-02$ $-6.82186 \mathrm{E}-02$ $-6.90835 \mathrm{E}-02$ $-6.99554 \mathrm{E}-02$

$-7.08345 \mathrm{E}-02$ $-7.17208 \mathrm{E}-02$ $-7.26143 \mathrm{E}-02$ $-7.35150 \mathrm{E}-02$ $-7.44231 \mathrm{E}-02$

$-7.53384 \mathrm{E}-02$ $-7.62610 \mathrm{E}-02$ $-7.71911 \mathrm{E}-02$ $-7.81285 \mathrm{E}-02$ $-7.90733 \mathrm{E}-02$

$-8.00255 \mathrm{E}-02$ $-8.09853 \mathrm{E}-02$ $-8.19525 \mathrm{E}-02$ $-8.29272 \mathrm{E}-02$ $-8.39095 \mathrm{E}-02$

$-8.48994 \mathrm{E}-02$ $-8.58969 \mathrm{E}-02$ $-8.69020 \mathrm{E}-02$ $-8.79148 \mathrm{E}-02$ $-8.89353 \mathrm{E}-02$

$-8.99635 \mathrm{E}-02$ $-9.09994 \mathrm{E}-02$ $-9.20431 \mathrm{E}-02$ $-9.30947 \mathrm{E}-02$ $-9.41540 \mathrm{E}-02$
$2.30060 \mathrm{E} \quad 01$ $2.30090 \mathrm{E} \quad 01$ $2.30120 \mathrm{E} \quad 01$ $2.30150 \mathrm{E} \quad 01$

$2.30179 \mathrm{E} \quad 01$

$2.30208 \mathrm{E} 01$ $2.30237 \mathrm{E} \quad 01$ $2.30265 \mathrm{E} \quad 01$ $2.30293 \mathrm{E} 01$

$2.30321 \mathrm{E} \quad 01$

$2.30348 \mathrm{E} \quad 01$ $2.30375 \mathrm{E} \quad 01$ $2.30402 \mathrm{E} \quad 01$

$2.30429 \mathrm{E} 01$

$2.30455 \mathrm{E} \quad 01$

$2.30481 \mathrm{E} \quad 01$

$2.30507 \mathrm{E} \quad 01$

$2.30532 \mathrm{E} \quad 01$

$2.30557 \mathrm{E} 01$

$2.30582 \mathrm{E} \quad 01$

$2.30607 \mathrm{E} \quad 01$

$2.30631 \mathrm{E} \quad 01$

$2.30655 \mathrm{E} \quad 01$

$2.30679 \mathrm{E} 01$

$2.30703 \mathrm{E} \quad 01$

$2.30726 \mathrm{E} \quad 01$

$2.30750 \mathrm{E} 01$

$2.30773 \mathrm{E} 01$

$2.30795 \mathrm{E} \quad 01$

$2.30818 \mathrm{E} \quad 01$

$2.30840 \mathrm{E} \quad 01$

$2.30862 \mathrm{E} \quad 01$

$2.30884 \mathrm{E} \quad 01$

$2.30906 \mathrm{E} \quad 01$

$2.30927 \mathrm{E} \quad 01$

$2.30948 \mathrm{E} \quad 01$

$2.30969 \mathrm{E} \quad 01$

$2.30990 \mathrm{E} \quad 01$

$2.31031 \mathrm{E} 01$

$2.31051 \mathrm{E} \quad 01$

$2.31072 \mathrm{E} \quad 01$

$2.31091 \mathrm{E}$ 01

$2.31111 \mathrm{E} 01$

$2.31131 \mathrm{E} \quad 01$

$2.31150 \mathrm{E} \quad 01$ $2.31169 \mathrm{E} \quad 01$ $2.31188 \mathrm{E} .01$ $2.31207 \mathrm{E} \quad 01$ $2.31225 \mathrm{E} \quad 01$

$2.31244 \mathrm{E} \quad 01$ $2.31262 \mathrm{E} \quad 01$ $2.31280 \mathrm{E} 01$ $2.31298 \mathrm{E} 01$ $2.31316 \mathrm{E} 01$

$2.31334 \mathrm{E} \quad 01$ $2.31351 \mathrm{E} 01$ $2.31369 \mathrm{E}$ ol $2.31386 \mathrm{E}$ 01 $2.31403 \mathrm{E} 01$
$2.31011 \mathrm{E} 01$

$-4.33532 \mathrm{E}-02$ $-4.39914 \mathrm{E}-02$ $-4.46357 \mathrm{E}-02$ $-4.52862 \mathrm{E}-02$ $-4.59429 \mathrm{E}-02$

$-4.66057 \mathrm{E}-02$ $-4.72749 \mathrm{E}-02$ $-4.79503 \mathrm{E}-02$ $-4.86320 \mathrm{E}-02$ $-4.93201 \mathrm{E}-02$

$-5.00145 \mathrm{E}-02$ $-5.07153 \mathrm{E}-02$ $-5.14225 \mathrm{E}-02$ $-5.21361 \mathrm{E}-02$ $-5.28562 \mathrm{E}-02$

$-5.35829 \mathrm{E}-02$ $-5.43160 \mathrm{E}-02$ $-5.50557 \mathrm{E}-02$ $-5.58020 \mathrm{E}-02$ $-5.65549 \mathrm{E}-02$

$-5.73145 \mathrm{E}-02$ $-5.80807 \mathrm{E}-02$ $-5.88536 \mathrm{E}-02$ $-5.96333 \mathrm{E}-02$ $-6.04197 \mathrm{E}-02$

$-6.12129 \mathrm{E}-02$ $-6.20129 \mathrm{E}-02$ $-6.28197 \mathrm{E}-02$ $-6.36334 \mathrm{E}-02$ $-6.44540 \mathrm{E}-02$

$-6.52815 \mathrm{E}-02$ $-6.61160 \mathrm{E}-02$ $-6.69574 \mathrm{E}-02$ $-6.78059 \mathrm{E}-02$ $-6.86614 \mathrm{E}-02$

$-6.95239 \mathrm{E}-02$ $-7.03936 \mathrm{E}-02$ $-7.12704 \mathrm{E}-02$ $-7.21543 \mathrm{E}-02$

$-7.30454 \mathrm{E}-02$

$-7.39437 \mathrm{E}-02$ 


$$
Z=9 \quad A=18
$$$$
\rho=1.2 A^{1 / 3} F
$$

p

24.10

24.20

24.30

24.40

24.50

24.60

24.70

24.80

24.90

25.00

25.10

25.20

25.30

25.40

25.50

25.60

25.70

25.80

25.90

26.00

26.10

26.20

26.30

26.40

26.50

26.60

26.70

26.80

26.90

27.00

27.10

27.20

27.30

27.40

27.50

27.60

27.70

27.80

27.90

28.00

28.10

28.20

28.30

28.40

28.50

28.60

28.70

28.80

28.90

29.00

29.10

29.20

29.30

29.40

29.50

29.60

29.70

29.80

29.90

30.00

$$
F_{0}
$$

$f_{1}$

$g_{1}$

$\tan \delta_{1}$

$f_{-1}$

$6.90412 \mathrm{E}-03 \quad-2.40235 \mathrm{E} \quad 00$ $6.90722 \mathrm{E}-03$ $6.91025 \mathrm{E}-03$ $6.91323 \mathrm{E}-03$ $6.91615 \mathrm{E}-03$

$6.91903 \mathrm{E}-03$ $6.92185 \mathrm{E}-03$ $6.92461 \mathrm{E}-03$ $6.92732 \mathrm{E}-03$ $6.92998 \mathrm{E}-03$

$6.93259 \mathrm{E}-03$ $6.93515 \mathrm{E}-03$ $6.93764 \mathrm{E}-03$ $6.94010 \mathrm{E}-03$ $6.94251 \mathrm{E}-03$

$1.23815 \mathrm{E} \quad 00$

$1.23813 \mathrm{E} \quad 00$ $1.23811 \mathrm{E} \quad 00$ $1.23809 \mathrm{E} \quad 00$ $1.23806 \mathrm{E} \quad 00$ $1.23804 \mathrm{E} \quad 00$

$1.23802 \mathrm{E} \quad 00$ $1.23800 \mathrm{E} \quad 00$ $1.23798 \mathrm{E} \quad 00$ $1.23795 \mathrm{E} \quad 00$ $1.23793 \mathrm{E} \quad 00$

$1.23791 \mathrm{E} \quad 00$ $1.23789 \mathrm{E} \quad 00$ $1.23787 \mathrm{E} \quad 00$ $1.23785 \mathrm{E} \quad 00$

$1.23783 \mathrm{E} \quad 00$

$1.23781 \mathrm{E} \quad 00$ $1.23779 \mathrm{E} \quad 00$ $1.23776 \mathrm{E} \quad 00$ $1.23774 \mathrm{E} \quad 00$ $1.23772 \mathrm{E} \quad 00$

$1.23770 \mathrm{E} \quad 00$ $1.23768 \mathrm{E} \quad 00$

$1.23766 \mathrm{E} \quad 00$

$1.23764 \mathrm{E} \quad 00$

$1.23762 \mathrm{E} \quad 00$

$1.23760 \mathrm{E} \quad 00$ $1.23758 \mathrm{E} \quad 00$ $1.23756 \mathrm{E} \quad 00$ $1.23754 \mathrm{E} \quad 00$

$1.23752 \mathrm{E} \quad 00$

$1.23750 \mathrm{E} \quad 00$

$1.23748 \mathrm{E} \quad 00$

$1.23746 \mathrm{E} \quad 00$

$1.23744 \mathrm{E} \quad 00$

$1.23742 \mathrm{E} \quad 00$

$1.23740 \mathrm{E} \quad 00$

$1.23739 \mathrm{E} \quad 00$

$1.23737 \mathrm{E} \quad 00$

$1.23735 \mathrm{E} \quad 00$

$1.23733 \mathrm{E} \quad 00$

$1.23731 \mathrm{E} \quad 00$ $1.23729 \mathrm{E} \quad 00$ $1.23727 \mathrm{E} \quad 00$ $1.23725 \mathrm{E} \quad 00$

$1.23723 \mathrm{E} \quad 00$
$-2.68214 \mathrm{E} \quad 01$ $-2.69274 \mathrm{E} 01$ $-2.70333 \mathrm{E} 01$ $-2.71392 \mathrm{E} \quad 01$ $-2.72451 \mathrm{E} 01$

$-2.73509 \mathrm{E}$ 0I $-2.74567 \mathrm{E} \quad 01$ $-2.75625 \mathrm{E} \quad 01$ $-2.76682 \mathrm{E} 01$

$-2.78796 \mathrm{E} 01$ $-2.79853 \mathrm{E} 01$ $-2.80909 \mathrm{E} \quad 01$ $-2.81965 \mathrm{E} 01$ $-2.83021 \mathrm{E} 01$

$-2.84076 \mathrm{E} \quad 01$ $-2.85131 \mathrm{E} 01$ $-2.86186 \mathrm{E} \quad 01$ $-2.87240 \mathrm{E}$ 01 $-2.88294 \mathrm{E} 01$

$-2.89348 \mathrm{E} \quad 01$ $-2.90401 \mathrm{E} 01$ $-2.91454 \mathrm{E} 01$ $-2.92507 \mathrm{E} 01$ $-2.93560 \mathrm{E} 01$

$-2.94612 \mathrm{E} 01$ $-2.97767 \mathrm{E} 01$ $-2.98817 \mathrm{E} \quad 01$

$-2.99868 \mathrm{E} \quad 01$ $-3.00918 \mathrm{E} 01$ $-3.01968 \mathrm{E} \quad 01$ $-3.03018 \mathrm{E} 0$ $-3.04067 \mathrm{E} 01$

$-3.05116 \mathrm{E} \quad 01$ $-3.06165 \mathrm{E} \quad 01$ $-3.07213 \mathrm{E} 01$ $-3.08262 \mathrm{E} 01$ $-3.09309 \mathrm{E} 01$

$-3.10357 \mathrm{E} 01$ $-3.11404 \mathrm{E} \quad 01$ $-3.12451 \mathrm{E} 01$ $-3.13497 \mathrm{E} 01$ $-3.14543 \mathrm{E} 01$

$-3.15589 \mathrm{E} \quad 01$ $-3.16634 \mathrm{E} 01$ $-3.17679 \mathrm{E} 01$ $-3.18724 \mathrm{E}$ 01 $-3.19769 \mathrm{E}$ 01 $-2.77739 \mathrm{E}$ 01 $-2.95664 \mathrm{E} 01$ $-2.96715 \mathrm{E} \quad 01$
$-2.62087 \mathrm{E} \quad 00$ $-2.63865 \mathrm{E} \quad 00$ $-2.65649 \mathrm{E} \quad 00$ $-2.67438 \mathrm{E} \quad 00$ $-2.69233 \mathrm{E} 00$

$-2.71034 \mathrm{E} \quad 00$ $-2.72840 \mathrm{E} \quad 00$ $-2.74652 \mathrm{E} \quad 00$ $-2.76469 \mathrm{E} \quad 00$ $-2.78292 \mathrm{E} \quad 00$

$-2.80121 \mathrm{E} \quad 00$ $-2.81955 \mathrm{E} 00$ $-2.83795 \mathrm{E} \quad 00$ $-2.85641 \mathrm{E} 00$ $-2.87492 \mathrm{E} 00$

$-2.89349 \mathrm{E} \quad 00$ $-2.91211 \mathrm{E} \quad 00$ $-2.93079 \mathrm{E} \quad 00$ $-2.94952 \mathrm{E} \quad 00$ $-2.96831 \mathrm{E} 00$

$-2.98716 \mathrm{E} \quad 00$ $-3.00606 \mathrm{E} \quad 00$ $-3.02502 \mathrm{E} 00$ $-3.04404 \mathrm{E} \quad 00$ $-3.06311 \mathrm{E} 00$

$-3.08223 \mathrm{E} \quad 00$ $-3.10141 \mathrm{E} 00$ $-3.12065 \mathrm{E} 00$ $-3.13995 \mathrm{E} 00$ $-3.15929 \mathrm{E} 00$

$-3.17870 \mathrm{E} \quad 00$ $-3.19816 \mathrm{E} \quad 00$ $-3.21767 \mathrm{E} \quad 00$ $-3.25687 \mathrm{E} 00$

$-3.27656 \mathrm{E} \quad 00$ $-3.29629 \mathrm{E} \quad 00$ $-3.31609 \mathrm{E} 00$ $-3.33594 \mathrm{E} \quad 00$

$-3.37580 \mathrm{E} \quad 00$ $-3.39582 \mathrm{E} \quad 00$ $-3.41589 \mathrm{E} \quad 00$ $-3.43602 \mathrm{E} \quad 00$ $-3.45620 \mathrm{E} \quad 00$

$-3.47643 \mathrm{E} \quad 00$ $-3.49673 \mathrm{E} 00$ $-3.51707 \mathrm{E} \quad 00$ $-3.53748 \mathrm{E} 00$ $-3.55794 \mathrm{E} \quad 00$ $-3.23725 \mathrm{E} \quad 00$ $-3.35584 \mathrm{E} 00$
$6.94485 \mathrm{E}-03$ $6.94716 \mathrm{E}-03$ $6.94942 \mathrm{E}-03$ $6.95162 \mathrm{E}-03$ $6.95379 \mathrm{E}-03$

$6.95590 \mathrm{E}-03$ $6.95797 \mathrm{E}-03$ $6.95999 \mathrm{E}-03$ $6.96196 \mathrm{E}-03$ $6.96390 \mathrm{E}-03$

$6.96578 \mathrm{E}-03$ $6.96762 \mathrm{E}-03$ $6.96942 \mathrm{E}-03$ $6.97116 \mathrm{E}-03$ $6.97287 \mathrm{E}-03$

$6.97453 \mathrm{E}-03$ $6.97615 \mathrm{E}-03$ $6.97772 \mathrm{E}-03$ $6.97926 \mathrm{E}-03$ $6.98076 \mathrm{E}-03$

$6.98221 \mathrm{E}-03$ $6.98362 \mathrm{E}-03$ $6.98500 \mathrm{E}-03$ $6.98633 \mathrm{E}-03$ $6.98761 \mathrm{E}-03$

$6.98887 \mathrm{E}-03$ $6.99007 \mathrm{E}-03$ $6.99124 \mathrm{E}-03$ $6.99237 \mathrm{E}-03$ $6.99347 \mathrm{E}-03$

$6.99452 \mathrm{E}-03$ $6.99554 \mathrm{E}-03$ $6.99652 \mathrm{E}-03$ $6.99746 \mathrm{E}-03$ $6.99836 \mathrm{E}-03$

$6.99924 \mathrm{E}-03$ $7.00007 \mathrm{E}-03$

$7.00086 \mathrm{E}-03$

$7.00162 \mathrm{E}-03$

$7.00234 \mathrm{E}-03$

$7.00303 \mathrm{E}-03$ $7.00368 \mathrm{E}-03$

$7.00430 \mathrm{E}-03$

$7.00489 \mathrm{E}-03$

$7.00543 \mathrm{E}-03$ $-2.41926 \mathrm{E} \quad 00$ $-2.43624 \mathrm{E} \quad 00$ $-2.45327 \mathrm{E} \quad 00$ $-2.47036 \mathrm{E} \quad 00$

$-2.48750 \mathrm{E} \quad 00$ $-2.50470 \mathrm{E} \quad 00$ $-2.52196 \mathrm{E} 00$ $-2.53927 \mathrm{E} \quad 00$ $-2.55664 \mathrm{E} \quad 00$

$-2.57406 \mathrm{E} \quad 00$ 
ELECTRON RADIAL FUNCTIONS

$Z=9 \quad A=18 \quad \rho=1.2 A^{1 / 3} F$

Electron

$\bar{F}$

$1.32188 \mathrm{E}-03$

$1.33286 \mathrm{E}-03$

$1.34388 \mathrm{E}-03$

$1.35495 \mathrm{E}-03$

$1.36607 \mathrm{E}-03$

$1.37723 \mathrm{E}-03$

$1.38843 \mathrm{E}-03$

$1.39968 \mathrm{E}-03$

$1.41098 \mathrm{E}-03$

$1.42232 \mathrm{E}-03$

$1.43371 \mathrm{E}-03$

$1.44514 \mathrm{E}-03$

$1.45662 \mathrm{E}-03$

$1.46814 \mathrm{E}-03$

$1.47971 \mathrm{E}-03$

$1.49132 \mathrm{E}-03$

$1.50298 \mathrm{E}-03$

$1.51469 \mathrm{E}-03$

$1.52644 \mathrm{E}-03$

$1.53823 \mathrm{E}-03$

$1.55007 \mathrm{E}-03$

$1.56196 \mathrm{E}-03$

$1.57389 \mathrm{E}-03$

$1.58587 \mathrm{E}-03$

$1.59789 \mathrm{E}-03$

$1.60996 \mathrm{E}-03$

$1.62207 \mathrm{E}-03$

$1.63423 \mathrm{E}-03$

$1.64643 \mathrm{E}-03$

$1.65868 \mathrm{E}-03$

$1.67097 \mathrm{E}-03$

$1.68331 \mathrm{E}-03$

$1.69570 \mathrm{E}-03$

$1.70813 \mathrm{E}-03$

$1.72061 \mathrm{E}-03$

$1.73313 \mathrm{E}-03$

$1.74569 \mathrm{E}-03$

$1.75831 \mathrm{E}-03$

$1.77096 \mathrm{E}-03$

$1.78366 \mathrm{E}-03$

$1.79641 \mathrm{E}-03$

$1.80921 \mathrm{E}-03$

$1.82204 \mathrm{E}-03$

$1.83493 \mathrm{E}-03$

$1.84786 \mathrm{E}-03$

$1.86083 \mathrm{E}-03$

$1.87385 \mathrm{E}-03$

$1.88692 \mathrm{E}-03$

$1.90003 \mathrm{E}-03$

$1.91318 \mathrm{E}-03$

$1.92638 \mathrm{E}-03$

$1.93963 \mathrm{E}-03$

$1.95292 \mathrm{E}-03$

$1.96626 \mathrm{E}-03$

$1.97964 \mathrm{E}-03$

$1.99307 \mathrm{E}-03$

$2.00654 \mathrm{E}-03$

2.02006 E-03

$2.03363 \mathrm{E}-03$

$2.04723 \mathrm{E}-03$ $f_{2}$

$-1.69885 \mathrm{E} \quad 00$

$-1.71303 \mathrm{E} 00$

$-1.72726 \mathrm{E} \quad 00$

$-1.74155 \mathrm{E} 00$

$-1.75590 \mathrm{E} \quad 00$

$-1.77030 \mathrm{E} \quad 00$

$-1.78477 \mathrm{E} \quad 00$

$-1.79929 \mathrm{E} \quad 00$

$-1.81386 \mathrm{E} \quad 00$

$-1.82850 \mathrm{E} 00$

$-1.84319 \mathrm{E} \quad 00$

$-1.85795 \mathrm{E} \quad 00$

$-1.87276 \mathrm{E} \quad 00$

$-1.88762 \mathrm{E} 00$

$-1.90255 \mathrm{E} \quad 00$

$-1.91753 \mathrm{E} \quad 00$

$-1.93257 \mathrm{E} 00$

$-1.94767 \mathrm{E} 00$

$-1.96282 \mathrm{E} \quad 00$

$-1.97803 \mathrm{E} \quad 00$

$-1.99330 \mathrm{E} \quad 00$

$-2.00863 \mathrm{E} \quad 00$

$-2.02402 \mathrm{E} 00$

$-2.03946 \mathrm{E} \quad 00$

$-2.05496 \mathrm{E} \quad 00$

$-2.07051 \mathrm{E} \quad 00$

$-2.08613 \mathrm{E} \quad 00$

$-2.10180 \mathrm{E} 00$

$-2.11753 \mathrm{E} \quad 00$

$-2.13332 \mathrm{E} \quad 00$

$-2.14916 \mathrm{E} \quad 00$

$-2.16506 \mathrm{E} \quad 00$

$-2.18102 \mathrm{E} \quad 00$

$-2.19704 \mathrm{E} \quad 00$

$-2.21311 \mathrm{E} \quad 00$

$-2.22924 \mathrm{E} \quad 00$

$-2.24543 \mathrm{E} \quad 00$

$-2.26167 \mathrm{E} \quad 00$

$-2.27797 \mathrm{E} \quad 00$

$-2.29433 \mathrm{E} 00$

$-2.31075 \mathrm{E} \quad 00$

$-2.32722 \mathrm{E} \quad 00$

$-2.34375 \mathrm{E} \quad 00$

$-2.36034 \mathrm{E} 00$

$-2.37699 \mathrm{E} \quad 00$

$-2.39369 \mathrm{E} \quad 00$

$-2.41045 \mathrm{E} \quad 00$

$-2.42726 \mathrm{E} \quad 00$

$-2.44414 \mathrm{E} \quad 00$

$-2.46107 \mathrm{E} \quad 00$

$-2.47805 \mathrm{E} \quad 00$

$-2.49510 \mathrm{E} \quad 00$

$-2.51220 \mathrm{E} \quad 00$

$-2.52936 \mathrm{E} \quad 00$

$-2.54657 \mathrm{E} \quad 00$

$-2.56384 \mathrm{E} \quad 00$

$-2.58117 \mathrm{E} \quad 00$

$-2.59856 \mathrm{E} \quad 00$

$-2.61600 \mathrm{E} \quad 00$

$-2.63350 \mathrm{E} 00$ $g_{2}$

$-9.52213 \mathrm{E}-02$

- $9.62964 \mathrm{E}-02$

$-9.73795 \mathrm{E}-02$

$-9.84704 \mathrm{E}-02$

$-9.95694 \mathrm{E}-02$

$-1.00676 \mathrm{E}-01$

$-1.01791 \mathrm{E}-01$

$-1.02914 \mathrm{E}-01$

$-1.04045 \mathrm{E}-01$

$-1.05185 \mathrm{E}-01$

$-1.06332 \mathrm{E}-01$

$-1.07488 \mathrm{E}-01$

$-1.08651 \mathrm{E}-01$

$-1.09823 \mathrm{E}-01$

$-1.11003 \mathrm{E}-01$

$-1.12192 \mathrm{E}-01$

$-1.13389 \mathrm{E}-01$

$-1.14594 \mathrm{E}-01$

$-1.15807 \mathrm{E}-01$

$-1.17029 \mathrm{E}-01$

$-1.18259 \mathrm{E}-01$

$-1.19498 \mathrm{E}-01$

$-1.20745 \mathrm{E}-01$

$-1.22001 \mathrm{E}-01$

$-1.23265 \mathrm{E}-01$

$-1.24538 \mathrm{E}-01$

$-1.25819 \mathrm{E}-01$

$-1.27109 \mathrm{E}-01$

$-1.28408 \mathrm{E}-01$

$-1.29715 \mathrm{E}-01$

$-1.31031 \mathrm{E}-01$

$-1.32355 \mathrm{E}-01$

$-1.33689 \mathrm{E}-01$

$-1.35031 \mathrm{E}-01$

$-1.36382 \mathrm{E}-01$

$-1.37742 \mathrm{E}-01$

$-1.39110 \mathrm{E}-01$

$-1.40488 \mathrm{E}-01$

$-1.41874 \mathrm{E}-01$

$-1.43270 \mathrm{E}-01$

$-1.44674 \mathrm{E}-01$

$-1.46088 \mathrm{E}-01$

$-1.47510 \mathrm{E}-01$

$-1.48941 \mathrm{E}-01$

$-1.50382 \mathrm{E}-01$

$-1.51831 \mathrm{E}-01$

$-1.53290 \mathrm{E}-01$

$-1.54758 \mathrm{E}-01$

$-1.56235 \mathrm{E}-01$

$-1.57721 \mathrm{E}-01$

$-1.59217 \mathrm{E}-01$

$-1.60722 \mathrm{E}-01$

$-1.62236 \mathrm{E}-01$

$-1.63759 \mathrm{E}-01$

$-1.65292 \mathrm{E}-01$

$-1.66834 \mathrm{E}-01$

$-1.68386 \mathrm{E}-01$

$-1.69947 \mathrm{E}-01$

$-1.71517 \mathrm{E}-01$

$-1.73097 \mathrm{E}-01$

$\tan \delta_{2}$

$f_{-2}$

$2.31420 \mathrm{E} \quad 01$

$-9.45220 \mathrm{E}-02$

$2.31453 \mathrm{E} \quad 01 \quad-9.55859 \mathrm{E}-02$

$2.31470 \mathrm{E} \quad 01 \quad-9.66576 \mathrm{E}-02$

$2.31486 \mathrm{E} \quad 01 \quad-9.77372 \mathrm{E}-02$

$2.31502 \mathrm{E} \quad 01$

$2.31518 \mathrm{E} \quad 01$

$2.31534 \mathrm{E} \quad 01$

$2.31550 \mathrm{E} \quad 01$

$2.31566 \mathrm{E} \quad 01$

$2.31581 \mathrm{E} \quad 01$

$2.31597 \mathrm{E} \quad 01$

$2.31612 \mathrm{E} \quad 01$

$2.31627 \mathrm{E} \quad 01$

$2.31642 \mathrm{E} \quad 01$

$2.31657 \mathrm{E} \quad 01$

$2.31672 \mathrm{E} \quad 01$

$2.31687 \mathrm{E} \quad 01$

$2.31701 \mathrm{E} \quad 01$

$2.31716 \mathrm{E} \quad 01$

$2.31730 \mathrm{E} \quad 01$

$2.31744 \mathrm{E} \quad 01$

$2.31758 \mathrm{E} \quad 01$

$2.31772 \mathrm{E} \quad 01$

$2.31786 \mathrm{E} \quad 01$

$2.31800 \mathrm{E} \quad 01$

$2.31814 \mathrm{E} \quad 01$

$2.31827 \mathrm{E} \quad 01$

$2.31841 \mathrm{E} \quad 01$

$2.31854 \mathrm{E} 01$

$2.31867 \mathrm{E} \quad 01$

$2.31880 \mathrm{E} \quad 01$

$2.31893 \mathrm{E} \quad 01$

$2.31906 \mathrm{E} \quad 01$

$2.31919 \mathrm{E} \quad 01$

$2.31932 \mathrm{E} \quad 01$ $2.31945 \mathrm{E} \quad 01$ $2.31957 \mathrm{E} \quad 01$

$2.31970 \mathrm{E} 01$

$2.31982 \mathrm{E} \quad 01$

$2.31994 \mathrm{E} \quad 01$ $2.32007 \mathrm{E} \quad 01$

$2.32019 \mathrm{E} 01$

$2.32031 \mathrm{E} \quad 01$

$2.32043 \mathrm{E} \quad 01$

$2.32054 \mathrm{E} \quad 01$

$2.32066 \mathrm{E} \quad 01$

$2.32078 \mathrm{E} 01$

$2.32089 \mathrm{E} \quad 01$

$2.32101 \mathrm{E} 01$

$-9.88247 \mathrm{E}-02$

$-9.99202 \mathrm{E}-02$

$-1.01024 \mathrm{E}-01$

$-1.02135 \mathrm{E}-01$

$-1.03254 \mathrm{E}-01$

$-1.04382 \mathrm{E}-01$

$-1.05517 \mathrm{E}-01$

$-1.06661 \mathrm{E}-01$

$-1.07813 \mathrm{E}-01$

$-1.08973 \mathrm{E}-01$

$-1.10141 \mathrm{E}-01$

$-1.11317 \mathrm{E}-01$

$-1.12501 \mathrm{E}-01$

$-1.13694 \mathrm{E}-01$

$-1.14895 \mathrm{E}-01$

$-1.16105 \mathrm{E}-01$ $-1.17322 \mathrm{E}-01$

$-1.18549 \mathrm{E}-01$

$-1.19783 \mathrm{E}-01$

$-1.21026 \mathrm{E}-01$

$-1.22277 \mathrm{E}-01$

$-1.23537 \mathrm{E}-01$

$-1.24806 \mathrm{E}-01$

$-1.26083 \mathrm{E}-01$

$-1.27368 \mathrm{E}-01$

$-1.28662 \mathrm{E}-01$

$-1.29965 \mathrm{E}-01$

$-1.31276 \mathrm{E}-01$

$-1.32596 \mathrm{E}-01$

$-1.33925 \mathrm{E}-01$

$-1.35263 \mathrm{E}-01$ $-1.36609 \mathrm{E}-01$ $-1.37964 \mathrm{E}-01$ $-1.39328 \mathrm{E}-01$

$-1.40701 \mathrm{E}-01$

$-1.42082 \mathrm{E}-01$ $-1.43473 \mathrm{E}-01$ $-1.44872 \mathrm{E}-01$ $-1.46281 \mathrm{E}-01$ $-1.47698 \mathrm{E}-01$

$2.32112 \mathrm{E} \quad 01$ $2.32124 \mathrm{E} \quad 01$ $2.32135 \mathrm{E} \quad 0$ $2.32146 \mathrm{E} \quad 01$ $2.32157 \mathrm{E} 01$

$-1.49124 \mathrm{E}-01$ $-1.50560 \mathrm{E}-01$ $-1.52004 \mathrm{E}-01$ $-1.53458 \mathrm{E}-01$ $-1.54920 \mathrm{E}-01$

$-1.56392 \mathrm{E}-01$ $-1.57873 \mathrm{E}-01$ $-1.59363 \mathrm{E}-01$ 
ELECTRON RADIAL FUNCTIONS

Electron

$p$

30.10

30.20

30.30

30.40

30.50

30.60

30.70

30.80

30.90

31.00

31.10

31.20

31.30

31.40

31.50

31.60

31.70

31.80

31.90

32.00

32.10

32.20

32.30

32.40

32.50

32.60

32.70

32.80

32.90

33.00

33.10

33.20

33.30

33.40

33.50

33.60

33.70

33.80

33.90

34.00

34.10

34.20

34.30

34.40

34.50

34.60

34.70

34.80

34.90

35.00

35.10

35.20

35.30

$35.4 \mathrm{C}$

35.50

35.60

35.70

35.80

35.90

36.00

$$
Z=9 \quad A=18
$$

$f_{1}$

$1.23722 \mathrm{E} \quad 00$ $1.23720 \mathrm{E} \quad 00$ $1.23718 \mathrm{E} \quad 00$ $1.23716 \mathrm{E} \quad 00$

$1.23714 \mathrm{E} \quad 00$

$1.23712 \mathrm{E} \quad 00$ $1.23710 \mathrm{E} \quad 00$ $1.23709 \mathrm{E} \quad 00$ $1.23707 \mathrm{E} \quad 00$ $1.23705 \mathrm{E} \quad 00$

$1.23703 \mathrm{E} \quad 00$ $1.23701 \mathrm{E} \quad 00$ $1.23700 \mathrm{E} \quad 00$ $1.23698 \mathrm{E} \quad 00$ $1.23696 \mathrm{E} \quad 00$

$1.23694 \mathrm{E} \quad 00$ $1.23693 \mathrm{E} \quad 00$ $1.23691 \mathrm{E} \quad 00$ $1.23689 \mathrm{E} \quad 00$

$1.23687 \mathrm{E} \quad 00$

$1.23686 \mathrm{E} \quad 00$

$1.23684 \mathrm{E} \quad 00$

$1.23682 \mathrm{E} \quad 00$

$1.23680 \mathrm{E} \quad 00$

$1.23679 \mathrm{E} \quad 00$

$1.23677 \mathrm{E} \quad 00$

$1.23675 \mathrm{E} \quad 00$

$1.23674 \mathrm{E} \quad 00$

$1.23672 \mathrm{E} \quad 00$

$1.23670 \mathrm{E} \quad 00$

$1.23668 \mathrm{E} \quad 00$

$1.23667 \mathrm{E} \quad 00$

$1.23665 \mathrm{E} \quad 00$

$1.23663 \mathrm{E} \quad 00$

$1.23662 \mathrm{E} \quad 00$

$1.23660 \mathrm{E} \quad 00$

$1.23658 \mathrm{E} \quad 00$

$1.23657 \mathrm{E} \quad 00$

$1.23655 \mathrm{E} \quad 00$

$1.23654 \mathrm{E} \quad 00$

$1.23652 \mathrm{E} \quad 00$

$1.23650 \mathrm{E} \quad 00$

$1.23649 \mathrm{E} \quad 00$

$1.23647 \mathrm{E} \quad 00$

$1.23645 \mathrm{E} \quad 00$

1.23644. E 00

$1.23642 \mathrm{E} \quad 00$

$1.23641 \mathrm{E} \quad 00$

$1.23639 \mathrm{E} \quad 00$

$1.23638 \mathrm{E} \quad 00$

$1.23636 \mathrm{E} \quad 00$

$1.23634 \mathrm{E} \quad 00$

$1.23633 \mathrm{E} \quad 00$

$1.23631 \mathrm{E} \quad 00$

$1.23630 \mathrm{E} \quad 00$

$1.23628 \mathrm{E} \quad 00$

$1.23627 \mathrm{E} \quad 00$

$1.23625 \mathrm{E} \quad 00$

$1.23623 \mathrm{E} \quad 00$

$1.23622 \mathrm{E} \quad 00$

\begin{abstract}
$g_{1}$
\end{abstract}
$\begin{array}{llll}-3.20813 \mathrm{E} & 01 & -3.57845 \mathrm{E} & 00\end{array}$ $-3.21857 \mathrm{E} \quad 01$ $-3.22900 \mathrm{E} 01$ $-3.23943 \mathrm{E} 01$

$-3.24986 \mathrm{E} 01$

$-3.26029 \mathrm{E} 01$ $-3.27071 \mathrm{E} \quad 01$ $-3.28113 \mathrm{E} 01$ $-3.29154 \mathrm{E}$ 01 $-3.30195 \mathrm{E} 01$

$-3.31236 \mathrm{E} \quad 01$ $-3.32277 \mathrm{E}$ 01 $-3.33317 \mathrm{E}$ 01 $-3.34356 \mathrm{E} 01$ $-3.35396 \mathrm{E} 01$

$-3.36435 \mathrm{E} 01$ $-3.37474 \mathrm{E} 01$ $-3.38512 \mathrm{E} 01$ $-3.39550 \mathrm{E} 01$ $-3.40588 \mathrm{E} 01$

\section{$-3.41626 \mathrm{E} 01$}

$-3.42663 \mathrm{E} 01$

$-3.43699 \mathrm{E} 01$

$-3.44736 \mathrm{E} 01$

$-3.45772 \mathrm{E} 01$

$-3.46807 \mathrm{E} \quad 01$ $-3.47843 \mathrm{E} 01$ $-3.48878 \mathrm{E} 01$ $-3.49913 \mathrm{E} 01$ $-3.50947 \mathrm{E} \quad 01$

$\begin{array}{ll}-3.51981 \mathrm{E} & 01\end{array}$ $-3.53014 \mathrm{E} 01$ $-3.54048 \mathrm{E} 01$ $-3.55081 \mathrm{E} 01$ $-3.56113 \mathrm{E}$ 0l

-3.57145 E 01 $-3.58177 \mathrm{E} \quad 01$ $-3.59209 \mathrm{E}$ 01. $-3.60240 \mathrm{E} \quad 01$ $-3.61271 \mathrm{E} 01$

$-3.62301 \mathrm{E} \quad 01$ $-3.63331 \mathrm{E} 01$ $-3.64361 \mathrm{E} 01$ $-3.65390 \mathrm{E} 01$ $-3.66419 \mathrm{E} 01$

$-3.67448 \mathrm{E} \quad 01$ $-3.68476 \mathrm{E} 01$ $-3.69504 \mathrm{E} 01$ $-3.70532 \mathrm{E} 01$ -3.71559 E 01

$-3.72586 \mathrm{E} 01$ $-3.73612 \mathrm{E} \quad 01$ $-3.74639 \mathrm{E} 01$ $-3.75664 \mathrm{E} \quad 01$ $-3.76690 \mathrm{E} 01$

$-3.77715 \mathrm{E} 01$ $-3.78740 \mathrm{E} 01$ $-3.79764 \mathrm{E} 01$ $-3.80788 \mathrm{E} 01$ -3.81811 E 01
$-3.59902 \mathrm{E} 00$

$-3.61964 \mathrm{E} \quad 00$

$-3.64032 \mathrm{E} \quad 00$

$-3.66106 \mathrm{E} 00$

$-3.68185 \mathrm{E} \quad 00$

$-3.70269 \mathrm{E} \quad 00$

$-3.72359 \mathrm{E} 00$

$-3.74454 \mathrm{E} \quad 00$

$-3.76555 \mathrm{E} 00$

$-3.78662 \mathrm{E} \quad 00$

$-3.80774 \mathrm{E} \quad 00$

$-3.82891 \mathrm{E} \quad 00$

$-3.85014 \mathrm{E} \quad 00$

$-3.87143 \mathrm{E} 00$

$-3.89277 \mathrm{E} \quad 00$ $-3.91416 \mathrm{E} 00$ $-3.93561 \mathrm{E} 00$ $-3.95711 \mathrm{E} 00$ $-3.97867 \mathrm{E} \quad 00$

$-4.00028 \mathrm{E} \quad 00$ $-4.02195 \mathrm{E} \quad 00$ $-4.04368 \mathrm{E} \quad 00$ $-4.06545 \mathrm{E} \quad 00$ $-4.08729 \mathrm{E} 00$

$-4.10917 \mathrm{E} \quad 00$ $-4.13112 \mathrm{E} \quad 00$ $-4.15311 \mathrm{E} 00$ $-4.17516 \mathrm{E} 00$ $-4.19727 \mathrm{E} 00$

$-4.21943 \mathrm{E} \quad 00$ $-4.24164 \mathrm{E} 00$ $-4.26391 \mathrm{E} 00$ $-4.28624 \mathrm{E} \quad 00$ -4.30861 E 00

$-4.33105 \mathrm{E} \quad 00$ $-4.35353 \mathrm{E} \quad 00$ -4.37607 E 00 $-4.39867 \mathrm{E} \quad 00$ $-4.42132 \mathrm{E} \quad 00$

$-4.44402 \mathrm{E} \quad 00$ $-4.46678 \mathrm{E} \quad 00$ $-4.48959 \mathrm{E} \quad 00$ $-4.51246 \mathrm{E} \quad 00$ $-4.53538 \mathrm{E} \quad 00$

$-4.55836 \mathrm{E} \quad 00$ $-4.58139 \mathrm{E} \quad 00$ $-4.60447 \mathrm{E} \quad 00$ $-4.62761 \mathrm{E} \quad 00$ -4.65080 E 00

$-4.67405 \mathrm{E} \quad 00$ $-4.69735 \mathrm{E} \quad 00$ $-4.72070 \mathrm{E} 00$ $-4.74411 \mathrm{E} \quad 00$ $-4.76757 \mathrm{E} \quad 00$

$-4.79109 \mathrm{E} \quad 00$ $-4.81466 \mathrm{E} \quad 00$ $-4.83828 \mathrm{E} \quad 00$ $-4.86196 \mathrm{E} 00$ $-4.88569 \mathrm{E} \quad 00$

$$
\rho=1.2 A^{1 / 3} F
$$

$\tan \delta_{1}$

$7.00595 \mathrm{E}-03$

$7.00642 \mathrm{E}-03$

$7.00687 \mathrm{E}-03$

$7.00729 \mathrm{E}-03$

$7.00766 \mathrm{E}-03$

7.00801 E-03

$7.00832 \mathrm{E}-03$

$7.00860 \mathrm{E}-03$

$7.00885 \mathrm{E}-03$

$7.00906 \mathrm{E}-03$

$7.00924 \mathrm{E}-03$

$7.00940 \mathrm{E}-03$

$7.00952 \mathrm{E}-03$

$7.00961 \mathrm{E}-03$

$7.00967 \mathrm{E}-03$

$7.00969 \mathrm{E}-03$

$7.00969 \mathrm{E}-03$

$7.00966 \mathrm{E}-03$

$7.00959 \mathrm{E}-03$

$7.00949 \mathrm{E}-03$

$7.00937 \mathrm{E}-03$

$7.00922 \mathrm{E}-03$

$7.00904 \mathrm{E}-03$

$7.00882 \mathrm{E}-03$

$7.00858 \mathrm{E}-03$

$7.00831 \mathrm{E}-03$

$7.00801 \mathrm{E}-03$

$7.00767 \mathrm{E}-03$

$7.00731 \mathrm{E}-03$

$7.00693 \mathrm{E}-03$

7.00651 E-03

$7.00607 \mathrm{E}-03$

$7.00560 \mathrm{E}-03$

$7.00510 \mathrm{E}-03$

$7.00457 \mathrm{E}-03$

$7.00402 \mathrm{E}-03$ 
$Z=9 \quad A=18 \quad \rho=1.2 A^{1 / 3} F$

$\bar{F}$

$2.06089 \mathrm{E}-03$

$2.07459 \mathrm{E}-03$

$2.08834 \mathrm{E}-03$

$2.10213 \mathrm{E}-03$

$2.11596 \mathrm{E}-03$

$2.12984 \mathrm{E}-03$

$2.14377 \mathrm{E}-03$

$2.15774 \mathrm{E}-03$

$2.17176 \mathrm{E}-03$

$2.18582 \mathrm{E}-03$

$2.19993 \mathrm{E}-03$

$2.21408 \mathrm{E}-03$

$2.22828 \mathrm{E}-03$

$2.24253 \mathrm{E}-03$

$2.25682 \mathrm{E}-03$

$2.27115 \mathrm{E}-03$

$2.28553 \mathrm{E}-03$

$2.29996 \mathrm{E}-03$

$2.31443 \mathrm{E}-03$

$2.32894 \mathrm{E}-03$

$2.34350 \mathrm{E}-03$

$2.35811 \mathrm{E}-03$

$2.37276 \mathrm{E}-03$

$2.38746 \mathrm{E}-03$

2.40220 E-03

$2.41699 \mathrm{E}-03$

$2.43182 \mathrm{E}-03$

$2.44670 \mathrm{E}-03$

$2.46163 \mathrm{E}-03$

$2.47660 \mathrm{E}-03$

33.00

33.10

33.20

33.30

33.40

33.50

33.60

33.70

33.80

33.90

34.00

34.10

34.20

34.30

34.40

34.50

34.60

34.70

34.80

34.90

35.00

35.10

35.20

35.30

35.40

35.50

35.60

35.70

35.80

35.90

36.00

2.49161 E-03

$2.50667 \mathrm{E}-03$

$2.52178 \mathrm{E}-03$

$2.53693 \mathrm{E}-03$

2.55212 E- 03

$2.56737 \mathrm{E}-03$

$2.58265 \mathrm{E}-03$

$2.59798 \mathrm{E}-03$

$2.61336 \mathrm{E}-03$

$2.62878 \mathrm{E}-03$

$2.64425 \mathrm{E}-03$

$2.65977 \mathrm{E}-03$

$2.67532 \mathrm{E}-03$

$2.69093 \mathrm{E}-03$

$2.70658 \mathrm{E}-03$

$2.72227 \mathrm{E}-03$

$2.73801 \mathrm{E}-03$

$2.75380 \mathrm{E}-03$

$2.76963 \mathrm{E}-03$

$2.78550 \mathrm{E}-03$

$2.80143 \mathrm{E}-03$

$2.81739 \mathrm{E}-03$

$2.83340 \mathrm{E}-03$

$2.84946 \mathrm{E}-03$

$2.86556 \mathrm{E}-03$

2.88171 E- 03 $2.89791 \mathrm{E}-03$

$2.91414 \mathrm{E}-03$

$2.93043 \mathrm{E}-03$

$2.94676 \mathrm{E}-03$ $f_{2}$

$-2.65105 \mathrm{E} \quad 00$

$-2.66866 \mathrm{E} \quad 00$

$-2.68633 \mathrm{E} 00$

$-2.70406 \mathrm{E} 00$

$-2.72184 \mathrm{E} 00$

$-2.73968 \mathrm{E} \quad 00$

$-2.75758 \mathrm{E} \quad 00$

$-2.77553 \mathrm{E} \quad 00$

$-2.79354 \mathrm{E} \quad 00$

$-2.81161 \mathrm{E} 00$

$-2.82973 \mathrm{E} \quad 00$

$-2.84791 \mathrm{E} \quad 00$

$-2.86614 \mathrm{E} \quad 00$

$-2.88444 \mathrm{E} \quad 00$

$-2.90279 \mathrm{E} 00$

$-2.92119 \mathrm{E} \quad 00$

$-2.93965 \mathrm{E} \quad 00$

$-2.95817 \mathrm{E} \quad 00$

$-2.97675 \mathrm{E} \quad 00$

$-2.99538 \mathrm{E} \quad 00$

$-3.01407 \mathrm{E} \quad 00$

-3.03281 E 00

$-3.05161 \mathrm{E} 00$

$-3.07047 \mathrm{E} 00$

$-3.08938 \mathrm{E} 00$

$-3.10835 \mathrm{E} \quad 00$

$-3.12738 \mathrm{E} 00$

$-3.14646 \mathrm{E} 00$

$-3.16560 \mathrm{E} \quad 00$

$-3.18479 \mathrm{E} 00$
$-3.20404 \mathrm{E} \quad 00$

$-3.22335 \mathrm{E} 00$

$-3.24271 \mathrm{E} 00$

$-3.26213 \mathrm{E} \quad 00$

$-3.28161 \mathrm{E} 00$

$-3.30114 \mathrm{E} \quad 00$

$-3.32073 \mathrm{E} \quad 00$

$-3.34037 \mathrm{E} \quad 00$

$-3.36007 \mathrm{E} 00$

$-3.37982 \mathrm{E} 00$

$-3.39964 \mathrm{E} \quad 00$

$-3.41950 \mathrm{E} \quad 00$

$-3.43943 \mathrm{E} \quad 00$

$-3.45941 \mathrm{E} 00$

$-3.47944 \mathrm{E} \quad 00$

$-3.49953 \mathrm{E} \quad 00$

$-3.51968 \mathrm{E} 00$

$-3.53989 \mathrm{E} \quad 00$

$-3.56015 \mathrm{E} \quad 00$

$-3.58046 \mathrm{E} 00$

$-3.60083 \mathrm{E} 00$

$-3.62126 \mathrm{E} \quad 00$

$-3.64174 \mathrm{E} 00$

$-3.66228 \mathrm{E} 00$

$-3.68287 \mathrm{E} 00$

$-3.70352 \mathrm{E} \quad 00$

$-3.72423 \mathrm{E} 00$

$-3.74499 \mathrm{E} 00$

$-3.76580 \mathrm{E} \quad 00$

$-3.78668 \mathrm{E} 00$ $g_{2}$

$-1.74687 \mathrm{E}-01$

$-1.76286 \mathrm{E}-01$

$-1.77894 \mathrm{E}-01$

$-1.79512 \mathrm{E}-01$

$-1.81140 \mathrm{E}-01$

$-1.82777 \mathrm{E}-01$

$-1.84425 \mathrm{E}-01$

$-1.86081 \mathrm{E}-01$

$-1.87748 \mathrm{E}-01$

$-1.89424 \mathrm{E}-01$

$-1.91110 \mathrm{E}-01$

$-1.92806 \mathrm{E}-01$

$-1.94512 \mathrm{E}-01$

$-1.96227 \mathrm{E}-01$

$-1.97953 \mathrm{E}-01$

$-1.99688 \mathrm{E}-01$

$-2.01434 \mathrm{E}-01$

$-2.03189 \mathrm{E}-01$

$-2.04954 \mathrm{E}-01$

$-2.06730 \mathrm{E}-01$

$-2.08515 \mathrm{E}-01$

$-2.10310 \mathrm{E}-01$

$-2.12116 \mathrm{E}-01$

$-2.13932 \mathrm{E}-0]$

$-2.15758 \mathrm{E}-01$

$-2.17594 \mathrm{E}-01$

$-2.19440 \mathrm{E}-01$

$-2.21297 \mathrm{E}-01$

$-2.23163 \mathrm{E}-01$

$-2.25040 \mathrm{E}-01$

$-2.26928 \mathrm{E}-01$

$-2.28826 \mathrm{E}-01$

$-2.30734 \mathrm{E}-01$

$-2.32652 \mathrm{E}-01$

$-2.34581 \mathrm{E}-01$

$-2.36521 \mathrm{E}-01$

$-2.38471 \mathrm{E}-01$

$-2.40431 \mathrm{E}-01$

$-2.42402 \mathrm{E}-01$

$-2.44384 \mathrm{E}-01$

$-2.46376 \mathrm{E}-01$

$-2.48379 \mathrm{E}-01$

$-2.50392 \mathrm{E}-01$

$-2.52416 \mathrm{E}-01$

$-2.54451 \mathrm{E}-01$

$-2.56497 \mathrm{E}-01$

$-2.58553 \mathrm{E}-01$

$-2.60620 \mathrm{E}-01$

$-2.62698 \mathrm{E}-01$

$-2.64787 \mathrm{E}-01$

$-2.66886 \mathrm{E}-01$

$-2.68997 \mathrm{E}-01$

$-2.71118 \mathrm{E}-01$

$-2.73250 \mathrm{E}-01$

$-2.75394 \mathrm{E}-01$

$-2.77548 \mathrm{E}-01$

$-2.79713 \mathrm{E}-01$

$-2.81890 \mathrm{E}-01$

$-2.84077 \mathrm{E}-01$

$-2.86275 \mathrm{E}-01$ $\tan \delta_{2}$

$f_{-2}$

$2.32222 \mathrm{E} \quad 01-1.71619 \mathrm{E}-01$

$2.32233 \mathrm{E}$ 01 $-1.73193 \mathrm{E}-01$

$2.32243 \mathrm{E}$ 01 $-1.74777 \mathrm{E}-01$

$2.32254 \mathrm{E} \quad 01-1.76370 \mathrm{E}-01$

$2.32264 \mathrm{E}$ 0l $-1.77973 \mathrm{E}-01$

$2.32275 \mathrm{E} \quad 01$ $2.32285 \mathrm{E} \quad 01$

$2.32295 \mathrm{E} \quad 01$

$2.32305 \mathrm{E} \quad 01$

$2.32315 \mathrm{E} \quad 01$

$2.32325 \mathrm{E} \quad 01$

$2.32335 \mathrm{E} \quad 01$

$2.32345 \mathrm{E} \quad 01$

$2.32354 \mathrm{E} \quad 01$

$2.32364 \mathrm{E} \quad 01$

$2.32374 \mathrm{E} \quad 01$

$2.32383 \mathrm{E} \quad 01$

$2.32393 \mathrm{E} 01$

$2.32402 \mathrm{E} 01$

$2.32412 \mathrm{E} \quad 01$

$2.32421 \mathrm{E} \quad 01$

$2.32430 \mathrm{E} \quad 01$

$2.32439 \mathrm{E} \quad 01$

$2.32449 \mathrm{E} \quad 01$

$2.32458 \mathrm{E} \quad 01$

$2.32467 \mathrm{E} \quad 01$

$2.32476 \mathrm{E} \quad 01$

$2.32485 \mathrm{E} 01$

$2.32493 \mathrm{E} \quad 01$

$2.32502 \mathrm{E} \quad 01$

$2.32511 \mathrm{E} \quad 01$ $2.32520 \mathrm{E} \quad 01$

$2.32528 \mathrm{E} \quad 01$

$2.32537 \mathrm{E} \quad 01$

$2.32545 \mathrm{E} \quad 01$

$2.32554 \mathrm{E} \quad 01$

$2.32562 \mathrm{E} \quad 01$

$2.32571 \mathrm{E} \quad 01$

$2.32579 \mathrm{E} \quad 01$

$2.32587 \mathrm{E} \quad 01$

$2.32595 \mathrm{E} \quad 01$

$2.32604 \mathrm{E} \quad 01$

$2.32612 \mathrm{E} \quad 01$

$2.32620 \mathrm{E} \quad 01$

$2.32628 \mathrm{E} 01$

$2.32636 \mathrm{E} \quad 01$

$2.32644 \mathrm{E} \quad 01$

$2.32652 \mathrm{E} \quad 01$

$2.32659 \mathrm{E} \quad 01$

$2.32667 \mathrm{E} \quad 01$

$2.32675 \mathrm{E} \quad 01$

$2.32683 \mathrm{E} \quad 01$

$2.32690 \mathrm{E} 01$

$2.32698 \mathrm{E} \quad 01$

2.32705 E 01

$2.32713 \mathrm{E} \quad 01$

$2.32720 \mathrm{E} 01$

$2.32728 \mathrm{E} 01$

$2.32735 \mathrm{E} 01$

$2.32742 \mathrm{E} \quad 01$

$-1.79585 \mathrm{E}-01$

$-1.81207 \mathrm{E}-01$

$-1.82838 \mathrm{E}-01$

$-1.84479 \mathrm{E}-01$

$-1.86130 \mathrm{E}-01$

$-1.87791 \mathrm{E}-01$

$-1.89461 \mathrm{E}-01$

$-1.91141 \mathrm{E}-01$

$-1.92830 \mathrm{E}-01$ 
$p$

36.10

36.20

36.30

36.40

36.50

36.60

36.70

36.80

36.90

37.00

37.10

37.20

37.30

37.40

37.50

37.60

37.70

37.80

37.90

38.00

38.10

38.20

38.30

38.40

38.50

38.60

38.70

38.80

38.90

39.00

39.10

39.20

39.30

39.40

39.50

39.60

39.70

39.80

39.90

40.00

40.10

40.20

40.30

40.40

40.50

40.60

40.70

40.80

40.90

41.00

41.10

41.20

41.30

41.40

41.50

41.60

41.70

41.80

41.90

42.00
$F_{0}$

$f_{1}$

$1.23620 \mathrm{E} \quad 00$

$1.23619 \mathrm{E} \quad 00$

$1.23617 \mathrm{E} \quad 00$

$1.23616 \mathrm{E} \quad 00$

$1.23614 \mathrm{E} \quad 00$

$1.23613 \mathrm{E} \quad 00$

$1.23611 \mathrm{E} \quad 00$

$1.23610 \mathrm{E} \quad 00$

$1.23608 \mathrm{E} \quad 00$

$1.23607 \mathrm{E} \quad 00$

$1.23605 \mathrm{E} \quad 00$

$1.23604 \mathrm{E} \quad 00$

$1.23602 \mathrm{E} \quad 00$

$1.23601 \mathrm{E} \quad 00$

$1.23599 \mathrm{E} \quad 00$

$1.23598 \mathrm{E} \quad 00$

$1.23597 \mathrm{E} \quad 00$

$1.23595 \mathrm{E} \quad 00$

$1.23593 \mathrm{E} \quad 00$

$1.23592 \mathrm{E} \quad 00$

$1.23591 \mathrm{E} \quad 00$ $1.23589 \mathrm{E} \quad 00$

$1.23588 \mathrm{E} \quad 00$

$1.23586 \mathrm{E} \quad 00$

$1.23585 \mathrm{E} \quad 00$

$1.23583 \mathrm{E} \quad 00$
0

$\begin{array}{ll}1.23582 \mathrm{E} & 00\end{array}$

$1.23581 \mathrm{E} \quad 00$

$1.23579 \mathrm{E} \quad 00$

$1.23578 \mathrm{E} \quad 00$

$\begin{array}{ll}1.23576 \mathrm{E} & 00\end{array}$

$1.23575 \mathrm{E} \quad 00$

$1.23574 \mathrm{E} \quad 00$

$1.23572 \mathrm{E} \quad 00$

$1.23571 \mathrm{E} \quad 00$

$1.23569 \mathrm{E} \quad 00$

$1.23568 \mathrm{E} \quad 00$

$1.23567 \mathrm{E} \quad 00$

$1.23565 \mathrm{E} \quad 00$

$1.23564 \mathrm{E} \quad 00$

$1.23563 \mathrm{E} \quad 00$

$1.23561 \mathrm{E} \quad 00$

$1.23560 \mathrm{E} \quad 00$

$1.23558 \mathrm{E} \quad 00$

$1.23557 \mathrm{E} \quad 00$

$1.23556 \mathrm{E} \quad 00$ $1.23554 \mathrm{E} \quad 00$ $1.23553 \mathrm{E} \quad 00$ I.23552 E 00

$1.23550 \mathrm{E} \quad 00$

$1.23549 \mathrm{E} \quad 00$

$1.23548 \mathrm{E} \quad 00$

$1.23546 \mathrm{E} \quad 00$

$1.23545 \mathrm{E} \quad 00$

$1.23544 \mathrm{E} \quad 00$

$1.23542 \mathrm{E} \quad 00$

$1.23541 \mathrm{E} \quad 00$

$1.23540 \mathrm{E} \quad 00$

$1.23539 \mathrm{E} \quad 00$

$1.23537 \mathrm{E} \quad 00$ $g_{1}$

$-3.82835 \mathrm{E} \quad 01$ $-3.83857 \mathrm{E}$ 0l $-3.84880 \mathrm{E} 01$ $-3.85902 \mathrm{E}$ 0l

$-3.86924 \mathrm{E} \quad 01$

$-3.87945 \mathrm{E} 01$ $-3.88966 \mathrm{E}$ 0I $-3.89987 \mathrm{E} 01$ $-3.91007 \mathrm{E}$ 01 $-3.92027 \mathrm{E}$ 01

$-3.93046 \mathrm{E} 01$ $-3.94066 \mathrm{E} 01$ $-3.95084 \mathrm{E} 01$ $-3.96103 \mathrm{E} 01$ $-3.97121 \mathrm{E} \quad 01$

$-3.98138 \mathrm{E} 01$ $-3.99156 \mathrm{E} 01$ $-4.00172 \mathrm{E} 01$ $-4.01189 \mathrm{E} 01$ $-4.02205 \mathrm{E} 01$

$-4.03221 \mathrm{E} 01$ $-4.04236 \mathrm{E} 01$ $-4.05251 \mathrm{E} 01$ $-4.06266 \mathrm{E} \quad 01$ $-4.07280 \mathrm{E} 01$

$-4.08294 \mathrm{E} \quad 01$ $-4.09307 \mathrm{E} \quad 01$ $-4.10320 \mathrm{E}$ 01 $-4.11333 \mathrm{E} 01$ $-4.12345 \mathrm{E} 01$

$-4.13357 \mathrm{E} \quad 01$ $-4.14369 \mathrm{E}$ 0l $-4.15380 \mathrm{E} 01$ $-4.16391 \mathrm{E} 01$ $-4.17401 \mathrm{E} 01$

$-4.18411 \mathrm{E} \quad 01$ $-4.19420 \mathrm{E} \quad 01$ $-4.20430 \mathrm{E} \quad 01$ $-4.21438 \mathrm{E} 01$ $-4.22447 \mathrm{E} 01$

$-4.23455 \mathrm{E} \quad 01$ $-4.24463 \mathrm{E} 01$ $-4.25470 \mathrm{E} 01$ $-4.26477 \mathrm{E} 01$ $-4.27483 \mathrm{E} 01$

$-4.28489 \mathrm{E} 01$ $-4.29495 \mathrm{E} 01$ $-4.30500 \mathrm{E} \quad 01$ $-4.31505 \mathrm{E} 01$ $-4.32509 \mathrm{E} 01$

-4.33513 E 01 $-4.34517 \mathrm{E} \quad 01$ $-4.35520 \mathrm{E} 01$ $-4.36523 \mathrm{E} 01$ $-4.37526 \mathrm{E} 01$

$-4.38528 \mathrm{E} 01$ $-4.39529 \mathrm{E} 01$ $-4.40531 \mathrm{E} 01$ $-4.41532 \mathrm{E} \quad 01$ $-4.42532 \mathrm{E} 01$

\section{$-4.90948 \mathrm{E} 00$} $-4.93331 \mathrm{E} 00$ $-4.95721 \mathrm{E} \quad 00$ $-4.98115 \mathrm{E} \quad 00$ $-5.00515 \mathrm{E} \quad 00$

$-5.02921 \mathrm{E} \quad 00$ $-5.05332 \mathrm{E} \quad 00$ $-5.07748 \mathrm{E} 00$ $-5.10169 \mathrm{E} 00$ $-5.12596 \mathrm{E} 00$

$-5.15028 \mathrm{E} 00$ $-5.17466 \mathrm{E} 00$ $-5.19909 \mathrm{E} \quad 00$ $-5.22357 \mathrm{E} 00$ $-5.24810 \mathrm{E} 00$

$-5.27269 \mathrm{E} 00$ $-5.29734 \mathrm{E} 00$ $-5.32203 \mathrm{E} 00$ $-5.34678 \mathrm{E} \quad 00$ $-5.37158 \mathrm{E} 00$

$-5.39644 \mathrm{E} \quad 00$ $-5.42135 \mathrm{E} 00$ $-5.44631 \mathrm{E} \quad 00$ $-5.47133 \mathrm{E} \quad 00$ $-5.49640 \mathrm{E} 00$

$-5.52152 \mathrm{E} \quad 00$ $-5.54669 \mathrm{E} \quad 00$ $-5.57192 \mathrm{E} 00$ $-5.59721 \mathrm{E} 00$

$-5.62254 \mathrm{E} \quad 00$

$-5.64793 \mathrm{E} 00$ $-5.67337 \mathrm{E} \quad 00$ $-5.69886 \mathrm{E} \quad 00$ $-5.72441 \mathrm{E} \quad 00$ $-5.75001 \mathrm{E} 00$

$-5.77566 \mathrm{E} \quad 00$ $-5.80137 \mathrm{E} \quad 00$ $-5.82713 \mathrm{E} 00$ $-5.85294 \mathrm{E} \quad 00$ $-5.87880 \mathrm{E} 00$

$-5.90472 \mathrm{E} \quad 00$ $-5.93069 \mathrm{E} \quad 00$ $-5.95671 \mathrm{E} 00$ $-5.98279 \mathrm{E} 00$ $-6.00892 \mathrm{E} 00$

$-6.03510 \mathrm{E} 00$ $-6.06133 \mathrm{E} 00$ $-6.08762 \mathrm{E} \quad 00$ $-6.11395 \mathrm{E} 00$ $-6.14034 \mathrm{E} 00$

$-6.16679 \mathrm{E} 00$ $-6.19328 \mathrm{E} 00$ $-6.21983 \mathrm{E} 00$ $-6.24643 \mathrm{E} 00$ $-6.27309 \mathrm{E} 00$

$-6.29979 \mathrm{E} \quad 00$ $-6.32655 \mathrm{E} 00$ $-6.35336 \mathrm{E} 00$ $-6.38022 \mathrm{E} 00$ $-6.40714 \mathrm{E} \quad 00$

$\tan \delta_{1}$

$f_{-1}$

$6.98172 \mathrm{E}-03$ $6.98050 \mathrm{E}-03$ $6.97927 \mathrm{E}-03$ $6.97800 \mathrm{E}-03$ $6.97672 \mathrm{E}-03$

$6.97541 \mathrm{E}-03$ $6.97408 \mathrm{E}-03$ $6.97272 \mathrm{E}-03$ $6.97134 \mathrm{E}-03$ $6.96994 \mathrm{E}-03$

$6.96851 \mathrm{E}-03$ $6.96707 \mathrm{E}-03$ $6.96560 \mathrm{E}-03$ $6.96411 \mathrm{E}-03$ $6.96259 \mathrm{E}-03$

$6.96105 \mathrm{E}-03$ $6.95950 \mathrm{E}-03$ $6.95792 \mathrm{E}-03$ $6.95632 \mathrm{E}-03$ $6.95469 \mathrm{E}-03$

$6.95304 \mathrm{E}-03$ $6.95137 \mathrm{E}-03$ $6.94969 \mathrm{E}-03$ $6.94798 \mathrm{E}-03$ $6.94624 \mathrm{E}-03$

$6.94448 \mathrm{E}-03$ $6.94271 \mathrm{E}-03$ $6.94091 \mathrm{E}-03$ $6.93909 \mathrm{E}-03$ $6.93725 \mathrm{E}-03$

$6.93539 \mathrm{E}-03$ $6.93351 \mathrm{E}-03$ $6.93161 \mathrm{E}-03$ $6.92969 \mathrm{E}-03$ $6.92774 \mathrm{E}-03$

$6.92579 \mathrm{E}-03$ $6.92380 \mathrm{E}-03$ $6.92180 \mathrm{E}-03$ 
$Z=9 \quad A=18 \quad \rho=1.2 A^{1 / 3} F$

$\bar{F}$

36.10

36.20

36.30

36.40

36.50

36.60

36.70

36.80

36.90

37.00

37.10

37.20

37.30

37.40

37.50

37.60

37.70

37.80

37.90

38.00

38.10

38.20

38.30

38.40

38.50

38.60

38.70

38.80

38.90

39.00

39.10

39.20

39.30

39.40

39.50

39.60

39.70

39.80

39.90

40.00

40.10

40.20

40.30

40.40

40.50

40.60

40.70

40.80

40.90

41.00

41.10

41.20

41.30

41.40

41.50

41.60

41.70

41.80

41.90

42.00

\section{$f_{2} \quad g_{2}$}

$2.96313 \mathrm{E}-03$ $2.97955 \mathrm{E}-03$ $2.99602 \mathrm{E}-03$ $3.01253 \mathrm{E}-03$ $3.02908 \mathrm{E}-03$

$3.04569 \mathrm{E}-03$ $3.06233 \mathrm{E}-03$

$3.07902 \mathrm{E}-03$

$3.09576 \mathrm{E}-03$

$3.11254 \mathrm{E}-03$

$3.12937 \mathrm{E}-03$ $3.14625 \mathrm{E}-03$ $3.16316 \mathrm{E}-03$

$3.18013 \mathrm{E}-03$

$3.19714 \mathrm{E}-03$

$3.21419 \mathrm{E}-03$ $3.23129 \mathrm{E}-03$ $3.24844 \mathrm{E}-03$ $3.26563 \mathrm{E}-03$

$3.28286 \mathrm{E}-03$

$3.30014 \mathrm{E}-03$ $3.31747 \mathrm{E}-03$ $3.33484 \mathrm{E}-03$ $3.35226 \mathrm{E}-03$

$3.36972 \mathrm{E}-03$

$3.38723 \mathrm{E}-03$

$3.40478 \mathrm{E}-03$

$3.42238 \mathrm{E}-03$

3.44002 E-03

$3.45771 \mathrm{E}-03$

$3.47545 \mathrm{E}-03$

$3.49323 \mathrm{E}-03$

$3.51105 \mathrm{E}-03$

$3.52892 \mathrm{E}-03$

$3.54684 \mathrm{E}-03$

$3.56480 \mathrm{E}-03$

$3.58280 \mathrm{E}-03$

$3.60086 \mathrm{E}-03$

$3.61895 \mathrm{E}-03$

$3.63709 \mathrm{E}-03$

$3.65528 \mathrm{E}-03$

$3.67351 \mathrm{E}-03$

$3.69179 \mathrm{E}-03$

$3.71012 \mathrm{E}-03$

$3.72848 \mathrm{E}-03$

$3.74690 \mathrm{E}-03$

$3.76536 \mathrm{E}-03$

$3.78386 \mathrm{E}-03$

$3.80241 \mathrm{E}-03$

3.82101 E-03

$3.83965 \mathrm{E}-03$ $3.85833 \mathrm{E}-03$

$3.87706 \mathrm{E}-03$

$3.89584 \mathrm{E}-03$

$3.91466 \mathrm{E}-03$

$3.93353 \mathrm{E}-03$ $3.95244 \mathrm{E}-03$

$3.97140 \mathrm{E}-03$

$3.99040 \mathrm{E}-03$

$4.00945 \mathrm{E}-03$
$-3.80761 \mathrm{E} \quad 00 \quad-2.88485 \mathrm{E}-01$ $-3.82859 \mathrm{E} \quad 00 \quad-2.90706 \mathrm{E}-01$ $-3.84963 \mathrm{E}$ 00 $-2.92938 \mathrm{E}-01$ $-3.87072 \mathrm{E} \quad 00 \quad-2.95181 \mathrm{E}-01$ $-3.89187 \mathrm{E} \quad 00 \quad-2.97436 \mathrm{E}-01$

$-3.91308 \mathrm{E} \quad 00$ $-3.93434 \mathrm{E} 00$ $-3.95566 \mathrm{E} \quad 00$ $-3.97703 \mathrm{E} 00$ $-3.99846 \mathrm{E} \quad 00$

$-2.99701 \mathrm{E}-01$ $-3.01978 \mathrm{E}-01$ $-3.04266 \mathrm{E}-01$ $-3.06566 \mathrm{E}-01$ $-3.08877 \mathrm{E}-01$

$-4.01994 \mathrm{E} \quad 00$ $-4.04148 \mathrm{E} \quad 00$ $-4.06307 \mathrm{E} \quad 00$ $-4.08472 \mathrm{E} 00$ $-4.10642 \mathrm{E} \quad 00$

$-3.11199 \mathrm{E}-01$ $-3.13533 \mathrm{E}-01$ $-3.15878 \mathrm{E}-01$ $-3.18235 \mathrm{E}-01$ $-3.20603 \mathrm{E}-01$

$-4.12818 \mathrm{E} \quad 00$

$-3.22983 \mathrm{E}-01$ $-4.15000 \mathrm{E} \quad 00$ $-4.17187 \mathrm{E} \quad 00$ $-4.19379 \mathrm{E} \quad 00$ $-4.21577 \mathrm{E} \quad 00$

. $-3.27777 \mathrm{E}-0$ $-3.30191 \mathrm{E}-01$ $-3.32617 \mathrm{E}-01$

$-4.23781 \mathrm{E} 00$ $-4.25990 \mathrm{E} \quad 00$ $-4.28204 \mathrm{E} \quad 00$ $-4.30425 \mathrm{E} \quad 00$ $-4.32650 \mathrm{E} 00$

$-4.34881 \mathrm{E} \quad 00$ $-4.37118 \mathrm{E} \quad 00$ $-4.39360 \mathrm{E} 00$ $-4.41607 \mathrm{E} \quad 00$ $-4.43861 \mathrm{E} \quad 00$ $-4.48383 \mathrm{E} \quad 00$ $-4.50653 \mathrm{E} 00$ $-4.52928 \mathrm{E} \quad 00$ $-4.55208 \mathrm{E} \quad 00$

$-3.35055 \mathrm{E}-01$ $-3.37505 \mathrm{E}-01$ $-3.39966 \mathrm{E}-01$ $-3.42438 \mathrm{E}-01$ $-3.44923 \mathrm{E}-01$

$-3.47419 \mathrm{E}-01$ $-3.49928 \mathrm{E}-0$ $-3.52448 \mathrm{E}-01$ $-3.54979 \mathrm{E}-01$ $-3.57523 \mathrm{E}-01$

$-3.60079 \mathrm{E}-01$ $-3.62646 \mathrm{E}-01$ $-3.65226 \mathrm{E}-01$ $-3.67818 \mathrm{E}-01$ -3.70421 E-01

$-4.57494 \mathrm{E} \quad 00$ $-4.59786 \mathrm{E} \quad 00$ $-4.62083 \mathrm{E} \quad 00$ $-4.64385 \mathrm{E} \quad 00$ $-4.66693 \mathrm{E} \quad 00$

$-4.69007 \mathrm{E} \quad 00$ $-4.71326 \mathrm{E} \quad 00$ $-4.73650 \mathrm{E} \quad 00$ $-4.75980 \mathrm{E} \quad 00$ $-4.78315 \mathrm{E} \quad 00$

$-4.80656 \mathrm{E} \quad 00$ $-4.83002 \mathrm{E} \quad 00$ $-4.85354 \mathrm{E} \quad 00$ $-4.87711 \mathrm{E} 00$ $-4.90073 \mathrm{E} \quad 00$

$-3.73037 \mathrm{E}-01$ $-3.75665 \mathrm{E}-01$ $-3.78304 \mathrm{E}-01$ $-3.80956 \mathrm{E}-01$ $-3.83620 \mathrm{E}-01$

$-3.86297 \mathrm{E}-01$ $-3.88985 \mathrm{E}-01$ $-3.91686 \mathrm{E}-01$ $-3.94399 \mathrm{E}-01$ $-3.97124 \mathrm{E}-01$

$-3.99862 \mathrm{E}-01$ $-4.02612 \mathrm{E}-01$ $-4.05374 \mathrm{E}-01$ $-4.08148 \mathrm{E}-01$ $-4.10935 \mathrm{E}-01$

$-4.92441 \mathrm{E} \quad 00$ $-4.94815 \mathrm{E} \quad 00$ $-4.97194 \mathrm{E} \quad 00$ $-4.99578 \mathrm{E} \quad 00$ $-5.01968 \mathrm{E} 00$

$-4.13735 \mathrm{E}-01$ $-4.16547 \mathrm{E}-01$ $-4.19371 \mathrm{E}-01$ $-4.22208 \mathrm{E}-01$ $-4.25057 \mathrm{E}-0$

$-5.04363 \mathrm{E} 00$ $-5.06764 \mathrm{E} \quad 00$ $-5.09170 \mathrm{E} \quad 00$ $-5.11581 \mathrm{E} \quad 00$ $-5.13998 \mathrm{E} 00$
$-4.46119 \mathrm{E} \quad 00$

$\tan \delta_{2}$

$f_{-2}$

$2.32750 \mathrm{E} \quad 01$ $2.32757 \mathrm{E} \quad 01$ $2.32764 \mathrm{E} \quad 0$ 2.32771 E 01 $2.32778 \mathrm{E} \quad 01$

$2.32785 \mathrm{E} \quad 01$ $2.32792 \mathrm{E} \quad 01$ $2.32799 \mathrm{E} \quad 01$ $2.32806 \mathrm{E} \quad 01$ $2.32813 \mathrm{E} 01$

$2.32820 \mathrm{E} \quad 01$ $2.32827 \mathrm{E}$ ol $2.32834 \mathrm{E} \quad 01$

$2.32841 \mathrm{E} \quad 01$ $2.32847 \mathrm{E} \quad 01$

$2.32854 \mathrm{E} \quad 01$ $2.32861 \mathrm{E} \quad 0$ $2.32867 \mathrm{E} \quad 01$

$2.32874 \mathrm{E} \quad 01$

$2.32880 \mathrm{E} \quad 01$

$2.32887 \mathrm{E} \quad 01$ $2.32893 \mathrm{E} \quad 01$ $2.32900 \mathrm{E} 01$ $2.32906 \mathrm{E} \quad 0$ $2.32913 \mathrm{E} \quad 01$

$2.32919 \mathrm{E} \quad 01$ $2.32925 \mathrm{E} \quad 01$ $2.32931 \mathrm{E} \quad 01$ $2.32938 \mathrm{E} \quad 01$

$2.32944 \mathrm{E} \quad 0$

$2.32950 \mathrm{E} \quad 01$ $2.32956 \mathrm{E} \quad 01$ $2.32962 \mathrm{E} \quad 01$ $2.32968 \mathrm{E} \quad 01$ $2.32974 \mathrm{E} \quad 01$

$2.32980 \mathrm{E} \quad 01$ $2.32986 \mathrm{E} \quad 01$ $2.32992 \mathrm{E} \quad 01$ $2.32998 \mathrm{E} \quad 01$ $2.33004 \mathrm{E} \quad 01$

$2.33010 \mathrm{E} \quad 01$ $2.33015 \mathrm{E} \quad 01$ $2.33021 \mathrm{E} 01$ $2.33027 \mathrm{E} \quad 01$ $2.33033 \mathrm{E} \quad 01$

$2.33038 \mathrm{E} \quad 01$ $2.33044 \mathrm{E} \quad 01$ $2.33050 \mathrm{E} \quad 01$

$2.33055 \mathrm{E} 01$

$2.33061 \mathrm{E} 01$

$2.33066 \mathrm{E} \quad 01$ $2.33072 \mathrm{E} \quad 01$ $2.33077 \mathrm{E} 01$ $2.33083 \mathrm{E} \quad 01$ 
ELECTRON RADIAL FUNCTIONS

Electron

\begin{tabular}{|c|c|c|c|c|}
\hline$p$ & $F_{0}$ & & $f_{1}$ & $g_{1}$ \\
\hline 0.10 & $4.86640 \mathrm{E}$ & 00 & $-1.92118 \mathrm{E}-02$ & $-6.69524 \mathrm{E}-04$ \\
\hline 0.20 & $2.69996 \mathrm{E}$ & 00 & $-4.87091 \mathrm{E}-02$ & $-1.69953 \mathrm{E}-03$ \\
\hline 0.30 & $2.08466 \mathrm{E}$ & 00 & $-9.14753 E-02$ & $-3.19795 \mathrm{E}-03$ \\
\hline 0.40 & $1.81102 \mathrm{E}$ & 00 & $-1.46223 \mathrm{E}-01$ & $-5.12550 \mathrm{E}-03$ \\
\hline 0.50 & $1.66099 \mathrm{E}$ & 00 & $-2.11425 \mathrm{E}-01$ & $-7.43544 \mathrm{E}-03$ \\
\hline 0.60 & $1.56830 \mathrm{E}$ & 00 & $-2.85499 \mathrm{E}-01$ & $-1.00792 \mathrm{E}-02$ \\
\hline 0.70 & $1.50645 \mathrm{E}$ & 00 & $-3.66941 \mathrm{E}-01$ & $-1.30108 \mathrm{E}-02$ \\
\hline 0.80 & $1.46290 \mathrm{E}$ & 00 & $-4.544 .02 \mathrm{E}-01$ & $-1.61887 \mathrm{E}-02$ \\
\hline 0.90 & $1.43099 \mathrm{E}$ & 00 & $-5.46727 \mathrm{E}-01$ & $-1.95776 \mathrm{E}-02$ \\
\hline 1.00 & $1.40687 \mathrm{E}$ & 00 & $-6.42952 \mathrm{E}-01$ & $-2.31481 \mathrm{E}-02$ \\
\hline 1.10 & $1.38818 \mathrm{E}$ & 00 & $-7.42296 \mathrm{E}-01$ & $-2.68761 \mathrm{E}-02$ \\
\hline 1.20 & $1.37338 \mathrm{E}$ & 00 & $-8.44129 \mathrm{E}-01$ & $-3.07426 \mathrm{E}-02$ \\
\hline 1.30 & $1.36146 \mathrm{E}$ & 00 & $-9.47951 E-01$ & $-3.47323 \mathrm{E}-02$ \\
\hline 1.40 & $1.35171 \mathrm{E}$ & 00 & $-1.05336 \mathbf{E} \quad 00$ & $-3.88331 \mathrm{E}-02$ \\
\hline 1.50 & $1.34363 \mathrm{E}$ & 00 & $-1.16006 \mathrm{E} \quad 00$ & $-4.30355 \mathrm{E}-02$ \\
\hline 1.60 & $1.33686 \mathrm{E}$ & 00 & $-1.26777 \mathrm{E} \quad 00$ & $-4.73318 \mathrm{E}-02$ \\
\hline 1.70 & $1.33112 \mathrm{E}$ & 00 & $-1.37633 \mathrm{E} \quad 00$ & $-5.17163 \mathrm{E}-02$ \\
\hline 1.80 & $1.32621 \mathrm{E}$ & 00 & $-1.48555 \mathrm{E}$ & $-5.61840 \mathrm{E}-02$ \\
\hline 1.90 & $1.32197 \mathrm{E}$ & 00 & $-1.59533 \mathrm{E}$ & $-6.07312 \mathrm{E}-02$ \\
\hline 2.00 & $1.31828 \mathrm{E}$ & 00 & $-1.70555 \mathrm{E} \quad 00$ & $-6.53550 \mathrm{E} \rightarrow 02$ \\
\hline 2.10 & $1.31506 \mathrm{E}$ & 00 & $-1.81614 \mathrm{E}$ & $-7.00528 \mathrm{E}-02$ \\
\hline 2.20 & $1.31221 \mathrm{E}$ & 00 & $-1.92703 \mathrm{E}$ & $-7.48226 \mathrm{E}-02$ \\
\hline 2.30 & $1.30969 \mathrm{E}$ & 00 & $-2.03817 \mathrm{E}$ & $-7.96630 \mathrm{E}-02$ \\
\hline 2.40 & $1.30744 \mathrm{E}$ & 00 & $-2.14951 \mathrm{E}$ & $-8.45725 \mathrm{E}-02$ \\
\hline 2.50 & $1.30543 \mathrm{E}$ & 00 & $-2.26103 \mathrm{E} \quad 00$ & $-8.95501 \mathrm{E}-02$ \\
\hline 2.60 & $1.30361 \mathrm{E}$ & 00 & $-2.37268 \mathrm{E}$ & $-9.45949 \mathrm{E}-02$ \\
\hline 2.70 & $1.30197 \mathrm{E}$ & 00 & $-2.48444 \mathrm{E}$ & $-9.97062 \mathrm{E}-02$ \\
\hline 2.80 & $1.30048 \mathrm{E}$ & 00 & $-2.59630 \mathrm{E}$ & $-1.04883 \mathrm{E}-01$ \\
\hline 2.90 & $1.29912 \mathrm{E}$ & 00 & $-2.70824 \mathrm{E}$ & $-1.10126 \mathrm{E}-01$ \\
\hline 3.00 & $1.29787 \mathrm{E}$ & 00 & $-2.82024 \mathrm{E} \quad 00$ & $-1.15433 \mathrm{E}-01$ \\
\hline 3.10 & $1.29673 \mathrm{E}$ & 00 & $-2.93229 \mathrm{E}$ & $-1.20805 \mathrm{E}-01$ \\
\hline 3.20 & $1.29568 \mathrm{E}$ & 00 & $-3.04438 \mathrm{E}$ & $-1.2624 .1 \mathrm{E}-01$ \\
\hline 3.30 & $1.29470 \mathrm{E}$ & 00 & $-3.15650 \mathrm{E}$ & $-1.31741 \mathrm{E}-01$ \\
\hline 3.40 & $1.29380 \mathrm{E}$ & 00 & $-3.26865 \mathrm{E}$ & $-1.37305 \mathrm{E}-01$ \\
\hline 3.50 & $1.29296 \mathrm{E}$ & 00 & $-3.38081 \mathrm{E} \quad 00$ & $-1.42933 \mathrm{E}-01$ \\
\hline 3.60 & $1.29218 \mathrm{E}$ & 00 & $-3.49299 \mathrm{E}$ & $-1.48624 \mathrm{E}-01$ \\
\hline 3.70 & $1.29145 \mathrm{E}$ & 00 & $-3.60518 \mathrm{E}$ & $-1.54378 \mathrm{E}-01$ \\
\hline 3.80 & $1.29077 \mathrm{E}$ & 00 & $-3.71737 \mathrm{E} \quad 00$ & $-1.60195 \mathrm{E}-01$ \\
\hline 3.90 & $1.29013 \mathrm{E}$ & 00 & $-3.82956 \mathrm{E} \quad 00$ & $-1.66075 \mathrm{E}-01$ \\
\hline 4.00 & $1.28952 \mathrm{E}$ & 00 & $-3.94175 \mathrm{E} \quad 00$ & $-1.72019 \mathrm{E}-01$ \\
\hline 4.10 & $1.28896 \mathrm{E}$ & 00 & $-4.05394 \mathrm{E} \quad 00$ & $-1.78025 \mathrm{E}-01$ \\
\hline 4.20 & $1.28842 \mathrm{E}$ & 00 & $-4.16611 \mathrm{E} \quad 00$ & $-1.84093 \mathrm{E}-01$ \\
\hline 4.30 & $1.28791 \mathrm{E}$ & 00 & $-4.27829 E$ & $-1.90225 \mathrm{E}-01$ \\
\hline 4.40 & $1.28743 \mathrm{E}$ & 00 & $-4.39044 \mathrm{E}$ & $-1.96419 \mathrm{E}-01$ \\
\hline 4.50 & $1.28698 \mathrm{E}$ & 00 & $-4.50259 \mathrm{E}$ & $-2.02676 \mathrm{E}-01$ \\
\hline 4.60 & $1.28655 \mathrm{E}$ & 00 & $-4.61473 \mathrm{E} \quad 00$ & $-2.08995 \mathrm{E}-01$ \\
\hline 4.70 & $1.28613 \mathrm{E}$ & 00 & $-4.72685 \mathrm{E}$ & $-2.15377 \mathrm{E}-01$ \\
\hline 4.80 & $1.28574 \mathrm{E}$ & 00 & $-4.83895 \mathrm{E}$ & $-2.21821 \mathrm{E}-01$ \\
\hline 4.90 & $1.28537 \mathrm{E}$ & 00 & $-4.95104 \mathrm{E}$ & $-2.28328 \mathrm{E}-01$ \\
\hline 5.00 & $1.28501 \mathrm{E}$ & 00 & $-5.06311 \mathrm{E}$ & $-2.34897 \mathrm{E}-01$ \\
\hline 5.10 & $1.28467 \mathrm{E}$ & 00 & $-5.17516 \mathrm{E}$ & $-2.41528 \mathrm{E}-01$ \\
\hline 5.20 & $1.28434 \mathrm{E}$ & 00 & $-5.28720 \mathrm{E}$ & $-2.48221 \mathrm{E}-01$ \\
\hline 5.30 & $1.28402 \mathrm{E}$ & 00 & $-5.39921 \mathrm{E}$ & $-2.54977 \mathrm{E}-01$ \\
\hline 5.40 & $1.28372 \mathrm{E}$ & 00 & $-5.51121 \mathrm{E}$ & $-2.61796 \mathrm{E}-01$ \\
\hline 5.50 & $1.28343 \mathrm{E}$ & 00 & $-5.62319 \mathrm{E}$ & $-2.68676 \mathrm{E}-01$ \\
\hline 5.60 & $1.28315 \mathrm{E}$ & 00 & $-5.73514 \mathrm{E} \quad 00$ & $-2.75618 \mathrm{E}-01$ \\
\hline 5.70 & $1.28288 \mathrm{E}$ & 00 & $-5.84708 \mathrm{E}$ & $-2.82623 \mathrm{E}-01$ \\
\hline 5.80 & $1.28262 \mathrm{E}$ & 00 & $-5.95900 \mathrm{E}$ & $-2.89690 \mathrm{E}-01$ \\
\hline 5.90 & $1.28237 \mathrm{E}$ & 00 & $-6.07089 \mathrm{E}$ & \\
\hline 6.00 & $1.28213 \mathrm{E}$ & & $-6.18277 \mathrm{E}$ & $-3.04011 \mathrm{E}-01$ \\
\hline
\end{tabular}

$$
Z=10 \quad A=20 \quad \rho=1.2 A^{1 / 3} F
$$

$\tan \delta_{1} \quad f_{-1}$

$-3.40276 \mathrm{E}-01$

$-1.53485 \mathrm{E}-01$

$-9.87980 \mathrm{E}-02$

$-7.21433 \mathrm{E}-02$

$-5.62443 \mathrm{E}-02$

$-4.56368 \mathrm{E}-02$

$-3.80324 \mathrm{E}-02$

$-3.23007 \mathrm{E}-02$

$-2.78180 \mathrm{E}-02$

$-2.42114 \mathrm{E}-02$

$-2.12439 \mathrm{E}-02$

$-1.87575 \mathrm{E}-02$

$-1.66429 \mathrm{E}-02$

$-1.48215 \mathrm{E}-02$

$-1.32358 \mathrm{E}-02$

$-1.18425 \mathrm{E}-02$

$-1.06083 \mathrm{E}-02$

$-9.50719 \mathrm{E}-03$

$-8.51866 \mathrm{E}-03$

$-7.62620 \mathrm{E}-03$

$-6.81639 \mathrm{E}-03$

$-6.07819 \mathrm{E}-03$

$-5.40250 \mathrm{E}-03$

$-4.78165 \mathrm{E}-03$

$-4.20922 \mathrm{E}-03$

$-3.67975 \mathrm{E}-03$

$-3.18858 \mathrm{E}-03$

$-2.73168 \mathrm{E}-03$

$-2.30558 \mathrm{E}-03$

$-1.90727 \mathrm{E}-03$

$-1.53412 \mathrm{E}-03$

$-1.18382 \mathrm{E}-03$

$-8.54324 \mathrm{E}-04$

$-5.43850 \mathrm{E}-04$

$-2.50772 \mathrm{E}-04$

$2.62915 \mathrm{E}-05$ $2.88660 \mathrm{E}-04$

$5.37435 \mathrm{E}-04$

$7.73650 \mathrm{E}-04$

$9.98244 \mathrm{E}-04$

$1.21204 \mathrm{E}-03$

$1.41579 \mathrm{E}-03$

$1.61020 \mathrm{E}-03$

$1.79589 \mathrm{E}-03$

$1.97342 \mathrm{E}-03$

$2.14332 \mathrm{E}-03$

$2.30607 \mathrm{E}-03$

$2.46211 \mathrm{E}-03$

$2.61185 \mathrm{E}-03$

$2.75565 \mathrm{E}-03$

$2.89385 \mathrm{E}-03$

$3.02678 \mathrm{E}-03$

$3.15473 \mathrm{E}-03$

$3.27798 \mathrm{E}-03$

$3.39676 \mathrm{E}-03$

$3.51133 \mathrm{E}-03$ $3.62189 \mathrm{E}-03$ $3.72866 \mathrm{E}-03$

$3.83181 \mathrm{E}-03$

$3.93154 \mathrm{E}-03$
$-9.08420 \mathrm{E}-03$

$-1.35030 \mathrm{E}-02$

$-1.77362 \mathrm{E}-02$

$-2.19449 \mathrm{E}-02$

$-2.61392 \mathrm{E}-02$

$-3.03188 \mathrm{E}-02$

$-3.44853 \mathrm{E}-02$

$-3.86431 \mathrm{E}-02$

$-4.27984 \mathrm{E}-02$

$-4.69580 \mathrm{E}-02$

$-5.11289 \mathrm{E}-02$

$-5.53176 \mathrm{E}-02$

$-5.95303 \mathrm{E}-02$

$-6.37721 \mathrm{E}-02$

$-6.80477 \mathrm{E}-02$

$-7.23610 \mathrm{E}-02$

$-7.67156 \mathrm{E}-02$

$-8.11142 \mathrm{E}-02$

$-8.55594 \mathrm{E}-02$

$-9.00533 \mathrm{E}-02$

$-9.45976 \mathrm{E}-02$

$-9.91939 \mathrm{E}-02$

$-1.03844 \mathrm{E}-01$

$-1.08547 \mathrm{E}-01$

$-1.13307 \mathrm{E}-01$

$-1.18122 \mathrm{E}-01$

$-1.22995 \mathrm{E}-01$

$-1.27924 \mathrm{E}-01$

$-1.32912 \mathrm{E}-01$

$-1.37959 \mathrm{E}-01$

$-1.43064 \mathrm{E}-01$

$-1.48228 \mathrm{E}-01$

$-1.53452 \mathrm{E}-01$

$-1.58736 \mathrm{E}-01$

$-1.64080 \mathrm{E}-01$

$-1.69484 \mathrm{E}-01$

$-1.74948 \mathrm{E}-01$

$-1.80473 \mathrm{E}-01$

$-1.86059 \mathrm{E}-01$

$-1.91706 \mathrm{E}-01$

$-1.97414 \mathrm{E}-01$

$-2.03182 \mathrm{E}-01$

$-2.09012 \mathrm{E}-01$

$-2.14903 \mathrm{E}-01$

$-2.20856 \mathrm{E}-01$

$-2.26870 \mathrm{E}-01$

$-2.32945 \mathrm{E}-01$

$-2.39082 \mathrm{E}-01$

$-2.45280 \mathrm{E}-01$

$-2.51540 \mathrm{E}-01$

$-2.57862 \mathrm{E}-01$

$-2.64245 \mathrm{E}-01$

$-2.70690 \mathrm{E}-01$

$-2.77196 \mathrm{E}-01$

$-2.83765 \mathrm{E}-01$

$-2.90394 \mathrm{E}-01$

$-2.97086 \mathrm{E}-01$

$-3.03840 \mathrm{E}-01$

$-3.10655 \mathrm{E}-01$

$-3.17532 \mathrm{E}-01$ $g_{-1}$

$\tan \delta_{-1}$

$3.10857 \mathrm{E}-01$ $4.61408 \mathrm{E}-01$ $6.04648 \mathrm{E}-01$

$7.45763 \mathrm{E}-01$

$8.84830 \mathrm{E}-01$

$1.02162 \mathrm{E} \quad 00$

$1.15604 \mathrm{E} \quad 00$

$1.28812 \mathrm{E} \quad 00$

$1.41801 \mathrm{E} \quad 00$

$1.54590 \mathrm{E} \quad 00$

$1.67198 \mathrm{E} \quad 00$

$1.79647 \mathrm{E} \quad 00$

$1.91956 \mathrm{E} \quad 00$

$2.04144 \mathrm{E} \quad 00$

$2.16225 \mathrm{E} \quad 00$

$2.28215 \mathrm{E} \quad 00$

$2.40123 \mathrm{E} \quad 00$

$2.51962 \mathrm{E} \quad 00$

$2.63739 \mathrm{~F} 00$

$2.75462 \mathrm{E} \quad 00$

$2.87138 \mathrm{E} \quad 00$

$2.98773 \mathrm{E} \quad 00$

$3.10370 \mathrm{E} \quad 00$

$3.21934 \mathrm{E} \quad 00$

$3.33468 \mathrm{E} \quad 00$

$3.44976 \mathrm{E} \quad 00$

$3.56460 \mathrm{E} \quad 00$

$3.67923 \mathrm{E} \quad 00$

$3.79367 \mathrm{E} \quad 00$

$3.90792 \mathrm{E} \quad 00$

$4.02202 \mathrm{E} \quad 00$

$4.13596 \mathrm{E} \quad 00$

$4.24977 \mathrm{E} \quad 00$

$4.36345 \mathrm{E} \quad 00$

$4.47702 \mathrm{E} \quad 00$

$4.59048 \mathrm{E} \quad 00$ $4.70384 \mathrm{E} \quad 00$ $4.81710 \mathrm{E} \quad 00$

$4.93027 \mathrm{E} \quad 00$ $5.04337 \mathrm{E} \quad 00$

$5.15638 \mathrm{~F} \quad 00$ $5.26932 \mathrm{E} \quad 00$ $5.38220 \mathrm{E} \quad 00$ $5.49500 \mathrm{E} \quad 00$ $5.60774 \mathrm{E} \quad 00$

$5.72042 \mathrm{E} \quad 00$ $5.83305 \mathrm{E} \quad 00$ $5.94562 \mathrm{E} \quad 00$ $6.05814 \mathrm{E} \quad 00$ $6.17060 \mathrm{E} 00$

$6.28302 \mathrm{E} \quad 00$ $6.39539 \mathrm{E} \quad 00$ $6.50771 \mathrm{E} \quad 00$ $6.61999 \mathrm{E} \quad 00$ $6.73223 \mathrm{E} \quad 00$

$6.84442 \mathrm{~F} \quad 00$ $6.95658 \mathrm{E} \quad 00$ $7.06869 \mathrm{E} \quad 00$ $7.18077 \mathrm{E} \quad 00$ $7.29281 \mathrm{E} \quad 00$
$-3.20527 \mathrm{E} \quad 00$ $-4.99575 \mathrm{E} \quad 00$ $-7.08942 \mathrm{E} 00$ $-9.18645 \mathrm{E} \quad 00$ $-1.12397 \mathrm{E} 01$

$-1.32337 \mathrm{E} \quad 01$ $-1.51625 \mathrm{E} 01$ $-1.70238 \mathrm{E} 01$ $-1.88175 \mathrm{E} 01$ $-2.05447 \mathrm{E} 01$

$-2.22073 \mathrm{E} 01$ $-2.38074 \mathrm{E} 01$ $-2.53475 \mathrm{E} 01$ $-2.68302 \mathrm{E} 01$ $-2.82581 \mathrm{E} 01$

$-2.96338 \mathrm{E} \quad 01$ $-3.09597 \mathrm{E} 01$ $-3.22383 \mathrm{E} 01$ $-3.34718 \mathrm{E} 01$ -3.46625 E 01

$-3.58125 \mathrm{E} \quad 01$ $-3.69236 \mathrm{E} 01$ $-3.79978 \mathrm{E}$ 01 $-3.90368 \mathrm{E} 01$ $-4.00423 \mathrm{E} 01$

$-4.10159 \mathrm{E} \quad 01$ $-4.19589 \mathrm{E} 01$ $-4.28729 \mathrm{E} \quad 01$ $-4.37592 \mathrm{E} \quad 01$ $-4.46189 \mathrm{E} 01$

$-4.54532 \mathrm{E} \quad 01$ $-4.62633 \mathrm{E} 01$ $-4.70501 \mathrm{E} 01$ $-4.78147 \mathrm{E} 01$ $-4.85580 \mathrm{E} 0 \mathrm{i}$

$-4.92809 \mathrm{E} 01$ $-4.99842 \mathrm{E} 01$ $-5.06687 \mathrm{E} 01$ $-5.13352 \mathrm{E} 01$ $-5.19844 \mathrm{E} 01$

$-5.26168 \mathrm{E} \quad 01$ $-5.32333 \mathrm{E} 01$ $-5.38344 \mathrm{E} \quad 01$ $-5.44206 \mathrm{E} 01$ $-5.49925 \mathrm{E} 01$

$-5.55508 \mathrm{E} \quad 01$ $-5.60957 \mathrm{E} 01$ $-5.66279 \mathrm{E} 01$ $-5.71477 \mathrm{E} \quad 01$ $-5.76556 \mathrm{E} 01$

$-5.81522 \mathrm{E} \quad 01$ $-5.86376 \mathrm{E} 01$ $-5.91122 \mathrm{E}$ 01 $-5.95765 \mathrm{E} 01$ $-6.00309 \mathrm{E} 01$

$-6.04756 \mathrm{E} 01$ $-6.09109 \mathrm{E} 01$ $-6.13371 \mathrm{E} 01$ $-6.17546 \mathrm{E} 01$ $-6.21636 \mathrm{E} 01$ 
$\bar{F}$

p.

0.10

0.20

0.30

0.40

0.50

0.60

0.70

0.80

0.90

1.00

1.10

1.20

1.30

1.40

1.50

1.60

1.70

1.80

1.90

2.00

2.10

2.20

2.30

2.40

2.50

2.60

2.70

2.80

2.90

3.00

3.10

3.20

3.30

3.40

3.50

3.60

3.70

3.80

3.90

4.00

4.10

4.20

4.30

4.40

4.50

4.60

4.70

4.80

4.90

5.00

5.10

5.20

5.30

5.40

5.50

5.60

5.70

5.80

5.90

6.00 $f_{2}$

$-5.69172 \mathrm{E}-06$ $-2.76228 \mathrm{E}-05$ $-7.71748 \mathrm{E}-05$ $-1.64039 \mathrm{E}-04$ $-2.96147 \mathrm{E}-04$

$-4.79636 \mathrm{E}-04$ $-7.19027 \mathrm{E}-04$ $-1.01751 \mathrm{E}-03$ $-1.37724 \mathrm{E}-03$ $-1.79964 \mathrm{E}-03$

$-2.28558 \mathrm{E}-03$ $-2.83561 \mathrm{E}-03$ $-3.45000 \mathrm{E}-03$ $-4.12888 \mathrm{E}-03$ $-4.87228 \mathrm{E}-03$

$-5.68019 \mathrm{E}-03$ $-6.55254 \mathrm{E}-03$ $-7.48926 \mathrm{E}-03$ $-8.49024 \mathrm{E}-03$ $-9.55541 \mathrm{E}-03$

.35349E-06

$1.13666 \mathrm{E}-05$ $1.24481 \mathrm{E}-05$ $1.35796 \mathrm{E}-05$

$1.47612 \mathrm{E}-05$ $1.59928 \mathrm{E}-0.5$

$1.72745 \mathrm{E}-05$

$1.86062 \mathrm{E}-05$ $1.99880 \mathrm{E}-05$ $2.14198 \mathrm{E}-05$ $2.29016 \mathrm{E}-05$

$2.44335 \mathrm{E}-0.5$ $2.60155 \mathrm{E}-0.5$ $2.76475 \mathrm{E}-05$ $2.93295 \mathrm{E}-05$ $3.10616 \mathrm{E}-05$

$3.28437 \mathrm{E}-05$ $3.46759 \mathrm{E}-05$ $3.65581 \mathrm{E}-05$ $3.84904 \mathrm{E}-05$ $4.04727 \mathrm{E}-05$

$4.25050 \mathrm{E}-05$ $4.45873 \mathrm{E}-0.5$ $4.67198 \mathrm{E}-0.5$ $4.89022 \mathrm{E}-05$ $5.11346 \mathrm{E}-05$

$5.34170 \mathrm{E}-05$ $5.57495 \mathrm{E}-05$ $5.81320 \mathrm{E}-05$ $6.05645 \mathrm{E}-05$ $6.30471 \mathrm{E}-0.5$

$6.55796 \mathrm{E}-0.5$ $6.81622 \mathrm{E}-05$ $7.07947 \mathrm{E}-0.5$ $7.34773 \mathrm{E}-05$ $7.62098 \mathrm{E}-05$

$-1.06847 \mathrm{E}-02$ $-1.18780 \mathrm{E}-02$ $-1.31352 \mathrm{E}-02$ $-1.44562 \mathrm{E}-02$ $-1.58411 \mathrm{E}-02$

$-1.72897 \mathrm{E}-02$ $-1.88019 \mathrm{E}-02$ $-2.03778 \mathrm{E}-02$ $-2.20172 \mathrm{E}-02$ $-2.37202 \mathrm{E}-02$

$-2.54866 \mathrm{E}-02$ $-2.73165 \mathrm{E}-02$ $-2.92098 \mathrm{E}-02$ $-3.11664 \mathrm{E}-02$ $-3.31864 \mathrm{E}-02$

$-3.52697 \mathrm{E}-02$ $-3.74163 \mathrm{E}-02$ $-3.96262 \mathrm{E}-02$ $-4.18992 \mathrm{E}-02$ $-4.42355 \mathrm{E}-02$

$-4.66350 \mathrm{E}-02$ $-4.90977 \mathrm{E}-02$ $-5.16235 \mathrm{E}-02$ $-5.42124 \mathrm{E}-02$ $-5.68644 \mathrm{E}-02$

$-5.95796 \mathrm{E}-02$ $-6.23578 \mathrm{E}-02$ $-6.51991 \mathrm{E}-02$ $-6.81034 \mathrm{E}-02$ $-7.10707 \mathrm{E}-02$

$-7.41011 \mathrm{E}-02$ $-7.71944 \mathrm{E}-02$ $-8.03508 \mathrm{E}-02$ $-8.35701 \mathrm{E}-02$ $-8.68524 \mathrm{E}-02$

$-9.01976 \mathrm{E}-02$ $-9.36058 \mathrm{E}-02$ $-9.70768 \mathrm{E}-02$ $-1.00611 \mathrm{E}-01$ $-1.04208 \mathrm{E}-01$ $g_{2}$

$-1.14217 \mathrm{E}-07$ $-5.55006 \mathrm{E}-07$

$-1.55378 \mathrm{E}-06$

$-3.31177 \mathrm{E}-06$

$-5.99941 \mathrm{E}-06$

$-9.75554 \mathrm{E}-06$

$-1.46908 \mathrm{E}-05$

$-2.08922 \mathrm{E}-05$

$-2.84290 \mathrm{E}-05$

$-3.73573 \mathrm{E}-05$

$-4.77242 \mathrm{E}-05$ $-5.95702 \mathrm{E}-05$ $-7.29319 \mathrm{E}-05$ $-8.78435 \mathrm{E}-05$

$-1.04337 \mathrm{E}-04$

$-1.22444 \mathrm{E}-04$ $-1.42194 \mathrm{E}-04$ $-1.63620 \mathrm{E}-04$ $-1.86750 \mathrm{E}-04$ $-2.11615 \mathrm{E}-04$

$-2.38245 \mathrm{E}-04$

$-2.66671 \mathrm{E}-04$ $-2.96924 \mathrm{E}-04$ $-3.29033 \mathrm{E}-04$ $-3.63031 \mathrm{E}-04$

$-3.98946 \mathrm{E}-04$ $-4.36811 \mathrm{E}-04$ $-4.76657 \mathrm{E}-04$ $-5.18513 \mathrm{E}-04$ $-5.62413 \mathrm{E}-04$

$-6.08386 \mathrm{E}-04$ $-6.56464 \mathrm{E}-04$ $-7.06678 \mathrm{E}-04$ $-7.59060 \mathrm{E}-04$ $-8.13641 \mathrm{E}-04$

$-8.70453 \mathrm{E}-04$ $-9.29526 \mathrm{E}-04$ $-9.90892 \mathrm{E}-04$ $-1.05458 \mathrm{E}-03$ $-1.12063 \mathrm{E}-03$

$-1.18906 \mathrm{E}-03$ $-1.25992 \mathrm{E}-03$ $-1.33322 \mathrm{E}-03$ $-1.40901 \mathrm{E}-03$ $-1.48731 \mathrm{E}-03$

$-1.56816 \mathrm{E}-03$ $-1.65158 \mathrm{E}-03$ $-1.73761 \mathrm{E}-03$ $-1.82628 \mathrm{E}-03$ $-1.91763 \mathrm{E}-03$

$-2.01167 \mathrm{E}-03$

$-2.10846 \mathrm{E}-03$

$-2.20800 \mathrm{E}-03$

$-2.31035 \mathrm{E}-03$

$-2.41553 \mathrm{E}-03$

$-2.52356 \mathrm{E}-03$

$-2.63449 \mathrm{E}-03$

$-2.74834 \mathrm{E}-03$

$-2.86515 \mathrm{E}-03$

$-2.98495 \mathrm{E}-03$ $\tan \delta_{2}$

$f_{-2}$

$1.22839 \mathrm{E} \quad 00$

$2.82175 \mathrm{E} \quad 00$

$4.27361 \mathrm{E} \quad 00$

$5.62966 \mathrm{E} \quad 00$

$6.88684 \mathrm{E} \quad 00$

$8.04069 \mathrm{E} \quad 00$ $9.09058 \mathrm{E} \quad 00$ $1.00396 \mathrm{E} \quad 01$ $1.08937 \mathrm{E} \quad 01$ $1.16603 \mathrm{E} 01$

$1.23476 \mathrm{E} \quad 01$ $1.29638 \mathrm{E} \quad 01$ $1.35167 \mathrm{E} \quad 01$ $1.40136 \mathrm{E} \quad 01$ $1.44611 \mathrm{E} \quad 01$

$1.48650 \mathrm{E} \quad 01$ $1.52304 \mathrm{E} \quad 01$ $1.55619 \mathrm{E} \quad 01$ $1.58634 \mathrm{E} \quad 01$ $1.61384 \mathrm{E} \quad 01$

$1.63898 \mathrm{E} \quad 01$

$1.66204 \mathrm{E} \quad 01$

$1.68323 \mathrm{E} 01$

$1.70275 \mathrm{E} \quad 01$

$1.72079 \mathrm{E} \quad 01$

$1.73748 \mathrm{E} \quad 01$

$1.75297 \mathrm{E} \quad 01$

$1.76737 \mathrm{E} \quad 01$

$1.78079 \mathrm{E} 01$

$1.79331 \mathrm{E} \quad 01$

$\begin{array}{ll}1.80502 \mathrm{E} & 01\end{array}$ $1.81599 \mathrm{E} \quad 01$ $1.82629 \mathrm{E} \quad 01$ $1.83597 \mathrm{E} \quad 01$ $1.84508 \mathrm{E} \quad 01$

$\begin{array}{ll}1.85367 \mathrm{E} & 01\end{array}$ $1.86178 \mathrm{E} \quad 01$ $1.86945 \mathrm{E} \quad 01$ $1.87671 \mathrm{E} \quad 01$ $1.88360 \mathrm{E} \quad 01$

$1.89013 \mathrm{E} \quad 01$ $1.89634 \mathrm{E} \quad 01$ $1.90225 \mathrm{E} \quad 01$ $1.90787 \mathrm{E} \quad 01$ $1.91323 \mathrm{E} \quad 01$

$1.91835 \mathrm{E} \quad 01$ $1.92324 \mathrm{E} \quad 01$ $1.92791 \mathrm{E} \quad 01$ $1.93239 \mathrm{E} 01$ $1.93667 \mathrm{E} \quad 01$

$1.94078 \mathrm{E} \quad 01$ $1.94471 \mathrm{E} 01$ $1.94850 \mathrm{E} \quad 01$ $1.95213 \mathrm{E} 01$ $1.95562 \mathrm{E} 01$

$1.95898 \mathrm{E} \quad 01$ $1.96222 \mathrm{E} \quad 01$ $1.96534 \mathrm{E} \quad 01$ $1.96834 \mathrm{E} 01$ $1.97124 \mathrm{E} \quad 01$

$-1.79345 \mathrm{E}-06$ $-4.58873 \mathrm{E}-06$ $-8.74647 \mathrm{E}-06$ $-1.42595 \mathrm{E}-05$ $-2.11180 \mathrm{E}-05$ 
p

6.10

6.20

6.30

6.40

6.50

6.60

6.70

6.80

6.90

7.00

7.10

7.20

7.30

7.40

7.50

7.60

7.70

7.80

7.90

8.00

8.10

8.20

8.30

8.40

8.50

8.60

8.70

8.80

8.90

9.00

9.10

9.20

9.30

9.40

9.50

9.60

9.70

9.80

9.90

10.00

10.10

10.20

10.30

10.40

10.50

10.60

10.70

10.80

10.90

11.00

11.10

11.20

11.30

11.40

11.50

11.60

11.70

11.80

11.90

12.00
$F_{0}$

$1.28190 \mathrm{E} \quad 00$ $1.28167 \mathrm{E} \quad 00$ $1.28145 \mathrm{E} \quad 00$ $1.28124 \mathrm{E} \quad 00$

$1.28103 \mathrm{E} \quad 00$

$1.28083 \mathrm{E} \quad 00$ $1.28064 \mathrm{E} \quad 00$ $1.28045 \mathrm{E} \quad 00$ $1.28027 \mathrm{E} \quad 00$ $1.28009 \mathrm{E} \quad 00$

$1.27992 \mathrm{E} \quad 00$ $1.27975 \mathrm{E} \quad 00$ $1.27958 \mathrm{E} \quad 00$ $1.27942 \mathrm{E} \quad 00$ $1.27927 \mathrm{E} \quad 00$

$1.27911 \mathrm{E} \quad 00$ $1.27896 \mathrm{E} \quad 00$ $1.27882 \mathrm{E} \quad 00$ $1.27868 \mathrm{E} \quad 00$ $1.27854 \mathrm{E} \quad 00$

$1.27840 \mathrm{E} \quad 00$ $1.27827 \mathrm{E} \quad 00$ $1.27814 \mathrm{E} \quad 00$ $1.27801 \mathrm{E} \quad 00$ $1.27788 \mathrm{E} \quad 00$

$1.27776 \mathrm{E} \quad 00$ $1.27764 \mathrm{E} \quad 00$ $1.27752 \mathrm{E} \quad 00$ $1.27741 \mathrm{E} \quad 00$ $1.27729 \mathrm{E} \quad 00$

$1.27718 \mathrm{E} \quad 00$ $1.27707 \mathrm{E} \quad 00$ $1.27697 \mathrm{E} \quad 00$ $1.27686 \mathrm{E} \quad 00$ $1.27676 \mathrm{E} \quad 00$

$1.27665 \mathrm{E} \quad 00$ $1.27655 \mathrm{E} \quad 00$ $1.27645 \mathrm{E} \quad 00$ $1.27636 \mathrm{E} \quad 00$ $1.27626 \mathrm{E} \quad 00$

$1.27617 \mathrm{E} \quad 00$ $1.27607 \mathrm{E} \quad 00$ $1.27598 \mathrm{E} \quad 00$ $1.27589 \mathrm{E} \quad 00$ $1.27580 \mathrm{E} \quad 00$

$1.27572 \mathrm{E} \quad 00$ $1.27563 \mathrm{E} \quad 00$ $1.27555 \mathrm{E} \quad 00$ $1.27546 \mathrm{E} \quad 00$ $1.27538 \mathrm{E} \quad 00$

$1.27530 \mathrm{E} \quad 00$ $1.27522 \mathrm{E} \quad 00$ $1.27514 \mathrm{E} \quad 00$ $1.27506 \mathrm{E} \quad 00$ $1.27498 \mathrm{E} \quad 00$

$1.27491 \mathrm{E} \quad 00$ $1.27483 \mathrm{E} \quad 00$ $1.27476 \mathrm{E} \quad 00$ $1.27468 \mathrm{E} \quad 00$ $1.27461 \mathrm{E} \quad 00$ $f_{1}$

$-6.29462 \mathrm{E} \quad 00$ $-6.40645 \mathrm{E} \quad 00$ $-6.51826 \mathrm{E} 00$ $-6.63005 \mathrm{E} 00$

$-6.74181 \mathrm{E} 00$

$-6.85355 \mathrm{E} \quad 00$ $-6.96527 \mathrm{E} \quad 00$ $-7.07697 \mathrm{E} \quad 00$ $-7.18865 \mathrm{E} 00$ $-7.30030 \mathrm{E} 00$

$-7.41192 \mathrm{E} \quad 00$ $-7.52353 \mathrm{E} 00$ $-7.63511 \mathrm{E} 00$ $-7.74668 \mathrm{E} 00$ $-7.85821 \mathrm{E} \quad 00$

\section{$-7.96972 \mathrm{E} \quad 00$}

$-8.08121 \mathrm{E} 00$ $-8.19268 \mathrm{E} 00$

$-8.30412 \mathrm{E} \quad 00$

$-8.41554 \mathrm{E} \quad 00$

$-8.52694 \mathrm{E} \quad 00$ $-8.63831 \mathrm{E} 00$ $-8.74966 \mathrm{E} \quad 00$ $-8.86098 \mathrm{E} \quad 00$ $-8.97228 \mathrm{E} 00$

$-9.08357 \mathrm{E} \quad 00$ $-9.19482 \mathrm{E} \quad 00$ $-9.30605 \mathrm{E} \quad 00$ $-9.41726 \mathrm{E} 00$ $-9.52844 \mathrm{E} \quad 00$

$-9.63960 \mathrm{E} \quad 00$ $-9.75074 \mathrm{E} \quad 00$ $-9.86184 \mathrm{E} \quad 00$ $-9.97293 \mathrm{E} \quad 00$ $-1.00840 \mathrm{E} 01$

$-1.01950 \mathrm{E} 01$ $-1.03060 \mathrm{E} 01$ $-1.04170 \mathrm{E} 01$ $-1.05280 \mathrm{E} 01$ $-1.06389 \mathrm{E} 01$

$-1.07499 \mathrm{E} \quad 01$ $-1.08608 \mathrm{E} \quad 01$ $-1.09716 \mathrm{E} \quad 01$ $-1.10825 \mathrm{E} 01$ $-1.11933 \mathrm{E} 01$

$\begin{array}{ll}-1.13041 \mathrm{E} & 01\end{array}$ $-1.14149 \mathrm{E} 01$ $-1.15256 \mathrm{E} 01$ $-1.16363 \mathrm{E} 01$ $-1.17470 \mathrm{E} 01$

$-1.18577 \mathrm{E} \quad 01$ $-1.19683 \mathrm{E} 01$ $-1.20790 \mathrm{E}$ 0I $-1.21895 \mathrm{E} 01$ $-1.23001 \mathrm{E} \quad 01$

$-1.24107 \mathrm{E} \quad 01$ $-1.25212 \mathrm{E} 01$ $-1.26317 \mathrm{E} \quad 01$ $-1.27422 \mathrm{E} 01$ $-1.28526 \mathrm{E} 01$ $g_{1}$

$-3.11264 \mathrm{E}-01$

$-3.18580 \mathrm{E}-01$

$-3.25957 \mathrm{E}-01$

$-3.33397 \mathrm{E}-01$

$-3.40898 \mathrm{E}-01$

$-3.48462 \mathrm{E}-01$

$-3.56088 \mathrm{E}-01$

$-3.63775 \mathrm{E}-01$

$-3.71525 \mathrm{E}-01$

$-3.79337 \mathrm{E}-01$

$-3.87210 \mathrm{E}-01$

$-3.95145 \mathrm{E}-01$

$-4.03143 \mathrm{E}-01$

$-4.11202 \mathrm{E}-01$

$-4.19323 \mathrm{E}-01$

$-4.27506 \mathrm{E}-01$

$-4.35751 \mathrm{E}-01$

$-4.44058 \mathrm{E}-01$

$-4.52426 \mathrm{E}-01$

$-4.60856 \mathrm{E}-01$

$-4.69348 \mathrm{E}-01$

$-4.77902 \mathrm{E}-01$

$-4.86518 \mathrm{E}-01$

$-4.95195 \mathrm{E}-01$

$-5.03934 \mathrm{E}-01$

$-5.12735 \mathrm{E}-01$

$-5.21597 \mathrm{E}-01$

$-5.30521 \mathrm{E}-01$

$-5.39507 \mathrm{E}-01$

$-5.48555 \mathrm{E}-01$

$-5.57664 \mathrm{E}-01$

$-5.66834 \mathrm{E}-01$

$-5.76067 \mathrm{E}-01$

$-5.85360 \mathrm{E}-01$

$-5.94716 \mathrm{E}-01$

$-6.04133 \mathrm{E}-01$

$-6.13611 \mathrm{E}-01$

$-6.23151 \mathrm{E}-01$

$-6.32753 \mathrm{E}-01$

$-6.42416 \mathrm{E}-01$

$-6.52140 \mathrm{E}-01$

$-6.61926 \mathrm{E}-01$

$-6.71773 \mathrm{E}-01$

$-6.81682 \mathrm{E}-01$

$-6.91653 \mathrm{E}-01$

$-7.01684 \mathrm{E}-01$

$-7.11777 \mathrm{E}-01$

$-7.21931 \mathrm{E}-01$

$-7.32147 \mathrm{E}-01$

$-7.42424 \mathrm{E}-01$

$-7.52762 \mathrm{E}-01$ $-7.63162 \mathrm{E}-01$

$-7.73623 \mathrm{E}-01$

$-7.84145 \mathrm{E}-01$

$-7.94728 \mathrm{E}-01$

$-8.05373 \mathrm{E}-01$

$-8.16078 \mathrm{E}-01$

$-8.26846 \mathrm{E}-01$

$-8.37674 \mathrm{E}-01$

$-8.48563 \mathrm{E}-01$

$\tan \delta_{1}$

$4.02798 \mathrm{E}-03$

$4.12134 \mathrm{E}-03$

$4.21173 \mathrm{E}-03$

$4.29929 \mathrm{E}-03$

$4.38414 \mathrm{E}-03$

$4.46642 \mathrm{E}-03$

$4.54623 \mathrm{E}-03$

$4.62368 \mathrm{E}-03$

$4.69889 \mathrm{E}-03$

$4.77192 \mathrm{E}-03$

$4.84289 \mathrm{E}-03$

$4.91187 \mathrm{E}-03$

$4.97894 \mathrm{E}-03$

$5.04419 \mathrm{E}-03$

$5.10767 \mathrm{E}-03$

$5.16946 \mathrm{E}-03$

$5.22963 \mathrm{E}-03$

$5.28823 \mathrm{E}-03$

$5.34532 \mathrm{E}-03$

$5.40097 \mathrm{E}-03$

$5.45522 \mathrm{E}-03$

$5.50811 \mathrm{E}-03$

$5.55970 \mathrm{E}-03$

$5.61005 \mathrm{E}-03$

$5.65919 \mathrm{E}-03$

$5.70715 \mathrm{E}-03$

$5.75399 \mathrm{E}-03$

$5.79973 \mathrm{E}-03$

$5.84442 \mathrm{E}-03$

$5.88808 \mathrm{E}-03$

$5.93076 \mathrm{E}-03$

$5.97247 \mathrm{E}-03$

$6.01327 \mathrm{E}-03$

$6.05316 \mathrm{E}-03$

$6.09218 \mathrm{E}-03$

$6.13036 \mathrm{E}-03$

$6.16772 \mathrm{E}-03$

$6.20430 \mathrm{E}-03$

$6.24008 \mathrm{E}-03$ 
$Z=10 \quad A=20 \quad \rho=1.2 A^{1 / 3} F$

6.10

6.20

6.30

6.40

6.50

6.60

6.70

6.80

6.90

7.00

7.10

7.20

7.30

7.40

7.50

7.60

7.70

7.80

7.90

8.00

8.10

8.20

8.30

8.40

8.50

8.60

8.70

8.80

8.90

9.00

9.10

9.20

9.30

9.40

9.50

9.60

9.70

9.80

9.90

10.00

10.10

10.20

10.30

10.40

10.50

10.60

10.70

10.80

10.90

11.00

11.10

11.20

11.30

11.40

11.50

11.60

11.70

11.80

11.90

12.00
$\bar{F}$

$f_{2}$

$9.36551 \mathrm{E}-05$ $9.67376 \mathrm{E}-05$ $9.98701 \mathrm{E}-05$ $1.03053 \mathrm{E}-04$ $1.06285 \mathrm{E}-04$

$1.09567 \mathrm{E}-04$ $1.12900 \mathrm{E}-04$ $1.16282 \mathrm{E}-04$ $1.19715 \mathrm{E}-04$ $1.23197 \mathrm{E}-04$

$1.26729 \mathrm{E}-04$ $1.30312 \mathrm{E}-04$ $1.33944 \mathrm{E}-04$ $1.37626 \mathrm{E}-04$ $1.41358 \mathrm{E}-04$

$1.45140 \mathrm{E}-04$ $1.48972 \mathrm{E}-04$ $1.52854 \mathrm{E}-04$ $1.56786 \mathrm{E}-04$ $1.60768 \mathrm{E}-04$

$1.64800 \mathrm{E}-04$ $1.68882 \mathrm{E}-04$ $1.73014 \mathrm{E}-04$ $1.77196 \mathrm{E}-04$ $1.81427 \mathrm{E}-04$

$1.85709 \mathrm{E}-04$ $1.90041 \mathrm{E}-04$ $1.94422 \mathrm{E}-04$ $1.98854 \mathrm{E}-04$ $2.03335 \mathrm{E}-04$

$2.07866 \mathrm{E}-04$ $2.12448 \mathrm{E}-04$ $2.17079 \mathrm{E}-04$ $2.21760 \mathrm{E}-04$ $2.26491 \mathrm{E}-04$

$2.31272 \mathrm{E}-04$ $2.36103 \mathrm{E}-04$ $2.40984 \mathrm{E}-04$ $2.45915 \mathrm{E}-04$ $2.50895 \mathrm{E}-04$

$2.55926 \mathrm{E}-04$ $2.61007 \mathrm{E}-04$ $2.66137 \mathrm{E}-04$ $2.71318 \mathrm{E}-04$ $2.76548 \mathrm{E}-04$

$2.81828 \mathrm{E}-04$ $2.87158 \mathrm{E}-04$ $2.92538 \mathrm{E}-04$ $2.97968 \mathrm{E}-04$ $3.03448 \mathrm{E}-04$

$3.08978 \mathrm{E}-04$

$3.14558 \mathrm{E}-04$ $3.20187 \mathrm{E}-04$

$3.25867 \mathrm{E}-04$

$3.31596 \mathrm{E}-04$

$3.37375 \mathrm{E}-04$ $3.43205 \mathrm{E}-04$ $3.49084 \mathrm{E}-04$

$3.55013 \mathrm{E}-04$ $3.60992 \mathrm{E}-04$
$-1.07867 \mathrm{E}-01$ $-1.11590 \mathrm{E}-01$ $-1.15376 \mathrm{E}-01$ $-1.19224 \mathrm{E}-01$ $-1.23135 \mathrm{E}-01$

$-1.27109 \mathrm{E}-01$ $-1.31146 \mathrm{E}-01$

$-1.35245 \mathrm{E}-01$ $-1.39408 \mathrm{E}-01$ $-1.43633 \mathrm{E}-01$

- 1.47921 E-01 $-1.52271 \mathrm{E}-01$ $-1.56685 \mathrm{E}-01$ $-1.61161 \mathrm{E}-01$

$-1.65700 \mathrm{E}-01$

$-1.70301 \mathrm{E}-01$ $-1.74965 \mathrm{E}-01$ $-1.79692 \mathrm{E}-01$ $-1.84482 \mathrm{E}-01$ $-1.89334 \mathrm{E}-01$

$-1.94249 \mathrm{E}-01$ $-1.99226 \mathrm{E}-01$ $-2.04266 \mathrm{E}-01$ $-2.09369 \mathrm{E}-01$ $-2.14534 \mathrm{E}-01$

$-2.19762 \mathrm{E}-01$ $-2.25053 \mathrm{E}-01$ $-2.30406 \mathrm{E}-01$ $-2.35821 \mathrm{E}-01$ $-2.41300 \mathrm{E}-01$

$-2.46840 \mathrm{E}-01$ $-2.52443 \mathrm{E}-01$ $-2.58109 \mathrm{E}-01$ $-2.63837 \mathrm{E}-01$ $-2.69628 \mathrm{E}-01$

$-2.75481 \mathrm{E}-01$ $-2.81397 \mathrm{E}-01$ $-2.87375 \mathrm{E}-01$ $-2.93415 \mathrm{E}-01$ $-2.99518 \mathrm{E}-01$

$-3.05683 \mathrm{E}-01$ $-3.11911 \mathrm{E}-01$ $-3.18201 \mathrm{E}-01$ $-3.24554 \mathrm{E}-01$ $-3.30969 \mathrm{E}-01$

$-3.37446 \mathrm{E}-01$ $-3.43985 \mathrm{E}-01$ $-3.50587 \mathrm{E}-01$ $-3.57252 \mathrm{E}-01$ $-3.63978 \mathrm{E}-01$

$-3.70767 \mathrm{E}-01$ $-3.77618 \mathrm{E}-01$

$-3.84531 \mathrm{E}-01$

$-3.91507 \mathrm{E}-01$

$-3.98545 \mathrm{E}-01$

$-4.05645 \mathrm{E}-01$ $-4.12807 \mathrm{E}-01$ $-4.20032 \mathrm{E}-01$ $-4.27318 \mathrm{E}-01$ $-4.34667 \mathrm{E}-01$ $g_{2}$

$\tan \delta_{2}$ $f_{-2}$

$g-2$

$-3.10777 \mathrm{E}-03$ $-3.23363 \mathrm{E}-03$ $-3.36258 \mathrm{E}-03$ $-3.49464 \mathrm{E}-03$ $-3.62985 \mathrm{E}-03$

$-3.76823 \mathrm{E}-03$ $-3.90982 \mathrm{E}-03$ $-4.05465 \mathrm{E}-03$ $-4.20276 \mathrm{E}-03$ $-4.35416 \mathrm{E}-03$

$-4.50890 \mathrm{E}-03$ $-4.66701 \mathrm{E}-03$ $-4.82851 \mathrm{E}-03$ $-4.99344 \mathrm{E}-03$ $-5.16184 \mathrm{E}-03$

$-5.33372 \mathrm{E}-03$ $-5.50913 \mathrm{E}-03$ $-5.68809 \mathrm{E}-03$ $-5.87064 \mathrm{E}-03$ $-6.05681 \mathrm{E}-03$

$-6.24662 \mathrm{E}-03$ $-6.44012 \mathrm{E}-03$ $-6.63733 \mathrm{E}-03$ $-6.83828 \mathrm{E}-03$

$-7.04301 \mathrm{E}-03$

$-7.25155 \mathrm{E}-03$

$-7.46392 \mathrm{E}-03$

$-7.68016 \mathrm{E}-03$

$-7.90030 \mathrm{E}-03$

$-8.12438 \mathrm{E}-03$

$-8.35242 \mathrm{E}-03$ $-8.58445 \mathrm{E}-03$

$-8.82051 \mathrm{E}-03$

$-9.06063 \mathrm{E}-03$

$-9.30484 \mathrm{E}-03$

$-9.55317 \mathrm{E}-03$

$-9.80564 \mathrm{E}-03$

$-1.00623 \mathrm{E}-02$

$-1.03232 \mathrm{E}-02$

$-1.05883 \mathrm{E}-02$

$-1.08577 \mathrm{E}-02$

$-1.11314 \mathrm{E}-02$

$-1.14095 \mathrm{E}-02$

$-1.16919 \mathrm{E}-02$

$-1.19787 \mathrm{E}-02$

$-1.22700 \mathrm{E}-02$

$-1.25657 \mathrm{E}-02$

$-1.28659 \mathrm{E}-02$

$-1.31707 \mathrm{E}-02$

$-1.34800 \mathrm{E}-02$

$-1.37939 \mathrm{E}-02$

$-1.41124 \mathrm{E}-02$

$-1.44356 \mathrm{E}-02$

$-1.47634 \mathrm{E}-02$

$-1.50960 \mathrm{E}-02$

$-1.54333 \mathrm{E}-02$

$-1.57754 \mathrm{E}-02$

$-1.61223 \mathrm{E}-02$

$-1.64741 \mathrm{E}-02$

$-1.68307 \mathrm{E}-02$

$1.97404 \mathrm{E}$ 01 $-3.22946 \mathrm{E}-03$ $1.97674 \mathrm{E} \quad 01-3.35406 \mathrm{E}-03$ $1.97935 \mathrm{E} \quad 01-3.48163 \mathrm{E}-03$ $1.98188 \mathrm{E} \quad 01 \quad-3.61221 \mathrm{E}-03$ $1.98432 \mathrm{E}$ ol $-3.74583 \mathrm{E}-03$

$1.98668 \mathrm{E} \quad 01$ $1.98897 \mathrm{E} \quad 01$

$1.99119 \mathrm{E} \quad 01$

$1.99334 \mathrm{E} \quad 01$

$1.99542 \mathrm{E} \quad 01$

$1.99744 \mathrm{E} \quad 01$ $1.99940 \mathrm{E} \quad 01$ $2.00131 \mathrm{E} \quad 01$

$2.00316 \mathrm{E} \quad 01$

$2.00495 \mathrm{E} \quad 01$

$2.00670 \mathrm{E} \quad 01$ $2.00840 \mathrm{E} \quad 01$ $2.01005 \mathrm{E} \quad 01$

$2.01166 \mathrm{E} \quad 01$

$2.01322 \mathrm{E} \quad 01$

$2.01475 \mathrm{E} \quad 01$

$2.01623 \mathrm{E} \quad 01$

$2.01768 \mathrm{E} \quad 01$

$2.01909 \mathrm{E} \quad 01$

$2.02047 \mathrm{E} \quad 0$

$2.02181 \mathrm{E} \quad 01$

$2.02312 \mathrm{E} \quad 01$

$2.02440 \mathrm{E} \quad 01$

$2.02564 \mathrm{E} 01$

$2.02686 \mathrm{E} \quad 01$

$2.02805 \mathrm{E} \quad 01$ $2.02921 \mathrm{E} 01$

$2.03035 \mathrm{E} \quad 01$

$2.03146 \mathrm{E} 01$

$2.03254 \mathrm{E} \quad 01$

$2.03360 \mathrm{E} \quad 01$

$2.03464 \mathrm{E} \quad 01$

$2.03566 \mathrm{E} \quad 01$

$2.03665 \mathrm{E} \quad 01$

$2.03762 \mathrm{E} 01$ 
p

12.10

12.20

12.30

12.40

12.50

12.60

12.70

12.80

12.90

13.00

13.10

13.20

13.30

13.40

13.50

13.60

13.70

13.80

13.90

14.00

14.10

14.20

14.30

14.40

14.50

14.60

14.70

14.80

14.90

15.00

15.10

15.20

15.30

15.40

15.50

15.60

15.70

15.80

15.90

16.00

16.10

16.20

16.30

16.40

16.50

16.60

16.70

16.80

16.90

17.00

17.10

17.20

17.30

17.40

17.50

17.60

17.70

17.80

17.90

18.00

\begin{abstract}
$F_{0}$
\end{abstract}
$f_{1}$

$1.27454 \mathrm{E} \quad 00 \quad-1.29630 \mathrm{E} \quad 01$ $1.27447 \mathrm{E} \quad 00$ $1.27440 \mathrm{E} \quad 00$ $1.27433 \mathrm{E} \quad 00$

$1.27426 \mathrm{E} \quad 00$

$1.27419 \mathrm{E} \quad 00$

$1.27413 \mathrm{E} \quad 00$

$1.27406 \mathrm{E} \quad 00$

$1.27399 \mathrm{E} \quad 00$

$1.27393 \mathrm{E} \quad 00$

$1.27387 \mathrm{E} \quad 00$

$1.27380 \mathrm{E} \quad 00$

$1.27374 \mathrm{E} \quad 00$

$1.27368 \mathrm{E} \quad 00$

$1.27361 \mathrm{E} \quad 00$

$1.27355 \mathrm{E} \quad 00$ $1.27349 \mathrm{E} \quad 00$ $1.27343 \mathrm{E} \quad 00$ $\begin{array}{ll}1.27338 \mathrm{E} & 00\end{array}$

$1.27332 \mathrm{E} \quad 00$

$1.27326 \mathrm{E} \quad 00$

$1.27320 \mathrm{E} \quad 00$

$1.27314 \mathrm{E} \quad 00$

$1.27309 \mathrm{E} \quad 00$

$1.27303 \mathrm{E} \quad 00$

$1.27298 \mathrm{E} \quad 00$

$1.27292 \mathrm{E} \quad 00$

$1.27287 \mathrm{E} \quad 00$

$1.27281 \mathrm{E} \quad 00$

$1.27276 \mathrm{E} \quad 00$

$1.27271 \mathrm{E} \quad 00$ $1.27265 \mathrm{E} \quad 00$ $1.27260 \mathrm{E} \quad 00$ $1.27255 \mathrm{E} \quad 00$

$1.27250 \mathrm{E} \quad 00$

$1.27245 \mathrm{E} \quad 00$

$1.27240 \mathrm{E} \quad 00$

$1.27235 \mathrm{E} \quad 00$

$1.27230 \mathrm{E} \quad 00$

$1.27225 \mathrm{E} \quad 00$

$1.27220 \mathrm{E} \quad 00$ $1.27215 \mathrm{E} \quad 00$ $1.27210 \mathrm{E} \quad 00$ $1.27206 \mathrm{E} \quad 00$ $1.27201 \mathrm{E} \quad 00$

$1.27196 \mathrm{E} \quad 00$ $1.27192 \mathrm{E} \quad 00$ $1.27187 \mathrm{E} \quad 00$ $1.27182 \mathrm{E} \quad 00$ $1.27178 \mathrm{E} \quad 00$

$1.27173 \mathrm{E} \quad 00$ $1.27169 \mathrm{E} \quad 00$ $1.27164 \mathrm{E} \quad 00$ $1.27160 \mathrm{E} \quad 00$ $1.27156 \mathrm{E} \quad 00$

$1.27151 \mathrm{E} \quad 00$ $1.27147 \mathrm{E} \quad 00$ $1.27143 \mathrm{E} \quad 00$ $1.27138 \mathrm{E} \quad 00$ $1.27134 \mathrm{E} \quad 00$ $g_{1}$

$-8.59514 \mathrm{E}-01$ $-8.70525 \mathrm{E}-01$ $-8.81598 \mathrm{E}-01$ $-8.92732 \mathrm{E}-01$ $-9.03927 \mathrm{E}-01$

$-9.15183 \mathrm{E}-01$ $-9.26500 \mathrm{E}-01$

$-9.37878 \mathrm{E}-01$ $-9.49316 \mathrm{E}-01$ $-9.60816 \mathrm{E}-01$

$-9.72377 \mathrm{E}-01$ $-9.83999 \mathrm{E}-01$

$-9.95682 \mathrm{E}-01$

$-1.00743 \mathrm{E} \quad 00$

$-1.01923 \mathrm{E} \quad 0 \Theta$

$-1.03110 \mathrm{E} 00$ $-1.04302 \mathrm{E} \quad 00$ $-1.05501 \mathrm{E} \quad 00$

$-1.06706 \mathrm{E} 00$

$-1.07917 \mathrm{E} 00$

$-1.09134 \mathrm{E} \quad 00$

$-1.10357 \mathrm{E} \quad 00$

$-1.11586 \mathrm{E} 00$

$-1.12821 \mathrm{E} \quad 00$

$-1.14062 \mathrm{E} 00$

$-1.15309 \mathrm{E} \quad 00$ $-1.16563 \mathrm{E} \quad 00$

$-1.17822 \mathrm{E} \quad 00$

$-1.19088 \mathrm{E} 00$

$-1.20359 \mathrm{E} \quad 00$

$-1.21637 \mathrm{E} \quad 00$

$-1.22921 \mathrm{E} 00$

$-1.24210 \mathrm{E} 00$

$-1.25506 \mathrm{E} 00$

$-1.26808 \mathrm{E} 00$

$-1.28116 \mathrm{E} \quad 00$

$-1.29430 \mathrm{E} \quad 00$

$-1.30750 \mathrm{E} 00$

$-1.32076 \mathrm{E} 00$

$-1.33408 \mathrm{E} 00$

$-1.34746 \mathrm{E} 00$ $-1.36090 \mathrm{E} 00$

$-1.37441 \mathrm{E} 00$

$-1.38797 \mathrm{E} \quad 00$

$-1.40159 \mathrm{E} 00$

$-1.41527 \mathrm{E} \quad 00$

$-1.42902 \mathrm{E} \quad 00$

$-1.44282 \mathrm{E} \quad 00$

$-1.45668 \mathrm{E} \quad 00$

$-1.47061 \mathrm{E} \quad 00$

$-1.48459 \mathrm{E} \quad 00$

$-1.49864 \mathrm{E} \quad 00$

$-1.51274 \mathrm{E} \quad 00$

$-1.52691 \mathrm{E} \quad 00$

$-1.54113 \mathrm{E} \quad 00$

$-1.55542 \mathrm{E} \quad 00$

$-1.56976 \mathrm{E} \quad 00$

$-1.58417 \mathrm{E} \quad 00$

$-1.59863 \mathrm{E} \quad 00$

$-1.61316 \mathrm{E} 00$ $\tan \delta_{1}$

$6.87050 \mathrm{E}-03$

$6.89338 \mathrm{E}-03$

$6.91585 \mathrm{E}-03$

$6.93793 \mathrm{E}-03$

6.95961 E-03

$6.98093 \mathrm{E}-03$

$7.00186 \mathrm{E}-03$

$7.02244 \mathrm{E}-03$

$7.04265 \mathrm{E}-03$

$7.06252 \mathrm{E}-03$

$7.08205 \mathrm{E}-03$

$7.10126 \mathrm{E}-03$

$7.12013 \mathrm{E}-03$

$7.13869 \mathrm{E}-03$

$7.15694 \mathrm{E}-03$

$7.17488 \mathrm{E}-03$

$7.19252 \mathrm{E}-03$

$7.20989 \mathrm{E}-03$

$7.22694 \mathrm{E}-03$

7.24.374 E-03

$7.26025 \mathrm{E}-03$

$7.27650 \mathrm{E}-03$

$7.29248 \mathrm{E}-03$

$7.30820 \mathrm{E}-03$

$7.32367 \mathrm{E}-03$

$7.33889 \mathrm{E}-03$

$7.35386 \mathrm{E}-03$

$7.36860 \mathrm{E}-03$

$7.38312 \mathrm{E}-03$

7.39738E-03

$7.41143 \mathrm{E}-03$

$7.42526 \mathrm{E}-03$

$7.43887 \mathrm{E}-03$

$7.45226 \mathrm{E}-03$

$7.46544 \mathrm{E}-03$

$7.47842 \mathrm{E}-03$

$7.49120 \mathrm{E}-03$

$7.50379 \mathrm{E}-03$

$7.51616 \mathrm{E}-03$

$7.52836 \mathrm{E}-03$

$7.54035 \mathrm{E}-03$

$7.55219 \mathrm{E}-03$

$7.56382 \mathrm{E}-03$

$7.57527 \mathrm{E}-03$

$7.58655 \mathrm{E}-03$

$7.59767 \mathrm{E}-03$

$7.60861 \mathrm{E}-03$

$7.61937 \mathrm{E}-03$

$7.62999 \mathrm{E}-03$

$7.64043 \mathrm{E}-03$

$7.65072 \mathrm{E}-03$

$7.66084 \mathrm{E}-03$

$7.67081 \mathrm{E}-03$

$7.68064 \mathrm{E}-03$

$7.69030 \mathrm{E}-03$

$7.69984 \mathrm{E}-03$

$7.70923 \mathrm{E}-03$

$7.71846 \mathrm{E}-03$

$7.72756 \mathrm{E}-03$

$7.73652 \mathrm{E}-03$

$f_{-1}$

$-8.53653 \mathrm{E}-01$

$-8.64346 \mathrm{E}-01$

$-8.75101 \mathrm{E}-01$

$-8.85916 \mathrm{E}-01$

$-8.96793 \mathrm{E}-01$

$-9.07730 \mathrm{E}-01$

$-9.18729 \mathrm{E}-01$

$-9.29789 \mathrm{E}-01$

$-9.40910 \mathrm{E}-01$

$-9.52091 \mathrm{E}-01$

$-9.63334 \mathrm{E}-01$

$-9.74638 \mathrm{E}-01$

$-9.86002 \mathrm{E}-01$

$-9.97428 \mathrm{E}-01$

$-1.00891 \mathrm{E} \quad 00$

$-1.02046 \mathrm{E} \quad 00$

$-1.03207 \mathrm{E} 00$

$-1.04374 \mathrm{E} \quad 00$

$-1.05547 \mathrm{E} \quad 00$

$-1.06726 \mathrm{E} 00$

$-1.07911 \mathrm{E} \quad 00$

$-1.09102 \mathrm{E} \quad 00$

$-1.10300 \mathrm{E} 00$

$-1.11503 \mathrm{E} 00$

$-1.12713 \mathrm{E} 00$

$-1.13928 \mathrm{E} \quad 00$

$-1.15150 \mathrm{E} \quad 00$

$-1.16377 \mathrm{E} \quad 00$

$-1.17611 \mathrm{E} \quad 00$

$-1.18851 \mathrm{E} \quad 00$

$-1.20097 \mathrm{E} \quad 00$

$-1.21349 \mathrm{E} 00$

$-1.22607 \mathrm{E} \quad 00$

$-1.23871 \mathrm{E} 00$

$-1.25141 \mathrm{E} \quad 00$

$-1.26417 \mathrm{E} \quad 00$

$-1.27699 \mathrm{E} 00$

$-1.28988 \mathrm{E} 00$

$-1.30282 \mathrm{E} \quad 00$ 
ELECTRON RADIAL FUNCTIONS

$Z=10 \quad A=20 \quad \rho=1.2 A^{1 / 3} F$

Electron

$\bar{F}$

$3.67021 \mathrm{E}-04$

$3.73100 \mathrm{E}-04$

$3.79228 \mathrm{E}-04$

$3.85407 \mathrm{E}-04$

$3.91635 \mathrm{E}-04$

$3.97914 \mathrm{E}-04$

$4.04242 \mathrm{E}-04$

$4.10620 \mathrm{E}-04$

$4.17048 \mathrm{E}-04$

$4.23526 \mathrm{E}-04$

$4.30053 \mathrm{E}-04$

$4.36631 \mathrm{E}-04$

$4.43259 \mathrm{E}-04$

$4.49936 \mathrm{E}-04$

$4.56663 \mathrm{E}-04$

$4.63441 \mathrm{E}-04$

$4.70268 \mathrm{E}-04$

$4.77145 \mathrm{E}-04$

$4.84071 \mathrm{E}-04$

$4.91048 \mathrm{E}-04$

$4.98075 \mathrm{E}-04$

$5.05151 \mathrm{E}-04$

$5.12277 \mathrm{E}-04$

$5.19454 \mathrm{E}-04$

$5.26680 \mathrm{E}-04$

$5.33956 \mathrm{E}-04$

$5.41281 \mathrm{E}-04$

$5.48657 \mathrm{E}-04$

$5.56083 \mathrm{E}-04$

$5.63558 \mathrm{E}-04$

$5.71084 \mathrm{E}-04$

$5.78659 \mathrm{E}-04$

$5.86284 \mathrm{E}-04$

$5.93959 \mathrm{E}-04$

$6.01683 \mathrm{E}-04$

$6.09458 \mathrm{E}-04$

$6.17283 \mathrm{E}-04$

$6.25157 \mathrm{E}-04$

6.33081 E-04

$6.41055 \mathrm{E}-04$

$6.49079 \mathrm{E}-04$

$6.57153 \mathrm{E}-04$

$6.65277 \mathrm{E}-04$

$6.73450 \mathrm{E}-04$

$6.81674 \mathrm{E}-04$

$6.89947 \mathrm{E}-04$

$6.98271 \mathrm{E}-04$

$7.06643 \mathrm{E}-04$

$7.15066 \mathrm{E}-04$

$7.23539 \mathrm{E}-04$

$7.32062 \mathrm{E}-04$

$7.40634 \mathrm{E}-04$

$7.49256 \mathrm{E}-04$

$7.57928 \mathrm{E}-04$

$7.66650 \mathrm{E}-04$

$7.75422 \mathrm{E}-04$

$7.84244 \mathrm{E}-04$

$7.93115 \mathrm{E}-04$

$8.02037 \mathrm{E}-04$

$8.11008 \mathrm{E}-04$ $f_{2}$

$-4.42079 \mathrm{E}-01$

$-4.49552 \mathrm{E}-01$

$-4.57087 \mathrm{E}-01$

$-4.64685 \mathrm{E}-01$

$-4.72344 \mathrm{E}-01$

$-4.80066 \mathrm{E}-01$

$-4.87850 \mathrm{E}-01$

$-4.95696 \mathrm{E}-01$

$-5.03604 \mathrm{E}-01$

$-5.11574 \mathrm{E}-01$

$-5.19606 \mathrm{E}-01$

$-5.27700 \mathrm{E}-01$

$-5.35856 \mathrm{E}-01$

$-5.44074 \mathrm{E}-01$

$-5.52354 \mathrm{E}-01$

$-5.60697 \mathrm{E}-01$

$-5.69101 \mathrm{E}-01$

$-5.77567 \mathrm{E}-01$

$-5.86095 \mathrm{E}-01$

$-5.94684 \mathrm{E}-01$

$-6.03336 \mathrm{E}-01$

$-6.12050 \mathrm{E}-01$

$-6.20825 \mathrm{E}-01$

$-6.29663 \mathrm{E}-01$

$-6.38562 \mathrm{E}-01$

$-6.47523 \mathrm{E}-01$

$-6.56546 \mathrm{E}-01$

$-6.65631 \mathrm{E}-01$

$-6.74777 \mathrm{E}-01$

-6.83986E-01

$-6.93256 \mathrm{E}-01$

$-7.02588 \mathrm{E}-01$

$-7.11982 \mathrm{E}-01$

$-7.21437 \mathrm{E}-01$

$-7.30954 \mathrm{E}-01$

$-7.40533 \mathrm{E}-01$

$-7.50173 \mathrm{E}-01$

$-7.59875 \mathrm{E}-01$

$-7.69639 \mathrm{E}-01$

$-7.79465 \mathrm{E}-01$

$-7.89352 \mathrm{E}-01$ $-7.99301 \mathrm{E}-01$ $-8.09311 \mathrm{E}-01$ $-8.19383 \mathrm{E}-01$

$-8.29516 \mathrm{E}-01$

$-8.39711 \mathrm{E}-01$

$-8.49968 \mathrm{E}-01$

$-8.60286 \mathrm{E}-01$

$-8.70666 \mathrm{E}-01$

$-8.81107 \mathrm{E}-01$

$-8.91610 \mathrm{E}-01$

$-9.02174 \mathrm{E}-01$

$-9.12799 \mathrm{E}-01$

$-9.23486 \mathrm{E}-01$

$-9.34235 \mathrm{E}-01$

$-9.45045 \mathrm{E}-01$

$-9.55916 \mathrm{E}-01$

$-9.66849 \mathrm{E}-01$

$-9.77843 \mathrm{E}-01$

$-9.88898 \mathrm{E}-01$ $\tan \delta_{2}$

$f_{-2}$

$-1.71922 \mathrm{E}-02$

$-1.75587 \mathrm{E}-02$

$-1.79301 \mathrm{E}-02$

$-1.83065 \mathrm{E}-02$

$-1.86880 \mathrm{E}-02$

$-1.90745 \mathrm{E}-02$

$-1.94661 \mathrm{E}-02$

$-1.98628 \mathrm{E}-02$

$-2.02647 \mathrm{E}-02$

$-2.06717 \mathrm{E}-02$

$-2.10840 \mathrm{E}-02$

$-2.15015 \mathrm{E}-02$

$-2.19243 \mathrm{E}-02$

$-2.23523 \mathrm{E}-02$

$-2.27858 \mathrm{E}-02$

$-2.32246 \mathrm{E}-02$

$-2.36688 \mathrm{E}-02$

$-2.41184 \mathrm{E}-02$

$-2.45735 \mathrm{E}-02$

$-2.50341 \mathrm{E}-02$

$-2.55002 \mathrm{E}-02$

$-2.59719 \mathrm{E}-02$

$-2.64491 \mathrm{E}-02$

$-2.69320 \mathrm{E}-02$

$-2.74205 \mathrm{E}-02$

$-2.79147 \mathrm{E}-02$

$-2.84147 \mathrm{E}-02$

-2.89203E-02

$-2.94318 \mathrm{E}-02$

$-2.99490 \mathrm{E}-02$

$-3.04721 \mathrm{E}-02$

$-3.10010 \mathrm{E}-02$

$-3.15358 \mathrm{E}-02$

$-3.20766 \mathrm{E}-02$

$-3.26233 \mathrm{E}-02$

$-3.31760 \mathrm{E}-02$

$-3.37347 \mathrm{E}-02$

$-3.42995 \mathrm{E}-02$

$-3.48704 \mathrm{E}-02$

$-3.54474 \mathrm{E}-02$

$-3.60305 \mathrm{E}-02$

$-3.66198 \mathrm{E}-02$

$-3.72153 \mathrm{E}-02$

$-3.78170 \mathrm{E}-02$

$-3.84250 \mathrm{E}-02$

$-3.90393 \mathrm{E}-02$

$-3.96599 \mathrm{E}-02$

$-4.02869 \mathrm{E}-02$

$-4.09203 \mathrm{E}-02$

$-4.15601 \mathrm{E}-02$

$-4.22064 \mathrm{E}-02$

$-4.28591 \mathrm{E}-02$

$-4.35183 \mathrm{E}-02$

$-4.41841 \mathrm{E}-02$

$-4.48565 \mathrm{E}-02$

$-4.55355 \mathrm{E}-02$

$-4.62211 \mathrm{E}-02$

$-4.69134 \mathrm{E}-02$

$-4.76124 \mathrm{E}-02$

$-4.83181 \mathrm{E}-02$
$2.05416 \mathrm{E} \quad 01 \quad-1.70481 \mathrm{E}-02$ $2.05480 \mathrm{E} \quad 01-1.74069 \mathrm{E}-02$ $2.05542 \mathrm{E} \quad 01-1.77705 \mathrm{E}-02$ $2.05604 \mathrm{E}$ 01 $-1.81390 \mathrm{E}-02$

$2.05665 \mathrm{E} \quad 01 \quad-1.85124 \mathrm{E}-02$

$2.05724 \mathrm{E} \quad 01$ $2.05783 \mathrm{E} 01$ $2.05841 \mathrm{E} \quad 01$ $2.05897 \mathrm{E} \quad 01$ $2.05953 \mathrm{E} \quad 01$

$2.06008 \mathrm{E} \quad 01$

$2.06062 \mathrm{E} \quad 01$

$2.06115 \mathrm{E} \quad 01$

$2.06168 \mathrm{E} \quad 01$

$2.06219 \mathrm{E} 01$

$2.06270 \mathrm{E} \quad 01$

$2.06320 \mathrm{E} \quad 01$

$2.06369 \mathrm{E} \quad 01$

$2.06418 \mathrm{E} \quad 01$

$2.06465 \mathrm{E} 01$

$2.06512 \mathrm{E} \quad 01$ $2.06559 \mathrm{E} \quad 01$

$2.06604 \mathrm{E} 01$

$2.06649 \mathrm{E} \quad 01$

$2.06694 \mathrm{E} \quad 01$

$2.06737 \mathrm{E} \quad 01$

$2.06780 \mathrm{E} 01$

$2.06823 \mathrm{E} \quad 01$

$2.06865 \mathrm{E} \quad 01$

$2.06906 \mathrm{E} \quad 01$

$2.06947 \mathrm{E} \quad 01$

$2.06987 \mathrm{E} \quad 01$

$2.07027 \mathrm{E} \quad 01$

$2.07066 \mathrm{E} \quad 01$

$2.07104 \mathrm{E} \quad 01$

$2.07142 \mathrm{E} \quad 01$ $2.07180 \mathrm{E} \quad 01$ $2.07217 \mathrm{E} \quad 01$

$2.07253 \mathrm{E} 01$

$2.07290 \mathrm{E} \quad 01$

$2.07325 \mathrm{E} \quad 01$ $2.07360 \mathrm{E} \quad 01$ $2.07395 \mathrm{E} \quad 01$

$2.07429 \mathrm{E} \quad 01$

$2.07463 \mathrm{E} \quad 01$

$2.07496 \mathrm{E} \quad 01$

$2.07529 \mathrm{E} \quad 01$

$2.07562 \mathrm{E} \quad 01$

$2.07594 \mathrm{E} \quad 01$

$2.07626 \mathrm{E} 01$

$2.07657 \mathrm{E} \quad 01$ $2.07688 \mathrm{E} \quad 01$ $2.07719 \mathrm{E} \quad 01$ $2.07749 \mathrm{E} \quad 01$ $2.07779 \mathrm{E} \quad 01$

2.07809 E 01 $2.07838 \mathrm{E} \quad 01$ $2.07895 \mathrm{E} \quad 01$ $2.07923 \mathrm{E} 01$

$-1.88908 \mathrm{E}-02$ $-1.92742 \mathrm{E}-02$ $-1.96626 \mathrm{E}-02$ $-2.00560 \mathrm{E}-02$ $-2.04546 \mathrm{E}-02$

$-2.08582 \mathrm{E}-02$ $-2.12669 \mathrm{E}-02$ $-2.16809 \mathrm{E}-02$ $-2.21000 \mathrm{E}-02$ $-2.25244 \mathrm{E}-02$

$-2.29540 \mathrm{E}-02$ $-2.33889 \mathrm{E}-02$ $-2.38292 \mathrm{E}-02$ $-2.42748 \mathrm{E}-02$ $-2.47258 \mathrm{E}-02$

$-2.51822 \mathrm{E}-02$ $-2.56441 \mathrm{E}-02$ $-2.61114 \mathrm{E}-02$ $-2.65843 \mathrm{E}-02$ -2.70627 E-02

$-2.75466 \mathrm{E}-02$ $-2.80362 \mathrm{E}-02$ $-2.85314 \mathrm{E}-02$ $-2.90323 \mathrm{E}-02$ $-2.95389 \mathrm{E}-02$

$-3.00512 \mathrm{E}-02$ $-3.05693 \mathrm{E}-02$ $-3.10931 \mathrm{E}-02$ $-3.16228 \mathrm{E}-02$ $-3.21584 \mathrm{E}-02$

$-3.26998 \mathrm{E}-02$ $-3.32471 \mathrm{E}-02$ $-3.38004 \mathrm{E}-02$ $-3.43596 \mathrm{E}-02$ $-3.49249 \mathrm{E}-02$

$-3.54962 \mathrm{E}-02$ $-3.60736 \mathrm{E}-02$ $-3.66571 \mathrm{E}-02$ $-3.72467 \mathrm{E}-02$ $-3.78424 \mathrm{E}-02$

$-3.84444 \mathrm{E}-02$ $-3.90526 \mathrm{E}-02$ $-3.96670 \mathrm{E}-02$ $2.07867 \mathrm{E} \quad 01$ 
ELECTRON RADIAL FUNCTIONS

Electron

$p$

18.10

18.20

18.30

18.40

18.50

18.60

18.70

18.80

18.90

19.00

19.10

19.20

19.30

19.40

19.50

19.60

19.70

19.80

19.90

20.00

20.10

20.20

20.30

20.40

20.50

20.60

20.70

20.80

20.90

21.00

21.10

21.20

21.30

21.40

21.50

21.60

21.70

21.80

21.90

22.00

22.10

22.20

22.30

22.40

22.50

22.60

22.70

22.80

22.90

23.00

23.10

23.20

23.30

23.40

23.50

23.60

23.70

23.80

23.90

24.00

$$
Z=10 \quad A=20
$$

\section{$f_{1}$}

$1.27130 \mathrm{E} \quad 00$ $1.27126 \mathrm{E} \quad 00$ $1.27121 \mathrm{E} \quad 00$ $1.27117 \mathrm{E} \quad 00$ $1.27113 \mathrm{E} \quad 00$

\section{$1.27109 \mathrm{E} \quad 00$} $1.27105 \mathrm{E} \quad 00$ $1.27101 \mathrm{E} \quad 00$ $1.27097 \mathrm{E} \quad 00$ $1.27093 \mathrm{E} \quad 00$

$1.27089 \mathrm{E} \quad 00$ $1.27085 \mathrm{E} \quad 00$ $1.27081 \mathrm{E} \quad 00$ $\begin{array}{ll}1.27077 \mathrm{E} & 00\end{array}$ $1.27074 \mathrm{E} \quad 00$

$1.27070 \mathrm{E} \quad 00$ $1.27066 \mathrm{E} \quad 00$ $1.27062 \mathrm{E} \quad 00$ $1.27058 \mathrm{E} \quad 00$ $1.27055 \mathrm{E} \quad 00$

\section{$1.27051 \mathrm{E} \quad 00$}

$1.27047 \mathrm{E} \quad 00$

$1.27044 \mathrm{E} \quad 00$

$1.27040 \mathrm{E} \quad 00$

$1.27036 \mathrm{E} \quad 00$

$1.27033 \mathrm{E} \quad 00$ $1.27029 \mathrm{E} \quad 00$ $1.27026 \mathrm{E} \quad 00$ $1.27022 \mathrm{E} \quad 00$ $1.27019 \mathrm{E} \quad 00$

$1.27015 \mathrm{E} \quad 00$ $1.27012 \mathrm{E} \quad 00$ $1.27008 \mathrm{E} \quad 00$ $1.27005 \mathrm{E} \quad 00$ $1.27001 \mathrm{E} \quad 00$

$1.26998 \mathrm{E} \quad 00$ $1.26994 \mathrm{E} \quad 00$ $1.26991 \mathrm{E} \quad 00$ $1.26988 \mathrm{E} \quad 00$ $1.26984 \mathrm{E} \quad 00$

$1.26981 \mathrm{E} \quad 00$ $1.26978 \mathrm{E} \quad 00$ $1.26974 \mathrm{E} \quad 00$ $1.26971 \mathrm{E}-00$ $1.26968 \mathrm{E} \quad 00$

$1.26965 \mathrm{E} \quad 00$ $1.26961 \mathrm{E} \quad 00$ $1.26958 \mathrm{E} \quad 00$ $1.26955 \mathrm{E} \quad 00$ $1.26952 \mathrm{E} \quad 00$

$1.26949 \mathrm{E} \quad 00$ $1.26946 \mathrm{E} \quad 00$ $1.26942 \mathrm{E} \quad 00$ $1.26939 \mathrm{E} \quad 00$ $1.26936 \mathrm{E} \quad 00$

$1.26933 \mathrm{E} \quad 00$ $1.26930 \mathrm{E} \quad 00$ $1.26927 \mathrm{E} \quad 00$ $1.26924 \mathrm{E} \quad 00$ $1.26921 \mathrm{E} \quad 00$

$$
g_{1}
$$

$\begin{array}{llll}-1.95400 \mathrm{E} & 01 & -1.62774 \mathrm{E} & 00\end{array}$ $\begin{array}{llll}-1.96488 \mathrm{E} & 01 & -1.64239 \mathrm{E} & 00\end{array}$ $\begin{array}{llll}-1.97575 \mathrm{E} & 01 & -1.65709 \mathrm{E} & 00\end{array}$ $\begin{array}{llll}-1.98663 \mathrm{E} & 01 & -1.67186 \mathrm{E} & 00\end{array}$ $-1.99750 \mathrm{E} 01$

$-1.68669 \mathrm{E} 00$

$\begin{array}{llll}-2.00836 \mathrm{E} & 01 & -1.70157 \mathrm{E} & 00\end{array}$ $-2.01922 \mathrm{E} \quad 01 \quad-1.71652 \mathrm{E} \quad 00$ $\begin{array}{llll}-2.03008 \mathrm{E} & 01 & -1.73152 \mathrm{E} & 00\end{array}$ $\begin{array}{llll}-2.04094 \mathrm{E} & 01 & -1.74658 \mathrm{E} & 00\end{array}$ $-2.05180 \mathrm{E} \quad 01$ $-1.76171 \mathrm{E} 00$

$-2.06265 \mathrm{E} \quad 01$ $-2.07350 \mathrm{E} \quad 01$ $-2.08434 \mathrm{E} \quad 01$ $-2.09518 \mathrm{E} \quad 01$ $-2.10602 \mathrm{E} \quad 01$

$-2.11686 \mathrm{E} \quad 01$ $-2.12769 \mathrm{E} 01$ $-2.13853 \mathrm{E} 01$ $-2.14935 \mathrm{E} \quad 01$ $-2.16018 \mathrm{E} 01$

$-2.17100 \mathrm{E} \quad 01$ $-2.18182 \mathrm{E} \quad 01$ $-2.19263 \mathrm{E} \quad 01$ $-2.20345 \mathrm{E} \quad 01$ $-2.21426 \mathrm{E} 01$

$-2.22506 \mathrm{E} \quad 01$ $\begin{array}{lll}-2.23587 \mathrm{E} & 01\end{array}$ $-2.24667 \mathrm{E} \quad 01$ $-2.25747 \mathrm{E} 01$ $-2.26826 \mathrm{E} 01$

-2.27905 E 01 $-2.28984 \mathrm{E} 01$ $-2.30062 \mathrm{E} \quad 01$ $-2.31141 \mathrm{E} 01$ $-2.32219 \mathrm{E} 01$

$-2.33296 \mathrm{E} \quad 01$ $-2.34373 \mathrm{E} 01$ $-2.35450 \mathrm{E} \quad 01$ $-2.36527 \mathrm{E} \quad 01$ $-2.37603 \mathrm{E} 01$

$\begin{array}{ll}-2.38679 \mathrm{E} & 01\end{array}$ $-2.39755 \mathrm{E} 01$ $-2.40831 \mathrm{E} 01$ $-2.41906 \mathrm{E} 01$ $-2.42981 \mathrm{E} \quad 01$

$-2.44055 \mathrm{E} \quad 01$ $-2.45129 \mathrm{E} 01$ $-2.46203 \mathrm{E} 01$ $-2.47276 \mathrm{E} 01$ $-2.48350 \mathrm{E} 01$

$-2.49423 \mathrm{E} \quad 01$ $-2.50495 \mathrm{E} \quad 01$ $-2.51567 \mathrm{E} 01$ $-2.52639 \mathrm{E} 01$ $-2.53711 \mathrm{E} 01$

$-2.54782 \mathrm{E} \quad 01$ $-2.55853 \mathrm{E} \quad 01$ $-2.56924 \mathrm{E} \quad 01$ $-2.57994 \mathrm{E} 01$ $-2.59064 \mathrm{E} 01$
$-1.77689 \mathrm{E} \quad 00$ $-1.79214 \mathrm{E} 00$ $-1.80744 \mathrm{E} \quad 00$ $-1.82280 \mathrm{E} \quad 00$ $-1.83823 \mathrm{E} \quad 00$

$-1.85371 \mathrm{E} \quad 00$ $-1.86925 \mathrm{E} 00$ $-1.88486 \mathrm{E} \quad 00$ $-1.90052 \mathrm{E} 00$ $-1.91624 \mathrm{E} \quad 00$

$-1.93202 \mathrm{E} \quad 00$ $-1.94786 \mathrm{E} \quad 00$ $-1.96376 \mathrm{E} \quad 00$ $-1.97972 \mathrm{E} \quad 00$ $-1.99574 \mathrm{E} 00$

$-2.01182 \mathrm{E} \quad 00$ $-2.02796 \mathrm{E} 00$ $-2.04416 \mathrm{E} \quad 00$ $-2.06042 \mathrm{E} \quad 00$ $-2.07673 \mathrm{E} \quad 00$

$-2.09311 \mathrm{E} \quad 00$ $-2.10954 \mathrm{E} \quad 00$ $-2.12604 \mathrm{E} \quad 00$ $-2.14259 \mathrm{E} 00$ $-2.15921 \mathrm{E} \quad 00$

$-2.17588 \mathrm{E} \quad 00$ $-2.19261 \mathrm{E} \quad 00$ $-2.20940 \mathrm{E} 00$ $-2.24316 \mathrm{E} 00$

$-2.26013 \mathrm{E} \quad 00$ $-2.27716 \mathrm{E} \quad 00$ $-2.29424 \mathrm{E} \quad 00$ $-2.31139 \mathrm{E} \quad 00$ $-2.32860 \mathrm{E} \quad 00$

$-2.34586 \mathrm{E} \quad 00$ $-2.38057 \mathrm{E} \quad 00$ $-2.39801 \mathrm{E} \quad 00$ $-2.41551 \mathrm{E} \quad 00$

$-2.43307 \mathrm{E} \quad 00$ $-2.45068 \mathrm{E} \quad 00$ $-2.46836 \mathrm{E} \quad 00$ $-2.48610 \mathrm{E} 00$ $-2.50389 \mathrm{E} 00$ $-2.53966 \mathrm{E} 00$ $-2.55763 \mathrm{E} 00$ $-2.57566 \mathrm{E} \quad 00$ $-2.59375 \mathrm{E} \quad 00$ $-2.22625 \mathrm{E} \quad 00$ $-2.36318 \mathrm{E} 00$

$-2.52174 \mathrm{E} \quad 00$

$\tan \delta_{1}$

$7.74535 \mathrm{E}-03$ $7.75404 \mathrm{E}-03$ $7.76259 \mathrm{E}-03$ $7.77103 \mathrm{E}-03$

$7.77933 \mathrm{E}-03$

$7.78750 \mathrm{E}-03$

7.79554 E- 03

$7.80347 \mathrm{E}-03$

$7.81127 \mathrm{E}-03$

7.81895 E-03

$7.82653 \mathrm{E}-03$

$7.83398 \mathrm{E}-03$

$7.84131 \mathrm{E}-03$

$7.84854 \mathrm{E}-03$

$7.85565 \mathrm{E}-03$

$7.86264 \mathrm{E}-03$

$7.86954 \mathrm{E}-03$

$7.87634 \mathrm{E}-03$

$7.88301 \mathrm{E}-03$

$7.88959 \mathrm{E}-03$

$7.89606 \mathrm{E}-03$

$7.90243 \mathrm{E}-03$

$7.90871 \mathrm{E}-03$

$7.91488 \mathrm{E}-03$

$7.92095 \mathrm{E}-03$

$7.92692 \mathrm{E}-03$

$7.93282 \mathrm{E}-03$

7.93861 E- 03

$7.94429 \mathrm{E}-03$

$7.94992 \mathrm{E}-03$

$7.95543 \mathrm{E}-03$

$7.96084 \mathrm{E}-03$

$7.96619 \mathrm{E}-03$

$7.97144 \mathrm{E}-03$

$7.97660 \mathrm{E}-03$

$7.98168 \mathrm{E}-03$

$7.98668 \mathrm{E}-03$

$7.99159 \mathrm{E}-03$

$7.99641 \mathrm{E}-03$

$8.00117 \mathrm{E}-03$

$8.00584 \mathrm{E}-03$ $8.01042 \mathrm{E}-03$ $8.01492 \mathrm{E}-03$

$8.01936 \mathrm{E}-03$ 
$Z=10 \quad A=20 \quad \rho=1.2 A^{1 / 3} F$

$\bar{F}$

$8.20029 \mathrm{E}-04$ $8.29100 \mathrm{E}-04$ 8.38221 E-04 $8.47391 \mathrm{E}-04$ $8.56612 \mathrm{E}-04$

$8.65882 \mathrm{E}-04$ 8.75202E-04 $8.84572 \mathrm{E}-04$ $8.93992 \mathrm{E}-04$ $9.03461 \mathrm{E}-04$

$9.12981 \mathrm{E}-04$ $9.22550 \mathrm{E}-04$ $9.32169 \mathrm{E}-04$ $9.41838 \mathrm{E}-04$ $9.51557 \mathrm{E}-04$

$9.61326 \mathrm{E}-04$ 9.71144 E-04 $9.81013 E-04$ $9.90931 \mathrm{E}-04$ $1.00090 \mathrm{E}-03$

$1.01092 \mathrm{E}-03$ $1.02098 \mathrm{E}-03$ $1.03110 \mathrm{E}-03$ $1.04127 \mathrm{E}-03$ $1.05149 \mathrm{E}-03$

20.40

20.50

20.60

20.70

20.80

20.90

21.00

21.10

21.20

21.30

21.40

21.50

21.60

21.70

21.80

21.90

22.00

22.10

22.20

22.30

22.40

22.50

22.60

22.70

22.80

22.90

23.00

23.10

23.20

23.30

23.40

23.50

23.60

23.70

23.80

23.90

24.00

$1.06175 \mathrm{E}-03$ $1.07207 \mathrm{E}-03$ $1.08244 \mathrm{E}-03$ $1.09285 \mathrm{E}-03$ $1.10332 \mathrm{E}-03$

$1.11383 \mathrm{E}-03$ $1.12440 \mathrm{E}-03$ $1.13502 \mathrm{E}-03$ $1.14568 \mathrm{E}-03$ $1.15640 \mathrm{E}-03$

$1.16716 \mathrm{E}-03$ $1.17798 \mathrm{E}-03$ $1.18884 \mathrm{E}-03$ $1.19976 \mathrm{E}-03$ $1.21072 \mathrm{E}-03$

$1.22174 \mathrm{E}-03$ $1.23280 \mathrm{E}-03$ $1.24391 \mathrm{E}-03$ $1.25508 \mathrm{E}-03$ $1.26629 \mathrm{E}-03$

$1.27755 \mathrm{E}-03$ $1.28887 \mathrm{E}-03$ $1.30023 \mathrm{E}-03$ $1.31164 \mathrm{E}-03$ $1.32311 \mathrm{E}-03$

$1.33462 E-03$ $1.34618 \mathrm{E}-03$ 1.35779E-03 $1.36945 \mathrm{E}-03$ $1.38117 \mathrm{E}-03$

$1.39293 E-03$ $1.40474 \mathrm{E}-03$ $1.41660 \mathrm{E}-03$ $1.42851 \mathrm{E}-03$ $1.44047 \mathrm{E}-03$ $f_{2}$

$-1.00002 \mathrm{E} 00$ $-1.01119 \mathrm{E} 00$ $-1.02243 \mathrm{E} 00$ $-1.03373 \mathrm{E} 00$ $-1.04510 \mathrm{E} 00$

$-1.05652 \mathrm{E} \quad 00$ $-1.06800 \mathrm{E} 00$ $-1.07955 \mathrm{E} 00$ $-1.09116 \mathrm{E} 00$ $-1.10282 \mathrm{E} \quad 00$

$-1.11455 \mathrm{E} \quad 00$ $-1.12634 \mathrm{E} 00$ $-1.13819 \mathrm{E} 00$ $-1.15011 \mathrm{E} 00$ $-1.16208 \mathrm{E} 00$

$-1.17412 \mathrm{E} \quad 00$ -1.18621 E 00 $-1.19837 \mathrm{E} \quad 00$ $-1.21059 \mathrm{E} 00$ $-1.22287 \mathrm{E} 00$

$-1.23521 \mathrm{E} \quad 00$ $-1.24761 \mathrm{E} \quad 00$ $-1.26007 \mathrm{E} 00$ $-1.27259 \mathrm{E} 00$ $-1.28518 \mathrm{E} \quad 00$
$-1.29782 \mathrm{E} \quad 00$

$-1.31053 \mathrm{E} 00$

$-1.32329 \mathrm{E} 00$

$-1.33612 \mathrm{E} \quad 00$

$-1.34901 \mathrm{E} 00$

$-1.36196 \mathrm{E} 00$ $-1.37497 \mathrm{E} 00$ $-1.38804 \mathrm{E} 00$ $-1.40117 \mathrm{E} 00$

$-1.42762 \mathrm{E} \quad 00$ $-1.44093 \mathrm{E} \quad 00$ $-1.45431 \mathrm{E} \quad 00$ $-1.46774 \mathrm{E} \quad 00$ $-1.48124 \mathrm{E} \quad 00$

$-1.49480 \mathrm{E} \quad 00$ $-1.50842 \mathrm{E} \quad 00$ $-1.52209 \mathrm{E} \quad 00$ $-1.53583 \mathrm{E} 00$ $-1.54963 \mathrm{E} 00$

$-1.56349 \mathrm{E} 00$ $-1.57741 \mathrm{E} \quad 00$ $-1.59139 \mathrm{E} \quad 00$ $-1.60544 \mathrm{E} \quad 00$ $-1.61954 \mathrm{E} \quad 00$

$-1.63370 \mathrm{E} 00$ $-1.64793 \mathrm{E} 00$ $-1.66221 \mathrm{E} \quad 00$ $-1.67655 \mathrm{E} \quad 00$ $-1.69096 \mathrm{E} 00$

$-1.70542 \mathrm{E} \quad 00$ $-1.71995 \mathrm{E} 00$ $-1.73454 \mathrm{E} 00$ $-1.74918 \mathrm{E} \quad 00$ $-1.41437 \mathrm{E} 00$ $-1.76389 \mathrm{E} 00$ $g_{2}$

$\tan \delta_{2}$ $f_{-2}$

$-4.90306 \mathrm{E}-02$ $-4.97498 \mathrm{E}-02$ $-5.04759 \mathrm{E}-02$ $-5.12088 \mathrm{E}-02$ -5.19486 E-02

$-5.26953 \mathrm{E}-02$ $-5.34490 \mathrm{E}-02$ $-5.42096 \mathrm{E}-02$ $-5.49773 \mathrm{E}-02$ $-5.57519 \mathrm{E}-02$

$-5.65337 \mathrm{E}-02$ $-5.73225 \mathrm{E}-02$ $-5.81185 \mathrm{E}-02$ $-5.89216 \mathrm{E}-02$ $-5.97319 \mathrm{E}-02$

$-6.05494 \mathrm{E}-02$ $-6.13742 \mathrm{E}-02$ $-6.22063 \mathrm{E}-02$ $-6.30456 \mathrm{E}-02$ $-6.38923 \mathrm{E}-02$

$-6.47464 \mathrm{E}-02$ $-6.56078 \mathrm{E}-02$ $-6.64768 \mathrm{E}-02$ $-6.73531 \mathrm{E}-02$ $-6.82370 \mathrm{E}-02$

$-6.91283 \mathrm{E}-02$ $-7.00273 \mathrm{E}-02$ $-7.09338 \mathrm{E}-02$ $-7.18479 \mathrm{E}-02$ $-7.27697 \mathrm{E}-02$

$-7.36992 \mathrm{E}-02$ $-7.46363 \mathrm{E}-02$ $-7.55812 \mathrm{E}-02$ $-7.65339 \mathrm{E}-02$ $-7.74943 \mathrm{E}-02$

$-7.84626 \mathrm{E}-02$ $-7.94387 \mathrm{E}-02$ $-8.04228 \mathrm{E}-02$ $-8.14147 \mathrm{E}-02$ $-8.24146 \mathrm{E}-02$

$-8.34224 \mathrm{E}-02$ $-8.44383 \mathrm{E}-02$ $-8.54622 \mathrm{E}-02$ $-8.64942 \mathrm{E}-02$ $-8.75343 \mathrm{E}-02$

$-8.85825 \mathrm{E}-02$ $-8.96389 \mathrm{E}-02$ $-9.07034 \mathrm{E}-02$ $-9.17762 \mathrm{E}-02$ $-9.28572 \mathrm{E}-02$

$-9.39465 \mathrm{E}-02$ $-9.50442 \mathrm{E}-02$ $-9.61501 \mathrm{E}-02$ $-9.72644 \mathrm{E}-02$ $-9.83872 \mathrm{E}-02$

$-9.95183 \mathrm{E}-02$ $-1.00658 \mathrm{E}-01$ $-1.01806 \mathrm{E}-01$ $-1.02963 \mathrm{E}-01$ $-1.04128 \mathrm{E}-01$

$2.07951 \mathrm{E} \quad 01 \quad-4.82378 \mathrm{E}-02$ $2.07979 \mathrm{E}$ 01 $-4.89430 \mathrm{E}-02$ $2.08006 \mathrm{E} 01 \quad-4.96550 \mathrm{E}-02$ $2.08033 \mathrm{E} \quad 01 \quad-5.03737 \mathrm{E}-02$ $2.08060 \mathrm{E} \quad 01 \quad-5.10991 \mathrm{E}-02$

$2.08086 \mathrm{E} \quad 01 \quad-5.18314 \mathrm{E}-02$ $2.08112 \mathrm{E} 01 \quad-5.25705 \mathrm{E}-02$ $2.08138 \mathrm{E} 01 \quad-5.33165 \mathrm{E}-02$ $2.08163 \mathrm{E} \quad 01 \quad-5.40693 \mathrm{E}-02$ $2.08188 \mathrm{E} 01 \quad-5.48291 \mathrm{E}-02$

$2.08213 \mathrm{E} \quad 01 \quad-5.55959 \mathrm{E}-02$ $2.08238 \mathrm{E} 01 \quad-5.63697 \mathrm{E}-02$ $2.08262 \mathrm{E} \quad 01 \quad-5.71504 \mathrm{E}-02$ $2.08286 \mathrm{E}$ 01 $-5.79383 \mathrm{E}-02$ $2.08310 \mathrm{E}$ 01 $-5.87332 \mathrm{E}-02$

$2.08334 \mathrm{E} \quad 01 \quad-5.95352 \mathrm{E}-02$ $2.08357 \mathrm{E} 01-6.03444 \mathrm{E}-02$ $2.08380 \mathrm{E} \quad 01 \quad-6.11607 \mathrm{E}-02$ $2.08403 \mathrm{E} \quad 01-6.19843 \mathrm{E}-02$ $2.08426 \mathrm{E} \quad 01 \quad-6.28150 \mathrm{E}-02$

$2.08448 \mathrm{E} \quad 01$ $2.08470 \mathrm{E} \quad 01$ $2.08492 \mathrm{E} \quad 01$ $2.08514 \mathrm{E} \quad 01$ $2.08536 \mathrm{E} \quad 01$

$2.08557 \mathrm{E} \quad 01$

$2.08578 \mathrm{E} \quad 01$

$2.08599 \mathrm{E} \quad 01$

$2.08619 \mathrm{E} \quad 01$

$2.08640 \mathrm{E} \quad 01$

$2.08660 \mathrm{E} \quad 01$ $2.08680 \mathrm{E} \quad 01$ $2.08700 \mathrm{E} \quad 01$ $2.08720 \mathrm{E} \quad 01$ $2.08739 \mathrm{E} 01$

$2.08758 \mathrm{E} \quad 01$ $2.08778 \mathrm{E} \quad 01$ $2.08796 \mathrm{E} \quad 01$ $2.08815 \mathrm{E} \quad 01$ $2.08834 \mathrm{E} \quad 01$

$2.08852 \mathrm{E} \quad 01$ $2.08870 \mathrm{E} \quad 01$ $2.08888 \mathrm{E} \quad 01$ $2.08906 \mathrm{E} \quad 01$ $2.08924 \mathrm{E}$ 0l

$2.08942 \mathrm{E} \quad 01$ $2.08959 \mathrm{E}$ 01 $2.08976 \mathrm{E} \quad 01$ $2.08993 \mathrm{E} \quad 01$ $2.09010 \mathrm{E} \quad 01$

$2.09027 \mathrm{E} \quad 01$ $2.09043 \mathrm{E} 01$ $2.09060 \mathrm{E} \quad 01$ $2.09076 \mathrm{E} \quad 01$ $2.09092 \mathrm{E} 01$

2.09109 E 01

$2.09124 \mathrm{E} \quad 01$

$2.09140 \mathrm{E} \quad 01$

$2.09156 \mathrm{E} \quad 01$

$-6.36531 \mathrm{E}-02$

$-6.44984 \mathrm{E}-02$

$-6.53511 \mathrm{E}-02$

$-6.62111 \mathrm{E}-02$

$-6.70785 \mathrm{E}-02$

$-6.79533 \mathrm{E}-02$ 
ELECTRON RADIAL FUNCTIONS

$p$

24.10

24.20

24.30

24.40

24.50

24.60

24.70

24.80

24.90

25.00

25.10

25.20

25.30

25.40

25.50

25.60

25.70

25.80

25.90

26.00

26.10

26.20

26.30

26.40

26.50

26.60

26.70

26.80

26.90

27.00

27.10

27.20

27.30

27.40

27.50

27.60

27.70

27.80

27.90

28.00

28.10

28.20

28.30

28.40

28.50

28.60

28.70

28.80

28.90

29.00

29.10

29.20

29.30

29.40

29.50

29.60

29.70

29.80

29.90

30.00
$F_{0}$

$1.26918 \mathrm{E} \quad 00$ $1.26915 \mathrm{E} \quad 00$ $1.26912 \mathrm{E} \quad 00$ $1.26909 \mathrm{E} \quad 00$ $1.26906 \mathrm{E} \quad 00$

$1.26903 \mathrm{E} \quad 00$ $1.26900 \mathrm{E} \quad 00$ $1.26897 \mathrm{E} \quad 00$ $1.26894 \mathrm{E} \quad 00$ $1.26892 \mathrm{E} \quad 00$

$1.26889 \mathrm{E} \quad 00$ $1.26886 \mathrm{E} \quad 00$ $1.26883 \mathrm{E} \quad 00$ $1.26880 \mathrm{E} \quad 00$ $1.26877 \mathrm{E} \quad 00$

$1.26875 \mathrm{E} \quad 00$ $1.26872 \mathrm{E} \quad 00$ $1.26869 \mathrm{E} \quad 00$ $1.26866 \mathrm{E} \quad 00$ $1.26863 \mathrm{E} \quad 00$

$1.26861 \mathrm{E} \quad 00$ $1.26858 \mathrm{E} \quad 00$ $1.26855 \mathrm{E} \quad 00$ $1.26853 \mathrm{E} \quad 00$ $1.26850 \mathrm{E} \quad 00$

$1.26847 \mathrm{E} \quad 00$ $1.26844 \mathrm{E} \quad 00$ $1.26842 \mathrm{E} \quad 00$ $1.26839 \mathrm{E} \quad 00$ $1.26836 \mathrm{E} \quad 00$

$1.26834 \mathrm{E} \quad 00$ $1.26831 \mathrm{E} \quad 00$ $1.26829 \mathrm{E} \quad 00$ $1.26826 \mathrm{E} \quad 00$ $1.26823 \mathrm{E} \quad 00$

$1.26821 \mathrm{E} \quad 00$ $1.26818 \mathrm{E} \quad 00$ $1.26816 \mathrm{E} \quad 00$ $1.26813 \mathrm{E} \quad 00$ $1.26811 \mathrm{E} \quad 00$

$1.26808 \mathrm{E} \quad 00$ $1.26806 \mathrm{E} \quad 00$ $1.26803 \mathrm{E} \quad 00$ $1.26801 \mathrm{E}-00$ $1.26798 \mathrm{E} \quad 00$

$1.26796 \mathrm{E} \quad 00$ $1.26793 \mathrm{E} 00$ $1.26791 \mathrm{E} \quad 00$ $1.26788 \mathrm{E} \quad 00$ $1.26786 \mathrm{E} \quad 00$

$1.26783 \mathrm{E} \quad 00$ $1: 26781 \mathrm{E} \quad 00$ $1.26778 \mathrm{E} \quad 00$ $1.26776 \mathrm{E} \quad 00$ $1.26774 \mathrm{E} \quad 00$

$1.26771 \mathrm{E} \quad 00$ $1.26769 \mathrm{E} \quad 00$ $1.26766 \mathrm{E} \quad 00$ $1.26764 \mathrm{E} \quad 00$ $1.26762 \mathrm{E} \quad 00$ $f_{1}$

$\begin{array}{llll}-2.60134 \mathrm{E} & 01 & -2.61189 \mathrm{E} & 00\end{array}$ $\begin{array}{llll}-2.61203 \mathrm{E} & 01 & -2.63010 \mathrm{E} & 00\end{array}$ $\begin{array}{lllll}-2.62272 \mathrm{E} & 01 & -2.64836 \mathrm{E} & 00\end{array}$ $\begin{array}{lllll}-2.63341 \mathrm{E} & 01 & -2.66669 \mathrm{E} & 00\end{array}$ -2.64409 E 01

$-2.65477 \mathrm{E} \quad 01$ $-2.66545 \mathrm{E} 01$ $-2.67612 \mathrm{E} 01$ $-2.68679 \mathrm{E} \quad 01$ $-2.69746 \mathrm{E} 01$

$-2.70812 \mathrm{E} \quad 01$ $-2.71878 \mathrm{E} 01$ $-2.72944 \mathrm{E} 01$ $-2.74009 \mathrm{E} 01$ $-2.75074 \mathrm{E} \quad 01$

$-2.76139 \mathrm{E} 01$ $-2.77203 \mathrm{E} \quad 01$ $-2.78267 \mathrm{E}$ 01 $-2.79331 \mathrm{E} \quad 01$ $-2.80394 \mathrm{E} 01$

$-2.81457 \mathrm{E} \quad 01$ $-2.82520 \mathrm{E} \quad 01$ $-2.83582 \mathrm{E} 01$ $-2.84644 \mathrm{E} 01$ $-2.85706 \mathrm{E} 01$

$-2.86767 \mathrm{E} \quad 01$ $-2.87828 \mathrm{E} 01$ $-2.88889 \mathrm{E} \quad 01$ $-2.89949 \mathrm{E} 01$ $-2.91009 \mathrm{E} 01$

$-2.92068 \mathrm{E} \quad 01$ $-2.93128 \mathrm{E} \quad 01$ $-2.94187 \mathrm{E} \quad 01$ $-2.95245 \mathrm{E} 01$ $-2.96303 \mathrm{E} 01$

$-2.97361 \mathrm{E} \quad 01$ $-2.98418 \mathrm{E} 01$ $-2.99476 \mathrm{E} \quad 01$ $-3.00532 \mathrm{E} 01$ $-3.01589 \mathrm{E} 01$

$-3.02645 \mathrm{E} 01$ $-3.03701 \mathrm{E} \quad 01$ $-3.04756 \mathrm{E} 01$ $-3.05811 \mathrm{E} 01$ $-3.06866 \mathrm{E} 01$

$-3.07920 \mathrm{E} 01$ $-3.08974 \mathrm{E} 01$ $-3.10027 \mathrm{E} 01$ $-3.11081 \mathrm{E} 01$ $-3.12133 \mathrm{E} 01$

$-3.13186 \mathrm{E} 01$ $-3.14238 \mathrm{E} 01$ $-3.15290 \mathrm{E} 01$ $-3.16341 \mathrm{E} 01$ $-3.17392 \mathrm{E} 01$

$-3.18443 \mathrm{E} \quad 01$ $-3.19493 \mathrm{E} 01$ $-3.20543 \mathrm{E} 01$ $-3.21593 \mathrm{E} 01$ $-3.22642 \mathrm{E} \quad 01$ $-2.91022 \mathrm{E} \quad 00$ $-3.30410 \mathrm{E} \quad 00$ $-3.65709 \mathrm{E} 00$ $-3.74256 \mathrm{E} 00$
-2.68507 E 00

$\tan \delta_{1}$

$-2.70351 \mathrm{E} 00$ $-2.72201 \mathrm{E} \quad 00$ $-2.74057 \mathrm{E} \quad 00$ $-2.75918 \mathrm{E} \quad 00$ $-2.77786 \mathrm{E} 00$

$-2.79659 \mathrm{E} \quad 00$ $-2.81538 \mathrm{E} \quad 00$ $-2.83423 \mathrm{E} \quad 00$ $-2.85314 \mathrm{E} 00$ $-2.87211 \mathrm{E} \quad 00$

$-2.89113 \mathrm{E} \quad 00$ $-2.92936 \mathrm{E} \quad 00$ $-2.94856 \mathrm{E} \quad 00$ $-2.96782 \mathrm{E} \quad 00$

$-2.98713 \mathrm{E} \quad 00$ $-3.00651 \mathrm{E} \quad 00$ $-3.02594 \mathrm{E} \quad 00$ $-3.04543 \mathrm{E} \quad 00$ $-3.06498 \mathrm{E} 00$

$-3.08459 \mathrm{E} \quad 00$ $-3.10425 \mathrm{E} 00$ $-3.12398 \mathrm{E} \quad 00$ $-3.14376 \mathrm{E} 00$ $-3.16360 \mathrm{E} 00$

$-3.18350 \mathrm{E} \quad 00$ $-3.20345 \mathrm{E} 00$ $-3.22346 \mathrm{E} \quad 00$ $-3.24354 \mathrm{E} 00$ $-3.26367 \mathrm{E} \quad 00$

$-3.28385 \mathrm{E} \quad 00$ $-3.32440 \mathrm{E} 00$ $-3.34476 \mathrm{E} 00$ $-3.36518 \mathrm{E} 00$

$-3.38566 \mathrm{E} \quad 00$ $-3.40619 \mathrm{E} 00$ $-3.42678 \mathrm{E} 00$ $-3.44743 \mathrm{E} \quad 00$ $-3.46814 \mathrm{E} \quad 00$

$-3.48890 \mathrm{E} \quad 00$ $-3.50972 \mathrm{E} \quad 00$ $-3.53060 \mathrm{E} \quad 00$ $-3.55154 \mathrm{E} 00$ $-3.57254 \mathrm{E} \quad 00$

$-3.59359 \mathrm{E} \quad 00$ $-3.61470 \mathrm{E} 00$ $-3.63587 \mathrm{E} 00$ $-3.67837 \mathrm{E} \quad 00$

$-3.69971 \mathrm{E} \quad 00$ $-3.72111 \mathrm{E} \quad 00$ $-3.76407 \mathrm{E} \quad 00$ $-3.78564 \mathrm{E} \quad 00$

$8.08361 \mathrm{E}-03$ $8.08677 \mathrm{E}-03$ $8.08989 \mathrm{E}-03$ $8.09293 \mathrm{E}-03$ $8.09591 \mathrm{E}-03$

8.09883 E- 03 $8.10168 \mathrm{E}-03$ $8.10448 \mathrm{E}-03$ $8.10721 \mathrm{E}-03$ $8.10989 \mathrm{E}-03$

8.11251 E- 03 $8.11507 \mathrm{E}-03$ $8.11758 \mathrm{E}-03$ $8.12003 \mathrm{E}-03$ $8.12242 \mathrm{E}-03$

$8.124 .75 \mathrm{E}-03$ $8.12702 \mathrm{E}-03$ $8.12925 \mathrm{E}-03$ $8.13142 \mathrm{E}-03$ $8.13354 \mathrm{E}-03$

$8.13558 \mathrm{E}-03$ $8.13760 \mathrm{E}-03$ 8.13956 E-03 $8.14146 \mathrm{E}-03$ $8.14331 \mathrm{E}-03$

$8.14511 \mathrm{E}-03$ $8.14685 \mathrm{E}-03$ $8.14855 \mathrm{E}-03$ $8.15020 \mathrm{E}-03$ $8.15179 \mathrm{E}-03$

$8.15336 \mathrm{E}-03$ $8.15485 \mathrm{E}-03$ $8.15631 \mathrm{E}-03$ $8.15771 \mathrm{E}-03$ $8.15907 \mathrm{E}-03$

$8.16039 \mathrm{E}-03$ $8.16165 \mathrm{E}-03$ $8.16287 \mathrm{E}-03$ $8.16403 \mathrm{E}-03$

$8.16516 \mathrm{E}-03$

$8.16625 \mathrm{E}-03$ $8.16727 \mathrm{E}-03$ $8.16826 \mathrm{E}-03$ $8.16921 \mathrm{E}-03$ 8.17011 E-03

$8.17097 \mathrm{E}-03$ $8.17179 \mathrm{E}-03$ 
$\bar{F}$

24.10

24.20

24.30

24.40

24.50

24.60

24.70

24.80

24.90

25.00

25.10

25.20

25.30

25.40

25.50

25.60

25.70

25.80

25.90

26.00

26.10

26.20

26.30

26.40

26.50

26.60

26.70

26.80

26.90

27.00

27.10

27.20

27.30

27.40

27.50

27.60

27.70

27.80

27.90

28.00

28.10

28.20

28.30

28.40

28.50

28.60

28.70

28.80

28.90

29.00

29.10

29.20

29.30

29.40

29.50

29.60

29.70

29.80

29.90

30.00 $f_{2}$

$g_{2}$

$\tan \delta_{2}$ $f_{-2}$

$1.45248 \mathrm{E}-03$

$1.46454 \mathrm{E}-03$

$1.47665 \mathrm{E}-03$

$1.48881 \mathrm{E}-03$

$1.50102 \mathrm{E}-03$

$-1.77866 \mathrm{E} \quad 00$

$-1.79348 \mathrm{E} 00$

$-1.80837 \mathrm{E} \quad 00$

$-1.82332 \mathrm{E} \quad 00$

$-1.83833 \mathrm{E} 00$

$-1.05302 \mathrm{E}-01$

$-1.06484 \mathrm{E}-01$

$-1.07675 \mathrm{E}-01$

$-1.08875 \mathrm{E}-01$

$-1.10083 \mathrm{E}-01$

$1.51328 \mathrm{E}-03$

$1.52559 \mathrm{E}-03$

$1.53795 \mathrm{E}-03$

$1.55036 \mathrm{E}-03$

$1.56282 \mathrm{E}-03$

$1.57533 \mathrm{E}-03$

$1.58789 \mathrm{E}-03$

$1.60049 \mathrm{E}-03$

$1.61315 \mathrm{E}-03$

$1.62586 \mathrm{E}-03$

$1.63862 \mathrm{E}-03$

$1.65142 \mathrm{E}-03$

$1.66428 \mathrm{E}-03$

$1.67719 \mathrm{E}-03$

$1.69014 \mathrm{E}-03$

$1.70315 \mathrm{E}-03$

$1.71621 \mathrm{E}-03$

$1.72931 \mathrm{E}-03$

$1.74247 \mathrm{E}-03$

$1.75567 \mathrm{E}-03$

$1.76893 \mathrm{E}-03$

$1.78224 \mathrm{E}-03$

$1.79559 \mathrm{E}-03$

$1.80900 \mathrm{E}-03$

$1.8224 .5 \mathrm{E}-03$

$1.83595 \mathrm{E}-03$

$1.84951 \mathrm{E}-03$

$1.86311 \mathrm{E}-03$

$1.87677 \mathrm{E}-03$

$1.89047 \mathrm{E}-03$

$1.90422 \mathrm{E}-03$

$1.91803 \mathrm{E}-03$

$1.93188 \mathrm{E}-03$

$1.94578 \mathrm{E}-03$

$1.95974 \mathrm{E}-03$

$1.97374 \mathrm{E}-03$

$1.98779 \mathrm{E}-03$

$2.00189 \mathrm{E}-03$

$2.01604 \mathrm{E}-03$

$2.03024 \mathrm{E}-03$

$-1.85340 \mathrm{E} \quad 00$

$-1.86852 \mathrm{E} \quad 00$

$-1.88371 \mathrm{E} \quad 00$

$-1.89896 \mathrm{E} \quad 00$

$-1.91427 \mathrm{E} \quad 00$

$-1.92964 \mathrm{E} \quad 00$

$-1.94507 \mathrm{E} \quad 00$

$-1.96056 \mathrm{E} 00$

-1.97611 E 00

$-1.99172 \mathrm{E} \quad 00$

$-2.00739 \mathrm{E} \quad 00$

$-2.02312 \mathrm{E} 00$

$-2.03891 \mathrm{E} 00$

$-2.05476 \mathrm{E} \quad 00$

$-2.07067 \mathrm{E} \quad 00$

$-2.08664 \mathrm{E} \quad 00$

$-2.10267 \mathrm{E} \quad 00$

$-2.11876 \mathrm{E} 00$

$-2.13491 \mathrm{E} 00$

$-2.15111 \mathrm{E} \quad 00$

$-2.16738 \mathrm{E} \quad 00$

$-2.18371 \mathrm{E} 00$

$-2.20010 \mathrm{E} \quad 00$

$-2.21655 \mathrm{E} \quad 00$

$-2.23306 \mathrm{E} 00$

$-2.24963 \mathrm{E} \quad 00$

$-2.26625 \mathrm{E} \quad 00$

$-2.28294 \mathrm{E} \quad 00$

$-2.29969 \mathrm{E} \quad 00$

$-2.31650 \mathrm{E} \quad 00$

$-2.33336 \mathrm{E} \quad 00$

$-2.35029 \mathrm{E} \quad 00$

$-2.36728 \mathrm{E} \quad 00$

$-2.38432 \mathrm{E} \quad 00$

$-2.40143 \mathrm{E} 00$

$-2.41859 \mathrm{E} \quad 00$

$-2.43582 \mathrm{E} \quad 00$

$-2.45310 \mathrm{E} \quad 00$

$-2.47044 \mathrm{E} \quad 00$

$-2.48785 \mathrm{E} \quad 00$

$-1.11300 \mathrm{E}-01$

$-1.12526 \mathrm{E}-01$

$-1.13761 \mathrm{E}-01$

$-1.15004 \mathrm{E}-01$

$-1.16257 \mathrm{E}-01$

$-1.17518 \mathrm{E}-01$

$-1.18788 \mathrm{E}-01$

$-1.20067 \mathrm{E}-01$

$-1.21355 \mathrm{E}-01$

$-1.22652 \mathrm{E}-01$

$-1.23958 \mathrm{E}-01$

$-1.25273 \mathrm{E}-01$

$-1.26598 \mathrm{E}-01$

$-1.27931 \mathrm{E}-01$

$-1.29273 \mathrm{E}-01$

$-1.30625 \mathrm{E}-01$

$-1.31986 \mathrm{E}-01$

$-1.33356 \mathrm{E}-01$

$-1.34735 \mathrm{E}-01$

$-1.36124 \mathrm{E}-01$

$-1.37522 \mathrm{E}-01$

$-1.38929 \mathrm{E}-01$

$-1.40346 \mathrm{E}-01$

- $1.41772 \mathrm{E}-01$

-1.43208 E-01

$-1.44653 \mathrm{E}-01$

$-1.46108 \mathrm{E}-01$

$-1.47572 \mathrm{E}-01$

$-1.49046 \mathrm{E}-01$

$-1.50529 \mathrm{E}-01$

$-1.52022 \mathrm{E}-01$

$-1.53524 \mathrm{E}-01$

$-1.55037 \mathrm{E}-01$

$-1.56559 \mathrm{E}-01$

$-1.58090 \mathrm{E}-01$

$-1.59632 \mathrm{E}-01$

$-1.61183 \mathrm{E}-01$

$-1.62744 \mathrm{E}-01$

$-1.64315 \mathrm{E}-01$

$-1.65896 \mathrm{E}-01$

$-2.50531 \mathrm{E} \quad 00$

$-1.67487 \mathrm{E}-01$

$-2.52283 \mathrm{E} \quad 00$

$-2.54041 \mathrm{E} \quad 00$

$-2.55805 \mathrm{E} \quad 00$

$-2.57575 \mathrm{E} \quad 00$

$-1.69088 \mathrm{E}-01$

$-1.70698 \mathrm{E}-01$

$-1.72319 \mathrm{E}-01$

$-1.73950 \mathrm{E}-01$

$-2.59351 \mathrm{E} \quad 00$

$-2.61133 \mathrm{E} \quad 00$

$-2.62921 \mathrm{E} \quad 00$

$-2.64714 \mathrm{E} \quad 00$

$-2.66514 \mathrm{E} \quad 00$

$-2.68319 \mathrm{E} \quad 00$

$-2.70131 \mathrm{E} 00$

$-2.71948 \mathrm{E} 00$

$-2.73772 \mathrm{E} \quad 00$

$-2.75601 \mathrm{E} \quad 00$

$2.09187 \mathrm{E} \quad 01 \quad-1.03482 \mathrm{E}-01$

$2.09202 \mathrm{E}$ 01 $-1.04644 \mathrm{E}-01$

$2.09217 \mathrm{E}$ 0l $-1.05814 \mathrm{E}-01$

$2.09232 \mathrm{E} \quad 01 \quad-1.06993 \mathrm{E}-01$

$2.09247 \mathrm{E} \quad 01-1.08181 \mathrm{E}-01$

$2.09261 \mathrm{E} \quad 01 \quad-1.09378 \mathrm{E}-01$ $2.09276 \mathrm{E} \quad 01-1.10583 \mathrm{E}-01$

$2.09291 \mathrm{E} \quad 01 \quad-1.11796 \mathrm{E}-01$

$2.09305 \mathrm{E}$ 01 $-1.13019 \mathrm{E}-01$

$2.09319 \mathrm{E} \quad 01 \quad-1.14250 \mathrm{E}-01$

$2.09333 \mathrm{E} \quad 01$

$2.09347 \mathrm{E} \quad 01$

$2.09361 \mathrm{E} \quad 01$

$2.09375 \mathrm{E} \quad 01$

$2.09388 \mathrm{E} \quad 01$

$2.09402 \mathrm{E} \quad 01$

$2.09415 \mathrm{E} \quad 01$

$2.09429 \mathrm{E} \quad 01$

$2.09442 \mathrm{E} \quad 01$

$2.09455 \mathrm{E} \quad 01$

$2.09468 \mathrm{E} \quad 01$

$2.09481 \mathrm{E} \quad 01$

$2.09494 \mathrm{E} \quad 01$

$2.09507 \mathrm{E} \quad 01$

$2.09519 \mathrm{E} \quad 01$

$2.09532 \mathrm{E} \quad 01$

$2.09544 \mathrm{E} \quad 01$

$2.09556 \mathrm{E} \quad 01$

$2.09569 \mathrm{E} \quad 01$

$2.09581 \mathrm{E} \quad 01$

$2.09593 \mathrm{E} \quad 01$

$2.09605 \mathrm{E} \quad 01$

$2.09617 \mathrm{E} \quad 01$

$2.09628 \mathrm{E} \quad 01$

$2.09640 \mathrm{E} \quad 01$

$2.09652 \mathrm{E} \quad 01$

$2.09663 \mathrm{E} \quad 01$

$2.09674 \mathrm{E} \quad 01$

$2.09686 \mathrm{E} \quad 01$

$2.09697 \mathrm{E} \quad 0$

$2.09708 \mathrm{E} \quad 01$

$2.09719 \mathrm{E} \quad 01$

$2.09730 \mathrm{E} \quad 01$

$2.09741 \mathrm{E} \quad 01$

$2.09752 \mathrm{E} \quad 01$

$2.09763 \mathrm{E} \quad 01$ $2.09773 \mathrm{E} \quad 01$ $2.09784 \mathrm{E} \quad 01$

$2.09795 \mathrm{E} \quad 01$

$2.09805 \mathrm{E} \quad 01$

$2.09815 \mathrm{E} \quad 01$

$2.09826 \mathrm{E} \quad 01$

$2.09836 \mathrm{E} \quad 01$

$2.09846 \mathrm{E} \quad 01$

$2.09856 \mathrm{E} \quad 01$

$-1.15490 \mathrm{E}-01$

$-1.16739 \mathrm{E}-01$

$-1.17996 \mathrm{E}-01$

$-1.19263 \mathrm{E}-01$

$-1.20538 \mathrm{E}-01$

$-1.21822 \mathrm{E}-01$

$-1.23116 \mathrm{E}-01$

$-1.24418 \mathrm{E}-01$

$-1.25729 \mathrm{E}-01$

$-1.28379 \mathrm{E}-01$

$-1.29717 \mathrm{E}-01$

$-1.31065 \mathrm{E}-01$

$-1.32422 \mathrm{E}-01$

$-1.33788 \mathrm{E}-01$

$-1.35163 \mathrm{E}-01$

$-1.36547 \mathrm{E}-01$ 
ELECTRON RADIAL FUNCTIONS

Electron

$F_{0}$

30.10

30.20

30.30

30.40

30.50

30.60

30.70

30.80

30.90

31.00

31.10

31.20

31.30

31.40

31.50

31.60

31.70

31.80

31.90

32.00

32.10

32.20

32.30

32.40

32.50

32.60

32.70

32.80

32.90

33.00

33.10

33.20

33.30

33.40

33.50

33.60

33.70

33.80

33.90

34.00

34.10

34.20

34.30

34.40

34.50

34.60

34.70

34.80

34.90

35.00

35.10

35.20

35.30

35.40

35.50

35.60

35.70

35.80

35.90

36.00
$Z=10 \quad A=20$

$g_{1}$

$1.26759 \mathrm{E} \quad 00$ $1.26757 \mathrm{E} \quad 00$ $1.26755 \mathrm{E} \quad 00$ $1.26752 \mathrm{E} \quad 00$

$1.26750 \mathrm{E} \quad 00$

$1.26748 \mathrm{E} \quad 00$

$1.26745 \mathrm{E} \quad 00$

$1.26743 \mathrm{E} \quad 00$

$1.26741 \mathrm{E} \quad 00$

$1.26739 \mathrm{E} \quad 00$

$1.26736 \mathrm{E} \quad 00$

$1.26734 \mathrm{E} \quad 00$

$1.26732 \mathrm{E} \quad 00$

$1.26730 \mathrm{E} \quad 00$

$1.26727 \mathrm{E} \quad 00$

$1.26725 \mathrm{E} \quad 00$

$1.26723 \mathrm{E} \quad 00$

$1.26721 \mathrm{E} \quad 00$

$1.26719 \mathrm{E} \quad 00$

$1.26716 \mathrm{E} \quad 00$

$1.26714 \mathrm{E} \quad 00$

$1.26712 \mathrm{E} \quad 00$

$1.26710 \mathrm{E} \quad 00$

$1.26708 \mathrm{E} \quad 00$

$1.26705 \mathrm{E} \quad 00$

$1.26703 \mathrm{E} \quad 00$

$1.26701 \mathrm{E} \quad 00$

$1.26699 \mathrm{E} \quad 00$

$1.26697 \mathrm{E} \quad 00$

$1.26695 \mathrm{E} \quad 00$

$1.26693 \mathrm{E} \quad 00$ $1.26691 \mathrm{E} \quad 00$

$1.26688 \mathrm{E} \quad 00$

$1.26686 \mathrm{E} \quad 00$

$1.26684 \mathrm{E} \quad 00$

$1.26682 \mathrm{E} \quad 00$ $1.26680 \mathrm{E} \quad 00$ $1.26678 \mathrm{E} \quad 00$

$1.26676 \mathrm{E} \quad 00$

$1.26674 \mathrm{E} \quad 00$

$1.26672 \mathrm{E} \quad 00$

$1.26670 \mathrm{E} \quad 00$

$1.26668 \mathrm{E}-00$

$1.26666 \mathrm{E} \quad 00$

$1.26664 \mathrm{E} \quad 00$

$1.26662 \mathrm{E} \quad 00$

$1.26660 \mathrm{E} \quad 00$

$1.26658 \mathrm{E} \quad 00$

$1.26656 \mathrm{E} \quad 00$

$1.26654 \mathrm{E} \quad 00$

$1.26652 \mathrm{E} \quad 00$ $1.26650 \mathrm{E} \quad 00$ $1.26648 \mathrm{E} \quad 00$ $1.26646 \mathrm{E} \quad 00$

$1.26644 \mathrm{E} \quad 00$

$1.26642 \mathrm{E} \quad 00$ $1.26640 \mathrm{E} \quad 00$ $1.26638 \mathrm{E} \quad 00$

$1.26636 \mathrm{E} \quad 00$

$1.26634 \mathrm{E} \quad 00$ $f_{1}$

$-3.23691 \mathrm{E} \quad 01$ $-3.24740 \mathrm{E} 01$ $-3.25788 \mathrm{E} 01$ $-3.26836 \mathrm{E} 01$

-3.27883 E 01

$-3.28930 \mathrm{E} 01$

$-3.29977 \mathrm{E} 01$

$-3.31023 \mathrm{E} 01$

$-3.32069 \mathrm{E} 01$

$-3.33114 \mathrm{E} 01$

$-3.34159 \mathrm{E} 01$

$-3.35204 \mathrm{E} 01$

$-3.36249 \mathrm{E} 01$

$-3.37293 \mathrm{E} 01$

$-3.38337 \mathrm{E} 01$

$-3.39380 \mathrm{E} 01$

$-3.40423 \mathrm{E} 01$

$-3.41465 \mathrm{E} \quad 01$

$-3.42508 \mathrm{E} 01$

$-3.43549 \mathrm{E} 01$

$-3.44591 \mathrm{E} 01$

$-3.45632 \mathrm{E} 01$

$-3.46672 \mathrm{E} 01$

$-3.47713 \mathrm{E}$ ol

$-3.48753 \mathrm{E} 01$

$-3.49792 \mathrm{E} \quad 01$

$-3.50831 \mathrm{E}$ 01

$-3.51870 \mathrm{E} 01$

$-3.52908 \mathrm{E} \quad 01$

$-3.53946 \mathrm{E} 01$

$-3.54984 \mathrm{E} \quad 01$

$-3.56021 \mathrm{E} 01$

$-3.57058 \mathrm{E}$ 0l

$-3.58094 \mathrm{E} 01$

$-3.59130 \mathrm{E}$ ol

$-3.60166 \mathrm{E} \quad 01$

$-3.61201 \mathrm{E} 01$

$-3.62236 \mathrm{E} 01$

$-3.63270 \mathrm{E} 01$

$-3.64304 \mathrm{E}$ ol

$-3.65338 \mathrm{E} 01$

$-3.66371 \mathrm{E} 01$

$-3.67404 \mathrm{E} 01$

$-3.68437 \mathrm{E}$ 0l

$-3.69469 \mathrm{E} 01$

$-3.70501 \mathrm{E} 01$

$-3.71532 \mathrm{E}$ 0l

$-3.72563 \mathrm{E} 01$

$-3.73593 \mathrm{E} 01$

$-3.74623 \mathrm{E} 01$

$-3.75653 \mathrm{E}$ 01

$-3.76682 \mathrm{E} 01$

$-3.77711 \mathrm{E} 01$

$-3.78740 \mathrm{E} 01$

$-3.79768 \mathrm{E} 01$

$-3.80796 \mathrm{E} 01$

$-3.81823 \mathrm{E} 01$

$-3.82850 \mathrm{E} 01$

$-3.83876 \mathrm{E}$ 01

$-3.84902 \mathrm{E} 01$
$-3.80727 \mathrm{E} 00$

$-3.82895 \mathrm{E} \quad 00$

$-3.85069 \mathrm{E} \quad 00$

$-3.89435 \mathrm{E} \quad 00$

$-3.91626 \mathrm{E} 00$

$-3.93823 \mathrm{E} 00$

$-3.96025 \mathrm{E} \quad 00$

$-3.98233 \mathrm{E} 00$

$-4.00447 \mathrm{E} 00$

$-4.04893 \mathrm{E} \quad 00$

$-4.07124 \mathrm{E} \quad 00$

$-4.09360 \mathrm{E} \quad 00$

$-4.11603 \mathrm{E} \quad 00$

$-4.13851 \mathrm{E} \quad 00$

$-4.16105 \mathrm{E} \quad 00$

$-4.18364 \mathrm{E} \quad 00$

$-4.20630 \mathrm{E} 00$

$-4.22900 \mathrm{E} 00$

$-4.25177 \mathrm{E} \quad 00$

$-4.27459 \mathrm{E} \quad 00$

$-4.29747 \mathrm{E} \quad 00$

$-4.32040 \mathrm{E} \quad 00$

$-4.34340 \mathrm{E} 00$

$-4.36644 \mathrm{E} \quad 00$

$-4.38955 \mathrm{E} \quad 00$

$-4.41271 \mathrm{E} 00$

$-4.43593 \mathrm{E} \quad 00$

$-4.45920 \mathrm{E} \quad 00$

$-4.48253 \mathrm{E} \quad 00$

$-4.50592 \mathrm{E} \quad 00$

$-4.52936 \mathrm{E} \quad 00$

$-4.55287 \mathrm{E} \quad 00$

$-4.57642 \mathrm{E} \quad 00$

$-4.60003 \mathrm{E} \quad 00$

$-4.62370 \mathrm{E} 00$

$-4.64743 \mathrm{E} \quad 00$

$-4.67121 \mathrm{E} \quad 00$

$-4.69505 \mathrm{E} \quad 00$

$-4.71894 \mathrm{E} \quad 00$

$-4.74289 \mathrm{E} \quad 00$

$-4.76690 \mathrm{E} \quad 00$

$-4.79096 \mathrm{E} \quad 00$

$-4.81508 \mathrm{E} \quad 00$

$-4.83925 \mathrm{E} \quad 00$

$-4.86348 \mathrm{E} \quad 00$

$-4.88777 \mathrm{E} \quad 00$

$-4.91211 \mathrm{E} 00$

$-4.93651 \mathrm{E} \quad 00$

$-4.96096 \mathrm{E} \quad 00$

$-4.98547 \mathrm{E} \quad 00$

$-5.01004 \mathrm{E} \quad 00$

$-5.03466 \mathrm{E} 00$

$-5.05934 \mathrm{E} \quad 00$

$-5.08407 \mathrm{E} \quad 00$

$-5.10886 \mathrm{E} \quad 00$

$-5.13370 \mathrm{E} 00$

$-5.15860 \mathrm{E} \quad 00$

$-5.18356 \mathrm{E} 00$
$-3.87249 \mathrm{E} 00$

$-4.02667 \mathrm{E} \quad 00$

$\rho=1.2 A^{1 / 3} F$

$\tan \delta_{1}$

$8.17885 \mathrm{E}-03$

$8.17903 \mathrm{E}-03$

$8.17920 \mathrm{E}-03$

8.17932 E-03

8.17941 E-03

$8.17946 \mathrm{E}-03$

$8.17948 \mathrm{E}-03$

$8.17945 \mathrm{E}-03$

$8.17939 \mathrm{E}-03$

$8.17930 \mathrm{E}-03$

$8.17916 \mathrm{E}-03$

$8.17900 \mathrm{E}-03$

$8.17878 \mathrm{E}-03$

$8.17854 \mathrm{E}-03$

$8.17826 \mathrm{E}-03$

$8.17795 \mathrm{E}-03$

$8.17760 \mathrm{E}-03$

$8.17722 \mathrm{E}-03$

$8.17679 \mathrm{E}-03$

$8.17635 \mathrm{E}-03$

$8.17585 \mathrm{E}-03$

$8.17534 \mathrm{E}-03$

$8.17479 \mathrm{E}-03$

$8.17419 \mathrm{E}-03$

$8.17356 \mathrm{E}-03$

$8.17292 \mathrm{E}-03$

$8.17222 \mathrm{E}-03$

$8.17150 \mathrm{E}-03$

$8.17074 \mathrm{E}-03$

$8.16995 \mathrm{E}-03$

8.16913E-03

$8.16829 \mathrm{E}-03$

$8.16741 \mathrm{E}-03$

8.16649E-03

8.16554E-03

$8.16457 \mathrm{E}-03$

$8.16355 \mathrm{E}-03$

$8.16251 \mathrm{E}-03$

$8.16144 \mathrm{E}-03$

$8.16034 \mathrm{E}-03$

$8.15920 \mathrm{E}-03$ 
ELECTRON RADIAL FUNCTIONS

$Z=10 \quad A=20 \quad \rho=1.2 A^{1 / 3} F$

Electron

$\bar{F}$

$2.26424 \mathrm{E}-03$

$2.27929 \mathrm{E}-03$

$2.29439 \mathrm{E}-03$

$2.30953 \mathrm{E}-03$

$2.32473 \mathrm{E}-03$

$2.33998 \mathrm{E}-03$

$2.35527 \mathrm{E}-03$

$2.37062 \mathrm{E}-03$

$2.38602 \mathrm{E}-03$

$2.40146 \mathrm{E}-03$

$2.41696 \mathrm{E}-03$

$2.43250 \mathrm{E}-03$

$2.44810 \mathrm{E}-03$

$2.46374 \mathrm{E}-03$

$2.47944 \mathrm{E}-03$

31.60

31.70

31.80

31.90

32.00

32.10

32.20

32.30

32.40

32.50

32.60

32.70

32.80

32.90

33.00

33.10

33.20

33.30

33.40

33.50

33.60

33.70

33.80

33.90

34.00

34.10

34.20

34.30

34.40

34.50

34.60

34.70

34.80

34.90

35.00

35.10

35.20

35.30

35.40

35.50

35.60

35.70

35.80

35.90

36.00

$2.49518 \mathrm{E}-03$

$2.51098 \mathrm{E}-03$

$2.52682 \mathrm{E}-03$

$2.54272 \mathrm{E}-03$

$2.55866 \mathrm{E}-03$

$2.57465 \mathrm{E}-03$

$2.59070 \mathrm{E}-03$

$2.60679 \mathrm{E}-03$

$2.62293 \mathrm{E}-03$

$2.63913 \mathrm{E}-03$

$2.65537 \mathrm{E}-03$

$2.67166 \mathrm{E}-03$

$2.68800 \mathrm{E}-03$

$2.70439 \mathrm{E}-03$

2.72083 E-03

$2.73733 \mathrm{E}-03$

$2.75387 \mathrm{E}-03$

$2.77046 \mathrm{E}-03$

$2.78710 \mathrm{E}-03$

2.82053 E- 03

$2.83732 \mathrm{E}-03$

$2.85416 \mathrm{E}-03$

$2.87105 \mathrm{E}-03$

2.88798 E- 03

$2.90497 \mathrm{E}-03$

$2.92201 \mathrm{E}-03$

$2.93910 \mathrm{E}-03$

$2.95624 \mathrm{E}-03$

2.97343 E- 03

$2.99066 \mathrm{E}-03$

$3.00795 \mathrm{E}-03$

$3.02529 \mathrm{E}-03$

$3.04267 \mathrm{E}-03$

$3.06011 \mathrm{E}-03$

$3.07760 \mathrm{E}-03$ $3.09513 \mathrm{E}-03$

$3.11272 \mathrm{E}-03$

$3.13036 \mathrm{E}-03$

3.14804 E-03

$3.16578 \mathrm{E}-03$

$3.18356 \mathrm{E}-03$

$3.20140 \mathrm{E}-03$

$3.21928 \mathrm{E}-03$

$3.23721 \mathrm{E}-03$ $f_{2}$

$-2.77436 \mathrm{E} \quad 00$ $-2.79277 \mathrm{E} \quad 00$

$-2.81124 \mathrm{E} 00$

$-2.82977 \mathrm{E} \quad 00$

$-2.84836 \mathrm{E} \quad 00$

$-2.86701 \mathrm{E} \quad 00$

$-2.88571 \mathrm{E} 00$

$-2.90448 \mathrm{E} \quad 00$

$-2.92330 \mathrm{E} 00$

$-2.94218 \mathrm{E} \quad 00$

$-2.96112 \mathrm{E} \quad 00$

$-2.98013 \mathrm{E} \quad 00$

$-2.99919 \mathrm{E} \quad 00$

$-3.03748 \mathrm{E} 00$

$2.80379 \mathrm{E}-03$

$-3.05672 \mathrm{E} \quad 00$

$-3.07601 \mathrm{E} 00$

$-3.09537 \mathrm{E} 00$

$-3.13425 \mathrm{E} 00$

$-3.15378 \mathrm{E} \quad 00$

$-3.17337 \mathrm{E} \quad 00$

$-3.19302 \mathrm{E} 00$

$-3.21272 \mathrm{E} 00$

$-3.23249 \mathrm{E} 00$

$-3.25231 \mathrm{E} \quad 00$

$-3.27219 \mathrm{E} 00$

$-3.29213 \mathrm{E} 00$

$-3.31213 \mathrm{E} 00$

$-3.33219 \mathrm{E} 00$

$-3.35230 \mathrm{E} 00$

$-3.37248 \mathrm{E} 00$

$-3.39271 \mathrm{E} 00$

$-3.41300 \mathrm{E} 00$

$-3.43335 \mathrm{E} 00$

$-3.45376 \mathrm{E} \quad 00$

$-3.47422 \mathrm{E} 00$

$-3.49475 \mathrm{E} 00$

$-3.51533 \mathrm{E} \quad 00$

$-3.53597 \mathrm{E} 00$

$-3.55667 \mathrm{~F} \quad 00$

$-3.57743 \mathrm{E} \quad 00$

$-3.59824 \mathrm{E} 00$

$-3.61912 \mathrm{E} \quad 00$

$-3.64005 \mathrm{E} \quad 00$

$-3.66104 \mathrm{E} \quad 00$

$-3.68209 \mathrm{E} 00$

$-3.70320 \mathrm{E} \quad 00$

$-3.72436 \mathrm{E} 00$

$-3.76686 \mathrm{E} \quad 00$

$-3.78820 \mathrm{E} 00$

$-3.80960 \mathrm{E} \quad 00$

$-3.83105 \mathrm{E} 00$

$-3.85257 \mathrm{E} \quad 00$

$-3.87414 \mathrm{E} \quad 00$

$-3.89576 \mathrm{E} \quad 00$

$-3.91745 \mathrm{E} \quad 00$

$-3.93919 \mathrm{E} 00$

$-3.96100 \mathrm{E} 00$
$-3.01830 \mathrm{E} 00$

$g_{2}$

$\tan \delta_{2}$

$f_{-2}$

$-1.92559 \mathrm{E}-01$

$-1.94313 \mathrm{E}-01$

$-1.96077 \mathrm{E}-01$

$-1.97851 \mathrm{E}-01$

$-1.99636 \mathrm{E}-01$

$-2.01432 \mathrm{E}-01$

$-2.03237 \mathrm{E}-01$

$-2.05054 \mathrm{E}-01$

$-2.06881 \mathrm{E}-01$

$-2.08719 \mathrm{E}-01$

$-3.11478 \mathrm{E} 00$

$-2.10567 \mathrm{E}-01$

$-2.12426 \mathrm{E}-01$

$-2.14296 \mathrm{E}-01$

$-2.16176 \mathrm{E}-01$

$-2.18068 \mathrm{E}-01$

$-2.19970 \mathrm{E}-01$

$-2.21883 \mathrm{E}-01$

$-2.23806 \mathrm{E}-01$

$-2.25741 \mathrm{E}-01$

$-2.27686 \mathrm{E}-01$

$-2.29643 \mathrm{E}-01$

$-3.74558 \mathrm{E} \quad 00$

$2.09915 \mathrm{E} \quad 01 \quad-1.89337 \mathrm{E}-01$ $2.09925 \mathrm{E} \quad 01 \quad-1.91064 \mathrm{E}-01$

$2.09934 \mathrm{E} \quad 01 \quad-1.92801 \mathrm{E}-01$

$2.09944 \mathrm{E} \quad 01-1.94549 \mathrm{E}-01$

$2.09953 \mathrm{E} 01 \quad-1.96307 \mathrm{E}-01$

$2.09963 \mathrm{E} \quad 01-1.98076 \mathrm{E}-01$.

$2.09972 \mathrm{E} \quad 01 \quad-1.99855 \mathrm{E}-01$

$2.09981 \mathrm{E} \quad 01 \quad-2.01644 \mathrm{E}-01$

$2.09990 \mathrm{E} \quad 01 \quad-2.03444 \mathrm{E}-01$

$2.09999 \mathrm{E} \quad 01-2.05254 \mathrm{E}-01$

$2.10008 \mathrm{E} \quad 01 \quad-2.07075 \mathrm{E}-01$

$2.10017 \mathrm{E} \quad 01 \quad-2.08906 \mathrm{E}-01$

$2.10026 \mathrm{E} \quad 01 \quad-2.10749 \mathrm{E}-01$

$2.10035 \mathrm{E}$ 01 $-2.12601 \mathrm{E}-01$

$2.10044 \mathrm{E} \quad 01 \quad-2.14465 \mathrm{E}-01$

$2.10053 \mathrm{E} \quad 01$

$2.10061 \mathrm{E} 01$

$2.10070 \mathrm{E} \quad 01$

$2.10078 \mathrm{E} 01$

$2.10087 \mathrm{E} \quad 01$

$2.10095 \mathrm{E} \quad 01$

$2.10104 \mathrm{E} \quad 01$

$2.10112 \mathrm{E} 01$

$2.10120 \mathrm{E} \quad 01$

$2.10129 \mathrm{E} \quad 0$

$2.10137 \mathrm{E} \quad 01$

$2.10145 \mathrm{E} \quad 01$

$2.10153 \mathrm{E} 01$

$2.10161 \mathrm{E} \quad 01$

$2.10169 \mathrm{E} \quad 01$

$2.10177 \mathrm{E} \quad 01$

$2.10185 \mathrm{E} \quad 01$

$2.10193 \mathrm{E} \quad 01$

$2.10201 \mathrm{E} \quad 01$

$2.10208 \mathrm{E} 01$

$2.10216 \mathrm{E} \quad 01$

$2.10224 \mathrm{E} \quad 01$

$2.10231 \mathrm{E} \quad 01$

$2.10239 \mathrm{E} \quad 01$

$2.10246 \mathrm{E} \quad 01$

(

$-2.64606 \mathrm{E}-01$

$-2.66764 \mathrm{E}-01$

$-2.68934 \mathrm{E}-01$

$-2.71116 \mathrm{E}-01$

$-2.73309 \mathrm{E}-01$

$-2.75513 \mathrm{E}-01$

$-2.77729 \mathrm{E}-01$

$-2.79957 \mathrm{E}-01$

$-2.82197 \mathrm{E}-01$

$-2.84448 \mathrm{E}-01$

$-2.86711 \mathrm{E}-01$

$-2.88986 \mathrm{E}-01$

$-2.91272 \mathrm{E}-01$

$-2.93570 \mathrm{E}-01$

$-2.95880 \mathrm{E}-01$

$-2.98202 \mathrm{E}-01$

$-3.00536 \mathrm{E}-01$

$-3.02882 \mathrm{E}-01$

$2.10254 \mathrm{E} \quad 01$

$2.10261 \mathrm{E} 01$

$2.10268 \mathrm{E} 01$

$2.10276 \mathrm{E}$ 0l

$2.10283 \mathrm{E} \quad 01$

$2.10290 \mathrm{E}$ 0]

$2.10297 \mathrm{E} \quad 01$

$2.10305 \mathrm{E} \quad 01$

$2.10312 \mathrm{E}$

$2.10319 \mathrm{E} \quad 01$

$2.10326 \mathrm{E} \quad 01$

$2.10333 \mathrm{E}$ 01

$2.10340 \mathrm{E} 01$

$-2.16339 \mathrm{E}-01$

$-2.18224 \mathrm{E}-01$

$-2.20119 \mathrm{E}-01$

$-2.22026 \mathrm{E}-01$

$-2.23943 \mathrm{E}-01$

$-2.25871 \mathrm{E}-01$

$-2.27810 \mathrm{E}-01$

$-2.29760 \mathrm{E}-01$

$-2.31720 \mathrm{E}-01$

$-2.33692 \mathrm{E}-01$

$-2.35675 \mathrm{E}-01$

$-2.37668 \mathrm{E}-01$

$-2.39673 \mathrm{E}-01$

$-2.41689 \mathrm{E}-01$

$-2.43716 \mathrm{E}-01$

$-2.45754 \mathrm{E}-01$

$-2.47803 \mathrm{E}-01$

$-2.49863 \mathrm{E}-01$

$-2.51935 \mathrm{E}-01$

$-2.54018 \mathrm{E}-01$

$-2.56112 \mathrm{E}-01$

$-2.58217 \mathrm{E}-01$

$-2.60334 \mathrm{E}-01$

$-2.62462 \mathrm{E}-01$

$-2.64602 \mathrm{E}-01$ 
$Z=10 \quad A=20$

$F_{0}$ $f_{1}$

$g_{1}$

36.10

36.20

36.30

36.40

36.50

36.60

36.70

36.80

36.90

37.00

37.10

37.20

37.30

37.40

37.50

37.60

37.70

37.80

37.90

38.00

38.10

38.20

38.30

38.40

38.50

38.60

38.70

38.80

38.90

39.00

39.10

39.20

39.30

39.40

39.50

39.60

39.70

39.80

39.90

40.00

40.10

40.20

40.30

40.40

40.50

40.60

40.70

40.80

40.90

41.00

41.10

41.20

41.30

41.40

41.50

41.60

41.70

41.80

41.90

42.00
$1.26632 \mathrm{E} \quad 00$ $1.26630 \mathrm{E} \quad 00$ $1.26628 \mathrm{E} \quad 00$ $1.26626 \mathrm{E} \quad 00$ $1.26624 \mathrm{E} \quad 00$

$1.26623 \mathrm{E} \quad 00$ $1.26621 \mathrm{E} \quad 00$ $1.26619 \mathrm{E} \quad 00$ $1.26617 \mathrm{E} \quad 00$ $1.26615 \mathrm{E} \quad 00$

$1.26613 \mathrm{E} \quad 00$ $1.26611 \mathrm{E} \quad 00$ $1.26609 \mathrm{E} \quad 00$ $1.26608 \mathrm{E} \quad 00$

$1.26606 \mathrm{E} \quad 00$

$1.26604 \mathrm{E} \quad 00$ $1.26602 \mathrm{E} \quad 00$ $1.26600 \mathrm{E} \quad 00$ $1.26598 \mathrm{E} \quad 00$ $1.26596 \mathrm{E} \quad 00$

$1.26595 \mathrm{E} \quad 00$ $1.26593 \mathrm{E} \quad 00$ $1.26591 \mathrm{E} \quad 00$ $1.26589 \mathrm{E} \quad 00$ $1.26587 \mathrm{E} \quad 00$

$1.26586 \mathrm{E} \quad 00$ $1.26584 \mathrm{E} \quad 00$ $1.26582 \mathrm{E} \quad 00$ $1.26580 \mathrm{E} \quad 00$ $1.26578 \mathrm{E} \quad 00$

$1.26577 \mathrm{E} \quad 00$ $1.26575 \mathrm{E} \quad 00$ $1.26573 \mathrm{E} \quad 00$

$1.26571 \mathrm{E} \quad 00$

$1.26570 \mathrm{E} \quad 00$

$1.26568 \mathrm{E} \quad 00$ $1.26566 \mathrm{E} \quad 00$ $1.26564 \mathrm{E} \quad 00$ $1.26563 \mathrm{E} \quad 00$ $1.26561 \mathrm{E} \quad 00$

$1.26559 \mathrm{E} \quad 00$ $1.26558 \mathrm{E} \quad 00$ $1.26556 \mathrm{E} \quad 00$ $1.26554 \mathrm{E}^{-} 00$

$1.26552 \mathrm{E} \quad 00$

$1.26551 \mathrm{E} \quad 00$ $1.26549 \mathrm{E} \quad 00$ $1.26547 \mathrm{E} \quad 00$ $1.26546 \mathrm{E} \quad 00$

$1.26544 \mathrm{E} \quad 00$

$1.26542 \mathrm{E} \quad 00$ $1.26541 \mathrm{E} \quad 00$ $1.26539 \mathrm{E} \quad 00$ $1.26537 \mathrm{E} \quad 00$ $1.26536 \mathrm{E} \quad 00$

$1.26534 \mathrm{E} \quad 00$ $1.26532 \mathrm{E} \quad 00$ $1.26531 \mathrm{E} \quad 00$ $1.26529 \mathrm{E} \quad 00$ $1.26527 \mathrm{E} \quad 00$
$-3.85928 \mathrm{E} 01$ $-3.86954 \mathrm{E} 01$ $-3.87978 \mathrm{E}$ 0l $-3.89003 \mathrm{E} 01$ $-3.90027 \mathrm{E} \quad 01$

$-3.91051 \mathrm{E} 01$ $-3.92074 \mathrm{E} 01$ $-3.93097 \mathrm{E} 01$ $-3.94119 \mathrm{E}$ ol $-3.95141 \mathrm{E} \quad 01$

$-3.96163 \mathrm{E} 01$ $-3.97184 \mathrm{E} 01$ $-3.98205 \mathrm{E} 01$ $-3.99225 \mathrm{E} 01$ $-4.00245 \mathrm{E} 01$

$-4.01264 \mathrm{E} \quad 01$ $-4.02283 \mathrm{E} 01$ $-4.03302 \mathrm{E} 01$ $-4.04320 \mathrm{E} 01$ $-4.05338 \mathrm{E} 01$

$-4.06356 \mathrm{E} \quad 01$ $-4.07373 \mathrm{E}$ 0l $-4.08389 \mathrm{E} 01$ $-4.09405 \mathrm{E} 01$ $-4.10421 \mathrm{E} 01$

$-4.11436 \mathrm{E} 01$ $-4.12451 \mathrm{E} 01$ $-4.13466 \mathrm{E} 01$ $-4.14480 \mathrm{E} 01$

$-4.15493 \mathrm{E} 01$

$-4.16507 \mathrm{E} \quad 01$ $-4.17519 \mathrm{E} 01$ $-4.18532 \mathrm{E}$ 01 $-4.19544 \mathrm{E}$ 0I -4.20555 E 01

$-4.21566 \mathrm{E} 01$ $-4.22577 \mathrm{E} 01$ $-4.23587 \mathrm{E} 01$ $-4.24597 \mathrm{E} \quad 01$ $-4.25606 \mathrm{E} 01$

$-4.26615 \mathrm{E} 01$ $-4.27623 \mathrm{E}$ 01 $-4.28631 \mathrm{E} 01$ $-4.29639 \mathrm{E} 01$ $-4.30646 \mathrm{E} \quad 01$

$-4.31653 \mathrm{E} 01$ $-4.32659 \mathrm{E} 01$ $-4.33665 \mathrm{E} 01$ $-4.34670 \mathrm{E} 01$ $-4.35675 \mathrm{E} \quad 01$

$-4.36680 \mathrm{E} \quad 01$ $-4.37684 \mathrm{E} 01$ $-4.38688 \mathrm{E} 01$ $-4.39691 \mathrm{E} 01$ $-4.40694 \mathrm{E} 01$

$-4.41696 \mathrm{E} \quad 01$ $-4.42698 \mathrm{E} 01$ $-4.43700 \mathrm{E} 01$ $-4.44701 \mathrm{E} 01$ $-4.45701 \mathrm{E} 01$
$-5.20857 \mathrm{E} \quad 00$ $-5.23364 \mathrm{E} \quad 00$ $-5.25876 \mathrm{E} 00$ $-5.28394 \mathrm{E} \quad 00$ $-5.30917 \mathrm{E} \quad 00$

$-5.33446 \mathrm{E} \quad 00$ $-5.35981 \mathrm{E} \quad 00$ $-5.38521 \mathrm{E} \quad 00$ $-5.41066 \mathrm{E} 00$ $-5.43617 \mathrm{E} \quad 00$

$-5.46174 \mathrm{E} \quad 00$ $-5.48736 \mathrm{E} \quad 00$ $-5.51304 \mathrm{E} \quad 00$ $-5.53877 \mathrm{E} \quad 00$ $-5.56455 \mathrm{E} \quad 00$ $-5.61629 \mathrm{E} \quad 00$ $-5.64225 \mathrm{E} \quad 00$ $-5.66825 \mathrm{E} \quad 00$ $-5.69432 \mathrm{E} \quad 00$

$-5.72043 \mathrm{E} \quad 00$ $-5.74660 \mathrm{E} \quad 00$ $-5.77283 \mathrm{E} \quad 00$ $-5.79911 \mathrm{E} \quad 00$ $-5.82545 \mathrm{E} \quad 00$

$-5.85184 \mathrm{E} \quad 00$ $-5.90479 \mathrm{E} \quad 00$ $-5.93135 \mathrm{E} \quad 00$ $-5.95796 \mathrm{E} \quad 00$

$-5.98463 \mathrm{E} \quad 00$ $-6.01135 \mathrm{E} \quad 00$ $-6.03812 \mathrm{E} \quad 00$ $-6.06495 \mathrm{E} \quad 00$ $-6.09184 \mathrm{E} \quad 00$

$-6.11878 \mathrm{E} \quad 00$ $-6.14577 \mathrm{E} \quad 00$ $-6.17282 \mathrm{E} \quad 00$ $-6.19992 \mathrm{E} 00$ $-6.22708 \mathrm{E} \quad 00$

$-6.25429 \mathrm{E} \quad 00$ $-6.28156 \mathrm{E} 00$ $-6.30888 \mathrm{E} \quad 00$ $-6.33626 \mathrm{E} 00$ $-6.36369 \mathrm{E} \quad 00$

$-6.39117 \mathrm{E} \quad 00$ $-6.41871 \mathrm{E} \quad 00$ $-6.44630 \mathrm{E} 00$ $-6.47394 \mathrm{E} \quad 00$ $-6.50165 \mathrm{E} 00$

$-6.52940 \mathrm{E} 00$ $-6.55721 \mathrm{E} \cdot 00$ $-6.58507 \mathrm{E} \quad 00$ $-6.61299 \mathrm{E} \quad 00$ $-6.64096 \mathrm{E} 00$

$-6.66898 \mathrm{E} \quad 00$ $-6.69706 \mathrm{E} 00$ $-6.72520 \mathrm{E} \quad 00$ $-6.78162 \mathrm{E} 00$
$-5.59040 \mathrm{E} \quad 00$ $-5.87829 \mathrm{E} \quad 00$ $-6.75338 \mathrm{E} 00$

$\tan \delta_{1}$ $f_{-1}$

$8.13037 \mathrm{E}-03$ $8.12864 \mathrm{E}-03$

$8.12686 \mathrm{E}-03$

$8.12507 \mathrm{E}-03$

$8.12325 \mathrm{E}-03$

$8.12140 \mathrm{E}-03$

$8.11952 \mathrm{E}-03$

$8.11763 \mathrm{E}-03$

$8.11569 \mathrm{E}-03$

$8.11374 \mathrm{E}-03$

$8.11176 \mathrm{E}-03$

$8.10976 \mathrm{E}-03$

$8.10772 \mathrm{E}-03$

$8.10567 \mathrm{E}-03$

$8.10358 \mathrm{E}-03$

8.10146E-03

$8.09933 \mathrm{E}-03$

$8.09717 \mathrm{E}-03$

$8.09499 \mathrm{E}-03$

$8.09277 \mathrm{E}-03$

$8.09053 \mathrm{E}-03$

$8.08827 \mathrm{E}-03$

$8.08599 \mathrm{E}-03$

$8.08368 \mathrm{E}-03$

$8.08133 \mathrm{E}-03$

$8.07897 \mathrm{E}-03$

$8.07659 \mathrm{E}-03$

$8.07418 \mathrm{E}-03$

$8.07174 \mathrm{E}-03$

$8.06928 \mathrm{E}-03$

$8.06680 \mathrm{E}-03$

$8.06429 \mathrm{E}-03$

$8.06176 \mathrm{E}-03$

$8.05920 \mathrm{E}-03$

$8.05663 \mathrm{E}-03$

$8.05401 \mathrm{E}-03$

$8.05138 \mathrm{E}-03$

$8.04874 \mathrm{E}-03$

$8.04608 \mathrm{E}-03$

$8.04337 \mathrm{E}-03$

$8.04066 \mathrm{E}-03$

$8.03792 \mathrm{E}-03$ 


\section{$\bar{F}$}

36.10

36.20

36.30

36.40

36.50

36.60

36.70

36.80

36.90

37.00

37.10

37.20

37.30

37.40

37.50

37.60

37.70

37.80

37.90

38.00

38.10

38.20

38.30

38.40

38.50

38.60

38.70

38.80

38.90

39.00

39.10

39.20

39.30

39.40

39.50

39.60

39.70

39.80

39.90

40.00

40.10

40.20

40.30

40.40

40.50

40.60

40.70

40.80

40.90

41.00

41.10

41.20

41.30

41.40

41.50

41.60

41.70

41.80

41.90

42.00 $f_{2}$

$3.25520 \mathrm{E}-03$

$3.27323 \mathrm{E}-03$

$3.29132 \mathrm{E}-03$

$3.30945 \mathrm{E}-03$

$3.32763 \mathrm{E}-03$

$3.34586 \mathrm{E}-03$

$3.36415 \mathrm{E}-03$

$3.38248 \mathrm{E}-03$

$3.40086 \mathrm{E}-03$

$3.41929 \mathrm{E}-03$

3.43777 E-03

3.45631 E- 03

3.47489 E-03

3.49352 E- 03

$3.51220 \mathrm{E}-03$

3.53093 E- 03

$3.54971 \mathrm{E}-03$

$3.56854 \mathrm{E}-03$

$3.58742 \mathrm{E}-03$

$3.60635 \mathrm{E}-03$

$3.62532 \mathrm{E}-03$

$3.64435 \mathrm{E}-03$

$3.66343 \mathrm{E}-03$

$3.68256 \mathrm{E}-03$

$3.70174 \mathrm{E}-03$

$3.72096 \mathrm{E}-03$

$3.74024 \mathrm{E}-03$

$3.75957 \mathrm{E}-03$

$3.77895 \mathrm{E}-03$

$3.79837 \mathrm{E}-03$

$3.81785 \mathrm{E}-03$

$3.83738 \mathrm{E}-03$

$3.85695 \mathrm{E}-03$

$3.87658 \mathrm{E}-03$

$3.89625 \mathrm{E}-03$

$3.91598 \mathrm{E}-03$

$3.93575 \mathrm{E}-03$

$3.95558 \mathrm{E}-03$

$3.97545 \mathrm{E}-03$

$3.99538 \mathrm{E}-03$

$4.01535 \mathrm{E}-03$

$4.03537 \mathrm{E}-03$

$4.05545 \mathrm{E}-03$

$4.07557 \mathrm{E}-03$

$4.09574 \mathrm{E}-03$

$4.11596 \mathrm{E}-03$

$4.13624 \mathrm{E}-03$

$4.15656 \mathrm{E}-03$

$4.17693 \mathrm{E}-03$

$4.19735 \mathrm{E}-03$

$4.21782 \mathrm{E}-03$

$4.23834 \mathrm{E}-03$

$4.25891 \mathrm{E}-03$

$4.27953 \mathrm{E}-03$

$4.30020 \mathrm{E}-03$

$4.32092 \mathrm{E}-03$

$4.34169 \mathrm{E}-03$

$4.36251 \mathrm{E}-03$

$4.38338 \mathrm{E}-03$

$4.40430 \mathrm{E}-03$ $g_{2}$

$-3.98286 \mathrm{E} \quad 00 \quad-3.17209 \mathrm{E}-01$

$-4.00477 \mathrm{E} \quad 00 \quad-3.19639 \mathrm{E}-01$

$-4.02675 \mathrm{E} \quad 00 \quad-3.22081 \mathrm{E}-01$

$-4.04878 \mathrm{E} \quad 00 \quad-3.24535 \mathrm{E}-01$

$\begin{array}{lll}-4.07087 \mathrm{E} & 00 & -3.27002 \mathrm{E}-01\end{array}$

$-4.09302 \mathrm{E} \quad 00$

$-4.11522 \mathrm{E} \quad 00$

$-4.13749 \mathrm{E} \quad 00$

$-4.15981 \mathrm{E} \quad 00$

$-4.18219 \mathrm{E} 00$

$-4.20462 \mathrm{E} \quad 00$

$-4.22712 \mathrm{E} \quad 00$

$-4.24967 \mathrm{E} 00$

$-4.27228 \mathrm{E} \quad 00$

$-4.29494 \mathrm{E} \quad 00$

$-4.31766 \mathrm{E} \quad 00$

$-4.34045 \mathrm{E} \quad 00$

$-4.36328 \mathrm{E} \quad 00$

$-4.38618 \mathrm{E} \quad 00$

$-4.40913 \mathrm{E} 00$

$-4.43214 \mathrm{E} \quad 00$

$-4.45521 \mathrm{E} 00$

$-4.47833 \mathrm{E} \quad 00$

$-4.50151 \mathrm{E} 00$

$-4.52475 \mathrm{E} \quad 00$

$-4.54805 \mathrm{E} \quad 00$

$-4.57140 \mathrm{E} \quad 00$

$-4.59481 \mathrm{E} \quad 00$

$-4.61828 \mathrm{E} \quad 00$

$-4.64181 \mathrm{E} \quad 00$

$-4.66539 \mathrm{E} \quad 00$

$-4.68903 \mathrm{E} \quad 00$

$-4.71272 \mathrm{E} \quad 00$

$-4.73647 \mathrm{E} \quad 00$

$-4.76028 \mathrm{E} \quad 00$

$-4.78415 \mathrm{E} \quad 00$

$-4.80807 \mathrm{E} \quad 00$

$-4.83205 \mathrm{E} \quad 00$

$-4.85609 \mathrm{E} \quad 00$

$-4.88018 \mathrm{E} \quad 00$

$-4.90433 \mathrm{E} \quad 00$

$-4.92854 \mathrm{E} \quad 00$

$-4.95280 \mathrm{E} 00$

$-4.97712 \mathrm{E} 00$

$-5.00150 \mathrm{E} 00$

$-5.02594 \mathrm{E} \quad 00$

$-5.05043 \mathrm{E} 00$

$-5.07497 \mathrm{E} \quad 00$

$-5.09958 \mathrm{E} 00$

$-5.12424 \mathrm{E} \quad 00$

$-5.14895 \mathrm{E} \quad 00$

$-5.17373 \mathrm{E} 00$

$-5.19856 \mathrm{E} \quad 00$

$-5.22344 \mathrm{E} \quad 00$

$-5.24839 \mathrm{E} \quad 00$

$-5.27338 \mathrm{E} \quad 00$

$-5.29844 \mathrm{E} \quad 00$

$-5.32355 \mathrm{E} 00$

$-5.34872 \mathrm{E} \quad 00$

$-5.37394 \mathrm{E} 00$

$-3.29481 \mathrm{E}-01$

$-3.31972 \mathrm{E}-01$

$-3.34475 \mathrm{E}-01$

$-3.36991 \mathrm{E}-01$

$-3.39519 \mathrm{E}-01$

$-3.42059 \mathrm{E}-01$

$-3.44612 \mathrm{E}-01$

$-3.47177 \mathrm{E}-01$

$-3.49755 \mathrm{E}-01$

$-3.52345 \mathrm{E}-01$

$-3.54948 \mathrm{E}-01$

$-3.57563 \mathrm{E}-01$

$-3.60191 \mathrm{E}-01$

$-3.65485 \mathrm{E}-01$

$-3.68150 \mathrm{E}-01$

$-3.70829 \mathrm{E}-01$

$-3.73520 \mathrm{E}-01$

$-3.76224 \mathrm{E}-01$

$-3.78940 \mathrm{E}-01$

$-3.81670 \mathrm{E}-01$

$-3.84412 \mathrm{E}-01$

$-3.87167 \mathrm{E}-01$

$-3.89935 \mathrm{E}-01$

$-3.92716 \mathrm{E}-01$

$-3.95510 \mathrm{E}-01$

$-3.98317 \mathrm{E}-01$

$-4.01137 \mathrm{E}-01$

$-4.03970 \mathrm{E}-01$

$-4.06816 \mathrm{E}-01$

$-4.09675 \mathrm{E}-01$

$-4.12547 \mathrm{E}-01$

$-4.15432 \mathrm{E}-01$

$-4.18331 \mathrm{E}-01$

$-4.21242 \mathrm{E}-01$

$-4.24167 \mathrm{E}-01$

$-4.27105 \mathrm{E}-01$

$-4.30056 \mathrm{E}-01$

$-4.33021 \mathrm{E}-01$

$-4.35999 \mathrm{E}-0 \mathrm{l}$

$-4.38990 \mathrm{E}-01$

$-4.41995 \mathrm{E}-01$

$-4.45013 \mathrm{E}-01$

$-4.48045 \mathrm{E}-01$

$-4.51090 \mathrm{E}-01$

$-4.54148 \mathrm{E}-01$

$-4.57220 \mathrm{E}-01$

$-4.60306 \mathrm{E}-01$

$-4.63405 \mathrm{E}-01$

$-4.66518 \mathrm{E}-01$

$-4.69645 \mathrm{E}-01$

$-4.72785 \mathrm{E}-01$

$-4.75938 \mathrm{E}-01$

$-4.79106 \mathrm{E}-01$

$-4.82287 \mathrm{E}-01$ $\tan \delta_{2}$

$f_{-2}$

$2.10394 \mathrm{E}$ 01 $-3.12214 \mathrm{E}-0 \mathrm{l}$

$2.10400 \mathrm{E} \quad 01-3.14612 \mathrm{E}-01$

$2.10407 \mathrm{E}$ 01 $-3.17021 \mathrm{E}-01$

$2.10413 \mathrm{E} 01 \quad-3.19443 \mathrm{E}-01$

$2.10420 \mathrm{E}$ 0l $-3.21876 \mathrm{E}-0 \mathrm{l}$

$-3.62831 \mathrm{E}-01$

$2.10426 \mathrm{E} \quad 01 \quad-3.24322 \mathrm{E}-01$

$2.10432 \mathrm{E} \quad 0 \mathrm{l}-3.26780 \mathrm{E}-01$

$2.10439 \mathrm{E} \quad 01 \quad-3.29250 \mathrm{E}-01$

$2.10445 \mathrm{E}$ 01 $-3.31732 \mathrm{E}-01$

$2.10451 \mathrm{E}$ 01 $-3.34227 \mathrm{E}-01$

$2.10457 \mathrm{E} \quad 01 \quad-3.36734 \mathrm{E}-01$

$2.10464 \mathrm{E}$ 01 $-3.39253 \mathrm{E}-01$

$2.10470 \mathrm{E} \quad 01 \quad-3.41784 \mathrm{E}-01$

$2.10476 \mathrm{E}$ 01 $-3.44328 \mathrm{E}-01$

$2.10482 \mathrm{E} \quad 01 \quad-3.46885 \mathrm{E}-01$

$2.10488 \mathrm{E} \quad 01 \quad-3.49453 \mathrm{E}-01$

$2.10494 \mathrm{E} \quad 01-3.52034 \mathrm{E}-01$

$2.10500 \mathrm{E} \quad 01 \quad-3.54628 \mathrm{E}-01$

$2.10506 \mathrm{E} \quad 01 \quad-3.57234 \mathrm{E}-01$

$2.10512 \mathrm{E} \quad 01 \quad-3.59853 \mathrm{E}-01$

$2.10518 \mathrm{E} \quad 01 \quad-3.62484 \mathrm{E}-01$

$2.10524 \mathrm{E} \quad 01 \quad-3.65128 \mathrm{E}-01$

$2.10530 \mathrm{E} \quad 01 \quad-3.67784 \mathrm{E}-01$

$2.10535 \mathrm{E} \quad 01-3.70453 \mathrm{E}-01$

$2.10541 \mathrm{E} \quad 01 \quad-3.73135 \mathrm{E}-01$

$2.10547 \mathrm{E} \quad 01 \quad-3.75829 \mathrm{E}-01$

$2.10553 \mathrm{E} \quad 01-3.78536 \mathrm{E}-01$

$2.10558 \mathrm{E} \quad 01 \quad-3.81256 \mathrm{E}-01$

$2.10564 \mathrm{E} \quad 01 \quad-3.83989 \mathrm{E}-01$

$2.10569 \mathrm{E} \quad 01-3.86734 \mathrm{E}-01$

$2.10575 \mathrm{E} \quad 01 \quad-3.89493 \mathrm{E}-01$

$2.10581 \mathrm{E} \quad 01 \quad-3.92264 \mathrm{E}-01$

$2.10586 \mathrm{E} \quad 01 \quad-3.95048 \mathrm{E}-01$

$2.10592 \mathrm{E} \quad 01-3.97845 \mathrm{E}-01$

$2.10597 \mathrm{E} \quad 01 \quad-4.00655 \mathrm{E}-01$

$2.10602 \mathrm{E} \quad 01 \quad-4.03478 \mathrm{E}-01$

$2.10608 \mathrm{E} \quad 01 \quad-4.06314 \mathrm{E}-01$

$2.10613 \mathrm{E} \quad 01-4.09163 \mathrm{E}-01$

$2.10618 \mathrm{E} \quad 01 \quad-4.12025 \mathrm{E}-01$

$2.10624 \mathrm{E} \quad 01-4.14900 \mathrm{E}-01$

$2.10629 \mathrm{E} \quad 01 \quad-4.17788 \mathrm{E}-01$

$2.10634 \mathrm{E}$ 01 $-4.20690 \mathrm{E}-01$

$2.10640 \mathrm{E}$ 01 $-4.23604 \mathrm{E}-01$

$2.10645 \mathrm{E} \quad 01-4.26532 \mathrm{E}-01$

$2.10650 \mathrm{E} 01 \quad-4.29473 \mathrm{E}-01$

$2.10655 \mathrm{E} \quad 01 \quad-4.32427 \mathrm{E}-01$ 
ELECTRON RADIAL FUNCTIONS

Positron

\begin{tabular}{|c|c|c|c|}
\hline$p$ & $F_{0}$ & $f_{1}$ & $g_{1}$ \\
\hline 0.10 & $7.87646 \mathrm{E}-01$ & $5.65969 \mathrm{E}-03$ & $-4.76575 \mathrm{E}-06$ \\
\hline 0.20 & $8.88084 \mathrm{E}-0 \mathrm{l}$ & $2.56318 \mathrm{E}-02$ & $-2.11899 \mathrm{E}-05$ \\
\hline 0.30 & $9.22765 \mathrm{E}-01$ & $5.85151 \mathrm{E}-02$ & $-4.69062 \mathrm{E}-05$ \\
\hline 0.40 & $9.39961 \mathrm{E}-01$ & $1.03013 \mathrm{E}-01$ & $-7.90544 \mathrm{E}-05$ \\
\hline 0.50 & $9.50027 \mathrm{E}-01$ & $1.57604 \mathrm{E}-01$ & $-1.14256 \mathrm{E}-04$ \\
\hline 0.60 & $9.56510 \mathrm{E}-01$ & $2.20723 \mathrm{E}-01$ & $-1.49003 \mathrm{E}-04$ \\
\hline 0.70 & $9.60956 \mathrm{E}-01$ & $2.90890 \mathrm{E}-01$ & $-1.79960 \mathrm{E}-04$ \\
\hline 0.80 & $9.64145 \mathrm{E}-01$ & $3.66782 \mathrm{E}-01$ & $-2.04126 \mathrm{E}-04$ \\
\hline 0.90 & $9.66511 \mathrm{E}-01$ & $4.47272 \mathrm{E}-01$ & $-2.18928 \mathrm{E}-04$ \\
\hline 1.00 & $9.68314 \mathrm{E}-01$ & $5.31426 \mathrm{E}-01$ & $-2.22217 \mathrm{E}-04$ \\
\hline 1.10 & $9.69718 \mathrm{E}-01$ & $6.18482 \mathrm{E}-01$ & $-2.12241 \mathrm{E}-04$ \\
\hline 1.20 & $9.70831 \mathrm{E}-01$ & $7.07835 \mathrm{E}-01$ & $-1.87588 \mathrm{E}-04$ \\
\hline 1.30 & $9.71728 \mathrm{E}-01$ & $7.98981 \mathrm{E}-01$ & $-1.47129 \mathrm{E}-04$ \\
\hline 1.40 & $9.72461 \mathrm{E}-01$ & $8.91563 \mathrm{E}-01$ & $-8.99781 \mathrm{E}-05$ \\
\hline 1.50 & $9.73066 \mathrm{E}-0 \mathrm{l}$ & $9.85253 \mathrm{E}-0 \mathrm{l}$ & $-1.54292 \mathrm{E}-05$ \\
\hline 1.60 & $9.73571 \mathrm{E}-01$ & $1.07980 \mathrm{E}$ & $7.70680 \mathrm{E}-05$ \\
\hline 1.70 & $9.73998 \mathrm{E}-01$ & $1.17496 \mathrm{E}$ & $1.87936 \mathrm{E}-04$ \\
\hline 1.80 & $9.74361 \mathrm{E}-01$ & $1.27054 \mathrm{E}$ & $3.17483 \mathrm{E}-04$ \\
\hline 1.90 & $9.74671 \mathrm{E}-01$ & $1.36625 \mathrm{E}$ & $4.65891 \mathrm{E}-04$ \\
\hline 2.00 & $9.74939 \mathrm{E}-01$ & $1.46169 \mathrm{E}$ & $6.33154 \mathrm{E}-04$ \\
\hline 2.10 & $9.75173 \mathrm{E}-01$ & $1.55582 \mathrm{E}$ & $8.18766 \mathrm{E}-04$ \\
\hline 2.20 & $9.75376 \mathrm{E}-01$ & $1.64436 \mathrm{E}$ & $1.01980 \mathrm{E}-03$ \\
\hline 2.30 & $9.75554 \mathrm{E}-0 \mathrm{l}$ & $1.61807 \mathrm{E}$ & $1.15666 \mathrm{E}-03$ \\
\hline 2.40 & $9.75712 \mathrm{E}-01$ & $1.88819 \mathrm{E}$ & $1.52971 \mathrm{E}-03$ \\
\hline 2.50 & $9.75852 \mathrm{E}-01$ & $1.97152 \mathrm{E} \quad 00$ & $1.78629 \mathrm{E}-03$ \\
\hline 2.60 & $9.75977 \mathrm{E}-01$ & $2.06555 \mathrm{E}$ & $2.07067 \mathrm{E}-03$ \\
\hline 2.70 & $9.76088 \mathrm{E}-01$ & $2.16201 \mathrm{E}$ & $2.37689 \mathrm{E}-03$ \\
\hline 2.80 & $9.76189 \mathrm{E}-01$ & $2.25943 \mathrm{E}$ & $2.70395 \mathrm{E}-03$ \\
\hline 2.90 & $9.76279 \mathrm{E}-01$ & $2.35735 \mathrm{E}$ & $3.05154 \mathrm{E}-03$ \\
\hline 3.00 & $9.76360 \mathrm{E}-01$ & $2.45557 \mathrm{E}$ & $3.41958 \mathrm{E}-03$ \\
\hline 3.10 & $9.76434 \mathrm{E}-01$ & $2.55399 \mathrm{E}$ & $3.80802 \mathrm{E}-03$ \\
\hline 3.20 & $9.76502 \mathrm{E}-01$ & $2.65255 \mathrm{E} \quad 00$ & $4.21686 \mathrm{E}-03$ \\
\hline 3.30 & $9.76563 \mathrm{E}-0 \mathrm{l}$ & $2.75122 \mathrm{E} \quad 00$ & $4.64610 \mathrm{E}-03$ \\
\hline 3.40 & $9.76619 \mathrm{E}-01$ & $2.84996 \mathrm{E}$ & $5.09573 \mathrm{E}-03$ \\
\hline 3.50 & $9.76670 \mathrm{E}-01$ & $2.94877 \mathrm{E}$ & $5.56577 \mathrm{E}-03$ \\
\hline 3.60 & $9.76717 \mathrm{E}-01$ & $3.04762 \mathrm{E}$ & $6.05622 \mathrm{E}-03$ \\
\hline 3.70 & $9.76761 \mathrm{E}-01$ & $3.14652 \mathrm{E}$ & $6.56709 \mathrm{E}-03$ \\
\hline 3.80 & $9.76801 \mathrm{E}-01$ & $3.24545 \mathrm{E}$ & $7.09839 \mathrm{E}-03$ \\
\hline 3.90 & $9.76838 \mathrm{E}-01$ & $3.34440 \mathrm{E}$ & $7.65012 \mathrm{E}-03$ \\
\hline 4.00 & $9.76872 \mathrm{E}-01$ & $3.44338 \mathrm{E}$ & $8.22230 \mathrm{E}-03$ \\
\hline 4.10 & $9.76904 \mathrm{E}-01$ & $3.54237 \mathrm{E}$ & $8.81492 \mathrm{E}-03$ \\
\hline 4.20 & $9.76933 \mathrm{E}-01$ & $3.64138 \mathrm{E}$ & $9.42799 \mathrm{E}-03$ \\
\hline 4.30 & $9.76961 \mathrm{E}-01$ & $3.74040 \mathrm{E}$ & $1.00615 \mathrm{E}-02$ \\
\hline 4.40 & $9.76986 \mathrm{E}-01$ & $3.83943 \mathrm{E}$ & $1.07155 \mathrm{E}-02$ \\
\hline 4.50 & $9.77010 \mathrm{E}-01$ & $3.93847 \mathrm{E}$ & $1.13900 \mathrm{E}-02$ \\
\hline 4.60 & $9.77032 \mathrm{E}-01$ & $4.03752 \mathrm{E}$ & $1.20849 \mathrm{E}-02$ \\
\hline 4.70 & $9.77053 \mathrm{E}-01$ & $4.13657 \mathrm{E}$ & $1.28003 \mathrm{E}-02$ \\
\hline 4.80 & $9.77072 \mathrm{E}-01$ & $4.23562 \mathrm{E}$ & $1.35361 \mathrm{~F}$ \\
\hline 4.90 & $9.77091 \mathrm{E}-01$ & $4.33468 \mathrm{E}$ & $1.42925 \mathrm{E}-02$ \\
\hline 5.00 & $9.77108 \mathrm{E}-01$ & $4.43375 \mathrm{E} \quad 00$ & $1.50693 \mathrm{E}-02$ \\
\hline 5.10 & $9.77124 \mathrm{E}-01$ & $4.53281 \mathrm{E}$ & $1.58666 \mathrm{E}-02$ \\
\hline 5.20 & $9.77139 \mathrm{E}-01$ & $4.63188 \mathrm{E}$ & $1.66843 \mathrm{E}-02$ \\
\hline 5.30 & $9.77154 \mathrm{E}-01$ & $4.73095 \mathrm{E}$ & $1.75226 \mathrm{E}-02$ \\
\hline 5.40 & $9.77167 \mathrm{E}-01$ & $4.83002 \mathrm{E}$ & $1.83813 \mathrm{E}-02$ \\
\hline 5.50 & $9.77180 \mathrm{E}-01$ & $4.92909 \mathrm{E} \quad 00$ & $1.92606 \mathrm{E}-02$ \\
\hline 5.60 & $9.77192 \mathrm{E}-01$ & $5.02816 \mathrm{E}$ & $2.01603 \mathrm{E}-02$ \\
\hline 5.70 & $9.77204 \mathrm{E}-01$ & $5.12723 \mathrm{E}$ & $2.10805 \mathrm{E}-02$ \\
\hline 5.80 & $9.77215 \mathrm{E}-01$ & $5.22630 \mathrm{E}$ & $2.20212 \mathrm{E}-02$ \\
\hline 5.90 & $9.77225 \mathrm{E}-01$ & $5.32537 \mathrm{E}$ & $2.29824 \mathrm{E}-02$ \\
\hline 6.00 & $9.77235 \mathrm{E}-01$ & $5.42444 \mathrm{E} \quad 00$ & $2.39641 \mathrm{E}-02$ \\
\hline
\end{tabular}

$\tan \delta_{1}$

$3.09096 \mathrm{E}-02$

$1.54211 \mathrm{E}-02$

$1.02480 \mathrm{E}-02$

$7.64988 \mathrm{E}-03$

$6.08220 \mathrm{E}-03$

$5.03042 \mathrm{E}-03$

$4.27409 \mathrm{E}-03$

$3.70299 \mathrm{E}-03$

$3.25581 \mathrm{E}-03$

$2.89576 \mathrm{E}-03$

$2.59937 \mathrm{E}-03$

$2.35094 \mathrm{E}-03$

$2.13959 \mathrm{E}-03$

$1.95752 \mathrm{E}-03$

$1.79899 \mathrm{E}-03$

$1.65968 \mathrm{E}-03$

$1.53626 \mathrm{E}-03$

$1.42615 \mathrm{E}-03$

$1.32729 \mathrm{E}-03$

$1.23803 \mathrm{E}-03$

$1.15703 \mathrm{E}-03$

$1.08319 \mathrm{E}-03$

$1.01560 \mathrm{E}-03$

$9.53491 \mathrm{E}-04$

$8.96225 \mathrm{E}-04$

$8.43252 \mathrm{E}-04$

$7.94106 \mathrm{E}-04$

$7.48389 \mathrm{E}-04$

$7.05749 \mathrm{E}-04$

$6.65888 \mathrm{E}-04$

$6.28542 \mathrm{E}-04$

$5.93479 \mathrm{E}-04$

$5.60496 \mathrm{E}-04$

$5.29413 \mathrm{E}-04$

$5.00070 \mathrm{E}-04$

$4.72326 \mathrm{E}-04$

4.46052 E-04

$4.21135 \mathrm{E}-04$

3.97475 E-0 4

$3.74973 \mathrm{E}-04$

$3.53551 \mathrm{E}-04$

$3.33132 \mathrm{E}-04$

$3.13645 \mathrm{E}-04$

$2.95032 \mathrm{E}-04$

2.77231 E-04

$2.60192 \mathrm{E}-04$

$2.43868 \mathrm{E}-04$

2.28214 E- 04

$2.13188 \mathrm{E}-04$

$1.98756 \mathrm{E}-04$

$1.84881 \mathrm{E}-04$

$1.71532 \mathrm{E}-04$

$1.58681 \mathrm{E}-04$

$1.46299 \mathrm{E}-04$

1.34362 E- 04

$1.22845 \mathrm{E}-04$

$1.11729 \mathrm{E}-04$

$1.00990 \mathrm{E}-04$

$9.06109 \mathrm{E}-05$

8.05744 E-05

$$
f_{-1}
$$

$3.65261 \mathrm{E}-04$

7.68815 E- 04

$1.15863 \mathrm{E}-03$

$1.52903 \mathrm{E}-03$

$1.87597 \mathrm{E}-03$

2.19681 E-03

$2.49009 \mathrm{E}-03$

$2.75520 \mathrm{E}-03$

$2.99215 \mathrm{E}-03$

$3.20131 \mathrm{E}-03$

$3.38321 \mathrm{E}-03$

$3.53853 \mathrm{E}-03$

$3.66790 \mathrm{E}-03$

$3.77195 \mathrm{E}-03$

$3.85127 \mathrm{E}-03$

$3.90636 \mathrm{E}-03$

$3.93769 \mathrm{E}-03$

$3.94566 \mathrm{E}-03$

$3.93061 \mathrm{E}-03$

$3.89285 \mathrm{E}-03$

$3.83264 \mathrm{E}-03$

3.75022 E-03

$3.64576 \mathrm{E}-03$

$3.51946 \mathrm{E}-03$

$3.37145 \mathrm{E}-03$

$3.20186 \mathrm{E}-03$

$3.01082 \mathrm{E}-03$

2.79841 E-03

$2.56472 \mathrm{E}-03$

$2.30983 \mathrm{E}-03$

2.03381 E-03

$1.73672 \mathrm{E}-03$

$1.41860 \mathrm{E}-03$

$1.07951 \mathrm{E}-03$

7.19489E-04

$3.38569 \mathrm{E}-04$

$-6.32124 \mathrm{E}-05$

$-4.85827 \mathrm{E}-04$

$-9.29248 \mathrm{E}-04$

$-1.39345 \mathrm{E}-03$

$-1.87841 \mathrm{E}-03$

$-2.38412 \mathrm{E}-03$

$-2.91054 \mathrm{E}-03$

$-3.45768 \mathrm{E}-03$

$-4.02550 \mathrm{E}-03$

-4.61401 E-03

$-5.22318 \mathrm{E}-03$

$-5.85301 \mathrm{E}-03$

$-6.50348 \mathrm{E}-03$

-7.1745 9 E-03

$-7.86633 \mathrm{E}-03$

$-8.57869 \mathrm{E}-03$

$-9.31166 \mathrm{E}-03$

$-1.00652 \mathrm{E}-02$

$-1.08394 \mathrm{E}-02$

$-1.16342 \mathrm{E}-02$

$-1.24496 \mathrm{E}-02$

$-1.32855 \mathrm{E}-02$

$-1.41420 \mathrm{E}-02$

$-1.50191 \mathrm{E}-02$ g-1

$\tan \delta_{-1}$

$1.25357 \mathrm{E}-01$

$2.65254 \mathrm{E}-01$

$4.03239 \mathrm{E}-01$

$5.38557 \mathrm{E}-01$

$6.70790 \mathrm{E}-01$

7.99779 E-01

$9.25561 \mathrm{E}-01$

$1.04831 \mathrm{E} \quad 00$

$1.16827 \mathrm{E} \quad 00$

$1.28574 \mathrm{E} \quad 00$

$1.40099 \mathrm{E} \quad 00$

$1.51431 \mathrm{E} \quad 00$

$1.62595 \mathrm{E} \quad 00$

$1.73613 \mathrm{E} \quad 00$

$1.84504 \mathrm{E} \quad 00$

$1.95286 \mathrm{E} \quad 00$ $2.05973 \mathrm{E} \quad 00$

$2.16578 \mathrm{E} \quad 00$

$2.27111 \mathrm{E} \quad 00$

$2.37581 \mathrm{E} 00$

$2.47997 \mathrm{E} \quad 00$

$2.58364 \mathrm{E} \quad 00$

$2.68690 \mathrm{E} \quad 00$

$2.78978 \mathrm{E} \quad 00$

$2.89232 \mathrm{E} \quad 00$

$2.99457 \mathrm{E} \quad 00$

$3.09656 \mathrm{E} \quad 00$

$3.19832 \mathrm{E} \quad 00$

$3.29986 \mathrm{E} \quad 00$

$3.40121 \mathrm{E} \quad 00$

$3.50240 \mathrm{E} \quad 00$

$3.60342 \mathrm{E} \quad 00$

$3.70431 \mathrm{E} \quad 00$

$3.80507 \mathrm{E} \quad 00$

$3.90571 \mathrm{E} \quad 00$

$4.00625 \mathrm{E} \quad 00$

$4.10669 \mathrm{E} \quad 00$

$4.20704 \mathrm{E} \quad 00$

$4.30731 \mathrm{E} \quad 00$

$4.40751 \mathrm{E} \quad 00$

$4.50763 \mathrm{E} \quad 00$ $4.60769 \mathrm{E} \quad 00$ $4.70770 \mathrm{E} 00$

$4.80764 \mathrm{E} \quad 00$

$4.90754 \mathrm{E} \quad 00$

$5.00739 \mathrm{E} \quad 00$ $5.10720 \mathrm{E} \quad 00$ $5.20697 \mathrm{E} \quad 00$

$5.30669 \mathrm{E} \quad 00$ $5.40639 \mathrm{E} \quad 00$

$5.50605 \mathrm{E} \quad 00$ $5.60568 \mathrm{E} \quad 00$ $5.70528 \mathrm{E} \quad 00$ $5.80485 \mathrm{E} \quad 00$ $5.90440 \mathrm{E} \quad 00$

$6.00392 \mathrm{E} \quad 00$ $6.10342 \mathrm{E} \quad 00$ $6.20290 \mathrm{E} \quad 00$ $6.30236 \mathrm{E} \quad 00$ $6.40180 \mathrm{E} \quad 00$
$2.38230 \mathrm{E}$ 0l $4.74895 \mathrm{E} \quad 01$ $7.10461 \mathrm{E} \quad 01$ $9.43946 \mathrm{E} \quad 01$ $1.17464 \mathrm{E} \quad 02$

$1.40200 \mathrm{E} \quad 02$ $1.62561 \mathrm{E} \quad 02$ $1.84518 \mathrm{E} \quad 02$ $2.06053 \mathrm{E} \quad 02$ $2.27152 \mathrm{E} \quad 02$

$2.47813 \mathrm{E} \quad 02$ $2.68034 \mathrm{E} \quad 02$ $2.87818 \mathrm{E} \quad 02$ $3.07172 \mathrm{E} \quad 02$ $3.26102 \mathrm{E} \quad 02$

$3.44618 \mathrm{E} \quad 02$ $3.62729 \mathrm{E} \quad 02$ $3.80445 \mathrm{E} \quad 02$ $3.97776 \mathrm{E} \quad 02$ $4.14733 \mathrm{E} \quad 02$

$4.31326 \mathrm{E} \quad 02$ $4.47566 \mathrm{E} \quad 02$ $4.63462 \mathrm{E} \quad 02$ $4.79025 \mathrm{E} \quad 02$ $4.94264 \mathrm{E} \quad 02$

$5.09189 \mathrm{E} \quad 02$ $5.23809 \mathrm{E} \quad 02$ $5.38132 \mathrm{E} \quad 02$ $5.52168 \mathrm{E} \quad 02$ $5.65924 \mathrm{E} \quad 02$

$5.79410 \mathrm{E} \quad 02$ $5.92631 \mathrm{E} \quad 02$ $6.05597 \mathrm{E} \quad 02$ $6.18313 \mathrm{E} \quad 02$ $6.30788 \mathrm{E} 02$

$6.43028 \mathrm{E} \quad 02$ $6.55038 \mathrm{E} \quad 02$ $6.66827 \mathrm{E} \quad 02$ $6.78400 \mathrm{E} \quad 02$ $6.89762 \mathrm{E} \quad 02$

$7.00919 \mathrm{E} \quad 02$ $7.11877 \mathrm{E} \quad 02$ $7.22641 \mathrm{E} \quad 02$ $7.33217 \mathrm{E} \quad 02$ $7.43608 \mathrm{E} \quad 02$

$7.53819 \mathrm{E} \quad 02$
.7 $7.63857 \mathrm{E} \quad 02$ $7.73723 \mathrm{E} \quad 02$ $7.83424 \mathrm{E} \quad 02$ $7.92963 \mathrm{E} \quad 02$

$8.02345 \mathrm{E} \quad 02$ $8.11572 \mathrm{E} \quad 02$ $8.20650 \mathrm{E} \quad 02$ $8.29581 \mathrm{E} 02$ $8.38368 \mathrm{E} \quad 02$

$8.47017 \mathrm{E} \quad 02$ $8.55529 \mathrm{E} \quad 02$ $8.63909 \mathrm{E} \quad 02$ $8.72158 \mathrm{E} \quad 02$ $8.80280 \mathrm{E} \quad 02$ 
p $\bar{F}$

$2.12366 \mathrm{E}-09$ $9.53997 \mathrm{E}-09$ $2.22869 \mathrm{E}-08$ $4.03494 \mathrm{E}-08$ $6.37139 \mathrm{E}-08$

0.4

0.50

0.60

0.70

0.80

0.90

1.00

1.10

1.20

1.30

1.40

1.50

$9.23685 \mathrm{E}-08$ $1.26304 \mathrm{E}-07$

$1.65512 \mathrm{E}-07$

$2.09987 \mathrm{E}-07$

$2.59725 \mathrm{E}-07$

$3.14720 \mathrm{E}-07$ $3.74971 \mathrm{E}-07$ $4.40475 \mathrm{E}-07$ $5.11231 \mathrm{E}-07$ $5.87237 \mathrm{E}-07$

$1.60 \quad 6.68492 \mathrm{E}-07$

$1.70 \quad 7.54994 \mathrm{E}-07$

$1.80 \quad 8.46744 \mathrm{E}-07$

$1.90 \quad 9.43741 \mathrm{E}-07$

$2.00 \quad 1.04598 \mathrm{E}-06$

$2.10 \quad 1.15347 \mathrm{E}-06$

$2.20 \quad 1.26621 \mathrm{E}-06$

$2.30 \quad 1.38419 \mathrm{E}-06$

$2.40 \quad 1.50741 \mathrm{E}-06$

$2.50 \quad 1.63588 \mathrm{E}-06$

2.60

2.70

2.80

2.90

3.00

$1.76960 \mathrm{E}-06$

$1.90856 \mathrm{E}-06$

$2.05276 \mathrm{E}-06$

$2.20221 \mathrm{E}-06$

$2.35690 \mathrm{E}-06$

3.10

$2.51684 \mathrm{E}-06$

3.20

3.30

3.40

3.50

$.68202 \mathrm{E}-06$

$2.85245 \mathrm{E}-06$

$3.02811 \mathrm{E}-06$

$3.20903 \mathrm{E}-06$

3.60

3.70

3.80

3.90

4.00

4.10

4.20

4.30

4.40

4.50

4.60

4.70

4.80

4.90

5.00

5.10

5.20

5.30

5.40

5.50

5.60

5.70

5.80

5.90

6.00

$3.39519 \mathrm{E}-06$

$3.58659 \mathrm{E}-06$

$3.78324 \mathrm{E}-06$

$3.98513 \mathrm{E}-06$

$4.19226 \mathrm{E}-06$

$4.40464 \mathrm{E}-06$

$4.62226 \mathrm{E}-06$

$4.84513 \mathrm{E}-06$

$5.07324 \mathrm{E}-06$

$5.30659 \mathrm{E}-06$

$5.54519 \mathrm{E}-06$ $5.78903 \mathrm{E}-06$ $6.03812 \mathrm{E}-06$ $6.29245 \mathrm{E}-06$ $6.55202 \mathrm{E}-06$

$6.81684 \mathrm{E}-06$ $7.08690 \mathrm{E}-06$

$7.36221 \mathrm{E}-06$

$7.64276 \mathrm{E}-06$

$7.92855 \mathrm{E}-06$

$8.21959 \mathrm{E}-06$

$8.51587 \mathrm{E}-06$

$8.81739 \mathrm{E}-06$

$9.12416 \mathrm{E}-06$

$9.43617 \mathrm{E}-06$ $f_{2}$

$9.47394 \mathrm{E}-08$ $4.24042 \mathrm{E}-06$

$1.64741 \mathrm{E}-05$

$4.02355 \mathrm{E}-05$

$7.83024 \mathrm{E}-05$

$1.32781 \mathrm{E}-04$

$2.05174 \mathrm{E}-04$

$2.96464 \mathrm{E}-04$

$4.07215 \mathrm{E}-04$

$5.37623 \mathrm{E}-04$

$6.87505 \mathrm{E}-04$

$8.56159 \mathrm{E}-04$

$1.04170 \mathrm{E}-03$

$1.23966 \mathrm{E}-03$

$1.43352 \mathrm{E}-03$

$1.49586 \mathrm{E}-03$

$2.54928 \mathrm{E}-03$

$2.52632 \mathrm{E}-03$

$2.79426 \mathrm{E}-03$

$3.11538 \mathrm{E}-03$

$3.46767 \mathrm{E}-03$

$3.84537 \mathrm{E}-03$

$4.24632 \mathrm{E}-03$

$4.66947 \mathrm{E}-03$

$5.11430 \mathrm{E}-03$

$5.58048 \mathrm{E}-03$

$6.06781 \mathrm{E}-03$

$6.57616 \mathrm{E}-03$

$7.10542 \mathrm{E}-03$

$7.65552 \mathrm{E}-03$

8.22641 E-03

$8.81805 \mathrm{E}-03$

$9.43040 \mathrm{E}-03$

$1.00634 \mathrm{E}-02$

$1.07172 \mathrm{E}-02$

$1.13915 \mathrm{E}-02$

$1.20865 \mathrm{E}-02$

$1.28021 \mathrm{E}-02$

$1.35383 \mathrm{E}-02$

1.42951 E-02

$1.50724 \mathrm{E}-02$

$1.58704 \mathrm{E}-02$

$1.66889 \mathrm{E}-02$

$1.75280 \mathrm{E}-02$

$1.83876 \mathrm{E}-02$

$1.92678 \mathrm{E}-02$

$2.01686 \mathrm{E}-02$

$2.10899 \mathrm{E}-02$

$2.20317 \mathrm{E}-02$

$2.29940 \mathrm{E}-02$

$2.39769 \mathrm{E}-02$

$2.49804 \mathrm{E}-02$

$2.60043 \mathrm{E}-02$

$2.70488 \mathrm{E}-02$

2.81138E-02

$2.91994 \mathrm{E}-02$

3.03054 E-02

$3.14319 \mathrm{E}-02$

$3.25790 \mathrm{E}-02$

$3.37466 \mathrm{E}-02$ $\tan \delta_{2}$

$f_{-2}$

$-3.99642 \mathrm{E}-11$ $-1.74970 \mathrm{E}-09$ $-6.54956 \mathrm{E}-09$ $-1.51710 \mathrm{E}-08$ $-2.75290 \mathrm{E}-08$

$-4.27082 \mathrm{E}-08$ $-5.90475 \mathrm{E}-08$

$-7.42703 \mathrm{E}-08$

$-8.56315 \mathrm{E}-08$

$-9.00487 \mathrm{E}-08$

$-8.42199 \mathrm{E}-08$

$-6.47359 \mathrm{E}-08$

$-2.82191 \mathrm{E}-08$

$2.83197 \mathrm{E}-08$

$1.06083 \mathrm{E}-07$

$1.88810 \mathrm{E}-07$

$4.57259 \mathrm{E}-07$

$5.89456 \mathrm{E}-07$

$8.04740 \mathrm{E}-07$

$1.06950 \mathrm{E}-06$

$1.38413 \mathrm{E}-06$

$1.75159 \mathrm{E}-06$

$2.17538 \mathrm{E}-06$

$2.65919 \mathrm{E}-06$

$3.20680 \mathrm{E}-06$

$3.82200 \mathrm{E}-06$

$4.50859 \mathrm{E}-06$

5.27041 E-06

$6.11127 \mathrm{E}-06$

$7.03502 \mathrm{E}-06$

$8.04546 \mathrm{E}-06$

$9.14645 \mathrm{E}-06$

$1.03418 \mathrm{E}-05$

$1.16353 \mathrm{E}-05$

$1.30309 \mathrm{E}-05$

$1.45323 \mathrm{E}-05$

$1.61434 \mathrm{E}-05$

$1.78680 \mathrm{E}-05$

$1.97099 \mathrm{E}-05$

$2.16729 \mathrm{E}-05$

$2.37610 \mathrm{E}-05$

$2.59778 \mathrm{E}-05$

$2.83273 \mathrm{E}-05$

$3.08132 \mathrm{E}-05$

$3.34394 \mathrm{E}-05$

$3.62097 \mathrm{E}-05$

$3.91280 \mathrm{E}-05$

$4.21980 \mathrm{E}-05$

$4.54235 \mathrm{E}-05$

$4.88084 \mathrm{E}-05$

$5.23566 \mathrm{E}-05$

$5.60718 \mathrm{E}-05$

$5.99579 \mathrm{E}-05$

$6.40186 \mathrm{E}-05$

$6.82579 \mathrm{E}-05$

$7.26795 \mathrm{E}-0.5$

$7.72872 \mathrm{E}-05$

$8.20849 \mathrm{E}-05$

$8.70764 \mathrm{E}-05$

$9.22655 \mathrm{E}-05$

$-1.47613 \mathrm{E} 01$ $-2.92348 \mathrm{E} 01$ $-4.30977 \mathrm{E} \quad 01$ $-5.61419 \mathrm{E} 01$ $-6.82290 \mathrm{E} 01$

$-7.92920 \mathrm{E}$ 0l $-8.93233 \mathrm{E} 01$ $-9.83586 \mathrm{E} \quad 01$ $-1.06462 \mathrm{E} \quad 02$ $-1.13712 \mathrm{E} \quad 02$

$-1.20192 \mathrm{E} \quad 02$ $-1.25986 \mathrm{E} 02$ $-1.31173 \mathrm{E} \quad 02$ $-1.35824 \mathrm{E} \quad 02$ $-1.40003 \mathrm{E} 02$

$-1.43769 \mathrm{E} \quad 02$ $-1.47170 \mathrm{E} 02$ $-1.50251 \mathrm{E} \quad 02$ $-1.53049 \mathrm{E} 02$ $\begin{array}{lll}-1.55598 \mathrm{E} & 02\end{array}$

$-1.57927 \mathrm{E} \quad 02$ $-1.60059 \mathrm{E} 02$ $-1.62017 \mathrm{E} \quad 02$ $-1.63820 \mathrm{E} \quad 02$

-1.65484 E 02

$\begin{array}{ll}-1.67023 \mathrm{E} & 02\end{array}$ $-1.68449 \mathrm{E} \quad 02$ $-1.69775 \mathrm{E} 02$ $-1.71009 \mathrm{E} \quad 02$ $-1.72161 \mathrm{E} 02$

$-1.73237 \mathrm{E} \quad 02$ $-1.74245 \mathrm{E} \quad 02$ $-1.75190 \mathrm{E} 02$ $-1.76078 \mathrm{E} 02$ $-1.76914 \mathrm{E} 02$

$-1.77702 \mathrm{E} \quad 02$ $-1.78446 \mathrm{E} \quad 02$ $-1.79148 \mathrm{E} 02$ $-1.79814 \mathrm{E} \quad 02$ $-1.80444 \mathrm{E} \quad 02$

$\begin{array}{ll}-1.81042 \mathrm{E} & 02\end{array}$ $-1.81610 \mathrm{E} 02$ $-1.82151 \mathrm{E} \quad 02$ $-1.82665 \mathrm{E} \quad 02$ $-1.83156 \mathrm{E} \quad 02$

$-1.83624 \mathrm{E} \quad 02$ $\begin{array}{ll}-1.84071 \mathrm{E} & 02\end{array}$ $-1.84498 \mathrm{E} \quad 02$ $-1.84906 \mathrm{E} 02$ $-1.85298 \mathrm{E} 02$

$-1.85673 \mathrm{E} \quad 02$ $-1.86032 \mathrm{E} \quad 02$ $-1.86378 \mathrm{E} 02$ $-1.86709 \mathrm{E} \quad 02$ $-1.87028 \mathrm{E} \quad 02$

$\begin{array}{ll}-1.87335 \mathrm{E} & 02\end{array}$ 
ELECTRON RADIAL FUNCTIONS

Positron

\begin{tabular}{|c|c|c|c|c|c|c|}
\hline$p$ & $F_{0}$ & $f_{1}$ & & $g_{1}$ & $\tan \delta_{1}$ & $f_{-1}$ \\
\hline 6.10 & $9.77244 \mathrm{E}-01$ & $5.52351 \mathrm{E}$ & 00 & $2.49663 \mathrm{E}-02$ & $7.08624 \mathrm{E}-05$ & $-1.59168 \mathrm{E}-02$ \\
\hline 6.20 & $9.77252 \mathrm{E}-01$ & $5.62258 \mathrm{E}$ & 00 & $2.59889 \mathrm{E}-02$ & $6.14615 \mathrm{E}-$ & $-1.68351 \mathrm{E}-02$ \\
\hline 6.30 & $9.77261 \mathrm{E}-01$ & $5.72165 \mathrm{E}$ & 00 & $2.70321 \mathrm{E}-02$ & $5.23553 \mathrm{E}$ & $.77739 E-02$ \\
\hline 6.40 & $9.77269 \mathrm{E}-01$ & $5.82072 \mathrm{E}$ & 00 & $2.80958 \mathrm{E}-02$ & $4.35293 \mathrm{E}-05$ & $-1.87333 \mathrm{E}-02$ \\
\hline 6.50 & $9.77276 \mathrm{E}-01$ & $5.91978 \mathrm{E}$ & 00 & $2.91799 \mathrm{E}-02$ & $3.49735 \mathrm{E}-05$ & $-1.97132 \mathrm{E}-02$ \\
\hline 6.60 & $9.77283 \mathrm{E}-01$ & $6.01885 \mathrm{E}$ & 00 & $3.02846 \mathrm{E}-02$ & $2.66743 \mathrm{E}-05$ & $-2.07137 \mathrm{E}-02$ \\
\hline 6.70 & $9.77290 \mathrm{E}-01$ & $6.11791 \mathrm{E}$ & 00 & $3.14097 \mathrm{E}-02$ & $1.86202 \mathrm{E}-05$ & $-2.17348 \mathrm{E}-02$ \\
\hline 6.80 & $77297 \mathrm{E}-01$ & $6.21698 \mathrm{E}$ & 00 & $3.25554 \mathrm{E}-02$ & $1.07999 \mathrm{E}-05$ & $-2.27764 \mathrm{E}-02$ \\
\hline 6.90 & $9.77303 \mathrm{E}-01$ & $6.31604 \mathrm{E}$ & 00 & $3.37216 \mathrm{E}-02$ & $3.20531 \mathrm{E}-06$ & $-2.38386 \mathrm{E}-02$ \\
\hline 7.00 & $9.77308 \mathrm{E}-01$ & $6.41510 \mathrm{E}$ & 00 & $3.49082 \mathrm{E}-02$ & $-4.17325 \mathrm{E}-06$ & $-2.49213 \mathrm{E}-02$ \\
\hline 7.10 & $9.77314 \mathrm{E}-01$ & $6.51417 \mathrm{E}$ & 00 & $3.61154 \mathrm{E}-02$ & $-1.13486 \mathrm{E}-05$ & $-2.60245 \mathrm{E}-02$ \\
\hline 7.20 & $9.77319 \mathrm{E}-01$ & $6.61323 \mathrm{E}$ & 00 & $3.73430 \mathrm{E}-02$ & $-1.83252 \mathrm{E}-05$ & $-2.71484 \mathrm{E}-02$ \\
\hline 7.30 & $9.77324 \mathrm{E}-01$ & $6.71229 \mathrm{E}$ & 00 & $3.85912 \mathrm{E}-02$ & $-2.51117 \mathrm{E}-05$ & $-2.82927 \mathrm{E}-02$ \\
\hline 7.40 & $9.77329 \mathrm{E}-01$ & $6.81135 \mathrm{E}$ & 00 & $3.98599 \mathrm{E}-02$ & $-3.17168 \mathrm{E}-05$ & $-2.94576 \mathrm{E}-02$ \\
\hline 7.50 & $9.77334 \mathrm{E}-01$ & $6.91041 \mathrm{E}$ & 00 & $4.11491 \mathrm{E}-02$ & $-3.81474 \mathrm{E}-05$ & $-3.06431 \mathrm{E}-02$ \\
\hline 7.60 & $9.77339 \mathrm{E}-01$ & $7.00946 \mathrm{E}$ & 00 & $4.24587 \mathrm{E}-02$ & $-4.44099 E-05$ & $-3.18491 \mathrm{E}-02$ \\
\hline 7.70 & $.77343 \mathrm{E}-01$ & $7.10852 \mathrm{E}$ & 00 & $4.37889 \mathrm{E}-02$ & $-5.05110 \mathrm{E}-05$ & $-3.30756 \mathrm{E}-02$ \\
\hline 7.80 & $9.77347 \mathrm{E}-01$ & $7.20758 \mathrm{E}$ & 00 & $4.51396 \mathrm{E}-02$ & $-5.64563 \mathrm{E}-($ & $-3.43227 \mathrm{E}-02$ \\
\hline 7.90 & $9.77351 \mathrm{E}-01$ & $7.30664 \mathrm{E}$ & 00 & $4.65108 \mathrm{E}-02$ & $-6.22532 \mathrm{E}-05$ & $-3.55903 \mathrm{E}-02$ \\
\hline 8.00 & $9.77355 \mathrm{E}-01$ & $7.40569 \mathrm{E}$ & 00 & $4.79025 \mathrm{E}-02$ & $-6.79042 \mathrm{E}-05$ & $-3.68785 \mathrm{E}-02$ \\
\hline 8.10 & $9.77358 \mathrm{E}-01$ & $7.50475 \mathrm{E}$ & 00 & $4.93148 \mathrm{E}-02$ & $-7.34184 \mathrm{E}-05$ & $-3.81872 \mathrm{E}-02$ \\
\hline 8.20 & $77362 \mathrm{E}-01$ & $7.60380 \mathrm{E}$ & 00 & $5.07475 \mathrm{E}$ & $-7.87984 \mathrm{E}-05$ & $-3.95164 \mathrm{E}-02$ \\
\hline 8.30 & $9.77365 \mathrm{E}-01$ & $7.70286 \mathrm{E}$ & 00 & $5.22007 \mathrm{E}-02$ & $-8.40495 \mathrm{E}-$ & $-4.08662 \mathrm{E}-02$ \\
\hline 8.40 & $9.77368 \mathrm{E}-01$ & $191 \mathrm{E}$ & 00 & $5745 \mathrm{E}-02$ & -8. & $-4.22365 \mathrm{E}-02$ \\
\hline 8.50 & $9.77372 \mathbf{E}-01$ & $7.90097 \mathrm{E}$ & 00 & $5.51687 \mathrm{E}-02$ & -9. & $-4.36273 \mathrm{E}-02$ \\
\hline 8.60 & $9.77375 \mathrm{E}-01$ & $8.00002 \mathrm{E}$ & 00 & $5.66835 \mathrm{E}-02$ & $-9.90756 \mathrm{E}-05$ & $-4.50387 \mathrm{E}-02$ \\
\hline 8.70 & $\mathrm{E}-01$ & $8.09907 \mathrm{E}$ & 00 & $2188 \mathrm{E}-02$ & -1.0 & $.64706 \mathrm{E}-02$ \\
\hline 8.80 & $\mathrm{E}-01$ & $13 \mathrm{E}$ & 00 & $E-02$ & $3525 \mathrm{E}-04$ & $-4.79231 \mathrm{E}-02$ \\
\hline 8.90 & $9.77383 \mathrm{E}-01$ & $8.29718 \mathrm{E}$ & 00 & $6.13509 \mathrm{E}-02$ & $-1.13093 \mathrm{E}-04$ & $-4.93961 \mathrm{E}-02$ \\
\hline 9.00 & $9.77385 \mathrm{E}-01$ & $8.39623 \mathrm{E}$ & 00 & $6.29478 \mathrm{E}-02$ & $-1.17558 \mathrm{E}-04$ & $-5.08896 \mathrm{E}-02$ \\
\hline 9.10 & $9.77388 \mathrm{E}-01$ & $8.49528 \mathrm{E}$ & 00 & $6.45651 \mathrm{E}-02$ & $27 \mathrm{E}-04$ & $4037 \mathrm{E}-02$ \\
\hline 9.20 & E-01 & $434 \mathrm{E}$ & 00 & 6.6203 & $-1.26199 \mathrm{E}-04$ & $-5.39383 \mathrm{E}-02$ \\
\hline 9.30 & $9.77392 \mathrm{E}-01$ & $8.69339 \mathrm{E}$ & 00 & $6.78614 \mathrm{E}-02$ & $-1.30383 \mathrm{E}-04$ & $-5.54935 \mathrm{E}-02$ \\
\hline 9.40 & $9.77395 \mathrm{E}-01$ & $8.79245 \mathrm{E}$ & 00 & $6.95403 \mathrm{E}$ & $-1.34475 \mathrm{E}-04$ & $-5.70692 \mathrm{E}-02$ \\
\hline 9.50 & $9.77397 \mathrm{E}-01$ & $8.89150 \mathrm{E}$ & 00 & $7.12397 \mathrm{E}-02$ & $-1.38483 \mathrm{E}-$ & $-5.86654 \mathrm{E}-02$ \\
\hline 9.60 & $9.77399 \mathrm{E}-01$ & $8.99055 \mathrm{E}$ & 00 & $7.29596 \mathrm{E}-02$ & $-1.42407 \mathrm{E}-04$ & $-6.02821 \mathrm{E}-02$ \\
\hline 9.70 & $9.77401 \mathrm{E}-01$ & $9.08961 \mathrm{E}$ & 00 & $7.47001 \mathrm{E}-02$ & $-1.46251 \mathrm{E}-04$ & $-6.19194 \mathrm{E}-02$ \\
\hline 9.80 & $9.77403 \mathrm{E}-01$ & $9.18866 \mathrm{E}$ & 00 & $7.64611 \mathrm{E}-02$ & $-1.50016 \mathrm{E}-04$ & $-6.35773 \mathrm{E}-02$ \\
\hline 9.90 & $9.77404 \mathbf{E}-$ & $9.28772 \mathrm{E}$ & 00 & 7.82426 & $-1.53706 \mathrm{E}$ & $-6.52556 \mathrm{E}-$ \\
\hline 10.00 & $9.77406 \mathrm{E}-01$ & $9.38677 \mathrm{E}$ & 00 & 8.004461 & -1.5 & $-6.69545 \mathrm{E}-02$ \\
\hline .10 & $9.77408 \mathrm{E}-01$ & $9.48583 \mathrm{E}$ & 00 & $8.18671 \mathrm{E}-02$ & $-1.60866 \mathrm{E}-04$ & $-6.86740 \mathrm{E}-02$ \\
\hline 20 & $9.77410 \mathrm{E}-01$ & $9.58488 \mathrm{E}$ & 00 & $8.37102 \mathrm{E}-02$ & $-1.64342 \mathrm{E}-04$ & $-7.04140 \mathrm{E}-02$ \\
\hline 30 & $9.77411 \mathrm{E}-$ & $9.68394 \mathrm{E}$ & 00 & $8.55738 \mathrm{~F}$ & $-1.67750 \mathrm{E}-04$ & $-7.21745 \mathrm{E}-02$ \\
\hline 40 & 9.77 & $9.78299 \mathrm{E}$ & 00 & 8.74 & $-1.7 \quad$ & $-7.39555 \mathrm{E}-02$ \\
\hline 10.50 & $9.77414 \mathrm{E}-01$ & $9.88205 \mathrm{E}$ & 00 & $8.93626 \mathrm{E}-02$ & $74371 \mathrm{E}-04$ & $-7.57571 \mathrm{E}-02$ \\
\hline .60 & $9.77416 \mathrm{E}-01$ & $9.98111 \mathrm{E}$ & 00 & $9.12878 \mathrm{E}-02$ & $-1.77588 \mathrm{E}-04$ & $-7.75793 \mathrm{E}-02$ \\
\hline & $9.77417 \mathrm{E}-$ & $1.00802 \mathrm{E}$ & 01 & $9.32335 \mathrm{I}$ & -1.8 & $-7.94220 \mathrm{E}-02$ \\
\hline & $9.77418 \mathrm{E}-$ & $1.01792 \mathrm{E}$ & 01 & 9.51997 & -1.8 & -8.128521 \\
\hline & -01 & $83 E$ & 01 & -02 & -1 & $31689 \mathrm{E}-02$ \\
\hline 11.00 & $9.77421 \mathrm{E}-01$ & $1.03773 \mathrm{E}$ & 01 & $9.91937 \mathrm{E}-02$ & & $-8.50733 \mathrm{E}-02$ \\
\hline .10 & $22 \mathbf{E}-01$ & $1.04764 \mathrm{E}$ & 01 & $2 \mathrm{E}-01$ & $2 \mathrm{E}-04$ & $1 \mathrm{E}-02$ \\
\hline & $9.77423 \mathrm{E}-$ & $1.05755 \mathrm{E}$ & 01 & & & $9435 \mathrm{E}$ \\
\hline & $24 \mathrm{E}-01$ & $1.06745 \mathrm{E}$ & 01 & $1.05339 \mathrm{E}-01$ & $8512 \mathrm{E}-04$ & $-9.09094 \mathrm{E}-02$ \\
\hline 11.40 & $425 \mathrm{E}-01$ & $1.07736 \mathrm{E}$ & 01 & $1.07428 \mathrm{E}-01$ & $-2.01290 \mathrm{E}-04$ & $-9.28959 \mathrm{E}-02$ \\
\hline 11.50 & $9.77426 \mathrm{E}-01$ & $1.08727 \mathrm{E}$ & 01 & $1.09538 \mathrm{E}-01$ & $-2.04023 \mathrm{E}-04$ & $-9.49029 \mathrm{E}-02$ \\
\hline 11.60 & $9.77427 \mathrm{E}-01$ & $1.09717 \mathrm{E}$ & 01 & $1.11669 \mathrm{E}-01$ & $-2.06706 \mathrm{E}-04$ & $-9.69305 \mathrm{E}-02$ \\
\hline 11.70 & $9.77428 \mathrm{E}-01$ & $1.10708 \mathrm{E}$ & 01 & $1.13820 \mathrm{E}-01$ & $-2.09343 \mathrm{E}-04$ & $-9.89786 \mathrm{E}-02$ \\
\hline & $9.77429 \mathrm{E}-01$ & $1.11699 \mathrm{E}$ & 01 & $1.15991 \mathrm{E}-01$ & $-2.11937 \mathrm{E}-04$ & $-1.01047 \mathrm{E}-01$ \\
\hline .90 & $9.77430 \mathrm{E}-01$ & $1.12689 \mathrm{E}$ & 01 & $1.18183 \mathrm{E}-01$ & $-2.14485 \mathrm{E}-04$ & $-1.03136 \mathrm{E}-01$ \\
\hline 00 & $9.77431 \mathrm{E}-01$ & $1.13680 \mathrm{E}$ & 01 & $1.20396 \mathrm{E}-01$ & $-2.16993 \mathrm{E}-04$ & $-1.05246 \mathrm{E}-01$ \\
\hline
\end{tabular}

$Z=1 \quad A=1 \quad \rho=1.2 A^{1 / 3} F$

$49663 \mathrm{~F}-02$

$2.70321 \mathrm{E}-02$

$2.80958 \mathrm{E}-02$

$3.02846 \mathrm{E}-02$

(3.

$37216 \mathrm{E}-02$

$49082 \mathrm{E}-0$

$65108 \mathrm{E}-02$

$79025 \mathrm{E}-02$

$4.93148 \mathrm{E}-02$

$6.95403 \mathrm{E}-02$

$82426 \mathrm{E}-02$

$446 \mathrm{E}-02$

$9.12878 \mathrm{E}-02$

$9.32335 \mathrm{E}-02$

.51997E-02

$9.71865 \mathrm{E}-02$

$1.03270 \mathrm{E}-01$

$1.05339 \mathrm{E}-01$

$1.07428 \mathrm{E}-01$
$1.20396 \mathrm{E}-0$ $g_{-1}$

$\tan \delta_{-1}$

$6.50123 \mathrm{E} \quad 00$ $6.60063 \mathrm{E} \quad 00$ $6.70003 \mathrm{E} \quad 00$ $6.79940 \mathrm{E} \quad 00$ $6.89876 \mathrm{E} \quad 00$

$6.99811 \mathrm{E} \quad 00$ $7.09745 \mathrm{E} \quad 00$ $7.19678 \mathrm{E} \quad 00$

$7.29609 \mathrm{E} \quad 00$

$7.39539 \mathrm{E} \quad 00$

$7.49469 \mathrm{E} \quad 00$ $7.59397 \mathrm{E} \quad 00$ $7.69324 \mathrm{E} \quad 00$ $7.79251 \mathrm{E} \quad 00$ $7.89177 \mathrm{E} \quad 00$

$7.99102 \mathrm{E} \quad 00$ $8.09026 \mathrm{E} \quad 00$ $8.18950 \mathrm{E} \quad 00$ 8.28873 E 00 $8.38795 \mathrm{E} \quad 00$

$8.48717 \mathrm{E} \quad 00$ $8.58638 \mathrm{E} \quad 00$ $8.68559 \mathrm{E} \quad 00$ $8.78479 \mathrm{E} \quad 00$ 8.88399 E 00

$8.98318 \mathrm{E} \quad 00$ $9.08237 \mathrm{E} \quad 00$ $9.18155 \mathrm{E} \quad 00$ $9.28073 \mathrm{E} \quad 00$ $9.37991 \mathrm{E} \quad 00$

$9.47908 \mathrm{E} \quad 00$ $9.57826 \mathrm{E} \quad 00$ $9.67742 \mathrm{E} \quad 00$ $9.77659 \mathrm{E} \quad 00$ $9.87575 \mathrm{E} \quad 00$

$9.97491 \mathrm{E} \quad 00$ $1.00741 \mathrm{E} \quad 01$ $1.01732 \mathrm{E} \quad 01$ $1.02724 \mathrm{E} \quad 01$ $1.03715 \mathrm{E} \quad 01$

$1.04707 \mathrm{E} \quad 01$ $1.05698 \mathrm{E} \quad 01$ $1.06690 \mathrm{E} \quad 01$ $1.07681 \mathrm{E} \quad 01$ $1.08673 \mathrm{E} \quad 01$

$1.09664 \mathrm{E} \quad 01$ $1.10655 \mathrm{E} \quad 01$ $1.11647 \mathrm{E} \quad 01$ $1.12638 \mathrm{E} \quad 01$ $1.13630 \mathrm{E} 01$

$1.14621 \mathrm{E} \quad 01$ $1.15612 \mathrm{E} \quad 01$ $1.16604 \mathrm{E} \quad 01$ $1.17595 \mathrm{E} \quad 01$ $1.18587 \mathrm{E} \quad 01$

$1.19578 \mathrm{E} \quad 01$ $1.20569 \mathrm{E} \quad 01$ $1.21561 \mathrm{E} \quad 01$ $1.22552 \mathrm{E} \quad 01$ $1.23543 \mathrm{E} 01$
$8.88278 \mathrm{E} \quad 02$ $8.96156 \mathrm{E} \quad 02$ $9.03915 \mathrm{E} \quad 02$ $9.11558 \mathrm{E} \quad 02$ $9.19088 \mathrm{E} \quad 02$

$9.26508 \mathrm{E} \quad 02$ $9.33819 \mathrm{E} \quad 02$ $9.41024 \mathrm{E} \quad 02$ $9.48126 \mathrm{E} \quad 02$ $9.55128 \mathrm{E} \quad 02$

$9.62028 \mathrm{E} \quad 02$ $9.68832 \mathrm{E} \quad 02$ $\begin{array}{lll}9.75542 \mathrm{E} & 02\end{array}$ $9.82159 \mathrm{E} \quad 02$ $9.88684 \mathrm{E} \quad 02$

$9.95120 \mathrm{E} \quad 02$ $1.00147 \mathrm{E} \quad 03$ $1.00773 \mathrm{E} \quad 03$ $1.01391 \mathrm{E} \quad 03$ $1.02001 \mathrm{E} \quad 03$

$1.02603 \mathrm{E} \quad 03$ $1.03197 \mathrm{E} \quad 03$ $1.03783 \mathrm{E} \quad 03$ $1.04361 \mathrm{E} \quad 03$ $1.04932 \mathrm{E} \quad 03$

$1.05496 \mathrm{E} \quad 03$ $1.06053 \mathrm{E} \quad 03$ $1.06603 \mathrm{E} \quad 03$ $1.07145 \mathrm{E} \quad 03$ $1.07682 \mathrm{E} \quad 03$

$1.08211 \mathrm{E} \quad 03$ $1.08734 \mathrm{E} \quad 03$ $1.09250 \mathrm{E} \quad 03$ $1.09761 \mathrm{E} \quad 03$ $1.10265 \mathrm{E} \quad 03$

$1.10763 \mathrm{E} \quad 03$ $1.11255 \mathrm{E} \quad 03$ $1.11742 \mathrm{E} \quad 03$ $1.12223 \mathrm{E} \quad 03$ $1.12698 \mathrm{E} \quad 03$

$1.13167 \mathrm{E} \quad 03$ $1.13632 \mathrm{E} \quad 03$ $1.14090 \mathrm{E} \quad 03$ $1.14544 \mathrm{E} \quad 03$ $1.14993 \mathrm{E} \quad 03$

$1.15436 \mathrm{E} \quad 03$ $1.15875 \mathrm{E} \quad 03$ $1.16309 \mathrm{E} \quad 03$ $1.16737 \mathrm{E} \quad 03$ $1.17162 \mathrm{E} \quad 03$

$1.17581 \mathrm{E} \quad 03$ $1.17996 \mathrm{E} \quad 03$ $1.18407 \mathrm{E} \quad 03$ $1.18813 \mathrm{E} \quad 03$ $1.19215 \mathrm{E} \quad 03$

$1.19612 \mathrm{E} \quad 03$ $1.20006 \mathrm{E} \quad 03$ $1.20395 \mathrm{E} \quad 03$ $1.20780 \mathrm{E} \quad 03$ $1.21162 \mathrm{E} \quad 03$ 
ELECTRON RADIAL FUNCTIONS

$Z=1 \quad A=1 \quad \rho=1.2 A^{1 / 3} F$

Pesitron

$p$

6.10

6.20

6.30

6.40

6.50

6.60

6.70

6.80

6.90

7.00

7.10

7.20

7.30

7.40

7.50

7.60

7.70

7.80

7.90

8.00

8.10

8.20

8.30

8.40

8.50

8.60

8.70

8.80

8.90

9.00

9.10

9.20

9.30

9.40

9.50

9.60

9.70

9.80

9.90

10.00

10.10

10.20

10.30

10.40

10.50

10.60

10.70

10.80

10.90

11.00

11.10

11.20

11.30

11.40

11.50

11.60

11.70

11.80

11.90

12.00
$\bar{F}$

$9.75343 \mathrm{E}-06$ $1.00759 \mathrm{E}-05$ $1.04037 \mathrm{E}-05$ $1.07367 \mathrm{E}-05$ $1.10749 \mathrm{E}-05$

$1.14184 \mathrm{E}-05$ $1.17671 \mathrm{E}-05$ $1.21210 \mathrm{E}-0.5$ $1.24803 \mathrm{E}-05$ $1.28447 \mathrm{E}-05$

$1.32144 \mathrm{E}-05$ $1.35893 \mathrm{E}-05$ $1.39695 \mathrm{E}-05$ $1.43549 \mathrm{E}-05$ $1.47456 \mathrm{E}-05$

$1.51415 \mathrm{E}-05$ $1.55427 \mathrm{E}-05$ $1.59491 \mathrm{E}-05$ $1.63607 \mathrm{E}-05$ $1.67776 \mathrm{E}-05$

$1.71997 \mathrm{E}-05$ $1.76271 \mathrm{E}-05$ $1.80597 \mathrm{E}-05$ $1.84976 \mathrm{E}-05$ $1.89407 \mathrm{E}-05$

$1.93891 \mathrm{E}-05$ $1.98426 \mathrm{E}-05$ $2.03015 \mathrm{E}-05$ $2.07656 \mathrm{E}-05$ $2.12349 \mathrm{E}-0.5$

$2.17095 \mathrm{E}-05$ $2.21893 \mathrm{E}-05$ $2.26743 \mathrm{E}-05$ $2.31646 \mathrm{E}-05$ $2.36602 \mathrm{E}-05$

$2.41610 \mathrm{E}-05$ $2.46670 \mathrm{E}-05$ $2.51783 \mathrm{E}-05$ $2.56948 \mathrm{E}-05$ $2.62165 \mathrm{E}-05$

$2.67435 \mathrm{E}-05$ $2.72758 \mathrm{E}-05$ $2.78133 \mathrm{E}-05$ $2.83560 \mathrm{E}-05$ $2.89040 \mathrm{E}-05$

$2.94572 \mathrm{E}-05$ $3.00157 \mathrm{E}-05$ $3.05794 \mathrm{E}-05$ $3.11484 \mathrm{E}-05$ $3.17226 \mathrm{E}-05$

$3.23020 \mathrm{E}-05$ $3.28867 \mathrm{E}-05$ $3.34766 \mathrm{E}-05$ $3.40718 \mathrm{E}-05$ $3.46722 \mathrm{E}-05$

$3.52779 \mathrm{E}-05$ $3.58888 \mathrm{E}-05$ $3.65049 \mathrm{E}-05$ $3.71263 \mathrm{E}-05$ $3.77529 \mathrm{E}-05$ $f_{2}$

$3.49347 \mathrm{E}-02$

$3.61433 \mathrm{E}-02$

$3.73725 \mathrm{E}-02$

$3.86221 \mathrm{E}-02$

$3.98922 \mathrm{E}-02$

4.11829E-02

$4.24941 \mathrm{E}-02$

$4.38257 \mathrm{E}-02$

$4.51779 \mathrm{E}-02$

$4.65506 \mathrm{E}-02$

$4.79437 \mathrm{E}-02$

$4.93574 \mathrm{E}-02$

$5.07916 \mathrm{E}-02$

$5.22463 \mathrm{E}-02$

$5.37214 \mathrm{E}-02$

$5.52171 \mathrm{E}-02$

$5.67333 \mathrm{E}-02$

$5.82699 \mathrm{E}-02$

$5.98271 \mathrm{E}-02$

6.14048E-02

$6.30029 \mathrm{E}-02$

$6.46216 \mathrm{E}-02$

6.62607E-02

$6.79203 \mathrm{E}-02$

$6.96005 \mathrm{E}-02$

$7.13011 \mathrm{E}-02$

7.30222 E-02

$7.47638 \mathrm{E}-02$

$7.65259 \mathrm{E}-02$

$7.83085 \mathrm{E}-02$

$8.01116 \mathrm{E}-02$

8.19352E-02

8.37793E-02

8.56438E-02

$8.75289 \mathrm{E}-02$

8.94344 E-02

$9.13605 \mathrm{E}-02$

$9.33070 \mathrm{E}-02$

$9.52740 \mathrm{E}-02$

9.72615E-02

$9.92695 \mathrm{E}-02$

$1.01298 \mathrm{E}-01$

$1.03347 \mathrm{E}-01$

$1.05416 \mathrm{E}-01$

$1.07506 \mathrm{E}-01$

$1.09617 \mathrm{E}-01$

$1.11748 \mathrm{E}-01$

$1.13899 \mathrm{E}-01$

$1.16071 \mathrm{E}-01$

$1.18263 \mathrm{E}-01$

$1.20476 \mathrm{E}-01$

$1.22709 \mathrm{E}-01$

$1.24963 \mathrm{E}-01$

$1.27238 \mathrm{E}-01$

$1.29532 \mathrm{E}-01$

$1.31848 \mathrm{E}-01$

$1.34183 \mathrm{E}-01$

$1.36540 \mathrm{E}-01$

$1.38916 \mathrm{E}-01$

$1.41314 \mathrm{E}-01$ $g_{2}$

$\tan \delta_{2}$

$f_{-2}$

$9.76561 \mathrm{E}-05$

$1.03252 \mathrm{E}-04$

$1.09057 \mathrm{E}-04$

$1.15075 \mathrm{E}-04$

$1.21309 \mathrm{E}-04$

$1.27764 \mathrm{E} \rightarrow 04$

$1.34444 \mathrm{E}-04$

$1.41351 \mathrm{E}-04$

$1.48491 \mathrm{E}-04$

$1.55866 \mathrm{E}-04$

$1.63481 \mathrm{E}-04$

$1.71340 \mathrm{E}-04$

$1.79445 \mathrm{E}-04$

$1.87802 \mathrm{E}-04$

$1.96414 \mathrm{E}-04$

$2.05285 \mathrm{E}-04$

$2.14418 \mathrm{E}-04$

$2.23818 \mathrm{E}-04$

$2.33487 \mathrm{E}-04$

2.43431 E- 04

$2.53653 \mathrm{E}-04$

$2.64156 \mathrm{E}-04$

2.74945 E- 04

$2.86023 \mathrm{E}-04$

$2.97394 \mathrm{E}-04$

$3.09062 \mathrm{E}-04$

$3.21031 \mathrm{E}-04$

$3.33304 \mathrm{E}-04$

$3.45886 \mathrm{E}-04$

$3.58780 \mathrm{E}-04$

$3.71991 \mathrm{E}-04$

$3.85521 \mathrm{E}-04$

$3.99374 \mathrm{E}-04$

$4.13555 \mathrm{E}-04$

$4.28068 \mathrm{E}-04$

4.42916E-04

$4.58102 \mathrm{E}-04$

$4.73632 \mathrm{E}-04$

$4.89508 \mathrm{E}-04$

$5.05734 \mathrm{E}-04$

$5.22315 \mathrm{E}-04$

$5.39254 \mathrm{E}-04$

$5.56554 \mathrm{E}-04$

5.74221 E-04

$5.92256 \mathrm{E}-04$

$6.10666 \mathrm{E}-04$ $6.29452 \mathrm{E}-04$ $6.48619 \mathrm{E}-04$ $6.68171 \mathrm{E}-04$

$6.88112 \mathrm{E}-04$

$7.08445 \mathrm{E}-04$

$7.29174 \mathrm{E}-04$

$7.50304 \mathrm{E}-04$

$7.71837 \mathrm{E}-04$

$7.93778 \mathrm{E}-04$

8.16131 E-04 $8.38899 \mathrm{E}-04$ $8.62085 \mathrm{E}-04$

$8.85696 \mathrm{E}-04$

$9.09732 \mathrm{E}-04$

$-1.88709 \mathrm{E} \quad 02 \quad-6.37535 \mathrm{E}-05$ $-1.88955 \mathrm{E} \quad 02 \quad-6.83890 \mathrm{E}-05$ $\begin{array}{lll}-1.89193 \mathrm{E} & 02 & -7.32207 \mathrm{E}-05\end{array}$ $\begin{array}{lll}-1.89423 \mathrm{E} & 02 & -7.82525 \mathrm{E}-05\end{array}$ $-1.89646 \mathrm{E} \quad 02 \quad-8.34882 \mathrm{E}-05$

$-1.89861 \mathrm{E} \quad 02$ $-1.90070 \mathrm{E} 02$ $\rightarrow 1.90272 \mathrm{E} \quad 02$ $-1.90468 \mathrm{E} \quad 02$ $-1.90658 \mathrm{E} \quad 02$

$\begin{array}{ll}-1.90842 \mathbf{E} & 02\end{array}$ $-1.91020 \mathrm{E} 02$ $-1.91194 \mathrm{E} \quad 02$ $-1.91362 \mathrm{E} \quad 02$

$-1.91526 \mathrm{E} \quad 02$

$-1.91685 \mathrm{E} \quad 02$

$-1.91840 \mathrm{E} \quad 02$

$-1.91991 \mathrm{E} \quad 02$

$-1.92137 \mathrm{E} \quad 02$

$-1.92280 \mathrm{E} \quad 02$

$-1.92418 \mathrm{E} \quad 02$

$-1.92554 \mathrm{E} \quad 02$

$-1.92685 \mathrm{E} \quad 02$

$\begin{array}{lll}-1.92814 \mathrm{E} & 02\end{array}$

$-1.92939 \mathrm{E} \quad 02$

$-1.93061 \mathrm{E} \quad 02$

$-1.93180 \mathrm{E} \quad 02$

$\begin{array}{ll}-1.93296 \mathrm{E} & 02\end{array}$

$-1.93410 \mathrm{E} \quad 02$

$-1.93521 \mathrm{E} \quad 02$

$-1.93629 \mathrm{E} \quad 02$

$-1.93734 \mathrm{E} \quad 02$

$-1.93838 \mathrm{E} \quad 02$

$-1.93939 \mathrm{E} \quad 02$

$-8.89316 \mathrm{E}-05$ $-9.45866 \mathrm{E}-05$ $-1.00457 \mathrm{E}-04$

$-1.06546 \mathrm{E}-04$

$-1.12859 \mathrm{E}-04$ 
ELECTRON RADIAL FUNCTIONS

$$
A=1
$$

$$
\rho=1.2 A^{1 / 3} F
$$

$p$

12.10

12.20

12.30

12.40

12.50

12.60

12.70

12.80

12.90

13.00

13.10

13.20

13.30

13.40

13.50

13.60

13.70

13.80

13.90

14.00

14.10

14.20

14.30

14.40

14.50

14.60

14.70

14.80

14.90

15.00

15.10

15.20

15.30

15.40

15.50

15.60

15.70

15.80

15.90

16.00

16.10

16.20

16.30

16.40

16.50

16.60

16.70

16.80

16.90

17.00

17.10

17.20

17.30

17.40

17.50

17.60

17.70

17.80

17.90

18.00 $f_{1}$

$9.77432 \mathrm{E}-01$ $9.77433 \mathrm{E}-01$ $9.77434 \mathrm{E}-01$ $9.77434 \mathrm{E}-01$ $9.77435 \mathrm{E}-01$

$9.77436 \mathrm{E}-01$ $9.77436 \mathrm{E}-01$ $9.77437 \mathrm{E}-01$ $9.77438 \mathrm{E}-01$ $9.77438 \mathrm{E}-01$

9.77439E-01 $9.77440 \mathrm{E}-01$ $9.77440 \mathrm{E}-01$ $9.77441 \mathrm{E}-01$ $9.77441 \mathrm{E}-01$

$9.77442 \mathrm{E}-01$ $9.77442 \mathrm{E}-01$ $9.77443 \mathrm{E}-01$ $9.77443 \mathrm{E}-01$ $9.77444 \mathrm{E}-01$

$9.77444 \mathrm{E}-01$ $9.77445 \mathrm{E}-01$ $9.77445 \mathrm{E}-01$ $9.77445 \mathrm{E}-01$ $9.77446 \mathrm{E}-01$

$9.77446 \mathrm{E}-01$ $9.77446 \mathrm{E}-01$ $9.77447 \mathrm{E}-01$ $9.77447 \mathrm{E}-01$ $9.77448 \mathrm{E}-01$

$9.77448 \mathrm{E}-01$ $9.77448 \mathrm{E}-01$ $9.77448 \mathrm{E}-01$ $9.77449 \mathrm{E}-01$ 9.77449 E-01

9.77449E-01 $9.77449 \mathrm{E}-01$ $9.77450 \mathrm{E}-01$ $9.77450 \mathrm{E}-01$ 9.77450 E- 01

$9.77450 \mathrm{E}-01$ 9.77451 E-01 9.77451 E- 01 $9.77451 \mathrm{E}-01$ $9.77451 \mathrm{E}-01$

$9.77451 \mathrm{E}-01$ $9.77452 \mathrm{E}-01$ $9.77452 \mathrm{E}-01$ $9.77452 \mathrm{E}-01$ $9.77452 \mathrm{E}-01$

$9.77452 \mathrm{E}-01$ $9.77452 \mathrm{E}-01$ $9.77452 \mathrm{E}-01$ $9.77453 \mathrm{E}-01$ $9.77453 \mathrm{E}-01$

$9.77453 \mathrm{E}-01$ $9.77453 \mathrm{E}-01$ $9.77453 \mathrm{E}-01$ $9.77453 \mathrm{E}-01$ $9.77453 \mathrm{E}-01$ $g_{1}$

$1.14671 \mathrm{E} \quad 01$

$1.15661 \mathrm{E} \quad 01$

$1.16652 \mathrm{E} 01$

$1.17643 \mathrm{E} 01$

$1.18634 \mathrm{E} \quad 01$

$1.19624 \mathrm{E} \quad 01$

$1.20615 \mathrm{E} 01$

$1.21606 \mathrm{E} \quad 01$

$1.22597 \mathrm{E} \quad 01$

$1.23587 \mathrm{E} \quad 01$

$1.24578 \mathrm{E} \quad 01$

$1.25569 \mathrm{E} 01$

$1.26560 \mathrm{E} 01$

$1.27551 \mathrm{E} 01$

$1.28542 \mathrm{E} \quad 01$

$1.29533 \mathrm{E} \quad 01$

$1.30523 \mathrm{E} 01$

$1.31514 \mathrm{E} 01$

$1.32505 \mathrm{E} 01$

$1.33496 \mathrm{E} 01$

$\begin{array}{ll}1.34487 \mathrm{E} & 01\end{array}$

$1.35478 \mathrm{E} \quad 01$

$1.36469 \mathrm{E} 01$

$1.37460 \mathrm{E} 01$

$1.38451 \mathrm{E} 01$

$1.39442 \mathrm{E} \quad 01$

$1.40433 \mathrm{E} \quad 01$

$1.41424 \mathrm{E} \quad 01$

$1.42416 \mathrm{E} \quad 01$

$1.43407 \mathrm{E} \quad 01$

$1.44398 \mathrm{E} \quad 01$

$1.45389 \mathrm{E} 01$

$1.46380 \mathrm{E} \quad 01$

$1.47371 \mathrm{E} \quad 01$

$1.48363 \mathrm{E} 01$

$1.49354 \mathrm{E} \quad 01$

$1.50345 \mathrm{E} \quad 01$

$1.51336 \mathrm{E} 01$

$1.52328 \mathrm{E} \quad 01$

$1.53319 \mathrm{E} 01$

$1.54310 \mathrm{E} 01$

$1.55302 \mathrm{E} \quad 01$

$1.56293 \mathrm{E} \quad 01$

$1.57284 \mathrm{E} \quad 01$

$1.58276 \mathrm{E} \quad 01$

$\begin{array}{lll}1.59267 \mathrm{E} & 01\end{array}$

$1.60259 \mathrm{E} 01$

$1.61250 \mathrm{E} 01$

$1.62242 \mathrm{E} 01$

$1.63233 \mathrm{E} 01$

$\begin{array}{ll}1.64225 \mathrm{E} & 01\end{array}$

$1.65216 \mathrm{E} 01$

$1.66208 \mathrm{E} \quad 01$

$1.67199 \mathrm{E} 01$

$1.68191 \mathrm{E} 01$

$1.69183 \mathrm{E} \quad 01$

$1.70174 \mathrm{E} \quad 01$

$1.71166 \mathrm{E} \quad 01$

$1.72158 \mathrm{E} \quad 01$

$1.73150 \mathrm{E} \quad 01$ $\tan \delta_{1}$

$1.22629 \mathrm{E}-01$

$1.24883 \mathrm{E}-01$

$1.27157 \mathrm{E}-01$

$1.29452 \mathrm{E}-01$

$1.31767 \mathrm{E}-01$

$1.34103 \mathrm{E}-01$

$1.36460 \mathrm{E}-01$

1.38837 E-01

$1.41234 \mathrm{E}-01$

$1.43652 \mathrm{E}-01$

$1.46091 \mathrm{E}-01$

$1.48550 \mathrm{E}-01$

1:51030 E-01

$1.53530 \mathrm{E}-01$

$1.56051 \mathrm{E}-01$

$1.58592 \mathrm{E}-01$

$1.61154 \mathrm{E}-01$

$1.63737 \mathrm{E}-01$

$1.66340 \mathrm{E}-01$

$1.68963 \mathrm{E}-01$

$1.71608 \mathrm{E}-01$

$1.74272 \mathrm{E}-01$

$1.76958 \mathrm{E}-01$

$1.79664 \mathrm{E}-01$

$1.82390 \mathrm{E}-01$

$1.85137 \mathrm{E}-01$

$1.87905 \mathrm{E}-01$

$1.90693 \mathrm{E}-01$

$1.93501 \mathrm{E}-01$

$1.96331 \mathrm{E}-01$

$1.99180 \mathrm{E}-01$

$2.02051 \mathrm{E}-01$

$2.04942 \mathrm{E}-01$

$2.07853 \mathrm{E}-01$

$2.10785 \mathrm{E}-01$

$2.13738 \mathrm{E}-01$

$2.16711 \mathrm{E}-01$

$2.19705 \mathrm{E}-01$

$2.22720 \mathrm{E}-01$

$2.25755 \mathrm{E}-01$

$2.28810 \mathrm{E}-01$ $2.31886 \mathrm{E}-01$ $2.34983 \mathrm{E}-01$

$2.38100 \mathrm{E}-01$

$2.41238 \mathrm{E}-01$

2.44397 E-01

$2.47576 \mathrm{E}-01$

$2.50776 \mathrm{E}-01$

$2.53996 \mathrm{E}-01$

$2.57237 \mathrm{E}-01$

$2.60498 \mathrm{E}-01$

$2.63780 \mathrm{E}-01$

$2.67083 \mathrm{E}-01$

$2.70406 \mathrm{E}-01$

$2.73750 \mathrm{E}-01$

$2.77114 \mathrm{E}-01$ $2.80499 \mathrm{E}-01$

2.83905 E-0]

$2.87331 \mathrm{E}-01$

$2.90778 \mathrm{E}-01$

$\tan \delta_{1}$
$-2.19457 \mathrm{E}-04$
$-2.21882 \mathrm{E}-04$
$-2.24267 \mathrm{E}-04$
$-2.26614 \mathrm{E}-04$
$-2.28923 \mathrm{E}-04$

$f_{-1}$

$-1.07377 \mathrm{E}-01$

$-1.09528 \mathrm{E}-01$

$-1.11699 \mathrm{E}-01$

$-1.13891 \mathrm{E}-01$

$-1.16103 \mathrm{E}-\emptyset 1$

$-2.31194 \mathrm{E}-04$

$-2.33430 \mathrm{E}-04$

-2.35631 E-04

$-2.37797 \mathrm{E}-04$

$-2.39929 \mathrm{E}-04$

$-2.42030 \mathrm{E}-04$

$-2.44098 \mathrm{E}-04$

$-2.46134 \mathrm{E}-04$

-2.48141 E-04

$-2.50117 \mathrm{E}-04$

$-2.52063 \mathrm{E}-04$

$-2.53982 \mathrm{E}-04$

$-2.55873 \mathrm{E}-04$

$-2.57735 \mathrm{E}-04$

-2.59571 E-04

$-2.6138 \theta \mathrm{E}-04$

$-2.63165 \mathrm{E}-04$

$-2.64923 \mathrm{E}-04$

$-2.66658 \mathrm{E}-04$

$-2.68367 \mathrm{E}-04$

$-2.70053 \mathrm{E}-04$

$-2.71716 \mathrm{E}-04$

$-2.73356 \mathrm{E}-04$

$-2.74973 \mathrm{E}-04$

$-2.76570 \mathrm{E}-04$

$-2.78144 \mathrm{E}-04$

$-2.79698 \mathrm{E}-04$

-2.81231 E-04

$-2.82743 \mathrm{E}-04$

$-2.84236 \mathrm{E}-04$

$-2.85710 \mathrm{E}-04$

-2.87164 E-04 
ELECTRON RADIAL FUNCTIONS

$Z=1 \quad A=1 \quad \rho=1.2 A^{1 / 3} \mathrm{~F}$

Positron

$\bar{F}$

$3.83848 \mathrm{E}-05$

$3.90220 \mathrm{E}-05$

$3.96643 \mathrm{E}-05$

$4.03119 \mathrm{E}-05$

$4.09648 \mathrm{E}-05$

12.40

$4.16229 \mathrm{E}-05$ $4.22862 \mathrm{E}-05$ $4.29548 \mathrm{E}-05$ $4.36287 \mathrm{E}-05$ 4.43077 E-05

12.80

12.90

13.00

13.10

13.20

13.30

13.40

13.50

13.60

13.70

13.80

13.90

14.00

14.10

14.20

14.30

14.40

14.50

14.60

14.70

14.80

14.90

15.00

15.10

15.20

15.30

15.40

15.50

15.60

15.70

15.80

15.90

16.00

16.10

16.20

16.30

16.40

16.50

16.60

16.70

16.80

16.90

17.00

17.10

17.20

17.30

17.40

17.50

17.60

17.70

17.80

17.90

18.00

$4.49920 \mathrm{E}-05$

$4.56816 \mathrm{E}-05$

4.63764 E-05

4.70764E-05

$4.77817 \mathrm{E}-05$

4.84923 E-05

$4.92080 \mathrm{E}-05$

$4.99291 \mathrm{E}-05$

$5.06553 \mathrm{E}-05$

$5.13868 \mathrm{E}-05$

$5.21236 \mathrm{E}-05$

$5.28656 \mathrm{E}-05$

$5.36128 \mathrm{E}-05$

$5.43653 \mathrm{E}-05$

$5.51230 \mathrm{E}-05$

$5.58860 \mathrm{E}-05$

$5.66542 \mathrm{E}-05$

$5.74277 \mathrm{E}-05$

$5.82064 \mathrm{E}-05$

$5.89903 \mathrm{E}-05$

$5.97795 \mathrm{E}-05$

$6.05740 \mathrm{E}-05$

$6.13736 \mathrm{E}-05$

$6.21785 \mathrm{E}-05$

$6.29887 \mathrm{E}-05$

$6.38041 \mathrm{E}-05$ $6.46248 \mathrm{E}-05$ $6.54507 \mathrm{E}-05$ $6.62818 \mathrm{E}-05$ $6.71182 \mathrm{E}-05$

$6.79598 \mathrm{E}-05$ $6.88067 \mathrm{E}-05$ $6.96588 \mathrm{E}-05$ 7.05161 E-05 $7.13787 \mathrm{E}-05$

7.22466 E- 05

$7.31197 \mathrm{E}-05$

$7.39980 \mathrm{E}-05$

$7.48816 \mathrm{E}-05$

$7.57704 \mathrm{E}-05$

7.66644 E-05

$7.75637 \mathrm{E}-05$

7.84683 E- 05

$7.93781 \mathrm{E}-05$

8.02931 E-05

8.12134 E-05

$8.21389 \mathrm{E}-05$

8.30697E-05

$8.40057 \mathrm{E}-05$

$8.49469 \mathrm{E}-05$ $f_{2}$
$1.43731 \mathrm{E}-01$

$1.46169 \mathrm{E}-01$

$1.48628 \mathrm{E}-01$

$1.51107 \mathrm{E}-01$

$1.53607 \mathrm{E}-01$

$1.56127 \mathrm{E}-01$

$1.58668 \mathrm{E}-01$

$1.61229 \mathrm{E}-01$

$1.63810 \mathrm{E}-01$

$1.66412 \mathrm{E}-01$

$g_{2}$

$9.34201 \mathrm{E}-04$

$9.59103 \mathrm{E}-04$

$9.84443 \mathrm{E}-04$

$1.01023 \mathrm{E}-03$

$1.03645 \mathrm{E}-03$

$1.06313 \mathrm{E}-03$

$1.09026 \mathrm{E}-03$

$1.11785 \mathrm{E}-03$

$1.14590 \mathrm{E}-03$

1.17442 E-03

$1.69035 \mathrm{E}-01$

$1.71678 \mathrm{E}-01$

$1.74341 \mathrm{E}-01$

$1.77025 \mathrm{E}-01$

$1.79729 \mathrm{E}-01$

1.82454 E- 01

$1.85200 \mathrm{E}-01$

$1.87965 \mathrm{E}-01$

$1.90752 \mathrm{E}-01$

$1.93558 \mathrm{E}-01$

$1.96386 \mathrm{E}-01$

$1.99233 \mathrm{E}-01$

$2.02102 \mathrm{E}-01$

$2.04990 \mathrm{E}-01$

$2.07900 \mathrm{E}-01$

$2.10829 \mathrm{E}-01$

$2.13779 \mathrm{E}-01$

$2.16750 \mathrm{E}-01$

$2.19741 \mathrm{E}-01$

$2.22752 \mathrm{E}-01$

2.25784 E-01

$2.28837 \mathrm{E}-01$

$2.31910 \mathrm{E}-01$

$2.35003 \mathrm{E}-01$

$2.38117 \mathrm{E}-01$

$2.41252 \mathrm{E}-01$

$2.44406 \mathrm{E}-01$

$2.47582 \mathrm{E}-01$

$2.50778 \mathrm{E}-01$

$2.53994 \mathrm{E}-01$

$2.57230 \mathrm{E}-01$

$2.60488 \mathrm{E}-01$

$2.63765 \mathrm{E}-01$

$2.67063 \mathrm{E}-0 \mathrm{l}$

$2.70382 \mathrm{E}-01$

$2.73721 \mathrm{E}-01$

$2.77081 \mathrm{E}-01$

$2.80460 \mathrm{E}-01$

2.83861 E- 01

$2.87282 \mathrm{E}-01$

$2.90723 \mathrm{E}-01$

$2.94185 \mathrm{E}-01$

$2.97667 \mathrm{E}-01$

$3.01170 \mathrm{E}-01$

$3.04693 \mathrm{E}-01$

$3.08237 \mathrm{E}-01$

$3.11801 \mathrm{E}-01$

$3.15386 \mathrm{E}-01$

$3.18991 \mathrm{E}-01$

$3.22617 \mathrm{E}-01$

$1.20340 \mathrm{E}-03$

$1.23285 \mathrm{E}-03$

$1.26279 \mathrm{E}-03$

$1.29320 \mathrm{E}-03$

$1.32410 \mathrm{E}-03$

$1.35548 \mathrm{E}-03$

$1.38736 \mathrm{E}-03$

$1.41973 \mathrm{E}-03$

$1.45260 \mathrm{E}-03$

$1.48598 \mathrm{E}-03$

$1.51986 \mathrm{E}-03$

$1.55425 \mathrm{E}-03$

$1.58916 \mathrm{E}-03$

$1.62458 \mathrm{E}-03$

$1.66053 \mathrm{E}-03$

$1.69700 \mathrm{E}-03$

$1.73401 \mathrm{E}-03$

$1.77154 \mathrm{E}-03$

$1.80962 \mathrm{E}-03$

$1.84823 \mathrm{E}-03$

$1.88739 \mathrm{E}-03$

$1.92710 \mathrm{E}-03$

$1.96737 \mathrm{E}-03$

$2.00819 \mathrm{E}-03$

$2.04957 \mathrm{E}-03$

2.09151 E- 03

$2.13402 \mathrm{E}-03$

2.17711 E-03

$2.22077 \mathrm{E}-03$

$2.26500 \mathrm{E}-03$

$2.30983 \mathrm{E}-03$

$2.35524 \mathrm{E}-03$

$2.40124 \mathrm{E}-03$

$2.44783 \mathrm{E}-03$

$2.49503 \mathrm{E}-03$

$2.54282 \mathrm{E}-03$

$2.59122 \mathrm{E}-03$

$2.64023 \mathrm{E}-03$

$2.68986 \mathrm{E}-03$

$2.74010 \mathrm{E}-03$

$2.79097 \mathrm{E}-03$

$2.84246 \mathrm{E}-03$

$2.89458 \mathrm{E}-03$

$2.94733 \mathrm{E}-03$

$3.05475 \mathrm{E}-03$

$3.10942 \mathrm{E}-03$

$3.16475 \mathrm{E}-03$

$3.22072 \mathrm{E}-03$

$3.27735 \mathrm{E}-03$ $\tan \delta_{2}$

$-1.96003 \mathrm{E} \quad 02$

$-\mathrm{I} .96061 \mathrm{E} \quad 02$

$-1.96118 \mathrm{E} 02$

$-1.96174 \mathrm{E} \quad 02$

$-1.96229 \mathrm{E} \quad 02$

$-1.96283 \mathrm{E} \quad 02$

$-1.96337 \mathrm{E} 02$

$-1.96389 \mathrm{E} \quad 02$

$-1.96441 \mathrm{E} \quad 02$

$-1.96491 \mathrm{E} \quad 02$

$3.00072 \mathrm{E}-03$

$-1.96541 \mathrm{E} \quad 02$

$-1.96590 \mathrm{E} \quad 02$

$\begin{array}{ll}-1.96639 \mathrm{E} & 02\end{array}$

$-1.96686 \mathrm{E} \quad 02$

$-1.96733 \mathrm{E} 02$

$\begin{array}{ll}-1.96779 \mathrm{E} & 02\end{array}$

$-1.96825 \mathrm{E} \quad 02$

$-1.96869 \mathrm{E} \quad 02$

$-1.96913 \mathrm{E} 02$

$\begin{array}{ll}-1.96957 \mathrm{E} & 02\end{array}$

$-1.96999 \mathrm{E} \quad 02$

-1.97042 E 02

$-1.97083 \mathrm{E} 02$

$-1.97124 \mathrm{E} \quad 02$

-1.97164 E 02

$-1.97204 \mathrm{E} \quad 02$

$-1.97243 \mathrm{E} \quad 02$

$-1.97282 \mathrm{E} \quad 02$

$\begin{array}{ll}-1.97320 \mathrm{E} & 02\end{array}$

$-1.97357 \mathrm{E} \quad 02$

$\begin{array}{lll}-1.97394 \mathrm{E} & 02\end{array}$

$\begin{array}{ll}-1.97431 \mathrm{E} & 02\end{array}$

$\begin{array}{lll}-1.97467 \mathrm{E} & 02\end{array}$

$-1.97502 \mathrm{E} 02$

$\begin{array}{lll}-1.97537 \mathrm{E} & 02\end{array}$

$\begin{array}{ll}-1.97572 \mathrm{E} & 02\end{array}$

$-1.97606 \mathrm{E} \quad 02$

$-1.97640 \mathrm{E} \quad 02$

$-1.97673 \mathrm{E} 02$

$-1.97706 \mathrm{E} \quad 02$

$-1.97738 \mathrm{E} \quad 02$

$-1.97770 \mathrm{E} 02$

$-1.97801 \mathrm{E} \quad 02$

$\begin{array}{ll}-1.97832 \mathrm{E} & 02\end{array}$

-1.97863 E 02

$-1.97893 \mathrm{E} \quad 02$

$\begin{array}{ll}-1.97923 \mathrm{E} & 02\end{array}$

$-1.97953 \mathrm{E} 02$

$-1.97982 \mathrm{E} \quad 02$ 


\begin{tabular}{|c|c|c|c|c|c|c|}
\hline$p$ & $F_{0}$ & $f_{1}$ & & $g_{1}$ & $\tan \delta_{1}$ & $f_{-1}$ \\
\hline $\begin{array}{l}18.10 \\
18.20 \\
18.30 \\
18.40 \\
18.50\end{array}$ & $\begin{array}{l}9.77453 \mathrm{E}-01 \\
9.77453 \mathrm{E}-01 \\
9.77454 \mathrm{E}-01 \\
9.77454 \mathrm{E}-01 \\
9.77454 \mathrm{E}-01\end{array}$ & $\begin{array}{l}1.74141 \mathrm{E} \\
1.75133 \mathrm{E} \\
1.76125 \mathrm{E} \\
1.77117 \mathrm{E} \\
1.78109 \mathrm{E}\end{array}$ & $\begin{array}{l}01 \\
01 \\
01 \\
01 \\
01\end{array}$ & $\begin{array}{l}2.94246 \mathrm{E}-01 \\
2.97734 \mathrm{E}-01 \\
3.01242 \mathrm{E}-01 \\
3.04772 \mathrm{E}-01 \\
3.08322 \mathrm{E}-01\end{array}$ & $\begin{array}{l}-3.17156 \mathrm{E}-04 \\
-3.18230 \mathrm{E}-04 \\
-3.19292 \mathrm{E}-04 \\
-3.20342 \mathrm{E}-04 \\
-3.21380 \mathrm{E}-04\end{array}$ & $\begin{array}{l}-2.72833 \mathrm{E}-01 \\
-2.76219 \mathrm{E}-01 \\
-2.79625 \mathrm{E}-01 \\
-2.83052 \mathrm{E}-01 \\
-2.86499 \mathrm{E}-01\end{array}$ \\
\hline $\begin{array}{l}18.60 \\
18.70 \\
18.80 \\
18.90 \\
19.00\end{array}$ & $\begin{array}{l}9.77454 \mathrm{E}-01 \\
9.77454 \mathrm{E}-01 \\
9.77454 \mathrm{E}-01 \\
9.77454 \mathrm{E}-01 \\
9.77454 \mathrm{E}-01\end{array}$ & $\begin{array}{l}1.79101 \mathrm{E} \\
1.80093 \mathrm{E} \\
1.81085 \mathrm{E} \\
1.82077 \mathrm{E} \\
1.83069 \mathrm{E}\end{array}$ & $\begin{array}{l}01 \\
01 \\
01 \\
01 \\
01\end{array}$ & $\begin{array}{l}3.11892 \mathrm{E}-01 \\
3.15483 \mathrm{E}-01 \\
3.19095 \mathrm{E}-01 \\
3.22727 \mathrm{E}-01 \\
3.26380 \mathrm{E}-01\end{array}$ & $\begin{array}{l}-3.22406 \mathrm{E}-04 \\
-3.234 .21 \mathrm{E}-04 \\
-3.24426 \mathrm{E}-04 \\
-3.254 .19 \mathrm{E}-04 \\
-3.26401 \mathrm{E}-04\end{array}$ & $\begin{array}{l}-2.89967 \mathrm{E}-01 \\
-2.93456 \mathrm{E}-01 \\
-2.96965 \mathrm{E}-01 \\
-3.00495 \mathrm{E}-01 \\
-3.04046 \mathrm{E}-01\end{array}$ \\
\hline $\begin{array}{l}19.10 \\
19.20 \\
19.30 \\
19.40 \\
19.50\end{array}$ & $\begin{array}{l}9.77454 \mathrm{E}-01 \\
9.77454 \mathrm{E}-01 \\
9.77454 \mathrm{E}-01 \\
9.77454 \mathrm{E}-01 \\
9.77454 \mathrm{E}-01\end{array}$ & $\begin{array}{l}1.84061 \mathrm{E} \\
1.85053 \mathrm{E} \\
1.86045 \mathrm{E} \\
1.87037 \mathrm{E} \\
1.88029 \mathrm{E}\end{array}$ & $\begin{array}{l}01 \\
01 \\
01 \\
01 \\
01\end{array}$ & $\begin{array}{l}3.30054 \mathrm{E}-01 \\
3.33748 \mathrm{E}-01 \\
3.37463 \mathrm{E}-01 \\
3.41199 \mathrm{E}-01 \\
3.44955 \mathrm{E}-01\end{array}$ & $\begin{array}{l}-3.27374 \mathrm{E}-04 \\
-3.28334 \mathrm{E}-04 \\
-3.29285 \mathrm{E}-04 \\
-3.30226 \mathrm{E}-04 \\
-3.31157 \mathrm{E}-04\end{array}$ & $\begin{array}{l}-3.07617 \mathrm{E}-01 \\
-3.11209 \mathrm{E}-01 \\
-3.14821 \mathrm{E}-01 \\
-3.18454 \mathrm{E}-01 \\
-3.22108 \mathrm{E}-01\end{array}$ \\
\hline $\begin{array}{l}19.60 \\
19.70 \\
19.80 \\
19.90 \\
20.00\end{array}$ & $\begin{array}{l}9.77454 \mathrm{E}-01 \\
9.77454 \mathrm{E}-01 \\
9.77455 \mathrm{E}-01 \\
9.77455 \mathrm{E}-01 \\
9.77455 \mathrm{E}-01\end{array}$ & $\begin{array}{l}1.89021 \mathrm{E} \\
1.90013 \mathrm{E} \\
1.91006 \mathrm{E} \\
1.91998 \mathrm{E} \\
1.92990 \mathrm{E}\end{array}$ & $\begin{array}{l}01 \\
01 \\
01 \\
01 \\
01\end{array}$ & $\begin{array}{l}3.48731 \mathrm{E}-01 \\
3.52529 \mathrm{E}-01 \\
3.56347 \mathrm{E}-01 \\
3.60185 \mathrm{E}-01 \\
3.64045 \mathrm{E}-01\end{array}$ & $\begin{array}{l}-3.32078 \mathrm{E}-04 \\
-3.32988 \mathrm{E}-04 \\
-3.33891 \mathrm{E}-04 \\
-3.34782 \mathrm{E}-04 \\
-3.35665 \mathrm{E}-04\end{array}$ & $\begin{array}{l}-3.25782 \mathrm{E}-01 \\
-3.29477 \mathrm{E}-01 \\
-3.33192 \mathrm{E}-01 \\
-3.36929 \mathrm{E}-01 \\
-3.40685 \mathrm{E}-01\end{array}$ \\
\hline $\begin{array}{l}20.10 \\
20.20 \\
20.30 \\
20.40 \\
20.50\end{array}$ & $\begin{array}{l}9.77455 \mathrm{E}-01 \\
9.77455 \mathrm{E}-01 \\
9.77455 \mathrm{E}-01 \\
9.77455 \mathrm{E}-01 \\
9.77455 \mathrm{E}-01\end{array}$ & $\begin{array}{l}1.93982 \mathrm{E} \\
1.94975 \mathrm{E} \\
1.95967 \mathrm{E} \\
1.96960 \mathrm{E} \\
1.97952 \mathrm{E}\end{array}$ & $\begin{array}{l}01 \\
01 \\
01 \\
01 \\
01\end{array}$ & $\begin{array}{l}3.67924 \mathrm{E}-01 \\
3.71825 \mathrm{E}-01 \\
3.75746 \mathrm{E}-01 \\
3.79688 \mathrm{E}-01 \\
3.83650 \mathrm{E}-01\end{array}$ & $\begin{array}{l}-3.36539 \mathrm{E}-04 \\
-3.37405 \mathrm{E}-04 \\
-3.38260 \mathrm{E}-04 \\
-3.39107 \mathrm{E}-04 \\
-3.39945 \mathrm{E}-04\end{array}$ & $\begin{array}{l}-3.44463 \mathrm{E}-01 \\
-3.48261 \mathrm{E}-01 \\
-3.52080 \mathrm{E}-01 \\
-3.55919 \mathrm{E}-01 \\
-3.59779 \mathrm{E}-01\end{array}$ \\
\hline $\begin{array}{l}20.60 \\
20.70 \\
20.80 \\
20.90 \\
21.00\end{array}$ & $\begin{array}{l}9.77455 \mathrm{E}-01 \\
9.77455 \mathrm{E}-01 \\
9.77455 \mathrm{E}-01 \\
9.77455 \mathrm{E}-01 \\
9.77455 \mathrm{E}-01\end{array}$ & $\begin{array}{l}1.98944 \mathrm{E} \\
1.99937 \mathrm{E} \\
2.00929 \mathrm{E} \\
2.01922 \mathrm{E} \\
2.02914 \mathrm{E}\end{array}$ & $\begin{array}{l}01 \\
01 \\
01 \\
01 \\
01\end{array}$ & $\begin{array}{l}3.87633 \mathrm{E}-01 \\
3.91637 \mathrm{E}-01 \\
3.95661 \mathrm{E}-01 \\
3.99707 \mathrm{E}-01 \\
4.03772 \mathrm{E}-01\end{array}$ & $\begin{array}{l}-3.40775 \mathrm{E}-04 \\
-3.41596 \mathrm{E}-04 \\
-3.42409 \mathrm{E}-04 \\
-3.43215 \mathrm{E}-04 \\
-3.44012 \mathrm{E}-04\end{array}$ & $\begin{array}{l}-3.63660 \mathrm{E}-01 \\
-3.67561 \mathrm{E}-01 \\
-3.71483 \mathrm{E}-01 \\
-3.75426 \mathrm{E}-01 \\
-3.79389 \mathrm{E}-01\end{array}$ \\
\hline $\begin{array}{l}21.10 \\
21.20 \\
21.30 \\
21.40 \\
21.50\end{array}$ & $\begin{array}{l}9.77455 \mathrm{E}-01 \\
9.77455 \mathrm{E}-01 \\
9.77455 \mathrm{E}-01 \\
9.77455 \mathrm{E}-01 \\
9.77455 \mathrm{E}-01\end{array}$ & $\begin{array}{l}2.03907 \mathrm{E} \\
2.04900 \mathrm{E} \\
2.05892 \mathrm{E} \\
2.06885 \mathrm{E} \\
2.07878 \mathrm{E}\end{array}$ & $\begin{array}{l}01 \\
01 \\
01 \\
01 \\
01\end{array}$ & $\begin{array}{l}4.07858 \mathrm{E}-01 \\
4.11965 \mathrm{E}-01 \\
4.16093 \mathrm{E}-01 \\
4.20241 \mathrm{E}-01 \\
4.24410 \mathrm{E}-01\end{array}$ & $\begin{array}{l}-3.44801 \mathrm{E}-04 \\
-3.45582 \mathrm{E}-04 \\
-3.46355 \mathrm{E}-04 \\
-3.47121 \mathrm{E}-04 \\
-3.47880 \mathrm{E}-04\end{array}$ & $\begin{array}{l}-3.83373 \mathrm{E}-01 \\
-3.87378 \mathrm{E}-01 \\
-3.91403 \mathrm{E}-01 \\
-3.95449 \mathrm{E}-01 \\
-3.99516 \mathrm{E}-01\end{array}$ \\
\hline $\begin{array}{l}21.60 \\
21.70 \\
21.80 \\
21.90 \\
22.00\end{array}$ & $\begin{array}{l}9.77455 \mathrm{E}-01 \\
9.77455 \mathrm{E}-01 \\
9.77454 \mathrm{E}-01 \\
9.77454 \mathrm{E}-01 \\
9.77454 \mathrm{E}-01\end{array}$ & $\begin{array}{l}2.08870 \mathrm{E} \\
2.09863 \mathrm{E} \\
2.10856 \mathrm{E} \\
2.11849 \mathrm{E} \\
2.12842 \mathrm{E}\end{array}$ & $\begin{array}{l}01 \\
01 \\
01 \\
01 \\
01\end{array}$ & $\begin{array}{l}4.28600 \mathrm{E}-01 \\
4.32810 \mathrm{E}-01 \\
4.37041 \mathrm{E}-01 \\
4.41293 \mathrm{E}-01 \\
4.45565 \mathrm{E}-01\end{array}$ & $\begin{array}{l}-3.48631 \mathrm{E}-04 \\
-3.49375 \mathrm{E}-04 \\
-3.50111 \mathrm{E}-04 \\
-3.50841 \mathrm{E}-04 \\
-3.51563 \mathrm{E}-04\end{array}$ & $\begin{array}{l}-4.03603 \mathrm{E}-01 \\
-4.07711 \mathrm{E}-01 \\
-4.11839 \mathrm{E}-01 \\
-4.15989 \mathrm{E}-01 \\
-4.20158 \mathrm{E}-01\end{array}$ \\
\hline $\begin{array}{l}22.10 \\
22.20 \\
22.30 \\
22.40 \\
22.50\end{array}$ & $\begin{array}{l}9.77454 \mathrm{E}-01 \\
9.77454 \mathrm{E}-01 \\
9.77454 \mathrm{E}-01 \\
9.77454 \mathrm{E}-01 \\
9.77454 \mathrm{E}-01\end{array}$ & $\begin{array}{l}2.13835 \mathrm{E} \\
2.14828 \mathrm{E} \\
2.15820 \mathrm{E} \\
2.16814 \mathrm{E} \\
2.17807 \mathrm{E}\end{array}$ & $\begin{array}{l}01 \\
01 \\
01 \\
01 \\
01\end{array}$ & $\begin{array}{l}4.49858 \mathrm{E}-01 \\
4.54171 \mathrm{E}-01 \\
4.58505 \mathrm{E}-01 \\
4.62860 \mathrm{E}-01 \\
4.67236 \mathrm{E}-01\end{array}$ & $\begin{array}{l}-3.52279 \mathrm{E}-04 \\
-3.52988 \mathrm{E}-04 \\
-3.53690 \mathrm{E}-04 \\
-3.54384 \mathrm{E}-04 \\
-3.55073 \mathrm{E}-04\end{array}$ & $\begin{array}{l}-4.24349 \mathrm{E}-01 \\
-4.28560 \mathrm{E}-01 \\
-4.32792 \mathrm{E}-01 \\
-4.37045 \mathrm{E}-01 \\
-4.41318 \mathrm{E}-01\end{array}$ \\
\hline $\begin{array}{l}22.60 \\
22.70 \\
22.80 \\
22.90 \\
23.00\end{array}$ & $\begin{array}{l}9.77454 \mathrm{E}-01 \\
9.77454 \mathrm{E}-01 \\
9.77454 \mathrm{E}-01 \\
9.77454 \mathrm{E}-01 \\
9.77454 \mathrm{E}-01\end{array}$ & $\begin{array}{l}2.18800 \mathrm{E} \\
2.19793 \mathrm{E} \\
2.20786 \mathrm{E} \\
2.21779 \mathrm{E} \\
2.22772 \mathrm{E}\end{array}$ & $\begin{array}{l}01 \\
01 \\
01 \\
01 \\
01\end{array}$ & $\begin{array}{l}4.71632 \mathrm{E}-01 \\
4.76049 \mathrm{E}-01 \\
4.80487 \mathrm{E}-01 \\
4.84945 \mathrm{E}-01 \\
4.89424 \mathrm{E}-01\end{array}$ & $\begin{array}{l}-3.55755 \mathrm{E}-04 \\
-3.56432 \mathrm{E}-04 \\
-3.57102 \mathrm{E}-04 \\
-3.57764 \mathrm{E}-04 \\
-3.58421 \mathrm{E}-04\end{array}$ & $\begin{array}{l}-4.45612 \mathrm{E}-01 \\
-4.49927 \mathrm{E}-01 \\
-4.54262 \mathrm{E}-01 \\
-4.58618 \mathrm{E}-01 \\
-4.62995 \mathrm{E}-01\end{array}$ \\
\hline $\begin{array}{l}23.10 \\
23.20 \\
23.30 \\
23.40 \\
23.50\end{array}$ & $\begin{array}{l}9.77454 \mathrm{E}-01 \\
9.77454 \mathrm{E}-01 \\
9.77454 \mathrm{E}-01 \\
9.77454 \mathrm{E}-01 \\
9.77454 \mathrm{E}-01\end{array}$ & $\begin{array}{l}2.23765 \mathrm{E} \\
2.24759 \mathrm{E} \\
2.25752 \mathrm{E} \\
2.26745 \mathrm{E} \\
2.27738 \mathrm{E}\end{array}$ & $\begin{array}{l}01 \\
01 \\
01 \\
01 \\
01\end{array}$ & $\begin{array}{l}4.93924 \mathrm{E}-01 \\
4.98444 \mathrm{E}-01 \\
5.02985 \mathrm{E}-01 \\
5.07547 \mathrm{E}-01 \\
5.12129 \mathrm{E}-01\end{array}$ & $\begin{array}{l}-3.59073 \mathrm{E}-04 \\
-3.59717 \mathrm{E}-04 \\
-3.60356 \mathrm{E}-04 \\
-3.60989 \mathrm{E}-04 \\
-3.61617 \mathrm{E}-04\end{array}$ & $\begin{array}{l}-4.67392 \mathrm{E}-01 \\
-4.71810 \mathrm{E}-01 \\
-4.76249 \mathrm{E}-01 \\
-4.80708 \mathrm{E}-01 \\
-4.85189 \mathrm{E}-01\end{array}$ \\
\hline $\begin{array}{l}23.60 \\
23.70 \\
23.80 \\
23.90 \\
24.00\end{array}$ & $\begin{array}{l}9.77454 \mathrm{E}-01 \\
9.77454 \mathrm{E}-01 \\
9.77454 \mathrm{E}-01 \\
9.77454 \mathrm{E}-01 \\
9.77454 \mathrm{E}-01\end{array}$ & $\begin{array}{l}2.28732 \mathrm{E} \\
2.29725 \mathrm{E} \\
2.30719 \mathrm{E} \\
2.31712 \mathrm{E} \\
2.32706 \mathrm{E}\end{array}$ & $\begin{array}{l}01 \\
01 \\
01 \\
01 \\
01\end{array}$ & $\begin{array}{l}5.16732 \mathrm{E}-01 \\
5.21356 \mathrm{E}-01 \\
5.26000 \mathrm{E}-01 \\
5.30665 \mathrm{E}-01 \\
5.35351 \mathrm{E}-01\end{array}$ & $\begin{array}{l}-3.62239 \mathrm{E}-04 \\
-3.62854 \mathrm{E}-04 \\
-3.63464 \mathrm{E}-04 \\
-3.64070 \mathrm{E}-04 \\
-3.64670 \mathrm{E}-04\end{array}$ & $\begin{array}{l}-4.89689 \mathrm{E}-01 \\
-4.94211 \mathrm{E}-01 \\
-4.98753 \mathrm{E}-01 \\
-5.03316 \mathrm{E}-01 \\
-5.07900 \mathrm{E}-01\end{array}$ \\
\hline
\end{tabular}

$g_{-1}$ $\tan \delta_{-1}$

$1.84037 \mathrm{E} \quad 01$ $1.85029 \mathrm{E}-01$ $1.86021 \mathrm{E} \quad 01$ $1.87014 \mathrm{E} \quad 01$ $1.88006 \mathrm{E} \quad 01$

$1.88999 \mathrm{E} \quad 01$ $1.89991 \mathrm{E} \quad 01$ $1.90983 \mathrm{E}$ ol $1.91976 \mathrm{E} \quad 01$ $1.92968 \mathrm{E} 01$

$1.93961 \mathrm{E} \quad 01$ $1.94953 \mathrm{E}$ 0I $1.95946 \mathrm{E}$ 01 $1.96938 \mathrm{E} \quad 01$ $1.97931 \mathrm{E} 01$

$1.98924 \mathrm{E} \quad 01$ $1.99916 \mathrm{E} \quad 01$ $2.00909 \mathrm{E} \quad 01$ $2.01902 \mathrm{E} \quad 01$ $2.02894 \mathrm{E} \quad 01$

$2.03887 \mathrm{E} \quad 01$ $2.04880 \mathrm{E} \quad 01$ $2.05873 \mathrm{E} 01$ $2.06866 \mathrm{E} \quad 01$ $2.07858 \mathrm{E}$ 01

$2.08851 \mathrm{E} \quad 01$ $2.09844 \mathrm{E} \quad 01$ $2.10837 \mathrm{E}$ 01 $2.11830 \mathrm{E} \quad 01$ $2.12823 \mathrm{E} 01$

$2.13816 \mathrm{E} \quad 01$ $2.14809 \mathrm{E} \quad 01$ $2.15803 \mathrm{E}$ ol $2.16796 \mathrm{E} \quad 01$ $2.17789 \mathrm{E}$ ol

$2.18782 \mathrm{E} \quad 01$ $2.19775 \mathrm{E} \quad 01$ $2.20769 \mathrm{E}$ 0l $2.21762 \mathrm{E} \quad 01$ $2.22755 \mathrm{E} \quad 01$

$2.23749 \mathrm{E} \quad 01$ $2.24742 \mathrm{E} \quad 0 \mathrm{l}$ $2.25735 \mathrm{E}$ 0l $2.26729 \mathrm{E} \quad 01$ $2.27722 \mathrm{E} 01$

$2.28716 \mathrm{E} \quad 01$ $2.29709 \mathrm{E} \quad 01$ $2.30703 \mathrm{E} \quad 01$ $2.31697 \mathrm{E} \quad 01$ $2.32690 \mathrm{E} 01$

$2.33684 \mathrm{E} \quad 01$ $2.34678 \mathrm{E} \quad 01$ $2.35672 \mathrm{E} \quad 01$ $2.36665 \mathrm{E} \quad 01$ $2.37659 \mathrm{E} 01$

$2.38653 \mathrm{E} \quad 01$ $2.39647 \mathrm{E} \quad 01$ $2.40641 \mathrm{E} \quad 01$ $2.41635 \mathrm{E} \quad 01$ $2.42629 \mathrm{E}$ 01
$1.38798 \mathrm{E} \quad 03$ $1.39018 \mathrm{E} \quad 03$ $1.39237 \mathrm{E} \quad 03$ $1.39454 \mathrm{E} \quad 03$ $1.39670 \mathrm{E} \quad 03$

$1.39884 \mathrm{E} \quad 03$ $1.40096 \mathrm{E} \quad 03$ $1.40307 \mathrm{E} \quad 03$ $1.40516 \mathrm{E} \quad 03$ $1.40724 \mathrm{E} \quad 03$

$1.40931 \mathrm{E} \quad 03$ $1.41136 \mathrm{E} \quad 03$ $1.41339 \mathrm{E} \quad 03$ $1.41541 \mathrm{E} \quad 03$ $1.41741 \mathrm{E} \quad 03$

$1.41941 \mathrm{E} \quad 03$ $1.42138 \mathrm{E} \quad 03$ $1.42335 \mathrm{E} \quad 03$ $1.42530 \mathrm{E} \quad 03$ $1.42723 \mathrm{E} \quad 03$

$1.42916 \mathrm{E} \quad 03$ $1.43107 \mathrm{E} \quad 03$ $1.43296 \mathrm{E} \quad 03$ $1.43485 \mathrm{E} \quad 03$ $1.43672 \mathrm{E} \quad 03$

$1.43858 \mathrm{E} \quad 03$ $1.44043 \mathrm{E} \quad 03$ $1.44226 \mathrm{E} \quad 03$ $1.44408 \mathrm{E} \quad 03$ $1.44589 \mathrm{E} \quad 03$

$1.44769 \mathrm{E} \quad 03$ $1.44948 \mathrm{E} \quad 03$ $1.45125 \mathrm{E} \quad 03$ $1.45302 \mathrm{E} \quad 03$ $1.45477 \mathrm{E} \quad 03$

$1.45651 \mathrm{E} \quad 03$ $1.45824 \mathrm{E} \quad 03$ $1.45996 \mathrm{E} \quad 03$ $1.46167 \mathrm{E} \quad 03$ $1.46336 \mathrm{E} \quad 03$

$1.46505 \mathrm{E} \quad 03$ $1.46672 \mathrm{E} \quad 03$ $1.46839 \mathrm{E} \quad 03$ $1.47005 \mathrm{E} \quad 03$ 1.47169E 03

$1.47333 \mathrm{E} \quad 03$ $1.47495 \mathrm{E} \quad 03$ $1.47657 \mathrm{E} \quad 03$ $1.47817 \mathrm{E} \quad 03$ $1.47977 \mathrm{E} \quad 03$

$1.48136 \mathrm{E} \quad 03$ $1.48293 \mathrm{E} \quad 03$ $1.48450 \mathrm{E} \quad 03$ $1.48606 \mathrm{E} \quad 03$ $1.48761 \mathrm{E} \quad 03$

$1.48915 \mathrm{E} \quad 03$ $1.49068 \mathrm{E} \quad 03$ $1.49221 \mathrm{E} \quad 03$ $1.49372 \mathrm{E} \quad 03$ $1.49522 \mathrm{E} \quad 03$ 
ELECTRON RADIAL FUNCTIONS

$Z=1 \quad A=1 \quad \rho=1.2 A^{1 / 3} F$

Positron

$\bar{F}$

$8.58935 \mathrm{E}-05$

$8.68452 \mathrm{E}-05$

$8.78022 \mathrm{E}-05$

$8.87645 \mathrm{E}-05$

$8.97319 \mathrm{E}-05$

18.40

$18.60 \quad 9.07047 \mathrm{E}-05$

$18.70 \quad 9.16826 \mathrm{E}-05$

$18.80 \quad 9.26658 \mathrm{E}-05$

$18.90 \quad 9.36543 \mathrm{E}-05$

$19.00 \quad 9.46480 \mathrm{E}-05$

19.10

19.20

19.30

19.40

19.50

19.60

19.70

19.80

19.90

20.00

20.10

20.20

20.30

20.40

20.50

20.60

20.70

20.80

20.90

21.00

21.10

21.20

21.30

21.40

21.50

21.60

21.70

21.80

21.90

22.00

22.10

22.20

22.30

22.40

22.50

22.60

22.70

22.80

22.90

23.00

23.10

23.20

23.30

23.40

23.50

23.60

23.70

23.80

23.90

24.00

$1.14524 \mathrm{E}-04$

$1.15623 \mathrm{E}-04$

$1.16727 \mathrm{E}-04$

$1.17836 \mathrm{E}-04$

$1.18950 \mathrm{E}-04$

$1.20070 \mathrm{E}-04$

$1.21194 \mathrm{E}-04$

$1.22324 \mathrm{E}-04$

$1.23460 \mathrm{E}-04$

$1.24600 \mathrm{E}-04$

$1.25746 \mathrm{E}-04$

$1.26897 \mathrm{E}-04$

$1.28053 \mathrm{E}-04$

$1.29215 \mathrm{E}-04$

$1.30381 \mathrm{E}-04$

$1.31553 \mathrm{E}-04$

$1.32731 \mathrm{E}-04$

$1.35101 \mathrm{E}-04$

$1.36294 \mathrm{E}-04$

$1.37492 \mathrm{E}-04$

$1.38695 \mathrm{E}-04$

$1.39904 \mathrm{E}-04$

$1.41118 \mathrm{E}-04$

$1.42337 \mathrm{E}-04$

$1.43561 \mathrm{E}-04$

1.44791 E-04

$1.46026 \mathrm{E}-04$

$1.47266 \mathrm{E}-04$

$1.48512 \mathrm{E}-04$.

$1.49762 \mathrm{E}-04$

$1.51018 \mathrm{E}-04$ $f_{2}$

$3.26263 \mathrm{E}-01$

$3.29929 \mathrm{E}-01$

$3.33616 \mathrm{E}-01$

$3.37324 \mathrm{E}-01$

$3.41052 \mathrm{E}-01$

$3.44800 \mathrm{E}-01$

$3.48569 \mathrm{E}-01$

$3.52358 \mathrm{E}-01$

$3.56168 \mathrm{E}-01$

$3.59998 \mathrm{E}-01$

$3.63849 \mathrm{E}-01$

$3.67720 \mathrm{E}-01$

$3.71611 \mathrm{E}-01$

$3.75523 \mathrm{E}-01$

$3.79456 \mathrm{E}-01$

$3.83409 \mathrm{E}-01$

$3.87382 \mathrm{E}-01$

$3.91376 \mathrm{E}-01$

$3.95390 \mathrm{E}-01$

$3.99425 \mathrm{E}-01$

$4.03480 \mathrm{E}-01$

$4.07556 \mathrm{E}-01$

$4.11652 \mathrm{E}-01$

4.15768E-01

$4.19906 \mathrm{E}-01$

$4.24063 \mathrm{E}-01$

$4.28241 \mathrm{E}-01$

$4.32439 \mathrm{E}-01$

$4.36658 \mathrm{E}-01$

$4.40897 \mathrm{E}-01$

$1.33913 \mathrm{E}-04$
$4.45157 \mathrm{E}-01$

$4.49437 \mathrm{E}-01$

$4.53738 \mathrm{E}-01$

$4.58059 \mathrm{E}-01$

$4.62401 \mathrm{E}-01$

$4.66763 \mathrm{E}-01$

$4.71145 \mathrm{E}-01$

4.75548E-01

$4.79971 \mathrm{E}-01$

$4.84415 \mathrm{E}-01$

$4.88879 \mathrm{E}-01$

$4.93364 \mathrm{E}-01$

$4.97869 \mathrm{E}-01$

$5.02394 \mathrm{E}-01$

$5.06940 \mathrm{E}-01$

$5.11507 \mathrm{E}-01$

$5.16094 \mathrm{E}-01$

$5.20701 \mathrm{E}-01$

$5.25329 \mathrm{E}-01$

$5.29977 \mathrm{E}-01$

$5.34646 \mathrm{E}-01$

$5.39335 \mathrm{E}-01$

$5.44044 \mathrm{E}-01$

$5.48774 \mathrm{E}-01$

$5.53525 \mathrm{E}-01$

$5.58295 \mathrm{E}-01$

$5.63087 \mathrm{E}-01$

$5.67898 \mathrm{E}-01$

5.72731 E-01

$5.77583 \mathrm{E}-01$ $\tan \delta_{2}$

$f_{2}$

$3.33464 \mathrm{E}-03$

$3.39259 \mathrm{E}-03$

$3.45121 \mathrm{E}-03$

$3.51051 \mathrm{E}-03$

$3.57047 \mathrm{E}-03$

$3.63112 \mathrm{E}-03$

$3.69244 \mathrm{E}-03$

$3.75446 \mathrm{E}-03$

$3.81716 \mathrm{E}-03$

$3.88056 \mathrm{E}-03$

$3.94465 \mathrm{E}-03$

$4.00945 \mathrm{E}-03$

$4.07495 \mathrm{E}-03$

$4.14116 \mathrm{E}-03$

$4.20809 \mathrm{E}-03$

$4.27573 \mathrm{E}-03$

4.34409E-03

$4.41318 \mathrm{E}-03$

$4.48299 \mathrm{E}-03$

$4.55354 \mathrm{E}-03$

$4.62482 \mathrm{E}-03$

$4.69684 \mathrm{E}-03$

$4.76961 \mathrm{E}-03$

$4.84312 \mathrm{E}-03$

$4.91738 \mathrm{E}-03$

4.99240E-03

$5.06818 \mathrm{E}-03$

$5.14472 \mathrm{E}-03$

$5.22203 \mathrm{E}-03$

$5.30010 \mathrm{E}-03$

$5.37895 \mathrm{E}-03$

$5.45858 \mathrm{E}-03$

$5.53899 \mathrm{E}-03$

$5.62019 \mathrm{E}-03$

$5.70217 \mathrm{E}-03$

$5.78495 \mathrm{E}-03$

$5.86852 \mathrm{E}-03$

$5.95290 \mathrm{E}-03$

$6.03808 \mathrm{E}-03$

$6.12407 \mathrm{E}-03$

$6.21087 \mathrm{E}-03$

$6.29848 \mathrm{E}-03$

$6.38692 \mathrm{E}-03$

$6.47618 \mathrm{E}-03$

$6.56627 \mathrm{E}-03$

$6.65719 \mathrm{E}-03$

$6.74 .894 \mathrm{E}-03$

$6.84153 \mathrm{E}-03$

$6.934 .96 \mathrm{E}-03$

7.02924 E-03

$7.12437 \mathrm{E}-03$

$7.22036 \mathrm{E}-03$

7.31720E-03

$7.414 .91 \mathrm{E}-03$

$7.51348 \mathrm{E}-03$

7.61292E-03

$7.71323 \mathrm{E}-03$

$7.81442 \mathrm{E}-03$

$7.91649 \mathrm{E}-03$

$8.01944 \mathrm{E}-03$

$-1.98307 \mathrm{E} \quad 02-3.09353 \mathrm{E}-03$

$\begin{array}{lll}-1.98332 \mathrm{E} & 02 & -3.14897 \mathrm{E}-03\end{array}$

$-1.98356 \mathrm{E} \quad 02 \quad-3.20507 \mathrm{E}-03$

$-1.98381 \mathrm{E} \quad 02 \quad-3.26182 \mathrm{E}-03$

$-1.98405 \mathrm{E} \quad 02 \quad-3.31924 \mathrm{E}-03$

$\begin{array}{lll}-1.98429 \mathrm{E} & 02 & -3.37732 \mathrm{E}-03\end{array}$

$\begin{array}{lll}-1.98453 \mathrm{E} & 02 & -3.43608 \mathrm{E}-03\end{array}$

$\begin{array}{lll}-1.98476 \mathrm{E} & 02 & -3.49550 \mathrm{E}-03\end{array}$

$\begin{array}{lll}-1.98499 \mathrm{E} & 02 & -3.55560 \mathrm{E}-03\end{array}$

$-1.98522 \mathrm{E} \quad 02 \quad-3.61639 \mathrm{E}-03$

$\begin{array}{lll}-1.98545 \mathrm{E} & 02 & -3.67786 \mathrm{E}-03\end{array}$

$-1.98567 \mathrm{E} \quad 02 \quad-3.74001 \mathrm{E}-03$

$-1.98589 \mathrm{E} \quad 02 \quad-3.80286 \mathrm{E}-03$

$-1.98611 \mathrm{E} \quad 02 \quad-3.86641 \mathrm{E}-03$

$\begin{array}{lll}-1.98633 \mathrm{E} & 02 & -3.93066 \mathrm{E}-03\end{array}$

$-1.98654 \mathrm{E} \quad 02$

$-1.98675 \mathrm{E} \quad 02$

$-1.98696 \mathrm{E} \quad 02$

$-1.98717 \mathrm{E} \quad 02$

$-1.98738 \mathrm{E} \quad 02$

$\begin{array}{ll}-1.98758 \mathrm{E} & 02\end{array}$

$-1.98778 \mathrm{E} \quad 02$

$-1.98798 \mathrm{E} \quad 02$

$-1.98818 \mathrm{E} \quad 02$

-1.98837 E 02

$-1.98857 \mathrm{E} \quad 02$

$-1.98876 \mathrm{E} \quad 02$

$-1.98895 \mathrm{E} \quad 02$

$-1.98913 \mathrm{E} 02$

$-1.98932 \mathrm{E} \quad 02$

$\begin{array}{ll}-1.98950 \mathrm{E} & 02\end{array}$

$-1.98969 \mathrm{E} \quad 02$

$-1.98987 \mathrm{E} \quad 02$

$-1.99004 \mathrm{E} \quad 02$

$-1.99022 \mathrm{E} \quad 02$

$-1.99040 \mathrm{E} \quad 02$ $-1.99057 \mathrm{E} 02$

$-1.99074 \mathrm{E} 02$

$-1.99091 \mathrm{E} \quad 02$

$-1.99108 \mathrm{E} 02$

$-1.99125 \mathrm{E} \quad 02$

$-1.99141 \mathrm{E} 02$

$-1.99158 \mathrm{E} 02$

$-1.99174 \mathrm{E} \quad 02$

$\begin{array}{ll}-1.99190 \mathrm{E} & 02\end{array}$

$-1.99206 \mathrm{E} \quad 02$

$-1.99222 \mathrm{E} \quad 02$

$-1.99237 \mathrm{E} 02$

$-1.99253 \mathrm{E} 02$

$-1.99268 \mathrm{E} 02$

$-3.99561 \mathrm{E}-03$

$-4.06126 \mathrm{E}-03$

$-4.12763 \mathrm{E}-03$

$-4.19471 \mathrm{E}-03$

$-4.26252 \mathrm{E}-03$

$-4.33104 \mathrm{E}-03$

$-4.40030 \mathrm{E}-03$

$-4.47028 \mathrm{E}-03$

$-4.54100 \mathrm{E}-03$

$-4.61245 \mathrm{E}-03$

$-4.68465 \mathrm{E}-03$

$-4.75759 \mathrm{E}-03$

$-4.83128 \mathrm{E}-03$

$-4.90573 \mathrm{E}-03$

$-4.98093 \mathrm{E}-03$

$-5.05690 \mathrm{E}-03$

$-5.13363 \mathrm{E}-03$

$-5.21112 \mathrm{E}-03$

$-5.28939 \mathrm{E}-03$

$-5.36844 \mathrm{E}-03$ 
ELECTRON RADIAL FUNCTIONS

Positron

$\begin{array}{cc}p & F_{0} \\ 24.10 & 9.77454 \mathrm{E}-01 \\ 24.20 & 9.77454 \mathrm{E}-01 \\ 24.30 & 9.77454 \mathrm{E}-01 \\ 24.40 & 9.77453 \mathrm{E}-01 \\ 24.50 & 9.77453 \mathrm{E}-01 \\ & \\ 24.60 & 9.77453 \mathrm{E}-01 \\ 24.70 & 9.77453 \mathrm{E}-01 \\ 24.80 & 9.77453 \mathrm{E}-01 \\ 24.90 & 9.77453 \mathrm{E}-01 \\ 25.00 & 9.77453 \mathrm{E}-01 \\ & \\ 25.10 & 9.77453 \mathrm{E}-01 \\ 25.20 & 9.77453 \mathrm{E}-01 \\ 25.30 & 9.77453 \mathrm{E}-01 \\ 25.40 & 9.77453 \mathrm{E}-01 \\ 25.50 & 9.77453 \mathrm{E}-01\end{array}$

25.60

25.70

25.80

25.90

26.00

26.10

26.20

26.30

26.40
26.50

26.60

26.70

26.80

26.90

27.00

27.10

27.20

27.30

27.40

27.50

27.60

27.70

27.80

27.90

28.00

28.10

28.20

28.30

28.40

28.50

28.60

28.70

28.80

28.90

29.00

29.10

29.20

29.30

29.40

29.50

29.60

29.70

29.80

29.90

30.00

$9.77453 \mathrm{E}-01$ 9.77453 E-01 $9.77453 \mathrm{E}-01$ $9.77452 \mathrm{E}-01$ $9.77452 \mathrm{E}-01$

$9.77452 \mathrm{E}-01$ $9.77452 \mathrm{E}-01$ $9.77452 \mathrm{E}-01$ $9.77452 \mathrm{E}-01$ 9.77452 E- 01

9.77452 E-01 $9.77452 \mathrm{E}-01$ $9.77452 \mathrm{E}-01$ 9.77452 E-01 $9.77452 \mathrm{E}-01$

$9.77452 \mathrm{E}-01$ 9.77451 E-01 $9.77451 \mathrm{E}-01$ 9.77451 E-01 9.77451 E-01

$9.77451 \mathrm{E}-01$ $9.77451 \mathrm{E}-01$ $9.77451 \mathrm{E}-01$ 9.77451 E-01 9.77451 E-01

9.77451 E-01 9.77451 E-01 $9.77451 \mathrm{E}-01$ $9.77450 \mathrm{E}-01$ $9.77450 \mathrm{E}-01$

$9.77450 \mathrm{E}-01$ $9.77450 \mathrm{E}-01$ $9.77450 \mathrm{E}-01$ $9.77450 \mathrm{E}-01$ 9.77450E-01

$9.77450 \mathrm{E}-01$ $9.77450 \mathrm{E}-01$ $9.77450 \mathrm{E}-01$ $9.77450 \mathrm{E}-01$ $9.77450 \mathrm{E}-01$

9.774449E-01 $9.77449 \mathrm{E}-01$ $9.77449 \mathrm{E}-01$ $9.77449 \mathrm{E}-01$ 9.77449E-01

$Z=1$

$$
A=1
$$$$
\rho=1.2 A^{1 / 3} F
$$

$f_{1}$

$2.33699 \mathrm{E}$ $2.34693 \mathrm{E}$ ol $2.35687 \mathrm{E}$ ol $2.36680 \mathrm{E}$ ol $2.37674 \mathrm{E} \quad 01$

$2.38668 \mathrm{E} \quad 01$ $2.39661 \mathrm{E} 01$ $2.40655 \mathrm{E} \quad 01$ $2.41649 \mathrm{E} 01$

$2.42643 \mathrm{E}$ ol

$2.43637 \mathrm{E} \quad 01$ $2.44631 \mathrm{E} 01$ $2.45625 \mathrm{E} \quad 01$ $2.46619 \mathrm{E} 01$ $2.47613 \mathrm{E} 01$
$2.48607 \mathrm{E} \quad 01$

$2.49601 \mathrm{E} 01$ $2.50595 \mathrm{E} 01$ $2.51589 \mathrm{E} \quad 01$ $2.52584 \mathrm{E} \quad 01$

$2.53578 \mathrm{E} \quad 01$ $2.54572 \mathrm{E} \quad 01$ $2.55566 \mathrm{E} \quad 01$ $2.56561 \mathrm{E} 01$ $2.57555 \mathrm{E} \quad 01$

$2.58550 \mathrm{E} \quad 01$ $2.59544 \mathrm{E}$ 01 $2.60539 \mathrm{E} \quad 01$ $2.61533 \mathrm{E} \quad 01$ $2.62528 \mathrm{E} 01$

$2.63523 \mathrm{E} \quad 01$ $2.64517 \mathrm{E} \quad 01$ $2.65512 \mathrm{E} \quad 01$ $2.66507 \mathrm{E} \quad 01$ 2.67502 E 01

$2.68496 \mathrm{E} \quad 01$ $2.69491 \mathrm{E} 01$ $2.70486 \mathrm{E} 01$ $2.71481 \mathrm{E} 01$ $2.72476 \mathrm{E} \quad 01$

$2.73471 \mathrm{E} 01$ $2.74466 \mathrm{E} \quad 01$ $2.77452 \mathrm{E} \quad 01$

$2.78447 \mathrm{E} \quad 01$ $2.79443 \mathrm{E} \quad 01$ $2.80438 \mathrm{E} \quad 01$ $2.81433 \mathrm{E} \quad 01$ $2.82429 \mathrm{E} \quad 01$

$2.83424 \mathrm{E} \quad 01$ $2.84420 \mathrm{E} \quad 01$ $2.85415 \mathrm{E}$ 01 $2.86411 \mathrm{E} 01$ $2.87406 \mathrm{E}$ ol

$2.88402 \mathrm{E} \quad 01$ $2.89398 \mathrm{E} 01$ $2.90394 \mathrm{E} \quad 01$ $2.91389 \mathrm{E} \quad 01$ $2.92385 \mathrm{E} 01$ $2.75462 \mathrm{E} \quad 01$ $2.76457 \mathrm{E} \quad 01$ $g_{1} \quad \tan \delta_{1}$

$5.40058 \mathrm{E}-01 \quad-3.65264 \mathrm{E}-04$

$5.44785 \mathrm{E}-01-3.65853 \mathrm{E}-04$

$5.49533 \mathrm{E}-01 \quad-3.66437 \mathrm{E}-04$

$5.54302 \mathrm{E}-01-3.67016 \mathrm{E}-04$

$5.59091 \mathrm{E}-01$

$-3.67588 \mathrm{E}-04$

$5.63901 \mathrm{E}-01$

$-3.68157 \mathrm{E}-04$

$5.68732 \mathrm{E}-01$

$5.73584 \mathrm{E}-01$

$5.78456 \mathrm{E}-01$

$5.83349 \mathrm{E}-01$

$-3.68720 \mathrm{E}-04$

$-3.69279 \mathrm{E}-04$

$-3.69833 \mathrm{E}-04$

$-3.70381 \mathrm{E}-04$

$5.88263 \mathrm{E}-01$

$5.93197 \mathrm{E}-01$

$5.98152 \mathrm{E}-01$

$6.03128 \mathrm{E}-01$

$6.08124 \mathrm{E}-01$

$-3.70926 \mathrm{E}-04$

$-3.71464 \mathrm{E}-04$

$-3.71999 \mathrm{E}-04$

$-3.72529 \mathrm{E}-04$

$-3.73054 \mathrm{E}-04$

$6.13142 \mathrm{E}-01$

$6.18180 \mathrm{E}-01$

$6.23238 \mathrm{E}-01$

$6.28318 \mathrm{E}-01$

$6.33418 \mathrm{E}-01$

$6.38539 \mathrm{E}-01$

$6.43681 \mathrm{E}-01$

$6.48843 \mathrm{E}-01$

$6.54026 \mathrm{E}-0]$

$6.59230 \mathrm{E}-01$

$6.64455 \mathrm{E}-01$

$6.69700 \mathrm{E}-01$

$6.74966 \mathrm{E}-01$

$6.80253 \mathrm{E}-01$

$6.85561 \mathrm{E}-01$

$6.90890 \mathrm{E}-01$

$6.96239 \mathrm{E}-01$

$7.01609 \mathrm{E}-01$

$7.06999 \mathrm{E}-01$

7.12411 E-01

$7.17843 \mathrm{E}-01$

$7.23296 \mathrm{E}-01$

$7.28770 \mathrm{E}-01$

7.34264E-01

$7.39780 \mathrm{E}-01$

$7.45316 \mathrm{E}-01$

$7.50873 \mathrm{E}-01$

$7.56450 \mathrm{E}-01$

$7.62049 \mathrm{E}-01$

$7.67668 \mathrm{E}-01$

$7.73308 \mathrm{E}-01$

$7.78969 \mathrm{E}-01$

$7.84651 \mathrm{E}-01$

$7.90353 \mathrm{E}-01$

$7.96076 \mathrm{E}-01$

$8.01820 \mathrm{E}-01$

$8.07585 \mathrm{E}-01$

$8.13370 \mathrm{E}-01$

8.19177E-01

8.25004E-01

$8.30852 \mathrm{E}-01$

8.3672l E-01

$8.42611 \mathrm{E}-01$

$8.48521 \mathrm{E}-01$

8.54452 E-01

$-3.73576 \mathrm{E}-04$

$-3.74092 \mathrm{E}-04$

$-3.74604 \mathrm{E}-04$

$-3.75112 \mathrm{E}-04$

$-3.75616 \mathrm{E}-04$

$-3.76114 \mathrm{E}-04$

$-3.76609 \mathrm{E}-04$

$-3.77100 \mathrm{E}-04$

$-3.77587 \mathrm{E}-04$

-3.78070 E-04

$-3.78549 \mathrm{E}-04$

$-3.79023 \mathrm{E}-04$

$-3.79493 \mathrm{E}-04$

$-3.79960 \mathrm{E}-04$

$-3.80423 \mathrm{E}-04$

$-3.80882 \mathrm{E}-04$ $-3.81337 \mathrm{E}-04$ $-3.81789 \mathrm{E}-04$ $-3.82237 \mathrm{E}-04$

$-3.82681 \mathrm{E}-04$

$-3.83122 \mathrm{E}-04$

$-3.83558 \mathrm{E}-04$

-3.83992 E-04

$-3.84423 E-04$

$-3.84849 \mathrm{E}-04$

-3.85272 E-04

$-3.85692 \mathrm{E}-04$

$-3.86108 \mathrm{E}-04$

$-3.86521 \mathrm{E}-04$

$-3.86930 \mathrm{E}-04$

$-3.87337 \mathrm{E}-04$

$-3.87740 \mathrm{E}-04$

$-3.88140 \mathrm{E}-04$

$-3.88537 \mathrm{E}-04$

$-3.88931 \mathrm{E}-04$

$-3.89321 \mathrm{E}-04$

$-3.89709 \mathrm{E}-04$

$-3.90093 \mathrm{E}-04$

$-3.90474 \mathrm{E}-04$

$-3.90853 \mathrm{E}-04$

$-3.91229 \mathrm{E}-04$

-3.91601 E-04

$-3.91971 \mathrm{E}-04$

$-3.92337 \mathrm{E}-04$

$f_{-1}$

$-5.12504 \mathrm{E}-01$

$-5.17129 \mathrm{E}-01$

$-5.21775 \mathrm{E}-01$

$-5.26442 \mathrm{E}-01$

$-5.31129 \mathrm{E}-01$

$-5.35837 \mathrm{E}-01$

$-5.40565 \mathrm{E}-01$

$-5.45315 \mathrm{E}-01$

$-5.50085 \mathrm{E}-01$

-5.54875 E-01

$-5.59687 \mathrm{E}-01$

$-5.64519 \mathrm{E}-01$

$-5.69372 \mathrm{E}-0 \mathrm{l}$

$-5.74246 \mathrm{E}-01$

-5.79140 E-01 
$\bar{F}$

$f_{2}$

$1.52279 \mathrm{E}-04$

$1.53546 \mathrm{E}-04$

$1.54817 \mathrm{E}-04$

$1.56094 \mathrm{E}-04$

$1.57376 \mathrm{E}-04$

24.40

$1.58663 \mathrm{E}-04$

$1.59956 \mathrm{E}-04$

$1.61254 \mathrm{E}-04$

$1.62557 \mathrm{E}-04$

$1.63865 \mathrm{E}-04$

24.90

25.00

$1.65179 \mathrm{E}-04$

$1.66498 \mathrm{E}-04$

$1.67822 \mathrm{E}-04$

$1.69151 \mathrm{E}-04$

$1.70485 \mathrm{E}-04$

25.40

25.50

$25.60 \quad 1.71825 \mathrm{E}-04$

$25.70 \quad 1.73170 \mathrm{E}-04$

$25.80 \quad 1.74520 \mathrm{E}-04$

$25.90 \quad 1.75876 \mathrm{E}-04$

$26.00 \quad 1.77237 \mathrm{E}-04$

26.10

26.20

26.30

26.40

26.50

26.60

26.70

26.80

26.90

27.00

27.10

27.20

27.30

27.40

27.50

27.60

27.70

27.80

27.90

28.00

28.10

28.20

28.30

28.40

28.50

28.60

28.70

28.80

28.90

29.00

29.10

29.20

29.30

29.40

29.50

29.60

29.70

29.80

29.90

30.00
$1.78603 \mathrm{E}-04$

$1.79974 \mathrm{E}-04$

$1.81350 \mathrm{E}-04$

$1.82732 \mathrm{E}-04$

$1.84119 \mathrm{E}-04$

$1.85511 \mathrm{E}-04$

$1.86909 \mathrm{E}-04$

$1.88311 \mathrm{E}-04$

$1.89719 \mathrm{E}-04$

$1.91132 \mathrm{E}-04$

1.92551 E- 04

$1.93975 \mathrm{E}-04$

$1.95403 \mathrm{E}-04$

$1.96838 \mathrm{E}-04$

$1.98277 \mathrm{E}-04$

$1.99722 \mathrm{E}-04$

$2.01171 \mathrm{E}-04$

$2.02627 \mathrm{E}-04$

$2.04087 \mathrm{E}-04$

2.05553 E- 04

$2.07023 \mathrm{E}-04$

$2.08500 \mathrm{E}-04$

$2.09981 \mathrm{E}-04$

$2.11467 \mathrm{E}-04$

$2.12959 \mathrm{E}-04$

2.14456 E- 04 2.15959 E-04

$2.17466 \mathrm{E}-04$

2.18979 E-04

2.20497 E-04

2.22020 E- 04

$2.23549 \mathrm{E}-04$

2.25083 E- 04

$2.26622 \mathrm{E}-04$

$2.28166 \mathrm{E}-04$

$2.29716 \mathrm{E}-04$

$2.31270 \mathrm{E}-04$

$2.32830 \mathrm{E}-04$

$2.34395 \mathrm{E}-04$

$2.35966 \mathrm{E}-04$
$5.82457 \mathrm{E}-01$

$5.87350 \mathrm{E}-01$

5.92264 E-01

$5.97198 \mathrm{E}-01$

6.02153 E-01

$6.07128 \mathrm{E}-01$

6.12124 E-01

$6.17140 \mathrm{E}-01$

$6.22176 \mathrm{E}-01$

$6.27233 \mathrm{E}-01$

$6.32310 \mathrm{E}-01$

$6.37408 \mathrm{E}-01$

$6.42527 \mathrm{E}-01$

$6.47665 \mathrm{E}-01$

$6.52824 \mathrm{E}-01$

6.58004 E- 01

$6.63204 \mathrm{E}-01$

$6.68424 \mathrm{E}-01$

$6.73665 \mathrm{E}-01$

$6.78926 \mathrm{E}-01$

$6.84208 \mathrm{E}-01$

$6.89510 \mathrm{E}-01$

$6.94832 \mathrm{E}-01$

$7.00175 \mathrm{E}-01$

$7.05539 \mathrm{E}-01$

$7.10922 \mathrm{E}-01$

$7.16326 \mathrm{E}-01$

$7.21751 \mathrm{E}-01$

$7.27196 \mathrm{E}-01$

$7.32662 \mathrm{E}-01$

$7.38147 \mathrm{E}-01$

$7.43654 \mathrm{E}-01$

$7.49180 \mathrm{E}-01$

$7.54728 \mathrm{E}-01$

$7.60295 \mathrm{E}-01$

$7.65883 \mathrm{E}-01$

$7.71491 \mathrm{E}-01$

$7.77120 \mathrm{E}-01$

$7.82769 \mathrm{E}-01$

$7.88439 \mathrm{E}-01$

$7.94129 \mathrm{E}-01$

$7.99840 \mathrm{E}-01$

$8.05570 \mathrm{E}-01$

$8.11322 \mathrm{E}-01$

$8.17093 \mathrm{E}-01$

$8.22885 \mathrm{E}-01$ $8.28698 \mathrm{E}-01$ 8.34531 E-01 $8.40384 \mathrm{E}-01$ $8.46258 \mathrm{E}-01$

$8.52152 \mathrm{E}-01$

$8.58067 \mathrm{E}-01$

$8.64002 \mathrm{E}-01$

$8.69957 \mathrm{E}-01$

$8.75933 \mathrm{E}-01$

$8.81929 \mathrm{E}-01$

$8.87946 \mathrm{E}-0 \mathrm{l}$

$8.93983 \mathrm{E}-01$

$9.00040 \mathrm{E}-01$

$9.06118 \mathrm{E}-01$ $g_{2} \quad \tan \delta_{2}$

$f_{-2}$

$8.12329 \mathrm{E}-03$

$8.22802 \mathrm{E}-03$

$8.33365 \mathrm{E}-03$

$8.44018 \mathrm{E}-03$

$8.54762 \mathrm{E}-03$

$8.65596 \mathrm{E}-03$

$8.76521 \mathrm{E}-03$

$8.87538 \mathrm{E}-03$

$8.98646 \mathrm{E}-03$

$9.09847 \mathrm{E}-03$

9.21141 E- 03

$9.32527 \mathrm{E}-03$

$9.44007 \mathrm{E}-03$

$9.55581 \mathrm{E}-03$

$9.67249 \mathrm{E}-03$

$9.79011 \mathrm{E}-03$

$9.90868 \mathrm{E}-03$

$1.00282 \mathrm{E}-02$

$1.01487 \mathrm{E}-02$

$1.02701 \mathrm{E}-02$

$1.03925 \mathrm{E}-02$

$1.05159 \mathrm{E}-02$

$1.06403 \mathrm{E}-02$

$1.07656 \mathrm{E}-02$

$1.08919 \mathrm{E}-02$

$1.10192 \mathrm{E}-02$

$1.11475 \mathrm{E}-02$

$1.12767 \mathrm{E}-02$

$1.14070 \mathrm{E}-02$

$1.15382 \mathrm{E}-02$

$1.16705 \mathrm{E}-02$

$1.18038 \mathrm{E}-02$

$1.19381 \mathrm{E}-02$

$1.20733 \mathrm{E}-02$

$1.22097 \mathrm{E}-02$

$1.23470 \mathrm{E}-02$

$1.24853 \mathrm{E}-02$

$1.26247 \mathrm{E}-02$

$1.27651 \mathrm{E}-02$

$1.29066 \mathrm{E}-02$

$1.30491 \mathrm{E}-02$

$1.31926 \mathrm{E}-02$

1.33372 E-02

$1.34829 \mathrm{E}-02$

$1.36296 \mathrm{E}-02$

$1.37773 \mathrm{E}-02$

$1.39261 \mathrm{E}-02$

$1.40760 \mathrm{E}-02$

$1.42270 \mathrm{E}-02$

$1.43790 \mathrm{E}-02$

$1.45321 \mathrm{E}-02$

$1.46863 \mathrm{E}-02$

$1.48416 \mathrm{E}-02$

$1.49980 \mathrm{E}-02$

$1.51554 \mathrm{E}-02$

$1.53140 \mathrm{E}-02$

$1.54737 \mathrm{E}-02$

$1.56344 \mathrm{E}-02$

$1.57963 \mathrm{E}-02$

$1.59593 \mathrm{E}-02$

$\begin{array}{ll}-1.99429 \mathrm{E} & 02 \\ -1.99443 \mathrm{E} & 02 \\ -1.99456 \mathrm{E} & 02 \\ -1.99470 \mathrm{E} & 02 \\ -1.99483 \mathrm{E} & 02\end{array}$

$-7.70857 \mathrm{E}-03$
$-7.81001 \mathrm{E}-03$
$-7.91234 \mathrm{E}-03$
$-8.01555 \mathrm{E}-03$
$-8.11966 \mathrm{E}-03$

$-1.99497 \mathrm{E} \quad 02$

$-1.99510 \mathrm{E} 02$

$-1.99523 \mathrm{E} \quad 02$

$-1.99536 \mathrm{E} \quad 02$

$-1.99549 \mathrm{E} \quad 02$

$-1.99562 \mathrm{E} \quad 02$

$-1.99575 \mathrm{E} \quad 02$

$-1.99587 \mathrm{E} \quad 02$

$-1.99600 \mathrm{E} 02$

-1.99612 E 02

$-1.99625 \mathrm{E} \quad 02$

$-1.99637 \mathrm{E} 02$

$-1.99649 \mathrm{E} 02$

-1.99673 E 02

$-1.99685 \mathrm{E} \quad 02$

$-1.99696 \mathrm{E} \quad 02$

$-1.99708 \mathrm{E} \quad 02$

$-1.99720 \mathrm{E} \quad 02$

-1.99731 E 02

$-1.99742 \mathrm{E} \quad 02$

$-1.99754 \mathrm{E} \quad 02$

$-1.99765 \mathrm{E} 02$

$-1.99776 \mathrm{E} \quad 02$

$\begin{array}{ll}-1.99787 \mathrm{E} & 02\end{array}$

$-1.99798 \mathrm{E} \quad 02$

$-1.99809 \mathrm{E} 02$

$-1.99820 \mathrm{E} 02$

$-1.99830 \mathrm{E} 02$

$-1.99841 \mathrm{E} 02$

-1.99852 E 02

$-1.99862 \mathrm{E} 02$

$-1.99872 \mathrm{E} 02$

-1.99883 E 02

$-1.99893 \mathrm{E} 02$

$-1.99903 \mathrm{E} 02$

$-1.99913 \mathrm{E} 02$

$-1.99923 \mathrm{E} \quad 02$

$-1.99933 \mathrm{E} 02$

-1.99943 E 02

-1.99953 E 02

$-1.99963 \mathrm{E} \quad 02$

$-1.99972 \mathrm{E} \quad 02$

$-1.99982 \mathrm{E} \quad 02$

$-1.99991 \mathrm{E} 02$

$-2.00001 \mathrm{E} \quad 02$

$-2.00010 \mathrm{E} \quad 02$

$-2.00019 \mathrm{E} \quad 02$

$-2.00029 \mathrm{E} \quad 02$

$-2.00038 \mathrm{E} 02$

$-2.00047 \mathrm{E} \quad 02$

$-2.00056 \mathrm{E} \quad 02$

-2.00065 E 02

$-2.00074 \mathrm{E} \quad 02$

$-2.00083 \mathrm{E} 02$
$-1.99661 \mathrm{E} 02$ 
ELECTRON RADIAL FUNCTIONS

Positron

$\begin{array}{cc}p & F_{0} \\ 30.10 & 9.77449 \mathrm{E}-01 \\ 30.20 & 9.77449 \mathrm{E}-01 \\ 30.30 & 9.77449 \mathrm{E}-01 \\ 30.40 & 9.77449 \mathrm{E}-01 \\ 30.50 & 9.77449 \mathrm{E}-01\end{array}$

30.60

30.70

30.80

30.90

31.00

31.10

31.20

31.30

31.40

31.50

31.60

31.70

31.80

31.90

32.00

32.10

32.20

32.30

32.40

32.50

32.60

32.70

32.80

32.90

33.00

33.10

33.20

33.30

33.40

33.50

33.60

33.70

33.80

33.90

34.00

34.10

34.20

34.30

34.40

34.50

34.60

34.70

34.80

34.90

35.00

35.10

35.20

35.30

35.40

35.50

35.60

35.70

35.80

35.90

36.00

$9.77448 \mathrm{E}-01$ $9.77448 \mathrm{E}-01$ $9.77448 \mathrm{E}-01$ $9.77448 \mathrm{E}-01$ $9.77448 \mathrm{E}-01$

$9.77448 \mathrm{E}-01$ $9.77448 \mathrm{E}-01$ $9.77448 \mathrm{E}-01$ $9.77448 \mathrm{E}-01$ $9.77448 \mathrm{E}-01$

$9.77448 \mathrm{E}-01$ $9.77447 \mathrm{E}-0 \mathrm{I}$ $9.77447 \mathrm{E}-01$ $9.77447 \mathrm{E}-01$ $9.77447 \mathrm{E}-01$

$9.77447 \mathrm{E}-01$ $9.77447 \mathrm{E}-01$ $9.77447 \mathrm{E}-01$ $9.77447 \mathrm{E}-01$ $9.77447 \mathrm{E}-01$

$9.77447 \mathrm{E}-01$ $9.77447 \mathrm{E}-01$ $9.77446 \mathrm{E}-01$ $9.77446 \mathrm{E}-01$

$9.77446 \mathrm{E}-01$ $9.77446 \mathrm{E}-01$ $9.77446 \mathrm{E}-01$ $9.77446 \mathrm{E}-01$ $9.77446 \mathrm{E}-01$

$9.77446 \mathrm{E}-01$ $9.77446 \mathrm{E}-01$ $9.77446 \mathrm{E}-01$ $9.77445 \mathrm{E}-01$ $9.77445 \mathrm{E}-01$

$9.77445 \mathrm{E}-01$ $9.77445 \mathrm{E}-01$ $9.77445 \mathrm{E}-01$ $9.77445 \mathrm{E}-01$ $9.77445 \mathrm{E}-01$

$9.77445 \mathrm{E}-01$ $9.77445 \mathrm{E}-01$ $9.77445 \mathrm{E}-01$ $9.77444 \mathrm{E}-01$ $9.77444 \mathrm{E}-01$

$9.77444 \mathrm{E}-01$ $9.77444 \mathrm{E}-01$ $9.77444 \mathrm{E}-01$ $9.77444 \mathrm{E}-01$ 9.77444 E-01

$9.77444 \mathrm{E}-01$ $9.77444 \mathrm{E}-01$ $9.77443 \mathrm{E}-01$ $9.77443 \mathrm{E}-01$

$Z=1 \quad A=1$

$\rho=1.2 A^{1 / 3} F$

$f_{1}$

$2.93381 \mathrm{E} \quad 01$ $2.94377 \mathrm{E}$ 01 $2.95373 \mathrm{E} 01$ $2.96369 \mathrm{E} 01$ $2.97365 \mathrm{E} 01$ 9.77446E-01 9.77444 E-01

$2.98361 \mathrm{E} \quad 01$ $2.99357 \mathrm{E} \quad 01$

$3.00354 \mathrm{E} \quad 01$

$3.01350 \mathrm{E} \quad 01$

$3.02346 \mathrm{E} 01$

$3.03343 \mathrm{E} \quad 01$ $3.04339 \mathrm{E} 01$ $3.05335 \mathrm{E} \quad 01$ $3.06332 \mathrm{E} \quad 01$ $3.07328 \mathrm{E} \quad 01$

$3.08325 \mathrm{E} \quad 01$ $3.09321 \mathrm{E} 01$

$3.10318 \mathrm{E} \quad 01$

$3.11315 \mathrm{E} 01$

$3.12312 \mathrm{E} 01$

$3.13308 \mathrm{E} \quad 01$ $3.14305 \mathrm{E} 01$

$3.15302 \mathrm{E} \quad 01$

$3.16299 \mathrm{E} 01$

$3.17296 \mathrm{E} \quad 01$

$3.18293 \mathrm{E} \quad 01$ $3.19290 \mathrm{E} 01$ $3.20287 \mathrm{E} \quad 01$ $3.21285 \mathrm{E} \quad 01$

$3.22282 \mathrm{E} \quad 01$

$3.23279 \mathrm{E} \quad 01$ $3.24276 \mathrm{E} \quad 01$

$3.25274 \mathrm{E} \quad 01$

$3.26271 \mathrm{E} 01$

$3.27269 \mathrm{E} 01$

$3.28266 \mathrm{E} \quad 01$ $3.29264 \mathrm{E} \quad 01$ $3.30261 \mathrm{E} 01$ $3.31259 \mathrm{E} 01$

$3.32257 \mathrm{E} \quad 01$

$3.33254 \mathrm{E} \quad 01$ $3.34252 \mathrm{E} 01$ $3.35250 \mathrm{E} 01$

$3.36248 \mathrm{E} \quad 01$

$3.37246 \mathrm{E} 01$

$3.38244 \mathrm{E} \quad 01$

$3.39242 \mathrm{E} 01$

$3.40240 \mathrm{E} 01$

$3.41238 \mathrm{E} 01$

$3.42236 \mathrm{E} 01$

$3.43235 \mathrm{E} \quad 01$

$3.44233 \mathrm{E} \quad 01$

$3.45231 \mathrm{E} 01$

$3.46230 \mathrm{E} 01$

$3.47228 \mathrm{E} 01$

$3.48227 \mathrm{E} \quad 01$ $3.49225 \mathrm{E} \quad 01$ $3.50224 \mathrm{E} \quad 01$

$3.51223 \mathrm{E} 01$ $3.52221 \mathrm{E} \quad 01$ $g_{1}$

8.60405 E-01

$8.66378 \mathrm{E}-01$

$8.72371 \mathrm{E}-01$

$8.78386 \mathrm{E}-01$

8.84421 E-01
$8.90478 \mathrm{E}-01$

$8.96555 \mathrm{E}-01$

$9.02653 \mathrm{E}-01$

$9.08771 \mathrm{E}-01$

$9.14911 \mathrm{E}-01$

$9.21071 \mathrm{E}-01$

$9.27253 \mathrm{E}-01$

9.33455E-01

$9.39678 \mathrm{E}-01$

$9.45922 \mathrm{E}-01$

$9.52186 \mathrm{E}-01$

$9.58472 \mathrm{E}-01$

$9.64778 \mathrm{E}-01$

$9.71105 \mathrm{E}-01$

$9.77453 \mathrm{E}-01$

$9.83822 \mathrm{E}-0 \mathrm{l}$

$9.90212 \mathrm{E}-01$

$9.96623 \mathrm{E}-01$

$1.00951 \mathrm{E} \quad 00$

$1.01598 \mathrm{E} \quad 00$

$1.02247 \mathrm{E} \quad 00$

$1.02899 \mathrm{E} \quad 00$

$1.03553 \mathrm{E} \quad 00$

$1.04208 \mathrm{E} \quad 00$

$1.04866 \mathrm{E} \quad 00$

$1.05526 \mathrm{E} \quad 00$

$1.06188 \mathrm{E} \quad 00$

$1.06852 \mathrm{E} \quad 00$

$1.07518 \mathrm{E} \quad 00$

$1.08186 \mathrm{E} \quad 00$

$1.08857 \mathrm{E} \quad 00$

$1.09529 \mathrm{E} \quad 00$

$1.10204 \mathrm{E} \quad 00$

$1.10880 \mathrm{E} \quad 00$

$1.11559 \mathrm{E} \quad 00$

$1.12240 \mathrm{E} \quad 00$

$1.12922 \mathrm{E} \quad 00$

$1.13607 \mathrm{E} \quad 00$

$1.14294 \mathrm{E} \quad 00$

$1.14984 \mathrm{E} \quad 00$

$1.15675 \mathrm{E} \quad 00$

$1.16368 \mathrm{E} \quad 00$

$1.17064 \mathrm{E} \quad 00$

$1.17761 \mathrm{E} \quad 00$

$1.18461 \mathrm{E} \quad 00$

$1.19162 \mathrm{E} \quad 00$

$1.19866 \mathrm{E} \quad 00$

$1.20572 \mathrm{E} \quad 00$

$1.21280 \mathrm{E} \quad 00$

$1.21990 \mathrm{E} \quad 00$

$1.22702 \mathrm{E} \quad 00$

$1.24133 \mathrm{E} \quad 00$

$1.24852 \mathrm{E} \quad 00$
$1.00305 \mathrm{E} \quad 00$

$1.23417 \mathrm{E} \quad 00$

$\tan \delta_{1}$

$-3.93062 \mathrm{E}-04$ $-3.93421 \mathrm{E}-04$

$-3.93776 \mathrm{E}-04$

$-3.94129 \mathrm{E}-04$

$-3.94479 E-04$

$-3.94827 \mathrm{E}-04$

$-3.95172 \mathrm{E}-04$

$-3.95513 \mathrm{E}-04$

$-3.95853 \mathrm{E}-04$

$-3.96190 \mathrm{E}-04$

$-3.96524 \mathrm{E}-04$

$-3.96856 \mathrm{E}-04$

$-3.97185 \mathrm{E}-04$

$-3.97512 \mathrm{E}-04$

$-3.97836 \mathrm{E}-04$

$-3.98158 \mathrm{E}-04$

$-3.98477 \mathrm{E}-04$

$-3.98793 \mathrm{E}-04$

$-3.99108 \mathrm{E}-04$

$-3.99420 \mathrm{E}-04$

$-3.99730 \mathrm{E}-04$

$-4.00038 \mathrm{E}-04$

$-4.00343 \mathrm{E}-04$

$-4.00646 \mathrm{E}-04$

$-4.00947 \mathrm{E}-04$

$-4.01245 \mathrm{E}-04$

$-4.01541 \mathrm{E}-04$

$-4.01835 \mathrm{E}-04$

$-4.02127 \mathrm{E}-04$

$-4.02416 \mathrm{E}-04$

$-4.02703 \mathrm{E}-04$

$-4.02988 \mathrm{E}-04$

-4.03271 E-04

$-4.03552 \mathrm{E}-04$

$-4.03832 \mathrm{E}-04$

$-4.04108 \mathrm{E}-04$

$-4.04384 \mathrm{E}-04$

$-4.04656 \mathrm{E}-04$

$-4.04927 \mathrm{E}-04$

$-4.05196 \mathrm{E}-04$

$-4.05462 \mathrm{E}-04$

$-4.05727 \mathrm{E}-04$

$-4.05990 \mathrm{E}-04$

$-4.06251 \mathrm{E}-04$

$-4.06510 \mathrm{E}-04$

$-4.06767 \mathrm{E}-04$

$-4.07022 \mathrm{E}-04$

$-4.07275 \mathrm{E}-04$

$-4.07528 \mathrm{E}-04$

$-4.07778 \mathrm{E}-04$

$-4.08025 \mathrm{E}-04$ 
$\bar{F}$

30.10

30.20

30.30

30.40

30.50

30.60

30.70

30.80

30.90

31.00

31.10

31.20

31.30

31.40

31.50

31.60

31.70

31.80

31.90

32.00

32.10

32.20

32.30

32.40

32.50

32.60

32.70

32.80

32.90

33.00

33.10

33.20

33.30

33.40

33.50

33.60

33.70

33.80

33.90

34.00

34.10

34.20

34.30

34.40

34.50

34.60

34.70

34.80

34.90

35.00

35.10

35.20

35.30

35.40

35.50

35.60

35.70

35.80

35.90

36.00 $f_{2}$

$2.37542 \mathrm{E}-04$

$2.39123 \mathrm{E}-04$

$2.40709 \mathrm{E}-04$

$2.42300 \mathrm{E}-04$

$2.43897 \mathrm{E}-04$

$2.45499 \mathrm{E}-04$

$2.47106 \mathrm{E}-04$

$2.48719 \mathrm{E}-04$

$2.50336 \mathrm{E}-04$

$2.51959 \mathrm{E}-04$

$2.53587 \mathrm{E}-04$

$2.55221 \mathrm{E}-04$

$2.56860 \mathrm{E}-04$

$2.58503 \mathrm{E}-04$

$2.60153 \mathrm{E}-04$

$2.61807 \mathrm{E}-04$

$2.63467 \mathrm{E}-04$

$2.65131 \mathrm{E}-04$

$2.66801 \mathrm{E}-04$

$2.68477 \mathrm{E}-04$

$2.70157 \mathrm{E}-04$

$2.71843 \mathrm{E}-04$

$2.73534 \mathrm{E}-04$

$2.75231 \mathrm{E}-04$

$2.76932 \mathrm{E}-04$

$2.78639 \mathrm{E}-04$

$2.80351 \mathrm{E}-04$

$2.82068 \mathrm{E}-04$

$2.83791 \mathrm{E}-04$

$2.85519 \mathrm{E}-04$

$2.87252 \mathrm{E}-04$

$2.88990 \mathrm{E}-04$

$2.90734 \mathrm{E}-04$

$2.92482 \mathrm{E}-04$

$2.94236 \mathrm{E}-04$

$2.95996 \mathrm{E}-04$

$2.97760 \mathrm{E}-04$

$2.99530 \mathrm{E}-04$

$3.01305 \mathrm{E}-04$

$3.03085 \mathrm{E}-04$

3.04871 E-04

$3.06661 \mathrm{E}-04$

$3.08457 \mathrm{E}-04$

$3.10258 \mathrm{E}-04$

3.12065 E-04

$3.13877 \mathrm{E}-04$

$3.15693 \mathrm{E}-04$

$3.17516 \mathrm{E}-04$

$3.19343 \mathrm{E}-04$

$3.21176 \mathrm{E}-04$

$3.23014 \mathrm{E}-04$

$3.24857 \mathrm{E}-04$

$3.26705 \mathrm{E}-04$

$3.28559 \mathrm{E}-04$

$3.30418 \mathrm{E}-04$

3.32282 E- 04

3.34151 E- 04

$3.36026 \mathrm{E}-04$

$3.37906 \mathrm{E}-04$

$3.39791 \mathrm{E}-04$
$9.12216 \mathrm{E}-01$

$9.18335 \mathrm{E}-01$

$9.24474 \mathrm{E}-01$

$9.30633 \mathrm{E}-01$

$9.36813 \mathrm{E}-01$

$9.43013 \mathrm{E}-01$

$9.49234 \mathrm{E}-01$

$9.55475 \mathrm{E}-01$

$9.61736 \mathrm{E}-01$

$9.68018 \mathrm{E}-01$

$9.74320 \mathrm{E}-01$

$9.80643 \mathrm{E}-01$

$9.86986 \mathrm{E}-01$

$9.93349 \mathrm{E}-01$

$9.99733 \mathrm{E}-01$

$1.00614 \mathrm{E} \quad 00$

$1.01256 \mathrm{E} \quad 00$

$1.01901 \mathrm{E} \quad 00$

$1.02547 \mathrm{E} \quad 00$

$1.03196 \mathrm{E} \quad 00$

$1.03846 \mathrm{E} \quad 00$

$1.04499 \mathrm{E} \quad 00$

$1.05154 \mathrm{E} \quad 00$

$1.05810 \mathrm{E} \quad 00$

$1.06469 \mathrm{E} \quad 00$

$1.07130 \mathrm{E} \quad 00$

$1.07793 \mathrm{E} \quad 00$

$1.08458 \mathrm{E} \quad 00$

$1.09125 \mathrm{E} \quad 00$

$1.09794 \mathrm{E} \quad 00$

$1.10465 \mathrm{E} \quad 00$

$1.11138 \mathrm{E} \quad 00$

$1.11813 \mathrm{E} \quad 00$

$1.12490 \mathrm{E} \quad 00$

$1.13169 \mathrm{E} \quad 00$

$1.13850 \mathrm{E} \quad 00$

$1.14533 \mathrm{E} \quad 00$

$1.15219 \mathrm{E} \quad 00$

$1.15906 \mathrm{E} \quad 00$

$1.16595 \mathrm{E} \quad 00$

$1.17287 \mathrm{E} \quad 00$

$1.17980 \mathrm{E} \quad 00$

$1.18675 \mathrm{E} \quad 00$

$1.19373 \mathrm{E} \quad 00$

$1.20072 \mathrm{E} \quad 00$

$1.20774 \mathrm{E} \quad 00$

$1.21478 \mathrm{E} \quad 00$

$1.22183 \mathrm{E} \quad 00$

$1.22891 \mathrm{E} \quad 00$

$1.23601 \mathrm{E} \quad 00$

$1.24312 \mathrm{E} \quad 00$

$1.25026 \mathrm{E} \quad 00$

$1.25742 \mathrm{E} \quad 00$

$1.26460 \mathrm{E} \quad 00$

$1.27180 \mathrm{E} \quad 00$

$1.27902 \mathrm{E} \quad 00$

$1.28626 \mathrm{E} \quad 00$

$1.29352 \mathrm{E} \quad 00$

$1.30080 \mathrm{E} \quad 00$

$1.30810 \mathrm{E} \quad 00$ $g_{2}$

$1.61234 \mathrm{E}-02$

$1.62886 \mathrm{E}-02$

$1.64550 \mathrm{E}-02$

$1.66225 \mathrm{E}-02$

$1.67911 \mathrm{E}-02$

$1.69608 \mathrm{E}-02$

$1.71317 \mathrm{E}-02$

$1.73037 \mathrm{E}-02$

$1.74769 \mathrm{E}-02$

$1.76512 \mathrm{E}-02$

$1.78267 \mathrm{E}-02$

$1.80034 \mathrm{E}-02$

$1.81812 \mathrm{E}-02$

$1.83601 \mathrm{E}-02$

$1.85403 \mathrm{E}-02$

$1.87216 \mathrm{E}-02$

$1.89041 \mathrm{E}-02$

$1.90877 \mathrm{E}-02$

$1.92726 \mathrm{E}-02$

$1.94586 \mathrm{E}-02$

$1.96459 \mathrm{E}-02$

$1.98343 \mathrm{E}-02$

$2.00239 \mathrm{E}-02$

$2.02148 \mathrm{E}-02$

$2.04068 \mathrm{E}-02$

$2.06001 \mathrm{E}-02$

$2.07945 \mathrm{E}-02$

$2.09902 \mathrm{E}-02$

$2.11872 \mathrm{E}-02$

$2.13853 \mathrm{E}-02$

$2.15847 \mathrm{E}-02$

$2.17853 \mathrm{E}-02$

$2.19871 \mathrm{E}-02$

$2.21902 \mathrm{E}-02$

$2.23945 \mathrm{E}-02$

$2.26001 \mathrm{E}-02$

$2.28069 \mathrm{E}-02$

$2.30150 \mathrm{E}-02$

$2.32244 \mathrm{E}-02$

2.34350 E- 02

$2.36469 \mathrm{E}-02$

$2.38600 \mathrm{E}-02$

2.40745 E-02

$2.42902 \mathrm{E}-02$

2.45072 E-02

$2.47255 \mathrm{E}-02$

$2.49450 \mathrm{E}-02$

$2.51659 \mathrm{E}-02$

$2.53881 \mathrm{E}-02$

$2.56116 \mathrm{E}-02$

$2.58363 \mathrm{E}-02$

$2.60624 \mathrm{E}-02$

$2.62898 \mathrm{E}-02$

$2.65186 \mathrm{E}-02$

$2.67486 \mathrm{E}-02$

$2.69800 \mathrm{E}-02$

$2.72127 \mathrm{E}-02$

$2.74467 \mathrm{E}-02$

$2.76821 \mathrm{E}-02$

$2.79188 \mathrm{E}-02$

$\tan \delta_{2}$

$-2.00092 \mathrm{E} \quad 02$ $-2.00101 \mathrm{E} \quad 02$ $-2.00109 \mathrm{E} \quad 02$

$-2.00118 \mathrm{E} \quad 02$

$-2.00126 \mathrm{E} \quad 02$

$-2.00135 \mathrm{E} \quad 02$

$-2.00143 \mathrm{E} \quad 02$

$-2.00152 \mathrm{E} \quad 02$

$-2.00160 \mathrm{E} \quad 02$

$-2.00168 \mathrm{E} 02$

$-2.00177 \mathrm{E} \quad 02$

$-2.00185 \mathrm{E} \quad 02$

$-2.00193 \mathrm{E} \quad 02$

$-2.00201 \mathrm{E} \quad 02$

$-2.00209 \mathrm{E} \quad 02$

$-2.00217 \mathrm{E} \quad 02$

$\begin{array}{ll}-2.00225 \mathrm{E} & 02\end{array}$

$-2.00233 \mathrm{E} \quad 02$

$-2.00241 \mathrm{E} \quad 02$

$-2.00249 \mathrm{E} \quad 02$

$-2.00256 \mathrm{E} \quad 02$

$-2.00264 \mathrm{E} \quad 02$

$-2.00272 \mathrm{E} \quad 02$

$-2.00279 \mathrm{E} \quad 02$

$\begin{array}{ll}-2.00287 \mathrm{E} & 02\end{array}$

$-2.00294 \mathrm{E} \quad 02$

$-2.00302 \mathrm{E} \quad 02$

$-2.00309 \mathrm{E} \quad 02$

$-2.00316 \mathrm{E} \quad 02$

$-2.00324 \mathrm{E} \quad 02$

$-2.00331 \mathrm{E} \quad 02$

$-2.00338 \mathrm{E} \quad 02$

$-2.00345 \mathrm{E} \quad 02$

$-2.00352 \mathrm{E} \quad 02$

$-2.00359 \mathrm{E} 02$

$-2.00366 \mathrm{E} \quad 02$

$-2.00373 \mathrm{E} \quad 02$

$-2.00380 \mathrm{E} \quad 02$

$-2.00387 \mathrm{E} \quad 02$

$-2.00394 \mathrm{E} \quad 02$

$-2.00401 \mathrm{E} \quad 02$

$-2.00408 \mathrm{E} \quad 02$

$-2.00414 \mathrm{E} \quad 02$ 
ELECTRON RADIAL FUNCTIONS

$$
A=1
$$$$
\rho=1.2 A^{1 / 3} F
$$

\begin{tabular}{|c|c|c|c|}
\hline$p$ & $F_{0}$ & $f_{1}$ & \\
\hline 36.10 & $9.77443 \mathrm{E}-01$ & $3.53220 \mathrm{E}$ & 01 \\
\hline 36.20 & $9.77443 \mathrm{E}-01$ & $3.54219 \mathrm{E}$ & 01 \\
\hline 36.30 & $9.77443 \mathrm{E}-01$ & $3.55218 \mathrm{E}$ & 01 \\
\hline 36.40 & $9.77443 \mathrm{E}-01$ & $3.56217 \mathrm{E}$ & 01 \\
\hline 36.50 & $9.77443 \mathrm{E}-01$ & $3.57216 \mathrm{E}$ & 01 \\
\hline 36.60 & $9.77443 \mathrm{E}-01$ & $3.58215 \mathrm{E}$ & 01 \\
\hline 36.70 & $9.77443 \mathrm{E}-01$ & $3.59214 \mathrm{E}$ & 01 \\
\hline 36.80 & $9.77443 \mathrm{E}-01$ & $3.60213 \mathrm{E}$ & 01 \\
\hline 36.90 & $9.77443 \mathrm{E}-01$ & $3.61212 \mathrm{E}$ & 01 \\
\hline 37.00 & $9.77442 \mathrm{E}-01$ & $3.62212 \mathrm{E}$ & 01 \\
\hline 37.10 & $9.77442 \mathrm{E}-01$ & $3.63211 \mathrm{E}$ & 01 \\
\hline 37.20 & $9.77442 \mathrm{E}-01$ & $3.64210 \mathrm{E}$ & 01 \\
\hline 37.30 & $9.77442 \mathrm{E}-01$ & $3.65210 \mathrm{E}$ & 01 \\
\hline 37.40 & $9.77442 \mathrm{E}-01$ & $3.66209 \mathrm{E}$ & 01 \\
\hline 37.50 & $9.77442 \mathrm{E}-01$ & $3.67209 \mathrm{E}$ & 01 \\
\hline 37.60 & $9.77442 \mathrm{E}-01$ & $3.68208 \mathrm{E}$ & 01 \\
\hline 37.70 & $9.77442 \mathrm{E}-01$ & $3.69208 \mathrm{E}$ & 01 \\
\hline 37.80 & $9.77442 \mathrm{E}-01$ & $3.70208 \mathrm{E}$ & 01 \\
\hline 37.90 & $9.77442 \mathrm{E}-01$ & $3.71207 \mathrm{E}$ & 01 \\
\hline 38.00 & $9.77441 \mathrm{E}-01$ & $3.72207 \mathrm{E}$ & 01 \\
\hline 38.10 & $9.77441 \mathrm{E}-01$ & $3.73207 \mathrm{E}$ & 01 \\
\hline 38.20 & $9.77441 \mathrm{E}-01$ & $3.74207 \mathrm{E}$ & 01 \\
\hline 38.30 & $9.77441 \mathrm{E}-01$ & $3.75207 \mathrm{E}$ & 01 \\
\hline 38.40 & $9.77441 \mathrm{E}-01$ & $3.76207 \mathrm{E}$ & 01 \\
\hline 38.50 & $9.77441 \mathrm{E}-01$ & $3.77207 \mathrm{E}$ & 01 \\
\hline 38.60 & $9.77441 \mathrm{E}-01$ & $3.78207 \mathrm{E}$ & 01 \\
\hline 38.70 & $9.77441 \mathrm{E}-01$ & $3.79207 \mathrm{E}$ & 01 \\
\hline 38.80 & $9.77441 \mathrm{E}-01$ & $3.80208 \mathrm{E}$ & 01 \\
\hline 38.90 & $9.77441 \mathrm{E}-01$ & $3.81208 \mathrm{E}$ & 01 \\
\hline 39.00 & $9.77441 \mathrm{E}-01$ & $3.82208 \mathrm{E}$ & 01 \\
\hline 39.10 & $9.77440 \mathrm{E}-01$ & $3.83209 \mathrm{E}$ & 01 \\
\hline 39.20 & $9.77440 \mathrm{E}-01$ & $3.84209 \mathrm{E}$ & 01 \\
\hline 39.30 & $9.77440 \mathrm{E}-01$ & $3.85210 \mathrm{E}$ & 01 \\
\hline 39.40 & $9.77440 \mathrm{E}-01$ & $3.86210 \mathrm{E}$ & 01 \\
\hline 39.50 & $9.77440 \mathrm{E}-01$ & $3.87211 \mathrm{E}$ & 01 \\
\hline 39.60 & $9.77440 \mathrm{E}-01$ & $3.88212 \mathrm{E}$ & 01 \\
\hline 39.70 & $9.77440 \mathrm{E}-01$ & $3.89213 \mathrm{E}$ & 01 \\
\hline 39.80 & $9.77440 \mathrm{E}-01$ & $3.90213 \mathrm{E}$ & 01 \\
\hline 39.90 & $9.77440 \mathrm{E}-01$ & $3.91214 \mathrm{E}$ & 01 \\
\hline 40.00 & $9.77440 \mathrm{E}-01$ & $3.92215 \mathrm{E}$ & $0 \mathrm{I}$ \\
\hline 40.10 & $9.77439 \mathrm{E}-01$ & $3.93216 \mathrm{E}$ & 01 \\
\hline 40.20 & $9.77439 \mathrm{E}-01$ & $3.94217 \mathrm{E}$ & 01 \\
\hline 40.30 & $9.77439 \mathrm{E}-01$ & $3.95218 \mathrm{E}$ & 01 \\
\hline 40.40 & $9.77439 \mathrm{E}-01$ & $3.96220 \mathrm{E}$ & 01 \\
\hline 40.50 & $9.77439 \mathrm{E}-01$ & $3.97221 \mathrm{E}$ & 01 \\
\hline 40.60 & $9.77439 \mathrm{E}-01$ & $3.98222 \mathrm{E}$ & 01 \\
\hline 40.70 & $9.77439 \mathrm{E}-01$ & $3.99224 \mathrm{E}$ & 01 \\
\hline 40.80 & $9.77439 \mathrm{E}-01$ & $4.00225 \mathrm{E}$ & 01 \\
\hline 40.90 & $9.77439 \mathrm{E}-01$ & $4.01226 \mathrm{E}$ & 01 \\
\hline 41.00 & $9.77439 \mathrm{E}-01$ & $4.02228 \mathrm{E}$ & 01 \\
\hline 41.10 & $9.77439 \mathrm{E}-01$ & $4.03230 \mathrm{E}$ & 01 \\
\hline 41.20 & $9.77438 \mathrm{E}-01$ & $4.04231 \mathrm{E}$ & 01 \\
\hline 41.30 & $9.77438 \mathrm{E}-01$ & $4.05233 \mathrm{E}$ & 01 \\
\hline 41.40 & $9.77438 \mathrm{E}-01$ & $4.06235 \mathrm{E}$ & 01 \\
\hline 41.50 & $9.77438 \mathrm{E}-01$ & $4.07237 \mathrm{E}$ & 01 \\
\hline 41.60 & $9.77438 \mathrm{E}-01$ & $4.08238 \mathrm{E}$ & 01 \\
\hline 41.70 & $9.77438 \mathrm{E}-01$ & $4.09240 \mathrm{E}$ & 01 \\
\hline 41.80 & $9.77438 \mathrm{E}-01$ & $4.10242 \mathrm{E}$ & 01 \\
\hline 41.90 & $9.77438 \mathrm{E}-01$ & $4.11244 \mathrm{E}$ & 01 \\
\hline 42.00 & $9.77438 \mathrm{E}-01$ & $4.12247 \mathrm{E}$ & \\
\hline
\end{tabular}

\section{$g_{1}$}

$\tan \delta_{1}$

$f_{-1}$

$\begin{array}{lll}1.25572 \mathrm{E} & 00 & -4.10406 \mathrm{E}-04\end{array}$ $1.26295 \mathrm{E} \quad 00 \quad-4.10635 \mathrm{E}-04$ $\begin{array}{lll}1.27020 \mathrm{E} & 00 & -4.10861 \mathrm{E}-04\end{array}$ $1.27747 \mathrm{E} \quad 00 \quad-4.11086 \mathrm{E}-04$ $\begin{array}{lll}1.28475 \mathrm{E} & 00 & -4.11310 \mathrm{E}-04\end{array}$

$1.29207 \mathrm{E} \quad 00 \quad-4.11531 \mathrm{E}-04$ $1.29940 \mathrm{E} \quad 00 \quad-4.11753 \mathrm{E}-04$ $1.30675 \mathrm{E} \quad 00 \quad-4.11971 \mathrm{E}-04$ $\begin{array}{lll}1.31412 \mathrm{E} & 00 & -4.12189 \mathrm{E}-04\end{array}$ $1.32152 \mathrm{E} \quad 00 \quad-4.12404 \mathrm{E}-04$

$\begin{array}{lll}1.32893 \mathrm{E} & 00 & -4.12619 \mathrm{E}-04\end{array}$ $\begin{array}{llll}1.33637 \mathrm{E} & 00 & -4.12830 \mathrm{E}-04\end{array}$ $\begin{array}{lll}1.34383 \mathrm{E} & 00 & -4.13042 \mathrm{E}-04\end{array}$ $1.35131 \mathrm{E} \quad 00 \quad-4.13251 \mathrm{E}-04$

$\begin{array}{lll}1.35881 \mathrm{E} & 00 & -4.13459 \mathrm{E}-04\end{array}$

$\begin{array}{lll}1.36633 \mathrm{E} & 00 & -4.13665 \mathrm{E}-04\end{array}$ $\begin{array}{lll}1.37387 \mathrm{E} & 00 & -4.13870 \mathrm{E}-04\end{array}$ $\begin{array}{lll}1.38143 \mathrm{E} & 00 & -4.14072 \mathrm{E}-04\end{array}$ $1.38901 \mathrm{E} \quad 00 \quad-4.14274 \mathrm{E}-04$ $\begin{array}{lll}1.39662 \mathrm{E} & 00 & -4.14475 \mathrm{E}-04\end{array}$

$1.40424 \mathrm{E} \quad 00 \quad-4.14674 \mathrm{E}-04$

$1.41189 \mathrm{E} \quad 00 \quad-4.14871 \mathrm{E}-04$

$\begin{array}{lll}1.41956 \mathrm{E} & 00 & -4.15068 \mathrm{E}-04\end{array}$

$\begin{array}{lll}1.42725 \mathrm{E} & 00 & -4.15262 \mathrm{E}-04\end{array}$

$1.43496 \mathrm{E} \quad 00 \quad-4.15455 \mathrm{E}-04$

$1.44269 \mathrm{E} \quad 00$

$1.45044 \mathrm{E} \quad 00$

$1.45821 \mathrm{E} \quad 00$

$1.46601 \mathrm{E} \quad 00$

$1.47382 \mathrm{E} \quad 00$

$1.48166 \mathrm{E} \quad 00$

$1.48952 \mathrm{E} \quad 00$

$1.49739 \mathrm{E} \quad 00$

$1.50529 \mathrm{E} \quad 00$

$1.51321 \mathrm{E} \quad 00$

$1.52115 \mathrm{E} \quad 00$

$1.52912 \mathrm{E} \quad 00$

$1.53710 \mathrm{E} \quad 00$

$1.54511 \mathrm{E} \quad 00$

$1.55313 \mathrm{E} \quad 00$

$1.56118 \mathrm{E} \quad 00$

$1.56925 \mathrm{E} \quad 00$

$1.57734 \mathrm{E} \quad 00$

$1.58545 \mathrm{E} \quad 00$

$1.59358 \mathrm{E} \quad 00$

$1.60173 \mathrm{E} \quad 00$ $1.60990 \mathrm{E} \quad 00$ $1.61810 \mathrm{E} \quad 00$ $1.62631 \mathrm{E} \quad 00$

$1.63455 \mathrm{E} \quad 00$

$1.64281 \mathrm{E} \quad 00$

$1.65109 \mathrm{E} \quad 00$

$1.65939 \mathrm{E} \quad 00$

$\begin{array}{ll}1.66771 \mathrm{E} & 00\end{array}$

$1.67605 \mathrm{E} \quad 00$

$1.68441 \mathrm{E} \quad 00$

$1.69280 \mathrm{E} \quad 00$

$1.70120 \mathrm{E} \quad 00$

$1.70963 \mathrm{E} \quad 00$

$1.71808 \mathrm{E} \quad 00$
$-4.15647 \mathrm{E}-04$ $-4.15837 \mathrm{E}-04$ $-4.16026 \mathrm{E}-04$ $-4.16214 \mathrm{E}-04$ $-4.16400 \mathrm{E}-04$

$-4.16586 \mathrm{E}-04$ $-4.16768 \mathrm{E}-04$ $-4.17131 \mathrm{E}-04$ $-4.17311 \mathrm{E}-04$

$-4.17489 \mathrm{E}-04$ $-4.17666 \mathrm{E}-04$ $-4.17842 \mathrm{E}-04$ $-4.18016 \mathrm{E}-04$

$-4.18361 \mathrm{E}-04$ $-4.18531 \mathrm{E}-04$ $-4.18700 \mathrm{E}-04$ $-4.18868 \mathrm{E}-04$ $-4.19035 \mathrm{E}-04$

$-4.19201 \mathrm{E}-04$ $-4.19365 \mathrm{E}-04$ $-4.19690 \mathrm{E}-04$ $-4.19850 \mathrm{E}-04$

$-4.20010 \mathrm{E}-04$ $-4.20168 \mathrm{E}-04$ $-4.20325 \mathrm{E}-04$ $-4.20481 \mathrm{E}-04$ $-4.20636 \mathrm{E}-04$

$-4.20789 \mathrm{E}-04$ $-4.20942 \mathrm{E}-04$ $-4.21093 \mathrm{E}-04$ $-4.21243 \mathrm{E}-04$ $-4.21392 \mathrm{E}-04$ $-4.16951 \mathrm{E}-04$ $-4.18189 \mathrm{E}-04$ $-4.19528 \mathrm{E}-04$
$-1.21594 \mathrm{E} \quad 00$ $-1.22306 \mathrm{E} \quad 00$ $-1.23021 \mathrm{E} 00$ $-1.23738 \mathrm{E} \quad 00$ $-1.24456 \mathrm{E} \quad 00$

$-1.25177 \mathrm{E} \quad 00$ $-1.25900 \mathrm{E} 00$ $-1.26625 \mathrm{E} \quad 00$ $-1.27353 \mathrm{E} 00$ $-1.28082 \mathrm{E} \quad 00$

$-1.28813 \mathrm{E} \quad 00$ $-1.29547 \mathrm{E} \quad 00$ $-1.30282 \mathrm{E} 00$ $-1.31020 \mathrm{E} 00$ $-1.31760 \mathrm{E} \quad 00$

$-1.32502 \mathrm{E} \quad 00$ $-1.33246 \mathrm{E} 00$ $-1.33992 \mathrm{E} 00$ $-1.34740 \mathrm{E} 00$ $-1.35490 \mathrm{E} 00$

$-1.36243 \mathrm{E} 00$ $-1.36997 \mathrm{E} 00$ $-1.37754 \mathrm{E} \quad 00$ $-1.38513 \mathrm{E} 00$ $-1.39273 \mathrm{E} 00$

$-1.40036 \mathrm{E} 00$ $-1.40801 \mathrm{E} 00$ $-1.41569 \mathrm{E} 00$ $-1.42338 \mathrm{E} 00$ $-1.43109 \mathrm{E} 00$

$-1.43883 \mathrm{E} \quad 00$ $-1.44658 \mathrm{E} 00$ $-1.45436 \mathrm{E} 00$ $-1.46216 \mathrm{E} 00$ $-1.46998 \mathrm{E} 00$

$-1.47782 \mathrm{E} 00$ $-1.48568 \mathrm{E} 00$ $-1.49356 \mathrm{E} \quad 00$ $-1.50146 \mathrm{E} \quad 00$ $-1.50939 \mathrm{E} 00$

$-1.51733 \mathrm{E} 00$ $-1.52530 \mathrm{E} 00$ $-1.53329 \mathrm{E} 00$ $-1.54129 \mathrm{E} 00$ $-1.54932 \mathrm{E} 00$

$-1.55737 \mathrm{E} \quad 00$ $-1.56545 \mathrm{E} 00$ $-1.57354 \mathrm{E} \quad 00$ $-1.58165 \mathrm{E} \quad 00$ $-1.58979 \mathrm{E} \quad 00$

$-1.59794 \mathrm{E} 00$ $-1.60612 \mathrm{E} 00$ $-1.61432 \mathrm{E} 00$ $-1.62254 \mathrm{E} 00$ $-1.63078 \mathrm{E} 00$

$-1.63904 \mathrm{E} \quad 00$ $-1.64733 \mathrm{E} \quad 00$ $-1.65563 \mathrm{E} 00$ $-1.66396 \mathrm{E} 00$ $-1.67230 \mathrm{E} 00$ $g_{-1}$

$\tan \delta_{-1}$

$3.63210 \mathrm{E} \quad 01$

$3.64210 \mathrm{E} 01$

$3.65209 \mathrm{E} 01$

$3.66209 \mathrm{E} \quad 01$

$3.67209 \mathrm{E} 01$

$3.68208 \mathrm{E} \quad 01$

$3.69208 \mathrm{E} 01$

$3.70208 \mathrm{E} \quad 01$

$3.71208 \mathrm{E} 01$

$3.72208 \mathrm{E} 01$

$3.73207 \mathrm{E} \quad 01$

$3.74207 \mathrm{E} \quad 01$

$3.75208 \mathrm{E} \quad 01$

$3.76208 \mathrm{E} \quad 01$

$3.77208 \mathrm{E} 01$

$3.78208 \mathrm{E} \quad 01$ $3.79208 \mathrm{E} \quad 01$ $3.80209 \mathrm{E} 01$ $3.81209 \mathrm{E} 01$ $3.82209 \mathrm{E} 01$

$3.83210 \mathrm{E} 01$ $3.84211 \mathrm{E} \quad 01$

$3.85211 \mathrm{E} 01$

$3.86212 \mathrm{E} \quad 01$

$3.87213 \mathrm{E} 01$

$3.88213 \mathrm{E} \quad 01$ $3.89214 \mathrm{E} 01$ $3.90215 \mathrm{E} 01$ $3.91216 \mathrm{E} \quad 01$ $3.92217 \mathrm{E} \quad 01$

$3.93218 \mathrm{E} \quad 01$ $3.94219 \mathrm{E} \quad 01$ $3.95221 \mathrm{E} 01$ $3.96222 \mathrm{E} 01$ $3.97223 \mathrm{E} 01$

$3.98224 \mathrm{E} \quad 01$ $3.99226 \mathrm{E}$ ol $4.00227 \mathrm{E} \quad 01$ $4.01229 \mathrm{E} 01$ $4.02231 \mathrm{E} 01$

$4.03232 \mathrm{E} \quad 01$ $4.04234 \mathrm{E}$ 01 $4.05236 \mathrm{E} \quad 01$ $4.06238 \mathrm{E} 01$ $4.07239 \mathrm{E} 01$

$4.08241 \mathrm{E} \quad 01$ $4.09243 \mathrm{E} \quad 01$ $4.10246 \mathrm{E} 01$ $4.11248 \mathrm{E} 01$ $4.12250 \mathrm{E} \quad 01$

$4.13252 \mathrm{E} \quad 01$ $4.14255 \mathrm{E}$ 01 $4.15257 \mathrm{E}$ 01 $4.16259 \mathrm{E} 01$ $4.17262 \mathrm{E} 01$

$4.18264 \mathrm{E} 01$ $4.19267 \mathrm{E} \quad 01$ $4.20270 \mathrm{E} 01$ $4.21273 \mathrm{E} \quad 01$ $4.22275 \mathrm{E} 01$
$1.63271 \mathrm{E} \quad 03$

$1.63359 \mathrm{E} \quad 03$

$1.63447 \mathrm{E} \quad 03$

$1.63535 \mathrm{E} \quad 03$

$1.63622 \mathrm{E} \quad 03$

$1.63709 \mathrm{E} \quad 03$

$1.63796 \mathrm{E} \quad 03$

$1.63883 \mathrm{E} \quad 03$

$1.63969 \mathrm{E} \quad 03$

$1.64055 \mathrm{E} \quad 03$

$1.64140 \mathrm{E} \quad 03$ $1.64226 \mathrm{E} \quad 03$ $1.64312 \mathrm{E} \quad 03$

$1.64397 \mathrm{E} \quad 03$

$1.64481 \mathrm{E} \quad 03$

$1.64566 \mathrm{E} \quad 03$ $1.64650 \mathrm{E} \quad 03$

$1.64734 \mathrm{E} \quad 03$

$1.64818 \mathrm{E} \quad 03$

$1.64901 \mathrm{E} \quad 03$

$1.64985 \mathrm{E} \quad 03$ $1.65068 \mathrm{E} \quad 03$

$1.65150 \mathrm{E} \quad 03$

$1.65233 \mathrm{E} \quad 03$

$1.65316 \mathrm{E} \quad 03$

$1.65398 \mathrm{E} \quad 03$ $1.65480 \mathrm{E} \quad 03$ $1.65561 \mathrm{E} \quad 03$ $1.65643 \mathrm{E} \quad 03$ $1.65724 \mathrm{E} \quad 03$

$1.65804 \mathrm{E} \quad 03$ $1.65886 \mathrm{E} \quad 03$ $1.65966 \mathrm{E} \quad 03$ $1.66046 \mathrm{E} \quad 03$ $1.66126 \mathrm{E} \quad 03$

$1.66206 \mathrm{E} \quad 03$ $1.66286 \mathrm{E} \quad 03$ $1.66365 \mathrm{E} \quad 03$ $1.66444 \mathrm{E} \quad 03$ $1.66524 \mathrm{E} \quad 03$

$1.66602 \mathrm{E} \quad 03$ $1.66681 \mathrm{E} \quad 03$ $1.66759 \mathrm{E} \quad 03$ $1.66838 \mathrm{E} \quad 03$ $1.66916 \mathrm{E} \quad 03$

$1.66993 \mathrm{E} \quad 03$ $-1.67071 \mathrm{E} \quad 03$ $1.67148 \mathrm{E} \quad 03$ $1.67226 \mathrm{E} \quad 03$ $1.67303 \mathrm{E} \quad 03$

$1.67380 \mathrm{E} \quad 03$ $1.67456 \mathrm{E} \quad 03$ $1.67533 \mathrm{E} \quad 03$ $1.67609 \mathrm{E} \quad 03$ $1.67685 \mathrm{E} \quad 03$

$1.67761 \mathrm{E} \quad 03$ $1.67837 \mathrm{E} \quad 03$ $1.67913 \mathrm{E} \quad 03$ $1.67988 \mathrm{E} \quad 03$ $1.68063 \mathrm{E} \quad 03$ 
ELECTRON RADIAL FUNCTIONS

$Z=1 \quad A=1 \quad \rho=1.2 A^{1 / 3} F$

Positron

$\bar{F}$

$3.41681 \mathrm{E}-04$

$3.43577 \mathrm{E}-04$

$3.45478 \mathrm{E}-04$

$3.47384 \mathrm{E}-04$

$3.49295 \mathrm{E}-04$

36.50

$36.60 \quad 3.51211 \mathrm{E}-04$

$36.70 \quad 3.53133 \mathrm{E}-04$

$36.80 \quad 3.55060 \mathrm{E}-04$

$36.90 \quad 3.56993 \mathrm{E}-04$

$37.00 \quad 3.58930 \mathrm{E}-04$

$37.10 \quad 3.60873 \mathrm{E}-04$

$37.20 \quad 3.62821 \mathrm{E}-04$

$37.30 \quad 3.64774 \mathrm{E}-04$

$37.40 \quad 3.66733 \mathrm{E}-04$

$37.50 \quad 3.68696 \mathrm{E}-04$

37.60

37.70

37.80

37.90

38.00

38.10

38.20

38.30

38.40

38.50

$3.70665 \mathrm{E}-04$

$3.72640 \mathrm{E}-04$

$3.74619 \mathrm{E}-04$

3.76604 E-04

3.78594 E-04

$3.80589 \mathrm{E}-04$

$3.82589 \mathrm{E}-04$

$3.84595 \mathrm{E}-04$

$3.86606 \mathrm{E}-04$

$3.88622 \mathrm{E}-04$

$38.60 \quad 3.90644 \mathrm{E}-04$

$38.70 \quad 3.92670 \mathrm{E}-04$

$38.80 \quad 3.94702$ E-04

$38.90 \quad 3.96739 \mathrm{E}-04$

$39.00 \quad 3.98782 \mathrm{E}-04$

$39.10 \quad 4.00830 \mathrm{E}-04$

$39.20 \quad 4.02882 \mathrm{E}-04$

$39.30 \quad 4.04941 \mathrm{E}-04$

$39.40 \quad 4.07004 \mathrm{E}-04$

$39.50 \quad 4.09073 \mathrm{E}-04$

$39.60 \quad 4.11146 \mathrm{E}-04$

$39.70 \quad 4.13226 \mathrm{E}-04$

$39.80 \quad 4.15310 \mathrm{E}-04$

$39.90 \quad 4.17399 \mathrm{E}-04$

$40.00 \quad 4.19494 \mathrm{E}-04$

$40.10 \quad 4.21594 \mathrm{E}-04$

$40.20 \quad 4.23700 \mathrm{E}-04$

$40.30 \quad 4.25810 \mathrm{E}-04$

$40.40 \quad 4.27926 \mathrm{E}-04$

$40.50 \quad 4.30047 \mathrm{E}-04$

40.60

40.70

40.80

40.90

41.00

4.32173 E-04

$4.34305 \mathrm{E}-04$

$4.36442 \mathrm{E}-04$

$4.38584 \mathrm{E}-04$

4.40731 E-04

41.10

41.20

41.30

41.40

4.42884 E-04

$4.45041 \mathrm{E}-04$

4.47204 E-04

$4.49373 \mathrm{E}-04$

$41.50 \quad 4.51546 \mathrm{E}-04$

$41.60 \quad 4.53725 \mathrm{E}-04$

$41.70 \quad 4.55909 \mathrm{E}-04$

$41.80 \quad 4.58098 \mathrm{E}-04$

$41.90 \quad 4.60292 \mathrm{E}-04$

42.00 $f_{2}$

$1.31542 \mathrm{E} \quad 00$

$1.32276 \mathrm{E} \quad 00$

$\begin{array}{ll}1.33012 \mathrm{E} & 00\end{array}$

$1.33750 \mathrm{E} \quad 00$

$1.34491 \mathrm{E} \quad 00$

$1.35233 \mathrm{E} \quad 00$

$\begin{array}{ll}1.35977 \mathrm{E} & 00\end{array}$

$1.36724 \mathrm{E} \quad 00$

$\begin{array}{ll}1.37472 \mathrm{E} & 00\end{array}$

$1.38222 \mathrm{E} \quad 00$

$1.38975 \mathrm{E} \quad 00$

$1.39729 \mathrm{E} \quad 00$

$1.40486 \mathrm{E} \quad 00$

$1.41244 \mathrm{E} \quad 00$

$1.42005 \mathrm{E} \quad 00$

$1.42768 \mathrm{E} \quad 00$

$1.43532 \mathrm{E} \quad 00$

$1.44299 \mathrm{E} \quad 00$

$1.45068 \mathrm{E} \quad 00$

$1.45838 \mathrm{E} \quad 00$

$1.46611 \mathrm{E} \quad 00$

$1.47386 \mathrm{E} \quad 00$

$1.48163 \mathrm{E} \quad 00$

$1.48942 \mathrm{E} \quad 00$

$1.49723 \mathrm{E} \quad 00$

$1.50506 \mathrm{E} \quad 00$

$1.51291 \mathrm{E} \quad 00$

$1.52078 \mathrm{E} \quad 00$

$\begin{array}{lll}1.52867 \mathrm{E} & 00\end{array}$

$1.53658 \mathrm{E} \quad 00$

$1.54451 \mathrm{E} \quad 00$

$1.55246 \mathrm{E} \quad 00$

$1.56044 \mathrm{E} \quad 00$

$1.56843 \mathrm{E} \quad 00$

$1.57644 \mathrm{E} \quad 00$

$\begin{array}{ll}1.58447 \mathrm{E} & 00\end{array}$

$1.59253 \mathrm{E} \quad 00$

$1.60060 \mathrm{E} \quad 00$

$1.60870 \mathrm{E} \quad 00$

$1.61681 \mathrm{E} \quad 00$

$1.62495 \mathrm{E} \quad 00$

$1.63310 \mathrm{E} \quad 00$

$1.64128 \mathrm{E} \quad 00$

$1.64947 \mathrm{E} \quad 00$

$1.65769 \mathrm{E} 00$

$1.66592 \mathrm{E} \quad 00$

$1.67418 \mathrm{E} \quad 00$

$1.68246 \mathrm{E} \quad 00$

$1.69076 \mathrm{E} \quad 00$

$1.69907 \mathrm{E} \quad 00$

$1.70741 \mathrm{E} \quad 00$

$1.71577 \mathrm{E} \quad 00$

$1.72415 \mathrm{E} \quad 00$

$1.73255 \mathrm{E} \quad 00$

$1.74097 \mathrm{E} \quad 00$

$\begin{array}{ll}1.74941 \mathrm{E} & 00\end{array}$

$1.75787 \mathrm{E} \quad 00$

$1.76635 \mathrm{E} \quad 00$

$1.77485 \mathrm{E} \quad 00$

$1.78337 \mathrm{E} \quad 00$ $g_{2}$

$\tan \delta_{2}$

$f_{-2}$

$2.81568 \mathrm{E}-02-2.00529 \mathrm{E} \quad 02$

$2.83962 \mathrm{E}-02 \quad-2.00535 \mathrm{E} \quad 02$

$2.86370 \mathrm{E}-02 \quad-2.00541 \mathrm{E} \quad 02$

$2.88791 \mathrm{E}-02 \quad-2.00547 \mathrm{E} \quad 02$

$2.91226 \mathrm{E}-02$

$-2.00553 \mathrm{E} \quad 02$

$2.93674 \mathrm{E}-02$

$2.96136 \mathrm{E}-02$

$2.98612 \mathrm{E}-02$

3.01101 E- 02

$3.03605 \mathrm{E}-02$

$3.06122 \mathrm{E}-02$

$3.08652 \mathrm{E}-02$

$3.11197 \mathrm{E}-02$

$3.13756 \mathrm{E}-02$

$3.16329 \mathrm{E}-02$

$3.18915 \mathrm{E}-02$

$3.21516 \mathrm{E}-02$

3.24131 E-02

$3.26760 \mathrm{E}-02$

$3.29403 \mathrm{E}-02$

$-2.00559 \mathrm{E} \quad 02$

$-2.00565 \mathrm{E} \quad 02$

$-2.00571 \mathrm{E} \quad 02$

$-2.00577 \mathrm{E} \quad 02$

$-2.00582 \mathrm{E} \quad 02$

$-2.00588 \mathrm{E} \quad 02$

$-2.00594 \mathrm{E} \quad 02$

$-2.00600 \mathrm{E} \quad 02$

-2.00605 E 02

$-2.00611 \mathrm{E} \quad 02$

$-2.00616 \mathrm{E} \quad 02$

$-2.00622 \mathrm{E} \quad 02$

$-2.00627 \mathrm{E} 02$

$-2.00633 \mathrm{E} \quad 02$

$-2.00638 \mathrm{E} \quad 02$

$3.32061 \mathrm{E}-02$

$3.34732 \mathrm{E}-02$

$3.37418 \mathrm{E}-02$

$3.40119 \mathrm{E}-02$

3.42833 E- 02

$3.45562 \mathrm{E}-02$

$3.48305 \mathrm{E}-02$

$3.51063 \mathrm{E}-02$

$3.53836 \mathrm{E}-02$

$3.56622 \mathrm{E}-02$

$3.59424 \mathrm{E}-02$

$3.62240 \mathrm{E}-02$

$3.65071 \mathrm{E}-02$

$3.67916 \mathrm{E}-02$

$3.70776 \mathrm{E}-02$

$3.73651 \mathrm{E}-02$

3.76541 E-02

$3.79446 \mathrm{E}-02$

$3.82365 \mathrm{E}-02$

$3.85300 \mathrm{E}-02$

3.88249 E-02

$3.91213 \mathrm{E}-02$

3.94193 E-02

$3.97187 \mathrm{E}-02$

$4.00197 \mathrm{E}-02$

$-2.00644 \mathrm{E} \quad 02$ $-2.00649 \mathrm{E} \quad 02$

$-2.00655 \mathrm{E} \quad 02$

$-2.00660 \mathrm{E} 02$

-2.00665 E 02

$-2.00671 \mathrm{E} \quad 02$

$-2.00676 \mathrm{E} \quad 02$

$-2.00681 \mathrm{E} 02$

$-2.00686 \mathrm{E} \quad 02$

$-2.00691 \mathrm{E} \quad 02$

$-2.00697 \mathrm{E} \quad 02$

$-2.00702 \mathrm{E} \quad 02$

$-2.00707 \mathrm{E} \quad 02$

$-2.00712 \mathrm{E} \quad 02$

$-2.00717 \mathrm{E} \quad 02$

$\begin{array}{ll}-2.00722 \mathrm{E} & 02\end{array}$

$-2.00727 \mathrm{E} \quad 02$

$\begin{array}{lll}-2.00732 \mathrm{E} & 02\end{array}$

$-2.00737 \mathrm{E} \quad 02$

$\begin{array}{ll}-2.00742 \mathrm{E} & 02\end{array}$

$-2.00747 \mathrm{E} \quad 02$

$-2.00752 \mathrm{E} \quad 02$

$-2.00756 \mathrm{E} 02$

$-2.00761 \mathrm{E} 02$

-2.00766 E 02

4.03222 E-02

$4.06262 \mathrm{E}-02$

$4.09317 \mathrm{E}-02$

$4.12387 \mathrm{E}-02$

$4.15473 \mathrm{E}-02$

4.18574 E- 02

$4.21690 \mathrm{E}-02$

4.24822 E-02

4.27969 E-02

$4.31132 \mathrm{E}-02$

$4.34311 \mathrm{E}-02$

4.37504 E-02

4.40714E-02

$4.43939 \mathrm{E}-02$

$4.47180 \mathrm{E}-02$
$-2.00771 \mathrm{E} \quad 02$

$\begin{array}{lll}-2.00776 \mathrm{E} & 02\end{array}$

$-2.00780 \mathrm{E} \quad 02$

-2.00785 E 02

$-2.00790 \mathrm{E} \quad 02$

$-2.00794 \mathrm{E} \quad 02$

$-2.00799 \mathrm{E} \quad 02$

-2.00803 E 02

$-2.00808 \mathrm{E} 02$

-2.00813 E 02

$-2.00817 \mathrm{E} \quad 02$

$-2.00822 \mathrm{E} \quad 02$

$-2.00826 \mathrm{E} \quad 02$

$-2.00831 \mathrm{E} 02$

-2.00835 E 02

$-2.72537 \mathrm{E}-02$

$-2.74883 \mathrm{E}-02$

$-2.77241 \mathrm{E}-02$

$-2.79614 \mathrm{E}-02$

$-2.81999 \mathrm{E}-02$

$-2.84398 \mathrm{E}-02$

$-2.86811 \mathrm{E}-02$

$-2.89237 \mathrm{E}-02$

-2.91677 E-02

$-2.94131 \mathrm{E}-02$

$-2.96598 \mathrm{E}-02$

$-2.99079 \mathrm{E}-02$

$-3.01574 \mathrm{E}-02$

$-3.04082 \mathrm{E}-02$

$-3.06605 \mathrm{E}-02$

$-3.09141 \mathrm{E}-02$

$-3.11691 \mathrm{E}-02$

$-3.14255 \mathrm{E}-02$

$-3.16833 \mathrm{E}-02$

$-3.19425 \mathrm{E}-02$

$-3.22032 \mathrm{E}-02$

$-3.24652 \mathrm{E}-02$

$-3.27286 \mathrm{E}-02$

$-3.29935 \mathrm{E}-02$

$-3.32598 \mathrm{E}-02$

$-3.35275 \mathrm{E}-02$ 
ELECTRON RADIAL FUNCTIONS

Positron

p $\quad F_{0}$

$0.10 \quad 6.10213 \mathrm{E}-01$

$0.20 \quad 7.85806 \mathrm{E}-01$

$0.30 \quad 8.50399 \mathrm{E}-01$

$0.40 \quad 8.83113 \mathrm{E}-01$

$0.50 \quad 9.02461 \mathrm{E}-01$

$0.60 \quad 9.14998 \mathrm{E}-01$

$0.70 \quad 9.23629 \mathrm{E}-01$

$0.80 \quad 9.29835 \mathrm{E}-01$

$0.90 \quad 9.34446 \mathrm{E}-01$

$1.00 \quad 9.37963 \mathrm{E}-01$

$1.10 \quad 9.40704 \mathrm{E}-01$

$1.20 \quad 9.42878 \mathrm{E}-01$

$1.30 \quad 9.44630 \mathrm{E}-01$

$1.40 \quad 9.46061 \mathrm{E}-01$

$1.50 \quad 9.47243 \mathrm{E}-01$

$1.60 \quad 9.48230 \mathrm{E}-01$

$1.70 \quad 9.49061 \mathrm{E}-01$

$1.80 \quad 9.49769 \mathrm{E}-01$

$1.90 \quad 9.50374 \mathrm{E}-01$

$2.00 \quad 9.50897 \mathrm{E}-01$

2.10

2.20

2.30

2.40

2.50

2.60

2.70

2.80

2.90

3.00

3.10

3.20

3.30

3.40

3.50

3.60

3.70

3.80

3.90

4.00

4.10

4.20

4.30

4.40

4.50

4.60

4.70

4.80

4.90

5.00

5.10

5.20

5.30

5.40

5.50

5.60

5.70

5.80

5.90

6.00
$Z=2$

$f_{1}$

5.30341 E-03

2.44802 E- 02

$5.65708 \mathrm{E}-02$

$1.00272 \mathrm{E}-01$

$1.54061 \mathrm{E}-01$

$2.16370 \mathrm{E}-01$

$2.85718 \mathrm{E}-01$

3.60780 E-01

$4.40436 \mathrm{E}-01$

5.23753 E-01

$6.09976 \mathrm{E}-01$

$6.98502 \mathrm{E}-01$

$7.88856 \mathrm{E}-01$

$8.80660 \mathrm{E}-01$

$9.73618 \mathrm{E}-01$

$1.06750 \mathrm{E} \quad 00$

$1.16212 \mathrm{E} \quad 00$

$1.25732 \mathrm{E} \quad 00$

$1.35301 \mathrm{E} \quad 00$

$1.44907 \mathrm{E} \quad 00$

$1.54543 \mathrm{E} \quad 00$

$1.64204 \mathrm{E} \quad 00$

$1.73882 \mathrm{E} \quad 00$

$1.83574 \mathrm{E} \quad 00$

$1.93275 \mathrm{E} 00$

$2.02979 \mathrm{E} \quad 00$

$2.12679 \mathrm{E} \quad 00$

$2.22365 \mathrm{E} \quad 00$

$2.32019 \mathrm{E} \quad 00$

$2.41599 \mathrm{E} \quad 00$

$2.50976 \mathrm{E} \quad 00$

$2.59455 \mathrm{E} \quad 00$

$2.92992 \mathrm{E} \quad 00$

$2.83524 \mathrm{E} \quad 00$

$2.92363 \mathrm{E} \quad 00$

$3.01819 \mathrm{E} \quad 00$

$3.11443 \mathrm{E} \quad 00$

$3.21137 \mathrm{E} \quad 00$

$3.30867 \mathrm{E} \quad 00$

$3.40618 \mathrm{E} \quad 00$

$3.50382 \mathrm{E} \quad 00$

$3.60156 \mathrm{E} \quad 00$

$3.69936 \mathrm{E} \quad 00$

$3.79720 \mathrm{E} \quad 00$

$3.89509 \mathrm{E} \quad 00$

$3.99300 \mathrm{E} \quad 00$

$4.09094 \mathrm{E} \quad 00$

$4.18889 \mathrm{E} \quad 00$

$4.28686 \mathrm{E} \quad 00$

$4.38484 \mathrm{E} \quad 00$

$4.48283 \mathrm{E} \quad 00$

$4.58082 \mathrm{E} \quad 00$

$4.67883 \mathrm{E} \quad 00$

$4.77684 \mathrm{E} \quad 00$

$4.87486 \mathrm{E} \quad 00$

$4.97288 \mathrm{E} \quad 00$

$5.07090 \mathrm{E} \quad 00$

$5.16893 \mathrm{E} \quad 00$

$5.26696 \mathrm{E} \quad 00$

$5.36499 \mathrm{E} \quad 00$ $g_{1}$

$-1.34762 \mathrm{E}-05$

$-6.16088 \mathrm{E}-05$

$-1.40117 \mathrm{E}-04$

$-2.42917 \mathrm{E}-04$

$-3.62838 \mathrm{E}-04$

$-4.92451 \mathrm{E}-04$

$-6.24696 \mathrm{E}-04$

$-7.53237 \mathrm{E}-04$

$-8.72659 \mathrm{E}-04$

$-9.78442 \mathrm{E}-04$

$-1.06691 \mathrm{E}-03$

$-1.13510 \mathrm{E}-03$

$-1.18065 \mathrm{E}-03$

$-1.20171 \mathrm{E}-03$

$-1.19678 \mathrm{E}-03$

$-1.16470 \mathrm{E}-03$

$-1.10452 \mathrm{E}-03$

$-1.01552 \mathrm{E}-03$

$-8.97102 \mathrm{E}-04$

$-7.48787 \mathrm{E}-04$

$-5.70199 \mathrm{E}-04$

$-3.61034 \mathrm{E}-04$

$-1.21049 \mathrm{E}-04$

$1.49948 \mathrm{E}-04$

$4.52103 \mathrm{E}-04$

$7.85516 \mathrm{E}-04$

$1.15024 \mathrm{E}-03$

$1.54624 \mathrm{E}-03$

$1.97336 \mathrm{E}-03$

$2.43107 \mathrm{E}-03$

$2.91757 \mathrm{E}-03$

$3.42276 \mathrm{E}-03$

$4.32564 \mathrm{E}-03$

4.63257E-03

$5.23872 \mathrm{E}-03$

$5.88586 \mathrm{E}-03$

$6.56744 \mathrm{E}-03$

$7.28208 \mathrm{E}-03$

$8.02928 \mathrm{E}-03$

$8.80886 \mathrm{E}-03$

$9.62072 \mathrm{E}-03$

$1.04648 \mathrm{E}-02$

$1.13411 \mathrm{E}-02$

$1.22496 \mathrm{E}-02$

$1.31902 \mathrm{E}-02$

$1.41631 \mathrm{E}-02$

$1.51681 \mathrm{E}-02$

$1.62053 \mathrm{E}-02$

$1.72747 \mathrm{E}-02$

$1.83762 \mathrm{E}-02$

$1.95100 \mathrm{E}-02$

$2.06759 \mathrm{E}-02$

$2.18740 \mathrm{E}-02$

$2.31044 \mathrm{E}-02$

2.43669E-02

$2.56616 \mathrm{E}-02$

$2.69885 \mathrm{E}-02$

$2.83476 \mathrm{E}-02$

$2.97389 \mathrm{E}-02$

$3.11624 \mathrm{E}-02$ $\tan \delta_{1}$

$6.21070 \mathrm{E}-02$

$3.09460 \mathrm{E}-02$

$2.05825 \mathrm{E}-02$

$1.53824 \mathrm{E}-02$

$1.22459 \mathrm{E}-02$

$1.01419 \mathrm{E}-02$

$8.62914 \mathrm{E}-03$

$7.48688 \mathrm{E}-03$

$6.59253 \mathrm{E}-03$

$5.87243 \mathrm{E}-03$

$5.27966 \mathrm{E}-03$

$4.78282 \mathrm{E}-03$

$4.36015 \mathrm{E}-03$

$3.99604 \mathrm{E}-03$

$3.67900 \mathrm{E}-03$

$3.40039 \mathrm{E}-03$

$3.15356 \mathrm{E}-03$

$2.93336 \mathrm{E}-03$

$2.73565 \mathrm{E}-03$

$2.55715 \mathrm{E}-03$

$2.39516 \mathrm{E}-03$

$2.24750 \mathrm{E}-03$

$2.11233 \mathrm{E}-03$

$1.98813 \mathrm{E}-03$

$1.87361 \mathrm{E}-03$

$1.76768 \mathrm{E}-03$

$1.66940 \mathrm{E}-03$

$1.57798 \mathrm{E}-03$

$1.49271 \mathrm{E}-03$

$1.41300 \mathrm{E}-03$

$1.33833 \mathrm{E}-03$

$1.26822 \mathrm{E}-03$

$1.20226 \mathrm{E}-03$

$1.14011 \mathrm{E}-03$

$1.08144 \mathrm{E}-03$

$1.02597 \mathrm{E}-03$

$9.73436 \mathrm{E}-04$

$9.23620 \mathrm{E}-04$

$8.76310 \mathrm{E}-04$

8.31324 E- 04

$7.88495 \mathrm{E}-04$

$7.47673 \mathrm{E}-04$

$7.08718 \mathrm{E}-04$

$6.71508 \mathrm{E}-04$

$6.35925 \mathrm{E}-04$

$6.01866 \mathrm{E}-04$

$5.69234 \mathrm{E}-04$

$5.37945 \mathrm{E}-04$

$5.07913 \mathrm{E}-04$

$4.79067 \mathrm{E}-04$

$4.51338 \mathrm{E}-04$

$4.24662 \mathrm{E}-04$

$3.98978 \mathrm{E}-04$

$3.74235 \mathrm{E}-04$

3.50381 E-04

$3.27369 \mathrm{E}-04$

$3.05158 \mathrm{E}-04$

2.83702 E- 04

2.62969 E-04

$2.42917 \mathrm{E}-04$

$f_{-1}$

$6.43251 \mathrm{E}-04$

$1.44854 \mathrm{E}-03$

$2.23191 \mathrm{E}-03$

$2.98144 \mathrm{E}-03$

$3.68993 \mathrm{E}-03$

$4.35297 \mathrm{E}-03$

$4.96829 \mathrm{E}<03$

$5.53521 \mathrm{E}-03$

$6.05408 \mathrm{E}-03$

$6.52586 \mathrm{E}-03$

$6.95184 \mathrm{E}-03$

$7.33340 \mathrm{E}-03$

$7.67192 \mathrm{E}-03$

$7.96869 \mathrm{E}-03$

$8.22489 \mathrm{E}-03$

$8.44156 \mathrm{E}-03$

8.61962 E-03

8.75987E-03

$8.86300 \mathrm{E}-03$

$8.92961 \mathrm{E}-03$

$8.96023 \mathrm{E}-03$

$8.95531 \mathrm{E}-03$

$8.91522 \mathrm{E}-03$

$8.84030 \mathrm{E}-03$

$8.73085 \mathrm{E}-03$

$8.58712 \mathrm{E}-03$

$8.40932 \mathrm{E}-03$

$8.19767 \mathrm{E}-03$

$7.95231 \mathrm{E}-03$

$7.67340 \mathrm{E}-03$

$7.36107 \mathrm{E}-03$

$7.01543 \mathrm{E}-03$

$6.63659 \mathrm{E}-03$

$6.22464 \mathrm{E}-03$

$5.77966 \mathrm{E}-03$

$5.30171 \mathrm{E}-03$

$4.79087 \mathrm{E}-03$

$4.24718 \mathrm{E}-03$

3.67071 E-03

$3.06150 \mathrm{E}-03$

$2.41958 \mathrm{E}-03$ 
ELECTRON RADIAL FUNCTIONS

$Z=2 \quad A=4 \quad \rho=1.2 A^{1 / 3} \mathrm{~F}$

Positron

$\bar{F}$ $f_{2}$

$4.20932 \mathrm{E}-09$ $2.13447 \mathrm{E}-08$ $5.18226 \mathrm{E}-08$ $9.55773 \mathrm{E}-08$ $1.52542 \mathrm{E}-07$

$2.22658 \mathrm{E}-07$ $3.05879 \mathrm{E}-07$ $4.02164 \mathrm{E}-07$ $5.11483 \mathrm{E}-07$ $6.33813 \mathrm{E}-07$

$7.69134 \mathrm{E}-07$ $9.17432 \mathrm{E}-07$ $1.07869 \mathrm{E}-06$ $1.25291 \mathrm{E}-06$ $1.44008 \mathrm{E}-06$

$1.64019 \mathrm{E}-06$ $1.85324 \mathrm{E}-06$ $2.07923 \mathrm{E}-06$ $2.31814 \mathrm{E}-06$ $2.56999 \mathrm{E}-06$

$2.83477 \mathrm{E}-06$ $3.11247 \mathrm{E}-06$ $3.40309 \mathrm{E}-06$ $3.70664 \mathrm{E}-06$ $4.02312 \mathrm{E}-06$

$4.35252 \mathrm{E}-06$ $4.69484 \mathrm{E}-06$ $5.05007 \mathrm{E}-06$ $5.41824 \mathrm{E}-06$ $5.79931 \mathrm{E}-06$

$6.19332 \mathrm{E}-06$ $6.60024 \mathrm{E}-06$ $7.02008 \mathrm{E}-06$ $7.45284 \mathrm{E}-06$ $7.89852 \mathrm{E}-06$

$8.35710 \mathrm{E}-06$ $8.82862 \mathrm{E}-06$ $9.31305 \mathrm{E}-06$ $9.81040 \mathrm{E}-06$ $1.03207 \mathrm{E}-05$

$1.08439 \mathrm{E}-05$

$1.13800 \mathrm{E}-05$

$1.19290 \mathrm{E}-05$

$1.24909 \mathrm{E}-05$

$1.30658 \mathrm{E}-05$

$1.36536 \mathrm{E}-05$ $1.42543 \mathrm{E}-05$ $1.48679 \mathrm{E}-05$ $1.54944 \mathrm{E}-05$ $1.61338 \mathrm{E}-05$

$1.67862 \mathrm{E}-05$ $1.74515 \mathrm{E}-05$ $1.81297 \mathrm{E}-05$ $1.88208 \mathrm{E}-05$ $1.95248 \mathrm{E}-05$

$2.02418 \mathrm{E}-05$ $2.09716 \mathrm{E}-05$ $2.17144 \mathrm{E}-05$ $2.24701 \mathrm{E}-05$ $2.32387 \mathrm{E}-05$
$8.32769 \mathrm{E}-07$ $7.95970 \mathrm{E}-06$ $2.77545 \mathrm{E}-05$ $6.57275 \mathrm{E}-05$ $1.26350 \mathrm{E}-04$

$2.13048 \mathrm{E}-04$ $3.28318 \mathrm{E}-04$ $4.73878 \mathrm{E}-04$ $6.50891 \mathrm{E}-04$ $8.60080 \mathrm{E}-04$

$1.10187 \mathrm{E}-03$

$1.37651 \mathrm{E}-03$

$1.68409 \mathrm{E}-03$

$2.02462 \mathrm{E}-03$

$2.39807 \mathrm{E}-03$

$2.80433 \mathrm{E}-03$ $3.24329 \mathrm{E}-03$

$3.71476 \mathrm{E}-03$

$4.21848 \mathrm{E}-03$

4.75404E-03

$5.32067 \mathrm{E}-03$ $5.91668 \mathrm{E}-03$ $6.53726 \mathrm{E}-03$

$7.15959 \mathrm{E}-03$ $6.36570 \mathrm{E}-03$

$8.80508 \mathrm{E}-03$ $9.51471 \mathrm{E}-03$ $1.02960 \mathrm{E}-02$

$1.11191 \mathrm{E}-02$

$1.19781 \mathrm{E}-02$

1.28714 E-02 $1.37980 \mathrm{E}-02$ $1.47577 \mathrm{E}-02$ $1.57501 \mathrm{E}-02$ $1.67751 \mathrm{E}-02$

$1.78326 \mathrm{E}-02$ $1.89225 \mathrm{E}-02$ $2.00449 \mathrm{E}-02$ $2.11997 \mathrm{E}-02$ $2.23868 \mathrm{E}-02$

2.36062E-02 $2.48579 \mathrm{E}-02$

$2.61419 \mathrm{E}-02$

$2.74582 \mathrm{E}-02$

$2.88067 \mathrm{E}-02$

$3.01875 \mathrm{E}-02$ $3.16005 \mathrm{E}-02$ $3.30458 \mathrm{E}-02$ $3.45233 \mathrm{E}-02$ 3.60331 E-02

$3.75750 \mathrm{E}-02$ $3.91492 \mathrm{E}-02$ $4.07555 \mathrm{E}-02$ $4.23941 \mathrm{E}-02$ $4.40649 \mathrm{E}-02$

4.57679 $\mathrm{E}-02$ $4.75031 \mathrm{E}-02$ $4.92704 \mathrm{E}-02$ $5.10700 \mathrm{E}-02$ $5.29018 \mathrm{E}-02$ $g_{2}$

$\tan \delta_{2}$

$-1.13075 \mathrm{E}-09 \quad-7.34373 \mathrm{E} \quad 00$ $-1.06915 \mathrm{E}-08 \quad-1.45950 \mathrm{E} \quad 01$ $-3.66165 \mathrm{E}-08 \quad-2.15249 \mathrm{E} \quad 0 \mathrm{l}$ $-8.45742 \mathrm{E}-08 \quad-2.80393 \mathrm{E} \quad 01$ $-1.57469 \mathrm{E}-07$

$-2.55398 \mathrm{E}-07$ $-3.75938 \mathrm{E}-07$ $-5.14573 \mathrm{E}-07$ $-6.65215 \mathrm{E}-07$

$-8.20584 \mathrm{E}-07$

$-9.72575 \mathrm{E}-07$ $-1.11253 \mathrm{E}-06$ $-1.23140 \mathrm{E}-06$ $-1.31992 \mathrm{E}-06$ $-1.36864 \mathrm{E}-06$

$-1.36805 \mathrm{E}-06$ $-1.30856 \mathrm{E}-06$ $-1.18060 \mathrm{E}-06$ $-9.74592 \mathrm{E}-07$ $-6.80995 \mathrm{E}-07$

$-2.90393 \mathrm{E}-07$ $2.06339 \mathrm{E}-07$ $8.17331 \mathrm{E}-07$

$1.54508 \mathrm{E}-06$

$1.95520 \mathrm{E}-06$

$3.51316 \mathrm{E}-06$ $4.67454 \mathrm{E}-06$ $6.01301 \mathrm{E}-06$

$7.52879 \mathrm{E}-06$

$9.22964 \mathrm{E}-06$

$1.11246 \mathrm{E}-05$ $1.32230 \mathrm{E}-05$ $1.55342 \mathrm{E}-05$ $1.80677 \mathrm{E}-05$ $2.08331 \mathrm{E}-05$

$2.38399 \mathrm{E}-05$ $2.70975 \mathrm{E}-05$ $3.06156 \mathrm{E}-05$ $3.44037 \mathrm{E}-05$

$3.84712 \mathrm{E}-05$

$4.28278 \mathrm{E}-05$ $4.74830 \mathrm{E}-05$

$5.24462 \mathrm{E}-05$

$5.77270 \mathrm{E}-05$

$6.33351 \mathrm{E}-05$

$6.92797 \mathrm{E}-05$ $7.55706 \mathrm{E}-05$ $8.22172 \mathrm{E}-05$ $8.92291 \mathrm{E}-05$ $9.66158 \mathrm{E}-05$

$1.04387 \mathrm{E}-04$ $1.12552 \mathrm{E}-04$ $1.21120 \mathrm{E}-04$ $1.30101 \mathrm{E}-04$ $1.39504 \mathrm{E}-04$

$1.49339 \mathrm{E}-04$ $1.59616 \mathrm{E}-04$ $1.70344 \mathrm{E}-04$ $1.81532 \mathrm{E}-04$ $1.93190 \mathrm{E}-04$

$f_{-2}$

$6.10514 \mathrm{E}-08$ $2.72757 \mathrm{E}-07$ $6.29262 \mathrm{E}-07$ $1.11968 \mathrm{E}-06$ $1.73073 \mathrm{E}-06$

$2.44795 \mathrm{E}-06$ $3.25661 \mathrm{E}-06$ 4.14227 E-06 $5.09110 \mathrm{E}-06$ $6.08999 \mathrm{E}-06$

$7.12653 \mathrm{E}-06$ $8.18889 \mathrm{E}-06$

$9.26577 \mathrm{E}-06$

$1.03463 \mathrm{E}-05$

$1.14198 \mathrm{E}-05$

$1.24760 \mathrm{E}-05$ $1.35049 \mathrm{E}-05$ $1.44963 \mathrm{E}-05$ $1.54404 \mathrm{E}-05$ $1.63274 \mathrm{E}-05$

$1.71477 \mathrm{E}-05$ $1.78915 \mathrm{E}-05$ $1.85492 \mathrm{E}-05$

$1.91112 \mathrm{E}-05$

$1.95678 \mathrm{E}-05$

$1.99097 \mathrm{E}-05$ $2.01271 \mathrm{E}-05$ $2.02105 \mathrm{E}-05$

$2.01505 \mathrm{E}-05$

$1.99375 \mathrm{E}-05$

$1.95619 \mathrm{E}-05$ $1.90143 \mathrm{E}-05$ $1.82851 \mathrm{E}-05$ $1.73647 \mathrm{E}-05$ $1.62438 \mathrm{E}-05$

$1.49128 \mathrm{E}-05$ $1.33621 \mathrm{E}-05$ $1.15823 \mathrm{E}-05$ $9.56380 \mathrm{E}-06$ $7.29715 \mathrm{E}-06$

$4.77283 \mathrm{E}-06$ 
ELECTRON RADIAL FUNCTIONS

Positron

\begin{tabular}{|c|c|}
\hline$p$ & $F_{0}$ \\
\hline 6.10 & $9.55319 \mathrm{E}-01$ \\
\hline 6.20 & $9.55335 \mathrm{E}-01$ \\
\hline 6.30 & $9.55350 \mathrm{E}-01$ \\
\hline 6.40 & $9.55363 \mathrm{E}-01$ \\
\hline 6.50 & $9.55377 \mathrm{E}-01$ \\
\hline 6.60 & $9.55389 \mathrm{E}-01$ \\
\hline 6.70 & $9.55401 \mathrm{E}-01$ \\
\hline 6.80 & $9.55413 \mathrm{E}-01$ \\
\hline 6.90 & $9.55423 \mathrm{E}-01$ \\
\hline 7.00 & $9.55434 \mathrm{E}-01$ \\
\hline 7.10 & $9.55443 \mathrm{E}-01$ \\
\hline 7.20 & $9.55453 \mathrm{E}-01$ \\
\hline 7.30 & $9.55461 \mathrm{E}-01$ \\
\hline 7.40 & $9.55470 \mathrm{E}-01$ \\
\hline 7.50 & $9.55478 \mathrm{E}-01$ \\
\hline 7.60 & $9.55485 \mathrm{E}-01$ \\
\hline 7.70 & $9.55492 \mathrm{E}-01$ \\
\hline 7.80 & $9.55499 \mathrm{E}-01$ \\
\hline 7.90 & $9.55506 \mathrm{E}-01$ \\
\hline 8.00 & $9.55512 \mathrm{E}-01$ \\
\hline 8.10 & $9.55518 \mathrm{E}-01$ \\
\hline 8.20 & $9.55523 E-01$ \\
\hline 8.30 & $9.55529 \mathrm{E}-01$ \\
\hline 8.40 & $9.55534 \mathrm{E}-01$ \\
\hline 8.50 & $9.55539 \mathrm{E}-01$ \\
\hline 8.60 & $9.55544 \mathrm{E}-01$ \\
\hline 8.70 & $9.55548 \mathrm{E}-01$ \\
\hline 8.80 & $9.55552 \mathrm{E}-01$ \\
\hline 8.90 & $9.55556 \mathrm{E}-01$ \\
\hline 9.00 & $9.55560 \mathrm{E}-01$ \\
\hline 9.10 & $9.55564 \mathrm{E}-01$ \\
\hline 9.20 & $9.55568 \mathrm{E}-01$ \\
\hline 9.30 & $9.55571 \mathrm{E}-01$ \\
\hline 9.40 & $9.55574 \mathrm{E}-01$ \\
\hline 9.50 & $9.55577 \mathrm{E}-0 \mathrm{l}$ \\
\hline 9.60 & $9.55580 \mathrm{E}-01$ \\
\hline 9.70 & $9.55583 \mathrm{E}-01$ \\
\hline 9.80 & $9.55586 \mathrm{E}-01$ \\
\hline 9.90 & $9.55588 \mathrm{E}-0 \mathrm{l}$ \\
\hline 0.00 & $9.55591 \mathrm{E}-01$ \\
\hline 0.10 & $9.55593 \mathrm{E}-01$ \\
\hline 0.20 & $9.55596 \mathrm{E}-01$ \\
\hline 0.30 & $9.55598 \mathrm{E}-01$ \\
\hline 0.40 & $9.55600 \mathrm{E}-01$ \\
\hline 0.50 & $9.55602 \mathrm{E}-01$ \\
\hline 0.60 & $9.55604 \mathrm{E}-01$ \\
\hline 0.70 & $9.55605 \mathrm{E}-01$ \\
\hline 0.80 & $9.55607 \mathrm{E}-01$ \\
\hline 0.90 & $9.55609 \mathrm{E}-01$ \\
\hline 1.00 & $9.55610 \mathrm{E}-01$ \\
\hline 1.10 & $9.55612 \mathrm{E}-01$ \\
\hline 1.20 & $9.55613 \mathrm{E}-01$ \\
\hline 1.30 & $9.55614 \mathrm{E}-01$ \\
\hline 1.40 & $9.55615 \mathrm{E}-01$ \\
\hline 1.50 & $9.55617 \mathrm{E}-01$ \\
\hline 1.60 & $9.55618 \mathrm{E}-01$ \\
\hline 1.70 & $9.55619 \mathrm{E}-01$ \\
\hline 1.80 & $9.55620 \mathrm{E}-01$ \\
\hline $\begin{array}{l}1.90 \\
2.00\end{array}$ & $\begin{array}{l}9.55621 \mathrm{E}-01 \\
9.55622 \mathrm{E}-01\end{array}$ \\
\hline
\end{tabular}

$Z=2 \quad A=4$

$f_{1}$

$5.46303 \mathrm{E} \quad 00$ $5.56106 \mathrm{E} \quad 00$ $5.65910 \mathrm{E} \quad 00$ $5.75714 \mathrm{E} \quad 00$ $5.85519 \mathrm{E} \quad 00$

$5.95323 \mathrm{E} \quad 00$ $6.05128 \mathrm{E} \quad 00$ $6.14932 \mathrm{E} \quad 00$ $6.24737 \mathrm{E} \quad 00$ $6.34542 \mathrm{E} \quad 00$

$6.4434 .7 \mathrm{E} \quad 00$ $6.54153 \mathrm{E} \quad 00$ $6.63958 \mathrm{E} \quad 00$ $6.73764 \mathrm{E} \quad 00$ $6.83569 \mathrm{E} \quad 00$

$6.93375 \mathrm{E} \quad 00$

$7.03181 \mathrm{E} \quad 00$

$7.12987 \mathrm{E} \quad 00$

$7.22794 \mathrm{E} \quad 00$

$7.32600 \mathrm{E} \quad 00$

$7.42407 \mathrm{E} \quad 00$

$7.52214 \mathrm{E} \quad 00$

$7.62021 \mathrm{E} \quad 00$

$\begin{array}{ll}7.71828 \mathrm{E} & 00\end{array}$

$7.81636 \mathrm{E} \quad 00$

$7.91444 \mathrm{E} \quad 00$ $8.01252 \mathrm{E} \quad 00$ $8.11060 \mathrm{E} \quad 00$ $8.20868 \mathrm{E} \quad 00$ $8.30677 \mathrm{E} \quad 00$

$8.40485 \mathrm{E} \quad 00$ $8.50295 \mathrm{E} \quad 00$ $8.60104 \mathrm{E} \quad 00$ $8.69914 \mathrm{E} \quad 00$ $8.79724 \mathrm{E} \quad 00$

$8.89533 \mathrm{E} \quad 00$ $8.99344 \mathrm{E} \quad 00$ $9.09155 \mathrm{E} \quad 00$ $9.18966 \mathrm{E} \quad 00$ $9.28777 \mathrm{E} \quad 00$

$9.38589 \mathrm{E} \quad 00$ $9.48401 \mathrm{E} \quad 00$ $9.58213 \mathrm{E} \quad 00$ $9.68025 \mathrm{E} \quad 00$ $9.77838 \mathrm{E} \quad 00$

$9.87652 \mathrm{E} \quad 00$ $9.97465 \mathrm{E} \quad 00$ $\begin{array}{lll}1.00728 \mathrm{E} & 01\end{array}$ $1.01709 \mathrm{E} \quad 01$ $1.02691 \mathrm{E} 01$

$\begin{array}{ll}1.03672 \mathrm{E} & 01\end{array}$ $1.04654 \mathrm{E} \quad 01$ $1.05635 \mathrm{E} \quad 01$ $1.06617 \mathrm{E} 01$ $1.07599 \mathrm{E} 01$

$1.08580 \mathrm{E} \quad 01$ $1.09562 \mathrm{E} 01$ $1.10544 \mathrm{E} \quad 01$ $1.11526 \mathrm{E} \quad 01$ $1.12507 \mathrm{E} \quad 01$ $g_{1}$

$3.26182 \mathrm{E}-02$

$3.41061 \mathrm{E}-02$

$3.56263 \mathrm{E}-02$

$3.71787 \mathrm{E}-02$

$3.87633 \mathrm{E}-02$

$4.03801 \mathrm{E}-02$

$4.20291 \mathrm{E}-02$

$4.37104 \mathrm{E}-02$

$4.54239 \mathrm{E}-02$

$4.71696 \mathrm{E}-02$

$4.89476 \mathrm{E}-02$

$5.07578 \mathrm{E}-02$

$5.26002 \mathrm{E}-02$

$5.44749 \mathrm{E}-02$

$5.63818 \mathrm{E}-02$

$5.83209 \mathrm{E}-02$

$6.02923 \mathrm{E}-02$

$6.22960 \mathrm{E}-02$

$6.43319 \mathrm{E}-02$

$6.64000 \mathrm{E}-02$

$6.85005 \mathrm{E}-02$

$7.06331 \mathrm{E}-02$

$7.27981 \mathrm{E}-02$

$7.49953 \mathrm{E}-02$

$7.72247 \mathrm{E}-02$

$7.94865 \mathrm{E}-02$ $8.17805 \mathrm{E}-02$ $8.41068 \mathrm{E}-02$ 8.64653E-02

$8.88561 \mathrm{E}-02$

$9.12792 \mathrm{E}-02$ $9.37347 \mathrm{E}-02$ $9.62224 \mathrm{E}-02$ $9.87423 \mathrm{E}-02$ $1.01295 \mathrm{E}-01$

$1.03879 \mathrm{E}-01$ $1.06496 \mathrm{E}-01$

$1.09145 \mathrm{E}-01$

$1.11827 \mathrm{E}-01$

$1.14540 \mathrm{E}-01$

$1.17287 \mathrm{E}-01$ $1.20065 \mathrm{E}-01$ $1.22876 \mathrm{E}-01$

$1.25719 \mathrm{E}-01$

$1.28594 \mathrm{E}-01$

$1.31502 \mathrm{E}-01$ $1.34442 \mathrm{E}-01$ $1.37414 \mathrm{E}-01$ $1.40419 \mathrm{E}-01$ $1.43456 \mathrm{E}-01$

$1.46525 \mathrm{E}-01$ $1.49627 \mathrm{E}-01$ $1.52761 \mathrm{E}-01$ $1.55927 \mathrm{E}-01$ $1.59126 \mathrm{E}-01$

$1.62357 \mathrm{E}-01$

$1.65621 \mathrm{E}-01$

$1.68917 \mathrm{E}-01$

$1.72245 \mathrm{E}-01$

$1.75605 \mathrm{E}-01$ $\tan \delta_{1}$

$f_{-1}$

$2.23518 \mathrm{E}-04-1.72474 \mathrm{E}-02$

$2.04738 \mathrm{E}-04-1.85711 \mathrm{E}-02$

$1.86549 \mathrm{E}-04-1.99271 \mathrm{E}-02$

$1.68922 \mathrm{E}-04-2.13154 \mathrm{E}-02$

$1.51836 \mathrm{E}-04 \quad-2.27361 \mathrm{E}-02$

$1.35261 \mathrm{E}-04$

$1.19179 \mathrm{E}-04$

$1.03566 \mathrm{E}-04$

$8.84019 \mathrm{E}-05$

$7.36702 \mathrm{E}-05$

$5.93491 \mathrm{E}-05$

$4.54238 \mathrm{E}-05$

$3.18769 \mathrm{E}-05$

$1.86967 \mathrm{E}-05$

$5.86292 \mathrm{E}-06$

$-6.63310 \mathrm{E}-06$

$-1.88036 \mathrm{E}-05$

$-3.06663 \mathrm{E}-05$

$-4.22292 \mathrm{E}-05$

$-5.35016 \mathrm{E}-05$

$-6.44992 \mathrm{E}-05$

$-7.52277 \mathrm{E}-05$

$-8.57001 \mathrm{E}-05$

$-9.59199 \mathrm{E}-05$

$-1.05904 \mathrm{E}-04$

$-1.15650 \mathrm{E}-04$ $-1.25176 \mathrm{E}-04$ $-1.34484 \mathrm{E}-04$

$-1.43585 \mathrm{E}-04$

$-1.52482 \mathrm{E}-04$

$-1.61182 \mathrm{E}-04$

$-1.69696 \mathrm{E}-04$

$-1.78023 \mathrm{E}-04$

$-1.86173 \mathrm{E}-04$

$-1.94154 \mathrm{E}-04$

$-2.01965 \mathrm{E}-04$

$-2.09616 \mathrm{E}-04$

$-2.17110 \mathrm{E}-04$

$-2.24449 \mathrm{E}-04$

$-2.31643 \mathrm{E}-04$

$-2.38692 \mathrm{E}-04$

$-2.45605 \mathrm{E}-04$

$-2.52380 \mathrm{E}-04$

$-2.59025 \mathrm{E}-04$

$-2.65540 \mathrm{E}-04$

$-2.71933 \mathrm{E}-04$

$-2.78207 \mathrm{E}-04$

$-2.84362 \mathrm{E}-04$

$-2.90403 \mathrm{E}-04$

$-2.96332 \mathrm{E}-04$

$-3.02156 \mathrm{E}-04$

$-3.07871 \mathrm{E}-04$

$-3.13487 \mathrm{E}-04$

$-3.19003 \mathrm{E}-04$

$-3.24419 \mathrm{E}-04$

$-3.29740 \mathrm{E}-04$

$-3.34971 \mathrm{E}-04$

$-3.40114 \mathrm{E}-04$

$-3.45163 \mathrm{E}-04$

$-3.50130 \mathrm{E}-04$
-2.41891 E- 02 $-2.56744 \mathrm{E}-02$ $-2.71921 \mathrm{E}-02$ $-2.87421 \mathrm{E}-02$

$-3.19390 \mathrm{E}-02$ $-3.35860 \mathrm{E}-02$ $-3.52652 \mathrm{E}-02$ $-3.69768 \mathrm{E}-02$ $-3.87207 \mathrm{E}-02$

$-4.04970 \mathrm{E}-02$ $-4.23055 \mathrm{E}-02$ $-4.41463 \mathrm{E}-02$ $-4.60195 \mathrm{E}-02$ $-4.79250 \mathrm{E}-02$

$-4.98628 \mathrm{E}-02$ $-5.18329 \mathrm{E}-02$ $-5.38353 \mathrm{E}-02$ $-5.58701 \mathrm{E}-02$ $-5.79371 \mathrm{E}-02$

$-6.00365 \mathrm{E}-02$ $-6.21682 \mathrm{E}-02$ $-6.43322 \mathrm{E}-02$ $-6.65285 \mathrm{E}-02$ $-6.87572 \mathrm{E}-02$

$-7.10182 \mathrm{E}-02$ $-7.56371 \mathrm{E}-02$ $-7.79951 \mathrm{E}-02$ $-8.03854 \mathrm{E}-02$

$-8.28080 \mathrm{E}-02$ $-8.52629 \mathrm{E}-02$ $-8.77502 \mathrm{E}-02$ $-9.02699 \mathrm{E}-02$ $-9.28218 \mathrm{E}-02$

$-9.54061 \mathrm{E}-02$ $-9.80228 \mathrm{E}-02$ $-1.00672 \mathrm{E}-01$ $-1.03353 \mathrm{E}-01$ $-1.06067 \mathrm{E}-01$

$-1.08813 \mathrm{E}-01$ $-1.11591 \mathrm{E}-01$ $-1.14402 \mathrm{E}-01$ $-1.17245 \mathrm{E}-01$ $-1.20121 \mathrm{E}-01$

$-1.23029 \mathrm{E}-01$ $-1.25969 \mathrm{E}-01$ $-1.28942 \mathrm{E}-01$ $-1.31947 \mathrm{E}-01$ $-1.34984 \mathrm{E}-01$

$-1.38054 \mathrm{E}-01$ $-1.41156 \mathrm{E}-01$ $-1.44290 \mathrm{E}-01$ $-1.47457 \mathrm{E}-01$ $-1.50657 \mathrm{E}-01$ $-3.03244 \mathrm{E}-02$ $-7.33115 \mathrm{E}-02$

$g_{-1}$

$6.43068 \mathrm{E} \quad 00$ $6.52909 \mathrm{E} \quad 00$ $6.62749 \mathrm{E} \quad 00$ $6.72588 \mathrm{E} \quad 00$ $6.82425 \mathrm{E} \quad 00$

$6.92262 \mathrm{E} \quad 00$ $7.02097 \mathrm{E} \quad 00$ $7.11932 \mathrm{E} \quad 00$ $7.21765 \mathrm{E} \quad 00$ $7.31598 \mathrm{E} \quad 00$

$7.41429 \mathrm{E} \quad 00$ $7.51260 \mathrm{E} \quad 00$ $7.61091 \mathrm{E} \quad 00$ $7.70920 \mathrm{E} \quad 00$ $7.80749 \mathrm{E} \quad 00$

$7.90578 \mathrm{E} \quad 00$ $8.00406 \mathrm{E} \quad 00$ $8.10234 \mathrm{E} \quad 00$ 8.20061 E $\quad 00$ $8.29888 \mathrm{E} \quad 00$

$8.39714 \mathrm{E} \quad 00$ $8.49540 \mathrm{E} \quad 00$ $8.59366 \mathrm{E} \quad 00$ $8.69192 \mathrm{E} \quad 00$ $8.79017 \mathrm{E} \quad 00$

$8.88843 \mathrm{E} \quad 00$ $8.98668 \mathrm{E} \quad 00$ $9.08493 \mathrm{E} \quad 00$ $9.18317 \mathrm{E} \quad 00$ $9.28142 \mathrm{E} \quad 00$

$9.37967 \mathrm{E} \quad 00$ $9.47791 \mathrm{E} \quad 00$ $9.57616 \mathrm{E} \quad 00$ $9.67440 \mathrm{E} \quad 00$ $9.77265 \mathrm{E} \quad 00$

$9.87089 \mathrm{E} \quad 00$ $9.96914 \mathrm{E} \quad 00$ $1.00674 \mathrm{E} \quad 01$ $1.01656 \mathrm{E} \quad 01$ $1.02639 \mathrm{E} \quad 01$

$\begin{array}{lll}1.03621 \mathrm{E} & 01\end{array}$ $1.04604 \mathrm{E} \quad 01$ $1.05586 \mathrm{E} \quad 01$ 
ELECTRON RADIAL FUNCTIONS

$Z=2 \quad A=4 \quad \rho=1.2 A^{1 / 3} F$

Positron

$\bar{F}$

2.40202 E- 05 $2.48147 \mathrm{E}-05$ $2.56221 \mathrm{E}-05$ $2.64423 \mathrm{E}-05$ $2.72755 \mathrm{E}-05$

$2.81216 \mathrm{E}-05$

6.60

6.70

6.80

6.90

7.00

$89807 \mathrm{E}-05$

$2.98526 \mathrm{E}-05$

$3.07375 \mathrm{E}-05$

$3.16353 \mathrm{E}-05$

$7.10 \quad 3.25460 \mathrm{E}-05$

$7.20 \quad 3.34696 \mathrm{E}-05$

$7.30 \quad 3.44061 \mathrm{E}-05$

$7.40 \quad 3.53556 \mathrm{E}-05$

$7.50 \quad 3.63179 \mathrm{E}-05$

$7.60 \quad 3.72932 \mathrm{E}-05$

$7.70 \quad 3.82814 \mathrm{E}-05$

$7.80 \quad 3.92825 \mathrm{E}-05$

$7.90 \quad 4.02965 \mathrm{E}-05$

$8.00 \quad 4.13235 \mathrm{E}-05$

$8.10 \quad 4.23633 \mathrm{E}-05$

$8.20 \quad 4.34161 \mathrm{E}-05$

$8.30 \quad 4.44818 \mathrm{E}-05$

$8.40 \quad 4.55604 \mathrm{E}-05$

$8.50 \quad 4.66519 \mathrm{E}-05$

$8.60 \quad 4.77564 \mathrm{E}-05$

$8.70 \quad 4.88737 \mathrm{E}-05$

$8.80 \quad 5.00040 \mathrm{E}-05$

$8.90 \quad 5.11472 \mathrm{E}-05$

$9.00 \quad 5.23033 \mathrm{E}-05$

$9.10 \quad 5.34723 \mathrm{E}-05$

$9.20 \quad 5.46542 \mathrm{E}-05$

$9.30 \quad 5.58491 \mathrm{E}-05$

$9.40 \quad 5.70568 \mathrm{E}-05$

$9.50 \quad 5.82775 \mathrm{E}-05$

$9.60 \quad 5.95111 \mathrm{E}-05$

9.70

9.80

9.90

10.00

10.10

10.20

10.30

10.40

10.50

10.60

10.70

10.80

10.90

11.00

11.10

11.20

11.30

11.40

11.50

11.60

11.70

11.80

11.90

12.00

$6.98449 \mathrm{E}-05$

$7.11947 \mathrm{E}-05$

$7.25575 \mathrm{E}-05$

$7.39332 \mathrm{E}-05$

$7.53217 \mathrm{E}-05$

$7.67232 \mathrm{E}-05$

$7.81377 \mathrm{E}-05$

$7.95650 \mathrm{E}-05$

$8.10052 \mathrm{E}-05$

$8.24584 \mathrm{E}-05$

$8.39245 \mathrm{E}-05$

$8.54035 \mathrm{E}-05$

$8.68954 \mathrm{E}-05$

$8.84003 \mathrm{E}-05$

$8.99180 E-05$

$9.14487 \mathrm{E}-05$

$9.29922 \mathrm{E}-05$ $f_{2}$

$5.47657 \mathrm{E}-02$ $5.66619 \mathrm{E}-02$

$5.85901 \mathrm{E}-02$

$6.05506 \mathrm{E}-02$

$6.25433 \mathrm{E}-02$

$6.45681 \mathrm{E}-02$

$6.66252 \mathrm{E}-02$

$6.87144 \mathrm{E}-02$

$7.08357 \mathrm{E}-02$

$7.29893 \mathrm{E}-02$

$7.51750 \mathrm{E}-02$

$7.73928 \mathrm{E}-02$

$7.96428 \mathrm{E}-02$

$8.19250 \mathrm{E}-02$

$8.42394 \mathrm{E}-02$

8.65859E-02

$8.89646 \mathrm{E}-02$

$9.13754 \mathrm{E}-02$

$9.38184 \mathrm{E}-02$

$9.62936 \mathrm{E}-02$

$9.88009 \mathrm{E}-02$

$1.01340 \mathrm{E}-01$

$1.03912 \mathrm{E}-01$

$1.06516 \mathrm{E}-01$

$1.09152 \mathrm{E}-01$

$1.11820 \mathrm{E}-01$

$1.14520 \mathrm{E}-01$

$1.17252 \mathrm{E}-01$

$1.20017 \mathrm{E}-01$

$1.22814 \mathrm{E}-01$

$1.25642 \mathrm{E}-01$

$1.28503 \mathrm{E}-01$

$1.31397 \mathrm{E}-01$

$1.34322 \mathrm{E}-01$

$1.37279 \mathrm{E}-01$

$1.40269 \mathrm{E}-01$

$1.43290 \mathrm{E}-01$

$1.46344 \mathrm{E}-01$

$1.49430 \mathrm{E}-01$

$1.52548 \mathrm{E}-01$

$1.55699 \mathrm{E}-01$

$1.58881 \mathrm{E}-01$

$1.62096 \mathrm{E}-01$

$1.65342 \mathrm{E}-01$

$1.68621 \mathrm{E}-01$
$1.71932 \mathrm{E}-01$

$1.75275 \mathrm{E}-01$

$1.78650 \mathrm{E}-01$

$1.82057 \mathrm{E}-01$

$1.85497 \mathrm{E}-01$

$1.88968 \mathrm{E}-01$

$1.92472 \mathrm{E}-01$

$1.96008 \mathrm{E}-01$

$1.99576 \mathrm{E}-01$

$2.03176 \mathrm{E}-01$

$2.06808 \mathrm{E}-01$

$2.10473 \mathrm{E}-01$

$2.14169 \mathrm{E}-01$

$2.17898 \mathrm{E}-01$

$2.21658 \mathrm{E}-01$ $g_{2}$

$\tan \delta_{2}$

$f_{-2}$

$2.05328 \mathrm{E}-04$

$2.17955 \mathrm{E}-04$

$2.31080 \mathrm{E}-04$

$2.44714 \mathrm{E}-04$

$2.58865 \mathrm{E}-04$

$2.73543 \mathrm{E}-04$

$2.88758 \mathrm{E}-04$

$3.04519 \mathrm{E}-04$

$3.20835 \mathrm{E}-04$

$3.37717 \mathrm{E}-04$

$3.55173 \mathrm{E}-04$

$3.73214 \mathrm{E}-04$

$3.91849 \mathrm{E}-04$

$4.11086 \mathrm{E}-04$

4.30937E-04

$4.51409 \mathrm{E}-04$

$4.72514 \mathrm{E}-04$

$4.94260 \mathrm{E}-04$

$5.16656 \mathrm{E}-04$

$5.39714 \mathrm{E}-04$

$5.63440 \mathrm{E}-04$

$5.87847 \mathrm{E}-04$

$6.12942 \mathrm{E}-04$

$6.38735 \mathrm{E}-04$

$6.65237 \mathrm{E}-04$

$6.92456 \mathrm{E}-04$

$7.20401 \mathrm{E}-04$

$7.49084 \mathrm{E}-04$

$7.78512 \mathrm{E}-04$

$8.08696 \mathrm{E}-04$

$8.39644 \mathrm{E}-04$

$8.71367 \mathrm{E}-04$

$9.03875 \mathrm{E}-04$

$9.37175 \mathrm{E}-04$

$9.71279 \mathrm{E}-04$

$1.00620 \mathrm{E}-03$

$1.04193 \mathrm{E}-03$

$1.07850 \mathrm{E}-03$

$1.11591 \mathrm{E}-03$

$1.15418 \mathrm{E}-03$

$1.19330 \mathrm{E}-03$

$1.23329 \mathrm{E}-03$

$1.27416 \mathrm{E}-03$

$1.31592 \mathrm{E}-03$

$1.35858 \mathrm{E}-03$

$1.40214 \mathrm{E}-03$

$1.44662 \mathrm{E}-03$

$1.49203 \mathrm{E}-03$

$1.53838 \mathrm{E}-03$

$1.58567 \mathrm{E}-03$

$1.63391 \mathrm{E}-03$

$1.68312 \mathrm{E}-03$

$1.73331 \mathrm{E}-03$

$1.78448 E-03$

$1.83664 \mathrm{E}-03$

$1.88980 \mathrm{E}-03$

$1.94398 \mathrm{E}-03$

$1.99918 \mathrm{E}-03$

$2.05541 \mathrm{E}-03$

$2.11268 \mathrm{E}-03$
$-9.40279 \mathrm{E} \quad 01$

$-9.41502 \mathrm{E} \quad 01$

$-9.42684 \mathrm{E} \quad 01$

$-9.43826 \mathrm{E}$ 01

$-9.44931 \mathrm{E}$ 01

$-9.46001 \mathrm{E} \quad 01$

$-9.47036 \mathrm{E} 01$

$-9.48039 \mathrm{E} 01$

$-9.49011 \mathrm{E} 01$

$-9.49953 \mathrm{E} 01$

$-9.50867 \mathrm{E}$ 01

$-9.51754 \mathrm{E} \quad 01$

$-9.52614 \mathrm{E} \quad 01$

$-9.53451 \mathrm{E} 01$

$-9.54263 \mathrm{E} \quad 01$

$-9.55053 \mathrm{E} \quad 01$

$-9.55821 \mathrm{E} \quad 01$

$-9.56568 \mathrm{E} 01$

$-9.57294 \mathrm{E} \quad 01$

$-9.58002 \mathrm{E} \quad 01$

$\begin{array}{ll}-9.58691 \mathrm{E} & 01\end{array}$

$-9.59362 \mathrm{E}$ ol

$-9.60015 \mathrm{E} 01$

$-9.60653 \mathrm{E} 01$

$-9.61274 \mathrm{E} \quad 01$

$-9.61880 \mathrm{E} \quad 01$

$-9.62471 \mathrm{E} 01$

$-9.63048 \mathrm{E} 01$

$-9.63611 \mathrm{E} 01$

$-9.64161 \mathrm{E} 01$

$-9.64698 \mathrm{E} \quad 01$

$-9.65222 \mathrm{E} \quad 01$

$-9.65735 \mathrm{E} \quad 01$

$-9.66236 \mathrm{E} 01$

$-9.66726 \mathrm{E} 01$

$-9.67205 \mathrm{E} \quad 01$

$-9.67673 \mathrm{E} 01$

$-9.68132 \mathrm{E} 01$

$-9.68580 \mathrm{E} 01$

$-9.69019 \mathrm{E} 01$

$-9.69449 \mathrm{E}$ ol

$-9.69870 \mathrm{E} 01$

$-9.70282 \mathrm{E} 01$

$-9.70686 \mathrm{E} 01$

$-9.71082 \mathrm{E}$ ol

$-9.71470 \mathrm{E} \quad 01$

$-9.71850 \mathrm{E}$ ol

$-9.72223 \mathrm{E} 01$

$-9.72588 \mathrm{E} 01$

$-9.72947 \mathrm{E} \quad 01$

$-9.73299 \mathrm{E} \quad 01$

$-9.73644 \mathrm{E}$ ol

$-9.73982 \mathrm{E} 01$

$-9.74315 \mathrm{E} 01$

$-9.74641 \mathrm{E} \quad 01$

$\begin{array}{lll}-9.74961 \mathrm{E} & 01\end{array}$

$-9.75276 \mathrm{E}$ ol

$-9.75585 \mathrm{E}$ 01

$-9.75888 \mathrm{E} 01$

$-9.76186 \mathrm{E} 01$

$-1.14474 \mathrm{E}-04$

$-1.24606 \mathrm{E}-04$

$-1.35206 \mathrm{E}-04$

$-1.46282 \mathrm{E}-04$

$-1.57844 \mathrm{E}-04$

$-1.69902 \mathrm{E}-04$

$-1.82464 \mathrm{E}-04$

$-1.95541 \mathrm{E}-04$

$-2.09142 \mathrm{E}-04$

$-2.23277 \mathrm{E}-04$

$-2.37954 \mathrm{E}-04$

$-2.53185 \mathrm{E}-04$

$-2.68977 \mathrm{E}-04$

$-2.85341 \mathrm{E}-04$

$-3.02285 \mathrm{E}-04$

$-3.19821 \mathrm{E}-04$ 


\begin{tabular}{|c|c|c|c|c|c|c|}
\hline$p$ & $F_{0}$ & $f_{1}$ & & $g_{1}$ & $\tan \delta_{1}$ & $f_{-1}$ \\
\hline $\begin{array}{l}12.10 \\
12.20 \\
12.30 \\
12.40 \\
12.50\end{array}$ & $\begin{array}{l}9.55622 \mathrm{E}-01 \\
9.55623 \mathrm{E}-01 \\
9.55624 \mathrm{E}-01 \\
9.55625 \mathrm{E}-01 \\
9.55625 \mathrm{E}-01\end{array}$ & $\begin{array}{l}1.13489 \mathrm{E} \\
1.14471 \mathrm{E} \\
1.15453 \mathrm{E} \\
1.16435 \mathrm{E} \\
1.17417 \mathrm{E}\end{array}$ & $\begin{array}{l}01 \\
01 \\
01 \\
01 \\
01\end{array}$ & $\begin{array}{l}1.78999 \mathrm{E}-01 \\
1.82424 \mathrm{E}-01 \\
1.85882 \mathrm{E}-01 \\
1.89372 \mathrm{E}-01 \\
1.92894 \mathrm{E}-01\end{array}$ & $\begin{array}{l}-3.55014 E-04 \\
-3.59818 E-04 \\
-3.64538 E-04 \\
-3.69182 E-04 \\
-3.73752 E-04\end{array}$ & $\begin{array}{l}-1.53888 \mathrm{E}-01 \\
-1.57153 \mathrm{E}-01 \\
-1.60449 \mathrm{E}-01 \\
-1.63778 \mathrm{E}-01 \\
-1.67139 \mathrm{E}-01\end{array}$ \\
\hline $\begin{array}{l}12.60 \\
12.70 \\
12.80 \\
12.90 \\
13.00\end{array}$ & $\begin{array}{l}9.55626 \mathrm{E}-01 \\
9.55627 \mathrm{E}-01 \\
9.55627 \mathrm{E}-01 \\
9.55628 \mathrm{E}-01 \\
9.55628 \mathrm{E}-01\end{array}$ & $\begin{array}{l}1.18399 \mathrm{E} \\
1.19381 \mathrm{E} \\
1.20364 \mathrm{E} \\
1.21346 \mathrm{E} \\
1.22328 \mathrm{E}\end{array}$ & $\begin{array}{l}01 \\
01 \\
01 \\
01 \\
01\end{array}$ & $\begin{array}{l}1.96449 \mathrm{E}-01 \\
2.00036 \mathrm{E}-01 \\
2.03656 \mathrm{E}-01 \\
2.07308 \mathrm{E}-01 \\
2.10993 \mathrm{E}-01\end{array}$ & $\begin{array}{l}-3.78246 \mathrm{E}-04 \\
-3.82669 \mathrm{E}-04 \\
-3.87021 \mathrm{E}-04 \\
-3.91303 \mathrm{E}-04 \\
-3.95519 \mathrm{E}-04\end{array}$ & $\begin{array}{l}-1.70533 \mathrm{E}-01 \\
-1.73959 \mathrm{E}-01 \\
-1.77418 \mathrm{E}-01 \\
-1.80909 \mathrm{E}-01 \\
-1.84432 \mathrm{E}-01\end{array}$ \\
\hline $\begin{array}{l}13.10 \\
13.20 \\
13.30 \\
13.40 \\
13.50\end{array}$ & $\begin{array}{l}9.55628 \mathrm{E}-01 \\
9.55629 \mathrm{E}-01 \\
9.55629 \mathrm{E}-01 \\
9.55630 \mathrm{E}-01 \\
9.55630 \mathrm{E}-01\end{array}$ & $\begin{array}{l}1.23310 \mathrm{E} \\
1.24293 \mathrm{E} \\
1.25275 \mathrm{E} \\
1.26258 \mathrm{E} \\
1.27240 \mathrm{E}\end{array}$ & $\begin{array}{l}01 \\
01 \\
01 \\
01 \\
01\end{array}$ & $\begin{array}{l}2.14710 \mathrm{E}-01 \\
2.18459 \mathrm{E}-01 \\
2.22241 \mathrm{E}-01 \\
2.26055 \mathrm{E}-01 \\
2.29902 \mathrm{E}-01\end{array}$ & $\begin{array}{l}-3.99668 \mathrm{E}-04 \\
-4.03755 \mathrm{E}-04 \\
-4.07776 \mathrm{E}-04 \\
-4.11735 \mathrm{E}-04 \\
-4.15636 \mathrm{E}-04\end{array}$ & $\begin{array}{l}-1.87988 \mathrm{E}-01 \\
-1.91577 \mathrm{E}-01 \\
-1.95197 \mathrm{E}-01 \\
-1.98851 \mathrm{E}-01 \\
-2.02536 \mathrm{E}-01\end{array}$ \\
\hline $\begin{array}{l}13.60 \\
13.70 \\
13.80 \\
13.90 \\
14.00\end{array}$ & $\begin{array}{l}9.55630 \mathrm{E}-01 \\
9.55630 \mathrm{E}-01 \\
9.55631 \mathrm{E}-01 \\
9.55631 \mathrm{E}-01 \\
9.55631 \mathrm{E}-01\end{array}$ & $\begin{array}{l}1.28223 \mathrm{E} \\
1.29205 \mathrm{E} \\
1.30188 \mathrm{E} \\
1.31171 \mathrm{E} \\
1.32154 \mathrm{E}\end{array}$ & $\begin{array}{l}01 \\
01 \\
01 \\
01 \\
01\end{array}$ & $\begin{array}{l}2.33781 \mathrm{E}-01 \\
2.37692 \mathrm{E}-01 \\
2.41636 \mathrm{E}-01 \\
2.45613 \mathrm{E}-01 \\
2.49621 \mathrm{E}-01\end{array}$ & $\begin{array}{l}-4.19476 \mathrm{E}-04 \\
-4.23259 \mathrm{E}-04 \\
-4.26987 \mathrm{E}-04 \\
-4.30659 \mathrm{E}-04 \\
-4.34276 \mathrm{E}-04\end{array}$ & $\begin{array}{l}-2.06254 \mathrm{E}-01 \\
-2.10005 \mathrm{E}-01 \\
-2.13788 \mathrm{E}-01 \\
-2.17603 \mathrm{E}-01 \\
-2.21451 \mathrm{E}-01\end{array}$ \\
\hline $\begin{array}{l}14.10 \\
14.20 \\
14.30 \\
14.40 \\
14.50\end{array}$ & $\begin{array}{l}9.55631 \mathrm{E}-01 \\
9.55631 \mathrm{E}-01 \\
9.55631 \mathrm{E}-01 \\
9.55632 \mathrm{E}-01 \\
9.55632 \mathrm{E}-01\end{array}$ & $\begin{array}{l}1.33136 \mathrm{E} \\
1.34119 \mathrm{E} \\
1.35102 \mathrm{E} \\
1.36085 \mathrm{E} \\
1.37068 \mathrm{E}\end{array}$ & $\begin{array}{l}01 \\
01 \\
01 \\
01 \\
01\end{array}$ & $\begin{array}{l}2.53663 \mathrm{E}-01 \\
2.57736 \mathrm{E}-01 \\
2.61843 \mathrm{E}-01 \\
2.65981 \mathrm{E}-01 \\
2.70152 \mathrm{E}-01\end{array}$ & $\begin{array}{l}-4.37840 \mathrm{E}-04 \\
-4.41351 \mathrm{E}-04 \\
-4.44815 \mathrm{E}-04 \\
-4.48227 \mathrm{E}-04 \\
-4.51589 \mathrm{E}-04\end{array}$ & $\begin{array}{l}-2.25332 \mathrm{E}-01 \\
-2.29245 \mathrm{E}-01 \\
-2.33190 \mathrm{E}-01 \\
-2.37168 \mathrm{E}-01 \\
-2.41178 \mathrm{E}-01\end{array}$ \\
\hline $\begin{array}{l}14.60 \\
14.70 \\
14.80 \\
14.90 \\
15.00\end{array}$ & $\begin{array}{l}9.55632 \mathrm{E}-01 \\
9.55632 \mathrm{E}-01 \\
9.55632 \mathrm{E}-01 \\
9.55632 \mathrm{E}-01 \\
9.55632 \mathrm{E}-01\end{array}$ & $\begin{array}{l}1.38051 \mathrm{E} \\
1.39034 \mathrm{E} \\
1.40017 \mathrm{E} \\
1.41001 \mathrm{E} \\
1.41984 \mathrm{E}\end{array}$ & $\begin{array}{l}01 \\
01 \\
01 \\
01 \\
01\end{array}$ & $\begin{array}{l}2.74356 \mathrm{E}-01 \\
2.78592 \mathrm{E}-01 \\
2.82861 \mathrm{E}-01 \\
2.87162 \mathrm{E}-01 \\
2.91495 \mathrm{E}-01\end{array}$ & $\begin{array}{l}-4.54903 \mathrm{E}-04 \\
-4.58171 \mathrm{E}-04 \\
-4.61395 \mathrm{E}-04 \\
-4.64571 \mathrm{E}-04 \\
-4.67704 \mathrm{E}-04\end{array}$ & $\begin{array}{l}-2.45221 \mathrm{E}-01 \\
-2.49297 \mathrm{E}-01 \\
-2.53404 \mathrm{E}-01 \\
-2.57545 \mathrm{E}-01 \\
-2.61718 \mathrm{E}-01\end{array}$ \\
\hline $\begin{array}{l}15.10 \\
15.20 \\
15.30 \\
15.40 \\
15.50\end{array}$ & $\begin{array}{l}9.55632 \mathrm{E}-01 \\
9.55631 \mathrm{E}-01 \\
9.55631 \mathrm{E}-01 \\
9.55631 \mathrm{E}-01 \\
9.55631 \mathrm{E}-01\end{array}$ & $\begin{array}{l}1.42967 \mathrm{E} \\
1.43951 \mathrm{E} \\
1.44934 \mathrm{E} \\
1.45918 \mathrm{E} \\
1.46901 \mathrm{E}\end{array}$ & $\begin{array}{l}01 \\
01 \\
01 \\
01 \\
01\end{array}$ & $\begin{array}{l}2.95861 \mathrm{E}-01 \\
3.00260 \mathrm{E}-01 \\
3.04691 \mathrm{E}-01 \\
3.09155 \mathrm{E}-01 \\
3.13651 \mathrm{E}-01\end{array}$ & $\begin{array}{l}-4.70794 \mathrm{E}-04 \\
-4.73841 \mathrm{E}-04 \\
-4.76848 \mathrm{E}-04 \\
-4.79813 \mathrm{E}-04 \\
-4.82738 \mathrm{E}-04\end{array}$ & $\begin{array}{l}-2.65923 \mathrm{E}-01 \\
-2.70161 \mathrm{E}-01 \\
-2.74431 \mathrm{E}-01 \\
-2.78734 \mathrm{E}-01 \\
-2.83070 \mathrm{E}-01\end{array}$ \\
\hline $\begin{array}{l}15.60 \\
15.70 \\
15.80 \\
15.90 \\
16.00\end{array}$ & $\begin{array}{l}9.55631 \mathrm{E}-01 \\
9.55631 \mathrm{E}-01 \\
9.55631 \mathrm{E}-01 \\
9.55631 \mathrm{E}-01 \\
9.55630 \mathrm{E}-01\end{array}$ & $\begin{array}{l}1.47885 \mathrm{E} \\
1.48869 \mathrm{E} \\
1.49852 \mathrm{E} \\
1.50836 \mathrm{E} \\
1.51820 \mathrm{E}\end{array}$ & $\begin{array}{l}01 \\
01 \\
01 \\
01 \\
01\end{array}$ & $\begin{array}{l}3.18179 \mathrm{E}-01 \\
3.22740 \mathrm{E}-01 \\
3.27334 \mathrm{E}-01 \\
3.31960 \mathrm{E}-01 \\
3.36619 \mathrm{E}-01\end{array}$ & $\begin{array}{l}-4.85624 \mathrm{E}-04 \\
-4.88471 \mathrm{E}-04 \\
-4.91281 \mathrm{E}-04 \\
-4.94054 \mathrm{E}-04 \\
-4.96790 \mathrm{E}-04\end{array}$ & $\begin{array}{l}-2.87438 \mathrm{E}-01 \\
-2.91838 \mathrm{E}-01 \\
-2.96272 \mathrm{E}-01 \\
-3.00737 \mathrm{E}-01 \\
-3.05236 \mathrm{E}-01\end{array}$ \\
\hline $\begin{array}{l}16.10 \\
16.20 \\
16.30 \\
16.40 \\
16.50\end{array}$ & $\begin{array}{l}9.55630 \mathrm{E}-01 \\
9.55630 \mathrm{E}-01 \\
9.55630 \mathrm{E}-01 \\
9.55630 \mathrm{E}-01 \\
9.55629 \mathrm{E}-01\end{array}$ & $\begin{array}{l}1.52804 \mathrm{E} \\
1.53788 \mathrm{E} \\
1.54772 \mathrm{E} \\
1.55756 \mathrm{E} \\
1.56740 \mathrm{E}\end{array}$ & $\begin{array}{l}01 \\
01 \\
01 \\
01 \\
01\end{array}$ & $\begin{array}{l}3.41310 \mathrm{E}-01 \\
3.46034 \mathrm{E}-01 \\
3.50790 \mathrm{E}-01 \\
3.55579 \mathrm{E}-01 \\
3.60401 \mathrm{E}-01\end{array}$ & $\begin{array}{l}-4.99491 \mathrm{E}-04 \\
-5.02155 \mathrm{E}-04 \\
-5.04786 \mathrm{E}-04 \\
-5.07379 \mathrm{E}-04 \\
-5.09944 \mathrm{E}-04\end{array}$ & $\begin{array}{l}-3.09766 \mathrm{E}-01 \\
-3.14330 \mathrm{E}-01 \\
-3.18926 \mathrm{E}-01 \\
-3.23554 \mathrm{E}-01 \\
-3.28215 \mathrm{E}-01\end{array}$ \\
\hline $\begin{array}{l}16.60 \\
16.70 \\
16.80 \\
16.90 \\
17.00\end{array}$ & $\begin{array}{l}9.55629 \mathrm{E}-01 \\
9.55629 \mathrm{E}-01 \\
9.55629 \mathrm{E}-01 \\
9.55628 \mathrm{E}-01 \\
9.55628 \mathrm{E}-01\end{array}$ & $\begin{array}{l}1.57724 \mathrm{E} \\
1.58709 \mathrm{E} \\
1.59693 \mathrm{E} \\
1.60677 \mathrm{E} \\
1.61662 \mathrm{E}\end{array}$ & $\begin{array}{l}01 \\
01 \\
01 \\
01 \\
01\end{array}$ & $\begin{array}{l}3.65255 \mathrm{E}-01 \\
3.70141 \mathrm{E}-01 \\
3.75061 \mathrm{E}-01 \\
3.80012 \mathrm{E}-01 \\
3.84997 \mathrm{E}-01\end{array}$ & $\begin{array}{l}-5.12474 \mathrm{E}-04 \\
-5.14972 \mathrm{E}-04 \\
-5.17439 \mathrm{E}-04 \\
-5.19875 \mathrm{E}-04 \\
-5.22280 \mathrm{E}-04\end{array}$ & $\begin{array}{l}-3.32909 \mathrm{E}-01 \\
-3.37635 \mathrm{E}-01 \\
-3.42394 \mathrm{E}-01 \\
-3.47185 \mathrm{E}-01 \\
-3.52010 \mathrm{E}-01\end{array}$ \\
\hline $\begin{array}{l}17.10 \\
17.20 \\
17.30 \\
17.40 \\
17.50\end{array}$ & $\begin{array}{l}9.55628 \mathrm{E}-01 \\
9.55627 \mathrm{E}-01 \\
9.55627 \mathrm{E}-01 \\
9.55627 \mathrm{E}-01 \\
9.55626 \mathrm{E}-01\end{array}$ & $\begin{array}{l}1.62647 \mathrm{E} \\
1.63631 \mathrm{E} \\
1.64616 \mathrm{E} \\
1.65601 \mathrm{E} \\
1.66585 \mathrm{E}\end{array}$ & $\begin{array}{l}01 \\
01 \\
01 \\
01 \\
01\end{array}$ & $\begin{array}{l}3.90014 \mathrm{E}-01 \\
3.95063 \mathrm{E}-01 \\
4.00145 \mathrm{E}-01 \\
4.05260 \mathrm{E}-01 \\
4.10408 \mathrm{E}-01\end{array}$ & $\begin{array}{l}-5.24655 \mathrm{E}-04 \\
-5.27000 \mathrm{E}-04 \\
-5.29318 \mathrm{E}-04 \\
-5.31604 \mathrm{E}-04 \\
-5.33864 \mathrm{E}-04\end{array}$ & $\begin{array}{l}-3.56866 \mathrm{E}-01 \\
-3.61755 \mathrm{E}-01 \\
-3.66677 \mathrm{E}-01 \\
-3.71632 \mathrm{E}-01 \\
-3.76619 \mathrm{E}-01\end{array}$ \\
\hline $\begin{array}{l}17.60 \\
17.70 \\
17.80 \\
17.90 \\
18.00\end{array}$ & $\begin{array}{l}9.55626 \mathrm{E}-01 \\
9.55626 \mathrm{E}-01 \\
9.55625 \mathrm{E}-01 \\
9.55625 \mathrm{E}-01 \\
9.55625 \mathrm{E}-01\end{array}$ & $\begin{array}{l}1.67570 \mathrm{E} \\
1.68555 \mathrm{E} \\
1.69540 \mathrm{E} \\
1.70525 \mathrm{E} \\
1.71510 \mathrm{E}\end{array}$ & $\begin{array}{l}01 \\
01 \\
01 \\
01 \\
01\end{array}$ & $\begin{array}{l}4.15587 \mathrm{E}-01 \\
4.20800 \mathrm{E}-01 \\
4.26045 \mathrm{E}-01 \\
4.31323 \mathrm{E}-01 \\
4.36634 \mathrm{E}-01\end{array}$ & $\begin{array}{l}-5.36097 \mathrm{E}-04 \\
-5.38303 \mathrm{E}-04 \\
-5.40483 \mathrm{E}-04 \\
-5.42636 \mathrm{E}-04 \\
-5.44760 \mathrm{E}-04\end{array}$ & $\begin{array}{l}-3.81639 \mathrm{E}-01 \\
-3.86691 \mathrm{E}-01 \\
-3.91776 \mathrm{E}-01 \\
-3.96894 \mathrm{E}-01 \\
-4.02045 \mathrm{E}-01\end{array}$ \\
\hline
\end{tabular}

$g_{-1}$

$1.23276 \mathrm{E} \quad 01$

$1.24259 \mathrm{E} 01$

$1.25242 \mathrm{E} \quad 01$

$1.26225 \mathrm{E} \quad 01$

$1.27208 \mathrm{E} 01$

$1.28191 \mathrm{E} \quad 01$

$1.29175 \mathrm{E} \quad 01$

$1.30158 \mathrm{E} \quad 01$

$1.31141 \mathrm{E} 01$

$1.32124 \mathrm{E} \quad 01$

$1.33108 \mathrm{E} \quad 01$

$1.34091 \mathrm{E} 01$

$1.35075 \mathrm{E} \quad 01$

$1.36058 \mathrm{E} \quad 01$

$1.37042 \mathrm{E} \quad 01$

$\begin{array}{lll}1.38025 \mathrm{E} & 01\end{array}$

$1.39009 \mathrm{E} \quad 01$

$1.39993 \mathrm{E} 01$

$1.40977 \mathrm{E} \quad 01$

$1.41960 \mathrm{E} \quad 01$

$1.42944 \mathrm{E} \quad 01$

$1.43928 \mathrm{E} \quad 01$

$1.44912 \mathrm{E} \quad 01$

$1.45896 \mathrm{E} 01$

$1.46880 \mathrm{E} 01$

$1.47864 \mathrm{E} \quad 01$

$1.48848 \mathrm{E} \quad 01$

$1.49833 \mathrm{E} \quad 01$

$1.50817 \mathrm{E} \quad 01$

$1.51801 \mathrm{E} \quad 01$

$\begin{array}{ll}1.52786 \mathrm{E} & 01\end{array}$ $1.53770 \mathrm{E} \quad 01$

$1.54755 \mathrm{E} 01$

$1.55739 \mathrm{E} \quad 01$

$1.56724 \mathrm{E} \quad 01$

$1.57708 \mathrm{E} \quad 01$

$1.58693 \mathrm{E} \quad 01$

$1.59678 \mathrm{E} \quad 01$

$1.60663 \mathrm{E} 01$

$1.61648 \mathrm{E} 01$

$1.62633 \mathrm{E} \quad 01$

$1.63618 \mathrm{E} 01$

$1.64603 \mathrm{E} 01$

$1.65588 \mathrm{E} \quad 01$

$1.66573 \mathrm{E} 01$

$1.67558 \mathrm{E} \quad 01$

$1.68544 \mathrm{E} \quad 01$

$1.69529 \mathrm{E} \quad 01$

$1.70515 \mathrm{E} 01$

$1.71500 \mathrm{E} \quad 01$

$1.72486 \mathrm{E} \quad 01$ $1.73472 \mathrm{E} \quad 01$

$1.74457 \mathrm{E} \quad 01$

$1.75443 \mathrm{E} \quad 01$

$1.76429 \mathrm{E} 01$

$1.77415 \mathrm{E} \quad 01$ $1.78401 \mathrm{E} \quad 01$ $\begin{array}{ll}1.79387 \mathrm{E} & 01\end{array}$

$1.80373 \mathrm{E} 01$ $1.81359 \mathrm{E} \quad 01$ $\tan \delta_{-1}$

$6.40673 \mathrm{E} \quad 02$ $6.42769 \mathrm{E} \quad 02$ $6.44847 \mathrm{E} \quad 02$ $6.46904 \mathrm{E} \quad 02$ $6.48941 \mathrm{E} \quad 02$

$6.50960 \mathrm{E} \quad 02$ $6.52960 \mathrm{E} \quad 02$ $6.54942 \mathrm{E} \quad 02$ $6.56905 \mathrm{E} \quad 02$ $6.58850 \mathrm{E} \quad 02$

$6.60777 \mathrm{E} \quad 02$ $6.62687 \mathrm{E} \quad 02$ $6.64580 \mathrm{E} \quad 02$ $6.66457 \mathrm{E} \quad 02$ $6.68316 \mathrm{E} \quad 02$

$6.70159 \mathrm{E} \quad 02$ $6.71986 \mathrm{E} \quad 02$ $6.73797 \mathrm{E} \quad 02$ $6.75592 \mathrm{E} \quad 02$ $6.77372 \mathrm{E} \quad 02$

$6.79136 \mathrm{E} \quad 02$ $6.80887 \mathrm{E} \quad 02$ $6.82621 \mathrm{E} \quad 02$ $6.84341 \mathrm{E} \quad 02$ $6.86047 \mathrm{E} \quad 02$

$6.87740 \mathrm{E} \quad 02$ $6.89418 \mathrm{E} \quad 02$ $6.91081 \mathrm{E} \quad 02$ $6.92732 \mathrm{E} \quad 02$ $6.94370 \mathrm{E} \quad 02$

$6.95993 \mathrm{E} \quad 02$ $6.97605 \mathrm{E} \quad 02$ $6.99203 \mathrm{E} \quad 02$ $\begin{array}{lll}7.00788 \mathrm{E} & 02\end{array}$ $7.02361 \mathrm{E} \quad 02$

$7.03922 \mathrm{E} \quad 02$ $7.05471 \mathrm{E} \quad 02$ $7.07007 \mathrm{E} \quad 02$ $7.08532 \mathrm{E} \quad 02$ $7.10045 \mathrm{E} \quad 02$

$7.11546 \mathrm{E} \quad 02$ $7.13037 \mathrm{E} \quad 02$ $7.14516 \mathrm{E} \quad 02$ $7.15986 \mathrm{E} \quad 02$ $\begin{array}{ll}7.17442 \mathrm{E} & 02\end{array}$

7.18889 E $\quad 02$ $7.20325 \mathrm{E} \quad 02$ $7.21751 \mathrm{E} \quad 02$ $7.23165 \mathrm{E} \quad 02$ $7.24571 \mathrm{E} \quad 02$

$7.25966 \mathrm{E} \quad 02$ $7.27351 \mathrm{E} \quad 02$ $7.28726 \mathrm{E} \quad 02$ $7.30093 \mathrm{E} \quad 02$ $7.31449 \mathrm{E} \quad 02$

$7.32796 \mathrm{E} \quad 02$ $7.34133 \mathrm{E} \quad 02$ $7.35461 \mathrm{E} \quad 02$ $7.36780 \mathrm{E} \quad 02$ $7.38092 \mathrm{E} \quad 02$ 
ELECTRON RADIAL FUNCTIONS

$Z=2 \quad A=4 \quad \rho=1.2 A^{1 / 3} \mathrm{~F}$

Positron

\section{$\bar{F}$}

$9.45488 \mathrm{E}-05$ $9.61182 \mathrm{E}-0.5$ $9.77005 \mathrm{E}-05$ $9.92958 \mathrm{E}-05$ $1.00904 \mathrm{E}-04$

$1.02525 \mathrm{E}-04$ $1.04159 \mathrm{E}-04$ $1.05806 \mathrm{E}-04$ $1.07466 \mathrm{E}-04$ $1.09138 \mathrm{E}-04$

12.70

12.80

12.90

13.00

13.10

13.20

13.30

13.40

13.50

$1.10824 \mathrm{E}-04$ $1.12523 \mathrm{E}-04$

$1.14234 \mathrm{E}-04$

$1.15959 \mathrm{E}-04$

$1.17696 \mathrm{E}-04$

13.60

13.70

13.80

13.90

14.00

$1.19446 \mathrm{E}-04$

$1.21209 \mathrm{E}-04$

$1.22985 \mathrm{E}-04$

$1.24774 \mathrm{E}-04$

$1.26576 \mathrm{E}-04$

$14.10 \quad 1.28391 \mathrm{E}-04$

$14.20 \quad 1.30219 \mathrm{E}-04$

$14.30 \quad 1.32059 \mathrm{E}-04$

$14.40 \quad 1.33913 \mathrm{E}-04$

$14.50 \quad 1.35779 \mathrm{E}-04$

$14.60 \quad 1.37659 \mathrm{E}-04$

$14.70 \quad 1.39551 \mathrm{E}-04$

$14.80 \quad 1.41456 \mathrm{E}-04$

$14.90 \quad 1.43374 \mathrm{E}-04$

$15.00 \quad 1.45305 \mathrm{E}-04$

$15.10 \quad 1.47249 \mathrm{E}-04$

$15.20 \quad 1.49206 \mathrm{E}-04$

$15.30 \quad 1.51176 \mathrm{E}-04$

$15.40 \quad 1.53159 \mathrm{E}-04$

$15.50 \quad 1.55154 \mathrm{E}-04$

$15.60 \quad 1.57163 \mathrm{E}-04$

$15.70 \quad 1.59184 \mathrm{E}-04$

$15.80 \quad 1.61219 \mathrm{E}-04$

$15.90 \quad 1.63266 \mathrm{E}-04$

$16.00 \quad 1.65326 \mathrm{E}-04$

$16.10 \quad 1.67399 \mathrm{E}-04$

$16.20 \quad 1.69485 \mathrm{E}-04$

$16.30 \quad 1.71584 \mathrm{E}-04$

$16.40 \quad 1.73696 \mathrm{E}-04$

$16.50 \quad 1.75821 \mathrm{E}-04$

$16.60 \quad 1.77958 \mathrm{E}-04$

$16.70 \quad 1.80109 \mathrm{E}-04$

$16.80 \quad 1.82272 \mathrm{E}-04$

$16.90 \quad 1.84449 \mathrm{E}-04$

$17.00 \quad 1.86638 \mathrm{E}-04$

17.10

17.20

17.30

17.40

17.50

$1.88841 \mathrm{E}-04$

$1.91056 \mathrm{E}-04$

$1.93284 \mathrm{E}-04$

$1.95525 \mathrm{E}-04$

$1.97779 \mathrm{E}-04$

17.60

17.70

17.80

17.90

$2.00046 \mathrm{E}-04$

$2.02325 \mathrm{E}-04$

$2.04618 \mathrm{E}-04$

$2.06924 \mathrm{E}-04$

18.00 $f_{2}$

$2.25451 \mathrm{E}-01$ $2.29276 \mathrm{E}-01$

$2.33134 \mathrm{E}-01$

$2.37023 \mathrm{E}-01$

2.40944 E-01

$2.44897 \mathrm{E}-01$

$2.48883 \mathrm{E}-01$

$2.52901 \mathrm{E}-01$

$2.56950 \mathrm{E}-01$

$2.61032 \mathrm{E}-01$

$2.65146 \mathrm{E}-01$

$2.69292 \mathrm{E}-01$

$2.73471 \mathrm{E}-01$

$2.77681 \mathrm{E}-01$

$2.81923 \mathrm{E}-01$

$2.86198 \mathrm{E}-01$

$2.90505 \mathrm{E}-01$

$2.94843 \mathrm{E}-01$

$2.99214 \mathrm{E}-01$

$3.03617 \mathrm{E}-01$

$3.08052 \mathrm{E}-01$

$3.12519 \mathrm{E}-01$

$3.17019 \mathrm{E}-01$

$3.21550 \mathrm{E}-01$

$3.26113 \mathrm{E}-01$

$3.30709 \mathrm{E}-01$

$3.35337 \mathrm{E}-01$

$3.39996 \mathrm{E}-01$

$3.44688 \mathrm{E}-01$

$3.49412 \mathrm{E}-01$

$3.54168 \mathrm{E}-01$

$3.58956 \mathrm{E}-01$

$3.63776 \mathrm{E}-01$

$3.68629 \mathrm{E}-01$

3:73513E-01

$3.78430 \mathrm{E}-01$

$3.83378 \mathrm{E}-01$

$3.88359 \mathrm{E}-01$

$3.93371 \mathrm{E}-01$

$3.98416 \mathrm{E}-01$

$4.03493 \mathrm{E}-01$

$4.08602 \mathrm{E}-01$

$4.13743 \mathrm{E}-01$

$4.18916 \mathrm{E}-01$

4.24121 E-01

$4.29359 \mathrm{E}-01$

$4.34628 \mathrm{E}-01$

$4.39929 \mathrm{E}-01$

$4.45263 \mathrm{E}-01$

$4.50628 \mathrm{E}-01$

$4.56026 \mathrm{E}-01$

$4.61455 \mathrm{E}-01$

$4.66917 \mathrm{E}-01$

4.72411 E-01

$4.77937 \mathrm{E}-01$

$4.83495 \mathrm{E}-01$ $4.89085 \mathrm{E}-01$

4.94707 E-01

$5.00361 \mathrm{E}-01$

$5.06047 \mathrm{E}-01$ $g_{2}$

$\tan \delta_{2}$

$f_{-2}$

$2.17100 \mathrm{E}-03$ $2.23038 \mathrm{E}-03$

$2.29083 \mathrm{E}-03$

$2.35236 \mathrm{E}-03$

$2.41498 \mathrm{E}-03$

$2.47869 \mathrm{E}-03$

$2.54351 \mathrm{E}-03$

$2.60945 \mathrm{E}-03$

$2.67652 \mathrm{E}-03$

$2.74472 \mathrm{E}-03$

$2.81406 \mathrm{E}-03$

$2.88456 \mathrm{E}-03$

$2.95623 \mathrm{E}-03$

$3.02907 \mathrm{E}-03$

$3.10309 \mathrm{E}-03$

$3.17830 \mathrm{E}-03$

$3.25472 \mathrm{E}-03$

$3.33235 \mathrm{E}-03$

$3.41120 \mathrm{E}-03$

$3.49128 \mathrm{E}-03$

$3.57260 \mathrm{E}-03$

$3.65517 \mathrm{E}-03$

$3.73901 \mathrm{E}-03$

$3.82410 \mathrm{E}-03$

$3.91048 \mathrm{E}-03$

$3.99815 \mathrm{E}-03$ $4.08711 \mathrm{E}-03$

$4.17738 \mathrm{E}-03$

$4.26897 \mathrm{E}-03$

$4.36188 \mathrm{E}-03$

$4.45612 \mathrm{E}-03$

$4.55171 \mathrm{E}-03$

$4.64866 \mathrm{E}-03$

$4.74697 \mathrm{E}-03$

$4.84665 \mathrm{E}-03$

$4.94772 \mathrm{E}-03$

$5.05018 \mathrm{E}-03$

5.15404 E- 03

$5.25931 \mathrm{E}-03$

$5.36600 \mathrm{E}-03$

$5.47412 \mathrm{E}-03$

$5.58368 \mathrm{E}-03$

$5.69469 \mathrm{E}-03$

$5.80716 \mathrm{E}-03$

$5.92110 \mathrm{E}-03$

$6.03651 \mathrm{E}-03$

6.15341 E-03

$6.27181 \mathrm{E}-03$

$6.39172 \mathrm{E}-03$

$6.51314 \mathrm{E}-03$

$6.63608 \mathrm{E}-03$

$6.76056 \mathrm{E}-03$

$6.88659 \mathrm{E}-03$

$7.01416 \mathrm{E}-03$

$7.14331 \mathrm{E}-03$

$7.27402 \mathrm{E}-03$

$7.40632 \mathrm{E}-03$

$7.54020 \mathrm{E}-03$

$7.67569 \mathrm{E}-03$

$7.81279 \mathrm{E}-03$
$-9.76479 \mathrm{E} \quad 01-1.87441 \mathrm{E}-03$ $-9.76767 \mathrm{E}$ 01 $-1.92940 \mathrm{E}-03$

$-9.77050 \mathrm{E} \quad 01-1.98542 \mathrm{E}-03$

$\begin{array}{lll}-9.77328 \mathrm{E} & 01 & -2.04249 \mathrm{E}-03\end{array}$

$-9.77601 \mathrm{E} \quad 01 \quad-2.10061 \mathrm{E}-03$

$-9.77870 \mathrm{E} \quad 01$

$-9.78135 \mathrm{E} \quad 01$

$-9.78395 \mathrm{E} \quad 01$

$-9.78650 \mathrm{E}$ ol

$-9.78902 \mathrm{E} 01$

$-9.79150 \mathrm{E} \quad 01$

$-9.79393 \mathrm{E} \quad 01$

$-9.79633 \mathrm{E} 01$

$-9.79869 \mathrm{E} \quad 01$

$-9.80102 \mathrm{E} \quad 01$

$\begin{array}{ll}-9.80330 \mathrm{E} & 01\end{array}$

$-9.80556 \mathrm{E} \quad 01$

$-9.80996 \mathrm{E} \quad 01$

$-9.81211 \mathrm{E} 01$

$\begin{array}{ll}-9.81424 \mathrm{E} & 01\end{array}$

$-9.81632 \mathrm{E} \quad 01$

$\begin{array}{lll}-9.81838 \mathrm{E} & 01\end{array}$

$-9.82041 \mathrm{E} \quad 01$

$-9.82241 \mathrm{E} 01$

$-9.82438 \mathrm{E} \quad 01$

$-9.82632 \mathrm{E} \quad 01$

$-9.82824 \mathrm{E} \quad 01$

$-9.83013 \mathrm{E} \quad 01$

$-9.83199 \mathrm{E} 01$

$\begin{array}{ll}-9.83382 \mathrm{E} & 01\end{array}$

$-9.83563 \mathrm{E} 01$

$-9.83742 \mathrm{E}$ ol

$-9.83918 \mathrm{E}$ 01

$-9.84092 \mathrm{E} 01$

$-9.84263 \mathrm{E} \quad 01$

$-9.84432 \mathrm{E} \quad 01$

$-9.84599 \mathrm{E} \quad 01$

$\begin{array}{lll}-9.84764 \mathrm{E} & 01\end{array}$

$\begin{array}{lll}-9.84926 \mathrm{E} & 01\end{array}$

$-9.85087 \mathrm{E} \quad 01$

$-9.85245 \mathrm{E}$ 0l

$\begin{array}{ll}-9.85402 \mathrm{E} & 01\end{array}$

$-9.85556 \mathrm{E} \quad 01$

$-9.85708 \mathrm{E} 01$

$\begin{array}{ll}-9.85859 \mathrm{E} & 01\end{array}$

$-9.86008 \mathrm{E} \quad 01$

$-9.86154 \mathrm{E} 01$

$-9.86299 \mathrm{E} \quad 01$

$-9.86442 \mathrm{E} 01$

$\begin{array}{ll}-9.86584 \mathrm{E} & 01\end{array}$

$-9.86724 \mathrm{E} \quad 01$

$-9.86862 \mathrm{E} 01$

$-9.86998 \mathrm{E} \quad 01$

$-9.87133 \mathrm{E} 01$

$-9.87266 \mathrm{E} 01$

$\begin{array}{lll}-9.87397 \mathrm{E} & 01\end{array}$

$-9.87528 \mathrm{E} 01$

$-9.87656 \mathrm{E} \quad 01$

-9.87783 E 01

$-2.15980 \mathrm{E}-03$

$-2.22007 \mathrm{E}-03$

$-2.28142 \mathrm{E}-03$

$-2.34387 \mathrm{E}-03$

$-2.40742 \mathrm{E}-03$

$-2.47208 \mathrm{E}-03$

$-2.53787 \mathrm{E}-03$

$-2.60479 \mathrm{E}-03$

$-2.67285 \mathrm{E}-03$

$-2.74206 \mathrm{E}-03$

$-2.81244 \mathrm{E}-03$

$-2.88398 \mathrm{E}-03$

$-2.95670 \mathrm{E}-03$

$-3.03062 \mathrm{E}-03$

$-3.10573 \mathrm{E}-03$
$-9.80778 \mathrm{E} \quad 01$ 
ELECTRON RADIAL FUNCTIONS

Positron

$Z=2 \quad A=4$

$\rho=1.2 A^{1 / 3} \mathrm{~F}$

p Fo

18.10

18.20

18.30

18.40

18.50

18.60

18.70

18.80

18.90

19.00

19.10

19.20

19.30

19.40

19.50

19.60

19.70

19.80

19.90

20.00

20.10

20.20

20.30

20.40

20.50

20.60

20.70

20.80

20.90

21.00

21.10

21.20

21.30

21.40

21.50

21.60

21.70

21.80

21.90

22.00

22.10

22.20

22.30

22.40

22.50

22.60

22.70

22.80

22.90

23.00

23.10

23.20

23.30

23.40

23.50

23.60

23.70

23.80

23.90

24.00 $f_{1}$

$9.55624 \mathrm{E}-01$ $9.55624 \mathrm{E}-01$ $9.55623 \mathrm{E}-01$ $9.55623 \mathrm{E}-01$ $9.55623 \mathrm{E}-01$

$9.55622 \mathrm{E}-01$ $9.55622 \mathrm{E}-01$ $9.55622 \mathrm{E}-01$ $9.55621 \mathrm{E}-01$ $9.55621 \mathrm{E}-01$

$9.55620 \mathrm{E}-01$ $9.55620 \mathrm{E}-01$ $9.55619 \mathrm{E}-01$ $9.55619 \mathrm{E}-01$ $9.55619 \mathrm{E}-01$

$9.55618 \mathrm{E}-01$ $9.55618 \mathrm{E}-01$ $9.55617 \mathrm{E}-01$ $9.55617 \mathrm{E}-01$ $9.55616 \mathrm{E}-01$

$9.55616 \mathrm{E}-01$ $9.55615 \mathrm{E}-01$ $9.55615 \mathrm{E}-01$ $9.55614 \mathrm{E}-01$ $9.55614 \mathrm{E}-01$

$9.55613 \mathrm{E}-01$ $9.55613 \mathrm{E}-01$ $9.55612 \mathrm{E}-01$ $9.55612 \mathrm{E}-01$ $9.55612 \mathrm{E}-01$

$9.55611 \mathrm{E}-01$ $9.55610 \mathrm{E}-01$ $9.55610 \mathrm{E}-01$ $9.55609 \mathrm{E}-01$ $9.55609 \mathrm{E}-01$

$9.55608 \mathrm{E}-01$ $9.55608 \mathrm{E}-01$ $9.55607 \mathrm{E}-01$ $9.55607 \mathrm{E}-01$ $9.55606 \mathrm{E}-01$

$9.55606 \mathrm{E}-01$ $9.55605 \mathrm{E}-01$ $9.55605 \mathrm{E}-01$ $9.55604 \mathrm{E}-01$ $9.55604 \mathrm{E}-01$

$9.55603 \mathrm{E}-01$ $9.55603 \mathrm{E}-01$ $9.55602 \mathrm{E}-01$ $9.55602 \mathrm{E}-01$ $9.55601 \mathrm{E}-01$

$9.55601 \mathrm{E}-01$ $9.55600 \mathrm{E}-01$ $9.55600 \mathrm{E}-01$ $9.55599 \mathrm{E}-01$ $9.55599 \mathrm{E}-01$

$9.55598 \mathrm{E}-01$ $9.55598 \mathrm{E}-01$ $9.55597 \mathrm{E}-01$ $9.55597 \mathrm{E}-01$ $9.55596 \mathrm{E}-01$ $g_{1}$

$\tan \delta_{1}$ $f-1$

$1.72496 \mathrm{E} \quad 01$ $1.73481 \mathrm{E} \quad 01$ $1.74466 \mathrm{E} \quad 01$ $1.75452 \mathrm{E} \quad 01$ $1.76437 \mathrm{E} \quad 01$

$1.77423 \mathrm{E} \quad 01$ $1.78408 \mathrm{E} \quad 01$ $1.79394 \mathrm{E} \quad 01$ $1.80380 \mathrm{E} \quad 01$ $1.81365 \mathrm{E} \quad 01$

$1.82351 \mathrm{E} \quad 01$

$1.83337 \mathrm{E} \quad 01$

$1.84323 \mathrm{E} \quad 01$

$1.85310 \mathrm{E} \quad 01$

$1.86296 \mathrm{E} 01$

$\begin{array}{ll}1.87282 \mathrm{E} & 01\end{array}$ $1.88268 \mathrm{E} \quad 01$ $1.89255 \mathrm{E} \quad 01$

$1.90241 \mathrm{E} \quad 01$

$1.91228 \mathrm{E} \quad 01$

$1.92214 \mathrm{E} \quad 01$ $1.93201 \mathrm{E} 01$ $1.94188 \mathrm{E} \quad 01$ $1.95175 \mathrm{E} \quad 01$

$1.96162 \mathrm{E} 01$

$1.97149 \mathrm{E} \quad 01$

$1.98136 \mathrm{E} \quad 01$

$1.99123 \mathrm{E} \quad 01$

$2.00110 \mathrm{E} 01$

$2.01098 \mathrm{E} 01$

$2.02085 \mathrm{E} \quad 01$

$2.03073 \mathrm{E} 01$

$2.04060 \mathrm{E} \quad 01$

$2.05048 \mathrm{E} \quad 01$

$2.06036 \mathrm{E} 01$

$2.07023 \mathrm{E} \quad 01$

$2.08011 \mathrm{E} \quad 01$

$2.08999 \mathrm{E} 01$

$2.09987 \mathrm{E} \quad 01$

$2.10976 \mathrm{E} 01$

$2.11964 \mathrm{E} \quad 0 \mathrm{I}$

$2.12952 \mathrm{E} \quad 01$

$2.13941 \mathrm{E} \quad 01$

$2.14929 \mathrm{E} 01$

$2.15918 \mathrm{E} 01$

$2.16906 \mathrm{E} \quad 01$

$2.17895 \mathrm{E} \quad 01$

$2.18884 \mathrm{E} \quad 01$

2.19873 E 01

$2.20862 \mathrm{E} 01$

$2.21851 \mathrm{E} \quad 01$

$2.22840 \mathrm{E} \quad 01$

$2.23829 \mathrm{E} 01$

$2.24819 \mathrm{E} \quad 01$

$2.25808 \mathrm{E} 01$

$2.26798 \mathrm{E} \quad 01$

$2.27787 \mathrm{E} \quad 01$

$2.28777 \mathrm{E} \quad 01$

$2.29767 \mathrm{E} 01$

$2.30757 \mathrm{E} 01$
4.41977 E-01 $4.47353 \mathrm{E}-01$ $4.52761 \mathrm{E}-01$ $4.58203 \mathrm{E}-01$ $4.63676 \mathrm{E}-01$

4.69183 E-01 4.74722 E-01 $4.80294 \mathrm{E}-01$ $4.85898 \mathrm{E}-01$ $4.91536 \mathrm{E}-01$

$4.97205 \mathrm{E}-01$

$5.02908 \mathrm{E}-01$

$5.08643 \mathrm{E}-01$

$5.14411 \mathrm{E}-01$

5.20212 E-01

$5.26046 \mathrm{E}-01$

$5.31912 \mathrm{E}-01$

$5.37811 \mathrm{E}-01$

$5.43742 \mathrm{E}-01$

$5.49707 \mathrm{E}-01$

$5.55704 \mathrm{E}-01$

$5.61734 \mathrm{E}-01$

$5.67797 \mathrm{E}-01$

$5.73892 \mathrm{E}-01$

$5.80021 \mathrm{E}-01$

$5.86182 \mathrm{E}-01$

$5.92375 \mathrm{E}-01$

$5.98602 \mathrm{E}-01$

$6.04862 \mathrm{E}-01$

$6.11154 \mathrm{E}-01$

$6.17479 \mathrm{E}-01$ $6.23837 \mathrm{E}-01$ $6.30228 \mathrm{E}-01$ $6.36651 \mathrm{E}-01$ $6.43108 \mathrm{E}-01$

$6.49597 \mathrm{E}-01$ $6.56119 \mathrm{E}-01$ $6.62674 \mathrm{E}-0 \mathrm{~F}$ $6.69262 \mathrm{E}-01$ $6.75883 \mathrm{E}-01$

$6.82537 \mathrm{E}-01$ $6.89223 \mathrm{E}-01$ $6.95943 \mathrm{E}-01$ $7.02695 \mathrm{E}-\mathrm{OI}$

$7.09480 \mathrm{E}-01$

$7.16298 \mathrm{E}-01$ $7.23149 \mathrm{E}-01$

$7.30034 \mathrm{E}-01$

$7.36950 \mathrm{E}-01$

$7.43900 \mathrm{E}-01$

$7.50883 \mathrm{E}-01$

$7.57899 \mathrm{E}-01$

$7.64948 \mathrm{E}-01$

$7.72030 \mathrm{E}-01$

$7.79144 \mathrm{E}-01$

$7.86292 \mathrm{E}-01$

$7.93473 \mathrm{E}-01$

$8.00686 \mathrm{E}-01$

$8.07933 \mathrm{E}-01$

8.152]3 E-01
$-5.46864 \mathrm{E}-04$

$-5.53019 \mathrm{E}-04$

$-5.55023 \mathrm{E}-04$

$-5.57004 \mathrm{E}-04$

$-5.58962 \mathrm{E}-04$

$-5.60894 \mathrm{E}-04$

$-5.62808 \mathrm{E}-04$

$-5.64698 \mathrm{E}-04$

$-5.66566 \mathrm{E}-04$

$-5.68413 \mathrm{E}-04$

$-5.70240 \mathrm{E}-04$

$=5.72045 \mathrm{E}-04$

$-5.73829 \mathrm{E}-04$

$-5.75594 \mathrm{E}-04$

$-5.77339 \mathrm{E}-04$

$-5.79063 \mathrm{E}-04$

$-5.80771 \mathrm{E}-04$

$-5.82456 \mathrm{E}-04$

$-5.84126 \mathrm{E}-04$

$-5.85776 \mathrm{E}-04$

$-5.87408 \mathrm{E}-04$

$-5.89020 \mathrm{E}-04$

$-5.90617 \mathrm{E}-04$

$-5.92197 \mathrm{E}-04$

$-5.93758 \mathrm{E}-04$

$-5.95301 \mathrm{E}-04$

$-5.96829 \mathrm{E}-04$

$-5.98341 \mathrm{E}-04$

$-5.99836 \mathrm{E}-04$ $-6.01314 \mathrm{E}-04$

$-6.02779 \mathrm{E}-04$

$-6.04227 \mathrm{E}-04$

$-6.05658 \mathrm{E}-04$

$-6.07075 \mathrm{E}-04$

$-6.08477 \mathrm{E}-04$

$-4.07228 \mathrm{E}-01$

$-4.12444 \mathrm{E}-01$

-4.17692 E-01

$-4.22973 \mathrm{E}-01$

$-4.28287 \mathrm{E}-01$ 
ELECTRON RADIAL FUNCTIONS

$Z=2 \quad A=4 \quad \rho=1.2 A^{1 / 3} F$

Positron

$\bar{F}$

$2.11574 \mathrm{E}-04$ $2.13918 \mathrm{E}-04$ $2.16275 \mathrm{E}-04$ $2.18645 \mathrm{E}-04$ $2.21028 \mathrm{E}-04$

$2.23424 \mathrm{E}-04$ $2.25833 \mathrm{E}-04$ $2.28255 \mathrm{E}-04$ $2.30690 \mathrm{E}-04$ $2.33137 \mathrm{E}-04$

$2.35598 \mathrm{E}-04$ $2.38072 \mathrm{E}-04$ $2.40558 \mathrm{E}-04$ $2.43057 \mathrm{E}-04$ $2.45569 \mathrm{E}-04$

$2.48095 \mathrm{E}-04$ $2.50633 \mathrm{E}-04$ $2.53184 \mathrm{E}-04$ $2.55747 \mathrm{E}-04$ $2.58324 \mathrm{E}-04$

$2.60914 \mathrm{E}-04$ $2.63517 \mathrm{E}-04$ $2.66132 \mathrm{E}-04$ 2.68761 E-04 $2.71402 \mathrm{E}-04$

$2.74056 \mathrm{E}-04$ $2.76723 \mathrm{E}-04$ $2.79404 \mathrm{E}-04$ $2.82097 \mathrm{E}-04$ $2.84803 \mathrm{E}-04$

$2.87521 \mathrm{E}-04$ $2.90253 \mathrm{E}-04$ $2.92998 \mathrm{E}-04$ $2.95755 \mathrm{E}-04$ $2.98526 \mathrm{E}-04$

$3.01309 \mathrm{E}-04$ $3.04106 \mathrm{E}-04$ $3.06915 \mathrm{E}-04$ $3.09737 \mathrm{E}-04$ $3.12572 \mathrm{E}-04$

$3.15420 \mathrm{E}-04$ 3.18281 E- 04 $3.21155 \mathrm{E}-04$ $3.24042 \mathrm{E}-04$ $3.26941 \mathrm{E}-04$

$3.29854 \mathrm{E}-04$ $3.32779 \mathrm{E}-04$ $3.35718 \mathrm{E}-04$ $3.38669 \mathrm{E}-04$ $3.41633 \mathrm{E}-04$

3.44611 E-04 $3.47601 \mathrm{E}-04$ 3.50604 E- 04 $3.53620 \mathrm{E}-04$ $3.56648 \mathrm{E}-04$

23.60

23.70

23.80

23.90

24.00

$3.59690 \mathrm{E}-04$ $3.62745 \mathrm{E}-04$ $3.65812 \mathrm{E}-04$ 3.68893 E- 04 $3.71986 \mathrm{E}-04$ $f_{2}$

$5.11765 \mathrm{E}-01$ 5.17515 E-01

$5.23298 \mathrm{E}-01$

$5.29112 \mathrm{E}-01$

$5.34958 \mathrm{E}-01$

$5.40837 \mathrm{E}-01$

$5.46747 \mathrm{E}-01$

$5.52690 \mathrm{E}-01$

$5.58664 \mathrm{E}-01$

5.64671 E-01

$5.70710 \mathrm{E}-01$

$5.76780 \mathrm{E}-01$

$5.82883 \mathrm{E}-01$

$5.89018 \mathrm{E}-01$

$5.95185 \mathrm{E}-01$

$6.01383 \mathrm{E}-01$

$6.07614 \mathrm{E}-01$

$6.13877 \mathrm{E}-01$

$6.20172 \mathrm{E}-01$

$6.26499 \mathrm{E}-01$

$6.32858 \mathrm{E}-01$

$6.39249 \mathrm{E}-01$

$6.45672 \mathrm{E}-01$

$6.52127 \mathrm{E}-01$

$6.58614 \mathrm{E}-01$

$6.65133 \mathrm{E}-01$

$6.71684 \mathrm{E}-01$

$6.78267 \mathrm{E}-01$

$6.84882 \mathrm{E}-01$

$6.91529 \mathrm{E}-01$

$6.98208 \mathrm{E}-01$

$7.04919 \mathrm{E}-01$

$7.11662 \mathrm{E}-01$

$7.18437 \mathrm{E}-01$

$7.25244 \mathrm{E}-01$

$7.32083 \mathrm{E}-01$

$7.45857 \mathrm{E}-01$

$7.52793 \mathrm{E}-01$

$7.59760 \mathrm{E}-01$

$7.66759 \mathrm{E}-01$

$7.73790 \mathrm{E}-01$

$7.80853 \mathrm{E}-01$

$7.87948 \mathrm{E}-01$

7.95075 E-01

8.02234 E-01

$8.09425 \mathrm{E}-01$

$8.16648 \mathrm{E}-01$

$8.23903 \mathrm{E}-01$

$8.31190 \mathrm{E}-01$

$8.38508 \mathrm{E}-01$

$8.45859 \mathrm{E}-01$

8.53242 E-01

$8.60657 \mathrm{E}-01$

$8.68104 \mathrm{E}-01$

8.75583 E-01 $8.83093 \mathrm{E}-01$

$8.90636 \mathrm{E}-01$

$8.98211 \mathrm{E}-01$

$9.05817 \mathrm{E}-01$
7.38954E-01

$g_{2}$

$\tan \delta_{2}$

$f_{-2}$

g-2

$\tan \delta_{-2}$

$7.95151 \mathrm{E}-03 \quad-9.87909 \mathrm{E} \quad 01 \quad-7.33495 \mathrm{E}-03$ $8.09186 \mathrm{E}-03-9.88033 \mathrm{E}$ 01 $-7.46899 \mathrm{E}-03$ $8.23385 \mathrm{E}-03 \quad-9.88155 \mathrm{E}$ 01 $-7.60465 \mathrm{E}-03$ $8.37748 \mathrm{E}-03 \quad-9.88277 \mathrm{E}$ 01 $-7.74192 \mathrm{E}-03$ $8.52278 \mathrm{E}-03-9.88397 \mathrm{E}$ 01 $-7.88082 \mathrm{E}-03$

$8.66974 \mathrm{E}-03$ $8.81837 \mathrm{E}-03$ $8.96870 \mathrm{E}-03$

$9.12072 \mathrm{E}-03$ $9.27444 \mathrm{E}-03$

$9.42988 \mathrm{E}-03$ $9.58704 \mathrm{E}-03$

$9.74594 \mathrm{E}-03$

$9.90658 \mathrm{E}-03$

$1.00690 \mathrm{E}-02$

$1.02331 \mathrm{E}-02$

$1.03991 \mathrm{E}-02$

$1.05668 \mathrm{E}-02$

$1.07363 \mathrm{E}-02$

$1.09076 \mathrm{E}-02$

1.10807E-02

$1.12556 \mathrm{E}-02$

$1.14323 \mathrm{E}-02$

$1.16109 \mathrm{E}-02$

$1.17914 \mathrm{E}-02$

$1.19737 \mathrm{E}-02$

$1.21578 \mathrm{E}-02$

$1.23439 \mathrm{E}-02$

$1.25318 \mathrm{E}-02$

$1.27216 \mathrm{E}-02$

$1.29134 \mathrm{E}-02$

$1.31070 \mathrm{E}-02$

$1.33025 \mathrm{E}-02$

$1.35000 \mathrm{E}-02$

$1.36995 \mathrm{E}-02$

$1.39009 \mathrm{E}-02$

$1.41042 \mathrm{E}-02$

$1.43095 \mathrm{E}-02$

$1.45168 \mathrm{E}-02$

$1.47261 \mathrm{E}-02$

$1.49374 \mathrm{E}-02$

$1.51507 \mathrm{E}-02$

$1.53660 \mathrm{E}-02$

$1.55833 \mathrm{E}-02$

$1.58027 \mathrm{E}-02$

$1.60241 \mathrm{E}-02$

$1.62476 \mathrm{E}-02$

$1.64732 \mathrm{E}-02$

$1.67008 \mathrm{E}-02$

$1.69305 \mathrm{E}-02$

$1.71623 \mathrm{E}-02$

$1.73962 \mathrm{E}-02$

$1.76322 \mathrm{E}-02$

$1.78703 \mathrm{E}-02$

$1.81106 \mathrm{E}-02$
$1.83530 \mathrm{E}-02$ $1.85975 \mathrm{E}-02$

$1.88443 \mathrm{E}-02$

$1.90931 \mathrm{E}-02$

$1.93442 \mathrm{E}-02$
$-9.88515 \mathrm{E} \quad 01$

$-9.88633 \mathrm{E} 01$

$-9.88749 \mathrm{E} 01$

$\begin{array}{lll}-9.88863 \mathrm{E} & 01\end{array}$

$-9.88977 \mathrm{E} \quad 01$

$-9.89089 \mathrm{E} 01$

$-9.89200 \mathrm{E} 01$

$-9.89310 \mathrm{E}$ 0l

$\begin{array}{ll}-9.89419 \mathrm{E} & 01\end{array}$

$-9.89526 \mathrm{E} 01$

$-8.02135 \mathrm{E}-03$

$-8.16353 \mathrm{E}-03$

$-8.30736 \mathrm{E}-03$

$-8.45285 \mathrm{E}-03$

$-8.60002 \mathrm{E}-03$

$-8.74887 \mathrm{E}-03$

$-8.89942 \mathrm{E}-03$

$-9.05167 \mathrm{E}-03$

$-9.20563 \mathrm{E}-03$

$-9.36130 \mathrm{E}-03$

$-9.89633 \mathrm{E} \quad 01$

$-9.89738 \mathrm{E} 01$

$-9.89842 \mathrm{E} \quad 01$

$\begin{array}{ll}-9.89945 \mathrm{E} & 01\end{array}$

$-9.90047 \mathrm{E} 01$

$-9.90148 \mathrm{E}$ ol

$-9.90248 \mathrm{E} 01$

$-9.90346 \mathrm{E} 01$

$-9.90444 \mathrm{E} 01$

$-9.90541 \mathrm{E} 01$

$-9.90637 \mathrm{E} 01$

$\begin{array}{lll}-9.90732 \mathrm{E} & 01\end{array}$

$-9.90826 \mathrm{E} 01$

$\begin{array}{lll}-9.90919 \mathrm{E} & 01\end{array}$

$-9.91011 \mathrm{E} 01$

$-9.51871 \mathrm{E}-03$

$-9.67786 \mathrm{E}-03$

$-9.83876 \mathrm{E}-03$

$-1.00014 \mathrm{E}-02$

$-1.01658 \mathrm{E}-02$

$-1.03321 \mathrm{E}-02$

$-1.05000 \mathrm{E}-02$

$-1.06698 \mathrm{E}-02$

$-1.08414 \mathrm{E}-02$

$-1.10148 \mathrm{E}-02$

$-1.11901 \mathrm{E}-02$

$-1.13671 \mathrm{E}-02$

$-1.15461 \mathrm{E}-02$

$-1.17268 \mathrm{E}-02$

$-1.19094 \mathrm{E}-02$

$-9.91102 \mathrm{E} 01$

$-1.20939 \mathrm{E}-02$

$-9.91193 \mathrm{E} 01$

$-9.91282 \mathrm{E} 01$

$-9.91371 \mathrm{E} 01$

$-9.91458 \mathrm{E} 01$

$-1.22803 \mathrm{E}-02$

$-1.24686 \mathrm{E}-02$

$-1.26588 \mathrm{E}-02$

$-1.28509 \mathrm{E}-02$

$\begin{array}{ll}-9.91545 \mathrm{E} & 01\end{array}$

$-9.91631 \mathrm{E} \quad 01$

$-9.91717 \mathrm{E} \quad 01$

$-9.91801 \mathrm{E} 01$

-9.91885 E 01

$-1.30449 \mathrm{E}-02$

$-1.32408 \mathrm{E}-02$

$-1.34387 \mathrm{E}-02$

$-1.36385 \mathrm{E}-02$

$-1.38403 \mathrm{E}-02$

$\begin{array}{lll}-9.91967 \mathrm{E} & 01\end{array}$

$-9.92050 \mathrm{E} \quad 01$

$-9.92131 \mathrm{E} \quad 01$

$-9.92211 \mathrm{E} 01$

$-1.40440 \mathrm{E}-02$

$-1.42498 \mathrm{E}-02$

$-1.44575 \mathrm{E}-02$

$-1.46672 \mathrm{E}-02$

$-1.48789 \mathrm{E}-02$

$\begin{array}{lll}-9.92370 \mathrm{E} & 01\end{array}$

$-9.92449 \mathrm{E} \quad 01$

$-9.92526 \mathrm{E} \quad 01$

$\begin{array}{lll}-9.92603 \mathrm{E} & 01\end{array}$

$-9.92680 \mathrm{E} \quad 01$

$-9.92755 \mathrm{E} \quad 01$

$-9.92830 \mathrm{E} 01$

$-9.92904 \mathrm{E} 01$

$-9.92978 \mathrm{E} 01$

$-9.93051 \mathrm{E} 01$

$-1.50926 \mathrm{E}-02$ $-1.53084 \mathrm{E}-02$ $-1.55262 \mathrm{E}-02$ $-1.57460 \mathrm{E}-02$ $-1.59679 \mathrm{E}-02$

$-9.93123 \mathrm{E} \quad 01$ 
ELECTRON RADIAL FUNCTIONS

Positron

\begin{tabular}{|c|c|c|c|}
\hline$p$ & $F_{0}$ & $f_{1}$ & \\
\hline 24.10 & $9.55596 \mathrm{E}-01$ & $2.31747 \mathrm{E}$ & 01 \\
\hline 24.20 & $9.55595 \mathrm{E}-01$ & $2.32737 \mathrm{E}$ & 01 \\
\hline 24.30 & $9.55594 \mathrm{E}-01$ & $2.33727 \mathrm{E}$ & 01 \\
\hline 24.40 & $9.55594 \mathrm{E}-01$ & $2.34717 \mathrm{E}$ & 01 \\
\hline 24.50 & $9.55593 \mathrm{E}-01$ & $2.35708 \mathrm{E}$ & 01 \\
\hline 24.60 & $9.55593 \mathrm{E}-01$ & $2.36698 \mathrm{E}$ & 01 \\
\hline 24.70 & $9.55592 \mathrm{E}-01$ & $2.37689 \mathrm{E}$ & 01 \\
\hline 24.80 & $9.55592 \mathrm{E}-01$ & $2.38679 \mathrm{E}$ & 01 \\
\hline 24.90 & $9.55591 \mathrm{E}-01$ & $2.39670 \mathrm{E}$ & 01 \\
\hline 25.00 & $9.55591 \mathrm{E}-01$ & $2.40661 \mathrm{E}$ & 01 \\
\hline 25.10 & $9.55590 \mathrm{E}-01$ & $2.41652 \mathrm{E}$ & 01 \\
\hline 25.20 & $9.55590 \mathrm{E}-01$ & $2.42643 \mathrm{E}$ & 01 \\
\hline 25.30 & $9.55589 \mathrm{E}-01$ & $2.43634 \mathrm{E}$ & 01 \\
\hline 25.40 & $9.55589 \mathrm{E}-01$ & $2.44625 \mathrm{E}$ & 01 \\
\hline 25.50 & $9.55588 \mathrm{E}-01$ & $2.45616 \mathrm{E}$ & 01 \\
\hline 25.60 & $9.55588 \mathrm{E}-01$ & $2.46608 \mathrm{E}$ & 01 \\
\hline 25.70 & $9.55587 \mathrm{E}-01$ & $2.47599 \mathrm{E}$ & 01 \\
\hline 25.80 & $9.55586 \mathrm{E}-01$ & $2.48591 \mathrm{E}$ & 01 \\
\hline 25.90 & $9.55586 \mathrm{E}-01$ & $2.49583 \mathrm{E}$ & 01 \\
\hline 26.00 & $9.55585 \mathrm{E}-01$ & $2.50574 \mathrm{E}$ & 01 \\
\hline 26.10 & $9.55585 \mathrm{E}-01$ & $2.51566 \mathrm{E}$ & 01 \\
\hline 26.20 & $9.55584 \mathrm{E}-01$ & $2.52558 \mathrm{E}$ & 01 \\
\hline 26.30 & $9.55584 \mathrm{E}-01$ & $2.53550 \mathrm{E}$ & 01 \\
\hline 26.40 & $9.55583 \mathrm{E}-01$ & $2.54543 \mathrm{E}$ & 01 \\
\hline 26.50 & $9.55583 \mathrm{E}-01$ & $2.55535 \mathrm{E}$ & 01 \\
\hline 26.60 & $9.55582 \mathrm{E}-01$ & $2.56527 \mathrm{E}$ & 01 \\
\hline 26.70 & $9.55582 \mathrm{E}-01$ & $2.57520 \mathrm{E}$ & 01 \\
\hline 26.80 & $9.55581 \mathrm{E}-01$ & $2.58512 \mathrm{E}$ & 01 \\
\hline 26.90 & $9.55581 \mathrm{E}-01$ & $2.59505 \mathrm{E}$ & 01 \\
\hline 27.00 & $9.55580 \mathrm{E}-01$ & $2.60498 \mathrm{E}$ & 01 \\
\hline 27.10 & $9.55580 \mathrm{E}-01$ & $2.61491 \mathrm{E}$ & 01 \\
\hline 27.20 & $9.55579 \mathrm{E}-01$ & $2.62484 \mathrm{E}$ & 01 \\
\hline 27.30 & $9.55579 \mathrm{E}-01$ & $2.63477 \mathrm{E}$ & 01 \\
\hline 27.40 & $9.55578 \mathrm{E}-01$ & $2.64470 \mathrm{E}$ & 01 \\
\hline 27.50 & $9.55578 \mathrm{E}-01$ & $2.65463 \mathrm{E}$ & 01 \\
\hline 27.60 & $9.55577 \mathrm{E}-01$ & $2.66457 \mathrm{E}$ & 01 \\
\hline 27.70 & $9.55576 \mathrm{E}-01$ & $2.67450 \mathrm{E}$ & 01 \\
\hline 27.80 & $9.55576 \mathrm{E}-01$ & $2.68444 \mathrm{E}$ & 01 \\
\hline 27.90 & $9.55575 \mathrm{E}-01$ & $2.69438 \mathrm{E}$ & 01 \\
\hline 28.00 & $9.55575 \mathrm{E}-01$ & $2.70431 \mathrm{E}$ & 01 \\
\hline 28.10 & $9.55574 \mathrm{E}-01$ & $2.71425 \mathrm{E}$ & 01 \\
\hline 28.20 & $9.55574 \mathrm{E}-01$ & $2.72419 \mathrm{E}$ & 01 \\
\hline 28.30 & $9.55573 \mathrm{E}-01$ & $2.73414 \mathrm{E}$ & 01 \\
\hline 28.40 & $9.55573 \mathrm{E}-01$ & $2.74408 \mathrm{E}$ & 01 \\
\hline 28.50 & $9.55572 \mathrm{E}-01$ & $2.75402 \mathrm{E}$ & 01 \\
\hline 28.60 & $9.55572 \mathrm{E}-01$ & $2.76397 \mathrm{E}$ & 01 \\
\hline 28.70 & $9.55571 \mathrm{E}-01$ & $2.77391 \mathrm{E}$ & 01 \\
\hline 28.80 & $9.55571 \mathrm{E}-01$ & $2.78386 \mathrm{E}$ & 01 \\
\hline 28.90 & $9.55570 \mathrm{E}-01$ & $2.79381 \mathrm{E}$ & 01 \\
\hline 29.00 & $9.55570 \mathrm{E}-01$ & $2.80376 \mathrm{E}$ & 01 \\
\hline 29.10 & $9.55569 \mathrm{E}-01$ & $2.81371 \mathrm{E}$ & 01 \\
\hline 29.20 & $9.55569 \mathrm{E}-01$ & $2.82366 \mathrm{E}$ & 01 \\
\hline 29.30 & $9.55568 \mathrm{E}-01$ & $2.83361 \mathrm{E}$ & 01 \\
\hline 29.40 & $9.55568 \mathrm{E}-01$ & $2.84356 \mathrm{E}$ & 01 \\
\hline 29.50 & $9.55567 \mathrm{E}-01$ & $2.85352 \mathrm{E}$ & 01 \\
\hline 29.60 & $9.55567 \mathrm{E}-01$ & $2.86348 \mathrm{E}$ & 01 \\
\hline 29.70 & $9.55566 \mathrm{E}-01$ & $2.87343 \mathrm{E}$ & 01 \\
\hline 29.80 & $9.55565 \mathrm{E}-$ & $2.88339 \mathrm{E}$ & 01 \\
\hline 29.90 & $9.55565 \mathrm{E}-01$ & $2.89335 \mathrm{E}$ & 01 \\
\hline 30.00 & $9.55564 \mathrm{E}-01$ & $2.90331 \mathrm{E}$ & 01 \\
\hline
\end{tabular}

$Z=2 \quad A=4 \quad \rho=1.2 A^{1 / 3} F$

$g_{1} \quad \tan \delta_{1}$

$8.22526 \mathrm{E}-01 \quad-6.38083 \mathrm{E}-04$

$8.29872 \mathrm{E}-01-6.39164 \mathrm{E}-04$

$8.37250 \mathrm{E}-01-6.40234 \mathrm{E}-04$

$8.44662 \mathrm{E}-01 \quad-6.41297 \mathrm{E}-04$

$8.52107 \mathrm{E}-01-6.42347 \mathrm{E}-04$

$8.59585 \mathrm{E}-01$

$8.67096 \mathrm{E}-01$

$8.74640 \mathrm{E}-01$

$8.82218 \mathrm{E}-01$

$8.89828 \mathrm{E}-01$

$-6.43386 \mathrm{E}-04$

$-6.44417 \mathrm{E}-04$

$-6.45435 \mathrm{E}-04$

$-6.46443 \mathrm{E}-04$

$-6.47441 \mathrm{E}-04$

$8.97471 \mathrm{E}-01$

$9.05148 \mathrm{E}-01$

$9.12857 \mathrm{E}-01$

$9.20600 \mathrm{E}-01$

$9.28376 \mathrm{E}-01$

$9.36185 \mathrm{E}-01$

$9.44027 \mathrm{E}-01$

$9.51902 \mathrm{E}-01$

$9.59810 \mathrm{E}-01$

$9.67752 \mathrm{E}-01$

$-6.48430 \mathrm{E}-04$

$-6.49407 \mathrm{E}-04$

$-6.50377 \mathrm{E}-04$

$-6.51338 \mathrm{E}-04$

$-6.52288 \mathrm{E}-04$

$-6.53226 \mathrm{E}-04$

$-6.54159 \mathrm{E}-04$

$-6.55082 \mathrm{E}-04$

$-6.55994 \mathrm{E}-04$

$-6.56898 \mathrm{E}-04$.

$9.75726 \mathrm{E}-01$

$9.83734 \mathrm{E}-01$

$9.91776 \mathrm{E}-01$

$9.99850 \mathrm{E}-01$

$1.00796 \mathrm{E} \quad 00$

$-6.57792 \mathrm{E}-04$

$-6.58678 \mathrm{E}-04$

$-6.59557 \mathrm{E}-04$

$-6.60427 \mathrm{E}-04$

$-6.61286 \mathrm{E}-04$

$1.01610 \mathrm{E} \quad 00$

$1.02427 \mathrm{E} \quad 00$

$1.03248 \mathrm{E} \quad 00$

$1.04072 \mathrm{E} \quad 00$

$1.04899 \mathrm{E} \quad 00$

$1.05730 \mathrm{E} \quad 00$

$1.06564 \mathrm{E} \quad 00$

$1.07401 \mathrm{E} \quad 00$

$1.08242 \mathrm{E} \quad 00$

$1.09086 \mathrm{E} \quad 00$

$1.09933 \mathrm{E} \quad 00$

$1.10784 \mathrm{E} \quad 00$

$1.11638 \mathrm{E} \quad 00$

$1.12495 \mathrm{E} \quad 00$

$1.13356 \mathrm{E} \quad 00$

$1.14220 \mathrm{E} \quad 00$

$1.15087 \mathrm{E} \quad 00$

$1.15958 \mathrm{E} \quad 00$

$1.16832 \mathrm{E} \quad 00$

$1.17709 \mathrm{E} \quad 00$

$-6.62139 \mathrm{E}-04$

$-6.62984 \mathrm{E}-04$

$-6.63817 \mathrm{E}-04$

$-6.64645 \mathrm{E}-04$

$-6.65462 \mathrm{E}-04$

$-6.66275 \mathrm{E}-04$ $-6.67075 \mathrm{E}-04$

$-6.67870 \mathrm{E}-04$

$-6.68658 \mathrm{E}-04$

$-6.69438 \mathrm{E}-04$

$-6.70210 \mathrm{E}-04$

$-6.70973 \mathrm{E}-04$

$-6.71732 \mathrm{E}-04$

$-6.72481 \mathrm{E}-04$

$-6.73222 \mathrm{E}-04$

$-7.78209 \mathrm{E}-01$

$-7.85396 \mathrm{E}-01$

$-7.92616 \mathrm{E}-01$

$-7.99869 \mathrm{E}-01$

-8.07155 E-01

$-8.14475 \mathrm{E}-01$

$-8.21827 \mathrm{E}-01$

$-8.29212 \mathrm{E}-01$

$-8.36631 \mathrm{E}-01$

$-8.44082 \mathrm{E}-01$

$-8.51567 \mathrm{E}-01$

$-8.59085 \mathrm{E}-01$

$-8.66636 \mathrm{E}-01$

$-8.74220 \mathrm{E}-01$

$-8.81838 \mathrm{E}-01$

$-8.89488 \mathrm{E}-01$

$-8.97172 \mathrm{E}-01$

$-9.04889 \mathrm{E}-01$

$-9.12639 \mathrm{E}-01$

$-9.20422 \mathrm{E}-01$

$-9.28238 \mathrm{E}-01$

$-9.36088 \mathrm{E}-01$

$-9.43970 \mathrm{E}-01$

$-9.51886 \mathrm{E}-01$

$-9.59836 \mathrm{E}-01$

$-9.67818 \mathrm{E}-01$

$-9.75834 \mathrm{E}-01$

$-9.83883 \mathrm{E}-01$

$-9.91965 \mathrm{E}-01$

$-1.00008 \mathrm{E} \quad 00$

$-1.00823 \mathrm{E} \quad 00$

$-1.01641 \mathrm{E} \quad 00$

-1.02463 E 00

$-1.03288 \mathrm{E} 00$

$-1.04116 \mathrm{E} 00$

-1.04947 E 00

$-1.05782 \mathrm{E} 00$

$-1.06620 \mathrm{E} \quad 00$

$-1.07462 \mathrm{E} 00$

$-1.08307 \mathrm{E} \quad 00$

$-6.73958 \mathrm{E}-04$

$-6.74685 \mathrm{E}-04$

$-6.75406 \mathrm{E}-04$

$-6.76119 \mathrm{E}-04$

$-6.76826 \mathrm{E}-04$

$1.18590 \mathrm{E} \quad 00$

$1.19474 \mathrm{E} \quad 00$

$1.20361 \mathrm{E} \quad 00$

$1.21252 \mathrm{E} \quad 00$

$1.22146 \mathrm{E} \quad 00$

$1.23043 \mathrm{E} \quad 00$

$1.23944 \mathrm{E} \quad 00$

$1.24848 \mathrm{E} \quad 00$

$1.25755 \mathrm{E} \quad 00$

$1.26666 \mathrm{E} \quad 00$

$-6.77524 \mathrm{E}-04$

$-6.78218 \mathrm{E}-04$

$-6.78903 \mathrm{E}-04$

$-6.79584 \mathrm{E}-04$

$-6.80256 \mathrm{E}-04$

$-6.80922 \mathrm{E}-04$

$-6.81580 \mathrm{E}-04$

$-6.82232 \mathrm{E}-04$

$-6.82878 \mathrm{E}-04$

$-6.83518 \mathrm{E}-04$

$-1.09155 \mathrm{E} \quad 00$

$-1.10006 \mathrm{E} \quad 00$

$-1.10861 \mathrm{E} 00$

$-1.11720 \mathrm{E} 00$

$-1.12581 \mathrm{E} 00$

$-1.13446 \mathrm{E} \quad 00$ $-1.14314 \mathrm{E} \quad 00$ $-1.15186 \mathrm{E} 00$ $-1.16061 \mathrm{E} 00$ $-1.16939 \mathrm{E} \quad 00$

$-1.17821 \mathrm{E} \quad 00$ $-1.18706 \mathrm{E} 00$ $-1.19594 \mathrm{E} \quad 00$ $-1.20485 \mathrm{E} 00$ $-1.21380 \mathrm{E} \quad 00$

$1.27580 \mathrm{E} \quad 00$

$1.28498 \mathrm{E} \quad 00$

$1.29418 \mathrm{E} \quad 00$

$-6.84151 \mathrm{E}-04$

$-6.84779 \mathrm{E}-04$

$-6.85401 \mathrm{E}-04$

$-6.86016 \mathrm{E}-04$

$-1.22279 \mathrm{E} 00$

$-1.23181 \mathrm{E} 00$

$-1.24086 \mathrm{E} 00$

$-1.24994 \mathrm{E} \quad 00$

$-1.25906 \mathrm{E} 00$ $g_{-1}$

$\tan \delta_{-1}$

$2.41668 \mathrm{E} \quad 01$ $2.42660 \mathrm{E} \quad 01$ $2.43651 \mathrm{E} 01$ $2.44643 \mathrm{E} \quad 01$ 2.45634E 01

$2.46626 \mathrm{E} \quad 01$ $2.47618 \mathrm{E} \quad 01$ $2.48610 \mathrm{E} \quad 01$ $2.49602 \mathrm{E} \quad 01$ $2.50594 \mathrm{E} \quad 01$

$2.51587 \mathrm{E} \quad 01$ $2.52579 \mathrm{E} 01$ $2.53571 \mathrm{E} \quad 01$ $2.54564 \mathrm{E} \quad 01$ $2.55557 \mathrm{E} \quad 01$

$2.56549 \mathrm{E} \quad 01$ $2.57542 \mathrm{E} \quad 01$ $2.58535 \mathrm{E} \quad 01$ $2.59528 \mathrm{E} \quad 01$ $2.60521 \mathrm{E} 01$

$2.61515 \mathrm{E} \quad 01$ $2.62508 \mathrm{E} \quad 01$ $2.63502 \mathrm{E} \quad 01$

$2.64495 \mathrm{E} \quad 01$

$2.65489 \mathrm{E} \quad 01$

$2.66483 \mathrm{E} \quad 01$ $2.67476 \mathrm{E} \quad 0 \mathrm{l}$ $2.68470 \mathrm{E} \quad 01$ $2.69465 \mathrm{E} \quad 01$ $2.70459 \mathrm{E} 01$

$2.71453 \mathrm{E} \quad 01$ $2.72448 \mathrm{E} \quad 01$ $2.73442 \mathrm{E} \quad 01$ $2.74437 \mathrm{E} \quad 01$ $2.75432 \mathrm{E} \quad 0 \mathrm{l}$

$2.76426 \mathrm{E} \quad 01$ $2.77421 \mathrm{E} 01$ $2.78416 \mathrm{E}$ 0l $2.79412 \mathrm{E} \quad 01$ $2.80407 \mathrm{E} 01$

$2.81402 \mathrm{E} \quad 01$ $2.82398 \mathrm{E} \quad 01$ $2.83393 \mathrm{E} 01$ $2.84389 \mathrm{E} \quad 01$ $2.85385 \mathrm{E} 01$

$2.86381 \mathrm{E} \quad 01$ $2.87377 \mathrm{E} \quad 01$ $2.88373 \mathrm{E} \quad 01$ $2.89370 \mathrm{E} 01$ $2.90366 \mathrm{E} \quad 01$

$2.91363 \mathrm{E} \quad 01$ $2.92359 \mathrm{E} 01$ $2.93356 \mathrm{E} \quad 01$ $2.94353 \mathrm{E} \quad 01$ $2.95350 \mathrm{E} \quad 01$

$2.96347 \mathrm{E} \quad 01$ $2.97344 \mathrm{E} \quad 01$ $2.98342 \mathrm{E} \quad 01$ $2.99339 \mathrm{E} 01$ $3.00337 \mathrm{E} 01$

$8.04804 \mathrm{E} \quad 02$ $8.05730 \mathrm{E} \quad 02$ $8.06652 \mathrm{E} \quad 02$ $8.07567 \mathrm{E} \quad 02$ 8.08480 E 02

$8.09388 \mathrm{E} \quad 02$ $8.10292 \mathrm{E} \quad 02$ $8.11193 \mathrm{E} \quad 02$ 8.12090 E 02 8.12983 E 02

$8.13873 \mathrm{E} \quad 02$ $8.14758 \mathrm{E} \quad 02$ $8.15639 \mathrm{E} \quad 02$ $8.16516 \mathrm{E} \quad 02$ $8.17390 \mathrm{E} \quad 02$

$8.18263 \mathrm{E} \quad 02$ $8.19128 \mathrm{E} \quad 02$ $8.19991 \mathrm{E} 02$ $8.20852 \mathrm{E} \quad 02$ $8.21708 \mathrm{E} \quad 02$

$8.22562 \mathrm{E} \quad 02$ $8.23412 \mathrm{E} \quad 02$ $8.24257 \mathrm{E} \quad 02$ $8.25100 \mathrm{E} \quad 02$ $8.25940 \mathrm{E} \quad 02$

$8.26776 \mathrm{E} \quad 02$ $8.27608 \mathrm{E} \quad 02$ $8.28440 \mathrm{E} \quad 02$ $8.29266 \mathrm{E} \quad 02$ $8.30091 \mathrm{E} \quad 02$

$8.30911 \mathrm{E} \quad 02$ $8.31730 \mathrm{E} \quad 02$ $8.32545 \mathrm{E} \quad 02$ $8.33357 \mathrm{E} \quad 02$ 8.34165 E 02

$8.34971 \mathrm{E} \quad 02$ 8.35775E 02 $8.36574 \mathrm{E} \quad 02$ $8.37372 \mathrm{E} \quad 02$ $8.38168 \mathrm{E} \quad 02$

$8.38959 \mathrm{E} \quad 02$ $8.39749 \mathrm{E} \quad 02$ $8.40535 \mathrm{E} \quad 02$ $8.41319 \mathrm{E} \quad 02$ 8.42101 E 02

$8.42880 \mathrm{E} \quad 02$ $8.43656 \mathrm{E} \quad 02$ $8.44431 \mathrm{E} \quad 02$ $8.45200 \mathrm{E} \quad 02$ 8.45970 E 02

$8.46736 \mathrm{E} \quad 02$ 8.47500 E 02 $8.48262 \mathrm{E} \quad 02$ 
$p$

24.10

24.20

24.30

24.40

24.50

24.60

24.70

24.80

24.90

25.00

25.10

25.20

25.30

25.40

25.50

25.60

25.70

25.80

25.90

26.00

26.10

26.20

26.30

26.40

26.50

26.60

26.70

26.80

26.90

27.00

27.10

27.20

27.30

27.40

27.50

27.60

27.70

27.80

27.90

28.00

28.10

28.20

28.30

28.40

28.50

28.60

28.70

28.80

28.90

29.00

29.10

29.20

29.30

29.40

29.50

29.60

29.70

29.80

29.90

30.00 $f_{2}$

$3.75093 \mathrm{E}-04$

$3.78212 \mathrm{E}-04$

$3.81344 \mathrm{E}-04$

$3.84489 \mathrm{E}-04$

$3.87647 \mathrm{E}-04$

$3.90818 \mathrm{E}-04$

$3.94002 \mathrm{E}-04$

$3.97198 \mathrm{E}-04$

$4.00408 \mathrm{E}-04$

$4.03630 \mathrm{E}-04$

$4.06866 \mathrm{E}-04$

4.10114 E-04

$4.13375 \mathrm{E}-04$

$4.16650 \mathrm{E}-04$

$4.19937 \mathrm{E}-04$

$4.23237 \mathrm{E}-04$

$4.26550 \mathrm{E}-04$

$4.29876 \mathrm{E}-04$

$4.33214 \mathrm{E}-04$

$4.36566 \mathrm{E}-04$

$4.39931 \mathrm{E}-04$

$4.43308 \mathrm{E}-04$

$4.46698 \mathrm{E}-04$

$4.50102 \mathrm{E}-04$

$4.53518 \mathrm{E}-04$

$4.56947 \mathrm{E}-04$

$4.60389 \mathrm{E}-04$

$4.63844 \mathrm{E}-04$

$4.67312 \mathrm{E}-04$

4.70793E-04

4.74287E-04

$4.77793 \mathrm{E}-04$

$4.81313 \mathrm{E}-04$

$4.84845 \mathrm{E}-04$

$4.88391 \mathrm{E}-04$

$4.91949 \mathrm{E}-04$

$4.95520 \mathrm{E}-04$

$4.99105 \mathrm{E}-04$

$5.02702 \mathrm{E}-04$

$5.06312 \mathrm{E}-04$

$5.09934 \mathrm{E}-04$

$5.13570 \mathrm{E}-04$

$5.17219 \mathrm{E}-04$

$5.20881 \mathrm{E}-04$

$5.24555 \mathrm{E}-04$

$5.28243 \mathrm{E}-04$

$5.31943 \mathrm{E}-04$

$5.35656 \mathrm{E}-04$

$5.39382 \mathrm{E}-04$

$5.43122 \mathrm{E}-04$

$5.46874 \mathrm{E}-04$

$5.50638 \mathrm{E}-04$

$5.54416 \mathrm{E}-04$

$5.58207 \mathrm{E}-04$

$5.62011 \mathrm{E}-04$

$5.65827 \mathrm{E}-04$

$5.69657 \mathrm{E}-04$

$5.73499 \mathrm{E}-04$

$5.77355 \mathrm{E}-04$

$5.81223 \mathrm{E}-04$ $g_{2}$

$\tan \delta_{2}$

$f_{-2}$

$9.13456 \mathrm{E}-01$

$9.21126 \mathrm{E}-01$

9.28829E-01

$9.36563 \mathrm{E}-01$

$9.44330 \mathrm{E}-01$

$9.52128 \mathrm{E}-01$

$9.59958 \mathrm{E}-01$

$9.67820 \mathrm{E}-01$

$9.75714 \mathrm{E}-01$

$9.83640 \mathrm{E}-01$

$9.91598 \mathrm{E}-01$

$9.99588 \mathrm{E}-01$

$1.00761 \mathrm{E} \quad 00$

$1.01566 \mathrm{E} \quad 00$

$1.02375 \mathrm{E} \quad 00$

$1.03187 \mathrm{E} \quad 00$

$1.04002 \mathrm{E} \quad 00$

$1.04820 \mathrm{E} \quad 00$

$1.05641 \mathrm{E} \quad 00$

$1.06466 \mathrm{E} \quad 00$

$1.07293 \mathrm{E} \quad 00$

$1.08124 \mathrm{E} \quad 00$

$1.08958 \mathrm{E} \quad 00$

$1.09796 \mathrm{E} \quad 00$

$1.10636 \mathrm{E} \quad 00$

$1.11480 \mathrm{E} \quad 00$

$1.12327 \mathrm{E} \quad 00$

$1.13177 \mathrm{E} \quad 00$

$1.14030 \mathrm{E} \quad 00$

$1.14886 \mathrm{E} \quad 00$

$1.15746 \mathrm{E} \quad 00$ $1.16609 \mathrm{E} \quad 00$

$1.17475 \mathrm{E} \quad 00$

$1.18344 \mathrm{E} \quad 00$

$1.19216 \mathrm{E} \quad 00$

$1.20092 \mathrm{E} \quad 00$

$1.20971 \mathrm{E} \quad 00$

$1.21853 \mathrm{E} \quad 00$

$1.22738 \mathrm{E} \quad 00$

$1.23626 \mathrm{E} \quad 00$

$1.24518 \mathrm{E} \quad 00$

$1.25412 \mathrm{E} \quad 00$

$1.26310 \mathrm{E} \quad 00$

$1.27211 \mathrm{E} \quad 00$

$1.28116 \mathrm{E} \quad 00$

$1.29023 \mathrm{E} \quad 00$

$1.29934 \mathrm{E} \quad 00$

$1.30847 \mathrm{E} \quad 00$

$1.31764 \mathrm{E} \quad 00$

$1.32685 \mathrm{E} \quad 00$

$1.33608 \mathrm{E} \quad 00$

$1.34535 \mathrm{E} \quad 00$

$1.35464 \mathrm{E} \quad 00$

$1.36397 \mathrm{E} \quad 00$

$1.37333 \mathrm{E} \quad 00$

$1.38273 \mathrm{E} \quad 00$

$1.39215 \mathrm{E} \quad 00$

$1.40161 \mathrm{E} 00$

$1.41110 \mathrm{E} \quad 00$

$1.42062 \mathrm{E} \quad 00$
$1.95975 \mathrm{E}-02$

$1.98529 \mathrm{E}-02$

$2.01106 \mathrm{E}-02$

$2.03704 \mathrm{E}-02$

$2.06325 \mathrm{E}-02$

$2.08969 \mathrm{E}-02$

$2.11634 \mathrm{E}-02$

$2.14323 \mathrm{E}-02$

$2.17033 \mathrm{E}-02$

$2.19767 \mathrm{E}-02$

$2.22524 \mathrm{E}-02$

$2.25303 \mathrm{E}-02$

$2.28105 \mathrm{E}-02$

$2.30931 \mathrm{E}-02$

$2.33780 \mathrm{E}-02$

$2.36652 \mathrm{E}-02$

$2.39547 \mathrm{E}-02$

$2.42466 \mathrm{E}-02$

$2.45408 \mathrm{E}-02$

2.48375 E-02

$2.51364 \mathrm{E}-02$

$2.54378 \mathrm{E}-02$

$2.57416 \mathrm{E}-02$

$2.60478 \mathrm{E}-02$

$2.63564 \mathrm{E}-02$

$2.66674 \mathrm{E}-02$

$2.69808 \mathrm{E}-02$

$2.72967 \mathrm{E}-02$

$2.76151 \mathrm{E}-02$

$2.79359 \mathrm{E}-02$

$2.82592 \mathrm{E}-02$

$2.85849 \mathrm{E}-02$

$2.89132 \mathrm{E}-02$

$2.92439 \mathrm{E}-02$

$2.95772 \mathrm{E}-02$

$2.99130 \mathrm{E}-02$

$3.02513 \mathrm{E}-02$

$3.05921 \mathrm{E}-02$

$3.09355 \mathrm{E}-02$

$3.12814 \mathrm{E}-02$

$3.16299 \mathrm{E}-02$

$3.19810 \mathrm{E}-02$

$3.23347 \mathrm{E}-02$

$3.26909 \mathrm{E}-02$

$3.30498 \mathrm{E}-02$

3.34113E-02

$3.37754 \mathrm{E}-02$

$3.41421 \mathrm{E}-02$

$3.48835 \mathrm{E}-02$

$3.52582 \mathrm{E}-02$

$3.56355 \mathrm{E}-02$

$3.60156 \mathrm{E}-02$

$3.63983 \mathrm{E}-02$

$3.67837 \mathrm{E}-02$

$3.71718 \mathrm{E}-02$

$3.75626 \mathrm{E}-02$

$3.79562 \mathrm{E}-02$

$3.83525 \mathrm{E}-02$

$3.87515 \mathrm{E}-02$
$3.45115 \mathrm{E}-02$

$\begin{array}{ccc}\tan \delta_{2} & & f_{-2} \\ -9.93475 \mathrm{E} & 01 & -1.85467 \mathrm{E}-02 \\ -9.93544 \mathrm{E} & 01 & -1.87939 \mathrm{E}-02 \\ -9.93612 \mathrm{E} & 01 & -1.90433 \mathrm{E}-02 \\ -9.93679 \mathrm{E} & 01 & -1.92949 \mathrm{E}-02 \\ -9.93746 \mathrm{E} & 01 & -1.95487 \mathrm{E}-02\end{array}$

$-9.93813 \mathrm{E} 01$

$-9.93878 \mathrm{E} 01$

$-9.93944 \mathrm{E} 01$

$-9.94009 \mathrm{E} \quad 01$

$\begin{array}{lll}-9.94073 \mathrm{E} & 01\end{array}$

$\begin{array}{lll}-9.94136 \mathrm{E} & 01\end{array}$

$-9.94199 \mathrm{E} 01$

$-9.94262 \mathrm{E} 01$

$-9.94324 \mathrm{E} 01$

$-9.94386 \mathrm{E} 01$

$-9.94447 \mathrm{E} 01$

$-9.94507 \mathrm{E} 01$

$-9.94567 \mathrm{E} 01$

$-9.94627 \mathrm{E} 01$

$-9.94686 \mathrm{E} 01$

$\begin{array}{ll}-9.94745 \mathrm{E} & 01\end{array}$

$-9.94803 \mathrm{E} 01$

$-9.94861 \mathrm{E} 01$

$-9.94918 \mathrm{E} 01$

$-9.94975 \mathrm{E} 01$

$\begin{array}{ll}-9.95031 \mathrm{E} & 01\end{array}$

$-9.95087 \mathrm{E}$ 01

$-9.95143 \mathrm{E} 01$

$-9.95198 \mathrm{E} 01$

$-9.95253 \mathrm{E} 01$

$-9.95307 \mathrm{E} \quad 01$

$-9.95361 \mathrm{E} 01$

$-9.95415 \mathrm{E} 01$

$-9.95468 \mathrm{E}$ 01

$-9.95520 \mathrm{E} 01$

$-9.95573 \mathrm{E} 01$

$-9.95625 \mathrm{E} 01$

$-9.95676 \mathrm{E} \quad 01$

$-9.95727 \mathrm{E} \quad 01$

$-9.95778 \mathrm{E} 01$

$-9.95828 \mathrm{E} 01$

$-9.95878 \mathrm{E} 01$

$-9.95928 \mathrm{E} 01$

$-9.95977 \mathrm{E} 01$

$-9.96026 \mathrm{E} 01$

$-9.96075 \mathrm{E} \quad 01$ 
ELECTRON RADIAL FUNCTIONS

Positron

p

30.10

30.20

30.30

30.40

30.50

30.60

30.70

30.80

30.90

31.00

31.10

31.20

31.30

31.40

31.50

31.60

31.70

31.80

31.90

32.00

32.10

32.20

32.30

32.40

32.50

32.60

32.70

32.80

32.90

33.00

33.10

33.20

33.30

33.40

33.50

33.60

33.70

33.80

33.90

34.00

34.10

34.20

34.30

34.40

34.50

34.60

34.70

34.80

34.90

35.00

35.10

35.20

35.30

35.40

35.50

35.60

35.70

35.80

35.90

36.00
$F_{0}$

$9.55564 \mathrm{E}-0]$

$9.55563 \mathrm{E}-01$

$9.55563 \mathrm{E}-01$

$9.55562 \mathrm{E}-01$

$9.55562 \mathrm{E}-01$

$9.55561 \mathrm{E}-01$

$9.55561 \mathrm{E}-01$

$9.55560 \mathrm{E}-01$

$9.55560 \mathrm{E}-01$

9.55559E-01

$9.55559 \mathrm{E}-01$

$9.55558 \mathrm{E}-01$

9.55558E-01

$9.55557 \mathrm{E}-01$

$9.55557 \mathrm{E}-01$

9.55556E-01

$9.55556 \mathrm{E}-01$

$9.55555 \mathrm{E}-01$

$9.55555 \mathrm{E}-01$

$9.55554 \mathrm{E}-01$

9.55554E-01

9.55553 E-01

$9.55553 \mathrm{E}-01$

9.55552 E-01

$9.55552 \mathrm{E}-01$

$9.55551 \mathrm{E}-01$

$9.55551 \mathrm{E}-01$

9.55550E-01

$9.55550 \mathrm{E}-01$

$9.55549 \mathrm{E}-01$

$9.55549 \mathrm{E}-01$

$9.55548 \mathrm{E}-0$

$9.55548 \mathrm{E}-01$

$9.55547 \mathrm{E}-01$

$9.55547 \mathrm{E}-01$

$9.55546 \mathrm{E}-01$

$9.55546 \mathrm{E}-01$

$9.55545 \mathrm{E}-01$

$9.55545 \mathrm{E}-01$

$9.55544 \mathrm{E}-01$

$9.55544 \mathrm{E}-01$

$9.55543 \mathrm{E}-01$

$9.55543 \mathrm{E}-01$

$9.55543 \mathrm{E}-01$

$9.55542 \mathrm{E}-01$

9.55542E-01

$9.55541 \mathrm{E}-01$

$9.55541 \mathrm{E}-01$

$9.55540 \mathrm{E}-01$

$9.55540 \mathrm{E}-01$

$9.55539 \mathrm{E}-01$

$9.55539 \mathrm{E}-01$

$9.55538 \mathrm{E}-01$

$9.55538 \mathrm{E}-01$

$9.55537 \mathrm{E}-01$

$9.55537 \mathrm{E}-01$

$9.55536 \mathrm{E}-01$

$9.55536 \mathrm{E}-01$

$9.55535 \mathrm{E}-01$

$9.55535 \mathrm{E}-01$

$Z=2 \quad A=4$

$f_{1}$

$2.91327 \mathrm{E} \quad 01$ $2.92323 \mathrm{E} \quad 01$ $2.93320 \mathrm{E} \quad 01$ $2.94316 \mathrm{E}$ 01

2.95313E 01

$2.96310 \mathrm{E} \quad 01$ $2.97306 \mathrm{E} 01$ $2.98303 \mathrm{E} \quad 01$ $2.99300 \mathrm{E} \quad 01$ $3.00298 \mathrm{E} 01$

$3.01295 \mathrm{E} 01$ $3.02292 \mathrm{E} 01$ $3.03290 \mathrm{E} \quad 01$ $3.04288 \mathrm{E} 01$

3.05285 E 01

$3.06283 \mathrm{E} \quad 01$ $3.07281 \mathrm{E} \quad 01$

$3.08279 \mathrm{E} \quad 01$

$3.09278 \mathrm{E} \quad 01$

$3.10276 \mathrm{E} \quad 01$

$3.11275 \mathrm{E} \quad 01$ $3.12273 \mathrm{E} \quad 01$ $3.13272 \mathrm{E} 01$ $3.14271 \mathrm{E} \quad 01$ $3.15270 \mathrm{E} 01$

$3.16269 \mathrm{E} \quad 01$ $3.17268 \mathrm{E} \quad 01$ $3.18267 \mathrm{E} \quad 01$ $3.19267 \mathrm{E} 01$ $3.20267 \mathrm{E} \quad 01$

$3.21266 \mathrm{E} \quad 01$ $3.22266 \mathrm{E} \quad 01$ $3.23266 \mathrm{E} 01$ $3.24266 \mathrm{E} 01$

$3.25266 \mathrm{E} 01$

$3.26267 \mathrm{E} \quad 01$ $3.27267 \mathrm{E} 01$ $3.28268 \mathrm{E} 01$ $3.29269 \mathrm{E} \quad 01$ $3.30270 \mathrm{E} 01$

$3.31271 \mathrm{E} \quad 01$ $3.32272 \mathrm{E} \quad 01$ $3.33273 \mathrm{E} 01$ $3.34274 \mathrm{E}$ ol $3.35276 \mathrm{E}$ 0I

$3.36278 \mathrm{E} \quad 01$ $3.37279 \mathrm{E} \quad 01$ $3.38281 \mathrm{E} 01$ $3.39283 \mathrm{E} 01$ $3.40286 \mathrm{E} 01$

$3.41288 \mathrm{E} \quad 01$ $3.42290 \mathrm{E} 01$ $3.43293 \mathrm{E} \quad 01$ $3.44296 \mathrm{E} 01$ $3.45299 \mathrm{E} 01$

$3.46302 \mathrm{E} \quad 01$ $3.47305 \mathrm{E} 01$ $3.48308 \mathrm{E} \quad 01$ $3.49311 \mathrm{E} \quad 01$

$3.50315 \mathrm{E} 01$ $g_{1} \quad \tan \delta_{1}$

$1.32201 \mathrm{E} \quad 00$

$1.33135 \mathrm{E} \quad 00$

$1.34073 \mathrm{E} \quad 00$

$1.35014 \mathrm{E} \quad 00$

$1.35958 \mathrm{E} \quad 00$

$1.36905 \mathrm{E} \quad 00$

$1.37856 \mathrm{E} \quad 00$

$1.38811 \mathrm{E} \quad 00$

$1.39768 \mathrm{E} \quad 00$

$1.40729 \mathrm{E} \quad 00$

$1.41694 \mathrm{E} \quad 00$

$1.42662 \mathrm{E} \quad 00$

$1.43633 \mathrm{E} \quad 00$

$1.44607 \mathrm{E} \quad 00$

$1.45585 \mathrm{E} \quad 00$

$1.46566 \mathrm{E} \quad 00$

$1.47551 \mathrm{E} \quad 00$

$1.48539 \mathrm{E} \quad 00$

$1.49530 \mathrm{E} \quad 00$

$1.50525 \mathrm{E} \quad 00$

$1.51523 \mathrm{E} \quad 00$

$1.52524 \mathrm{E} \quad 00$

$1.53529 \mathrm{E} \quad 00$

$1.54537 \mathrm{E} \quad 00$

$1.55549 \mathrm{E} \quad 00$

$1.56564 \mathrm{E} \quad 00$

$1.57582 \mathrm{E} \quad 00$

$1.58604 \mathrm{E} \quad 00$

$\begin{array}{lll}1.59629 \mathrm{E} & 00\end{array}$

$1.60657 \mathrm{E} \quad 00$

$1.61689 \mathrm{E} \quad 00$

$1.62724 \mathrm{E} \quad 00$

$1.63763 \mathrm{E} \quad 00$

$1.64805 \mathrm{E} \quad 00$

$1.65850 \mathrm{E} \quad 00$

$1.66899 \mathrm{E} \quad 00$

$1.67951 \mathrm{E} \quad 00$

$1.69006 \mathrm{E} \quad 00$

$1.70065 \mathrm{E} \quad 00$

$1.71127 \mathrm{E} \quad 00$

$1.72193 \mathrm{E} \quad 00$

$1.73262 \mathrm{E} \quad 00$

$1.74334 \mathrm{E} \quad 00$

$1.75410 \mathrm{E} \quad 00$

$1.76490 \mathrm{E} \quad 00$

$1.77572 \mathrm{E} \quad 00$

$1.78658 \mathrm{E} \quad 00$

$1.79748 \mathrm{E} \quad 00$

$1.80840 \mathrm{E} \quad 00$

$1.81937 \mathrm{E} \quad 00$

$\begin{array}{lll}1.83036 \mathrm{E} & 00\end{array}$

$1.84139 \mathrm{E} \quad 00$

$1.85246 \mathrm{E} \quad 00$

$1.86355 \mathrm{E} \quad 00$

$1.87469 \mathrm{E} \quad 00$

$1.88585 \mathrm{E} \quad 00$

$1.89705 \mathrm{E} \quad 00$

$1.90829 \mathrm{E} \quad 00$

$1.91956 \mathrm{E} \quad 00$

$1.93086 \mathrm{E} \quad 00$

$-6.87229 \mathrm{E}-04$

$-6.87823 \mathrm{E}-04$

$-6.88413 \mathrm{E}-04$

$-6.89000 \mathrm{E}-04$

$-6.89578 \mathrm{E}-04$

$-6.90153 \mathrm{E}-04$

$-6.90719 \mathrm{E}-04$

$-6.91281 \mathrm{E}-04$

$-6.91838 \mathrm{E}-04$

$-6.92389 \mathrm{E}-04$

$-6.92933 \mathrm{E}-04$

$-6.93473 \mathrm{E}-04$

$-6.94006 \mathrm{E}-04$

$-6.94535 \mathrm{E}-04$

$-6.95059 \mathrm{E}-04$

$-6.95577 \mathrm{E}-04$

$-6.96091 \mathrm{E}-04$

$-6.96598 \mathrm{E}-04$

$-6.97100 \mathrm{E}-04$

$-6.97598 \mathrm{E}-04$

$-6.98090 \mathrm{E}-04$

$-6.98578 \mathrm{E}-04$

$-6.99060 \mathrm{E}-04$

$-6.99535 \mathrm{E}-04$

$-7.00009 \mathrm{E}-04$

$-7.00476 \mathrm{E}-04$

$-7.00939 \mathrm{E}-04$

$-7.01396 \mathrm{E}-04$

$-7.01850 \mathrm{E}-04$

$-7.02297 \mathrm{E}-04$

$-7.02739 \mathrm{E}-04$

$-7.03179 \mathrm{E}-04$

$-7.03613 \mathrm{E}-04$

$-7.04043 \mathrm{E}-04$

$-7.04467 \mathrm{E}-04$

$-7.04887 \mathrm{E}-04$ $-7.05302 \mathrm{E}-04$

$-7.05712 \mathrm{E}-04$

$-7.06118 \mathrm{E}-04$

$-7.06523 \mathrm{E}-04$ 
$\bar{F}$

$f_{2}$

$5.85104 \mathrm{E}-04$

$5.88998 \mathrm{E}-04$

$5.92905 \mathrm{E}-04$

$5.96825 \mathrm{E}-04$

$6.00758 \mathrm{E}-04$

$6.04704 \mathrm{E}-04$

$6.08662 \mathrm{E}-04$

$6.12634 \mathrm{E}-04$

$6.16618 \mathrm{E}-04$

$6.20616 \mathrm{E}-04$

$6.24626 \mathrm{E}-04$

$6.28649 \mathrm{E}-04$

$6.32686 \mathrm{E}-04$

$6.36735 \mathrm{E}-04$

$6.40797 \mathrm{E}-04$

$6.44871 \mathrm{E}-04$

$6.48959 \mathrm{E}-04$

$6.53060 \mathrm{E}-04$

$6.57174 \mathrm{E}-04$

$6.61300 \mathrm{E}-04$

$6.65440 \mathrm{E}-04$

$6.69592 \mathrm{E}-04$

$6.73757 \mathrm{E}-04$

$6.77935 \mathrm{E}-04$

$6.82127 \mathrm{E}-04$

$6.86331 \mathrm{E}-04$

$6.90547 \mathrm{E}-04$

$6.94777 \mathrm{E}-04$

$6.99020 \mathrm{E}-04$

$7.03276 \mathrm{E}-04$

$7.07544 \mathrm{E}-04$

$7.11826 \mathrm{E}-04$

$7.16120 \mathrm{E}-04$

7.20428 E-04

$7.24748 \mathrm{E}-04$

$7.29081 \mathrm{E}-04$

$7.33427 \mathrm{E}-04$

$7.37786 \mathrm{E}-04$

$7.42158 \mathrm{E}-04$

$7.46543 \mathrm{E}-04$

$7.50941 \mathrm{E}-04$

7.55351 E- 04

$7.59775 \mathrm{E}-04$

$7.64211 \mathrm{E}-04$

$7.68661 \mathrm{E}-04$

$7.73123 \mathrm{E}-04$

$7.77598 \mathrm{E}-04$

$7.82086 \mathrm{E}-04$

$7.86587 \mathrm{E}-04$

7.91101 E-04

$7.95628 \mathrm{E}-04$

$8.00168 \mathrm{E}-04$

$8.04721 \mathrm{E}-04$

$8.09286 \mathrm{E}-04$

$8.13865 \mathrm{E}-04$

$8.18456 \mathrm{E}-04$ $8.23061 \mathrm{E}-04$ $8.27678 \mathrm{E}-04$ $8.32308 \mathrm{E}-04$

$8.36951 \mathrm{E}-04$
$1.43017 \mathrm{E} \quad 00$

$1.43975 \mathrm{E} \quad 00$

$1.44937 \mathrm{E} \quad 00$

$1.45901 \mathrm{E} \quad 00$

$1.46869 \mathrm{E} \quad 00$

$1.47841 \mathrm{E} \quad 00$ $1.48815 \mathrm{E} \quad 00$ $\begin{array}{ll}1.49792 \mathrm{E} & 00\end{array}$ $1.50773 \mathrm{E} \quad 00$ $\begin{array}{lll}1.51757 \mathrm{E} & 00\end{array}$

$1.52744 \mathrm{E} \quad 00$ $1.53734 \mathrm{E} \quad 00$ $1.54727 \mathrm{E} \quad 00$ $1.55724 \mathrm{E} \quad 00$ $1.56724 \mathrm{E} \quad 00$

$\begin{array}{ll}1.57726 \mathrm{E} & 00\end{array}$ $1.58733 \mathrm{E} \quad 00$ $1.59742 \mathrm{E} \quad 00$ $1.60754 \mathrm{E} \quad 00$ $1.61770 \mathrm{E} \quad 00$

$1.62789 \mathrm{E} \quad 00$ $1.63811 \mathrm{E} \quad 00$ $1.64836 \mathrm{E} \quad 00$ $1.65864 \mathrm{E} \quad 00$ $1.66896 \mathrm{E} \quad 00$

$1.67930 \mathrm{E} \quad 00$ $1.68968 \mathrm{E} \quad 00$ $1.70009 \mathrm{E} \quad 00$ $1.71053 \mathrm{E} \quad 00$ $1.72101 \mathrm{E} \quad 00$

$1.73151 \mathrm{E} \quad 00$ $1.74205 \mathrm{E} \quad 00$ $1.75262 \mathrm{E} \quad 00$ $1.76322 \mathrm{E} \quad 00$ $1.77385 \mathrm{E} \quad 00$

$1.78452 \mathrm{E} \quad 00$ $1.79521 \mathrm{E} \quad 00$ $1.80594 \mathrm{E} \quad 00$ $1.81670 \mathrm{E} \quad 00$ $1.82749 \mathrm{E} \quad 00$

$1.83832 \mathrm{E} \quad 00$ $1.84917 \mathrm{E} \quad 00$ $1.86006 \mathrm{E} \quad 00$ $1.87097 \mathrm{E} \quad 00$ $1.88192 \mathrm{E} \quad 00$

$1.89291 \mathrm{E} \quad 00$ $1.90392 \mathrm{E} \quad 00$ $1.91496 \mathrm{E} \quad 00$ $1.92604 \mathrm{E} \quad 00$ $1.93715 \mathrm{E} \quad 00$

$1.94829 \mathrm{E} \quad 00$ $1.95946 \mathrm{E} \quad 00$ $1.97066 \mathrm{E} \quad 00$ $1.98190 \mathrm{E} \quad 00$ $1.99316 \mathrm{E} \quad 00$

$2.00446 \mathrm{E} \quad 00$ $2.01579 \mathrm{E} \quad 00$ $2.02715 \mathrm{E} \quad 00$ $2.03855 \mathrm{E} \quad 00$ $2.04997 \mathrm{E} \quad 00$ $\begin{array}{cr}g_{2} & \tan \delta_{2} \\ 3.91533 \mathrm{E}-02 & -9.96764 \mathrm{E}\end{array}$

$3.95579 \mathrm{E}-02$

$3.99652 \mathrm{E}-02$

$4.03753 \mathrm{E}-02$

$4.07882 \mathrm{E}-02$

$4.12039 \mathrm{E}-02$

$4.16225 \mathrm{E}-02$

$4.20438 \mathrm{E}-02$

$4.24679 \mathrm{E}-02$

$4.28950 \mathrm{E}-02$

$4.33248 \mathrm{E}-02$

$4.37575 \mathrm{E}-02$

$4.41931 \mathrm{E}-02$

$4.46315 \mathrm{E}-02$

$4.50728 \mathrm{E}-02$

$4.55171 \mathrm{E}-02$

$4.59642 \mathrm{E}-02$

$4.64142 \mathrm{E}-02$

$4.68672 \mathrm{E}-02$

4.73231 E-02

$4.77819 \mathrm{E}-02$

$4.82437 \mathrm{E}-02$

$4.87085 \mathrm{E}-02$

$4.91762 \mathrm{E}-02$

$4.96469 \mathrm{E}-02$

$5.01206 \mathrm{E}-02$ $5.05973 \mathrm{E}-02$ $5.10770 \mathrm{E}-02$ $5.15597 \mathrm{E}-02$

$5.20454 \mathrm{E}-02$

$5.25342 \mathrm{E}-02$

$5.30260 \mathrm{E}-02$

$5.35209 \mathrm{E}-02$

$5.40188 \mathrm{E}-02$

$5.45198 \mathrm{E}-02$

$5.50239 \mathrm{E}-02$

$5.55311 \mathrm{E}-02$

$5.60413 \mathrm{E}-02$

$5.65547 \mathrm{E}-02$

$5.70712 \mathrm{E}-02$

$5.75909 \mathrm{E}-02$

$5.81137 \mathrm{E}-02$

$5.86396 \mathrm{E}-02$

$5.91687 \mathrm{E}-02$

$5.97009 \mathrm{E}-02$

$6.02363 \mathrm{E}-02$ $6.07750 \mathrm{E}-02$ $6.13168 \mathrm{E}-02$ $6.18618 \mathrm{E}-02$ $6.24100 \mathrm{E}-02$

$6.29614 \mathrm{E}-02$ $6.35161 \mathrm{E}-02$ $6.40740 \mathrm{E}-02$ $6.46352 \mathrm{E}-02$ $6.51996 \mathrm{E}-02$

$6.57673 \mathrm{E}-02$ $6.63383 \mathrm{E}-02$ $6.69126 \mathrm{E}-02$ $6.74902 \mathrm{E}-02$ $6.80710 \mathrm{E}-02$ $-9.96807 \mathrm{E}$ 0l $-9.96850 \mathrm{E} \quad 0 \mathrm{I}$ $-9.96893 \mathrm{E}$ 0I

$-9.96978 \mathrm{E} 01$ $-9.97020 \mathrm{E} 01$ $-9.97062 \mathrm{E}$ ol $-9.97103 \mathrm{E} \quad 01$ $-9.97144 \mathrm{E} \quad 01$

$-9.97185 \mathrm{E} \quad 01$ $-9.97226 \mathrm{E} \quad 01$ $-9.97266 \mathrm{E} 0 \mathrm{l}$ $-9.97306 \mathrm{E}$ 0l $-9.97346 \mathrm{E} \quad 01$

$-9.97385 \mathrm{E}$ 01 $-9.97425 \mathrm{E}$ 01 $-9.97464 \mathrm{E} 01$

$-9.97503 \mathrm{E}$ ol

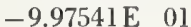

$\begin{array}{lll}-9.97579 \mathrm{E} & 01\end{array}$

$-9.97618 \mathrm{E} 01$ $-9.97655 \mathrm{E}$ 0l

$-9.97693 \mathrm{E}$ ol

$-9.97730 \mathrm{E} 01$

$\begin{array}{lll}-9.97767 \mathrm{E} & 01\end{array}$ $-9.97804 \mathrm{E}$ 01 $-9.97841 \mathrm{E} 01$ $-9.97877 \mathrm{E} \quad 01$ $-9.97913 \mathrm{E} \quad 01$

$-9.97949 \mathrm{E} \quad 0]$ $-9.97985 \mathrm{E}$ 01 $-9.98021 \mathrm{E} \quad 01$ $-9.98056 \mathrm{E}$ 0]

$-9.98126 \mathrm{E} \quad 01$ $-9.98160 \mathrm{E} 01$ $-9.98195 \mathrm{E} \quad 01$ $\begin{array}{lll}-9.98229 \mathrm{E} & 01\end{array}$

$-9.98263 \mathrm{E} 01$

$\begin{array}{ll}-9.98297 \mathrm{E} & 01\end{array}$ $-9.98330 \mathrm{E} \quad 01$ $-9.98364 \mathrm{E} 01$ $-9.98397 \mathrm{E} \quad 01$ $-9.98430 \mathrm{E} 01$

$-9.98463 \mathrm{E} \quad 01$ $-9.98496 \mathrm{E} \quad 01$ $-9.98528 \mathrm{E} 01$ $-9.98560 \mathrm{E} 01$ $\begin{array}{rr}-9.98592 \mathrm{E} & 01\end{array}$

$-9.98624 \mathrm{E} \quad 01$ $-9.98656 \mathrm{E} 0 \mathrm{l}$

$\begin{array}{lll}-9.98687 \mathrm{E} & 01\end{array}$

$-9.98719 \mathrm{E} 01$

$-9.98750 \mathrm{E} \quad 01$

$-9.98781 \mathrm{E} \quad 01$

$\begin{array}{rr}-9.98812 \mathrm{E} & 01\end{array}$

$-9.98842 \mathrm{E}$ 0l

$\begin{array}{rl}-9.98873 \mathrm{E} & 01\end{array}$

$-9.98903 \mathrm{E} \quad 01$
E-02 $-9.96764 \mathrm{E}$ 0l $-3.75543 \mathrm{E}-02$ $-9.96936 \mathrm{E} 01$

$-3.79487 \mathrm{E}-02$ $-3.83459 \mathrm{E}-02$ $-3.87459 \mathrm{E}-02$ $-3.91486 \mathrm{E}-02$

$-3.95540 \mathrm{E}-02$ $-9.98091 \mathrm{E}$ ol

$-3.99623 \mathrm{E}-02$

$-4.03733 \mathrm{E}-02$

$-4.07872 \mathrm{E}-02$

$-4.12038 \mathrm{E}-02$

$-4.16233 \mathrm{E}-02$

$-4.20455 \mathrm{E}-02$

$-4.24707 \mathrm{E}-02$

$-4.28986 \mathrm{E}-02$

$-4.33294 \mathrm{E}-02$

$-4.37631 \mathrm{E}-02$

$-4.41997 \mathrm{E}-02$

$-4.46391 \mathrm{E}-02$

$-4.50814 \mathrm{E}-02$

$-4.55266 \mathrm{E}-02$

$-4.59748 \mathrm{E}-02$

$-4.64258 \mathrm{E}-02$

$-4.68798 \mathrm{E}-02$

$-4.73367 \mathrm{E}-02$

$-4.77966 \mathrm{E}-02$

$-4.82594 \mathrm{E}-02$

$-4.87252 \mathrm{E}-02$

$-4.91940 \mathrm{E}-02$

$-4.96657 \mathrm{E}-02$

$-5.01405 \mathrm{E}-02$

$-5.06182 \mathrm{E}-02$

$-5.10990 \mathrm{E}-02$

$-5.15828 \mathrm{E}-02$

$-5.20696 \mathrm{E}-02$

$-5.25595 \mathrm{E}-02$

$-5.30524 \mathrm{E}-02$

$-5.35484 \mathrm{E}-02$

$-5.40474 \mathrm{E}-02$

$-5.45495 \mathrm{E}-02$

$-5.50547 \mathrm{E}-02$

$-5.55630 \mathrm{E}-02$

$-5.60744 \mathrm{E}-02$

$-5.65890 \mathrm{E}-02$

$-5.71066 \mathrm{E}-02$ 
ELECTRON RADIAL FUNCTIONS

Positron

\begin{tabular}{|c|c|c|c|}
\hline$p$ & $F_{0}$ & $f_{1}$ & \\
\hline 36.10 & $9.55534 \mathrm{E}-01$ & $3.51319 \mathrm{E}$ & 01 \\
\hline 36.20 & $9.55534 \mathrm{E}-01$ & $3.52322 \mathrm{E}$ & 01 \\
\hline 36.30 & $9.55533 \bar{E}-01$ & $3.53326 \mathrm{E}$ & 01 \\
\hline 36.40 & $9.55533 \mathrm{E}-01$ & $3.54330 \mathrm{E}$ & 01 \\
\hline 36.50 & $9.55533 \mathrm{E}-01$ & $3.55335 \mathrm{E}$ & 01 \\
\hline 36.60 & $9.55532 \mathrm{E}-01$ & $3.56339 \mathrm{E}$ & 01 \\
\hline 36.70 & $9.55532 \mathrm{E}-01$ & $3.57343 \mathrm{E}$ & 01 \\
\hline 36.80 & $9.55531 \mathrm{E}-01$ & $3.58348 \mathrm{E}$ & 01 \\
\hline 36.90 & $9.55531 \mathrm{E}-01$ & $3.59353 \mathrm{E}$ & 01 \\
\hline 37.00 & $9.55530 \mathrm{E}-01$ & $3.60358 \mathrm{E}$ & 01 \\
\hline 37.10 & $9.55530 \mathrm{E}-01$ & $3.61363 \mathrm{E}$ & 01 \\
\hline 37.20 & $9.55529 \mathrm{E}-01$ & $3.62368 \mathrm{E}$ & 01 \\
\hline 37.30 & $9.55529 \mathrm{E}-01$ & $3.63373 \mathrm{E}$ & 01 \\
\hline 37.40 & $9.55528 \mathrm{E}-01$ & $3.64379 \mathrm{E}$ & 01 \\
\hline 37.50 & $9.55528 \mathrm{E}-01$ & $3.65385 \mathrm{E}$ & 01 \\
\hline 37.60 & $9.55527 \mathrm{E}-01$ & $3.66390 \mathrm{E}$ & 01 \\
\hline 37.70 & $9.55527 \mathrm{E}-01$ & $3.67396 \mathrm{E}$ & 01 \\
\hline 37.80 & $9.55526 \mathrm{E}-01$ & $3.68402 \mathrm{E}$ & 01 \\
\hline 37.90 & $9.55526 \mathrm{E}-01$ & $3.69409 \mathrm{E}$ & 01 \\
\hline 38.00 & $9.55526 \mathrm{E}-01$ & $3.70415 \mathrm{E}$ & 01 \\
\hline 38.10 & $9.55525 \mathrm{E}-01$ & $3.71421 \mathrm{E}$ & 01 \\
\hline 38.20 & $9.55525 \mathrm{E}-01$ & $3.72428 \mathrm{E}$ & 01 \\
\hline 38.30 & $9.55524 \mathrm{E}-01$ & $3.73435 \mathrm{E}$ & 01 \\
\hline 38.40 & $9.55524 \mathrm{E}-01$ & $3.74442 \mathrm{E}$ & 01 \\
\hline 38.50 & $9.55523 \mathrm{E}-01$ & $3.75449 \mathrm{E}$ & 01 \\
\hline 38.60 & $9.55523 \mathrm{E}-01$ & $3.76456 \mathrm{E}$ & 01 \\
\hline 38.70 & $9.55523 \mathrm{E}-01$ & $3.77463 \mathrm{E}$ & 01 \\
\hline 38.80 & $9.55522 \mathrm{E}-01$ & $3.78471 \mathrm{E}$ & 01 \\
\hline 38.90 & $9.55522 \mathrm{E}-01$ & $3.79478 \mathrm{E}$ & 01 \\
\hline 39.00 & $9.55521 \mathrm{E}-01$ & $3.80486 \mathrm{E}$ & 01 \\
\hline 39.10 & $9.55521 \mathrm{E}-01$ & $3.81494 \mathrm{E}$ & 01 \\
\hline 39.20 & $9.55520 \mathrm{E}-01$ & $3.82502 \mathrm{E}$ & 01 \\
\hline 39.30 & $9.55520 \mathrm{E}-01$ & $3.83511 \mathrm{E}$ & 01 \\
\hline 39.40 & $9.55519 \mathrm{E}-01$ & $3.84519 \mathrm{E}$ & 01 \\
\hline 39.50 & $9.55519 \mathrm{E}-01$ & $3.85528 \mathrm{E}$ & 01 \\
\hline 39.60 & $9.55519 \mathrm{E}-01$ & $3.86536 \mathrm{E}$ & 01 \\
\hline 39.70 & $9.55518 \mathrm{E}-01$ & $3.87545 \mathrm{E}$ & 01 \\
\hline 39.80 & $9.55518 \mathrm{E}-01$ & $3.88554 \mathrm{E}$ & 01 \\
\hline 39.90 & $9.55517 \mathrm{E}-01$ & $3.89563 \mathrm{E}$ & 01 \\
\hline 40.00 & $9.55517 \mathrm{E}-01$ & $3.90573 \mathrm{E}$ & 01 \\
\hline 40.10 & $9.55516 \mathrm{E}-01$ & $3.91582 \mathrm{E}$ & 01 \\
\hline 40.20 & $9.55516 \mathrm{E}-01$ & $3.92592 \mathrm{E}$ & 01 \\
\hline 40.30 & $9.55515 \mathrm{E}-01$ & $3.93601 \mathrm{E}$ & 01 \\
\hline 40.40 & $9.55515 \mathrm{E}-01$ & $3.94611 \mathrm{E}$ & 01 \\
\hline 40.50 & $9.55515 \mathrm{E}-01$ & $3.95621 \mathrm{E}$ & 01 \\
\hline 40.60 & $9.55514 \mathrm{E}-01$ & $3.96632 \mathrm{E}$ & 01 \\
\hline 40.70 & $9.55514 \mathrm{E}-01$ & $3.97642 \mathrm{E}$ & 01 \\
\hline 40.80 & $9.55513 \mathrm{E}-01$ & $3.98653 \mathrm{E}$ & 01 \\
\hline 40.90 & $9.55513 \mathrm{E}-01$ & $3.99663 \mathrm{E}$ & 01 \\
\hline 41.00 & $9.55512 \mathrm{E}-01$ & $4.00674 \mathrm{E}$ & 01 \\
\hline 41.10 & $9.55512 \mathrm{E}-01$ & $4.01685 \mathrm{E}$ & 01 \\
\hline 41.20 & $9.55511 \mathrm{E}-01$ & $4.02697 \mathrm{E}$ & 01 \\
\hline 41.30 & $9.55511 \mathrm{E}-01$ & $4.03708 \mathrm{E}$ & 01 \\
\hline 41.40 & $9.55511 \mathrm{E}-01$ & $4.04719 \mathrm{E}$ & 01 \\
\hline 41.50 & $9.55510 \mathrm{E}-01$ & $4.05731 \mathrm{E}$ & 01 \\
\hline 41.60 & $9.55510 \mathrm{E}-01$ & $4.06743 \mathrm{E}$ & 01 \\
\hline 41.70 & $9.55509 \mathrm{E}-01$ & $4.07755 \mathrm{E}$ & 01 \\
\hline 41.80 & $9.55509 \mathrm{E}-01$ & $4.08767 \mathrm{E}$ & 01 \\
\hline 41.90 & $9.55509 \mathrm{E}-$ & $4.09779 \mathrm{E}$ & 01 \\
\hline & & & \\
\hline
\end{tabular}

$Z=2 \quad A=4 \quad \rho=1.2 A^{1 / 3} F$

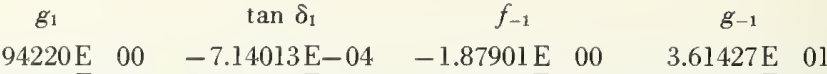
$\begin{array}{lllllll}1.95357 \mathrm{E} & 00 & -7.14323 \mathrm{E}-04 & -1.89023 \mathrm{E} & 00 & 3.62433 \mathrm{E} & 01\end{array}$ $\begin{array}{lllll}1.96497 \mathrm{E} & 00 & -7.14632 \mathrm{E}-04 & -1.90148 \mathrm{E} & 00\end{array}$ $\begin{array}{llllll}1.97641 \mathrm{E} & 00 & -7.14939 \mathrm{E}-04 & -1.91276 \mathrm{E} & 00\end{array}$ $\begin{array}{lllll}1.98789 \mathrm{E} & 00 & -7.15241 \mathrm{E}-04 & -1.92408 \mathrm{E} & 00\end{array}$

$\begin{array}{lllll}1.99939 \mathrm{E} & 00 & -7.15540 \mathrm{E}-04 & -1.93543 \mathrm{E} & 00\end{array}$ $2.01093 \mathrm{E} \quad 00 \quad-7.15835 \mathrm{E}-04 \quad-1.94682 \mathrm{E} \quad 00$ $2.02251 \mathrm{E} \quad 00 \quad-7.16125 \mathrm{E}-04 \quad-1.95824 \mathrm{E} \quad 00$ $2.03412 \mathrm{E} \quad 00 \quad-7.16414 \mathrm{E}-04 \quad-1.96969 \mathrm{E} \quad 00$ $\begin{array}{lllll}2.04577 \mathrm{E} & 00 & -7.16697 \mathrm{E}-04 & -1.98118 \mathrm{E} & 00\end{array}$

$2.05744 \mathrm{E} \quad 00 \quad-7.16977 \mathrm{E}-04 \quad-1.99271 \mathrm{E} \quad 00$ $2.06916 \mathrm{E} \quad 00 \quad-7.17254 \mathrm{E}-04 \quad-2.00426 \mathrm{E} \quad 00$ $2.08090 \mathrm{E} \quad 00 \quad-7.17527 \mathrm{E}-04 \quad-2.01586 \mathrm{E} \quad 00$ $2.09268 \mathrm{E} \quad 00 \quad-7.17797 \mathrm{E}-04 \quad-2.02748 \mathrm{E} \quad 00$ $2.10450 \mathrm{E} \quad 00 \quad-7.18063 \mathrm{E}-04 \quad-2.03914 \mathrm{E} \quad 00$

$2.11635 \mathrm{E} \quad 00 \quad-7.18328 \mathrm{E}-04 \quad-2.05084 \mathrm{E} \quad 00$ $\begin{array}{lllll}2.12823 \mathrm{E} & 00 & -7.18588 \mathrm{E}-04 & -2.06257 \mathrm{E} & 00\end{array}$ $2.14015 \mathrm{E} \quad 00 \quad-7.18844 \mathrm{E}-04 \quad-2.07433 \mathrm{E} \quad 00$ $\begin{array}{lllll}2.15211 \mathrm{E} & 00 & -7.19095 \mathrm{E}-04 & -2.08613 \mathrm{E} & 00\end{array}$ $2.16409 \mathrm{E} \quad 00 \quad-7.19344 \mathrm{E}-04 \quad-2.09796 \mathrm{E} \quad 00$

$2.17611 \mathrm{E} \quad 00 \quad-7.19590 \mathrm{E}-04 \quad-2.10983 \mathrm{E} \quad 00$ $2.18817 \mathrm{E} \quad 00 \quad-7.19833 \mathrm{E}-04 \quad-2.12173 \mathrm{E} \quad 00$ $\begin{array}{lllll}2.20026 \mathrm{E} & 00 & -7.20073 \mathrm{E}-04 & -2.13366 \mathrm{E} & 00\end{array}$ $2.21238 \mathrm{E} \quad 00 \quad-7.20311 \mathrm{E}-04 \quad-2.14563 \mathrm{E} \quad 00$ $\begin{array}{lllll}2.22454 \mathrm{E} & 00 & -7.20543 \mathrm{E}-04 & -2.15764 \mathrm{E} & 00\end{array}$

$2.23674 \mathrm{E} \quad 00 \quad-7.20774 \mathrm{E}-04 \quad-2.16968 \mathrm{E} \quad 00$ $2.24896 \mathrm{E} \quad 00 \quad-7.21000 \mathrm{E}-04 \quad-2.18175 \mathrm{E} \quad 00$ $2.26123 \mathrm{E} \quad 00 \quad-7.21224 \mathrm{E}-04 \quad-2.19386 \mathrm{E} \quad 00$ $2.27352 \mathrm{E} \quad 00 \quad-7.21444 \mathrm{E}-04 \quad-2.20600 \mathrm{E} \quad 00$ $\begin{array}{lllll}2.28585 \mathrm{E} & 00 & -7.21662 \mathrm{E}-04 & -2.21818 \mathrm{E} & 00\end{array}$

$\begin{array}{lllll}2.29822 \mathrm{E} & 00 & -7.21874 \mathrm{E}-04 & -2.23039 \mathrm{E} & 00\end{array}$ $\begin{array}{llllll}2.31062 \mathrm{E} & 00 & -7.22087 \mathrm{E}-04 & -2.24263 \mathrm{E} & 00\end{array}$ $2.32306 \mathrm{E} \quad 00 \quad-7.22295 \mathrm{E}-04 \quad-2.25491 \mathrm{E} \quad 00$ $\begin{array}{lllll}2.33552 \mathrm{E} & 00 & -7.22499 \mathrm{E}-04 & -2.26723 \mathrm{E} & 00\end{array}$ $2.34803 \mathrm{E} \quad 00 \quad-7.22701 \mathrm{E}-04 \quad-2.27958 \mathrm{E} \quad 00$

$\begin{array}{lllll}2.36057 \mathrm{E} & 00 & -7.22901 \mathrm{E}-04 & -2.29196 \mathrm{E} & 00\end{array}$ $2.37314 \mathrm{E} \quad 00 \quad-7.23094 \mathrm{E}-04 \quad-2.30438 \mathrm{E} \quad 00$ $\begin{array}{llllll}2.38575 \mathrm{E} & 00 & -7.23287 \mathrm{E}-04 & -2.31683 \mathrm{E} & 00\end{array}$ $\begin{array}{lllll}2.39839 \mathrm{E} & 00 & -7.23478 \mathrm{E}-04 & -2.32932 \mathrm{E} & 00\end{array}$ $\begin{array}{lllll}2.41106 \mathrm{E} & 00 & -7.23664 \mathrm{E}-04 & -2.34184 \mathrm{E} & 00\end{array}$

$2.42377 \mathrm{E} \quad 00 \quad-7.23848 \mathrm{E}-04 \quad-2.35440 \mathrm{E} \quad 00$ $\begin{array}{llllll}2.43652 \mathrm{E} & 00 & -7.24028 \mathrm{E}-04 & -2.36699 \mathrm{E} & 00\end{array}$ $\begin{array}{lllll}2.44930 \mathrm{E} & 00 & -7.24207 \mathrm{E}-04 & -2.37961 \mathrm{E} & 00\end{array}$ $2.46211 \mathrm{E} \quad 00 \quad-7.24382 \mathrm{E}-04 \quad-2.39228 \mathrm{E} \quad 00$ $\begin{array}{lllll}2.47496 \mathrm{E} & 00 & -7.24554 \mathrm{E}-04 & -2.40497 \mathrm{E} & 00\end{array}$

$2.48785 \mathrm{E} \quad 00 \quad-7.24723 \mathrm{E}-04 \quad-2.41770 \mathrm{E} \quad 00$ $\begin{array}{lllll}2.50077 \mathrm{E} & 00 & -7.24890 \mathrm{E}-04 & -2.43047 \mathrm{E} & 00\end{array}$ $\begin{array}{lllll}2.51372 \mathrm{E} & 00 & -7.25055 \mathrm{E}-04 & -2.44326 \mathrm{E} & 00\end{array}$ $\begin{array}{lllll}2.52671 \mathrm{E} & 00 & -7.25215 \mathrm{E}-04 & -2.45610 \mathrm{E} & 00\end{array}$ $\begin{array}{lllll}2.53973 \mathrm{E} & 00 & -7.25373 \mathrm{E}-04 & -2.46897 \mathrm{E} & 00\end{array}$

$\begin{array}{lllll}2.55279 \mathrm{E} & 00 & -7.25528 \mathrm{E}-04 & -2.48187 \mathrm{E} & 00\end{array}$ $2.56588 \mathrm{E} \quad 00 \quad-7.25679 \mathrm{E}-04 \quad-2.49481 \mathrm{E} \quad 00$ $\begin{array}{llllll}2.57901 \mathrm{E} & 00 & -7.25830 \mathrm{E}-04 & -2.50778 \mathrm{E} & 00\end{array}$ $\begin{array}{llllll}2.59217 \mathrm{E} & 00 & -7.25977 \mathrm{E}-04 & -2.52079 \mathrm{E} & 00\end{array}$ $\begin{array}{lllll}2.60537 \mathrm{E} & 00 & -7.26123 \mathrm{E}-04 & -2.53383 \mathrm{E} & 00\end{array}$

$\begin{array}{lllll}2.61860 \mathrm{E} & 00 & -7.26265 \mathrm{E}-04 & -2.54691 \mathrm{E} & 00\end{array}$ $\begin{array}{llllll}2.63186 \mathrm{E} & 00 & -7.26402 \mathrm{E}-04 & -2.56002 \mathrm{E} & 00\end{array}$ $2.64516 \mathrm{E} \quad 00 \quad-7.26536 \mathrm{E}-04 \quad-2.57317 \mathrm{E} \quad 00$

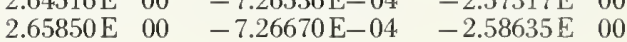
$2.67187 \mathrm{E} \quad 00 \quad-7.26800 \mathrm{E}-04 \quad-2.59957 \mathrm{E} \quad 00$
$3.63438 \mathrm{E} \quad 01$

$3.64444 \mathrm{E} 01$

$3.65450 \mathrm{E} \quad 01$

$3.66456 \mathrm{E} \quad 01$ $3.67463 \mathrm{E} \quad 01$ $3.68469 \mathrm{E} \quad 01$ $3.69476 \mathrm{E} \quad 01$ $3.70483 \mathrm{E} \quad 01$

$3.71490 \mathrm{E} \quad 01$ $3.72497 \mathrm{E} \quad 01$ $3.73504 \mathrm{E} \quad 01$ $3.74511 \mathrm{E} \quad 01$ $3.75519 \mathrm{E} 01$

$3.76526 \mathrm{E} \quad 01$ $3.77534 \mathrm{E} \quad 01$ $3.78542 \mathrm{E} \quad 01$ $3.79550 \mathrm{E} \quad 01$ $3.80558 \mathrm{E} \quad 01$

$3.81567 \mathrm{E} \quad 01$ $3.82575 \mathrm{E} \quad 01$ $3.83584 \mathrm{E}$ 01 $3.84593 \mathrm{E} \quad 01$ $3.85602 \mathrm{E} \quad 01$

$3.86611 \mathrm{E} \quad 01$ $3.87620 \mathrm{E} \quad 01$ $3.88630 \mathrm{E} \quad 01$ $3.89639 \mathrm{E} 01$ $3.90649 \mathrm{E} 01$

$3.91659 \mathrm{E} \quad 01$ $3.92669 \mathrm{E} 01$ $3.93679 \mathrm{E} \quad 01$ $3.94690 \mathrm{E} 01$ $3.95700 \mathrm{E} \quad 01$

$3.96711 \mathrm{E} \quad 01$ $3.97722 \mathrm{E} \quad 01$ $3.98733 \mathrm{E} 01$ $3.99744 \mathrm{E} \quad 01$ $4.00755 \mathrm{E} \quad 01$

$4.01767 \mathrm{E} \quad$ 01 $4.02778 \mathrm{E} \quad 01$ $4.03790 \mathrm{E} \quad 01$ $4.04802 \mathrm{E} \quad 01$ $4.05814 \mathrm{E} \cdot 01$

$4.06826 \mathrm{E} \quad 01$ $4.07839 \mathrm{E} \quad 01$ $4.08851 \mathrm{E} 01$ $4.09864 \mathrm{E} \quad 01$ $4.10877 \mathrm{E} \quad 01$

$4.11890 \mathrm{E} \quad 01$ $4.12903 \mathrm{E} 01$ $4.13916 \mathrm{E}$ 01 $4.14930 \mathrm{E} \quad 01$ $4.15944 \mathrm{E} \quad 01$

$4.16957 \mathrm{E} \quad 01$ $4.17971 \mathrm{E} \quad 01$ $4.18986 \mathrm{E}$ ol $4.20000 \mathrm{E} 01$ $4.21015 \mathrm{E} \quad 01$ $\tan \delta_{-1}$

$8.95720 \mathrm{E} \quad 02$ $8.96372 \mathrm{E} \quad 02$ 8.97021 E $\quad 02$ $8.97668 \mathrm{E} \quad 02$ $8.98316 \mathrm{E} \quad 02$

8.98962 E 02 $8.99606 \mathrm{E} \quad 02$ $9.00251 \mathrm{E} \quad 02$ $9.00894 \mathrm{E} \quad 02$ $9.01537 \mathrm{E} \quad 02$

$9.02179 \mathrm{E} \quad 02$ $9.02819 \mathrm{E} \quad 02$ $9.03459 \mathrm{E} \quad 02$ $9.04098 \mathrm{E} \quad 02$ $9.04736 \mathrm{E} \quad 02$

$9.05372 \mathrm{E} \quad 02$ $9.06008 \mathrm{E} \quad 02$ $9.06644 \mathrm{E} \quad 02$ $9.07280 \mathrm{E} \quad 02$ $9.07914 \mathrm{E} \quad 02$

$9.08548 \mathrm{E} \quad 02$ $9.09180 \mathrm{E} \quad 02$ $9.09811 \mathrm{E} \quad 02$ $9.10441 \mathrm{E} \quad 02$ $9.11072 \mathrm{E} \quad 02$

$9.11701 \mathrm{E} \quad 02$ $9.12331 \mathrm{E} \quad 02$ $9.12958 \mathrm{E} .02$ $9.13586 \mathrm{E} \quad 02$ 9.14213 E 02

$9.14840 \mathrm{E} \quad 02$ $9.15464 \mathrm{E} \quad 02$ $9.16089 \mathrm{E} \quad 02$ $9.16714 \mathrm{E} \quad 02$ $9.17338 \mathrm{E} \quad 02$

$9.17960 \mathrm{E} \quad 02$ $9.18584 \mathrm{E} \quad 02$ $9.19206 \mathrm{E} \quad 02$ $9.19826 \mathrm{E} \quad 02$ $9.20448 \mathrm{E} \quad 02$

$9.21068 \mathrm{E} \quad 02$ $9.21689 \mathrm{E} \quad 02$ $9.22308 \mathrm{E} \quad 02$ $9.22926 \mathrm{E} \quad 02$ $9.23545 \mathrm{E} \quad 02$

$9.24163 \mathrm{E} \quad 02$ $9.24780 \mathrm{E} \quad 02$ $9.25396 \mathrm{E} \quad 02$ $9.26013 \mathrm{E} \quad 02$ $9.26629 \mathrm{E} \quad 02$

$9.27245 \mathrm{E} \quad 02$ $9.27862 \mathrm{E} \quad 02$ $9.28475 \mathrm{E} \quad 02$ $9.29090 \mathrm{E} \quad 02$ $9.29702 \mathrm{E} \quad 02$

$9.30316 \mathrm{E} \quad 02$ $9.30930 \mathrm{E} \quad 02$ $9.31544 \mathrm{E} \quad 02$ $9.32157 \mathrm{E} \quad 02$ $9.32769 \mathrm{E} \quad 02$ 
$\bar{F}$

$8.41608 \mathrm{E}-04$

$8.46276 \mathrm{E}-04$

$8.50958 \mathrm{E}-04$

$8.55653 \mathrm{E}-04$

$8.60361 \mathrm{E}-04$

$8.65081 \mathrm{E}-04$

$8.69815 \mathrm{E}-04$

$8.74561 \mathrm{E}-04$

$8.79321 \mathrm{E}-04$

$8.84093 \mathrm{E}-04$

$8.88878 \mathrm{E}-04$

$8.93676 \mathrm{E}-04$

$8.98487 \mathrm{E}-04$

$9.03311 \mathrm{E}-04$

$9.08148 \mathrm{E}-04$

$9.12997 \mathrm{E}-04$

$9.17860 \mathrm{E}-04$

$9.22736 \mathrm{E}-04$

$9.27624 \mathrm{E}-04$

$9.32525 \mathrm{E}-04$

$9.37440 \mathrm{E}-04$

$9.47307 \mathrm{E}-04$

$9.52260 \mathrm{E}-04$

$9.57226 \mathrm{E}-04$

$9.62205 \mathrm{E}-04$

$9.67197 \mathrm{E}-04$

$9.72202 \mathrm{E}-04$

$9.77219 \mathrm{E}-04$

$9.82250 \mathrm{E}-04$

$9.87293 \mathrm{E}-04$

$9.92350 \mathrm{E}-04$

$9.97419 \mathrm{E}-04$

$1.00250 \mathrm{E}-03$

$1.00760 \mathrm{E}-03$

$1.01270 \mathrm{E}-03$

$1.01782 \mathrm{E}-03$

$1.02296 \mathrm{E}-03$

$1.02811 \mathrm{E}-03$

$1.03327 \mathrm{E}-03$

$1.03844 \mathrm{E}-03$ $1.04362 \mathrm{E}-03$ $1.04882 \mathrm{E}-03$ $1.05403 \mathrm{E}-03$ $1.05926 \mathrm{E}-03$

$1.06449 \mathrm{E}-03$ $1.07501 \mathrm{E}-03$ $1.08028 \mathrm{E}-03$

$1.09087 \mathrm{E}-03$ $1.09619 \mathrm{E}-03$ $1.10152 \mathrm{E}-03$ $1.10686 \mathrm{E}-03$ $1.11221 \mathrm{E}-03$

41.60

41.70

41.80

41.90

42.00

$1.13375 \mathrm{E}-03$

$1.13917 \mathrm{E}-03$
$9.42367 \mathrm{E}-04$ $1.06974 \mathrm{E}-03$ $1.08557 \mathrm{E}-03$ $f_{2}$ $2.07292 \mathrm{E} \quad 00$

$2.08444 \mathrm{E} \quad 00$

$2.09599 \mathrm{E} \quad 00$

$2.10757 \mathrm{E} \quad 00$

$2.11919 \mathrm{E} \quad 00$ $2.13084 \mathrm{E} \quad 00$ $2.14251 \mathrm{E} \quad 00$ $2.15422 \mathrm{E} \quad 00$ $2.16597 \mathrm{E} \quad 00$

$2.17774 \mathrm{E} \quad 00$ $2.18954 \mathrm{E} \quad 00$ $2.20138 \mathrm{E} \quad 00$ $2.21325 \mathrm{E} \quad 00$ $2.22515 \mathrm{E} \quad 00$

$2.23708 \mathrm{E} \quad 00$ $2.24904 \mathrm{E} \quad 00$ $2.26104 \mathrm{E} \quad 00$ $2.27306 \mathrm{E} \quad 00$ $2.28512 \mathrm{E} \quad 00$

$2.29721 \mathrm{E} \quad 00$ $2.30933 \mathrm{E} \quad 00$ $2.32148 \mathrm{E} \quad 00$ $2.33367 \mathrm{E} \quad 00$ $2.34588 \mathrm{E} \quad 00$

$2.35813 \mathrm{E} \quad 00$ $2.37041 \mathrm{E} \quad 00$ $2.38272 \mathrm{E} \quad 00$ $2.39506 \mathrm{E} \quad 00$ $2.40744 \mathrm{E} \quad 00$

$2.41984 \mathrm{E} \quad 00$ $2.43228 \mathrm{E} \quad 00$ $2.44475 \mathrm{E} \quad 00$ $2.45725 \mathrm{E} \quad 00$ $2.46978 \mathrm{E} \quad 00$

$2.48234 \mathrm{E} \quad 00$ $2.49493 \mathrm{E} \quad 00$ $2.50756 \mathrm{E} \quad 00$ $2.52022 \mathrm{E} \quad 00$ $2.53291 \mathrm{E} \quad 00$

$2.54563 \mathrm{E} \quad 00$ $2.55838 \mathrm{E} \quad 00$ $2.57116 \mathrm{E} \quad 00$ $2.58398 \mathrm{E} \quad 00$ $2.59683 \mathrm{E} \quad 00$

$2.60970 \mathrm{E} \quad 00$ $2.62262 \mathrm{E} \quad 00$ $2.63556 \mathrm{E} \quad 00$ $2.64853 \mathrm{E} \quad 00$

$2.66153 \mathrm{E} \quad 00$

$2.67457 \mathrm{E} \quad 00$ $2.68764 \mathrm{E} \quad 00$ $2.70074 \mathrm{E} \quad 00$ $2.71387 \mathrm{E} \quad 00$ $2.72703 \mathrm{E} \quad 00$

$2.74022 \mathrm{E} \quad 00$ $2.75345 \mathrm{E} \quad 00$ $2.76670 \mathrm{E} \quad 00$ $2.77999 \mathrm{E} \quad 00$ $2.79331 \mathrm{E} \quad 00$
$2.06143 \mathrm{E} \quad 00$

$g_{2}$

$\tan \delta_{2}$

$g-2$

$6.86552 \mathrm{E}-02 \quad-9.98933 \mathrm{E} \quad 01 \quad-6.63940 \mathrm{E}-02$ $6.92428 \mathrm{E}-02-9.98963 \mathrm{E}$ 01 $-6.69696 \mathrm{E}-02$ $6.98336 \mathrm{E}-02 \quad-9.98993 \mathrm{E} \quad 01 \quad-6.75484 \mathrm{E}-02$ $\begin{array}{llll}7.04278 \mathrm{E}-02 & -9.99022 \mathrm{E} & 01 & -6.81305 \mathrm{E}-02\end{array}$ $\begin{array}{llll}7.10253 \mathrm{E}-02 & -9.99051 \mathrm{E} & 01 & -6.87160 \mathrm{E}-02\end{array}$

$7.16263 \mathrm{E}-02$ $7.22306 \mathrm{E}-02$ $7.28382 \mathrm{E}-02$

$7.34493 \mathrm{E}-02$

$7.40637 \mathrm{E}-02$

$7.46816 \mathrm{E}-02$

$7.53029 \mathrm{E}-02$

$7.59276 \mathrm{E}-02$

$7.65557 \mathrm{E}-02$

$7.71873 \mathrm{E}-02$

7.78224. E- 02

$7.84609 \mathrm{E}-02$

$7.91029 \mathrm{E}-02$

$7.97484 \mathrm{E}-02$

$8.03973 \mathrm{E}-02$

$8.10498 \mathrm{E}-02$

$8.17058 \mathrm{E}-02$

$8.23653 \mathrm{E}-02$

$8.30284 \mathrm{E}-02$

$8.36950 \mathrm{E}-02$

$8.43651 \mathrm{E}-02$

$8.50388 \mathrm{E}-02$

8.57161 E-02

$8.63969 \mathrm{E}-02$

$8.70813 \mathrm{E}-02$

$8.77693 \mathrm{E}-02$

$8.84610 \mathrm{E}-02$

$8.91562 \mathrm{E}-02$

8.98551 E-02

$9.05576 \mathrm{E}-02$

$9.12637 \mathrm{E}-02$

$9.19735 \mathrm{E}-02$

$9.26870 \mathrm{E}-02$

$9.34041 \mathrm{E}-02$

$9.41249 \mathrm{E}-02$

$9.48494 \mathrm{E}-02$

$9.55776 \mathrm{E}-02$

$9.63095 \mathrm{E}-02$

$9.70451 \mathrm{E}-02$

$9.77845 \mathrm{E}-02$

$9.85275 \mathrm{E}-02$

$9.92744 \mathrm{E}-02$

$1.00025 \mathrm{E}-01$

$1.00779 \mathrm{E}-01$

$1.01537 \mathrm{E}-01$

$1.02299 \mathrm{E}-01$

$1.03065 \mathrm{E}-01$

$1.03835 \mathrm{E}-01$

$1.04608 \mathrm{E}-01$

$1.05385 \mathrm{E}-01$

$1.06166 \mathrm{E}-01$

$1.06951 \mathrm{E}-01$

$1.07739 \mathrm{E}-01$

$1.08532 \mathrm{E}-01$
$1.09328 \mathrm{E}-01$

\section{$-9.99081 \mathrm{E} \quad 01$ $-9.99110 \mathrm{E} 01$ $-9.99139 \mathrm{E} 01$ $-9.99168 \mathrm{E} 01$ $\begin{array}{lll}-9.99196 \mathrm{E} & 01\end{array}$ \\ $-9.99225 \mathrm{E} \quad 01$ $-9.99253 \mathrm{E} 01$ $-9.99281 \mathrm{E} 01$ $-9.99309 \mathrm{E} 01$ $-9.99337 \mathrm{E} \quad 01$ \\ $-6.93048 \mathrm{E}-02$ $-6.98969 \mathrm{E}-02$ $-7.04924 \mathrm{E}-02$ $-7.10912 \mathrm{E}-02$ $-7.16934 \mathrm{E}-02$ \\ $-7.22990 \mathrm{E}-02$ $-7.29080 \mathrm{E}-02$ $-7.35203 \mathrm{E}-02$ $-7.41361 \mathrm{E}-02$ $-7.47553 \mathrm{E}-02$}

$-9.99364 \mathrm{E} \quad 01$ $-9.99419 \mathrm{E} \quad 01$ $-9.99446 \mathrm{E} 01$ $-9.99473 \mathrm{E} \quad 01$

$-9.99500 \mathrm{E} \quad 01$ $-9.99527 \mathrm{E} \quad 01$ $-9.99554 \mathrm{E} \quad 01$ $-9.99580 \mathrm{E} 01$ $-9.99607 \mathrm{E} 01$

$-9.99633 \mathrm{E} \quad 01$ $-9.996 .59 \mathrm{E} 01$ $-9.99685 \mathrm{E} 01$ $-9.99711 \mathrm{E} \quad 01$ $-9.99736 \mathrm{E} 01$

$-9.99762 \mathrm{E} 01$ $-9.99787 \mathrm{E} \quad 01$ $-9.99812 \mathrm{E} \quad 01$ $-9.99838 \mathrm{E} \quad 01$ $-9.99863 \mathrm{E} 01$

$-9.99887 \mathrm{E} \quad 01$ $-9.99912 \mathrm{E} 01$ $-9.99937 \mathrm{E} \quad 01$ $-9.99961 \mathrm{E} \quad 01$ $-9.99986 \mathrm{E} \quad 01$

$-1.00001 \mathrm{E} \quad 02$ $-1.00003 \mathrm{E} 02$ $-1.00006 \mathrm{E} \quad 02$ $-1.00008 \mathrm{E} \quad 02$ $-1.00011 \mathrm{E} \quad 02$

$-1.00013 \mathrm{E} \quad 02$ $-1.00015 \mathrm{E} \quad 02$ $-1.00018 \mathrm{E} \quad 02$ $-1.00020 \mathrm{E} 02$

$-1.00022 \mathrm{E} \quad 02$

$-1.00025 \mathrm{E} \quad 02$ $-1.00027 \mathrm{E} \quad 02$ $-1.00029 \mathrm{E} 02$ $-1.00031 \mathrm{E} 02$ $-1.00034 \mathrm{E} \quad 02$

$-1.00036 \mathrm{E} \quad 02$ $\begin{array}{ll}-1.00038 \mathrm{E} & 02\end{array}$ $-1.00040 \mathrm{E} \quad 02$ $-1.00043 \mathrm{E} 02$ $-1.00045 \mathrm{E} \quad 02$

$-7.53779 \mathrm{E}-02$ $-7.60039 \mathrm{E}-02$ $-7.66334 \mathrm{E}-02$ $-7.72664 \mathrm{E}-02$ $-7.79028 \mathrm{E}-02$

$-7.85427 \mathrm{E}-02$ $-7.91860 \mathrm{E}-02$ $-7.98329 \mathrm{E}-02$ $-8.04832 \mathrm{E}-02$

$-8.11371 \mathrm{E}-02$

$-8.17945 \mathrm{E}-02$ $-8.24554 \mathrm{E}-02$ $-8.31198 \mathrm{E}-02$ $-8.37878 \mathrm{E}-02$ $-8.44594 \mathrm{E}-02$

$-8.51345 \mathrm{E}-02$ $-8.58132 \mathrm{E}-02$ $-8.64954 \mathrm{E}-02$ $-8.71813 \mathrm{E}-02$ $-8.78707 \mathrm{E}-02$

$-8.85638 \mathrm{E}-02$ $-8.92605 \mathrm{E}-02$ $-8.99608 \mathrm{E}-02$ $-9.06648 \mathrm{E}-02$ $-9.13724 \mathrm{E}-02$

$-9.20837 \mathrm{E}-02$ $-9.27986 \mathrm{E}-02$ $-9.35172 \mathrm{E}-02$ $-9.42395 \mathrm{E}-02$ $-9.49655 \mathrm{E}-02$

$-9.56952 \mathrm{E}-02$ $-9.64287 \mathrm{E}-02$ $-9.99392 \mathrm{E} 01$ 
ELECTRON RADIAL FUNCTIONS

Positron

\begin{tabular}{|c|c|c|c|}
\hline$p$ & $F_{0}$ & $f_{1}$ & $g_{1}$ \\
\hline $\begin{array}{l}0.10 \\
0.20 \\
0.30 \\
0.40 \\
0.50\end{array}$ & $\begin{array}{l}4.65245 \mathrm{E}-01 \\
6.92790 \mathrm{E}-01 \\
7.82710 \mathrm{E}-01 \\
8.29322 \mathrm{E}-01 \\
8.57199 \mathrm{E}-01\end{array}$ & $\begin{array}{l}4.83539 \mathrm{E}-03 \\
2.32381 \mathrm{E}-02 \\
5.45456 \mathrm{E}-02 \\
9.74576 \mathrm{E}-02 \\
1.50449 \mathrm{E}-01\end{array}$ & $\begin{array}{l}-2.40947 \mathrm{E}-05 \\
-1.15147 \mathrm{E}-04 \\
-2.67792 \mathrm{E}-04 \\
-4.72414 \mathrm{E}-04 \\
-7.17671 \mathrm{E}-04\end{array}$ \\
\hline $\begin{array}{l}0.60 \\
0.70 \\
0.80 \\
0.90 \\
1.00\end{array}$ & $\begin{array}{l}8.75378 \mathrm{E}-01 \\
8.87944 \mathrm{E}-01 \\
8.97001 \mathrm{E}-01 \\
9.03742 \mathrm{E}-01 \\
9.08890 \mathrm{E}-01\end{array}$ & $\begin{array}{l}2.11949 \mathrm{E}-01 \\
2.80474 \mathrm{E}-01 \\
3.54704 \mathrm{E}-01 \\
4.33513 \mathrm{E}-01 \\
5.15971 \mathrm{E}-01\end{array}$ & $\begin{array}{l}-9.91824 \mathrm{E}-04 \\
-1.28374 \mathrm{E}-03 \\
-1.58345 \mathrm{E}-03 \\
-1.88243 \mathrm{E}-03 \\
-2.17362 \mathrm{E}-03\end{array}$ \\
\hline $\begin{array}{l}1.10 \\
1.20 \\
1.30 \\
1.40 \\
1.50\end{array}$ & $\begin{array}{l}9.12905 \mathrm{E}-01 \\
9.16090 \mathrm{E}-01 \\
9.18659 \mathrm{E}-01 \\
9.20755 \mathrm{E}-01 \\
9.22486 \mathrm{E}-01\end{array}$ & $\begin{array}{l}6.01323 \mathrm{E}-01 \\
6.88970 \mathrm{E}-01 \\
7.78437 \mathrm{E}-01 \\
8.69348 \mathrm{E}-01 \\
9.61409 \mathrm{E}-01\end{array}$ & $\begin{array}{l}-2.45124 \mathrm{E}-03 \\
-2.71068 \mathrm{E}-03 \\
-2.94828 \mathrm{E}-03 \\
-3.16112 \mathrm{E}-03 \\
-3.34691 \mathrm{E}-03\end{array}$ \\
\hline $\begin{array}{l}1.60 \\
1.70 \\
1.80 \\
1.90 \\
2.00\end{array}$ & $\begin{array}{l}9.23932 \mathrm{E}-01 \\
9.25151 \mathrm{E}-01 \\
9.26185 \mathrm{E}-01 \\
9.27070 \mathrm{E}-01 \\
9.27834 \mathrm{E}-01\end{array}$ & $\begin{array}{ll}1.05439 \mathrm{E} & 00 \\
1.14811 \mathrm{E} & 00 \\
1.24242 \mathrm{E} & 00 \\
1.33721 \mathrm{E} & 00 \\
1.43240 \mathrm{E} & 00\end{array}$ & $\begin{array}{l}-3.50385 \mathrm{E}-03 \\
-3.63048 \mathrm{E}-03 \\
-3.72567 \mathrm{E}-03 \\
-3.78851 \mathrm{E}-03 \\
-3.81827 \mathrm{E}-03\end{array}$ \\
\hline $\begin{array}{l}2.10 \\
2.20 \\
2.30 \\
2.40 \\
2.50\end{array}$ & $\begin{array}{l}9.28496 \mathrm{E}-01 \\
9.29074 \mathrm{E}-01 \\
9.29579 \mathrm{E}-01 \\
9.30025 \mathrm{E}-01 \\
9.30421 \mathrm{E}-01\end{array}$ & $\begin{array}{ll}1.52789 \mathrm{E} & 00 \\
1.62365 \mathrm{E} & 00 \\
1.71962 \mathrm{E} & 00 \\
1.81576 \mathrm{E} & 00 \\
1.91205 \mathrm{E} & 00\end{array}$ & $\begin{array}{l}-3.81437 \mathrm{E}-03 \\
-3.77632 \mathrm{E}-03 \\
-3.70375 \mathrm{E}-03 \\
-3.59634 \mathrm{E}-03 \\
-3.45383 \mathrm{E}-03\end{array}$ \\
\hline $\begin{array}{l}2.60 \\
2.70 \\
2.80 \\
2.90 \\
3.00\end{array}$ & $\begin{array}{l}9.30771 \mathrm{E}-01 \\
9.31085 \mathrm{E}-01 \\
9.31365 \mathrm{E}-01 \\
9.31617 \mathrm{E}-01 \\
9.31844 \mathrm{E}-01\end{array}$ & $\begin{array}{ll}2.00845 \mathrm{E} & 00 \\
2.10495 \mathrm{E} & 00 \\
2.20153 \mathrm{E} & 00 \\
2.29817 \mathrm{E} & 00 \\
2.39487 \mathrm{E} & 00\end{array}$ & $\begin{array}{l}-3.27602 \mathrm{E}-03 \\
-3.06273 \mathrm{E}-03 \\
-2.81382 \mathrm{E}-03 \\
-2.52916 \mathrm{E}-03 \\
-2.20866 \mathrm{E}-03\end{array}$ \\
\hline $\begin{array}{l}3.10 \\
3.20 \\
3.30 \\
3.40 \\
3.50\end{array}$ & $\begin{array}{l}9.32050 \mathrm{E}-01 \\
9.32236 \mathrm{E}-01 \\
9.32405 \mathrm{E}-01 \\
9.32559 \mathrm{E}-01 \\
9.32700 \mathrm{E}-01\end{array}$ & $\begin{array}{ll}2.49161 \mathrm{E} & 00 \\
2.58838 \mathrm{E} & 00 \\
2.68517 \mathrm{E} & 00 \\
2.78198 \mathrm{E} & 00 \\
2.87881 \mathrm{E} & 00\end{array}$ & $\begin{array}{l}-1.85224 \mathrm{E}-03 \\
-1.45981 \mathrm{E}-03 \\
-1.03133 \mathrm{E}-03 \\
-5.66754 \mathrm{E}-04 \\
-6.60344 \mathrm{E}-05\end{array}$ \\
\hline $\begin{array}{l}3.60 \\
3.70 \\
3.80 \\
3.90 \\
4.00\end{array}$ & $\begin{array}{l}9.32828 \mathrm{E}-01 \\
9.32946 \mathrm{E}-01 \\
9.33055 \mathrm{E}-01 \\
9.33155 \mathrm{E}-01 \\
9.33247 \mathrm{E}-01\end{array}$ & $\begin{array}{ll}2.97563 \mathrm{E} & 00 \\
3.07244 \mathrm{E} & 00 \\
3.16924 \mathrm{E} & 00 \\
3.26600 \mathrm{E} & 00 \\
3.36272 \mathrm{E} & 00\end{array}$ & $\begin{array}{l}4.70855 \mathrm{E}-04 \\
1.04393 \mathrm{E}-03 \\
1.65321 \mathrm{E}-03 \\
2.29869 \mathrm{E}-03 \\
2.98035 \mathrm{E}-03\end{array}$ \\
\hline $\begin{array}{l}4.10 \\
4.20 \\
4.30 \\
4.40 \\
4.50\end{array}$ & $\begin{array}{l}9.33332 \mathrm{E}-01 \\
9.33410 \mathrm{E}-01 \\
9.33483 \mathrm{E}-01 \\
9.33551 \mathrm{E}-01 \\
9.33614 \mathrm{E}-01\end{array}$ & $\begin{array}{ll}3.45934 \mathrm{E} & 00 \\
3.55583 \mathrm{E} & 00 \\
3.65206 \mathrm{E} & 00 \\
3.74779 \mathrm{E} & 00 \\
3.84230 \mathrm{E} & 00\end{array}$ & $\begin{array}{l}3.69814 \mathrm{E}-03 \\
4.45196 \mathrm{E}-03 \\
5.24159 \mathrm{E}-03 \\
6.06648 \mathrm{E}-03 \\
6.92508 \mathrm{E}-03\end{array}$ \\
\hline $\begin{array}{l}4.60 \\
4.70 \\
4.80 \\
4.90 \\
5.00\end{array}$ & $\begin{array}{l}9.33671 \mathrm{E}-01 \\
9.33726 \mathrm{E}-01 \\
9.33777 \mathrm{E}-01 \\
9.33824 \mathrm{E}-01 \\
9.33868 \mathrm{E}-01\end{array}$ & $\begin{array}{ll}3.93223 \mathrm{E} & 00 \\
3.88748 \mathrm{E} & 00 \\
4.15974 \mathrm{E} & 00 \\
4.24701 \mathrm{E} & 00 \\
4.34103 \mathrm{E} & 00\end{array}$ & $\begin{array}{l}7.81008 \mathrm{E}-03 \\
8.43655 \mathrm{E}-03 \\
9.79358 \mathrm{E}-03 \\
1.07820 \mathrm{E}-02 \\
1.18216 \mathrm{E}-02\end{array}$ \\
\hline $\begin{array}{l}5.10 \\
5.20 \\
5.30 \\
5.40 \\
5.50\end{array}$ & $\begin{array}{l}9.33909 \mathrm{E}-01 \\
9.33948 \mathrm{E}-01 \\
9.33983 \mathrm{E}-01 \\
9.34017 \mathrm{E}-01 \\
9.34049 \mathrm{E}-01\end{array}$ & $\begin{array}{ll}4.43661 \mathrm{E} & 00 \\
4.53279 \mathrm{E} & 00 \\
4.62926 \mathrm{E} & 00 \\
4.72589 \mathrm{E} & 00 \\
4.82264 \mathrm{E} & 00\end{array}$ & $\begin{array}{l}1.29011 \mathrm{E}-02 \\
1.40183 \mathrm{E}-02 \\
1.51726 \mathrm{E}-02 \\
1.63637 \mathrm{E}-02 \\
1.75915 \mathrm{E}-02\end{array}$ \\
\hline $\begin{array}{l}5.60 \\
5.70 \\
5.80 \\
5.90 \\
6.00\end{array}$ & $\begin{array}{l}9.34079 \mathrm{E}-01 \\
9.34107 \mathrm{E}-01 \\
9.34133 \mathrm{E}-01 \\
9.34158 \mathrm{E}-01 \\
9.34182 \mathrm{E}-01\end{array}$ & $\begin{array}{ll}4.91945 \mathrm{E} & 00 \\
5.01631 \mathrm{E} & 00 \\
5.11320 \mathrm{E} & 00 \\
5.21013 \mathrm{E} & 00 \\
5.30707 \mathrm{E} & 00\end{array}$ & $\begin{array}{l}1.88560 \mathrm{E}-02 \\
2.01570 \mathrm{E}-02 \\
2.14946 \mathrm{E}-02 \\
2.28687 \mathrm{E}-02 \\
2.42793 \mathrm{E}-02\end{array}$ \\
\hline
\end{tabular}

$\tan \delta_{1}$

$\rho=1.2 A^{1 / 3} F$
$9.38084 \mathrm{E}-02$

$4.66011 \mathrm{E}-02$

$3.10113 \mathrm{E}-02$

$2.32013 \mathrm{E}-02$

$1.84933 \mathrm{E}-02$

$1.53360 \mathrm{E}-02$

$1.30663 \mathrm{E}-02$

$1.13527 \mathrm{E}-02$

$1.00110 \mathrm{E}-02$

$8.93085 \mathrm{E}-03$

$8.04167 \mathrm{E}-03$

$7.29642 \mathrm{E}-03$

$6.66242 \mathrm{E}-03$

$6.11626 \mathrm{E}-03$

$5.64071 \mathrm{E}-03$

$5.22282 \mathrm{E}-03$

$4.85260 \mathrm{E}-03$

$4.52230 \mathrm{E}-03$

$4.22575 \mathrm{E}-03$

$3.95801 \mathrm{E}-03$

$3.71506 \mathrm{E}-03$

$3.49357 \mathrm{E}-03$

$3.29083 \mathrm{E}-03$

$3.10454 \mathrm{E}-03$

$2.93278 \mathrm{E}-03$

$2.77389 \mathrm{E}-03$

$2.62649 \mathrm{E}-03$

$2.48937 \mathrm{E}-03$

$2.36148 \mathrm{E}-03$

$2.24194 \mathrm{E}-03$

$2.12993 \mathrm{E}-03$

$2.02477 \mathrm{E}-03$

$1.92585 \mathrm{E}-03$

$1.83264 \mathrm{E}-03$

$1.74465 \mathrm{E}-03$

$1.66145 \mathrm{E}-03$

$1.58266 \mathrm{E}-03$

$1.50795 \mathrm{E}-03$

$1.43700 \mathrm{E}-03$

$1.36953 \mathrm{E}-03$

$1.30530 \mathrm{E}-03$

$1.24408 \mathrm{E}-03$

$1.18566 \mathrm{E}-03$

$1.12986 \mathrm{E}-03$

$1.07650 \mathrm{E}-03$

$1.02542 \mathrm{E}-03$

$9.76490 \mathrm{E}-04$

$9.29570 \mathrm{E}-04$

$8.84537 \mathrm{E}-04$

$8.41280 \mathrm{E}-04$

$7.99700 \mathrm{E}-04$

$7.59699 \mathrm{E}-04$

$7.21195 \mathrm{E}-04$

$6.84094 \mathrm{E}-04$

$6.48327 \mathrm{E}-04$

$6.13830 \mathrm{E}-04$

$5.80524 \mathrm{E}-04$

$5.48359 \mathrm{E}-04$

$5.17276 \mathrm{E}-04$

4.87215E-04 $f_{-1}$

$8.42791 \mathrm{E}-04$

$2.04289 \mathrm{E}-03$

$3.22144 \mathrm{E}-03$

$4.35664 \mathrm{E}-03$

$5.43833 \mathrm{E}-03$

$6.46092 \mathrm{E}-03$

$7.42190 \mathrm{E}-03$

$8.32089 \mathrm{E}-03$

$9.15888 \mathrm{E}-03$

$9.93766 \mathrm{E}-03$

$1.06594 \mathrm{E}-02$

$1.13262 \mathrm{E}-02$

$1.19404 \mathrm{E}-02$

$1.25038 \mathrm{E}-02$

$1.30183 \mathrm{E}-02$

$1.34855 \mathrm{E}-02$

$1.39068 \mathrm{E}-02$

$1.42833 \mathrm{E}-02$

$1.46161 \mathrm{E}-02$

$1.49060 \mathrm{E}-02$

$1.51539 \mathrm{E}-02$

$1.53605 \mathrm{E}-02$

$1.55262 \mathrm{E}-02$

$1.56516 \mathrm{E}-02$

$1.57371 \mathrm{E}-02$

$1.57831 \mathrm{E}-02$

$1.57899 \mathrm{E}-02$

$1.57579 \mathrm{E}-02$

$1.56871 \mathrm{E}-02$

$1.55779 \mathrm{E}-02$

$1.54305 \mathrm{E}-02$

$1.52450 \mathrm{E}-02$

$1.50216 \mathrm{E}-02$

$1.47603 \mathrm{E}-02$

$1.44614 \mathrm{E}-02$

$1.41249 \mathrm{E}-02$ $1.37509 \mathrm{E}-02$

$1.33396 \mathrm{E}-02$

$1.28909 \mathrm{E}-02$

$1.24049 \mathrm{E}-02$

$1.18817 \mathrm{E}-02$

$1.13215 \mathrm{E}-02$

$1.07241 \mathrm{E}-02$

$1.00896 \mathrm{E}-02$

$9.41820 \mathbf{E}-03$

8.70978E-03

$7.96443 \mathbf{E}-03$

$7.18217 \mathrm{E}-03$

$6.36303 \mathrm{E}-03$

$5.50703 \mathrm{E}-03$

$4.61420 \mathrm{E}-03$

$3.68455 \mathrm{E}-03$

$2.71810 \mathrm{E}-03$

$1.71488 \mathrm{E}-03$

$6.74891 \mathrm{E}-04$

$-4.01845 \mathrm{E}-04$

$-1.51532 \mathrm{E}-03$

$-2.66552 \mathrm{E}-03$

$-3.85243 \mathrm{E}-03$

$-5.07605 \mathrm{E}-03$
-03 $g_{-1}$

9.63452 E-02

$2.34284 \mathrm{E}-01$

$3.71386 \mathrm{E}-01$

$5.05880 \mathrm{E}-01$

6.37194E-01

$7.65135 \mathrm{E}-01$

8.89742 E-01

$1.01120 \mathrm{E} \quad 00$

$1.12977 \mathrm{E} \quad 00$

$1.24575 \mathrm{E} \quad 00$

$1.35944 \mathrm{E} \quad 00$

$1.47113 \mathrm{E} \quad 00$

$1.58108 \mathrm{E} \quad 00$

$1.68953 \mathrm{E} \quad 00$

$1.79666 \mathrm{E} \quad 00$

$1.90267 \mathrm{E} \quad 00$

$2.00770 \mathrm{E} \quad 00$

$2.11188 \mathrm{E} \quad 00$

$2.21533 \mathrm{E} \quad 00$

$2.31812 \mathrm{E} \quad 00$

$2.42035 \mathrm{E} \quad 00$

$2.52209 \mathrm{E} \quad 00$

$2.62339 \mathrm{E} \quad 00$

$2.72430 \mathrm{E} \quad 00$

$2.82488 \mathrm{E} \quad 00$

$2.92515 \mathrm{E} \quad 00$

$3.02515 \mathrm{E} \quad 00$

$3.12491 \mathrm{E} \quad 00$

$3.22445 \mathrm{E} \quad 00$

$3.32380 \mathrm{E} \quad 00$

$3.42297 \mathrm{E} \quad 00$

$3.52199 \mathrm{E} \quad 00$

$3.62086 \mathrm{E} \quad 00$

$3.71960 \mathrm{E} \quad 00$

$3.81822 \mathrm{E} \quad 00$

$3.91674 \mathrm{E} \quad 00$ $4.01515 \mathrm{E} \quad 00$

$4.11348 \mathrm{E} \quad 00$

$4.21173 \mathrm{E}$

$4.30990 \mathrm{E} \quad 00$

$4.40800 \mathrm{E} \quad 00$

$4.50604 \mathrm{E} \quad 00$

$4.60402 \mathrm{E} \quad 00$

$4.70195 \mathrm{E} \quad 00$

$4.79983 \mathrm{E} \quad 00$

$4.89765 \mathrm{E} \quad 00$

$4.99544 \mathrm{E} \quad 00$

$5.09319 \mathrm{E} \quad 00$

$5.19090 \mathrm{E} \quad 00$

$5.28858 \mathrm{E} \quad 00$

$5.38623 \mathrm{E} \quad 00$

$5.48385 \mathrm{E} \quad 00$

$5.58144 \mathrm{E} \quad 00$

$5.67900 \mathrm{E} \quad 00$

$5.77655 \mathrm{E} \quad 00$

$5.87407 \mathrm{E} \quad 00$

$5.97157 \mathrm{E} \quad 00$

$6.06905 \mathrm{E} \quad 00$

$6.16652 \mathrm{E} \quad 00$

$6.26397 \mathrm{E} \quad 00$ $\tan \delta_{-1}$

$8.15667 \mathrm{E} \quad 00$ $1.59892 \mathrm{E} \quad 01$ $2.38841 \mathrm{E} \quad 01$ $3.17561 \mathrm{E} 01$ $3.95706 \mathrm{E} \quad 01$

$\begin{array}{ll}4.73045 \mathrm{E} & 01\end{array}$ $5.49413 \mathrm{E}$ 01 $6.24689 \mathrm{E} \quad 01$ $6.98789 \mathrm{E} \quad 01$ $7.71658 \mathrm{E} \quad 01$

$8.43263 \mathrm{E} \quad 01$ $9.13587 \mathrm{E} \quad 01$ $9.82627 \mathrm{E} \quad 01$ $\begin{array}{ll}1.05039 \mathrm{E} & 02\end{array}$ $1.11688 \mathrm{E} \quad 02$

$1.18212 \mathrm{E} \quad 02$ $1.24614 \mathrm{E} \quad 02$ $1.30895 \mathrm{E} \quad 02$ $1.37058 \mathrm{E} \quad 02$ $1.43105 \mathrm{E} \quad 02$

$1.49040 \mathrm{E} \quad 02$ $\begin{array}{ll}1.54865 \mathrm{E} & 02\end{array}$ $1.60582 \mathrm{E} \quad 02$ $\begin{array}{ll}1.66195 \mathrm{E} & 02\end{array}$ $\begin{array}{ll}1.71705 \mathrm{E} & 02\end{array}$

$1.77116 \mathrm{E} \quad 02$ $\begin{array}{ll}1.82429 \mathrm{E} & 02\end{array}$ $1.87648 \mathrm{E} \quad 02$ $1.92775 \mathrm{E} \quad 02$ $1.97812 \mathrm{E} \quad 02$

$2.02761 \mathrm{E} \quad 02$ $2.07625 \mathrm{E} \quad 02$ $2.12406 \mathrm{E} \quad 02$ $2.17106 \mathrm{E} \quad 02$ $2.21726 \mathrm{E} \quad 02$

$2.26269 \mathrm{E} \quad 02$ $2.30737 \mathrm{E} \quad 02$ $\begin{array}{ll}2.35132 \mathrm{E} & 02\end{array}$ $2.39455 \mathrm{E} \quad 02$ $2.43708 \mathrm{E} \quad 02$

$2.47893 \mathrm{E} \quad 02$ $2.52011 \mathrm{E} \quad 02$ $2.56064 \mathrm{E} \quad 02$ $2.60055 \mathrm{E} \quad 02$ $2.63982 \mathrm{E} \quad 02$

$2.67850 \mathrm{E} \quad 02$ $2.71658 \mathrm{E} \quad 02$ $2.75409 \mathrm{E} \quad 02$ $2.79103 \mathrm{E} \quad 02$ $2.82742 \mathrm{E} \quad 02$

$2.86327 \mathrm{E} \quad 02$ $2.89859 \mathrm{E} \quad 02$ $2.93340 \mathrm{E} \quad 02$ $2.96771 \mathrm{E} \quad 02$ $3.00152 \mathrm{E} \quad 02$

$3.03485 \mathrm{E} \quad 02$ $3.06771 \mathrm{E} \quad 02$ $3.10010 \mathrm{E} \quad 02$ $3.13206 \mathrm{E} \quad 02$ $3.16356 \mathrm{E} \quad 02$ 


\section{$\bar{F}$}

$4.31105 \mathrm{E}-09$ $2.48014 \mathrm{E}-08$ $6.26374 \mathrm{E}-08$ $1.17722 \mathrm{E}-07$ $1.89929 \mathrm{E}-07$

$2.79146 \mathrm{E}-07$ $3.85281 \mathrm{E}-07$ $5.08258 \mathbf{E}-07$ $6.48014 \mathrm{E}-07$ $8.04505 \mathrm{E}-07$

$9.77691 \mathrm{E}-07$ $1.16754 \mathrm{E}-06$ $1.37404 \mathrm{E}-06$ $1.59717 \mathrm{E}-06$ $1.83690 \mathrm{E}-06$

$2.09324 \mathrm{E}-06$ $2.36617 \mathrm{E}-06$ $2.65569 \mathrm{E}-06$ $2.96179 \mathrm{E}-06$ $3.28446 \mathrm{E}-06$

$3.62370 \mathrm{E}-06$ $3.97951 \mathrm{E}-06$ $4.35188 \mathrm{E}-06$ $4.74082 \mathrm{E}-06$ $5.14632 \mathrm{E}-06$

$5.56838 \mathrm{E}-06$ $6.00700 \mathrm{E}-06$ $6.46217 \mathrm{E}-06$ $6.93391 \mathrm{E}-06$

$7.42219 \mathrm{E}-06$

7.92704 E-06 $8.44843 \mathrm{E}-06$ $8.98638 \mathrm{E}-06$ $9.54089 \mathrm{E}-06$ $1.01119 \mathrm{E}-05$

$1.06995 \mathrm{E}-05$ $1.13037 \mathrm{E}-05$ $1.19244 \mathrm{E}-05$ $1.25617 \mathrm{E}-05$ $1.32155 \mathrm{E}-05$

$1.38858 \mathrm{E}-05$ $1.45727 \mathrm{E}-05$ $1.52762 \mathrm{E}-05$ $1.59962 \mathrm{E}-05$ $1.67328 \mathrm{E}-05$

$1.74859 \mathrm{E}-05$ $1.82555 \mathrm{E}-05$ $1.90417 \mathrm{E}-05$ $1.98445 \mathrm{E}-05$ $2.06638 \mathrm{E}-05$

$2.14997 \mathrm{E}-05$ $2.23521 \mathrm{E}-05$ $2.32210 \mathrm{E}-05$ $2.41065 \mathrm{E}-05$ $2.50086 \mathrm{E}-05$

$2.59271 \mathrm{E}-0.5$ $2.68623 \mathrm{E}-05$ $2.78140 \mathrm{E}-05$ $2.87822 \mathrm{E}-0.5$ $2.97670 \mathrm{E}-05$ $f_{2}$

$8.35901 \mathrm{E}-07$ $8.56283 \mathrm{E}-06$ $3.04873 \mathrm{E}-05$ $7.29123 \mathrm{E}-05$ $1.40950 \mathrm{E}-04$

$2.38512 \mathrm{E}-04$ $3.68444 \mathrm{E}-04$ $5.32723 \mathrm{E}-04$ $7.32658 \mathrm{E}-04$ $9.69079 \mathrm{E}-04$

$1.24248 \mathrm{E}-03$ $1.55314 \mathrm{E}-03$ $1.90117 \mathrm{E}-03$

$2.28662 \mathrm{E}-03$

2.70947 E-03

$3.16964 \mathrm{E}-03$ $3.66708 \mathrm{E}-03$

$4.20169 \mathrm{E}-03$

$4.77339 \mathrm{E}-03$

$5.38209 \mathrm{E}-03$

$6.02768 \mathrm{E}-03$ $6.71008 \mathrm{E}-03$ $7.42919 \mathrm{E}-03$ $8.18490 \mathrm{E}-03$ $8.97708 \mathrm{E}-03$

$9.80559 \mathrm{E}-03$

$1.06703 \mathrm{E}-02$

$1.15708 \mathrm{E}-02$

$1.25070 \mathrm{E}-02$

$1.34782 \mathrm{E}-02$

$1.44836 \mathrm{E}-02$

$1.55219 \mathrm{E}-02$

$1.65901 \mathrm{E}-02$

$1.76821 \mathrm{E}-02$

$1.87796 \mathrm{E}-02$

$1.98016 \mathrm{E}-02$

$1.84350 \mathrm{E}-02$

$2.31942 \mathrm{E}-02$

$2.42284 \mathrm{E}-02$

$2.54912 \mathrm{E}-02$

$2.68328 \mathrm{E}-02$

$2.82271 \mathrm{E}-02$

$2.96660 \mathrm{E}-02$

$3.11457 \mathrm{E}-02$

$3.26648 \mathrm{E}-02$

$3.42221 \mathrm{E}-02$ $3.58171 \mathrm{E}-02$ $3.74496 \mathrm{E}-02$ $3.91193 \mathrm{E}-02$ $4.08259 \mathrm{E}-02$

$4.25694 \mathrm{E}-02$

$4.43497 \mathrm{E}-02$

$4.61667 \mathrm{E}-02$

4.80204E-02

$4.99107 \mathrm{E}-02$

$5.18376 \mathrm{E}-02$

$5.38011 \mathrm{E}-02$

$5.58011 \mathrm{E}-02$

$5.78377 \mathrm{E}-02$

$5.99108 \mathrm{E}-02$ $g_{2}$

$-2.28999 \mathrm{E}-09$ $-2.33150 \mathrm{E}-08$ $-8.21770 \mathrm{E}-08$ $-1.93814 \mathrm{E}-07$ $-3.68142 \mathrm{E}-07$

$-6.09990 \mathrm{E}-07$ $-9.19626 \mathrm{E}-07$ $-1.29358 \mathrm{E}-06$ $-1.72550 \mathrm{E}-06$ $-2.20695 \mathrm{E}-06$

$-2.72800 \mathrm{E}-06$ $-3.27774 \mathrm{E}-06$ $-3.84461 \mathrm{E}-06$ $-4.41660 \mathrm{E}-06$ $-4.98144 \mathrm{E}-06$

$-5.52673 \mathrm{E}-06$ $-6.03993 \mathrm{E}-06$ $-6.50849 \mathrm{E}-06$ $-6.91985 \mathrm{E}-06$ $-7.26142 \mathrm{E}-06$

$-7.52064 \mathrm{E}-06$ $-7.68496 \mathrm{E}-06$ $-7.74185 \mathrm{E}-06$ $-7.67882 \mathrm{E}-06$ $-7.48338 \mathrm{E}-06$

$-7.14309 \mathrm{E}-06$ $-6.64554 \mathrm{E}-06$ $-5.97834 \mathrm{E}-06$ $-5.12922 \mathrm{E}-06$ $-4.08594 \mathrm{E}-06$

$-2.83645 \mathrm{E}-06$ $-1.36898 \mathrm{E}-06$ $3.27554 \mathrm{E}-07$ $2.26261 \mathrm{E}-06$ $4.44007 \mathrm{E}-06$

$6.83424 \mathrm{E}-06$ $8.37053 \mathrm{E}-06$ $1.30625 \mathrm{E}-05$ $1.62933 \mathrm{E}-05$ $1.99333 \mathrm{E}-05$

$2.39244 \mathrm{E}-05$ $2.82668 \mathrm{E}-05$ $3.29689 \mathrm{E}-05$ $3.80415 \mathrm{E}-05$ $4.34961 \mathrm{E}-05$

$4.93447 \mathrm{E}-0.5$ $5.55991 \mathrm{E}-05$ $6.22719 \mathrm{E}-05$ $6.93752 \mathrm{E}-05$

$7.69212 \mathrm{E}-05$

$8.49222 \mathrm{E}-05$ $9.33906 \mathrm{E}-05$ $1.02339 \mathrm{E}-04$

$1.11779 \mathrm{E}-04$

$1.21723 \mathrm{E}-04$

$1.32184 \mathrm{E}-04$ $1.43174 \mathrm{E}-04$ $1.54705 \mathrm{E}-04$ $1.66790 \mathrm{E}-04$ 1.79441 E-04 $\tan \delta_{2}$

$-4.85287 \mathrm{E} 00$ $-9.70328 \mathrm{E} \quad 00$

$-1.43231 \mathrm{E} 01$

$-1.86606 \mathrm{E} 01$

$-2.26739 \mathrm{E} 01$

$-2.63430 \mathrm{E} 01$

$-2.96667 \mathbf{E} 01$

$-3.26578 \mathrm{E} 01$

$-3.53384 \mathrm{E} \quad 0$

$-3.77351 \mathrm{E} 01$

$-3.98761 \mathrm{E} 01$

$-4.17895 \mathrm{E} 01$

$-4.35015 \mathrm{E} \quad 01$

$-4.50359 \mathrm{E} \quad 01$

$-4.64144 \mathrm{E} \quad 01$

$-4.76559 \mathrm{E} \quad 01$

$-4.87769 \mathrm{E} \quad 01$

$-4.97921 \mathrm{E} \quad 01$

$-5.07141 \mathrm{E} 01$

$-5.15537 \mathrm{E} \quad 01$

$-5.23204 \mathrm{E}$ 0I

$-5.30225 \mathrm{E} 01$

$-5.36671 \mathrm{E} 01$

$-5.42604 \mathrm{E} 01$

$-5.48079 \mathrm{E} 0 \mathrm{l}$

$-5.53142 \mathrm{E} 0 \mathrm{I}$

$-5.57836 \mathrm{E} 01$

$-5.62197 \mathrm{E} 01$

$-5.66257 \mathrm{E} 01$

$-5.70044 \mathrm{E} 01$

$-5.73583 \mathrm{E} 01$

$-5.76897 \mathrm{E}$ 0l

$-5.80005 \mathrm{E} 01$

$-5.82925 \mathrm{E}$ 0l

$-5.85673 \mathrm{E} 01$

$-5.88263 \mathrm{E} 0 \mathrm{l}$

$-5.90707 \mathrm{E} 01$

$-5.93017 \mathrm{E} \quad 01$

$-5.95203 \mathrm{E} 01$

$-5.97275 \mathrm{E}$ 0I

$-5.99241 \mathrm{E} \quad 01$

$-6.01109 \mathrm{E} 01$

$-6.02884 \mathrm{E} 01$

$-6.04575 \mathrm{E} 01$

$-6.06187 \mathrm{E} \quad 01$

$-6.07724 \mathrm{E} 01$

-6.09192 E 01

$-6.10595 \mathrm{E} 01$

$-6.11938 \mathrm{E} 01$

$-6.13223 \mathrm{E} 01$

-6.14455 E 01

$-6.15637 \mathrm{E} 01$

$-6.16771 \mathrm{E} 01$

$-6.17861 \mathrm{E} 01$

$-6.18908 \mathrm{E} 01$

$-6.19915 \mathrm{E} 0 \mathrm{I}$ $-6.20885 \mathrm{E} 01$ $-6.21820 \mathrm{E} 01$ $-6.22720 \mathrm{E} 01$ $-6.23588 \mathrm{E} 01$

$f_{-2}$

$9.271] 1 \mathrm{E}-08$ $4.41645 \mathrm{E}-07$ $1.04098 \mathrm{E}-06$ $1.87428 \mathrm{E}-06$ $2.92169 \mathrm{E}-06$

4.16183E-06 $5.57319 \mathrm{E}-06$ $7.13495 \mathrm{E}-06$ $8.82748 \mathrm{E}-06$

$1.06324 \mathrm{E}-05$

$1.25325 \mathrm{E}-05$

$1.45116 \mathrm{E}-05$

$1.65546 \mathrm{E}-05$

$1.86466 \mathrm{E}-05$

$2.07737 \mathrm{E}-05$

$2.29222 \mathbf{E}-05$

$2.50788 \mathrm{E}-05$

$2.72304 \mathrm{E}-05$

$2.93640 \mathrm{E}-05$

$3.14669 \mathrm{E}-05$

$3.35265 \mathrm{E}-05$

$3.55302 \mathrm{E}-05$

$3.74653 \mathrm{E}-05$

$3.93195 \mathrm{E}-05$

$4.10804 \mathrm{E}-05$

$4.27355 \mathrm{E}-05$

$4.42724 \mathrm{E}-05$

$4.56788 \mathrm{E}-05$

$4.69423 \mathrm{E}-05$

$4.80505 \mathrm{E}-05$

$4.89912 \mathrm{E}-05$

$4.97519 \mathrm{E}-05$

$5.03204 \mathrm{E}-05$

$5.06843 \mathrm{E}-05$

$5.08313 \mathrm{E}-05$

$5.07490 \mathrm{E}-05$

$5.04252 \mathrm{E}-05$

$4.98475 \mathrm{E}-05$

$4.90036 \mathrm{E}-05$

$4.78811 \mathrm{E}-05$

$4.64679 \mathrm{E}-05$ 
ELECTRON RADIAL FUNCTIONS

Positron

\begin{tabular}{|c|c|c|c|}
\hline$p$ & $F_{0}$ & $f_{1}$ & \\
\hline 6.10 & $9.34203 \mathrm{E}-01$ & $5.40403 \mathrm{E}$ & 00 \\
\hline 6.20 & $9.34224 \mathrm{E}-01$ & $5.50100 \mathrm{E}$ & 00 \\
\hline 6.30 & $9.34243 \mathrm{E}-01$ & $5.59799 \mathrm{E}$ & 00 \\
\hline 6.40 & $9.34262 \mathrm{E}-01$ & $5.69498 \mathrm{E}$ & 00 \\
\hline 6.50 & $9.34279 \mathrm{E}-01$ & $5.79198 \mathrm{E}$ & 00 \\
\hline 6.60 & $9.34295 \mathrm{E}-01$ & $5.88899 \mathrm{E}$ & 00 \\
\hline 6.70 & $9.34311 \mathrm{E}-01$ & $5.98601 \mathrm{E}$ & 00 \\
\hline 6.80 & $9.34325 \mathrm{E}-01$ & $6.08303 \mathrm{E}$ & 00 \\
\hline 6.90 & $9.34339 \mathrm{E}-01$ & $6.18005 \mathrm{E}$ & 00 \\
\hline .00 & $9.34352 \mathrm{E}-01$ & $0.27708 \mathrm{E}$ & 00 \\
\hline 7.10 & $9.34364 \mathrm{E}-01$ & $6.37411 \mathrm{E}$ & 00 \\
\hline 7.20 & $9.34376 \mathrm{E}-01$ & $6.47116 \mathrm{E}$ & 00 \\
\hline 7.30 & $9.34386 \mathrm{E}-01$ & $6.56819 \mathrm{E}$ & 00 \\
\hline 7.40 & $9.34397 \mathrm{E}-01$ & $6.66524 \mathrm{E}$ & 00 \\
\hline 7.50 & $9.34407 \mathrm{E}-01$ & $6.76230 \mathrm{E}$ & 00 \\
\hline 7.60 & $9.34416 \mathrm{E}-01$ & $6.85935 \mathrm{E}$ & 00 \\
\hline 7.70 & $9.34424 \mathrm{E}-01$ & $6.95641 \mathrm{E}$ & 00 \\
\hline 7.80 & $9.34433 \mathrm{E}-01$ & $7.05347 \mathrm{E}$ & 00 \\
\hline 7.90 & $9.34441 \mathrm{E}-01$ & $7.15053 \mathrm{E}$ & 00 \\
\hline 8.00 & $9.34448 \mathrm{E}-01$ & $7.24760 \mathrm{E}$ & 00 \\
\hline 8.10 & $9.34455 \mathrm{E}-01$ & $7.34467 \mathrm{E}$ & 00 \\
\hline 8.20 & $9.34461 \mathrm{E}-01$ & $7.44175 \mathrm{E}$ & 00 \\
\hline 8.30 & $9.34467 \mathrm{E}-01$ & $7.53883 \mathrm{E}$ & 00 \\
\hline 8.40 & $9.34473 \mathrm{E}-01$ & $7.63591 \mathrm{E}$ & 00 \\
\hline 8.50 & $9.34479 \mathrm{E}-01$ & $7.73300 \mathrm{E}$ & 00 \\
\hline 8.60 & $9.34484 \mathrm{E}-01$ & $7.83009 \mathrm{E}$ & 00 \\
\hline 8.70 & $9.34489 \mathrm{E}-01$ & $7.92719 \mathrm{E}$ & 00 \\
\hline 8.80 & $9.34493 \mathrm{E}-01$ & $8.02429 \mathrm{E}$ & 00 \\
\hline 8.90 & $9.34498 \mathrm{E}-01$ & $8.12139 \mathrm{E}$ & 00 \\
\hline 9.00 & $9.34502 \mathrm{E}-01$ & $8.21850 \mathrm{E}$ & 00 \\
\hline 9.10 & $9.34506 \mathrm{E}-01$ & $8.31561 \mathrm{E}$ & 00 \\
\hline 9.20 & $9.34510 \mathrm{E}-01$ & $8.41272 \mathrm{E}$ & 00 \\
\hline 9.30 & $9.34513 \mathrm{E}-01$ & $8.50985 \mathrm{E}$ & 00 \\
\hline 9.40 & $9.34517 \mathrm{E}-01$ & $8.60697 \mathrm{E}$ & 00 \\
\hline 9.50 & $9.34520 \mathrm{E}-01$ & $8.70409 \mathrm{E}$ & 00 \\
\hline 9.60 & $9.34522 \mathrm{E}-01$ & $8.80123 \mathrm{E}$ & 00 \\
\hline 9.70 & $9.34525 \mathrm{E}-01$ & $8.89836 \mathrm{E}$ & 00 \\
\hline 9.80 & $9.34527 \mathrm{E}-01$ & $8.99550 \mathrm{E}$ & 00 \\
\hline 9.90 & $9.34530 \mathrm{E}-01$ & $9.09265 \mathrm{E}$ & 00 \\
\hline 10.00 & $9.34532 \mathrm{E}-01$ & $9.18980 \mathrm{E}$ & 00 \\
\hline 10.10 & $9.34534 \mathrm{E}-01$ & $9.28696 \mathrm{E}$ & 00 \\
\hline 10.20 & $9.34536 \mathrm{E}-01$ & $9.38412 \mathrm{E}$ & 00 \\
\hline 10.30 & $9.34537 \mathrm{E}-01$ & $9.48128 \mathrm{E}$ & 00 \\
\hline 10.40 & $9.34539 \mathrm{E}-01$ & $9.57845 \mathrm{E}$ & 00 \\
\hline & $9.34540 \mathrm{E}-01$ & $9.67563 \mathrm{E}$ & 00 \\
\hline 10.60 & $9.34542 \mathrm{E}-01$ & $9.77281 \mathrm{E}$ & 00 \\
\hline 10.70 & $9.34543 \mathrm{E}-01$ & $9.86999 \mathrm{E}$ & 00 \\
\hline 10.80 & $9.34544 \mathrm{E}-01$ & $9.96718 \mathrm{E}$ & 00 \\
\hline 10.90 & $9.34545 \mathrm{E}-01$ & $1.00644 \mathrm{E}$ & 01 \\
\hline 11.00 & $9.34546 \mathrm{E}-01$ & $1.01616 \mathrm{E}$ & 01 \\
\hline 11.10 & $9.34547 \mathrm{E}-01$ & $1.02588 \mathrm{E}$ & 01 \\
\hline 11.20 & $9.34547 \mathrm{E}-01$ & $1.03560 \mathrm{E}$ & 01 \\
\hline 11.30 & $9.34548 \mathrm{E}-01$ & $1.04532 \mathrm{E}$ & 01 \\
\hline 11.40 & $9.34548 \mathrm{E}-01$ & $1.05504 \mathrm{E}$ & 01 \\
\hline 11.50 & $9.34549 \mathrm{E}-01$ & $1.06477 \mathrm{E}$ & 01 \\
\hline 11.60 & $9.34549 \mathrm{E}-01$ & $1.07449 \mathrm{E}$ & 01 \\
\hline & $9.34550 \mathrm{E}-01$ & $1.08421 \mathrm{E}$ & 01 \\
\hline & $9.34550 \mathrm{E}-01$ & $1.09394 \mathrm{E}$ & 01 \\
\hline 11.90 & $9.34550 \mathrm{E}-01$ & $1.10366 \mathrm{E}$ & 01 \\
\hline 12.00 & $9.34550 \mathrm{E}-01$ & $1.11339 \mathrm{E}$ & 01 \\
\hline
\end{tabular}

$$
Z=3 \quad A=6 \quad \rho=1.2 A^{1 / 3} F
$$

$g_{1}$
2.57265 E-02
2.72102 E-02
2.87304 E-02
3.02871 E-02
3.18803 E-02

$3.35101 \mathrm{E}-02$

$3.51764 \mathrm{E}-02$

$3.68792 \mathrm{E}-02$

$3.86185 \mathrm{E}-02$

$4.03943 \mathrm{E}-02$

$4.22067 \mathrm{E}-02$

4.40556 E-02

4.59411 E-02

$4.78630 \mathrm{E}-02$

$4.98216 \mathrm{E}-02$

$5.18167 \mathrm{E}-02$

$5.38483 \mathrm{E}-02$

$5.59164 \mathrm{E}-02$

$5.80211 \mathrm{E}-02$

$6.01624 \mathrm{E}-02$

$6.23402 \mathrm{E}-02$ $6.45546 \mathrm{E}-02$ $6.68056 \mathrm{E}-02$ $6.90931 \mathrm{E}-02$

$7.14172 \mathrm{E}-02$

7.37779 E-02

$7.61752 \mathrm{E}-02$ $7.86090 \mathrm{E}-02$ $8.10795 \mathrm{E}-02$ $8.35865 \mathrm{E}-02$

$8.61302 \mathrm{E}-02$ $8.87104 \mathrm{E}-02$ $9.13273 \mathrm{E}-02$ $9.39807 \mathrm{E}-02$ $9.66707 \mathrm{E}-02$

$9.93974 \mathrm{E}-02$ $1.02161 \mathrm{E}-01$ $1.04961 \mathrm{E}-01$

$1.07797 \mathrm{E}-01$

$1.10670 \mathrm{E}-01$

$1.13580 \mathrm{E}-01$

$1.16527 \mathrm{E}-01$

$1.19510 \mathrm{E}-01$

$1.22530 \mathrm{E}-01$

$1.25586 \mathrm{E}-01$

$1.28679 \mathrm{E}-01$

$1.31809 \mathrm{E}-01$

$1.34975 \mathrm{E}-01$

$1.38178 \mathrm{E}-01$

$1.41418 \mathrm{E}-01$

$1.44695 \mathrm{E}-01$ $1.48008 \mathrm{E}-01$ $1.51358 \mathrm{E}-01$

$1.54744 \mathrm{E}-01$
$1.58167 \mathrm{E}-01$

$1.61627 \mathrm{E}-01$ $1.65124 \mathrm{E}-01$

$1.68658 \mathrm{E}-01$

$1.72228 \mathrm{E}-01$

$1.75835 \mathrm{E}-01$ $\tan \delta_{1}$

$4.58132 \mathrm{E}-04$ $4.29981 \mathrm{E}-04$

$4.02715 \mathrm{E}-04$

$3.76296 \mathrm{E}-04$

$3.50680 \mathrm{E}-04$

$3.25836 \mathrm{E}-04$

$3.01733 \mathrm{E}-04$

$2.78334 \mathrm{E}-04$

$2.55604 \mathrm{E}-04$

2.33525 E-04

$2.12065 \mathrm{E}-04$

$1.91195 \mathrm{E}-04$

$1.70894 \mathrm{E}-04$

$1.51145 \mathrm{E}-04$

$1.31917 \mathrm{E}-04$

1.13195E-04

$9.49579 \mathrm{E}-05$

$7.71865 \mathrm{E}-05$

$5.98662 \mathrm{E}-05$

$4.29732 \mathrm{E}-05$

$2.65007 \mathrm{E}-05$

$1.04300 \mathrm{E}-05$

$-5.25215 \mathrm{E}-06$

$-2.05627 \mathrm{E}-05$

$-3.55101 \mathrm{E}-05$

$-5.01104 \mathrm{E}-05$

$-6.43751 \mathrm{E}-05$

$-7.83133 \mathrm{E}-05$

$-9.19424 \mathrm{E}-05$

$-1.05261 \mathrm{E}-04$

$-1.18286 \mathrm{E}-04$

$-1.31030 \mathrm{E}-04$

$-1.43498 \mathrm{E}-04$

$-1.55699 \mathrm{E}-04$

$-1.67640 \mathrm{E}-04$

$-1.79330 \mathrm{E}-04$

$-1.90779 \mathrm{E}-04$

$-2.01989 \mathrm{E}-04$

$-2.12976 \mathrm{E}-04$

$-2.23739 \mathrm{E}-04$

$-2.34286 \mathrm{E}-04$

$-2.44627 \mathrm{E}-04$

$-2.54761 \mathrm{E}-04$

$-2.64697 \mathrm{E}-04$

$-2.74445 \mathrm{E}-04$

$-2.84005 \mathrm{E}-04$

$-2.93384 \mathrm{E}-04$

$-3.02589 \mathrm{E}-04$

$-3.11620 \mathrm{E}-04$

$-3.20484 \mathrm{E}-04$

$-3.29186 \mathrm{E}-04$

$-3.37735 \mathrm{E}-04$

$-3.46122 \mathrm{E}-04$

$-3.54364 \mathrm{E}-04$

$-3.62458 \mathrm{E}-04$

$-3.70408 \mathrm{E}-04$

$-3.78223 \mathrm{E}-04$

$-3.85897 \mathrm{E}-04$

$-3.93445 \mathrm{E}-04$

$-4.00862 \mathrm{E}-04$ $f_{-1}$

$-6.33637 \mathrm{E}-03$ $-7.63338 \mathrm{E}-03$ $-8.96706 \mathrm{E}-03$ $-1.03374 \mathrm{E}-02$

$-1.17444 \mathrm{E}-02$

$-1.31881 \mathrm{E}-02$ $-1.46685 \mathrm{E}-02$ $-1.61855 \mathrm{E}-02$ $-1.77392 \mathrm{E}-02$ $-1.93295 \mathrm{E}-02$

$-2.09564 \mathrm{E}-02$ $-2.26200 \mathrm{E}-02$ $-2.43203 \mathrm{E}-02$ $-2.60571 \mathrm{E}-02$ $-2.78307 \mathrm{E}-02$

$-2.96408 \mathrm{E}-02$ $-3.14876 \mathrm{E}-02$ $-3.33710 \mathrm{E}-02$ $-3.52911 \mathrm{E}-02$ $-3.72478 \mathrm{E}-02$

$-3.92412 \mathrm{E}-02$ $-4.12712 \mathrm{E}-02$ $-4.33378 \mathrm{E}-02$ $-4.54410 \mathrm{E}-02$ $-4.75810 \mathrm{E}-02$

$-4.97575 \mathrm{E}-02$ $-5.19707 \mathrm{E}-02$ $-5.42205 \mathrm{E}-02$ $-5.65070 \mathrm{E}-02$ $-5.88302 \mathrm{E}-02$

$-6.11900 \mathrm{E}-02$ $-6.35864 \mathrm{E}-02$ $-6.60195 \mathrm{E}-02$ $-6.84893 \mathrm{E}-02$ $-7.09957 \mathrm{E}-02$

$-7.35388 \mathrm{E}-02$ $-7.61186 \mathrm{E}-02$ $-7.87350 \mathrm{E}-02$ $-8.13881 \mathrm{E}-02$ $-8.40779 \mathrm{E}-02$

$-8.68044 \mathrm{E}-02$ $-8.95675 \mathrm{E}-02$ $-9.23674 \mathrm{E}-02$ $-9.52039 \mathrm{E}-02$ $-9.80771 \mathrm{E}-02$

$-1.00987 \mathrm{E}-01$ $-1.03934 \mathrm{E}-01$ $-1.06917 \mathrm{E}-01$ $-1.09937 \mathrm{E}-01$ $-1.12994 \mathrm{E}-01$

$-1.16087 \mathrm{E}-01$ $-1.19218 \mathrm{E}-01$ $-1.22385 \mathrm{E}-01$ $-1.25588 \mathrm{E}-01$ $-1.28829 \mathrm{E}-01$

$-1.32106 \mathrm{E}-01$ $-1.35420 \mathrm{E}-01$ $-1.38770 \mathrm{E}-01$ $-1.42158 \mathrm{E}-01$ $-1.45582 \mathrm{E}-01$ $g_{-1}$

$6.36140 \mathrm{E} \quad 00$ $6.45882 \mathrm{E} \quad 00$ $6.55623 \mathrm{E} \quad 00$ $6.65362 \mathrm{E} \quad 00$ $6.75101 \mathrm{E} \quad 00$

$6.84838 \mathrm{E} \quad 00$ $6.94575 \mathrm{E} \quad 00$ $7.04310 \mathrm{E} \quad 00$ $7.14045 \mathrm{E} \quad 00$ $7.23779 \mathrm{E} \quad 00$

$7.33512 \mathrm{E} \quad 00$ $7.43245 \mathrm{E} \quad 00$ $7.52977 \mathrm{E} \quad 00$ $7.62709 \mathrm{E} \quad 00$ $7.72441 \mathrm{E} \quad 00$

$7.82171 \mathrm{E} \quad 00$ $7.91902 \mathrm{E} \quad 00$ $8.01632 \mathrm{E} \quad 00$ $8.11362 \mathrm{E} \quad 00$ $8.21092 \mathrm{E} \quad 00$

8.30821 E $\quad 00$ $8.40551 \mathrm{E} \quad 00$ $8.50280 \mathrm{E} \quad 00$ $8.60009 \mathrm{E} 00$ $8.69738 \mathrm{E} \quad 00$

$8.79467 \mathrm{E} \quad 00$ $8.89196 \mathrm{E} \quad 00$ $8.98925 \mathrm{E} \quad 00$ $9.08654 \mathrm{E} \quad 00$ $9.18383 \mathrm{E} \quad 00$

$9.28113 \mathrm{E} \quad 00$ $9.37842 \mathrm{E} \quad 00$ $9.47572 \mathrm{E} \quad 00$ $9.57301 \mathrm{E} \quad 00$ $9.67031 \mathrm{E} \quad 00$

$9.76761 \mathrm{E} \quad 00$ $9.86491 \mathrm{E} \quad 00$ $9.96222 \mathrm{E} \quad 00$ $1.00595 \mathrm{E} \quad 01$ $1.01568 \mathrm{E} \quad 01$

$\begin{array}{ll}1.02542 \mathrm{E} & 01\end{array}$ $1.03515 \mathrm{E} \quad 01$ $\begin{array}{ll}1.04488 \mathrm{E} & 01\end{array}$ $1.05461 \mathrm{E} \quad 01$ $\begin{array}{ll}1.06434 \mathrm{E} & 01\end{array}$

$1.07408 \mathrm{E} \quad 01$ $\begin{array}{ll}1.08381 \mathrm{E} & 01\end{array}$ $1.09354 \mathrm{E} \quad 01$ $1.10328 \mathrm{E} \quad 01$ $1.11301 \mathrm{E} \quad 01$

$\begin{array}{ll}1.12275 \mathrm{E} & 01\end{array}$ $1.13248 \mathrm{E} 01$ $\begin{array}{ll}1.14222 \mathrm{E} & 01\end{array}$ $\begin{array}{ll}1.15196 \mathrm{E} & 01\end{array}$ $\begin{array}{ll}1.16169 \mathrm{E} & 01\end{array}$

$\begin{array}{ll}1.17143 \mathrm{E} & 01\end{array}$ $\begin{array}{lll}1.18117 \mathrm{E} & 01\end{array}$ $\begin{array}{lll}1.19091 \mathrm{E} & 01\end{array}$ $\begin{array}{ll}1.20065 \mathrm{E} & 01\end{array}$ $\begin{array}{lll}1.21039 \mathrm{E} & 01\end{array}$

$\tan \delta_{-1}$

$3.19463 \mathrm{E} \quad 02$ $3.22528 \mathrm{E} \quad 02$ $3.25551 \mathrm{E} \quad 02$ $3.28534 \mathrm{E} \quad 02$ $3.31477 \mathrm{E} \quad 02$

$3.34381 \mathrm{E} \quad 02$ $3.37247 \mathrm{E} \quad 02$ $3.40076 \mathrm{E} \quad 02$ $3.42868 \mathrm{E} \quad 02$ $3.45624 \mathrm{E} \quad 02$

$3.48345 \mathrm{E} \quad 02$ $3.51032 \mathrm{E} \quad 02$ $3.53684 \mathrm{E} \quad 02$ $3.56304 \mathrm{E} \quad 02$ $3.58891 \mathrm{E} \quad 02$

$3.61446 \mathrm{E} \quad 02$ $3.63970 \mathrm{E} \quad 02$ $3.66463 \mathrm{E} \quad 02$ $3.68927 \mathrm{E} \quad 02$

$3.71360 \mathrm{E} \quad 02$

$3.73764 \mathrm{E} \quad 02$ $3.76141 \mathrm{E} \quad 02$ $3.78489 \mathrm{E} \quad 02$ $3.80810 \mathrm{E} \quad 02$ $3.83105 \mathrm{E} \quad 02$

$3.85372 \mathrm{E} \quad 02$ $3.87614 \mathrm{E} \quad 02$ $3.89831 \mathrm{E} \quad 02$ $3.92022 \mathrm{E} \quad 02$ $3.94189 \mathrm{E} \quad 02$

$3.96332 \mathrm{E} \quad 02$ $3.98451 \mathrm{E} \quad 02$ $4.00547 \mathrm{E} \quad 02$ $4.02620 \mathrm{E} \quad 02$ $4.04671 \mathrm{E} \quad 02$

$4.06700 \mathrm{E} \quad 02$ $4.08707 \mathrm{E} \quad 02$ $4.10693 \mathrm{E} \quad 02$ $4.12657 \mathrm{E} \quad 02$ $4.14602 \mathrm{E} \quad 02$

$4.16526 \mathrm{E} \quad 02$ 
ELECTRON RADIAL FUNCTIONS

$Z=3 \quad A=6 \quad \rho=1.2 A^{1 / 3} \mathrm{~F}$

Positron

$\bar{F}$

$6.10 \quad 3.07683 \mathrm{E}-05$

$6.20 \quad 3.17862 \mathrm{E}-05$

$6.30 \quad 3.28206 \mathrm{E}-05$

$6.40 \quad 3.38716 \mathrm{E}-05$

$6.50 \quad 3.49391 \mathrm{E}-05$

$6.60 \quad 3.60232 \mathrm{E}-05$

$6.70 \quad 3.71238 \mathrm{E}-05$

$6.80 \quad 3.82410 \mathrm{E}-05$

$6.90 \quad 3.93747 \mathrm{E}-05$

$7.00 \quad 4.05249 \mathrm{E}-05$

$7.10 \quad 4.16917 \mathrm{E}-05$

$7.20 \quad 4.28751 \mathrm{E}-05$

$7.30 \quad 4.40750 \mathrm{E}-05$

$7.40 \quad 4.52914 \mathrm{E}-05$

$7.50 \quad 4.65244 \mathrm{E}-05$

$7.60 \quad 4.77740 \mathrm{E}-05$

$7.70 \quad 4.90400 \mathrm{E}-05$

$7.80 \quad 5.03227 \mathrm{E}-05$

$7.90 \quad 5.16218 \mathrm{E}-05$

$8.00 \quad 5.29376 \mathrm{E}-05$

$8.10 \quad 5.42698 \mathrm{E}-05$

$8.20 \quad 5.56186 \mathrm{E}-05$

$8.30 \quad 5.69840 \mathrm{E}-05$

$8.40 \quad 5.83659 \mathrm{E}-05$

$8.50 \quad 5.97643 \mathrm{E}-05$

$8.60 \quad 6.11793 \mathrm{E}-05$

$8.70 \quad 6.26108 \mathrm{E}-05$

$8.80 \quad 6.40589 \mathrm{E}-05$

$8.90 \quad 6.55235 \mathrm{E}-05$

$9.00 \quad 6.70048 \mathrm{E}-05$

$9.10 \quad 6.85025 \mathrm{E}-05$

$9.20 \quad 7.00167 \mathrm{E}-05$

$9.30 \quad 7.15475 \mathrm{E}-05$

$9.40 \quad 7.30949 \mathrm{E}-05$

$9.50 \quad 7.46588 \mathrm{E}-05$

$9.60 \quad 7.62393 \mathrm{E}-05$

$9.70 \quad 7.78362 \mathrm{E}-05$

$9.80 \quad 7.94498 \mathrm{E}-05$

$9.90 \quad 8.10799 \mathrm{E}-05$

$10.00 \quad 8.27265 \mathrm{E}-05$

$10.10 \quad 8.43897 \mathrm{E}-05$

$10.20 \quad 8.60694 \mathrm{E}-05$

$10.30 \quad 8.77657 \mathrm{E}-05$

$10.40 \quad 8.94785 \mathrm{E}-05$

$10.50 \quad 9.12078 \mathrm{E}-05$

$10.60 \quad 9.29537 \mathrm{E}-05$

10.70

10.80

10.90

11.00

$9.47162 \mathrm{E}-05$

$9.64952 \mathrm{E}-05$

$9.82907 \mathrm{E}-05$

$11.10 \quad 1.01931 \mathrm{E}-04$

$11.20 \quad 1.03777 \mathrm{E}-04$

$11.30 \quad 1.05638 \mathrm{E}-04$

$11.40 \quad 1.07517 \mathrm{E}-04$

$11.50 \quad 1.09411 \mathrm{E}-04$

$11.60 \quad 1.11323 \mathrm{E}-04$

$11.70 \quad 1.13251 \mathrm{E}-04$

$11.80 \quad 1.15195 \mathrm{E}-04$

$11.90 \quad 1.17156 \mathrm{E}-04$

$12.00 \quad 1.19133 \mathrm{E}-04$ $f_{2}$

$6.20204 \mathrm{E}-02$ $6.41666 \mathrm{E}-02$

$6.63491 \mathrm{E}-02$

$6.85682 \mathrm{E}-02$

$7.08237 \mathrm{E}-02$

$7.31157 \mathrm{E}-02$

$7.54442 \mathrm{E}-02$

$7.78091 \mathrm{E}-02$

$8.02104 \mathrm{E}-02$

$8.26483 \mathrm{E}-02$

$8.51225 \mathrm{E}-02$

$8.76332 \mathrm{E}-02$

$9.01803 \mathrm{E}-02$

$9.27639 \mathrm{E}-02$

$9.53839 \mathrm{E}-02$

$9.80403 \mathrm{E}-02$

$1.00733 \mathrm{E}-01$

$1.03462 \mathrm{E}-01$

$1.06228 \mathrm{E}-01$

$1.09030 \mathrm{E}-01$

$1.11869 \mathrm{E}-01$

$1.14744 \mathrm{E}-01$

$1.17655 \mathrm{E}-01$

$1.20603 \mathrm{E}-01$

$1.23587 \mathrm{E}-01$

$1.26607 \mathrm{E}-01$

$1.29664 \mathrm{E}-01$

$1.32758 \mathrm{E}-01$

$1.35888 \mathrm{E}-01$

$1.39054 \mathrm{E}-01$

$1.42256 \mathrm{E}-01$

$1.45495 \mathrm{E}-01$

$1.48771 \mathrm{E}-01$

$1.52082 \mathrm{E}-01$

$1.55431 \mathrm{E}-01$

$1.58815 \mathrm{E}-01$

$1.62236 \mathrm{E}-01$

$1.65693 \mathrm{E}-01$

$1.69187 \mathrm{E}-01$

$1.72717 \mathrm{E}-01$

$1.76284 \mathrm{E}-01$

$1.79887 \mathrm{E}-01$

$1.83526 \mathrm{E}-01$

$1.87202 \mathrm{E}-01$

$1.90914 \mathrm{E}-01$

$1.94662 \mathrm{E}-01$

$1.98447 \mathrm{E}-01$

$2.02268 \mathrm{E}-01$

$2.06126 \mathrm{E}-01$

$2.10020 \mathrm{E}-01$

$2.13950 \mathrm{E}-01$

$2.17917 \mathrm{E}-01$

$2.21920 \mathrm{E}-01$

$2.25959 \mathrm{E}-01$

2.30035 E-01

$2.34147 \mathrm{E}-01$

$2.38296 \mathrm{E}-01$

$2.42481 \mathrm{E}-01$

$2.46702 \mathrm{E}-01$

2.50960 E-01 $\tan \delta_{2}$

$f_{-2}$

$1.92670 \mathrm{E}-04$

$2.06490 \mathrm{E}-04$

$2.20913 \mathrm{E}-04$

$2.35951 \mathrm{E}-04$

$2.51617 \mathrm{E}-04$

$2.67922 \mathrm{E}-04$

$2.84880 \mathrm{E}-04$

$3.02503 \mathrm{E}-04$

$3.20803 \mathrm{E}-04$

$3.39792 \mathrm{E}-04$

$3.59483 \mathrm{E}-04$

$3.79887 \mathrm{E}-04$

$4.01018 \mathrm{E}-04$

$4.22888 \mathrm{E}-04$

$4.45509 \mathrm{E}-04$

$4.68893 \mathrm{E}-04$

$4.93053 \mathrm{E}-04$

$5.18000 \mathrm{E}-04$

$5.43748 \mathrm{E}-04$

$5.70309 \mathrm{E}-04$

$5.97695 \mathrm{E}-04$

$6.25918 \mathrm{E}-04$

$6.54990 \mathrm{E}-04$

$6.84925 \mathrm{E}-04$

$7.15734 \mathrm{E}-04$

$7.47429 \mathrm{E}-04$

$7.80024 \mathrm{E}-04$

$8.13530 \mathrm{E}-04$

$8.47959 \mathrm{E}-04$

$8.83325 \mathrm{E}-04$

$9.19639 \mathrm{E}-04$

$9.56913 \mathrm{E}-04$

$9.95160 \mathrm{E}-04$

$1.03439 \mathrm{E}-03$

$1.07462 \mathrm{E}-03$

$1.11586 \mathrm{E}-03$

$1.15812 \mathrm{E}-03$

$1.20142 \mathrm{E}-03$

$1.24577 \mathrm{E}-03$

$1.29117 \mathrm{E}-03$

$1.33764 \mathrm{E}-03$

$1.38520 \mathrm{E}-03$

$1.43386 \mathrm{E}-03$

$1.48362 \mathrm{E}-03$

$1.53450 \mathrm{E}-03$

$1.58652 \mathrm{E}-03$

$1.63968 \mathrm{E}-03$

$1.69401 \mathrm{E}-03$

$1.74950 \mathrm{E}-03$

$1.80617 \mathrm{E}-03$

$1.86404 \mathrm{E}-03$

$1.92311 \mathrm{E}-03$

$1.98341 \mathrm{E}-03$

$2.04493 \mathrm{E}-03$

$2.10770 \mathrm{E}-03$

$2.17173 \mathrm{E}-03$

$2.23703 \mathrm{E}-03$

$2.30360 \mathrm{E}-03$

$2.37147 \mathrm{E}-03$

$2.44065 \mathrm{E}-03$

$-6.24427 \mathrm{E} \quad 01 \quad-6.18553 \mathrm{E}-05$ $-6.25236 \mathrm{E} 01 \quad-7.22234 \mathrm{E}-05$

$-6.26018 \mathrm{E}$ 0l $-8.31534 \mathrm{E}-0.5$

$-6.26774 \mathrm{E} \quad 01 \quad-9.46578 \mathrm{E}-05$

$-6.27505 \mathrm{E} \quad 01-1.06749 \mathrm{E}-04$

$-6.28212 \mathrm{E} \quad 01$

$-6.28897 \mathrm{E} \quad 01$

$-6.29561 \mathrm{E} 01$

$-6.30204 \mathrm{E} 01$

$-6.30827 \mathrm{E} \quad 01$

$-6.31432 \mathrm{E} 01$

$-6.32018 \mathrm{E} 01$

$-6.32588 \mathrm{E} \quad 01$

$-6.33141 \mathrm{E} \quad 01$

$-6.33679 \mathrm{E} 01$

$-6.34201 \mathrm{E} \quad 01$

$-6.34709 \mathrm{E} \quad 01$

$-6.35203 \mathrm{E} 01$

$-6.35684 \mathrm{E} \quad 01$

$-6.36152 \mathrm{E} 01$

$-6.36608 \mathrm{E} 01$

$-6.37051 \mathrm{E} 01$

$-6.37484 \mathrm{E} 01$

$-6.37905 \mathrm{E} \quad 01$

$-6.38316 \mathrm{E} 01$

$\begin{array}{ll}-6.38717 \mathrm{E} & 01\end{array}$

$-6.39108 \mathrm{E} 01$

$-6.39490 \mathrm{E} 01$

$-6.39862 \mathrm{E} 01$

$-6.40226 \mathrm{E} 01$

$\begin{array}{lll}-6.40581 \mathrm{E} & 01\end{array}$

$-6.40928 \mathrm{E} 01$

$-6.41267 \mathrm{E} 01$

$-6.41599 \mathrm{E} 01$

$-6.41923 \mathrm{E} 01$

$-6.42240 \mathrm{E} 0 \mathrm{I}$

$-6.42550 \mathrm{E} 01$

$-6.42853 \mathrm{E} 01$

$-6.43149 \mathrm{E} 01$

$-6.43440 \mathrm{E} 01$

$-6.43724 \mathrm{E} \quad 01$

$-6.44003 \mathrm{E} \quad 01$

$-6.44275 \mathrm{E} 01$

$-6.44543 \mathrm{E} \quad 01$

$-6.44804 \mathrm{E} 01$

$-6.45061 \mathrm{E} 01$

$-6.45313 \mathrm{E} 01$

$-6.45559 \mathrm{E} 01$

$-6.45801 \mathrm{E} 01$

$-6.46038 \mathrm{E} 01$

$-6.46271 \mathrm{E} \quad 01$

$-6.46499 \mathrm{E} 01$

$-6.46723 \mathrm{E} 01$

$-6.46943 \mathrm{E} 01$

$-6.47158 \mathrm{E} 01$

$-1.19439 \mathrm{E}-04$

$-1.32740 \mathrm{E}-04$

$-1.46665 \mathrm{E}-04$

$-1.61226 \mathrm{E}-04$

$-1.76435 \mathrm{E}-04$

$-1.92305 \mathrm{E}-04$

$-2.08848 \mathrm{E}-04$

$-2.26076 \mathrm{E}-04$

$-2.44002 \mathrm{E}-04$

$-2.62638 \mathrm{E}-04$

$-2.81996 \mathrm{E}-04$

$-3.02088 \mathrm{E}-04$

$-3.22928 \mathrm{E}-04$

$-3.44526 \mathrm{E}-04$

$-3.66896 \mathrm{E}-04$

$-3.90050 \mathrm{E}-04$

$-4.14001 \mathrm{E}-04$

$-4.38760 \mathrm{E}-04$

$-4.64339 \mathrm{E}-04$

$-4.90752 \mathrm{E}-04$

$-5.18011 \mathrm{E}-04$

$-5.46127 \mathrm{E}-04$

$-5.75114 \mathrm{E}-04$

$-6.04983 \mathrm{E}-04$

$-6.35747 \mathrm{E}-04$

$-6.67418 \mathrm{E}-04$

$-7.00009 \mathrm{E}-04$

$-7.33531 \mathrm{E}-04$

$-7.67998 \mathrm{E}-04$

$-8.03421 \mathrm{E}-04$

$-8.39812 \mathrm{E}-04$

$-8.77185 \mathrm{E}-04$

$-9.15552 \mathrm{E}-04$

$-9.54924 \mathrm{E}-04$

$-9.9531+\mathrm{E}-04$

$-1.03673 \mathrm{E}-03$

$-1.07920 \mathrm{E}-03$

$-1.12272 \mathrm{E}-03$

$-1.16730 \mathrm{E}-03$

$-1.21297 \mathrm{E}-03$

$-1.25973 \mathrm{E}-03$

$-1.30759 \mathrm{E}-03$

$-1.35657 \mathrm{E}-03$

$-1.40667 \mathrm{E}-03$

$-1.45792 \mathrm{E}-03$

$-1.51032 \mathrm{E}-03$

$-1.56389 \mathrm{E}-03$ 
ELECTRON RADIAL FUNCTIONS

Positron

\begin{tabular}{|c|c|c|c|}
\hline$p$ & $F_{0}$ & $f_{1}$ & \\
\hline 12,10 & $9.34550 \mathrm{E}-01$ & $1.12312 \mathrm{E}$ & 01 \\
\hline 12.20 & $9.34550 \mathrm{E}-01$ & $1.13284 \mathrm{E}$ & 01 \\
\hline 12.30 & $9.34550 \mathrm{E}-01$ & $1.14257 \mathrm{E}$ & 01 \\
\hline 12.40 & $9.34549 \mathrm{E}-01$ & $1.15230 \mathrm{E}$ & 01 \\
\hline 12.50 & $9.34549 \mathrm{E}-01$ & $1.16203 \mathrm{E}$ & 01 \\
\hline 12.60 & $9.34549 \mathrm{E}-01$ & $1.17176 \mathrm{E}$ & 01 \\
\hline 12.70 & $9.34549 \mathrm{E}-01$ & $1.18149 \mathrm{E}$ & 01 \\
\hline 12.80 & $9.34548 \mathrm{E}-01$ & $1.19122 \mathrm{E}$ & 01 \\
\hline 12.90 & $9.34548 \mathrm{E}-01$ & $1.20095 \mathrm{E}$ & 01 \\
\hline 13.00 & $9.34547 \mathrm{E}-01$ & $1.21068 \mathrm{E}$ & 01 \\
\hline 13.10 & $9.34547 \mathrm{E}-01$ & $1.22041 \mathrm{E}$ & 01 \\
\hline 13.20 & $9.34546 \mathrm{E}-01$ & $1.23015 \mathrm{E}$ & 01 \\
\hline 13.30 & $9.34546 \mathrm{E}-01$ & $1.23988 \mathrm{E}$ & 01 \\
\hline 13.40 & $9.34545 \mathrm{E}-01$ & $1.24962 \mathrm{E}$ & 01 \\
\hline 13.50 & $9.34545 \mathrm{E}-01$ & $1.25935 \mathrm{E}$ & 01 \\
\hline 13.60 & $9.34544 \mathrm{E}-01$ & $1.26909 \mathrm{E}$ & 01 \\
\hline 13.70 & $9.34543 \mathrm{E}-01$ & $1.27883 \mathrm{E}$ & 01 \\
\hline 13.80 & $9.34542 \mathrm{E}-01$ & $1.28856 \mathrm{E}$ & 01 \\
\hline 13.90 & $9.34542 \mathrm{E}-01$ & $1.29830 \mathrm{E}$ & 01 \\
\hline 14.00 & $9.34541 \mathrm{E}-01$ & $1.30804 \mathrm{E}$ & 01 \\
\hline 14.10 & $9.34540 \mathrm{E}-01$ & $1.31778 \mathrm{E}$ & 01 \\
\hline 14.20 & $9.34539 \mathrm{E}-01$ & $1.32752 \mathrm{E}$ & 01 \\
\hline 14.30 & $9.34538 \mathrm{E}-01$ & $1.33726 \mathrm{E}$ & 01 \\
\hline 14.40 & $9.34537 \mathrm{E}-01$ & $1.34700 \mathrm{E}$ & 01 \\
\hline 14.50 & $9.34537 \mathrm{E}-01$ & $1.35675 \mathrm{E}$ & 01 \\
\hline 14.60 & $9.34536 \mathrm{E}-01$ & $1.36649 \mathrm{E}$ & 01 \\
\hline 14.70 & $9.34535 \mathrm{E}-01$ & $1.37623 \mathrm{E}$ & 01 \\
\hline 14.80 & $9.34534 \mathrm{E}-01$ & $1.38598 \mathrm{E}$ & 01 \\
\hline 14.90 & $9.34532 \mathrm{E}-01$ & $1.39572 \mathrm{E}$ & 01 \\
\hline 15.00 & $9.34532 \mathrm{E}-01$ & $1.40547 \mathrm{E}$ & 01 \\
\hline 15.10 & $9.34530 \mathrm{E}-01$ & $1.41522 \mathrm{E}$ & 01 \\
\hline 15.20 & $9.34529 \mathrm{E}-01$ & $1.42497 \mathrm{E}$ & 01 \\
\hline 15.30 & $9.34528 \mathrm{E}-01$ & $1.43472 \mathrm{E}$ & 01 \\
\hline 15.40 & $9.34527 \mathrm{E}-01$ & $1.44446 \mathrm{E}$ & 01 \\
\hline 15.50 & $9.34526 \mathrm{E}-01$ & $1.45422 \mathrm{E}$ & 01 \\
\hline 15.60 & $9.34525 \mathrm{E}-01$ & $1.46397 \mathrm{E}$ & 01 \\
\hline 15.70 & $9.34524 \mathrm{E}-01$ & $1.47372 \mathrm{E}$ & 01 \\
\hline 15.80 & $9.34523 \mathrm{E}-01$ & $1.48347 \mathrm{E}$ & 01 \\
\hline 15.90 & $9.34521 \mathrm{E}-01$ & $1.49323 \mathrm{E}$ & 01 \\
\hline 16.00 & $9.34520 \mathrm{E}-01$ & $1.50298 \mathrm{E}$ & 01 \\
\hline 16.10 & $9.34519 \mathrm{E}-01$ & $1.51274 \mathrm{E}$ & 01 \\
\hline 16.20 & $9.34518 \mathrm{E}-01$ & $1.52249 \mathrm{E}$ & 01 \\
\hline 16.30 & $9.34517 \mathrm{E}-01$ & $1.53225 \mathrm{E}$ & 01 \\
\hline 16.40 & $9.34515 \mathrm{E}-01$ & $1.54201 \mathrm{E}$ & 01 \\
\hline 16.50 & $9.34514 \mathrm{E}-01$ & $1.55177 \mathrm{E}$ & 01 \\
\hline 16.60 & $9.34513 \mathrm{E}-01$ & $1.56153 \mathrm{E}$ & 01 \\
\hline 16.70 & $9.34512 \mathrm{E}-01$ & $1.57129 \mathrm{E}$ & 01 \\
\hline 16.80 & $9.34510 \mathrm{E}-01$ & $1.58105 \mathrm{E}$ & 01 \\
\hline 16.90 & $9.34509 \mathrm{E}-01$ & $1.59081 \mathrm{E}$ & 01 \\
\hline 17.00 & $9.34508 \mathrm{E}-01$ & $1.60057 \mathrm{E}$ & 01 \\
\hline 17.10 & $9.34506 \mathrm{E}-01$ & $1.61034 \mathrm{E}$ & 01 \\
\hline 17.20 & $9.34505 \mathrm{E}-01$ & $1.62010 \mathrm{E}$ & 01 \\
\hline 17.30 & $9.34504 \mathrm{E}-01$ & $1.62987 \mathrm{E}$ & 01 \\
\hline 17.40 & $9.34502 \mathrm{E}-01$ & $1.63963 \mathrm{E}$ & 01 \\
\hline 17.50 & $9.34501 \mathrm{E}-01$ & $1.64940 \mathrm{E}$ & 01 \\
\hline 17.60 & $9.34500 \mathrm{E}-01$ & $1.65917 \mathrm{E}$ & 01 \\
\hline 17.70 & $9.34498 \mathrm{E}-01$ & $1.66894 \mathrm{E}$ & 01 \\
\hline 17.80 & $9.34497 \mathrm{E}-01$ & $1.67871 \mathrm{E}$ & 01 \\
\hline 17.90 & $9.34496 \mathrm{E}-01$ & $1.68848 \mathrm{E}$ & 01 \\
\hline 18.00 & $9.34494 \mathrm{E}-01$ & $1.69825 \mathrm{E}$ & 01 \\
\hline
\end{tabular}

$Z=3 \quad A=6 \quad \rho=1.2 A^{1 / 3} F$

$\begin{array}{ccccc}g_{1} & \tan \delta_{1} & f_{-1} & g_{-1} & \\ 1.79478 \mathrm{E}-01 & -4.08151 \mathrm{E}-04 & -1.49043 \mathrm{E}-01 & 1.22013 \mathrm{E} & 01 \\ 1.83159 \mathrm{E}-01 & -4.15323 \mathrm{E}-04 & -1.52541 \mathrm{E}-01 & 1.22987 \mathrm{E} & 01 \\ 1.86876 \mathrm{E}-01 & -4.22370 \mathrm{E}-04 & -1.56075 \mathrm{E}-01 & 1.23961 \mathrm{E} & 01 \\ 1.90630 \mathrm{E}-01 & -4.29305 \mathrm{E}-04 & -1.59647 \mathrm{E}-01 & 1.24935 \mathrm{E} & 01 \\ 1.94420 \mathrm{E}-01 & -4.36124 \mathrm{E}-04 & -1.63255 \mathrm{E}-01 & 1.25909 \mathrm{E} & 01\end{array}$

$\begin{array}{lllll}1.98248 \mathrm{E}-01 & -4.42831 \mathrm{E}-04 & -1.66900 \mathrm{E}-01 & 1.26884 \mathrm{E} & 01\end{array}$

$2.02112 \mathrm{E}-01-4.49430 \mathrm{E}-04 \quad-1.70581 \mathrm{E}-01$

$2.06013 \mathrm{E}-01 \quad-4.55922 \mathrm{E}-04 \quad-1.74300 \mathrm{E}-01$

$2.09950 \mathrm{E}-01-4.62309 \mathrm{E}-04-1.78055 \mathrm{E}-01$

$2.13925 \mathrm{E}-01 \quad-4.68598 \mathrm{E}-04 \quad-1.81847 \mathrm{E}-01$

$2.17936 \mathrm{E}-01$

$2.21984 \mathrm{E}-01$

$2.26069 \mathrm{E}-01$

$2.30191 \mathrm{E}-01$

$2.34349 \mathrm{E}-01$

$2.38544 \mathrm{E}-01$

$2.42777 \mathrm{E}-01$

$2.47046 \mathrm{E}-01$

$2.51351 \mathrm{E}-01$

2.55694 E- 01

$2.60073 \mathrm{E}-01$

$2.64490 \mathrm{E}-01$

$2.68943 \mathrm{E}-01$

$2.73433 \mathrm{E}-01$

$2.77960 \mathrm{E}-01$

$2.82524 \mathrm{E}-01$

$2.87125 \mathrm{E}-01$

$2.91763 \mathrm{E}-01$

$2.96437 \mathrm{E}-01$

$3.01149 \mathrm{E}-01$

$3.05897 \mathrm{E}-01$

$3.10682 \mathrm{E}-01$

$3.15505 \mathrm{E}-01$

$3.20364 \mathrm{E}-01$

$3.25260 \mathrm{E}-01$

3.30193E-01

$3.35163 \mathrm{E}-01$

$3.40170 \mathrm{E}-01$

$3.45214 \mathrm{E}-01$

$3.50295 \mathrm{E}-01$

$3.55413 \mathrm{E}-01$

$3.60568 \mathrm{E}-01$

$3.65760 \mathrm{E}-01$

$3.70989 \mathrm{E}-01$

$3.76255 \mathrm{E}-01$

$3.81558 \mathrm{E}-01$ $3.86898 \mathrm{E}-01$ $3.92275 \mathrm{E}-01$ $3.97689 \mathrm{E}-01$

$4.03140 \mathrm{E}-01$

4.08629 E-01 $4.14154 \mathrm{E}-01$ $4.19716 \mathrm{E}-01$ $4.25316 \mathrm{E}-01$ $4.30952 \mathrm{E}-01$

4.36626E-01 $4.42337 \mathrm{E}-01$ $4.48085 \mathrm{E}-01$ $4.53870 \mathrm{E}-01$ $4.59692 \mathrm{E}-01$
$-4.74785 \mathrm{E}-04$

$-4.80874 \mathrm{E}-04$

$-4.86870 \mathrm{E}-04$

$-4.92774 \mathrm{E}-04$

$-4.98589 \mathrm{E}-04$

$-5.04311 \mathrm{E}-04$

$-5.09950 \mathrm{E}-04$

$-5.15502 \mathrm{E}-04$

$-5.20968 \mathrm{E}-04$

$-5.26356 \mathrm{E}-04$

$-5.31662 \mathrm{E}-04$

$-5.36890 \mathrm{E}-04$

$-5.42045 \mathrm{E}-04$

$-5.47122 \mathrm{E}-04$

$-5.52124 \mathrm{E}-04$

$-5.57059 \mathrm{E}-04$

$-5.61921 \mathrm{E}-04$

$-5.66715 \mathrm{E}-04$

$-5.71438 \mathrm{E}-04$

$-5.76098 \mathrm{E}-04$

$-5.80686 \mathrm{E}-04$

$-5.85218 \mathrm{E}-04$

$-5.89686 \mathrm{E}-04$

$-5.94090 \mathrm{E}-04$

$-5.98435 \mathrm{E}-04$

$-6.02721 \mathrm{E}-04$

$-6.06945 \mathrm{E}-04$

$-6.11116 \mathrm{E}-04$

$-6.15233 \mathrm{E}-04$

$-6.19289 \mathrm{E}-04$

$-6.23296 \mathrm{E}-04$

$-6.27250 \mathrm{E}-04$

$-6.31150 \mathrm{E}-04$

$-6.38794 \mathrm{E}-04$

$-6.42547 \mathrm{E}-04$

$-6.46246 \mathrm{E}-04$

$-6.49897 \mathrm{E}-04$

$-6.53505 \mathrm{E}-04$

$-6.57065 \mathrm{E}-04$

$-6.60582 \mathrm{E}-04$

$-6.64053 \mathrm{E}-04$

$-6.67476 \mathrm{E}-04$

$-6.70863 \mathrm{E}-04$

$-6.74204 \mathrm{E}-04$

$-6.77505 \mathrm{E}-04$

$-6.80763 \mathrm{E}-04$

$-6.83983 \mathrm{E}-04$

$-6.87160 \mathrm{E}-04$
$-6.34998 \mathrm{E}-04$

$-6.90302 \mathrm{E}-04$
$-1.85676 \mathrm{E}-01$

$-1.89542 \mathrm{E}-01$

$-1.93444 \mathrm{E}-01$

$-1.97384 \mathrm{E}-01$

$-2.01360 \mathrm{E}-01$

$-2.05373 \mathrm{E}-01$

$-2.09423 \mathrm{E}-01$

$-2.13510 \mathrm{E}-01$

$-2.17633 \mathrm{E}-01$

$-2.21794 \mathrm{E}-01$

$-2.25992 \mathrm{E}-01$

$-2.30226 \mathrm{E}-01$

$-2.34497 \mathrm{E}-01$

$-2.38805 \mathrm{E}-01$

$-2.43150 \mathrm{E}-01$

$-2.47532 \mathrm{E}-01$

$-2.51951 \mathrm{E}-01$

$-2.56407 \mathrm{E}-01$

$-2.60900 \mathrm{E}-01$

$-2.65429 \mathrm{E}-01$

$-2.69996 \mathrm{E}-01$

$-2.74599 \mathrm{E}-01$

$-2.79240 \mathrm{E}-01$

$-2.83917 \mathrm{E}-01$

$-2.88632 \mathrm{E}-01$

$-2.98172 \mathrm{E}-01$

$-3.02997 \mathrm{E}-01$

$-3.07859 \mathrm{E}-01$

$-3.12759 \mathrm{E}-01$

$-3.17695 \mathrm{E}-01$ $-3.22669 \mathrm{E}-01$ $-3.27679 \mathrm{E}-01$ $-3.37811 \mathrm{E}-01$

$-3.42933 \mathrm{E}-01$ $-3.48092 \mathrm{E}-01$ $-3.53287 \mathrm{E}-01$ $-3.58520 \mathrm{E}-01$ $-3.63790 \mathrm{E}-01$

$-3.69097 \mathrm{E}-01$ $-3.74441 \mathrm{E}-01$ $-3.79822 \mathrm{E}-01$ $-3.85241 \mathrm{E}-01$ $-3.90696 \mathrm{E}-01$

$-3.96189 \mathrm{E}-01$ $-4.01718 \mathrm{E}-01$

$-4.07285 \mathrm{E}-01$ $-4.12889 \mathrm{E}-01$ $-4.18530 \mathrm{E}-01$
$-2.93383 \mathrm{E}-01$ $-3.32727 \mathrm{E}-01$
$1.27858 \mathrm{E} \quad 01$

$1.28832 \mathrm{E} 01$

$1.29807 \mathrm{E} \quad 01$

$1.30781 \mathrm{E} 01$

$1.31756 \mathrm{E} \quad 01$

$1.32731 \mathrm{E} \quad 01$

$1.33705 \mathrm{E} \quad 01$

$1.34680 \mathrm{E} 01$

$1.35655 \mathrm{E} 01$

$1.36630 \mathrm{E} \quad 01$ $1.37605 \mathrm{E} \quad 01$

$1.38580 \mathrm{E} 01$

$1.39555 \mathrm{E} \quad 01$

$1.40531 \mathrm{E} 01$

$1.41506 \mathrm{E} \quad 01$ $1.42481 \mathrm{E} 01$ $1.43457 \mathrm{E} \quad 01$

$1.44432 \mathrm{E} 01$

$1.45408 \mathrm{E} \quad 01$

$1.46384 \mathrm{E} \quad 01$

$1.47359 \mathrm{E} \quad 01$

$1.48335 \mathrm{E} 01$

$1.49311 \mathrm{E} 01$

$1.50287 \mathrm{E} 01$

$1.51263 \mathrm{E} \quad 01$

$1.52239 \mathrm{E} \quad 01$

$1.53216 \mathrm{E} 01$

$1.54192 \mathrm{E} 01$

$1.55168 \mathrm{E} 01$

$1.56145 \mathrm{E} \quad 01$

$1.57122 \mathrm{E} \quad 01$

$1.58098 \mathrm{E} 01$

$\begin{array}{ll}1.59075 \mathrm{E} & 01\end{array}$

$1.60052 \mathrm{E} 01$

$1.61029 \mathrm{E} \quad 01$ $1.62006 \mathrm{E} 01$ $1.62983 \mathrm{E} 01$ $1.63960 \mathrm{E} 01$ $1.64937 \mathrm{E} \quad 01$

$\begin{array}{ll}1.65915 \mathrm{E} & 01\end{array}$ $1.66892 \mathrm{E} 01$ $1.67870 \mathrm{E} 01$

$\begin{array}{lll}1.68847 \mathrm{E} & 01\end{array}$

$1.69825 \mathrm{E} 01$

$1.70803 \mathrm{E} 01$ $1.71781 \mathrm{E} 01$ $1.72759 \mathrm{E} \quad 01$ $\begin{array}{lll}1.73737 \mathrm{E} & 01\end{array}$

$1.74715 \mathrm{E} 01$

$\begin{array}{ll}1.75693 \mathrm{E} & 01\end{array}$

$1.76672 \mathrm{E} \quad 01$

$1.77650 \mathrm{E} 01$

$1.78629 \mathrm{E} 01$

$1.79607 \mathrm{E} 01$ $\tan \delta_{-1}$

$4.51234 \mathrm{E} \quad 02$ $4.52801 \mathrm{E} \quad 02$ $4.54356 \mathrm{E} \quad 02$ $4.55895 \mathrm{E} \quad 02$ $4.57421 \mathrm{E} \quad 02$

$4.58934 \mathrm{E} \quad 02$ $4.60433 \mathrm{E} \quad 02$ $4.61919 \mathrm{E} \quad 02$ $4.63393 \mathrm{E} \quad 02$ $4.64853 \mathrm{E} \quad 02$

$4.66301 \mathrm{E} \quad 02$ $4.67737 \mathrm{E} \quad 02$ $4.69161 \mathrm{E} \quad 02$ $\begin{array}{ll}4.70572 \mathrm{E} & 02\end{array}$ $4.71971 \mathrm{E} \quad 02$

$4.73360 \mathrm{E} \quad 02$ $4.74736 \mathrm{E} \quad 02$ $4.76101 \mathrm{E} \quad 02$ $4.77456 \mathrm{E} \quad 02$ $4.78799 \mathrm{E} \quad 02$

$4.80132 \mathrm{E} \quad 02$ $4.81454 \mathrm{E} \quad 02$ $4.82764 \mathrm{E} \quad 02$ $4.84066 \mathrm{E} \quad 02$ $4.85357 \mathrm{E} \quad 02$

$4.86637 \mathrm{E} \quad 02$ $4.87907 \mathrm{E} \quad 02$ $4.89168 \mathrm{E} \quad 02$ $4.90420 \mathrm{E} \quad 02$ $4.91661 \mathrm{E} \quad 02$

$4.92894 \mathrm{E} \quad 02$ $4.94117 \mathrm{E} \quad 02$ $4.95330 \mathrm{E} \quad 02$ $4.96536 \mathrm{E} \quad 02$ $4.97732 \mathrm{E} \quad 02$

$4.98919 \mathrm{E} \quad 02$ $5.00099 \mathrm{E} \quad 02$ $5.01269 \mathrm{E} \quad 02$ $5.02430 \mathrm{E} \quad 02$ $5.03584 \mathrm{E} \quad 02$

$5.04729 \mathrm{E} \quad 02$ $5.05866 \mathrm{E} \quad 02$ $5.06995 \mathrm{E} \quad 02$ $5.08117 \mathrm{E} \quad 02$ $5.09231 \mathrm{E} \quad 02$

$5.10337 \mathrm{E} \quad 02$ $5.11436 \mathrm{E} \quad 02$ $5.12528 \mathrm{E} \quad 02$ $5.13611 \mathrm{E} \quad 02$ $5.14688 \mathrm{E} \quad 02$

$5.15757 \mathrm{E} \quad 02$ $5.16819 \mathrm{E} \quad 02$ $5.17875 \mathrm{E} \quad 02$ 5.18923 E 02 $5.19965 \mathrm{E} \quad 02$

$5.21000 \mathrm{E} \quad 02$ $5.22028 \mathrm{E} \quad 02$ $5.23050 \mathrm{E} \quad 02$ $5.24066 \mathrm{E} \quad 02$ $5.25075 \mathrm{E} \quad 02$ 
ELECTRON RADIAL FUNCTIONS

$Z=3 \quad A=6 \quad \rho=1.2 A^{1 / 3} \mathrm{~F}$

Pusitron

$\bar{F}$

$f_{2}$

$1.21128 \mathrm{E}-04$

$1.23138 \mathrm{E}-04$

$1.25165 \mathrm{E}-04$

$1.27209 \mathrm{E}-04$

$1.29269 \mathrm{E}-04$

12.40

12.50

12.60

12.70

12.80

12.90

13.00

13.10

13.20

13.30

13.40

13.50

13.60

13.70

13.80

13.90

14.00

14.10

14.20

14.30

14.40

14.50

14.60

14.70

14.80

14.90

15.00

15.10

15.20

15.30

15.40

15.50

15.60

15.70

15.80

15.90

16.00

16.10

16.20

16.30

16.40

16.50

16.60

16.70

16.80

16.90

17.00

17.10

17.20

17.30

17.40

17.50

17.60

17.70

17.80

17.90

18.00
$1.31346 \mathrm{E}-04$

$1.33440 \mathrm{E}-04$

$1.35549 \mathrm{E}-04$

$1.37676 \mathrm{E}-04$

$1.39819 \mathrm{E}-04$

$1.41978 \mathrm{E}-04$

$1.44154 \mathrm{E}-04$

$1.46347 \mathrm{E}-04$

$1.48556 \mathrm{E}-04$

$1.50782 \mathrm{E}-04$

$1.53024 \mathrm{E}-04$

$1.55283 \mathrm{E}-04$

$1.57558 \mathrm{E}-04$

$1.59850 \mathrm{E}-04$

$1.62158 \mathrm{E}-04$

$1.64483 \mathrm{E}-04$

$1.66825 \mathrm{E}-04$

$1.69183 \mathrm{E}-04$

$1.71557 \mathrm{E}-04$

$1.73949 \mathrm{E}-04$

$1.76356 \mathrm{E}-04$

$1.78780 \mathrm{E}-04$

$1.81221 \mathrm{E}-04$

$1.83679 \mathrm{E}-04$

$1.86152 \mathrm{E}-04$

$1.88643 \mathrm{E}-04$

$1.91150 \mathrm{E}-04$

$1.93673 \mathrm{E}-04$

$1.96213 \mathrm{E}-04$

$1.98770 \mathrm{E}-04$

$2.01343 \mathrm{E}-04$

$2.03932 \mathrm{E}-04$

$2.06539 \mathrm{E}-04$

$2.09161 \mathrm{E}-04$

2.11801 E- 04

$2.14456 \mathrm{E}-04$

$2.17129 \mathrm{E}-04$

$2.19818 \mathrm{E}-04$

$2.22523 \mathrm{E}-04$

$2.25245 \mathrm{E}-04$

$2.27984 \mathrm{E}-04$

$2.30739 \mathrm{E}-04$

$2.33510 \mathrm{E}-04$

$2.36299 \mathrm{E}-04$

$2.39103 \mathrm{E}-04$

$2.41925 \mathrm{E}-04$

$2.44762 \mathrm{E}-04$

$2.47617 \mathrm{E}-04$

$2.50488 \mathrm{E}-04$

$2.53375 \mathrm{E}-04$

$2.56279 \mathrm{E}-04$

$2.59199 \mathrm{E}-04$

$2.62136 \mathrm{E}-04$

$2.65090 \mathrm{E}-04$

$2.68060 \mathrm{E}-04$ $g_{2}$

$2.55254 \mathrm{E}-01$

$2.59584 \mathrm{E}-01$

$2.63951 \mathrm{E}-01$

$2.68354 \mathrm{E}-01$

$2.72793 \mathrm{E}-01$

$2.77269 \mathrm{E}-01$

$2.81782 \mathrm{E}-01$

$2.86330 \mathrm{E}-01$

$2.90915 \mathrm{E}-01$

$2.95536 \mathrm{E}-01$

$3.00194 \mathrm{E}-01$

$3.04888 \mathrm{E}-01$

$3.09618 \mathrm{E}-01$

$3.14384 \mathrm{E}-01$

$3.19188 \mathrm{E}-01$

$3.24027 \mathrm{E}-01$

$3.28902 \mathrm{E}-01$

$3.33814 \mathrm{E}-01$

$3.38763 \mathrm{E}-01$

$3.43747 \mathrm{E}-01$

$3.48769 \mathrm{E}-01$

$3.53826 \mathrm{E}-01$

$3.58920 \mathrm{E}-01$

$3.64050 \mathrm{E}-01$

$3.69216 \mathrm{E}-01$

$3.74419 \mathrm{E}-01$

$3.79658 \mathrm{E}-01$

$3.84933 \mathrm{E}-01$

$3.90245 \mathrm{E}-01$

$3.95593 \mathrm{E}-01$

$4.00977 \mathrm{E}-01$

$4.06397 \mathrm{E}-01$

$4.11854 \mathrm{E}-01$

$4.17348 \mathrm{E}-01$

$4.22877 \mathrm{E}-01$

$4.28443 \mathrm{E}-01$

$4.34045 \mathrm{E}-01$

4.39684 E-01

$4.45359 \mathrm{E}-01$

$4.51070 \mathrm{E}-01$

$4.56817 \mathrm{E}-01$

$4.62601 \mathrm{E}-01$

$4.68421 \mathrm{E}-01$

$4.74277 \mathrm{E}-0$

$4.80170 \mathrm{E}-01$

$4.86099 \mathrm{E}-01$

4.92064 E-0l

$4.98066 \mathrm{E}-01$

$5.04104 \mathrm{E}-01$

5.10178 E-01

$5.16288 \mathrm{E}-01$

$5.22435 \mathrm{E}-01$

$5.28618 \mathrm{E}-01$

$5.34837 \mathrm{E}-01$

$5.41093 \mathrm{E}-01$

$5.47384 \mathrm{E}-01$

$5.53713 \mathrm{E}-01$

$5.60077 \mathrm{E}-01$

$5.66478 \mathrm{E}-01$

$5.72914 \mathrm{E}-01$ $\tan \delta_{2}$

$f_{-2}$

$2.51115 \mathrm{E}-03$

$2.58297 \mathrm{E}-03$

$2.65614 \mathrm{E}-03$

$2.73066 \mathrm{E}-0.3$

$2.80655 \mathrm{E}-03$

$2.88382 \mathrm{E}-03$

$2.96248 \mathrm{E}-03$

$3.04254 \mathrm{E}-03$

$3.12402 \mathrm{E}-03$

$3.20693 \mathrm{E}-0.3$

$3.29129 \mathrm{E}-03$

$3.37709 \mathrm{E}-0.3$

$3.46436 \mathrm{E}-03$

$3.55311 \mathrm{E}-03$

$3.64335 \mathrm{E}-03$

$3.73509 \mathrm{E}-03$

$3.82835 \mathrm{E}-0.3$

$3.92313 \mathrm{E}-03$

$4.01946 \mathrm{E}-03$

$4.11733 \mathrm{E}-03$

$4.21677 \mathrm{E}-03$

$4.31779 \mathrm{E}-03$

$4.42039 \mathrm{E}-03$

$4.52460 \mathrm{E}-03$

$4.63042 \mathrm{E}-03$

$4.73787 \mathrm{E}-03$

$4.84695 \mathrm{E}-03$

$4.95768 \mathrm{E}-03$

$5.07008 \mathrm{E}-03$

$5.18415 \mathrm{E}-03$

$5.29991 \mathrm{E}-03$

$5.41737 \mathrm{E}-03$

$5.53654 \mathrm{E}-03$

$5.65744 \mathrm{E}-03$

$5.78007 \mathrm{E}-03$

$5.90445 \mathrm{E}-03$

$6.03059 \mathrm{E}-03$

$6.15851 \mathrm{E}-03$

$6.28821 \mathrm{E}-03$

$6.41971 \mathrm{E}-03$

$6.55302 \mathrm{E}-03$

$6.68815 \mathrm{E}-03$

$6.82512 \mathrm{E}-03$

$6.96393 \mathrm{E}-03$

$7.10461 \mathrm{E}-03$

$7.24715 \mathrm{E}-03$

$7.39158 \mathrm{E}-03$

$7.53791 \mathrm{E}-03$

$7.68614 \mathrm{E}-03$

$7.83630 \mathrm{E}-03$

$7.98839 \mathrm{E}-03$

$8.14242 \mathrm{E}-03$

$8.29841 \mathrm{E}-03$

$8.45638 \mathrm{E}-03$

$8.6+632 \mathrm{E}-03$

$8.77826 \mathrm{E}-03$

$8.94221 \mathrm{E}-03$

$9.10817 \mathrm{E}-03$

$9.27617 \mathrm{E}-03$

$9.44621 \mathrm{E}-03$

$-6.48374 \mathrm{E} \quad 01 \quad-2.10051 \mathrm{E}-03$ $-6.48565 \mathrm{E}$ 01 $-2.16642 \mathrm{E}-03$ $-6.48752 \mathrm{E}$ ol $-2.23363 \mathrm{E}-03$ $-6.48936 \mathrm{E}$ 01 $-2.30215 \mathrm{E}-03$

$-6.49117 \mathrm{E}$ 01 $-2.37199 \mathrm{E}-03$

$-6.49294 \mathrm{E} 01$ $-6.49469 \mathrm{E} 01$ $-6.49641 \mathrm{E} \quad 01$ $-6.49810 \mathrm{E} 01$ $-6.49977 \mathrm{E} \quad 01$

$-6.50141 \mathrm{E} \quad 01$

$-6.50302 \mathrm{E} \quad 01$

$-6.50460 \mathrm{E} \quad 01$

$-6.50617 \mathrm{E} 01$

$-6.50770 \mathrm{E} 01$

$-6.50922 \mathrm{E} 01$ $-6.51071 \mathrm{E} \quad 01$

$-6.51217 \mathrm{E} \quad 01$

$-6.51362 \mathrm{E} 01$

$-6.51504 \mathrm{E} \quad 01$

$-6.51645 \mathrm{E} 01$

$-6.51783 \mathrm{E} \quad 01$

$-6.51919 \mathrm{E} \quad 01$

$-6.52053 \mathrm{E} 01$

$-6.52185 \mathrm{E} 01$

$-6.52316 \mathrm{E} 01$

$-6.52444 \mathrm{E} \quad 01$

$-6.52571 \mathrm{E} 01$

$-6.52696 \mathrm{E} \quad 01$

$-6.52819 \mathrm{E} 01$

$-6.52940 \mathrm{E} 01$

$-6.53060 \mathrm{E} 01$

$-6.53178 \mathrm{E} \quad 01$

$-6.53295 \mathrm{E} 01$

$-6.53409 \mathrm{E} 01$

$-2.44318 \mathrm{E}-03$ $-2.51572 \mathrm{E}-03$

$-2.58961 \mathrm{E}-03$

$-2.66489 \mathrm{E}-03$

$-2.74155 \mathrm{E}-03$

$-2.81961 \mathrm{E}-03$

$-2.89909 \mathrm{E}-03$

$-2.97999 \mathrm{E}-03$

$-3.06232 \mathrm{E}-03$

$-3.14611 \mathrm{E}-03$ 
ELECTRON RADIAL FUNCTIONS

Positron

$p-F_{0}$

18.10

18.20

18.30

18.40

18.50

18.60

18.70

18.80

18.90

19.00

19.10

19.20

19.30

19.40

19.50

19.60

19.70

19.80

19.90

20.00

20.10

20.20

20.30

20.40

20.50

20.60

20.70

20.80

20.90

21.00

21.10

21.20

21.30

21.40

21.50

21.60

21.70

21.80

21.90

22.00

22.10

22.20

22.30

22.40

22.50

22.60

22.70

22.80

22.90

23.00

23.10

23.20

23.30

23.40

23.50

23.60

23.70

23.80

23.90

24.00
$Z=3$

$f_{1}$

9.34493E-0]

9.34492E-01

$9.34490 \mathrm{E}-01$

$9.34489 \mathrm{E}-01$

$9.34487 \mathrm{E}-01$

$9.34486 \mathrm{E}-01$

$9.34485 \mathrm{E}-01$

$9.34483 \mathrm{E}-01$

$9.34482 \mathrm{E}-01$

$9.34481 \mathrm{E}-01$

$9.34479 \mathrm{E}-01$

$9.34478 \mathrm{E}-01$

$9.34476 \mathrm{E}-01$

$9.34475 \mathrm{E}-01$

$9.34473 \mathrm{E}-01$

$9.34472 \mathrm{E}-01$

$9.34471 \mathrm{E}-01$

$9.34469 \mathrm{E}-01$

$9.34468 \mathrm{E}-01$

$9.34466 \mathrm{E}-01$

9.34465 E-01

$9.34463 \mathrm{E}-01$

$9.34462 \mathrm{E}-01$

$9.34461 \mathrm{E}-01$

$9.34459 \mathrm{E}-01$

9.34458E-01

$9.34456 \mathrm{E}-01$

$9.34455 \mathrm{E}-01$

9.34454 E-01

9.34452 E-01

$9.34451 \mathrm{E}-01$

9.34449E-01

$9.34448 \mathrm{E}-01$

$9.34446 \mathrm{E}-01$

9.34445 E-01

9.34444 E-01

9.34442E-01

$9.34441 \mathrm{E}-01$

9.34439 E-01

9.34438E-01

9.34436E-01

$9.34435 \mathrm{E}-01$

$9.34434 \mathrm{E}-01$

$9.34432 \mathrm{E}-01$

9.34431 E-01

$9.34429 \mathrm{E}-01$

$9.34428 \mathrm{E}-01$

$9.34427 \mathrm{E}-01$

$9.34425 \mathrm{E}-01$

$9.34424 \mathrm{E}-01$

$9.34422 \mathrm{E}-01$

$9.34421 \mathrm{E}-01$

$9.34420 \mathrm{E}-01$

$9.34418 \mathrm{E}-01$

$9.34417 \mathrm{E}-01$

$9.34415 \mathrm{E}-01$ $9.34414 \mathrm{E}-01$

$9.34413 \mathrm{E}-01$

$9.34411 \mathrm{E}-01$

$9.34410 \mathrm{E}-01$
$1.70803 \mathrm{E} \quad 01$

$1.71780 \mathrm{E} \quad 0$

$1.72758 \mathrm{E} \quad 01$

$1.73735 \mathrm{E} \quad 01$

$1.75691 \mathrm{E} \quad 01$

$1.76669 \mathrm{E} \quad 01$

$\begin{array}{lll}1.77647 \mathrm{E} & 01\end{array}$

$1.78625 \mathrm{E} \quad 01$

$1.79603 \mathrm{E} \quad 01$

$1.80581 \mathrm{E} \quad 01$

$1.81560 \mathrm{E} \quad 01$

$1.82538 \mathrm{E} \quad 01$

$1.83517 \mathrm{E} \quad 01$

$1.84495 \mathrm{E} \quad 01$

$1.85474 \mathrm{E} \quad 01$

$1.86453 \mathrm{E} \quad 01$

$1.87432 \mathrm{E} \quad 01$

$1.88411 \mathrm{E} \quad 01$

$189390 \mathrm{E} 01$

$1.90370 \mathrm{E} \quad 01$

$1.91349 \mathrm{E} \quad 01$

$\begin{array}{lll}1.92329 \mathrm{E} & 01\end{array}$

$1.93308 \mathrm{E} \quad 01$

$1.94288 \mathrm{E} \quad 01$

$1.95268 \mathrm{E} \quad 01$

$1.96248 \mathrm{E} \quad 01$

$1.97228 \mathrm{E} 01$

$1.98208 \mathrm{E} \quad 01$

$1.99188 \mathrm{E} 01$

$2.00169 \mathrm{E} \quad 01$

$2.01149 \mathrm{E} \quad 01$

$2.02130 \mathrm{E} \quad 01$

$2.03110 \mathrm{E} \quad 01$

$2.04091 \mathrm{E} \quad 01$

$2.05072 \mathrm{E} \quad 01$

$2.06053 \mathrm{E} \quad 01$

$2.07034 \mathrm{E} 01$

$2.08016 \mathrm{E} \quad 01$

$2.08997 \mathrm{E} \quad 01$

$2.09978 \mathrm{E} \quad 01$

$2.10960 \mathrm{E} \quad 01$

$2.11942 \mathrm{E} \quad 01$

$2.12924 \mathrm{E} \quad 01$

$2.13906 \mathrm{E} 01$

2.14888 E 01

$2.15870 \mathrm{E} \quad 01$

$2.17835 \mathrm{E} \quad 01$

$2.18817 \mathrm{E} \quad 01$

$2.19800 \mathrm{E} \quad 01$

$2.20783 \mathrm{E} \quad 01$

$2.21766 \mathrm{E} \quad 01$

$2.22749 \mathrm{E} \quad 01$

$2.23732 \mathrm{E} \quad 01$

$2.24715 \mathrm{E} \quad 01$

$2.25698 \mathrm{E} \quad 01$

$2.26682 \mathrm{E} \quad 01$

$2.27665 \mathrm{E} \quad 01$

$2.28649 \mathrm{E} \quad 01$
$1.74713 \mathrm{E} \quad 01$

$2.16852 \mathrm{E} \quad 01$ $g_{1}$

$4.65551 \mathrm{E}-01$

$4.71448 \mathrm{E}-01$

$4.77382 \mathrm{E}-01$

$4.83352 \mathrm{E}-01$

$4.89360 \mathrm{E}-01$

$4.95405 \mathrm{E}-01$

$5.01488 \mathrm{E}-01$

$5.07607 \mathrm{E}-01$

$5.13764 \mathrm{E}-01$

$5.19958 \mathrm{E}-01$

$5.26189 \mathrm{E}-01$

$5.32457 \mathrm{E}-01$

$5.38763 \mathrm{E}-01$

$5.45106 \mathrm{E}-01$

$5.51486 \mathrm{E}-01$

$5.57903 \mathrm{E}-01$

$5.64358 \mathrm{E}-01$

$5.70850 \mathrm{E}-01$

$5.77379 \mathrm{E}-01$

$5.83946 \mathrm{E}-01$

$5.90550 \mathrm{E}-01$

$5.97191 \mathrm{E}-01$

$6.03869 \mathrm{E}-01$

$6.10585 \mathrm{E}-01$

$6.17339 \mathrm{E}-01$

$6.24129 \mathrm{E}-01$

$6.30957 \mathrm{E}-01$

$6.37822 \mathrm{E}-01$

$6.44725 \mathrm{E}-01$

$6.51665 \mathrm{E}-01$

$6.58643 \mathrm{E}-01$

$6.65657 \mathrm{E}-01$

$6.72710 \mathrm{E}-01$

$6.79799 \mathrm{E}-01$

$6.86927 \mathrm{E}-01$

$6.94091 \mathrm{E}-01$

$7.01294 \mathrm{E}-01$

$7.08533 \mathrm{E}-01$

$7.15810 \mathrm{E}-01$

$7.23125 \mathrm{E}-01$

7.30477 E-01

$7.37866 \mathrm{E}-01$

$7.45294 \mathrm{E}-01$

$7.52758 \mathrm{E}-01$

$7.60260 \mathrm{E}-01$

$7.67800 \mathrm{E}-01$

$7.75377 \mathrm{E}-01$

$7.82992 \mathrm{E}-01$

$7.90645 \mathrm{E}-01$

$7.98335 \mathrm{E}-01$

$8.06062 \mathrm{E}-01$

$8.13828 \mathrm{E}-01$

$8.21630 \mathrm{E}-01$

$8.29471 \mathrm{E}-01$

$8.37349 \mathrm{E}-01$

$8.45265 \mathrm{E}-01$

$8.53218 \mathrm{E}-01$

$8.61209 \mathrm{E}-01$

$8.69238 \mathrm{E}-01$

8.77305E-01

$\tan \delta_{1}$

$-6.93404 \mathrm{E}-04$

$-6.96465 \mathrm{E}-04$

$-6.99492 \mathrm{E}-04$

$-7.02485 \mathrm{E}-04$

$-7.05436 \mathrm{E}-04$

$-7.08356 \mathrm{E}-04$

$-7.11240 \mathrm{E}-04$

$-7.14088 \mathrm{E}-04$

$-7.16904 \mathrm{E}-04$

-7.19687 E-04

$-7.22435 \mathrm{E}-04$

$-7.25151 \mathrm{E}-04$

$-7.27840 \mathrm{E}-04$

$-7.30493 \mathrm{E}-04$

$-7.33114 \mathrm{E}-04$

$-7.35705 \mathrm{E}-04$

$-7.38269 \mathrm{E}-04$

$-7.40802 \mathrm{E}-04$

$-7.43308 \mathrm{E}-04$

$-7.45780 \mathrm{E}-04$

$-7.48226 \mathrm{E}-04$

$-7.50646 \mathrm{E}-04$

$-7.53036 \mathrm{E}-04$

$-7.55402 \mathrm{E}-04$

$-7.57737 \mathrm{E}-04$

$-7.60046 \mathrm{E}-04$

$-7.62327 \mathrm{E}-04$

$-7.64588 \mathrm{E}-04$

$-7.66822 \mathrm{E}-04$

$-7.69029 \mathrm{E}-04$

$-7.71212 \mathrm{E}-04$

$-7.73372 \mathrm{E}-04$

$-7.75509 \mathrm{E}-04$

$-7.77619 \mathrm{E}-04$

$-7.79707 \mathrm{E}-04$

$-7.81774 \mathrm{E}-04$

$-7.83813 \mathrm{E}-04$

$-7.85833 \mathrm{E}-04$

$-7.87833 \mathrm{E}-04$

$-7.89807 \mathrm{E}-04$

$-7.91762 \mathrm{E}-04$

$-7.93692 \mathrm{E}-04$

$-7.95607 \mathrm{E}-04$ 


$\begin{array}{cc}p & \bar{F} \\ 18.10 & 2.71047 \mathrm{E}-04 \\ 18.20 & 2.74050 \mathrm{E}-04 \\ 18.30 & 2.77070 \mathrm{E}-04 \\ 18.40 & 2.80106 \mathrm{E}-04 \\ 18.50 & 2.83159 \mathrm{E}-04 \\ & \\ 18.60 & 2.86229 \mathrm{E}-04 \\ 18.70 & 2.89315 \mathrm{E}-04 \\ 18.80 & 2.92417 \mathrm{E}-04 \\ 18.90 & 2.95536 \mathrm{E}-04 \\ 19.00 & 2.98672 \mathrm{E}-04\end{array}$

$19.10-3.01824 \mathrm{E}-04$

$19.20 \quad 3.04992 \mathrm{E}-04$

$19.30 \quad 3.08177 \mathrm{E}-04$

$19.40 \quad 3.11379 \mathrm{E}-04$

$19.50 \quad 3.14598 \mathrm{E}-04$

$19.60 \quad 3.17832 \mathrm{E}-04$

$19.70 \quad 3.21084 \mathrm{E}-04$

$19.80 \quad 3.24352 \mathrm{E}-04$

$19.90 \quad 3.27636 \mathrm{E}-04$

$20.00 \quad 3.30937 \mathrm{E}-04$

20.10

20.20

20.30

20.40

20.50

20.60

20.70

20.80

20.90

21.00

21.10

21.20

21.30

21.40

21.50

21.60

21.70

21.80

21.90

22.00

22.10

22.20

22.30

22.40

22.50

22.60

22.70

22.80

22.90

23.00

23.10

23.20

23.30

23.40

23.50

23.60

23.70

23.80

23.90

24.00

$3.34255 \mathrm{E}-04$ $3.37589 \mathrm{E}-04$ $3.40939 \mathrm{E}-04$ $3.44306 \mathrm{E}-04$ $3.47690 \mathrm{E}-04$

$3.51090 \mathrm{E}-04$

$3.54507 \mathrm{E}-04$ $3.57940 \mathrm{E}-04$ $3.61390 \mathrm{E}-04$ $3.64857 \mathrm{E}-04$

$3.68340 \mathrm{E}-04$ $3.71839 \mathrm{E}-04$ $3.75355 \mathrm{E}-04$ $3.78888 \mathrm{E}-04$ $3.82437 \mathrm{E}-04$

$3.86003 \mathrm{E}-04$ $3.89585 \mathrm{E}-04$ 3.93183 E-04 $3.96799 \mathrm{E}-04$ 4.00431 E-04

$4.04079 \mathrm{E}-04$ $4.07744 \mathrm{E}-04$ $4.11425 \mathrm{E}-04$ $4.15123 \mathrm{E}-04$ $4.18838 \mathrm{E}-04$

$4.22569 \mathrm{E}-04$ $4.26317 \mathrm{E}-04$ $4.30081 \mathrm{E}-04$ $4.33861 \mathrm{E}-04$ $4.37659 \mathrm{E}-04$

$4.41472 \mathrm{E}-04$ $4.45303 \mathrm{E}-04$ $4.49150 \mathrm{E}-04$ $4.53013 \mathrm{E}-04$ $4.56893 \mathrm{E}-04$

$4.60790 \mathrm{E}-04$ $4.64703 \mathrm{E}-04$ $4.68632 \mathrm{E}-04$ $4.72578 \mathrm{E}-04$ $4.76541 \mathrm{E}-04$ $f_{2}$

$5.79388 \mathrm{E}-01$ $5.85898 \mathrm{E}-01$ $5.92443 \mathrm{E}-01$ $5.99025 \mathrm{E}-01$ $6.05643 \mathrm{E}-0]$

$6.12298 \mathrm{E}-01$ $6.18989 \mathrm{E}-01$ $6.25716 \mathrm{E}-01$ $6.32479 \mathrm{E}-01$ $6.39279 \mathrm{E}-01$

$6.46114 \mathrm{E}-01$ $6.52987 \mathrm{E}-01$ $6.59895 \mathrm{E}-01$ $6.66839 \mathrm{E}-01$ $6.73820 \mathrm{E}-01$

$6.80837 \mathrm{E}-01$ $6.87890 \mathrm{E}-01$ $6.94980 \mathrm{E}-01$ $7.02106 \mathrm{E}-01$ $7.09268 \mathrm{E}-01$

$7.16466 \mathrm{E}-01$ $7.23700 \mathrm{E}-01$ $7.30971 \mathrm{E}-01$ $7.38278 \mathrm{E}-01$

7.45621 E-01

$7.53000 \mathrm{E}-01$

$7.60416 \mathrm{E}-0]$

$7.67868 \mathrm{E}-01$

$7.75356 \mathrm{E}-01$

$7.82880 \mathrm{E}-01$

$7.90440 \mathrm{E}-01$

$7.98037 \mathrm{E}-01$

$8.05670 \mathrm{E}-01$

$8.13339 \mathrm{E}-01$

$8.21044 \mathrm{E}-01$

$8.28785 \mathrm{E}-01$

$8.36563 \mathrm{E}-01$

$8.44376 \mathrm{E}-01$

$8.52226 \mathrm{E}-01$

8.60113E-01

$8.68035 \mathrm{E}-01$

$8.75993 \mathrm{E}-01$

8.83988E-01

$8.92019 \mathrm{E}-01$

$9.00086 \mathrm{E}-01$

$9.08189 \mathrm{E}-01$

$9.16329 \mathrm{E}-01$

$9.24504 \mathrm{E}-01$

$9.32716 \mathrm{E}-01$

$9.40964 \mathrm{E}-01$

$9.49248 \mathrm{E}-01$ $9.65924 \mathrm{E}-01$ $9.74317 \mathrm{E}-01$ $9.82746 \mathrm{E}-01$

$9.91211 \mathrm{E}-01$ $9.99712 \mathrm{E}-01$ $1.00825 \mathrm{E} \quad 00$ $g_{2}$

$9.61832 \mathrm{E}-03$ $9.79249 \mathrm{E}-03$ $9.96874 \mathrm{E}-03$ $1.01471 \mathrm{E}-02$ $1.03275 \mathrm{E}-02$

$1.05101 \mathrm{E}-02$ $1.06948 \mathrm{E}-02$ $1.08816 \mathrm{E}-02$

$1.10706 \mathrm{E}-02$

$1.12618 \mathrm{E}-02$

1.14551 E-02 $1.16506 \mathrm{E}-02$ $1.18483 \mathrm{E}-02$ $1.20483 \mathrm{E}-02$ $1.22505 \mathrm{E}-02$

$1.24549 \mathrm{E}-02$ $1.26615 \mathrm{E}-02$ $1.28705 \mathrm{E}-02$ $1.30817 \mathrm{E}-02$ $1.32952 \mathrm{E}-02$ $9.57568 \mathrm{E}-01$ $1.01682 \mathrm{E} \quad 00$ $1.02543 \mathrm{E} \quad 00$

$1.35110 \mathrm{E}-02$ $1.37291 \mathrm{E}-02$

$1.39495 \mathrm{E}-02$

$1.43974 \mathrm{E}-02$

$1.46249 \mathrm{E}-02$

$1.48548 \mathrm{E}-02$

$1.50871 \mathrm{E}-02$

$1.53217 \mathrm{E}-02$

$1.55588 \mathrm{E}-02$

$1.57983 \mathrm{E}-02$ $1.60402 \mathrm{E}-02$

$1.62846 \mathrm{E}-02$

$1.65314 \mathrm{E}-02$

$1.67807 \mathrm{E}-02$

$1.70325 \mathrm{E}-02$ $1.72868 \mathrm{E}-02$

$1.75436 \mathrm{E}-02$

$1.78029 \mathrm{E}-02$

$1.80648 \mathrm{E}-02$

$1.83292 \mathrm{E}-02$

$1.85962 \mathrm{E}-02$

$1.88657 \mathrm{E}-02$

$1.91378 \mathrm{E}-02$

$1.94126 \mathrm{E}-02$

$1.96899 \mathrm{E}-02$ $1.99698 \mathrm{E}-02$ $2.02524 \mathrm{E}-02$ $2.05376 \mathrm{E}-02$ $2.08255 \mathrm{E}-02$

$2.11161 \mathrm{E}-02$ $2.14093 \mathrm{E}-02$ $2.17052 \mathrm{E}-02$ $2.20039 \mathrm{E}-02$ $2.23052 \mathrm{E}-02$

$2.26093 \mathrm{E}-02$ $2.29161 \mathrm{E}-02$ $2.32257 \mathrm{E}-02$ $2.35380 \mathrm{E}-02$ $2.38532 \mathrm{E}-02$ $\tan \delta_{2}$

$f_{-2}$

$6-2$

$-6.55934 \mathrm{E}$ 01 $-8.77969 \mathrm{E}-03$ $-6.56016 \mathrm{E} \quad 01 \quad-8.91546 \mathrm{E}-03$ $-6.56097 \mathrm{E}$ 01 $-9.11328 \mathrm{E}-03$ $-6.56177 \mathrm{E}$ 01 $-9.28315 \mathrm{E}-03$ $-6.56257 \mathrm{E} 01 \quad-9.45508 \mathrm{E}-03$

$-6.56335 \mathrm{E} 01$ $-6.56413 \mathrm{E} 01$ $-6.56489 \mathrm{E} 01$ $-6.56565 \mathrm{E} 01$ $-6.56640 \mathrm{E} 01$

$-9.62909 \mathrm{E}-03$ $-9.80519 \mathrm{E}-03$ $-9.98339 \mathrm{E}-03$ $-1.01637 \mathrm{E}-02$ $-6.56715 \mathrm{E} \quad 01 \quad-1.05307 \mathrm{E}-02$ $-6.56788 \mathrm{E} \quad 01 \quad-1.07174 \mathrm{E}-02$ $-6.56861 \mathrm{E} \quad 01 \quad-1.09063 \mathrm{E}-02$ $-6.56933 \mathrm{E}$ 01 $-1.10974 \mathrm{E}-02$ $-6.57004 \mathrm{E}$ 01 $-1.12906 \mathrm{E}-02$

$-6.57074 \mathrm{E} \quad 01 \quad-1.14861 \mathrm{E}-02$ $-6.57144 \mathrm{E}$ 01 $-1.16837 \mathrm{E}-02$ $-6.57212 \mathrm{E}$ 01 $-1.18836 \mathrm{E}-02$ $-6.57281 \mathrm{E}$ 01 $-1.20857 \mathrm{E}-02$ $-6.57348 \mathrm{E} \quad 01 \quad-1.22901 \mathrm{E}-02$

$1.41723 \mathrm{E}-02$

$-6.57415 \mathrm{E} \quad 01$ $-6.57481 \mathrm{E} 01$ $-6.57546 \mathrm{E} 01$ $-6.57611 \mathrm{E} 01$ $-6.57675 \mathrm{E} \quad 01$

$-6.57738 \mathrm{E} \quad 01$ $-6.57801 \mathrm{E} 01$ $-6.57863 \mathrm{E} 01$ $-6.57925 \mathrm{E} 01$ $-6.57986 \mathrm{E} 01$

$-6.58046 \mathrm{E} 0 \mathrm{l}$ $-6.58106 \mathrm{E} 01$ $-6.58165 \mathrm{E} 01$ $-6.58223 \mathrm{E}$ 0l -6.58281 E 01

$-6.58339 \mathrm{E} \quad 01$ $-6.58396 \mathrm{E} \quad 01$ $-6.58452 \mathrm{E}$ ol $-6.58508 \mathrm{E} 01$ $-6.58563 \mathrm{E} 01$

$-1.24967 \mathrm{E}-02$ $-1.27056 \mathrm{E}-02$ $-1.29168 \mathrm{E}-02$ $-1.31303 \mathbf{E}-02$ $-1.33461 \mathrm{E}-02$

$-1.35642 \mathrm{E}-02$ $-1.37847 \mathrm{E}-02$ $-1.40075 \mathrm{E}-02$ $-1.42326 \mathrm{E}-02$ $-1.44601 \mathrm{E}-02$

$-1.46901 \mathrm{E}-02$ $-1.49224 E-02$ $-1.51571 \mathrm{E}-02$ $-1.53942 \mathrm{E}-02$ $-1.56338 \mathrm{E}-02$

$-1.58758 \mathrm{E}-02$ $-1.61202 \mathrm{E}-02$ $-1.63672 \mathrm{E}-02$ $-1.66166 \mathrm{E}-02$ $-1.68685 \mathrm{E}-02$

$-6.58618 \mathrm{E}$ 01 $-6.58672 \mathrm{E}$ 01 $-6.58726 \mathrm{E} 01$ $-6.58780 \mathrm{E} 01$ $-6.58832 \mathrm{E} 01$

$-1.71229 \mathrm{E}-02$ $-1.73798 \mathrm{E}-02$ 
ELECTRON RADIAL FUNCTIONS

Positron

$\begin{array}{cc}p & F_{0} \\ 24.10 & 9.34408 \mathrm{E}-01 \\ 24.20 & 9.34407 \mathrm{E}-01 \\ 24.30 & 9.34406 \mathrm{E}-01 \\ 24.40 & 9.34404 \mathrm{E}-01 \\ 24.50 & 9.34403 \mathrm{E}-01\end{array}$

24.60

24.70

24.80

24.90

25.00

25.10

25.20

25.30

25.40

25.50

25.60

25.70

25.80

25.90

26.00

26.10

26.20

26.30

26.40

26.50

26.60

26.70

26.80

26.90

27.00

27.10

27.20

27.30

27.40

27.50

27.60

27.70

27.80

27.90

28.00

28.10

28.20

28.30

28.40

28.50

28.60

28.70

28.80

28.90

29.00

29.10

29.20

29.30

29.40

29.50

29.60

29.70

29.80

29.90

30.00

$9.34401 \mathrm{E}-01$ $9.34400 \mathrm{E}-01$ $9.34399 \mathrm{E}-01$ $9.34397 \mathrm{E}-01$ $9.34396 \mathrm{E}-01$

9.34395E-01 $9.34393 \mathrm{E}-01$ 9.34392E-01 $9.34390 \mathrm{E}-01$ $9.34389 \mathrm{E}-01$

$9.34388 \mathrm{E}-01$ $9.34386 \mathrm{E}-01$ $9.34385 \mathrm{E}-01$ $9.34384 \mathrm{E}-01$ $9.34382 \mathrm{E}-01$

$9.34381 \mathrm{E}-01$ $9.34380 \mathrm{E}-01$ $9.34378 \mathrm{E}-01$ $9.34377 \mathrm{E}-01$ $9.34376 \mathrm{E}-01$

9.34374 E-01 $9.34373 \mathrm{E}-01$ $9.34372 \mathrm{E}-01$ $9.34371 \mathrm{E}-01$ $9.34369 \mathrm{E}-01$

$9.34368 \mathrm{E}-01$ $9.34367 \mathrm{E}-01$ $9.34365 \mathrm{E}-01$ $9.34364 \mathrm{E}-01$ $9.34363 \mathrm{E}-01$

$9.34362 \mathrm{E}-01$ $9.34360 \mathrm{E}-01$ $9.34359 \mathrm{E}-01$ $9.34358 \mathrm{E}-01$ $9.34356 \mathrm{E}-01$ 9.34354 E-01 $9.34352 \mathrm{E}-01$ $9.34351 \mathrm{E}-01$ $9.34350 \mathrm{E}-01$

$9.34348 \mathrm{E}-01$ $9.34347 \mathrm{E}-01$ $9.34346 \mathrm{E}-01$ $9.34345 \mathrm{E}-01$ $9.34343 \mathrm{E}-01$

$9.34342 \mathrm{E}-01$ 9.34341 E-01 $9.34340 \mathrm{E}-0 \mathrm{I}$ $9.34338 \mathrm{E}-01$ $9.34337 \mathrm{E}-01$

9.34336 E- 01 9.34335 E-01 9.34333 E-01 9.34332 E-01 $9.34331 \mathrm{E}-01$

$Z=3 \quad A=6$

$$
\rho=1.2 A^{1 / 3} F
$$

$f_{1}$

$2.29633 \mathrm{E} \quad 01$ $2.30617 \mathrm{E} \quad 01$ $2.31601 \mathrm{E} 01$ $2.32586 \mathrm{E} 01$ $2.33570 \mathrm{E} \quad 01$

$9.34355 \mathrm{E}-01$
$2.34555 \mathrm{E} \quad 01$

$2.35539 \mathrm{E} 01$

$2.36524 \mathrm{E} \quad 01$

$2.37509 \mathrm{E} \quad 01$

$2.38494 \mathrm{E} \quad 01$

$2.39479 \mathrm{E} \quad 01$ $2.40464 \mathrm{E} \quad 01$

$2.41450 \mathrm{E} \quad 01$

$2.42435 \mathrm{E} 01$

$2.43421 \mathrm{E} \quad 01$

$2.44407 \mathrm{E} \quad 01$

$2.45393 \mathrm{E} \quad 01$

$2.46379 \mathrm{E} \quad 01$

$2.47365 \mathrm{E} \quad 01$

$2.48351 \mathrm{E} \quad 01$

$2.49338 \mathrm{E} \quad 01$ $2.50324 \mathrm{E} \quad 01$

$2.51311 \mathrm{E} \quad 01$

$2.52298 \mathrm{E} \quad 01$

$2.53285 \mathrm{E} 01$

$2.54272 \mathrm{E} \quad 01$ $2.55259 \mathrm{E} \quad 01$ $2.56247 \mathrm{E} \quad 01$ $2.57234 \mathrm{E} \quad 01$ $2.58222 \mathrm{E} \quad 01$

$2.59210 \mathrm{E} \quad 01$ $2.60198 \mathrm{E} 01$ $2.61186 \mathrm{E} \quad 01$ $2.62174 \mathrm{E} \quad 01$ $2.63163 \mathrm{E} \quad 01$

$2.64151 \mathrm{E} \quad 01$ $2.66129 \mathrm{E} \quad 01$ $2.67118 \mathrm{E} \quad 01$ $2.68107 \mathrm{E} \quad 01$

$2.69096 \mathrm{E} \quad 01$ $2.70086 \mathrm{E} \quad 01$ $2.71075 \mathrm{E} \quad 01$ $2.72065 \mathrm{E} \quad 01$ $2.73055 \mathrm{E} \quad 01$

$2.74045 \mathrm{E} \quad 01$ $2.75035 \mathrm{E} \quad 01$ $2.76025 \mathrm{E} \quad 01$ $2.77015 \mathrm{E} \quad 01$ $2.78006 \mathrm{E} \quad 01$

$2.78997 \mathrm{E} \quad 01$ $2.79988 \mathrm{E} \quad 01$ $2.80979 \mathrm{E} \quad 01$ $2.81970 \mathrm{E} \quad 01$ $2.82961 \mathrm{E} \quad 01$

$2.83953 \mathrm{E} \quad 01$ $2.84944 \mathrm{E} \quad 01$ $2.85936 \mathrm{E} \quad 01$ $2.86928 \mathrm{E} \quad 01$ $2.87920 \mathrm{E} \quad 01$ $2.65140 \mathrm{E} \quad 01$ $g_{1}$ $8.85410 \mathrm{E}-01$
$8.93551 \mathrm{E}-01$
$9.01731 \mathrm{E}-01$
$9.09949 \mathrm{E}-01$
$9.18204 \mathrm{E}-01$

$9.26497 \mathrm{E}-01$

9.34828E-01

9.43196E-01

9.51603E-01

$9.60047 \mathrm{E}-01$

$9.68529 \mathrm{E}-01$

$9.77048 \mathrm{E}-01$

$9.85606 \mathrm{E}-01$

9.94201 E-01

$1.00283 \mathrm{E} \quad 00$

$1.01151 \mathrm{E} \quad 00$

$1.02022 \mathrm{E} \quad 00$

$1.02896 \mathrm{E} \quad 00$

$1.03775 \mathrm{E} \quad 00$

$1.04657 \mathrm{E} \quad 00$

$1.05543 \mathrm{E} \quad 00$

$1.06433 \mathrm{E} \quad 00$

$1.07327 \mathrm{E} \quad 00$

$1.08224 \mathrm{E} \quad 00$

$1.09125 \mathrm{E} \quad 00$

$1.10030 \mathrm{E} \quad 00$ $1.10939 \mathrm{E} \quad 00$

$1.11852 \mathrm{E} \quad 00$

$1.12768 \mathrm{E} \quad 00$

$1.13689 \mathrm{E} \quad 00$

$1.14613 \mathrm{E} \quad 00$

$1.15541 \mathrm{E} \quad 00$

$1.16472 \mathrm{E} \quad 00$

$1.17408 \mathrm{E} \quad 00$

$1.18347 \mathrm{E} \quad 00$

$1.19290 \mathrm{E} \quad 00$

$1.20237 \mathrm{E} \quad 00$

$1.21188 \mathrm{E} \quad 00$

$1.22143 \mathrm{E} \quad 00$

$1.23101 \mathrm{E} \quad 00$

$1.24063 \mathrm{E} \quad 00$

$1.25029 \mathrm{E} \quad 00$

$1.25999 \mathrm{E} \quad 00$

$1.26973 \mathrm{E} \quad 00$

$1.27950 \mathrm{E} \quad 00$

$1.28932 \mathrm{E} \quad 00$

$1.29917 \mathrm{E} \quad 00$

$1.30906 \mathrm{E} \quad 00$

$1.31898 \mathrm{E} \quad 00$

$1.32895 \mathrm{E} \quad 00$

$1.33896 \mathrm{E} \quad 00$ $1.34900 \mathrm{E} \quad 00$ $1.35908 \mathrm{E} \quad 00$

$1.36920 \mathrm{E} \quad 00$

$1.37936 \mathrm{E} \quad 00$

$1.38955 \mathrm{E} \quad 00$ $1.39979 \mathrm{E} \quad 00$

$1.41006 \mathrm{E} \quad 00$

$1.42037 \mathrm{E} \quad 00$

$1.43072 \mathrm{E} \quad 00$

$\tan \delta_{1}$

$-8.26677 \mathrm{E}-04$

$-8.28234 \mathrm{E}-04$

$-8.29775 \mathrm{E}-04$

$-8.31297 \mathrm{E}-04$

-8.32801 E-04

$-8.34292 \mathrm{E}-04$

$-8.35766 \mathrm{E}-04$

$-8.37224 \mathrm{E}-04$

$-8.38667 \mathrm{E}-04$

$-8.40094 \mathrm{E}-04$

$-8.41509 E-04$

$-8.42906 \mathrm{E}-04$

$-8.44288 \mathrm{E}-04$

$-8.45656 \mathrm{E}-04$

$-8.47009 \mathrm{E}-04$

$-8.48349 E-04$

$-8.49673 \mathrm{E}-04$

$-8.50983 \mathrm{E}-04$

$-8.52279 \mathrm{E}-04$

$-8.53563 \mathrm{E}-04$

$-8.54834 \mathrm{E}-04$

$-8.56088 \mathrm{E}-04$

$-8.57333 \mathrm{E}-04$

$-8.58559 \mathrm{E}-04$

$-8.59777 \mathrm{E}-04$

$-8.60978 \mathrm{E}-04$

$-8.62169 \mathrm{E}-04$

$-8.63346 \mathrm{E}-04$

$-8.64512 \mathrm{E}-04$

$-8.65665 \mathrm{E}-04$

$-8.66803 \mathrm{E}-04$

$-8.67930 \mathrm{E}-04$

$-8.69048 \mathrm{E}-04$

$-8.70151 \mathrm{E}-04$

$-8.71243 \mathrm{E}-04$

$-8.72324 \mathrm{E}-04$

$-8.73391 \mathrm{E}-04$

$-8.74451 \mathrm{E}-04$

$-8.75495 \mathrm{E}-04$

$-8.76530 \mathrm{E}-04$

$-8.77555 \mathrm{E}-04$

$-8.78567 \mathrm{E}-04$

$-8.79566 \mathrm{E}-04$

$-8.80557 \mathrm{E}-04$

$-8.81537 \mathrm{E}-04$

$-8.82505 \mathrm{E}-04$

$-8.83463 \mathrm{E}-04$

$-8.84409 \mathrm{E}-04$

$-8.85347 \mathrm{E}-04$

$-8.86275 \mathrm{E}-04$

$-8.87192 \mathrm{E}-04$ $-8.88099 \mathrm{E}-04$

$-8.88997 \mathrm{E}-04$

$-8.89884 \mathrm{E}-04$ 


\section{$\bar{F}$}

$4.80520 \mathrm{E}-04$ $4.84516 \mathrm{E}-04$ $4.88528 \mathrm{E}-04$ $4.92557 \mathrm{E}-04$ $+.96603 \mathrm{E}-04$

$5.00665 \mathrm{E}-04$ $5.04743 \mathrm{E}-04$ $5.08838 \mathrm{E}-0.4$ $5.12950 \mathrm{E}-04$ $5.17078 \mathrm{E}-04$

$5.21222 \mathrm{E}-04$ $5.25383 \mathrm{E}-04$ $5.29561 \mathrm{E}-04$ $5.33755 \mathrm{E}-04$ $5.37966 \mathrm{E}-04$

$5.42193 \mathrm{E}-04$ $5.46437 \mathrm{E}-04$ $5.50698 \mathrm{E}-04$ $5.54975 \mathrm{E}-04$ $5.59268 \mathrm{E}-04$

$5.63578 \mathrm{E}-04$ $5.67905 \mathrm{E}-04$ $5.72248 \mathrm{E}-04$ $5.76608 \mathrm{E}-04$ $5.80984 \mathrm{E}-04$

$5.85377 \mathrm{E}-04$

$5.89786 \mathrm{E}-04$

$5.94212 \mathrm{E}-04$

$5.98654 \mathrm{E}-04$

$6.03113 \mathrm{E}-04$

$6.07589 \mathrm{E}-04$

$6.12081 \mathrm{E}-04$

$6.16589 \mathrm{E}-04$

$6.21114 \mathrm{E}-04$

$6.25656 \mathrm{E}-04$

$6.30214 \mathrm{E}-04$ $6.34789 \mathrm{E}-04$ $6.39380 \mathrm{E}-04$ $6.43988 \mathrm{E}-04$ $6.48612 \mathrm{E}-04$

$6.53253 \mathrm{E}-04$ $6.57910 \mathrm{E}-04$ $6.62584 \mathrm{E}-04$ $6.67275 \mathrm{E}-04$ $6.71982 \mathrm{E}-04$

$6.76705 \mathrm{E}-04$ $6.81445 \mathrm{E}-04$ $6.86202 \mathrm{E}-04$ $6.90975 \mathrm{E}-04$ $6.95765 \mathrm{E}-04$

$7.00571 \mathrm{E}-04$ $7.05394 \mathrm{E}-04$ $7.10233 \mathrm{E}-04$ $7.15089 \mathrm{E}-04$ $7.19962 \mathrm{E}-04$

7.24851 E- 04 $7.29756 \mathrm{E}-04$ $7.34678 \mathrm{E}-04$ $7.39617 \mathrm{E}-04$ $7.44572 \mathrm{E}-04$ $f_{2}$

$1.03408 \mathrm{E} \quad 00$ $1.0+276 \mathrm{E} \quad 00$ $1.05148 \mathrm{E} \quad 00$ $1.06023 \mathrm{E} \quad 00$ $1.06902 \mathrm{E} \quad 00$

$1.07785 \mathrm{E} \quad 00$ $1.08671 \mathrm{E} \quad 00$ $1.09561 \mathrm{E} \quad 00$ $1.10454 \mathrm{E} \quad 00$ $1.11351 \mathrm{E} \quad 00$

$1.12252 \mathrm{E} \quad 00$ $1.13156 \mathrm{E} \quad 00$ $1.14064 \mathrm{E} \quad 00$ $1.14976 \mathrm{E} \quad 00$ $1.15891 \mathrm{E} \quad 00$

$1.16809 \mathrm{E} \quad 00$ $1.17732 \mathrm{E} \quad 00$ $1.18658 \mathrm{E} \quad 00$ $1.19587 \mathrm{E} \quad 00$ $1.20520 \mathrm{E} \quad 00$

$1.21457 \mathrm{E} \quad 00$ $1.22398 \mathrm{E} \quad 00$ $1.23342 \mathrm{E} \quad 00$ $1.24289 \mathrm{E} \quad 00$ $1.25240 \mathrm{E} \quad 00$

$1.26195 \mathrm{E} \quad 00$

$1.27154 \mathrm{E} \quad 00$

$1.28116 \mathrm{E} \quad 00$

$1.29081 \mathrm{E} \quad 00$

$1.30050 \mathrm{E} \quad 00$

$1.31023 \mathrm{E} \quad 00$ $1.32000 \mathrm{E} \quad 00$ $1.32980 \mathrm{E} \quad 00$ $1.33963 \mathrm{E} \quad 00$ $1.34950 \mathrm{E} \quad 00$

$1.35941 \mathrm{E} \quad 00$ $1.36936 \mathrm{E} \quad 00$ $1.37934 \mathrm{E} \quad 00$ $1.38936 \mathrm{E} \quad 00$ $1.39941 \mathrm{E} \quad 00$

$1.40950 \mathrm{E} \quad 00$ $1.41962 \mathrm{E} \quad 00$ $1.42978 \mathrm{E} \quad 00$ $1.43998 \mathrm{E} \quad 00$ $1.45021 \mathrm{E} \quad 00$

$1.46048 \mathrm{E} \quad 00$ $1.47078 \mathrm{E} \quad 00$ $1.48112 \mathrm{E} \quad 00$ $1.49150 \mathrm{E} \quad 00$ $1.50191 \mathrm{E} \quad 00$

$1.51236 \mathrm{E} \quad 00$ $1.52285 \mathrm{E} \quad 00$ $1.53337 \mathrm{E} \quad 00$ $1.54392 \mathrm{E} \quad 00$ $1.55452 \mathrm{E} \quad 00$

$1.56514 \mathrm{E} \quad 00$ $1.57581 \mathrm{E} \quad 00$ $1.58651 \mathrm{E} \quad 00$ $1.59725 \mathrm{E} \quad 00$ $1.60802 \mathrm{E} \quad 00$ $g_{2}$

$\tan \delta_{2}$

$f_{-2}$

$2.41711 \mathrm{E}-02$ $2.44918 \mathrm{E}-02$

$2.48154 \mathrm{E}-02$

$2.51417 \mathrm{E}-02$

$2.54709 \mathrm{E}-02$

$2.58030 \mathrm{E}-02$

$2.61379 \mathrm{E}-02$

$2.6+757 \mathrm{E}-02$

$2.68164 \mathrm{E}-02$

$2.71600 \mathrm{E}-02$

$2.75065 \mathrm{E}-02$

$2.78559 \mathrm{E}-02$

$2.82083 \mathrm{E}-02$

$2.85636 \mathrm{E}-02$

$2.89219 \mathrm{E}-02$

$2.92832 \mathrm{E}-02$

$2.96474 \mathrm{E}-02$

$3.00147 \mathrm{E}-02$

$3.03849 \mathrm{E}-02$

$3.07582 \mathrm{E}-02$

$3.11345 \mathrm{E}-02$

$3.15139 \mathrm{E}-02$

$3.18963 \mathrm{E}-02$

$3.22818 \mathrm{E}-02$

$3.26704 \mathrm{E}-02$

$3.30621 \mathrm{E}-02$

$3.34569 \mathrm{E}-02$

$3.38548 \mathrm{E}-02$

$3.42559 \mathrm{E}-02$

$3.46600 \mathrm{E}-02$

$3.50674 \mathrm{E}-02$ $3.54779 \mathrm{E}-02$

$3.58916 \mathrm{E}-02$

$3.63085 \mathrm{E}-02$

$3.67286 \mathrm{E}-02$

$3.71519 \mathrm{E}-02$ $3.75785 \mathrm{E}-02$ $3.80083 \mathrm{E}-02$ $3.84414 \mathrm{E}-02$ $3.88777 \mathrm{E}-02$

$3.93173 \mathrm{E}-02$ $3.97602 \mathrm{E}-02$ $4.02064 \mathrm{E}-62$ $4.06559 \mathrm{E}-02$ $4.11087 \mathrm{E}-02$

$4.15649 \mathrm{E}-02$ $4.20245 \mathrm{E}-02$ $4.24874 \mathrm{E}-02$ $4.29537 \mathrm{E}-02$ $4.34233 \mathrm{E}-02$

$4.38964 \mathrm{E}-02$ $4.43729 \mathrm{E}-02$ $4.48528 \mathrm{E}-02$ $4.53362 \mathrm{E}-02$ $4.58230 \mathrm{E}-02$

$4.63132 \mathrm{E}-02$ $4.68070 \mathrm{E}-02$ $4.73042 \mathrm{E}-02$ $4.78049 \mathrm{E}-02$ $4.83092 \mathrm{E}-02$
$-6.59615 \mathrm{E} 0 \mathrm{I}$ $-6.59661 \mathrm{E} 01$ $-6.59706 \mathrm{E} 01$ $-6.59750 \mathrm{E} 01$ $-6.59795 \mathrm{E} 01$

$-6.59838 \mathrm{E} \quad 01$ $-6.59882 \mathrm{E} 0 \mathrm{I}$ $-6.59925 \mathrm{E} 01$ $-6.59968 \mathrm{E} 01$ $-6.60010 \mathrm{E} 01$

$-6.60052 \mathrm{E} \quad 01$ $-6.60094 \mathrm{E} 01$ $-6.60135 \mathrm{E} 01$

$-6.60176 \mathrm{E} 01$

$-6.60217 \mathrm{E} 01$

$-6.60258 \mathrm{E} 01$

$-6.60298 \mathrm{E} 01$

$-6.60337 \mathrm{E} 01$

$-6.60377 \mathrm{E} 01$

$-6.60416 \mathrm{E} 01$

$-6.60455 \mathrm{E} \quad 01$

$-6.60493 \mathrm{E} 01$

$-6.60531 \mathrm{E} 01$

$-6.60569 \mathrm{E} \quad 01$

$-6.60607 \mathrm{E} 01$

$-6.60644 \mathrm{E} \quad 01$

$-6.60681 \mathrm{E} 01$

$-6.60718 \mathrm{E} 01$

$-6.60755 \mathrm{E} 01$

$-6.60791 \mathrm{E} 01$

$-6.60827 \mathrm{E} \quad 01$

$-6.60862 \mathrm{E} 01$

$-6.60898 \mathrm{E} 01$

$-6.60933 \mathrm{E} 01$

$-6.60967 \mathrm{E} \quad 01$

$-6.61002 \mathrm{E} 01$ $-6.61036 \mathrm{E} 01$

$-6.61070 \mathrm{E} 01$

$-6.61104 \mathrm{E} 01$

$-6.61138 \mathrm{E} 01$

$-6.61171 \mathrm{E} 01$

$-6.61204 \mathrm{E} 01$

$-6.61237 \mathrm{E} \quad 01$

$-6.61270 \mathrm{E} 01$

$-6.61302 \mathrm{E} \quad 01$

$-6.61334 \mathrm{E} \quad 01$

$-6.61366 \mathrm{E} 01$

$-6.61398 \mathrm{E} 01$

$-6.61429 \mathrm{E} 01$

$-6.61460 \mathrm{E} 01$

$-6.61491 \mathrm{E} \quad 01$

$-6.61522 \mathrm{E} 01$

$-6.61553 \mathrm{E} 01$

$-6.61583 \mathrm{E} \quad 01$

-6.61613 E 01

$-6.61643 \mathrm{E} \quad 01$

$-6.61673 \mathrm{E} 01$

$-6.61702 \mathrm{E} 01$

$-6.61732 \mathrm{E} \quad 01$

$-6.61761 \mathrm{E} 01$

$-2.27563 \mathrm{E}-02$ $-2.30661 \mathrm{E}-02$ $-2.33788 \mathrm{E}-02$ $-2.36942 \mathbf{E}-02$ $-2.40124 \mathrm{E}-02$

$-2.43335 \mathrm{E}-02$ $-2.46573 \mathrm{E}-02$ $-2.49840 \mathrm{E}-02$ $-2.53136 \mathrm{E}-02$ $-2.56460 \mathrm{E}-02$

$-2.59812 \mathrm{E}-02$ $-2.63194 \mathrm{E}-02$ $-2.66605 \mathrm{E}-02$ $-2.70044 \mathrm{E}-02$ $-2.73513 \mathrm{E}-02$

$-2.77012 \mathrm{E}-02$ $-2.80539 \mathrm{E}-02$ $-2.84097 \mathrm{E}-02$ $-2.87684 \mathrm{E}-02$

$-2.91301 \mathrm{E}-02$

$-2.94947 \mathrm{E}-02$ $-2.98624 \mathrm{E}-02$ $-3.02331 \mathrm{E}-02$ $-3.06069 \mathrm{E}-02$ $-3.09836 \mathrm{E}-02$

$-3.13635 \mathrm{E}-02$

$-3.17464 \mathrm{E}-02$

$-3.21324 \mathrm{E}-02$

$-3.25215 \mathrm{E}-02$

$-3.29137 \mathrm{E}-02$

$-3.33090 \mathrm{E}-02$ $-3.37074 \mathrm{E}-02$

$-3.41090 \mathrm{E}-02$

$-3.45137 \mathrm{E}-02$

$-3.49216 \mathrm{E}-02$

$-3.53326 \mathrm{E}-02$ $-3.57469 \mathrm{E}-02$

$-3.61644 \mathrm{E}-02$

$-3.65850 \mathrm{E}-02$

$-3.70090 \mathrm{E}-02$

$-3.74361 \mathrm{E}-02$ 
ELECTRON RADIAL FUNCTIONS

Positron

$\begin{array}{cc}p & F_{0} \\ 30.10 & 9.34330 \mathrm{E}-01 \\ 30.20 & 9.34328 \mathrm{E}-01 \\ 30.30 & 9.34327 \mathrm{E}-01 \\ 30.40 & 9.34326 \mathrm{E}-01 \\ 30.50 & 9.34325 \mathrm{E}-01 \\ & \\ 30.60 & 9.34323 \mathrm{E}-01 \\ 30.70 & 9.34322 \mathrm{E}-01 \\ 30.80 & 9.34321 \mathrm{E}-01 \\ 30.90 & 9.34320 \mathrm{E}-01 \\ 31.00 & 9.34318 \mathrm{E}-01\end{array}$

31.10

31.20

31.30

31.40

31.50

31.60

31.70

31.80

31.90

32.00

32.10

32.20

32.30

32.40

32.50

32.60

32.70

32.80

32.90

33.00

33.10

33.20

33.30

33.40

33.50

33.60

33.70

33.80

33.90

34.00

34.80

34.20

34.30

34.40

34.50

34.60

34.70

34.80

34.90

35.00

35.10

35.20

35.30

35.40

35.50

35.60

35.70

35.80

35.90

36.00

$9.34317 \mathrm{E}-01$ $9.34316 \mathrm{E}-01$ $9.34315 \mathrm{E}-01$ $9.34313 \mathrm{E}-01$ $9.34312 \mathrm{E}-01$

$9.34311 \mathrm{E}-01$ $9.34309 \mathrm{E}-01$ $9.34307 \mathrm{E}-01$

$9.34306 \mathrm{E}-01$

9.34305E-01 9.34304E-01 $9.34303 \mathrm{E}-01$ $9.34301 \mathrm{E}-01$ $9.34300 \mathrm{E}-01$

9.34299E-01 $9.34298 \mathrm{E}-01$ $9.34297 \mathrm{E}-01$ $9.34296 \mathrm{E}-01$ $9.34294 \mathrm{E}-01$

9.34293E-01 $9.34292 \mathrm{E}-01$ 9.34291 E-01 $9.34290 \mathrm{E}-01$ $9.34289 \mathrm{E}-01$

9.34287 E-01 $9.34286 \mathrm{E}-01$ 9.34284 E-01 $9.34283 \mathrm{E}-01$

$9.34282 \mathrm{E}-01$ 9.34281 E-01 $9.34279 \mathrm{E}-01$ $9.34278 \mathrm{E}-01$ 9.34277E-01

9.34276E-01 9.34275E-01 $9.34274 \mathrm{E}-01$ $9.34273 \mathrm{E}-01$ 9.34271 E-01

$9.34270 \mathrm{E}-01$ 9.34.269E-01 9.34268 E-01 $9.34267 \mathrm{E}-01$ $9.34266 \mathrm{E}-01$

$9.34265 \mathrm{E}-01$ 9.34264 E-01 9.34263E-01 $9.34261 \mathrm{E}-01$ $9.34260 \mathrm{E}-01$ $f_{1}$

$2.88912 \mathrm{E} \quad 01$ $2.89905 \mathrm{E} \quad 01$ $2.90897 \mathrm{E} \quad 01$ $2.91890 \mathrm{E} \quad 01$ $2.92883 \mathrm{E} 01$

$2.93876 \mathrm{E} \quad 01$ $2.94869 \mathrm{E} \quad 01$ $2.95863 \mathrm{E} \quad 01$ $2.96856 \mathrm{E} \quad 01$ $2.97850 \mathrm{E} \quad 01$ 9.34310E-01 $9.34285 \mathrm{E}-01$

2.98843 E 01

$2.99837 \mathrm{E} \quad 01$

$\begin{array}{lll}3.00832 & \mathbf{E} & 01\end{array}$

$\begin{array}{lll}3.01826 \mathrm{E} & 01\end{array}$

$3.02820 \mathrm{E} \quad 01$

$3.03815 \mathrm{E} \quad 01$

$3.04810 \mathrm{E} 01$

$3.05805 \mathrm{E} \quad 01$

$3.06800 \mathrm{E} \quad 01$

$3.07795 \mathrm{E} \quad 01$

$3.08791 \mathrm{E} \quad 01$ $3.09786 \mathrm{E} \quad 01$ $3.10782 \mathrm{E} 01$ $3.11778 \mathrm{E} \quad 01$ $3.12774 \mathrm{E} \quad 01$

$3.13770 \mathrm{E} \quad 01$ $3.14767 \mathrm{E} \quad 01$ $3.15763 \mathrm{E} \quad 01$ $3.16760 \mathrm{E}$ ol $3.17757 \mathrm{E} \quad 01$

$3.18754 \mathrm{E} \quad 01$ $3.19751 \mathrm{E} \quad 01$ $3.20749 \mathrm{E} \quad 01$ $3.21746 \mathrm{E} \quad 01$ $3.22744 \mathrm{E} \quad 01$

$3.23742 \mathrm{E} 01$ $3.24740 \mathrm{E} \quad 01$ $3.25738 \mathrm{E}$ 01 $3.26737 \mathrm{E} \quad 01$ $3.27736 \mathrm{E} \quad 01$

$3.28734 \mathrm{E} \quad 01$ $3.29733 \mathrm{E} \quad 01$ $3.30733 \mathrm{E} 01$ $3.31732 \mathrm{E} 01$ $3.32731 \mathrm{E} \quad 01$

$3.33731 \mathrm{E} \quad 01$ $3.34731 \mathrm{E} \quad 01$ $3.35731 \mathrm{E} \quad 01$ $3.36731 \mathrm{E} \quad 01$ $3.37732 \mathrm{E} \quad 01$

$3.38732 \mathrm{E} \quad 01$ $3.39733 \mathrm{E} \quad 01$ $3.40734 \mathrm{E} \quad 01$ $3.41735 \mathrm{E} \quad 01$ $3.42737 \mathrm{E} \quad 01$

$3.43738 \mathrm{E} \quad 01$ $3.44740 \mathrm{E} \quad 01$ $3.46744 \mathrm{E}$ ol $3.47746 \mathrm{E} \quad 01$
$Z=3 \quad A=6$

$g_{1}$

$\tan \delta_{\mathrm{i}}$ $f_{-1}$

$1.44111 \mathrm{E} \quad 00 \quad-8.95819 \mathrm{E}-04 \quad-1.37830 \mathrm{E} \quad 00$ $\begin{array}{lllll}1.45154 \mathrm{E} & 00 & -8.96627 \mathrm{E}-04 & -1.38855 \mathrm{E} & 00\end{array}$

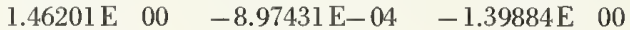
$\begin{array}{lllll}1.47251 \mathrm{E} & 00 & -8.98223 \mathrm{E}-04 & -1.40917 \mathrm{E} & 00\end{array}$

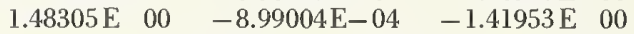

$1.49363 \mathrm{E} \quad 00$ $1.50425 \mathrm{E} \quad 00$

$1.51491 \mathrm{E} \quad 00$

$1.52561 \mathrm{E} \quad 00$

$1.53634 \mathrm{E} \quad 00$ $3.45742 \mathrm{E} \quad 01$ $\begin{array}{lll}1.54712 \mathrm{E} & 00 & -9.03516 \mathrm{E}-04\end{array}$

$\begin{array}{lll}1.55793 \mathrm{E} & 00 & -9.04237 \mathrm{E}-04\end{array}$

$\begin{array}{lll}1.56878 \mathrm{E} & 00 & -9.04949 \mathrm{E}-04\end{array}$

$\begin{array}{lll}1.57967 \mathrm{E} & 00 & -9.05654 \mathrm{E}-04\end{array}$

$1.59060 \mathrm{E} \quad 00 \quad-9.06352 \mathrm{E}-04$

$1.60156 \mathrm{E} \quad 00$

$1.61257 \mathrm{E} \quad 00$

$1.62361 \mathrm{E} \quad 00$

$1.63470 \mathrm{E} \quad 00$

$1.64582 \mathrm{E} \quad 00$

$\begin{array}{ll}1.65698 \mathrm{E} & 00\end{array}$

$1.66818 \mathrm{E} \quad 00$

$\begin{array}{ll}1.67942 \mathrm{E} & 00\end{array}$

$1.69069 \mathrm{E} \quad 00$

$\begin{array}{ll}1.70201 \mathrm{E} & 00\end{array}$

$1.71336 \mathrm{E} \quad 00$

$1.72476 \mathrm{E} \quad 00$

$1.73619 \mathrm{E} \quad 00$

$1.74766 \mathrm{E} \quad 00$

$1.75917 \mathrm{E} \quad 00$

1.77072 E 00

$1.78231 \mathrm{E} \quad 00$

$1.79393 \mathrm{E} \quad 00$

$\begin{array}{ll}1.80560 \mathrm{E} & 00\end{array}$

$1.81730 \mathrm{E} \quad 00$

$1.82904 \mathrm{E} \quad 00$

$1.84083 \mathrm{E} \quad 00$

$1.85265 \mathrm{E} \quad 00$

$1.86451 \mathrm{E} \quad 00$

$1.87641 \mathrm{E} \quad 00$

$1.88835 \mathrm{E} \quad 00$ $1.90032 \mathrm{E} \quad 00$

$1.91234 \mathrm{E} \quad 00$

$1.92440 \mathrm{E} \quad 00$

$1.93649 \mathrm{E} \quad 00$

$\begin{array}{ll}1.94862 \mathrm{E} & 00\end{array}$

$1.96080 \mathrm{E} \quad 00$

$1.97301 \mathrm{E} \quad 00$

$1.98526 \mathrm{E} \quad 00$

$1.99755 \mathrm{E} \quad 00$

$2.00988 \mathrm{E} \quad 00$ $2.02225 \mathrm{E} \quad 00$

$2.03466 \mathrm{E} \quad 00$

$2.04710 \mathrm{E} \quad 00$

$2.05959 \mathrm{E} \quad 00$

$2.07212 \mathrm{E} \quad 00$

$2.08468 \mathrm{E} \quad 00$

$2.09729 \mathrm{E} \quad 00$

$2.10993 \mathrm{E} \quad 00$

$2.12261 \mathrm{E} \quad 00$

$-9.07036 \mathrm{E}-04$ $-9.07718 \mathrm{E}-04$ $-9.08389 \mathrm{E}-04$ $-9.09052 \mathrm{E}-04$

$-9.09711 \mathrm{E}-04$

$-9.10357 \mathrm{E}-04$ $-9.10998 \mathrm{E}-04$ $-9.11630 \mathrm{E}-04$ $-9.12254 \mathrm{E}-04$

$-9.12871 \mathrm{E}-04$

$-9.13480 \mathrm{E}-04$ $-9.14079 \mathrm{E}-04$ $-9.14675 \mathrm{E}-04$ $-9.15259 \mathrm{E}-04$ $-9.15840 \mathrm{E}-04$

$-9.16412 \mathrm{E}-04$ $-9.16974 \mathrm{E}-04$ $-9.17531 \mathrm{E}-04$ $-9.18082 \mathrm{E}-04$

$-9.18625 \mathrm{E}-04$

$-9.19160 \mathrm{E}-04$ $-9.19691 \mathrm{E}-04$ $-9.20211 \mathrm{E}-04$ $-9.20727 \mathrm{E}-04$

$-9.21736 \mathrm{E}-04$ $-9.22228 \mathrm{E}-04$

$-9.22717 \mathrm{E}-04$

$-9.23196 \mathrm{E}-04$

$-9.23672 \mathrm{E}-04$

$-9.24140 \mathrm{E}-04$ $-9.24600 \mathrm{E}-04$

$-9.25055 \mathrm{E}-04$

$-9.25504 \mathrm{E}-04$

$-9.25949 \mathrm{E}-04$

$-9.26381 \mathrm{E}-04$ $-9.26811 \mathrm{E}-04$

$-9.27231 \mathrm{E}-04$

$-9.27648 \mathrm{E}-04$

$-9.28059 \mathrm{E}-04$

$-9.28460 \mathrm{E}-04$

$-9.28858 \mathrm{E}-04$

$-9.29252 \mathrm{E}-04$

$-9.30016 \mathrm{E}-04$
$-8.99776 \mathrm{E}-04$ $-9.00542 \mathrm{E}-04$ $-9.01297 \mathrm{E}-04$ $-9.02047 \mathrm{E}-04$

$-1.42994 \mathrm{E} 00$ $-1.44038 \mathrm{E} 00$ $-1.45086 \mathrm{E} 00$ $-1.46138 \mathrm{E} 00$ $-1.47194 \mathrm{E} 00$

$-1.48254 \mathrm{E} \quad 00$ $-1.49318 \mathrm{E} \quad 00$ $-1.50385 \mathrm{E} \quad 00$ $-1.51457 \mathrm{E} 00$ $-1.52532 \mathrm{E} \quad 00$

$-9.21234 \mathrm{E}-04$

$-9.29637 \mathrm{E}-04$

$-1.53611 \mathrm{E} \quad 00$ 
$\bar{F}$

$7.49544 \mathrm{E}-04$

$7.54532 \mathrm{E}-04$

$7.59537 \mathrm{E}-04$

$7.64558 \mathrm{E}-04$

$7.69596 \mathrm{E}-04$

$7.74650 \mathrm{E}-04$

$7.79721 \mathrm{E}-04$

$7.84809 \mathrm{E}-04$

$7.89913 \mathrm{E}-04$

$7.95033 \mathrm{E}-04$

$8.00170 \mathrm{E}-04$

$8.05324 \mathrm{E}-04$

$8.10494 \mathrm{E}-04$

8.15681 E-04

$8.20884 \mathrm{E}-04$

$8.26104 \mathrm{E}-04$

$8.31340 \mathrm{E}-04$

$8.36593 \mathrm{E}-04$

$8.41863 \mathrm{E}-04$

$8.47148 \mathrm{E}-04$

8.52451 E- 04

$8.57770 \mathrm{E}-04$

$8.63105 \mathrm{E}-04$

$8.68458 \mathrm{E}-04$

$8.73826 \mathrm{E}-04$

$8.79211 \mathrm{E}-04$

8.84613E-04

8.90031 E-04

$8.95466 \mathrm{E}-04$

$9.00918 \mathrm{E}-04$

$9.06386 \mathrm{E}-04$

$9.11870 \mathrm{E}-04$

$9.17371 \mathrm{E}-04$

$9.22889 \mathrm{E}-04$

$9.28423 \mathrm{E}-04$

$9.33973 \mathrm{E}-04$ $9.39540 \mathrm{E}-04$

$9.45124 \mathrm{E}-04$

9.50724. E-04

$9.56341 \mathrm{E}-04$

$9.61974 \mathrm{E}-04$

$9.67624 \mathrm{E}-04$

$9.73291 \mathrm{E}-04$

$9.78973 \mathrm{E}-04$

$9.84673 \mathrm{E}-04$

$9.90389 \mathrm{E}-04$

$9.96121 \mathrm{E}-04$

$1.00187 \mathrm{E}-03$

$1.00764 \mathrm{E}-03$

$1.01342 \mathrm{E}-03$

$1.01922 \mathrm{E}-03$

$1.02503 \mathrm{E}-03$

$1.03086 \mathrm{E}-03$

$1.03671 \mathrm{E}-03$

$1.04258 \mathrm{E}-03$

$1.04846 \mathrm{E}-03$

$1.05436 \mathrm{E}-03$

$1.06027 \mathrm{E}-03$

$1.06620 \mathrm{E}-03$

$1.07215 \mathrm{E}-03$ $\int_{2}$

$g_{2}$

$\tan \delta_{2}$

$f_{-2}$

$1.61883 \mathrm{E} \quad 00$ $1.62967 \mathrm{E} \quad 00$ $1.64055 \mathrm{E} \quad 00$

$1.65147 \mathrm{E} \quad 00$

$1.66242 \mathrm{E} \quad 00$

$1.67341 \mathrm{E} \quad 00$

$1.68443 \mathrm{E} \quad 00$

$1.69549 \mathrm{E} \quad 00$

$1.70659 \mathrm{E} 00$

$1.71772 \mathrm{E} \quad 00$

$1.72888 \mathrm{E} \quad 00$ $1.74009 \mathrm{E} \quad 00$

$1.75133 \mathrm{E} \quad 00$

$1.76260 \mathrm{E} \quad 00$

$1.77391 \mathrm{E} \quad 00$

$\begin{array}{ll}1.78526 \mathrm{E} & 00\end{array}$

$1.79664 \mathrm{E} \quad 00$

$1.80806 \mathrm{E} \quad 00$

$1.81952 \mathrm{E} \quad 00$

$1.83101 \mathrm{E} \quad 00$

$1.84253 \mathrm{E} \quad 00$

$1.85410 \mathrm{E} \quad 00$

$1.86569 \mathrm{E} \quad 00$

$1.87733 \mathrm{E} \quad 00$

].88900E 00

$1.90070 \mathrm{E} \quad 00$

$1.91245 \mathrm{E} \quad 00$

$1.92422 \mathrm{E} \quad 00$

$1.93604 \mathrm{E} \quad 00$

$1.94789 \mathrm{E} \quad 00$

$1.95977 \mathrm{E} \quad 00$

$1.97169 \mathrm{E} \quad 00$

$1.98365 \mathrm{E} \quad 00$

$1.99564 \mathrm{E} \quad 00$

$2.00767 \mathrm{E} \quad 00$

$2.01973 \mathrm{E} \quad 00$

$2.03183 \mathrm{E} \quad 00$

$2.04397 \mathrm{E} \quad 00$

$2.05614 \mathrm{E} \quad 00$

$2.06835 \mathrm{E} \quad 00$

$2.08059 \mathrm{E} \quad 00$

$2.09287 \mathrm{E} \quad 00$

$2.10519 \mathrm{E} \quad 00$

$2.11754 \mathrm{E} \quad 00$

$2.12992 \mathrm{E} \quad 00$

$2.14235 \mathrm{E} \quad 00$

$2.15480 \mathrm{E} \quad 00$

$2.16730 \mathrm{E} \quad 00$

$2.17983 \mathrm{E} \quad 00$

$2.19239 \mathrm{E} \quad 00$

$2.20499 \mathrm{E} \quad 00$

$2.21763 \mathrm{E} \quad 00$

$2.23030 \mathrm{E} \quad 00$

$2.24301 \mathrm{E} \quad 00$

$2.25575 \mathrm{E} \quad 00$

$2.26853 \mathrm{E} \quad 00$

$2.28135 \mathrm{E} \quad 00$

$2.29420 \mathrm{E} \quad 00$

$2.30709 \mathrm{E} \quad 00$

$2.32001 \mathrm{E} \quad 00$
$4.88170 \mathrm{E}-02$

$4.93283 \mathrm{E}-02$

$4.98431 \mathrm{E}-02$

$5.03615 \mathrm{E}-02$

$5.08835 \mathrm{E}-02$

$5.14091 \mathrm{E}-02$

$5.19382 \mathrm{E}-02$

$5.247 \mathrm{i} 0 \mathrm{E}-02$

$5.30074 \mathrm{E}-02$

$5.35474 \mathrm{E}-02$

$5.40911 \mathrm{E}-02$

$5.46384 \mathrm{E}-02$

$5.51894 \mathrm{E}-02$

$5.57440 \mathrm{E}-02$

$5.63024 \mathrm{E}-02$

$5.68645 \mathrm{E}-02$

$5.74303 \mathrm{E}-02$

$5.79998 \mathrm{E}-02$

$5.85731 \mathrm{E}-02$

5.91501 E- 02

$5.97309 \mathrm{E}-02$ $6.03154 \mathrm{E}-02$

$6.09038 \mathrm{E}-02$

$6.14960 \mathrm{E}-02$

$6.20919 \mathrm{E}-02$

$6.26917 \mathrm{E}-02$ $6.32954 \mathrm{E}-02$ $6.39029 \mathrm{E}-02$

$6.45142 \mathrm{E}-02$

$6.51295 \mathrm{E}-02$

$6.57486 \mathrm{E}-02$

$6.63716 \mathrm{E}-02$

$6.69985 \mathrm{E}-02$

$6.76294 \mathrm{E}-02$

$6.82642 \mathrm{E}-02$

$6.89029 \mathrm{E}-02$

$6.95456 \mathrm{E}-02$

$7.01923 \mathrm{E}-02$

$7.08429 \mathrm{E}-02$

$7.14976 \mathrm{E}-02$

$7.21562 \mathrm{E}-02$

$7.28189 \mathrm{E}-02$

$7.34856 \mathrm{E}-02$

$7.41564 \mathrm{E}-02$

$7.48312 \mathrm{E}-02$

$7.55101 \mathrm{E}-02$

7.61931 E- 02

$7.68801 \mathrm{E}-02$

$7.75713 \mathrm{E}-02$

$7.82666 \mathrm{E}-02$

$7.89660 \mathrm{E}-02$ $7.96696 \mathrm{E}-02$ $8.03773 \mathrm{E}-02$

$8.10891 \mathrm{E}-02$

8.18052 E-02

$8.25255 \mathrm{E}-02$ $8.32499 \mathrm{E}-02$

$8.39786 \mathrm{E}-02$

$8.47115 \mathrm{E}-02$

$8.54486 \mathrm{E}-02$
$-6.61790 \mathrm{E} \quad 01 \quad-4.66780 \mathrm{E}-02$ $-6.61818 \mathrm{E} 01 \quad-4.71759 \mathrm{E}-02$

$-6.61847 \mathrm{E}$ 01 $-4.76774 \mathrm{E}-02$

$-6.61875 \mathrm{E}$ 01 $-4.81824 \mathrm{E}-02$

$-6.61903 \mathrm{E} 01-4.86910 \mathrm{E}-02$

$-6.61931 \mathrm{E} \quad 01$

$-6.61987 \mathrm{E} 01 \quad-5.02379 \mathrm{E}-02$

$-6.62014 \mathrm{E}$ 01 $-5.07606 \mathrm{E}-02$

$-6.62041 \mathrm{E} \quad 0 \mathrm{l}-5.12870 \mathrm{E}-02$

$-6.62068 \mathrm{E} \quad 0 \mathrm{l}$

$-6.62095 \mathrm{E} \quad 0 \mathrm{l}$

$-6.62122 \mathrm{E} 01$

$-6.62148 \mathrm{E} \quad 0$

$-6.62174 \mathrm{E} \quad 01$

$-6.62201 \mathrm{E} \quad 01$

$-6.62227 \mathrm{E} \quad 01$

$-6.62252 \mathrm{E} \quad 01$

$-6.62278 \mathrm{E} \quad 01$

$-6.62304 \mathrm{E} 01$

$-6.62329 \mathrm{E} 01$

$-6.62354 \mathrm{E} \quad 01$

$-6.62404 \mathrm{E} 0 \mathrm{O}$

$-6.62428 \mathrm{E} 01$

$-6.62453 \mathrm{E} 01$

$-6.62477 \mathrm{E}$ 0l

$-6.62502 \mathrm{E} 0 \mathrm{I}$

$-6.62526 \mathrm{E} 01$

$-6.62573 \mathrm{E} \quad 01$

$-6.62597 \mathrm{E} 01$

$-6.62620 \mathrm{E} 01$

$-6.62644 \mathrm{E} 01$

$-6.62667 \mathrm{E} 01$

$-6.62690 \mathrm{E} \quad 0 \mathrm{l}$

$-6.62713 \mathrm{E} 01$

$-6.62736 \mathrm{E} 01$

$-6.62758 \mathrm{E} 01$

-6.62781 E 01

$-6.62803 \mathrm{E} 01$

$-6.62825 \mathrm{E} 01$

$-6.62847 \mathrm{E} 01$

$-6.62869 \mathrm{E}$ 01

$-6.62891 \mathrm{E} 01$

$-6.62913 \mathrm{E} \quad 01$

$-6.62934 \mathrm{E}$ 01

$-6.62956 \mathrm{E} 01$

$-6.62977 \mathrm{E} 01$

$-6.62998 \mathrm{E} 01$

$-6.63019 \mathrm{E} 01$

$-6.63040 \mathrm{E} 01$

$-6.63061 \mathrm{E} 01$

$-6.63082 \mathrm{E} 01$

$-6.63102 \mathrm{E} 01$

$-6.63123 \mathrm{E} \quad 0 \mathrm{I}$

$-6.63143 \mathrm{E} 01$

$-6.63163 \mathrm{E} 01$

$-6.63183 \mathrm{E}$ 01

$-6.63204 \mathrm{E} 01$

$-5.18170 \mathrm{E}-02$

$-5.23506 \mathrm{E}-02$

$-5.28878 \mathrm{E}-02$

$-5.34287 \mathrm{E}-02$

$-5.39732 \mathrm{E}-02$

$-5.45214 \mathrm{E}-02$

$-5.50732 \mathrm{E}-02$

$-5.56288 \mathrm{E}-02$

$-5.61880 \mathrm{E}-02$

$-5.67510 \mathrm{E}-02$

$-5.73177 \mathrm{E}-02$

$-5.78881 \mathrm{E}-02$

$-5.84623 \mathrm{E}-02$

$-5.90402 \mathrm{E}-02$

$-5.96219 \mathrm{E}-02$

$-6.02074 \mathrm{E}-02$

$-6.07967 \mathrm{E}-02$

$-6.13898 \mathrm{E}-02$

$-6.19867 \mathrm{E}-02$

$-6.25875 \mathrm{E}-02$

$-6.31921 \mathrm{E}-02$

$-6.38006 \mathrm{E}-02$

$-6.44129 \mathrm{E}-02$

$-6.50291 \mathrm{E}-02$

$-6.56492 \mathrm{E}-02$

$-6.62733 \mathrm{E}-02$

$-6.69012 \mathrm{E}-02$

$-6.75331 \mathrm{E}-02$

$-6.81689 \mathrm{E}-02$

$-6.88087 \mathrm{E}-02$

$-6.94524 \mathrm{E}-02$

$-7.01002 \mathrm{E}-02$

$-7.07519 \mathrm{E}-02$

$-7.14076 \mathrm{E}-02$

$-7.20673 \mathrm{E}-02$

$-7.27311 \mathrm{E}-02$

$-7.33989 \mathrm{E}-02$

$-7.40707 \mathrm{E}-02$

$-7.47467 \mathrm{E}-02$

$-7.54267 \mathrm{E}-02$

$-7.61108 \mathrm{E}-02$
$-6.62379 \mathrm{E} 0 \mathrm{I}$

$-6.62550 \mathrm{E} \quad 01$ 
p $\quad F_{0}$

36.10

36.20

36.30

36.40

36.50

36.60

36.70

36.80

36.90

37.00

37.10

37.20

37.30

37.40

37.50

37.60

37.70

37.80

37.90

38.00

38.10

38.20

38.30

38.40

38.50

38.60

38.70

38.80

38.90

39.00

39.10

39.20

39.30

39.40

39.50

39.60

39.70

39.80

39.90

40.00

40.10

40.20

40.30

40.40

40.50

40.60

40.70

40.80

40.90

41.00

41.10

41.20

41.30

41.40

41.50

41.60

41.70

41.80

41.90

42.00 $f_{1}$

$9.34259 \mathrm{E}-01$ $9.34258 \mathrm{E}-01$ $9.34257 \mathrm{E}-01$ $9.34256 \mathrm{E}-01$ $9.34255 \mathrm{E}-01$

9.34254E-01 $9.34253 \mathrm{E}-01$ $9.34252 \mathrm{E}-01$ $9.34250 \mathrm{E}-01$ 9.34249E-01

$9.34248 \mathrm{E}-01$ $9.34247 \mathrm{E}-01$ $9.34246 \mathrm{E}-01$ $9.34245 \mathrm{E}-01$ $9.34244 \mathrm{E}-01$

$9.34243 \mathrm{E}-01$ 9.34242 E-01 $9.34241 \mathrm{E}-01$ $9.34240 \mathrm{E}-01$ 9.34239E-01

9.34238E-01 $9.34237 \mathrm{E}-01$ $9.34235 \mathrm{E}-01$ $9.34234 \mathrm{E}-01$ 9.34233E-01

9.34232E-01 $9.34231 \mathrm{E}-01$ 9.34230E-01 $9.34229 \mathrm{E}-01$ $9.34228 \mathrm{E}-01$

9.34227 E-01 $9.34226 \mathrm{E}-01$ $9.34225 \mathrm{E}-01$ $9.34224 \mathrm{E}-01$ $9.34223 \mathrm{E}-01$

9.34222E-01 $9.34221 \mathrm{E}-01$ $9.34220 \mathrm{E}-01$ $9.34219 \mathrm{E}-01$ $9.34218 \mathrm{E}-01$

$9.34217 \mathrm{E}-01$ $9.34216 \mathrm{E}-01$ $9.34215 \mathrm{E}-01$ $9.34214 \mathrm{E}-01$ $9.34213 \mathrm{E}-01$

$9.34212 \mathrm{E}-01$ $9.34211 \mathrm{E}-01$ $9.34210 \mathrm{E}-01$ $9.34209 \mathrm{E}-01$ $9.34208 \mathrm{E}-01$

$9.34207 \mathrm{E}-01$ $9.34206 \mathrm{E}-01$ $9.34205 \mathrm{E}-01$ $9.34204 \mathrm{E}-01$ $9.34203 \mathrm{E}-01$

9.34202E-01 $9.34201 \mathrm{E}-01$ $9.34200 \mathrm{E}-01$ $9.34199 \mathrm{E}-01$ 9.34198E-01 $g_{1}$

$\tan \delta_{1}$

$f_{-1}$

$g_{-1}$

$3.48748 \mathrm{E} \quad 01$ $3.49751 \mathrm{E} 01$ $3.50754 \mathrm{E} \quad 01$

$3.51757 \mathrm{E} \quad 01$

$3.52760 \mathrm{E} \quad 01$

$3.53763 \mathrm{E} \quad 01$ $3.54767 \mathrm{E} \quad 01$ $3.55771 \mathrm{E} \quad 01$ $3.56775 \mathrm{E} \quad 01$

$3.57779 \mathrm{E} \quad 01$

$3.58783 \mathrm{E} \quad 01$

$3.59788 \mathrm{E} \quad 01$

$3.60792 \mathrm{E} \quad 01$

$3.61797 \mathrm{E} \quad 01$

$3.62802 \mathrm{E} 01$

$3.63807 \mathrm{E} \quad 01$

$3.64813 \mathrm{E} 01$

$3.65819 \mathrm{E} \quad 01$

$3.66824 \mathrm{E} \quad 01$

$3.67830 \mathrm{E} \quad 01$

$3.68837 \mathrm{E} \quad 01$

$3.69843 \mathrm{E} \quad 01$

$3.70850 \mathrm{E} \quad 01$

$3.71857 \mathrm{E} \quad 01$

$3.72864 \mathrm{E} \quad 01$

$3.73871 \mathrm{E} \quad 01$

$3.74878 \mathrm{E} \quad 01$

$3.75886 \mathrm{E} \quad 01$

$3.76894 \mathrm{E} 01$

$3.77902 \mathrm{E} \quad 01$

$3.78910 \mathrm{E} \quad 01$

$3.79918 \mathrm{E} 01$

$3.80927 \mathrm{E} \quad 01$

$3.81936 \mathrm{E} \quad 01$

$3.82945 \mathrm{E} \quad 01$

$3.83954 \mathrm{E} \quad 01$ $3.84963 \mathrm{E} \quad 01$ $3.85973 \mathrm{E} 01$

$3.86983 \mathrm{E} 01$

$3.87993 \mathrm{E} \quad 01$

$3.89003 \mathrm{E} \quad 01$ $3.90014 \mathrm{E} \quad 01$ $3.91024 \mathrm{E} \quad 01$ $3.92035 \mathrm{E} 01$ $3.93046 \mathrm{E} \quad 01$

$3.94058 \mathrm{E} \quad 01$ $3.95069 \mathrm{E} \quad 01$ $3.96081 \mathrm{E} \quad 01$ $3.97093 \mathrm{E} \quad 01$ $3.98105 \mathrm{E} \quad 01$

$3.99117 \mathrm{E} \quad 01$ $4.00130 \mathrm{E} \quad 01$

$4.01143 \mathrm{E} \quad 01$

$4.02156 \mathrm{E} 01$

$4.03169 \mathrm{E} \quad 01$

$4.04182 \mathrm{E} \quad 01$ $4.05196 \mathrm{E} \quad 01$ $4.06210 \mathrm{E} \quad 01$

$4.07224 \mathrm{E} \quad 01$

$4.08238 \mathrm{E} \quad 01$
$2.13534 \mathrm{~F}$ $2.14810 \mathrm{E}$ $2.16090 \mathrm{E} \quad 00$ $2.17374 \mathrm{E} \quad 00$ $2.18662 \mathrm{E} \quad 00$

$2.19954 \mathrm{E} \quad 00$ $2.21250 \mathrm{E} \quad 00$ $2.22550 \mathrm{E} \quad 00$ $2.23854 \mathrm{E} \quad 00$

$2.25161 \mathrm{E} \quad 00$

$2.26473 \mathrm{E} \quad 00$ $2.27789 \mathrm{E} \quad 00$

$2.29108 \mathrm{E} \quad 00$

$2.30432 \mathrm{E}-00$

$2.31760 \mathrm{E} \quad 00$

$2.33091 \mathrm{E} \quad 00$ $2.34427 \mathrm{E} \quad 00$ $2.35766 \mathrm{E} \quad 00$ $2.37109 \mathrm{E} \quad 00$ $2.38457 \mathrm{E} \quad 00$

$2.39808 \mathrm{E} \quad 00$ $2.41164 \mathrm{E} \quad 00$ $2.42523 \mathbf{E} \quad 00$ $2.43886 \mathrm{E} \quad 00$ 2.45253 E 00

$2.46625 \mathrm{E} \quad 00$ $2.48000 \mathrm{E} \quad 00$ $2.49379 \mathrm{E} \quad 00$ $2.50762 \mathrm{E} \quad 00$ $2.52149 \mathrm{E} \quad 00$

$2.53541 \mathrm{E} \quad 00$ $2.54936 \mathrm{E} \quad 00$ $2.56335 \mathrm{E} \quad 00$

$2.57738 \mathrm{E} \quad 00$

$2.59145 \mathrm{E} \quad 00$

$2.60556 \mathrm{E} \quad 00$ $2.61971 \mathrm{E} \quad 00$ $2.63391 \mathrm{E} 00$

$2.64814 \mathrm{E} \quad 00$

$2.66241 \mathrm{E} \quad 00$ $2.69107 \mathrm{E} \quad 00$ $2.70546 \mathrm{E} \quad 00$ $2.71990 \mathrm{E} \quad 00$

$2.74888 \mathrm{E} \quad 00$ $2.76343 \mathrm{E} \quad 00$ $2.77803 \mathrm{E} \quad 00$ $2.79266 \mathrm{E} \quad 00$ $2.80733 \mathrm{E} \quad 00$

$2.82205 \mathrm{E} \quad 00$ $2.83680 \mathrm{E} \quad 00$ $2.85160 \mathrm{E} \quad 00$

$2.86643 \mathrm{E} \quad 00$

$2.88131 \mathrm{E} \quad 00$

$2.89622 \mathrm{E} \quad 00$ $2.91118 \mathrm{E} \quad 00$ $2.92617 \mathrm{E} \quad 00$ $2.94121 \mathrm{E} \quad 00$ $2.95629 \mathrm{E} \quad 00$
$2.67672 \mathrm{E} \quad 00$ $2.73437 \mathrm{E} \quad 00$

$-9.30394 \mathrm{E}-04$

$-9.30761 \mathrm{E}-04$

$-9.31123 \mathrm{E}-04$

$-9.31476 \mathrm{E}-04$

$-9.31827 \mathrm{E}-04$

$-9.32169 \mathrm{E}-04$

$-9.32509 \mathrm{E}-04$

$-9.32842 \mathrm{E}-04$

$-9.33171 \mathrm{E}-04$

$-9.33489 \mathrm{E}-04$

$-9.33806 \mathrm{E}-04$

$-9.34118 \mathrm{E}-04$

$-9.34422 \mathrm{E}-04$

$-9.34722 \mathrm{E}-04$

$-9.35016 \mathrm{E}-04$

$-9.35305 \mathrm{E}-04$

$-9.35588 \mathrm{E}-04$

$-9.35863 \mathrm{E}-04$

$-9.36137 \mathrm{E}-04$

$-9.36405 \mathrm{E}-04$

$-9.36667 \mathrm{E}-04$

$-9.36924 \mathrm{E}-04$

$-9.37176 \mathrm{E}-04$

$-9.37420 \mathrm{E}-04$

$-9.37662 \mathrm{E}-04$

$-9.37898 \mathrm{E}-04$

$-9.38129 \mathrm{E}-04$

$-9.38354 \mathrm{E}-04$

$-9.38574 \mathrm{E}-04$

$-9.38794 \mathrm{E}-04$

$-9.39003 \mathrm{E}-04$

$-9.39209 \mathrm{E}-04$

$-9.39408 \mathrm{E}-04$

$-9.39605 \mathrm{E}-04$

$-9.39796 \mathrm{E}-04$

$-9.39984 \mathrm{E}-04$

$-9.40164 \mathrm{E}-04$

$-9.40341 \mathrm{E}-04$

$-9.40513 \mathrm{E}-04$

$-9.40680 \mathrm{E}-04$ 
ELECTRON RADIAL FUNCTIONS

$Z=3 \quad A=6 \quad \rho=1.2 A^{1 / 3} F$

Pesitron

$\bar{F}$

$1.07811 \mathrm{E}-03$

$1.08409 \mathrm{E}-03$

1.09009 E-03

$1.09611 \mathrm{E}-03$

$1.10214 \mathrm{E}-03$

$1.10818 \mathrm{E}-03$

$1.11425 \mathrm{E}-03$

$1.12033 \mathrm{E}-03$

$1.12642 \mathrm{E}-03$

$1.13253 \mathrm{E}-03$

$1.13866 \mathrm{E}-03$

$1.14481 \mathrm{E}-03$

$1.15097 \mathrm{E}-03$

$1.15715 \mathrm{E}-03$

$1.16335 \mathrm{E}-03$

$1.16956 \mathrm{E}-03$

$1.17579 \mathrm{E}-03$

$1.18203 \mathrm{E}-03$

$1.18830 \mathrm{E}-03$

$1.19457 \mathrm{E}-03$

$1.20087 \mathrm{E}-03$

$1.20718 \mathrm{E}-03$

$1.21351 \mathrm{E}-03$

$1.21985 \mathrm{E}-03$

$1.22621 \mathrm{E}-03$

$1.23259 \mathrm{E}-03$

$1.23899 \mathrm{E}-03$

$1.24540 \mathrm{E}-03$

$1.25182 \mathrm{E}-03$

$1.25827 \mathrm{E}-03$

$1.26473 \mathrm{E}-03$

$1.27120 \mathrm{E}-03$

$1.27770 \mathrm{E}-03$

$1.28421 \mathrm{E}-03$

$1.29073 \mathrm{E}-03$

$1.29728 \mathrm{E}-03$

$1.30384 \mathrm{E}-03$

$1.31041 \mathrm{E}-03$

$1.31701 \mathrm{E}-03$

$1.32361 \mathrm{E}-03$

$1.33024 \mathrm{E}-03$

$1.33688 \mathrm{E}-03$

$1.34354 \mathrm{E}-03$

$1.35022 \mathrm{E}-03$

$1.35691 \mathrm{E}-03$

$1.36362 \mathrm{E}-03$

$1.37034 \mathrm{E}-03$

$1.37708 \mathrm{E}-03$

$1.38384 \mathrm{E}-03$

$1.39062 \mathrm{E}-03$

$1.39741 \mathrm{E}-03$

$1.40421 \mathrm{E}-03$

$1.41104 \mathrm{E}-03$

$1.41788 \mathrm{E}-03$

$1.42474 \mathrm{E}-03$

$1.43161 \mathrm{E}-03$

$1.43850 \mathrm{E}-03$

$1.44541 \mathrm{E}-03$

$1.45233 \mathrm{E}-03$

$1.45927 \mathrm{E}-03$ $f_{2}$

$2.33297 \mathrm{E} \quad 00$

$2.34596 \mathrm{E} \quad 00$

$2.35899 \mathrm{E} \quad 00$

$2.37206 \mathrm{E} \quad 00$

$2.38516 \mathrm{E} \quad 00$

$2.39830 \mathrm{E} \quad 00$

$2.41147 \mathrm{E} \quad 00$

$2.42468 \mathrm{E} \quad 00$

$2.43792 \mathrm{E} \quad 00$

$2.45120 \mathrm{E} \quad 00$

$2.46452 \mathrm{E} \quad 00$

$2.47787 \mathrm{E} \quad 00$

$2.49126 \mathrm{E} \quad 00$

$2.50468 \mathrm{E} \quad 00$

$2.51814 \mathrm{E} \quad 00$

$2.53163 \mathrm{E} \quad 00$

$2.54516 \mathrm{E} \quad 00$

$2.55873 \mathrm{E} \quad 00$

$2.57233 \mathrm{E} \quad 00$

$2.58596 \mathrm{E} \quad 00$

$2.59963 \mathrm{E} \quad 00$

$2.61334 \mathrm{E} \quad 00$

$2.62709 \mathrm{E} \quad 00$

$2.64086 \mathrm{E} \quad 00$

$2.65468 \mathrm{E} \quad 00$

$2.66853 \mathrm{E} \quad 00$

$2.68241 \mathrm{E} \quad 00$

$2.69634 \mathrm{E} \quad 00$

$2.71029 \mathrm{E} \quad 00$

$2.72429 \mathrm{E} \quad 00$

$2.73831 \mathrm{E} \quad 00$

$2.75238 \mathrm{E} \quad 00$

$2.76648 \mathrm{E} \quad 00$

$2.78061 \mathrm{E} \quad 00$

$2.79478 \mathrm{E} \quad 00$

$2.80899 \mathrm{E} \quad 00$

$2.82323 \mathrm{E} \quad 00$

$2.83751 \mathrm{E} \quad 00$

$2.85182 \mathrm{E} \quad 00$

$2.86617 \mathrm{E} \quad 00$

$2.88055 \mathrm{E} \quad 00$

$2.89497 \mathrm{E} \quad 00$

$2.90943 \mathrm{E} \quad 00$

$2.92392 \mathrm{E} \quad 00$

$2.93844 \mathrm{E} \quad 00$

$2.95300 \mathrm{E} \quad 00$

$2.96760 \mathrm{E} \quad 00$

$2.98223 \mathrm{E} \quad 00$

$2.99690 \mathrm{E} \quad 00$

$3.01160 \mathrm{E} \quad 00$

$3.02634 \mathrm{E} \quad 00$

$3.04112 \mathrm{E} \quad 00$

$3.05593 \mathrm{E} \quad 00$

$3.07077 \mathrm{E} \quad 00$

$3.08565 \mathrm{E} \quad 00$

$3.10057 \mathrm{E} \quad 00$

$3.11552 \mathrm{E} \quad 00$

$3.13051 \mathrm{E} \quad 00$

$3.14553 \mathrm{E} \quad 00$

$3.16059 \mathrm{E} \quad 00$ $g_{2}$

$8.61900 \mathrm{E}-02$

$8.69357 \mathrm{E}-02$

$8.76856 \mathrm{E}-02$

$8.84398 \mathrm{E}-02$

$8.91983 \mathrm{E}-02$

$8.99611 \mathrm{E}-02$

$9.07282 \mathrm{E}-02$

$9.14997 \mathrm{E}-02$

$9.22755 \mathrm{E}-02$

$9.30557 \mathrm{E}-02$

$9.38402 \mathrm{E}-02$

$9.46292 \mathrm{E}-02$

$9.54225 \mathrm{E}-02$

$9.62202 \mathrm{E}-02$

$9.70224 \mathrm{E}-02$

$9.78289 \mathrm{E}-02$

$9.86400 \mathrm{E}-02$

$9.94555 \mathrm{E}-02$

$1.00275 \mathrm{E}-01$

$1.01100 \mathrm{E}-01$

$1.01929 \mathrm{E}-01$

$1.02762 \mathrm{E}-01$

$1.03600 \mathrm{E}-01$

$1.04443 \mathrm{E}-01$

$1.05290 \mathrm{E}-01$

$1.06141 \mathrm{E}-01$

$1.06997 \mathrm{E}-01$

$1.07858 \mathrm{E}-01$

$1.08723 \mathrm{E}-01$

$1.09593 \mathrm{E}-01$

$1.10468 \mathrm{E}-01$

$1.11347 \mathrm{E}-01$

$1.12231 \mathrm{E}-01$

$1.13119 \mathrm{E}-01$

$1.14012 \mathrm{E}-01$

$1.14910 \mathrm{E}-01$

$1.15812 \mathrm{E}-0$

$1.16719 \mathrm{E}-01$

$1.17631 \mathrm{E}-01$

$1.18548 \mathrm{E}-01$

$1.19469 \mathrm{E}-01$

$1.20395 \mathrm{E}-01$

$1.21326 \mathrm{E}-01$

$1.22261 \mathrm{E}-01$

$1.23202 \mathbf{E}-01$

$1.24147 \mathrm{E}-01$

$1.25097 \mathrm{E}-01$

$1.26052 \mathrm{E}-01$

$1.27011 \mathrm{E}-01$

$1.27976 \mathrm{E}-01$

$1.28945 \mathrm{E}-01$

$1.29919 \mathrm{E}-01$

$1.30898 \mathrm{E}-01$

$1.31882 \mathrm{E}-01$

$1.32871 \mathrm{E}-01$

$1.33865 \mathrm{E}-01$ $1.34863 \mathrm{E}-01$

$1.35867 \mathrm{E}-01$

$1.36875 \mathrm{E}-01$

$1.37889 \mathrm{E}-01$

$\tan \delta_{2}$

$f_{2}$

$-6.63223 \mathrm{E} \quad 01 \quad-8.31792 \mathrm{E}-02$

$-6.63243 \mathrm{E} 01 \quad-8.39091 \mathrm{E}-02$

$-6.63263 \mathrm{E} \quad 01 \quad-8.46432 \mathrm{E}-02$

$-6.63282 \mathrm{E} \quad 01 \quad-8.53815 \mathrm{E}-02$

$-6.63302 \mathrm{E} 01 \quad-8.61241 \mathrm{E}-02$

$-6.63321 \mathrm{E} \quad 01 \quad-8.68710 \mathrm{E}-02$

$-6.63340 \mathrm{E}$ 01 $-8.76222 \mathrm{E}-02$

$\begin{array}{lll}-6.63359 \mathrm{E} & 01 & -8.83776 \mathrm{E}-02\end{array}$

$-6.63378 \mathrm{E} \quad 01 \quad-8.91374 \mathrm{E}-02$

$-6.63397 \mathrm{E}$ 01 $-8.99015 \mathrm{E}-02$

$-6.63416 \mathrm{E} 01$

$-6.63435 \mathrm{E} 01$

$-6.63453 \mathrm{E} 01$

$-6.63472 \mathrm{E} \quad 01$

$-6.63490 \mathrm{E} 01$

$-6.63508 \mathrm{E} \quad 01$

$-6.63527 \mathrm{E} \quad 01$

$-6.63545 \mathrm{E} \quad 01$

$-6.63563 \mathrm{E} \quad 01$

$-6.63581 \mathrm{E} 01$

$-6.63598 \mathrm{E} \quad 01$

$-6.63616 \mathrm{E} 01$

$-6.63634 \mathrm{E} \quad 01$

$\begin{array}{lll}-6.63651 \mathrm{E} & 01\end{array}$

$-6.63669 \mathrm{E} 01$

$-6.63686 \mathrm{E} \quad 01$

$-6.63703 \mathrm{E} 01$

$-6.63720 \mathrm{E} 01$

$-6.63737 \mathrm{E}$ 01

$-6.63754 \mathrm{E} \quad 01$

$\begin{array}{lll}-6.63771 \mathrm{E} & 01\end{array}$

$-6.63788 \mathrm{E} 01$

$-6.63804 \mathrm{E} \quad 01$

$-6.63821 \mathrm{E} \quad 01$

$-6.63838 \mathrm{E} 01$

$-9.06699 \mathrm{E}-02$

$-9.14426 \mathrm{E}-02$

$-9.22197 \mathrm{E}-02$

$-9.30012 \mathrm{E}-02$

$-9.37870 \mathrm{E}-02$

$-9.45773 \mathrm{E}-02$

$-9.53719 \mathrm{E}-02$

$-9.61710 \mathrm{E}-02$

$-9.69745 \mathrm{E}-02$

$-9.77824 \mathrm{E}-02$

$-9.859+7 \mathrm{E}-02$

$-9.94116 \mathrm{E}-02$

$-1.00233 \mathrm{E}-01$

$-1.01059 \mathrm{E}-01$

$-1.01889 \mathrm{E}-01$

$-1.02724 \mathrm{E}-01$

$-1.03563 \mathrm{E}-01$

$-1.04407 \mathrm{E}-01$

$-1.05255 \mathrm{E}-01$

$-1.06108 \mathrm{E}-01$

$-1.06966 \mathrm{E}-01$

$-1.07828 \mathrm{E}-01$

$-1.08695 \mathrm{E}-01$

$-1.09566 \mathrm{E}-01$

$-1.10442 \mathrm{E}-01$

$-6.63854 \mathrm{E} \quad 01$

$-6.63870 \mathrm{E} 01$

$-6.63887 \mathrm{E} 01$

$-6.63903 \mathrm{E} \quad 01$

$-6.63919 \mathrm{E} 01$

$-6.63935 \mathrm{E} \quad 01$

$-6.63951 \mathrm{E} \quad 01$

$-6.63967 \mathrm{E} 01$

$-6.63982 \mathrm{E} 01$

$-6.63998 \mathrm{E} 01$

$-6.64014 \mathrm{E} \quad 01$

$-6.64029 \mathrm{E} 01$

$-6.64044 \mathrm{E} 01$

$-6.64060 \mathrm{E} 01$

$-6.64075 \mathrm{E} 01$

$-1.11323 \mathrm{E}-01$

$-1.12208 \mathrm{E}-01$

$-1.13098 \mathrm{E}-01$

$-1.13992 \mathrm{E}-01$

$-1.14892 \mathrm{E}-01$

$-1.15795 \mathrm{E}-01$

$-1.16704 \mathrm{E}-01$

$-1.17617 \mathrm{E}-01$

$-1.18535 \mathrm{E}-01$

$-1.19458 \mathrm{E}-01$

$-1.20386 \mathrm{E}-01$

$-1.21318 \mathrm{E}-01$

$-1.22255 \mathrm{E}-01$

$-1.23197 \mathrm{E}-01$

$-1.24144 \mathrm{E}-01$

$-6.64090 \mathrm{E} 01 \quad-1.25095 \mathrm{E}-01$

$-6.64106 \mathrm{E} 01$

$-6.64121 \mathrm{E} \quad 01$

$-6.64136 \mathrm{E} 01$

$-6.64151 \mathrm{E} 01$

$-1.26052 \mathrm{E}-01$

$-1.27013 \mathrm{E}-01$

$-1.27979 \mathrm{E}-01$

$-1.28950 \mathrm{E}-01$

$-6.6+165 \mathrm{E} \quad 01$

$-6.64180 \mathrm{E} 01$

$-6.6+195 \mathrm{E} 01$

$-6.64209 \mathrm{E} 01$

$-6.6+224 \mathrm{E} 01$ 
ELECTRON RADIAL FUNCTIONS

Positron

$\begin{array}{cc}p & F_{0} \\ 0.10 & 3.49363 \mathrm{E}-01 \\ 0.20 & 6.08592 \mathrm{E}-01 \\ 0.30 & 7.19481 \mathrm{E}-01 \\ 0.40 & 7.78431 \mathrm{E}-01 \\ 0.50 & 8.14112 \mathrm{E}-01 \\ & \\ 0.60 & 8.37538 \mathrm{E}-01 \\ 0.70 & 8.53798 \mathrm{E}-01 \\ 0.80 & 8.65550 \mathrm{E}-01 \\ 0.90 & 8.74313 \mathrm{E}-01 \\ 1.00 & 8.81012 \mathrm{E}-01\end{array}$

1.10

1.20

]. 30

1.40

1.50

I. 60

1.70

1.80

1.90

2.00

2.10

2.20

2.30

2.40

2.50

2.60

2.70

2.80

2.90

3.00

3.10

3.20

3.30

3.40

3.50

3.60

3.70

3.80

3.90

4.00

4.10

4.20

4.30

4.40

4.50

4.60

4.70

4.80

4.90

5.00

5.10

5.20

5.30

5.40

5.50

5.60

5.70

5.80

5.90

6.00
$Z=4 \quad A=8$

$f_{1}$

$4.31631 \mathrm{E}-03$

$2.19495 \mathrm{E}-02$

$5.24811 \mathrm{E}-02$

$9.46134 \mathrm{E}-02$

$1.46818 \mathrm{E}-01$

$2.07521 \mathrm{E}-01$

$2.75237 \mathrm{E}-0 \mathrm{I}$

$3.48644 \mathrm{E}-01$

$4.26617 \mathrm{E}-0 \mathrm{I}$

5.08224 E-01

$5.92712 \mathrm{E}-01$ $6.79484 \mathrm{E}-01$

$7.68063 \mathrm{E}-01$

$8.58078 \mathrm{E}-01$

$9.49233 \mathrm{E}-01$

$1.04130 \mathrm{E} \quad 00$

$1.13410 \mathrm{E} \quad 00$

$\begin{array}{ll}1.22748 \mathrm{E} & 00\end{array}$

$1.32134 \mathrm{E} \quad 00$

$1.41559 \mathrm{E} \quad 00$

$1.51015 \mathrm{E} \quad 00$

$1.60496 \mathrm{E} \quad 00$

$\begin{array}{ll}1.60496 \mathrm{E} & 00 \\ 1.69998 \mathrm{E} & 00\end{array}$

$1.79518 \mathrm{E} \quad 00$

$1.89052 \mathrm{E} \quad 00$

$1.98597 \mathrm{E} \quad 00$

$2.08152 \mathrm{E} \quad 00$

$2.17716 \mathrm{E} \quad 00$

$2.27286 \mathrm{E} \quad 00$

$2.36861 \mathrm{E} \quad 00$

$2.46441 \mathrm{E} \quad 00$

$2.56025 \mathrm{E} \quad 00$

$2.65613 \mathrm{E} \quad 00$

$2.75203 \mathrm{E} \quad 00$

$2.84795 \mathrm{E} \quad 00$

$2.94389 \mathrm{E} \quad 00$ $3.03984 \mathrm{E} \quad 00$ $3.13581 \mathrm{E} \quad 00$ $3.23179 \mathrm{E} \quad 00$ $3.32778 \mathrm{E} \quad 00$.

$3.42377 \mathrm{E} \quad 00$

$3.51976 \mathrm{E} \quad 00$

$3.61576 \mathrm{E} \quad 00$

$3.71177 \mathrm{E} \quad 00$

$3.80777 \mathrm{E} \quad 00$

$3.90377 \mathrm{E} \quad 00$

$3.99977 \mathrm{E} \quad 00$

$4.09576 \mathrm{E} \quad 00$

$4.19175 \mathrm{E} \quad 00$

$4.28773 \mathrm{E} \quad 00$

$4.38369 \mathrm{E} \quad 00$ $4.47964 \mathrm{E} \quad 00$

$4.57555 \mathrm{E} \quad 00$

$4.67142 \mathrm{E} \quad 00$

$4.76721 \mathrm{E} \quad 00$

$4.86284 \mathrm{E} \quad 00$

$4.95816 \mathrm{E} \quad 00$

$5.05256 \mathrm{E} \quad 00$

$5.14170 \mathrm{E} \quad 00$

$5.26718 \mathrm{E} \quad 00$ $g_{1}$

$-3.24690 \mathrm{E}-05$
$-1.64440 \mathrm{E}-04$
$-3.90539 \mathrm{E}-04$
$-6.97597 \mathrm{E}-04$
$-1.07003 \mathrm{E}-03$
$-1.49174 \mathrm{E}-03$
$-1.94745 \mathrm{E}-03$
$-2.42353 \mathrm{E}-03$
$-2.90831 \mathrm{E}-03$
$-3.39214 \mathrm{E}-03$

$-4.32708 \mathrm{E}-03$

$-4.76693 \mathrm{E}-03$

$-5.18276 \mathrm{E}-03$

$-5.57145 \mathrm{E}-03$

$-5.93056 \mathrm{E}-03$

$-6.55266 \mathrm{E}-03$

$-6.81290 \mathrm{E}-03$

$-7.03786 \mathrm{E}-03$

$-7.22678 \mathrm{E}-03$

$-7.37901 \mathrm{E}-03$

$-7.57148 \mathrm{E}-03$

$-7.61095 \mathrm{E}-03$

$-7.61219 \mathrm{E}-03$

$-7.57497 \mathrm{E}-03$

$-7.49909 \mathrm{E}-03$

$-7.38439 \mathrm{E}-03$

$-7.23076 \mathrm{E}-03$

$-7.03806 \mathrm{E}-03$

$-6.80621 \mathrm{E}-03$

$-6.53513 \mathrm{E}-03$

$-6.22475 \mathrm{E}-03$

$-5.87502 \mathrm{E}-03$

$-5.48588 \mathrm{E}-03$

$-5.05730 \mathrm{E}-03$

$-4.58924 \mathrm{E}-03$

$-4.08167 \mathrm{E}-03$

$-3.53456 \mathrm{E}-03$

$-2.94789 \mathrm{E}-03$

$-2.32164 \mathrm{E}-03$

$-1.65579 \mathrm{E}-03$

$-9.50327 \mathrm{E}-04$

$-2.05242 \mathrm{E}-04$

$5.79478 \mathrm{E}-04$

$1.40384 \mathrm{E}-03$

$2.26785 \mathrm{E}-03$

$3.17152 \mathrm{E}-03$

4.11484 E-03

$5.09780 \mathrm{E}-03$ $6.12040 \mathrm{E}-03$

$7.18262 \mathrm{E}-03$

$8.28441 \mathrm{E}-03$

$9.42570 \mathrm{E}-03$

$1.06062 \mathrm{E}-02$ $1.18256 \mathrm{E}-02$ $1.30820 \mathrm{E}-02$ $1.43628 \mathrm{E}-02$ $1.57894 \mathrm{E}-02$
$-3.86715 \mathrm{E}-03$

$-6.25814 \mathrm{E}-03$

$-7.49405 \mathrm{E}-03$ $\tan \delta_{1}$ $\rho=1.2 \AA^{1 / 3} \mathrm{~F}$

$1.26237 \mathrm{E}-01$

$6.24136 \mathrm{E}-02$

$4.15425 \mathrm{E}-02$

$3.11095 \mathrm{E}-02$

$2.48257 \mathrm{E}-02$

$2.06135 \mathrm{E}-02$

$1.75859 \mathrm{E}-02$

$1.53005 \mathrm{E}-02$

$1.35113 \mathrm{E}-02$

$1.20709 \mathrm{E}-02$

$1.08852 \mathrm{E}-02$

$9.89147 \mathrm{E}-03$

$9.04610 \mathrm{E}-03$

$8.31788 \mathrm{E}-03$

$7.68381 \mathrm{E}-03$

$7.12661 \mathrm{E}-03$

$6.63299 \mathrm{E}-03$

$6.19260 \mathrm{E}-03$

$5.79721 \mathrm{E}-03$

$5.44023 \mathrm{E}-03$

$5.11629 \mathrm{E}-03$

$4.82099 \mathrm{E}-03$

$4.55068 \mathrm{E}-03$

$4.30230 \mathrm{E}-03$

$4.07329 \mathrm{E}-03$

$3.86145 \mathrm{E}-03$

$3.66492 \mathrm{E}-03$

$3.48211 \mathrm{E}-03$

$3.31160 \mathrm{E}-03$

$3.15221 \mathrm{E}-03$

$3.00288 \mathrm{E}-03$

$2.86268 \mathrm{E}-03$

$2.73081 \mathrm{E}-03$

$2.60653 \mathrm{E}-03$

$2.48922 \mathrm{E}-03$

$2.37830 \mathrm{E}-03$

$2.27326 \mathrm{E}-03$

$2.17366 \mathrm{E}-03$

$2.07907 \mathrm{E}-03$

$1.98913 \mathrm{E}-03$

$1.90350 \mathrm{E}-03$

$1.82189 \mathrm{E}-03$

$1.74400 \mathrm{E}-03$

$1.66961 \mathrm{E}-03$

$1.59848 \mathrm{E}-03$

$1.53039 \mathrm{E}-03$

$1.46517 \mathrm{E}-03$

$1.40262 \mathrm{E}-03$

$1.34259 \mathrm{E}-03$

$1.28493 \mathrm{E}-03$

$1.22950 \mathrm{E}-03$

$1.17618 \mathrm{E}-03$

$1.12486 \mathrm{E}-03$

$1.07541 \mathrm{E}-03$

$1.02774 \mathrm{E}-03$

$9.81758 \mathrm{E}-04$

$9.37368 \mathrm{E}-04$

$8.94505 \mathrm{E}-04$

$8.53069 \mathrm{E}-04$

$8.13010 \mathrm{E}-04$

$f_{-1}$

$9.73945 \mathrm{E}-04$

$2.55486 \mathrm{E}-03$

$4.12496 \mathrm{E}-03$

$5.64432 \mathrm{E}-03$

$7.09863 \mathrm{E}-03$

$8.48083 \mathrm{E}-03$

$9.78813 \mathrm{E}-03$

$1.10205 \mathrm{E}-02$

$1.21797 \mathrm{E}-02$

$1.32682 \mathrm{E}-02$

$1.42892 \mathrm{E}-02$

$1.52458 \mathrm{E}-02$

$1.61409 \mathrm{E}-02$

$1.69772 \mathrm{E}-02$

$1.77572 \mathrm{E}-02$

$1.84829 \mathrm{E}-02$

$1.91564 \mathrm{E}-02$

$1.97790 \mathrm{E}-02$

$2.03524 \mathrm{E}-02$

$2.08776 \mathrm{E}-02$

$2.13556 \mathrm{E}-02$

$2.17875 \mathrm{E}-02$

$2.21738 \mathrm{E}-02$

$2.25154 \mathrm{E}-02$

$2.28127 \mathrm{E}-02$

$2.30663 \mathrm{E}-02$

$2.32765 \mathrm{E}-02$

$2.34438 \mathrm{E}-02$

$2.35685 \mathrm{E}-02$

$2.36509 \mathrm{E}-02$

$2.36913 \mathrm{E}-02$

$2.36897 \mathrm{E}-02$

$2.36466 \mathrm{E}-02$

$2.35619 \mathrm{E}-02$

$2.34360 \mathrm{E}-02$

$2.32689 \mathrm{E}-02$

$2.30607 \mathrm{E}-02$

$2.28116 \mathrm{E}-02$

$2.25216 \mathrm{E}-02$

$2.21909 \mathrm{E}-02$

$2.18195 \mathrm{E}-02$

$2.14075 \mathrm{E}-02$

$2.09550 \mathrm{E}-02$

$2.04620 \mathrm{E}-02$

$1.99286 \mathrm{E}-02$

$1.93548 \mathrm{E}-02$

$1.87406 \mathrm{E}-02$

I. $80862 \mathrm{E}-02$

$1.73915 \mathrm{E}-02$

$1.66566 \mathrm{E}-02$

$1.58815 \mathrm{E}-02$

$1.50662 \mathrm{E}-02$

$1.42108 \mathrm{E}-02$

$1.33152 \mathrm{E}-02$

$1.23796 \mathrm{E}-02$

I.14038E-02 $1.03880 \mathrm{E}-02$

$9.33218 \mathrm{E}-03$

$8.23629 \mathrm{E}-03$

$7.10038 \mathrm{E}-03$ 
ELECTRON RADIAL FUNCTIONS

$Z=4 \quad A=8 \quad \rho=1.2 A^{1 / 3} F$

Positren

$\bar{F}$

$4.05602 \mathrm{E}-09$

$2.66059 \mathrm{E}-08$

$6.99628 \mathrm{E}-08$

$1.34033 \mathrm{E}-07$

$2.18630 \mathrm{E}-07$

$3.23575 \mathrm{E}-07$

$4.48720 \mathrm{E}-07$

$5.93942 \mathrm{E}-07$

$7.59144 \mathrm{E}-07$

$9.44250 \mathrm{E}-07$

$1.14920 \mathrm{E}-06$

$1.37394 \mathrm{E}-06$

$1.61845 \mathrm{E}-06$

$1.88269 \mathrm{E}-06$

$2.16663 \mathrm{E}-06$

$2.47026 \mathrm{E}-06$

$2.79357 \mathrm{E}-06$

$3.13654 \mathrm{E}-06$

$3.49917 \mathrm{E}-06$

$3.88144 \mathrm{E}-06$

$4.28336 \mathrm{E}-06$

$4.70490 \mathrm{E}-06$

$5.14608 \mathrm{E}-06$

$5.60689 \mathrm{E}-06$

$6.08732 \mathrm{E}-06$

$6.58737 \mathrm{E}-06$

$7.10704 \mathrm{E}-06$

$7.64633 \mathrm{E}-06$

$8.20523 \mathrm{E}-06$

$8.78376 \mathrm{E}-06$

$9.38189 \mathrm{E}-06$

$9.99962 \mathrm{E}-06$

$1.06370 \mathrm{E}-05$

$1.12939 \mathrm{E}-05$

$1.19705 \mathrm{E}-05$

$1.26667 \mathrm{E}-05$

$1.33825 \mathrm{E}-05$

$1.41179 \mathrm{E}-05$

$1.48729 \mathrm{E}-05$

$1.56475 \mathrm{E}-05$

$1.64417 \mathrm{E}-05$

$1.72555 \mathrm{E}-05$

$1.80889 \mathrm{E}-0.5$

$1.89420 \mathrm{E}-05$

$1.98146 \mathrm{E}-05$

$2.07068 \mathrm{E}-05$

$2.16187 \mathrm{E}-0.5$

$2.25501 \mathrm{E}-0.5$

$2.35011 \mathrm{E}-05$

$2.44718 \mathrm{E}-05$

$2.54620 \mathrm{E}-05$

$2.64719 \mathrm{E}-05$

$2.75013 \mathrm{E}-05$

$2.85504 \mathrm{E}-05$

$2.96190 \mathrm{E}-05$

$3.07073 \mathrm{E}-05$

$3.18151 \mathrm{E}-05$

$3.29426 \mathrm{E}-05$

$3.40896 \mathrm{E}-05$

$3.52563 \mathrm{E}-05$ $f_{2}$

8.71831 E-07 $9.02756 \mathrm{E}-06$ $3.24843 \mathrm{E}-05$

$7.81764 \mathrm{E}-05$

$1.51725 \mathrm{E}-04$

$2.57430 \mathrm{E}-04$

$3.98415 \mathrm{E}-04$.

$5.76847 \mathrm{E}-04$

$7.94161 \mathrm{E}-04$

$1.05127 \mathrm{E}-03$

$1.34871 \mathrm{E}-03$

$1.68678 \mathrm{E}-03$

$2.06562 \mathrm{E}-03$

$2.48528 \mathrm{E}-03$

$2.94572 \mathrm{E}-03$

$3.44690 \mathrm{E}-03$ $3.98873 \mathrm{E}-03$ $4.57115 \mathrm{E}-03$ $5.19406 \mathrm{E}-03$

$5.85738 \mathrm{E}-03$

$6.56104 \mathrm{E}-03$

$7.30497 \mathrm{E}-03$

$8.08909 \mathrm{E}-03$

$8.91334 \mathrm{E}-03$

$9.77767 \mathrm{E}-03$

$1.06820 \mathrm{E}-02$

$1.16264 \mathrm{E}-02$

$1.26106 \mathrm{E}-02$

$1.36347 \mathrm{E}-02$

$1.46987 \mathrm{E}-02$

$1.58024 \mathrm{E}-02$

$1.69459 \mathrm{E}-02$

$1.81290 \mathrm{E}-02$

$1.93518 \mathrm{E}-02$

$2.06141 \mathrm{E}-02$

$2.19158 \mathrm{E}-02$

$2.32569 \mathrm{E}-02$

$2.46371 \mathrm{E}-02$

$2.60562 \mathrm{E}-02$

$2.75140 \mathrm{E}-02$

$2.90098 \mathrm{E}-02$

$3.05427 \mathrm{E}-02$

$3.21111 \mathrm{E}-02$

$3.37115 \mathrm{E}-02$

$3.53350 \mathrm{E}-02$

$3.69522 \mathrm{E}-02$

$3.83567 \mathrm{E}-02$

$4.18589 \mathrm{E}-02$

$4.28081 \mathrm{E}-02$

$4.45461 \mathrm{E}-02$

$4.63977 \mathrm{E}-02$

$4.83115 \mathrm{E}-02$

$5.02748 \mathrm{E}-02$

$5.22830 \mathrm{E}-02$

$5.43339 \mathrm{E}-02$

$5.64266 \mathrm{E}-02$

$5.85602 \mathrm{E}-02$

$6.07345 \mathrm{E}-02$

$6.29491 \mathrm{E}-02$

$6.52038 \mathrm{E}-02$ $g_{2}$

$\tan \delta_{2}$

$f_{-2}$

$g-2$

$\tan \delta_{-2}$

$-3.64397 \mathrm{E}-09 \quad-3.59507 \mathrm{E} \quad 00 \quad 1.19928 \mathrm{E}-07$

$-3.75660 \mathrm{E}-08$

$-1.34197 \mathrm{E}-07$

$-3.19751 \mathrm{E}-07$

$-6.12840 \mathrm{E}-07$

$-1.02439 \mathrm{E}-06$

$-1.55343 \mathrm{E}-06$

$-2.21338 \mathrm{E}-06$

$-2.98331 \mathrm{E}-06$

$-3.85917 \mathrm{E}-06$

$-4.82969 \mathrm{E}-06$

$-5.88213 \mathrm{E}-06$

$-7.00276 \mathrm{E}-06$

$-8.17724 \mathrm{E}-06$

$-9.39082 \mathrm{E}-06$

$-1.06286 \mathrm{E}-0.5$

$-1.18753 \mathrm{E}-05$

$-1.31160 \mathrm{E}-05$

$-1.43354 \mathrm{E}-05$

$-1.55183 \mathrm{E}-05$

$-1.66495 \mathrm{E}-05$

$-1.77140 \mathrm{E}-05$

$-1.86966 \mathrm{E}-05$

$-1.95823 \mathrm{E}-05$

$-2.03559 \mathrm{E}-05$

$-2.10025 \mathrm{E}-0.5$

$-2.15071 \mathrm{E}-05$

$-2.18547 \mathrm{E}-05$

$-2.20303 \mathrm{E}-05$

$-2.20190 \mathrm{E}-05$

$-2.18059 \mathrm{E}-05$

$-2.13761 \mathrm{E}-05$

$-2.07147 \mathrm{E}-05$

$-1.98069 \mathrm{E}-05$

$-1.86378 \mathrm{E}-05$

$-1.71926 \mathrm{E}-05$

$-1.54565 \mathrm{E}-05$

$-1.34148 \mathrm{E}-05$

$-1.10527 \mathrm{E}-05$

$-8.35561 \mathrm{E}-06$

$-5.30903 \mathrm{E}-06$

$-1.89870 \mathrm{E}-06$

$1.88918 \mathrm{E}-06$

$6.06721 \mathrm{E}-06$

$1.06448 \mathrm{E}-05$

$1.56181 \mathrm{E}-05$

$2.08730 \mathrm{E}-05$

$2.78703 \mathrm{E}-05$

$3.37138 \mathrm{E}-05$

$4.05101 \mathrm{E}-05$

$4.78514 \mathrm{E}-05$

$5.57203 \mathrm{E}-05$

$6.41235 \mathrm{E}-05$

$7.30731 \mathrm{E}-05$

$8.25825 \mathrm{E}-05$

$9.26659 \mathrm{E}-05$

$1.03338 \mathrm{E}-04$

$1.14612 \mathrm{E}-04$

$1.26504 \mathrm{E}-04$

$1.39028 \mathrm{E}-04$
$-1.07187 \mathrm{E} 01$

$-1.39689 \mathrm{E} 01$

$-1.69735 \mathrm{E} 01$

$-1.97185 \mathrm{E} 01$

$-2.22037 \mathrm{E} \quad 01$

$-2.44392 \mathrm{E} 01$

$-2.64419 \mathrm{E} 01$

$-2.82318 \mathrm{E} \quad 01$

$-2.98303 \mathrm{E} 01$

$-3.25360 \mathrm{E} 01$

$-3.36808 \mathrm{E} 01$

$-3.47090 \mathrm{E} 01$

$-3.56349 \mathrm{E} 01$

$-3.64709 \mathrm{E} \quad 01$

$-3.72278 \mathrm{E} \quad 01$

$-3.79151 \mathrm{E} 01$

$-3.85409 \mathrm{E} 01$

$-3.91124 \mathrm{E} \quad 01$

$-3.96356 \mathrm{E} \quad 01$

$-4.01160 \mathrm{E} \quad 01$

$-4.05581 \mathrm{E} 01$

$-4.09660 \mathrm{E} 01$

$-4.16930 \mathrm{E}$ 0]

$-4.20178 \mathrm{E} 01$

$-4.23202 \mathrm{E} 01$

$-4.26023 \mathrm{E} 01$

$-4.28659 \mathrm{E} 01$

$-4.31127 \mathrm{E} \quad 01$

$-4.35617 \mathrm{E} 01$

$-4.37663 \mathrm{E} 01$

$-4.39592 \mathrm{E} 01$

$-4.41412 \mathrm{E} 01$

$-4.43132 \mathrm{E} 01$

$-4.44760 \mathrm{E} \quad 01$

$-4.46303 \mathrm{E} 01$

$-4.47767 \mathrm{E} \quad 01$

$-4.49157 \mathrm{E} 01$

$-4.50479 \mathrm{E} \quad 01$

$-4.51738 \mathrm{E} \quad 01$

$-4.52938 \mathrm{E} 01$

$-4.54082 \mathrm{E} \quad 01$

$-4.55175 \mathrm{E} 01$

$-4.56220 \mathrm{E} \quad 01$

$-4.57219 \mathrm{E} 01$

$-4.58176 \mathrm{E} \quad 01$

$-4.59094 \mathrm{E}$ 01

$-4.59973 \mathrm{E} \quad 01$

$-4.60817 \mathrm{E} \quad 01$

$-4.61629 \mathrm{E} 01$

$-4.62408 \mathrm{E} 01$

$-4.63158 \mathrm{E}$ 01

$-4.63880 \mathrm{E} \quad 01$

$-4.64575 \mathrm{E} 01$

$-4.65246 \mathrm{E} \quad 01$

$-4.65892 \mathrm{E} 01$
$-3.12585 \mathrm{E} 01$

$-4.13433 \mathrm{E} 01$

$-4.33442 \mathrm{E} 01$

$6.10393 \mathrm{E}-07$

$1.46948 \mathrm{E}-06$

$2.67484 \mathrm{E}-06$

4.19962E-06

$6.01516 \mathrm{E}-06$

$8.09291 \mathrm{E}-06$

$1.04 .055 \mathrm{E}-05$

$1.29276 \mathrm{E}-05$

$1.56353 \mathrm{E}-05$

$1.85070 \mathrm{E}-05$

$2.15224 \mathrm{E}-05$

$2.46623 \mathrm{E}-05$

$2.79087 \mathrm{E}-05$

$3.12444 \mathrm{E}-05$

$3.46527 \mathrm{E}-05$

$3.81172 \mathrm{E}-05$

$4.16223 \mathrm{E}-05$

$4.51523 \mathrm{E}-05$

4.86918E-05

$5.22255 \mathrm{E}-05$

$5.57384 \mathrm{E}-05$

$5.92153 \mathbf{E}-05$

$6.26414 \mathrm{E}-05$

$6.60016 \mathrm{E}-05$

$6.92812 \mathrm{E}-05$

$7.24652 \mathrm{E}-05$

$7.55389 \mathrm{E}-05$

$7.84874 \mathrm{E}-0.5$

$8.12960 \mathrm{E}-05$

$8.39497 \mathrm{E}-05$

$8.64339 E-05$

$8.87338 \mathrm{E}-05$

$9.08347 \mathrm{E}-05$

$9.27217 \mathrm{E}-05$

$9.43800 \mathrm{E}-05$

$9.57950 \mathrm{E}-05$

$9.69519 \mathrm{E}-05$

$9.78358 \mathrm{E}-05$

$9.84321 \mathrm{E}-05$

$9.87260 \mathrm{E}-05$

$9.87027 \mathrm{E}-05$ 
ELECTRON RADIAL FUNCTIONS

Positron

$\begin{array}{cc}p & F_{0} \\ 6.10 & 9.13838 \mathrm{E}-01 \\ 6.20 & 9.13862 \mathrm{E}-01 \\ 6.30 & 9.13884 \mathrm{E}-01 \\ 6.40 & 9.13905 \mathrm{E}-01 \\ 6.50 & 9.13925 \mathrm{E}-01 \\ & \\ 6.60 & 9.13944 \mathrm{E}-01 \\ 6.70 & 9.13961 \mathrm{E}-01 \\ 6.80 & 9.13978 \mathrm{E}-01 \\ 6.90 & 9.13993 \mathrm{E}-01 \\ 7.00 & 9.14006 \mathrm{E}-01 \\ & \\ 7.10 & 9.14019 \mathrm{E}-01 \\ 7.20 & 9.14032 \mathrm{E}-01 \\ 7.30 & 9.14044 \mathrm{E}-01 \\ 7.40 & 9.14055 \mathrm{E}-01 \\ 7.50 & 9.14065 \mathrm{E}-01\end{array}$

$7.60 \quad 9.14075 \mathrm{E}-01$

$7.70 \quad 9.14084 \mathrm{E}-01$

$7.80 \quad 9.14092 \mathrm{E}-01$

$7.90 \quad 9.14100 \mathrm{E}-01$

$8.00 \quad 9.14107 \mathrm{E}-01$

$8.10 \quad 9.14114 \mathrm{E}-01$

$8.20 \quad 9.14120 \mathrm{E}-01$

$8.30 \quad 9.14126 \mathrm{E}-01$

$8.40 \quad 9.14131 \mathrm{E}-01$

$8.50 \quad 9.14137 \mathrm{E}-01$

$8.60 \quad 9.14141 \mathrm{E}-01$

$8.70 \quad 9.14145 \mathrm{E}-01$

$8.80 \quad 9.14149 \mathrm{E}-01$

$8.90 \quad 9.14153 \mathrm{E}-01$

$9.00 \quad 9.14157 \mathrm{E}-01$

9.10

9.20

9.30

9.40

9.50

9.60

9.70

9.80

9.90

10.00

10.10

10.20

10.30

10.40

10.50

10.60

10.70

10.80

10.90

11.00

11.10

11.20

11.30

11.40

11.50

11.60

11.70

11.80

11.90

12,00
$Z=4$

$f_{1}$

$5.35010 \mathrm{E} \quad 00$

$5.44404 \mathrm{E} \quad 00$

$5.53925 \mathrm{E} \quad 00$

$5.63486 \mathrm{E} \quad 00$

$5.73064 \mathrm{E} \quad 00$

$5.82651 \mathrm{E} \quad 00$

$5.92244 \mathrm{E} \quad 00$

$6.01841 \mathrm{E} \quad 00$

$6.11440 \mathrm{E} 00$

$6.21041 \mathrm{E} \quad 00$

$6.30644 \mathrm{E} \quad 00$

$6.40248 \mathrm{E} \quad 00$

$6.49853 \mathrm{E} \quad 00$

$6.59459 \mathrm{E} \quad 00$

$6.69066 \mathrm{E} \quad 00$

$6.78674 \mathrm{E} \quad 00$ $6.88281 \mathrm{E} \quad 00$ $6.97890 \mathrm{E} \quad 00$

$7.07500 \mathrm{E} \quad 00$

$7.17110 \mathrm{E} \quad 00$

$7.26721 \mathrm{E} \quad 00$

$7.36332 \mathrm{E} \quad 00$

$7.45943 \mathrm{E} \quad 00$

$7.55555 \mathrm{E} \quad 00$

$7.65168 \mathrm{E} \quad 00$

$7.74782 \mathrm{E} \quad 00$

$7.84395 \mathrm{E} \quad 00$

$7.94010 \mathrm{E} \quad 00$

$8.03625 \mathrm{E} \quad 00$

$8.13240 \mathrm{E} \quad 00$

$8.22856 \mathrm{E} \quad 00$ $8.32473 \mathrm{E} \quad 00$ $8.42090 \mathrm{E} \quad 00$ $8.51708 \mathrm{E} \quad 00$ $8.61326 \mathrm{E} \quad 00$

$8.70945 \mathrm{E} \quad 00$ $8.80564 \mathrm{E} \quad 00$ $8.90185 \mathrm{E} \quad 00$ $8.99805 \mathrm{E} \quad 00$ $9.09426 \mathrm{E} \quad 00$

$9.19049 \mathrm{E} \quad 00$ $9.28671 \mathrm{E} \quad 00$ $9.38295 \mathrm{E} \quad 00$ $9.47919 \mathrm{E} \quad 00$ $9.57543 \mathrm{E} \quad 00$

$9.67168 \mathrm{E} 00$ $9.76794 \mathrm{E} \quad 00$ $9.86421 \mathrm{E} \quad 00$ $9.96048 \mathrm{E} \quad 00$ $1.00568 \mathrm{E} 01$

$1.01530 \mathrm{E} \quad 01$ $1.02493 \mathrm{E} \quad 01$ $\begin{array}{ll}1.03456 \mathrm{E} & 01\end{array}$ $1.04420 \mathrm{E} \quad 01$ $\begin{array}{ll}1.05383 \mathrm{E} & 01\end{array}$

$\begin{array}{ll}1.06346 \mathrm{E} & 01\end{array}$ $1.07309 \mathrm{E} 01$ $\begin{array}{lll}1.08273 \mathrm{E} & 01\end{array}$ $\begin{array}{lll}1.09236 \mathrm{E} & 01\end{array}$ $1.10200 \mathrm{E} 01$

$$
A=8
$$

$g_{1}$

$1.71316 \mathrm{E}-02$

$1.85456 \mathrm{E}-02$

$2.00031 \mathrm{E}-02$

$2.15015 \mathrm{E}-02$

2.30402 E-02

$2.46189 \mathrm{E}-02$

$2.62376 \mathrm{E}-02$

$2.78961 \mathrm{E}-02$

$2.95945 \mathrm{E}-02$

$3.13327 \mathrm{E}-02$

$3.31108 \mathrm{E}-02$

$3.49287 \mathrm{E}-02$

$3.67864 \mathrm{E}-02$

$3.86840 \mathrm{E}-02$

$4.06213 \mathrm{E}-02$

$4.25985 \mathrm{E}-02$

$4.46155 \mathrm{E}-02$

4.66724.E-02

$4.87690 \mathrm{E}-02$

$5.09056 \mathrm{E}-02$

$5.30820 \mathrm{E}-02$

$5.52982 \mathrm{E}-02$

$5.75542 \mathrm{E}-02$

$5.98502 \mathrm{E}-02$

$6.21859 \mathrm{E}-02$

$6.45616 \mathrm{E}-02$

$6.69771 \mathrm{E}-02$

$6.94325 \mathrm{E}-02$

$7.19278 \mathrm{E}-02$

7.44629 E- 02

7.70380 E- 02

$7.96529 \mathrm{E}-02$

8.23078E-02

$8.50025 \mathrm{E}-02$

$8.77371 \mathrm{E}-02$

$9.05117 \mathrm{E}-02$

$9.33262 \mathrm{E}-02$

$9.61807 \mathrm{E}-02$

$9.90750 \mathrm{E}-02$

$1.02009 \mathrm{E}-01$

1.04984. E-01

$1.07998 \mathrm{E}-01$

$1.11052 \mathrm{E}-01$

$1.14146 \mathrm{E}-01$

$1.17280 \mathrm{E}-01$

1.20454 E- 01

$1.23668 \mathrm{E}-01$

$1.26922 \mathrm{E}-01$

$1.30216 \mathrm{E}-01$

$1.33550 \mathrm{E}-01$

$1.36924 \mathrm{E}-01$

$1.40338 \mathrm{E}-01$

$1.43792 \mathrm{E}-01$

$1.47286 \mathrm{E}-01$

$1.50820 \mathrm{E}-01$

$1.54395 \mathrm{E}-01$

$1.58009 \mathrm{E}-01$

$1.61663 \mathrm{E}-01$

$1.65357 \mathrm{E}-01$

$1.69092 \mathrm{E}-01$ $\tan \delta_{1}$

$\rho=1.2 A^{1 / 3} F$

$7.74251 \mathrm{E}-0$

$7.36731 \mathrm{E}-04$

$7.00399 \mathrm{E}-04$

$6.65192 \mathrm{E}-04$

$6.31060 \mathrm{E}-04$

$5.97959 \mathrm{E}-04$

$5.65835 \mathrm{E}-04$

$5.34658 \mathrm{E}-04$

$5.04376 \mathrm{E}-04$

$4.74959 \mathrm{E}-04$

$4.46362 \mathrm{E}-04$

$4.18558 \mathrm{E}-04$

$3.91516 \mathrm{E}-04$

$3.65202 \mathrm{E}-04$

$3.39589 \mathrm{E}-04$

$3.14647 \mathrm{E}-04$

$2.90354 \mathrm{E}-04$

2.66684 E-04

$2.43613 \mathrm{E}-04$

$2.21119 \mathrm{E}-04$

$1.99181 \mathrm{E}-04$

$1.77776 \mathrm{E}-04$

$1.56892 \mathrm{E}-04$

$1.36505 \mathrm{E}-04$

$1.16597 \mathrm{E}-04$

$9.71591 \mathrm{E}-05$

$7.81680 \mathrm{E}-05$

$5.96074 \mathrm{E}-05$

$4.14672 \mathrm{E}-05$

$2.37377 \mathrm{E}-05$

$6.39192 \mathrm{E}-06$

$-1.05685 \mathrm{E}-05$

$-2.71642 \mathrm{E}-05$

$-4.34015 \mathrm{E}-05$

$-5.92954 \mathrm{E}-05$

$-7.48542 \mathrm{E}-05$

$-9.00894 \mathrm{E}-05$

$-1.05012 \mathrm{E}-04$

$-1.19625 \mathrm{E}-04$

$-1.33945 \mathrm{E}-04$

$-1.47971 \mathrm{E}-04$

$-1.61725 \mathrm{E}-04$

$-1.75214 \mathrm{E}-04$

$-1.88431 \mathrm{E}-04$

$-2.01394 \mathrm{E}-04$

$-2.14108 \mathrm{E}-04$

$-2.26581 \mathrm{E}-04$

$-2.38818 \mathrm{E}-04$

$-2.50825 \mathrm{E}-04$

$-2.62613 \mathrm{E}-04$

$-2.74183 \mathrm{E}-04$

$-2.85541 \mathrm{E}-04$

$-2.96690 \mathrm{E}-04$

$-3.07642 \mathrm{E}-04$

$-3.18398 \mathrm{E}-04$

$-3.28962 \mathrm{E}-04$

$-3.3934 .7 \mathrm{E}-04$

$-3.49548 \mathrm{E}-04$

$-3.59571 \mathrm{E}-04$

$-3.69424 \mathrm{E}-04$

$f_{-1}$

$5.92444 \mathrm{E}-03$

$4.70851 \mathrm{E}-03$

$3.45258 \mathrm{E}-03$

$2.15668 \mathrm{E}-03$

$8.20790 \mathrm{E}-04$

$-5.55068 \mathrm{E}-04$

$-1.97089 \mathrm{E}-03$

$-3.42667 \mathrm{E}-03$

$-4.92241 \mathrm{E}-03$

$-6.45810 \mathrm{E}-03$

$-8.03373 \mathrm{E}-03$

$-9.64932 \mathrm{E}-03$

$-1.13048 \mathrm{E}-02$

$-1.30003 \mathrm{E}-02$

$-1.47357 \mathrm{E}-02$

$-1.65111 \mathrm{E}-02$

$-1.83263 \mathrm{E}-02$

$-2.01815 \mathrm{E}-02$

$-2.20767 \mathrm{E}-02$

$-2.40118 \mathrm{E}-02$

$-2.59868 \mathrm{E}-02$

$-2.80018 \mathrm{E}-02$

$-3.00567 \mathrm{E}-02$

$-3.21515 \mathrm{E}-02$

$-3.42863 \mathrm{E}-02$

$-3.64611 \mathrm{E}-02$

$-3.86757 \mathrm{E}-02$

$-4.09304 \mathrm{E}-02$

$-4.32249 \mathrm{E}-02$

$-4.55595 \mathrm{E}-02$

$-4.79340 \mathrm{E}-02$

$-5.03484 \mathrm{E}-02$

$-5.28029 \mathrm{E}-02$

$-5.52972 \mathrm{E}-02$

$-5.78316 \mathrm{E}-02$

$-6.04059 \mathrm{E}-02$

$-6.30203 \mathrm{E}-02$

$-6.56745 \mathrm{E}-02$

$-6.83688 \mathrm{E}-02$

-7.11031 E-02

$-7.38774 \mathrm{E}-02$

$-7.66916 \mathrm{E}-02$

$-7.95459 \mathrm{E}-02$

$-8.24402 \mathrm{E}-02$

$-8.53745 \mathrm{E}-02$

$-8.83489 \mathrm{E}-02$

$-9.13632 \mathrm{E}-02$

$-9.44176 \mathrm{E}-02$

$-9.75121 \mathrm{E}-02$

$-1.00647 \mathrm{E}-01$

$-1.03821 \mathrm{E}-01$

$-1.07036 \mathrm{E}-01$

$-1.10290 \mathrm{E}-01$

$-1.13585 \mathrm{E}-01$ 
ELECTRON RADIAL FUNCTIONS

$Z=4 \quad A=8 \quad \rho=1.2 A^{1 / 3} F$

Prositron

$\bar{F}$

$3.64425 \mathrm{E}-05$

$3.76484 \mathrm{E}-05$

$3.88738 \mathrm{E}-05$

$4.01189 \mathrm{E}-05$

$4.13835 \mathrm{E}-05$

$4.26678 \mathrm{E}-05$

$4.39716 \mathrm{E}-05$

4.5295 I E-05

$4.66382 \mathrm{E}-05$

$4.80008 \mathrm{E}-05$

$4.93830 \mathrm{E}-05$

$5.07849 \mathrm{E}-05$

$5.22063 \mathrm{E}-05$

$5.36474 \mathrm{E}-0.5$

$5.51080 \mathrm{E}-05$

$5.65882 \mathrm{E}-05$

$5.80880 \mathrm{E}-05$

$5.96075 \mathrm{E}-05$

$6.11465 \mathrm{E}-05$

$6.27051 \mathrm{E}-05$

$6.42834 \mathrm{E}-05$

$6.58812 \mathrm{E}-05$

$6.74986 \mathrm{E}-05$

$6.91356 \mathrm{E}-05$

$7.07922 \mathrm{E}-05$

$7.24684 \mathrm{E}-05$

$7.41642 \mathrm{E}-05$

$7.58796 \mathrm{E}-05$

$7.76146 \mathrm{E}-05$

$7.93692 \mathrm{E}-05$

$8.11434 \mathrm{E}-05$

$8.29372 \mathrm{E}-05$

$8.47505 \mathrm{E}-05$

$8.65835 \mathrm{E}-05$

8.84.361 E-05

$9.03082 \mathrm{E}-05$

$9.22000 \mathrm{E}-05$

$9.41114 \mathrm{E}-05$

$9.60423 \mathrm{E}-05$

$9.79929 \mathrm{E}-05$

$9.99630 \mathrm{E}-05$

$1.01953 \mathrm{E}-04$

$1.03962 \mathrm{E}-04$

$1.05991 \mathrm{E}-04$

$1.08040 \mathrm{E}-04$

$1.10108 \mathrm{E}-04$

$1.12195 \mathrm{E}-04$

$1.14303 \mathrm{E}-04$

$1.164 .30 \mathrm{E}-04$

$1.18576 \mathrm{E}-04$

$1.20742 \mathrm{E}-04$

$1.22928 \mathrm{E}-04$

$1.25133 \mathrm{E}-04$

$1.27358 \mathrm{E}-04$

$1.29602 \mathrm{E}-04$

$1.31866 \mathrm{E}-04$

$1.34150 \mathrm{E}-04$

$1.36453 \mathrm{E}-04$

$1.38776 \mathrm{E}-04$

$1.41119 \mathrm{E}-04$ $f_{2}$

$6.74987 \mathrm{E}-02$

$6.98335 \mathrm{E}-02$

7.22083 E-02

$7.46229 \mathrm{E}-02$

7.70774E-02

$7.95717 \mathrm{E}-02$

$8.21057 \mathrm{E}-02$

$8.46795 \mathrm{E}-02$

$8.72930 \mathrm{E}-02$

$8.99463 \mathrm{E}-02$

$9.26392 \mathrm{E}-02$

$9.53719 \mathrm{E}-02$

$9.81443 \mathrm{E}-02$

$1.00956 \mathrm{E}-01$

$1.03808 \mathrm{E}-01$

$1.06700 \mathrm{E}-01$

$1.09631 \mathrm{E}-01$

$1.12601 \mathrm{E}-01$

$1.15612 \mathrm{E}-01$

$1.18662 \mathrm{E}-01$

$1.21752 \mathrm{E}-01$

$1.24881 \mathrm{E}-01$

$1.28050 \mathrm{E}-01$

$1.31259 \mathrm{E}-01$

$1.34507 \mathrm{E}-01$

$1.37795 \mathrm{E}-01$

$1.41123 \mathrm{E}-01$

$1.44490 \mathrm{E}-01$

$1.47897 \mathrm{E}-01$

$1.51343 \mathrm{E}-01$

$1.54829 \mathrm{E}-01$

$1.58355 \mathrm{E}-01$

$1.61920 \mathrm{E}-01$

$1.65525 \mathrm{E}-01$

$1.69170 \mathrm{E}-01$

$1.72854 \mathrm{E}-01$

$1.76578 \mathrm{E}-01$

$1.80342 \mathrm{E}-01$

$1.84145 \mathrm{E}-01$

$1.87987 \mathrm{E}-01$

$1.91870 \mathrm{E}-01$

$1.95792 \mathrm{E}-01$

$1.99753 \mathrm{E}-01$

$2.03754 \mathrm{E}-01$

$2.07795 \mathrm{E}-01$

$2.11876 \mathrm{E}-01$

$2.15996 \mathrm{E}-01$

$2.20155 \mathrm{E}-01$

$2.24354 \mathrm{E}-01$

$2.28593 \mathrm{E}-01$

2.32872 E-01

$2.37190 \mathrm{E}-01$

$2.41547 \mathrm{E}-01$

$2.45945 \mathrm{E}-01$

$2.50381 \mathrm{E}-01$

$2.54858 \mathrm{E}-01$

$2.59374 \mathrm{E}-01$

$2.63929 \mathrm{E}-01$

$2.68524 \mathrm{E}-01$

$2.73159 \mathrm{E}-01$ $g_{2}$

$1.52199 \mathrm{E}-04$

$1.66032 \mathrm{E}-04$

$1.80541 \mathrm{E}-04$

$1.95741 \mathrm{E}-04$

$2.11647 \mathrm{E}-04$

$2.28274 \mathbf{E}-04$

$2.45636 \mathrm{E}-04$

$2.63749 \mathrm{E}-04$

$2.82626 \mathrm{E}-04$

$3.02283 \mathrm{E}-04$

$3.22734 \mathrm{E}-04$

$3.43995 \mathrm{E}-04$

$3.66080 \mathrm{E}-04$

$3.89004 \mathrm{E}-04$

4.12781 E-04

$4.37427 \mathrm{E}-04$

$4.62956 \mathrm{E}-04$

$4.89383 \mathrm{E}-04$

$5.16722 \mathrm{E}-04$

$5.44990 \mathrm{E}-04$

$5.74199 \mathrm{E}-04$

$6.04365 \mathrm{E}-04$

$6.35504 \mathrm{E}-04$

$6.67628 \mathrm{E}-04$

$7.00755 \mathrm{E}-04$

$7.34897 \mathrm{E}-04$ $7.70071 \mathrm{E}-04$ $8.06290 \mathrm{E}-04$

$8.43570 \mathrm{E}-04$

8.81925 E-04

$9.21369 \mathrm{E}-04$

$9.61919 \mathrm{E}-04$

$1.00359 \mathrm{E}-03$

$1.04639 \mathrm{E}-03$

$1.09034 \mathrm{E}-03$

$1.13546 \mathrm{E}-03$

$1.18176 \mathrm{E}-03$

$1.22925 \mathrm{E}-03$

$1.27794 \mathrm{E}-03$

$1.32786 \mathrm{E}-03$

$1.37902 \mathrm{E}-03$

$1.43143 \mathrm{E}-03$

$1.48511 \mathrm{E}-03$

$1.54007 \mathrm{E}-03$

$1.59632 \mathrm{E}-03$

$1.65389 \mathrm{E}-03$

$1.71279 \mathrm{E}-03$

$1.77302 \mathrm{E}-03$

$1.83461 \mathrm{E}-03$

$1.89757 \mathrm{E}-03$

$1.96192 \mathrm{E}-03$ $2.02767 \mathrm{E}-03$

$2.09483 \mathrm{E}-03$

$2.16342 \mathrm{E}-03$

$2.23346 \mathrm{E}-03$

$2.30495 \mathrm{E}-03$

$2.37793 \mathrm{E}-03$

$2.45238 \mathrm{E}-03$

$2.52835 \mathrm{E}-03$

$2.60583 \mathrm{E}-0.3$ $\tan \delta_{2}$

$f_{-2}$

$-4.66516 \mathrm{E} \quad 01$ $-4.67118 \mathrm{E} \quad 01$

$-4.67700 \mathrm{E} \quad 01$

$-4.68263 \mathrm{E} 01$

$-4.68807 \mathrm{E} \quad 01$

$-4.69334 \mathrm{E} 01$ $-4.69844 \mathrm{E} \quad 01$ $-4.70337 \mathrm{E} \quad 01$ $-4.70816 \mathrm{E} \quad 01$

$-4.71280 \mathrm{E} 01$

$-4.71730 \mathrm{E} \quad 01$

$-4.72166 \mathrm{E} \quad 01$

$-4.72590 \mathrm{E} 01$

$-4.73002 \mathrm{E} \quad 01$

$-4.73402 \mathrm{E} 0 \mathrm{I}$

-4.73791 E 01

$-4.74169 \mathrm{E} \quad 01$

$-4.74537 \mathrm{E} 01$

$-4.74895 \mathrm{E} 01$

$-4.75243 \mathrm{E} 01$

$-4.75582 \mathrm{E} \quad 01$

$-4.75912 \mathrm{E} \quad 01$

$-4.76234 \mathrm{E} 01$

$-4.76548 \mathrm{E} \quad 01$

$-4.76854 \mathrm{E} \quad 01$

$-4.77152 \mathrm{E} \quad 01$ $-4.77443 \mathrm{E}$ 01

$-4.77727 \mathrm{E} \quad 01$

$-4.78004 \mathrm{E} \quad 01$

$-4.78275 \mathrm{E} \quad 01$

$-4.78539 \mathrm{E}$ 0l

$-4.78797 \mathrm{E} \quad 01$

$-4.79050 \mathrm{E}$ 01

$-4.79296 \mathrm{E} 01$

$-4.79538 \mathrm{E} 01$

$-4.79773 \mathrm{E} \quad 01$

$-4.80004 \mathrm{E} 01$

$-4.80230 \mathrm{E} 01$

$-4.80450 \mathrm{E} \quad 01$

$-4.80667 \mathrm{E} 01$

$-4.80878 \mathrm{E} \quad 01$

-4.81085 E 01

$-4.81288 \mathrm{E} \quad 01$

$-4.81487 \mathrm{E}$ ol

$-4.81682 \mathrm{E} 01$

$-4.81873 \mathrm{E} 01$

$-4.82060 \mathrm{E}$ 01

$-4.82244 \mathrm{E} 01$

$-4.82423 \mathrm{E} 01$

$-4.82600 \mathrm{E} 01$

$-4.82773 \mathrm{E} 01$ $-4.82943 \mathrm{E} 01$

$-4.83110 \mathrm{E} \quad 01$

$-4.83273 \mathrm{E}$ 01

$-4.83434 \mathrm{E} \quad 01$

-4.83591 E 01

$-4.83746 \mathrm{E} \quad 01$

$-4.83898 \mathrm{E} 01$

$-4.84047 \mathrm{E} \quad 01$

$-4.84194 \mathrm{E} 01$

$1.83589 \mathrm{E}-05$

$8.89136 \mathrm{E}-06$

$-1.20347 \mathrm{E}-06$

$-1.19404 \mathrm{E}-05$

$-2.33341 \mathrm{E}-0.5$

$-3.53993 \mathrm{E}-05$

$-4.81510 \mathrm{E}-05$

$-6.16038 \mathrm{E}-05$

$-7.57724 \mathrm{E}-05$

$-9.06717 \mathrm{E}-05$

$-1.06316 \mathrm{E}-04$

$-1.22721 \mathrm{E}-04$

$-1.39901 \mathrm{E}-04$

$-1.5787 \mathrm{I} \mathrm{E}-04$

$-1.76645 E-04$

$-1.96239 \mathrm{E}-04$

$-2.16667 \mathrm{E}-04$

$-2.37943 \mathrm{E}-04$

$-2.60083 \mathrm{E}-04$

$-2.83102 \mathrm{E}-04$

$-3.07013 \mathrm{E}-04$

$-3.31833 \mathrm{E}-04$

$-3.57575 \mathrm{E}-04$

$-3.84255 \mathrm{E}-04$

$-4.11887 \mathrm{E}-04$

$-4.40486 \mathrm{E}-04$

$-4.70066 \mathrm{E}-04$

$-5.00643 \mathrm{E}-04$

$-5.32232 \mathrm{E}-04$

$-5.64847 \mathrm{E}-04$

$-5.98502 \mathrm{E}-04$

$-6.33214 \mathrm{E}-04$

$-6.68995 \mathrm{E}-04$

$-7.05862 \mathrm{E}-04$

$-7.43829 \mathrm{E}-04$

$-7.82910 \mathrm{E}-04$

$-8.23121 \mathrm{E}-04$

$-8.64476 \mathrm{E}-04$

$-9.06990 \mathrm{E}-04$

$-9.50678 \mathrm{E}-04$

$-9.95554 \mathrm{E}-04$

$-1.04163 \mathrm{E}-03$ 
$p$

$F_{0}$

12.10

12.20

12.30

12.40

12.50

12.60

12.70

12.80

12.90

13.00

13.10

13.20

13.30

13.40

13.50

13.60

13.70

13.80

13.90

14.00

14.10

14.20

14.30

14.40

14.50

14.60

14.70

14.80

14.90

15.00

15.10

15.20

15.30

15.40

15.50

15.60

15.70

15.80

15.90

16.00

16.10

16.20

16.30

16.40

16.50

16.60

16.70

16.80

16.90

17.00

17.10

17.20

17.30

17.40

17.50

17.60

17.70

17.80

17.90

18.00

\section{$f_{1}$}

$9.14163 \mathrm{E}-01$

$9.14161 \mathrm{E}-01$

$9.14159 \mathrm{E}-01$

$9.14158 \mathrm{E}-01$

$9.14156 \mathrm{E}-01$

9.14154E-01 $9.14153 \mathrm{E}-01$ $9.14151 \mathrm{E}-01$ $9.14149 \mathrm{E}-01$ $9.14146 \mathrm{E}-01$

9.14144E-01 $9.14142 \mathrm{E}-01$ $9.14140 \mathrm{E}-01$ $9.14138 \mathrm{E}-01$ $9.14136 \mathrm{E}-01$

9.14133E-01 $9.14131 \mathrm{E}-01$ $9.14129 \mathrm{E}-01$ $9.14126 \mathrm{E}-01$ 9.14124E-01

$9.14121 \mathrm{E}-01$ $9.14119 \mathrm{E}-01$ $9.14116 \mathrm{E}-01$ $9.14114 \mathrm{E}-01$ $9.14111 \mathrm{E}-01$

$9.14109 \mathrm{E}-01$ $9.14106 \mathrm{E}-01$ $9.14104 \mathrm{E}-0 \mathrm{I}$ $9.14101 \mathrm{E}-01$ $9.14098 \mathrm{E}-01$

$9.14096 \mathrm{E}-01$ $9.14093 \mathrm{E}-01$ $9.14090 \mathrm{E}-01$ $9.14088 \mathrm{E}-01$ $9.14085 \mathrm{E}-01$

$9.14082 \mathrm{E}-01$ $9.14079 \mathrm{E}-01$ $9.14076 \mathrm{E}-01$ $9.14074 \mathrm{E}-01$ $9.14071 \mathrm{E}-01$

$9.14068 \mathrm{E}-01$ $9.14065 \mathrm{E}-01$ 9.14062 E-01 $9.14060 \mathrm{E}-01$ 9.14057E-01

9.14054 E-01 $9.14051 \mathrm{E}-01$ $9.14048 \mathrm{E}-01$ $9.14046 \mathrm{E}-01$ $9.14043 \mathrm{E}-01$

$9.14040 \mathrm{E}-01$ $9.14038 \mathrm{E}-01$ $9.14035 \mathrm{E}-01$ $9.14032 \mathrm{E}-01$ $9.14029 \mathrm{E}-01$

$9.14026 \mathrm{E}-01$ $9.14023 \mathrm{E}-01$ $9.14020 \mathrm{E}-01$ $9.14018 \mathrm{E}-01$ $9.14015 \mathrm{E}-01$ $g_{1}$

$1.11163 \mathrm{E} \quad 01$

$1.12127 \mathrm{E} \quad 01$

$1.13091 \mathrm{E} 01$

$1.14055 \mathrm{E} \quad 01$

$1.15019 \mathrm{E} 01$

$1.15983 \mathrm{E} 01$

I.16947E 01

$1.17911 \mathrm{E}$ 0I

$1.18875 \mathrm{E} \quad 01$

$1.19840 \mathrm{E} \quad 01$

$1.20804 \mathrm{E} \quad 01$

$1.21768 \mathrm{E} \quad 01$

$1.22733 \mathrm{E} 01$

$1.23698 \mathrm{E} \quad 01$

$1.24663 \mathrm{E} 01$

$1.25628 \mathrm{E} \quad 01$

$1.26592 \mathrm{E} 01$

$1.27558 \mathrm{E} \quad 01$

$1.28523 \mathrm{E} 01$

$1.29488 \mathrm{E} 01$

$1.30453 \mathrm{E} 01$

$1.31419 \mathrm{E} 01$

$1.32384 \mathrm{E} 01$

$1.33350 \mathrm{E} 01$

$1.34316 \mathrm{E} 01$

$1.35281 \mathrm{E} \quad 01$

$\begin{array}{ll}1.36247 \mathrm{E} & 01\end{array}$

$\begin{array}{lll}1.37213 \mathrm{E} & 01\end{array}$

$1.38179 \mathrm{E} \quad 01$

$1.39146 \mathrm{E} \quad 01$

$1.40112 \mathrm{E} \quad 01$

$1.41078 \mathrm{E} 01$

$1.42045 \mathrm{E} \quad 01$

$1.43011 \mathrm{E} 01$

$\begin{array}{ll}1.43978 \mathrm{E} & 01\end{array}$

$1.44945 \mathrm{E} \quad 01$ $\begin{array}{ll}1.45912 \mathrm{E} & 01\end{array}$ $1.46879 \mathrm{E} \quad 01$

$1.47846 \mathrm{E} \quad 01$

$1.48813 \mathrm{E} \quad 01$

$1.49781 \mathrm{E} \quad 01$ $\begin{array}{ll}1.50748 \mathrm{E} & 01\end{array}$ $\begin{array}{ll}1.51716 \mathrm{E} & 01\end{array}$ $\begin{array}{ll}1.52683 \mathrm{E} & 01\end{array}$ $1.53651 \mathrm{E} \quad 01$

$\begin{array}{ll}1.54619 \mathrm{E} \quad 01 & 01\end{array}$ $1.55587 \mathrm{E} \quad 01$ $1.56555 \mathrm{E} 01$ $1.57523 \mathrm{E} \quad 01$ $\begin{array}{ll}1.58491 \mathrm{E} & 01\end{array}$

$1.59460 \mathrm{E} \quad 01$ $\begin{array}{ll}1.60428 \mathrm{E} & 01\end{array}$ $\begin{array}{ll}1.61397 \mathrm{E} & 01\end{array}$ $1.62366 \mathrm{E} \quad 01$ $1.63335 \mathrm{E} \quad 01$

$1.64304 \mathrm{E} \quad 01$ $1.65273 \mathrm{E} \quad 01$ $\begin{array}{ll}1.66242 \mathrm{E} & 01\end{array}$ $1.67211 \mathrm{E} \quad 01$ $1.68181 \mathrm{E} \quad 01$ $\tan \delta_{1}$ $f_{-1}$

$1.72866 \mathrm{E}-01 \quad-3.79109 \mathrm{E}-04 \quad-1.37771 \mathrm{E}-01$

$1.76681 \mathrm{E}-01 \quad-3.88631 \mathrm{E}-04 \quad-1.41386 \mathrm{E}-01$

$1.80535 \mathrm{E}-01 \quad-3.97993 \mathrm{E}-04 \quad-1.45042 \mathrm{E}-01$

$1.84430 \mathrm{E}-01-4.07196 \mathrm{E}-04-1.48737 \mathrm{E}-01$

$1.88365 \mathrm{E}-01-4.16250 \mathrm{E}-04 \quad-1.52473 \mathrm{E}-01$

$1.92340 \mathrm{E}-01$

$1.96355 \mathrm{E}-01$

$2.00410 \mathrm{E}-01$

$2.04505 \mathrm{E}-01$

$2.08641 \mathrm{E}-01$

$2.12816 \mathrm{E}-01$

$2.17032 \mathrm{E}-01$

$2.21288 \mathrm{E}-01$

$2.25584 \mathrm{E}-01$

$2.29920 \mathrm{E}-01$

$2.34297 \mathrm{E}-01$

$2.38714 \mathrm{E}-01$

$2.43170 \mathrm{E}-01$

$2.47667 \mathrm{E}-01$

$2.52205 \mathrm{E}-01$

$2.56782 \mathrm{E}-01$

$2.61400 \mathrm{E}-01$

$2.66058 \mathrm{E}-01$

$2.70756 \mathrm{E}-01$

$2.75495 \mathrm{E}-01$

$2.80274 \mathrm{E}-01$

$2.85093 \mathrm{E}-01$

$2.89952 \mathrm{E}-01$

$2.94852 \mathrm{E}-01$

$2.99792 \mathrm{E}-01$

$3.04772 \mathrm{E}-01$

$3.09793 \mathrm{E}-01$

$3.14854 \mathrm{E}-01$

$3.19955 \mathrm{E}-01$

$3.25097 \mathrm{E}-01$

$3.30279 \mathrm{E}-01$

$3.35501 \mathrm{E}-01$

$3.40764 \mathrm{E}-01$

$3.46067 \mathrm{E}-01$

$3.51410 \mathrm{E}-01$

$3.56794 \mathrm{E}-01$

$3.62219 \mathrm{E}-01$

$3.67684 \mathrm{E}-01$

$3.73189 \mathrm{E}-01$

$3.78735 \mathrm{E}-01$

$3.84321 \mathrm{E}-01$

$3.89947 \mathrm{E}-01$

$3.95614 \mathrm{E}-01$

$4.01322 \mathrm{E}-01$

$4.07070 \mathrm{E}-01$

$4.12850 \mathrm{E}-01$ $4.18688 \mathrm{E}-01$

$4.24558 \mathrm{E}-01$

$4.30468 \mathrm{E}-01$

$4.36419 \mathrm{E}-01$

$4.42410 \mathrm{E}-0 \mathrm{I}$

$4.48442 \mathrm{E}-01$

$4.54514 \mathrm{E}-01$

$4.60628 \mathrm{E}-01$

$4.66781 \mathrm{E}-01$

$-4.25151 \mathrm{E}-04$

$-4.33910 \mathrm{E}-04$

$-4.42527 \mathrm{E}-04$

$-4.51007 \mathrm{E}-04$

$-4.59348 \mathrm{E}-04$

$-4.67556 \mathrm{E}-04$

$-4.75636 \mathrm{E}-04$

$-4.83588 \mathrm{E}-04$

$-4.91420 \mathrm{E}-04$

$-4.99127 \mathrm{E}-04$

$-5.06716 \mathrm{E}-04$

$-5.14187 \mathrm{E}-04$

$-5.21549 \mathrm{E}-04$

$-5.28799 \mathrm{E}-04$

$-5.35933 \mathrm{E}-04$

$-5.42966 \mathrm{E}-04$

$-5.49896 \mathrm{E}-04$

$-5.56721 \mathrm{E}-04$

$-5.63449 \mathrm{E}-04$

$-5.70077 \mathrm{E}-04$

$-5.76604 \mathrm{E}-04$

$-5.83040 \mathrm{E}-04$

$-5.89387 \mathrm{E}-04$

$-5.95636 \mathrm{E}-04$

$-6.01801 \mathrm{E}-04$

$-6.07876 \mathrm{E}-04$

$-6.13869 \mathrm{E}-04$

$-6.19778 \mathrm{E}-04$

$-6.25601 \mathrm{E}-04$

$-6.31346 \mathrm{E}-04$

$-6.37012 \mathrm{E}-04$

$-6.42599 \mathrm{E}-04$

$-6.48112 \mathrm{E}-04$

$-6.53546 \mathrm{E}-04$

$-6.58911 \mathrm{E}-04$

$-6.64199 \mathrm{E}-04$

$-6.694 .17 \mathrm{E}-04$

$-6.74569 \mathrm{E}-04$

$-6.79651 \mathrm{E}-04$

$-6.84662 \mathrm{E}-04$ 
p

12.10

12.20

12.30

12.40

12.50

12.60

12.70

12.80

12.90

13.00

13.10

13.20

13.30

13.40

13.50

13.60

13.70

13.80

13.90

14.00

14.10

14.20

14.30

14.40

14.50

14.60

14.70

14.80

14.90

15.00

15.10

15.20

15.30

15.40

15.50

15.60

15.70

15.80

15.90

16.00

16.10

16.20

16.30

16.40

16.50

16.60

16.70

16.80

16.90

17.00

17.10

17.20

17.30

17.40

17.50

17.60

17.70

17.80

17.90

18.00
$\bar{F}$

$1.43481 \mathrm{E}-04$

$1.45862 \mathrm{E}-04$

$1.48264 \mathrm{E}-04$

$1.50684 \mathrm{E}-04$

$1.53125 \mathrm{E}-04$

$1.55585 \mathrm{E}-04$

$1.58064 \mathrm{E}-04$

$1.60564 \mathrm{E}-04$

$1.63082 \mathrm{E}-04$

$1.65621 \mathrm{E}-04$

$1.68179 \mathrm{E}-04$

$1.70756 \mathrm{E}-04$

$1.73353 \mathrm{E}-04$

$1.75970 \mathrm{E}-04$

$1.78606 \mathrm{E}-04$

$1.81262 \mathrm{E}-04$

$1.83938 \mathrm{E}-04$

$1.86633 \mathrm{E}-04$

$1.89348 \mathrm{E}-04$

$1.92082 \mathrm{E}-04$

$1.94836 \mathrm{E}-04$

$1.97609 \mathrm{E}-04$

$2.00402 \mathrm{E}-04$

$2.03215 \mathrm{E}-04$

$2.06047 \mathrm{E}-04$

$2.08899 \mathrm{E}-04$

$2.11771 \mathrm{E}-04$

$2.14662 \mathrm{E}-04$

$2.17572 \mathrm{E}-04$

2.20503 E-04

$2.23452 \mathrm{E}-04$

$2.26422 \mathrm{E}-04$

$2.29411 \mathrm{E}-04$

$2.32419 \mathrm{E}-04$

$2.35447 \mathrm{E}-04$

$2.38495 \mathrm{E}-04$

$2.41563 \mathrm{E}-04$

$2.44650 \mathrm{E}-04$

$2.47756 \mathrm{E}-04$

$2.50882 \mathrm{E}-04$

$2.54028 \mathrm{E}-04$

$2.57193 \mathrm{E}-04$

$2.60378 \mathrm{E}-04$

$2.63583 \mathrm{E}-04$

2.66807 E-04

$2.70050 \mathrm{E}-04$

$2.73314 \mathrm{E}-04$

$2.76596 \mathrm{E}-04$

$2.79899 \mathrm{E}-04$

$2.83221 \mathrm{E}-04$

$2.86563 \mathrm{E}-04$

$2.89924 \mathrm{E}-04$

$2.93305 \mathrm{E}-04$

$2.96705 \mathrm{E}-04$

$3.00125 \mathrm{E}-04$

$3.03565 \mathrm{E}-04$

$3.07024 \mathrm{E}-04$

$3.10503 \mathrm{E}-04$

$3.14001 \mathrm{E}-04$

$3.17519 \mathrm{E}-04$ $f_{2}$

$2.77834 \mathrm{E}-01$

$2.82548 \mathrm{E}-01$

$2.87301 \mathrm{E}-01$

$2.92094 \mathrm{E}-01$

$2.96927 \mathrm{E}-01$

$3.01799 \mathrm{E}-01$

$3.06711 \mathrm{E}-01$

$3.11662 \mathrm{E}-01$

$3.16653 \mathrm{E}-01$

$3.21684 \mathrm{E}-01$

$3.26754 \mathbf{E}-01$

$3.31864 \mathrm{E}-01$

$3.37013 \mathrm{E}-01$

3.42202 E-01

$3.47430 \mathrm{E}-01$

$3.52698 \mathrm{E}-01$

$3.58005 \mathrm{E}-01$

$3.63352 \mathrm{E}-01$

$3.68739 \mathrm{E}-01$

$3.74165 \mathrm{E}-01$

$3.79631 \mathrm{E}-01$

$3.85136 \mathrm{E}-01$

$3.90681 \mathrm{E}-01$

$3.96265 \mathrm{E}-01$

$4.01889 \mathrm{E}-01$

$4.07553 \mathrm{E}-01$

$4.13256 \mathrm{E}-01$

$4.18998 \mathrm{E}-01$

$4.24780 \mathrm{E}-01$

$4.30602 \mathrm{E}-01$

$4.36463 \mathrm{E}-01$

4.42364 E-01

4.48304 E-01

$4.54284 \mathrm{E}-01$

$4.60303 \mathrm{E}-01$

$4.66362 \mathrm{E}-01$

$4.72460 \mathrm{E}-01$

$4.78598 \mathrm{E}-01$

$4.84776 \mathrm{E}-01$

$4.90993 \mathrm{E}-01$

$4.97249 \mathrm{E}-01$

$5.03545 \mathrm{E}-01$

$5.09880 \mathrm{E}-01$

$5.16255 \mathrm{E}-01$

$5.22670 \mathrm{E}-01$

$5.29124 \mathrm{E}-01$

$5.35617 \mathrm{E}-01$

$5.42150 \mathrm{E}-01$

$5.48723 \mathrm{E}-01$

$5.55335 \mathrm{E}-01$

$5.61986 \mathrm{E}-01$

$5.68677 \mathrm{E}-01$

$5.75408 \mathrm{E}-01$

$5.82178 \mathrm{E}-01$

$5.88987 \mathrm{E}-01$

$5.95836 \mathrm{E}-01$

$6.02724 \mathrm{E}-0$

$6.09652 \mathrm{E}-01$

$6.16620 \mathrm{E}-01$

$6.23626 \mathrm{E}-01$ $g_{2}$

$\tan \delta_{2}$

$f$.

$2.68484 \mathrm{E}-03$

$2.76541 \mathrm{E}-03$

$2.84753 \mathrm{E}-03$

$2.93123 \mathrm{E}-03$

$3.01652 \mathrm{E}-03$

$3.10342 \mathrm{E}-03$

$3.19195 \mathrm{E}-03$

$3.28210 \mathrm{E}-03$

$3.37391 \mathrm{E}-03$

$3.46739 \mathrm{E}-03$

$3.56254 \mathrm{E}-03$

$3.65940 \mathrm{E}-03$

$3.75796 \mathrm{E}-03$

$3.85824 \mathrm{E}-03$

$3.96027 \mathrm{E}-03$

$4.06405 \mathrm{E}-03$

$4.16960 \mathrm{E}-03$

$4.27693 \mathrm{E}-03$

$4.38606 \mathrm{E}-03$

$4.49701 \mathrm{E}-03$

$4.60978 \mathrm{E}-03$

$4.72440 \mathrm{E}-03$

$4.84087 \mathrm{E}-03$

$4.95922 \mathrm{E}-03$

$5.07945 \mathrm{E}-03$

$5.20159 \mathrm{E}-03$

$5.32564 \mathrm{E}-03$

$5.45162 \mathrm{E}-03$

$5.57955 \mathrm{E}-03$

$5.70944 \mathrm{E}-03$

$5.84131 \mathrm{E}-03$

$5.97516 \mathrm{E}-03$

$6.11102 \mathrm{E}-03$

$6.24890 \mathrm{E}-03$

$6.38882 \mathrm{E}-03$

$6.53078 \mathrm{E}-03$

$6.67481 \mathrm{E}-03$

$6.82092 \mathrm{E}-03$

$6.96912 \mathrm{E}-03$

$7.11943 \mathrm{E}-03$

$7.27186 \mathrm{E}-03$

$7.42643 \mathrm{E}-03$

$7.58316 \mathrm{E}-03$

$7.74204 \mathrm{E}-03$

$7.90312 \mathrm{E}-03$

$8.06638 \mathrm{E}-03$

$8.23186 \mathrm{E}-03$

$8.39957 \mathrm{E}-03$

$8.56951 \mathrm{E}-03$

$8.74171 \mathrm{E}-03$

$8.91619 \mathrm{E}-03$

$9.09294 \mathrm{E}-03$

$9.27200 \mathrm{E}-03$

$9.45337 \mathrm{E}-03$

$9.63707 \mathrm{E}-03$

$9.82311 \mathrm{E}-03$

$1.00115 \mathrm{E}-02$

$1.02023 \mathrm{E}-02$

$1.03955 \mathrm{E}-02$

$1.05910 \mathrm{E}-02$

$-4.84338 \mathrm{E} \quad 01 \quad-2.16542 \mathrm{E}-03$

$-4.84480 \mathrm{E}$ 01 $-2.23867 \mathrm{E}-0.3$

$-4.84619 \mathrm{E}$ 0l $-2.31343 \mathrm{E}-03$

$-4.84756 \mathrm{E}$ 01 $-2.38972 \mathrm{E}-03$

$\begin{array}{lll}-4.84891 \mathrm{E} & 01 & -2.46755 \mathrm{E}-03\end{array}$

$-4.85023 \mathrm{E} \quad 01 \quad-2.54694 \mathrm{E}-03$

$-4.85153 \mathrm{E}$ 01 $-2.62790 \mathrm{E}-03$

$-4.85281 \mathrm{E}$ 01 $-2.71945 \mathrm{E}-03$

$-4.85407 \mathrm{E}$ ol $-2.79460 \mathrm{E}-03$

$-4.85531 \mathrm{E} \quad 01 \quad-2.88036 \mathrm{E}-03$

$-4.85653 \mathrm{E} \quad 01$

$-4.85773 \mathrm{E} \quad 01$

$-4.85891 \mathrm{E} \quad 01$

$-4.86007 \mathrm{E} \quad 01$

$-4.86121 \mathrm{E} \quad 01$

$-4.86234 \mathrm{E} \quad 01$

$-4.86345 \mathrm{E} \quad 01$

$-4.86454 \mathrm{E} \quad 01$

$-4.86561 \mathrm{E} \quad 01$

$-4.86667 \mathrm{E} 01$

$-4.86772 \mathrm{E} \quad 01$

$-4.86875 \mathrm{E} \quad 01$

$-4.86976 \mathrm{E} \quad 01$

$-4.87076 \mathrm{E} \quad 01$

$-4.87174 \mathrm{E} \quad 01$

$-4.87271 \mathrm{E} \quad 01$

$-4.87367 \mathrm{E} \quad 01$

$-4.87461 \mathrm{E} \quad 01$

$-4.87554 \mathrm{E} \quad 01$

$-4.87645 \mathrm{E} \quad 01$

$-2.96776 \mathrm{E}-03$

$-3.05681 \mathrm{E}-03$

$-3.14751 \mathrm{E}-03$

$-3.23989 \mathrm{E}-03$

$-3.33396 \mathrm{E}-03$

$-3.42974 \mathrm{E}-03$

$-3.52724 \mathrm{E}-03$

$-3.62647 \mathrm{E}-03$

$-3.72745 \mathrm{E}-03$

$-3.83019 \mathrm{E}-03$ 
ELECTRON RADIAL FUNCTIONS

Positron

$\begin{array}{cc}p & F_{0} \\ 18.10 & 9.14012 \mathrm{E}-01 \\ 18.20 & 9.14009 \mathrm{E}-01 \\ 18.30 & 9.14006 \mathrm{E}-01 \\ 18.40 & 9.14003 \mathrm{E}-01 \\ 18.50 & 9.14000 \mathrm{E}-01 \\ & \\ 18.60 & 9.13998 \mathrm{E}-01 \\ 18.70 & 9.13995 \mathrm{E}-01 \\ 18.80 & 9.13992 \mathrm{E}-01 \\ 18.90 & 9.13989 \mathrm{E}-01 \\ 19.00 & 9.13986 \mathrm{E}-01\end{array}$

19.10

19.20

19.30

19.40

19.50

19.60

19.70

19.80

19.90

20.00

20.10

20.20

20.30

20.40

20.50

20.60

20.70

20.80

20.90

21.00

21.10

21.20

21.30

21.40

21.50

21.60

21.70

21.80

21.90

22.00

22.10

22.20

22.30

22.40

22.50

22.60

22.70

22.80

22.90

23.00

23.10

23.20

23.30

23.40

23.50

23.60

23.70

23.80

23.90

24.00

$Z=4$

$A=8$

$\rho=1.2 A^{1 / 3} F$

$f_{1}$

$1.69151 \mathrm{E} \quad 01$

$1.70120 \mathrm{E} \quad 01$

$1.71090 \mathrm{E} 01$

$1.72060 \mathrm{E} 01$

$1.73030 \mathrm{E}$ ol

$1.74000 \mathrm{E} \quad 01$

$1.74971 \mathrm{E} \quad 01$

$1.75941 \mathrm{E} \quad 01$

$1.76912 \mathrm{E} 01$

$1.77882 \mathrm{E} 01$

$1.78853 \mathrm{E} \quad 01$ $1.79824 \mathrm{E} \quad 01$ $1.80795 \mathrm{E} \quad 01$

$1.81766 \mathrm{E} \quad 01$

$1.82738 \mathrm{E} 01$

$1.83709 \mathrm{E} \quad 01$

$1.84681 \mathrm{E} \quad 01$

$1.85652 \mathrm{E}$ 01

$1.86624 \mathrm{E} 01$

$1.87596 \mathrm{E} \quad 01$

$\begin{array}{ll}1.88568 \mathrm{E} & 01\end{array}$ $1.89541 \mathrm{E} \quad 01$

$1.90513 \mathrm{E}$ 01

$1.91485 \mathrm{E} \quad 01$

$1.92458 \mathrm{E} 01$

$\begin{array}{lll}1.93431 \mathrm{E} & 01\end{array}$

$1.94404 \mathrm{E} \quad 01$

$1.95377 \mathrm{E} \quad 01$

$1.96350 \mathrm{E} 01$

$1.97323 \mathrm{E} \quad 01$

$1.98297 \mathrm{E} \quad 01$

$1.99270 \mathrm{E} 01$

$2.00244 \mathrm{E} \quad 01$

$2.01218 \mathrm{E} 01$

$2.02192 \mathrm{E} 01$

$2.03166 \mathrm{E} \quad 01$ $2.04140 \mathrm{E} \quad 01$

$2.05115 \mathrm{E} \quad 01$

$2.06089 \mathrm{E} 01$

$2.07064 \mathrm{E} \quad 01$

$2.08039 \mathrm{E} \quad 01$ $2.09014 \mathrm{E} \quad 01$ $2.09989 \mathrm{E} 01$

$2.10964 \mathrm{E} \quad 01$

$2.11940 \mathrm{E} 01$

$2.12915 \mathrm{E} \quad 01$

$2.13891 \mathrm{E} 01$

$2.14867 \mathrm{E} \quad 01$

$2.15843 \mathrm{E} \quad 01$

$2.16819 \mathrm{E} \quad 01$

$2.17795 \mathrm{E} 01$

$2.18772 \mathrm{E} \quad 01$

$2.19748 \mathrm{E} 01$

$2.20725 \mathrm{E} \quad 01$

$2.21702 \mathrm{E} 01$

$\begin{array}{lll}2.22679 \mathrm{E} & 01\end{array}$

$2.23656 \mathrm{E} \quad 01$

$2.24634 \mathrm{E} \quad 01$

$2.25611 \mathrm{E} \quad 01$

$2.26589 \mathrm{E} \quad 01$ $g_{1}$

$4.72976 \mathrm{E}-01$
$4.79211 \mathrm{E}-01$
$4.85486 \mathrm{E}-01$
$4.91803 \mathrm{E}-01$
$4.98159 \mathrm{E}-01$

$5.04557 \mathrm{E}-01$

$5.10995 \mathrm{E}-01$

$5.17474 \mathrm{E}-01$

$5.23994 \mathrm{E}-01$
$5.30554 \mathrm{E}-01$

$5.37155 \mathrm{E}-01$

$5.43797 \mathrm{E}-01$

$5.50479 \mathrm{E}-01$

$5.57203 \mathrm{E}-01$

$5.63967 \mathrm{E}-01$

$5.70772 \mathrm{E}-01$

$5.77617 \mathrm{E}-01$

$5.84504 \mathrm{E}-01$

5.91431 E-01

$5.98399 \mathrm{E}-01$

$6.05408 \mathrm{E}-01$

$6.12458 \mathrm{E}-01$

$6.19549 \mathrm{E}-01$

$6.26680 \mathrm{E}-01$

$6.33853 \mathrm{E}-01$

$6.41066 \mathrm{E}-01$ $6.48320 \mathrm{E}-01$ $6.55616 \mathrm{E}-01$ $6.62952 \mathrm{E}-01$ $6.70329 \mathrm{E}-01$

$6.77747 \mathrm{E}-01$ $6.85206 \mathrm{E}-01$ $6.92706 \mathrm{E}-01$

$7.00247 \mathrm{E}-01$

$7.07829 \mathrm{E}-01$

$7.15453 \mathrm{E}-01$

$7.23117 \mathrm{E}-01$

$7.30822 \mathrm{E}-01$

$7.38568 \mathrm{E}-01$

$7.46356 \mathrm{E}-01$

$7.54184 \mathrm{E}-01$

$7.62054 \mathrm{E}-01$

$7.69964 \mathrm{E}-01$

$7.77916 \mathrm{E}-01$

$7.85909 \mathrm{E}-01$

$7.93944 \mathrm{E}-01$ $8.02019 \mathrm{E}-01$ $8.10136 \mathrm{E}-01$ $8.18293 \mathrm{E}-01$ 8.26492E-01

8.34733E-01

$8.43014 \mathrm{E}-01$

$8.51337 \mathrm{E}-01$

$8.59701 \mathrm{E}-01$

$8.68106 \mathrm{E}-01$

$8.76553 \mathrm{E}-01$ $8.85041 \mathrm{E}-01$

$8.93570 \mathrm{E}-01$

$9.02141 \mathrm{E}-01$

$9.10753 \mathrm{E}-01$ $\tan \delta_{1}$

$-7.56596 \mathrm{E}-04$ $-7.60623 \mathrm{E}-04$ $-7.64601 \mathrm{E}-04$ $-7.68531 \mathrm{E}-04$

$-7.72411 \mathrm{E}-04$

$-7.76245 \mathrm{E}-04$

$-7.80033 \mathrm{E}-04$

$-7.83774 \mathrm{E}-04$

$-7.87469 \mathrm{E}-04$

$-7.91117 \mathrm{E}-04$

$-7.94723 \mathrm{E}-04$

$-7.98280 \mathrm{E}-04$

$-8.01799 \mathrm{E}-04$

$-8.05277 \mathrm{E}-04$

$-8.08714 \mathrm{E}-04$

$-8.12110 \mathrm{E}-04$

$-8.15465 \mathrm{E}-04$

$-8.18782 \mathrm{E}-04$

$-8.22051 \mathrm{E}-04$

$-8.25291 \mathrm{E}-04$

$-8.28492 \mathrm{E}-04$

$-8.31650 \mathrm{E}-04$

$-8.34774 \mathrm{E}-04$

$-8.37860 \mathrm{E}-04$

$-8.40912 \mathrm{E}-04$

$-8.43928 \mathrm{E}-04$

$-8.46907 \mathrm{E}-04$

$-8.49854 \mathrm{E}-04$

$-8.52765 \mathrm{E}-04$

$-8.55642 \mathrm{E}-04$

$-8.58490 \mathrm{E}-04$

$-8.61300 \mathrm{E}-04$

$-8.64077 \mathrm{E}-04$

$-8.66827 \mathrm{E}-04$

$-8.69545 \mathrm{E}-04$

$-8.72227 \mathrm{E}-04$

$-8.74883 \mathrm{E}-04$

$-8.77507 \mathrm{E}-04$

$-8.80102 \mathrm{E}-04$

$-8.82667 \mathrm{E}-04$

$-8.85202 \mathrm{E}-04$

$-8.87706 \mathrm{~F}-04$

$-8.90187 \mathrm{E}-04$

$-8.92638 \mathrm{E}-04$

$-8.95056 \mathrm{E}-04$

$-8.97450 \mathrm{E}-04$

$-8.99817 \mathrm{E}-04$

$-9.02156 \mathrm{E}-04$

$-9.04472 \mathrm{E}-04$

$-9.06761 \mathrm{E}-04$

$-9.09021 \mathrm{E}-04$

$-9.11261 \mathrm{E}-04$

$-9.13470 \mathrm{E}-04$

$-9.15656 \mathrm{E}-04$

$-9.17820 \mathrm{E}-04$

$-9.19960 \mathrm{E}-04$

$-9.22072 \mathrm{E}-04$

$-9.24160 \mathrm{E}-04$

$-9.26227 \mathrm{E}-04$

$-9.28274 \mathrm{E}-04$

$f_{-1}$

$-4.25999 \mathrm{E}-01$

$-4.32037 \mathrm{E}-01$

$-4.38116 \mathrm{E}-01$

$-4.44235 \mathrm{E}-01$

$-4.50395 \mathrm{E}-01$

$-4.56596 \mathrm{E}-01$

$-4.62837 \mathrm{E}-01$

$-4.69119 \mathrm{E}-01$

$-4.75442 \mathrm{E}-01$

$-4.81806 \mathrm{E}-01$

$-4.88210 \mathrm{E}-01$

$-4.94656 \mathrm{E}-01$

$-5.01141 \mathrm{E}-01$

$-5.07668 \mathrm{E}-01$

$-5.14236 \mathrm{E}-01$

$-5.20844 \mathrm{E}-01$

$-5.27494 \mathrm{E}-01$

$-5.341 .84 \mathrm{E}-01$

$-5.40915 \mathrm{E}-01$

$-5.47687 \mathrm{E}-01$

$-5.54499 \mathrm{E}-01$

$-5.61353 \mathrm{E}-01$

$-5.68247 \mathrm{E}-01$

$-5.75183 \mathrm{E}-01$

$-5.82159 \mathrm{E}-01$

$-5.89177 \mathrm{E}-01$

$-5.96235 \mathrm{E}-01$

$-6.03334 \mathrm{E}-01$

$-6.10475 \mathrm{E}-01$

$-6.17656 \mathrm{E}-01$

$-6.24878 \mathrm{E}-01$

$-6.32141 \mathrm{E}-01$

$-6.39446 \mathrm{E}-01$

$-6.46791 \mathrm{E}-01$

$-6.54178 \mathrm{E}-01$

$-6.61605 \mathrm{E}-01$

$-6.69074 \mathrm{E}-01$

$-6.76584 \mathrm{E}-01$

$-6.84134 \mathrm{E}-01$

$-6.91726 \mathrm{E}-01$

$-6.99359 \mathrm{E}-01$

$-7.07034 \mathrm{E}-01$

$-7.14749 \mathrm{E}-01$

$-7.22506 \mathrm{E}-01$

$-7.30304 \mathrm{E}-01$

$-7.38143 \mathrm{E}-01$

$-7.46023 \mathrm{E}-01$

$-7.53944 \mathrm{E}-01$

$-7.61907 \mathrm{E}-01$

$-7.69911 \mathrm{E}-01$

$-7.77956 \mathrm{E}-01$

$-7.86043 \mathrm{E}-01$

$-7.94171 \mathrm{E}-01$ 
ELECTRON RADIAL FUNCTIONS

$Z=4 \quad A=8 \quad \rho=1.2 A^{1 / 3} F$

Prsitron

$\bar{F}$

$3.21057 \mathrm{E}-0$

$3.24614 \mathrm{E}-04$

$3.28190 \mathrm{E}-04$

$3.31787 \mathrm{E}-04$

$3.35403 \mathrm{E}-04$

$3.39038 \mathrm{E}-04$

$3.42693 \mathrm{E}-04$

$3.46368 \mathrm{E}-04$

$3.50062 \mathrm{E}-04$

$3.53776 \mathrm{E}-04$

$3.57509 \mathrm{E}-04$

$3.61262 \mathrm{E}-04$

$3.65035 \mathrm{E}-04$

$3.68827 \mathrm{E}-04$

$3.72639 \mathrm{E}-04$

$3.764 .70 \mathrm{E}-04$

$3.80321 \mathrm{E}-04$

$3.84192 \mathrm{E}-04$

$3.88082 \mathrm{E}-04$

$3.91992 \mathrm{E}-04$

$3.95921 \mathrm{E}-04$

$3.99870 \mathrm{E}-04$

$4.03838 \mathrm{E}-04$

$4.07826 \mathrm{E}-04$

$4.11834 \mathrm{E}-04$

$4.15861 \mathrm{E}-04$

$4.19908 \mathrm{E}-04$

$4.23975 \mathrm{E}-04$

$4.28061 \mathrm{E}-04$

$4.32166 \mathrm{E}-04$

$4.36292 \mathrm{E}-04$

$4.40436 \mathrm{E}-04$

$4.44601 \mathrm{E}-04$

$4.48785 \mathrm{E}-04$

$4.52988 \mathrm{E}-04$

$4.57211 \mathrm{E}-04$

$4.61454 \mathrm{E}-04$

$4.65716 \mathrm{E}-04$

$4.69998 \mathrm{E}-04$

$4.74300 \mathrm{E}-04$

$4.78621 \mathrm{E}-04$

$4.82962 \mathrm{E}-04$

$4.87322 \mathrm{E}-04$

$4.91702 \mathrm{E}-04$

$4.96101 \mathrm{E}-04$

$5.00520 \mathrm{E}-04$

$5.04959 \mathrm{E}-04$

5.09417 E-04

$5.13895 \mathrm{E}-04$

5.18392 E-04

$5.22909 \mathrm{E}-04$

$5.27446 \mathrm{E}-04$

$5.32002 \mathrm{E}-04$

$5.36578 \mathrm{E}-04$

$5.41173 \mathrm{E}-04$

$5.45788 \mathrm{E}-04$

$5.50423 \mathrm{E}-04$

$5.55077 \mathrm{E}-04$

$5.59751 \mathrm{E}-04$

$5.64444 \mathrm{E}-04$ $f_{2}$

$g_{2}$

$\tan \delta_{2}$

$f_{-}$

$6.30673 \mathrm{E}-01$ $6.37759 \mathrm{E}-01$ $6.44884 \mathrm{E}-01$ $6.52049 \mathrm{E}-01$ $6.59253 \mathrm{E}-01$

$6.66497 \mathrm{E}-01$ $6.73779 \mathrm{E}-01$ $6.81102 \mathrm{E}-01$ $6.88464 \mathrm{E}-01$ $6.95866 \mathrm{E}-01$

$7.03307 \mathrm{E}-01$ $7.10787 \mathrm{E}-01$ $7.18307 \mathrm{E}-01$ $7.25866 \mathrm{E}-01$ $7.33465 \mathrm{E}-01$

$7.41103 \mathrm{E}-01$ $7.48781 \mathrm{E}-0$ $7.56497 \mathrm{E}-01$ $7.64254 \mathrm{E}-01$

$7.72050 \mathrm{E}-01$

$7.79885 \mathrm{E}-01$ $7.87760 \mathrm{E}-01$ $7.95674 \mathrm{E}-01$ $8.03627 \mathrm{E}-01$

$8.11620 \mathrm{E}-01$

8.19653E-01 $8.27725 \mathrm{E}-01$ $8.35836 \mathrm{E}-01$ $8.43986 \mathrm{E}-01$ $8.52176 \mathrm{E}-0$

8.60406E-01 $8.68674 \mathrm{E}-01$ $8.76983 \mathrm{E}-0$ $8.85330 \mathrm{E}-01$ $8.93717 \mathrm{E}-01$

$9.02143 \mathrm{E}-01$ $9.10609 \mathrm{E}-01$ $9.19114 \mathrm{E}-01$ $9.27659 \mathrm{E}-01$ $9.36242 \mathrm{E}-01$

$9.44866 \mathrm{E}-01$ $9.53528 \mathrm{E}-01$ $9.62230 \mathrm{E}-01$ $9.70971 \mathrm{E}-01$ $9.79752 \mathrm{E}-01$

$9.88572 \mathrm{E}-01$ $9.97431 \mathrm{E}-01$ $1.00633 \mathrm{E} \quad 00$ $1.01527 \mathrm{E} \quad 00$ $1.02425 \mathrm{E} \quad 00$

$1.03326 \mathrm{E} \quad 00$ $1.04232 \mathrm{E} \quad 00$ $1.05141 \mathrm{E} 00$ $1.06055 \mathrm{E} \quad 00$ $1.06972 \mathrm{E} \quad 00$

$1.07894 \mathrm{E} \quad 00$ $1.08819 \mathrm{E} \quad 00$ $1.09748 \mathrm{E} \quad 00$ $1.10681 \mathrm{E} \quad 00$ $1.11618 \mathrm{E} \quad 00$
$1.07890 \mathrm{E}-02$ $1.09894 \mathrm{E}-02$ $1.11923 \mathrm{E}-02$

$1.13976 \mathrm{E}-02$

$1.16054 \mathrm{E}-02$

$1.18157 \mathrm{E}-02$

$1.20285 \mathrm{E}-02$

1. $22438 \mathrm{E}-02$

$1.24617 \mathrm{E}-02$

$1.26821 \mathrm{E}-02$

$1.29051 \mathrm{E}-02$

$1.31306 \mathrm{E}-02$

$1.33587 \mathrm{E}-02$

$1.35895 \mathrm{E}-02$

1. $38229 \mathrm{E}-02$

$1.40589 \mathrm{E}-02$

$1.42975 \mathrm{E}-02$

$1.45389 \mathrm{E}-02$

$1.47829 \mathrm{E}-02$

$1.50296 \mathrm{E}-02$

$1.52790 \mathrm{E}-02$

$1.55312 \mathrm{E}-02$

$1.57860 \mathrm{E}-02$

$1.60437 \mathrm{E}-02$

$1.6304 .1 \mathrm{E}-02$

$1.65673 \mathrm{E}-02$ $1.68333 \mathrm{E}-02$ $1.71021 \mathrm{E}-02$ $1.73738 \mathrm{E}-02$ $1.76483 \mathrm{E}-02$

$1.79256 \mathrm{E}-02$ $1.82058 \mathrm{E}-02$ $1.84889 \mathrm{E}-02$

$1.87749 \mathrm{E}-02$ $1.90639 \mathrm{E}-02$

$1.93557 \mathrm{E}-02$ $1.96505 \mathrm{E}-02$ $1.99483 \mathrm{E}-02$ $2.02490 \mathrm{E}-02$ $2.05528 \mathrm{E}-02$

$2.08595 \mathrm{E}-02$ $2.11693 \mathrm{E}-02$ $2.14821 \mathrm{E}-02$ $2.17979 \mathrm{E}-02$ $2.21168 \mathrm{E}-02$

$2.24388 \mathrm{E}-02$ $2.27639 \mathrm{E}-02$ $2.30921 \mathrm{E}-02$ $2.34234 \mathrm{E}-02$ $2.37578 \mathrm{E}-02$

$2.40954 \mathrm{E}-02$

$2.44362 \mathrm{E}-02$

$2.47801 \mathrm{E}-02$

$2.51272 \mathrm{E}-02$

$2.58312 \mathrm{E}-02$ $2.61880 \mathrm{E}-02$

$2.65481 \mathrm{E}-02$

$2.69114 \mathrm{E}-02$

$2.72780 \mathrm{E}-02$
$2.54776 \mathrm{E}-02$ $\begin{array}{ll}-4.89963 \mathrm{E} & 01\end{array}$ $-4.90024 \mathrm{E} 01$ $-4.90085 \mathrm{E} \quad 01$ $-4.90144 \mathrm{E} \quad 01$ $-4.90203 \mathrm{E} 01$

$-4.90262 \mathrm{E} \quad 01$ $-4.90319 \mathrm{E} 01$ $-4.90377 \mathrm{E} 01$ $-4.90433 \mathrm{E} 01$ $-4.90489 \mathrm{E} 0 \mathrm{I}$

$-4.90544 \mathrm{E} \quad 01$ $-4.90599 \mathrm{E} 01$ $-4.90653 \mathrm{E} 01$ $-4.90706 \mathrm{E} 01$ $-4.90759 \mathrm{E} 0 \mathrm{I}$

$-4.90811 \mathrm{E} \quad 01$ $-4.90863 \mathrm{E} \quad 01$ $-4.90914 \mathrm{E} \quad 01$ $-4.90965 \mathrm{E} \quad 01$

$-4.91015 \mathrm{E} 01$

$-4.91065 \mathrm{E} \quad 01$ $-4.91114 \mathrm{E}$ 0l $-4.91163 \mathrm{E} \quad 01$ $-4.91211 \mathrm{E} \quad 01$ $-4.91258 \mathrm{E} \quad 01$

$-4.91306 \mathrm{E} 01$ $-4.91352 \mathrm{E} \quad 01$ $\begin{array}{lll}-4.91399 \mathrm{E} & 01\end{array}$ $-4.91444 \mathrm{E} 01$ $-4.91490 \mathrm{E} \quad 01$

$-4.91535 \mathrm{E} \quad 01$ $\begin{array}{lll}-4.91579 \mathrm{E} & 01\end{array}$

$-4.91623 \mathrm{E} \quad 01$

$-4.91667 \mathrm{E} 01$

$-4.91710 \mathrm{E} 01$

$-4.91753 \mathrm{E} \quad 01$

$-4.91795 \mathrm{E} 01$

$-4.91837 \mathrm{E} \quad 01$

$-4.91919 \mathrm{E} \quad 01$

$-4.91960 \mathrm{E} \quad 01$

$-4.92001 \mathrm{E} \quad 01$

$-4.92041 \mathrm{E} 01$

$-4.92080 \mathrm{E} \quad 01$

$-4.92120 \mathrm{E} \quad 01$

$-4.92158 \mathrm{E} \quad 01$

$-4.92197 \mathrm{E} 01$

$-4.92235 \mathrm{E} 01$

$-4.92273 \mathrm{E} \quad 01$

$-4.92311 \mathrm{E} 01$

$-4.92348 \mathrm{E} \quad 01$

$-4.92385 \mathrm{E} 01$

$-4.92421 \mathrm{E} \quad 01$

$-4.92457 \mathrm{E} \quad 01$

$-4.92529 \mathrm{E} \quad 01$

$-4.92564 \mathrm{E}$ 01

$-4.92634 \mathrm{E}$ 0I

$-4.92668 \mathrm{E} 01$
$-4.91878 \mathrm{E}$ ol

$-4.92493 \mathrm{E} 01$

$-4.92599 \mathrm{E} \quad 01$

$-9.74363 \mathrm{E}-03$ $-9.93378 \mathrm{E}-03$ $-1.01263 \mathrm{E}-02$ $-1.03213 \mathrm{E}-02$ $-1.05187 \mathrm{E}-02$

$-1.07185 \mathrm{E}-02$ $-1.09208 \mathrm{E}-02$

$-1.11255 \mathrm{E}-02$

$-1.13328 \mathrm{E}-02$

$-1.15425 \mathrm{E}-02$

$-1.17548 \mathrm{E}-02$ $-1.19696 \mathrm{E}-02$ $-1.21869 \mathrm{E}-02$ $-1.24068 \mathrm{E}-02$ $-1.26293 \mathrm{E}-02$

$-1.28543 \mathrm{E}-02$ $-1.30820 \mathrm{E}-02$ $-1.33123 \mathrm{E}-02$ $-1.35452 \mathrm{E}-02$ $-1.37807 \mathrm{E}-02$

$-1.40190 \mathrm{E}-02$ $-1.42599 \mathrm{E}-02$

$-1.45034 \mathrm{E}-02$ $-1.47497 \mathrm{E}-02$

$-1.49988 \mathrm{E}-02$

$-1.52505 \mathrm{E}-02$ $-1.55050 \mathrm{E}-02$ $-1.57623 \mathrm{E}-02$ $-1.60223 \mathrm{E}-02$ $-1.62852 \mathrm{E}-02$

$-1.65508 \mathrm{E}-02$ $-1.68193 \mathrm{E}-02$ $-1.70906 \mathrm{E}-02$ $-1.73648 \mathrm{E}-02$ $-1.76418 \mathrm{E}-02$

$-1.79218 \mathrm{E}-02$ $-1.82046 \mathrm{E}-02$ $-1.84903 \mathrm{E}-02$ $-1.87790 \mathrm{E}-02$ $-1.90706 \mathrm{E}-02$

$-1.93651 \mathrm{E}-02$ $-1.96627 \mathrm{E}-02$ 
Positron

\begin{tabular}{|c|c|c|c|}
\hline$p$ & $F_{0}$ & $f_{1}$ & \\
\hline 24.10 & $9.13846 \mathbf{E}-01$ & $2.27567 \mathrm{E}$ & 01 \\
\hline 24.20 & $9.13844 \mathrm{E}-01$ & $2.28545 \mathrm{E}$ & 01 \\
\hline 24.30 & $9.13841 \mathrm{E}-01$ & $2.29523 \mathrm{E}$ & 01 \\
\hline 24.40 & $9.13838 \mathrm{E}-01$ & $2.30501 \mathrm{E}$ & 01 \\
\hline 24.50 & $9.13836 \mathrm{E}-01$ & $2.31480 \mathrm{E}$ & 01 \\
\hline 24.60 & $9.13833 \mathrm{E}-01$ & $2.32458 \mathrm{E}$ & 01 \\
\hline 24.70 & $9.13831 \mathrm{E}-01$ & $2.33437 \mathrm{E}$ & 01 \\
\hline 24.80 & $9.13828 \mathrm{E}-01$ & $2.34416 \mathrm{E}$ & 01 \\
\hline 24.90 & $9.13826 \mathrm{E}-01$ & $2.35395 \mathrm{E}$ & 01 \\
\hline 25.00 & $9.13823 \mathrm{E}-01$ & $2.36375 \mathrm{E}$ & 01 \\
\hline 25.10 & $9.13820 \mathrm{E}-01$ & $2.37354 \mathrm{E}$ & 01 \\
\hline 25.20 & $9.13818 \mathrm{E}-01$ & $2.38334 \mathrm{E}$ & 01 \\
\hline 25.30 & $9.13815 \mathrm{E}-01$ & $2.39313 \mathrm{E}$ & 01 \\
\hline 5.40 & $9.13813 \mathrm{E}-01$ & $2.40293 \mathrm{E}$ & 01 \\
\hline 25.50 & $9.13810 \mathrm{E}-01$ & $2.41273 \mathrm{E}$ & 01 \\
\hline 25.60 & $9.13808 \mathrm{E}-01$ & $2.42254 \mathbf{E}$ & 01 \\
\hline 25.70 & $9: 13805 \mathrm{E}-01$ & $2.43234 \mathrm{E}$ & 01 \\
\hline 25.80 & $9.13803 \mathrm{E}-01$ & $2.44215 \mathrm{E}$ & 01 \\
\hline 5.90 & $9.13800 \mathrm{E}-01$ & $2.45195 \mathrm{E}$ & 01 \\
\hline 26.00 & $9.13798 \mathrm{E}-0 \mathrm{l}$ & $2.46176 \mathrm{E}$ & 01 \\
\hline 26.10 & $9.13795 \mathrm{E}-01$ & $2.47157 \mathrm{E}$ & 01 \\
\hline 26.20 & $9.13793 \mathrm{E}-01$ & $2.48139 \mathrm{E}$ & 01 \\
\hline 26.30 & $9.13790 \mathrm{E}-01$ & $2.49120 \mathrm{E}$ & 01 \\
\hline 26.40 & $9.13788 \mathrm{E}-01$ & $2.50102 \mathrm{E}$ & 01 \\
\hline 26.50 & $9.13785 \mathrm{E}-01$ & $2.51083 \mathrm{E}$ & 01 \\
\hline 26.60 & $9.13783 \mathrm{E}-01$ & $2.52065 \mathrm{E}$ & 01 \\
\hline 26.70 & $9.13780 \mathrm{E}-01$ & $2.53047 \mathrm{E}$ & 01 \\
\hline 26.80 & $9.13778 \mathrm{E}-01$ & $2.54030 \mathrm{E}$ & 01 \\
\hline 26.90 & $9.13775 \mathrm{E}-01$ & $2.55012 \mathrm{E}$ & 01 \\
\hline 27.00 & $9.13773 \mathrm{E}-01$ & $2.55995 \mathrm{E}$ & 01 \\
\hline 27.10 & $9.13770 \mathrm{E}-01$ & $2.56977 \mathrm{E}$ & 01 \\
\hline 27.20 & $9.13768 \mathrm{E}-01$ & $2.57960 \mathrm{E}$ & 01 \\
\hline 27.30 & $9.13765 \mathrm{E}-01$ & $2.58944 \mathrm{E}$ & 01 \\
\hline 27.40 & $9.13763 \mathrm{E}-01$ & $2.59927 \mathrm{E}$ & 01 \\
\hline 27.50 & $9.13761 \mathrm{E}-01$ & $2.60910 \mathrm{E}$ & 01 \\
\hline 27.60 & $9.13758 \mathrm{E}-01$ & $2.61894 \mathrm{E}$ & 01 \\
\hline 27.70 & $9.13756 \mathrm{E}-01$ & $2.62878 \mathrm{E}$ & 01 \\
\hline 27.80 & $9.13753 \mathrm{E}-01$ & $2.63862 \mathrm{E}$ & 01 \\
\hline 27.90 & $9.13751 \mathrm{E}-01$ & $2.64846 \mathrm{E}$ & 01 \\
\hline 28.00 & $9.13749 \mathrm{E}-01$ & $2.65830 \mathrm{E}$ & 01 \\
\hline 28.10 & $9.13746 \mathrm{E}-01$ & $2.66815 \mathrm{E}$ & 01 \\
\hline 28.20 & $9.13744 \mathrm{E}-01$ & $2.67800 \mathrm{E}$ & 01 \\
\hline 28.30 & $9.13742 \mathrm{E}-01$ & $2.68785 \mathrm{E}$ & 01 \\
\hline 28.40 & $9.13739 \mathrm{E}-01$ & $2.69770 \mathrm{E}$ & 01 \\
\hline 28.50 & $9.13737 \mathrm{E}-01$ & $2.70755 \mathrm{E}$ & 01 \\
\hline 28.60 & $9.13734 \mathrm{E}-01$ & $2.71741 \mathrm{E}$ & 01 \\
\hline 28.70 & $9.13732 \mathrm{E}-01$ & $2.72726 \mathrm{E}$ & 01 \\
\hline 28.80 & $9.13730 \mathrm{E}-01$ & $2.73712 \mathrm{E}$ & 01 \\
\hline 28.90 & $9.13727 \mathrm{E}-01$ & $2.74698 \mathrm{E}$ & 01 \\
\hline 29.00 & $9.13725 \mathrm{E}-01$ & $2.75684 \mathrm{E}$ & 01 \\
\hline 29.10 & $9.13723 \mathrm{E}-01$ & $2.76671 \mathrm{E}$ & 01 \\
\hline 29.20 & $9.13720 \mathrm{E}-01$ & $2.77657 \mathrm{E}$ & 01 \\
\hline 29.30 & $9.13718 \mathrm{E}-01$ & $2.78644 \mathrm{E}$ & 01 \\
\hline 29.40 & $9.13716 \mathrm{E}-01$ & $2.79631 \mathrm{E}$ & 01 \\
\hline 29.50 & $9.13713 \mathrm{E}-01$ & $2.80618 \mathrm{E}$ & 01 \\
\hline 29.60 & $9.13711 \mathrm{E}-01$ & $2.81606 \mathrm{E}$ & 01 \\
\hline 29.70 & $9.13709 \mathrm{E}-01$ & $2.82593 \mathrm{E}$ & 01 \\
\hline 29.80 & $9.13707 \mathrm{E}-01$ & $2.83581 \mathrm{E}$ & 01 \\
\hline 29.90 & $9.13704 \mathrm{E}-01$ & $2.84569 \mathrm{E}$ & 01 \\
\hline 30.00 & $9.13702 \mathrm{E}-01$ & $2.85557 \mathrm{E}$ & 01 \\
\hline
\end{tabular}

$Z=4 \quad A=8 \quad \rho=1.2 A^{1 / 3} F$

\begin{tabular}{|c|c|c|c|}
\hline$g_{1}$ & $\tan \delta_{1}$ & $f_{-1}$ & $g_{-1}$ \\
\hline $\begin{array}{l}9.19407 \mathrm{E}-01 \\
9.28101 \mathrm{E}-01 \\
9.36838 \mathrm{E}-01 \\
9.45615 \mathrm{E}-01 \\
9.54434 \mathrm{E}-01\end{array}$ & $\begin{array}{l}-9.30289 \mathrm{E}-04 \\
-9.32289 \mathrm{E}-04 \\
-9.34263 \mathrm{E}-04 \\
-9.36217 \mathrm{E}-04 \\
-9.38153 \mathrm{E}-04\end{array}$ & $\begin{array}{l}-8.60684 \mathrm{E}-01 \\
-8.69184 \mathrm{E}-01 \\
-8.77726 \mathrm{E}-01 \\
-8.86309 \mathrm{E}-01 \\
-8.94934 \mathrm{E}-01\end{array}$ & $\begin{array}{l}2.37401 \mathrm{E} \\
2.38381 \mathrm{E} \\
2.39361 \mathrm{E} \\
2.40342 \mathrm{E} \\
2.41323 \mathrm{E}\end{array}$ \\
\hline $\begin{array}{l}9.63295 \mathrm{E}-01 \\
9.72197 \mathrm{E}-01 \\
9.81140 \mathrm{E}-01 \\
9.90125 \mathrm{E}-01 \\
9.99151 \mathrm{E}-01\end{array}$ & $\begin{array}{l}-9.40056 \mathrm{E}-04 \\
-9.41947 \mathrm{E}-04 \\
-9.43814 \mathrm{E}-04 \\
-9.45657 \mathrm{E}-04 \\
-9.47483 \mathrm{E}-04\end{array}$ & $\begin{array}{l}-9.03600 \mathrm{E}-01 \\
-9.12308 \mathrm{E}-01 \\
-9.21057 \mathrm{E}-01 \\
-9.29848 \mathrm{E}-01 \\
-9.38681 \mathrm{E}-01\end{array}$ & $\begin{array}{l}2.42304 \mathrm{E} \\
2.43285 \mathrm{E} \\
2.44266 \mathrm{E} \\
2.45247 \mathrm{E} \\
2.46229 \mathrm{E}\end{array}$ \\
\hline $\begin{array}{ll}1.00822 \mathrm{E} & 00 \\
1.01733 \mathrm{E} & 00 \\
1.02648 \mathrm{E} & 00 \\
1.03567 \mathrm{E} & 00 \\
1.04491 \mathrm{E} & 00\end{array}$ & $\begin{array}{r}-9.49284 \mathrm{E}-04 \\
-9.51068 \mathrm{E}-04 \\
-9.52829 \mathrm{E}-04 \\
-9.54571 \mathrm{E}-04 \\
-9.56301 \mathrm{E}-04\end{array}$ & $\begin{array}{l}-9.47555 \mathrm{E}-01 \\
-9.56470 \mathrm{E}-01 \\
-9.65428 \mathrm{E}-01 \\
-9.74427 \mathrm{E}-01 \\
-9.83467 \mathrm{E}-01\end{array}$ & $\begin{array}{l}2.47210 \mathrm{E} \\
2.48192 \mathrm{E} \\
2.49174 \mathrm{E} \\
2.50156 \mathrm{E} \\
2.51139 \mathrm{E}\end{array}$ \\
\hline $\begin{array}{ll}1.05418 \mathrm{E} & 00 \\
1.06350 \mathrm{E} & 00 \\
1.07286 \mathrm{E} & 00 \\
1.08226 \mathrm{E} & 00 \\
1.09170 \mathrm{E} & 00\end{array}$ & $\begin{array}{l}-9.58003 \mathrm{E}-04 \\
-9.59689 \mathrm{E}-04 \\
-9.61354 \mathrm{E}-04 \\
-9.63000 \mathrm{E}-04 \\
-9.64628 \mathrm{E}-04\end{array}$ & $\begin{array}{ll}-9.92549 \mathrm{E}-01 \\
-1.00167 \mathrm{E} & 00 \\
-1.01084 \mathrm{E} & 00 \\
-1.02005 \mathrm{E} & 00 \\
-1.02929 \mathrm{E} & 00\end{array}$ & $\begin{array}{l}2.52121 \mathrm{E} \\
2.53104 \mathrm{E} \\
2.54087 \mathrm{E} \\
2.55070 \mathrm{E} \\
2.56053 \mathrm{E}\end{array}$ \\
\hline $\begin{array}{ll}1.10119 \mathrm{E} & 00 \\
1.11071 \mathrm{E} & 00 \\
1.12028 \mathrm{E} & 00 \\
1.12989 \mathrm{E} & 00 \\
1.13954 \mathrm{E} & 00\end{array}$ & $\begin{array}{l}-9.66238 \mathrm{E}-04 \\
-9.67826 \mathrm{E}-04 \\
-9.69399 \mathrm{E}-04 \\
-9.70956 \mathrm{E}-04 \\
-9.72493 \mathrm{E}-04\end{array}$ & $\begin{array}{ll}-1.03859 \mathrm{E} & 00 \\
-1.04792 \mathrm{E} & 00 \\
-1.05729 \mathrm{E} & 00 \\
-1.06671 \mathrm{E} & 00 \\
-1.07617 \mathrm{E} & 00\end{array}$ & $\begin{array}{l}2.57037 \mathrm{E} \\
2.58020 \mathrm{E} \\
2.59004 \mathrm{E} \\
2.59988 \mathrm{E} \\
2.60972 \mathrm{E}\end{array}$ \\
\hline
\end{tabular}

$\begin{array}{lll}1.14923 \mathrm{E} & 00 & -9.74014 \mathrm{E}-04\end{array}$

$\begin{array}{lll}1.15897 \mathrm{E} & 00 & -9.75514 \mathrm{E}-04\end{array}$

$\begin{array}{lll}1.16874 \mathrm{E} & 00 & -9.76999 \mathrm{E}-04\end{array}$

$\begin{array}{lll}1.17856 \mathrm{E} & 00 & -9.78471 \mathrm{E}-04\end{array}$

$\begin{array}{lll}1.18842 \mathrm{E} & 00 & -9.79918 \mathrm{E}-04\end{array}$

$\begin{array}{lll}1.19832 \mathrm{E} & 00 & -9.81355 \mathrm{E}-04\end{array}$

$\begin{array}{lll}1.20827 \mathrm{E} & 00 & -9.82770 \mathrm{E}-04\end{array}$

$\begin{array}{lll}1.21825 \mathrm{E} & 00 & -9.84172 \mathrm{E}-04\end{array}$

$\begin{array}{lll}1.22828 \mathrm{E} & 00 & -9.85557 \mathrm{E}-04\end{array}$

$\begin{array}{lll}1.23835 \mathrm{E} & 00 & -9.86928 \mathrm{E}-04\end{array}$

$\begin{array}{lll}1.24846 \mathrm{E} & 00 & -9.88281 \mathrm{E}-04\end{array}$

$\begin{array}{lll}1.25861 \mathrm{E} & 00 & -9.89614 \mathrm{E}-04\end{array}$

$\begin{array}{lll}1.26881 \mathrm{E} & 00 & -9.90940 \mathrm{E}-04\end{array}$

$\begin{array}{lll}1.27905 \mathrm{E} & 00 & -9.92243 \mathrm{E}-04\end{array}$

$\begin{array}{lll}1.28933 \mathrm{E} & 00 & -9.93533 \mathrm{E}-04\end{array}$

$1.29965 \mathrm{E} \quad 00 \quad-9.94808 \mathrm{E}-04$

$1.31001 \mathrm{E} \quad 00 \quad-9.96072 \mathrm{E}-04$

$\begin{array}{lll}1.32042 \mathrm{E} & 00 & -9.97315 \mathrm{E}-04\end{array}$

$\begin{array}{lll}1.33086 \mathrm{E} & 00 & -9.98544 \mathrm{E}-04\end{array}$

$\begin{array}{lll}1.34135 \mathrm{E} & 00 & -9.99759 \mathrm{E}-04\end{array}$

$\begin{array}{lll}1.35188 \mathrm{E} & 00 & -1.00096 \mathrm{E}-03\end{array}$

$\begin{array}{lll}1.36246 \mathrm{E} & 00-1.00215 \mathrm{E}-03\end{array}$

$\begin{array}{lll}1.37307 \mathrm{E} & 00 & -1.00332 \mathrm{E}-03\end{array}$

$\begin{array}{lll}1.38373 \mathrm{E} & 00 & -1.00448 \mathrm{E}-03\end{array}$

$\begin{array}{lll}1.39443 \mathrm{E} & 00 & -1.00562 \mathrm{E}-03\end{array}$

$1.40517 \mathrm{E} \quad 00-1.00676 \mathrm{E}-03$

$\begin{array}{lll}1.41596 \mathrm{E} & 00 & -1.00787 \mathrm{E}-03\end{array}$

$\begin{array}{lll}1.42679 \mathrm{E} & 00 & -1.00897 \mathrm{E}-03\end{array}$

$\begin{array}{lll}1.43766 \mathrm{E} & 00 & -1.01006 \mathrm{E}-03\end{array}$

$\begin{array}{lll}1.44857 \mathrm{E} & 00 & -1.01113 \mathrm{E}-03\end{array}$

$\begin{array}{lll}1.45952 \mathrm{E} & 00 & -1.01219 \mathrm{E}-03\end{array}$

$\begin{array}{lll}1.47052 \mathrm{E} & 00 & -1.01324 \mathrm{E}-03\end{array}$

$\begin{array}{lll}1.48155 \mathrm{E} & 00 & -1.01428 \mathrm{E}-03\end{array}$

$\begin{array}{lll}1.49263 \mathrm{E} & 00 & -1.01530 \mathrm{E}-03\end{array}$

$\begin{array}{lll}1.50376 \mathrm{E} & 00 & -1.01631 \mathrm{E}-03\end{array}$
$-1.08567 \mathrm{E} \quad 00$ $-1.09521 \mathrm{E} 00$ $-1.10479 \mathrm{E} 00$ $-1.11442 \mathrm{E} 00$ $-1.12408 \mathrm{E} 00$

$-1.13379 \mathrm{E} \quad 00$ $-1.14354 \mathrm{E} \quad 00$ $-1.15334 \mathrm{E} \quad 00$ $-1.16317 \mathrm{E} \quad 00$ $-1.17305 \mathrm{E} \quad 00$

$-1.18297 \mathrm{E} 00$ $-1.19293 \mathrm{E} 00$ $-1.20293 \mathrm{E} 00$ $-1.21297 \mathrm{E} 00$ $-1.22306 \mathrm{E} 00$

$-1.23319 \mathrm{E} \quad 00$ $-1.24336 \mathrm{E} \cdot 00$ $-1.25357 \mathrm{E} \quad 00$ $-1.26383 \mathrm{E} \quad 00$ $-1.27413 \mathrm{E} 00$

$-1.28447 \mathrm{E} \quad 00$ $-1.29485 \mathrm{E} \quad 00$ $-1.30527 \mathrm{E} 00$ $-1.31574 \mathrm{E} \quad 00$ $-1.32625 \mathrm{E} \quad 00$

$-1.33680 \mathrm{E} \quad 00$ $-1.34739 \mathrm{E} 00$ $-1.35803 \mathrm{E} \quad 00$ $-1.36870 \mathrm{E} \quad 00$ $-1.37942 \mathrm{E} 00$

$-1.39019 \mathrm{E} 00$ $-1.40099 \mathrm{E} 00$ $-1.41184 \mathrm{E} 00$ $-1.42273 \mathrm{E} 00$ $-1.43366 \mathrm{E} 00$ $\begin{array}{ll}2.61957 \mathrm{E} & 01\end{array}$ $2.62941 \mathrm{E} \quad 01$ $2.63926 \mathrm{E} \quad 01$ $2.64911 \mathrm{E} \quad 01$ $2.65896 \mathrm{E} \quad 01$

$2.66881 \mathrm{E} \quad 01$ $2.67866 \mathrm{E} \quad 01$ $2.68852 \mathrm{E} \quad 01$ $2.69838 \mathrm{E} \quad 01$ $2.70824 \mathrm{E} \quad 01$

$2.71810 \mathrm{E} \quad 01$ $2.72796 \mathrm{E} \quad 01$ $2.73783 \mathrm{E} \quad 01$ $2.74769 \mathrm{E} \quad 01$ $2.75756 \mathrm{E} \quad 01$

$2.76743 \mathrm{E} \quad 01$ $2.77731 \mathrm{E} \quad 01$ $2.78718 \mathrm{E} \quad 01$ $2.79706 \mathrm{E} \quad 01$ $2.80694 \mathrm{E} \quad 01$

$2.81682 \mathrm{E} \quad 01$ $2.82670 \mathrm{E} \quad 01$ $2.83658 \mathrm{E} \quad 01$ $2.84647 \mathrm{E}$ 0] $2.85636 \mathrm{E} \quad 01$

$2.86625 \mathrm{E} \quad 01$ $2.87614 \mathrm{E} \quad 01$ $2.88603 \mathbf{E} 01$ $2.89593 \mathrm{E} 01$ $2.90583 \mathrm{E} \quad 01$

$2.91573 \mathrm{E} \quad 01$ $2.92563 \mathrm{E} \quad 01$ $2.93553 \mathrm{E} \quad 01$ $2.94544 \mathrm{E} \quad 01$ $2.95535 \mathrm{E} \quad 01$ $\tan \delta_{-1}$

$4.68032 \mathrm{E} \quad 02$ $4.68696 \mathrm{E} \quad 02$ $4.69358 \mathrm{E} \quad 02$ $4.70018 \mathrm{E} \quad 02$ $4.70674 \mathrm{E} \quad 02$

$4.71331 \mathrm{E} \quad 02$ 4.71984E 02 $4.72635 \mathrm{E} \quad 02$ $4.73284 \mathrm{E} \quad 02$ $4.73931 \mathrm{E} \quad 02$

$\begin{array}{ll}4.74577 \mathrm{E} & 02\end{array}$ 4. $75220 \mathrm{E} \quad 02$ $4.75862 \mathrm{E} \quad 02$ $4.76501 \mathrm{E} \quad 02$ $4.77138 \mathrm{E} \quad 02$

$4.77773 \mathrm{E} \quad 02$ $4.78407 \mathrm{E} \quad 02$ $4.79039 \mathrm{E} \quad 02$ $4.79669 \mathrm{E} \quad 02$ $4.80298 \mathrm{E} \quad 02$

4.80924E 02 $4.81549 \mathrm{E} \quad 02$ $4.82173 \mathrm{E} \quad 02$ $4.82794 \mathrm{E} \quad 02$ $4.83413 \mathrm{E} \quad 02$

$4.84031 \mathrm{E} \quad 02$ $4.84648 \mathrm{E} \quad 02$ $\begin{array}{lll}4.85263 \mathrm{E} & 02\end{array}$ $4.85876 \mathrm{E} \quad 02$ $4.86489 \mathrm{E} \quad 02$

$4.87098 \mathrm{E} \quad 02$ $4.87708 \mathrm{E} \quad 02$ $4.88315 \mathrm{E} \quad 02$ $4.88921 \mathrm{E} \quad 02$ $4.89525 \mathrm{E} \quad 02$

$4.90128 \mathrm{E} \quad 02$ $4.90730 \mathrm{E} \quad 02$ $4.91330 \mathrm{E} \quad 02$ $4.91929 \mathrm{E} \quad 02$ $4.92527 \mathrm{E} \quad 02$

$4.93123 \mathrm{E} \quad 02$ $4.93717 \mathrm{E} \quad 02$ $4.94312 \mathrm{E} \quad 02$ $4.94904 \mathrm{E} \quad 02$ $4.95496 \mathrm{E} \quad 02$

$4.96086 \mathrm{E} \quad 02$ $4.96675 \mathrm{E} \quad 02$ $4.97263 \mathrm{E} \quad 02$ $4.97849 \mathrm{E} \quad 02$ $4.98435 \mathrm{E} \quad 02$

$4.99018 \mathrm{E} \quad 02$ $4.99603 \mathrm{E} \quad 02$ $5.00185 \mathrm{E} \quad 02$ $5.00766 \mathrm{E} \quad 02$ $5.01346 \mathrm{E} 02$.

$5.01925 \mathrm{E} \quad 02$ $5.02503 \mathrm{E} \quad 02$ $5.03080 \mathrm{E} \cdot 02$ $5.03657 \mathrm{E} \quad 02$ $5.04232 \mathrm{E} \quad 02$ 
ELECTRON RADIAL FUNCTIONS

$Z=4 \quad A=8 \quad \rho=1.2 A^{1 / 3} F$

Positron

$\bar{F}$

$5.69157 \mathrm{E}-04$

$5.73889 \mathrm{E}-04$

$5.78641 \mathrm{E}-04$

$5.83413 \mathbf{E}-04$

$5.88204 \mathrm{E}-04$

$5.93015 \mathrm{E}-04$

$5.97845 \mathrm{E}-04$

$6.02695 \mathrm{E}-04$

$6.07565 \mathrm{E}-04$

$6.12454 \mathrm{E}-04$

$6.17363 \mathrm{E}-04$

$6.22291 \mathrm{E}-04$

$6.27239 \mathrm{E}-04$

$6.32207 \mathrm{E}-04$

$6.37194 \mathbf{E}-04$

$6.42200 \mathrm{E}-04$

$6.47227 \mathrm{E}-04$

$6.52272 \mathrm{E}-04$

$6.57338 \mathrm{E}-04$

$6.62423 \mathrm{E}-04$

$6.67528 \mathrm{E}-04$

$6.72652 \mathrm{E}-04$

$6.77796 \mathrm{E}-04$

$6.82959 \mathrm{E}-04$

$6.88142 \mathrm{E}-04$

$6.93344 \mathrm{E}-04$

$6.98567 \mathrm{E}-04$

$7.03808 \mathrm{E}-04$

$7.09070 \mathrm{E}-04$

$7.14350 \mathrm{E}-04$

$7.19651 \mathrm{E}-04$

7.24 .971 E-04

$7.30311 \mathrm{E}-04$

$7.35670 \mathrm{E}-04$

$7.41049 \mathrm{E}-04$

$7.46447 \mathrm{E}-04$

$7.51865 \mathrm{E}-04$

$7.57303 \mathbf{E}-04$

$7.62760 \mathrm{E}-04$

$7.68237 \mathrm{E}-04$

$7.73733 \mathrm{E}-04$ $7.79249 \mathrm{E}-04$

$7.84784 \mathrm{E}-04$

$7.90340 \mathrm{E}-04$

$7.95914 \mathrm{E}-04$

$8.01509 \mathrm{E}-04$

8.07123 E-04

$8.12756 \mathrm{E}-04$

$8.18409 \mathrm{E}-04$

8.24082 E- 04

$8.29774 \mathrm{E}-04$

$8.35486 \mathrm{E}-04$

$8.41217 \mathrm{E}-04$

$8.46968 \mathrm{E}-04$

$8.52739 \mathrm{E}-04$

$8.58529 \mathrm{E}-04$

$8.64338 \mathrm{E}-04$

$8.70168 \mathrm{E}-04$

$8.76017 \mathrm{E}-04$

8.81885 E- 04 . $f_{2}$

$g_{2}$

$\tan \delta_{2}$

$f_{-2}$

$1.12559 \mathrm{E} \quad 00$

$1.13504 \mathrm{E} \quad 00$

$1.14453 \mathrm{E} \quad 00$

$1.15406 \mathrm{E} \quad 00$

$1.16362 \mathrm{E} \quad 00$

$1.17323 \mathrm{E} \quad 00$

$1.18288 \mathrm{E} \quad 00$

$1.19256 \mathrm{E} \quad 00$

$1.20228 \mathrm{E} \quad 00$

$1.21205 \mathrm{E} \quad 00$

$1.22185 \mathrm{E} \quad 00$

$1.23169 \mathrm{E} \quad 00$

$1.24157 \mathrm{E} \quad 00$

$1.25149 \mathrm{E} \quad 00$

$1.26145 \mathrm{E} \quad 00$

$1.27145 \mathrm{E} \quad 00$

$1.28149 \mathrm{E} \quad 00$

$1.29157 \mathrm{E} \quad 00$

$\begin{array}{lll}1.30169 \mathrm{E} \quad 00 & 0\end{array}$

$1.31184 \mathrm{E} \quad 00$

$1.32204 \mathrm{E} \quad 00$

$1.33227 \mathrm{E} \quad 00$

$1.34255 \mathrm{E} \quad 00$

$1.35286 \mathrm{E} \quad 00$

$1.36321 \mathrm{E} \quad 00$

$1.37360 \mathrm{E} \quad 00$

$1.38403 \mathrm{E} \quad 00$

$1.39450 \mathrm{E} \quad 00$

$1.40501 \mathrm{E} \quad 00$

$1.41556 \mathrm{E} \quad 00$

$1.42615 \mathrm{E} \quad 00$

$1.43677 \mathrm{E} \quad 00$

$1.44744 \mathrm{E} \quad 00$

$1.45814 \mathrm{E} \quad 00$

$1.46889 \mathrm{E} \quad 00$

$1.47967 \mathrm{E} \quad 00$

$1.49049 \mathrm{E} \quad 00$

$1.50135 \mathrm{E} \quad 00$

$1.51225 \mathrm{E} \quad 00$

$1.52320 \mathrm{E} \quad 00$

$\begin{array}{lll}1.53417 \mathrm{E} \quad 00 & 0\end{array}$

$1.54519 \mathrm{E} \quad 00$

$1.55625 \mathrm{E} \quad 00$

$1.56735 \mathrm{E} \quad 00$

$1.57848 \mathrm{E} \quad 00$

$1.58966 \mathrm{E} \quad 00$

$1.60087 \mathrm{E} \quad 00$

$\begin{array}{lll}1.61212 \mathrm{E} & 00\end{array}$

$1.62342 \mathrm{E} \quad 00$

$1.63475 \mathrm{E} \quad 00$

$1.64612 \mathrm{E} \quad 00$ $1.65753 \mathrm{E} \quad 00$

$1.66898 \mathrm{E} \quad 00$

$1.68046 \mathrm{E} \quad 00$

$1.69199 \mathrm{E} \quad 00$

$1.70356 \mathrm{E} \quad 00$

$1.71516 \mathrm{E} \quad 00$

$\begin{array}{lll}1.72681 \mathrm{E} & 00\end{array}$

$\begin{array}{lll}1.73849 \mathrm{E} & 00\end{array}$

$1.75021 \mathrm{E} \quad 00$
$2.76480 \mathrm{E}-02$

$2.80212 \mathrm{E}-02$

$2.83978 \mathrm{E}-02$

$2.87777 \mathrm{E}-02$

$2.91609 \mathrm{E}-02$

$2.95476 \mathrm{E}-02$

$2.99376 \mathrm{E}-02$

$3.03310 \mathrm{E}-02$

$3.07278 \mathrm{E}-02$

$3.11281 \mathrm{E}-02$

$3.15318 \mathrm{E}-02$

$3.19390 \mathrm{E}-02$

$3.23496 \mathrm{E}-02$

$3.27638 \mathrm{E}-02$

$3.31814 \mathrm{E}-02$

$3.36025 \mathrm{E}-02$

$3.40272 \mathrm{E}-02$

$3.44555 \mathrm{E}-02$

$3.48873 \mathrm{E}-02$

$3.53226 \mathrm{E}-02$

$3.57616 \mathrm{E}-02$

$3.62042 \mathrm{E}-02$

$3.66504 \mathrm{E}-02$

$3.71002 \mathrm{E}-02$

$3.75536 \mathrm{E}-02$

$3.80108 \mathrm{E}-02$

$3.84716 \mathrm{E}-02$

$3.89361 \mathrm{E}-02$

$3.94043 \mathrm{E}-02$

$3.98763 \mathrm{E}-02$

$4.03520 \mathrm{E}-02$

$4.08314 \mathrm{E}-02$

$4.13146 \mathrm{E}-02$

$4.18015 \mathrm{E}-02$

$4.22923 \mathrm{E}-02$

$4.27869 \mathrm{E}-02$

$4.32853 \mathrm{E}-02$

$4.37875 \mathrm{E}-02$

$4.42936 \mathrm{E}-02$

$4.48036 \mathrm{E}-02$

$4.53175 \mathrm{E}-02$

$4.58352 \mathrm{E}-02$

$4.63569 \mathrm{E}-02$

$4.68825 \mathrm{E}-02$

$4.74120 \mathrm{E}-02$

$4.79455 \mathrm{E}-02$

$4.84829 \mathrm{E}-02$

4.90244 E-02

$4.95698 \mathrm{E}-02$

$5.01193 \mathrm{E}-02$

$5.06727 \mathrm{E}-02$

$5.12303 \mathrm{E}-02$

$5.17918 \mathrm{E}-02$

$5.23575 \mathrm{E}-02$

$5.29272 \mathrm{E}-02$

$5.35011 \mathrm{E}-02$

$5.40790 \mathrm{E}-02$

$5.46611 \mathrm{E}-02$

$5.52474 \mathrm{E}-02$

$5.58378 \mathrm{E}-02$ $\begin{array}{lll}-4.92702 \mathrm{E} & 01 & -2.58996 \mathrm{E}-02\end{array}$ $\begin{array}{lll}-4.92736 \mathrm{E} & 0 \mathrm{l} & -2.62597 \mathrm{E}-02\end{array}$ $-4.92769 \mathrm{E} \quad 01-2.66230 \mathrm{E}-02$ $-4.92802 \mathrm{E} \quad 01 \quad-2.69896 \mathrm{E}-02$ $\begin{array}{lll}-4.92835 \mathrm{E} & 01 & -2.73595 \mathrm{E}-02\end{array}$

$-4.92868 \mathrm{E} \quad 01$

$-4.92900 \mathrm{E} \quad 01$

$-4.92964 \mathrm{E}$ 0I

$-4.92996 \mathrm{E} \quad 01$

$-2.77327 \mathrm{E}-02$

$-2.81092 \mathrm{E}-02$

$-2.84892 \mathrm{E}-02$

$-2.88724 \mathrm{E}-02$

$-2.92591 \mathrm{E}-02$

$-4.93027 \mathrm{E}$ 0I

$-2.96492 \mathrm{E}-02$

$-4.93058 \mathrm{E} \quad 01$

$-4.93089 \mathrm{E} \quad 01$

$-4.93120 \mathrm{E} 01$

$-4.93150 \mathrm{E} 01$

$-3.04395 \mathrm{E}-02$

$-3.08398 \mathrm{E}-02$

$-3.12436 \mathrm{E}-02$

$-4.93180 \mathrm{E} \quad 01$

$-4.93210 \mathrm{E}$ 0l

$-4.93239 \mathrm{E} 0 \mathrm{l}$

$\begin{array}{ll}-4.93269 \mathrm{E} & 01\end{array}$

$-4.93298 \mathrm{E} \quad 01$

$-4.93327 \mathrm{E} \quad 01$

$-4.93355 \mathrm{E} 01$

$-4.93384 \mathrm{E} 01$

$-4.93412 \mathrm{E} \quad 01$

$-4.93440 \mathrm{E}$ 01

$-4.93467 \mathrm{E} \quad 01$

$-4.93495 \mathrm{E} 01$

$-4.93522 \mathrm{E} 01$

$-4.93549 \mathrm{E} \quad 0 \mathrm{I}$

$-4.93576 \mathrm{E}$ ol

$-4.93603 \mathrm{E} \quad 01$

$-4.93630 \mathrm{E}$ 01

$-4.93656 \mathrm{E} 01$

$-4.93682 \mathrm{E} 01$

$-4.93708 \mathrm{E} 01$

$-4.93734 \mathrm{E} \quad 01$

$-4.93759 \mathrm{E}$ 01

$-4.93785 \mathrm{E} 01$

$-4.93810 \mathrm{E} 01$

-4.93835 E 01

$-3.16509 \mathrm{E}-02$

$-3.20616 \mathrm{E}-02$

$-3.24759 \mathrm{E}-02$

$-3.28936 \mathrm{E}-02$

$-3.33149 \mathrm{E}-02$

$-3.37397 \mathrm{E}-02$

$-3.41681 \mathrm{E}-02$

$-3.46000 \mathrm{E}-02$

$-3.50356 \mathrm{E}-02$

$-3.54747 \mathrm{E}-02$

$-3.59175 \mathrm{E}-02$

$-3.63638 \mathrm{E}-02$

$-3.68139 \mathrm{E}-02$

$-3.72675 \mathrm{E}-02$

$-3.77249 \mathrm{E}-02$

$-3.81859 \mathrm{E}-02$

$-3.86507 \mathrm{E}-02$

$-3.91191 \mathrm{E}-02$

$-3.95913 \mathrm{E}-02$

$-4.00673 \mathrm{E}-02$

$-4.05470 \mathrm{E}-02$

$-4.10305 \mathrm{E}-02$

$-4.15177 \mathrm{E}-02$

$-4.20088 \mathrm{E}-02$

$-4.25037 \mathrm{E}-02$

$-4.93859 \mathrm{E} \quad 01$

$-4.93884 \mathrm{E} 01$

$-4.93908 \mathrm{E} 01$

$-4.93933 \mathrm{E} 01$

$-4.93957 \mathrm{E} \quad 01$

$-4.93981 \mathrm{E} 01$

$-4.94004 \mathrm{E} \quad 01$

$-4.94028 \mathrm{E} 01$

$-4.94051 \mathrm{E} 01$

$-4.94075 \mathrm{E} \quad 01$

$\begin{array}{ll}-4.94098 \mathrm{E} & 01\end{array}$

$-4.94120 \mathrm{E} \quad 01$

$-4.94143 \mathrm{E} 01$

$-4.94166 \mathrm{E} \quad 01$

$-4.94188 \mathrm{E} \quad 01$

$-4.94210 \mathrm{E} \quad 01$

$-4.94232 \mathrm{E}$ 0l

$-4.94254 \mathrm{E}$ ol

$-4.94298 \mathrm{E} 01$

$-4.30024 \mathrm{E}-02$

$-4.35050 \mathrm{E}-02$

$-4.40114 \mathrm{E}-02$

$-4.45218 \mathrm{E}-02$

$-4.50360 \mathrm{E}-02$

$-4.55541 \mathrm{E}-02$

$-4.60761 \mathrm{E}-02$
$-4.92932 \mathrm{E} \quad 01$

$-4.94276 \mathrm{E} 01$ 
ELECTRON RADIAL FUNCTIONS

Positron

p

30.10

30.20

30.30

30.40

30.50

30.60

30.70
30.80

30.90

31.00

31.10

31.20

31.30

31.40

31.50

31.60

31.70

31.80

31.90

32.00

32.10

32.20

32.30

32.40

32.50

32.60

32.70

32.80

32.90

33.00

33.10

33.20

33.30

33.40

33.50

33.60

33.70

33.80

33.90

34.00

34.10

34.20

34.30

34.40

34.50

34.60

34.70

34.80

34.90

35.00

35.10

35.20

35.30

35.40

35.50

35.60

35.70

35.80

35.90

36.00
$F_{0}$

$9.13700 \mathrm{E}-01$

$9.13697 \mathrm{E}-01$

$9.13695 \mathrm{E}-01$

$9.13693 \mathrm{E}-01$

$9.13691 \mathrm{E}-01$

$9.13689 \mathrm{E}-01$

$9.13686 \mathrm{E}-01$

$9.13684 \mathrm{E}-01$

$9.13682 \mathrm{E}-01$

$9.13680 \mathrm{E}-01$

$9.13677 \mathrm{E}-01$

$9.13675 \mathrm{E}-01$

$9.13673 \mathrm{E}-01$

$9.13671 \mathrm{E}-01$

$9.13668 \mathrm{E}-01$

$9.13666 \mathrm{E}-01$

$9.13664 \mathrm{E}-01$

$9.13662 \mathrm{E}-01$

$9.13660 \mathrm{E}-01$

$9.13657 \mathrm{E}-01$

$9.13655 \mathrm{E}-01$

$9.13653 \mathrm{E}-01$

$9.13651 \mathrm{E}-01$

$9.13649 \mathrm{E}-01$

9.13647E-01

$9.13645 \mathrm{E}-01$

$9.13643 \mathrm{E}-01$

$9.13640 \mathrm{E}-01$

$9.13638 \mathrm{E}-01$

$9.13636 \mathrm{E}-01$

9.13634 E-01

$9.13632 \mathrm{E}-01$

$9.13630 \mathrm{E}-01$

$9.13628 \mathrm{E}-01$

$9.13626 \mathrm{E}-01$

$9.13623 \mathrm{E}-01$

$9.13621 \mathrm{E}-01$

$9.13619 \mathrm{E}-01$

$9.13617 \mathrm{E}-01$

$9.13615 \mathrm{E}-01$

$9.13613 \mathrm{E}-01$

$9.13611 \mathrm{E}-01$

$9.13609 \mathrm{E}-01$

$9.13607 \mathrm{E}-01$

$9.13605 \mathrm{E}-01$

9.13603E-01

$9.13601 \mathrm{E}-01$

$9.13599 \mathrm{E}-01$

9.13597E-01

9.13595E-01

$9.13593 \mathrm{E}-01$

$9.13591 \mathrm{E}-01$

$9.13589 \mathrm{E}-01$

$9.13587 \mathrm{E}-01$

$9.13585 \mathrm{E}-01$

$9.13583 \mathrm{E}-01$

9.13581 E-01

9.13579E-01

$9.13577 \mathrm{E}-01$

$9.13575 \mathrm{E}-01$ $f_{1}$

$2.86545 \mathrm{E}$ 0]

$2.87534 \mathrm{E} \quad 01$

$2.88522 \mathrm{E} \quad 01$

$2.89511 \mathrm{E} 01$

$2.90500 \mathrm{E} \quad 01$

$2.91489 \mathrm{E} \quad 01$

$2.92479 \mathrm{E} \quad 01$

$2.93468 \mathrm{E} \quad 01$

$2.94458 \mathrm{E} \quad 01$

$2.95448 \mathrm{E} 01$

$2.96439 \mathrm{E} \quad 01$

$2.97429 \mathrm{E} \quad 01$

$2.98420 \mathrm{E} \quad 01$

$2.99411 \mathrm{E} 01$

$3.00402 \mathrm{E} \quad 01$

$3.01393 \mathrm{E} \quad 01$

$3.02384 \mathrm{E} 01$

$3.03376 \mathrm{E} \quad 01$

$3.04368 \mathrm{E} 01$

$3.05360 \mathrm{E}$ ol

$3.06352 \mathrm{E} \quad 01$

$3.07345 \mathrm{E} \quad 01$

$3.08337 \mathrm{E} \quad 01$

$3.09330 \mathrm{E} \quad 01$

$3.10323 \mathrm{E}$ ol

$3.11317 \mathrm{E} \quad 01$

$3.12310 \mathrm{E} 01$

$3.13304 \mathrm{E}$ ol

$3.14298 \mathrm{E} 01$

$3.15292 \mathrm{E} \quad 01$

$3.16286 \mathrm{E} \quad 01$

$3.17281 \mathrm{E} \quad 01$

$3.18276 \mathrm{E}$ 01

$3.19271 \mathrm{E} \quad 01$

$3.20266 \mathrm{E} 01$

$3.21261 \mathrm{E} \quad 01$

$3.22257 \mathrm{E} \quad 01$

$3.23253 \mathrm{E}$ 01

$3.24249 \mathrm{E} \quad 01$

3.25245 E 01

$3.26242 \mathrm{E} \quad 01$

$3.27238 \mathrm{E}$ ol

$3.28235 \mathrm{E} \quad 01$

$3.29233 \mathrm{E}$ 01

$3.30230 \mathrm{E} \quad 01$

$3.31227 \mathrm{E} \quad 01$

3.32225 E 01

$3.33223 \mathrm{E} 01$

$3.34222 \mathrm{E} 01$

$3.35220 \mathrm{E} 01$

$3.36219 \mathrm{E} \quad 01$

$3.37218 \mathrm{E} \quad 01$

$3.38217 \mathrm{E}$ 01

$3.39216 \mathrm{E} 01$

$3.40216 \mathrm{E} \quad 01$

$3.41216 \mathrm{E} \quad 01$

$3.42216 \mathrm{E}$ 01

$3.43216 \mathrm{E}$ 0l

$3.44217 \mathrm{E} \quad 01$

$Z=4 \quad A=8 \quad \rho=1.2 A^{1 / 3} F$

$\begin{array}{ccc}g_{1} & \tan \delta_{1} & f_{-1}\end{array}$

$\begin{array}{lll}1.51492 \mathrm{E} & 00 & -1.01731 \mathrm{E}-03\end{array}$

$1.52613 \mathrm{E} \quad 00-1.01829 \mathrm{E}-03$

$1.53738 \mathrm{E} \quad 00-1.01926 \mathrm{E}-03$

$1.54867 \mathrm{E} \quad 00-1.02022 \mathrm{E}-03$

$\begin{array}{lll}1.56001 \mathrm{E} & 00 & -1.02117 \mathrm{E}-03\end{array}$

$\begin{array}{lll}1.57139 \mathrm{E} & 00 & -1.02210 \mathrm{E}-03\end{array}$

$1.58281 \mathrm{E} \quad 00 \quad-1.02302 \mathrm{E}-03$

$\begin{array}{lll}1.59427 \mathrm{E} & 00 & -1.02394 \mathrm{E}-03\end{array}$

$\begin{array}{lll}1.60577 \mathrm{E} & 00 & -1.02484 \mathrm{E}-03\end{array}$

$\begin{array}{lll}1.61732 \mathrm{E} & 00 & -1.02572 \mathrm{E}-03\end{array}$

$\begin{array}{lll}1.62891 \mathrm{E} & 00 & -1.02659 \mathrm{E}-03\end{array}$

$1.64054 \mathrm{E} \quad 00-1.02746 \mathrm{E}-03$

$1.65222 \mathrm{E} \quad 00 \quad-1.02831 \mathrm{E}-03$

$\begin{array}{lll}1.66394 \mathrm{E} & 00-1.02915 \mathrm{E}-03\end{array}$

$\begin{array}{lll}1.67570 \mathrm{E} & 00-1.02998 \mathrm{E}-03\end{array}$

$1.68750 \mathrm{E} \quad 00$

$\begin{array}{lll}1.69935 \mathrm{E} & 00 & -1.03160 \mathrm{E}-03\end{array}$

$\begin{array}{lll}1.71123 \mathrm{E} & 00 & -1.03239 \mathrm{E}-03\end{array}$

$1.72317 \mathrm{E} \quad 00 \quad-1.03318 \mathrm{E}-03$

$1.73514 \mathrm{E} \quad 00 \quad-1.03395 \mathrm{E}-03$

$1.74716 \mathrm{E} \quad 00 \quad-1.03471 \mathrm{E}-03$

$1.75922 \mathrm{E} \quad 00$

$1.77132 \mathrm{E} \quad 00$

$1.78346 \mathrm{E} \quad 00$

$1.79565 \mathrm{E} \quad 00$

$1.80788 \mathrm{E} \quad 00$

$1.82016 \mathrm{E} \quad 00$

$1.83247 \mathrm{E} \quad 00$

$1.84483 \mathrm{E} \quad 00$

$1.85723 \mathrm{E} \quad 00$

$1.86968 \mathrm{E} \quad 00$

$1.88217 \mathrm{E} \quad 00$

$1.89470 \mathrm{E} \quad 00$

$1.90727 \mathrm{E} \quad 00$

$1.91989 \mathrm{E} \quad 00$

$1.93255 \mathrm{E} \quad 00$

$1.94526 \mathrm{E} \quad 00$

$1.95800 \mathrm{E} \quad 00$

$1.97079 \mathrm{E} 00$

$1.98363 \mathrm{E} \quad 00$

$1.99650 \mathrm{E} \quad 00$

$2.00942 \mathrm{E} \quad 00$

$2.02238 \mathrm{E} \quad 00$

$2.03539 \mathrm{E} \quad 00$

$2.04844 \mathrm{E} \quad 00$

$2.06153 \mathrm{E} \quad 00$

$2.07467 \mathrm{E} \quad 00$

$2.08785 \mathrm{E} \quad 00$

$2.10107 \mathrm{E} \quad 00$

$2.11433 \mathrm{E} \quad 00$

$2.12764 \mathrm{E} \quad 00$

$2.14100 \mathrm{E} \quad 00$

$2.15439 \mathrm{E} \quad 00$

$2.16783 \mathrm{E} \quad 00$

$2.18131 \mathrm{E} \quad 00$

$2.19484 \mathrm{E} \quad 00$

$2.20841 \mathrm{E} \quad 00$

$2.22202 \mathrm{E} \quad 00$

$2.23568 \mathrm{E} \quad 00$

$2.24938 \mathrm{E} \quad 00$

$-1.44463 \mathrm{E} \quad 00$

$-1.45565 \mathrm{E} 00$

$-1.46671 \mathrm{E} 00$

$-1.47781 \mathrm{E} \quad 00$

$-1.48895 \mathrm{E} 00$

$-1.50014 \mathrm{E} \quad 00$

$-1.51137 \mathrm{E} 00$

$-1.52264 \mathrm{E} 00$

$-1.53396 \mathrm{E} 00$

$-1.54531 \mathrm{E} 00$

$-1.55671 \mathrm{E} \quad 00$

$-1.56816 \mathrm{E} 00$

$-1.57964 \mathrm{E} \quad 00$

$-1.59117 \mathrm{E} \quad 00$

$-1.60274 \mathrm{E} 00$

$-1.61435 \mathrm{E} \quad 00$

$-1.62601 \mathrm{E} \quad 00$

$-1.63771 \mathrm{E} \quad 00$

$-1.64945 \mathrm{E} \quad 00$

$-1.66123 \mathrm{E} 00$

$-1.67306 \mathrm{E} \quad 00$

$-1.684 .93 \mathrm{E} 00$

$-1.69684 \mathrm{E} \quad 00$

$-1.70880 \mathrm{E} \quad 00$

$-1.72080 \mathrm{E} 00$

$-1.73284 \mathrm{E} \quad 00$

$-1.74492 \mathrm{E} 00$

$-1.75705 \mathrm{E} 00$

$-1.76922 \mathrm{E} 00$

$-1.78143 \mathrm{E} 00$

$-1.79369 \mathrm{E} \quad 00$

$-1.80599 \mathrm{E} 00$

$-1.81833 \mathrm{E} 00$

$-1.83072 \mathrm{E} 00$

$-1.84315 \mathrm{E} 00$

$-1.85562 \mathrm{E} \quad 00$ $-1.86813 \mathrm{E} 00$

$-1.88069 \mathrm{E} 00$

$-1.89329 \mathrm{E} 00$

$-1.90594 \mathrm{E} \quad 00$

$-1.91863 \mathrm{E} \quad 00$

$-1.93136 \mathrm{E} 00$

$-1.94413 \mathrm{E} 00$ 
$\bar{F}$

$8.87774 \mathrm{E}-04$ $8.93681 \mathrm{E}-04$ $8.99608 \mathrm{E}-04$ $9.05555 \mathrm{E}-04$ $9.11522 \mathrm{E}-04$

$9.17508 \mathrm{E}-04$ $9.23513 \mathrm{E}-04$ $9.29538 \mathrm{E}-04$ $9.35583 \mathrm{E}-04$ $9.41648 \mathrm{E}-04$

9.47731 E- 04 $9.53835 \mathrm{E}-04$ $9.59958 \mathrm{E}-04$ $9.66101 \mathrm{E}-04$ $9.72263 \mathrm{E}-04$

$9.78445 \mathrm{E}-04$ $9.84646 \mathrm{E}-04$ $9.90867 \mathrm{E}-04$ $9.97108 \mathrm{E}-04$ $1.00337 \mathrm{E}-03$

$1.00965 \mathrm{E}-03$ $1.01595 \mathrm{E}-03$ $1.02227 \mathrm{E}-03$ $1.02860 \mathrm{E}-03$ $1.03496 \mathrm{E}-03$

$1.04134 \mathrm{E}-03$ $1.04774 \mathrm{E}-03$ $1.05415 \mathrm{E}-03$ $1.06059 \mathrm{E}-03$ $1.06705 \mathrm{E}-03$

$1.07352 \mathrm{E}-03$ $1.08002 \mathrm{E}-03$ $1.08653 \mathrm{E}-03$ $1.09307 \mathrm{E}-03$ $1.09962 \mathrm{E}-03$

33.40

33.50

33.60

33.70

33.80

33.90

34.00

34.10

34.20

34.30

34.40

34.50

34.60

34.70

34.80

34.90

35.00

$1.10619 \mathrm{E}-03$

$1.11279 \mathrm{E}-03$

$1.11940 \mathrm{E}-03$

$1.12603 \mathrm{E}-03$

$1.13268 \mathrm{E}-03$

$1.13936 \mathrm{E}-03$

$1.14605 \mathrm{E}-03$

$1.15276 \mathrm{E}-03$

$1.15949 \mathrm{E}-03$

$1.16624 \mathrm{E}-03$

$1.17301 \mathrm{E}-03$

$1.17980 \mathrm{E}-03$

1.18661 E-03

$1.19343 \mathrm{E}-03$

$1.20028 \mathrm{E}-03$

35.10

35.20

35.30

35.40

35.50

35.60

35.70

35.80

35.90

36.00 $f_{2}$

$\begin{array}{lll}1.76197 \mathrm{E} & 00\end{array}$ $1.77377 \mathrm{E} \quad 00$ $1.78561 \mathrm{E} \quad 00$ $1.79749 \mathrm{E} \quad 00$ $1.80941 \mathrm{E} \quad 00$

$1.82137 \mathrm{E} \quad 00$ $1.83336 \mathrm{E} \quad 00$ $1.84540 \mathrm{E} \quad 00$ $1.85747 \mathrm{E} \quad 00$ $1.86958 \mathrm{E} \quad 00$

$1.88173 \mathrm{E} \quad 00$ $1.89392 \mathrm{E} \quad 00$ $1.90615 \mathrm{E} \quad 00$ $1.91842 \mathrm{E} \quad 00$ $1.93073 \mathrm{E} \quad 00$

$1.94308 \mathrm{E} \quad 00$ $1.95546 \mathrm{E} \quad 00$ $1.96789 \mathrm{E} \quad 00$ $1.98035 \mathrm{E} \quad 00$ $1.99286 \mathrm{E} \quad 00$

$2.00540 \mathrm{E} \quad 00$ $2.01798 \mathrm{E} \quad 00$ $2.03060 \mathrm{E} \quad 00$ $2.04326 \mathrm{E} \quad 00$ $2.05595 \mathrm{E} \quad 00$

$2.06869 \mathrm{E} \quad 00$ $2.08147 \mathrm{E} \quad 00$ $2.09428 \mathrm{E} \quad 00$ $2.10713 \mathrm{E} \quad 00$ $2.12003 \mathrm{E} \quad 00$

$2.13296 \mathrm{E} \quad 00$ $2.14593 \mathrm{E} \quad 00$ $2.15894 \mathrm{E} \quad 00$ $2.17199 \mathrm{E} \quad 00$ $2.18507 \mathrm{E} \quad 00$

$2.19820 \mathrm{E} \quad 00$ $2.21136 \mathrm{E} \quad 00$ $2.22457 \mathrm{E} \quad 00$ $2.23781 \mathrm{E} \quad 00$ $2.25109 \mathrm{E} \quad 00$

$2.26441 \mathrm{E} \quad 00$ $2.27777 \mathrm{E} \quad 00$ $2.29117 \mathrm{E} \quad 00$ $2.30461 \mathrm{E} \quad 00$ $2.31808 \mathrm{E} \quad 00$

$2.33160 \mathrm{E} \quad 00$ $2.34515 \mathrm{E} \quad 00$ $2.35874 \mathrm{E} \quad 00$ $2.37237 \mathrm{E} \quad 00$ $2.38605 \mathrm{E} \quad 00$

$2.39975 \mathrm{E} \quad 00$ $2.41350 \mathrm{E} \quad 00$ $2.42729 \mathrm{E} \quad 00$ $2.44111 \mathrm{E} \quad 00$ $2.45498 \mathrm{E} \quad 00$

$2.46888 \mathrm{E} \quad 00$ $2.48282 \mathrm{E} \quad 00$ $2.49680 \mathrm{E} \quad 00$ $2.51082 \mathrm{E} \quad 00$ $2.52488 \mathrm{E} \quad 00$ $g_{2}$

$\tan \delta_{2}$

$f_{-2}$

$5.64324 \mathrm{E}-02 \quad-4.94319 \mathrm{E} \quad 01 \quad-5.38046 \mathrm{E}-02$ $5.70311 \mathrm{E}-02$ $5.76341 \mathrm{E}-02$

$5.82413 \mathrm{E}-02$

$5.88527 \mathrm{E}-02$

$5.94683 \mathrm{E}-02$ $6.00883 \mathrm{E}-02$ $6.07125 \mathrm{E}-02$ $6.13409 \mathrm{E}-02$ $6.19737 \mathrm{E}-02$

$6.26109 \mathrm{E}-02$ $6.32523 \mathrm{E}-02$ $6.38981 \mathrm{E}-02$ $6.45482 \mathrm{E}-02$ $6.52028 \mathrm{E}-02$

$6.58617 \mathrm{E}-02$ $6.65251 \mathrm{E}-02$ $6.71928 \mathrm{E}-02$ $6.78650 \mathrm{E}-02$ $6.85417 \mathrm{E}-02$

$6.92228 \mathrm{E}-02$ $6.99083 \mathrm{E}-02$ $7.05984 \mathrm{E}-02$ $7.12930 \mathrm{E}-02$ 7.19921 E-02

$7.26958 \mathrm{E}-02$ $7.34040 \mathrm{E}-02$

$7.41168 \mathrm{E}-02$

7.48341 E-02

7.55561 E- 02

$7.62826 \mathrm{E}-02$ $7.70138 \mathrm{E}-02$ $7.77496 \mathrm{E}-02$

$7.84901 \mathrm{E}-02$

$7.92352 \mathrm{E}-02$

$7.99850 \mathrm{E}-02$

$8.07396 \mathrm{E}-02$

$8.14988 \mathrm{E}-02$

$8.22627 \mathrm{E}-02$

$8.30314 \mathrm{E}-02$

$8.38049 \mathrm{E}-02$ $8.45831 \mathrm{E}-02$ $8.53661 \mathrm{E}-02$ $8.61539 \mathrm{E}-02$ $8.69465 \mathrm{E}-02$

$8.77439 \mathrm{E}-02$ $8.85462 \mathrm{E}-02$ $8.93534 \mathrm{E}-02$ $9.01654 \mathrm{E}-02$ $9.09823 \mathrm{E}-02$

$9.18041 \mathrm{E}-02$ $9.26307 \mathrm{E}-02$ $9.34624 \mathrm{E}-02$ $9.42990 \mathrm{E}-02$ $9.51405 \mathrm{E}-02$

$9.59870 \mathrm{E}-02$ $9.68385 \mathrm{E}-02$ $9.76950 \mathrm{E}-02$ $9.85565 \mathrm{E}-02$ $9.94231 \mathrm{E}-02$

$-4.94341 \mathrm{E} \quad 01$

$-5.43873 \mathrm{E}-02$

$-4.94362 \mathrm{E}$ ol $-5.49740 \mathrm{E}-02$

$-4.94383 \mathrm{E}$ 01 $-5.55649 \mathrm{E}-02$

$-4.94404 \mathrm{E}$ 01 $-5.61601 \mathrm{E}-02$

$-4.94425 \mathrm{E} \cdot 01$

$-5.67594 \mathrm{E}-02$

$-4.94445 \mathrm{E}$ 01

$-4.94466 \mathrm{E} \quad 01$

$-4.94486 \mathrm{E}$ 01

$-4.94506 \mathrm{E} \quad 01$

$-5.73629 \mathrm{E}-02$

$-5.79707 \mathrm{E}-02$

$-5.85827 \mathrm{E}-02$

$-5.91989 \mathrm{E}-02$

$-4.94526 \mathrm{E} \quad 01$

$-4.94546 \mathrm{E} \quad 01$

$-4.94566 \mathrm{E} 01$

$-4.94586 \mathrm{E} \quad 01$

$-4.94606 \mathrm{E} 01$

$-5.98195 \mathrm{E}-02$

$-6.04443 \mathrm{E}-02$

$-6.10734 \mathrm{E}-02$

$-6.17068 \mathrm{E}-02$

$-6.23446 \mathrm{E}-02$

$-4.94625 \mathrm{E} \quad 01$

$-6.29867 \mathrm{E}-02$

$-4.94644 \mathrm{E} \quad 01$

$-4.94664 \mathrm{E} 01$

$-4.94683 \mathrm{E} 01$

$-4.94702 \mathrm{E} 01$

$-6.36331 \mathrm{E}-02$

$-6.42839 \mathrm{E}-02$

$-6.49392 \mathrm{E}-02$

$-6.55988 \mathrm{E}-02$

$-4.94720 \mathrm{E} \quad 0 \mathrm{l}$

$-4.94739 \mathrm{E} 01$

$-4.94758 \mathrm{E} \quad 01$

$-4.94776 \mathrm{E} 01$

$-4.94794 \mathrm{E} \quad 01$

$-6.62628 \mathrm{E}-02$

$-6.69313 \mathrm{E}-02$

$-6.76042 \mathrm{E}-02$

$-6.82815 \mathrm{E}-02$

$-6.89634 \mathrm{E}-02$

$-4.94813 \mathrm{E} \quad 01$

$-4.94831 \mathrm{E} \quad 01$

$-6.96497 \mathrm{E}-02$

$-4.94849 \mathrm{E}$ 0l

$-4.94867 \mathrm{E}$ 0l

$-4.94885 \mathrm{E} \quad 0 \mathrm{l}$

$-4.94902 \mathrm{E} \quad 01$

$-4.94920 \mathrm{E} 01$

$-4.94937 \mathrm{E}$ 0l

$-4.94955 \mathrm{E} 01$

$-4.94972 \mathrm{E} \quad 01$

$-4.94989 \mathrm{E} \quad 01$

$-4.95006 \mathrm{E}$ 01

$-4.95023 \mathrm{E} \quad 01$

$-4.95040 \mathrm{E}$ 0l

$-4.95056 \mathrm{E} \quad 01$

$-4.95073 \mathrm{E} 01$

$-4.95090 \mathrm{E}$ 01

$-4.95106 \mathrm{E} 01$

$-4.95122 \mathrm{E} 01$

$-4.95139 \mathrm{E} 01$

$-4.95155 \mathrm{E} 01$

$-4.95171 \mathrm{E} 01$

$-4.95187 \mathrm{E}$ ol

$-4.95202 \mathrm{E} 01$

$-4.95218 \mathrm{E} 01$

$\begin{array}{lll}-4.95234 \mathrm{E} & 01\end{array}$

-4.95249 E 01

$-4.95265 \mathrm{E} \quad 01$

$-4.95280 \mathrm{E} 01$

$-4.95296 \mathrm{E} \quad 01$

$-7.03406 \mathrm{E}-02$

$-7.10359 \mathrm{E}-02$

$-7.17358 \mathrm{E}-02$

$-7.24402 \mathrm{E}-02$

$-7.31492 \mathrm{E}-02$

$-7.38628 \mathrm{E}-02$

$-7.45810 \mathrm{E}-02$

$-7.53037 \mathrm{E}-02$

$-7.60311 \mathrm{E}-02$

$-7.67631 \mathrm{E}-02$

$-7.74998 \mathrm{E}-02$ 
$p$

36.10

36.20

36.30

36.40

36.50

36.60

36.70

36.80

36.90

37.00

37.10

37.20

37.30

37.40

37.50

37.60

37.70

37.80

37.90

38.00

38.10

38.20

38.30

38.40

38.50

38.60

38.70

38.80

38.90

39.00

39.10

39.20

39.30

39.40

39.50

39.60

39.70

39.80

39.90

40.00

40.10

40.20

40.30

40.40

40.50

40.60

40.70

40.80

40.90

41.00

41.10

41.20

41.30

41.40

41.50

41.60

4.1 .70

41.80

41.90

42.00
$F_{0}$

9.13573E-01 $9.13571 \mathrm{E}-01$

$9.13569 \mathrm{E}-01$ $9.13567 \mathrm{E}-01$

9.13565 E-01

$9.13563 \mathrm{E}-01$

$9.13561 \mathrm{E}-01$

$9.13559 \mathrm{E}-01$

$9.13557 \mathrm{E}-01$

$9.13555 \mathrm{E}-01$

$9.13553 \mathrm{E}-01$

9.13551 E-01

$9.13549 \mathrm{E}-01$

$9.13547 \mathrm{E}-01$

$9.13545 \mathrm{E}-01$

$9.13544 \mathrm{E}-01$

$9.13542 \mathrm{E}-0 \mathrm{I}$

$9.13540 \mathrm{E}-01$

$9.13538 \mathrm{E}-01$

$9.13536 \mathrm{E}-01$

$9.13534 \mathrm{E}-01$

$9.13532 \mathrm{E}-01$

$9.13530 \mathrm{E}-01$

$9.13528 \mathrm{E}-01$

$9.13527 \mathrm{E}-01$

$9.13525 \mathrm{E}-01$

$9.13523 \mathrm{E}-01$

$9.13521 \mathrm{E}-01$

$9.13519 \mathrm{E}-01$

$9.13517 \mathrm{E}-01$

$9.13516 \mathrm{E}-01$

$9.13514 \mathrm{E}-01$

$9.13512 \mathrm{E}-01$

$9.13510 \mathrm{E}-01$

$9.13508 \mathrm{E}-01$

$9.13506 \mathrm{E}-01$

$9.13504 \mathrm{E}-01$

9.13503E-01

$9.13501 \mathrm{E}-01$

$9.13499 \mathrm{E}-01$

$9.134 .97 \mathrm{E}-01$

$9.13495 \mathrm{E}-01$

$9.13494 \mathrm{E}-01$

9.13492E-01

$9.13490 \mathrm{E}-01$

$9.13488 \mathrm{E}-01$

$9.13486 \mathrm{E}-01$

$9.13485 \mathrm{E}-01$

$9.13483 \mathrm{E}-01$

9.134.81 E-01

$9.13479 \mathrm{E}-01$ $9.13478 \mathrm{E}-01$ $9.13476 \mathrm{E}-01$ $9.13474 \mathrm{E}-01$

$9.13472 \mathrm{E}-01$

$9.13470 \mathrm{E}-01$ $9.13469 \mathrm{E}-01$ $9.13467 \mathrm{E}-01$ $9.13465 \mathrm{E}-0 \mathrm{I}$ $9.134 .64 \mathrm{E}-01$ $f_{1}$

$3.46219 \mathrm{E} \quad 01$

$3.47220 \mathrm{E}$ 01

$3.48221 \mathrm{E} \quad 01$

$3.49223 \mathrm{E} \quad 01$

$3.50225 \mathrm{E} 01$

$3.51227 \mathrm{E} \quad 01$

$3.52229 \mathrm{E} 01$

$3.53232 \mathrm{E} 01$

$3.54235 \mathrm{E} \quad 01$

$3.55238 \mathrm{E} 01$

$3.56241 \mathrm{E} \quad 01$

$3.57245 \mathrm{E} 01$

$3.58249 \mathrm{E} 01$

$3.59253 \mathrm{E} 01$

$3.60257 \mathrm{E} 01$

$3.61262 \mathrm{E} 01$

$3.62266 \mathrm{E} 01$

$3.63271 \mathrm{E} 01$

$3.64277 \mathrm{E} \quad 01$

$3.65282 \mathrm{E} 01$

$3.66288 \mathrm{E} 01$

$3.67294 \mathrm{E} \quad 01$

$3.68300 \mathrm{E} 01$

$3.69307 \mathrm{E} \quad 01$

$3.70314 \mathrm{E} 01$

$3.71320 \mathrm{E} \quad 01$

$3.72328 \mathrm{E}$ 0l

$3.73335 \mathrm{E} \quad 01$

$3.74343 \mathrm{E} 01$

$3.75351 \mathrm{E} 01$

$3.76359 \mathrm{E} \quad 01$

$3.77368 \mathrm{E}$ 01

$3.78376 \mathrm{E} \quad 01$

$3.79385 \mathrm{E} \quad 01$

$3.80395 \mathrm{E} 01$

$3.81404 \mathrm{E} \quad 01$

$3.82414 \mathrm{E} 01$

$3.83424 \mathrm{E}$ ol

$3.84434 \mathrm{E}$ 01

$3.85445 \mathrm{E} 01$

$3.86455 \mathrm{E} \quad 01$

$3.87466 \mathrm{E} 01$

$3.88478 \mathrm{E} 01$

$3.89489 \mathrm{E} \quad 01$

$3.90501 \mathrm{E} 01$

$3.91513 \mathrm{E} \quad 01$

$3.92526 \mathrm{E} \quad 01$

$3.93538 \mathrm{E}$ 0l

$3.94551 \mathrm{E} 01$

$3.95564 \mathrm{E} 01$

$3.96577 \mathrm{E} \quad 01$

$3.97591 \mathrm{E} 01$

$3.98605 \mathrm{E} \quad 01$

$3.99619 \mathrm{E}$ 01

$4.00634 \mathrm{E} \quad 01$

$4.01648 \mathrm{E} \quad 01$ $4.02663 \mathrm{E} 01$ $4.03679 \mathrm{E} 01$

$4.04694 \mathrm{E} \quad 01$

$4.05710 \mathrm{E} 01$ $g_{1}$

$2.26313 \mathrm{E} \quad 00$

$\tan \delta_{1}$

$f_{-1}$

$00-1.05744 \mathrm{E}-03$

$2.29075 \mathrm{E} \quad 00 \quad-1.05781 \mathrm{E}-03$

$2.30462 \mathrm{E} \quad 00-1.05817 \mathrm{E}-03$

$2.31854 \mathrm{E} \quad 00-1.05852 \mathrm{E}-03$

$2.33250 \mathrm{E} \quad 00-1.05886 \mathrm{E}-03$

$2.34651 \mathrm{E} \quad 00-1.05920 \mathrm{E}-03$

$2.36056 \mathrm{E} \quad 00-1.05952 \mathrm{E}-03$

$2.37465 \mathrm{E} \quad 00-1.05984 \mathrm{E}-03$

$2.38879 \mathrm{E} \quad 00-1.06015 \mathrm{E}-03$

$2.40297 \mathrm{E} \quad 00 \quad-1.06045 \mathrm{E}-03$

$2.41720 \mathrm{E} \quad 00-1.06075 \mathrm{E}-03$

$2.43147 \mathrm{E} \quad 00 \quad-1.06104 \mathrm{E}-03$

$2.44578 \mathrm{E} \quad 00-1.06132 \mathrm{E}-03$

$2.46014 \mathrm{E} \quad 00 \quad-1.06159 \mathrm{E}-03$

$2.47454 \mathrm{E} \quad 00-1.06186 \mathrm{E}-03$

$2.48899 \mathrm{E} \quad 00-1.06212 \mathrm{E}-03$

$2.50348 \mathrm{E} \quad 00 \quad-1.06237 \mathrm{E}-03$

$2.51801 \mathrm{E} \quad 00 \quad-1.06261 \mathrm{E}-03$

$2.53259 \mathrm{E} \quad 00 \quad-1.06284 \mathrm{E}-03$

$2.54721 \mathrm{E} \quad 00 \quad-1.06307 \mathrm{E}-03$

$2.56187 \mathrm{E} \quad 00 \quad-1.06329 \mathrm{E}-03$

$2.57658 \mathrm{E} \quad 00-1.06350 \mathrm{E}-03$

$2.59134 \mathrm{E} \quad 00 \quad-1.06371 \mathrm{E}-03$

$2.60614 \mathrm{E} \quad 00-1.06391 \mathrm{E}-03$

$2.62098 \mathrm{E} \quad 00-1.06410 \mathrm{E}-03$

$2.63586 \mathrm{E} \quad 00-1.06428 \mathrm{E}-03$

$2.65080 \mathrm{E} \quad 00-1.06446 \mathrm{E}-03$

$2.66577 \mathrm{E} \quad 00 \quad-1.06463 \mathrm{E}-03$

$2.68079 \mathrm{E} \quad 00-1.06479 \mathrm{E}-03$

$2.69585 \mathrm{E} \quad 00 \quad-1.06495 \mathrm{E}-03$

$2.71096 \mathrm{E} \quad 00$

$2.72611 \mathrm{E} \quad 00$

$2.74131 \mathrm{E} \quad 00$

$2.75655 \mathrm{E} \quad 00$

$2.77184 \mathrm{E} \quad 00$

2.78717 E $\quad 00$

$2.80254 \mathrm{E} \quad 00$

$2.81796 \mathrm{E} \quad 00$

$2.83343 \mathrm{E} \quad 00$

$2.84894 \mathrm{E} \quad 00$

$2.86449 \mathrm{E} \quad 00$

$2.88009 \mathrm{E} \quad 00$

$2.89573 \mathrm{E} \quad 00$

$2.91142 \mathrm{E} \quad 00$

$2.92715 \mathrm{E} \quad 00$

$2.94292 \mathrm{E} \quad 00$

$2.95874 \mathrm{E} \quad 00$

$2.97461 \mathrm{E} \quad 00$

$2.99052 \mathrm{E} \quad 00$

$3.00648 \mathrm{E} \quad 00$

$3.02248 \mathrm{E} \quad 00$

$3.03852 \mathrm{E} \quad 00$

$3.05461 \mathrm{E} \quad 00$

$3.07075 \mathrm{E} \quad 00$

$3.08693 \mathrm{E} \quad 00$

$3.10316 \mathrm{E} \quad 00$

$3.11943 \mathrm{E} \quad 00$

$3.13574 \mathrm{E} \quad 00$

$3.15210 \mathrm{E} \quad 00$
$-1.06510 \mathrm{E}-03$

$-1.06524 \mathrm{E}-03$

$-1.06538 \mathrm{E}-03$

$-1.06551 \mathrm{E}-03$

$-1.06563 \mathrm{E}-03$

$-1.06574 \mathrm{E}-03$

$-1.06585 \mathrm{E}-03$

$-1.06595 \mathrm{E}-03$

$-1.06605 \mathrm{E}-03$

$-1.06614 \mathrm{E}-03$

$-1.06622 \mathrm{E}-03$

$-1.06630 \mathrm{E}-03$

$-1.06637 \mathrm{E}-03$

$-1.06648 \mathrm{E}-03$

$-1.06654 \mathrm{E}-03$

$-1.06658 \mathrm{E}-03$

$-1.06662 \mathrm{E}-03$

$-1.06665 \mathrm{E}-03$

$-1.06667 \mathrm{E}-03$

$-1.06669 \mathrm{E}-03$

$-1.06671 \mathrm{E}-03$

$-1.06671 \mathrm{E}-03$

$-1.06671 \mathrm{E}-03$

$-1.06670 \mathrm{E}-03$

$-1.06669 \mathrm{E}-03$

$-1.06667 \mathrm{E}-03$

$-1.06665 \mathrm{E}-03$

$-1.06662 \mathrm{E}-03$
$-1.06643 \mathrm{E}-03$

$-2.18150 \mathrm{E} \quad 00$

$-2.19510 \mathrm{E} \quad 00$

$-2.20875 \mathrm{E} \quad 00$ $-2.22244 \mathrm{E} \quad 00$

$-2.23617 \mathrm{E} 00$

$-2.24995 \mathrm{E} \quad 00$

$-2.26377 \mathrm{E} \quad 00$

$-2.27763 \mathrm{E} 00$ 
ELECTRON RADIAL FUNCTIONS

$Z=4 \quad A=8 \quad \rho=1.2 A^{1 / 3} F$

Positron

$\bar{F}$

$1.27690 \mathrm{E}-03$

$1.28398 \mathrm{E}-03$

$1.29108 \mathrm{E}-03$

$1.29821 \mathrm{E}-03$

$1.30535 \mathrm{E}-03$

36.40

36.50

$1.31251 \mathrm{E}-03$

$1.31969 \mathrm{E}-03$

$1.32689 \mathrm{E}-03$

$1.33411 \mathrm{E}-03$

$1.34135 \mathrm{E}-03$

37.10

$1.34861 \mathrm{E}-03$

$1.35589 \mathrm{E}-03$

$1.36319 \mathrm{E}-03$

$1.37050 \mathrm{E}-03$

$1.37784 \mathrm{E}-03$

37.40

37.50

37.60

37.70

37.80

37.90

38.00

$1.38520 \mathrm{E}-03$

$1.39257 \mathrm{E}-03$

$1.39997 \mathrm{E}-03$

$1.40739 \mathrm{E}-03$

$1.41482 \mathrm{E}-03$

38.10

38.20

$1.42228 \mathrm{E}-03$

$1.42975 \mathrm{E}-03$

$1.43724 \mathrm{E}-03$

$1.44476 \mathrm{E}-03$

$1.45229 \mathrm{E}-03$

38.50

$1.45984 \mathrm{E}-03$

38.60

38.70

38.80

38.90

39.00

$1.46742 \mathrm{E}-03$

$1.47501 \mathrm{E}-03$

$1.48262 \mathrm{E}-03$

$1.49025 \mathrm{E}-03$

39.10

39.20

39.30

39.40

39.50

$1.49790 \mathrm{E}-03$

$1.50557 \mathrm{E}-03$

$1.51326 \mathrm{E}-03$

$1.52097 \mathrm{E}-03$

$1.52870 \mathrm{E}-03$

39.60

39.70

39.80

39.90

40.00

$1.53645 \mathrm{E}-03$

$1.54422 \mathrm{E}-03$

$1.55200 \mathrm{E}-03$

$1.55981 \mathrm{E}-0.3$

$1.56764 \mathrm{E}-03$

40. 10

$1.57549 \mathrm{E}-03$

$40.20 \quad 1.58335 \mathrm{E}-03$

$40.30 \quad 1.59124 \mathrm{E}-03$

$40.40 \quad 1.59914 \mathrm{E}-03$

$1.60707 \mathrm{E}-03$

40.60

40.70

40.80

40.90

41.00

$1.61501 \mathrm{E}-03$

$1.62298 \mathrm{E}-03$

$1.63096 \mathrm{E}-03$

$1.63896 \mathrm{E}-03$

41.10

$1.64699 \mathrm{E}-03$

$1.66309 \mathrm{E}-03$

$1.67117 \mathrm{E}-03$

$41.40 \quad 1.67927 \mathrm{E}-03$

$41.50 \quad 1.68739 \mathrm{E}-03$

$41.60 \quad 1.69553 \mathrm{E}-03$

$41.70 \quad 1.70369 \mathrm{E}-03$

$41.80 \quad 1.71187 \mathrm{E}-03$

$41.90 \quad 1.72007 \mathrm{E}-03$

42.00 $f_{2}$

$2.53898 \mathrm{E} \quad 00$

$2.55312 \mathrm{E} \quad 00$

$2.56729 \mathrm{E} \quad 00$

$2.58151 \mathrm{E} \quad 00$

$2.59576 \mathrm{E} \quad 00$

$2.61005 \mathrm{E} \quad 00$

$2.62438 \mathrm{E} \quad 00$

$2.63875 \mathrm{E} \quad 00$

$2.65315 \mathrm{E} \quad 00$

$2.66760 \mathrm{E} \quad 00$

$2.68208 \mathrm{E} \quad 00$

$2.69661 \mathrm{E} \quad 00$

$2.71117 \mathrm{E} \quad 00$

$2.72577 \mathrm{E} \quad 00$

$2.74041 \mathrm{E} \quad 00$

$2.75509 \mathrm{E} \quad 00$

$2.76981 \mathrm{E} \quad 00$

$2.78456 \mathrm{E} \quad 00$

$2.79936 \mathrm{E} \quad 00$

$2.81419 \mathrm{E} \quad 00$

$2.82906 \mathrm{E} \quad 00$

$2.84397 \mathrm{E} \quad 00$

$2.85892 \mathrm{E} \quad 00$

$2.87391 \mathrm{E} \quad 00$

$2.88893 \mathrm{E} \quad 00$

$2.90400 \mathrm{E} \quad 00$

$2.91910 \mathrm{E} \quad 00$

$2.93424 \mathrm{E} \quad 00$

$2.94943 \mathrm{E} \quad 00$

$2.96464 \mathrm{E} \quad 00$

$2.97990 \mathrm{E} \quad 00$

$2.99520 \mathrm{E} \quad 00$

$3.01053 \mathrm{E} \quad 00$

$3.02591 \mathrm{E} \quad 00$

$3.04132 \mathrm{E} \quad 00$

$3.05677 \mathrm{E} \quad 00$

$3.07226 \mathrm{E} \quad 00$

$3.08779 \mathrm{E} \quad 00$

$3.10336 \mathrm{E} \quad 00$

$3.11896 \mathrm{E} \quad 00$

$3.13461 \mathrm{E} \quad 00$

$3.15029 \mathrm{E} \quad 00$

$3.16601 \mathrm{E} \quad 00$

$3.18177 \mathrm{E} \quad 00$

$3.19757 \mathrm{E} \quad 00$

$3.21340 \mathrm{E} \quad 00$

$3.22928 \mathrm{E} \quad 00$

$3.24519 \mathrm{E} \quad 00$

$3.26115 \mathrm{E} \quad 00$

$3.27714 \mathrm{E} \quad 00$

$3.29317 \mathrm{E} \quad 00$

$3.30923 \mathrm{E} \quad 00$

$3.32534 \mathrm{E} \quad 00$

$3.34148 \mathrm{E} \quad 00$

$3.35767 \mathrm{E} \quad 00$

$3.37389 \mathrm{E} \quad 00$

$3.39015 \mathrm{E} \quad 00$

$3.40645 \mathrm{E} \quad 00$

$3.42279 \mathrm{E} \quad 00$

$3.43916 \mathrm{E} \quad 00$ $g_{2}$

$\tan \delta_{2}$

$f_{-2}$

$1.00295 \mathrm{E}-01 \quad-4.95385 \mathrm{E}$ ol $-9.66119 \mathrm{E}-02$

$1.01171 \mathrm{E}-01 \quad-4.95400 \mathrm{E} \quad 01 \quad-9.74695 \mathrm{E}-02$

$1.02053 \mathrm{E}-01 \quad-4.95415 \mathrm{E} \quad 01 \quad-9.83320 \mathrm{E}-02$

$1.02940 \mathrm{E}-01 \quad-4.95429 \mathrm{E}$ 01 $-9.91997 \mathrm{E}-02$

$1.03832 \mathrm{E}-01$

$1.04729 \mathrm{E}-01$

$1.05631 \mathrm{E}-01$

$1.06538 \mathrm{E}-01$

$1.07451 \mathrm{E}-01$

$1.08368 \mathrm{E}-01$

$1.09291 \mathrm{E}-01$

$1.10219 \mathrm{E}-01$

$1.11152 \mathrm{E}-01$

$1.12091 \mathrm{E}-01$

$1.13035 \mathrm{E}-01$

$1.13983 \mathrm{E}-01$

$1.14938 \mathrm{E}-01$

$1.15897 \mathrm{E}-01$

$1.16862 \mathrm{E}-01$

$1.17832 \mathrm{E}-01$

$1.18808 \mathrm{E}-01$

$1.19789 \mathrm{E}-01$

$1.20775 \mathrm{E}-01$

$1.21766 \mathrm{E}-01$

$1.22763 \mathrm{E}-01$

$1.23766 \mathrm{E}-01$

$1.24773 \mathrm{E}-01$

$1.25787 \mathrm{E}-01$

$1.26805 \mathrm{E}-01$

$1.27829 \mathrm{E}-01$

$1.28859 \mathrm{E}-01$

$1.29894 \mathrm{E}-01$

$1.30935 \mathrm{E}-01$

$1.31981 \mathrm{E}-01$

$1.33032 \mathrm{E}-01$

$1.34090 \mathrm{E}-01$

$1.35152 \mathrm{E}-01$

$1.36221 \mathrm{E}-01$

$1.37295 \mathrm{E}-01$

$1.38374 \mathrm{E}-01$

$1.39459 \mathrm{E}-01$

$1.40550 \mathrm{E}-01$

$1.41646 \mathrm{E}-01$

$1.42748 \mathrm{E}-01$

$1.43856 \mathrm{E}-01$

$1.44970 \mathrm{E}-01$

$1.46089 \mathrm{E}-01$

$1.47214 \mathrm{E}-01$

$1.48344 \mathrm{E}-01$

$1.49481 \mathrm{E}-01$

$1.50623 \mathrm{E}-01$

$1.51771 \mathrm{E}-01$

$1.52924 \mathrm{E}-01$

$1.54084 \mathrm{E}-01$

$1.55249 \mathrm{E}-01$

$1.56420 \mathrm{E}-01$

$1.57597 \mathrm{E}-01$

$1.58780 \mathrm{E}-01$

$1.59969 \mathrm{E}-01$

$1.61164 \mathrm{E}-01$
$-4.95444 \mathrm{E} \quad 01$

$-4.95458 \mathrm{E} \quad 01$

$-4.95472 \mathrm{E} \quad 01$

$-4.95487 \mathrm{E}$ 01

$-4.95501 \mathrm{E} 01$

$-4.95515 \mathrm{E} \quad 01$

$-4.95529 \mathrm{E} \quad 01$

$-4.95543 \mathrm{E} \quad 01$

$-4.95556 \mathrm{E} \quad 01$

$-4.95570 \mathrm{E}$ 0I

$-4.95584 \mathrm{E} \quad 01$

$-4.95597 \mathrm{E} \quad 01$

$-4.95611 \mathrm{E} 01$

$-4.95624 \mathrm{E} \quad 01$

$-4.95638 \mathrm{E} \quad 01$

$-4.95651 \mathrm{E} \quad 01$

$-4.95664 \mathrm{E} \quad 0 \mathrm{l}$

$-4.95677 \mathrm{E} \quad 01$

$-4.95690 \mathrm{E} \quad 01$

$-4.95703 \mathrm{E} 01$

$-4.95716 \mathrm{E} 01$

$-4.95729 \mathrm{E} \quad 01$

$-4.95742 \mathrm{E} 01$

$-4.95755 \mathrm{E} \quad 01$

$-4.95767 \mathrm{E} \quad 01$

$-4.95780 \mathrm{E} 01$

$-4.95792 \mathrm{E} 01$

$-4.95817 \mathrm{E} \quad 01$

$-4.95830 \mathrm{E} \quad 01$

$-4.95842 \mathrm{E} 01$

$-4.95854 \mathrm{E} \quad 01$

$-4.95866 \mathrm{E} \quad 01$

$-4.95878 \mathrm{E} \quad 01$

$-4.95890 \mathrm{E} \quad 01$

$-4.95902 \mathrm{E} 01$

$-4.95914 \mathrm{E} \quad 01$

$-4.95926 \mathrm{E} \quad 01$

$-4.95938 \mathrm{E} 01$

$-4.95949 \mathrm{E} 01$

$-4.95961 \mathrm{E} 01$

$-4.95973 \mathrm{E} \quad 01$

$-4.95984 \mathrm{E} \quad 01$

$-4.95996 \mathrm{E} 01$

$-4.96007 \mathrm{E} 01$

$-4.96018 \mathrm{E} 0 \mathrm{l}$

$-4.96030 \mathrm{E} \quad 01$

$-4.96041 \mathrm{E} 01$

$-4.96052 \mathrm{E} \quad 01$

$-4.96063 \mathrm{E} \quad 01$

$-4.96074 \mathrm{E} 01$

$-4.96085 \mathrm{E} \quad 01$

$-4.96096 \mathrm{E} \quad 01$

$-4.96107 \mathrm{E} 01$

$-4.96118 \mathrm{E}$ 0l

$-4.96129 \mathrm{E} 01$
$-4.95805 \mathrm{E} 01$

$-1.00072 \mathrm{E}-01$

$-1.00950 \mathrm{E}-01$

$-1.01833 \mathrm{E}-01$

$-1.02721 \mathrm{E}-01$

$-1.03614 \mathrm{E}-01$

$-1.04512 \mathrm{E}-01$

$-1.05415 \mathrm{E}-01$

$-1.06324 \mathrm{E}-01$

$-1.07238 \mathrm{E}-01$ 
ELECTRON RADIAL FUNCTIONS

Positron

$\begin{array}{cc}p & F_{0} \\ 0.10 & 2.58641 \mathrm{E}-01 \\ 0.20 & 5.32718 \mathrm{E}-01 \\ 0.30 & 6.60481 \mathrm{E}-01 \\ 0.40 & 7.30275 \mathrm{E}-01 \\ 0.50 & 7.73061 \mathrm{E}-01\end{array}$

$\begin{array}{ll}0.60 & 8.01354 \mathrm{E}-01 \\ 0.70 & 8.21077 \mathrm{E}-01 \\ 0.80 & 8.35372 \mathrm{E}-01 \\ 0.90 & 8.46052 \mathrm{E}-01\end{array}$

$\begin{array}{ll}0.00 & 8.54227 \mathrm{E}-01\end{array}$

$1.10 \quad 8.60611 \mathrm{E}-01$

$1.20 \quad 8.65682 \mathrm{E}-01$

$1.30 \quad 8.69771 \mathrm{E}-01$

$1.40 \quad 8.73110 \mathrm{E}-01$

$1.50 \quad 8.75867 \mathrm{E}-01$

$1.60 \quad 8.78168 \mathrm{E}-01$

$1.70 \quad 8.80103 \mathrm{E}-01$

$1.80 \quad 8.81747 \mathrm{E}-01$

$1.90 \quad 8.83151 \mathrm{E}-01$

$2.00 \quad 8.84360 \mathrm{E}-01$

$2.10 \quad 8.85407 \mathrm{E}-01$

$2.20 \quad 8.86319 \mathrm{E}-01$

$2.30 \quad 8.87116 \mathrm{E}-01$

$2.40 \quad 8.87818 \mathrm{E}-01$

$2.50 \quad 8.88438 \mathrm{E}-01$

$2.60 \quad 8.88987 \mathrm{E}-01$

$2.70 \quad 8.89476 \mathrm{E}-01$

$2.80 \quad 8.89913 \mathrm{E}-01$

$2.90 \quad 8.90304 \mathrm{E}-01$

$3.00 \quad 8.90656 \mathrm{E}-01$

3.10

3.20

3.30

3.40

3.50

3.60

3.70

3.80

3.90

4.00

4.10

4.20

4.30

4.40

4.50

4.60

4.70

4.80

4.90

8.93632E-01

$5.10 \quad 8.93752 \mathrm{E}-01$

$5.20 \quad 8.93805 \mathrm{E}-01$

$5.30 \quad 8.93855 \mathrm{E}-01$

$5.40 \quad 8.93901 \mathrm{E}-01$

$5.50 \quad 8.93944 \mathrm{E}-01$

$5.60 \quad 8.93984 \mathrm{E}-01$

$5.70 \quad 8.94021 \mathrm{E}-01$

$5.80 \quad 8.94056 \mathrm{E}-01$

$5.90 \quad 8.94087 \mathrm{E}-01$

$6.00 \quad 8.94117 \mathrm{E}-01$

$$
Z=5 \quad A=11
$$

$f_{1}$

$3.79845 \mathrm{E}-03$

$2.06605 \mathrm{E}-02$

$5.04215 \mathrm{E}-02$

$9.17837 \mathrm{E}-02$

$1.43214 \mathrm{E}-01$

$2.03134 \mathrm{E}-01$

$2.70057 \mathrm{E}-01$

$3.42659 \mathrm{E}-01$

$4.19811 \mathrm{E}-01$

$5.00586 \mathrm{E}-01$

$5.84228 \mathrm{E}-01$

$6.70141 \mathrm{E}-01$

$7.57851 \mathrm{E}-01$

$8.46985 \mathrm{E}-01$

$9.37251 \mathrm{E}-01$

$1.02842 \mathrm{E} \quad 00$

$1.12031 \mathrm{E} \quad 00$

$1.21278 \mathrm{E} \quad 00$

$1.30572 \mathrm{E} \quad 00$

$1.39904 \mathrm{E} \quad 00$

$1.49267 \mathrm{E} \quad 00$

$1.58655 \mathrm{E} \quad 00$

$1.68063 \mathrm{E} 00$

$1.77489 \mathrm{E} \quad 00$

$1.86928 \mathrm{E} \quad 00$

$1.96379 \mathrm{E} \quad 00$

$2.05839 \mathrm{E} \quad 00$

$2.15307 \mathrm{E} \quad 00$

$2.24781 \mathrm{E} \quad 00$

$2.34261 \mathrm{E} \quad 00$

$2.43746 \mathrm{E} \quad 00$

$2.53234 \mathrm{E} \quad 00$

$2.62726 \mathrm{E} \quad 00$

$2.72220 \mathrm{E} \quad 00$

$2.81716 \mathrm{E} \quad 00$

$2.91214 \mathrm{E} \quad 00$

$3.00714 \mathrm{E} \quad 00$

$3.10215 \mathrm{E} \quad 00$

$3.19717 \mathrm{E} \quad 00$

$3.29220 \mathrm{E} \quad 00$

$3.38723 \mathrm{E} \quad 00$

$3.48227 \mathrm{E} \quad 00$

$3.57732 \mathrm{E} \quad 00$

$3.67237 \mathrm{E} \quad 00$

$3.76743 \mathrm{E} \quad 00$

$3.86248 \mathrm{E} 00$ $3.95754 \mathrm{E} \quad 00$ $4.05260 \mathrm{E} \quad 00$

$4.14766 \mathrm{E} \quad 00$

$4.24273 \mathrm{E} \quad 00$

$4.33779 \mathrm{E} \quad 00$

$4.43285 \mathrm{E} \quad 00$

$4.52791 \mathrm{E} \quad 00$

$4.62297 \mathrm{E} \quad 00$

$4.71803 \mathrm{E} \quad 00$

$4.81308 \mathrm{E} \quad 00$ $4.90813 \mathrm{E} \quad 00$

$5.00317 \mathrm{E} \quad 00$

$5.09820 \mathrm{E} \quad 00$

5.19321 E 00 $g_{1}$

$-3.78961 \mathrm{E}-05$

$-2.05418 \mathrm{E}-04$

$-4.98505 \mathrm{E}-04$

$-9.00462 \mathrm{E}-04$

$-1.39150 \mathrm{E}-03$

$-1.95115 \mathrm{E}-03$

$-2.56008 \mathrm{E}-03$

$-3.20097 \mathrm{E}-03$

$-3.85907 \mathrm{E}-03$

$-4.52216 \mathrm{E}-03$

$-5.18032 \mathrm{E}-03$

$-5.82561 \mathrm{E}-03$

$-6.45175 \mathrm{E}-03$

$-7.05377 \mathrm{E}-03$

$-7.62775 \mathrm{E}-03$

$-8.17063 \mathrm{E}-03$

$-8.67996 \mathrm{E}-03$

$-9.15381 \mathrm{E}-03$

$-9.59066 \mathrm{E}-03$

$-9.98928 \mathrm{E}-03$

$-1.03487 \mathrm{E}-02$

$-1.06681 \mathrm{E}-02$

$-1.09469 \mathrm{E}-02$

$-1.11846 \mathrm{E}-02$

$-1.13807 \mathrm{E}-02$

$-1.15348 \mathrm{E}-02$

$-1.16468 \mathrm{E}-02$

$-1.17164 \mathrm{E}-02$

$-1.17433 \mathrm{E}-02$

$-1.17274 \mathrm{E}-02$

$-1.16686 \mathrm{E}-02$

$-1.15667 \mathrm{E}-02$

$-1.14217 \mathrm{E}-02$

$-1.12335 \mathrm{E}-02$

$-1.10020 \mathrm{E}-02$

$-1.07272 \mathrm{E}-02$

$-1.04090 \mathrm{E}-02$

$-1.00473 \mathrm{E}-02$

$-9.64212 \mathrm{E}-03$

$-9.19346 \mathrm{E}-03$

$-8.70124 \mathrm{E}-03$

$-8.16547 \mathrm{E}-03$

$-7.58612 \mathrm{E}-03$

$-6.96316 \mathrm{E}-03$

$-6.29659 \mathrm{E}-03$

$-5.58638 \mathrm{E}-03$

$-4.83252 \mathrm{E}-03$

$-4.03501 \mathrm{E}-03$

$-3.19383 \mathrm{E}-03$

$-2.30897 \mathrm{E}-03$

$-1.38043 \mathrm{E}-03$

$-4.08200 \mathrm{E}-04$

$6.07730 \mathrm{E}-04$

$1.66736 \mathrm{E}-03$

$2.77070 \mathrm{E}-03$

$3.91775 \mathrm{E}-03$

5.10851 E-03

6.34296 E-03

$7.62113 \mathrm{E}-03$

$8.94298 \mathrm{E}-03$ $\tan \delta_{1}$

$1.59622 \mathrm{E}-01$

$7.84112 \mathrm{E}-02$

$5.21840 \mathrm{E}-02$

$3.91105 \mathrm{E}-02$

$3.12448 \mathrm{E}-02$

$2.59750 \mathrm{E}-02$

$2.21884 \mathrm{E}-02$

$1.93305 \mathrm{E}-02$

$1.70934 \mathrm{E}-02$

$1.52925 \mathrm{E}-02$

$1.38101 \mathrm{E}-02$

$1.25678 \mathrm{E}-02$

$1.15110 \mathrm{E}-02$

$1.06007 \mathrm{E}-02$

$9.80804 \mathrm{E}-03$

$9.11151 \mathrm{E}-03$

$8.49447 \mathrm{E}-03$

$7.94396 \mathrm{E}-03$

7.44971 E-03

$7.00348 \mathrm{E}-03$

$6.59855 \mathrm{E}-03$

$6.22943 \mathrm{E}-03$

$5.89153 \mathrm{E}-03$

$5.58107 \mathrm{E}-03$

5.29481 E-03

$5.03002 \mathrm{E}-03$

$4.78437 \mathrm{E}-03$

$4.55586 \mathrm{E}-03$

4.34273 E-03

$4.14350 \mathrm{E}-03$

$3.95685 \mathrm{E}-03$

$3.78162 \mathrm{E}-03$

$3.61679 \mathrm{E}-03$

$3.46145 \mathrm{E}-03$

3.31482 E- 03

3.17619 E-03

$3.04491 \mathrm{E}-03$

$2.92042 \mathrm{E}-03$

$2.80220 \mathrm{E}-03$

$2.68979 \mathrm{E}-03$

$2.58278 \mathrm{E}-03$

$2.48078 \mathrm{E}-03$

$2.38345 \mathrm{E}-03$

$2.29047 \mathrm{E}-03$

$2.20158 \mathrm{E}-03$

$2.11650 \mathrm{E}-03$

$2.03498 \mathrm{E}-03$

$1.95682 \mathrm{E}-03$

$1.88180 \mathrm{E}-03$

$1.80975 \mathrm{E}-03$

$1.74050 \mathrm{E}-03$

$1.67387 \mathrm{E}-03$

$1.60974 \mathrm{E}-03$

$1.54795 \mathrm{E}-03$

$1.48839 \mathrm{E}-03$

$1.43094 \mathrm{E}-03$

$1.37548 \mathrm{E}-03$

$1.32192 \mathrm{E}-03$

$1.27017 \mathrm{E}-03$

$1.22012 \mathrm{E}-03$

$f_{-1}$

$1.04759 \mathrm{E}-03$

$2.98900 \mathrm{E}-03$

$4.94452 \mathrm{E}-03$

$6.84410 \mathrm{E}-03$

$8.66708 \mathrm{E}-03$

$1.04043 \mathrm{E}-02$

$1.20525 \mathrm{E}-02$

$1.36118 \mathrm{E}-02$

$1.50847 \mathrm{E}-02$

$1.64746 \mathrm{E}-02$

$1.77856 \mathrm{E}-02$

$1.90215 \mathrm{E}-02$

$2.01862 \mathrm{E}-02$

$2.12832 \mathrm{E}-02$

$2.23155 \mathrm{E}-02$

$2.32858 \mathrm{E}-02$

$2.41966 \mathrm{E}-02$

$2.50498 \mathrm{E}-02$

$2.58472 \mathrm{E}-02$

$2.65902 \mathrm{E}-02$

$2.72803 \mathrm{E}-02$

$2.79185 \mathrm{E}-02$

$2.85058 \mathrm{E}-02$

$2.90430 \mathrm{E}-02$

$2.95307 \mathrm{E}-02$

$2.99698 \mathrm{E}-02$

$3.03606 \mathrm{E}-02$

$3.07037 \mathrm{E}-02$

3.09994 E-02

$3.12482 \mathrm{E}-02$

$3.14503 \mathrm{E}-02$

3.16061 E-02

$3.17157 \mathrm{E}-02$

$3.17795 \mathrm{E}-02$

3.17975E-02

3.17701 E-02

3.16972 E-02

3.15791 E-02

$3.14159 \mathrm{E}-02$

$3.12077 \mathrm{E}-02$

$3.09546 \mathrm{E}-02$

$3.06566 \mathrm{E}-02$

$3.03140 \mathrm{E}-02$

$2.99267 \mathrm{E}-02$

$2.94948 \mathrm{E}-02$

$2.90183 \mathrm{E}-02$

$284974 \mathrm{E}-02$

2.79321 E-02 
$\bar{F}$

0.10
0.20

0.30

0.40

0.50

0.60

0.70

0.80

0.90

1.00

1.10

1.20

1.30

1.40

1.50

1.60

1.70

1.80

1.90

2.00

2.10

2.20

2.30

2.40

2.50

2.60

2.70

2.80

2.90

3.00

3.10

3.20

3.30

3.40

3.50

3.60

3.70

3.80

3.90

4.00

4.10

4.20

4.30

4.40

4.50

4.60

4.70

4.80

4.90

5.00

5.10

5.20

5.30

5.40

5.50

5.60

5.70

5.80

5.90

6.00 $f_{2}$

$3.87081 \mathrm{E}-09$

$2.90936 \mathrm{E}-08$

$7.97292 \mathrm{E}-08$

$1.55749 \mathrm{E}-07$

$2.56889 \mathrm{E}-07$

$3.82884 \mathrm{E}-07$

$5.33507 \mathrm{E}-07$

$7.08570 \mathrm{E}-07$

$9.07924 \mathrm{E}-07$

$1.13145 \mathrm{E}-06$

$1.37906 \mathrm{E}-06$

$1.65067 \mathrm{E}-06$

$1.94623 \mathrm{E}-06$

$2.26570 \mathrm{E}-06$

$2.60903 \mathrm{E}-06$

$2.97621 \mathrm{E}-06$

$3.36721 \mathrm{E}-06$

$3.78200 \mathrm{E}-06$

$4.22059 \mathrm{E}-06$

4.68294E-06

$5.16906 \mathrm{E}-06$ $5.67894 \mathrm{E}-06$

$6.21257 \mathrm{E}-06$

$6.76994 \mathrm{E}-06$

$7.35105 \mathrm{E}-06$

$7.95589 \mathrm{E}-06$

$8.58446 \mathrm{E}-06$

$9.23676 \mathrm{E}-06$

$9.91279 \mathrm{E}-06$

$1.06125 \mathrm{E}-05$

$1.13360 \mathrm{E}-05$

$1.20832 \mathrm{E}-05$

$1.28541 \mathrm{E}-05$

$1.36487 \mathrm{E}-05$

$1.44670 \mathrm{E}-05$

$1.53091 \mathrm{E}-05$

$1.61748 \mathrm{E}-05$

$1.70643 \mathrm{E}-05$

$1.79775 \mathrm{E}-05$

$1.89144 \mathrm{E}-05$

$1.98750 \mathrm{E}-05$

$2.08593 \mathrm{E}-05$

$2.18673 \mathrm{E}-05$

$2.28990 \mathrm{E}-05$

$2.39544 \mathrm{E}-05$

$2.50336 \mathrm{E}-05$

$2.61364 \mathrm{E}-05$

$2.72629 \mathrm{E}-05$

$2.84132 \mathrm{E}-05$

$2.95871 \mathrm{E}-05$

$3.07847 \mathrm{E}-05$

$3.20061 \mathrm{E}-05$

$3.32511 \mathrm{E}-05$

$3.45199 \mathrm{E}-05$

$3.58123 \mathrm{E}-05$

$3.71285 \mathrm{E}-05$

$3.84683 \mathrm{E}-05$

$3.98318 \mathrm{E}-05$

$4.12191 \mathrm{E}-05$

$4.26300 \mathrm{E}-0.5$ $g_{2}$

$8.85042 \mathrm{E}-07$

$9.53315 \mathrm{E}-06$

$3.48346 \mathrm{E}-05$

$8.44968 \mathrm{E}-05$

$1.64764 \mathrm{E}-04$

$2.80406 \mathrm{E}-04$

$4.34893 \mathrm{E}-04$

$6.30619 \mathrm{E}-04$

$8.69172 \mathrm{E}-04$

$1.15156 \mathrm{E}-03$

$1.47836 \mathrm{E}-03$

$1.84993 \mathrm{E}-03$

$2.26639 \mathrm{E}-03$

$2.72780 \mathrm{E}-03$

$3.23413 \mathrm{E}-03$

$3.78531 \mathrm{E}-03$

$4.38127 \mathrm{E}-03$

$5.02190 \mathrm{E}-03$

$5.70713 \mathrm{E}-03$

$6.43686 \mathrm{E}-03$

$7.21100 \mathrm{E}-03$

$8.02948 \mathrm{E}-03$

$8.89223 \mathrm{E}-03$

$9.79919 \mathrm{E}-03$

$1.07503 \mathrm{E}-02$

$1.17455 \mathrm{E}-02$

$1.27847 \mathrm{E}-02$

$1.38680 \mathrm{E}-02$

$1.49952 \mathrm{E}-02$

$1.61663 \mathrm{E}-02$

$1.73814 \mathrm{E}-02$

$1.86403 \mathrm{E}-02$

1.99431 E- 02

$2.12897 \mathrm{E}-02$

$2.26800 \mathrm{E}-02$

$2.41142 \mathrm{E}-02$

$2.55921 \mathrm{E}-02$

$2.71138 \mathrm{E}-02$

$2.86791 \mathrm{E}-02$

$3.02882 \mathrm{E}-02$

$3.19410 \mathrm{E}-02$

$3.36374 \mathrm{E}-02$

$3.53774 \mathrm{E}-02$

$3.71610 \mathrm{E}-02$

$3.89882 \mathrm{E}-02$

$4.08588 \mathrm{E}-02$

$4.27728 \mathrm{E}-02$

$4.47303 \mathrm{E}-02$

$4.67307 \mathrm{E}-02$

$4.87738 \mathrm{E}-02$

$5.08591 \mathrm{E}-02$

$5.29849 \mathrm{E}-02$

$5.51466 \mathrm{E}-02$

$5.73230 \mathrm{E}-02$

$5.85939 \mathrm{E}-02$

$6.21011 \mathrm{E}-02$ $6.43943 \mathrm{E}-02$

$6.67719 \mathrm{E}-02$

$6.92024 \mathrm{E}-02$

$7.16798 \mathrm{E}-02$
$-4.92848 \mathrm{E}-09$

$-5.28914 \mathrm{E}-08$

$-1.92101 \mathrm{E}-07$

$-4.62117 \mathrm{E}-07$

$-8.91763 \mathrm{E}-07$

$-1.49900 \mathrm{E}-06$

$-2.29211 \mathrm{E}-06$

$-3.27141 \mathrm{E}-06$

$-4.43115 \mathrm{E}-06$

$-5.76117 \mathrm{E}-06$

$-7.24823 \mathrm{E}-06$

$-8.87704 \mathrm{E}-06$

$-1.06309 \mathrm{E}-05$

$-1.24923 \mathrm{E}-05$

$-1.44431 \mathrm{E}-05$

$-1.64649 \mathrm{E}-05$

$-1.85392 \mathrm{E}-05$

$-2.06474 \mathrm{E}-05$

$-2.27707 \mathrm{E}-05$

$-2.48906 \mathrm{E}-05$

$-2.69882 \mathrm{E}-05$

$-2.90452 \mathrm{E}-05$

$-3.10428 \mathrm{E}-05$

$-3.29627 \mathrm{E}-05$

$-3.47863 \mathrm{E}-05$

$-3.64951 \mathrm{E}-05$

$-3.80708 \mathrm{E}-05$

$-3.94951 \mathrm{E}-05$

$-4.07495 \mathrm{E}-05$

$-4.18158 \mathrm{E}-05$

$-4.26756 \mathrm{E}-05$

$-4.33108 \mathrm{E}-05$

$-4.37030 \mathrm{E}-05$

$-4.38341 \mathrm{E}-05$

$-4.36858 \mathrm{E}-05$

$-4.32400 \mathrm{E}-05$

$-4.24783 \mathrm{E}-05$

$-4.13828 \mathrm{E}-05$

$-3.99351 \mathrm{E}-05$

$-3.81172 \mathrm{E}-05$

$-3.59109 \mathrm{E}-05$

$-3.32980 \mathrm{E}-05$

$-3.02604 \mathrm{E}-05$

$-2.67801 \mathrm{E}-05$

$-2.28389 \mathrm{E}-05$

$-1.84188 \mathrm{E}-05$

$-1.35015 \mathrm{E}-05$

$-8.06929 \mathrm{E}-06$

$-2.10398 \mathrm{E}-06$

$4.41219 \mathrm{E}-06$

$1.14968 \mathrm{E}-05$

$1.91668 \mathrm{E}-05$

$2.74366 \mathrm{E}-05$

$3.63079 \mathrm{E}-05$

$4.50789 \mathrm{E}-05$

$5.62249 \mathrm{E}-05$

$6.70656 \mathrm{E}-05$

$7.86347 \mathrm{E}-05$

$9.09256 \mathrm{E}-05$

$1.03951 \mathrm{E}-04$

$\tan \delta_{2}$

$f_{-2}$

$-2.83007 \mathrm{E} \quad 00$

$-5.77556 \mathrm{E} \quad 00$

$-8.55258 \mathrm{E} \quad 00$

$-1.11511 \mathrm{E} 01$

$-1.35509 \mathrm{E} 01$

$-1.57418 \mathrm{E} 01$

$-1.77241 \mathrm{E} 01$

$-1.95065 \mathrm{E} 01$

$-2.11025 \mathrm{E} 01$

$-2.25284 \mathrm{E} 01$

$-2.38015 \mathrm{E} \quad 01$

$-2.49386 \mathrm{E} \quad 01$

$-2.59555 \mathrm{E} \quad 01$

$-2.68666 \mathrm{E} \quad 01$

$-2.76848 \mathrm{E} \quad 01$

$-2.84213 \mathrm{E} 01$

$-2.90863 \mathrm{E} \quad 01$

$-2.96883 \mathrm{E} 01$

$-3.02348 \mathrm{E} \quad 01$

$-3.07324 \mathrm{E} \quad 01$

$-3.11868 \mathrm{E} \quad 01$

$-3.16027 \mathrm{E} 01$

$-3.19846 \mathrm{E} \quad 01$

$-3.23360 \mathrm{E}$ 01

$-3.26602 \mathrm{E} 01$

$-3.29600 \mathrm{E} \quad 01$

$-3.32379 \mathrm{E} \quad 0 \mathrm{I}$

$-3.34960 \mathrm{E} 01$

$-3.37363 \mathrm{E} 01$

$-3.39605 \mathrm{E} 01$

$-3.41699 \mathrm{E} \quad 01$

$-3.43660 \mathrm{E} \quad 01$

$-3.45499 \mathrm{E} \quad 01$

$-3.47227 \mathrm{E} \quad 01$

$-3.48852 \mathrm{E} 01$

$-3.50384 \mathrm{E} \quad 01$

$-3.51830 \mathrm{E} \quad 01$

$-3.53196 \mathrm{E} \quad 01$

$-3.54489 \mathrm{E} 01$

$-3.55715 \mathrm{E} 01$

$-3.56877 \mathrm{E} \quad 01$

$-3.57982 \mathrm{E} 01$ 
ELECTRON RADIAL FUNCTIONS

Positroñ

\begin{tabular}{|c|c|c|c|c|c|c|}
\hline$p$ & $F_{0}$ & $f_{1}$ & & $g_{1}$ & $\tan \delta_{1}$ & $f_{-1}$ \\
\hline 6.10 & $8.94144 \mathrm{E}-01$ & $5.28821 \mathrm{E}$ & 00 & $1.03085 \mathrm{E}-02$ & $1.17170 \mathrm{E}-03$ & $1.65555 \mathrm{E}-02$ \\
\hline 6.20 & $8.94171 \mathrm{E}-01$ & $5.38317 \mathrm{E}$ & 00 & $1.17177 \mathrm{E}-02$ & $1.12483 \mathrm{E}-03$ & $1.53714 \mathrm{E}-02$ \\
\hline 6.30 & $8.94194 \mathrm{E}-01$ & $5.47807 \mathrm{E}$ & 00 & $1.31704 \mathrm{E}-02$ & $1.07944 \mathrm{E}-03$ & $1.41433 \mathrm{E}-02$ \\
\hline 6.40 & $8.94217 \mathrm{E}-01$ & $5.57288 \mathrm{E}$ & 00 & $1.46665 \mathrm{E}-02$ & $1.03547 \mathrm{E}-03$ & $1.28711 \mathrm{E}-02$ \\
\hline 6.50 & $8.94238 \mathrm{E}-01$ & $5.66753 \mathrm{E}$ & 00 & $1.62059 \mathrm{E}-02$ & $9.92832 \mathrm{E}-04$ & $1.15549 \mathrm{E}-02$ \\
\hline 6.60 & $8.94257 \mathrm{E}-01$ & $5.76174 \mathrm{E}$ & 00 & $1.77874 \mathrm{E}-02$ & $9.51488 \mathrm{E}-04$ & $1.01947 \mathrm{E}-02$ \\
\hline 6.70 & $8.94275 \mathrm{E}-0 \mathrm{l}$ & $5.85475 \mathrm{E}$ & 00 & $1.94083 \mathrm{E}-02$ & $9.11374 \mathrm{E}-04$ & $8.79043 \mathrm{E}-03$ \\
\hline 6.80 & $8.94291 \mathrm{E}-01$ & $5.93903 \mathrm{E}$ & 00 & $2.10411 \mathrm{E}-02$ & $8.72430 \mathrm{E}-04$ & $7.34215 \mathrm{E}-03$ \\
\hline 6.90 & $8.94307 \mathrm{E}-01$ & $6.06439 \mathrm{E}$ & 00 & $2.28676 \mathrm{E}-02$ & $8.34613 \mathrm{E}-04$ & $5.84986 \mathrm{E}-03$ \\
\hline 7.00 & $8.94321 \mathrm{E}-01$ & $6.15031 \mathrm{E}$ & 00 & $2.45940 \mathrm{E}-02$ & $7.97869 \mathrm{E}-04$ & $4.31356 \mathrm{E}-03$ \\
\hline 7.10 & 8.94334E-01 & $6.24353 \mathrm{E}$ & 00 & $2.63909 \mathrm{E}-02$ & $7.62160 \mathrm{E}-04$ & $2.73326 \mathrm{E}-03$ \\
\hline 7.20 & $8.94347 \mathrm{E}-01$ & $6.33781 \mathrm{E}$ & 00 & $2.82354 \mathrm{E}-02$ & $7.27447 \mathrm{E}-04$ & $1.10896 \mathrm{E}-03$ \\
\hline 7.30 & $8.94358 \mathrm{E}-01$ & $6.43251 \mathrm{E}$ & 00 & $3.01253 \mathrm{E}-02$ & $6.93683 \mathrm{E}-04$ & $-5.59337 \mathrm{E}-04$ \\
\hline 7.40 & $8.94368 \mathrm{E}-01$ & $6.52737 \mathrm{E}$ & 00 & $3.20595 \mathrm{E}-02$ & $6.60828 \mathrm{E}-04$ & $-2.27163 \mathrm{E}-03$ \\
\hline 7.50 & $8.94378 \mathrm{E}-01$ & $6.62232 \mathrm{E}$ & 00 & $3.40380 \mathrm{E}-02$ & $6.28847 \mathrm{E}-04$ & $-4.02792 \mathrm{E}-03$ \\
\hline 7.60 & $8.94386 \mathrm{E}-01$ & $6.71734 \mathrm{E}$ & 00 & $3.60605 \mathrm{E}-02$ & $5.97711 \mathrm{E}-04$ & $-5.82819 \mathrm{E}-03$ \\
\hline 7.70 & $8.94394 \mathrm{E}-01$ & $6.81240 \mathrm{E}$ & 00 & $3.81270 \mathrm{E}-$ & $5.67380 \mathrm{E}-04$ & $-7.67247 \mathrm{E}-03$ \\
\hline 7.80 & $8.94402 \mathrm{E}-01$ & $6.90748 \mathrm{E}$ & 00 & $4.02373 \mathrm{E}-02$ & $5.37830 \mathrm{E}-04$ & $-9.56073 \mathrm{E}-03$ \\
\hline 7.90 & $8.94409 \mathrm{E}-01$ & $7.00260 \mathrm{E}$ & 00 & $4.23917 \mathrm{E}-02$ & $5.09032 \mathrm{E}-04$ & $-1.14930 \mathrm{E}-02$ \\
\hline 8.00 & $8.94415 \mathrm{E}-01$ & $7.09773 \mathrm{E}$ & 00 & $4.45900 \mathrm{E}-02$ & $4.80958 \mathrm{E}-04$ & $-1.34692 \mathrm{E}-02$ \\
\hline 8.10 & $8.94420 \mathrm{E}-01$ & $7.19288 \mathrm{E}$ & 00 & $4.68322 \mathrm{E}-02$ & $4.53576 \mathrm{E}-04$ & $-1.54895 \mathrm{E}-02$ \\
\hline 8.20 & $8.94425 \mathrm{E}-01$ & $7.28803 \mathrm{E}$ & 00 & $4.91182 \mathrm{E}-02$ & $4.26860 \mathrm{E}-04$ & $-1.75537 \mathrm{E}-02$ \\
\hline 8.30 & $8.94429 \mathrm{E}-01$ & $7.38320 \mathrm{E}$ & 00 & $5.14482 \mathrm{E}-02$ & $4.00800 \mathrm{E}-04$ & $-1.96620 \mathrm{E}-02$ \\
\hline 8.40 & $8.94433 \mathrm{E}-01$ & $7.47839 \mathrm{E}$ & 00 & $5.38222 \mathrm{E}-02$ & $3.75351 \mathrm{E}-04$ & $-2.18142 \mathrm{E}-02$ \\
\hline 8.50 & $8.94437 \mathrm{E}-01$ & $7.57358 \mathrm{E}$ & 00 & $5.62400 \mathrm{E}-02$ & $3.50514 \mathrm{E}-04$ & $-2.40105 \mathrm{E}-02$ \\
\hline 8.60 & $8.94440 \mathrm{E}-01$ & $7.66878 \mathrm{E}$ & 00 & $5.87017 \mathrm{E}-02$ & $3.26259 \mathrm{E}-04$ & $-2.62507 \mathrm{E}-02$ \\
\hline 8.70 & $8.94442 \mathrm{E}-01$ & $7.76399 \mathrm{E}$ & 00 & $6.12074 \mathrm{E}-02$ & $3.02561 \mathrm{E}-04$ & $-2.85350 \mathrm{E}-02$ \\
\hline 8.80 & $8.94445 \mathrm{E}-01$ & $7.85922 \mathrm{E}$ & 00 & $6.37571 \mathrm{E}-02$ & $2.79407 \mathrm{E}-04$ & $-3.08633 \mathrm{E}-02$ \\
\hline 8.90 & $8.94446 \mathrm{E}-01$ & $7.95444 \mathrm{E}$ & 00 & $6.63506 \mathrm{E}-02$ & $2.56780 \mathrm{E}-04$ & $-3.32356 \mathrm{E}-02$ \\
\hline 9.00 & $8.94448 \mathrm{E}-01$ & $8.04968 \mathrm{E}$ & 00 & $6.89881 \mathrm{E}-02$ & $2.34660 \mathrm{E}-04$ & $-3.56519 \mathrm{E}-02$ \\
\hline 9.10 & $8.94449 \mathrm{E}-01$ & $8.14492 \mathrm{E}$ & 00 & $7.16695 \mathrm{E}-02$ & $2.13027 \mathrm{E}-04$ & $-3.81123 \mathrm{E}-02$ \\
\hline 9.20 & $8.94450 \mathrm{E}-01$ & $8.24018 \mathrm{E}$ & 00 & $7.43949 \mathrm{E}-02$ & $1.91872 \mathrm{E}-04$ & $-4.06167 \mathrm{E}-02$ \\
\hline 9.30 & $8.94450 \mathrm{E}-01$ & $8.33544 \mathrm{E}$ & 00 & $7.71643 \mathrm{E}-02$ & $1.71180 \mathrm{E}-04$ & $-4.31651 \mathrm{E}-02$ \\
\hline 9.40 & $8.94451 \mathrm{E}-$ & $8.43071 \mathrm{E}$ & 00 & $76 \mathrm{E}-02$ & $1.50931 \mathrm{E}-04$ & $-4.57575 \mathrm{E}-02$ \\
\hline 9.50 & $8.94450 \mathrm{E}-01$ & $8.52599 \mathrm{E}$ & 00 & $8.28349 \mathrm{E}-02$ & $1.31106 \mathrm{E}-04$ & $-4.83941 \mathrm{E}-02$ \\
\hline 9.60 & $8.94450 \mathrm{E}-01$ & $8.62128 \mathrm{E}$ & 00 & $8.57362 \mathrm{E}-02$ & $1.11707 \mathrm{E}-04$ & $-5.10746 \mathrm{E}-02$ \\
\hline 9.70 & $8.94449 \mathrm{E}-01$ & $8.71657 \mathrm{E}$ & 00 & 8.86 & 9.271 & $-5.37992 \mathrm{E}-02$ \\
\hline 9.80 & $8.94449 \mathrm{E}-01$ & $8.81: 88 \mathrm{E}$ & 00 & $9.16707 \mathrm{E}-02$ & $7.41139 \mathrm{E}-05$ & $-5.65679 \mathrm{E}-02$ \\
\hline 9.90 & $8.94448 \mathrm{E}-01$ & $8.90719 \mathrm{E}$ & 00 & $9.47040 \mathrm{E}-$ & $5.59003 \mathrm{E}-($ & $-5.93807 \mathrm{E}-02$ \\
\hline 10.00 & $8.94447 \mathrm{E}-01$ & $9.00251 \mathrm{E}$ & 00 & $9.77813 \mathrm{E}-02$ & $3.80472 \mathrm{E}-05$ & $-6.22375 \mathrm{E}-02$ \\
\hline 10.10 & $8.94445 \mathrm{E}-01$ & $9.09784 \mathrm{E}$ & 00 & $1.00903 \mathrm{E}-01$ & $2.05612 \mathrm{E}-05$ & $-6.51385 \mathrm{E}-02$ \\
\hline .20 & $8.94443 \mathrm{E}-01$ & $9.19318 \mathrm{E}$ & 00 & $1.04068 \mathrm{E}-01$ & $3.42488 \mathrm{E}-06$ & $-6.80835 \mathrm{E}-02$ \\
\hline 10.30 & $8.94442 \mathrm{E}-$ & $9.28853 \mathrm{E}$ & 00 & $1.07277 \mathrm{E}$ & $-1.33733 \mathrm{E}-$ & $-7.10726 \mathrm{E}-02$ \\
\hline 10.40 & $8.94440 \mathrm{E}-01$ & $9.38388 \mathrm{E}$ & 00 & $1.10531 \mathrm{E}-01$ & $-2.98431 \mathrm{E}-$ & $-7.41058 \mathrm{E}-02$ \\
\hline 10.50 & $8.94438 \mathrm{E}-01$ & $9.47924 \mathrm{E}$ & 00 & $1.13828 \mathrm{E}-01$ & $-4.59934 \mathrm{E}-05$ & $-7.71831 \mathrm{E}-02$ \\
\hline 10.60 & $8.94435 \mathrm{E}-01$ & $9.57462 \mathrm{E}$ & 00 & $1.17170 \mathrm{E}-01$ & $-6.18360 E-05$ & $-8.03045 \mathrm{E}-02$ \\
\hline 10.70 & $8.94433 \mathrm{E}-$ & $9.67000 \mathrm{E}$ & 00 & & $-7.73681 \mathrm{E}-$ & $-8.34701 \mathrm{E}-02$ \\
\hline 10.80 & $8.94431 \mathrm{E}-$ & $9.76540 \mathrm{E}$ & 00 & $1.23985 \mathrm{E}-01$ & $26120 \mathrm{E}-05$ & $-8.66798 \mathrm{E}-02$ \\
\hline 10.90 & $8.94428 \mathrm{E}-$ & $9.86080 \mathrm{E}$ & 00 & $1.27459 \mathrm{E}-01$ & $-1.07570 \mathrm{E}-04$ & $-8.99337 \mathrm{E}-02$ \\
\hline 11.00 & $8.94425 \mathrm{E}-0$ & $9.95621 \mathrm{E}$ & 00 & $1.30977 \mathrm{E}-0$ & $-1.22239 \mathrm{E}-04$ & $-9.32317 \mathrm{E}-02$ \\
\hline 11.10 & $8.94422 \mathrm{E}-01$ & $1.00516 \mathrm{E}$ & 01 & $1.34539 \mathrm{E}-01$ & $-1.36643 \mathrm{E}-04$ & $-9.65738 \mathrm{E}-02$ \\
\hline 11.20 & $8.94419 \mathrm{E}-01$ & $1.01471 \mathrm{E}$ & 01 & $1.38145 \mathrm{E}-01$ & $-1.50786 \mathrm{E}-04$ & $-9.99602 \mathrm{E}-02$ \\
\hline 11.30 & $8.94416 \mathrm{E}-01$ & $1.02425 \mathrm{E}$ & 01 & $1.41795 \mathrm{E}-01$ & $-1.64672 \mathrm{E}-04$ & $-1.03391 \mathrm{E}-01$ \\
\hline 11.40 & $8.94413 \mathrm{E}-01$ & $1.03379 \mathrm{E}$ & 01 & $1.45489 \mathrm{E}-01$ & $-1.78299 \mathrm{E}-04$ & $-1.06865 \mathrm{E}-01$ \\
\hline 11.50 & $8.94409 \mathrm{E}-01$ & $1.04334 \mathrm{E}$ & 01 & $1.49228 \mathrm{E}-01$ & $-1.91686 \mathrm{E}-04$ & $-1.10384 \mathrm{E}-01$ \\
\hline 11.60 & $8.94406 \mathrm{E}-01$ & $1.05289 \mathrm{E}$ & 01 & $1.53010 \mathrm{E}-01$ & $-2.04830 \mathrm{E}-04$ & $-1.13947 \mathrm{E}-01$ \\
\hline & $8.94402 \mathrm{E}-01$ & $1.06243 \mathrm{E}$ & 01 & $1.56837 \mathrm{E}-01$ & $-2.17747 \mathrm{E}-04$ & $-1.17555 \mathrm{E}-01$ \\
\hline & $8.94398 \mathrm{E}-01$ & $1.07198 \mathrm{E}$ & 01 & $1.60708 \mathrm{E}-01$ & $-2.30438 \mathrm{E}-04$ & $-1.21206 \mathrm{E}-01$ \\
\hline 11.90 & $8.94394 \mathrm{E}-01$ & $1.08153 \mathrm{E}$ & 01 & $1.64623 \mathrm{E}-01$ & $-2.42904 \mathrm{E}-04$ & $-1.24902 \mathrm{E}-01$ \\
\hline 12.00 & $8.94390 \mathrm{E}-01$ & $1.09108 \mathrm{E}$ & 01 & $1.68583 \mathrm{E}-01$ & $-2.55160 \mathrm{E}-04$ & $-1.28642 \mathrm{E}-01$ \\
\hline
\end{tabular}

$$
Z=5 \quad A=11 \quad \rho=1.2 A^{1 / 3} F
$$

$\begin{array}{ccccc}g_{1} & \tan \delta_{1} & f_{-1} & g_{-1} & \\ 1.03085 \mathrm{E}-02 & 1.17170 \mathrm{E}-03 & 1.65555 \mathrm{E}-02 & 6.22840 \mathrm{E} & 00 \\ 1.17177 \mathrm{E}-02 & 1.12483 \mathrm{E}-03 & 1.53714 \mathrm{E}-02 & 6.32392 \mathrm{E} & 00 \\ 1.31704 \mathrm{E}-02 & 1.07944 \mathrm{E}-03 & 1.41433 \mathrm{E}-02 & 6.41943 \mathrm{E} & 00 \\ 1.46665 \mathrm{E}-02 & 1.03547 \mathrm{E}-03 & 1.28711 \mathrm{E}-02 & 6.51492 \mathrm{E} & 00 \\ 1.62059 \mathrm{E}-02 & 9.92832 \mathrm{E}-04 & 1.15549 \mathrm{E}-02 & 6.61041 \mathrm{E} & 00\end{array}$

$6.70589 \mathrm{E} \quad 00$ $6.80136 \mathrm{E} \quad 00$ $6.89683 \mathrm{E} \quad 00$ $6.99229 \mathrm{E} \quad 00$ $7.08775 \mathrm{E} \quad 00$

$7.18320 \mathrm{E} \quad 00$ $7.27865 \mathrm{E} \quad 00$ $7.37410 \mathrm{E} \quad 00$ $7.46954 \mathrm{E} \quad 00$ $7.56499 \mathrm{E} \quad 00$

$7.66043 \mathrm{E} \quad 00$ $7.75587 \mathrm{E} \quad 00$ $7.85132 \mathrm{E} \quad 00$ $7.94676 \mathrm{E} \quad 00$ $8.04220 \mathrm{E} \quad 00$

$8.13765 \mathrm{E} \quad 00$ $8.23309 \mathrm{E} \quad 00$ $8.32854 \mathrm{E} \quad 00$ $8.42399 \mathrm{E} \quad 00$ 8.51945 E 00

$8.61490 \mathrm{E} \quad 00$ $8.71036 \mathrm{E} \quad 00$ $8.80583 \mathrm{E} \quad 00$ 8.90129 E 00 $8.99677 \mathrm{E} \quad 00$

$9.09224 \mathrm{E} \quad 00$ $9.18773 \mathrm{E} \quad 00$ $9.28321 \mathrm{E} \quad 00$ $9.37870 \mathrm{E} \quad 00$ $9.47420 \mathrm{E} \quad 00$

$9.56970 \mathrm{E} \quad 00$ $9.66521 \mathrm{E} \quad 00$ $9.76073 \mathrm{E} \quad 00$ $\begin{array}{lll}9.85625 \mathrm{E} & 00\end{array}$ $9.95178 \mathrm{E} \quad 00$

$1.00473 \mathrm{E} \quad 01$ $1.01429 \mathrm{E} \quad 01$ $1.02384 \mathrm{E} \quad 01$ $1.03340 \mathrm{E} 01$ $1.04295 \mathrm{E} 01$

$1.05251 \mathrm{E} \quad 01$ $1.06207 \mathrm{E} 01$ $1.07163 \mathrm{E} \quad 01$ $1.08119 \mathrm{E} 01$ $1.09075 \mathrm{E} \quad 01$

$1.10031 \mathrm{E} \quad 01$ $1.10987 \mathrm{E} 01$ $1.11943 \mathrm{E} 01$ $1.12900 \mathrm{E} \quad 01$ $1.13856 \mathrm{E} 01$

$1.14813 \mathrm{E} \quad 01$ $1.15770 \mathrm{E}$ 0l $1.16726 \mathrm{E} 01$ $1.17683 \mathrm{E} 01$ $1.18640 \mathrm{E} \quad 01$ $\tan \delta_{-1}$

$2.07991 \mathrm{E} \quad 02$ $2.10161 \mathrm{E} \quad 02$ $2.12305 \mathrm{E} \quad 02$ $2.14424 \mathrm{E} \quad 02$ $2.16518 \mathrm{E} \quad 02$

$2.18587 \mathrm{E} \quad 02$ $2.20634 \mathrm{E} \quad 02$ $2.22656 \mathrm{E} \quad 02$ $2.24656 \mathrm{E} \quad 02$ $2.26633 \mathrm{E} \quad 02$

$2.28588 \mathrm{E} \quad 02$ $2.30521 \mathrm{E} \quad 02$ $2.32433 \mathrm{E} \quad 02$ $2.34324 \mathrm{E} \quad 02$ $2.36194 \mathrm{E} \quad 02$

$2.38044 \mathrm{E} \quad 02$ $2.39874 \mathrm{E} \quad 02$ $2.41685 \mathrm{E} \quad 02$ $2.43476 \mathrm{E} \quad 02$ $2.45249 \mathrm{E} \quad 02$

$2.47003 \mathrm{E} \quad 02$ $2.48739 \mathrm{E} \quad 02$ $2.50457 \mathrm{E} \quad 02$ $2.52157 \mathrm{E} \quad 02$ $2.53840 \mathrm{E} \quad 02$

$2.55506 \mathrm{E} \quad 02$ $2.57155 \mathrm{E} \quad 02$ $2.58788 \mathrm{E} \quad 02$ $2.60404 \mathrm{E} \quad 02$ 2.62005 E 02

$2.63590 \mathrm{E} \quad 02$ $2.65160 \mathrm{E} \quad 02$ $2.66714 \mathrm{E} \quad 02$ $2.68254 \mathrm{E} \quad 02$ 2.69778 E 02

$2.71289 \mathrm{E} \quad 02$ $2.72786 \mathrm{E} \quad 02$ $2.74268 \mathrm{E} \quad 02$ $2.75737 \mathrm{E} \quad 02$ $2.77192 \mathrm{E} \quad 02$

$2.78633 \mathrm{E} \quad 02$ $2.80062 \mathrm{E} \quad 02$ $2.81478 \mathrm{E} \quad 02$ $2.82882 \mathrm{E} \quad 02$ $2.84272 \mathrm{E} \quad 02$

$2.85650 \mathrm{E} \quad 02$ $2.87017 \mathrm{E} \quad 02$ $2.88371 \mathrm{E} \quad 02$ $2.89713 \mathrm{E} \quad 02$ 2.91045 E 02

$2.92365 \mathrm{E} \quad 02$ $2.93673 \mathrm{E} \quad 02$ $2.94970 \mathrm{E} \quad 02$ $2.96257 \mathrm{E} \quad 02$ $2.97533 \mathrm{E} \quad 02$

$2.98799 \mathrm{E} \quad 02$ $3.00054 \mathrm{E} \quad 02$ $3.01299 \mathrm{E} \quad 02$ $3.02534 \mathrm{E} \quad 02$ $3.03759 \mathrm{E} \quad 02$ 
$\bar{F}$

$f_{2}$

$4.40647 \mathrm{E}-05$

$4.55230 \mathrm{E}-05$

$4.70051 \mathrm{E}-05$

$4.85108 \mathrm{E}-05$

$5.00402 \mathrm{E}-05$

6.50

6.60

6.70

6.80

6.90

7.00

7.10

7.20

7.30

7.40

7.50

7.60

7.70

7.80

7.90

8.00

8.10

8.20

8.30

8.40

8.50

8.60

8.70

8.80

8.90

9.00

9.10

9.20

9.30

9.40

9.50

9.60

9.70

9.80

9.90

10.00

10.10

10.20

10.30

10.40

10.50

10.60

10.70

10.80

10.90

11.00

11.10

11.20

11.30

11.40

11.50

11.60

11.70

11.80

11.90

12.00
$5.15934 \mathrm{E}-05$

$5.31702 \mathrm{E}-05$

$5.47707 \mathrm{E}-05$

$5.63949 \mathrm{E}-05$

$5.80429 \mathrm{E}-05$

$5.97145 \mathrm{E}-05$ $6.14098 \mathrm{E}-05$

$6.31288 \mathrm{E}-05$

$6.48715 \mathrm{E}-05$

$6.66379 \mathrm{E}-05$

$6.84280 \mathrm{E}-05$

$7.02417 \mathrm{E}-05$

$7.20792 \mathrm{E}-05$

$7.39404 \mathrm{E}-05$

$7.58253 \mathrm{E}-05$

$7.77338 \mathrm{E}-05$

$7.96661 \mathrm{E}-05$

$8.16220 \mathrm{E}-05$

$8.36017 \mathrm{E}-05$

$8.56050 \mathrm{E}-05$

$8.76320 \mathrm{E}-05$

$8.96827 \mathrm{E}-05$

$9.17571 \mathrm{E}-05$

$9.38552 \mathrm{E}-05$

$9.59770 \mathrm{E}-05$

$9.81225 \mathrm{E}-05$

$1.00292 \mathrm{E}-04$

$1.02485 \mathrm{E}-04$

$1.04701 \mathrm{E}-04$

$1.06941 \mathrm{E}-04$

$1.09205 \mathrm{E}-04$

$1.11493 \mathrm{E}-04$

$1.13804 \mathrm{E}-04$

$1.16139 \mathrm{E}-04$

$1.18498 \mathrm{E}-04$

$1.20880 \mathrm{E}-04$

$1.23286 \mathrm{E}-04$

$1.25716 \mathrm{E}-04$

$1.28169 \mathrm{E}-04$

$1.30647 \mathrm{E}-04$

$1.33147 \mathrm{E}-04$

$1.38220 \mathrm{E}-04$

$1.40792 \mathrm{E}-04$

$1.43388 \mathrm{E}-04$

$1.46007 \mathrm{E}-04$

$1.48650 \mathrm{E}-04$

$1.51317 \mathrm{E}-04$

$1.54007 \mathrm{E}-04$

$1.56721 \mathrm{E}-04$

$1.59459 \mathrm{E}-04$

$1.62220 \mathrm{E}-04$

$1.65005 \mathrm{E}-04$

$1.67814 \mathrm{E}-04$

$1.70646 \mathrm{E}-04$
$1.35672 \mathrm{E}-04$ $g_{2}$

$1.17730 \mathrm{E}-04$

$1.32278 \mathrm{E}-04$

$1.47613 \mathrm{E}-04$

$1.63753 \mathrm{E}-04$

$1.80717 \mathrm{E}-04$

$93826 \mathrm{E}-02$

8.20386 E-02

$8.47385 \mathrm{E}-02$

8.74822 E-02 $9.02698 \mathrm{E}-02$

$9.31010 \mathrm{E}-02$

$9.59760 \mathrm{E}-02$

$9.88946 \mathrm{E}-02$

$1.01857 \mathrm{E}-01$

$1.04863 \mathrm{E}-01$

$1.07913 \mathrm{E}-01$

$1.11006 \mathrm{E}-01$

$1.14143 \mathrm{E}-01$

$1.17323 \mathrm{E}-01$

$1.20547 \mathrm{E}-01$

$1.23815 \mathrm{E}-01$

$1.27126 \mathrm{E}-01$

$1.30481 \mathrm{E}-01$

$1.33880 \mathrm{E}-01$

$1.37322 \mathrm{E}-01$

$1.40808 \mathrm{E}-01$

$1.44337 \mathrm{E}-01$

$1.47910 \mathrm{E}-01$

$1.51527 \mathrm{E}-01$

$1.55187 \mathrm{E}-01$

$1.58891 \mathrm{E}-01$

$1.62638 \mathrm{E}-01$

$1.66429 \mathrm{E}-01$

$1.70264 \mathrm{E}-01$

$1.74142 \mathrm{E}-01$

$1.78064 \mathrm{E}-01$

$1.82029 \mathrm{E}-01$

$1.86038 \mathrm{E}-01$

$1.90090 \mathrm{E}-01$

$1.94186 \mathrm{E}-01$

$1.98326 \mathrm{E}-01$

$2.02509 \mathrm{E}-01$

$2.06736 \mathrm{E}-01$

$2.11006 \mathrm{E}-01$

$2.15320 \mathrm{E}-01$

$2.19677 \mathrm{E}-01$

$2.24078 \mathrm{E}-01$

$2.28523 \mathrm{E}-01$

$2.33011 \mathrm{E}-01$

$2.37543 \mathrm{E}-01$

$2.42118 \mathrm{E}-01$

$2.46737 \mathrm{E}-01$

$2.51399 \mathrm{E}-01$

$2.56105 \mathrm{E}-01$

$2.60855 \mathrm{E}-01$

$2.65648 \mathrm{E}-01$

$2.70484 \mathrm{E}-01$

$2.75364 \mathrm{E}-01$

$2.80288 \mathrm{E}-01$

$2.85255 \mathrm{E}-01$

$2.90266 \mathrm{E}-01$

$2.95320 \mathrm{E}-01$

$3.00418 \mathrm{E}-01$

$1.98522 \mathrm{E}-04$

$2.17186 \mathrm{E}-04$

$2.36728 \mathrm{E}-04$

$2.57165 \mathrm{E}-04$

$2.78516 \mathrm{E}-04$

$3.00798 \mathrm{E}-04$

$3.24030 \mathrm{E}-04$

$3.48229 \mathrm{E}-04$

$3.73415 \mathrm{E}-04$

$3.99604 \mathrm{E}-04$

$4.26815 \mathrm{E}-04$

$4.55066 \mathrm{E}-04$

$4.84375 \mathrm{E}-04$

5.14761 E-04

$5.46240 \mathrm{E}-04$

$5.78833 \mathrm{E}-04$

$6.12555 \mathrm{E}-04$

$6.47427 \mathrm{E}-04$

$6.83464 \mathrm{E}-04$

$7.20686 \mathrm{E}-04$

$7.59111 \mathrm{E}-04$

$7.98757 \mathrm{E}-04$

$8.39641 \mathrm{E}-04$

$8.81783 \mathrm{E}-04$

9.25199E-04

$9.69909 \mathrm{E}-04$

$1.01593 \mathrm{E}-03$

$1.06328 \mathrm{E}-03$

$1.11198 \mathrm{E}-03$

$1.16204 \mathrm{E}-03$

$1.21349 \mathrm{E}-03$

$1.26633 \mathrm{E}-03$

$1.32060 \mathrm{E}-03$

$1.37630 \mathrm{E}-03$

$1.43346 \mathrm{E}-03$

$1.49210 \mathrm{E}-03$

$1.55223 \mathrm{E}-03$

$1.61386 \mathrm{E}-03$

$1.67702 \mathrm{E}-03$

$1.74173 \mathrm{E}-03$

$1.80801 \mathrm{E}-03$

$1.94532 \mathrm{E}-03$

$2.01639 \mathrm{E}-03$

$2.08910 \mathrm{E}-03$

$2.16347 \mathrm{E}-03$

$2.23951 \mathrm{E}-03$

$2.31723 \mathrm{E}-03$

$2.39667 \mathrm{E}-03$

$2.47783 \mathrm{E}-03$

$2.56074 \mathrm{E}-03$

$2.64541 \mathrm{E}-03$

$2.73187 \mathrm{E}-03$

$2.82012 \mathrm{E}-03$

$2.91018 \mathrm{E}-03$ $\tan \delta_{2}$

$f_{-2}$

$-3.71767 \mathrm{E} 01 \quad 1.02866 \mathrm{E}-04$

$-3.72245 \mathrm{E} 01$

$-3.72707 \mathrm{E} 01$

$-3.73154 \mathrm{E} 01$

$-3.73586 \mathrm{E} 01$

$1.87586 \mathrm{E}-03$

$-3.74004 \mathrm{E} \quad 01$ $-3.74408 \mathrm{E} \quad 01$ $-3.74800 \mathrm{E} 01$ $-3.75180 \mathrm{E} \quad 01$ $-3.75549 \mathrm{E} 01$

$-3.75906 \mathrm{E} 01$

$-3.76253 \mathrm{E} \quad 01$

$-3.76589 \mathrm{E} 01$

$-3.76916 \mathrm{E} 01$

$-3.77234 \mathrm{E} 01$

$-3.77542 \mathrm{E} 01$

$-3.77843 \mathrm{E} \quad 01$

$-3.78134 \mathrm{E}$ 01

$-3.78419 \mathrm{E} 01$

$-3.78695 \mathrm{E} 01$

$-3.78964 \mathrm{E} \quad 01$ $-3.79226 \mathrm{E} 01$

$-3.79482 \mathrm{E} \quad 01$

$-3.79731 \mathrm{E} 01$

$-3.79974 \mathrm{E} \quad 01$

$-3.80211 \mathrm{E} 01$

$-3.80442 \mathrm{E} \quad 01$

$-3.80667 \mathrm{E} \quad 01$

$-3.80887 \mathrm{E} 01$

$-3.81102 \mathrm{E} 01$

$-3.81312 \mathrm{E} 01$

$-3.81517 \mathrm{E} 01$

$-3.81717 \mathrm{E} 01$

$-3.81913 \mathrm{E} \quad 01$

$-3.82104 \mathrm{E} 01$

$-3.82291 \mathrm{E} \quad 01$

$-3.82475 \mathrm{E} 01$

$-3.82654 \mathrm{E} 01$

$-3.82829 \mathrm{E} 01$

$-3.83001 \mathrm{E} 01$

$-3.83169 \mathrm{E} 01$

$-3.83333 \mathrm{E} 01$

$-3.83494 \mathrm{E} 01$

$-3.83652 \mathrm{E} 01$

$-3.83807 \mathrm{E} 01$

$-3.83958 \mathrm{E} 01$

$-3.84107 \mathrm{E} 01$

$-3.84252 \mathrm{E} 01$ 
ELECTRON RADIAL FUNCTIONS

Positron

p $\quad F_{0}$

12.10

12.20

12.30

12.40

12.50

12.60

12.70

12.80

12.90

13.00

13.10

13.20

13.30

13.40

13.50

13.60

13.70

13.80

13.90

14.00

14.10

14.20

14.30

14.40

14.50

14.60

14.70

14.80

14.90

15.00

15.10

15.20

15.30

15.40

15.50

15.60

15.70

15.80

15.90

16.00

16.10

16.20

16.30

16.40

16.50

16.60

16.70

16.80

16.90

17.00

17.10

17.20

17.30

17.40

17.50

17.60

17.70

17.80

17.90

18.00

$Z=5$

$f_{1}$

$8.94387 \mathrm{E}-01$ $8.94383 \mathrm{E}-01$ $8.94379 \mathrm{E}-01$ $8.94375 \mathrm{E}-01$ 8.94371 E-01

$8.94367 \mathrm{E}-01$ $8.94363 \mathrm{E}-01$ $8.94359 \mathrm{E}-01$ $8.94354 \mathrm{E}-01$ $8.94350 \mathrm{E}-01$

$8.94346 \mathrm{E}-01$ $8.94341 \mathrm{E}-01$ $8.94337 \mathrm{E}-01$ $8.94332 \mathrm{E}-01$ $8.94328 \mathrm{E}-01$

$8.94323 \mathrm{E}-01$ $8.94319 \mathrm{E}-01$ $8.94314 \mathrm{E}-01$ $8.94310 \mathrm{E}-01$ 8.94305E-01

$8.94300 \mathrm{E}-01$ $8.94296 \mathrm{E}-01$ $8.94291 \mathrm{E}-01$ $8.94286 \mathrm{E}-01$ 8.94281 E-01

8.94277E-01 $8.94272 \mathrm{E}-01$ $8.94268 \mathrm{E}-01$ $8.94263 \mathrm{E}-01$ $8.94258 \mathrm{E}-01$

$8.94253 \mathrm{E}-01$ $8.94248 \mathrm{E}-01$ $8.94244 \mathrm{E}-01$ $8.94239 \mathrm{E}-01$ $8.94234 \mathrm{E}-01$

$8.94229 \mathrm{E}-01$ $8.94224 \mathrm{E}-01$ 8.94219E-01 $8.94215 \mathrm{E}-01$ $8.94210 \mathrm{E}-01$

$8.94205 \mathrm{E}-01$ $8.94200 \mathrm{E}-01$ 8.94195E-01 $8.94190 \mathrm{E}-01$ $8.94185 \mathrm{E}-01$

8.94181 E- 01 $8.94176 \mathrm{E}-01$ 8.94171 E-01 $8.94166 \mathrm{E}-01$ 8.94161 E-01

$8.94156 \mathrm{E}-01$ $8.94151 \mathrm{E}-01$ $8.94147 \mathrm{E}-01$ 8.94142 E-01 $8.94137 \mathrm{E}-01$

$8.94132 \mathrm{E}-01$ $8.94127 \mathrm{E}-01$ $8.94122 \mathrm{E}-01$ 8.94118E-01 $8.94113 \mathrm{E}-01$
$1.10064 \mathrm{E} \quad 01$ $1.11019 \mathrm{E} \quad 01$ $1.11974 \mathrm{E} \quad 01$

$1.12930 \mathrm{E} \quad 01$

$1.13885 \mathrm{E} \quad 01$

$1.14841 \mathrm{E} \quad 01$ $1.15797 \mathrm{E} \quad 01$ $1.16753 \mathrm{E} \quad 01$ $1.17709 \mathrm{E} \quad 01$

$1.18665 \mathrm{E} \quad 01$

$1.19621 \mathrm{E} \quad 01$ $1.20578 \mathrm{E} \quad 01$ $1.21534 \mathrm{E} \quad 01$ $1.22491 \mathrm{E} \quad 01$ $1.23447 \mathrm{E} \quad 01$

$1.24404 \mathrm{E} \quad 01$ $1.25361 \mathrm{E} \quad 01$ $1.26318 \mathrm{E} \quad 01$

$1.27275 \mathrm{E} \quad 01$

$1.28233 \mathrm{E} 01$

$1.29190 \mathrm{E} \quad 01$ $1.30148 \mathrm{E} \quad 01$ $1.31105 \mathrm{E} \quad 01$ $1.32063 \mathrm{E} \quad 01$

$1.33021 \mathrm{E} \quad 01$

$1.33979 \mathrm{E} \quad 01$ $1.34937 \mathrm{E} \quad 01$ $1.35895 \mathrm{E} \quad 01$ $1.36854 \mathrm{E} \quad 01$

$1.37812 \mathrm{E} \quad 01$

$1.38771 \mathrm{E} \quad 01$ $1.39730 \mathrm{E} \quad 01$ $1.40689 \mathrm{E} \quad 01$

$1.41648 \mathrm{E} \quad 01$

$1.42607 \mathrm{E} \quad 01$

$1.43566 \mathrm{E} \quad 01$ $1.44526 \mathrm{E} \quad 01$ $1.45485 \mathrm{E} \quad 01$ $1.46445 \mathrm{E} \quad 0$

$1.47405 \mathrm{E} \quad 01$

$1.48365 \mathrm{E} \quad 01$ $1.49325 \mathrm{E} 01$ $1.50285 \mathrm{E} \quad 01$ $1.51245 \mathrm{E} \quad 0$

$1.52206 \mathrm{E} \quad 01$

$1.53167 \mathrm{E} \quad 01$ $1.54127 \mathrm{E} \quad 01$

$1.55088 \mathrm{E} \quad 01$

$1.56049 \mathrm{E} \quad 01$

$1.57011 \mathrm{E} \quad 01$

$1.57972 \mathrm{E} \quad 01$ $1.58934 \mathrm{E} \quad 01$ $1.59895 \mathrm{E} \quad 01$ $1.60857 \mathrm{E} \quad 01$ $1.61819 \mathrm{E} \quad 01$

$1.62781 \mathrm{E} \quad 01$ $1.63743 \mathrm{E} \quad 01$ $1.64706 \mathrm{E} \quad 01$ $1.65668 \mathrm{E} \quad 01$ $1.66631 \mathrm{E} \quad 01$
$A=11$

$\rho=1.2 A^{1 / 3} F$ $\begin{array}{lll}g_{1} & \tan \delta_{1} & f_{-1}\end{array}$

$1.72586 \mathrm{E}-01$ $1.76634 \mathrm{E}-01$

$1.80726 \mathrm{E}-0$

$1.84862 \mathrm{E}-01$

$1.89043 \mathrm{E}-01$

$1.93267 \mathrm{E}-01$ $1.97536 \mathrm{E}-01$ $2.01850 \mathrm{E}-01$

$2.06207 \mathrm{E}-01$

$2.10609 \mathrm{E}-01$

$2.15055 \mathrm{E}-01$

$2.19545 \mathrm{E}-01$

$2.24080 \mathrm{E}-01$

$2.28659 \mathrm{E}-01$

$2.33283 \mathrm{E}-01$

$2.37950 \mathrm{E}-01$

$2.42663 \mathrm{E}-01$

$2.47419 \mathrm{E}-01$

$2.52220 \mathrm{E}-01$

$2.57065 \mathrm{E}-01$

$2.61955 \mathrm{E}-01$

$2.66889 \mathrm{E}-01$

$2.71868 \mathrm{E}-01$

$2.76891 \mathrm{E}-01$

$2.81958 \mathrm{E}-01$

$2.87070 \mathrm{E}-01$ $2.92227 \mathrm{E}-01$

$2.97428 \mathrm{E}-01$

$3.02673 \mathrm{E}-01$

$3.07963 \mathrm{E}-01$

$3.13298 \mathrm{E}-01$

$3.18677 \mathrm{E}-01$

$3.24100 \mathrm{E}-01$

$3.29568 \mathrm{E}-01$

$3.35081 \mathrm{E}-01$

$3.40639 \mathrm{E}-01$

$3.46241 \mathrm{E}-01$

$3.51887 \mathrm{E}-01$

$3.57578 \mathrm{E}-01$

$3.63314 \mathrm{E}-01$

$3.69095 \mathrm{E}-01$

$3.74920 \mathrm{E}-0$

$3.80790 \mathrm{E}-01$

$3.86705 \mathrm{E}-01$

$3.92664 \mathrm{E}-01$

$3.98668 \mathrm{E}-01$ $4.04717 \mathrm{E}-01$

$4.10810 \mathrm{E}-01$

$4.16949 \mathrm{E}-01$

$4.23132 \mathrm{E}-01$

$4.29360 \mathrm{E}-01$ $4.35632 \mathrm{E}-01$ $4.41950 \mathrm{E}-01$ $4.48313 \mathrm{E}-01$ $4.54720 \mathrm{E}-01$

$4.61172 \mathrm{E}-01$

$4.67669 \mathrm{E}-0 \mathrm{l}$

$4.74211 \mathrm{E}-01$

$4.80798 \mathrm{E}-01$

$4.87429 \mathrm{E}-0 \mathrm{l}$

$-2.67201 \mathrm{E}-04$

$-2.79044 \mathrm{E}-04$

$-2.90678 \mathrm{E}-04$

$-3.02116 \mathrm{E}-04$

$-3.13370 \mathrm{E}-04$

$-3.24433 \mathrm{E}-04$

$-3.35320 \mathrm{E}-04$

$-3.46024 \mathrm{E}-04$

$-3.56549 \mathrm{E}-04$

$-3.66908 \mathrm{E}-04$

$-3.77106 \mathrm{E}-04$

$-3.87138 \mathrm{E}-04$

$-3.97006 \mathrm{E}-04$

$-4.06724 \mathrm{E}-04$

$-4.16287 \mathrm{E}-04$

$-4.25704 \mathrm{E}-04$

$-4.34969 \mathrm{E}-04$

$-4.44098 \mathrm{E}-04$

$-4.53087 \mathrm{E}-04$

$-4.61936 \mathrm{E}-04$

$-4.70654 \mathrm{E}-04$

$-4.79239 \mathrm{E}-04$

$-4.87694 \mathrm{E}-04$

$-4.96027 \mathrm{E}-04$

$-5.04231 \mathrm{E}-04$

$-5.12320 \mathrm{E}-04$

$-5.20292 \mathrm{E}-04$

$-5.28144 \mathrm{E}-04$

$-5.35882 \mathrm{E}-04$

$-5.43508 \mathrm{E}-04$

$-5.51018 \mathrm{E}-04$

$-5.58431 \mathrm{E}-04$

$-5.65738 \mathrm{E}-04$

$-5.72943 \mathrm{E}-04$

$-5.80037 \mathrm{E}-04$

$-5.87033 \mathrm{E}-04$

$-5.93939 \mathrm{E}-04$

$-6.00740 \mathrm{E}-04$ 
ELECTRON RADIAL FUNCTIONS

$Z=5 \quad A=11 \quad \rho=1.2 A^{1 / 3} \mathrm{~F}$

Positron

$\bar{F}$

$1.73503 \mathrm{E}-04$

$1.76382 \mathrm{E}-04$

$1.79286 \mathrm{E}-04$

$1.82213 \mathrm{E}-04$

$1.85164 \mathrm{E}-04$

$1.88139 \mathrm{E}-04$

$1.91137 \mathrm{E}-04$

$1.94159 \mathrm{E}-04$

$1.97205 \mathrm{E}-04$

$2.00274 \mathrm{E}-04$

$2.03367 \mathrm{E}-04$

$2.06484 \mathrm{E}-04$

$2.09624 \mathrm{E}-04$

$2.12788 \mathrm{E}-04$

$2.15976 \mathrm{E}-04$

$2.19187 \mathrm{E}-04$

$2.22422 \mathrm{E}-04$

$2.25681 \mathrm{E}-04$

$2.28964 \mathrm{E}-04$

$2.32270 \mathrm{E}-04$

$2.35600 \mathrm{E}-04$

$2.38953 \mathrm{E}-04$

$2.42330 \mathrm{E}-04$

$2.45731 \mathrm{E}-04$

$2.49156 \mathrm{E}-04$

$2.52604 \mathrm{E}-04$

$2.56076 \mathrm{E}-04$

$2.59572 \mathrm{E}-04$

$2.63091 \mathrm{E}-04$

$2.66634 \mathrm{E}-04$

$2.70201 \mathrm{E}-04$

$2.73791 \mathrm{E}-04$

$2.77405 \mathrm{E}-04$

$2.81043 \mathrm{E}-04$

$2.84704 \mathrm{E}-04$

$2.88390 \mathrm{E}-04$

$2.92098 \mathrm{E}-04$

2.95831 E-04

$2.99587 \mathrm{E}-04$

$3.03367 \mathrm{E}-04$

$3.07170 \mathrm{E}-04$

$3.10998 \mathrm{E}-04$

$3.14848 \mathrm{E}-04$

$3.18723 \mathrm{E}-04$

3.22621 E- 04

$3.26543 \mathrm{E}-04$

$3.30489 \mathrm{E}-04$

$3.34458 \mathrm{E}-04$

$3.38451 \mathrm{E}-04$

$3.42468 \mathrm{E}-04$

$3.46508 \mathrm{E}-04$

$3.50572 \mathrm{E}-04$

$3.54660 \mathrm{E}-04$

$3.58771 \mathrm{E}-04$

$3.62906 \mathrm{E}-04$

$3.67065 \mathrm{E}-04$

$3.71247 \mathrm{E}-04$

$3.75453 \mathrm{E}-04$

$3.79683 \mathrm{E}-04$

$3.83937 \mathrm{E}-04$ $f_{2}$

$g_{2}$

$\tan \delta_{2}$

$f_{-2}$

$3.05560 \mathrm{E}-01$

$3.10745 \mathrm{E}-01$

$3.15973 \mathrm{E}-01$

$3.21245 \mathrm{E}-01$

$3.26561 \mathrm{E}-01$

$3.31920 \mathrm{E}-01$

$3.37322 \mathrm{E}-01$

$3.42768 \mathrm{E}-01$

$3.48258 \mathrm{E}-.01$

3.53791 E-01

$3.59368 \mathrm{E}-01$

$3.64988 \mathrm{E}-01$

$3.70651 \mathrm{E}-01$

$3.76359 \mathrm{E}-01$

$3.82109 \mathrm{E}-01$

$3.87904 \mathrm{E}-01$

$3.93741 \mathrm{E}-01$

$3.99623 \mathrm{E}-01$

$4.05547 \mathrm{E}-01$

$4.11516 \mathrm{E}-01$

$4.17527 \mathrm{E}-01$

$4.23583 \mathrm{E}-01$

4.29681 E-01

$4.35824 \mathrm{E}-01$

$4.42009 \mathrm{E}-01$

$4.48238 \mathrm{E}-01$

$4.54511 \mathrm{E}-01$

$4.60827 \mathrm{E}-01$

$4.67187 \mathrm{E}-01$

$4.73590 \mathrm{E}-01$

$4.80037 \mathrm{E}-01$

$4.86527 \mathrm{E}-01$

$4.93060 \mathrm{E}-01$

$4.99637 \mathrm{E}-0]$

$5.06258 \mathrm{E}-01$

$5.12922 \mathrm{E}-01$

$5.19629 \mathrm{E}-01$

$5.26380 \mathrm{E}-01$

$5.33174 \mathrm{E}-01$

$5.40012 \mathrm{E}-01$

$5.46894 \mathrm{E}-01$

$5.53818 \mathrm{E}-01$

$5.60786 \mathrm{E}-01$

$5.67798 \mathrm{E}-01$

$5.74853 \mathrm{E}-01$

$5.81951 \mathrm{E}-01$

$5.89093 \mathrm{E}-01$

$5.96279 \mathrm{E}-01$

$6.03507 \mathrm{E}-01$

$6.10780 \mathrm{E}-01$

$6.18095 \mathrm{E}-01$

$6.25454 \mathrm{E}-01$

$6.32857 \mathrm{E}-01$

$6.40303 \mathrm{E}-01$

$6.47792 \mathrm{E}-01$

$6.55325 \mathrm{E}-01$

$6.62901 \mathrm{E}-01$

$6.70520 \mathrm{E}-01$

$6.78183 \mathrm{E}-01$

$6.85890 \mathrm{E}-01$
$3.00209 \mathrm{E}-03$

$3.09584 \mathrm{E}-03$

$3.19147 \mathrm{E}-03$

$3.28899 \mathrm{E}-03$

$3.38841 \mathrm{E}-03$

$3.48976 \mathrm{E}-03$

$3.59305 \mathrm{E}-03$

$3.69830 \mathrm{E}-03$

$3.80553 \mathrm{E}-03$

$3.91476 \mathrm{E}-03$

$4.02600 \mathrm{E}-03$

$4.13928 \mathrm{E}-03$

$4.25461 \mathrm{E}-03$

$4.37201 \mathrm{E}-03$

$4.49150 \mathrm{E}-03$

$4.61309 \mathrm{E}-03$

$4.73681 \mathrm{E}-03$

$4.86267 \mathrm{E}-03$

$4.99068 \mathrm{E}-03$

$5.12088 \mathrm{E}-03$

$5.25327 \mathrm{E}-03$

$5.38788 \mathrm{E}-03$

$5.52471 \mathrm{E}-03$

$5.66380 \mathrm{E}-03$

$5.80516 \mathrm{E}-03$

$5.94880 \mathrm{E}-03$ $6.09475 \mathrm{E}-03$ $6.24301 \mathrm{E}-03$

$6.39362 \mathrm{E}-03$

$6.54659 \mathrm{E}-03$

$6.70193 \mathrm{E}-03$

$6.85966 \mathrm{E}-03$

$7.01981 \mathrm{E}-03$

$7.18239 \mathrm{E}-03$

$7.34742 \mathrm{E}-03$

$7.51491 \mathrm{E}-03$

$7.68489 \mathrm{E}-03$

$7.85737 \mathrm{E}-03$

$8.03237 \mathrm{E}-03$

8.20991 E-03

$8.39001 \mathrm{E}-03$

$8.57267 \mathrm{E}-03$

8.75794.E-03

$8.94581 \mathrm{E}-03$

$9.13631 \mathrm{E}-03$

$9.32946 \mathrm{E}-03$

$9.52527 \mathrm{E}-03$

$9.72377 \mathrm{E}-03$

$9.92496 \mathrm{E}-03$

$1.01289 \mathrm{E}-02$

$1.03355 \mathrm{E}-02$

$1.05449 \mathrm{E}-02$

$1.07571 \mathrm{E}-02$

$1.09721 \mathrm{E}-02$

$1.11899 \mathrm{E}-02$

$1.14105 \mathrm{E}-02$

$1.16339 \mathrm{E}-02$

$1.18602 \mathrm{E}-02$

$1.20894 \mathrm{E}-02$

$1.23215 \mathrm{E}-02$
$-3.85915 \mathrm{E} 01$

$-3.86028 \mathrm{E} 01$

$-3.86138 \mathrm{E} \quad 01$

$-3.86247 \mathrm{E} \quad 01$

$-3.86353 \mathrm{E} 01$

$\begin{array}{lll}-3.86459 \mathrm{E} & 01\end{array}$

$-3.86562 \mathrm{E} \quad 01$

$-3.86663 \mathrm{E} 01$

$-3.86763 \mathrm{E} 01$

$\begin{array}{lll}-3.86862 \mathrm{E} & 01\end{array}$

$-3.86958 \mathrm{E} 01$

$-3.87054 \mathrm{E} 01$

$-3.87147 \mathrm{E}$ 01

$-3.87240 \mathrm{E} 01$

$-3.87330 \mathrm{E} 01$

$-3.87420 \mathrm{E} \quad 01$

$-3.87508 \mathrm{E} \quad 01$

$-3.87594 \mathrm{E} \quad 01$

$-3.87680 \mathrm{E}$ 01

$-3.87764 \mathrm{E}$ 01

$-3.87847 \mathrm{E} \quad 01$

$-3.87928 \mathrm{E} \quad 01$

$-3.88009 \mathrm{E} \quad 01$

$-3.88088 \mathrm{E} 01$

$-3.88166 \mathrm{E} 01$

$-3.88243 \mathrm{E} \quad 01$

$-3.88319 \mathrm{E}$ 01

$-3.88394 \mathrm{E} \quad 01$

$-3.88467 \mathrm{E} \quad 01$

$-3.88540 \mathrm{E} 01$

$-3.88612 \mathrm{E} \quad 01$

$-3.88682 \mathrm{E} \quad 01$

$-3.88752 \mathrm{E} 01$

$-3.88821 \mathrm{E} 01$

$-3.88889 \mathrm{E} 01$

$-3.88956 \mathrm{E} 01$ $-3.89022 \mathrm{E} 01$

$-3.89087 \mathrm{E} 01$

$-3.89151 \mathrm{E}$ 01

$-3.89215 \mathrm{E} 01$

$\begin{array}{lll}-3.89277 \mathrm{E} & 01\end{array}$

$-3.89339 \mathrm{E} 01$

$-3.89400 \mathrm{E}$ 01

$-3.89461 \mathrm{E} 01$

$-3.89520 \mathrm{E} 01$

$\begin{array}{lll}-3.89579 \mathrm{E} & 01\end{array}$

$-3.89637 \mathrm{E} 01$

$-3.89694 \mathrm{E} 01$

$-3.89751 \mathrm{E} 01$

$-3.89807 \mathrm{E} \quad 01$

$\begin{array}{lll}-3.89862 \mathrm{E} & 01\end{array}$

$-3.89917 \mathrm{E}$ 01

$-3.89971 \mathrm{E} 01$

$-3.90024 \mathrm{E} \quad 01$

$-3.90076 \mathrm{E}$ ol

$-3.90128 \mathrm{E} \quad 01$

$-3.90180 \mathrm{E} \quad 01$

$-3.90231 \mathrm{E} 01$

$-3.90281 \mathrm{E} 01$

$-3.90330 \mathrm{E} 01$

$-2.34296 \mathrm{E}-03$

$-2.42757 \mathrm{E}-0.3$

$-2.51400 \mathrm{E}-03$

$-2.60225 \mathrm{E}-03$

$-2.69235 \mathrm{E}-03$

$-2.784 .32 \mathrm{E}-03$

$-2.87817 \mathrm{E}-03$

$-2.97392 \mathrm{E}-03$

$-3.07159 \mathrm{E}-03$

$-3.17120 \mathrm{E}-03$

$-3.27276 \mathbf{E}-03$

$-3.37630 \mathrm{E}-03$

$-3.48183 \mathrm{E}-03$

$-3.58936 \mathrm{E}-03$

$-3.69893 \mathrm{E}-03$

$-3.81054 \mathrm{E}-03$

$-3.92421 \mathrm{E}-03$

$-4.03997 \mathrm{E}-03$

$-4.15782 \mathrm{E}-03$

$-4.27780 \mathrm{E}-03$

$-4.39991 \mathrm{E}-03$

$-4.52417 \mathrm{E}-03$

$-4.65060 \mathrm{E}-03$

$-4.77923 \mathrm{E}-03$

$-4.91006 \mathrm{E}-03$

$-5.04312 \mathrm{E}-03$

$-5.17843 \mathrm{E}-03$

$-5.31599 \mathrm{E}-03$

$-5.45584 \mathrm{E}-03$

$-5.59798 \mathrm{E}-03$

$-5.74244 \mathrm{E}-03$

$-5.88923 \mathrm{E}-03$

$-6.03838 \mathrm{E}-03$

$-6.18989 \mathrm{E}-03$

$-6.34380 \mathrm{E}-03$

$-6.50011 \mathrm{E}-03$

$-6.65884 \mathrm{E}-03$

$-6.82002 \mathrm{E}-03$

$-6.98366 \mathrm{E}-03$

$-7.14977 \mathrm{E}-03$

$-7.31838 \mathrm{E}-03$

$-7.48951 \mathrm{E}-03$ 
ELECTRON RADIAL FUNCTIONS

Positron

p $\quad F_{0}$

18.10

18.20

18.30

18.40

18.50

18.60

18.70

18.80

18.90

19.00

19.10

19.20

19.30

19.40

19.50

19.60

19.70

19.80

19.90

20.00

20.10

20.20

20.30

20.40

20.50

20.60

20.70

20.80

20.90

21.00

21.10

21.20

21.30

21.40

21.50

21.60

21.70

21.80

21.90

22.00

22.10

22.20

22.30

22.40

22.50

22.60

22.70

22.80

22.90

23.00

23.10

23.20

23.30

23.40

23.50

23.60

23.70

23.80

23.90

24.00
$Z=5 \quad A=11$

$g_{1}$

$\tan \delta_{1}$

$f_{-1}$

$g_{-1}$

$\tan \delta_{-1}$

$1.67594 \mathrm{E} \quad 01$

$1.68557 \mathrm{E} \quad 01$

$1.69520 \mathrm{E} \quad 01$

$1.70483 \mathrm{E} \quad 01$

$1.7144 .7 \mathrm{E} \quad 01$

$8.94094 \mathrm{E}-0$

$8.94089 \mathrm{E}-01$

$1.72411 \mathrm{E} \quad 01$

$1.73374 \mathrm{E} \quad 01$

$1.74338 \mathrm{E} \quad 01$

$1.75302 \mathrm{E} \quad 01$

$1.76267 \mathrm{E} \quad 01$

$1.77231 \mathrm{E} \quad 01$

$1.78196 \mathrm{E} \quad 01$

$1.79160 \mathrm{E} \quad 01$

$1.80125 \mathrm{E} \quad 01$

$1.81090 \mathrm{E} \quad 01$

$1.82056 \mathrm{E} \quad 01$

$1.83021 \mathrm{E} \quad 01$

$1.83986 \mathrm{E} \quad 01$

$1.84952 \mathrm{E} \quad 01$

$1.85918 \mathrm{E} \quad 01$

$1.86884 \mathrm{E} \quad 01$

$1.87850 \mathrm{E} \quad 01$

$1.88817 \mathrm{E} \quad 01$

$1.89783 \mathrm{E} \quad 01$

$1.90750 \mathrm{E} \quad 01$

$1.91717 \mathrm{E} \quad 01$

$1.92684 \mathrm{E} \quad 01$

$1.93651 \mathrm{E} \quad 01$

$1.94619 \mathrm{E} \quad 01$

$1.95586 \mathrm{E} \quad 01$

$1.96554 \mathrm{E} \quad 01$

$1.97522 \mathrm{E} \quad 01$

$1.98490 \mathrm{E} \quad 01$

$1.99458 \mathrm{E}$ 01

$2.00427 \mathrm{E} \quad 01$

$2.01395 \mathrm{E} \quad 01$

$2.02364 \mathrm{E} \quad 01$

$2.03333 \mathrm{E} 01$

$2.04302 \mathrm{E} \quad 01$

$2.05272 \mathrm{E} \quad 01$

$2.06241 \mathrm{E} \quad 01$

$2.07211 \mathrm{E} \quad 01$

$2.08181 \mathrm{E} \quad 01$

$2.09151 \mathrm{E} \quad 01$

$2.10121 \mathrm{E} 01$

$2.11092 \mathrm{E} \quad 01$

$2.12062 \mathrm{E} \quad 01$

$2.13033 \mathrm{E} \quad 01$

$2.14004 \mathrm{E} \quad 01$

$2.14975 \mathrm{E} \quad 01$

$2.15947 \mathrm{E} \quad 01$

$2.16918 \mathrm{E} \quad 01$

$2.17890 \mathrm{E} \quad 01$

$2.18862 \mathrm{E} \quad 01$

$2.19834 \mathrm{E} \quad 01$

$2.20807 \mathrm{E} \quad 01$

$2.21779 \mathrm{E} \quad 01$

$2.22752 \mathrm{E} 01$

$2.23725 \mathrm{E} \quad 01$

$2.24698 \mathrm{E} \quad 01$
$5.00828 \mathrm{E}-01$

$5.07595 \mathrm{E}-01$

$5.14407 \mathrm{E}-01$

$5.21263 \mathrm{E}-01$

$5.28165 \mathrm{E}-01$

$5.35111 \mathrm{E}-01$

$5.42103 \mathrm{E}-01$

$5.49140 \mathrm{E}-01$

$5.56222 \mathrm{E}-01$

$5.63349 \mathrm{E}-01$

$5.70521 \mathrm{E}-01$

$5.77738 \mathrm{E}-01$

$5.85001 \mathrm{E}-01$

$5.92308 \mathrm{E}-01$

$5.99661 \mathrm{E}-01$

$6.07059 \mathrm{E}-01$

$6.14502 \mathrm{E}-01$

$6.21991 \mathrm{E}-01$

$6.29524 \mathrm{E}-01$

$6.37103 \mathrm{E}-01$

$6.44727 \mathrm{E}-01$

$6.52397 \mathrm{E}-01$

$6.60112 \mathrm{E}-01$

$6.67872 \mathrm{E}-01$

$6.75677 \mathrm{E}-01$

$6.83528 \mathrm{E}-01$

$6.91424 \mathrm{E}-01$

$6.99366 \mathrm{E}-01$

$7.07353 \mathrm{E}-01$

$7.15385 \mathrm{E}-01$

$7.23463 \mathrm{E}-01$

$7.31587 \mathrm{E}-01$

$7.39756 \mathrm{E}-01$

$7.47970 \mathrm{E}-01$

$7.56230 \mathrm{E}-01$

$7.64535 \mathrm{E}-01$

$7.72887 \mathrm{E}-01$

$7.81283 \mathrm{E}-01$

$7.89725 \mathrm{E}-01$ $8.06746 \mathrm{E}-01$

$8.15325 \mathrm{E}-01$

$8.23950 \mathrm{E}-0]$

$8.32621 \mathrm{E}-01$

$8.41337 \mathrm{E}-01$ $8.50099 \mathrm{E}-01$

$8.58906 \mathrm{E}-01$

$8.67760 \mathrm{E}-01$

$8.76659 \mathrm{E}-01$

$8.85604 \mathrm{E}-01$ $8.94595 \mathrm{E}-01$ 9.03632E-01

$9.12714 \mathrm{E}-01$

$9.21843 \mathrm{E}-01$

$9.31017 \mathrm{E}-01$ $9.40237 \mathrm{E}-01$ $9.49503 \mathrm{E}-01$ $9.58815 \mathrm{E}-01$ $9.68174 \mathrm{E}-01$
$4.94107 \mathrm{E}-01$

$7.98213 \mathrm{E}-01$

$$
\begin{array}{ll}
-7.34287 \mathrm{E}-04 & -4.40866 \mathrm{E}-01 \\
-7.39225 \mathrm{E}-04 & -4.47371 \mathrm{E}-01 \\
-7.44101 \mathrm{E}-04 & -4.53921 \mathrm{E}-01 \\
-7.48912 \mathrm{E}-04 & -4.60516 \mathrm{E}-01 \\
-7.53664 \mathrm{E}-04 & -4.67156 \mathrm{E}-01
\end{array}
$$

$-7.58361 \mathrm{E}-04$

$-7.62993 \mathrm{E}-04$

$-7.67572 \mathrm{E}-04$

$-7.72085 \mathrm{E}-04$

$-7.76548 \mathrm{E}-04$

$-7.80957 \mathrm{E}-04$

$-7.85304 \mathrm{E}-04$

$-7.89602 \mathrm{E}-04$

$-7.93847 \mathrm{E}-04$

$-7.98037 \mathrm{E}-04$

$-8.02180 \mathrm{E}-04$

$-8.06265 \mathrm{E}-04$

$-8.10298 \mathrm{E}-04$.

$-8.14291 \mathrm{E}-04$

$-8.18231 \mathrm{E}-04$

$-8.22122 \mathrm{E}-04$

$-8.25967 \mathrm{E}-04$

$-8.29759 \mathrm{E}-04$

$-8.33518 \mathrm{E}-04$

$-8.37218 \mathrm{E}-04$

$-8.40880 \mathrm{E}-04$

$-8.44492 \mathrm{E}-04$

$-8.48064 \mathrm{E}-04$

$-8.51593 \mathrm{E}-04$

$-8.55079 \mathrm{E}-04$

$-8.58523 \mathrm{E}-04$

$-8.61923 \mathrm{E}-04$

$-8.65285 \mathrm{E}-04$

$-8.68609 \mathrm{E}-04$

$-8.71885 \mathrm{E}-04$

$-8.75132 \mathrm{E}-04$

$-8.78332 \mathrm{E}-04$

$-8.81495 \mathrm{E}-04$

$-8.84621 \mathrm{E}-04$

$-8.87703 \mathrm{E}-04$

$-8.90761 \mathrm{E}-04$

$-8.93774 \mathrm{E}-04$

$-8.96751 \mathrm{E}-04$

$-8.99698 \mathrm{E}-04$

$-9.02602 \mathrm{E}-04$

$-9.05475 \mathrm{E}-04$

$-9.08311 \mathrm{E}-04$

$-9.11117 \mathrm{E}-04$

$-9.13886 \mathrm{E}-04$

$-9.16620 \mathrm{E}-04$

$-9.19331 \mathrm{E}-04$

$-9.22003 \mathrm{E}-04$

$-9.24643 \mathrm{E}-04$

$-9.27253 \mathrm{E}-04$

$-9.29832 \mathrm{E}-04$

$-9.32378 \mathrm{E}-04$

$-9.34891 \mathrm{E}-04$

$-9.39829 \mathrm{E}-04$

$-9.42259 \mathrm{E}-04$

$-4.73841 \mathrm{E}-01$

$-4.80572 \mathrm{E}-01$

$-4.87347 \mathrm{E}-01$

$-4.94168 \mathrm{E}-01$

$-5.01033 \mathrm{E}-01$

$-5.07944 \mathrm{E}-01$

$-5.14900 \mathrm{E}-01$

$-5.21901 \mathrm{E}-01$

$-5.28948 \mathrm{E}-01$

$-5.36039 \mathrm{E}-01$

$-5.43176 \mathrm{E}-01$

$-5.50358 \mathrm{E}-01$

$-5.57586 \mathrm{E}-01$

$-5.64858 \mathrm{E}-01$

$-5.72176 \mathrm{E}-01$

$-5.79539 \mathrm{E}-01$

$-5.86948 \mathrm{E}-01$

$-5.94402 \mathrm{E}-01$

$-6.01901 \mathrm{E}-01$

$-6.09446 \mathrm{E}-01$

$-6.17036 \mathrm{E}-01$

$-6.24671 \mathrm{E}-01$

$-6.32352 \mathrm{E}-01$

$-6.40078 \mathrm{E}-01$

$-6.47850 \mathrm{E}-01$

$-6.55668 \mathrm{E}-01$

$-6.63530 \mathrm{E}-01$

$-6.71439 \mathrm{E}-0 \mathrm{l}$

$-6.79393 \mathrm{E}-01$

$-6.87392 \mathrm{E}-01$

$-6.95437 \mathrm{E}-01$

$-7.03528 \mathrm{E}-01$

$-7.11664 \mathrm{E}-01$
$-9.37379 \mathrm{E}-04$ 
$\bar{F}$

$f_{2}$

18.10

18.20

18.30

18.40

18.50

18.60

18.70

18.80

18.90

19.00

19.10

19.20

19.30

19.40

19.50

19.60

19.70

19.80

19.90

20.00

20.10

20.20

20.30

20.40

20.50

20.60

20.70

20.80

20.90

21.00

21.10

21.20

21.30

21.40

21.50

21.60

21.70

21.80

21.90

22.00

22.10

22.20

22.30

22.40

22.50

22.60

22.70

22.80

22.90

23.00

23.10

23.20

23.30

23.40

23.50

23.60

23.70

23.80

23.90

24.00
$3.88214 \mathrm{E}-04$ $3.92515 \mathrm{E}-04$ $3.96839 \mathrm{E}-04$ $4.01188 \mathrm{E}-04$ $4.05559 \mathrm{E}-04$

$4.09955 \mathrm{E}-04$ 4.14374 E-04 $4.18817 \mathrm{E}-04$ $4.23284 \mathrm{E}-04$ $4.27774 \mathrm{E}-04$

$4.32288 \mathrm{E}-04$ $4.36826 \mathrm{E}-04$ $4.41387 \mathrm{E}-04$ $4.45972 \mathrm{E}-04$ $4.50581 \mathrm{E}-04$

$4.55213 \mathrm{E}-04$ $4.59869 \mathrm{E}-04$ $4.64549 \mathrm{E}-04$ $4.69252 \mathrm{E}-04$ $4.73979 \mathrm{E}-04$

$4.78730 \mathrm{E}-04$ $4.83504 \mathrm{E}-04$ $4.88302 \mathrm{E}-04$ $4.93124 \mathrm{E}-04$ $4.97970 \mathrm{E}-04$

$5.02839 \mathrm{E}-04$ $5.07732 \mathrm{E}-04$ $5.12648 \mathrm{E}-04$ $5.17588 \mathrm{E}-04$ $5.22552 \mathrm{E}-04$

$5.27540 \mathrm{E}-04$ $5.32551 \mathrm{E}-04$ $5.37586 \mathrm{E}-04$ $5.42644 \mathrm{E}-04$ $5.47727 \mathrm{E}-04$

$5.52832 \mathrm{E}-04$ $5.57962 \mathrm{E}-04$ $5.63115 \mathrm{E}-04$ $5.68292 \mathrm{E}-04$ $5.73493 \mathrm{E}-04$

$5.78717 \mathrm{E}-04$ $5.83965 \mathrm{E}-04$ $5.89237 \mathrm{E}-04$ $5.94532 \mathrm{E}-04$ $5.99851 \mathrm{E}-04$

$6.05194 \mathrm{E}-04$ $6.10560 \mathrm{E}-04$ $6.15950 \mathrm{E}-04$ $6.21364 \mathrm{E}-04$ $6.26801 \mathrm{E}-04$

$6.32262 \mathrm{E}-04$ $6.37747 \mathrm{E}-04$ $6.43255 \mathrm{E}-04$ $6.48788 \mathrm{E}-04$ $6.54343 \mathrm{E}-04$

$6.59923 \mathrm{E}-04$ $6.65526 \mathrm{E}-04$ $6.71153 \mathrm{E}-04$ $6.76803 \mathrm{E}-04$ $6.82477 \mathrm{E}-04$
$6.93640 \mathrm{E}-01$

$7.01433 \mathrm{E}-01$

$7.09269 \mathrm{E}-01$

$7.17149 \mathrm{E}-01$

$7.25073 \mathrm{E}-01$

$7.33039 \mathrm{E}-01$

$741049 \mathrm{E}-01$

$7.49103 \mathrm{E}-01$

$7.57200 \mathrm{E}-01$

$7.65340 \mathrm{E}-01$

$7.73523 \mathrm{E}-01$

$7.81750 \mathrm{E}-01$

$7.90021 \mathrm{E}-01$

$7.98334 \mathrm{E}-01$

$8.06691 \mathrm{E}-01$

8.15092E-01 $8.23535 \mathrm{E}-01$

8.32022E-01

$8.40553 \mathrm{E}-01$

$8.49126 \mathrm{E}-01$

8.57743E-01

$8.66404 \mathrm{E}-01$

$8.75108 \mathrm{E}-01$

$8.83854 \mathrm{E}-01$

$8.92645 \mathrm{E}-01$

$9.01478 \mathrm{E}-01$

$9.10356 \mathrm{E}-01$

$9.19276 \mathrm{E}-01$

$9.28239 \mathrm{E}-01$

$9.37246 \mathrm{E}-01$

$9.46296 \mathrm{E}-01$

$9.55389 \mathrm{E}-01$

$9.64526 \mathrm{E}-01$

$9.73706 \mathrm{E}-01$

$9.82930 \mathrm{E}-01$

$9.92196 \mathrm{E}-01$

$1.00151 \mathrm{E} \quad 00$

$1.01086 \mathrm{E} \quad 00$

$1.02026 \mathrm{E} \quad 00$

$1.02970 \mathrm{E} \quad 00$

$1.03918 \mathrm{E} \quad 00$

$1.04870 \mathrm{E} \quad 00$

$1.05827 \mathrm{E} \quad 00$

$1.06789 \mathrm{E} \quad 00$

$1.07754 \mathrm{E} \quad 00$

$1.08724 \mathrm{E} \quad 00$ $1.09698 \mathrm{E} \quad 00$

$1.10677 \mathrm{E} \quad 00$

$1.11660 \mathrm{E} \quad 00$

$1.12647 \mathrm{E} \quad 00$

$1.13639 \mathrm{E} \quad 00$

$1.14634 \mathrm{E} \quad 00$

$1.15635 \mathrm{E} \quad 00$

$1.16639 \mathrm{E} \quad 00$

$1.17648 \mathrm{E} \quad 00$

$1.18661 \mathrm{E} \quad 00$

$1.19678 \mathrm{E} \quad 00$

$1.20700 \mathrm{E} \quad 00$

$1.21726 \mathrm{E} \quad 00$

$1.22757 \mathrm{E} \quad 00$ $g_{2}$

$\tan \delta_{2}$

$f_{2}$

$1.25565 \mathrm{E}-02$

$1.27945 \mathrm{E}-02$

$1.30354 \mathrm{E}-02$

$1.32792 \mathrm{E}-02$

$1.35261 \mathrm{E}-02$

$1.37759 \mathrm{E}-02$

$1.40288 \mathrm{E}-02$

$1.42847 \mathrm{E}-02$

$1.45437 \mathrm{E}-02$

$1.48058 \mathrm{E}-02$

$1.50709 \mathrm{E}-02$

$1.53392 \mathrm{E}-02$

$1.56106 \mathrm{E}-02$

$1.58852 \mathrm{E}-02$

$1.61629 \mathrm{E}-02$

$1.64438 \mathrm{E}-02$

$1.67279 \mathrm{E}-02$

$1.70153 \mathrm{E}-02$

$1.73059 \mathrm{E}-02$

$1.75997 \mathrm{E}-02$

$1.78968 \mathrm{E}-02$

$1.81972 \mathrm{E}-02$

$1.85009 \mathrm{E}-02$

$1.88080 \mathrm{E}-02$

$1.91184 \mathrm{E}-02$

$1.94321 \mathrm{E}-02$

$1.97493 \mathrm{E}-02$

$2.00698 \mathrm{E}-02$

$2.03938 \mathrm{E}-02$

$2.07212 \mathrm{E}-02$

$2.10521 \mathrm{E}-02$

$2.13864 \mathrm{E}-02$

$2.17242 \mathrm{E}-02$

$2.20656 \mathrm{E}-02$

$2.24104 \mathrm{E}-02$

$2.27588 \mathrm{E}-02$

$2.31108 \mathrm{E}-02$

$2.34664 \mathrm{E}-02$

$2.38255 \mathrm{E}-02$

$2.41883 \mathrm{E}-02$

$2.45547 \mathrm{E}-02$

$2.49248 \mathrm{E}-02$

$2.52985 \mathrm{E}-02$

$2.56759 \mathrm{E}-02$

$2.60570 \mathrm{E}-02$

$2.64419 \overrightarrow{\mathrm{E}}-02$

$2.68305 \mathrm{E}-02$

$2.72228 \mathrm{E}-02$

$2.76189 \mathrm{E}-02$

$2.80189 \mathrm{E}-02$

$2.84226 \mathrm{E}-02$

$2.88302 \mathrm{E}-02$

$2.92416 \mathrm{E}-02$

$2.96569 \mathrm{E}-02$

$3.00761 \mathrm{E}-02$

$3.04991 \mathrm{E}-02$

$3.09261 \mathrm{E}-02$

$3.13571 \mathrm{E}-02$

$3.17920 \mathrm{E}-02$

$3.22309 \mathrm{E}-02$

$-3.90379 \mathrm{E}$ 01 $-1.12426 \mathrm{E}-02$ $-3.90428 \mathrm{E} 01$ $-3.90476 \mathrm{E} 01$

$-3.90523 \mathrm{E} \quad 01$

$-3.90570 \mathrm{E} 01$

\section{$-3.90616 \mathrm{E} \quad 01$}

$-3.90662 \mathrm{E} 01$

$-3.90708 \mathrm{E} 01$

$-3.90752 \mathrm{E} 01$

$-3.90797 \mathrm{E} 01$

$-3.90840 \mathrm{E} \quad 01$

$-3.90884 \mathrm{E}$ 0l

$-3.90927 \mathrm{E} 01$

$-3.90969 \mathrm{E} 01$

$-3.91011 \mathrm{E} 01$

$-3.91053 \mathrm{E} 01$

$-3.91094 \mathrm{E} 01$

$-3.91134 \mathrm{E} 01$

$-3.91175 \mathrm{E} \quad 01$

$-3.91214 \mathrm{E} 01$

$-3.91254 \mathrm{E} \quad 01$

$-3.91293 \mathrm{E} 01$

$-3.91331 \mathrm{E} 01$

$-3.91370 \mathrm{E} 01$

$-3.91407 \mathrm{E}$ 0l

$-3.91445 \mathrm{E} \quad 01$

$-3.91482 \mathrm{E} 01$

$-3.91519 \mathrm{E}$ 01

$-3.91555 \mathrm{E} 01$

$-3.91591 \mathrm{E} 01$

$-3.91627 \mathrm{E} \quad 01$

$-3.91662 \mathrm{E} 01$

$-3.91697 \mathrm{E} 01$

$-3.91731 \mathrm{E} 01$

$-3.91766 \mathrm{E} 01$

$-3.91800 \mathrm{E} 01$

$-3.91833 \mathrm{E}$ 01

$-3.91866 \mathrm{E}$ 01

$-3.91899 \mathrm{E} 01$

$\begin{array}{ll}-3.91932 \mathrm{E} & 01\end{array}$

$-3.91964 \mathrm{E} 01$ 
ELECTRON RADIAL FUNCTIONS

Positron

\begin{tabular}{|c|c|c|c|}
\hline$p$ & $F_{0}$ & $f_{1}$ & \\
\hline 24.10 & $8.93838 \mathrm{E}-01$ & $2.25672 \mathrm{E}$ & 01 \\
\hline 24.20 & $8.93834 \mathrm{E}-0 \mathrm{l}$ & $2.26645 \mathrm{E}$ & 01 \\
\hline 24.30 & $8.93829 \mathrm{E}-01$ & $2.27619 \mathrm{E}$ & 01 \\
\hline 24.40 & $8.93825 \mathrm{E}-01$ & $2.28593 \mathrm{E}$ & 01 \\
\hline 24.50 & $8.93821 \mathrm{E}-01$ & $2.29567 \mathrm{E}$ & 01 \\
\hline 24.60 & $8.93817 \mathrm{E}-01$ & $2.30541 \mathrm{E}$ & 01 \\
\hline 24.70 & $8.93813 \mathrm{E}-01$ & $2.31516 \mathrm{E}$ & 01 \\
\hline 24.80 & $8.93809 \mathrm{E}-01$ & $2.32491 \mathrm{E}$ & 01 \\
\hline 24.90 & $8.93805 \mathrm{E}-01$ & $2.33466 \mathrm{E}$ & 01 \\
\hline 25.00 & $8.93801 \mathrm{E}-01$ & $2.34441 \mathrm{E}$ & 01 \\
\hline 25.10 & $8.93797 \mathrm{E}-01$ & $2.35416 \mathrm{E}$ & 01 \\
\hline 25.20 & $8.93792 \mathrm{E}-01$ & $2.36392 \mathrm{E}$ & 01 \\
\hline 25.30 & $8.93788 \mathrm{E}-01$ & $2.37367 \mathrm{E}$ & 01 \\
\hline 25.40 & $8.93784 \mathrm{E}-01$ & $2.38343 \mathrm{E}$ & 01 \\
\hline 25.50 & $8.93780 \mathrm{E}-01$ & $2.39320 \mathrm{E}$ & 01 \\
\hline 25.60 & $8.93776 \mathrm{E}-01$ & $2.40296 \mathrm{E}$ & 01 \\
\hline 25.70 & $8.93772 \mathrm{E}-01$ & $2.41273 \mathrm{E}$ & 01 \\
\hline 25.80 & $8.93768 \mathrm{E}-01$ & $2.42249 \mathrm{E}$ & 01 \\
\hline 25.90 & $8.93764 \mathrm{E}-01$ & $2.43226 \mathrm{E}$ & 01 \\
\hline 26.00 & $.8 .93760 \mathrm{E}-01$ & $2.44204 \mathrm{E}$ & 01 \\
\hline 26.10 & $8.93756 \mathrm{E}-01$ & $2.45181 \mathrm{E}$ & 01 \\
\hline 26.20 & $8.93752 \mathrm{E}-01$ & $2.46159 \mathrm{E}$ & 01 \\
\hline 26.30 & $8.93749 \mathrm{E}-01$ & $2.47137 \mathrm{E}$ & 01 \\
\hline 26.40 & $8.93745 \mathrm{E}-01$ & $2.48115 \mathrm{E}$ & 01 \\
\hline 26.50 & $8.93741 \mathrm{E}-01$ & $2.49093 \mathrm{E}$ & 01 \\
\hline 26.60 & $8.93737 \mathrm{E}-01$ & $2.50072 \mathrm{E}$ & 01 \\
\hline 26.70 & $8.93733 \mathrm{E}-01$ & $2.51050 \mathrm{E}$ & 01 \\
\hline 26.80 & $8.93729 \mathrm{E}-01$ & $2.52029 \mathrm{E}$ & 01 \\
\hline 26.90 & $8.93725 \mathrm{E}-01$ & $2.53008 \mathrm{E}$ & 01 \\
\hline 27.00 & $8.93721 \mathrm{E}-01$ & $2.53988 \mathrm{E}$ & 01 \\
\hline 27.10 & $8.93717 \mathrm{E}-01$ & $2.54967 \mathrm{E}$ & 01 \\
\hline 27.20 & $8.93713 \mathrm{E}-01$ & $2.55947 \mathrm{E}$ & 01 \\
\hline 27.30 & $8.93710 \mathrm{E}-01$ & $2.56927 \mathrm{E}$ & 01 \\
\hline 27.40 & $8.93706 \mathrm{E}-01$ & $2.57908 \mathrm{E}$ & 01 \\
\hline 27.50 & $8.93702 \mathrm{E}-01$ & $2.58888 \mathrm{E}$ & 01 \\
\hline 27.60 & $8.93698 \mathrm{E}-01$ & $2.59869 \mathrm{E}$ & 01 \\
\hline 27.70 & $8.93694 \mathrm{E}-01$ & $2.60850 \mathrm{E}$ & 01 \\
\hline 27.80 & $8.93691 \mathrm{E}-01$ & $2.61831 \mathrm{E}$ & 01 \\
\hline 27.90 & $8.93687 \mathrm{E}-01$ & $2.62812 \mathrm{E}$ & 01 \\
\hline 28.00 & $8.93683 \mathrm{E}-01$ & $2.63794 \mathrm{E}$ & 01 \\
\hline 28.10 & $8.93679 \mathrm{E}-01$ & $2.64776 \mathrm{E}$ & 01 \\
\hline 28.20 & $8.93675 \mathrm{E}-01$ & $2.65758 \mathrm{E}$ & 01 \\
\hline 28.30 & $8.93672 \mathrm{E}-01$ & $2.66740 \mathrm{E}$ & 01 \\
\hline 28.40 & $8.93668 \mathrm{E}-01$ & $2.67723 \mathrm{E}$ & 01 \\
\hline 28.50 & $8.93664 \mathrm{E}-01$ & $2.68706 \mathrm{E}$ & 01 \\
\hline 28.60 & $8.93660 \mathrm{E}-01$ & $2.69689 \mathrm{E}$ & 01 \\
\hline 28.70 & $8.93657 \mathrm{E}-01$ & $2.70672 \mathrm{E}$ & 01 \\
\hline 28.80 & $8.93653 \mathrm{E}-01$ & $2.71656 \mathrm{E}$ & 01 \\
\hline 28.90 & $8.93649 \mathrm{E}-01$ & $2.72639 \mathrm{E}$ & 01 \\
\hline 29.00 & $8.93646 \mathrm{E}-01$ & $2.73623 \mathrm{E}$ & 01 \\
\hline 29.10 & $8.93642 \mathrm{E}-01$ & $2.74607 \mathrm{E}$ & 01 \\
\hline 29.20 & $8.93638 E-01$ & $2.75592 \mathrm{E}$ & 01 \\
\hline 29.30 & $8.93635 \mathrm{E}-01$ & $2.76577 \mathrm{E}$ & 01 \\
\hline 29.40 & $8.93631 \mathrm{E}-01$ & $2.77561 \mathrm{E}$ & 01 \\
\hline 29.50 & $8.93628 \mathrm{E}-01$ & $2.78547 \mathrm{E}$ & 01 \\
\hline 29.60 & $8.93624 \mathrm{E}-01$ & $2.79532 \mathrm{E}$ & 01 \\
\hline 29.70 & $8.93620 \mathrm{E}-01$ & $2.80518 \mathrm{E}$ & 01 \\
\hline 29.80 & $8.93617 \mathrm{E}-01$ & $2.81504 \mathrm{E}$ & 01 \\
\hline 29.90 & $8.93613 \mathrm{E}-01$ & $2.82490 \mathrm{E}$ & 01 \\
\hline 30.00 & $8.93610 \mathrm{E}-01$ & $2.83476 \mathrm{E}$ & 01 \\
\hline
\end{tabular}

$Z=5 \quad A=11 \quad \rho=1.2 A^{1 / 3} F$

\begin{tabular}{|c|c|c|c|}
\hline$g_{1}$ & $\tan \delta_{1}$ & $f_{-1}$ & $g_{-1}$ \\
\hline $9.77578 \mathrm{E}-01$ & $-9.44656 \mathrm{E}-04$ & $-9.11433 \mathrm{E}-01$ & $2.35474 \mathrm{E}$ \\
\hline $9.87028 \mathrm{E}-01$ & $-9.47023 \mathrm{E}-04$ & $-9.20670 \mathrm{E}-01$ & $2.36450 \mathrm{E}$ \\
\hline $9.96525 \mathrm{E}-01$ & $-9.49359 E-04$ & $-9.29953 \mathrm{E}-01$ & $2.37426 \mathrm{E}$ \\
\hline $1.00607 \mathrm{E} \quad 00$ & $-9.51670 \mathrm{E}-04$ & $-9.39282 \mathrm{E}-01$ & $2.38403 \mathrm{E}$ \\
\hline $1.01566 \mathrm{E}$ & $-9.53953 \mathrm{E}-04$ & $-9.48658 \mathrm{E}-01$ & $2.39380 \mathrm{E}$ \\
\hline $1.02529 \mathrm{E} \quad 00$ & $-9.56204 \mathrm{E}-04$ & $-9.58079 \mathrm{E}-01$ & $2.40357 \mathrm{E}$ \\
\hline $1.03497 \mathrm{E}$ & $-9.58433 \mathrm{E}-04$ & $-9.67547 \mathrm{E}-01$ & $2.41334 \mathrm{E}$ \\
\hline $1.04470 \mathrm{E}$ & $-9.60635 \mathrm{E}-04$ & $-9.77061 \mathrm{E}-01$ & $2.42312 \mathrm{E}$ \\
\hline $1.05447 \mathrm{E}$ & $-9.62810 \mathrm{E}-04$ & $-9.86621 \mathrm{E}-01$ & $2.43290 \mathrm{E}$ \\
\hline & $-9.64954 \mathrm{E}-04$ & $-9.96227 \mathrm{E}-01$ & $2.44268 \mathrm{E}$ \\
\hline
\end{tabular}

$1.07415 \mathrm{E} \quad 00 \quad-9.67076 \mathrm{E}-04$

$1.08407 \mathrm{E} \quad 00 \quad-9.69170 \mathrm{E}-04$.

$1.09402 \mathrm{E} \quad 00 \quad-9.71243 \mathrm{E}-04$

$\begin{array}{lll}1.10403 \mathrm{E} & 00 & -9.73285 \mathrm{E}-04\end{array}$

$1.11408 \mathrm{E} \quad 00 \quad-9.75301 \mathrm{E}-04$.

$1.12417 \mathrm{E} \quad 00 \quad-9.77298 \mathrm{E}-04$

$1.13432 \mathrm{E} \quad 00 \quad-9.79264 \mathrm{E}-04$

$1.14451 \mathrm{E} \quad 00 \quad-9.81207 \mathrm{E}-04$

$1.15474 \mathrm{E} \quad 00 \quad-9.83131 \mathrm{E}-04$

$1.16503 \mathrm{E} \quad 00 \quad-9.85026 \mathrm{E}-04$

$1.17535 \mathrm{E} \quad 00 \quad-9.86896 \mathrm{E}-04$

$1.18573 \mathrm{E} \quad 00 \quad-9.88744 \mathrm{E}-04$.

$1.19615 \mathrm{E} \quad 00 \quad-9.90571 \mathrm{E}-04$

$1.20662 \mathrm{E} \quad 00 \quad-9.92374 \mathrm{E}-04$

$1.21713 \mathrm{E} \quad 00 \quad-9.94153 \mathrm{E}-04$

$1.22769 \mathrm{E} \quad 00 \quad-9.95910 \mathrm{E}-04$

$1.23830 \mathrm{E} \quad 00 \quad-9.97647 \mathrm{E}-04$

$1.24896 \mathrm{E} \quad 00 \quad-9.99356 \mathrm{E}-04$

$1.25966 \mathrm{E} \quad 00-1.00105 \mathrm{E}-03$

$1.27040 \mathrm{E} \quad 00-1.00272 \mathrm{E}-03$

$1.28120 \mathrm{E} \quad 00-1.00436 \mathrm{E}-03$

$1.29204 \mathrm{E} \quad 00 \quad-1.00599 \mathrm{E}-03$

$1.30293 \mathrm{E} \quad 00-1.00760 \mathrm{E}-03$

$1.31386 \mathrm{E} \quad 00-1.00918 \mathrm{E}-03$

$1.32484 \mathrm{E} \quad 00-1.01074 \mathrm{E}-03$

$1.33587 \mathrm{E} \quad 00 \quad-1.01229 \mathrm{E}-03$

$1.34694 \mathrm{E} \quad 00-1.01381 \mathrm{E}-03$

$1.35806 \mathrm{E} \quad 00 \quad-1.01531 \mathrm{E}-03$

$1.36923 \mathrm{E} \quad 00-1.01680 \mathrm{E}-03$

$\begin{array}{lll}1.38045 \mathrm{E} & 00 & -1.01826 \mathrm{E}-03\end{array}$

$1.39171 \mathrm{E} \quad 00 \quad-1.01970 \mathrm{E}-03$

$\begin{array}{lll}1.40302 \mathrm{E} & 00 & -1.02112 \mathrm{E}-03\end{array}$

$\begin{array}{lll}1.41437 \mathrm{E} & 00 & -1.02253 \mathrm{E}-03\end{array}$

$1.42578 \mathrm{E} \quad 00-1.02392 \mathrm{E}-03$

$1.43723 \mathrm{E} \quad 00-1.02528 \mathrm{E}-03$

$1.44872 \mathrm{E} \quad 00 \quad-1.02663 \mathrm{E}-03$

$1.46027 \mathrm{E} \quad 00-1.02796 \mathrm{E}-03$

$\begin{array}{lll}1.47186 \mathrm{E} & 00 & -1.02927 \mathrm{E}-03\end{array}$

$1.48350 \mathrm{E} \quad 00-1.03056 \mathrm{E}-03$

$1.49518 \mathrm{E} \quad 00-1.03183 \mathrm{E}-03$

$1.50691 \mathrm{E} \quad 00 \quad-1.03309 \mathrm{E}-03$

$1.51869 \mathrm{E} \quad 00-1.03433 \mathrm{E}-03$

$\begin{array}{lll}1.53052 \mathrm{E} & 00-1.03555 \mathrm{E}-03\end{array}$

$1.54239 \mathrm{E} \quad 00-1.03675 \mathrm{E}-03$

$\begin{array}{lll}1.55431 \mathrm{E} & 00 & -1.03794 \mathrm{E}-03\end{array}$

$1.56628 \mathrm{E} \quad 00-1.03910 \mathrm{E}-03$

$1.57830 \mathrm{E} \quad 00-1.04026 \mathrm{E}-03$

$\begin{array}{lll}1.59036 \mathrm{E} & 00 & -1.04139 \mathrm{E}-03\end{array}$

$1.60247 \mathrm{E} \quad 00 \quad-1.04251 \mathrm{E}-03$

$1.61463 \mathrm{E} \quad 00-1.04361 \mathrm{E}-03$
$-1.00588 \mathrm{E} 00$

$-1.01558 \mathrm{E} \quad 00$

$-1.02532 \mathrm{E} 00$

$-1.03512 \mathrm{E} \quad 00$

$-1.04495 \mathrm{E} 00$

$-1.05484 \mathrm{E} \quad 00$

$-1.06477 \mathrm{E} \quad 00$

$-1.07475 \mathrm{E} \quad 00$

$-1.08477 \mathrm{E} 00$

$-1.09484 \mathrm{E} \quad 00$

$-1.10496 \mathrm{E} \quad 00$ $-1.11512 \mathrm{E} 00$

$-1.12533 \mathrm{E} \quad 00$

$-1.13559 \mathrm{E} 00$

-1.14589 E 00

$-1.15624 \mathrm{E} \quad 00$

$-1.16663 \mathrm{E} \quad 00$

$-1.17708 \mathrm{E} 00$

$-1.18757 \mathrm{E} 00$

$-1.19810 \mathrm{E} 00$

$-1.20869 \mathrm{E} \quad 00$

$-1.21932 \mathrm{E} \quad 00$

$-1.22999 \mathrm{E} 00$

$-1.24071 \mathrm{E} 00$

$-1.25149 \mathrm{E} 00$

$-1.26230 \mathrm{E} \quad 00$ $-1.27317 \mathrm{E} 00$ $-1.28408 \mathrm{E} 00$ $-1.29503 \mathrm{E} 00$ $-1.30604 \mathrm{E} 00$

$-1.31709 \mathrm{E} 00$ $-1.32819 \mathrm{E} 00$ $-1.33933 \mathrm{E} 00$ $-1.35053 \mathrm{E} 00$ $-1.36177 \mathrm{E} 00$

$-1.37305 \mathrm{E} \quad 00$ $-1.38439 \mathrm{E} 00$ $-1.39577 \mathrm{E} 00$ $-1.40720 \mathrm{E} 00$ -1.41867 E 00

$-1.43019 \mathrm{E} \quad 00$ $-1.44176 \mathrm{E} \quad 00$ $-1.45338 \mathrm{E} 00$ $-1.46505 \mathrm{E} \quad 00$ $-1.47676 \mathrm{E} 00$

$-1.48852 \mathrm{E} \quad 00$ $-1.50032 \mathrm{E} \quad 00$ $-1.51218 \mathrm{E} 00$ $-1.52408 \mathrm{E} \quad 00$ $-1.53603 \mathrm{E} 00$
$2.45246 \mathrm{E} \quad 01$ $2.46224 \mathrm{E} \quad 01$ $2.47203 \mathrm{E}$ 01 $2.48182 \mathrm{E} 01$ $2.49161 \mathrm{E} 01$

$2.50140 \mathrm{E} \quad 01$ $2.51120 \mathrm{E} \quad 01$ $2.52099 \mathrm{E} \quad 01$ $2.53079 \mathrm{E} 01$ $2.54060 \mathrm{E} \quad 01$

$2.55040 \mathrm{E} \quad 01$ $2.56021 \mathrm{E} 01$ $2.57001 \mathrm{E} \quad 01$ $2.57982 \mathrm{E} \quad 01$ $2.58964 \mathrm{E} \quad 01$

$2.59945 \mathrm{E} \quad 01$ $2.60927 \mathrm{E} \quad 01$ $2.61909 \mathrm{E} 01$ $2.62891 \mathrm{E} \quad 01$ $2.63874 \mathrm{E}$ 0l

$2.64856 \mathrm{E} \quad 01$ $2.65839 \mathrm{E} \quad 01$ $2.66822 \mathrm{E} \quad 01$ $2.67806 \mathrm{E}$ 01 $2.68789 \mathrm{E} \quad 01$

$2.69773 \mathrm{E} \quad 01$ $2.70757 \mathrm{E} \quad 01$ $2.71742 \mathrm{E} \quad 01$ $2.72726 \mathrm{E} \quad 01$ $2.73711 \mathrm{E} \quad 01$

$2.74696 \mathrm{E} \quad 01$ $2.75681 \mathrm{E} \quad 01$ $2.76667 \mathrm{E} \quad 01$ $2.77652 \mathrm{E} \quad 01$ $2.78638 \mathrm{E} \quad 01$

$2.79625 \mathrm{E} \quad 01$ $2.80611 \mathrm{E} 01$ $2.81598 \mathrm{E} \quad 01$ $2.82585 \mathrm{E} \quad 01$ 2.83572 E 01

$2.84559 \mathrm{E} \quad 01$ $2.85547 \mathrm{E} \quad 01$ $2.86535 \mathrm{E} \quad 01$ $2.87523 \mathrm{E} 01$ $2.88512 \mathrm{E}$ ol

$2.89500 \mathrm{E} \quad 01$ $2.90489 \mathrm{E} \quad 01$ $2.91479 \mathrm{E} \quad 01$ $2.92468 \mathrm{E} \quad 01$ $2.93458 \mathrm{E} \quad 01$ $\tan \delta_{-1}$

$\begin{array}{lll}4.07961 \mathrm{E} & 02\end{array}$ $4.08615 \mathrm{E} \quad 02$ $4.09267 \mathrm{E} \quad 02$ $4.09917 \mathrm{E} \quad 02$ $4.10565 \mathrm{E} \quad 02$

$4.11212 \mathrm{E} \quad 02$ $4.11857 \mathrm{E} \quad 02$ $4.12500 \mathrm{E} \quad 02$ $4.13142 \mathrm{E} \quad 02$ 4.13783E 02

$4.14421 \mathrm{E} \quad 02$ $4.15059 \mathrm{E} \quad 02$ $4.15694 \mathrm{E} \quad 02$ $4.16328 \mathrm{E} \quad 02$ $4.16962 \mathrm{E} \quad 02$

$4.17593 \mathrm{E} \quad 02$ $4.18223 \mathrm{E} \quad 02$ $4.18853 \mathrm{E} \quad 02$ 4.19479E 02 $4.20106 \mathrm{E} \quad 02$

$4.20731 \mathrm{E} \quad 02$ $4.21355 \mathrm{E} \quad 02$ $4.21977 \mathrm{E} \quad 02$ $4.22598 \mathrm{E} \quad 02$ $4.23218 \mathrm{E} \quad 02$

$4.23837 \mathrm{E} \quad 02$ $4.24455 \mathrm{E} \quad 02$ $4.25072 \mathrm{E} \quad 02$ $4.25687 \mathrm{E} \quad 02$ $4.26301 \mathrm{E} \quad 02$

$4.26915 \mathrm{E} \quad 02$ $4.27527 \mathrm{E} \quad 02$ $4.28139 \mathrm{E} \quad 02$ $4.28748 \mathrm{E} \quad 02$ $4.29358 \mathrm{E} \quad 02$

$4.29966 \mathrm{E} \quad 02$ 4.30574. E 02 $4.31181 \mathrm{E} \quad 02$ $4.31786 \mathrm{E} \quad 02$ $4.32390 \mathrm{E} \quad 02$

$4.32995 \mathrm{E} \quad 02$ $4.33598 \mathrm{E} \quad 02$ $4.34200 \mathrm{E} \quad 02$ $4.34801 \mathrm{E} \quad 02$ $4.35402 \mathrm{E} \quad 02$

$4.36002 \mathrm{E} \quad 02$ $4.36601 \mathrm{E} \quad 02$ $4.37199 \mathrm{E} \quad 02$ $4.37797 \mathrm{E} \quad 02$ 4.38394E 02

$4.38990 \mathrm{E} \quad 02$ $4.39586 \mathrm{E} \quad 02$ $4.40181 \mathrm{E} \quad 02$ $4.40776 \mathrm{E} \quad 02$ $4.41369 \mathrm{E} \quad 02$

$4.41963 \mathrm{E} \quad 02$ $4.42555 \mathrm{E} \quad 02$ $4.43148 \mathrm{E} \quad 02$ $4.43739 \mathrm{E} \quad 02$ $4.44330 \mathrm{E} \quad 02$ 
$\bar{F}$

$6.88175 \mathrm{E}-04$ $6.93897 \mathrm{E}-04$ $6.99642 \mathrm{E}-04$ $7.05411 \mathrm{E}-04$.

$7.11203 \mathrm{E}-04$

$7.17020 \mathrm{E}-04$ $7.22860 \mathrm{E}-04$ $7.28723 \mathrm{E}-04$

$7.34610 \mathrm{E}-04$

$7.40521 \mathrm{E}-04$

$7.46456 \mathrm{E}-04$

$7.52414 \mathrm{E}-04$

$7.58396 \mathrm{E}-04$

$7.64402 \mathrm{E}-04$

7.70431 E-04

$7.76484 \mathrm{E}-04$

$7.82560 \mathrm{E}-04$

$7.88661 \mathrm{E}-04$

$7.94785 \mathrm{E}-04$

$8.00932 \mathrm{E}-04$

8.07103E-04

$8.13298 \mathrm{E}-04$

$8.19517 \mathrm{E}-04$

$8.25759 \mathrm{E}-04$

8.32025 E-04

$8.38315 \mathrm{E}-04$

$8.44628 \mathrm{E}-04$

$8.50966 \mathrm{E}-04$

$8.57326 \mathrm{E}-04$

$8.63711 \mathrm{E}-04$

$8.70119 \mathrm{E}-04$

8.76551 E-04

$8.83006 \mathrm{E}-04$

$8.89485 \mathrm{E}-04$

$8.95988 \mathrm{E}-04$

$9.02514 \mathrm{E}-04$

$9.09064 \mathrm{E}-04$

$9.15638 \mathrm{E}-04$

$9.22236 \mathrm{E}-04$

$9.28857 \mathrm{E}-04$

$9.35501 \mathrm{E}-04$

$9.42170 \mathrm{E}-04$

$9.48862 \mathrm{E}-04$

$9.55578 \mathrm{E}-04$

$9.62317 \mathrm{E}-04$

$9.69081 \mathrm{E}-04$

$9.75867 \mathrm{E}-04$

$9.82678 \mathrm{E}-04$

$9.89512 \mathrm{E}-04$

$9.96370 \mathrm{E}-04$

$1.00325 \mathrm{E}-03$

$1.01016 \mathrm{E}-03$

$1.01709 \mathrm{E}-03$

$1.02404 \mathrm{E}-03$

$1.03101 \mathrm{E}-03$

$1.03801 \mathrm{E}-03$

$1.04504 \mathrm{E}-03$

$1.05209 \mathrm{E}-03$

$1.05916 \mathrm{E}-03$

$1.06625 \mathrm{E}-03$ $f_{2}$

$\tan \delta_{2}$

$f_{-2}$

b

$\tan \delta$

$1.23792 \mathrm{E} \quad 00$

$1.24831 \mathrm{E} \quad 00$

$1.25874 \mathrm{E} \quad 00$

$1.26922 \mathrm{E} \quad 00$

$1.27974 \mathrm{E} \quad 00$

$1.29030 \mathrm{E} \quad 00$

$1.30091 \mathrm{E} \quad 00$

$1.31155 \mathrm{E} \quad 00$

$1.32225 \mathrm{E} \quad 00$

$1.33298 \mathrm{E} \quad 00$

$1.34376 \mathrm{E} \quad 00$

$1.35458 \mathrm{E} \quad 00$

$1.36545 \mathrm{E} \quad 00$

$1.37636 \mathrm{E} \quad 00$

$1.38731 \mathrm{E} \quad 00$

$1.39830 \mathrm{E} \quad 00$ $1.40934 \mathrm{E} \quad 00$

$1.42042 \mathrm{E} \quad 00$

$1.43154 \mathrm{E} \quad 00$

$1.44271 \mathrm{E} \quad 00$

$1.45392 \mathrm{E} \quad 00$

$1.46517 \mathrm{E} \quad 00$

$\begin{array}{ll}1.47647 \mathrm{E} \quad 00 & 00\end{array}$

$1.48781 \mathrm{E} \quad 00$

$1.49919 \mathrm{E} \quad 00$

$1.51061 \mathrm{E} \quad 00$

$1.52208 \mathrm{E} \quad 00$

$1.53359 \mathrm{E} \quad 00$

$1.54515 \mathrm{E} \quad 00$

$1.55675 \mathrm{E} \quad 00$

$1.56839 \mathrm{E} \quad 00$

$1.58007 \mathrm{E} \quad 00$

$1.59180 \mathrm{E} \quad 00$

$1.60357 \mathrm{E} \quad 00$

$1.61538 \mathrm{E} \quad 00$

$1.62723 \mathrm{E} \quad 00$ $1.63913 \mathrm{E} \quad 00$ $1.65107 \mathrm{E} \quad 00$ $1.66306 \mathrm{E} \quad 00$ $1.67509 \mathrm{E} \quad 00$

$1.68716 \mathrm{E} \quad 00$ $1.69927 \mathrm{E} \quad 00$

$1.71143 \mathrm{E} \quad 00$ $1.72363 \mathrm{E} \quad 00$ $1.73587 \mathrm{E} \quad 00$

$1.74815 \mathrm{E} \quad 00$ $1.76048 \mathrm{E} \quad 00$ $1.77285 \mathrm{E} \quad 00$ $1.78527 \mathrm{E} \quad 00$ $1.79772 \mathrm{E} \quad 00$

$1.81022 \mathrm{E} \quad 00$ $1.82277 \mathrm{E} \quad 00$ $1.83535 \mathrm{E} \quad 00$ $1.84798 \mathrm{E} \quad 00$ $1.86065 \mathrm{E} \quad 00$

$\begin{array}{ll}1.87337 \mathrm{E} & 00\end{array}$ $1.88612 \mathrm{E} \quad 00$ $1.89892 \mathrm{E} \quad 00$ $1.91177 \mathrm{E} \quad 00$ $1.92465 \mathrm{E} \quad 00$
$3.26738 \mathrm{E}-02$

$3.31206 \mathrm{E}-02$

$3.35715 \mathrm{E}-02$

$3.40265 \mathrm{E}-02$

$3.44855 \mathrm{E}-02$

$3.49486 \mathrm{E}-02$

$3.54158 \mathrm{E}-02$

$3.58871 \mathrm{E}-02$

$3.63626 \mathrm{E}-02$

$3.68422 \mathrm{E}-02$

$3.73260 \mathrm{E}-02$

$3.78139 \mathrm{E}-02$

$3.83061 \mathrm{E}-02$

$3.88025 \mathrm{E}-02$

$3.93032 \mathrm{E}-02$

$3.98081 \mathrm{E}-02$

$4.03173 \mathrm{E}-02$

$4.08308 \mathrm{E}-02$

$4.13486 \mathrm{E}-02$

$4.18707 \mathrm{E}-02$

$4.23972 \mathrm{E}-02$ $4.29280 \mathrm{E}-02$

$4.34633 \mathrm{E}-02$

$4.40029 \mathrm{E}-02$

$4.45470 \mathrm{E}-02$

$4.50955 \mathrm{E}-02$

$4.56485 \mathrm{E}-02$

$4.62059 \mathrm{E}-02$

$4.67678 \mathrm{E}-02$

$4.73343 \mathrm{E}-02$

$4.79052 \mathrm{E}-02$

$4.84808 \mathrm{E}-02$

$4.90608 \mathrm{E}-02$

$4.96455 \mathrm{E}-02$

$5.02348 \mathrm{E}-02$

$5.08287 \mathrm{E}-02$

$5.14272 \mathrm{E}-02$

$5.20303 \mathrm{E}-02$

$5.26382 \mathrm{E}-02$

$5.32507 \mathrm{E}-02$

$5.38680 \mathrm{E}-02$

$5.44900 \mathrm{E}-02$

$5.51166 \mathrm{E}-02$

$5.57481 \mathrm{E}-02$

$5.63844 \mathrm{E}-02$

$5.70254 \mathrm{E}-02$

$5.76713 \mathrm{E}-02$

$5.83220 \mathrm{E}-02$

$5.89775 \mathrm{E}-02$

$5.96379 \mathrm{E}-02$

$6.03032 \mathrm{E}-02$ $6.09734 \mathrm{E}-02$ $6.16485 \mathrm{E}-02$ $6.23286 \mathrm{E}-02$ $6.30136 \mathrm{E}-02$

$6.37035 \mathrm{E}-02$ $6.43985 \mathrm{E}-02$ $6.50985 \mathrm{E}-02$ $6.58035 \mathrm{E}-02$ $6.65136 \mathrm{E}-02$

\begin{abstract}
$-3.92553 \mathrm{E} \quad 01$ $-3.92580 \mathrm{E} 01$ $-3.92606 \mathrm{E} 01$ $-3.92633 \mathrm{E} 01$ $-3.92659 \mathrm{E} 01$
\end{abstract}

$-3.04892 \mathrm{E}-02$ $-3.09198 \mathrm{E}-02$ $-3.13543 \mathrm{E}-02$ $-3.17928 \mathrm{E}-02$ $-3.22353 \mathrm{E}-02$

$-3.92685 \mathrm{E}$ 01 $-3.92710 \mathrm{E}$ 0I $-3.92736 \mathrm{E}$ 01 $-3.92761 \mathrm{E}$ 01 $-3.92786 \mathrm{E}$ 0I

$-3.92811 \mathrm{E}$ 01

$-3.92836 \mathrm{E} 01$

$-3.92860 \mathrm{E}$ 01

$-3.92884 \mathrm{E}$ 0l

$-3.92908 \mathrm{E}$ 0l

$-3.92932 \mathrm{E} \quad 01$ $-3.92956 \mathrm{E}$ 0l $-3.92979 \mathrm{E}$ ol $-3.93002 \mathrm{E}$ 0l $-3.93026 \mathrm{E} \quad 01$

$-3.93049 \mathrm{E} 01$ $-3.93071 \mathrm{E} 01$ $-3.93094 \mathrm{E}$ 0I $-3.93116 \mathrm{E}$ OI $-3.93138 \mathrm{E}$ ol

$-3.93160 \mathrm{E}$ 01 $-3.93182 \mathrm{E}$ 01

$-3.93204 \mathrm{E}$ ol

$-3.93225 \mathrm{E} 01$

$-3.93247 \mathrm{E}$ 01

$-3.93268 \mathrm{E} 01$

$-3.93289 \mathrm{E}$ ol

$-3.93310 \mathrm{E}$ 0I

$-3.93331 \mathrm{E}$ ol

$-3.93351 \mathrm{E}$ 0l

$\begin{array}{ll}-3.93372 \mathrm{E} & 01\end{array}$ $-3.93392 \mathrm{E}$ 0l $-3.93412 \mathrm{E}$ ol $-3.93432 \mathrm{E}$ 0l $-3.93452 \mathrm{E} 01$

$-3.93471 \mathrm{E} 01$

$-3.93491 \mathrm{E}$ 01

$-3.93510 \mathrm{E}$ ol

$-3.93529 \mathrm{E}$ 0I

$-3.93548 \mathrm{E}$ ol

$-3.93567 \mathrm{E} \quad 01$

$-3.93586 \mathrm{E}$ 0I

$-3.93605 \mathrm{E}$ 01

$-3.93623 \mathrm{E}$ 01

$-3.93642 \mathrm{E} 01$

$-3.26818 \mathrm{E}-02$

$-3.31324 \mathrm{E}-02$

$-3.35870 \mathrm{E}-02$

$-3.40457 \mathrm{E}-02$

$-3.45085 \mathrm{E}-02$

$-3.49754 \mathrm{E}-02$

$-3.54464 \mathrm{E}-02$

$-3.59216 \mathrm{E}-02$

$-3.64010 \mathrm{E}-02$

$-3.68845 \mathrm{E}-02$

$-3.73723 \mathrm{E}-02$

$-3.78642 \mathrm{E}-02$

$-3.83604 \mathrm{E}-02$

$-3.88609 \mathrm{E}-02$

$-3.93656 \mathrm{E}-02$

$-3.98746 \mathrm{E}-02$

$-4.03879 \mathrm{E}-02$

$-4.09056 \mathrm{E}-02$

$-4.14276 \mathrm{E}-02$

$-4.19539 \mathrm{E}-02$

$-4.24847 \mathrm{E}-02$

$-4.30198 \mathrm{E}-02$

$-4.35594 \mathrm{E}-02$

$-4.41033 \mathrm{E}-02$

$-4.46518 \mathrm{E}-02$

$-4.52047 \mathrm{E}-02$

$-4.57621 \mathrm{E}-02$

$-4.63240 \mathrm{E}-02$

$-4.68904 \mathrm{E}-02$

$-4.74614 \mathrm{E}-02$

$-4.80369 \mathrm{E}-02$

$-4.86170 \mathrm{E}-02$

$-4.92017 \mathrm{E}-02$

$-4.97910 \mathrm{E}-02$

$-5.03849 \mathrm{E}-02$

$-5.09835 \mathrm{E}-02$

$-5.15867 \mathrm{E}-02$

$-5.21947 \mathrm{E}-02$

$-5.28073 \mathrm{E}-02$

$-5.34247 \mathrm{E}-02$

$-5.40467 \mathrm{E}-02$

$-5.46736 \mathrm{E}-02$

$-5.53052 \mathrm{E}-02$

$-5.59416 \mathrm{E}-02$

$-5.65828 \mathrm{E}-02$

$-3.93660 \mathrm{E} 01$ 
ELECTRON RADIAL FUNCTIONS

Positron

$\begin{array}{cc}p & F_{0} \\ 30.10 & 8.93606 \mathrm{E}-01 \\ 30.20 & 8.93602 \mathrm{E}-01 \\ 30.30 & 8.93599 \mathrm{E}-01 \\ 30.40 & 8.93595 \mathrm{E}-01 \\ 30.50 & 8.93592 \mathrm{E}-01\end{array}$

30.60

30.70

30.80

30.90

31.00

31.10

31.20

31.30

31.40

31.50

31.60

31.70

31.80

31.90

32.00

32.10

32.20

32.30

32.40

32.50

32.60

32.70

32.80

32.90

33.00

33.10

33.20

33.30

33.40

33.50

33.60

33.70

33.80

33.90

34.00

34.10

34.20

34.30

34.40

34.50

34.60

34.70

34.80

34.90

35.00

35.10

35.20

35.30

35.40

35.50

35.60

35.70

35.80

35.90

36.00

$8.93588 \mathrm{E}-01$ $8.93585 \mathrm{E}-01$ $8.93581 \mathrm{E}-01$ $8.93578 \mathrm{E}-\mathrm{cl}$ $8.93574 \mathrm{E}-01$

$8.93571 \mathrm{E}-01$ $8.93567 \mathrm{E}-01$ $8.93564 \mathrm{E}-01$ $8.93560 \mathrm{E}-01$ $8.93557 \mathrm{E}-01$

$8.93554 \mathrm{E}-01$ $8.93550 \mathrm{E}-01$ $8.93547 \mathrm{E}-01$ $8.93543 \mathrm{E}-01$ $8.93540 \mathrm{E}-01$

$8.93536 \mathrm{E}-01$ $8.93533 \mathrm{E}-01$ $8.93530 \mathrm{E}-01$ $8.93526 \mathrm{E}-01$ 8.93523 E-01

$8.93520 \mathrm{E}-0 \mathrm{I}$ $8.93516 \mathrm{E}-01$ $8.93513 \mathrm{E}-01$ $8.93510 \mathrm{E}-01$ $8.93506 \mathrm{E}-01$

$8.93503 \mathrm{E}-01$ $8.93500 \mathrm{E}-01$ $8.93496 \mathrm{E}-01$ $8.93493 \mathrm{E}-01$ $8.93490 \mathrm{E}-01$

$8.93487 \mathrm{E}-01$ $8.93483 \mathrm{E}-01$ $8.93480 \mathrm{E}-01$ $8.93477 \mathrm{E}-01$ $8.93474 \mathrm{E}-01$

$8.93470 \mathrm{E}-01$ $8.93467 \mathrm{E}-01$ $8.93464 \mathrm{E}-01$ $8.934 .61 \mathrm{E}-01$ 8.93458E-01

8.93454:E-01 $8.93451 \mathrm{E}-01$ $8.93448 \mathrm{E}-01$ 8.93445 E-01 $8.93442 \mathrm{E}-01$

$8.93439 \mathrm{E}-01$ 8.93435 E-01 $8.93432 \mathrm{E}-01$ $8.93429 \mathrm{E}-01$ $8.93426 \mathrm{E}-01$

$8.93423 \mathrm{E}-01$ $8.93420 \mathrm{E}-01$ $8.93417 \mathrm{E}-01$ $8.93414 \mathrm{E}-01$ $8.93411 \mathrm{E}-01$

$Z=5$

$$
A=11
$$

$$
\rho=1.2 A^{1 / 3} F
$$

$$
f_{\mathrm{I}}
$$

$2.84463 \mathrm{E} \quad 01$ $2.85449 \mathrm{E} \quad 01$ $2.86437 \mathrm{E} \quad 01$ $2.87424 \mathrm{E} \quad 01$ 2.88411 E 01
$2.89399 \mathrm{E} \quad 01$ $2.91376 \mathrm{E} \quad 01$ $2.92364 \mathrm{E} 01$ $2.93353 \mathrm{E} \quad 01$

$2.94342 \mathrm{E} \quad 01$ $2.95332 \mathrm{E} \quad 01$ $2.96321 \mathrm{E} 01$ $2.97311 \mathrm{E} \quad 01$ 2.98301 E 01

$2.99292 \mathrm{E} \quad 01$ $3.00282 \mathrm{E} \quad 01$

$3.01273 \mathrm{E} \quad 01$

$3.02264 \mathrm{E} \quad 01$

$3.03256 \mathrm{E} \quad 01$

$3.04247 \mathrm{E} 01$ $3.05239 \mathrm{E} 01$ $3.06231 \mathrm{E} 01$ $3.07224 \mathrm{E} \quad 01$

$3.08217 \mathrm{E} \quad 01$

$3.09210 \mathrm{E} \quad 01$ $3.10203 \mathrm{E} \quad 01$ $3.11196 \mathrm{E} 01$ $3.12190 \mathrm{E} \quad 01$ $3.13184 \mathrm{E} \quad 01$

$3.14178 \mathrm{E} \quad 01$ $3.15173 \mathrm{E} 01$ $3.16168 \mathrm{E} \quad 01$ $3.17163 \mathrm{E} 01$ $3.18158 \mathrm{E} \quad 01$

$3.19154 \mathrm{E} \quad 01$ $3.20150 \mathrm{E} \quad 01$ $3.21146 \mathrm{E} \quad 01$ $3.22143 \mathrm{E} \cdot 01$ $3.23139 \mathrm{E}$ 0I

$3.24136 \mathrm{E} \quad 01$ $3.25134 \mathrm{E} \quad 01$ $3.26131 \mathrm{E} 01$ $3.27129 \mathrm{E} \quad 01$

$3.29126 \mathrm{E} \quad 01$ $3.30125 \mathrm{E} \quad 01$ $3.31124 \mathrm{E} \quad 01$ $3.32123 \mathrm{E} \quad 01$ $3.33122 \mathrm{E} \quad 01$

$3.34122 \mathrm{E} \quad 01$ $3.35122 \mathrm{E} \quad 01$ $3.36123 \mathrm{E} \quad 01$ $3.37124 \mathrm{E} \quad 01$ $3.38125 \mathrm{E} \quad 01$

$3.39126 \mathrm{E} \quad 01$ $3.40127 \mathrm{E} \quad 01$ $3.41129 \mathrm{E} \quad 01$ $3.42131 \mathrm{E} \quad 01$ $3.43134 \mathrm{E} \quad 01$ $2.90387 \mathrm{E} \quad 01$ $3.28127 \mathrm{E} \quad 01$ $g_{1} \quad \tan \delta_{1}$

$\begin{array}{lll}1.62683 \mathrm{E} & 00 & -1.04469 \mathrm{E}-03\end{array}$ $1.63909 \mathrm{E} \quad 00-1.04576 \mathrm{E}-03$ $\begin{array}{lll}1.65139 \mathrm{E} & 00-1.04681 \mathrm{E}-03\end{array}$ $\begin{array}{lll}1.66373 \mathrm{E} & 00-1.04785 \mathrm{E}-03\end{array}$ $1.67613 \mathrm{E} \quad 00-1.04887 \mathrm{E}-03$

$1.68857 \mathrm{E} \quad 00-1.04987 \mathrm{E}-03$ $1.70106 \mathrm{E} \quad 00-1.05086 \mathrm{E}-03$

$1.71360 \mathrm{E} \quad 00-1.05183 \mathrm{E}-03$

$\begin{array}{lll}1.72618 \mathrm{E} & 00 & -1.05279 \mathrm{E}-03\end{array}$

$1.73881 \mathrm{E} \quad 00 \quad-1.05373 \mathrm{E}-03$

$1.75149 \mathrm{E} \quad 00$

$1.76422 \mathrm{E} \quad 00$

$1.77700 \mathrm{E} \quad 00$

$1.78982 \mathrm{E} \quad 00$

$1.80269 \mathrm{E} \quad 00$

$1.81561 \mathrm{E} \quad 00$

$1.82858 \mathrm{E} \quad 00$

$\begin{array}{ll}1.84159 \mathrm{E} & 00\end{array}$

$1.85465 \mathrm{E} \quad 00$

$1.86776 \mathrm{E} \quad 00$

$1.88092 \mathrm{E} \quad 00$

$1.89413 \mathrm{E} \quad 00$

$1.90738 \mathrm{E} \quad 00$

$1.92069 \mathrm{E} \quad 00$

$1.93404 \mathrm{E} \quad 00$

$1.94743 \mathrm{E} \quad 00$

$1.96088 \mathrm{E} \quad 00$

$\begin{array}{ll}1.97437 \mathrm{E} & 00\end{array}$

$1.98792 \mathrm{E} \quad 00$

$2.00151 \mathrm{E} \quad 00$

$2.01515 \mathrm{E} \quad 00$

$2.02883 \mathrm{E} \quad 00$

$2.04257 \mathrm{E} \quad 00$

$2.05635 \mathrm{E} \quad 00$

$2.07019 \mathrm{E} \quad 00$

$2.08407 \mathrm{E} \quad 00$

$2.09800 \mathrm{E} \quad 00$

$2.11197 \mathrm{E} \quad 00$

$2.12600 \mathrm{E} \quad 00$

$2.14007 \mathrm{E} \quad 00$

$2.15420 \mathrm{E} \quad 00$

$2.16837 \mathrm{E} \quad 00$

$2.18259 \mathrm{E} \quad 00$

$2.19686 \mathrm{E} \quad 00$

$2.21117 \mathbf{E} \quad 00$

$2.22554 \mathrm{E} \quad 00$

$2.23995 \mathrm{E} \quad 00$

$2.25442 \mathrm{E} \quad 00$

$2.26893 \mathrm{E} \quad 00$

$2.28349 \mathrm{E} \quad 00$

$2.29810 \mathrm{E} \quad 00$

$2.31276 \mathrm{E} \quad 00$

$2.32746 \mathrm{E} \quad 00$

$2.34222 \mathrm{E} \quad 00$

$2.35702 \mathrm{E} \quad 00$

$2.37188 \mathrm{E} \quad 00$ $2.38678 \mathrm{E} \quad 00$

$2.40173 \mathrm{E} \quad 00$

$2.41673 \mathrm{E} \quad 00$ $2.43179 \mathrm{E} \quad 00$

$-1.05466 \mathrm{E}-03$ $-1.05557 \mathrm{E}-03$ $-1.05646 \mathrm{E}-03$ $-1.05735 \mathrm{E}-03$ $-1.05821 \mathrm{E}-03$

$-1.05906 \mathrm{E}-03$ $-1.05990 \mathrm{E}-03$ $-1.06072 \mathrm{E}-03$ $-1.06153 \mathrm{E}-03$ $-1.06232 \mathrm{E}-03$

$-1.06310 \mathrm{E}-03$ $-1.06387 \mathrm{E}-03$ $-1.06462 \mathrm{E}-03$ $-1.06536 \mathrm{E}-03$

$-1.06608 \mathrm{E}-03$

$-1.06679 \mathrm{E}-03$ $-1.06749 \mathrm{E}-03$ $-1.06817 \mathrm{E}-03$ $-1.06884 \mathrm{E}-03$ $-1.06950 \mathrm{E}-03$

$-1.07014 \mathrm{E}-03$ $-1.07077 \mathrm{E}-03$ $-1.07139 \mathrm{E}-03$ $-1.07199 \mathrm{E}-03$ $-1.07258 \mathrm{E}-03$

$-1.07316 \mathrm{E}-03$ $-1.07372 \mathrm{E}-03$ $-1.07428 \mathrm{E}-03$ $-1.07481 \mathrm{E}-03$

$-1.07535 \mathrm{E}-03$

$-1.07586 \mathrm{E}-03$ $-1.07636 \mathrm{E}-03$ $-1.07685 \mathrm{E}-03$ $-1.07734 \mathrm{E}-03$ $-1.07780 \mathrm{E}-03$

$-1.07826 \mathrm{E}-03$ $-1.07869 \mathrm{E}-03$ $-1.07913 \mathrm{E}-03$ $-1.07955 \mathrm{E}-03$ $-1.07995 \mathrm{E}-03$

$-1.08035 \mathrm{E}-03$ $-1.08074 \mathrm{E}-03$ $-1.08111 \mathrm{E}-03$ $-1.08147 \mathrm{E}-03$ $-1.08182 \mathrm{E}-03$

$-1.08216 \mathrm{E}-03$ $-1.08249 \mathrm{E}-03$ $-1.08281 \mathrm{E}-03$ 
ELECTRON RADIAL FUNCTIONS

$$
Z=5 \quad A=11 \quad \rho=1.2 A^{1 / 3} F
$$

$\bar{F}$

30.10

30.20

30.30

30.40

30.50

30.60

30.70

30.80

30.90

31.00

31.10

31.20

31.30

31.40

31.50

31.60

31.70

31.80

31.90

32.00

32.10

32.20

32.30

32.40

32.50

32.60

32.70

32.80

32.90

33.00

33.10

33.20

33.30

33.40

33.50

33.60

33.70

33.80

33.90

34.00

34.10

34.20

34.30

34.40

34.50

34.60

34.70

34.80

34.90

35.00

35.10

35.20

35.30

35.40

35.50

35.60

35.70

35.80

35.90

36.00 $f_{2}$

$1.07337 \mathrm{E}-03$

$1.08051 \mathrm{E}-03$

$1.08768 \mathrm{E}-03$

$1.09487 \mathrm{E}-03$

$1.10208 \mathrm{E}-03$

$1.10932 \mathrm{E}-03$

$1.11658 \mathrm{E}-03$

$1.12386 \mathrm{E}-03$

$1.13117 \mathrm{E}-03$

$1.13850 \mathrm{E}-03$

$1.14585 \mathrm{E}-03$

$1.15323 \mathrm{E}-03$

$1.16063 \mathrm{E}-03$

$1.16806 \mathrm{E}-03$

$1.17551 \mathrm{E}-03$

$1.18298 \mathrm{E}-03$

$1.19048 \mathrm{E}-03$

$1.19800 \mathrm{E}-03$

$1.20555 \mathrm{E}-03$

$1.21311 \mathrm{E}-03$

$1.22071 \mathrm{E}-03$

$1.22832 \mathrm{E}-03$

$1.23596 \mathrm{E}-03$

$1.24362 \mathrm{E}-03$

$1.25131 \mathrm{E}-03$

$1.25902 \mathrm{E}-03$

$1.26675 \mathrm{E}-03$

$1.27451 \mathrm{E}-03$

$1.28229 \mathrm{E}-03$

$1.29010 \mathrm{E}-03$

$1.29792 \mathrm{E}-03$

$1.30578 \mathrm{E}-03$

$1.31365 \mathrm{E}-03$

$1.32155 \mathrm{E}-03$

$1.32947 \mathrm{E}-03$

$1.33742 \mathrm{E}-03$

$1.34539 \mathrm{E}-03$

$1.35339 \mathrm{E}-03$

$1.36140 \mathrm{E}-03$

$1.36945 \mathrm{E}-03$

$1.37751 \mathrm{E}-03$

$1.38560 \mathrm{E}-03$

$1.39371 \mathrm{E}-03$

$1.40185 \mathrm{E}-03$

$1.41001 \mathrm{E}-03$

$1.41819 \mathrm{E}-03$

$1.42640 \mathrm{E}-03$

$1.43463 \mathrm{E}-03$

$1.44288 \mathrm{E}-03$

$1.45116 \mathrm{E}-03$

$1.45946 \mathrm{E}-03$

$1.46779 \mathrm{E}-03$

$1.47614 \mathrm{E}-03$

$1.48451 \mathrm{E}-03$

$1.49291 \mathrm{E}-03$

$1.50133 \mathrm{E}-03$

$1.50977 \mathrm{E}-03$

$1.51824 \mathrm{E}-03$

$1.52673 \mathrm{E}-03$

$1.53524 \mathrm{E}-03$ $g_{2}$

$1.93758 \mathrm{E} \quad 00$

$1.95056 \mathrm{E} \quad 00$

$1.96357 \mathrm{E} \quad 00$

$1.97663 \mathrm{E} \quad 00$

$1.98973 \mathrm{E} \quad 00$

$2.00287 \mathrm{E} \quad 00$

$2.01606 \mathrm{E} \quad 00$

$2.02929 \mathrm{E} \quad 00$

$2.04256 \mathrm{E} \quad 00$

$2.05587 \mathrm{E} \quad 00$

$2.06923 \mathrm{E} \quad 00$

$2.08263 \mathrm{E} \quad 00$

$2.09607 \mathrm{E} \quad 00$

$2.10956 \mathrm{E} \quad 00$

$2.12309 \mathrm{E} \quad 00$

$2.13666 \mathrm{E} \quad 00$

$2.15027 \mathrm{E} \quad 00$

$2.16393 \mathrm{E} \quad 00$

$2.17763 \mathrm{E} \quad 00$

$2.19137 \mathrm{E} \quad 00$

$2.20516 \mathrm{E} \quad 00$

$2.21899 \mathrm{E} \quad 00$

$2.23286 \mathrm{E} \quad 00$

$2.24677 \mathrm{E} \quad 00$

$2.26073 \mathrm{E} \quad 00$

$2.27472 \mathrm{E} \quad 00$

$2.28877 \mathrm{E} \quad 00$

$2.30285 \mathrm{E} \quad 00$

$2.31698 \mathrm{E} \quad 00$

$2.33115 \mathrm{E} \quad 00$

$2.34536 \mathrm{E} \quad 00$

$2.35962 \mathrm{E} \quad 00$

$2.37391 \mathrm{E} \quad 00$

$2.38825 \mathrm{E} \quad 00$

$2.40264 \mathrm{E} \quad 00$

$2.41706 \mathrm{E} \quad 00$

$2.43153 \mathrm{E} \quad 00$

$2.44604 \mathrm{E} \quad 00$

$2.46060 \mathrm{E} \quad 00$

$2.47519 \mathrm{E} \quad 00$

$2.48983 \mathrm{E} \quad 00$

$2.50451 \mathrm{E} \quad 00$

$2.51924 \mathrm{E} \quad 00$

$2.53401 \mathrm{E} \quad 00$

$2.54881 \mathrm{E} \quad 00$

$2.56367 \mathrm{E} \quad 00$

$2.57856 \mathrm{E} \quad 00$

$2.59350 \mathrm{E} \quad 00$

$2.60848 \mathrm{E} \quad 00$

$2.62350 \mathrm{E} \quad 00$

$2.63857 \mathrm{E} \quad 00$

$2.65367 \mathrm{E} \quad 00$

$2.66882 \mathrm{E} \quad 00$

$2.68402 \mathrm{E} \quad 00$

$2.69925 \mathrm{E} \quad 00$

$2.71453 \mathrm{E} \quad 00$

$2.72985 \mathrm{E} \quad 00$

$2.74521 \mathrm{E} \quad 00$

$2.76062 \mathrm{E} \quad 00$

$2.77607 \mathrm{E} \quad 00$
$6.72287 \mathrm{E}-02$

$6.79489 \mathrm{E}-02$

$6.86742 \mathrm{E}-02$

$6.94046 \mathrm{E}-02$

$7.01402 \mathrm{E}-02$

$7.08809 \mathrm{E}-02$

$7.16267 \mathrm{E}-02$

$7.23778 \mathrm{E}-02$

$7.31340 \mathrm{E}-02$

$7.38955 \mathrm{E}-02$

7.46622 E- 02

$7.54342 \mathrm{E}-02$

$7.62115 \mathrm{E}-02$

$7.69940 \mathrm{E}-02$

$7.77819 \mathrm{E}-02$

$7.85751 \mathrm{E}-02$

$7.93736 \mathrm{E}-02$

$8.01775 \mathrm{E}-02$

$8.09868 \mathrm{E}-02$

$8.18015 \mathrm{E}-02$

$8.26216 \mathrm{E}-02$

$8.34471 \mathrm{E}-02$

8.42781 E-02

$8.51146 \mathrm{E}-02$

$8.59565 \mathrm{E}-02$

8.68039E-02

$8.76569 \mathrm{E}-02$

$8.85154 \mathrm{E}-02$

$8.93795 \mathrm{E}-02$

$9.02491 \mathrm{E}-02$

$9.11244 \mathrm{E}-02$

$9.20052 \mathrm{E}-02$

$9.28917 \mathrm{E}-02$

$9.37839 \mathrm{E}-02$

$9.46816 \mathrm{E}-02$

$9.55851 \mathrm{E}-02$

9.64943 E-02

$9.74092 \mathrm{E}-02$

$9.83298 \mathrm{E}-02$

$9.92562 \mathbf{E}-02$

$1.00188 \mathrm{E}-01$

$1.01126 \mathrm{E}-01$

$1.02070 \mathrm{E}-01$

$1.03020 \mathrm{E}-01$

$1.03975 \mathrm{E}-01$

$1.04936 \mathrm{E}-01$

$1.05903 \mathrm{E}-01$

$1.06877 \mathrm{E}-01$

$1.07855 \mathrm{E}-01$

$1.08840 \mathrm{E}-01$

$1.09831 \mathrm{E}-01$

$1.10828 \mathrm{E}-01$

$1.11831 \mathrm{E}-01$

$1.12840 \mathrm{E}-01$

$1.13854 \mathrm{E}-01$

$1.14875 \mathrm{E}-01$

$1.15902 \mathrm{E}-01$

$1.16935 \mathrm{E}-01$

$1.17974 \mathrm{E}-01$

$1.19019 \mathrm{E}-01$

$\tan \delta_{2}$

$f_{2}$

$-3.93836 \mathrm{E} 01 \quad-6.39586 \mathrm{E}-02$ $-3.93853 \mathrm{E} \quad 0] \quad-6.46589 \mathrm{E}-02$ $-3.93870 \mathrm{E}$ 01 $-6.53642 \mathrm{E}-02$ $-3.93887 \mathrm{E} \quad 01-6.60746 \mathrm{E}-02$

$-3.93903 \mathrm{E} 01 \quad-6.67900 \mathrm{E}-02$

$-3.93920 \mathrm{E} \quad 01 \quad-6.75106 \mathrm{E}-02$

$-3.93936 \mathrm{E}$ 01 $-6.82362 \mathrm{~F}-02$

$\begin{array}{lll}-3.93952 \mathrm{E} & 01 & -6.89670 \mathrm{E}-02\end{array}$

$-3.93968 \mathrm{E} \quad 01 \quad-6.97030 \mathrm{E}-02$

$-3.93985 \mathrm{E} \quad 01 \quad-7.04441 \mathrm{E}-02$

$-3.94000 \mathrm{E} 01$

$-7.11903 \mathrm{E}-02$

$-3.94016 \mathrm{E} \quad 01$

$-3.94032 \mathrm{E} 01$

$-3.94048 \mathrm{E} \quad 01$

$-3.94063 \mathrm{E} 01$

$-3.94079 \mathrm{E} 01$

$-3.94094 \mathrm{E} 01$

$-3.94109 \mathrm{E} \quad 01$

$-3.94124 \mathrm{E} \quad 01$

$-3.94139 \mathrm{E} \quad 01$

$-3.94154 \mathrm{E} \quad 01$

$\begin{array}{lll}-3.94169 \mathrm{E} & 01\end{array}$

$-3.94184 \mathrm{E} 01$

$-3.94198 \mathrm{E} 01$

$-3.94213 \mathrm{E} 01$

$-7.19418 \mathrm{E}-02$

$-7.26985 \mathrm{E}-02$

$-7.34604 \mathrm{E}-02$

$-7.42276 \mathrm{E}-02$

$\begin{array}{lll}-3.94227 \mathbf{E} & 01\end{array}$

$-3.94242 \mathrm{E} 01$

$-3.94256 \mathrm{E} 01$

$-3.94270 \mathrm{E} 01$

$-3.94284 \mathrm{E} \quad 01$

$\begin{array}{ll}-3.94298 \mathrm{E} & 01\end{array}$

$-3.94312 \mathrm{E} 01$

$-3.94326 \mathrm{E} 01$

$-3.94340 \mathrm{E} 01$

$-3.94354 \mathrm{E} 01$

$\begin{array}{lll}-3.94367 \mathrm{E} & 01\end{array}$ 
ELECTRON RADIAL FUNCTIONS

Positron

$F_{0}$

36.10

36.20

36.30

36.40

36.50

36.60

36.70

36.80

36.90

37.00

37.10

37.20

37.30

37.40

37.50

37.60

37.70

37.80

37.90

38.00

38.10

38.20

38.30

38.40

38.50

38.60

38.70

38.80

38.90

39.00

39.10

39.20

39.30

39.40

39.50

39.60

39.70

39.80

39.90

40.00

40.10

40.20

40.30

40.40

40.50

40.60

40.70

40.80

40.90

41.00

41.10

41.20

41.30

41.40

41.50

41.60

41.70

41.80

41.90

42.00 $f_{1}$

93407 E-01 $8.93405 \mathrm{E}-01$ $8.93401 \mathrm{E}-01$ $8.93398 \mathrm{E}-01$ $8.93395 \mathrm{E}-01$

$8.93392 \mathrm{E}-01$ $8.93389 \mathrm{E}-01$ $8.93386 \mathrm{E}-01$ $8.93383 \mathrm{E}-01$ $8.93380 \mathrm{E}-01$

$8.93377 \mathrm{E}-01$ $8.93374 \mathrm{E}-01$ $8.93371 \mathrm{E}-01$ $8.93368 \mathrm{E} \quad 01$ $8.93365 \mathrm{E}-01$

$8.93363 \mathrm{E}-01$ $8.93360 \mathrm{E}-01$ $8.93357 \mathrm{E}-01$ $8.93354 \mathrm{E}-01$ $8.93351 \mathrm{E}-01$

8.93348E-01 $8.93345 \mathrm{E}-01$ $8.93342 \mathrm{E}-01$ $8.93339 \mathrm{E}-01$ $8.93336 \mathrm{E}-01$

8.93333E-01 $8.93331 \mathrm{E}-01$ $8.93328 \mathrm{E}-01$ $8.93325 \mathrm{E}-01$ $8.93322 E-01$

$8.93319 \mathrm{E}-01$ $8.93316 \mathrm{E}-01$ $8.93313 \mathrm{E}-01$ $8.93310 \mathrm{E}-01$ $8.93308 \mathrm{E}-01$

8.93305E-01 $8.93302 \mathrm{E}-01$ $8.93299 \mathrm{E}-01$ 8.93296E-01 8.93293E-01

$8.93291 \mathrm{E}-01$ $8.93288 \mathrm{E}-0$ $8.93285 \mathrm{E}-01$ $8.93282 \mathrm{E}-01$ $8.93279 \mathrm{E}-01$

$8.93277 \mathrm{E}-01$ $8.93274 \mathrm{E}-01$ $8.93271 \mathrm{E}-01$ $8.93268 \mathrm{E}-01$ $8.93266 \mathrm{E}-01$

8.93263E-01 $8.93260 \mathrm{E}-01$ $8.93257 \mathrm{E}-01$ $8.93255 \mathrm{E}-0]$ $8.93252 \mathrm{E}-01$

$8.93249 \mathrm{E}-01$ $8.93247 \mathrm{E}-01$ $8.93244 \mathrm{E}-01$ $8.93241 \mathrm{E}-01$ $8.93238 \mathrm{E}-01$

$Z=5$

$A=11$

$\rho=1.2 \mathrm{~A}^{1 / 3} \mathrm{~F}$

$3.44137 \mathrm{E} 01$ $3.45140 \mathrm{E} \quad 01$ $3.46143 \mathrm{E} \quad 01$ $3.47147 \mathrm{E} \quad 01$ $3.48151 \mathrm{E} 01$

$3.49155 \mathrm{E} \quad 01$ $3.50159 \mathrm{E} \quad 01$ $3.51164 \mathrm{E} \quad 01$ $3.52169 \mathrm{E} \quad 01$ 3.53175 E 01

$3.54181 \mathrm{E} \quad 01$ $3.55187 \mathrm{E} 01$ $3.56193 \mathrm{E} \quad 01$

$3.57200 \mathrm{E} \quad 01$ $3.58207 \mathrm{E} \quad 01$

$3.59214 \mathrm{E} \quad 01$ $3.60222 \mathrm{E} \quad 01$ $3.61229 \mathrm{E} 01$ $3.62238 \mathrm{E} \quad 01$

$3.63246 \mathrm{E} \quad 01$

$3.64255 \mathrm{E} \quad 01$

$3.65264 \mathrm{E} \quad 01$

$3.66274 \mathrm{E} \quad 01$

$3.67283 \mathrm{E} 01$

3.68293 E 01

$3.69304 \mathrm{E} \quad 01$

$3.70314 \mathrm{E} \quad 01$

$3.71325 \mathrm{E} \quad 01$

$3.72337 \mathrm{E} \quad 01$

$3.73348 \mathrm{E} \quad 01$

$3.74360 \mathrm{E} \quad 01$ $3.75373 \mathrm{E} \quad 01$ $3.76385 \mathrm{E} \quad 01$ $3.77398 \mathrm{E} \quad 01$

$3.78412 \mathrm{E} \quad 01$

$3.79425 \mathrm{E} \quad 01$ $3.80439 \mathrm{E} 01$ $3.81453 \mathrm{E} \quad 01$ $3.82468 \mathrm{E} \quad 01$ $3.83483 \mathrm{E} \quad 01$

$3.84498 \mathrm{E} \quad 01$

$3.85513 \mathrm{E} 01$

$3.86529 \mathrm{E} \quad 01$

$3.87546 \mathrm{E} \quad 01$

$3.88562 \mathrm{E} 01$

$3.89579 \mathrm{E} \quad 01$ $3.90596 \mathrm{E} \quad 01$ $3.91614 \mathrm{E} \quad 01$ $3.92632 \mathrm{E} \quad 01$ $3.93650 \mathrm{E} \quad 01$

$3.94668 \mathrm{E} \quad 01$ $3.95687 \mathrm{E} \quad 01$ $3.96707 \mathrm{E} 01$ $3.97726 \mathrm{E} 01$ $3.98746 \mathrm{E} \quad 01$

$3.99766 \mathrm{E} \quad 01$ $4.00787 \mathrm{E} \quad 01$ $4.01808 \mathrm{E} \quad 01$ $4.02829 \mathrm{E} \quad 01$ $4.03851 \mathrm{E} \quad 01$ $\tan \delta_{1}$

$f_{-1}$

$2.44689 \mathrm{E} \quad 00$

$2.46203 \mathrm{E} \quad 00-1.08397 \mathrm{E}-03$

$2.47723 \mathrm{E} \quad 00-1.08423 \mathrm{E}-03$

$2.49248 \mathrm{E} \quad 00-1.08449 \mathrm{E}-03$

$2.50777 \mathrm{E} \quad 00-1.08472 \mathrm{E}-03$

$2.52312 \mathrm{E} \quad 00$

$2.53851 \mathrm{E} \quad 00$

$2.55396 \mathrm{E} \quad 00$

$2.56945 \mathrm{E} \quad 00$

$2.58500 \mathrm{E} \quad 00$

$2.60059 \mathrm{E} \quad 00$

$2.61623 \mathrm{E} \quad 00$

$2.63192 \mathrm{E} \quad 00$

$2.64766 \mathrm{E} \quad 00$

$2.66345 \mathrm{E} \quad 00$

$2.67929 \mathrm{E} \quad 00$

$2.69518 \mathrm{E} \quad 00$

$2.71112 \mathrm{E} \quad 00$

$2.72711 \mathrm{E} \quad 00$

$2.74315 \mathrm{E} \quad 00$

$2.75924 \mathrm{E} \quad 00$

$2.77538 \mathrm{E} \quad 00$

$2.79157 \mathrm{E} \quad 00$

$2.80780 \mathrm{E} \quad 00$

$2.82409 \mathrm{E} \quad 00$

$2.84043 \mathrm{E} \quad 00$

$2.85682 \mathrm{E} \quad 00$

$2.87326 \mathrm{E} \quad 00$

$2.88975 \mathrm{E} \quad 00$

$2.90628 \mathrm{E} \quad 00$

$2.92287 \mathrm{E} \quad 00$ $2.93951 \mathrm{E} \quad 00$ $2.95620 \mathrm{E} \quad 00$ $2.97294 \mathrm{E} \quad 00$

$2.98973 \mathrm{E} \quad 00$

$3.00657 \mathrm{E} \quad 00$

$3.02346 \mathrm{E} \quad 00$

$3.04040 \mathrm{E} \quad 00$

$3.05739 \mathrm{E} 00$

$3.07443 \mathrm{E} \quad 00$

$3.09152 \mathrm{E} \quad 00$

$3.10867 \mathrm{E} \quad 00$

$3.12586 \mathrm{E} \quad 00$

$3.14310 \mathrm{E} \quad 00$

$3.16040 \mathrm{E} \quad 00$

$3.17774 \mathrm{E} \quad 00$

$3.19514 \mathrm{E} \quad 00$

$3.21259 \mathrm{E} \quad 00$

$3.23008 \mathrm{E} \quad 00$

$3.24763 \mathrm{E} \quad 00$

$3.26523 \mathrm{E}^{\circ} 00$

$3.28288 \mathrm{E} \quad 00$

$3.30058 \mathrm{E} \quad 00$

$3.31833 \mathrm{E} \quad 00$

$3.33613 \mathrm{E} \quad 00$

$3.35399 \mathrm{E} \quad 00$

$3.37189 \mathrm{E} \quad 00$

$3.38985 \mathrm{E} \quad 00$

$3.40786 \mathrm{E} \quad 00$

$3.42592 \mathrm{E} \quad 00$

$-1.08496 \mathrm{E}-03$

$-1.08518 \mathrm{E}-03$

$-1.08539 \mathrm{E}-03$

$-1.08559 \mathrm{E}-03$

$-1.08578 \mathrm{E}-03$

$-1.08595 \mathrm{E}-03$

$-1.08612 \mathrm{E}-03$

$-1.08628 \mathrm{E}-03$

$-1.08643 \mathrm{E}-03$

$-1.08657 \mathrm{E}-03$

$-1.08670 \mathrm{E}-03$

$-1.08682 \mathrm{E}-03$

$-1.08693 \mathrm{E}-03$

$-1.08702 \mathrm{E}-03$

-1.08711 E-03

$-1.08719 \mathrm{E}-03$

$-1.08726 \mathrm{E}-03$

$-1.08732 \mathrm{E}-03$

$-1.08737 \mathrm{E}-03$

$-1.08741 \mathrm{E}-03$

$-1.08744 \mathrm{E}-03$

$-1.08746 \mathrm{E}-03$

$-1.08747 \mathrm{E}-03$

$-1.08748 \mathrm{E}-03$

$-1.08747 \mathrm{E}-03$

$-1.08746 \mathrm{E}-03$

$-1.08743 \mathrm{E}-03$

$-1.08740 \mathrm{E}-03$

$-1.08735 \mathrm{E}-03$

$-1.08730 \mathrm{E}-03$

$-1.08724 \mathrm{E}-03$

$-1.08717 \mathrm{E}-03$

$-1.08709 \mathrm{E}-03$

$-1.08700 \mathrm{E}-03$

$-1.08691 \mathrm{E}-03$

$-1.08680 \mathrm{E}-03$

$-1.08669 \mathrm{E}-03$

$-1.08656 \mathrm{E}-03$ 
ELECTRON RADIAL FUNCTIONS

$Z=5 \quad A=11 \quad \rho=1.2 A^{1 / 3} F$

Position

$\bar{F}$

$1.54378 \mathrm{E}-03$ $1.55235 \mathrm{E}-03$ $1.56093 \mathrm{E}-03$ $1.56954 \mathrm{E}-03$ $1.57817 \mathrm{E}-03$

$1.58683 \mathrm{E}-03$

$1.59551 \mathrm{E}-0.3$

$1.60422 \mathrm{E}-03$

$1.61294 \mathrm{E}-03$

$1.62170 \mathrm{E}-03$

$1.63047 \mathrm{E}-03$

$1.63927 \mathrm{E}-03$

$1.64809 \mathrm{E}-03$

$1.65694 \mathrm{E}-03$

$1.66581 \mathrm{E}-03$

$1.67470 \mathrm{E}-03$

$1.68362 \mathrm{E}-03$

$1.69256 \mathrm{E}-03$

$1.70153 \mathrm{E}-03$

$1.71051 \mathrm{E}-03$

37.90

38.10

38.20

$1.71953 \mathrm{E}-03$

$1.72856 \mathrm{E}-03$

$1.73762 \mathrm{E}-03$

$1.74670 \mathrm{E}-03$

$1.75581 \mathrm{E}-03$

38.40

$1.76494 \mathrm{E}-03$

$1.77409 \mathrm{E}-03$

$1.78327 \mathrm{E}-03$

$1.79247 \mathrm{E}-03$

$1.80170 \mathrm{E}-03$

39.00

39.10

39.20

39.30

39.40

39.50

39.60

39.70

39.80

39.90

40.00

$1.81095 \mathrm{E}-03$

$1.82022 \mathrm{E}-03$

$1.82951 \mathrm{E}-03$

$1.83883 \mathrm{E}-03$

$1.84818 \mathrm{E}-03$

$1.85754 \mathrm{E}-03$

$1.86693 \mathrm{E}-03$

$1.87635 \mathrm{E}-03$

$1.88579 \mathrm{E}-03$

$1.89525 \mathrm{E}-03$

40.10

40.20

40.30

40.40

40.50

40.60

40.70

40.80

40.90

$1.90473 \mathrm{E}-03$

$1.91424 \mathrm{E}-03$

$1.92378 \mathrm{E}-03$

$1.93333 \mathrm{E}-03$

$1.94291 \mathrm{E}-03$

$1.95251 \mathrm{E}-03$

$1.96214 \mathrm{E}-03$

$1.97179 \mathrm{E}-03$

$1.98147 \mathrm{E}-03$

$1.99117 \mathrm{E}-03$

41.10

41.20

41.30

41.40

41.50

41.60

41.70

41.80

41.90

42.00 $f_{2}$

$2.79156 \mathrm{E} \quad 00$

$2.80709 \mathrm{E} \quad 00$

$2.82266 \mathrm{E} \quad 00$

$2.83828 \mathrm{E} \quad 00$

$2.85394 \mathrm{E} \quad 00$

$2.86965 \mathrm{E} \quad 00$

$2.88539 \mathrm{E} \quad 00$

$2.90118 \mathrm{E} \quad 00$

$2.91701 \mathrm{E} \quad 00$

$2.93288 \mathrm{E} \quad 00$

$2.94880 \mathrm{E} \quad 00$

$2.96475 \mathrm{E} \quad 00$

$2.98075 \mathrm{E} \quad 00$

$2.99679 \mathrm{E} \quad 00$

$3.01288 \mathrm{E} \quad 00$

$3.02901 \mathrm{E} \quad 00$

$3.04518 \mathrm{E} \quad 00$

$3.06139 \mathrm{E} \quad 00$

$3.07764 \mathrm{E} \quad 00$

$3.09394 \mathrm{E} \quad 00$

$3.11028 \mathrm{E} \quad 00$

$3.12666 \mathrm{E} \quad 00$

$3.14308 \mathrm{E} \quad 00$

$3.15955 \mathrm{E} 00$

$3.17605 \mathrm{E} \quad 00$

$3.19260 \mathrm{E} \quad 00$

$3.20920 \mathrm{E} \quad 00$

$3.22583 \mathrm{E} \quad 00$

$3.24251 \mathrm{E} \quad 00$

$3.25923 \mathrm{E} \quad 00$

$3.27599 \mathrm{E} \quad 00$

$3.29279 \mathrm{E} \quad 00$

$3.30964 \mathrm{E} \quad 00$

$3.32653 \mathrm{E} \quad 00$

$3.34346 \mathrm{E} \quad 00$

$3.36043 \mathrm{E} \quad 00$

$3.37745 \mathrm{E} \quad 00$

$3.39451 \mathrm{E} \quad 00$

$3.41161 \mathrm{E} \quad 00$

$3.42875 \mathrm{E} \quad 00$

$3.44593 \mathrm{E} \quad 00$

$3.46316 \mathrm{E} \quad 00$

$3.48043 \mathrm{E} \quad 00$

$3.49774 \mathrm{E} \quad 00$

$3.51509 \mathrm{E} \quad 00$

$3.53249 \mathrm{E} \quad 00$

$3.54992 \mathrm{E} \quad 00$

$3.56740 \mathrm{E} \quad 00$

$3.58492 \mathrm{E} \quad 00$

$3.60249 \mathrm{E} \quad 00$

$3.62009 \mathrm{E} \quad 00$

$3.63774 \mathrm{E} \quad 00$

$3.65543 \mathrm{E} \quad 00$

$3.67316 \mathrm{E} \quad 00$

$3.69094 \mathrm{E} \quad 00$

$3.70875 \mathrm{E} \quad 00$

$3.72661 \mathrm{E} \quad 00$

$3.74451 \mathrm{E} \quad 00$

$3.76246 \mathrm{E} \quad 00$

$3.78044 \mathrm{E} \quad 00$ $g_{2}$

$1.20070 \mathrm{E}-01$

$1.21128 \mathrm{E}-01$

$1.22191 \mathrm{E}-01$

$1.23261 \mathrm{E}-01$

$1.24337 \mathrm{E}-01$

$1.25419 \mathrm{E}-01$

$1.26508 \mathrm{E}-01$

$1.27602 \mathrm{E}-01$

$1.28703 \mathrm{E}-01$

$1.29810 \mathrm{E}-01$

$1.30924 \mathrm{E}-01$

$1.32044 \mathrm{E}-01$

$1.33170 \mathrm{E}-01$

$1.34302 \mathrm{E}-01$

$1.35441 \mathrm{E}-01$

$1.36586 \mathrm{E}-01$

$1.37738 \mathrm{E}-01$

$1.38896 \mathrm{E}-01$

$1.40060 \mathrm{E}-01$

$1.41231 \mathrm{E}-01$

$1.42409 \mathrm{E}-01$

$1.43593 \mathrm{E}-01$

$1.44783 \mathrm{E}-01$

$1.45980 \mathrm{E}-01$

$1.47183 \mathrm{E}-01$

$1.48393 \mathrm{E}-01$

$1.49610 \mathrm{E}-01$

$1.50833 \mathrm{E}-01$

$1.52063 \mathrm{E}-01$

$1.53300 \mathrm{E}-01$

$1.54543 \mathrm{E}-01$

$1.55792 \mathrm{E}-01$

$1.57049 \mathrm{E}-01$

$1.58312 \mathrm{E}-01$

$1.59582 \mathrm{E}-01$

$1.60859 \mathrm{E}-01$

$1.62142 \mathrm{E}-01$

$1.63432 \mathrm{E}-01$

$1.64729 \mathrm{E}-01$

$1.66033 \mathrm{E}-01$

$1.67343 \mathrm{E}-01$

$1.68661 \mathrm{E}-01$

$1.69985 \mathrm{E}-01$

$1.71316 \mathrm{E}-01$

$1.72654 \mathrm{E}-01$

$1.73999 \mathrm{E}-01$

$1.75351 \mathrm{E}-01$

$1.76710 \mathrm{E}-01$

$1.78076 \mathrm{E}-01$

$1.79448 \mathrm{E}-01$

$1.80828 \mathrm{E}-01$

$1.82215 \mathrm{E}-01$

$1.83609 \mathrm{E}-01$

$1.85010 \mathrm{E}-01$

$1.86417 \mathrm{E}-01$

$1.87832 \mathrm{E}-01$

$1.89255 \mathrm{E}-01$

$1.90684 \mathrm{E}-01$

$1.92120 \mathrm{E}-01$

$1.93564 \mathrm{E}-01$ $\tan \delta_{2}$

$-3.94681 \mathrm{E} \quad 01$

$-3.94693 \mathrm{E} 01$

$-3.94705 \mathrm{E} 01$

$-3.94716 \mathrm{E} 01$

$-3.94728 \mathrm{E} 01$

$-3.94739 \mathrm{E} \quad 01$

$-3.94750 \mathrm{E}$ 01

$-3.94762 \mathrm{E} 01$

$-3.94773 \mathrm{E} \quad 01$

$-3.94784 \mathrm{E} 01$

$-3.94795 \mathrm{E} 01$

$-3.94806 \mathrm{E} 01$

$-3.94817 \mathrm{E}$ 01

$-3.94828 \mathrm{E} 01$

$-3.94838 \mathrm{E} 01$

$-3.94849 \mathrm{E} \quad 01$

$-3.94860 \mathrm{E} 01$

$-3.94871 \mathrm{E} \quad 01$

$-3.94881 \mathrm{E} 01$

$-3.94892 \mathrm{E} 01$

$-3.94902 \mathrm{E} \quad 01$

$-3.94913 \mathrm{E} 01$

$-3.94923 \mathrm{E} \quad 01$

$-3.94933 \mathrm{E} 01$

$-3.94943 \mathrm{E} \quad 01$

$-3.94954 \mathrm{E} \quad 01$

$-3.94964 \mathrm{E} 01$

$-3.94974 \mathrm{E}$ 0l

$-3.94984 \mathrm{E} 01$

$-3.94994 \mathrm{E} 01$

$-3.95004 \mathrm{E} 01$

$-3.95014 \mathrm{E} 01$

$-3.95023 \mathrm{E} 01$

$-3.95033 \mathrm{E}$ 0l

$-3.95043 \mathrm{E} 01$

$-3.95053 \mathrm{E} \quad 01$

$-3.95062 \mathrm{E} 01$

$-3.95072 \mathrm{E} 01$

$-3.95081 \mathrm{E} 01$

$-3.95091 \mathrm{E} 01$

$-3.95100 \mathrm{E} \quad 01$

$-3.95110 \mathrm{E} 01$

$-3.95119 \mathrm{E} 01$

$-3.95128 \mathrm{E} 01$

$-3.95137 \mathrm{E} 01$

$-3.95146 \mathrm{E} \quad 01$

$-3.95156 \mathrm{E} \quad 01$

$-3.95165 \mathrm{E} 01$

$-3.95174 \mathrm{E}$ 0l

$-3.95183 \mathrm{E}$ 0I

$-3.95192 \mathrm{E}$ 0l

$-3.95201 \mathrm{E} 01$

$-3.95209 \mathrm{E} \quad 01$

$-3.95218 \mathrm{E} 01$

$-3.95227 \mathrm{E}$ 0l

$\begin{array}{ll}-3.95236 \mathrm{E} & 01\end{array}$

$-3.95244 \mathrm{E}$ 0l

$-3.95253 \mathrm{E} \quad 01$

$-3.95261 \mathrm{E} 01$

$-3.95270 \mathrm{E} 01$

$f_{-2}$

$-1.15501 \mathrm{E}-0$

$-1.16535 \mathrm{E}-01$

$-1.17575 \mathrm{E}-01$

$-1.18621 \mathrm{E}-01$

$-1.19673 \mathrm{E}-01$

$-1.20732 \mathrm{E}-01$

$-1.21796 \mathrm{E}-01$

$-1.22867 \mathrm{E}-01$

$-1.23944 \mathrm{E}-01$

$-1.25028 \mathrm{E}-01$

$-1.26117 \mathrm{E}-01$

$-1.27213 \mathrm{E}-01$

$-1.28315 \mathrm{E}-01$

$-1.29423 \mathrm{E}-01$

$-1.30538 \mathrm{E}-01$

$-1.31659 \mathrm{E}-01$

$-1.32786 \mathrm{E}-01$

$-1.33920 \mathrm{E}-01$ 
ELECTRON RADIAL FUNCTIONS

$\begin{array}{cc}p & F_{0} \\ 0.10 & 1.89022 \mathrm{E}-01 \\ 0.20 & 4.64738 \mathrm{E}-01 \\ 0.30 & 6.05600 \mathrm{E}-01 \\ 0.40 & 6.84821 \mathrm{E}-01 \\ 0.50 & 7.34047 \mathrm{E}-01 \\ & \\ 0.60 & 7.66845 \mathrm{E}-01 \\ 0.70 & 7.89813 \mathrm{E}-01 \\ 0.80 & 8.06511 \mathrm{E}-01 \\ 0.90 & 8.19008 \mathrm{E}-01 \\ 1.00 & 8.28587 \mathrm{E}-01\end{array}$

$1.10 \quad 8.36075 \mathrm{E}-01$

$1.20 \quad 8.42026 \mathrm{E}-01$

$1.30 \quad 8.46824 \mathrm{E}-01$

$1.40 \quad 8.50743 \mathrm{E}-01$

$1.50 \quad 8.53978 \mathrm{E}-01$

$1.60 \quad 8.56677 \mathrm{E}-01$

$1.70 \quad 8.58948 \mathrm{E}-01$

$1.80 \quad 8.60873 \mathrm{E}-01$

$1.90 \quad 8.62519 \mathrm{E}-01$

$2.00 \quad 8.63934 \mathrm{E}-01$

$2.10 \quad 8.65159 \mathrm{E}-01$

$2.20 \quad 8.66223 \mathrm{E}-01$

$2.30 \quad 8.67155 \mathrm{E}-01$

$2.40 \quad 8.67973 \mathrm{E}-01$

$2.50 \quad 8.68695 \mathrm{E}-01$

$2.60 \quad 8.69334 \mathrm{E}-01$

$2.70 \quad 8.69902 \mathrm{E}-01$

$2.80 \quad 8.70409 \mathrm{E}-01$

$2.90 \quad 8.70863 \mathrm{E}-01$

$3.00 \quad 8.71270 \mathrm{E}-01$

3.10

3.20

3.30

3.40

3.50

3.60

3.70

3.80

3.90

4.00

4.10

4.20

4.30

4.40

4.50

4.60

4.70

4.80

4.90

5.00

5.10

5.20

5.30

5.40

5.50

5.60

5.70

5.80

5.90

6.00
$8.71636 \mathrm{E}-01$

$8.71966 \mathrm{E}-01$

$8.72265 \mathrm{E}-01$

$8.72535 \mathrm{E}-01$

$8.72782 \mathrm{E}-01$

$8.73005 \mathrm{E}-01$ $8.73209 \mathrm{E}-01$ $8.73394 \mathrm{E}-01$ $8.73564 \mathrm{E}-01$ $8.73720 \mathrm{E}-01$

$8.73863 \mathrm{E}-01$ $8.73994 \mathrm{E}-01$ $8.74114 \mathrm{E}-01$ $8.74225 \mathrm{E}-01$ $8.74326 \mathrm{E}-01$

$8.74420 \mathrm{E}-01$ $8.74506 \mathrm{E}-01$ $8.74586 \mathrm{E}-01$ $8.74660 \mathrm{E}-01$ 8.74727 E-01

$8.74790 \mathrm{E}-01$ $8.74848 \mathrm{E}-01$ $8.74902 \mathrm{E}-01$ 8.74951 E-01 8.74997E-01

$8.75040 \mathrm{E}-01$ 8.75079E-01 $8.75114 \mathrm{E}-01$ $8.75148 \mathrm{E}-01$ $8.75179 \mathrm{E}-01$

\section{$f_{1}$}

$3.32774 \mathrm{E}-03$

$1.94286 \mathrm{E}-02$

4.84302 E-02

$8.90368 \mathrm{E}-02$

$1.39711 \mathrm{E}-01$

$1.98868 \mathrm{E}-01$

$2.65020 \mathrm{E}-01$

$3.36838 \mathrm{E}-01$

$4.13195 \mathrm{E}-01$

$4.93161 \mathrm{E}-01$

$5.75983 \mathrm{E}-01$

$6.61063 \mathrm{E}-01$

$7.47928 \mathrm{E}-01$

$8.36206 \mathrm{E}-01$

$9.25607 \mathrm{E}-01$

$1.01590 \mathrm{E} \quad 00$

$1.10691 \mathrm{E} \quad 00$

$1.19849 \mathrm{E} \quad 00$

$1.29054 \mathrm{E} \quad 00$

$1.38295 \mathrm{E} \quad 00$

$1.47567 \mathrm{E} \quad 00$

$1.56864 \mathrm{E} \quad 00$

$1.66180 \mathrm{E} \quad 00$

$1.75513 \mathrm{E} 00$

$1.84860 \mathrm{E} \quad 00$

$1.94218 \mathrm{E} \quad 00$

$2.03585 \mathrm{E} 00$

$2.12959 \mathrm{E} \quad 00$

$2.22340 \mathrm{E} \quad 00$

$2.31726 \mathrm{E} \quad 00$

$2.41117 \mathrm{E} \quad 00$ $2.50511 \mathrm{E} \quad 00$ $2.59908 \mathrm{E} \quad 00$ $2.69307 \mathrm{E} \quad 00$ $2.78708 \mathrm{E} \quad 00$

$2.88112 \mathrm{E} \quad 00$ $2.97516 \mathrm{E} \quad 00$

$3.06922 \mathrm{E} \quad 00$

$3.16329 \mathrm{E} \quad 00$

$3.25737 \mathrm{E} \quad 00$

$3.35145 \mathrm{E} \quad 00$ $3.44554 \mathrm{E} \quad 00$ $3.53963 \mathrm{E} \quad 00$

$3.63373 \mathrm{E} \quad 00$

$3.72784 \mathrm{E} \quad 00$

$3.82194 \mathrm{E} \quad 00$ $3.91605 \mathrm{E} \quad 00$ $4.01016 \mathrm{E} \quad 00$ $4.10427 \mathrm{E} 00$ $4.19839 \mathrm{E} 00$

$4.29250 \mathrm{E} \quad 00$ $4.38662 \mathrm{E} 00$ $4.48074 \mathrm{E} \quad 00$ $4.57486 \mathrm{E} \quad 00$ $4.66898 \mathrm{E} \quad 00$

$4.76310 \mathrm{E} \quad 00$ $4.85723 \mathrm{E} \quad 00$

$4.95135 \mathrm{E} \quad 00$

$5.04548 \mathrm{E} \quad 00$

$5.13960 \mathrm{E} \quad 00$ $g_{1}$

$-4.24641 \mathrm{E}-05$ $-2.47239 \mathrm{E}-04$ $-6.13516 \mathrm{E}-04$ $-1.12095 \mathrm{E}-03$ $-1.74533 \mathrm{E}-03$

$-2.46164 \mathrm{E}-03$ $-3.24623 \mathrm{E}-03$ $-4.07801 \mathrm{E}-03$ $-4.93899 \mathrm{E}-03$ $-5.81429 \mathrm{E}-03$

$-6.69182 \mathrm{E}-03$ $-7.56197 \mathrm{E}-03$ $-8.41711 \mathrm{E}-03$ $-9.25120 \mathrm{E}-03$ $-1.00595 \mathrm{E}-02$

$-1.08384 \mathrm{E}-02$ $-1.15848 \mathrm{E}-02$ $-1.22964 \mathrm{E}-02$ $-1.29715 \mathrm{E}-02$ $-1.36085 \mathrm{E}-02$

$-1.42063 \mathrm{E}-02$ $-1.47639 \mathrm{E}-02$ $-1.52806 \mathrm{E}-02$ $-1.57557 \mathrm{E}-02$ $-1.61888 \mathrm{E}-02$

$-1.65794 \mathrm{E}-02$ $-1.69272 \mathrm{E}-02$ $-1.72319 \mathrm{E}-02$ $-1.74934 \mathrm{E}-02$ $-1.77113 \mathrm{E}-02$

$-1.78856 \mathrm{E}-02$ $-1.80161 \mathrm{E}-02$ $-1.81027 \mathrm{E}-02$ $-1.81453 \mathrm{E}-02$ $-1.81439 \mathrm{E}-02$

$-1.80983 \mathrm{E}-02$ $-1.80085 \mathrm{E}-02$ $-1.78744 \mathrm{E}-02$ $-1.76961 \mathrm{E}-02$ $-1.74735 \mathrm{E}-02$

$-1.72065 \mathrm{E}-02$ $-1.68951 \mathrm{E}-02$ $-1.65393 \mathrm{E}-02$ $-1.61391 \mathrm{E}-02$ $-1.56944 \mathrm{E}-02$

$-1.52053 \mathrm{E}-02$ $-1.46717 \mathrm{E}-02$ $-1.40936 \mathrm{E}-02$ $-1.34711 \mathrm{E}-02$ $-1.28040 \mathrm{E}-02$

$-1.20924 \mathrm{E}-02$ $-1.13362 \mathrm{E}-02$ $-1.05356 \mathrm{E}-02$ $-9.69040 \mathrm{E}-03$ $-8.80067 \mathrm{E}-03$

$-7.86638 \mathrm{E}-03$ $-6.88753 \mathrm{E}-03$ $-5.86412 \mathrm{E}-03$ $-4.79615 \mathrm{E}-03$ $-3.68360 \mathrm{E}-03$ $\tan \delta_{1}$

1.94195E-01 9.46184 E-02 $6.29416 \mathrm{E}-02$ $4.72058 \mathrm{E}-02$ $3.77508 \mathrm{E}-02$

3.14202E-02

$2.68731 \mathrm{E}-02$

$2.34419 \mathrm{E}-02$

$2.07564 \mathrm{E}-02$

$1.85947 \mathrm{E}-02$

$1.68156 \mathrm{E}-02$

$1.53246 \mathrm{E}-02$

$1.40562 \mathrm{E}-02$

$1.29637 \mathrm{E}-02$

$1.20125 \mathrm{E}-02$

$1.11765 \mathrm{E}-02$

$1.04361 \mathrm{E}-02$

$9.77541 \mathrm{E}-03$

$9.18229 \mathrm{E}-03$

8.64678E-03

$8.16086 \mathrm{E}-03$

$7.71788 \mathrm{E}-03$

$7.31241 \mathrm{E}-03$

$6.93984 \mathrm{E}-03$

$6.59631 \mathrm{E}-03$

$6.27856 \mathrm{E}-03$

$5.98377 \mathrm{E}-03$

$5.70955 \mathrm{E}-03$

$5.45380 \mathrm{E}-03$

$5.21472 \mathrm{E}-03$

$4.99074 \mathrm{E}-03$

$4.78045 \mathrm{E}-03$

$4.58265 \mathrm{E}-03$

$4.39626 \mathrm{E}-03$

$4.22030 \mathrm{E}-03$

$4.05395 \mathrm{E}-03$

$3.89641 \mathrm{E}-03$

$3.74702 \mathrm{E}-03$

$3.60516 \mathrm{E}-03$

$3.47027 \mathrm{E}-03$

$3.34185 \mathrm{E}-03$

$3.21946 \mathrm{E}-03$

$3.10267 \mathrm{E}-03$

$2.99111 \mathrm{E}-03$

$2.88443 \mathrm{E}-03$

$2.78233 \mathrm{E}-03$

$2.68451 \mathrm{E}-03$

$2.59072 \mathrm{E}-03$

$2.50071 \mathrm{E}-03$

$2.41427 \mathrm{E}-03$

$2.33115 \mathrm{E}-03$

$2.25122 \mathrm{E}-03$

$2.17426 \mathrm{E}-03$

$2.10012 \mathrm{E}-03$

$2.02866 \mathrm{E}-03$

$1.95972 \mathrm{E}-03$

$1.89318 \mathrm{E}-03$

$1.82891 \mathrm{E}-03$

$1.76680 \mathrm{E}-03$

$1.70675 \mathrm{E}-03$

$f_{-1}$

$1.07475 \mathrm{E}-03$

$3.35147 \mathrm{E}-03$

$5.68695 \mathrm{E}-03$

$7.96677 \mathrm{E}-03$

$1.01616 \mathrm{E}-02$

$1.22597 \mathrm{E}-02$

$1.42573 \mathrm{E}-02$

$1.61548 \mathrm{E}-02$

$1.79555 \mathrm{E}-02$

$1.96639 \mathrm{E}-02$

$2.12847 \mathrm{E}-02$

$2.28231 \mathrm{E}-02$

$2.42836 \mathrm{E}-02$

$2.56705 \mathrm{E}-02$

$2.69875 \mathrm{E}-02$

$2.82379 \mathrm{E}-02$

$2.94247 \mathrm{E}-02$

$3.05501 \mathrm{E}-02$ 
$f_{2}$

$3.14664 \mathrm{E}-09$

$2.72298 \mathrm{E}-08$

$7.78383 \mathrm{E}-08$

$1.55096 \mathrm{E}-07$

$2.58705 \mathrm{E}-07$

$3.88343 \mathrm{E}-07$

$5.43726 \mathrm{E}-07$

$7.24616 \mathrm{E}-07$

$9.30825 \mathrm{E}-07$

$1.16220 \mathrm{E}-06$

$1.4 .1863 \mathrm{E}-06$

$1.70002 \mathrm{E}-06$

$2.00629 \mathrm{E}-06$

$2.33739 \mathrm{E}-06$

$2.69327 \mathrm{E}-06$

$3.07390 \mathrm{E}-06$

$3.47925 \mathrm{E}-06$

$3.90929 \mathrm{E}-06$

$4.36401 \mathrm{E}-06$

$4.84339 \mathrm{E}-06$

$5.34741 \mathrm{E}-06$

$5.87607 \mathrm{E}-06$

$6.42937 \mathrm{E}-06$

$7.00728 \mathrm{E}-06$

$7.60981 \mathrm{E}-06$

$8.23695 \mathrm{E}-06$ $8.88869 \mathrm{E}-06$ $9.56503 \mathrm{E}-06$ $1.02660 \mathrm{E}-05$ $1.09915 \mathrm{E}-05$

$1.17416 \mathrm{E}-05$

$1.25163 \mathrm{E}-05$

$1.33156 \mathrm{E}-05$

$1.41395 \mathrm{E}-05$

$1.49880 \mathrm{E}-05$

$1.58610 \mathrm{E}-05$

$1.67587 \mathrm{E}-05$

$1.76809 \mathrm{E}-05$

$1.86276 \mathrm{E}-05$

$1.95990 \mathrm{E}-05$

$2.05949 \mathrm{E}-05$

$2.16154 \mathrm{E}-05$

$2.26605 \mathrm{E}-05$

$2.37302 \mathrm{E}-05$

$2.48244 \mathrm{E}-05$

$2.59431 \mathrm{E}-05$

$2.70865 \mathrm{E}-05$

$2.82544 \mathrm{E}-05$

$2.94469 \mathrm{E}-05$

$3.06640 \mathrm{E}-05$

$3.19056 \mathrm{E}-05$

$3.31718 \mathrm{E}-05$

$3.44625 \mathrm{E}-05$

$3.57778 \mathrm{E}-05$

$3.71177 \mathrm{E}-05$

$3.84822 \mathrm{E}-05$

$3.98712 \mathrm{E}-05$

$4.12847 \mathrm{E}-05$

$4.27229 \mathrm{E}-05$

$4.41856 \mathrm{E}-05$
$7.96937 \mathrm{E}-07$

$9.21914 \mathrm{E}-06$

$3.44122 \mathrm{E}-05$

8.43084.E-05

$1.65329 \mathrm{E}-04$

$2.82376 \mathrm{E}-04$

$4.39009 \mathrm{E}-04$

$6.37681 \mathrm{E}-04$

$8.80018 \mathrm{E}-04$

$1.16704 \mathrm{E}-03$

$1.49936 \mathrm{E}-03$

$1.87730 \mathrm{E}-03$

2.30101 E-03

$2.77052 \mathrm{E}-03$

3.28581 E-03

3.84681 E-03

$4.45343 \mathrm{E}-03$

5.10557 E-03

$5.80314 \mathrm{E}-03$

6.54604 E-03

7.33419E-03

$8.16749 \mathrm{E}-03$

$9.04589 \mathrm{E}-03$

$9.96932 \mathrm{E}-03$

$1.09377 \mathrm{E}-02$

$1.19510 \mathrm{E}-02$

$1.30091 \mathrm{E}-02$

$1.41121 \mathrm{E}-02$

$1.52599 \mathrm{E}-02$

$1.64523 \mathrm{E}-02$

$1.76895 \mathrm{E}-02$

$1.89714 \mathrm{E}-02$

$2.02979 \mathrm{E}-02$

$2.16690 \mathrm{E}-02$

2.30848 E- 02

2.45451 E- 02

$2.60500 \mathrm{E}-02$

$2.75994 \mathrm{E}-02$

$2.91933 \mathrm{E}-02$

$3.08318 \mathrm{E}-02$

$3.25147 \mathrm{E}-02$

$3.42422 \mathrm{E}-02$

$3.60140 \mathrm{E}-02$

$3.78304 \mathrm{E}-02$

$3.96911 \mathrm{E}-02$

4.15962 E-02

$4.35457 \mathrm{E}-02$

$4.55396 \mathrm{E}-02$

$4.75779 \mathrm{E}-02$

$4.96604 \mathrm{E}-02$

5.17871 E-02 $5.39581 \mathrm{E}-02$ $5.61732 \mathrm{E}-02$ $5.84324 \mathrm{E}-02$ $6.07355 \mathrm{E}-02$

$6.30824 \mathrm{E}-02$ $6.54730 \mathrm{E}-02$ $6.79067 \mathrm{E}-02$

$7.03834 \mathrm{E}-02$

$7.29019 \mathrm{E}-02$ $\tan \delta_{2}$ $f_{-2}$ $-5.70229 \mathrm{E}-09$ $-6.57709 \mathrm{E}-08$ $-2.44316 \mathrm{E}-07$ $-5.94 .604 \mathrm{E}-07$

$-1.15637 \mathrm{E}-06$

$-1.95569 \mathrm{E}-06$ $-3.00648 \mathrm{E}-06$ $-4.31263 \mathrm{E}-06$ $-5.87048 \mathrm{E}-06$ $-7.67083 \mathrm{E}-06$

$-9.70064 \mathrm{E}-06$ $-1.19443 \mathrm{E}-05$

$-1.43845 \mathrm{E}-05$

$-1.70029 \mathrm{E}-05$

$-1.97803 \mathrm{E}-05$

$-2.26976 \mathrm{E}-05$

$-2.57349 \mathrm{E}-05$

$-2.88726 \mathrm{E}-05$

$-3.20910 \mathrm{E}-05$

$-3.53704 \mathrm{E}-05$

$-3.86910 \mathrm{E}-05$

$-4.20333 \mathrm{E}-05$

$-4.53776 \mathrm{E}-05$

$-4.87045 \mathrm{E}-05$

$-5.19945 \mathrm{E}-05$

$-5.52281 \mathrm{E}-05$ $-5.83861 \mathrm{E}-05$ $-6.14491 \mathrm{E}-05$

$-6.43978 \mathrm{E}-05$

$-6.72130 \mathrm{E}-05$

$-6.98754 \mathrm{E}-05$

$-7.23658 \mathrm{E}-05$

$-7.46652 \mathrm{E}-05$

$-7.67542 \mathrm{E}-05$

$-7.86139 \mathrm{E}-05$

$-8.02251 \mathrm{E}-05$ $-8.15687 \mathrm{E}-05$

$-8.26257 \mathrm{E}-05$

$-8.33769 \mathrm{E}-05$

$-8.38033 \mathrm{E}-05$

$-8.38859 \mathrm{E}-05$

$-8.36057 \mathrm{E}-05$

$-8.29435 \mathrm{E}-05$

$-8.18805 \mathrm{E}-05$

$-8.03975 \mathrm{E}-05$

$-7.84756 \mathrm{E}-05$

$-7.60958 \mathrm{E}-05$

$-7.32392 \mathrm{E}-05$

$-6.98866 \mathrm{E}-05$

$-6.60193 \mathrm{E}-05$

$-6.16181 \mathrm{E}-05$ $-5.66641 \mathrm{E}-05$ $-5.11385 \mathrm{E}-05$ $-4.50223 \mathrm{E}-05$ $-3.82966 \mathrm{E}-05$

$-3.09426 \mathrm{E}-05$ $-2.29416 \mathrm{E}-05$ $-1.42747 \mathrm{E}-05$ $-4.92360 \mathrm{E}-06$ $5.13002 \mathrm{E}-06$
$-2.31124 \mathrm{E} \quad 00$

$-4.78710 \mathrm{E} \quad 00$

$-7.10542 \mathrm{E} \quad 00$

$-9.27020 \mathrm{E}$ 00

$-1.12671 \mathrm{E} 01$

$-1.30887 \mathrm{E}$ 0l

$-1.47360 \mathrm{E} 01$

$-1.62163 \mathrm{E} 01$

$-1.75413 \mathrm{E} \quad 01$

$-1.87247 \mathrm{E} 01$

$-1.97809 \mathrm{E} \quad 01$

$-2.07240 \mathrm{E} \quad 0 \mathrm{I}$

$-2.15673 \mathrm{E}$ 01

$-2.23226 \mathrm{E}$ 01

$-2.30007 \mathrm{E} 01$

$-2.36111 \mathrm{E} 01$

$-2.41621 \mathrm{E} \quad 01$

$-2.46609 \mathrm{E} 01$

$-2.51136 \mathrm{E} 01$

$-2.55258 \mathrm{E}$ 0l

$-2.59021 \mathrm{E} \quad 01$

$-2.62465 \mathrm{E} \quad 01$

$\begin{array}{lll}-2.65627 \mathrm{E} & 01\end{array}$

$-2.68537 \mathrm{E}$ 01

$\begin{array}{lll}-2.71221 \mathrm{E} & 01\end{array}$

$-2.73703 \mathrm{E} \quad 01$

$-2.76003 \mathrm{E} 01$

$-2.78140 \mathrm{E} \quad 01$

$-2.80129 \mathrm{E} 01$

$-2.81984 \mathrm{E} \quad 01$

$-2.83718 \mathrm{E} \quad 01$

$-2.85341 \mathrm{E} 01$

$-2.86863 \mathrm{E} 01$

$-2.88292 \mathrm{E} \quad 01$

$-2.89638 \mathrm{E} 01$

$-2.90905 \mathrm{E} \quad 01$

$-2.92102 \mathrm{E} \quad 01$

$-2.93232 \mathrm{E}$ 01

$-2.94302 \mathrm{E} \quad 01$

$-2.95316 \mathrm{E}$ 01

$-2.96278 \mathrm{E} \quad 01$

$\begin{array}{lll}-2.97192 \mathrm{E} & 01\end{array}$

$-2.98061 \mathrm{E} \quad 01$

$-2.98888 \mathrm{E}$ 01

$-2.99676 \mathrm{E} \quad 01$

$-3.00428 \mathrm{E} 01$

$-3.01146 \mathrm{E}$ 01

$-3.01832 \mathrm{E} \quad 01$

$-3.02489 \mathrm{E} 01$

$-3.03118 \mathrm{E}$ 0l

$\begin{array}{ll}-3.03720 \mathrm{E} & 0 \mathrm{I}\end{array}$

$-3.04298 \mathrm{E} 01$

$-3.04852 \mathrm{E} \quad 01$

$-3.05385 \mathrm{E}$ 0l

$-3.05897 \mathrm{E} 01$

$-3.06390 \mathrm{E} \quad 01$

$-3.06864 \mathrm{E} \quad 01$

$-3.07321 \mathrm{E} \quad 01$

$-3.07761 \mathrm{E} 01$

$-3.08185 \mathrm{E} 01$

$1.58488 \mathrm{E}-07$

$9.27104 \mathrm{E}-07$

$2.32961 \mathrm{E}-06$

$4.33104 \mathrm{E}-06$

$6.88925 \mathrm{E}-06$

$9.95981 \mathrm{E}-06$

$1.34991 \mathrm{E}-0.5$

$1.74661 \mathrm{E}-05$

$2.18232 \mathrm{E}-0.5$

$2.65362 \mathrm{E}-05$

$3.15739 \mathrm{E}-05$

$3.69079 \mathrm{E}-05$

$4.25121 \mathrm{E}-05$

$4.83618 \mathrm{E}-05$

$5.44339 \mathrm{E}-05$

$6.07062 \mathrm{E}-05$

$6.71574 \mathrm{E}-05$

$7.37667 \mathrm{E}-05$

$8.05136 \mathrm{E}-05$

$8.73784 \mathrm{E}-05$

$9.43412 \mathrm{E}-05$

$1.01382 \mathrm{E}-04$

$1.08483 \mathrm{E}-04$

$1.15623 \mathrm{E}-04$

$1.22784 \mathrm{E}-04$

$1.29947 \mathrm{E}-04$

$1.37092 \mathrm{E}-04$

$1.44200 \mathrm{E}-04$

$1.51253 \mathrm{E}-04$

$1.58231 \mathrm{E}-04$

$1.65116 \mathrm{E}-04$

$1.71889 \mathrm{E}-04$

$1.78530 \mathrm{E}-04$

$1.85021 \mathrm{E}-04$

$1.91342 \mathrm{E}-04$

$1.97476 \mathrm{E}-04$

$2.03402 \mathrm{E}-04$

$2.09103 \mathrm{E}-04$

$2.14558 \mathrm{E}-04$

$2.19750 \mathrm{E}-04$

$2.24659 \mathrm{E}-04$

$2.29266 \mathrm{E}-04$

$2.33553 \mathrm{E}-04$ 
ELECTRON RADIAL FUNCTIONS

\begin{tabular}{|c|c|c|c|c|c|c|}
\hline$p$ & $F_{0}$ & $f_{1}$ & & $g_{1}$ & $\tan \delta_{1}$ & $f_{-1}$ \\
\hline 6.10 & $8.75207 \mathrm{E}-01$ & $5.23373 \mathrm{E}$ & 00 & $-2.52647 \mathrm{E}-03$ & $1.64866 \mathrm{E}-03$ & $3.22102 \mathrm{E}-02$ \\
\hline 6.20 & $8.75233 \mathrm{E}-01$ & $5.32785 \mathrm{E}$ & 00 & $-1.32477 \mathrm{E}-03$ & $1.59243 \mathrm{E}-03$ & $3.12394 \mathrm{E}-02$ \\
\hline 6.30 & $8.75257 \mathrm{E}-01$ & $5.42198 \mathrm{E}$ & 00 & $-7.84830 \mathrm{E}-05$ & $1.53797 \mathrm{E}-03$ & $3.02238 \mathrm{E}-02$ \\
\hline 6.40 & $8.75280 \mathrm{E}-01$ & $5.51611 \mathrm{E}$ & 00 & $1.21239 \mathrm{E}-03$ & $1.48521 \mathrm{E}-03$ & $2.91632 \mathrm{E}-02$ \\
\hline 6.50 & $8.75300 \mathrm{E}-01$ & $5.61023 \mathrm{E}$ & 00 & $2.54785 \mathrm{E}-03$ & $1.43405 \mathrm{E}-03$ & $2.80577 \mathrm{E}-02$ \\
\hline 6.60 & $8.75319 \mathrm{E}-01$ & $5.70436 \mathrm{E}$ & 00 & $3.92790 \mathrm{E}-03$ & $1.38444 \mathrm{E}-03$ & $2.69073 \mathrm{E}-02$ \\
\hline 6.70 & $8.75336 \mathrm{E}-01$ & $5.79848 \mathrm{E}$ & 00 & $5.35253 \mathrm{E}-03$ & $1.33631 \mathrm{E}-03$ & $2.57120 \mathrm{E}-02$ \\
\hline 6.80 & $8.75352 \mathrm{E}-01$ & $5.89259 \mathrm{E}$ & 00 & $6.82176 \mathrm{E}-03$ & $1.28959 \mathrm{E}-03$ & $2.44718 \mathrm{E}-02$ \\
\hline 6.90 & $8.75366 \mathrm{E}-01$ & $5.98669 \mathrm{E}$ & 00 & $8.33556 \mathrm{E}-03$ & $1.24422 \mathrm{E}-03$ & $2.31868 \mathrm{E}-02$ \\
\hline 7.00 & $8.75379 \mathrm{E}-01$ & $6.08080 \mathrm{E}$ & 00 & $9.89396 \mathrm{E}-03$ & $1.20014 \mathrm{E}-03$ & $2.18569 \mathrm{E}-02$ \\
\hline 7.10 & $8.75391 \mathrm{E}-01$ & $6.17488 \mathrm{E}$ & 00 & $1.14969 \mathrm{E}-02$ & $1.15730 \mathrm{E}-03$ & $2.04822 \mathrm{E}-02$ \\
\hline 7.20 & $8.75401 \mathrm{E}-01$ & $6.26895 \mathrm{E}$ & 00 & $1.31444 \mathrm{E}-02$ & $1.11565 \mathrm{E}-03$ & $1.90626 \mathrm{E}-02$ \\
\hline 7.30 & $8.75410 \mathrm{E}-01$ & $6.36299 \mathrm{E}$ & 00 & $1.48364 \mathrm{E}-02$ & $1.07514 \mathrm{E}-03$ & $1.75982 \mathrm{E}-02$ \\
\hline 7.40 & $8.75419 \mathrm{E}-01$ & $6.45699 \mathrm{E}$ & 00 & $1.65729 \mathrm{E}-02$ & $1.03572 \mathrm{E}-03$ & $1.60889 \mathrm{E}-02$ \\
\hline 7.50 & $8.75427 \mathrm{E}-01$ & $6.55093 \mathbf{E}$ & 00 & $1.83538 \mathrm{E}-02$ & $9.97353 \mathrm{E}-04$ & $1.45348 \mathrm{E}-02$ \\
\hline 7.60 & $8.75434 \mathrm{E}-01$ & $6.64479 \mathrm{E}$ & 00 & $2.01789 \mathrm{E}-02$ & $9.60003 \mathrm{E}-04$ & $1.29358 \mathrm{E}-02$ \\
\hline 7.70 & $8.75440 \mathrm{E}-01$ & $6.73849 \mathrm{E}$ & 00 & $2.20480 \mathrm{E}-02$ & $9.23621 \mathrm{E}-04$ & $1.12920 \mathrm{E}-02$ \\
\hline 7.80 & $8.75445 \mathrm{E}-01$ & $6.83193 \mathrm{E}$ & 00 & $2.39606 \mathrm{E}-02$ & $8.88163 \mathrm{E}-04$ & $9.60341 \mathrm{E}-03$ \\
\hline 7.90 & $8.75450 \mathrm{E}-01$ & $6.92476 \mathrm{E}$ & 00 & $2.59152 \mathrm{E}-02$ & $8.53620 \mathrm{E}-04$ & $7.86994 \mathrm{E}-03$ \\
\hline 8.00 & $8.75453 \mathrm{E}-01$ & $7.01592 \mathrm{E}$ & 00 & $2.79072 \mathrm{E}-02$ & $8.19931 \mathrm{E}-04$ & $6.09163 \mathrm{E}-03$ \\
\hline 8.10 & $8.75456 \mathrm{E}-01$ & $7.09846 \mathrm{E}$ & 00 & $2.99061 \mathrm{E}-02$ & $7.87080 \mathrm{E}-04$ & $4.26848 \mathrm{E}-03$ \\
\hline 8.20 & $8.75459 \mathrm{E}-01$ & $7.26117 \mathrm{E}$ & 00 & $3.23008 \mathrm{E}-02$ & $7.55039 \mathrm{E}-04$ & $2.40050 \mathrm{E}-03$ \\
\hline 8.30 & $8.75460 \mathrm{E}-01$ & $7.31796 \mathrm{E}$ & 00 & $3.42763 \mathrm{E}-02$ & $7.23770 \mathrm{E}-04$ & $4.87667 \mathrm{E}-04$ \\
\hline 8.40 & $8.75462 \mathrm{E}-01$ & $7.40745 \mathrm{E}$ & 00 & $3.64397 \mathrm{E}-02$ & $6.93260 \mathrm{E}-04$ & $-1.47001 \mathrm{E}-03$ \\
\hline 8.50 & $8.75463 \mathrm{E}-01$ & $7.49989 \mathrm{E}$ & 00 & $3.86608 \mathrm{E}-02$ & $6.63463 \mathrm{E}-04$ & $-3.47253 \mathrm{E}-03$ \\
\hline 8.60 & $8.75463 \mathrm{E}-01$ & $7.59319 \mathrm{E}$ & 00 & $4.09303 \mathrm{E}-02$ & $6.34362 \mathrm{E}-04$ & $-5.51989 \mathrm{E}-03$ \\
\hline 8.70 & $8.75463 \mathbf{E}-01$ & $7.68684 \mathrm{E}$ & 00 & $4.32460 \mathrm{E}-02$ & $6.05935 \mathrm{E}-04$ & $61212 \mathrm{E}-03$ \\
\hline 8.80 & $8.75463 \mathrm{E}-01$ & $7.78071 \mathrm{E}$ & 00 & $4.56075 \mathrm{E}-02$ & $5.78163 \mathrm{E}-04$ & $-9.74919 \mathrm{E}-03$ \\
\hline 8.90 & $8.75462 \mathbf{E}-01$ & $7.87473 \mathrm{E}$ & 00 & $4.80143 \mathrm{E}-02$ & $5.51022 \mathrm{E}-04$ & $-1.19311 \mathrm{E}-02$ \\
\hline 9.00 & $8.75460 \mathrm{E}-01$ & $7.96881 \mathrm{E}$ & 00 & $5.04661 \mathrm{E}-02$ & $5.24487 \mathrm{E}-04$ & $-1.41579 \mathrm{E}-02$ \\
\hline 9.10 & $8.75459 \mathrm{E}-01$ & $8.06295 \mathrm{E}$ & 00 & $5.29629 \mathrm{E}-02$ & $4.98540 \mathrm{E}-04$ & $-1.64296 \mathrm{E}-02$ \\
\hline 9.20 & $8.75457 \mathrm{E}-01$ & $8.15714 \mathrm{E}$ & 00 & $5.55047 \mathrm{E}-02$ & $4.73163 \mathrm{E}-04$ & $-1.87461 \mathrm{E}-02$ \\
\hline 9.30 & $8.75454 \mathrm{E}-01$ & $8.25136 \mathrm{E}$ & 00 & $5.80914 \mathrm{E}-02$ & $4.48343 \mathrm{E}-04$ & $-2.11076 \mathrm{E}-02$ \\
\hline 9.40 & $8.75452 \mathrm{E}-01$ & $8.34561 \mathrm{E}$ & 00 & $6.07230 \mathrm{E}-02$ & $4.24056 \mathrm{E}-04$ & $-2.35139 \mathrm{E}-02$ \\
\hline 9.50 & $8.75449 \mathrm{E}-01$ & $8.43988 \mathrm{E}$ & 00 & $6.33993 \mathrm{E}-02$ & $4.00287 \mathrm{E}-04$ & $-2.59651 \mathrm{E}-02$ \\
\hline 9.60 & $8.75446 \mathrm{E}-01$ & $8.53418 \mathrm{E}$ & 00 & $6.61207 \mathrm{E}-02$ & $3.77020 \mathrm{E}-04$ & $-2.84612 \mathrm{E}-02$ \\
\hline 9.70 & $8.75442 \mathrm{E}-01$ & $8.62848 \mathrm{E}$ & 00 & $6.88868 \mathrm{E}-02$ & $3.54240 \mathrm{E}-04$ & $-3.10021 \mathrm{E}-02$ \\
\hline 9.80 & $8.75439 \mathrm{E}-01$ & $8.72282 \mathrm{E}$ & 00 & $7.16979 \mathrm{E}-02$ & $3.3194 .0 \mathrm{E}-04$ & $-3.35880 \mathrm{E}-02$ \\
\hline 9.90 & $8.75435 \mathrm{E}-$ & $8.81716 \mathrm{E}$ & 00 & $7.45538 \mathrm{E}-02$ & $3.10089 \mathrm{E}-04$ & $-3.62188 \mathrm{E}-02$ \\
\hline 10.00 & $8.75430 \mathrm{E}-01$ & $8.91151 \mathrm{E}$ & 00 & $7.74545 \mathrm{E}-02$ & $2.88683 \mathrm{E}-04$ & $-3.88946 \mathrm{E}-02$ \\
\hline 10.10 & $8.75426 \mathrm{E}-01$ & $9.00589 \mathrm{E}$ & 00 & $8.04003 \mathrm{E}-02$ & $2.67711 \mathrm{E}-04$ & $-4.16152 \mathrm{E}-02$ \\
\hline 10.20 & $8.75422 \mathrm{E}-01$ & $9.10028 \mathrm{E}$ & 00 & $8.33908 \mathrm{E}-02$ & $2.47154 \mathrm{E}-04$ & $-4.43808 \mathrm{E}-02$ \\
\hline 10.30 & $8.75417 \mathrm{E}-01$ & $9.19467 \mathrm{E}$ & 00 & $8.64262 \mathrm{E}-02$ & $2.27013 \mathrm{E}$ & $-4.71914 \mathrm{E}-02$ \\
\hline 10.40 & 8.75412 E-01 & $9.28908 \mathrm{E}$ & 00 & $8.95066 \mathrm{E}-02$ & $2.07258 \mathrm{E}-04$ & $-5.00469 \mathrm{E}-0$ \\
\hline 10.50 & $8.75407 \mathrm{E}-01$ & $9.38350 \mathrm{E}$ & 00 & $9.26318 \mathrm{E}-02$ & $1.87894 \mathrm{E}-04$ & $-5.29473 \mathrm{E}-02$ \\
\hline 10.60 & $8.75402 \mathrm{E}-01$ & $9.47794 \mathrm{E}$ & 00 & $9.58020 \mathrm{E}-02$ & $1.68906 \mathrm{E}-04$ & $-5.58927 \mathrm{E}-02$ \\
\hline 10.70 & $8.75396 \mathrm{E}-01$ & $9.57239 \mathrm{E}$ & 00 & $9.90171 \mathrm{E}-02$ & $1.50277 \mathrm{E}-04$ & $-5.88831 \mathrm{E}-02$ \\
\hline 10.80 & $8.75391 \mathrm{E}-01$ & $9.66684 \mathrm{E}$ & 00 & $1.02277 \mathrm{E}-01$ & $1.32002 \mathrm{E}-04$ & $-6.19184 \mathrm{E}-02$ \\
\hline 10.90 & $8.75385 \mathrm{E}-01$ & $9.76131 \mathrm{E}$ & 00 & $1.05582 \mathrm{E}-01$ & $1.14070 \mathrm{E}-04$ & $-6.49988 \mathrm{E}-02$ \\
\hline 11.00 & $8.75379 \mathrm{E}-01$ & $9.85579 \mathrm{E}$ & 00 & $1.08932 \mathrm{E}-01$ & $9.64758 \mathrm{E}-05$ & $-6.81241 \mathrm{E}-02$ \\
\hline 11.10 & $8.75374 \mathrm{E}-01$ & $9.95029 \mathrm{E}$ & 00 & $1.12327 \mathrm{E}-01$ & $7.92062 \mathrm{E}-05$ & $-7.12945 \mathrm{E}-02$ \\
\hline 11.20 & $8.75368 \mathrm{E}-01$ & $1.00448 \mathrm{E}$ & 01 & $1.15767 \mathrm{E}-01$ & $6.22583 \mathrm{E}-05$ & $-7.45098 \mathrm{E}-02$ \\
\hline 11.30 & $8.75362 \mathrm{E}-01$ & $1.01393 \mathrm{E}$ & 01 & $1.19251 \mathrm{E}-01$ & $4.56149 \mathrm{E}-05$ & $-7.77702 \mathrm{E}-02$ \\
\hline 11.40 & $8.75356 \mathrm{E}-01$ & $1.02338 \mathrm{E}$ & 01 & $1.22781 \mathrm{E}-01$ & $2.92718 \mathrm{E}-05$ & $-8.10756 \mathrm{E}-02$ \\
\hline 11.50 & $8.75349 \mathrm{E}-01$ & $1.03284 \mathrm{E}$ & 01 & $1.26356 \mathrm{E}-01$ & $1.32262 \mathrm{E}-05$ & $-8.44261 \mathrm{E}-02$ \\
\hline 11.60 & $8.75343 \mathrm{E}-01$ & $1.04229 \mathrm{E}$ & 01 & $1.29976 \mathrm{E}-01$ & $-2.54526 \mathrm{E}-06$ & $-8.78216 \mathrm{E}-02$ \\
\hline & $8.75336 \mathrm{E}-01$ & $1.05175 \mathrm{E}$ & 01 & $1.33640 \mathrm{E}-$ & $-1.80225 \mathrm{E}-$ & $-9.12622 \mathrm{E}-02$ \\
\hline & $8.75330 \mathrm{E}-01$ & $1.06120 \mathrm{E}$ & 01 & $1.37350 \mathrm{E}-$ & $3.32360 \mathrm{E}-\mathrm{C}$ & $-9.47478 \mathrm{E}-0$ \\
\hline 11.90 & $8.75323 \mathrm{E}-01$ & $1.07066 \mathrm{E}$ & 01 & $1.41105 \mathrm{E}-01$ & $-4.81815 \mathrm{E}-05$ & $-9.82786 \mathrm{E}-02$ \\
\hline 12.00 & $8.75316 \mathrm{E}-01$ & $1.08012 \mathrm{E}$ & 01 & $1.44905 \mathrm{E}-01$ & $-6.28659 \mathrm{E}-05$ & $-1.01854 \mathrm{E}-01$ \\
\hline
\end{tabular}

$g_{-1} \quad \tan \delta_{-1}$

$6.16377 \mathrm{E} \quad 00$ $6.25834 \mathrm{E} \quad 00$

$6.35290 \mathrm{E} \quad 00$

$6.44745 \mathrm{E} 00$

$6.54199 \mathrm{E} \quad 00$

$6.63653 \mathrm{E} \quad 00$

$6.73106 \mathrm{E} \quad 00$

$6.82558 \mathrm{E} \quad 00$

$6.92010 \mathrm{E} \quad 00$

$7.01462 \mathrm{E} \quad 00$

$7.10913 \mathrm{E} \quad 00$

$7.20364 \mathrm{E} \quad 00$

$7.29815 \mathrm{E} \quad 00$

$7.39265 \mathrm{E} \quad 00$

$7.48716 \mathrm{E} \quad 00$

$7.58167 \mathrm{E} \quad 00$

$7.67617 \mathrm{E} \quad 00$

$7.77068 \mathrm{E} \quad 00$

$7.86519 \mathrm{E} \quad 00$

$7.95970 \mathrm{E} \quad 00$

$8.05421 \mathrm{E} \quad 00$

$8.14872 \mathrm{E} \quad 00$

8.24324. E 00

$8.33776 \mathrm{E} \quad 00$

$8.43228 \mathrm{E} \quad 00$

$8.52681 \mathrm{E} \quad 00$

$8.62134 \mathrm{E} \quad 00$

$8.71588 \mathrm{E} \quad 00$

$8.81042 \mathrm{E} \quad 00$

$8.90496 \mathbf{E} \quad 00$

$8.99952 \mathrm{E} \quad 00$ $9.09407 \mathrm{E} \quad 00$

$9.18864 \mathrm{E} \quad 00$

$9.28321 \mathrm{E} \quad 00$

$9.37778 \mathrm{E} \quad 00$

$9.47237 \mathrm{E} \quad 00$

$9.56696 \mathrm{E} \quad 00$

$9.66156 \mathrm{E} \quad 00$

$9.75616 \mathrm{E} \quad 00$

$9.85077 \mathrm{E} \quad 00$

$9.94540 \mathrm{E} \quad 00$ $1.00400 \mathrm{E} \quad 01$ $1.01347 \mathrm{E} 01$ $1.02293 \mathrm{E} 01$

$1.03240 \mathrm{E} \quad 01$

$1.04186 \mathrm{E} \quad 01$ $1.05133 \mathrm{E} 01$ $1.06080 \mathrm{E} \quad 01$ $1.07027 \mathrm{E} \quad 01$ $1.07974 \mathrm{E} 01$

$1.08921 \mathrm{E} \quad 01$ $1.09868 \mathrm{E} \quad 01$ $1.10815 \mathrm{E} \quad 01$ $1.11763 \mathrm{E} 01$ $1.12710 \mathrm{E} \quad 01$

$1.13658 \mathrm{E} \quad 01$ $1.14606 \mathrm{E} \quad 01$ $1.15553 \mathrm{E} 01$ $1.16501 \mathrm{E} 01$ $1.17449 \mathrm{E} 01$
$1.80936 \mathrm{E} \quad 02$

$1.82908 \mathrm{E} \quad 02$

$\begin{array}{ll}1.84857 \mathrm{E} & 02\end{array}$

$1.86786 \mathrm{E} \quad 02$

$\begin{array}{ll}1.88694 \mathrm{E} & 02\end{array}$

$1.90582 \mathrm{E} \quad 02$

$1.92450 \mathrm{E} \quad 02$

$1.94297 \mathrm{E} \quad 02$

$1.96126 \mathrm{E} \quad 02$

$1.97935 \mathrm{E} \quad 02$

$1.99726 \mathrm{E} \quad 02$ $2.01498 \mathrm{E} \quad 02$ $2.03252 \mathrm{E} \quad 02$

$2.04988 \mathrm{E} \quad 02$

$2.06707 \mathrm{E} \quad 02$

$2.08408 \mathrm{E} \quad 02$

$2.10093 \mathrm{E} \quad 02$

$2.11760 \mathrm{E} \quad 02$

$2.13412 \mathrm{E} \quad 02$

$2.15047 \mathrm{E} \quad 02$

$2.16667 \mathrm{E} \quad 02$

$2.18271 \mathrm{E} \quad 02$

$2.19859 \mathrm{E} \quad 02$

$2.21433 \mathrm{E} \quad 02$

$2.22991 \mathrm{E} \quad 02$

$2.24535 \mathrm{E} \quad 02$

$2.26065 \mathrm{E} \quad 02$

$2.27580 \mathrm{E} \quad 02$

$2.29081 \mathrm{E} \quad 02$

$2.30569 \mathrm{E} \quad 02$

$2.32043 \mathrm{E} \quad 02$

$2.33504 \mathrm{E} \quad 02$

$2.34952 \mathrm{E} \quad 02$

$2.36387 \mathrm{E} \quad 02$

$2.37809 \mathrm{E} \quad 02$

$2.39219 \mathrm{E} \quad 02$

$2.40616 \mathrm{E} \quad 02$

$2.42002 \mathrm{E} \quad 02$

$2.43375 \mathrm{E} \quad 02$

$2.44737 \mathrm{E} \quad 02$

$2.46087 \mathrm{E} \quad 02$

$2.47425 \mathrm{E} \quad 02$

$2.48753 \mathrm{E} \quad 02$

$2.50069 \mathrm{E} \quad 02$

$2.51375 \mathrm{E} \quad 02$

$2.52670 \mathrm{E} \quad 02$ $2.53954 \mathrm{E} \quad 02$

$2.55228 \mathrm{E} \quad 02$

$2.56491 \mathrm{E} \quad 02$

$2.57745 \mathrm{E} \quad 02$

$2.58988 \mathrm{E} \quad 02$

$2.60222 \mathrm{E} \quad 02$

$2.61446 \mathrm{E} \quad 02$

$2.62660 \mathrm{E} \quad 02$

$2.63866 \mathrm{E} \quad 02$

$2.65061 \mathrm{E} \quad 02$

$2.66248 \mathrm{E} \quad 02$

$2.67426 \mathrm{E} \quad 02$

$2.68595 \mathrm{E} \quad 02$

$2.69755 \mathrm{E} \quad 02$ 
ELECTRON RADIAL FUNCTIONS

$Z=6 \quad A=12 \quad \rho=1.2 A^{1 / 3} F$

Pusitron

p

6.10

6.20

6.30

6.40

6.50

6.60

6.70

6.80

6.90

7.00

7.10

7.20

7.30

7.40

7.50

7.60

7.70

7.80

7.90

8.00

8.10

8.20

8.30

8.40

8.50

8.60

8.70

8.80

8.90

9.00

9.10

9.20

9.30

9.40

9.50

9.60

9.70

9.80

9.90

10.00

10.10

10.20

10.30

10.40

10.50

10.60

10.70

10.80

10.90

11.00

11.10

11.20

11.30

11.40

11.50

11.60

11.70

11.80

11.90

12.00

\section{$\bar{F}$}

$4.56729 \mathrm{E}-05$

$4.71847 \mathrm{E}-05$

$4.87211 \mathrm{E}-05$

$5.02820 \mathrm{E}-05$

$5.18674 \mathrm{E}-05$

$5.34775 \mathrm{E}-05$ $5.51121 \mathrm{E}-05$ $5.67713 \mathrm{E}-05$ $5.84550 \mathrm{E}-05$ $6.01633 \mathrm{E}-05$

$6.18961 \mathrm{E}-05$ $6.36535 \mathrm{E}-05$ $6.54355 \mathrm{E}-05$ $6.72420 \mathrm{E}-05$ $6.90730 \mathrm{E}-05$

$7.09287 \mathrm{E}-05$ $7.28088 \mathrm{E}-05$

$7.47135 \mathrm{E}-05$

$7.66428 \mathrm{E}-0.5$

$7.85966 \mathrm{E}-05$

$8.05750 \mathrm{E}-05$ $8.25780 \mathrm{E}-05$ $8.46055 \mathrm{E}-05$ $8.66575 \mathrm{E}-05$ $8.87341 \mathrm{E}-05$

$9.08352 \mathrm{E}-0.5$ $9.29609 \mathrm{E}-05$ $9.51111 \mathrm{E}-05$ $9.72859 \mathrm{E}-05$ $9.94853 \mathrm{E}-05$

$1.01709 \mathrm{E}-04$ $1.03958 \mathrm{E}-04$ $1.06231 \mathrm{E}-04$ $1.08528 \mathrm{E}-04$

$1.10850 \mathrm{E}-04$

$1.13197 \mathrm{E}-04$ $1.15568 \mathrm{E}-04$ $1.17964 \mathrm{E}-04$ $1.20384 \mathrm{E}-04$ $1.22829 \mathrm{E}-04$

$1.25298 \mathrm{E}-04$ $1.27792 \mathrm{E}-04$ $1.30311 \mathrm{E}-04$ $1.32854 \mathrm{E}-04$ $1.35421 \mathrm{E}-04$

$1.38013 \mathbf{E}-04$

$1.40630 \mathrm{E}-04$

$1.43271 \mathrm{E}-04$

$1.45937 \mathrm{E}-04$

$1.48627 \mathrm{E}-04$.

$1.51342 \mathrm{E}-04$

$1.54082 \mathrm{E}-04$

$1.56846 \mathrm{E}-04$

$1.59634 \mathrm{E}-04$

$1.62447 \mathrm{E}-04$

$1.6528 .5 \mathrm{E}-04$ $1.68147 \mathrm{E}-04$ $1.71033 \mathrm{E}-04$ $1.73945 \mathrm{E}-04$ $1.76880 \mathrm{E}-04$ $f_{2}$

$g_{2}$

$\tan \delta_{2}$

$f_{-2}$

7.54611 E-02

$7.80581 \mathrm{E}-02$

$8.06866 \mathrm{E}-02$

8.33281 E-02

$8.5904 .9 \mathrm{E}-02$

$8.65776 \mathrm{E}-02$

$9.24616 \mathrm{E}-02$

$9.50476 \mathrm{E}-02$

$9.78890 \mathrm{E}-02$

$1.00821 \mathrm{E}-01$

$1.03814 \mathrm{E}-01$

$1.06860 \mathrm{E}-01$

$1.09955 \mathrm{E}-01$

$1.13096 \mathrm{E}-01$

$1.16285 \mathrm{E}-01$

$1.19519 \mathrm{E}-01$

$1.22799 \mathrm{E}-01$

$1.26123 \mathrm{E}-01$

$1.29493 \mathrm{E}-01$

$1.32908 \mathrm{E}-01$

$1.36367 \mathrm{E}-01$

$1.39871 \mathrm{E}-01$

$1.43419 \mathrm{E}-01$

$1.47012 \mathrm{E}-01$

$1.50650 \mathrm{E}-01$

$1.54332 \mathrm{E}-01$

$1.58059 \mathrm{E}-01$

$1.61830 \mathrm{E}-01$

$1.65645 \mathrm{E}-01$

$1.69506 \mathrm{E}-01$

$1.73410 \mathrm{E}-01$

$1.77359 \mathrm{E}-01$

$1.81353 \mathrm{E}-01$

$1.85391 \mathrm{E}-01$

$1.89473 \mathrm{E}-01$

$1.93599 \mathrm{E}-01$

$1.97771 \mathrm{E}-01$

$2.01986 \mathrm{E}-01$

$2.06246 \mathrm{E}-01$

$2.10551 \mathrm{E}-01$

2.14899E-01 $2.19293 \mathrm{E}-01$ $2.23730 \mathrm{E}-01$ $2.28212 \mathrm{E}-01$

$2.32739 \mathrm{E}-01$

2.37309E-01

$2.41924 \mathrm{E}-01$

$2.46584 \mathrm{E}-01$

$2.51288 \mathrm{E}-01$

$2.56036 \mathrm{E}-01$

$2.60829 \mathrm{E}-01$

$2.65666 \mathrm{E}-01$

$2.70547 \mathrm{E}-01$

$2.75473 \mathrm{E}-01$

2.80443 E-01

$2.85457 \mathrm{E}-01$ $2.90516 \mathrm{E}-01$

$2.95619 \mathrm{E}-01$

$3.00767 \mathrm{E}-01$

$3.05959 \mathrm{E}-01$
$1.59039 \mathrm{E}-05$

$2.74142 \mathrm{E}-05$

$3.96740 \mathrm{E}-05$

$5.26853 \mathrm{E}-05$

$6.63937 \mathrm{E}-05$

$7.90917 \mathrm{E}-05$

$9.74770 \mathrm{E}-05$

$1.13582 \mathrm{E}-04$

$1.30760 \mathrm{E}-04$

$1.48875 \mathrm{E}-04$

$1.67920 \mathrm{E}-04$

$1.87905 \mathrm{E}-04$

$2.08847 \mathrm{E}-04$

$2.30760 \mathrm{E}-04$

$2.53664 \mathrm{E}-04$

$2.77579 \mathrm{E}-04$

$3.02522 \mathrm{E}-04$

$3.28511 \mathrm{E}-04$

$3.55565 \mathrm{E}-04$

$3.83705 \mathrm{E}-04$

4.12947E-04

$4.43312 \mathrm{E}-04$

$4.74818 \mathrm{E}-04$

$5.07483 \mathrm{E}-04$

$5.41328 \mathrm{E}-04$.

5.76371 E-04 $6.12630 \mathrm{E}-04$

$6.50126 \mathrm{E}-04$

$6.88875 \mathrm{E}-04$

$7.28899 \mathrm{E}-04$

$7.70215 \mathrm{E}-04$ $8.12842 \mathrm{E}-04$

$8.56800 \mathrm{E}-04$

$9.02108 \mathrm{E}-04$

$9.48783 \mathrm{E}-04$

$9.96846 \mathrm{E}-04$ $1.04632 \mathrm{E}-03$ $1.09721 \mathrm{E}-03$

$1.14955 \mathrm{E}-03$

$1.20335 \mathrm{E}-03$

$1.25863 \mathrm{E}-03$ $1.31542 \mathrm{E}-03$ $1.37372 \mathrm{E}-03$ $1.43357 \mathrm{E}-03$ $1.49497 \mathrm{E}-03$

$1.55794 \mathrm{E}-03$

$1.62252 \mathrm{E}-03$

$1.68871 \mathrm{E}-03$

$1.75653 \mathrm{E}-03$

$1.82600 \mathrm{E}-03$

$1.89715 \mathrm{E}-03$

$1.96998 \mathrm{E}-03$

$2.04453 \mathrm{E}-03$

$2.12080 \mathrm{E}-03$

$2.19882 \mathrm{E}-03$

$2.27860 \mathrm{E}-03$ $2.36018 \mathrm{E}-03$

$2.44355 \mathrm{E}-03$

$2.52875 \mathrm{E}-03$

$2.61579 \mathrm{E}-03$
$-3.08595 \mathrm{E} 01$ $-3.08991 \mathrm{E} 01$

$-3.09373 \mathrm{E} 01$

$-3.09742 \mathrm{E} 01$

$-3.10100 \mathrm{E} 01$

$-3.10445 \mathrm{E} 01$

$-3.10780 \mathrm{E} 01$

$-3.11104 \mathrm{E} 01$

$-3.11419 \mathrm{E} 01$

$-3.11723 \mathrm{E} \quad 01$

$-3.12019 \mathrm{E} \quad 01$

$-3.12305 \mathrm{E} 01$

$-3.12584 \mathrm{E} 01$

$-3.12854 \mathrm{E} 01$

$-3.13117 \mathrm{E} 01$

$-3.13372 \mathrm{E} 01$

$-3.13620 \mathrm{E} \quad 01$

$-3.13862 \mathrm{E} \quad 01$

$-3.14097 \mathrm{E} \quad 01$

$-3.14325 \mathrm{E} 01$

$-3.14548 \mathrm{E} 01$ $-3.14765 \mathrm{E} \quad 01$

$-3.14976 \mathrm{E} 01$

$-3.15182 \mathrm{E} 01$

$-3.15383 \mathrm{E} 01$

$-3.15579 \mathrm{E} 01$

$-3.15770 \mathrm{E} 01$

$-3.15956 \mathrm{E} 01$

$-3.16138 \mathrm{E} 01$

$-3.16316 \mathrm{E} 01$

$-3.16489 \mathrm{E} \quad 01$

$-3.16659 \mathrm{E} \quad 01$

$-3.16824 \mathrm{E} 01$

$-3.16986 \mathrm{E} 01$

$-3.17145 \mathrm{E} 01$

$-3.17300 \mathrm{E} \quad 01$

$-3.17451 \mathrm{E} 01$

$-3.17599 \mathrm{E} 01$

$-3.17744 \mathrm{E} 01$

$-3.17886 \mathrm{E} 01$ 
ELECTRON RADIAL FUNCITIONS

Positron

p $F_{0}$

12.10

12.20

12.30

12.40

12.50

12.60

12.70

12.80

12.90

13.00

13.10

13.20

13.30

13.40

13.50

13.60

13.70

13.80

13.90

14.00

14.10

14.20

14.30

14.40

14.50

14.60

14.70

14.80

14.90

15.00

15.10

15.20

15.30

15.40

15.50

15.60

15.70

15.80

15.90

16.00

16.10

16.20

16.30

16.40

16.50

16.60

16.70

16.80

16.90

17.00

17.10

17.20

17.30

17.40

17.50

17.60

17.70

17.80

17.90

18.00
$8.75309 \mathrm{E}-01$

$8.75302 \mathrm{E}-01$

$8.75295 \mathrm{E}-01$

$8.75288 \mathrm{E}-01$

$8.75281 \mathrm{E}-01$

$8.75274 \mathrm{E}-01$

$8.75267 \mathrm{E}-01$

$8.75260 \mathrm{E}-01$

$8.75253 \mathrm{E}-01$

$8.75246 \mathrm{E}-01$

8.75239E-01

$8.75231 \mathrm{E}-01$

$8.75224 \mathrm{E}-01$

$8.75217 \mathrm{E}-01$

$8.75209 \mathrm{E}-01$

8.75202E-01

$8.75195 \mathrm{E}-01$

$8.75188 \mathrm{E}-01$

$8.75180 \mathrm{E}-01$

$8.75173 \mathrm{E}-01$

$8.75165 \mathrm{E}-01$

$8.75158 \mathrm{E}-01$

$8.75151 \mathrm{E}-01$

$8.75143 \mathrm{E}-01$

$8.75136 \mathrm{E}-01$

$8.75128 \mathrm{E}-01$

$8.75121 \mathrm{E}-0$

$8.75113 \mathrm{E}-01$

$8.75106 \mathrm{E}-01$

$8.75098 \mathrm{E}-0$

8.75091 E-01

$8.75083 \mathrm{E}-01$

$8.75076 \mathrm{E}-01$

$8.75068 \mathrm{E}-01$

8.75061 E-0

$8.75053 \mathrm{E}-01$

$8.75046 \mathrm{E}-01$

$8.75039 \mathrm{E}-01$

$8.75031 \mathrm{E}-01$

$8.75024 \mathrm{E}-01$

$8.75016 \mathrm{E}-01$

$8.75009 \mathrm{E}-01$

$8.75001 \mathrm{E}-01$

$8.74994 \mathrm{E}-01$

$8.74987 \mathrm{E}-01$

$8.74979 \mathrm{E}-01$

$8.74972 \mathrm{E}-01$

$8.74965 \mathrm{E}-0 \mathrm{l}$

$8.74957 \mathrm{E}-01$

$8.74950 \mathrm{E}-01$

8.74943E-01 $8.74935 \mathrm{E}-01$ $8.74928 \mathrm{E}-01$ $8.74921 \mathrm{E}-01$

$8.74914 \mathrm{E}-01$

$8.74906 \mathrm{E}-01$ $8.74899 \mathrm{E}-01$ $8.74892 \mathrm{E}-01$ $8.74885 \mathrm{E}-01$ $8.74877 \mathrm{E}-01$

$Z=6 \quad A=12$

$$
\rho=1.2 A^{1 / 3} F
$$

$f_{1}$

$1.08958 \mathrm{E} \quad 01$

$1.09905 \mathrm{E} \quad 01$

$1.10851 \mathrm{E} \quad 01$

$1.11797 \mathrm{E} \quad 01$

$1.12744 \mathrm{E} \quad 01$

$1.13691 \mathrm{E} \quad 01$

$1.14637 \mathrm{E} \quad 01$

$1.15584 \mathrm{E}$ 0l

$1.16531 \mathrm{E} \quad 01$

$1.17478 \mathrm{E} \quad 01$

$1.18426 \mathrm{E} \quad 01$

$1.19373 \mathrm{E} 01$

$1.20321 \mathrm{E} \quad 01$

$1.21268 \mathrm{E} 01$

$1.22216 \mathrm{E} \quad 01$

$1.23164 \mathrm{E} \quad 01$

$1.24112 \mathrm{E} \quad 01$

$1.25060 \mathrm{E} \quad 01$

$1.26009 \mathrm{E} \quad 01$

$1.26957 \mathrm{E} \quad 01$

$1.27906 \mathrm{E} \quad 01$

$1.28854 \mathrm{E} \quad 01$

$1.29803 \mathrm{E} \quad 01$

$1.30752 \mathrm{E} 01$

$1.31701 \mathrm{E} \quad 01$

$1.32651 \mathrm{E} \quad 01$

$1.33600 \mathrm{E}$ ol

$1.34549 \mathrm{E} \quad 01$

$1.35499 \mathrm{E} 01$

$1.36449 \mathrm{E} 01$

$1.37399 \mathrm{E} \quad 01$

$1.38349 \mathrm{E}$ 0l

$1.39299 \mathrm{E} 01$

$1.40250 \mathrm{E} \quad 01$

$1.41200 \mathrm{E} \quad 01$

$1.42151 \mathrm{E} \quad 01$

$1.43102 \mathrm{E} \quad 01$

$1.44053 \mathrm{E}$ 01

$1.45004 \mathrm{E} \quad 01$

$1.45955 \mathrm{E} \quad 01$

$1.46907 \mathrm{E} \quad 01$

$1.47858 \mathrm{E}$ 0l

$1.48810 \mathrm{E} \quad 01$

$1.49762 \mathrm{E} \quad 01$

$1.50714 \mathrm{E} \quad 01$

$1.51666 \mathrm{E} \quad 01$

$1.52618 \mathrm{E} \quad 01$

$1.53571 \mathrm{E}$ 01

$1.54523 \mathrm{E} \quad 01$

$1.55476 \mathrm{E} \quad 01$

$1.56429 \mathrm{E} \quad 01$ $1.57382 \mathrm{E} \quad 01$

$1.58336 \mathrm{E} \quad 01$

$1.59289 \mathrm{E} \quad 01$

$1.60243 \mathrm{E} \quad 01$

$1.61196 \mathrm{E} \quad 01$ $1.62150 \mathrm{E} \quad 01$

$1.63104 \mathrm{E} \quad 01$

$1.64059 \mathrm{E} 01$

$1.65013 \mathrm{E} 01$ $g_{1}$

$\tan \delta_{1}$

$f_{-1}$

$1.48750 \mathrm{E}-0 \mathrm{l}$

$-7.73091 \mathrm{E}-05$

$1.52640 \mathrm{E}-01 \quad-9.14933 \mathrm{E}-05$

$1.56575 \mathrm{E}-01-1.05440 \mathrm{E}-04$

$1.60555 \mathrm{E}-01-1.19156 \mathrm{E}-04$

$1.64580 \mathrm{E}-01$

$-1.32642 \mathrm{E}-04$

$1.68651 \mathrm{E}-01$

$1.72766 \mathrm{E}-01$

$1.76927 \mathrm{E}-01$

$1.81132 \mathrm{E}-01$

$1.85383 \mathrm{E}-01$

$1.89679 \mathrm{E}-01$

$1.94021 \mathrm{E}-01$

$1.98407 \mathrm{E}-01$

$2.02839 \mathrm{E}-01$

$2.07315 \mathrm{E}-01$

$2.11838 \mathrm{E}-01$

$2.16405 \mathrm{E}-01$

$2.21017 \mathrm{E}-01$

$2.25675 \mathrm{E}-01$

$2.30378 \mathrm{E}-01$

$2.35127 \mathrm{E}-01$

$2.39920 \mathrm{E}-01$

$2.44759 \mathrm{E}-01$

$2.49644 \mathrm{E}-01$

2.54573 E-01

$2.59548 \mathrm{E}-01$

$2.64569 \mathrm{E}-0 \mathrm{l}$

$2.69634 \mathrm{E}-01$

$2.74745 \mathrm{E}-01$

2.79902 E-0l

$2.85104 \mathrm{E}-01$

$2.90351 \mathrm{E}-01$

$2.95644 \mathrm{E}-01$

$3.00982 \mathrm{E}-01$

$3.06366 \mathrm{E}-01$

$3.11795 \mathrm{E}-01$

$3.17270 \mathrm{E}-01$

$3.22790 \mathrm{E}-01$

$3.28356 \mathrm{E}-01$

$3.33967 \mathrm{E}-01$

$3.39624 \mathrm{E}-01$

$3.45327 \mathrm{E}-01$

$3.51075 \mathrm{E}-01$

$3.56869 \mathrm{E}-01$

$3.62708 \mathrm{E}-01$

$3.68593 \mathrm{E}-01$

$3.74523 \mathrm{E}-01$

$3.80499 \mathrm{E}-01$

$3.86521 \mathrm{E}-01$

$3.92589 \mathrm{E}-01$

$3.98702 \mathrm{E}-01$

$4.04861 \mathrm{E}-0$

$4.11066 \mathrm{E}-01$

$4.17316 \mathrm{E}-01$

$4.23613 \mathrm{E}-01$

$4.29955 \mathrm{E}-01$ $4.36342 \mathrm{E}-01$

$4.42776 \mathrm{E}-01$

$4.49256 \mathrm{E}-01$

$4.55781 \mathrm{E}-01$

$-1.45903 \mathrm{E}-04$

$-1.58941 \mathrm{E}-04$

$-1.71763 \mathrm{E}-04$

$-1.84393 \mathrm{E}-04$

$-1.96799 \mathrm{E}-04$

$-2.09014 \mathrm{E}-04$

$-2.21036 \mathrm{E}-04$

$-2.32867 \mathrm{E}-04$

$-2.44509 \mathrm{E}-04$

$-2.55962 \mathrm{E}-04$

$-2.67240 \mathrm{E}-04$

$-2.78349 \mathrm{E}-04$

$-2.89287 \mathrm{E}-04$

$-3.00045 \mathrm{E}-04$

$-3.10650 \mathrm{E}-04$

$-3.21092 \mathrm{E}-04$

$-3.31372 \mathrm{E}-04$

$-3.41511 \mathrm{E}-04$

$-3.51483 \mathrm{E}-04$

$-3.61309 \mathrm{E}-04$

$-3.70995 \mathrm{E}-04$

$-3.80540 \mathrm{E}-04$

$-3.89942 \mathrm{E}-04$

$-3.99201 \mathrm{E}-04$

$-4.08337 \mathrm{E}-04$

$-4.17330 \mathrm{E}-04$

$-4.26205 \mathrm{E}-04$

$-4.34949 \mathrm{E}-04$

$-4.43567 \mathrm{E}-04$

$-4.52062 \mathrm{E}-04$

$-4.60444 \mathrm{E}-04$

$-4.68698 \mathrm{E}-04$

$-4.76851 \mathrm{E}-04$

$-4.84879 \mathrm{E}-04$

$-4.92796 \mathrm{E}-04$

$-5.00612 \mathrm{E}-04$ 
ELECTRON RADIAL FUNCTIONS

$Z=6 \quad A=12 \quad \rho=1.2 A^{1 / 3} F$

Positron

$\bar{F}$

$1.79841 \mathrm{E}-04$

$1.82826 \mathrm{E}-04$

$1.85835 \mathrm{E}-04$

$1.88869 \mathrm{E}-04$

$1.91928 \mathrm{E}-04$

$1.95010 \mathrm{E}-04$

$1.98118 \mathrm{E}-04$

$2.01250 \mathrm{E}-04$

$2.04407 \mathrm{E}-04$

$2.07588 \mathrm{E}-04$

$2.10794 \mathrm{E}-04$

$2.14024 \mathrm{E}-04$

$2.17279 \mathrm{E}-04$

$2.20558 \mathrm{E}-04$

$2.23862 \mathrm{E}-04$

$2.27190 \mathrm{E}-04$

$2.30543 \mathrm{E}-04$

$2.33921 \mathrm{E}-04$

$2.37323 \mathrm{E}-04$

$2.40749 \mathrm{E}-04$

$2.44201 \mathrm{E}-04$

$2.47676 \mathrm{E}-04$

$2.51176 \mathrm{E}-04$

2.54701 E- 04

$2.58250 \mathrm{E}-04$

$2.61824 \mathrm{E}-04$

$2.65423 \mathrm{E}-04$

$2.69046 \mathrm{E}-04$

$2.72693 \mathrm{E}-04$

$2.76365 \mathrm{E}-04$

$2.80061 \mathrm{E}-04$

$2.83782 \mathrm{E}-04$

$2.87528 \mathrm{E}-04$

$2.91298 \mathrm{E}-04$

$2.95093 \mathrm{E}-04$

$2.98912 \mathrm{E}-04$

$3.02756 \mathrm{E}-04$

$3.06624 \mathrm{E}-04$

$3.10517 \mathrm{E}-04$

$3.14434 \mathrm{E}-04$

$3.18376 \mathrm{E}-04$

$3.22342 \mathrm{E}-04$

$3.26333 \mathrm{E}-04$

$3.30349 \mathrm{E}-04$

$3.34389 \mathrm{E}-04$

$3.38454 \mathrm{E}-04$

$3.42543 \mathrm{E}-04$

$3.46656 \mathrm{E}-04$

$3.50794 \mathrm{E}-04$

$3.54957 \mathrm{E}-04$

$3.59144 \mathrm{E}-04$

$3.63356 \mathrm{E}-04$

$3.67592 \mathrm{E}-04$

$3.71853 \mathrm{E}-04$

$3.76139 \mathrm{E}-04$

17.60

17.70

17.80

17.90

18.00 $f_{2}$

$g_{2}$

$3.11195 \mathrm{E}-01$

$3.16476 \mathrm{E}-01$

$3.21801 \mathrm{E}-01$

$3.27170 \mathrm{E}-01$

$3.32584 \mathrm{E}-01$

$3.38042 \mathrm{E}-01$

$3.43544 \mathrm{E}-01$

$3.49091 \mathrm{E}-01$

$3.54682 \mathrm{E}-01$

$3.60317 \mathrm{E}-01$

$3.65997 \mathrm{E}-01$

$3.71721 \mathrm{E}-01$

$3.77489 \mathrm{E}-01$

$3.83302 \mathrm{E}-01$

$3.89159 \mathrm{E}-01$

$3.95060 \mathrm{E}-01$

$4.01006 \mathrm{E}-01$

$4.06996 \mathrm{E}-0 \mathrm{l}$

$4.13030 \mathrm{E}-01$

$4.19109 \mathrm{E}-01$

$4.25232 \mathrm{E}-0 \mathrm{l}$

$4.31399 \mathrm{E}-01$

$4.37611 \mathrm{E}-01$

$4.43867 \mathrm{E}-01$

$4.50167 \mathrm{E}-01$

$4.56511 \mathrm{E}-01$

$4.62900 \mathrm{E}-01$

$4.69333 \mathrm{E}-01$

$4.75810 \mathrm{E}-01$

$4.82332 \mathrm{E}-01$

$4.88898 \mathrm{E}-01$

$4.95508 \mathrm{E}-01$

$5.02163 \mathrm{E}-01$

$5.08862 \mathrm{E}-01$

5.15605 E-01

$5.22392 \mathrm{E}-01$

$5.29224 \mathrm{E}-01$

$5.36100 \mathrm{E}-01$

$5.43020 \mathrm{E}-01$

$5.49984 \mathrm{E}-01$

$5.56993 \mathrm{E}-01$

$5.64046 \mathrm{E}-01$

$5.71143 \mathrm{E}-01$

$5.78285 \mathrm{E}-01$

$5.85471 \mathrm{E}-01$

5.92701 E-01

$5.99975 \mathrm{E}-01$

$6.07294 \mathrm{E}-01$

$6.14657 \mathrm{E}-01$

$6.22064 \mathrm{E}-01$

$6.29515 \mathrm{E}-01$

$6.37010 \mathrm{E}-01$

$6.44550 \mathrm{E}-01$

$6.52134 \mathrm{E}-01$

$6.59762 \mathrm{E}-01$

$6.67435 \mathrm{E}-01$

$6.75152 \mathrm{E}-01$

$6.82912 \mathrm{E}-01$

$6.90717 \mathrm{E}-01$

$6.98567 \mathrm{E}-01$ $\tan \delta_{2}$

$f_{-2}$

$2.70470 \mathrm{E}-03$

$2.79548 \mathrm{E}-03$

$2.88816 \mathrm{E}-03$

$2.98275 \mathrm{E}-03$

$3.07928 \mathrm{E}-03$

$3.17777 \mathrm{E}-03$

$3.27823 \mathrm{E}-03$

$3.38069 \mathrm{E}-03$

$3.48516 \mathrm{E}-03$

$3.59166 \mathrm{E}-03$

$3.70021 \mathrm{E}-03$

$3.81083 \mathrm{E}-03$

$3.92353 \mathrm{E}-03$

$4.03835 \mathrm{E}-03$

$4.15529 \mathrm{E}-03$

$4.27437 \mathrm{E}-03$

$4.39562 \mathrm{E}-03$

$4.51905 \mathrm{E}-03$

$4.64468 \mathrm{E}-03$

$4.77253 \mathrm{E}-03$

$4.90262 \mathrm{E}-03$

$5.03497 \mathrm{E}-03$

$5.1696 \theta \mathrm{E}-03$

$5.30652 \mathrm{E}-03$

$5.44576 \mathrm{E}-03$

$5.58733 \mathrm{E}-03$

$5.73125 \mathrm{E}-03$

$5.87754 \mathrm{E}-03$

$6.02623 \mathrm{E}-03$

$6.17733 \mathrm{E}-03$

$6.33085 \mathrm{E}-03$

$6.48682 \mathrm{E}-03$

$6.64526 \mathrm{E}-03$

$6.80618 \mathrm{E}-03$

$6.96961 \mathrm{E}-03$

$7.13556 \mathrm{E}-03$

$7.30405 \mathrm{E}-03$

$7.47510 \mathrm{E}-03$

$7.64873 \mathrm{E}-03$

$7.82496 \mathrm{E}-03$

8.00381 E- 03

$8.18529 \mathrm{E}-03$

$8.36943 \mathrm{E}-03$

$8.55625 \mathrm{E}-03$

$8.74575 \mathrm{E}-03$

$8.93797 \mathrm{E}-03$

$9.13292 \mathrm{E}-03$

$9.33062 \mathrm{E}-03$

$9.53109 \mathrm{E}-03$

$9.73434 \mathrm{E}-03$

$9.94040 \mathrm{E}-03$

$1.01493 \mathrm{E}-02$

$1.03610 \mathrm{E}-02$

$1.05756 \mathrm{E}-02$

$1.07931 \mathrm{E}-02$

$1.10135 \mathrm{E}-02$

$1.12368 \mathrm{E}-02$

$1.14630 \mathrm{E}-02$

$1.16922 \mathrm{E}-02$

$1.19244 \mathrm{E}-02$
$-3.20296 \mathrm{E} \quad 01 \quad-1.97543 \mathrm{E}-03$

$-3.20389 \mathrm{E}$ ol $-2.05631 \mathrm{E}-03$

$-3.20480 \mathrm{E}$ 01 $-2.13903 \mathrm{E}-03$

$-3.20570 \mathrm{E} \quad 01-2.22359 \mathrm{E}-03$

$-3.20658 \mathrm{E}$ 01 $-2.31003 \mathrm{E}-03$

$-3.20745 \mathrm{E} \quad 01$

$-3.20831 \mathrm{E} 01$

$-3.20915 \mathrm{E} 01$

$-3.20997 \mathrm{E} \quad 01$

$-3.21079 \mathrm{E} 01$

$-3.21159 \mathrm{E} 01$

$-3.21237 \mathrm{E}$ 01

$-3.21315 \mathrm{E} 01$

$-3.21391 \mathrm{E} 01$

$-3.21466 \mathrm{E} \quad 01$

$-3.21540 \mathrm{E} \quad 01$

$-3.21613 \mathrm{E} 01$

$-3.21684 \mathrm{E} \quad 01$

$-3.21755 \mathrm{E} 01$

$-3.21824 \mathrm{E} \quad 01$

$-3.21893 \mathrm{E} \quad 01$

$-3.21960 \mathrm{E} 01$

$-3.22027 \mathrm{E} 01$

$-3.22092 \mathrm{E} 01$

$-3.22157 \mathrm{E} 01$

$-3.22221 \mathrm{E} \quad 01$

$-3.22283 \mathrm{E} 01$

$-3.22345 \mathrm{E} \quad 01$

$-3.22466 \mathrm{E} 01$

$-3.22525 \mathrm{E} \quad 01$

$-3.22584 \mathrm{E} \quad 01$

$-3.22642 \mathrm{E} \quad 01$

$-3.22699 \mathrm{E} \quad 01$

$-3.22755 \mathrm{E} \quad 01$

$-3.22810 \mathrm{E} \quad 01$

$-3.22865 \mathrm{E}$ 0l

$-3.22919 \mathrm{E} 01$

$-3.22972 \mathrm{E} 01$

$-3.23024 \mathrm{E} 01$

$-3.23076 \mathrm{E} 01$

$-3.23127 \mathrm{E} \quad 01$

$-3.23178 \mathrm{E} 01$

$-3.23227 \mathrm{E} 01$

$-3.23277 \mathrm{E} \quad 01$

$-3.23325 \mathrm{E} \quad 01$

$-3.23373 \mathrm{E} 01$

$-3.23421 \mathrm{E} 01$

$-3.23467 \mathrm{E} 01$

$-3.23514 \mathrm{E} 01$

$-3.23559 \mathrm{E} \quad 01$ $-3.23604 \mathrm{E} 01$ $-3.23649 \mathrm{E} 01$ $-3.23693 \mathrm{E} 01$

$-3.23737 \mathrm{E} 01$

$-3.23780 \mathrm{E} \quad 01$

$-3.23822 \mathrm{E} 01$

$-3.23864 \mathrm{E}$ 01

$-3.23906 \mathrm{E} \quad 01$

$-3.23947 \mathrm{E} \quad 01$

$-2.39837 \mathrm{E}-03$

$-2.48861 \mathrm{E}-03$

$-2.58079 \mathrm{E}-03$

$-2.67491 \mathrm{E}-03$

$-2.77100 \mathrm{E}-03$

$-2.86908 \mathrm{E}-03$

$-2.96917 \mathrm{E}-03$

$-3.07128 \mathrm{E}-03$

$-3.17543 \mathrm{E}-03$

$-3.28165 \mathrm{E}-03$

$-3.38995 \mathrm{E}-03$

$-3.50035 \mathrm{E}-03$

$-3.61287 \mathrm{E}-03$

$-3.72752 \mathrm{E}-03$

$-3.84434 \mathrm{E}-03$

$-3.96333 \mathrm{E}-03$

$-4.08452 \mathrm{E}-03$

$-4.20792 \mathrm{E}-03$

$-4.33355 \mathrm{E}-03$

$-4.46143 \mathrm{E}-03$

$-4.59159 \mathrm{E}-03$

$-4.72403 \mathrm{E}-03$

$-4.85879 \mathrm{E}-03$

$-4.99587 \mathrm{E}-03$

$-5.13530 \mathrm{E}-03$

$-5.27709 \mathrm{E}-03$

$-5.42127 \mathrm{E}-03$

$-5.56785 \mathrm{E}-03$

$-5.71685 \mathrm{E}-03$

$-5.86830 \mathrm{E}-03$

$-6.02220 \mathrm{E}-03$

$-6.17859 \mathrm{E}-03$

$-6.33747 \mathrm{E}-03$

$-6.49887 \mathrm{E}-03$

$-6.66280 \mathrm{E}-03$

$-6.82928 \mathrm{E}-03$

$-6.99835 \mathrm{E}-03$

$-7.17000 \mathrm{E}-03$

$-7.34426 \mathrm{E}-03$

$-7.52116 \mathrm{E}-03$

$-7.70070 \mathrm{E}-03$

$-7.88291 \mathrm{E}-03$
$-3.22406 \mathrm{E} 01$ 


\begin{tabular}{|c|c|c|c|c|c|c|}
\hline$p$ & $F_{0}$ & $f_{1}$ & & $g_{1}$ & $\tan \delta_{1}$ & $f_{-1}$ \\
\hline 18.10 & $8.74870 \mathrm{E}-01$ & $1.65968 \mathrm{E}$ & 01 & $4.62353 \mathrm{E}-01$ & $-6.36555 \mathrm{E}-04$ & $-4.05719 \mathrm{E}-01$ \\
\hline 18.20 & $8.74863 \mathrm{E}-01$ & $1.66923 \mathrm{E}$ & 01 & $4.68970 \mathrm{E}-01$ & $-6.42459 E-04$ & $-4.12115 \mathrm{E}-01$ \\
\hline 18.30 & $8.74856 \mathrm{E}-01$ & $1.67878 \mathrm{E}$ & 01 & $4.75633 \mathrm{E}-01$ & $-6.48280 \mathrm{E}-04$ & $-4.18557 \mathrm{E}-01$ \\
\hline 18.40 & $8.74849 \mathrm{E}-01$ & $1.68833 \mathrm{E}$ & 01 & $4.82342 \mathrm{E}-01$ & $-6.54032 \mathrm{E}-04$ & $-4.25045 \mathrm{E}-01$ \\
\hline 18.50 & $8.74842 \mathrm{E}-01$ & $1.69788 \mathrm{E}$ & 01 & $4.89096 \mathrm{E}-01$ & $-6.59710 \mathrm{E}-04$ & $-4.31578 \mathrm{E}-01$ \\
\hline 18.60 & $8.74835 \mathrm{E}-01$ & $1.70743 \mathrm{E}$ & 01 & $4.95897 \mathrm{E}-01$ & $-6.65315 \mathrm{E}-04$ & $-4.38158 \mathrm{E}-01$ \\
\hline 18.70 & $8.74828 \mathrm{E}-01$ & $1.71699 \mathrm{E}$ & 01 & $5.02744 \mathrm{E}-01$ & $-6.70845 E-04$ & $-4.44784 \mathrm{E}-01$ \\
\hline 18.80 & $8.74821 \mathrm{E}-01$ & $1.72655 \mathrm{E}$ & 01 & $5.09637 \mathrm{E}-01$ & $-6.76316 \mathrm{E}-04$ & $-4.51456 \mathrm{E}-01$ \\
\hline 18.90 & $8.74814 \mathrm{E}-01$ & $1.73611 \mathrm{E}$ & 01 & $5.16576 \mathrm{E}-01$ & $-6.81707 \mathrm{E}-04$ & $-4.58174 \mathrm{E}-01$ \\
\hline 19.00 & $8.74807 \mathrm{E}-01$ & $1.74567 \mathrm{E}$ & 01 & $5.23560 \mathrm{E}-01$ & $-6.87036 \mathrm{E}-04$ & $-4.64937 \mathrm{E}-01$ \\
\hline 19.10 & $8.74800 \mathrm{E}-01$ & $1.75523 \mathrm{E}$ & 01 & $5.30591 \mathrm{E}-01$ & $-6.92299 \mathrm{E}-04$ & $-4.71747 \mathrm{E}-01$ \\
\hline 19.20 & $8.74793 \mathrm{E}-01$ & $1.76480 \mathrm{E}$ & 01 & $5.37668 \mathrm{E}-01$ & $-6.97490 \mathrm{E}-04$ & $-4.78604 \mathrm{E}-01$ \\
\hline 19.30 & $8.74786 \mathrm{E}-01$ & $1.77436 \mathrm{E}$ & 01 & $5.44791 \mathrm{E}-01$ & $-7.02619 \mathrm{E}-04$ & $-4.85506 \mathrm{E}-01$ \\
\hline 19.40 & $8.74779 \mathrm{E}-01$ & $1.78393 \mathrm{E}$ & 01 & $5.51960 \mathrm{E}-01$ & $-7.07687 \mathrm{E}-04$ & $-4.92454 \mathrm{E}-01$ \\
\hline 19.50 & $8.74772 \mathrm{E}-01$ & $1.79350 \mathrm{E}$ & 01 & $5.59175 \mathrm{E}-01$ & $-7.12689 \mathrm{E}-04$ & $-4.99449 \mathrm{E}-01$ \\
\hline 19.60 & $8.74765 \mathrm{E}-01$ & $1.80307 \mathrm{E}$ & 01 & $5.66437 \mathrm{E}-01$ & $-7.17632 \mathrm{E}-04$ & $-5.06490 \mathrm{E}-01$ \\
\hline 19.70 & $8.74758 \mathrm{E}-01$ & $1.81265 \mathrm{E}$ & 01 & $5.73744 \mathrm{E}-01$ & $-7.22508 \mathrm{E}-04$ & $-5.13576 \mathrm{E}-01$ \\
\hline 19.80 & $8.74752 \mathbf{E}-01$ & $1.82222 \mathrm{E}$ & 01 & $5.81098 \mathrm{E}-01$ & $-7.27323 \mathrm{E}-04$ & $-5.20710 \mathrm{E}-01$ \\
\hline 19.90 & $8.74745 \mathrm{E}-01$ & $1.83180 \mathrm{E}$ & 01 & $5.88497 \mathrm{E}-01$ & $-7.32086 \mathrm{E}-04$ & $-5.27889 \mathrm{E}-01$ \\
\hline 20.00 & $8.74738 \mathrm{E}-01$ & $1.84138 \mathrm{E}$ & 01 & $5.95943 \mathrm{E}-01$ & $-7.36781 \mathrm{E}-04$ & $-5.35115 \mathrm{E}-01$ \\
\hline 20.10 & $8.74731 \mathrm{E}-01$ & $1.85096 \mathrm{E}$ & 01 & $6.03436 \mathrm{E}-01$ & $-7.41425 \mathrm{E}-04$ & $-5.42386 \mathrm{E}-01$ \\
\hline 20.20 & $8.74724 \mathrm{E}-01$ & $1.86054 \mathrm{E}$ & 01 & $6.10974 \mathrm{E}-01$ & $-7.46009 \mathrm{E}-04$ & $-5.49705 \mathrm{E}-01$ \\
\hline 20.30 & $8.74717 \mathrm{E}-01$ & $1.87013 \mathrm{E}$ & 01 & $6.18559 \mathrm{E}-01$ & $-7.50534 \mathrm{E}-04$ & $-5.57069 \mathrm{E}-01$ \\
\hline 20.40 & $8.74711 \mathrm{E}-01$ & $1.87971 \mathrm{E}$ & 01 & $6.26189 \mathrm{E}-01$ & $-7.55015 \mathrm{E}-04$ & $-5.64480 \mathrm{E}-01$ \\
\hline 20.50 & $8.74704 \mathbf{E}-01$ & $1.88930 \mathrm{E}$ & 01 & $6.33867 \mathrm{E}-01$ & $-7.59424 \mathrm{E}-04$ & $-5.71937 \mathrm{E}-01$ \\
\hline 20.60 & $8.74697 \mathbf{E}-01$ & $1.89889 \mathrm{E}$ & 01 & $6.41590 \mathrm{E}-01$ & $-7.63784 \mathrm{E}-04$ & $-5.79441 \mathrm{E}-01$ \\
\hline 20.70 & $8.74691 \mathrm{E}-01$ & $1.90848 \mathrm{E}$ & 01 & $6.49360 \mathrm{E}-01$ & $-7.68102 \mathrm{E}-04$ & $-5.86990 \mathrm{E}-01$ \\
\hline 20.80 & $8.74684 \mathrm{E}-01$ & $1.91808 \mathrm{E}$ & 01 & $6.57176 \mathrm{E}-01$ & $-7.72355 \mathrm{E}-04$ & $-5.94587 \mathrm{E}-01$ \\
\hline 20.90 & $8.74677 \mathbf{E}-01$ & $1.92767 \mathrm{E}$ & 01 & $6.65039 \mathrm{E}-01$ & $-7.76556 \mathrm{E}-04$ & $-6.02229 \mathrm{E}-01$ \\
\hline 21.00 & $8.74671 \mathrm{E}-01$ & $1.93727 \mathrm{E}$ & 01 & $6.72948 \mathrm{E}-01$ & $-7.80715 \mathrm{E}-04$ & $-6.09918 \mathrm{E}-01$ \\
\hline 21.10 & $8.74664 \mathrm{E}-01$ & $1.94687 \mathrm{E}$ & 01 & $6.80903 \mathrm{E}-01$ & $-7.84819 \mathrm{E}-04$ & $-6.17654 \mathrm{E}-01$ \\
\hline 21.20 & $8.74657 \mathrm{E}-01$ & $1.95647 \mathrm{E}$ & 01 & $6.88905 \mathrm{E}-01$ & $-7.88865 \mathrm{E}-04$ & $-6.25436 \mathrm{E}-01$ \\
\hline 21.30 & $8.74651 \mathrm{E}-01$ & $1.96608 \mathrm{E}$ & 01 & $6.96953 \mathrm{E}-0 \mathrm{l}$ & $-7.92876 \mathrm{E}-04$ & $-6.33265 \mathrm{E}-01$ \\
\hline 21.40 & $8.74644 \mathbf{E}-01$ & $1.97568 \mathrm{E}$ & 01 & $7.05048 \mathrm{E}-01$ & $-7.96825 \mathrm{E}-04$ & $-6.41140 \mathrm{E}-01$ \\
\hline 21.50 & $8.74638 \mathrm{E}-01$ & $1.98529 \mathrm{E}$ & 01 & $7.13189 \mathrm{E}-01$ & $-8.00732 \mathrm{E}-04$ & $-6.49062 \mathrm{E}-01$ \\
\hline 21.60 & $8.74631 \mathrm{E}-01$ & $1.99490 \mathrm{E}$ & 01 & $7.21377 \mathrm{E}-01$ & $-8.04596 \mathrm{E}-04$ & $-6.57030 \mathrm{E}-01$ \\
\hline 21.70 & $8.74625 \mathrm{E}-01$ & $2.00451 \mathrm{E}$ & 01 & $7.29611 \mathrm{E}-01$ & $-8.08403 \mathrm{E}-04$ & $-6.65045 \mathrm{E}-01$ \\
\hline 21.80 & $8.74618 \mathrm{E}-$ & $2.01412 \mathrm{E}$ & 01 & $7.37891 \mathrm{E}$ & $-8.12168 \mathrm{E}-04$ & $-6.73106 \mathrm{E}-01$ \\
\hline 21.90 & $8.74612 \mathrm{E}-01$ & $2.02374 \mathrm{E}$ & 01 & $9 \mathrm{E}-01$ & $-8.15891 \mathrm{E}-04$ & $-6.81214 \mathrm{E}-01$ \\
\hline 22.00 & $8.74605 \mathrm{E}-01$ & $2.03336 \mathrm{E}$ & 01 & $7.54592 \mathrm{E}-01$ & $-8.19570 \mathrm{E}-04$ & $-6.89369 \mathrm{E}-01$ \\
\hline 22.10 & $8.74599 \mathrm{E}-01$ & $2.04298 \mathrm{E}$ & 01 & $7.63013 \mathrm{E}-01$ & $-8.23200 \mathrm{E}-04$ & $-6.97570 \mathrm{E}-01$ \\
\hline 22.20 & $8.74592 \mathrm{E}-01$ & $2.05260 \mathrm{E}$ & 01 & $7.71480 \mathrm{E}-01$ & $-8.26787 \mathrm{E}-04$ & $-7.05818 \mathbf{E}-01$ \\
\hline 22.30 & $8.74586 \mathrm{E}-01$ & $2.06222 \mathrm{E}$ & 01 & -01 & $30321 \mathrm{E}-04$ & $-7.14113 \mathrm{E}-01$ \\
\hline 22.40 & $8.74579 \mathrm{E}-01$ & $2.07184 \mathrm{E}$ & 01 & $7.88554 \mathrm{E}-01$ & $-8.33829 \mathrm{E}-04$ & $-7.22454 \mathrm{E}-01$ \\
\hline 22.50 & $8.74573 \mathrm{E}-01$ & $2.08147 \mathrm{E}$ & 01 & $7.97161 \mathrm{E}-01$ & $-8.37284 \mathrm{E}-04$ & $-7.30842 \mathrm{E}-01$ \\
\hline 22.60 & $8.74567 \mathrm{E}-01$ & $2.09110 \mathrm{E}$ & 01 & $8.05815 \mathrm{E}-01$ & $-8.40696 \mathrm{E}-04$ & $-7.39277 \mathrm{E}-01$ \\
\hline 22.70 & $8.74560 \mathrm{E}-01$ & $2.10073 \mathrm{E}$ & 01 & $8.14516 \mathrm{E}-01$ & $-8.44074 \mathrm{E}-04$ & $-7.47759 \mathrm{E}-01$ \\
\hline 22.80 & $8.74554 \mathrm{E}-01$ & $2.11037 \mathrm{E}$ & 01 & $8.23263 \mathrm{E}-01$ & $-8.47401 \mathrm{E}-04$ & $-7.56287 \mathrm{E}-01$ \\
\hline 22.90 & $8.74548 \mathrm{E}-01$ & $2.12000 \mathrm{E}$ & 01 & $8.32057 \mathrm{E}-01$ & $-8.50697 \mathrm{E}-04$ & $-7.64863 \mathrm{E}-01$ \\
\hline 23.00 & $8.74542 \mathrm{E}-01$ & $2.12964 \mathrm{E}$ & 01 & $8.40898 \mathrm{E}-01$ & $-8.53951 \mathrm{E}-04$ & $-7.73485 \mathrm{E}-01$ \\
\hline 23.10 & $8.74535 \mathrm{E}-01$ & $2.13928 \mathrm{E}$ & 01 & $8.49785 \mathrm{E}-01$ & $-8.57163 \mathrm{E}-04$ & $-7.82154 \mathrm{E}-01$ \\
\hline 23.20 & $8.74529 \mathrm{E}-01$ & $2.14892 \mathrm{E}$ & 01 & $8.58719 \mathrm{E}-01$ & $-8.60332 \mathrm{E}-04$ & $-7.90870 \mathrm{E}-01$ \\
\hline 23.30 & $8.74523 \mathrm{E}-01$ & $2.15856 \mathrm{E}$ & 01 & $8.67701 \mathrm{E}-01$ & $-8.63475 \mathrm{E}-04$ & $-7.99633 \mathrm{E}-01$ \\
\hline 23.40 & $8.74517 \mathrm{E}-01$ & $2.16821 \mathrm{E}$ & 01 & $8.76729 \mathrm{E}-01$ & $-8.66570 \mathrm{E}-04$ & $-8.08443 \mathrm{E}-01$ \\
\hline 23.50 & $8.74511 \mathrm{E}-01$ & $2.17786 \mathrm{E}$ & 01 & $8.85804 \mathrm{E}-01$ & $-8.69630 \mathrm{E}-04$ & $-8.17300 \mathrm{E}-0 \mathrm{l}$ \\
\hline 23.60 & $8.74504 \mathrm{E}-01$ & $2.18751 \mathrm{E}$ & 01 & $8.94926 \mathrm{E}-01$ & $-8.72645 \mathrm{E}-04$ & $-8.26203 \mathrm{E}-01$ \\
\hline 23.70 & $8.74498 \mathrm{E}-01$ & $2.19716 \mathrm{E}$ & 01 & $9.04095 \mathrm{E}-$ & $-8.75638 \mathrm{E}-04$ & $-8.35154 \mathrm{E}-01$ \\
\hline 23.80 & $8.74492 \mathrm{E}-$ & $2.20682 \mathrm{E}$ & 01 & $9.13311 \mathrm{E}-01$ & $-8.78581 \mathrm{E}-04$ & $-8.44152 \mathrm{E}-01$ \\
\hline 23.90 & $8.74486 \mathrm{E}-01$ & $2.21647 \mathrm{E}$ & 01 & $9.22574 \mathrm{E}-01$ & $-8.81498 \mathrm{E}-04$ & $-8.53197 \mathrm{E}-01$ \\
\hline 24.00 & $8.74480 \mathrm{E}-01$ & $2.22613 \mathrm{E}$ & 01 & $9.31884 \mathrm{E}-01$ & $-8.84376 \mathrm{E}-04$ & $-8.62289 \mathrm{E}-01$ \\
\hline
\end{tabular}

$g_{-1}$ $\tan \delta_{-1}$

$1.75537 \mathrm{E} \quad 01$ $1.76494 \mathrm{E} \quad 01$ $1.77452 \mathrm{E} \quad 01$ $1.78409 \mathrm{E} \quad 01$ $1.79367 \mathrm{E} \quad 01$

$1.80325 \mathrm{E} \quad 01$ $1.81283 \mathrm{E} \quad 01$ $1.82241 \mathrm{E} \quad 01$ $1.83199 \mathrm{E} 01$ $1.84158 \mathrm{E} \quad 01$

$1.85117 \mathrm{E} \quad 01$ $1.86075 \mathrm{E}$ 01 $\begin{array}{ll}1.87035 \mathrm{E} & 01\end{array}$ $1.87994 \mathrm{E} \quad 01$ $1.88953 \mathrm{E} \quad 01$

$1.89913 \mathrm{E} \quad 01$ $1.90873 \mathrm{E} \quad 01$ $1.91833 \mathrm{E} .01$ $1.92793 \mathrm{E} \quad 01$ $1.93754 \mathrm{E} \quad 01$

$1.94714 \mathrm{E} \quad 01$ $1.95675 \mathrm{E} \quad 01$ $1.96636 \mathrm{E} \quad 01$ $1.97597 \mathrm{E} \quad 01$ $1.98559 \mathrm{E} \quad 01$

$1.99521 \mathrm{E} \quad 01$ $2.00482 \mathrm{E} \quad 01$ $2.01444 \mathrm{E} \quad 01$ $2.02407 \mathrm{E} \quad 01$ $2.03369 \mathrm{E} \quad 01$

$2.04332 \mathrm{E} \quad 01$ $2.05294 \mathrm{E} \quad 01$ $2.06257 \mathrm{E} \quad 01$ $2.07221 \mathrm{E} \quad 01$ $2.08184 \mathrm{E} \quad 01$

$2.09148 \mathrm{E} \quad 01$ $2.10111 \mathrm{E} 01$ $2.11075 \mathrm{E} \quad 01$ $2.12040 \mathrm{E}$ 01 $2.13004 \mathrm{E} \quad 01$

$2.13969 \mathrm{E} \quad 01$ $2.14934 \mathrm{E} \quad 01$ $2.15899 \mathrm{E} 01$ $2.16864 \mathrm{E} \quad 01$ $2.17830 \mathrm{E} \quad 01$

$2.18795 \mathrm{E} \quad 01$ $2.19761 \mathrm{E}$ 01 $2.20727 \mathrm{E} \quad 01$ $2.21694 \mathrm{E} \quad 01$ $2.22660 \mathrm{E} 01$

$2.23627 \mathrm{E} \quad 01$ $2.24594 \mathrm{E} \cdot 01$ $2.25561 \mathrm{E}$ 0I $2.26529 \mathrm{E} \quad 01$ $2.27496 \mathrm{E} 01$

$2.28464 \mathrm{E} \quad 01$ $2.29433 \mathrm{E} 01$ $2.30401 \mathrm{E} \quad 01$ $2.31369 \mathrm{E} \quad 01$ $2.32338 \mathrm{E} 01$
$3.28007 \mathrm{E} \quad 02$ $3.28808 \mathrm{E} \quad 02$ $3.29606 \mathrm{E} \quad 02$ $3.30400 \mathrm{E} \quad 02$ $3.31190 \mathrm{E} \quad 02$

$3.31977 \mathrm{E} \quad 02$ $3.32761 \mathrm{E} \quad 02$ $3.33541 \mathrm{E} \quad 02$ $3.34318 \mathrm{E} \quad 02$ $3.35091 \mathrm{E} \quad 02$

$3.35861 \mathrm{E} \quad 02$ $3.36628 \mathrm{E} \quad 02$ $3.37392 \mathrm{E} \quad 02$ $3.38153 \mathrm{E} \quad 02$ $\begin{array}{ll}3.38910 \mathrm{E} & 02\end{array}$

3.39665 E 02 $3.40416 \mathrm{E} \quad 02$ $3.41165 \mathrm{E} \quad 02$ $3.41911 \mathrm{E} \quad 02$ $3.42654 \mathrm{E} \quad 02$

$3.43394 \mathrm{E} \quad 02$ $3.44131 \mathrm{E} \quad 02$ $3.44866 \mathrm{E} \quad 02$ $3.45596 \mathrm{E} \quad 02$ $3.46326 \mathrm{E} \quad 02$

$3.47053 \mathrm{E} \quad 02$ $3.47776 \mathrm{E} \quad 02$ $3.48498 \mathrm{E} \quad 02$ $3.49217 \mathrm{E} \quad 02$ 3.49932 E 02

$3.50646 \mathrm{E} \quad 02$ $3.51358 \mathrm{E} \quad 02$ $3.52067 \mathrm{E} \quad 02$ $3.52774 \mathrm{E} \quad 02$ $3.53478 \mathrm{E} \quad 02$

$3.54180 \mathrm{E} \quad 02$ $3.54880 \mathrm{E} \quad 02$ $3.55578 \mathrm{E} \quad 02$ $3.56273 \mathrm{E} \quad 02$ $3.56966 \mathrm{E} \quad 02$

$3.57657 \mathrm{E} \quad 02$ $3.58346 \mathrm{E} \quad 02$ $3.59034 \mathrm{E} \quad 02$ $3.59718 \mathrm{E} \quad 02$ $3.60402 \mathrm{E} \quad 02$

$3.61084 \mathrm{E} \quad 02$ $3.61762 \mathrm{E} \quad 02$ $3.62440 \mathrm{E} \quad 02$ $3.63115 \mathrm{E} \quad 02$ 3.63789 E 02

$3.64461 \mathrm{E} \quad 02$ $3.65131 \mathrm{E} \quad 02$ $3.65798 \mathrm{E} \quad 02$ $3.66465 \mathrm{E} \quad 02$ $3.67130 \mathrm{E} \quad 02$

$3.67794 \mathrm{E} \quad 02$ $3.68455 \mathrm{E} \quad 02$ $3.69115 \mathrm{E} \quad 02$ $3.69773 \mathrm{E} \quad 02$ $3.70430 \mathrm{E} \quad 02$ 
ELECTRON RADIAL FUNCTIONS

$Z=6 \quad A=12 \quad \rho=1.2 A^{1 / 3} F$

Positron

$\bar{F}$

$4.02366 \mathrm{E}-04$

$4.06823 \mathrm{E}-04$

$4.11305 \mathrm{E}-04$

$4.15811 \mathrm{E}-04$

4.20342 E- 04

$4.24897 \mathrm{E}-04$

$4.29477 \mathrm{E}-04$

$4.34081 \mathrm{E}-04$

$4.38710 \mathrm{E}-04$

4.43364. E-04

4.48042 E-04

$4.52744 \mathrm{E}-04$

$4.57471 \mathrm{E}-04$

$4.62223 \mathrm{E}-04$

$4.66999 \mathrm{E}-04$

$4.71799 \mathrm{E}-04$

$4.76624 \mathrm{E}-04$

$4.81474 \mathrm{E}-04$

$4.86348 \mathrm{E}-04$

$4.91247 \mathrm{E}-04$

$4.96170 \mathrm{E}-04$

$5.01118 \mathrm{E}-04$

$5.06090 \mathrm{E}-04$

$5.11087 \mathrm{E}-04$

$5.16108 \mathrm{E}-04$

$5.21154 \mathrm{E}-04$

$5.26225 \mathrm{E}-04$

$5.31320 \mathrm{E}-04$

$5.36439 \mathrm{E}-04$

5.41583 E- 04

$5.46752 \mathrm{E}-04$

$5.51945 \mathrm{E}-04$

$5.57162 \mathrm{E}-04$

$5.62404 \mathrm{E}-04$

5.67671 E-04

$5.72962 \mathrm{E}-04$

$5.78278 \mathrm{E}-04$

$5.83618 \mathrm{E}-04$

$5.88983 \mathrm{E}-04$

5.94372 E- 04

$5.99786 \mathrm{E}-04$

$6.05224 \mathrm{E}-04$

$6.10687 \mathrm{E}-04$

$6.16175 \mathrm{E}-04$

$6.21687 \mathrm{E}-04$

$6.27223 \mathrm{E}-04$

$6.32784 \mathrm{E}-04$

$6.38370 \mathrm{E}-04$

$6.43980 \mathrm{E}-04$

$6.49614 \mathrm{E}-04$

$6.55274 \mathrm{E}-04$

$6.60957 \mathrm{E}-04$

$6.66665 \mathrm{E}-04$

$6.72398 \mathrm{E}-04$

$6.78155 \mathrm{E}-04$

$6.83937 \mathrm{E}-04$

$6.89743 \mathrm{E}-04$

$6.95574 \mathrm{E}-04$

$7.01429 \mathrm{E}-04$

$7.07309 \mathrm{E}-04$ $f_{2}$

$7.06461 \mathrm{E}-01$

$7.14399 \mathrm{E}-01$

$7.22381 \mathrm{E}-01$

$7.30407 \mathrm{E}-01$

$7.38477 \mathrm{E}-01$

7.46592E-01

$7.54750 \mathrm{E}-01$

$7.62953 \mathrm{E}-01$

$7.71201 \mathrm{E}-01$

$7.79492 \mathrm{E}-01$

$7.87828 \mathrm{E}-01$

$7.96207 \mathrm{E}-01$

$8.04631 \mathrm{E}-01$

8.13099E-01

$8.21611 \mathrm{E}-01$

$8.30168 \mathrm{E}-01$

$8.38768 \mathrm{E}-01$

$8.47413 \mathrm{E}-01$

$8.56102 \mathrm{E}-0 \mathrm{l}$

$8.64835 \mathrm{E}-01$

$8.73612 \mathrm{E}-01$

$8.82433 \mathrm{E}-01$

$8.91299 \mathrm{E}-01$

$9.00208 \mathrm{E}-01$

$9.09162 \mathrm{E}-01$

$9.18160 \mathrm{E}-01$

$9.27201 \mathrm{E}-01$

$9.36288 \mathrm{E}-01$

$9.45418 \mathrm{E}-01$

$9.54592 \mathrm{E}-01$

$9.63811 \mathrm{E}-01$

$9.73073 \mathrm{E}-01$

$9.82380 \mathrm{E}-01$

$9.91730 \mathrm{E}-01$

$1.00113 \mathrm{E} \quad 00$

$1.01056 \mathrm{E} \quad 00$

$1.02005 \mathrm{E} \quad 00$

$1.02957 \mathrm{E} \quad 00$

$1.03915 \mathrm{E} \quad 00$

$1.04876 \mathrm{E} \quad 00$

$1.05842 \mathrm{E} \quad 00$

$1.06812 \mathrm{E} \quad 00$

$1.07787 \mathrm{E} \quad 00$

$1.08766 \mathrm{E} \quad 00$

$1.09750 \mathrm{E} \quad 00$

$1.10738 \mathrm{E} \quad 00$

$1.11730 \mathrm{E} \quad 00$

$1.12727 \mathrm{E} \quad 00$

$1.13728 \mathrm{E} \quad 00$

$1.14734 \mathrm{E} \quad 00$

$1.15744 \mathrm{E} \quad 00$

$1.16758 \mathrm{E} \quad 00$

$1.17777 \mathrm{E} \quad 00$

$1.18800 \mathrm{E} \quad 00$

$1.19828 \mathrm{E} \quad 00$

$1.20860 \mathrm{E} \quad 00$

$1.21896 \mathrm{E} \quad 00$

$1.22937 \mathrm{E} \quad 00$

$1.23982 \mathrm{E} 00$

$1.25032 \mathbf{E} \quad 00$ $g_{2}$

$\tan \delta_{2}$

$f_{-2}$

$1.21595 \mathrm{E}-02$

$1.23977 \mathrm{E}-02$

$1.26389 \mathrm{E}-02$

$1.28832 \mathrm{E}-02$

$1.31305 \mathrm{E}-02$

$1.33809 \mathrm{E}-02$

$1.36345 \mathrm{E}-02$

$1.38911 \mathrm{E}-02$

$1.41509 \mathrm{E}-02$

$1.44139 \mathrm{E}-02$

$1.46801 \mathrm{E}-02$

$1.49494 \mathrm{E}-02$

$1.52220 \mathrm{E}-02$

$1.54978 \mathrm{E}-02$

$1.57769 \mathrm{E}-02$

$1.60592 \mathrm{E}-02$

$1.63449 \mathrm{E}-02$

$1.66339 \mathrm{E}-02$

$1.69262 \mathrm{E}-02$

$1.72218 \mathrm{E}-02$

$1.75208 \mathrm{E}-02$

$1.78233 \mathrm{E}-02$

$1.81291 \mathrm{E}-02$

$1.84383 \mathrm{E}-02$

$1.87510 \mathrm{E}-02$

$1.90672 \mathrm{E}-02$

$1.93869 \mathrm{E}-02$

$1.97100 \mathrm{E}-02$

$2.00367 \mathrm{E}-02$

$2.03669 \mathrm{E}-02$

$2.07007 \mathrm{E}-02$

$2.10381 \mathrm{E}-02$

$2.13791 \mathrm{E}-02$

$2.17236 \mathrm{E}-02$

$2.20719 \mathrm{E}-02$

$2.24237 \mathrm{E}-02$

$2.27793 \mathrm{E}-02$

$2.31385 \mathrm{E}-02$

$2.35015 \mathrm{E}-02$

$2.38681 \mathrm{E}-02$

$2.42386 \mathrm{E}-02$

$2.46128 \mathrm{E}-02$

$2.49908 \mathrm{E}-02$

$2.53725 \mathrm{E}-02$

$2.57581 \mathrm{E}-02$

$2.61476 \mathrm{E}-02$

$2.65409 \mathrm{E}-02$

$2.69381 \mathrm{E}-02$

$2.73392 \mathrm{E}-02$

$2.77442 \mathrm{E}-02$

$2.81532 \mathrm{E}-02$

$2.85661 \mathrm{E}-02$

$2.89830 \mathrm{E}-02$

$2.94038 \mathrm{E}-02$

$2.98287 \mathrm{E}-02$

$3.02576 \mathrm{E}-02$

$3.06906 \mathrm{E}-02$

$3.11276 \mathrm{E}-02$

$3.15688 \mathrm{E}-02$

$3.20140 \mathrm{E}-02$

$-3.23987 \mathrm{E} 01$

$-3.24027 \mathrm{E}$ 01 $-1.09491 \mathrm{E}-02$

$-3.24067 \mathrm{E}$ 01 $-1.11765 \mathrm{E}-02$

$-3.24106 \mathrm{E}$ 01 $-1.14070 \mathrm{E}-02$

$-3.24145 \mathrm{E} \quad 01 \quad-1.16405 \mathrm{E}-02$

$-3.24183 \mathrm{E} \quad 01$

$-3.24221 \mathrm{E} \quad 01$

$-3.24258 \mathrm{E} 01$

$-3.24295 \mathrm{E} 01$

$-3.24332 \mathrm{E} 01$

$-3.24368 \mathrm{E} \quad 01$

$-3.24404 \mathrm{E} \quad 01$

$-3.24440 \mathrm{E} 01$

$-3.24475 \mathrm{E} \quad 01$

$-3.24509 \mathrm{E} 01$

$-3.24544 \mathrm{E} \quad 01$

$-3.24578 \mathrm{E} 01$

$-3.24611 \mathrm{E} 01$

$-3.24645 \mathrm{E} \quad 01$

$-3.24678 \mathrm{E} 01$

$-3.24710 \mathrm{E} 01$

$-3.24742 \mathrm{E} 01$

$-3.24774 \mathrm{E} 01$

$-3.24806 \mathrm{E} 01$

$-3.24837 \mathrm{E} 01$

$-3.24868 \mathrm{E} \quad 01$

$-3.24899 \mathrm{E} 01$

$-3.24929 \mathrm{E} 01$

$-3.24959 \mathrm{E} 01$

$-3.24989 \mathrm{E} 01$

$-3.25018 \mathrm{E} 01$

$-3.25047 \mathrm{E} 01$

$-3.25076 \mathrm{E} 01$

$-3.25105 \mathrm{E} \quad 01$

$-3.25133 \mathrm{E} 01$

$-3.25161 \mathrm{E} 01$

$-3.25189 \mathrm{E} 01$

$-3.25216 \mathrm{E} 01$

$-3.25244 \mathrm{E} \quad 01$

$-3.25271 \mathrm{E} 01$

$-3.25297 \mathrm{E} \quad 01$

$-3.25324 \mathrm{E} 01$

$-3.25350 \mathrm{E} 01$

$-3.25376 \mathrm{E} 01$

$-3.25402 \mathrm{E}$ ol $-2.36617 \mathrm{E}-02$

$-3.25427 \mathrm{E} \quad 01$

$-3.25453 \mathrm{E} 01$

$-3.25478 \mathrm{E} 01$

$-3.25503 \mathrm{E} 01$

$-3.25527 \mathrm{E} 01$

$-3.25552 \mathrm{E} \quad 01$

$-3.25576 \mathrm{E} \quad 01$

$-3.25600 \mathrm{E} 01$

$-3.25624 \mathrm{E} 01$

$-3.25647 \mathrm{E} 01$

$-3.25670 \mathrm{E} 01$

$-3.25694 \mathrm{E} 01$

$-3.25716 \mathrm{E} 01$

$-1.18769 \mathrm{E}-02$

$-1.21165 \mathrm{E}-02$

$-1.23591 \mathrm{E}-02$

$-1.26048 \mathrm{E}-02$

$-1.28535 \mathrm{E}-02$

$-1.31054 \mathrm{E}-02$

$-1.33605 \mathrm{E}-02$

$-1.36187 \mathrm{E}-02$

$-1.38801 \mathrm{E}-02$

$-1.41447 \mathrm{E}-02$

$-1.44124 \mathrm{E}-02$

$-1.46835 \mathrm{E}-02$

$-1.49577 \mathrm{E}-02$

$-1.52353 \mathrm{E}-02$

$-1.55161 \mathrm{E}-02$

$-1.58003 \mathrm{E}-02$

$-1.60878 \mathrm{E}-02$

$-1.63786 \mathrm{E}-02$

$-1.66728 \mathrm{E}-02$

$-1.69704 \mathrm{E}-02$

$-1.72713 \mathrm{E}-02$

$-1.75757 \mathrm{E}-02$

$-1.78836 \mathrm{E}-02$

$-1.81949 \mathrm{E}-02$

$-1.85097 \mathrm{E}-02$

$-1.88280 \mathrm{E}-02$

$-1.91498 \mathrm{E}-02$

$-1.94751 \mathrm{E}-02$

$-1.98040 \mathrm{E}-02$

$-2.01364 \mathrm{E}-02$

$-2.04725 \mathrm{E}-02$

$-2.08122 \mathrm{E}-02$

$-2.11554 \mathrm{E}-02$

$-2.15024 \mathrm{E}-02$

$-2.18530 \mathrm{E}-02$

$-2.22073 \mathrm{E}-02$

$-2.25653 \mathrm{E}-02$

$-2.29270 \mathrm{E}-02$

$-2.32925 \mathrm{E}-02$

$-2.40347 \mathrm{E}-\mathrm{O} 02$

$-2.44115 \mathrm{E}-02$

$-2.47921 \mathrm{E}-02$

$-2.51766 \mathrm{E}-02$

$-2.55649 \mathrm{E}-02$

$-2.59571 \mathrm{E}-02$ 
ELECTRON RADIAL FUNCTIONS

Positron

\begin{tabular}{|c|c|c|c|}
\hline$p$ & $F_{0}$ & $f_{1}$ & \\
\hline 24.10 & $8.74474 \mathrm{E}-01$ & $2.23579 \mathrm{E}$ & 01 \\
\hline 24.20 & $8.74468 \mathrm{E}-01$ & $2.24545 \mathrm{E}$ & 01 \\
\hline 24.30 & $8.74462 \mathrm{E}-01$ & $2.25512 \mathrm{E}$ & 01 \\
\hline 24.40 & $8.74456 \mathrm{E}-01$ & $2.26479 \mathrm{E}$ & 01 \\
\hline 24.50 & $8.74450 \mathrm{E}-01$ & $2.27446 \mathrm{E}$ & 01 \\
\hline 24.60 & $8.74444 \mathrm{E}-01$ & $2.28413 \mathrm{E}$ & 01 \\
\hline 24.70 & $8.74438 \mathrm{E}-01$ & $2.29380 \mathrm{E}$ & 01 \\
\hline 24.80 & $8.74432 \mathrm{E}-01$ & $2.30348 \mathrm{E}$ & 01 \\
\hline 24.90 & $8.74426 \mathrm{E}-01$ & $2.31316 \mathrm{E}$ & 01 \\
\hline 25.00 & $8.74420 \mathrm{E}-01$ & $2.32284 \mathrm{E}$ & 01 \\
\hline 25.10 & $8.74414 \mathrm{E}-01$ & $2.33252 \mathrm{E}$ & 01 \\
\hline 25.20 & $8.74408 \mathrm{E}-01$ & $2.34220 \mathrm{E}$ & 01 \\
\hline 25.30 & $8.74402 \mathrm{E}-01$ & $2.35189 \mathrm{E}$ & 01 \\
\hline 25.40 & $8.74396 \mathrm{E}-01$ & $2.36158 \mathrm{E}$ & 01 \\
\hline 25.50 & $8.74391 \mathrm{E}-01$ & $2.37127 \mathrm{E}$ & 01 \\
\hline 25.60 & $8.74385 \mathrm{E}-01$ & $2.38097 \mathrm{E}$ & 01 \\
\hline 25.70 & $8.74379 \mathrm{E}-01$ & $2.39066 \mathrm{E}$ & 01 \\
\hline 25.80 & $8.74373 \mathrm{E}-01$ & $2.40036 \mathrm{E}$ & 01 \\
\hline 25.90 & $8.74367 \mathrm{E}-01$ & $2.41006 \mathrm{E}$ & 01 \\
\hline 26.00 & $8.74362 \mathrm{E}-01$ & $2.41977 \mathrm{E}$ & 01 \\
\hline 26.10 & $8.74356 \mathrm{E}-01$ & $2.42947 \mathrm{E}$ & 01 \\
\hline 26.20 & $8.74350 \mathrm{E}-01$ & $2.43918 \mathrm{E}$ & 01 \\
\hline 26.30 & $8.74345 \mathrm{E}-01$ & $2.44889 \mathrm{E}$ & 01 \\
\hline 26.40 & $8.74339 \mathrm{E}-01$ & $2.45860 \mathrm{E}$ & 01 \\
\hline 26.50 & $8.74333 \mathrm{E}-01$ & $2.46832 \mathrm{E}$ & 01 \\
\hline 26.60 & $8.74327 \mathrm{E}-01$ & $2.47804 \mathrm{E}$ & 01 \\
\hline 26.70 & $8.74322 \mathrm{E}-01$ & $2.48775 \mathrm{E}$ & 01 \\
\hline 26.80 & $8.74316 \mathrm{E}-01$ & $2.49748 \mathrm{E}$ & 01 \\
\hline 26.90 & $8.74310 \mathrm{E}-01$ & $2.50720 \mathrm{E}$ & 01 \\
\hline 27.00 & $8.74305 \mathrm{E}-01$ & $2.51693 \mathrm{E}$ & 01 \\
\hline 27.10 & $8.74299 \mathrm{E}-01$ & $2.52666 \mathrm{E}$ & 01 \\
\hline 27.20 & $8.74294 \mathrm{E}-01$ & $2.53639 \mathrm{E}$ & 01 \\
\hline 27.30 & $8.74288 \mathrm{E}-01$ & $2.54612 \mathrm{E}$ & 01 \\
\hline 27.40 & $8.74283 \mathrm{E}-01$ & $2.55586 \mathrm{E}$ & 01 \\
\hline 27.50 & $8.74278 \mathrm{E}-01$ & $2.56560 \mathrm{E}$ & 01 \\
\hline 27.60 & $8.74272 \mathrm{E}-01$ & $2.57534 \mathrm{E}$ & 01 \\
\hline 27.70 & $8.74267 \mathrm{E}-01$ & $2.58509 \mathrm{E}$ & 01 \\
\hline 27.80 & $8.74261 \mathrm{E}-01$ & $2.59483 \mathrm{E}$ & 01 \\
\hline 27.90 & $8.74256 \mathrm{E}-01$ & $2.60458 \mathrm{E}$ & 01 \\
\hline 28.00 & $8.74250 \mathrm{E}-01$ & $2.61433 \mathrm{E}$ & 01 \\
\hline 28.10 & $8.74245 \mathrm{E}-01$ & $2.62409 \mathrm{E}$ & 01 \\
\hline 28.20 & $8.74239 \mathrm{E}-01$ & $2.63384 \mathrm{E}$ & 01 \\
\hline 28.30 & $8.74234 \mathrm{E}-01$ & $2.64360 \mathrm{E}$ & 01 \\
\hline 28.40 & $8.74229 \mathrm{E}-01$ & $2.65336 \mathrm{E}$ & 01 \\
\hline 28.50 & $8.74223 \mathrm{E}-01$ & $2.66313 \mathrm{E}$ & 01 \\
\hline 28.60 & $8.74218 \mathrm{E}-01$ & $2.67289 \mathrm{E}$ & 01 \\
\hline 28.70 & $8.74213 \mathrm{E}-01$ & $2.68266 \mathrm{E}$ & 01 \\
\hline 28.80 & $8.74207 \mathrm{E}-01$ & $2.69243 \mathrm{E}$ & 01 \\
\hline 28.90 & $8.74202 \mathrm{E}-01$ & $2.70221 \mathrm{E}$ & 01 \\
\hline 29.00 & $8.74197 \mathrm{E}-01$ & $2.71198 \mathrm{E}$ & 01 \\
\hline 29.10 & $8.74192 \mathrm{E}-01$ & $2.72176 \mathrm{E}$ & 01 \\
\hline 29.20 & $8.74186 \mathrm{E}-01$ & $2.73154 \mathrm{E}$ & 01 \\
\hline 29.30 & $8.74181 \mathrm{E}-01$ & $2.74133 \mathrm{E}$ & 01 \\
\hline 29.40 & $8.74176 \mathrm{E}-01$ & $2.75112 \mathrm{E}$ & 01 \\
\hline 29.50 & $8.74171 \mathrm{E}-01$ & $2.76091 \mathrm{E}$ & 01 \\
\hline 29.60 & $8.74165 \mathrm{E}-01$ & $2.77070 \mathrm{E}$ & 01 \\
\hline 29.70 & $8.74160 \mathrm{E}-01$ & $2.78049 \mathrm{E}$ & 01 \\
\hline 29.80 & $8.74155 \mathrm{E}-01$ & $2.79029 \mathrm{E}$ & 01 \\
\hline 29.90 & $8.74150 \mathrm{E}-01$ & $2.80009 \mathrm{E}$ & 01 \\
\hline 30.00 & $8.74145 \mathrm{E}-01$ & $2.80989 \mathrm{E}$ & 01 \\
\hline
\end{tabular}

$Z=6 \quad A=12 \quad \rho=1.2 A^{1 / 3} F$

\begin{tabular}{|c|c|c|c|c|c|c|}
\hline$g_{1}$ & $\tan \delta_{1}$ & $f_{-1}$ & $g_{-1}$ & & $\tan \delta_{-1}$ & \\
\hline $9.41241 \mathrm{E}-01$ & $-8.87214 \mathrm{E}-04$ & $-8.71428 \mathrm{E}-01$ & $2.33307 \mathrm{E}$ & 01 & $3.71086 \mathrm{E}$ & 02 \\
\hline $9.50645 \mathrm{E}-01$ & $-8.90018 \mathrm{E}-04$ & $-8.80614 \mathrm{E}-01$ & $2.34277 \mathrm{E}$ & 01 & $3.71740 \mathrm{E}$ & 02 \\
\hline $9.60096 \mathrm{E}-01$ & $-8.92793 \mathrm{E}-04$ & $-8.89848 \mathrm{E}-01$ & $2.35246 \mathrm{E}$ & 01 & $3.72392 \mathrm{E}$ & 02 \\
\hline $9.69594 \mathrm{E}-01$ & $-8.95532 \mathrm{E}-04$ & $-8.99128 \mathrm{E}-01$ & $2.36216 \mathrm{E}$ & 01 & $3.73043 \mathrm{E}$ & 02 \\
\hline $9.79139 \mathrm{E}-01$ & $-8.98235 \mathrm{E}-04$ & $-9.08456 \mathrm{E}-01$ & $2.37186 \mathrm{E}$ & 01 & $3.73692 \mathrm{E}$ & 02 \\
\hline $9.88732 \mathrm{E}-01$ & $-9.00904 \mathrm{E}-04$ & $-9.17831 \mathrm{E}-01$ & $2.38156 \mathrm{E}$ & 01 & $3.74341 \mathrm{E}$ & 02 \\
\hline $9.98371 \mathrm{E}-01$ & $-9.03548 \mathrm{E}-04$ & $-9.27253 \mathrm{E}-01$ & $2.39126 \mathrm{E}$ & 01 & $3.74986 \mathrm{E}$ & 02 \\
\hline $1.00806 \mathrm{E} \quad 00$ & $-9.06147 \mathrm{E}-04$ & $-9.36722 \mathrm{E}-01$ & $2.40097 \mathrm{E}$ & 01 & $3.75633 \mathrm{E}$ & 02 \\
\hline $1.01779 \mathrm{E} \quad 00$ & $-9.08725 \mathrm{E}-04$ & $-9.46239 \mathrm{E}-01$ & $2.41068 \mathrm{E}$ & 01 & $3.76276 \mathrm{E}$ & 02 \\
\hline $1.02757 \mathrm{E} \quad 00$ & $-9.11265 \mathrm{E}-04$ & $-9.55803 \mathrm{E}-01$ & $2.42039 \mathrm{E}$ & 01 & $3.76919 \mathrm{E}$ & 02 \\
\hline $1.03740 \mathrm{E}$ & $-9.13768 \mathrm{E}-04$ & $-9.65414 \mathrm{E}-01$ & $2.43010 \mathrm{E}$ & 01 & $3.77561 \mathrm{E}$ & 02 \\
\hline $1.04728 \mathrm{E}$ & $-9.16253 \mathrm{E}-04$ & $-9.75073 \mathrm{E}-01$ & $2.43982 \mathrm{E}$ & 01 & $3.78200 \mathrm{E}$ & 02 \\
\hline $1.05720 \mathrm{E}$ & $-9.18698 \mathrm{E}-04$ & $-9.84778 \mathrm{E}-01$ & $2.44953 \mathrm{E}$ & 01 & $3.78840 \mathrm{E}$ & 02 \\
\hline $1.06717 \mathrm{E}$ & $-9.21118 \mathrm{E}-04$ & $-9.94532 \mathrm{E}-01$ & $2.45926 \mathrm{E}$ & 01 & $3.79477 \mathrm{E}$ & 02 \\
\hline $1.07719 \mathrm{E}$ & $-9.23504 \mathrm{E}-04$ & $-1.00433 \mathrm{E} \quad 00$ & $2.46898 \mathrm{E}$ & 01 & $3.80113 \mathrm{E}$ & 02 \\
\hline $1.08725 \mathrm{E}$ & $-9.25858 \mathrm{E}-04$ & $-1.01418 \mathrm{E}$ & $2.47870 \mathrm{E}$ & 01 & $3.80749 \mathrm{E}$ & 02 \\
\hline $1.09737 \mathrm{E}$ & $-9.28186 \mathrm{E}-04$ & $-1.02408 \mathrm{E}$ & $2.48843 \mathrm{E}$ & 01 & $3.81383 \mathrm{E}$ & 02 \\
\hline $1.10752 \mathrm{E}$ & $-9.30481 \mathrm{E}-04$ & $-1.03402 \mathrm{E}$ & $2.49816 \mathrm{E}$ & 01 & $3.82016 \mathrm{E}$ & 02 \\
\hline $1773 \mathrm{E}$ & $-9.32749 \mathrm{E}-04$ & $-1.04401 \mathrm{E} \quad 00$ & $2.50789 \mathrm{E}$ & 01 & $3.82648 \mathrm{E}$ & 02 \\
\hline $1.12799 \mathrm{E}$ & $-9.34989 \mathrm{E}-04$ & $-1.05405 \mathrm{E} \quad 00$ & $2.51763 \mathrm{E}$ & 01 & $3.83279 \mathrm{E}$ & 02 \\
\hline $1.13829 \mathrm{E} \quad 00$ & $-9.37193 \mathrm{E}-04$ & $-1.06413 \mathbf{E} \quad 00$ & $2.52737 \mathrm{E}$ & 01 & $3.83910 \mathrm{E}$ & 02 \\
\hline $1.14864 \mathrm{E} \quad 00$ & $-9.39379 \mathrm{E}-04$ & $-1.07427 \mathrm{E} \quad 00$ & $2.53710 \mathrm{E}$ & 01 & $3.84538 \mathrm{E}$ & 02 \\
\hline $1.15904 \mathrm{E} \quad 00$ & $-9.41527 \mathrm{E}-04$ & $-1.08445 \mathrm{E} \quad 00$ & $2.54685 \mathrm{E}$ & 01 & $3.85167 \mathrm{E}$ & 02 \\
\hline $1.16948 \mathrm{E} \quad 00$ & $-9.43657 \mathrm{E}-04$ & $-1.09468 E$ & $2.55659 \mathrm{E}$ & 01 & $3.85793 \mathrm{E}$ & 02 \\
\hline $1.17997 \mathrm{E} \quad 00$ & $-9.45750 \mathrm{E}-04$ & $-1.10495 \mathrm{E} \quad 00$ & $2.56634 \mathrm{E}$ & 01 & $3.86420 \mathrm{E}$ & 02 \\
\hline $1.19051 \mathrm{E} \quad 00$ & $-9.47821 \mathrm{E}-04$ & $-1.11528 \mathrm{E} \quad 00$ & $2.57609 \mathrm{E}$ & 01 & $3.87045 \mathrm{E}$ & 02 \\
\hline $1.20110 \mathrm{E} \quad 00$ & $-9.49868 \mathrm{E}-04$ & $-1.12565 \mathrm{E} \quad 00$ & $2.58584 \mathrm{E}$ & 01 & $3.87669 \mathrm{E}$ & 02 \\
\hline $1.21174 \mathrm{E} \quad 00$ & $-9.51886 \mathrm{E}-04$ & $-1.13607 \mathrm{E} \quad 00$ & $2.59559 \mathrm{E}$ & 01 & $3.88292 \mathrm{E}$ & 02 \\
\hline $1.22242 \mathrm{E} \quad 00$ & $-9.53874 \mathrm{E}-04$ & $-1.14654 \mathrm{E}$ & $2.60535 \mathrm{E}$ & 01 & $3.88915 \mathrm{E}$ & 02 \\
\hline $1.23315 \mathrm{E}$ & $-9.55837 \mathrm{E}-04$ & $-1.15705 \mathrm{E}$ & $2.61511 \mathrm{E}$ & 01 & $3.89537 \mathrm{E}$ & 02 \\
\hline $1.24393 \mathrm{E} \quad 00$ & $-9.57777 \mathrm{E}-04$ & $-1.16761 \mathrm{E} \quad 00$ & $2.62487 \mathrm{E}$ & 01 & $3.90157 \mathrm{E}$ & 02 \\
\hline $1.25475 \mathrm{E} \quad 00$ & $-9.59691 \mathrm{E}-04$ & $-1.17822 \mathrm{E} \quad 00$ & $2.63464 \mathrm{E}$ & 01 & $3.90777 \mathrm{E}$ & 02 \\
\hline $1.26563 \mathrm{E}$ & $-9.61581 \mathrm{E}-04$ & $-1.18888 \mathrm{E} \quad 00$ & $2.64440 \mathrm{E}$ & 01 & $3.91395 \mathrm{E}$ & 02 \\
\hline $1.27655 \mathrm{E}$ & $-9.63439 \mathrm{E}-04$ & $-1.19959 \mathrm{E} \quad 00$ & $2.65417 \mathrm{E}$ & 01 & $3.92014 \mathrm{E}$ & 02 \\
\hline $1.28752 \mathrm{E}$ & $-9.65279 \mathrm{E}-04$ & $-1.21034 \mathrm{E} \quad 00$ & $2.66395 \mathrm{E}$ & 01 & $3.92631 \mathrm{E}$ & 02 \\
\hline $1.29854 \mathrm{E}$ & $-9.67091 \mathrm{E}-04$ & $-1.22115 \mathrm{E}$ & $2.67372 \mathrm{E}$ & 01 & $3.93248 \mathrm{E}$ & 02 \\
\hline $1.30960 \mathrm{E}$ & $-9.68874 \mathrm{E}-04$ & $-1.23200 \mathrm{E}$ & $2.68350 \mathrm{E}$ & 01 & $3.93865 \mathrm{E}$ & 02 \\
\hline $1.32072 \mathrm{E}$ & $-9.70642 \mathbf{E}-04$ & $-1.24290 \mathrm{E}$ & $2.69328 \mathrm{E}$ & 01 & $3.94480 \mathrm{E}$ & 02 \\
\hline $1.33188 \mathrm{E}$ & $-9.72383 \mathrm{E}-04$ & $-1.25384 \mathrm{E} \quad 00$ & $2.70306 \mathrm{E}$ & 01 & $3.95095 \mathrm{E}$ & 02 \\
\hline $1.34309 \mathrm{E} \quad 00$ & $-9.74103 \mathrm{E}-04$ & $-1.26484 \mathrm{E} \quad 00$ & $2.71285 \mathrm{E}$ & 01 & $3.95708 \mathrm{E}$ & 02 \\
\hline $1.35434 \mathrm{E}$ & $-9.75786 \mathrm{E}-04$ & $-1.27588 \mathrm{E} \quad 00$ & $2.72263 \mathrm{E}$ & 01 & $3.96323 \mathrm{E}$ & 02 \\
\hline $1.36565 \mathrm{E} \quad 00$ & $-9.77459 \mathrm{E}-04$ & $-1.28697 \mathrm{E} \quad 00$ & $2.73242 \mathrm{E}$ & 01 & $3.96935 \mathrm{E}$ & 02 \\
\hline $1.37700 \mathrm{E} \quad 00$ & $-9.79104 \mathrm{E}-04$ & $-1.29811 \mathrm{E} \quad 00$ & $2.74222 \mathrm{E}$ & 01 & $3.97547 \mathrm{E}$ & 02 \\
\hline $1.38840 \mathrm{E}$ & $-9.80720 \mathrm{E}-04$ & $-1.30930 \mathrm{E}$ & $2.75201 \mathrm{E}$ & 01 & $3.98160 \mathrm{E}$ & 02 \\
\hline $1.39985 \mathrm{E}$ & $-9.82318 \mathrm{E}-04$ & $-1.32053 \mathrm{E}$ & $2.76181 \mathrm{E}$ & 01 & $3.98771 \mathrm{E}$ & 02 \\
\hline $1.41135 \mathrm{E}$ & $-9.83898 \mathbf{E}-04$ & $-1.33181 \mathrm{E} \quad 00$ & $2.77161 \mathrm{E}$ & 01 & $3.99381 \mathrm{E}$ & 02 \\
\hline $1.42290 \mathrm{E}$ & $-9.85451 \mathrm{E}-04$ & $-1.34315 \mathrm{E} \quad 00$ & $2.78141 \mathrm{E}$ & 01 & $3.99992 \mathrm{E}$ & 02 \\
\hline $1.43449 \mathrm{E}$ & $-9.86983 \bar{E}-04$ & $-1.35452 \mathrm{E}$ & $2.79122 \mathrm{E}$ & 01 & $4.00601 \mathrm{E}$ & 02 \\
\hline $1.44613 \mathrm{E}$ & $-9.88493 \mathrm{E}-04$ & $-1.36595 \mathrm{E} \quad 00$ & $2.80103 \mathrm{E}$ & 01 & $4.01210 \mathrm{E}$ & 02 \\
\hline $1.45782 \mathrm{E}$ & $-9.89983 \mathrm{E}-04$ & $-1.37743 \mathrm{E}$ & $2.81084 \mathrm{E}$ & 01 & $4.01819 \mathrm{E}$ & 02 \\
\hline $1.46956 \mathrm{E}$ & $-9.91448 \mathrm{E}-04$ & $-1.38895 \mathrm{E} \quad 00$ & $2.82065 \mathrm{E}$ & 01 & $4.02427 \mathrm{E}$ & 02 \\
\hline $1.48135 \mathrm{E}$ & $-9.92890 \mathrm{E}-04$ & $-1.40053 \mathrm{E} \quad 00$ & $2.83047 \mathrm{E}$ & 01 & $4.03035 \mathrm{E}$ & 02 \\
\hline $1.49319 \mathrm{E}$ & $-9.94309 \mathrm{E}-04$ & $-1.41215 \mathrm{E} \quad 00$ & $2.84029 \mathrm{E}$ & 01 & $4.03643 \mathrm{E}$ & 02 \\
\hline $1.50507 \mathrm{E}$ & $-9.95715 \mathrm{E}-04$ & $-1.42382 \mathrm{E} \quad 00$ & $2.85011 \mathrm{E}$ & 01 & $4.04249 \mathrm{E}$ & 02 \\
\hline $1.51700 \mathrm{E} \quad 00$ & $-9.97097 \mathrm{E}-04$ & $-1.43554 \mathbf{E} \quad 00$ & $2.85994 \mathrm{E}$ & 01 & $4.04856 \mathrm{E}$ & 02 \\
\hline $1.52898 \mathrm{E}$ & $-9.98458 \mathrm{E}-04$ & $-1.44730 \mathrm{E} \quad 00$ & $2.86977 \mathrm{E}$ & 01 & $4.05462 \mathrm{E}$ & 02 \\
\hline $1.54101 \mathrm{E}$ & $-9.99793 \mathrm{E}-04$ & $-1.45912 \mathrm{E} \quad 00$ & $2.87960 \mathrm{E}$ & 01 & $4.06069 \mathrm{E}$ & 02 \\
\hline $1.55309 \mathrm{E}$ & $-1.00112 \mathrm{E}-03$ & $-1.47098 \mathrm{E} \quad 00$ & $2.88943 \mathrm{E}$ & 01 & $4.06673 \mathrm{E}$ & 02 \\
\hline $1.56521 \mathrm{E} \quad 00$ & $-1.00241 \mathrm{E}-03$ & $-1.48290 \mathrm{E} \quad 00$ & $2.89927 \mathrm{E}$ & 01 & $4.07280 \mathrm{E}$ & 02 \\
\hline $1.57739 \mathrm{E} \quad 00$ & $-1.00370 \mathrm{E}-03$ & $-1.49486 \mathbf{E} \quad 00$ & $2.90910 \mathrm{E}$ & 01 & $4.07883 \mathrm{E}$ & 02 \\
\hline
\end{tabular}


ELECTRON RADIAL FUNCTIONS

$Z=6 \quad A=12 \quad \rho=1.2 A^{1 / 3} F$

Pesitron

$\bar{F}$

$7.13214 \mathrm{E}-04$

$7.19143 \mathrm{E}-04$

$7.25096 \mathrm{E}-04$

$7.31074 \mathrm{E}-04$

7.37077 E-04

$7.43104 \mathrm{E}-04$

$7.49155 \mathrm{E}-04$

$7.55232 \mathrm{E}-04$

$7.61332 \mathrm{E}-04$

$7.67457 \mathrm{E}-04$

$7.73607 \mathrm{E}-04$

$7.79781 \mathrm{E}-04$

$7.85980 \mathrm{E}-04$

$7.92203 \mathrm{E}-04$

$7.98451 \mathrm{E}-04$

8.04723E-04

$8.11020 \mathrm{E}-04$

$8.17341 \mathrm{E}-04$

$8.23687 \mathrm{E}-04$

$8.30058 \mathrm{E}-04$

$8.36452 \mathrm{E}-04$

$8.42872 \mathrm{E}-04$

$8.49316 \mathrm{E}-04$

$8.55784 \mathrm{E}-04$

$8.62277 \mathrm{E}-04$

$8.68795 \mathrm{E}-04$

$8.75337 \mathrm{E}-04$

8.81904 E-04

$8.88495 \mathrm{E}-04$

8.95111 E-04

$9.01751 \mathrm{E}-04$

$9.08416 \mathrm{E}-04$

$9.15105 \mathrm{E}-04$

$9.21818 \mathrm{E}-04$

$9.28557 \mathrm{E}-04$

$9.35320 \mathrm{E}-04$

$9.42107 \mathrm{E}-04$

$9.48919 \mathrm{E}-04$

$9.55755 \mathrm{E}-04$

$9.62616 \mathrm{E}-04$

$9.69501 \mathrm{E}-04$

$9.76411 \mathrm{E}-04$

$9.83346 \mathrm{E}-04$

$9.90305 \mathrm{E}-04$

$9.97288 \mathrm{E}-04$

$1.00430 \mathrm{E}-03$

$1.01133 \mathrm{E}-03$

$1.01839 \mathrm{E}-03$

$1.02547 \mathrm{E}-03$

$1.03257 \mathrm{E}-03$

29.10

29.20

29.30

29.40

29.50

29.60

29.70

29.80

29.90

30.00

$1.06124 \mathrm{E}-03$

$1.06847 \mathrm{E}-03$

$1.07573 \mathrm{E}-03$

$1.08300 \mathrm{E}-03$

$1.09031 \mathrm{E}-03$

$1.09763 \mathrm{E}-03$

$1.10498 \mathrm{E}-03$ $f_{2}$

$1.26086 \mathrm{E} \quad 00$

$1.27144 \mathrm{E} \quad 00$

$1.28207 \mathrm{E} \quad 00$

$1.29274 \mathrm{E} \quad 00$

$1.30346 \mathrm{E} \quad 00$

$1.31421 \mathrm{E} \quad 00$

$1.32502 \mathrm{E} \quad 00$

$1.33587 \mathrm{E} \quad 00$

$1.34676 \mathrm{E} \quad 00$

$1.35769 \mathrm{E} \quad 00$

$1.36867 \mathrm{E} \quad 00$

$1.37970 \mathrm{E} \quad 00$

$1.39076 \mathrm{E} \quad 00$

$1.40188 \mathrm{E} \quad 00$

$1.41303 \mathrm{E} \quad 00$

$1.42423 \mathrm{E} \quad 00$

$1.43547 \mathrm{E} \quad 00$

$1.44676 \mathrm{E} \quad 00$

$1.45809 \mathrm{E} \quad 00$

$1.46947 \mathrm{E} \quad 00$

$1.49235 \mathrm{E} \quad 00$

$1.50385 \mathrm{E} \quad 00$

$1.51540 \mathrm{E} \quad 00$

$1.52700 \mathrm{E} \quad 00$

$1.53864 \mathrm{E} \quad 00$

$1.55032 \mathrm{E} \quad 00$

$1.56204 \mathrm{E} \quad 00$

$1.57381 \mathrm{E} \quad 00$

$1.58563 \mathrm{E} \quad 00$

$1.59748 \mathrm{E} \quad 00$

$1.60939 \mathrm{E} \quad 00$

$1.62133 \mathrm{E} \quad 00$

$1.63332 \mathrm{E} \quad 00$

$1.64535 \mathrm{E} \quad 00$

$1.65743 \mathrm{E} \quad 00$

$1.66955 \mathrm{E} \quad 00$

$1.68171 \mathrm{E} \quad 00$

$1.69392 \mathrm{E} \quad 00$

$1.70617 \mathrm{E} \quad 00$

$1.71847 \mathrm{E} \quad 00$

$1.73081 \mathrm{E} \quad 00$

$1.74319 \mathrm{E} \quad 00$

$1.75562 \mathrm{E} \quad 00$

$1.76809 \mathrm{E} \quad 00$

$1.78060 \mathrm{E} \quad 00$

$1.79316 \mathrm{E} \quad 00$

$1.80576 \mathrm{E} \quad 00$

$1.81841 \mathrm{E} \quad 00$

$1.83110 \mathrm{E} \quad 00$

$1.84383 \mathrm{E} \quad 00$

$1.85661 \mathrm{E} \quad 00$

$1.86943 \mathrm{E} \quad 00$

$1.88229 \mathrm{E} \quad 00$

$1.89520 \mathrm{E} \quad 00$

$1.90815 \mathrm{E} \quad 00$

$1.92115 \mathrm{E} \quad 00$

$1.93419 \mathrm{E} \quad 00$

$1.94727 \mathrm{E} \quad 00$

$1.96040 \mathrm{E} \quad 00$
$1.48088 \mathrm{E} \quad 00$

$g_{2}$

$\tan \delta_{2}$

$f_{-2}$

$3.24634 \mathrm{E}-02$

$3.29169 \mathrm{E}-02$

$3.33745 \mathrm{E}-02$

$3.38364 \mathrm{E}-02$

$3.43024 \mathrm{E}-02$

$3.47727 \mathrm{E}-02$

$3.52472 \mathrm{E}-02$

$3.57259 \mathrm{E}-02$

$3.62089 \mathrm{E}-02$

$3.66962 \mathrm{E}-02$

$3.71879 \mathrm{E}-02$

$3.76838 \mathrm{E}-02$

$3.81841 \mathrm{E}-02$

$3.86888 \mathrm{E}-02$

$3.91979 \mathrm{E}-02$

$3.97114 \mathrm{E}-02$

$4.02293 \mathrm{E}-02$

$4.07516 \mathrm{E}-02$

4.12784 E-02

$4.18097 \mathrm{E}-02$

$4.23455 \mathrm{E}-02$ $4.28858 \mathrm{E}-02$

$4.34306 \mathrm{E}-02$

$4.39800 \mathrm{E}-02$

4.45340E-02

$4.50925 \mathrm{E}-02$

$4.56557 \mathrm{E}-02$

$4.62235 \mathrm{E}-02$

$4.67960 \mathrm{E}-02$

$4.73731 \mathrm{E}-02$

$4.79549 \mathrm{E}-02$

4.85414 E-02

$4.91327 \mathrm{E}-02$

4.97286E-02

$5.03294 \mathrm{E}-02$

$5.09349 \mathrm{E}-02$

$5.15452 \mathrm{E}-02$

$5.21604 \mathrm{E}-02$

$5.27803 \mathrm{E}-02$

$5.34052 \mathrm{E}-02$

$5.40349 \mathrm{E}-02$

$5.46695 \mathrm{E}-02$

$5.53090 \mathrm{E}-02$

$5.59534 \mathrm{E}-02$

$5.66028 \mathrm{E}-02$

$5.72571 \mathrm{E}-02$

$5.79165 \mathrm{E}-02$

$5.85808 \mathrm{E}-02$

$5.92502 \mathrm{E}-02$

$5.99246 \mathrm{E}-02$

6.06041 E-02 $6.12886 \mathrm{E}-02$ $6.19783 \mathrm{E}-02$ $6.26730 \mathrm{E}-02$ $6.33729 \mathrm{E}-02$

$-3.25784 \mathrm{E}$ 01 $-3.00962 \mathrm{E}-02$ $-3.25806 \mathrm{E}$ 01 $-3.05323 \mathrm{E}-02$ $-3.25828 \mathrm{E}$ 01 $-3.09724 \mathrm{E}-02$ $-3.25850 \mathrm{E}$ 01 $-3.14167 \mathrm{E}-02$ $-3.25872 \mathrm{E}$ 0I $-3.18651 \mathrm{E}-02$

$-3.25893 \mathrm{E} \quad 01 \quad-3.23177 \mathrm{E}-02$ $-3.25914 \mathrm{E}$ 01 $-3.27744 \mathrm{E}-02$ $-3.25935 \mathrm{E}$ 01 $-3.32353 \mathrm{E}-02$ $-3.25956 \mathrm{E}$ 01 $-3.37004 \mathrm{E}-02$ $-3.25977 \mathrm{E} \quad 01 \quad-3.41698 \mathrm{E}-02$

$-3.25997 \mathrm{E} \quad 01 \quad-3.46434 \mathrm{E}-02$ $-3.26018 \mathrm{E}$ 01 $-3.51213 \mathrm{E}-02$ $-3.26038 \mathrm{E}$ 01 $-3.56035 \mathrm{E}-02$ $-3.26058 \mathrm{E}$ 01 $-3.60900 \mathrm{E}-02$

$-3.26078 \mathrm{E}$ 01 $-3.65808 \mathrm{E}-02$

$-3.26098 \mathrm{E} \quad 01 \quad-3.70759 \mathrm{E}-02$

$-3.26117 \mathrm{E}$ 01 $-3.75754 \mathrm{E}-02$

$-3.26136 \mathrm{E}$ 01 $-3.80793 \mathrm{E}-02$

$-3.26156 \mathrm{E} \quad 01 \quad-3.85876 \mathrm{E}-02$

$\begin{array}{lll}-3.26175 \mathrm{E} & 01 & -3.91003 \mathrm{E}-02\end{array}$

$-3.26194 \mathrm{E} \quad 01$

$-3.96174 \mathrm{E}-02$

$-3.26212 \mathrm{E} 01$

$-3.26231 \mathrm{E} 01$

$-3.26250 \mathrm{E} 01$

$-3.26268 \mathrm{E} 01$

$-4.01390 \mathrm{E}-02$

$-4.06651 \mathrm{E}-02$

$-4.11957 \mathrm{E}-02$

$-4.17308 \mathrm{E}-02$

$-3.26286 \mathrm{E} 01$

$-3.26304 \mathrm{E} 01$

$-3.26322 \mathrm{E} 01$

$-3.26340 \mathrm{E} 01$

$-3.26358 \mathrm{E} \quad 01$

$-3.26375 \mathrm{E} 01$

$-3.26393 \mathrm{E} 01$

$-3.26410 \mathrm{E} 01$

$-3.26427 \mathrm{E} 01$

$-3.26444 \mathrm{E} 01$

$-4.22704 \mathrm{E}-02$

$-4.28145 \mathrm{E}-02$

$-4.33633 \mathrm{E}-02$

$-4.39166 \mathrm{E}-02$

$-4.44745 \mathrm{E}-02$

$-3.26461 \mathrm{E} \quad 01$

$-3.26478 \mathrm{E} 01$

$-3.26494 \mathrm{E} 01$

$-3.26511 \mathrm{E} 01$

$-3.26527 \mathrm{E} 01$

$-4.50370 \mathrm{E}-02$

$-4.56042 \mathrm{E}-02$

$-4.61761 \mathrm{E}-02$

$-4.67526 \mathrm{E}-02$

$-4.73338 \mathrm{E}-02$

$-3.26543 \mathrm{E} 01$

$-3.26559 \mathrm{E} 01$

$-3.26575 \mathrm{E} 0$

$-3.26591 \mathrm{E} 01$

$-3.26607 \mathrm{E} 01$

$-3.26623 \mathrm{E} 01$

$-4.79198 \mathrm{E}-02$

$-4.85105 \mathrm{E}-02$

$-4.91059 \mathrm{E}-02$

$-4.97061 \mathrm{E}-02$

$-5.03111 \mathrm{E}-02$

$6.40779 \mathrm{E}-02$

$6.47882 \mathrm{E}-02$

$6.55035 \mathrm{E}-02$

$6.62242 \mathrm{E}-02$

$6.69500 \mathrm{E}-02$

b-2

$1.31424 \mathrm{E} \quad 00$

$1.32505 \mathrm{E} \quad 00$

$1.33590 \mathrm{E} 00$

$1.34679 \mathrm{E} 00$

$1.35773 \mathrm{E} \quad 00$

$1.36871 \mathrm{E} \quad 00$

$1.37973 \mathrm{E} \quad 00$

$1.39080 \mathrm{E} \quad 00$

$1.40192 \mathrm{E} \quad 00$

$1.41307 \mathrm{E} \quad 00$

$1.42427 \mathrm{E} \quad 00$

$1.43552 \mathrm{E} \quad 00$

$1.44681 \mathrm{E} \quad 00$

$1.45814 \mathrm{E} \quad 00$

$1.46952 \mathrm{E} \quad 00$

$1.48094 \mathrm{E} \quad 00$

$1.49240 \mathrm{E} \quad 00$

$1.50391 \mathrm{E} \quad 00$

$1.51546 \mathrm{E} \quad 00$

$1.52706 \mathrm{E} \quad 00$

$1.53870 \mathrm{E} \quad 00$

$1.55038 \mathrm{E} \quad 00$

$1.56211 \mathrm{E} \quad 00$

$1.57388 \mathrm{E} \quad 00$

$1.58570 \mathrm{E} \quad 00$

$1.59756 \mathrm{E} \quad 00$

$1.60946 \mathrm{E} \quad 00$

$1.62141 \mathrm{E} \quad 00$ 
ELECTRON RADIAL FUNCTIONS

Positron

$\begin{array}{cc}p & F_{0} \\ 30.10 & 8.74140 \mathrm{E}-0 \\ 30.20 & 8.74135 \mathrm{E}-0 \\ 30.30 & 8.74130 \mathrm{E}-0 \\ 30.40 & 8.74125 \mathrm{E}-0 \\ 30.50 & 8.74119 \mathrm{E}-0\end{array}$

30.60

30.70

30.80

30.90

31.00

31.10

31.20

31.30

31.40

31.50

31.60

31.70

31.80

31.90

32.00

32.10

32.20

32.30

32.40

32.50

32.60

32.70

32.80

32.90

33.00

33.10

33.20

33.30

33.40

33.50

33.60

33.70

33.80

33.90

34.00

34.10

34.20

34.30

34.40

34.50

34.60

34.70

34.80

34.90

35.00

35.10

35.20

35.30

35.40

35.50

35.60

35.70

35.80

35.90

36.00

$8.74109 \mathrm{E}-01$

$8.73941 \mathrm{E}-01$ $f_{1}$

$2.81970 \mathrm{E} \quad 01$

$2.82951 \mathrm{E} \quad 01$

$2.83932 \mathrm{E} \quad 01$

$2.84913 \mathrm{E} \quad 01$

$2.85895 \mathrm{E} \quad 01$

$8.74114 \mathrm{E}-01$

$8.74104 \mathrm{E}-01$

$8.74099 \mathrm{E}-01$

$8.74094 \mathrm{E}-01$

$8.74089 \mathrm{E}-01$

$8.74084 \mathrm{E}-01$

$8.74080 \mathrm{E}-01$

$8.74074 \mathrm{E}-01$

$8.74070 \mathrm{E}-01$

$8.74065 \mathrm{E}-01$

$8.74060 \mathrm{E}-01$

$8.74055 \mathrm{E}-01$

$8.74050 \mathrm{E}-01$

$8.74045 \mathrm{E}-01$

$8.74040 \mathrm{E}-01$

$8.74035 \mathrm{E}-01$

$8.74031 \mathrm{E}-01$

8.74026E-01

$8.74021 \mathrm{E}-01$

$8.74016 \mathrm{E}-01$

$8.74011 \mathrm{E}-01$

$8.74007 \mathrm{E}-01$

8.74002 E-01

8.73997 E-01

8.73993 E-01

$8.73988 \mathrm{E}-01$

$8.73983 \mathrm{E}-01$

$8.73978 \mathrm{E}-01$

8.73974E-01

8.73969E-01

$8.73964 \mathrm{E}-01$

$8.73960 \mathrm{E}-01$

$8.73955 \mathrm{E}-01$

$8.73951 \mathrm{E}-01$

$8.73946 \mathrm{E}-01$

$8.73937 \mathrm{E}-01$

$8.73932 \mathrm{E}-01$

8.73928E-01

$8.73923 \mathrm{E}-01$

$8.73919 \mathrm{E}-01$

$8.73914 \mathrm{E}-01$

$8.73910 \mathrm{E}-01$

$8.73905 \mathrm{E}-01$

$8.73900 \mathrm{E}-01$

$8.73896 \mathrm{E}-01$

8.73892 E-01

$8.73887 \mathrm{E}-01$

$8.73883 \mathrm{E}-01$

$8.73878 \mathrm{E}-01$

$8.73874 \mathrm{E}-01$

$8.73869 \mathrm{E}-01$

$8.73865 \mathrm{E}-01$

$8.73861 \mathrm{E}-01$

$2.86877 \mathrm{E} \quad 01$

$2.87859 \mathrm{E} \quad 01$

$2.88841 \mathrm{E}$ 01

$2.89824 \mathrm{E} 01$

$2.90807 \mathrm{E} \quad 01$

$2.91790 \mathrm{E} \quad 01$

$2.92774 \mathrm{E} \quad 01$

$2.94741 \mathrm{E} \quad 01$

$2.95726 \mathrm{E} \quad 01$

$2.96710 \mathrm{E} \quad 01$

$2.97695 \mathrm{E} \quad 01$

$2.98680 \mathrm{E} \quad 01$

$2.99666 \mathrm{E} \quad 01$

$3.00652 \mathrm{E} 01$

$3.01638 \mathrm{E} \quad 01$

$3.02624 \mathrm{E} \quad 01$

$3.03611 \mathrm{E} \quad 01$

$3.04598 \mathrm{E} \quad 01$

$3.05585 \mathrm{E} \quad 01$

$3.06572 \mathrm{E} \quad 01$

$3.07560 \mathrm{E} \quad 01$

$3.08548 \mathrm{E} \quad 01$

$3.09536 \mathrm{E} \quad 01$

$3.10525 \mathrm{E} 01$

$3.11514 \mathrm{E} \quad 01$

$3.13493 \mathrm{E} \quad 01$

$3.14482 \mathrm{E} \quad 01$

$3.15473 \mathrm{E} \quad 01$

$3.16463 \mathrm{E} \quad 01$

$3.17454 \mathrm{E} \quad 01$

$3.18445 \mathrm{E} \quad 01$

$3.20428 \mathrm{E} \quad 01$

$3.21420 \mathrm{E} \quad 01$

$3.22412 \mathrm{E} 01$

$3.23404 \mathrm{E} 01$

$3.24397 \mathrm{E} \quad 01$

$3.25390 \mathrm{E} 01$

$3.26384 \mathrm{E} \quad 01$

$3.28371 \mathrm{E} 01$

$3.29366 \mathrm{E} \quad 01$

$3.30360 \mathrm{E} 01$

$3.31355 \mathrm{E} \quad 01$

$3.32350 \mathrm{E} \quad 01$

$3.33346 \mathrm{E} 01$

$3.34342 \mathrm{E} 01$

$3.36335 \mathrm{E} \quad 01$

$3.37332 \mathrm{E} 01$

$3.38329 \mathrm{E} \quad 01$

$3.39326 \mathrm{E} \quad 01$

$3.40324 \mathrm{E} \quad 01$
$Z=6$

$A=12$

$\rho=1.2 A^{1 / 3} F$

$2.93757 \mathrm{E} \quad 01$

$3.12503 \mathrm{E} \quad 01$

$3.19436 \mathrm{E} \quad 01$

$3.27377 \mathrm{E} \quad 01$

$3.35338 \mathrm{E} 01$ $\begin{array}{lll}g_{1} & \tan \delta_{1} & f_{-1}\end{array}$

$1.58961 \mathrm{E} \quad 00$

$-1.00619 \mathrm{E}-03$

$\begin{array}{lll}1.62657 \mathrm{E} & 00 & -1.00861 \mathrm{E}-03\end{array}$

$1.63899 \mathrm{E} \quad 00-1.00978 \mathrm{E}-03$

$1.65146 \mathrm{E} \quad 00 \quad-1.01095 \mathrm{E}-03$

$1.66397 \mathrm{E} \quad 00-1.01209 \mathrm{E}-03$

$\begin{array}{lll}1.67653 \mathrm{E} & 00 & -1.01321 \mathrm{E}-03\end{array}$

$1.68915 \mathrm{E} \quad 00 \quad-1.01431 \mathrm{E}-03$

$1.70181 \mathrm{E} \quad 00-1.01539 \mathrm{E}-03$

$1.71452 \mathrm{E} \quad 00-1.01647 \mathrm{E}-03$

$1.72728 \mathrm{E} \quad 00=-1.01751 \mathrm{E}-03$

$1.74008 \mathrm{E} \quad 00-1.01854 \mathrm{E}-03$

$1.75294 \mathrm{E} \quad 00-1.01956 \mathrm{E}-03$

$1.76585 \mathrm{E} \quad 00-1.02055 \mathrm{E}-03$

$1.77880 \mathrm{E} \quad 00$

$1.79181 \mathrm{E} \quad 00$

$1.80486 \mathrm{E} \quad 00$

$1.81796 \mathrm{E} \quad 00$

$1.83111 \mathrm{E} \quad 00$

$1.84431 \mathrm{E} \quad 00$

$1.85756 \mathrm{E} \quad 00$

$1.87086 \mathrm{E} \quad 00$

$1.88421 \mathrm{E} \quad 00$

$1.89760 \mathrm{E} \quad 00$

$-1.02152 \mathrm{E}-03$

$-1.02248 \mathrm{E}-03$

$-1.02343 \mathrm{E}-03$

$-1.02434 \mathrm{E}-03$

$-1.02525 \mathrm{E}-03$

$-1.02614 \mathrm{E}-03$

$-1.02701 \mathrm{E}-03$

$-1.02787 \mathrm{E}-03$

$-1.02871 \mathrm{E}-03$

$-1.02953 \mathrm{E}-03$

$1.91105 \mathrm{E} \quad 00$

$1.92454 \mathrm{E} \quad 00$

$1.93809 \mathrm{E} \quad 00$

$1.95168 \mathrm{E} \quad 00$

$1.96533 \mathrm{E} \quad 00$

$1.97902 \mathrm{E} \quad 00$

$1.99276 \mathrm{E} \quad 00$

$2.00655 \mathrm{E} \quad 00$

$2.02040 \mathrm{E} \quad 00$

$2.03429 \mathrm{E} \quad 00$

$2.04823 \mathrm{E} \quad 00$

$2.06222 \mathrm{E} \quad 00$

$2.07626 \mathrm{E} \quad 00$

$2.09035 \mathrm{E} \quad 00$

$2.10449 \mathrm{E} \quad 00$

$-1.03033 \mathrm{E}-03$

$-1.03112 \mathrm{E}-03$

$-1.03190 \mathrm{E}-03$

$-1.03265 \mathrm{E}-03$

$-1.03339 \mathrm{E}-03$

$-1.03412 \mathrm{E}-03$

$-1.03482 \mathrm{E}-03$

$-1.03551 \mathrm{E}-03$

$-1.03619 \mathrm{E}-03$

$-1.03685 \mathrm{E}-03$

$-1.03749 \mathrm{E}-03$

$-1.03813 \mathrm{E}-03$

$-1.03874 \mathrm{E}-03$

$-1.03934 \mathrm{E}-03$

$2.11868 \mathrm{E} \quad 00$ $2.13292 \mathrm{E} \quad 00$

$2.14721 \mathrm{E} \quad 00$

$2.16154 \mathrm{E} \quad 00$

2.17593 E 00

$2.19037 \mathrm{E} \quad 00$

$2.20486 \mathrm{E} \quad 00$

$2.21940 \mathrm{E} \quad 00$

$2.23399 \mathrm{E} \quad 00$

2.24863 E 00

$2.26332 \mathrm{E} \quad 00$

$2.27805 \mathrm{E} \quad 00$

$2.29284 \mathrm{E} \quad 00$

$2.30768 \mathrm{E} \quad 00$

$2.32257 \mathrm{E} \quad 00$

$-1.04050 \mathrm{E}-03$

$-1.04106 \mathrm{E}-03$

$-1.04159 \mathrm{E}-03$

$-1.04212 \mathrm{E}-03$

$-1.04263 \mathrm{E}-03$

$-1.04312 \mathrm{E}-03$

$-1.04360 \mathrm{E}-03$

$-1.04407 \mathrm{E}-03$

$-1.04453 \mathrm{E}-03$

$-1.04496 \mathrm{E}-03$

$-1.04539 \mathrm{E}-03$

$-1.04580 \mathrm{E}-03$

$-1.04620 \mathrm{E}-03$

$-1.04659 \mathrm{E}-03$

$-1.04695 \mathrm{E}-03$

$2.33751 \mathrm{E} \quad 00$

$2.35250 \mathrm{E} \quad 00$

$2.36754 \mathrm{E} \quad 00$

$2.38264 \mathrm{E} \quad 00$

$-1.04731 \mathrm{E}-03$

$-1.04765 \mathrm{E}-03$

$-1.04798 \mathrm{E}-03$

$-1.04829 \mathrm{E}-03$

$2.39778 \mathrm{E} \quad 00$
$-1.03992 \mathrm{E}-03$

$-1.50687 \mathrm{E} 00$

$-1.51893 \mathrm{E} \quad 00$

$-1.53103 \mathrm{E} 00$

$-1.54319 \mathrm{E} \quad 00$

$-1.55540 \mathrm{E} \quad 00$

$-1.56765 \mathrm{E} \quad 00$

$-1.57995 \mathrm{E} \quad 00$

$-1.59230 \mathrm{E} \quad 00$ 
ELECTRON RADIAL FUNCTIONS

$$
Z=6 \quad A=12 \quad \rho=1.2 A^{1 / 3} F
$$

$\bar{F}$

$f_{2}$

$1.11236 \mathrm{E}-03$

30.10

30.20

30.30

30.40

30.50

$1.11976 \mathrm{E}-03$

$1.12718 \mathrm{E}-03$

$1.13463 \mathrm{E}-03$

$1.14211 \mathrm{E}-03$

30.60

$1.14961 \mathrm{E}-03$

$1.15713 \mathrm{E}-03$

$1.16468 \mathrm{E}-03$

$1.17225 \mathrm{E}-03$

30.80

30.90

$1.17985 \mathrm{E}-03$

31.10

31.20

31.30

31.40

31.50

$1.18747 \mathrm{E}-03$

$1.19511 \mathrm{E}-03$

$1.20278 \mathrm{E}-03$

$1.21048 \mathrm{E}-03$

$1.21820 \mathrm{E}-03$

31.60

31.70

31.80

31.90

32.00

$1.22594 \mathrm{E}-03$

$1.23371 \mathrm{E}-03$

$1.24150 \mathrm{E}-03$

$1.24932 \mathrm{E}-03$

$1.25716 \mathrm{E}-03$

$32.10 \quad 1.26503 \mathrm{E}-03$

$32.20 \quad 1.27292 \mathrm{E}-03$

$32.30 \quad 1.28083 \mathrm{E}-03$

$32.40 \quad 1.28877 \mathrm{E}-03$

$32.50 \quad 1.29674 \mathrm{E}-03$

$32.60 \quad 1.30473 \mathrm{E}-03$

$32.70 \quad 1.31274 \mathrm{E}-03$

32.80

32.90

33.00

33.10

33.20

33.30

33.40

33.50

33.60

33.70

33.80

33.90

34.00

$1.32078 \mathrm{E}-03$

$1.32884 \mathrm{E}-03$

$1.33693 \mathrm{E}-03$

$1.34504 \mathrm{E}-03$

$1.35317 \mathrm{E}-03$

$1.36133 \mathrm{E}-03$

$1.36952 \mathrm{E}-03$

$1.37773 \mathrm{E}-03$

$1.38596 \mathrm{E}-03$

$1.39422 \mathrm{E}-03$

$1.40250 \mathrm{E}-03$

$1.41081 \mathrm{E}-03$

$1.41914 \mathrm{E}-03$

34.10

34.20

34.30

34.40

34.50

34.60

34.70

34.80

34.90

35.00

35.10

35.20

35.30

35.40

35.50

$1.42750 \mathrm{E}-03$

$1.43588 \mathrm{E}-03$

$1.44429 \mathrm{E}-03$

$1.45272 \mathrm{E}-03$

$1.46117 \mathrm{E}-03$

$1.46965 \mathrm{E}-03$

$1.47816 \mathrm{E}-03$

$1.48668 \mathrm{E}-03$

$1.49524 \mathrm{E}-03$

$1.50381 \mathrm{E}-03$

$1.51242 \mathrm{E}-03$

$1.52104 \mathrm{E}-03$

$1.52969 \mathrm{E}-03$

$1.53837 \mathrm{E}-03$

$1.54707 \mathrm{E}-03$

35.60

35.70

35.80

35.90

36.00
$1.97357 \mathrm{E} \quad 00$

$1.98678 \mathrm{E} \quad 00$

$2.00004 \mathrm{E} \quad 00$

$2.01334 \mathrm{E} \quad 00$

$2.02668 \mathrm{E} \quad 00$

$2.04007 \mathrm{E} \quad 00$

$2.05351 \mathrm{E} \quad 00$

$2.06698 \mathrm{E} \quad 00$

$2.08050 \mathrm{E} \cdot 00$

$2.09406 \mathrm{E} \quad 00$

$2.10767 \mathrm{E} \quad 00$

$2.12132 \mathrm{E} \quad 00$

$2.13501 \mathrm{E} \quad 00$

$2.14875 \mathrm{E} \quad 00$

$2.16253 \mathrm{E} \quad 00$

$2.17636 \mathrm{E} \quad 00$

$2.19023 \mathrm{E} \quad 00$

$2.20414 \mathrm{E} \quad 00$

$2.21809 \mathrm{E} \quad 00$

$2.23209 \mathrm{E} \quad 00$

$2.24613 \mathrm{E} \quad 00$

$2.26022 \mathrm{E} \quad 00$

$2.27435 \mathrm{E} \quad 00$

$2.28852 \mathrm{E} \quad 00$

$2.30274 \mathrm{E} \quad 00$

$2.31700 \mathrm{E} \quad 00$

$2.33130 \mathrm{E} \quad 00$

$2.34565 \mathrm{E} \quad 00$

$2.36004 \mathrm{E} \quad 00$

$2.37448 \mathrm{E} \quad 00$

$2.38895 \mathrm{E} \quad 00$

$2.40347 \mathrm{E} \quad 00$

$2.41804 \mathrm{E} \quad 00$

$2.43265 \mathrm{E} \quad 00$

$2.44730 \mathrm{E} \quad 00$

$2.46199 \mathrm{E} \quad 00$

$2.47673 \mathrm{E} \quad 00$

$2.49152 \mathrm{E} \quad 00$

$2.50634 \mathrm{E} \quad 00$

$2.52121 \mathrm{E} \quad 00$

$2.53612 \mathrm{E} \quad 00$

$2.55108 \mathrm{E} \quad 00$

$2.56608 \mathrm{E} \quad 00$

$2.58112 \mathrm{E} \quad 00$

$2.59620 \mathrm{E} \quad 00$

$2.61133 \mathrm{E} \quad 00$

$2.62651 \mathrm{E} \quad 00$

$2.64172 \mathrm{E} \quad 00$

$2.65698 \mathrm{E} \quad 00$

$2.67228 \mathrm{E} \quad 00$

$2.68763 \mathrm{E} \quad 00$

$2.70302 \mathrm{E} \quad 00$

$2.71845 \mathrm{E} \quad 00$

$2.73393 \mathrm{E} \quad 00$

$2.74945 \mathrm{E} \quad 00$

$2.76501 \mathrm{E} \quad 00$ $2.78062 \mathrm{E} \quad 00$

$2.79627 \mathrm{E} \quad 00$

$2.81196 \mathrm{E} \quad 00$

$2.82770 \mathrm{E} \quad 00$ $g_{2}$

$6.76812 \mathrm{E}-02$

$6.84175 \mathrm{E}-02$

$6.91592 \mathrm{E}-02$

$6.99061 \mathrm{E}-02$

$7.06584 \mathrm{E}-02$

$7.14160 \mathrm{E}-02$

$7.21789 \mathrm{E}-02$

$7.29473 \mathrm{E}-02$

$7.37210 \mathrm{E}-02$

$7.45002 \mathrm{E}-02$

$7.52848 \mathrm{E}-02$

$7.60748 \mathrm{E}-02$

$7.68703 \mathrm{E}-02$

$7.76713 \mathrm{E}-02$

7.84778 E-02

$7.92898 \mathrm{E}-02$

$8.01074 \mathrm{E}-02$

$8.09305 \mathrm{E}-02$

$8.17592 \mathrm{E}-02$

$8.25936 \mathrm{E}-02$

$8.34335 \mathrm{E}-02$

$8.42791 \mathrm{E}-02$

$8.51303 \mathrm{E}-02$

8.59872 E-02

$8.68498 \mathrm{E}-02$

$8.77181 \mathrm{E}-02$

8.85921 E-02

$8.94719 \mathrm{E}-02$

$9.03575 \mathrm{E}-02$

$9.12488 \mathrm{E}-02$

$9.21460 \mathrm{E}-02$

$9.30489 \mathrm{E}-02$

$9.39577 \mathrm{E}-02$

$9.48724 \mathrm{E}-02$

$9.57929 \mathrm{E}-02$

$9.67194 \mathrm{E}-02$

$9.76517 \mathrm{E}-02$

$9.85900 \mathrm{E}-02$

$9.95343 \mathrm{E}-02$

$1.00484 \mathrm{E}-01$

$1.01441 \mathrm{E}-01$

$1.02403 \mathrm{E}-01$

$1.03371 \mathrm{E}-01$

$1.04345 \mathrm{E}-01$

$1.05326 \mathrm{E}-01$

$1.06312 \mathrm{E}-01$

$1.07305 \mathrm{E}-01$

$1.08303 \mathrm{E}-01$

$1.09308 \mathrm{E}-01$

$1.10319 \mathrm{E}-01$

$1.11336 \mathrm{E}-01$

$1.12359 \mathrm{E}-01$

$1.13389 \mathrm{E}-01$

$1.14425 \mathrm{E}-01$

$1.15467 \mathrm{E}-01$

$1.16515 \mathrm{E}-01$

$1.17570 \mathrm{E}-01$

$1.18630 \mathrm{E}-01$

$1.19698 \mathrm{E}-01$

$1.20771 \mathrm{E}-01$

$\tan \delta_{2}$

$f_{-2}$

$-3.26845 \mathrm{E} \quad 01 \quad-6.41563 \mathrm{E}-02$ $-3.26859 \mathrm{E} \quad 01$

$-3.26873 \mathrm{E} 01$

$-3.26887 \mathrm{E} \quad 01$

$-3.26900 \mathrm{E} \quad 01$

$-3.26914 \mathrm{E} 01$

$-3.26927 \mathrm{E}$ 01

$-3.26941 \mathrm{E} 01$

$-3.26954 \mathrm{E} 01$

$-3.26967 \mathrm{E} 01$

$-3.26981 \mathrm{E} 01$

$-3.26994 \mathrm{E} 01$

$-3.27007 \mathrm{E} 01$

$-3.27020 \mathrm{E}$ 01

$-3.27032 \mathrm{E} 01$

$-3.27045 \mathrm{E} 01$

$-3.27058 \mathrm{E} \quad 01$

$-3.27070 \mathrm{E} 01$

$-3.27083 \mathrm{E} 01$

$-3.27095 \mathrm{E} 01$

$-3.27108 \mathrm{E} 01$

$-3.27120 \mathrm{E} 01$

$-3.27132 \mathrm{E} 01$

$-3.27144 \mathrm{E} 01$

$-3.27156 \mathrm{E} 01$

$-3.27168 \mathrm{E} 0 \mathrm{l}$

$-3.27180 \mathrm{E} 01$

$-3.27192 \mathrm{E} \quad 01$

$-3.27204 \mathrm{E} 01$

$-3.27215 \mathrm{E} \quad 01$

$-3.27227 \mathrm{E} \quad 01$

$-3.27238 \mathrm{E} 01$

$-3.27250 \mathrm{E}$ 01

$-3.27261 \mathrm{E} 01$

$-3.27272 \mathrm{E} 01$

$-3.27284 \mathrm{E} \quad 01$

$-3.27295 \mathrm{E} \quad 01$

$-3.27306 \mathrm{E} 01$

$-3.27317 \mathrm{E} 01$

$-3.27328 \mathrm{E} 01$

$-3.27339 \mathrm{E} 01$

$-3.27350 \mathrm{E}$ 01

$-3.27360 \mathrm{E} 01$

$-3.27371 \mathrm{E} \quad 01$

$-3.27382 \mathrm{E} 01$

$-3.27392 \mathrm{E} 01$

$-3.27403 \mathrm{E} \quad 01$

$-3.27413 \mathrm{E} \quad 01$

$-3.27424 \mathrm{E} \quad 01$

$-3.27434 \mathrm{E} 01$

$-3.27444 \mathrm{E} \quad 01$

$-3.27454 \mathrm{E} 01$

$-3.27464 \mathrm{E} 01$

$-3.27475 \mathrm{E} 01$

$-3.27485 \mathrm{E} 01$

$-3.27494 \mathrm{E} \quad 01$

$-3.27504 \mathrm{E} \quad 01$ 
ELECTRON RADIAL FUNCTIONS

Positron

\begin{tabular}{|c|c|c|c|}
\hline$p$ & $F_{0}$ & $f_{1}$ & \\
\hline 36.10 & $8.73856 \mathrm{E}-01$ & $3.41322 \mathrm{E}$ & 01 \\
\hline 36.20 & $8.73852 \mathrm{E}-01$ & $3.42321 \mathrm{E}$ & 01 \\
\hline 36.30 & $8.73847 \mathrm{E}-01$ & $3.43319 \mathrm{E}$ & 01 \\
\hline 36.40 & $8.73843 \mathbf{E}-01$ & $3.44318 \mathrm{E}$ & 01 \\
\hline 36.50 & $8.73839 \mathrm{E}-01$ & $3.45318 \mathrm{E}$ & 01 \\
\hline 36.60 & $8.73834 \mathrm{E}-01$ & $3.46318 \mathrm{E}$ & 01 \\
\hline 36.70 & $8.73830 \mathrm{E}-01$ & $3.47318 \mathrm{E}$ & 01 \\
\hline 36.80 & $8.73826 \mathrm{E}-01$ & $3.48318 \mathrm{E}$ & 01 \\
\hline 36.90 & $8.73821 \mathrm{E}-01$ & $3.49319 \mathrm{E}$ & 01 \\
\hline 37.00 & $8.73817 \mathrm{E}-01$ & $3.50320 \mathrm{E}$ & 01 \\
\hline 37.10 & $8.73813 \mathrm{E}-01$ & $3.51321 \mathrm{E}$ & 01 \\
\hline 37.20 & $8.73809 \mathrm{E}-01$ & $3.52323 \mathrm{E}$ & 01 \\
\hline 37.30 & $8.73804 \mathrm{E}-01$ & $3.53325 \mathrm{E}$ & 01 \\
\hline 37.40 & $8.73800 \mathrm{E}-01$ & $3.54327 \mathrm{E}$ & 01 \\
\hline 37.50 & $8.73796 \mathrm{E}-01$ & $3.55330 \mathrm{E}$ & 01 \\
\hline 37.60 & $8.73792 \mathrm{E}-01$ & $3.56333 \mathrm{E}$ & 01 \\
\hline 37.70 & $8.73787 \mathrm{E}-01$ & $3.57336 \mathrm{E}$ & 01 \\
\hline 37.80 & $8.73783 \mathrm{E}-01$ & $3.58340 \mathrm{E}$ & 01 \\
\hline 37.90 & $8.73779 \mathrm{E}-01$ & $3.59344 \mathrm{E}$ & 01 \\
\hline 38.00 & $8.73775 \mathrm{E}-01$ & $3.60348 \mathrm{E}$ & 01 \\
\hline 38.10 & $8.73771 \mathrm{E}-01$ & $3.61353 \mathrm{E}$ & 01 \\
\hline 38.20 & $8.73767 \mathrm{E}-01$ & $3.62358 \mathrm{E}$ & 01 \\
\hline 38.30 & $8.73762 \mathrm{E}-01$ & $3.63363 \mathrm{E}$ & 01 \\
\hline 38.40 & $8.73758 \mathrm{E}-01$ & $3.64369 \mathrm{E}$ & 01 \\
\hline 38.50 & $8.73754 \mathrm{E}-01$ & $3.65375 \mathrm{E}$ & 01 \\
\hline 38.60 & $8.73750 \mathrm{E}-01$ & $3.66382 \mathrm{E}$ & 01 \\
\hline 38.70 & $8.73746 \mathrm{E}-01$ & $3.67388 \mathrm{E}$ & 01 \\
\hline 38.80 & $8.73742 \mathrm{E}-01$ & $3.68395 \mathrm{E}$ & 01 \\
\hline 38.90 & $8.73738 \mathrm{E}-01$ & $3.69403 \mathrm{E}$ & 01 \\
\hline 39.00 & $8.73734 \mathrm{E}-01$ & $3.70411 \mathrm{E}$ & 01 \\
\hline 39.10 & $8.73730 \mathrm{E}-01$ & $3.71419 \mathrm{E}$ & 01 \\
\hline 39.20 & $8.73725 \mathrm{E}-01$ & $3.72427 \mathrm{E}$ & 01 \\
\hline 39.30 & $8.73721 \mathrm{E}-01$ & $3.73436 \mathrm{E}$ & 01 \\
\hline 39.40 & $8.73717 \mathrm{E}-01$ & $3.74445 \mathrm{E}$ & 01 \\
\hline 39.50 & $8.73713 \mathrm{E}-01$ & $3.75455 \mathrm{E}$ & 01 \\
\hline 39.60 & $8.73709 \mathrm{E}-01$ & $3.76465 \mathrm{E}$ & 01 \\
\hline 39.70 & $8.73705 \mathrm{E}-01$ & $3.77475 \mathrm{E}$ & 01 \\
\hline 39.80 & $8.73701 \mathrm{E}-01$ & $3.78486 \mathrm{E}$ & 01 \\
\hline 39.90 & $8.73697 \mathrm{E}-01$ & $3.79497 \mathrm{E}$ & 01 \\
\hline 40.00 & $8.73693 \mathrm{E}-01$ & $3.80508 \mathrm{E}$ & 01 \\
\hline 40.10 & $8.73689 \mathrm{E}-01$ & $3.81520 \mathrm{E}$ & 01 \\
\hline 40.20 & $8.73685 \mathrm{E}-01$ & $3.82532 \mathrm{E}$ & 01 \\
\hline 40.30 & $8.73681 \mathrm{E}-01$ & $3.83544 \mathrm{E}$ & 01 \\
\hline 40.40 & $8.73677 \mathrm{E}-01$ & $3.84557 \mathrm{E}$ & 01 \\
\hline 40.50 & $8.73673 \mathrm{E}-01$ & $3.85570 \mathrm{E}$ & 01 \\
\hline 40.60 & $8.73669 \mathrm{E}-01$ & $3.86583 \mathrm{E}$ & 01 \\
\hline 40.70 & $8.73665 \mathrm{E}-01$ & $3.87597 \mathrm{E}$ & 01 \\
\hline 40.80 & $8.73662 \mathrm{E}-01$ & $3.88611 \mathrm{E}$ & 01 \\
\hline 40.90 & $8.73658 \mathrm{E}-01$ & $3.89626 \mathrm{E}$ & 01 \\
\hline 41.00 & $8.73654 \mathrm{E}-01$ & $3.90641 \mathrm{E}$ & 01 \\
\hline 41.10 & $8.73650 \mathrm{E}-01$ & $3.91656 \mathrm{E}$ & 01 \\
\hline 41.20 & $8.73646 \mathrm{E}-01$ & $3.92672 \mathrm{E}$ & 01 \\
\hline 41.30 & $8.73642 \mathrm{E}-01$ & $3.93688 \mathrm{E}$ & 01 \\
\hline 41.40 & $8.73638 \mathrm{E}-01$ & $3.94704 \mathrm{E}$ & 01 \\
\hline 41.50 & $8.73634 \mathrm{E}-01$ & $3.95721 \mathrm{E}$ & 01 \\
\hline 41.60 & $8.73630 \mathrm{E}-01$ & $3.96738 \mathrm{E}$ & 01 \\
\hline 41.70 & $8.73627 \mathrm{E}-01$ & $3.97756 \mathrm{E}$ & 01 \\
\hline 41.80 & $8.73623 \mathrm{E}-01$ & $3.98774 \mathrm{E}$ & 01 \\
\hline 41.90 & $8.73619 \mathrm{E}-01$ & $3.99792 \mathrm{E}$ & 01 \\
\hline 42.00 & $8.73615 \mathrm{E}-01$ & $4.00811 \mathrm{E}$ & 01 \\
\hline
\end{tabular}

$Z=6 \quad A=12 \quad \rho=1.2 A^{1 / 3} \mathrm{~F}$

$\begin{array}{lllll}g_{1} & \tan \delta_{1} & f_{-1} & g_{-1} & \tan \delta_{-1}\end{array}$

$\begin{array}{lllllll}2.41297 \mathrm{E} & 00 & -1.04889 \mathrm{E}-03 & -2.31763 \mathrm{E} & 00 & 3.51486 \mathrm{E} & 01\end{array}$ $2.42821 \mathrm{E} \quad 00 \quad-1.04916 \mathrm{E}-03 \quad-2.33267 \mathrm{E} \quad 00$

$2.44350 \mathrm{E} \quad 00-1.04943 \mathrm{E}-03 \quad-2.34775 \mathrm{E} \quad 00$

$\begin{array}{lllll}2.45885 \mathrm{E} & 00 & -1.04968 \mathrm{E}-03 & -2.36289 \mathrm{E} & 00\end{array}$

$2.47424 \mathrm{E} \quad 00 \quad-1.04991 \mathrm{E}-03 \quad-2.37808 \mathrm{E} \quad 00$

$\begin{array}{lllll}2.48969 \mathrm{E} & 00 & -1.05013 \mathrm{E}-03 & -2.39332 \mathrm{E} & 00\end{array}$

$\begin{array}{lllll}2.50518 \mathrm{E} & 00 & -1.05035 \mathrm{E}-03 & -2.40861 \mathrm{E} & 00\end{array}$

$\begin{array}{llllll}2.52073 \mathrm{E} & 00 & -1.05055 \mathrm{E}-03 & -2.42395 \mathrm{E} & 00\end{array}$

$\begin{array}{lllll}2.53633 \mathrm{E} & 00 & -1.05073 \mathrm{E}-03 & -2.43934 \mathrm{E} & 00\end{array}$

$2.55197 \mathrm{E} \quad 00 \quad-1.05091 \mathrm{E}-03 \quad-2.45478 \mathrm{E} \quad 00$

$\begin{array}{lllll}2.56767 \mathrm{E} & 00 & -1.05107 \mathrm{E}-03 & -2.47028 \mathrm{E} & 00\end{array}$

$\begin{array}{lllll}2.58342 \mathrm{E} & 00 & -1.05121 \mathrm{E}-03 & -2.48582 \mathrm{E} & 00\end{array}$

$\begin{array}{lllll}2.59922 \mathrm{E} & 00 & -1.05135 \mathrm{E}-03 & -2.50141 \mathrm{E} & 00\end{array}$

$\begin{array}{llllll}2.61507 \mathrm{E} & 00 & -1.05147 \mathrm{E}-03 & -2.51706 \mathrm{E} & 00\end{array}$

$\begin{array}{lllll}2.63097 \mathrm{E} & 00 & -1.05158 \mathrm{E}-03 & -2.53276 \mathrm{E} & 00\end{array}$

$2.64693 \mathrm{E} \quad 00 \quad-1.05168 \mathrm{E}-03$

$2.66293 \mathrm{E} \quad 00-1.05178 \mathrm{E}-03$

$2.67899 \mathrm{E} \quad 00-1.05185 \mathrm{E}-03$

$2.69509 \mathrm{E} \quad 00-1.05191 \mathrm{E}-03$

$2.71125 \mathrm{E} \quad 00 \quad-1.05196 \mathrm{E}-03$

$\begin{array}{lll}2.72746 \mathrm{E} & 00 & -1.05200 \mathrm{E}-03\end{array}$

$2.74372 \mathrm{E} \quad 00-1.05203 \mathrm{E}-03$

$2.76003 \mathrm{E} \quad 00-1.05205 \mathrm{E}-03$

$2.77639 \mathrm{E} \quad 00-1.05205 \mathrm{E}-03$

$2.79281 \mathrm{E} \quad 00 \quad-1.05204 \mathrm{E}-03$

$2.80927 \mathrm{E} \quad 00-1.05203 \mathrm{E}-03$

$2.82579 \mathrm{E} \quad 00-1.05200 \mathrm{E}-03$

$2.84236 \mathrm{E} \quad 00-1.05196 \mathrm{E}-03$

$2.85898 \mathrm{E} \quad 00-1.05190 \mathrm{E}-03$

$\begin{array}{lll}2.87565 \mathrm{E} & 00-1.05184 \mathrm{E}-03\end{array}$

$-2.54851 \mathrm{E} \quad 00$

$-2.56431 \mathrm{E} \quad 00$

$-2.58016 \mathrm{E} \quad 00$

$-2.59606 \mathrm{E} \quad 00$

$-2.61201 \mathrm{E} \quad 00$

$-2.62802 \mathrm{E} \quad 00$

$-2.64407 \mathrm{E} \quad 00$

$-2.66018 \mathrm{E} \quad 00$

$-2.67634 \mathrm{E} \quad 00$

$-2.69255 \mathrm{E} \quad 00$

$-2.70881 \mathrm{E} \quad 00$

$-2.72513 \mathrm{E} \quad 00$

$-2.74149 \mathrm{E} \quad 00$

$-2.75791 \mathrm{E} \quad 00$

$-2.77438 \mathrm{E} 00$

$2.89237 \mathrm{E} \quad 00 \quad-1.05176 \mathrm{E}-03$

$2.90914 \mathrm{E} \quad 00 \quad-1.05168 \mathrm{E}-03$

$2.92597 \mathrm{E} \quad 00-1.05158 \mathrm{E}-03$

$2.94285 \mathrm{E} \quad 00-1.05147 \mathrm{E}-03$

$2.95978 \mathrm{E} \quad 00-1.05135 \mathrm{E}-03$

$2.97676 \mathrm{E} \quad 00-1.05122 \mathrm{E}-03$

$2.99379 \mathrm{E} \quad 00 \quad-1.05108 \mathrm{E}-03$

$3.01088 \mathrm{E} \quad 00-1.05092 \mathrm{E}-03$

$3.02801 \mathrm{E} \quad 00 \quad-1.05077 \mathrm{E}-03$

$3.04520 \mathrm{E} \quad 00-1.05059 \mathrm{E}-03$

$3.06244 \mathrm{E} \quad 00-1.05040 \mathrm{E}-03$

$3.07973 \mathrm{E} \quad 00-1.05020 \mathrm{E}-03$

$3.09708 \mathrm{E}$ 00 $-1.05000 \mathrm{E}-03$

$3.11448 \mathrm{E} \quad 00-1.04978 \mathrm{E}-03$

$3.13192 \mathrm{E} \quad 00-1.04956 \mathrm{E}-03$

$3.14942 \mathrm{E} \quad 00-1.04932 \mathrm{E}-03$ $3.16698 \mathrm{E} \quad 00 \quad-1.04908 \mathrm{E}-03$ $3.18458 \mathrm{E} \quad 00-1.04882 \mathrm{E}-03$ $3.20224 \mathrm{E} \quad 00-1.04855 \mathrm{E}-03$ $3.21995 \mathrm{E} \quad 00 \quad-1.04827 \mathrm{E}-03$

$-2.79090 \mathrm{E} \quad 00$ $-2.80747 \mathrm{E} \quad 00$ $-2.82409 \mathrm{E} 00$ $-2.84077 \mathrm{E} \quad 00$ $-2.85749 \mathrm{E} \quad 00$

$-2.87427 \mathrm{E} \quad 00$ $-2.89110 \mathrm{E} 00$ $-2.90799 \mathrm{E} \quad 00$ $-2.92492 \mathrm{E} 00$ $-2.94191 \mathrm{E} \quad 00$

$-2.95895 \mathrm{E} \quad 00$ $-2.97604 \mathrm{E} \quad 00$ $-2.99318 \mathrm{E} \quad 00$ $-3.01038 \mathrm{E} \quad 00$ $-3.02763 \mathrm{E} 00$

$-3.04493 \mathrm{E} \quad 00$ $-3.06228 \mathrm{E} \quad 00$ $-3.07969 \mathrm{E} \quad 00$ $-3.09714 \mathrm{E} \quad 00$ $-3.11465 \mathrm{E} \quad 00$

$\begin{array}{lllll}3.23771 \mathrm{E} & 00 & -1.04798 \mathrm{E}-03 & -3.13222 \mathrm{E} & 00\end{array}$ $\begin{array}{lllll}3.25553 \mathrm{E} & 00 & -1.04769 \mathrm{E}-03 & -3.14983 \mathrm{E} & 00\end{array}$ $3.27340 \mathrm{E} \quad 00 \quad-1.04737 \mathrm{E}-03 \quad-3.16750 \mathrm{E} \quad 00$ $\begin{array}{lllll}3.29132 \mathrm{E} & 00 & -1.04705 \mathrm{E}-03 & -3.18522 \mathrm{E} & 00\end{array}$ $\begin{array}{lllll}3.30929 \mathrm{E} & 00 & -1.04672 \mathrm{E}-03 & -3.20299 \mathrm{E} & 00\end{array}$

$\begin{array}{lllll}3.32731 \mathrm{E} & 00 & -1.04638 \mathrm{E}-03 & -3.22082 \mathrm{E} & 00\end{array}$ $\begin{array}{lllll}3.34539 \mathrm{E} & 00 & -1.04604 \mathrm{E}-03 & -3.23870 \mathrm{E} & 00\end{array}$ $\begin{array}{lllll}3.36352 \mathrm{E} & 00 & -1.04568 \mathrm{E}-03 & -3.25663 \mathrm{E} & 00\end{array}$ $3.38170 \mathrm{E} \quad 00 \quad-1.04531 \mathrm{E}-03 \quad-3.27461 \mathrm{E} \quad 00$ $\begin{array}{lllll}3.39994 \mathrm{E} & 00 & -1.04492 \mathrm{E}-03 & -3.29265 \mathrm{E} & 00\end{array}$
$3.52488 \mathrm{E}$ 01 $3.53491 \mathrm{E} \quad 01$ $3.54495 \mathrm{E} 01$ $3.55499 \mathrm{E} \quad 01$

$3.56503 \mathrm{E} \quad 01$ $3.57507 \mathrm{E} \quad 01$ $3.58512 \mathrm{E} \quad 01$ $3.59517 \mathrm{E} \quad 01$ $3.60523 \mathrm{E} \quad 01$

$3.61529 \mathrm{E} \quad 01$ $3.62535 \mathrm{E} \quad 01$ $3.63541 \mathrm{E} \quad 01$ $3.64548 \mathrm{E} \quad 01$ $3.65555 \mathrm{E} \quad 01$

$3.66563 \mathrm{E} \quad 01$ $3.67571 \mathrm{E} \quad 01$ $3.68579 \mathrm{E} \quad 01$ $3.69588 \mathrm{E} \quad 01$ $3.70597 \mathrm{E} \quad 01$

$3.71606 \mathrm{E} \quad 01$ $3.72615 \mathrm{E} \quad 01$ $3.73625 \mathrm{E} \quad 01$ $\begin{array}{ll}3.74636 \mathrm{E} & 01\end{array}$ $3.75647 \mathrm{E} \quad 01$

$3.76658 \mathrm{E} \quad 01$ $3.77669 \mathrm{E} \quad 01$ $3.78681 \mathrm{E} \quad 01$ $3.79693 \mathrm{E} \quad 01$ $3.80705 \mathrm{E} \quad 01$

$3.81718 \mathrm{E} \quad 01$ $3.82732 \mathrm{E} \quad 01$ $3.83745 \mathrm{E} \quad 01$ $\begin{array}{lll}3.84759 \mathrm{E} & 01\end{array}$ $3.85773 \mathrm{E} \quad 01$

$3.86788 \mathrm{E} \quad 01$ $3.87803 \mathrm{E}$ 0] $3.88819 \mathrm{E} \quad 01$ $3.89834 \mathrm{E} \quad 01$ $3.90850 \mathrm{E} \quad 01$

$3.91867 \mathrm{E} \quad 01$ $3.92884 \mathrm{E} \quad 01$ $3.93901 \mathrm{E} 01$ $3.94919 \mathrm{E} 01$ $3.95937 \mathrm{E} 01$

$3.96955 \mathrm{E} \quad 01$ $3.97974 \mathrm{E} \quad 01$ $3.98993 \mathrm{E} 01$ $4.00013 \mathrm{E} \quad 01$ $4.01033 \mathrm{E} \quad 01$

$4.02053 \mathrm{E} \quad 01$ $4.03074 \mathrm{E} \quad 01$ $4.04095 \mathrm{E} \quad 01$ $4.05116 \mathrm{E} \quad 01$ $4.06138 \mathrm{E} 01$

$4.07160 \mathrm{E} \quad 01$ $4.08183 \mathrm{E} \quad 01$ $4.09206 \mathrm{E} \quad 01$ $4.10229 \mathrm{E} \quad 01$ $4.11253 \mathrm{E} 01$
$4.44860 \mathrm{E} \quad 02$ $4.45478 \mathrm{E} \quad 02$ $4.46095 \mathrm{E} \quad 02$ $4.46713 \mathrm{E} \quad 02$ $4.47332 \mathrm{E} \quad 02$

$4.47953 \mathrm{E} \quad 02$ $4.48572 \mathrm{E} \quad 02$ $4.49193 \mathrm{E} \quad 02$ $4.49815 \mathrm{E} \quad 02$ $4.50437 \mathrm{E} \quad 02$

$4.51061 \mathrm{E} \quad 02$ $4.51686 \mathrm{E} \quad 02$ $4.52310 \mathrm{E} \quad 02$ $4.52935 \mathrm{E} \quad 02$ $4.53562 \mathrm{E} \quad 02$

$4.54189 \mathrm{E} \quad 02$ $4.54816 \mathrm{E} \quad 02$ $4.55446 \mathrm{E} \quad 02$ $4.56075 \mathrm{E} \quad 02$ $4.56707 \mathrm{E} \quad 02$

$4.57338 \mathrm{E} \quad 02$ $4.57969 \mathrm{E} \quad 02$ $4.58603 \mathrm{E} \quad 02$ $\begin{array}{ll}4.59237 \mathrm{E} & 02\end{array}$ $4.59872 \mathrm{E} \quad 02$

$4.60507 \mathrm{E} \quad 02$ $4.61145 \mathrm{E} \quad 02$ $4.61783 \mathrm{E} \quad 02$ $4.62423 \mathrm{E} \quad 02$ $\begin{array}{lll}4.63062 \mathrm{E} & 02\end{array}$

4.63702E 02 $4.64345 \mathrm{E} \quad 02$ $4.64988 \mathrm{E} \quad 02$ $4.65632 \mathrm{E} \quad 02$ $4.66277 \mathrm{E} \quad 02$

$4.66924 \mathrm{E} \quad 02$ $4.67571 \mathrm{E} \quad 02$ $4.68220 \mathrm{E} \quad 02$ $4.68868 \mathrm{E} \quad 02$ $4.69519 \mathrm{E} \quad 02$

$4.70172 \mathrm{E} \quad 02$ $4.70826 \mathrm{E} \quad 02$ $4.71479 \mathrm{E} \quad 02$ 4.72134E 02 $4.72791 \mathrm{E} \quad 02$

$\begin{array}{lll}4.73449 \mathrm{E} \quad 02 & 0\end{array}$ $4.74106 \mathrm{E} \quad 02$ $4.74767 \mathrm{E} \quad 02$ $4.75429 \mathrm{E} \quad 02$ $4.76091 \mathrm{E} \quad 02$

$4.76756 \mathrm{E} \quad 02$ $4.77420 \mathrm{E} \quad 02$ $4.78088 \mathrm{E} \quad 02$ $4.78757 \mathrm{E} \quad 02$ $4.79425 \mathrm{E} \quad 02$

$4.80096 \mathrm{E} \quad 02$ $4.80768 \mathrm{E} \quad 02$ $4.81441 \mathrm{E} \quad 02$ $4.82117 \mathrm{E} \quad 02$ $4.82794 \mathrm{E} \quad 02$ 
ELECTRON RADIAL FUNCTIONS

$Z=6 \quad A=12 \quad \rho=1.2 A^{1 / 3} F$

Positron

$\bar{F}$

$1.59978 \mathrm{E}-03$

$1.60865 \mathrm{E}-03$

$1.61755 \mathrm{E}-03$

$1.62647 \mathrm{E}-03$

$1.63542 \mathrm{E}-03$

$1.64439 \mathrm{E}-03$

$1.65338 \mathrm{E}-03$

$1.66240 \mathrm{E}-03$

$1.67144 \mathrm{E}-03$

$1.68051 \mathrm{E}-03$

36.90

37.00

37.10

37.20

37.30

37.40

37.50

37.60

37.70

37.80

37.90

38.00

$1.68960 \mathrm{E}-03$

$1.69872 \mathrm{E}-03$

$1.70786 \mathrm{E}-03$

$1.71703 \mathrm{E}-03$

$1.72622 \mathrm{E}-03$

$1.73543 \mathrm{E}-03$

$1.74467 \mathrm{E}-03$

$1.75393 \mathrm{E}-03$

$1.76322 \mathrm{E}-03$

$1.77253 \mathrm{E}-03$

38.10

38.20

38.30

38.40

38.50

38.60

38.70

38.80

38.90

39.00

39.10

39.20

39.30

39.40

39.50

39.60

39.70

39.80

39.90

$40.00^{\circ}$

$1.78187 \mathrm{E}-03$

$1.79123 \mathrm{E}-03$

$1.80062 \mathrm{E}-03$

$1.81003 \mathrm{E}-03$

$1.81947 \mathrm{E}-03$

$1.82893 \mathrm{E}-03$

$1.83841 \mathrm{E}-03$

$1.84792 \mathrm{E}-03$

$1.85745 \mathrm{E}-03$

$1.86701 \mathrm{E}-03$

$1.87659 \mathrm{E}-03$

$1.88620 \mathrm{E}-03$

$1.89583 \mathrm{E}-03$

$1.90549 \mathrm{E}-03$

$1.91517 \mathrm{E}-03$

$1.92487 \mathrm{E}-03$

$1.93460 \mathrm{E}-03$

$1.94436 \mathrm{E}-03$

$1.95414 \mathrm{E}-03$

$1.96394 \mathrm{E}-03$

$40.10 \quad 1.97377 \mathrm{E}-03$

$40.20 \quad 1.98362 \mathrm{E}-03$

$40.30 \quad 1.99350 \mathrm{E}-03$

$40.40 \quad 2.00340 \mathrm{E}-03$

$40.50 \quad 2.01332 \mathrm{E}-03$

$40.60 \quad 2.02327 \mathrm{E}-03$

$40.70 \quad 2.03325 \mathrm{E}-03$

$40.80 \quad 2.04325 \mathrm{E}-03$

$40.90 \quad 2.05327 \mathrm{E}-03$

$41.00 \quad 2.06332 \mathrm{E}-03$

$41.10 \quad 2.07339 \mathrm{E}-03$

$41.20 \quad 2.08349 \mathrm{E}-03$

$41.30 \quad 2.09361 \mathrm{E}-03$

$41.40 \quad 2.10376 \mathrm{E}-03$

$41.50 \quad 2.11393 \mathrm{E}-03$

$41.60 \quad 2.12412 \mathrm{E}-03$

$41.70 \quad 2.13434 \mathrm{E}-03$

$41.80 \quad 2.14459 \mathrm{E}-03$

$41.90 \quad 2.15486 \mathrm{E}-03$

$42.00 \quad 2.16515 \mathrm{E}-03$ $f_{2}$

$2.84348 \mathrm{E} \quad 00$ $2.85930 \mathrm{E} \quad 00$

$2.87517 \mathrm{E} \quad 00$

$2.89107 \mathrm{E} \quad 00$

$2.90703 \mathrm{E} \quad 00$

$2.92302 \mathrm{E} \quad 00$

$2.93906 \mathrm{E} \quad 00$

$2.95514 \mathrm{E} \quad 00$

$2.97127 \mathrm{E} \quad 00$

$2.98744 \mathrm{E} \quad 00$

$3.00365 \mathrm{E} \quad 00$

$3.01990 \mathrm{E} \quad 00$

$3.03620 \mathrm{E} \quad 00$

$3.05254 \mathrm{E} \quad 00$

$3.06893 \mathrm{E} \quad 00$

$3.08536 \mathrm{E} \quad 00$

$3.10183 \mathrm{E} \quad 00$

$3.11834 \mathrm{E} \quad 00$

$3.13490 \mathrm{E} \quad 00$

$3.15150 \mathrm{E} \quad 00$

$3.16814 \mathrm{E} \quad 00$

$3.18483 \mathrm{E} \quad 00$

$3.20156 \mathrm{E} \quad 00$

$3.21833 \mathrm{E} \quad 00$

$3.23515 \mathrm{E} \quad 00$

$3.25201 \mathrm{E} \quad 00$

$3.26891 \mathrm{E} \quad 00$

$\begin{array}{ll}3.28586 \mathrm{E} & 00\end{array}$

$3.30284 \mathrm{E} \quad 00$

$3.31987 \mathrm{E} \quad 00$

$3.33695 \mathrm{E} \quad 00$

$3.35407 \mathrm{E} \quad 00$

$3.37123 \mathrm{E} \quad 00$

$3.38843 \mathrm{E} \quad 00$

$3.40568 \mathrm{E} \quad 00$

$3.42297 \mathrm{E} \quad 00$

$3.44030 \mathrm{E} \quad 00$

$3.45768 \mathrm{E} \quad 00$

$3.47509 \mathrm{E} \quad 00$

$3.49256 \mathrm{E} \quad 00$

$3.51006 \mathrm{E} \quad 00$

$3.52761 \mathrm{E} \quad 00$

$3.54520 \mathrm{E} \quad 00$

$3.56283 \mathrm{E} \quad 00$

$3.58051 \mathrm{E} \quad 00$

3.59823 E 00

$3.61599 \mathrm{E} \quad 00$

$3.63380 \mathrm{E} \quad 00$

$3.65164 \mathrm{E} \quad 00$

$3.66954 \mathrm{E} \quad 00$

$3.68747 \mathrm{E} \quad 00$

$3.70545 \mathrm{E} \quad 00$

$3.72347 \mathrm{E} \quad 00$

$3.74153 \mathrm{E} \quad 00$

$3.75963 \mathrm{E} \quad 00$

$3.77778 \mathrm{E} \quad 00$

$3.79597 \mathrm{E} \quad 00$

$3.81421 \mathrm{E} \quad 00$

$3.83249 \mathrm{E} \quad 00$

$3.85081 \mathrm{E} \quad 00$ $g_{2}$

$1.21851 \mathrm{E}-01$

$1.22937 \mathrm{E}-01$

$1.24030 \mathrm{E}-01$

$1.25129 \mathrm{E}-01$

$1.26234 \mathrm{E}-01$

$1.27346 \mathrm{E}-01$

$1.28465 \mathrm{E}-01$

$1.29590 \mathrm{E}-01$

$1.30721 \mathrm{E}-01$

$1.31859 \mathrm{E}-01$

$1.33004 \mathrm{E}-01$

$1.34155 \mathrm{E}-01$

$1.35312 \mathrm{E}-01$

$1.36476 \mathrm{E}-01$

$1.37647 \mathrm{E}-01$

$1.38825 \mathrm{E}-01$

$1.40009 \mathrm{E}-01$

$1.41200 \mathrm{E}-01$

$1.42397 \mathrm{E}-01$

$1.43601 \mathrm{E}-01$

$1.44812 \mathrm{E}-01$

$1.46030 \mathrm{E}-01$

$1.47254 \mathrm{E}-01$

$1.48485 \mathrm{E}-0]$

$1.49724 \mathrm{E}-01$

$1.50968 \mathrm{E}-01$

$1.52220 \mathrm{E}-01$

$1.53479 \mathrm{E}-01$

$1.54744 \mathrm{E}-01$

$1.56016 \mathrm{E}-01$

$1.57295 \mathrm{E}-01$

$1.58582 \mathrm{E}-01$

$1.59875 \mathrm{E}-01$

$1.61175 \mathrm{E}-01$

$1.62482 \mathrm{E}-01$

$1.63796 \mathrm{E}-01$

$1.65117 \mathrm{E}-01$

$1.66445 \mathrm{E}-01$

$1.67780 \mathrm{E}-01$

$1.69122 \mathrm{E}-01$

$1.70472 \mathrm{E}-01$

$1.71828 \mathrm{E}-01$

$1.73192 \mathrm{E}-01$

$1.74562 \mathrm{E}-01$

$1.75940 \mathrm{E}-01$

$1.77325 \mathrm{E}-01$

$1.78717 \mathrm{E}-01$

$1.80117 \mathrm{E}-01$

$1.81524 \mathrm{E}-01$

$1.82938 \mathrm{E}-01$

$1.84359 \mathrm{E}-01$

$1.85787 \mathrm{E}-01$

$1.87223 \mathrm{E}-01$

$1.88667 \mathrm{E}-01$

1.90117 E-01

$1.91575 \mathrm{E}-01$

$1.93040 \mathrm{E}-01$

$1.94513 \mathrm{E}-01$

$1.95993 \mathrm{E}-01$

$1.97481 \mathrm{E}-01$

$\tan \delta_{2}$

$f_{-2}$

$-3.27543 \mathrm{E} 01-1.16944 \mathrm{E}-01$

$-3.27553 \mathrm{E} \quad 01$

$-3.27563 \mathrm{E} 01$

$-3.27572 \mathrm{E} \quad 01$

$-3.27582 \mathrm{E}$ 01

$-1.18006 \mathrm{E}-01$

$-1.19073 \mathrm{E}-01$

$-1.20147 \mathrm{E}-01$

$-1.21228 \mathrm{E}-01$

$-3.27591 \mathrm{E} \quad 01$

$-3.27600 \mathrm{E} \quad 01$

$-3.27610 \mathrm{E} 01$

$-3.27619 \mathrm{E} \quad 01$

$-3.27628 \mathrm{E} 01$

$-1.22314 \mathrm{E}-01$

$-1.23408 \mathrm{E}-01$

$-1.24507 \mathrm{E}-01$

$-1.25613 \mathrm{E}-01$

$-1.26726 \mathrm{E}-01$

$-3.27637 \mathrm{E} \quad 01$

$-3.27646 \mathrm{E} \quad 01$

$-3.27655 \mathrm{E} 01$

$-3.27664 \mathrm{E} \quad 01$

$-3.27673 \mathrm{E} 01$

$-3.27682 \mathrm{E} 01$

$-3.27691 \mathrm{E} 01$

$-3.27700 \mathrm{E} \quad 01$

$-3.27709 \mathrm{E} 01$

$-3.27717 \mathrm{E} \quad 01$

$-3.27726 \mathrm{E} \quad 01$

$-3.27734 \mathrm{E} 01$

$-3.27743 \mathrm{E} 01$

$-3.27751 \mathrm{E} 01$

$-3.27760 \mathrm{E} 01$

$-3.27768 \mathrm{E} \quad 01$

$-3.27777 \mathbf{E} 01$

$-3.27785 \mathrm{E} 01$

$-3.27793 \mathrm{E} 01$

$-3.27802 \mathrm{E} 01$

$-3.27810 \mathrm{E} 01$

$-3.27818 \mathrm{E} \quad 01$

$-3.27826 \mathrm{E} 01$

$-3.27834 \mathrm{E} \quad 01$

$-3.27842 \mathrm{E} 01$

$-3.27850 \mathrm{E} \quad 01$

$-3.27858 \mathrm{E}$ 01

$-3.27866 \mathrm{E} 01$

$-3.27874 \mathrm{E} \quad 01$

$-3.27882 \mathrm{E} 01$

$-3.27889 \mathrm{E} \quad 01$

$-3.27897 \mathrm{E} 01$

$-3.27905 \mathrm{E} \quad 01$

$-3.27912 \mathrm{E} 01$

$-3.27920 \mathrm{E} 01$

$\begin{array}{lll}-3.27928 \mathrm{E} & 01\end{array}$

$-3.27935 \mathrm{E} 01$

$-3.27943 \mathrm{E} \quad 01$

$-3.27950 \mathrm{E} 01$

$-3.27958 \mathrm{E} \quad 01$

$-3.27965 \mathrm{E} \quad 01$

$-3.27972 \mathrm{E} 01$

$-3.27980 \mathrm{E} 01$

$\begin{array}{lll}-3.27987 \mathrm{E} & 01\end{array}$

$-3.27994 \mathrm{E} 01$

$-1.27845 \mathrm{E}-01$

$-1.28970 \mathrm{E}-01$

$-1.30102 \mathrm{E}-01$

$-1.31241 \mathrm{E}-01$

$-1.32386 \mathrm{E}-01$

$-1.33538 \mathrm{E}-01$

$-1.34696 \mathrm{E}-01$

$-1.35861 \mathrm{E}-01$ 
ELECTRON RADIAL FUNCTIONS

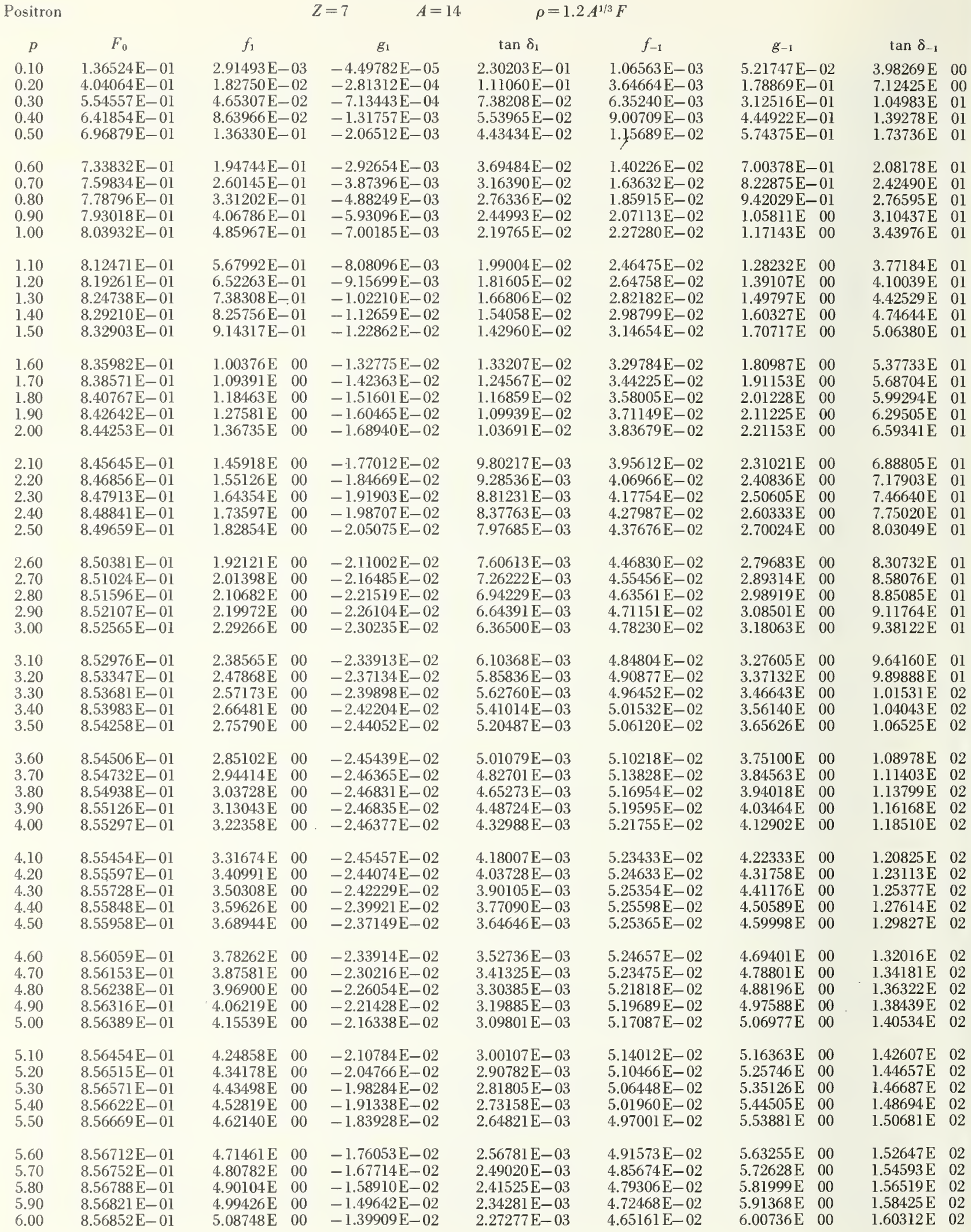


$\bar{F}$

0.10

0.20

0.30

0.40

0.50

0.60

0.70

0.80

0.90

1.00

1.10

1.20

1.30

1.40

1.50

1.60

1.70

1.80

1.90

2.00

2.10

2.20

2.30

2.40

2.50

2.60

2.70

2.80

2.90

3.00

3.10

3.20

3.30

3.40

3.50

3.60

3.70

3.80

3.90

4.00

4.10

4.20

4.30

4.40

4.50

4.60

4.70

4.80

4.90

5.00

5.10

5.20

5.30

5.40

5.50

5.60

5.70

5.80

5.90

6.00 $f_{2}$

$2.65897 \mathrm{E}-09$

$2.66160 \mathrm{E}-08$

$7.94353 \mathrm{E}-08$

$1.61494 \mathrm{E}-07$

$2.72463 \mathrm{E}-07$

4.11945 E-07

$5.79578 \mathrm{E}-07$

$7.75063 \mathrm{E}-07$

$9.98154 \mathrm{E}-07$

$1.24866 \mathrm{E}-06$

$1.52643 \mathrm{E}-06$

$1.83133 \mathrm{E}-06$

$2.16329 \mathrm{E}-06$

$2.52222 \mathrm{E}-06$

$2.90806 \mathrm{E}-06$

$3.32077 \mathrm{E}-06$

$3.76031 \mathrm{E}-06$

$4.22665 \mathrm{E}-06$

$4.71977 \mathrm{E}-06$

$5.23964 \mathrm{E}-06$

$5.78625 \mathrm{E}-06$ $6.35958 \mathrm{E}-06$ $6.95963 \mathrm{E}-06$

$7.58638 \mathrm{E}-06$

$8.23982 \mathrm{E}-06$

$8.91996 \mathrm{E}-06$

$9.62677 \mathrm{E}-06$

$1.03603 \mathrm{E}-05$

$1.11204 \mathrm{E}-05$

$1.19073 \mathrm{E}-05$

$1.27207 \mathrm{E}-05$

$1.35609 \mathrm{E}-05$

$1.44277 \mathrm{E}-05$

$1.53212 \mathrm{E}-05$

$1.62413 \mathrm{E}-05$

$1.71881 \mathrm{E}-05$

$1.81615 \mathrm{E}-05$

$1.91615 \mathrm{E}-05$

$2.01882 \mathrm{E}-05$

2.12416 E- 05

$2.23215 \mathrm{E}-05$

$2.34282 \mathrm{E}-05$

$2.45614 \mathrm{E}-05$

$2.57213 \mathrm{E}-05$

$2.69079 \mathrm{E}-05$

$2.81210 \mathrm{E}-05$

$2.93608 \mathrm{E}-05$

$3.06273 \mathrm{E}-05$

$3.19203 \mathrm{E}-05$

$3.32400 \mathrm{E}-05$

$3.45863 \mathrm{E}-05$

$3.59593 \mathrm{E}-05$

$3.73589 \mathrm{E}-05$

$3.87851 \mathrm{E}-05$

$4.02379 \mathrm{E}-05$

$4.17174 \mathrm{E}-05$

$4.32234 \mathrm{E}-05$

$4.47561 \mathrm{E}-05$

$4.63155 \mathrm{E}-05$

$4.79014 \mathrm{E}-05$
$7.45573 \mathrm{E}-07$

$9.15658 \mathrm{E}-06$

$3.48367 \mathrm{E}-05$

$8.61357 \mathrm{E}-05$

$1.69808 \mathrm{E}-04$

$2.91006 \mathrm{E}-04$

$4.53465 \mathrm{E}-04$

$6.59760 \mathrm{E}-04$

$9.11590 \mathrm{E}-04$

$1.21002 \mathrm{E}-03$

$1.55568 \mathrm{E}-03$

$1.94891 \mathrm{E}-03$

$2.38987 \mathrm{E}-03$

$2.87858 E-03$

$3.41500 \mathrm{E}-03$

$3.99907 \mathrm{E}-03$

$4.63068 \mathrm{E}-03$

$5.30974 \mathrm{E}-03$

$6.03614 \mathrm{E}-03$

$6.80977 \mathrm{E}-03$

$7.63056 \mathrm{E}-03$

$8.49840 \mathrm{E}-03$

$9.41322 \mathrm{E}-03$

$1.03750 \mathrm{E}-02$

$1.13835 \mathrm{E}-02$

$1.24389 \mathrm{E}-02$

$1.35410 \mathrm{E}-02$

$1.46898 \mathrm{E}-02$

$1.58853 \mathrm{E}-02$

$1.71274 \mathrm{E}-02$

$1.84160 \mathrm{E}-02$

$1.97512 \mathrm{E}-02$

$2.11329 \mathrm{E}-02$

$2.25611 \mathrm{E}-02$

$2.40358 \mathrm{E}-02$

$2.55570 \mathrm{E}-02$

$2.71246 \mathrm{E}-02$

$2.87386 \mathrm{E}-02$

$3.03990 \mathrm{E}-02$

$3.21058 \mathrm{E}-02$

$3.38590 \mathrm{E}-02$

$3.56586 \mathrm{E}-02$

$3.75045 \mathrm{E}-02$

$3.93968 \mathrm{E}-02$

4.13354 E-02

$4.33203 \mathrm{E}-02$

$4.53515 \mathrm{E}-02$

$4.74291 \mathrm{E}-02$

$4.95529 \mathrm{E}-02$

5.17231 E-02

$5.39395 \mathrm{E}-02$

$5.62022 \mathrm{E}-02$

$5.85112 \mathrm{E}-02$

$6.08664 \mathrm{E}-02$

$6.32679 \mathrm{E}-02$

$6.57156 \mathrm{E}-02$

$6.82095 \mathrm{E}-02$

$7.07497 \mathrm{E}-02$

$7.33359 \mathrm{E}-02$

$7.59684 \mathrm{E}-02$ $g_{2}$

$\tan \delta_{2}$

$f_{-2}$

$-6.46642 \mathrm{E}-09$

$-7.92126 \mathrm{E}-08$

$-3.00104 \mathrm{E}-07$

$-7.37765 \mathrm{E}-07$

$-1.44400 \mathrm{E}-06$

$-2.45364 \mathrm{E}-06$

$-3.78642 \mathrm{E}-06$

$-5.44969 \mathrm{E}-06$

$-7.44142 \mathrm{E}-06$

$-9.75274 \mathrm{E}-06$

$-1.23700 \mathrm{E}-05$

$-1.52765 \mathrm{E}-05$

$-1.84534 \mathrm{E}-05$

$-2.18805 \mathrm{E}-05$

$-2.55368 \mathrm{E}-05$

$-2.94010 \mathrm{E}-05$

$-3.34513 \mathrm{E}-05$

$-3.76661 \mathrm{E}-05$

$-4.20235 \mathrm{E}-05$

$-4.65018 \mathrm{E}-05$

$-5.10792 \mathrm{E}-05$

$-5.57342 \mathrm{E}-05$

$-6.04452 \mathrm{E}-05$

$-6.51907 \mathrm{E}-05$

$-6.99492 \mathrm{E}-05$

$-7.46994 \mathrm{E}-05$

$-7.94200 \mathrm{E}-05$

$-8.40897 \mathrm{E}-05$

$-8.86874 \mathrm{E}-05$

$-9.31919 \mathrm{E}-05$

$-9.75820 \mathrm{E}-05$

$-1.01837 \mathrm{E}-04$

$-1.05935 \mathrm{E}-04$

$-1.09856 \mathrm{E}-04$

$-1.13578 \mathrm{E}-04$

$-1.17081 \mathrm{E}-04$

$-1.20344 \mathrm{E}-04$

$-1.23345 \mathrm{E}-04$

$-1.26064 \mathrm{E}-04$

$-1.28479 \mathrm{E}-04$

- 1.30571 E-04

$-1.32317 \mathrm{E}-04$

$-1.33698 \mathrm{E}-04$

$-1.34692 \mathrm{E}-04$

$-1.35279 \mathrm{E}-04$

$-1.35437 \mathrm{E}-04$

$-1.35145 \mathrm{E}-04$

$-1.34384 \mathrm{E}-04$

$-1.33132 \mathrm{E}-04$

$-1.31368 \mathrm{E}-04$

$-1.29071 \mathrm{E}-04$

$-1.26221 \mathrm{E}-04$

$-1.22796 \mathrm{E}-04$

$-1.18777 \mathrm{E}-04$

$-1.14141 \mathrm{E}-04$

$-1.08869 \mathrm{E}-04$

$-1.02940 \mathrm{E}-04$

$-9.63320 \mathrm{E}-05$

$-8.90251 \mathrm{E}-05$

$-8.09984 \mathrm{E}-05$

$\begin{array}{ll}-1.93299 \mathrm{E} & 00 \\ -4.07750 \mathrm{E} & 00 \\ -6.06943 \mathrm{E} & 00 \\ -7.92500 \mathrm{E} & 00 \\ -9.63454 \mathrm{E} & 00\end{array}$

$-1.11927 \mathrm{E} \quad 01$

$-1.26008 \mathrm{E} 01$

$-1.38656 \mathrm{E} 01$

$-1.49972 \mathrm{E} \quad 01$

$-1.60074 \mathrm{E} \quad 01$

$-1.69088 \mathrm{E} 01$

$-1.77135 \mathrm{E} 01$

$-1.84327 \mathrm{E} \quad 01$

$-1.90768 \mathrm{E} \quad 01$

$-1.96550 \mathrm{E} \quad 0 \mathrm{l}$

$-2.01754 \mathrm{E} \quad 01$

$-2.06450 \mathrm{E} \quad 01$

$-2.10700 \mathrm{E} \quad 01$

$-2.14558 \mathrm{E} 01$

$-2.18070 \mathrm{E} \quad 01$

$-2.21276 \mathrm{E} \quad 01$

$-2.24210 \mathrm{E} 01$

$-2.26903 \mathrm{E} 01$

$-2.29381 \mathrm{E} 01$

$-2.31667 \mathrm{E} 01$

$-2.33781 \mathrm{E} \quad 01$

$-2.35740 \mathrm{E} \quad 01$

$-2.37559 \mathrm{E} \quad 0 \mathrm{l}$

$-2.39253 \mathrm{E} 01$

$-2.40832 \mathrm{E} \quad 01$

$-2.42308 \mathrm{E} \quad 01$

$-2.43690 \mathrm{E} \quad 01$

$-2.44985 \mathrm{E} \quad 01$

$-2.46202 \mathrm{E} \quad 01$

$-2.47347 \mathrm{E} 01$

$-2.48426 \mathrm{E} \quad 01$

$-2.49445 \mathrm{E} \quad 01$

$-2.50407 \mathrm{E} \quad 01$

$-2.51318 \mathrm{E} 01$

-2.52181 E 01

$-2.52999 \mathrm{E} \quad 01$

$-2.53777 \mathrm{E} 01$

$-2.54516 \mathrm{E} 01$

$-2.55220 \mathrm{E} 01$

$-2.55891 \mathrm{E} 01$

$-2.56531 \mathrm{E} \quad 01$ 
ELECTRON RADIAL FUNCTIONS

Positron

p $F_{0}$

$6.10 \quad 8.56879 \mathrm{E}-01$

$6.20 \quad 8.56905 \mathrm{E}-01$

$6.30 \quad 8.56927 \mathrm{E}-01$

$6.40 \quad 8.56948 \mathrm{E}-01$

$6.50 \quad 8.56966 \mathrm{E}-01$

6.60

6.70

6.80

6.90

7.00

7.10

7.20

7.30

7.40

7.50

7.60

7.70

7.80

7.90

8.00

8.10

8.20

8.30

8.40

8.50

8.60

8.70

8.80

8.90

9.00

9.10

9.20

9.30

9.40

9.50

9.60

9.70

9.80

9.90

10.00

10.10

10.20

10.30

10.40

10.50

10.60

10.70

10.80

10.90

11.00

11.10

11.20

11.30

11.40

11.50

11.60

11.70

11.80

11.90

12.00

$8.56983 \mathrm{E}-01$ $8.56998 \mathrm{E}-01$ $8.57012 \mathrm{E}-01$ $8.57024 \mathrm{E}-01$ $8.57035 \mathrm{E}-01$ $8.57052 \mathrm{E}-01$ $8.57059 \mathrm{E}-01$ $8.57064 \mathrm{E}-01$ $8.57069 \mathrm{E}-01$

$8.57072 \mathrm{E}-01$ $8.57075 \mathrm{E}-01$ $8.57077 \mathrm{E}-01$ $8.57079 \mathrm{E}-01$ $8.57079 \mathrm{E}-01$

$8.57079 \mathrm{E}-01$ $8.57078 \mathrm{E}-01$ $8.57077 \mathrm{E}-01$ $8.57075 \mathrm{E}-01$ $8.57072 \mathrm{E}-01$

$8.57069 \mathrm{E}-01$ $8.57065 \mathrm{E}-01$ $8.57061 \mathrm{E}-01$ $8.57057 \mathrm{E}-01$ $8.57052 \mathrm{E}-01$

$8.57046 \mathrm{E}-01$ $8.57041 \mathrm{E}-01$ $8.57035 \mathrm{E}-01$ $8.57028 \mathrm{E}-01$ $8.57022 \mathrm{E}-01$

$8.57015 \mathrm{E}-01$ $8.57008 \mathrm{E}-01$ $8.57000 \mathrm{E}-01$ $8.56992 \mathrm{E}-01$ $8.56985 \mathrm{E}-01$

$8.56976 \mathrm{E}-01$ $8.56968 \mathrm{E}-01$ $8.56960 \mathrm{E}-01$ $8.56951 \mathrm{E}-01$ $8.56942 \mathrm{E}-01$

$8.56934 \mathrm{E}-01$ $8.56925 \mathrm{E}-01$ $8.56915 \mathrm{E}-01$ $8.56906 \mathrm{E}-01$ $8.56896 \mathrm{E}-01$

$8.56887 \mathrm{E}-01$ $8.56878 \mathrm{E}-01$ $8.56868 \mathrm{E}-01$ $8.56858 \mathrm{E}-01$ $8.56848 \mathrm{E}-01$

$8.56838 \mathrm{E}-01$ $8.56828 \mathrm{E}-01$ $8.56818 \mathrm{E}-01$ $8.56808 \mathrm{E}-01$ $8.56798 \mathrm{E}-01$
$Z=7 \quad A=14$

$g_{1}$

$\tan \delta_{1}$

$\rho=1.2 A^{1 / 3} F$

$8.57044 \mathrm{E}-01$

$$
f_{1}
$$

$5.18071 \mathrm{E} \quad 00$ $5.27394 \mathrm{E} \quad 00$

$5.36717 \mathrm{E} \quad 00$

$5.46040 \mathrm{E} \quad 00$

$5.55364 \mathrm{E} \quad 00$

$5.64688 \mathrm{E} \quad 00$ $5.74013 \mathrm{E} \quad 00$

$5.83339 \mathrm{E} \quad 00$

$6.01990 \mathrm{E} \quad 00$

$6.11316 \mathrm{E} \quad 00$ $6.20642 \mathrm{E} \quad 00$ $6.29970 \mathrm{E} \quad 00$ $6.39297 \mathrm{E} \quad 00$ $6.48624 \mathrm{E} \quad 00$

$6.57952 \mathrm{E} \quad 00$ $6.67280 \mathrm{~F} \quad 00$ $6.76608 \mathrm{E} \quad 00$ $6.85937 \mathrm{E} \quad 00$ $6.95264 \mathrm{E} \quad 00$

$7.04591 \mathrm{E} \quad 00$ $7.13918 \mathrm{E} \quad 00$

$7.23243 \mathrm{E} \quad 00$

$7.32566 \mathrm{E} \quad 00$

$7.41885 \mathrm{E} \quad 00$

$7.51199 \mathrm{E} \quad 00$

$7.60503 \mathrm{E} \quad 00$

$7.69789 \mathrm{E} \quad 00$

$7.79036 \mathrm{E} \quad 00$

$7.88179 \mathrm{E} \quad 00$

$7.96800 \mathrm{E} \quad 00$ $8.11653 \mathrm{E} \quad 00$ $8.17568 \mathrm{E} \quad 00$ $8.26554 \mathrm{E} \quad 00$ $8.35768 \mathrm{E} \quad 00$

$8.45044 \mathrm{E} \quad 00$

$8.54350 \mathrm{E} \quad 00$

$8.63669 \mathrm{E} \quad 00$

$8.72997 \mathrm{E} \quad 00$

$8.82334 \mathrm{E} \quad 00$

$8.91674 \mathrm{E} \quad 00$ $9.01018 \mathrm{E} \quad 00$ $9.10365 \mathrm{E} \quad 00$ $9.19714 \mathrm{E} \quad 00$ $9.29066 \mathrm{E} \quad 00$

$9.38420 \mathrm{E} \quad 00$ $9.47775 \mathrm{E} \quad 00$ $9.57132 \mathrm{E} \quad 00$ $9.66490 \mathrm{E} \quad 00$

$9.75850 \mathrm{E} \quad 00$

$9.85213 \mathrm{E} \quad 00$ $9.94576 \mathrm{E} \quad 00$ $1.00394 \mathrm{E} \quad 01$ $1.01331 \mathrm{E} \quad 01$ $1.02267 \mathrm{E} \quad 01$

$1.03204 \mathrm{E} \quad 01$

$1.04141 \mathrm{E} \quad 01$

$1.05079 \mathrm{E} \quad 01$

$1.06016 \mathrm{E} 01$

$1.06953 \mathrm{E} \quad 01$
$5.92664 \mathrm{E} \quad 00$
$-1.19049 \mathrm{E}-02$

$-1.07922 \mathrm{E}-02$

$-9.63299 \mathrm{E}-03$

$-8.42732 \mathrm{E}-03$

$-7.17515 \mathrm{E}-03$

$-5.87648 \mathrm{E}-03$

$-4.53131 \mathrm{E}-03$

$-3.13963 \mathrm{E}-03$

$-1.70143 \mathrm{E}-03$

$-2.16720 \mathrm{E}-04$

$1.31452 \mathrm{E}-03$

$2.89229 \mathrm{E}-03$

$4.51658 \mathrm{E}-03$

$6.18742 \mathrm{E}-03$

$7.90479 \mathrm{E}-03$

$9.66871 \mathrm{E}-03$

$1.14792 \mathrm{E}-02$

$1.33362 \mathrm{E}-02$

$1.52397 \mathrm{E}-02$

$1.71898 \mathrm{E}-02$

$1.91864 \mathrm{E}-02$

$2.12295 \mathrm{E}-02$

$2.33191 \mathrm{E}-02$

2.54551 E-02

$2.76373 \mathrm{E}-02$

$2.98657 \mathrm{E}-02$

$3.21398 \mathrm{E}-02$

$3.44585 \mathrm{E}-02$

$3.68185 \mathrm{E}-02$

$3.91984 \mathrm{E}-02$

4.19435 E-02

$4.42785 \mathrm{E}-02$

$4.68170 \mathrm{E}-02$

$4.94139 \mathrm{E}-02$

$5.20606 \mathrm{E}-02$

$5.47556 \mathrm{E}-02$

$5.74978 \mathrm{E}-02$

$6.02872 \mathrm{E}-02$

$6.31239 \mathrm{E}-02$

$6.60073 \mathrm{E}-02$

$6.89378 \mathrm{E}-02$

$7.19150 \mathrm{E}-02$

$7.49392 \mathrm{E}-02$

$7.80103 \mathrm{E}-02$

$8.11282 \mathrm{E}-02$ $8.42930 \mathrm{E}-02$ $8.75046 \mathrm{E}-02$

$9.07631 \mathrm{E}-02$

$9.40685 \mathrm{E}-02$

9.74209E-02 $1.00820 \mathrm{E}-01$

$1.04266 \mathrm{E}-01$

$1.07759 \mathrm{E}-01$

$1.11299 \mathrm{E}-01$

$1.14886 \mathrm{E}-01$

$1.18520 \mathrm{E}-01$

$1.22201 \mathrm{E}-01$

$1.25929 \mathrm{E}-01$

$1.29703 \mathrm{E}-01$
$-1.29711 \mathrm{E}-02$

2.20502 E- 03

$2.13943 \mathrm{E}-03$

$2.07592 \mathrm{E}-03$

$2.01438 \mathrm{E}-03$

$1.95472 \mathrm{E}-03$

$1.89686 \mathrm{E}-03$

$1.84074 \mathrm{E}-03$

$1.78625 \mathrm{E}-03$

$1.73335 \mathrm{E}-03$

$1.68194 \mathrm{E}-03$

$1.63199 \mathrm{E}-03$

$1.58342 \mathrm{E}-03$

$1.53618 \mathrm{E}-03$

$1.49023 \mathrm{E}-03$

$1.44549 \mathrm{E}-03$

$1.40193 \mathrm{E}-03$

$1.35951 \mathrm{E}-03$

$1.31819 \mathrm{E}-03$

$1.27790 \mathrm{E}-03$

$1.23864 \mathrm{E}-03$

$1.20035 \mathrm{E}-03$

$1.16298 \mathrm{E}-03$

$1.12654 \mathrm{E}-03$

$1.09097 \mathrm{E}-03$

$1.05624 \mathrm{E}-03$

$1.02232 \mathrm{E}-03$

$9.89190 \mathrm{E}-04$

$9.56821 \mathrm{E}-04$

$9.25189 \mathrm{E}-04$

$8.94257 \mathrm{E}-04$

$8.64028 \mathrm{E}-04$

$8.34451 \mathrm{E}-04$

$8.05528 \mathrm{E}-04$

$7.77230 \mathrm{E}-04$

$7.49536 \mathrm{E}-04$

$7.22421 \mathrm{E}-04$

$6.95878 \mathrm{E}-04$

$6.69888 \mathrm{E}-04$

$6.44431 \mathrm{E}-04$

$6.19503 \mathrm{E}-04$

$5.95072 \mathrm{E}-04$

$5.71130 \mathrm{E}-04$

$5.47670 \mathrm{E}-04$

$5.24655 \mathrm{E}-04$

$5.02107 \mathrm{E}-04$ 
$\bar{F}$

$6.10 \quad 4.95140 \mathrm{E}-05$

$6.20 \quad 5.11532 \mathrm{E}-05$

$6.30 \quad 5.28190 \mathrm{E}-05$

$6.40 \quad 5.45114 \mathrm{E}-05$

$6.50 \quad 5.62304 \mathrm{E}-05$

$6.60 \quad 5.79761 \mathrm{E}-05$

$6.70 \quad 5.97484 \mathrm{E}-05$

$6.80 \quad 6.15473 \mathrm{E}-05$

$6.90 \quad 6.33728 \mathrm{E}-05$

$7.00 \quad 6.52249 \mathrm{E}-05$

$7.10 \quad 6.71037 \mathrm{E}-05$

$7.20 \quad 6.90091 \mathrm{E}-05$

$7.30 \quad 7.09410 \mathrm{E}-05$

$7.40 \quad 7.28996 \mathrm{E}-05$

$7.50 \quad 7.48848 \mathrm{E}-05$

$7.60 \quad 7.68966 \mathrm{E}-05$

$7.70 \quad 7.89351 \mathrm{E}-05$

$7.80 \quad 8.10001 \mathrm{E}-05$

$7.90 \quad 8.30917 \mathrm{E}-05$

$8.00 \quad 8.52100 \mathrm{E}-05$

$8.10 \quad 8.73549 \mathrm{E}-05$

$8.20 \quad 8.95264 \mathrm{E}-05$

$8.30 \quad 9.17244 \mathrm{E}-05$

$8.40 \quad 9.39491 \mathrm{E}-05$

$8.50 \quad 9.62005 \mathrm{E}-05$

$8.60 \quad 9.84784 \mathrm{E}-05$

$8.70 \quad 1.00783 \mathrm{E}-04$

$8.80 \quad 1.03114 \mathrm{E}-04$

$8.90 \quad 1.05472 \mathrm{E}-04$

$9.00 \quad 1.07856 \mathrm{E}-04$

$9.10 \quad 1.10267 \mathrm{E}-04$

$9.20 \quad 1.12705 \mathrm{E}-04$

$9.30 \quad 1.15169 \mathrm{E}-04$

$9.40 \quad 1.17660 \mathrm{E}-04$

$9.50 \quad 1.20177 \mathrm{E}-04$

9.60

9.70

9.80

9.90

10.00

10.10

10.20

10.30

10.40

10.50

10.60

10.70

10.80

10.90

11.00

11.10

11.20

11.30

11.40

11.50

11.60

11.70

11.80

11.90

12.00

$1.30512 \mathrm{E}-$

$1.33163 \mathrm{E}-04$

$1.35840 \mathrm{E}-04$

$1.38543 \mathrm{E}-04$

$1.41274 \mathrm{E}-04$

$1.44030 \mathrm{E}-04$

1.46814 E-04

$1.49624 \mathrm{E}-04$

$1.52460 \mathrm{E}-04$

$1.55324 \mathrm{E}-04$

$1.58213 \mathrm{E}-04$

$1.61130 \mathrm{E}-04$

$1.64073 \mathrm{E}-04$

$1.67042 \mathrm{E}-04$

$1.70039 \mathrm{E}-04$

$1.73061 \mathrm{E}-04$

$1.76111 \mathrm{E}-04$

$1.79187 \mathrm{E}-04$

$1.82289 \mathrm{E}-04$

$1.85418 F_{-}-04$

$1.88574 \mathrm{E}-04$

$1.91757 \mathrm{E}-04$ $f_{2}$

$7.86471 \mathrm{E}-02$

$8.13719 \mathrm{E}-02$

$8.41424 \mathrm{E}-02$

$8.69592 \mathrm{E}-02$

$8.98217 \mathrm{E}-02$

$9.27300 \mathrm{E}-02$

$9.56839 \mathrm{E}-02$

$9.86828 \mathrm{E}-02$

$1.01726 \mathrm{E}-01$

$1.04814 \mathrm{E}-01$

$1.07942 \mathrm{E}-01$

$1.11105 \mathrm{E}-01$

$1.14284 \mathrm{E}-01$

$1.17314 \mathrm{E}-01$

$1.21606 \mathrm{E}-01$

$1.24580 \mathrm{E}-01$

$1.27931 \mathrm{E}-01$

$1.31368 \mathrm{E}-01$

$1.34866 \mathrm{E}-01$

$1.38415 \mathrm{E}-01$

$1.42013 \mathrm{E}-01$

$1.45659 \mathrm{E}-01$

$1.49353 \mathrm{E}-01$

$1.53094 \mathrm{E}-01$

$1.56881 \mathrm{E}-01$

$1.60716 \mathrm{E}-01$

$1.64597 \mathrm{E}-01$

$1.68524 \mathrm{E}-01$

$1.72498 \mathrm{E}-01$

$1.76518 \mathrm{E}-01$

$1.80584 \mathrm{E}-01$

$1.84698 \mathrm{E}-01$

$1.88857 \mathrm{E}-01$

$1.93062 \mathrm{E}-01$

$1.97314 \mathrm{E}-01$

$2.01612 \mathrm{E}-01$

$2.05956 \mathrm{E}-01$

$2.10347 \mathrm{E}-01$

$2.14784 \mathrm{E}-01$

$2.19267 \mathrm{E}-01$
$2.23797 \mathrm{E}-01$

$2.28372 \mathrm{E}-01$

$2.32994 \mathrm{E}-01$

$2.37662 \mathrm{E}-01$

2.42377 E-01

$2.47137 \mathrm{E}-01$

$2.51944 \mathrm{E}-01$

$2.56797 \mathrm{E}-01$

$2.61697 \mathrm{E}-01$

$2.66642 \mathrm{E}-01$

$2.76672 \mathrm{E}-01$

$2.81756 \mathrm{E}-01$

$2.86887 \mathrm{E}-01$

$2.92063 \mathrm{E}-01$

$2.97286 \mathrm{E}-01$

$3.02555 \mathrm{E}-01$

$3.07870 \mathrm{E}-01$

$3.13232 \mathrm{E}-01$

$3.18640 \mathrm{E}-01$
$2.71634 \mathrm{E}-01$ $g_{2}$

$-7.22312 \mathrm{E}-05$

$-6.27026 \mathrm{E}-05$

$-5.23918 \mathrm{E}-05$

$-4.12782 \mathrm{E}-05$

$-2.93412 \mathrm{E}-05$

$-1.65599 \mathrm{E}-05$

$-2.91395 \mathrm{E}-06$

$1.16172 \mathrm{E}-05$

$2.70535 \mathrm{E}-05$

$4.34150 \mathrm{E}-05$

$6.07197 \mathrm{E}-05$

$7.89814 \mathrm{E}-05$

$9.81990 \mathrm{E}-05$

$1.18215 \mathrm{E}-04$

$1.40594 \mathrm{E}-04$

$1.62532 \mathrm{E}-04$

$1.85905 \mathrm{E}-04$

$2.10417 \mathrm{E}-04$

$2.36059 \mathrm{E}-04$

$2.62842 \mathrm{E}-04$

$2.90785 \mathrm{E}-04$

$3.19907 \mathrm{E}-04$

$3.50229 \mathrm{E}-04$

$3.81771 \mathrm{E}-04$

$4.14553 \mathrm{E}-04$

$4.48597 \mathrm{E}-04$

$4.83922 \mathrm{E}-04$

$5.20549 \mathrm{E}-04$

$5.58499 \mathrm{E}-04$

$5.97792 \mathrm{E}-04$

$6.38451 \mathrm{E}-04$

$6.80496 \mathrm{E}-04$

$7.23945 \mathrm{E}-04$

$7.68822 \mathrm{E}-04$

$8.15146 \mathrm{E}-04$

8.62939 E- 04

$9.12221 \mathrm{E}-04$

$9.63013 \mathrm{E}-04$

$1.01533 \mathrm{E}-03$

$1.06921 \mathrm{E}-03$

$1.12466 \mathrm{E}-03$

$1.18169 \mathrm{E}-03$

$1.24035 \mathrm{E}-03$

$1.30063 \mathrm{E}-03$

$1.36258 \mathrm{E}-03$

$1.42620 \mathrm{E}-03$

$1.49151 \mathrm{E}-03$

$1.55854 \mathrm{E}-03$

$1.62732 \mathrm{E}-03$

$1.69785 \mathrm{E}-03$

$1.77016 \mathrm{E}-03$

$1.84427 \mathrm{E}-03$

$1.92020 \mathrm{E}-03$

$1.99798 \mathrm{E}-03$

$2.07761 \mathrm{E}-03$

$2.15914 \mathrm{E}-03$

$2.24256 \mathrm{E}-03$

$2.32791 \mathrm{E}-03$

$2.41521 \mathrm{E}-03$

$2.50447 \mathrm{E}-03$ $\tan \delta_{2}$

$f$.

$-2.63480 \mathrm{E}$ 0l

$-2.63817 \mathrm{E} \quad 01$

$-2.64142 \mathrm{E}$ 0I

$-2.64456 \mathrm{E}$ 0I

$-2.64760 \mathrm{E} 01$

$-2.65055 \mathrm{E} \quad 01$

$-2.65339 \mathrm{E} \quad 01$

$-2.65615 \mathrm{E} \quad 01$

$-2.65882 \mathrm{E} \quad 01$

$-2.66142 \mathrm{E} 01$

$-2.66393 \mathrm{E} 01$

$-2.66637 \mathrm{E} \quad 01$

$-2.66874 \mathrm{E} \quad 01$

$-2.67104 \mathrm{E} \quad 01$

$-2.67327 \mathrm{E} 01$

$-2.67544 \mathrm{E} \quad 01$

$-2.67756 \mathrm{E} 01$

$-2.67961 \mathrm{E} 01$

$-2.68161 \mathrm{E} 01$

-2.68355 E 01

$-2.68545 \mathrm{E} \quad 01$

$-2.68729 \mathrm{E} 01$

$-2.68909 \mathrm{E} \quad 0 \mathrm{I}$

$-2.69084 \mathrm{E} 01$

$-2.69255 \mathrm{E} \quad 01$

$\begin{array}{lll}-2.69421 \mathrm{E} & 01\end{array}$

$-2.69584 \mathrm{E} 01$

$-2.69743 \mathrm{E} \quad 01$

$-2.69897 \mathrm{E} \quad 01$

$-2.70048 \mathrm{E} 01$

$-2.70196 \mathrm{E} 01$

$-2.70340 \mathrm{E} 01$

$-2.70481 \mathrm{E} 01$

$-2.70619 \mathrm{E} \quad 01$

$-2.70754 \mathrm{E} 01$

$-2.70885 \mathrm{E} \quad 01$

$-2.71014 \mathrm{E} \quad 01$

$-2.71140 \mathrm{E} 01$

$-2.71263 \mathrm{E} \quad 01$

$-2.71384 \mathrm{E} \quad 01$

$-2.71502 \mathrm{E} \quad 01$

$-2.71618 \mathrm{E} 01$

$-2.71731 \mathrm{E} \quad 01$

$-2.71842 \mathrm{E} \quad 01$

$-2.71951 \mathrm{E} 01$

$-2.72057 \mathrm{E} \quad 01$

$-2.72162 \mathrm{E} \quad 01$

$-2.72264 \mathrm{E} 01$

$-2.72365 \mathrm{E} \quad 01$

$-2.72463 \mathrm{E} 01$

$-2.72560 \mathrm{E} \quad 01$

$-2.72655 \mathrm{E}$ 0I

$-2.72748 \mathrm{E} \quad 01$

$-2.72839 \mathrm{E} 01$

$-2.72929 \mathrm{E} \quad 01$

$-2.73017 \mathrm{E} \quad 01$

$-2.73103 \mathrm{E} \quad 01$

$-2.73188 \mathrm{E} \quad 01$

$-2.73271 \mathrm{E} 01$

$-2.73353 \mathrm{E}$ 01

$3.62472 \mathrm{E}-04$

$3.59927 \mathrm{E}-04$

$3.56669 \mathrm{E}-04$

$3.52676 \mathrm{E}-04$

$3.47928 \mathrm{E}-04$

$3.42403 \mathrm{E}-04$

$3.36081 \mathrm{E}-04$

$3.28942 \mathrm{E}-04$

$3.20964 \mathrm{E}-04$

$3.12127 \mathrm{E}-04$.

$3.02410 \mathrm{E}-04$

$2.91792 \mathrm{E}-04$

$2.80252 \mathrm{E}-04$

$2.67770 \mathrm{E}-04$

$2.54325 \mathrm{E}-04$

$2.39896 \mathrm{E}-04$

$2.24463 \mathrm{E}-04$

$2.08004 \mathrm{E}-04$ 
Positron

$\begin{array}{cc}p & F_{0} \\ 12.10 & 8.56787 \mathrm{E}-01 \\ 12.20 & 8.56777 \mathrm{E}-01 \\ 12.30 & 8.56766 \mathrm{E}-01 \\ 12.40 & 8.56756 \mathrm{E}-01 \\ 12.50 & 8.56745 \mathrm{E}-01 \\ 12.60 & 8.56734 \mathrm{E}-01 \\ 12.70 & 8.56724 \mathrm{E}-01 \\ 12.80 & 8.56713 \mathrm{E}-01 \\ 12.90 & 8.56703 \mathrm{E}-01 \\ 13.00 & 8.56693 \mathrm{E}-01\end{array}$

13.10

13.20

13.30

13.40

13.50

13.60

13.70

13.80

13.90

14.00

14.10

14.20

14.30

14.40

14.50

14.60

14.70

14.80

14.90

15.00

15.10

15.20

15.30

15.40

15.50

15.60

15.70

15.80

15.90

16.00

16.10

16.20

16.30

16.40

16.50

16.60

16.70

16.80

16.90

17.00

17.10

17.20

17.30

17.40

17.50

17.60

17.70

17.80

17.90

18.00

$8.56682 \mathrm{E}-01$

$8.56671 \mathrm{E}-01$

$8.56661 \mathrm{E}-01$

$8.56650 \mathrm{E}-01$

$8.56639 \mathrm{E}-01$

$8.56628 \mathrm{E}-01$

$8.56618 \mathrm{E}-01$

$8.56607 \mathrm{E}-01$

$8.56596 \mathrm{E}-01$

$8.56586 \mathrm{E}-01$

8.56575 E-01

$8.56564 \mathrm{E}-01$

$8.56554 \mathrm{E}-01$

$8.56543 \mathrm{E}-01$

$8.56532 \mathrm{E}-01$

$8.56521 \mathrm{E}-01$

$8.56511 \mathrm{E}-01$

$8.56500 \mathrm{E}-01$

$8.56489 \mathrm{E}-01$

$8.56479 \mathrm{E}-01$

$8.56468 \mathrm{E}-01$

$8.56457 \mathrm{E}-01$

$8.56447 \mathrm{E}-01$

$8.56436 \mathrm{E}-0 \mathrm{l}$

$8.56426 \mathrm{E}-01$

$8.56415 \mathrm{E}-01$

$8.56404 \mathrm{E}-01$

$8.56394 \mathrm{E}-01$

8.56384 E-01

$8.56373 \mathrm{E}-01$

$8.56363 \mathrm{E}-01$

$8.56353 \mathrm{E}-01$

8.56342E-01

$8.56332 \mathrm{E}-0 \mathrm{l}$

$8.56321 \mathrm{E}-01$

$8.56311 \mathrm{E}-01$

$8.56301 \mathrm{E}-01$

$8.56291 \mathrm{E}-01$

$8.56280 \mathrm{E}-01$

$8.56270 \mathrm{E}-01$

$8.56260 \mathrm{E}-01$

$8.56250 \mathrm{E}-0 \mathrm{l}$

$8.56239 \mathrm{E}-01$

$8.56229 \mathrm{E}-01$

$8.56219 \mathrm{E}-01$

$8.56209 \mathrm{E}-01$

$8.56199 \mathrm{E}-01$

$8.56189 \mathrm{E}-01$

$8.56179 \mathrm{E}-01$

$8.56169 \mathrm{E}-01$

$$
Z=7 \quad A=14 \quad \rho=1.2 A^{1 / 3} F
$$

$$
f_{1}
$$

$\begin{array}{ll}1.07891 \mathrm{E} & 01\end{array}$

$1.08829 \mathrm{E} \quad 01$

$\begin{array}{ll}1.09767 \mathrm{E} & 01\end{array}$

$1.10704 \mathrm{E} \quad 01$

$1.11643 \mathrm{E} \quad 01$

$\begin{array}{ll}1.12581 \mathrm{E} & 01\end{array}$

$1.13519 \mathrm{E} \quad 01$

$\begin{array}{ll}1.14458 \mathrm{E} & 01\end{array}$

$\begin{array}{ll}1.15396 \mathrm{E} & 01\end{array}$

$1.16335 \mathrm{E} 01$
$1.17274 \mathrm{E} \quad 01$

$1.18213 \mathrm{E} \quad 01$

$1.19153 \mathrm{E} \quad 01$

$1.20092 \mathrm{E} \quad 01$

$1.21032 \mathrm{E} \quad 01$

$\begin{array}{ll}1.21971 \mathrm{E} & 01\end{array}$

$1.22911 \mathrm{E} \quad 01$

$\begin{array}{ll}1.23851 \mathrm{E} & 01\end{array}$

$1.24791 \mathrm{E} \quad 01$

$\begin{array}{ll}1.26672 \mathrm{E} & 01\end{array}$

$1.27613 \mathrm{E} \quad 01$

$1.28554 \mathrm{E} \quad 01$

$1.29495 \mathrm{E} \quad 01$

$1.30436 \mathrm{E} \quad 01$

$1.31377 \mathrm{E} \quad 01$

$1.32318 \mathrm{E} \quad 01$

$1.33260 \mathrm{E} \quad 01$

$\begin{array}{ll}1.34202 \mathrm{E} & 01\end{array}$

$1.35144 \mathrm{E} \quad 01$

$1.36086 \mathrm{E} \quad 01$

$1.37028 \mathrm{E} \quad 01$

$1.37970 \mathrm{E} \quad 01$

$1.38913 \mathrm{E} \quad 01$

$\begin{array}{ll}1.39855 \mathrm{E} & 01\end{array}$

$\begin{array}{ll}1.40798 \mathrm{E} & 01\end{array}$

$1.41741 \mathrm{E} \quad 01$

$\begin{array}{ll}1.42685 \mathrm{E} & 01\end{array}$

$1.43628 \mathrm{E} \quad 01$

$1.44571 \mathrm{E} \quad 01$

$1.45515 \mathrm{E} \quad 01$

$\begin{array}{ll}1.46459 \mathrm{E} & 01\end{array}$

$1.47403 \mathrm{E} \quad 01$

$1.48347 \mathrm{E} \quad 01$

$1.49292 \mathrm{E} \quad 01$

$1.50236 \mathrm{E} \quad 01$

$1.51181 \mathrm{E} \quad 01$

$1.52126 \mathrm{E} 01$

$1.53071 \mathrm{E} \quad 01$

$\begin{array}{lll}1.54016 \mathrm{E} & 01\end{array}$

$\begin{array}{ll}1.54962 \mathrm{E} & 01\end{array}$

$\begin{array}{lll}1.55908 \mathrm{E} & 01\end{array}$

$1.56853 \mathrm{E} \quad 01$

$\begin{array}{lll}1.57799 \mathrm{E} & 01\end{array}$

$1.58746 \mathrm{E} \quad 01$

$\begin{array}{ll}1.59692 \mathrm{E} & 01\end{array}$

$1.60639 \mathrm{E} \quad 01$

$\begin{array}{ll}1.61585 \mathrm{E} & 01\end{array}$

$\begin{array}{ll}1.62532 \mathrm{E} & 01\end{array}$

$1.63479 \mathrm{E} 01$ $\begin{array}{ll}1.25732 \mathrm{E} & 01\end{array}$ $g_{1}$

$1.33526 \mathrm{E}-01$

$1.37394 \mathrm{E}-01$

$1.41310 \mathrm{E}-01$

$1.45273 \mathrm{E}-01$

$1.49283 \mathrm{E}-01$

$1.53340 \mathrm{E}-01$

$1.57444 \mathrm{E}-01$

$1.61595 \mathrm{E}-01$

$1.65793 \mathrm{E}-01$

$1.70039 \mathrm{E}-01$

$1.74331 \mathrm{E}-01$

$1.78671 \mathrm{E}-01$

$1.83058 \mathrm{E}-01$

$1.87492 \mathrm{E}-01$

$1.91973 \mathrm{E}-01$

$1.96501 \mathrm{E}-01$

$2.01077 \mathrm{E}-01$

$2.05700 \mathrm{E}-01$

$2.10370 \mathrm{E}-01$

$2.15087 \mathrm{E}-01$

$2.19852 \mathrm{E}-01$ $2.24663 \mathrm{E}-01$

$2.29523 \mathrm{E}-01$

$2.34429 \mathrm{E}-01$

2.39383E-01

2.44384 E-01

$2.49433 \mathrm{E}-01$

$2.54528 \mathrm{E}-01$

$2.59672 \mathrm{E}-01$

2.64862 E-01

$2.70100 \mathrm{E}-01$

$2.75386 \mathrm{E}-01$

$2.80719 \mathrm{E}-01$

$2.86100 \mathrm{E}-01$

$2.91527 \mathrm{E}-01$

$2.97003 \mathrm{E}-01$

$3.02526 \mathrm{E}-01$

$3.08096 \mathrm{E}-01$

$3.13714 \mathrm{E}-01$

$3.19380 \mathrm{E}-01$

$3.25093 \mathrm{E}-01$

$3.30854 \mathrm{E}-01$

$3.36663 \mathrm{E}-01$

$3.42519 \mathrm{E}-01$

$3.48422 \mathrm{E}-01$

$3.54374 \mathrm{E}-01$

$3.60373 \mathrm{E}-01$

$3.66420 \mathrm{E}-01$

$3.72514 \mathrm{E}-01$

$3.78657 \mathrm{E}-01$

$3.84847 \mathrm{E}-01$

$3.91084 \mathrm{E}-01$

$3.97370 \mathrm{E}-01$

$4.03703 \mathrm{E}-01$

$4.10085 \mathrm{E}-01$

$4.16514 \mathrm{E}-01$

$4.22991 \mathrm{E}-01$

$4.29516 \mathrm{E}-01$

$4.36089 \mathrm{E}-01$

$4.42709 \mathrm{E}-01$

$\tan \delta_{1}$

$1.93380 \mathrm{E}-04$

$1.76872 \mathrm{E}-04$

$1.60649 \mathrm{E}-04$

$1.44695 \mathrm{E}-04$

$1.29006 \mathrm{E}-04$

$1.13582 \mathrm{E}-04$

$9.84164 \mathrm{E}-05$

$8.35013 \mathrm{E}-05$

$6.88287 \mathrm{E}-05$

$5.43944 \mathrm{E}-05$

4.01917 E-05

$2.62186 \mathrm{E}-05$

$1.24678 \mathrm{E}-05$

$-1.06706 \mathrm{E}-06$

$-1.43871 \mathrm{E}-05$

$-2.74913 \mathrm{E}-05$

$-4.03964 \mathrm{E}-05$

$-5.30938 \mathrm{E}-05$

$-6.56129 \mathrm{E}-05$

$-7.79233 \mathrm{E}-05$

$-9.00592 \mathrm{E}-05$

$-1.01993 \mathrm{E}-04$

$-1.13759 \mathrm{E}-04$

$-1.25348 \mathrm{E}-04$

$-1.36764 \mathrm{E}-04$

$-1.47999 \mathrm{E}-04$

$-1.59078 \mathrm{E}-04$

$-1.69995 \mathrm{E}-04$

$-1.80745 \mathrm{E}-04$

$-1.91344 \mathrm{E}-04$

$-2.01788 \mathrm{E}-04$

$-2.12082 \mathrm{E}-04$

$-2.22219 \mathrm{E}-04$

$-2.32215 \mathrm{E}-04$

$-2.42074 \mathrm{E}-04$

$-2.51785 \mathrm{E}-04$

$-2.61367 \mathrm{E}-04$

$-2.70806 \mathrm{E}-04$

$-2.80121 \mathrm{E}-04$

$-2.89299 \mathrm{E}-04$

$-2.98351 \mathrm{E}-04$

$-3.07272 \mathrm{E}-04$

$-3.16082 \mathrm{E}-04$

$-3.24759 \mathrm{E}-04$

$-3.33324 \mathrm{E}-04$

$-3.41766 \mathrm{E}-04$

$-3.50099 \mathrm{E}-04$

$-3.58308 \mathrm{E}-04$

$-3.66419 \mathrm{E}-04$

$-3.74413 \mathrm{E}-04$

$-3.82301 \mathrm{E}-04$

$-3.90096 \mathrm{E}-04$

$-3.97765 \mathrm{E}-04$

$-4.05350 \mathrm{E}-04$

$-4.12825 \mathrm{E}-04$

$-4.20199 \mathrm{E}-04$

$-4.27487 \mathrm{E}-04$

$-4.34658 \mathrm{E}-04$

$-4.41747 \mathrm{E}-04$

$-4.48749 \mathrm{E}-04$ 
ELECTRON RADIAL FUNCTIONS

$Z=7 \quad A=14 \quad \rho=1.2 A^{1 / 3} F$

Positron

$\bar{F}$

$f_{2}$

$1.94966 \mathrm{E}-04$

$1.98201 \mathrm{E}-04$

$2.01463 \mathrm{E}-04$

$2.04752 \mathrm{E}-04$

$2.08067 \mathrm{E}-04$

$2.11409 \mathrm{E}-04$

$2.14778 \mathrm{E}-04$

$2.18173 \mathrm{E}-04$

$2.21595 \mathrm{E}-04$

$2.25043 \mathrm{E}-04$

$2.28518 \mathrm{E}-04$

$2.32020 \mathrm{E}-04$

$2.35548 \mathrm{E}-04$

$2.39102 \mathrm{E}-04$

$2.42684 \mathrm{E}-04$

$2.46291 \mathrm{E}-04$

$2.49926 \mathrm{E}-04$

$2.53587 \mathrm{E}-04$

$2.57275 \mathrm{E}-04$

$2.60989 \mathrm{E}-04$

$2.64730 \mathrm{E}-04$

$2.68497 \mathrm{E}-04$

$2.72291 \mathrm{E}-04$

$2.76112 \mathrm{E}-04$

$2.79959 \mathrm{E}-04$

$2.83833 \mathrm{E}-04$

$2.87733 \mathrm{E}-04$

$2.91660 \mathrm{E}-04$

$2.95613 \mathrm{E}-04$

$2.99594 \mathrm{E}-04$

$3.03600 \mathrm{E}-04$

$3.07634 \mathrm{E}-04$

$3.11694 \mathrm{E}-04$

$3.15780 \mathrm{E}-04$

$3.19893 \mathrm{E}-04$

$3.24033 \mathrm{E}-04$

$3.28199 \mathrm{E}-04$

$3.32392 \mathrm{E}-04$

$3.36611 \mathrm{E}-04$

$3.40857 \mathrm{E}-04$

$3.45130 \mathrm{E}-04$

$3.49429 \mathrm{E}-04$

3.53755 E-04

$3.58107 \mathrm{E}-04$

$3.62486 \mathrm{E}-04$

$3.66892 \mathrm{E}-04$

$3.71324 \mathrm{E}-04$

$3.75782 \mathrm{E}-04$

$3.80268 \mathrm{E}-04$

$3.84780 \mathrm{E}-04$

$3.89318 \mathrm{E}-04$

$3.93883 \mathrm{E}-04$

$3.98475 \mathrm{E}-04$

$4.03093 \mathrm{E}-04$

$4.07737 \mathrm{E}-04$

17.60

17.70

17.80

17.90

18.00

$4.12409 \mathrm{E}-04$

$4.17107 \mathrm{E}-04$

4.21831 E-04

$4.26582 \mathrm{E}-04$

$4.31360 \mathrm{E}-04$ $g_{2}$

$3.24094 \mathrm{E}-01$

$3.29594 \mathrm{E}-0$

$3.35140 \mathrm{E}-01$

$3.40733 \mathrm{E}-01$

$3.46371 \mathrm{E}-01$

$3.52056 \mathrm{E}-01$

$3.57787 \mathrm{E}-01$

$3.63564 \mathrm{E}-01$

$3.69388 \mathrm{E}-01$

$3.75257 \mathrm{E}-01$

$3.81173 \mathrm{E}-01$

$3.87135 \mathrm{E}-01$

$3.93143 \mathrm{E}-01$

$3.99198 \mathrm{E}-01$

$4.05298 \mathrm{E}-01$

$4.11445 \mathrm{E}-01$

$4.17638 \mathrm{E}-01$

$4.23877 \mathrm{E}-01$

$4.30162 \mathrm{E}-01$

$4.36493 \mathrm{E}-01$

$4.42870 \mathrm{E}-01$

$4.49294 \mathrm{E}-01$

$4.55764 \mathrm{E}-01$

$4.62280 \mathrm{E}-01$

$4.68842 \mathrm{E}-01$

$4.75450 \mathrm{E}-01$

$4.82105 \mathrm{E}-01$

$4.88805 \mathrm{E}-01$

$4.95552 \mathrm{E}-01$

$5.02345 \mathrm{E}-01$

$5.09184 \mathrm{E}-01$

$5.16069 \mathrm{E}-01$

$5.23000 \mathrm{E}-01$

$5.29977 \mathrm{E}-01$

5.37001 E-01

$5.44070 \mathrm{E}-01$

$5.51186 \mathrm{E}-01$

$5.58348 \mathrm{E}-01$

$5.65556 \mathrm{E}-01$

$5.72810 \mathrm{E}-01$

$5.80110 \mathrm{E}-01$

$5.87457 \mathrm{E}-01$

$5.94849 \mathrm{E}-01$

$6.02288 \mathrm{E}-01$

$6.09772 \mathrm{E}-01$

$6.17303 \mathrm{E}-01$ $6.24880 \mathrm{E}-01$

$6.32503 \mathrm{E}-01$

$6.40171 \mathrm{E}-01$

$6.47886 \mathrm{E}-01$

$6.55648 \mathrm{E}-01$

$6.63455 \mathrm{E}-01$

$6.71308 \mathrm{E}-01$

$6.79208 \mathrm{E}-01$

$6.87153 \mathrm{E}-01$
$6.95145 \mathrm{E}-01$

$7.03182 \mathrm{E}-01$

$7.11266 \mathrm{E}-01$

$7.19396 \mathrm{E}-01$

$7.27571 \mathrm{E}-01$ $\tan \delta_{2}$

$f_{-2}$

$2.59573 \mathrm{E}-03$

$2.68899 \mathrm{E}-03$

$2.78428 \mathrm{E}-03$

$2.88162 \mathrm{E}-03$

$2.98103 \mathrm{E}-03$

$3.08253 \mathrm{E}-03$

$3.18614 \mathrm{E}-03$

$3.29189 \mathrm{E}-03$

$3.39979 \mathrm{E}-03$

$3.50987 \mathrm{E}-03$

$3.62214 \mathrm{E}-03$

$3.73662 \mathbf{E}-03$

$3.85334 \mathrm{E}-03$

$3.97232 \mathbf{E}-03$

$4.09358 \mathrm{E}-03$

$4.21714 \mathrm{E}-03$

$4.34301 \mathrm{E}-03$

$4.47123 \mathrm{E}-03$

4.60181 E-03

$4.73477 \mathrm{E}-03$

$4.87013 \mathrm{E}-03$

$5.00791 \mathrm{E}-03$

$5.14814 \mathrm{E}-03$

$5.29084 \mathrm{E}-03$

$5.43602 \mathrm{E}-03$

$5.58371 \mathrm{E}-03$

$5.73392 \mathrm{E}-03$

$5.88668 \mathrm{E}-03$

$6.04201 \mathrm{E}-03$

$6.19993 \mathrm{E}-03$

$6.36047 \mathrm{E}-03$

$6.52363 \mathrm{E}-03$

$6.68944 \mathrm{E}-03$

$6.85792 \mathrm{E}-03$

$7.02910 \mathrm{E}-03$

$7.20300 \mathrm{E}-03$

$7.37962 \mathrm{E}-03$

$7.55901 \mathrm{E}-03$

$7.74117 \mathrm{E}-03$

$7.92612 \mathrm{E}-03$

8.11389E-03

$8.30450 \mathrm{E}-03$

$8.49797 \mathrm{E}-03$

$8.69432 \mathrm{E}-03$

$8.89357 \mathrm{E}-03$

$9.09573 \mathrm{E}-03$

$9.30085 \mathrm{E}-03$

$9.50892 \mathrm{E}-03$

$9.71998 \mathrm{E}-03$

$9.93404 \mathrm{E}-03$

$1.01511 \mathrm{E}-02$

$1.03713 \mathrm{E}-02$

$1.05944 \mathrm{E}-02$

$1.08207 \mathrm{E}-02$

$1.10501 \mathrm{E}-02$

$1.12826 \mathrm{E}-02$

$1.15183 \mathrm{E}-02$

$1.17572 \mathrm{E}-02$

$1.19992 \mathrm{E}-02$

$1.22444 \mathrm{E}-02$
$-2.73434 \mathrm{E} \quad 01 \quad-1.76453 \mathrm{E}-03$

$-2.73513 \mathrm{E}$ 01 $-1.84667 \mathrm{E}-03$

$-2.73591 \mathrm{E}$ 01 $-1.93077 \mathrm{E}-03$

$-2.73667 \mathrm{E} 01-2.01685 \mathrm{E}-03$

$\begin{array}{lll}-2.73742 \mathrm{E} & 01 & -2.10493 \mathrm{E}-03\end{array}$

$\begin{array}{lll}-2.73816 \mathrm{E} & 0\end{array} \quad-2.19504 \mathrm{E}-03$

$-2.73889 \mathrm{E} \quad 01 \quad-2.28719 \mathrm{E}-03$

$-2.73960 \mathrm{E}$ 01 $-2.38140 \mathrm{E}-03$

$-2.74030 \mathrm{E}$ 01 $-2.47770 \mathrm{E}-03$

$-2.74100 \mathrm{E}$ 01 $-2.57610 \mathrm{E}-03$

$-2.74168 \mathrm{E} \quad 01$

$-2.74234 \mathrm{E}$ 0l

$-2.74300 \mathrm{E} 01$

$-2.74365 \mathrm{E} \quad 01$

$-2.74429 \mathrm{E} \quad 01$

$-2.74492 \mathbf{E} 01$

$-2.74554 \mathrm{E} \quad 01$

$-2.74615 \mathrm{E} \quad 01$

$-2.74675 \mathrm{E} 01$

$-2.74734 \mathrm{E} \quad 01$

$-2.67663 \mathrm{E}-03$

$-2.77930 \mathrm{E}-0.3$

$-2.88415 \mathrm{E}-03$

$-2.99118 \mathrm{E}-03$

$-3.10042 \mathrm{E}-03$

$-2.74792 \mathrm{E} \quad 01$

$-2.74850 \mathrm{E} 01$

$-2.74906 \mathrm{E} 01$

$-2.74962 \mathrm{E} 01$

$-2.75017 \mathrm{E} \quad 01$

$-3.21189 \mathrm{E}-03$

$-3.32561 \mathrm{E}-03$

$-3.44160 \mathrm{E}-03$

$-3.55989 \mathrm{E}-03$

$-3.68048 \mathrm{E}-03$

$-2.75071 \mathrm{E} \quad 01$

$-2.75124 \mathrm{E} \quad 01$

$-2.75177 \mathrm{E} \quad 01$

$-2.75229 \mathrm{E} \quad 01$

$-2.75280 \mathrm{E} \quad 01$

$-3.80342 \mathrm{E}-03$

$-3.92870 \mathrm{E}-03$

$-4.05636 \mathrm{E}-03$

$-4.18642 \mathrm{E}-03$

$-4.31889 \mathrm{E}-03$

$-2.75330 \mathrm{E} \quad 01$

$-2.75380 \mathrm{E} \quad 01$

$-2.75429 \mathrm{E} \quad 01$

$-2.75477 \mathrm{E}$ 01

$-2.75525 \mathrm{E} 01$

$\begin{array}{ll}-2.75572 \mathrm{E} & 01\end{array}$

$-2.75619 \mathrm{E}$ 01

$-2.75664 \mathrm{E} \quad 01$

$-2.75710 \mathrm{E}$ 01

$\begin{array}{ll}-2.75754 \mathrm{E} & 01\end{array}$

$-4.45380 \mathrm{E}-03$

$-4.59117 \mathrm{E}-03$

$-4.73102 \mathrm{E}-03$

$-4.87336 \mathrm{E}-03$

$-5.01823 \mathrm{E}-03$

$-2.75798 \mathrm{E} \quad 01$

$-2.75842 \mathrm{E}$ 0l

$-2.75885 \mathrm{E} \quad 01$

$-2.75927 \mathrm{E} \quad 01$

$-2.75969 \mathrm{E} 01$

$-2.76010 \mathrm{E} \quad 01$

$-2.76051 \mathrm{E} 01$

$-2.76092 \mathrm{E} 01$

$-2.76131 \mathrm{E} \quad 01$

$-2.76171 \mathrm{E} 01$

$\begin{array}{ll}-2.76209 \mathrm{E} & 01\end{array}$

$-2.76248 \mathrm{E} 01$

$-2.76286 \mathrm{E} \quad 01$

$-2.76323 \mathrm{E} 01$

$-2.76360 \mathrm{E} \quad 0 \mathrm{I}$

$-5.16564 \mathrm{E}-03$

$-5.31561 \mathrm{E}-03$

$-5.46816 \mathrm{E}-03$

$-5.62332 \mathrm{E}-03$

$-5.78110 \mathrm{E}-03$

$-5.94152 \mathrm{E}-03$

$-6.10461 \mathrm{E}-03$

$-6.27039 \mathrm{E}-03$

$-6.43888 \mathrm{E}-03$

$-6.61009 \mathrm{E}-03$

$-6.78405 \mathrm{E}-03$

$-6.96078 \mathrm{E}-03$

$-7.14029 \mathrm{E}-03$

$-7.32262 \mathrm{E}-03$

$-7.50778 \mathrm{E}-03$

$-2.76397 \mathrm{E} 01$

$-2.76469 \mathrm{E} \quad 01$

$-2.76504 \mathrm{E} 01$

$-2.76539 \mathrm{E} \quad 01$
$-2.76433 \mathrm{E} 01$ 
ELECTRON RADIAL FUNCTIONS

$p$

$F_{0}$

18.10

18.20

18.30

18.40

18.50

18.60

18.70

18.80

18.90

19.00

19.10

19.20

19.30

19.40

19.50

19.60

19.70

19.80

19.90

20.00

20.10

20.20

20.30

20.40

20.50

20.60

20.70

20.80

20.90

21.00

21.10

21.20

21.30

21.40

21.50

21.60

21.70

21.80

21.90

22.00

22.10

22.20

22.30

22.40

22.50

22.60

22.70

22.80

22.90

23.00

23.10

23.20

23.30

23.40

23.50

23.60

23.70

23.80

23.90

24.00

\section{$f_{1}$}

$8.56159 \mathrm{E}-01$ $8.56149 \mathrm{E}-01$ $8.56139 \mathrm{E}-01$ $8.56130 \mathrm{E}-01$ $8.56120 \mathrm{E}-01$

$8.56110 \mathrm{E}-01$ $8.56101 \mathrm{E}-01$ 8.56091 E-01 $8.56081 \mathrm{E}-01$ $8.56072 \mathrm{E}-01$

$8.56062 \mathrm{E}-01$ $8.56052 \mathrm{E}-01$ $8.56043 \mathrm{E}-01$ $8.56033 \mathrm{E}-01$ $8.56024 \mathrm{E}-01$

$8.56014 \mathrm{E}-01$ $8.56005 \mathrm{E}-01$ $8.55995 \mathrm{E}-01$ $8.55986 \mathrm{E}-01$ $8.55976 \mathrm{E}-01$

$8.55967 \mathrm{E}-01$ $8.55958 \mathrm{E}-01$ $8.55948 \mathrm{E}-01$ $8.55939 \mathrm{E}-01$ $8.55930 \mathrm{E}-01$

$8.55921 \mathrm{E}-01$ $8.55911 \mathrm{E}-01$ $8.55902 \mathrm{E}-01$ $8.55893 \mathrm{E}-01$ 8.55884E-01

$8.55875 \mathrm{E}-01$ $8.55866 \mathrm{E}-01$ $8.55857 \mathrm{E}-01$ $8.55848 \mathrm{E}-01$ $8.55839 \mathrm{E}-01$

$8.55830 \mathrm{E}-01$ $8.55821 \mathrm{E}-01$ $8.55812 \mathrm{E}-01$ $8.55803 \mathrm{E}-01$ $8.55795 \mathrm{E}-01$

$8.55786 \mathrm{E}-01$ $8.55777 \mathrm{E}-01$ 8.55768E-01 $8.55760 \mathrm{E}-01$ $8.55751 \mathrm{E}-01$

$8.55743 \mathrm{E}-01$ $8.55734 \mathrm{E}-01$ $8.55725 \mathrm{E}-01$ $8.55717 \mathrm{E}-01$ $8.55708 \mathrm{E}-01$

$8.55700 \mathrm{E}-01$ $8.55691 \mathrm{E}-01$ $8.55683 \mathrm{E}-01$ $8.55674 \mathrm{E}-01$ $8.55666 \mathrm{E}-01$

$8.55658 \mathrm{E}-01$ $8.55649 \mathrm{E}-01$ 8.55641 E-01 $8.55633 \mathrm{E}-01$ $8.55624 \mathrm{E}-01$ $g_{1}$

$1.64427 \mathrm{E} \quad 01$

$1.65374 \mathrm{E} \quad 01$

$1.66322 \mathrm{E} \quad 01$

$1.67270 \mathrm{E} \quad 01$

$1.68218 \mathrm{E} 01$

$1.69166 \mathrm{E} \quad 01$

$1.70115 \mathrm{E} \quad 01$

$1.71063 \mathrm{E} \quad 01$

$1.72012 \mathrm{E} \quad 01$

$1.72961 \mathrm{E} \quad 01$

$1.73911 \mathrm{E} \quad 01$

$1.74860 \mathrm{E} \quad 01$

$1.75810 \mathrm{E} \quad 01$

$1.76760 \mathrm{E} \quad 01$

$1.77710 \mathrm{E} \quad 01$

$1.78660 \mathrm{E} \quad 01$ $1.79610 \mathrm{E} \quad 01$ $1.80561 \mathrm{E} \quad 01$

$1.81512 \mathrm{E} \quad 01$

$1.82463 \mathrm{E} \quad 01$

$1.83414 \mathrm{E} \quad 01$

$1.84366 \mathrm{E} \quad 01$

$1.85318 \mathrm{E} 01$

$1.86270 \mathrm{E} \quad 01$

$1.87222 \mathrm{E} \quad 01$

$1.88174 \mathrm{E} \quad 01$

$1.89127 \mathrm{E} \quad 01$

$1.90079 \mathrm{E} \quad 01$

$1.91033 \mathrm{E} 01$

$1.91986 \mathrm{E} \quad 01$

$1.92939 \mathrm{E} \quad 01$ $1.93893 \mathrm{E} \quad 01$ $1.94847 \mathrm{E} \quad 01$

$1.95801 \mathrm{E} \quad 01$

$1.96755 \mathrm{E} \quad 01$

$1.97710 \mathrm{E} \quad 01$

$1.98664 \mathrm{E} \quad 01$

$1.99619 \mathrm{E} \quad 01$

$2.00575 \mathrm{E} \quad 01$

$2.01530 \mathrm{E} 01$

$2.02486 \mathrm{E} \quad 01$

$2.03442 \mathrm{E} \quad 01$

$2.04398 \mathrm{E} \quad 01$

$2.05354 \mathrm{E} \quad 01$

$2.06311 \mathrm{E} \quad 01$

$2.07267 \mathrm{E} \quad 01$ $2.08224 \mathrm{E} \quad 01$ $2.09182 \mathrm{E} \quad 01$ $2.10139 \mathrm{E} \quad 01$

$2.11097 \mathrm{E} \quad 01$

$2.12055 \mathrm{E} \quad 01$

$2.13013 \mathrm{E} \quad 01$

$2.13971 \mathrm{E} 01$

$2.14930 \mathrm{E} \quad 01$

$2.15889 \mathrm{E} \quad 01$

2.16848 E 01 $2.17808 \mathrm{E} \quad 01$

$2.18767 \mathrm{E} \quad 01$

$2.19727 \mathrm{E} \quad 01$

$2.20687 \mathrm{E} \quad 01$
$4.49378 \mathrm{E}-01$

$4.56095 \mathrm{E}-01$

$4.62860 \mathrm{E}-01$

$4.69672 \mathrm{E}-01$

$4.76533 \mathrm{E}-01$

4.83442 E-01 $4.90399 \mathrm{E}-01$ $4.97403 \mathrm{E}-01$

$5.04456 \mathrm{E}-01$

$5.11558 \mathrm{E}-01$

$5.18707 \mathrm{E}-01$

$5.25904 \mathrm{E}-01$

$5.33150 \mathrm{E}-01$

5.40444 E-01

$5.47786 \mathrm{E}-0 \mathrm{l}$

$5.55176 \mathrm{~F}-01$

$5.62614 \mathrm{E}-01$

$5.70101 \mathrm{E}-01$

$5.77636 \mathrm{E}-01$

$5.85220 \mathrm{E}-01$

$5.92852 \mathrm{E}-01$ $6.00532 \mathrm{E}-01$

$6.08261 \mathrm{E}-01$

$6.16038 \mathrm{E}-01$

$6.23863 \mathrm{E}-01$

$6.31737 \mathrm{E}-01$ $6.39660 \mathrm{E}-01$

$6.47631 \mathrm{E}-01$

$6.55650 \mathrm{E}-01$

$6.63718 \mathrm{E}-01$

$6.71835 \mathrm{E}-01$ $6.80000 \mathrm{E}-01$ $6.88214 \mathrm{E}-01$

$6.96476 \mathrm{E}-01$

$7.04787 \mathrm{E}-01$

$7.13147 \mathrm{E}-01$

$7.21556 \mathrm{E}-01$

$7.30013 \mathrm{E}-01$

$7.38519 \mathrm{E}-01$

$7.47074 \mathrm{E}-01$

$7.55678 \mathrm{E}-01$

$7.64330 \mathrm{E}-01$

$7.73032 \mathrm{E}-01$

$7.81782 \mathrm{E}-01$

$7.90581 \mathrm{E}-01$

7.99429E-01 $8.08326 \mathrm{E}-01$ $8.17272 \mathrm{E}-01$ $8.26267 \mathrm{E}-01$ $8.35311 \mathrm{E}-01$

$8.44404 \mathrm{E}-01$ $8.53546 \mathrm{E}-01$ $8.62737 \mathrm{E}-01$ $8.71978 \mathrm{E}-01$ $8.81268 \mathrm{E}-01$

$8.90606 \mathrm{E}-01$ $8.99994 \mathrm{E}-01$ $9.09431 \mathrm{E}-01$ $9.18917 \mathrm{E}-01$ $9.28453 \mathrm{E}-01$

\section{$\tan \delta_{1}$ \\ $-4.55656 \mathrm{E}-04$ $-4.62464 \mathrm{E}-04$ $-4.69203 \mathrm{E}-04$ $-4.75837 \mathrm{E}-04$ \\ $-4.82387 \mathrm{E}-04$ \\ $-4.88856 \mathrm{E}-04$ \\ $-4.95239 \mathrm{E}-04$ \\ $-5.01551 \mathrm{E}-04$ \\ $-5.07769 \mathrm{E}-04$ \\ $-5.13912 \mathrm{E}-04$ \\ $-5.19978 \mathrm{E}-04$ \\ $-5.25962 \mathrm{E}-04$ \\ $-5.31878 \mathrm{E}-04$ \\ $-5.37712 \mathrm{E}-04$ \\ $-5.43472 \mathrm{E}-04$}

$-5.49159 \mathrm{E}-04$

$-5.54775 \mathrm{E}-04$

$-5.60326 \mathrm{E}-04$

$-5.65802 \mathrm{E}-04$

$-5.71200 \mathrm{E}-04$

$-5.76544 \mathrm{E}-04$

$-5.81814 \mathrm{E}-04$

$-5.87017 \mathrm{E}-04$

$-5.92159 \mathrm{E}-04$

$-5.97228 \mathrm{E}-04$

$-6.02243 \mathrm{E}-04$

$-6.07195 \mathrm{E}-04$

$-6.12084 \mathrm{E}-04$

$-6.16906 \mathrm{E}-04$

$-6.21670 \mathrm{E}-04$

$-6.26381 \mathrm{E}-04$

$-6.31025 \mathrm{E}-04$

$-6.35616 \mathrm{E}-04$

$-6.40147 \mathrm{E}-04$

$-6.44620 \mathrm{E}-04$

$-6.49046 \mathrm{E}-04$

$-6.53409 \mathrm{E}-04$

$-6.57721 \mathrm{E}-04$

$-6.61971 \mathrm{E}-04$ 
ELECTRON RADIAL FUNCTIONS

$Z=7 \quad A=14 \quad \rho=1.2 A^{1 / 3} F$

Positron

$\bar{F}$

$4.36165 \mathrm{E}-04$

$4.40996 \mathrm{E}-04$

$4.45853 \mathrm{E}-04$

$4.50737 \mathrm{E}-04$

$4.55648 \mathrm{E}-04$

$4.60585 \mathrm{E}-04$

4. $65549 \mathrm{E}-04$

$4.70539 \mathrm{E}-04$

$4.75556 \mathrm{E}-04$

$4.80599 \mathrm{E}-04$

$4.85669 \mathrm{E}-04$

$4.90766 \mathrm{E}-04$

$4.95889 \mathrm{E}-04$

$5.01039 \mathrm{E}-04$

$5.06215 \mathrm{E}-04$

$5.11418 \mathrm{E}-04$

$5.16648 \mathrm{E}-04$

$5.21904 \mathrm{E}-04$

$5.27187 \mathrm{E}-04$

$5.32496 \mathrm{E}-04$

$5.37832 \mathrm{E}-04$

$5.43194 \mathrm{E}-04$

$5.48583 \mathrm{E}-04$

$5.53999 \mathrm{E}-04$

$5.59441 \mathrm{E}-04$

$5.64910 \mathrm{E}-04$

$5.70405 \mathrm{E}-04$

$5.75927 \mathrm{E}-04$

$5.81475 \mathrm{E}-04$

5.87051 E-04

$5.92652 \mathrm{E}-04$

$5.98280 \mathrm{E}-04$

$6.03935 \mathrm{E}-04$

$6.09616 \mathrm{E}-04$

$6.15324 \mathrm{E}-04$

$6.21059 \mathrm{E}-04$

$6.26820 \mathrm{E}-04$

$6.32608 \mathrm{E}-04$

$6.38422 \mathrm{E}-04$

$6.44263 \mathrm{E}-04$

$6.50130 \mathrm{E}-04$

$6.56024 \mathrm{E}-04$

$6.61945 \mathrm{E}-04$

$6.67892 \mathrm{E}-04$

$6.73866 \mathrm{E}-04$

$6.79866 \mathrm{E}-04$

$6.85893 \mathrm{E}-04$

$6.91946 \mathrm{E}-04$

$6.98026 \mathrm{E}-04$

$7.04133 \mathrm{E}-04$

$7.10266 \mathrm{E}-04$

$7.16426 \mathrm{E}-04$

$7.22612 \mathrm{E}-04$

$7.28825 \mathrm{E}-04$

$7.35064 \mathrm{E}-04$

$7.41330 \mathrm{E}-04$

$7.47623 \mathrm{E}-04$

$7.53942 \mathrm{E}-04$

$7.60287 \mathrm{E}-04$

$7.66660 \mathrm{E}-04$ $f_{2}$

$g_{2}$

$\tan \delta_{2}$

$f_{-2}$

$7.35793 \mathrm{E}-01$

$7.44062 \mathrm{E}-01$

$7.52376 \mathrm{E}-01$

$7.60736 \mathrm{E}-01$

$7.69142 \mathrm{E}-01$

$7.77593 \mathrm{E}-01$

$7.86092 \mathrm{E}-01$

$7.94636 \mathrm{E}-01$

$8.03226 \mathrm{E}-01$

$8.11862 \mathrm{E}-01$

8.20544E-01

$8.29272 \mathrm{E}-01$

$8.38046 \mathrm{E}-01$

$8.46867 \mathrm{E}-01$

$8.55733 \mathrm{E}-01$

$8.64645 \mathrm{E}-01$

$8.73603 \mathrm{E}-01$

$8.82607 \mathrm{E}-01$

$8.91658 \mathrm{E}-01$

$9.00753 \mathrm{E}-01$

$9.09896 \mathrm{E}-01$

9.19084 E-01

$9.28318 \mathrm{E}-01$

$9.37598 \mathrm{E}-01$

$9.46924 \mathrm{E}-01$

$9.56296 \mathrm{E}-01$

$9.65714 \mathrm{E}-01$

$9.75178 \mathrm{E}-01$

$9.84688 \mathrm{E}-01$

$9.94243 \mathrm{E}-01$

$1.00384 \mathrm{E} \quad 00$

$1.01349 \mathrm{E} \quad 00$

$1.02319 \mathrm{E} \quad 00$

$1.03293 \mathrm{E} \quad 00$

$1.04271 \mathrm{E} \quad 00$

$1.05254 \mathrm{E} \quad 00$

$1.06242 \mathrm{E} \quad 00$

$1.07234 \mathrm{E} \quad 00$

$1.08231 \mathrm{E} \quad 00$

$1.09233 \mathrm{E} \quad 00$

$1.10239 \mathrm{E} \quad 00$

$1.11249 \mathrm{E} \quad 00$

$1.12265 \mathrm{E} \quad 00$

$1.13285 \mathrm{E} \quad 00$

$1.14309 \mathrm{E} \quad 00$

$1.15338 \mathrm{E} \quad 00$

$1.16372 \mathrm{E} \quad 00$

$1.17410 \mathrm{E} \quad 00$

$1.18453 \mathrm{E} \quad 00$

$1.19500 \mathrm{E} 00$

$1.20552 \mathrm{E} \quad 00$

$1.21609 \mathrm{E} \quad 00$

$1.22670 \mathrm{E} 00$

$1.23735 \mathrm{E} \quad 00$

$1.24806 \mathrm{E} \quad 00$

$1.25881 \mathrm{E} \quad 00$

$1.26960 \mathrm{E} \quad 00$

$1.28044 \mathrm{E} \quad 00$

$1.29133 \mathrm{E} \quad 00$

$1.30226 \mathrm{E} \quad 00$
$1.24929 \mathrm{E}-02$

$1.27446 \mathrm{E}-02$

$1.29996 \mathrm{E}-02$

$1.32579 \mathrm{E}-02$

$1.35195 \mathrm{E}-02$

$1.37845 \mathrm{E}-02$

$1.40528 \mathrm{E}-02$

$1.43244 \mathrm{E}-02$

$1.45995 \mathrm{E}-02$

$1.48780 \mathrm{E}-02$

$1.51599 \mathrm{E}-02$

$1.54453 \mathrm{E}-02$

$1.57342 \mathrm{E}-02$

$1.60265 \mathrm{E}-02$

$1.63224 \mathrm{E}-02$

$1.66218 \mathrm{E}-02$

$1.69248 \mathrm{E}-02$

$1.72314 \mathrm{E}-02$

$1.75416 \mathrm{E}-02$

$1.78554 \mathrm{E}-02$

$1.81728 \mathrm{E}-02$

$1.84939 \mathrm{E}-02$

$1.88187 \mathrm{E}-02$

$1.91473 \mathrm{E}-02$

$1.94795 \mathrm{E}-02$

$1.98155 \mathrm{E}-02$

$2.01552 \mathrm{E}-02$

$2.04988 \mathrm{E}-02$

$2.08461 \mathrm{E}-02$

$2.11973 \mathrm{E}-02$

$2.15523 \mathrm{E}-02$

$2.19112 \mathrm{E}-02$

$2.22740 \mathrm{E}-02$

$2.26408 \mathrm{E}-02$

$2.30114 \mathrm{E}-02$

$2.33860 \mathrm{E}-02$

$2.37646 \mathrm{E}-02$

$2.41472 \mathrm{E}-02$

$2.45338 \mathrm{E}-02$

2.49244 E- 02

$2.53191 \mathrm{E}-02$

$2.57179 \mathrm{E}-02$

$2.61207 \mathrm{E}-02$

$2.65277 \mathrm{E}-02$

$2.69389 \mathrm{E}-02$

$2.73541 \mathrm{E}-02$

$2.77736 \mathrm{E}-02$

$2.81973 \mathrm{E}-02$

$2.86252 \mathrm{E}-02$

$2.90573 \mathrm{E}-02$

$2.94937 \mathrm{E}-02$

$2.99344 \mathrm{E}-02$

$3.03794 \mathrm{E}-02$

$3.08287 \mathrm{E}-02$

$3.12824 \mathrm{E}-02$

$3.17405 \mathrm{E}-02$

$3.22029 \mathrm{E}-02$

$3.26697 \mathrm{E}-02$

$3.31410 \mathrm{E}-02$

$3.36167 \mathrm{E}-02$

\section{$-2.76573 \mathrm{E}$ 01 $-1.08723 \mathrm{E}-02$ $-2.76607 \mathrm{E}$ 01 $-1.11088 \mathrm{E}-02$ \\ $-2.76641 \mathrm{E}$ 01 $-1.13485 \mathrm{E}-02$ \\ $-2.76674 \mathrm{E}$ 01 $-1.15914 \mathrm{E}-02$}

$-2.76707 \mathrm{E}$ ol $-1.18375 \mathrm{E}-02$

$-2.76740 \mathrm{E} \quad 01$

$-2.76772 \mathrm{E} \quad 01$

$-2.76804 \mathrm{E} \quad 0 \mathrm{l}$

$-2.76835 \mathrm{E} \quad 01$

$-2.76867 \mathrm{E} \quad 01$

$-2.76897 \mathrm{E} \quad 01$

$-2.76928 \mathrm{E} 01$

$-2.76958 \mathrm{E} \quad 01$

$-2.76988 \mathrm{E}$ ol

$-2.77017 \mathrm{E} \quad 01$

$-2.77047 \mathrm{E} \quad 01$

$-2.77075 \mathrm{E} \quad 01$

$-2.77104 \mathrm{E} \quad 01$

$-2.77132 \mathrm{E} \quad 01$

$-2.77160 \mathrm{E} \quad 01$

$-2.77188 \mathrm{E} \quad 01$

$-2.77215 \mathrm{E} \quad 01$

$-2.77243 \mathrm{E}$ 01

$-2.77269 \mathrm{E} \quad 01$

$-2.77296 \mathrm{E} \quad 01$

$-2.77322 \mathrm{E} \quad 01$

$-2.77348 \mathrm{E}$ 0l

$-2.77374 \mathrm{E}$ 01

$-2.77400 \mathrm{E}$ 0I

$-2.77425 \mathrm{E} 01$

$-2.77450 \mathrm{E} \quad 01$

$-2.77475 \mathrm{E} 01$

$-2.77499 \mathrm{E}$ 0I

$-2.77524 \mathrm{E}$ ol

$-2.77548 \mathrm{E} \quad 01$

$-2.77572 \mathrm{E} \quad 01$

$-2.77595 \mathrm{E}$ 0l

$-2.77619 \mathrm{E} \quad 01$

$-2.77642 \mathrm{E} \quad 01$

$-2.77665 \mathrm{E} \quad 01$

$-2.77688 \mathrm{E} \quad 01$

$-2.77710 \mathrm{E}$ 01

$-2.77732 \mathrm{E} 01$

$-2.77755 \mathrm{E}$ 0l

$-2.77776 \mathrm{E}$ ol

$-2.77798 \mathrm{E} \quad 01$

$-2.77820 \mathrm{E} \quad 0 \mathrm{I}$

$-2.77841 \mathrm{E} \quad 01$

$-2.77862 \mathrm{E} 01$

$-2.77883 \mathrm{E}$ 0l

$-2.77904 \mathrm{E} \quad 01$

$-2.77924 \mathrm{E} 01$

$-2.77945 \mathrm{E}$ ol

$-2.77965 \mathrm{E} 01$

$-2.77985 \mathrm{E} \quad 01$

$-2.78005 \mathrm{E} \quad 01$

$-2.78024 \mathrm{E} \quad 01$

$-1.20869 \mathrm{E}-02$

$-1.23396 \mathrm{E}-02$

$-1.25956 \mathrm{E}-02$

$-1.28549 \mathrm{E}-02$

$-1.31176 \mathrm{E}-02$

$-1.33836 \mathrm{E}-02$

$-1.36531 \mathrm{E}-02$

$-1.39259 \mathrm{E}-02$

$-1.42022 \mathrm{E}-02$

$-1.44819 \mathrm{E}-02$

$-1.47651 \mathrm{E}-02$

$-1.50518 \mathrm{E}-02$

$-1.53420 \mathrm{E}-02$

$-1.56358 \mathrm{E}-02$

$-1.59331 \mathrm{E}-02$

$-1.62340 \mathrm{E}-02$

$-1.65384 \mathrm{E}-02$

$-1.68465 \mathrm{E}-02$

$-1.71583 \mathrm{E}-02$

$-1.74736 \mathrm{E}-02$

$-1.77927 \mathrm{E}-02$

$-1.81155 \mathrm{E}-02$

$-1.84420 \mathrm{E}-02$

$-1.87722 \mathrm{E}-02$

$-1.91062 \mathrm{E}-02$

$-1.94440 \mathrm{E}-02$

$-1.97856 \mathrm{E}-02$

$-2.01310 \mathrm{E}-02$

$-2.04802 \mathrm{E}-02$

$-2.08333 \mathrm{E}-02$

$-2.11903 \mathrm{E}-02$

$-2.15513 \mathrm{E}-02$

$-2.19161 \mathrm{E}-02$

$-2.22849 \mathrm{E}-02$

$-2.26576 \mathrm{E}-02$

$-2.30344 \mathrm{E}-02$

$-2.34151 \mathrm{E}-02$

$-2.37999 \mathrm{E}-02$

$-2.41888 \mathrm{E}-02$

$-2.45817 \mathrm{E}-02$

$-2.49787 \mathrm{E}-02$

$-2.53798 \mathrm{E}-02$

$-2.57850 \mathrm{E}-02$

$-2.61944 \mathrm{E}-02$

$-2.66080 \mathrm{E}-02$

$-2.70258 \mathrm{E}-02$ 
ELECTRON RADIAL FUNCTIONS

Positron

\begin{tabular}{|c|c|c|c|}
\hline$\rho$ & $F_{0}$ & $f_{1}$ & \\
\hline 24.10 & $8.55616 \mathrm{E}-01$ & $2.21648 \mathrm{E}$ & 01 \\
\hline 24.20 & $8.55608 \bar{E}-01$ & $2.22608 \mathrm{E}$ & 01 \\
\hline 24.30 & $8.55600 \mathrm{E}-01$ & $2.23569 \mathrm{E}$ & 01 \\
\hline 24.40 & $8.55592 \mathrm{E}-01$ & $2.24530 \mathrm{E}$ & 01 \\
\hline 24.50 & $8.55583 \mathrm{E}-01$ & $2.25492 \mathrm{E}$ & 01 \\
\hline 24.60 & $8.55575 \mathrm{E}-01$ & $2.26453 \mathrm{E}$ & 01 \\
\hline 24.70 & $8.55567 \mathrm{E}-01$ & $2.27415 \mathrm{E}$ & 01 \\
\hline 24.80 & $8.55559 \mathrm{E}-01$ & $2.28377 \mathrm{E}$ & 01 \\
\hline 24.90 & $8.55551 \mathrm{E}-01$ & $2.29340 \mathrm{E}$ & 01 \\
\hline 25.00 & $8.55543 \mathrm{E}-01$ & $2.30302 \mathrm{E}$ & 01 \\
\hline 25.10 & $8.55535 \mathrm{E}-01$ & $2.31265 \mathrm{E}$ & 01 \\
\hline 25.20 & $8.55527 \mathrm{E}-01$ & $2.32228 \mathrm{E}$ & 01 \\
\hline 25.30 & $8.55519 \mathrm{E}-01$ & $2.33191 \mathrm{E}$ & 01 \\
\hline 25.40 & $8.55511 \mathrm{E}-01$ & $2.34155 \mathrm{E}$ & 01 \\
\hline 25.50 & $8.55503 \mathrm{E}-01$ & $2.35119 \mathrm{E}$ & 01 \\
\hline 25.60 & $8.55495 \mathrm{E}-01$ & $2.36083 \mathrm{E}$ & 01 \\
\hline 25.70 & $8.55487 \mathrm{E}-01$ & $2.37048 \mathrm{E}$ & 01 \\
\hline 25.80 & $8.55480 \mathrm{E}-01$ & $2.38012 \mathrm{E}$ & 01 \\
\hline 25.90 & $8.55472 \mathrm{E}-01$ & $2.38977 \mathrm{E}$ & 01 \\
\hline 26.00 & $8.55464 \mathrm{E}-01$ & $2.39942 \mathrm{E}$ & 01 \\
\hline 26.10 & $8.55456 \mathrm{E}-01$ & $2.40908 \mathrm{E}$ & 01 \\
\hline 26.20 & $8.55448 \mathrm{E}-01$ & $2.41874 \mathrm{E}$ & 01 \\
\hline 26.30 & $8.55441 \mathrm{E}-01$ & $2.42840 \mathrm{E}$ & 01 \\
\hline 26.40 & $8.55433 \mathrm{E}-01$ & $2.43806 \mathrm{E}$ & 01 \\
\hline 26.50 & $8.55426 \overline{\mathrm{E}}-01$ & $2.44772 \mathrm{E}$ & 01 \\
\hline 26.60 & $8.55418 \mathrm{E}-01$ & $2.45739 \mathrm{E}$ & 01 \\
\hline 26.70 & $8.55410 \mathrm{E}-01$ & $2.46706 \mathrm{E}$ & 01 \\
\hline 26.80 & $8.55403 \mathrm{E}-01$ & $2.47673 \mathrm{E}$ & 01 \\
\hline 26.90 & $8.55395 \bar{E}-01$ & $2.48641 \mathrm{E}$ & 01 \\
\hline 27.00 & $8.55388 \mathrm{E}-01$ & $2.49609 \mathrm{E}$ & 01 \\
\hline 27.10 & $8.55380 \mathrm{E}-01$ & $2.50577 \mathrm{E}$ & 01 \\
\hline 27.20 & $8.55372 \mathrm{E}-01$ & $2.51545 \mathrm{E}$ & 01 \\
\hline 27.30 & $8.55365 \mathrm{E}-01$ & $2.52514 \mathrm{E}$ & 01 \\
\hline 27.40 & $8.55358 \mathrm{E}-01$ & $2.53483 \mathrm{E}$ & 01 \\
\hline 27.50 & $8.55350 \mathrm{E}-01$ & $2.54452 \mathrm{E}$ & 01 \\
\hline 27.60 & $8.55343 E-01$ & $2.55422 \mathrm{E}$ & 01 \\
\hline 27.70 & $8.55335 \overline{\mathrm{E}}-01$ & $2.56392 \mathrm{E}$ & 01 \\
\hline 27.80 & $8.55328 E-01$ & $2.57362 \mathrm{E}$ & 01 \\
\hline 27.90 & $8.55321 \mathrm{E}-01$ & $2.58332 \mathrm{E}$ & 01 \\
\hline 28.00 & $8.55313 \mathrm{E}-01$ & $2.59303 \mathrm{E}$ & 01 \\
\hline 28.10 & $8.55306 \mathrm{E}-01$ & $2.60274 \mathrm{E}$ & 01 \\
\hline 28.20 & $8.55299 \mathrm{E}-01$ & $2.61245 \mathrm{E}$ & 01 \\
\hline 28.30 & $8.55291 \mathrm{E}-01$ & $2.62216 \mathrm{E}$ & 01 \\
\hline 28.40 & $8.55284 \mathrm{E}-01$ & $2.63188 \mathrm{E}$ & 01 \\
\hline 28.50 & $8.55277 \mathrm{E}-01$ & $2.64160 \mathrm{E}$ & 01 \\
\hline 28.60 & $8.55270 \mathrm{E}-01$ & $2.65133 \mathrm{E}$ & 01 \\
\hline 28.70 & $8.55263 \mathrm{E}-01$ & $2.66105 \mathrm{E}$ & 01 \\
\hline 28.80 & $8.55255 \mathrm{E}-01$ & $2.67078 \mathrm{E}$ & 01 \\
\hline 28.90 & $8.55248 \mathrm{E}-01$ & $2.68051 \mathrm{E}$ & 01 \\
\hline 29.00 & $8.55241 \mathrm{E}-01$ & $2.69025 \mathrm{E}$ & 01 \\
\hline 29.10 & 8.55234.E-01 & $2.69999 \mathrm{E}$ & 01 \\
\hline 29.20 & $8.55227 \mathrm{E}-01$ & $2.70973 \mathrm{E}$ & 01 \\
\hline 29.30 & $8.55220 \mathrm{E}-01$ & $2.71947 \mathrm{E}$ & 01 \\
\hline 29.40 & $8.55213 \mathrm{E}-01$ & $2.72922 \mathrm{E}$ & 01 \\
\hline 29.50 & $8.55206 \mathrm{E}-01$ & $2.73897 \mathrm{E}$ & 01 \\
\hline 29.60 & $8.55199 \mathrm{E}-01$ & $2.74872 \mathrm{E}$ & 01 \\
\hline 29.70 & $8.55192 \mathrm{E}-01$ & $2.75848 \mathrm{E}$ & 01 \\
\hline 29.80 & $8.55185 \mathrm{E}-01$ & $2.76823 \mathrm{E}$ & 01 \\
\hline 29.90 & $8.55178 \mathbf{E}-01$ & $2.77800 \mathrm{E}$ & 01 \\
\hline 30.00 & $8.55171 \mathrm{E}-01$ & $2.78776 \mathrm{E}$ & 01 \\
\hline
\end{tabular}

$Z=7 \quad A=14 \quad \rho=1.2 A^{1 / 3} F$

$\begin{array}{ccr}g_{1} & \tan \delta_{1} & f_{-1} \\ 9.38039 \mathrm{E}-01 & -7.43241 \mathrm{E}-04 & -8.63376\end{array}$

$9.47673 \mathrm{E}-01 \quad-7.46425 \mathrm{E}-04$

$9.57357 \mathrm{E}-01 \quad-7.49571 \mathrm{E}-04$

$9.67090 \mathrm{E}-01-7.52668 \mathrm{E}-04$

$9.76872 \mathrm{E}-01 \quad-7.55725 \mathrm{E}-04$

$-8.63376 \mathrm{E}-01$

$-8.72784 \mathrm{E}-01$

$-8.82240 \mathrm{E}-01$

$-8.91747 \mathrm{E}-01$

$-9.01302 \mathrm{E}-01$

$9.86704 \mathrm{E}-01$

$-7.58745 \mathrm{E}-04$

$9.96585 \mathrm{E}-01 \quad-7.61736 \mathrm{E}-04$

$\begin{array}{lll}1.00652 \mathrm{E} & 00 & -7.64683 \mathrm{E}-04\end{array}$

$1.01650 \mathrm{E} \quad 00 \quad-7.67584 \mathrm{E}-04$

$\begin{array}{lll}1.02653 \mathrm{E} & 00 \quad-7.70454 \mathrm{E}-04\end{array}$

$-9.10907 \mathrm{E}-01$

$-9.20562 \mathrm{E}-01$

$-9.30266 \mathrm{E}-01$

$-9.40020 \mathrm{E}-01$

$-9.49824 \mathrm{E}-01$

$1.03661 \mathrm{E} \quad 00$

$1.04674 \mathrm{E} \quad 00$

$1.05691 \mathrm{E} \quad 00$

$1.06714 \mathrm{E} \quad 00$

$1.07742 \mathrm{E} \quad 00$

$1.08775 \mathrm{E} \quad 00$

$1.09813 \mathrm{E} \quad 00$

$1.10855 \mathrm{E} \quad 00$

$1.11903 \mathrm{E} \quad 00$

$1.12956 \mathrm{E} \quad 00$

$-7.73275 \mathrm{E}-04$

$-7.76072 \mathrm{E}-04$

$-7.78834 \mathrm{E}-04$

$-7.81555 \mathrm{E}-04$

$-7.84243 \mathrm{E}-04$

$-7.86885 \mathrm{E}-04$

$-7.89506 \mathrm{E}-04$

$-7.92087 \mathrm{E}-04$

$-7.94632 \mathrm{E}-04$

$1.14013 \mathrm{E} \quad 00$

$-7.97139 \mathrm{E}-04$

$\begin{array}{lll}1.15076 \mathrm{E} & 00 & -8.02064 \mathrm{E}-04\end{array}$

$1.16144 \mathrm{E} \quad 00 \quad-8.04467 \mathrm{E}-04$

$\begin{array}{lll}1.17216 \mathrm{E} & 00 & -8.06846 \mathrm{E}-04\end{array}$

$1.18294 \mathrm{E} \quad 00 \quad-8.09194 \mathrm{E}-04$

$\begin{array}{lll}1.19377 \mathrm{E} & 00 \quad-8.11511 \mathrm{E}-04\end{array}$

$1.20464 \mathrm{E} \quad 00 \quad-8.13793 \mathrm{E}-04$

$\begin{array}{lll}1.21557 \mathrm{E} & 00 & -8.16036 \mathrm{E}-04\end{array}$

$\begin{array}{lll}1.22655 \mathrm{E} & 00 & -8.18258 \mathrm{E}-04\end{array}$

$\begin{array}{lll}1.23757 \mathrm{E} & 00 & -8.20445 \mathrm{E}-04\end{array}$

$1.24865 \mathrm{E} \quad 00$

$1.25978 \mathrm{E} \quad 00$

$1.27095 \mathrm{E} \quad 00$

$1.28218 \mathrm{E} \quad 00$

$1.29346 \mathrm{E} \quad 00$

$1.30479 \mathrm{E} \quad 00$

$1.31617 \mathrm{E} \quad 00$

$1.32759 \mathrm{E} \quad 00$

$1.33907 \mathrm{E} \quad 00$

$1.35060 \mathrm{E} \quad 00$

$-8.22600 \mathrm{E}-04$

$-8.24731 \mathrm{E}-04$

$-8.26825 \mathrm{E}-04$

$-8.28897 \mathrm{E}-04$

$-8.30932 \mathrm{E}-04$

$-8.32936 \mathrm{E}-04$

$-8.34921 \mathrm{E}-04$

$-8.36867 \mathrm{E}-04$

$-8.38784 \mathrm{E}-04$

$-8.40683 \mathrm{E}-04$

$1.36218 \mathrm{E} \quad 00$

$1.37381 \mathrm{E} \quad 00$

$-8.42547 \mathrm{E}-04$

$-9.59677 \mathrm{E}-01$

$-9.69580 \mathrm{E}-01$

$-9.79533 \mathrm{E}-01$

$-9.89535 \mathrm{E}-01$

$-9.99587 \mathrm{E}-01$

$-1.00969 \mathrm{E} 00$

$-1.01984 \mathrm{E} \quad 00$

$-1.03004 \mathrm{E} 00$

$-1.04029 \mathrm{E} 00$

$-1.05060 \mathrm{E} 00$

$-1.06095 \mathrm{E} 00$

$-1.07135 \mathrm{E} 00$

$-1.08180 \mathrm{E} \quad 00$

$-1.09230 \mathrm{E} 00$

$-1.10285 \mathrm{E} \quad 00$

$-1.11345 \mathrm{E} 00$

$-1.12410 \mathrm{E} 00$

$-1.13481 \mathrm{E} \quad 00$

$-1.14556 \mathrm{E} \quad 00$

$-1.15636 \mathrm{E} 00$

$-1.16721 \mathrm{E} 00$

$-1.17812 \mathrm{E} \quad 00$

$-1.18907 \mathrm{E} 00$

$-1.20007 \mathrm{E} \quad 00$

$-1.21112 \mathrm{E} \quad 00$

$-1.22223 \mathrm{E} 00$

$-1.23338 \mathrm{E} 00$

$-1.24459 \mathrm{E} \quad 00$

$-1.25584 \mathrm{E} 00$

$-1.26715 \mathrm{E} \quad 00$

$1.38549 \mathrm{E} \quad 00 \quad-8.46202 \mathrm{E}-04$

$\begin{array}{lll}1.39723 \mathrm{E} & 00 & -8.47979 \mathrm{E}-04\end{array}$

$\begin{array}{lll}1.40901 \mathrm{E} & 00 & -8.49723 \mathrm{E}-04\end{array}$

$1.42084 \mathrm{E} \quad 00 \quad-8.51460 \mathrm{E}-04$

$\begin{array}{lll}1.43272 \mathrm{E} & 00 & -8.53162 \mathrm{E}-04\end{array}$

$\begin{array}{lll}1.44466 \mathrm{E} & 00 & -8.54838 \mathrm{E}-04\end{array}$

$\begin{array}{lll}1.45664 \mathrm{E} & 00 & -8.56482 \mathrm{E}-04\end{array}$

$\begin{array}{lll}1.46868 \mathrm{E} & 00 & -8.58106 \mathrm{E}-04\end{array}$

$1.48076 \mathrm{E} \quad 00$

$1.49290 \mathrm{E} \quad 00$

$1.50509 \mathrm{E} \quad 00$

$1.51733 \mathrm{E} \quad 00$

$1.52962 \mathrm{E} \quad 00$

$-8.59705 \mathrm{E}-04$ $-8.61279 \mathrm{E}-04$ $-8.62823 \mathrm{E}-04$

$-8.64347 \mathrm{E}-04$

$-8.65841 \mathrm{E}-04$

$1.54196 \mathrm{E} \quad 00$

$1.55435 \mathrm{E} \quad 00$

$1.56679 \mathrm{E} \quad 00$

$1.57929 \mathrm{E} \quad 00$

$-8.67320 \mathrm{E}-04$

$-8.68764 \mathrm{E}-04$

$-8.70194 \mathrm{E}-04$

$-8.71592 \mathrm{E}-04$

$1.59183 \mathrm{E} \quad 00$
$-1.27851 \mathrm{E} \quad 00$

$-1.28991 \mathrm{E} \quad 00$

$-1.30137 \mathrm{E} 00$

$-1.31288 \mathrm{E} \quad 00$

$-1.32444 \mathrm{E} 00$

$-1.33605 \mathrm{E} 00$

$-1.34771 \mathrm{E} \quad 00$

$-1.35942 \mathrm{E} \quad 00$

$-1.37118 \mathrm{E} 00$

$-1.38299 \mathrm{E} \quad 00$

$-1.39486 \mathrm{E} \quad 00$ $-1.40677 \mathrm{E} 00$ $-1.41874 \mathrm{E} \quad 00$ $-1.43075 \mathrm{E} \quad 00$ $-1.44282 \mathrm{E} 00$

$-1.45494 \mathrm{E} \quad 00$ $-1.46711 \mathrm{E} \quad 00$ $-1.47933 \mathrm{E} 00$ $-1.49160 \mathrm{E} \quad 00$ $-1.50392 \mathrm{E} \quad 00$ $g_{-1}$

$2.31325 \mathrm{E} \quad 01$ $2.32289 \mathrm{E} \quad 01$ $2.33253 \mathrm{E} \quad 01$ $2.34217 \mathrm{E} \quad 01$ $2.35182 \mathrm{E} 01$

$2.36147 \mathrm{E} \quad 01$ $2.37112 \mathrm{E} 01$ $2.38078 \mathrm{E} \quad 01$ $2.39044 \mathrm{E} \quad 01$ $2.40010 \mathrm{E} \quad 01$

$2.40976 \mathrm{E} \quad 01$ $2.41942 \mathrm{E} 01$ $2.42909 \mathrm{E} 01$ $2.43876 \mathrm{E} \quad 01$ $2.44844 \mathrm{E} \quad 01$

$2.45811 \mathrm{E} \quad 01$ $2.46779 \mathrm{E} \quad 01$ $2.47747 \mathrm{E} \quad 01$ $2.48716 \mathrm{E} \quad 01$ 2.49685 E 01

$2.50654 \mathrm{E} \quad 01$ $2.51623 \mathrm{E} \quad 01$ $2.52592 \mathrm{E} \quad 01$ $2.53562 \mathrm{E} 01$ $2.54532 \mathrm{E} 01$

$2.55503 \mathrm{E} 01$ $2.56473 \mathrm{E} 01$ $2.57444 \mathrm{E} \quad 01$ $2.58416 \mathrm{E} \quad 01$ $2.59387 \mathrm{E} \quad 01$

$2.60359 \mathrm{E} \quad 01$ $2.61331 \mathrm{E} 01$ $2.62303 \mathrm{E} 01$ $2.63276 \mathrm{E} 01$ $2.64249 \mathrm{E} \quad 01$

$2.65222 \mathrm{E} \quad 01$ $2,66196 \mathrm{E} 01$ $2.67170 \mathrm{E} 01$ $2.68144 \mathrm{E} 01$ $2.69118 \mathrm{E} \quad 01$

$2.70093 \mathrm{E} \quad 01$ $2.71068 \mathrm{E} \quad 01$ $2.72043 \mathrm{E} 01$ $2.73019 \mathrm{E} \quad 01$ $\begin{array}{ll}2.73995 \mathrm{E} & 01\end{array}$

$2.74971 \mathrm{E} \quad 01$ $2.75948 \mathrm{E} \quad 01$ 2.76924 E 01 $2.77901 \mathrm{E} \quad 01$ $2.78879 \mathrm{E} \quad 01$

$2.79857 \mathrm{E} \quad 01$ $2.80835 \mathrm{E} \quad 01$ $2.81813 \mathrm{E} \quad 01$ $\begin{array}{lll}2.82792 \mathrm{E} & 01\end{array}$ $\begin{array}{ll}2.83771 \mathrm{E} & 01\end{array}$

$2.84750 \mathrm{E} \quad 01$ $2.85729 \mathrm{E} \quad 01$ $2.86709 \mathrm{E} \quad 01$ $2.87689 \mathrm{E} \quad 01$ $2.88670 \mathrm{E} 01$ $\tan \delta_{-1}$

$3.50751 \mathrm{E} \quad 02$ $3.51445 \mathrm{E} \quad 02$ $3.52136 \mathrm{E} \quad 02$ $3.52828 \mathrm{E} \quad 02$ $3.53519 \mathrm{E} \quad 02$

$3.54208 \mathrm{E} \quad 02$ $3.54895 \mathrm{E} \quad 02$ $3.55582 \mathrm{E} \quad 02$ $3.56269 \mathrm{E} \quad 02$ $3.56953 \mathrm{E} \quad 02$

$3.57639 \mathrm{E} \quad 02$ $3.58322 \mathrm{E} \quad 02$ $3.59003 \mathrm{E} \quad 02$ $3.59685 \mathrm{E} \quad 02$ $3.60365 \mathrm{E} \quad 02$

$3.61046 \mathrm{E} \quad 02$ $3.61724 \mathrm{E} \quad 02$ $3.62402 \mathrm{E} \quad 02$ $3.63080 \mathrm{E} \quad 02$ $3.63757 \mathrm{E} \quad 02$

$3.64433 \mathrm{E} \quad 02$ $3.65108 \mathrm{E} \quad 02$ $3.65783 \mathrm{E} \quad 02$ 3.66457 E 02 $3.67130 \mathrm{E} \quad 02$

$3.67803 \mathrm{E} \quad 02$ $3.68475 \mathrm{E} \quad 02$ $3.69147 \mathrm{E} \quad 02$ $3.69818 \mathrm{E} \quad 02$ 3.70489 E 02

$3.71159 \mathrm{E} \quad 02$ $3.71829 \mathrm{E} \quad 02$ $3.72498 \mathrm{E} \quad 02$ $3.73166 \mathrm{E} \quad 02$ 3.73835 E 02

$3.74504 \mathrm{E} \quad 02$ $3.75170 \mathrm{E} \quad 02$ $3.75838 \mathrm{E} \quad 02$ $3.76506 \mathrm{E} \quad 02$ $3.77172 \mathrm{E} \quad 02$

$3.77839 \mathrm{E} \quad 02$ $3.78505 \mathrm{E} \quad 02$ $3.79170 \mathrm{E} \quad 02$ $3.79836 \mathrm{E} \quad 02$ $3.80503 \mathrm{E} \quad 02$

$3.81167 \mathrm{E} \quad 02$ $3.81833 \mathrm{E} \quad 02$ $3.82498 \mathrm{E} \quad 02$ $3.83164 \mathrm{E} \quad 02$ $3.83828 \mathrm{E} \quad 02$

$3.84493 \mathrm{E} \quad 02$ $3.85157 \mathrm{E} \quad 02$ $3.85823 \mathrm{E} \quad 02$ $3.86487 \mathrm{E} \quad 02$ $3.87152 \mathrm{E} \quad 02$

$3.87816 \mathrm{E} \quad 02$ $3.88481 \mathrm{E} \quad 02$ $3.89145 \mathrm{E} \quad 02$ $3.89810 \mathrm{E} \quad 02$ 3.90475 E 02 
$f_{2}$

$7.73059 \mathrm{E}-04$

$7.79484 \mathrm{E}-04$

$7.85936 \mathrm{E}-04$

$7.92415 \mathrm{E}-04$

$7.98920 \mathrm{E}-04$

$8.05452 \mathrm{E}-04$

$8.12010 \mathrm{E}-04$

$8.18595 \mathrm{E}-04$

$8.25206 \mathrm{E}-04$

$8.31844 \mathrm{E}-04$

8.38509E-04

$8.45200 \mathrm{E}-04$

$8.51918 \mathrm{E}-04$

$8.58662 \mathrm{E}-04$

$8.65433 \mathrm{E}-04$

8.72230E-04 8.79054 E-04 $8.85905 \mathrm{E}-04$ 8.92782 E-04 $8.99686 \mathrm{E}-04$

$9.06616 \mathrm{E}-04$ $9.13573 \mathrm{E}-04$ $9.20556 \mathrm{E}-04$ $9.27566 \mathrm{E}-04$ 9.34602 E- 04

$9.41665 \mathrm{E}-04$ $9.48755 \mathrm{E}-04$ 9.55871 E-04 $9.63014 \mathrm{E}-04$ $9.70183 \mathrm{E}-04$

$9.77379 \mathrm{E}-04$ $9.84602 \mathrm{E}-04$ $9.91851 \mathrm{E}-04$ $9.99126 \mathrm{E}-04$ $1.00643 \mathrm{E}-03$

$1.01376 \mathrm{E}-03$

$1.02111 \mathrm{E}-03$

$1.02849 \mathrm{E}-03$

$1.03590 \mathrm{E}-03$

$1.04334 \mathrm{E}-03$

$1.05080 \mathrm{E}-03$ $1.05829 \mathrm{E}-03$ $1.06580 \mathrm{E}-03$ $1.07334 \mathrm{E}-03$ $1.08091 \mathrm{E}-03$

$1.08851 \mathrm{E}-03$ $1.09613 \mathrm{E}-03$

$1.10377 \mathrm{E}-03$

$1.11145 \mathrm{E}-03$

$1.11915 \mathrm{E}-03$

$1.12688 \mathrm{E}-03$

$1.13463 \mathrm{E}-03$

$1.14241 \mathrm{E}-03$

$1.15022 \mathrm{E}-03$

$1.15805 \mathrm{E}-03$

$1.16591 \mathrm{E}-03$

$1.17380 \mathrm{E}-03$

$1.18171 \mathrm{E}-03$

$1.18965 \mathrm{E}-03$

$1.19762 \mathrm{E}-03$
$1.31324 \mathrm{E} \quad 00$

$1.32426 \mathrm{E} \quad 00$

$1.33533 \mathrm{E} \quad 00$

$1.34645 \mathrm{E} \quad 00$

$1.35761 \mathrm{E} 00$

$1.36882 \mathrm{E} \quad 00$ $1.38007 \mathrm{E} \quad 00$

$1.39137 \mathrm{E} \quad 00$

$1.40271 \mathrm{E} \quad 00$

$1.41410 \mathrm{E} \quad 00$

$1.42554 \mathrm{E} \quad 00$

$1.43702 \mathrm{E} \quad 00$

$1.44855 \mathrm{E} \quad 00$

$1.46012 \mathrm{E} \quad 00$
00

$1.47174 \mathrm{E} \quad 00$

$1.48340 \mathrm{E} \quad 00$

$1.49511 \mathrm{E} \quad 00$

$1.50687 \mathrm{E} \quad 00$

$\begin{array}{ll}1.51867 \mathrm{E} & 00\end{array}$

$1.53052 \mathrm{E} \quad 00$

$1.54241 \mathrm{E} \quad 00$

$1.55435 \mathrm{E} \quad 00$

$1.56633 \mathrm{E} \quad 00$

$1.57836 \mathrm{E} \quad 00$

$1.59044 \mathrm{E} \quad 00$

$1.60256 \mathrm{E} \quad 00$

$1.61473 \mathrm{E} \quad 00$

$1.62694 \mathrm{E} \quad 00$

$1.63920 \mathrm{E} \quad 00$

$1.65150 \mathrm{E} \quad 00$

$1.66385 \mathrm{E} \quad 00$ $1.67625 \mathrm{E} \quad 00$ $1.68869 \mathrm{E} \quad 00$

$1.70117 \mathrm{E} \quad 00$

$1.71371 \mathrm{E} \quad 00$

$1.72628 \mathrm{E} \quad 00$ $1.73891 \mathrm{E} \quad 00$ $1.75158 \mathrm{E} \quad 00$ $1.76429 \mathrm{E} \quad 00$

$1.77705 \mathrm{E} \quad 00$

$1.78986 \mathrm{E} \quad 00$ $1.80271 \mathrm{E} \quad 00$ $1.81561 \mathrm{E} \quad 00$ $1.82855 \mathrm{E} \quad 00$

$1.84154 \mathrm{E} \quad 00$

$1.85457 \mathrm{E} \quad 00$

$1.86765 \mathrm{E} \quad 00$

$1.88077 \mathrm{E} \quad 00$

$1.89394 \mathrm{E} \quad 00$

$1.90716 \mathrm{E} \quad 00$

$1.92042 \mathrm{E} \quad 00$ $1.93373 \mathrm{E} \quad 00$ $1.94708 \mathrm{E} \quad 00$ $1.96048 \mathrm{E} \quad 00$ $1.97392 \mathrm{E} \quad 00$

$1.98741 \mathrm{E} \quad 00$ $2.00095 \mathrm{E} \quad 00$ $2.01453 \mathrm{E} \quad 00$ $2.02815 \mathrm{E} \quad 00$ $2.04182 \mathrm{E} \quad 00$ $g_{2} \quad \tan \delta_{2}$

$f_{-2}$

$40969 \mathrm{E}-02$

$3.45816 \mathrm{E}-02$

$3.50708 \mathrm{E}-02$

$3.55645 \mathrm{E}-02$

$3.60627 \mathrm{E}-02$

$3.65656 \mathrm{E}-02$

$3.70730 \mathrm{E}-02$

$3.75851 \mathrm{E}-02$

$3.81017 \mathrm{E}-02$

3.86231 E-02

$3.91491 \mathrm{E}-02$

$3.96798 \mathrm{E}-02$

$4.02152 \mathrm{E}-02$

4.07554 E-02

$4.13003 \mathrm{E}-02$

$4.18500 \mathrm{E}-02$ $4.24045 \mathrm{E}-02$ $4.29639 \mathrm{E}-02$

$4.35280 \mathrm{E}-02$

$4.40970 \mathrm{E}-02$

$4.46709 \mathrm{E}-02$

$4.52498 \mathrm{E}-02$

$4.58335 \mathrm{E}-02$

4.64221 E- 02

$4.70158 \mathrm{E}-02$

$4.76144 \mathrm{E}-02$ $4.82180 \mathrm{E}-02$

$4.88267 \mathrm{E}-02$

$4.94403 \mathrm{E}-02$

$5.00591 \mathrm{E}-02$

$5.06830 \mathrm{E}-02$ $5.13119 \mathrm{E}-02$

$5.19460 \mathrm{E}-02$

$5.25852 \mathrm{E}-02$

$5.32296 \mathrm{E}-02$

$5.38792 \mathrm{E}-02$

$5.45340 \mathrm{E}-02$

$5.51940 \mathrm{E}-02$

$5.58593 \mathrm{E}-02$

$5.65299 \mathrm{E}-02$

$5.72057 \mathrm{E}-02$ $5.78868 \mathrm{E}-02$ $5.85733 \mathrm{E}-02$ $5.92652 \mathrm{E}-02$ $5.99624 \mathrm{E}-02$

$6.06649 \mathrm{E}-02$ $6.13730 \mathrm{E}-02$ $6.20864 \mathrm{E}-02$ $6.28053 \mathrm{E}-02$ $6.35297 \mathrm{E}-02$

$6.42596 \mathrm{E}-02$ $6.49950 \mathrm{E}-02$ $6.57359 \mathrm{E}-02$ $6.64824 \mathrm{E}-02$ $6.72345 \mathrm{E}-02$

$6.79921 \mathrm{E}-02$ $6.87554 \mathrm{E}-02$ $6.95244 \mathrm{E}-02$

$7.02989 \mathrm{E}-02$

$7.10792 \mathrm{E}-02$

$$
\begin{array}{lll}
-2.78101 \mathrm{E} & 01 & -3.14388 \mathrm{E}-02 \\
-2.78120 \mathrm{E} & 01 & -3.19041 \mathrm{E}-02 \\
-2.78139 \mathrm{E} & 01 & -3.23739 \mathrm{E}-02 \\
-2.78157 \mathrm{E} & 01 & -3.28481 \mathrm{E}-02 \\
-2.78176 \mathrm{E} & 01 & -3.33267 \mathrm{E}-02
\end{array}
$$

$\begin{array}{ll}-2.78194 \mathrm{E} & 01\end{array}$

$\begin{array}{lll}-2.78212 \mathrm{E} & 01\end{array}$

$-2.78230 \mathrm{E} \quad 01$

$-2.78248 \mathrm{E} \quad 01$

$-2.78265 \mathrm{E} \quad 01$

$-2.78283 \mathrm{E}$ 0]

$-2.78300 \mathrm{E} \quad 01$

$-2.78317 \mathrm{E} \quad 01$

$-2.78334 \mathrm{E} \quad 01$

$\begin{array}{lll}-2.78351 \mathrm{E} & 01\end{array}$

$\begin{array}{lll}-2.78368 \mathrm{E} & 01\end{array}$

$\begin{array}{ll}-2.78385 \mathrm{E} & 01\end{array}$

$-2.78401 \mathrm{E} \quad 01$

$-2.78417 \mathrm{E} \quad 01$

$-2.78434 \mathrm{E} \quad 01$

$-2.78450 \mathrm{E} \quad 01$

$-2.78466 \mathrm{E} \quad 01$

$-2.78482 \mathrm{E} \quad 01$

$-2.78497 \mathrm{E} \quad 01$

$-2.78513 \mathrm{E} 01$

$-2.78528 \mathrm{E} \quad 01$

$-2.78544 \mathrm{E} \quad 01$

$-2.78559 \mathrm{E} \quad 01$

$-2.78574 \mathrm{E} 01$

$-2.78589 \mathrm{E} 01$

$-2.78604 \mathrm{E} \quad 01$

$-2.78619 \mathrm{E} 01$

$-2.78633 \mathrm{E} \quad 01$

$-2.78648 \mathrm{E} 01$

$-2.78662 \mathrm{E} 0 \mathrm{l}$

$-2.78677 \mathrm{E} \quad 01$

$-2.78691 \mathrm{E} \quad 01$

$-2.78705 \mathrm{E} \quad 01$

$-2.78719 \mathrm{E} 01$

$-2.78733 \mathrm{E} \quad 01$

$-2.78747 \mathrm{E} \quad 01$ 
$p$

$F_{0}$

30.10

30.20

30.30

30.40

30.50

30.60

30.70

30.80

30.90

31.00

31.10

31.20

31.30

31.40

31.50

31.60

31.70

31.80

31.90

32.00

32.10

32.20

32.30

32.40

32.50

32.60

32.70

32.80

32.90

33.00

33.10

33.20

33.30

33.40

33.50

33.60

33.70

33.80

33.90

34.00

34.10

34.20

34.30

34.40

34.50

34.60

34.70

34.80

34.90

35.00

35.10

35.20

35.30

35.40

35.50

35.60

35.70

35.80

35.90

36.00 $f_{1}$

$8.55164 \mathrm{E}-01$

$8.55157 \mathrm{E}-01$

$8.55150 \mathrm{E}-01$

$8.55144 \mathrm{E}-01$

$8.55137 \mathrm{E}-01$

$8.55130 \mathrm{E}-01$

$8.55123 \mathrm{E}-01$

$8.55116 \mathrm{E}-01$

$8.55110 \mathrm{E}-01$

$8.55103 \mathrm{E}-01$

$8.55096 \mathrm{E}-0 \mathrm{I}$

$8.55090 \mathrm{E}-01$

$8.55083 \mathrm{E}-0$

$8.55076 \mathrm{E}-01$

$8.55070 \mathrm{E}-01$

$8.55063 \mathrm{E}-01$

$8.55056 \mathrm{E}-01$

$8.55050 \mathrm{E}-01$

$8.55043 \mathrm{E}-01$

$8.55037 \mathrm{E}-01$

$8.55031 \mathrm{E}-01$

$8.55024 \mathrm{E}-01$

$8.55018 \mathrm{E}-01$

$8.55011 \mathrm{E}-01$

$8.55005 \mathrm{E}-0$

$8.54998 \mathrm{E}-01$

$8.54992 \mathrm{E}-01$

$8.54985 \mathrm{E}-01$

$8.54979 \mathrm{E}-01$

$8.54973 \mathrm{E}-01$

$8.54966 \mathrm{E}-01$

$8.54960 \mathrm{E}-01$

$8.54954 \mathrm{E}-01$

$8.54947 \mathrm{E}-01$

$8.54941 \mathrm{E}-01$

$8.54935 \mathrm{E}-01$

$8.54928 \mathrm{E}-01$

$8.54922 \mathrm{E}-01$

$8.54916 \mathrm{E}-0]$

$8.54910 \mathrm{E}-01$

$8.54904 \mathrm{E}-01$

$8.54897 \mathrm{E}-01$

8.54891 E-01

$8.54885 \mathrm{E}-0 \mathrm{l}$

$8.54879 \mathrm{E}-01$

$8.54873 \mathrm{E}-01$

$8.54867 \mathrm{E}-01$

$8.54861 \mathrm{E}-01$

$8.54855 \mathrm{E}-01$

$8.54849 \mathrm{E}-01$

$8.54842 \mathrm{E}-01$

$8.54836 \mathrm{E}-01$

$8.54830 \mathrm{E}-01$

$8.54824 \mathrm{E}-01$

$8.54819 \mathrm{E}-01$

$8.54813 \mathrm{E}-01$

$8.54807 \mathrm{E}-01$

8.54801 E- 01

$8.54795 \mathrm{E}-01$

$8.54789 \mathrm{E}-01$ $g_{1}$

$2.79753 \mathrm{E} \quad 01$ $2.80730 \mathrm{E} \quad 01$

$2.81707 \mathrm{E} \quad 01$

$2.82685 \mathrm{E} \quad 01$

$2.83663 \mathrm{E} \quad 01$

$2.84641 \mathrm{E} \quad 01$

$2.85620 \mathrm{E} \quad 01$

$2.86599 \mathrm{E} 01$

$2.87578 \mathrm{E} \quad 01$

$2.88558 \mathrm{E} \quad 0 \mathrm{l}$

$2.89538 \mathrm{E} \quad 01$

$2.90518 \mathrm{E} \quad 01$

$2.91498 \mathrm{E} \quad 01$

$2.92479 \mathrm{E} \quad 0 \mathrm{l}$

$2.93460 \mathrm{E} 01$

$2.94441 \mathrm{E} \quad 01$

$2.95423 \mathrm{E} 01$

$2.96405 \mathrm{E} \quad 01$

$2.97388 \mathrm{E} \quad 01$

$2.98370 \mathrm{E} \quad 01$

$2.99353 \mathrm{E} \quad 01$

$3.00337 \mathrm{E}$ 01

$3.01320 \mathrm{E} 01$

$3.02304 \mathrm{E} \quad 01$

$3.03289 \mathrm{E} 01$

$3.04273 \mathrm{E} 01$

$3.05258 \mathrm{E}$ 01

$3.06244 \mathrm{E} 01$

$3.07229 \mathrm{E} \quad 01$

$3.08215 \mathrm{E} 01$

$3.09201 \mathrm{E} \quad 01$

$3.10188 \mathrm{E} \quad 01$

$3.11175 \mathrm{E} 01$

$3.12162 \mathrm{E} 01$

$3.13150 \mathrm{E} \quad 01$

$3.14138 \mathrm{E} \quad 01$

$3.15126 \mathrm{E} \quad 01$

$3.16115 \mathrm{E}$ ol

$3.17104 \mathrm{E}$ 01

$3.18093 \mathrm{E}$ 01.

$3.19083 \mathrm{E} 01$

$3.20073 \mathrm{E} 01$

$3.21063 \mathrm{E}$ 01

$3.22054 \mathrm{E} 01$

$3.23045 \mathrm{E} 01$

$3.24036 \mathrm{E} \quad 01$

$3.25028 \mathrm{E} 01$

$3.26020 \mathrm{E} 01$

$3.27013 \mathrm{E} \quad 01$

$3.28005 \mathrm{E} 01$

$3.28998 \mathrm{E} 01$

$3.29992 \mathrm{E} 01$

$3.30986 \mathrm{E} 01$

$3.31980 \mathrm{E} 01$

$3.32975 \mathrm{E} 01$

$3.33970 \mathrm{E} \quad 01$

$3.34965 \mathrm{E} 01$

$3.35960 \mathrm{E} 01$

$3.36956 \mathrm{E}$ 01

$3.37953 \mathrm{E} 01$ $\tan \delta_{1}$

$f_{-1}$

$g_{-1}$

$1.60443 \mathrm{E} \quad 00 \quad-8.74314 \mathrm{E}-04$

$1.61707 \mathrm{E} \quad 00 \quad-8.75643 \mathrm{E}-04$

$\begin{array}{lll}1.62977 \mathrm{E} & 00 & -8.76946 \mathrm{E}-04\end{array}$

$\begin{array}{lll}1.64252 \mathrm{E} & 00 & -8.78217 \mathrm{E}-04\end{array}$

$1.65532 \mathrm{E} \quad 00 \quad-8.79482 \mathrm{E}-04$

$1.66817 \mathrm{E} \quad 00$

$1.68108 \mathrm{E} \quad 00$

$1.69403 \mathrm{E} \quad 00$

$1.70704 \mathrm{E} \quad 00$

$1.72009 \mathrm{E} \quad 00$

$1.73320 \mathrm{E} \quad 00$

$1.74636 \mathrm{E} \quad 00$

$1.75957 \mathrm{E} \quad 00$

$1.77283 \mathrm{E} \quad 00$

$1.78615 \mathrm{E} \quad 00$

$1.79952 \mathrm{E} \quad 00$

$1.81293 \mathrm{E} \quad 00$

$1.82640 \mathrm{E} \quad 00$

$1.83992 \mathrm{E} \quad 00$

$1.85350 \mathrm{E} \quad 00$

$1.86712 \mathrm{E} \quad 00$

$1.88080 \mathrm{E} \quad 00$

$1.89453 \mathrm{E} \quad 00$

$1.90831 \mathrm{E} \quad 00$

$1.92214 \mathrm{E} \quad 00$

$1.93602 \mathrm{E} \quad 00$

$1.94996 \mathrm{E} \quad 00$

$1.96395 \mathrm{E} \quad 00$

$1.97799 \mathrm{E} \quad 00$

$1.99208 \mathrm{E} \quad 00$

$2.00623 \mathrm{E} \quad 00$

$2.02042 \mathrm{E} \quad 00$

$2.03467 \mathrm{E} \quad 00$

$2.04897 \mathrm{E} \quad 00$

$2.06333 \mathrm{E} \quad 00$

$2.07773 \mathrm{E} \quad 00$

$2.09219 \mathrm{E} \quad 00$

$2.10670 \mathrm{E} \quad 00$

$2.12127 \mathrm{E} \quad 00$

$2.13588 \mathrm{E} \quad 00$

$2.15055 \mathrm{E} \quad 00$

$2.16527 \mathrm{E} \quad 00$

$2.18005 \mathrm{E} \quad 00$

$2.19487 \mathrm{E} \quad 00$

$2.20975 \mathrm{E} \quad 00$

$2.22468 \mathrm{E} \quad 00$

$2.23967 \mathrm{E} \quad 00$

$2.25470 \mathrm{E} \quad 00$

$2.26979 \mathrm{E} \quad 00$

$2.28494 \mathrm{E} \quad 00$

$2.30013 \mathrm{E} \quad 00$

$2.31538 \mathrm{E} \quad 00$

$2.33068 \mathrm{E} \quad 00$

$2.34604 \mathrm{E} \quad 00$

$2.36145 \mathrm{E} \quad 00$

$2.37691 \mathrm{E} \quad 00$

$2.39242 \mathrm{E} \quad 00$

$2.40799 \mathrm{E} \quad 00$

$2.42361 \mathrm{E} \quad 00$

$2.43929 \mathrm{E} \quad 00$

$-8.80721 \mathrm{E}-04$

$-8.81932 \mathrm{E}-04$

$-8.83123 \mathrm{E}-04$

$-8.84290 \mathrm{E}-04$

$-8.85440 \mathrm{E}-04$

$-8.86562 \mathrm{E}-04$

$-8.87662 \mathrm{E}-04$

$-8.88741 \mathrm{E}-04$

$-8.89803 \mathrm{E}-04$

$-8.90839 \mathrm{E}-04$

$-8.91854 \mathrm{E}-04$

$-8.92854 \mathrm{E}-04$

$-8.93828 \mathrm{E}-04$

$-8.94785 \mathrm{E}-04$

$-8.95715 \mathrm{E}-04$

$-8.96629 \mathrm{E}-04$

$-8.97521 \mathrm{E}-04$

$-8.98392 \mathrm{E}-04$

$-8.99247 \mathrm{E}-04$

$-9.00075 \mathrm{E}-04$

$-9.00893 \mathrm{E}-04$

$-9.01687 \mathrm{E}-04$

$-9.02464 \mathrm{E}-04$

$-9.03217 \mathrm{E}-04$

$-9.03949 \mathrm{E}-04$

$-9.04664 \mathrm{E}-04$

$-9.05355 \mathrm{E}-04$

$-9.06037 \mathrm{E}-04$

$-9.06695 \mathrm{E}-04$

$-9.07342 \mathrm{E}-04$

$-9.07962 \mathrm{E}-04$

$-9.08569 \mathrm{E}-04$

$-9.09158 \mathrm{E}-04$

$-9.09722 \mathrm{E}-04$

$-9.10267 \mathrm{E}-04$

$-9.10806 \mathrm{E}-04$

$-9.11319 \mathrm{E}-04$

$-9.11818 \mathrm{E}-04$

$-9.12294 \mathrm{E}-04$

$-9.12757 \mathrm{E}-04$ 
$\bar{F}$

$1.20561 \mathrm{E}-03$

$1.21363 \mathrm{E}-03$

$1.22168 \mathrm{E}-03$

$1.22975 \mathrm{E}-03$

$1.23785 \mathrm{E}-03$

$1.24597 \mathrm{E}-03$

$1.25413 \mathrm{E}-03$

$1.26231 \mathrm{E}-03$

$1.27051 \mathrm{E}-03$

$1.27874 \mathrm{E}-03$

$1.28700 \mathrm{E}-03$

$1.29529 \mathrm{E}-03$

$1.30360 \mathrm{E}-03$

$1.31194 \mathrm{E}-03$

$1.32030 \mathrm{E}-03$

$1.32869 \mathrm{E}-03$

$1.33711 \mathrm{E}-03$

$1.34556 \mathrm{E}-03$

$1.35403 \mathrm{E}-03$

$1.36252 \mathrm{E}-03$

$1.37105 \mathrm{E}-03$

$1.37960 \mathrm{E}-03$

$1.38818 \mathrm{E}-03$

$1.39678 \mathrm{E}-03$

$1.40541 \mathrm{E}-03$

$1.41407 \mathrm{E}-03$

$1.42275 \mathrm{E}-03$

$1.43146 \mathrm{E}-03$

$1.44020 \mathrm{E}-03$

$1.44896 \mathrm{E}-03$

$1.45775 \mathrm{E}-03$ $1.46657 \mathrm{E}-03$

$1.47541 \mathrm{E}-03$

$1.48428 \mathrm{E}-03$

$1.49318 \mathrm{E}-03$

$1.50210 \mathrm{E}-03$

$1.51105 \mathrm{E}-03$

$1.52002 \mathrm{E}-03$

$1.52903 \mathrm{E}-03$

$1.53805 \mathrm{E}-03$

$1.54711 \mathrm{E}-03$

$1.55619 \mathrm{E}-03$

$1.56530 \mathrm{E}-03$

$1.57444 \mathrm{E}-03$

$1.58360 \mathrm{E}-03$

$1.59278 \mathrm{E}-03$

$1.60200 \mathrm{E}-03$

$1.61124 \mathrm{E}-03$

$1.62051 \mathrm{E}-03$

$1.62980 \mathrm{E}-03$

$1.63912 \mathbf{E}-03$

$1.64847 \mathrm{E}-03$

$1.65785 \mathrm{E}-03$

$1.66725 \mathrm{E}-03$

$1.67667 \mathrm{E}-03$

$1.68613 \mathrm{E}-03$

$1.69561 \mathrm{E}-03$

$1.70511 \mathrm{E}-03$

$1.71465 \mathrm{E}-03$

1.72421 E-03 $f_{2}$

$2.05554 \mathrm{E} \quad 00$

$2.06930 \mathrm{E} \quad 00$

$2.08311 \mathrm{E} \quad 00$

$2.09696 \mathrm{E} \quad 00$

$2.11086 \mathrm{E} \quad 00$

$2.12480 \mathrm{E} \quad 00$

$2.13879 \mathrm{E} \quad 00$

$2.15283 \mathrm{E} \quad 00$

$2.16691 \mathrm{E} \quad 00$

$2.18103 \mathrm{E} \quad 00$

$2.19520 \mathrm{E} \quad 00$ $2.20942 \mathrm{E} \quad 00$

$2.22368 \mathrm{E} \quad 00$

$2.23799 \mathrm{E} \quad 00$

$2.25234 \mathrm{E} \quad 00$

$2.26674 \mathrm{E} \quad 00$

$2.28118 \mathrm{E} \quad 00$

$2.29567 \mathrm{E} \quad 00$

$2.31020 \mathrm{E} \quad 00$

$2.32478 \mathrm{E} \quad 00$

$2.33941 \mathrm{E} \quad 00$

$2.35408 \mathrm{E} \quad 00$

$2.36879 \mathrm{E} \quad 00$

$2.38355 \mathrm{E} \quad 00$

$2.39836 \mathrm{E} \quad 00$

$2.41321 \mathrm{E} \quad 00$

$2.42810 \mathrm{E} \quad 00$

$2.44304 \mathrm{E} \quad 00$

$2.45803 \mathrm{E} \quad 00$

$2.47306 \mathrm{E} \quad 00$

$2.48814 \mathrm{E} \quad 00$

$2.50326 \mathrm{E} \quad 00$

$2.51843 \mathrm{E} \quad 00$

$2.53364 \mathrm{E} \quad 00$

$2.54890 \mathrm{E} \quad 00$

$2.56420 \mathrm{E} \quad 00$ $2.57955 \mathrm{E} \quad 00$ $2.59495 \mathrm{E} \quad 00$ $2.61039 \mathrm{E} \quad 00$ $2.62587 \mathrm{E} \quad 00$

$2.64140 \mathrm{E} \quad 00$ $2.65697 \mathrm{E} \quad 00$

$2.67259 \mathrm{E} \quad 00$

$2.68826 \mathrm{E} \quad 00$

$\begin{array}{lll}2.70397 \mathrm{E} & 00\end{array}$

$2.71972 \mathrm{E} \quad 00$ $2.73552 \mathrm{E} \quad 00$ $2.75137 \mathrm{E} \cdot 00$ $2.76726 \mathrm{E} \quad 00$ $2.78319 \mathrm{E} \quad 00$

$2.79918 \mathrm{E} \quad 00$ $2.81520 \mathrm{E} \quad 00$ $2.83127 \mathrm{E} \quad 00$

$2.84739 \mathrm{E} \quad 00$

$2.86355 \mathrm{E} \quad 00$

$2.87976 \mathrm{E} \quad 00$ $2.89601 \mathrm{E} \quad 00$ $2.91230 \mathrm{E} \quad 00$ $2.92864 \mathrm{E} \quad 00$ $2.94503 \mathrm{E} \quad 00$ $g_{2}$

$7.18652 \mathrm{E}-02$

$7.26569 \mathrm{E}-02$

$7.34543 \mathrm{E}-02$

$7.42575 \mathrm{E}-02$

$7.50665 \mathrm{E}-02$

$7.58812 \mathrm{E}-02$

$7.67018 \mathrm{E}-02$

$7.75283 \mathrm{E}-02$

$7.83606 \mathrm{E}-02$

$7.91988 \mathrm{E}-02$

$8.00429 \mathrm{E}-02$ $8.08929 \mathrm{E}-02$

$8.17488 \mathrm{E}-02$

$8.26108 \mathrm{E}-02$

$8.34787 \mathrm{E}-02$

$8.43526 \mathrm{E}-02$ $8.52325 \mathrm{E}-02$

$8.61185 \mathrm{E}-02$

$8.70106 \mathrm{E}-02$

$8.79087 \mathrm{E}-02$

$8.88130 \mathrm{E}-02$

$8.97233 \mathrm{E}-02$

$9.06399 \mathrm{E}-02$

$9.15626 \mathrm{E}-02$

$9.24915 \mathrm{E}-02$

$9.34265 \mathrm{E}-02$

$9.43679 \mathrm{E}-02$

$9.53154 \mathrm{E}-02$

$9.62693 \mathrm{E}-02$

$9.72294 \mathrm{E}-02$

$9.81959 \mathrm{E}-02$

$9.91687 \mathrm{E}-02$

$1.00148 \mathrm{E}-01$

$1.01133 \mathrm{E}-01$

$1.02125 \mathrm{E}-01$

$1.03124 \mathrm{E}-01$

$1.04128 \mathrm{E}-01$

$1.05139 \mathrm{E}-01$

$1.06157 \mathrm{E}-01$

$1.07181 \mathrm{E}-01$

$1.08212 \mathrm{E}-01$

$1.09249 \mathrm{E}-01$

$1.10293 \mathrm{E}-01$

$1.11343 \mathrm{E}-01$

$1.12400 \mathrm{E}-01$

$1.13464 \mathrm{E}-01$

$1.14534 \mathrm{E}-01$

$1.15611 \mathrm{E}-01$

$1.16694 \mathrm{E}-01$

$1.17785 \mathrm{E}-01$

$1.18882 \mathrm{E}-01$

$1.19985 \mathrm{E}-01$

$1.21096 \mathrm{E}-01$

$1.22213 \mathrm{E}-01$

].23337 E-01

$1.24468 \mathrm{E}-01$

$1.25605 \mathrm{E}-01$

$1.26750 \mathrm{E}-01$

$1.27901 \mathrm{E}-01$

$1.29060 \mathrm{E}-01$ $\tan \delta_{2}$

$f_{-2}$

4

$-2.79003 \mathrm{E} \quad 01 \quad-6.79227 \mathrm{E}-02$ $-2.79015 \mathrm{E}$ 01 $-6.86909 \mathrm{E}-02$ $-2.79027 \mathrm{E} \quad 01 \quad-6.94648 \mathrm{E}-02$ $-2.79039 \mathrm{E}$ 01 $-7.02444 \mathrm{E}-02$ $-2.79050 \mathrm{E}$ 01 $-7.10296 \mathrm{E}-02$

$-2.79062 \mathrm{E}$ 01 $-7.18206 \mathrm{E}-02$ $-2.79073 \mathrm{E}$ 01 $-7.26174 \mathrm{E}-02$ $-2.79085 \mathrm{E} \quad 01 \quad-7.34199 \mathrm{E}-02$ $-2.79096 \mathrm{E} \quad 01 \quad-7.42283 \mathrm{E}-02$ $-2.79108 \mathrm{E} \quad 01 \quad-7.50424 \mathrm{E}-02$

$-2.79119 \mathrm{E}$ 01 $\quad-7.58624 \mathrm{E}-02$ $-2.79130 \mathrm{E}$ 01 $-7.66883 \mathrm{E}-02$ $-2.79141 \mathrm{E}$ 01 $-7.75200 \mathrm{E}-02$ $-2.79152 \mathrm{E}$ 01 $-7.83576 \mathrm{E}-02$ $-2.79163 \mathrm{E} \quad 01 \quad-7.92011 \mathrm{E}-02$

$-2.79174 \mathrm{E} \quad 01 \quad-8.00506 \mathrm{E}-02$ $-2.79184 \mathrm{E} \quad 01 \quad-8.09060 \mathrm{E}-02$ $-2.79195 \mathrm{E} \quad 01 \quad-8.17674 \mathrm{E}-02$ $-2.79206 \mathrm{E}$ 01 $-8.26348 \mathrm{E}-02$ $-2.79216 \mathrm{E} \quad 01 \quad-8.35083 \mathrm{E}-02$

$\begin{array}{lll}-2.79227 \mathrm{E} & 01\end{array}$ $-2.79237 \mathrm{E} \quad 01$ $-2.79248 \mathrm{E} \quad 01$

$-2.79258 \mathrm{E} \quad 01$ $-2.79268 \mathrm{E} 01$

$-2.79278 \mathrm{E} \quad 01$ $-2.79288 \mathrm{E} 01$ $-2.79298 \mathrm{E} \quad 01$ $-2.79308 \mathrm{E} \quad 01$ $-2.79318 \mathrm{E} \quad 01$

$-8.43877 \mathrm{E}-02$ $-8.52733 \mathrm{E}-02$ $-8.61649 \mathrm{E}-02$ $-8.70626 \mathrm{E}-02$ $-8.79664 \mathrm{E}-02$

$-8.88764 \mathrm{E}-02$ $-8.97925 \mathrm{E}-02$ $-9.07148 \mathrm{E}-02$ $-9.16434 \mathrm{E}-02$ $-9.25781 \mathrm{E}-02$

$\begin{array}{ll}-2.79328 \mathrm{E} & 0 \mathrm{l}\end{array}$ $-2.79338 \mathrm{E} 01$ $-2.79347 \mathrm{E} \quad 01$ $-2.79357 \mathrm{E} \quad 01$ $-2.79367 \mathrm{E} \quad 01$

$\begin{array}{lll}-2.79376 \mathrm{E} & 01\end{array}$ $-2.79386 \mathrm{E} \quad 01$ $-2.79395 \mathrm{E} 01$ $-2.79405 \mathrm{E} \quad 01$

$-2.79414 \mathrm{E} \quad 01$

$-2.79423 \mathrm{E} \quad 01$ $-2.79432 \mathrm{E}$ 01 $-2.79441 \mathrm{E} \quad 01$ $\begin{array}{lll}-2.79451 \mathrm{E} & 01\end{array}$ $-2.79460 \mathrm{E} 01$

$-9.35191 \mathrm{E}-02$ $-9.44663 \mathrm{E}-02$ $-9.54199 \mathrm{E}-02$ $-9.63797 \mathrm{E}-02$ $-9.73459 \mathrm{E}-02$

$-9.83184 \mathrm{E}-02$ $-9.92973 \mathrm{E}-02$ $-1.00283 \mathrm{E}-01$ $-1.01274 \mathrm{E}-01$

$-1.02272 \mathrm{E}-01$

$-1.03277 \mathrm{E}-01$

$-1.0+288 \mathrm{E}-01$

$-1.05305 \mathrm{E}-01$

$-1.06329 \mathrm{E}-01$

$-1.07360 \mathrm{E}-01$

$\begin{array}{lll}-2.79469 \mathrm{E} & 01\end{array}$

$-1.08397 \mathrm{E}-01$

$\begin{array}{lll}-2.79477 \mathrm{E} & 01\end{array}$

$-2.79486 \mathrm{E} 01$

$\begin{array}{lll}-2.79495 \mathrm{E} & 01\end{array}$

$-2.79504 \mathrm{E} 01$

$\begin{array}{lll}-2.79513 \mathrm{E} & 01\end{array}$

$-2.79521 \mathrm{E}$ 01

$-2.79530 \mathrm{E} 01$

$-2.79538 \mathrm{E}$ 01

$-2.79547 \mathrm{E}$ ol

$-2.79555 \mathrm{E} \quad 01$ $-2.79564 \mathrm{E} \quad 01$ $-2.79572 \mathrm{E} 01$ $-2.79580 \mathrm{E} \quad 01$ $-2.79589 \mathrm{E} 01$

$2.12502 \mathrm{E} \quad 00$

$2.13901 \mathrm{E} \quad 00$

$2.1530+\mathrm{E} 00$

$2.16713 \mathrm{E} \quad 00$

$2.18126 \mathrm{E} \quad 00$

$2.19543 \mathrm{E} \quad 00$

$2.20965 \mathrm{E} \quad 00$

$2.22391 \mathrm{E} \quad 00$

$2.23822 \mathrm{E} \quad 00$

$2.25258 \mathrm{E} \quad 00$

$2.26698 \mathrm{E} \quad 00$

$2.28143 \mathrm{E} \quad 00$

$2.29592 \mathrm{E} \quad 00$

$2.31045 \mathrm{E} \quad 00$

$2.32504 \mathrm{E} \quad 00$ 
ELECTRON RADIAL FUNCTIONS

,

$F_{0}$

36.10

36.20

36.30

36.40

36.50

36.60

36.70

36.80

36.90

37.00

37.10

37.20

37.30

37.40

37.50

37.60

37.70

37.80

37.90

38.00

38.10

38.20

38.30

38.40

38.50

38.60

38.70

38.80

38.90

39.00

39.10

39.20

39.30

39.40

39.50

39.60

39.70

39.80

39.90

40.00

40.10

40.20

40.30

40.40

40.50

40.60

40.70

40.80

40.90

41.00

41.10

41.20

41.30

41.40

41.50

41.60

41.70

41.80

41.90

42.00 $f_{1}$

$8.54783 \mathrm{E}-01$ $8.54777 \mathrm{E}-01$ 8.54771 E-01 $8.54765 \mathrm{E}-01$ $8.54760 \mathrm{E}-01$

$8.54754 \mathrm{E}-01$ $8.54748 \mathrm{E}-01$ 8.54742 E-01 $8.54736 \mathrm{E}-01$ 8.54731 E-01

8.54725E-01 $8.54719 \mathrm{E}-01$ $8.54713 \mathrm{E}-01$ $8.54707 \mathrm{E}-01$ $8.54701 \mathrm{E}-01$

8.54696E-01 $8.54690 \mathrm{E}-01$ $8.54684 \mathrm{E}-01$ $8.54679 \mathrm{E}-01$ $8.54673 \mathrm{E}-01$

$8.54668 \mathrm{E}-01$ $8.54662 \mathrm{E}-01$ $8.54656 \mathrm{E}-01$ $8.54651 \mathrm{E}-01$ $8.54645 \mathrm{E}-01$

8.54640E-01 $8.54634 \mathrm{E}-01$ 8.54629E-01 $8.54623 \mathrm{E}-01$ $8.54618 \mathrm{E}-01$

8.54612E-01 $8.54607 \mathrm{E}-01$ $8.54601 \mathrm{E}-01$ $8.54596 \mathrm{E}-01$ $8.54590 \mathrm{E}-01$

$8.54585 \mathrm{E}-01$ $8.54580 \mathrm{E}-01$ $8.54574 \mathrm{E}-01$ $8.54569 \mathrm{E}-01$ 8.54564E-01

$8.54558 \mathrm{E}-01$ $8.54553 \mathrm{E}-01$ $8.54548 \mathrm{E}-01$ $8.54542 \mathrm{E}-01$ $8.54537 \mathrm{E}-01$

8.54532E-01 $8.54526 \mathrm{E}-01$ $8.54521 \mathrm{E}-01$ $8.54516 \mathrm{E}-01$ 8.54511 E-01

$8.54505 \mathrm{E}-01$ $8.54500 \mathrm{E}-01$ 8.54495 E-01 $8.54490 \mathrm{E}-01$ 8.54485 E-01

$8.54479 \mathrm{E}-01$ $8.54474 \mathrm{E}-01$ $8.54469 \mathrm{E}-01$ $8.54464 \mathrm{E}-01$ $8.54459 \mathrm{E}-0 \mathrm{l}$
$3.38950 \mathrm{E} \quad 01$ $3.39947 \mathrm{E} \quad 01$ $3.40944 \mathrm{E} \quad 01$ $3.41942 \mathrm{E} \quad 01$

$3.42940 \mathrm{E} 01$

$3.43939 \mathrm{E} \quad 01$ $3.44938 \mathrm{E} 01$ $3.45937 \mathrm{E}$ ol $3.46937 \mathrm{E} \quad 01$ $3.47937 \mathrm{E} 01$

$3.48937 \mathrm{E} \quad 01$ $3.49938 \mathrm{E} \quad 01$ $3.50939 \mathrm{E} 01$ $3.51940 \mathrm{E} 01$ $3.52942 \mathrm{E} \quad 01$

$3.53944 \mathrm{E} \quad 01$ $3.54947 \mathrm{E} 01$ $3.55950 \mathrm{E} 01$ $3.56954 \mathrm{E} \quad 01$

$3.57957 \mathrm{E} \quad 01$

$3.58962 \mathrm{E} \quad 01$ $3.59966 \mathrm{E} \quad 01$ $3.60971 \mathrm{E} 01$ $3.61977 \mathrm{E} \quad 01$ $3.62982 \mathrm{E} 01$

$3.63988 \mathrm{E} 01$ $3.64995 \mathrm{E} 01$ $3.66002 \mathrm{E} \quad 01$ $3.67009 \mathrm{E} \quad 01$ 3.68017 E 01

$3.69025 \mathrm{E} \quad 01$ $3.70034 \mathrm{E} \quad 01$ $3.71043 \mathrm{E} 01$ $3.72052 \mathrm{E} \quad 01$ $3.73062 \mathrm{E} 01$

$3.74072 \mathrm{E} \quad 01$ $3.75082 \mathrm{E} \quad 01$ $3.76093 \mathrm{E} \quad 01$ $3.77104 \mathrm{E} \quad 01$ $3.78116 \mathrm{E} \quad 01$

$3.79128 \mathrm{E} \quad 01$ $3.80141 \mathrm{E} \quad 01$ $3.81154 \mathrm{E} \quad 01$ $3.82167 \mathrm{E} \quad 01$

3.83181 E 01

$3.84195 \mathrm{E} \quad 01$ $3.85210 \mathrm{E} \quad 01$ $3.86225 \mathrm{E} 01$ $3.87240 \mathrm{E} \quad 01$

$3.88256 \mathrm{E} \quad 01$

$3.89272 \mathrm{E} \quad 01$ $3.90289 \mathrm{E} \quad 01$ $3.91306 \mathrm{E} 01$ $3.92324 \mathrm{E} \quad 01$ $3.93342 \mathrm{E} 01$

$3.94360 \mathrm{E} \quad 01$ $3.95379 \mathrm{E}$ ol $3.96398 \mathrm{E} \quad 01$ $3.97418 \mathrm{E}$ 0l $3.98438 \mathrm{E} \quad 01$ $g_{1}$

$2.45501 \mathrm{E} \quad 00$ $2.47080 \mathrm{E} \quad 00$ $2.48663 \mathrm{E} \quad 00$ $2.50252 \mathrm{E} \quad 00$ $2.51846 \mathrm{E} \quad 00$

$2.53445 \mathrm{E} \quad 00$ $2.55050 \mathrm{E} \quad 00$ $2.56661 \mathrm{E} \quad 00$ $2.58276 \mathrm{E} \quad 00$ $2.59897 \mathrm{E} \quad 00$

$2.61523 \mathrm{E} \quad 00$ $2.63155 \mathrm{E} \quad 00$ $2.64792 \mathrm{E} \quad 00$

$2.66435 \mathrm{E} \quad 00$

$2.68083 \mathrm{E} \quad 00$

$2.69736 \mathrm{E} \quad 00$ $2.71395 \mathrm{E} \quad 00$ $2.73059 \mathrm{E} \quad 00$ $2.74 .728 \mathrm{E} \quad 00$

$2.76403 \mathrm{E} \quad 00$

$2.78083 \mathrm{E} \quad 00$ $2.79769 \mathrm{E} \quad 00$ $2.81460 \mathrm{E} \quad 00$ $2.83157 \mathrm{E} \quad 00$

$2.84859 \mathrm{E} \quad 00$

$2.86567 \mathrm{E} \quad 00$ $2.88280 \mathrm{E} \quad 00$ $2.89998 \mathrm{E} \quad 00$ $2.91722 \mathrm{E} \quad 00$ $2.93451 \mathrm{E} \quad 00$

$2.95186 \mathrm{E} \quad 00$ $2.96927 \mathrm{E} \quad 00$ $2.98672 \mathrm{E} \quad 00$ $3.00424 \mathrm{E} \quad 00$

$3.02180 \mathrm{E} \quad 00$

$3.03943 \mathrm{E} \quad 00$ $3.05710 \mathrm{E} \quad 00$ $3.07483 \mathrm{E} \quad 00$ $3.09262 \mathrm{E} \quad 00$ $3.11046 \mathrm{E} \quad 00$

$3.12836 \mathrm{E} \quad 00$ $3.14631 \mathrm{E} \quad 00$ $3.16432 \mathrm{E} \quad 00$

$3.18239 \mathrm{E} \quad 00$

$3.20050 \mathrm{E} \quad 00$

$3.21868 \mathrm{E} \quad 00$ $3.23691 \mathrm{E} \quad 00$ $3.25519 \mathrm{E} \quad 00$ $3.27353 \mathrm{E} \quad 00$

$3.29193 \mathrm{E} \quad 00$

$3.31038 \mathrm{E} \quad 00$ $3.32889 \mathrm{E} \quad 00$ $3.34745 \mathrm{E} \quad 00$ $3.36607 \mathrm{E} \quad 00$ $3.38474 \mathrm{E} \quad 00$

$3.40348 \mathrm{E} \quad 00$ $3.42226 \mathrm{E} \quad 00$

$3.44110 \mathrm{E} \quad 00$

$3.46000 \mathrm{E} \quad 00$

$3.47896 \mathrm{E} \quad 00$

$\tan \delta_{1}$

$f_{-1}$

$-9.17858 \mathrm{E}-04 \quad-2.35381 \mathrm{E} \quad 00$ $-9.18045 \mathrm{E}-04 \quad-2.36938 \mathrm{E} \quad 00$ $-9.18205 \mathrm{E}-04 \quad-2.38500 \mathrm{E} \quad 00$ $-9.18360 \mathrm{E}-04 \quad-2.40067 \mathrm{E} \quad 00$ $-9.18494 \mathrm{E}-04$

$-9.18616 \mathrm{E}-04$ $-9.18710 \mathrm{E}-04$ $-9.18805 \mathrm{E}-04$ $-9.18871 \mathrm{E}-04$ $-9.18940 \mathrm{E}-04$

$-9.18979 \mathrm{E}-04$

$-9.19005 \mathrm{E}-04$

$-9.19020 \mathrm{E}-04$

$-9.19021 \mathrm{E}-04$

$-9.19005 \mathrm{E}-04$

$-9.18977 \mathrm{E}-04$ $-9.18931 \mathrm{E}-04$ $-9.18872 \mathrm{E}-04$ $-9.18799 \mathrm{E}-04$

$-9.18707 \mathrm{E}-04$

$-9.18610 \mathrm{E}-04$

$-9.18490 \mathrm{E}-04$

$-9.18361 \mathrm{E}-04$

$-9.18218 \mathrm{E}-04$

$-9.18051 \mathrm{E}-04$

$-9.17890 \mathrm{E}-04$

$-9.17699 \mathrm{E}-04$

$-9.17508 \mathrm{E}-04$

$-9.17289 \mathrm{E}-04$

$-9.17062 \mathrm{E}-04$

$-9.16823 \mathrm{E}-04$

$-9.16573 \mathrm{E}-04$

$-9.16302 \mathrm{E}-04$

$-9.16018 \mathrm{E}-04$

$-9.15726 \mathrm{E}-04$

$-9.15419 \mathrm{E}-04$

$-9.15103 \mathrm{E}-04$

$-9.14773 \mathrm{E}-04$

$-9.14415 \mathrm{E}-04$ 
$\bar{F}$

36.10

36.20

36.30

36.40

36.50

36.60

36.70

36.80

36.90

37.00

37.10

37.20

37.30

37.40

37.50

37.60

37.70

37.80

37.90

38.00

38.10

38.20

38.30

38.40

38.50

38.60

38.70

38.80

38.90

39.00

39.10

39.20

39.30

39.40

39.50

39.60

39.70

39.80

39.90

40.00

40.10

40.20

40.30

40.40

40.50

40.60

40.70

40.80

40.90

41.00

41.10

41.20

41.30

41.40

41.50

41.60

41.70

41.80

41.90

42.00 $f_{2}$

$1.73380 \mathrm{E}-03$

$1.74341 \mathrm{E}-03$

$1.75305 \mathrm{E}-03$

$1.76271 \mathrm{E}-03$

$1.77241 \mathrm{E}-03$

$1.78213 \mathrm{E}-03$

$1.79187 \mathrm{E}-03$

$1.80164 \mathrm{E}-03$

$1.81144 \mathrm{E}-03$

$1.82127 \mathrm{E}-03$

$1.83112 \mathrm{E}-03$

$1.84100 \mathrm{E}-03$

$1.85091 \mathrm{E}-03$

$1.86084 \mathrm{E}-03$

$1.87080 \mathrm{E}-03$

$1.88078 \mathrm{E}-03$

$1.89079 \mathrm{E}-03$

$1.90083 \mathrm{E}-03$

$1.91089 \mathrm{E}-03$

$1.92099 \mathrm{E}-03$

$1.93110 \mathrm{E}-03$

$1.94125 \mathrm{E}-03$

$1.95142 \mathrm{E}-03$

$1.96162 \mathrm{E}-03$

$1.97184 \mathrm{E}-03$

$1.98209 \mathrm{E}-03$

$1.99237 \mathrm{E}-03$

$2.00267 \mathrm{E}-03$

$2.01300 \mathrm{E}-03$

$2.02336 \mathrm{E}-03$

$2.03374 \mathrm{E}-03$

$2.04415 \mathrm{E}-03$

$2.05459 \mathrm{E}-03$

$2.06505 \mathrm{E}-03$

$2.07554 \mathrm{E}-03$

$2.08606 \mathrm{E}-03$

$2.09660 \mathrm{E}-03$

$2.10717 \mathrm{E}-03$

$2.11776 \mathrm{E}-03$

$2.12839 \mathrm{E}-03$

$2.13903 \mathrm{E}-03$

$2.14971 \mathrm{E}-03$

$2.16041 \mathrm{E}-03$

$2.17114 \mathrm{E}-03$

$2.18189 \mathrm{E}-03$

$2.19268 \mathrm{E}-03$

$2.20348 \mathrm{E}-03$

$2.21432 \mathrm{E}-03$

$2.22518 \mathrm{E}-03$

$2.23607 \mathrm{E}-03$

$2.24698 \mathrm{E}-03$

$2.25792 \mathrm{E}-03$

$2.26889 \mathrm{E}-03$

$2.27988 \mathrm{E}-03$

$2.29091 \mathrm{E}-03$

$2.30195 \mathrm{E}-03$

$2.31303 \mathrm{E}-03$

$2.32413 \mathrm{E}-03$

$2.33525 \mathrm{E}-03$

$2.34641 \mathrm{E}-03$
$2.96146 \mathrm{E} \quad 00$

$2.97794 \mathrm{E} \quad 00$ $2.99446 \mathrm{E} \quad 00$

$3.01103 \mathrm{E} \quad 00$

$3.02764 \mathrm{E} \quad 00$

$3.04430 \mathrm{E} \quad 00$ $3.06100 \mathrm{E} \quad 00$

$3.07774 \mathrm{E} \quad 00$

$3.09453 \mathrm{E} \quad 00$

$3.11137 \mathrm{E} \quad 00$

$3.12825 \mathrm{E} \quad 00$

$3.14518 \mathrm{E} \quad 00$

$3.16215 \mathrm{E} \quad 00$

$3.17917 \mathrm{E} \quad 00$

$3.19623 \mathrm{E} \quad 00$

$3.21333 \mathrm{E} \quad 00$

$3.23048 \mathrm{E} \quad 00$

$3.24768 \mathrm{E} \quad 00$

$3.26492 \mathrm{E} \quad 00$

$3.28220 \mathrm{E} \quad 00$

$3.29953 \mathrm{E} \quad 00$

$3.31691 \mathrm{E} \quad 00$

$3.33433 \mathrm{E} \quad 00$

$3.35179 \mathrm{E} \quad 00$

$3.36930 \mathrm{E} \quad 00$

$3.38686 \mathrm{E} \quad 00$

$3.40446 \mathrm{E} \quad 00$

$3.42210 \mathrm{E} \quad 00$

$3.43979 \mathrm{E} \quad 00$

$3.45752 \mathrm{E} \quad 00$

$3.47530 \mathrm{E} \quad 00$

$3.49312 \mathrm{E} \quad 00$

$3.51099 \mathrm{E} \quad 00$

$3.52891 \mathrm{E} \quad 00$

$3.54686 \mathrm{E} \quad 00$

$3.56487 \mathrm{E} \quad 00$

$3.58291 \mathrm{E} \quad 00$

$3.60100 \mathrm{E} \quad 00$

$3.61914 \mathrm{E} \quad 00$

$3.63732 \mathrm{E} \quad 00$

$3.65555 \mathrm{E} \quad 00$

$3.67382 \mathrm{E} \quad 00$

$3.69213 \mathrm{E} 00$

$3.71049 \mathrm{E} \quad 00$

$3.72890 \mathrm{E} \quad 00$

$3.74734 \mathrm{E} \quad 00$

$3.76584 \mathrm{E} \quad 00$

$3.78438 \mathrm{E} \quad 00$

$3.80296 \mathrm{E} \quad 00$

$3.82159 \mathrm{E} \quad 00$

$3.84026 \mathrm{E} \quad 00$

$3.85898 \mathrm{E} \quad 00$

$3.87774 \mathrm{E} \quad 00$

$3.89654 \mathrm{E} \quad 00$

$3.91539 \mathrm{E} \quad 00$

$3.93429 \mathrm{E} \quad 00$

$3.95323 \mathrm{E} \quad 00$

$3.97221 \mathrm{E} \quad 00$

$3.99124 \mathrm{E} \quad 00$

$4.01031 \mathrm{E} \quad 00$ $\tan \delta_{2}$

$f_{-2}$

$1.30225 \mathrm{E}-01$

$1.31397 \mathrm{E}-01$

$1.32576 \mathrm{E}-01$

$1.33762 \mathrm{E}-01$

$1.34955 \mathrm{E}-01$

$1.36155 \mathrm{E}-01$

$1.37363 \mathrm{E}-01$

$1.38577 \mathrm{E}-01$

$1.39798 \mathrm{E}-01$

$1.41027 \mathrm{E}-01$

$1.42262 \mathrm{E}-01$

$1.43505 \mathrm{E}-01$

$1.44755 \mathrm{E}-01$

$1.46012 \mathrm{E}-01$

$1.47276 \mathrm{E}-01$

$1.48547 \mathrm{E}-01$

$1.49826 \mathrm{E}-01$

$1.51112 \mathrm{E}-01$

$1.52405 \mathrm{E}-01$

$1.53706 \mathrm{E}-01$

$1.55013 \mathrm{E}-01$

$1.56329 \mathrm{E}-01$

$1.57651 \mathrm{E}-01$

$1.58981 \mathrm{E}-01$

$1.60318 \mathrm{E}-01$

$1.61663 \mathrm{E}-01$

$1.63015 \mathrm{E}-01$

$1.64375 \mathrm{E}-01$

$1.65742 \mathrm{E}-01$

$1.67117 \mathrm{E}-01$

$1.68499 \mathrm{E}-0 \mathrm{I}$

$1.69889 \mathrm{E}-01$

$1.71286 \mathrm{E}-01$

$1.72691 \mathrm{E}-01$

$1.74103 \mathrm{E}-01$

$1.75523 \mathrm{E}-01$

$1.76951 \mathrm{E}-01$

$1.78386 \mathrm{E}-01$

$1.79830 \mathrm{E}-01$

$1.81280 \mathrm{E}-01$

$1.82739 \mathrm{E}-01$

$1.84205 \mathrm{E}-01$

$1.85679 \mathrm{E}-01$

$1.87161 \mathrm{E}-01$

$1.88651 \mathrm{E}-01$

$1.90148 \mathrm{E}-01$

$1.91653 \mathrm{E}-01$

$1.93166 \mathrm{E}-01$

$1.94687 \mathrm{E}-01$

$1.96216 \mathrm{E}-01$

$1.97753 \mathrm{E}-01$

$1.99298 \mathrm{E}-01$

$2.00851 \mathrm{E}-01$

$2.02412 \mathrm{E}-01$

$2.03980 \mathrm{E}-01$

$2.05557 \mathrm{E}-01$

$2.07142 \mathrm{E}-01$

$2.08735 \mathrm{E}-01$

$2.10336 \mathrm{E}-01$

$2.11945 \mathrm{E}-01$

$$
-2.79597 \mathrm{E} \quad 01
$$

01 $-1.25897 \mathrm{E}-01$

$-2.79613 \mathrm{E}$ 01 $-1.27049 \mathrm{E}-01$

$-2.79621 \mathrm{E}$ 01 $-1.28207 \mathrm{E}-01$

$-2.79629 \mathrm{E} \quad 01 \quad-1.29372 \mathrm{E}-01$

$-2.79637 \mathrm{E} \quad 01$

$-2.79645 \mathrm{E} \quad 01$

$-2.79653 \mathrm{E} \quad 01$

$-2.79661 \mathrm{E} \quad 01$

\section{$-2.79677 \mathrm{E} \quad 01$}

$-2.79684 \mathrm{E} \quad 01$

$-2.79692 \mathrm{E} \quad 01$

$-2.79700 \mathrm{E}$ 0I

$-2.79707 \mathrm{E} 01$

$-2.79715 \mathrm{E} \quad 01$

$-2.79722 \mathrm{E} \quad 01$

$-2.79730 \mathrm{E} \quad 01$

$-2.79737 \mathrm{E} \quad 01$

$-2.79745 \mathrm{E} \quad 01$

$-2.79752 \mathrm{E} \quad 01$

$-2.79759 \mathrm{E} \quad 01$

$-2.79767 \mathrm{E}$ 01

$-2.79774 \mathrm{E}$ 0I

$-2.79781 \mathrm{E} \quad 01$

$-2.79788 \mathrm{E} 01$

$-2.79795 \mathrm{E}$ 01

$-2.79802 \mathrm{E} \quad 01$

$-2.79809 \mathrm{E}$ 01

$-2.79816 \mathrm{E}$ 01

$-2.79823 \mathrm{E} \quad 01$

$-2.79830 \mathrm{E}$ 01

$-2.79837 \mathrm{E} 01$

$-2.79844 \mathrm{E} \quad 01$

-2.79851 E 01

$-1.30545 \mathrm{E}-0$

$-1.31724 \mathrm{E}-01$

$-1.32910 \mathrm{E}-01$

$-1.34103 \mathrm{E}-01$

$-1.35304 \mathrm{E}-01$

$-1.36511 \mathrm{E}-01$ 
ELECTRON RADIAL FUNCTIONS

Positron

$p$

0.10

0.20

0.30

0.40

0.50

0.60

0.70

0.80

0.90

1.00

1.10

1.20

1.30

1.40

1.50

1.60

1.70

1.80

1.90

2.00

2.10

2.20

2.30

2.40

2.50

2.60

2.70

2.80

2.90

3.00

3.10

3.20

3.30

3.40

3.50

3.60

3.70

3.80

3.90

4.00

4.10

4.20

4.30

4.40

4.50

4.60

4.70

4.80

4.90

5.00

5.10

5.20

5.30

5.40

5.50

5.60

5.70

5.80

5.90

6.00
$F_{0}$

$9.75707 \mathrm{E}-02$

$3.50158 \mathbf{E}-01$

$5.07156 \mathrm{E}-01$

$6.01250 \mathrm{E}-01$

$6.61460 \mathrm{E}-01$

$7.02229 \mathrm{E}-01$

$7.31062 \mathrm{E}-01$

$7.52155 \mathrm{E}-01$

$7.68010 \mathrm{E}-01$

$7.80195 \mathrm{E}-01$

$7.89736 \mathrm{E}-01$

$7.97327 \mathrm{E}-01$

$8.03452 \mathrm{E}-01$

$8.08454 \mathrm{E}-01$

$8.12583 \mathrm{E}-01$

$8.16025 \mathrm{E}-01$ $8.18919 \mathrm{E}-01$

8.21371 E-01

$8.23463 \mathrm{E}-01$

$8.25260 \mathrm{E}-01$

$8.26812 \mathrm{E}-01$

$8.28160 \mathrm{E}-01$

$8.29337 \mathrm{E}-01$

$8.30368 \mathrm{E}-01$

$8.31276 \mathrm{E}-01$

8.32078 E- 01

$8.32789 \mathrm{E}-01$

$8.33421 \mathrm{E}-01$

$8.33985 \mathrm{E}-01$

8.34490 E-01

$8.34943 \mathrm{E}-01$

$8.35349 \mathrm{E}-01$

$8.35716 \mathrm{E}-01$

$8.36047 \mathrm{E}-01$

8.36346E-01

$8.36617 \mathrm{E}-01$ $8.36862 \mathrm{E}-01$ $8.37085 \mathrm{E}-01$ $8.37287 \mathrm{E}-01$ 8.37472E-01

8.37641 E-01 $8.37794 \mathrm{E}-01$ $8.37934 \mathrm{E}-01$ $8.38061 \mathrm{E}-01$ $8.38178 \mathrm{E}-01$

$8.38285 \mathrm{E}-01$ $8.38382 \mathrm{E}-01$ $8.38471 \mathrm{E}-01$ $8.38552 \mathrm{E}-01$ $8.38627 \mathrm{E}-01$

8.38695E-01 $8.38756 \mathrm{E}-01$ $8.38813 \mathrm{E}-01$ $8.38864 \mathrm{E}-01$ 8.38911 E-01

$8.38953 \mathrm{E}-01$ $8.38992 \mathrm{E}-01$ $8.39027 \mathrm{E}-0 \mathrm{l}$ $8.39058 \mathrm{E}-01$ $8.39087 \mathrm{E}-01$

$$
Z=8 \quad A=17
$$$$
\rho=1.2 A^{1 / 3} \mathrm{~F}
$$

$f_{1}$
$2.54533 \mathrm{E}-03$
$1.71795 \mathrm{E}-02$
$4.47000 \mathrm{E}-02$
$8.38370 \mathrm{E}-02$
$1.33045 \mathrm{E}-01$
$1.90732 \mathrm{E}-01$
$2.55398 \mathrm{E}-01$
$3.25712 \mathrm{E}-01$
$4.00544 \mathrm{E}-01$
$4.78961 \mathrm{E}-01$

$5.60209 \mathrm{E}-01$
$6.43693 \mathrm{E}-01$
$7.28939 \mathrm{E}-01$
$8.15579 \mathrm{E}-01$
$9.03322 \mathrm{E}-01$

$g_{1}$
$-4.58576 \mathrm{E}-05$
$-3.08834 \mathrm{E}-04$
$-8.00681 \mathrm{E}-04$
$-1.49434 \mathrm{E}-03$
$-2.35690 \mathrm{E}-03$

$\tan \delta_{1}$

$2.67914 \mathrm{E}-01$

$1.27763 \mathrm{E}-01$

8.48294E-02

6.36857 E-02

$5.10239 \mathrm{E}-02$

$4.25602 \mathrm{E}-02$

$3.64865 \mathrm{E}-02$

$3.19057 \mathrm{E}-02$

$2.83218 \mathrm{E}-02$

$2.54377 \mathrm{E}-02$

$2.30642 \mathrm{E}-02$

$2.10755 \mathrm{E}-02$

$1.93838 \mathrm{E}-02$

$1.79267 \mathrm{E}-02$

$1.66581 \mathrm{E}-02$

$9.91941 \mathrm{E}-01$

$1.08126 \mathrm{E} \quad 00$

$1.17113 \mathrm{E} \quad 00$

$1.26146 \mathrm{E} \quad 00$

$1.35214 \mathrm{E} \quad 00$

$\begin{array}{ll}1.44312 \mathrm{E} & 00\end{array}$

$1.53434 \mathrm{E} \quad 00$

$1.62574 \mathrm{E} \quad 00$

$1.71731 \mathrm{E} \quad 00$

$1.80900 \mathrm{E} \quad 00$

$1.90079 \mathrm{E} \quad 00$

$1.99268 \mathrm{E} \quad 00$

$2.08463 \mathrm{E} \quad 00$

$2.17664 \mathrm{E} \quad 00$

$2.26870 \mathrm{E} \quad 00$

$2.36080 \mathrm{E} \quad 00$

$2.45293 \mathrm{E} \quad 00$

$2.54509 \mathrm{E} \quad 00$

$2.63727 \mathrm{E} \quad 00$

$2.72947 \mathrm{E} \quad 00$

$2.82169 \mathrm{E} \quad 00$ $2.91391 \mathrm{E} \quad 00$

$3.00615 \mathrm{E} \quad 00$

$3.09840 \mathrm{E} \quad 00$

$3.19066 \mathrm{E} \quad 00^{\circ}$

$3.28292 \mathrm{E} \quad 00$

$3.37519 \mathrm{E} \quad 00$

$3.46747 \mathrm{E} \quad 00$

$3.55974 \mathrm{E} \quad 00$

$3.65203 \mathrm{E} \quad 00$

$3.74431 \mathrm{E} \quad 00$

$3.83660 \mathrm{E} \quad 00$

$3.92889 \mathrm{E} \quad 00$

$4.02119 \mathrm{E} \quad 00$

$4.11349 \mathrm{E} \quad 00$

$4.20579 \mathrm{E} \quad 00$

$4.29809 \mathrm{E} \quad 00$

$4.39040 \mathrm{E} \quad 00$

$4.48271 \mathrm{E} \quad 00$

$4.57503 \mathrm{E} \quad 00$

$4.66735 \mathrm{E} \quad 00$

$4.75967 \mathrm{E} \quad 00$

$4.85200 \mathrm{E} \quad 00$

$4.94433 \mathrm{E} \quad 00$

$5.03667 \mathrm{E} \quad 00$
-9.37381 E-03

$-1.06413 \mathrm{E}-02$

$-1.31385 \mathrm{E}-02$

$-1.43538 \mathrm{E}-02$

$-1.55399 \mathrm{E}-02$

$-1.66927 \mathrm{E}-02$

$-1.78093 \mathrm{E}-02$

$-1.88871 \mathrm{E}-02$

$-1.99243 \mathrm{E}-02$

$-2.09192 \mathrm{E}-02$

$-2.18707 \mathrm{E}-02$

$-2.27778 \mathrm{E}-02$

$-2.36396 \mathrm{E}-02$

$-2.44555 \mathrm{E}-02$

$-2.52249 \mathrm{E}-02$

$-2.59475 \mathrm{E}-02$

$-2.66228 \mathrm{E}-02$

$-2.72507 \mathrm{E}-02$

$-2.78307 \mathrm{E}-02$

$-2.83628 \mathrm{E}-02$

$-2.88467 \mathrm{E}-02$

$-2.92824 \mathrm{E}-02$

$-2.96697 \mathrm{E}-02$

$-3.00085 \mathrm{E}-02$

$-3.02987 \mathrm{E}-02$ $-3.05403 \mathrm{E}-02$

$-3.07332 \mathrm{E}-02$

$-3.08773 \mathrm{E}-02$

$-3.09726 \mathrm{E}-02$

$-3.10191 \mathrm{E}-02$

$-3.10167 \mathrm{E}-02$

$-3.09655 \mathrm{E}-02$

$-3.08653 \mathrm{E}-02$

$-3.07162 \mathrm{E}-02$

$-3.05182 \mathrm{E}-02$

$-3.02711 \mathrm{E}-02$

$-2.99751 \mathrm{E}-02$

$-2.92362 \mathrm{E}-02$

$-2.87931 \mathrm{E}-02$ $-2.83011 \mathrm{E}-02$

$-2.77600 \mathrm{E}-02$

$-2.71699 \mathrm{E}-02$

$-2.65308 \mathrm{E}-02$

$-2.58425 \mathrm{E}-02$

$-2.51053 \mathrm{E}-02$

$-2.43189 \mathrm{E}-02$

$-2.34835 \mathrm{E}-02$

$-2.25990 \mathrm{E}-02$
$-2.96302 \mathrm{E}-02$
$1.55434 \mathrm{E}-02$

$1.45560 \mathrm{E}-02$

$1.36750 \mathrm{E}-02$

$1.28841 \mathrm{E}-02$

$1.21700 \mathrm{E}-02$

$1.15221 \mathrm{E}-02$

$1.09314 \mathrm{E}-02$

$1.03908 \mathrm{E}-02$

$9.89398 \mathrm{E}-03$

$9.43595 \mathrm{E}-03$

$9.01227 \mathrm{E}-03$

$8.61924 \mathrm{E}-03$

$8.25360 \mathrm{E}-03$

$7.91261 \mathrm{E}-03$

$7.59385 \mathrm{E}-03$

$7.29522 \mathrm{E}-03$

$7.01485 \mathrm{E}-03$

$6.75113 \mathrm{E}-03$

$6.50263 \mathrm{E}-03$

$6.26804 \mathrm{E}-03$

$6.04626 \mathrm{E}-03$ $5.83623 \mathrm{E}-03$

$5.63708 \mathrm{E}-03$

$5.44795 \mathrm{E}-03$

$5.26813 \mathrm{E}-03$

$5.09695 \mathrm{E}-03$

$4.93378 \mathrm{E}-03$

$4.77810 \mathrm{E}-03$

$4.62939 \mathrm{E}-03$

$4.48719 \mathrm{E}-03$

$4.35109 \mathrm{E}-03$

$4.09571 \mathrm{E}-03$

$3.97574 \mathrm{E}-03$

$3.86051 \mathrm{E}-03$

$3.74975 \mathrm{E}-03$

$3.64322 \mathrm{E}-03$

$3.54065 \mathrm{E}-03$

$3.44185 \mathrm{E}-03$

3.34661 E-03

$3.25475 \mathrm{E}-03$

$3.16608 \mathrm{E}-03$

$3.08045 \mathrm{E}-03$

$2.99771 \mathrm{E}-03$

$2.91770 \mathrm{E}-03$
$4.22071 \mathrm{E}-03$

$f_{-1}$

$1.02954 \mathrm{E}-03$

$3.88003 \mathrm{E}-03$ 


\section{$\bar{F}$}

0.10

0.20

0.30

0.40

0.50

0.60

0.70

0.80

0.90

1.00

1.10

1.20

1.30

1.40

1.50

1.60

1.70

1.80

1.90

2.00

2.10

2.20

2.30

2.40

2.50

2.60

2.70

2.80

2.90

3.00

3.10

3.20

3.30

3.40

3.50

3.60

3.70

3.80

3.90

4.00

4.10

4.20

4.30

4.40

4.50

4.60

4.70

4.80

4.90

5.00

5.10

5.20

5.30

5.40

5.50

5.60

5.70

5.80

5.90

6.00
$2.29406 \mathrm{E}-09$ $2.66829 \mathrm{E}-08$ $8.32183 \mathrm{E}-08$ $1.72676 \mathrm{E}-07$ $2.94708 \mathrm{E}-07$

$4.48824 \mathrm{E}-07$ $6.34566 \mathrm{E}-07$ $8.51547 \mathrm{E}-07$ $1.09945 \mathrm{E}-06$ $1.37803 \mathrm{E}-06$

$1.68709 \mathrm{E}-06$ $2.02647 \mathrm{E}-06$ $2.39605 \mathrm{E}-06$ $2.79573 \mathrm{E}-06$ $3.22543 \mathrm{E}-06$

$3.68510 \mathrm{E}-06$ $4.17468 \mathrm{E}-06$ $4.69414 \mathrm{E}-06$

$5.24344 \mathrm{E}-06$ $5.82256 \mathrm{E}-06$

$6.43147 \mathrm{E}-06$ $7.07016 \mathrm{E}-06$ $7.73861 \mathrm{E}-06$ $8.43681 \mathrm{E}-06$ $9.16474 \mathrm{E}-06$

$9.92241 \mathrm{E}-06$ $1.07098 \mathrm{E}-05$ $1.15269 \mathrm{E}-05$ $1.23737 \mathrm{E}-05$ $1.32502 \mathrm{E}-05$

$1.41564 \mathrm{E}-05$ $1.50923 \mathrm{E}-05$ $1.60578 \mathrm{E}-05$ $1.70531 \mathrm{E}-05$ $1.80780 \mathrm{E}-05$

$1.91326 \mathrm{E}-05$ $2.02169 \mathrm{E}-05$ $2.13309 \mathrm{E}-05$ $2.24745 \mathrm{E}-05$ $2.36478 \mathrm{E}-05$

$2.48507 \mathrm{E}-05$

$2.60833 \mathrm{E}-05$

$2.73456 \mathrm{E}-05$

$2.86375 \mathrm{E}-05$

$2.99591 \mathrm{E}-05$

$3.13103 \mathrm{E}-05$ $3.26912 \mathrm{E}-05$ $3.41018 \mathrm{E}-05$ $3.55420 \mathrm{E}-05$ $3.70118 \mathrm{E}-05$

$3.85113 \mathrm{E}-05$ $4.00404 \mathrm{E}-05$

$4.15992 \mathrm{E}-05$

$4.31876 \mathrm{E}-05$

$4.48057 \mathrm{E}-05$

$4.64534 \mathrm{E}-05$ $4.81307 \mathrm{E}-05$ $4.98377 \mathrm{E}-05$ $5.15744 \mathrm{E}-05$ $5.33406 \mathrm{E}-05$ $f_{2}$

$g_{2}$

$\tan \delta_{2}$

$f_{-2}$

$6.99883 \mathrm{E}-07$ $9.19372 \mathrm{E}-06$ $3.57020 \mathrm{E}-05$ $8.91335 \mathrm{E}-05$ $1.76689 \mathrm{E}-04$

$3.03860 \mathrm{E}-04$ $4.74618 \mathrm{E}-04$ $6.91699 \mathrm{E}-04$ $9.56906 \mathrm{E}-04$ $1.27137 \mathrm{E}-03$

1.63574 E-03 $2.05039 \mathrm{E}-03$ $2.51546 \mathrm{E}-03$ $3.03099 \mathrm{E}-03$

$3.59692 \mathrm{E}-03$

$4.21319 \mathrm{E}-03$

$4.87967 \mathrm{E}-03$

$5.59626 \mathrm{E}-03$

$6.36285 \mathrm{E}-03$

$7.17932 \mathrm{E}-03$

$8.04558 \mathrm{E}-03$ $8.96153 \mathrm{E}-03$ $9.92709 \mathrm{E}-03$ $1.09422 \mathrm{E}-02$

$1.20067 \mathrm{E}-02$

$1.31207 \mathrm{E}-02$ $1.42840 \mathrm{E}-02$ $1.54966 \mathrm{E}-02$ $1.67584 \mathrm{E}-02$ $1.80695 \mathrm{E}-02$

$1.94297 \mathrm{E}-02$ $2.08391 \mathrm{E}-02$ $2.22976 \mathrm{E}-02$

$2.38052 \mathrm{E}-02$

$2.53619 \mathrm{E}-02$

$2.69676 \mathrm{E}-02$ $2.86223 \mathrm{E}-02$ $3.03260 \mathrm{E}-02$

$3.20787 \mathrm{E}-02$

$3.38804 \mathrm{E}-02$

$3.57311 \mathrm{E}-02$

$3.76307 \mathrm{E}-02$

$3.95792 \mathrm{E}-02$

$4.15767 \mathrm{E}-02$

4.36232 E-02

$4.57185 \mathrm{E}-02$ $4.78627 \mathrm{E}-02$

$5.00558 \mathrm{E}-02$ $5.22978 \mathrm{E}-02$ $5.45887 \mathrm{E}-02$

$5.69285 \mathrm{E}-02$ $5.93171 \mathrm{E}-02$ $6.17546 \mathrm{E}-02$ $6.42409 \mathrm{E}-02$ $6.67762 \mathrm{E}-02$

$6.93602 \mathrm{E}-02$ $7.19931 \mathrm{E}-02$ $7.46748 \mathrm{E}-02$ $7.74053 \mathrm{E}-02$ $8.01847 \mathrm{E}-02$
$-7.09727 \mathrm{E}-09-1.64235 \mathrm{E} \quad 00$

$-9.30126 \mathrm{E}-08$

$-3.59813 \mathrm{E}-07$

$-8.93607 \mathrm{E}-07$

$-1.75982 \mathrm{E}-06$

$-3.00304 \mathrm{E}-06$

$-4.64931 \mathrm{E}-06$

$-6.70951 \mathrm{E}-06$

$-9.18301 \mathrm{E}-06$

$-1.20608 \mathrm{E}-05$

$-1.53282 \mathrm{E}-05$

$-1.89665 \mathrm{E}-05$

$-2.29546 \mathrm{E}-05$

$-2.72697 \mathrm{E}-05$

$-3.18881 \mathrm{E}-05$

$-3.67857 \mathrm{E}-05$

$-4.19378 \mathrm{E}-05$

$-4.73199 \mathrm{E}-05$

$-5.29072 \mathrm{E}-05$

$-5.86752 \mathrm{E}-05$

$-6.45992 \mathrm{E}-05$

$-7.06547 \mathrm{E}-05$

$-7.68175 \mathrm{E}-05$

$-8.30632 \mathrm{E}-05$

$-8.93676 \mathrm{E}-05$

$-9.57066 \mathrm{E}-05$

$-1.02056 \mathrm{E}-04$

$-1.08392 \mathrm{E}-04$

$-1.14691 \mathrm{E}-04$

$-1.20928 \mathrm{E}-04$

$-1.27081 \mathrm{E}-04$

$-1.33124 \mathrm{E}-04$

$-1.39035 \mathrm{E}-04$

$-1.44790 \mathrm{E}-04$

$-1.50364 \mathrm{E}-04$

$-1.55736 \mathrm{E}-04$

$-1.60879 \mathrm{E}-04$

$-1.65773 \mathrm{E}-04$

$-1.70391 \mathrm{E}-04$

$-1.74712 \mathrm{E}-04$

$-1.78711 \mathrm{E}-04$

$-1.82365 \mathrm{E}-04$

$-1.85651 \mathrm{E}-04$

$-1.88545 \mathrm{E}-04$

$-1.91023 \mathrm{E}-04$

$-1.93061 \mathrm{E}-04$

$-1.94638 \mathrm{E}-04$

$-1.95728 \mathrm{E}-04$

$-1.96309 \mathrm{E}-04$

$-1.96356 \mathrm{E}-04$

$-1.95848 \mathrm{E}-04$

$-1.94759 \mathrm{E}-04$

$-1.93068 \mathrm{E}-04$

$-1.90749 \mathrm{E}-04$

$-1.87780 \mathrm{E}-04$

$-1.84137 \mathrm{E}-04$

$-1.79798 \mathrm{E}-04$

$-1.74738 \mathrm{E}-04$

$-1.68934 \mathrm{E}-04$

$-1.62362 \mathrm{E}-04$

$-3.54207 \mathrm{E} \quad 00$

$-5.29028 \mathrm{E} \quad 00$

$-6.91446 \mathrm{E} \quad 00$

$-8.40878 \mathrm{E} \quad 00$

$-9.76956 \mathrm{E} \quad 00$

$-1.09985 \mathrm{E} \quad 01$

$-1.21017 \mathrm{E} 01$

$-1.30883 \mathrm{E} \quad 01$

$\begin{array}{lll}-1.39688 \mathrm{E} & 01\end{array}$

$-1.47541 \mathrm{E} \quad 01$

$-1.54550 \mathrm{E} \quad 01$

$-1.60813 \mathrm{E} \quad 01$

$-1.66420 \mathrm{E} \quad 01$

$-1.71453 \mathrm{E} 01$.

$\begin{array}{ll}-1.75982 \mathrm{E} & 01\end{array}$

$-1.80068 \mathrm{E} \quad 01$

$-1.83766 \mathrm{E} \quad 01$

$-1.87122 \mathrm{E} 01$

$-1.90177 \mathrm{E} \quad 01$

$-1.92965 \mathrm{E} \quad 01$

$-1.95517 \mathrm{E} \quad 01$

$-1.97859 \mathrm{E} \quad 01$

$-2.00013 \mathrm{E} 01$

$-2.02001 \mathrm{E} \quad 01$

$-2.03839 \mathrm{E} \quad 01$

$-2.05542 \mathrm{E} \quad 01$

$-2.07123 \mathrm{E} 01$

$-2.08595 \mathrm{E} \quad 01$

$-2.09968 \mathrm{E} \quad 01$

$-2.11251 \mathrm{E} \quad 01$

$-2.12452 \mathrm{E}$ 0I

$-2.13578 \mathrm{E} 01$

$-2.14635 \mathrm{E} 01$

$-2.15630 \mathrm{E} \quad 01$

$-2.16568 \mathrm{E} \quad 01$

$-2.17453 \mathrm{E} 01$

$-2.18289 \mathrm{E} \quad 01$

$-2.19080 \mathrm{E} \quad 01$

$-2.19830 \mathrm{E} 01$

$\begin{array}{ll}-2.20541 \mathrm{E} & 01\end{array}$

$-2.21217 \mathrm{E} \quad 01$ 
ELECTRON RADIAL FUNCTIONS

Positron

\begin{tabular}{|c|c|c|c|c|}
\hline$p$ & $F_{0}$ & $f_{1}$ & & $g_{1}$ \\
\hline 6.10 & $8.39113 \mathrm{E}-01$ & $5.12901 \mathrm{E}$ & 00 & $-2.16654 \mathrm{E}-02$ \\
\hline 6.20 & $8.39136 \mathrm{E}-01$ & $5.22136 \mathrm{E}$ & 00 & $-2.06827 \mathrm{E}-02$ \\
\hline 6.30 & $8.39156 \mathrm{E}-01$ & $5.31371 \mathrm{E}$ & 00 & $-1.96509 \mathrm{E}-02$ \\
\hline 6.40 & $8.39174 \mathrm{E}-01$ & $5.40606 \mathrm{E}$ & 00 & $-1.85700 \mathrm{E}-02$ \\
\hline 6.50 & $8.39190 \mathrm{E}-01$ & $5.49843 \mathrm{E}$ & 00 & $-1.74400 \mathrm{E}-02$ \\
\hline 6.60 & $8.39203 \mathrm{E}-01$ & $5.59079 \mathrm{E}$ & 00 & $-1.62608 \mathrm{E}-02$ \\
\hline 6.70 & $8.39215 \mathrm{E}-01$ & $5.68317 \mathrm{E}$ & 00 & $-1.50325 \mathrm{E}-02$ \\
\hline 6.80 & $8.39226 \mathrm{E}-01$ & $5.77555 \mathrm{E}$ & 00 & $-1.37551 \mathrm{E}-02$ \\
\hline 6.90 & $8.39234 \mathrm{E}-01$ & $5.86793 \mathrm{E}$ & 00 & $-1.24286 \mathrm{E}-02$ \\
\hline 7.00 & $8.39241 \mathrm{E}-01$ & $5.96032 \mathrm{E}$ & 00 & $-1.10529 \mathrm{E}-02$ \\
\hline 7.10 & $8.39247 \mathrm{E}-01$ & $6.05272 \mathrm{E}$ & 00 & $-9.62802 \mathrm{E}-03$ \\
\hline 7.20 & $8.39251 \mathrm{E}-01$ & $6.14513 \mathbf{E}$ & 00 & $-8.15401 \mathrm{E}-03$ \\
\hline 7.30 & $8.39253 \mathrm{E}-01$ & $6.23754 \mathrm{E}$ & 00 & $-6.63082 \mathrm{E}-03$ \\
\hline 7.40 & $8.39255 \mathrm{E}-01$ & $6.32996 \mathrm{E}$ & 00 & $-5.05845 \mathrm{E}-03$ \\
\hline 7.50 & $8.39256 \mathrm{E}-01$ & $6.42239 \mathrm{E}$ & 00 & $-3.43690 \mathrm{E}-03$ \\
\hline 7.60 & $8.39256 \mathrm{E}-01$ & $6.51482 \mathrm{E}$ & 00 & $-1.76616 \mathrm{E}-03$ \\
\hline 7.70 & $8.39255 \mathrm{E}-01$ & $6.60727 \mathrm{E}$ & 00 & $-4.62193 \mathrm{E}-05$ \\
\hline 7.80 & $8.39253 \mathrm{E}-01$ & $6.69972 \mathrm{E}$ & 00 & $1.72293 \mathrm{E}-03$ \\
\hline 7.90 & $8.39250 \mathrm{E}-01$ & $6.79218 \mathrm{E}$ & 00 & $3.54130 \mathrm{E}-03$ \\
\hline 8.00 & $8.39246 \mathrm{E}-01$ & $6.88464 \mathrm{E}$ & 00 & $5.40888 \mathrm{E}-03$ \\
\hline 8.10 & $8.39242 \mathrm{E}-01$ & $6.97712 \mathrm{E}$ & 00 & $7.32571 \mathrm{E}-03$ \\
\hline 8.20 & $8.39236 \mathrm{E}-01$ & $7.06960 \mathrm{E}$ & 00 & $9.29177 \mathrm{E}-03$ \\
\hline 8.30 & $8.39231 \mathrm{E}-01$ & $7.16210 \mathrm{E}$ & 00 & $1.13071 \mathrm{E}-02$ \\
\hline 8.40 & $8.39224 \mathrm{E}-01$ & $7.25459 \mathrm{E}$ & 00 & $1.33716 \mathrm{E}-02$ \\
\hline 8.50 & $8.39217 \mathrm{E}-01$ & $7.34710 \mathrm{E}$ & 00 & $1.54855 \mathrm{E}-02$ \\
\hline 8.60 & $8.39210 \mathrm{E}-01$ & $7.43961 \mathrm{E}$ & 00 & $1.76486 \mathrm{E}-02$ \\
\hline 8.70 & $8.39202 \mathrm{E}-01$ & $7.53214 \mathrm{E}$ & 00 & $1.98609 \mathrm{E}-02$ \\
\hline 8.80 & $8.39193 \mathrm{E}-01$ & $7.62466 \mathrm{E}$ & 00 & $2.21226 \mathrm{E}-02$ \\
\hline 8.90 & $8.39184 \mathrm{E}-01$ & $7.71720 \mathrm{E}$ & 00 & $2.44335 \mathrm{E}-02$ \\
\hline 9.00 & $8.39175 \mathrm{E}-01$ & $7.80974 \mathrm{E}$ & 00 & $2.67938 \mathrm{E}-02$ \\
\hline 9.10 & $8.39166 \mathrm{E}-01$ & $7.90229 \mathrm{E}$ & 00 & $2.92033 \mathrm{E}-02$ \\
\hline 9.20 & $8.39155 \mathrm{E}-01$ & $7.99482 \mathrm{E}$ & 00 & $3.16621 \mathrm{E}-02$ \\
\hline 9.30 & $8.39145 \mathrm{E}$ & $8.08735 \mathrm{E}$ & 00 & $3.41701 \mathrm{E}-02$ \\
\hline 9.40 & $8.39134 \mathrm{E}-01$ & $8.17987 \mathrm{E}$ & 00 & $3.67273 \mathrm{E}-02$ \\
\hline 9.50 & $8.39123 \mathrm{E}-01$ & $8.27240 \mathrm{E}$ & 00 & $3.93339 \mathrm{E}-02$ \\
\hline 9.60 & $8.39112 \mathrm{E}-01$ & $8.36479 \mathrm{E}$ & 00 & $4.19891 \mathrm{E}-02$ \\
\hline 9.70 & 8.3 & $697 \mathrm{E}$ & 00 & $3 E-02$ \\
\hline 9.80 & $8.39088 \mathrm{E}-01$ & $8.54832 \mathrm{E}$ & 00 & $4.74400 \mathrm{E}-02$ \\
\hline 9.90 & $8.39076 \mathrm{E}-01$ & $8.61239 \mathrm{E}$ & 00 & $5.00777 \mathrm{E}-02$ \\
\hline 10.00 & $8.39064 \mathrm{E}-01$ & $8.73977 \mathrm{E}$ & 00 & $5.31346 \mathrm{E}-02$ \\
\hline 10.10 & $8.39051 \mathrm{E}-01$ & $8.83072 \mathrm{E}$ & 00 & $5.60281 \mathrm{E}-02$ \\
\hline 10.20 & $8.39039 \mathrm{E}-01$ & $8.92287 \mathrm{E}$ & 00 & $5.89780 \mathrm{E}-02$ \\
\hline 10.30 & $8.39026 \mathrm{E}-01$ & $9.01531 \mathrm{E}$ & 00 & $6.19789 \mathrm{E}-02$ \\
\hline 10.40 & $8.39013 \mathrm{E}$ & $9.10790 \mathrm{E}$ & 00 & $6.50301 \mathrm{E}-02$ \\
\hline 10.50 & $8.39000 \mathrm{E}-01$ & $9.20056 \mathrm{E}$ & 00 & $6.81312 \mathrm{E}-02$ \\
\hline 10.60 & $8.38987 \mathrm{E}-01$ & $9.29324 \mathrm{E}$ & 00 & $7.12819 \mathrm{E}-02$ \\
\hline 10.70 & $8.38973 \mathrm{E}$ & $9.38597 \mathrm{E}$ & 00 & $7.44822 \mathrm{E}-02$ \\
\hline 10.80 & $8.38960 \mathrm{E}-$ & & 00 & $7.77322 \mathrm{E}-02$ \\
\hline 10.90 & $8.38946 \mathrm{E}-01$ & $9.57149 \mathrm{E}$ & 00 & $8.10316 \mathrm{E}-02$ \\
\hline 11.00 & $8.38932 \mathrm{E}-01$ & $9.66428 \mathrm{E}$ & 00 & $8.43807 \mathrm{E}-02$ \\
\hline 11.10 & $8.38918 \mathrm{E}-01$ & $9.75708 \mathrm{E}$ & 00 & $8.77794 \mathrm{E}-02$ \\
\hline 11.20 & $8.38904 \mathrm{E}-01$ & & 00 & $9.12278 \mathrm{E}-02$ \\
\hline 11.30 & $8.38890 \mathrm{E}-01$ & $9.94276 \mathrm{E}$ & 00 & $9.47257 \mathrm{E}-02$ \\
\hline 11.40 & $8.38876 \mathrm{E}-01$ & $1.00356 \mathrm{E}$ & 01 & $9.82732 \mathrm{E}-02$ \\
\hline 11.50 & $8.38862 \mathrm{E}-01$ & $1.01285 \mathrm{E}$ & 01 & $1.01871 \mathrm{E}-01$ \\
\hline 11.60 & $8.38848 \mathrm{E}-01$ & $1.02214 \mathrm{E}$ & 01 & $1.05517 \mathrm{E}-01$ \\
\hline & $8.38834 \mathrm{E}-01$ & $1.03143 \mathrm{E}$ & 01 & $1.09214 \mathrm{E}-01$ \\
\hline & $8.38819 \mathrm{E}-01$ & & 01 & $1.12960 \mathrm{E}-01$ \\
\hline 11.90 & $8.38805 \mathrm{E}-01$ & $1.05001 \mathrm{E}$ & 01 & $1.16756 \mathrm{E}-01$ \\
\hline & $8.38791 \mathrm{E}-01$ & $1.05931 \mathrm{E}$ & 0] & $1.20602 \mathrm{E}-01$ \\
\hline
\end{tabular}

$\tan \delta_{1}$ $\rho=1.2 A^{1 / 3} F$
$2.84029 \mathrm{E}-03$

$2.76538 \mathrm{E}-03$

$2.69283 \mathrm{E}-03$

$2.62254 \mathrm{E}-03$

$2.55440 \mathrm{E}-03$

$2.48831 \mathrm{E}-03$

$2.42421 \mathrm{E}-03$

$2.36198 \mathrm{E}-03$

$2.30155 \mathrm{E}-03$

$2.24284 \mathrm{E}-03$

$2.18580 \mathrm{E}-03$

$2.13033 \mathrm{E}-03$

$2.07639 \mathrm{E}-03$

$2.02391 \mathrm{E}-03$

$1.97283 \mathrm{E}-03$

$1.92310 \mathrm{E}-03$

$1.87467 \mathrm{E}-03$

$1.82748 \mathrm{E}-03$

$1.78149 \mathrm{E}-03$

$1.73666 \mathrm{E}-03$

$1.69295 \mathrm{E}-03$

$1.65030 \mathrm{E}-03$

$1.60870 \mathrm{E}-03$

$1.56809 \mathrm{E}-03$

$1.52845 \mathrm{E}-03$

$1.48974 \mathrm{E}-03$

$1.45193 \mathrm{E}-03$

$1.41499 \mathrm{E}-03$

$1.37889 \mathrm{E}-03$

$1.34360 \mathrm{E}-03$

$1.30910 \mathrm{E}-03$

$1.27536 \mathrm{E}-03$

$1.24236 \mathrm{E}-03$

$1.21007 \mathrm{E}-03$

$1.17848 \mathrm{E}-03$

$1.14755 \mathrm{E}-03$

$1.11729 \mathrm{E}-03$

$1.08765 \mathrm{E}-03$

$1.05862 \mathrm{E}-03$

$1.03018 \mathrm{E}-03$

$1.00232 \mathrm{E}-03$

$9.75019 \mathrm{E}-04$

$9.48266 \mathrm{E}-04$

$9.22047 \mathrm{E}-04$

$8.96325 \mathrm{E}-04$

$8.71114 \mathrm{E}-04$ 8.46385 E- 04 $8.22140 \mathrm{E}-04$

$7.98343 \mathrm{E}-04$

7.74993 E-04

$7.52088 \mathrm{E}-04$

$7.29601 \mathrm{E}-04$

$7.07525 \mathrm{E}-04$

$6.85855 \mathrm{E}-04$

$6.64575 \mathrm{E}-04$

$6.43679 \mathrm{E}-04$

$6.23163 \mathrm{E}-04$

$6.03003 \mathrm{E}-04$

$5.83200 \mathrm{E}-04$
$5.63739 \mathrm{E}-04$ $f_{-1}$

$5.76893 \mathrm{E}-02$

$5.69631 \mathrm{E}-02$

$5.61873 \mathrm{E}-02$

$5.53619 \mathrm{E}-02$

5.44871 E-02

$5.35629 \mathrm{E}-02$

$5.25891 \mathrm{E}-02$

$5.15660 \mathrm{E}-02$

$5.04933 \mathrm{E}-02$

4.93713 E- 02

$4.81998 \mathrm{E}-02$

$4.69789 \mathrm{E}-02$

$4.57086 \mathrm{E}-02$

$4.43888 \mathrm{E}-02$

$4.30197 \mathrm{E}-02$

4.16012E-02

$4.01333 \mathrm{E}-02$

$3.86159 \mathrm{E}-02$

$3.70492 \mathrm{E}-02$

$3.54330 \mathrm{E}-02$

$3.37675 \mathrm{E}-02$

$3.20525 \mathrm{E}-02$

$3.02882 \mathrm{E}-02$

$2.84744 \mathrm{E}-02$

$2.66112 \mathrm{E}-02$

$2.46986 \mathrm{E}-02$ $2.27365 \mathrm{E}-02$

$2.07251 \mathrm{E}-02$

$1.86642 \mathrm{E}-02$

$1.65538 \mathrm{E}-02$

$1.43941 \mathrm{E}-02$

$1.21848 \mathrm{E}-02$

$9.92611 \mathrm{E}-03$

$7.61794 \mathrm{E}-03$

$5.26029 \mathrm{E}-03$

$2.85316 \mathrm{E}-03$

$3.96537 \mathrm{E}-04$

$-2.10959 \mathrm{E}-03$

$-4.66523 \mathrm{E}-03$

$-7.27039 \mathrm{E}-03$

$-9.92508 \mathrm{E}-03$

$-1.26293 \mathrm{E}-02$

$-1.53831 \mathrm{E}-02$

$-1.81864 \mathrm{E}-02$

$-2.10394 \mathrm{E}-02$

$-2.39419 \mathrm{E}-02$ $-2.68939 \mathrm{E}-02$ $-2.98957 \mathrm{E}-02$ $-3.29470 \mathrm{E}-02$

$-3.60479 \mathrm{E}-02$

$-3.91985 \mathrm{E}-02$ $-4.23987 \mathrm{E}-02$

$-4.56487 \mathrm{E}-02$

$-4.89483 \mathrm{E}-02$

$-5.22976 \mathrm{E}-02$

$-5.56966 \mathrm{E}-02$

$-5.91453 \mathrm{E}-02$

$-6.26437 \mathrm{E}-02$

$-6.61919 \mathrm{E}-02$

$-6.97899 \mathrm{E}-02$ g-1

$\tan \delta_{-1}$

$6.04008 \mathrm{E} \quad 00$ $6.13287 \mathrm{E} \quad 00$ $6.22564 \mathrm{E} \quad 00$

$6.31841 \mathrm{E} \quad 00$

$6.41118 \mathrm{E} \quad 00$

$6.50393 \mathrm{E} \quad 00$

$6.59669 \mathrm{E} \quad 00$

$6.68944 \mathrm{E} \quad 00$

$6.78219 \mathrm{E} \quad 00$

$6.87494 \mathrm{E} \quad 00$

$6.96768 \mathrm{E} \quad 00$

$7.06043 \mathrm{E} \quad 00$

$7.15318 \mathrm{E} \quad 00$

$7.24593 \mathrm{E} \quad 00$

$7.33868 \mathrm{E} \quad 00$

$7.43143 \mathrm{E} \quad 00$

$7.52419 \mathrm{E} \quad 00$

$7.61695 \mathrm{E} \quad 00$

$7.70971 \mathrm{E} \quad 00$

$7.80248 \mathrm{E} \quad 00$

$7.89525 \mathrm{E} \quad 00$

$7.98803 \mathrm{E} \quad 00$

$8.08082 \mathrm{E} \quad 00$

$8.17360 \mathrm{E} \quad 00$

$8.26640 \mathrm{E} \quad 00$

$8.35921 \mathrm{E} \quad 00$

$8.45202 \mathrm{E} \quad 00$

$8.54484 \mathrm{E} \quad 00$

$8.63767 \mathrm{E} 00$

8.73050 E 00

$8.82335 \mathrm{E} \quad 00$

$8.91620 \mathrm{E} \quad 00$

$9.00906 \mathrm{E} \quad 00$

$9.10194 \mathrm{E} \quad 00$

$9.19482 \mathrm{E} \quad 00$

$9.28771 \mathrm{E} \quad 00$

$9.38062 \mathrm{E} \quad 00$

$9.47353 \mathrm{E} \quad 00$

$9.56646 \mathrm{E} \quad 00$

$9.65940 \mathrm{E} \quad 00$

$9.75234 \mathrm{E} \quad 00$

$9.84530 \mathrm{E} \quad 00$

$9.93828 \mathrm{E} \quad 00$

$1.00313 \mathrm{E} 01$

$1.01243 \mathrm{E} \quad 01$

$1.02173 \mathrm{E} \quad 01$

$1.03103 \mathrm{E} \quad 01$

$1.04033 \mathrm{E} \quad 01$

$1.04964 \mathrm{E} \quad 01$

$1.05894 \mathrm{E} \quad 01$

$1.06825 \mathrm{E} \quad 01$ $1.07756 \mathrm{E} \quad 01$

$1.08687 \mathrm{E} \quad 01$

$1.09618 \mathrm{E} \quad 01$

$1.10550 \mathrm{E} \quad 01$

$1.11481 \mathrm{E} \quad 01$

$1.12413 \mathrm{E} 01$

$1.13344 \mathrm{E} \quad 01$

$1.14276 \mathrm{E} \quad 01$

$1.15208 \mathrm{E} 01$
$1.48677 \mathrm{E} \quad 02$ $1.50456 \mathrm{E} \quad 02$ $1.52218 \mathrm{E} \quad 02$ $1.53966 \mathrm{E} \quad 02$ $1.55697 \mathrm{E} \quad 02$

$1.57414 \mathrm{E} \quad 02$ $1.59117 \mathrm{E} \quad 02$ $1.60804 \mathrm{E} \quad 02$ $1.62478 \mathrm{E} \quad 02$ $1.64137 \mathrm{E} \quad 02$

$1.65783 \mathrm{E} \quad 02$ $1.67414 \mathrm{E} \quad 02$

$1.69033 \mathrm{E} \quad 02$ $1.70638 \mathrm{E} \quad 02$ $1.72230 \mathrm{E} \quad 02$

$\begin{array}{ll}1.73809 \mathrm{E} & 02\end{array}$ $1.75375 \mathrm{E} \quad 02$ $1.76929 \mathrm{E} \quad 02$ $1.78470 \mathrm{E} \quad 02$ $1.79999 \mathrm{E} \quad 02$

$1.81516 \mathrm{E} \quad 02$ $1.83021 \mathrm{E} \quad 02$ $1.84515 \mathrm{E} \quad 02$ $\begin{array}{ll}1.85997 \mathrm{E} & 02\end{array}$ $1.87468 \mathrm{E} \quad 02$

$\begin{array}{ll}1.88927 \mathrm{E} & 02\end{array}$ $\begin{array}{lll}1.90376 \mathrm{E} & 02\end{array}$ $\begin{array}{lll}1.91814 \mathrm{E} & 02\end{array}$ $1.93241 \mathrm{E} \quad 02$ $1.94657 \mathrm{E} \quad 02$

$1.96064 \mathrm{E} \quad 02$ $1.97459 \mathrm{E} \quad 02$ $1.98845 \mathrm{E} \quad 02$ $2.00220 \mathrm{E} \quad 02$ $2.01587 \mathrm{E} \quad 02$

$2.02942 \mathrm{E} \quad 02$ $2.04289 \mathrm{E} \quad 02$ $2.05627 \mathrm{E} \quad 02$ $2.06954 \mathrm{E} \quad 02$ $\begin{array}{ll}2.08273 \mathrm{E} & 02\end{array}$

$2.09583 \mathrm{E} \quad 02$ $2.10883 \mathrm{E} \quad 02$ $2.12175 \mathrm{E} \quad 02$ $2.13459 \mathrm{E} \quad 02$ $2.14733 \mathrm{E} \quad 02$

$2.16000 \mathrm{E} \quad 02$ 2.17257 E 02 $2.18508 \mathrm{E} \quad 02$ $2.19749 \mathrm{E} \quad 02$ 2.20983 E 02

$2.22209 \mathrm{E} \quad 02$ $2.23427 \mathrm{E} \quad 02$ $2.24637 \mathrm{E} \quad 02$ $2.25840 \mathrm{E} \quad 02$ $2.27035 \mathrm{E} \quad 02$

$2.28223 \mathrm{E} \quad 02$ $2.29404 \mathrm{E} \quad 02$ $2.30578 \mathrm{E} \quad 02$ $2.31745 \mathrm{E} \quad 02$ $2.32904 \mathrm{E} \quad 02$ 
6.10

6.20

6.30

6.40

6.50

6.60

6.70

6.80

6.90

7.00

7.10

7.20

7.30

7.40

7.50

7.60

7.70

7.80

7.90

8.00

8.10

8.20

8.30

8.40

8.50

8.60

8.70

8.80

8.90

9.00

9.10

9.20

9.30

9.40

9.50

9.60

9.70

9.80

9.90

10.00

10.10

10.20

10.30

10.40

10.50

10.60

10.70

10.80

10.90

11.00

11.10

11.20

11.30

11.40

11.50

11.60

11.70

11.80

11.90

12.00
$\bar{F}$

$f_{2}$

$5.51365 \mathrm{E}-05$

$5.69620 \mathrm{E}-05$

$5.88172 \mathrm{E}-05$

$6.07020 \mathrm{E}-05$

$6.26164 \mathrm{E}-05$

$6.45605 \mathrm{E}-05$ $6.65342 \mathrm{E}-05$

$6.85376 \mathrm{E}-05$

$7.05705 \mathrm{E}-05$

$7.26330 \mathrm{E}-05$

$7.47253 \mathrm{E}-05$

$7.68471 \mathrm{E}-05$

$7.89986 \mathrm{E}-05$

$8.11797 \mathrm{E}-05$

8.33904 E- 05

$8.56307 \mathrm{E}-05$ $8.79007 \mathrm{E}-05$ $9.02003 \mathrm{E}-05$ $9.25295 \mathrm{E}-05$

$9.48883 \mathrm{E}-05$

$9.72768 \mathrm{E}-05$

$9.96949 \mathrm{E}-05$

$1.02143 \mathrm{E}-04$

$1.04620 \mathrm{E}-04$

$1.07127 \mathrm{E}-04$

$1.09663 \mathrm{E}-04$

$1.12229 \mathrm{E}-04$

$1.14825 \mathrm{E}-04$

$1.17451 \mathrm{E}-04$

$1.20106 \mathrm{E}-04$

$1.22790 \mathrm{E}-04$

$1.25504 \mathrm{E}-04$

$1.28248 \mathrm{E}-04$

$1.31022 \mathrm{E}-04$

$1.33825 \mathrm{E}-04$

$1.36658 \mathrm{E}-04$

$1.39520 \mathrm{E}-04$

$1.42412 \mathrm{E}-04$

$1.45333 \mathrm{E}-04$

$1.48284 \mathrm{E}-04$

$1.51265 \mathrm{E}-04$

$1.54276 \mathrm{E}-04$

$1.57316 \mathrm{E}-04$

$1.60385 \mathrm{E}-04$

$1.63484 \mathrm{E}-04$

$1.66613 \mathrm{E}-04$

$1.69771 \mathrm{E}-04$

$1.72959 \mathrm{E}-04$

$1.76177 \mathrm{E}-04$

$1.79424 \mathrm{E}-04$

$1.82701 \mathrm{E}-04$

$1.86007 \mathrm{E}-04$

$1.89343 \mathrm{E}-04$

$1.92709 \mathrm{E}-04$

$1.96104 \mathrm{E}-04$

$1.99529 \mathrm{E}-04$

$2.02983 \mathrm{E}-04$

$2.06467 \mathrm{E}-04$

2.09981 E-04

$2.13524 \mathrm{E}-04$ $g_{2}$

$\tan \delta_{2}$

$f_{-2}$

$8.30128 \mathrm{E}-02$

$8.58897 \mathrm{E}-02$

$8.88155 \mathrm{E}-02$

$9.17899 \mathrm{E}-02$

$9.48131 \mathrm{E}-02$

$9.78852 \mathrm{E}-02$

$1.01006 \mathrm{E}-01$

$1.04175 \mathrm{E}-01$

$1.07393 \mathrm{E}-01$

$1.10659 \mathrm{E}-01$

$1.13974 \mathrm{E}-01$

$1.17337 \mathrm{E}-01$

$1.20749 \mathrm{E}-01$

$1.24208 \mathrm{E}-01$

$1.27714 \mathrm{E}-01$

$1.31266 \mathrm{E}-01$

$1.34862 \mathrm{E}-01$

$1.38498 \mathrm{E}-01$

$1.42154 \mathrm{E}-01$

$1.45710 \mathrm{E}-01$

$1.50696 \mathrm{E}-01$

$1.53899 \mathrm{E}-01$

$1.57720 \mathrm{E}-01$

$1.61639 \mathrm{E}-01$

$1.65623 \mathrm{E}-01$

$1.69662 \mathrm{E}-01$

$1.73753 \mathrm{E}-01$

$1.77895 \mathrm{E}-01$

$1.82087 \mathrm{E}-01$

$1.86329 \mathrm{E}-01$

$1.90620 \mathrm{E}-01$

$1.94961 \mathrm{E}-01$

$1.99350 \mathrm{E}-01$

$2.03789 \mathrm{E}-01$

$2.08276 \mathrm{E}-01$

$2.12813 \mathrm{E}-01$

$2.17398 \mathrm{E}-01$

$2.22033 \mathrm{E}-01$

$2.26716 \mathrm{E}-01$

$2.31449 \mathrm{E}-01$

$2.36230 \mathrm{E}-01$

$2.41060 \mathrm{E}-01$

$2.45938 \mathrm{E}-01$

$2.50866 \mathrm{E}-01$

$2.55842 \mathrm{E}-01$

$2.60868 \mathrm{E}-01$

$2.65942 \mathrm{E}-01$

$2.71065 \mathrm{E}-01$

$2.76236 \mathrm{E}-01$

$2.81457 \mathrm{E}-01$

$2.86726 \mathrm{E}-01$

$2.92045 \mathrm{E}-01$

$2.97412 \mathrm{E}-01$

$3.02827 \mathrm{E}-01$

$3.08292 \mathrm{E}-01$

$3.13805 \mathrm{E}-01$

$3.19367 \mathrm{E}-01$

$3.24978 \mathrm{E}-01$

$3.30638 \mathrm{E}-01$

$3.36346 \mathrm{E}-01$
$-1.46823 \mathrm{E}-04$

$-1.37809 \mathrm{E}-04$

$-1.27934 \mathrm{E}-04$

$-1.17174 \mathrm{E}-04$

$-1.05507 \mathrm{E}-04$

$-9.29084 \mathrm{E}-05$

$-7.93551 \mathrm{E}-05$

$-6.48239 \mathrm{E}-05$

$-4.92913 \mathrm{E}-05$

$-3.27343 \mathrm{E}-05$

$-1.51294 \mathrm{E}-05$

$3.54647 \mathrm{E}-06$

$2.33163 \mathrm{E}-05$

$4.42029 \mathrm{E}-05$

$6.62282 \mathrm{E}-05$

$8.94129 \mathrm{E}-05$

$1.13775 \mathrm{E}-04$

$1.39313 \mathrm{E}-04$

$1.65902 \mathrm{E}-04$

$1.95477 \mathrm{E}-04$

$2.24043 \mathrm{E}-04$

$2.54627 \mathrm{E}-04$

$2.86603 \mathrm{E}-04$

$3.19950 \mathrm{E}-04$

$3.54684 \mathrm{E}-04$

$3.90819 \mathrm{E}-04$

4.28381 E- 04

$4.67391 \mathrm{E}-04$

$5.07871 \mathrm{E}-04$

$5.49846 \mathrm{E}-04$

$5.93339 \mathrm{E}-04$

$6.38370 \mathrm{E}-04$

$6.84967 \mathrm{E}-04$

7.33149 E- 04

$7.82943 \mathrm{E}-04$

$8.34370 \mathrm{E}-04$

$8.87456 \mathrm{E}-04$

$9.42220 \mathrm{E}-04$

$9.98691 \mathrm{E}-04$

$1.05689 \mathrm{E}-03$

$1.11684 \mathrm{E}-03$

$1.17856 \mathrm{E}-03$

$1.24208 \mathrm{E}-03$

$1.30741 \mathrm{E}-03$

$1.37460 \mathrm{E}-03$

$1.44365 \mathrm{E}-03$

$1.51460 \mathrm{E}-03$

$1.58745 \mathrm{E}-03$

$1.66225 \mathrm{E}-03$

$1.73900 \mathrm{E}-03$

$1.81775 \mathrm{E}-03$

$1.89850 \mathrm{E}-03$

$1.98128 \mathrm{E}-03$

2.06611 E-03

$2.15302 \mathrm{E}-03$

$2.24204 \mathrm{E}-03$

$2.33318 \mathrm{E}-03$

$2.42647 \mathrm{E}-03$

$2.52193 \mathrm{E}-03$ .

$-2.30221 \mathrm{E} 0$

$-2.30494 \mathrm{E} 01$

$-2.30758 \mathrm{E} \quad 01$

$-2.31014 \mathrm{E} 01$

$-2.31261 \mathrm{E} \quad 01$

$-2.31501 \mathrm{E} 01$

$-2.31733 \mathrm{E} 01$

$-2.31958 \mathrm{E} 01$

$-2.32176 \mathrm{E} \quad 01$

$-2.32388 \mathrm{E} 01$

$-2.32594 \mathrm{E} \quad 01$

$-2.32794 \mathrm{E} 01$

$-2.32988 \mathrm{E} 01$

$-2.33176 \mathrm{E} \quad 01$

$-2.33360 \mathrm{E} 01$

$-2.33538 \mathrm{E} 01$

$-2.33712 \mathrm{E} \quad 01$

$-2.33881 \mathrm{E} 01$

$-2.34045 \mathrm{E} 01$

$-2.34205 \mathrm{E} \quad 0 \mathrm{l}$

$-2.34361 \mathrm{E} 01$

$-2.34513 \mathrm{E} \quad 01$

$-2.34662 \mathrm{E} 01$

$-2.34806 \mathrm{E} \quad 01$

$-2.34948 \mathrm{E} 01$

$-2.35085 \mathrm{E} 01$

$-2.35220 \mathrm{E} 0 \mathrm{l}$

$-2.35351 \mathrm{E} 01$

$-2.35479 \mathrm{E} 01$

$-2.35604 \mathrm{E} 01$

$-2.35727 \mathrm{E} \quad 01$

$-2.35846 \mathrm{E} 01$

$-2.35963 \mathrm{E} 01$

$-2.36078 \mathrm{E} \quad 01$

$-2.36189 \mathrm{E} 01$

$-2.36299 \mathrm{E} 01$

$-2.36406 \mathrm{E} 01$

$-2.36511 \mathrm{E} 01$

$-2.36613 \mathrm{E} \quad 0 \mathrm{l}$ 
ELECTRON RADIAL FUNCTIONS

$p$

12.10

12.20

12.30

12.40

12.50

12.60

12.70

12.80

12.90

13.00

13.10

13.20

13.30

13.40

13.50

13.60

13.70

13.80

13.90

14.00

14.10

14.20

14.30

14.40

14.50

14.60

14.70

14.80

14.90

15.00

15.10

15.20

15.30

15.40

15.50

15.60

15.70

15.80

15.90

16.00

16.10

16.20

16.30

16.40

16.50

16.60

16.70

16.80

16.90

17.00

17.10

17.20

17.30

17.40

17.50

17.60

17.70

17.80

17.90

18.00
$F_{0}$

$8.38776 \mathrm{E}-01$ $8.38762 \mathrm{E}-01$ $8.38747 \mathrm{E}-01$ $8.38733 \mathrm{E}-01$ 8.38719E-01

$8.38704 \mathrm{E}-01$ $8.38689 \mathrm{E}-01$ $8.38675 \mathrm{E}-0 \mathrm{l}$ $8.38660 \mathrm{E}-0 \mathrm{I}$ $8.38646 \mathrm{E}-01$

$8.38631 \mathrm{E}-01$ $8.38616 \mathrm{E}-01$ $8.38601 \mathrm{E}-01$ $8.38587 \mathrm{E}-01$ $8.38572 \mathrm{E}-01$

$8.38558 \mathrm{E}-01$ 8.38543E-01 $8.38528 \mathrm{E}-01$ $8.38514 \mathrm{E}-01$ 8.38499E-01

$8.38485 \mathrm{E}-01$ $8.38470 \mathrm{E}-01$ $8.38456 \mathrm{E}-01$ $8.38442 \mathrm{E}-01$ $8.38427 \mathrm{E}-01$

8.38413E-01 $8.38399 \mathrm{E}-01$ $8.38384 \mathrm{E}-01$ $8.38370 \mathrm{E}-01$ $8.38356 \mathrm{E}-01$

$8.38341 \mathrm{E}-01$ $8.38327 \mathrm{E}-01$ $8.38313 \mathrm{E}-01$ $8.38299 \mathrm{E}-01$ 8.38285E-01

$8.38271 \mathrm{E}-01$ $8.38257 \mathrm{E}-01$ $8.38243 \mathrm{E}-01$ $8.38229 \mathrm{E}-01$ $8.38215 \mathrm{E}-01$

$8.38201 \mathrm{E}-01$ $8.38187 \mathrm{E}-01$ $8.38173 \mathrm{E}-01$ $8.38160 \mathrm{E}-01$ $8.38146 \mathrm{E}-01$

8.38132E-01 $8.38118 \mathrm{E}-01$ $8.38105 \mathrm{E}-01$ $8.38091 \mathrm{E}-01$ $8.38078 \mathrm{E}-01$

$8.38064 \mathrm{E}-01$ $8.38051 \mathrm{E}-01$ $8.38037 \mathrm{E}-01$ $8.38024 \mathrm{E}-01$ $8.38011 \mathrm{E}-01$

8.37998E-01 $8.37985 \mathrm{E}-01$ 8.37971 E-01 $8.37958 \mathrm{E}-01$ 8.37945 E-01 $f_{1}$

$1.06861 \mathrm{E} \quad 01$ $\begin{array}{lll}1.07791 \mathrm{E} & 01\end{array}$

$1.08721 \mathrm{E} \quad 01$

$1.09651 \mathrm{E} \quad 01$

$1.10581 \mathrm{E} 01$

$1.11512 \mathrm{E} \quad 01$ $1.12442 \mathrm{E} \quad 01$

$1.13373 \mathrm{E} 01$

$1.14304 \mathrm{E} \quad 01$

$1.15235 \mathrm{E} 01$

$1.16167 \mathrm{E} \quad 01$

$1.17098 \mathrm{E} 01$

$\begin{array}{lll}1.18030 \mathrm{E} & 01\end{array}$

$\begin{array}{ll}1.18962 \mathrm{E} & 01\end{array}$

$1.19894 \mathrm{E} \quad 01$

$\begin{array}{lll}1.20826 \mathrm{E} & 01\end{array}$

$1.21758 \mathrm{E} \quad 01$

$1.22691 \mathrm{E} \quad 01$

$1.23623 \mathrm{E} \quad 01$

$1.24556 \mathrm{E} \quad 01$

$1.25489 \mathrm{E} \quad 01$

$1.26422 \mathrm{E} \quad 01$

$1.27356 \mathrm{E} \quad 01$

$1.28289 \mathrm{E} \quad 01$

$1.29223 \mathrm{E} \quad 01$

$\begin{array}{ll}1.30157 \mathrm{E} & 01\end{array}$ $1.31091 \mathrm{E} \quad 01$ $\begin{array}{ll}1.32026 \mathrm{E} & 01\end{array}$ $1.32960 \mathrm{E} \quad 01$ $1.33895 \mathrm{E} \quad 01$

$1.34830 \mathrm{E} \quad 01$ $1.35765 \mathrm{E} \quad 01$ $1.36700 \mathrm{E} \quad 01$ $\begin{array}{ll}1.37635 \mathrm{E} & 01\end{array}$ $1.38571 \mathrm{E} 01$

$1.39507 \mathrm{E} \quad 01$ $1.40443 \mathrm{E} \quad 01$ $\begin{array}{lll}1.41379 \mathrm{E} & 01\end{array}$ $1.42315 \mathrm{E} \quad 01$ $\begin{array}{ll}1.43252 \mathrm{E} & 01\end{array}$

\begin{tabular}{ll}
$1.44189 \mathrm{E} \quad 01$ \\
\hline
\end{tabular} $1.45126 \mathrm{E} \quad 01$ $1.46063 \mathrm{E} \quad 01$ $1.47000 \mathrm{E} \quad 01$ $1.47938 \mathrm{E} \quad 01$

$\begin{array}{ll}1.48876 \mathrm{E} & 01\end{array}$ $1.49814 \mathrm{E} \quad 01$ $1.50752 \mathrm{E} \quad 01$ $1.51690 \mathrm{E} 01$ $1.52629 \mathrm{E} \quad 01$

$\begin{array}{ll}1.53568 \mathrm{E} & 01\end{array}$ $1.54507 \mathrm{E} \quad 01$ $1.55446 \mathrm{E} \quad 01$ $1.56386 \mathrm{E} \quad 01$ $\begin{array}{lll}1.57325 \mathrm{E} & 01\end{array}$

$1.58265 \mathrm{E} \quad 01$ $\begin{array}{lll}1.59205 \mathrm{E} & 01\end{array}$ $1.60146 \mathrm{E} \quad 01$ $1.61086 \mathrm{E} \quad 01$ $\begin{array}{ll}1.62027 \mathrm{E} & 01\end{array}$ $g_{1}$

$1.24497 \mathrm{E}-01$

$1.28442 \mathrm{E}-01$

$1.32437 \mathrm{E}-01$

$1.36481 \mathrm{E}-01$

$1.40576 \mathrm{E}-01$

$1.44720 \mathrm{E}-01$

$1.48914 \mathrm{E}-01$

$1.53158 \mathrm{E}-01$

$1.57452 \mathrm{E}-01$

$1.61795 \mathrm{E}-01$

$1.66188 \mathrm{E}-01$

$1.70632 \mathrm{E}-01$

$1.75125 \mathrm{E}-01$

$1.79668 \mathrm{E}-01$

$1.84261 \mathrm{E}-01$

$1.88904 \mathrm{E}-01$

$1.93597 \mathrm{E}-01$

$1.98340 \mathrm{E}-01$

$2.03133 \mathrm{E}-01$

$2.07976 \mathrm{E}-01$

$2.12869 \mathrm{E}-01$

$2.17812 \mathrm{E}-01$

$2.22806 \mathrm{E}-01$

$2.27849 \mathrm{E}-01$

$2.32943 \mathrm{E}-01$

$2.38086 \mathrm{E}-01$

$2.43280 \mathrm{E}-01$

$2.48524 \mathrm{E}-01$

$2.53818 \mathrm{E}-01$

$2.59162 \mathrm{E}-01$

$2.64557 \mathrm{E}-01$

$2.70002 \mathrm{E}-01$

$2.75497 \mathrm{E}-01$

$2.81042 \mathrm{E}-01$

$2.86638 \mathrm{E}-01$

$2.92284 \mathrm{E}-01$

$2.97981 \mathrm{E}-01$

$3.03727 \mathrm{E}-01$

$3.09524 \mathrm{E}-01$

$3.15372 \mathrm{E}-01$

$3.21270 \mathrm{E}-01$

$3.27219 \mathrm{E}-01$

$3.33217 \mathrm{E}-01$

$3.39267 \mathrm{E}-01$

$3.45367 \mathrm{E}-01$

$3.51517 \mathrm{E}-01$

$3.57718 \mathrm{E}-01$

$3.63970 \mathrm{E}-01$

$3.70272 \mathrm{E}-01$

$3.76625 \mathrm{E}-01$

$3.83029 \mathrm{E}-01$

$3.89483 \mathrm{E}-01$

$3.95988 \mathrm{E}-01$

$4.02544 \mathrm{E}-01$

$4.09150 \mathrm{E}-01$

4.15807E-01 $4.22515 \mathrm{E}-01$

$4.29274 \mathrm{E}-01$

$4.36083 \mathrm{E}-01$

4.42943 E-01

$\tan \delta_{1}$

$5.44616 \mathrm{E}-04$ $5.25825 \mathrm{E}-04$

$5.07357 \mathrm{E}-04$

$4.89199 \mathrm{E}-04$

4.71357E-04

$4.53809 \mathrm{E}-04$ $4.36558 \mathrm{E}-04$

$4.19586 \mathrm{E}-04$

$4.02902 \mathrm{E}-04$

$3.86483 \mathrm{E}-04$

$3.70335 \mathrm{E}-04$

$3.54445 \mathrm{E}-04$

$3.38818 \mathrm{E}-04$

$3.23432 \mathrm{E}-04$

$3.08292 \mathrm{E}-04$

$2.93408 \mathrm{E}-04$

$2.78745 \mathrm{E}-04$

$2.64308 \mathrm{E}-04$

$2.50102 \mathrm{E}-04$

$2.36115 \mathrm{E}-04$

$2.22340 \mathrm{E}-04$

$2.08783 \mathrm{E}-04$

$1.95423 \mathrm{E}-04$

$1.82271 \mathrm{E}-04$

$1.69329 \mathrm{E}-04$

$1.56561 \mathrm{E}-04$ $1.43994 \mathrm{E}-04$

$1.31620 \mathrm{E}-04$

$1.19425 \mathrm{E}-04$

$1.07406 \mathrm{E}-04$

$9.55737 \mathrm{E}-05$ $8.39055 \mathrm{E}-05$

$7.24046 \mathrm{E}-05$

$6.10745 \mathrm{E}-05$

$4.99157 \mathrm{E}-05$

$3.89082 \mathrm{E}-05$

$2.80629 \mathrm{E}-05$

$1.73645 \mathrm{E}-05$

$6.82891 \mathrm{E}-06$

$-3.55979 \mathrm{E}-06$ 
ELECTRON RADIAL FUNCTIONS

$$
Z=8 \quad A=17 \quad \rho=1.2 A^{1 / 3} F
$$

$\bar{F}$

$f_{2}$

12.10

12.20

12.30

12.40

12.50

12.60

12.70

12.80

12.90

13.00

13.10

13.20

13.30

13.40

13.50

13.60

13.70

13.80

13.90

14.00

14.10

14.20

14.30

14.40

14.50

14.60

14.70

14.80

14.90

15.00

15.10

15.20

15.30

15.40

15.50

15.60

15.70

15.80

15.90

16.00

16.10

16.20

16.30

16.40

16.50

16.60

16.70

16.80

16.90

17.00

17.10

17.20

17.30

17.40

17.50

17.60

17.70

17.80

17.90

18.00
$2.17097 \mathrm{E}-04$ $2.20699 \mathrm{E}-04$

$2.24331 \mathrm{E}-04$ $2.27993 \mathrm{E}-04$

$2.31684 \mathrm{E}-04$

$2.35405 \mathrm{E}-04$

$2.39156 \mathrm{E}-04$

$2.42936 \mathrm{E}-04$

$2.46745 \mathrm{E}-04$

2.50584 E- 04

$2.54453 \mathrm{E}-04$

$2.58352 \mathrm{E}-04$

$2.62280 \mathrm{E}-04$

$2.66237 \mathrm{E}-04$

2.70224 E-04

2.74241 E-04

$2.78287 \mathrm{E}-04$

$2.82363 \mathrm{E}-04$

$2.86469 \mathrm{E}-04$

$2.90604 \mathbf{E}-04$

$2.94769 \mathrm{E}-04$ $2.98963 \mathrm{E}-04$ $3.03187 \mathrm{E}-04$ 3.07441 E-04

$3.11724 \mathrm{E}-04$

$3.16036 \mathrm{E}-04$

$3.20379 \mathrm{E}-04$

$3.24750 \mathrm{E}-04$

$3.29152 \mathrm{E}-04$

$3.33583 \mathrm{E}-04$

$3.38043 \mathrm{E}-04$

$3.42534 \mathrm{E}-04$

$3.47053 \mathrm{E}-04$

3.5 I603 E-04

$3.56182 \mathrm{E}-04$

$3.60790 \mathrm{E}-04$

$3.65429 \mathrm{E}-04$

$3.70096 \mathrm{E}-04$

$3.74794 \mathrm{E}-04$

3.79521 E-04

$3.84277 \mathrm{E}-04$

$3.89063 \mathrm{E}-04$

$3.93879 \mathrm{E}-04$

$3.98724 \mathrm{E}-04$

$4.03599 \mathrm{E}-04$

$4.08503 \mathrm{E}-04$

$4.13437 \mathrm{E}-04$

$4.18401 \mathrm{E}-04$

4.23394 E-04

$4.28417 \mathrm{E}-04$

$4.33469 \mathrm{E}-04$

4.38551 E-04

$4.43663 \mathrm{E}-04$

4.48804E-04

$4.53974 \mathrm{E}-04$

$4.59175 \mathrm{E}-04$

$4.64404 \mathrm{E}-04$

$4.69664 \mathrm{E}-04$

$4.74953 \mathrm{E}-04$

$4.80271 \mathrm{E}-04$ $g_{2}$

$\tan \delta_{2}$

$f_{-2}$

$3.42104 \mathrm{E}-01$

$3.47910 \mathrm{E}-01$

$3.53765 \mathrm{E}-01$

$3.59668 \mathrm{E}-01$

$3.65621 \mathrm{E}-01$

$3.71622 \mathrm{E}-01$

$3.77671 \mathrm{E}-01$

$3.83770 \mathrm{E}-01$

$3.89917 \mathrm{E}-01$

$3.96114 \mathbf{E}-01$

$4.02358 \mathrm{E}-01$

$4.08652 \mathbf{E}-01$

$4.14994 \mathrm{E}-01$

$4.21385 \mathrm{E}-01$

$4.27825 \mathrm{E}-01$

4.34314 E-01

4.40851 E-01

$4.47437 \mathrm{E}-01$

$4.54072 \mathrm{E}-01$

$4.60756 \mathrm{E}-01$

4.67488E-01

$4.74269 \mathrm{E}-01$

4.81099E-01

$4.87977 \mathrm{E}-01$

4.94904.E-01

$5.01881 \mathrm{E}-01$

$5.08905 \mathrm{E}-01$

$5.15978 \mathrm{E}-01$

$5.23100 \mathrm{E}-01$

$5.30271 \mathrm{E}-01$

$5.37490 \mathrm{E}-01$

$5.44758 \mathrm{E}-01$

$5.52076 \mathrm{E}-01$

$5.59441 \mathrm{E}-01$

$5.66855 \mathrm{E}-01$

$5.74318 \mathrm{E}-01$

$5.81830 \mathrm{E}-01$

$5.89390 \mathrm{E}-01$

$5.96999 \mathrm{E}-01$

$6.04656 \mathrm{E}-01$

$6.12363 \mathrm{E}-01$

$6.20118 \mathrm{E}-01$

$6.27922 \mathrm{E}-01$

$6.35774 \mathrm{E}-01$

$6.43675 \mathrm{E}-01$

$6.51624 \mathrm{E}-01$

$6.59623 \mathrm{E}-01$

$6.67669 \mathrm{E}-01$

$6.75765 \mathrm{E}-01$

$6.83909 \mathrm{E}-01$

$6.92102 \mathrm{E}-01$

$7.00344 \mathrm{E}-01$

$7.08634 \mathrm{E}-01$

$7.16973 \mathrm{E}-01$

$7.25360 \mathrm{E}-01$

$7.33796 \mathrm{E}-01$

$7.42281 \mathrm{E}-01$

$7.50814 \mathrm{E}-01$

$7.59396 \mathrm{E}-01$

$7.68026 \mathrm{E}-01$
$2.61959 \mathrm{E}-03$

$2.71946 \mathrm{E}-03$

$2.82158 \mathrm{E}-03$

$2.92596 \mathrm{E}-03$

$3.03263 \mathrm{E}-03$

$3.14162 \mathrm{E}-03$ $3.25293 \mathrm{E}-03$

$3.36661 \mathrm{E}-03$

$3.48267 \mathrm{E}-03$

$3.60113 \mathrm{E}-03$

$3.72202 \mathrm{E}-03$

$3.84537 \mathrm{E}-03$

$3.97119 \mathrm{E}-03$

$4.09951 \mathrm{E}-03$

$4.23036 \mathrm{E}-03$

4.36374. E-03

$4.49970 \mathrm{E}-03$

$4.63825 \mathrm{E}-03$

$4.77941 \mathrm{E}-03$

$4.92322 \mathrm{E}-03$

$5.06969 \mathrm{E}-03$

$5.21884 \mathrm{E}-03$

$5.37071 \mathrm{E}-03$

$5.52531 \mathrm{E}-03$

$5.68266 \mathrm{E}-03$

$5.84280 \mathrm{E}-03$

$6.00573 \mathrm{E}-03$

$6.17149 \mathrm{E}-03$

$6.34010 \mathrm{E}-03$

$6.51158 \mathrm{E}-03$

$6.68597 \mathrm{E}-03$ $6.86326 \mathrm{E}-03$

$7.04351 \mathrm{E}-03$

$7.22672 \mathrm{E}-03$

7.41292E-03

$7.60213 \mathrm{E}-03$

$7.79438 \mathrm{E}-03$

$7.98969 \mathrm{E}-03$

$8.18807 \mathrm{E}-03$

8.38957 E-03

8.59420E-03

$8.80199 \mathrm{E}-03$

$9.01294 \mathrm{E}-03$

$9.22710 \mathrm{E}-03$

$9.44448 \mathrm{E}-03$

$9.66511 \mathrm{E}-03$

$9.88900 \mathrm{E}-03$

$1.01162 \mathrm{E}-02$

$1.03467 \mathrm{E}-02$

$1.05805 \mathrm{E}-02$

$1.08178 \mathrm{E}-02$

$1.10584 \mathrm{E}-02$

$1.13024 \mathrm{E}-02$

$1.15498 \mathrm{E}-02$

$1.18007 \mathrm{E}-02$

$1.20551 \mathrm{E}-02$

$1.23129 \mathrm{E}-02$

$1.25743 \mathrm{E}-02$

$1.28393 \mathrm{E}-02$

$1.31078 \mathrm{E}-02$
$-2.38291 \mathrm{E} \quad 01 \quad-1.65743 \mathrm{E}-03$

$-2.38359 \mathrm{E}$ 01 $-1.74457 \mathrm{E}-03$

$-2.38427 \mathrm{E}$ 01 $-1.83387 \mathrm{E}-03$

$-2.38493 \mathrm{E}$ 01 $-1.92535 \mathrm{E}-03$

$\begin{array}{lll}-2.38558 \mathrm{E} & 01 & -2.01905 \mathrm{E}-03\end{array}$

$-2.38623 \mathrm{E} \quad 01 \quad-2.11498 \mathrm{E}-03$

$-2.38686 \mathrm{E} \quad 01 \quad-2.21317 \mathrm{E}-03$

$-2.38748 \mathrm{E} \quad 01 \quad-2.31364 \mathrm{E}-03$

$-2.38809 \mathrm{E} \quad 01 \quad-2.41641 \mathrm{E}-03$

$-2.38869 \mathrm{E} \quad 01 \quad-2.52152 \mathrm{E}-03$

$-2.38928 \mathrm{E} \quad 01$

$-2.39043 \mathrm{E} \quad 01 \quad-2.85103 \mathrm{E}-03$

$-2.39099 \mathrm{E} \quad 01 \quad-2.96567 \mathrm{E}-03$

$-2.39155 \mathrm{E} \quad 01 \quad-3.08277 \mathrm{E}-03$

$-2.39209 \mathrm{E} \quad 01 \quad-3.20233 \mathrm{E}-03$

$-2.39263 \mathrm{E}$ 01 $-3.32438 \mathrm{E}-03$

$-2.39316 \mathrm{E}$ 01 $-3.44895 \mathrm{E}-03$

$-2.39368 \mathrm{E}$ 01 $-3.57605 \mathrm{E}-03$

$-2.39420 \mathrm{E}$ 01 $-3.70572 \mathrm{E}-03$

$-2.39470 \mathrm{E}$ 01 $-3.83797 \mathrm{E}-03$

$-2.39520 \mathrm{E}$ 01 $-3.97283 \mathrm{E}-03$

$-2.39569 \mathrm{E} \quad 01 \quad-4.11032 \mathrm{E}-03$

$-2.39617 \mathrm{E}$ 01 $-4.25047 \mathrm{E}-03$

$-2.39665 \mathrm{E} \quad 01 \quad-4.39329 \mathrm{E}-03$

$-2.39712 \mathrm{E}$ 01 $-4.53882 \mathrm{E}-03$

$-2.39758 \mathrm{E} \quad 01 \quad-4.68707 \mathrm{E}-03$

$-2.39804 \mathrm{E} \quad 01 \quad-4.83807 \mathrm{E}-03$

$-2.39849 \mathrm{E} \quad 01 \quad-4.99184 \mathrm{E}-03$

$-2.39894 \mathrm{E} \quad 01 \quad-5.14841 \mathrm{E}-03$

$-2.39937 \mathrm{E} \quad 01 \quad-5.30779 \mathrm{E}-03$

$-2.39980 \mathrm{E}$ 01 $-5.47002 \mathrm{E}-03$

$-2.40023 \mathrm{E}$ 01 $-5.63512 \mathrm{E}-03$

$-2.40065 \mathrm{E} \quad 01 \quad-5.80310 \mathrm{E}-03$

$-2.40107 \mathrm{E}$ 01 $-5.97399 \mathrm{E}-03$

$-2.40147 \mathrm{E} \quad 01 \quad-6.14782 \mathrm{E}-03$

$-2.40188 \mathrm{E} \quad 01 \quad-6.32461 \mathrm{E}-03$

$-2.40228 \mathrm{E} \quad 01 \quad-6.50438 \mathrm{E}-03$

$-2.40267 \mathrm{E}$ 01 $-6.68716 \mathrm{E}-03$

$-2.40306 \mathrm{E} \quad 01 \quad-6.87296 \mathrm{E}-03$ 
ELECTRON RADIAL FUNCTIONS

Positron

p $\quad F_{0}$

18.10

18.20

18.30

18.40

18.50

18.60

18.70

18.80

18.90

19.00

19.10

19.20

19.30

19.40

19.50

19.60

19.70

19.80

19.90

20.00

20.10

20.20

20.30

20.40

20.50

20.60

20.70

20.80

20.90

21.00

21.10

21.20

21.30

21.40

21.50

21.60

21.70

21.80

21.90

22.00

22.10

22.20

22.30

22.40

22.50

22.60

22.70

22.80

22.90

23.00

23.10

23.20

23.30

23.40

23.50

23.60

23.70

23.80

23.90

24.00 $f_{1}$

8.37932E-01

$8.37919 \mathrm{E}-01$

$8.37906 \mathrm{E}-01$

$8.37893 \mathrm{E}-01$

$8.37880 \mathrm{E}-01$

$8.37867 \mathrm{E}-01$

$8.37854 \mathrm{E}-01$

$8.37842 \mathrm{E}-01$

$8.37829 \mathrm{E}-01$

$8.37816 \mathrm{E}-01$

8.37804E-01

$8.37791 \mathrm{E}-01$

$8.37778 \mathrm{E}-01$

$8.37766 \mathrm{E}-01$

$8.37753 \mathrm{E}-01$

$8.37741 \mathrm{E}-01$

$8.37729 \mathrm{E}-01$

$8.37717 \mathrm{E}-01$

$8.37704 \mathrm{E}-01$

$8.37692 \mathrm{E}-01$

$8.37680 \mathrm{E}-01$

$8.37668 \mathrm{E}-01$

$8.37655 \mathrm{E}-01$

$8.37643 \mathrm{E}-01$

8.37631 E-01

$8.37619 \mathrm{E}-01$

$8.37607 \mathrm{E}-01$

$8.37595 \mathrm{E}-01$

$8.37583 \mathrm{E}-01$

$8.37571 \mathrm{E}-01$

$8.37560 \mathrm{E}-01$

$8.37548 \mathrm{E}-01$

$8.37536 \mathrm{E}-01$

$8.37524 \mathrm{E}-01$

$8.37513 \mathrm{E}-01$

$8.37501 \mathrm{E}-01$

$8.374 .89 \mathrm{E}-01$

$8.37478 \mathrm{E}-01$

$8.37466 \mathrm{E}-01$

$8.37455 \mathrm{E}-01$

$8.37444 \mathrm{E}-01$

$8.37432 \mathrm{E}-01$

8.37421 E-01

$8.374 .09 \mathrm{E}-01$

$8.37398 \mathrm{E}-01$

$8.37387 \mathrm{E}-01$

$8.37376 \mathrm{E}-01$

$8.37365 \mathrm{E}-01$

$8.37353 \mathrm{E}-01$

8.37342E-01

8.37331 E-01

$8.37320 \mathrm{E}-01$

$8.37309 \mathrm{E}-01$

$8.37298 \mathrm{E}-01$

$8.37287 \mathrm{E}-01$

$8.37276 \mathrm{E}-01$

$8.37266 \mathrm{E}-01$

$8.37255 \mathrm{E}-01$

$8.37244 \mathrm{E}-01$

$8.37234 \mathrm{E}-01$

$Z=8$

$$
A=17
$$$$
\rho=1.2 A^{1 / 3} F
$$

$1.62968 \mathrm{E} \quad 01$

$1.63909 \mathrm{E} 01$

$1.64851 \mathrm{E} \quad 01$

$1.65792 \mathrm{E} \quad 01$

$1.66734 \mathrm{E} \quad 01$

$1.67676 \mathrm{E} \quad 01$

$1.68619 \mathrm{E} \quad 01$

$1.69561 \mathrm{E} \quad 01$

$1.70504 \mathrm{E} 01$

$1.71447 \mathrm{E} \quad 01$

$1.72390 \mathrm{E} \quad 01$

$1.73334 \mathrm{E} \quad 01$

$1.74277 \mathrm{E} \quad 01$

$1.75221 \mathrm{E} \quad 01$

$1.76165 \mathrm{E} 01$

$1.77 .110 \mathrm{E} \quad 01$

$1.78054 \mathrm{E}$ 01

$1.78999 \mathrm{E} \quad 01$

$1.79944 \mathrm{E} \quad 01$

$1.80890 \mathrm{E} \quad 01$

$\begin{array}{ll}1.81835 \mathrm{E} & 01\end{array}$

$1.82781 \mathrm{E} 01$

$1.83727 \mathrm{E} \quad 01$

$1.84674 \mathrm{E} \quad 01$

$1.85620 \mathrm{E}$ 0I

$1.86567 \mathrm{E} \quad 01$

$1.87514 \mathrm{E} \quad 01$

$1.88461 \mathrm{E} \quad 01$

$1.89409 \mathrm{E} \quad 01$

$1.90357 \mathrm{E} \quad 01$

$1.91305 \mathrm{E} \quad 01$ $1.92253 \mathrm{E} \quad 01$

$1.93202 \mathrm{E} \quad 01$

$1.94151 \mathrm{E} \quad 01$

$1.95100 \mathrm{E} 01$

$1.96049 \mathrm{E} \quad 01$ $1.96999 \mathrm{E} \quad 01$ $1.97949 \mathrm{E} \quad 01$ $1.98899 \mathrm{E} \quad 01$ $1.9984 .9 \mathrm{E} \quad 01$

$2.00800 \mathrm{E} \quad 01$ $2.01751 \mathrm{E} \quad 01$ $2.02702 \mathrm{E} \quad 01$ $2.03653 \mathrm{E} \quad 01$

$2.04605 \mathrm{E} 01$

$2.05557 \mathrm{E} \quad 01$

$2.06509 \mathrm{E} \quad 01$

$2.07462 \mathrm{E} 01$

$2.08414 \mathrm{E} \quad 01$

$2.09367 \mathrm{E} \quad 01$

$2.10321 \mathrm{E} \quad 01$ $2.11274 \mathrm{E} \quad 01$ $2.12228 \mathrm{E} \quad 01$ $2.13182 \mathrm{E} \quad 01$ $2.14137 \mathrm{E} \quad 01$

$2.15091 \mathrm{E} \quad 01$ $2.16046 \mathrm{E} \quad 01$ $2.17002 \mathrm{E} \quad 01$ $2.17957 \mathrm{E} \quad 01$

$2.18913 \mathrm{E} 01$
$4.49855 \mathrm{E}-01$

$4.56817 \mathrm{E}-01$

$4.63830 \mathrm{E}-01$

$4.70894 \mathrm{E}-01$

$4.78009 \mathrm{E}-01$

$4.85175 \mathrm{E}-01$

$4.92392 \mathrm{E}-01$

$4.99660 \mathrm{E}-01$

$5.06979 \mathrm{E}-01$

5.14349 E-01

5.21771 E-01

$5.29243 \mathrm{E}-01$

$5.36767 \mathrm{E}-01$

5.44342 E-01

$5.51968 \mathrm{E}-01$

$5.59645 \mathrm{E}-01$

$5.67373 \mathrm{E}-01$

$5.75153 \mathrm{E}-01$

$5.82984 \mathrm{E}-01$

$5.90867 \mathrm{E}-01$

$5.98801 \mathrm{E}-01$

$6.06786 \mathrm{E}-01$

$6.14823 \mathrm{E}-01$

$6.22911 \mathrm{E}-01$

$6.31050 \mathrm{E}-0 \mathrm{l}$

$6.39242 \mathrm{E}-01$

$6.47484 \mathrm{E}-01$

$6.55778 \mathrm{E}-01$

$6.64124 \mathrm{E}-01$

$6.72522 \mathrm{E}-0 \mathrm{I}$

$6.80971 \mathrm{E}-01$

$6.89471 \mathrm{E}-01$

$6.98024 \mathrm{E}-01$

$7.06628 \mathrm{E}-01$

$7.15284 \mathrm{E}-01$

$7.23991 \mathrm{E}-01$

$7.32751 \mathrm{E}-01$

$7.41562 \mathrm{E}-01$

$7.50425 \mathrm{E}-0 \mathrm{l}$

7.59340 E-01

$7.68307 \mathrm{E}-0 \mathrm{l}$

$7.77326 \mathrm{E}-01$

$7.86397 \mathrm{E}-01$

$7.95520 \mathrm{E}-01$

$8.04695 \mathrm{E}-01$

$8.13922 \mathrm{E}-01$

$8.23201 \mathrm{E}-01$

$8.32533 \mathrm{E}-01$

$8.41916 \mathrm{E}-01$

$8.51352 \mathrm{E}-01$

$8.60839 \mathrm{E}-01$

$8.70380 \mathrm{E}-01$

$8.79972 \mathrm{E}-01$

$8.89616 \mathrm{E}-01$

8.99314E-01

$9.09063 \mathrm{E}-01$

$9.18865 \mathrm{E}-01$

$9.28719 \mathrm{E}-01$

$9.38626 \mathrm{E}-01$

$9.48585 \mathrm{E}-01$

$-3.93056 \mathrm{E}-04$

$-4.22351 \mathrm{E}-04$

$\tan \delta_{1}$
$-1.91418 \mathrm{E}-04$

$-1.91418 \mathrm{E}-04$
$-1.99085 \mathrm{E}-04$

$-2.06661 \mathrm{E}-04$

$-2.14117 \mathrm{E}-04$

$-2.21496 \mathrm{E}-04$

$-2.28781 \mathrm{E}-04$

$-2.35955 \mathrm{E}-04$

$-2.43034 \mathrm{E}-04$

$-2.50024 \mathrm{E}-04$

$-2.56924 \mathrm{E}-04$

$-2.63736 \mathrm{E}-04$

$-2.70468 \mathrm{E}-04$

$-2.77090 \mathrm{E}-04$

$-2.83635 \mathrm{E}-04$

$-2.90103 \mathrm{E}-04$

$-2.96473 \mathrm{E}-04$

$-3.02773 \mathrm{E}-04$

$-3.08983 \mathrm{E}-04$

$-3.15112 \mathrm{E}-04$

$-3.21170 \mathrm{E}-04$

$-3.27139 \mathrm{E}-04$

$-3.33033 \mathrm{E}-04$

$-3.38850 \mathrm{E}-04$

$-3.44593 \mathrm{E}-04$

$-3.50259 \mathrm{E}-04$

$-3.55865 \mathrm{E}-04$

$-3.61391 \mathrm{E}-04$

$-3.66826 \mathrm{E}-04$

$-3.72219 \mathrm{E}-04$

$-3.77530 \mathrm{E}-04$

$-3.82769 \mathrm{E}-04$

$-3.87951 \mathrm{E}-04$

$-3.98099 \mathrm{E}-04$

$-4.03076 \mathrm{E}-04$

$-4.07994 \mathrm{E}-04$

$-4.12841 \mathrm{E}-04$

$-4.17629 \mathrm{E}-04$

$-4.27019 \mathrm{E}-04$ 
ELECTRON RADIAL FUNCTIONS

$Z=8 \quad A=17 \quad \rho=1.2 A^{1 / 3} F$

Positron

$\bar{F}$

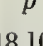

18.20

18.30

18.40

18.50

18.60

18.70

18.80

18.90

19.00

19.10

19.20

19.30

19.40

19.50

19.60

19.70

19.80

19.90

20.00

20.10

20.20

20.30

20.40

20.50

20.60

20.70

20.80

20.90

21.00

21.10

21.20

21.30

21.40

21.50

21.60

21.70

21.80

21.90

22.00

22.10

22.20

22.30

22.40

22.50

22.60

22.70

22.80

22.90

23.00

23.10

23.20

23.30

23.40

23.50

23.60

23.70

23.80

23.90

24.00 $f_{2}$

$4.85620 \mathrm{E}-04$

$4.90997 \mathrm{E}-04$

$4.96404 \mathrm{E}-04$

$5.01841 \mathrm{E}-04$

$5.07308 \mathrm{E}-04$

$5.12804 \mathrm{E}-04$

$5.18329 \mathrm{E}-04$

$5.23885 \mathrm{E}-04$

$5.29469 \mathrm{E}-04$

$5.35084 \mathrm{E}-04$

$5.40727 \mathrm{E}-04$

$5.46401 \mathrm{E}-04$

$5.52104 \mathrm{E}-04$

$5.57837 \mathrm{E}-04$

$5.63599 \mathrm{E}-04$

$5.69391 \mathrm{E}-04$

$5.75212 \mathrm{E}-04$

$5.81063 \mathrm{E}-04$

$5.86944 \mathrm{E}-04$

5.92854 E-04

$5.98793 \mathrm{E}-04$ $6.04763 \mathrm{E}-04$ $6.10761 \mathrm{E}-04$

$6.16790 \mathrm{E}-04$

$6.22848 \mathrm{E}-04$

$6.28935 \mathrm{E}-04$

$6.35052 \mathrm{E}-04$

$6.41199 \mathrm{E}-04$

$6.47375 \mathrm{E}-04$

$6.53581 \mathrm{E}-04$

$6.59816 \mathrm{E}-04$

$6.66081 \mathrm{E}-04$

$6.72376 \mathrm{E}-04$

$6.78700 \mathrm{E}-04$

$6.85054 \mathrm{E}-04$

$6.91437 \mathrm{E}-04$

$6.97850 \mathrm{E}-04$

7.04292 E-04

$7.10764 \mathrm{E}-04$

$7.17266 \mathrm{E}-04$

$7.23797 \mathrm{E}-04$

7.30357E-04

$7.36947 \mathrm{E}-04$

$7.43567 \mathrm{E}-04$

$7.50217 \mathrm{E}-04$

$7.56895 \mathrm{E}-04$

$7.63604 \mathrm{E}-04$

$7.70342 \mathrm{E}-04$

$7.77110 \mathrm{E}-04$

$7.83907 \mathrm{E}-04$

$7.90734 \mathrm{E}-04$

$7.97590 \mathrm{E}-04$

$8.04476 \mathrm{E}-04$

$8.11392 \mathrm{E}-04$

$8.18337 \mathrm{E}-04$

8.25311 E- 04

$8.32315 \mathrm{E}-04$

$8.39349 \mathrm{E}-04$

8.46412 E-04

$8.53505 \mathrm{E}-04$
$7.76706 \mathrm{E}-01$

$7.85434 \mathrm{E}-01$

$7.94210 \mathrm{E}-01$

$8.03035 \mathrm{E}-01$

$8.11908 \mathrm{E}-01$

$8.20831 \mathrm{E}-01$

$8.29801 \mathrm{E}-01$

$8.38820 \mathrm{E}-01$

$8.47888 \mathrm{E}-01$

$8.57004 \mathrm{E}-01$

$8.66169 \mathrm{E}-01$

$8.75383 \mathrm{E}-01$

$8.84645 \mathrm{E}-0$

$8.93956 \mathrm{E}-01$

$9.03315 \mathrm{E}-01$

$9.12723 \mathrm{E}-01$

$9.22179 \mathrm{E}-01$

$9.31683 \mathrm{E}-01$

$9.41237 \mathrm{E}-01$

$9.50839 \mathrm{E}-01$

$9.60489 E-01$

$9.70188 \mathrm{E}-01$

$9.79936 \mathrm{E}-01$

$9.89732 \mathrm{E}-01$

$9.99576 \mathrm{E}-01$

$1.00947 \mathrm{E} \quad 00$

$1.01941 \mathrm{E} \quad 00$

$1.02940 \mathrm{E} \quad 00$

$1.03944 \mathrm{E} \quad 00$

$1.04953 \mathrm{E} \quad 00$

$1.05966 \mathrm{E} \quad 00$

$1.06985 \mathrm{E} \quad 00$

$1.08008 \mathrm{E} \quad 00$

$1.09036 \mathrm{E} \quad 00$

$1.10069 \mathrm{E} \quad 00$

$1.11107 \mathrm{E} \quad 00$

$1.12149 \mathrm{E} \quad 00$

$1.13197 \mathrm{E} \quad 00$

$1.14249 \mathrm{E} \quad 00$

$1.15306 \mathrm{E} \quad 00$

$1.16368 \mathrm{E} \quad 00$

$1.17435 \mathrm{E} \quad 00$

$1.18507 \mathrm{E} \quad 00$

$1.19583 \mathrm{E} \quad 00$

$1.20664 \mathrm{E} \quad 00$

$1.21751 \mathrm{E} \quad 00$

$1.22842 \mathrm{E} \quad 00$

$1.23938 \mathrm{E} \quad 00$

$1.25038 \mathrm{E} \quad 00$

$1.26144 \mathrm{E} \quad 00$

$1.27254 \mathrm{E} \quad 00$

$1.28369 \mathrm{E} \quad 00$

$1.29490 \mathrm{E} \quad 00$

$1.30614 \mathrm{E} \quad 00$

$1.31744 \mathrm{E} \quad 00$

$1.32879 \mathrm{E} \quad 00$

$1.34018 \mathrm{E} \quad 00$

$1.35162 \mathrm{E} \quad 00$

$1.36311 \mathrm{E} \quad 00$

$1.37465 \mathrm{E} \quad 00$ $\tan \delta_{2}$

$f_{-2}$

$1.33800 \mathrm{E}-02-2.41017 \mathrm{E}$ ol $-1.15158 \mathrm{E}-02$

$1.36557 \mathrm{E}-02-2.41046 \mathrm{E} \quad 01-1.17742 \mathrm{E}-02$

$1.39351 \mathrm{E}-02 \quad-2.41075 \mathrm{E} \quad 01 \quad-1.20361 \mathrm{E}-02$

$1.42182 \mathrm{E}-02-2.41104 \mathrm{E}$ 01 $-1.23016 \mathrm{E}-02$

$1.45049 \mathrm{E}-02 \quad-2.41133 \mathrm{E}$ 01 $-1.25707 \mathrm{E}-02$

$1.47953 \mathrm{E}-02$

$1.50895 \mathrm{E}-02$

$1.53875 \mathrm{E}-02$

$1.56892 \mathrm{E}-02$

$1.59948 \mathrm{E}-02$

$1.63041 \mathrm{E}-02$

$1.66173 \mathrm{E}-02$

$1.69344 \mathrm{E}-02$

$1.72554 \mathrm{E}-02$

$1.75804 \mathrm{E}-02$

$1.79092 \mathrm{E}-02$

$1.82421 \mathrm{E}-02$

$1.85789 \mathrm{E}-02$

$1.89197 \mathrm{E}-02$

$1.92646 \mathrm{E}-02$

$1.96135 \mathrm{E}-02$

$1.99666 \mathrm{E}-02$

$2.03237 \mathrm{E}-02$

$2.06850 \mathrm{E}-02$

$2.10504 \mathrm{E}-02$

$2.14200 \mathrm{E}-02$

$2.17938 \mathrm{E}-02$

$2.21718 \mathrm{E}-02$

$2.25541 \mathrm{E}-02$

$2.29407 \mathrm{E}-02$

$2.33315 \mathrm{E}-02$

$2.37267 \mathrm{E}-02$

$2.41262 \mathrm{E}-02$

$2.45300 \mathrm{E}-02$

$2.49383 \mathrm{E}-02$

$2.53510 \mathrm{E}-02$

$2.57680 \mathrm{E}-02$

$2.61896 \mathrm{E}-02$

$2.66156 \mathrm{E}-02$

$2.70462 \mathrm{E}-02$

$2.74813 \mathrm{E}-02$

$2.79209 \mathrm{E}-02$

$2.83651 \mathrm{E}-02$

$2.88138 \mathrm{E}-02$

$2.92673 \mathrm{E}-02$

$2.97253 \mathrm{E}-02$

$3.01880 \mathrm{E}-02$

$3.06554 \mathrm{E}-02$

$3.11275 \mathrm{E}-02$

$3.16044 \mathrm{E}-02$

$3.20860 \mathrm{E}-02$

$3.25724 \mathrm{E}-02$

$3.30636 \mathrm{E}-02$

$3.35597 \mathrm{E}-02$

3.40605 E- 02

$3.45663 \mathrm{E}-02$

$3.50770 \mathrm{E}-02$

$3.55925 \mathrm{E}-02$

$3.61131 \mathrm{E}-02$

$3.66385 \mathrm{E}-02$

$-2.41161 \mathrm{E} \quad 01$

$-2.41189 \mathrm{E} \quad 01$

$-2.41217 \mathrm{E} 01$

$-2.41244 \mathrm{E} \quad 01$

$-2.41271 \mathrm{E} 01$

$-2.41298 \mathrm{E} 01$

$-2.41324 \mathrm{E} \quad 01$

$-2.41351 \mathrm{E} 01$

$-2.41377 \mathrm{E} \quad 01$

$-2.41402 \mathrm{E} 01$

$-2.41428 \mathrm{E} \quad 01$

$-2.41453 \mathrm{E} \quad 01$

$-2.41477 \mathrm{E}$ 0l

$-2.41502 \mathrm{E} 01$

$-2.41526 \mathrm{E} \quad 01$

$-2.41550 \mathrm{E} \quad 01$

$-2.41574 \mathrm{E}$ 01

$-2.41598 \mathrm{E} \quad 01$

$-2.41621 \mathrm{E} \quad 01$

$-2.41644 \mathrm{E} \quad 0 \mathrm{l}$

$-1.28434 \mathrm{E}-02$

$-1.31198 \mathrm{E}-02$

$-1.33999 \mathrm{E}-02$

$-1.36837 \mathrm{E}-02$

$-1.39712 \mathrm{E}-02$

$-1.42625 \mathrm{E}-02$

$-1.45575 \mathrm{E}-02$

$-1.48564 \mathrm{E}-02$

$-1.51590 \mathrm{E}-02$

$-1.54656 \mathrm{E}-02$

$-1.57759 \mathrm{E}-02$

$-1.60902 \mathrm{E}-02$

$-1.64084 \mathrm{E}-02$

$-1.67305 \mathrm{E}-02$

$-1.70566 \mathrm{E}-02$

$-1.73867 \mathrm{E}-02$

$-1.77207 \mathrm{E}-02$

$-1.80588 \mathrm{E}-02$

$-1.84010 \mathrm{E}-02$

$-1.87472 \mathrm{E}-02$

$-2.41667 \mathrm{E} 01$

$-2.41690 \mathrm{E} 01$ 
ELECTRON RADIAL FUNCTIONS

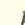

24.10

24.20

24.30

24.40

24.50

24.60

24.70

24.80

24.90

25.00

25.10

25.20

25.30

25.40

25.50

25.60

25.70

25.80

25.90

26.00

26.10

26.20

26.30

26.40

26.50

26.60

26.70

26.80

26.90

27.00

27.10

27.20

27.30

27.40

27.50

27.60

27.70

27.80

27.90

28.00

28.10

28.20

28.30

28.40

28.50

28.60

28.70

28.80

28.90

29.00

29.10

29.20

29.30

29.40

29.50

29.60

29.70

29.80

29.90

30.00
$F_{0}$

$8.37223 \mathrm{E}-0$

$8.37212 \mathrm{E}-01$

$8.37202 \mathrm{E}-01$

$8.37191 \mathrm{E}-01$

$8.37180 \mathrm{E}-01$

$8.37170 \mathrm{E}-01$

$8.37159 \mathrm{E}-01$

$8.37149 \mathrm{E}-01$

$8.37138 \mathrm{E}-01$

$8.37128 \mathrm{E}-01$

$8.37118 \mathrm{E}-01$

$8.37107 \mathrm{E}-01$

$8.37097 \mathrm{E}-01$

$8.37087 \mathrm{E}-01$

$8.37076 \mathrm{E}-01$

$8.37066 \mathrm{E}-01$

$8.37056 \mathrm{E}-01$

$8.37046 \mathrm{E}-01$

$8.37036 \mathrm{E}-01$

$8.37026 \mathrm{E}-01$

$8.37016 \mathrm{E}-01$

$8.37006 \mathrm{E}-01$

$8.36996 \mathrm{E}-01$

$8.36986 \mathrm{E}-01$

$8.36976 \mathrm{E}-0 \mathrm{l}$

$8.36966 \mathrm{E}-0 \mathrm{l}$

$8.36956 \mathrm{E}-01$

$8.36946 \mathrm{E}-01$

$8.36937 \mathrm{E}-01$

$8.36927 \mathrm{E}-01$

$8.36917 \mathrm{E}-01$

$8.36907 \mathrm{E}-01$

$8.36898 \mathrm{E}-01$

$8.36888 \mathrm{E}-0 \mathrm{l}$

$8.36878 \mathrm{E}-01$

$8.36869 \mathrm{E}-01$

$8.36859 \mathrm{E}-01$

$8.36850 \mathrm{E}-01$

$8.36840 \mathrm{E}-0 \mathrm{l}$

8.36831 E-01

$8.36821 \mathrm{E}-01$

$8.36812 \mathrm{E}-01$

$8.36802 \mathrm{E}-01$

$8.36793 \mathrm{E}-01$

8.36784 E-01

8.36774 E-01

$8.36765 \mathrm{E}-01$

$8.36756 \mathrm{E}-01$

$8.36746 \mathrm{E}-01$

$8.36737 \mathrm{E}-01$

8.36728E-01

$8.36719 \mathrm{E}-01$

$8.36710 \mathrm{E}-01$

$8.36701 \mathrm{E}-01$

$8.36692 \mathrm{E}-0 \mathrm{l}$

$8.36683 \mathrm{E}-01$

$8.36674 \mathrm{E}-01$

$8.36665 \mathrm{E}-01$

$8.36656 \mathrm{E}-01$

$8.36647 \mathrm{E}-01$ $f_{1}$

$2.19869 \mathrm{E} \quad 0 \mathrm{I}$ $2.20826 \mathrm{E} \quad 0$ $2.21782 \mathrm{E}$ ol $2.22739 \mathrm{E}$ 0I

$2.23697 \mathrm{E} \quad 01$

$2.24654 \mathrm{E} \quad 01$ $2.25612 \mathrm{E} \quad 01$

$2.26570 \mathrm{E} \quad 01$

$2.27528 \mathrm{E} \quad 01$

$2.28487 \mathrm{E} \quad 01$

$2.29446 \mathrm{E} \quad 01$

$2.30406 \mathrm{E}$ ol

$2.31365 \mathrm{E}$ ol

$2.32325 \mathrm{E}$ 01

$2.33285 \mathrm{E} \quad 01$

$2.34246 \mathrm{E} \quad 01$ $2.35207 \mathrm{E} \quad 0$ $2.36168 \mathrm{E} 01$ $2.37129 \mathrm{E} \quad 0$

$2.38091 \mathrm{E} \quad 01$

$2.39053 \mathrm{E} \quad 01$ $2.40015 \mathrm{E} \quad 01$ $2.40978 \mathrm{E} \quad 0$ $2.41941 \mathrm{E} \quad 01$ $2.42904 \mathrm{E} \quad 01$

$2.43868 \mathrm{E} \quad 01$ $2.44831 \mathrm{E} \quad 01$ $2.45796 \mathrm{E} \quad 01$

$2.46760 \mathrm{E} \quad 01$

$2.47725 \mathrm{E} \quad 01$

$2.48690 \mathrm{E} \quad 01$ $2.49655 \mathrm{E} \quad 01$ $2.50621 \mathrm{E} \quad 01$ $2.51587 \mathrm{E} \quad 0$

$2.52554 \mathrm{E} \quad 01$

$2.53520 \mathrm{E} \quad 01$ $2.54488 \mathrm{E} \quad 01$ $2.55455 \mathrm{E} \quad 01$ $2.56423 \mathrm{E} \quad 01$ $2.57391 \mathrm{E} \quad 01$

$2.58359 \mathrm{E} \quad 01$ $2.59328 \mathrm{E} \quad 01$ $2.60297 \mathrm{E} \quad 01$

$2.61266 \mathrm{E} \quad 01$

$2.62236 \mathrm{E} \quad 01$

$2.63206 \mathrm{E} \quad 01$ $2.64176 \mathrm{E}$ 01

$2.65147 \mathrm{E} \quad 01$

$2.66118 \mathrm{E} \quad 01$

$2.67089 \mathrm{E}$ ol

$2.68061 \mathrm{E} \quad 01$ $2.69033 \mathrm{E}$ 01 $2.70005 \mathrm{E} \quad 01$ $2.70978 \mathrm{E} \quad 01$ $2.71951 \mathrm{E}$ 0I

$2.72925 \mathrm{E} \quad 01$ $2.73898 \mathrm{E} \quad 01$ $2.74872 \mathrm{E} \quad 01$ $2.75847 \mathrm{E} \quad 01$ $2.76822 \mathrm{E}$ 0I $g_{1}$

$9.58597 \mathrm{E}-01$

$9.68661 \mathrm{E}-01$

$9.78778 \mathrm{E}-01$

$9.88948 \mathrm{E}-01$

$9.99170 \mathrm{E}-01$

$1.00945 \mathrm{E} \quad 00$

$1.01977 \mathrm{E} \quad 00$

$1.03015 \mathrm{E} 00$

$1.04059 \mathrm{E} \quad 00$

$1.05107 \mathrm{E} \quad 00$

$1.06161 \mathrm{E} \quad 00$

$1.07220 \mathrm{E} \quad 00$

$1.08285 \mathrm{E} \quad 00$

$1.09355 \mathrm{E} \quad 00$

$1.10430 \mathrm{E} \quad 00$

$1.11510 \mathrm{E} \quad 00$

$1.12596 \mathrm{E} \quad 00$

$1.13687 \mathrm{E} \quad 00$

$1.14783 \mathrm{E} \quad 00$

$1.15885 \mathrm{E} \quad 00$

$1.16991 \mathrm{E} \quad 00$

$1.18104 \mathrm{E} \quad 00$

$1.19221 \mathrm{E} \quad 00$

$1.20344 \mathrm{E} \quad 00$

$1.21472 \mathrm{E} \quad 00$

$1.22606 \mathrm{E} \quad 00$

$1.23745 \mathrm{E} \quad 00$

$1.24889 \mathrm{E} \quad 00$

$1.26039 \mathrm{E} \quad 00$

$1.27194 \mathrm{E} \quad 00$

$1.28354 \mathrm{E} \quad 00$

$1.29520 \mathrm{E} \quad 00$

$1.30691 \mathrm{E} \quad 00$

$1.31867 \mathrm{E} \quad 00$

$1.33049 \mathrm{E} \quad 00$

$1.34236 \mathrm{E} \quad 00$

$1.35429 \mathrm{E} \quad 00$

$1.36627 \mathrm{E} \quad 00$

1.37830 E 00

$1.39039 \mathrm{E} \quad 00$

$1.40253 \mathrm{E} \quad 00$

$1.41472 \mathrm{E} \quad 00$

$1.42697 \mathrm{E} \quad 00$

$1.43928 \mathrm{E} \quad 00$

$1.45163 \mathrm{E} \quad 00$

$1.46404 \mathrm{E} \quad 00$

$1.47651 \mathrm{E} \quad 00$

$1.48903 \mathrm{E} \quad 00$

$1.50160 \mathrm{E} \quad 00$

$1.51423 \mathrm{E} 00$

$1.52691 \mathrm{E} \quad 00$

$\begin{array}{lll}1.53965 \mathrm{E} & 00\end{array}$

$1.55244 \mathrm{E} \quad 00$

$1.56529 \mathrm{E} \quad 00$

$1.57819 \mathrm{E} \quad 00$

$1.59114 \mathrm{E} \quad 00$

$1.60415 \mathrm{E} \quad 00$

$1.61721 \mathrm{E} 00$

$1.63033 \mathrm{E} \quad 00$

$1.64350 \mathrm{E} \quad 00$

$\tan \delta_{1}$

$-5.11985 \mathrm{E}-04$

$-5.15461 \mathrm{E}-04$

$-5.18900 \mathrm{E}-04$

$-5.22280 \mathrm{E}-04$

$-5.25627 \mathrm{E}-04$

$-5.28919 \mathrm{E}-04$

$-5.32175 \mathrm{E}-04$

$-5.35381 \mathrm{E}-04$

$-5.38540 \mathrm{E}-04$

$-5.41659 \mathrm{E}-04$

$-5.44731 \mathrm{E}-04$

$-5.47765 \mathrm{E}-04$

$-5.50753 \mathrm{E}-04$

$-5.53705 \mathrm{E}-04$

$-5.56609 \mathrm{E}-04$

$-5.59464 \mathrm{E}-04$

$-5.62288 \mathrm{E}-04$

$-5.65074 \mathrm{E}-04$

$-5.67814 \mathrm{E}-04$

-5.70524 E- 04

$-5.73186 \mathrm{E}-04$

$-5.75809 \mathrm{E}-04$

$-5.78399 \mathrm{E}-04$

$-5.80947 \mathrm{E}-04$

$-5.83450 \mathrm{E}-04$

$-5.85930 \mathrm{E}-04$

$-5.88363 \mathrm{E}-04$

$-5.90762 \mathrm{E}-04$

$-5.93125 \mathrm{E}-04$

$-5.95452 \mathrm{E}-04$

$-5.97741 \mathrm{E}-04$

$-5.99994 \mathrm{E}-04$

$-6.02224 \mathrm{E}-04$

$-6.04402 \mathrm{E}-04$

$-6.06562 \mathrm{E}-04$

$-6.08671 \mathrm{E}-04$

$-6.10753 \mathrm{E}-04$

$-6.12808 \mathrm{E}-04$

$-6.14822 \mathrm{E}-04$

$-6.16804 \mathrm{E}-04$

$-6.18759 \mathrm{E}-04$

$-6.20676 \mathrm{E}-04$ 
ELECTRON RADIAL FUNCTIONS

$Z=8 \quad A=17 \quad \rho=1.2 A^{1 / 3} \mathrm{~F}$

Positron

$\bar{F}$

$8.60628 \mathrm{E}-04$ $8.67780 \mathrm{E}-04$

$8.74961 \mathrm{E}-04$

$8.82172 \mathrm{E}-04$

$8.89413 \mathrm{E}-04$

$8.96683 \mathrm{E}-04$ $9.03983 \mathrm{E}-04$

$9.11312 \mathrm{E}-04$

$9.18671 \mathrm{E}-04$

$9.26060 \mathrm{E}-04$

$9.33478 \mathrm{E}-04$

$9.40925 \mathrm{E}-04$

$9.48403 \mathrm{E}-04$

$9.55909 \mathrm{E}-04$

$9.63446 \mathrm{E}-04$

$9.71011 \mathrm{E}-04$

$9.78607 \mathrm{E}-04$

$9.86232 \mathrm{E}-04$

$9.93886 \mathrm{E}-04$

$1.00157 \mathrm{E}-03$

$1.00928 \mathrm{E}-03$

$1.01703 \mathrm{E}-03$

$1.02480 \mathrm{E}-03$

$1.03260 \mathrm{E}-03$

$1.04043 \mathrm{E}-03$

$1.04830 \mathrm{E}-03$

$1.05619 \mathrm{E}-03$

$1.06411 \mathrm{E}-03$

$1.07206 \mathrm{E}-03$

$1.08004 \mathrm{E}-03$

$1.08805 \mathrm{E}-03$

$1.09609 \mathrm{E}-03$

$1.10415 \mathrm{E}-03$

$1.11225 \mathrm{E}-03$

$1.12038 \mathrm{E}-03$

$1.12854 \mathrm{E}-03$

$1.13672 \mathrm{E}-03$

$1.14494 \mathrm{E}-03$

$1.15318 \mathrm{E}-03$

$1.16146 \mathrm{E}-03$

$1.16976 \mathrm{E}-03$

$1.17810 \mathrm{E}-03$

$1.18646 \mathrm{E}-03$

$1.19486 \mathrm{E}-03$

$1.20328 \mathrm{E}-03$

$1.21173 \mathrm{E}-03$

$1.22021 \mathrm{E}-03$

$1.22873 \mathrm{E}-03$

$1.23727 \mathrm{E}-03$

$1.24584 \mathrm{E}-03$

$1.25444 \mathrm{E}-03$

$1.26307 \mathrm{E}-03$

$1.27173 \mathrm{E}-03$

$1.28042 \mathrm{E}-03$

$1.28914 \mathrm{E}-03$

$1.29788 \mathrm{E}-03$

$1.30666 \mathrm{E}-03$

$1.31547 \mathrm{E}-03$

$1.32431 \mathrm{E}-03$

$1.33317 \mathrm{E}-03$ $f_{2}$

$1.38624 \mathrm{E} \quad 00$ $1.39788 \mathrm{E} \quad 00$

$1.40956 \mathrm{E} \quad 00$

$1.42129 \mathrm{E} \quad 00$

$1.43308 \mathrm{E} \quad 00$

$1.44490 \mathrm{E} \quad 00$

$1.45678 \mathrm{E} \quad 00$

$1.46871 \mathrm{E} \quad 00$

$1.48068 \mathrm{E} \quad 00$

$1.49270 \mathrm{E} \quad 00$

$1.50477 \mathrm{E} \quad 00$

$1.51689 \mathrm{E} \quad 00$

$1.52906 \mathrm{E} \quad 00$

$1.54127 \mathrm{E} \quad 00$

$1.55354 \mathrm{E} \quad 00$

$1.56585 \mathrm{E} \quad 00$

$1.57821 \mathrm{E} \quad 00$

$1.59062 \mathrm{E} \quad 00$

$1.60307 \mathrm{E} \quad 00$

$1.61558 \mathrm{E} \quad 00$

$1.62813 \mathrm{E} \quad 00$

$1.64073 \mathrm{E} \quad 00$

$1.65338 \mathrm{E} \quad 00$

$1.66608 \mathrm{E} \quad 00$

$1.67882 \mathrm{E} \quad 00$

$1.69162 \mathrm{E} \quad 00$

$1.70446 \mathrm{E} \quad 00$

$1.71735 \mathrm{E} \quad 00$

$1.73029 \mathrm{E} \quad 00$

$1.74327 \mathrm{E} \quad 00$

$1.75631 \mathrm{E} \quad 00$

$1.76939 \mathrm{E} \quad 00$

$1.78252 \mathrm{E} \quad 00$

$1.79570 \mathrm{E} \quad 00$

$1.80893 \mathrm{E} \quad 00$

$1.82220 \mathrm{E} \quad 00$

$1.83552 \mathrm{E} \quad 00$

$1.84889 \mathrm{E} \quad 00$

$1.86231 \mathrm{E} \quad 00$

$1.87578 \mathrm{E} \quad 00$

$1.88930 \mathrm{E} \quad 00$

$1.90286 \mathrm{E} \quad 00$

$1.91647 \mathrm{E} \quad 00$

$1.93013 \mathrm{E} \quad 00$

$1.94384 \mathrm{E} \quad 00$

$1.95760 \mathrm{E} \quad 00$

$1.97140 \mathrm{E} \quad 00$

$1.98525 \mathrm{E} \quad 00$

$1.99915 \mathrm{E} \quad 00$

$2.01310 \mathrm{E} \quad 00$

$2.02709 \mathrm{E} \quad 00$

$2.04114 \mathrm{E} \quad 00$

$2.05523 \mathrm{E} \quad 00$

$2.06937 \mathrm{E} \quad 00$

$2.08356 \mathrm{E} \quad 00$

$2.09779 \mathrm{E} \quad 00$

$2.11208 \mathrm{E} \quad 00$

$2.12641 \mathrm{E} \quad 00$

$2.14079 \mathrm{E} \quad 00$

$2.15521 \mathrm{E} \quad 00$ $g_{2}$

$\tan \delta_{2}$

$3.71691 \mathrm{E}-02$

$3.77046 \mathrm{E}-02$

$3.82451 \mathrm{E}-02$

$3.87907 \mathrm{E}-02$

$3.93413 \mathrm{E}-02$

$3.98971 \mathrm{E}-02$

$4.04580 \mathrm{E}-02$

$4.10241 \mathrm{E}-02$

$4.15954 \mathrm{E}-02$

$4.21718 \mathrm{E}-02$

$4.27535 \mathrm{E}-02$

$4.33404 \mathbf{E}-02$

$4.39326 \mathrm{E}-02$

$4.45300 \mathrm{E}-02$

$4.51328 \mathrm{E}-02$

$4.57409 \mathrm{E}-02$

$4.63544 \mathrm{E}-02$

$4.69733 \mathrm{E}-02$

$4.75976 \mathrm{E}-02$

$4.82273 \mathbf{E}-02$

$4.88624 \mathrm{E}-02$

$4.95031 \mathrm{E}-02$

$5.01492 \mathrm{E}-02$

$5.08008 \mathrm{E}-02$

$5.14580 \mathrm{E}-02$

$5.21208 \mathrm{E}-02$

$5.27892 \mathrm{E}-02$

$5.34632 \mathrm{E}-02$

$5.41428 \mathrm{E}-02$

$5.48281 \mathrm{E}-02$

$5.55191 \mathrm{E}-02$

$5.62157 \mathrm{E}-02$

$5.69181 \mathrm{E}-02$

$5.76263 \mathrm{E}-02$

$5.83402 \mathrm{E}-02$

$5.90600 \mathrm{E}-02$

$5.97855 \mathrm{E}-02$

$6.05169 \mathrm{E}-02$

$6.12542 \mathrm{E}-02$

$6.19974 \mathrm{E}-02$

$6.27465 \mathrm{E}-02$

$6.35015 \mathrm{E}-02$

$6.42625 \mathrm{E}-02$

$6.50294 \mathrm{E}-02$

$6.58024 \mathrm{E}-02$

$6.65814 \mathrm{E}-02$

$6.73665 \mathrm{E}-02$

$6.81576 \mathrm{E}-02$

$6.89549 \mathrm{E}-02$

$6.97582 \mathbf{E}-02$

$7.05677 \mathrm{E}-02$

$7.13834 \mathrm{E}-02$

$7.22052 \mathrm{E}-02$

$7.30333 \mathrm{E}-02$

$7.38676 \mathrm{E}-02$

$7.47082 \mathrm{E}-02$

$7.55551 \mathrm{E}-02$

$7.64082 \mathrm{E}-02$

$7.72677 \mathrm{E}-02$

7.81335 E-02

$$
\begin{array}{lll}
-2.42343 \mathrm{E} & 01 & -3.41237 \mathrm{E}-02 \\
-2.42360 \mathrm{E} & 01 & -3.46372 \mathrm{E}-02 \\
-2.42376 \mathrm{E} & 01 & -3.51556 \mathrm{E}-02 \\
-2.42392 \mathrm{E} & 01 & -3.56790 \mathrm{E}-02 \\
-2.42408 \mathrm{E} & 01 & -3.62074 \mathrm{E}-02
\end{array}
$$

$\begin{array}{lll}-2.42424 \cdot \mathrm{E} & 01 & -3.67408 \mathrm{E}-02\end{array}$

$-2.42439 \mathrm{E}$ 01 $-3.72793 \mathrm{E}-02$

$-2.42455 \mathrm{E}$ 01 $-3.78228 \mathrm{E}-02$

$-2.42470 \mathrm{E}$ 01 $-3.83715 \mathrm{E}-02$

$-2.42485 \mathrm{E} \quad 01 \quad-3.89253 \mathrm{E}-02$

$-2.42501 \mathrm{E} \quad 01 \quad-3.94842 \mathrm{E}-02$

$-2.42516 \mathrm{E}$ 01 $-4.00483 \mathrm{E}-02$

$-2.42531 \mathrm{E}$ 01 $-4.06176 \mathrm{E}-02$

$-2.42545 \mathrm{E} \quad 01 \quad-4.11921 \mathrm{E}-02$

$-2.42560 \mathrm{E} \quad 01 \quad-4.17718 \mathrm{E}-02$

$\begin{array}{lll}-2.42575 \mathrm{E} & 01 & -4.23568 \mathrm{E}-02 \\ -2.42589 \mathrm{E} & 01 & -4.29471 \mathrm{E}-02 \\ -2.42603 \mathrm{E} & 01 & -4.35427 \mathrm{E}-02 \\ -2.42618 \mathrm{E} & 01 & -4.41436 \mathrm{E}-02 \\ -2.42632 \mathrm{E} & 01 & -4.47499 \mathrm{E}-02\end{array}$

$-2.42646 \mathrm{E} \quad 01$

$-2.42659 \mathrm{E} 01$

$-2.42673 \mathrm{E} \quad 01$

$-2.42687 \mathrm{E} \quad 01$

$-2.42700 \mathrm{E} 01$

$-2.42714 \mathrm{E} \quad 01$

$-2.42727 \mathrm{E} \quad 01$

$-2.42740 \mathrm{E} \quad 01$

$-2.42753 \mathrm{E} 01$

$-2.42767 \mathrm{E} \quad 01$

$-2.42779 \mathrm{E} \quad 01$

$-2.42792 \mathrm{E} 01$

$-2.42805 \mathrm{E} \quad 01$

$-2.42818 \mathrm{E} 01$

$-2.42830 \mathrm{E} \quad 01$

$-2.42843 \mathbf{E} 01$

$-2.42855 \mathrm{E} \quad 01$

$-2.42867 \mathrm{E} \quad 01$

$-2.42879 \mathrm{E} \quad 01$

$-2.42891 \mathrm{E} \quad 01$

$-2.42903 \mathrm{E} \quad 01$

$-2.42915 \mathrm{E} \quad 01$

$-2.42927 \mathrm{E} 01$

$-2.42939 \mathrm{E} 01$

$-2.42951 \mathrm{E} 01$

$-2.42962 \mathrm{E} \quad 01$

$-2.42974 \mathrm{E} 01$

$-2.42985 \mathrm{E} \quad 01$

$-2.42996 \mathrm{E} 01$

$-2.43008 \mathrm{E} 01$

$-2.43019 \mathrm{E} 01$

$-2.43030 \mathrm{E} \quad 01$

$-2.43041 \mathrm{E} \quad 01$

$-2.43052 \mathrm{E} \quad 01$

$-2.43063 \mathrm{E} 01$

$-2.43073 \mathrm{E} \quad 01$

$-2.43084 \mathrm{E} 01$

$-2.43095 \mathrm{E} \quad 01$

$-2.43105 \mathrm{E} 01$

$-2.43116 \mathrm{E} 01$
$-4.53615 \mathrm{E}-02$

$-4.59786 \mathrm{E}-02$

$-4.66010 \mathrm{E}-02$

$-4.72290 \mathrm{E}-02$

$-4.78623 \mathbf{E}-02$

$-4.85012 \mathrm{E}-02$

$-4.91456 \mathrm{E}-02$

$-5.04510 \mathrm{E}-02$

$-5.11121 \mathrm{E}-02$

$-5.17788 \mathrm{E}-02$

$-5.24511 \mathrm{E}-02$

$-5.31291 \mathrm{E}-02$

$-5.38128 \mathrm{E}-02$

$-5.45021 \mathrm{E}-02$

$-5.51972 \mathrm{E}-02$

$-5.58980 \mathrm{E}-02$

$-5.66046 \mathrm{E}-02$

$-5.73170 \mathrm{E}-02$

$-5.80352 \mathrm{E}-02$

$-5.87592 \mathrm{E}-02$

$-5.94891 \mathrm{E}-02$

$-6.02249 \mathrm{E}-02$

$-6.09666 \mathrm{E}-02$

$-6.17142 \mathrm{E}-02$

$-6.24678 \mathrm{E}-02$

$-6.32273 \mathrm{E}-02$

$-6.39928 \mathrm{E}-02$

$-6.47644 \mathrm{E}-02$

$-6.55420 \mathrm{E}-02$

$-6.63257 \mathrm{E}-02$

$-6.71155 \mathrm{E}-02$

$-6.79114 \mathrm{E}-02$

$-6.87134 \mathrm{E}-02$

$-6.95216 \mathrm{E}-02$

$-7.03360 \mathrm{E}-02$

$-7.11566 \mathrm{E}-02$

$-7.19834 \mathrm{E}-02$

$-7.28164 \mathrm{E}-02$

$-7.36558 \mathrm{E}-02$
$-4.97956 \mathrm{E}-02$

$1.44499 \mathrm{E} \quad 00$

$1.45687 \mathrm{E} \quad 00$

$1.46880 \mathrm{E} \quad 00$

$1.48077 \mathrm{E} \quad 00$ 
p

30.10
30.20

30.20
30.30

30.40

30.50

30.60

30.70

30.80

30.90

31.00

31.10

31.20

31.30

31.40

31.50

31.60

31.70

31.80

31.90

32.00

32.10

32.20

32.30

32.40

32.50

32.60

32.70

32.80

32.90

33.00

33.10

33.20

33.30

33.40

33.50

33.60

33.70

33.80

33.90

34.00

34.10

34.20

34.30

34.40

34.50

34.60

34.70

34.80

34.90

35.00

35.10

35.20

35.30

35.40

35.50

35.60

35.70

35.80

35.90

36.00
$F_{0}$

8.36638E-0 $8.36629 \mathrm{E}-01$ $8.36620 \mathrm{E}-01$ 8.36611 E-01 $8.36603 \mathrm{E}-0$

$8.36594 \mathrm{E}-01$ $8.36585 \mathrm{E}-01$ $8.36576 \mathrm{E}-01$ $8.36568 \mathrm{E}-0$ 8.36559E-01

8.36550E-01 $8.36542 \mathrm{E}-01$ $8.36533 \mathrm{E}-01$ $8.36525 \mathrm{E}-01$ $8.36516 \mathrm{E}-01$

$8.36508 \mathrm{E}-01$ $8.36499 \mathrm{E}-01$ 8.36491 E-01 $8.36482 \mathrm{E}-01$ 8.36474 E-01

8.36466E-01 $8.36457 \mathrm{E}-01$ $8.36449 \mathrm{E}-01$ $8.36441 \mathrm{E}-01$ $8.36432 \mathrm{E}-01$

$8.36424 \mathrm{E}-0$ $8.36416 \mathrm{E}-0$ 8.36407 E-01 8.36399E-01 8.36391 E-0]

8.36383E-01 $8.36375 \mathrm{E}-01$ $8.36366 \mathrm{E}-01$ $8.36358 \mathrm{E}-01$ $8.36350 \mathrm{E}-01$

8.36342E-01 $8.36334 \mathrm{E}-01$ $8.36326 \mathrm{E}-01$ $8.36318 \mathrm{E}-01$ $8.36310 \mathrm{E}-01$

8.36302 E-01 8.36294 E-01 $8.36286 \mathrm{E}-01$ $8.36278 \mathrm{E}-01$ $8.36270 \mathrm{E}-01$

$8.36263 \mathrm{E}-01$ $8.36255 \mathrm{E}-01$ 8.36247E-0 $8.36239 \mathrm{E}-01$ $8.36231 \mathrm{E}-01$

$8.36224 \mathrm{E}-01$ $8.36216 \mathrm{E}-01$ 8.36208E-01 $8.36200 \mathrm{E}-01$ $8.36193 \mathrm{E}-01$

$8.36185 \mathrm{E}-01$ $8.36177 \mathrm{E}-01$ $8.36170 \mathrm{E}-01$ 8.36l62E-01 $8.36155 \mathrm{E}-01$ $f_{1}$

$2.78772 \mathrm{E} 01$

$2.79748 \mathrm{E}$ 01

$2.80725 \mathrm{E} \quad 01$

$2.81701 \mathrm{E} \quad 01$

$2.82678 \mathrm{E} \quad 01$

$2.83655 \mathrm{E} \quad 01$

$2.84633 \mathrm{E} 01$

$2.85611 \mathrm{E} \quad 01$

$2.86589 \mathrm{E} \quad 01$

$2.87568 \mathrm{E} \quad 01$

$2.88547 \mathrm{E} \quad 01$

$2.89527 \mathrm{E} \quad 01$

$2.90506 \mathrm{E}$ 0l

$2.91487 \mathrm{E} \quad 01$

$2.92467 \mathrm{E} \quad 01$

$2.93448 \mathrm{E}$ 0l

$2.94429 \mathrm{E} \quad 01$

$2.95411 \mathrm{E} \quad 01$

$2.96393 \mathrm{E} 01$

$2.97376 \mathrm{E} \quad 01$

$2.98358 \mathrm{E}$ 01

$2.99342 \mathrm{E} 01$

$3.00325 \mathrm{E} \quad 01$

$3.01309 \mathrm{E} \quad 01$

$3.02293 \mathrm{E} \quad 01$

$3.03278 \mathrm{E} 01$

$3.04263 \mathrm{E} \quad 01$

$3.05249 \mathrm{E}$ 01

$3.06234 \mathrm{E} 01$

$3.07221 \mathrm{E} \quad 01$

$3.08207 \mathrm{E}$ 01

$3.09194 \mathrm{E}$ ol

$3.10182 \mathrm{E} \quad 01$

$3.11170 \mathrm{E} \quad 01$

$3.12158 \mathrm{E} \quad 01$

$3.13146 \mathrm{E} \quad 01$

$3.14135 \mathrm{E} \quad 01$

$3.15125 \mathrm{E} 01$

$3.16114 \mathrm{E} \quad 0 \mathrm{l}$.

$3.17105 \mathrm{E} 01$ $3.18095 \mathrm{E} \quad 01$ $3.19086 \mathrm{E} 01$ $3.20077 \mathrm{E} \quad 01$ $3.21069 \mathrm{E} 01$

$3.22061 \mathrm{E} \quad 01$ $3.23054 \mathrm{E} \quad 01$ $3.24047 \mathrm{E} 01$ $3.25040 \mathrm{E}$ 01 $3.26034 \mathrm{E} \quad 01$

$3.27028 \mathrm{E} \quad 01$ $3.28023 \mathrm{E} 01$ $3.29018 \mathrm{E} \quad 01$

$3.30013 \mathrm{E} \quad 01$

$3.31009 \mathrm{E} 01$

$3.32006 \mathrm{E} \quad 01$ $3.33002 \mathrm{E} 01$ $3.33999 \mathrm{E} \quad 01$

$3.34997 \mathrm{E} \quad 01$ $3.35995 \mathrm{E} 01$ $g_{1}$

$1.65673 \mathrm{E} \quad 00$

$1.67002 \mathrm{E} \quad 00$

$1.68335 \mathrm{E} \quad 00$

$1.69675 \mathrm{E} \quad 00$

$1.71019 \mathrm{E} \quad 00$

$1.72369 \mathrm{E} \quad 00$

$1.73725 \mathrm{E} \quad 00$

$1.75086 \mathrm{E} \quad 00$

$1.76453 \mathrm{E} \quad 00$

$1.77825 \mathrm{E} \quad 00$

$1.79203 \mathrm{E} \quad 00$

$1.80586 \mathrm{E} \quad 00$

$1.81975 \mathrm{E} \quad 00$

$1.83369 \mathrm{E} \quad 00$

$\begin{array}{ll}1.84769 \mathrm{E} & 00\end{array}$

$\begin{array}{lll}1.86175 \mathrm{E} & 00\end{array}$

$1.87585 \mathrm{E} \quad 00$

$1.89002 \mathrm{E} \quad 00$

$1.90424 \mathrm{E} \quad 00$

$1.91851 \mathrm{E} \quad 00$

$1.93285 \mathrm{E} \quad 00$

$1.94723 \mathrm{E} \quad 00$

$1.96168 \mathrm{E} \quad 00$

$1.97617 \mathrm{E} \quad 00$

$1.99073 \mathrm{E} \quad 00$

$2.00534 \mathrm{E} \quad 00$

$2.02000 \mathrm{E} \quad 00$

$2.03472 \mathrm{E} \quad 00$

$2.04950 \mathrm{E} \quad 00$

$2.06434 \mathrm{E} \quad 00$

$2.07922 \mathrm{E} \quad 00$

$2.09417 \mathrm{E} \quad 00$

$2.10917 \mathrm{E} \quad 00$

$2.12423 \mathrm{E} \quad 00$

$2.13934 \mathrm{E} \quad 00$

$2.15451 \mathrm{E} \quad 00$

$2.16974 \mathrm{E} \quad 00$

$2.18502 \mathrm{E} \quad 00$

$2.20036 \mathrm{E} \quad 00$

$2.21576 \mathrm{E} \quad 00$

$2.23121 \mathrm{E} \quad 00$ $2.24672 \mathrm{E} \quad 00$

$2.26229 \mathrm{E} \quad 00$

$2.27791 \mathrm{E} \quad 00$

$2.29359 \mathrm{E} \quad 00$

$2.30932 \mathrm{E} \quad 00$

$2.32512 \mathrm{E} \quad 00$

$2.34097 \mathrm{E} \quad 00$

$2.35687 \mathrm{E} \quad 00$

$2.37283 \mathrm{E} \quad 00$

$2.38886 \mathrm{E} \quad 00$

$2.40493 \mathrm{E} \quad 00$

$2.42107 \mathrm{E} \quad 00$

$2.43726 \mathrm{E} \quad 00$

$2.45351 \mathrm{E} \quad 00$

$2.46981 \mathrm{E} \quad 00$ $2.48618 \mathrm{E} \quad 00$ $2.50260 \mathrm{E} \quad 00$ $2.51908 \mathrm{E} \quad 00$ $2.53561 \mathrm{E} \quad 00$

$\tan \delta_{1}$

$-6.51308 \mathrm{E}-04$ $-6.52630 \mathrm{E}-04$

$-6.53919 \mathrm{E}-04$

$-6.55195 \mathrm{E}-04$

$-6.56441 \mathrm{E}-04$

$-6.57652 \mathrm{E}-04$

$-6.58837 \mathrm{E}-04$

$-6.60002 \mathrm{E}-04$

$-6.61138 \mathrm{E}-04$

$-6.62242 \mathrm{E}-04$

$-6.63322 \mathrm{E}-04$

$-6.64386 \mathrm{E}-04$

$-6.65413 \mathrm{E}-04$

$-6.66417 \mathrm{E}-04$

$-6.67399 \mathrm{E}-04$

$-6.68359 \mathrm{E}-04$

$-6.69298 \mathrm{E}-04$

$-6.70207 \mathrm{E}-04$

$-6.71093 \mathrm{E}-04$

$-6.71954 \mathrm{E}-04$

$-6.72784 \mathrm{E}-04$

$-6.73598 \mathrm{E}-04$

$-6.74390 \mathrm{E}-04$

$-6.75147 \mathrm{E}-04$

$-6.75887 \mathrm{E}-04$

$-6.76606 \mathrm{E}-04$

$-6.77301 \mathrm{E}-04$

$-6.77971 \mathrm{E}-04$

$-6.78627 \mathrm{E}-04$

-6.79251 E-04

$-6.79854 \mathrm{E}-04$

$-6.80436 \mathrm{E}-04$

$-6.80994 \mathrm{E}-04$

$-6.81542 \mathrm{E}-04$

$-6.82048 \mathrm{E}-04$

$-6.82542 \mathrm{E}-04$

$-6.83014 \mathrm{E}-04$

$-6.83470 \mathrm{E}-04$

$-6.83902 \mathrm{E}-04$

$-6.84300 \mathrm{E}-04$ 
ELECTRON RADIAL FUNCTIONS

$Z=8 \quad A=17 \quad \rho=1.2 A^{1 / 3} F$

Positron

$\bar{F}$

$1.34207 \mathrm{E}-03$ $1.35099 \mathrm{E}-03$ $1.35995 \mathrm{E}-03$ $1.36893 \mathrm{E}-03$ $1.37795 \mathrm{E}-03$

$1.38699 \mathrm{E}-03$ $1.39607 \mathrm{E}-03$ $1.40517 \mathrm{E}-03$ $1.41430 \mathrm{E}-03$ $1.42346 \mathrm{E}-03$

$1.43265 \mathrm{E}-03$ $1.44188 \mathrm{E}-03$ $1.45113 \mathrm{E}-03$ $1.4604 .1 \mathrm{E}-03$ $1.46972 \mathrm{E}-03$

$1.47905 \mathrm{E}-03$ $1.48842 \mathrm{E}-03$ $1.49782 \mathrm{E}-03$ $1.50725 \mathrm{E}-03$ $1.51671 \mathrm{E}-03$

$1.52619 \mathrm{E}-03$ $1.53571 \mathrm{E}-03$ $1.54526 \mathrm{E}-03$ $1.55483 \mathrm{E}-03$ $1.56444 \mathrm{E}-03$

$1.57407 \mathrm{E}-03$

$1.58374 \mathrm{E}-03$

$1.59343 \mathrm{E}-03$

$1.60315 \mathrm{E}-03$

$1.61291 \mathrm{E}-03$

$1.62269 \mathrm{E}-03$

$1.63250 \mathrm{E}-03$

$1.64234 \mathrm{E}-03$

$1.65221 \mathrm{E}-03$

$1.66211 \mathrm{E}-03$

$1.67204 \mathrm{E}-03$

$1.68200 \mathrm{E}-03$

$1.69199 \mathrm{E}-03$

$1.70201 \mathrm{E}-03$

$1.71206 \mathrm{E}-03$

$1.72214 \mathrm{E}-03$

$1.73225 \mathrm{E}-03$

$1.74238 \mathrm{E}-03$

$1.75255 \mathrm{E}-03$

$1.76275 \mathrm{E}-03$

$1.77297 \mathrm{E}-03$ $1.78323 \mathrm{E}-03$

$1.79351 \mathrm{E}-03$ $1.80383 \mathrm{E}-03$

$1.81417 \mathrm{E}-03$

$1.82454 \mathrm{E}-03$

$1.83495 \mathrm{E}-03$

$1.84538 \mathrm{E}-03$

$1.85584 \mathrm{E}-03$

$1.86633 \mathrm{E}-03$

35.60

35.70

35.80

35.90

36.00

$1.87685 \mathrm{E}-03$ $1.88740 \mathrm{E}-03$ $1.89798 \mathrm{E}-03$ $1.90859 \mathrm{E}-03$ $1.91923 \mathrm{E}-03$ $f_{2}$

$2.16969 \mathrm{E} \quad 00$ $2.18421 \mathrm{E} \quad 00$ $2.19879 \mathrm{E} \quad 00$ $2.21340 \mathrm{E} \quad 00$ $2.22807 \mathrm{E} \quad 00$

$2.24279 \mathrm{E} \quad 00$ $2.25755 \mathrm{E} \quad 00$ $2.27236 \mathrm{E} \quad 00$ $2.28722 \mathrm{E} \quad 00$ $2.30212 \mathrm{E} \quad 00$

$2.31708 \mathrm{E} \quad 00$ $2.33208 \mathrm{E} \quad 00$ $2.34713 \mathrm{E} \quad 00$ $2.36223 \mathrm{E} \quad 00$ $2.37737 \mathrm{E} \quad 00$

$2.39257 \mathrm{E} \quad 00$ $2.40781 \mathrm{E} \quad 00$ $2.42310 \mathrm{E} \quad 00$ $2.43843 \mathrm{E} \quad 00$ $2.45382 \mathrm{E} \quad 00$

$2.46925 \mathrm{E} \quad 00$ $2.48473 \mathrm{E} \quad 00$ $2.50026 \mathrm{E} \quad 00$ $2.51584 \mathrm{E} \quad 00$ $2.53146 \mathrm{E} \quad 00$

$2.54713 \mathrm{E} \quad 00$ $2.56285 \mathrm{E} \quad 00$ $2.57862 \mathrm{E} \quad 00$ $2.59443 \mathrm{E} \quad 00$ $2.61029 \mathrm{E} \quad 00$

$2.62620 \mathrm{E} \quad 00$ $2.64216 \mathrm{E} \quad 00$ $2.65816 \mathrm{E} \quad 00$ $2.67422 \mathrm{E} \quad 00$ $2.69031 \mathrm{E} \quad 00$

$2.70646 \mathrm{E} \quad 00$ $2.72266 \mathrm{E} \quad 00$ $2.73890 \mathrm{E} \quad 00$ $2.75519 \mathrm{E} \quad 00$ 2.77153 E $\quad 00$

$2.78792 \mathrm{E} \quad 00$ $2.80435 \mathrm{E} \quad 00$ $2.82083 \mathrm{E} \quad 00$ $2.83736 \mathrm{E} \quad 00$ $2.85394 \mathrm{E} \quad 00$

$2.87056 \mathrm{E} \quad 00$ $2.88723 \mathrm{E} \quad 00$ $2.90395 \mathrm{E} \quad 00$ $2.92072 \mathrm{E} \quad 00$ $2.93753 \mathrm{E} \quad 00$

$2.95439 \mathrm{E} \quad 00$ $2.97130 \mathrm{E} \quad 00$ $2.98826 \mathrm{E} \quad 00$ $3.00526 \mathrm{E} \quad 00$ $3.02231 \mathrm{E} \quad 00$ (.07375E $3.10828 \mathrm{E} \quad 00$ $g_{2}$

$7.90058 \mathrm{E}-02$ $7.98845 \mathrm{E}-02$ $8.07695 \mathrm{E}-02$ $8.16610 \mathrm{E}-02$ $8.25589 \mathrm{E}-02$

$8.34634 \mathrm{E}-02$ $8.43743 \mathrm{E}-02$ $8.52918 \mathrm{E}-02$ $8.62159 \mathrm{E}-02$ $8.71465 \mathrm{E}-02$

$8.80837 \mathrm{E}-02$ $8.90276 \mathrm{E}-02$ 8.99781 E-02

$9.09353 \mathrm{E}-02$

$9.18992 \mathrm{E}-02$

$9.28698 \mathrm{E}-02$

$9.38471 \mathrm{E}-02$

$9.48312 \mathrm{E}-02$

$9.58221 \mathrm{E}-02$

$9.68198 \mathrm{E}-02$

$9.78244 \mathrm{E}-02$

$9.88358 \mathrm{E}-02$

$9.98541 \mathrm{E}-02$

$1.00879 \mathrm{E}-01$

$1.01911 \mathrm{E}-01$

$1.02950 \mathrm{E}-01$

$1.03996 \mathrm{E}-01$

$1.05049 \mathrm{E}-01$

$1.06109 \mathrm{E}-01$

$1.07177 \mathrm{E}-01$

$1.08251 \mathrm{E}-01$ $1.09332 \mathrm{E}-01$

$1.10420 \mathrm{E}-01$

$1.11516 \mathrm{E}-01$

1.12618E-01

$1.13728 \mathrm{E}-01$

$1.14845 \mathrm{E}-01$

$1.15969 \mathrm{E}-01$

$1.17101 \mathrm{E}-01$

$1.18240 \mathrm{E}-01$

$1.19386 \mathrm{E}-01$

$1.20539 \mathrm{E}-01$

$1.21700 \mathrm{E}-01$

I. $22868 \mathrm{E}-01$

$1.24043 \mathrm{E}-01$

$1.25226 \mathrm{E}-01$

$1.26416 \mathrm{E}-01$

$1.27614 \mathrm{E}-01$

$1.28819 \mathrm{E}-01$

$1.30032 \mathrm{E}-01$

$1.31252 \mathrm{E}-0 \mathrm{l}$

$1.32479 \mathrm{E}-01$

$1.33715 \mathrm{E}-01$

$1.34958 \mathrm{E}-01$

$1.36208 \mathrm{E}-01$

$1.37466 \mathrm{E}-01$ $1.38732 \mathrm{E}-01$ $1.40005 \mathrm{E}-01$

$1.41287 \mathrm{E}-01$

$1.42575 \mathrm{E}-01$ $\tan \delta_{2}$

$f_{-2}$

$g-2$

$-2.43126 \mathrm{E} \quad 01 \quad-7.45014 \mathrm{E}-02$ $-2.43136 \mathrm{E}$ 01 $-7.53534 \mathrm{E}-02$ $-2.43147 \mathrm{E}$ 01 $-7.62117 \mathrm{E}-02$ $-2.43157 \mathrm{E}$ 01 $-7.70764 \mathrm{E}-02$ $-2.43167 \mathrm{E} \quad 01 \quad-7.79475 \mathrm{E}-02$

$-2.43177 \mathrm{E}$ 01 $-7.88249 \mathrm{E}-02$ $-2.43187 \mathrm{E}$ 01 $-7.97088 \mathrm{E}-02$ $-2.43197 \mathrm{E}$ 01 $-8.05992 \mathrm{E}-02$ $-2.43207 \mathrm{E}$ 01 $-8.14961 \mathrm{E}-02$ $\begin{array}{lll}-2.43216 \mathrm{E} & 01 & -8.23994 \mathrm{E}-02\end{array}$

$-2.43226 \mathrm{E} \quad 01$ $-2.43236 \mathrm{E} 01$ $-2.43245 \mathrm{E} \quad 01$ $-2.43255 \mathrm{E} \quad 01$ $-2.43264 \mathrm{E} \quad 01$

$-2.43274 \mathrm{E} \quad 01$ $-2.43283 \mathrm{E} 01$ $-2.43292 \mathrm{E}$ 01 $-2.43302 \mathrm{E} \quad 01$ $-2.43311 \mathrm{E} \quad 01$

$-8.33093 \mathrm{E}-02$ $-8.42257 \mathrm{E}-02$ $-8.51488 \mathrm{E}-02$ $-8.60784 \mathrm{E}-02$ $-8.70146 \mathrm{E}-02$

$-2.43320 \mathrm{E} \quad 01$ $-2.43329 \mathrm{E}$ 01 $-2.43338 \mathrm{E} \quad 01$ $-2.43347 \mathrm{E} \quad 01$ $-2.43356 \mathrm{E}$ 0l

$-8.79575 \mathrm{E}-02$ $-8.89070 \mathrm{E}-02$ $-8.98632 \mathrm{E}-02$ $-9.08262 \mathrm{E}-02$ $-9.17958 \mathrm{E}-02$

$-2.43364 \mathrm{E} \quad 01$

$-2.43373 \mathrm{E} \quad 01$

$-2.43382 \mathrm{E}$ 0I

$-2.43391 \mathrm{E} 01$

$-2.43399 \mathrm{E}$ ol

$-2.43408 \mathrm{E} 01$ $-2.43416 \mathrm{E} \quad 01$ $-2.43425 \mathrm{E} 01$ $-2.43433 \mathrm{E} \quad 01$ $-2.43441 \mathrm{E} 01$

$-9.27723 \mathrm{E}-02$ $-9.37555 \mathrm{E}-02$ $-9.47455 \mathrm{E}-02$ $-9.57423 \mathrm{E}-02$ $-9.67460 \mathrm{E}-02$

$-2.43450 \mathrm{E}$ 0I $-2.43458 \mathrm{E} \quad 01$ $-2.43466 \mathrm{E}$ 0l $-2.43474 \mathrm{E}$ ol -2.43482 E 01

$-9.77566 \mathrm{E}-02$ $-9.87740 \mathrm{E}-02$ $-9.97984 \mathrm{E}-02$ $-1.00830 \mathrm{E}-01$ $-1.01868 \mathrm{E}-01$

$-1.02913 \mathrm{E}-01$ $-1.03966 \mathrm{E}-01$ $-1.05025 \mathrm{E}-01$ $-1.06091 \mathrm{E}-01$ $-1.07165 \mathrm{E}-01$

$-2.43490 \mathrm{E} \quad 01$ $-2.43498 \mathrm{E}$ 0l $-2.43506 \mathrm{E} 01$ $-2.43514 \mathrm{E}$ 01 $-2.43522 \mathrm{E}$ ol

$-1.08245 \mathrm{E}-01$ $-1.09333 \mathrm{E}-01$ $-1.10428 \mathrm{E}-01$ $-1.11530 \mathrm{E}-01$ $-1.12639 \mathrm{E}-0 \mathrm{l}$

$-1.13755 \mathrm{E}-01$ $-1.14879 \mathrm{E}-01$ $-1.16010 \mathrm{E}-01$ $-1.17148 \mathrm{E}-01$ $-1.18293 \mathrm{E}-01$

$-2.43530 \mathrm{E} \quad 01$ $-2.43537 \mathrm{E} 01$ $-2.43545 \mathrm{E} \quad 01$ $-2.43553 \mathrm{E}$ 01 $-2.43560 \mathrm{E} \quad 01$

\section{$-2.43568 \mathrm{E} 01$}

$-2.43575 \mathrm{E} \quad 01$ $-2.43583 \mathrm{E} 01$ $-2.43590 \mathrm{E} \quad 01$ $-2.43598 \mathrm{E} 01$

$-1.19446 \mathrm{E}-\dot{0} 01$ $-1.20606 \mathrm{E}-01$ $-1.21774 \mathrm{E}-01$ $-1.22949 \mathrm{E}-01$ $-1.24131 \mathrm{E}-01$

$2.24305 \mathrm{E} \quad 00$ 
(1)

36.10

36.20

36.30

36.40

36.50

36.60

36.70

36.80

36.90

37.00

37.10

37.20

37.30

37.40

37.50

37.60

37.70

37.80

37.90

38.00

38.10

38.20

38.30

38.40

38.50

38.60

38.70

38.80

38.90

39.00

39.10

39.20

39.30

39.40

39.50

39.60

39.70

39.80

39.90

40.00

40.10

40.20

40.30

40.40

40.50

40.60

40.70

40.80

40.90

41.00

41.10

41.20

41.30

41.40

41.50

41.60

41.70

41.80

41.90

42.00
$F_{0}$

8.3614.7E-01

$8.36139 \mathrm{E}-01$

$8.36132 \mathrm{E}-01$

$8.36124 \mathrm{E}-01$

$8.36117 \mathrm{E}-01$

$8.36109 \mathrm{E}-01$

$8.36102 \mathrm{E}-01$

8.36095E-01

$8.36087 \mathrm{E}-01$

$8.36080 \mathrm{E}-01$

8.36072 E-01 $8.36065 \mathrm{E}-01$ 8.36058E-01 $8.36050 \mathrm{E}-01$ 8.36043 E-01

$8.36036 \mathrm{E}-01$ $8.36028 \mathrm{E}-01$ $8.36021 \mathrm{E}-01$ $8.36014 \mathrm{E}-01$ 8.36007E-01

$8.36000 \mathrm{E}-01$ $8.35992 \mathrm{E}-01$ $8.35085 \mathrm{E}-01$ $8.35978 \mathrm{E}-01$ $8.35971 E-01$

8.35964.E-01 8.35957E-01 $8.35950 \mathrm{E}-01$ 8.35943E-01 8.35936 E-01

8.35929 E-01 8.35922E-01 $8.35915 \mathrm{E}-01$ $8.35908 \mathrm{E}-01$ 8.35901 E-01

8.35894E-01 $8.35887 \mathrm{E}-01$ $8.35880 \mathrm{E}-01$ $8.35873 \mathrm{E}-01$ $8.35866 \mathrm{E}-01$

$8.35859 \mathrm{E}-01$ 8.35852E-01 $8.35845 \mathrm{E}-01$ $8.35839 \mathrm{E}-01$ 8.35832E-01

8.35825 E-01 8.35818E-01 $8.35812 \mathrm{E}-01$ $8.35805 \mathrm{E}-01$ $8.35798 \mathrm{E}-01$

$8.35791 \mathrm{E}-01$ $8.35785 \mathrm{E}-01$ 8.35778E-01 8.35771 E-01 $8.35765 \mathrm{E}-01$

$8.35758 \mathrm{E}-01$ $8.35751 \mathrm{E}-01$ $8.35745 \mathrm{E}-01$ $8.35738 \mathrm{E}-01$ 8.35732 E-01 $f_{1}$

$3.36993 \mathrm{E} \quad 01$

$3.37992 \mathrm{E} \quad 01$

$3.38991 \mathrm{E} \quad 01$

$3.39991 \mathrm{E} \quad 01$

$3.40991 \mathrm{E} \quad 01$

$3.41992 \mathrm{E} \quad 01$

$3.42993 \mathrm{E} \quad 01$

$3.43994 \mathrm{E} \quad 01$

$3.44996 \mathrm{E} \quad 0 \mathrm{I}$

$3.45998 \mathrm{E} 01$

$3.47001 \mathrm{E} \quad 01$ $3.48004 \mathrm{E} \quad 01$

$3.49008 \mathrm{E} \quad 01$

$3.50012 \mathrm{E} \quad 01$

$3.51016 \mathrm{E} \quad 01$

$3.52021 \mathrm{E} \quad 01$

$3.53026 \mathrm{E} \quad 01$

$3.54032 \mathrm{E}$ 01

$3.55038 \mathrm{E}$ 0l

$3.56045 \mathrm{E} \quad 0$

$3.57052 \mathrm{E} \quad 01$

$3.58060 \mathrm{E} \quad 01$

$3.59068 \mathrm{E} \quad 01$

$3.60076 \mathrm{E} \quad 01$

$3.61085 \mathrm{E} \quad 01$

$3.62095 \mathrm{E} \quad 01$

$3.63104 \mathrm{E} \quad 01$

$3.64115 \mathrm{E}$ 01

$3.65126 \mathrm{E} \quad 01$

$3.66137 \mathrm{E} \quad 01$

$3.67148 \mathrm{E} \quad 01$

$3.68161 \mathrm{E} \quad 01$

$3.69173 \mathrm{E} 01$

$3.70186 \mathrm{E} \quad 01$

$3.71200 \mathrm{E} 01$

$3.72214 \mathrm{E} \quad 01$

$3.73228 \mathrm{E} \quad 01$

$3.74243 \mathrm{E}$ 01

$3.75259 \mathrm{E} \quad 01$

$3.76275 \mathrm{E} \quad 01$

$3.77291 \mathrm{E} \quad 01$

$3.78308 \mathrm{E} \quad 01$

$3.79325 \mathrm{E} \quad 01$

$3.80343 \mathrm{E}$ ol

$3.81362 \mathrm{E} \quad 01$

$3.82380 \mathrm{E} \quad 01$

$3.83400 \mathrm{E} \quad 01$

$3.84419 \mathrm{E}$ ol

$3.85440 \mathrm{E}$ 01

$3.86460 \mathrm{E} \quad 01$

$3.87482 \mathrm{E} \quad 01$

$3.88503 \mathrm{E} \quad 01$

$3.89525 \mathrm{E} \quad 01$

$3.90548 \mathrm{E} \quad 01$

$3.91571 \mathrm{E} \quad 0 \mathrm{i}$

$3.92595 \mathrm{E} \quad 01$ $3.93619 \mathrm{E} \quad 01$

$3.94644 \mathrm{E}$ 01

$3.95669 \mathrm{E} \quad 01$

$3.96695 \mathrm{E} 01$ $g_{1} \quad \tan \delta_{1}$

$2.55220 \mathrm{E} \quad 00$

$2.56886 \mathrm{E} \quad 00$

$2.58556 \mathrm{E} \quad 00$

$2.60233 \mathrm{E} \quad 00$

$2.61915 \mathrm{E} \quad 00$

$2.63603 \mathrm{E} \quad 00$

$2.65297 \mathrm{E} \quad 00$

$2.66997 \mathrm{E} \quad 00$

$2.68703 \mathrm{E} \quad 00$

$2.70414 \mathrm{E} \quad 00$

$2.72131 \mathrm{E} \quad 00$

$2.73854 \mathrm{E} \quad 00$

$2.75583 \mathrm{E} \quad 00$

$2.77317 \mathrm{E} \quad 00$

$2.79058 \mathrm{E}$

$2.80804 \mathrm{E} \quad 00$

$2.82556 \mathrm{E} \quad 00$

$2.84314 \mathrm{E} \quad 00$

$2.86077 \mathrm{E} \quad 00$

$2.87847 \mathrm{E} \quad 00$

$2.89622 \mathrm{E} \quad 00$

$2.91404 \mathrm{E} \quad 00$

$2.93191 \mathrm{E} \quad 00$

$2.94984 \mathrm{E} \quad 00$

$2.96783 \mathrm{E} \quad 00$

$2.98588 \mathrm{E} \quad 00$

$3.00398 \mathrm{E} \quad 00$

$3.02215 \mathrm{E} \quad 00$

$3.04038 \mathrm{E} \quad 00$

$3.05866 \mathrm{E} \quad 00$

$3.07700 \mathrm{E} \quad 00$

$3.09540 \mathrm{E} \quad 00$

$3.11387 \mathrm{E} \quad 00$

$3.13239 \mathrm{E} \quad 00$

$3.15097 \mathrm{E} \quad 00$

$3.16961 \mathrm{E} \quad 00$

$3.18831 \mathrm{E} \quad 00$

$3.20707 \mathrm{E} \quad 00$

$3.22589 \mathrm{E} \quad 00$

$3.24476 \mathrm{E} \quad 00$

$3.26370 \mathrm{E} \quad 00$

$3.28270 \mathrm{E} \quad 00$

$3.30176 \mathrm{E} \quad 00$

$3.32087 \mathrm{E} \quad 00$

$3.34005 \mathrm{E} \quad 00$

$3.35929 \mathrm{E} \quad 00$

$3.37859 \mathrm{E} \quad 00$

$3.39794 \mathrm{E} \quad 00$

$3.41736 \mathrm{E} \quad 00$

$3.43684 \mathrm{E} \quad 00$

$3.45638 \mathrm{E} \quad 00$

$3.47598 \mathrm{E} \quad 00$

$3.49564 \mathrm{E} \quad 00$

$3.51536 \mathrm{E} \quad 00$

$3.53514 \mathrm{E} \quad 00$

$3.55498 \mathrm{E} \quad 00$

$3.57488 \mathrm{E} \quad 00$

$3.59484 \mathrm{E} \quad 00$

$3.61487 \mathrm{E} \quad 00$

$3.63495 \mathrm{E} \quad 00$

$-6.88199 \mathrm{E}-04$

$-6.88167 \mathrm{E}-04$

$-6.88130 \mathrm{E}-04$

$-6.88066 \mathrm{E}-04$

$-6.87979 \mathrm{E}-04$

$-6.87871 \mathrm{E}-04$

$-6.87754 \mathrm{E}-04$

$-6.87623 \mathrm{E}-04$

$-6.87463 \mathrm{E}-04$

$-6.87289 \mathrm{E}-04$

$-6.87096 \mathrm{E}-04$

$-6.86888 \mathrm{E}-04$

$-6.86661 \mathrm{E}-04$

$-6.86421 \mathrm{E}-04$

$-6.86157 \mathrm{E}-04$

$-6.85876 \mathrm{E}-04$

$-6.85581 \mathrm{E}-04$

$-6.85267 \mathrm{E}-04$

$-6.84939 \mathrm{E}-04$

$-6.84587 \mathrm{E}-04$

$-6.84222 \mathrm{E}-04$

$-6.83841 \mathrm{E}-04$

$-6.83444 \mathrm{E}-04$

$-6.83033 \mathrm{E}-04$

$-6.82610 \mathrm{E}-04$

$-6.82159 \mathrm{E}-04$

$-6.81691 \mathrm{E}-04$

$-6.81204 \mathrm{E}-04$

$-6.80715 \mathrm{E}-04$

$-6.80199 \mathrm{E}-04$

$-6.79669 \mathrm{E}-04$

$-6.79125 \mathrm{E}-04$

$-6.78571 \mathrm{E}-04$

$-6.77995 \mathrm{E}-04$

$-6.77404 \mathrm{E}-04$

$-6.76789 \mathrm{E}-04$

$-6.76155 \mathrm{E}-04$

$-6.75526 \mathrm{E}-04$

$-6.74872 \mathrm{E}-04$

$-6.74199 \mathrm{E}-04$

$-6.73515 \mathrm{E}-04$ 
$f_{2}$

$1.92990 \mathrm{E}-03$ $1.94060 \mathrm{E}-03$ $1.95133 \mathrm{E}-03$ $1.96209 \mathrm{E}-03$ $1.97287 \mathrm{E}-03$

$1.98369 \mathrm{E}-03$ $1.99454 \mathrm{E}-03$ $2.00541 \mathrm{E}-03$ $2.01632 \mathrm{E}-03$ $2.02725 \mathrm{E}-03$

$2.03822 \mathrm{E}-03$ $2.04921 \mathrm{E}-03$ $2.06023 \mathrm{E}-03$ $2.07129 \mathrm{E}-03$ $2.08237 \mathrm{E}-03$

$2.09348 \mathrm{E}-03$ $2.10462 \mathrm{E}-03$ $2.11579 \mathrm{E}-03$ $2.12699 \mathrm{E}-03$ $2.13822 \mathrm{E}-03$

$2.14948 \mathrm{E}-03$ $2.16077 \mathrm{E}-03$ $2.17209 \mathrm{E}-03$ $2.18344 \mathrm{E}-03$ $2.19482 \mathrm{E}-03$

$2.20623 \mathrm{E}-03$ $2.21766 \mathrm{E}-03$ $2.22913 \mathrm{E}-03$ $2.24063 \mathrm{E}-03$ $2.25215 \mathrm{E}-03$

$2.26371 \mathrm{E}-03$ $2.27529 \mathrm{E}-03$ $2.28690 \mathrm{E}-03$ $2.29855 \mathrm{E}-03$ $2.31022 \mathrm{E}-03$

$2.32192 \mathrm{E}-03$ $2.33366 \mathrm{E}-03$ $2.34542 \mathrm{E}-03$ $2.35721 \mathrm{E}-03$

$2.36903 \mathrm{E}-03$

$2.38088 \mathrm{E}-03$ $2.39276 \mathrm{E}-03$ $2.40467 \mathrm{E}-03$ $2.41661 \mathrm{E}-03$ $2.42858 \mathrm{E}-03$

$2.44058 \mathrm{E}-03$ $2.45261 \mathrm{E}-03$ $2.46466 \mathrm{E}-03$ $2.47675 \mathrm{E}-03$ $2.48887 \mathrm{E}-03$

$2.50101 \mathrm{E}-03$ $2.51319 \mathrm{E}-03$ $2.52539 \mathrm{E}-03$ $2.53763 \mathrm{E}-03$ $2.54989 \mathrm{E}-03$

$2.56218 \mathrm{E}-03$ $2.57451 \mathrm{E}-03$ $2.58686 \mathrm{E}-03$ $2.59924 \mathrm{E}-03$ $2.61165 \mathrm{E}-03$ $g_{2}$

$\tan \delta_{2}$ $f_{-2}$

$3.12562 \mathrm{E} \quad 00$ $3.14300 \mathrm{E} \quad 00$ $3.16043 \mathrm{E} \quad 00$ $3.17791 \mathrm{E} \quad 00$ $3.19544 \mathrm{E} \quad 00$

$3.21301 \mathrm{E} \quad 00$ $3.23063 \mathrm{E} \quad 00$ $3.24830 \mathrm{E} \quad 00$ $3.26602 \mathrm{E} \quad 00$ $3.28378 \mathrm{E} \quad 00$

$3.30159 \mathrm{E} \quad 00$ $3.31944 \mathrm{E} \quad 00$ $3.33735 \mathrm{E} \quad 00$ $3.35530 \mathrm{E} \quad 00$ $3.37330 \mathrm{E} \quad 00$

$3.39135 \mathrm{E} \quad 00$ $3.40944 \mathrm{E} \quad 00$ $3.42758 \mathrm{E} \quad 00$

$3.44577 \mathrm{E} \quad 00$

$3.46400 \mathrm{E} \quad 00$

$3.48229 \mathrm{E} \quad 00$ $3.50062 \mathrm{E} \quad 00$ $3.51899 \mathrm{E} \quad 00$ $3.53742 \mathrm{E} \quad 00$ $3.55589 \mathrm{E} \quad 00$

$3.57441 \mathrm{E} \quad 00$ $3.59297 \mathrm{E} \quad 00$ $3.61159 \mathrm{E} \quad 00$ $3.63025 \mathrm{E} \quad 00$ $3.64895 \mathrm{E} \quad 00$

$3.66771 \mathrm{E} \quad 00$ 3.68651 E 00 $3.70536 \mathrm{E} \quad 00$ $3.72425 \mathrm{E} \quad 00$ $3.74320 \mathrm{E} \quad 00$

$3.76219 \mathrm{E} \quad 00$ $3.78122 \mathrm{E} \quad 00$ $3.80031 \mathrm{E} \quad 00$ $3.81944 \mathrm{E} \quad 00$ $3.83862 \mathrm{E} \quad 00$

$3.85784 \mathrm{E} \quad 00$ $3.87711 \mathrm{E} \quad 00$ $3.89643 \mathrm{E} \quad 00$ $3.91580 \mathrm{E} \quad 00$ $3.93521 \mathrm{E} \quad 00$

$3.95467 \mathrm{E} \quad 00$ $3.97418 \mathrm{E} \quad 00$ $3.99373 \mathrm{E} \quad 00$ $4.01333 \mathrm{E} \quad 00$ $4.03298 \mathrm{E} \quad 00$

$4.05268 \mathrm{E} \quad 00$ $4.07242 \mathrm{E} \quad 00$ $4.09221 \mathrm{E} \quad 00$ $4.11204 \mathrm{E} \quad 00$ $4.13192 \mathrm{E} \quad 00$

$4.15185 \mathrm{E} \quad 00$ $4.17183 \mathrm{E} \quad 00$ $4.19185 \mathrm{E} \quad 00$ $4.21192 \mathrm{E} \quad 00$ $4.23204 \mathrm{E} \quad 00$

$1.43872 \mathrm{E}-01$ $1.80377 \mathrm{E}-01$
$1.45177 \mathrm{E}-01$

$1.46489 \mathrm{E}-0 \mathrm{I}$

$1.47809 \mathrm{E}-01$

$1.49137 \mathrm{E}-01$

$1.50472 \mathrm{E}-01$

$1.51816 \mathrm{E}-01$

$1.53167 \mathrm{E}-01$

$1.54527 \mathrm{E}-01$

$1.55894 \mathrm{E}-01$

$1.57269 \mathrm{E}-01$

$1.58653 \mathrm{E}-01$

$1.60044 \mathrm{E}-01$

$1.61443 \mathrm{E}-01$

$1.62851 \mathrm{E}-01$

$1.64266 \mathrm{E}-01$

$1.65690 \mathrm{E}-01$

$1.67122 \mathrm{E}-01$

$1.68562 \mathrm{E}-01$

$1.70010 \mathrm{E}-01$

$1.71466 \mathrm{E}-01$

$1.72931 \mathrm{E}-01$

$1.74403 \mathrm{E}-01$

$1.75884 \mathrm{E}-01$

$1.77374 \mathrm{E}-01$

$1.78871 \mathrm{E}-01$ $1.81892 \mathrm{E}-01$ $1.83414 \mathrm{E}-01$ $1.84945 \mathrm{E}-01$

$-2.43641 \mathrm{E} \quad 01$ $-2.43648 \mathrm{E} \quad 01$ $-2.43655 \mathrm{E} \quad 01$ $-2.43662 \mathrm{E} \quad 01$ $-2.43669 \mathrm{E} \quad 01$

$-2.43676 \mathrm{E} \quad$ U1 $\begin{array}{lll}-2.43683 \mathrm{E} & 01\end{array}$ $-2.43690 \mathrm{E} \quad 01$ $-2.43696 \mathrm{E} \quad 01$ -2.43703 E 01

$-2.43710 \mathrm{E} \quad 01$ $-2.43717 \mathrm{E} \quad 01$ $-2.43723 \mathrm{E} \quad 01$ $-2.43730 \mathrm{E} 01$ $-2.43736 \mathrm{E} \quad 01$

$-2.43743 \mathrm{E} \quad 01$ $-2.43750 \mathrm{E} \quad 01$ $-2.43756 \mathrm{E} \quad 01$ $-2.43762 \mathrm{E} \quad 01$ $-2.43769 \mathrm{E} \quad 01$

$-2.43775 \mathrm{E} \quad 01$ $-2.43782 \mathrm{E} \quad 01$ $-2.43788 \mathrm{E} \quad 01$ $-2.43794 \mathrm{E} \quad 01$ $-2.43800 \mathrm{E} \quad 01$

$-2.43806 \mathrm{E} \quad 01$ $-2.43813 \mathrm{E} \quad 01$ $-2.43819 \mathrm{E} 01$ $-2.43825 \mathrm{E} 01$

$-2.43831 \mathrm{E}$ 0l

$1.86485 \mathrm{E}-01$

$1.88033 \mathrm{E}-01$

$1.89589 \mathrm{E}-01$

$1.91154 \mathrm{E}-01$

$1.92728 \mathrm{E}-01$

$1.94310 \mathrm{E}-01$

$1.95900 \mathrm{E}-01$

$1.97499 \mathrm{E}-01$

$1.99107 \mathrm{E}-01$

$2.00724 \mathrm{E}-01$

$\begin{array}{lll}-2.43837 \mathrm{E} & 01\end{array}$

$-2.43843 \mathrm{E} \quad 01$

$\begin{array}{lll}-2.43849 \mathrm{E} & 01\end{array}$

$-2.43855 \mathrm{E} \quad 01$

-2.43861 E 01

$\begin{array}{ll}-2.43867 \mathrm{E} & 01\end{array}$

$-2.43872 \mathrm{E} \quad 01$

$-2.43878 \mathrm{E} 01$

$-2.43884 \mathrm{E} \quad 01$

$-2.43890 \mathrm{E} 01$

$2.02349 \mathrm{E}-01$ $2.03982 \mathrm{E}-01$ $2.05625 \mathrm{E}-01$ $2.07276 \mathrm{E}-01$ $2.08936 \mathrm{E}-01$

$-2.43895 \mathrm{E} \quad 01$ $\begin{array}{lll}-2.43901 \mathrm{E} & 01\end{array}$ $-2.43907 \mathrm{E} \quad 01$ $-2.43913 \mathrm{E} \quad 01$ $-2.43918 \mathrm{E} \quad 01$

$2.10604 \mathrm{E}-01$ $2.12282 \mathrm{E}-01$ $2.13968 \mathrm{E}-01$

$2.15663 \mathrm{E}-01$

$2.17367 \mathrm{E}-01$

$2.19080 \mathrm{E}-01$ $2.20801 \mathrm{E}-01$ $2.22532 \mathrm{E}-01$

$2.24271 \mathrm{E}-01$

$2.26020 \mathrm{E}-01$

$2.27777 \mathrm{E}-01$ $2.29544 \mathrm{E}-01$ $2.31319 \mathrm{E}-01$ $2.33104 \mathrm{E}-01$

$-2.43924 \mathrm{E} \quad 01$ $-2.43929 \mathrm{E} \quad 01$ $-2.43935 \mathrm{E} \quad 01$ $-2.43940 \mathrm{E} \quad 01$ $-2.43946 \mathrm{E} \quad 01$

$\begin{array}{lll}-2.43951 \mathrm{E} & 01\end{array}$ $-2.43956 \mathrm{E} \quad 01$ $-2.43962 \mathrm{E} \quad 0 \mathrm{I}$ $-2.43967 \mathrm{E} \quad 01$ $-2.43972 \mathrm{E} \quad 01$

$-2.43978 \mathrm{E} \quad 0 \mathrm{I}$ $-2.43983 \mathrm{E} \quad 0 \mathrm{l}$ $-2.43988 \mathrm{E} \quad 01$
$2.34898 \mathrm{E}-01$

$-1.37633 \mathrm{~F}-0]$ $-1.38906 \mathrm{E}-01$ $-1.40187 \mathrm{E}-01$ $-1.41476 \mathrm{E}-01$ $-1.42772 \mathrm{E}-01$

$-1.44076 \mathrm{E}-0]$ $-1.45388 \mathrm{E}-01$ $-1.46708 \mathrm{E}-01$ $-1.48036 \mathrm{E}-01$ $-1.49371 \mathrm{E}-01$

$-1.50714 \mathrm{E}-01$ $-1.52066 \mathrm{E}-01$ $-1.53425 \mathrm{E}-01$ $-1.54792 \mathrm{E}-01$ $-1.56167 \mathrm{E}-01$

$-1.57551 \mathrm{E}-01$ $-1.58942 \mathrm{E}-01$ $-1.60341 \mathrm{E}-01$ $-1.61749 \mathrm{E}-01$ $-1.63164 \mathrm{E}-01$

$-1.64588 \mathrm{E}-01$ $-1.66020 \mathrm{E}-01$ $-1.67460 \mathrm{E}-01$ $-1.68908 \mathrm{E}-01$ $-1.70364 \mathrm{E}-01$

$-1.71829 \mathrm{E}-01$ $-1.73301 \mathrm{E}-01$ $-1.74783 \mathrm{E}-01$ $-1.76272 \mathrm{E}-01$ $-1.77770 \mathrm{E}-01$

$-1.79276 \mathrm{E}-01$ $-1.80790 \mathrm{E}-01$ $-1.82313 \mathbf{E}-01$ $-1.83844 \mathrm{E}-0 \mathrm{l}$ $-1.85384 \mathrm{E}-01$

$-1.86932 \mathrm{E}-01$ $-1.88489 \mathrm{E}-01$ $-1.90054 \mathrm{E}-01$ $-1.91628 \mathrm{E}-01$ $-1.93210 \mathrm{E}-01$

$-1.94801 \mathrm{E}-01$ $-1.96401 \mathrm{E}-01$ $-1.98009 \mathrm{E}-01$ $-1.99625 \mathrm{E}-01$ $-2.01251 \mathrm{E}-01$ 
ELECTRON RADIAL FUNCTIONS

$\begin{array}{cc}p & F_{0} \\ 0.10 & 6.90970 \mathrm{E}-02 \\ 0.20 & 3.02548 \mathrm{E}-01 \\ 0.30 & 4.63310 \mathrm{E}-01 \\ 0.40 & 5.63023 \mathrm{E}-01 \\ 0.50 & 6.27843 \mathrm{E}-01 \\ & \\ 0.60 & 6.72114 \mathrm{E}-01 \\ 0.70 & 7.03589 \mathrm{E}-01 \\ 0.80 & 7.26691 \mathrm{E}-01 \\ 0.90 & 7.44095 \mathrm{E}-01 \\ 1.00 & 7.57489 \mathrm{E}-01 \\ & \\ 1.10 & 7.67988 \mathrm{E}-01 \\ 1.20 & 7.76346 \mathrm{E}-01 \\ 1.30 & 7.83091 \mathrm{E}-01 \\ 1.40 & 7.88601 \mathrm{E}-01 \\ 1.50 & 7.93147 \mathrm{E}-01 \\ & \\ 1.60 & 7.96938 \mathrm{E}-01 \\ 1.70 & 8.00122 \mathrm{E}-01 \\ 1.80 & 8.02819 \mathrm{E}-01 \\ 1.90 & 8.05120 \mathrm{E}-01\end{array}$

$\begin{array}{ll}1.90 & 8.05120 \mathrm{E}-01 \\ 2.00 & 8.07094 \mathrm{E}-01\end{array}$

$2.10 \quad 8.08798 \mathrm{E}-01$

$2.20 \quad 8.10276 \mathrm{E}-01$

$2.30 \quad 8.11565 \mathrm{E}-01$

$2.40 \quad 8.12694 \mathrm{E}-01$

$2.50 \quad 8.13686 \mathrm{E}-01$

$2.60 \quad 8.14562 \mathrm{E}-01$

$2.70 \quad 8.15337 \mathrm{E}-01$

$2.80 \quad 8.16025 \mathrm{E}-01$

$2.90 \quad 8.16639 \mathrm{E}-01$

$3.00 \quad 8.17186 \mathrm{E}-01$

$3.10 \quad 8.17677 \mathrm{E}-01$

$3.20 \quad 8.18117 \mathrm{E}-01$

$3.30 \quad 8.18512 \mathrm{E}-01$

$3.40 \quad 8.18868 \mathrm{E}-01$

$3.50 \quad 8.19189 \mathrm{E}-01$

$3.60 \quad 8.19480 \mathrm{E}-01$

$3.70 \quad 8.19742 \mathrm{E}-01$

$3.80 \quad 8.19980 \mathrm{E}-01$

$3.90 \quad 8.20195 \mathrm{E}-01$

$4.00 \quad 8.20391 \mathrm{E}-01$

$4.10 \quad 8.20568 \mathrm{E}-01$

$4.20 \quad 8.20729 \mathrm{E}-01$

$4.30 \quad 8.20877 \mathrm{E}-01$

$4.40 \quad 8.21009 \mathrm{E}-01$

$4.50 \quad 8.21131 \mathrm{E}-01$

$4.60 \quad 8.21240 \mathrm{E}-01$

$4.70 \quad 8.21341 \mathrm{E}-01$

$4.80 \quad 8.21432 \mathrm{E}-01$

$4.90 \quad 8.21514 \mathrm{E}-01$

$5.00 \quad 8.21590 \mathrm{E}-01$

$5.10 \quad 8.21657 \mathrm{E}-01$

$5.20 \quad 8.21719 \mathrm{E}-01$

$5.30 \quad 8.21774 \mathrm{E}-01$

$5.40 \quad 8.21824 \mathrm{E}-01$

$5.50 \quad 8.21869 \mathrm{E}-01$

$5.60 \quad 8.21910 \mathrm{E}-01$

$5.70 \quad 8.21946 \mathrm{E}-01$

$5.80 \quad 8.21979 \mathrm{E}-01$

$5.90 \quad 8.22007 \mathrm{E}-01$

$6.00 \quad 8.22033 \mathrm{E}-01$

\section{$2.21242 \mathrm{E}-03$}

$1.61325 \mathrm{E}-02$

$4.29280 \mathrm{E}-02$

$8.13495 \mathrm{E}-02$

$1.29848 \mathrm{E}-01$

$1.86826 \mathrm{E}-01$

$2.50780 \mathrm{E}-01$

$3.20374 \mathrm{E}-01$

$3.94476 \mathrm{E}-01$

$4.72152 \mathrm{E}-01$

$5.52648 \mathrm{E}-01$

$6.35369 \mathrm{E}-01$

$7.19842 \mathrm{E}-01$

$8.05697 \mathrm{E}-01$

$8.92645 \mathrm{E}-01$

$9.80461 \mathrm{E}-01$

$1.06897 \mathrm{E} \quad 00$

$1.15802 \mathrm{E} \quad 00$

$1.24752 \mathrm{E} \quad 00$

$1.33737 \mathrm{E} \quad 00$

$1.42751 \mathrm{E} \quad 00$

$1.51788 \mathrm{E} \quad 00$

$1.60844 \mathrm{E} \quad 00$

$1.69915 \mathrm{E} \quad 00$

$1.78998 \mathrm{E} \quad 00$

$1.88092 \mathrm{E} \quad 00$

$1.97193 \mathrm{E} \quad 00$

$2.06302 \mathrm{E} \quad 00$

$2.15416 \mathrm{E} \quad 00$

$2.24535 \mathrm{E} \quad 00$

$2.33657 \mathrm{E} \quad 00$

$2.42782 \mathrm{E} \quad 00$

$2.51910 \mathrm{E} \quad 00$

$2.61040 \mathrm{E} \quad 00$

$2.70172 \mathrm{E} \quad 00$

$2.79305 \mathrm{E} \quad 00$

$2.88439 \mathrm{E} \quad 00$

$2.97574 \mathrm{E} \quad 00$

$3.06710 \mathrm{E} \quad 00$

$3.15847 \mathrm{E} \quad 00$

$3.24984 \mathrm{E} \quad 00$

$3.34122 \mathrm{E} \quad 00$

$3.43260 \mathrm{E} \quad 00$

$3.52398 \mathrm{E} \quad 00$

$3.61537 \mathrm{E} \quad 00$

$3.70676 \mathrm{E} \quad 00$

$3.79816 \mathrm{E} \quad 00$

$3.88955 \mathrm{E} \quad 00$

$3.98095 \mathrm{E} \quad 00$

$4.07236 \mathrm{E} \quad 00$

$4.16376 \mathrm{E} \quad 00$

$4.25517 \mathrm{E} \quad 00$

$4.34658 \mathrm{E} \quad 00$

$4.43800 \mathrm{E} \quad 00$

$4.52942 \mathrm{E} \quad 00$

$4.62084 \mathrm{E} \quad 00$

$4.71227 \mathrm{E} \quad 00$

$4.80370 \mathrm{E} \quad 00$

$4.89514 \mathrm{E} \quad 00$

$4.98658 \mathrm{E} \quad 00$ $g_{1}$

$-4.60963 \mathrm{E}-05$
$-3.35475 \mathrm{E}-04$
$-8.89859 \mathrm{E}-04$
$-1.67901 \mathrm{E}-03$
$-2.66552 \mathrm{E}-03$

$-2.66552 \mathrm{E}-03$

$-3.81072 \mathrm{E}-03$

$-5.07808 \mathrm{E}-03$

$-6.43510 \mathrm{E}-03$

$-7.85413 \mathrm{E}-03$

$-9.31235 \mathrm{E}-03$

$-1.07913 \mathrm{E}-02$

$-1.22763 \mathrm{E}-02$

$-1.37557 \mathrm{E}-02$

$-1.52205 \mathrm{E}-02$

$-1.66634 \mathrm{E}-02$

$-1.80789 \mathrm{E}-02$

$-1.94625 \mathrm{E}-02$

$-2.08108 \mathrm{E}-02$

$-2.21211 \mathrm{E}-02$

$-2.33911 \mathrm{E}-02$

$-2.46191 \mathrm{E}-02$

$-2.58038 \mathrm{E}-02$

$-2.69439 \mathrm{E}-02$

$-2.80388 \mathrm{E}-02$

$-2.90875 \mathrm{E}-02$

$-3.00895 \mathrm{E}-02$

$-3.10444 \mathrm{E}-02$

$-3.19517 \mathrm{E}-02$

$-3.28112 \mathrm{E}-02$

$-3.36225 \mathrm{E}-02$

$-3.43854 \mathrm{E}-02$

$-3.50998 \mathrm{E}-02$

$-3.57655 \mathrm{E}-02$

$-3.63824 \mathrm{E}-02$

$-3.69503 \mathrm{E}-02$

$-3.74692 \mathrm{E}-02$

$-3.79391 \mathrm{E}-02$

$-3.83598 \mathrm{E}-02$

$-3.87312 \mathrm{E}-02$

$-3.90534 \mathrm{E}-02$

$-3.93264 \mathrm{E}-02$

$-3.95500 \mathrm{E}-02$

$-3.97243 \mathrm{E}-02$

$-3.98491 \mathrm{E}-02$

$-3.99247 \mathrm{E}-02$

$-3.99507 \mathrm{E}-02$

$-3.99274 \mathrm{E}-02$

$-3.98546 \mathrm{E}-02$

$-3.97324 \mathrm{E}-02$

$-3.95607 \mathrm{E}-02$

$-3.93396 \mathrm{E}-02$

$-3.90690 \mathrm{E}-02$

$-3.87489 \mathrm{E}-02$

$-3.83793 \mathrm{E}-02$

$-3.79602 \mathrm{E}-02$

$-3.74915 \mathrm{E}-02$

$-3.69734 \mathrm{E}-02$

$-3.64058 \mathrm{E}-02$

$-3.57886 \mathrm{E}-02$

$-3.51220 \mathrm{E}-02$ $\tan \delta_{1}$

$f_{-1}$

$3.07623 \mathrm{E}-01$

$1.44759 \mathrm{E}-01$

$9.59756 \mathrm{E}-02$

$7.20766 \mathrm{E}-02$

$5.77939 \mathrm{E}-02$

$4.82563 \mathrm{E}-02$

$4.14156 \mathrm{E}-02$

$3.62583 \mathrm{E}-02$

$3.22241 \mathrm{E}-02$

$2.89780 \mathrm{E}-02$

$2.63070 \mathrm{E}-02$

$2.40690 \mathrm{E}-02$

$2.21655 \mathrm{E}-02$

$2.05260 \mathrm{E}-02$

$1.90987 \mathrm{E}-02$

$1.78445 \mathrm{E}-02$

$1.67334 \mathrm{E}-02$

$1.57423 \mathrm{E}-02$

$1.48524 \mathrm{E}-02$

$1.40490 \mathrm{E}-02$

$1.33200 \mathrm{E}-02$

$1.26555 \mathrm{E}-02$

$1.20472 \mathrm{E}-02$

$1.14883 \mathrm{E}-02$

$1.09730 \mathrm{E}-02$

$1.04963 \mathrm{E}-02$

$1.00542 \mathrm{E}-02$

$9.64280 \mathrm{E}-03$

$9.25918 \mathrm{E}-03$

$8.90057 \mathrm{E}-03$

$8.56460 \mathrm{E}-03$

8.24918

$7.95248 \mathrm{E}-03$

$7.67290 \mathrm{E}-03$

$7.40899 \mathrm{E}-03$

$7.15948 \mathrm{E}-03$

$6.92320 \mathrm{E}-03$

$6.69914 \mathrm{E}-03$

$6.48637 \mathrm{E}-03$

$6.28407 \mathrm{E}-03$

$6.09149 \mathrm{E}-03$

$5.90794 \mathrm{E}-03$

$5.73278 \mathrm{E}-03$

$5.56547 \mathrm{E}-03$

$5.40552 \mathrm{E}-03$

$5.25241 \mathrm{E}-03$

$5.10573 \mathrm{E}-03$

$4.96510 \mathrm{E}-03$

$4.83013 \mathrm{E}-03$

$4.70051 \mathrm{E}-03$

$4.57591 \mathrm{E}-03$

$4.45605 \mathrm{E}-03$

4.34067 E-03

$4.22953 \mathrm{E}-03$

$4.12238 \mathrm{E}-03$

$4.01905 \mathrm{E}-03$

$3.91930 \mathrm{E}-03$

$3.82297 \mathrm{E}-03$

$3.72988 \mathrm{E}-03$

$3.63987 \mathrm{E}-03$ $g_{-1}$

$9.74640 \mathrm{E}-04$

$4.05790 \mathrm{E}-03$

7.47031 E-03

$1.08604 \mathrm{E}-02$

$1.41483 \mathrm{E}-02$

$1.73072 \mathrm{E}-02$

$2.03289 \mathrm{E}-02$

$2.32140 \mathrm{E}-02$

$2.59673 \mathrm{E}-02$

$2.85962 \mathrm{E}-02$

$3.11085 \mathrm{E}-02$

$3.35120 \mathrm{E}-02$

$3.58142 \mathrm{E}-02$

$3.80217 \mathrm{E}-02$

$4.01404 \mathrm{E}-02$

$4.21755 \mathrm{E}-02$

$4.41314 \mathrm{E}-02$

$4.60119 \mathrm{E}-02$

$4.78204 \mathrm{E}-02$

$4.95597 \mathrm{E}-02$

$5.12323 \mathrm{E}-02$

$5.28402 \mathrm{E}-02$

5.43851 E-02

$5.58688 \mathrm{E}-02$

$5.72924 \mathrm{E}-02$

$5.86573 \mathrm{E}-02$

$5.99643 \mathrm{E}-02$

$6.12144 \mathrm{E}-02$

$6.24083 \mathrm{E}-02$

$6.35468 \mathrm{E}-02$

$6.46303 \mathrm{E}-02$

$6.56595 \mathrm{E}-02$

$6.66348 \mathrm{E}-02$

$6.75566 \mathrm{E}-02$

$6.84252 \mathrm{E}-02$

$6.92412 \mathrm{E}-02$

$7.00045 \mathrm{E}-02$

$7.07156 \mathrm{E}-02$

$7.13747 \mathrm{E}-02$

$7.19819 \mathrm{E}-02$

$7.25376 \mathrm{E}-02$

$7.30418 \mathrm{E}-02$

$7.34947 \mathrm{E}-02$

7.38964.E-02

$7.42471 \mathrm{E}-02$

$7.45469 \mathrm{E}-02$

$7.47958 \mathrm{E}-02$

$7.49940 \mathrm{E}-02$

$7.51416 \mathrm{E}-02$

$7.52386 \mathrm{E}-02$

$7.52851 \mathrm{E}-02$

$7.52812 \mathrm{E}-02$

$7.52269 \mathrm{E}-02$

$7.51223 \mathrm{E}-02$

$7.49675 \mathrm{E}-02$

$7.47624 \mathrm{E}-02$

$7.45071 \mathrm{E}-02$

$7.42017 \mathrm{E}-02$

$7.38462 \mathrm{E}-02$

$7.34406 \mathrm{E}-02$ 
$Z=9 \quad A=18 \quad \rho=1.2 A^{1 / 3} F$

$\bar{F}$

$1.79636 \mathrm{E}-09$

$2.43848 \mathrm{E}-08$

$7.95434 \mathrm{E}-08$

$1.68509 \mathrm{E}-07$

$2.90973 \mathrm{E}-07$

4. $4.6397 \mathrm{E}-07$

$6.34261 \mathrm{E}-07$

$8.54120 \mathrm{E}-07$

$1.10561 \mathrm{E}-06$

$1.38844 \mathrm{E}-06$

$1.70238 \mathrm{E}-06$

$2.04724 \mathrm{E}-06$

$2.42288 \mathrm{E}-06$

$2.82920 \mathrm{E}-06$

$3.26610 \mathrm{E}-06$

$3.73350 \mathrm{E}-06$

$4.23136 \mathrm{E}-06$

4.75962 E-06

$5.31825 \mathrm{E}-06$

$5.90722 \mathrm{E}-06$

$6.52649 \mathrm{E}-06$

$7.17605 \mathrm{E}-06$

$7.85588 \mathrm{E}-06$

$8.56596 \mathrm{E}-06$

$9.30629 \mathrm{E}-06$

$1.00768 \mathrm{E}-05$

$1.08776 \mathrm{E}-05$

$1.17086 \mathrm{E}-05$

$1.25698 \mathrm{E}-05$

$1.34612 \mathrm{E}-05$

$1.43827 \mathrm{E}-05$

$1.53345 \mathrm{E}-05$

$1.63164 \mathrm{E}-05$

$1.73285 \mathrm{E}-05$

$1.83708 \mathrm{E}-05$

$1.94433 \mathrm{E}-05$

$2.05459 \mathrm{E}-05$

$2.16787 \mathrm{E}-05$

$2.28416 \mathrm{E}-05$

$2.40347 \mathrm{E}-05$

$2.52579 \mathrm{E}-05$

$2.65113 \mathrm{E}-05$

$2.77949 \mathrm{E}-0.5$

$2.91085 \mathrm{E}-05$

$3.04524 \mathrm{E}-05$

$3.18263 \mathrm{E}-05$

$3.32305 \mathrm{E}-05$

$3.46647 \mathrm{E}-05$

$3.61291 \mathrm{E}-05$

$3.76236 \mathrm{E}-05$

$3.91482 \mathrm{E}-05$

4.07030E-05

$4.22879 \mathrm{E}-05$

$4.39029 \mathrm{E}-05$

$4.55480 \mathrm{E}-05$

4.72233 E-05

$4.89288 \mathrm{E}-05$

$5.06643 \mathrm{E}-05$

$5.24299 \mathrm{E}-05$

$5.42257 \mathrm{E}-05$ $f_{2}$

$6.26647 \mathrm{E}-07$

$8.81659 \mathrm{E}-06$

$3.49549 \mathrm{E}-05$

$8.81243 \mathrm{E}-0.5$

$1.75662 \mathrm{E}-04$

$3.03155 \mathrm{E}-04$

$4.74645 \mathrm{E}-04$

$6.92908 \mathrm{E}-04$

$9.59770 \mathrm{E}-04$

$1.27637 \mathrm{E}-03$

$1.64338 \mathrm{E}-03$

$2.06114 \mathrm{E}-03$

$2.52982 \mathrm{E}-03$

$3.04942 \mathrm{E}-03$

$3.61991 \mathrm{E}-03$

$4.24120 \mathrm{E}-03$

$4.91317 \mathrm{E}-03$

$5.63570 \mathrm{E}-03$

$6.40868 \mathrm{E}-03$

$7.23199 \mathrm{E}-03$

8.10554E-03

$9.02922 \mathrm{E}-03$

$1.00029 \mathrm{E}-02$

$1.10266 \mathrm{E}-02$

$1.21002 \mathrm{E}-02$

$1.32237 \mathrm{E}-02$

$1.43969 \mathrm{E}-02$

$1.56198 \mathrm{E}-02$

$1.68924 \mathrm{E}-02$

$1.82146 \mathrm{E}-02$

$1.95865 \mathrm{E}-02$

$2.10079 \mathrm{E}-02$

$2.24789 \mathrm{E}-02$

$2.39993 \mathrm{E}-02$

$2.55693 \mathrm{E}-02$

$2.71887 \mathrm{E}-02$

$2.88576 \mathrm{E}-02$

$3.05759 \mathrm{E}-02$

$3.23436 \mathrm{E}-02$

$3.41607 \mathrm{E}-02$

$3.60272 \mathrm{E}-02$

$3.79431 \mathrm{E}-02$

$3.99084 \mathrm{E}-02$

4.19229E-02

$4.39869 \mathrm{E}-02$

$4.61002 \mathrm{E}-02$

$5.04 .747 \mathrm{E}-02$

$5.27360 \mathrm{E}-02$

$5.50465 \mathrm{E}-02$

$5.74064 \mathrm{E}-02$

$5.98155 \mathrm{E}-02$

$6.22740 \mathrm{E}-02$

$6.47817 \mathrm{E}-02$

$6.73388 \mathrm{E}-02$

$6.99451 \mathrm{E}-02$

$7.26006 \mathrm{E}-02$

$7.53055 \mathrm{E}-02$

$7.80596 \mathrm{E}-02$

$8.08630 \mathrm{E}-02$
$4.82628 \mathrm{E}-02$ $g_{2}$

$-7.36158 \mathrm{E}-09$

$-1.03361 \mathrm{E}-07$

$-4.08411 \mathrm{E}-07$

$-1.02490 \mathrm{E}-06$

$-2.03124 \mathrm{E}-06$

$-3.48170 \mathrm{E}-06$

$-5.40912 \mathrm{E}-06$

$-7.82876 \mathrm{E}-06$

$-1.07427 \mathrm{E}-05$

$-1.41432 \mathrm{E}-05$

$-1.80160 \mathrm{E}-05$

$-2.23425 \mathrm{E}-05$

$-2.71011 \mathrm{E}-05$

$-3.22683 \mathrm{E}-05$

$-3.78196 \mathrm{E}-05$

$-4.37299 \mathrm{E}-05$

$-4.99739 \mathrm{E}-05$

$-5.65259 \mathrm{E}-05$

$-6.33604 \mathrm{E}-05$

$-7.04518 \mathrm{E}-05$

$-7.77747 \mathrm{E}-05$

$-8.53039 \mathrm{E}-05$

$-9.30140 \mathrm{E}-05$

$-1.00880 \mathrm{E}-04$

$-1.08877 \mathrm{E}-04$

$-1.16980 \mathrm{E}-04$

$-1.25164 \mathrm{E}-04$

$-1.33404 \mathrm{E}-04$

$-1.41676 \mathrm{E}-04$

$-1.49955 \mathrm{E}-04$

$-1.58217 \mathrm{E}-04$

$-1.66437 \mathrm{E}-04$

$-1.74591 \mathrm{E}-04$

$-1.82654 \mathrm{E}-04$

$-1.90602 \mathrm{E}-04$

$-1.98410 \mathrm{E}-04$

$-2.06055 \mathrm{E}-04$

$-2.13511 \mathrm{E}-04$

$-2.20755 \mathrm{E}-04$

$-2.27763 \mathrm{E}-04$

$-2.34510 \mathrm{E}-04$

$-2.40972 \mathrm{E}-04$

$-2.47124 \mathrm{E}-04$

$-2.52943 \mathrm{E}-04$

$-2.58405 \mathrm{E}-04$

$-2.63485 \mathrm{E}-04$

$-2.68159 \mathrm{E}-04$

$-2.724 .03 \mathrm{E}-04$

$-2.76193 \mathrm{E}-04$

$-2.79505 \mathrm{E}-04$

$-2.82314 \mathrm{E}-04$

$-2.84597 \mathrm{E}-04$

$-2.86329 \mathrm{E}-04$

$-2.87486 \mathrm{E}-04$

$-2.88045 \mathrm{E}-04$

$-2.87980 \mathrm{E}-04$

$-2.87269 \mathrm{E}-04$

$-2.85886 \mathrm{E}-04$

$-2.83808 \mathrm{E}-04$

$-2.81011 \mathrm{E}-04$ $\tan \delta_{2}$

$-1.40990 \mathrm{E} \quad 00$

$-3.12274 \mathrm{E} \quad 00$

$-4.68238 \mathrm{E} \quad 00$

$-6.12707 \mathrm{E} \quad 00$

$-7.45429 \mathrm{E} \quad 00$

$-8.66175 \mathrm{E} \quad 00$

$-9.75143 \mathrm{E} 00$

$-1.07291 \mathrm{E} 01$

$-1.16030 \mathrm{E} 01$

$-1.23827 \mathrm{E} 01$

$-1.30778 \mathrm{E} 01$

$-1.36980 \mathrm{E}$ ol

$-1.42521 \mathrm{E} \quad 01$

$-1.47481 \mathrm{E} 01$

$-1.51931 \mathrm{E} 01$

$-1.55935 \mathrm{E} \quad 01$

$-1.59548 \mathrm{E} 01$

$-1.62817 \mathrm{E} 01$

$-1.65783 \mathrm{E} 01$

$-1.68482 \mathrm{E} 01$

$-1.70945 \mathrm{E} \quad 01$

$-1.73200 \mathrm{E} \quad 01$

$-1.75269 \mathrm{E} 01$

$-1.77172 \mathrm{E} 01$

$-1.78928 \mathrm{E} 01$

$-1.80551 \mathrm{E} 01$

$-1.83452 \mathrm{E}$ 0I

$-1.84752 \mathrm{E} \quad 01$

$-1.85964 \mathrm{E} \quad 01$

$\begin{array}{lll}-1.87097 \mathrm{E} & 01\end{array}$

$-1.88157 \mathrm{E} \quad 01$

$-1.89151 \mathrm{E} 01$

$-1.90085 \mathrm{E} \quad 01$

$-1.90963 \mathrm{E} 01$

$-1.91791 \mathrm{E} 01$

$-1.92572 \mathrm{E} 01$

$-1.93311 \mathrm{E} 01$

$-1.94009 \mathrm{E} 01$

$-1.94671 \mathrm{E} \cdot 01$

$-1.95299 \mathrm{E} \quad 01$

$-1.95895 \mathrm{E} \quad 01$

$-1.96462 \mathrm{E} \quad 01$

$-1.97002 \mathrm{E} \quad 01$

$-1.97516 \mathrm{E} \quad 01$

$-1.98007 \mathrm{E} \quad 01$

$-1.98476 \mathrm{E} \quad 01$

$-1.98923 \mathrm{E} 01$

$-1.99352 \mathrm{E} 01$

$-1.99762 \mathrm{E} 01$

$-2.00155 \mathrm{E} \quad 01$

$-2.00532 \mathrm{E} \quad 01$

$-2.00894 \mathrm{E} \quad 01$

$-2.01575 \mathrm{E} 01$

$-2.01896 \mathrm{E} \quad 01$

$-2.02206 \mathrm{E} \quad 01$

$-2.02504 \mathrm{E} 01$

$-2.02791 \mathrm{E} 01$

$-2.03068 \mathrm{E} 01$
$-1.82055 \mathrm{E} \quad 01$

$-2.01241 \mathrm{E} \quad 01$

g-2

$1.79659 \mathrm{E}-07$

$1.31693 \mathrm{E}-06$

$3.53790 \mathrm{E}-06$

$6.78974 \mathrm{E}-06$

$1.10043 \mathrm{E}-05$

$1.61102 \mathrm{E}-0.5$

$2.20387 \mathrm{E}-05$

$2.87262 \mathrm{E}-05$

$3.61152 \mathrm{E}-05$

$4.41548 \mathrm{E}-05$

$5.27997 \mathrm{E}-05$

$6.20091 \mathrm{E}-05$

$7.17462 \mathrm{E}-05$

$8.19775 \mathrm{E}-05$

$9.26713 \mathrm{E}-05$

$1.03798 \mathrm{E}-04$

$1.15329 \mathrm{E}-04$

$1.27238 \mathrm{E}-04$

$1.39498 \mathrm{E}-04$

$1.52083 \mathrm{E}-04$

$1.64968 \mathrm{E}-04$

$1.78128 \mathrm{E}-04$

$1.91538 \mathrm{E}-04$

$2.05173 \mathrm{E}-04$

$2.19008 \mathrm{E}-04$

$2.33021 \mathrm{E}-04$

$2.47186 \mathrm{E}-04$

$2.61478 \mathrm{E}-04$

$2.75875 \mathrm{E}-04$

$2.90352 \mathrm{E}-04$

$3.04884 \mathrm{E}-04$

$3.19449 \mathrm{E}-04$

$3.34021 \mathrm{E}-04$

$3.48576 \mathrm{E}-04$

$3.63092 \mathrm{E}-04$

$3.77543 \mathrm{E}-04$

$3.91906 \mathrm{E}-04$

$4.06157 \mathrm{E}-04$

$4.20271 \mathrm{E}-04$

$4.34226 \mathrm{E}-04$

$4.47995 \mathrm{E}-04$

$4.61557 \mathrm{E}-04$

$4.74887 \mathrm{E}-04$ 
ELECTRON RADIAL FUNCTIONS

Positron

$\begin{array}{cc}p & F_{0} \\ 6.10 & 8.22055 \mathrm{E}-01 \\ 6.20 & 8.22074 \mathrm{E}-01 \\ 6.30 & 8.22091 \mathrm{E}-01 \\ 6.40 & 8.22104 \mathrm{E}-01 \\ 6.50 & 8.22117 \mathrm{E}-01\end{array}$

6.70

6.80

6.90

7.00

7.10

7.20

7.30

7.40

7.50

7.60

7.70

7.80

7.90

8.00

8.10

8.20

8.30

8.40

8.50

8.60

8.70

8.80

8.90

9.00

9.10

9.20

9.30

9.40

9.50

9.60

9.70

9.80

9.90

10.00

10.10

10.20

10.30

10.40

10.50

10.60

10.70

10.80

10.90

11.00

11.10

11.20

11.30

11.40

11.50

11.60

11.70

11.80

11.90

12.00

$8.22127 \mathrm{E}-01$

$8.22134 \mathrm{E}-01$

$8.22139 \mathrm{E}-01$

$8.22143 \mathrm{E}-01$

$8.22146 \mathrm{E}-01$

$8.22147 \mathrm{E}-01$

$8.22147 \mathrm{E}-01$

$8.22145 \mathrm{E}-01$

$8.22142 \mathrm{E}-01$

$8.22137 \mathrm{E}-01$

8.22132 E-01

$8.22126 \mathrm{E}-01$

$8.22119 \mathrm{E}-01$

$8.22111 \mathrm{E}-01$

$8.22102 \mathrm{E}-01$

8.22092 E-01

$8.22082 \mathrm{E}-01$

$8.22071 \mathrm{E}-01$

$8.22059 \mathrm{E}-01$

$8.22047 \mathrm{E}-01$

$8.22035 \mathrm{E}-01$

$8.22022 \mathrm{E}-01$

$8.22008 \mathrm{E}-01$

$8.21994 \mathrm{E}-0 \mathrm{I}$

$8.21980 \mathrm{E}-01$

$8.21965 \mathrm{E}-01$

$8.21949 \mathrm{E}-01$

8.21934 E-01

$8.21918 \mathrm{E}-01$

$8.21901 \mathrm{E}-01$

$8.21885 \mathrm{E}-01$

$8.21868 \mathrm{E}-01$

$8.21851 \mathrm{E}-01$

$8.21834 \mathrm{E}-01$

$8.21816 \mathrm{E}-01$

$8.21799 \mathrm{E}-01$

$8.21781 \mathrm{E}-01$

$8.21763 \mathrm{E}-01$

$8.21745 \mathrm{E}-01$

$8.21728 \mathrm{E}-01$

$8.21709 \mathrm{E}-01$

$8.21691 \mathrm{E}-01$

$8.21672 \mathrm{E}-01$

$8.21654 \mathrm{E}-01$

$8.21635 \mathrm{E}-01$

$8.21617 \mathrm{E}-01$

$8.21598 \mathrm{E}-01$

$8.21579 \mathrm{E}-01$

$8.21560 \mathrm{E}-01$

8.21541 E-01

$8.21522 \mathrm{E}-01$

$8.21503 \mathrm{E}-01$

$8.21484 \mathrm{E}-01$

$8.21465 \mathrm{E}-01$

$8.21446 \mathrm{E}-01$
$Z=9 \quad A=18$

$g_{1}$

$5.07802 \mathrm{E} \quad 00$

$g_{1}$
$-3.44057 \mathrm{E}-02$
$-3.36399 \mathrm{E}-02$
$-3.28246 \mathrm{E}-02$
$-3.19598 \mathrm{E}-02$
$-3.10453 \mathrm{E}-02$

$5.16947 \mathrm{E} \quad 00$

$5.26093 \mathrm{E} \quad 00$

$535239 \mathrm{~F} 00$

$5.44386 \mathrm{E} \quad 00$

$5.53533 \mathrm{E} \quad 00$

$5.62681 \mathrm{E} \quad 00$

$5.71829 \mathrm{E} \quad 00$

$5.80979 \mathrm{E} \quad 00$

$5.90129 \mathrm{E} \quad 00$

$5.99280 \mathrm{E} \quad 00$

$6.08431 \mathrm{E} \quad 00$

$6.17583 \mathrm{E} \quad 00$

$6.26736 \mathrm{E} \quad 00$

$6.35890 \mathrm{E} \quad 00$

$6.45045 \mathrm{E} \quad 00$

$6.54200 \mathrm{E} \quad 00$

$6.63357 \mathrm{E} \quad 00$

$6.72513 \mathrm{E} \quad 00$

$6.81671 \mathrm{E} \quad 00$

$6.90831 \mathrm{E} \quad 00$ $6.99990 \mathrm{E} \quad 00$

$7.09151 \mathrm{E} \quad 00$

$7.18313 \mathrm{E} \quad 00$

$7.27476 \mathrm{E} \quad 00$

$7.36640 \mathrm{E} \quad 00$

$7.45804 \mathrm{E} \quad 00$

$7.54970 \mathrm{E} \quad 00$

$7.64137 \mathrm{E} \quad 00$

$7.73305 \mathrm{E} \quad 00$

$7.82474 \mathrm{E} \quad 00$

$7.91643 \mathrm{E} \quad 00$

$8.00815 \mathrm{E} \quad 00$

$8.09986 \mathrm{E} \quad 00$

$8.19159 \mathrm{E} \quad 00$

$8.28332 \mathrm{E} \quad 00$ $8.37506 \mathrm{E} \quad 00$ 8.46681 E $\quad 00$ $8.55857 \mathrm{E} \quad 00$ $8.65033 \mathrm{E} \quad 00$

$8.74208 \mathrm{E} \quad 00$

8.83385 E 00

$8.92560 \mathrm{E} \quad 00$

$9.01734 \mathrm{E} \quad 00$

$9.10902 \mathrm{E} \quad 00$

$9.20067 \mathrm{E} \quad 00$ $9.29213 \mathrm{E} \quad 00$ $9.38328 \mathrm{E} \quad 00$ $9.47348 \mathrm{E} \quad 00$ $9.55686 \mathrm{E} \quad 00$

$9.67602 \mathrm{E} \quad 00$ $9.75844 \mathrm{E} \quad 00$ $9.84857 \mathrm{E} \quad 00$ $9.93978 \mathrm{E} \quad 00$ $1.00314 \mathrm{E} 01$

$\begin{array}{lll}1.01231 \mathrm{E} & 01\end{array}$ $1.02150 \mathrm{E} \quad 01$ $\begin{array}{ll}1.03070 \mathrm{E} & 01\end{array}$ $1.03989 \mathrm{E} \quad 01$ $1.04910 \mathrm{E} \quad 01$
$-3.00813 \mathrm{E}-02$

$-2.90678 \mathrm{E}-02$

$-2.80046 \mathrm{E}-02$

$-2.68919 \mathrm{E}-02$

$-2.57296 \mathrm{E}-02$

$-2.45177 \mathrm{E}-02$

$-2.32562 \mathrm{E}-02$

$-2.19450 \mathrm{E}-02$

$-2.05843 \mathrm{E}-02$

$-1.91739 \mathrm{E}-02$

$-1.77139 \mathrm{E}-02$

$-1.62043 \mathrm{E}-02$

$-1.46450 \mathrm{E}-02$

$-1.30360 \mathrm{E}-02$

$-1.13774 \mathrm{E}-02$

$-9.66912 \mathrm{E}-03$

$-7.91115 \mathrm{E}-03$

$-6.10349 \mathrm{E}-03$

$-4.24613 \mathrm{E}-03$

$-2.33906 \mathrm{E}-03$.

$-3.82275 \mathrm{E}-04$

$1.62424 \mathrm{E}-03$

$3.68048 \mathrm{E}-03$

$5.78648 \mathrm{E}-03$

$7.94223 \mathrm{E}-03$

$1.01477 \mathrm{E}-02$

$1.24030 \mathrm{E}-02$

$1.47081 \mathrm{E}-02$

$1.70630 \mathrm{E}-02$

$1.94676 \mathrm{E}-02$

$2.19221 \mathrm{E}-02$

$2.44264 \mathrm{E}-02$

$2.69804 \mathrm{E}-02$

$2.95844 \mathrm{E}-02$

$3.22381 \mathrm{E}-02$

$3.49416 \mathrm{E}-02$

$3.76950 \mathrm{E}-02$

4.04981 E-02

$4.33509 \mathrm{E}-02$

$4.62533 \mathrm{E}-02$

$4.92053 \mathrm{E}-02$

$5.22060 \mathrm{E}-02$

$5.52544 \mathrm{E}-02$

$5.83465 \mathrm{E}-02$

$6.48260 \mathrm{E}-02$

$6.80169 \mathrm{E}-02$

7.13082 E-02

$7.46567 \mathrm{E}-02$

7.80579 E-02

$8.15098 \mathrm{E}-02$

$8.50124 \mathrm{E}-02$

$8.85659 \mathrm{E}-02$

$9.58234 \mathrm{E}-02$
$6.14438 \mathrm{E}-02$

$9.21696 \mathrm{E}-02$

$\tan \delta_{1}$

$3.55280 \mathrm{E}-0.3$

$3.46853 \mathrm{E}-03$

$3.38691 \mathrm{E}-0.3$

$3.30784 \mathrm{E}-03$

$3.23119 \mathrm{E}-03$

$3.15686 \mathrm{E}-03$

$3.08475 \mathrm{E}-03$

$3.01474 \mathrm{E}-03$

$2.94677 \mathrm{E}-03$

$2.88072 \mathrm{E}-03$

$2.81656 \mathrm{E}-03$

$2.75416 \mathrm{E}-03$

$2.69349 \mathrm{E}-03$

$2.63445 \mathrm{E}-03$

$2.57700 \mathrm{E}-03$

$2.52106 \mathrm{E}-0.3$

$2.46657 \mathrm{E}-03$

$2.41351 \mathrm{E}-03$

$2.36178 \mathrm{E}-03$

$2.31135 \mathrm{E}-03$

$2.26218 \mathrm{E}-03$

$2.21422 \mathrm{E}-03$

$2.16742 \mathrm{E}-03$

$2.12175 \mathrm{E}-03$

$2.07716 \mathrm{E}-03$

$2.03362 \mathrm{E}-03$

$1.99110 \mathrm{E}-03$

$1.94954 \mathrm{E}-03$

$1.90895 \mathrm{E}-03$

$1.86926 \mathrm{E}-03$

$1.83045 \mathrm{E}-03$

$1.79251 \mathrm{E}-03$

$1.75540 \mathrm{E}-03$

$1.71908 \mathrm{E}-03$

$1.68355 \mathrm{E}-03$

$1.64877 \mathrm{E}-03$

$1.61473 \mathrm{E}-03$

$1.58140 \mathrm{E}-03$

$1.54874 \mathrm{E}-03$

$1.51677 \mathrm{E}-03$

$1.48544 \mathrm{E}-03$

$1.45474 \mathrm{E}-03$

$1.42465 \mathrm{E}-03$

$1.39516 \mathrm{E}-03$

$1.36625 \mathrm{E}-03$

$1.33789 \mathrm{E}-03$

$1.31009 \mathrm{E}-03$

$1.28282 \mathrm{E}-03$

$1.22981 \mathrm{E}-03$

$1.20405 \mathrm{E}-03$

$1.17877 \mathrm{E}-03$

$1.15395 \mathrm{E}-03$

$1.12958 \mathrm{E}-03$

$1.10566 \mathrm{E}-03$

$1.08216 \mathrm{E}-03$

$1.05909 \mathrm{E}-03$

$1.03642 \mathrm{E}-03$ 
ELECTRON RADIAL FUNCTIONS

$Z=9 \quad A=18 \quad \rho=1.2 A^{1 / 3} \mathrm{~F}$

\section{$\bar{F}$}

$p$

6.10

6.20

6.30

6.40

6.50

6.60

6.70

6.80

6.90

7.00

7.10

7.20

7.30

7.40

7.50

7.60

7.70

7.80

7.90

8.00

8.10

8.20

8.30

8.40

8.50

8.60

8.70

8.80

8.90

9.00

9.10

9.20

9.30

9.40

9.50

9.60

9.70

9.80

9.90

10.00

10.10

10.20

10.30

10.40

10.50

10.60

10.70

10.80

10.90

11.00

11.10

11.20

11.30

11.40

11.50

11.60

11.70

11.80

11.90

12.00
$5.60516 \mathrm{E}-05$ $5.79076 \mathrm{E}-05$ $5.97936 \mathrm{E}-05$ $6.17099 \mathrm{E}-05$ $6.36563 \mathrm{E}-05$

$6.56327 \mathrm{E}-05$ $6.76393 \mathrm{E}-05$ $6.96759 \mathrm{E}-05$ $7.17427 \mathrm{E}-05$ $7.38396 \mathrm{E}-05$

$7.59666 \mathrm{E}-05$ $7.81237 \mathrm{E}-0.5$ $8.03110 \mathrm{E}-05$ $8.25282 \mathrm{E}-05$ $8.47756 \mathrm{E}-05$

8.70531 E-05 $8.93608 \mathrm{E}-05$ $9.16985 \mathrm{E}-05$ $9.40663 \mathrm{E}-05$ $9.64642 \mathrm{E}-05$

$9.88923 \mathrm{E}-05$ $1.01350 \mathrm{E}-04$ $1.03839 \mathrm{E}-04$ $1.06357 \mathrm{E}-04$ $1.08905 \mathrm{E}-04$

$1.11484 \mathrm{E}-04$ $1.14092 \mathrm{E}-04$ $1.16731 \mathrm{E}-04$ $1.19400 \mathrm{E}-04$ 1.22099 E-04

$1.24828 \mathrm{E}-04$ $1.27587 \mathrm{E}-04$ $1.30376 \mathrm{E}-04$ $1.33195 \mathrm{E}-04$ $1.36044 \mathrm{E}-04$

$1.38924 \mathrm{E}-04$ $1.41833 \mathrm{E}-04$ $1.44773 \mathrm{E}-04$ $1.47742 \mathrm{E}-04$ $1.50742 \mathrm{E}-04$

$1.53772 \mathrm{E}-04$ $1.56832 \mathrm{E}-04$ $1.59922 \mathrm{E}-04$ $1.63042 \mathrm{E}-04$ $1.66192 \mathrm{E}-04$

$1.69372 \mathrm{E}-04$ $1.72582 \mathrm{E}-04$ $1.75823 \mathrm{E}-04$ $1.79093 \mathrm{E}-04$ $1.82394 \mathrm{E}-04$

$1.85724 \mathrm{E}-04$ $1.89085 \mathrm{E}-04$ $1.92476 \mathrm{E}-04$ $1.95897 \mathrm{E}-04$ $1.99347 \mathrm{E}-04$

$2.02828 \mathrm{E}-04$ $2.06340 \mathrm{E}-04$ $2.09881 \mathrm{E}-04$ $2.13452 \mathrm{E}-04$

$2.17053 \mathrm{E}-04$ $f_{2}$

$8.37156 \mathrm{E}-02$ $8.66175 \mathrm{E}-02$ $8.95686 \mathrm{E}-02$ $9.25691 \mathrm{E}-02$ $9.56188 \mathrm{E}-02$

$9.87176 \mathrm{E}-02$ $1.01866 \mathrm{E}-01$ $1.05063 \mathrm{E}-01$ $1.08310 \mathrm{E}-01$ $1.11605 \mathrm{E}-01$

$1.14950 \mathrm{E}-01$ $1.18345 \mathrm{E}-01$ $1.21788 \mathrm{E}-01$ $1.25280 \mathrm{E}-01$ $1.28822 \mathrm{E}-01$

$1.32413 \mathrm{E}-01$

$1.36053 \mathrm{E}-01$ $1.39742 \mathrm{E}-01$

$1.43479 \mathrm{E}-01$

$1.47266 \mathrm{E}-01$

$1.51102 \mathrm{E}-01$

$1.54987 \mathrm{E}-01$

$1.58919 \mathrm{E}-01$

$1.62901 \mathrm{E}-01$

$1.66928 \mathrm{E}-01$

$1.71003 \mathrm{E}-01$

$1.75120 \mathrm{E}-01$

$1.79272 \mathrm{E}-01$

$1.83425 \mathrm{E}-01$

$1.87004 \mathrm{E}-01$

$1.92513 \mathrm{E}-01$

$1.96723 \mathrm{E}-01$

$2.01104 \mathrm{E}-01$

$2.05561 \mathrm{E}-01$

$2.10077 \mathrm{E}-01$

$2.14645 \mathrm{E}-01$

$2.19265 \mathrm{E}-01$

$2.23935 \mathrm{E}-01$

$2.28656 \mathrm{E}-01$

$2.33427 \mathrm{E}-01$

$2.38248 \mathrm{E}-01$

$2.43118 \mathrm{E}-01$

$2.48038 \mathrm{E}-01$

$2.53007 \mathrm{E}-01$

$2.58026 \mathrm{E}-01$

$2.63093 \mathrm{E}-01$

$2.68211 \mathrm{E}-01$

$2.73377 \mathrm{E}-01$

$2.78593 \mathrm{E}-01$

$2.83858 E-01$

$2.89173 \mathrm{E}-01$

$2.94536 \mathrm{E}-01$

$2.99949 \mathrm{E}-01$

$3.05411 \mathrm{E}-01$

$3.10923 \mathrm{E}-01$

$3.16483 \mathrm{E}-01$

$3.22093 \mathrm{E}-01$

$3.27752 \mathrm{E}-01$

$3.33461 \mathrm{E}-01$

$3.39218 \mathrm{E}-01$ $g_{2}$

$\tan \delta_{2}$

$-2.77470 \mathrm{E}-04$

$-2.73162 \mathrm{E}-04$

$-2.68062 \mathrm{E}-04$

$-2.62147 \mathrm{E}-04$

$-2.55393 \mathrm{E}-04$

$-2.47773 \mathrm{E}-04$

$-2.39267 \mathrm{E}-04$

$-2.29848 \mathrm{E}-04$

$-2.19494 \mathrm{E}-04$

$-2.08179 \mathrm{E}-04$

$-1.95880 \mathrm{E}-04$

$-1.82573 \mathrm{E}-04$

$-1.68234 \mathrm{E}-04$

$-1.52838 \mathrm{E}-04$

$-1.36362 \mathrm{E}-04$

$-1.18782 \mathrm{E}-04$

$-1.00073 \mathrm{E}-04$

$-8.02122 \mathrm{E}-05$

$-5.91746 \mathrm{E}-05$

$-3.69367 \mathrm{E}-05$

$-1.34745 \mathrm{E}-05$

$1.12359 \mathrm{E}-0.5$

$3.72181 \mathrm{E}-05$

$6.44959 \mathrm{E}-0.5$

$9.30919 \mathrm{E}-05$

$1.23029 \mathrm{E}-04$ $1.54326 \mathrm{E}-04$

$1.86997 \mathrm{E}-04$

$2.21016 \mathrm{E}-04$

$2.55599 \mathrm{E}-04$

$2.94296 \mathrm{E}-04$

$3.32585 \mathrm{E}-04$

$3.72559 \mathrm{E}-04$

$4.14108 \mathrm{E}-04$

$4.57233 \mathrm{E}-04$

$5.01947 \mathrm{E}-04$

$5.48275 \mathrm{E}-04$

$5.96238 \mathrm{E}-04$

$6.45862 \mathrm{E}-04$

$6.97168 \mathrm{E}-04$

$7.50182 \mathrm{E}-04$ $8.04925 \mathrm{E}-04$

8.61424E-04

$9.19703 \mathrm{E}-04$

$9.79782 \mathrm{E}-04$

$1.04169 \mathrm{E}-03$

$1.10544 \mathrm{E}-03$

$1.17108 \mathrm{E}-03$

$1.23861 \mathrm{E}-03$

$1.30806 \mathrm{E}-03$

$1.37946 \mathrm{E}-03$

$1.45283 \mathrm{E}-03$

$1.52820 \mathrm{E}-03$

$1.60558 \mathrm{E}-03$

$1.68501 \mathrm{E}-03$

$1.76650 \mathrm{E}-03$

$1.85009 \mathrm{E}-03$

$1.93579 \mathrm{E}-03$

$2.02363 \mathrm{E}-03$

$2.11363 \mathrm{E}-03$

$\begin{array}{ll}-2.03335 \mathrm{E} & 0 \\ -2.03593 \mathrm{E} & 0 \\ -2.03842 \mathrm{E} & 0 \\ -2.04083 \mathrm{E} & 0 \\ -2.04316 \mathrm{E} & 0\end{array}$

$-2.04541 \mathrm{E} \quad 01$ $-2.04760 \mathrm{E} \quad 01$ $-2.04971 \mathrm{E} \quad 01$ $-2.05176 \mathrm{E} \quad 01$

$-2.05374 \mathrm{E} \quad 0 \mathrm{l}$

$\begin{array}{lll}-2.05567 \mathrm{E} & 01\end{array}$ $-2.05754 \mathrm{E} \quad 01$ $-2.05935 \mathrm{E} \quad 01$ $-2.06112 \mathrm{E} \quad 01$ $-2.06283 \mathrm{E} 01$

$\begin{array}{ll}-2.06449 \mathrm{E} & 01\end{array}$ $-2.06611 \mathrm{E} 01$ $-2.06769 \mathrm{E} \quad 01$ $-2.06922 \mathrm{E} \quad 01$ $-2.07071 \mathrm{E} \quad 01$

$\begin{array}{lll}-2.07216 \mathrm{E} & 01\end{array}$ $-2.07357 \mathrm{E} 01$ $-2.07495 \mathrm{E} \quad 01$ $-2.07629 \mathrm{E} 01$ $-2.07760 \mathrm{E} 01$

$-2.07888 \mathrm{E} \quad 01$ $-2.08012 \mathrm{E} 0 \mathrm{l}$ $-2.08134 \mathrm{E} 01$ $\begin{array}{lll}-2.08252 \mathrm{E} & 01\end{array}$ $-2.08368 \mathrm{E} 01$

$-2.08481 \mathrm{E} \quad 01$ $-2.08592 \mathrm{E} \quad 01$ $-2.08700 \mathrm{E} \quad 01$ $-2.08805 \mathrm{E} \quad 0 \mathrm{l}$ $-2.08908 \mathrm{E} \quad 01$

$-2.09009 \mathrm{E} \quad 01$ $-2.09108 \mathrm{E} \quad 01$ $-2.09204 \mathrm{E} \quad 01$ $-2.09299 \mathrm{E} 01$ $-2.09391 \mathrm{E} 01$

$-2.09482 \mathrm{E} \quad 01$ $-2.09570 \mathrm{E} 01$ $\begin{array}{ll}-2.09657 \mathrm{E} & 01\end{array}$ $-2.09742 \mathrm{E} 01$ $-2.09826 \mathrm{E} 01$ 
ELECTRON RADIAL FUNCTIONS

$\begin{array}{cc}p & F_{0} \\ 12.10 & 8.21427 \mathrm{E}-01 \\ 12.20 & 8.21407 \mathrm{E}-01 \\ 12.30 & 8.21388 \mathrm{E}-01 \\ 12.40 & 8.21369 \mathrm{E}-01 \\ 12.50 & 8.21350 \mathrm{E}-01\end{array}$

12.60

12.70

12.80

12.90

13.00

13.10

13.20

13.30

13.40

13.50

13.60

13.70

13.80

13.90

14.00

14.10

14.20

14.30

14.40

14.50

14.60

14.70

14.80

14.90

15.00

15.10

15.20

15.30

15.40

15.50

15.60

15.70

15.80

15.90

16.00

16.10

16.20

16.30

16.40

16.50

16.60

16.70

16.80

16.90

17.00

17.10

17.20

17.30

17.40

17.50

17.60

17.70

17.80

17.90

18.00

$8.21330 \mathrm{E}-01$

$8.21311 \mathrm{E}-01$

8.21292 E-01

$8.21274 \mathrm{E}-01$

$8.21254 \mathrm{E}-01$

$8.21235 \mathrm{E}-01$

$8.21216 \mathrm{E}-01$

$8.21197 \mathrm{E}-01$

$8.21178 \mathrm{E}-01$

$8.21160 \mathrm{E}-01$

$8.21141 \mathrm{E}-01$

$8.21122 \mathrm{E}-01$

$8.21103 \mathrm{E}-01$

$8.21084 \mathrm{E}-01$

$8.21065 \mathrm{E}-01$

$8.21047 \mathrm{E}-01$

$8.21028 \mathrm{E}-01$

$8.21009 \mathrm{E}-01$

8.20991 E-01

$8.20972 \mathrm{E}-01$

8.20954E-01 $8.20935 \mathrm{E}-01$ $8.20917 \mathrm{E}-01$ $8.20899 \mathrm{E}-01$ $8.20880 \mathrm{E}-01$

$8.20862 \mathrm{E}-01$ $8.20844 \mathrm{E}-01$ $8.20826 \mathrm{E}-01$ $8.20808 \mathrm{E}-01$ $8.20789 \mathrm{E}-01$

8.20771 E-01 $8.20753 \mathrm{E}-01$ $8.20736 \mathrm{E}-01$ $8.20718 \mathrm{E}-01$ $8.20700 \mathrm{E}-01$

8.20682E-01 $8.20665 \mathrm{E}-01$ 8.20647 E-01 $8.20629 \mathrm{E}-01$ $8.20612 \mathrm{E}-01$

8.20595E-01 $8.20577 \mathrm{E}-01$ $8.20560 \mathrm{E}-01$ $8.20543 \mathrm{E}-01$ $8.20526 \mathrm{E}-01$

$8.20508 \mathrm{E}-01$ $8.20491 \mathrm{E}-01$ 8.20474 E-01 $8.20457 \mathrm{E}-01$ $8.20440 \mathrm{E}-01$

8.20424 E-01 $8.20407 \mathrm{E}-01$ $8.20390 \mathrm{E}-01$ 8.20373E-01 8.20356E-01 $f_{1}$

$\begin{array}{lll}1.05830 \mathrm{E} & 01\end{array}$

$1.06751 \mathrm{E} 01$

$\begin{array}{ll}1.07672 \mathrm{E} & 01\end{array}$

$1.08594 \mathrm{E} \quad 01$

$1.09515 \mathrm{E} 01$
$1.10437 \mathrm{E} \quad 01$

$1.11359 \mathrm{E} 01$

$\begin{array}{lll}1.12281 \mathrm{E} & 01\end{array}$

$1.13204 \mathrm{E} \quad 01$

$\begin{array}{ll}1.14126 \mathrm{E} & 01\end{array}$

$1.15049 \mathrm{E} \quad 01$

$1.15972 \mathrm{E} 01$

$\begin{array}{lll}1.16895 \mathrm{E} & 01\end{array}$

$1.17819 \mathrm{E} 01$

$1.18742 \mathrm{E} 01$

$1.19666 \mathrm{E} \quad 01$ $1.20590 \mathrm{E} \quad 01$ $1.21514 \mathrm{E} \quad 01$ $1.22438 \mathrm{E} \quad 01$

$1.23363 \mathrm{E} 01$

$\begin{array}{lll}1.24287 \mathrm{E} & 01\end{array}$

$1.25212 \mathrm{E} 01$

$1.26137 \mathrm{E} 01$

$1.27063 \mathrm{E} 01$

$1.27988 \mathrm{E} \quad 01$

$1.28914 \mathrm{E} \quad 01$

$1.29840 \mathrm{E} \quad 01$

$\begin{array}{ll}1.30766 \mathrm{E} & 01\end{array}$

$1.31692 \mathrm{E} \quad 01$

$1.32619 \mathrm{E} 01$

$1.33545 \mathrm{E} \quad 01$

$\begin{array}{lll}1.34472 \mathrm{E} & 01\end{array}$

$1.35399 \mathrm{E} \quad 01$

$\begin{array}{ll}1.36326 \mathrm{E} & 01\end{array}$

$1.37254 \mathrm{E} \quad 01$

$\begin{array}{ll}1.38182 \mathrm{E} & 01\end{array}$

$1.39110 \mathrm{E} 01$

$\begin{array}{ll}1.40038 \mathrm{E} & 01\end{array}$

$1.40966 \mathrm{E} 01$

$1.41894 \mathrm{E} \quad 01$.

$1.42823 \mathrm{E} \quad 01$

$1.43752 \mathrm{E} \quad 01$

$1.44681 \mathrm{E} \quad 01$

$1.45611 \mathrm{E} \quad 01$

$1.46540 \mathrm{E} 01$

$1.47470 \mathrm{E} \quad 01$

$1.48400 \mathrm{E} \quad 01$

$1.49330 \mathrm{E} \quad 01$

$\begin{array}{lll}1.50261 \mathrm{E} & 01\end{array}$

$1.51192 \mathrm{E} \quad 01$

$\begin{array}{lll}1.52123 \mathrm{E} & 01\end{array}$

$1.53054 \mathrm{E} \quad 01$

$1.54917 \mathrm{E} \quad 01$

$1.55848 \mathrm{E} 01$

$\begin{array}{lll}1.56781 \mathrm{E} & 01\end{array}$

$1.57713 \mathrm{E} \quad 01$

$1.58645 \mathrm{E} 01$

$1.59578 \mathrm{E} 01$

$1.60511 \mathrm{E} 01$
$1.53985 \mathrm{E} 01$ $g_{1}$

$9.95279 \mathrm{E}-02$

$1.03283 \mathrm{E}-01$

$1.07087 \mathrm{E}-01$

$1.10942 \mathrm{E}-01$

$1.14848 \mathrm{E}-01$

$1.18803 \mathrm{E}-01$

$1.22809 \mathrm{E}-01$

$1.26865 \mathrm{E}-01$

$1.30972 \mathrm{E}-01$

$1.35129 \mathrm{E}-01$

$1.39336 \mathrm{E}-01$

$1.43593 \mathrm{E}-01$

$1.47901 \mathrm{E}-01$

$1.52260 \mathrm{E}-01$

$1.56668 \mathrm{E}-01$

$1.61128 \mathrm{E}-01$

$1.65637 \mathrm{E}-01$

$1.70198 \mathrm{E}-01$

$1.74808 \mathrm{E}-01$

$1.79469 \mathrm{E}-01$

$1.84181 \mathrm{E}-01$

$1.88943 \mathrm{E}-01$

$1.93756 \mathrm{E}-01$

$1.98619 \mathrm{E}-01$

$2.03533 \mathrm{E}-01$

$2.08498 \mathrm{E}-01$

$2.13513 \mathrm{E}-01$

$2.18579 \mathrm{E}-01$

$2.23696 \mathrm{E}-01$

$2.28863 \mathrm{E}-01$

2.34081 E- 01

$2.39349 \mathrm{E}-01$

$2.44669 \mathrm{E}-01$

$2.50039 \mathrm{E}-01$

$2.55460 \mathrm{E}-01$

$2.60932 \mathrm{E}-01$

$2.66455 \mathrm{E}-01$

$2.72028 \mathrm{E}-01$

$2.77653 \mathrm{E}-01$

$2.83328 \mathrm{E}-01$

$2.89055 \mathrm{E}-01$ $2.94832 \mathrm{E}-01$

$3.00660 \mathrm{E}-01$

$3.06539 \mathrm{E}-01$

$3.12469 \mathrm{E}-01$

$3.18451 \mathrm{E}-01$

$3.24483 \mathrm{E}-01$

$3.30567 \mathrm{E}-01$

$3.36701 \mathrm{E}-01$

$3.42887 \mathrm{E}-01$

$3.49124 \mathrm{E}-01$

$3.55412 \mathrm{E}-01$

$3.61751 \mathrm{E}-01$

$3.68142 \mathrm{E}-01$

$3.74583 \mathrm{E}-01$

$3.81076 \mathrm{E}-01$ $3.87621 \mathrm{E}-01$

$3.94216 \mathrm{E}-01$

$4.00863 \mathrm{E}-01$

$4.07561 \mathrm{E}-01$

$\tan \delta_{1}$

$9.70796 \mathrm{E}-04$ $9.49663 \mathrm{E}-04$ $9.28908 \mathrm{E}-04$ $9.08494 \mathrm{E}-04$ $8.88432 \mathrm{E}-04$

3.68715 E-04

$8.49308 \mathrm{E}-04$

$8.30232 \mathrm{E}-04$

$8.11480 \mathrm{E}-04$

$7.93033 \mathrm{E}-04$

$7.74880 \mathrm{E}-04$

$7.57018 \mathrm{E}-04$

$7.39460 \mathrm{E}-04$

$7.22165 \mathrm{E}-04$

$7.05148 \mathrm{E}-04$

$6.88412 \mathrm{E}-04$

$6.71932 \mathrm{E}-04$

$6.55711 \mathrm{E}-04$

$6.39744 \mathrm{E}-04$

$6.24032 \mathrm{E}-04$

$6.08552 \mathrm{E}-04$

$5.93315 \mathrm{E}-04$

$5.78309 \mathrm{E}-04$

$5.63532 \mathrm{E}-04$

$5.48977 \mathrm{E}-04$

$5.34642 \mathrm{E}-04$

$5.20528 \mathrm{E}-04$

$5.06619 \mathrm{E}-04$

$4.92911 \mathrm{E}-04$

4.79419E-04

$4.66115 \mathrm{E}-04$

$4.53015 \mathrm{E}-04$

$4.40100 \mathrm{E}-04$

$4.27372 \mathrm{E}-04$

$4.14827 \mathrm{E}-04$

$4.02473 \mathrm{E}-04$

$3.90282 \mathrm{E}-04$

$3.78282 \mathrm{E}-04$

$3.66442 \mathrm{E}-04$

$3.54777 \mathrm{E}-04$

$3.43269 \mathrm{E}-04$

$3.31926 \mathrm{E}-04$

$3.20747 \mathrm{E}-04$

$3.09732 \mathrm{E}-04$

$2.98849 \mathrm{E}-04$

$2.88130 \mathrm{E}-04$

$2.77558 \mathrm{E}-04$

$2.67136 \mathrm{E}-04$

$2.56857 \mathrm{E}-04$

$2.46719 \mathrm{E}-04$

$2.36701 \mathrm{E}-04$ 
ELECTRON RADIAL FUNCTIONS

$Z=9 \quad A=18 \quad \rho=1.2 A^{1 / 3} F$

Positron

$\bar{F}$

$2.20684 \mathrm{E}-04$

$2.24346 \mathrm{E}-04$

$2.28037 \mathrm{E}-04$

$2.31759 \mathrm{E}-04$

$2.35511 \mathrm{E}-04$

$2.39292 \mathrm{E}-04$

$2.43104 \mathrm{E}-04$

$2.46946 \mathrm{E}-04$

$2.50818 \mathrm{E}-04$

$2.54720 \mathrm{E}-04$

$2.58652 \mathrm{E}-04$

$2.62614 \mathrm{E}-04$

$2.66606 \mathrm{E}-04$

$2.70628 \mathrm{E}-04$

$2.74680 \mathrm{E}-04$

$2.78763 \mathrm{E}-04$

$2.82875 \mathrm{E}-04$

$2.87018 \mathrm{E}-04$

$2.91190 \mathrm{E}-04$

$2.95393 \mathrm{E}-04$

$2.99625 \mathrm{E}-04$

$3.03888 \mathrm{E}-04$

$3.08181 \mathrm{E}-04$

$3.12504 \mathrm{E}-04$

$3.16857 \mathrm{E}-04$

$3.21240 \mathrm{E}-04$

$3.25653 \mathrm{E}-04$

$3.30096 \mathrm{E}-04$

$3.34569 \mathrm{E}-04$

$3.39072 \mathrm{E}-04$

$3.43605 \mathrm{E}-04$

$3.48169 \mathrm{E}-04$

$3.52762 \mathrm{E}-04$

$3.57385 \mathrm{E}-04$

$3.62039 \mathrm{E}-04$

$3.66722 \mathrm{E}-04$

$3.71436 \mathrm{E}-04$

$3.76179 \mathrm{E}-04$

$3.80953 \mathrm{E}-04$

$3.85757 \mathrm{E}-04$

$3.90590 \mathrm{E}-04$

$3.95454 \mathrm{E}-04$

$4.00348 \mathrm{E}-04$

$4.05272 \mathrm{E}-04$

$4.10226 \mathrm{E}-04$

$4.15210 \mathrm{E}-04$

$4.20224 \mathrm{E}-04$

$4.25268 \mathrm{E}-04$

$4.30342 \mathrm{E}-04$

$4.35446 \mathrm{E}-04$

4.40581 E- 04

$4.45745 \mathrm{E}-04$

$4.50939 \mathrm{E}-04$

$4.56164 \mathrm{E}-04$

$4.61418 \mathrm{E}-04$

4.66703 E-04

$4.72017 \mathrm{E}-04$

$4.77362 \mathrm{E}-04$

$4.82737 \mathrm{E}-04$

4.88141 E-04 $f_{2}$

$3.45025 \mathrm{E}-01$

$3.50881 \mathrm{E}-01$

$3.56787 \mathrm{E}-01$

$3.62741 \mathrm{E}-01$

3.68744 E- 01

$3.74797 \mathrm{E}-01$

$3.80899 \mathrm{E}-01$

$3.87050 \mathrm{E}-01$

$3.93251 \mathrm{E}-01$

$3.99500 \mathrm{E}-01$

$4.05799 \mathrm{E}-01$

$4.12147 \mathrm{E}-01$

$4.18544 \mathrm{E}-01$

$4.24990 \mathrm{E}-01$

$4.31485 \mathrm{E}-01$

$4.38030 \mathrm{E}-01$

$4.44624 \mathrm{E}-01$

$4.51267 \mathrm{E}-01$

$4.57959 \mathrm{E}-01$

$4.64700 \mathrm{E}-01$

$4.71491 \mathrm{E}-01$

$4.78331 \mathrm{E}-01$

$4.85220 \mathrm{E}-01$

$4.92158 \mathrm{E}-01$

$4.99145 \mathrm{E}-01$

$5.06181 \mathrm{E}-01$

$5.13266 \mathrm{E}-01$

5.20401 E-01

$5.27585 \mathrm{E}-01$

$5.34818 \mathrm{E}-01$

$5.42099 \mathrm{E}-01$

$5.49431 \mathrm{E}-01$

$5.56811 \mathrm{E}-01$

$5.64240 \mathrm{E}-01$

$5.71719 \mathrm{E}-01$

$5.79247 \mathrm{E}-01$

$5.86823 \mathrm{E}-01$

$5.94449 \mathrm{E}-01$

$6.02124 \mathrm{E}-01$

$6.09848 \mathrm{E}-01$

$6.17622 \mathrm{E}-01$

$6.25444 \mathrm{E}-01$

$6.33315 \mathrm{E}-01$

$6.41236 \mathrm{E}-01$

$6.49206 \mathrm{E}-01$

$6.57224 \mathrm{E}-01$

$6.65292 \mathrm{E}-01$

$6.73409 \mathrm{E}-01$

$6.81575 \mathrm{E}-01$

$6.89790 \mathrm{E}-01$

$6.98054 \mathrm{E}-01$

$7.06367 \mathrm{E}-01$

$7.14729 \mathrm{E}-01$

$7.23141 \mathrm{E}-01$

$7.31601 \mathrm{E}-01$

$7.40111 \mathrm{E}-01$

$7.48669 \mathrm{E}-01$

$7.57277 \mathrm{E}-01$

$7.65934 \mathrm{E}-01$

$7.74639 \mathrm{E}-01$ $g_{2}$

$2.20582 \mathrm{E}-03$

$2.30022 \mathrm{E}-03$

$2.39686 \mathrm{E}-03$

$2.49576 \mathrm{E}-03$

$2.59693 \mathrm{E}-03$

$2.70042 \mathrm{E}-03$

$2.80624 \mathrm{E}-03$

$2.91441 \mathrm{E}-03$

$3.02496 \mathrm{E}-03$

$3.13792 \mathrm{E}-03$

$3.25330 \mathrm{E}-03$

$3.37114 \mathrm{E}-03$

$3.49145 \mathrm{E}-03$

$3.61426 \mathrm{E}-03$

$3.73959 \mathrm{E}-03$

$3.86748 \mathrm{E}-03$

$3.99793 \mathrm{E}-03$

4.13098E-03

$4.26665 \mathrm{E}-03$

$4.40496 \mathrm{E}-03$

$4.54595 \mathrm{E}-03$

$4.68962 \mathrm{E}-03$

$4.83601 \mathrm{E}-03$

$4.98515 \mathrm{E}-03$

$5.13704 \mathrm{E}-03$

$5.29172 \mathrm{E}-03$

$5.44921 \mathrm{E}-03$

$5.60955 \mathrm{E}-03$

$5.77273 \mathrm{E}-03$

$5.93881 \mathrm{E}-03$

$6.10779 \mathrm{E}-03$

$6.27971 \mathrm{E}-03$

$6.45458 \mathrm{E}-03$

$6.63243 \mathrm{E}-03$

$6.81329 \mathrm{E}-03$

$6.99717 \mathrm{E}-03$

$7.18411 \mathrm{E}-03$

$7.37412 \mathrm{E}-03$

$7.56724 \mathrm{E}-03$

$7.76347 \mathrm{E}-03$

$7.96286 \mathrm{E}-03$

$8.16541 \mathrm{E}-03$

$8.37117 \mathrm{E}-03$

$8.58015 \mathrm{E}-03$

$8.79237 \mathrm{E}-03$

$9.00786 \mathrm{E}-03$

$9.22664 \mathrm{E}-03$

$9.44873 \mathrm{E}-03$

$9.67417 \mathrm{E}-03$

$9.90297 \mathrm{E}-03$

$1.01352 \mathrm{E}-02$

$1.03708 \mathrm{E}-02$

$1.06098 \mathrm{E}-02$

$1.08523 \mathrm{E}-02$

$1.10983 \mathrm{E}-02$

$1.13478 \mathrm{E}-02$

$1.16008 \mathrm{E}-02$

$1.18574 \mathrm{E}-02$

$1.21175 \mathrm{E}-02$

$1.23813 \mathrm{E}-02$

$\tan \delta_{2}$

$f_{-2}$

$-2.10962 \mathrm{E} \quad 01$

$11022 \mathrm{E} 01$

$-2.11082 \mathrm{E} \quad 01$

$-2.11140 \mathrm{E} 01$

$-2.11198 \mathrm{E} 01$

$-2.11254 \mathrm{E} \quad 01$

$-2.11310 \mathrm{E} \quad 01$

$-2.11365 \mathrm{E} \quad 01$

$-2.11418 \mathrm{E} \quad 01$

$-2.11471 \mathrm{E} 01$

$-2.11524 \mathrm{E} \quad 01$

$-2.11575 \mathrm{E} 01$

$-2.11625 \mathrm{E} 01$

$-2.11675 \mathrm{E} \quad 01$

$-2.11724 \mathrm{E} 01$

$-2.11772 \mathrm{E} \quad 01$

$-2.11819 \mathrm{E} \quad 01$

$-2.11866 \mathrm{E} \quad 01$

$-2.11912 \mathrm{E} 01$

$-2.11957 \mathrm{E} 01$

$-2.12002 \mathrm{E} \quad 01$

$-2.12046 \mathrm{E} \quad 01$

$-2.12089 \mathrm{E} 01$

$-2.12132 \mathrm{E} \quad 01$

$-2.12174 \mathrm{E} 01$

$-2.12215 \mathrm{E} \quad 01$

$-2.12256 \mathrm{E} \quad 01$

$-2.12297 \mathrm{E} 01$

$-2.12336 \mathrm{E} \quad 01$

$-2.12375 \mathrm{E} 01$

$-2.12414 \mathrm{E} \quad 01$

$-2.12452 \mathrm{E} \quad 01$

$-2.12490 \mathrm{E} \quad 01$

$-2.12527 \mathrm{E} 01$

$-2.12563 \mathrm{E} \quad 01$

$\begin{array}{lll}-2.12599 \mathrm{E} & 01\end{array}$

$-2.12635 \mathrm{E} \quad 01$

$-2.12670 \mathrm{E} 01$

$-2.12705 \mathrm{E} 01$

$\begin{array}{lll}-2.12739 \mathrm{E} & 01\end{array}$

$-2.12773 \mathrm{E} \quad 01$

$-2.12806 \mathrm{E} \quad 01$

$-2.12839 \mathrm{E}$ 01

$-2.12871 \mathrm{E} \quad 01$

$-2.12903 \mathrm{E}$ ol

$-1.17490 \mathrm{E}-03$

$-1.33897 \mathrm{E}-03$

$-1.42426 \mathrm{E}-03$

$-1.51176 \mathrm{E}-03$

$-1.60148 \mathrm{E}-03$

$-1.69345 \mathrm{E}-03$

$-1.78770 \mathrm{E}-03$

$-1.88425 \mathrm{E}-03$

$-1.98312 \mathrm{E}-03$

$-2.08434 \mathrm{E}-03$

$-2.18794 \mathrm{E}-03$

$-2.29392 \mathrm{E}-03$

$-2.40233 \mathrm{E}-03$

$-2.51318 \mathrm{E}-03$

$-2.62650 \mathrm{E}-03$

$-2.74231 \mathrm{E}-03$

$-2.86064 \mathrm{E}-03$

$-2.98151 \mathrm{E}-03$

$-3.10494 \mathrm{E}-03$

$-3.23095 \mathrm{E}-03$

$-3.35959 \mathrm{E}-03$

$-3.49085 \mathrm{E}-03$

$-3.62478 \mathrm{E}-03$

$-3.76139 \mathrm{E}-03$

$-3.90071 \mathrm{E}-03$

$-4.04276 \mathrm{E}-03$

$-4.18757 \mathrm{E}-03$

$-4.33516 \mathrm{E}-03$

$-4.48555 \mathrm{E}-03$

$-4.63877 \mathrm{E}-03$

$-4.79484 \mathrm{E}-03$

$-4.95379 \mathrm{E}-03$

$-5.11564 \mathrm{E}-03$

$-5.28042 \mathrm{E}-03$

$-5.44814 \mathrm{E}-03$

$-5.61884 \mathrm{E}-03$

$-5.79253 \mathrm{E}-03$

$-5.96924 \mathbf{E}-03$

$-6.14900 \mathrm{E}-03$

$-6.33182 \mathrm{E}-03$

$-6.51774 \mathrm{E}-03$

$-6.70677 \mathrm{E}-03$

$-6.89895 \mathrm{E}-03$

$-7.09429 \mathrm{E}-03$

$-2.12935 \mathrm{E} 01$

$-7.29281 \mathrm{E}-03$

$-7.49455 \mathrm{E}-03$

$-7.69953 \mathrm{E}-03$

$-7.90776 \mathrm{E}-03$

$-8.11929 \mathrm{E}-03$

$-8.33412 \mathrm{E}-03$

$-8.55228 \mathrm{E}-03$

$-8.77380 \mathrm{E}-03$

$-8.99870 \mathrm{E}-03$

$-9.22700 \mathrm{E}-03$

$-2.13231 \mathrm{E} \quad 01$

$-2.13259 \mathrm{E} \quad 01$

$-9.45873 \mathrm{E}-03$ 
ELECTRON RADIAL FUNCTIONS

Positron

$p$

18.10

18.20

18.30

18.40

18.50

18.60

18.70

18.80

18.90

19.00

19.10

19.20

19.30

19.40

19.50

19.60

19.70

19.80

19.90

20.00

20.10

20.20

20.30

20.40

20.50

20.60

20.70

20.80

20.90

21.00

21.10

21.20

21.30

21.40

21.50

21.60

21.70

21.80

21.90

22.00

22.10

22.20

22.30

22.40

22.50

22.60

22.70

22.80

22.90

23.00

23.10

23.20

23.30

23.40

23.50

23.60

23.70

23.80

23.90

24.00
$F_{0}$

$8.20340 \mathrm{E}-01$

8.20324 E-01

$8.20307 \mathrm{E}-01$

8.20291 E-01

$8.20275 \mathrm{E}-01$

8.20258 E-01 8.20242 E-01

8.20226 E-01

$8.20210 \mathrm{E}-01$

8.20194E-01

$8.20178 \mathrm{E}-01$

$8.20162 \mathrm{E}-01$

8.20146E-01

$8.20130 \mathrm{E}-01$

8.20114E-01

$8.20099 \mathrm{E}-01$

$8.20083 \mathrm{E}-01$

$8.20067 \mathrm{E}-01$

$8.20052 \mathrm{E}-01$

$8.20036 \mathrm{E}-01$

$8.20021 \mathrm{E}-01$ $3.20006 \mathrm{E}-01$ 8.19991 E-01 $8.19975 \mathrm{E}-01$ $8.19960 \mathrm{E}-01$

$8.19945 \mathrm{E}-01$ 8.19930 E-01 $8.19915 \mathrm{E}-01$ $8.19900 \mathrm{E}-01$ 8.19885 E-01

$8.19870 \mathrm{E}-01$ $8.19855 \mathrm{E}-01$ 8.19841 E-01 $8.19826 \mathrm{E}-01$ 8.19811 E-01

$8.19796 \mathrm{E}-01$ $8.19782 \mathrm{E}-01$ $8.19767 \mathrm{E}-01$ $8.19753 \mathrm{E}-01$ 8.19738E-01

8.19724E-01 $8.19710 \mathrm{E}-01$ $8.19695 \mathrm{E}-01$ $8.19681 \mathrm{E}-01$ $8.19667 \mathrm{E}-01$

8.19653E-01 $8.19639 \mathrm{E}-01$ $8.19625 \mathrm{E}-01$ $8.19611 \mathrm{E}-01$ $8.19597 \mathrm{E}-01$

8.19583 E-01 $8.19569 \mathrm{E}-01$ $8.19556 \mathrm{E}-01$ $8.19542 \mathrm{E}-01$ $8.19528 \mathrm{E}-01$

$8.19515 \mathrm{E}-01$ $8.19501 \mathrm{E}-01$ $8.19488 \mathrm{E}-01$ $8.19474 \mathrm{E}-01$ 8.19461 E-01

$Z=9 \quad A=18$

$$
\rho=1.2 A^{1 / 3} F
$$

$f_{1}$

$1.61444 \mathrm{E} \quad 01$

$1.62378 \mathrm{E} \quad 01$

$1.63312 \mathrm{E} \quad 01$

$\begin{array}{ll}1.64245 \mathrm{E} & 01\end{array}$

$1.65180 \mathrm{E} 01$

$1.66114 \mathrm{E} \quad 01$ $1.67049 \mathrm{E} 01$ $1.67984 \mathrm{E} \quad 01$ $1.68919 \mathrm{E} \quad 01$

$1.69854 \mathrm{E} \quad 01$

$1.70790 \mathrm{E} \quad 01$

$1.71726 \mathrm{E} \quad 01$ $1.72662 \mathrm{E} \quad 01$ $1.73598 \mathrm{E} \quad 01$ $1.74535 \mathrm{E} \quad 01$

$1.75471 \mathrm{E} \quad 01$ $1.76409 \mathrm{E} \quad 01$ $1.77346 \mathrm{E} \quad 01$ $1.78284 \mathrm{E}$ 01 $1.79221 \mathrm{E} \quad 01$

$1.80160 \mathrm{E} \quad 01$ $1.81098 \mathrm{E} \quad 01$ $1.82037 \mathrm{E} \quad 01$ $1.82976 \mathrm{E}$ ol $1.83915 \mathrm{E} \quad 01$

$1.84854 \mathrm{E} \quad 01$ $1.85794 \mathrm{E} \quad 01$ $1.86734 \mathrm{E} \quad 01$ $1.87674 \mathrm{E} \quad 01$ $1.88615 \mathrm{E} \quad 01$

$1.89555 \mathrm{E} \quad 01$ $1.90496 \mathrm{E}$ 01 $1.91438 \mathrm{E} \quad 01$ $1.92379 \mathrm{E} \quad 01$ $1.93321 \mathrm{E} \quad 01$

$1.94263 \mathrm{E} \quad 01$ $1.95206 \mathrm{E} \quad 01$ $1.96148 \mathrm{E} \quad 01$ $1.97091 \mathrm{E} \quad 01$ $1.98034 \mathrm{E} \quad 01$

$1.98978 \mathrm{E} \quad 01$ $1.99922 \mathrm{E} \quad 01$ $2.00866 \mathrm{E} \quad 01$ $2.01810 \mathrm{E} \quad 01$ $2.02755 \mathrm{E} \quad 01$

$2.03700 \mathrm{E} \quad 01$ $2.04645 \mathrm{E} \quad 01$ $2.05590 \mathrm{E} \quad 01$ $2.06536 \mathrm{E} \quad 01$

$2.07482 \mathrm{E} \quad 01$

$2.08429 \mathrm{E} \quad 01$ $2.09375 \mathrm{E} \quad 01$ $2.10322 \mathrm{E} \quad 01$ $2.11269 \mathrm{E} \quad 01$ $2.12217 \mathrm{E} 01$

$2.13165 \mathrm{E} \quad 01$ $2.14113 \mathrm{E} \quad 01$ $2.15061 \mathrm{E} \quad 01$ $2.16010 \mathrm{E} 01$ $2.16959 \mathrm{E}$ 01 $g_{1}$

4.14312 E-01

$4.21113 \mathrm{E}-01$

$4.27965 \mathrm{E}-01$

$4.34869 \mathrm{E}-01$

$4.41825 \mathrm{E}-01$

$4.48832 \mathrm{E}-01$

$4.55891 \mathrm{E}-01$

$4.63001 \mathrm{E}-01$

$4.70163 \mathrm{E}-01$

$4.77376 \mathrm{E}-01$

4.84641 E-01

$4.91958 \mathrm{E}-01$

$4.99326 \mathrm{E}-01$

$5.06747 \mathrm{E}-01$

$5.14219 \mathrm{E}-01$

$5.21742 \mathrm{E}-0 \mathrm{l}$

$5.29318 \mathrm{E}-01$

$5.36945 \mathrm{E}-01$

$5.44625 \mathrm{E}-01$

$5.52356 \mathrm{E}-01$

$5.60139 \mathrm{E}-01$

$5.67974 \mathrm{E}-01$

5.75861 E-01

$5.83800 \mathrm{E}-01$

$5.91791 \mathrm{E}-01$

$5.99834 \mathrm{E}-01$

$6.07930 \mathrm{E}-01$

$6.16077 \mathrm{E}-01$

$6.24276 \mathrm{E}-01$

$6.32528 \mathrm{E}-01$

$6.40832 \mathrm{E}-01$

$6.49188 \mathrm{E}-01$

$6.57596 \mathrm{E}-01$

$6.66057 \mathrm{E}-01$

$6.74570 \mathrm{E}-01$

$6.83135 \mathrm{E}-01$ $6.91753 \mathrm{E}-0 \mathrm{l}$

$7.00423 \mathrm{E}-01$

$7.09146 \mathrm{E}-01$

$7.17921 \mathrm{E}-01$

$7.2674 .9 \mathrm{E}-01$

$7.35629 \mathrm{E}-01$

7.44561 E-01

$7.53547 \mathrm{E}-01$

$7.62585 \mathrm{E}-01$

$7.71676 \mathrm{E}-01$

$7.80819 \mathrm{E}-01$

$7.90015 \mathrm{E}-01$

$7.99264 \mathrm{E}-01$

$8.08565 \mathrm{E}-01$

$8.17920 \mathrm{E}-01$

$8.27327 \mathrm{E}-01$

$8.36787 \mathrm{E}-01$

$8.46300 \mathrm{E}-01$

$8.55866 \mathrm{E}-01$

$8.65485 \mathrm{E}-01$

$8.75157 \mathrm{E}-01$

$8.84882 \mathrm{E}-01$

$8.94661 \mathrm{E}-01$

$9.04492 \mathrm{E}-01$

$\tan \delta_{1}$

$1.43889 \mathrm{E}-04$

$1.35287 \mathrm{E}-04$

$1.26790 \mathrm{E}-04$

$1.18403 \mathrm{E}-04$

$1.10136 \mathrm{E}-04$

$1.01979 \mathrm{E}-04$

$9.39314 \mathrm{E}-05$

$8.59817 \mathrm{E}-05$

$7.81298 \mathrm{E}-05$

$7.04000 \mathrm{E}-05$

$6.27703 \mathrm{E}-05$

$5.52202 \mathrm{E}-05$

$4.77955 \mathrm{E}-05$

$4.04513 \mathrm{E}-05$

$3.32082 \mathrm{E}-05$

$2.60618 \mathrm{E}-05$

$1.90078 \mathrm{E}-05$

$1.20393 \mathrm{E}-05$

$5.17868 \mathrm{E}-06$

$-1.60771 \mathrm{E}-06$

$-8.29928 \mathrm{E}-06$

$-1.49089 \mathrm{E}-05$

$-2.14362 \mathrm{E}-05$

$-2.78662 \mathrm{E}-05$

$-3.42138 \mathrm{E}-05$

$-4.04894 \mathrm{E}-05$

$-4.66713 \mathrm{E}-05$

$-5.27907 \mathrm{E}-05$

$-5.88060 \mathrm{E}-05$

$-6.47575 \mathrm{E}-05$

$-7.06298 \mathrm{E}-05$

$-7.64264 \mathrm{E}-05$

$-8.21429 \mathrm{E}-05$

$-8.77969 \mathrm{E}-05$

$-9.33642 \mathrm{E}-05$

$-9.88632 \mathrm{E}-05$

$-1.04287 \mathrm{E}-04$

$-1.09650 \mathrm{E}-04$

$-1.14939 \mathrm{E}-04$ 
ELECTRON RADIAL FUNCTIONS

$Z=9 \quad A=18 \quad \rho=1.2 A^{1 / 3} F$

Positron

$\bar{F}$

$4.93576 \mathrm{E}-04$

$4.99041 \mathrm{E}-04$

$5.04536 \mathrm{E}-04$

$5.10061 \mathrm{E}-04$

$5.15616 \mathrm{E}-04$

$5.21201 \mathrm{E}-04$

$5.26816 \mathrm{E}-04$

$5.32461 \mathrm{E}-04$

$5.38136 \mathrm{E}-04$

$5.43841 \mathrm{E}-04$

$5.49576 \mathrm{E}-04$

$5.55341 \mathrm{E}-04$

$5.61136 \mathrm{E}-04$

$5.66962 \mathrm{E}-04$

$5.72817 \mathrm{E}-04$

5.78702 E-04

$5.84618 \mathrm{E}-04$

$5.90563 \mathrm{E}-04$

$5.96539 \mathrm{E}-04$

6.02544 E-04

$6.08580 \mathrm{E}-04$

$6.14646 \mathrm{E}-04$

$6.20741 \mathrm{E}-04$

$6.26867 \mathrm{E}-04$

$6.33023 \mathrm{E}-04$

$6.39208 \mathrm{E}-04$

$6.45424 \mathrm{E}-04$

$6.51670 \mathrm{E}-04$

$6.57946 \mathrm{E}-04$

$6.64252 \mathrm{E}-04$

$6.70588 \mathrm{E}-04$

$6.76953 \mathrm{E}-04$

$6.83349 \mathrm{E}-04$

$6.89775 \mathrm{E}-04$

$6.96231 \mathrm{E}-04$

$7.02717 \mathrm{E}-04$

$7.09234 \mathrm{E}-04$

$7.15780 \mathrm{E}-04$

$7.22356 \mathrm{E}-04$

7.28962 E- 04

$7.35598 \mathrm{E}-04$

$7.42265 \mathrm{E}-04$

$7.48961 \mathrm{E}-04$

$7.55687 \mathrm{E}-04$

7.62444 E-04

$7.69230 \mathrm{E}-04$

$7.76047 \mathrm{E}-04$

$7.82893 \mathrm{E}-04$

$7.89770 \mathrm{E}-04$

$7.96676 \mathrm{E}-04$

$8.03613 \mathrm{E}-04$

$8.10579 \mathrm{E}-04$

$8.17576 \mathrm{E}-04$

$8.24603 \mathrm{E}-04$

8.31659 E- 04 .

$8.3874 .6 \mathrm{E}-04$

$8.45863 \mathrm{E}-04$

$8.53009 \mathrm{E}-04$

$8.60186 \mathrm{E}-04$

$8.67393 \mathrm{E}-04$ $f_{2}$

$7.83395 \mathrm{E}-01$

$7.92198 \mathrm{E}-01$

8.01051 E-01

$8.09953 \mathrm{E}-01$

8.18904E-01

8.27904E-01

$8.36953 \mathrm{E}-01$

$8.46051 \mathrm{E}-01$

$8.55198 \mathrm{E}-01$

8.64393 E-01

8.73639E-01

$8.82933 \mathrm{E}-01$

$8.92276 \mathrm{E}-01$

$9.01668 \mathrm{E}-01$

$9.11109 \mathrm{E}-01$

$9.20599 \mathrm{E}-01$

$9.30138 \mathrm{E}-01$

$9.39726 \mathrm{E}-01$

$9.49363 \mathrm{E}-01$

$9.59049 \mathrm{E}-01$

$9.68783 \mathrm{E}-01$

$9.78567 \mathrm{E}-01$

$9.88400 \mathrm{E}-01$

$9.98282 \mathrm{E}-01$

$1.00821 \mathrm{E} \quad 00$

$1.01819 \mathrm{E} \quad 00$

$1.02822 \mathrm{E} \quad 00$

$1.03830 \mathrm{E} \quad 00$

$1.04842 \mathrm{E} \quad 00$

$1.05860 \mathrm{E} \quad 00$

$1.06882 \mathrm{E} \quad 00$

$1.07910 \mathrm{E} \quad 00$

$1.08942 \mathrm{E} \quad 00$

$1.09979 \mathrm{E} \quad 00$

$1.11021 \mathrm{E} \quad 00$

$1.12068 \mathrm{E} \quad 00$

$1.13120 \mathrm{E} \quad 00$

$1.14176 \mathrm{E} \quad 00$

$1.15238 \mathrm{E} \quad 00$

$1.16304 \mathrm{E} \quad 00$

$1.17376 \mathrm{E} \quad 00$

$1.18452 \mathrm{E} \quad 00$

$1.19533 \mathrm{E} \quad 00$

$1.20619 \mathrm{E} \quad 00$

$1.21710 \mathrm{E} \quad 00$

$1.22806 \mathrm{E} \quad 00$

$1.23906 \mathrm{E} \quad 00$

$1.25012 \mathrm{E} \quad 00$

$1.26122 \mathrm{E} \quad 00$

$1.27237 \mathrm{E} \quad 00$

$1.28358 \mathrm{E} \quad 00$

$1.29483 \mathrm{E} \quad 00$

$1.30613 \mathrm{E} \quad 00$

$\begin{array}{ll}1.31747 \mathrm{E} & 00\end{array}$

$1.32887 \mathrm{E} \quad 00$

$1.34032 \mathrm{E} \quad 00$

$1.35181 \mathrm{E} \quad 00$

$1.36335 \mathrm{E} \quad 00$

$1.37495 \mathrm{E} \quad 00$
0

$\begin{array}{ll}1.38659 \mathrm{E} & 00\end{array}$ $g_{2}$

$1.26487 \mathrm{E}-02$

$1.29197 \mathrm{E}-02$

$1.31945 \mathrm{E}-02$

$1.34729 \mathrm{E}-02$

$1.37550 \mathrm{E}-02$

$1.40409 \mathrm{E}-02$

$1.43306 \mathrm{E}-02$

$1.46241 \mathrm{E}-02$

$1.49213 \mathrm{E}-02$

$1.52224 \mathrm{E}-02$

$1.55274 \mathrm{E}-02$

$1.58363 \mathrm{E}-02$

$1.61491 \mathrm{E}-02$

$1.64659 \mathrm{E}-02$

$1.67866 \mathrm{E}-02$

$1.71113 \mathrm{E}-02$

$1.74400 \mathrm{E}-02$

$1.77727 \mathrm{E}-02$

$1.81095 \mathrm{E}-02$

$1.84504 \mathrm{E}-02$

$1.87954 \mathrm{E}-02$

$1.91445 \mathrm{E}-02$

$1.94978 \mathrm{E}-02$

$1.98552 \mathrm{E}-02$

$2.02169 \mathrm{E}-02$

$2.05828 \mathrm{E}-02$

$2.09529 \mathrm{E}-02$

$2.13273 \mathrm{E}-02$

$2.17060 \mathrm{E}-02$

$2.20890 \mathbf{E}-02$

2.24764E- 02

2.28682 E- 02

$2.32643 \mathrm{E}-02$

$2.36649 \mathrm{E}-02$

2.40699E-02

$2.44793 \mathrm{E}-02$

$2.48933 \mathrm{E}-02$

$2.53118 \mathrm{E}-02$

$2.57348 \mathrm{E}-02$

$2.61624 \mathrm{E}-02$

$2.65945 \mathrm{E}-02$

$2.70313 \mathrm{E}-02$

$2.74727 \mathrm{E}-02$

$2.79187 \mathrm{E}-02$

$2.83695 \mathrm{E}-02$

$2.8824 .9 \mathrm{E}-02$

$2.92851 \mathrm{E}-02$

$2.97500 \mathrm{E}-02$

$3.02197 \mathrm{E}-02$

$3.06942 \mathrm{E}-02$

$3.11736 \mathrm{E}-02$

$3.16577 \mathrm{E}-02$

$3.21468 \mathrm{E}-02$

$3.26407 \mathrm{E}-02$

$3.31396 \mathbf{E}-02$

$3.36434 \mathrm{E}-02$

$3.41522 \mathrm{E}-02$

$3.46659 \mathrm{E}-02$

$3.51847 \mathrm{E}-02$

$3.57085 \mathrm{E}-02$ $\tan \delta_{2}$

$-2.13366 \mathrm{E} \quad 01$

$-2.13418 \mathrm{E}$ 01 $-1.11789 \mathrm{E}-02$

$-2.13444 \mathrm{E}$ 01 $-1.14389 \mathrm{E}-02$

$-2.13469 \mathrm{E} \quad 01 \quad-1.17026 \mathrm{E}-02$

$-2.13494 \mathrm{E} \quad 01-1.19699 \mathrm{E}-02$

$-2.13518 \mathrm{E}$ 01 $-1.22409 \mathrm{E}-02$

$-2.13543 \mathrm{E} \quad 01 \quad-1.25157 \mathrm{E}-02$

$-2.13567 \mathrm{E}$ 01 $-1.27942 \mathrm{E}-02$

$-2.13591 \mathrm{E} \quad 01 \quad-1.30764 \mathrm{E}-02$

$-2.13614 \mathrm{E} \quad 01$

$-2.13638 \mathrm{E} \quad 01$

$-2.13661 \mathrm{E} \quad 01$

$-2.13684 \mathrm{E} \quad 01$

$-2.13706 \mathrm{E} 01$

$-2.13729 \mathrm{E} \quad 01$

$-2.13751 \mathrm{E} \quad 01$

$-2.13773 \mathrm{E}$ 01

$-2.13794 \mathrm{E}$ 0l

$-2.13816 \mathrm{E} \quad 01$

$-2.13837 \mathrm{E} \quad 01$

$-2.13858 \mathrm{E}$ ol

$\begin{array}{lll}-2.13879 \mathrm{E} & 01\end{array}$

$-2.13899 \mathrm{E} \quad 01$

$-2.13920 \mathrm{E} 01$

$-2.13940 \mathrm{E} \quad 01$

$-2.13960 \mathrm{E} 01$

$-2.13980 \mathrm{E} \quad 0 \mathrm{l}$

$-2.13999 \mathrm{E} 01$

$-2.14018 \mathrm{E} \quad 01$

$-2.14038 \mathrm{E} \quad 01$

$\begin{array}{lll}-2.14057 \mathrm{E} & 01\end{array}$

$-2.14075 \mathrm{E} \quad 01$

$-2.14094 \mathrm{E} \quad 01$

$-2.14113 \mathrm{E} 01$

$-2.14131 \mathrm{E} \quad 01$

$-2.14149 \mathrm{E} \quad 01$

$-2.14167 \mathrm{E} 01$

$-2.14185 \mathrm{E} \quad 01$

$-2.14202 \mathrm{E} 01$

$-2.14219 \mathrm{E} \quad 01$

$-2.14237 \mathrm{E} 01$

$-2.14254 \mathrm{E} \quad 01$

$-2.14271 \mathrm{E} \quad 01$

$-2.14288 \mathrm{E} 01$

$-2.14304 \mathrm{E} \quad 01$

$-2.14321 \mathrm{E} 01$

$-2.14337 \mathrm{E} \quad 01$

$-2.14353 \mathrm{E}$ 01

$-2.14369 \mathrm{E} 01$

$-2.14385 \mathrm{E} \quad 01$

$-2.14401 \mathrm{E} 01$

$-2.14416 \mathrm{E} \quad 01$

$-2.14432 \mathrm{E} 01$

$-2.14447 \mathrm{E} 01$

$-2.14462 \mathrm{E} 01$

$-2.14477 \mathrm{E} \quad 01$

$-2.14492 \mathrm{E} \quad 0$

$-2.14507 \mathrm{E} 01$

$-2.14522 \mathrm{E} 01$

$-1.33625 \mathrm{E}-02$

$-1.36523 \mathrm{E}-02$

$-1.39460 \mathrm{E}-02$

$-1.42435 \mathrm{E}-02$

$-1.45450 \mathrm{E}-02$

$-1.48503 \mathrm{E}-02$

$-1.51596 \mathrm{E}-02$

$-1.54728 \mathrm{E}-02$

$-1.57900 \mathrm{E}-02$

$-1.61112 \mathrm{E}-02$

$-1.64365 \mathrm{E}-02$

$-1.67657 \mathrm{E}-02$

$-1.70991 \mathrm{E}-02$

$-1.74366 \mathrm{E}-02$

$-1.77782 \mathrm{E}-02$

$-1.81239 \mathrm{E}-02$

$-1.84738 \mathrm{E}-02$

$-1.88279 \mathrm{E}-02$

$-1.91862 \mathrm{E}-02$

$-1.95488 \mathrm{E}-02$

$-1.99156 \mathrm{E}-02$

$-2.02867 \mathrm{E}-02$

$-2.06622 \mathrm{E}-02$

$-2.10419 \mathrm{E}-02$

$-2.14261 \mathrm{E}-02$

$-2.18146 \mathrm{E}-02$

$-2.22075 \mathrm{E}-02$

$-2.26049 \mathrm{E}-02$

$-2.30067 \mathrm{E}-02$

$-2.34130 \mathrm{E}-02$

$-2.38239 \mathrm{E}-02$

$-2.42392 \mathrm{E}-02$

$-2.46591 \mathrm{E}-02$

$-2.50836 \mathrm{E}-02$

$-2.55126 \mathrm{E}-02$

$-2.59464 \mathrm{E}-02$

$-2.63847 \mathrm{E}-02$

$-2.68277 \mathrm{E}-02$

$-2.72755 \mathrm{E}-02$

$-2.77279 \mathrm{E}-02$

$-2.81851 \mathrm{E}-02$

$-2.86471 \mathrm{E}-02$ 
ELECTRON RADIAL FUNCTIONS

Positron

p

24.10

24.20

24.30

24.40

24.50

24.60

24.70

24.80

24.90

25.00

25.10

25.20

25.30

25.40

25.50

25.60

25.70

25.80

25.90

26.00

26.10

26.20

26.30

26.40

26.50

26.60

26.70

26.80

26.90

27.00

27.10

27.20

27.30

27.40

27.50

27.60

27.70

27.80

27.90

28.00

28.10

28.20

28.30

28.40

28.50

28.60

28.70

28.80

28.90

29.00

29.10

29.20

29.30

29.40

29.50

29.60

29.70

29.80

29.90

30.00
$F_{0}$

$8.19447 \mathrm{E}-01$ $8.19434 \mathrm{E}-01$ $8.19420 \mathrm{E}-01$ $8.19407 \mathrm{E}-01$ 8.19394 E-01

$8.19381 \mathrm{E}-01$ $8.19368 \mathrm{E}-01$ $8.19354 \mathrm{E}-01$ $8.19341 \mathrm{E}-01$ $8.19328 \mathrm{E}-01$

8.19315E-01 $8.19302 \mathrm{E}-01$ $8.19290 \mathrm{E}-01$ $8.19277 \mathrm{E}-01$ 8.19264E-01

8.19251E-01 $8.19238 \mathrm{E}-01$ $8.19226 \mathrm{E}-01$ $8.19213 \mathrm{E}-01$ $8.19200 \mathrm{E}-01$

8.19188E-01 $8.19175 \mathrm{E}-01$ $8.19163 \mathrm{E}-01$ $8.19151 \mathrm{E}-01$ 8.19138E-01

8.19126E-01 $8.19113 \mathrm{E}-01$ 8.19101 E-01 $8.19089 \mathrm{E}-01$ $8.19077 \mathrm{E}-01$

$8.19065 \mathrm{E}-01$ $8.19052 \mathrm{E}-01$ $8.19040 \mathrm{E}-01$ $8.19028 \mathrm{E}-01$ $8.19016 \mathrm{E}-01$

8.19004E-01 $8.18992 \mathrm{E}-01$ $8.18980 \mathrm{E}-01$ $8.18969 \mathrm{E}-01$ $8.18957 \mathrm{E}-01$

$8.18945 \mathrm{E}-01$ 8.18933 E-01 $8.18922 \mathrm{E}-01$ $8.18910 \mathrm{E}-01$ $8.18898 \mathrm{E}-01$

$8.18887 \mathrm{E}-01$ $8.18875 \mathrm{E}-01$ $8.18864 \mathrm{E}-01$ $8.18852 \mathrm{E}-01$ $8.18841 \mathrm{E}-01$

8.18829E-01 $8.18818 \mathrm{E}-01$ 8.18807 E-01 $8.18795 \mathrm{E}-01$ 8.18784 E-01

8.18773E-01 8.18761 E-01 $8.18750 \mathrm{E}-01$ 8.18739E-01 $8.18728 \mathrm{E}-01$
$Z=9$

$f_{1}$

$2.17908 \mathrm{E} \quad 0 \mathrm{l}$ $2.18858 \mathrm{E} \quad 01$ $2.19808 \mathrm{E} \quad 01$ $2.20758 \mathrm{E} \quad 01$ $2.21709 \mathrm{E} \quad 01$

$2.22660 \mathrm{E} \quad 01$ $2.23611 \mathrm{E} \quad 01$ $2.24562 \mathrm{E}$ 01 $2.25514 \mathrm{E} \quad 01$

$2.26466 \mathrm{E} \quad 01$

$2.27419 \mathrm{E} \quad 01$ $2.28371 \mathrm{E} \quad 01$ $2.29324 \mathrm{E} \quad 01$ $2.30278 \mathrm{E} 01$ $2.31231 \mathrm{E} \quad 01$

$2.32185 \mathrm{E} \quad 01$ $2.33139 \mathrm{E} \quad 01$ $2.34094 \mathrm{E} \quad 01$ $2.35049 \mathrm{E} \quad 01$

$2.36004 \mathrm{E} \quad 01$

$2.36960 \mathrm{E} \quad 01$ $2.37916 \mathrm{E} \quad 01$ $2.38872 \mathrm{E} 01$ $2.39829 \mathrm{E} \quad 01$

$2.40786 \mathrm{E} 01$

$2.41743 \mathrm{E} \quad 01$

$2.42700 \mathrm{E}$ 01

$2.43658 \mathrm{E} \quad 01$

$2.44617 \mathrm{E} \quad 01$

$2.45575 \mathrm{E} \quad 01$

$2.46534 \mathrm{E} \quad 01$ $2.47493 \mathrm{E} \quad 01$

$2.48453 \mathrm{E} \quad 01$

$2.49413 \mathrm{E} \quad 01$

$2.50373 \mathrm{E} 01$

$2.51334 \mathrm{E} \quad 01$ $2.52295 \mathrm{E} \quad 01$ $2.53256 \mathrm{E} \quad 01$ $2.54218 \mathrm{E} \quad 01$ $2.55180 \mathrm{E} \quad 01$

$2.56142 \mathrm{E} \quad 01$ $2.57105 \mathrm{E} \quad 01$ $2.58068 \mathrm{E} \quad 01$ $2.59031 \mathrm{E} 01$ $2.59995 \mathrm{E} \quad 01$

$2.60959 \mathrm{E} \quad 01$

$2.61923 \mathrm{E} \quad 01$

$2.62888 \mathrm{E} 01$

$2.63853 \mathrm{E} \quad 01$

$2.64819 \mathrm{E} 01$

$2.65785 \mathrm{E} \quad 01$

$2.66751 \mathrm{E} \quad 01$

$2.67718 \mathrm{E} \quad 01$

$2.68685 \mathrm{E} \quad 01$

$2.69652 \mathrm{E} 01$

$2.70620 \mathrm{E} \quad 01$

$2.71588 \mathrm{E} \quad 01$

$2.72556 \mathrm{E} \quad 01$

$2.73525 \mathrm{E} \quad 01$

$2.74494 \mathrm{E} 01$
$A=18$

$\rho=1.2 A^{1 / 3} F$

$g_{1}$

$\tan \delta_{1}$
$-2.15116 \mathrm{E}-04$

$f_{-1}$

$9.14377 \mathrm{E}-01$

$9.24314 \mathrm{E}-01$

$9.34306 \mathrm{E}-01$

$9.44350 \mathrm{E}-01$

9.54447 E-01

$9.64598 \mathrm{E}-01$

$9.74802 \mathrm{E}-01$

$9.85060 \mathrm{E}-01$

$9.95371 \mathrm{E}-01$

$\begin{array}{ll}1.00573 \mathrm{E} & 00\end{array}$

$1.01615 \mathrm{E} \quad 00$

$1.02662 \mathrm{E} \quad 00$

$1.03715 \mathrm{E} \quad 00$

$1.04773 \mathrm{E} \quad 00$

$1.05836 \mathrm{E} \quad 00^{\circ}$

$1.06905 \mathrm{E} \quad 00$

$1.07979 \mathrm{E} \quad 00$

$1.09058 \mathrm{E} \quad 00$

$1.10143 \mathrm{E} \quad 00$

$1.11233 \mathrm{E} \quad 00$

$1.12328 \mathrm{E} \quad 00$

$1.13429 \mathrm{E} \quad 00$

$1.14535 \mathrm{E} \quad 00$

$1.15647 \mathrm{E} \quad 00$

$1.16764 \mathrm{E} \quad 00$

$1.17887 \mathrm{E} \quad 00$

$1.19015 \mathrm{E} \quad 00$

$1.20148 \mathrm{E} \quad 00$

$1.21287 \mathrm{E} \quad 00$

$1.22431 \mathrm{E} \quad 00$

$1.23580 \mathrm{E} \quad 00$

$1.24735 \mathrm{E} \quad 00$

$1.25896 \mathrm{E} \quad 00$

$1.27062 \mathrm{E} \quad 00$

$1.28233 \mathrm{E} \quad 00$

$1.29410 \mathrm{E} \quad 00$ $1.30592 \mathrm{E} \quad 00$

$1.31780 \mathrm{E} \quad 00$

$1.32973 \mathrm{E} \quad 00$

$1.34171 \mathrm{E} \quad 00$

$1.35375 \mathrm{E} \quad 00$

$1.36585 \mathrm{E} \quad 00$

$1.37800 \mathrm{E} \quad 00$

$1.39020 \mathrm{E} \quad 00$

$1.40246 \mathrm{E} \quad 00$

$1.41478 \mathrm{E} \quad 00$

$1.42715 \mathrm{E} \quad 00$

$1.43957 \mathrm{E} \quad 00$

$1.45205 \mathrm{E} \quad 00$

$1.46458 \mathrm{E} \quad 00$

$1.47717 \mathrm{E} \quad 00$

$1.48982 \mathrm{E} \quad 00$

$1.50251 \mathrm{E} \quad 00$

$1.51527 \mathrm{E} \quad 00$

$1.52808 \mathrm{E} \quad 00$

$1.54094 \mathrm{E} \quad 00$

$1.55386 \mathrm{E} \quad 00$

$1.56684 \mathrm{E} \quad 00$

$\begin{array}{ll}1.57987 \mathrm{E} & 00\end{array}$

$1.59296 \mathrm{E} \quad 00$

$-2.19003 \mathrm{E}-04$

$-2.22839 \mathrm{E}-04$

$-2.26615 \mathrm{E}-04$

$-2.30333 \mathrm{E}-04$

$-2.34014 \mathrm{E}-04$.

$-2.37645 \mathrm{E}-04$

$-2.41211 \mathrm{E}-04$

$-2.44739 \mathrm{E}-04$

$-2.48208 \mathrm{E}-04$

$-2.51641 \mathrm{E}-04$

$-2.55022 \mathrm{E}-04$

$-2.58342 \mathrm{E}-04$

$-2.61626 \mathrm{E}-04$

$-2.64868 \mathrm{E}-04$

$-2.68044 \mathrm{E}-04$

$-2.71185 \mathrm{E}-04$

$-2.74285 \mathrm{E}-04$

$-2.77333 \mathrm{E}-04$

$-2.80350 \mathrm{E}-04$

$-2.83304 \mathrm{E}-04$

$-2.86221 \mathrm{E}-04$

$-2.89096 \mathrm{E}-04$

$-2.91930 \mathrm{E}-04$

$-2.94724 \mathrm{E}-04$

$-2.97472 \mathrm{E}-04$

$-3.00176 \mathrm{E}-04$

$-3.02846 \mathrm{E}-04$

$-3.05463 \mathrm{E}-04$

$-3.08046 \mathrm{E}-04$

$-3.10580 \mathrm{E}-04$

$-3.13089 \mathrm{E}-04$

$-3.15542 \mathrm{E}-04$

$-3.17966 \mathrm{E}-04$

$-3.20352 \mathrm{E}-04$

$-3.22697 \mathrm{E}-04$

$-3.25005 \mathrm{E}-04$

$-3.27283 \mathrm{E}-04$ 
ELECTRON RADIAL FUNCTIONS

$Z=9 \quad A=18 \quad \rho=1.2 A^{1 / 3} F$

Positron

$\bar{F}$

$8.74630 \mathrm{E}-04$ $8.81897 \mathrm{E}-04$ $8.89194 \mathrm{E}-04$ 8.96521 E-04 $9.03878 \mathrm{E}-04$

$9.11265 \mathrm{E}-04$ $9.18681 \mathrm{E}-04$ $9.26128 \mathrm{E}-04$ $9.33605 \mathrm{E}-04$ $9.41112 \mathrm{E}-04$

$9.48650 \mathrm{E}-04$ $9.56217 \mathrm{E}-04$ $9.63814 \mathrm{E}-04$ $9.71441 \mathrm{E}-04$ $9.79098 \mathrm{E}-04$

$9.86785 \mathrm{E}-04$ $9.94502 \mathrm{E}-04$ $1.00225 \mathrm{E}-03$ $1.01003 \mathrm{E}-03$ $1.01783 \mathrm{E}-03$

$1.02567 \mathrm{E}-03$ $1.03354 \mathrm{E}-03$ $1.04144 \mathrm{E}-03$ $1.04936 \mathrm{E}-03$ $1.05732 \mathrm{E}-03$

$1.06531 \mathrm{E}-03$ $1.07333 \mathrm{E}-03$ $1.08137 \mathrm{E}-03$ $1.08945 \mathrm{E}-03$ $1.09756 \mathrm{E}-03$

$1.10570 \mathrm{E}-03$

$1.11386 \mathrm{E}-03$

$1.12206 \mathrm{E}-03$

$1.13029 \mathrm{E}-03$

$1.13855 \mathrm{E}-03$

$1.14683 \mathrm{E}-03$

$1.15515 \mathrm{E}-03$

$1.16350 \mathrm{E}-03$

$1.17188 \mathrm{E}-03$

$1.18028 \mathrm{E}-03$

$1.18872 \mathrm{E}-03$ $1.19719 \mathrm{E}-03$ $1.20569 \mathrm{E}-03$ $1.21421 \mathrm{E}-03$ $1.22277 \mathrm{E}-03$

$1.23136 \mathrm{E}-03$ $1.23998 \mathrm{E}-03$ $1.24863 \mathrm{E}-03$ $1.25730 \mathrm{E}-03$ $1.26601 \mathrm{E}-03$

$1.27475 \mathrm{E}-03$ $1.28352 \mathrm{E}-03$ $1.29231 \mathrm{E}-03$ $1.30114 \mathrm{E}-03$ $1.31000 \mathrm{E}-03$

$1.31889 \mathrm{E}-03$ $1.32780 \mathrm{E}-03$ $1.33675 \mathrm{E}-03$ $1.34573 \mathrm{E}-03$ $1.35474 \mathrm{E}-03$ $f_{2}$

$1.39828 \mathrm{E} \quad 00$ $1.41002 \mathrm{E} \quad 00$ $1.42180 \mathrm{E} \quad 00$ $1.43364 \mathrm{E} \quad 00$ $1.44552 \mathrm{E} \quad 00$

$1.45746 \mathrm{E} \quad 00$ $1.46944 \mathrm{E} \quad 00$ $1.48147 \mathrm{E} \quad 00$ $1.49355 \mathrm{E} \quad 00$ $1.50568 \mathrm{E} \quad 00$

$1.51785 \mathrm{E} \quad 00$ $1.53008 \mathrm{E} \quad 00$ $1.54235 \mathrm{E} \quad 00$ $1.55468 \mathrm{E} \quad 00$ $1.56705 \mathrm{E} \quad 00$

$1.57947 \mathrm{E} \quad 00$ $1.59194 \mathrm{E} \quad 00$ $1.60446 \mathrm{E} \quad 00$ $1.61702 \mathrm{E} \quad 00$

$1.62964 \mathrm{E} \quad 00$

$1.64230 \mathrm{E} \quad 00$ $1.65501 \mathrm{E} \quad 00$ $1.66777 \mathrm{E} \quad 00$ $1.68058 \mathrm{E} \quad 00$ $1.69344 \mathrm{E} \quad 00$

$1.70635 \mathrm{E} \quad 00$ $1.71930 \mathrm{E} \quad 00$ $1.73231 \mathrm{E} \quad 00$

$1.74536 \mathrm{E} \quad 00$

$1.75846 \mathrm{E} \quad 00$

$1.77161 \mathrm{E} \quad 00$ $1.78481 \mathrm{E} \quad 00$ $1.79806 \mathrm{E} \quad 00$ $1.81135 \mathrm{E} \quad 00$ $1.82470 \mathrm{E} \quad 00$

$1.83809 \mathrm{E} \quad 00$ $1.85153 \mathrm{E} \quad 00$ $\begin{array}{lll}1.86502 \mathrm{E} & 00\end{array}$ $1.87856 \mathrm{E} \quad 00$ $1.89215 \mathrm{E} \quad 00$

$1.90578 \mathrm{E} \quad 00$ $1.91947 \mathrm{E} \quad 00$ $1.93320 \mathrm{E} \quad 00$ $1.94698 \mathrm{E} \quad 00$ $1.96081 \mathrm{E} \quad 00$

$1.97469 \mathrm{E} \quad 00$ $1.98861 \mathrm{E} \quad 00$ $2.00259 \mathrm{E} \quad 00$ $2.01661 \mathrm{E} \quad 00$ $2.03068 \mathrm{E} \quad 00$

$2.04480 \mathrm{E} \quad 00$ $2.05897 \mathrm{E} \quad 00$ $2.07319 \mathrm{E} \quad 00$ $2.08745 \mathrm{E} \quad 00$ $2.10177 \mathrm{E} \quad 00$

$2.11613 \mathrm{E} \quad 00$ $2.13054 \mathrm{E} \quad 00$ $2.14500 \mathrm{E} \quad 00$ $2.15951 \mathrm{E} \quad 00$ $2.17406 \mathrm{E} \quad 00$ $\tan \delta_{2}$

$f_{-2}$

$3.62375 \mathrm{E}-02$

$3.67714 \mathrm{E}-02$

$3.73105 \mathrm{E}-02$

$3.78547 \mathrm{E}-02$

$3.84041 \mathrm{E}-02$

$3.89586 \mathrm{E}-02$

$3.95184 \mathrm{E}-02$

$4.00834 \mathrm{E}-02$

$4.06537 \mathrm{E}-02$

4.12292 E-02

4.18100 E-02

4.23961 E-02

$4.29876 \mathrm{E}-02$

4.35845 E-02

$4.41868 \mathrm{E}-02$

4.47945 E-02

$4.54076 \mathrm{E}-02$

$4.60262 \mathrm{E}-02$

4.66502 E-02

$4.72798 \mathrm{E}-02$

4.79150 E-02 $4.85557 \mathrm{E}-02$

$4.92020 \mathrm{E}-02$

$4.98539 \mathrm{E}-02$

$5.05114 \mathrm{E}-02$

$5.11746 \mathrm{E}-02$

5.18435 E-02

5.25181 E-02

5.31984 E-02

$5.38844 \mathrm{E}-02$

$5.45763 \mathrm{E}-02$

$5.52739 \mathrm{E}-02$

$5.59774 \mathrm{E}-02$

$5.66867 \mathrm{E}-02$

$5.74019 \mathrm{E}-02$

$5.81230 \mathrm{E}-02$

$5.88500 \mathrm{E}-02$

$5.95829 \mathrm{E}-02$

$6.03218 \mathrm{E}-02$

$6.10667 \mathrm{E}-02$

$6.18176 \mathrm{E}-02$ $6.25746 \mathrm{E}-02$ $6.33376 \mathrm{E}-02$ $6.41067 \mathrm{E}-02$ $6.48819 \mathrm{E}-02$

$6.56632 \mathrm{E}-02$ $6.64507 \mathrm{E}-02$ $6.72444 \mathrm{E}-02$ 6.80443 E-02 $6.88504 \mathrm{E}-02$

$6.96627 \mathrm{E}-02$

$7.04814 \mathrm{E}-02$

$7.13063 \mathrm{E}-02$

$7.21375 \mathrm{E}-02$

$7.29751 \mathrm{E}-02$

$7.38191 \mathrm{E}-02$

$7.46694 \mathrm{E}-02$

$7.55262 \mathrm{E}-02$

$7.63894 \mathrm{E}-02$

$7.72591 \mathrm{E}-02$
$-2.14536 \mathrm{E}$ 01 $-3.30232 \mathrm{E}-02$ $-2.14551 \mathrm{E} 01-3.35342 \mathrm{E}-02$ $-2.14565 \mathrm{E}$ 01 $-3.40502 \mathrm{E}-02$ $-2.14579 \mathrm{E} 01-3.45712 \mathrm{E}-02$ $-2.14593 \mathrm{E}$ 01 $-3.50974 \mathrm{E}-02$

$-2.14607 \mathrm{E} \quad 01 \quad-3.56286 \mathrm{E}-02$ $-2.14621 \mathrm{E}$ 01 $-3.61649 \mathrm{E}-02$ $-2.14635 \mathrm{E}$ 01 $-3.67064 \mathrm{E}-02$ $-2.14648 \mathrm{E}$ 01 $-3.72531 \mathrm{E}-02$ $-2.14662 \mathrm{E} \quad 01-3.78050 \mathrm{E}-02$

$-2.14675 \mathrm{E} \quad 01 \quad-3.83621 \mathrm{E}-02$ $-2.14688 \mathrm{E} \quad 01 \quad-3.89244 \mathrm{E}-02$ $-2.14702 \mathrm{E} \quad 01 \quad-3.94920 \mathrm{E}-02$ $-2.14715 \mathrm{E} \quad 01 \quad-4.00649 \mathrm{E}-02$ $-2.14728 \mathrm{E}$ 01 $-4.06432 \mathrm{E}-02$

$-2.14740 \mathrm{E} \quad 01 \quad-4.12267 \mathrm{E}-02$ $-2.14753 \mathrm{E} \quad 01 \quad-4.18157 \mathrm{E}-02$ $-2.14766 \mathrm{E}$ 01 $-4.24100 \mathrm{E}-02$ $-2.14778 \mathrm{E} \quad 01 \quad-4.30097 \mathrm{E}-02$ $-2.14791 \mathrm{E} \quad 01 \quad-4.36149 \mathrm{E}-02$

$-2.14803 \mathrm{E} 01$ $-2.14815 \mathrm{E} \quad 01$ $-2.14827 \mathrm{E} \quad 01$ $-2.14839 \mathrm{E} \quad 01$ $-2.14851 \mathrm{E} \quad 01$

$-4.42255 \mathrm{E}-02$ $-4.48416 \mathrm{E}-02$ $-4.54632 \mathrm{E}-02$ $-4.60904 \mathrm{E}-02$ $-4.67231 \mathrm{E}-02$

$-2.14863 \mathrm{E} \quad 01$ $-2.14875 \mathrm{E} \quad 01$ $-2.14887 \mathrm{E} 01$ $-2.14898 \mathrm{E} \quad 01$ $-2.14910 \mathrm{E} 01$

$-2.14921 \mathrm{E} 01$ $-2.14932 \mathrm{E} 01$ $-2.14944 \mathrm{E} \quad 01$ $-2.14955 \mathrm{E} 01$ $-2.14966 \mathrm{E} 01$

$-2.14977 \mathrm{E} 01$ $-2.14988 \mathrm{E} 01$ $-2.14998 \mathrm{E} 01$ $-2.15009 \mathrm{E} 01$ $-2.15020 \mathrm{E} 01$

$-4.73614 \mathrm{E}-02$ $-4.80052 \mathrm{E}-02$ $-4.86547 \mathrm{E}-02$ $-4.93099 \mathrm{E}-02$ $-4.99707 \mathrm{E}-02$

$-5.06373 \mathrm{E}-02$ $-5.13095 \mathrm{E}-02$ $-5.19875 \mathrm{E}-02$ $-5.26713 \mathrm{E}-02$ $-5.33609 \mathrm{E}-02$

$-5.40562 \mathrm{E}-02$ $-5.47575 \mathrm{E}-02$ $-5.54645 \mathrm{E}-02$ $-5.61775 \mathrm{E}-02$ $-5.68964 \mathrm{E}-02$

$-2.15030 \mathrm{E}$ 01 $-5.76212 \mathrm{E}-02$ $-2.15041 \mathrm{E}$ 01 $-5.83520 \mathrm{E}-02$ $-2.15051 \mathrm{E} \quad 01 \quad-5.90887 \mathrm{E}-02$ $-2.15062 \mathrm{E} \quad 01 \quad-5.98315 \mathrm{E}-02$ $-2.15072 \mathrm{E} \quad 01 \quad-6.05803 \mathrm{E}-02$

$-2.15082 \mathrm{E} \quad 01-6.13351 \mathrm{E}-02$ $-2.15092 \mathrm{E} 01 \quad-6.20960 \mathrm{E}-02$ $-2.15102 \mathrm{E} \quad 01 \quad-6.28631 \mathrm{E}-02$ $-2.15112 \mathrm{E} \quad 01-6.36362 \mathrm{E}-02$ $-2.15122 \mathrm{E} \quad 01 \quad-6.44155 \mathrm{E}-02$

$-2.15132 \mathrm{E} \quad 01$

$-2.15142 \mathrm{E} \quad 01$

$-2.15151 \mathrm{E} 01$

$-2.15161 \mathrm{E} 01$

$-2.15171 \mathrm{E} 01$

$-6.52010 \mathrm{E}-02$

$-6.59926 \mathrm{E}-02$

$-6.67905 \mathrm{E}-02$ $-6.75946 \mathrm{E}-02$ $-6.84050 \mathrm{E}-02$

$-2.15180 \mathrm{E} \quad 01$ $-2.15190 \mathrm{E} 01$ $-2.15199 \mathrm{E} 01$ $-2.15208 \mathrm{E} 01$

$-6.92217 \mathrm{E}-02$ $-7.00447 \mathrm{E}-02$ $-7.08740 \mathrm{E}-02$ $-7.17097 \mathrm{E}-02$ $-7.25518 \mathrm{E}-02$

$\tan \delta_{-2}$

$1.45755 \mathrm{E} \quad 00$

$1.46953 \mathrm{E} \quad 00$

$1.48156 \mathrm{E} \quad 00$

1.49365 E 00

$1.50578 \mathrm{E} \quad 00$

$1.51796 \mathrm{E} \quad 00$

$1.53018 \mathrm{E} \quad 00$

$1.54246 \mathrm{E} \quad 00$

$1.55479 \mathrm{E} \quad 00$

$1.56716 \mathrm{E} 00$

$\begin{array}{ll}1.57958 \mathrm{E} & 00\end{array}$ $1.59205 \mathrm{E} \quad 00$ $1.60457 \mathrm{E} \quad 00$ 
ELECTRON RADIAL FUNCTIONS

Positron

$F_{0}$

30.10

30.20

30.30

30.40

30.50

30.60

30.70

30.80

30.90

31.00

31.10

31.20

31.30

31.40

31.50

31.60

31.70

31.80

31.90

32.00

32.10

32.20

32.30

32.40

32.50

32.60

32.70

32.80

32.90

33.00

33.10

33.20

33.30

33.40

33.50

33.60

33.70

33.80

33.90

34.00

34.10

34.20

34.30

34.40

34.50

34.60

34.70

34.80

34.90

35.00

35.10

35.20

35.30

35.40

35.50

35.60

35.70

35.80

35.90

36.00 $f_{1}$

$8.18717 \mathrm{E}-01$

$8.18706 \mathrm{E}-01$

8.18695 E-01

8.18684 E-01

8.18673 E-01

8.18662 E-01

8.18651 E-01

$8.18640 \mathrm{E}-01$

$8.18629 \mathrm{E}-01$

8.18618 E-01

$8.18608 \mathrm{E}-01$

$8.18597 \mathrm{E}-01$

$8.18586 \mathrm{E}-01$

8.18575 E-01

8.18565 E-01

$8.18554 \mathrm{E}-01$ $8.18544 \mathrm{E}-01$

$8.18533 \mathrm{E}-01$

8.18522 E-01

$8.18512 \mathrm{E}-01$

$8.18501 \mathrm{E}-01$

$8.18491 \mathrm{E}-01$

8.18481 E-01

$8.18470 \mathrm{E}-01$

$8.18460 \mathrm{E}-01$

$8.18449 \mathrm{E}-01$

$8.18439 \mathrm{E}-01$

$8.18429 \mathrm{E}-01$

$8.18419 \mathrm{E}-01$

$8.18408 \mathrm{E}-01$

$8.18398 \mathrm{E}-01$

$8.18388 \mathrm{E}-01$

$8.18378 \mathrm{E}-01$

$8.18368 \mathrm{E}-01$

$8.18358 \mathrm{E}-01$

$8.18348 \mathrm{E}-01$

$8.18338 \mathrm{E}-01$

$8.18328 \mathrm{E}-01$

$8.18318 \mathrm{E}-01$

$8.18308 \mathrm{E}-01$

$8.18298 \mathrm{E}-01$ $8.18288 \mathrm{E}-01$ $8.18278 \mathrm{E}-01$ $8.18268 \mathrm{E}-01$

$8.18258 \mathrm{E}-01$

$8.18249 \mathrm{E}-01$ $8.18239 \mathrm{E}-01$ $8.18229 \mathrm{E}-01$ $8.18219 \mathrm{E}-01$ $8.18210 \mathrm{E}-01$

$8.18200 \mathrm{E}-01$ $8.18190 \mathrm{E}-01$ 8.18181 E-01 $8.18171 \mathrm{E}-01$ $8.18162 \mathrm{E}-01$

$8.18152 \mathrm{E}-01$ $8.18143 \mathrm{E}-01$ $8.18133 \mathrm{E}-01$ $8.18124 \mathrm{E}-01$ $8.18114 \mathrm{E}-01$

$Z=9 \quad A=18 \quad \rho=1.2 A^{1 / 3} F$

$2.75464 \mathrm{E} \quad 01$

$2.76434 \mathrm{E} \quad 01$

$2.77404 \mathrm{E} \quad 01$

$2.78375 \mathrm{E} \quad 01$

$2.79346 \mathrm{E} \quad 0]$.

$2.80317 \mathrm{E} \quad 01$ $2.81289 \mathrm{E} \quad 01$ $2.82261 \mathrm{E} \quad 01$ $2.83234 \mathrm{E} \quad 01$ $2.84207 \mathrm{E} \quad 01$

$2.85180 \mathrm{E} \quad 01$ $2.86154 \mathrm{E} \quad 01$ $2.87128 \mathrm{E} \quad 01$ $2.88102 \mathrm{E} \quad 01$ 2.89077 E 01

$2.90053 \mathrm{E} \quad 01$ $2.91028 \mathrm{E} 01$ $2.92004 \mathrm{E} \quad 01$ $2.92981 \mathrm{E} \quad 01$ $2.93958 \mathrm{E} 01$

$2.94935 \mathrm{E} \quad 01$ $2.95913 \mathrm{E} \quad 01$ $2.96891 \mathrm{E} \quad 01$ $2.97869 \mathrm{E} \quad 01$ 2.98848 E 01

$2.99827 \mathrm{E} \quad 01$ $3.00807 \mathrm{E} \quad 01$ $3.01787 \mathrm{E} \quad 01$ $3.02768 \mathrm{E} \quad 01$ $3.03748 \mathrm{E} \quad 01$

$3.04730 \mathrm{E} \quad 01$ $3.05712 \mathrm{E} \quad 01$ $3.06694 \mathrm{E} \quad 01$ $3.07676 \mathrm{E} \quad 01$ $3.08659 \mathrm{E} 01$

$3.09643 \mathrm{E} \quad 01$ $3.10626 \mathrm{E} \quad 01$ $3.11611 \mathrm{E} \quad 01$ $3.12595 \mathrm{E} \quad 01$ $3.13580 \mathrm{E} \quad 01$

$3.14566 \mathrm{E} \quad 01$ $3.15552 \mathrm{E} \quad 01$ $3.16538 \mathrm{E} \quad 01$ $3.17525 \mathrm{E} \quad 01$ $3.18512 \mathrm{E} \quad 01$

$3.19500 \mathrm{E} \quad 01$ $3.20488 \mathrm{E} 01$ $3.21476 \mathrm{E} \quad 01$ $3.22465 \mathrm{E} \quad 01$ $3.23454 \mathrm{E} 01$

$3.24444 \mathrm{E} \quad 01$ $3.25434 \mathrm{E} 01$ $3.26425 \mathrm{E} 01$ $3.27416 \mathrm{E} \quad 01$ $3.28408 \mathrm{E} \quad 01$

$3.29400 \mathrm{E} \quad 01$ $3.30392 \mathrm{E} \quad 01$ $3.31385 \mathrm{E} 01$ $3.32378 \mathrm{E} \quad 01$ $3.33372 \mathrm{E} \quad 01$ $\tan \delta_{1}$

$f_{-1}$

$1.60610 \mathrm{E} \quad 00 \quad-3.69706 \mathrm{E}-04$ $1.61930 \mathrm{E} \quad 00-3.71150 \mathrm{E}-04$ $1.63255 \mathrm{E} \quad 00 \quad-3.72572 \mathrm{E}-04$

$1.64586 \mathrm{E} \quad 00 \quad-3.73961 \mathrm{E}-04$

$\begin{array}{lll}1.65922 \mathrm{E} & 00 & -3.75305 \mathrm{E}-04\end{array}$

$1.67264 \mathrm{E} \quad 00$ $1.68612 \mathrm{E} \quad 00$

$1.69965 \mathrm{E} \quad 00$

$1.71324 \mathrm{E} \quad 00$

$1.72688 \mathrm{E} \quad 00$

$1.74058 \mathrm{E} \quad 00$

$1.75434 \mathrm{E} \quad 00$

$1.76815 \mathrm{E} \quad 00$

$1.78202 \mathrm{E} \quad 00$

$1.79594 \mathrm{E} \quad 00$

$1.80992 \mathrm{E} \quad 00$

$1.82395 \mathrm{E} \quad 00$

$1.83805 \mathrm{E} \quad 00$

$1.85219 \mathrm{E} \quad 00$

$1.86640 \mathrm{E} \quad 00$

$1.88066 \mathrm{E} \quad 00$

$1.89498 \mathrm{E} \quad 00$

$1.90935 \mathrm{E} \quad 00$

$1.92378 \mathrm{E} \quad 00$

$1.93827 \mathrm{E} \quad 00$

$1.95281 \mathrm{E} \quad 00$

].96742E 00

$1.98207 \mathrm{E} \quad 00$

$1.99679 \mathrm{E} \quad 00$

$2.01156 \mathrm{E} \quad 00$

$2.02639 \mathrm{E} \quad 00$

$2.04127 \mathrm{E} \quad 00$

$2.05621 \mathrm{E} \quad 00$

$2.07121 \mathrm{E} \quad 00$

$2.08627 \mathrm{E} \quad 00$

$2.10138 \mathrm{E} \quad 00$

$2.11655 \mathrm{E} \quad 00$

$2.13178 \mathrm{E} \quad 00$

$2.14706 \mathrm{E} \quad 00$

$2.16240 \mathrm{E} \quad 00$

$2.17780 \mathrm{E} \quad 00$

$2.19326 \mathrm{E} \quad 00$

$2.20878 \mathrm{E} \quad 00$

$2.22435 \mathrm{E} \quad 00$

2.23998 E 00

$2.25566 \mathrm{E} \quad 00$

$2.27141 \mathrm{E} \quad 00$

$2.28721 \mathrm{E} \quad 00$

$2.30307 \mathrm{E} \quad 00$

$2.31899 \mathrm{E} \quad 00$

$2.33497 \mathrm{E} \quad 00$

$2.35100 \mathrm{E} \quad 00$

$2.36709 \mathrm{E} \quad 00$

$2.38324 \mathrm{E} \quad 00$

$2.39945 \mathrm{E} \quad 00$

$2.41572 \mathrm{E} \quad 00$

$2.43204 \mathrm{E} \quad 00$

$2.44843 \mathrm{E} \quad 00$

$2.46487 \mathrm{E} \quad 00$

$2.48137 \mathrm{E} \quad 00$

$-3.76638 \mathrm{E}-04$

$-3.77938 \mathrm{E}-04$

$-3.79195 \mathrm{E}-04$

$-3.80438 \mathrm{E}-04$

$-3.81638 \mathrm{E}-04$

$-3.82809 \mathrm{E}-04$

$-3.83969 \mathrm{E}-04$

$-3.85088 \mathrm{E}-04$

$-3.86176 \mathrm{E}-04$

$-3.87241 \mathrm{E}-04$

$-3.88273 \mathrm{E}-04$

$-3.89289 \mathrm{E}-04$

$-3.90266 \mathrm{E}-04$

$-3.91215 \mathrm{E}-04$

$-3.92137 \mathrm{E}-04$

$-3.93038 \mathrm{E}-04$

$-3.93910 \mathrm{E}-04$

$-3.94755 \mathrm{E}-04$

$-3.95565 \mathrm{E}-04$

$-3.96358 \mathrm{E}-04$

$-3.97129 \mathrm{E}-04$

$-3.97865 \mathrm{E}-04$

$-3.98574 \mathrm{E}-04$

$-3.99256 \mathrm{E}-04$

$-3.99919 \mathrm{E}-04$

$-4.00550 \mathrm{E}-04$

$-4.01161 \mathrm{E}-04$

$-4.01748 \mathrm{E}-04$

$-4.02308 \mathrm{E}-04$

$-4.02841 \mathrm{E}-04$

$-4.03355 \mathrm{E}-04$

$-4.03842 \mathrm{E}-04$

$-4.04298 \mathrm{E}-04$

$-4.04741 \mathrm{E}-04$

$-4.05157 \mathrm{E}-04$

$-4.05551 \mathrm{E}-04$

$-4.05909 \mathrm{E}-04$

$-4.06246 \mathrm{E}-04$

$-4.06571 \mathrm{E}-04$

$-4.06862 \mathrm{E}-04$

$-4.07137 \mathrm{E}-04$

$-4.07388 \mathrm{E}-04$ 
ELECTRON RADIAL FUNCTIONS

$Z=9 \quad A=18 \quad \rho=1.2 A^{1 / 3} F$

Positron

$\bar{F}$

$f_{2}$

$1.36378 \mathrm{E}-03$

$1.37284 \mathrm{E}-03$

$1.38194 \mathrm{E}-03$

$1.39107 \mathrm{E}-03$

$1.40023 \mathrm{E}-03$

$1.40942 \mathrm{E}-03$

$1.41863 \mathrm{E}-03$

$1.42788 \mathrm{E}-03$

$1.43716 \mathrm{E}-03$

$1.44647 \mathrm{E}-03$

$1.45581 \mathrm{E}-03$

$1.46517 \mathrm{E}-03$

$1.47457 \mathrm{E}-03$

$1.48400 \mathrm{E}-03$

$1.49346 \mathrm{E}-03$

$1.50295 \mathrm{E}-03$

$1.51246 \mathrm{E}-03$

$1.52201 \mathrm{E}-03$

$1.53159 \mathrm{E}-03$

$1.54120 \mathrm{E}-03$

$1.55084 \mathrm{E}-03$

$1.56051 \mathrm{E}-03$

$1.57020 \mathrm{E}-03$

$1.57993 \mathrm{E}-03$

$1.58969 \mathrm{E}-03$

$1.59948 \mathrm{E}-03$

$1.60930 \mathrm{E}-03$

$1.61914 \mathrm{E}-03$

$1.62902 \mathrm{E}-03$

$1.63893 \mathrm{E}-03$

$1.64887 \mathrm{E}-03$

$1.65884 \mathrm{E}-03$

$1.66883 \mathrm{E}-03$

$1.67886 \mathrm{E}-03$

$1.68892 \mathrm{E}-03$

$1.69901 \mathrm{E}-03$

$1.70913 \mathrm{E}-03$

$1.71928 \mathrm{E}-03$

$1.72945 \mathrm{E}-03$

$1.73966 \mathrm{E}-03$

$1.74990 \mathrm{E}-03$

$1.76017 \mathrm{E}-03$

$1.77047 \mathrm{E}-03$

$1.78079 \mathrm{E}-03$

$1.79115 \mathrm{E}-03$

$1.80154 \mathrm{E}-03$

$1.81196 \mathrm{E}-03$

$1.82241 \mathrm{E}-03$

$1.83289 \mathrm{E}-03$

$1.84339 \mathrm{E}-03$

$1.85393 \mathrm{E}-03$

$1.86450 \mathrm{E}-03$

$1.87510 \mathrm{E}-03$

$1.88573 \mathrm{E}-03$

$1.89639 \mathrm{E}-03$

$1.90707 \mathrm{E}-03$

$1.91779 \mathrm{E}-03$

$1.92854 \mathrm{E}-03$

$1.93932 \mathrm{E}-03$

$1.95013 \mathrm{E}-03$ $g_{2}$

$\tan \delta_{2}$

$f_{-2}$

$2.18867 \mathrm{E} \quad 00$ $2.20332 \mathrm{E} \quad 00$ $2.21802 \mathrm{E} \quad 00$ $2.23277 \mathrm{E} \quad 00$ $2.24757 \mathrm{E} \quad 00$

$2.26241 \mathrm{E} \quad 00$ $2.27731 \mathrm{E} \quad 00$ $2.29225 \mathrm{E} \quad 00$ $2.30724 \mathrm{E} \quad 00$ $2.32228 \mathrm{E} \quad 00$

$2.33737 \mathrm{E} \quad 00$ $2.35250 \mathrm{E} \quad 00$ $2.36769 \mathrm{E} \quad 00$ $2.38292 \mathrm{E} \quad 00$ $2.39820 \mathrm{E} \quad 00$

$2.41353 \mathrm{E} \quad 00$ $2.42891 \mathrm{E} \quad 00$ $2.44433 \mathrm{E} \quad 00$ $2.45980 \mathrm{E} \quad 00$ $2.47532 \mathrm{E} \quad 00$

$2.49090 \mathrm{E} \quad 00$ $2.50651 \mathrm{E} \quad 00$ $2.52218 \mathrm{E} \quad 00$ $2.53789 \mathrm{E} \quad 00$ $2.55366 \mathrm{E} \quad 00$

$2.56947 \mathrm{E} \quad 00$ $2.58533 \mathrm{E} \quad 00$ $2.60123 \mathrm{E} \quad 00$ $2.61719 \mathrm{E} \quad 00$ $2.63319 \mathrm{E} \quad 00$

$2.64924 \mathrm{E} \quad 00$ $2.66534 \mathrm{E} \quad 00$ $2.68149 \mathrm{E} \quad 00$ $2.69769 \mathrm{E} \quad 00$ $2.71393 \mathrm{E} \quad 00$

$2.73022 \mathrm{E} \quad 00$ $2.74656 \mathrm{E} \quad 00$ $2.76295 \mathrm{E} \quad 00$ $2.77939 \mathrm{E} \quad 00$ $2.79587 \mathrm{E} \quad 00$

$2.81241 \mathrm{E} \quad 00$ $2.82899 \mathrm{E} \quad 00$ $2.84561 \mathrm{E} \quad 00$ $2.86229 \mathrm{E} \quad 00$ $2.87902 \mathrm{E} \quad 00$

$2.89579 \mathrm{E} \quad 00$ $2.91261 \mathrm{E} \quad 00$ $2.92948 \mathrm{E} \quad 00$ $2.94639 \mathrm{E} \quad 00$ $2.96336 \mathrm{E} \quad 00$

$2.98037 \mathrm{E} \quad 00$ $2.99743 \mathrm{E} \quad 00$ $3.01454 \mathrm{E} \quad 00$ $3.03170 \mathrm{E} \quad 00$ $3.04890 \mathrm{E} \quad 00$

$3.06615 \mathrm{E} \quad 00$ $3.08345 \mathrm{E} \quad 00$ $3.10080 \mathrm{E} \quad 00$ $3.11820 \mathrm{E} \quad 00$ $3.13564 \mathrm{E} \quad 00$
$7.81353 \mathrm{E}-02$

$7.90180 \mathrm{E}-02$

$7.99072 \mathrm{E}-02$

$8.08029 \mathrm{E}-02$

$8.17053 \mathrm{E}-02$

$8.26143 \mathrm{E}-02$

$8.35298 \mathrm{E}-02$

$8.44521 \mathrm{E}-02$

$8.53810 \mathrm{E}-02$

$8.63166 \mathrm{E}-02$

$8.72590 \mathrm{E}-02$

$8.82080 \mathrm{E}-02$

$8.91639 \mathrm{E}-02$

$9.01265 \mathrm{E}-02$

$9.10960 \mathrm{E}-02$

$9.20723 \mathrm{E}-02$

$9.30555 \mathrm{E}-02$

$9.40455 \mathrm{E}-02$

$9.50425 \mathrm{E}-02$

$9.60464 \mathrm{E}-02$

9.70573 E-02

$9.80751 \mathrm{E}-02$

$9.91000 \mathrm{E}-02$

$1.00132 \mathrm{E}-01$

$1.01171 \mathrm{E}-01$

$1.02217 \mathrm{E}-01$

$1.03270 \mathrm{E}-01$

$1.04330 \mathrm{E}-01$

$1.05398 \mathrm{E}-01$

$1.06472 \mathrm{E}-01$

$1.07554 \mathrm{E}-01$

$1.08643 \mathrm{E}-01$

$1.09739 \mathrm{E}-01$

$1.10843 \mathrm{E}-01$

$1.11953 \mathrm{E}-01$

$1.13071 \mathrm{E}-01$

$1.14197 \mathrm{E}-01$

$1.15330 \mathrm{E}-01$

$1.16470 \mathrm{E}-01$

$1.17617 \mathrm{E}-01$

$1.18772 \mathrm{E}-01$ $1.19935 \mathrm{E}-01$

$1.21105 \mathrm{E}-01$

$1.22282 \mathrm{E}-01$

$1.23467 \mathrm{E}-01$

$1.24660 \mathrm{E}-01$

$1.25860 \mathrm{E}-01$

$1.27067 \mathrm{E}-01$

$1.28283 \mathrm{E}-01$

$1.29505 \mathrm{E}-01$

$1.30736 \mathrm{E}-01$

$1.31974 \mathrm{E}-01$

$1.33220 \mathrm{E}-01$

$1.34474 \mathrm{E}-01$

$1.35735 \mathrm{E}-01$

$1.37005 \mathrm{E}-01$ $1.38282 \mathrm{E}-01$ 1.39567 E-01 $1.40859 \mathrm{E}-01$

$1.42160 \mathrm{E}-01$
$-2.15227 \mathrm{E} \quad 01 \quad-7.34003 \mathrm{E}-02$ $-2.15236 \mathrm{E}$ 01 $-7.42552 \mathrm{E}-02$ $-2.15245 \mathrm{E} \quad 01 \quad-7.51166 \mathrm{E}-02$ $-2.15254 \mathrm{E}$ ol $-7.59845 \mathrm{E}-02$ $-2.15263 \mathrm{E} \quad 01 \quad-7.68588 \mathrm{E}-02$

$-2.15272 \mathrm{E} \quad 01$ $-2.15280 \mathrm{E} \quad 01$ $-2.15289 \mathrm{E} \quad 01$ $-2.15298 \mathrm{E} 01$ $-2.15306 \mathrm{E}$ 01

$-2.15315 \mathrm{E} \quad 01$ $-2.15323 \mathrm{E} \quad 01$ $-2.15332 \mathrm{E} \quad 01$ $-2.15340 \mathrm{E} \quad 01$ $-2.15349 \mathrm{E} 01$

$-7.77397 \mathrm{E}-02$ $-7.86271 \mathrm{E}-02$ $-7.95211 \mathrm{E}-02$ $-8.04218 \mathrm{E}-02$ $-8.13290 \mathrm{E}-02$

$-8.22429 \mathrm{E}-02$ $-8.31634 \mathrm{E}-02$ $-8.40906 \mathrm{E}-02$

$-8.50246 \mathrm{E}-02$

$-8.59653 \mathrm{E}-02$

$\begin{array}{lll}-2.15357 \mathrm{E} & 01\end{array}$ $-2.15365 \mathrm{E} 01$ $-2.15373 \mathrm{E} \quad 01$ $-2.15382 \mathrm{E} 01$ $-2.15390 \mathrm{E} 01$

$-8.69127 \mathrm{E}-02$ $-8.78669 \mathrm{E}-02$ $-8.88280 \mathrm{E}-02$ $-8.97959 \mathrm{E}-02$ $-9.07706 \mathrm{E}-02$

$-2.15398 \mathrm{E} \quad 01$ $-2.15406 \mathrm{E} \quad 01$ $-2.15413 \mathrm{E}$ 01 $-2.15421 \mathrm{E} 01$ $-2.15429 \mathrm{E} 01$

$-2.15437 \mathrm{E} \quad 01$ $-2.15445 \mathrm{E} \quad 01$ $-2.15452 \mathrm{E} \quad 01$ $-2.15460 \mathrm{E} \quad 01$

$-2.15468 \mathrm{E} 01$

$-2.15475 \mathrm{E} 01$

$-2.15483 \mathrm{E} \quad 0 \mathrm{l}$

$-2.15490 \mathrm{E} \quad 01$

$-2.15497 \mathrm{E} \quad 01$

$-2.15505 \mathrm{E} 01$

$-2.15512 \mathrm{E} \quad 01$ $-2.15519 \mathrm{E} \quad 01$ $-2.15526 \mathrm{E}$ ol $-2.15534 \mathrm{E}$ ol

-2.15541 E 01

$-2.15548 \mathrm{E} \quad 01$ $-2.15555 \mathrm{E} \quad 01$ $-2.15569 \mathrm{E} \quad 01$ $-2.15576 \mathrm{E} \quad 01$

$-2.15583 \mathrm{E}$ 01 $-2.15589 \mathrm{E} \quad 01$

$-2.15596 \mathrm{E} \quad 01$

$-2.15603 \mathrm{E}$ 01

$-2.15610 \mathrm{E} 01$

$-2.15616 \mathrm{E} 01$ $-2.15629 \mathrm{E}$ 01 $-2.15636 \mathrm{E} 01$ $-2.15642 \mathrm{E} 01$

$-2.15649 \mathrm{E} \quad 01$ $-2.15655 \mathrm{E} \quad 01$ $-2.15662 \mathrm{E} \quad 01$ $-2.15668 \mathrm{E} \quad 01$ $-2.15674 \mathrm{E} \quad 01$

$-9.17522 \mathrm{E}-02$ $-9.27408 \mathrm{E}-02$ $-9.37362 \mathrm{E}-02$ $-9.47386 \mathrm{E}-02$ $-9.57480 \mathrm{E}-02$

$-9.67644 \mathrm{E}-02$ $-9.77878 \mathrm{E}-02$ $-9.88182 \mathrm{E}-02$ $-9.98557 \mathrm{E}-02$

$-1.00900 \mathrm{E}-01$

$-1.01952 \mathrm{E}-01$ $-1.03011 \mathrm{E}-\mathrm{0l}$ $-1.04077 \mathrm{E}-01$ $-1.05150 \mathrm{E}-01$ $-1.06231 \mathrm{E}-01$

$-1.07318 \mathrm{E}-01$ $-1.08413 \mathrm{E}-01$ $-1.09515 \mathrm{E}-01$ $-1.10625 \mathrm{E}-01$ $-1.11742 \mathrm{E}-01$

$-1.12866 \mathrm{E}-01$ $-1.13997 \mathrm{E}-01$ $-1.15136 \mathrm{E}-01$ $-1.16283 \mathrm{E}-01$ $-1.17437 \mathrm{E}-01$

$-1.18598 \mathrm{E}-01$ $-1.19767 \mathrm{E}-01$ $-2.15562 \mathrm{E} \quad 01$ $-2.15623 \mathrm{E} 01$ 
ELECTRON RADIAL FUNCTIONS

Positron

p $\quad F_{0}$

36.10

36.20

36.30

36.40

36.50

36.60

36.70

36.80

36.90

37.00

37.10

37.20

37.30

37.40

37.50

37.60

37.70

37.80

37.90

38.00

38.10

38.20

38.30

38.40

38.50

38.60

38.70

38.80

38.90

39.00

39.10

39.20

39.30

39.40

39.50

39.60

39.70

39.80

39.90

40.00

40.10

40.20

40.30

40.40

40.50

40.60

40.70

40.80

40.90

41.00

41.10

41.20

41.30

41.40

41.50

41.60

41.70

41.80

41.90

42.00
$F_{0}$

$8.18105 \mathrm{E}-0$ $8.18095 \mathrm{E}-0$ $8.18086 \mathrm{E}-01$ $8.18077 \mathrm{E}-01$ $8.18067 \mathrm{E}-01$

$8.18058 \mathrm{E}-01$ $8.18049 \mathrm{E}-01$ $8.18040 \mathrm{E}-01$ $8.18030 \mathrm{E}-01$ 8.18021 E-01

$8.18012 \mathrm{E}-01$ $8.18003 \mathrm{E}-01$ $8.17994 \mathrm{E}-01$ $8.17984 \mathrm{E}-01$ $8.17975 \mathrm{E}-01$

8.17966 E-01 $8.17957 \mathrm{E}-0$ $8.17948 \mathrm{E}-01$ $8.17938 \mathrm{E}-0$ $8.17930 \mathrm{E}-0$

8.17921 E-01 $8.17912 \mathrm{E}-01$ $8.17903 \mathrm{E}-01$ $8.17894 \mathrm{E}-01$ $8.17885 \mathrm{E}-01$

$8.17876 \mathrm{E}-01$ $8.17867 \mathrm{E}-01$ $8.17858 \mathrm{E}-01$ $8.17850 \mathrm{E}-0$ $8.17841 \mathrm{E}-01$

$8.17832 \mathrm{E}-0$ $8.17823 \mathrm{E}-0$ $8.17815 \mathrm{E}-01$ $8.17806 \mathrm{E}-01$ 8.17797E-01

$8.17789 \mathrm{E}-01$ $8.17780 \mathrm{E}-01$ $8.17772 \mathrm{E}-01$ $8.17763 \mathrm{E}-01$ $8.17755 \mathrm{E}-01$

$8.17746 \mathrm{E}-01$ $8.17738 \mathrm{E}-0$ $8.17729 \mathrm{E}-01$ 8.17721 E-0 ㅓㅏ $8.17712 \mathrm{E}-01$

$8.17704 \mathrm{E}-01$ $8.17695 \mathrm{E}-01$ $8.17687 \mathrm{E}-0$ $8.17679 \mathrm{E}-01$ $8.17670 \mathrm{E}-01$

$8.17662 \mathrm{E}-01$ $8.17654 \mathrm{E}-01$ $8.17645 \mathrm{E}-01$ $8.17637 \mathrm{E}-01$ $8.17629 \mathrm{E}-01$

8.17621 E- 01 $8.17612 \mathrm{E}-01$ 8.17604 E-01 $8.17596 \mathrm{E}-01$ $8.17588 \mathrm{E}-01$

$Z=9 \quad A=18$ $\rho=1.2 A^{1 / 3} F$

$f_{1}$

$3.34366 \mathrm{E} \quad 01$ $3.35361 \mathrm{E} \quad 01$ $3.36356 \mathrm{E}$ 01 $3.37352 \mathrm{E}$ 01 $3.38348 \mathrm{E} \quad 01$

$3.39344 \mathrm{E} \quad 01$ $3.40341 \mathrm{E} \quad 01$ $3.41339 \mathrm{E} \quad 01$ $3.42336 \mathrm{E} \quad 01$ $3.43335 \mathrm{E} \quad 01$

$3.44334 \mathrm{E} \quad 01$ $3.45333 \mathrm{E} \quad 01$ $3.46333 \mathrm{E} \quad 01$ $3.47333 \mathrm{E} 01$ $3.48333 \mathrm{E} \quad 01$

$3.49335 \mathrm{E} \quad 01$ $3.50336 \mathrm{E} \quad 01$ $3.51338 \mathrm{E} \quad 01$ $3.52341 \mathrm{E} \quad 01$

$3.53344 \mathrm{E} 01$

$3.54347 \mathrm{E} \quad 01$ $3.55351 \mathrm{E} \quad 01$ $3.56356 \mathrm{E}$ 01 $3.57361 \mathrm{E} 01$ $3.58366 \mathrm{E} \quad 01$

$3.59372 \mathrm{E} \quad 01$ $3.60378 \mathrm{E}$ 01 $3.61385 \mathrm{E} 01$ $3.62393 \mathrm{E} \quad 01$

$3.63400 \mathrm{E} \quad 01$

$3.64409 \mathrm{E} \quad 01$ $3.65418 \mathrm{E}$ ol $3.66427 \mathrm{E} 01$ $3.67437 \mathrm{E} 01$ $3.68447 \mathrm{E} \quad 01$

$3.69458 \mathrm{E} \quad 01$ $3.70469 \mathrm{E} \quad 01$ $3.71481 \mathrm{E} \quad 01$ $3.72493 \mathrm{E} \quad 01$ $3.73506 \mathrm{E} \quad 01$

$3.74520 \mathrm{E} \quad 01$ $3.75534 \mathrm{E}$ 01 $3.76548 \mathrm{E}$ 01 $3.77563 \mathrm{E} \quad 01$ $3.78578 \mathrm{E} \quad 01$

$3.79594 \mathrm{E}$ 01 $3.80611 \mathrm{E} \quad 01$ $3.81627 \mathrm{E} \quad 01$ $3.82645 \mathrm{E} \quad 01$ $3.83663 \mathrm{E} \quad 01$

$3.84681 \mathrm{E} \quad 01$ $3.85700 \mathrm{E} 01$ $3.86720 \mathrm{E} 01$ $3.87740 \mathrm{E}$ ol $3.88760 \mathrm{E} \quad 01$

$3.89782 \mathrm{E} \quad 01$ $3.90803 \mathrm{E} \quad 01$ $3.91825 \mathrm{E} 01$ $3.92848 \mathrm{E} \quad 01$ $3.93871 \mathrm{E} \quad 01$ $\tan \delta_{1}$

$g_{1}$

$2.49793 \mathrm{E} \quad 00 \quad-4.08539 \mathrm{E}-04$

$2.51455 \mathrm{E} \quad 00 \quad-4.08457 \mathrm{E}-04$

$2.53122 \mathrm{E} \quad 00 \quad-4.08361 \mathrm{E}-04$

$2.54795 \mathrm{E} \quad 00 \quad-4.08237 \mathrm{E}-04$

$2.56475 \mathrm{E} \quad 00 \quad-4.08099 \mathrm{E}-04$

$2.58160 \mathrm{E} \quad 00$

$2.59851 \mathrm{E} \quad 00$

$2.61548 \mathrm{E} \quad 00$

$2.63251 \mathrm{E} \quad 00$

$2.64959 \mathrm{E} \quad 00$

$2.66674 \mathrm{E} \quad 00$

$2.68394 \mathrm{E} \quad 00$

$2.70121 \mathrm{E} \quad 00$

$2.71853 \mathrm{E} \quad 00$

$2.73591 \mathrm{E} \quad 00$

$2.75336 \mathrm{E} \quad 00$

$2.77086 \mathrm{E} \quad 00$

$2.78842 \mathrm{E} \quad 00$

$2.80604 \mathrm{E} \quad 00$

$2.82372 \mathrm{E} \quad 00$

$2.84146 \mathrm{E} \quad 00$

$2.85926 \mathrm{E} \quad 00$

$2.87712 \mathrm{E} \quad 00$

$2.89503 \mathrm{E} \quad 00$

$2.91301 \mathrm{E} \quad 00$

$2.93105 \mathrm{E} \quad 00$

$2.94915 \mathrm{E} \quad 00$

$2.96731 \mathrm{E} \quad 00$

$2.98553 \mathrm{E} \quad 00$

$3.00380 \mathrm{E} \quad 00$

$3.02214 \mathrm{E} \quad 00$

$3.04054 \mathrm{E} \quad 00$

$3.05900 \mathrm{E} \quad 00$

$3.07752 \mathrm{E} \quad 00$

$3.09610 \mathrm{E} \quad 00$

$3.11474 \mathrm{E} \quad 00$

$3.13344 \mathrm{E} \quad 00$

$3.15220 \mathrm{E} \quad 00$

$3.17103 \mathrm{E} \quad 00$

$3.18991 \mathrm{E} \quad 00$

$3.20886 \mathrm{E} \quad 00$

$3.22786 \mathrm{E} \quad 00$

$3.24693 \mathrm{E} \quad 00$

$3.26605 \mathrm{E} \quad 00$

$3.28524 \mathrm{E} \quad 00$

$3.30449 \mathrm{E} \quad 00$

$3.32380 \mathrm{E} \quad 00$

$3.34317 \mathrm{E} \quad 00$

$3.36261 \mathrm{E} \quad 00$

$3.38210 \mathrm{E} \quad 00$

$3.40166 \mathrm{E} \quad 00$

$3.42128 \mathrm{E} \quad 00$

$3.44096 \mathrm{E} \quad 00$

$3.46070 \mathrm{E} \quad 00$

$3.48050 \mathrm{E} \quad 00$

$3.50036 \mathrm{E} \quad 00$

$3.52029 \mathrm{E} \quad 00$

$3.54028 \mathrm{E} \quad 00$

$3.56033 \mathrm{E} \quad 00$

$3.58044 \mathrm{E} \quad 00$

$-4.07932 \mathrm{E}-04$ $-4.07752 \mathrm{E}-04$ $-4.07541 \mathrm{E}-04$ $-4.07318 \mathrm{E}-04$ $-4.07068 \mathrm{E}-04$

$-4.06804 \mathrm{E}-04$ $-4.06523 \mathrm{E}-04$ $-4.06221 \mathrm{E}-04$ $-4.05899 \mathrm{E}-04$ $-4.05540 \mathrm{E}-04$

$-4.05178 \mathrm{E}-04$ $-4.04798 \mathrm{E}-04$ $-4.04393 \mathrm{E}-04$ $-4.03983 \mathrm{E}-04$ $-4.03529 \mathrm{E}-04$

$-4.03069 \mathrm{E}-04$ $-4.02586 \mathrm{E}-04$ $-4.02092 \mathrm{E}-04$ $-4.01576 \mathrm{E}-04$ $-4.01032 \mathrm{E}-04$

$-4.00473 \mathrm{E}-04$ $-3.99907 \mathrm{E}-04$ $-3.99307 \mathrm{E}-04$ $-3.98701 \mathrm{E}-04$

$-3.98068 \mathrm{E}-04$

$-3.97421 \mathrm{E}-04$ $-3.96749 \mathrm{E}-04$ $-3.96070 \mathrm{E}-04$ $-3.95373 \mathrm{E}-04$ $-3.94640 \mathrm{E}-04$

$-3.93910 \mathrm{E}-04$ $-3.93155 \mathrm{E}-04$ $-3.92379 \mathrm{E}-04$ $-3.91586 \mathrm{E}-04$ $-3.90782 \mathrm{E}-04$

$-3.89951 \mathrm{E}-04$ $-3.89115 \mathrm{E}-04$ $-3.88256 \mathrm{E}-04$ $-3.87370 \mathrm{E}-04$ $-3.86474 \mathrm{E}-04$ 
ELECTRON RADIAL FUNCTIONS

$$
Z=9 \quad A=18 \quad \rho=1.2 A^{1 / 3} F
$$

$\bar{F}$

$f_{2}$

$1.96097 \mathrm{E}-03$

$1.97183 \mathrm{E}-03$

$1.98273 \mathrm{E}-03$

$1.99366 \mathrm{E}-03$

$2.00462 \mathrm{E}-03$

$2.01561 \mathrm{E}-03$

$2.02662 \mathrm{E}-03$

$2.03767 \mathrm{E}-03$

$2.04875 \mathrm{E}-03$

$2.05986 \mathrm{E}-03$

$2.07100 \mathrm{E}-03$

$2.08217 \mathrm{E}-03$

$2.09336 \mathrm{E}-03$

$2.10459 \mathrm{E}-03$

$2.11585 \mathrm{E}-03$

$2.12714 \mathrm{E}-03$

$2.13846 \mathrm{E}-03$

$2.14981 \mathrm{E}-03$

$2.16118 \mathrm{E}-03$

$2.17259 \mathrm{E}-03$

$2.18403 \mathrm{E}-03$

$2.19550 \mathrm{E}-03$

$2.20700 \mathrm{E}-03$

$2.21852 \mathrm{E}-03$

$2.23008 \mathrm{E}-03$

$2.24167 \mathrm{E}-03$

$2.25329 \mathrm{E}-03$

$2.26494 \mathrm{E}-03$

$2.27662 \mathrm{E}-03$

$2.28832 \mathrm{E}-03$

$2.30006 \mathrm{E}-03$

$2.31183 \mathrm{E}-03$

$2.32363 \mathrm{E}-03$

$2.33546 \mathrm{E}-03$

2.34731 E-03

$2.35920 \mathrm{E}-03$

$2.37112 \mathrm{E}-03$

$2.38307 \mathrm{E}-03$

$2.39505 \mathrm{E}-03$

$2.40705 \mathrm{E}-03$

$2.41909 \mathrm{E}-03$

$2.43116 \mathrm{E}-03$

$2.44326 \mathrm{E}-03$

$2.45539 \mathrm{E}-03$

$2.46755 \mathrm{E}-03$

$2.47973 \mathrm{E}-03$

2.49195 E-03

2.50420 E-03

$2.51648 \mathrm{E}-03$

$2.52878 \mathrm{E}-03$

$2.54112 \mathrm{E}-03$

$2.55349 \mathrm{E}-03$

$2.56589 \mathrm{E}-03$

$2.57832 \mathrm{E}-03$

$2.59078 \mathrm{E}-03$

$2.60326 \mathrm{E}-03$

$2.61578 \mathrm{E}-03$

$2.62833 \mathrm{E}-03$

$2.64091 \mathrm{E}-03$

$2.65351 \mathrm{E}-03$ $g_{2}$

$\tan \delta_{2}$

$f_{-2}$

$3.15313 \mathrm{E} \quad 00$

$3.17067 \mathrm{E} \quad 00$

$3.18826 \mathrm{E} \quad 00$

$3.20589 \mathrm{E} \quad 00$

$3.22358 \mathrm{E} \quad 00$

$3.24131 \mathrm{E} \quad 00$

$3.25909 \mathrm{E} \quad 00$

$3.27691 \mathrm{E} \quad 00$

$3.29478 \mathrm{E} \quad 00$

$3.31271 \mathrm{E} \quad 00$

$3.33068 \mathrm{E} \quad 00$

$3.34869 \mathrm{E} \quad 00$

$3.36676 \mathrm{E} \quad 00$

$3.38487 \mathrm{E} \quad 00$

$3.40303 \mathrm{E} \quad 00$

$3.42124 \mathrm{E} \quad 00$

$3.43950 \mathrm{E} \quad 00$

$3.45780 \mathrm{E} \quad 00$

$3.47615 \mathrm{E} \quad 00$

$3.49455 \mathrm{E} \quad 00$

$3.51300 \mathrm{E} \quad 00$

$3.53149 \mathrm{E} \quad 00$

$3.55003 \mathrm{E} \quad 00$

$3.56862 \mathrm{E} \quad 00$

$3.58726 \mathrm{E} \quad 00$

$3.60594 \mathrm{E} \quad 00$

$3.62468 \mathrm{E} \quad 00$

$3.64346 \mathrm{E} \quad 00$

$3.66228 \mathrm{E} \quad 00$

$3.68116 \mathrm{E} \quad 00$

$3.70008 \mathrm{E} \quad 00$

$3.71905 \mathrm{E} \quad 00$

$3.73807 \mathrm{E} \quad 00$

$3.75714 \mathrm{E} \quad 00$

$3.77625 \mathrm{E} \quad 00$

$3.79541 \mathrm{E} \quad 00$

$3.81462 \mathrm{E} \quad 00$

$3.83387 \mathrm{E} \quad 00$

$3.85318 \mathrm{E} \quad 00$

$3.87253 \mathrm{E} \quad 00$

$3.89192 \mathrm{E} \quad 00$

$3.91137 \mathrm{E} \quad 00$

$3.93086 \mathrm{E} \quad 00$

$3.95040 \mathrm{E} \quad 00$

$3.96999 \mathrm{E} \quad 00$

$3.98963 \mathrm{E} \quad 00$ $4.00931 \mathrm{E} \quad 00$

$4.02904 \mathrm{E} \quad 00$

$4.04881 \mathrm{E} \quad 00$

$4.06864 \mathrm{E} \quad 00$

$4.08851 \mathrm{E} \quad 00$

$4.10843 \mathrm{E} \quad 00$

$4.1284 .0 \mathrm{E} \quad 00$

4.1484.1 E 00

$4.1684 .7 \mathrm{E} \quad 00$

$4.18858 \mathrm{E} \quad 00$

4.20874.E 00

$4.22894 \mathrm{E} \quad 00$

$4.24919 \mathrm{E} \quad 00$

$4.26949 \mathrm{E} \quad 00$
$1.43469 \mathrm{E}-01$

$1.44785 \mathrm{E}-01$

$1.46109 \mathrm{E}-01$

$1.47442 \mathrm{E}-01$

$1.48782 \mathrm{E}-01$

$1.50130 \mathrm{E}-01$

$1.51487 \mathrm{E}-01$

$1.52851 \mathrm{E}-01$

$1.54224 \mathrm{E}-01$

$1.55604 \mathrm{E}-01$

$1.56993 \mathrm{E}-01$

$1.58390 \mathrm{E}-01$

$1.59795 \mathrm{E}-01$

$1.61209 \mathrm{E}-01$

$1.62630 \mathrm{E}-01$

$1.64060 \mathrm{E}-01$

$1.65498 \mathrm{E}-01$

$1.66944 \mathrm{E}-01$

$1.68399 \mathrm{E}-01$

$1.69862 \mathrm{E}-01$

$1.71333 \mathrm{E}-01$

$1.72813 \mathrm{E}-01$

$1.74301 \mathrm{E}-01$

$1.75798 \mathrm{E}-01$

$1.77303 \mathrm{E}-01$

$1.78816 \mathrm{E}-01$

$1.80338 \mathrm{E}-01$

$1.81869 \mathrm{E}-01$

$1.834 .08 \mathrm{E}-01$

$1.84956 \mathrm{E}-01$

$-2.15681 \mathrm{E} \quad 01$ $-2.15687 \mathrm{E} 01$

$-2.15693 \mathrm{E}$ 01

$-2.15699 \mathrm{E} 01$

$-2.15706 \mathrm{E} 01$

$-2.15712 \mathrm{E} 01$

$-2.15718 \mathrm{E} \quad 01$

$-2.15724 \mathrm{E} \quad 01$

$-2.15730 \mathrm{E} 01$

$-2.15736 \mathrm{E} 01$

$-2.15742 \mathrm{E} \quad 01$

$-2.15747 \mathrm{E}$ 01

$-2.15753 \mathrm{E} \quad 01$

$-2.15759 \mathrm{E} 01$

$-2.15765 \mathrm{E} 01$

$-2.15771 \mathrm{E} \quad 01$

$-2.15776 \mathrm{E} 01$

$-2.15782 \mathrm{E} \quad 0 \mathrm{l}$

$-2.15788 \mathrm{E} 01$

$-2.15793 \mathrm{E} 01$

$-2.15799 \mathrm{E} 01$

$-2.15805 \mathrm{E}$ 0l

$-2.15810 \mathrm{E}$ 01

$-2.15816 \mathrm{E} \quad 01$

-2.15821 E 01

$-2.15827 \mathrm{E} 01$

$-2.15832 \mathrm{E} \quad 01$

$-2.15838 \mathrm{E}$ 01

$-2.15843 \mathrm{E} \quad 01$

$-2.15848 \mathrm{E} 01$

$1.86512 \mathrm{E}-01$

$1.88077 \mathrm{E}-01$

$1.89651 \mathrm{E}-01$

$1.91233 \mathrm{E}-01$

$1.92824 \mathrm{E}-01$

$-2.15854 \mathrm{E} \quad 01$

$-2.15859 \mathrm{E} 01$

$-2.15864 \mathrm{E} 01$

$-2.15869 \mathrm{E}$ ol

$-2.15875 \mathrm{E} \quad 01$

$1.94423 \mathrm{E}-01$

$1.96032 \mathrm{E}-01$

$1.97649 \mathrm{E}-01$

$1.99275 \mathrm{E}-01$

$2.00910 \mathrm{E}-01$

$2.02553 \mathrm{E}-01$

$2.04205 \mathrm{E}-01$

$2.05867 \mathrm{E}-01$

$2.07537 \mathrm{E}-01$

$2.09216 \mathrm{E}-01$

$-2.15880 \mathrm{E} \quad 01$

$-2.15885 \mathrm{E} \quad 01$

$-2.15890 \mathrm{E} \quad 01$

$-2.15895 \mathrm{E} \quad 01$

$-2.15900 \mathrm{E} 01$

$-2.15905 \mathrm{E} 01$

$-2.15910 \mathrm{E} \quad 01$

$-2.15915 \mathrm{E} 01$

$-2.15920 \mathrm{E} \quad 01$

$-2.15925 \mathrm{E} 01$

2.10904 E-01

$2.12601 \mathrm{E}-01$

$2.14307 \mathrm{E}-01$

$2.16022 \mathrm{E}-01$

$2.17746 \mathrm{E}-01$

$-2.15930 \mathrm{E} \quad 01$

$-2.15935 \mathrm{E} \quad 01$

$-2.15940 \mathrm{E} 01$

$-2.15944 \mathrm{E} 01$

$-2.15949 \mathrm{E} 01$

$2.19479 \mathrm{E}-01$

$2.21221 \mathrm{E}-01$

$2.22973 \mathrm{E}-01$

$2.24733 \mathrm{E}-01$

2.26503 E-01

$-2.15954 \mathrm{E} \quad 01$

$-2.15959 \mathrm{E} 01$

$-2.15964 \mathrm{E} \quad 01$

$-2.15968 \mathrm{E} 01$

-2.15973 E 01

$2.28281 \mathrm{E}-01$

$-2.15978 \mathrm{E}$ 0l

$2.30069 \mathrm{E}-01$

$2.31867 \mathrm{E}-01$

$2.33673 \mathrm{E}-01$

$-2.15982 \mathrm{E} 0 \mathrm{l}$

$-2.15987 \mathrm{E} \quad 01$

$-2.15991 \mathrm{E} 01$

$-2.15996 \mathrm{E} 01$

\begin{abstract}
$-1.36929 \mathrm{E}-01$
$-1.38213 \mathrm{E}-01$

$-1.39505 \mathrm{E}-01$

$-1.40805 \mathrm{E}-01$

$-1.42113 \mathrm{E}-01$

$-1.43428 \mathrm{E}-01$

$-1.44752 \mathrm{E}-01$

$-1.46083 \mathrm{E}-01$

$-1.47423 \mathrm{E}-01$

$-1.48770 \mathrm{E}-01$
\end{abstract}

$-1.50126 \mathrm{E}-01$

$-1.51490 \mathrm{E}-01$

$-1.52861 \mathrm{E}-01$

$-1.54241 \mathrm{E}-01$

$-1.55629 \mathrm{E}-01$

$-1.57026 \mathrm{E}-01$

$-1.58430 \mathrm{E}-01$

$-1.59843 \mathrm{E}-01$

$-1.61264 \mathrm{E}-01$

$-1.62693 \mathrm{E}-01$

$-1.64130 \mathrm{E}-01$

$-1.65576 \mathrm{E}-01$

$-1.67030 \mathrm{E}-01$

$-1.68492 \mathrm{E}-01$

$-1.69963 \mathrm{E}-01$

$-1.71442 \mathrm{E}-01$

$-1.72930 \mathrm{E}-01$

$-1.74426 \mathrm{E}-01$

$-1.75931 \mathrm{E}-01$

-1.77444 E-01

$-1.78965 \mathrm{E}-01$

$-1.80496 \mathrm{E}-01$

$-1.82034 \mathrm{E}-01$

$-1.83582 \mathrm{E}-01$

$-1.85137 \mathrm{E}-01$

$-1.86702 \mathrm{E}-01$

$-1.88275 \mathrm{E}-01$

$-1.89857 \mathrm{E}-01$

$-1.91448 \mathrm{E}-01$

$-1.93047 \mathrm{E}-01$

$-1.94655 \mathrm{E}-01$

$-1.96272 \mathrm{E}-01$

$-1.97897 \mathrm{E}-01$ 
Positron

\begin{tabular}{|c|c|c|c|}
\hline$p$ & $F_{0}$ & $f_{1}$ & $g_{1}$ \\
\hline 0.10 & $4.85317 \mathrm{E}-02$ & $1.91966 \mathrm{E}-03$ & $-4.52327 \mathrm{E}-05$ \\
\hline 0.20 & $2.60625 \mathrm{E}-01$ & $1.51456 \mathrm{E}-02$ & $-3.56243 \mathrm{E}-04$ \\
\hline 0.30 & $4.22727 \mathrm{E}-01$ & $4.12275 \mathrm{E}-02$ & $-9.66906 \mathrm{E}-04$ \\
\hline 0.40 & $5.26941 \mathrm{E}-01$ & $7.89456 \mathrm{E}-02$ & $-1.84417 \mathrm{E}-03$ \\
\hline 0.50 & $5.95814 \mathrm{E}-01$ & $1.26749 \mathrm{E}-01$ & $-2.94622 \mathrm{E}-03$ \\
\hline 0.60 & $6.43278 \mathrm{E}-01$ & $1.83033 \mathrm{E}-01$ & $-4.22972 \mathrm{E}-03$ \\
\hline 0.70 & $6.77207 \mathrm{E}-01$ & $2.46291 \mathrm{E}-01$ & $-5.65379 \mathrm{E}-03$ \\
\hline 0.80 & $7.02198 \mathrm{E}-01$ & $3.15184 \mathrm{E}-01$ & $-7.18205 \bar{E}-03$ \\
\hline 0.90 & $7.21067 \mathrm{E}-01$ & $3.88574 \mathrm{E}-01$ & $-8.78358 \mathrm{E}-03$ \\
\hline 1.00 & $7.35613 \mathrm{E}-01$ & $4.65529 \mathrm{E}-01$ & $-1.04329 \mathrm{E}-02$ \\
\hline 1.10 & $7.47024 \mathrm{E}-01$ & $5.45294 \mathrm{E}-01$ & $-1.21094 \mathrm{E}-02$ \\
\hline 1.20 & $7.56114 \mathrm{E}-01$ & $6.27272 \mathrm{E}-01$ & $-1.37967 \mathrm{E}-02$ \\
\hline 1.30 & $7.63453 \mathrm{E}-01$ & $7.10992 \mathrm{E}-01$ & $-1.54818 \mathrm{E}-02$ \\
\hline 1.40 & $7.69447 \mathrm{E}-01$ & $7.96084 \mathrm{E}-01$ & $-1.71546 \mathrm{E}-02$ \\
\hline 1.50 & $7.74395 \mathrm{E}-0 \mathrm{l}$ & $8.82259 \mathrm{E}-01$ & $-1.88072 \mathrm{E}-02$ \\
\hline 1.60 & $7.78516 \mathrm{E}-01$ & $9.69293 \mathrm{E}-01$ & $-2.04333 \mathrm{E}-02$ \\
\hline 1.70 & $7.81980 \mathrm{E}-01$ & $1.05701 \mathrm{E} \quad 00$ & $-2.20280 \mathrm{E}-02$ \\
\hline 1.80 & $7.84911 \mathrm{E}-01$ & $1.14527 \mathrm{E} \quad 00$ & $-2.35874 \mathrm{E}-02$ \\
\hline 1.90 & $7.87409 \mathrm{E}-0 \mathrm{l}$ & $1.23396 \mathrm{E} \quad 00$ & $-2.51086 \mathrm{E}-02$ \\
\hline 2.00 & $7.89551 \mathrm{E}-0 \mathrm{l}$ & $1.32300 \mathrm{E} \quad 00$ & $-2.65891 \mathrm{E}-02$ \\
\hline 2.10 & $7.91399 \mathrm{E}-01$ & $1.41232 \mathrm{E} \quad 00$ & $-2.80271 \mathrm{E}-02$ \\
\hline 20 & $7.93001 \mathrm{E}-01$ & $1.50187 \mathrm{E} \quad 00$ & $-2.94209 \mathrm{E}-02$ \\
\hline 2.30 & $7.94396 \mathrm{E}-01$ & $1.59160 \mathrm{E} \quad 00$ & $-3.07693 \mathrm{E}-02$ \\
\hline 2.40 & $7.95616 \mathrm{E}-01$ & $1.68148 \mathrm{E} \quad 00$ & $-3.20715 \mathrm{E}-02$ \\
\hline 2.50 & $7.96688 \mathrm{E}-01$ & $1.77148 \mathrm{E} \quad 00$ & $-3.33265 \mathrm{E}-02$ \\
\hline 2.60 & $7.97632 \mathrm{E}-01$ & $1.86157 \mathrm{E} \quad 00$ & $-3.45337 \mathrm{E}-02$ \\
\hline 70 & $7.98467 \mathrm{E}-01$ & $1.95175 \mathrm{E} \quad 00$ & $-3.56927 \mathrm{E}-02$ \\
\hline 2.80 & $7.99208 \mathrm{E}-01$ & $2.04199 \mathrm{E}$ & $-3.68030 \mathrm{E}-02$ \\
\hline 2.90 & $7.99866 \mathrm{E}-01$ & $2.13228 \bar{E}$ & $-3.78642 \mathrm{E}-02$ \\
\hline 3.00 & $8.00453 \mathrm{E}-01$ & $2.22262 \mathrm{E}$ & $-3.88760 \mathrm{E}-02$ \\
\hline 3.10 & $8.00977 \mathrm{E}-01$ & $2.31299 \mathrm{E} \quad 00$ & $-3.98382 \mathrm{E}-02$ \\
\hline 3.20 & $8.01447 \mathrm{E}-01$ & $2.40338 \mathrm{E} \quad 00$ & $-4.07507 \mathrm{E}-02$ \\
\hline 3.30 & $8.01869 \mathrm{E}-01$ & $2.49381 \mathrm{E}$ & $-4.16132 \mathrm{E}-02$ \\
\hline 3.40 & $8.02247 \mathrm{E}-01$ & $2.58425 \mathrm{E}$ & $-4.24256 \mathrm{E}-02$ \\
\hline 50 & $8.02588 \mathrm{E}-0 \mathrm{l}$ & $2.67470 \mathrm{E}$ & $-4.31879 \mathrm{E}-02$ \\
\hline 3.60 & $8.02895 \mathrm{E}-01$ & $2.76517 \mathrm{E} \quad 00$ & $-4.38998 \mathrm{E}-02$ \\
\hline 3.70 & $8.03171 \mathrm{E}-01$ & $2.85565 \mathrm{E}$ & $-4.45614 \mathrm{E}-02$ \\
\hline 3.80 & $8.03421 \mathrm{E}-01$ & $2.94614 \mathrm{E} \quad 00$ & $-4.51725 \mathrm{E}-02$ \\
\hline 3.90 & $8.03647 \mathrm{E}-01$ & $3.03664 \mathrm{E}$ & $-4.57332 \mathrm{E}-02$ \\
\hline 4.00 & $8.03852 \mathrm{E}-01$ & $3.12714 \mathrm{E} \quad 00$ & $-4.62433 \mathrm{E}-02$ \\
\hline 4.10 & $8.04037 \mathrm{E}-01$ & $3.21764 \mathrm{E}$ & $-4.67029 \mathrm{E}-02$ \\
\hline 4.20 & $8.04204 \mathrm{E}-01$ & $3.30815 \mathrm{E}$ & $-4.71118 \mathrm{E}-02$ \\
\hline 4.30 & $8.04355 \mathrm{E}-01$ & $3.39867 \mathrm{E}$ & $-4.74702 \mathrm{E}-02$ \\
\hline 4.40 & $8.04492 \mathrm{E}-01$ & $3.48918 \mathrm{E}$ & $-4.77779 \mathrm{E}-02$ \\
\hline 4.50 & $8.04616 \mathrm{E}-01$ & $3.57971 \mathrm{E} \quad 00$ & $-4.80349 \mathrm{E}-02$ \\
\hline 4.60 & $8.04728 \mathrm{E}-01$ & $3.67023 \mathrm{E} \quad 00$ & $-4.82412 \mathrm{E}-02$ \\
\hline 4.70 & $8.04830 \mathrm{E}-0 \mathrm{l}$ & $3.76076 \mathrm{E} \quad 00$ & $-4.83968 \mathrm{E}-02$ \\
\hline 4.8 & $8.04920 \mathrm{E}-01$ & $3.85128 \mathrm{E} \quad 00$ & $-4.85017 \mathrm{E}-02$ \\
\hline 4.90 & $8.05003 \mathrm{E}-0 \mathrm{l}$ & $3.94181 \mathrm{E}$ & $-4.85558 \mathrm{E}-02$ \\
\hline 5.00 & $8.05077 \mathrm{E}-01$ & $4.03235 \mathrm{E} \quad 00$ & $-4.85593 \mathrm{E}-02$ \\
\hline 5.10 & $8.05143 \mathrm{E}-01$ & $4.12289 \mathrm{E} \quad 00$ & $-4.85119 \mathrm{E}-02$ \\
\hline & $8.05203 \mathrm{E}-01$ & $4.21343 \mathrm{E} \quad 00$ & $-4.84139 \mathrm{E}-02$ \\
\hline 5. & $8.05256 \mathrm{E}-01$ & $4.30397 \mathrm{E}$ & $-4.82650 \mathrm{E}-02$ \\
\hline & $8.05304 \mathrm{E}-01$ & $4.39452 \mathrm{E} \quad 00$ & $-4.80654 \mathrm{E}-02$ \\
\hline & $8.05346 \mathrm{E}-01$ & $4.48507 \mathrm{E} \quad 00$ & $-4.78150 \mathrm{E}-02$ \\
\hline 5.60 & $8.05383 \mathrm{E}-01$ & $4.57563 \mathrm{E}$ & $-4.75139 \mathrm{E}-02$ \\
\hline 7 & $8.05416 \mathrm{E}-01$ & $4.66619 \mathrm{E} \quad 00$ & $-4.71620 \mathrm{E}-02$ \\
\hline 5.8 & $8.05444 \mathrm{E}-01$ & $4.75675 \mathrm{E}$ & $-4.67592 \mathrm{E}-02$ \\
\hline & $8.05469 \mathrm{E}-01$ & $4.84732 \mathrm{E} \quad 00$ & $-4.63057 \mathrm{E}-02$ \\
\hline 6.00 & $8.05490 \mathrm{E}-01$ & $4.93790 \mathrm{E} \quad 00$ & $-4.58014 \mathrm{E}-02$ \\
\hline
\end{tabular}

$\tan \delta_{1}$

$$
\rho=1.2 A^{1 / 3} F
$$

$3.49651 \mathrm{E}-0$

$1.62072 \mathrm{E}-01$

$1.07265 \mathrm{E}-01$

$8.05706 \mathrm{E}-02$

$6.46534 \mathrm{E}-02$

$5.40361 \mathrm{E}-02$

$4.64258 \mathrm{E}-02$

$4.06903 \mathrm{E}-02$

$3.62049 \mathrm{E}-02$

$3.25965 \mathrm{E}-02$

$2.96276 \mathrm{E}-02$

$2.71402 \mathrm{E}-02$

$2.50247 \mathrm{E}-02$

$2.32027 \mathrm{E}-02$

$2.16165 \mathrm{E}-02$

$2.02227 \mathrm{E}-02$

$1.89881 \mathrm{E}-02$

$1.78867 \mathrm{E}-02$

$1.68979 \mathrm{E}-02$

$1.60052 \mathrm{E}-02$

$1.51951 \mathrm{E}-02$

$1.44567 \mathrm{E}-02$

$1.37808 \mathrm{E}-02$

$1.31598 \mathrm{E}-02$

$1.25872 \mathrm{E}-02$

$1.20575 \mathrm{E}-02$

$1.15662 \mathrm{E}-02$

$1.11091 \mathrm{E}-02$

$1.06829 \mathrm{E}-02$

$1.02844 \mathrm{E}-02$

$9.91110 \mathrm{E}-03$ $9.56063 \mathrm{E}-03$ $9.23097 \mathrm{E}-03$

$8.92033 \mathrm{E}-03$

$8.62709 \mathrm{E}-03$

$8.34985 \mathrm{E}-03$ $8.08731 \mathrm{E}-03$ $7.83837 \mathrm{E}-03$

$7.60198 \mathrm{E}-03$

$7.37720 \mathrm{E}-03$

$7.16322 \mathrm{E}-03$ $6.95928 \mathrm{E}-03$

$6.76469 \mathrm{E}-03$ $6.57880 \mathrm{E}-03$ $6.40107 \mathrm{E}-03$

$6.23097 \mathrm{E}-03$ $6.06801 \mathrm{E}-03$

$5.91176 \mathrm{E}-03$

$5.76181 \mathrm{E}-03$

$5.61779 \mathrm{E}-03$

$5.47937 \mathrm{E}-03$ $5.34621 \mathrm{E}-03$ $5.21802 \mathrm{E}-03$ $5.09454 \mathrm{E}-03$

$4.97552 \mathrm{E}-03$

$4.86071 \mathrm{E}-03$ $4.74990 \mathrm{E}-03$ $4.64287 \mathrm{E}-03$ $4.53947 \mathrm{E}-03$ 4.43948E-03 $f_{-1}$

$9.07512 \mathrm{E}-04$

$4.18488 \mathrm{E}-03$

$7.93011 \mathrm{E}-03$

$1.16792 \mathrm{E}-02$

$1.53254 \mathrm{E}-02$

$1.88338 \mathrm{E}-02$

$2.21934 \mathrm{E}-02$

$2.54043 \mathrm{E}-02$

$2.84720 \mathrm{E}-02$

$3.14045 \mathrm{E}-02$

$3.42106 \mathrm{E}-02$

$3.68993 \mathrm{E}-02$

$3.94788 \mathrm{E}-02$

$4.19567 \mathrm{E}-02$

$4.43396 \mathrm{E}-02$

$4.66334 \mathrm{E}-02$

$4.88429 \mathrm{E}-02$

$5.09727 \mathrm{E}-02$

$5.30263 \mathrm{E}-02$

$5.50070 \mathrm{E}-02$

$5.69175 \mathrm{E}-02$

$5.87601 \mathrm{E}-02$

$6.05369 \mathrm{E}-02$

$6.22496 \mathrm{E}-02$

$6.38998 \mathrm{E}-02$

$6.54887 \mathrm{E}-02$

$6.70175 \mathrm{E}-02$

$6.84872 \mathrm{E}-02$

$6.98986 \mathrm{E}-02$

$7.12525 \mathrm{E}-02$

$7.25496 \mathrm{E}-02$

$7.37904 \mathrm{E}-02$

$7.49756 \mathrm{E}-02$

$7.61054 \mathrm{E}-02$

$7.71805 \mathrm{E}-02$

$7.82010 \mathrm{E}-02$ $7.91674 \mathrm{E}-02$ $8.00799 \mathrm{E}-02$ 8.09388E-02 $8.17444 \mathrm{E}-02$

$8.24969 \mathrm{E}-02$ $8.31963 \mathrm{E}-02$ $8.38430 \mathrm{E}-02$ 8.44371 E-02 8.49786 E- 02

$8.54678 \mathrm{E}-02$ $8.59048 \mathrm{E}-02$ $8.62896 \mathrm{E}-02$ $8.66224 \mathrm{E}-02$ $8.69032 \mathrm{E}-02$

8.71322E-02 8.73093 E-02 $8.74347 \mathrm{E}-02$ $8.75085 \mathrm{E}-02$ $8.75306 \mathrm{E}-02$

$8.75012 \mathrm{E}-02$ $8.74202 \mathrm{E}-02$ 8.72878 E-02 $8.71039 \mathrm{E}-02$ 8.68686 E- 02 g-1

$3.10916 \mathrm{E}-02$

$1.43580 \mathrm{E}-01$

$2.72715 \mathrm{E}-01$

4.02932 E-01

5.30842 E-01

$6.55442 \mathrm{E}-01$

$7.76506 \mathrm{E}-01$

$8.94132 \mathrm{E}-01$

$1.00857 \mathrm{E} \quad 00$

$1.12014 \mathrm{E} \quad 00$

$1.22917 \mathrm{E} \quad 00$

$1.33597 \mathrm{E} \quad 00$

$1.44083 \mathrm{E} \quad 00$

$1.54400 \mathrm{E} \quad 00$

$1.64573 \mathrm{E} \quad 00$

$1.74619 \mathrm{E} \quad 00$

$1.84557 \mathrm{E} \quad 00$

$1.94399 \mathrm{E} \quad 00$

$2.04159 \mathrm{E} \quad 00$

$2.13847 \mathrm{E} \quad 00$

$2.23472 \mathrm{E} \quad 00$

$2.33042 \mathrm{E} \quad 00$

$2.42563 \mathrm{E} \quad 00$

$2.52041 \mathrm{E} \quad 00$

$2.61481 \mathrm{E} \quad 00$

2.70888 E $\quad 00$ $2.80264 \mathrm{E} \quad 00$

$2.89613 \mathrm{E} \quad 00$

$2.98939 \mathrm{E} \quad 00$

$3.08243 \mathrm{E} \quad 00$

$3.17527 \mathrm{E} \quad 00$

$3.26794 \mathrm{E} \quad 00$

$3.36045 \mathrm{E} \quad 00$

$3.45282 \mathrm{E} 00$

$3.54506 \mathrm{E} \quad 00$

$3.63719 \mathrm{E} \quad 00$ $3.72921 \mathrm{E} \quad 00$ $3.82113 \mathrm{E} \quad 00$

$3.91296 \mathrm{E} \quad 00$

$4.00471 \mathrm{E} \quad 00$

$4.09639 \mathrm{E} \quad 00$

$4.18801 \mathrm{E} \quad 00$

$4.27956 \mathrm{E} \quad 00$

$4.37106 \mathrm{E} \quad 00$

$4.46250 \mathrm{E} \quad 00$

$4.55390 \mathrm{E} \quad 00$

$4.64526 \mathrm{E} \quad 00$

$4.73657 \mathrm{E} \quad 00$

$4.82786 \mathrm{E} \quad 00$

$4.91911 \mathrm{E} \quad 00$

$5.01033 \mathrm{E} \quad 00$

$5.10153 \mathrm{E}$ 00

$5.19269 \mathrm{E} \quad 00$

$5.28384 \mathrm{E} \quad 00$

$\begin{array}{lll}5.37497 \mathrm{E} & 00\end{array}$

$5.46608 \mathrm{E} \quad 00$ $5.55718 \mathrm{E} \quad 00$ $5.64826 \mathrm{E} \quad 00$ $5.73933 \mathrm{E} \quad 00$ $5.83039 \mathrm{E} \quad 00$ $\tan \delta_{-1}$

$3.30233 \mathrm{E} \quad 00$ $5.22273 \mathrm{E} \quad 00$ $7.54600 \mathrm{E} \quad 00$ $9.96151 \mathrm{E} \quad 00$ $1.24173 \mathrm{E} \quad 01$

$1.48933 \mathrm{E} \quad 01$ $1.73784 \mathrm{E} \quad 01$ $1.98654 \mathrm{E} \quad 01$ $2.23492 \mathrm{E} \quad 01$ $2.48262 \mathrm{E} \quad 01$

$2.72935 \mathrm{E} \quad 01$ $2.97493 \mathrm{E} \quad 01$ $3.21919 \mathrm{E} 01$ $3.46202 \mathrm{E} 01$ $3.70334 \mathrm{E} \quad 01$

$3.94310 \mathrm{E} \quad 01$ $4.18124 \mathrm{E} \quad 01$ $4.41774 \mathrm{E} \quad 01$ $4.65257 \mathrm{E} \quad 01$ $4.88573 \mathrm{E} \quad 01$

$5.11720 \mathrm{E} \quad 01$ $5.34699 \mathrm{E} \quad 01$ $5.57511 \mathrm{E} \quad 01$ $5.80154 \mathrm{E} \quad 01$ $6.02631 \mathrm{E} \quad 01$

$6.24942 \mathrm{E} \quad 01$ $6.47089 \mathrm{E} \quad 01$ $6.69073 \mathrm{E} \quad 01$ $6.90895 \mathrm{E} 01$ $7.12557 \mathrm{E} \quad 01$

$7.34059 \mathrm{E} \quad 01$ $7.55405 \mathrm{E} \quad 01$ $7.76595 \mathrm{E} \quad 0$ $7.97631 \mathrm{E} \quad 01$ $8.18513 \mathrm{E} \quad 01$

$8.39246 \mathrm{E} \quad 01$ $8.59829 \mathrm{E} \quad 01$ $8.80265 \mathrm{E} \quad 01$ $9.00555 \mathrm{E} \quad 01$ $9.20701 \mathrm{E} 01$

$\begin{array}{lll}9.40704 \mathrm{E} & 01\end{array}$ $9.60567 \mathrm{E} \quad 01$ $9.80290 \mathrm{E} \quad 01$ $9.99875 \mathrm{E} \quad 01$ $1.01932 \mathrm{E} \quad 02$

$1.03864 \mathrm{E} \quad 02$ $1.05782 \mathrm{E} \quad 02$ $\begin{array}{lll}1.07687 \mathrm{E} & 02\end{array}$ $1.09580 \mathrm{E} \quad 02$ $1.11459 \mathrm{E} \quad 02$

$1.13326 \mathrm{E} \quad 02$ $1.15180 \mathrm{E} \quad 02$ $1.17021 \mathrm{E} \quad 02$ $1.18851 \mathrm{E} \quad 02$ $1.20668 \mathrm{E} \quad 02$

$1.22474 \mathrm{E} \quad 02$ $1.24268 \mathrm{E} \quad 02$ $1.26049 \mathrm{E} \quad 02$ $1.27820 \mathrm{E} \quad 02$ $1.29579 \mathrm{E} \quad 02$ 
ELECTRON RADIAL FUNCTIONS

$Z=10 \quad A=20 \quad \rho=1.2 A^{1 / 3} F$

Positron

$\bar{F}$

$0.10 \quad 1.44456 \mathrm{E}-09$

0.20

0.30

0.40

0.50

0.60

0.70

0.80

0.90

1.00

1.10

1.20

1.30

1.40

1.50

1.60

1.70

1.80

1.90

2.00

2.10

2.20

2.30

2.40

2.50

2.60

2.70

2.80

2.90

3.00

3.10

3.20

3.30

3.40

3.50

3.60

3.70

3.80

3.90

4.00

4.10

4.20

4.30

4.40

4.50

4.60

4.70

4.80

4.90

5.00

5.10

5.20

5.30

5.40

5.50

5.60

5.70

5.80

5.90

6.00

$2.29816 \mathrm{E}-08$

$7.84778 \mathrm{E}-08$

$1.69788 \mathrm{E}-07$

$2.96665 \mathrm{E}-07$

$4.58512 \mathrm{E}-07$

$6.54730 \mathrm{E}-07$

$8.84796 \mathrm{E}-07$

$1.14828 \mathrm{E}-06$

$1.44483 \mathrm{E}-06$

$1.77419 \mathrm{E}-06$

$2.13613 \mathrm{E}-06$

$2.53047 \mathrm{E}-06$

$2.95710 \mathrm{E}-06$

$3.41589 \mathrm{E}-06$

$3.90676 \mathrm{E}-06$

$4.42965 \mathrm{E}-06$

$4.98450 \mathrm{E}-06$

$5.57125 \mathrm{E}-06$

$6.18989 \mathrm{E}-06$

$6.84037 \mathrm{E}-06$

$8.23676 \mathrm{E}-06$

$8.98263 \mathrm{E}-06$

$9.76026 \mathrm{E}-06$

$1.05696 \mathrm{E}-05$

$1.14107 \mathrm{E}-05$

$1.22836 \mathrm{E}-05$

$1.31881 \mathrm{E}-05$

$1.41244 \mathrm{E}-05$

$1.50924 \mathrm{E}-05$

$1.60920 \mathrm{E}-05$

$1.71233 \mathrm{E}-05$

$1.81864 \mathrm{E}-05$

$1.92810 \mathrm{E}-05$

$2.04074 \mathrm{E}-05$

$2.15655 \mathrm{E}-05$

$2.27551 \mathrm{E}-05$

$2.39765 \mathrm{E}-05$

2.52295 E- 05

$2.65141 \mathrm{E}-05$

$2.78305 \mathrm{E}-05$

$2.91784 \mathrm{E}-05$

$3.05580 \mathrm{E}-05$

$3.19693 \mathrm{E}-05$

$3.34121 \mathrm{E}-05$

$3.48866 \mathrm{E}-05$

$3.63928 \mathrm{E}-05$

$3.79305 \mathrm{E}-05$

$3.95000 \mathrm{E}-05$

$4.11010 \mathrm{E}-05$ $4.27336 \mathrm{E}-05$ $4.43979 \mathrm{E}-05$ $4.60938 \mathrm{E}-05$

$4.78213 \mathrm{E}-05$

$4.95804 \mathrm{E}-05$

$5.13712 \mathrm{E}-05$

$5.31935 \mathrm{E}-05$

$5.50475 \mathrm{E}-05$

$5.69331 \mathrm{E}-05$ $f_{2}$

$7.52267 \mathrm{E}-06$ $g_{2}$

$\tan \delta_{2}$

$f_{-2}$

B -2

$5.68008 \mathrm{E}-07$

$8.58414 \mathrm{E}-06$

$3.47662 \mathrm{E}-05$

$8.85254 \mathrm{E}-05$

$1.77460 \mathrm{E}-04$

$3.07350 \mathrm{E}-04$

$4.82372 \mathrm{E}-04$

$7.05391 \mathrm{E}-04$

$9.78287 \mathrm{E}-04$

$1.30223 \mathrm{E}-03$

$1.67790 \mathrm{E}-03$

$2.10566 \mathrm{E}-03$

$2.58565 \mathrm{E}-03$

$3.11790 \mathrm{E}-03$

$3.70234 \mathrm{E}-03$

4.33888E-03

$5.02741 \mathrm{E}-03$

$5.76779 \mathrm{E}-03$

$6.55990 \mathrm{E}-03$

$7.40362 \mathrm{E}-03$

$8.29885 \mathrm{E}-03$

$9.24548 \mathrm{E}-03$

$1.02434 \mathrm{E}-02$

$1.12926 \mathrm{E}-02$

$1.23929 \mathrm{E}-02$

$1.35443 \mathrm{E}-02$

$1.47468 \mathrm{E}-02$

$1.60002 \mathrm{E}-02$

$1.73045 \mathrm{E}-02$

$1.86597 \mathrm{E}-02$

$2.00658 \mathrm{E}-02$

$2.15226 \mathrm{E}-02$

$2.30303 \mathrm{E}-02$

$2.45887 \mathrm{E}-02$

$2.61978 \mathrm{E}-02$

$2.78576 \mathrm{E}-02$

$2.95682 \mathrm{E}-02$

$3.13293 \mathrm{E}-02$

$3.31411 \mathrm{E}-02$

$3.50036 \mathrm{E}-02$

$3.69167 \mathrm{E}-02$

$3.88804 \mathrm{E}-02$

$4.08947 \mathrm{E}-02$

$4.29596 \mathrm{E}-02$

$4.50750 \mathrm{E}-02$

4.72410E-02

$4.94576 \mathrm{E}-02$

$5.17247 \mathrm{E}-02$

$5.40424 \mathrm{E}-02$

$5.64106 \mathrm{E}-02$

$5.88294 \mathrm{E}-02$

$6.12986 \mathrm{E}-02$

$6.38185 \mathrm{E}-02$

$6.63888 \mathrm{E}-02$

$6.90096 \mathrm{E}-02$

$7.16810 \mathrm{E}-02$

$7.44029 \mathrm{E}-02$

$7.71752 \mathrm{E}-02$

$7.99981 \mathrm{E}-02$

$8.28714 \mathrm{E}-02$
$-7.55422 \mathrm{E}-09$

$-1.13950 \mathrm{E}-07$

$-4.60081 \mathrm{E}-07$

$-1.16658 \mathrm{E}-06$

$-2.32626 \mathrm{E}-06$

$-4.00398 \mathrm{E}-06$

$-6.23972 \mathrm{E}-06$

$-9.05318 \mathrm{E}-06$

$-1.24487 \mathrm{E}-05$

$-1.64196 \mathrm{E}-05$

$-2.09513 \mathrm{E}-05$

$-2.60245 \mathrm{E}-05$

$-3.16161 \mathrm{E}-05$

$-3.77014 \mathrm{E}-05$

$-4.42541 \mathrm{E}-05$

$-5.12474 \mathrm{E}-05$

$-5.86541 \mathrm{E}-05$

$-6.64470 \mathrm{E}-05$

$-7.45985 \mathrm{E}-05$

$-8.30816 \mathrm{E}-05$

$-9.18689 \mathrm{E}-05$

$-1.00933 \mathrm{E}-04$

$-1.10248 \mathrm{E}-04$

$-1.19787 \mathrm{E}-04$

$-1.29522 \mathrm{E}-04$

$-1.39428 \mathrm{E}-04$

$-1.49478 \mathrm{E}-04$

$-1.59646 \mathrm{E}-04$

$-1.69904 \mathrm{E}-04$

$-1.80228 \mathrm{E}-04$

$-1.90592 \mathrm{E}-04$

$-2.00968 \mathrm{E}-04$

$-2.11331 \mathrm{E}-04$

$-2.21655 \mathrm{E}-04$

$-2.31915 \mathrm{E}-04$

$-2.42084 \mathrm{E}-04$

$-2.52136 \mathrm{E}-04$

$-2.62046 \mathrm{E}-04$

$-2.71788 \mathrm{E}-04$

$-2.81336 \mathrm{E}-04$

$-2.90664 \mathrm{E}-04$

$-2.99748 \mathrm{E}-04$

$-3.08560 \mathrm{E}-04$

$-3.17075 \mathrm{E}-04$

$-3.25268 \mathrm{E}-04$

$-3.33113 \mathrm{E}-04$

$-3.40585 \mathrm{E}-04$

$-3.47657 \mathrm{E}-04$

$-3.54304 \mathrm{E}-04$

$-3.60501 \mathrm{E}-04$

$-3.66221 \mathrm{E}-04$

$-3.71439 \mathrm{E}-04$

$-3.76131 \mathrm{E}-04$

$-3.80269 \mathrm{E}-04$

$-3.83828 \mathrm{E}-04$

$-3.86784 \mathrm{E}-04$

$-3.89109 \mathrm{E}-04$

$-3.90779 \mathrm{E}-04$

$-3.91769 \mathrm{E}-04$

$-3.92052 \mathrm{E}-04$
$-1.21794 \mathrm{E} \quad 00$

$-2.78465 \mathrm{E} \quad 00$

$-4.19433 \mathrm{E} 00$

$-5.49586 \mathrm{E} \quad 00$

$-6.68966 \mathrm{E} 00$

$-7.77463 \mathrm{E} \quad 00$

$-8.75306 \mathrm{E} \quad 00$

$-9.63042 \mathrm{E} \quad 00$

$-1.04143 \mathrm{E} 01$

$-1.11133 \mathrm{E} 01$

$-1.17364 \mathrm{E} \quad 01$

$-1.22921 \mathrm{E} \quad 01$

$-1.27884 \mathrm{E} \quad 01$

$-1.32327 \mathrm{E} 01$

$-1.36312 \mathrm{E} 01$

$-1.39896 \mathrm{E} \quad 01$

$-1.43130 \mathrm{E} 01$

$-1.46055 \mathrm{E} \quad 01$

$-1.48710 \mathrm{E} \quad 01$

$-1.51125 \mathrm{E} 01$

$-1.53329 \mathrm{E} 01$

$-1.55346 \mathrm{E} 01$

$-1.57197 \mathrm{E} \quad 01$

$-1.58899 \mathrm{E} 01$

$-1.60469 \mathrm{E} 01$

$-1.61921 \mathrm{E} 01$

$-1.63266 \mathrm{E} \quad 01$

$-1.64515 \mathrm{E} 01$

$-1.65678 \mathrm{E} 01$

$-1.66762 \mathrm{E} 01$

$-1.67774 \mathrm{E} \quad 01$

$-1.68722 \mathrm{E} 01$

$-1.69611 \mathrm{E} 01$

$-1.70446 \mathrm{E} 01$

$-1.71231 \mathrm{E} 01$

$-1.71971 \mathrm{E} \quad 01$

$-1.72670 \mathrm{E} \quad 01$

$-1.73329 \mathrm{E} 01$

$-1.73954 \mathrm{E} 01$

$-1.74545 \mathrm{E} 01$

$-1.75107 \mathrm{E} 01$

$-1.75640 \mathrm{E} 01$ 
ELECTRON RADIAL FUNCTIONS

Positron

$\begin{array}{cc}p & F_{0} \\ 6.10 & 8.05508 \mathrm{E}-01 \\ 6.20 & 8.05522 \mathrm{E}-01 \\ 6.30 & 8.05534 \mathrm{E}-01 \\ 6.40 & 8.05543 \mathrm{E}-01 \\ 6.50 & 8.05550 \mathrm{E}-01 \\ & \\ 6.60 & 8.05554 \mathrm{E}-01 \\ 6.70 & 8.05556 \mathrm{E}-01 \\ 6.80 & 8.05557 \mathrm{E}-01 \\ 6.90 & 8.05555 \mathrm{E}-01 \\ 7.00 & 8.05552 \mathrm{E}-01\end{array}$

7.10

7.20

7.30

7.40

7.50

7.60

7.70

7.80

7.90

8.00

8.10

8.20

8.30

8.40

8.50

8.60

8.70

8.80

8.90

9.00

9.10

9.20

9.30

9.40

9.50

9.60

9.70

9.80

9.90

10.00

10.10

10.20

10.30

10.40

10.50

10.60

10.70

10.80

10.90

11.00

11.10

11.20

11.30

11.40

11.50

11.60

11.70

11.80

11.90

12.00
$Z=10 \quad A=20$

$\begin{array}{lll}f_{1} & g_{1}\end{array}$

$5.02848 \mathrm{E} \quad 00 \quad-4.52462 \mathrm{E}-02$

$5.11906 \mathrm{E} \quad 00 \quad-4.46403 \mathrm{E}-02$

$5.20966 \mathrm{E} \quad 00 \quad-4.39835 \mathrm{E}-02$

$5.30025 \mathrm{E} \quad 00-4.32759 \mathrm{E}-02$

$5.39086 \mathrm{E} \quad 00 \quad-4.25175 \mathrm{E}-02$

$5.48147 \mathrm{E} \quad 00 \quad-4.17083 \mathrm{E}-02$

$5.57209 \mathrm{E} \quad 00-4.08482 \mathrm{E}-02$

$\begin{array}{lll}5.66272 \mathrm{E} & 00 & -3.99372 \mathrm{E}-02\end{array}$

$5.75335 \mathrm{E} \quad 00 \quad-3.89754 \mathrm{E}-02$

$5.84399 \mathrm{E} \quad 00-3.79628 \mathrm{E}-02$

$5.93464 \mathrm{E} \quad 00$ $6.02530 \mathrm{E} \quad 00$

$6.11596 \mathrm{E} \quad 00$

$6.20664 \mathrm{E} \quad 00$

$6.29732 \mathrm{E} \quad 00$

$6.38802 \mathrm{E} \quad 00$

$6.47872 \mathrm{E} \quad 00$

$6.56943 \mathrm{E} \quad 00$

$6.66016 \mathrm{E} \quad 00$

$6.75089 \mathrm{E} \quad 00$

$6.84163 \mathrm{E} \quad 00$

$6.93239 \mathrm{E} \quad 00$

$7.02316 \mathrm{E} \quad 00$

$7.11393 \mathrm{E} \quad 00$

$7.20472 \mathrm{E} \quad 00$

$7.29552 \mathrm{E} \quad 00$

$7.38633 \mathrm{E} \quad 00$

$7.47715 \mathrm{E} \quad 00$

$7.56799 \mathrm{E} \quad 00$

$7.65884 \mathrm{E} \quad 00$

$7.74970 \mathrm{E} \quad 00$

$7.84057 \mathrm{E} \quad 00$

$7.93146 \mathrm{E} \quad 00$

$8.02236 \mathrm{E} \quad 00$

$8.11327 \mathrm{E} \quad 00$

$8.204 .19 \mathrm{E} \quad 00$

8.29513 E 00

$8.38608 \mathrm{E} \quad 00$

$8.47705 \mathrm{E} \quad 00$

$8.56802 \mathrm{E} \quad 00$

$8.65901 \mathrm{E} \quad 00$

$8.75002 \mathrm{E} \quad 00$

$8.84104 \mathrm{E} \quad 00$

$8.93207 \mathrm{E} \quad 00$

$9.02311 \mathrm{E} \quad 00$

$9.11418 \mathrm{E} \quad 00$

$9.20525 \mathrm{E} \quad 00$

$9.29632 \mathrm{E} \quad 00$

$9.38743 \mathrm{E} \quad 00$

$9.47853 \mathrm{E} \quad 00$

$9.56963 \mathrm{E} \quad 00$

$9.66078 \mathrm{E} \quad 00$

$9.75189 \mathrm{E} \quad 00$

$9.84302 \mathrm{E} \quad 00$

$9.93409 \mathrm{E} \quad 00$

$1.00251 \mathrm{E} \quad 01$

$\begin{array}{lll}1.01160 \mathrm{E} & 01\end{array}$

$1.02061 \mathrm{E} \quad 01$

$1.02908 \mathrm{E} \quad 01$

$\begin{array}{lll}1.03963 \mathrm{E} & 01\end{array}$
$-3.68993 \mathrm{E}-02$

$-3.57849 \mathrm{E}-02$

$-3.46196 \mathrm{E}-02$

$-3.34035 \mathrm{E}-02$

$-3.21364 \mathrm{E}-02$

$-2.94495 \mathrm{E}-02$

$-2.80297 \mathrm{E}-02$

$-2.65590 \mathrm{E}-02$

$-2.50373 \mathrm{E}-02$

$-2.34647 \mathrm{E}-02$

$-2.18412 \mathrm{E}-02$

$-2.01666 \mathrm{E}-02$

$-1.84411 \mathrm{E}-02$

$-1.66646 \mathrm{E}-02$

$-1.48371 \mathrm{E}-02$

$-1.29586 \mathrm{E}-02$

$-1.10291 \mathrm{E}-02$

$-9.04855 \mathrm{E}-03$

$-7.01696 \mathrm{E}-03$

$-4.93432 \mathrm{E}-03$

$-2.80062 \mathrm{E}-03$

$-6.15840 \mathrm{E}-04$

$1.62002 \mathrm{E}-03$

$3.90699 \mathrm{E}-03$

$6.24506 \mathrm{E}-03$

$8.63424 \mathrm{E}-03$

$1.10746 \mathrm{E}-02$

$1.61087 \mathrm{E}-02$

$1.87025 \mathrm{E}-02$ $2.13475 \mathrm{E}-02$

$2.40437 \mathrm{E}-02$

$2.67911 \mathrm{E}-02$

2.95897 E-02

$3.24395 \mathrm{E}-02$

$3.53406 \mathrm{E}-02$

$3.82930 \mathrm{E}-02$

$4.12966 \mathrm{E}-02$

$4.43515 \mathrm{E}-02$

$4.74575 \mathrm{E}-02$

$5.06151 \mathrm{E}-02$

$5.38237 \mathrm{E}-02$

$5.70836 \mathrm{E}-02$

$6.03945 \mathrm{E}-02$

$6.37560 \mathrm{E}-02$

$6.71681 \mathrm{E}-02$

$7.06261 \mathrm{E}-02$

$7.40955 \mathrm{E}-02$

$7.77683 \mathrm{E}-02$
$-3.08184 \mathrm{E}-02$

$1.35661 \mathrm{E}-02$

$$
\rho=1.2 A^{1 / 3} F
$$

$\tan \delta_{1}$

$4.34277 \mathrm{E}-03$

$4.24914 \mathrm{E}-03$

$4.15847 \mathrm{E}-03$

$4.07064 \mathrm{E}-03$

$3.98550 \mathrm{E}-03$

$3.90293 \mathrm{E}-03$

$3.82282 \mathrm{E}-03$

$3.74508 \mathrm{E}-03$

$3.66957 \mathrm{E}-03$

$3.59623 \mathrm{E}-03$

$3.52495 \mathrm{E}-03$

$3.45565 \mathrm{E} \rightarrow 03$

$3.38826 \mathrm{E}-03$

$3.32269 \mathrm{E}-03$

$3.25887 \mathrm{E}-03$

$3.19675 \mathrm{E}-03$

$3.13624 \mathrm{E}-03$

$3.07730 \mathrm{E}-03$

$3.01986 \mathrm{E}-03$

$2.96386 \mathrm{E}-03$

$2.90925 \mathrm{E}-03$

$2.85600 \mathrm{E}-03$

$2.80403 \mathrm{E}-03$

$2.75332 \mathrm{E}-03$

$2.70380 \mathrm{E}-03$

$2.65547 \mathrm{E}-03$

$2.60824 \mathrm{E}-03$

$2.56211 \mathrm{E}-03$

$2.51703 \mathrm{E}-03$

$2.47296 \mathrm{E}-03$

$2.42989 \mathrm{E}-03$

$2.38776 \mathrm{E}-03$

$2.34656 \mathrm{E}-03$

$2.30625 \mathrm{E}-03$

$2.26681 \mathrm{E}-03$

$2.22820 \mathrm{E}-03$

2.19041 E-03

$2.15340 \mathrm{E}-03$

$2.11717 \mathrm{E}-03$

$2.08168 \mathrm{E}-03$

$2.04692 \mathrm{E}-03$

$2.01284 \mathrm{E}-03$

$1.97945 \mathrm{E}-03$

$1.94672 \mathrm{E}-03$

$1.91463 \mathrm{E}-03$

$1.88318 \mathrm{E}-03$

$1.85232 \mathrm{E}-03$

$1.82205 \mathrm{E}-03$

$1.79237 \mathrm{E}-03$

$1.76324 \mathrm{E}-03$

$1.73467 \mathrm{E}-03$

$1.70660 \mathrm{E}-03$

$1.67909 \mathrm{E}-03$

$1.65206 \mathrm{E}-03$

$1.62552 \mathrm{E}-03$

$1.59947 \mathrm{E}-03$

$1.57387 \mathrm{E}-03$

$1.54874 \mathrm{E}-03$

$1.52404 \mathrm{E}-03$

$1.49979 \mathrm{E}-03$

$f_{-1}$

$8.65820 \mathrm{E}-02$

$8.62439 \mathrm{E}-02$

$8.58546 \mathrm{E}-02$

$8.54140 \mathrm{E}-02$

8.49221 E-02

$8.43790 \mathrm{E}-02$

$8.37846 \mathrm{E}-02$

$8.31390 \mathrm{E}-02$

$8.24422 \mathrm{E}-02$

$8.16942 \mathrm{E}-02$

$8.08950 \mathrm{E}-02$

$8.00446 \mathrm{E}-02$

7.91431 E-02

$7.81905 \mathrm{E}-02$

$7.71866 \mathrm{E}-02$

$7.61316 \mathrm{E}-02$

$7.50254 \mathrm{E}-02$

$7.38682 \mathrm{E}-02$

$7.26597 \mathrm{E}-02$

7.14002 E-02

7.00895 E-02

$6.87276 \mathrm{E}-02$

$6.73146 \mathrm{E}-02$

$6.58505 \mathrm{E}-02$

6.43351 E-02

$6.27687 \mathrm{E}-02$

$6.11511 \mathrm{E}-02$

$5.94823 \mathrm{E}-02$

$5.77624 \mathrm{E}-02$

$5.59913 \mathrm{E}-02$

$5.41690 \mathrm{E}-02$

$5.22955 \mathrm{E}-02$

$5.03708 \mathrm{E}-02$

$4.83949 \mathrm{E}-02$

$4.63678 \mathrm{E}-02$

4.42895E-02

$4.21599 \mathrm{E}-02$

$3.99791 \mathrm{E}-02$

$3.77471 \mathrm{E}-02$

$3.54638 \mathrm{E}-02$

$3.31292 \mathrm{E}-02$ $3.07433 \mathrm{E}-02$

$2.83062 \mathrm{E}-02$

$2.58177 \mathrm{E}-02$

$2.32779 \mathrm{E}-02$

$2.06868 \mathrm{E}-02$

$1.80443 \mathrm{E}-02$

$1.53505 \mathrm{E}-02$

$1.26053 \mathrm{E}-02$

$9.80875 \mathrm{E}-03$

$6.96078 \mathrm{E}-03$

$4.06140 \mathrm{E}-03$

$1.11058 \mathrm{E}-03$

$-1.89168 \mathrm{E}-03$

$-4.94539 \mathrm{E}-03$

$-8.05058 \mathrm{E}-03$

$-1.12073 \mathrm{E}-02$

$-1.44154 \mathrm{E}-02$

$-1.76751 \mathrm{E}-02$

$-2.09864 \mathrm{E}-02$ 
$Z=10 \quad A=20 \quad \rho=1.2 A^{1 / 3} F$

$\bar{F}$

$5.88503 \mathrm{E}-05$ $6.07991 \mathrm{E}-05$ $6.27795 \mathrm{E}-05$ $6.47915 \mathrm{E}-05$ $6.68351 \mathrm{E}-05$

$6.89102 \mathrm{E}-05$ $7.10171 \mathrm{E}-05$ $7.31555 \mathrm{E}-05$ $7.53255 \mathrm{E}-05$ $7.75271 \mathrm{E}-05$

$7.97603 \mathrm{E}-05$ $8.20251 \mathrm{E}-05$ $8.43215 \mathrm{E}-05$ $8.66494 \mathrm{E}-05$ $8.90090 \mathrm{E}-05$

7.60

7.70

7.80

7.90

8.00

8.10

8.20

8.30

8.40

8.50

8.60

8.70

8.80

8.90

9.00

9.10

9.20

9.30

9.40

9.50

9.60

9.70

9.80

9.90

10.00

10.10

10.20

10.30

10.40

10.50

10.60

10.70

10.80

10.90

11.00

11.10

11.20

11.30

11.40

11.50

11.60

11.70

11.80

11.90

12.00

$1.11666 \mathrm{E}-04$

$1.14342 \mathrm{E}-04$

$1.17049 \mathrm{E}-04$ $1.19787 \mathrm{E}-04$ $1.22557 \mathrm{E}-04$

$1.25359 \mathrm{E}-04$

$1.28192 \mathrm{E}-04$

$1.31057 \mathrm{E}-04$

$1.33954 \mathrm{E}-04$

$1.36881 \mathrm{E}-04$

$1.39841 \mathrm{E}-04$

$1.42832 \mathrm{E}-04$

$1.45855 \mathrm{E}-04$

$1.48909 \mathrm{E}-04$

$1.51995 \mathrm{E}-04$

$1.55112 \mathrm{E}-04$

$1.58261 \mathrm{E}-04$

$1.61442 \mathrm{E}-04$ $1.64654 \mathrm{E}-04$ $1.67897 \mathrm{E}-04$ $1.71173 \mathrm{E}-04$ $1.74479 \mathrm{E}-04$

$1.77818 \mathrm{E}-04$ $1.81187 \mathrm{E}-04$ $1.84589 \mathrm{E}-04$ $1.88022 \mathrm{E}-04$ $1.91486 \mathrm{E}-04$

$1.94983 \mathrm{E}-04$ $1.98510 \mathrm{E}-04$ $2.02069 \mathrm{E}-04$ $2.05660 \mathrm{E}-04$ $2.09282 \mathrm{E}-04$

$2.12936 \mathrm{E}-04$ $2.16622 \mathrm{E}-04$ $2.20339 \mathrm{E}-04$ $2.24087 \mathrm{E}-04$ $2.27867 \mathrm{E}-04$ $f_{2}$

$8.57953 \mathrm{E}-02$ $8.87697 \mathrm{E}-02$ $9.17945 \mathrm{E}-02$ $9.48699 \mathrm{E}-02$ $9.79956 \mathrm{E}-02$

$1.01172 \mathrm{E}-01$ $1.04399 \mathrm{E}-01$

$1.07676 \mathrm{E}-01$

$1.11003 \mathrm{E}-01$

$1.14382 \mathrm{E}-0$

$1.17810 \mathrm{E}-01$

$1.21289 \mathrm{E}-01$

$1.24819 \mathrm{E}-01$

$1.28399 \mathrm{E}-01$

$1.32029 \mathrm{E}-01$

$1.35710 \mathrm{E}-01$

$1.39441 \mathrm{E}-01$

$1.43222 \mathrm{E}-01$

$1.47054 \mathrm{E}-0$

$1.50937 \mathrm{E}-01$

$1.54869 \mathrm{E}-01$

$1.58852 \mathrm{E}-01$

$1.62886 \mathrm{E}-01$

$1.66969 \mathrm{E}-01$

$1.71103 \mathrm{E}-01$

$3.41795 \mathrm{E}-0$ $3.47696 \mathrm{E}-01$ $g_{2}$

$\tan \delta_{2}$

$f_{-2}$

$-3.91603 \mathrm{E}-04$ $-3.90396 \mathrm{E}-04$ $-3.88406 \mathrm{E}-04$ $-3.85607 \mathrm{E}-04$ $-3.81974 \mathrm{E}-04$

$-3.77481 \mathrm{E}-04$ $-3.72102 \mathrm{E}-04$ $-3.65813 \mathrm{E}-04$ $-3.58587 \mathrm{E}-04$

$-3.50398 \mathrm{E}-04$

$-3.41223 \mathrm{E}-04$ $-3.31033 \mathrm{E}-04$ $-3.19806 \mathrm{E}-04$ $-3.07513 \mathrm{E}-04$

$-2.94131 \mathrm{E}-04$

$-2.79634 \mathrm{E}-04$ $-2.63996 \mathrm{E}-04$

$-2.47191 \mathrm{E}-04$ $-2.29194 \mathrm{E}-04$

$-2.09980 \mathrm{E}-04$

$-1.89523 \mathrm{E}-04$

$-1.67797 \mathrm{E}-04$

$-1.44777 \mathrm{E}-04$

$-1.20437 \mathrm{E}-04$

$-9.47530 \mathrm{E}-05$

$-6.76982 \mathrm{E}-05$ $-3.92477 \mathrm{E}-05$

$-9.37610 \mathrm{E}-06$

$2.19418 \mathrm{E}-05$

$5.47310 \mathrm{E}-05$

$8.90165 \mathrm{E}-05$

$1.24822 \mathrm{E}-04$

$1.62170 \mathrm{E}-04$

$2.01081 \mathrm{E}-04$

$2.41567 \mathrm{E}-04$

$2.83606 \mathrm{E}-04$

$3.26856 \mathrm{E}-04$

$3.74852 \mathrm{E}-04$

$4.20930 \mathrm{E}-04$

$4.69677 \mathrm{E}-04$

$5.20296 \mathrm{E}-04$

$5.72724 \mathrm{E}-04$ $6.26972 \mathrm{E}-04$

$6.83062 \mathrm{E}-04$

7.41011 E-04

$8.00844 \mathrm{E}-04$

$8.62587 \mathrm{E}-04$

$9.26265 \mathrm{E}-04$

$9.91900 \mathrm{E}-04$

$1.05952 \mathrm{E}-03$

$1.12916 \mathrm{E}-03$

$1.20082 \mathrm{E}-03$

$1.27455 \mathrm{E}-03$

$1.35037 \mathrm{E}-03$

$1.42829 \mathrm{E}-03$

$1.50836 \mathrm{E}-03$

$1.59059 \mathrm{E}-03$

$1.67500 \mathrm{E}-03$

$1.76163 \mathrm{E}-03$

$1.85050 \mathrm{E}-03$ $-1.82518 \mathrm{E} \quad 01$ $-1.82741 \mathrm{E} 01$ $-1.82956 \mathrm{E} \quad 01$ $-1.83165 \mathrm{E} 01$

$\begin{array}{lll}-1.83366 \mathrm{E} & 01\end{array}$ $-1.83561 \mathrm{E} 01$ $-1.83750 \mathrm{E} 01$ $-1.83933 \mathrm{E} 01$ $-1.84110 \mathrm{E} 01$

$-1.84283 \mathrm{E} \quad 01$ $-1.84450 \mathrm{E} 01$ $-1.84612 \mathrm{E} 01$ $-1.84769 \mathrm{E} 01$ $-1.84922 \mathrm{E} 01$

$\begin{array}{lll}-1.85071 \mathrm{E} & 01\end{array}$ $-1.85215 \mathrm{E} 01$ $-1.85356 \mathrm{E} 01$ $-1.85493 \mathrm{E} 01$ $-1.85626 \mathrm{E} 01$

$\begin{array}{ll}-1.85756 \mathrm{E} & 01\end{array}$ $-1.85882 \mathrm{E} 01$ $-1.86125 \mathrm{E} \quad 01$

$-1.86242 \mathrm{E} 01$

$-1.86356 \mathrm{E} 01$ $-1.86467 \mathrm{E} 01$ $-1.86576 \mathrm{E} 01$ $-1.86681 \mathrm{E} \quad 01$ $-1.86785 \mathrm{E} \quad 01$

$-1.86886 \mathrm{E} 01$ $-1.86985 \mathrm{E} 01$ -1.87081 E 01 $-1.87175 \mathrm{E} \quad 01$ $-1.87268 \mathrm{E} 01$

$-1.87358 \mathrm{E} \quad 01$ $-1.87446 \mathrm{E} 01$ $-1.87532 \mathrm{E} 01$ $-1.87616 \mathrm{E} 01$ $-1.87699 \mathrm{E} \quad 01$

$\begin{array}{lll}-1.87780 \mathrm{E} & 01\end{array}$ $-1.87859 \mathrm{E} 01$ $\begin{array}{lll}-1.87937 \mathrm{E} & 01\end{array}$ $-1.88012 \mathrm{E} 01$ $-1.88087 \mathrm{E} \quad 01$

$-1.88160 \mathrm{E} 01$ $-1.88302 \mathrm{E} \quad 01$ $-1.88370 \mathrm{E} 01$

-1.88438 E 01

$-1.88504 \mathrm{E} \quad 01$ $-1.88569 \mathrm{E} 01$ $-1.88632 \mathrm{E} 01$ $-1.88695 \mathrm{E} \quad 01$ $-1.88756 \mathrm{E} 01$

$-1.88816 \mathrm{E} \quad 01$ $-1.88876 \mathrm{E} \quad 01$ $-1.88934 \mathrm{E} 01$ $-1.88991 \mathrm{E} 01$ $\begin{array}{lll}-1.89047 \mathrm{E} & 01\end{array}$
$-1.82288 \mathrm{E} 01$ $-1.86005 \mathrm{E} \quad 01$ $-1.88231 \mathrm{E} 01$

Positron

$\tan \delta_{-2}$

$8.01838 \mathrm{E}-04$ $8.10117 \mathrm{E}-04$

$8.17697 \mathrm{E}-04$

$8.24552 \mathrm{E}-04$

$8.30656 \mathrm{E}-04$

$8.35985 \mathrm{E}-04$

$8.40512 \mathrm{E}-04$

$8.44212 \mathrm{E}-04$

$8.47060 \mathrm{E}-04$

$8.49029 \mathrm{E}-04$

$8.50096 \mathrm{E}-04$

$8.50233 \mathrm{E}-04$

$8.49416 \mathrm{E}-04$

$8.47618 \mathrm{E}-04$

$8.44815 \mathrm{E}-04$

$8.40980 \mathrm{E}-04$

$8.36089 \mathrm{E}-04$

$8.30116 \mathrm{E}-04$

$8.23035 \mathrm{E}-04$

$8.14821 \mathrm{E}-04$

$8.05449 \mathrm{E}-04$

$7.94892 \mathrm{E}-04$

$7.83125 \mathrm{E}-04$

$7.70123 \mathrm{E}-04$

$7.55860 \mathrm{E}-04$

$7.40310 \mathrm{E}-04$

$7.23449 \mathrm{E}-04$

$7.05250 \mathrm{E}-04$

$6.85688 \mathrm{E}-04$

$6.64738 \mathrm{E}-04$

$6.42374 \mathrm{E}-04$

$6.18570 \mathrm{E}-04$ 
ELECTRON RADIAL FUNCTIONS

Positron

$F_{0}$

12.10

12.20

12.30

12.40

12.50

12.60

12.70

12.80

12.90

13.00

13.10

13.20

13.30

13.40

13.50

13.60

13.70

13.80

13.90

14.00

14.10

14.20

14.30

14.40

14.50

14.60

14.70

14.80

14.90

15.00

15.10

15.20

15.30

15.40

15.50

15.60

15.70

15.80

15.90

16.00

16.10

16.20

16.30

16.40

16.50

16.60

16.70

16.80

16.90

17.00

17.10

17.20

17.30

17.40

17.50

17.60

17.70

17.80

17.90

18.00 $f_{1}$

8.0454.3E-0 $8.04519 \mathrm{E}-01$ $8.044 .94 \mathrm{E}-01$ $8.04470 \mathrm{E}-01$ $8.04446 \mathrm{E}-01$

$8.04422 \mathrm{E}-01$ 8.04398E-01 $8.04374 \mathrm{E}-01$ $8.04350 \mathrm{E}-01$ $8.04326 \mathrm{E}-01$

$8.04302 \mathrm{E}-01$ $8.04278 \mathrm{E}-01$ $8.04254 \mathrm{E}-0$ $8.04230 \mathrm{E}-01$ $8.04206 \mathrm{E}-01$

$8.04183 \mathrm{E}-01$ $8.04159 \mathrm{E}-01$ $8.04 .136 \mathrm{E}-01$ $8.04112 \mathrm{E}-01$ $8.04089 \mathrm{E}-01$

$8.04065 \mathrm{E}-01$ 8.04042 E-0 $8.04019 \mathrm{E}-01$ $8.03995 \mathrm{E}-0]$ $8.03972 \mathrm{E}-01$

8.03949 E-01 $8.03926 \mathrm{E}-01$ $8.03903 \mathrm{E}-01$ $8.03880 \mathrm{E}-01$ $8.03858 \mathrm{E}-01$

8.03835 E-0 $8.03812 \mathrm{E}-01$ $8.03790 \mathrm{E}-01$ $8.03767 \mathrm{E}-01$ $8.03745 \mathrm{E}-01$

$8.03723 \mathrm{E}-01$ $8.03700 \mathrm{E}-01$ $8.03678 \mathrm{E}-01$ $8.03657 \mathrm{E}-01$ $8.03635 \mathrm{E}-01$

$8.03613 \mathrm{E}-01$ $8.03591 \mathrm{E}-01$ $8.03569 \mathrm{E}-01$ $8.03547 \mathrm{E}-01$ $8.03526 \mathrm{E}-01$

$8.03504 \mathrm{E}-01$ $8.03483 \mathrm{E}-01$ $8.03461 \mathrm{E}-01$ $8.03440 \mathrm{E}-01$ $8.034 .19 \mathrm{E}-01$

$8.03398 \mathrm{E}-01$ $8.03377 \mathrm{E}-01$ $8.03356 \mathrm{E}-01$ $8.03335 \mathrm{E}-01$ $8.03314 \mathrm{E}-01$

$8.03293 \mathrm{E}-01$ $8.03273 \mathrm{E}-01$ $8.03252 \mathrm{E}-01$ $8.03232 \mathrm{E}-01$ $8.03211 \mathrm{E}-01$
$Z=10 \quad A=20$

$g_{1}$

$1.04847 \mathrm{E} \quad 01$ $\begin{array}{ll}1.05752 \mathrm{E} & 01\end{array}$ $1.06662 \mathrm{E} \quad 01$ $1.07574 \mathrm{E} \quad 01$ $1.08486 \mathrm{E} 01$

$1.09400 \mathrm{E} \quad 01$ $1.10314 \mathrm{E} \quad 01$ $\begin{array}{ll}1.11227 \mathrm{E} & 01\end{array}$ $\begin{array}{ll}1.12142 \mathrm{E} & 01\end{array}$ $1.13057 \mathrm{E} \quad 01$

$\begin{array}{ll}1.13971 \mathrm{E} & 01\end{array}$ $1.14887 \mathrm{E} \quad 01$ $1.15802 \mathrm{E} \quad 01$ $1.16717 \mathrm{E} \quad 01$ $1.17633 \mathrm{E} \quad 01$

$1.1854 .9 \mathrm{E} \quad 01$ $1.19465 \mathrm{E} \quad 01$ $1.20382 \mathrm{E} \quad 01$ $1.21298 \mathrm{E} \quad 01$ $1.22215 \mathrm{E} \quad 01$

$1.23132 \mathrm{E} \quad 01$ $1.24049 \mathrm{E}$ 01 $1.24967 \mathrm{E} \quad 01$ $1.25885 \mathrm{E} \quad 01$ $1.26803 \mathrm{E} 01$

$1.27721 \mathrm{E} \quad 01$ $1.28639 \mathrm{E} \quad 01$ $1.29558 \mathrm{E} \quad 01$ $1.30476 \mathrm{E}$ 01 $1.31395 \mathrm{E} \quad 01$

$1.32315 \mathrm{E} \quad 01$ $1.33234 \mathrm{E}$ 01 $1.34 .154 \mathrm{E} \quad 01$ $1.35073 \mathrm{E}$ 01 $1.35994 \mathrm{E} \quad 01$

$1.36914 \mathrm{E} \quad 01$ $1.37835 \mathrm{E}$ 01 $1.38755 \mathrm{E} \quad 01$ $1.39676 \mathrm{E} \quad 01$ $1.40598 \mathrm{E} \quad 01$

$1.41519 \mathrm{E}$ 01 $1.4244 .1 \mathrm{E} 01$ $1.43363 \mathrm{E}$ ol $1.44285 \mathrm{E} \quad 01$ $1.45207 \mathrm{E} \quad 01$

$1.46130 \mathrm{E} \quad 01$ $1.47053 \mathrm{E} \quad 01$ $1.47976 \mathrm{E}$ 01 $1.48899 \mathrm{E} \quad 01$ $1.49823 \mathrm{E} 01$

$1.50747 \mathrm{E} \quad 01$ $1.51671 \mathrm{E} \quad 01$ $1.52595 \mathrm{E} \quad 01$ $1.53520 \mathrm{E} \quad 01$ $1.54445 \mathrm{E} \quad 01$

$1.55370 \mathrm{E} \quad 01$ $\begin{array}{ll}1.56295 \mathrm{E} & 01\end{array}$ $1.57221 \mathrm{E}$ 0I $1.58147 \mathrm{E} \quad 01$ $1.59073 \mathrm{E} \quad 01$

$8.13674 \mathrm{E}-02$
$8.50333 \mathrm{E}-02$
$8.87545 \mathrm{E}-02$
$9.25280 \mathrm{E}-02$
$9.63539 \mathrm{E}-02$

$9.63539 \mathrm{E}-02$

$1.00232 \mathrm{E}-01$

$1.04161 \mathrm{E}-01$

$1.08142 \mathrm{E}-01$

$1.12175 \mathrm{E}-01$

$1.20396 \mathrm{E}-01$

$1.24584 \mathrm{E}-01$

$1.28824 \mathrm{E}-01$

$1.33116 \mathrm{E}-01$

$1.37459 \mathrm{E}-01$

1.4.1854 E-01

$1.46301 \mathrm{E}-01$

$1.50800 \mathrm{E}-01$

$1.55350 \mathrm{E}-01$

$1.59953 \mathrm{E}-01$

$1.64607 \mathrm{E}-01$

$1.69313 \mathrm{E}-01$

$1.74072 \mathrm{E}-01$

$1.78882 \mathrm{E}-01$

$1.83744 \mathrm{E}-01$

$1.88658 \mathrm{E}-01$

$1.93624 \mathrm{E}-01$

$1.98642 \mathrm{E}-01$

$2.03712 \mathrm{E}-01$

2.08834.E-01

$2.14008 \mathrm{E}-01$ $2.19235 \mathrm{E}-01$

$2.24513 \mathrm{E}-01$

$2.35227 \mathrm{E}-01$

$2.46149 \mathrm{E}-01$

$2.51689 \mathrm{E}-01$

$2.57281 \mathrm{E}-01$

2.62925 E-01

$2.68622 \mathrm{E}-01$

$2.74370 \mathrm{E}-01$

$2.80172 \mathrm{E}-01$

$2.86025 \mathrm{E}-01$

2.91931 E-01

$2.97890 \mathrm{E}-01$

$3.09964 \mathrm{E}-01$

$3.16080 \mathrm{E}-01$

$3.28470 \mathrm{E}-01$

$3.34744 \mathrm{E}-01$

$3.41070 \mathrm{E}-01$

$3.47449 \mathrm{E}-01$

3.53881 E-01

$3.60366 \mathrm{E}-01$

$3.66903 \mathrm{E}-01$

$3.73493 \mathrm{E}-01$

$3.80136 \mathrm{E}-01$

$3.86831 \mathrm{E}-01$
$1.16260 \mathrm{E}-01$

$2.29844 \mathrm{E}-01$

$2.40662 \mathrm{E}-01$

$3.03901 \mathrm{E}-01$

$3.22249 \mathrm{E}-01$

$\tan \delta_{1}$

$1.47595 \mathrm{E}-03$

$1.45253 \mathrm{E}-03$

$1.42951 \mathrm{E}-03$

$1.40688 \mathrm{E}-03$

$1.38464 \mathrm{E}-03$

$1.36277 \mathrm{E}-03$

$1.34 .128 \mathrm{E}-03$

$1.32012 \mathrm{E}-03$

$1.29934 \mathrm{E}-03$

$1.27889 \mathrm{E}-03$

$1.25878 \mathrm{E}-03$

$1.23898 \mathrm{E}-03$

$1.21952 \mathrm{E}-03$

$1.20036 \mathrm{E}-03$

$1.18151 \mathrm{E}-03$

$1.16297 \mathrm{E}-03$

$1.14472 \mathrm{E}-03$

$1.12674 \mathrm{E}-03$

$1.10907 \mathrm{E}-03$

$1.09165 \mathrm{E}-03$

$1.07451 \mathrm{E}-03$

$1.05763 \mathrm{E}-03$

$1.04102 \mathrm{E}-03$

$1.02466 \mathrm{E}-03$

$1.00855 \mathrm{E}-03$

$9.92676 \mathrm{E}-04$

$9.77056 \mathrm{E}-04$

$9.61658 \mathrm{E}-04$

$9.46478 \mathrm{E}-04$

$9.31553 \mathbf{E}-04$

9.16829 E-04 $9.02329 \mathrm{E}-04$ $8.88045 \mathrm{E}-04$ $8.73969 \mathrm{E}-04$ $8.60092 \mathrm{E}-04$

$8.46421 \mathrm{E}-04$ 8.32955 E-04 $8.19663 \mathrm{E}-04$ $8.06590 \mathrm{E}-04$ $7.93678 \mathrm{E}-04$

$7.80973 \mathrm{E}-04$

$7.68423 \mathrm{E}-04$

$7.56068 \mathrm{E}-04$

$7.43890 \mathrm{E}-04$

$7.31879 \mathrm{E}-04$ 
$Z=10 \quad A=20 \quad \rho=1.2 A^{1 / 3} /$

$\bar{F}$

$2.31679 \mathrm{E}-04$ $2.35522 \mathrm{E}-04$ $2.39397 \mathrm{E}-04$ $2.43303 \mathrm{E}-04$ $2.47241 \mathrm{E}-04$

$2.51210 \mathrm{E}-04$ $2.55211 \mathrm{E}-04$ $2.59244 \mathrm{E}-04$ $2.63308 \mathrm{E}-04$ $2.67403 \mathrm{E}-04$

$2.71530 \mathrm{E}-04$ $2.75689 \mathrm{E}-04$ $2.79879 \mathrm{E}-04$ $2.84101 \mathrm{E}-04$ $2.88354 \mathrm{E}-04$

$2.92639 \mathrm{E}-04$ $2.96955 \mathrm{E}-04$ $3.01303 \mathrm{E}-04$ $3.05682 \mathrm{E}-04$ $3.10093 \mathrm{E}-04$

$3.14535 \mathrm{E}-04$ $3.19009 \mathrm{E}-04$ $3.23514 \mathrm{E}-04$ $3.28052 \mathrm{E}-04$ $3.32620 \mathrm{E}-04$

$3.37220 \mathrm{E}-04$ $3.41852 \mathrm{E}-04$ $3.46515 \mathrm{E}-04$

$3.51210 \mathrm{E}-04$

$3.55936 \mathrm{E}-04$

3.60694. $\mathrm{E}-04$ $3.65483 \mathrm{E}-04$ $3.70304 \mathrm{E}-04$ $3.75156 \mathrm{E}-04$ $3.80040 \mathrm{E}-04$

$3.84955 \mathrm{E}-04$ $3.89902 \mathrm{E}-04$ $3.94880 \mathrm{E}-04$ $3.99890 \mathrm{E}-04$ $4.04932 \mathrm{E}-04$

16.10

16.20

16.30

16.40

16.50

$4.10005 \mathrm{E}-04$ $4.15109 \mathrm{E}-04$ $4.20245 \mathrm{E}-04$ $4.25413 \mathrm{E}-04$ $4.30612 \mathrm{E}-04$

16.60

16.70

16.80

16.90

17.00

17.10

17.20

17.30

17.40

17.50

17.60

17.70

17.80

17.90

18.00

4.35843E-04 $4.41105 \mathrm{E}-04$ $4.46399 \mathrm{E}-04$ $4.51724 \mathrm{E}-04$ $4.57080 \mathrm{E}-04$

4.62469 E-04 $4.67888 \mathrm{E}-04$ $4.73340 \mathrm{E}-04$ $4.78822 \mathrm{E}-04$ $4.84337 \mathrm{E}-04$

$4.89882 \mathrm{E}-04$ $4.95460 \mathrm{E}-04$ $5.01068 \mathrm{E}-04$ $5.06709 \mathrm{E}-04$ $5.12381 \mathrm{E}-04$ $f_{2}$

$3.53648 \mathrm{E}-01$ $3.59649 \mathrm{E}-01$

$3.71805 \mathrm{E}-01$

$3.77959 \mathrm{E}-01$

$3.84163 \mathrm{E}-01$ $3.90417 \mathrm{E}-01$ $3.96722 \mathrm{E}-01$ $4.03077 \mathrm{E}-01$ $4.09483 \mathrm{E}-01$

$4.15939 \mathrm{E}-01$ $4.22446 \mathrm{E}-01$ $4.29003 \mathrm{E}-01$ $4.35611 \mathrm{E}-01$ $4.42269 \mathrm{E}-01$

$4.48977 \mathrm{E}-01$ $4.55736 \mathrm{E}-01$ $4.62545 \mathrm{E}-01$ $4.69405 \mathrm{E}-01$ $4.76315 \mathrm{E}-01$

$4.83275 \mathrm{E}-01$ $4.90286 \mathrm{E}-01$ $4.97347 \mathrm{E}-01$ $5.04459 \mathrm{E}-01$ $5.11621 \mathrm{E}-01$

$5.18833 \mathrm{E}-01$ $5.26096 \mathrm{E}-01$ $5.33410 \mathrm{E}-01$ $5.40773 \mathrm{E}-01$ $5.48187 \mathrm{E}-01$

$5.55652 \mathrm{E}-01$ $5.63166 \mathrm{E}-01$ $5.70732 \mathrm{E}-01$ $5.78347 \mathrm{E}-01$ $5.86013 \mathrm{E}-01$

$5.93729 \mathrm{E}-01$ $6.01496 \mathrm{E}-01$ $6.09313 \mathrm{E}-01$ $6.17180 \mathrm{E}-01$ $6.25098 \mathrm{E}-01$

$6.33066 \mathrm{E}-01$ $6.41084 \mathrm{E}-01$ $6.49153 \mathrm{E}-01$ $6.57272 \mathrm{E}-01$ $6.65441 \mathrm{E}-01$

$6.73661 \mathrm{E}-01$

$6.81931 \mathrm{E}-01$

$6.90251 \mathrm{E}-01$

$6.98622 \mathrm{E}-01$

$7.07043 \mathrm{E}-01$

7.15514E-01

$7.24036 \mathrm{E}-01$

$7.32608 \mathrm{E}-01$

$7.41230 \mathrm{E}-01$

$7.49903 \mathrm{E}-01$

$7.58625 \mathrm{E}-01$

$7.67399 \mathrm{E}-01$

$7.76222 \mathrm{E}-01$

$7.85096 \mathrm{E}-01$

$7.94020 \mathrm{E}-01$
$3.65702 \mathrm{E}-01$

$g_{2}$

$\tan \delta_{2}$

$f_{-2}$

$1.94164 \mathrm{E}-03$

$2.03506 \mathrm{E}-03$

$2.13081 \mathrm{E}-03$

$2.22889 \mathrm{E}-03$

$2.32934 \mathrm{E}-03$

$2.43218 \mathrm{E}-03$

$2.53744 \mathrm{E}-03$

$2.64514 \mathrm{E}-03$

$2.75532 \mathrm{E}-03$

$2.86799 \mathrm{E}-03$

$2.98318 \mathrm{E}-03$

$3.10091 \mathrm{E}-03$

$3.22122 \mathrm{E}-03$

$3.34412 \mathrm{E}-03$

$3.46965 \mathrm{E}-03$

$3.59782 \mathrm{E}-03$

$3.72866 \mathrm{E}-03$

$3.86221 \mathrm{E}-03$

$3.99848 \mathrm{E}-03$

$4.13750 \mathrm{E}-03$

$4.27929 \mathrm{E}-03$

$4.42389 \mathrm{E}-03$

$4.57131 \mathrm{E}-03$

$4.72159 \mathrm{E}-03$

$4.87474 \mathrm{E}-03$

$5.03080 \mathrm{E}-03$

$5.18978 \mathrm{E}-03$

$5.35172 \mathrm{E}-03$

$5.51664 \mathrm{E}-03$

$5.68456 \mathrm{E}-03$

5.85551 E- 03 $6.02952 \mathrm{E}-03$

$6.20661 \mathrm{E}-03$

$6.38681 \mathrm{E}-03$

$6.57014 \mathrm{E}-03$

$6.75663 \mathrm{E}-03$ $6.94630 \mathrm{E}-03$

$7.13918 \mathrm{E}-03$

$7.33530 \mathrm{E}-03$

$7.53468 \mathrm{E}-03$

$7.73734 \mathrm{E}-03$

$7.94331 \mathrm{E}-03$

$8.15262 \mathrm{E}-03$

$8.36530 \mathrm{E}-03$

$8.58135 \mathrm{E}-03$

$-1.89102 \mathrm{E} \quad 01 \quad-8.1274 .7 \mathrm{E}-04$

$-1.89156 \mathrm{E} \quad 01 \quad-8.91613 \mathrm{E}-04$

$-1.89209 \mathrm{E} \quad 01 \quad-9.72709 \mathrm{E}-04$

$-1.89261 \mathrm{E} \quad 01 \quad-1.05606 \mathrm{E}-03$

$-1.89313 \mathrm{E}$ 01 $-1.14170 \mathrm{E}-03$

$-1.89363 \mathrm{E} \quad 01$

$-1.89413 \mathrm{E} \quad 01$

$-1.89462 \mathrm{E} \quad 01$

$-1.89510 \mathrm{E} \quad 01$

$-1.89557 \mathrm{E} \quad 01$

$-1.89604 \mathrm{E} \quad 01$

$-1.89650 \mathrm{E} \quad 01$

$-1.89695 \mathrm{E} \quad 01$

$-1.89739 \mathrm{E} \quad 01$

$-1.89783 \mathrm{E} 01$

$-1.89826 \mathrm{E} \quad 01$

$-1.89868 \mathrm{E} 01$

$-1.89910 \mathrm{E} 01$

$-1.89951 \mathrm{E} 01$

$-1.89991 \mathrm{E} 01$

$-1.90031 \mathrm{E} 01$

$-1.90070 \mathrm{E} 01$

$-1.90109 \mathrm{E} 01$

$-1.90147 \mathrm{E} \quad 01$

$-1.90185 \mathrm{E} \quad 01$

$-1.90222 \mathrm{E} \quad 01$

$-1.90258 \mathrm{E} 01$

$-1.90294 \mathrm{E} 01$

$-1.90330 \mathrm{E} 01$

$-1.90365 \mathrm{E} 01$

$-1.90399 \mathrm{E} \quad 01$

$-1.90433 \mathrm{E} 01$

$-1.90467 \mathrm{E} 01$

$-1.90500 \mathrm{E} 01$

$-1.90533 \mathrm{E} 01$

$-1.90565 \mathrm{E} \quad 01$ $-1.90597 \mathrm{E} \quad 01$

$-1.90628 \mathrm{E} 01$

$-1.90659 \mathrm{E} 01$

$-1.90689 \mathrm{E} 01$

$-1.90720 \mathrm{E} \quad 01$

$-1.90749 \mathrm{E} 01$

$-1.90779 \mathrm{E} 01$

$-1.90808 \mathrm{E} 01$

$-1.90836 \mathrm{E} 01$

$8.80083 \mathrm{E}-03$

$9.02374 \mathrm{E}-03$

$9.25011 \mathrm{E}-03$

$9.47998 \mathrm{E}-03$

$9.71336 \mathrm{E}-03$

$9.95028 \mathrm{E}-03$

$1.01908 \mathrm{E}-02$

$1.04348 \mathrm{E}-02$

$1.06825 \mathrm{E}-02$

$1.09339 \mathrm{E}-02$

$1.11889 \mathrm{E}-02$

$1.14476 \mathrm{E}-02$

$1.17100 \mathrm{E}-02$

$1.19762 \mathrm{E}-02$

$-1.22964 \mathrm{E}-03$ $-1.31992 \mathrm{E}-03$

$-1.41255 \mathrm{E}-03$

$-1.50757 \mathrm{E}-03$

$-1.60500 \mathrm{E}-03$

$-1.70487 \mathrm{E}-03$

$-1.80719 \mathrm{E}-03$

$-1.91200 \mathrm{E}-03$

$-2.01933 \mathrm{E}-03$

$-2.12919 \mathrm{E}-03$

$-2.24161 \mathrm{E}-03$

$-2.35663 \mathrm{E}-03$

$-2.47425 \mathrm{E}-03$

$-2.59452 \mathrm{E}-03$

$-2.71745 \mathrm{E}-03$

$-2.84308 \mathrm{E}-03$

$-2.97141 \mathrm{E}-03$

$-3.10249 \mathrm{E}-03$

$-3.23634 \mathrm{E}-03$

$-3.37298 \mathrm{E}-03$

$-3.51244 \mathrm{E}-03$

$-3.65474 \mathrm{E}-03$

$-3.79991 \mathrm{E}-03$

$-3.94798 \mathrm{E}-03$

$-4.09897 \mathrm{E}-03$

$-4.25290 \mathrm{E}-03$

$-4.40980 \mathrm{E}-03$

$-4.56970 \mathrm{E}-03$

$-4.73262 \mathrm{E}-03$

$-4.89859 \mathrm{E}-03$

$-5.06764 \mathrm{E}-03$

$-5.23978 \mathrm{E}-03$

$-5.41505 \mathrm{E}-03$

$-5.59346 \mathrm{E}-03$

$-5.77506 \mathrm{E}-03$

$-5.95985 \mathrm{E}-03$

$-6.14787 \mathrm{E}-03$

$-6.33914 \mathrm{E}-03$

$-6.53369 \mathrm{E}-03$

$-6.73154 \mathrm{E}-03$ 
ELECTRON RADIAL FUNCTIONS

Positron

(1)

18.10

18.20

18.30

18.40

18.50

18.60

18.70

18.80

18.90

19.00

19.10

19.20

19.30

19.40

19.50

19.60

19.70

19.80

19.90

20.00

20.10

20.20

20.30

20.40

20.50

20.60

20.70

20.80

20.90

21.00

21.10

21.20

21.30

21.40

21.50

21.60

21.70

21.80

21.90

22.00

22.10

22.20

22.30

22.40

22.50

22.60

22.70

22.80

22.90

23.00

23.10

23.20

23.30

23.40

23.50

23.60

23.70

23.80

23.90

24.00
$Z=10 \quad A=20$

$g_{1}$

$8.03190 \mathrm{E}-01$

$8.03170 \mathrm{E}-01$

$8.03150 \mathrm{E}-01$

$8.03130 \mathrm{E}-01$

$8.03110 \mathrm{E}-01$

$8.03090 \mathrm{E}-01$

$8.03070 \mathrm{E}-01$

$8.03050 \mathrm{E}-01$

$8.03031 \mathrm{E}-01$

8.03011 E-01

8.02991 E- 01

$8.02972 \mathrm{E}-01$

$8.02952 \mathrm{E}-01$

$8.02933 \mathrm{E}-01$

$8.02913 \mathrm{E}-01$

$8.02894 \mathrm{E}-01$

$8.02875 \mathrm{E}-01$

$8.02856 \mathrm{E}-01$

$8.02837 \mathrm{E}-01$

$8.02818 \mathrm{E}-01$

$8.02799 \mathrm{E}-01$

$8.02781 \mathrm{E}-01$

$8.02762 \mathrm{E}-01$

$8.02743 \mathrm{E}-01$

8.02724 E-01

$8.02706 \mathrm{E}-01$

$8.02687 \mathrm{E}-01$

$8.02669 \mathrm{E}-01$

$8.02651 \mathrm{E}-01$

$8.02632 \mathrm{E}-01$

8.02614E-01

$8.02596 \mathrm{E}-01$

$8.02578 \mathrm{E}-01$

$8.02560 \mathrm{E}-01$

$8.02542 \mathrm{E}-01$

$8.02524 \mathrm{E}-01$ $8.02506 \mathrm{E}-01$ $8.02488 \mathrm{E}-01$ $8.02470 \mathrm{E}-01$ $8.02453 \mathrm{E}-01$

$8.02435 \mathrm{E}-01$ $8.02418 \mathrm{E}-01$

$8.02401 \mathrm{E}-01$

$8.02383 \mathrm{E}-01$

$8.02366 \mathrm{E}-01$

$8.02349 \mathrm{E}-01$

8.02331 E-01

8.02314 E-01

$8.02297 \mathrm{E}-01$

8.02280 E- 01

$8.02263 \mathrm{E}-01$

$8.02246 \mathrm{E}-01$

$8.02230 \mathrm{E}-01$

8.02213E-01

$8.02196 \mathrm{E}-01$

$8.02179 \mathrm{E}-01$

8.02163 E-01

$8.02146 \mathrm{E}-01$

$8.02130 \mathrm{E}-01$

8.02113 E-01 $f_{1}$

$1.59999 \mathrm{E} \quad 01$

$1.60926 \mathrm{E} \quad 0$

$1.61853 \mathrm{E} 01$

$1.62780 \mathrm{E} 01$

$1.63707 \mathrm{E} \quad 01$

$1.64635 \mathrm{E} \quad 01$

$1.65563 \mathrm{E} 0$

$1.66491 \mathrm{E} 01$

$1.67420 \mathrm{E} \quad 01$

$1.68348 \mathrm{E} \quad 01$

$1.69277 \mathrm{E} \quad 01$

$1.70207 \mathrm{E} \quad 01$

$1.71136 \mathrm{E} \quad 01$

$1.72996 \mathrm{E} 01$

$\begin{array}{ll}1.73927 \mathrm{E} & 01\end{array}$

$1.74857 \mathrm{E} \quad 01$

$1.75788 \mathrm{E} \quad 01$

$1.76719 \mathrm{E} \quad 01$

$1.77651 \mathrm{E} 01$

$1.78583 \mathrm{E} \quad 01$

$1.79515 \mathrm{E} \quad 01$

$1.80447 \mathrm{E} \quad 01$

$1.81380 \mathrm{E} 01$

$1.82312 \mathrm{E} 01$

$1.83246 \mathrm{E} \quad 01$

$1.84179 \mathrm{E} 01$

$1.85113 \mathrm{E} 01$

$1.86047 \mathrm{E} \quad 01$

$1.86981 \mathrm{E} \quad 01$

$1.87916 \mathrm{E} \quad 01$

$1.88851 \mathrm{E} \quad 01$

$1.89786 \mathrm{E}$ 0l

$1.90721 \mathrm{E} \quad 01$

$1.91657 \mathrm{E} \quad 01$

$1.92593 \mathrm{E} \quad 01$

$1.93530 \mathrm{E} \quad 01$

$1.94466 \mathrm{E} \quad 01$

$1.95403 \mathrm{E} \quad 01$

$1.96341 \mathrm{E} \quad 01$

$1.97278 \mathrm{E} \quad 01$

$1.98216 \mathrm{E} \quad 01$

$1.99155 \mathrm{E} \quad 01$

$2.00093 \mathrm{E} \quad 01$

$2.01032 \mathrm{E} \quad 01$

$2.01971 \mathrm{E} \quad 01$

$2.02911 \mathrm{E} \quad 01$

$2.03850 \mathrm{E} 01$

$2.04790 \mathrm{E} 01$

$2.05731 \mathrm{E} 01$

$2.06672 \mathrm{E} \quad 01$

$2.07613 \mathrm{E} \quad 01$

$2.08554 \mathrm{E} \quad 01$

$2.09496 \mathrm{E} \quad 01$

$2.10438 \mathrm{E} 01$

$2.11380 \mathrm{E} \quad 01$

$2.12323 \mathrm{E} \quad 01$

$2.13266 \mathrm{E} \quad 01$

$2.14209 \mathrm{E} \quad 01$

$2.15153 \mathrm{E} \quad 01$
$1.72066 \mathrm{E} \quad 01$
$3.93580 \mathrm{E}-01$

$4.00381 \mathrm{E}-01$

$4.07235 \mathrm{E}-01$

$4.14143 \mathrm{E}-01$

$4.21103 \mathrm{E}-01$

$4.28116 \mathrm{E}-01$

$4.35182 \mathrm{E}-01$

$4.42301 \mathrm{E}-01$

$4.49473 \mathrm{E}-01$

$4.56699 \mathrm{E}-01$

$4.63977 \mathrm{E}-01$

$4.71309 \mathrm{E}-01$

$4.78693 \mathbf{E}-01$

$4.86131 \mathrm{E}-01$

$4.93623 \mathrm{E}-01$

$5.01167 \mathrm{E}-01$

$5.08765 \mathrm{E}-01$

$5.16416 \mathrm{E}-01$

$5.24121 \mathrm{E}-01$

$5.31879 \mathrm{E}-01$

$5.39690 \mathrm{E}-01$

$5.47555 \mathrm{E}-01$

$5.55473 \mathrm{E}-01$

$5.63445 \mathrm{E}-01$

5.71471 E-01

$5.79550 \mathrm{E}-01$

$5.87683 \mathrm{E}-01$

$5.95869 \mathrm{E}-01$

$6.04109 \mathrm{E}-01$

$6.12403 \mathrm{E}-01$

$6.20750 \mathrm{E}-01$

6.29151 E-01

$6.37606 \mathrm{E}-01$

$6.46115 \mathrm{E}-01$

$6.54678 \mathrm{E}-01$

$6.63295 \mathrm{E}-01$

$6.71966 \mathrm{E}-01$

$6.80690 \mathrm{E}-01$

$6.89469 \mathrm{E}-01$

6.98301 E-01

$7.07188 \mathrm{E}-01$

$7.16129 \mathrm{E}-01$

$7.25124 \mathrm{E}-01$

$7.34173 \mathrm{E}-01$

$7.43277 \mathrm{E}-01$

$7.52435 \mathrm{E}-01$

$7.61647 \mathrm{E}-01$

$7.70913 \mathrm{E}-01$

$7.80234 \mathrm{E}-01$

7.89609 E-01 $8.08523 \mathrm{E}-01$

8.18061 E-01

$8.27654 \mathrm{E}-01$

$8.37302 \mathrm{E}-01$

$8.47005 \mathrm{E}-01$

$8.56761 \mathrm{E}-01$

8.66573E-01

$8.86360 \mathrm{E}-01$
$7.99039 \mathrm{E}-01$

$8.76439 \mathrm{E}-01$

$$
\rho=1.2 A^{1 / 3} F
$$

$\tan \delta_{1}$

$f_{-1}$

$5.60826 \mathrm{E}-04$

$5.51331 \mathrm{E}-04$

$5.41975 \mathrm{E}-04$

$5.32725 \mathrm{E}-04$

$5.23616 \mathrm{E}-04$

$5.14620 \mathrm{E}-04$

$5.05753 \mathrm{E}-04$

$4.96995 \mathrm{E}-04$

$4.88366 \mathrm{E}-04$

$4.79847 \mathrm{E}-04$

$4.71436 \mathrm{E}-04$

$4.63139 \mathrm{E}-04$

$4.54960 \mathrm{E}-04$

$4.46877 \mathrm{E}-04$

$4.38911 \mathrm{E}-04$

$4.31056 \mathrm{E}-04$

$4.23296 \mathrm{E}-04$

$4.15626 \mathrm{E}-04$

$4.08078 \mathrm{E}-04$

$4.00625 \mathrm{E}-04$

$3.93266 \mathrm{E}-04$

$3.86015 \mathrm{E}-04$

$3.78850 \mathrm{E}-04$

$3.71784 \mathrm{E}-04$

$3.64814 \mathrm{E}-04$

$3.57936 \mathrm{E}-04$

$3.51138 \mathrm{E}-04$

$3.44433 \mathrm{E}-04$

$3.37833 \mathrm{E}-04$

3.31291 E- 04

3. $24857 \mathrm{~F}-04$

$3.18520 \mathrm{E}-04$

$3.12237 \mathrm{E}-04$

$3.06060 \mathrm{E}-04$

$2.99956 \mathrm{E}-04$

$2.93929 \mathrm{E}-04$

$2.87987 \mathrm{E}-04$

$2.82124 \mathrm{E}-04$

$2.76343 \mathrm{E}-04$ 
ELECTRON RADIAL FUNCTIONS

$Z=10 \quad A=20 \quad \rho=1.2 A^{1 / 3} F$

Positron

$p$

18.10

18.20

18.30

18.40

18.50

18.60

18.70

18.80

18.90

19.00

19.10

19.20

19.30

19.40

19.50

19.60

19.70

19.80

19.90

20.00

20.10

20.20

20.30

20.40

20.50

20.60

20.70

20.80

20.90

21.00

21.10

21.20

21.30

21.40

21.50

21.60

21.70

21.80

21.90

22.00

22.10

22.20

22.30

22.40

22.50

22.60

22.70

22.80

22.90

23.00

23.10

23.20

23.30

23.40

23.50

23.60

23.70

23.80

23.90

24.00

\section{$\bar{F}$}

$5.18085 \mathrm{E}-04$

$5.23819 \mathrm{E}-04$

$5.29586 \mathrm{E}-04$

$5.35384 \mathrm{E}-04$

$5.41213 \mathrm{E}-04$

$5.47074 \mathrm{E}-04$

$5.52966 \mathrm{E}-04$

$5.58890 \mathrm{E}-04$

$5.64846 \mathrm{E}-04$

$5.70833 \mathrm{E}-04$

$5.76851 \mathrm{E}-04$

5.82901 E- 04

$5.88983 \mathrm{E}-04$

$5.95096 \mathrm{E}-04$

$6.01240 \mathrm{E}-04$

$6.07416 \mathrm{E}-04$

$6.13624 \mathrm{E}-04$

$6.19863 \mathrm{E}-04$

$6.26134 \mathrm{E}-04$

$6.32436 \mathrm{E}-04$

$6.38769 \mathrm{E}-04$

$6.45134 \mathrm{E}-04$

$6.51531 \mathrm{E}-04$

$6.57959 \mathrm{E}-04$

$6.64418 \mathrm{E}-04$

$6.70909 \mathrm{E}-04$

6.77432 E-04

$6.83986 \mathrm{E}-04$

$6.90571 \mathrm{E}-04$

$6.97188 \mathrm{E}-04$

$7.03837 \mathrm{E}-04$

$7.10517 \mathrm{E}-04$

$7.17228 \mathrm{E}-04$

$7.23972 \mathrm{E}-04$

$7.30746 \mathrm{E}-04$

$7.37552 \mathrm{E}-04$

$7.44390 \mathrm{E}-04$

$7.51259 \mathrm{E}-04$

$7.58160 \mathrm{E}-04$

$7.65092 \mathrm{E}-04$

$7.72055 \mathrm{E}-04$

$7.79050 \mathrm{E}-04$

$7.86077 \mathrm{E}-04$

$7.93135 \mathrm{E}-04$

$8.00224 \mathrm{E}-04$

$8.07345 \mathrm{E}-04$

$8.14498 \mathrm{E}-04$

$8.21682 \mathrm{E}-04$

$8.28897 \mathrm{E}-04$

$8.36145 \mathrm{E}-04$

$8.43423 \mathrm{E}-04$

$8.50733 \mathrm{E}-04$

$8.58074 \mathrm{E}-04$

$8.65447 \mathrm{E}-04$

$8.72852 \mathrm{E}-04$

$8.80288 \mathrm{E}-04$

$8.87755 \mathrm{E}-04$

8.95254 E-04

$9.02785 \mathrm{E}-04$

$9.10347 \mathrm{E}-04$ $f_{2}$

8.02995E-01

$8.12020 \mathrm{E}-01$

$8.21094 \mathrm{E}-01$

$8.30219 \mathrm{E}-01$

$8.39395 \mathrm{E}-01$

$8.48621 \mathrm{E}-01$

$8.57897 \mathrm{E}-0$

$8.67223 \mathrm{E}-01$

$8.76599 \mathrm{E}-01$

$8.86026 \mathrm{E}-01$

$8.95503 \mathrm{E}-01$

$9.05030 \mathrm{E}-01$

$9.14607 \mathrm{E}-01$

$9.24235 \mathrm{E}-01$

$9.33913 \mathrm{E}-01$

$9.43641 \mathrm{E}-01$

$9.53419 \mathrm{E}-0$

$9.63248 \mathrm{E}-01$

$9.73127 \mathrm{E}-01$

$9.83056 \mathrm{E}-01$

$9.93035 \mathrm{E}-01$

$1.00306 \mathrm{E} \quad 00$

$1.01314 \mathrm{E} \quad 00$

$1.02327 \mathrm{E} \quad 00$

$1.03345 \mathrm{E} \quad 00$

$1.04368 \mathrm{E} \quad 00$ $1.05396 \mathrm{E} \quad 00$

$1.06429 \mathrm{E} \quad 00$

$1.07467 \mathrm{E} \quad 00$

$1.08510 \mathrm{E} \quad 00$

$1.09559 \mathrm{E} \quad 00$

$1.10612 \mathrm{E} \quad 00$

$1.11670 \mathrm{E} \quad 00$

$1.12733 \mathrm{E} \quad 00$

$1.13801 \mathrm{E} \quad 00$

$1.14874 \mathrm{E} \quad 00$

$1.15952 \mathrm{E} \quad 00$

$1.17036 \mathrm{E} \quad 00$

$1.18124 \mathrm{E} \quad 00$

$1.19217 \mathrm{E} \quad 00$

$1.20315 \mathrm{E} \quad 00$

$1.21418 \mathrm{E} \quad 00$

$1.22527 \mathrm{E} \quad 00$

$1.23640 \mathrm{E} 00$

$1.24758 \mathrm{E} \quad 00$

$1.25881 \mathrm{E} \quad 00$

$1.27010 \mathrm{E} \quad 00$

$1.28143 \mathrm{E} \quad 00$

$1.29281 \mathrm{E} \quad 00$

$1.30425 \mathrm{E} \quad 00$

$1.31573 \mathrm{E} \quad 00$

$1.32726 \mathrm{E} \quad 00$

$1.33884 \mathrm{E} \quad 00$

$1.35048 \mathrm{E} \quad 00$

$1.36216 \mathrm{E} \quad 00$

$1.37389 \mathrm{E} \quad 00$

$1.38568 \mathrm{E} \quad 00$

$1.39751 \mathrm{E} \quad 00$

$1.40939 \mathrm{E} \quad 00$

$1.42133 \mathrm{E} 00$ $g_{2}$

$\tan \delta_{2}$

$f_{-2}$

g-2

$\tan \delta_{-2}$

$1.25198 \mathrm{E}-02-1.91250 \mathrm{E} \quad 01-1.03671 \mathrm{E}-02$

$1.27974 \mathrm{E}-02-1.91273 \mathrm{E}$ 01 $-1.06250 \mathrm{E}-02$

$1.30788 \mathrm{E}-02-1.91296 \mathrm{E} \quad 01-1.08867 \mathrm{E}-02$

$1.33640 \mathrm{E}-02-1.91319 \mathrm{E}$ 01 $-1.11522 \mathrm{E}-02$

$1.36532 \mathrm{E}-02-1.91341 \mathrm{E}$ 01 $-1.14214 \mathrm{E}-02$

$1.39463 \mathrm{E}-02$

$1.42433 \mathrm{E}-02$

$1.45443 \mathrm{E}-02$

$1.48493 \mathrm{E}-02$

$1.51583 \mathrm{E}-02$

$1.54714 \mathrm{E}-02$

$1.57886 \mathrm{E}-02$

$1.61098 \mathrm{E}-02$

$1.64352 \mathrm{E}-02$

$1.67647 \mathrm{E}-02$

$1.70985 \mathrm{E}-02$

$1.74364 \mathrm{E}-02$

$1.77785 \mathrm{E}-02$

$1.81249 \mathrm{E}-02$

$1.84756 \mathrm{E}-02$

$1.88305 \mathrm{E}-02$

$1.91898 \mathrm{E}-02$

$1.95535 \mathrm{E}-02$

$1.99215 \mathrm{E}-02$

$2.02940 \mathrm{E}-02$

$2.06708 \mathrm{E}-02$

$2.10522 \mathrm{E}-02$

$2.14380 \mathrm{E}-02$

$2.18283 \mathrm{E}-02$

2.22232 E- 02

$2.26226 \mathrm{E}-02$

$2.30266 \mathrm{E}-02$

$2.34352 \mathrm{E}-02$

2.38484.E-02

$2.42663 \mathrm{E}-02$

$2.46889 \mathrm{E}-02$

$2.51162 \mathrm{E}-02$

$2.55482 \mathrm{E}-02$

$2.59850 \mathrm{E}-02$

$2.64266 \mathrm{E}-02$

$2.68730 \mathrm{E}-02$

$2.73242 \mathrm{E}-02$

$2.77803 \mathrm{E}-02$

$2.82413 \mathrm{E}-02$

$2.87072 \mathrm{E}-02$

$2.91780 \mathrm{E}-02$

$2.96538 \mathrm{E}-02$

$3.01346 \mathrm{E}-02$

$3.06204 \mathrm{E}-02$

$3.11112 \mathrm{E}-02$

$3.16071 \mathrm{E}-02$

$3.21081 \mathrm{E}-02$

$3.26143 \mathrm{E}-02$

$3.31255 \mathrm{E}-02$

$3.36419 \mathrm{E}-02$

$3.41635 \mathrm{E}-02$

$3.46904 \mathrm{E}-02$

$3.52224 \mathrm{E}-02$

$3.57598 \mathrm{E}-02$

$3.63024 \mathrm{E}-02$

$-1.91364 \mathrm{E} \quad 01$

$-1.91386 \mathrm{E}$ 01

$-1.91407 \mathrm{E} 01$

$-1.91429 \mathrm{E} 01$

$-1.91450 \mathrm{E} 01$

$-1.16945 \mathrm{E}-02$

$-1.19715 \mathrm{E}-02$

$-1.22523 \mathrm{E}-02$

$-1.25371 \mathrm{E}-02$

$-1.28258 \mathrm{E}-02$

$-1.91471 \mathrm{E} \quad 01$

$-1.91492 \mathrm{E} 01$

$-1.91513 \mathrm{E} \quad 01$

$-1.91533 \mathrm{E} 01$

$-1.91553 \mathrm{E} 01$

$-1.31184 \mathrm{E}-02$

$-1.34151 \mathrm{E}-02$

$-1.37158 \mathrm{E}-02$

$-1.40205 \mathrm{E}-02$

$-1.43293 \mathrm{E}-02$

$-1.91573 \mathrm{E} 01$

$-1.91593 \mathrm{E} 01$

$-1.91613 \mathrm{E} 01$

$-1.91632 \mathrm{E} 01$

$-1.91651 \mathrm{E} 01$

$-1.91670 \mathrm{E}$ ol

$-1.91689 \mathrm{E} 01$

$-1.91707 \mathrm{E} 01$

$-1.91726 \mathrm{E} 01$

$-1.91744 \mathrm{E} 01$

$-1.46422 \mathrm{E}-02$

$-1.49591 \mathrm{E}-02$

$-1.52803 \mathrm{E}-02$

$-1.56056 \mathrm{E}-02$

$-1.59351 \mathrm{E}-02$

$-1.62688 \mathrm{E}-02$

$-1.66068 \mathrm{E}-02$

$-1.69490 \mathrm{E}-02$ 
$p \quad F_{0}$

24.10

24.20

24.30

24.40

24.50

24.60

24.70

24.80

24.90

25.00

25.10

25.20

25.30

25.40

25.50

25.60

25.70

25.80

25.90

26.00

26.10

26.20

26.30

26.40

26.50

26.60

26.70

26.80

26.90

27.00

27.10

27.20

27.30

27.40

27.50

27.60

27.70

27.80

27.90

28.00

28.10

28.20

28.30

28.40

28.50

28.60

28.70

28.80

28.90

29.00

29.10

29.20

29.30

29.40

29.50

29.60

29.70

29.80

29.90

30.00 $f_{1}$

$8.02097 \mathrm{E}-01$

$8.02081 \mathrm{E}-01$

$8.02064 \mathrm{E}-01$

$8.02048 \mathrm{E}-01$

$8.02032 \mathrm{E}-01$

8.02016E-01

$8.02000 \mathrm{E}-01$

$8.01984 \mathrm{E}-01$

$8.01968 \mathrm{E}-01$

$8.01952 \mathrm{E}-01$

$8.01936 \mathrm{E}-01$

$8.01921 \mathrm{E}-01$

$8.01905 \mathrm{E}-01$

$8.01889 \mathrm{E}-01$

$8.01874 \mathrm{E}-01$

$8.01858 \mathrm{E}-01$

$8.01843 \mathrm{E}-01$

$8.01827 \mathrm{E}-01$

$8.01812 \mathrm{E}-01$

$8.01797 \mathrm{E}-01$

$8.01781 \mathrm{E}-01$

$8.01766 \mathrm{E}-01$

$8.01751 \mathrm{E}-01$

$8.01736 \mathrm{E}-01$

$8.01721 \mathrm{E}-01$

8.01706E-01

$8.01691 \mathrm{E}-01$

$8.01676 \mathrm{E}-01$

$8.01661 \mathrm{E}-01$

$8.01646 \mathrm{E}-01$

8.01631 E-01

$8.01616 \mathrm{E}-01$

$8.01602 \mathrm{E}-01$

8.01587 E-01

$8.01572 \mathrm{E}-01$

$8.01558 \mathrm{E}-01$

$8.01543 \mathrm{E}-01$

$8.01529 \mathrm{E}-01$

$8.01514 \mathrm{E}-01$

$8.01500 \mathrm{E}-01$

$8.01486 \mathrm{E}-01$ $8.01471 \mathrm{E}-01$

$8.01457 \mathrm{E}-01$

8.01443 E-01

$8.01429 \mathrm{E}-01$

$8.01415 \mathrm{E}-01$

$8.01400 \mathrm{E}-01$

$8.01387 \mathrm{E}-01$

$8.01373 \mathrm{E}-01$

$8.01359 \mathrm{E}-01$

$8.01345 \mathrm{E}-01$

$8.01331 \mathrm{E}-01$

$8.01317 \mathrm{E}-01$

$8.01303 \mathrm{E}-01$

$8.01289 \mathrm{E}-01$

$8.01276 \mathrm{E}-01$ $8.01262 \mathrm{E}-01$ $8.01249 \mathrm{E}-01$

$8.01235 \mathrm{E}-01$

$8.01221 \mathrm{E}-01$ $g_{1}$

$2.16097 \mathrm{E} \quad 01$

$2.17041 \mathrm{E} 01$

$2.17986 \mathrm{E} \quad 01$

$2.18931 \mathrm{E} \quad 01$

$2.19876 \mathrm{E} 01$

$2.20822 \mathrm{E} \quad 01$

$2.21768 \mathrm{E} \quad 01$

$2.22714 \mathrm{E} \quad 01$

$2.23661 \mathrm{E} \quad 01$

$2.24608 \mathrm{E} \quad 01$

$2.25555 \mathrm{E} \quad 01$

$2.26503 \mathrm{E} \quad 01$

$2.27451 \mathrm{E} 01$

$2.28399 \mathrm{E} 01$

$2.29348 \mathrm{E}$ ol

$2.30297 \mathrm{E} \quad 01$

$2.31247 \mathrm{E} \quad 01$

$2.32196 \mathrm{E} 01$

$2.33146 \mathrm{E} \quad 01$

$2.34097 \mathrm{E} 01$

$2.35048 \mathrm{E} \quad 01$

$2.35999 \mathrm{E} 01$

$2.36951 \mathrm{E} 01$

$2.37902 \mathrm{E} 01$

$2.38855 \mathrm{E} 01$

$2.39807 \mathrm{E} \quad 01$

$2.40760 \mathrm{E} \quad 01$

$2.41714 \mathrm{E} \quad 01$

$2.42668 \mathrm{E} \quad 01$

$2.43622 \mathrm{E} \quad 01$

$2.44576 \mathrm{E} \quad 01$

$2.45531 \mathrm{E} \quad 01$

$2.46486 \mathrm{E} \quad 01$

$2.47442 \mathrm{E} 01$

2.48398 E 01

$2.49354 \mathrm{E} \quad 01$

$2.50311 \mathrm{E} 01$

$2.51268 \mathrm{E} 01$

$2.52225 \mathrm{E} \quad 01$

$2.53183 \mathrm{E} 01$

2.54141 E 01

$2.55100 \mathrm{E} 01$

$2.56059 \mathrm{E} \quad 01$

$2.57018 \mathrm{E} 01$

$2.57978 \mathrm{E} \quad 01$

$2.58938 \mathrm{E} \quad 01$

$2.59899 \mathrm{E} 01$

$2.60860 \mathrm{E} 01$

$2.61821 \mathrm{E} 01$

$2.62783 \mathrm{E} 01$

$2.63745 \mathrm{E} \quad 01$

$2.64707 \mathrm{E} 01$

$2.65670 \mathrm{E} 01$

$2.66633 \mathrm{E} 01$

$2.67597 \mathrm{E} \quad 01$

$2.68561 \mathrm{E} \quad 01$

$2.69526 \mathrm{E} \quad 01$

$2.70491 \mathrm{E} \quad 01$

$2.71456 \mathrm{E} \quad 01$

2.72422 E 01 $\tan \delta_{1}$

$f_{-1}$

$8.96337 \mathrm{E}-01$

$9.06368 \mathrm{E}-01$

$9.16454 \mathrm{E}-01$

$9.26594 \mathrm{E}-01$

$9.36790 \mathrm{E}-01$

9.47041 E-01

$9.57346 \mathrm{E}-01$

9.67707E-01

$9.78123 \mathrm{E}-01$

$9.88594 \mathrm{E}-01$

$9.99120 \mathrm{E}-01$

$1.00970 \mathrm{E} \quad 00$

$1.02034 \mathrm{E} \quad 00$

$1.03103 \mathrm{E} \quad 00$

$1.04178 \mathrm{E} \quad 00$

$1.05258 \mathrm{E} \quad 00$

$1.06344 \mathrm{E} \quad 00$

$1.07435 \mathrm{E} \quad 00$

$1.08532 \mathrm{E} \quad 00$

$1.09635 \mathrm{E} \quad 00$

$1.10743 \mathrm{E} \quad 00$

$1.11856 \mathrm{E} \quad 00$

$1.12975 \mathrm{E} 00$

$1.14100 \mathrm{E} \quad 00$

$1.15231 \mathrm{E} \quad 00$

$1.16366 \mathrm{E} \quad 00$

$1.17508 \mathrm{E} \quad 00$

$1.18655 \mathrm{E} \quad 00$

$1.19808 \mathrm{E} \quad 00$

$1.20966 \mathrm{E} \quad 00$

$1.22130 \mathrm{E} \quad 00$

$1.23299 \mathrm{E} \quad 00$

$1.24474 \mathrm{E} \quad 00$

$1.25655 \mathrm{E} \quad 00$

$1.26841 \mathrm{E} \quad 00$

$1.28033 \mathrm{E} \quad 00$

$1.29231 \mathrm{E} \quad 00$

$1.30434 \mathrm{E} \quad 00$

$1.31643 \mathrm{E} \quad 00$

$1.32858 \mathrm{E} \quad 00$

$1.34078 \mathrm{E} \quad 00$

$1.35303 \mathrm{E} \quad 00$

$1.36535 \mathrm{E} \quad 00$

$1.37772 \mathrm{E} \quad 00$

$1.39015 \mathrm{E} \quad 00$

$1.40263 \mathrm{E} \quad 00$

$1.41517 \mathrm{E} \quad 00$

$1.42777 \mathrm{E} \quad 00$

$1.44043 \mathrm{E} \quad 00$

$1.45314 \mathrm{E} \quad 00$

$1.46591 \mathrm{E} \quad 00$

$1.47873 \mathrm{E} \quad 00$

$1.49162 \mathrm{E} \quad 00$

$1.50455 \mathrm{E} \quad 00$

$1.51755 \mathrm{E} 00$

$1.53061 \mathrm{E} \quad 00$

$1.54372 \mathrm{E} \quad 00$

$1.55689 \mathrm{E} \quad 00$

$1.57011 \mathrm{E} \quad 00$

$1.58340 \mathrm{E} \quad 00$

$\begin{array}{cccc}1.67109 \mathrm{E}-04 & -8.08846 \mathrm{E}-01 & 2.25622 \mathrm{E} & 01 \\ 1.62891 \mathrm{E}-04 & -8.18627 \mathrm{E}-01 & 2.26570 \mathrm{E} & 01 \\ 1.58725 \mathrm{E}-04 & -8.28464 \mathrm{E}-01 & 2.27519 \mathrm{E} & 01 \\ 1.54631 \mathrm{E}-04 & -8.38355 \mathrm{E}-01 & 2.28469 \mathrm{E} & 01 \\ 1.50588 \mathrm{E}-04 & -8.48302 \mathrm{E}-01 & 2.29418 \mathrm{E} & 01\end{array}$

$2.30368 \mathrm{E} \quad 01$ $2.31319 \mathrm{E} \quad 01$

$2.32269 \mathrm{E} 01$

$2.33220 \mathrm{E} 01$

$2.34172 \mathrm{E} 01$

$2.35124 \mathrm{E} \quad 01$ $2.36076 \mathrm{E} 01$ $2.37028 \mathrm{E} \quad 01$ $2.37981 \mathrm{E} 01$ $2.38935 \mathrm{E} 01$

$2.39888 \mathrm{E} \quad 01$ $2.40842 \mathrm{E} \quad 01$ $2.41797 \mathrm{E} \quad 01$ $2.42751 \mathrm{E} \quad 01$ $2.43706 \mathrm{E} 01$

$2.44662 \mathrm{E} \quad 01$ $2.45618 \mathrm{E} \quad 01$ $2.46574 \mathrm{E} \quad 01$ $2.47531 \mathrm{E} \quad 01$ $2.48488 \mathrm{E} 01$

$2.49445 \mathrm{E} \quad 01$ $2.50403 \mathrm{E} \quad 01$ $2.51361 \mathrm{E} \quad 01$ $2.52319 \mathrm{E} 01$ $2.53278 \mathrm{E} \quad 01$

$2.54237 \mathrm{E} \quad 01$ $2.55197 \mathrm{E} 01$ $2.56157 \mathrm{E} 01$ $2.57118 \mathrm{E} 01$ $2.58078 \mathrm{E} 01$

$2.59040 \mathrm{E} \quad 01$ $2.60001 \mathrm{E} \quad 01$ $2.60963 \mathrm{E} 01$ $261926 \mathrm{E} 01$

$2.62888 \mathrm{E} 01$

$2.63852 \mathrm{E} \quad 01$ $2.64815 \mathrm{E} 01$ $2.65779 \mathrm{E} \quad 01$

$2.66744 \mathrm{E} \quad 01$ $2.67708 \mathrm{E} \quad 01$ 
ELECTRON RADIAL FUNCTIONS

$Z=10 \quad A=20 \quad \rho=1.2 A^{1 / 3} F$

Pesitron

$\bar{F}$

$9.17940 \mathrm{E}-04$ $9.25565 \mathrm{E}-04$ $9.33222 \mathrm{E}-04$ $9.40909 \mathrm{E}-04$ $9.48629 \mathrm{E}-04$

$9.56380 \mathrm{E}-04$ $9.64163 \mathrm{E}-04$ $9.71976 \mathrm{E}-04$ $9.79822 \mathrm{E}-04$ $9.87698 \mathrm{E}-04$

$9.95607 \mathrm{E}-04$ $1.00355 \mathrm{E}-03$ $1.01152 \mathrm{E}-03$ $1.01952 \mathrm{E}-03$ $1.02755 \mathrm{E}-03$

$1.03562 \mathrm{E}-03$ $1.04372 \mathrm{E}-03$ $1.05185 \mathrm{E}-03$ $1.06001 \mathrm{E}-03$ $1.06820 \mathrm{E}-03$

$1.07642 \mathrm{E}-03$ $1.08467 \mathrm{E}-03$ $1.09296 \mathrm{E}-03$ $1.10128 \mathrm{E}-03$ $1.10963 \mathrm{E}-03$

$1.11801 \mathrm{E}-03$ $1.12642 \mathrm{E}-03$ $1.13486 \mathrm{E}-03$ $1.14334 \mathrm{E}-03$ $1.15184 \mathrm{E}-03$

$1.16038 \mathrm{E}-03$ $1.16895 \mathrm{E}-03$ $1.17755 \mathrm{E}-03$ $1.18619 \mathrm{E}-03$ $1.19485 \mathrm{E}-03$

27.40

27.50

27.60

27.70

27.80

27.90

28.00

28.10

28.20

28.30

28.40

28.50

28.60

28.70

28.80

28.90

29.00

29.10

29.20

29.30

29.40

29.50

29.60

29.70

29.80

29.90

30.00

$1.20354 \mathrm{E}-03$ $1.21227 \mathrm{E}-03$ $1.22103 \mathrm{E}-03$ $1.22982 \mathrm{E}-03$ $1.23864 \mathrm{E}-03$

$1.24749 \mathrm{E}-03$ $1.25638 \mathrm{E}-03$ $1.26529 \mathrm{E}-03$ 1.27424. E- 03 $1.28322 \mathrm{E}-03$

$1.29223 \mathrm{E}-03$ $1.30127 \mathrm{E}-03$ $1.31034 \mathrm{E}-03$ $1.31945 \mathrm{E}-03$ $1.32858 \mathrm{E}-03$

$1.33775 \mathrm{E}-03$ $1.34695 \mathrm{E}-03$ $1.35618 \mathrm{E}-03$ $1.36544 \mathrm{E}-03$ $1.37474 \mathrm{E}-03$

$1.38406 \mathrm{E}-03$ $1.39342 \mathrm{E}-03$ $1.40281 \mathrm{E}-03$ $1.41223 \mathrm{E}-03$ $1.42168 \mathrm{E}-03$ $f_{2}$
$1.43331 \mathrm{E} \quad 00$

$1.44534 \mathrm{E} \quad 00$

$1.45743 \mathrm{E} \quad 00$

$1.46956 \mathrm{E} \quad 00$

$1.48174 \mathrm{E} \quad 00$

$1.49397 \mathrm{E} \quad 00$

$1.50626 \mathrm{E} \quad 00$

$1.51859 \mathrm{E} \quad 00$

$1.53097 \mathrm{E} \quad 00$

$1.54341 \mathrm{E} \quad 00$

$1.55589 \mathrm{E} \quad 00$

$1.56842 \mathrm{E} \quad 00$

$1.58100 \mathrm{E} \quad 00$

$1.59364 \mathrm{E} \quad 00$

$1.60632 \mathrm{E} \quad 00$

$1.61905 \mathrm{E} \quad 00$ $1.63183 \mathrm{E} \quad 00$

$1.64466 \mathrm{E} \quad 00$

$1.65755 \mathrm{E} \quad 00$

$1.67048 \mathrm{E} \quad 00$

$1.68346 \mathrm{E} \quad 00$ $1.69649 \mathrm{E} \quad 00$

$1.70957 \mathrm{E} \quad 00$

$1.72270 \mathrm{E} \quad 00$

$1.73588 \mathrm{E} \quad 00$

$1.74911 \mathrm{E} \quad 00$

$1.76240 \mathrm{E} \quad 00$

$1.77573 \mathrm{E} \quad 00$

$1.78911 \mathrm{E} \quad 00$

$1.80254 \mathrm{E} \quad 00$

$1.81602 \mathrm{E} \quad 00$ $1.82955 \mathrm{E} \quad 00$ $1.84313 \mathrm{E} \quad 00$ $1.85676 \mathrm{E} \quad 00$ $1.87043 \mathrm{E} \quad 00$

$g_{2}$

$3.68504 \mathrm{E}-02$ $3.74037 \mathrm{E}-02$ $3.79624 \mathrm{E}-02$

$3.90959 \mathrm{E}-02$

$3.96708 \mathrm{E}-02$

$4.02512 \mathrm{E}-02$

$4.08371 \mathrm{E}-02$

4.20255 E-02

$4.26280 \mathrm{E}-02$ 4.32362 E-02 $4.38499 \mathrm{E}-02$ $4.44693 \mathrm{E}-02$ 4.50944 E-02

4.57252 E- 02 $4.63617 \mathrm{E}-02$ 4.70039 E-02 $4.76519 \mathrm{E}-02$ $4.83057 \mathrm{E}-02$

4.89654 E-02 $4.96309 \mathrm{E}-02$

$5.03023 \mathrm{E}-02$

$5.09795 \mathrm{E}-02$

$5.16627 \mathrm{E}-02$

$5.23519 \mathrm{E}-02$ $5.30470 \mathrm{E}-02$ $5.37481 \mathrm{E}-02$

$5.44553 \mathrm{E}-02$

$5.51685 \mathrm{E}-02$

$5.58877 \mathrm{E}-02$ 5.66131 E-02 $5.73446 \mathrm{E}-02$

$5.88261 \mathrm{E}-02$

$1.88416 \mathrm{E} \quad 00$

$1.89794 \mathrm{E} \quad 00$

$1.91177 \mathrm{E} \quad 00$

$1.92565 \mathrm{E} \quad 00$

$1.93958 \mathrm{E} \quad 00$

$1.95355 \mathrm{E} \quad 00$

$1.96758 \mathrm{E} \quad 00$

$1.98166 \mathrm{E} \quad 00$

$1.99578 \mathrm{E} \quad 00$

$2.00996 \mathrm{E} \quad 00$

$2.02419 \mathrm{E} \quad 00$ $2.03846 \mathrm{E} \quad 00$

$2.05279 \mathrm{E} \quad 00$

$2.06716 \mathrm{E} \quad 00$

$2.08159 \mathrm{E} \quad 00$

$2.09606 \mathrm{E} \quad 00$

$2.11059 \mathrm{E} \quad 00$

$2.12516 \mathrm{E} \quad 00$

$2.13978 \mathrm{E} \quad 00$

$2.15446 \mathrm{E} \quad 00$

$2.16918 \mathrm{E} \quad 00$

$2.18395 \mathrm{E} \quad 00$

$2.19877 \mathrm{E} \quad 00$

$2.21365 \mathrm{E} \quad 00$

$2.22857 \mathrm{E} \quad 00$

$5.95762 \mathrm{E}-02$

$6.03325 \mathrm{E}-02$

$6.10950 \mathrm{E}-02$

$6.18638 \mathrm{E}-02$

$6.26389 \mathrm{E}-02$

$6.34203 \mathrm{E}-02$

$6.42081 \mathrm{E}-02$

$6.50023 \mathrm{E}-02$

$6.58028 \mathrm{E}-02$

$6.66098 \mathrm{E}-02$

6.74233 E-02 $6.82432 \mathrm{E}-02$ $6.90697 \mathrm{E}-02$

$6.99026 \mathrm{E}-02$

7.07421 E-02

7.15883 E- 02

$7.24410 \mathrm{E}-02$

$7.33003 \mathrm{E}-02$

$7.41663 \mathrm{E}-02$

$7.50390 \mathrm{E}-02$

$7.59184 \mathrm{E}-02$

$7.68045 \mathrm{E}-02$

$7.76974 \mathrm{E}-02$

$7.85970 \mathrm{E}-02$

$7.95035 \mathrm{E}-02$
$3.85264 \mathrm{E}-02$

$4.14286 \mathrm{E}-02$ $5.80823 \mathrm{E}-02$

$\tan \delta_{2}$

$f_{-2}$

$-1.92295 \mathrm{E}$ 0I

$-1.92308 \mathrm{E} 0$

$-1.92320 \mathrm{E} 01$

$-1.92333 \mathrm{E} 01$

$-1.92345 \mathrm{E} \quad 01$

$-1.92358 \mathrm{E} \quad 01$

$-1.92370 \mathrm{E}$ 0I

$-1.92383 \mathrm{E} 01$

$-1.92395 \mathrm{E}$ 0I

$-1.92407 \mathrm{E} 01$

$-1.92419 \mathrm{E} \quad 01$

$-1.92430 \mathrm{E} \quad 01$

$-1.92442 \mathrm{E} 01$

$-1.92454 \mathrm{E} \quad 01$

$-1.92465 \mathrm{E} 01$

$\begin{array}{ll}-1.92477 \mathrm{E} & 01\end{array}$

$-1.92488 \mathrm{E} 01$

$-1.92499 \mathrm{E} \quad 01$

$-1.92511 \mathrm{E} 01$

$-1.92522 \mathrm{E} 01$

$-1.92533 \mathrm{E} 01$

$-1.92544 \mathrm{E} 01$

$-1.92555 \mathrm{E} \quad 01$

$-1.92565 \mathrm{E} \quad 01$

$-1.92576 \mathrm{E} \quad 01$

$\begin{array}{lll}-1.92587 \mathrm{E} & 01\end{array}$

$-1.92597 \mathrm{E} \quad 01$

$-1.92607 \mathrm{E} 01$

$-1.92618 \mathrm{E} 01$

$-1.92628 \mathrm{E}$ 0I

$-1.92638 \mathrm{E} \quad 0 \mathrm{I}$ $-1.92648 \mathrm{E} 01$ $-1.92658 \mathrm{E} 01$ $-1.92668 \mathrm{E} \quad 01$

$-1.92678 \mathrm{E} 01$
$-1.92688 \mathrm{E} 01$

$-1.92698 \mathrm{E} 01$

$-1.92707 \mathrm{E} 01$

$-1.92717 \mathrm{E} \quad 01$

$-1.92726 \mathrm{E} 01$

$-1.92736 \mathrm{E} \quad 01$

$-1.92745 \mathrm{E} \quad 01$

$-1.92755 \mathrm{E} \quad 01$

$-1.92764 \mathrm{E} \quad 01$

$-1.92773 \mathrm{E} 01$

$-1.92782 \mathrm{E} \quad 01$

$-1.92791 \mathrm{E} 01$

$-1.92809 \mathrm{E} 01$

$-1.92818 \mathrm{E} 01$

$-1.92827 \mathrm{E} \quad 01$

$-1.92835 \mathrm{E}$ 01

$-1.92844 \mathrm{E}$ 01

$-1.92853 \mathrm{E} 01$

$-1.92861 \mathrm{E} 01$

$-1.92870 \mathrm{E} \quad 01$ $-1.92878 \mathrm{E} 01$ $-1.92886 \mathrm{E} 01$

$-1.92895 \mathrm{E} 01$

$-1.92903 \mathrm{E} \quad 01$
$-1.92800 \mathrm{E} 0 \mathrm{l}$

$-3.33692 \mathrm{E}-02$

$-3.38977 \mathrm{E}-02$

$-3.44316 \mathrm{E}-02$

$-3.49708 \mathrm{E}-02$

$-3.55153 \mathrm{E}-02$

$-3.60652 \mathrm{E}-02$

$-3.66204 \mathrm{E}-02$

$-3.71811 \mathrm{E}-02$

$-3.77472 \mathrm{E}-02$

$-3.83188 \mathrm{E}-02$

$-3.88959 \mathrm{E}-02$

$-3.94784 \mathbf{E}-02$

$-4.00665 \mathrm{E}-02$ 
ELECTRON RADIAL 'UNCTIONS

Positron

$p$

$F_{0}$

30.10

30.20

30.30

30.40

30.50

30.60

30.70

30.80

30.90

31.00

31.10

31.20

31.30

31.40

31.50

31.60

31.70

31.80

31.90

32.00

32.10

32.20

32.30

32.40

32.50

32.60

32.70

32.80

32.90

33.00

33.10

33.20

33.30

33.40

33.50

33.60

33.70

33.80

33.90

34.00

34.10

34.20

34.30

34.40

34.50

34.60

34.70

34.80

34.90

35.00

35.10

35.20

35.30

35.40

35.50

35.60

35.70

35.80

35.90

36.00 $f_{1}$

$8.01208 \mathrm{E}-01$ $8.01195 \mathrm{E}-01$ $8.01181 \mathrm{E}-01$ $8.01168 \mathrm{E}-01$ 8.01154 E- 01

8.01141 E-01 $8.01128 \mathrm{E}-01$ $8.01115 \mathrm{E}-01$ $8.01101 \mathrm{E}-01$ $8.01088 \mathrm{E}-01$

$8.01075 \mathrm{E}-01$ $8.01063 \mathrm{E}-01$ $8.01049 \mathrm{E}-01$ $8.01037 \mathrm{E}-01$ $8.01024 \mathrm{E}-01$

$8.01011 \mathrm{E}-01$ $8.00998 \mathrm{E}-01$ $8.00985 \mathrm{E}-01$ $8.00972 \mathrm{E}-01$ $8.00959 \mathrm{E}-01$

$8.00947 \mathrm{E}-01$ $8.00934 \mathrm{E}-01$ $8.00921 \mathrm{E}-01$ $8.00909 \mathrm{E}-01$ $8.00896 \mathrm{E}-01$

8.00884 E-01 $8.00871 \mathrm{E}-01$ $8.00859 \mathrm{E}-01$ $8.00846 \mathrm{E}-01$ $8.00834 \mathrm{E}-01$

8.00821 E-01 $8.00809 \mathrm{E}-01$ $8.00797 \mathrm{E}-01$ $8.00784 \mathrm{E}-0 \mathrm{I}$ $8.00772 \mathrm{E}-01$

$8.00760 \mathrm{E}-01$ $8.00748 \mathrm{E}-01$ $8.00736 \mathrm{E}-01$ $8.00724 \mathrm{E}-01$ $8.00711 \mathrm{E}-01$

$8.00699 \mathrm{E}-01$ $8.00687 \mathrm{E}-01$ $8.00675 \mathrm{E}-01$ $8.00664 \mathrm{E}-01$ $8.00652 \mathrm{E}-01$

$8.00640 \mathrm{E}-0 \mathrm{I}$ $8.00628 \mathrm{E}-01$ $8.00616 \mathrm{E}-01$ $8.00604 \mathrm{E}-01$ $8.00592 \mathrm{E}-01$

$8.00581 \mathrm{E}-01$ $8.00569 \mathrm{E}-01$ $8.00557 \mathrm{E}-01$ $8.00546 \mathrm{E}-01$ $8.00534 \mathrm{E}-01$

$8.00523 \mathrm{E}-01$ $8.00511 \mathrm{E}-01$ $8.00500 \mathrm{E}-01$ $8.00488 \mathrm{E}-01$ $8.00477 \mathrm{E}-01$

$Z=8$

A $=17$

$2.73388 \mathrm{E} \quad 01$ $2.74354 \mathrm{E} \quad 01$ $2.75321 \mathrm{E} \quad 01$ $2.76289 \mathrm{E} \quad 01$ $2.77256 \mathrm{E} 01$

$2.78225 \mathrm{E} \quad 01$ $2.79193 \mathrm{E} \quad 01$ $2.80162 \mathrm{E} \quad 01$ $2.81132 \mathrm{E} \quad 01$ $2.82101 \mathrm{E} 01$

$2.83072 \mathrm{E} \quad$ ol $2.84043 \mathrm{E} \quad 01$ $2.85014 \mathrm{E} \quad 01$

$2.85985 \mathrm{E} \quad 01$

$2.86957 \mathrm{E} \quad 01$

$2.87930 \mathrm{E} \quad 01$ $2.88902 \mathrm{E} \quad 01$ $2.89876 \mathrm{E} \quad 01$ $2.90849 \mathrm{E} \quad 01$ $2.91823 \mathrm{E} \quad 01$

$2.92798 \mathrm{E} \quad 01$ $2.93773 \mathrm{E} \quad 01$ $2.94748 \mathrm{E} \quad 01$ $2.95724 \mathrm{E} \quad 01$ $2.96701 \mathrm{E} 01$

$2.97677 \mathrm{E} \quad 01$ $2.98655 \mathrm{E} \quad 01$ $2.99632 \mathrm{E} \quad 01$

$3.00610 \mathrm{E} \quad 01$

$3.01589 \mathrm{E} \quad 01$

$3.02568 \mathrm{E} \quad 01$ $3.03547 \mathrm{E} \quad 01$ $3.04527 \mathrm{E} 01$ $3.05508 \mathrm{E}$ ol $3.06488 \mathrm{E} \quad 01$

$3.07470 \mathrm{E} \quad 01$ $3.08451 \mathrm{E} 01$ $3.09433 \mathrm{E} 01$ $3.10416 \mathrm{E} \quad 01$ $3.11399 \mathrm{E}$ 0I

$3.12383 \mathrm{E} \quad 01$ $3.13367 \mathrm{E} \quad 01$ $3.14351 \mathrm{E}$ 0I $3.15336 \mathrm{E} 01$ $3.16322 \mathrm{E}$ 0I

$3.17308 \mathrm{E} \quad 01$ $3.18294 \mathrm{E} \quad 01$ $3.19281 \mathrm{E} 01$ $3.20268 \mathrm{E}$ ol

$3.21256 \mathrm{E} \quad 01$

$3.22244 \mathrm{E} \quad 01$ $3.23233 \mathrm{E} \quad 01$ $3.24222 \mathrm{E} 01$ $3.25212 \mathrm{E} 01$ $3.26202 \mathrm{E} 01$

$3.27193 \mathrm{E} \quad 01$ $3.28184 \mathrm{E} \quad 01$ $3.29176 \mathrm{E} 01$ $3.30168 \mathrm{E} \quad 01$ $3.31160 \mathrm{E} \quad 01$ $\begin{array}{ccr}g_{1} & & \tan \delta_{1} \\ 59674 \mathrm{~F} & 00 & 206129 \mathrm{~F}\end{array}$

$4 \mathrm{E} \quad 00$ $1.61013 \mathrm{E} \quad 00$ $1.62359 \mathrm{E} \quad 00$ $1.63710 \mathrm{E} \quad 00$

$1.65067 \mathrm{E} \quad 00$

$1.66430 \mathrm{E} \quad 00$ $1.67799 \mathrm{E} \quad 00$

$1.69173 \mathrm{E} \quad 00$

$1.70553 \mathrm{E} \quad 00$

$1.71939 \mathrm{E} \quad 00$

$1.73331 \mathrm{E} \quad 00$ $1.74729 \mathrm{E} \quad 00$

$1.76132 \mathrm{E} \quad 00$

$1.77541 \mathrm{E} \quad 00$

$1.78956 \mathrm{E} \quad 00$

$1.80377 \mathrm{E} \quad 00$ $1.81804 \mathrm{E} \quad 00$ $1.83236 \mathrm{E} \quad 00$ $1.84674 \mathrm{E} \quad 00$

$1.86119 \mathrm{E} \quad 00$

$1.87568 \mathrm{E} \quad 00$ $1.89024 \mathrm{E} \quad 00$ $1.90486 \mathrm{E} \quad 00$ $1.91953 \mathrm{E} \quad 00$

$1.93427 \mathrm{E} \quad 00$

$1.94906 \mathrm{E} \quad 00$ $1.96391 \mathrm{E} \quad 00$ $1.97882 \mathrm{E} \quad 00$ $1.99379 \mathrm{E} \quad 00$ $2.00882 \mathrm{E} \quad 00$

$2.02391 \mathrm{E} \quad 00$ $2.03905 \mathrm{E} \quad 00$ $2.05426 \mathrm{E} \quad 00$ $2.06952 \mathrm{E} \quad 00$

$2.08485 \mathrm{E} \quad 00$

$2.10023 \mathrm{E} \quad 00$ $2.11567 \mathrm{E} \quad 00$ $2.13117 \mathrm{E} \quad 00$ $2.14673 \mathrm{E} \quad 00$ $2.16235 \mathrm{E} \quad 00$

$2.17803 \mathrm{E} \quad 00$ $2.19377 \mathrm{E} \quad 00$ $2.20957 \mathrm{E} \quad 00$

$2.22543 \mathrm{E} \quad 00$

$2.24135 \mathrm{E} \quad 00$

$2.25733 \mathrm{E} \quad 00$ $2.27337 \mathrm{E} \quad 00$ $228947 \mathrm{E} \quad 00$ $2.30563 \mathrm{E} \quad 00$

$2.32184 \mathrm{E} \quad 00$

$2.33812 \mathrm{E} \quad 00$ $2.35446 \mathrm{E} \quad 00$ $2.37086 \mathrm{E} \quad 00$ $2.38732 \mathrm{E} \quad 00$ 2.40384 E 00

$2.42042 \mathrm{E} \quad 00$ $2.43706 \mathrm{E} \quad 00$ $2.45376 \mathrm{E} \quad 00$ $2.47052 \mathrm{E} \quad 00$ $2.48735 \mathrm{E} \quad 00$

$\tan \delta$

$2.06129 \mathrm{E}-06$ $5.98204 \mathrm{E}-07$ $-8.53634 \mathrm{E}-07$ $-2.26760 \mathrm{E}-06$

$-3.64786 \mathrm{E}-06$

$-4.99433 \mathrm{E}-06$ $-6.30988 \mathrm{E}-06$ $-7.58586 \mathrm{E}-06$ $-8.83190 \mathrm{E}-06$ $-1.00492 \mathrm{E}-05$

$-1.12290 \mathrm{E}-05$ $-1.23790 \mathrm{E}-05$ $-1.34797 \mathrm{E}-05$ $-1.45691 \mathrm{E}-05$

$-1.56158 \mathrm{E}-05$

$-1.66418 \mathrm{E}-05$ $-1.76286 \mathrm{E}-05$ $-1.85857 \mathrm{E}-05$ $-1.94978 \mathrm{E}-05$

$-2.04057 \mathrm{E}-05$

$-2.12596 \mathrm{E}-0.5$ $-2.20987 \mathrm{E}-05$ $-2.29075 \mathrm{E}-05$ $-2.36688 \mathrm{E}-05$ $-2.44117 \mathrm{E}-05$

$-2.51330 \mathrm{E}-0.5$ $-2.58111 \mathrm{E}-05$ $-2.64698 \mathrm{E}-05$ $-2.70911 \mathrm{E}-05$ $-2.76815 \mathrm{E}-05$

$-2.82547 \mathrm{E}-05$ $-2.87932 \mathrm{E}-05$ $-2.93090 \mathrm{E}-05$ $-2.97942 \mathrm{E}-05$

$-3.02466 \mathrm{E}-05$

$-3.06747 \mathrm{E}-05$ $-3.10727 \mathrm{E}-05$ $-3.14458 \mathrm{E}-05$ 
$\bar{F}$

$1.43116 \mathrm{E}-03$

$1.44067 \mathrm{E}-03$

$1.45022 \mathrm{E}-03$

$1.45979 \mathrm{E}-03$

$1.46940 \mathrm{E}-03$

$1.47904 \mathrm{E}-03$

$1.48871 \mathrm{E}-03$

$1.49841 \mathrm{E}-03$

$1.50815 \mathrm{E}-03$

$1.51791 \mathrm{E}-03$

$1.52771 \mathrm{E}-03$

$1.53754 \mathrm{E}-03$

$1.54740 \mathrm{E}-03$

$1.55729 \mathrm{E}-03$

$1.56721 \mathrm{E}-03$

$1.57717 \mathrm{E}-03$

$1.58715 \mathrm{E}-03$

$1.59717 \mathrm{E}-03$

$1.60722 \mathrm{E}-03$

$1.61730 \mathrm{E}-03$

$1.6274 .1 \mathrm{E}-03$

$1.63755 \mathrm{E}-03$

$1.64773 \mathrm{E}-03$

$1.65793 \mathrm{E}-03$

$1.66817 \mathrm{E}-03$

$1.67844 \mathrm{E}-03$

$1.68874 \mathrm{E}-03$

$1.69907 \mathrm{E}-03$

$1.70944 \mathrm{E}-03$

$1.71983 \mathrm{E}-03$

$1.73026 \mathrm{E}-03$

$1.74071 \mathrm{E}-03$

$1.75120 \mathrm{E}-03$

$1.76172 \mathrm{E}-03$

$1.77228 \mathrm{E}-03$

$1.78286 \mathrm{E}-03$

$1.79347 \mathrm{E}-03$

$1.80412 \mathrm{E}-03$

$1.81480 \mathrm{E}-03$

$1.82551 \mathrm{E}-03$

$1.83625 \mathrm{E}-03$

$1.84702 \mathrm{E}-03$

$1.85782 \mathrm{E}-03$

$1.86866 \mathrm{E}-03$

$1.87953 \mathrm{E}-03$

$1.89042 \mathrm{E}-03$

$1.90135 \mathrm{E}-03$

$1.91232 \mathrm{E}-03$

$1.92331 \mathrm{E}-03$

$1.93433 \mathrm{E}-03$

$1.94539 \mathrm{E}-03$

$1.95647 \mathrm{E}-03$

$1.96759 \mathrm{E}-03$

$1.97874 \mathrm{E}-03$

$1.98992 \mathrm{E}-03$

35.60

35.70

35.80

35.90

36.00 $f_{2}$

$2.24354 \mathrm{E} \quad 00$ $2.25856 \mathrm{E} \quad 00$ $2.27363 \mathrm{E} \quad 00$ $2.28875 \mathrm{E} \quad 00$ $2.30392 \mathrm{E} \quad 00$

$2.31913 \mathrm{E} \quad 00$ $2.33440 \mathrm{E} \quad 00$ $2.34972 \mathrm{E} \quad 00$ $2.36509 \mathrm{E} \quad 00$ $2.38050 \mathrm{E} \quad 00$

$2.39597 \mathrm{E} \quad 00$ $2.41148 \mathrm{E} \quad 00$ $2.42705 \mathrm{E} \quad 00$ $2.44266 \mathrm{E} \quad 00$ $2.45833 \mathrm{E} \quad 00$

$2.47404 \mathrm{E} \quad 00$ $2.48980 \mathrm{E} \quad 00$ $2.50561 \mathrm{E} \quad 00$ $2.52148 \mathrm{E} \quad 00$ $2.53739 \mathrm{E} \quad 00$

$2.55335 \mathrm{E} \quad 00$ $2.56936 \mathrm{E} \quad 00$ $2.58542 \mathrm{E} \quad 00$ $2.60152 \mathrm{E} \quad 00$ $2.61768 \mathrm{E} \quad 00$

$2.63389 \mathrm{E} \quad 00$ $2.65015 \mathrm{E} \quad 00$ $2.66645 \mathrm{E} \quad 00$ $2.68281 \mathrm{E} \quad 00$ $2.69921 \mathrm{E} \quad 00$

$2.71567 \mathrm{E} \quad 00$ $2.73217 \mathrm{E} \quad 00$ $2.74872 \mathrm{E} \quad 00$ $2.76532 \mathrm{E} \quad 00$ $2.78197 \mathrm{E} \quad 00$

$2.79867 \mathrm{E} \quad 00$ $2.81542 \mathrm{E} \quad 00$ $2.83222 \mathrm{E} \quad 00$ $2.84907 \mathrm{E} \quad 00$ $2.86597 \mathrm{E} \quad 00$

$2.88291 \mathrm{E} \quad 00$ $2.89991 \mathrm{E} \quad 00$ $2.91696 \mathrm{E} \quad 00$ $2.93405 \mathrm{E} \quad 00$ $2.95119 \mathrm{E} \quad 00$

$2.96838 \mathrm{E} \quad 00$ $2.98563 \mathrm{E} \quad 00$ $3.00292 \mathrm{E} \quad 00$ $3.02026 \mathrm{E} \quad 00$ $3.03765 \mathrm{E} \quad 00$

$3.05509 \mathrm{E} \quad 00$ $3.07257 \mathrm{E} \quad 00$ $3.09011 \mathrm{E} \quad 00$ $3.10770 \mathrm{E} \quad 00$ $3.12533 \mathrm{E} \quad 00$

$3.14301 \mathrm{E} \quad 00$ $3.16075 \mathrm{E} \quad 00$ $3.17853 \mathrm{E} \quad 00$ $3.19636 \mathrm{E} \quad 00$ $3.21424 \mathrm{E} \quad 00$ $g_{2}$

$8.04168 \mathrm{E}-02$ $8.13370 \mathrm{E}-02$ $8.22640 \mathrm{E}-02$ $8.31980 \mathrm{E}-02$ $8.41388 \mathrm{E}-02$

$8.50867 \mathrm{E}-02$ $8.60415 \mathrm{E}-02$ $8.70033 \mathrm{E}-02$ $8.79721 \mathrm{E}-02$ 8.89480 E-02

$8.99310 \mathrm{E}-02$ $9.09212 \mathrm{E}-02$ $9.19183 \mathrm{E}-02$ $9.29227 \mathrm{E}-02$ $9.39343 \mathrm{E}-02$

$9.49531 \mathrm{E}-02$ $9.59791 \mathrm{E}-02$ $9.70124 \mathrm{E}-02$ $9.80529 \mathrm{E}-02$

$9.91008 \mathrm{E}-02$

$1.00156 \mathrm{E}-01$

$1.01219 \mathrm{E}-01$

$1.02288 \mathrm{E}-01$

$1.03366 \mathrm{E}-01$

$1.04451 \mathrm{E}-01$

$1.05543 \mathrm{E}-01$

$1.06642 \mathrm{E}-01$

$1.07750 \mathrm{E}-01$

$1.08864 \mathrm{E}-01$

$1.09987 \mathrm{E}-01$

$1.11117 \mathrm{E}-01$

$1.12254 \mathrm{E}-01$

$1.13399 \mathrm{E}-01$

$1.14552 \mathrm{E}-01$

$1.15713 \mathrm{E}-01$

$1.16881 \mathrm{E}-01$

$1.18057 \mathrm{E}-01$

$1.19240 \mathrm{E}-01$

$1.20432 \mathrm{E}-01$

$1.21631 \mathrm{E}-01$

$1.22838 \mathrm{E}-01$

$1.24053 \mathrm{E}-01$

$1.25276 \mathrm{E}-01$

$1.26507 \mathrm{E}-01$

$1.27745 \mathrm{E}-01$

$1.28992 \mathrm{E}-0 \mathrm{l}$

$1.30247 \mathrm{E}-01$

$1.31509 \mathrm{E}-01$

$1.32780 \mathrm{E}-01$

$1.34059 \mathrm{E}-01$

$1.35345 \mathrm{E}-01$

$1.36640 \mathrm{E}-01$

$1.37943 \mathrm{E}-01$

$1.39254 \mathrm{E}-01$

$1.40574 \mathrm{E}-01$

$1.41901 \mathrm{E}-01$

$1.43237 \mathrm{E}-01$

$1.44581 \mathrm{E}-01$

$1.45933 \mathrm{E}-01$

$1.47294 \mathrm{E}-01$ $\tan \delta_{2}$

$f_{-2}$

$-1.92911 \mathrm{E} \quad 01 \quad-7.53039 \mathrm{E}-02$

$-1.92919 \mathrm{E}$ 01 $-7.61943 \mathrm{E}-02$

$-1.92927 \mathrm{E}$ 01 $-7.70915 \mathrm{E}-02$

$-1.92935 \mathrm{E} \quad 01 \quad-7.79955 \mathrm{E}-02$

$-1.92943 \mathrm{E}$ 01 $\quad-7.89064 \mathrm{E}-02$

$-1.92951 \mathrm{E} \quad 01$

$-1.92959 \mathrm{E} 01$

$-1.92967 \mathrm{E}$ 01

$-1.92975 \mathrm{E} 01$

$-1.92982 \mathrm{E} 01$

$-1.92990 \mathrm{E} \quad 01$

$-1.92998 \mathrm{E} \quad 01$

$-1.93005 \mathrm{E} \quad 01$

$-1.93013 \mathrm{E} \quad 01$

$-1.93020 \mathrm{E} 01$

$-1.93027 \mathrm{E} \quad 01$

$-1.93035 \mathrm{E} \quad 01$

$-1.93042 \mathrm{E} 01$

$-1.93049 \mathrm{E} 01$

$-1.93057 \mathrm{E} \quad 01$

$-1.93064 \mathrm{E} \quad 01$

$-1.93071 \mathrm{E} 01$

$-1.93078 \mathrm{E} \quad 01$

$-1.93085 \mathrm{E} 01$

$-1.93092 \mathrm{E} 01$

$-1.93099 \mathrm{E} \quad 01$

$-1.93106 \mathrm{E} 01$

$-1.93113 \mathrm{E} 01$

$-1.93119 \mathrm{E} 01$

$-1.93126 \mathrm{E} 01$

$-1.93133 \mathrm{E} \quad 01$

$-1.93139 \mathrm{E}$ 0l

$-1.93146 \mathrm{E}$ 01

$-1.93153 \mathrm{E} \quad 01$

$-1.93159 \mathrm{E} 01$

$-1.93166 \mathrm{E} \quad 01$

$-1.93172 \mathrm{E} \quad 01$

$-1.93179 \mathrm{E} 01$

$-1.93185 \mathrm{E}$ 0l

$-1.93191 \mathrm{E} 01$

$-1.93198 \mathrm{E} 01$ $-1.93204 \mathrm{E} \quad 01$

$-1.93210 \mathrm{E} 01$

$-1.93216 \mathrm{E} 01$

$-1.93223 \mathrm{E} 01$

$-1.93229 \mathrm{E}$ 0l

$-1.93235 \mathrm{E}$ ol

$-1.93241 \mathrm{E} 01$

$-1.93247 \mathrm{E}$ 01

$-1.93253 \mathrm{E} 01$

$\begin{array}{lll}-1.93259 \mathrm{E} & 01\end{array}$

$-1.93265 \mathrm{E} 01$

$-1.93271 \mathrm{E} 01$

$-1.93276 \mathrm{E} 01$

-1.93282 E 01

$-1.93288 \mathrm{E} \quad 01$

$-1.93294 \mathrm{E} 01$

$-1.93299 \mathrm{E} 01$

$-1.93305 \mathrm{E} 01$

$-1.93311 \mathrm{E} 01$

$-7.98241 \mathrm{E}-02$

$-8.07488 \mathrm{E}-02$

$-8.16803 \mathrm{E}-02$

$-8.26188 \mathrm{E}-02$

$-8.35643 \mathrm{E}-02$

$-8.45168 \mathrm{E}-02$

$-8.54763 \mathrm{E}-02$

$-8.64429 \mathrm{E}-02$

$-8.74165 \mathrm{E}-02$

$-8.83972 \mathrm{E}-02$

$-8.93851 \mathrm{E}-02$

$-9.03801 \mathrm{E}-02$

$-9.13823 \mathrm{E}-02$

$-9.23917 \mathrm{E}-02$

$-9.34083 \mathrm{E}-02$

$-9.44321 \mathrm{E}-02$

$-9.54633 \mathrm{E}-02$

$-9.65017 \mathrm{E}-02$

$-9.75474 \mathrm{E}-02$

$-9.86006 \mathrm{E}-02$

$-9.96610 \mathrm{E}-02$

$-1.00729 \mathrm{E}-01$

$-1.01804 \mathrm{E}-01$

$-1.02887 \mathrm{E}-01$

$-1.03977 \mathrm{E}-01$

$-1.05075 \mathrm{E}-01$

$-1.06180 \mathrm{E}-01$

$-1.07293 \mathrm{E}-01$

$-1.08413 \mathrm{E}-01$

$-1.09541 \mathrm{E}-01$

$-1.10677 \mathrm{E}-01$

$-1.11820 \mathrm{E}-01$

$-1.12971 \mathrm{E}-01$

$-1.14130 \mathrm{E}-01$

$-1.15296 \mathrm{E}-01$

$-1.16470 \mathrm{E}-01$

$-1.17652 \mathrm{E}-01$

$-1.18842 \mathrm{E}-01$

$-1.20039 \mathrm{E}-01$

$-1.21245 \mathrm{E}-01$

$-1.22458 \mathrm{E}-01$

$-1.23679 \mathrm{E}-01$ 


\section{$F_{0}$}

36.10

36.20

36.30

36.40

36.50

36.60

36.70

36.80

36.90

37.00

37.10

37.20

37.30

37.40

37.50

37.60

37.70

37.80

37.90

38.00

38.10

38.20

38.30

38.40

38.50

38.60

38.70

38.80

38.90

39.00

39.10

39.20

39.30

39.40

39.50

39.60

39.70

39.80

39.90

40.00

40.10

40.20

40.30

40.40

40.50

40.60

40.70

40.80

40.90

41.00

41.10

41.20

41.30

41.40

41.50

41.60

41.70

41.80

41.90

42.00

$$
f_{1}
$$

$g_{1}$

$\tan \delta_{1}$

$f_{-1}$

$8.00465 \mathrm{E}-01$

$8.00454 \mathrm{E}-01$

$8.00442 \mathrm{E}-01$

$8.00431 \mathrm{E}-01$

8.00420 E-01

$8.00408 \mathrm{E}-01$

$8.00397 \mathrm{E}-01$

$8.00386 \mathrm{E}-0 \mathrm{I}$

$8.00375 \mathrm{E}-01$

8.00364 E-01

$8.00353 \mathrm{E}-01$

8.00341 E-01

$8.00330 \mathrm{E}-01$

$8.00319 \mathrm{E}-01$

$8.00308 \mathrm{E}-01$

$8.00297 \mathrm{E}-01$

$8.00286 \mathrm{E}-01$

$8.00275 \mathrm{E}-01$

$8.00265 \mathrm{E}-01$

$8.00254 \mathrm{E}-01$

$8.00243 \mathrm{E}-01$

$8.00232 \mathrm{E}-01$

$8.00221 \mathrm{E}-01$

$8.00210 \mathrm{E}-01$

$8.00200 \mathrm{E}-01$

$8.00189 \mathrm{E}-01$

$8.00178 \mathrm{E}-01$

$8.00168 \mathrm{E}-01$

$8.00157 \mathrm{E}-01$

$8.00146 \mathrm{E}-01$

$8.00136 \mathrm{E}-01$

$8.00125 \mathrm{E}-01$

$8.00115 \mathrm{E}-01$

$8.00104 \mathrm{E}-01$

$8.00094 \mathrm{E}-01$

$8.00083 \mathrm{E}-01$ $8.00073 \mathrm{E}-01$

$8.00062 \mathrm{E}-01$

$8.00052 \mathrm{E}-01$

8.00041 E- 01

$8.00031 \mathrm{E}-01$

$8.00021 \mathrm{E}-01$

$8.00010 \mathrm{E}-01$

$8.00000 \mathrm{E}-01$

$7.99990 \mathrm{E}-01$

$7.99980 \mathrm{E}-01$

$7.99970 \mathrm{E}-01$

$7.99959 \mathrm{E}-01$

$7.99949 \mathrm{E}-01$

$7.99939 \mathrm{E}-01$

$7.99929 \mathrm{E}-01$

$7.99919 \mathrm{E}-01$

$7.99909 \mathrm{E}-01$

$7.99899 \mathrm{E}-01$

$7.99889 \mathrm{E}-01$

$7.99879 \mathrm{E}-01$

$7.99869 \mathrm{E}-01$

$7.99859 \mathrm{E}-01$

$7.99849 \mathrm{E}-01$

$7.99839 \mathrm{E}-01$
$3.32154 \mathrm{E} \quad 01$ $3.33147 \mathrm{E} \quad 01$ $3.34141 \mathrm{E} 01$ $3.35136 \mathrm{E} \quad 01$ $3.36131 \mathrm{E} 01$

$3.37127 \mathrm{E} \quad 01$ $3.38123 \mathrm{E} \quad 01$ $3.39120 \mathrm{E} \quad 01$ $3.40117 \mathrm{E} \quad 01$ $3.41114 \mathrm{E} \quad 01$

$3.42113 \mathrm{E} \quad 01$

$3.44110 \mathrm{E} \quad 01$

$3.45110 \mathrm{E} \quad 01$

$3.46110 \mathrm{E} 01$

$3.47111 \mathrm{E} \quad 01$ $3.48112 \mathrm{E} \quad 01$ $3.49114 \mathrm{E} \quad 01$ $3.50116 \mathrm{E} \quad 01$ $3.51119 \mathrm{E}$ ol

$3.52122 \mathrm{E} \quad 01$ $3.53126 \mathrm{E} \quad 01$ $3.54131 \mathrm{E} \quad 01$ $3.55136 \mathrm{E} \quad 01$

$3.56141 \mathrm{E} \quad 01$

$3.57147 \mathrm{E} \quad 01$ $3.58154 \mathrm{E} \quad 01$ $3.59161 \mathrm{E} \quad 01$ $3.60168 \mathrm{E} \quad 01$

$3.61176 \mathrm{E} \quad 01$

$3.62185 \mathrm{E} \quad 01$ $3.63194 \mathrm{E} \quad 01$

$3.64204 \mathrm{E} \quad 01$

$3.65214 \mathrm{E} \quad 01$

$3.66225 \mathrm{E} 01$

$3.67236 \mathrm{E} \quad 01$ $3.68248 \mathrm{E} 01$ $3.69261 \mathrm{E} \quad 01$

$3.70274 \mathrm{E} \quad 01$

$3.71287 \mathrm{E} \quad 01$

$3.72301 \mathrm{E} \quad 01$ $3.73316 \mathrm{E} \quad 01$ $3.74331 \mathrm{E} \quad 01$ $3.75347 \mathrm{E} \quad 01$ $3.76364 \mathrm{E} \quad 01$

$3.77380 \mathrm{E} \quad 01$ $3.78398 \mathrm{E} \quad 01$ $3.79416 \mathrm{E} \quad 01$ $3.80435 \mathrm{E} \quad 01$

$3.81454 \mathrm{E} \quad 01$

$3.82474 \mathrm{E} \quad 01$ $3.83494 \mathrm{E} \quad 01$ $3.84515 \mathrm{E} 01$ $3.85537 \mathrm{E} \quad 01$ $3.86559 \mathrm{E}$ 0l

$3.87581 \mathrm{E} \quad 01$ $3.88604 \mathrm{E}$ 01 $3.89628 \mathrm{E} \quad 01$ $3.90653 \mathrm{E} \quad 01$ $3.91678 \mathrm{E} \quad 01$ $3.43111 \mathrm{E} \quad 01$
$2.50423 \mathrm{E} \quad 00 \quad-3.27885 \mathrm{E}-05$ $2.52118 \mathrm{E} \quad 00 \quad-3.25538 \mathrm{E}-05$ $2.53818 \mathrm{E} \quad 00 \quad-3.22801 \mathrm{E}-05$ $2.55525 \mathrm{E} \quad 00 \quad-3.19944 \mathrm{E}-0.5$ $2.57237 \mathrm{E} \quad 00 \quad-3.16860 \mathrm{E}-05$

$2.58956 \mathrm{E} \quad 00$ $2.60681 \mathrm{E} \quad 00 \quad-3.09894 \mathrm{E}-05$ $2.62412 \mathrm{E} \quad 00 \quad-3.06093 \mathrm{E}-05$

$2.64149 \mathrm{E} \quad 00 \quad-3.02003 \mathrm{E}-05$

$2.65893 \mathrm{E} \quad 00 \quad-2.97754 \mathrm{E}-05$

$2.67642 \mathrm{E} \quad 00$

$2.69398 \mathrm{E} \quad 00$

$2.71160 \mathrm{E} \quad 00$

$2.72928 \mathbf{E} \quad 00$

$2.74702 \mathrm{E} \quad 00$

$2.76482 \mathrm{E} \quad 00$ $2.78268 \mathrm{E} \quad 00$

$2.80061 \mathrm{E} \quad 00$

$2.81860 \mathrm{E} \quad 00$

$2.83665 \mathrm{E} \quad 00$

$2.85476 \mathrm{E} \quad 00$

$2.87293 \mathrm{E} \quad 00$

$2.89117 \mathrm{E} \quad 00$

$2.90947 \mathrm{E} \quad 00$

2.92783 E 00

$2.94625 \mathrm{E} \quad 00$

$2.96474 \mathrm{E} \quad 00$

$2.98329 \mathrm{E} \quad 00$

$3.00190 \mathrm{E} \quad 00$

$3.02057 \mathrm{E} \quad 00$

$3.03931 \mathrm{E} \quad 00$

$3.05810 \mathrm{E} \quad 00$

$3.07697 \mathrm{E} \quad 00$

$3.09589 \mathrm{E} \quad 00$

$3.11488 \mathrm{E} \quad 00$

$3.13393 \mathrm{E} \quad 00$

$3.15304 \mathrm{E} \quad 00$

$3.17222 \mathrm{E} \quad 00$

$3.21076 \mathrm{E} \quad 00$

$3.23013 \mathrm{E} \quad 00$

$3.24956 \mathrm{E} \quad 00$

$3.26906 \mathrm{E} \quad 00$

$3.28862 \mathrm{E} \quad 00$

$3.30824 \mathrm{E} \quad 00$

$3.32792 \mathrm{E} \quad 00$

$3.34767 \mathrm{E} \quad 00$

$3.36749 \mathrm{E} \quad 00$

$3.38736 \mathrm{E} \quad 00$

$3.40730 \mathrm{E} \quad 00$

$3.42731 \mathrm{E} \quad 00$

$3.44738 \mathrm{E} \quad 00$

$3.46751 \mathrm{E} \quad 00$

$3.48771 \mathrm{E} \quad 00$

$3.50798 \mathrm{E} \quad 00$

$3.52830 \mathrm{E} \quad 00$

$3.54870 \mathrm{E} \quad 00$

$3.56915 \mathrm{E} \quad 00$

$3.58967 \mathrm{E} \quad 00$

$3.61026 \mathrm{E} \quad 00$

$-2.93280 \mathrm{E}-05$

$-2.88576 \mathrm{E}-05$

$-2.83563 \mathrm{E}-05$

$-2.78432 \mathrm{E}-05$

$-2.73007 \mathrm{E}-05$

$-2.67308 \mathrm{E}-05$

$-2.61469 \mathrm{E}-05$

$-2.55360 \mathrm{E}-05$

$-2.49189 \mathrm{E}-0.5$

$-2.42635 \mathrm{E}-05$

$-2.35904 \mathrm{E}-05$

$-2.28975 \mathrm{E}-05$

$-2.21799 \mathrm{E}-05$

$-2.14471 \mathrm{E}-05$

$-2.06836 \mathrm{E}-05$

$-1.99069 \mathrm{E}-05$

$-1.91062 \mathrm{E}-05$

$-1.82839 \mathrm{E}-05$

$-1.74375 \mathrm{E}-05$

$-1.65788 \mathrm{E}-05$

$-1.56973 \mathrm{E}-05$

$-1.47847 \mathrm{E}-05$

$-1.38587 \mathrm{E}-05$

$-1.29153 \mathrm{E}-05$

$-1.19491 \mathrm{E}-05$

$-1.09537 \mathrm{E}-05$

$-9.94391 \mathrm{E}-06$

$-8.92043 \mathrm{E}-06$

$-7.88513 \mathrm{E}-06$

$-6.80761 \mathrm{E}-06$

$-5.72264 \mathrm{E}-06$

$-4.62286 \mathrm{E}-06$

$-3.49084 \mathrm{E}-06$

$-2.34663 \mathrm{E}-06$

$-1.19263 \mathrm{E}-06$

$-4.07465 \mathrm{E}-09$

$1.20405 \mathrm{E}-06$

$2.42965 \mathrm{E}-06$

$3.66743 \mathrm{E}-06$
$3.19146 \mathrm{E} \quad 00$ 
$Z=10 \quad A=20 \quad \rho=1.2 A^{1 / 3} F$

$\bar{F}$

$2.05767 \mathrm{E}-03$

$2.06907 \mathrm{E}-03$

$2.08051 \mathrm{E}-03$

$2.09197 \mathrm{E}-03$

$2.10347 \mathrm{E}-03$

$2.11499 \mathrm{E}-03$

$2.12655 \mathrm{E}-03$

$2.13814 \mathrm{E}-03$

$2.14976 \mathrm{E}-03$

$2.16142 \mathrm{E}-03$

$2.17310 \mathrm{E}-03$

$2.18482 \mathrm{E}-03$

$2.19656 \mathrm{E}-03$

$2.20834 \mathrm{E}-03$

$2.22015 \mathrm{E}-03$

$2.23200 \mathrm{E}-03$

$2.24387 \mathrm{E}-03$

$2.25577 \mathrm{E}-03$

$2.26771 \mathrm{E}-03$

$2.27968 \mathrm{E}-03$

$2.29168 \mathrm{E}-03$

$2.30371 \mathrm{E}-03$

$2.31577 \mathrm{E}-03$

$2.32786 \mathrm{E}-03$

$2.33999 \mathrm{E}-03$

$2.35214 \mathrm{E}-03$

$2.36433 \mathrm{E}-03$

$2.37655 \mathrm{E}-03$

$2.38880 \mathrm{E}-03$

$2.40108 \mathrm{E}-03$

$2.41340 \mathrm{E}-03$

$2.42574 \mathrm{E}-03$

$2.43812 \mathrm{E}-03$

$2.45053 \mathrm{E}-03$

$2.46297 \mathrm{E}-03$

$2.47544 \mathrm{E}-03$

$2.48794 \mathrm{E}-03$

$2.50047 \mathrm{E}-03$

$2.51304 \mathrm{E}-03$

$2.52563 \mathrm{E}-03$

$2.53826 \mathrm{E}-03$

$2.55092 \mathrm{E}-03$

$2.56361 \mathrm{E}-03$

$2.57633 \mathrm{E}-03$

$2.58909 \mathrm{E}-03$

$2.60187 \mathrm{E}-03$

$2.61469 \mathrm{E}-03$

$2.62754 \mathrm{E}-03$

$2.64042 \mathrm{E}-03$

$2.65333 \mathrm{E}-03$

41.10

$2.66627 \mathrm{E}-03$

$2.67924 \mathrm{E}-03$

$2.69225 \mathrm{E}-03$

$2.70529 \mathrm{E}-03$

$2.71836 \mathrm{E}-03$

41.60

41.70

41.80

41.90

42.00 $f_{2}$

$3.23217 \mathrm{E} \quad 00$

$3.25015 \mathrm{E} \quad 00$

$3.26818 \mathrm{E} \quad 00$

$3.28625 \mathrm{E} \quad 00$

$3.30438 \mathrm{E} \quad 00$

$3.32255 \mathbf{E} \quad 00$

$3.34077 \mathrm{E} \quad 00$

$3.35905 \mathbf{E} \quad 00$

$3.37737 \mathrm{E} \quad 00$

$3.39574 \mathrm{E} \quad 00$

$3.41416 \mathrm{E} \quad 00$

$3.43262 \mathrm{E} \quad 00$

$3.45114 \mathrm{E} \quad 00$

$3.46971 \mathrm{E} \quad 00$

$3.48832 \mathrm{E} \quad 00$

$3.50698 \mathrm{E} \quad 00$

$3.52570 \mathrm{E} \quad 00$

$3.54446 \mathrm{E} \quad 00$

$3.56327 \mathrm{E} \quad 00$

$3.58213 \mathrm{E} \quad 00$

$3.60103 \mathrm{E} \quad 00$ $3.61999 \mathrm{E} \quad 00$

$3.63900 \mathrm{E} \quad 00$

$3.65805 \mathrm{E} \quad 00$

$3.67715 \mathrm{E} \quad 00$

$3.69630 \mathrm{E} \quad 00$ $3.71551 \mathrm{E} \quad 00$

$3.73475 \mathrm{E} \quad 00$

$3.75405 \mathrm{E} \quad 00$

$3.77340 \mathrm{E} \quad 00$

$3.79280 \mathrm{E} \quad 00$

$3.81224 \mathrm{E} \quad 00$

$3.83173 \mathrm{E} \quad 00$

$3.85127 \mathrm{E} \quad 00$

$3.87086 \mathrm{E} \quad 00$

$3.89050 \mathrm{E} \quad 00$

$3.91019 \mathrm{E} \quad 00$

$3.92993 \mathrm{E} \quad 00$

$3.94971 \mathrm{E} \quad 00$

$.3 .96954 \mathrm{E} \quad 00$

$3.98943 \mathrm{E} \quad 00$

$4.00936 \mathrm{E} \quad 00$

$4.02934 \mathrm{E} \quad 00$

$4.04936 \mathrm{E} \quad 00$

$4.06944 \mathrm{E} \quad 00$

$4.08957 \mathrm{E} \quad 00$

$4.10974 \mathrm{E} \quad 00$

$4.12996 \mathrm{E} \quad 00$

$4.15023 \mathrm{E} \quad 00$

$4.17055 \mathrm{E} \quad 00$

$4.19092 \mathrm{E} \quad 00$

$4.21133 \mathrm{E} \quad 00$

$4.23180 \mathrm{E} \quad 00$

$4.25231 \mathrm{E} \quad 00$

$4.27287 \mathrm{E} \quad 00$

$4.29348 \mathrm{E} \quad 00$ $4.31414 \mathrm{E} \quad 00$ $4.33485 \mathrm{E} \quad 00$ $4.35560 \mathrm{E} \quad 00$ $4.37641 \mathrm{E} \quad 00$ $g_{2}$

$\tan \delta_{2}$

$f_{-2}$

$1.48663 \mathrm{E}-01$

$1.50040 \mathrm{E}-01$

$1.51426 \mathrm{E}-01$

$1.52820 \mathrm{E}-01$

$1.54223 \mathrm{E}-01$

$-1.93316 \mathrm{E} 01$

$-1.93322 \mathrm{E} 01$

$-1.93327 \mathrm{E} 01$

$-1.93333 \mathrm{E} 01$

$-1.93338 \mathrm{E} 01$

$-1.41618 \mathrm{E}-01$

$-1.42960 \mathrm{E}-01$

$-1.44311 \mathrm{E}-01$

$-1.45670 \mathrm{E}-01$

$1.55634 \mathrm{E}-01$

$1.57053 \mathrm{E}-01$

$1.58481 \mathrm{E}-01$

$1.59918 \mathrm{E}-01$

$1.61363 \mathrm{E}-01$

$\begin{array}{lll}-1.93344 \mathrm{E} & 01\end{array}$

$-1.93349 \mathrm{E} 01$

$-1.93355 \mathrm{E} 01$

$-1.93360 \mathrm{E} 01$

$-1.93365 \mathrm{E} 01$

$1.62816 \mathrm{E}-01$

$1.64279 \mathrm{E}-01$

$1.65749 \mathrm{E}-01$

$1.67229 \mathrm{E}-01$

$1.68717 \mathrm{E}-01$

$1.70214 \mathrm{E}-01$

$1.71720 \mathrm{E}-01$

$1.73234 \mathrm{E}-01$

$1.74757 \mathrm{E}-01$

$1.76289 \mathrm{E}-01$

$1.77830 \mathrm{E}-01$

$1.79379 \mathrm{E}-01$

$1.80938 \mathrm{E}-01$

$1.82505 \mathrm{E}-01$

$1.84082 \mathrm{E}-01$

$1.85667 \mathrm{E}-01$

$1.87261 \mathrm{E}-01$

$1.88864 \mathrm{E}-01$

$1.90476 \mathrm{E}-01$

$1.92098 \mathrm{E}-01$

$1.93728 \mathrm{E}-01$

$1.95368 \mathrm{E}-01$

$1.97016 \mathrm{E}-01$

$1.98674 \mathrm{E}-01$

$2.00341 \mathrm{E}-0]$

$2.02017 \mathrm{E}-01$

$2.03702 \mathrm{E}-01$

$2.05397 \mathrm{E}-01$

$2.07101 \mathrm{E}-01$

$2.08814 \mathrm{E}-01$

$2.10536 \mathrm{E}-01$

$2.12268 \mathrm{E}-01$

$2.14009 \mathrm{E}-01$

$2.15760 \mathrm{E}-01$

$2.17520 \mathrm{E}-01$

2.19289E-01

$2.21068 \mathrm{E}-01$

$2.22856 \mathrm{E}-01$

$2.24654 \mathrm{E}-01$

$2.26462 \mathrm{E}-01$

$2.28279 \mathrm{E}-01$

$2.30105 \mathrm{E}-01$

$2.31941 \mathrm{E}-01$

$2.33787 \mathrm{E}-01$

$2.35643 \mathrm{E}-01$

$2.37508 \mathrm{E}-01$

$2.39383 \mathrm{E}-01$

$2.41267 \mathrm{E}-01$

$2.43162 \mathrm{E}-01$

$2.45066 \mathrm{E}-01$
$-1.93371 \mathrm{E} \quad 01$ $-1.93376 \mathrm{E} 01$ $-1.93381 \mathrm{E} 01$ $-1.93386 \mathrm{E} 01$

-1.93392 E 01

$-1.93397 \mathrm{E} 01$

$-1.93402 \mathrm{E} 01$

$-1.93407 \mathrm{E} 01$

$-1.93412 \mathrm{E} 01$

$-1.93417 \mathrm{E} 01$

$-1.93422 \mathrm{E} 01$

$-1.93427 \mathrm{E} 01$

$-1.93432 \mathrm{E} 01$

$-1.93437 \mathrm{E} 01$

$-1.93442 \mathrm{E} 01$

$-1.93447 \mathrm{E} \quad 01$

$-1.93451 \mathrm{E} 01$

$-1.93456 \mathrm{E} 01$

$-1.93461 \mathrm{E} \quad 01$

$-1.93466 \mathrm{E} 01$

$-1.93471 \mathrm{E} \quad 01$

$-1.93475 \mathrm{E} 01$

$-1.93480 \mathrm{E} 01$

$-1.93485 \mathrm{E} 01$

$-1.93489 \mathrm{E} 01$

$-1.93494 \mathrm{E} \quad 01$

$-1.93498 \mathrm{E} 01$

$-1.93503 \mathrm{E} 01$

$-1.93508 \mathrm{E} 01$

$-1.93512 \mathrm{E} 01$

$-1.93517 \mathrm{E} \quad 01$

$-1.93521 \mathrm{E} 01$

$-1.93525 \mathrm{E} 01$

$-1.93530 \mathrm{E} 01$

$-1.93534 \mathrm{E} 01$

$-1.93539 \mathrm{E} \quad 01$

$-1.93543 \mathbf{E} 01$

$-1.93547 \mathrm{E} 01$

$-1.93552 \mathrm{E} 01$

$-1.93556 \mathrm{E} 01$

$\begin{array}{ll}-1.93560 \mathrm{E} & 01\end{array}$

$-1.93564 \mathrm{E} 01$

$-1.93569 \mathrm{E} 01$

$-1.93573 \mathrm{E} 01$

$-1.93577 \mathrm{E} 01$

-1.93581 E 01

$-1.93585 \mathrm{E} 01$

$-1.93589 \mathrm{E} 01$

$-1.93593 \mathrm{E} 01$

$-1.93598 \mathrm{E} 01$
$-1.47038 \mathrm{E}-01$

$-1.48414 \mathrm{E}-01$

$-1.49798 \mathrm{E}-01$

$-1.51191 \mathrm{E}-01$

$-1.52592 \mathrm{E}-01$

$-1.54002 \mathrm{E}-01$

$-1.55420 \mathrm{E}-01$

$-1.56847 \mathrm{E}-01$

$-1.58282 \mathrm{E}-01$

$-1.59726 \mathrm{E}-01$

$-1.61178 \mathrm{E}-01$

$-1.62639 \mathrm{E}-01$

$-1.64109 \mathrm{E}-01$

$-1.65587 \mathrm{E}-01$

$-1.67074 \mathrm{E}-01$

$-1.68570 \mathrm{E}-01$

$-1.70074 \mathrm{E}-01$

$-1.71587 \mathrm{E}-01$

$-1.73109 \mathrm{E}-01$

$-1.74640 \mathrm{E}-01$

$-1.76180 \mathrm{E}-01$

$-1.77728 \mathrm{E}-01$

$-1.79286 \mathrm{E}-01$

$-1.80852 \mathrm{E}-01$

$-1.82427 \mathrm{E}-01$

$-1.84012 \mathrm{E}-01$

$-1.85605 \mathrm{E}-01$

$-1.87207 \mathrm{E}-01$

$-1.88818 \mathrm{E}-01$

$-1.90439 \mathrm{E}-01$

$-1.92068 \mathrm{E}-01$

$-1.93707 \mathrm{E}-01$

$-1.95354 \mathrm{E}-01$

$-1.97011 \mathrm{E}-01$

$-1.98677 \mathrm{E}-01$

$-2.00353 \mathrm{E}-01$

$-2.02037 \mathrm{E}-01$ 


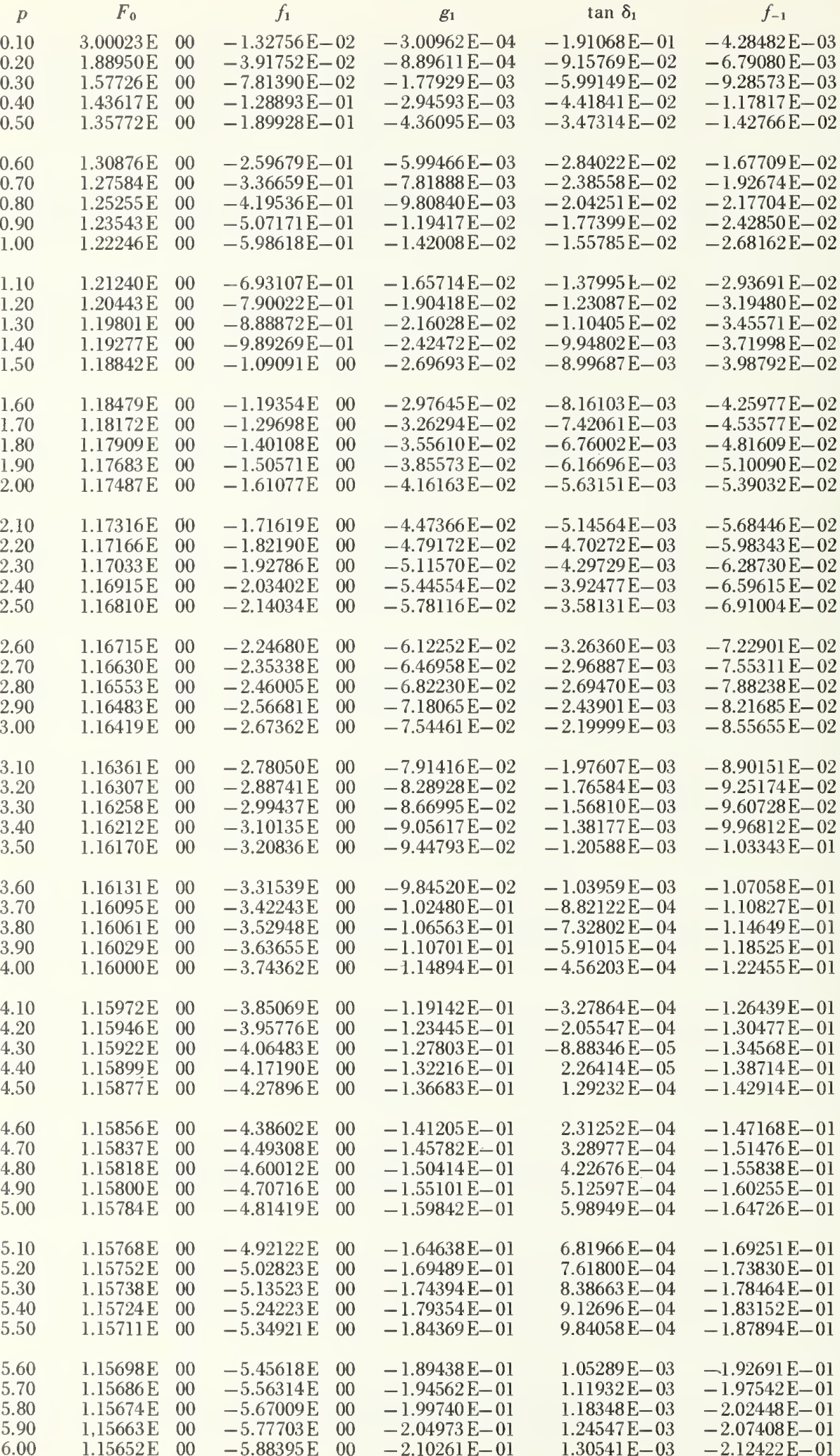

$g_{-1}$ $\tan \delta_{-1}$

$2.44414 \mathrm{E}-01$ $3.86520 \mathrm{E}-01$ $5.26660 \mathrm{E}-01$ $6.65023 \mathrm{E}-01$ $8.01082 \mathrm{E}-01$

$9.34548 \mathrm{E}-01$ $1.06535 \mathrm{E} \quad 00$ $1.19357 \mathrm{E} \quad 00$ $1.31940 \mathrm{E} \quad 00$ $1.44304 \mathrm{E} \quad 00$

$1.56474 \mathrm{E} \quad 00$ $1.68473 \mathrm{E} \quad 00$ $1.80323 \mathrm{E} \quad 00$ $1.92043 \mathrm{E} \quad 00$ $2.03650 \mathrm{E} \quad 00$

$2.15159 \mathrm{E} \quad 00$ $2.26583 \mathrm{E} \quad 00$ $2.37933 \mathrm{E} \quad 00$ $2.49218 \mathrm{E} \quad 00$ $2.60447 \mathrm{E} \quad 00$

$2.71626 \mathrm{E} \quad 00$ $2.82761 \mathrm{E} \quad 00$ $2.93858 \mathrm{E} \quad 00$ $3.04921 \mathrm{E} \quad 00$ $3.15953 \mathrm{E} \quad 00$

$3.26958 \mathrm{E} \quad 00$ $3.37939 \mathrm{E} \quad 00$ $3.48898 \mathrm{E} \quad 00$ $3.59837 \mathrm{E} \quad 00$ $3.70758 \mathrm{E} \quad 00$

$3.81664 \mathrm{E} \quad 00$ $3.92554 \mathrm{E} \quad 00$ $4.03431 \mathrm{E} \quad 00$ $4.14296 \mathrm{E} \quad 00$ $4.25149 \mathrm{E} \quad 00$

$4.35992 \mathrm{E} \quad 00$ $4.46825 \mathrm{E} \quad 00$ $4.57649 \mathrm{E} \quad 00$ $4.68465 \mathrm{E} \quad 00$ $4.79273 \mathrm{E} \quad 00$

4.90074 E 00 $5.00869 \mathrm{E} \quad 00$ $5.11656 \mathrm{E} \quad 00$ $5.22438 \mathrm{E} \quad 00$ $5.33214 \mathrm{E} \quad 00$

$5.43985 \mathrm{E} \quad 00$ $5.54751 \mathrm{E} \quad 00$ $5.65513 \mathrm{E} \quad 00$ $5.76269 \mathrm{E} \quad 00$ $5.87021 \mathrm{E} \quad 00$

$5.97770 \mathrm{E} \quad 00$ $6.08514 \mathrm{E} \quad 00$ $6.19254 \mathrm{E} \quad 00$ $6.29991 \mathrm{E} \quad 00$ $6.40725 \mathrm{E} \quad 00$

$6.51455 \mathrm{E} \quad 00$ $6.62182 \mathrm{E} \quad 00$ $6.72905 \mathrm{E} \quad 00$ $6.83626 \mathrm{E} \quad 00$ $6.94344 \mathrm{E} \quad 00$
$-4.39128 \mathrm{E} \quad 00$ $-8.01018 \mathrm{E} \quad 00$ $-1.17252 \mathrm{E} 01$ $-1.53936 \mathrm{E} 01$ $-1.89838 \mathrm{E} \quad 01$

$-2.24825 \mathrm{E} \quad 01$ $-2.58833 \mathrm{E}$ 01 $-2.91831 \mathrm{E} 01$ $-3.23813 \mathrm{E} 01$ $-3.54787 \mathrm{E} 01$

$-3.84772 \mathrm{E} \quad 01$ $-4.13793 \mathrm{E} \quad 01$ $-4.41880 \mathrm{E} 01$ $-4.69065 \mathrm{E} 01$ $-4.95381 \mathrm{E} 01$ $-5.20862 \mathrm{E} 01$ $-5.45543 \mathrm{E} 01$ $-5.69454 \mathrm{E} \quad 01$ $-5.92630 \mathrm{E} 01$ -6.15101 E 01

$-6.36896 \mathrm{E} \quad 01$ $-6.58043 \mathrm{E} 01$ $-6.78571 \mathrm{E} 01$ $-6.98505 \mathrm{E} 01$ $-7.17869 \mathrm{E} 01$

$-7.36687 \mathrm{E} \quad 01$ $-7.54981 \mathrm{E} 01$ $-7.72773 \mathrm{E} \quad 01$ $-7.90082 \mathrm{E} 01$ $-8.06928 \mathrm{E} 01$

$-8.23329 \mathrm{E} 01$ $-8.39303 \mathrm{E} 01$ $-8.54865 \mathrm{E} 01$ $-8.70032 \mathrm{E} \quad 01$ -8.84817 E 01

$-8.99238 \mathrm{E} \quad 01$ $-9.13304 \mathrm{E} \quad 01$ $-9.27030 \mathrm{E} 01$ $-9.40429 \mathrm{E} 01$ $-9.53512 \mathrm{E} 01$

$-9.66289 \mathrm{E} 01$ $-9.78773 \mathrm{E}$ 01 $-9.90972 \mathrm{E} 01$ $-1.00290 \mathrm{E} \quad 02$ $-1.01456 \mathrm{E} 02$ $-1.02596 \mathrm{E} \quad 02$ $-1.03711 \mathrm{E} 02$ $-1.04803 \mathrm{E} 02$ $-1.05871 \mathrm{E} \quad 02$ $-1.06918 \mathrm{E} 02$

$-1.07942 \mathrm{E} \quad 02$ $-1.08945 \mathrm{E} \quad 02$ $-1.09928 \mathrm{E} 02$ $-1.10891 \mathrm{E} 02$ $-1.11835 \mathrm{E} 02$

$-1.12760 \mathrm{E} 02$ $-1.13667 \mathrm{E} \quad 02$ $-1.14557 \mathrm{E} 02$ $-1.15430 \mathrm{E} \quad 02$ $-1.16287 \mathrm{E} 02$ 
$\bar{F}$

$5.86209 \mathrm{E}-08$

1.2994.1 E-07

$2.37944 \mathrm{E}-07$

$3.81762 \mathrm{E}-07$

$5.61636 \mathrm{E}-07$

$7.77910 \mathrm{E}-07$

$1.03090 \mathrm{E}-06$

$1.32086 \mathrm{E}-06$

$1.64801 \mathrm{E}-06$

$2.01252 \mathrm{E}-06$

$2.41452 \mathrm{E}-06$

$2.85411 \mathrm{E}-06$

$3.33137 \mathrm{E}-06$

$3.84636 \mathrm{E}-06$

$4.39913 \mathrm{E}-06$

$4.98972 \mathrm{E}-06$

$5.61815 \mathrm{E}-06$

$6.28446 \mathrm{E}-06$

$6.98865 \mathrm{E}-06$

$7.73075 \mathrm{E}-06$

$8.51077 \mathrm{E}-06$

$9.32870 \mathrm{E}-06$

$1.01846 \mathrm{E}-05$

$1.10784 \mathrm{E}-05$

$1.20101 \mathrm{E}-05$

$1.29798 \mathrm{E}-05$

$1.39875 \mathrm{E}-05$

$1.50331 \mathrm{E}-05$

$1.61166 \mathrm{E}-05$

$1.72381 \mathrm{E}-05$

$1.83976 \mathrm{E}-05$

$1.95950 \mathrm{E}-05$

$2.08304 \mathrm{E}-05$

$2.21037 \mathrm{E}-05$

$2.34150 \mathrm{E}-05$

$2.47643 \mathrm{E}-0.5$

$2.61515 \mathrm{E}-05$

$2.75767 \mathrm{E}-05$

$2.90398 \mathrm{E}-0.5$

$3.05409 \mathrm{E}-0.5$

$3.20799 \mathrm{E}-05$

$3.36569 \mathrm{E}-05$

$3.52719 \mathrm{E}-05$

$3.69248 \mathrm{E}-05$

$3.86156 \mathrm{E}-05$

4.03444 E-05

$4.21112 \mathrm{E}-05$

$4.39160 \mathrm{E}-05$

$4.57586 \mathrm{E}-05$

$4.76393 \mathrm{E}-05$

$4.95579 \mathrm{E}-05$

$5.15143 \mathrm{E}-05$

$5.35089 \mathrm{E}-05$

$5.55413 \mathrm{E}-05$

$5.76117 \mathrm{E}-05$

$5.97200 \mathrm{E}-05$

$6.18664 \mathrm{E}-05$

$6.40506 \mathrm{E}-05$

$6.62728 \mathrm{E}-0.5$

$6.85329 \mathrm{E}-05$ $f_{2}$

$g_{2}$

$-3.46119 \mathrm{E}-06$

$-2.01665 \mathrm{E}-05$

$-6.01954 \mathrm{E}-05$

$-1.32294 \mathrm{E}-04$

$-2.43601 \mathrm{E}-04$

$-3.99626 \mathrm{E}-04$

$-6.04412 \mathrm{E}-04$

$-8.60796 \mathrm{E}-04$

$-1.17069 \mathrm{E}-03$

$-1.53535 \mathrm{E}-03$

$-1.95553 \mathrm{E}-03$

$-2.43168 \mathrm{E}-03$

$-2.96404 \mathrm{E}-03$

$-3.55272 \mathrm{E}-03$

$-4.19773 \mathrm{E}-03$

$-4.89904 \mathrm{E}-03$

$-5.65658 \mathrm{E}-03$

$-6.47028 \mathrm{E}-03$

$-7.34005 \mathrm{E}-03$

$-8.26582 \mathrm{E}-03$

$-9.24749 \mathrm{E}-03$

$-1.02850 \mathrm{E}-02$

$-1.13783 \mathrm{E}-02$

$-1.25272 \mathrm{E}-02$

$-1.37318 \mathrm{E}-02$

$-1.49920 \mathrm{E}-02$

$-1.63077 \mathrm{E}-02$

$-1.76788 \mathrm{E}-02$

$-1.91055 \mathrm{E}-02$

$-2.05875 \mathrm{E}-02$

$-2.21249 \mathrm{E}-02$

$-2.37176 \mathrm{E}-02$

$-2.53656 \mathrm{E}-02$

$-2.70689 \mathrm{E}-02$

$-2.88274 \mathrm{E}-02$

$-3.06412 \mathrm{E}-02$

$-3.25102 \mathrm{E}-02$

$-3.44344 \mathrm{E}-02$

$-3.64137 \mathrm{E}-02$

$-3.84482 \mathrm{E}-02$

$-4.05379 \mathrm{E}-02$

$-4.26826 \mathrm{E}-02$

$-4.48825 \mathrm{E}-02$

$-4.71375 \mathrm{E}-02$

$-4.94475 \mathrm{E}-02$

$-5.18126 \mathrm{E}-02$

$-5.42328 \mathrm{E}-02$

$-5.67080 \mathrm{E}-02$

$-5.92382 \mathrm{E}-02$

$-6.18235 \mathrm{E}-02$

$-6.44638 \mathrm{E}-02$

$-6.71590 \mathrm{E}-02$

$-6.99093 \mathrm{E}-02$

$-7.27146 \mathrm{E}-02$

$-7.55748 \mathrm{E}-02$

$-7.84901 \mathrm{E}-02$

$-8.14603 \mathrm{E}-02$

$-8.44854 \mathrm{E}-02$

$-8.75655 \mathrm{E}-02$

$-9.07005 \mathrm{E}-02$ $\tan \delta_{2}$

$f_{-2}$

$-4.53407 \mathrm{E}-08$

$-2.64636 \mathrm{E}-07$

$-7.92168 \mathrm{E}-07$

$-1.74771 \mathrm{E}-06$

$-3.23357 \mathrm{E}-06$

$-5.33433 \mathrm{E}-06$

$-8.11864 \mathrm{E}-06$

-1.16421 E-05

$-1.59502 \mathrm{E}-05$

$-2.10815 \mathrm{E}-05$

$-2.70692 \mathrm{E}-05$

$-3.39431 \mathrm{E}-05$

$-4.17311 \mathrm{E}-05$

$-5.04594 \mathrm{E}-05$

$-6.01533 \mathrm{E}-05$

$-7.08378 \mathrm{E}-05$

$-8.25374 \mathrm{E}-05$

$-9.52767 \mathrm{E}-05$

$-1.09080 \mathrm{E}-04$

-1.23972 E-04

- 1.39977 E-04

$-1.57119 \mathrm{E}-04$

$-1.75424 \mathrm{E}-04$

$-1.94916 \mathrm{E}-04$

$-2.15619 \mathrm{E}-04$

$-2.37559 \mathrm{E}-04$

$-2.60760 \mathrm{E}-04$

$-2.85248 \mathrm{E}-04$

$-3.11047 \mathrm{E}-04$

$-3.38182 \mathrm{E}-04$

$-3.66678 \mathrm{E}-04$

$-3.96561 \mathrm{E}-04$

$-4.27855 \mathrm{E}-04$

$-4.60586 \mathrm{E}-04$

$-4.94779 \mathrm{E}-04$

$-5.30459 \mathrm{E}-04$

$-5.67651 \mathrm{E}-04$

$-6.06380 \mathrm{E}-04$

$-6.46672 \mathrm{E}-04$

$-6.88552 \mathrm{E}-04$

$-7.32045 \mathrm{E}-04$

$-7.77176 \mathrm{E}-04$

$-8.23971 \mathrm{E}-04$

$-8.72455 \mathrm{E}-04$

$-9.22652 \mathrm{E}-04$

$-9.74589 \mathrm{E}-04$

$-1.02829 \mathrm{E}-03$

$-1.08378 \mathrm{E}-03$

$-1.14109 \mathrm{E}-03$

$-1.20024 \mathrm{E}-03$

$-1.26125 \mathrm{E}-03$

$-1.32415 \mathrm{E}-03$

$-1.38898 \mathrm{E}-03$

$-1.45574 \mathrm{E}-03$

$-1.52447 \mathrm{E}-03$

$-1.59519 \mathrm{E}-03$

$-1.66793 \mathrm{E}-03$

$-1.74271 \mathrm{E}-03$

$-1.81956 \mathrm{E}-03$

$-1.89851 \mathrm{E}-03$
$2.32085 \mathrm{E} \quad 00 \quad-6.84880 \mathrm{E}-07$

$4.82351 \mathrm{E} \quad 00 \quad-2.03657 \mathrm{E}-06$

$7.18406 \mathrm{E} \quad 00 \quad-4.12533 \mathrm{E}-06$

$9.40344 \mathrm{E} \quad 00 \quad-6.94963 \mathrm{E}-06$

$1.14638 \mathrm{E} 01-1.05084 \mathrm{E}-0.5$

$1.33544 \mathrm{E} \quad 01$

$1.50732 \mathrm{E} \quad 01$

$1.66252 \mathrm{E} 01$

$1.80205 \mathrm{E} \quad 01$

$1.92715 \mathrm{E} \quad 01$

$-1.48023 \mathrm{E}-05$ $-1.98351 \mathrm{E}-05$

$-2.56140 \mathrm{E}-05$

$-3.21499 \mathrm{E}-05$

$-3.94566 \mathrm{E}-05$

$2.03920 \mathrm{E} \quad 01$

$2.13956 \mathrm{E} \quad 01$

$2.22954 \mathrm{E} \quad 01$

$2.31034 \mathrm{E} \quad 01$

$2.38305 \mathrm{E} \quad 01$

$-4.75507 \mathrm{E}-05$

$-5.64510 \mathrm{E}-05$

$-6.61780 \mathrm{E}-05$

$-7.67533 \mathrm{E}-05$

$-8.81996 \mathrm{E}-05$

$2.44863 \mathrm{E} \quad 01$

$2.50793 \mathrm{E} \quad 01$

$-1.00540 \mathrm{E}-04$

$-1.13800 \mathrm{E}-04$

$2.56170 \mathrm{E} \quad 01-1.28002 \mathrm{E}-04$

$2.61059 \mathrm{E} \quad 01-1.43172 \mathrm{E}-04$

$2.65515 \mathrm{E} \quad 01-1.59334 \mathrm{E}-04$

$2.69588 \mathrm{E} \quad 01$

$2.73321 \mathrm{E} \quad 01$

$2.76751 \mathrm{E} \quad 01$

$2.79911 \mathrm{E} \quad 01$

$2.82828 \mathrm{E} \quad 01$

$2.85528 \mathrm{E} \quad 01$

$2.88032 \mathrm{E} \quad 01$

$2.90360 \mathrm{E} \quad 01$

$2.92528 \mathrm{E} \quad 01$

$2.94551 \mathrm{E} \quad 01$

$2.96443 \mathrm{E} \quad 01$

$2.98215 \mathrm{E} \quad 01$

$2.99877 \mathrm{E} \quad 01$

$3.01440 \mathrm{E} \quad 01$

$3.02911 \mathrm{E} 01$

$-1.76514 \mathrm{E}-04$

$-1.94736 \mathrm{E}-04$

$-2.14027 \mathrm{E}-04$

$-2.34411 \mathrm{E}-04$

$-2.55913 \mathrm{E}-04$

$3.04298 \mathrm{E} \quad 01$

$3.05607 \mathrm{E} \quad 01$

$3.08016 \mathrm{E} \quad 01$

$3.09127 \mathrm{E} \quad 01$

$-2.78560 \mathrm{E}-04$

$-3.02376 \mathrm{E}-04$

$-3.27387 \mathrm{E}-04$

$-3.53618 \mathrm{E}-04$

$-3.81095 \mathrm{E}-04$

$3.10181 \mathrm{E} \quad 01$

$3.11182 \mathrm{E} \quad 01$

$3.12135 \mathrm{E} \quad 01$

$3.13042 \mathrm{E} \quad 01$

$3.13907 \mathrm{E} \quad 01$

$-4.09843 \mathrm{E}-04$

$-4.39889 \mathrm{E}-04$

$-4.71256 \mathrm{E}-04$

$-5.03971 \mathrm{E}-04$

$-5.38058 \mathrm{E}-04$

$-5.73545 \mathrm{E}-04$

$-6.10455 \mathrm{E}-04$

$-6.48815 \mathrm{E}-04$

$-6.88649 \mathrm{E}-04$

$-7.29984 \mathrm{E}-04$

$-7.72844 \mathrm{E}-04$

$-8.17255 \mathrm{E}-04$

$-8.63243 \mathrm{E}-04$

$-9.10832 \mathrm{E}-04$

$-9.60049 \mathrm{E}-04$

$3.14732 \mathrm{E} \quad 01$

$-1.01092 \mathrm{E}-03$

$3.15520 \mathrm{E} 01-1.06346 \mathrm{E}-03$

$3.16273 \mathrm{E} 01-1.11772 \mathrm{E}-03$

$3.16994 \mathrm{E}$ 01 $-1.17370 \mathrm{E}-03$

$3.17684 \mathrm{E} \quad 01-1.23143 \mathrm{E}-03$

$3.18346 \mathrm{E} \quad 01 \quad-1.29094 \mathrm{E}-03$

$3.18981 \mathrm{E}$ 01 $-1.35226 \mathrm{E}-03$

$3.19590 \mathrm{E} \quad 01 \quad-1.41541 \mathrm{E}-03$

$3.20176 \mathrm{E} \quad 01 \quad-1.48041 \mathrm{E}-03$

$3.20739 \mathrm{E} \quad 01-1.54730 \mathrm{E}-03$

$3.21280 \mathrm{E} \quad 01-1.61609 \mathrm{E}-03$

$3.21802 \mathrm{E} \quad 01-1.68681 \mathrm{E}-03$

$3.22304 \mathrm{E} \quad 01-1.75949 \mathrm{E}-03$

$3.22788 \mathrm{E}$ 01 $-1.83415 \mathrm{E}-03$

$3.23255 \mathrm{E}$ 01 $-1.91082 \mathrm{E}-03$
$3.06844 \mathrm{E} \quad 01$

g-2

$6.83754 \mathrm{E}-05$

$2.02860 \mathrm{E}-04$

$4.09394 \mathrm{E}-04$ 
ELECTRON RADIAL FUNCTIONS

\begin{tabular}{|c|c|c|c|c|c|c|c|c|c|c|c|}
\hline Electro & & & & & $=6$ & $\rho=$ & $A^{1 / 3} F$ & & & & \\
\hline$p$ & $F_{0}$ & & $f_{1}$ & & $g_{1}$ & $\tan \delta_{1}$ & $f_{-1}$ & $g_{-1}$ & & $\tan \delta_{-1}$ & \\
\hline $\begin{array}{l}6.10 \\
6.20 \\
6.30 \\
6.40 \\
6.50\end{array}$ & $\begin{array}{l}1.15642 \mathrm{E} \\
1.15631 \mathrm{E} \\
1.15622 \mathrm{E} \\
1.15613 \mathrm{E} \\
1.15603 \mathrm{E}\end{array}$ & $\begin{array}{l}00 \\
00 \\
00 \\
00 \\
00\end{array}$ & $\begin{array}{l}-5.99086 \mathrm{E} \\
-6.09776 \mathrm{E} \\
-6.20465 \mathrm{E} \\
-6.31153 \mathrm{E} \\
-6.41839 \mathrm{E}\end{array}$ & $\begin{array}{l}00 \\
00 \\
00 \\
00 \\
00\end{array}$ & $\begin{array}{l}-2.15603 \mathrm{E}-01 \\
-2.21000 \mathrm{E}-01 \\
-2.26452 \mathrm{E}-01 \\
-2.31958 \mathrm{E}-01 \\
-2.37518 \mathrm{E}-01\end{array}$ & $\begin{array}{l}1.36339 \mathrm{E}-03 \\
1.41950 \mathrm{E}-03 \\
1.47384 \mathrm{E}-03 \\
1.52648 \mathrm{E}-03 \\
1.57750 \mathrm{E}-03\end{array}$ & $\begin{array}{l}-2.17491 \mathrm{E}-01 \\
-2.22614 \mathrm{E}-01 \\
-2.27791 \mathrm{E}-01 \\
-2.33023 \mathrm{E}-01 \\
-2.38310 \mathrm{E}-01\end{array}$ & $\begin{array}{l}7.05059 \mathrm{E} \\
7.15771 \mathrm{E} \\
7.26481 \mathrm{E} \\
7.37188 \mathrm{E} \\
7.47892 \mathrm{E}\end{array}$ & $\begin{array}{l}00 \\
00 \\
00 \\
00 \\
00\end{array}$ & $\begin{array}{l}-1.17127 \mathrm{E} \\
-1.17952 \mathrm{E} \\
-1.18762 \mathrm{E} \\
-1.19557 \mathrm{E} \\
-1.20338 \mathrm{E}\end{array}$ & $\begin{array}{l}02 \\
02 \\
02 \\
02 \\
02\end{array}$ \\
\hline $\begin{array}{l}6.60 \\
6.70 \\
6.80 \\
6.90 \\
7.00\end{array}$ & $\begin{array}{l}1.15595 \mathrm{E} \\
1.15586 \mathrm{E} \\
1.15578 \mathrm{E} \\
1.15570 \mathrm{E} \\
1.15563 \mathrm{E}\end{array}$ & $\begin{array}{l}00 \\
00 \\
00 \\
00 \\
00\end{array}$ & $\begin{array}{l}-6.52523 \mathrm{E} \\
-6.63207 \mathrm{E} \\
-6.73889 \mathrm{E} \\
-6.84570 \mathrm{E} \\
-6.95250 \mathrm{E}\end{array}$ & $\begin{array}{l}00 \\
00 \\
00 \\
00 \\
00\end{array}$ & $\begin{array}{l}-2.43133 \mathrm{E}-01 \\
-2.48803 \mathrm{E}-01 \\
-2.54527 \mathrm{E}-01 \\
-2.60306 \mathrm{E}-01 \\
-2.66139 \mathrm{E}-01\end{array}$ & $\begin{array}{l}1.62699 \mathrm{E}-03 \\
1.67499 \mathrm{E}-03 \\
1.72158 \mathrm{E}-03 \\
1.76682 \mathrm{E}-03 \\
1.81077 \mathrm{E}-03\end{array}$ & $\begin{array}{l}-2.43650 \mathrm{E}-01 \\
-2.49045 \mathrm{E}-01 \\
-2.54495 \mathrm{E}-01 \\
-2.59999 \mathrm{E}-01 \\
-2.65558 \mathrm{E}-01\end{array}$ & $\begin{array}{l}7.58594 \mathrm{E} \\
7.69294 \mathrm{E} \\
7.79991 \mathrm{E} \\
7.90686 \mathrm{E} \\
8.01379 \mathrm{E}\end{array}$ & $\begin{array}{l}00 \\
00 \\
00 \\
00 \\
00\end{array}$ & $\begin{array}{l}-1.21105 \mathrm{E} \\
-1.21859 \mathrm{E} \\
-1.22600 \mathrm{E} \\
-1.23328 \mathrm{E} \\
-1.24043 \mathrm{E}\end{array}$ & $\begin{array}{l}02 \\
02 \\
02 \\
02 \\
02\end{array}$ \\
\hline $\begin{array}{l}7.10 \\
7.20 \\
7.30 \\
7.40 \\
7.50\end{array}$ & $\begin{array}{l}1.15555 \mathrm{E} \\
1.15548 \mathrm{E} \\
1.15541 \mathrm{E} \\
1.15534 \mathrm{E} \\
1.15528 \mathrm{E}\end{array}$ & $\begin{array}{l}00 \\
00 \\
00 \\
00 \\
00\end{array}$ & $\begin{array}{l}-7.05928 \mathrm{E} \\
-7.16604 \mathrm{E} \\
-7.27280 \mathrm{E} \\
-7.37954 \mathrm{E} \\
-7.48626 \mathrm{E}\end{array}$ & $\begin{array}{l}00 \\
00 \\
00 \\
00 \\
00\end{array}$ & $\begin{array}{l}-2.72027 \mathrm{E}-01 \\
-2.77969 \mathrm{E}-01 \\
-2.83965 \mathrm{E}-01 \\
-2.90016 \mathrm{E}-01 \\
-2.96122 \mathrm{E}-01\end{array}$ & $\begin{array}{l}1.85347 \mathrm{E}-03 \\
1.89498 \mathrm{E}-03 \\
1.93535 \mathrm{E}-03 \\
1.97463 \mathrm{E}-03 \\
2.01285 \mathrm{E}-03\end{array}$ & $\begin{array}{l}-2.71170 \mathrm{E}-01 \\
-2.76838 \mathrm{E}-01 \\
-2.82559 \mathrm{E}-01 \\
-2.88335 \mathrm{E}-01 \\
-2.94166 \mathrm{E}-01\end{array}$ & $\begin{array}{l}8.12069 \mathrm{E} \\
8.22757 \mathrm{E} \\
8.33443 \mathrm{E} \\
8.44127 \mathrm{E} \\
8.54808 \mathrm{E}\end{array}$ & $\begin{array}{l}00 \\
00 \\
00 \\
00 \\
00\end{array}$ & $\begin{array}{l}-1.24746 \mathrm{E} \\
-1.25438 \mathrm{E} \\
-1.26118 \mathrm{E} \\
-1.26787 \mathrm{E} \\
-1.27445 \mathrm{E}\end{array}$ & $\begin{array}{l}02 \\
02 \\
02 \\
02 \\
02\end{array}$ \\
\hline $\begin{array}{l}7.60 \\
7.70 \\
7.80 \\
7.90 \\
8.00\end{array}$ & $\begin{array}{l}1.15521 \mathrm{E} \\
1.15515 \mathrm{E} \\
1.15509 \mathrm{E} \\
1.15503 \mathrm{E} \\
1.15497 \mathrm{E}\end{array}$ & $\begin{array}{l}00 \\
00 \\
00 \\
00 \\
00\end{array}$ & $\begin{array}{l}-7.59298 \mathrm{E} \\
-7.69968 \mathrm{E} \\
-7.80636 \mathrm{E} \\
-7.91304 \mathrm{E} \\
-8.01969 \mathrm{E}\end{array}$ & $\begin{array}{l}00 \\
00 \\
00 \\
00 \\
00\end{array}$ & $\begin{array}{l}-3.02282 \mathrm{E}-01 \\
-3.08497 \mathrm{E}-01 \\
-3.14766 \mathrm{E}-01 \\
-3.21089 \mathrm{E}-01 \\
-3.27467 \mathrm{E}-01\end{array}$ & $\begin{array}{l}2.05005 \mathrm{E}-03 \\
2.08628 \mathrm{E}-03 \\
2.12159 \mathrm{E}-03 \\
2.15598 \mathrm{E}-03 \\
2.18951 \mathrm{E}-03\end{array}$ & $\begin{array}{l}-3.00051 \mathrm{E}-01 \\
-3.05990 \mathrm{E}-01 \\
-3.11984 \mathrm{E}-01 \\
-3.18032 \mathrm{E}-01 \\
-3.24135 \mathrm{E}-01\end{array}$ & $\begin{array}{l}8.65488 \mathrm{E} \\
8.76166 \mathrm{E} \\
8.86842 \mathrm{E} \\
8.97516 \mathrm{E} \\
9.08187 \mathrm{E}\end{array}$ & $\begin{array}{l}00 \\
00 \\
00 \\
00 \\
00\end{array}$ & $\begin{array}{l}-1.28093 \mathrm{E} \\
-1.28730 \mathrm{E} \\
-1.29357 \mathrm{E} \\
-1.29974 \mathrm{E} \\
-1.30582 \mathrm{E}\end{array}$ & $\begin{array}{l}02 \\
02 \\
02 \\
02 \\
02\end{array}$ \\
\hline $\begin{array}{l}8.10 \\
8.20 \\
8.30 \\
8.40 \\
8.50\end{array}$ & $\begin{array}{l}1.15491 \mathrm{E} \\
1.15486 \mathrm{E} \\
1.15481 \mathrm{E} \\
1.15475 \mathrm{E} \\
1.15470 \mathrm{E}\end{array}$ & $\begin{array}{l}00 \\
00 \\
00 \\
00 \\
00\end{array}$ & $\begin{array}{l}-8.12633 \mathrm{E} \\
-8.23297 \mathrm{E} \\
-8.33958 \mathrm{E} \\
-8.44618 \mathrm{E} \\
-8.55277 \mathrm{E}\end{array}$ & $\begin{array}{l}00 \\
00 \\
00 \\
00 \\
00\end{array}$ & $\begin{array}{l}-3.33899 \mathrm{E}-01 \\
-3.40386 \mathrm{E}-01 \\
-3.46927 \mathrm{E}-01 \\
-3.53523 \mathrm{E}-01 \\
-3.60173 \mathrm{E}-01\end{array}$ & $\begin{array}{l}2.22220 \mathrm{E}-03 \\
2.25409 \mathrm{E}-03 \\
2.28519 \mathrm{E}-03 \\
2.31554 \mathrm{E}-03 \\
2.34517 \mathrm{E}-03\end{array}$ & $\begin{array}{l}-3.30292 \mathrm{E}-01 \\
-3.36503 \mathrm{E}-01 \\
-3.42768 \mathrm{E}-01 \\
-3.49088 \mathrm{E}-01 \\
-3.55463 \mathrm{E}-01\end{array}$ & $\begin{array}{l}9.18857 \mathrm{E} \\
9.29525 \mathrm{E} \\
9.40191 \mathrm{E} \\
9.50855 \mathrm{E} \\
9.61517 \mathrm{E}\end{array}$ & $\begin{array}{l}00 \\
00 \\
00 \\
00 \\
00\end{array}$ & $\begin{array}{l}-1.31180 \mathrm{E} \\
-1.31770 \mathrm{E} \\
-1.32350 \mathrm{E} \\
-1.32922 \mathrm{E} \\
-1.33486 \mathrm{E}\end{array}$ & $\begin{array}{l}02 \\
02 \\
02 \\
02 \\
02\end{array}$ \\
\hline $\begin{array}{l}8.60 \\
8.70 \\
8.80 \\
8.90 \\
9.00\end{array}$ & $\begin{array}{l}1.15465 \mathrm{E} \\
1.15460 \mathrm{E} \\
1.15456 \mathrm{E} \\
1.15451 \mathrm{E} \\
1.15446 \mathrm{E}\end{array}$ & $\begin{array}{l}00 \\
00 \\
00 \\
00 \\
00\end{array}$ & $\begin{array}{l}-8.65934 \mathrm{E} \\
-8.76590 \mathrm{E} \\
-8.87244 \mathrm{E} \\
-8.97897 \mathrm{E} \\
-9.08548 \mathrm{E}\end{array}$ & $\begin{array}{l}00 \\
00 \\
00 \\
00 \\
00\end{array}$ & $\begin{array}{l}-3.66877 \mathrm{E}-01 \\
-3.73636 \mathrm{E}-01 \\
-3.80449 \mathrm{E}-01 \\
-3.87316 \mathrm{E}-01 \\
-3.94238 \mathrm{E}-01\end{array}$ & $\begin{array}{l}2.37411 \mathrm{E}-03 \\
2.40236 \mathrm{E}-03 \\
2.42996 \mathrm{E}-03 \\
2.45693 \mathrm{E}-03 \\
2.48329 \mathrm{E}-03\end{array}$ & $\begin{array}{l}-3.61892 \mathrm{E}-01 \\
-3.68375 \mathrm{E}-01 \\
-3.74912 \mathrm{E}-01 \\
-3.81504 \mathrm{E}-01 \\
-3.88150 \mathrm{E}-01\end{array}$ & $\begin{array}{l}9.72178 \mathrm{E} \\
9.82837 \mathrm{E} \\
9.93493 \mathrm{E} \\
1.00415 \mathrm{E} \\
1.01480 \mathrm{E}\end{array}$ & $\begin{array}{l}00 \\
00 \\
00 \\
01 \\
01\end{array}$ & $\begin{array}{l}-1.34041 \mathrm{E} \\
-1.34588 \mathrm{E} \\
-1.35127 \mathrm{E} \\
-1.35658 \mathrm{E} \\
-1.36182 \mathrm{E}\end{array}$ & $\begin{array}{l}02 \\
02 \\
02 \\
02 \\
02\end{array}$ \\
\hline $\begin{array}{l}9.10 \\
9.20 \\
9.30 \\
9.40 \\
9.50\end{array}$ & $\begin{array}{l}1.15442 \mathrm{E} \\
1.15437 \mathrm{E} \\
1.15433 \mathrm{E} \\
1.15429 \mathrm{E} \\
1.15425 \mathrm{E}\end{array}$ & $\begin{array}{l}00 \\
00 \\
00 \\
00 \\
00\end{array}$ & $\begin{array}{l}-9.19198 \mathrm{E} \\
-9.29847 \mathrm{E} \\
-9.40494 \mathrm{E} \\
-9.51140 \mathrm{E} \\
-9.61784 \mathrm{E}\end{array}$ & $\begin{array}{l}00 \\
00 \\
00 \\
00 \\
00\end{array}$ & $\begin{array}{l}-4.01214 \mathrm{E}-01 \\
-4.08244 \mathrm{E}-01 \\
-4.15329 \mathrm{E}-01 \\
-4.22468 \mathrm{E}-01 \\
-4.29661 \mathrm{E}-01\end{array}$ & $\begin{array}{l}2.50906 \mathrm{E}-03 \\
2.53426 \mathrm{E}-03 \\
2.55890 \mathrm{E}-03 \\
2.58300 \mathrm{E}-03 \\
2.60658 \mathrm{E}-03\end{array}$ & $\begin{array}{l}-3.94850 \mathrm{E}-01 \\
-4.01605 \mathrm{E}-01 \\
-4.08414 \mathrm{E}-01 \\
-4.15278 \mathrm{E}-01 \\
-4.22195 \mathrm{E}-01\end{array}$ & $\begin{array}{l}1.02545 \mathrm{E} \\
1.03610 \mathrm{E} \\
1.04675 \mathrm{E} \\
1.05740 \mathrm{E} \\
1.06804 \mathrm{E}\end{array}$ & $\begin{array}{l}01 \\
01 \\
01 \\
01 \\
01\end{array}$ & $\begin{array}{l}-1.36699 \mathrm{E} \\
-1.37208 \mathrm{E} \\
-1.37711 \mathrm{E} \\
-1.38207 \mathrm{E} \\
-1.38696 \mathrm{E}\end{array}$ & $\begin{array}{l}02 \\
02 \\
02 \\
02 \\
02\end{array}$ \\
\hline $\begin{array}{r}9.60 \\
9.70 \\
9.80 \\
9.90 \\
10.00\end{array}$ & $\begin{array}{l}1.15421 \mathrm{E} \\
1.15417 \mathrm{E} \\
1.15413 \mathrm{E} \\
1.15409 \mathrm{E} \\
1.15405 \mathrm{E}\end{array}$ & $\begin{array}{l}00 \\
00 \\
00 \\
00 \\
00\end{array}$ & $\begin{array}{l}-9.72427 \mathrm{E} \\
-9.83068 \mathrm{E} \\
-9.93708 \mathrm{E} \\
-1.00435 \mathrm{E} \\
-1.01498 \mathrm{E}\end{array}$ & $\begin{array}{l}00 \\
00 \\
00 \\
01 \\
01 .\end{array}$ & $\begin{array}{l}-4.36909 \mathrm{E}-01 \\
-4.44211 \mathrm{E}-01 \\
-4.51567 \mathrm{E}-01 \\
-4.58978 \mathrm{E}-01 \\
-4.66443 \mathrm{E}-01\end{array}$ & $\begin{array}{l}2.62966 \mathrm{E}-03 \\
2.65225 \mathrm{E}-03 \\
2.67436 \mathrm{E}-03 \\
2.69601 \mathrm{E}-03 \\
2.71722 \mathrm{E}-03\end{array}$ & $\begin{array}{l}-4.29167 \mathrm{E}-01 \\
-4.36193 \mathrm{E}-01 \\
-4.43274 \mathrm{E}-01 \\
-4.50409 \mathrm{E}-01 \\
-4.57598 \mathrm{E}-01\end{array}$ & $\begin{array}{l}1.07868 \mathrm{E} \\
1.08932 \mathrm{E} \\
1.09996 \mathrm{E} \\
1.11060 \mathrm{E} \\
1.12124 \mathrm{E}\end{array}$ & $\begin{array}{l}01 \\
01 \\
01 \\
01 \\
01\end{array}$ & $\begin{array}{l}-1.39178 \mathrm{E} \\
-1.39654 \mathrm{E} \\
-1.40124 \mathrm{E} \\
-1.40587 \mathrm{E} \\
-1.41045 \mathrm{E}\end{array}$ & $\begin{array}{l}02 \\
02 \\
02 \\
02 \\
02\end{array}$ \\
\hline $\begin{array}{l}10.10 \\
10.20 \\
10.30 \\
10.40 \\
10.50\end{array}$ & $\begin{array}{l}1.15402 \mathrm{E} \\
1.15398 \mathrm{E} \\
1.15395 \mathrm{E} \\
1.15391 \mathrm{E} \\
1.15388 \mathrm{E}\end{array}$ & $\begin{array}{l}00 \\
00 \\
00 \\
00 \\
00\end{array}$ & $\begin{array}{l}-1.02562 \mathrm{E} \\
-1.03625 \mathrm{E} \\
-1.04688 \mathrm{E} \\
-1.05752 \mathrm{E} \\
-1.06814 \mathrm{E}\end{array}$ & $\begin{array}{l}01 \\
01 \\
01 \\
01 \\
01\end{array}$ & $\begin{array}{l}-4.73962 \mathrm{E}-01 \\
-4.81535 \mathrm{E}-01 \\
-4.89163 \mathrm{E}-01 \\
-4.96844 \mathrm{E}-01 \\
-5.04580 \mathrm{E}-01\end{array}$ & $\begin{array}{l}2.73800 \mathrm{E}-03 \\
2.75835 \mathrm{E}-03 \\
2.77829 \mathrm{E}-03 \\
2.79784 \mathrm{E}-03 \\
2.81700 \mathrm{E}-03\end{array}$ & $\begin{array}{l}-4.64841 \mathrm{E}-01 \\
-4.72139 \mathrm{E}-01 \\
-4.79491 \mathrm{E}-01 \\
-4.86897 \mathrm{E}-01 \\
-4.94357 \mathrm{E}-01\end{array}$ & $\begin{array}{l}1.13187 \mathrm{E} \\
1.14250 \mathrm{E} \\
1.15313 \mathrm{E} \\
1.16376 \mathrm{E} \\
1.17439 \mathrm{E}\end{array}$ & $\begin{array}{l}01 \\
01 \\
01 \\
01 \\
01\end{array}$ & $\begin{array}{l}-1.41497 \mathrm{E} \\
-1.41943 \mathrm{E} \\
-1.42383 \mathrm{E} \\
-1.42818 \mathrm{E} \\
-1.43248 \mathrm{E}\end{array}$ & $\begin{array}{l}02 \\
02 \\
02 \\
02 \\
02\end{array}$ \\
\hline $\begin{array}{l}10.60 \\
10.70 \\
10.80 \\
10.90 \\
11.00\end{array}$ & $\begin{array}{l}1.15384 \mathrm{E} \\
1.15381 \mathrm{E} \\
1.15378 \mathrm{E} \\
1.15374 \mathrm{E} \\
1.15371 \mathrm{E}\end{array}$ & $\begin{array}{l}00 \\
00 \\
00 \\
00 \\
00\end{array}$ & $\begin{array}{l}-1.07877 \mathrm{E} \\
-1.08940 \mathrm{E} \\
-1.10002 \mathrm{E} \\
-1.11065 \mathrm{E} \\
-1.12127 \mathrm{E}\end{array}$ & $\begin{array}{l}01 \\
01 \\
01 \\
01 \\
01\end{array}$ & $\begin{array}{l}-5.12371 \mathrm{E}-01 \\
-5.20215 \mathrm{E}-01 \\
-5.28114 \mathrm{E}-01 \\
-5.36067 \mathrm{E}-01 \\
-5.44073 \mathrm{E}-01\end{array}$ & $\begin{array}{l}2.83578 \mathrm{E}-03 \\
2.85420 \mathrm{E}-03 \\
2.87226 \mathrm{E}-03 \\
2.88998 \mathrm{E}-03 \\
2.90736 \mathrm{E}-03\end{array}$ & $\begin{array}{l}-5.01871 \mathrm{E}-01 \\
-5.09440 \mathrm{E}-01 \\
-5.17063 \mathrm{E}-01 \\
-5.24740 \mathrm{E}-01 \\
-5.32471 \mathrm{E}-01\end{array}$ & $\begin{array}{l}1.18502 \mathrm{E} \\
1.19564 \mathrm{E} \\
1.20626 \mathrm{E} \\
1.21688 \mathrm{E} \\
1.22750 \mathrm{E}\end{array}$ & $\begin{array}{l}01 \\
01 \\
01 \\
01 \\
01\end{array}$ & $\begin{array}{l}-1.43672 \mathrm{E} \\
-1.44091 \mathrm{E} \\
-1.44505 \mathrm{E} \\
-1.44914 \mathrm{E} \\
-1.45318 \mathrm{E}\end{array}$ & $\begin{array}{l}02 \\
02 \\
02 \\
02 \\
02\end{array}$ \\
\hline $\begin{array}{l}11.10 \\
11.20 \\
11.30 \\
11.40 \\
11.50\end{array}$ & $\begin{array}{l}1.15368 \mathrm{E} \\
1.15365 \mathrm{E} \\
1.15362 \mathrm{E} \\
1.15359 \mathrm{E} \\
1.15356 \mathrm{E}\end{array}$ & $\begin{array}{l}00 \\
00 \\
00 \\
00 \\
00\end{array}$ & $\begin{array}{l}-1.13189 \mathrm{E} \\
-1.14251 \mathrm{E} \\
-1.15312 \mathrm{E} \\
-1.16374 \mathrm{E} \\
-1.17435 \mathrm{E}\end{array}$ & $\begin{array}{l}01 \\
01 \\
01 \\
01 \\
01\end{array}$ & $\begin{array}{l}-5.52135 \mathrm{E}-01 \\
-5.60250 \mathrm{E}-01 \\
-5.68420 \mathrm{E}-01 \\
-5.76643 \mathrm{E}-01 \\
-5.84921 \mathrm{E}-01\end{array}$ & $\begin{array}{l}2.92441 \mathrm{E}-03 \\
2.94114 \mathrm{E}-03 \\
2.95756 \mathrm{E}-03 \\
2.97368 \mathrm{E}-03 \\
2.98950 \mathrm{E}-03\end{array}$ & $\begin{array}{l}-5.40257 \mathrm{E}-01 \\
-5.48096 \mathrm{E}-01 \\
-5.55990 \mathrm{E}-01 \\
-5.63938 \mathrm{E}-01 \\
-5.71940 \mathrm{E}-01\end{array}$ & $\begin{array}{l}1.23812 \mathrm{E} \\
1.24873 \mathrm{E} \\
1.25935 \mathrm{E} \\
1.26996 \mathrm{E} \\
1.28057 \mathrm{E}\end{array}$ & $\begin{array}{l}01 \\
01 \\
01 \\
01 \\
01\end{array}$ & $\begin{array}{l}-1.45718 \mathrm{E} \\
-1.46113 \mathrm{E} \\
-1.46503 \mathrm{E} \\
-1.46889 \mathrm{E} \\
-1.47271 \mathrm{E}\end{array}$ & $\begin{array}{l}02 \\
02 \\
02 \\
02 \\
02\end{array}$ \\
\hline $\begin{array}{l}11.60 \\
11.70 \\
11.80 \\
11.90 \\
12.00\end{array}$ & $\begin{array}{l}1.15353 \mathrm{E} \\
1.15350 \mathrm{E} \\
1.15348 \mathrm{E} \\
1.15345 \mathrm{E} \\
1.15342 \mathrm{E}\end{array}$ & $\begin{array}{l}00 \\
00 \\
00 \\
00 \\
00\end{array}$ & $\begin{array}{l}-1.18497 \mathrm{E} \\
-1.19558 \mathrm{E} \\
-1.20619 \mathrm{E} \\
-1.21680 \mathrm{E} \\
-1.22740 \mathrm{E}\end{array}$ & $\begin{array}{l}01 \\
01 \\
01 \\
01 \\
01\end{array}$ & $\begin{array}{l}-5.93253 \mathrm{E}-01 \\
-6.01639 \mathrm{E}-01 \\
-6.10079 \mathrm{E}-01 \\
-6.18573 \mathrm{E}-01 \\
-6.27121 \mathrm{E}-01\end{array}$ & $\begin{array}{l}3.00504 \mathrm{E}-03 \\
3.02029 \mathrm{E}-03 \\
3.03527 \mathrm{E}-03 \\
3.04998 \mathrm{E}-03 \\
3.06443 \mathrm{E}-03\end{array}$ & $\begin{array}{l}-5.79996 \mathrm{E}-01 \\
-5.88106 \mathrm{E}-01 \\
-5.96271 \mathrm{E}-01 \\
-6.04489 \mathrm{E}-01 \\
-6.12762 \mathrm{E}-01\end{array}$ & $\begin{array}{l}1.29118 \mathrm{E} \\
1.30178 \mathrm{E} \\
1.31239 \mathrm{E} \\
1.32299 \mathrm{E} \\
1.33359 \mathrm{E}\end{array}$ & $\begin{array}{l}01 \\
01 \\
01 \\
01 \\
01\end{array}$ & $\begin{array}{l}-1.47648 \mathrm{E} \\
-1.48021 \mathrm{E} \\
-1.48390 \mathrm{E} \\
-1.48754 \mathrm{E} \\
-1.49115 \mathrm{E}\end{array}$ & $\begin{array}{l}02 \\
02 \\
02 \\
02 \\
02\end{array}$ \\
\hline
\end{tabular}


$\bar{F}$

6.10

6.20

6.30

6.40

6.50

6.60

6.70

6.80

6.90

7.00

7.10

7.20

7.30

7.40

7.50

7.60

7.70

7.80

7.90

8.00

8.10

8.20

8.30

8.40

8.50

8.60

8.70

8.80

8.90

9.00

9.10

9.20

9.30

9.40

9.50

9.60

9.70

9.80

9.90

10.00

10.10

10.20

10.30

10.40

10.50

10.60

10.70

10.80

10.90

11.00

11.10

11.20

11.30

11.40

11.50

11.60

11.70

11.80

11.90

12.00 $f_{2}$

$7.08310 \mathrm{E}-05$

$7.31671 \mathrm{E}-05$

$7.55411 \mathrm{E}-05$

$7.79529 \mathrm{E}-05$

$8.04028 \mathrm{E}-05$

$8.28906 \mathrm{E}-05$

$8.54163 \mathrm{E}-05$

$8.79800 \mathrm{E}-05$

$9.05817 \mathrm{E}-05$

$9.32213 \mathrm{E}-05$

$9.58988 \mathrm{E}-05$

$9.86143 \mathrm{E}-05$

$1.01368 \mathrm{E}-04$

$1.04159 \mathrm{E}-04$

$1.06988 \mathrm{E}-04$

$1.09856 \mathrm{E}-04$

$1.12761 \mathrm{E}-04$

$1.15704 \mathrm{E}-04$

$1.18685 \mathrm{E}-04$

$1.21704 \mathrm{E}-04$.

$1.24761 \mathrm{E}-04$

$1.27856 \mathrm{E}-04$

$1.30988 \mathrm{E}-04$

$1.34159 \mathrm{E}-04$

$1.37368 \mathrm{E}-04$

$1.40614 \mathrm{E}-04$

$1.43899 \mathrm{E}-04$

$1.47221 \mathrm{E}-04$

$1.50582 \mathrm{E}-04$

$1.53980 \mathrm{E}-04$

$1.57416 \mathrm{E}-04$

$1.60891 \mathrm{E}-04$

$1.64403 \mathrm{E}-04$

$1.67953 \mathrm{E}-04$

1.71541 E-04

$1.75167 \mathrm{E}-04$ $1.78831 \mathrm{E}-04$

$1.82532 \mathrm{E}-04$

$1.86272 \mathrm{E}-04$

$1.90050 \mathrm{E}-04$

$1.93865 \mathrm{E}-04$

$1.97719 \mathrm{E}-04$

$2.01610 \mathrm{E}-04$

$2.05540 \mathrm{E}-04$

$2.09507 \mathrm{E}-04$

$2.13512 \mathrm{E}-04$

$2.17555 \mathrm{E}-04$

$2.21636 \mathrm{E}-04$

$2.25755 \mathrm{E}-04$

$2.29912 \mathrm{E}-04$

$2.34107 \mathrm{E}-04$

$2.38340 \mathrm{E}-04$

$2.42611 \mathrm{E}-04$

$2.46919 \mathrm{E}-04$

$2.51266 \mathrm{E}-04$

$2.55650 \mathrm{E}-04$

$2.60073 \mathrm{E}-04$

$2.64533 \mathrm{E}-04$

2.69031 E- 04

$2.73568 \mathrm{E}-04$ $g_{2}$

$-9.38904 \mathrm{E}-02$

$-9.71353 \mathrm{E}-02$

$-1.00435 \mathrm{E}-01$

$-1.03790 \mathrm{E}-01$

$-1.07199 \mathrm{E}-01$

$-1.10664 \mathrm{E}-01$

$-1.14183 \mathrm{E}-01$

$-1.17757 \mathrm{E}-01$

$-1.21386 \mathrm{E}-01$

$-1.25070 \mathrm{E}-01$

$-1.28809 \mathrm{E}-01$

$-1.32603 \mathrm{E}-01$

$-1.36451 \mathrm{E}-01$

$-1.40355 \mathrm{E}-01$

$-1.44313 \mathrm{E}-01$

$-1.48326 \mathrm{E}-01$

$-1.52394 \mathrm{E}-01$

$-1.56516 \mathrm{E}-01$

$-1.60694 \mathrm{E}-01$

$-1.64926 \mathrm{E}-01$

$-1.69213 \mathrm{E}-01$

$-1.73555 \mathrm{E}-01$

$-1.77951 \mathrm{E}-01$

$-1.82403 \mathrm{E}-01$

$-1.86909 \mathrm{E}-01$

$-1.91470 \mathrm{E}-01$

$-1.96085 \mathrm{E}-01$

$-2.00756 \mathrm{E}-01$

$-2.05481 \mathrm{E}-01$

$-2.10260 \mathrm{E}-01$

$-2.15095 \mathrm{E}-01$

$-2.19984 \mathrm{E}-01$

$-2.24928 \mathrm{E}-01$

$-2.29927 \mathrm{E}-01$

$-2.34980 \mathrm{E}-01$

$-2.40088 \mathrm{E}-01$

$-2.45251 \mathrm{E}-01$

$-2.50468 \mathrm{E}-01$

$-2.55740 \mathrm{E}-01$

$-2.61067 \mathrm{E}-01$

$-2.66448 \mathrm{E}-01$

$-2.71884 \mathrm{E}-01$

$-2.77375 \mathrm{E}-01$

$-2.82920 \mathrm{E}-01$

$-2.88520 \mathrm{E}-01$

$-2.94175 \mathrm{E}-01$

$-2.99884 \mathrm{E}-01$

$-3.05647 \mathrm{E}-01$

$-3.11466 \mathrm{E}-01$

$-3.17339 \mathrm{E}-01$

$-3.23266 \mathrm{E}-01$

$-3.29248 \mathrm{E}-01$

$-3.35285 \mathrm{E}-01$

$-3.41376 \mathrm{E}-01$

$-3.47522 \mathrm{E}-01$

$-3.53722 \mathrm{E}-01$

$-3.59977 \mathrm{E}-01$

$-3.66286 \mathrm{E}-01$

$-3.72650 \mathrm{E}-01$

$-3.79068 \mathrm{E}-01$
$-1.97957 \mathrm{E}-03$

$-2.06278 \mathrm{E}-03$

$-2.14815 \mathrm{E}-03$

$-2.23572 \mathrm{E}-03$

-2.3255 I E-03

$-2.41755 \mathrm{E}-03$

$-2.51185 \mathrm{E}-03$

$-2.60845 \mathrm{E}-03$

$-2.70738 \mathrm{E}-03$

$-2.80864 \mathrm{E}-03$

$-2.91228 \mathrm{E}-03$

$-3.01832 \mathrm{E}-03$

$-3.12678 \mathrm{E}-03$

$-3.23769 \mathrm{E}-03$

$-3.35107 \mathrm{E}-03$

$-3.46694 \mathrm{E}-03$

$-3.58534 \mathrm{E}-03$

$-3.70629 \mathrm{E}-03$

$-3.82981 \mathrm{E}-03$

$-3.95593 \mathrm{E}-03$

$-4.08467 \mathrm{E}-03$

$-4.21607 \mathrm{E}-03$

$-4.35013 \mathrm{E}-03$

$-4.48690 \mathrm{E}-03$

$-4.62639 \mathrm{E}-03$

$-4.76864 \mathrm{E}-03$

$-4.91365 \mathrm{E}-03$

$-5.06147 \mathrm{E}-03$

$-5.21212 \mathrm{E}-03$

$-5.36561 \mathrm{E}-03$

$-5.52198 \mathrm{E}-03$

$-5.68125 \mathrm{E}-03$

$-5.84345 \mathrm{E}-03$

$-6.00860 \mathrm{E}-03$

$-6.17673 \mathrm{E}-03$

$-6.34786 \mathrm{E}-03$

$-6.52202 \mathrm{E}-03$

$-6.69923 \mathrm{E}-03$

$-6.87952 \mathrm{E}-03$

$-7.06291 \mathrm{E}-03$

$-7.24943 \mathrm{E}-03$

$-7.43910 \mathrm{E}-03$

$-7.63195 \mathrm{E}-03$

$-7.82801 \mathrm{E}-03$

$-8.02729 \mathrm{E}-03$

$-8.22983 \mathrm{E}-03$

$-8.43565 \mathrm{E}-03$

$-8.64477 \mathrm{E}-03$

$-8.85722 \mathrm{E}-03$

$-9.07302 \mathrm{E}-03$

$-9.29221 \mathrm{E}-03$

$-9.51480 \mathrm{E}-03$

$-9.74081 \mathrm{E}-03$

$-9.97029 \mathrm{E}-03$

$-1.02032 \mathrm{E}-02$

$-1.04397 \mathrm{E}-02$

$-1.06797 \mathrm{E}-02$

$-1.09232 \mathrm{E}-02$

$-1.11704 \mathrm{E}-02$

$-1.14211 \mathrm{E}-02$

$\tan \delta_{2}$

$f_{-2}$

g

$\tan \delta_{2}$

$3.23706 \mathrm{E}$ 0l $-1.98953 \mathrm{E}-03$

$3.24141 \mathrm{E}$ 01 $-2.07029 \mathrm{E}-03$

$3.24562 \mathrm{E}$ ol $-2.15313 \mathrm{E}-03$

$3.24968 \mathrm{E}$ 01 $-2.23808 \mathrm{E}-03$

$3.25362 \mathrm{E}$ ol $-2.32517 \mathrm{E}-03$

$3.25742 \mathrm{E} \quad 01 \quad-2.41442 \mathrm{E}-03$

$3.26111 \mathrm{E}$ 01 $-2.50585 \mathrm{E}-03$

$3.26468 \mathrm{E}$ ol $-2.59950 \mathrm{E}-03$

$3.26814 \mathrm{E} 01 \quad-2.69537 \mathrm{E}-03$

$3.27149 \mathrm{E} \quad 01-2.79351 \mathrm{E}-03$

$3.27475 \mathrm{E}$ ol $-2.89394 \mathrm{E}-03$

$3.27790 \mathrm{E}$ ol $-2.99667 \mathrm{E}-03$

$3.28097 \mathrm{E}$ 01 $-3.10175 \mathrm{E}-03$

$3.28395 \mathrm{E}$ ol $-3.20918 \mathrm{E}-03$

$3.28684 \mathrm{E}$ ol $-3.31901 \mathrm{E}-03$

$3.28966 \mathrm{E} \quad 01-3.43124 \mathrm{E}-03$

$3.29239 \mathrm{E}$ ol $-3.54591 \mathrm{E}-03$

$3.29505 \mathrm{E}$ 01 $-3.66305 \mathrm{E}-03$

$3.29764 \mathrm{E}$ 01 $-3.78267 \mathrm{E}-03$

$3.30016 \mathrm{E}$ 01 $-3.90481 \mathrm{E}-03$

$3.30261 \mathrm{E} \quad 01 \quad-4.02949 \mathrm{E}-03$

$3.30500 \mathrm{E}$ 01 $-4.15673 \mathrm{E}-03$

$3.30733 \mathrm{E}$ 01 $-4.28656 \mathrm{E}-03$

$3.30961 \mathrm{E}$ 01 $-4.41900 \mathrm{E}-03$

$3.31182 \mathrm{E}$ 0I $-4.55409 \mathrm{E}-03$

$3.31398 \mathrm{E}$ 01 $-4.69184 \mathrm{E}-03$

$3.31609 \mathrm{E}$ 01 $-4.83228 \mathrm{E}-03$

$3.31814 \mathrm{E}$ 01 $-4.97544 \mathrm{E}-03$

$3.32015 \mathrm{E}$ 01 $-5.12133 \mathrm{E}-03$

$3.32211 \mathrm{E}$ 01 $-5.26999 \mathrm{E}-03$

$3.32402 \mathrm{E} \quad 01 \quad-5.42145 \mathrm{E}-03$

$3.32589 \mathrm{E}$ 0l $-5.57572 \mathrm{E}-03$

$3.32772 \mathrm{E}$ 01 $-5.73283 \mathrm{E}-03$

$3.32950 \mathrm{E}$ 01 $-5.89281 \mathrm{E}-03$

$3.33125 \mathrm{E}$ ol $-6.05568 \mathrm{E}-03$

$3.33296 \mathrm{E}$ 01 $-6.22147 \mathrm{E}-03$

$3.33463 \mathrm{E}$ 01 $-6.39020 \mathrm{E}-03$

$3.33626 \mathrm{E} 01-6.56190 \mathrm{E}-03$

$3.33786 \mathrm{E}$ 01 $-6.73659 \mathrm{E}-03$ 
$F_{0}$

12.10

12.20

12.30

12.40

12.50

12.60

12.70

12.80

12.90

13.00

13.10

13.20

13.30

13.40

13.50

13.60

13.70

13.80

13.90

14.00

14.10

14.20

14.30

14.40

14.50

14.60

14.70

14.80

14.90

15.00

15.10

15.20

15.30

15.40

15.50

15.60

15.70

15.80

15.90

16.00

16.10

16.20

16.30

16.40

16.50

16.60

16.70

16.80

16.90

17.00

17.10

17.20

17.30

17.40

17.50

17.60

17.70

17.80

17.90

18.00

\section{$f_{1}$}

$1.15339 \mathrm{E} \quad 00$

$1.15337 \mathrm{E} \quad 00$

$1.15334 \mathrm{E} \quad 00$

$1.15331 \mathrm{E} \quad 00$

$1.15329 \mathrm{E} \quad 00$

$1.15326 \mathrm{E} \quad 00$

$1.15324 \mathrm{E} \quad 00$

$1.15321 \mathrm{E} \quad 00$

$1.15319 \mathrm{E} \quad 00$

$1.15316 \mathrm{E} \quad 00$

$1.15314 \mathrm{E} \quad 00$

$1.15312 \mathrm{E} \quad 00$

$1.15309 \mathrm{E} \quad 00$

$\begin{array}{ll}1.15307 \mathrm{E} & 00\end{array}$

$1.15305 \mathrm{E} \quad 00$

$1.15303 \mathrm{E} \quad 00$

$1.15300 \mathrm{E} \quad 00$

$1.15298 \mathrm{E} \quad 00$

$1.15296 \mathrm{E} \quad 00$

$1.15294 \mathrm{E} \quad 00$

$1.15292 \mathrm{E} \quad 00$ $1.15290 \mathrm{E} \quad 00$

$1.15288 \mathrm{E} \quad 00$

$1.15286 \mathrm{E} \quad 00$

$1.15284 \mathrm{E} \quad 00$

$1.15282 \mathrm{E} \quad 00$

$1.15280 \mathrm{E} \quad 00$

$1.15278 \mathrm{E} \quad 00$

$1.15276 \mathrm{E} \quad 00$

$1.15274 \mathrm{E} \quad 00$

$1.15272 \mathrm{E} \quad 00$

$1.15270 \mathrm{E} \quad 00$

$1.15268 \mathrm{E} \quad 00$

$\begin{array}{lll}1.15266 \mathrm{E} & 00\end{array}$

$1.15264 \mathrm{E} \quad 00$

$1.15262 \mathrm{E} \quad 00$ $1.15261 \mathrm{E} \quad 00$

$1.15259 \mathrm{E} \quad 00$

$1.15257 \mathrm{E} \quad 00$

$1.15255 \mathrm{E} \quad 00$

$1.15254 \mathrm{E} \quad 00$

$1.15252 \mathrm{E} \quad 00$

$1.15250 \mathrm{E} \quad 00$

$1.15249 \mathrm{E} \quad 00$

$1.15247 \mathrm{E}$ od

$1.15245 \mathrm{E} \quad 00$

$1.15244 \mathrm{E} \quad 00$

$1.15242 \mathrm{E} \quad 00$

$1.15240 \mathrm{E} \quad 00$

$1.15239 \mathrm{E} \quad 00$

$1.15237 \mathrm{E} \quad 00$

$1.15235 \mathrm{E} \quad 00$

$1.15234 \mathrm{E} \quad 00$

$1.15232 \mathrm{E} \quad 00$

$1.15231 \mathrm{E} \quad 00$

$1.15229 \mathrm{E} \quad 00$

$1.15228 \mathrm{E} \quad 00$

$1.15226 \mathrm{E} \quad 00$

$1.15225 \mathrm{E} \quad 00$

$1.15223 \mathrm{E} \quad 00$ $g_{1}$

$-1.23801 \mathrm{E} \quad 01$ $-1.24861 \mathrm{E} \quad 01$ $-1.25921 \mathrm{E} 01$ $-1.26981 \mathrm{E} 01$ $-1.28041 \mathrm{E} 01$

$-1.29101 \mathrm{E} \quad 01$ $-1.30160 \mathrm{E} 01$

$-1.31219 \mathrm{E} \quad 01$

$-1.32279 \mathrm{E}$ 01

$-1.33338 \mathrm{E}$ 0l

$-1.34397 \mathrm{E} 01$

$-1.35455 \mathrm{E} \quad 01$

$-1.36514 \mathrm{E} \quad 01$

$-1.37572 \mathrm{E} \quad 01$

$-1.38630 \mathrm{E} 01$

$-1.39689 \mathrm{E} 01$

$-1.40746 \mathrm{E} 01$

$-1.41804 \mathrm{E} \quad 01$

$-1.42862 \mathrm{E} 01$

$-1.43919 \mathrm{E} 01$

$-1.44976 \mathrm{E} \quad 01$

$-1.46033 \mathrm{E} 01$

$-1.47090 \mathrm{E} 01$

$-1.48147 \mathrm{E} \quad 01$

$-1.49204 \mathrm{E} 01$

$-1.50260 \mathrm{E} 01$

$-1.51316 \mathrm{E} 01$

$-1.52372 \mathrm{E} 01$

$-1.53428 \mathrm{E} 01$

-1.54484 E 01

$-1.55539 \mathrm{E} \quad 0 \mathrm{l}$

$-1.56595 \mathrm{E} \quad 01$

$-1.57650 \mathrm{E} 01$

$-1.58705 \mathrm{E} \quad 01$

$-1.59760 \mathrm{E} 01$

$-1.60814 \mathrm{E} \quad 01$

$-1.61869 \mathrm{E}$ 01

$-1.62923 \mathrm{E} 01$

$-1.63977 \mathrm{E} \quad 01$

$-1.65031 \mathrm{E}$ 0I

$-1.66085 \mathrm{E} 01$

$-1.67139 \mathrm{E} 01$

$-1.68192 \mathrm{E} 01$

$-1.69246 \mathrm{E} 01$

-1.70299 E 01

$-1.71352 \mathrm{E} 01$

$-1.72404 \mathrm{E} \quad 01$

$-1.73457 \mathrm{E} 01$

$-1.74509 \mathrm{E} 01$

$-1.75561 \mathrm{E} 01$

$-1.76613 \mathrm{E} 01$

$-1.77665 \mathrm{E} 01$

$-1.78717 \mathrm{E} 01$

$-1.79768 \mathrm{E} 01$

$-1.80819 \mathrm{E} 01$

$-1.81871 \mathrm{E} \quad 01$

$-1.82921 \mathrm{E} 01$

$-1.83972 \mathrm{E} \quad 01$

$-1.85023 \mathrm{E} 01$

$-1.86073 \mathrm{E} 01$
$-6.35724 \mathrm{E}-01$

$-6.44380 \mathrm{E}-01$

$-6.53090 \mathrm{E}-01$

$-6.61855 \mathrm{E}-01$

$-6.70673 \mathrm{E}-01$

$-6.79546 \mathrm{E}-01$

$-6.884 .72 \mathrm{E}-01$

$-6.97453 \mathrm{E}-01$

$-7.06488 \mathrm{E}-01$

$-7.15576 \mathrm{E}-0 \mathrm{I}$

$-7.24719 \mathrm{E}-01$

$-733915 \mathrm{E}-01$

$-7.43165 \mathrm{E}-01$

$-7.52470 \mathrm{E}-01$

$-7.61828 \mathrm{E}-01$

$-7.71241 \mathrm{E}-01$

$-7.80707 \mathrm{E}-01$

$-7.90227 \mathrm{E}-01$

$-7.99801 \mathrm{E}-01$

$-8.09429 \mathrm{E}-01$

$-8.19110 \mathrm{E}-0 \mathrm{I}$

$-8.28846 \mathrm{E}-0 \mathrm{I}$

$-8.38635 \mathrm{E}-01$

$-8.48478 \mathrm{E}-01$

$-8.58376 \mathrm{E}-01$

$-8.68326 \mathrm{E}-01$

$-8.78331 \mathrm{E}-0 \mathrm{I}$

$-8.88390 \mathrm{E}-0 \mathrm{I}$

$-8.98502 \mathrm{E}-01$

$-9.08668 \mathrm{E}-0 \mathrm{I}$

$-9.18888 \mathrm{E}-01$

$-9.29161 \mathrm{E}-01$

$-9.39489 \mathrm{E}-01$

$-9.49870 \mathrm{E}-01$

$-9.60304 \mathrm{E}-01$

$-9.70793 \mathrm{E}-01$

$-9.81335 \mathrm{E}-01$

$-9.91931 \mathrm{E}-01$

$-1.00258 \mathrm{E} \quad 00$

$-1.01328 \mathrm{E} 00$

$-1.02404 \mathrm{E} 00$

$-1.03485 \mathrm{E} \quad 00$

$-1.04572 \mathrm{E} 00$

$-1.05663 \mathrm{E} 00$

$-1.06760 \mathrm{E} \quad 00$

$-1.07863 \mathrm{E} \quad 00$

$-1.08971 \mathrm{E} 00$

$-1.10084 \mathrm{E} \quad 00$

$-1.11203 \mathrm{E} 00$

$-1.12327 \mathrm{E} 00$

$-1.13456 \mathrm{E} \quad 00$ $-1.14590 \mathrm{E} 00$ $-1.15730 \mathrm{E} 00$ $-1.16876 \mathrm{E} 00$

$-1.18026 \mathrm{E} 00$

-1.19182 E 00 $-1.20344 \mathrm{E} 00$ $-1.21511 \mathrm{E} 00$ $-1.22683 \mathrm{E} 00$

$-1.23860 \mathrm{E} 00$

$\tan \delta_{1}$

$3.07863 \mathrm{E}-03$

$3.09257 \mathrm{E}-03$

$3.10628 \mathrm{E}-03$

$3.11975 \mathrm{E}-03$

$3.13299 \mathrm{E}-03$

$3.14600 \mathrm{E}-03$

$3.15879 \mathrm{E}-03$

$3.17136 \mathrm{E}-03$

$3.18374 \mathrm{E}-03$

$3.19588 \mathrm{E}-03$

$3.20784 \mathrm{E}-03$

$3.21960 \mathrm{E}-03$

$3.23117 \mathrm{E}-03$

$3.24255 \mathrm{E}-03$

$3.25373 \mathrm{E}-03$

$3.26474 \mathrm{E}-03$

$3.27558 \mathrm{E}-03$

$3.28625 \mathrm{E}-03$

$3.29673 \mathrm{E}-03$

$3.30706 \mathrm{E}-03$

$3.31722 \mathrm{E}-03$

$3.32722 \mathrm{E}-03$

$3.33708 \mathrm{E}-03$

$3.34676 \mathrm{E}-03$

3.35631 E-03

$3.36570 \mathrm{E}-03$

$3.37495 \mathrm{E}-03$

$3.38406 \mathrm{E}-03$

$3.39303 \mathrm{E}-03$

$3.40187 \mathrm{E}-03$

$3.41056 \mathrm{E}-03$

$3.41914 \mathrm{E}-03$

$3.42758 \mathrm{E}-03$

$3.43589 \mathrm{E}-03$

$3.44408 \mathrm{E}-03$

$3.45216 \mathrm{E}-03$

$3.46010 \mathrm{E}-03$

$3.46794 \mathrm{E}-03$

$3.47565 \mathrm{E}-03$

$3.48325 \mathrm{E}-03$

$3.49075 \mathrm{E}-03$ 
14.10

15.10

15.20

15.30

15.40

15.50

15.60

15.70

15.80

15.90

16.00

16.10

16.20

16.30

16.40

16.50

16.60

16.70

16.80

16.90

17.00

17.10

17.20

17.30 $f_{2}$

$2.78142 \mathrm{E}-04$ $2.82754 \mathrm{E}-04$ $2.874 .04 \mathrm{E}-04$ $2.92092 \mathrm{E}-04$ $2.96818 \mathrm{E}-04$

3.01581 E-04 $3.06383 \mathrm{E}-04$

$3.11222 \mathrm{E}-04$.

$3.16100 \mathrm{E}-04$

$3.21015 \mathrm{E}-04$

$3.25969 \mathrm{E}-04$ $3.30960 \mathrm{E}-04$ $3.35989 \mathrm{E}-04$ $3.41056 \mathrm{E}-04$ $3.46161 \mathrm{E}-04$

$3.51304 \mathrm{E}-04$ $3.56485 \mathrm{E}-04$ $3.61704 \mathrm{E}-04$ $3.66960 \mathrm{E}-04$ $3.72255 \mathrm{E}-04$

$3.77587 \mathrm{E}-04$ $3.82958 \mathrm{E}-04$

$3.88366 \mathrm{E}-04$

$3.93812 \mathrm{E}-04$

3.99297 E- 04

$4.04819 \mathrm{E}-04$

$4.10379 \mathrm{E}-04$

$4.15977 \mathrm{E}-04$

$4.21612 \mathrm{E}-04$

$4.27286 \mathrm{E}-04$

$4.32998 \mathrm{E}-04$ $4.38747 \mathrm{E}-04$ $4.44535 \mathrm{E}-04$ $4.50360 \mathrm{E}-04$ $4.56224 \mathrm{E}-04$

$4.62125 \mathrm{E}-04$ $4.68064 \mathrm{E}-04$ 4.74041 E-04 $4.80056 \mathrm{E}-04$ $4.86109 \mathrm{E}-04$

$4.92200 \mathrm{E}-04$ $4.98328 \mathrm{E}-04$ $5.04495 \mathrm{E}-04$ $5.10699 \mathrm{E}-04$ $5.16942 \mathrm{E}-04$

$5.23222 \mathrm{E}-04$ 5.29541 E-04. $5.35897 \mathrm{E}-04$ 5.42291 E-04 5.48723 E-04

$5.55193 \mathrm{E}-04$ $5.61701 \mathrm{E}-04$ $5.68246 \mathrm{E}-04$ $5.74830 \mathrm{E}-04$ 5.81451 E- 04

5.88111 E-04. $5.94808 \mathrm{E}-04$ $6.01544 \mathrm{E}-04$ $6.08317 \mathrm{E}-04$ $6.15128 \mathrm{E}-04$
$-3.85542 \mathrm{E}-01$ $-3.92069 \mathrm{E}-01$ $-3.98651 \mathrm{E}-01$ $-4.05287 \mathrm{E}-01$

$-4.11978 \mathrm{E}-01$

$-4.18723 \mathrm{E}-01$ $-4.25523 \mathrm{E}-01$ $-4.32377 \mathrm{E}-01$ $-4.39286 \mathrm{E}-01$ $-4.46249 \mathrm{E}-01$

$-4.53267 \mathrm{E}-01$ $-4.60339 \mathrm{E}-01$ $-4.67465 \mathrm{E}-01$ $-4.74646 \mathrm{E}-01$ $-4.81881 \mathrm{E}-01$

$-4.89171 \mathrm{E}-01$ $-4.96515 \mathrm{E}-01$ $-5.03913 \mathrm{E}-01$ $-5.11366 \mathrm{E}-01$ $-5.18873 \mathrm{E}-01$

$-5.26434 \mathrm{E}-01$ $-5.34050 \mathrm{E}-01$ $-5.41720 \mathrm{E}-01$ $-5.49444 \mathrm{E}-01$ $-5.57223 \mathrm{E}-01$

$-5.65056 \mathrm{E}-01$ $-5.72943 \mathrm{E}-01$ $-5.80885 \mathrm{E}-01$ $-5.88881 \mathrm{E}-01$ $-5.96931 \mathrm{E}-01$

$-6.05035 \mathrm{E}-01$ $-6.13194 \mathrm{E}-01$ $-6.21407 \mathrm{E}-01$ $-6.29674 \mathrm{E}-01$ $-6.37996 \mathrm{E}-01$

$-6.46371 \mathrm{E}-01$ $-6.54801 \mathrm{E}-01$ $-6.63285 E-01$ $-6.71824 \mathrm{E}-01$ $-6.80416 \mathrm{E}-01$

$-6.89062 \mathrm{E}-01$ $-6.97763 \mathrm{E}-01$ $-7.06518 \mathrm{E}-01$ $-7.15327 \mathrm{E}-01$ $-7.24190 \mathrm{E}-01$

$-7.33108 \mathrm{E}-01$ $-7.42079 \mathrm{E}-01$ $-7.51105 \mathrm{E}-01$ $-7.60185 \mathrm{E}-01$ $-7.69318 \mathrm{E}-01$

$-7.78506 \mathrm{E}-01$ $-7.87748 \mathrm{E}-01$ $-7.97044 \mathrm{E}-01$ $-8.06394 \mathrm{E}-01$ $-8.15798 \mathrm{E}-01$

$-8.25257 \mathrm{E}-01$ $-8.34769 \mathrm{E}-01$ $-8.44335 \mathrm{E}-01$ $-8.53955 \mathrm{E}-01$ $-8.63629 \mathrm{E}-01$ $g_{2}$

$-1.16755 \mathrm{E}-02$ $-1.19335 \mathrm{E}-02$ $-1.21952 \mathrm{E}-02$ $-1.24606 \mathrm{E}-02$ $-1.27297 \mathrm{E}-02$

$-1.30026 \mathrm{E}-02$ $-1.32793 \mathrm{E}-02$ $-1.35598 \mathrm{E}-02$ $-1.38441 \mathrm{E}-02$ $-1.41322 \mathrm{E}-02$

$-1.44243 \mathrm{E}-02$ $-1.47202 \mathrm{E}-02$ $-1.50201 \mathrm{E}-02$ $-1.53240 \mathrm{E}-02$ $-1.56318 \mathrm{E}-02$

$-1.59436 \mathrm{E}-02$ $-1.62595 \mathrm{E}-02$ $-1.65794 \mathrm{E}-02$ $-1.69034 \mathrm{E}-02$ $-1.72315 \mathrm{E}-02$

$-1.75638 \mathrm{E}-02$ $-1.79002 \mathrm{E}-02$ $-1.82407 \mathrm{E}-02$ $-1.85855 \mathrm{E}-02$ $-1.89345 \mathrm{E}-02$

$-1.92878 \mathrm{E}-02$ $-1.96454 \mathrm{E}-02$ $-2.00072 \mathrm{E}-02$ $-2.03734 \mathrm{E}-02$ $-2.07440 \mathrm{E}-02$

$-2.11189 \mathrm{E}-02$ $-2.14982 \mathrm{E}-02$ $-2.18820 \mathrm{E}-02$ $-2.22702 \mathrm{E}-02$ $-2.26629 \mathrm{E}-02$

$-2.30601 \mathrm{E}-02$ $-2.34619 \mathrm{E}-02$ $-2.38682 \mathrm{E}-02$ $-2.42791 \mathrm{E}-02$ $-2.46945 \mathrm{E}-02$

$-2.51147 \mathrm{E}-02$ $-2.55394 \mathrm{E}-02$ $-2.59689 \mathrm{E}-02$ $-2.64030 \mathrm{E}-02$ $-2.68419 \mathrm{E}-02$

$-2.72856 \mathrm{E}-02$ $-2.77340 \mathrm{E}-02$ $-2.81872 \mathrm{E}-02$ $-2.86453 \mathrm{E}-02$ $-2.91082 \mathrm{E}-02$

$-2.95760 \mathrm{E}-02$ $-3.00487 \mathrm{E}-02$ $-3.05263 \mathrm{E}-02$ $-3.10089 \mathrm{E}-02$ $-3.14964 \mathrm{E}-02$

$-3.19890 \mathrm{E}-02$ $-3.24866 \mathrm{E}-02$ $-3.29892 \mathrm{E}-02$ $-3.34970 \mathrm{E}-02$ $-3.40098 \mathrm{E}-02$

$\tan \delta_{2}$

$3.36603 \mathrm{E} \quad 01$ $3.36706 \mathrm{E} \quad 01$ $3.36807 \mathrm{E} \quad 01$ $3.36906 \mathrm{E} \quad 0$ $3.37003 \mathrm{E} 01$

$3.37099 \mathrm{E} \quad 01$ $3.37194 \mathrm{E}$ 01

$3.37286 \mathrm{E} \quad 01$

$3.37378 \mathrm{E} \quad 01$

$3.37467 \mathrm{E}$ 01

$3.37556 \mathrm{E} \quad 01$

$3.37643 \mathrm{E} 01$

$3.37728 \mathrm{E} 01$

$3.37813 \mathrm{E}$ 01

$3.37895 \mathrm{E}$ ol

$3.37977 \mathrm{E} \quad 01$ $3.38058 \mathrm{E} \quad 01$ $3.38137 \mathrm{E} \quad 01$

$3.38215 \mathrm{E} \quad 01$

$3.38291 \mathrm{E} 01$

$3.38367 \mathrm{E} \quad 01$ $3.38442 \mathrm{E} \quad 01$ $3.38515 \mathrm{E} \quad 01$

$3.38588 \mathrm{E} 01$

$3.38659 \mathrm{E} \quad 01$

$3.38729 \mathrm{E} \quad 01$ $3.38798 \mathrm{E}$ 01 $3.38867 \mathrm{E} 01$

$3.38934 \mathrm{E}$ ol

$3.39001 \mathrm{E} 01$

$3.39066 \mathrm{E} \quad 01$ $3.39131 \mathrm{E} \quad 01$ $3.39194 \mathrm{E}$ 01 $3.39257 \mathrm{E} \quad 01$ $3.39319 \mathrm{E} \quad 01$

$3.39381 \mathrm{E} \quad 01$ $3.39441 \mathrm{E} \quad 01$ $3.39500 \mathrm{E} 01$ $3.39559 \mathrm{E} \quad 01$

$3.39617 \mathrm{E} 01$

$3.39675 \mathrm{E}$ 01 $3.39731 \mathrm{E} 01$ 
$$
p
$$

$F_{0}$

18.10

18.20

18.30

18.40

18.50

18.60

18.70

18.80

18.90

19.00

19.10

19.20

19.30

19.40

19.50

19.60

19.70

19.80

19.90

20.00

20.10

20.20

20.30

20.40

20.50

20.60

20.70

20.80

20.90

21.00

21.10

21.20

21.30

21.40

21.50

21.60

21.70

21.80

21.90

22.00

22.10

22.20

22.30

22.40

22.50

22.60

22.70

22.80

22.90

23.00

23.10

23.20

23.30

23.40

23.50

23.60

23.70

23.80

23.90

24.00 $f_{1}$

$1.15222 \mathrm{E} \quad 00$ $1.15220 \mathrm{E} \quad 00$ $1.15219 \mathrm{E} \quad 00$ $1.15217 \mathrm{E} \quad 00$ $1.15216 \mathrm{E} \quad 00$

$1.15214 \mathrm{E} \quad 00$ $1.15213 \mathrm{E} \quad 00$ $1.15212 \mathrm{E} \quad 00$ $\begin{array}{ll}1.15210 \mathrm{E} & 00\end{array}$ $1.15209 \mathrm{E} \quad 00$

$1.15207 \mathrm{E} \quad 00$ $1.15206 \mathrm{E} \quad 00$ $1.15205 \mathrm{E} \quad 00$ $1.15203 \mathrm{E} \quad 00$ $\begin{array}{ll}1.15202 \mathrm{E} & 00\end{array}$

$1.15201 \mathrm{E} \quad 00$ $1.15199 \mathrm{E} \quad 00$ $1.15198 \mathrm{E} \quad 00$ $1.15197 \mathrm{E} 00$ $1.15196 \mathrm{E} \quad 00$

$1.15194 \mathrm{E} \quad 00$ $1.15193 \mathrm{E} \quad 00$ $1.15192 \mathrm{E} \quad 00$ $1.15190 \mathrm{E} \quad 00$ $1.15189 \mathrm{E} \quad 00$

$1.15188 \mathrm{E} \quad 00$ $1.15187 \mathrm{E} \quad 00$ $1.15185 \mathrm{E} \quad 00$ $\begin{array}{ll}1.15184 \mathrm{E} & 00\end{array}$ $1.15183 \mathrm{E} 00$

$1.15182 \mathrm{E} \quad 00$
0 $\begin{array}{lll}1.15181 \mathrm{E} & 00\end{array}$ $1.15179 \mathrm{E} \quad 00$ $1.15178 \mathrm{E} \quad 00$ $1.15177 \mathrm{E} \quad 00$

$1.15176 \mathrm{E} \quad 00$ $1.15175 \mathrm{E} \quad 00$ $1.15173 \mathrm{E} \quad 00$ $\begin{array}{ll}1.15172 \mathrm{E} & 00\end{array}$ $1.15171 \mathrm{E} \quad 00$

$1.15170 \mathrm{E} \quad 00$ $1.15169 \mathrm{E} \quad 00$ $1.15168 \mathrm{E} \quad 00$ $1.15167 \mathrm{E} \quad 00$ $1.15166 \stackrel{\mathrm{E}}{00} 00$

$1.15164 \mathrm{E} \quad 00$ $1.15163 \mathrm{E} \quad 00$ $1.15162 \mathrm{E} \quad 00$
0 $1.15161 \mathrm{E} \quad 00$ $1.15160 \mathrm{E} \quad 00$

$1.15159 \mathrm{E} \quad 00$ $1.15158 \mathrm{E} \quad 00$ $1.15157 \mathrm{E} \quad 00$ $1.15156 \mathrm{E} \quad 00$ $1.15155 \mathrm{E} \quad 00$

$1.15154 \mathrm{E} \quad 00$ $\begin{array}{ll}1.15153 \mathrm{E} & 00\end{array}$ $\begin{array}{ll}1.15152 \mathrm{E} & 00\end{array}$ $1.15150 \mathrm{E} \quad 00$ $1.15149 \mathrm{E} 00$
$-1.87123 \mathrm{E} \quad 01$ -1.88173 E 01 $-1.89223 \mathrm{E} 01$ $-1.90273 \mathrm{E} 01$ -1.91322 E 01

$-1.92371 \mathrm{E} \quad 01$ $-1.93420 \mathrm{E} 01$ $-1.94469 \mathrm{E} \quad 01$ $-1.95518 \mathrm{E} \quad 01$ $-1.96566 \mathrm{E} 01$

-1.97614 E 01 $-1.98662 \mathrm{E} \quad 01$ $-1.99710 \mathrm{E} 01$ $-2.00758 \mathrm{E} \quad 01$ $-2.01805 \mathrm{E} \quad 01$

$-2.02853 \mathrm{E} \quad 01$ $-2.03900 \mathrm{E} 01$ $-2.04946 \mathrm{E} \quad 01$ $-2.05993 \mathrm{E} 01$ $-2.07040 \mathrm{E} \quad 01$

$-2.08086 \mathrm{E} \quad 01$ $-2.09132 \mathrm{E} \quad 01$ $-2.10178 \mathrm{E} \quad 01$ $-2.11224 \mathrm{E} \quad 01$ $-2.12269 \mathrm{E} \quad 01$

$-2.13314 \mathrm{E} \quad 01$ $-2.14359 \mathrm{E} \quad 01$ $-2.15404 \mathrm{E} 01$ $-2.16449 \mathrm{E} 01$ $-2.17493 \mathrm{E} 01$

$-2.18538 \mathrm{E} \quad 01$ $-2.19582 \mathrm{E} 01$ $-2.20626 \mathrm{E} \quad 01$ $-2.21669 \mathrm{E} \quad 01$ $-2.22713 \mathrm{E} \quad 01$

$-2.23756 \mathrm{E} \quad 01$ $-2.24799 \mathrm{E} 01$ $-2.25842 \mathrm{E} 01$ $-2.26884 \mathrm{E} 01$ $-2.27927 \mathrm{E} \quad 01$

$-2.28969 \mathrm{E} \quad 01$ $-2.30011 \mathrm{E} \quad 01$ $-2.31053 \mathrm{E}$ 01 $-2.32094 \mathrm{E} \quad 01$ $-2.33135 \mathrm{E} \quad 01$

$-2.34177 \mathrm{E} \quad 01$ $-2.35217 \mathrm{E}$ 01 $-2.36258 \mathrm{E} 01$ $-2.37299 \mathrm{E}$ ol $-2.38339 \mathrm{E} \quad 01$

$-2.39379 \mathrm{E} \quad 01$ $-2.40419 \mathrm{E} 01$ $-2.41458 \mathrm{E} \quad 01$ $-2.42498 \mathrm{E} 01$ $-2.43537 \mathrm{E} \quad 01$

$-2.44576 \mathrm{E} \quad 01$ $-2.45615 \mathrm{E} \quad 01$ $-2.46653 \mathrm{E} 01$ $-2.47692 \mathrm{E} \quad 01$ $-2.48730 \mathrm{E} \quad 01$ $g_{1}$

$-1.25043 \mathrm{E} \quad 00$ $-1.26231 \mathrm{E} \quad 00$ $-1.27424 \mathrm{E} \quad 00$ $-1.28623 \mathrm{E} \quad 00$ -1.29827 E 00

$-1.31037 \mathrm{E} \quad 00$ $-1.32252 \mathrm{E} 00$ $-1.33472 \mathrm{E} \quad 00$ $-1.34697 \mathrm{E} 00$ $-1.35928 \mathrm{E} \quad 00$

$-1.37164 \mathrm{E} \quad 00$ $-1.38405 \mathrm{E} 00$ $-1.39652 \mathrm{E} \quad 00$ $-1.40904 \mathrm{E} \quad 00$ $-1.42162 \mathrm{E} 00$

$-1.43424 \mathrm{E} \quad 00$ $-1.44692 \mathrm{E} \quad 00$ $-1.45966 \mathrm{E} \quad 00$ $-1.47245 \mathrm{E} \quad 00$ $-1.48529 \mathrm{E} \quad 00$

$-1.49818 \mathrm{E} \quad 00$ $-1.51113 \mathrm{E} 00$ $-1.52413 \mathrm{E} 00$

$-1.53718 \mathrm{E} 00$

$-1.55028 \mathrm{E} 00$

$-1.56344 \mathrm{E} \quad 00$

$-1.57665 \mathrm{E} 00$

$-1.58992 \mathrm{E} 00$

$-1.60324 \mathrm{E} \quad 00$

$-1.61661 \mathrm{E} 00$

$-1.63003 \mathrm{E} \quad 00$

$-1.64351 \mathrm{E} \quad 00$

$-1.65704 \mathrm{E} \quad 00$

$-1.67063 \mathrm{E} \quad 00$

$-1.68426 \mathrm{E} \quad 00$

$-1.69795 \mathrm{E} \quad 00$

$-1.71169 \mathrm{E} \quad 00$

$-1.72549 \mathrm{E} \quad 00$

$-1.73934 \mathrm{E} \quad 00$

$-1.75324 \mathrm{E} \quad 00$

$-1.76719 \mathrm{E} \quad 00$

$-1.78120 \mathrm{E} 00$

$-1.79526 \mathrm{E} 00$

$-1.80937 \mathrm{E} \quad 00$

$-1.82354 \mathrm{E} \quad 00$

$-1.83776 \mathrm{E} \quad 00$ $-1.85203 \mathrm{E} 00$

$-1.86635 \mathrm{E} \quad 00$

$-1.88073 \mathrm{E} \quad 00$

$-1.89516 \mathrm{E} \quad 00$

$-1.90964 \mathrm{E} \quad 00$

$-1.92417 \mathrm{E} \quad 00$

$-1.93876 \mathrm{E} \quad 00$

$-1.95340 \mathrm{E} 00$

$-1.96810 \mathrm{E} 00$

$-1.98284 \mathrm{E} \quad 00$

$-1.99764 \mathrm{E} \quad 00$

$-2.01249 \mathrm{E} \quad 00$

$-2.02740 \mathrm{E} \quad 00$

$-2.04235 \mathrm{E} \quad 00$

$\tan \delta_{1}$

$f_{-1}$

$3.61992 \mathrm{E}-03$ $3.62546 \mathrm{E}-03$ $3.63092 \mathrm{E}-03$

$3.63631 \mathrm{E}-03$

$3.64162 \mathrm{E}-03$

$3.64686 \mathrm{E}-03$

$3.65202 \mathrm{E}-03$

$3.65712 \mathrm{E}-03$

$3.66214 \mathrm{E}-03$

$3.66709 \mathrm{E}-03$

$3.67198 \mathrm{E}-03$

$3.67679 \mathrm{E}-03$

$3.68154 \mathrm{E}-03$

$3.68622 \mathrm{E}-03$

$3.69084 \mathrm{E}-03$

$3.69540 \mathrm{E}-03$ $3.69989 \mathrm{E}-03$

$3.70431 \mathrm{E}-03$

$3.70868 \mathrm{E}-03$

$3.71298 \mathrm{E}-03$

$3.71723 \mathrm{E}-03$

$3.72142 \mathrm{E}-03$

$3.72554 \mathrm{E}-03$

$3.72962 \mathrm{E}-03$

$3.73362 \mathrm{E}-03$

$3.73758 \mathrm{E}-03$

$3.74149 \mathrm{E}-03$

$3.74533 \mathrm{E}-03$

$3.74912 \mathrm{E}-03$

$3.75287 \mathrm{E}-03$

$3.75655 \mathrm{E}-03$

$3.76018 \mathrm{E}-03$

$3.76377 \mathrm{E}-03$

$3.76730 \mathrm{E}-03$

$3.77079 \mathrm{E}-03$

$3.77422 \mathrm{E}-03$

$3.77760 \mathrm{E}-03$ 
$\bar{F}$

$6.21977 \mathrm{E}-04$ $6.28864 \mathrm{E}-04$ $6.35789 \mathrm{E}-04$ $6.42752 \mathrm{E}-04$ $6.49752 \mathrm{E}-04$

$6.56791 \mathrm{E}-04$ $6.63867 \mathrm{E}-04$ $6.70982 \mathrm{E}-04$ $6.78134 \mathrm{E}-04$ $6.85324 \mathrm{E}-04$

$6.92552 \mathrm{E}-04$ $6.99818 \mathrm{E}-04$ $7.07122 \mathrm{E}-04$

$7.14464 \mathrm{E}-04$ $7.21844 \mathrm{E}-04$

$7.29262 \mathrm{E}-04$ $7.36717 \mathrm{E}-04$ $7.44210 \mathrm{E}-04$ $7.51742 \mathrm{E}-04$ $7.59311 \mathrm{E}-04$

$7.66918 \mathrm{E}-04$ $7.74563 \mathrm{E}-04$ $7.82246 \mathrm{E}-04$ $7.89967 \mathrm{E}-04$ $7.97726 \mathrm{E}-04$

$8.05522 \mathrm{E}-04$ $8.13357 \mathrm{E}-04$ $8.21229 \mathrm{E}-04$ $8.29140 \mathrm{E}-04$ $8.37088 \mathrm{E}-04$

$8.45074 \mathrm{E}-04$ $8.53098 \mathrm{E}-04$ $8.61160 \mathrm{E}-04$ $8.69260 \mathrm{E}-04$ $8.77398 \mathrm{E}-04$

$8.85573 \mathrm{E}-04$ $8.93787 \mathrm{E}-04$ $9.02039 \mathrm{E}-04$ $9.10328 \mathrm{E}-04$ $9.18655 \mathrm{E}-04$

$9.27020 \mathrm{E}-04$ $9.35423 \mathrm{E}-04$ $9.43864 \mathrm{E}-04$ $9.52343 \mathrm{E}-04$ $9.60860 \mathrm{E}-04$

$9.69415 \mathrm{E}-04$ $9.78007 \mathrm{E}-04$ $9.86638 \mathrm{E}-04$ $9.95306 \mathrm{E}-04$ $1.00401 \mathrm{E}-03$

$1.01276 \mathrm{E}-03$ $1.02154 \mathrm{E}-03$ $1.03036 \mathrm{E}-03$ $1.03922 \mathrm{E}-03$ $1.04811 \mathrm{E}-03$

23.60 23.70 23.80 23.90 24.00

$1.05705 \mathrm{E}-03$ $1.06602 \mathrm{E}-03$ $1.07503 \mathrm{E}-03$ $1.08407 \mathrm{E}-03$ $1.09316 \mathrm{E}-03$ $f_{2}$

$-8.73358 \mathrm{E}-01 \quad-3.45278 \mathrm{E}-02$

$-8.83140 \mathrm{E}-01 \quad-3.50509 \mathrm{E}-02$

$-8.92977 \mathrm{E}-01 \quad-3.55792 \mathrm{E}-02$

$-9.02867 \mathrm{E}-01 \quad-3.61126 \mathrm{E}-02$

$-9.12811 \mathrm{E}-01 \quad-3.66514 \mathrm{E}-02$

$-9.22809 \mathrm{E}-0 \mathrm{l}$

$-9.32861 \mathrm{E}-01$

$-9.42967 \mathrm{E}-01$

$-9.53127 \mathrm{E}-01$

$-9.63340 \mathrm{E}-01$

$-9.73608 \mathrm{E}-01$

$-9.83930 \mathrm{E}-01$

$-9.94305 \mathrm{E}-01$

$-1.00473 \mathrm{E} \quad 00$

$-1.01522 \mathrm{E} \quad 00$

$-1.02575 \mathrm{E} \quad 00$

$-1.03634 \mathrm{E} \quad 00$

$-1.04699 \mathrm{E} \quad 00$

$-1.05769 \mathrm{E} 00$

$-1.06844 \mathrm{E} \quad 00$

$-1.07925 \mathrm{E} \quad 00$ $-1.09011 \mathrm{E} \quad 00$ $-1.10102 \mathrm{E} \quad 00$ $-1.11199 \mathrm{E} \quad 00$ $-1.12301 \mathrm{E} 00$

$-1.13408 \mathrm{E} \quad 00$ $-1.14521 \mathrm{E} \quad 00$ $-1.15639 \mathrm{E} \quad 00$ $-1.16763 \mathrm{E} 00$ $-1.17892 \mathrm{E} \quad 00$

$-1.19026 \mathrm{E} \quad 00$ $-1.20166 \mathrm{E} \quad 00$ $-1.21311 \mathrm{E} \quad 00$ $-1.22461 \mathrm{E} \quad 00$ $-1.23617 \mathrm{E} \quad 00$

$-1.24778 \mathrm{E} \quad 00$ $-1.25945 \mathrm{E} \quad 00$ $-1.27117 \mathrm{E} 00$ $-1.28294 \mathrm{E} 00$ $-1.29477 \mathrm{E} \quad 00$

$-1.30665 \mathrm{E} \quad 00$ $-1.31858 \mathrm{E} \quad 00$ $-1.33056 \mathrm{E} 00$ $-1.34261 \mathrm{E} \quad 00$ $-1.35470 \mathrm{E} 00$

$-3.71953 \mathrm{E}-02$ $-3.77446 \mathrm{E}-02$ $-3.82991 \mathrm{E}-02$ $-3.88590 \mathrm{E}-02$ -3.94242 E-02

$-3.99948 \mathrm{E}-02$ $-4.05708 \mathrm{E}-02$ $-4.11522 \mathrm{E}-02$ $-4.17391 \mathrm{E}-02$ $-4.23314 \mathrm{E}-02$

$-4.29292 \mathrm{E}-02$ $-4.35326 \mathrm{E}-02$ $-4.41415 \mathrm{E}-02$ $-4.47560 \mathrm{E}-02$ $-4.53761 \mathrm{E}-02$

$-4.60018 \mathrm{E}-02$ $-4.66331 \mathrm{E}-02$ $-4.72702 \mathrm{E}-02$ $-4.79129 \mathrm{E}-02$ $-4.85613 \mathrm{E}-02$

$-4.92155 \mathrm{E}-02$ $-4.98755 \mathrm{E}-02$ $-5.05412 \mathrm{E}-02$ $-5.12128 \mathrm{E}-02$ $-5.18902 \mathrm{E}-02$

$-5.25735 \mathrm{E}-02$ $-5.32627 \mathrm{E}-02$ $-5.39578 \mathrm{E}-02$ $-5.46589 \mathrm{E}-02$ $-5.53659 \mathrm{E}-02$

$-5.60789 \mathrm{E}-02$ $-5.67979 \mathrm{E}-02$ $-5.75230 \mathrm{E}-02$ $-5.82541 \mathrm{E}-02$ $-5.89913 \mathrm{E}-02$

$-5.97346 \mathrm{E}-02$ $-6.04841 \mathrm{E}-02$ $-6.12397 \mathrm{E}-02$ $-6.20016 \mathrm{E}-02$ $-6.27696 \mathrm{E}-02$

$-1.36685 \mathrm{E} \quad 00$ $-1.37905 \mathrm{E} \quad 00$ $-1.39130 \mathrm{E} \quad 00$ $-1.40361 \mathrm{E} \quad 00$ $-1.41597 \mathrm{E} \quad 00$

$-6.35439 \mathrm{E}-02$ $-6.43244 \mathrm{E}-02$ $-6.51112 \mathrm{E}-02$ $-6.59044 \mathrm{E}-02$ $-6.67038 \mathrm{E}-02$

$-1.42838 \mathrm{E} \quad 00$ $-1.44085 \mathrm{E} \quad 00$ $-1.45337 \mathrm{E} \quad 00$ $-1.46595 \mathrm{E} \quad 00$ $-1.47858 \mathrm{E} \quad 00$

$-6.75097 \mathrm{E}-02$ $-6.83219 \mathrm{E}-02$ $-6.91405 \mathrm{E}-02$ $-6.99656 \mathrm{E}-02$ $-7.07971 \mathrm{E}-02$
$-1.49126 \mathrm{E} \quad 00$ $-1.50399 \mathrm{E} 00$ $-1.51678 \mathrm{E} 00$ $-1.52963 \mathrm{E} 00$ $-1.54252 \mathrm{E} 00$ $\tan \delta_{2}$

$3.40682 \mathrm{E} \quad 01$

$3.40726 \mathrm{E} \quad 01$

$3.40770 \mathrm{E} \quad 01$

$3.40813 \mathrm{E} \quad 0$

$3.40856 \mathrm{E} \quad 0$

$3.40898 \mathrm{E} \quad 01$

$3.40940 \mathrm{E} 01$

$3.40982 \mathrm{E} \quad 01$

$3.41022 \mathrm{E} \quad 01$

$3.41063 \mathrm{E} 01$

$3.41103 \mathrm{E} \quad 01$

$3.41143 \mathrm{E} \quad 01$

$3.41182 \mathrm{E} \quad 01$

$3.41221 \mathrm{E} \quad 01$

$3.41259 \mathrm{E} \quad 01$

$3.41297 \mathrm{E} \quad 01$

$3.41335 \mathrm{E} \quad 01$

$3.41372 \mathrm{E} \quad 01$

$3.41409 \mathrm{E} 01$

$3.41445 \mathrm{E} \quad 01$

$3.41481 \mathrm{E} \quad 01$

$3.41517 \mathrm{E} \quad 01$

$3.41552 \mathrm{E} \quad 01$

$3.41587 \mathrm{E} \quad 01$

$3.41621 \mathrm{E} \quad 01$

$3.41656 \mathrm{E} \quad 01$ $3.41689 \mathrm{E} \quad 01$ $3.41723 \mathrm{E} \quad 01$ $3.41756 \mathrm{E} \quad 01$ $3.41789 \mathrm{E} \quad 01$

$3.41822 \mathrm{E} \quad 01$ $3.41854 \mathrm{E} \quad 01$ $3.41886 \mathrm{E} \quad 01$ $3.41917 \mathrm{E} \quad 01$

$3.41949 \mathrm{E} 01$

$3.41980 \mathrm{E} \quad 01$

$3.42010 \mathrm{E} \quad 01$

$3.42041 \mathrm{E} 01$

$3.42071 \mathrm{E} \quad 01$

$3.42101 \mathrm{E} 01$

$3.42130 \mathrm{E} \quad 01$ $3.42160 \mathrm{E} \quad 01$ $3.42189 \mathrm{E} 01$ $3.42217 \mathrm{E} \quad 01$ $3.42246 \mathrm{E} \quad 01$

$3.42274 \mathrm{E} \quad 01$ $3.42302 \mathrm{E} \quad 01$ $3.42330 \mathrm{E}$ 01 $3.42357 \mathrm{E} \quad 01$ $3.42385 \mathrm{E} 01$

$3.42412 \mathrm{E} \quad 01$ $3.42438 \mathrm{E} \quad 01$ $3.42465 \mathrm{E} \quad 01$

$3.42491 \mathrm{E} 01$ $3.42517 \mathrm{E} \quad 01$

$3.42543 \mathrm{E} \quad 01$ $3.42568 \mathrm{E}$ 01 $3.42594 \mathrm{E} \quad 01$ $3.42619 \mathrm{E}$ 01 $3.42644 \mathrm{E} \quad 01$

\section{$f_{-2}$}

$-3.36381 \mathrm{E}-02$

$-3.41486 \mathrm{E}-02$

$-3.46642 \mathrm{E}-02$

$-3.51849 \mathrm{E}-02$

$-3.57107 \mathrm{E}-02$

$-3.62417 \mathrm{E}-02$

$-3.67780 \mathrm{E}-02$

$-3.73194 \mathrm{E}-02$

$-3.78661 \mathrm{E}-02$

$-3.84180 \mathrm{E}-02$

$-3.89752 \mathrm{E}-02$

$-3.95378 \mathrm{E}-02$

$-4.01057 \mathrm{E}-02$

$-4.06789 \mathrm{E}-02$

$-4.12576 \mathrm{E}-02$

$-4.18416 \mathrm{E}-02$

$-4.24311 \mathrm{E}-02$

$-4.30261 \mathrm{E}-02$

$-4.36265 \mathrm{E}-02$

$-4.42325 \mathrm{E}-02$

$-4.48440 \mathrm{E}-02$

$-4.54611 \mathrm{E}-02$

$-4.60837 \mathrm{E}-02$

$-4.67120 \mathrm{E}-02$

$-4.73459 \mathrm{E}-02$

$-4.79855 E-02$

$-4.86307 \mathrm{E}-02$

$-4.92817 \mathrm{E}-02$

$-4.99384 \mathrm{E}-02$

$-5.06009 \mathrm{E}-02$

$-5.12691 \mathrm{E}-02$

$-5.19432 \mathrm{E}-02$

$-5.26231 \mathrm{E}-02$

$-5.33088 \mathrm{E}-02$

$-5.40005 \mathrm{E}-02$

$-5.46980 \mathrm{E}-02$

$-5.54015 \mathrm{E}-02$

$-5.61109 \mathrm{E}-02$

$-5.68263 \mathrm{E}-02$

$-5.75478 \mathrm{E}-02$

$-5.82752 \mathrm{E}-02$ $-5.90087 \mathrm{E}-02$ $-5.97483 \mathrm{E}-02$ $-6.04940 \mathrm{E}-02$ $-6.12458 \mathrm{E}-02$

$-6.20038 \mathrm{E}-02$ $-6.27680 \mathrm{E}-02$ 


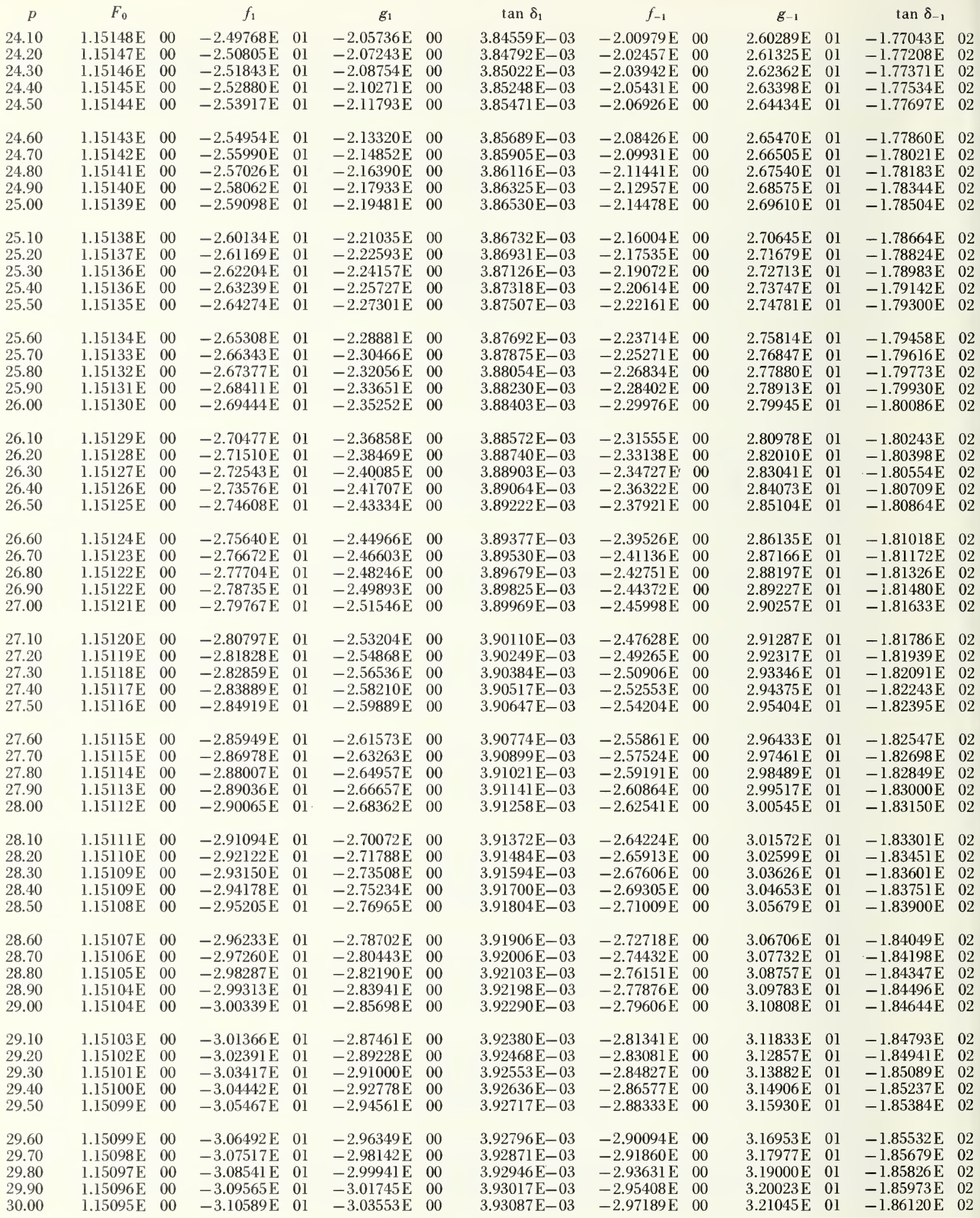


$Z=6 \quad A=12 \quad \rho=1.3 A^{1 / 3} F$

\section{$\bar{F}$}

$1.10228 \mathrm{E}-03$

$1.11144 \mathrm{E}-03$

$1.12064 \mathrm{E}-03$

$1.12988 \mathrm{E}-03$

$1.13916 \mathrm{E}-03$

$1.14847 \mathrm{E}-03$

$1.15782 \mathrm{E}-03$

$1.16721 \mathrm{E}-03$

$1.17663 \mathrm{E}-03$

$1.18610 \mathrm{E}-03$

$1.19560 \mathrm{E}-03$

$1.20514 \mathrm{E}-03$

$1.21472 \mathrm{E}-03$

$1.22433 \mathrm{E}-03$

$1.23399 \mathrm{E}-03$

$1.24368 \mathrm{E}-03$

$1.25341 \mathrm{E}-03$

$1.26318 \mathrm{E}-03$

$1.28282 \mathrm{E}-03$

$1.29271 \mathrm{E}-03$

$1.30262 \mathrm{E}-03$

$1.31258 \mathrm{E}-03$

$1.32258 \mathrm{E}-03$

$1.33261 \mathrm{E}-03$

$1.34268 \mathrm{E}-03$

$1.35279 \mathrm{E}-03$

$1.36293 \mathrm{E}-03$

$1.37312 \mathrm{E}-03$

$1.38334 \mathrm{E}-03$

$1.39360 \mathrm{E}-03$

$1.40390 \mathrm{E}-03$

$1.41423 \mathrm{E}-03$

$1.42461 \mathrm{E}-03$

$1.43502 \mathrm{E}-03$

$1.44547 \mathrm{E}-03$

$1.45596 \mathrm{E}-03$

$1.46648 \mathrm{E}-03$

$1.47705 \mathrm{E}-03$

$1.48765 \mathrm{E}-03$

$1.49829 \mathrm{E}-03$

$1.50896 \mathrm{E}-03$

$1.51968 \mathrm{E}-03$

$1.53043 \mathrm{E}-03$

$1.54122 \mathrm{E}-03$

28.60

28.70

28.80

28.90

29.00

29.10

29.20

29.30

29.40

29.50

29.60

29.70

29.80

29.90

30.00

$1.55205 \mathrm{E}-03$ $1.56292 \mathrm{E}-03$ $1.57382 \mathrm{E}-03$ $1.58476 \mathrm{E}-03$ $1.59574 \mathrm{E}-03$

$1.60676 \mathrm{E}-03$ $1.61782 \mathrm{E}-03$ $1.62891 \mathrm{E}-03$ $1.64004 \mathrm{E}-03$ $1.65121 \mathrm{E}-03$

$1.66242 \mathrm{E}-03$ $1.67366 \mathrm{E}-03$ $1.68495 \mathrm{E}-03$ $1.69627 \mathrm{E}-03$
$1.27298 \mathrm{E}-03$

$f_{2} \quad g_{2}$

$\tan \delta_{2}$

$f_{-2}$

$-1.55547 \mathrm{E} \quad 00 \quad-7.59232 \mathrm{E}-02$ $-1.56847 \mathrm{E} \quad 00 \quad-7.68006 \mathrm{E}-02$ $-1.58153 \mathrm{E} \quad 00 \quad-7.76847 \mathrm{E}-02$ $-1.59464 \mathrm{E} \quad 00 \quad-7.85754 \mathrm{E}-02$ $-1.60780 \mathrm{E} \quad 00 \quad-7.94729 \mathrm{E}-02$ $1.70763 \mathrm{E}-03$
$-1.62101 \mathrm{E} \quad 00$ $1.63428 \mathrm{E} \quad 00$

$-1.64760 \mathrm{E} \quad 00$

$-1.66098 \mathrm{E} \quad 00$

$-1.67441 \mathrm{E} \quad 00$

$-1.68789 \mathrm{E} \quad 00$ $-1.70142 \mathrm{E} \quad 00$ $-1.71501 \mathrm{E} \quad 00$ $-1.72865 \mathrm{E} \quad 00$

$-1.74235 \mathrm{E} \quad 00$

$-1.75609 \mathrm{E} \quad 00$ $-1.76989 \mathrm{E} \quad 00$ $-1.78375 \mathrm{E} \quad 00$ $-1.79765 \mathrm{E} \quad 00$

$-1.81161 \mathrm{E} \quad 00$

$-1.82563 \mathrm{E} \quad 00$ $-1.83969 \mathrm{E} \quad 00$ $-1.85381 \mathrm{E} \quad 00$ $-1.86798 \mathrm{E} 00$ $-1.88221 \mathrm{E} \quad 00$

$-1.89649 \mathrm{E} \quad 00$ $-1.91082 \mathrm{E} \quad 00$ $-1.92520 \mathrm{E} \quad 00$ $-1.93964 \mathrm{E} \quad 00$ $-1.95413 \mathrm{E} 00$

$-1.96867 \mathrm{E} \quad 00$ $-1.98327 \mathrm{E} \quad 00$ $-1.99792 \mathrm{E} 00$ $-2.01262 \mathrm{E} \quad 00$ $-2.02738 \mathrm{E} \quad 00$

$-2.04218 \mathrm{E} \quad 00$ $-2.05704 \mathrm{E} \quad 00$ $-2.07196 \mathrm{E} \quad 00$ $-2.08692 \mathrm{E} \quad 00$ $-2.10194 \mathrm{E} \quad 00$

$-2.11702 \mathrm{E} \quad 00$ $-2.13214 \mathrm{E} \quad 00$ $-2.14732 \mathrm{E} 00$ $-2.16255 \mathrm{E} \quad 00$ $-2.17783 \mathrm{E} 00$

$-8.03770 \mathrm{E}-02$
$-8.12880 \mathrm{E}-02$ $-8.22056 \mathrm{E}-02$ $-8.31301 \mathrm{E}-02$ $-8.40615 \mathrm{E}-02$

$-8.49996 \mathrm{E}-02$ $-8.59447 \mathrm{E}-02$ $-8.68966 \mathrm{E}-02$ $-8.78555 \mathrm{E}-02$ $-8.88213 \mathrm{E}-02$

$-8.97940 \mathrm{E}-02$ $-9.07738 \mathrm{E}-02$ $-9.17606 \mathrm{E}-02$ $-9.27544 \mathrm{E}-02$ $-9.37554 \mathrm{E}-02$

$-9.47634 \mathrm{E}-02$ $-9.57785 \mathrm{E}-02$ $-9.68007 \mathrm{E}-02$ $-9.78301 \mathrm{E}-02$ $-9.88667 \mathrm{E}-02$

$-9.99106 \mathrm{E}-02$ $-1.00962 \mathrm{E}-01$ $-1.03086 \mathrm{E}-01$ $-1.04158 \mathrm{E}-01$

$-1.05239 \mathrm{E}-01$ $-1.06326 \mathrm{E}-01$ $-1.07421 \mathrm{E}-01$ $-1.08524 \mathrm{E}-01$ $-1.09634 \mathrm{E}-01$

$-1.10751 \mathrm{E}-01$ $-1.11876 \mathrm{E}-01$ $-1.13008 \mathrm{E}-01$ $-1.14148 \mathrm{E}-01$ $-1.15295 \mathrm{E}-01$

$-1.16450 \mathrm{E}-01$ $-1.17612 \mathrm{E}-01$ $-1.18783 \mathrm{E}-01$ $-1.19960 \mathrm{E}-01$ $-1.21146 \mathrm{E}-01$

$-2.19317 \mathrm{E} \quad 00$ $-2.20856 \mathrm{E} \quad 00$ $-2.22400 \mathrm{E} \quad 00$ $-2.23949 \mathrm{E} \quad 00$ $-2.25504 \mathrm{E} 00$

$-1.22339 \mathrm{E}-01$ $-1.23540 \mathrm{E}-01$ $-1.24749 \mathrm{E}-01$ $-1.25965 \mathrm{E}-01$ $-1.27189 \mathrm{E}-01$

$\begin{array}{lll}-2.27064 \mathrm{E} & 00 & -1.28421 \mathrm{E}-01\end{array}$ $-2.28629 \mathrm{E} \quad 00 \quad-1.29661 \mathrm{E}-01$ $-2.30200 \mathrm{E} \quad 00-1.30909 \mathrm{E}-01$ $-2.31776 \mathrm{E} \quad 00 \quad-1.32164 \mathrm{E}-01$ $-2.33357 \mathrm{E} \quad 00 \quad-1.33428 \mathrm{E}-01$

$-2.34943 \mathrm{E} \quad 00 \quad-1.34699 \mathrm{E}-01$ $-2.36535 \mathrm{E} \quad 00 \quad-1.35979 \mathrm{E}-0 \mathrm{l}$ $-2.38132 \mathrm{E} \quad 00 \quad-1.37266 \mathrm{E}-01$ $-2.39734 \mathrm{E} \quad 00 \quad-1.38562 \mathrm{E}-01$ $-2.41341 \mathrm{E} \quad 00 \quad-1.39865 \mathrm{E}-01$ $-1.02020 \mathrm{E}-01$ $3.42693 \mathrm{E} \quad 01$ $3.42717 \mathrm{E} \quad 01$ $3.42741 \mathrm{E} \quad 01$ $3.42765 \mathrm{E} \quad 01$

$3.42789 \mathrm{E} \quad 01$

$3.42812 \mathrm{E} \quad 01$

$3.42836 \mathrm{E} \quad 01$

$3.42859 \mathrm{E}$ 01

$3.42882 \mathrm{E} \quad 01$

$3.42904 \mathrm{E} \quad 01$ $3.42927 \mathrm{E} \quad 01$ $3.42949 \mathrm{E} \quad 01$ $3.42971 \mathrm{E}$ 01 $3.42993 \mathrm{E} 01$

$3.43015 \mathrm{E}$ 01 $3.43037 \mathrm{E} \quad 01$ $3.43058 \mathrm{E} \quad 01$ $3.43079 \mathrm{E} \quad 01$

$3.43100 \mathrm{E} \quad 01$

$3.43121 \mathrm{E} \quad 01$

$3.43142 \mathrm{E} \quad 01$

$3.43163 \mathrm{E} 01$

$3.43183 \mathrm{E} \quad 01$

$3.43244 \mathrm{E} \quad 01$

$3.43263 \mathrm{E} 01$

$3.43283 \mathrm{E} \quad 01$

$3.43303 \mathrm{E} 01$

$3.43322 \mathrm{E} 01$

$3.43341 \mathrm{E} \quad 01$

$3.43360 \mathrm{E} \quad 01$

$3.43379 \mathrm{E} \quad 01$

$3.43398 \mathrm{E} 01$

$3.43417 \mathrm{E} \quad 01$

$3.43435 \mathrm{E} \quad 01$

$3.43454 \mathrm{E} \quad 01$

$3.43472 \mathrm{E} \quad 01$

$3.43490 \mathrm{E} \quad 01$

$3.43508 \mathrm{E} \quad 01$

$3.43526 \mathrm{E}$ ol

$3.43543 \mathrm{E} \quad 01$

$3.43561 \mathrm{E} 01$

$3.43578 \mathrm{E} \quad 01$

$3.43596 \mathrm{E} \quad 01$

$3.43613 \mathrm{E}$ 01

$3.43630 \mathrm{E} \quad 01$

$3.43647 \mathrm{E} 01$

$3.43664 \mathrm{E} \quad 01$

$3.43680 \mathrm{E} \quad 01$

$3.43697 \mathrm{E} \quad 01$

$3.43713 \mathrm{E} \quad 01$

$3.43730 \mathrm{E}$ 01

$3.43746 \mathrm{E} \quad 01$

$3.43762 \mathrm{E} 01$

$3.43778 \mathrm{E} \quad 01$

$3.43794 \mathrm{E}$ ol

$3.43810 \mathrm{E} \quad 01$

$3.43825 \mathrm{E} \quad 01$
$3.42669 \mathrm{E} \quad 01$

$3.43204 \mathrm{E} \quad 01$

$3.43224 \mathrm{E} \quad 01$

$-7.41287 \mathrm{E}-02$ $-7.49885 \mathrm{E}-02$ $-7.58549 \mathrm{E}-02$ $-7.67278 \mathrm{E}-02$ $-7.76074 \mathrm{E}-02$

$-7.84936 \mathrm{E}-02$ $-7.93864 \mathrm{E}-02$ $-8.02860 \mathrm{E}-02$ $-8.11923 \mathrm{E}-02$ $-8.21053 \mathrm{E}-02$

$-8.30251 \mathrm{E}-02$ $-8.39517 \mathrm{E}-02$ $-8.48851 \mathrm{E}-02$ $-8.58253 \mathrm{E}-02$ $-8.67724 \mathrm{E}-02$

$-8.77264 \mathrm{E}-02$ $-8.86873 \mathrm{E}-02$ $-8.96551 \mathrm{E}-02$ $-9.06299 \mathrm{E}-02$ $-9.16116 \mathrm{E}-02$

$-9.26004 \mathrm{E}-02$ $-9.35962 \mathrm{E}-02$ $-9.45991 \mathrm{E}-02$ $-9.56091 \mathrm{E}-02$ $-9.66261 \mathrm{E}-02$

$-9.76503 \mathrm{E}-02$ $-9.86817 \mathrm{E}-02$ $-9.97202 \mathrm{E}-02$ $-1.00766 \mathrm{E}-01$ $-1.01819 \mathrm{E}-01$

$-1.02879 \mathrm{E}-01$ $-1.03947 \mathrm{E}-01$ $-1.05021 \mathrm{E}-01$ $-1.06103 \mathrm{E}-01$ $-1.07193 \mathrm{E}-01$

$-1.08290 \mathrm{E}-01$ $-1.09394 \mathrm{E}-01$ $-1.10506 \mathrm{E}-01$ $-1.11625 \mathrm{E}-01$ $-1.12751 \mathrm{E}-01$

$-1.13885 \mathrm{E}-01$ $-1.15027 \mathrm{E}-01$ 
ELECTRON RADIAL FUNCTIONS

Electron

30.10

30.20

30.30

30.40

30.50

30.60

30.70

30.80

30.90

31.00

31.10

31.20

31.30

31.40

31.50

31.60

31.70

31.80

31.90

32.00

32.10

32.20

32.30

32.40

32.50

32.60

32.70

32.80

32.90

33.00

33.10

33.20

33.30

33.40

33.50

33.60

33.70

33.80

33.90

34.00

34.10

34.20

34.30

34.40

34.50

34.60

34.70

34.80

34.90

35.00

35.10

35.20

35.30

35.40

35.50

35.60

35.70

35.80

35.90

36.00
$Z=6 \quad A=12$

\begin{abstract}
$F_{0}$
\end{abstract}
$1.15095 \mathrm{E} \quad 00$ $1.15094 \mathrm{E} \quad 00$ $1.15093 \mathrm{E} \quad 00$ $1.15092 \mathrm{E} \quad 00$ $1.15092 \mathrm{E} \quad 00$

$1.15091 \mathrm{E} \quad 00$ $1.15090 \mathrm{E} \quad 00$ $1.15089 \mathrm{E} \quad 00$ $1.15088 \mathrm{E} \quad 00$ $1.15088 \mathrm{E} \quad 00$

$1.15087 \mathrm{E} \quad 00$ $1.15086 \mathrm{E} \quad 00$ $1.15085 \mathrm{E} \quad 00$ $1.15085 \mathrm{E} \quad 00$ $1.15084 \mathrm{E} \quad 00$

$1.15083 \mathrm{E} \quad 00$ $1.15082 \mathrm{E} \quad 00$ $1.15082 \mathrm{E} \quad 00$ $1.15081 \mathrm{E} \quad 00$ $1.15080 \mathrm{E} \quad 00$

$1.15079 \mathrm{E} \quad 00$. $1.15079 \mathrm{E} \quad 00$ $1.15078 \mathrm{E} \quad 00$

$1.15077 \mathrm{E} \quad 00$

$1.15077 \mathrm{E} \quad 00$

$1.15076 \mathrm{E} \quad 00$ $1.15075 \mathrm{E} \quad 00$ $1.15074 \mathrm{E} \quad 00$ $1.15074 \mathrm{E} \quad 00$ $1.15073 \mathrm{E} \quad 00$

$1.15072 \mathrm{E} \quad 00$ $1.15072 \mathrm{E} \quad 00$ $1.15071 \mathrm{E} \quad 00$ $1.15070 \mathrm{E} \quad 00$ $1.15069 \mathrm{E} \quad 00$

$1.15069 \mathrm{E} \quad 00$ $1.15068 \mathrm{E} \quad 00$ $1.15067 \mathrm{E} \quad 00$ $1.15067 \mathrm{E} \quad 00$

$1.15066 \mathrm{E} \quad 00$

$1.15065 \mathrm{E} \quad 00$ $1.15065 \mathrm{E} \quad 00$ $1.15064 \mathrm{E} \quad 00$ $1.15063 \mathrm{E} \quad 00$ $1.15063 \mathrm{E} \quad 00$

$1.15062 \mathrm{E} \quad 00$ $1.15061 \mathrm{E} \quad 00$ $1.15061 \mathrm{E} \quad 00$ $1.15060 \mathrm{E} \quad 00$ $1.15059 \mathrm{E} \quad 00$

$1.15059 \mathrm{E} \quad 00$ $1.15058 \mathrm{E} \quad 00$ $1.15057 \mathrm{E} \quad 00$ $1.15057 \mathrm{E} \quad 00$

$1.15056 \mathrm{E} \quad 00$

$1.15055 \mathrm{E} \quad 00$ $1.15055 \mathrm{E} \quad 00$ $1.15054 \mathrm{E} \quad 00$ $1.15053 \mathrm{E} \quad 00$ $1.15053 \mathrm{E} \quad 00$ $f_{1}$

$-3.11612 \mathrm{E} \quad 01$ $-3.12635 \mathrm{E} 01$ $\begin{array}{ll}-3.13658 \mathrm{E} & 01\end{array}$ $-3.14681 \mathrm{E} 01$ $-3.15704 \mathrm{E} 01$

$-3.16726 \mathrm{E} 01$ $-3.17748 \mathrm{E}-01$ $-3.18770 \mathrm{E} 01$ $\begin{array}{ll}-3.19791 \mathrm{E} & 01\end{array}$ $-3.20812 \mathbf{E} 01$

$-3.21833 \mathbf{E} 01$ $-3.22854 \mathrm{E} \quad 01$ $-3.23874 \mathrm{E} \quad 01$ $-3.24894 \mathrm{E} 01$ $-3.25914 \mathrm{E} \quad 01$

$-3.26934 \mathrm{E} 01$ $-3.27953 \mathrm{E} 01$ $-3.28972 \mathrm{E} 01$ $-3.29991 \mathrm{E} 01$ $-3.31010 \mathrm{E} 01$

$-3.32028 \mathrm{E} 01$ $-3.33046 \mathrm{E} 01$ $-3.34064 \mathrm{E} 01$ $-3.35081 \mathrm{E} 01$ $-3.36098 \mathrm{E} 01$

$-3.37115 \mathrm{E} \quad 01$ $-3.38132 \mathrm{E} 01$ $-3.39148 \mathrm{E} 01$ $-3.40165 \mathrm{E} 01$ -3.41180 E 01

$\begin{array}{lll}-3.42196 \mathrm{E} & 01\end{array}$ $-3.43211 \mathrm{E} 01$ $-3.44226 \mathrm{E} 01$ $-3.45241 \mathrm{E} 01$ $-3.46255 \mathrm{E} 01$

$-3.47270 \mathrm{E} \quad 01$ $-3.48284 \mathrm{E} 01$ $-3.49297 \mathrm{E} 01$ $-3.50311 \mathrm{E} 01$ $-3.51324 \mathrm{E} 01$

$-3.52336 \mathrm{E} 01$ $-3.53349 \mathrm{E} 01$ -3.54361 E 01 $-3.55373 \mathrm{E} \quad 01$ -3.56385 E 01

$-3.57396 \mathrm{E} 01$ $-3.58407 \mathrm{E} 01$ $-3.59418 \mathrm{E} 01$ $-3.60429 \mathrm{E} 01$ $-3.61439 \mathrm{E} 01$

$-3.62449 \mathrm{E} 01$ $-3.63459 \mathrm{E} 01$ $-3.64468 \mathrm{E} 01$ $-3.65477 \mathrm{E} 01$ $-3.66486 \mathrm{E} 01$

$-3.67495 \mathrm{E} \quad 01$ $-3.68503 \mathrm{E} 01$ $-3.69511 \mathrm{E} 01$ $-3.70519 \mathrm{E} 01$ $-3.71526 \mathrm{E} 01$ $g_{1}$

$-3.05367 \mathrm{E} \quad 00$ $-3.07186 \mathrm{E} 00$ $-3.09011 \mathrm{E} 00$ -3.10840 E 00

$-3.12675 \mathrm{E} \quad 00$

$-3.14515 \mathrm{E} \quad 00$

$-3.16360 \mathrm{E} 00$

$-3.18210 \mathrm{E} 00$

$-3.20065 \mathrm{E} 00$

$-3.21925 \mathrm{E} 00$

$-3.25662 \mathrm{E} 00$

$-3.27538 \mathrm{E} 00$

$-3.29419 \mathrm{E} \quad 00$

$-3.31305 \mathrm{E} 00$

$-3.33196 \mathrm{E} 00$

$-3.35093 \mathrm{E} 00$

$-3.36994 \mathrm{E} 00$

$-3.38901 \mathrm{E} 00$

$-3.40813 \mathrm{E} 00$

$-3.42730 \mathrm{E} 00$

$-3.44652 \mathrm{E} 00$

$-3.46579 \mathrm{E} 00$

$-3.48512 \mathrm{E} 00$

$-3.50449 \mathrm{E} 00$

$-3.52392 \mathrm{E} \quad 00$

$-3.54340 \mathrm{E} 00$

$-3.56293 \mathrm{E} 00$

$-3.58251 \mathrm{E} 00$

$-3.60214 \mathrm{E} \quad 00$

$-3.62182 \mathrm{E} 00$

$-3.64156 \mathrm{E} 00$

$-3.66134 \mathrm{E} 00$

$-3.68118 \mathrm{E} \quad 00$

$-3.70107 \mathrm{E} 00$

$-3.72101 \mathrm{E} \quad 00$

$-3.74100 \mathrm{E} 00$

$-3.76104 \mathrm{E} 00$

$-3.78113 \mathrm{E} 00$

$-3.80127 \mathrm{E} 00$

$-3.82146 \mathrm{E} 00$

$-3.84171 \mathrm{E} 00$

$-3.86201 \mathrm{E} 00$

$-3.88235 \mathrm{E} \quad 00$

$-3.90275 \mathrm{E} 00$

$-3.92320 \mathrm{E} \quad 00$

$-3.94370 \mathrm{E} 00$

$-3.96425 \mathrm{E} 00$

$-3.98485 \mathrm{E} 00$

$-4.00550 \mathrm{E} \quad 00$

$-4.02621 \mathrm{E} \quad 00$

$-4.04696 \mathrm{E} 00$

$-4.06777 \mathrm{E} 00$

$-4.08862 \mathrm{E} 00$

$-4.10953 \mathrm{E} 00$

$-4.13048 \mathrm{E} 00$

$-4.15149 \mathrm{E} 00$

$-4.17255 \mathrm{E} 00$

$-4.19366 \mathrm{E} \quad 00$

$-4.21482 \mathrm{E} \quad 00$
$-3.23791 \mathrm{E} 00$

$\tan \delta_{1}$

$3.93154 \mathrm{E}-03$

$3.93220 \mathrm{E}-03$

$3.93283 \mathrm{E}-03$

$3.93344 \mathrm{E}-03$

$3.93402 \mathbf{E}-03$

$3.93459 \mathrm{E}-03$

$3.93514 \mathrm{E}-03$

$3.93566 \mathrm{E}-03$

$3.93616 \mathrm{E}-03$

$3.93665 \mathrm{E}-03$

$3.93712 \mathrm{E}-03$

$3.93756 \mathrm{E}-03$

$3.93798 \mathrm{E}-03$

$3.93839 \mathrm{E}-03$

$3.93877 \mathrm{E}-03$

$3.93913 \mathrm{E}-03$

$3.93948 \mathrm{E}-03$

$3.93981 \mathrm{E}-03$

$3.94011 \mathrm{E}-03$

$3.94040 \mathrm{E}-03$

$3.94067 \mathrm{E}-03$

$3.94092 \mathrm{E}-03$

$3.94115 \mathrm{E}-03$

$3.94136 \mathrm{E}-03$

$3.94156 \mathrm{E}-03$

$3.94173 \mathrm{E}-03$

$3.94189 \mathrm{E}-03$

$3.94203 \mathrm{E}-03$

$3.94215 \mathrm{E}-03$

$3.94225 \mathrm{E}-03$

$3.94234 \mathrm{E}-03$

$3.94240 \mathrm{E}-03$

$3.94246 \mathrm{E}-03$

$3.94249 \mathrm{E}-03$

$3.94250 \mathrm{E}-03$

$3.94250 \mathrm{E}-03$

$3.94248 \mathrm{E}-03$

$3.94244 \mathrm{E}-03$

$3.94239 \mathrm{E}-03$

$3.94232 \mathrm{E}-03$

$3.94224 \mathrm{E}-03$ 
ELECTRON RADIAL FUNCTIONS

$Z=6 \quad A=12 \quad \rho=1.3 A^{1 / 3} F$

Eirecesen

$\bar{F}$

$1.71902 \mathrm{E}-03$

$1.73046 \mathrm{E}-03$

$1.74193 \mathrm{E}-03$

$1.75344 \mathrm{E}-03$

$1.76499 \mathrm{E}-03$

$1.77658 \mathrm{E}-03$ $1.78820 \mathrm{E}-03$ $1.79986 \mathrm{E}-03$ $1.81156 \mathrm{E}-03$ $1.82330 \mathrm{E}-03$

$1.83508 \mathrm{E}-03$ $1.84689 \mathrm{E}-03$ $1.85874 \mathrm{E}-03$ $1.87063 \mathrm{E}-03$ $1.88256 \mathrm{E}-03$

$1.89452 \mathrm{E}-03$

$1.90652 \mathrm{E}-03$ $1.91857 \mathrm{E}-03$

$1.93064 \mathrm{E}-03$ $1.94276 \mathrm{E}-03$

$1.95492 \mathrm{E}-03$ $1.96711 \mathrm{E}-03$ $1.97934 \mathrm{E}-03$ $1.99161 \mathrm{E}-03$ $2.00391 \mathrm{E}-03$

$2.01626 \mathrm{E}-03$ $2.02864 \mathrm{E}-03$ $2.04106 \mathrm{E}-03$ $2.05352 \mathrm{E}-03$ $2.06601 \mathrm{E}-03$

$2.07855 \mathrm{E}-03$ $2.09112 \mathrm{E}-03$ $2.10373 \mathrm{E}-03$ $2.11637 \mathrm{E}-03$ $2.12906 \mathrm{E}-03$

$2.14178 \mathrm{E}-03$ $2.15454 \mathrm{E}-03$ $2.16734 \mathrm{E}-03$ $2.18018 \mathrm{E}-03$ $2.19305 \mathrm{E}-03$

$2.20596 \mathrm{E}-03$ $2.21891 \mathrm{E}-03$ $2.23190 \mathrm{E}-03$ $2.24493 \mathrm{E}-03$ $2.25799 \mathrm{E}-03$

$2.27109 \mathrm{E}-03$ $2.28423 \mathrm{E}-03$ $2.29741 \mathrm{E}-03$ $2.31063 E-03$ $2.32388 \mathrm{E}-03$

$2.33717 \mathrm{E}-03$ $2.35050 \mathrm{E}-03$ $2.36387 \mathrm{E}-03$ $2.37727 \mathrm{E}-03$ $2.39071 \mathrm{E}-03$

$2.40419 \mathrm{E}-03$ $2.41771 \mathrm{E}-03$ $2.43127 \mathrm{E}-03$

$2.44486 \mathrm{E}-03$ $2.45850 \mathrm{E}-03$ $f_{2}$

$-2.42954 \mathrm{E} \quad 00$ $-2.44572 \mathrm{E} \quad 00$ $-2.46195 \mathrm{E} \quad 00$ $-2.47823 \mathrm{E} \quad 00$

$-2.49457 \mathrm{E} \quad 00$

$-2.51096 \mathrm{E} \quad 00$ $-2.52740 \mathrm{E} \quad 00$ $-2.54389 \mathrm{E} \quad 00$ $-2.56044 \mathrm{E} \quad 00$ $-2.57703 \mathrm{E} \quad 00$

$-2.59369 \mathrm{E} \quad 00$ $-2.61039 \mathrm{E} \quad 00$ $-2.62714 \mathrm{E} \quad 00$ $-2.64395 \mathrm{E} \quad 00$ $-2.66081 \mathrm{E} \quad 00$

$-2.67773 \mathrm{E} \quad 00$ $-2.69469 \mathrm{E} \quad 00$ $-2.71171 \mathrm{E} \quad 00$ $-2.72878 \mathrm{E} \quad 00$ $-2.74590 \mathrm{E} \quad 00$

$-2.76307 \mathrm{E} \quad 00$ $-2.78030 \mathrm{E} \quad 00$ $-2.79758 \mathrm{E} \quad 00$ $-2.81491 \mathrm{E} \quad 00$ $-2.83229 \mathrm{E} \quad 00$

$-2.84973 \mathrm{E} \quad 00$ $-2.86722 \mathrm{E} \quad 00$ $-2.88476 \mathrm{E} \quad 00$ $-2.90235 \mathrm{E} \quad 00$ $-2.91999 \mathrm{E} 00$

$-2.93769 \mathrm{E} \quad 00$ $-2.95544 \mathrm{E} \quad 00$ $-2.97324 \mathrm{E} \quad 00$ $-2.99109 \mathrm{E} 00$ $-3.00900 \mathrm{E} \quad 00$

$-3.02695 \mathrm{E} \quad 00$ $-3.04496 \mathrm{E} 00$ $-3.06302 \mathrm{E} \quad 00$ $-3.08113 \mathrm{E} \quad 00$ $-3.09930 \mathrm{E} 00$

$-3.11752 \mathrm{E} \quad 00$ $-3.13578 \mathrm{E} \quad 00$ $-3.15410 \mathrm{E} \quad 00$ $-3.17248 \mathrm{E} \quad 00$ $-3.19090 \mathrm{E} 00$

$-3.20938 \mathrm{E} \quad 00$ $-3.22791 \mathrm{E} \quad 00$ $-3.24649 \mathrm{E} 00$ $-3.26512 \mathrm{E} \quad 00$ $-3.28380 \mathrm{E} 00$

$-3.30254 \mathrm{E} \quad 00$ $-3.32132 \mathrm{E} \quad 00$ $-3.34016 \mathrm{E} \quad 00$ $-3.35905 \mathrm{E} \quad 00$ $-3.37800 \mathrm{E} \quad 00$

$-3.39699 \mathrm{E} \quad 00$ $-3.41604 \mathrm{E} \quad 00$ $-3.43513 \mathrm{E} 00$ $-3.45428 \mathrm{E} \quad 00$ $-3.47348 \mathrm{E} \quad 00$ $g_{2}$

$-1.41177 \mathrm{E}-01$ $-1.42496 \mathrm{E}-01$ $-1.43824 \mathrm{E}-01$ $-1.45160 \mathrm{E}-01$ $-1.46504 \mathrm{E}-01$

$-1.47856 \mathrm{E}-01$ $-1.49216 \mathrm{E}-01$ $-1.50585 \mathrm{E}-01$ $-1.51962 \mathrm{E}-01$ $-1.53347 \mathrm{E}-01$

$-1.54740 \mathrm{E}-01$ $-1.56142 \mathrm{E}-01$ $-1.57552 \mathrm{E}-01$ $-1.58970 \mathrm{E}-01$ $-1.60397 \mathrm{E}-0 \mathrm{H}$

$-1.61832 \mathrm{E}-01$ $-1.63276 \mathrm{E}-01$ $-1.64728 \mathrm{E}-01$ $-1.66189 \mathrm{E}-01$ $-1.67658 \mathrm{E}-01$

$-1.69136 \mathrm{E}-01$ $-1.70622 \mathrm{E}-01$ $-1.72117 \mathrm{E}-01$ $-1.73620 \mathrm{E}-01$ $-1.75132 \mathrm{E}-01$

$-1.76653 \mathrm{E}-01$ $-1.78182 \mathrm{E}-01$ $-1.797 .20 \mathrm{E}-01$ $-1.81267 \mathrm{E}-01$ $-1.82823 \mathrm{E}-01$

$-1.84387 \mathrm{E}-01$ $-1.85960 \mathrm{E}-01$ $-1.87542 \mathrm{E}-01$ $-1.89133 \mathrm{E}-01$ $-1.90733 \mathrm{E}-01$

$-1.92341 \mathrm{E}-01$ $-1.93959 \mathrm{E}-01$ $-1.95585 \mathrm{E}-01$ $-1.97220 \mathrm{E}-01$ $-1.98865 \mathrm{E}-01$

$-2.00518 \mathrm{E}-01$ $-2.02181 \mathrm{E}-01$ $-2.03852 \mathrm{E}-01$ $-2.05533 \mathrm{E}-01$ $-2.07222 \mathrm{E}-01$

$-2.08921 \mathrm{E}-01$ $-2.10629 \mathrm{E}-01$ $-2.12346 \mathrm{E}-01$ $-2.14073 \mathrm{E}-01$ $-2.15808 \mathrm{E}-01$

$-2.17553 \mathrm{E}-01$ $-2.19307 \mathrm{E}-01$ $-2.21070 \mathrm{E}-01$ $-2.22843 \mathrm{E}-01$ $-2.24625 \mathrm{E}-01$

$-2.26416 \mathrm{E}-01$ $-2.28217 \mathrm{E}-01$ $-2.30027 \mathrm{E}-01$ $-2.31847 \mathrm{E}-01$ $-2.33676 \mathrm{E}-01$ $\tan \delta 2$

$$
f_{\cdot 2}
$$

3.43841 E 01

E $01-1.39474 \mathrm{E}-0$

3.43872 E 01

$3.43887 \mathrm{E} \quad$ ()l

3.43902 E 01

$-1.40779 \mathrm{E}-01$

$-1.42092 \mathrm{E}-01$

$3.43917 \mathrm{E}$ 0l $3.43932 \mathrm{E} 01$ $3.43947 \mathrm{E}$ 01 $3.43962 \mathrm{E}$ 01

$3.43977 \mathrm{E}$ 0l

$3.43991 \mathrm{E} \quad 01$

$3.44006 \mathrm{E} \quad 01$

$3.44020 \mathrm{E} 01$

$3.44034 \mathrm{E} \quad 0$

$3.44048 \mathrm{E} 01$

$3.44062 \mathrm{E} \quad 01$

$3.44076 \mathrm{E} 01$

$3.44090 \mathrm{E} \quad 01$

$3.44104 \mathrm{E} \quad 01$

$3.44118 \mathrm{E}$ 0l

3.44131 E 01 $3.44145 \mathrm{E} \quad 01$ $3.44158 \mathrm{E} \quad 01$ $3.44172 \mathrm{E} \quad 01$ $3.44185 \mathrm{E} \quad 01$

$3.44198 \mathrm{E} \quad 01$ $3.44211 \mathrm{E} \quad 01$

$3.44224 \mathrm{E} \quad 01$

$3.44237 \mathrm{E} \quad 01$

$3.44250 \mathrm{E} \quad 0$

$3.44263 \mathrm{E} \quad 01$ $3.44276 \mathrm{E}$ 0l $3.44288 \mathrm{E} \quad 01$ $3.44301 \mathrm{E} 01$ $3.44313 \mathrm{E} \quad 01$

$3.44326 \mathrm{E} \quad 01$ $3.44338 \mathrm{E} \quad 01$ $3.44350 \mathrm{E} \quad 01$ $3.44362 \mathrm{E} \quad 01$ 3.44375 E 01

$3.44387 \mathrm{E} \quad 01$

$3.44399 \mathrm{E} \quad 01$

$3.44410 \mathrm{E} \quad 01$

$3.44422 \mathrm{E} \quad 01$

$3.44434 \mathrm{E} 01$

$3.44446 \mathrm{E} \quad 01$ $3.44457 \mathrm{E} \quad 01$ $3.44469 \mathrm{E} \quad 01$ $3.44480 \mathrm{E} \quad 01$ $3.44491 \mathrm{E} \quad 01$

$3.44503 \mathrm{E} \quad 01$ $3.44514 \mathrm{E} \quad 01$ $3.44525 \mathrm{E} \quad 01$

$3.44536 \mathrm{E} \quad 01$

$3.44547 \mathrm{E} \quad 01$

$3.44558 \mathrm{E} \quad 01$ $3.44569 \mathrm{E} \quad 0 \mathrm{l}$

$3.44580 \mathrm{E} \quad 01$

$3.44591 \mathrm{E}$ 01

$3.44602 \mathrm{E} \quad 01$

$-1.44742 \mathrm{E}-01$

$-1.46080 \mathrm{E}-01$ $-1.47425 \mathrm{E}-01$ $-1.48779 \mathrm{E}-01$

$-1.50141 \mathrm{E}-01$

$-1.51511 \mathrm{E}-01$ $-1.52889 \mathrm{E}-0 \mathrm{l}$ $-1.54276 \mathrm{E}-01$ $-1.55670 \mathrm{E}-01$

$-1.57074 \mathrm{E}-01$

$-1.58485 \mathrm{E}-01$ $-1.59905 \mathrm{E}-01$ $-1.61333 \mathrm{E}-01$ $-1.62770 \mathrm{E}-01$ $-1.64215 \mathrm{E}-01$

$-1.65668 \mathrm{E}-01$ $-1.67130 \mathrm{E}-01$ $-1.68601 \mathrm{E}-01$ $-1.70080 \mathrm{E}-01$ $-1.71567 \mathrm{E}-01$

$-1.73063 \mathrm{E}-01$ $-1.74568 \mathrm{E}-01$ $-1.76081 \mathrm{E}-01$ $-1.77603 \mathrm{E}-01$ $-1.79134 \mathrm{E}-01$

$-1.80673 \mathrm{E}-01$ $-1.82222 \mathrm{E}-01$ $-1.83778 \mathrm{E}-01$ $-1.85344 \mathrm{E}-01$ $-1.86918 \mathrm{E}-01$

$-1.88501 \mathrm{E}-01$ $-1.90093 \mathrm{E}-01$ $-1.91694 \mathrm{E}-01$ $-1.93304 \mathrm{E}-01$ $-1.94923 \mathbf{E}-01$

$-1.96550 \mathrm{E}-01$ $-1.98187 \mathrm{E}-01$ $-1.99832 \mathrm{E}-01$ 


\begin{tabular}{|c|c|c|c|c|c|c|c|c|}
\hline$p$ & $F_{0}$ & & $f_{1}$ & & $g_{1}$ & & $\tan \delta_{1}$ & $f_{-1}$ \\
\hline 36.10 & $1.15052 \mathrm{E}$ & 00 & $-3.72533 \mathrm{E}$ & 01 & $-4.23603 \mathrm{E}$ & 00 & $3.93713 \mathrm{E}-03$ & $-4.15591 \mathrm{E}$ \\
\hline 6.20 & $1.15051 \mathrm{E}$ & 00 & $-3.73540 \mathbf{E}$ & 01 & $-4.25729 \mathrm{E}$ & 00 & $3.93670 \mathrm{E}-03$ & $-4.17690 \mathrm{E}$ \\
\hline 36.30 & $1.15051 \mathrm{E}$ & 00 & $-3.74546 \mathrm{E}$ & 01 & $-4.27861 \mathrm{E}$ & 00 & $3.93628 \mathrm{E}-$ & $4.19794 \mathrm{E}$ \\
\hline 36.40 & $1.15050 \mathrm{E}$ & 00 & $-3.75553 \mathbf{E}$ & 01 & $-4.29997 \mathrm{E}$ & 00 & $3.93583 \mathrm{E}-$ & $-4.21904 \mathrm{E}$ \\
\hline 36.50 & $1.15049 \mathrm{E}$ & 00 & $-3.76559 \mathrm{E}$ & 01 & $-4.32138 \mathrm{E}$ & 00 & $3.93537 \mathrm{E}-$ & $-4.24018 \mathrm{E}$ \\
\hline 60 & $1.15049 \mathrm{E}$ & 00 & $-3.77564 \mathrm{E}$ & 01 & $-4.34285 \mathrm{E}$ & 00 & $3.93488 \mathrm{E}-03$ & $-4.26138 \mathrm{E}$ \\
\hline .70 & $1.15048 \mathrm{E}$ & 00 & $-3.78570 \mathrm{E}$ & 01 & $-4.36436 \mathrm{E}$ & 00 & $40 E-03$ & $-4.28262 \mathrm{E}$ \\
\hline .80 & $1.15048 \mathrm{E}$ & 00 & $-3.79575 \mathrm{E}$ & 01 & $-4.38592 \mathrm{E}$ & 00 & $3.93389 \mathrm{E}-$ & $-4.30392 \mathrm{E}$ \\
\hline .90 & $1.15047 \mathrm{E}$ & 00 & $-3.80579 \mathrm{E}$ & 01 & $-4.40754 \mathrm{E}$ & 00 & $3.93337 \mathrm{E}-03$ & $-4.32526 \mathrm{E}$ \\
\hline .00 & $1.15046 \mathrm{E}$ & 00 & $-3.81584 \mathrm{E}$ & 01 & $-4.42921 \mathrm{E}$ & 00 & $3.93284 \mathrm{E}-03$ & $-4.34666 \mathrm{E}$ \\
\hline .10 & $1.15046 \mathrm{E}$ & 00 & $-3.82588 \mathrm{E}$ & 01 & $-4.45092 \mathrm{E}$ & 00 & $229 \mathrm{E}-03$ & $-4.36811 \mathrm{E}$ \\
\hline .20 & $1.15045 \mathrm{E}$ & 00 & $-3.83592 \mathrm{E}$ & 01 & $-4.47269 \mathrm{E}$ & 00 & $72 \mathrm{E}-03$ & $38961 \mathrm{E}$ \\
\hline .30 & $1.15044 \mathrm{E}$ & 00 & $-3.84595 \mathrm{E}$ & 01 & $-4.49450 \mathrm{E}$ & 00 & $3.93115 \mathrm{E}-$ & $4.41116 \mathrm{E}$ \\
\hline .40 & $1.15044 \mathrm{E}$ & 00 & $-3.85599 \mathrm{E}$ & 01 & $-4.51637 \mathrm{E}$ & 00 & $3.93056 \mathrm{E}-03$ & $43275 \mathrm{E}$ \\
\hline .50 & $1.15043 \mathrm{E}$ & 00 & $-3.86602 \mathrm{E}$ & 01 & $-4.53829 \mathrm{E}$ & 00 & $3.92995 \mathrm{E}-03$ & $-4.45440 \mathrm{E}$ \\
\hline .60 & $1.15043 \mathrm{E}$ & 00 & $-3.87604 \mathrm{E}$ & 01 & $-4.56026 \mathrm{E}$ & 00 & $33 \mathrm{E}-03$ & $-4.47610 \mathrm{E}$ \\
\hline .70 & $1.15042 \mathrm{E}$ & 00 & $-3.88607 \mathrm{E}$ & 01 & $-4.58228 \mathrm{E}$ & 00 & $71 \mathrm{E}-$ & $.49785 \mathrm{E}$ \\
\hline .80 & $1.15041 \mathrm{E}$ & 00 & $-3.89609 \mathrm{E}$ & 01 & $-4.60435 \mathrm{E}$ & 00 & $06 \mathrm{E}-$ & $.51965 \mathrm{E}$ \\
\hline .90 & $1.15041 \mathrm{E}$ & 00 & $-3.90611 \mathrm{E}$ & 01 & $-4.62646 \mathrm{E}$ & 00 & $40 E-03$ & $.54150 \mathrm{E}$ \\
\hline 38.00 & $1.15040 \mathrm{E}$ & 00 & $-3.91612 \mathrm{E}$ & 01 & $-4.64863 \mathrm{E}$ & 00 & $672 \mathrm{E}-03$ & $-4.56341 \mathrm{E}$ \\
\hline 10 & $1.15039 \mathrm{E}$ & 00 & $-3.92613 \mathrm{E}$ & 01 & $-4.67085 \mathrm{E}$ & 00 & $03 \mathbf{E}-03$ & $-4.58536 \mathrm{E}$ \\
\hline & $1.15039 \mathrm{E}$ & 00 & $-3.93614 \mathrm{E}$ & 01 & $-4.69312 \mathrm{E}$ & 00 & $34 \mathrm{E}-$ & $60736 \mathrm{E}$ \\
\hline 30 & $5038 \mathrm{E}$ & 00 & $-3.94615 \mathrm{E}$ & 01 & $-4.71544 \mathrm{E}$ & 00 & $62 \mathrm{E}-03$ & $62941 \mathrm{E}$ \\
\hline 40 & $38 \mathrm{E}$ & 00 & $-3.95615 \mathrm{E}$ & 01 & $73781 \mathrm{E}$ & 00 & $89 \mathrm{E}-03$ & $65151 \mathrm{E}$ \\
\hline 38.50 & $1.15037 \mathrm{E}$ & 00 & $-3.96615 \mathrm{E}$ & 01 & $-4.76024 \mathrm{E}$ & 00 & $3.92315 \mathrm{E}-03$ & $-4.67367 \mathrm{E}$ \\
\hline 3.60 & $1.15037 \mathrm{E}$ & 00 & $-3.97615 \mathrm{E}$ & 01 & $-4.78271 \mathrm{E}$ & 00 & $3.92241 \mathrm{E}-03$ & $69587 \mathrm{E}$ \\
\hline .70 & $1.15036 \mathrm{E}$ & 00 & $8614 \mathrm{E}$ & 01 & $9523 \mathrm{E}$ & 00 & $3 E-03$ & $1812 \mathrm{E}$ \\
\hline .80 & $35 \mathrm{E}$ & 00 & $-3.99613 \mathrm{E}$ & 01 & $82780 \mathrm{E}$ & 00 & $85 \mathrm{E}-03$ & .74043E \\
\hline 3.90 & $35 \mathrm{E}$ & 00 & $-4.00612 \mathrm{E}$ & 01 & $-4.85042 \mathrm{E}$ & 00 & $3.92006 \mathrm{E}-03$ & $-4.76278 \mathrm{E}$ \\
\hline 9.00 & $1.15034 \mathrm{E}$ & 00 & $-4.01610 \mathrm{E}$ & 01 & $-4.87309 \mathrm{E}$ & 00 & $3.91925 \mathrm{E}-03$ & $-4.78518 \mathrm{E}$ \\
\hline .10 & $1.15034 \mathrm{E}$ & 00 & $-4.02608 \mathrm{E}$ & 01 & $-4.89581 \mathrm{E}$ & 00 & $43 E-03$ & $80764 \mathrm{E}$ \\
\hline .20 & $33 \mathrm{E}$ & 00 & $-4.03606 \mathrm{E}$ & 01 & $1859 \mathrm{E}$ & 00 & $0 \mathrm{E}-03$ & $83014 \mathrm{E}$ \\
\hline .30 & $1.15032 \mathrm{E}$ & 00 & $-4.04604 \mathrm{E}$ & 01 & $-4.94141 \mathrm{E}$ & 00 & $3.91675 \mathrm{E}-$ & $-4.85269 \mathrm{E}$ \\
\hline 39.40 & $1.15032 \mathrm{E}$ & 00 & $-4.05601 \mathrm{E}$ & 01 & $-4.96428 \mathrm{E}$ & 00 & $90 \mathrm{E}-$ & $87530 \mathrm{E}$ \\
\hline 39.50 & $1.15031 \mathrm{E}$ & 00 & $-4.06598 \mathrm{E}$ & 01 & $-4.98720 \mathrm{E}$ & 00 & $3.91502 \mathrm{E}-03$ & $-4.89795 \mathrm{E}$ \\
\hline .60 & $1.15031 \mathrm{E}$ & 00 & $-4.07594 \mathrm{E}$ & 01 & $-5.01017 \mathrm{E}$ & 00 & $4 \mathrm{E}-03$ & $2066 \mathrm{E}$ \\
\hline .70 & $1.15030 \mathrm{E}$ & 00 & $-4.08591 \mathrm{E}$ & 01 & $-5.03319 \mathrm{E}$ & 00 & $5 \mathrm{E}-$ & $94341 \mathrm{E}$ \\
\hline 39.80 & $29 \mathrm{E}$ & 00 & $9587 \mathrm{E}$ & 01 & $526 \mathrm{E}$ & 00 & $3 \mathrm{E}-$ & $96621 \mathrm{E}$ \\
\hline 9.90 & $29 \mathrm{E}$ & 00 & $-4.10582 \mathrm{E}$ & 01 & $7939 \mathrm{E}$ & 00 & $2 \mathrm{E}-$ & $98907 \mathrm{E}$ \\
\hline 40.00 & $1.15028 \mathrm{E}$ & 00 & $-4.11577 \mathrm{E}$ & 01. & $-5.10256 \mathrm{E}$ & 00 & $3.91048 \mathrm{E}-03$ & $-5.01197 \mathrm{E}$ \\
\hline 40.10 & $5028 \mathrm{E}$ & 00 & $-4.12573 \mathrm{E}$ & 01 & & 00 & $4 \mathrm{E}-03$ & $03492 \mathrm{E}$ \\
\hline & $27 \mathrm{E}$ & 00 & $-4.13567 \mathrm{E}$ & 01 & $1905 \mathrm{E}$ & 00 & $7 \mathrm{E}-$ & $05793 \mathrm{E}$ \\
\hline & $27 \mathrm{E}$ & 00 & -4.1 & 01 & & 00 & & $8098 \mathrm{E}$ \\
\hline .40 & $26 \mathrm{E}$ & 00 & $-4.15556 \mathrm{E}$ & 01 & -5 & 00 & $2 \mathrm{E}-03$ & $0409 \mathrm{E}$ \\
\hline 4( & 1.15025 & 00 & $-4.16549 \mathrm{E}$ & & & 00 & $3.90563 \mathrm{E}-03$ & $-5.12724 \mathrm{E}$ \\
\hline 40.60 & $1.15025 \mathrm{E}$ & 00 & $-4.17543 \mathrm{E}$ & 01 & $-5.24263 \mathrm{E}$ & 00 & $2 \mathrm{E}-03$ & $-5.15044 \mathrm{E}$ \\
\hline & & 00 & -4.18 & 01 & & 00 & & $7369 \mathrm{E}$ \\
\hline 40.80 & $1.15024 \mathrm{E}$ & 00 & $-4.19529 \mathrm{E}$ & 01 & -5 . & 00 & $7 \mathrm{E}-03$ & $-5.19700 \mathrm{E}$ \\
\hline & $1.15023 \mathrm{E}$ & 00 & $-4.20521 \mathrm{E}$ & 01 & -5 & 00 & $153 \mathrm{E}-0$ & $22035 \mathrm{E}$ \\
\hline 41.00 & $1.15023 \mathrm{E}$ & 00 & $-4.21513 \mathrm{E}$ & 01 & $-5.33701 \mathrm{E}$ & 00 & $3.90048 \mathrm{E}-03$ & $.24375 \mathrm{E}$ \\
\hline 41.10 & $1.15022 \mathrm{E}$ & 00 & $-4.22505 \mathrm{E}$ & 01 & $-5.36073 \mathrm{E}$ & 00 & $3.89941 \mathrm{E}-03$ & $-5.26720 \mathrm{E}$ \\
\hline & & 00 & $-4.23497 \mathrm{E}$ & 01 & -5 & $0 c$ & & \\
\hline & $5021 \mathrm{E}$ & 00 & $-4.24488 \mathrm{E}$ & 01 & & 00 & 03 & $31426 \mathrm{E}$ \\
\hline & $1.15020 \mathrm{E}$ & 00 & $-4.25479 \mathrm{E}$ & 01 & & 00 & $3 \mathrm{E}-03$ & $-5.33786 \mathrm{E}$ \\
\hline 41.50 & $1.15020 \mathrm{E}$ & 00 & $-4.26470 \mathrm{E}$ & 01 & $-5.45610 \mathrm{E}$ & 00 & $3.89502 \mathrm{E}-03$ & $-5.36151 \mathrm{E}$ \\
\hline t & $1.15019 \mathrm{E}$ & 00 & $-4.27460 \mathrm{E}$ & 01 & $-5.48007 \mathrm{E}$ & 00 & $389 \mathrm{E}-03$ & $-5.38521 \mathrm{E}$ \\
\hline & $1.15019 \mathrm{E}$ & 00 & $-4.28450 \mathrm{E}$ & 01 & $-5.50409 \mathrm{E}$ & 00 & $275 \mathrm{E}-03$ & $-5.40896 \mathrm{E}$ \\
\hline & $1.15018 \mathrm{E}$ & 00 & $-4.29439 \mathrm{E}$ & 01 & $-5.52815 \mathrm{E}$ & 00 & $9160 \mathrm{E}-03$ & $-5.43276 \mathrm{E}$ \\
\hline & $1.15018 \mathrm{E}$ & 00 & $-4.30429 \mathrm{E}$ & 01 & $-5.55227 \mathrm{E}$ & 00 & $3.89044 \mathrm{E}-03$ & $-5.45661 \mathrm{E}$ \\
\hline & $1.15017 \mathrm{E}$ & 00 & $-4.31418 \mathrm{E}$ & 01 & $-5.57643 \mathrm{E}$ & 00 & $3.88926 \mathrm{E}-03$ & $-5.48051 \mathrm{E}$ \\
\hline
\end{tabular}

$g_{-1} \quad \tan \delta_{-1}$

$3.82916 \mathrm{E} \quad 01 \quad-1.94970 \mathrm{E} \quad 02$

$3.83921 \mathrm{E} \quad 01 \quad-1.95115 \mathrm{E} \quad 02$

$\begin{array}{llll}3.84926 \mathrm{E} & 01 & -1.95261 \mathrm{E} & 02\end{array}$

$\begin{array}{llll}3.85931 \mathrm{E} & 01 & -1.95406 \mathrm{E} & 02\end{array}$

$\begin{array}{llll}3.86936 \mathrm{E} & 01 & -1.95552 \mathrm{E} & 02\end{array}$

$\begin{array}{lllll}3.87940 \mathrm{E} & 01 & -1.95698 \mathrm{E} & 02\end{array}$

$\begin{array}{llll}3.88944 \mathrm{E} & 01 & -1.95844 \mathrm{E} & 02\end{array}$

$3.89948 \mathrm{E} \quad 01-1.95990 \mathrm{E} \quad 02$

$\begin{array}{llll}3.90952 \mathrm{E} & 01 & -1.96136 \mathrm{E} & 02\end{array}$

$3.91955 \mathrm{E} \quad 01 \quad-1.96282 \mathrm{E} \quad 02$

$\begin{array}{lllll}3.92958 \mathrm{E} & 01 & -1.96429 \mathrm{E} & 02\end{array}$ $3.93960 \mathrm{E} \quad 01 \quad-1.96575 \mathrm{E} \quad 02$ $\begin{array}{llll}3.94963 \mathrm{E} & 01 & -1.96722 \mathrm{E} & 02\end{array}$ $3.95965 \mathrm{E} \quad 01 \quad-1.96869 \mathrm{E} \quad 02$ $\begin{array}{llll}3.96966 \mathrm{E} & 01 & -1.97015 \mathrm{E} & 02\end{array}$

$3.97968 \mathrm{E} \quad 01$ $3.98969 \mathrm{E} \quad 01$ $3.99969 \mathrm{E} \quad 01$ $4.00970 \mathrm{E} \quad 01$ $4.01970 \mathrm{E} \quad 01$

$-1.97162 \mathrm{E} \quad 02$ $-1.97309 \mathrm{E} 02$ $-1.97456 \mathrm{E} \quad 02$ $-1.97603 \mathrm{E} 02$ $-1.97751 \mathrm{E} \quad 02$

$4.02970 \mathrm{E} \quad 01$ $4.03969 \mathrm{E} \quad 01$ $4.04969 \mathrm{E} 01$ $4.05968 \mathrm{E} 01$ $4.06966 \mathrm{E} \quad 01$

$4.07965 \mathrm{E} \quad 01$ $4.08963 \mathrm{E} \quad 01$ $4.09960 \mathrm{E} \quad 01$ $4.10958 \mathrm{E} \quad 01$ $4.11955 \mathrm{E} 01$

$4.12952 \mathrm{E} \quad 01$ $4.13948 \mathrm{E} 01$ $4.14944 \mathrm{E} \quad 01$ $4.15940 \mathrm{E} 01$ $4.16936 \mathrm{E} 01$

$-1.97898 \mathrm{E} \quad 02$ $-1.98046 \mathrm{E} 02$ $-1.98194 \mathrm{E} 02$ -1.98341 E 02
-1.98489 $-1.98489 \mathrm{E} 02$

$\begin{array}{ll}-1.98637 \mathrm{E} & 02\end{array}$ $-1.98786 \mathrm{E} \quad 02$ $-1.98934 \mathrm{E} \quad 02$ $-1.99083 \mathrm{E} 02$ $\begin{array}{ll}-1.99232 \mathrm{E} & 02\end{array}$

$-1.99380 \mathrm{E} \quad 02$ $-1.99530 \mathrm{E} 02$ $-1.99679 \mathrm{E} 02$ $-1.99828 \mathrm{E} 02$ $-1.99978 \mathrm{E} \quad 02$

$4.17931 \mathrm{E} \quad 01$ $4.18926 \mathrm{E} 01$ $4.19920 \mathrm{E}$ 0l $4.20915 \mathrm{E} \quad 01$ $4.21909 \mathrm{E} 01$

$4.22902 \mathrm{E} \quad 01$ $4.23896 \mathrm{E} \quad 01$ $4.24889 \mathrm{E}$ 01

$4.25881 \mathrm{E} 01$

$4.26874 \mathrm{E} \quad 01$

$4.27866 \mathrm{E} \quad 01$ $4.28858 \mathrm{E} 01$ $4.29849 \mathrm{E} \quad 01$ $4.30840 \mathrm{E} \quad 01$ $4.31831 \mathrm{E} \quad 01$

$4.32821 \mathrm{E} \quad 01$ $4.33812 \mathrm{E} \quad 01$ $4.34801 \mathrm{E} 01$ $4.35791 \mathrm{E} 01$ $4.36780 \mathbf{E} 01$

$4.37769 \mathrm{E} \quad 01$ $4.38758 \mathrm{E} 01$ $4.39746 \mathrm{E} \quad 01$ $4.40734 \mathrm{E} \quad 01$ $4.41721 \mathrm{E} 01$
$-2.00127 \mathrm{E} \quad 02$ $-2.00277 \mathrm{E} \quad 02$ $-2.00427 \mathrm{E} 02$ $-2.00577 \mathrm{E} \quad 02$ $-2.00727 \mathrm{E} \quad 02$

$-2.00878 \mathrm{E} \quad 02$ $-2.01029 \mathrm{E} 02$ $-2.01180 \mathrm{E} \quad 02$ $-2.01331 \mathrm{E} \quad 02$ $\begin{array}{ll}-2.01482 \mathrm{E} & 02\end{array}$

$\begin{array}{ll}-2.01633 \mathrm{E} & 02\end{array}$ $-2.01785 \mathrm{E} \quad 02$ $-2.01937 \mathrm{E} \quad 02$ $-2.02089 \mathrm{E} \quad 02$ $-2.02241 \mathrm{E} \quad 02$

$-2.02393 \mathrm{E} 02$ $-2.02546 \mathrm{E} 02$ $-2.02698 \mathrm{E} \quad 02$ $-2.02852 \mathrm{E} \quad 02$ $-2.03005 \mathrm{E} \quad 02$

$-2.03158 \mathrm{E} \quad 02$ $-2.03312 \mathrm{E} \quad 02$ $-2.03465 \mathrm{E} 02$ $-2.03620 \mathrm{E} \quad 02$ $\begin{array}{ll}-2.03774 \mathrm{E} & 02\end{array}$ 
ELECTRON RADIAL FUNCTIONS

$$
Z=6 \quad A=12 \quad \rho=1.3 A^{1 / 3} F
$$

$p$

36.10

36.20

36.30

36.40

36.50

36.60

36.70

36.80

36.90

37.00

37.10

37.20

37.30

37.40

37.50

37.60

37.70

37.80

37.90

38.00

38.10

38.20

38.30

38.40

38.50

38.60

38.70

38.80

38.90

39.00

39.10

39.20

39.30

39.40

39.50

39.60

39.70

39.80

39.90

40.00

40.10

40.20

40.30

40.40

40.50

40.60

40.70

40.80

40.90

41.00

41.10

41.20

41.30

41.40

41.50

41.60

41.70

41.80

41.90

42.00
$\bar{F}$

$2.47217 \mathrm{E}-03$

$2.48587 \mathrm{E}-03$

$2.49962 \mathrm{E}-03$

$2.51340 \mathrm{E}-03$

$2.52722 \mathrm{E}-03$

$2.54108 \mathrm{E}-03$

$2.55498 \mathrm{E}-03$

$2.56892 \mathrm{E}-03$

$2.58289 \mathrm{E}-03$

$2.59690 \mathrm{E}-03$

$2.61095 \mathrm{E}-03$

$2.62504 \mathrm{E}-03$

$2.63916 \mathrm{E}-03$

$2.65332 \mathrm{E}-03$

$2.66752 \mathrm{E}-03$

$2.68176 \mathrm{E}-03$

$2.69604 \mathrm{E}-03$

$2.71035 \mathrm{E}-03$

$2.72470 \mathrm{E}-03$

$2.73909 E-03$

2.75352E-03 $2.76799 \mathrm{E}-03$

$2.78249 \mathrm{E}-03$

$2.79703 \mathrm{E}-03$

2.81161 E-03

$2.82623 \mathrm{E}-03$

$2.84088 \mathrm{E}-03$

$2.85557 \mathrm{E}-03$

$2.87031 \mathrm{E}-03$

$2.88507 \mathrm{E}-03$

$2.89988 \mathrm{E}-03$

$2.91472 \mathrm{E}-03$

2.92961 E- 03

$2.94453 \mathrm{E}-03$

$2.95948 \mathrm{E}-03$

$2.97448 \mathrm{E}-03$

$2.98951 \mathrm{E}-03$

$3.00459 \mathrm{E}-03$

3:01970E-03

$3.03484 \mathrm{E}-03$

$3.05003 \mathrm{E}-03$

$3.06525 \mathrm{E}-03$

$3.08051 \mathrm{E}-03$

$3.09581 \mathrm{E}-03$

$3.11115 \mathrm{E}-03$

$3.12652 \mathrm{E}-03$ $3.14193 \mathrm{E}-03$ $3.15738 \mathrm{E}-03$

$3.17287 \mathrm{E}-03$

$3.18840 \mathrm{E}-03$

$3.20396 \mathrm{E}-03$

$3.21957 \mathrm{E}-03$

$3.23520 \mathrm{E}-03$

$3.25088 \mathrm{E}-03$

$3.26660 \mathrm{E}-03$

$3.28235 \mathrm{E}-03$ $3.29814 \mathrm{E}-03$

$3.31397 \mathrm{E}-03$

$3.32984 \mathrm{E}-03$

$3.34574 \mathrm{E}-03$ $f_{2}$

$g_{2}$

$-3.49274 \mathrm{E} \quad 00 \quad-2.35514 \mathrm{E}-01$

$-3.51204 \mathrm{E} \quad 00 \quad-2.37362 \mathrm{E}-01$

$-3.53140 \mathrm{E} \quad 00 \quad-2.39220 \mathrm{E}-01$

$-3.55080 \mathrm{E} \quad 00 \quad-2.41087 \mathrm{E}-01$

$-3.57026 \mathrm{E} 00 \quad-2.42964 \mathrm{E}-01$

$-3.58977 \mathrm{E} \quad 00$

$-2.44850 \mathrm{E}-01$

$-3.60934 \mathrm{E} \quad 00$

$-3.62895 \mathrm{E} 00$

$-3.64862 \mathrm{E} \quad 00$

$-3.66833 \mathrm{E} 00$

$-2.46746 \mathrm{E}-0$

$-2.48652 \mathrm{E}-01$

$-2.50567 \mathrm{E}-01$

$-2.52492 \mathrm{E}-01$

$-3.68810 \mathrm{E} \quad 00$

$-2.54426 \mathrm{E}-01$

$-3.70792 \mathrm{E} \quad 00$

$-3.72779 \mathrm{E} \quad 00$

$-3.74771 \mathrm{E} \quad 00$

$-3.76769 \mathrm{E} \quad 00$

$-2.56371 \mathrm{E}-01$

$-2.58325 \mathrm{E}-01$

$-2.60289 \mathrm{E}-01$

$-2.62263 \mathrm{E}-01$

$-3.78771 \mathrm{E} \quad 00$

$-3.80779 \mathrm{E} \quad 00$

$-3.82792 \mathrm{E} \quad 00$

$-3.84810 \mathrm{E} \quad 00$

$-3.86833 \mathrm{E} 00$

$-2.64246 \mathrm{E}-01$

$-2.66240 \mathrm{E}-01$

$-2.68243 \mathrm{E}-01$

$-2.70256 \mathrm{E}-01$

$-2.72279 \mathrm{E}-01$

$-3.88861 \mathrm{E} 00$

$-2.74312 \mathrm{E}-01$

$-3.90894 \mathrm{E} 00$

$-3.92933 \mathrm{E} \quad 00$

$-3.94976 \mathrm{E} 00$

$-3.97025 \mathrm{E} \quad 00$

$-3.99079 \mathrm{E} 00$

$-4.01138 \mathrm{E} \quad 00$

$-4.03202 \mathrm{E} \quad 00$

$-4.05271 \mathrm{E} \quad 00$

$-4.07345 \mathrm{E} \quad 00$

$-4.09425 \mathrm{E} \quad 00$

$-4.11509 \mathrm{E} \quad 00$

$-4.13599 \mathrm{E} 00$

$-4.15694 \mathrm{E} \quad 00$

$-4.17794 \mathrm{E} 00$

$-4.19898 \mathrm{E} \quad 00$

$-2.76355 \mathrm{E}-01$

$-2.78408 \mathrm{E}-01$

$-2.80471 \mathrm{E}-01$

$-2.82544 \mathrm{E}-01$

$-4.22009 \mathrm{E} \quad 00$

$-4.24124 \mathrm{E} \quad 00$

$-4.26244 \mathrm{E} \quad 00$

$-4.28369 \mathrm{E} \quad 00$

$-2.84628 \mathrm{E}-01$

$-2.86721 \mathrm{E}-01$

$-2.88824 \mathrm{E}-01$

$-2.90938 \mathrm{E}-01$

$-2.93061 \mathrm{E}-01$

$-4.30500 \mathrm{E} \quad 00$

$-4.32635 \mathrm{E} \quad 00$

$-4.34776 \mathrm{E} \quad 00$

$-4.36922 \mathrm{E} \quad 00$

$-4.39073 \mathrm{E} 00$

$-4.41229 \mathrm{E} \quad 00$

$-4.43390 \mathrm{E} \quad 00$

$-4.45556 \mathrm{E} \quad 00$

$-4.47727 \mathrm{E} \quad 00$

$-4.49903 \mathrm{E} \quad 00$

$-4.52084 \mathrm{E} \quad 00$

$-4.54271 \mathrm{E} \quad 00$

$-4.56462 \mathrm{E} \quad 00$

$-4.58659 \mathrm{E} \quad 00$

$-4.60860 \mathrm{E} \quad 00$

$-4.63067 \mathrm{E} \quad 00$

$-4.65279 \mathrm{E} \quad 00$

$-4.67496 \mathrm{E} \quad 00$

$-4.69718 \mathrm{E} \quad 00$

$-4.71945 \mathrm{E} \quad 00$

$-2.95195 \mathrm{E}-01$

$-2.97339 \mathrm{E}-01$

$-2.99494 \mathrm{E}-01$

$-3.01658 \mathrm{E}-01$

$-3.03833 \mathrm{E}-01$

$-3.06018 \mathrm{E}-01$

$-3.08213 \mathrm{E}-01$

$-3.10419 \mathrm{E}-01$

$-3.12635 \mathrm{E}-01$

$-3.14862 \mathrm{E}-01$

$-3.17099 \mathrm{E}-01$

$-3.19346 \mathrm{E}-01$

$-3.21604 \mathrm{E}-01$

$-3.23872 \mathrm{E}-01$

$-3.26151 \mathrm{E}-01$

$\tan \delta_{2}$

$f_{-2}$

$3.44612 \mathrm{E}$ 0l $-2.31013 \mathrm{E}-01$

$3.44623 \mathrm{E}$ 01 $-2.32833 \mathrm{E}-01$

$3.44633 \mathrm{E}$ 0l $-2.34663 \mathrm{E}-01$

$3.44644 \mathrm{E}$ 01 $-2.36503 \mathrm{E}-01$

$3.44654 \mathrm{E}$ ol $-2.38351 \mathrm{E}-01$

$3.44665 \mathrm{E} \quad 01$

$3.44675 \mathrm{E} \quad 01$

$3.44685 \mathrm{E} 01$

$3.44696 \mathrm{E} \quad 01$

$3.44706 \mathrm{E}$ 0l

$-2.40210 \mathrm{E}-01$

$-2.42078 \mathrm{E}-01$

$-2.43955 \mathrm{E}-01$

$-2.45842 \mathrm{E}-01$

$-2.47739 \mathrm{E}-01$

$3.44716 \mathrm{E} \quad 01$

$3.44726 \mathrm{E} \quad 01$

$3.44736 \mathrm{E} \quad 01$

$3.44746 \mathrm{E} \quad 01$

$3.44755 \mathrm{E} 01$

$3.44765 \mathrm{E} 01$

$3.44775 \mathrm{E} \quad 01$

$3.44785 \mathrm{E} \quad 01$

$3.44794 \mathrm{E} \quad 01$

$3.44804 \mathrm{E} \quad 01$

$3.44813 \mathrm{E} \quad 01$

$3.44823 \mathrm{E} \quad 01$

$3.44832 \mathrm{E} \quad 01$

$3.44842 \mathrm{E} 01$

3.44851 E 01

$3.44860 \mathrm{E} \quad 01$

$3.44869 \mathrm{E} \quad 01$

$3.44879 \mathrm{E} \quad 01$

$3.44888 \mathrm{E} 01$

$3.44897 \mathrm{E} \quad 01$

$3.44906 \mathrm{E} \quad 01$

$3.44915 \mathrm{E} \quad 01$

$3.44924 \mathrm{E} 01$

$3.44933 \mathrm{E}$ 01

$3.44941 \mathrm{E} \quad 01$

$3.44950 \mathrm{E} \quad 01$

$3.44959 \mathrm{E} \quad 01$

$3.44968 \mathrm{E} \quad 01$

$3.44976 \mathrm{E} \quad 01$

$3.44985 \mathrm{E} \quad 01$

$-2.49646 \mathrm{E}-01$

$-2.51562 \mathrm{E}-01$

$-2.53487 \mathrm{E}-01$

$-2.55423 \mathrm{E}-01$

$-2.57368 \mathrm{E}-01$

$-2.59323 \mathrm{E}-01$

$-2.61287 \mathrm{E}-01$

$-2.63262 \mathrm{E}-01$

$-2.65246 \mathrm{E}-01$

$-2.67240 \mathrm{E}-01$

$-2.69244 \mathrm{E}-01$

$-2.71258 \mathrm{E}-01$

$-2.73282 \mathrm{E}-01$

$-2.75316 \mathrm{E}-01$

$-2.77359 \mathrm{E}-01$

$3.44993 \mathrm{E} \quad 01$

$3.45002 \mathrm{E} \quad 01$ 


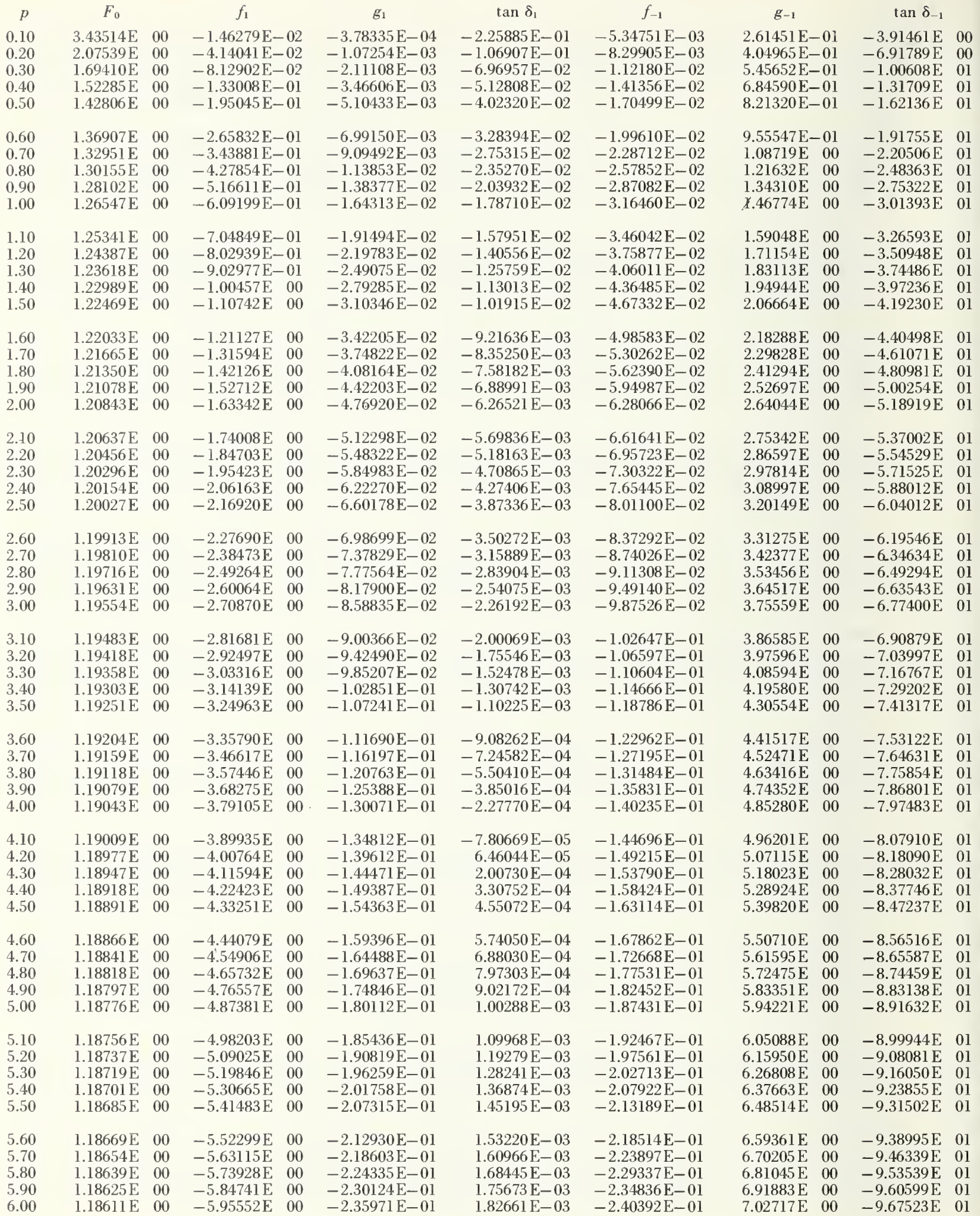


ELECTRON RADIAL FUNCTIONS

$Z=7 \quad A=14 \quad \rho=1.3 A^{1 / 3} F$

Electron

$\bar{F}$

$7.85280 \mathrm{E}-08$ $1.604 .60 \mathrm{E}-07$ $2.84826 \mathrm{E}-07$ $4.49730 \mathrm{E}-07$ $6.55347 \mathrm{E}-07$

$9.02077 \mathrm{E}-07$ $1.19031 \mathrm{E}-06$ $1.52037 \mathrm{E}-06$ $1.89254 \mathrm{E}-06$ $2.30702 \mathrm{E}-06$

$2.76399 \mathrm{E}-06$ $3.26357 \mathrm{E}-06$ $3.80587 \mathrm{E}-06$ $4.39096 \mathrm{E}-06$ 5.01891 E-06

$5.68977 \mathrm{E}-06$ $6.40357 \mathrm{E}-06$ $7.16033 \mathrm{E}-06$ $7.96010 \mathrm{E}-06$ $8.80288 \mathrm{E}-06$

$9.68869 \mathrm{E}-06$ $1.06175 \mathrm{E}-05$ $1.15894 \mathrm{E}-0.5$ $1.26044 \mathrm{E}-05$ $1.36624 \mathrm{E}-05$

$1.47635 \mathrm{E}-05$ $1.59076 \mathrm{E}-05$ $1.70948 \mathrm{E}-05$ $1.83251 \mathrm{E}-05$ $1.95985 \mathrm{E}-05$

$2.09149 \mathrm{E}-05$ $2.22745 \mathrm{E}-05$ $2.36771 \mathrm{E}-05$ $2.51227 \mathrm{E}-05$ $2.66115 \mathrm{E}-05$

$2.81434 \mathrm{E}-05$ $2.97183 \mathrm{E}-05$ $3.13363 \mathrm{E}-05$ $3.29973 \mathrm{E}-05$ $3.47014 \mathrm{E}-05$

$3.64487 \mathrm{E}-05$ $3.82390 \mathrm{E}-05$ $4.00723 \mathrm{E}-05$ $4.19488 \mathrm{E}-05$ $4.38683 \mathrm{E}-05$

$4.58309 \mathrm{E}-0.5$ $4.78365 \mathrm{E}-05$ $4.98852 \mathrm{E}-0.5$ $5.19770 \mathrm{E}-05$ $5.41119 \mathrm{E}-05$

$5.62898 \mathrm{E}-0.5$ $5.85109 \mathrm{E}-05$ $6.07749 \mathrm{E}-05$ $6.30820 \mathrm{E}-0.5$ $6.54323 \mathrm{E}-05$

$6.78255 \mathrm{E}-05$ $7.02618 \mathrm{E}-05$ $7.27412 \mathrm{E}-0.5$ $7.52636 \mathrm{E}-05$ $7.78291 \mathrm{E}-0.5$ $f_{2}$

$g_{2}$

$\tan \delta_{2}$ $f_{-2}$

$-4.05978 \mathrm{E}-06$ $-2.24872 \mathrm{E}-05$ $-6.59604 \mathrm{E}-05$ $-1.43712 \mathrm{E}-04$ $-2.63285 \mathrm{E}-04$

$-4.30500 \mathrm{E}-04$ $-6.49637 \mathrm{E}-04$ $-9.23702 \mathrm{E}-04$ $-1.25473 \mathrm{E}-03$

$-1.64403 \mathrm{E}-03$

$-2.09244 . \mathrm{E}-03$ $-2.60042 \mathrm{E}-03$ $-3.16824 \mathrm{E}-03$ $-3.79602 \mathrm{E}-03$

$-4.48376 \mathrm{E}-03$

$-5.23144 \mathrm{E}-03$ $-6.03900 \mathrm{E}-03$ $-6.90635 \mathrm{E}-03$ $-7.83341 \mathrm{E}-03$ $-8.82008 \mathrm{E}-03$

$-9.86629 \mathrm{E}-03$ $-1.09720 \mathrm{E}-02$

$-1.21370 \mathrm{E}-02$

$-1.33613 \mathrm{E}-02$

$-1.46449 \mathrm{E}-02$

$-1.59877 \mathrm{E}-02$ $-1.73896 \mathrm{E}-02$ $-1.88506 \mathrm{E}-02$ $-2.03706 \mathrm{E}-02$

$-2.19497 \mathrm{E}-02$

$-2.35876 \mathrm{E}-02$

$-2.52845 \mathrm{E}-02$

$-2.70403 \mathrm{E}-02$

$-2.88550 \mathrm{E}-02$

$-3.07285 \mathrm{E}-02$

$-3.26608 \mathrm{E}-02$

$-3.46518 \mathrm{E}-02$

$-3.67017 \mathrm{E}-02$

$-3.88103 \mathrm{E}-02$

$-4.09776 \mathrm{E}-02$

$-4.32036 \mathrm{E}-02$

$-4.54883 \mathrm{E}-02$

$-4.78317 \mathrm{E}-02$

$-5.02338 \mathrm{E}-02$

$-5.26944 \mathrm{E}-02$

$-5.52137 \mathrm{E}-02$

$-5.77917 \mathrm{E}-02$

$-6.04282 \mathrm{E}-02$

$-6.31233 \mathrm{E}-02$

$-6.58770 \mathrm{E}-02$

$-6.86893 \mathrm{E}-02$

$-7.15601 \mathrm{E}-02$

$-7.44895 \mathrm{E}-02$

$-7.74774 \mathrm{E}-02$

$-8.05238 \mathrm{E}-02$

$-8.36287 \mathrm{E}-02$

$-8.67922 \mathrm{E}-02$

$-9.00141 \mathrm{E}-02$

$-9.32945 \mathrm{E}-02$

$-9.66334 \mathrm{E}-02$
$-6.06179 \mathrm{E}-08$

$-3.36304 \mathrm{E}-07$

$-9.89057 \mathrm{E}-07$

$-2.16262 \mathrm{E}-06$

$-3.97951 \mathrm{E}-06$

$-6.54061 \mathrm{E}-06$

$-9.92741 \mathrm{E}-06$

$-1.42055 \mathrm{E}-05$

$-1.94281 \mathrm{E}-0.5$

$-2.56399 \mathrm{E}-05$

$-3.28790 \mathrm{E}-05$

$-4.11796 \mathrm{E}-05$

$-5.05730 \mathrm{E}-05$

$-6.10890 \mathrm{E}-05$

$-7.27560 \mathrm{E}-05$

$-8.56020 \mathrm{E}-05$

$-9.96545 \mathrm{E}-0.5$

$-1.14941 \mathrm{E}-04$

$-1.31489 \mathrm{E}-04$

$-1.49325 \mathrm{E}-04$

$-1.68477 \mathrm{E}-04$

$-1.88973 \mathrm{E}-04$

$-2.10839 \mathrm{E}-04$

$-2.34104 \mathrm{E}-04$

$-2.58796 \mathrm{E}-04$

$-2.84941 \mathrm{E}-04$

$-3.12568 \mathrm{E}-04$

$-3.4 .1705 \mathrm{E}-04$

$-3.72380 \mathrm{E}-04$

$-4.04621 \mathrm{E}-04$

$-4.38455 \mathrm{E}-04$

$-4.73911 \mathrm{E}-04$

$-5.11017 \mathrm{E}-04$

$-5.49800 \mathrm{E}-04$

$-5.90290 \mathrm{E}-04$

$-6.32515 \mathrm{E}-04$

$-6.76502 \mathrm{E}-04$

$-7.22280 \mathrm{E}-04$

$-7.69877 \mathrm{E}-04$

$-8.19321 \mathrm{E}-04$

$-8.70641 \mathrm{E}-04$

$-9.23865 \mathrm{E}-04$

$-9.79021 \mathrm{E}-04$

$-1.03614 \mathbf{E}-03$

$-1.09524 \mathrm{E}-03$

$-1.15636 \mathrm{E}-03$

$-1.21953 \mathrm{E}-03$

$-1.28477 \mathrm{E}-03$

$-1.35211 \mathrm{E}-03$

$-1.49322 \mathrm{E}-03$

$-1.56704 \mathrm{E}-03$

$-1.64307 \mathrm{E}-03$

$-1.72135 \mathrm{E}-03$

$-1.80190 \mathrm{E}-03$

$-1.88475 \mathrm{E}-03$

$-1.96993 \mathrm{E}-03$

$-2.05746 \mathrm{E}-03$

$-2.14738 \mathrm{E}-03$

$-2.23971 \mathrm{E}-03$
$-1.42159 \mathrm{E}-03$ $\begin{array}{lll}1.94277 \mathrm{E} & 00 \quad-9.24682 \mathrm{E}-07\end{array}$ $4.11403 \mathrm{E} \quad 00 \quad-2.63940 \mathrm{E}-06$ $6.14817 \mathrm{E} \quad 00 \quad-5.26198 \mathrm{E}-06$ $8.05829 \mathrm{E} \quad 00 \quad-8.78957 \mathrm{E}-06$ $9.83124 \mathrm{E} \quad 00-1.32193 \mathrm{E}-05$

$1.14583 \mathrm{E} \quad 01 \quad-1.85502 \mathrm{E}-05$ $1.29379 \mathrm{E} \quad 01 \quad-2.47846 \mathrm{E}-0.5$ $1.42744 \mathrm{E}$ 0l $-3.19289 \mathrm{E}-0.5$ $\begin{array}{lll}1.54762 \mathrm{E} & 01 & -3.99940 \mathrm{E}-0.5\end{array}$ $1.65540 \mathrm{E} \quad 01 \quad-4.89941 \mathrm{E}-0.5$

$1.75196 \mathrm{E} \quad 01 \quad-5.89470 \mathrm{E}-05$ $1.83847 \mathrm{E}$ 01 $-6.98730 \mathrm{E}-05$ $1.91605 \mathrm{E} \quad 01 \quad-8.17944 \mathrm{E}-05$ $1.98573 \mathrm{E} \quad 01 \quad-9.47352 \mathrm{E}-05$ $2.04844 \mathrm{E} 01-1.08720 \mathrm{E}-04$

$2.10502 \mathrm{E} \quad 01 \quad-1.23776 \mathrm{E}-04$ $2.15618 \mathrm{E} \quad 01 \quad-1.39930 \mathrm{E}-04$ $2.20258 \mathrm{E} \quad 01-1.57207 \mathrm{E}-04$ $2.24476 \mathrm{E}$ ol $-1.75637 \mathrm{E}-04$ $2.28322 \mathrm{E}$ 01 $-1.95247 \mathrm{E}-04$

$2.31838 \mathrm{E} \quad 01 \quad-2.16064 \mathrm{E}-04$ $2.35061 \mathrm{E}$ 0l $-2.38118 \mathrm{E}-04$ $2.38022 \mathrm{E}$ 0l $-2.61436 \mathrm{E}-04$ $2.40750 \mathrm{E}$ 01 $-2.86047 \mathrm{E}-04$ $2.43269 \mathrm{E} 01 \quad-3.11980 \mathrm{E}-04$

$2.45600 \mathrm{E} \quad 01 \quad-3.39261 \mathrm{E}-04$ $2.47763 \mathrm{E}$ 01 $-3.67922 \mathrm{E}-04$ $2.49773 \mathrm{E} \quad 01 \quad-3.97988 \mathrm{E}-04$ $2.51646 \mathrm{E}$ 01 $-4.29490 \mathrm{E}-04$ $2.53393 \mathrm{E} 01 \quad-4.62456 \mathrm{E}-04$

$2.55027 \mathrm{E} \quad 01 \quad-4.96914 \mathrm{E}-04$ $2.56558 \mathrm{E}$ ol $-5.32892 \mathrm{E}-04$ $2.57994 \mathrm{E}$ ol $-5.70420 \mathrm{E}-04$ $2.59344 \mathrm{E}$ 01 $-6.09525 \mathrm{E}-04$ $2.60615 \mathrm{E} 01 \quad-6.50237 \mathrm{E}-04$

$2.61813 \mathrm{E} \quad 01 \quad-6.92584 \mathrm{E}-04$ $2.62944 \mathrm{E}$ 0l $-7.36593 \mathrm{E}-04$ $2.64013 \mathrm{E}$ 01 $-7.82295 \mathrm{E}-04$ $2.65026 \mathrm{E}$ 0l $-8.29716 \mathrm{E}-04$ $2.65985 \mathrm{E} \quad 01 \quad-8.78887 \mathrm{E}-04$

$2.66896 \mathrm{E} \quad 01 \quad-9.29834 \mathrm{E}-04$ $2.67761 \mathrm{E} \quad 01 \quad-9.82587 \mathrm{E}-04$ $2.68585 \mathrm{E} \quad 01-1.03717 \mathrm{E}-03$ $2.69368 \mathrm{E} \quad 01-1.09363 \mathrm{E}-03$

$2.70116 \mathrm{E} \quad 01-1.15197 \mathrm{E}-03$

$2.70829 \mathrm{E} \quad 01-1.21223 \mathrm{E}-03$

$2.71510 \mathrm{E} \quad 01-1.27443 \mathrm{E}-03$

$2.72161 \mathrm{E} 01-1.33862 \mathrm{E}-03$

$2.72784 \mathrm{E} \quad 01-1.40481 \mathrm{E}-03$

$2.73381 \mathrm{E}$ ol $-1.47303 \mathrm{E}-03$

$2.73952 \mathrm{E} \quad 01 \quad-1.54332 \mathrm{E}-03$ $2.74501 \mathrm{E}$ ol $-1.61569 \mathrm{E}-03$ $2.75028 \mathrm{E}$ 01 $-1.69018 \mathrm{E}-03$ $2.75534 \mathrm{E}$ 0l $-1.76682 \mathrm{E}-03$ $2.76020 \mathrm{E} \quad 01 \quad-1.84564 \mathrm{E}-03$

$2.76488 \mathrm{E}$ ol $-1.92666 \mathrm{E}-03$ $2.76939 \mathrm{E} \quad 01 \quad-2.00991 \mathrm{E}-03$ $2.77373 \mathrm{E}$ ol $-2.09542 \mathrm{E}-03$ $2.77792 \mathrm{E} \quad 01 \quad-2.18322 \mathrm{E}-03$ $2.78195 \mathrm{E} \quad 01 \quad-2.27334 \mathrm{E}-03$

\section{g-2}

$\tan \delta$

$7.91313 \mathrm{E}-0.5$ $2.25407 \mathrm{E}-04$

$4.47874 \mathrm{E}-04$

$7.44729 \mathrm{E}-04$

$1.11377 \mathrm{E}-03$

$1.55269 \mathrm{E}-0.3$ $2.05928 \mathrm{E}-03$

$2.63160 \mathrm{E}-03$

$3.26802 \mathrm{E}-03$

$3.96722 \mathrm{E}-03$

$4.72814 \mathrm{E}-03$ $5.54 .999 \mathrm{E}-03$ $6.43214 \mathrm{E}-03$

7.374.14 E-03

$8.37563 \mathrm{E}-03$

$9.43635 \mathrm{E}-03$

$1.05561 \mathrm{E}-02$

$1.17347 \mathrm{E}-02$

$1.29721 \mathrm{E}-02$

1. $4.2682 \mathrm{E}-02$

$1.56229 \mathrm{E}-02$ $1.70362 \mathrm{E}-02$ $1.85081 \mathrm{E}-02$

$2.00385 \mathrm{E}-02$

$2.16273 \mathrm{E}-02$

$2.3274 .7 \mathrm{E}-02$ $2.49806 \mathrm{E}-02$ $2.67449 \mathrm{E}-02$ $2.85677 \mathrm{E}-02$

$3.04 .490 \mathrm{E}-02$

$3.23887 \mathrm{E}-02$ $3.43869 \mathrm{E}-02$

$3.64436 \mathrm{E}-02$

$3.85587 \mathrm{E}-02$

$4.07323 \mathrm{E}-02$

$4.29643 \mathrm{E}-02$ $4.52547 \mathrm{E}-02$

$4.76037 \mathrm{E}-02$

$5.00110 \mathrm{E}-02$ 
$p$

$$
F_{0}
$$

6.10

6.20

6.30

6.40

6.50

6.60

6.70

6.80

6.90

7.00

7.10

7.20
7.30

7.30
7.40

7.50

7.60

7.70

7.80

7.90

8.00

8.10

8.20

8.30

8.40

8.50

8.60

8.70

8.80

8.90

9.00

9.10

9.20

9.30

9.40

9.50

9.60

9.70

9.80

9.90

10.00

10.10

10.20

10.30

10.40

10.50

10.60

10.70

10.80

10.90

11.00

11.10

11.20

11.30

11.40

11.50

11.60

11.70

11.80

11.90

12.00
$1.18598 \mathrm{E} \quad 00$

$1.18585 \mathrm{E} \quad 00$

$\begin{array}{ll}1.18573 \mathrm{E} & 00\end{array}$

$1.18561 \mathrm{E} \quad 00$

$1.18550 \mathrm{E} \quad 00$

$1.18539 \mathrm{E} \quad 00$

$1.18528 \mathrm{E} \quad 00$

$1.18518 \mathrm{E} \quad 00$

$1.18508 \mathrm{E} \quad 00$

$1.18498 \mathrm{E} \quad 00$

$1.18488 \mathrm{E} \quad 00$

$1.18479 \mathrm{E} \quad 00$

$1.18470 \mathrm{E} \quad 00$

$1.18461 \mathrm{E} \quad 00$

$1.18453 \mathrm{E} \quad 00$

$1.18445 \mathrm{E} \quad 00$

$1.18437 \mathrm{E} \quad 00$

$1.18429 \mathrm{E} \quad 00$

$\begin{array}{ll}1.18421 \mathrm{E} & 00\end{array}$

$1.18414 \mathrm{E} \quad 00$

$1.18407 \mathrm{E} \quad 00$

$1.18400 \mathrm{E} \quad 00$

$1.18393 \mathrm{E} \quad 00$

$1.18386 \mathrm{E} \quad 00$

$1.18379 \mathrm{E} \quad 00$

$1.18373 \mathrm{E} \quad 00$

$1.18366 \mathrm{E} \quad 00$

$1.18360 \mathrm{E} \quad 00$

$1.18354 \mathrm{E} \quad 00$

$1.18348 \mathrm{E} \quad 00$

$1.18342 \mathrm{E} \quad 00$

$\begin{array}{ll}1.18337 \mathrm{E} & 00\end{array}$

$1.18331 \mathrm{E} \quad 00$

$1.18326 \mathrm{E} \quad 00$

$1.18320 \mathrm{E} \quad 00$

$1.18315 \mathrm{E} \quad 00$

$1.18310 \mathrm{E} \quad 00$

$1.18304 \mathrm{E} \quad 00$

$1.18299 \mathrm{E} \quad 00$

$1.18295 \mathrm{E} \quad 00$

$1.18290 \mathrm{E} \quad 00$

$1.18285 \mathrm{E} \quad 00$

$1.18280 \mathrm{E} \quad 00$

$1.18276 \mathrm{E} \quad 00$

$1.18271 \mathrm{E} \quad 00$

$1.18267 \mathrm{E} \quad 00$

$1.18262 \mathrm{E} \quad 00$

$1.18258 \mathrm{E} \quad 00$

$\begin{array}{ll}1.18254 \mathrm{E} \quad 00 & 00\end{array}$

$1.18250 \mathrm{E} \quad 00$

$1.18246 \mathrm{E} \quad 00$

$1.18242 \mathrm{E} \quad 00$

$1.18238 \mathrm{E} \quad 00$

$1.18234 \mathrm{E} \quad 00$

$1.18230 \mathrm{E} 00$

$1.18226 \mathrm{E} \quad 00$

$1.18222 \mathrm{E} \quad 00$

$1.18218 \mathrm{E} \quad 00$

$1.18215 \mathrm{E} \quad 00$

$1.18211 \mathrm{E} \quad 00$ $f_{1}$

$g_{1}$

$-6.06361 \mathrm{E} \quad 00 \quad-2.41876 \mathrm{E}-01$

$-6.17169 \mathrm{~F} 00-2.47840 \mathrm{~F}-01$

$-6.27975 \mathrm{E} \quad 00 \quad-2.53861 \mathrm{E}-01$

$-6.38780 \mathrm{E} \quad 00 \quad-2.59940 \mathrm{E}-01$

$-6.49584 \mathrm{E} \quad 00 \quad-2.66078 \mathrm{E}-01$

$-6.60385 \mathrm{E} \quad 00$

$-6.71186 \mathrm{E} 00$

$-6.81984 \mathrm{E} \quad 00$

$-6.92782 \mathrm{E} \quad 00$

$-7.03577 \mathrm{E} \quad 00$

$-7.14371 \mathrm{E} \quad 00$

$-7.25163 \mathrm{E} \quad 00$

$-7.35954 \mathrm{E} \quad 00$

$-7.46743 \mathrm{E} 00$

$-7.57530 \mathrm{E} \quad 00$

$-7.68316 \mathrm{E} \quad 00$

$-7.79100 \mathrm{E} \quad 00$

$-7.89883 \mathrm{E} \quad 00$

$-8.00664 \mathrm{E} \quad 00$

$-8.11443 \mathrm{E} \quad 00$

$-8.22220 \mathrm{E} \quad 00$

$-8.32996 \mathrm{E} \quad 00$

$-8.43770 \mathrm{E} \quad 00$

$-8.54543 \mathrm{E} \quad 00$

$-8.65313 \mathrm{E} \quad 00$

$-8.76082 \mathrm{E} \quad 00$

$-8.86850 \mathrm{E} \quad 00$

$-8.97616 \mathrm{E} \quad 00$

$-9.08380 \mathrm{E} 00$

$-9.19142 \mathrm{E} \quad 00$

$-9.29902 \mathrm{E} \quad 00$

$-9.40661 \mathrm{E} \quad 00$

$-9.51418 \mathrm{E} 00$

$-9.62173 \mathrm{E} 00$

$-9.72927 \mathrm{E} \quad 00$

$-9.83679 \mathrm{E} \quad 00$

$-9.94429 \mathrm{E} \quad 00$

$-1.00518 \mathrm{E} \quad 01$

$-1.01592 \mathrm{E} \quad 01$

$-1.02667 \mathrm{E} 01$

$-1.03741 \mathrm{E} \quad 01$

$-1.04815 \mathrm{E} 01$

$-1.05889 \mathrm{E} 01$

$-1.06963 \mathrm{E} 01$

$-1.08037 \mathrm{E} 01$

$-1.09110 \mathrm{E} 01$

$-1.10183 \mathrm{E} 01$

$-1.11256 \mathrm{E} 01$

$-1.12329 \mathrm{E} 01$

$-1.13402 \mathrm{E} 01$

$-1.14475 \mathrm{E} 01$

$-1.15547 \mathrm{E} 01$

$-1.16619 \mathrm{E} 01$

$-1.17691 \mathrm{E} 01$

$-1.18763 \mathrm{E} 01$

$-1.19835 \mathrm{E} \quad 01$

$-1.20906 \mathrm{E} 01$

$-1.21977 \mathrm{E} 01$

$-1.23048 \mathrm{E} \quad 01$

$-1.24119 \mathrm{E} 01$
$-2.72273 \mathrm{E}-01$

$-2.78526 \mathrm{E}-01$

$-2.84837 \mathrm{E}-01$

$-2.91206 \mathrm{E}-01$

$-2.97633 \mathrm{E}-01$

$-3.04118 \mathrm{E}-01$

$-3.10661 \mathrm{E}-01$

$-3.17262 \mathrm{E}-01$

$-3.23920 \mathrm{E}-01$

$-3.30637 \mathrm{E}-01$

$-3.37411 \mathrm{E}-01$

$-3.44243 \mathrm{E}-01$

$-3.51133 \mathrm{E}-01$

$-3.58081 \mathrm{E}-01$

$-3.65087 \mathrm{E}-01$

$-3.72151 \mathrm{E}-01$

$-3.79272 \mathrm{E}-01$

-3.86451 E-01

$-3.93688 \mathrm{E}-01$

$-4.00983 \mathrm{E}-01$

$-4.08335 \mathrm{E}-01$

$-4.15745 \mathrm{E}-01$

$-4.23213 \mathrm{E}-01$

$-4.30739 \mathrm{E}-01$

$-4.38322 \mathrm{E}-01$

$-4.45963 \mathrm{E}-01$

-4. 53662 E-01

$-4.61419 \mathrm{E}-01$

$-4.77105 \mathrm{E}-01$

$-4.85034 \mathrm{E}-01$

$-4.93021 \mathrm{E}-01$

$-5.01066 \mathrm{E}-01$

$-5.09168 \mathrm{E}-01$

$-5.17328 \mathrm{E}-01$

$-5.25546 \mathrm{E}-01$

$-5.33821 \mathrm{E}-01$

$-5.42154 \mathrm{E}-01$

$-5.50544 \mathrm{E}-01$

$-5.58992 \mathrm{E}-01$

$-5.67498 \mathrm{E}-01$

$-5.76061 \mathrm{E}-01$

$-5.84681 \mathrm{E}-01$

$-5.93359 \mathrm{E}-01$

$-6.02095 \mathrm{E}-01$

$-6.10888 \mathrm{E}-01$

$-6.19738 \mathrm{E}-01$

$-6.28646 \mathrm{E}-01$

$-6.37612 \mathrm{E}-01$

$-6.46634 \mathrm{E}-01$

$-6.55715 \mathrm{E}-01$

$-6.64852 \mathrm{E}-01$

$-6.74047 \mathrm{E}-01$

$-6.83300 \mathrm{E}-01$

$-6.92610 \mathrm{E}-01$
$-4.69233 \mathrm{E}-01$

$\tan \delta_{1}$

$f_{-1}$

$1.89419 \mathrm{E}-03$

$1.95961 \mathrm{E}-03$

$2.02295 \mathrm{E}-03$

$2.08431 \mathrm{E}-03$

$2.14379 \mathrm{E}-03$

$2.20147 \mathrm{E}-03$

$2.25741 \mathrm{E}-03$

$2.31171 \mathrm{E}-03$

$2.36442 \mathbf{E}-03$

$2.41563 \mathbf{E}-03$

$2.46539 \mathrm{E}-03$

$2.51376 \mathrm{E}-03$

$2.56080 \mathrm{E}-03$

$2.60654 \mathrm{E}-03$

$2.65108 \mathrm{E}-03$

2.69442 E-03

$2.73663 \mathrm{E}-03$

$2.77774 \mathrm{E}-03$

$2.81780 \mathrm{E}-03$

2.85684 E-03

$2.89491 \mathrm{E}-03$

$2.93204 \mathrm{E}-03$

$2.96826 \mathrm{E}-03$

$3.00360 \mathrm{E}-03$

$3.03809 \mathrm{E}-03$

$3.07177 \mathrm{E}-03$

$3.10465 \mathrm{E}-03$

$3.13678 \mathrm{E}-03$

$3.16816 \mathrm{E}-03$

3.19884 E-03

$3.22882 \mathrm{E}-03$

$3.25814 \mathrm{E}-03$

$3.28680 \mathrm{E}-03$

$3.31484 \mathrm{E}-03$

$3.34226 \mathrm{E}-03$

$3.36911 \mathrm{E}-03$

$3.39538 \mathrm{E}-03$

$3.42109 \mathrm{E}-03$

$3.44627 \mathrm{E}-03$

$3.47092 \mathrm{E}-03$

$3.49507 \mathrm{E}-03$

$3.51872 \mathrm{E}-03$

$3.54189 \mathrm{E}-03$ 
p

6.10

6.20

6.30

6.40

6.50

6.60

6.70

6.80

6.90

7.00

7.10

7.20

7.30

7.40

7.50

7.60

7.70

7.80

7.90

8.00

8.10

8.20

8.30

8.40

8.50

8.60

8.70

8.80

8.90

9.00

9.10

9.20

9.30

9.40

9.50

9.60

9.70

9.80

9.90

10.00

10.10

10.20

10.30

10.40

10.50

10.60

10.70

10.80

10.90

11.00

11.10

11.20

11.30

11.40

11.50

11.60

11.70

11.80

11.90

12.00
$\bar{F}$

$f_{2}$

$8.04377 \mathrm{E}-05$ $8.30893 \mathrm{E}-05$ $8.57840 \mathrm{E}-05$ $8.85217 \mathrm{E}-05$

$9.13025 \mathrm{E}-05$

$9.41263 \mathrm{E}-05$ $9.69932 \mathrm{E}-05$ $9.99031 \mathrm{E}-05$ $1.02856 \mathrm{E}-04$ $1.05852 \mathrm{E}-04$

$1.08891 \mathrm{E}-04$ $1.11973 \mathrm{E}-04$ $1.15099 \mathrm{E}-04$ $1.18267 \mathrm{E}-04$ $1.21478 \mathrm{E}-04$

$1.24733 \mathrm{E}-04$ $1.28030 \mathrm{E}-04$ $1.31370 \mathrm{E}-04$ $1.34754 \mathrm{E}-04$ $1.38180 \mathrm{E}-04$

$1.41650 \mathrm{E}-04$ $1.45163 \mathrm{E}-04$ $1.48718 \mathrm{E}-04$ $1.52317 \mathrm{E}-04$ $1.55959 \mathrm{E}-04$

$1.59643 \mathrm{E}-04$ $1.63371 \mathrm{E}-04$ $1.67142 \mathrm{E}-04$ $1.70956 \mathrm{E}-04$ $1.74813 \mathrm{E}-04$

$1.78713 \mathrm{E}-04$ $1.82656 \mathrm{E}-04$ $1.86642 \mathrm{E}-04$ $1.90671 \mathrm{E}-04$ $1.94743 \mathrm{E}-04$

$1.98858 \mathrm{E}-04$ $2.03016 \mathrm{E}-04$ $2.07218 \mathrm{E}-04$ $2.11462 \mathrm{E}-04$ $2.15749 \mathrm{E}-04$

$2.20080 \mathrm{E}-04$ $2.24453 \mathrm{E}-04$ $2.28869 \mathrm{E}-04$ $2.33329 \mathrm{E}-04$ $2.37831 \mathrm{E}-04$

$2.42377 \mathrm{E}-04$ $2.46965 \mathrm{E}-04$ $2.51597 \mathrm{E}-04$ $2.56271 \mathrm{E}-04$ $2.60989 \mathrm{E}-04$

$2.65749 \mathrm{E}-04$ $2.70553 \mathrm{E}-04$ $2.75400 \mathrm{E}-04$ $2.80289 \mathrm{E}-04$ $2.85222 \mathrm{E}-04$

$2.90198 \mathrm{E}-04$ $2.95216 \mathrm{E}-04$ $3.00278 \mathrm{E}-04$ $3.05383 \mathrm{E}-04$ 3.10531 E-04 $g_{2}$

$-1.00031 \mathrm{E}-01$ $-1.03487 \mathrm{E}-01$ $-1.07001 \mathrm{E}-01$ $-1.10574 \mathrm{E}-01$ $-1.14205 \mathrm{E}-01$

$-1.17894 \mathrm{E}-01$ $-1.21642 \mathrm{E}-01$ $-1.25449 \mathrm{E}-01$ $-1.29313 \mathrm{E}-01$ $-1.33237 \mathrm{E}-01$

$-1.37218 \mathrm{E}-01$ $-1.41258 \mathrm{E}-01$ $-1.45356 \mathrm{E}-01$ $-1.49513 \mathrm{E}-01$ $-1.53728 \mathrm{E}-01$

-1.58001 E-01 $-1.62333 \mathrm{E}-01$ $-1.66723 \mathrm{E}-01$ $-1.71171 \mathrm{E}-01$ $-1.75677 \mathrm{E}-01$

$-1.80242 \mathrm{E}-01$ $-1.84865 \mathrm{E}-01$ $-1.89547 \mathrm{E}-01$ $-1.94287 \mathrm{E}-01$ $-1.99085 \mathrm{E}-01$

$-2.03941 \mathrm{E}-01$ $-2.08855 \mathrm{E}-01$ $-2.13828 \mathrm{E}-01$ $-2.18859 \mathrm{E}-01$ $-2.23948 \mathrm{E}-01$

$-2.29095 \mathrm{E}-01$ $-2.34301 \mathrm{E}-01$ $-2.39565 \mathrm{E}-01$ $-2.44887 \mathrm{E}-01$ $-2.50267 \mathrm{E}-01$

$-2.55705 \mathrm{E}-01$ $-2.61201 \mathrm{E}-01$ $-2.66756 \mathrm{E}-01$ $-2.72369 \mathrm{E}-01$ $-2.78040 \mathrm{E}-01$

$-2.83769 \mathrm{E}-01$ $-2.89556 \mathrm{E}-01$ $-2.95401 \mathrm{E}-01$ $-3.01304 \mathrm{E}-01$ $-3.07266 \mathrm{E}-01$

$-3.13285 \mathrm{E}-01$ $-3.19363 \mathrm{E}-01$ $-3.25499 \mathrm{E}-01$ $-3.31692 \mathrm{E}-01$ $-3.37944 \mathrm{E}-01$

$-3.44254 \mathrm{E}-01$ $-3.50622 \mathrm{E}-01$ $-3.57048 \mathrm{E}-01$ $-3.63531 \mathrm{E}-01$ $-3.70073 \mathrm{E}-01$

$-3.76673 \mathrm{E}-01$ $-3.83331 \mathrm{E}-01$ $-3.90047 \mathrm{E}-01$ $-3.96821 \mathrm{E}-01$ $-4.03652 \mathrm{E}-01$ $\tan \delta_{2}$

$f_{-2}$

$-2.33449 \mathrm{E}-03$

$-2.43174 \mathrm{E}-03$

$-2.53148 \mathrm{E}-03$

$-2.63375 \mathrm{E}-03$

$-2.73857 \mathrm{E}-03$

$-2.84598 \mathrm{E}-03$

$-2.95600 \mathrm{E}-03$

$-3.06866 \mathrm{E}-03$

$-3.18399 \mathrm{E}-03$

$-3.30201 \mathrm{E}-03$

$-3.42276 \mathrm{E}-03$

$-3.54626 \mathrm{E}-03$

$-3.67255 \mathrm{E}-03$

$-3.80164 \mathrm{E}-03$

$-3.93357 \mathrm{E}-03$

$-4.06836 \mathrm{E}-03$

$-4.20606 \mathrm{E}-03$

$-4.34667 \mathrm{E}-03$

$-4.49023 \mathrm{E}-03$

$-4.63678 \mathrm{E}-03$

$-4.78633 \mathrm{E}-03$

$-4.93891 \mathrm{E}-03$

$-5.09456 \mathrm{E}-03$

$-5.25331 \mathrm{E}-03$

$-5.41517 \mathrm{E}-03$

$-5.58018 \mathrm{E}-03$

$-5.74837 \mathrm{E}-03$

$-5.91976 \mathrm{E}-03$

$-6.09439 \mathrm{E}-03$

$-6.27227 \mathrm{E}-03$

$-6.45345 \mathrm{E}-03$

$-6.63794 \mathrm{E}-03$

$-6.82579 \mathrm{E}-03$

$-7.01700 \mathrm{E}-03$

$-7.21162 \mathrm{E}-03$

$-7.40967 \mathrm{E}-03$

$-7.61117 \mathrm{E}-03$

$-7.81616 \mathrm{E}-03$

$-8.02467 \mathrm{E}-03$

$-8.23673 \mathrm{E}-03$

$-8.45235 \mathrm{E}-03$

$-8.67157 \mathrm{E}-03$

$-8.89442 \mathrm{E}-03$

$-9.12093 \mathrm{E}-03$

$-9.35112 \mathrm{E}-03$

$-9.58502 \mathrm{E}-03$ $-9.82267 \mathrm{E}-03$

$-1.00641 \mathrm{E}-02$

$-1.03093 \mathrm{E}-02$

$-1.05583 \mathrm{E}-02$

$-1.08112 \mathrm{E}-02$ $-1.10680 \mathrm{E}-02$ $-1.13286 \mathrm{E}-02$ $-1.15933 \mathrm{E}-02$ $-1.18618 \mathrm{E}-02$

$-1.21344 \mathrm{E}-02$ $-1.24110 \mathrm{E}-02$ $-1.26916 \mathrm{E}-02$ $-1.29763 \mathrm{E}-02$ $-1.32652 \mathrm{E}-02$

$2.78585 \mathrm{E} \quad 01 \quad-2.36580 \mathrm{E}-03$

$2.78961 \mathrm{E}$ ol $-2.46064 \mathrm{E}-03$

$2.79325 \mathrm{E}$ 01 $-2.55787 \mathrm{E}-03$

$2.79676 \mathrm{E}$ 01 $-2.65754 \mathrm{E}-03$

$2.80016 \mathrm{E}$ ol $-2.75967 \mathrm{E}-03$

$2.80345 \mathrm{E}$ 01 $-2.86429 \mathrm{E}-03$

$2.80664 \mathrm{E}$ 01 $-2.97142 \mathrm{E}-03$

$2.80972 \mathrm{E}$ 01 $-3.08109 \mathrm{E}-03$

$2.81271 \mathrm{E}$ 01 $-3.19334 \mathrm{E}-03$

$2.81561 \mathrm{E} 01 \quad-3.30819 \mathrm{E}-03$

$2.81843 \mathrm{E} \quad 01$

$2.82116 \mathrm{E} 01$

$2.82381 \mathrm{E} \quad 01$

$2.82638 \mathrm{E} \quad 01$

$2.82888 \mathrm{E} \quad 01$

$2.83131 \mathrm{E} \quad 01$

$2.83368 \mathrm{E} \quad 01$

$2.83598 \mathrm{E} \quad 01$

$2.83822 \mathrm{E} \quad 01$

$2.84040 \mathrm{E} 01$

$2.84252 \mathrm{E} \quad 01$

$2.84458 \mathrm{E} \quad 01$

$2.84660 \mathrm{E} 01$

$2.84856 \mathrm{E} \quad 01$

2.85048 E 01

$2.85234 \mathrm{E} \quad 01$

$2.85416 \mathrm{E} \quad 01$

$2.85594 \mathrm{E} \quad 01$

$2.85768 \mathrm{E} \quad 01$

$2.85937 \mathrm{E} \quad 01$

$-3.42566 \mathrm{E}-03$

$-3.54580 \mathrm{E}-03$

$-3.66862 \mathrm{E}-03$

$-3.79415 \mathrm{E}-03$

$-3.92243 \mathrm{E}-03$

$-4.05347 \mathrm{E}-03$

$-4.18732 \mathrm{E}-03$

$-4.32399 \mathrm{E}-03$

$-4.46352 \mathrm{E}-03$

$-4.60593 \mathrm{E}-03$ 


\begin{tabular}{|c|c|c|c|c|c|}
\hline$p$ & $F_{0}$ & & $f_{1}$ & & $g_{1}$ \\
\hline 12.10 & $1.18208 \mathrm{E}$ & 00 & $-1.25190 \mathrm{E}$ & 01 & $-7.01977 \mathrm{E}-0$ \\
\hline 12.20 & $1.18204 \mathrm{E}$ & 00 & $-1.26260 \mathrm{E}$ & 01 & $-7.11402 \mathrm{E}-$ \\
\hline 30 & $1.18200 \mathrm{E}$ & 00 & $-1.27331 \mathrm{E}$ & 01 & $7.20884 \mathrm{E}-$ \\
\hline & $1.18197 \mathrm{E}$ & 00 & $-1.28401 \mathrm{E}$ & 01 & $-7.30423 \mathrm{E}$ \\
\hline .50 & $1.18194 \mathrm{E}$ & 00 & $-1.29471 \mathrm{E}$ & 01 & $-7.40020 \mathrm{E}$ \\
\hline .60 & $1.18190 \mathrm{E}$ & 00 & $-1.30541 \mathrm{E}$ & 01 & $-7.49673 \mathrm{E}-$ \\
\hline .70 & $1.18187 \mathrm{E}$ & 00 & $-1.31610 \mathrm{E}$ & 01 & $-7.59385 \mathrm{E}$ \\
\hline .80 & $1.18184 \mathrm{E}$ & 00 & $-1.32680 \mathrm{E}$ & 01 & $-7.69153 \mathrm{E}$ \\
\hline .90 & $1.18180 \mathrm{E}$ & 00 & $-1.33749 \mathrm{E}$ & 01 & $-7.78979 \mathrm{E}$ \\
\hline .00 & $1.18177 \mathrm{E}$ & 00 & $-1.34818 \mathrm{E}$ & 01 & $-7.88862 \mathrm{E}$ \\
\hline .10 & $1.18174 \mathrm{E}$ & 00 & $-1.35887 \mathrm{E}$ & 01 & $-7.98802 \mathrm{E}$ \\
\hline .20 & $1.18171 \mathrm{E}$ & 00 & $-1.36955 \mathrm{E}$ & 01 & $-8.08800 \mathrm{E}$ \\
\hline .30 & $1.18168 \mathrm{E}$ & 00 & $-1.38024 \mathrm{E}$ & 01 & $-8.18854 \mathrm{E}$ \\
\hline .40 & $1.18165 \mathrm{E}$ & 00 & $-1.39092 \mathrm{E}$ & 01 & $-8.28966 \mathrm{E}$ \\
\hline 3.50 & $1.18162 \mathrm{E}$ & 00 & $-1.40160 \mathrm{E}$ & 01 & $-8.39136 \mathrm{E}$ \\
\hline 60 & $1.18159 \mathrm{E}$ & 00 & $-1.41228 \mathrm{E}$ & 01 & $-8.49362 \mathrm{E}$ \\
\hline .60 & $1.18156 \mathrm{E}$ & 00 & $-1.42296 \mathrm{E}$ & 01 & $-8.59645 \mathrm{E}$ \\
\hline 30 & $1.18153 \mathrm{E}$ & 00 & $-1.43363 \mathrm{E}$ & 01 & $-8.69986 \mathrm{E}$ \\
\hline .90 & $1.18150 \mathrm{E}$ & 00 & $.44430 \mathrm{E}$ & 01 & $-8.80384 \mathrm{E}$ \\
\hline 1.00 & $1.18147 \mathrm{E}$ & 00 & $-1.45497 \mathrm{E}$ & 01 & $-8.90838 \mathrm{E}$ \\
\hline 10 & $1.18144 \mathrm{E}$ & 00 & $-1.46564 \mathrm{E}$ & 01 & $-9.01350 \mathrm{E}-$ \\
\hline .20 & $1.18141 \mathrm{E}$ & 00 & $47631 \mathrm{E}$ & 01 & $-9.11920 \mathrm{E}$ \\
\hline 30 & $1.18139 \mathrm{E}$ & 00 & $-1.48697 \mathrm{E}$ & 01 & $2546 \mathrm{E}$ \\
\hline 40 & $1.18136 \mathrm{E}$ & 00 & $-1.49764 \mathrm{E}$ & 01 & $-9.33229 \mathrm{E}$ \\
\hline .50 & $1.18133 \mathrm{E}$ & 00 & $-1.50830 \mathrm{E}$ & 01 & $-9.43970 \mathrm{E}$ \\
\hline .60 & $1.18130 \mathrm{E}$ & 00 & $-1.51896 \mathrm{E}$ & 01 & $-9.54767 \mathrm{E}-$ \\
\hline .70 & $1.18128 \mathrm{E}$ & 00 & $52961 \mathrm{E}$ & 01 & $621 \mathrm{E}-$ \\
\hline 4.80 & $1.18125 \mathrm{E}$ & 00 & $-1.54027 \mathrm{E}$ & 01 & $-9.76533 \mathrm{E}$ \\
\hline 4.90 & $1.18122 \mathrm{E}$ & 00 & $-1.55092 \mathrm{E}$ & 01 & $-9.87501 \mathrm{E}-$ \\
\hline .00 & $1.18120 \mathrm{E}$ & 00 & $.56157 \mathrm{E}$ & 01 & $-9.98527 \mathrm{E}-$ \\
\hline .10 & $1.18117 \mathrm{E}$ & 00 & $-1.57222 \mathrm{E}$ & 01 & $-1.00961 \mathrm{E}$ \\
\hline .20 & $1.18115 \mathrm{E}$ & 00 & $-1.58287 \mathrm{E}$ & 01 & $-1.02075 \mathrm{E}$ \\
\hline .3 & $112 \mathrm{E}$ & 00 & $-1.59351 \mathrm{E}$ & 01 & $-1.03194 \mathrm{E}$ \\
\hline 0 & $1.18110 \mathrm{E}$ & 00 & $04.16 \mathrm{E}$ & 01 & $-1.04320 \mathrm{E}$ \\
\hline .50 & $1.18107 \mathrm{E}$ & 00 & $-1.61480 \mathrm{E}$ & 01 & $-1.05451 \mathrm{E}$ \\
\hline 60 & $1.18105 \mathrm{E}$ & 00 & $-1.62544 \mathrm{E}$ & 01 & $-1.06588 \mathrm{E}$ \\
\hline .70 & $02 \mathrm{E}$ & 00 & $-1.63607 \mathrm{E}$ & 01 & $-1.07730 \mathrm{E}$ \\
\hline & $00 \mathrm{E}$ & 00 & $4671 \mathrm{E}$ & 01 & $878 \mathrm{E}$ \\
\hline .90 & $097 \mathrm{E}$ & 00 & $734 \mathrm{E}$ & 01 & $-1.10032 \mathrm{E}$ \\
\hline 0.00 & $1.18095 \mathrm{E}$ & 00 & $-1.66797 \mathrm{E}$ & 01 & $-1.11191 \mathrm{E}$ \\
\hline 6.10 & $1.18093 \mathrm{E}$ & 00 & $-1.67860 \mathrm{E}$ & 01 & $-1.12356 \mathrm{E}$ \\
\hline 0 & & 00 & -1 & 01 & $527 \mathrm{E}$ \\
\hline .30 & $1.18088 \mathrm{E}$ & 00 & $9985 \mathrm{E}$ & 01 & $-1.14704 \mathrm{E}$ \\
\hline 6.40 & $1.18086 \mathrm{E}$ & 00 & $-1.71047 \mathrm{E}$ & 01 & $-1.15886 \mathrm{E}$ \\
\hline .50 & $1.18084 \mathrm{E}$ & 00 & $2109 \mathrm{E}$ & 01 & $-1.17074 \mathrm{E}$ \\
\hline 16.60 & $1.18081 \mathrm{E}$ & 00 & $-1.73171 \mathrm{E}$ & 01 & $-1.18267 \mathrm{E}$ \\
\hline 670 & $079 \mathrm{E}$ & 00 & $-1.74233 \mathrm{E}$ & 01 & $-1.19467 \mathrm{E}$ \\
\hline 68 & $18077 \mathrm{E}$ & 00 & $-1.75294 \mathrm{E}$ & 01 & $-1.20672 \mathrm{E}$ \\
\hline & $75 \mathrm{E}$ & 00 & $6355 \mathrm{E}$ & 01 & $-1.21882 \mathrm{E}$ \\
\hline 800 & $1.18072 \mathrm{E}$ & 00 & $77416 \mathrm{E}$ & 01 & $-1.23098 \mathrm{E}$ \\
\hline 1 & $1.18070 \mathrm{E}$ & 00 & $-1.78477 \mathrm{E}$ & 01 & $-1.24320 \mathrm{E}$ \\
\hline & $68 \mathrm{E}$ & 00 & $9537 \mathrm{E}$ & 01 & $548 \mathrm{E}$ \\
\hline & & 00 & $80598 \mathrm{E}$ & 01 & $-1.26781 \mathrm{E}$ \\
\hline ?. & & 00 & $-1.81658 E$ & 01 & $-1.28020 \mathrm{E}$ \\
\hline & $1.18062 \mathrm{E}$ & 00 & $-1.82718 \mathrm{E}$ & 01 & $-1.29265 \mathrm{E}$ \\
\hline & $1.18060 \mathrm{E}$ & 00 & $-1.83778 \mathrm{E}$ & 01 & $-1.30515 \mathrm{E}$ \\
\hline & & 00 & $-1.84837 \mathrm{E}$ & 01 & $-1.31771 \mathrm{E}$ \\
\hline & $1.18056 \mathrm{E}$ & 00 & $-1.85896 \mathrm{E}$ & 01 & $-1.33032 \mathrm{E}$ \\
\hline & $53 \mathrm{E}$ & 00 & $-1.86955 \mathrm{E}$ & 01 & $-1.34300 \mathrm{E}$ \\
\hline & $1.18051 \mathrm{E}$ & 00 & $-1.88014 \mathrm{E}$ & 01 & $-1.35573 \mathrm{E} \quad 00$ \\
\hline
\end{tabular}

$\tan \delta_{1}$

\section{$3.89048 \mathrm{E}-03$}

$3.90665 \mathrm{E}-03$

$3.92252 \mathrm{E}-03$

$3.93813 \mathrm{E}-03$

$3.95346 \mathrm{E}-03$

$3.96853 \mathrm{E}-03$

$3.98334 \mathrm{E}-03$

$3.99790 \mathrm{E}-03$

$4.01220 \mathrm{E}-03$

$4.02627 \mathrm{E}-03$

$4.04011 \mathrm{E}-03$

$4.05371 \mathrm{E}-03$

$4.06708 \mathrm{E}-03$

$4.08024 \mathrm{E}-03$

$4.09318 \mathrm{E}-03$

$4.10589 \mathrm{E}-03$

$4.11841 \mathrm{E}-03$

4.13072 E-03

$4.14285 \mathrm{E}-03$

$4.15476 \mathrm{E}-03$

$4.16650 \mathrm{E}-03$

4.17804E-03

$4.18939 \mathrm{E}-03$

$4.20058 \mathrm{E}-03$

$4.21159 \mathrm{E}-03$

$4.22241 \mathrm{E}-03$

$4.23307 \mathrm{E}-03$

$4.24357 \mathrm{E}-03$

$4.25390 \mathrm{E}-03$

$4.26407 \mathrm{E}-03$

$4.27409 \mathrm{E}-03$

$4.28396 \mathrm{E}-03$

$4.29366 \mathrm{E}-03$

$4.30322 \mathrm{E}-03$

$4.31264 \mathrm{E}-03$

4.32192E-03

$4.33105 \mathrm{E}-03$

$4.34005 \mathrm{E}-03$

$4.34891 \mathrm{E}-03$

$4.35764 \mathrm{E}-03$

$4.36624 \mathrm{E}-03$ $4.37470 \mathrm{E}-03$ 4. $38305 \mathrm{E}-03$ $4.39127 \mathrm{E}-03$ $4.39936 \mathrm{E}-03$

$4.40734 \mathrm{E}-03$ $4.41520 \mathrm{E}-03$ 4. $42293 \mathrm{E}-03$ 4. $43057 \mathrm{E}-03$ 4.43809 $\mathrm{E}-03$

$4.44549 \mathrm{E}-03$ $4.45280 \mathrm{E}-03$ $4.45998 \mathrm{E}-03$ 4.46707 E-03 $4.47405 \mathrm{E}-03$

4.48093 E-03 $4.48772 \mathrm{E}-03$ $4.49439 \mathrm{E}-03$ $4.50097 \mathrm{E}-03$ $4.50747 \mathrm{E}-03$ $f_{-1}$

$-6.88493 \mathrm{E}-01$ $-6.97623 \mathrm{E}-01$ $-7.06811 \mathrm{E}-01$ $-7.16057 \mathrm{E}-01$ $-7.25359 \mathrm{E}-01$

$-7.34719 \mathrm{E}-01$ $-7.44137 \mathrm{E}-01$ $-7.53612 \mathrm{E}-01$ $-7.63144 \mathrm{E}-01$ $-7.72733 \mathrm{E}-01$

$-7.82380 \mathrm{E}-01$ $-7.92083 \mathrm{E}-01$ $-8.01844 \mathrm{E}-01$ $-8.11662 \mathrm{E}-01$ $-8.21538 \mathrm{E}-01$

$-8.31470 \mathrm{E}-01$

$-8.41460 \mathrm{E}-01$

$-8.51507 \mathrm{E}-01$

$-8.61611 \mathrm{E}-01$

$-8.71773 \mathrm{E}-01$

$-8.81991 \mathrm{E}-01$ $-8.92267 \mathrm{E}-01$

$-9.02600 \mathrm{E}-01$

$-9.12989 \mathrm{E}-01$

$-9.23436 \mathrm{E}-01$

$-9.33940 \mathrm{E}-01$

$-9.44501 \mathrm{E}-01$

$-9.55119 \mathrm{E}-01$

$-9.65794 \mathrm{E}-01$

$-9.76526 \mathrm{E}-01$

$-9.87315 \mathrm{E}-01$

$-9.98161 \mathrm{E}-01$

$-1.00906 \mathrm{E} 00$

$-1.02002 \mathrm{E} 00$

$-1.03104 \mathrm{E} 00$

$-1.04212 \mathrm{E} 00$

$-1.05325 \mathrm{E} 00$

$-1.06443 \mathrm{E} \quad 00$

$-1.07568 \mathrm{E} 00$

$-1.08698 \mathrm{E} 00$

$-1.09834 \mathrm{E} \quad 00$ $-1.10975 \mathrm{E} 00$ $-1.12123 \mathrm{E} 00$ $-1.13276 \mathrm{E} \quad 00$ $-1.14434 \mathrm{E} \quad 00$

$-1.15598 \mathrm{E} \quad 00$ $-1.16768 \mathrm{E} 00$ $-1.17944 \mathrm{E} 00$ $-1.19125 \mathrm{E} \quad 00$ $-1.20312 \mathrm{E} 00$

$-1.21505 \mathrm{E} \quad 00$ $-1.22703 \mathrm{E} 00$ $-1.23907 \mathrm{E} \quad 00$ $-1.25117 \mathrm{E} 00$ $-1.26332 \mathrm{E} \quad 00$

$-1.27553 \mathrm{E} \quad 00$ $-1.28780 \mathrm{E} \quad 00$ $-1.30012 \mathrm{E} 00$ $-1.31250 \mathrm{E} 00$ $-1.32494 \mathrm{E} 00$ $g_{-1}$

$1.35918 \mathrm{E} \quad 01$

$1.36988 \mathrm{E} 01$

$1.38058 \mathrm{E} \quad 01$

$1.39127 \mathrm{E} \quad 01$

$1.40197 \mathrm{E} 01$

$1.41266 \mathrm{E} \quad 01$ $1.42334 \mathrm{E} \quad 01$

$1.43403 \mathrm{E} 01$

$1.44472 \mathrm{E} 01$

$1.45540 \mathrm{E} \quad 01$

$1.46608 \mathrm{E} \quad 01$ $1.47676 \mathrm{E} \quad 01$ $1.48743 \mathrm{E} \quad 01$

$\begin{array}{lll}1.49811 \mathrm{E} & 01\end{array}$

$1.50878 \mathrm{E} 01$

$1.51945 \mathrm{E} \quad 01$

$1.53012 \mathrm{E} 01$

$1.54079 \mathrm{E} 01$

$1.55145 \mathrm{E} \quad 01$

$1.56212 \mathrm{E} \quad 01$

$\begin{array}{ll}1.57278 \mathrm{E} & 01\end{array}$ $1.58344 \mathrm{E} \quad 01$ $1.59409 \mathrm{E} \quad 01$ $1.60475 \mathrm{E} 01$ $1.61540 \mathrm{E} \quad 01$

$1.62605 \mathrm{E} \quad 01$ $1.63670 \mathrm{E} \quad 01$ $1.64734 \mathrm{E} \quad 01$ $1.65799 \mathrm{E} \quad 01$ $1.66863 \mathrm{E} 01$

$1.67927 \mathrm{E} \quad 01$ $1.68991 \mathrm{E} 01$ $1.70054 \mathrm{E} \quad 01$ $1.71118 \mathrm{E} \quad 01$ $1.72181 \mathrm{E} \quad 01$

$1.73244 \mathrm{E} \quad 01$ $1.74307 \mathrm{E} \quad 01$ $1.75369 \mathrm{E} 01$ $1.76431 \mathrm{E} 01$ $1.77494 \mathrm{E} \quad 01$

$1.78555 \mathrm{E} \quad 01$ $1.79617 \mathrm{E} \quad 01$ $1.80679 \mathrm{E} \quad 01$ $1.81740 \mathrm{E} \quad 01$ $1.82801 \mathrm{E} 01$

$1.83862 \mathrm{E} \quad 01$ $1.84922 \mathrm{E} 0$ $1.85983 \mathrm{E} 01$ $1.87043 \mathrm{E} \quad 01$ $1.88103 \mathrm{E} \quad 01$

$1.89163 \mathrm{E} \quad 01$ $1.90222 \mathrm{E} \quad 01$ $1.91281 \mathrm{E} \quad 01$ $1.92341 \mathrm{E} 01$ $1.93399 \mathrm{E} 01$

$1.94458 \mathrm{E} \quad 01$ $1.95517 \mathrm{E} \quad 01$ $1.96575 \mathrm{E} 01$ $1.97633 \mathrm{E} \quad 01$ $1.98691 \mathrm{E} 01$ $\tan \delta_{-1}$

$-1.23414 \mathrm{E} \quad 02$

$-1.23697 \mathrm{E} 02$

$-1.23977 \mathrm{E} \quad 02$

$-1.24254 \mathrm{E} \quad 02$

$-1.24527 \mathrm{E} 02$

$-1.24798 \mathrm{E} \quad 02$ $-1.25067 \mathrm{E} 02$ $-1.25332 \mathrm{E} 02$ $-1.25595 \mathrm{E} \quad 02$ $-1.25856 \mathrm{E} 02$

$-1.26113 \mathrm{E} \quad 02$ $-1.26369 \mathrm{E} 02$ $-1.26622 \mathrm{E} \quad 02$ $-1.26872 \mathrm{E} \quad 02$ $-1.27120 \mathrm{E} 02$

$-1.27366 \mathrm{E} \quad 02$ $-1.27609 \mathrm{E} \quad 02$ $-1.27850 \mathrm{E} 02$ $-1.28089 \mathrm{E} 02$ $-1.28326 \mathrm{E} 02$

$-1.28561 \mathrm{E} \quad 02$ $-1.28793 \mathrm{E} 02$ $-1.29024 \mathrm{E} \quad 02$ $-1.29253 \mathrm{E} \quad 02$ $-1.29479 \mathrm{E} 02$

$-1.29704 \mathrm{E} \quad 02$ $-1.29927 \mathrm{E} 02$ $\begin{array}{ll}-1.30147 \mathrm{E} & 02\end{array}$ $-1.30366 \mathrm{E} \quad 02$ $-1.30584 \mathrm{E} 02$

$-1.30799 \mathrm{E} \quad 02$ $-1.31013 \mathrm{E} 02$ $-1.31225 \mathrm{E} 02$ $-1.31435 \mathrm{E} 02$ $-1.31644 \mathrm{E} 02$

$-1.31851 \mathrm{E} \quad 02$ $-1.32056 \mathrm{E} 02$ $-1.32260 \mathrm{E} 02$ $-1.32463 \mathrm{E} 02$ $-1.32664 \mathrm{E} 02$

$-1.32863 \mathrm{E} \quad 02$ $-1.33061 \mathrm{E} \quad 02$ $-1.33257 \mathrm{E} \quad 02$ $-1.33452 \mathrm{E} \quad 02$ $-1.33646 \mathrm{E} 02$

$\begin{array}{ll}-1.33838 \mathrm{E} & 02\end{array}$ $-1.34029 \mathrm{E} 02$ $-1.34219 \mathrm{E} 02$ $-1.34407 \mathrm{E} 02$ $-1.34594 \mathrm{E} \quad 02$

$-1.34780 \mathrm{E} \quad 02$ $-1.34965 \mathrm{E} 02$ $-1.35148 \mathrm{E} 02$ $-1.35330 \mathrm{E} 02$ $-1.35511 \mathrm{E} 02$

$-1.35691 \mathrm{E} \quad 02$ $-1.35870 \mathrm{E} 02$ $-1.36048 \mathrm{E} 02$ $-1.36224 \mathrm{E} \quad 02$ $-1.36400 \mathrm{E} 02$ 


\begin{tabular}{|c|c|c|c|c|c|c|c|c|}
\hline$p$ & $\bar{F}$ & $f_{2}$ & $g_{2}$ & $\tan \delta_{2}$ & & $f_{-2}$ & $g-2$ & $\tan \delta_{2}$ \\
\hline $\begin{array}{l}12.10 \\
12.20 \\
12.30 \\
12.40 \\
12.50\end{array}$ & $\begin{array}{l}3.15722 \mathrm{E}-04 \\
3.20956 \mathrm{E}-04 \\
3.26232 \mathrm{E}-04 \\
3.31552 \mathrm{E}-04 \\
3.36915 \mathrm{E}-04\end{array}$ & $\begin{array}{l}-4.10542 \mathrm{E}-01 \\
-4.17490 \mathrm{E}-01 \\
-4.24496 \mathrm{E}-01 \\
-4.31559 \mathrm{E}-01 \\
-4.38680 \mathrm{E}-01\end{array}$ & $\begin{array}{l}-1.35581 \mathrm{E}-02 \\
-1.38553 \mathrm{E}-02 \\
-1.41566 \mathrm{E}-02 \\
-1.44621 \mathrm{E}-02 \\
-1.47719 \mathrm{E}-02\end{array}$ & $\begin{array}{l}2.89735 \mathrm{E} \\
2.89823 \mathrm{E} \\
2.89911 \mathrm{E} \\
2.89996 \mathrm{E} \\
2.90081 \mathrm{E}\end{array}$ & $\begin{array}{l}01 \\
01 \\
01 \\
01 \\
01\end{array}$ & $\begin{array}{l}-1.32758 \mathrm{E}-02 \\
-1.35648 \mathrm{E}-02 \\
-1.38579 \mathrm{E}-02 \\
-1.41551 \mathrm{E}-02 \\
-1.44565 \mathrm{E}-02\end{array}$ & $\begin{array}{l}4.45839 \mathrm{E}-01 \\
4.53078 \mathrm{E}-01 \\
4.60375 \mathrm{E}-01 \\
4.67730 \mathrm{E}-01 \\
4.75143 \mathrm{E}-01\end{array}$ & $\begin{array}{l}-3.24011 \mathrm{E}-02 \\
-3.24078 \mathrm{E}-02 \\
-3.24145 \mathrm{E}-02 \\
-3.24210 \mathrm{E}-02 \\
-3.24275 \mathrm{E}-02\end{array}$ \\
\hline $\begin{array}{l}12.60 \\
12.70 \\
12.80 \\
12.90 \\
13.00\end{array}$ & $\begin{array}{l}3.42321 \mathrm{E}-04 \\
3.47770 \mathrm{E}-04 \\
3.53262 \mathrm{E}-04 \\
3.58797 \mathrm{E}-04 \\
3.64375 \mathrm{E}-04\end{array}$ & $\begin{array}{l}-4.45860 \mathrm{E}-01 \\
-4.53097 \mathrm{E}-01 \\
-4.60392 \mathrm{E}-01 \\
-4.67745 \mathrm{E}-01 \\
-4.75156 \mathrm{E}-01\end{array}$ & $\begin{array}{l}-1.50859 \mathrm{E}-02 \\
-1.54043 \mathrm{E}-02 \\
-1.57269 \mathrm{E}-02 \\
-1.60540 \mathrm{E}-02 \\
-1.63854 \mathrm{E}-02\end{array}$ & $\begin{array}{l}2.90164 \mathrm{E} \\
2.90245 \mathrm{E} \\
2.90325 \mathrm{E} \\
2.90404 \mathrm{E} \\
2.90482 \mathrm{E}\end{array}$ & $\begin{array}{l}01 \\
01 \\
01 \\
01 \\
01\end{array}$ & $\begin{array}{l}-1.47620 \mathrm{E}-02 \\
-1.50718 \mathrm{E}-02 \\
-1.53857 \mathrm{E}-02 \\
-1.57040 \mathrm{E}-02 \\
-1.60265 \mathrm{E}-02\end{array}$ & $\begin{array}{l}4.82613 \mathrm{E}-01 \\
4.90142 \mathrm{E}-01 \\
4.97728 \mathrm{E}-01 \\
5.05372 \mathrm{E}-01 \\
5.13074 \mathrm{E}-01\end{array}$ & $\begin{array}{l}-3.24339 \mathrm{E}-02 \\
-3.24402 \mathrm{E}-02 \\
-3.24464 \mathrm{E}-02 \\
-3.24525 \mathrm{E}-02 \\
-3.24585 \mathrm{E}-02\end{array}$ \\
\hline $\begin{array}{l}13.10 \\
13.20 \\
13.30 \\
13.40 \\
13.50\end{array}$ & $\begin{array}{l}3.69996 \mathrm{E}-04 \\
3.75660 \mathrm{E}-04 \\
3.81367 \mathrm{E}-04 \\
3.87117 \mathrm{E}-04 \\
3.92910 \mathrm{E}-04\end{array}$ & $\begin{array}{l}-4.82624 \mathrm{E}-01 \\
-4.90151 \mathrm{E}-01 \\
-4.97735 \mathrm{E}-01 \\
-5.05377 \mathrm{E}-01 \\
-5.13077 \mathrm{E}-01\end{array}$ & $\begin{array}{l}-1.67212 \mathrm{E}-02 \\
-1.70615 \mathrm{E}-02 \\
-1.74062 \mathrm{E}-02 \\
-1.77555 \mathrm{E}-02 \\
-1.81092 \mathrm{E}-02\end{array}$ & $\begin{array}{l}2.90558 \mathrm{E} \\
2.90634 \mathrm{E} \\
2.90708 \mathrm{E} \\
2.90780 \mathrm{E} \\
2.90852 \mathrm{E}\end{array}$ & $\begin{array}{l}01 \\
01 \\
01 \\
01 \\
01\end{array}$ & $\begin{array}{l}-1.63534 \mathrm{E}-02 \\
-1.66846 \mathrm{E}-02 \\
-1.70201 \mathrm{E}-02 \\
-1.73601 \mathrm{E}-02 \\
-1.77045 \mathrm{E}-02\end{array}$ & $\begin{array}{l}5.20834 \mathrm{E}-01 \\
5.28651 \mathrm{E}-01 \\
5.36527 \mathrm{E}-01 \\
5.44460 \mathrm{E}-01 \\
5.52451 \mathrm{E}-01\end{array}$ & $\begin{array}{l}-3.24645 \mathrm{E}-02 \\
-3.24704 \mathrm{E}-02 \\
-3.24762 \mathrm{E}-02 \\
-3.24819 \mathrm{E}-02 \\
-3.24876 \mathrm{E}-02\end{array}$ \\
\hline $\begin{array}{l}13.60 \\
13.70 \\
13.80 \\
13.90 \\
14.00\end{array}$ & $\begin{array}{l}3.98746 \mathrm{E}-04 \\
4.04625 \mathrm{E}-04 \\
4.10547 \mathrm{E}-04 \\
4.16513 \mathrm{E}-04 \\
4.22521 \mathrm{E}-04\end{array}$ & $\begin{array}{l}-5.20835 \mathrm{E}-01 \\
-5.28651 \mathrm{E}-01 \\
-5.36524 \mathrm{E}-01 \\
-5.44455 \mathrm{E}-01 \\
-5.52444 \mathrm{E}-01\end{array}$ & $\begin{array}{l}-1.84676 \mathrm{E}-02 \\
-1.88305 \mathrm{E}-02 \\
-1.91980 \mathrm{E}-02 \\
-1.95701 \mathrm{E}-02 \\
-1.99469 \mathrm{E}-02\end{array}$ & $\begin{array}{l}2.90923 \mathrm{E} \\
2.90992 \mathrm{E} \\
2.91061 \mathrm{E} \\
2.91128 \mathrm{E} \\
2.91195 \mathrm{E}\end{array}$ & $\begin{array}{l}01 \\
01 \\
01 \\
01 \\
01\end{array}$ & $\begin{array}{l}-1.80534 \mathrm{E}-02 \\
-1.84067 \mathrm{E}-02 \\
-1.87646 \mathrm{E}-02 \\
-1.91270 \mathrm{E}-02 \\
-1.94940 \mathrm{E}-02\end{array}$ & $\begin{array}{l}5.60499 \mathrm{E}-01 \\
5.68606 \mathrm{E}-01 \\
5.76770 \mathrm{E}-01 \\
5.84992 \mathrm{E}-01 \\
5.93272 \mathrm{E}-01\end{array}$ & $\begin{array}{l}-3.24932 \mathrm{E}-02 \\
-3.24987 \mathrm{E}-02 \\
-3.25041 \mathrm{E}-02 \\
-3.25095 \mathrm{E}-02 \\
-3.25148 \mathrm{E}-02\end{array}$ \\
\hline $\begin{array}{l}14.10 \\
14.20 \\
14.30 \\
14.40 \\
14.50\end{array}$ & $\begin{array}{l}4.28572 \mathrm{E}-04 \\
4.34666 \mathrm{E}-04 \\
4.40803 \mathrm{E}-04 \\
4.46984 \mathrm{E}-04 \\
4.53207 \mathrm{E}-04\end{array}$ & $\begin{array}{l}-5.60491 \mathrm{E}-01 \\
-5.68595 \mathrm{E}-01 \\
-5.76758 \mathrm{E}-01 \\
-5.84977 \mathrm{E}-01 \\
-5.93255 \mathrm{E}-01\end{array}$ & $\begin{array}{l}-2.03285 \mathrm{E}-02 \\
-2.07147 \mathrm{E}-02 \\
-2.11057 \mathrm{E}-02 \\
-2.15014 \mathrm{E}-02 \\
-2.19020 \mathrm{E}-02\end{array}$ & $\begin{array}{l}2.91260 \mathrm{E} \\
2.91324 \mathrm{E} \\
2.91388 \mathrm{E} \\
2.91451 \mathrm{E} \\
2.91512 \mathrm{E}\end{array}$ & $\begin{array}{l}01 \\
01 \\
01 \\
01 \\
01\end{array}$ & $\begin{array}{l}-1.98656 \mathrm{E}-02 \\
-2.02418 \mathrm{E}-02 \\
-2.06227 \mathrm{E}-02 \\
-2.10082 \mathrm{E}-02 \\
-2.13985 \mathrm{E}-02\end{array}$ & $\begin{array}{l}6.01609 \mathrm{E}-01 \\
6.10004 \mathrm{E}-01 \\
6.18457 \mathrm{E}-01 \\
6.26967 \mathrm{E}-01 \\
6.35535 \mathrm{E}-01\end{array}$ & $\begin{array}{l}-3.25200 \mathrm{E}-02 \\
-3.25252 \mathrm{E}-02 \\
-3.25303 \mathrm{E}-02 \\
-3.25354 \mathrm{E}-02 \\
-3.25404 \mathrm{E}-02\end{array}$ \\
\hline $\begin{array}{l}4.60 \\
4.70 \\
4.80 \\
4.90 \\
5.00\end{array}$ & $\begin{array}{l}4.59473 \mathrm{E}-04 \\
4.65782 \mathrm{E}-04 \\
4.72135 \mathrm{E}-04 \\
4.78530 \mathrm{E}-04 \\
4.84968 \mathrm{E}-04\end{array}$ & $\begin{array}{l}-6.01590 \mathrm{E}-01 \\
-6.09983 \mathrm{E}-01 \\
-6.18434 \mathrm{E}-01 \\
-6.26942 \mathrm{E}-01 \\
-6.35509 \mathrm{E}-01\end{array}$ & $\begin{array}{l}-2.23074 \mathrm{E}-02 \\
-2.27176 \mathrm{E}-02 \\
-2.31328 \mathrm{E}-02 \\
-2.35529 \mathrm{E}-02 \\
-2.39779 \mathrm{E}-02\end{array}$ & $\begin{array}{l}2.91573 \mathrm{E} \\
2.91633 \mathrm{E} \\
2.91692 \mathrm{E} \\
2.91750 \mathrm{E} \\
2.91808 \mathrm{E}\end{array}$ & $\begin{array}{l}01 \\
01 \\
01 \\
01 \\
01\end{array}$ & $\begin{array}{l}-2.17935 \mathrm{E}-02 \\
-2.21932 \mathrm{E}-02 \\
-2.25978 \mathrm{E}-02 \\
-2.30072 \mathrm{E}-02 \\
-2.34214 \mathrm{E}-02\end{array}$ & $\begin{array}{l}6.44161 \mathrm{E}-01 \\
6.52845 \mathrm{E}-01 \\
6.61586 \mathrm{E}-01 \\
6.70384 \mathrm{E}-01 \\
6.79241 \mathrm{E}-01\end{array}$ & $\begin{array}{l}-3.25453 \mathrm{E}-02 \\
-3.25502 \mathrm{E}-02 \\
-3.25550 \mathrm{E}-02 \\
-3.25597 \mathrm{E}-02 \\
-3.25644 \mathrm{E}-02\end{array}$ \\
\hline $\begin{array}{l}15.10 \\
15.20 \\
15.30 \\
15.40 \\
15.50\end{array}$ & $\begin{array}{l}4.91449 \mathrm{E}-04 \\
4.97974 \mathrm{E}-04 \\
5.04541 \mathrm{E}-04 \\
5.11151 \mathrm{E}-04 \\
5.17804 \mathrm{E}-04\end{array}$ & $\begin{array}{l}-6.44132 \mathrm{E}-01 \\
-6.52814 \mathrm{E}-01 \\
-6.61552 \mathrm{E}-01 \\
-6.70349 \mathrm{E}-01 \\
-6.79203 \mathrm{E}-01\end{array}$ & $\begin{array}{l}-2.44078 \mathrm{E}-02 \\
-2.48428 \mathrm{E}-02 \\
-2.52828 \mathrm{E}-02 \\
-2.57279 \mathrm{E}-02 \\
-2.61780 \mathrm{E}-02\end{array}$ & $\begin{array}{l}2.91864 \mathrm{E} \\
2.91920 \mathrm{E} \\
2.91975 \mathrm{E} \\
2.92030 \mathrm{E} \\
2.92083 \mathrm{E}\end{array}$ & $\begin{array}{l}01 \\
01 \\
01 \\
01 \\
01\end{array}$ & $\begin{array}{l}-2.38405 \mathrm{E}-02 \\
-2.42645 \mathrm{E}-02 \\
-2.46934 \mathrm{E}-02 \\
-2.51273 \mathrm{E}-02 \\
-2.55662 \mathrm{E}-02\end{array}$ & $\begin{array}{l}6.88155 \mathrm{E}-01 \\
6.97126 \mathrm{E}-01 \\
7.06155 \mathrm{E}-01 \\
7.15242 \mathrm{E}-01 \\
7.24387 \mathrm{E}-01\end{array}$ & $\begin{array}{l}-3.25690 \mathrm{E}-02 \\
-3.25736 \mathrm{E}-02 \\
-3.25782 \mathrm{E}-02 \\
-3.25826 \mathrm{E}-02 \\
-3.25871 \mathrm{E}-02\end{array}$ \\
\hline $\begin{array}{l}15.60 \\
15.70 \\
15.80 \\
15.90 \\
16.00\end{array}$ & $\begin{array}{l}5.24501 \mathrm{E}-04 \\
5.31240 \mathrm{E}-04 \\
5.38023 \mathrm{E}-04 \\
5.44848 \mathrm{E}-04 \\
5.51716 \mathrm{E}-04\end{array}$ & $\begin{array}{l}-6.88115 \mathrm{E}-01 \\
-6.97084 \mathrm{E}-01 \\
-7.06111 \mathrm{E}-01 \\
-7.15196 \mathrm{E}-01 \\
-7.24338 \mathrm{E}-01\end{array}$ & $\begin{array}{l}-2.66333 \mathrm{E}-02 \\
-2.70937 \mathrm{E}-02 \\
-2.75593 \mathrm{E}-02 \\
-2.80300 \mathrm{E}-02 \\
-2.85060 \mathrm{E}-02\end{array}$ & $\begin{array}{l}2.92136 \mathrm{E} \\
2.92188 \mathrm{E} \\
2.92240 \mathrm{E} \\
2.92291 \mathrm{E} \\
2.92341 \mathrm{E}\end{array}$ & $\begin{array}{l}01 \\
01 \\
01 \\
01 \\
01\end{array}$ & $\begin{array}{l}-2.60102 \mathrm{E}-02 \\
-2.64591 \mathrm{E}-02 \\
-2.69132 \mathrm{E}-02 \\
-2.73723 \mathrm{E}-02 \\
-2.78365 \mathrm{E}-02\end{array}$ & $\begin{array}{l}7.33588 \mathrm{E}-01 \\
7.42848 \mathrm{E}-01 \\
7.52165 \mathrm{E}-01 \\
7.61539 \mathrm{E}-01 \\
7.70971 \mathrm{E}-01\end{array}$ & $\begin{array}{l}-3.25914 \mathrm{E}-02 \\
-3.25958 \mathrm{E}-02 \\
-3.26000 \mathrm{E}-02 \\
-3.26043 \mathrm{E}-02 \\
-3.26084 \mathrm{E}-02\end{array}$ \\
\hline $\begin{array}{l}16.10 \\
16.20 \\
16.30 \\
16.40 \\
16.50\end{array}$ & $\begin{array}{l}5.58627 \mathrm{E}-04 \\
5.65582 \mathrm{E}-04 \\
5.72579 \mathrm{E}-04 \\
5.79620 \mathrm{E}-04 \\
5.86703 \mathrm{E}-04\end{array}$ & $\begin{array}{l}-7.33538 \mathrm{E}-01 \\
-7.42795 \mathrm{E}-01 \\
-7.52109 \mathrm{E}-01 \\
-7.61482 \mathrm{E}-01 \\
-7.70911 \mathrm{E}-01\end{array}$ & $\begin{array}{l}-2.89872 \mathrm{E}-02 \\
-2.94738 \mathrm{E}-02 \\
-2.99656 \mathrm{E}-02 \\
-3.04627 \mathrm{E}-02 \\
-3.09652 \mathrm{E}-02\end{array}$ & $\begin{array}{l}2.92390 \mathrm{E} \\
2.92439 \mathrm{E} \\
2.92488 \mathrm{E} \\
2.92535 \mathrm{E} \\
2.92582 \mathrm{E}\end{array}$ & $\begin{array}{l}01 \\
01 \\
01 \\
01 \\
01\end{array}$ & $\begin{array}{l}-2.83060 \mathrm{E}-02 \\
-2.87806 \mathrm{E}-02 \\
-2.92604 \mathrm{E}-02 \\
-2.97454 \mathrm{E}-02 \\
-3.02358 \mathrm{E}-02\end{array}$ & $\begin{array}{l}7.80461 \mathrm{E}-01 \\
7.90008 \mathrm{E}-01 \\
7.99612 \mathrm{E}-01 \\
8.09274 \mathrm{E}-01 \\
8.18994 \mathrm{E}-01\end{array}$ & $\begin{array}{l}-3.26126 \mathrm{E}-02 \\
-3.26167 \mathrm{E}-02 \\
-3.26207 \mathrm{E}-02 \\
-3.26247 \mathrm{E}-02 \\
-3.26287 \mathrm{E}-02\end{array}$ \\
\hline $\begin{array}{l}16.60 \\
16.70 \\
16.80 \\
16.90 \\
17.00\end{array}$ & $\begin{array}{l}5.93829 \mathrm{E}-04 \\
6.00999 \mathrm{E}-04 \\
6.08211 \mathrm{E}-04 \\
6.15466 \mathrm{E}-04 \\
6.22765 \mathrm{E}-04\end{array}$ & $\begin{array}{l}-7.80399 \mathrm{E}-01 \\
-7.89943 \mathrm{E}-01 \\
-7.99545 \mathrm{E}-01 \\
-8.09205 \mathrm{E}-01 \\
-8.18922 \mathrm{E}-01\end{array}$ & $\begin{array}{l}-3.14732 \mathrm{E}-02 \\
-3.19865 \mathrm{E}-02 \\
-3.25053 \mathrm{E}-02 \\
-3.30295 \mathrm{E}-02 \\
-3.35592 \mathrm{E}-02\end{array}$ & $\begin{array}{l}2.92629 \mathrm{E} \\
2.92675 \mathrm{E} \\
2.92720 \mathrm{E} \\
2.92765 \mathrm{E} \\
2.92809 \mathrm{E}\end{array}$ & $\begin{array}{l}01 \\
01 \\
01 \\
01 \\
01\end{array}$ & $\begin{array}{l}-3.07314 \mathrm{E}-02 \\
-3.12323 \mathrm{E}-02 \\
-3.17386 \mathrm{E}-02 \\
-3.22503 \mathrm{E}-02 \\
-3.27674 \mathrm{E}-02\end{array}$ & $\begin{array}{l}8.28771 \mathrm{E}-01 \\
8.38605 \mathrm{E}-01 \\
8.48497 \mathrm{E}-01 \\
8.58446 \mathrm{E}-01 \\
8.68453 \mathrm{E}-01\end{array}$ & $\begin{array}{l}-3.26326 \mathrm{E}-02 \\
-3.26364 \mathrm{E}-02 \\
-3.26403 \mathrm{E}-02 \\
-3.26440 \mathrm{E}-02 \\
-3.26478 \mathrm{E}-02\end{array}$ \\
\hline $\begin{array}{l}17.10 \\
17.20 \\
17.30 \\
17.40 \\
17.50\end{array}$ & $\begin{array}{l}6.30106 \mathrm{E}-04 \\
6.37490 \mathrm{E}-04 \\
6.44918 \mathrm{E}-04 \\
6.52388 \mathrm{E}-04 \\
6.59902 \mathrm{E}-04\end{array}$ & $\begin{array}{l}-8.28696 \mathrm{E}-01 \\
-8.38528 \mathrm{E}-01 \\
-8.48418 \mathrm{E}-01 \\
-8.58364 \mathrm{E}-01 \\
-8.68368 \mathrm{E}-01\end{array}$ & $\begin{array}{l}-3.40945 \mathrm{E}-02 \\
-3.46353 \mathrm{E}-02 \\
-3.51818 \mathrm{E}-02 \\
-3.57338 \mathrm{E}-02 \\
-3.62914 \mathrm{E}-02\end{array}$ & $\begin{array}{l}2.92852 \mathrm{E} \\
2.92896 \mathrm{E} \\
2.92938 \mathrm{E} \\
2.92980 \mathrm{E} \\
2.93022 \mathrm{E}\end{array}$ & $\begin{array}{l}01 \\
01 \\
01 \\
01 \\
01\end{array}$ & $\begin{array}{l}-3.32899 \mathrm{E}-02 \\
-3.38178 \mathrm{E}-02 \\
-3.43513 \mathrm{E}-02 \\
-3.48903 \mathrm{E}-02 \\
-3.54348 \mathrm{E}-02\end{array}$ & $\begin{array}{l}8.78517 \mathrm{E}-01 \\
8.88638 \mathrm{E}-01 \\
8.98817 \mathrm{E}-01 \\
9.09053 \mathrm{E}-01 \\
9.19346 \mathrm{E}-01\end{array}$ & $\begin{array}{l}-3.26515 \mathrm{E}-02 \\
-3.26552 \mathrm{E}-02 \\
-3.26588 \mathrm{E}-02 \\
-3.26624 \mathrm{E}-02 \\
-3.26659 \mathrm{E}-02\end{array}$ \\
\hline $\begin{array}{l}17.60 \\
17.70 \\
17.80 \\
17.90 \\
18.00\end{array}$ & $\begin{array}{l}6.67458 \mathrm{E}-04 \\
6.75057 \mathrm{E}-04 \\
6.82700 \mathrm{E}-04 \\
6.90385 \mathrm{E}-04 \\
6.98113 \mathrm{E}-04\end{array}$ & $\begin{array}{l}-8.78430 \mathrm{E}-01 \\
-8.88549 \mathrm{E}-01 \\
-8.98725 \mathrm{E}-01 \\
-9.08958 \mathrm{E}-01 \\
-9.19249 \mathrm{E}-01\end{array}$ & $\begin{array}{l}-3.68548 \mathrm{E}-02 \\
-3.74238 \mathrm{E}-02 \\
-3.79986 \mathrm{E}-02 \\
-3.85790 \mathrm{E}-02 \\
-3.91653 \mathrm{E}-02\end{array}$ & $\begin{array}{l}2.93063 \mathrm{E} \\
2.93103 \mathrm{E} \\
2.93144 \mathrm{E} \\
2.93183 \mathrm{E} \\
2.93222 \mathrm{E}\end{array}$ & $\begin{array}{l}01 \\
01 \\
01 \\
01 \\
01\end{array}$ & $\begin{array}{l}-3.59849 \mathrm{E}-02 \\
-3.65406 \mathrm{E}-02 \\
-3.71019 \mathrm{E}-02 \\
-3.76689 \mathrm{E}-02 \\
-3.82416 \mathrm{E}-02\end{array}$ & $\begin{array}{l}9.29697 \mathrm{E}-01 \\
9.40105 \mathrm{E}-01 \\
9.50571 \mathrm{E}-01 \\
9.61093 \mathrm{E}-01 \\
9.71674 \mathrm{E}-01\end{array}$ & $\begin{array}{l}-3.26695 \mathrm{E}-02 \\
-3.26729 \mathrm{E}-02 \\
-3.26764 \mathrm{E}-02 \\
-3.26798 \mathrm{E}-02 \\
-3.26832 \mathrm{E}-02\end{array}$ \\
\hline
\end{tabular}


ELECTRON RADIAL FUNCTIONS

Electron

$Z=7 \quad A=14$

$\rho=1.3 A^{1 / 3} F$

18.10

18.20

18.30

18.40

18.50

18.60

18.70

18.80

18.90

19.00

19.10

19.20

19.30

19.40

19.50

19.60

19.70

19.80

19.90

20.00

20.10

20.20

20.30

20.40

20.50

20.60

20.70

20.80

20.90

21.00

21.10

21.20

21.30

21.40

21.50

21.60

21.70

21.80

21.90

22.00

22.10

22.20

22.30

22.40

22.50

22.60

22.70

22.80

22.90

23.00

23.10

23.20

23.30

23.40

23.50

23.60

23.70

23.80

23.90

24.00
$F_{0}$

$1.18049 \mathrm{E} \quad 00$ $1.18047 \mathrm{E} \quad 00$

$1.18045 \mathrm{E} \quad 00$

$1.18044 \mathrm{E} \quad 00$

$1.18042 \mathrm{E} \quad 00$

$1.18040 \mathrm{E} \quad 00$ $1.18038 \mathrm{E} \quad 00$ $1.18036 \mathrm{E} \quad 00$ $1.18034 \mathrm{E} \quad 00$

$1.18032 \mathrm{E} \quad 00$

$1.18030 \mathrm{E} \quad 00$ $1.18028 \mathrm{E} \quad 00$ $1.18026 \mathrm{E} \quad 00$ $1.18025 \mathrm{E} \quad 00$ $1.18023 \mathrm{E} \quad 00$

$1.18021 \mathrm{E} \quad 00$ $1.18019 \mathrm{E} \quad 00$ $1.18017 \mathrm{E} \quad 00$ $1.18016 \mathrm{E} \quad 00$ $1.18014 \mathrm{E} \quad 00$

$1.18012 \mathrm{E} \quad 00$ $1.18010 \mathrm{E} \quad 00$ $1.18009 \mathrm{E} \quad 00$ $1.18007 \mathrm{E} \quad 00$ $1.18005 \mathrm{E} \quad 00$

$1.18003 \mathrm{E} \quad 00$ $1.18002 \mathrm{E} \quad 00$ $1.18000 \mathrm{E} \quad 00$ $1.17998 \mathrm{E} \quad 00$ $1.17997 \mathrm{E} \quad 00$

$1.17995 \mathrm{E} \quad 00$ $1.17993 \mathrm{E} \quad 00$ $1.17992 \mathrm{E} \quad 00$ $1.17990 \mathrm{E} \quad 00$ $1.17988 \mathrm{E} \quad 00$

$1.17987 \mathrm{E} \quad 00$ $1.17985 \mathrm{E} \quad 00$ $1.17984 \mathrm{E} \quad 00$ $1.17982 \mathrm{E} \quad 00$ $1.17980 \mathrm{E} \quad 00$

$1.17979 \mathrm{E} \quad 00$ $1.17977 \mathrm{E} \quad 00$ $1.17976 \mathrm{E} \quad 00$ $1.17974 \mathrm{E} \quad 00$ $1.17973 \mathrm{E} \quad 00$

$1.17971 \mathrm{E} \quad 00$ $1.17970 \mathrm{E} \quad 00$ $1.17968 \mathrm{E} \quad 00$ $1.17967 \mathrm{E} \quad 00$ $1.17965 \mathrm{E} \quad 00$

$1.17964 \mathrm{E} \quad 00$ $1.17962 \mathrm{E} \quad 00$ $1.17961 \mathrm{E} \quad 00$ $1.17959 \mathrm{E} \quad 00$ $1.17958 \mathrm{E} \quad 00$

$1.17956 \mathrm{E} \quad 00$ $1.17955 \mathrm{E} \quad 00$ $1.17954 \mathrm{E} \quad 00$ $1.17952 \mathrm{E} \quad 00$ $1.17951 \mathrm{E} \quad 00$ $f_{1}$

$\begin{array}{ll}-1.89073 \mathrm{E} & 01\end{array}$ $-1.90131 \mathrm{E} 01$ $-1.91189 \mathrm{E} \quad 01$ $-1.92247 \mathrm{E}$ 01 $-1.93305 \mathrm{E} 01$

$-1.94362 \mathrm{E} 01$ $-1.95419 \mathrm{E} 01$ $-1.96476 \mathrm{E} 01$ $-1.97533 \mathrm{E} \quad 01$ $-1.98590 \mathrm{E} \quad 01$

$-1.99646 \mathrm{E} \quad 01$ $\begin{array}{ll}-2.00702 \mathbf{E} & 01\end{array}$ $-2.01758 \mathrm{E} \quad 01$ $\begin{array}{lll}-2.02814 \mathrm{E} & 01\end{array}$ $-2.03869 \mathrm{E} 01$

$\begin{array}{ll}-2.04925 \mathrm{E} & 01\end{array}$ $-2.05980 \mathrm{E} \quad 01$ $-2.07034 \mathrm{E} 01$ $-2.08089 \mathrm{E} \quad 01$ $-2.09143 \mathrm{E} \quad 01$

$\begin{array}{lll}-2.10197 \mathrm{E} & 01\end{array}$ $-2.11251 \mathrm{E} 01$ $-2.12305 \mathrm{E} \quad 01$ $-2.13358 \mathrm{E} \quad 01$ $-2.14411 \mathrm{E} \quad 01$

$-2.15464 \mathrm{E} \quad 01$ $-2.16517 \mathrm{E} 01$ $-2.17569 \mathrm{E} 01$ $-2.18621 \mathrm{E} 01$ $-2.19673 \mathrm{E} 01$

$-2.20725 \mathrm{E} \quad 01$ $-2.21776 \mathrm{E}$ ol $-2.22828 \mathrm{E} \quad 01$ $-2.23879 \mathrm{E} 01$ $-2.24929 \mathrm{E} 01$

$-2.25980 \mathrm{E} 01$ $-2.27030 \mathrm{E} 01$ $-2.28080 \mathrm{E} 01$ $-2.29130 \mathrm{E} 01$ $-2.30180 \mathrm{E} 01$.

$-2.31229 \mathrm{E} \quad 01$ $-2.32278 \mathrm{E} 01$ $-2.33327 \mathrm{E}$ 0l $-2.34375 \mathrm{E} 01$ $-2.35424 \mathrm{E}$ 0l

$-2.36472 \mathrm{E} 01$ $-2.37520 \mathrm{E}$ 01 $-2.38567 \mathrm{E} 01$ $-2.39615 \mathrm{E} 01$ $-2.40662 \mathrm{E} 01$

\section{$-2.41708 \mathrm{E} 01$} $-2.42755 \mathrm{E} \quad 01$ $-2.43801 \mathrm{E} 01$ $-2.44847 \mathrm{E} \quad 01$ $-2.45893 \mathrm{E} 01$

$-2.46939 \mathrm{E} \quad 01$ $-2.47984 \mathrm{E} \quad 01$ $-2.49029 \mathrm{E} 01$ $-2.50074 \mathrm{E} 01$ $-2.51119 \mathrm{E} 01$ $g_{1}$

$-1.36851 \mathrm{E} \quad 00$ $-1.38135 \mathrm{E} \quad 00$ $-1.39425 \mathrm{E} 00$ $-1.40721 \mathrm{E} 00$

$-1.42022 \mathrm{E} 00$

$-1.43329 \mathrm{E} \quad 00$

$-1.44641 \mathrm{E} \quad 00$

$-1.45959 \mathrm{E} \quad 00$

$-1.47283 \mathrm{E} 00$

$-1.48612 \mathrm{E} \quad 00$

$-1.49947 \mathrm{E} \quad 00$ $-1.51288 \mathrm{E} \quad 00$

$-1.52634 \mathrm{E} \quad 00$

$-1.53986 \mathrm{E} \quad 00$

$-1.55344 \mathrm{E} \quad 00$

$-1.56707 \mathrm{E} \quad 00$

$-1.58076 \mathrm{E} \quad 00$

$-1.59450 \mathrm{E} \quad 00$

$-1.60830 \mathrm{E} \quad 00$

$-1.62216 \mathrm{E} \quad 00$

$-1.63608 \mathrm{E} \quad 00$

$-1.65004 \mathrm{E} \quad 00$

$-1.66407 \mathrm{E} \quad 00$

$-1.67815 \mathrm{E} \quad 00$

$-1.69229 \mathrm{E} 00$

$-1.70649 \mathrm{E} \quad 00$ $-1.72074 \mathrm{E} \quad 00$

$-1.73504 \mathrm{E} \quad 00$

$-1.74941 \mathrm{E} \quad 00$

$-1.76382 \mathrm{E} \quad 00$

$-1.77830 \mathrm{E} \quad 00$

$-1.79283 \mathrm{E} 00$

$-1.80742 \mathrm{E} \quad 00$

$-1.82206 \mathrm{E} \quad 00$

$-1.83676 \mathrm{E} 00$

$-1.85152 \mathrm{E} \quad 00$

$-1.86633 \mathrm{E} \quad 00$

$-1.88119 \mathrm{E} \quad 00$

$-1.89612 \mathrm{E} 00$

$-1.91110 \mathrm{E} 00$

$-1.92613 \mathrm{E} \quad 00$ $-1.94122 \mathrm{E} 00$

$-1.95637 \mathrm{E} 00$

$-1.97157 \mathrm{E} \quad 00$

$-1.98683 \mathrm{E} 00$

$-2.00215 \mathrm{E} \quad 00$

$-2.01752 \mathrm{E} \quad 00$

$-2.03294 \mathrm{E} \quad 00$

$-2.04842 \mathrm{E} \quad 00$

$-2.06396 \mathrm{E} \quad 00$

$-2.07956 \mathrm{E} \quad 00$

$-2.09520 \mathrm{E} \quad 00$

$-2.11091 \mathrm{E} 00$

$-2.12667 \mathrm{E} \quad 00$

$-2.14249 \mathrm{E} 00$

$-2.15836 \mathrm{E} \quad 00$

$-2.17429 \mathrm{E} \quad 00$

$-2.19027 \mathrm{E} \quad 00$

$-2.20631 \mathrm{E} \quad 00$

$-2.22240 \mathrm{E} \quad 00$

$\tan \delta_{1}$

$4.51386 \mathrm{E}-03$

$4.52016 \mathrm{E}-03$

$4.52638 \mathrm{E}-03$

$4.53250 \mathrm{E}-03$

$4.53853 \mathrm{E}-03$

$4.54447 \mathrm{E}-03$

$4.55033 \mathrm{E}-03$

$4.55611 \mathrm{E}-03$

$4.56179 \mathrm{E}-03$

$4.56740 \mathrm{E}-03$

$4.57293 \mathrm{E}-03$

$4.57837 \mathrm{E}-03$

$4.58375 \mathrm{E}-03$

$4.58903 \mathrm{E}-03$

$4.59424 \mathrm{E}-03$

$4.59938 \mathrm{E}-03$

$4.60444 \mathrm{E}-03$

$4.60943 \mathrm{E}-03$

$4.61435 \mathrm{E}-03$

$4.61919 \mathrm{E}-03$

$4.62396 \mathrm{E}-03$ $4.62867 \mathrm{E}-03$

$4.63330 \mathrm{E}-03$

$4.63787 \mathrm{E}-03$

$4.64236 \mathrm{E}-03$

$4.64680 \mathrm{E}-03$

$4.65117 \mathrm{E}-03$

$4.65547 \mathrm{E}-03$

$4.65970 \mathrm{E}-03$

4.66388E-03

$4.66800 \mathrm{E}-03$

$4.67204 \mathrm{E}-03$

$4.67604 \mathrm{E}-03$

$4.67997 \mathrm{E}-03$

$4.68384 \mathrm{E}-03$

$4.68766 \mathrm{E}-03$

$4.69141 \mathrm{E}-03$

$4.69511 \mathrm{E}-03$

$4.69875 \mathrm{E}-03$

4.70234 E-03 
ELECTRON RADIAL FUNCTIONS

$Z=7 \quad A=14 \quad \rho=1.3 A^{1 / 3} \mathrm{~F}$

Electron

$\bar{F}$

$7.05885 \mathrm{E}-04$

$7.13700 \mathrm{E}-04$

$7.21557 \mathrm{E}-04$

$7.29458 \mathrm{E}-04$

$7.37401 \mathrm{E}-04$

$7.45387 \mathrm{E}-04$

$7.53417 \mathrm{E}-04$

$7.61489 \mathrm{E}-04$

$7.69604 \mathrm{E}-04$

$7.77763 \mathrm{E}-04$

7.85964. E- 04

$7.94208 \mathrm{E}-04$

$8.02496 \mathrm{E}-04$

$8.10826 \mathrm{E}-04$

$8.19199 \mathrm{E}-04$.

$8.27616 \mathrm{E}-04$ $8.36075 \mathrm{E}-04$

$8.44577 \mathrm{E}-04$

$8.53123 \mathrm{E}-04$

8.61711 E-04

$8.70342 \mathrm{E}-04$

$8.79016 \mathrm{E}-04$

$8.87734 \mathrm{E}-04$

$8.96494 \mathrm{E}-04$

$9.05297 \mathrm{E}-04$

$9.14144 \mathrm{E}-04$ $9.23033 \mathrm{E}-04$

$9.31965 \mathrm{E}-04$

$9.40940 \mathrm{E}-04$

$9.49958 \mathrm{E}-04$

$9.59019 \mathrm{E}-04$

$9.68124 \mathrm{E}-04$

$9.77271 \mathrm{E}-04$

$9.86461 \mathrm{E}-04$

$9.95694 \mathrm{E}-04$

$1.00497 \mathrm{E}-03$

$1.01429 \mathrm{E}-03$

$1.02365 \mathrm{E}-03$

$1.03306 \mathrm{E}-03$

$1.04250 \mathrm{E}-03$

$1.05200 \mathrm{E}-03$

$1.06153 \mathrm{E}-03$

$1.07111 \mathrm{E}-03$

$1.08073 \mathrm{E}-03$

$1.09039 \mathrm{E}-03$

$1.10010 \mathrm{E}-03$

$1.10985 \mathrm{E}-03$

$1.11964 \mathrm{E}-03$

$1.12947 \mathrm{E}-03$

$1.13935 \mathrm{E}-03$

$1.14927 \mathrm{E}-03$

$1.15924 \mathrm{E}-03$

$1.16924 \mathrm{E}-03$

$1.17929 \mathrm{E}-03$

$1.18939 \mathrm{E}-03$

$1.19952 \mathrm{E}-03$

$1.20970 \mathrm{E}-03$

$1.21992 \mathrm{E}-03$

$1.23019 \mathrm{E}-03$

$1.24049 \mathrm{E}-03$ $f_{2}$

$-9.29598 \mathrm{E}-01$

$-9.40004 \mathrm{E}-01$

$-9.50466 \mathrm{E}-01$

$-9.60987 \mathrm{E}-01$

$-9.71564 \mathrm{E}-01$

$-9.82199 \mathrm{E}-01$

$-9.92890 \mathrm{E}-01$

$-1.00364 \mathrm{E} \quad 00$

$-1.01445 \mathrm{E} \quad 00$

$-1.02531 \mathrm{E} 00$

$-1.03623 \mathrm{E} \quad 00$

$-1.04721 \mathrm{E} 00$

$-1.05824 \mathrm{E} \quad 00$

$-1.06934 \mathrm{E} 00$

$-1.08048 \mathrm{E} \quad 00$

$-1.09169 \mathrm{E} \quad 00$

$-1.10295 \mathrm{E} \quad 00$

$-1.11428 \mathrm{E} \quad 00$

$-1.12565 \mathrm{E} \quad 00$

$-1.13709 \mathrm{E} 00$

$-1.14858 \mathrm{E} \quad 00$

$-1.16013 \mathrm{E} 00$

$-1.17174 \mathrm{E} 00$

$-1.18340 \mathrm{E} \quad 00$

$-1.19512 \mathrm{E} \quad 00$

$-1.20690 \mathrm{E} \quad 00$

$-1.21873 \mathrm{E} \quad 00$

$-1.23062 \mathrm{E} 00$

$-1.24257 \mathrm{E} 00$

$-1.25457 \mathrm{E} 00$

$-1.26664 \mathrm{E} \quad 00$

$-1.27876 \mathrm{E} 00$

$-1.29093 \mathrm{E} 00$

$-1.30316 \mathrm{E} 00$

$-1.31545 \mathrm{E} \quad 00$

$-1.32780 \mathrm{E} \quad 00$

$-1.34020 \mathrm{E} \quad 00$

$-1.35266 \mathrm{E} 00$

$-1.36518 \mathrm{E} \quad 00$

$-1.37776 \mathrm{E} 00$

$-1.39039 \mathrm{E} \quad 00$

$-1.40308 \mathrm{E} \quad 00$

$-1.41582 \mathrm{E} \quad 00$

$-1.42862 \mathrm{E} \quad 00$

$-1.44148 \mathrm{E} \quad 00$

$-1.45439 \mathrm{E} \quad 00$

$-1.46736 \mathrm{E} 00$

$-1.48039 \mathrm{E} \quad 00$

$-1.49348 \mathrm{E} 00$

$-1.50662 \mathrm{E} \quad 00$

$-1.51982 \mathrm{E} \quad 00$

$-1.53307 \mathrm{E} \quad 00$

$-1.54638 \mathrm{E} 00$

$-1.55975 \mathrm{E} \quad 00$

$-1.57318 \mathrm{E} 00$

$-1.58666 \mathrm{E} \quad 00$

$-1.60020 \mathrm{E} 00$

$-1.61379 \mathrm{E} 00$

$-1.62744 \mathrm{E} 00$

$-1.64115 \mathrm{E} 00$ $g_{2}$

$-3.97574 \mathrm{E}-02$

$-4.03554 \mathrm{E}-02$

$-4.09591 \mathrm{E}-02$

$-4.15688 \mathrm{E}-02$

$-4.21844 \mathrm{E}-02$

$-4.28059 \mathrm{E}-02$

$-4.34334 \mathrm{E}-02$

$-4.40669 \mathrm{E}-02$

$-4.47064 \mathrm{E}-02$

$-4.53520 \mathrm{E}-02$

$-4.60037 \mathrm{E}-02$

$-4.66615 \mathrm{E}-02$

$-4.73254 \mathrm{E}-02$

$-4.79954 \mathrm{E}-02$

$-4.86717 \mathrm{E}-02$

$-4.93542 \mathrm{E}-02$

$-5.00429 \mathrm{E}-02$

$-5.07379 \mathrm{E}-02$

$-5.14392 \mathrm{E}-02$

$-5.21469 \mathrm{E}-02$

$-5.28609 \mathrm{E}-02$

$-5.35813 \mathrm{E}-02$

$-5.43081 \mathrm{E}-02$

$-5.50413 \mathrm{E}-02$

$-5.57810 \mathrm{E}-02$

$-5.65272 \mathrm{E}-02$

$-5.72799 \mathrm{E}-02$

$-5.80392 \mathrm{E}-02$

$-5.88051 \mathrm{E}-02$

$-5.95775 \mathrm{E}-02$

$-6.03566 \mathrm{E}-02$

$-6.11424 \mathrm{E}-02$

$-6.19348 \mathrm{E}-02$

$-6.27339 \mathrm{E}-02$

$-6.35398 \mathrm{E}-02$

$-6.43525 \mathrm{E}-02$

$-6.51720 \mathrm{E}-02$

$-6.59982 \mathrm{E}-02$

$-6.68314 \mathrm{E}-02$

$-6.76714 \mathrm{E}-02$

$-6.85183 \mathrm{E}-02$

$-6.93722 \mathrm{E}-02$

$-7.02330 \mathrm{E}-02$

$-7.11008 \mathrm{E}-02$

$-7.19757 \mathrm{E}-02$

$-7.28575 \mathrm{E}-02$

$-7.37465 \mathrm{E}-02$

$-7.46425 \mathrm{E}-02$

$-7.55457 \mathrm{E}-02$

$-7.64560 \mathrm{E}-02$

$-7.73735 \mathrm{E}-02$

$-7.82982 \mathrm{E}-02$

$-7.92301 \mathrm{E}-02$

$-8.01693 \mathrm{E}-02$

$-8.11158 \mathrm{E}-02$

$-8.20696 \mathrm{E}-02$

$-8.30307 \mathrm{E}-02$

$-8.39993 \mathrm{E}-02$

$-8.49752 \mathrm{E}-02$

$-8.59585 \mathrm{E}-02$ $\tan \delta_{2}$

$f_{-2}$

$2.93261 \mathrm{E} \quad 01 \quad-3.88200 \mathrm{E}-02$

$2.93300 \mathrm{E} \quad 01 \quad-3.94041 \mathrm{E}-02$

$2.93337 \mathrm{E}$ 01 $-3.99940 \mathrm{E}-02$

$2.93375 \mathrm{E} \quad 01 \quad-4.05897 \mathrm{E}-02$

$2.93412 \mathrm{E} 01 \quad-4.11912 \mathrm{E}-02$

$2.93448 \mathrm{E} \quad 01$

$2.93485 \mathrm{E}$ 01

$2.93521 \mathrm{E} \quad 01$

$2.93556 \mathrm{E} \quad 01$

$2.93591 \mathrm{E} \quad 01$

$2.93626 \mathrm{E} \quad 01$

$2.93660 \mathrm{E} \quad 01$

$2.93694 \mathrm{E} \quad 01$

$2.93727 \mathrm{E} \quad 01$

$2.93760 \mathrm{E} \quad 01$

$2.93793 \mathrm{E} \quad 01$

$2.93826 \mathrm{E} \quad 01$

$2.93858 \mathrm{E} \quad 01$

$2.93890 \mathrm{E} \quad 01$

$2.93921 \mathrm{E} \quad 01$

$2.93952 \mathrm{E} \quad 01$

$2.93983 \mathrm{E} \quad 01$

$2.94014 \mathrm{E} \quad 01$

$2.94044 \mathrm{E} \quad 01$

$2.94074 \mathrm{E} \quad 01$

$2.94103 \mathrm{E} \quad 01$

$2.94133 \mathrm{E} 01$

$2.94162 \mathrm{E} \quad 01$

$2.94190 \mathrm{E} \quad 01$

$2.94219 \mathrm{E} \quad 01$

$2.94247 \mathrm{E} \quad 01$ $2.94275 \mathrm{E} 01$ $2.94302 \mathrm{E} \quad 01$

$2.94330 \mathrm{E} \quad 01$

$2.94357 \mathrm{E} \quad 01$

2.94384E 01

$2.94410 \mathrm{E} \quad 01$

$2.94436 \mathrm{E} \quad 01$

$2.94463 \mathrm{E} 01$

$2.94488 \mathrm{E} 01$

$2.94514 \mathrm{E} \quad 01$ $2.94539 \mathrm{E} \quad 01$

$2.94564 \mathrm{E} \quad 01$

$2.94589 \mathrm{E} \quad 01$

$2.94614 \mathrm{E} \quad 01$

$2.94638 \mathrm{E} \quad 01$

$2.94662 \mathrm{E} 01$

$2.94686 \mathrm{E} 01$

$2.94710 \mathrm{E} \quad 01$

$2.94734 \mathrm{E} \quad 01$

$2.94757 \mathrm{E} \quad 01$

$2.94780 \mathrm{E} \quad 01$

$2.94803 \mathrm{E} \quad 01$

$2.94826 \mathrm{E} \quad 01$

$2.94848 \mathrm{E} 01$

$2.94871 \mathrm{E} \quad 01$ $2.94893 \mathrm{E} 01$

$2.94914 \mathrm{E} \quad 01$

$2.94936 \mathrm{E} \quad 01$

$2.94958 \mathrm{E} 0 \mathrm{l}$

$$
\begin{aligned}
& -4.17986 \mathrm{E}-02 \\
& -4.24118 \mathrm{E}-02 \\
& -4.30309 \mathrm{E}-02 \\
& -4.36560 \mathrm{E}-02 \\
& -4.42870 \mathrm{E}-02
\end{aligned}
$$

$-4.49240 \mathrm{E}-02$

$-4.55671 \mathrm{E}-02$

$-4.62162 \mathrm{E}-02$

$-4.68713 \mathrm{E}-02$

$-4.75326 \mathrm{E}-02$

$-4.81999 \mathrm{E}-02$

$-4.88735 \mathrm{E}-02$

$-4.95532 \mathrm{E}-02$

$-5.02391 \mathrm{E}-02$

$-5.09312 \mathrm{E}-02$

$-5.16297 \mathrm{E}-02$

$-5.23344 \mathrm{E}-02$

$-5.30454 \mathrm{E}-02$

$-5.37628 \mathrm{E}-02$

$-5.44865 \mathrm{E}-02$

$-5.52167 \mathrm{E}-02$

$-5.59532 \mathrm{E}-02$

$-5.66963 \mathrm{E}-02$

$-5.74458 \mathrm{E}-02$

$-5.82018 \mathrm{E}-02$

$-5.89644 \mathrm{E}-02$

$-5.97335 \mathrm{E}-02$

$-6.05093 \mathrm{E}-02$

$-6.12916 \mathrm{E}-02$

$-6.20806 \mathrm{E}-02$

$-6.28763 \mathrm{E}-02$

$-6.36787 \mathrm{E}-02$

$-6.44878 \mathrm{E}-02$

$-6.53037 \mathrm{E}-02$

$-6.61263 \mathrm{E}-02$

$-6.69558 \mathrm{E}-02$

$-6.77921 \mathrm{E}-02$

$-6.86353 \mathrm{E}-02$

$-6.94853 \mathrm{E}-02$

$-7.03423 \mathrm{E}-02$

$-7.12063 \mathrm{E}-02$ 
ELECTRON RADIAL FUNCTIONS

Electron

$$
Z=7 \quad A=14 \quad \rho=1.3 A^{1 / 3} F
$$

p $\quad F_{0}$

24.10

24.20

24.30

24.40

24.50

24.60

24.70

24.80

24.90

25.00

25.10

25.20

25.30

25.40

25.50

25.60

25.70

25.80

25.90

26.00

26.10

26.20

26.30

26.40

26.50

26.60

26.70

26.80

26.90

27.00

27.10

27.20

27.30

27.40

27.50

27.60

27.70

27.80

27.90

28.00

28.10

28.20

28.30

28.40

28.50

28.60

28.70

28.80

28.90

29.00

29.10

29.20

29.30

29.40

29.50

29.60

29.70

29.80

29.90

30.00 $f_{1}$

$g_{1}$

$1.17949 \mathrm{E} \quad 00$ $1.17948 \mathrm{E} \quad 00$ $1.17947 \mathrm{E} \quad 00$ $1.17945 \mathrm{E} \quad 00$ $1.17944 \mathrm{E} \quad 00$

$1.17942 \mathrm{E} \quad 00$ $1.17941 \mathrm{E} \quad 00$ $1.17940 \mathrm{E} \quad 00$ $1.17938 \mathrm{E} \quad 00$ $1.17937 \mathrm{E} \quad 00$

$1.17936 \mathrm{E} \quad 00$ $1.17934 \mathrm{E} \quad 00$ $1.17933 \mathrm{E} \quad 00$ $1.17932 \mathrm{E} \quad 00$ $1.17930 \mathrm{E} \quad 00$

$1.17929 \mathrm{E} \quad 00$ $1.17928 \mathrm{E} \quad 00$ $1.17926 \mathrm{E} \quad 00$ $1.17925 \mathrm{E} \quad 00$ $1.17924 \mathrm{E} \quad 00$

$1.17923 \mathrm{E} \quad 00$ $1.17921 \mathrm{E} \quad 00$ $1.17920 \mathrm{E} \quad 00$ $1.17919 \mathrm{E} \quad 00$ $1.17917 \mathrm{E} \quad 00$

$1.17916 \mathrm{E} \quad 00$ $1.17915 \mathrm{E} \quad 00$ $1.17914 \mathrm{E} \quad 00$ $1.17912 \mathrm{E} \quad 00$ $1.17911 \mathrm{E} \quad 00$

$1.17910 \mathrm{E} \quad 00$ $1.17909 \mathrm{E} \quad 00$ $1.17908 \mathrm{E} \quad 00$ $1.17906 \mathrm{E} \quad 00$ $1.17905 \mathrm{E} \quad 00$

$1.17904 \mathrm{E} \quad 00$ $1.17903 \mathrm{E} \quad 00$ $1.17902 \mathrm{E} \quad 00$ $1.17900 \mathrm{E} \quad 00$ $1.17899 \mathrm{E} \quad 00$

$1.17898 \mathrm{E} \quad 00$ $1.17897 \mathrm{E} \quad 00$ $1.17896 \mathrm{E} \quad 00$ $1.17895 \mathrm{E} \quad 00$
0 $1.17893 \mathrm{E} \quad 00$

$1.17892 \mathrm{E} \quad 00$ $1.17891 \mathrm{E} \quad 00$ $1.17890 \mathrm{E} \quad 00$ $1.17889 \mathrm{E} \quad 00$ $1.17888 \mathrm{E} \quad 00$

$1.17886 \mathrm{E} \quad 00$ $1.17885 \mathrm{E} \quad 00$ $1.17884 \mathrm{E} \quad 00$ $1.17883 \mathrm{E} \quad 00$ $1.17882 \mathrm{E} \quad 00$

$1.17881 \mathrm{E} \quad 00$ $\begin{array}{ll}1.17880 \mathrm{E} & 00\end{array}$ $1.17879 \mathrm{E} \quad 00$ $1.17878 \mathrm{E} \quad 00$ $1.17876 \mathrm{E} \quad 00$ $-2.52163 \mathrm{E} \quad 01$ $-2.53207 \mathrm{E} 01$ $-2.54251 \mathrm{E} 01$ $-2.55294 \mathrm{E} 01$ $-2.56337 \mathrm{E} 01$

$-2.57380 \mathrm{E} \quad 01$ $-2.58423 \mathrm{E} 01$ $-2.59466 \mathrm{E} 01$ $-2.60508 \mathrm{E} 01$ $-2.61550 \mathrm{E} 01$

$-2.62592 \mathrm{E} 01$ $-2.63633 \mathrm{E} 01$ $-2.64674 \mathrm{E} \quad 01$ $-2.65715 \mathrm{E} 01$ $-2.66756 \mathrm{E} 01$

$\begin{array}{ll}-2.67796 \mathrm{E} & 01\end{array}$ $-2.68836 \mathrm{E} \quad 01$ $-2.69876 \mathrm{E} \quad 01$ $-2.70915 \mathrm{E} \quad 01$ $-2.71955 \mathrm{E} 01$

$\begin{array}{lll}-2.72994 \mathrm{E} & 01\end{array}$ $\begin{array}{lll}-2.74033 \mathrm{E} & 01\end{array}$ $-2.75071 \mathrm{E} 01$ $-2.76109 \mathrm{E} \quad 01$ $-2.77147 \mathrm{E} \quad 01$

$-2.78185 \mathrm{E} 01$ $-2.79222 \mathrm{E} \quad 01$ $-2.80259 \mathrm{E}$ 0l $-2.81296 \mathrm{E} 01$ $\begin{array}{lll}-2.82333 \mathrm{E} & 01\end{array}$

$-2.83369 \mathrm{E} 01$ $-2.84405 \mathrm{E} \quad 01$ $-2.85441 \mathrm{E} 01$ $-2.86476 \mathrm{E} 01$ $-2.87511 \mathrm{E} 01$

$-2.88546 \mathrm{E} \quad 01$ $\begin{array}{lll}-2.89581 \mathrm{E} & 01\end{array}$ $-2.90615 \mathrm{E} \quad 01$ $-2.91649 \mathrm{E} \quad 01$

$\begin{array}{cc}g_{1} & \\ -2.23855 \mathrm{E} & 00 \\ -2.25476 \mathrm{E} & 00 \\ -2.27102 \mathrm{E} & 00 \\ -2.28734 \mathrm{E} & 00 \\ -2.30371 \mathrm{E} & 00\end{array}$

$-2.32014 \mathrm{E} \quad 00$ $-2.33662 \mathrm{E} \quad 00$ $-2.35316 \mathrm{E} \quad 00$ $-2.36975 \mathrm{E} \quad 00$ $-2.38640 \mathrm{E} \quad 00$

$-2.40311 \mathrm{E} \quad 00$ $-2.41987 \mathrm{E} \quad 00$ $-2.43668 \mathrm{E} \quad 00$ $-2.45355 \mathrm{E} \quad 00$ $-2.47048 \mathrm{E} \quad 00$

$-2.48746 \mathrm{E} \quad 00$ $-2.50450 \mathrm{E} \quad 00$ $-2.52159 \mathrm{E} \quad 00$ $-2.53874 \mathrm{E} \quad 00$ $-2.55594 \mathrm{E} \quad 00$

$-2.57320 \mathrm{E} \quad 00$ $-2.59051 \mathrm{E} \quad 00$ $-2.60788 \mathrm{E} \quad 00$ $-2.62531 \mathrm{E} \quad 00$ $-2.64278 \mathrm{E} \quad 00$

$-2.66032 \mathrm{E} \quad 00$ $-2.67791 \mathrm{E} \quad 00$ $-2.69555 \mathrm{E} \quad 00$ $-2.71325 \mathrm{E} \quad 00$ $-2.73101 \mathrm{E} \quad 00$

$-2.74881 \mathrm{E} \quad 00$ $-2.76668 \mathrm{E} \quad 00$ $-2.78460 \mathrm{E} \quad 00$ $-2.80257 \mathrm{E} \quad 00$ $-2.82060 \mathrm{E} \quad 00$

$-2.83869 \mathrm{E} \quad 00$ $-2.85683 \mathrm{E} \quad 00$ $-2.87502 \mathrm{E} \quad 00$ $-2.89327 \mathrm{E} \quad 00$ $-2.91157 \mathrm{E} \quad 00$

$-2.93717 \mathrm{E} 01$ $-2.94750 \mathrm{E} 01$ $-2.95783 \mathrm{E} 01$ $-2.96815 \mathrm{E} 01$ $-2.97848 \mathrm{E} 01$

$-2.92993 \mathrm{E} \quad 00$ $-2.94835 \mathrm{E} \quad 00$ $-2.96681 \mathrm{E} \quad 00$ $-2.98534 \mathrm{E} \quad 00$ $-3.00391 \mathrm{E} \quad 00$

$\begin{array}{llll}-2.98880 \mathrm{E} & 01 & -3.02255 \mathrm{E} & 00\end{array}$ $\begin{array}{llll}-2.99912 \mathrm{E} & 01 & -3.04123 \mathrm{E} & 00\end{array}$ $\begin{array}{llll}-3.00943 \mathrm{E} & 01 & -3.05998 \mathrm{E} & 00\end{array}$ $\begin{array}{llll}-3.01974 \mathrm{E} & 01 & -3.07877 \mathrm{E} & 00\end{array}$ $-3.03005 \mathrm{E} \quad 01$ $-3.09763 \mathrm{E} \quad 00$

$-3.04036 \mathrm{E} \quad 01$ $-3.05066 \mathrm{E} 01$ $-3.06097 \mathrm{E} \quad 01$ $-3.07126 \mathrm{E} \quad 01$

$-3.11653 \mathrm{E} \quad 00$ $-3.13549 \mathrm{E} \quad 00$ $-3.15451 \mathrm{E} \quad 00$ $-3.17358 \mathrm{E}$ 00 $-3.19270 \mathrm{E} \quad 00$

$\begin{array}{llll}-3.09185 \mathrm{E} & 01 & -3.21188 \mathrm{E} & 00\end{array}$ $-3.10214 \mathrm{E} \quad 01 \quad-3.23112 \mathrm{E} \quad 00$ $-3.11242 \mathrm{E}$ 01 $-3.25040 \mathrm{E} \quad 00$ $\begin{array}{llll}-3.12271 \mathrm{E} & 01 & -3.26975 \mathrm{E} & 00\end{array}$ $\begin{array}{llll}-3.13299 \mathrm{E} & 01 & -3.28914 \mathrm{E} & 00\end{array}$

$\tan \delta_{1}$

$f_{-1}$

$4.76581 \mathrm{E}-03$ $4.76832 \mathrm{E}-03$ $4.77079 \mathrm{E}-03$

$4.77320 \mathrm{E}-03$

$4.77558 \mathrm{E}-03$

$4.77791 \mathrm{E}-03$

4.78021 E-03

$4.78247 \mathrm{E}-03$

$4.78467 \mathrm{E}-03$

4.78684 E-03

$4.78896 \mathrm{E}-03$

$4.79106 \mathrm{E}-03$

$4.79311 \mathrm{E}-03$

$4.79513 \mathrm{E}-03$

$4.79710 \mathrm{E}-03$

4. $79903 \mathbf{E}-03$

$4.80094 \mathrm{E}-03$

$4.80280 \mathrm{E}-03$

$4.80462 \mathrm{E}-03$

$4.80641 \mathrm{E}-03$

$4.80816 \mathrm{E}-03$

$4.80987 \mathrm{E}-03$

$4.81154 \mathrm{E}-03$

$4.81319 \mathrm{E}-03$

$4.81480 \mathrm{E}-03$

$4.81637 \mathrm{E}-03$

$4.81791 \mathrm{E}-03$

$4.81942 \mathrm{E}-03$

$4.82089 \mathrm{E}-03$

$4.82232 \mathrm{E}-03$

$4.82372 \mathrm{E}-03$

$4.82510 \mathrm{E}-03$

$4.82644 \mathrm{E}-03$

$4.82775 \mathrm{E}-03$

$4.82902 \mathrm{E}-03$

$4.83026 \mathrm{E}-03$

$4.8314 .7 \mathrm{E}-03$

$4.83265 \mathrm{E}-03$

$4.83379 \mathrm{E}-03$

$4.83491 \mathrm{E}-03$

$4.83599 \mathrm{E}-03$

$4.83705 \mathrm{E}-03$

$4.83808 \mathrm{E}-03$

$4.83907 \mathrm{E}-03$

$4.84003 \mathrm{E}-03$

$4.84097 \mathrm{E}-03$

$4.84188 \mathrm{E}-03$

$4.84276 \mathrm{E}-03$

$4.84360 \mathrm{E}-03$

$4.84442 \mathrm{E}-03$

$4.84522 \mathrm{E}-03$

$4.84598 \mathrm{E}-03$

$4.84671 \mathrm{E}-03$

$4.84743 \mathrm{E}-03$

$4.84810 \mathrm{E}-03$

$4.84876 \mathrm{E}-03$ $4.84939 \mathrm{E}-03$

$4.84999 \mathrm{E}-03$ 
ELECTRON RADIAL FUNCTIONS

$Z=7 \quad A=14 \quad \rho=1.3 A^{1 / 3} F$

Electron

$\bar{F}$

$1.25085 \mathrm{E}-03$

$1.26124 \mathrm{E}-03$

$1.27168 \mathrm{E}-03$

$1.28216 \mathrm{E}-03$

$1.29268 \mathrm{E}-03$

$1.30324 \mathrm{E}-03$

$1.31385 \mathrm{E}-03$

$1.32450 \mathrm{E}-03$

$1.33520 \mathrm{E}-03$

$1.34594 \mathrm{E}-03$

$1.35672 \mathrm{E}-03$

$1.36754 \mathrm{E}-03$

$1.37841 \mathrm{E}-03$

$1.38932 \mathrm{E}-03$

$1.40027 \mathrm{E}-03$

$1.41126 \mathrm{E}-03$

$1.42230 \mathrm{E}-03$

$1.43338 \mathrm{E}-03$

$1.44451 \mathrm{E}-03$

$1.45568 \mathrm{E}-03$

$1.46689 \mathrm{E}-03$

$1.47814 \mathrm{E}-03$

$1.48944 \mathrm{E}-03$

$1.50078 \mathrm{E}-03$

$1.51216 \mathrm{E}-03$

$1.52358 \mathrm{E}-03$ $1.53505 \mathrm{E}-03$

$1.54656 \mathrm{E}-03$

$1.55812 \mathrm{E}-03$

$1.56971 \mathrm{E}-03$

$1.58135 \mathrm{E}-03$

$1.59304 \mathrm{E}-03$

$1.60476 \mathrm{E}-03$

$1.61653 \mathrm{E}-03$

$1.62834 \mathrm{E}-03$

$1.64020 \mathrm{E}-03$

$1.65210 \mathrm{E}-03$

$1.66404 \mathrm{E}-03$

$1.67602 \mathrm{E}-03$

$1.68805 \mathrm{E}-03$

$1.70012 \mathrm{E}-03$

$1.71223 \mathrm{E}-03$

$1.72439 \mathrm{E}-03$

$1.73659 \mathrm{E}-03$

$1.74883 \mathrm{E}-03$

$1.76111 \mathrm{E}-03$

$1.77344 \mathrm{E}-03$

1.78581 E-03

$1.79823 \mathrm{E}-03$

$1.81068 \mathrm{E}-03$

$1.82318 \mathrm{E}-03$

$1.83572 \mathrm{E}-03$

$1.84831 \mathrm{E}-03$

$1.86094 \mathrm{E}-03$

$1.87361 \mathrm{E}-03$

$1.88632 \mathrm{E}-03$

$1.89908 \mathrm{E}-03$

$1.91188 \mathrm{E}-03$

$1.92473 \mathrm{E}-03$

$1.93761 \mathrm{E}-03$ $f_{2}$

$-1.65491 \mathrm{E} \quad 00$

$-1.66874 \mathrm{E} \quad 00$

$-1.68261 \mathrm{E} \quad 00$

$-1.69655 \mathrm{E} \quad 00$

$-1.71054 \mathrm{E} \quad 00$

$-1.72458 \mathrm{E} \quad 00$

$-1.73869 \mathrm{E} 00$

$-1.75285 \mathrm{E} 00$

$-1.76706 \mathrm{E} 00$

$-1.78133 \mathrm{E} 00$

$-1.79566 \mathrm{E} \quad 00$

$-1.81005 \mathrm{E} \quad 00$

$-1.82449 \mathrm{E} \quad 00$

$-1.83899 \mathrm{E} \quad 00$

$-1.85354 \mathrm{E} \quad 00$

$-1.86815 \mathrm{E} \quad 00$

$-1.88282 \mathrm{E} 00$

$-1.89754 \mathrm{E} 00$

$-1.91232 \mathrm{E} 00$

$-1.92716 \mathrm{E} 00$

$-1.94205 \mathrm{E} \quad 00$

$-1.95700 \mathrm{E} 00$

$-1.97200 \mathrm{E} 00$

$-1.98706 \mathrm{E} 00$

$-2.00218 \mathrm{E} 00$

$-2.01735 \mathrm{E} \quad 00$

$-2.03258 \mathrm{E} \quad 00$

$-2.04786 \mathrm{E} \quad 00$

$-2.06321 \mathrm{E} \quad 00$

$-2.07860 \mathrm{E} \quad 00$

$-2.09406 \mathrm{E} \quad 00$

$-2.10956 \mathrm{E} \quad 00$

$-2.12513 \mathrm{E} \quad 00$

$-2.14075 \mathrm{E} \quad 00$

$-2.15643 \mathrm{E} \quad 00$

$-2.17216 \mathrm{E} \quad 00$

$-2.18795 \mathrm{E} \quad 00$

$-2.20380 \mathrm{E} 00$

$-2.21970 \mathrm{E} \quad 00$

$-2.23566 \mathrm{E} \quad 00$

$-2.25167 \mathrm{E} \quad 00$

$-2.26774 \mathrm{E} \quad 00$

$-2.28386 \mathrm{E} \quad 00$

$-2.30005 \mathrm{E} \quad 00$

$-2.31628 \mathrm{E} \quad 00$

$-2.33257 \mathrm{E} \quad 00$

$-2.34892 \mathrm{E} \quad 00$

$-2.36533 \mathrm{E} 00$

$-2.38179 \mathrm{E} \quad 00$

$-2.39830 \mathrm{E} \quad 00$

$-2.41488 \mathrm{E} \quad 00$

$-2.43150 \mathrm{E} \quad 00$

$-2.44819 \mathrm{E} 00$

$-2.46492 \mathrm{E} \quad 00$

$-2.48172 \mathrm{E} \quad 00$

$\begin{array}{lll}-2.49857 \mathrm{E} & 00\end{array}$

$-2.51548 \mathrm{E} \quad 00$

$-2.53244 \mathrm{E} \quad 00$

$-2.54945 \mathrm{E} \quad 00$

$-2.56653 \mathrm{E} 00$ $g_{2}$

$-8.69494 \mathrm{E}-02$

$-8.79476 \mathrm{E}-02$

$-8.89534 \mathrm{E}-02$

$-8.99667 \mathrm{E}-02$

$-9.09876 \mathrm{E}-02$

$-9.20161 \mathrm{E}-02$

$-9.30522 \mathrm{E}-02$

$-9.40959 \mathrm{E}-02$

$-9.51473 \mathrm{E}-02$

$-9.62064 \mathrm{E}-02$

$-9.72732 \mathrm{E}-02$

$-9.83477 \mathrm{E}-02$

$-9.94301 \mathrm{E}-02$

$-1.00520 \mathrm{E}-01$

$-1.01618 \mathrm{E}-01$

$-1.02724 \mathrm{E}-01$

$-1.03838 \mathrm{E}-01$

$-1.04959 \mathrm{E}-01$

$-1.06089 \mathrm{E}-01$

$-1.07227 \mathrm{E}-01$

$-1.08372 \mathrm{E}-01$

$-1.09526 \mathrm{E}-01$

$-1.10687 \mathrm{E}-01$

$-1.11857 \mathrm{E}-01$

$-1.13035 \mathrm{E}-01$

$-1.14221 \mathrm{E}-01$

$-1.15415 \mathrm{E}-01$

$-1.16617 \mathrm{E}-01$

$-1.17827 \mathrm{E}-01$

$-1.19046 \mathrm{E}-01$

$-1.20273 \mathrm{E}-01$

$-1.21508 \mathrm{E}-01$

$-1.22752 \mathrm{E}-01$

$-1.24004 \mathrm{E}-01$

$-1.25264 \mathrm{E}-01$

$-1.26533 \mathrm{E}-01$

$-1.27810 \mathrm{E}-01$

$-1.29096 \mathrm{E}-01$

$-1.30390 \mathrm{E}-01$

$-1.31692 \mathrm{E}-01$

-1.33003E-01

$-1.34323 \mathrm{E}-01$

$-1.35651 \mathrm{E}-01$

$-1.36988 \mathrm{E}-01$

$-1.38334 \mathrm{E}-01$

$-1.39688 \mathrm{E}-01$

$-1.41051 \mathrm{E}-01$

$-1.42422 \mathrm{E}-01$

$-1.43802 \mathrm{E}-01$

$-1.45192 \mathrm{E}-01$

$-1.46589 \mathrm{E}-01$

$-1.47996 \mathrm{E}-01$

$-1.49412 \mathrm{E}-01$

$-1.50836 \mathrm{E}-01$

$-1.52270 \mathrm{E}-01$

$-1.53712 E-01$

$-1.55163 \mathrm{E}-01$

$-1.56623 \mathrm{E}-01$

$-1.58093 \mathrm{E}-01$

$-1.59571 \mathrm{E}-01$ $\tan \delta_{2}$

$f_{-2}$

$2.94979 \mathrm{E}$ ol $\quad-8.50181 \mathrm{E}-02$ $2.95000 \mathrm{E} \quad 01 \quad-8.59969 \mathrm{E}-02$ $2.95021 \mathrm{E}$ 01 $-8.69832 \mathrm{E}-02$ $2.95042 \mathrm{E} \quad 01 \quad-8.79769 \mathrm{E}-02$

$2.95063 \mathrm{E}$ ol $-8.89781 \mathrm{E}-02$

$2.95083 \mathrm{E} \quad 01 \quad-8.99868 \mathrm{E}-02$ $2.95103 \mathrm{E}$ 01 $-9.10029 \mathrm{E}-02$ $2.95124 \mathrm{E}$ o1 $-9.20267 \mathrm{E}-02$ $2.95144 \mathrm{E}$ 01 $-9.30580 \mathrm{E}-02$

$2.95163 \mathrm{E}$ 01 $-9.40969 \mathrm{E}-02$

$2.95183 \mathrm{E} \quad 01 \quad-9.51435 \mathrm{E}-02$ $2.95202 \mathrm{E} \quad 01 \quad-9.61977 \mathrm{E}-02$ $2.95222 \mathrm{E}$ ol $-9.72596 \mathrm{E}-02$ $2.95241 \mathrm{E} \quad 01 \quad-9.83292 \mathrm{E}-02$ $2.95260 \mathrm{E} \quad 01 \quad-9.94066 \mathrm{E}-02$

$2.95279 \mathrm{E} \quad 01-1.00492 \mathrm{E}-01$ $2.95297 \mathrm{E} \quad 01 \quad-1.01585 \mathrm{E}-01$ $2.95316 \mathrm{E} \quad 01-1.02685 \mathrm{E}-01$ $2.95334 \mathrm{E} \quad 01 \quad-1.03794 \mathrm{E}-01$ $2.95353 \mathrm{E}$ ol $-1.04910 \mathrm{E}-0 \mathrm{l}$

$2.95371 \mathrm{E} \quad 01 \quad-1.06035 \mathrm{E}-01$ $2.95389 \mathrm{E}$ 01 $-1.07167 \mathrm{E}-01$ $2.95406 \mathrm{E}$ 01 $-1.08307 \mathrm{E}-01$ $2.95424 \mathrm{E}$ ol $-1.09455 \mathrm{E}-01$ $2.95442 \mathrm{E}$ ol $-1.10612 \mathrm{E}-01$

$2.95459 \mathrm{E} 01 \quad-1.11776 \mathrm{E}-01$ $2.95476 \mathrm{E}$ 01 $-1.12948 \mathrm{E}-01$ $2.95493 \mathrm{E}$ 01 $-1.14129 \mathrm{E}-01$ $2.95510 \mathrm{E}$ 01 $-1.15317 \mathrm{E}-01$ $2.95527 \mathrm{E}$ 01 $-1.16514 \mathrm{E}-01$

$2.95544 \mathrm{E} \quad 01-1.17719 \mathrm{E}-01$ $2.95561 \mathrm{E}$ 0l $-1.18932 \mathrm{E}-01$ $2.95577 \mathrm{E}$ 0l $-1.20153 \mathrm{E}-01$ $2.95593 \mathrm{E}$ 01 $-1.21383 \mathrm{E}-01$ $2.95610 \mathrm{E} \quad 01-1.22620 \mathrm{E}-01$

$2.95626 \mathrm{E}$ ol $-1.23867 \mathrm{E}-01$ $2.95642 \mathrm{E} \quad 01 \quad-1.25121 \mathrm{E}-01$ $2.95658 \mathrm{E} \quad 01-1.26384 \mathrm{E}-01$ $2.95673 \mathrm{E} \quad 01 \quad-1.27655 \mathrm{E}-01$ $2.95689 \mathrm{E}$ 01 $-1.28935 \mathrm{E}-01$

$2.95705 \mathrm{E} \quad 01$ $2.95720 \mathrm{E} \quad 01$ $2.95735 \mathrm{E} \quad 01$ $2.95751 \mathrm{E} 01$ $2.95766 \mathrm{E} \quad 01$

$2.95781 \mathrm{E} \quad 01$ $2.95795 \mathrm{E} \quad 01$ $2.95810 \mathrm{E} \quad 01$ $2.95825 \mathrm{E} \quad 01$

$2.95839 \mathrm{E} \quad 01$

$2.95854 \mathrm{E} \quad 01$ $2.95868 \mathrm{E} \quad 01$ $2.95882 \mathrm{E} 01$ $2.95896 \mathrm{E} \quad 01$ $2.95910 \mathrm{E} 01$

2.95924. E 01 $2.95938 \mathrm{E} \quad 01$ $2.95952 \mathrm{E} \quad 01$ $2.95966 \mathrm{E} \quad 01$ $2.95979 \mathrm{E} \quad 01$

$g-2$

$1.72490 \mathrm{E} \quad 00$

$\begin{array}{lll}1.73901 \mathrm{E} & 00\end{array}$

$1.75317 \mathrm{E} \quad 00$

$1.76739 \mathrm{E} \quad 00$

$1.78167 \mathrm{E} \quad 00$

$\begin{array}{ll}1.79600 \mathrm{E} & 00\end{array}$

$1.81039 \mathrm{E} \quad 00$

$1.82484 \mathrm{E} \quad 00$

$1.83934 \mathrm{E} \quad 00$

$1.85390 \mathrm{E} \quad 00$

$1.86851 \mathrm{E} \quad 00$

$1.88319 \mathrm{E} \quad 00$

$1.89791 \mathrm{E} \quad 00$

$1.91270 \mathrm{E} \quad 00$

$1.92754 \mathrm{E} \quad 00$

$1.94243 \mathrm{E} \quad 00$ $1.95739 \mathrm{E} \quad 00$

$1.97239 \mathrm{E} \quad 00$

$1.98746 \mathrm{E} \quad 00$

$2.00258 \mathrm{E} \quad 00$

$2.01776 \mathrm{E} \quad 00$

$2.03299 \mathrm{E} \quad 00$

$2.04828 \mathrm{E} \quad 00$

$2.06363 \mathrm{E} 00$

$2.07903 \mathrm{E} \quad 00$

$2.09449 \mathrm{E} \quad 00$

$2.11000 \mathrm{E} \quad 00$

$2.12557 \mathrm{E} \quad 00$

$2.14120 \mathrm{E} 00$

$2.15688 \mathrm{E} \quad 00$

$2.17262 \mathrm{E} \quad 00$

$2.18842 \mathrm{E} \quad 00$

$2.20427 \mathrm{E} \quad 00$

$2.22017 \mathrm{E} \quad 00$

$2.23614 \mathrm{E} \quad 00$

$2.25215 \mathrm{E} \quad 00$ $2.26823 \mathrm{E} \quad 00$ $2.28436 \mathrm{E} \quad 00$ $2.30055 \mathrm{E} \quad 00$

$2.31679 \mathrm{E} \quad 00$

$2.33309 \mathrm{E} \quad 00$ 
ELECTRON RADIAL FUNCTIONS

Electron

\begin{tabular}{|c|c|c|c|c|c|c|}
\hline$p$ & $F_{0}$ & & $f_{1}$ & & $g_{1}$ & \\
\hline 30.10 & $1.17875 \mathrm{E}$ & 00 & $-3.14327 \mathrm{E}$ & 01 & $-3.30860 \mathrm{E}$ & 00 \\
\hline 30.20 & $1.17874 \mathrm{E}$ & 00 & $-3.15354 \mathrm{E}$ & 01 & $-3.32810 \mathrm{E}$ & 00 \\
\hline 30.30 & $1.17873 \mathrm{E}$ & 00 & $-3.16381 \mathrm{E}$ & 01 & $-3.34766 \mathrm{E}$ & 00 \\
\hline 30.40 & $1.17872 \mathrm{E}$ & 00 & $-3.17408 \mathrm{E}$ & 01 & $-3.36728 \mathrm{E}$ & 00 \\
\hline 30.50 & $1.17871 \mathrm{E}$ & 00 & $-3.18435 \mathrm{E}$ & 01 & $-3.38695 \mathrm{E}$ & 00 \\
\hline 30.60 & $1.17870 \mathrm{E}$ & 00 & $-3.19461 \mathrm{E}$ & 01 & $-3.40667 \mathrm{E}$ & 00 \\
\hline 30.70 & $1.17869 \mathrm{E}$ & 00 & $-3.20487 \mathrm{E}$ & 01 & $-3.42645 \mathrm{E}$ & 00 \\
\hline 30.80 & $1.17868 \mathrm{E}$ & 00 & $-3.21512 \mathrm{E}$ & 01 & $-3.44628 \mathrm{E}$ & 00 \\
\hline 30.90 & $1.17867 \mathrm{E}$ & 00 & $-3.22538 \mathrm{E}$ & 01 & $-3.46617 \mathrm{E}$ & 00 \\
\hline 31.00 & $1.17866 \mathrm{E}$ & 00 & $-3.23563 \mathrm{E}$ & 01 & $-3.48611 \mathrm{E}$ & 00 \\
\hline 31.10 & $1.17865 \mathrm{E}$ & 00 & $-3.24587 \mathrm{E}$ & 01 & $-3.50611 \mathrm{E}$ & 00 \\
\hline 31.20 & $1.17864 \mathrm{E}$ & 00 & $-3.25612 \mathrm{E}$ & 01 & $-3.52615 \mathrm{E}$ & 00 \\
\hline 31.30 & $1.17863 \mathrm{E}$ & 00 & $-3.26636 \mathrm{E}$ & 01 & $-3.54626 \mathrm{E}$ & 00 \\
\hline 31.40 & $1.17862 \mathrm{E}$ & 00 & $-3.27660 \mathrm{E}$ & 01 & $-3.56642 \mathrm{E}$ & 00 \\
\hline 31.50 & $1.17861 \mathrm{E}$ & 00 & $-3.28683 \mathrm{E}$ & 01 & $-3.58663 \mathbf{E}$ & 00 \\
\hline 31.60 & $1.17860 \mathrm{E}$ & 00 & $-3.29707 \mathbf{E}$ & 01 & $-3.60689 \mathrm{E}$ & 00 \\
\hline 31.70 & $1.17858 \mathrm{E}$ & 00 & $-3.30730 \mathrm{E}$ & 01 & $-3.62722 \mathbf{E}$ & 00 \\
\hline 31.80 & $1.17857 \mathrm{E}$ & 00 & $-3.31752 \mathrm{E}$ & 01 & $-3.64759 \mathbf{E}$ & 00 \\
\hline 31.90 & $1.17856 \mathrm{E}$ & 00 & $-3.32775 \mathbf{E}$ & 01 & $-3.66802 \mathrm{E}$ & 00 \\
\hline 32.00 & $1.17855 \mathrm{E}$ & 00 & $-3.33797 \mathrm{E}$ & 01 & $-3.68850 \mathrm{E}$ & 00 \\
\hline 32.10 & $1.17854 \mathrm{E}$ & 00 & $-3.34818 \mathrm{E}$ & 01 & $-3.70904 \mathrm{E}$ & 00 \\
\hline 32.20 & $1.17853 \mathrm{E}$ & 00 & $-3.35840 \mathbf{E}$ & 01 & $-3.72963 \mathrm{E}$ & 00 \\
\hline 32.30 & $1.17852 \mathrm{E}$ & 00 & $-3.36861 \mathrm{E}$ & 01 & $-3.75027 \mathrm{E}$ & 00 \\
\hline 32.40 & $1.17851 \mathrm{E}$ & 00 & $-3.37882 \mathrm{E}$ & 01 & $-3.77097 \mathrm{E}$ & 00 \\
\hline 32.50 & $1.17850 \mathrm{E}$ & 00 & $-3.38902 \mathrm{E}$ & 01 & $-3.79173 \mathrm{E}$ & 00 \\
\hline 32.60 & $1.17849 \mathrm{E}$ & 00 & $-3.39922 \mathrm{E}$ & 01 & $-3.81253 \mathbf{E}$ & 00 \\
\hline 32.70 & $1.17848 \mathrm{E}$ & 00 & $-3.40942 \mathrm{E}$ & 01 & $-3.83339 \mathrm{E}$ & 00 \\
\hline 32.80 & $1.17847 \mathrm{E}$ & 00 & $-3.41962 \mathbf{E}$ & 01 & $-3.85431 \mathrm{E}$ & 00 \\
\hline 32.90 & $1.17846 \mathrm{E}$ & 00 & $-3.42981 \mathrm{E}$ & 01 & $-3.87528 \mathrm{E}$ & 00 \\
\hline 33.00 & $1.17845 \mathrm{E}$ & 00 & $-3.44000 \mathrm{E}$ & 01 & $-3.89630 \mathrm{E}$ & 00 \\
\hline 33.10 & $1.17844 \mathrm{E}$ & 00 & $-3.45018 \mathrm{E}$ & 01 & $-3.91737 \mathrm{E}$ & 00 \\
\hline 33.20 & $1.17844 \mathrm{E}$ & 00 & $-3.46036 \mathrm{E}$ & 01 & $-3.93850 \mathrm{E}$ & 00 \\
\hline 33.30 & $1.17843 \mathrm{E}$ & 00 & $-3.47054 \mathrm{E}$ & 01 & $-3.95969 E$ & 00 \\
\hline 33.40 & $1.17842 \mathrm{E}$ & 00 & $-3.48072 \mathrm{E}$ & 01 & $-3.98093 \mathrm{E}$ & 00 \\
\hline 33.50 & $1.17841 \mathrm{E}$ & 00 & $-3.49089 \mathrm{E}$ & 01 & $-4.00222 \mathrm{E}$ & 00 \\
\hline 33.60 & $1.17840 \mathrm{E}$ & 00 & $-3.50106 \mathrm{E}$ & 01 & $-4.02 \overline{3} 56 \mathrm{E}$ & 00 \\
\hline 33.70 & $1.17839 \mathrm{E}$ & 00 & $-3.51123 \mathrm{E}$ & 01 & $-4.04496 \mathrm{E}$ & 00 \\
\hline 33.80 & $1.17838 \mathrm{E}$ & 00 & $-3.52140 \mathrm{E}$ & 01 & $-4.06641 \mathrm{E}$ & 00 \\
\hline 33.90 & $1.17837 \mathrm{E}$ & 00 & $-3.53156 \mathrm{E}$ & 01 & $-4.08792 \mathrm{E}$ & 00 \\
\hline 34.00 & $1.17836 \mathrm{E}$ & 00 & $-3.54171 \mathrm{E}$ & 01 & $-4.10948 \mathrm{E}$ & 00 \\
\hline 34.10 & $1.17835 \mathrm{E}$ & 00 & $-3.55187 \mathrm{E}$ & 01 & $-4.13109 \mathrm{E}$ & 00 \\
\hline 34.20 & $1.17834 \mathrm{E}$ & 00 & $-3.56202 \mathrm{E}$ & 01 & $-4.15276 \mathrm{E}$ & 00 \\
\hline 34.30 & $1.17833 \mathrm{E}$ & 00 & $-3.57217 \mathrm{E}$ & 01 & $-4.17448 \mathrm{E}$ & 00 \\
\hline 34.40 & $1.17832 \mathrm{E}$ & 00 & $-3.58231 \mathrm{E}$ & 01 & $-4.19626 \mathrm{E}$ & 00 \\
\hline 34.50 & $1.17831 \mathrm{E}$ & 00 & $-3.59245 \mathrm{E}$ & 01 & $-4.21808 \mathrm{E}$ & 00 \\
\hline 34.60 & $1.17830 \mathrm{E}$ & 00 & $-3.60259 \mathrm{E}$ & 01 & $-4.23996 \mathrm{E}$ & 00 \\
\hline 34.70 & $1.17829 \mathrm{E}$ & 00 & $-3.61272 \mathrm{E}$ & 01 & $-4.26190 \mathrm{E}$ & 00 \\
\hline 34.80 & $1.17828 \mathrm{E}$ & 00 & $-3.62286 \mathrm{E}$ & 01 & $-4.28389 \mathrm{E}$ & 00 \\
\hline 34.90 & $1.17827 \mathrm{E}$ & 00 & $-3.63298 \mathrm{E}$ & 01 & $-4.30593 \mathrm{E}$ & 00 \\
\hline 35.00 & $1.17826 \mathrm{E}$ & 00 & $-3.64311 \mathrm{E}$ & 01 & $-4.32802 \mathrm{E}$ & 00 \\
\hline 35.10 & $1.17826 \mathrm{E}$ & 00 & $-3.65323 \mathrm{E}$ & 01 & $-4.35017 \mathrm{E}$ & 00 \\
\hline 35.20 & $1.17825 \mathrm{E}$ & 00 & $-3.66335 \mathrm{E}$ & 01 & $-4.37237 \mathrm{E}$ & 00 \\
\hline 35.30 & $1.17824 \mathrm{E}$ & 00 & $-3.67347 \mathrm{E}$ & 01 & $-4.39463 \mathrm{E}$ & 00 \\
\hline 35.40 & $1.17823 \mathrm{E}$ & 00 & $-3.68358 \mathrm{E}$ & 01 & $-4.41694 \mathrm{E}$ & 00 \\
\hline 35.50 & $1.17822 \mathrm{E}$ & 00 & $-3.69369 \mathrm{E}$ & 01 & $-4.43930 \mathrm{E}$ & 00 \\
\hline 35.60 & $1.17821 \mathrm{E}$ & 00 & $-3.70379 \mathrm{E}$ & 01 & $-4.46172 \mathrm{E}$ & 00 \\
\hline 35.70 & $1.17820 \mathrm{E}$ & 00 & $-3.71389 \mathrm{E}$ & 01 & $-4.48418 \mathrm{E}$ & 00 \\
\hline 35.80 & $1.17819 \mathrm{E}$ & 00 & $-3.72399 \mathrm{E}$ & 01 & $-4.50671 \mathrm{E}$ & 00 \\
\hline 35.90 & $1.17818 \mathrm{E}$ & 00 & $-3.73409 \mathbf{E}$ & 01 & $-4.52928 \mathrm{E}$ & 00 \\
\hline 36.00 & $1.17817 \mathrm{E}$ & 00 & $-3.74418 \mathrm{E}$ & 01 & $-4.55191 \mathrm{E}$ & \\
\hline
\end{tabular}

$\tan \delta$

$\rho=1.3 A^{1 / 3} F$
$4.85163 \mathrm{E}-03 \quad-3.24263 \mathrm{E} \quad 00$

$4.85212 \mathrm{E}-03 \quad-3.26185 \mathrm{E} \quad 00$

$4.85259 \mathrm{E}-03-3.28112 \mathrm{E} \quad 00$

$4.85302 \mathrm{E}-03 \quad-3.30044 \mathrm{E} \quad 00$

$\begin{array}{lll}4.85344 \mathrm{E}-03 & -3.31983 \mathrm{E} & 00\end{array}$

$4.85384 \mathrm{E}-03 \quad-3.33926 \mathrm{E} \quad 00$

$4.85420 \mathrm{E}-03$

$4.85455 \mathrm{E}-03$

$4.85486 \mathrm{E}-03$

$4.85516 \mathrm{E}-03$

$4.85542 \mathrm{E}-03$

$4.85567 \mathrm{E}-03$

$4.85588 \mathrm{E}-03$

$4.85608 \mathrm{E}-03$

$4.85625 \mathrm{E}-03$

$4.85639 \mathrm{E}-03$

$4.85652 \mathrm{E}-03$

$4.85662 \mathrm{E}-03$

$4.85670 \mathrm{E}-03$

$4.85675 \mathrm{E}-03$

$4.85678 \mathrm{E}-03$

$4.85679 \mathrm{E}-03$

$4.85677 \mathrm{E}-03$

$4.85673 \mathrm{E}-03$

$4.85667 \mathrm{E}-03$

$4.85659 \mathrm{E}-03$

$4.85649 \mathrm{E}-03$

$4.85637 \mathrm{E}-03$

$4.85622 \mathrm{E}-03$

$4.85604 \mathrm{E}-03$

$4.85585 \mathrm{E}-03$

$4.85563 \mathrm{E}-03$

$4.85540 \mathrm{E}-03$

$4.85514 \mathrm{E}-03$

$4.85487 \mathrm{E}-03$

$4.85457 \mathrm{E}-03$

$4.85426 \mathrm{E}-03$

$4.85392 \mathrm{E}-03$

$4.85356 \mathrm{E}-03$

$4.85317 \mathrm{E}-03$

$4.85278 \mathrm{E}-03$

$4.85235 \mathrm{E}-03$

$4.85191 \mathrm{E}-03$

$4.85145 \mathrm{E}-03$

$4.85097 \mathrm{E}-03$

$4.85047 \mathrm{E}-03$ $4.84995 \mathrm{E}-03$

$4.84941 \mathrm{E}-03$

$4.84886 \mathrm{E}-03$

$4.84828 \mathrm{E}-03$

$4.84767 \mathrm{E}-03$

$4.84706 \mathrm{E}-03$

$4.84642 \mathrm{E}-03$

$4.84576 \mathrm{E}-03$

$4.84508 \mathrm{E}-03$

$4.84439 \mathrm{E}-03$

$4.84368 \mathrm{E}-03$

$4.84294 \mathrm{E}-03$

$4.84220 \mathrm{E}-03$

$4.84143 \mathrm{E}-03$
$-3.35875 \mathrm{E} 00$

$-3.37829 \mathrm{E} 00$

$-3.39789 \mathrm{E} 00$

$-3.41755 \mathrm{E} 00$

$-3.43725 \mathrm{E} \quad 00$ $-3.45701 \mathrm{E} 00$ $-3.47683 \mathrm{E} \quad 00$ $-3.49670 \mathrm{E} 00$ $-3.51662 \mathrm{E} 00$

$-3.53660 \mathrm{E} 00$ $-3.55663 \mathrm{E} 00$ $-3.57672 \mathrm{E} 00$ $-3.59686 \mathrm{E} \quad 00$ $-3.61706 \mathrm{E} 00$

$-3.63731 \mathrm{E} \quad 00$ $-3.65761 \mathrm{E} \quad 00$ $-3.67797 \mathrm{E} \quad 00$ $-3.69838 \mathrm{E} 00$ $-3.71884 \mathrm{E} 00$

$-3.73936 \mathrm{E} \quad 00$ $-3.75994 \mathrm{E} \quad 00$ $-3.78056 \mathrm{E} \quad 00$ $-3.80124 \mathrm{E} \quad 00$ $-3.82198 \mathrm{E} 00$

$-3.84277 \mathrm{E} 00$ $-3.86361 \mathrm{E} 00$ $-3.88451 \mathrm{E} 00$ $-3.90546 \mathrm{E} \quad 00$ $-3.92646 \mathrm{E} 00$

$-3.94752 \mathrm{E} 00$ $-3.96863 \mathrm{E} 00$ $-3.98980 \mathrm{E} 00$ $-4.01102 \mathrm{E} \quad 00$ $-4.03229 \mathrm{E} 00$

$-4.05362 \mathrm{E} 00$ $-4.07500 \mathrm{E} \quad 00$ $-4.09643 \mathrm{E} \quad 00$ $-4.13946 \mathrm{E} \quad 00$

$-4.16105 \mathrm{E} \quad 00$ $-4.18270 \mathrm{E} \quad 00$ $-4.20440 \mathrm{E} \quad 00$ $-4.22616 \mathrm{E} \quad 00$ $-4.24797 \mathrm{E} 00$

$-4.26983 \mathrm{E} 00$ $-4.29174 \mathrm{E} \quad 00$ $-4.31371 \mathrm{E} \quad 00$ $-4.33574 \mathrm{E} \quad 00$ $-4.35781 \mathrm{E} \quad 00$

$-4.37994 \mathrm{E} \quad 00$ $-4.40212 \mathrm{E} \quad 00$ $-4.42436 \mathrm{E} \quad 00$ $-4.44665 \mathrm{E} \quad 00$ $-4.46899 \mathrm{E} 00$ $-4.11792 \mathrm{E} \quad 00$ $g_{-1}$

$3.24856 \mathrm{E} \quad 01$ $3.25882 \mathrm{E} \quad 01$ $3.26908 \mathrm{E} 01$ $3.27934 \mathrm{E} \quad 01$

$3.28959 \mathrm{E} 01$

$3.29984 \mathrm{E} \quad 01$ $3.31008 \mathrm{E} \quad 01$ $3.32032 \mathrm{E} \quad 01$

$3.33056 \mathrm{E} \quad 01$

$3.34080 \mathrm{E} 01$

$3.35103 \mathrm{E} \quad 01$ $3.36126 \mathrm{E} \quad 01$ $3.37149 \mathrm{E} 01$

$3.38172 \mathrm{E} \quad 01$

$3.39194 \mathrm{E} \quad 01$

$3.40216 \mathrm{E} \quad 01$

$3.41237 \mathrm{E} 01$

$3.42258 \mathrm{E} \quad 01$

$3.43279 \mathrm{E} \quad 01$

$3.44300 \mathrm{E} \quad 01$

$3.45320 \mathrm{E} \quad 01$ $3.46340 \mathrm{E} \quad 01$

$3: 47360 \mathrm{E} \quad 01$

$3.48379 \mathrm{E} \quad 01$

$3.49398 \mathrm{E} 01$

$3.50417 \mathrm{E} \quad 01$ $3.51435 \mathrm{E} \quad 01$ $3.52453 \mathrm{E} \quad 01$

$3.53471 \mathrm{E} \quad 01$

$3.54489 \mathrm{E} \quad 01$

$3.55506 \mathrm{E} \quad 01$ $3.56523 \mathrm{E} \quad 01$ $3.57539 \mathrm{E} \quad 01$ $3.58555 \mathrm{E} 01$

$3.59571 \mathrm{E} 01$

$3.60587 \mathrm{E} \quad 01$ $3.61602 \mathrm{E}$ 0I

$3.62617 \mathrm{E} \quad 01$

$3.63632 \mathrm{E} 01$

$3.64646 \mathrm{E} \quad 01$

$3.65660 \mathrm{E} \quad 01$ $3.66674 \mathrm{E} \quad 01$ $3.67687 \mathrm{E} \quad 01$

$3.68700 \mathrm{E}$ 0l

$3.69713 \mathrm{E} 01$

$3.70725 \mathrm{E} \quad 01$ $3.71737 \mathrm{E} \quad 01$ $3.72749 \mathrm{E} \quad 01$ $3.73760 \mathrm{E} \quad 01$ $3.74771 \mathrm{E} 01$

$3.75782 \mathrm{E} \quad 01$ $3.76792 \mathrm{E} \quad 01$ $3.77802 \mathrm{E} \quad 01$ $3.78812 \mathrm{E} \quad 01$ $3.79821 \mathrm{E} 01$

$3.80830 \mathrm{E} \quad 01$ $3.81839 \mathrm{E} 01$ $3.82847 \mathrm{E} \quad 01$ $3.83855 \mathrm{E} 01$ $3.84863 \mathrm{E} 01$ $\tan \delta_{-1}$

$-1.53245 \mathrm{E} \quad 02$ $-1.53368 \mathrm{E} 02$ $-1.53490 \mathrm{E} 02$ $-1.53612 \mathrm{E} 02$ $-1.53734 \mathrm{E} \quad 02$

$\begin{array}{ll}-1.53856 \mathrm{E} & 02\end{array}$ $-1.53978 \mathrm{E} 02$ $-1.54100 \mathrm{E} 02$ $-1.54222 \mathrm{E} \quad 02$ $-1.54343 \mathrm{E} 02$

$-1.54465 \mathrm{E} 02$ $-1.54587 \mathrm{E} \quad 02$ $-1.54709 \mathrm{E} 02$ $-1.54830 \mathrm{E} 02$ $-1.54952 \mathrm{E} \quad 02$

$-1.55074 \mathrm{E} \quad 02$ $-1.55195 \mathrm{E} \quad 02$ $-1.55317 \mathrm{E} \quad 02$ $-1.55438 \mathrm{E} \quad 02$ $-1.55560 \mathrm{E} 02$

$-1.55681 \mathrm{E} \quad 02$ $\begin{array}{lll}-1.55803 \mathrm{E} & 02\end{array}$ $-1.55924 \mathrm{E} \quad 02$ $-1.56046 \mathrm{E} 02$ $-1.56167 \mathrm{E} \quad 02$

$-1.56289 \mathrm{E} 02$ $-1.56410 \mathrm{E} 02$ $-1.56532 \mathrm{E} \quad 02$ -1.56653 E 02 $-1.56775 \mathrm{E} \quad 02$

$-1.56897 \mathrm{E} 02$ $-157018 \mathrm{E} \quad 02$ $-1.57140 \mathrm{E} \quad 02$ $-1.57262 \mathrm{E} \quad 02$ $-1.57383 \mathrm{E} \quad 02$

$-1.57505 \mathrm{E} \quad 02$ $-1.57627 \mathrm{E} 02$ $-1.57749 \mathrm{E} 02$ $-1.57870 \mathrm{E} \quad 02$ $-1.57993 \mathrm{E} 02$

$-1.58114 \mathrm{E} \quad 02$ $-1.58236 \mathrm{E} 02$ $-1.58358 \mathrm{E} \quad 02$ -1.58481 E 02 $-1.58603 \mathrm{E} 02$

$-1.58725 \mathrm{E} \quad 02$ $-1.58847 \mathrm{E} \quad 02$ $-1.58970 \mathrm{E} 02$ $\begin{array}{ll}-1.59092 \mathrm{E} & 02\end{array}$ $-1.59214 \mathrm{E} \quad 02$ $-1.59337 \mathrm{E} \quad 02$ $-1.59460 \mathrm{E} \quad 02$ $-1.59582 \mathrm{E} \quad 02$ $\begin{array}{ll}-1.59705 \mathrm{E} & 02\end{array}$ $-1.59828 \mathrm{E} 02$ $-1.59951 \mathrm{E} \quad 02$ $-1.60074 \mathrm{E} \quad 02$ $-1.60197 \mathrm{E} 02$ $-1.60321 \mathrm{E} 02$ $\begin{array}{ll}-1.60444 \mathrm{E} & 02\end{array}$ 
ELECTRON RADIAL FUNCTIONS

$Z=7 \quad A=14 \quad \rho=1.3 A^{1 / 3} F$

Electron

$\bar{F}$

$1.95054 \mathrm{E}-03$

$1.96351 \mathrm{E}-03$

$1.97653 \mathrm{E}-03$

$1.98959 \mathrm{E}-03$

$2.00269 \mathrm{E}-03$

$2.01583 \mathrm{E}-03$

$2.02902 \mathrm{E}-03$

$2.04225 \mathrm{E}-03$

$2.05552 \mathrm{E}-03$

$2.06884 \mathrm{E}-03$

$2.08220 \mathrm{E}-03$

$2.09560 \mathrm{E}-03$

$2.10905 \mathrm{E}-03$

$2.12253 \mathrm{E}-03$

$2.13607 \mathrm{E}-03$

$2.14964 \mathrm{E}-03$

$2.16326 \mathrm{E}-03$

$2.17692 \mathrm{E}-03$

$2.19062 \mathrm{E}-03$

$2.20436 \mathrm{E}-03$

$2.21815 \mathrm{E}-03$

$2.23198 \mathrm{E}-03$

$2.24586 \mathrm{E}-03$

$2.25978 \mathrm{E}-03$

$2.27374 \mathrm{E}-03$

$2.28774 \mathrm{E}-03$

$2.30179 \mathrm{E}-03$

$2.31588 \mathrm{E}-03$

$2.33001 \mathrm{E}-03$

$2.34418 \mathrm{E}-03$

$2.35840 \mathrm{E}-03$

$2.37266 \mathrm{E}-03$

$2.38697 \mathrm{E}-03$

$2.40132 \mathrm{E}-03$

$2.41571 \mathrm{E}-03$

$2.43014 \mathrm{E}-03$

$2.44462 \mathrm{E}-03$

$2.45913 \mathrm{E}-03$

$2.47370 \mathrm{E}-03$

$2.48830 \mathrm{E}-03$

$2.50295 \mathrm{E}-03$

$2.51764 \mathrm{E}-03$

$2.53237 \mathrm{E}-03$

$2.54715 \mathrm{E}-03$

$2.56197 \mathrm{E}-03$

$2.57683 \mathrm{E}-03$

$2.59174 \mathrm{E}-03$

$2.60669 \mathrm{E}-03$

$2.62168 \mathrm{E}-03$

$2.63671 \mathrm{E}-03$

$2.65179 \mathrm{E}-03$

$2.66691 \mathrm{E}-03$

$2.68208 \mathrm{E}-03$

$2.69728 \mathrm{E}-03$

$2.71253 \mathrm{E}-03$

$2.72782 \mathrm{E}-03$ $2.74316 \mathrm{E}-03$ $2.75854 \mathrm{E}-03$ $2.77396 \mathrm{E}-03$ $2.789+2 \mathrm{E}-03$ $f_{2} \quad g_{2}$

$-2.58366 \mathrm{E} \quad 00$

$-1.61059 \mathrm{E}-01$

$-2.60084 \mathrm{E} \quad 00 \quad-1.62555 \mathrm{E}-01$

$-2.61808 \mathrm{~F} \quad 00 \quad-1.64061 \mathrm{E}-01$

$-2.63537 \mathrm{E} \quad 00-1.65575 \mathrm{E}-0 \mathrm{I}$

$-2.65272 \mathrm{E} \quad 00 \quad-1.67099 \mathrm{E}-01$

$-2.67013 \mathrm{E} \quad 00-1.68632 \mathrm{E}-01$

$-2.68759 \mathrm{E} \quad 00 \quad-1.70175 \mathrm{E}-01$

$-2.70511 \mathrm{E} \quad 00 \quad-1.71726 \mathrm{E}-01$

$-2.72268 \mathrm{E} \quad 00 \quad-1.73287 \mathrm{E}-01$

$-2.74031 \mathrm{E} \quad 00 \quad-1.74857 \mathrm{E}-01$

$-2.75799 \mathrm{E} \quad 00$

$-2.77573 \mathrm{E} \quad 00$

$-2.79353 \mathrm{E} \quad 00$

$-2.81138 \mathrm{E} \quad 00$

$-2.82928 \mathrm{E} \quad 00$

$-2.84724 \mathrm{E} \quad 00$

$-2.86526 \mathrm{E} \quad 00$

$-2.88333 \mathrm{E} \quad 00$

$-2.90145 \mathrm{E} \quad 00$

$-2.91963 \mathrm{E} 00$

$-2.93787 \mathrm{E} \quad 00$

$-2.95616 \mathrm{E} \quad 00$

$-2.97451 \mathrm{E} \quad 00$

$-2.99291 \mathrm{E} \quad 00$

-3.01137 E 00

$-3.02988 \mathrm{E} 00$

-3.04845 E 00

$-3.06707 \mathrm{E} 00$

$-3.08575 \mathrm{E} 00$

$-3.10448 \mathrm{E} 00$

$-3.12327 \mathrm{E} \quad 00$

$-3.14211 \mathrm{E} 00$

$-3.16101 \mathrm{E} 00$

$-3.17997 \mathrm{E} 00$

$-3.19897 \mathrm{E} \quad 00$

$-3.21804 \mathrm{E} \quad 00$

$-3.23716 \mathrm{E} 00$

$-3.25633 \mathrm{E} \quad 00$

$-3.27556 \mathrm{E} 00$

$-3.29484 \mathrm{E} 00$

$-3.31418 \mathrm{E} \quad 00$

$-3.33357 \mathrm{E} \quad 00$

$-3.35302 \mathrm{E} 00$

$-3.37252 \mathrm{E} 00$

$-3.39208 \mathrm{E} 00$

$-3.41169 \mathrm{E} \quad 00$

$-3.43135 \mathrm{E} \quad 00$

$-3.45108 \mathrm{E} 00$

$-3.47085 \mathrm{E} \quad 00$

$-3.49068 \mathrm{E} 00$

$-3.51057 \mathrm{E} \quad 00$

$-3.53051 \mathrm{E} 00$

$-3.55050 \mathrm{E} \quad 00$

$-3.57055 \mathrm{E} 00$

$-3.59066 \mathrm{E} 00$

$-1.76437 \mathrm{E}-01$

$-1.78026 \mathrm{E}-01$

$-1.79624 \mathrm{E}-01$

$-1.81232 \mathrm{E}-01$

$-1.82849 \mathrm{E}-01$

$-1.84476 \mathrm{E}-01$

$-1.86112 \mathrm{E}-01$

$-1.87757 \mathrm{E}-01$

$-1.89412 \mathrm{E}-01$

$-1.91077 \mathrm{E}-01$

$-1.92751 \mathrm{E}-01$

$-1.94435 \mathrm{E}-01$

$-1.96129 \mathrm{E}-01$

$-1.97832 \mathrm{E}-01$

$-1.99545 \mathrm{E}-01$

$-2.01268 \mathrm{E}-01$

$-2.03000 \mathrm{E}-01$

$-2.04743 \mathrm{E}-01$

$-2.06495 \mathrm{E}-01$

$-2.08257 \mathrm{E}-01$

$-2.10028 \mathrm{E}-01$

$-2.11810 \mathrm{E}-01$

$-2.13602 \mathrm{E}-01$

$-2.15403 \mathrm{E}-01$

$-2.17215 \mathrm{E}-01$

$-2.19036 \mathrm{E}-01$

$-2.20868 \mathrm{E}-0 \mathrm{l}$

$-2.22709 \mathrm{E}-01$

$-2.24561 \mathrm{E}-01$

$-2.26422 \mathrm{E}-01$

$-2.28294 \mathrm{E}-01$

$-2.30176 \mathrm{E}-01$

$-2.32068 \mathrm{E}-01$

$-2.33971 \mathrm{E}-01$

$-2.35883 \mathrm{E}-01$

$-2.37806 \mathrm{E}-01$

$-2.39739 \mathrm{E}-01$

$-2.41683 \mathrm{E}-01$

$-2.43637 \mathrm{E}-01$

$-2.45601 \mathrm{E}-01$

$-2.47575 \mathrm{E}-01$

$-2.49560 \mathrm{E}-01$

$-2.51556 \mathrm{E}-01$

$-2.53561 \mathrm{E}-01$

$-3.61081 \mathrm{E} \quad 00$

$-3.63103 \mathrm{E} \quad 00$

$-3.65129 \mathrm{E} \quad 00$

$-3.67162 \mathrm{E} 00$

$-3.69199 \mathrm{E} 00$ $\tan \delta_{2}$

$2.95993 \mathrm{E}$ 0l

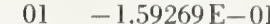

$2.96019 \mathrm{E}$ 01 $-1.60750 \mathrm{E}-01$

$2.96032 \mathrm{E}$ 0l $-1.62239 \mathrm{E}-01$

$2.96046 \mathrm{E} 01-1.63738 \mathrm{E}-01$

$2.96059 \mathrm{E} \quad 01-1.65246 \mathrm{E}-01$

$2.96071 \mathrm{E}$ 01 $-1.66763 \mathrm{E}-01$

$2.96084 \mathrm{E}$ 0l $-1.68289 \mathrm{E}-0 \mathrm{l}$

$2.96097 \mathrm{E}$ 01 $-1.69824 \mathrm{E}-01$

$2.96110 \mathrm{E} \quad 01-1.71368 \mathrm{E}-01$

$2.96122 \mathrm{E} \quad 01-1.72922 \mathrm{E}-01$

$2.96135 \mathrm{E}$ 01 $-1.74485 \mathrm{E}-01$

$2.96147 \mathrm{E}$ 0l $-1.76057 \mathrm{E}-01$

$2.96160 \mathrm{E} 01-1.77639 \mathrm{E}-01$

$2.96172 \mathrm{E}$ ol $-1.79230 \mathrm{E}-01$

$2.96184 \mathrm{E} 01-1.80830 \mathrm{E}-01$

$2.96196 \mathrm{E} \quad 01-1.82440 \mathrm{E}-01$

$2.96208 \mathrm{E}$ 01 $-1.84059 \mathrm{E}-01$

$2.96220 \mathrm{E}$ ol $-1.85688 \mathrm{E}-01$

$2.96232 \mathrm{E} \quad 01-1.87326 \mathrm{E}-01$

$2.96244 \mathrm{E}$ 01 $-1.88973 \mathrm{E}-01$

$2.96255 \mathrm{E}$ 01 $-1.90631 \mathrm{E}-01$

$2.96267 \mathrm{E}$ ol $-1.92297 \mathrm{E}-01$

$2.96278 \mathrm{E}$ 01 $-1.93974 \mathrm{E}-01$

$2.96290 \mathrm{E} 01-1.95659 \mathrm{E}-01$

$2.96301 \mathrm{E} 01 \quad-1.97355 \mathrm{E}-01$

$2.96313 \mathrm{E} 01-1.99060 \mathrm{E}-01$

$2.96324 \mathrm{E}$ 0l $-2.00775 \mathrm{E}-01$

$2.96335 \mathrm{E}$ 01 $-2.02500 \mathrm{E}-01$

$2.96346 \mathrm{E}$ 01 $-2.04234 \mathrm{E}-01$

$2.96357 \mathrm{E}$ 01 $-2.05978 \mathrm{E}-01$

$2.96368 \mathrm{E}$ 01 $-2.07732 \mathrm{E}-01$

$2.96379 \mathrm{E}$ 01 $-2.09496 \mathrm{E}-01$

$2.96390 \mathrm{E} \quad 01 \quad-2.11269 \mathrm{E}-01$

$2.96401 \mathrm{E} \quad 01 \quad-2.13053 \mathrm{E}-01$

$2.96411 \mathrm{E}$ 01 $-2.14846 \mathrm{E}-01$

$2.96422 \mathrm{E}$ ol $-2.16649 \mathrm{E}-01$

$2.96433 \mathrm{E}$ 0l $-2.18463 \mathrm{E}-01$

$2.96443 \mathrm{E} 01 \quad-2.20286 \mathrm{E}-01$

$2.96453 \mathrm{E}$ 01 $-2.22119 \mathrm{E}-01$

$2.96464 \mathrm{E}$ 0l $-2.23962 \mathrm{E}-0 \mathrm{l}$

$2.96474 \mathrm{E} \quad 01 \quad-2.25816 \mathrm{E}-01$

$2.96484 \mathrm{E}$ 01 $-2.27679 \mathrm{E}-01$

$2.96495 \mathrm{E} \quad 01 \quad-2.29553 \mathrm{E}-01$

$2.96505 \mathrm{E} \quad 01 \quad-2.31436 \mathrm{E}-01$

$2.96515 \mathrm{E} \quad 01-2.33330 \mathrm{E}-\dot{0} 1$

$2.96525 \mathrm{E}$ 01 $-2.35234 \mathrm{E}-01$

$2.96535 \mathrm{E}$ 0l $-2.37148 \mathrm{E}-01$

$2.96545 \mathrm{E}$ 01 $-2.39073 \mathrm{E}-01$

$2.96554 \mathrm{E}$ 01 $-2.41008 \mathrm{E}-01$

$2.96564 \mathrm{E}$ 01 $-2.42953 \mathrm{E}-01$

$2.96574 \mathrm{E}$ ol $-2.44908 \mathrm{E}-01$

$2.96584 \mathrm{E}$ 01 $-2.46874 \mathrm{E}-01$

$2.96593 \mathrm{E}$ 01 $-2.48850 \mathrm{E}-01$

$2.96603 \mathrm{E} \quad 01 \quad-2.50836 \mathrm{E}-01$

$2.96612 \mathrm{E} \quad 01 \quad-2.52833 \mathrm{E}-01$

$2.96622 \mathrm{E} \quad 01 \quad-2.548+1 \mathrm{E}-01$

$2.96631 \mathrm{E}$ ol $-2.56858 \mathrm{E}-0$

$2.96640 \mathrm{E}$ 01 $-2.58887 \mathrm{E}-01$

$2.96650 \mathrm{E}$ 01 $-2.60926 \mathrm{E}-01$

$\tan \delta_{2}$

$2.67076 \mathrm{E} \quad 00 \quad-3.29351 \mathrm{E}-02$

$2.68823 \mathrm{E} \quad 00 \quad-3.29363 \mathrm{E}-02$

$2.70575 \mathrm{E} \quad 00 \quad-3.29376 \mathrm{E}-02$

$2.72333 \mathrm{E} \quad 00-3.29389 \mathrm{E}-02$

$2.74096 \mathrm{E} \quad 00 \quad-3.29402 \mathrm{E}-02$

$2.75865 \mathrm{E} \quad 00$

$2.77640 \mathrm{E} \quad 00$

$2.79 .120 \mathrm{E} \quad 00$

$2.81205 \mathrm{E} \quad 00$

$2.82996 \mathrm{E} \quad 00$

$2.84793 \mathrm{E} \quad 00$

$2.86595 \mathrm{E} \quad 00$

$2.88403 \mathrm{E} \quad 00$

$2.90216 \mathrm{E} \quad 00$

2.92035 E 00

$2.93860 \mathrm{E} \quad 00$

$2.95689 \mathrm{E} \quad 00$

$2.97525 \mathrm{E} \quad 00$

$2.99366 \mathrm{E} \quad 00$

$3.01212 \mathrm{E} \quad 00$

$-3.29414 \mathrm{E}-02$

$-3.29427 \mathrm{E}-02$

$-3.29439 \mathrm{E}-02$

$-3.29451 \mathrm{E}-02$

$-3.2946 ! \mathrm{E}-02$

$3.03064 \mathrm{E} \quad 00$

$3.04921 \mathrm{E} \quad 00$

$3.06784 \mathrm{E} \quad 00$

$3.08653 \mathrm{E} \quad 00$

3.10527 E 00

$3.12406 \mathrm{E} \quad 00$

$3.1+291 \mathrm{E} \quad 00$

$3.16182 \mathrm{E} \quad 00$

$3.18078 \mathrm{E} \quad 00$

$3.19980 \mathrm{E} \quad 00$

$3.21887 \mathrm{E} \quad 00$

$3.23799 \mathrm{E} \quad 00$

$3.25717 \mathrm{E} \quad 00$

$3.27641 \mathrm{E} \quad 00$

$3.29570 \mathrm{E} \quad 00$

$3.31504 \mathrm{E} \quad 00$

$3.33444 \mathrm{E} \quad 00$

$3.35390 \mathrm{E} \quad 00$

$3.37341 \mathrm{E} \quad 00$

$3.39297 \mathrm{E} \quad 00$

$3.41259 \mathrm{E} \quad 00$

$3.43227 \mathrm{E} \quad 00$

$3.45199 \mathrm{E} \quad 00$

$3.47178 \mathrm{E} \quad 00$

$3.49162 \mathrm{E} \quad 00$

$3.51151 \mathrm{E} \quad 00$

$3.53146 \mathrm{E} \quad 00$

$3.55146 \mathrm{E} \quad 00$

$3.57152 \mathrm{E} \quad 00$

$3.59163 \mathrm{E} \quad 00$

$3.61180 \mathrm{E} \quad 00$

$3.63202 \mathrm{E} \quad 00$

$3.65229 \mathrm{E} \quad 00$

$3.67262 \mathrm{E} \quad 00$ 
ELECTRON RADIAL FUNCTIONS

Electron

$F_{0}$

36.10

36.20

36.30

36.40

36.50

36.60

36.70

36.80

36.90

37.00

37.10

37.20

37.30

37.40

37.50

37.60

37.70

37.80

37.90

38.00

38.10

38.20

38.30

38.40

38.50

38.60

38.70

38.80

38.90

39.00

39.10

39.20

39.30

39.40

39.50

39.60

39.70

39.80

39.90

40.00

40.10

40.20

40.30

40.40

40.50

40.60

40.70

40.80

40.90

41.00

41.10

41.20

41.30

41.40

41.50

41.60

41.70

41.80

41.90

42.00

$$
Z=7 \quad A=14
$$

$f_{1}$

$1.17816 \mathrm{E} \quad 00$

$1.17816 \mathrm{E} \quad 00$

$1.17815 \mathrm{E} \quad 00$

$1.17814 \mathrm{E} \quad 00$

$1.17813 \mathrm{E} \quad 00$

$1.17812 \mathrm{E} \quad 00$ $1.17811 \mathrm{E} \quad 00$ $\begin{array}{ll}1.17810 \mathrm{E} & 00\end{array}$ $1.17809 \mathrm{E} \quad 00$ $1.17809 \mathrm{E} \quad 00$

$1.17808 \mathrm{E} \quad 00$ $\begin{array}{ll}1.17807 \mathrm{E} & 00\end{array}$ $1.17806 \mathrm{E} \quad 00$ $\begin{array}{ll}1.17805 \mathrm{E} & 00\end{array}$ $1.17804 \mathrm{E} \quad 00$

$1.17803 \mathrm{E} \quad 00$ $\begin{array}{ll}1.17802 \mathrm{E} & 00\end{array}$ $1.17802 \mathrm{E} \quad 00$ $1.17801 \mathrm{E} \quad 00$ $1.17800 \mathrm{E} \quad 00$

$1.17799 \mathrm{E} \quad 00$ $1.17798 \mathrm{E} \quad 00$ $\begin{array}{ll}1.17797 \mathrm{E} & 00\end{array}$ $1.17797 \mathrm{E} \quad 00$ $1.17796 \mathrm{E} \quad 00$

$1.17795 \mathrm{E} \quad 00$ $1.17794 \mathrm{E} \quad 00$ $1.17793 \mathrm{E} \quad 00$ $1.17792 \mathrm{E} \quad 00$ $1.17792 \mathrm{E} \quad 00$

$1.17791 \mathrm{E} \quad 00$ $1.17790 \mathrm{E} \quad 00$ $1.17789 \mathrm{E} \quad 00$ $1.17788 \mathrm{E} \quad 00$ $1.17788 \mathrm{E} \quad 00$

$1.17787 \mathrm{E} \quad 00$ $1.17786 \mathrm{E} \quad 00$ $\begin{array}{ll}1.17785 \mathrm{E} & 00\end{array}$ $1.17784 \mathrm{E} \quad 00$ $1.17784 \mathrm{E} \quad 00$

$1.17783 \mathrm{E} \quad 00$ $1.17782 \mathrm{E} \quad 00$ $1.17781 \mathrm{E} \quad 00$ $1.17780 \mathrm{E} \quad 00$ $1.17780 \mathrm{E} \quad 00$

$1.17779 \mathrm{E} \quad 00$
0 $1.17778 \mathrm{E} \quad 00$ $\begin{array}{ll}1.17777 \mathrm{E} & 00\end{array}$ $\begin{array}{ll}1.17776 \mathrm{E} & 00\end{array}$ $1.17776 \mathrm{E} \quad 00$

$1.17775 \mathrm{E} \quad 00$ $1.17774 \mathrm{E} \quad 00$ $1.17773 \mathrm{E} \quad 00$ $1.17773 \mathrm{E} \quad 00$ $1.17772 \mathrm{E} \quad 00$

$1.17771 \mathrm{E} \quad 00$
0 $1.17770 \mathrm{E} \quad 00$ $1.17770 \mathrm{E} \quad 00$ $1.17769 \mathrm{E} \quad 00$

$1.17768 \mathrm{E} \quad 00$ $g_{1}$ $\begin{array}{ll}-3.75427 \mathrm{E} & 01 \\ -3.76435 \mathrm{E} & 01\end{array}$ $-3.77444 \mathrm{E} 01$ $-3.78452 \mathrm{E}$ 0l $-3.79459 \mathrm{E} 01$

$-3.80466 \mathrm{E} 01$ $-3.81473 \mathrm{E} 01$ $-3.82480 \mathrm{E} 01$ $-3.83486 \mathrm{E} \quad 01$ $-3.84492 \mathrm{E}$ 01

$-3.85497 \mathrm{E} 01$ $-3.86502 \mathrm{E} 01$ $-3.87507 \mathrm{E}$ 0I $-3.88512 \mathrm{E} 01$ $-3.89516 \mathrm{E} 01$

$-3.90520 \mathrm{E} 01$ $-3.91523 \mathrm{E} 01$ $-3.92526 \mathrm{E} 01$ $-3.93529 \mathrm{E} \quad 01$ $-3.94531 \mathrm{E} 01$

$-3.95534 \mathrm{E} \quad 01$ $-3.96535 \mathrm{E} \quad 01$ $-3.97537 \mathrm{E} 01$ $-3.98538 \mathrm{E}$ 01 $-3.99538 \mathrm{E} 01$

$-4.00539 \mathrm{E} \quad 01$ $-4.01539 \mathrm{E} 01$ $-4.02538 \mathrm{E}$ 01 $-4.03538 \mathrm{E} 01$ $-4.04537 \mathrm{E} 01$

$-4.05535 \mathrm{E} \quad 01$ $-4.06534 \mathrm{E} 01$ $-4.07532 \mathrm{E} \quad 0 \mathrm{I}$ $-4.08529 \mathrm{E}$ 0I $-4.09526 \mathrm{E} 0 \mathrm{I}$

$-4.10523 \mathrm{E} 01$ $-4.11520 \mathrm{E}$ 0I $-4.12516 \mathrm{E} 01$ $-4.13512 \mathrm{E} 01$ $-4.14507 \mathrm{E} \quad 01$

$-4.15502 \mathrm{E} 01$ $-4.16497 \mathrm{E} 01$ $-4.17491 \mathrm{E} 01$ $-4.18485 \mathrm{E} 01$ $-4.19479 \mathrm{E} 01$

$-4.20472 \mathrm{E} \quad 01$ $-4.21465 \mathrm{E} \quad 01$ $-4.22457 \mathrm{E} 01$ $-4.23450 \mathrm{E} \quad 01$ -4.24441 E 01

$-4.25433 \mathrm{E}$ 0I $-4.26424 \mathrm{E} 01$ $-4.27415 \mathrm{E} 01$ $-4.28405 \mathrm{E} 01$ $-4.29395 \mathrm{E} 01$

$-4.30385 \mathrm{E} \quad 01$ $-4.31374 \mathrm{E} \quad 01$ $-4.32363 \mathrm{E} 01$ $-4.33351 \mathrm{E} \quad 01$ $-4.34339 \mathrm{E} 01$
$-4.57459 \mathrm{E} \quad 00$ $-4.59733 \mathrm{E} \quad 00$ $-4.62012 \mathrm{E} 00$ $-4.64296 \mathrm{E} \quad 00$ $-4.66585 \mathrm{E} \quad 00$

$-4.68880 \mathrm{E} \quad 00$ $-4.71180 \mathrm{E} 00$ $-4.73485 \mathrm{E} \quad 00$ $-4.75796 \mathrm{E} \quad 00$ $-4.78112 \mathrm{E} \quad 00$

$-4.80433 \mathrm{E} \quad 00$ $-4.82759 \mathrm{E} \quad 00$ $-4.85091 \mathrm{E} \quad 00$

$-4.87428 \mathrm{E} \quad 00$

$-4.89770 \mathrm{E} 00$

$-4.92118 \mathrm{E} \quad 00$

$-4.94471 \mathrm{E} \quad 00$

$-4.96829 \mathrm{E} \quad 00$

$-4.99193 \mathrm{E} 00$

-5.01562 E 00

$-5.03936 \mathrm{E} \quad 00$ $-5.06315 \mathrm{E} 00$ $-5.08699 \mathrm{E} 00$ $-5.11089 \mathrm{E} \quad 00$ $-5.13484 \mathrm{E} \quad 00$

$-5.15885 \mathrm{E} \quad 00$ $-5.18290 \mathrm{E} \quad 00$ $-5.20701 \mathrm{E} \quad 00$ $-5.23118 \mathrm{E} \quad 00$ $-5.25539 \mathrm{E} \quad 00$

$-5.27966 \mathrm{E} \quad 00$ $-5.30398 \mathrm{E} 00$ $-5.32835 \mathrm{E} \quad 00$ $-5.35277 \mathrm{E} \quad 00$ $-5.37725 \mathrm{E} 00$

$-5.40178 \mathrm{E} \quad 00$ $-5.42636 \mathrm{E} 00$ $-5.45099 \mathrm{E} \quad 00$ $-5.47568 \mathrm{E} \quad 00$ $-5.50041 \mathrm{E} \quad 00$

$-5.52521 \mathrm{E} \quad 00$ $-5.55005 \mathrm{E} \quad 00$ $-5.57494 \mathrm{E} \quad 00$ $-5.59989 \mathrm{E} 00$

$-5.62489 \mathrm{E} \quad 00$

$-5.64994 \mathrm{E} 00$

$-5.67504 \mathrm{E} \quad 00$

$-5.70020 \mathrm{E} \quad 00$

$-5.72541 \mathrm{E} 00$

$-5.75067 \mathrm{E} \quad 00$

$-5.77598 \mathrm{E} \quad 00$

$-5.80134 \mathrm{E} \quad 00$

$-5.82676 \mathrm{E} \quad 00$

$-5.85222 \mathrm{E} \quad 00$

$-5.87774 \mathrm{E} \quad 00$

$-5.90332 \mathrm{E} \quad 00$ $-5.92894 \mathrm{E} \quad 00$

$-5.95461 \mathrm{E} \quad 00$

$-5.98034 \mathrm{E} \quad 00$

$-6.00612 \mathrm{E} 00$

$\tan \delta_{1}$

$4.84065 \mathrm{E}-03$ $4.83985 \mathrm{E}-03$ $4.83902 \mathrm{E}-03$

$4.83819 \mathrm{E}-03$

4.83733 E- 03

$4.83646 \mathrm{E}-03$

$4.83555 \mathrm{E}-03$

$4.83465 \mathrm{E}-03$

$4.83371 \mathrm{E}-03$

$4.83277 \mathrm{E}-03$

$4.83180 \mathrm{E}-03$

$4.83082 \mathrm{E}-03$

$4.82982 \mathrm{E}-03$

4.82881 E-03

$4.82778 \mathrm{E}-03$

$4.82673 \mathrm{E}-03$

$4.82566 \mathrm{E}-03$

$4.82458 \mathrm{E}-03$

$4.82348 \mathrm{E}-03$

$4.82236 \mathrm{E}-03$

$4.82123 \mathrm{E}-03$ $4.82007 \mathrm{E}-03$

$4.81891 \mathrm{E}-03$

$4.81773 \mathrm{E}-03$

$4.81652 \mathrm{E}-03$

$4.81531 \mathrm{E}-03$

$4.81408 \mathrm{E}-03$

$4.81284 \mathrm{E}-03$

$4.81157 \mathrm{E}-03$

$4.81029 \mathrm{E}-03$

$4.80899 \mathrm{E}-03$

$4.80768 \mathrm{E}-03$

$4.80635 \mathrm{E}-03$

$4.80500 \mathrm{E}-03$

$4.80364 \mathrm{E}-03$

$4.80227 \mathrm{E}-03$ $4.80088 \mathrm{E}-03$

$4.79948 \mathrm{E}-03$

$4.79805 \mathrm{E}-03$

$4.79662 \mathrm{E}-03$

$4.79516 \mathrm{E}-03$

$4.79370 \mathrm{E}-03$ 
$\bar{F}$

$2.80493 \mathrm{E}-03$ $2.82048 \mathrm{E}-03$

$2.83608 \mathrm{E}-03$

$2.85171 \mathrm{E}-03$

$2.86739 \mathrm{E}-03$

$2.88311 \mathrm{E}-03$

$2.89888 \mathrm{E}-03$

$2.91469 \mathrm{E}-03$

$2.93054 \mathrm{E}-03$

$2.94643 \mathrm{E}-03$

$2.96237 \mathrm{E}-03$

$2.97835 \mathrm{E}-03$

$2.99437 \mathrm{E}-03$

$3.01044 \mathrm{E}-03$

$3.02654 \mathrm{E}-03$

$3.04270 \mathrm{E}-03$

$3.05889 \mathrm{E}-03$

$3.07513 \mathrm{E}-03$

$3.09141 \mathrm{E}-03$

3.10773E-03

37.90

38.00

38.10

38.20

38.30

38.40

38.50

38.60

38.70

38.80

38.90

39.00

39.10

39.20

39.30

39.40

39.50

39.60

39.70

39.80

39.90

40.00

40.10

40.20

40.30

40.40

40.50

40.60

40.70

40.80

40.90

41.00

41.10

41.20

41.30

41.40

41.50

41.60

41.70

41.80

41.90

42.00
$3.12410 \mathrm{E}-03$

$3.14051 \mathrm{E}-03$

$3.15696 \mathrm{E}-03$

$3.17345 \mathrm{E}-03$

$3.18999 \mathrm{E}-03$

3.20657 E-03

$3.22320 \mathrm{E}-03$

$3.23987 \mathrm{E}-03$

$3.25658 \mathrm{E}-03$

$3.27333 \mathrm{E}-03$

3.29012 E-03

$3.30696 \mathrm{E}-03$

$3.32384 \mathrm{E}-03$

$3.34077 \mathrm{E}-03$

$3.35774 \mathrm{E}-03$

$3.37475 \mathrm{E}-03$ $3.39180 \mathrm{E}-03$ $3.40890 \mathrm{E}-03$

$3.42604 \mathrm{E}-03$

$3.44322 \mathrm{E}-03$

$3.46045 \mathrm{E}-03$

$3.47771 \mathrm{E}-03$

$3.49503 \mathrm{E}-03$

$3.51238 \mathrm{E}-03$

$3.52978 \mathrm{E}-03$

$3.54722 \mathrm{E}-03$

$3.56470 \mathrm{E}-03$

$3.58223 \mathrm{E}-03$

$3.59980 \mathrm{E}-03$

$3.6174 .1 \mathrm{E}-03$

$3.63506 \mathrm{E}-03$

$3.67050 \mathrm{E}-03$

$3.68829 \mathrm{E}-03$

$3.70611 \mathrm{E}-03$

3.72398E-03

$3.74190 \mathrm{E}-03$

$3.75985 \mathrm{E}-03$

$3.77785 \mathrm{E}-03$

3.79589E-03 $3.65276 \mathrm{E}-03$ $g_{2}$

$\tan \delta_{2}$

$f_{-2}$

$-3.71243 \mathrm{E} \quad 00 \quad-2.67897 \mathrm{E}-01$

$-3.73291 \mathrm{E} \quad 00 \quad-2.69988 \mathrm{E}-01$

$-3.75345 \mathrm{E} \quad 00 \quad-2.72089 \mathrm{E}-01$

$-3.77405 \mathrm{E} \quad 00 \quad-2.74201 \mathrm{E}-01$

$-3.79470 \mathrm{E} \quad 00 \quad-2.76324 \mathrm{E}-01$

$-3.81540 \mathrm{E} \quad 00$

$-3.83616 \mathrm{E} 00$

$-3.85697 \mathrm{E} \quad 00$

$-3.87784 \mathrm{E} \quad 00$

$-3.89876 \mathrm{E} \quad 00$

$-3.91973 \mathrm{E} \quad 00$

$-3.94076 \mathrm{E} 00$

$-3.96184 \mathrm{E} 00$

$-3.98298 \mathrm{E} \quad 00$

$-4.00417 \mathrm{E} \quad 00$

$-4.02542 \mathrm{E} \quad 00$

$-4.04672 \mathrm{E} \quad 00$

$-4.06808 \mathrm{E} \quad 00$

$-4.08948 \mathrm{E} \quad 00$

$-4.11095 \mathrm{E} \quad 00$

$-2.78457 \mathrm{E}-01$

$-2.80601 \mathrm{E}-01$

$-2.82757 \mathrm{E}-01$

$-2.84922 \mathrm{E}-01$

$-2.87099 \mathrm{E}-01$

$-4.13246 \mathrm{E} \quad 00$

$-4.15404 \mathrm{E} \quad 00$

$-4.17566 \mathrm{E} \quad 00$

$-4.19734 \mathrm{E} \quad 00$

$-4.21907 \mathrm{E} \quad 00$

$-4.24086 \mathrm{E} \quad 00$

$-4.26270 \mathrm{E} \quad 00$

$-4.28460 \mathrm{E} \quad 00$

$-4.30655 \mathrm{E} \quad 00$

$-4.32855 \mathrm{E} \quad 00$

$-4.35060 \mathrm{E} \quad 00$

$-4.37272 \mathrm{E} \quad 00$

$-4.39488 \mathrm{E} \quad 00$

$-4.41710 \mathrm{E} \quad 00$

$-4.43937 \mathrm{E} \quad 00$

$-2.89287 \mathrm{E}-01$

$-2.91486 \mathrm{E}-01$

$-2.93695 \mathrm{E}-01$

$-2.95916 \mathrm{E}-01$

$-2.98147 \mathrm{E}-01$

$-3.00390 \mathrm{E}-01$

$-3.02644 \mathrm{E}-01$

$-3.04909 \mathrm{E}-01$

$-3.07184 \mathrm{E}-01$

$-3.09471 \mathrm{E}-01$

$-3.11770 \mathrm{E}-01$

$-3.14079 \mathrm{E}-01$

$-3.16400 \mathrm{E}-01$

$-3.18732 \mathrm{E}-01$

$-3.21075 \mathrm{E}-01$

$-3.23429 \mathrm{E}-01$

$-3.25795 \mathrm{E}-01$

$-3.28172 \mathrm{E}-01$

$-3.30560 \mathrm{E}-01$

$-3.32960 \mathrm{E}-01$

$-3.35371 \mathrm{E}-01$

$-3.37794 \mathrm{E}-01$

$-3.40228 \mathrm{E}-01$

$-3.42674 \mathrm{E}-01$

$-4.46170 \mathrm{E} \quad 00$

$-4.48408 \mathrm{E} \quad 00$

$-4.50651 \mathrm{E} \quad 00$

$-4.52900 \mathrm{E} 00$

$-4.55154 \mathrm{E} \quad 00$

$-4.57413 \mathrm{E} 00$

$-4.59678 \mathrm{E} \quad 00$

$-4.61948 \mathrm{E} 00$

$-4.64224 \mathrm{E} \quad 00$

$-4.66505 \mathrm{E} 00$

$-4.68791 \mathrm{E} \quad 00$

$-4.71083 \mathrm{E} \quad 00$

$-4.73380 \mathrm{E} \quad 00$

$-4.75682 \mathrm{E} \quad 00$

$-4.77990 \mathrm{E} \quad 00$

$-4.80303 \mathrm{E} \quad 00$

$-4.82621 \mathrm{E} \quad 00$

$-4.84945 \mathrm{E} \quad 00$

$-4.87274 \mathrm{E} \quad 00$

$-4.89608 \mathrm{E} \quad 00$

$-4.91948 \mathrm{E} \quad 00$

$-4.94293 \mathrm{E} \quad 00$

$-4.96643 \mathrm{E} 00$

$-4.98999 \mathrm{E} \quad 00$

$-5.01360 \mathrm{E} 00$

$2.96659 \mathrm{E} \quad 01 \quad-2.62975 \mathrm{E}-0 \mathrm{]}$

$2.96668 \mathrm{E} \quad 01 \quad-2.65035 \mathrm{E}-01$

$2.96677 \mathrm{E} \quad 01-2.67106 \mathrm{E}-01$

$2.96686 \mathrm{E} \quad 01 \quad-2.69187 \mathrm{E}-01$

$2.96695 \mathrm{E} 01 \quad-2.71279 \mathrm{E}-01$

$2.96704 \mathrm{E} \quad 01 \quad-2.73382 \mathrm{E}-01$

$2.96713 \mathrm{E}$ 01 $-2.75495 \mathrm{E}-01$

$2.96722 \mathrm{E} \quad 01 \quad-2.77619 \mathrm{E}-01$

$2.96731 \mathrm{E} \quad 01 \quad-2.79754 \mathrm{E}-01$

$2.96739 \mathrm{E} \quad 01 \quad-2.81899 \mathrm{E}-01$

$2.96748 \mathrm{E} \quad 01 \quad-2.84056 \mathrm{E}-01$

2.96757 E $01 \quad-2.86223 \mathrm{E}-01$

$2.96765 \mathrm{E}$ 0l $-2.88401 \mathrm{E}-01$

$2.96774 \mathrm{E} \quad 01 \quad-2.90590 \mathrm{E}-01$

$2.96782 \mathrm{E}$ 01 $-2.92790 \mathrm{E}-01$

$2.96791 \mathrm{E} \quad 01 \quad-2.95001 \mathrm{E}-01$

$2.96799 \mathrm{E} \quad 01 \quad-2.97223 \mathrm{E}-01$

$2.96807 \mathrm{E} \quad 01-2.99456 \mathrm{E}-01$

$2.96816 \mathrm{E} \quad 01 \quad-3.01700 \mathrm{E}-01$

$2.96824 \mathrm{E} \quad 01-3.03955 \mathrm{E}-01$

$2.96832 \mathrm{E} \quad 01 \quad-3.06221 \mathrm{E}-01$

$2.96840 \mathrm{E} \quad 01-3.08498 \mathrm{E}-01$

$2.96848 \mathrm{E} \quad 01 \quad-3.10786 \mathrm{E}-01$

$2.96857 \mathrm{E}$ 0l $-3.13085 \mathrm{E}-01$

$2.96865 \mathrm{E} \quad 01 \quad-3.15396 \mathrm{E}-01$

$2.96873 \mathrm{E} \quad 01$

$2.96881 \mathrm{E} \quad 01$

$2.96888 \mathrm{E} \quad 01$

$2.96896 \mathrm{E} \quad 01$

$2.96904 \mathrm{E} \quad 01$

$2.96912 \mathrm{E} \quad 01$

$2.96920 \mathrm{E} \quad 01$

$2.96927 \mathrm{E} \quad 01$

$2.96935 \mathrm{E} 01$

$2.96943 \mathrm{E} 01$

$-3.17718 \mathrm{E}-01$

$-3.20050 \mathrm{E}-01$

$-3.22395 \mathrm{E}-01$

$-3.24750 \mathrm{E}-01$

$-3.27117 \mathrm{E}-01$

$-3.29495 \mathrm{E}-01$

$-3.31885 \mathrm{E}-01$

$-3.34286 \mathrm{E}-01$

$-3.36698 \mathrm{E}-01$

$-3.47600 \mathrm{E}-01$

$-3.50080 \mathrm{E}-01$

$-3.52572 \mathrm{E}-01$

$-3.55076 \mathrm{E}-01$

$-3.57591 \mathrm{E}-01$

$-3.60118 \mathrm{E}-01$

$-3.62656 \mathrm{E}-01$

$-3.65207 \mathrm{E}-01$

$-3.67769 \mathrm{E}-01$

$-3.70343 \mathrm{E}-01$

$-3.72928 \mathrm{E}-01$

$-3.75526 \mathrm{E}-01$

$-3.78135 \mathrm{E}-01$

$-3.80756 \mathrm{E}-01$

$-3.83389 \mathrm{E}-01$

$2.96950 \mathrm{E} \quad 01$

$2.96958 \mathrm{E} 01$

$2.96965 \mathrm{E} \quad 01$

$2.96973 \mathrm{E} \quad 01$

$2.96980 \mathrm{E} 01$

$2.96987 \mathrm{E} \quad 01$

$2.96995 \mathrm{E} \quad 01$

$2.97002 \mathrm{E} \quad 01$

$2.97009 \mathrm{E} \quad 01$

$2.97016 \mathrm{E} 01$

$2.97024 \mathrm{E} \quad 01$

$2.97031 \mathrm{E} \quad 01$

$2.97038 \mathrm{E} \quad 01$

$2.97045 \mathrm{E} \quad 01$

$2.97052 \mathrm{E} \quad 01$

$2.97059 \mathrm{E} \quad 01$

2.97066 E 01

$2.97073 \mathrm{E} \quad 01$

$2.97080 \mathrm{E} \quad 01$

$2.97086 \mathrm{E} \quad 01$

$2.97093 \mathrm{E} \quad 01$

$2.97100 \mathrm{E} \quad 01$

$2.97107 \mathrm{E} \quad 01$

$2.97114 \mathrm{E} \quad 01$

$2.97120 \mathrm{E} 01$

g-2

$\tan \delta$,

$3.81646 \mathrm{E} \quad 00$

$3.83723 \mathrm{E} \quad 00$

$3.85805 \mathrm{E} \quad 00$

$3.87893 \mathrm{E} \quad 00$

$3.89986 \mathrm{E} \quad 00$

$3.92084 \mathrm{E} \quad 00$ 
ELECTRON RADIAL FUNCTIONS

Positron

$\begin{array}{cc}p & F_{0} \\ 0.10 & 1.88993 \mathrm{E}-01 \\ 0.20 & 4.64667 \mathrm{E}-01 \\ 0.30 & 6.05507 \mathrm{E}-01 \\ 0.40 & 6.84715 \mathrm{E}-01 \\ 0.50 & 7.33934 \mathrm{E}-01 \\ & \\ 0.60 & 7.66727 \mathrm{E}-01 \\ 0.70 & 7.89692 \mathrm{E}-01 \\ 0.80 & 8.06387 \mathrm{E}-01 \\ 0.90 & 8.18882 \mathrm{E}-01 \\ 1.00 & 8.28460 \mathrm{E}-01\end{array}$

$1.10 \quad 8.35946 \mathrm{E}-01$

$1.20 \quad 8.41897 \mathrm{E}-01$

$1.30 \quad 8.46694 \mathrm{E}-01$

$1.40 \quad 8.50612 \mathrm{E}-01$

$1.50 \quad 8.53847 \mathrm{E}-01$

$1.60 \quad 8.56545 \mathrm{E}-01$

$1.70 \quad 8.58816 \mathrm{E}-01$

$1.80 \quad 8.60741 \mathrm{E}-01$

$1.90 \quad 8.62386 \mathrm{E}-01$

$2.00 \quad 8.63801 \mathrm{E}-01$

$2.10 \quad 8.65026 \mathrm{E}-01$

$2.20 \quad 8.66090 \mathrm{E}-01$

$2.30 \quad 8.67022 \mathrm{E}-01$

$2.40 \quad 8.67840 \mathrm{E}-01$

$2.50 \quad 8.68561 \mathrm{E}-01$

$2.60 \quad 8.69200 \mathrm{E}-01$

$2.70 \quad 8.69769 \mathrm{E}-01$

$2.80 \quad 8.70275 \mathrm{E}-01$

$2.90 \quad 8.70729 \mathrm{E}-01$

$3.00 \quad 8.71136 \mathrm{E}-01$

$3.10 \quad 8.71502 \mathrm{E}-01$

$3.20 \quad 8.71832 \mathrm{E}-01$

$3.30 \quad 8.72131 \mathrm{E}-01$

$3.40 \quad 8.72401 \mathrm{E}-01$

$3.50 \quad 8.72648 \mathrm{E}-01$

$3.60 \quad 8.72871 \mathrm{E}-01$

$3.70 \quad 8.73075 \mathrm{E}-01$

$3.80 \quad 8.73260 \mathrm{E}-01$

$3.90 \quad 8.73430 \mathrm{E}-01$

$4.00 \quad 8.73586 \mathrm{E}-01$

$4.10 \quad 8.73729 \mathrm{E}-01$

$4.20 \quad 8.73860 \mathrm{E}-01$

$4.30 \quad 8.73980 \mathrm{E}-01$

$4.40 \quad 8.74090 \mathrm{E}-01$

$4.50 \quad 8.74192 \mathrm{E}-01$

$4.60 \quad 8.74286 \mathrm{E}-01$

$4.70 \quad 8.74372 \mathrm{E}-01$

$4.80 \quad 8.74452 \mathrm{E}-0 \mathrm{l}$

$4.90 \quad 8.74526 \mathrm{E}-01$

$5.00 \quad 8.74593 \mathrm{E}-01$

$5.10 \quad 8.74656 \mathrm{E}-01$

$5.20 \quad 8.74714 \mathrm{E}-01$

$5.30 \quad 8.74767 \mathrm{E}-01$

$5.40 \quad 8.74817 \mathrm{E}-01$

$5.50 \quad 8.74863 \mathrm{E}-01$

$5.60 \quad 8.74905 \mathrm{E}-01$

$5.70 \quad 8.74944 \mathrm{E}-01$

$5.80 \quad 8.74980 \mathrm{E}-01$

$5.90 \quad 8.75013 \mathrm{E}-01$

$6.00 \quad 8.75044 \mathrm{E}-01$

$Z=6 \quad A=12$

$f_{1}$

$3.32716 \mathrm{E}-03$

$1.94269 \mathrm{E}-02$

$4.84267 \mathrm{E}-02$

$8.90309 \mathrm{E}-02$

$1.39702 \mathrm{E}-01$

$1.98856 \mathrm{E}-01$

$2.65005 \mathrm{E}-01$

$3.36819 \mathrm{E}-01$

$4.13173 \mathrm{E}-01$

$4.93136 \mathrm{E}-01$

$5.75955 \mathrm{E}-01$ $6.61032 \mathrm{E}-01$

$7.47894 \mathrm{E}-01$

$8.36170 \mathrm{E}-01$

$9.25569 \mathrm{E}-01$

$1.01586 \mathrm{E} \quad 00$

$1.10687 \mathrm{E} \quad 00$

$1.19845 \mathrm{E} \quad 00$

$1.29050 \mathrm{E} \quad 00$

$1.38291 \mathrm{E} \quad 00$

$1.47563 \mathrm{E} \quad 00$

$1.56860 \mathrm{E} \quad 00$

$1.66177 \mathrm{E} \quad 00$

$1.75510 \mathrm{E} \quad 00$

$1.84858 \mathrm{E} \quad 00$

$1.94216 \mathrm{E} \quad 00$ $2.03583 \mathrm{E} \quad 00$ $2.12958 \mathrm{E} \quad 00$ $2.22340 \mathrm{E} \quad 00$ $2.31726 \mathrm{E} \quad 00$

$2.41117 \mathrm{E} \quad 00$ $2.50512 \mathrm{E} \quad 00$ $2.59910 \mathrm{E} \quad 00$

$2.69310 \mathrm{E} \quad 00$

$2.78713 \mathrm{E} \quad 00$

$2.88117 \mathrm{E} \quad 00$ $2.97523 \mathrm{E} \quad 00$ $3.06930 \mathrm{E} \quad 00$

$3.16338 \mathrm{E} \quad 00$

$3.25747 \mathrm{E} \quad 00$

$3.35157 \mathrm{E} \quad 00$

$3.44567 \mathrm{E} \quad 00$

$3.53978 \mathrm{E} \quad 00$

$3.63390 \mathrm{E} \quad 00$

$3.72802 \mathrm{E} \quad 00$

$3.82214 \mathrm{E} \quad 00$ $3.91626 \mathrm{E} \quad 00$ $4.01039 \mathrm{E} \quad 00$

$4.10453 \mathrm{E} \quad 00$

$4.19865 \mathrm{E} \quad 00$

$4.29279 \mathrm{E} \quad 00$ $4.38693 \mathrm{E} \quad 00$ $4.48106 \mathrm{E} \quad 00$ $4.57520 \mathrm{E} \quad 00$ $4.66934 \mathrm{E} \quad 00$

$4.76348 \mathrm{E} \quad 00$ $4.85762 \mathrm{E} \quad 00$ $4.95176 \mathrm{E} \quad 00$

$5.04590 \mathrm{E} \quad 00$

$5.14004 \mathrm{E} \quad 00$ $g_{1}$

$-4.11380 \mathrm{E}-05$

$-2.39460 \mathrm{E}-04$

$-5.93903 \mathrm{E}-04$

$-1.08432 \mathrm{E}-03$

$-1.68673 \mathrm{E}-03$

$-2.37633 \mathrm{E}-03$

$-3.12971 \mathrm{E}-03$

$-3.92593 \mathrm{E}-03$

$-4.74716 \mathrm{E}-03$

$-5.57863 \mathrm{E}-03$

$-6.40836 \mathrm{E}-03$

$-7.22681 \mathrm{E}-03$

$-8.02639 \mathrm{E}-03$

$-8.80111 \mathrm{E}-03$

$-9.54630 \mathrm{E}-03$

$-1.02582 \mathrm{E}-02$

$-1.09340 \mathrm{E}-02$

$-1.15713 \mathrm{E}-02$

$-1.21683 \mathrm{E}-02$

$-1.27235 \mathrm{E}-02$

$-1.32358 \mathrm{E}-02$

$-1.37042 \mathrm{E}-02$

$-1.41279 \mathrm{E}-02$

$-1.45065 \mathrm{E}-02$

$-1.48392 \mathrm{E}-02$

$-1.51258 \mathrm{E}-02$

$-1.53660 \mathrm{E}-02$

$-1.55593 \mathrm{E}-02$

$-1.57057 \mathrm{E}-02$

$-1.58048 \mathrm{E}-02$

$-1.58566 \mathrm{E}-02$

$-1.58609 \mathrm{E}-02$

$-1.58177 \mathrm{E}-02$

$-1.57267 \mathrm{E}-02$

$-1.55879 \mathrm{E}-02$

$-1.54013 \mathrm{E}-02$

$-1.51668 \mathrm{E}-02$

$-1.48844 \mathrm{E}-02$

$-1.45539 \mathrm{E}-02$

- 1.41755 E-02

$-1.37489 \mathrm{E}-02$

$-1.32743 \mathrm{E}-02$

$-1.27516 \mathrm{E}-02$

$-1.21807 \mathrm{E}-02$

$-1.15616 \mathrm{E}-02$

$-1.08944 \mathrm{E}-02$

$-1.01790 \mathrm{E}-02$

$-9.41539 \mathrm{E}-03$

$-8.60357 \mathrm{E}-03$

$-7.74351 \mathrm{E}-03$

$-6.83522 \mathrm{E}-03$

$-5.87868 \mathrm{E}-03$

$-4.87389 \mathrm{E}-03$

$-3.82083 \mathrm{E}-03$

$-2.71951 \mathrm{E}-03$

$-1.56991 \mathrm{E}-03$

$-3.72025 \mathrm{E}-04$

$8.74142 \mathrm{E}-04$

$2.16860 \mathrm{E}-03$

$3.51135 \mathrm{E}-03$ $\tan \delta_{1}$

$1.94195 \mathrm{E}-01$

9.46184E-02

$6.29416 \mathrm{E}-02$

$4.72058 \mathrm{E}-02$

$3.77508 \mathrm{E}-02$

$3.14202 \mathrm{E}-02$

$2.68731 \mathrm{E}-02$

$2.34419 \mathrm{E}-02$

$2.07564 \mathrm{E}-02$

$1.85947 \mathrm{E}-02$

$1.68156 \mathrm{E}-02$

$1.53246 \mathrm{E}-02$

$1.40563 \mathrm{E}-02$

$1.29637 \mathrm{E}-02$

$1.20125 \mathrm{E}-02$

$1.11766 \mathrm{E}-02$

$1.04361 \mathrm{E}-02$

$9.77547 \mathrm{E}-03$

$9.18236 \mathrm{E}-03$

$8.64686 \mathrm{E}-03$

$8.16095 \mathrm{E}-03$

$7.71799 \mathrm{E}-03$

$7.31252 \mathrm{E}-03$

$6.93997 \mathrm{E}-03$

$6.59646 \mathrm{E}-03$

$6.27871 \mathrm{E}-03$

$5.98394 \mathrm{E}-03$

$5.70974 \mathrm{E}-03$

$5.45401 \mathrm{E}-03$

$5.21494 \mathrm{E}-03$

$4.99097 \mathrm{E}-03$

$4.78071 \mathrm{E}-03$

$4.58293 \mathrm{E}-03$

$4.39655 \mathrm{E}-03$

$4.22061 \mathrm{E}-03$

$4.05428 \mathrm{E}-03$

$3.89676 \mathrm{E}-03$

$3.74739 \mathrm{E}-03$

$3.60555 \mathrm{E}-03$

$3.47069 \mathrm{E}-03$

$3.34230 \mathrm{E}-03$

$3.21993 \mathrm{E}-03$

$3.10316 \mathrm{E}-03$

$2.99163 \mathrm{E}-03$

$2.88498 \mathrm{E}-03$

$2.78290 \mathrm{E}-03$

$2.68512 \mathrm{E}-03$

$2.59136 \mathrm{E}-03$

$2.50137 \mathrm{E}-03$

$2.41496 \mathrm{E}-03$

$2.33188 \mathrm{E}-03$

$2.25197 \mathrm{E}-03$

$2.17504 \mathrm{E}-03$

2.10094E-03

$2.02951 \mathrm{E}-03$

$1.96060 \mathrm{E}-03$

$1.89410 \mathrm{E}-03$

$1.82987 \mathrm{E}-03$

$1.76780 \mathrm{E}-03$

$1.70778 \mathrm{E}-03$

$f_{-1}$

$1.07464 \mathrm{E}-03$

$3.35058 \mathrm{E}-03$

$5.68386 \mathrm{E}-03$

$7.95945 \mathrm{E}-03$

$1.01474 \mathrm{E}-02$

1. $22357 \mathrm{E}-02$

$1.42201 \mathrm{E}-02$

$1.61011 \mathrm{E}-02$

$1.78815 \mathrm{E}-02$

$1.95659 \mathrm{E}-02$

$2.11590 \mathrm{E}-02$

$2.26658 \mathrm{E}-02$

$2.40910 \mathrm{E}-02$

$2.54387 \mathrm{E}-02$

$2.67127 \mathrm{E}-02$

$2.79163 \mathrm{E}-02$

$2.90525 \mathrm{E}-02$

$3.01236 \mathrm{E}-02$

$3.11318 \mathrm{E}-02$

$3.20789 \mathrm{E}-02$

$3.29665 \mathrm{E}-02$

$3.37960 \mathrm{E}-02$

$3.45685 \mathrm{E}-02$

$3.52850 \mathrm{E}-02$

$3.59464 \mathrm{E}-02$

$3.65534 \mathrm{E}-02$

$3.71068 \mathrm{E}-02$

$3.76070 \mathrm{E}-02$

3.80547 E- 02

$3.84501 \mathrm{E}-02$

$3.87938 \mathrm{E}-02$

$3.90860 \mathrm{E}-02$

$3.93270 \mathrm{E}-02$

$3.95171 \mathrm{E}-02$

$3.96566 \mathrm{E}-02$

3.97457 E- 02

$3.97845 \mathrm{E}-02$

$3.97731 \mathrm{E}-02$

$3.97119 \mathrm{E}-02$

$3.96009 \mathrm{E}-02$

3.94401 E-02

$3.92298 \mathrm{E}-02$

$3.89700 \mathrm{E}-02$

$3.86609 \mathrm{E}-02$

$3.83024 \mathrm{E}-02$

$3.78946 \mathrm{E}-02$

$3.74377 \mathrm{E}-02$

$3.69317 \mathrm{E}-02$

$3.63766 \mathrm{E}-02$

$3.57725 \mathrm{E}-02$

$3.51195 \mathrm{E}-02$ 
ELECTRON RADIAL FUNCTIONS

$Z=6 \quad A=12 \quad \rho=1.3 A^{1 / 3} F$

Positron

$\bar{F}$

$p$

0.10

0.20

0.30

0.40

0.50

0.60

0.70

0.80

0.90

1.00

1.10

1.20

1.30

1.40

1.50

1.60

1.70

1.80

1.90

2.00

2.10

2.20

2.30

2.40

2.50

2.60

2.70

2.80

2.90

3.00

3.10

3.20

3.30

3.40

3.50

3.60

3.70

3.80

3.90

4.00

4.10

4.20

4.30

4.40

4.50

4.60

4.70

4.80

4.90

5.00

5.10

5.20

5.30

5.40

5.50

5.60

5.70

5.80

5.90

6.00 $f_{2}$

$3.69269 \mathrm{E}-09$

3.19551 E- 08

$9.13459 \mathrm{E}-08$

$1.82010 \mathrm{E}-07$

$3.03599 \mathrm{E}-07$

$4.55734 \mathrm{E}-07$

$6.38080 \mathrm{E}-07$

$8.50361 \mathrm{E}-07$

$1.09235 \mathrm{E}-06$

$1.36388 \mathrm{E}-06$

$1.66481 \mathrm{E}-06$

$1.99503 \mathrm{E}-06$

$2.35445 \mathrm{E}-06$

2.74301 E- 06

$3.16064 \mathrm{E}-06$

$3.60733 \mathrm{E}-06$

$4.08301 \mathrm{E}-06$

$4.58768 \mathrm{E}-06$

$5.12131 \mathrm{E}-06$

$5.68388 \mathrm{E}-06$

$6.27537 \mathrm{E}-06$

$6.89577 \mathrm{E}-06$

$7.54508 \mathrm{E}-06$

$8.22329 \mathrm{E}-06$

$8.93037 \mathrm{E}-06$

$9.66633 \mathrm{E}-06$

$1.04312 \mathrm{E}-0.5$

$1.12249 \mathrm{E}-0.5$

$1.20475 \mathrm{E}-05$

$1.28989 \mathrm{E}-0.5$

$1.37792 \mathrm{E}-05$

$1.46884 \mathrm{E}-05$

$1.56263 \mathrm{E}-05$

$1.65932 \mathrm{E}-0.5$

$1.75889 \mathrm{E}-05$

$1.86135 \mathrm{E}-05$

$1.96669 \mathrm{E}-05$

$2.07491 \mathrm{E}-05$

$2.18602 \mathrm{E}-05$

2.30001 E- 05

$2.41688 \mathrm{E}-05$

$2.53664 \mathrm{E}-05$

$2.65929 \mathrm{E}-05$

$2.78481 \mathrm{E}-05$

$2.91322 \mathrm{E}-05$

$3.04451 \mathrm{E}-0.5$

$3.17869 \mathrm{E}-0.5$

$3.31575 \mathrm{E}-05$

$3.45569 \mathrm{E}-0.5$

$3.59852 \mathrm{E}-05$

$3.74423 \mathrm{E}-0.5$ $3.89282 \mathrm{E}-05$

$4.04429 \mathrm{E}-05$

$4.19865 \mathrm{E}-05$

4.35589E-0.5

$4.51601 \mathrm{E}-0.5$

$4.67902 \mathrm{E}-0.5$

$4.84490 \mathrm{E}-05$

$5.01368 \mathrm{E}-05$

$5.18533 \mathrm{E}-05$ $g_{2}$

$8.62934 \mathrm{E}-07$

$9.98602 \mathrm{E}-06$

$3.72770 \mathrm{E}-0.5$

$9.13292 \mathrm{E}-0.5$

$1.79099 \mathrm{E}-04$

$3.05896 \mathrm{E}-04$

$4.75576 \mathrm{E}-04$

$6.90798 \mathrm{E}-04$

$9.53324 \mathrm{E}-04$

$1.26426 \mathrm{E}-03$

$1.62426 \mathrm{E}-03$

$2.03368 \mathrm{E}-03$

$2.49269 \mathrm{E}-03$

$3.00132 \mathrm{E}-03$

$3.55954 \mathrm{E}-03$

$4.16728 \mathrm{E}-03$

$4.82444 \mathrm{E}-03$

$5.53091 \mathrm{E}-03$

$6.28659 \mathrm{E}-03$

$7.09139 \mathrm{E}-03$

$7.94519 \mathrm{E}-03$

$8.84792 \mathrm{E}-03$

$9.79950 \mathrm{E}-03$

$1.07999 \mathrm{E}-02$

$1.18489 \mathrm{E}-02$

$1.29466 \mathrm{E}-02$

$1.40929 \mathrm{E}-02$

$1.52878 \mathrm{E}-02$

$1.65311 \mathrm{E}-02$

$1.78229 \mathrm{E}-02$

$1.91632 \mathrm{E}-02$

$2.05518 \mathrm{E}-02$

$2.19888 \mathrm{E}-02$

$2.34741 \mathrm{E}-02$

$2.50078 \mathrm{E}-02$

$2.65897 \mathrm{E}-02$

$2.82199 \mathrm{E}-02$

$2.98983 \mathrm{E}-02$

$3.16249 \mathrm{E}-02$

$3.33997 \mathrm{E}-02$

$3.52227 \mathrm{E}-02$

$3.70938 \mathrm{E}-02$

$3.90130 \mathrm{E}-02$

$4.09803 \mathrm{E}-02$

$4.29956 \mathrm{E}-02$

$4.50589 \mathrm{E}-02$

$4.71702 \mathrm{E}-02$

$4.93292 \mathrm{E}-02$

$5.15362 \mathrm{E}-02$

$5.37906 \mathbf{E}-02$

$5.60927 \mathrm{E}-02$

$5.84419 \mathrm{E}-02$

$6.08379 \mathrm{E}-02$

$6.32800 \mathrm{E}-02$

$6.57669 \mathrm{E}-02$

$6.82964 \mathrm{E}-02$

$7.08636 \mathrm{E}-02$

$7.34530 \mathrm{E}-02$

$7.60009 \mathrm{E}-02$

$7.70543 \mathrm{E}-02$
$-5.96932 \mathrm{E}-09$ $-6.88498 \mathrm{E}-08$ $-2.55619 \mathrm{E}-07$

$-6.21622 \mathrm{E}-07$

$-1.20770 \mathrm{E}-06$

$-2.04000 \mathrm{E}-06$

$-3.13165 \mathrm{E}-06$

$-4.48502 \mathrm{E}-06$

$-6.09433 \mathrm{E}-06$

$-7.94785 \mathrm{E}-06$

$-1.00297 \mathrm{E}-05$

$-1.23214 \mathrm{E}-05$

$-1.48023 \mathrm{E}-0.5$

$-1.74510 \mathrm{E}-05$

$-2.02450 \mathrm{E}-05$

$-2.31618 \mathrm{E}-0.5$

$-2.61782 \mathrm{E}-05$

$-2.92714 \mathrm{E}-05$

$-3.24182 \mathrm{E}-05$

$-3.55956 \mathrm{E}-05$

$-3.87805 \mathrm{E}-0.5$

$-4.19500 \mathrm{E}-05$

$-4.50812 \mathrm{E}-05$

$-4.81513 \mathrm{E}-05$

$-5.11374 \mathrm{E}-05$

$-5.40168 \mathrm{E}-05$

$-5.67669 \mathrm{E}-05$

$-5.93650 \mathrm{E}-05$

$-6.17885 \mathrm{E}-05$

$-6.40149 \mathrm{E}-05$

$-6.60215 \mathrm{E}-0.5$

$-6.77860 \mathrm{E}-0.5$

$-6.92859 \mathrm{E}-05$

$-7.04987 \mathrm{E}-0.5$

$-7.14019 \mathrm{E}-05$

$-7.19732 \mathrm{E}-05$

$-7.21902 \mathrm{E}-05$

$-7.20305 \mathrm{E}-05$

$-7.14717 \mathrm{E}-05$

$-7.04916 \mathrm{E}-05$

$-6.90678 \mathrm{E}-05$

$-6.71778 \mathrm{E}-05$

$-6.47996 \mathrm{E}-05$

$-6.19106 \mathrm{E}-05$

$-5.84887 \mathrm{E}-05$

$-5.45115 \mathrm{E}-05$

$-4.99569 \mathrm{E}-05$

$-4.48025 \mathrm{E}-05$

$-3.90263 \mathrm{E}-05$

$-3.26059 \mathrm{E}-05$

$-2.55193 \mathrm{E}-05$

$-1.77445 \mathrm{E}-05$

$-9.25947 \mathrm{E}-06$

$-4.26607 \mathrm{E}-08$

$9.92712 \mathrm{E}-06$

$2.06699 \mathrm{E}-05$

$3.22032 \mathrm{E}-05$

$4.45352 \mathrm{E}-0.5$

$5.76280 \mathrm{E}-05$

$7.01403 \mathrm{E}-05$

$\begin{array}{rcc}\tan \delta_{2} & f_{-2} \\ -2.31124 \mathrm{E} & 00 & 1.71681 \mathrm{E}-0 \\ -4.78710 \mathrm{E} & 00 & 1.00410 \mathrm{E}-0 \\ -7.10542 \mathrm{E} & 00 & 2.52237 \mathrm{E}-0 \\ -9.27020 \mathrm{E} & 00 & 4.68754 \mathrm{E}-0 \\ -1.12671 \mathrm{E} & 01 & 7.45258 \mathrm{E}-0\end{array}$

$1.07678 \mathrm{E}-0.5$

$1.45843 \mathrm{E}-05$

$1.88558 \mathrm{E}-05$

$2.35396 \mathrm{E}-05$

$2.85971 \mathrm{E}-05$

$3.39925 \mathrm{E}-05$

$3.96935 \mathrm{E}-0.5$

$4.56697 \mathrm{E}-05$

$5.18928 \mathrm{E}-0.5$

$5.83360 \mathrm{E}-05$

$6.49734 \mathrm{E}-05$

$7.17802 \mathrm{E}-05$

$7.87322 \mathrm{E}-05$

$8.58055 \mathrm{E}-05$

$9.29768 \mathrm{E}-05$

$1.00223 \mathrm{E}-04$

$1.07521 \mathrm{E}-04$

$1.14849 \mathrm{E}-04$

$1.22183 \mathrm{E}-04$

$1.29502 \mathrm{E}-04$

$1.36782 \mathrm{E}-04$

$1.44002 \mathrm{E}-04$

$1.51139 \mathrm{E}-04$

$1.58171 \mathrm{E}-04$

$1.65075 \mathrm{E}-04$

$1.71829 \mathrm{E}-04$

$1.78412 \mathrm{E}-04$

$1.84800 \mathrm{E}-04$

$1.90972 \mathrm{E}-04$

$1.96906 \mathrm{E}-04$

$2.02578 \mathrm{E}-04$

$2.07968 \mathrm{E}-04$

$2.13052 \mathrm{E}-04$

$2.17809 \mathrm{E}-04$

$2.22216 \mathrm{E}-04$

$2.26251 \mathrm{E}-04$

$2.29893 \mathrm{E}-04$

$2.33117 \mathrm{E}-04$

$2.35904 \mathrm{E}-04$

$2.38230 \mathrm{E}-04$

2.40072E-04 
ELECTRON RADIAL FUNCTIONS

Positron

$\begin{array}{cc}p & F_{0} \\ 6.10 & 8.75072 \mathrm{E}-01 \\ 6.20 & 8.75099 \mathrm{E}-01 \\ 6.30 & 8.75123 \mathrm{E}-01 \\ 6.40 & 8.75145 \mathrm{E}-01 \\ 6.50 & 8.75166 \mathrm{E}-01 \\ 6.60 & 8.75185 \mathrm{E}-01 \\ 6.70 & 8.75201 \mathrm{E}-01 \\ 6.80 & 8.75217 \mathrm{E}-01 \\ 6.90 & 8.75231 \mathrm{E}-01 \\ 7.00 & 8.75245 \mathrm{E}-01\end{array}$

7.10

7.20

7.30

7.40

7.50

7.60

7.70

7.80

7.90

8.00

8.10

8.20

8.30

8.40

8.50

8.60

8.70

8.80

8.90

9.00

9.10

9.20

9.30

9.40

9.50

9.60

9.70

9.80

9.90

10.00

10.10

10.20

10.30

10.40

10.50

10.60

10.70

10.80

10.90

11.00

11.10

11.20

11.30

11.40

11.50

11.60

11.70

11.80

11.90

12.00
$8.75256 \mathrm{E}-01$

$8.75266 \mathrm{E}-01$

$8.75276 \mathrm{E}-01$

$8.75285 \mathrm{E}-01$

$8.75292 \mathrm{E}-01$

$8.75299 \mathrm{E}-01$

$8.75305 \mathrm{E}-01$

$8.75315 \mathrm{E}-01$

$8.75319 \mathrm{E}-01$

$8.75322 \mathrm{E}-01$

$8.75325 \mathrm{E}-01$

$8.75326 \mathrm{E}-0]$

$8.75327 \mathrm{E}-01$

$8.75328 \mathrm{E}-01$

$8.75329 \mathrm{E}-01$

$8.75329 \mathrm{E}-01$

$8.75328 \mathrm{E}-01$

$8.75327 \mathrm{E}-01$

$8.75326 \mathrm{E}-01$

$8.75324 \mathrm{E}-01$

$8.75322 \mathrm{E}-01$

$8.75320 \mathrm{E}-01$

$8.75317 \mathrm{E}-01$

$8.75314 \mathrm{E}-01$

$8.75312 \mathrm{E}-01$

$8.75308 \mathrm{E}-01$

$8.75304 \mathrm{E}-01$

$8.75300 \mathrm{E}-01$

$8.75296 \mathrm{E}-01$

$8.75292 \mathrm{E}-01$

$8.75287 \mathrm{E}-01$

$8.75283 \mathrm{E}-01$

$8.75278 \mathrm{E}-01$

$8.75273 \mathrm{E}-01$

$8.75267 \mathrm{E}-01$

$8.75262 \mathrm{E}-01$

$8.75256 \mathrm{E}-01$

$8.75251 \mathrm{E}-01$

$8.75240 \mathrm{E}-01$

$8.75233 \mathrm{E}-01$

$8.75227 \mathrm{E}-01$

$8.75221 \mathrm{E}-01$

$8.75215 \mathrm{E}-01$

$8.75208 \mathrm{E}-01$

$8.75202 \mathrm{E}-01$

$8.75195 \mathrm{E}-01$

$8.75189 \mathrm{E}-01$

$8.75182 \mathrm{E}-01$
$8.75310 \mathrm{E}-01$

$8.75245 \mathrm{E}-01$
$Z=6$

$A=12$

$\rho=1.3 A^{1 / 3} F$ $f_{1}$

$5.23417 \mathrm{E} \quad 00$

$5.32830 \mathrm{~F}$

$5.42242 \mathrm{E} \quad 00$

$5.51653 \mathrm{E} \quad 00$

$5.61062 \mathrm{E} \quad 00$

$5.70469 \mathrm{E} \quad 00$

$5.79872 \mathrm{E} \quad 00$

$5.89271 \mathrm{E} \quad 00$

$5.98661 \mathrm{E} \quad 00$

$6.08037 \mathrm{E} \quad 00$

$6.17387 \mathrm{E} \quad 00$

$6.26678 \mathrm{E} \quad 00$

$6.35797 \mathrm{E} \quad 00$

$6.43803 \mathrm{E} \quad 00$

$6.58082 \mathrm{E} \quad 00$

$6.65691 \mathrm{E} \quad 00$

$6.74779 \mathrm{E} \quad 00$

$6.84064 \mathrm{E} \quad 00$

$6.93418 \mathrm{E} \quad 00$

$7.02798 \mathrm{E} \quad 00$

$7.12195 \mathrm{E} \quad 00$

$7.21602 \mathrm{E} \quad 00$

$7.31017 \mathrm{E} \quad 00$

$7.40435 \mathrm{E} \quad 00$

$7.49857 \mathrm{E} \quad 00$

$7.59284 \mathrm{E} \quad 00$

$7.68711 \mathrm{E} \quad 00$

$7.78142 \mathrm{E} \quad 00$

$7.87573 \mathrm{E} \quad 00$

$7.97007 \mathrm{E} \quad 00$

$8.06441 \mathrm{E} \quad 00$ $8.15879 \mathrm{E} \quad 00$ $8.25317 \mathrm{E} \quad 00$ $8.34757 \mathrm{E} \quad 00$ $8.44197 \mathrm{E} \quad 00$

$8.53640 \mathrm{E} \quad 00$ $8.63083 \mathrm{E} \cdot 00$ $8.72527 \mathrm{E} \quad 00$ $8.81973 \mathrm{E} \quad 00$ $8.91420 \mathrm{E} \quad 00$

$9.00869 \mathrm{E} \quad 00$ $9.10318 \mathrm{E} \quad 00$ $9.19769 \mathrm{E} \quad 00$ $9.29221 \mathrm{E} \quad 00$ $9.38674 \mathrm{E} \quad 00$

$9.48128 \mathrm{E} \quad 00$ $9.57583 \mathrm{E} \quad 00$ $9.67040 \mathrm{E} \quad 00$ $9.76498 \mathrm{E} \quad 00$ $9.85958 \mathrm{E} \quad 00$

$9.95418 \mathrm{E} \quad 00$ $1.00488 \mathrm{E} 01$ $1.01434 \mathrm{E} \quad 01$ $1.02381 \mathrm{E} 01$ $1.03327 \mathrm{E} \quad 01$

$1.04274 \mathrm{E} \quad 01$ $\begin{array}{lll}1.05221 \mathrm{E} & 01\end{array}$ $1.06168 \mathrm{E} \quad 01$ $\begin{array}{ll}1.07115 \mathrm{E} & 01\end{array}$ $1.08062 \mathrm{E} \quad 01$ $g_{1}$

$4.90241 \mathrm{E}-03$

$6.34 .174 \mathrm{E}-03$

$7.82937 \mathrm{E}-03$

$9.36528 \mathrm{E}-03$

$1.09494 \mathrm{E}-02$

$1.25819 \mathrm{E}-02$

$1.42625 \mathrm{E}-02$

$1.59912 \mathrm{E}-02$

$1.77679 \mathrm{E}-02$

$1.95925 \mathrm{E}-02$

$2.14642 \mathrm{E}-02$

$2.33817 \mathrm{E}-02$

$2.53401 \mathrm{E}-02$

$2.72981 \mathrm{E}-02$

$2.95792 \mathrm{E}-02$

$3.16167 \mathrm{E}-02$

$3.37673 \mathrm{E}-02$

$3.59750 \mathrm{E}-02$

$3.82342 \mathrm{E}-02$

$4.05429 \mathrm{E}-02$

$4.29008 \mathrm{E}-02$

$4.53076 \mathrm{E}-02$

$4.77632 \mathrm{E}-02$

$5.02674 \mathrm{E}-02$

$5.28203 \mathrm{E}-02$

$5.54219 \mathrm{E}-02$

$5.80720 \mathrm{E}-02$

$6.07708 \mathrm{E}-02$

$6.35181 \mathrm{E}-02$

$6.63140 \mathrm{E}-02$

$6.91585 \mathrm{E}-02$

$7.20517 \mathrm{E}-02$

$7.49934 \mathrm{E}-02$

$7.79838 \mathrm{E}-02$

$8.10227 \mathrm{E}-02$

$8.41104 \mathrm{E}-02$

$8.72467 \mathrm{E}-02$

$9.04316 \mathrm{E}-02$

$9.36652 \mathrm{E}-02$

$9.69475 \mathrm{E}-02$

$1.00278 \mathrm{E}-01$

$1.03658 \mathrm{E}-01$

$1.07086 \mathrm{E}-01$

$1.10564 \mathrm{E}-01$

$1.14089 \mathrm{E}-01$

$1.17664 \mathrm{E}-01$

$1.21287 \mathrm{E}-01$

$1.24959 \mathrm{E}-01$

$1.28680 \mathrm{E}-01$

$1.32450 \mathrm{E}-01$

$1.36268 \mathrm{E}-01$

$1.40135 \mathrm{E}-01$

$1.44051 \mathrm{E}-01$

$1.48016 \mathrm{E}-01$

$1.52030 \mathrm{E}-01$

$1.56093 \mathrm{E}-01$

$1.60204 \mathrm{E}-01$

$1.64365 \mathrm{E}-01$

$1.68574 \mathrm{E}-01$

$1.72832 \mathrm{E}-01$

$\tan \delta_{1}$

$1.64972 \mathrm{E}-03$

$1.59353 \mathrm{E}-03$

$1.53911 \mathrm{E}-03$

$1.48639 \mathrm{E}-03$

$1.43528 \mathrm{E}-03$

$1.38571 \mathrm{E}-03$

$1.33762 \mathrm{E}-03$

$1.29093 \mathrm{E}-03$

$1.24561 \mathrm{E}-03$

$1.20157 \mathrm{E}-03$

$1.15878 \mathrm{E}-03$

$1.11717 \mathrm{E}-03$

$1.07671 \mathrm{E}-03$

$1.03733 \mathrm{E}-03$

$9.99016 \mathrm{E}-04$

$9.61714 \mathrm{E}-04$

$9.25381 \mathrm{E}-04$

$8.89971 \mathrm{E}-04$

$8.55478 \mathrm{E}-04$

$8.21839 \mathrm{E}-04$

$7.89040 \mathrm{E}-04$

$7.57050 \mathrm{E}-04$

$7.25834 \mathrm{E}-04$

$6.95377 \mathrm{E}-04$

$6.65634 \mathrm{E}-04$

$6.36588 \mathrm{E}-04$

$6.08216 \mathrm{E}-04$

$5.80499 \mathrm{E}-04$

$5.53415 \mathrm{E}-0$.

$5.26937 \mathrm{E}-04$

$5.01048 \mathrm{E}-04$

$4.75729 \mathrm{E}-04$

$4.50969 \mathrm{E}-04$

$4.26741 \mathrm{E}-04$

$4.03033 \mathrm{E}-04$

$3.79827 \mathrm{E}-04$

$3.57110 \mathrm{E}-04$

$3.34872 \mathrm{E}-04$

$3.13084 \mathrm{E}-04$

$2.91742 \mathrm{E}-04$

$2.70835 \mathrm{E}-04$

$2.50343 \mathrm{E}-04$

$2.30268 \mathrm{E}-04$

$2.10580 \mathrm{E}-04$

$1.91283 \mathrm{E}-04$

$1.72363 \mathrm{E}-04$

$1.53802 \mathrm{E}-04$

$1.35597 \mathrm{E}-04$

$1.17736 \mathrm{E}-04$

$1.00212 \mathrm{E}-04$

$8.30137 \mathrm{E}-05$

$6.61379 \mathrm{E}-05$

$4.95672 \mathrm{E}-05$

$3.32976 \mathrm{E}-05$

$1.73262 \mathrm{E}-05$

$1.62951 \mathrm{E}-06$

$-1.37722 \mathrm{E}-05$

$-2.89096 \mathrm{E}-05$

$-4.37782 \mathrm{E}-05$ 
ELECTRON RADIAL FUNCTIONS

$Z=6 \quad A=12 \quad \rho=1.3 A^{1 / 3} F$

Prositron

$\bar{F}$

$5.35986 \mathrm{E}-0.5$

$5.53728 \mathrm{E}-05$

$5.71758 \mathrm{E}-0.5$

$5.90076 \mathrm{E}-0.5$

$6.08682 \mathrm{E}-05$

$6.27577 \mathrm{E}-05$ $6.46759 \mathrm{E}-0.5$

$6.66230 \mathrm{E}-05$

$6.85989 \mathrm{E}-05$

$7.06036 \mathrm{E}-05$

$7.26372 \mathrm{E}-05$

$7.46995 \mathrm{E}-05$

$7.67907 \mathrm{E}-05$

$7.89107 \mathrm{E}-05$

$8.10596 \mathrm{E}-05$

$8.32372 \mathrm{E}-05$ $8.54436 \mathrm{E}-05$ $8.76788 \mathrm{E}-05$ $8.99429 \mathrm{E}-05$ $9.22358 \mathrm{E}-05$

$9.45575 \mathrm{E}-05$

$9.69080 \mathrm{E}-05$

$9.92874 \mathrm{E}-05$

$1.01695 \mathrm{E}-04$

$1.04132 \mathrm{E}-04$

$1.06598 \mathrm{E}-04$

$1.09093 \mathrm{E}-04$

$1.11616 \mathrm{E}-04$

$1.14168 \mathrm{E}-04$

$1.16749 \mathrm{E}-04$

$1.19359 \mathrm{E}-04$ $1.21998 \mathrm{E}-04$ $1.24665 \mathrm{E}-04$ $1.27361 \mathrm{E}-04$ $1.30086 \mathrm{E}-04$

9.60

9.70

9.80

9.90

10.00

$1.32840 \mathrm{E}-04$

$1.35623 \mathrm{E}-04$

$1.38435 \mathrm{E}-04$

$1.41275 \mathrm{E}-04$

$1.44144 \mathrm{E}-04$

10.10

10.20

10.30

10.40

10.50

$1.47042 \mathrm{E}-04$ $1.49969 \mathrm{E}-04$ $1.52924 \mathrm{E}-04$

$1.55908 \mathrm{E}-04$

$1.58922 \mathrm{E}-04$

10.60

10.70

10.80

10.90

$1.61963 \mathrm{E}-04$

$1.65034 \mathrm{E}-04$

$1.68134 \mathrm{E}-04$

$1.71262 \mathrm{E}-04$

$1.74419 \mathrm{E}-04$

11.10

11.20

11.30

11.40

11.50

$1.77605 \mathrm{E}-04$ $1.80820 \mathrm{E}-04$

$1.84064 \mathrm{E}-04$

$1.87336 \mathrm{E}-04$

$1.90637 \mathrm{E}-04$

11.60

11.70

11.80

11.90

$1.93967 \mathrm{E}-04$

$1.97326 \mathrm{E}-04$

$2.00714 \mathrm{E}-04$

$2.04130 \mathrm{E}-04$

$2.07575 \mathrm{E}-04$ $f_{2}$

$8.22986 \mathrm{E}-02$

$8.48830 \mathrm{E}-02$

8.76935 E- 02

$9.05906 \mathrm{E}-02$

$9.35505 \mathrm{E}-02$

$9.65653 \mathrm{E}-02$

$9.96320 \mathrm{E}-02$

$1.02749 \mathrm{E}-01$

$1.05917 \mathrm{E}-01$

$1.09133 \mathrm{E}-01$

$1.12398 \mathrm{E}-01$

$1.15712 \mathrm{E}-01$

$1.19075 \mathrm{E}-01$

$1.22486 \mathrm{E}-01$

$1.25945 \mathrm{E}-01$

$1.29453 \mathrm{E}-01$

$1.33009 \mathrm{E}-01$

$1.36613 \mathrm{E}-01$

$1.40265 \mathrm{E}-01$

$1.43966 \mathrm{E}-01$

$1.47715 \mathrm{E}-01$

$1.51512 \mathrm{E}-01$

$1.55357 \mathrm{E}-01$

$1.59251 \mathrm{E}-01$

$1.63192 \mathrm{E}-01$

$1.67182 \mathrm{E}-01$

$1.71220 \mathrm{E}-01$

$1.75306 \mathrm{E}-01$

$1.79440 \mathrm{E}-01$

$1.83622 \mathrm{E}-01$

$1.87852 \mathrm{E}-01$

$1.92131 \mathrm{E}-01$

$1.96457 \mathrm{E}-01$

$2.00832 \mathrm{E}-01$

$2.05254 \mathrm{E}-01$

$2.09725 \mathrm{E}-01$

$2.14244 \mathrm{E}-01$

2.18811 E- 01

$2.23426 \mathrm{E}-01$

2.28089 E-01

$2.32800 \mathrm{E}-01$

$2.37560 \mathrm{E}-01$

$2.42367 \mathrm{E}-01$

$2.47222 \mathrm{E}-01$

$2.52126 \mathrm{E}-01$

$2.57077 \mathrm{E}-01$

$2.62077 \mathrm{E}-01$

$2.67125 \mathrm{E}-01$

$2.72220 \mathrm{E}-01$

$2.77364 \mathrm{E}-01$

$2.82556 \mathrm{E}-01$

$2.87796 \mathrm{E}-01$

$2.93084 \mathrm{E}-01$

2.98420 E- 01

$3.03804 \mathrm{E}-01$

$3.09236 \mathrm{E}-01$

$3.14716 \mathrm{E}-01$

$3.20244 \mathrm{E}-01$

$3.25820 \mathrm{E}-01$

$3.31445 \mathrm{E}-01$ $\tan \delta$

$f_{-2}$

$8.74305 \mathrm{E}-0.5$

$1.03091 \mathrm{E}-04$

$1.19853 \mathrm{E}-04$

$1.37606 \mathrm{E}-04$

$1.56353 \mathrm{E}-04$

$1.76107 \mathrm{E}-04$

$1.96887 \mathrm{E}-04$

$2.18715 \mathrm{E}-04$

$2.41612 \mathrm{E}-04$

$2.65600 \mathrm{E}-04$

$2.90702 \mathrm{E}-04$

$3.16937 \mathrm{E}-04$

$3.44330 \mathrm{E}-04$

$3.72903 \mathrm{E}-04$

$4.02677 \mathrm{E}-04$

$4.33674 \mathrm{E}-04$

$4.65917 \mathrm{E}-04$

$4.99428 \mathrm{E}-04$

$5.34229 \mathrm{E}-04$

$5.70343 \mathrm{E}-04$

$6.07792 \mathrm{E}-04$

$6.46598 \mathrm{E}-04$

$6.86782 \mathrm{E}-04$

$7.28368 \mathrm{E}-04$

$7.71377 \mathrm{E}-04$

$8.15833 \mathrm{E}-04$

$8.61757 \mathrm{E}-04$

$9.09171 \mathrm{E}-04$

$9.58097 \mathrm{E}-04$

$1.00856 \mathrm{E}-03$

$1.06058 \mathrm{E}-03$

$1.11417 \mathrm{E}-03$

$1.16937 \mathrm{E}-03$

$1.22620 \mathrm{E}-03$

$1.28466 \mathrm{E}-03$

$1.34480 \mathrm{E}-03$

$1.40663 \mathrm{E}-03$

$1.47017 \mathrm{E}-03$

$1.53544 \mathrm{E}-03$

$1.60247 \mathrm{E}-03$

$1.67129 \mathrm{E}-03$

$1.74190 \mathrm{E}-03$

$1.81434 \mathrm{E}-03$

$1.88862 \mathrm{E}-03$

$1.96477 \mathrm{E}-03$

$2.04281 \mathrm{E}-03$

$2.12276 \mathrm{E}-03$

$2.20465 \mathrm{E}-03$

$2.28850 \mathrm{E}-03$

$2.374 .32 \mathrm{E}-03$

$2.46215 \mathrm{E}-03$

$2.55200 \mathrm{E}-0.3$

$2.64390 \mathrm{E}-03$

$2.73786 \mathrm{E}-03$

$2.83392 \mathrm{E}-03$

$2.93209 \mathrm{E}-03$

$3.03239 \mathrm{E}-0.3$

$3.13485 \mathrm{E}-03$

$3.23949 \mathrm{E}-03$

$3.34633 \mathrm{E}-03$

\begin{abstract}
$-3.08595 \mathrm{E} \quad 01$ $-3.08991 \mathrm{E} 01$ $-3.09373 \mathrm{E} \quad 01$ $-3.09742 \mathrm{E} 01$

$-3.10100 \mathrm{E} 01$
\end{abstract}

$-3.10445 \mathrm{E} \quad 01$

$-3.10780 \mathrm{E} 01$

$-3.11104 \mathrm{E} 01$

$-3.11419 \mathrm{E}$ 0l

$-3.11723 \mathrm{E} 01$

$-3.12019 \mathrm{E} 01$

$-3.12305 \mathrm{E} 01$

$-3.12584 \mathrm{E} \quad 01$

$-3.12854 \mathrm{E} 01$

$-3.13117 \mathrm{E} 01$

$-3.13372 \mathrm{E} 01$

$-3.13620 \mathrm{E} 01$

$-3.13862 \mathrm{E} \quad 01$

$-3.14097 \mathrm{E} 01$

$-3.14325 \mathrm{E} 01$

$-3.14548 \mathrm{E} \quad 01$

$-3.14765 \mathrm{E}$ 01

$-3.14976 \mathrm{E} 01$

$-3.15182 \mathrm{E} 01$

$-3.15383 \mathrm{E} 01$

$-3.15579 \mathrm{E} \quad 01$

$-3.15770 \mathrm{E}$ 0l

$-3.15956 \mathrm{E} 01$

$-3.16138 \mathrm{E} 01$

$-3.16316 \mathrm{E} 01$

$-3.16489 \mathrm{E} 01$

$-3.16659 \mathrm{E} 01$

$-3.16824 \mathrm{E} 01$

$-3.16986 \mathrm{E} 01$

$-3.17145 \mathrm{E} 01$

$-3.17300 \mathrm{E} 01$

$-3.17451 \mathrm{E} 01$

$-3.17599 \mathrm{E}$ 01

$-3.17744 \mathrm{E} 01$

$-3.17886 \mathrm{E} 01$

$-3.18025 \mathrm{E} \quad 01$

$-3.18161 \mathrm{E} 01$

$-3.18294 \mathrm{E} 01$

$-3.18424 \mathrm{E}$ 0l

$-3.18552 \mathrm{E} 01$

$-3.18678 \mathrm{E} 01$

$-3.18800 \mathrm{E} 01$

-3.18921 E 01

$-3.19039 \mathrm{E} 01$

$-3.19155 \mathrm{E} \quad 01$

$-3.19268 \mathrm{E} 01$ $-3.19380 \mathrm{E} 01$ $-3.19489 \mathrm{E}$ ol

$-3.19597 \mathrm{E} 01$

$-3.19702 \mathrm{E} 01$

$-3.19805 \mathrm{E} \quad 01$

$-3.19907 \mathrm{E} \quad 01$

$-3.20007 \mathrm{E} 01$

$-3.20105 \mathrm{E} \quad 01$

$-3.20201 \mathrm{E} 01$

g

$1.94657 \mathrm{E}-04$

$1.85751 \mathrm{E}-04$

$1.75985 \mathrm{E}-04$

$1.65336 \mathrm{E}-04$

$1.53782 \mathrm{E}-04$

$1.41300 \mathrm{E}-04$

$1.27869 \mathrm{E}-04$

$1.13466 \mathrm{E}-04$

$9.80694 \mathrm{E}-05$

$8.16562 \mathrm{E}-05$

$6.42045 \mathrm{E}-05$

$4.56920 \mathrm{E}-05$

$2.60966 \mathrm{E}-0.5$

$5.39599 \mathrm{E}-06$

$-1.64320 \mathrm{E}-0.5$

$-3.94097 \mathrm{E}-0.5$

$-6.35593 \mathrm{E}-05$

$-8.89030 \mathrm{E}-05$ 


\begin{tabular}{|c|c|c|c|c|c|c|}
\hline$p$ & $F_{0}$ & $f_{1}$ & & $g_{1}$ & $\tan \delta_{1}$ & $f_{-1}$ \\
\hline 12.10 & $8.75174 \mathrm{E}-01$ & $1.09009 \mathrm{E}$ & 01 & $1.77139 \mathrm{E}-01$ & $-7.27500 \mathrm{E}-05$ & $-1.31628 \mathrm{E}-01$ \\
\hline 12.20 & $8.75168 \mathrm{E}-01$ & $1.09957 \mathrm{E}$ & 01 & $1.81495 \mathrm{E} \sim 01$ & $-8.68553 \mathrm{E}-05$ & $-1.35742 \mathrm{E}-01$ \\
\hline 12.30 & $8.75161 \mathrm{E}-01$ & $1.10904 \mathrm{E}$ & 01 & $1.85900 \mathrm{E}-01$ & $-1.00722 \mathrm{E}-04$ & $1.39904 \mathrm{E}-01$ \\
\hline 12.40 & $8.75154 \mathrm{E}-01$ & $1.11852 \mathrm{E}$ & 01 & $1.90354 \mathrm{E}-01$ & $-1.14358 \mathrm{E}-04$ & $-1.44116 \mathrm{E}-01$ \\
\hline 12.50 & $8.75147 \mathrm{E}-01$ & $1.12800 \mathrm{E}$ & 01 & $1.94857 \mathrm{E}-01$ & $-1.27763 \mathrm{E}-04$ & $-1.48377 \mathrm{E}-01$ \\
\hline 12.60 & $8.75140 \mathrm{E}-01$ & $1.13748 \mathrm{E}$ & 01 & $1.99409 \mathrm{E}-01$ & $-1.40943 \mathrm{E}-04$ & $-1.52687 \mathrm{E}-01$ \\
\hline 12.70 & $8.75133 \mathrm{E}-01$ & $1.14696 \mathrm{E}$ & 01 & $2.04010 \mathrm{E}-01$ & $-1.53899 \mathrm{E}-04$ & $-1.57046 \mathrm{E}-01$ \\
\hline 12.80 & $8.75126 \mathrm{E}-01$ & $1.15644 \mathrm{E}$ & 01 & $2.08660 \mathrm{E}-01$ & $-1.66638 \mathrm{E}-04$ & $-1.61454 \mathrm{E}-01$ \\
\hline 12.90 & $8.75119 \mathrm{E}-01$ & $1.16593 \mathrm{E}$ & 01 & $2.13359 \mathrm{E}-01$ & $-1.79184 \mathrm{E}-04$ & $-1.65911 \mathrm{E}-01$ \\
\hline 13.00 & $8.75111 \mathrm{E}-01$ & $1.17541 \mathrm{E}$ & 01 & $2.18108 \mathrm{E}-01$ & $-1.91505 \mathrm{E}-04$ & $-1.70417 \mathrm{E}-01$ \\
\hline 13.10 & $8.75104 \mathrm{E}-01$ & $1.18490 \mathrm{E}$ & 01 & $2.22905 \mathrm{E}-01$ & $-2.03635 \mathrm{E}-04$ & $-1.74973 \mathrm{E}-01$ \\
\hline 13.20 & $8.75097 \mathrm{E}-01$ & $1.19439 \mathrm{E}$ & 01 & $2.27751 \mathrm{E}-01$ & $-2.15571 \mathrm{E}-04$ & $-1.79577 \mathrm{E}-01$ \\
\hline 30 & $8.75090 \mathrm{E}-01$ & $1.20388 \mathrm{E}$ & 01 & $2.32646 \mathrm{E}-01$ & $-2.27317 \mathrm{E}-04$ & $-1.84231 \mathrm{E}-01$ \\
\hline 13.40 & $8.75082 \mathrm{E}-0 \mathrm{l}$ & $1.21337 \mathrm{E}$ & 01 & $2.37591 \mathrm{E}-01$ & $-2.38871 \mathrm{E}-04$ & $-1.88934 \mathrm{E}-01$ \\
\hline 13.50 & $8.75075 \mathrm{E}-01$ & $1.22287 \mathbf{E}$ & 01 & $2.42585 \mathrm{E}-01$ & $-2.50237 \mathrm{E}-04$ & $-1.93686 \mathrm{E}-01$ \\
\hline 13.60 & $8.75068 \mathrm{E}-01$ & $1.23236 \mathrm{E}$ & 01 & $2.47627 \mathrm{E}-01$ & $-2.61426 \mathrm{E}-04$ & $-1.98487 \mathrm{E}-01$ \\
\hline .70 & $8.75061 \mathrm{E}-01$ & $1.24186 \mathrm{E}$ & 01 & $2.52719 \mathrm{E}-01$ & $-2.72447 \mathrm{E}-04$ & $-2.03337 \mathrm{E}-01$ \\
\hline 13.80 & $8.75053 \mathrm{E}-01$ & $1.25135 \mathrm{E}$ & 01 & $2.57861 \mathrm{E}-01$ & $95 \mathrm{E}-04$ & $-2.08237 \mathrm{E}-01$ \\
\hline 13.90 & $8.75046 \mathrm{E}-01$ & $1.26085 \mathrm{E}$ & 01 & $2.63051 \mathrm{E}-01$ & $-2.93962 \mathrm{E}-04$ & $2.13186 \mathrm{E}-01$ \\
\hline 14.00 & $8.75039 \mathrm{E}-01$ & $1.27035 \mathrm{E}$ & 01 & $2.68290 \mathrm{E}-01$ & $476 \mathrm{E}-04$ & $-2.18184 \mathrm{E}-01$ \\
\hline 14.10 & $8.75031 \mathrm{E}-01$ & $1.27986 \mathrm{E}$ & 01 & $2.73579 \mathrm{E}-\mathbf{0 1}$ & $-3.14826 \mathrm{E}-04$ & $-2.23231 \mathrm{E}-01$ \\
\hline 1.20 & $8.75024 \mathrm{E}-0 \mathrm{l}$ & $1.28936 \mathrm{E}$ & 01 & $2.78917 \mathrm{E}-01$ & $-3.25014 \mathrm{E}-04$ & $-2.28328 \mathrm{E}-0 \mathrm{l}$ \\
\hline 14.30 & $8.75016 \mathrm{E}-01$ & $1.29886 \mathrm{E}$ & 01 & $2.84304 \mathrm{E}-01$ & $-3.35059 \mathrm{E}-04$ & $-2.33474 \mathrm{E}-01$ \\
\hline 14.40 & $8.75009 \mathrm{E}-01$ & $1.30837 \mathrm{E}$ & 01 & $2.89741 \mathrm{E}-01$ & $-3.44938 \mathrm{E}-04$ & $-2.38670 \mathrm{E}-01$ \\
\hline 14.50 & $8.75001 \mathrm{E}-01$ & $1.31788 \mathrm{E}$ & 01 & $2.95227 \mathrm{E}-01$ & $-3.54669 \mathrm{E}-04$ & $-2.43914 \mathrm{E}-01$ \\
\hline 14.60 & $8.74994 \mathrm{E}-01$ & $1.32739 \mathrm{E}$ & 01 & $3.00762 \mathrm{E}-01$ & $-3.64260 \mathrm{E}-04$ & $-2.49208 \mathrm{E}-01$ \\
\hline 14.70 & $5 E-01$ & $1.33690 \mathrm{E}$ & 01 & $3.06346 \mathrm{E}$ & $-3.73709 \mathrm{E}-04$ & $54552 \mathrm{E}-01$ \\
\hline 14.80 & $8.74979 \mathrm{E}-01$ & $1.34641 \mathrm{E}$ & 01 & $3.11980 \mathrm{E}-01$ & $-3.83014 \mathrm{E}-04$ & $-2.59945 \mathrm{E}-01$ \\
\hline 14.90 & $8.74971 \mathrm{E}-01$ & $1.35593 \mathrm{E}$ & 01 & $3.17663 \mathrm{E}-01$ & $-3.92176 \mathrm{E}-04$ & $-2.65387 \mathrm{E}-01$ \\
\hline 15.00 & $8.74964 \mathrm{E}-01$ & $1.36544 \mathrm{E}$ & 01 & $3.23396 \mathrm{E}-01$ & -4.01 & $-2.70879 \mathrm{E}-01$ \\
\hline 15.10 & $8.74956 \mathrm{E}-01$ & $1.37496 \mathrm{E}$ & 01 & $3.29178 \mathrm{E}-01$ & $-4.10109 \mathrm{E}-04$ & $76420 \mathrm{E}-01$ \\
\hline 15.20 & $8.74949 \mathrm{E}-01$ & $1.38448 \mathrm{E}$ & 01 & $3.35009 \mathrm{E}-01$ & $-4.18885 \mathrm{E}-04$ & $-2.82011 \mathrm{E}-01$ \\
\hline 15.30 & $8.74941 \mathrm{E}-01$ & $1.39400 \mathrm{E}$ & 01 & $3.40890 \mathrm{E}-01$ & $-4.27528 \mathrm{E}-04$ & $-2.87651 \mathrm{E}-01$ \\
\hline 15.40 & $8.74934 \mathrm{E}-01$ & $1.40353 \mathrm{E}$ & 01 & $3.46820 \mathrm{E}-0 \mathrm{l}$ & $-4.36045 \mathrm{E}-04$ & $-2.93340 \mathrm{E}-01$ \\
\hline .50 & $8.74926 \mathrm{E}-01$ & $1.41305 \mathrm{E}$ & 01 & $3.52800 \mathrm{E}-01$ & $39 \mathrm{E}-04$ & $-2.99079 \mathrm{E}-01$ \\
\hline 15.60 & $8.74919 \mathrm{E}-01$ & $1.42258 \mathrm{E}$ & 01 & $3.58829 \mathrm{E}-01$ & $-4.52719 \mathrm{E}-04$ & $-3.04868 \mathrm{E}-01$ \\
\hline 15.70 & $8.74912 \mathrm{E}-01$ & $1.43211 \mathrm{E}$ & 01 & 3.6490 & $70 \mathrm{E}-04$ & $-3.10706 \mathrm{E}-01$ \\
\hline 15.80 & $8.74904 \mathrm{E}-01$ & $1.44164 \mathrm{E}$ & 01 & $3.71036 \mathrm{E}$ & -4.68 & $-3.16594 \mathrm{E}-01$ \\
\hline 15.90 & 8.748971 & $117 \mathrm{E}$ & 01 & $\mathrm{E}-01$ & $5 E-04$ & $22532 \mathrm{E}-01$ \\
\hline 16.00 & $8.74889 \mathrm{E}-01$ & $1.46070 \mathrm{E}$ & 01 & $3.83441 \mathrm{E}-01$ & $56 \mathrm{E}-04$ & $-3.28519 \mathrm{E}-01$ \\
\hline 16.10 & $8.74882 \mathrm{E}-01$ & $1.47024 \mathrm{E}$ & 01 & $3.89718 \mathrm{E}-01$ & $-4.92368 \mathrm{E}-04$ & $-3.34555 \mathrm{E}-01$ \\
\hline 16.20 & $8.74875 \mathrm{E}$ & $7977 \mathbf{E}$ & 01 & & $8 \mathrm{E}-04$ & $.40642 \mathrm{E}-01$ \\
\hline 16.30 & $67 \mathrm{E}-01$ & $3931 \mathrm{E}$ & 01 & & $4 E-04$ & $46778 \mathrm{E}-01$ \\
\hline 16.40 & $8.74860 \mathrm{E}-01$ & $1.49885 \mathrm{E}$ & 01 & 4.0884 & $39 \mathrm{E}-04$ & $-3.52963 \mathrm{E}-01$ \\
\hline 16.50 & $8.74852 \mathrm{E}-01$ & $1.50839 \mathrm{E}$ & 01 & 4.1532 & $2 \mathrm{E}-04$ & $-3.59198 \mathrm{E}-01$ \\
\hline 16.60 & $8.74845 \mathrm{E}-01$ & $1.51794 \mathrm{E}$ & 01 & $4.21846 \mathrm{E}-01$ & $-5.29306 \mathrm{E}-04$ & $-3.65484 \mathrm{E}-01$ \\
\hline 16.70 & $8.74838 \mathrm{E}-01$ & $1.52748 \mathrm{E}$ & 01 & -01 & -5.36 & $-3.71818 \mathrm{E}-01$ \\
\hline 16.80 & $8.74830 \mathrm{E}-01$ & $1.53703 \mathrm{E}$ & 01 & 4.3504 & $382 \mathrm{E}-04$ & $-3.78203 \mathrm{E}-01$ \\
\hline 16.90 & $8.74823 \mathrm{E}-$ & $1.54658 \mathrm{E}$ & 01 & 4171 & $70 \mathrm{E}-04$ & $-3.84637 \mathrm{E}-01$ \\
\hline 17.0 & $8.74816 \mathrm{E}-01$ & $1.55613 \mathrm{E}$ & 01 & & $0 \mathrm{E}-04$ & $-3.91121 \mathrm{E}-01$ \\
\hline 17.10 & $8.74808 \mathrm{E}-01$ & $1.56568 \mathrm{E}$ & 01 & $4.55216 \mathrm{E}-01$ & $-5.63774 \mathrm{E}-04$ & $-3.97655 \mathrm{E}-01$ \\
\hline 17.20 & 7480 & $1.57524 \mathrm{E}$ & 01 & 4. & -5.70 & $-4.04239 \mathrm{E}-01$ \\
\hline 17.3 & $8.74794 \mathrm{E}-$ & $1.58479 \mathrm{E}$ & 01 & & -5.76 & $-4.10873 \mathrm{E}-01$ \\
\hline 17.40 & $8.74786 \mathrm{E}-01$ & $1.59435 \mathrm{E}$ & 01 & $6 \mathrm{E}-01$ & $8 \mathrm{E}-04$ & $-4.17556 \mathrm{E}-01$ \\
\hline 17.50 & $8.74779 \mathrm{E}-01$ & $1.60391 \mathrm{E}$ & 01 & $4.82809 \mathrm{E}-0 \mathrm{l}$ & $-5.89703 \mathrm{E}-04$ & $-4.24290 \mathrm{E}-01$ \\
\hline 17.60 & $8.74772 \mathrm{E}-01$ & $1.61347 \mathrm{E}$ & 01 & $4.89832 \mathrm{E}-01$ & $-5.95966 \mathrm{E}-04$ & $-4.31073 \mathrm{E}-01$ \\
\hline 17.70 & $8.74765 \mathrm{E}-01$ & $1.62304 \mathrm{E}$ & 01 & $4.96904 \mathrm{E}-01$ & $-6.02151 \mathrm{E}-04$ & $-4.37907 \mathrm{E}-01$ \\
\hline 17.8 & $8.74758 \mathrm{E}-0 \mathrm{l}$ & $1.63260 \mathrm{E}$ & 01 & $5.04027 \mathrm{E}-01$ & $-6.08250 \mathrm{E}-04$ & $-4.44790 \mathrm{E}-01$ \\
\hline 17.9 & $8.74750 \mathrm{E}-0 \mathrm{~J}$ & $1.64217 \mathrm{E}$ & 01 & $5.11199 \mathrm{E}-01$ & $-6.14262 \mathrm{E}-04$ & $-4.51723 \mathrm{E}-01$ \\
\hline & $8.74743 \mathrm{E}-01$ & $1.65174 \mathrm{E}$ & 01 & $5.18422 \mathrm{E}-01$ & $-6.20201 \mathrm{E}-04$ & $-4.58707 \mathrm{E}-01$ \\
\hline
\end{tabular}

$g_{-1}$

$1.18466 \mathrm{E} \quad 01$ $1.19416 \mathrm{E} \quad 01$ $1.20366 \mathrm{E} \quad 01$ $1.21316 \mathrm{E} \quad 01$ $1.22266 \mathrm{E} 01$

$1.23216 \mathrm{E} \quad 01$ $1.24167 \mathrm{E} \quad 01$ $1.25117 \mathrm{E} \quad 01$ $1.26068 \mathrm{E} \quad 01$ $1.27019 \mathrm{E} \quad 01$

$1.27970 \mathrm{E}$ ol $1.28921 \mathrm{E} \quad 01$ $1.29873 \mathrm{E} 01$ $1.30824 \mathrm{E} 01$ $1.31776 \mathrm{E} \quad 01$

$1.32728 \mathrm{E} \quad 01$ $1.33680 \mathrm{E} 01$ $1.34632 \mathrm{E} \quad 01$ $1.35584 \mathrm{E} \quad 0$ $1.36536 \mathrm{E} \quad 01$

$1.37489 \mathrm{E} \quad 01$ $1.38442 \mathrm{E} \quad 01$ $1.39395 \mathrm{E} \quad 01$ $1.40348 \mathrm{E} \quad 01$ $1.41301 \mathrm{E} \quad 01$

$1.42254 \mathrm{E} \quad 01$ $1.43208 \mathrm{E} 01$ $1.44162 \mathrm{E} \quad 01$ $1.45115 \mathrm{E} \quad 01$ $1.46069 \mathrm{E} \quad 01$

$\begin{array}{ll}1.47024 \mathrm{E} & 01\end{array}$ $\begin{array}{ll}1.47978 \mathrm{E} & 01\end{array}$ $1.48933 \mathrm{E} \quad 01$ $1.49887 \mathrm{E} \quad 01$ $1.50842 \mathrm{E} \quad 01$

$1.51797 \mathrm{E} \quad 01$ $1.52753 \mathrm{E} \quad 01$ $1.53708 \mathrm{E} 01$ $1.54664 \mathrm{E} \quad 01$ $1.55620 \mathrm{E} \quad 01$

$1.56576 \mathrm{E} \quad 01$ $1.57532 \mathrm{E} \quad 01$ $1.58488 \mathrm{E} \quad 01$ $1.59445 \mathrm{E} \quad 01$ $1.60402 \mathrm{E} \cdot 01$

$1.61359 \mathrm{E} \quad 01$ $1.62316 \mathrm{E} \quad 01$ $1.63273 \mathrm{E} \quad 01$ $1.64230 \mathrm{E} \quad 01$ $1.65188 \mathrm{E} \quad 01$

$1.66146 \mathrm{E} \quad 01$ $1.67104 \mathrm{E} \quad 01$ $1.68062 \mathrm{E} \quad 01$ $1.69021 \mathrm{E} \quad 01$ $1.69980 \mathrm{E} \quad 01$

$1.70938 \mathrm{E} \quad 01$ $1.71898 \mathrm{E} 01$ $1.72857 \mathrm{E}$ 0l $1.73816 \mathrm{E} \quad 01$ $\begin{array}{ll}1.74776 \mathrm{E} & 01\end{array}$ $\tan \delta_{-1}$

$2.71301 \mathrm{E} \quad 02$ $2.72454 \mathrm{E} \quad 02$ $2.73599 \mathrm{E} \quad 02$ $2.74735 \mathrm{E} \quad 02$ $2.75864 \mathrm{E} \quad 02$

$2.76984 \mathrm{E} \quad 02$ $2.78098 \mathrm{E} \quad 02$ $2.79203 \mathrm{E} \quad 02$ $2.80300 \mathrm{E} \quad 02$ $2.81391 \mathrm{E} \quad 02$

$2.82474 \mathrm{E} \quad 02$ $2.83550 \mathrm{E} \quad 02$ $2.84618 \mathrm{E} \quad 02$ $2.85680 \mathrm{E} \quad 02$ $2.86736 \mathrm{E} \quad 02$

$2.87784 \mathrm{E} \quad 02$ $2.88825 \mathrm{E} \quad 02$ $2.89860 \mathrm{E} \quad 02$ $2.90888 \mathrm{E} \quad 02$ $2.91910 \mathrm{E} \quad 02$

$2.92926 \mathrm{E} \quad 02$ $2.93935 \mathrm{E} \quad 02$ $2.94938 \mathrm{E} \quad 02$ $2.95935 \mathrm{E} \quad 02$ $2.96927 \mathrm{E} \quad 02$

$2.97912 \mathrm{E} \quad 02$ $2.98891 \mathrm{E} \quad 02$ $2.99865 \mathrm{E} \quad 02$ $3.00834 \mathrm{E} \quad 02$ $3.01796 \mathrm{E} \quad 02$

$3.02754 \mathrm{E} \quad 02$ $3.03705 \mathrm{E} \quad 02$ $3.04651 \mathrm{E} \quad 02$ $3.05593 \mathrm{E} \quad 02$ $3.06529 \mathrm{E} \quad 02$

$3.07459 \mathrm{E} \quad 02$ $3.08386 \mathrm{E} \quad 02$ $3.09306 \mathrm{E} \quad 02$

$3.10222 \mathrm{E} \quad 02$

$3.11134 \mathrm{E} \quad 02$

$3.12040 \mathrm{E} \quad 02$ $3.12943 \mathrm{E} \quad 02$ $3.13839 \mathrm{E} \quad 02$

$3.14732 \mathrm{E} \quad 02$

$3.15620 \mathrm{E} \quad 02$

$3.16504 \mathrm{E} \quad 02$ $3.17383 \mathrm{E} \quad 02$ $3.18259 \mathrm{E} \quad 02$ $3.19130 \mathrm{E} \quad 02$ $3.19996 \mathrm{E} \quad 02$

$3.20859 \mathrm{E} \quad 02$ $3.21718 \mathrm{E} \quad 02$ $3.22573 \mathrm{E} \quad 02$ $3.23423 \mathrm{E} \quad 02$ $3.24270 \mathrm{E} \quad 02$

$3.25113 \mathrm{E} \quad 02$ $3.25952 \mathrm{E} \quad 02$ $3.26787 \mathrm{E} \quad 02$ $3.27620 \mathrm{E} \quad 02$ $3.28448 \mathrm{E} \quad 02$ 
ELECTRON RADIAL FUNCTIONS

$Z=6 \quad A=12 \quad \rho=1.3 A^{1 / 3} F$

l'ositron

$\bar{F}$

$2.11049 \mathrm{E}-04$ $2.14552 \mathrm{E}-04$ $2.18084 \mathrm{E}-04$ $2.21644 \mathrm{E}-04$ $2.25233 \mathrm{E}-04$

$2.28851 \mathrm{E}-04$ $2.32498 \mathrm{E}-04$ $2.36174 \mathrm{E}-04$ $2.39878 \mathrm{E}-04$

$2.43611 \mathrm{E}-04$

$2.47373 \mathrm{E}-04$

$2.51164 \mathrm{E}-04$ $2.54984 \mathrm{E}-04$ $2.58832 \mathrm{E}-04$ $2.62710 \mathrm{E}-04$

13.40

13.50

13.60

13.70

13.80

13.90

14.00

$2.66616 \mathrm{E}-04$ $2.70550 \mathrm{E}-04$ $2.74514 \mathrm{E}-04$ $2.78506 \mathrm{E}-04$ $2.82528 \mathrm{E}-04$

14.10

14.20

14.30

14.40

$2.86578 \mathrm{E}-04$ $2.90656 \mathrm{E}-04$ $2.94764 \mathrm{E}-04$ $2.98900 \mathrm{E}-04$ $3.03066 \mathrm{E}-04$

14.60

14.70

14.80

14.90

15.00

$3.07260 \mathrm{E}-04$ $3.11482 \mathrm{E}-04$ $3.15734 \mathrm{E}-04$ $3.20014 \mathrm{E}-04$ $3.24324 \mathrm{E}-04$

15.10

15.20

15.30

15.40

15.50

15.60

15.70

15.80

15.90

16.00

$3.28662 \mathrm{E}-04$ $3.33028 \mathrm{E}-04$ $3.37424 \mathrm{E}-04$ $3.41848 \mathrm{E}-04$ $3.46301 \mathrm{E}-04$

$3.50784 \mathrm{E}-04$

$3.55294 \mathrm{E}-04$

$3.59834 \mathrm{E}-04$

$3.64402 \mathrm{E}-04$

$3.68999 \mathrm{E}-04$

16.10

16.20

16.30

16.40

16.50

$3.73625 \mathrm{E}-04$

$3.78280 \mathrm{E}-04$

$3.82963 \mathrm{E}-04$

$3.87676 \mathrm{E}-04$

$3.92417 \mathrm{E}-04$

16.60

16.70

16.80

16.90

$3.97187 \mathrm{E}-04$

$4.01985 \mathrm{E}-04$

$4.06813 \mathrm{E}-04$

$4.11669 \mathrm{E}-04$

$4.16554 \mathrm{E}-04$

17.10

17.20

17.30

17.40

17.50

17.60

17.70

17.80

17.90

18.00

$4.21468 \mathrm{E}-04$

$4.26411 \mathrm{E}-04$

$4.31382 \mathrm{E}-04$

$4.36382 \mathrm{E}-04$

$4.41411 \mathrm{E}-04$

$4.46469 \mathrm{E}-04$ $4.51556 \mathrm{E}-04$

4.56671 E-04

$4.61815 \mathrm{E}-04$

$4.66988 \mathrm{E}-04$ $f_{2}$

$3.37117 \mathrm{E}-01$

$3.42838 \mathrm{E}-01$

$3.48606 \mathrm{E}-01$

$3.54422 \mathrm{E}-01$

$3.60287 \mathrm{E}-01$

$3.66199 \mathrm{E}-01$

$3.72159 \mathrm{E}-01$

$3.78168 \mathrm{E}-01$

$3.84224 \mathrm{E}-01$

$3.90328 \mathrm{E}-01$

$3.96481 \mathrm{E}-01$

$4.02681 \mathrm{E}-01$

$4.08930 \mathrm{E}-01$

$4.15226 \mathrm{E}-01$

$4.21571 \mathrm{E}-01$

$4.27963 \mathrm{E}-01$

4.34404E-01

$4.40892 \mathrm{E}-01$

$4.47428 \mathrm{E}-01$

$4.54013 \mathrm{E}-01$

$4.60645 \mathrm{E}-01$

$4.67325 \mathrm{E}-01$

$4.74054 \mathrm{E}-01$

$4.80830 \mathrm{E}-01$

$4.87654 \mathrm{E}-01$

$4.94526 \mathrm{E}-01$

$5.01447 \mathrm{E}-01$

$5.08415 \mathrm{E}-01$

$5.15431 \mathrm{E}-01$

5.22495E-01

$5.29607 \mathrm{E}-01$

$5.36767 \mathrm{E}-01$

$5.43975 \mathrm{E}-01$

$5.51231 \mathrm{E}-01$

$5.58534 \mathrm{E}-01$

$5.65886 \mathrm{E}-01$

$5.73286 \mathrm{E}-01$

$5.80733 \mathrm{E}-01$

$5.88229 \mathrm{E}-01$

$5.95772 \mathrm{E}-01$

$6.03364 \mathrm{E}-01$

$6.11003 \mathrm{E}-01$

$6.18690 \mathrm{E}-01$

$6.26425 \mathrm{E}-01$

6.34208E-01

$6.42039 \mathrm{E}-01$

$6.49918 \mathrm{E}-01$

$6.57844 \mathrm{E}-01$

$6.65819 \mathrm{E}-01$

$6.73841 \mathrm{E}-01$

$6.81912 \mathrm{E}-01$

$6.90030 \mathrm{E}-01$

$6.98196 \mathrm{E}-01$

$7.06410 \mathrm{E}-01$

$7.14672 \mathrm{E}-01$

$7.22982 \mathrm{E}-01$

$7.31339 \mathrm{E}-01$

$7.39745 \mathrm{E}-01$

$7.48198 \mathrm{E}-01$

$7.56699 \mathrm{E}-01$ $g_{2}$

$3.45540 \mathrm{E}-03$

$3.56672 \mathrm{E}-03$

$3.68029 \mathrm{E}-03$

$3.79616 \mathrm{E}-03$

$3.91435 \mathrm{E}-03$

$4.03486 \mathrm{E}-03$

$4.15774 \mathrm{E}-03$

$4.28299 \mathrm{E}-03$

$4.41064 \mathrm{E}-03$

$4.54072 \mathrm{E}-03$

$4.67324 \mathrm{E}-03$

$4.80824 \mathrm{E}-03$

$4.94572 \mathrm{E}-03$

$5.08571 \mathrm{E}-03$

$5.22824 \mathrm{E}-03$

$5.37333 \mathrm{E}-03$

$5.52099 \mathrm{E}-03$

$5.82415 \mathrm{E}-03$

$5.97968 \mathrm{E}-03$

$6.13788 \mathrm{E}-03$ $6.29878 \mathrm{E}-03$

$6.46239 \mathrm{E}-03$

$6.62872 \mathrm{E}-03$

$6.79782 \mathrm{E}-03$

$6.96970 \mathrm{E}-03$

$7.14437 \mathrm{E}-03$

$7.32187 \mathrm{E}-03$

$7.68543 \mathrm{E}-03$

$7.87153 \mathrm{E}-03$

$8.06054 \mathrm{E}-03$

$8.25249 \mathrm{E}-03$

$8.44739 \mathrm{E}-03$

$8.64527 \mathrm{E}-03$

$8.84616 \mathrm{E}-03$

$9.05006 \mathrm{E}-03$

$9.25701 \mathrm{E}-03$

$9.46703 \mathrm{E}-03$

$9.68013 \mathrm{E}-03$

$9.89634 \mathrm{E}-03$

$1.01157 \mathrm{E}-02$

$1.03382 \mathrm{E}-02$

$1.05639 \mathrm{E}-02$

$1.07928 \mathrm{E}-02$

$1.10249 \mathrm{E}-02$

$1.12602 \mathrm{E}-02$

$1.14988 \mathrm{E}-02$

$1.17407 \mathrm{E}-02$

$1.19860 \mathrm{E}-02$

$1.22345 \mathrm{E}-02$ $1.24864 \mathrm{E}-02$

$1.27417 \mathrm{E}-02$

$1.30004 \mathrm{E}-02$

$1.32625 \mathrm{E}-02$

$1.35280 \mathrm{E}-02$ $1.37970 \mathrm{E}-02$ $1.40695 \mathrm{E}-02$

$1.43455 \mathrm{E}-02$

$1.46251 \mathrm{E}-02$
$5.67126 \mathrm{E}-03$

$7.50222 \mathrm{E}-03$

$\tan \delta_{2}$

$f_{-2}$ $-3.20296 \mathrm{E} 01 \quad-2.62376 \mathrm{E}-03$ $-3.20389 \mathrm{E}$ ol $-2.72365 \mathrm{E}-03$ $-3.20480 \mathrm{E}$ 0I $-2.82574 \mathrm{E}-03$ $-3.20570 \mathrm{E}$ 0l $-2.93004 \mathrm{E}-03$ $-3.20658 \mathrm{E}$ 0l $-3.03658 \mathrm{E}-03$

\section{$-3.20745 \mathrm{E} \quad 01 \quad-3.14538 \mathrm{E}-03$}

$-3.20831 \mathrm{E}$ 01 $-3.25646 \mathrm{E}-03$

$-3.20915 \mathrm{E}$ 01 $-3.36985 \mathrm{E}-03$

$-3.20997 \mathrm{E}$ 01 $-3.48557 \mathrm{E}-03$

$-3.21078 \mathrm{E} 01 \quad-3.60364 \mathrm{E}-03$

$-3.21158 \mathrm{E} \quad 01$ $-3.21237 \mathrm{E} 01$ $-3.21315 \mathrm{E} \quad 01$ $-3.21391 \mathrm{E} 01$ $-3.21466 \mathrm{E} 01$

$-3.21540 \mathrm{E} \quad 01$ $-3.21613 \mathrm{E} 01$ $-3.21684 \mathrm{E} \quad 01$ $-3.21755 \mathrm{E} \quad 01$ $-3.21824 \mathrm{E} 01$

$-3.21893 \mathrm{E} \quad 01$

$-3.21960 \mathrm{E} \quad 01$

$-3.22027 \mathrm{E}$ 01

$-3.22092 \mathrm{E} \quad 01$

$-3.22157 \mathrm{E} \quad 01$

$-3.22221 \mathrm{E}$ 01 $-3.22283 \mathrm{E} 01$ $-3.22345 \mathrm{E} \quad 01$ $-3.22406 \mathrm{E}$ 0l

$-3.22466 \mathrm{E} 01$

$-3.22525 \mathrm{E} \quad 01$ $-3.22584 \mathrm{E} \quad 01$ $-3.22642 \mathrm{E} 01$ $-3.22698 \mathrm{E}$ 0l

$-3.22755 \mathrm{E} \quad 01$

$-3.22810 \mathrm{E} 01$ $-3.22865 \mathrm{E} \quad 01$ $-3.22918 \mathrm{E} 01$ $-3.22972 \mathrm{E} \quad 01$ $-3.23024 \mathrm{E}$ ol

$-3.23076 \mathrm{E} \quad 01$ $-3.23127 \mathrm{E} \quad 01$ $-3.23178 \mathrm{E} \quad 01$ $-3.23227 \mathrm{E}$ 0I $-3.23277 \mathrm{E} \quad 01$

$-3.23325 \mathrm{E} \quad 01$ $-3.23373 \mathrm{E}$ 0l $-3.23421 \mathrm{E} \quad 01$ $-3.23467 \mathrm{E}$ 01 $-3.23514 \mathrm{E} \quad 01$

$-3.72407 \mathrm{E}-03$

$-3.84691 \mathrm{E}-03$

$-3.97216 \mathrm{E}-03$

$-4.09984 \mathrm{E}-03$

$-4.22999 \mathrm{E}-03$

$-4.36262 \mathrm{E}-03$ 
p

$F_{0}$

18.10

18.20

18.30

18.40

18.50

18.60

18.70

18.80

18.90

19.00

19.10

19.20

19.30

19.40

19.50

19.60

19.70

19.80

19.90

20.00

20.10

20.20

20.30

20.40

20.50

20.60

20.70

20.80

20.90

21.00

21.10

21.20

21.30

21.40

21.50

21.60

21.70

21.80

21.90

22.00

22.10

22.20

22.30

22.40

22.50

22.60

22.70

22.80

22.90

23.00

23.10

23.20

23.30

23.40

23.50

23.60

23.70

23.80

23.90

24.00 $f_{1}$

8.74736E-01 $8.74729 \mathrm{E}-01$ $8.74722 \mathrm{E}-01$ $8.74715 \mathrm{E}-01$ $8.74707 \mathrm{E}-01$

$8.74701 \mathrm{E}-01$ $8.74693 \mathrm{E}-01$ $8.74686 \mathrm{E}-01$ 8.74679E-01 8.74672 E-01

8.74665E-01 $8.74658 \mathrm{E}-01$ $8.74652 \mathrm{E}-01$ $8.74645 \mathrm{E}-01$ 8.74638E-01

8.74631 E-01 $8.74624 \mathrm{E}-01$ $8.74617 \mathrm{E}-01$ $8.74610 \mathrm{E}-01$ $8.74604 \mathrm{E}-01$

$8.74597 \mathrm{E}-01$ $8.74590 \mathrm{E}-01$ $8.74583 \mathrm{E}-01$ $8.74576 \mathrm{E}-01$ $8.74570 \mathrm{E}-01$

$8.74563 \mathrm{E}-01$ $8.74556 \mathrm{E}-01$ $8.74550 \mathrm{E}-01$ $8.74543 \mathrm{E}-01$ $8.74536 \mathrm{E}-01$

$8.74530 \mathrm{E}-01$ $8.74523 \mathrm{E}-01$ $8.74516 \mathrm{E}-01$ $8.74510 \mathrm{E}-01$ $8.74503 \mathrm{E}-01$

8.74497E-01 $8.74490 \mathrm{E}-01$ 8.74484 E-01 $8.74477 \mathrm{E}-01$ 8.74471 E-01

8.74464 E-01 $8.74458 \mathrm{E}-01$ $8.74452 \mathrm{E}-01$ $8.74445 \mathrm{E}-01$ 8.74439E-01

8.74433E-01 $8.74426 \mathrm{E}-01$ $8.74420 \mathrm{E}-01$ $8.74414 \mathrm{E}-01$ 8.74407E-0]

$8.74401 \mathrm{E}-01$ $8.74395 \mathrm{E}-01$ $8.74389 \mathrm{E}-01$ 8.74382 E-01 $8.74376 \mathrm{E}-01$

$8.74370 \mathrm{E}-01$ $8.74364 \mathrm{E}-01$ $8.74358 \mathrm{E}-01$ $8.74352 \mathrm{E}-01$ $8.74346 \mathrm{E}-01$ $g_{1}$

$\tan \delta_{1}$

$f_{-1}$

$1.66131 \mathrm{E} \quad 01$ $1.67089 \mathrm{E} \quad 01$ $1.68046 \mathrm{E} \quad 01$

$1.69004 \mathrm{E} \quad 01$

$1.69962 \mathrm{E} \quad 01$

$1.70920 \mathrm{E} \quad 01$ $1.71878 \mathrm{E} \quad 01$ $1.72837 \mathrm{E} \quad 01$ $1.73796 \mathrm{E} \quad 01$

$1.74754 \mathrm{E} \quad 01$

$1.75714 \mathrm{E} \quad 01$

$1.76673 \mathrm{E} \quad 01$

$1.77633 \mathrm{E} 01$

$1.78592 \mathrm{E} \quad 01$

$\begin{array}{lll}1.79552 \mathrm{E} & 01\end{array}$

$1.80512 \mathrm{E} \quad 01$

$1.81473 \mathrm{E} \quad 01$

$1.82433 \mathrm{E} \quad 01$

$1.83394 \mathrm{E} \quad 01$

$1.84355 \mathrm{E} \quad 01$

$1.85316 \mathrm{E} \quad 01$ $1.86278 \mathrm{E} \quad 01$ $1.87239 \mathrm{E} \quad 01$ $1.88201 \mathrm{E} \quad 01$

$1.89163 \mathrm{E} \quad 01$

$1.90126 \mathrm{E} \quad 01$ $1.91088 \mathrm{E} \quad 01$ $1.92051 \mathrm{E} \quad 01$ $1.93014 \mathrm{E} \quad 01$ $1.93977 \mathrm{E} \quad 01$

$1.94940 \mathrm{E} \quad 01$ $1.95904 \mathrm{E} \quad 01$ $1.96868 \mathrm{E} \quad 01$ $1.97832 \mathrm{E} \quad 01$

$1.98796 \mathrm{E} \quad 01$

$1.99761 \mathrm{E} \quad 01$ $2.00725 \mathrm{E} \quad 01$ $2.01690 \mathrm{E} \quad 01$ $2.02656 \mathrm{E} \quad 01$ $2.03621 \mathrm{E} \quad 01$

$2.04587 \mathrm{E} \quad 01$ $2.05552 \mathrm{E} \quad 01$ $2.06519 \mathrm{E} 01$ $2.07485 \mathrm{E} \quad 01$ $2.08451 \mathrm{E} 01$

$2.09418 \mathrm{E} \quad 01$ $2.10385 \mathrm{E} \quad 01$ $2.11353 \mathrm{E} 01$ $2.12320 \mathrm{E} \quad 01$ $2.13288 \mathrm{E} \quad 01$

$2.14256 \mathrm{E} \quad 01$ $2.15224 \mathrm{E} \quad 01$ $2.16193 \mathrm{E} 01$ $2.17162 \mathrm{E} \quad 01$

$2.18131 \mathrm{E} 01$

$2.19100 \mathrm{E} 01$ $2.20069 \mathrm{E} \quad 01$ $2.21039 \mathrm{E} \quad 01$

$2.22009 \mathrm{E} \quad 01$ $2.22979 \mathrm{E} 01$
$5.25694 \mathrm{E}-01$

$5.33017 \mathrm{E}-01$

$5.40389 \mathrm{E}-01$

$5.47811 \mathrm{E}-01$

$5.55284 \mathrm{E}-01$

$5.62806 \mathrm{E}-01$ $5.70379 \mathrm{E}-01$ $5.78002 \mathrm{E}-01$ $5.85674 \mathrm{E}-01$

$5.93397 \mathrm{E}-01$

$6.01171 \mathrm{E}-01$ $6.08994 \mathrm{E}-01$ $6.16868 \mathrm{E}-01$ $6.24792 \mathrm{E}-01$ $6.32766 \mathrm{E}-01$

$6.40790 \mathrm{E}-01$ $6.48865 \mathrm{E}-01$ $6.56990 \mathrm{E}-01$ $6.65166 \mathrm{E}-01$ $6.73392 \mathrm{E}-01$

$6.81668 \mathrm{E}-01$ $6.89995 \mathrm{E}-01$ $6.98372 \mathrm{E}-01$ $7.06799 \mathrm{E}-01$

$7.15277 \mathrm{E}-01$

$7.23806 \mathrm{E}-01$

$7.32385 \mathrm{E}-01$

$7.41015 \mathrm{E}-01$

$7.49696 \mathrm{E}-01$

$7.58427 \mathrm{E}-01$

$7.67208 \mathrm{E}-01$

$7.76041 \mathrm{E}-01$

$7.84924 \mathrm{E}-01$

$7.93857 \mathrm{E}-01$

$8.02842 \mathrm{E}-01$

$8.11877 \mathrm{E}-01$ $8.20963 \mathrm{E}-01$ $8.30100 \mathrm{E}-01$ 8.39288E-01

$8.48527 \mathrm{E}-01$

$8.57816 \mathrm{E}-01$ $8.67157 \mathrm{E}-01$ $8.76548 \mathrm{E}-01$ $8.85991 \mathrm{E}-01$ $8.95484 \mathrm{E}-01$

$9.05029 \mathrm{E}-01$ $9.14624 \mathrm{E}-01$ $9.24271 \mathrm{E}-01$ $9.33968 \mathrm{E}-01$ $9.43717 \mathrm{E}-01$

$9.53518 \mathrm{E}-01$ $9.63369 \mathrm{E}-01$ $9.73271 \mathrm{E}-01$ $9.83225 \mathrm{E}-01$ $9.93230 \mathrm{E}-01$

$1.00329 \mathrm{E} \quad 00$ $1.01339 \mathrm{E} \quad 00$ $1.02355 \mathrm{E} \quad 00$ $1.03376 \mathrm{E} \quad 00$ $1.04403 \mathrm{E} \quad 00$

$-6.26060 \mathrm{E}-04$
$-6.31844 \mathrm{E}-04$
$-6.37545 \mathrm{E}-04$
$-6.43175 \mathrm{E}-04$
$-6.48732 \mathrm{E}-04$

$-6.54215 \mathrm{E}-04$ $-6.59622 \mathrm{E}-04$ $-6.64968 \mathrm{E}-04$ $-6.70235 \mathrm{E}-04$

$-6.75439 \mathrm{E}-04$

$-6.80576 \mathrm{E}-04$ $-6.85642 \mathrm{E}-04$

$-6.90643 \mathrm{E}-04$ $-6.95583 \mathrm{E}-04$

$-7.00456 \mathrm{E}-04$

$-7.05271 \mathrm{E}-04$

$-7.10017 \mathrm{E}-04$

$-7.14701 \mathrm{E}-04$

$-7.19333 \mathrm{E}-04$

$-7.23896 \mathrm{E}-04$

$-7.28408 \mathrm{E}-04$ $-7.32858 \mathrm{E}-04$

$-7.37249 \mathrm{E}-04$

$-7.41596 \mathrm{E}-04$

$-7.45869 \mathrm{E}-04$

$-7.50094 \mathrm{E}-04$

$-7.54275 \mathrm{E}-04$

$-7.58390 \mathrm{E}-04$

$-7.62453 \mathrm{E}-04$

$-7.66473 \mathrm{E}-04$

$-7.70439 \mathrm{E}-04$

$-7.74345 \mathrm{E}-04$

$-7.78214 \mathrm{E}-04$

$-7.82022 \mathrm{E}-04$

$-7.85787 \mathrm{E}-04$

$-7.89508 \mathrm{E}-04$

$-7.93172 \mathrm{E}-04$

$-7.96792 \mathrm{E}-04$

$-8.00371 \mathrm{E}-04$

$-8.03904 \mathrm{E}-04$

$-8.07388 \mathrm{E}-04$

$-8.10828 \mathrm{E}-04$ 
$Z=6 \quad A=12 \quad \rho=1.3 A^{1 / 3} F$

$\bar{F}$

18.10

18.20

18.30

18.40

18.50

18.60

18.70

18.80

18.90

19.00

19.10

19.20

19.30

19.40

19.50

19.60

19.70

19.80

19.90

20.00

20.10

20.20

20.30

20.40

20.50

20.60

20.70

20.80

20.90

21.00

21.10

21.20

21.30

21.40

21.50

21.60

21.70

21.80

21.90

22.00

22.10

22.20

22.30

22.40

22.50

22.60

22.70

22.80

22.90

23.00

23.10

23.20

23.30

23.40

23.50

23.60

23.70

23.80

23.90

24.00 $f_{2}$

$7.65248 \mathrm{E}-01$

$7.73845 \mathrm{E}-01$

$7.82490 \mathrm{E}-01$

$7.91182 \mathrm{E}-01$

$7.99923 \mathrm{E}-01$

$8.08711 \mathrm{E}-01$

$8.17547 \mathrm{E}-01$

8.26431 E-01

$8.35362 \mathrm{E}-01$

8.44341 E-01

8.53369E-01

$8.62444 \mathrm{E}-01$

$8.71567 \mathrm{E}-01$

$8.80738 \mathrm{E}-01$

$8.89956 \mathrm{E}-01$

$8.99222 \mathrm{E}-01$

$9.08536 \mathrm{E}-01$

$9.17898 \mathrm{E}-01$

$9.27307 \mathrm{E}-01$

$9.36764 \mathrm{E}-01$

$5.70746 \mathrm{E}-04$

$5.76495 \mathrm{E}-04$

$5.82272 \mathrm{E}-04$ $5.88079 \mathrm{E}-04$ $5.93914 \mathrm{E}-04$ $5.99778 \mathrm{E}-04$ $6.05671 \mathrm{E}-04$

$6.11592 \mathrm{E}-04$ $6.17542 \mathrm{E}-04$ $6.23521 \mathrm{E}-04$ $6.29529 \mathrm{E}-04$ $6.35566 \mathrm{E}-04$

6.41631 E- 04 $6.47726 \mathrm{E}-04$ $6.53849 \mathrm{E}-04$ $6.60001 \mathrm{E}-04$ $6.66181 \mathrm{E}-04$

$6.72390 \mathrm{E}-04$ $6.78629 \mathrm{E}-04$ $6.84896 \mathrm{E}-04$ $6.91191 \mathrm{E}-04$ $6.97516 \mathrm{E}-04$

$7.03869 \mathrm{E}-04$ 7.10251 E-04 $7.16662 \mathrm{E}-04$ $7.23102 \mathrm{E}-04$ 7.29570 E-04

$7.36068 \mathrm{E}-04$ $7.42594 \mathrm{E}-04$ $7.49148 \mathrm{E}-04$ $7.55732 \mathrm{E}-04$ 7.62344 E- 04

$7.68986 \mathrm{E}-04$ $7.75656 \mathrm{E}-04$ $7.82354 \mathrm{E}-04$ $7.89082 \mathrm{E}-04$ $7.95838 \mathrm{E}-04$

$8.02623 \mathrm{E}-04$ $8.09437 \mathrm{E}-04$ $8.16280 \mathrm{E}-04$ 8.23151 E-04 8.30051 E-04

$9.46269 \mathrm{E}-01$

$9.55822 \mathrm{E}-01$

$9.65423 \mathrm{E}-0$

$9.75071 \mathrm{E}-01$

$9.84767 \mathrm{E}-01$

$9.94511 \mathrm{E}-01$

$1.00430 \mathrm{E} \quad 00$

$1.01414 \mathrm{E} \quad 00$

$1.02403 \mathrm{E} \quad 00$

$1.03396 \mathrm{E} \quad 00$

$1.07418 \mathrm{E} \quad 00$

$1.12552 \mathrm{E} \quad 00$

$1.13593 \mathrm{E} \quad 00$

$1.14639 \mathrm{E} \quad 00$ $1.17806 \mathrm{E} \quad 00$ $1.18871 \mathrm{E} \quad 00$ $1.21015 \mathrm{E} \quad 00$ $1.22094 \mathrm{E} \quad 00$ $1.23178 \mathrm{E} \quad 00$ $1.24267 \mathrm{E} \quad 00$

$1.25360 \mathrm{E} \quad 00$ $1.26459 \mathrm{E} \quad 00$ $1.28670 \mathrm{E} \quad 00$ $133149 \mathrm{E} \quad 00$ $1.35417 \mathrm{E} \quad 00$ $g_{2}$

$\tan \delta_{2}$

$f_{-2}$

$1.49082 \mathrm{E}-02$

$1.51948 \mathrm{E}-02$

$1.54851 \mathrm{E}-02$

$1.57790 \mathrm{E}-02$

$1.60765 \mathrm{E}-02$

$1.63777 \mathrm{E}-02$

$1.66826 \mathrm{E}-02$

$1.69912 \mathrm{E}-02$

$1.73035 \mathrm{E}-02$

$1.76196 \mathrm{E}-02$

$1.79394 \mathrm{E}-02$ $1.82631 \mathrm{E}-02$

$1.85906 \mathrm{E}-02$

$1.89219 \mathrm{E}-02$

$1.92570 \mathrm{E}-02$

$1.95961 \mathrm{E}-02$

$1.99391 \mathrm{E}-02$

$2.02860 \mathrm{E}-02$

$2.06368 \mathrm{E}-02$

$2.09916 \mathrm{E}-02$

$1.04395 \mathrm{E} \quad 00$ $1.05398 \mathrm{E} \quad 00$

$1.06405 \mathrm{E} \quad 00$

$1.08435 \mathrm{E} \quad 00$

$1.09457 \mathrm{E} \quad 00$

$1.10484 \mathrm{E} \quad 00$

$1.11516 \mathrm{E} \quad 00$ $1.15690 \mathrm{E} \quad 00$ $1.16745 \mathrm{E} \quad 00$

$1.19940 \mathrm{E} \quad 00$ $\begin{array}{ll}1.27562 \mathrm{E} & 00\end{array}$ $1.29782 \mathrm{E} \quad 00$

$1.30900 \mathrm{E} \quad 00$ $1.32022 \mathrm{E} \quad 00$ $1.34280 \mathrm{E} \quad 00$

$-3.23987 \mathrm{E} \quad 01-1.32605 \mathrm{E}-02$ $-3.24027 \mathrm{E}$ 01 $-1.35313 \mathrm{E}-02$ $-3.24067 \mathrm{E}$ 01 $-1.38057 \mathrm{E}-02$ $-3.24106 \mathrm{E}$ 01 $-1.40835 \mathrm{E}-02$ $-3.24145 \mathrm{E}$ ol $-1.43650 \mathrm{E}-02$

$-3.24183 \mathrm{E}$ 01 $-1.46501 \mathrm{E}-02$ $-3.24221 \mathrm{E}$ 01 $-1.49387 \mathrm{E}-02$ $-3.24258 \mathrm{E}$ 01 $-1.52310 \mathrm{E}-02$ $-3.24295 \mathrm{E}$ 01 $-1.55270 \mathrm{E}-02$ $-3.24332 \mathrm{E} 01-1.58266 \mathrm{E}-02$

$-3.24368 \mathrm{E} \quad 01$ $-3.24404 \mathrm{E} 01$ $-3.24439 \mathrm{E} 01$ $-3.24475 \mathrm{E} \quad 01$ $-3.24509 \mathrm{E} 01$

$-3.24544 \mathrm{E} \quad 01$ $-3.24578 \mathrm{E} 01$ $-3.24611 \mathrm{E} 01$ $-3.24644 \mathrm{E} 01$ $-3.24677 \mathrm{E} 01$

$2.13505 \mathrm{E}-02$ $2.17133 \mathrm{E}-02$ $2.20802 \mathrm{E}-02$ $2.24511 \mathrm{E}-02$ $2.28261 \mathrm{E}-02$

$2.32053 \mathrm{E}-02$ $2.35885 \mathrm{E}-02$ $2.39759 \mathrm{E}-02$ $2.43675 \mathrm{E}-02$

$2.47633 \mathrm{E}-02$

$2.51633 \mathrm{E}-02$ $2.55675 \mathrm{E}-02$ $2.59760 \mathrm{E}-02$ $2.63888 \mathrm{E}-02$

$2.68059 \mathrm{E}-02$

$2.72273 \mathrm{E}-02$ $2.76530 \mathrm{E}-02$ $2.80832 \mathrm{E}-02$

$2.85177 \mathrm{E}-02$

$2.89566 \mathrm{E}-02$

$2.94000 \mathrm{E}-02$ $2.98479 \mathrm{E}-02$ $3.03002 \mathrm{E}-02$ $3.07570 \mathrm{E}-02$

$3.12183 \mathrm{E}-02$

$3.16842 \mathrm{E}-02$ $3.21547 \mathrm{E}-02$

$3.26297 \mathrm{E}-02$ $3.31094 \mathrm{E}-02$

3.35937E-02

$3.40827 \mathrm{E}-02$

$3.45763 \mathrm{E}-02$

$3.50746 \mathrm{E}-02$

$3.55777 \mathrm{E}-02$

$3.60855 \mathrm{E}-02$

$3.65981 \mathrm{E}-02$ $3.71155 \mathrm{E}-02$ $3.76376 \mathrm{E}-02$ $3.81647 \mathrm{E}-02$

$3.86965 \mathrm{E}-02$

$-1.61299 \mathrm{E}-02$

$-1.64370 \mathrm{E}-02$

$-1.67478 \mathrm{E}-02$

$-1.70624 \mathrm{E}-02$

$-1.73807 \mathrm{E}-02$

$-1.77029 \mathrm{E}-02$

$-1.80289 \mathrm{E}-02$

$-1.83588 \mathrm{E}-02$

$-1.86925 \mathrm{E}-02$

$-1.90302 \mathrm{E}-02$

$-3.24710 \mathrm{E}$ 01 $-1.93717 \mathrm{E}-02$ $-3.24742 \mathrm{E} 01$ $-3.24774 \mathrm{E} \quad 01$ $-3.24806 \mathrm{E} 01$

$-3.24837 \mathrm{E} \quad 01$

$-3.24868 \mathrm{E} 01$

$-3.24899 \mathrm{E} 01$

$-3.24929 \mathrm{E} 01$

$-3.24959 \mathrm{E} 01$

$-3.24989 \mathrm{E} 01$

$-3.25018 \mathrm{E} 01$

$-3.25047 \mathrm{E} \quad 01$

$-3.25076 \mathrm{E} 01$

$-3.25105 \mathrm{E} 01$

$-3.25133 \mathrm{E} 01$

$-3.25161 \mathrm{E} \quad 01$

$-3.25189 \mathrm{E} 01$

$-3.25216 \mathrm{E} 01$

$-3.25243 \mathrm{E} 01$

$-3.25271 \mathrm{E} 01$

$\begin{array}{lll}-3.25297 \mathrm{E} & 01\end{array}$

$-3.25324 \mathrm{E} 01$

$-3.25350 \mathrm{E}$ 0l

$-3.25376 \mathrm{E} 01$

$-3.25402 \mathrm{E} 01$

$-3.25427 \mathrm{E} \quad 01$

$-3.25453 \mathrm{E} 01$

$-3.25478 \mathrm{E}$ 0l

$-3.25502 \mathrm{E}$ 0l

$-3.25527 \mathrm{E} 01$ 
ELECTRON RADIAL FUNCTIONS

Positron

$F_{0}$

24.10

24.20

24.30

24.40

24.50

24.60

24.70

24.80

24.90

25.00

25.10

25.20

25.30

25.40

25.50

25.60

25.70

25.80

25.90

26.00

26.10

26.20

26.30

26.40

26.50

26.60

26.70

26.80

26.90

27.00

27.10

27.20

27.30

27.40

27.50

27.60

27.70

27.80

27.90

28.00

28.10

28.20

28.30

28.40

28.50

28.60

28.70

28.80

28.90

29.00

29.10

29.20

29.30

29.40

29.50

29.60

29.70

29.80

29.90

30.00

$Z=6$

$f_{1}$

$8.74340 \mathrm{E}-01$

$8.74334 \mathrm{E}-01$

$8.74327 \mathrm{E}-01$

$8.74321 \mathrm{E}-01$

$8.74316 \mathrm{E}-01$

$8.74310 \mathrm{E}-01$

$8.74304 \mathrm{E}-01$

$8.74298 \mathrm{E}-0 \mathrm{l}$

$8.74292 \mathrm{E}-01$

$8.74286 \mathrm{E}-01$

$8.74280 \mathrm{E}-01$

$8.74274 \mathrm{E}-0 \mathrm{l}$

$8.74268 \mathrm{E}-0]$

$8.74262 \mathrm{E}-01$

$8.74256 \mathrm{E}-01$

$8.74251 \mathrm{E}-01$

$8.74245 \mathrm{E}-01$

$8.74239 \mathrm{E}-01$

$8.74233 \mathrm{E}-01$

8.74227E-01

$8.74222 \mathrm{E}-0$

$8.74216 \mathrm{E}-01$

$8.74210 \mathrm{E}-01$

$8.74205 \mathrm{E}-0$

$8.74199 \mathrm{E}-01$

$8.74193 \mathrm{E}-01$

$8.74187 \mathrm{E}-01$

$8.74182 \mathrm{E}-01$

$8.74176 \mathrm{E}-01$

$8.74171 \mathrm{E}-01$

$8.74165 \mathrm{E}-01$

$8.74159 \mathrm{E}-01$

$8.74154 \mathrm{E}-01$

$8.74149 \mathrm{E}-01$

$8.74143 \mathrm{E}-01$

$8.74138 \mathrm{E}-01$

$8.74132 \mathrm{E}-01$

$8.74127 \mathrm{E}-01$

$8.74121 \mathrm{E}-01$

$8.74116 \mathrm{E}-01$

8.74111 E-01

$8.74105 \mathrm{E}-01$

$8.74100 \mathrm{E}-01$

$8.74094 \mathrm{E}-01$

8.74089 E-01

8.74084E-01

$8.74078 \mathrm{E}-01$

$8.74073 \mathrm{E}-01$

$8.74068 \mathrm{E}-01$

$8.74063 \mathrm{E}-01$

$8.74057 \mathrm{E}-01$

$8.74052 \mathrm{E}-01$

$8.74047 \mathrm{E}-01$

8.74042 E-01

$8.74037 \mathrm{E}-01$

$8.74031 \mathrm{E}-01$

$8.74026 \mathrm{E}-01$

$8.74021 \mathrm{E}-01$

$8.74016 \mathrm{E}-01$

$8.74011 \mathrm{E}-01$ $g_{1}$

$2.23950 \mathrm{E} \quad 01$

$2.24920 \mathrm{E} 01$

$2.25891 \mathrm{E}$ 0I

$2.26863 \mathrm{E} \quad 01$

$2.27834 \mathrm{E} \quad 01$

$2.28806 \mathrm{E} \quad 01$

$2.29778 \mathrm{E} 01$

$2.30750 \mathrm{E} \quad 01$

$2.31723 \mathrm{E} 01$

$2.32695 \mathrm{E} \quad 01$

$2.33669 \mathrm{E} \quad 01$

$2.34642 \mathrm{E} \quad 01$

$2.35615 \mathrm{E} 01$

$2.36589 \mathrm{E} \quad 01$

2.37563 E 01

$2.38538 \mathrm{E} \quad 01$

$2.39512 \mathrm{E} \quad 01$

$2.40487 \mathrm{E} \quad 01$

$2.41462 \mathrm{E} \quad 01$

$2.42438 \mathrm{E} 01$

2.43413E 01

$2.44389 \mathrm{E} 01$

$2.45366 \mathrm{E} 01$

$2.46342 \mathrm{E}$ 0I

$2.47319 \mathrm{E} \quad 01$

$2.48296 \mathrm{E} \quad 01$

$2.49273 \mathrm{E} 01$

$2.50251 \mathrm{E} \quad 01$

$2.51229 \mathrm{E} \quad 01$

$2.52207 \mathrm{E} \quad 01$

$2.53186 \mathrm{E} \quad 01$

$2.54164 \mathrm{E} 01$

$2.55143 \mathrm{E}$ 01

$2.56123 \mathrm{E}, 01$

$2.57102 \mathrm{E} \quad 01$

$2.58082 \mathrm{E} \quad 01$

$2.59062 \mathrm{E} \quad 01$

$2.60043 \mathrm{E} 01$

$2.61023 \mathrm{E} 01$

$2.62005 \mathrm{E} \quad 01$.

2.62986 E 01 $2.63967 \mathrm{E} \quad 01$ $2.64949 \mathrm{E} \quad 01$

$2.65932 \mathrm{E} 01$

2.66914 E 01

$2.67897 \mathrm{E} \quad 01$

$2.68880 \mathrm{E} \quad 01$

$2.69863 \mathrm{E} \quad 01$

$2.70847 \mathrm{E}$ 01

$2.71831 \mathrm{E} 01$

$2.72815 \mathrm{E} \quad 01$

$2.73800 \mathrm{E} \quad 01$

$2.74785 \mathrm{E} 01$

$2.75770 \mathrm{E} \quad 01$

2.76755 E 01

$2.77741 \mathrm{E} \quad 01$

$2.78727 \mathrm{E} \quad 01$

$2.79714 \mathrm{E} \quad 01$

$2.80700 \mathrm{E} \quad 01$

2.81688 E 01

$$
A=12
$$$$
\rho=1.3 A^{1 / 3} F
$$

$\tan \delta_{1}$

$f_{-1}$

$1.05434 \mathrm{E} \quad 00 \quad-8.68336 \mathrm{E}-04 \quad-9.80175 \mathrm{E}-01$

$\begin{array}{llll}1.06470 \mathrm{E} & 00 & -8.70980 \mathrm{E}-04 & -9.90305 \mathrm{E}-01\end{array}$

$\begin{array}{lllll}1.07512 \mathrm{E} & 00 & -8.73594 \mathrm{E}-04 & -1.00049 \mathrm{E} & 00\end{array}$

$\begin{array}{lllll}1.08559 \mathrm{E} & 00 & -8.76171 \mathrm{E}-04 & -1.01072 \mathrm{E} & 00\end{array}$

$\begin{array}{lllll}1.09611 \mathrm{E} & 00 & -8.78712 \mathrm{E}-04 & -1.02101 \mathrm{E} & 00\end{array}$

$1.10668 \mathrm{E} \quad 00 \quad-8.81218 \mathrm{E}-04$

$1.11730 \mathrm{E} \quad 00 \quad-8.83697 \mathrm{E}-04$

$\begin{array}{lll}1.12797 \mathrm{E} & 00 & -8.86132 \mathrm{E}-04\end{array}$

$1.13870 \mathrm{E} \quad 00-8.88544 \mathrm{E}-04$

$1.14948 \mathrm{E} \quad 00$

$1.16031 \mathrm{E} \quad 00$

$1.17119 \mathrm{E} \quad 00$

$1.18212 \mathrm{E} \quad 00$

$1.19311 \mathrm{E} \quad 00$

$1.20414 \mathrm{E} \quad 00$

$1.21523 \mathrm{E} \quad 00$

$\begin{array}{ll}1.22637 \mathrm{E} & 00\end{array}$

$1.23756 \mathrm{E} \quad 00$

$1.24881 \mathrm{E} \quad 00$

$1.26010 \mathrm{E} \quad 00$

$1.27145 \mathrm{E} \quad 00$

$1.28285 \mathrm{E} \quad 00$

$1.29430 \mathrm{E} \quad 00$

$\begin{array}{ll}1.30581 \mathrm{E} & 00\end{array}$

$1.31737 \mathrm{E} \quad 00$

$1.32898 \mathrm{E} \quad 00$

$1.34064 \mathrm{E} \quad 00$

$1.35235 \mathrm{E} \quad 00$

$1.36412 \mathrm{E} \quad 00$

$1.37593 \mathrm{E} \quad 00$

$1.38780 \mathrm{E} \quad 00$

$1.39973 \mathrm{E} \quad 00$

$1.41170 \mathrm{E} \quad 00$

$\begin{array}{ll}1.42373 \mathrm{E} & 00\end{array}$

$1.43581 \mathrm{E} \quad 00$

1.44794. $\mathrm{E} \quad 00$

$1.46013 \mathrm{E} \quad 00$

$1.47237 \mathrm{E} \quad 00$

$1.48466 \mathrm{E} \quad 00$

$1.49700 \mathrm{E} \quad 00$

$1.50940 \mathrm{E} \quad 00$

$1.52185 \mathrm{E} \quad 00$

$1.53435 \mathrm{E} \quad 00$

$1.54690 \mathrm{E} \quad 00$

$1.55951 \mathrm{E} \quad 00$

$\begin{array}{ll}1.57217 \mathrm{E} & 00\end{array}$

$1.58488 \mathrm{E} \quad 00$

$\begin{array}{ll}1.59765 \mathrm{E} & 00\end{array}$

$\begin{array}{ll}1.61046 \mathrm{E} & 00\end{array}$

$1.62334 \mathrm{E} \quad 00$

$\begin{array}{lll}1.63626 \mathrm{E} & 00\end{array}$

$1.64924 \mathrm{E} \quad 00$

$1.66227 \mathrm{E} \quad 00$

$1.67535 \mathrm{E} \quad 00$

$1.68849 \mathrm{E} 00$

$1.70168 \mathrm{E} \quad 00$

$1.71493 \mathrm{E} \quad 00$

$1.72822 \mathrm{E} \quad 00$

$1.74157 \mathrm{E} \quad 00$

$1.75498 \mathrm{E} \quad 00$

$-8.90919 \mathrm{E}-04$

$-8.93255 \mathrm{E}-04$

$-8.95573 \mathrm{E}-04$

$-8.97850 \mathrm{E}-04$

$-9.00101 \mathrm{E}-04$

$-9.02318 \mathrm{E}-04$

$-9.04503 \mathrm{E}-04$

$-9.06660 \mathrm{E}-04$

$-9.08783 \mathrm{E}-04$

$-9.10880 \mathrm{E}-04$

$-9.12947 \mathrm{E}-04$

$-9.14978 \mathrm{E}-04$

$-9.16989 \mathrm{E}-04$

$-9.18963 \mathrm{E}-04$

$-9.20917 \mathrm{E}-04$

$-9.22834 \mathrm{E}-04$

$-9.24728 \mathrm{E}-04$

$-9.26598 \mathrm{E}-04$

$-9.28438 \mathrm{E}-04$

$-9.3024 .7 \mathrm{E}-04$

$-9.32031 \mathrm{E}-04$

$-9.33791 \mathrm{E}-04$

$-9.35524 \mathrm{E}-04$

$-9.37233 \mathrm{E}-04$

$-9.38909 \mathrm{E}-04$

$-9.40566 \mathrm{E}-04$

$-9.42194 \mathrm{E}-04$

$-9.43793 \mathrm{E}-04$

$-9.45376 \mathrm{E}-04$

$-9.46932 \mathrm{E}-04$

$-9.48466 \mathrm{E}-04$

$-9.49961 \mathrm{E}-04$

$-9.51447 \mathrm{E}-04$

$-9.52903 \mathrm{E}-04$

$-9.54331 \mathrm{E}-04$

$-9.55739 \mathrm{E}-04$

$-9.57128 \mathrm{E}-04$ 
ELECTRON RADIAL FUNCTIONS

$Z=6 \quad A=12 \quad \rho=1.3 A^{1 / 3} F$

l'ositrun

$\bar{F}$

$8.36981 \mathrm{E}-04$

$8.43939 \mathrm{E}-04$

$8.50925 \mathrm{E}-04$

$8.57940 \mathrm{E}-04$

$8.64985 \mathrm{E}-04$

24.40

24.50

$8.72058 \mathrm{E}-04$

$8.79159 \mathrm{E}-04$

$8.86290 \mathrm{E}-04$

$8.93449 \mathrm{E}-04$

$9.00637 \mathrm{E}-04$

24.70

24.80

25.10

25.20

25.30

25.40

25.50

$9.07854 \mathrm{E}-04$

$9.15100 \mathrm{E}-04$

9.22374E-04

$9.29677 \mathrm{E}-04$

$9.37009 \mathrm{E}-04$

25.60

25.70

25.80

25.90

26.00

26.10

26.20

26.30

26.40

26.50

$9.44370 \mathrm{E}-04$

$9.51759 \mathrm{E}-04$

$9.59178 \mathrm{E}-04$

$9.66625 \mathrm{E}-04$

$9.74101 \mathrm{E}-04$

$9.81605 \mathrm{E}-04$

$9.89139 \mathrm{E}-04$

$9.96701 \mathrm{E}-04$

$1.00429 \mathrm{E}-03$

$1.01191 \mathrm{E}-03$

26.60

$1.01956 \mathrm{E}-03$

$1.02724 \mathrm{E}-03$

$1.03494 \mathrm{E}-03$

$1.04268 \mathrm{E}-03$

$1.05044 \mathrm{E}-03$

26.90

27.00

27.10

27.20

27.30

27.40

27.50

$1.05824 \mathrm{E}-03$

$1.06606 \mathrm{E}-03$

$1.07391 \mathrm{E}-03$

$1.08179 \mathrm{E}-03$

$1.08969 \mathrm{E}-03$

27.60

27.70

27.80

27.90

28.00

28.10

28.20

28.30

28.40

28.50

$1.09763 \mathrm{E}-03$

$1.10559 \mathrm{E}-03$

$1.11359 \mathrm{E}-03$

$1.12161 \mathrm{E}-03$

$1.12966 \mathrm{E}-03$

$1.13774 \mathrm{E}-03$

$1.14585 \mathrm{E}-03$

$1.15399 \mathrm{E}-03$

$1.16216 \mathrm{E}-03$

$1.17035 \mathrm{E}-03$

28.60

$1.17858 \mathrm{E}-03$

28.70

28.80

28.90

29.00

$1.18683 \mathrm{E}-03$

$1.19511 \mathrm{E}-03$

$1.20342 \mathrm{E}-03$

$1.21176 \mathrm{E}-03$

29.10

29.20

29.30

29.40

29.50

$1.22013 \mathrm{E}-03$

$1.22852 \mathrm{E}-03$

$1.23695 \mathrm{E}-03$

$1.24540 \mathrm{E}-03$

29.60

29.70

29.80

29.90

30.00 $f_{2}$

$g_{2}$

$\tan \delta_{2}$

$f$.

$1.36558 \mathrm{E} \quad 00$

$1.37704 \mathrm{E} \quad 00$

$1.38854 \mathrm{E} \quad 00$

$1.40010 \mathrm{E} \quad 00$

$1.41170 \mathrm{E} \quad 00$

$1.42335 \mathrm{E} \quad 00$

$1.43505 \mathrm{E} \quad 00$

$1.44679 \mathrm{E} \quad 00$

$1.45858 \mathrm{E} \quad 00$

$1.47042 \mathrm{E} \quad 00$

$1.48231 \mathrm{E} \quad 00$

$1.49424 \mathrm{E} \quad 00$

$1.50622 \mathrm{E} \quad 00$

$1.51825 \mathrm{E} \quad 00$

$1.53033 \mathrm{E} \quad 00$

$1.54245 \mathrm{E} \quad 00$

$1.55462 \mathrm{E} \quad 00$

$1.56684 \mathrm{E} \quad 00$

$1.57911 \mathrm{E} \quad 00$

$1.59142 \mathrm{E} \quad 00$

$1.60378 \mathrm{E} \quad 00$

$1.61619 \mathrm{E} \quad 00$

$1.62865 \mathrm{E} \quad 00$

$1.64115 \mathrm{E} \quad 00$

$1.65370 \mathrm{E} \quad 00$

$1.66630 \mathrm{E} \quad 00$

$1.67895 \mathrm{E} \quad 00$

$1.69164 \mathrm{E} \quad 00$

$1.70438 \mathrm{E} \quad 00$

$1.71717 \mathrm{E} \quad 00$

$1.73000 \mathrm{E} \quad 00$

$1.74288 \mathrm{E} \quad 00$

$1.75581 \mathrm{E} \quad 00$

$1.76879 \mathrm{E} \quad 00$

$1.78182 \mathrm{E} \quad 00$

$1.79489 \mathrm{E} \quad 00$

$1.80801 \mathrm{E} \quad 00$

$1.82117 \mathrm{E} \quad 00$

$1.83439 \mathrm{E} \quad 00$

$1.84765 \mathrm{E} \quad 00$

$1.86096 \mathrm{E} \quad 00$

$1.87431 \mathrm{E} \quad 00$

$\begin{array}{ll}1.88772 \mathrm{E} & 00\end{array}$

$1.90117 \mathrm{E} \quad 00$

$1.91466 \mathrm{E} \quad 00$

$1.92821 \mathrm{E} \quad 00$

$1.94180 \mathrm{E} \quad 00$

$1.95544 \mathrm{E} \quad 00$

$1.96913 \mathrm{E} \quad 00$

$1.98286 \mathrm{E} \quad 00$

$1.99664 \mathrm{E} \quad 00$

$2.01047 \mathrm{E} \quad 00$

$2.02434 \mathrm{E} \quad 00$

$2.03827 \mathrm{E} \quad 00$

$2.05224 \mathrm{E} \quad 00$

$206625 \mathrm{E} \quad 00$

$2.08032 \mathrm{E} \quad 00$

$2.09443 \mathrm{E} \quad 00$

$2.10859 \mathrm{E} \quad 00$

$2.12279 \mathrm{E} \quad 00$
$3.92333 \mathrm{E}-02$

$3.97749 \mathrm{E}-02$

$4.03215 \mathrm{E}-02$

$4.08730 \mathrm{E}-02$

4.14295E-02

4.19910E-02

$4.25574 \mathbf{E}-02$

$4.31289 \mathrm{E}-02$

$4.37055 \mathrm{E}-02$

$4.42871 \mathrm{E}-02$

$4.48738 \mathrm{E}-02$

$4.54657 \mathrm{E}-02$

$4.60627 \mathrm{E}-02$

$4.66648 \mathrm{E}-02$

$4.72722 \mathrm{E}-02$

$4.78847 \mathrm{E}-02$

$4.85025 \mathrm{E}-02$

$4.91255 \mathrm{E}-02$

$4.97538 \mathrm{E}-02$

$5.03874 \mathrm{E}-02$

$5.10263 \mathrm{E}-02$

$5.23201 \mathrm{E}-02$

$5.29751 \mathrm{E}-02$

$5.36355 \mathrm{E}-02$

$5.43013 \mathrm{E}-02$

$5.49726 \mathrm{E}-02$

$5.56493 \mathrm{E}-02$

$5.63315 \mathrm{E}-02$

$5.70193 \mathrm{E}-02$

$5.77126 \mathrm{E}-02$

$5.84114 \mathrm{E}-02$

$5.91158 \mathrm{E}-02$

$5.98258 \mathrm{E}-02$

$6.05414 \mathrm{E}-02$

$6.12627 \mathrm{E}-02$

$6.19896 \mathrm{E}-02$

$6.27223 \mathrm{E}-02$

$6.34606 \mathrm{E}-02$

$6.42047 \mathrm{E}-02$

$6.49545 \mathrm{E}-02$

$6.57101 \mathrm{E}-02$

$6.64715 \mathrm{E}-02$

$6.72387 \mathrm{E}-02$

$6.80118 \mathrm{E}-02$

$6.87907 \mathrm{E}-02$ $6.95755 \mathrm{E}-02$

$7.03663 \mathrm{E}-02$

$7.11629 \mathrm{E}-02$

$7.19655 \mathrm{E}-02$

$7.27741 \mathrm{E}-02$

$7.35886 \mathrm{E}-02$

$7.44092 \mathrm{E}-02$

$7.52358 \mathrm{E}-02$

$7.60685 \mathrm{E}-02$

$7.69073 \mathrm{E}-02$

$7.77521 \mathrm{E}-02$

$7.86031 \mathrm{E}-02$

$7.94602 \mathrm{E}-02$

$8.03235 \mathrm{E}-02$
$5.16705 \mathrm{E}-02$

$-3.25784 \mathrm{E}$ 01 $-3.65041 \mathrm{E}-02$ $-3.25806 \mathrm{E}$ 01 $-3.70255 \mathrm{E}-02$ $-3.25828 \mathrm{E}$ 01 $-3.75517 \mathrm{E}-02$ $-3.25850 \mathrm{E}$ 01 $-3.80828 \mathrm{E}-02$ $-3.25871 \mathrm{E}$ 0l $-3.86188 \mathrm{E}-02$

$-3.25893 \mathrm{E}$ ol $-3.91597 \mathrm{E}-02$ $-3.25914 \mathrm{E}$ 01 $-3.97055 \mathrm{E}-02$ $-3.25935 \mathrm{E}$ 01 $-4.02563 \mathrm{E}-02$ $-3.25956 \mathrm{E} \quad 01 \quad-4.08121 \mathrm{E}-02$ $-3.25976 \mathrm{E} 01 \quad-4.13728 \mathrm{E}-02$

$-3.25997 \mathrm{E} 01$ $-3.26017 \mathrm{E} 01 \quad-4.25095 \mathrm{E}-02$ $-3.26038 \mathrm{E}$ 01 $-4.30854 \mathrm{E}-02$ $-3.26058 \mathrm{E}$ 01 $-4.36664 \mathrm{E}-02$ $-3.26077 \mathrm{E}$ 01 $-4.42525 \mathrm{E}-02$

$-3.26097 \mathrm{E}$ 01 $-4.48438 \mathrm{E}-02$ $-3.26117 \mathrm{E}$ 01 $-4.54402 \mathrm{E}-02$ $-3.26136 \mathrm{E}$ 01 $-4.60417 \mathrm{E}-02$ $-3.26155 \mathrm{E} 01-4.66485 \mathrm{E}-02$

$-3.26174 \mathrm{E} 01 \quad-4.72605 \mathrm{E}-02$

$-3.26193 \mathrm{E}$ 01 $-4.78777 \mathrm{E}-02$ $-3.26212 \mathrm{E}$ ol $-4.85003 \mathrm{E}-02$ $-3.26231 \mathrm{E}$ 01 $-4.91281 \mathrm{E}-02$ $-3.26249 \mathrm{E} 01 \quad-4.97612 \mathrm{E}-02$ $-3.26268 \mathrm{E}$ 01 $-5.03996 \mathrm{E}-02$

$-3.26286 \mathrm{E}$ 01 $-5.10434 \mathrm{E}-02$ $-3.26304 \mathrm{E}$ 01 $-5.16925 \mathrm{E}-02$ $-3.26322 \mathrm{E}$ 01 $-5.23471 \mathrm{E}-02$ $-3.26339 \mathrm{E} \quad 01 \quad-5.30071 \mathrm{E}-02$ $-3.26357 \mathrm{E} \quad 01 \quad-5.36725 \mathrm{E}-02$

$-3.26375 \mathrm{E}$ 01 $-5.43434 \mathrm{E}-02$ $-3.26392 \mathrm{E}$ 01 $-5.50197 \mathrm{E}-02$ $-3.26409 \mathrm{E}$ 01 $-5.57016 \mathrm{E}-02$ $-3.26426 \mathrm{E}$ 01 $-5.63890 \mathrm{E}-02$ $-3.26443 \mathrm{E}$ 01 $-5.70819 \mathrm{E}-02$

$-3.26460 \mathrm{E}$ 0l $-5.77805 \mathrm{E}-02$ $-3.26477 \mathrm{E}$ 01 $-5.84846 \mathrm{E}-02$ $-3.26494 \mathrm{E}$ 01 $-5.91943 \mathrm{E}-02$ $-3.26510 \mathrm{E}$ 01 $-5.99097 \mathrm{E}-02$

$-3.26526 \mathrm{E}$ 01 $-6.06307 \mathrm{E}-02$

$-3.26543 \mathrm{E} 01 \quad-6.13574 \mathrm{E}-02$

$-3.26559 \mathrm{E}$ 01 $-6.20898 \mathrm{E}-02$

$-3.26575 \mathrm{E}$ 01 $-6.28279 \mathrm{E}-02$

$-3.26591 \mathrm{E}$ 01 $-6.35718 \mathrm{E}-02$

$-3.26606 \mathrm{E} \quad 01 \quad-6.43214 \mathrm{E}-02$

$-3.26622 \mathrm{E} \quad 01$

$-3.26638 \mathrm{E} 01$

$-3.26653 \mathrm{E} 01$

$-3.26668 \mathrm{E} \quad 01$

$-3.26684 \mathrm{E} 01$

$-3.26699 \mathrm{E} 01$

$-3.26714 \mathrm{E} 01$

$-3.26729 \mathrm{E} 01$

$-3.26743 \mathrm{E} 01$

$-3.26758 \mathrm{E} 01$

$-3.26773 \mathrm{E} \quad 01$

$-4.19386 \mathrm{E}-02$ 
$\begin{array}{cc}p & F_{0} \\ 30.10 & 8.74006 \mathrm{E}-0 \\ 30.20 & 8.74001 \mathrm{E}-0 \\ 30.30 & 8.73995 \mathrm{E}-0 \\ 30.40 & 8.73990 \mathrm{E}-0 \\ 30.50 & 8.73985 \mathrm{E}-0 \\ & \\ 30.60 & 8.73980 \mathrm{E}-0 \\ 30.70 & 8.73975 \mathrm{E}-0 \\ 30.80 & 8.73970 \mathrm{E}-0 \\ 30.90 & 8.73965 \mathrm{E}-0 \\ 31.00 & 8.73960 \mathrm{E}-0\end{array}$

31.10

31.20

31.30

31.40

31.50

31.60

31.70

31.80

31.90

32.00

32.10

32.20

32.30

32.40

32.50

32.60

32.70

32.80

32.90

33.00

33.10

33.20

33.30

33.40

33.50

33.60

33.70

33.80

33.90

34.00

34.10

34.20

34.30

34.40

34.50

34.60

34.70

34.80

34.90

35.00

35.10

35.20

35.30

35.40

35.50

35.60

35.70

35.80

35.90

36.00

8.73950E-01 $f_{1}$

$2.82675 \mathrm{E} \quad 01$ $2.83663 \mathrm{E} \quad 01$ $2.84651 \mathrm{E} \quad 01$

$2.85639 \mathrm{E} \quad 01$

$2.86627 \mathrm{E} \quad 01$

$2.87616 \mathrm{E} \quad 01$

$2.88606 \mathrm{E} \quad 01$

$2.89595 \mathrm{E} \quad 01$

$2.90585 \mathrm{E} \quad 01$

$2.91575 \mathrm{E} \quad 01$

8.73955 E-01

$8.73945 \mathrm{E}-01$

$8.73940 \mathrm{E}-01$

$8.73935 \mathrm{E}-01$

$8.73930 \mathrm{E}-01$

$8.73926 \mathrm{E}-01$

$8.73921 \mathrm{E}-01$

$8.73916 \mathrm{E}-01$

8.73911 E-01

$8.73906 \mathrm{E}-01$

8.73901 E-01

$8.73896 \mathrm{E}-01$

$8.73892 \mathrm{E}-01$

$8.73887 \mathrm{E}-01$

$8.73882 \mathrm{E}-01$

$8.73877 \mathrm{E}-01$

$8.73872 \mathrm{E}-01$

$8.73868 \mathrm{E}-01$

$8.73863 \mathrm{E}-01$

$8.73858 \mathrm{E}-01$

$8.73854 \mathrm{E}-01$

$8.73849 \mathrm{E}-01$

$8.73844 \mathrm{E}-01$

8.73840 E-01

$8.73835 \mathrm{E}-01$

$8.73830 \mathrm{E}-01$

$8.73826 \mathrm{E}-01$

8.73821 E-01

$8.73816 \mathrm{E}-01$

8.73812E-01

$8.73807 \mathrm{E}-01$

$8.73803 \mathrm{E}-01$

$8.73798 \mathrm{E}-01$

$8.73794 \mathrm{E}-01$

$8.73789 \mathrm{E}-01$

$8.73784 \mathrm{E}-01$

$8.73780 \mathrm{E}-01$

8.73775 E-01

8.73771 E-01

$8.73766 \mathrm{E}-01$

$8.73762 \mathrm{E}-01$

$8.73757 \mathrm{E}-01$

8.73753E-01

8.73749 E-01

8.73744 E-01

8.73740E-01

$8.73735 \mathrm{E}-01$

8.73731 E-01

$8.73726 \mathrm{E}-01$

$2.92566 \mathrm{E} \quad 01$

$2.93557 \mathrm{E} \quad 01$

$2.94548 \mathrm{E} 01$

$2.95539 \mathrm{E} \quad 01$

$2.96531 \mathrm{E} 01$

$2.98516 \mathrm{E} \quad 01$

$2.99508 \mathrm{E} \quad 01$

$3.00502 \mathrm{E} \quad 01$

$3.01495 \mathrm{E} 01$

$3.02489 \mathrm{E} \quad 01$

$3.03483 \mathrm{E} \quad 01$

$3.04477 \mathrm{E} 01$

$3.05472 \mathrm{E} \quad 01$

$3.06467 \mathrm{E} 01$

$3.07463 \mathrm{E} \quad 01$

$3.08459 \mathrm{E} \quad 01$

$3.09455 \mathrm{E} \quad 01$

$3.10451 \mathrm{E} \quad 01$

$3.11448 \mathrm{E} \quad 01$

$3.12445 \mathrm{E} \quad 01$

$3.13443 \mathrm{E} \quad 01$

$3.14441 \mathrm{E} \quad 01$

$3.15439 \mathrm{E} \quad 01$

$3.16437 \mathrm{E} \quad 01$

$3.17436 \mathrm{E}$ 0]

$3.18436 \mathrm{E} 01$

$3.19435 \mathrm{E} 01$

$3.20435 \mathrm{E} \quad 01$

$3.21435 \mathrm{E} 01$

$3.22436 \mathrm{E} 01$

$3.23437 \mathrm{E} \quad 01$

$3.24439 \mathrm{E} 01$

$3.25440 \mathrm{E} \quad 01$

$3.26442 \mathrm{E} \quad 01$

$3.27445 \mathrm{E} \quad 01$

$3.28448 \mathrm{E} \quad 01$

$3.29451 \mathrm{E} 01$

$3.30454 \mathrm{E} \quad 01$

$3.31458 \mathrm{E} \quad 01$

$3.32463 \mathrm{E} 01$

$3.33467 \mathrm{E} 01$

$3.34472 \mathrm{E} 01$

$\begin{array}{ll}3.35478 \mathrm{E} & 01\end{array}$

$3.36483 \mathrm{E} 01$

$3.37489 \mathrm{E} 01$

$3.38496 \mathrm{E} 01$

$3.39503 \mathrm{E} \quad 01$

$3.40510 \mathrm{E} 01$

$3.41518 \mathrm{E} \quad 01$ $g_{1}$

$\tan \delta_{1}$

$f_{-1}$

$g_{-1}$

$\tan \delta_{-1}$

$\begin{array}{lllll}1.76843 \mathrm{E} & 00 & -9.75236 \mathrm{E}-04 & -1.68039 \mathrm{E} & 00\end{array}$

$\begin{array}{lllll}1.78194 \mathrm{E} & 00 & -9.76281 \mathrm{E}-04 & -1.69367 \mathrm{E} & 00\end{array}$

$\begin{array}{lllll}1.79551 \mathrm{E} & 00 & -9.77297 \mathrm{E}-04 & -1.70700 \mathrm{E} & 00\end{array}$

$\begin{array}{lllll}1.80913 \mathrm{E} & 00 & -9.78298 \mathrm{E}-04 & -1.72039 \mathrm{E} & 00\end{array}$

$\begin{array}{lllll}1.82280 \mathrm{E} & 00 & -9.79269 \mathrm{E}-04 & -1.73384 \mathrm{E} & 00\end{array}$

$\begin{array}{lllll}1.83652 \mathrm{E} & 00 & -9.80228 \mathrm{E}-04 & -1.74734 \mathrm{E} & 00\end{array}$

$\begin{array}{lllll}1.85030 \mathrm{E} & 00 & -9.81164 \mathrm{E}-04 & -1.76089 \mathrm{E} & 00\end{array}$

$\begin{array}{lllll}1.86413 \mathrm{E} & 00 & -9.82079 \mathrm{E}-04 & -1.77449 \mathrm{E} & 00\end{array}$

$\begin{array}{lllll}1.87802 \mathrm{E} & 00 & -9.82972 \mathrm{E}-04 & -1.78815 \mathrm{E} & 00\end{array}$

$\begin{array}{lllll}1.89196 \mathrm{E} & 00 & -9.83853 \mathrm{E}-04 & -1.80186 \mathrm{E} & 00\end{array}$

$2.97523 \mathrm{E} \quad 01$
$1.90595 \mathrm{E} \quad 00 \quad-9.84719 \mathrm{E}-04$

$\begin{array}{lll}1.92000 \mathrm{E} & 00 & -9.85559 \mathrm{E}-04\end{array}$

$1.93410 \mathrm{E} \quad 00 \quad-9.86377 \mathrm{E}-04$

$\begin{array}{lll}1.94825 \mathrm{E} & 00 & -9.87183 \mathrm{E}-04\end{array}$

$1.96246 \mathrm{E}$ 00 $-9.87964 \mathrm{E}-04$

$1.97672 \mathrm{E} \quad 00 \quad-9.88727 \mathrm{E}-04$

$\begin{array}{lll}1.99104 \mathrm{E} & 00 & -9.89475 \mathrm{E}-04\end{array}$

$2.00541 \mathrm{E} \quad 00 \quad-9.90212 \mathrm{E}-04$

$2.01983 \mathrm{~F} \quad 00 \quad-9.90915 \mathrm{E}-04$

$2.03431 \mathrm{E} \quad 00 \quad-9.91608 \mathrm{E}-04$

$2.04885 \mathrm{E} \quad 00$

$2.06343 \mathrm{E} \quad 00$

$2.07807 \mathrm{E} \quad 00$

$2.09277 \mathrm{E} \quad 00$

$2.10752 \mathrm{E} \quad 00$

$2.12232 \mathrm{E} \quad 00$

$2.13718 \mathrm{E} \quad 00$

$2.15209 \mathrm{E} \quad 00$

$2.16706 \mathrm{E} \quad 00$

$2.18208 \mathrm{E} \quad 00$

$2.19716 \mathrm{E} \quad 00$

$2.21229 \mathrm{E} \quad 00$

$2.22748 \mathrm{E} \quad 00$

$2.24272 \mathrm{E} \quad 00$

$2.25801 \mathrm{E} \quad 00$

$2.27336 \mathrm{E} \quad 00$

$2.28877 \mathrm{E} \quad 00$

$2.30423 \mathbf{E} 00$

$2.31974 \mathrm{E} \quad 00$

$2.33531 \mathrm{E} \quad 00$

$2.35094 \mathrm{E} \quad 00$

$2.36662 \mathrm{E} \quad 00$

$2.38235 \mathrm{E} \quad 00$

$2.39814 \mathrm{E} \quad 00$

$2.41399 \mathrm{E} \quad 00$

$2.42989 \mathrm{E} \quad 00$

$2.44584 \mathrm{E} \quad 00$

$2.46185 \mathrm{E} \quad 00$

$2.47792 \mathrm{E} \quad 00$

$2.49404 \mathrm{E} \quad 00$

$2.51022 \mathrm{E} \quad 00$

$2.52645 \mathrm{E} \quad 00$

$2.54274 \mathrm{E} \quad 00$

$2.55908 \mathrm{E} \quad 00$

$2.57548 \mathrm{E} \quad 00$

$2.59193 \mathrm{E} \quad 00$

$2.60844 \mathrm{E} \quad 00$

$2.62501 \mathrm{E} \quad 00$

$2.64163 \mathrm{E} \quad 00$

$2.65831 \mathrm{E} 00$
$-9.92286 \mathrm{E}-04$

$-9.92943 \mathrm{E}-04$

$-9.93582 \mathrm{E}-04$

$-9.94207 \mathrm{E}-04$

$-9.94814 \mathrm{E}-04$

$-9.95397 \mathrm{E}-04$

$-9.95969 \mathrm{E}-04$

$-9.96523 \mathrm{E}-04$

$-9.97061 \mathrm{E}-04$

$-9.97577 \mathrm{E}-04$

$-9.98082 \mathrm{E}-04$

$-9.98563 \mathrm{E}-04$

$-9.99037 \mathrm{E}-04$

$-9.99493 \mathrm{E}-04$

$-9.99927 \mathrm{E}-04$

$-1.00035 \mathrm{E}-03$

$-1.00114 \mathrm{E}-03$

$-1.00152 \mathrm{E}-03$

$-1.00187 \mathrm{E}-03$

$-1.00222 \mathrm{E}-03$ $-1.00255 \mathrm{E}-03$

$-1.00286 \mathrm{E}-03$

$-1.00316 \mathrm{E}-03$

$-1.00343 \mathrm{E}-03$

$-1.00370 \mathrm{E}-03$

$-1.00395 \mathrm{E}-03$

$-1.00418 \mathrm{E}-03$

$-1.00441 \mathrm{E}-03$

$-1.00461 \mathrm{E}-03$

$-1.00480 \mathrm{E}-03$

$-1.00498 \mathrm{E}-03$

$-1.00514 \mathrm{E}-03$

$-1.00529 \mathrm{E}-03$

$-1.00542 \mathrm{E}-03$ $-1.00565 \mathrm{E}-03$

$-1.00573 \mathrm{E}-03$

$-1.00581 \mathrm{E}-03$

$-1.00588 \mathrm{E}-03$
$-1.00075 \mathrm{E}-03$

$-1.00554 \mathrm{E}-03$
$-1.81563 \mathrm{E} \quad 00$

$-1.82945 \mathrm{E} \quad 00$

$-1.84332 \mathrm{E} \quad 00$

$-1.85725 \mathrm{E} \quad 00$

$-1.87123 \mathrm{E} 00$

$-1.88527 \mathrm{E} \quad 00$

$-1.89936 \mathrm{E} \quad 00$

$-1.91350 \mathrm{E} 00$

$-1.92770 \mathrm{E} \quad 00$

$-1.94196 \mathrm{E} 00$

$-1.95626 \mathrm{E} \quad 00$

$-1.97062 \mathrm{E} \quad 00$

$-1.98504 \mathrm{E} 00$

$-1.99951 \mathrm{E} 00$

$-2.01404 \mathrm{E} \quad 00$

$-2.02862 \mathrm{E} \quad 00$ 
ELECTRON RADIAL FUNCTIONS

$$
Z=6 \quad A=12 \quad \rho=1.3 A^{1 / 3} F
$$

$\bar{F}$

$f_{2}$

$1.30539 \mathrm{E}-03$

$1.31408 \mathrm{E}-03$

$1.32279 \mathrm{E}-03$

$1.33153 \mathrm{E}-03$

$1.34030 \mathrm{E}-03$

$1.34910 \mathrm{E}-03$

$1.35793 \mathrm{E}-03$

$1.36679 \mathrm{E}-03$

$1.37567 \mathrm{E}-03$

$1.38459 \mathrm{E}-03$

30.90

31.00

31.10

31.20

31.30

31.40

31.50

31.60

31.70

31.80

31.90

32.00

32.10

32.20

32.30

32.40

32.50

32.60

32.70

32.80

32.90

33.00

33.10

33.20

33.30

33.40

33.50

33.60

33.70

33.80

33.90

34.00

34.10

34.20

34.30

34.40

34.50

34.60

34.70

34.80

34.90

35.00

35.10

35.20

35.30

35.40

35.50

35.60

35.70

35.80

35.90

36.00
$1.39353 \mathrm{E}-03$

$1.40250 \mathrm{E}-03$

$1.41151 \mathrm{E}-03$

$1.42054 \mathrm{E}-03$

$1.42959 \mathrm{E}-03$

$1.43868 \mathrm{E}-03$

$1.44780 \mathrm{E}-03$

$1.45694 \mathrm{E}-03$

$1.46612 \mathrm{E}-03$

$1.47532 \mathrm{E}-03$

$1.48455 \mathrm{E}-03$

$1.49381 \mathrm{E}-03$

$1.50310 \mathrm{E}-03$

$1.51242 \mathrm{E}-03$

$1.52176 \mathrm{E}-03$

$1.53114 \mathrm{E}-03$

$1.54054 \mathrm{E}-03$

$1.54998 \mathrm{E}-03$

$1.55944 \mathrm{E}-03$

$1.56893 \mathrm{E}-03$

$1.57845 \mathrm{E}-03$

$1.58799 \mathrm{E}-03$

$1.59757 \mathrm{E}-03$

$1.60718 \mathrm{E}-03$

$1.61681 \mathrm{E}-03$

$1.62647 \mathrm{E}-03$

$1.63617 \mathrm{E}-03$

$1.64589 \mathrm{E}-03$

$1.65564 \mathrm{E}-03$

$1.66541 \mathrm{E}-03$

$1.67522 \mathrm{E}-03$

$1.68506 \mathrm{E}-03$

$1.69492 \mathrm{E}-03$

$1.70481 \mathrm{E}-03$

$1.71474 \mathrm{E}-03$

$1.72469 \mathrm{E}-03$

$1.73467 \mathrm{E}-03$

$1.74467 \mathrm{E}-03$

$1.75471 \mathrm{E}-03$

$1.76478 \mathrm{E}-03$

$1.77487 \mathrm{E}-03$

$1.78499 \mathrm{E}-03$

$1.79515 \mathrm{E}-03$

$1.80533 \mathrm{E}-03$

$1.81554 \mathrm{E}-03$

$1.82578 \mathrm{E}-03$

$1.83604 \mathrm{E}-03$

$1.84634 \mathrm{E}-03$

$1.85666 \mathrm{E}-03$

$1.86702 \mathrm{E}-03$ $g_{2}$

$\tan \delta_{2}$

$f_{-2}$

$2.13705 \mathrm{E} \quad 00$

$2.15135 \mathrm{E} \quad 00$

$2.16569 \mathrm{E} \quad 00$

$2.18009 \mathrm{E} \quad 00$

$2.19453 \mathrm{E} \quad 00$

$2.20902 \mathrm{E} \quad 00$

$2.22355 \mathrm{E} \quad 00$

$2.23814 \mathrm{E} \quad 00$

$2.25277 \mathrm{E} \quad 00$

$2.26744 \mathrm{E} \quad 00$

$2.28217 \mathrm{E} \quad 00$

$2.29694 \mathrm{E} \quad 00$

$2.31176 \mathrm{E} \quad 00$

$2.32662 \mathrm{E} \quad 00$

$2.34153 \mathrm{E} \quad 00$

$2.35649 \mathrm{E} \quad 00$

$2.37150 \mathrm{E} \quad 00$

$2.38655 \mathrm{E} \quad 00$

$2.40165 \mathrm{E} \quad 00$

$2.41680 \mathrm{E} \quad 00$

$2.43200 \mathrm{E} \quad 00$

$2.44724 \mathrm{E} \quad 00$

$2.46252 \mathrm{E} \quad 00$

$2.47786 \mathrm{E} \quad 00$

$2.49324 \mathrm{E} \quad 00$

$2.50867 \mathrm{E} \quad 00$

$2.52415 \mathrm{E} \quad 00$

$2.53967 \mathrm{E} \quad 00$

$2.55524 \mathrm{E} \quad 00$

$2.57086 \mathrm{E} \quad 00$

$2.58652 \mathrm{E} \quad 00$

$2.60223 \mathrm{E} \quad 00$

$2.61799 \mathrm{E} \quad 00$

$2.63379 \mathrm{E} \quad 00$

$2.64965 \mathrm{E} \quad 00$

$2.66554 \mathrm{E} \quad 00$

$2.68149 \mathrm{E} \quad 00$

$2.69748 \mathrm{E} \quad 00$

$2.71352 \mathrm{E} \quad 00$

$2.72961 \mathrm{E} \quad 00$

$2.74574 \mathrm{E} \quad 00$ $2.76192 \mathrm{E} \quad 00$

$2.77814 \mathrm{E} \quad 00$

$2.79442 \mathrm{E} \quad 00$

$2.81074 \mathrm{E} \quad 00$

$2.82710 \mathrm{E} \quad 00$

$2.84352 \mathrm{E} \quad 00$

$2.85998 \mathrm{E} \quad 00$

$2.87648 \mathrm{E} \quad 00$

$2.89304 \mathrm{E} \quad 00$

$2.90964 \mathrm{E} \quad 00$

$2.92628 \mathrm{E} \quad 00$

$2.94298 \mathrm{E} \quad 00$

$2.95972 \mathrm{E} \quad 00$

$2.97650 \mathrm{E} \quad 00$

$2.99334 \mathrm{E} \quad 00$ $3.01022 \mathrm{E} \quad 00$

$3.02715 \mathrm{E} \quad 00$

$3.04412 \mathrm{E} \quad 00$

$3.06114 \mathrm{E} \quad 00$

8.11931 E-02

$8.20688 \mathrm{E}-02$

$8.29507 \mathrm{E}-02$

$8.38389 \mathrm{E}-02$

$8.47334 \mathrm{E}-02$

$8.56342 \mathrm{E}-02$

$8.65412 \mathrm{E}-02$

$8.74547 \mathrm{E}-02$

$8.83745 \mathrm{E}-02$

$8.93006 \mathrm{E}-02$

$9.02332 \mathrm{E}-02$

$9.11723 \mathrm{E}-02$

$9.21177 \mathrm{E}-02$

$9.30697 \mathrm{E}-02$

$9.40281 \mathrm{E}-02$

$9.49931 \mathrm{E}-02$

$9.59646 \mathrm{E}-02$

$9.69426 \mathrm{E}-02$

$9.79272 \mathrm{E}-02$

$9.89185 \mathrm{E}-02$

9.99163E-02

$1.00921 \mathrm{E}-01$

$1.01932 \mathrm{E}-01$

$1.02950 \mathrm{E}-01$

$1.03974 \mathrm{E}-01$

$1.05006 \mathrm{E}-01$

$1.06044 \mathrm{E}-01$

$1.07089 \mathrm{E}-01$

$1.08140 \mathrm{E}-01$

$1.09199 \mathrm{E}-01$

$-3.26844 \mathrm{E} \quad 01$

$-3.26858 \mathrm{E} 01$

$-3.26872 \mathrm{E} \quad 01$

$-3.26886 \mathrm{E} 01$

$-3.26899 \mathrm{E} 01$

$-3.26913 \mathrm{E} 01$

$-3.26927 \mathrm{E}$ 01

$-3.26940 \mathrm{E}$ 0l

$-3.26953 \mathrm{E} 01$

$-3.26967 \mathrm{E} 01$

$-3.26980 \mathrm{E} \quad 01$

$-3.26993 \mathrm{E} \quad 01$

$-3.27006 \mathrm{E} 01$

$-3.27019 \mathrm{E}$ 01

$-3.27032 \mathrm{E} 01$

$-3.27044 \mathrm{E} \quad 01$

$-3.27057 \mathrm{E} \quad 01$

$-3.27070 \mathrm{E} \quad 01$

$-3.27082 \mathrm{E} 01$

$-3.27094 \mathrm{E} \quad 01$

$-3.27107 \mathrm{E} \quad 01$

$-3.27119 \mathrm{E} 01$

$-3.27131 \mathrm{E} \quad 01$

$-3.27143 \mathrm{E} \quad 01$

$-3.27155 \mathrm{E} 01$

$-3.27167 \mathrm{E} 01$

$-3.27179 \mathrm{E} \quad 01$

$-3.27191 \mathrm{E} 01$

$-3.27203 \mathrm{E} 01$

$-3.27214 \mathrm{E} 01$

$1.10264 \mathrm{E}-01$

$1.11336 \mathrm{E}-01$

$1.12415 \mathrm{E}-01$

$1.13501 \mathrm{E}-01$

$1.14594 \mathrm{E}-01$

$\begin{array}{lll}-3.27226 \mathrm{E} & 01\end{array}$

$-3.27237 \mathrm{E} \quad 01$

$-3.27249 \mathrm{E} 01$

$-3.27260 \mathrm{E} 01$

$\begin{array}{lll}-3.27271 \mathrm{E} & 01\end{array}$

$1.15694 \mathrm{E}-0 \mathrm{I}$

$1.16801 \mathrm{E}-01$

$1.17915 \mathrm{E}-01$

$1.19035 \mathrm{E}-01$

$1.20163 \mathrm{E}-01$

$1.21298 \mathrm{E}-01$

$-7.71181 \mathrm{E}-02$

$-7.79692 \mathrm{E}-02$

$-7.88264 \mathrm{E}-02$

$-7.96897 \mathrm{E}-02$

$-8.05593 \mathrm{E}-02$

$-8.14352 \mathrm{E}-02$

$-8.23172 \mathrm{E}-02$

$-8.32056 \mathrm{E}-02$

$-8.41002 \mathrm{E}-02$

$-8.50011 \mathrm{E}-02$

$-8.59084 \mathrm{E}-02$

$-8.68220 \mathrm{E}-02$

$-8.77421 \mathrm{E}-02$

$-8.86685 \mathrm{E}-02$

$-8.96013 \mathrm{E}-02$

$-9.05406 \mathrm{E}-02$

$-9.14863 \mathrm{E}-02$

$-9.24385 \mathrm{E}-02$

$-9.33973 \mathrm{E}-02$

$-9.43626 \mathrm{E}-02$

$-9.53344 \mathrm{E}-02$

$-9.63128 \mathrm{E}-02$

$-9.72978 \mathrm{E}-02$

$-9.82894 \mathrm{E}-02$

$-9.92876 \mathrm{E}-02$

$-1.00293 \mathrm{E}-01$

$-1.01304 \mathrm{E}-01$

$-1.02322 \mathrm{E}-01$

$-1.03347 \mathrm{E}-01$

$-1.04379 \mathrm{E}-01$

$-1.05418 \mathrm{E}-01$

$-1.06463 \mathrm{E}-01$

$-1.07515 \mathrm{E}-01$

$-1.08574 \mathrm{E}-01$

$-1.09640 \mathrm{E}-01$ 


$\begin{array}{cc}p & F_{0} \\ 36.10 & 8.73722 \mathrm{E}-01 \\ 36.20 & 8.73718 \mathrm{E}-01 \\ 36.30 & 8.73713 \mathrm{E}-01 \\ 36.40 & 8.73709 \mathrm{E}-01 \\ 36.50 & 8.73705 \mathrm{E}-01 \\ & \\ 36.60 & 8.73700 \mathrm{E}-01 \\ 36.70 & 8.73696 \mathrm{E}-01 \\ 36.80 & 8.73692 \mathrm{E}-01 \\ 36.90 & 8.73687 \mathrm{E}-01 \\ 37.00 & 8.73683 \mathrm{E}-01 \\ & \\ 37.10 & 8.73679 \mathrm{E}-01 \\ 37.20 & 8.73675 \mathrm{E}-01 \\ 37.30 & 8.73670 \mathrm{E}-01 \\ 37.40 & 8.73666 \mathrm{E}-01 \\ 37.50 & 8.73662 \mathrm{E}-01\end{array}$

37.60

37.70

37.80

37.90

38.00

38.10

38.20

38.30

38.40

38.50

38.60

38.70

38.80

38.90

39.00

39.10

39.20

39.30

39.40

39.50

39.60

39.70

39.80

39.90

40.00

40.10

40.20

40.30

40.40

40.50

40.60

40.70

40.80

40.90

41.00

41.10

41.20

41.30

41.40

41.50

41.60

41.70

41.80

41.90

42.00

$8.73658 \mathrm{E}-01$

$8.73653 \mathrm{E}-01$

$8.73649 \mathrm{E}-01$

$8.73645 \mathrm{E}-01$

$8.73641 \mathrm{E}-01$

$8.73637 \mathrm{E}-01$

8.73632E-01

$8.73628 \mathrm{E}-01$

$8.73624 \mathrm{E}-01$

$8.73620 \mathrm{E}-01$

$8.73616 \mathrm{E}-01$

$8.73612 \mathrm{E}-01$

$8.73608 \mathrm{E}-01$

$8.73604 \mathrm{E}-01$

$8.73600 \mathrm{E}-01$

8.73595E-01

$8.73591 \mathrm{E}-01$

$8.73587 \mathrm{E}-01$

$8.73583 \mathrm{E}-01$

$8.73579 \mathrm{E}-01$

$8.73575 \mathrm{E}-01$

$8.73571 \mathrm{E}-01$

$8.73567 \mathrm{E}-01$

$8.73563 \mathrm{E}-01$

$8.73559 \mathrm{E}-01$

8.73555E-01

$8.73551 \mathrm{E}-01$

$8.73547 \mathrm{E}-01$

$8.73543 \mathrm{E}-01$

8.73539E-01

$8.73535 \mathrm{E}-01$

$8.73531 \mathrm{E}-01$

$8.73527 \mathrm{E}-01$

$8.73524 \mathrm{E}-01$

$8.73520 \mathrm{E}-01$

$8.73516 \mathrm{E}-01$

$8.73512 \mathrm{E}-01$

$8.73508 \mathrm{E}-01$

$8.73504 \mathrm{E}-01$

$8.73500 \mathrm{E}-01$

$8.73496 \mathrm{E}-01$

$8.734 .92 \mathrm{E}-01$

$8.73489 \mathrm{E}-01$

$8.73485 \mathrm{E}-01$

8.73481 E-01 $f_{1}$

$3.42526 \mathrm{E} \quad 01$

$3.43534 \mathrm{E} \quad 01$

$3.44543 \mathrm{E} \quad 01$

$3.45552 \mathrm{E} 01$

$3.46561 \mathrm{E} \quad 01$

$3.47571 \mathrm{E} \quad 01$

$3.48581 \mathrm{E} \quad 01$

$3.49592 \mathrm{E} \quad 01$

$3.50603 \mathrm{E} \quad 01$

$3.51614 \mathrm{E} \quad 01$

$3.52626 \mathrm{E} \quad 01$

$3.53639 \mathrm{E} \quad 01$

$3.54651 \mathrm{E} \quad 01$

$3.55664 \mathrm{E} \quad 01$

$3.56677 \mathrm{E} \quad 01$
$3.57691 \mathrm{E} \quad 01$

$3.58705 \mathrm{E} \quad 01$

$3.59720 \mathrm{E} \quad 01$

$3.60735 \mathrm{E} \quad 01$

$3.61750 \mathrm{E} \quad 01$

$3.62766 \mathrm{E} \quad 01$

$3.63782 \mathrm{E} \quad 01$

$3.64799 \mathrm{E} \quad 01$

$3.65815 \mathrm{E} \quad 01$

$3.66833 \mathrm{E} 01$

$3.67851 \mathrm{E} \quad 01$

$3.68869 \mathrm{E} \quad 01$

$3.69887 \mathrm{E} \quad 01$

$3.70906 \mathrm{E} \quad 01$

$3.71926 \mathrm{E} \quad 01$

$3.73966 \mathrm{E} \quad 01$

$3.74986 \mathrm{E} \quad 01$

$3.76007 \mathrm{E} \quad 01$

$3.77029 \mathrm{E} \quad 01$

$3.78051 \mathrm{E} \quad 01$

$3.79073 \mathrm{E} \quad 01$

$3.80096 \mathrm{E} \quad 01$

$3.81119 \mathrm{E} \quad 01$

$3.82142 \mathrm{E} 01$.

$3.83166 \mathrm{E} \quad 01$

$3.84191 \mathrm{E} \quad 01$

$3.85216 \mathrm{E} 01$

$3.86241 \mathrm{E} \quad 01$

$3.87267 \mathrm{E} \quad 01$

$3.88293 \mathrm{E} \quad 01$

$3.89319 \mathrm{E} \quad 01$

$3.90346 \mathrm{E} \quad 01$

$3.91374 \mathrm{E} \quad 01$

$3.92401 \mathrm{E} \quad 01$

$3.93430 \mathrm{E} \quad 0 \mathrm{l}$

$3.94458 \mathrm{E} \quad 01$

$3.95488 \mathrm{E} \quad 01$

$3.96517 \mathrm{E} \quad 01$

$3.97547 \mathrm{E} \quad 01$

$3.98578 \mathrm{E} \quad 01$ $3.99609 \mathrm{E} \quad 01$

$4.00640 \mathrm{E} \quad 01$

$4.01672 \mathrm{E} \quad 01$

$4.02704 \mathrm{E} \quad 01$
$3.72946 \mathrm{E} \quad 01$ $\tan \delta_{1}$

$2.67504 \mathrm{E} \quad 00-1.00592 \mathrm{E}-03$

$2.69183 \mathrm{E} \quad 00-1.00595 \mathrm{E}-03$

$2.70868 \mathrm{E} \quad 00 \quad-1.00598 \mathrm{E}-03$

$2.72558 \mathrm{E} \quad 00-1.00598 \mathrm{E}-03$

$2.74254 \mathrm{E} \quad 00 \quad-1.00597 \mathrm{E}-03$

$2.75955 \mathrm{E} \quad 00 \quad-1.00595 \mathrm{E}-03$

$\begin{array}{lll}2.77662 \mathrm{E} & 00 & -1.00592 \mathrm{E}-03\end{array}$

$2.79374 \mathrm{E} \quad 00-1.00587 \mathrm{E}-03$

$2.81093 \mathrm{E} \quad 00 \quad-1.00581 \mathrm{E}-03$

$2.82816 \mathrm{E} \quad 00$

$-1.00574 \mathrm{E}-03$

$2.84546 \mathrm{E} \quad 00$

$2.86281 \mathrm{E} \quad 00$

$2.88022 \mathrm{E} \quad 00$

$2.89768 \mathrm{E} \quad 00$

$2.91520 \mathrm{E} \quad 00$

$2.93278 \mathrm{E} \quad 00$

$2.95041 \mathrm{E} \quad 00$

$2.96810 \mathrm{E} \quad 00$

$2.98585 \mathrm{E} \quad 00$

$3.00365 \mathrm{E} \quad 00$

$3.02151 \mathrm{E} \quad 00$

$3.03943 \mathrm{E} \quad 00$

$3.05740 \mathrm{E} \quad 00$

$3.07544 \mathrm{E} \quad 00$

$3.09352 \mathrm{E} \quad 00$

$3.11167 \mathrm{E} \quad 00$

$3.12987 \mathrm{E} \quad 00$

$3.14813 \mathrm{E} \quad 00$

$3.16645 \mathrm{E} \quad 00$

$3.18482 \mathrm{E} \quad 00$

$3.20325 \mathrm{E} \quad 00$

$3.22174 \mathrm{E} \quad 00$

$3.24029 \mathrm{E} \quad 00$

$3.25889 \mathrm{E} \quad 00$

$3.27755 \mathrm{E} \quad 00$

$3.29627 \mathrm{E} \quad 00$

$3.31505 \mathrm{E} \quad 00$

$3.33388 \mathrm{E} \quad 00$

$3.35277 \mathrm{E} \quad 00$

$3.37172 \mathrm{E} \quad 00$

$3.39073 \mathrm{E} \quad 00$

$3.40980 \mathrm{E} \quad 00$

$3.42892 \mathrm{E} \quad 00$

$3.44810 \mathrm{E} \quad 00$

$3.46734 \mathrm{E} \quad 00$

$3.48664 \mathrm{E} \quad 00$

$3.50599 \mathrm{E} \quad 00$

$3.52540 \mathrm{E} \quad 00$

$3.54488 \mathrm{E} \quad 00$

3.56441 E 00

$3.58400 \mathrm{E} \quad 00$

$3.60364 \mathrm{E} \quad 00$

$3.62335 \mathrm{E} \quad 00$

$3.64311 \mathrm{E} \quad 00$

$3.66293 \mathrm{E} 00$

$3.68282 \mathrm{E} \quad 00$

$3.70276 \mathrm{E} \quad 00$

$3.72275 \mathrm{E} \quad 00$

$3.74281 \mathrm{E} \quad 00$

$3.76293 \mathrm{E} \quad 00$
$-1.00565 \mathrm{E}-03$

$-1.00555 \mathrm{E}-03$

$-1.00544 \mathrm{E}-03$

$-1.00531 \mathrm{E}-03$

$-1.00517 \mathrm{E}-03$

$-1.00502 \mathrm{E}-03$

$-1.00486 \mathrm{E}-03$

$-1.00468 \mathrm{E}-03$

$-1.00449 \mathrm{E}-03$

$-1.00428 \mathrm{E}-03$

$-1.00407 \mathrm{E}-03$

$-1.00385 \mathrm{E}-03$

$-1.00361 \mathrm{E}-03$

$-1.00335 \mathrm{E}-03$

$-1.00309 \mathrm{E}-03$

$-1.00282 \mathrm{E}-03$

$-1.00253 \mathrm{E}-03$

$-1.00223 \mathrm{E}-03$

$-1.00191 \mathrm{E}-03$

$-1.00159 \mathrm{E}-03$

$-1.00125 \mathrm{E}-03$

$-1.00090 \mathrm{E}-03$

$-1.00054 \mathrm{E}-03$

$-1.00017 \mathrm{E}-03$

$-9.99787 \mathrm{E}-04$

$-9.99392 \mathrm{E}-04$

$-9.98565 \mathrm{E}-04$

$-9.98142 \mathrm{E}-04$

$-9.97700 \mathrm{E}-04$

$-9.97244 \mathrm{E}-04$

$-9.96776 \mathrm{E}-04$

$-9.96304 \mathrm{E}-04$

$-9.95819 \mathrm{E}-04$

$-9.95322 \mathrm{E}-04$

$-9.94811 \mathrm{E}-04$

$-9.94300 \mathrm{E}-04$

$-9.93766 \mathrm{E}-04$

$-9.92674 \mathrm{E}-04$

$-9.92108 \mathrm{E}-04$

$-9.90949 \mathrm{E}-04$

$-9.90347 \mathrm{E}-04$

$-9.89746 \mathrm{E}-04$

$-9.89127 \mathrm{E}-04$

$-9.88502 \mathrm{E}-04$

$-9.87863 \mathrm{E}-04$

$-9.87212 \mathrm{E}-04$

$-9.86549 \mathrm{E}-04$
$-9.98985 \mathrm{E}-04$

$-9.93223 \mathrm{E}-04$

$-9.91538 \mathrm{E}-04$

$f_{-1}$

$-2.57356 \mathrm{E} \quad 00$

$-2.59013 \mathrm{E} \quad 00$

$-2.60675 \mathrm{E} \quad 00$

$-2.62344 \mathrm{E} \quad 00$

$-2.64018 \mathrm{E} \quad 00$

$-2.65697 \mathrm{E} \quad 00$

$-2.67382 \mathrm{E} \quad 00$

$-2.69073 \mathrm{E} \quad 00$

$-2.70769 \mathrm{E} \quad 00$

$-2.72471 \mathrm{E} \quad 00$

$-2.74179 \mathrm{E} \quad 00$

$-2.75892 \mathrm{E} \quad 00$

$-2.77611 \mathrm{E} \quad 00$

$-2.79336 \mathrm{E} \quad 00$

$-2.81066 \mathrm{E} \quad 00$

$-2.82802 \mathrm{E} \quad 00$

$-2.84544 \mathrm{E} 00$

$-2.86291 \mathrm{E} \quad 00$ 
ELECTRON RADIAL FUNCTIONS

$Z=6 \quad A=12 \quad \rho=1.3 A^{1 / 3} F$

Positron

$\bar{F}$

$1.87740 \mathrm{E}-03$

$1.88781 \mathrm{E}-03$

$1.89825 \mathrm{E}-03$

$1.90872 \mathrm{E}-03$

$1.91922 \mathrm{E}-03$

$1.92974 \mathrm{E}-03$

$1.94030 \mathrm{E}-03$

$1.95088 \mathrm{E}-03$

$1.96149 \mathrm{E}-03$

$1.97213 \mathrm{E}-03$

$1.98280 \mathrm{E}-03$

$1.99350 \mathrm{E}-03$

$2.00423 \mathrm{E}-03$

$2.01499 \mathrm{E}-03$

$2.02577 \mathrm{E}-03$

$2.03659 \mathrm{E}-03$

$2.04743 \mathrm{E}-03$

$2.05830 \mathrm{E}-03$

$2.06920 \mathrm{E}-03$

$2.08013 \mathrm{E}-03$

$2.09109 \mathrm{E}-03$

$2.10207 \mathrm{E}-03$

$2.11309 \mathrm{E}-03$

$2.12413 \mathrm{E}-03$

$2.13521 \mathrm{E}-03$

2.14631 E-03

$2.15744 \mathrm{E}-03$

$2.16860 \mathrm{E}-03$

$2.17978 \mathrm{E}-03$

$2.19100 \mathrm{E}-03$

$2.20225 \mathrm{E}-03$

$2.21352 \mathrm{E}-03$

$2.22482 \mathrm{E}-03$

$2.23616 \mathrm{E}-03$

$2.24752 \mathrm{E}-03$

$2.25891 \mathrm{E}-03$

$2.27032 \mathrm{E}-03$

$2.28177 \mathrm{E}-03$

$2.29325 \mathrm{E}-03$

$2.30475 \mathrm{E}-03$

$2.31628 \mathrm{E}-03$

$2.32784 \mathrm{E}-03$

$2.33944 \mathrm{E}-03$

$2.35106 \mathrm{E}-03$

$2.36270 \mathrm{E}-03$

$2.37438 \mathrm{E}-03$

$2.38609 \mathrm{E}-03$

$2.39782 \mathrm{E}-03$

$2.40958 \mathrm{E}-03$

$2.42138 \mathrm{E}-03$

$2.43320 \mathrm{E}-03$

$2.44505 \mathrm{E}-03$

$2.45692 \mathrm{E}-03$

$2.46883 \mathrm{E}-03$

$2.48077 \mathrm{E}-03$

$2.49273 \mathrm{E}-03$

$2.50473 \mathrm{E}-03$

$2.51675 \mathrm{E}-03$

$2.52880 \mathrm{E}-03$

$2.54088 \mathrm{E}-03$ $f_{2}$

$g_{2}$

$\tan \delta_{2}$

$f$,

$3.07821 \mathrm{E} \quad 00$

$3.09532 \mathrm{E} \quad 00$

$3.11248 \mathrm{E} \quad 00$

$3.12969 \mathrm{E} \quad 00$

$3.14694 \mathrm{E} \quad 00$

$3.16425 \mathrm{E} \quad 00$

$3.18159 \mathrm{E} \quad 00$

$3.19899 \mathrm{E} \quad 00$

$3.21643 \mathrm{E} \quad 00$

$3.23391 \mathrm{E} \quad 00$

$3.25145 \mathrm{E} \quad 00$

$3.26902 \mathrm{E} \quad 00$

$3.28665 \mathrm{E} \quad 00$

$3.30432 \mathrm{E} \quad 00$

$3.3220+\mathrm{E} \quad 00$

$3.33981 \mathrm{E} \quad 00$

$3.35762 \mathrm{E} \quad 00$

$3.37548 \mathrm{E} \quad 00$

$3.39339 \mathrm{E} \quad 00$

$3.41134 \mathrm{E} \quad 00$

$3.42934 \mathrm{E} \quad 00$

$3.44738 \mathrm{E} \quad 00$

$3.46547 \mathrm{E} \quad 00$

$3.48361 \mathrm{E} \quad 00$

$3.50179 \mathrm{E} \quad 00$

$3.52002 \mathrm{E} \quad 00$

$3.53830 \mathrm{E} \quad 00$

$3.55662 \mathrm{E} \quad 00$

$3.57499 \mathrm{E} \quad 00$

$3.59341 \mathrm{E} \quad 00$

$3.61187 \mathrm{E} \quad 00$

$3.63038 \mathrm{E} \quad 00$

$3.64894 \mathrm{E} \quad 00$

$3.66754 \mathrm{E} \quad 00$

$3.68618 \mathrm{E} \quad 00$

$3.70488 \mathrm{E} \quad 00$

$3.72362 \mathrm{E} \quad 00$

$3.7+241 \mathrm{E} \quad 00$

$3.76124 \mathrm{E} \quad 00$

$3.78012 \mathrm{E} \quad 00$

$3.79904 \mathrm{E} \quad 00$ $3.81802 \mathrm{E} \quad 00$

$3.83703 \mathrm{E} \quad 00$

$3.85610 \mathrm{E} \quad 00$

$3.87521 \mathrm{E} \quad 00$

$3.89437 \mathrm{E} \quad 00$

$3.91357 \mathrm{E} \quad 00$

$3.93282 \mathrm{E} \quad 00$

$3.95211 \mathrm{E} \quad 00$

$3.97145 \mathrm{E} \quad 00$

$3.99084 \mathrm{E} \quad 00$

$4.01027 \mathrm{E} \quad 00$

$4.02975 \mathrm{E} 00$

$4.04928 \mathrm{E} \quad 00$

$4.06885 \mathrm{E} \quad 00$

$4.08847 \mathrm{E} \quad 00$

$4.10813 \mathrm{E} \quad 00$

$4.12784 \mathrm{E} \quad 00$

$4.14760 \mathrm{E} \quad 00$

$4.16740 \mathrm{E} \quad 00$
$1.45512 \mathrm{E}-01$

$46800 \mathrm{E}-0$

$1.48096 \mathrm{E}-01$

$1.49399 \mathrm{E}-01$

$1.50710 \mathrm{E}-01$

$1.52028 \mathrm{E}-01$

$1.53354 \mathrm{E}-01$

$1.54688 \mathrm{E}-01$

$1.56029 \mathrm{E}-01$

$1.57378 \mathrm{E}-01$

$1.58735 \mathrm{E}-01$

$1.60099 \mathrm{E}-01$

$1.61471 \mathrm{E}-01$

$1.62851 \mathrm{E}-01$

$1.64239 \mathrm{E}-01$

$1.6563+\mathrm{E}-01$

$1.67038 \mathrm{E}-01$

$1.68449 \mathrm{E}-01$

$1.69868 \mathrm{E}-0 \mathrm{l}$

$1.71295 \mathrm{E}-01$

$1.72730 \mathrm{E}-01$

$1.74173 \mathrm{E}-01$

$1.75624 \mathrm{E}-01$

$1.77082 \mathrm{E}-01$

$1.78549 \mathrm{E}-01$

$1.80024 \mathrm{E}-01$

$1.81507 \mathrm{E}-01$

$1.82998 \mathrm{E}-01$

$1.84497 \mathrm{E}-0]$

$1.86004 \mathrm{E}-01$

$1.87519 \mathrm{E}-01$

$1.89043 \mathrm{E}-01$

$1.90574 \mathrm{E}-01$

$1.92114 \mathrm{E}-01$

$1.93662 \mathrm{E}-01$

$1.95219 \mathrm{E}-01$

$1.96783 \mathrm{E}-01$

$1.98356 \mathrm{E}-01$

$1.99937 \mathrm{E}-01$

$2.01526 \mathrm{E}-01$

$2.03124 \mathrm{E}-01$

$2.04730 \mathrm{E}-01$

$2.06345 \mathrm{E}-01$

$2.07968 \mathrm{E}-01$

$2.09599 \mathrm{E}-01$

$2.11239 \mathrm{E}-01$

$2.12887 \mathrm{E}-01$

$2.14544 \mathrm{E}-01$

$2.16210 \mathrm{E}-01$

$2.17883 \mathrm{E}-01$

$2.19566 \mathrm{E}-01$

$2.21257 \mathrm{E}-01$

$2.22956 \mathrm{E}-01$

$2.24665 \mathrm{E}-01$

$2.26382 \mathrm{E}-0]$

$2.28107 \mathrm{E}-01$

$2.29841 \mathrm{E}-01$

$2.31584 \mathrm{E}-01$

$2.33336 \mathrm{E}-01$

$2.35096 \mathrm{E}-01$
$-3.27542 \mathrm{E}$ 0l $-1.39829 \mathrm{E}-01$

$-3.27551 \mathrm{E} \quad 01$

$-3.27561 \mathrm{E} \quad 01$

$-3.27570 \mathrm{E}$ 0l

$-3.27580 \mathrm{E} 01$

$-1.41088 \mathrm{E}-0$

$-1.42355 \mathrm{E}-0 \mathrm{l}$

$-1.43629 \mathrm{E}-01$

$-1.44910 \mathrm{E}-01$

$-3.27589 \mathrm{E} \quad 01$

$-3.27599 \mathrm{E}$ 0]

$-3.27608 \mathrm{E} \quad 01$

$-3.27617 \mathrm{E}$ 0l

$-3.27626 \mathrm{E} \quad 01$

$-1.46199 \mathrm{E}-01$

$-1.47496 \mathrm{E}-0$

$-1.48800 \mathrm{E}-01$

$-1.50112 \mathrm{E}-01$

$-1.51431 \mathrm{E}-01$

$-3.27635 \mathrm{E} \quad 01$

$-3.27645 \mathrm{E} 01$

$-3.27654 \mathrm{E} 01$

$-3.27662 \mathrm{E} \quad 01$

$-3.27671 \mathrm{E} \quad 01$

$-3.27680 \mathrm{E} \quad 01$

$-3.27689 \mathrm{E} \quad 01$

$-3.27698 \mathrm{E} 01$

$-3.27715 \mathrm{E} 01$

$-3.27724 \mathrm{E} \quad 01$

$-3.27733 \mathrm{E} 01$

$-3.27741 \mathrm{E} 01$

$-3.27749 \mathrm{E} 01$

$-3.27758 \mathrm{E} 01$

$-3.27766 \mathrm{E} 01$

$-3.27775 \mathrm{E} 01$

$-3.27783 \mathrm{E} 01$

$-3.27791 \mathrm{E} 01$

$-3.27799 \mathrm{E} 01$

$-3.27808 \mathrm{E} 01$

$-3.27816 \mathrm{E} 01$

$-3.27824 \mathrm{E} 01$

$-3.27832 \mathrm{E} 01$

$-3.27840 \mathrm{E} \quad 0 \mathrm{l}$

$-3.27848 \mathrm{E}$ 0l

$-3.27856 \mathrm{E} \quad 01$

$-3.27864 \mathrm{E}$ 0l

$-3.27871 \mathrm{E} 01$

$-3.27879 \mathrm{E} 01$

$-3.27887 \mathrm{E} \quad 01$

$-3.27895 \mathrm{E} 01$

$-3.27902 \mathrm{E}$ 0l

$-3.27910 \mathrm{E} 01$

$-3.27925 \mathrm{E} \quad 01$

$-3.27933 \mathrm{E}$ 0I

$-3.27940 \mathrm{E} 01$

$-3.27948 \mathrm{E} 01$

$-3.27955 \mathrm{E} 01$

$-3.27962 \mathrm{E} 01$

$-3.27970 \mathrm{E}$ 0I

$-3.27977 \mathrm{E} 01$

$-3.27984 \mathrm{E}$ 0I

$-3.27991 \mathrm{E} 01$

$-3.27999 \mathrm{E} \quad 01$

$-3.28006 \mathrm{E} 01$

$-3.28013 \mathrm{E} 01$

$-3.28020 \mathrm{E} 01$

$-3.28027 \mathrm{E} 01$

$-1.52758 \mathrm{E}-01$

$-1.54093 \mathrm{E}-01$

$-1.55435 \mathrm{E}-01$

$-1.56785 \mathrm{E}-01$

$-1.58143 \mathrm{E}-01$

$-1.59509 \mathrm{E}-01$

$-1.60882 \mathrm{E}-01$

$-1.62263 \mathrm{E}-01$

$-1.63652 \mathrm{E}-01$

$-1.65048 \mathrm{E}-01$

$-1.66453 \mathrm{E}-01$

$-1.67865 \mathrm{E}-01$

$-1.69286 \mathrm{E}-01$

$-1.70714 \mathrm{E}-01$

$-1.72150 \mathrm{E}-01$

$-1.73594 \mathrm{E}-01$

$-1.75046 \mathrm{E}-01$

$-1.76506 \mathrm{E}-01$

$-1.77974 \mathrm{E}-01$

$-1.79450 \mathrm{E}-01$

$-1.80935 \mathrm{E}-01$

$-1.82427 \mathrm{E}-01$

$-1.83927 \mathrm{E}-01$

$-1.85436 \mathrm{E}-01$

$-1.86952 \mathrm{E}-01$

$-1.88+77 \mathrm{E}-01$

$-1.90010 \mathrm{E}-01$

$-1.91551 \mathrm{E}-01$

$-1.93101 \mathrm{E}-01$

$-1.94659 \mathrm{E}-01$

$-1.96224 \mathrm{E}-01$

$-1.97799 \mathrm{E}-01$

$-1.99381 \mathrm{E}-01$
$-3.27707 \mathrm{E} \quad 01$

$-3.27918 \mathrm{E} 01$ 
Positron

$\begin{array}{cc}p & F_{0} \\ 0.10 & 1.36495 \mathrm{E}-01 \\ 0.20 & 4.03980 \mathrm{E}-01 \\ 0.30 & 5.54442 \mathrm{E}-01 \\ 0.40 & 6.41720 \mathrm{E}-01 \\ 0.50 & 6.96734 \mathrm{E}-01 \\ & \\ 0.60 & 7.33678 \mathrm{E}-01 \\ 0.70 & 7.59675 \mathrm{E}-01 \\ 0.80 & 7.78633 \mathrm{E}-01 \\ 0.90 & 7.92852 \mathrm{E}-01 \\ 1.00 & 8.03764 \mathrm{E}-01\end{array}$

$1.10 \quad 8.12301 \mathrm{E}-01$

$1.20 \quad 8.19090 \mathrm{E}-01$

$1.30 \quad 8.24566 \mathrm{E}-01$

$1.40 \quad 8.29037 \mathrm{E}-01$

$1.50 \quad 8.32729 \mathrm{E}-01$

$1.60 \quad 8.35807 \mathrm{E}-01$

$1.70 \quad 8.38396 \mathrm{E}-01$

$1.80 \quad 8.40591 \mathrm{E}-01$

$1.90 \quad 8.42466 \mathrm{E}-01$

$2.00 \quad 8.44076 \mathrm{E}-01$

$2.10 \quad 8.45468 \mathrm{E}-01$

$2.20 \quad 8.46679 \mathrm{E}-01$

$2.30 \quad 8.47736 \mathrm{E}-01$

$2.40 \quad 8.48663 \mathrm{E}-01$

$2.50 \quad 8.49481 \mathrm{E}-01$

2.60

2.70

2.80

2.90

3.00

3.10

3.20

3.30

3.40

3.50

3.60

3.70

3.80

3.90

4.00

4.10

4.20

4.30

4.40

4.50

4.60

4.70

4.80

4.90

5.00

5.10

5.20

5.30

5.40

5.50

5.60

5.70

5.80

5.90

6.00

8.50846

$8.51418 \mathrm{E}-01$

$8.51929 \mathrm{E}-01$

$8.52387 \mathrm{E}-01$

$8.52798 \mathrm{E}-01$

$8.53168 \mathrm{E}-01$

$8.53503 \mathrm{E}-01$

$8.53805 \mathrm{E}-01$

$8.54079 \mathrm{E}-01$

$8.54328 \mathrm{E}-01$

$8.54554 \mathrm{E}-01$

$8.54759 \mathrm{E}-01$

$8.54947 \mathrm{E}-01$

$8.55119 \mathrm{E}-01$

$8.55275 \mathrm{E}-01$

$8.55418 \mathrm{E}-01$

$8.55549 \mathrm{E}-01$

$8.55669 \mathrm{E}-01$

$8.55779 \mathrm{E}-01$

$8.55881 \mathrm{E}-01$

$8.55974 \mathrm{E}-01$

$8.56059 \mathrm{E}-01$

$8.56137 \mathrm{E}-01$

$8.56210 \mathrm{E}-01$

$8.56275 \mathrm{E}-01$

$8.56336 \mathrm{E}-01$

$8.56392 \mathrm{E}-01$

$8.56443 \mathrm{E}-01$

$8.56490 \mathrm{E}-01$

$8.56533 \mathrm{E}-01$

$8.56573 \mathrm{E}-01$

$8.56609 \mathrm{E}-01$

$8.56642 \mathrm{E}-01$

$8.56672 \mathrm{E}-01$
$Z=7 \quad A=14$

$f_{1} \quad g_{1}$

$-4.37557 \mathrm{E}-05$

$1.82732 \mathrm{E}-02-2.73602 \mathrm{E}-04$

$4.65266 \mathrm{E}-02-6.93586 \mathrm{E}-04$

$8.63893 \mathrm{E}-02-1.28012 \mathrm{E}-03$

$1.36319 \mathrm{E}-01-2.00484 \mathrm{E}-03$

$1.94729 \mathrm{E}-01$

$2.60124 \mathrm{E}-01$

$3.31177 \mathrm{E}-01$

$4.06756 \mathrm{E}-01$

$4.85932 \mathrm{E}-01$

$-2.83851 \mathrm{E}-03$

$-3.75342 \mathrm{E}-03$

$-4.72492 \mathrm{E}-03$

$-5.73196 \mathrm{E}-03$

$-6.75717 \mathrm{E}-03$

$5.67953 \mathrm{E}-01$

$6.52219 \mathrm{E}-01$

$-7.78645 \mathrm{E}-03$

$7.38261 \mathrm{E}-01$

$8.25705 \mathrm{E}-01$

$-8.80858 \mathrm{E}-03$

$-9.81466 \mathrm{E}-03$

$-1.07977 \mathrm{E}-02$

$9.14263 \mathrm{E}-01$

-1.17521 E-02

$1.00371 \mathrm{E} \quad 00$

$1.09386 \mathrm{E} \quad 00$

$1.18457 \mathrm{E} \quad 00$

$1.27575 \mathrm{E} \quad 00$

$1.36729 \mathrm{E} \quad 00$

$-1.26737 \mathrm{E}-02$

$-1.35589 \mathrm{E}-02$

$-1.44051 \mathrm{E}-02$

$-1.52102 \mathrm{E}-02$

$-1.59724 \mathrm{E}-02$

$1.45912 \mathrm{E} \quad 00$

$\begin{array}{ll}1.55120 \mathrm{E} & 00\end{array}$

$1.64348 \mathrm{E} \quad 00$

$1.73592 \mathrm{E} \quad 00$

$1.82849 \mathrm{E} \quad 00$

$1.92117 \mathrm{E} \quad 00$

$2.01394 \mathrm{E} \quad 00$

$2.10678 \mathrm{E} \quad 00$

$2.19969 \mathrm{E} \quad 00$

$2.29265 \mathrm{E} \quad 00$

$-1.66905 \mathrm{E}-02$

$-1.73632 \mathrm{E}-02$

$-1.79898 \mathrm{E}-02$

$-1.85695 \mathrm{E}-02$

$-1.91018 \mathrm{E}-02$

$2.38564 \mathrm{E} \quad 00$

$2.47868 \mathrm{E} \quad 00$

$2.57174 \mathrm{E} \quad 00$

$2.66483 \mathrm{E} \quad 00$

$2.75794 \mathrm{E} \quad 00$

$2.85106 \mathrm{E} \quad 00$

$2.94420 \mathrm{E} \quad 00$

$3.03735 \mathrm{E} \quad 00$

$3.13051 \mathrm{E} \quad 00$

$3.22368 \mathrm{E} 00$

$3.31686 \mathrm{E} \quad 00$

$3.41005 \mathrm{E} \quad 00$

$3.50324 \mathrm{E} \quad 00$

$3.59644 \mathrm{E} \quad 00$

$3.68964 \mathrm{E} \quad 00$

$3.78284 \mathrm{E} \quad 00$

$3.87605 \mathrm{E} \quad 00$

$3.96926 \mathrm{E} \quad 00$

$4.15570 \mathrm{E} \quad 00$

$4.24892 \mathrm{E} \quad 00$

$4.34215 \mathrm{E} \quad 00$

$4.43538 \mathrm{E} \quad 00$

$4.52862 \mathrm{E} \quad 00$

$4.62186 \mathrm{E} \quad 00$

$4.71510 \mathrm{E} \quad 00$

$4.80834 \mathrm{E} \quad 00$

$4.99485 \mathrm{E} \quad 00$

$5.08810 \mathrm{E} \quad 00$
$95861 \mathrm{E}-02$

$-2.04096 \mathrm{E}-02$

$-2.07481 \mathrm{E}-02$

$-2.10375 \mathrm{E}-02$

$-2.12776 \mathrm{E}-02$

$-2.14682 \mathrm{E}-02$

$-2.16093 \mathrm{E}-02$

$-2.17007 \mathrm{E}-02$

$-2.17424 \mathrm{E}-02$

$-2.17342 \mathrm{E}-02$

$-2.16761 \mathrm{E}-02$

$-2.15680 \mathrm{E}-02$

$-2.14099 \mathrm{E}-02$

$-2.12018 \mathrm{E}-02$

$-2.09436 \mathrm{E}-02$

$-2.06353 \mathrm{E}-02$

$-2.02768 \mathrm{E}-02$

$-1.98681 \mathrm{E}-02$

$-1.94093 \mathrm{E}-02$

$-1.89002 \mathrm{E}-02$

$-1.83409 \mathrm{E}-02$

$-1.77314 \mathrm{E}-02$

$-1.70716 \mathrm{E}-02$

$-1.63616 \mathrm{E}-02$

$-1.56012 \mathrm{E}-02$

$-1.39297 \mathrm{E}-02$

$-1.30185 \mathrm{E}-02$

$-1.20570 \mathrm{E}-02$

-1.10451 E- 02

$-9.98293 \mathrm{E}-03$

$-8.87040 \mathrm{E}-03$

$-7.70752 \mathrm{E}-03$

$-6.49429 \mathrm{E}-03$
$-1.47906 \mathrm{E}-02$
$2.91453 \mathrm{E}-03$

$\tan \delta_{i}$

$\rho=1.3 A^{1 / 3} F$

2.30203E-01

$1.11060 \mathrm{E}-01$

7.38208E-02

$5.53965 \mathrm{E}-02$

4.43434 E-02

$3.69484 \mathrm{E}-02$

$3.16391 \mathrm{E}-02$

$2.76336 \mathrm{E}-02$

$2.44993 \mathrm{E}-02$

$2.19766 \mathrm{E}-02$

$1.99004 \mathrm{E}-02$

$1.81606 \mathrm{E}-02$

$1.66806 \mathrm{E}-02$

$1.54059 \mathrm{E}-02$

$1.42960 \mathrm{E}-02$

$1.33207 \mathrm{E}-02$

$1.24568 \mathrm{E}-02$

$1.16860 \mathrm{E}-02$

$1.09940 \mathrm{E}-02$

$1.03692 \mathrm{E}-02$

$9.80229 \mathrm{E}-03$

$9.28550 \mathrm{E}-03$

$8.81245 \mathrm{E}-03$

$8.37779 \mathrm{E}-03$

$7.97703 \mathrm{E}-03$

$7.60633 \mathrm{E}-03$

$7.26244 \mathrm{E}-03$

$6.94252 \mathrm{E}-03$

$6.64417 \mathrm{E}-03$

$6.36528 \mathrm{E}-03$

$4.06248 \mathrm{E} \quad 00$

$4.90159 \mathrm{E} \quad 00$
$6.10398 \mathrm{E}-03$

$5.85868 \mathrm{E}-03$

$5.62794 \mathrm{E}-03$

5.41051 E-03

$5.20526 \mathrm{E}-03$

$5.01121 \mathrm{E}-03$

$4.82746 \mathrm{E}-03$

$4.6532] \mathrm{E}-03$

$4.33041 \mathrm{E}-03$

$4.18063 \mathrm{E}-03$

$4.03788 \mathrm{E}-03$

$3.90167 \mathrm{E}-03$

$3.77156 \mathrm{E}-03$

$3.64715 \mathrm{E}-03$

$3.52809 \mathrm{E}-03$

$3.41402 \mathrm{E}-03$

$3.30465 \mathrm{E}-03$

$3.19969 \mathrm{E}-03$

$3.09888 \mathrm{E}-03$

$3.00198 \mathrm{E}-03$

$2.90877 \mathrm{E}-03$

$2.81905 \mathrm{E}-03$

$2.73261 \mathrm{E}-03$

$2.64929 \mathrm{E}-03$

$2.56894 \mathrm{E}-03$

$2.49136 \mathrm{E}-03$

$2.34407 \mathrm{E}-03$

$2.27407 \mathrm{E}-03$
$4.48774 \mathrm{E}-03$

$2.41646 \mathrm{E}-03$

$f_{-1}$

$1.06550 \mathrm{E}-03$

$3.64567 \mathrm{E}-03$

$6.34914 \mathrm{E}-0.3$

$8.99940 \mathrm{E}-03$

1.15541 E-02

$1.39976 \mathrm{E}-02$

$1.63245 \mathrm{E}-02$

$1.85355 \mathrm{E}-02$

$2.06342 \mathrm{E}-02$

$2.26259 \mathrm{E}-02$

$2.45165 \mathrm{E}-02$

$2.63119 \mathrm{E}-02$

$2.80175 \mathrm{E}-02$

$2.96384 \mathrm{E}-02$

$3.11791 \mathrm{E}-02$

$3.26434 \mathrm{E}-02$

$3.40348 \mathrm{E}-02$

$3.53561 \mathrm{E}-02$

$3.66099 \mathrm{E}-02$

$3.77984 \mathrm{E}-02$

$3.89233 \mathrm{E}-02$ 
ELECTRON RADIAL FUNCTIONS

$$
Z=7 \quad A=14 \quad \rho=1.3 A^{1 / 3} F
$$

Positron

\section{$\bar{F}$}

$3.12030 \mathrm{E}-09$

$3.12339 \mathrm{E}-08$

$9.32175 \mathrm{E}-08$

$1.89513 \mathrm{E}-07$

$3.19736 \mathrm{E}-07$

$4.83418 \mathrm{E}-07$

$6.80136 \mathrm{E}-07$

$9.09537 \mathrm{E}-07$

$1.17134 \mathrm{E}-06$

$1.46530 \mathrm{E}-06$

$1.79126 \mathrm{E}-06$

$2.14907 \mathrm{E}-06$

$2.53862 \mathrm{E}-06$

$2.95982 \mathrm{E}-06$

$3.41261 \mathrm{E}-06$

$3.89693 \mathrm{E}-06$

4.41273 E-06

$4.95998 \mathrm{E}-06$

$5.53865 \mathrm{E}-06$

$6.14872 \mathrm{E}-06$

$6.79017 \mathrm{E}-06$

$7.46298 \mathrm{E}-06$

$8.16713 \mathrm{E}-06$

$8.90262 \mathrm{E}-06$

9.66944 E-06

$1.04676 \mathrm{E}-05$

$1.12970 \mathrm{E}-05$

$1.21578 \mathrm{E}-05$

$1.30498 \mathrm{E}-05$

$1.39732 \mathrm{E}-05$

$1.49278 \mathrm{E}-05$

$1.59137 \mathrm{E}-05$

$1.69309 \mathrm{E}-05$

$1.79794 \mathrm{E}-05$

$1.90592 \mathrm{E}-05$

$2.01702 \mathrm{E}-05$

$2.13125 \mathrm{E}-05$

$2.24861 \mathrm{E}-05$

$2.36909 \mathrm{E}-05$

$2.49270 \mathrm{E}-05$

$2.61944 \mathrm{E}-0.5$

$2.74930 \mathrm{E}-05$

$2.88229 \mathrm{E}-05$

$3.01840 \mathrm{E}-05$

$3.15764 \mathrm{E}-05$

$3.30001 \mathrm{E}-05$

$3.44550 \mathrm{E}-05$

$3.59411 \mathrm{E}-05$

$3.74585 \mathrm{E}-05$

$3.90072 \mathrm{E}-05$

4.05871 E-05

4.21983 E-0 05

$4.38407 \mathrm{E}-05$

4.55143 E- 05

$4.72193 \mathrm{E}-05$

4.89554 E-05

$5.07228 \mathrm{E}-05$

$5.25214 \mathrm{E}-05$

$5.43513 \mathrm{E}-05$

$5.62124 \mathrm{E}-05$ $f_{2}$

$8.07554 \mathrm{E}-07$

9.91894 E-06

$3.77379 \mathrm{E}-05$

$9.33097 \mathrm{E}-05$

1.83951 E-04

$3.15245 \mathrm{E}-04$

$4.91236 \mathrm{E}-04$

$7.14715 \mathrm{E}-04$

$9.87522 \mathrm{E}-04$

$1.31081 \mathrm{E}-03$

$1.68527 \mathrm{E}-03$

$2.11126 \mathrm{E}-03$

$2.58895 \mathrm{E}-03$

$3.11837 \mathrm{E}-03$

$3.69948 \mathrm{E}-03$

$4.33221 \mathrm{E}-03$

$5.01645 \mathrm{E}-03$

$5.75208 \mathrm{E}-03$

$6.53899 \mathrm{E}-03$

$7.37708 \mathrm{E}-03$

$8.26625 \mathrm{E}-03$

$9.20640 \mathrm{E}-03$

$1.01974 \mathrm{E}-02$

$1.12393 \mathrm{E}-02$

$1.23319 \mathrm{E}-02$

$1.34752 \mathrm{E}-02$

$1.46692 \mathrm{E}-02$

$1.59137 \mathrm{E}-02$

$1.72088 \mathrm{E}-02$

$1.85543 \mathrm{E}-02$

$1.99504 \mathrm{E}-02$

$2.13968 \mathrm{E}-02$

$2.28937 \mathrm{E}-02$

$2.44409 \mathrm{E}-02$

$2.60385 \mathrm{E}-02$

$2.76864 \mathrm{E}-02$

$2.93846 \mathrm{E}-02$

3.11331 E-02

$3.29318 \mathrm{E}-02$

$3.47809 \mathrm{E}-02$

$3.66801 \mathrm{E}-02$ $3.86296 \mathrm{E}-02$ $4.06293 \mathrm{E}-02$ $4.26792 \mathrm{E}-02$ 4.47793 E-02

$4.69296 \mathrm{E}-02$ $4.91300 \mathrm{E}-02$

$5.13806 \mathrm{E}-02$

$5.36813 \mathrm{E}-02$

$5.6032 \mathrm{I}$ E- 02

$5.84331 \mathrm{E}-02$ $6.08841 \mathrm{E}-02$ $6.33852 \mathrm{E}-02$ $6.59364 \mathrm{E}-02$ $6.85376 \mathrm{E}-02$

$7.11887 \mathrm{E}-02$ $7.38897 \mathrm{E}-02$ $7.66407 \mathrm{E}-02$ 7.94412 E-02 8.22915 E-02 $g_{2}$

$-6.80183 \mathrm{E}-09$

$-8.33062 \mathrm{E}-08$

$-3.15466 \mathrm{E}-07$

$-7.75014 \mathrm{E}-07$

$-1.51562 \mathrm{E}-06$

$-2.57275 \mathrm{E}-06$

$-3.96561 \mathrm{E}-06$

$-5.70012 \mathrm{E}-06$

$-7.77209 \mathrm{E}-06$

$-1.01700 \mathrm{E}-05$

$-1.28772 \mathrm{E}-05$

$-1.58737 \mathrm{E}-0.5$

$-1.91372 \mathrm{E}-05$

$-2.26440 \mathrm{E}-0.5$

$-2.63695 \mathrm{E}-05$

$-3.02888 \mathrm{E}-05$

$-3.43764 \mathrm{E}-05$

$-3.86070 \mathrm{E}-05$

$-4.29551 \mathrm{E}-05$

$-4.73952 \mathrm{E}-0.5$

$-5.19021 \mathrm{E}-0.5$

$-5.64504 \mathrm{E}-0.5$

$-6.10148 \mathrm{E}-05$

$-6.55702 \mathrm{E}-0.5$

$-7.00916 \mathrm{E}-05$

$-7.45539 \mathrm{E}-05$

$-7.89323 \mathrm{E}-0.5$

$-8.32017 \mathrm{E}-05$

$-8.73374 \mathrm{E}-05$

$-9.13146 \mathrm{E}-05$

$-9.51084 \mathrm{E}-05$

$-9.86944 \mathrm{E}-0.5$

$-1.02048 \mathrm{E}-04$

$-1.05144 \mathrm{E}-04$

$-1.07958 \mathrm{E}-04$

$-1.10465 \mathrm{E}-04$

$-1.12642 \mathrm{E}-04$

$-1.14462 \mathrm{E}-04$

$-1.15903 \mathrm{E}-04$

$-1.16939 \mathrm{E}-04$

$-1.17545 \mathrm{E}-04$

$-1.17698 \mathrm{E}-04$

$-1.17373 \mathrm{E}-04$

$-1.16545 \mathrm{E}-04$

$-1.15190 \mathrm{E}-04$

$-1.13283 \mathrm{E}-04$

$-1.10799 \mathrm{E}-04$

$-1.07716 \mathrm{E}-04$

$-1.04007 \mathrm{E}-04$

$-9.96490 \mathrm{E}-05$

$-9.46169 \mathrm{E}-05$

$-8.88867 \mathrm{E}-05$

$-8.24337 \mathrm{E}-05$

$-7.52336 \mathrm{E}-05$

$-6.72621 \mathrm{E}-05$

$-5.84945 \mathrm{E}-05$

$-4.89067 \mathrm{E}-05$

$-3.84743 \mathrm{E}-05$

$-2.71730 \mathrm{E}-05$

$-1.49786 \mathrm{E}-05$ $\tan \delta_{2}$

$-1.93299 \mathrm{E} \quad 00$ $-4.07750 \mathrm{E} \quad 00$ $-6.06943 \mathbf{E} 00$ $-7.92500 \mathrm{E} 00$ $-9.63454 \mathbf{E} 00$

$-1.11927 \mathrm{E} 01$ $-1.26008 \mathrm{E} \quad 01$ $-1.38656 \mathrm{E} 01$ $-1.49972 \mathrm{E} 01$ $-1.60074 \mathrm{E} 01$

$-1.69088 \mathrm{E} \quad 01$ $-1.77135 \mathrm{E} \quad 01$ $-1.84327 \mathrm{E} 01$ $-1.90768 \mathrm{E} 01$ $-1.96550 \mathrm{E} 01$

$-2.01754 \mathrm{E} 01$ $-2.06450 \mathrm{E} \quad 01$ $-2.10700 \mathrm{E} 01$ $-2.14558 \mathrm{E}$ 01 $-2.18070 \mathrm{E} 01$

$\begin{array}{lll}-2.21276 \mathrm{E} & 01\end{array}$ $-2.24210 \mathrm{E} 01$ $-2.26903 \mathrm{E} 01$ $-2.29381 \mathrm{E} 01$ $\begin{array}{lll}-2.31667 \mathrm{E} & 01\end{array}$

$\begin{array}{lll}-2.33781 \mathrm{E} & 01\end{array}$ $-2.35740 \mathrm{E} 01$ $\begin{array}{lll}-2.37559 \mathrm{E} & 01\end{array}$ $-2.39253 \mathrm{E} 01$ $-2.40832 \mathrm{E} \quad 01$

$-2.42308 \mathrm{E}$ 01 $-2.43690 \mathrm{E} 0 \mathrm{l}$ $-2.44985 \mathrm{E} \quad 01$ $-2.46202 \mathrm{E} 01$ $-2.47347 \mathrm{E} 01$

$-2.48426 \mathrm{E} \quad 01$ $-2.49445 \mathrm{E}$ 0I $-2.50407 \mathrm{E} 01$ $-2.51318 \mathrm{E} 01$ $-2.52181 \mathrm{E} 01$

$-2.52999 \mathrm{E} \quad 01$ $-2.53777 \mathrm{E} \quad 01$ $-2.54516 \mathrm{E} \quad 01$ $-2.55220 \mathrm{E} \quad 01$ $-2.55891 \mathrm{E} 01$

$-2.56531 \mathrm{E} \quad 01$ $-2.57142 \mathrm{E} \quad 01$ $-2.57726 \mathrm{E}$ 0I $-2.58285 \mathrm{E} 01$ $-2.58820 \mathrm{E} 01$

$-2.59332 \mathrm{E} \quad 01$ $-2.59824 \mathrm{E}$ 01 $-2.60296 \mathrm{E} \quad 01$ $-2.60749 \mathrm{E} 01$ $-2.61185 \mathrm{E} \quad 01$

$-2.61604 \mathrm{E} 01$ $-2.62007 \mathrm{E} 01$ $-2.62396 \mathrm{E} 01$ $-2.62770 \mathrm{E} \quad 01$ $-2.63132 \mathrm{E} 01$

$f_{-2}$

$1.84135 \mathrm{E}-07$

$1.15853 \mathrm{E}-06$

$2.97479 \mathrm{E}-06$

$5.58702 \mathrm{E}-06$

$8.93907 \mathrm{E}-06$

$1.29719 \mathrm{E}-05$

$1.76276 \mathrm{E}-05$

$2.28519 \mathbf{E}-05$

$2.85953 \mathbf{E}-05$

$3.48127 \mathrm{E}-05$

$4.14635 \mathrm{E}-05$ $4.85104 \mathrm{E}-05$

$5.59192 \mathrm{E}-05$

$6.36582 \mathrm{E}-05$

$7.16972 \mathbf{E}-05$

$8.00074 \mathrm{E}-05$

$8.85613 \mathrm{E}-05$

$9.73320 \mathrm{E}-05$

$1.06293 \mathrm{E}-04$

$1.15419 \mathrm{E}-04$

$1.24685 \mathrm{E}-04$

$1.34065 \mathrm{E}-04$

$1.43533 \mathrm{E}-04$

$1.53067 \mathrm{E}-04$

$1.62640 \mathrm{E}-04$

$1.72228 \mathrm{E}-04$

$1.81806 \mathrm{E}-04$

$1.91351 \mathrm{E}-04$

$2.00837 \mathrm{E}-04$

2.10241 E-04

$2.19537 \mathrm{E}-04$

$2.28702 \mathrm{E}-04$

$2.37711 \mathrm{E}-04$

$2.46540 \mathrm{E}-04$

$2.55164 \mathrm{E}-04$

$2.63559 \mathrm{E}-04$

$2.71700 \mathrm{E}-04$

$2.79564 \mathrm{E}-04$

$2.87126 \mathrm{E}-04$

$2.94361 \mathrm{E}-04$ 
ELECTRON RADIAL FL TCTIONS

\section{p}

6.10

6.20

6.30

6.40

6.50

6.60

6.70

6.80

6.90

7.00

7.10

7.20

7.30

7.40

7.50

7.60

7.70

7.80

7.90

8.00

8.10

8.20

8.30

8.40

8.50

8.60

8.70

8.80

8.90

9.00

9.10

9.20

9.30

9.40

9.50

9.60

9.70

9.80

9.90

10.00

10.10

10.20

10.30

10.40

10.50

10.60

10.70

10.80

10.90

11.00

11.10

11.20

11.30

11.40

11.50

11.60

11.70

11.80

11.90

12.00
$F_{0}$

$8.56700 \mathrm{E}-0$ $8.56726 \mathrm{E}-0$ $8.56748 \mathrm{E}-01$ $8.56769 \mathrm{E}-01$ $8.56787 \mathrm{E}-01$

$8.56804 \mathrm{E}-01$ $8.56819 \mathrm{E}-01$ $8.56833 \mathrm{E}-01$ $8.56845 \mathrm{E}-01$ $8.56856 \mathrm{E}-01$

$8.56865 \mathrm{E}-01$ $8.56872 \mathrm{E}-01$ $8.56880 \mathrm{E}-01$ $8.56885 \mathrm{E}-01$ $8.56889 \mathrm{E}-0 \mathrm{l}$

$8.56893 \mathrm{E}-01$ $8.56896 \mathrm{E}-01$ $8.56898 \mathrm{E}-01$ $8.56900 \mathrm{E}-01$ $8.56900 \mathrm{E}-01$

$8.56900 \mathrm{E}-01$ $8.56899 \mathrm{E}-01$ $8.56898 \mathrm{E}-01$ $8.56895 \mathrm{E}-01$ $8.56893 \mathrm{E}-01$

$8.56890 \mathrm{E}-01$ $8.56886 \mathrm{E}-01$ $8.56882 \mathrm{E}-01$ $8.56878 \mathrm{E}-01$ $8.56873 \mathrm{E}-01$

$8.56867 \mathrm{E}-01$ $8.56862 \mathrm{E}-01$ $8.56856 \mathrm{E}-0]$ $8.56849 \mathrm{E}-01$ $8.56843 \mathrm{E}-01$

$8.56836 \mathrm{E}-01$ $8.56829 \mathrm{E}-01$ $8.56821 \mathrm{E}-01$ $8.56813 \mathrm{E}-0]$ $8.56805 \mathrm{E}-01$

$8.56797 \mathrm{E}-01$ $8.56789 \mathrm{E}-0 \mathrm{I}$ $8.56781 \mathrm{E}-0$ $8.56772 \mathrm{E}-01$ $8.56763 \mathrm{E}-01$

$8.56755 \mathrm{E}-01$ $8.56746 \mathrm{E}-01$ $8.56736 \mathrm{E}-01$ $8.56727 \mathrm{E}-01$ $8.56717 \mathrm{E}-01$

$8.56708 \mathrm{E}-01$ $8.56699 \mathrm{E}-01$ $8.56689 \mathrm{E}-01$ $8.56679 \mathrm{E}-01$ $8.56669 \mathrm{E}-01$

$8.56659 \mathrm{E}-01$ $8.56649 \mathrm{E}-01$ $8.56639 \mathrm{E}-01$ $8.56629 \mathrm{E}-01$ $8.56619 \mathrm{E}-01$ $f_{1}$

$g_{1}$

$5.18136 \mathrm{E} \quad 00 \quad-5.23068 \mathrm{E}-03$

$5.27463 \mathrm{E} \quad 00 \quad-3.91672 \mathrm{E}-03$

$5.36790 \mathrm{E} \quad 00 \quad-2.55238 \mathrm{E}-03$

$5.46117 \mathrm{E} \quad 00-1.13766 \mathrm{E}-03$

$5.55444 \mathrm{E} \quad 00 \quad 3.27454 \mathrm{E}-04$

$5.64772 \mathrm{E} \quad 00$

$5.74101 \mathrm{E} \quad 00$

$5.83429 \mathrm{E} \quad 00$

$5.92758 \mathrm{E} \quad 00$

$6.02087 \mathrm{E} \quad 00$

$6.11416 \mathrm{E} \quad 00$

$6.20745 \mathrm{E} \quad 00$

$6.30073 \mathrm{E} \quad 00$

$6.39402 \mathrm{E} \quad 00$

$6.48729 \mathrm{E} \quad 00$

$6.58054 \mathrm{E} \quad 00$

$6.67376 \mathrm{E} \quad 00$

$6.76696 \mathrm{E} \quad 00$

$6.86006 \mathrm{E} \quad 00$

$6.95301 \mathrm{E} \quad 00$

$7.04567 \mathrm{E} \quad 00$

$7.13751 \mathrm{E} \quad 00$

$7.22563 \mathrm{E} \quad 00$

$7.38302 \mathrm{E} \quad 00$

$7.42963 \mathrm{E} \quad 00$

$7.51974 \mathrm{E} \quad 00$

$7.61196 \mathrm{E} \quad 00$

$7.70484 \mathrm{E} \quad 00$

$7.79793 \mathrm{E} \quad 00$

$7.89118 \mathrm{E} \quad 00$

$7.98451 \mathrm{E} \quad 00$ $8.07790 \mathrm{E} \quad 00$ $8.17132 \mathrm{E} \quad 00$ $8.26479 \mathrm{E} \quad 00$ $8.35827 \mathrm{E} \quad 00$

$8.45179 \mathrm{E} \quad 00$

$8.54532 \mathrm{E} \quad 00$

$8.63887 \mathrm{E} \quad 00$

$8.73244 \mathrm{E} 00$

$8.82603 \mathrm{E} \quad 00$

$8.91964 \mathrm{E} \quad 00$ $9.01325 \mathrm{E} \quad 00$ $9.10689 \mathrm{E} \quad 00$

$9.20054 \mathrm{E} \quad 00$

$9.29421 \mathrm{E} \quad 00$

$9.38788 \mathrm{E} \quad 00$ $9.48158 \mathrm{E} \quad 00$ $9.57529 \mathrm{E} \quad 00$ $9.66901 \mathrm{E} \quad 00$

$9.76275 \mathrm{E} \quad 00$

$9.85651 \mathrm{E} \quad 00$ $9.95028 \mathrm{E} \quad 00$ $1.00441 \mathrm{E} \quad 01$ $1.01379 \mathrm{E} \quad 01$ $1.02317 \mathrm{E} \quad 01$

$1.03255 \mathrm{E} \quad 01$

$1.04193 \mathrm{E} \quad 01$

$1.05132 \mathrm{E} \quad 01$

$1.06071 \mathrm{E} \quad 01$

$1.07009 \mathrm{E} \quad 01$
$1.84296 \mathrm{E}-03$

$3.40887 \mathrm{E}-03$

$5.02518 \mathrm{E}-03$

$6.69190 \mathrm{E}-03$

$8.40903 \mathrm{E}-03$

$1.01766 \mathrm{E}-02$

$1.19945 \mathrm{E}-02$

$1.38629 \mathrm{E}-02$

$1.57817 \mathrm{E}-02$

$1.77509 \mathrm{E}-02$

$1.97704 \mathrm{E}-02$

$2.18403 \mathrm{E}-02$

$2.39604 \mathrm{E}-02$

$2.61306 \mathrm{E}-02$

$2.83504 \mathrm{E}-02$

$3.06193 \mathrm{E}-02$

$3.29345 \mathrm{E}-02$

$3.52812 \mathrm{E}-02$

$3.80324 \mathrm{E}-02$

$4.02680 \mathrm{E}-02$

4.27765 E-02

$4.53462 \mathrm{E}-02$

4.79699E-02

$5.06453 \mathrm{E}-02$

$5.33720 \mathrm{E}-02$

$5.61498 \mathrm{E}-02$

$5.89784 \mathrm{E}-02$

$6.18579 \mathrm{E}-02$

$6.47881 \mathrm{E}-02$

$6.77691 \mathrm{E}-02$

$7.08009 \mathrm{E}-02$

$7.38835 \mathrm{E}-02$

$7.70168 \mathrm{E}-02$

$8.02008 \mathrm{E}-02$

$8.34358 \mathrm{E}-02$

$8.67214 \mathrm{E}-02$

$9.00578 \mathrm{E}-02$

$9.34451 \mathrm{E}-02$

$9.68832 \mathrm{E}-02$

$1.00372 \mathrm{E}-01$

$1.03912 \mathrm{E}-0$

$1.07502 \mathrm{E}-01$

$1.11144 \mathrm{E}-01$

$1.14836 \mathrm{E}-01$

$1.18579 \mathrm{E}-01$

$1.22374 \mathrm{E}-01$

$1.26219 \mathrm{E}-0$

$1.30115 \mathrm{E}-01$

$1.34062 \mathrm{E}-01$

$1.38060 \mathrm{E}-01$

$1.42108 \mathrm{E}-01$

$1.46208 \mathrm{E}-01$

$1.50359 \mathrm{E}-01$

$1.54561 \mathrm{E}-01$

$1.58814 \mathrm{E}-01$

$\tan \delta_{1}$

$2.20637 \mathrm{E}-03$

$2.14083 \mathrm{E}-03$

$2.07736 \mathrm{E}-03$

$2.01588 \mathrm{E}-03$

$1.95627 \mathrm{E}-03$

$1.89846 \mathrm{E}-03$

$1.84239 \mathrm{E}-03$

$1.78796 \mathrm{E}-03$

$1.73511 \mathrm{E}-03$

$1.68376 \mathrm{E}-03$

$1.63386 \mathrm{E}-03$

$1.58535 \mathrm{E}-03$

$1.53817 \mathrm{E}-0.3$

$1.49228 \mathrm{E}-03$

$1.44759 \mathrm{E}-03$

$1.40410 \mathrm{E}-03$

$1.36174 \mathrm{E}-03$

$1.32048 \mathrm{E}-03$

$1.28026 \mathrm{E}-03$

$1.24106 \mathrm{E}-03$

$1.20283 \mathrm{E}-03$

$16553 \mathrm{E}-03$

$1.12916 \mathrm{E}-03$

$1.09365 \mathrm{E}-03$

$1.05899 \mathrm{E}-03$

$1.02514 \mathrm{E}-03$

$9.92079 \mathrm{E}-04$

$9.59780 \mathrm{E}-04$

$9.28220 \mathrm{E}-04$

$8.97360 \mathrm{E}-04$

$8.67204 \mathrm{E}-04$

$8.37702 \mathrm{E}-04$

$8.08854 \mathrm{E}-04$

$7.80631 \mathrm{E}-04$

$7.53015 \mathrm{E}-04$

$7.25977 \mathrm{E}-04$ $6.99513 \mathrm{E}-04$

$6.73602 \mathrm{E}-04$

$6.48226 \mathrm{E}-04$

$6.23378 \mathrm{E}-04$

$5.99029 \mathrm{E}-04$

$5.75170 \mathrm{E}-04$

$5.51793 \mathrm{E}-04$

$5.28863 \mathrm{E}-04$ 
$p$ $\bar{F}$

6.10

6.20

6.30

6.40

6.50

6.60

6.70

6.80

6.90

7.00

7.10

7.20

7.30

7.40

7.50

7.60

7.70

7.80

7.90

8.00

8.10

8.20

8.30

8.40

8.50

8.60

8.70

8.80

8.90

9.00

9.10

9.20

9.30

9.40

9.50

9.60

9.70

9.80

9.90

10.00

10.10

10.20

10.30

10.40

10.50

10.60

10.70

10.80

10.90

11.00

11.10

11.20

11.30

11.40

11.50

11.60

11.70

11.80

11.90

12.00 $f_{2}$

$8.51911 \mathrm{E}-02$

$8.81398 \mathrm{E}-02$

$9.11364 \mathrm{E}-02$

$9.41798 \mathrm{E}-02$

$9.72676 \mathrm{E}-02$

$1.00387 \mathrm{E}-01$

$1.03488 \mathrm{E}-01$

$1.12409 \mathrm{E}-01$

$1.10438 \mathrm{E}-01$

$1.13700 \mathrm{E}-01$

$7.65416 \mathrm{E}-05$

$7.87463 \mathrm{E}-05$

$8.09822 \mathrm{E}-05$

$8.32494 \mathrm{E}-05$

$8.55478 \mathrm{E}-05$

$8.78774 \mathrm{E}-05$

$9.02383 \mathrm{E}-05$

$9.26304 \mathrm{E}-05$

$9.50537 \mathrm{E}-05$

$9.75083 \mathrm{E}-05$

$9.99940 \mathrm{E}-05$

$1.02511 \mathrm{E}-04$

$1.05059 \mathrm{E}-04$

$1.07639 \mathrm{E}-04$

$1.10249 \mathrm{E}-04$

$1.12891 \mathrm{E}-04$

$1.15564 \mathrm{E}-04$

$1.18269 \mathrm{E}-04$

$1.21004 \mathrm{E}-04$

$1.23771 \mathrm{E}-04$

$1.26569 \mathrm{E}-04$

$1.29399 \mathrm{E}-04$

$1.32259 \mathrm{E}-04$

$1.35151 \mathrm{E}-04$

$1.38074 \mathrm{E}-04$

$1.41028 \mathrm{E}-04$

$1.44013 \mathrm{E}-04$

$1.47030 \mathrm{E}-04$

$1.50077 \mathrm{E}-04$

$1.53156 \mathrm{E}-04$

$1.56267 \mathrm{E}-04$

$1.59408 \mathrm{E}-04$

$1.62581 \mathrm{E}-04$

$1.65785 \mathrm{E}-04$

$1.69020 \mathrm{E}-04$

$1.72286 \mathrm{E}-04$

$1.75584 \mathrm{E}-04$

$1.78912 \mathrm{E}-04$

$1.82272 \mathrm{E}-04$

$1.85664 \mathrm{E}-04$

$1.89086 \mathrm{E}-04$

$1.92540 \mathrm{E}-04$

$1.96025 \mathrm{E}-04$

$1.99541 \mathrm{E}-04$

$2.03088 \mathrm{E}-04$

$2.06666 \mathrm{E}-04$

$2.10276 \mathrm{E}-04$

$2.13917 \mathrm{E}-04$

$2.17589 \mathrm{E}-04$

$2.21292 \mathrm{E}-04$

$2.25027 \mathrm{E}-04$
$1.17073 \mathrm{E}-01$

$1.20511 \mathrm{E}-01$

$1.24006 \mathrm{E}-01$

$1.27554 \mathrm{E}-01$

$1.31155 \mathrm{E}-01$

$1.34806 \mathrm{E}-01$

$1.38508 \mathrm{E}-01$

$1.42262 \mathrm{E}-01$

$1.46065 \mathrm{E}-01$

$1.49919 \mathrm{E}-01$

$1.53823 \mathrm{E}-01$

$1.57778 \mathrm{E}-01$

$1.61782 \mathrm{E}-01$

$1.65837 \mathrm{E}-01$

$1.69942 \mathrm{E}-01$

$1.74098 \mathrm{E}-01$

$1.78303 \mathrm{E}-01$

$1.82559 \mathrm{E}-01$

$1.86865 \mathrm{E}-01$

$1.91220 \mathrm{E}-0 \mathrm{~B}$

$1.95626 \mathrm{E}-01$

$2.00082 \mathrm{E}-01$

$2.04589 \mathrm{E}-01$

$2.09145 \mathrm{E}-01$

2.13751 E-01

$2.18408 \mathrm{E}-01$

$2.23115 \mathrm{E}-01$

$2.27871 \mathrm{E}-01$

$2.32678 \mathrm{E}-01$

$2.37535 \mathrm{E}-01$

$2.42442 \mathrm{E}-01$

$2.47399 \mathrm{E}-01$

$2.52406 \mathrm{E}-01$

$2.57463 \mathrm{E}-01$

$2.62570 \mathrm{E}-01$

$2.67728 \mathrm{E}-01$

$2.72935 \mathrm{E}-01$

$2.78193 \mathrm{E}-01$

$2.83500 \mathrm{E}-01$

$2.88858 \mathrm{E}-01$

$2.94265 \mathrm{E}-01$

$2.99723 \mathrm{E}-01$

$3.05231 \mathrm{E}-01$

$3.10788 \mathrm{E}-01$

$3.16396 \mathrm{E}-01$

$3.22054 \mathrm{E}-01$

$3.27762 \mathrm{E}-01$

$3.33520 \mathrm{E}-01$

$3.39328 \mathrm{E}-01$

$3.45186 \mathrm{E}-01$ $g_{2}$

$\tan \delta_{2}$

$f_{-2}$

$-1.86674 \mathrm{E}-06$

$1.21863 \mathrm{E}-05$

$2.72042 \mathrm{E}-05$

$4.32098 \mathrm{E}-05$

$6.02245 \mathrm{E}-05$

$7.82599 \mathrm{E}-05$

$9.72842 \mathrm{E}-05$

$1.23715 \mathrm{E}-04$

$1.39280 \mathrm{E}-04$

$1.61655 \mathrm{E}-04$

$1.85261 \mathrm{E}-04$

$2.10069 \mathrm{E}-04$

$2.36096 \mathrm{E}-04$

$2.63362 \mathrm{E}-04$

$2.91889 \mathrm{E}-04$

$3.21703 \mathrm{E}-04$

$3.52825 \mathrm{E}-04$

3.85281 E- 04

$4.19095 \mathrm{E}-04$

$4.54291 \mathrm{E}-04$

$4.90893 \mathrm{E}-04$

$5.28927 \mathrm{E}-04$

$5.68414 \mathrm{E}-04$

$6.09382 \mathrm{E}-04$

$6.51853 \mathrm{E}-04$

$6.95853 \mathrm{E}-04$

7.41405 E- 04

$7.88534 \mathrm{E}-04$

$8.37265 \mathrm{E}-04$

$8.87621 \mathrm{E}-04$

$9.39628 \mathrm{E}-04$

$9.93308 \mathrm{E}-04$

$1.04869 \mathrm{E}-03$

$1.10579 \mathrm{E}-03$

$1.16464 \mathrm{E}-03$

$1.22526 \mathrm{E}-03$

$1.28768 \mathrm{E}-03$

$1.35192 \mathrm{E}-03$

$1.41801 \mathrm{E}-03$

$1.48596 \mathrm{E}-03$

$1.55581 \mathrm{E}-03$

$1.62757 \mathrm{E}-03$

$1.70128 \mathrm{E}-03$

$1.77696 \mathrm{E}-03$

$1.85463 \mathrm{E}-03$

$1.93431 \mathrm{E}-03$

$2.01603 \mathrm{E}-03$

$2.09982 \mathrm{E}-03$

$2.18570 \mathrm{E}-03$

$2.27368 \mathrm{E}-03$

$2.36381 \mathrm{E}-03$

$2.45610 \mathrm{E}-03$

$2.55058 \mathrm{E}-03$

$2.64726 \mathrm{E}-03$

$2.74618 \mathrm{E}-03$

$2.84736 \mathrm{E}-03$

$2.95083 \mathrm{E}-03$

$3.05660 \mathrm{E}-03$

$3.16471 \mathrm{E}-03$

$3.27517 \mathrm{E}-03$

$\begin{array}{ll}-2.63480 \mathrm{E} & 01 \\ -2.63817 \mathrm{E} & 01 \\ -2.64142 \mathrm{E} & 01 \\ -2.64456 \mathrm{E} & 01 \\ -2.64760 \mathrm{E} & 01\end{array}$

$-2.65054 \mathrm{E} 01$

$-2.65339 \mathrm{E} 0 \mathrm{I}$

$-2.65615 \mathrm{E} \quad 01$

$-2.65882 \mathrm{E} \quad 01$

$-2.66142 \mathrm{E} 01$

$-2.66393 \mathrm{E} \quad 01$

$-2.66637 \mathrm{E} 01$

$-2.66874 \mathrm{E} \quad 01$

$-2.67104 \mathrm{E} 01$

$-2.67327 \mathrm{E} 01$

$-2.67544 \mathrm{E} \quad 01$

$-2.67755 \mathrm{E} 01$

$-2.67961 \mathrm{E} 01$

$-2.68161 \mathrm{E} 01$

$-2.68355 \mathrm{E} 01$

$-2.68545 \mathrm{E} 01$

$-2.68729 \mathrm{E} \quad 01$

$-2.68909 \mathrm{E} 01$

$-2.69084 \mathrm{E} \quad 01$

$-2.69255 \mathrm{E} 01$

$-2.69421 \mathrm{E} \quad 01$

$-2.69584 \mathrm{E} 01$

$-2.69743 \mathrm{E} 01$

$-2.69897 \mathrm{E} 01$

$-2.70048 \mathrm{E} \quad 01$

$-2.70196 \mathrm{E} \quad 01$

$-2.70340 \mathrm{E}$ 01

$-2.70481 \mathrm{E} 01$

$-2.70619 \mathrm{E} 01$

$-2.70754 \mathrm{E} \quad 01$

$-2.70885 \mathrm{E} \quad 01$

$-2.71014 \mathrm{E} \quad 01$

$-2.71140 \mathrm{E} 01$

$-2.71263 \mathrm{E} 01$

$-2.71384 \mathrm{E} \quad 01$

$-2.71502 \mathrm{E} \quad 01$

$-2.71618 \mathrm{E} 01$

$-2.71731 \mathrm{E} 01$

$-2.71842 \mathrm{E} \quad 01$

$-2.71951 \mathrm{E} 01$

$-2.72057 \mathrm{E} \quad 01$

$-2.72162 \mathrm{E} \quad 01$

$-2.72264 \mathrm{E} 01$

$-2.72365 \mathrm{E} \quad 01$

$-2.72463 \mathrm{E} 01$

$-2.72560 \mathrm{E} \quad 01$

$-2.72655 \mathrm{E} \quad 01$

$-2.72748 \mathrm{E} \quad 01$ 
ELECTRON RADIAL FUNCTIONS

$p$

12.10

12.20

12.30

12.40

12.50

12.60

12.70

12.80

12.90

13.00

13.10

13.20

13.30

13.40

13.50

13.60

13.70

13.80

13.90

14.00

14.10

14.20

14.30

14.40

14.50

14.60

14.70

14.80

14.90

15.00

15.10

15.20

15.30

15.40

15.50

15.60

15.70

15.80

15.90

16.00

16.10

16.20

16.30

16.40

16.50

16.60

16.70

16.80

16.90

17.00

17.10

17.20

17.30

17.40

17.50

17.60

17.70

17.80

17.90

18.00
$F_{0}$

$8.56608 \mathrm{E}-01$

$8.56598 \mathrm{E}-01$

$8.56587 \mathrm{E}-01$

8.56577 E-01

$8.56566 \mathrm{E}-01$

$8.56555 \mathrm{E}-01$

$8.56545 \mathrm{E}-01$

$8.56534 \mathrm{E}-01$

$8.56524 \mathrm{E}-01$

$8.56514 \mathrm{E}-01$

$8.56503 \mathrm{E}-01$

$8.56492 \mathrm{E}-01$

$8.56482 \mathrm{E}-01$

$8.56471 \mathrm{E}-01$

$8.56460 \mathrm{E}-01$

8.56449E-01

$8.56439 \mathrm{E}-01$

$8.56428 \mathrm{E}-01$

$8.56417 \mathrm{E}-01$

$8.56407 \mathrm{E}-01$

$8.56396 \mathrm{E}-01$

$8.56385 \mathrm{E}-01$

$8.56375 \mathrm{E}-01$

$8.56364 \mathrm{E}-01$

$8.56353 \mathrm{E}-01$

$8.56342 \mathrm{E}-01$

$8.56332 \mathrm{E}-01$

$8.56321 \mathrm{E}-01$

$8.56310 \mathrm{E}-01$

$8.56300 \mathrm{E}-0 \mathrm{l}$

$8.56289 \mathrm{E}-01$

$8.56278 \mathrm{E}-01$

$8.56268 \mathrm{E}-01$

$8.56257 \mathrm{E}-01$

$8.56247 \mathrm{E}-01$

$8.56236 \mathrm{E}-01$

$8.56225 \mathrm{E}-01$

$8.56215 \mathrm{E}-01$

$8.56205 \mathrm{E}-01$

$8.56194 \mathrm{E}-01$

$8.56184 \mathrm{E}-01$

$8.56174 \mathrm{E}-01$

$8.56163 \mathrm{E}-01$

$8.56153 \mathrm{E}-01$

$8.56142 \mathrm{E}-01$

$8.56132 \mathrm{E}-01$

$8.56122 \mathrm{E}-01$

$8.56112 \mathrm{E}-01$

$8.56101 \mathrm{E}-01$

$8.56091 \mathrm{E}-01$

$8.56081 \mathrm{E}-01$

$8.56071 \mathrm{E}-01$

$8.56061 \mathrm{E}-01$

$8.56050 \mathrm{E}-01$

$8.56040 \mathrm{E}-01$

$8.56030 \mathrm{E}-01$

$8.56020 \mathrm{E}-01$

$8.56010 \mathrm{E}-01$

$8.56000 \mathrm{E}-01$

$8.55990 \mathrm{E}-01$ $f_{1}$

$1.07949 \mathrm{E} \quad 01$

$1.08888 \mathrm{E} \quad 01$

$1.09827 \mathrm{E} \quad 01$

$1.10767 \mathrm{E} \quad 01$

$1.11706 \mathrm{E} \quad 01$

$1.12646 \mathrm{E} \quad 01$

$1.13586 \mathrm{E} \quad 01$

$1.14526 \mathrm{E} \quad 01$

$1.15466 \mathrm{E} 01$

$1.16407 \mathrm{E} \quad 01$

$1.17348 \mathrm{E} \quad 01$

$1.18288 \mathrm{E} \quad 01$

$1.19229 \mathrm{E} \quad 01$

$1.20170 \mathrm{E} \quad 01$

$1.21112 \mathrm{E} \quad 01$

$1.22053 \mathrm{E} \quad 01$

$1.22995 \mathrm{E} \quad 01$

$1.23937 \mathrm{E} \quad 01$

$\begin{array}{lll}1.24879 \mathrm{E} & 01\end{array}$

$1.25821 \mathrm{E} \quad 01$

$1.26763 \mathrm{E} \quad 01$

$1.27706 \mathrm{E} \quad 01$

$1.28648 \mathrm{E} \quad 01$

$1.29591 \mathrm{E} \quad 01$

$1.30534 \mathrm{E} \quad 01$

$1.31477 \mathrm{E} \quad 01$

$1.32421 \mathrm{E} 01$

$1.33364 \mathrm{E} \quad 01$

$\begin{array}{lll}1.34308 \mathrm{E} & 01\end{array}$

$1.35252 \mathrm{E} 01$

$\begin{array}{ll}1.36196 \mathrm{E} & 01\end{array}$

$\begin{array}{lll}1.37141 \mathrm{E} & 01\end{array}$

$1.38085 \mathrm{E} \quad 01$

$1.39030 \mathrm{E} \quad 01$

$1.39975 \mathrm{E} \quad 01$

$1.40920 \mathrm{E} \quad 01$

$1.41865 \mathrm{E} \quad 01$

$1.42810 \mathrm{E} \quad 01$

$1.43756 \mathrm{E} \quad 01$

$\begin{array}{lll}1.44702 \mathrm{E} & 01\end{array}$

$1.45648 \mathrm{E} \quad 01$

$1.46594 \mathrm{E} \quad 01$

$\begin{array}{lll}1.47541 \mathrm{E} & 01\end{array}$

$1.48487 \mathrm{E} \quad 01$

$1.49434 \mathrm{E} \quad 01$

$1.50381 \mathrm{E} \quad 01$

$1.51329 \mathrm{E} \quad 01$

$1.52276 \mathrm{E} \quad 01$

$\begin{array}{lll}1.53224 \mathrm{E} & 01\end{array}$

$1.54172 \mathrm{E} \quad 01$

$1.55120 \mathrm{E} \quad 01$

$1.56068 \mathrm{E} \quad 01$

$1.57017 \mathrm{E} \quad 01$

$1.57965 \mathrm{E} \quad 01$

$1.58914 \mathrm{E} \quad 01$

$1.59863 \mathrm{E} \quad 01$

$1.60813 \mathrm{E} \quad 01$

$1.61762 \mathrm{E} \quad 01$

$1.62712 \mathrm{E} 01$

$1.63662 \mathrm{E} \quad 01$ $g_{1}$

$1.63118 \mathrm{E}-01$

$1.67474 \mathrm{E}-01$

$1.71880 \mathrm{E}-01$

$1.76337 \mathrm{E}-01$

$1.80845 \mathrm{E}-01$

$1.85405 \mathrm{E}-01$

$1.90016 \mathrm{E}-01$

$1.94678 \mathrm{E}-01$

$1.99391 \mathrm{E}-01$

$2.04155 \mathrm{E}-01$

$2.08970 \mathrm{E}-01$

$2.13837 \mathrm{E}-01$

$2.18755 \mathrm{E}-01$

$2.23725 \mathrm{E}-01$

$2.28745 \mathrm{E}-01$

$2.33817 \mathrm{E}-01$

$2.38940 \mathrm{E}-01$

$2.44115 \mathrm{E}-01$

$2.49341 \mathrm{E}-01$

$2.54618 \mathrm{E}-01$

$2.59947 \mathrm{E}-01$

$2.65327 \mathrm{E}-01$

$2.70759 \mathrm{E}-01$

$2.76242 \mathrm{E}-01$

$2.81776 \mathrm{E}-01$

2.87362E-01

$2.93000 \mathrm{E}-01$

$2.98689 \mathrm{E}-01$

$3.04429 \mathrm{E}-01$

$3.10222 \mathrm{E}-01$

$3.16065 \mathrm{E}-01$

$3.21961 \mathrm{E}-01$

$3.27908 \mathrm{E}-01$

$3.33907 \mathrm{E}-01$

$3.39957 \mathrm{E}-01$

$3.46059 \mathrm{E}-01$

$3.52213 \mathrm{E}-01$

$3.58419 \mathrm{E}-01$

$3.64676 \mathrm{E}-01$

$3.70985 \mathrm{E}-0 \mathrm{l}$

$3.77346 \mathrm{E}-01$

$3.83759 \mathrm{E}-01$

$3.90223 \mathrm{E}-01$

$3.96740 \mathrm{E}-0$

$4.03308 \mathrm{E}-01$

4.09929E-01

$4.16601 \mathrm{E}-01$

$4.23325 \mathrm{E}-0 \mathrm{l}$

$4.30101 \mathrm{E}-01$

$4.36930 \mathrm{E}-01$

$4.43810 \mathrm{E}-01$

$4.50742 \mathrm{E}-01$

$4.57726 \mathrm{E}-01$

$4.64763 \mathrm{E}-01$

$4.71851 \mathrm{E}-01$

$4.78992 \mathrm{E}-01$

$4.86185 \mathrm{E}-01$

$4.93430 \mathrm{E}-01$

$5.00728 \mathrm{E}-01$

$5.08077 \mathrm{E}-01$

$\tan \delta_{1}$

$1.99156 \mathrm{E}-04-1.13816 \mathrm{E}-01$

$1.82748 \mathrm{E}-04-1.17918 \mathrm{E}-01$

$1.66625 \mathrm{E}-04-1.22071 \mathrm{E}-01$

$1.50774 \mathrm{E}-04-1.26275 \mathrm{E}-01$

$1.35186 \mathrm{E}-04-1.30531 \mathrm{E}-01$

$1.19866 \mathrm{E}-04$

$1.04805 \mathrm{E}-04$

$8.99948 \mathrm{E}-05$

$7.54282 \mathrm{E}-0.5$

$6.11008 \mathrm{E}-05$

4.70057 E-05

$3.31413 \mathrm{E}-05$

$1.94999 \mathrm{E}-05$

$6.07531 \mathrm{E}-06$

$-7.13356 \mathrm{E}-06$

$-2.01258 \mathrm{E}-05$

$-3.29179 \mathrm{E}-05$

$-4.55016 \mathrm{E}-05$

$-5.79061 \mathrm{E}-05$

$-7.01010 \mathrm{E}-05$

$-8.21206 \mathrm{E}-05$

$-9.39376 \mathrm{E}-05$

$-1.05585 \mathrm{E}-04$

$-1.17055 \mathrm{E}-04$

$-1.28352 \mathrm{E}-04$

$-1.39466 \mathrm{E}-04$

$-1.50424 \mathrm{E}-04$

$-1.61218 \mathrm{E}-04$

$-1.71845 \mathrm{E}-04$

$-1.82319 \mathrm{E}-04$

$-1.92639 \mathrm{E}-04$

$-2.02807 \mathrm{E}-04$

$-2.12818 \mathrm{E}-04$

$-2.22686 \mathrm{E}-04$

$-2.32416 \mathrm{E}-04$

$-2.41998 \mathrm{E}-04$

$-2.51450 \mathrm{E}-04$

$-2.60758 \mathrm{E}-04$

$-2.69941 \mathrm{E}-04$

$-2.78986 \mathrm{E}-04$ 
ELECTRON RADIAL FUNCTIONS

$Z=7 \quad A=14 \quad \rho=1.3 A^{1 / 3} F$

Positron

$\bar{F}$

$2.28792 \mathrm{E}-04$

$2.32589 \mathrm{E}-0.0$

$2.36418 \mathrm{E}-04$

$2.40277 \mathrm{E}-04$

$2.44167 \mathrm{E}-0.4$

12.40

$2.48089 \mathrm{E}-04$

12.60

12.70

12.80

12.90

13.00

13.10

13.20

13.30

13.40

13.50

13.60

13.70

13.80

13.90

14.00

$14.10 \quad 3.10661 \mathrm{E}-04$

$14.20 \quad 3.15082 \mathrm{E}-04$

$14.30 \quad 3.19534 \mathrm{E}-04$

$14.40 \quad 3.24017 \mathrm{E}-04$

$14.50 \quad 3.28532 \mathrm{E}-04$

14.60

14.70

14.80

14.90

15.00

15.10

15.20

15.30

15.40

15.50

15.60

15.70

15.80

15.90

16.00

16.10

16.20

16.30

16.40

16.50

16.60

16.70

16.80

16.90

17.00

17.10

17.20

17.30

17.40

17.50

17.60

17.70

17.80

17.90

18.00

$3.33078 \mathrm{E}-04$

$3.37655 \mathrm{E}-04$

$3.42263 \mathrm{E}-04$

$3.51574 \mathrm{E}-04$

$3.56275 \mathrm{E}-0.4$

$3.61008 \mathrm{E}-04$

$3.65773 \mathrm{E}-04$

$3.70568 \mathrm{E}-04$

$3.75395 \mathrm{E}-04$

$3.80253 \mathrm{E}-04$

$3.85142 \mathrm{E}-04$

$3.90062 \mathrm{E}-04$

$3.95014 \mathrm{E}-04$

3.99997 E-0

$4.05010 \mathrm{E}-04$

$4.10055 \mathrm{E}-04$

$4.15132 \mathrm{E}-04$

$4.20239 \mathrm{E}-04$

$4.25378 \mathrm{E}-04$

$4.30548 \mathrm{E}-0.4$

$4.35749 \mathrm{E}-04$

$4.40981 \mathrm{E}-04$

$4.46245 \mathrm{E}-04$

$4.51539 \mathrm{E}-0.4$

$4.56865 \mathrm{E}-0.4$

$4.62222 \mathrm{E}-0.1$

$4.67610 \mathrm{E}-04$

$4.73030 \mathrm{E}-04$

$4.78480 \mathrm{E}-04$

$4.83962 \mathrm{E}-0.1$

$4.89475 \mathrm{E}-01$

$4.95020 \mathrm{E}-0.4$

$5.00595 \mathrm{E}-04$

$5.06202 E-01$ $f_{2}$

$3.51094 \mathrm{E}-01$

$3.57052 \mathrm{E}-01$

$3.63060 \mathrm{E}-01$

$3.69118 \mathrm{E}-01$

$3.75226 \mathrm{E}-01$

$3.81385 \mathrm{E}-01$

$3.87593 \mathrm{E}-01$

$3.93851 \mathrm{E}-01$

$4.00159 \mathrm{E}-01$

$4.06517 \mathrm{E}-01$

4.12925 E-01

$4.19383 \mathrm{E}-01$

$4.25892 \mathrm{E}-01$

$4.32450 \mathrm{E}-01$

$4.39058 \mathrm{E}-01$

$4.45716 \mathrm{E}-01$

$4.52425 \mathrm{E}-01$

$4.59183 \mathrm{E}-01$

$4.65991 \mathrm{E}-01$

$4.72849 \mathrm{E}-01$

$4.79757 \mathrm{E}-01$

$4.86715 \mathrm{E}-01$

$4.93723 \mathrm{E}-01$

$5.00781 \mathrm{E}-01$

$5.07889 \mathrm{E}-01$

$3.46903 \mathrm{E}-04$
$5.15018 \mathrm{E}-01$

$5.22255 \mathrm{E}-01$

$5.29513 \mathrm{E}-01$

$5.36821 \mathrm{E}-01$

$5.44179 \mathrm{E}-01$

$5.51587 \mathrm{E}-01$

$5.59044 \mathrm{E}-01$

$5.66552 \mathrm{E}-01$

$5.74110 \mathrm{E}-01$

$5.81717 \mathrm{E}-01$

$5.89375 \mathrm{E}-01$

$5.97082 \mathrm{E}-01$

$6.0+839 \mathrm{E}-01$

$6.12646 \mathrm{E}-01$

$6.20503 \mathrm{E}-01$

$6.28410 \mathrm{E}-01$

$6.36368 \mathrm{E}-01$

$6.44374 \mathrm{E}-01$

$6.52431 \mathrm{E}-01$

$6.60538 \mathrm{E}-01$

$6.68694 \mathrm{E}-01$

$6.76901 \mathrm{E}-01$

$6.85157 \mathrm{E}-01$

$6.93463 \mathrm{E}-01$

$7.01819 \mathrm{E}-01$

$7.10225 \mathrm{E}-01$

$7.18681 \mathrm{E}-01$

$7.27187 \mathrm{E}-01$

$7.35742 \mathrm{E}-01$

$7.44348 \mathrm{E}-01$

$7.53003 \mathrm{E}-01$

$7.61708 \mathrm{E}-01$

$7.70463 \mathrm{E}-01$

$7.79268 \mathrm{E}-01$

$7.88122 \mathrm{E}-01$ $g_{2}$

$\tan \delta$

$f$.

$3.38802 \mathrm{E}-03$

$3.5032 \omega \mathrm{E}-03$

$3.62094 \mathrm{E}-03$

$3.74108 \mathrm{E}-03$

$3.86369 \mathrm{E}-03$

$3.98880 \mathrm{E}-03$

$4.11644 \mathrm{E}-03$

$4.2466+\mathrm{E}-03$

4.37941 E-03

$4.51478 \mathrm{E}-03$

$4.65277 \mathrm{E}-03$

$4.79342 \mathbf{E}-03$

$4.93673 \mathrm{E}-03$

$5.08275 \mathrm{E}-03$

$5.23149 \mathrm{E}-03$

$5.38297 \mathrm{E}-03$

$5.53723 \mathrm{E}-03$

$5.69428 \mathrm{E}-03$

$5.85415 \mathrm{E}-03$

$6.01686 \mathbf{E}-03$

$6.18241 \mathrm{E}-03$

$6.35092 \mathrm{E}-03$

$6.52231 \mathrm{E}-03$

$6.69664 \mathrm{E}-03$

$6.87394 \mathrm{E}-03$

$7.05423 \mathrm{E}-03$

$7.23753 \mathrm{E}-03$

$7.42387 \mathrm{E}-03$

$7.61327 \mathrm{E}-03$

$7.80576 \mathrm{E}-03$

$8.00137 \mathrm{E}-03$

$8.20010 \mathrm{E}-03$

$8.40200 \mathrm{E}-03$

$8.60708 \mathrm{E}-03$

8.81537E-03

$9.02690 \mathrm{E}-03$

$9.24168 \mathrm{E}-03$

$9.45975 \mathrm{E}-03$

$9.68111 \mathrm{E}-03$

$9.90581 \mathrm{E}-03$

$1.01339 \mathrm{E}-02$

$1.03653 \mathrm{E}-02$

$1.06001 \mathrm{E}-02$

$1.08384 \mathrm{E}-02$

$1.10801 \mathrm{E}-02$

$1.13253 \mathrm{E}-02$

$1.15740 \mathrm{E}-02$

$1.18262 \mathrm{E}-02$

$1.20820 \mathrm{E}-02$

$1.23413 \mathrm{E}-02$

$1.26043 \mathrm{E}-02$

$1.28708 \mathrm{E}-02$

$1.31410 \mathrm{E}-02$

$1.34149 \mathrm{E}-02$

$1.36925 \mathrm{E}-02$

$1.39737 \mathrm{E}-02$

$1.42588 \mathrm{E}-02$

$1.45476 \mathrm{E}-02$

$1.48402 \mathrm{E}-02$

$1.51366 \mathrm{E}-02$

\begin{abstract}
$-2.73434 \mathrm{E} \quad 01$
-2.73513 E 01

$-2.73591 \mathrm{E}$ 0l

$-2.73667 \mathrm{E}$ 01

$-2.73742 \mathrm{E} \quad 01$

$-2.73816 \mathrm{E} \quad 01$

$-2.73889 \mathrm{E} \quad 01$

$-2.73960 \mathrm{E} \quad 01$

$-2.74030 \mathrm{E} \quad 01$

$-2.74100 \mathrm{E} \quad 01$
\end{abstract}

$-2.74167 \mathrm{E} \quad 01$

$-2.74234 \mathrm{E} \quad 01$

$-2.74300 \mathrm{E}$ ol

$-2.74365 \mathrm{E}$ 0l

$-2.74429 \mathrm{E} 01$

$-2.74492 \mathrm{E} \quad 01$

$-2.74554 \mathrm{E} \quad 01$

$-2.74615 \mathrm{E} 01$

$-2.74675 \mathrm{E} \quad 01$

$-2.74734 \mathrm{E} \quad 01$

$-2.74792 \mathrm{E} 01$

$-2.74850 \mathrm{E} \quad 01$

$-2.74906 \mathrm{E} \quad 01$

$-2.74962 \mathrm{E} \quad 01$

$-2.75017 \mathrm{E} \quad 01$

$-2.75071 \mathrm{E} \quad 01$

$-2.75124 \mathrm{E} 01$

$-2.75177 \mathrm{E} \quad 01$

$-2.75229 \mathrm{E} 01$

$-2.75280 \mathrm{E}$ 0I

$-2.75330 \mathrm{E} \quad 01$

$-2.75380 \mathrm{E} \quad 01$

$-2.75429 \mathrm{E} 01$

$-2.75477 \mathrm{E} \quad 01$

$-2.75525 \mathrm{E} 01$

$-2.75572 \mathrm{E} \quad 01$

$-2.75619 \mathrm{E} \quad 01$

$-2.75664 \mathrm{E} \quad 01$

$-2.75710 \mathrm{E} 01$

$-2.75754 \mathrm{E} \quad 01$

$\begin{array}{lll}-2.75798 E & 01\end{array}$

-2.75842 E 01

$-2.75885 \mathrm{E} \quad 01$

$-2.75927 \mathrm{E}$ 01

$-2.75969 \mathrm{E}$ ol

$-2.76010 \mathrm{E} \quad 01$

$-2.76051 \mathrm{E} 01$

$-2.76091 \mathrm{E} \quad 01$

$-2.76131 \mathrm{E} \quad 0 \mathrm{r}$

$-2.76171 \mathrm{E} \quad 01$

$-2.76209 \mathrm{E} \quad 01$

$-2.76248 \mathrm{E} \quad 01$

$-2.76286 \mathrm{E} 01$

$-2.76323 \mathrm{E} 01$

$-2.76360 \mathrm{E} 01$

$\begin{array}{lll}-2.76397 \mathrm{E} & 01\end{array}$

$-2.76433 \mathrm{E} 01$

$-2.76469 \mathrm{E} \quad 01$

$-2.76504 \mathrm{E} \quad 01$

$-2.76539 \mathrm{E} 01$

$-2.14192 \mathrm{E}-03$

$-2.5+437 \mathrm{E}-03$ 
p

18.10

18.20

18.30

18.40

18.50

18.60

18.70

18.80

18.90

19.00

19.10

19.20

19.30

19.40

19.50

19.60

19.70

19.80

19.90

20.00

20.10

20.20

20.30

20.40

20.50

20.60

20.70

20.80

20.90

21.00

21.10

21.20

21.30

21.40

21.50

21.60

21.70

21.80

21.90

22.00

22.10

22.20

22.30

22.40

22.50

22.60

22.70

22.80

22.90

23.00

23.10

23.20

23.30

23.40

23.50

23.60

23.70

23.80

23.90

24.00
$F_{0}$

$8.55980 \mathrm{E}-01$

$8.55970 \mathrm{E}-01$

8.55961 E-01

$8.55951 \mathrm{E}-01$

$8.55941 \mathrm{E}-01$

8.55932 E-01

$8.55922 \mathrm{E}-01$

$8.55912 \mathrm{E}-01$

$8.55902 \mathrm{E}-01$

$8.55893 \mathrm{E}-01$

$8.55883 \mathrm{E}-01$

$8.55873 \mathrm{E}-01$

$8.55864 \mathrm{E}-01$

$8.55854 \mathrm{E}-01$

$8.55845 \mathrm{E}-01$

$8.55835 \mathrm{E}-01$

$8.55826 \mathrm{E}-01$

$8.55816 \mathrm{E}-01$

$8.55807 \mathrm{E}-01$

$8.55798 \mathrm{E}-01$

$8.55788 \mathrm{E}-01$

$8.55779 \mathrm{E}-01$

$8.55769 \mathrm{E}-01$

$8.55760 \mathrm{E}-0 \mathrm{l}$

$8.55751 \mathrm{E}-01$

$8.55742 \mathrm{E}-01$

$8.55733 \mathrm{E}-01$

$8.55723 \mathrm{E}-01$

$8.55714 \mathrm{E}-01$

$8.55705 \mathrm{E}-01$

$8.55696 \mathrm{E}-01$

$8.55687 \mathrm{E}-01$

$8.55678 \mathrm{E}-01$

$8.55669 \mathrm{E}-01$

$8.55660 \mathrm{E}-01$

$8.55651 \mathrm{E}-01$

$8.55642 \mathrm{E}-01$

$8.55633 \mathrm{E}-01$

$8.55625 \mathrm{E}-01$

$8.55616 \mathrm{E}-01$

$8.55607 \mathrm{E}-01$

$8.55598 \mathrm{E}-01$

$8.55589 \mathrm{E}-01$

$8.55581 \mathrm{E}-01$

$8.55572 \mathrm{E}-01$

$8.55564 \mathrm{E}-01$

$8.55555 \mathrm{E}-01$

$8.55547 \mathrm{E}-01$

$8.55538 \mathrm{E}-01$

$8.55529 \mathrm{E}-01$

$8.55521 \mathrm{E}-01$

$8.55512 \mathrm{E}-01$

$8.55504 \mathrm{E}-01$

$8.55496 \mathrm{E}-01$

$8.55487 \mathrm{E}-01$

$8.55479 \mathrm{E}-01$

$8.55471 \mathrm{E}-01$

$8.55462 \mathrm{E}-01$

$8.55454 \mathrm{E}-01$

$8.55446 \mathrm{E}-01$ $f_{1}$

$\begin{array}{ll}1.64612 \mathrm{E} & 01\end{array}$

$1.65563 \mathrm{E} \quad 01$

$1.66514 \mathrm{E} \quad 01$

$1.67465 \mathrm{E} 01$

$1.68416 \mathrm{E} \quad 01$

$\begin{array}{ll}1.69367 \mathrm{E} & 01\end{array}$

$1.70319 \mathrm{E} \quad 01$

$1.71270 \mathrm{E} \quad 01$

$1.72222 \mathrm{E} \quad 01$

$1.73175 \mathrm{E} \quad 01$

$\begin{array}{ll}1.74127 \mathrm{E} & 01\end{array}$

$\begin{array}{lll}1.75080 \mathrm{E} & 01\end{array}$

$1.76033 \mathrm{E} \quad 01$

$\begin{array}{ll}1.76986 \mathrm{E} & 01\end{array}$

$1.77939 \mathrm{E} \quad 01$

$1.78893 \mathrm{E}$ 0l

$1.79847 \mathrm{E} \quad 01$

$1.80801 \mathrm{E} \quad 01$

$1.81755 \mathrm{E} \quad$ ol

$1.82710 \mathrm{E} \quad 01$

$1.83665 \mathrm{E} \quad 01$

$1.84620 \mathrm{E} 01$

$\begin{array}{lll}1.85575 \mathrm{E} & 01\end{array}$

$1.86531 \mathrm{E} 01$

$1.87487 \mathrm{E} \quad 01$

$\begin{array}{ll}1.88443 \mathrm{E} & 01\end{array}$

$1.89399 \mathrm{E} \quad 01$

$1.90356 \mathrm{E} \quad 01$

$1.91312 \mathrm{E} \quad 01$

$1.92269 \mathrm{E} \quad 01$

$1.93227 \mathrm{E} \quad 01$

$1.94184 \mathrm{E} \quad 01$

$1.95142 \mathrm{E} \quad 01$

$1.96100 \mathrm{E} \quad 01$

$1.97059 \mathrm{E} \quad 01$

$1.98017 \mathrm{E} \quad 01$

$1.98976 \mathrm{E} \quad 01$

$1.99935 \mathrm{E} \quad 01$

$2.00894 \mathrm{E} \quad 01$

$2.01854 \mathrm{E} \quad 01$

$2.02814 \mathrm{E} \quad 01$

$2.03774 \mathrm{E} \quad 01$

$2.04734 \mathrm{E} \quad 01$

$2.05695 \mathrm{E} \quad 01$

$2.06656 \mathrm{E} \quad 01$

$2.07617 \mathrm{E} \quad 01$

$2.08579 \mathrm{E} \quad 01$

$2.09541 \mathrm{E} \quad 01$

$2.10503 \mathrm{E} \quad 01$

$2.11465 \mathrm{E} \quad 01$

$2.12427 \mathrm{E} \quad 01$

$2.13390 \mathrm{E} \quad 01$

$2.14353 \mathrm{E} 01$

$2.15317 \mathrm{E} \quad 01$

$2.16280 \mathrm{E} 01$

$2.17244 \mathrm{E} \quad 01$

$2.18209 \mathrm{E} \quad 01$

$2.19173 \mathrm{E} \quad 01$

$2.20138 \mathrm{E} \quad 01$

$2.21103 \mathrm{E} 01$ $g_{1}$

5.154.79E-01

$5.22933 \mathrm{E}-01$

$5.30440 \mathrm{E}-01$

$5.37999 \mathrm{E}-01$

$5.45610 \mathrm{E}-01$

$5.53274 \mathrm{E}-01$

$5.60990 \mathrm{E}-01$

$5.68758 \mathrm{E}-01$

$5.76579 \mathrm{E}-01$

5.844 .52 E-01

$5.92378 \mathrm{E}-01$

$6.00357 \mathrm{E}-01$

$6.08388 \mathrm{E}-01$

$6.16471 \mathrm{E}-01$

$6.24608 \mathrm{E}-01$

$6.32796 \mathrm{E}-01$

$6.41038 \mathrm{E}-01$

$6.49332 \mathrm{E}-01$

$6.57679 \mathrm{E}-01$

$6.66079 \mathrm{E}-01$

$6.74532 \mathrm{E}-01$

$6.83037 \mathrm{E}-01$

$6.91595 \mathrm{E}-01$

7.00207E-01

$7.08871 \mathrm{E}-01$

$7.17588 \mathrm{E}-01$

$7.26357 \mathrm{E}-01$

$7.35180 \mathrm{E}-01$

$7.44056 \mathrm{E}-01$

$7.52985 \mathrm{E}-01$

$7.61967 \mathrm{E}-01$

$7.71002 \mathbf{E}-01$

$7.80091 \mathrm{E}-01$

$7.89232 \mathbf{E}-01$

$7.98427 \mathrm{E}-01$

$8.07674 \mathrm{E}-01$

$8.16975 \mathrm{E}-01$

$8.26330 \mathrm{E}-01$

$8.35737 \mathrm{E}-01$

$8.45198 \mathrm{E}-01$

$8.54713 \mathrm{E}-01$

$8.64280 \mathrm{E}-01$

$8.73901 \mathrm{E}-01$

$8.83576 \mathrm{E}-01$

$8.93304 \mathrm{E}-01$

$9.03086 \mathrm{E}-01$

$9.12921 \mathrm{E}-01$

$9.22810 \mathrm{E}-01$

$9.32752 \mathrm{E}-01$

$9.42748 \mathrm{E}-01$

$9.52798 \mathrm{E}-01$

$9.62901 \mathrm{E}-01$

$9.73058 \mathrm{E}-01$

$9.83269 \mathrm{E}-01$

$9.93534 \mathrm{E}-01$

$1.00385 \mathrm{E} \quad 00$

$1.01423 \mathrm{E} \quad 00$

$1.02465 \mathrm{E} \quad 00$

$1.03513 \mathrm{E} \quad 00$

$1.04567 \mathrm{E} \quad 00$

$\tan \delta_{1}$

$-4.42357 \mathrm{E}-04$
$-4.49014 \mathrm{E}-04$
$-4.55601 \mathrm{E}-04$
$-4.62081 \mathrm{E}-04$
$-4.68477 \mathrm{E}-04$

$-4.74791 \mathrm{E}-04$
$-4.81017 \mathrm{E}-04$
$-4.87173 \mathrm{E}-04$
$-4.93233 \mathrm{E}-04$
$-4.99217 \mathrm{E}-04$

$-5.05124 \mathrm{E}-04$

$-5.10947 \mathrm{E}-04$

$-5.16702 \mathrm{E}-04$

$-5.22375 \mathrm{E}-04$

$-5.27972 \mathrm{E}-04$

$-5.33495 \mathrm{E}-04$

$-5.38947 \mathrm{E}-04$

$-5.44332 \mathrm{E}-04$

$-5.49641 \mathrm{E}-04$

$-5.54872 \mathrm{E}-04$

$-5.60048 \mathrm{E}-04$

$-5.65150 \mathrm{E}-04$

$-5.70182 \mathrm{E}-04$

$-5.75154 \mathrm{E}-04$

$-5.80051 \mathrm{E}-04$

$-5.84895 \mathrm{E}-04$

$-5.89673 \mathrm{E}-04$

$-5.94387 \mathrm{E}-04$

$-5.99035 \mathrm{E}-04$

$-6.03623 \mathrm{E}-04$

$-6.08157 \mathrm{E}-04$

$-6.12624 \mathrm{E}-04$

$-6.17036 \mathrm{E}-04$

$-6.21388 \mathrm{E}-04$

$-6.25681 \mathrm{E}-04$

$-6.29926 \mathrm{E}-04$

$-6.34107 \mathrm{E}-04$

$-6.38236 \mathrm{E}-04$

$-6.42303 \mathrm{E}-04$

$-6.46328 \mathrm{E}-04$

$-6.50290 \mathrm{E}-04$.

$-6.54196 \mathrm{E}-04$

$-6.58060 \mathrm{E}-04$

$-6.61872 \mathrm{E}-04$

$-6.65633 \mathrm{E}-04$

$-6.69341 \mathrm{E}-04$

$-6.73005 \mathrm{E}-04$

$-6.76608 \mathrm{E}-04$

$-6.80164 \mathrm{E}-04$

$-6.83688 \mathrm{E}-04$

$-6.87149 \mathrm{E}-04$ 
ELECTRON RADIAL FUYCTIONS

$Z=7 \quad A=14 \quad \rho=1.3 A^{1 / 3} F$

Pusitron

$\bar{F}$

$P$
18.1

18.20

18.30

18.40

18.50

18.60

18.70

18.80

18.90

19.00

19.10

19.20

19.30

19.40

19.50

19.60

19.70

19.80

19.90

20.00

20.10

20.20

20.30

20.40

20.50

20.60

20.70

20.80

20.90

21.00

21.10

21.20

21.30

21.40

21.50

21.60

21.70

21.80

21.90

22.00

22.10

22.20

22.30

22.40

22.50

22.60

22.70

22.80

22.90

23.00

23.10

23.20

23.30

23.40

23.50

23.60

23.70

23.80

23.90

24.00 $f_{2}$

$7.97028 \mathrm{E}-01$ $8.05982 \mathrm{E}-01$

$8.14986 \mathrm{E}-01$

$8.24040 \mathrm{E}-01$

$8.33144 \mathrm{E}-01$

8.42298E-01

$8.51501 \mathrm{E}-01$

$8.60754 \mathrm{E}-01$

$8.70057 \mathrm{E}-01$

$8.79410 \mathrm{E}-0 \mathrm{l}$

$8.88812 \mathrm{E}-01$

$8.98265 E-01$

9.07767E-01

9.17319E-01

9.26921 E-01

$9.36572 \mathrm{E}-01$

$9.46273 \mathrm{E}-01$

$9.56024 \mathrm{E}-01$

$9.65825 \mathrm{E}-01$

$9.75675 \mathrm{E}-01$

$9.85576 \mathrm{E}-01$

$9.95526 \mathrm{E}-01$

$1.00553 \mathrm{E} \quad 00$

$1.01557 \mathrm{E} \quad 00$

$1.02567 \mathrm{E} \quad 00$

$6.50118 \mathrm{E}-0$

$6.56505 \mathrm{E}-04$

$6.62922 \mathrm{E}-04$

$6.69371 \mathrm{E}-04$

$6.75851 \mathrm{E}-04$

$6.82362 \mathrm{E}-04$

$6.88905 \mathrm{E}-04$

$6.95+78 \mathrm{E}-04$

$7.02083 \mathrm{E}-0$.

$7.08719 \mathrm{E}-04$

$7.15386 \mathrm{E}-04$

$7.2208+\mathrm{E}-0.1$

$7.28813 \mathrm{E}-04$

$7.35574 \mathrm{E}-01$

$7.42366 \mathrm{E}-0.1$

$7.49189 \mathrm{E}-0$

$7.56043 \mathrm{E}-04$

$7.62928 \mathrm{E}-04$

$7.69845 \mathrm{E}-04$

$7.76793 \mathrm{E}-0$.

$7.83772 \mathrm{E}-0.4$

$7.90782 \mathrm{E}-01$

$7.97824 \mathrm{E}-04$

$8.04896 \mathrm{E}-04$

$8.12000 \mathrm{E}-04$

$8.19135 \mathrm{E}-04$

$8.26301 \mathrm{E}-04$

8.33198E-04

8.40726 E-0.4

$8.47986 \mathrm{E}-04$

$8.55277 \mathrm{E}-04$

$8.62599 \mathrm{E}-04$

$8.69952 \mathrm{E}-04$

$8.77336 \mathrm{E}-04$

$8.8+752 \mathrm{E}-0+4$

$8.92198 \mathrm{E}-01$

$8.99676 \mathrm{E}-04$
$1.03582 \mathrm{E} \quad 00$

$1.04602 \mathrm{E} \quad 00$

$1.05627 \mathrm{E} \quad 00$

$1.06657 \mathrm{E} \quad 00$

$1.07691 \mathrm{E} \quad 00$

$1.08731 \mathrm{E} \quad 00$ $1.09776 \mathrm{E} \quad 00$

$1.10826 \mathrm{E} \quad 00$

$1.11880 \mathrm{E} \quad 00$

$1.12940 \mathrm{E} \quad 00$

$1.14004 \mathrm{E} \quad 00$

$1.15074 \mathrm{E} \quad 00$

$1.17228 \mathrm{E} \quad 00$

$1.18312 \mathrm{E} \quad 00$

$1.19402 \mathrm{E} \quad 00$

$1.20496 \mathrm{E} \quad 00$

$1.21595 \mathrm{E} \quad 00$

$1.22700 \mathrm{E} \quad 00$

$1.23809 \mathrm{E} \quad 00$

$1.24923 \mathrm{E} \quad 00$

$1.26042 \mathrm{E} \quad 00$

$1.27166 \mathrm{E} \quad 00$

$1.28296 \mathrm{E} \quad 00$

$1.29430 \mathrm{E} \quad 00$

$1.30569 \mathrm{E} \quad 00$

$1.31712 \mathrm{E} \quad 00$

$1.32861 \mathrm{E} \quad 00$

$1.34015 \mathrm{E} \quad 00$

$1.3517+\mathrm{E} \quad 00$

$1.36338 \mathrm{E} \quad 00$

$1.37507 \mathrm{E} \quad 00$

$1.38680 \mathrm{E} \quad 00$

$1.39859 \mathrm{E} \quad 00$

$1.41042 \mathrm{E} \quad 00$
$1.16148 \mathrm{E} \quad 00$ g.

$1.54368 \mathrm{E}-02$

$1.57410 \mathrm{E}-02$

$1.60489 \mathrm{E}-02$

$1.63609 \mathrm{E}-02$

$1.66767 \mathrm{E}-02$

$1.69965 \mathrm{E}-02$

$1.73203 \mathrm{E}-02$

1. $76481 \mathrm{E}-02$

$1.79800 \mathrm{E}-02$

$1.83159 \mathrm{E}-02$

$1.86559 \mathrm{E}-02$

$1.89999 \mathrm{E}-02$

$1.93+82 \mathrm{E}-02$

1.97005E-02

2.00571 E-02

$2.04179 \mathrm{E}-02$

$2.07828 \mathrm{E}-02$

$2.11521 \mathrm{E}-02$

$2.15256 \mathrm{E}-02$

$2.1903+E-02$

$2.22856 \mathrm{E}-02$

$2.26721 \mathrm{E}-02$

$2.30629 \mathrm{E}-02$

$2.34582 \mathrm{E}-02$

$2.38579 \mathrm{E}-02$

2.42620 E- 02

$2.46706 \mathrm{E}-02$

$2.50837 \mathrm{E}-02$

$2.55013 E-02$

$2.59235 \mathrm{E}-02$

$2.63502 \mathrm{E}-02$

$2.67815 \mathrm{E}-02$

$2.72174 \mathrm{E}-02$

$2.76580 \mathrm{E}-02$

2.81033 E- 02

2.85532 E- 02

$2.90078 \mathrm{E}-02$

$2.94672 \mathrm{E}-02$

$2.99313 \mathrm{E}-02$

$3.04002 \mathrm{E}-02$

$3.08739 \mathrm{E}-02$

$3.13525 \mathrm{E}-02$

$3.18359 \mathrm{E}-02$

$3.23242 \mathrm{E}-02$

$3.28174 \mathrm{E}-02$

$3.33156 \mathrm{E}-02$

$3.38186 \mathrm{E}-02$

$3.4326 \tau \mathrm{E}-02$

$3.48398 \mathrm{E}-02$

$3.53578 \mathrm{E}-02$

$3.58810 \mathrm{E}-02$

$3.61092 \mathrm{E}-02$

$3.69+25 \mathrm{E}-02$

$3.74809 \mathrm{E}-02$

$3.80245 \mathrm{E}-02$

$3.85733 \mathrm{E}-02$

$3.91272 \mathrm{E}-02$

$3.9686+E-02$

$4.02508 \mathrm{E}-02$

$+.08205 \mathrm{E}-02$

$\tan \delta_{z}$

$-2.76573 \mathrm{E} \quad 01$

$-2.76607 \mathrm{E} 01-1.38656 \mathrm{E}-02$

$-2.76641 \mathrm{E}$ 01 $-1.41558 \mathrm{E}-02$

$-2.76674 \mathrm{E}$ 01 $-1.44499 \mathrm{E}-02$

$-2.76707 \mathrm{E} 01-1.47479 \mathrm{E}-02$

$-2.76740 \mathrm{E} \quad 01$

$-2.76772 \mathrm{E} \quad 01$

$-2.76804 \mathrm{E} 01$

$-2.76835 \mathrm{E}$ 0l

$-2.76866 \mathrm{E} \quad 01$

$-2.76897 \mathrm{E} \quad 01$

$-2.76928 \mathrm{E} \quad 01$

$-2.76958 \mathrm{E} \quad 01$

$-2.76988 \mathrm{E} 01$

$-2.77017 \mathrm{E} \quad 01$

$\begin{array}{lll}-2.77046 E & 01\end{array}$

$-2.77075 \mathrm{E} \quad 01$

$-2.77104 \mathrm{E} \quad 01$

$-2.77132 \mathrm{E} 01$

$-1.50497 \mathrm{E}-02$

$-1.53554 \mathrm{E}-02$

$-1.56651 \mathrm{E}-02$

$-1.59787 \mathrm{E}-02$

$-1.62963 \mathrm{E}-02$

$-1.66179 E-02$

$-1.69435 \mathrm{E}-02$

$-1.72732 \mathrm{E}-02$

$-1.76069 \mathrm{E}-02$

$-1.79+48 \mathrm{E}-02$

$-1.82867 \mathrm{E}-02$

$-1.86329 \mathrm{E}-02$

$-1.89831 \mathrm{E}-02$

$-1.93376 \mathrm{E}-02$

$-1.96963 \mathrm{E}-02$

$-2.77188 \mathrm{E} \quad 01 \quad-2.00593 \mathrm{E}-02$

$-2.77215 \mathrm{E} 01 \quad-2.04265 \mathrm{E}-02$

$-2.77242 \mathrm{E}$ 01 $-2.07980 \mathrm{E}-02$

$-2.77269 \mathrm{E}$ 01 $-2.11738 \mathrm{E}-02$

$-2.7 \mathrm{~F} 296 \mathrm{E}$ 01 $-2.15540 \mathrm{E}-02$

$-2.77322 \mathrm{E}$ 01 $-2.19385 \mathrm{E}-02$

$-2.73348 \mathrm{E} 01 \quad-2.23274 \mathrm{E}-02$

$-2.7737+\mathrm{E}$ 01 $-2.27208 \mathrm{E}-02$

$-2.77400 \mathrm{E}$ 01 $-2.31185 \mathrm{E}-02$

$-2.77425 \mathrm{E} 01 \quad-2.35208 \mathrm{E}-02$

$-2.77450 \mathrm{E} \quad 01$

$-2.77475 \mathrm{E} \quad 01$

$-2.77499 \mathrm{E} \quad 01$

$-2.77524 \mathrm{E}$ ol

$-2.77548 \mathrm{E} 01$

$-2.77572 \mathrm{E} \quad 01$

$-2.77595 \mathrm{E} \quad 01$

$\begin{array}{lll}-2.77619 \mathrm{E} & 01\end{array}$

$-2.77642 \mathrm{E} \quad 01$

$-2.77665 \mathrm{E} \quad 01$

$-2.77687 \mathrm{E} \quad 01$

$-2.77710 \mathrm{E} 01$

$-2.77732 \mathrm{E} \quad 01$

$-2.77754 \mathrm{E} 01$

$-2.77776 \mathrm{E} 01$

$-2.77798 \mathrm{E} 01$

$-2.78819 \mathrm{E} 01$

$-2.77841 \mathrm{E}$ 0l

$-2.77862 \mathrm{E} 01$

$-2.77883 \mathrm{E} 01$

$-2.77904 \mathrm{E} \quad 01$ 


\begin{tabular}{|c|c|c|c|}
\hline$p$ & $F_{0}$ & $f_{1}$ & \\
\hline 24.10 & $8.55437 \mathrm{E}-01$ & $2.22068 \mathrm{E}$ & 01 \\
\hline 24.20 & $8.55429 \mathrm{E}-01$ & $2.23034 \mathrm{E}$ & 01 \\
\hline 24.30 & $8.55421 \mathrm{E}-01$ & $2.24000 \mathrm{E}$ & 01 \\
\hline 24.40 & $8.55413 \mathrm{E}-01$ & $2.24966 \mathrm{E}$ & 01 \\
\hline 24.50 & $8.55405 \mathrm{E}-01$ & $2.25933 \mathrm{E}$ & 01 \\
\hline 24.60 & $8.55396 \mathrm{E}-01$ & $2.26899 \mathrm{E}$ & 01 \\
\hline 24.70 & $8.55388 \mathrm{E}-01$ & $2.27867 \mathrm{E}$ & 01 \\
\hline 24.80 & $8.55380 \mathrm{E}-01$ & $2.28834 \mathrm{E}$ & 01 \\
\hline 24.90 & $8.55372 \mathrm{E}-01$ & $2.29802 \mathrm{E}$ & 01 \\
\hline 25.00 & $8.55364 \mathrm{E}-01$ & $2.30770 \mathrm{E}$ & 01 \\
\hline 25.10 & $8.55356 \mathrm{E}-01$ & $2.31738 \mathrm{E}$ & 01 \\
\hline 25.20 & $8.55348 \mathrm{E}-01$ & $2.32706 \mathrm{E}$ & 01 \\
\hline 25.30 & $8.55340 \mathrm{E}-01$ & $2.33675 \mathrm{E}$ & 01 \\
\hline 25.40 & $8.55332 \mathrm{E}-01$ & $2.34644 \mathrm{E}$ & 01 \\
\hline 25.50 & $8.55324 \mathrm{E}-01$ & $2.35614 \mathrm{E}$ & 01 \\
\hline 25.60 & $8.55317 \mathrm{E}-01$ & $2.36584 \mathrm{E}$ & 01 \\
\hline 25.70 & $8.55309 \mathrm{E}-0 \mathrm{l}$ & $2.37554 \mathrm{E}$ & 01 \\
\hline 25.80 & $8.55301 \mathrm{E}-01$ & $2.38524 \mathrm{E}$ & 01 \\
\hline 25.90 & $8.55293 \mathrm{E}-01$ & $2.39495 \mathrm{E}$ & 01 \\
\hline 26.00 & $8.55285 \mathrm{E}-01$ & $2.40466 \mathrm{E}$ & 01 \\
\hline 26.10 & $8.55277 \mathrm{E}-01$ & $2.41437 \mathrm{E}$ & 01 \\
\hline 26.20 & $8.55270 \mathrm{E}-0 \mathrm{l}$ & $2.42409 \mathrm{E}$ & 01 \\
\hline 26.30 & $8.55262 \mathrm{E}-01$ & $2.43381 \mathrm{E}$ & 01 \\
\hline 26.40 & $8.55254 \mathrm{E}-01$ & $2.44353 \mathrm{E}$ & 01 \\
\hline 26.50 & $8.55247 \mathrm{E}-01$ & $2.45325 \mathrm{E}$ & 01 \\
\hline 26.60 & $8.55239 \mathrm{E}-01$ & $2.46298 \mathrm{E}$ & 01 \\
\hline 26.70 & $8.55232 \mathrm{E}-01$ & $2.47271 \mathrm{E}$ & 01 \\
\hline 26.80 & $8.55224 \mathrm{E}-01$ & $2.48245 \mathrm{E}$ & 01 \\
\hline 26.90 & $8.55216 \mathrm{E}-01$ & $2.49219 \mathrm{E}$ & 01 \\
\hline 27.00 & $8.55209 \mathrm{E}-01$ & $2.50193 \mathrm{E}$ & 01 \\
\hline 27.10 & $8.55201 \mathrm{E}-0 \mathrm{I}$ & $2.51167 \mathrm{E}$ & 01 \\
\hline 27.20 & $8.55194 \mathrm{E}-01$ & $2.52142 \mathrm{E}$ & 01 \\
\hline 27.30 & $8.55186 \mathrm{E}-01$ & $2.53117 \mathrm{E}$ & 01 \\
\hline 27.40 & $8.55179 \mathrm{E}-01$ & $2.54092 \mathrm{E}$ & 01 \\
\hline 27.50 & $8.55171 \mathrm{E}-01$ & $2.55068 \mathrm{E}$ & 01 \\
\hline 27.60 & $8.55164 \mathrm{E}-01$ & $2.56044 \mathrm{E}$ & 01 \\
\hline 27.70 & $8.55157 \mathrm{E}-01$ & $2.57020 \mathrm{E}$ & 01 \\
\hline 27.80 & $8.55149 \mathrm{E}-01$ & $2.57997 \mathrm{E}$ & 01 \\
\hline 27.90 & $8.55142 \mathrm{E}-01$ & $2.58974 \mathrm{E}$ & 01 \\
\hline 28.00 & $8.55134 \mathrm{E}-01$ & $2.59951 \mathrm{E}$ & 01 . \\
\hline 28.10 & $8.55127 \mathrm{E}-01$ & $2.60929 \mathrm{E}$ & 01 \\
\hline 28.20 & $8.55120 \mathrm{E}-01$ & $2.61907 \mathrm{E}$ & 01 \\
\hline 28.30 & $8.55113 \mathrm{E}-01$ & $2.62885 \mathrm{E}$ & 01 \\
\hline 28.40 & $8.55105 \mathrm{E}-01$ & $2.63864 \mathrm{E}$ & 01 \\
\hline 28.50 & $8.55098 \mathrm{E}-01$ & $2.64843 \mathrm{E}$ & 01 \\
\hline 28.60 & $8.55091 \mathrm{E}-01$ & $2.65822 \mathrm{E}$ & 01 \\
\hline 28.70 & $8.55084 \mathrm{E}-01$ & $2.66802 \mathrm{E}$ & 01 \\
\hline 28.80 & $8.55077 \mathrm{E}-01$ & $2.67782 \mathrm{E}$ & 01 \\
\hline 28.90 & $8.55070 \mathrm{E}-01$ & $2.68762 \mathrm{E}$ & 01 \\
\hline 29.00 & $8.55062 \mathrm{E}-01$ & $2.69743 \mathrm{E}$ & 01 \\
\hline 29.10 & $8.55055 \mathrm{E}-01$ & $2.70724 \mathrm{E}$ & 01 \\
\hline 29.20 & $8.55048 \mathrm{E}-01$ & $2.71705 \bar{E}$ & 01 \\
\hline 29.30 & $8.55041 \mathrm{E}-01$ & $2.72687 \mathrm{E}$ & 01 \\
\hline 29.40 & $8.55034 \mathrm{E}-01$ & $2.73669 \mathrm{E}$ & 01 \\
\hline 29.50 & $8.55027 \mathrm{E}-01$ & $2.74651 \mathrm{E}$ & 01 \\
\hline 29.60 & $8.55020 \mathrm{E}-01$ & $2.75634 \mathrm{E}$ & 01 \\
\hline & $8.55013 \mathrm{E}-01$ & $2.76617 \mathrm{E}$ & 01 \\
\hline 29.80 & $8.55006 \mathrm{E}-01$ & $2.77601 \mathrm{E}$ & 01 \\
\hline 29.90 & $8.54999 \mathrm{E}-01$ & $2.78585 \mathrm{E}$ & 01 \\
\hline 30.00 & $8.54992 \mathrm{E}-01$ & $2.79569 \mathrm{E}$ & 01 \\
\hline
\end{tabular}

$\begin{array}{ccc}g_{1} & \tan \delta_{1}\end{array}$

$1.06690 \mathrm{E} \quad 00 \quad-7.22296 \mathrm{E}-04$

$\begin{array}{lll}1.07759 \mathrm{E} & 00 & -7.25238 \mathrm{E}-04\end{array}$

$\begin{array}{lll}1.08834 \mathrm{E} & 00 & -7.28131 \mathrm{E}-04\end{array}$

$1.09915 \mathrm{E} \quad 00 \quad-7.30982 \mathrm{E}-04$

$1.11001 \mathrm{E} \quad 00$

$1.12092 \mathrm{E} \quad 00$

$1.13189 \mathrm{E} \quad 00$

$1.14291 \mathrm{E} \quad 00$

$1.15398 \mathrm{E} \quad 00$

$1.16512 \mathrm{E} \quad 00$

$1.17630 \mathrm{E} \quad 00$

$1.18754 \mathrm{E} \quad 00$

$1.19883 \mathrm{E} \quad 00$

$1.21018 \mathrm{E} \quad 00$

$1.22158 \mathrm{E} \quad 00$

$1.23304 \mathrm{E} \quad 00$

$1.24455 \mathrm{E} \quad 00$

$1.25612 \mathrm{E} \quad 00$

$1.26774 \mathrm{E} \quad 00$

$1.27941 \mathrm{E} \quad 00$

$1.29114 \mathrm{E} \quad 00$

$1.30293 \mathrm{E} \quad 00$

$1.31477 \mathrm{E} \quad 00$

$1.32666 \mathrm{E} \quad 00$

$1.33861 \mathrm{E} \quad 00$

$1.35062 \mathrm{E} \quad 00$

$1.36268 \mathrm{E} \quad 00$

$1.37479 \mathrm{E} \quad 00$

$1.38696 \mathrm{E} \quad 00$

$1.39919 \mathrm{E} \quad 00$

$1.41147 \mathrm{E} \quad 00$

$1.42380 \mathrm{E} \quad 00$

$1.43619 \mathrm{E} \quad 00$

$1.44864 \mathrm{E} \quad 00$

$1.46114 \mathrm{E} \quad 00$

$1.47369 \mathrm{E} \quad 00$

$1.48630 \mathrm{E} \quad 00$

$1.49897 \mathrm{E} \quad 00$

$1.51169 \mathrm{E} \quad 00$

$1.52447 \mathrm{E} \quad 00$

$\begin{array}{ll}1.52447 \mathrm{E} & 00 \\ 1.53730 \mathrm{E} & 00\end{array}$

$1.55019 \mathrm{E} \quad 00$

$1.56314 \mathrm{E} \quad 00$

$1.57614 \mathrm{E} \quad 00$

$1.58919 \mathrm{E} \quad 00$

$1.60230 \mathrm{E} \quad 00$

$1.61547 \mathrm{E} \quad 00$

$1.62869 \mathrm{E} \quad 00$

$1.64197 \mathrm{E} \quad 00$

$1.65531 \mathrm{E} \quad 00$

$1.66870 \mathrm{E} \quad 00$

$1.68214 \mathrm{E} \quad 00$

$1.69565 \mathrm{E} \quad 00$

$1.70921 \mathrm{E} \quad 00$

$1.72282 \mathrm{E} \quad 00$ $1.73649 \mathrm{E} \quad 00$ $1.75022 \mathrm{E} \quad 00$ $1.76400 \mathrm{E} \quad 00$

$1.77784 \mathrm{E} \quad 00$

$-7.33795 \mathrm{E}-04$

$-7.36577 \mathrm{E}-04$

$-7.42008 \mathrm{E}-04$

$-7.44668 \mathrm{E}-04$

$-7.47277 \mathrm{E}-04$

$-7.47277 \mathrm{E}-04$
$-7.49862 \mathrm{E}-04$

$-7.52412 \mathrm{E}-04$

$-7.54918 \mathrm{E}-04$

$-7.57392 \mathrm{E}-04$

$-7.59819 \mathrm{E}-04$

$-7.62223 \mathrm{E}-04$

$-7.64587 \mathrm{E}-04$

$-7.66914 \mathrm{E}-04$

$-7.69202 \mathrm{E}-04$

$-7.71458 \mathrm{E}-04$

$-7.73686 \mathrm{E}-04$

$-7.75868 \mathrm{E}-04$

$-7.78024 \mathrm{E}-04$

$-7.80150 \mathrm{E}-04$

$-7.82242 \mathrm{E}-04$

$-7.84300 \mathrm{E}-04$

$-7.86317 \mathrm{E}-04$

$-7.90272 \mathrm{E}-04$

$-7.92198 \mathrm{E}-04$

$-7.94099 \mathrm{E}-04$

$-7.95963 \mathrm{E}-04$

$-7.97804 \mathrm{E}-04$

$-7.99608 \mathrm{E}-04$

$-8.01379 \mathrm{E}-04$

$-8.03130 \mathrm{E}-04$

$-8.04842 \mathrm{E}-04$

$-8.06524 \mathrm{E}-04$

$-8.08187 \mathrm{E}-04$

$-8.09813 \mathrm{E}-04$

$-8.11415 \mathrm{E}-04$

$-8.12992 \mathrm{E}-04$

$-8.14530 \mathrm{E}-04$

$-8.16033 \mathrm{E}-04$

$-8.17529 \mathrm{E}-04$

$-8.18989 \mathrm{E}-04$

$-8.20421 \mathrm{E}-04$

$-8.21822 \mathrm{E}-04$

$-8.23201 \mathrm{E}-04$

$-8.24554 \mathrm{E}-04$

$-8.25882 \mathrm{E}-04$

$-8.27179 \mathrm{E}-04$

$-8.28454 \mathrm{E}-04$

$-8.29699 \mathrm{E}-04$.

$-8.30928 \mathrm{E}-04$

-8.32121 E-04

$-8.33300 \mathrm{E}-04$

$-8.34446 \mathrm{E}-04$

$-8.35565 \mathrm{E}-04$ $f_{-1}$

$-9.77058 \mathrm{E}-01$

$-9.87456 \mathrm{E}-01$

$-9.97908 \mathrm{E}-01$

$-1.00841 \mathrm{E} 00$

$-1.01898 \mathrm{E} 00$

$-7.39316 \mathrm{E}-04$

$-7.88312 \mathrm{E}-04$
$-1.02959 \mathrm{E} \quad 00$

$-1.04026 \mathrm{E} \quad 00$

$-1.05098 \mathrm{E} 00$

$-1.06176 \mathrm{E} 00$

$-1.07259 \mathrm{E} 00$

$-1.08348 \mathrm{E} \quad 00$

$-1.09442 \mathrm{E} 00$

$-1.10542 \mathrm{E} 00$

$-1.11647 \mathrm{E} 00$

$-1.12757 \mathrm{E} \quad 00$

$-1.13873 \mathrm{E} \quad 00$

$-1.14995 \mathrm{E} 00$

$-1.16122 \mathrm{E} 00$

$-1.17254 \mathrm{E} 00$

$-1.18392 \mathrm{E} 00$

$-1.19535 \mathrm{E} \quad 00$

$-1.20684 \mathrm{E} \quad 00$

$-1.21838 \mathrm{E} 00$

$-1.22998 \mathrm{E} 00$

$-1.24164 \mathrm{E} \quad 00$

$-1.25334 \mathrm{E} \quad 00$

$-1.26511 \mathrm{E} 00$

$-1.27693 \mathrm{E} 00$

$-1.28880 \mathrm{E} 00$

$-1.30073 \mathrm{E} 00$

$-1.31271 \mathrm{E} 00$

$-1.32475 \mathrm{E} 00$

$-1.33684 \mathrm{E} 00$

$-1.34899 \mathrm{E} 00$

$-1.36120 \mathrm{E} 00$

$-1.37346 \mathrm{E} \quad 00$

$-1.38578 \mathrm{E} 00$

$-1.39815 \mathrm{E} 00$

$-1.41057 \mathrm{E} 00$

$-1.42306 \mathrm{E} 00$

$-1.43559 \mathrm{E} \quad 00$

$-1.44819 \mathrm{E} 00$

$-1.46084 \mathrm{E} 00$

$-1.47354 \mathrm{E} 00$

$-1.48630 \mathrm{E} \quad 00$

$-1.49912 \mathrm{E} \quad 00$

$-1.51199 \mathrm{E} 00$

$-1.52492 \mathrm{E} 00$

$-1.53790 \mathrm{E} \quad 00$

$-1.55094 \mathrm{E} 00$

$-1.56404 \mathrm{E} \quad 00$

$-1.57719 \mathrm{E} 00$

$-1.59040 \mathrm{E} 00$

$-1.60367 \mathrm{E} \quad 00$

$-1.61699 \mathrm{E} 00$

$-1.63036 \mathrm{E} \quad 00$

$-1.64380 \mathrm{E} 00$

$-1.65729 \mathrm{E} 00$

$-1.67083 \mathrm{E} \quad 00$

$-1.68443 \mathrm{E} 00$ $g_{-1}$

$2.31814 \mathrm{E} \quad 01$ $2.32783 \mathrm{E} \quad 01$

$2.33753 \mathrm{E} \quad 01$

$2.34723 \mathrm{E} \quad 01$

$2.35694 \mathrm{E} 01$

$2.36664 \mathrm{E} \quad 01$

$2.37635 \mathrm{E} 01$

$2.38607 \mathrm{E} \quad 01$

$2.39578 \mathrm{E} \quad 01$

$2.40550 \mathrm{E} 01$

$2.41523 \mathrm{E} \quad 01$

$2.42495 \mathrm{E} \quad 01$

$2.43468 \mathrm{E} \quad 01$

$2.44441 \mathrm{E} \quad 01$

$2.45415 \mathrm{E} \quad 01$

$2.46388 \mathrm{E} \quad 01$

$2.47363 \mathrm{E} \quad 01$

$2.48337 \mathrm{E} \quad 01$

$2.49312 \mathrm{E} \quad 01$

$2.50287 \mathrm{E} 01$

$2.51262 \mathrm{E} \quad 01$ $2.52238 \mathrm{E} 01$

$2.53214 \mathrm{E} \quad 01$

$2.54191 \mathrm{E} 01$

$2.55167 \mathrm{E} \quad 01$

$2.56144 \mathrm{E} \quad 01$

$2.57122 \mathrm{E} \quad 01$

$2.58100 \mathrm{E} \quad 01$

$2.59078 \mathrm{E} \quad 01$

$2.60056 \mathrm{E} 01$

$2.61035 \mathrm{E} \quad 01$

$2.62014 \mathrm{E} \quad 01$

$2.62993 \mathrm{E} 01$

$2.63973 \mathrm{E} \quad 01$

$2.64953 \mathrm{E} 01$

$2.65933 \mathrm{E} 01$

$2.66914 \mathrm{E} 01$

$2.67895 \mathrm{E} \quad 01$

$2.68876 \mathrm{E} 01$

$2.69858 \mathrm{E} 01$

$2.70840 \mathrm{E} \quad 01$

$2.71823 \mathrm{E} \quad 01$

$2.72806 \mathrm{E} \quad 01$

$2.73789 \mathrm{E} \quad 01$

$2.74772 \mathrm{E} \quad 01$

$2.75756 \mathrm{E} \quad 01$

$2.76740 \mathrm{E} \quad 01$

$2.77725 \mathrm{E} 01$

$2.78710 \mathrm{E} 01$

$2.79695 \mathrm{E}$ 0l

$2.80681 \mathrm{E} \quad 01$

$2.81667 \mathrm{E} \quad 01$

$2.82653 \mathrm{E} \quad 01$

$2.83640 \mathrm{E} 01$

$2.84627 \mathrm{E} \quad 01$

$2.85614 \mathrm{E} \quad 01$ $2.86602 \mathrm{E} \quad 01$

$2.87590 \mathrm{E} 01$

$2.88579 \mathrm{E} \quad 01$

$2.89568 \mathrm{E} 01$

$\tan \delta_{-1}$

$3.53979 \mathrm{E} \quad 02$ $3.54712 \mathrm{E} \quad 02$ $3.55443 \mathrm{E} \quad 02$ $3.56175 \mathrm{E} \quad 02$ $3.56906 \mathrm{E} \quad 02$

$3.57636 \mathrm{E} \quad 02$ $3.58365 \mathrm{E} \quad 02$ $3.59093 \mathrm{E} \quad 02$ $3.59821 \mathrm{E} \quad 02$ $3.60548 \mathrm{E} \quad 02$

$3.61276 \mathrm{E} \quad 02$ $3.62002 \mathrm{E} \quad 02$ $3.62727 \mathrm{E} \quad 02$ $3.63452 \mathrm{E} \quad 02$ $3.64176 \mathrm{E} \quad 02$

$3.64901 \mathrm{E} \quad 02$ $3.65624 \mathrm{E} \quad 02$ $3.66347 \mathrm{E} \quad 02$ $3.67070 \mathrm{E} \quad 02$ $3.67793 \mathrm{E} \quad 02$

$3.68516 \mathrm{E} \quad 02$ $3.69237 \mathrm{E} \quad 02$ $3.69959 \mathrm{E} \quad 02$ $3.70680 \mathrm{E} \quad 02$ $3.71401 \mathrm{E} \quad 02$

$3.72121 \mathrm{E} \quad 02$ $3.72842 \mathrm{E} \quad 02$ $3.73563 \mathrm{E} \quad 02$ $3.74283 \mathrm{E} \quad 02$ $3.75003 \mathrm{E} \quad 02$

$3.75724 \mathrm{E} \quad 02$ $3.76443 \mathrm{E} \quad 02$ $3.77164 \mathrm{E} \quad 02$ $3.77882 \mathrm{E} \quad 02$ 3.78603 E 02 $3.79323 \mathrm{E} \quad 02$ $3.80042 \mathrm{E} \quad 02$ $3.80763 \mathrm{E} \quad 02$ $3.81484 \mathrm{E} \quad 02$ $3.82203 \mathrm{E} \quad 02$

$3.82924 \mathrm{E} \quad 02$ $3.83644 \mathrm{E} \quad 02$ $3.84364 \mathrm{E} \quad 02$ $3.85085 \mathrm{E} \quad 02$ $3.85808 \mathrm{E} \quad 02$

$3.86529 \mathrm{E} \quad 02$ 
$\bar{F}$ $f_{2}$

$9.07186 \mathrm{E}-04$ $9.14726 \mathrm{E}-04$ $9.22297 \mathrm{E}-04$ $9.29900 \mathrm{E}-04$ $9.37534 \mathrm{E}-04$

$9.45199 \mathrm{E}-04$ $9.52895 \mathrm{E}-04$ $9.60622 \mathrm{E}-04$ $9.68381 \mathrm{E}-04$ $9.76170 \mathrm{E}-04$

$9.83991 \mathrm{E}-04$ $9.91843 \mathrm{E}-04$ $9.99726 \mathrm{E}-04$ $1.00764 \mathrm{E}-03$ $1.01559 \mathrm{E}-03$

$1.02356 \mathrm{E}-03$ $1.03157 \mathrm{E}-03$ $1.03961 \mathrm{E}-03$ $1.04768 \mathrm{E}-03$ $1.05578 \mathrm{E}-03$

$1.06391 \mathrm{E}-03$ $1.07208 \mathrm{E}-03$ $1.08027 \mathrm{E}-03$ $1.08850 \mathrm{E}-03$ $1.09676 \mathrm{E}-03$.

$1.10505 \mathrm{E}-03$ $1.11337 \mathrm{E}-03$ $1.12172 \mathrm{E}-03$ $1.13010 \mathrm{E}-03$ $1.13851 \mathrm{E}-03$

$1.14696 \mathrm{E}-03$ $1.15543 \mathrm{E}-03$ $1.16394 \mathrm{E}-03$ $1.17248 \mathrm{E}-03$ $1.18105 \mathrm{E}-03$

$1.18965 \mathrm{E}-03$ $1.19828 \mathrm{E}-03$ $1.20694 \mathrm{E}-03$ $1.21563 \mathrm{E}-03$ $1.22436 \mathrm{E}-03$

$1.23311 \mathrm{E}-03$ $1.24190 \mathrm{E}-03$ $1.25072 \mathrm{E}-03$ $1.25957 \mathrm{E}-03$ $1.26845 \mathrm{E}-03$

$1.27736 \mathrm{E}-03$ $1.28631 \mathrm{E}-03$ $1.29528 \mathrm{E}-03$ $1.30429 \mathrm{E}-03$ $1.31332 \mathrm{E}-03$

$1.32239 \mathrm{E}-03$ $1.33149 \mathrm{E}-03$ $1.34062 \mathrm{E}-03$ $1.34978 \mathrm{E}-03$ $1.35898 \mathrm{E}-03$

$1.36820 \mathrm{E}-03$ $1.37745 \mathrm{E}-03$ $1.38674 \mathrm{E}-03$ $1.39606 \mathrm{E}-03$ $1.40541 \mathrm{E}-03$ $g_{2}$

$1.42231 \mathrm{E} \quad 00$

$1.43425 \mathrm{E} \cdot 00$

$1.44623 \mathrm{E} \quad 00$

$1.45826 \mathrm{E} \quad 00$

$1.47035 \mathrm{E} \quad 00$

$1.48248 \mathrm{E} \quad 00$ $1.49466 \mathrm{E} \quad 00$ $1.50689 \mathrm{E} \quad 00$ $1.51918 \mathrm{E}, 00$ $1.53151 \mathrm{E} \quad 00$

$1.54389 \mathrm{E} \quad 00$ $1.55632 \mathrm{E} \quad 00$ $1.56879 \mathrm{E} \quad 00$ $1.58132 \mathrm{E} \quad 00$ $1.59390 \mathrm{E} \quad 00$

$1.60653 \mathrm{E} \quad 00$ $1.61920 \mathrm{E} \quad 00$ $1.63193 \mathrm{E} \quad 00$ $1.64471 \mathrm{E} \quad 00$ $1.65753 \mathrm{E} \quad 00$

$1.67040 \mathrm{E} \quad 00$ $1.68333 \mathrm{E} \quad 00$ $1.69630 \mathrm{E} \quad 00$ $1.70932 \mathrm{E} \quad 00$ $1.72239 \mathrm{E} \quad 00$

$1.73552 \mathrm{E} \quad 00$ $1.74869 \mathrm{E} \quad 00$ $1.76191 \mathrm{E} \quad 00$ $1.77518 \mathrm{E} \quad 00$ $1.78849 \mathrm{E} \quad 00$

$1.80186 \mathrm{E} \quad 00$
0 $1.81528 \mathrm{E} \quad 00$ $1.82874 \mathrm{E} \quad 00$ $1.84226 \mathrm{E} \quad 00$ $1.85582 \mathrm{E} \quad 00$

$1.86944 \mathrm{E} \quad 00$ $1.88310 \mathrm{E} \quad 00$ $1.89681 \mathrm{E} \quad 00$ $1.91058 \mathrm{E} \quad 00$ $1.92439 \mathrm{E} \quad 00$

$1.93825 \mathrm{E} \quad 00$ $1.95216 \mathrm{E} \quad 00$ $1.96612 \mathrm{E} \quad 00$ $1.98012 \mathrm{E} \quad 00$ $1.99418 \mathrm{E} \quad 00$

$2.00829 \mathrm{E} \quad 00$ $2.02244 \mathrm{E} \quad 00$ $2.03665 \mathrm{E} \quad 00$ $2.05090 \mathrm{E} \quad 00$ $2.06520 \mathrm{E} \quad 00$

$2.07955 \mathrm{E} \quad 00$ $2.09396 \mathrm{E} \quad 00$ $2.10841 \mathrm{E} \quad 00$ $2.12291 \mathrm{E} \quad 00$ $2.13746 \mathrm{E} \quad 00$

$2.15205 \mathrm{E} \quad 00$ $2.16670 \mathrm{E} \quad 00$ $2.18140 \mathrm{E} \quad 00$ $2.19614 \mathrm{E} \quad 00$ $2.21093 \mathrm{E} \quad 00$ $\tan \delta_{2}$

$f_{-2}$

4.13955E-02

4.19758E-02

$4.25614 \mathrm{E}-02$

$4.31524 \mathrm{E}-02$

4.37488E-02

4.43506 E-02 4.49578E-02

$4.55704 \mathrm{E}-02$

$4.61886 \mathrm{E}-02$

$4.68123 \mathrm{E}-02$

$4.74415 \mathrm{E}-02$

$4.80762 \mathrm{E}-02$

$4.87166 \mathrm{E}-02$

$4.93625 \mathrm{E}-02$

$5.00141 \mathrm{E}-02$

$5.06713 \mathrm{E}-02$

$5.13342 \mathrm{E}-02$

$5.20028 \mathrm{E}-02$

$5.26772 \mathrm{E}-\mathrm{\theta} 2$

$5.33572 \mathrm{E}-02$

$5.40431 \mathrm{E}-02$

$5.47347 \mathrm{E}-02$

$5.54322 \mathrm{E}-02$

$5.61355 \mathrm{E}-02$

$5.68447 \mathrm{E}-02$

$5.75597 \mathrm{E}-02$

$5.82807 \mathrm{E}-02$

$5.90076 \mathrm{E}-02$

$5.97405 \mathrm{E}-02$

$6.04794 \mathrm{E}-02$

$6.12243 \mathrm{E}-02$

$6.19752 \mathrm{E}-02$

$6.27322 \mathrm{E}-02$

$6.34953 \mathrm{E}-02$

$6.42645 \mathrm{E}-02$

$6.50398 \mathrm{E}-02$

$6.58213 \mathrm{E}-02$

$6.66089 \mathrm{E}-02$

$6.74028 \mathrm{E}-02$

$6.82029 \mathrm{E}-02$

$6.90092 \mathrm{E}-02$ $6.98218 \mathrm{E}-02$

$7.06407 \mathrm{E}-02$

$7.14659 \mathrm{E}-02$

$7.22976 \mathrm{E}-02$

$7.31355 \mathrm{E}-02$

$7.39799 \mathrm{E}-02$

$7.48306 \mathrm{E}-02$

$7.56879 \mathrm{E}-02$

$7.65515 \mathrm{E}-02$

$7.74217 \mathrm{E}-02$

$7.82984 \mathrm{E}-02$

$7.91816 \mathrm{E}-02$

$8.00714 \mathrm{E}-02$

$8.09678 \mathrm{E}-02$

$8.18708 \mathrm{E}-02$ $8.27804 \mathrm{E}-02$ 8.36967E-02 $8.46197 \mathrm{E}-02$

$8.55494 \mathrm{E}-02$
$-2.78101 \mathrm{E} \quad 01 \quad-3.83353 \mathrm{E}-02$ $-2.78120 \mathrm{E}$ 01 $-3.88931 \mathrm{E}-02$ $-2.78139 \mathrm{E}$ 01 $-3.94561 \mathrm{E}-02$ $-2.78157 \mathrm{E} \quad 01 \quad-4.00244 \mathrm{E}-02$ $-2.78175 \mathrm{E} \quad 01 \quad-4.05981 \mathrm{E}-02$

$-2.78194 \mathrm{E} \quad 01 \quad-4.11770 \mathrm{E}-02$ $-2.78212 \mathrm{E} \quad 01 \quad-4.17614 \mathrm{E}-02$ $-2.78230 \mathrm{E} \quad 01 \quad-4.23511 \mathrm{E}-02$ $-2.78247 \mathrm{E}$ 0l $-4.29462 \mathrm{E}-02$ $-2.78265 \mathrm{E}$ 01 $-4.35467 \mathrm{E}-02$

$-2.78282 \mathrm{E} 01$ $-2.78300 \mathrm{E} \quad 01$ $-2.78317 \mathrm{E} \quad 01$ $-2.78334 \mathrm{E} .01$ $-2.78351 \mathrm{E} 01$

$\begin{array}{lll}-2.78367 \mathrm{E} & 01\end{array}$

$-2.78384 \mathrm{E} 01$

$-2.78401 \mathrm{E} \quad 01$

$-2.78417 \mathrm{E} \quad 01$

$-2.78433 \mathrm{E} \quad 01$

$\begin{array}{lll}-2.78449 \mathrm{E} & 01\end{array}$

$-2.78465 \mathrm{E} \quad 01$

$-2.78481 \mathrm{E} 01$

$-2.78497 \mathrm{E} 01$

-2.78512 E 01

$-2.78528 \mathrm{E} \quad 01$ $\begin{array}{ll}-2.78543 \mathrm{E} & 01\end{array}$ $-2.78558 \mathrm{E} 01$ $-2.78574 \mathrm{E} 01$

$-2.78589 \mathrm{E} \quad 01$

$-2.78603 \mathrm{E} \quad 01$

$-2.78618 \mathrm{E} \quad 01$

$-2.78633 \mathrm{E} \quad 01$

$-2.78647 \mathrm{E} \quad 01$

-2.78662 E 01

$-2.78676 \mathrm{E} 01$

$-2.78690 \mathrm{E} 01$

$-2.78705 \mathrm{E} 01$

$-2.78719 \mathrm{E} 01$

$-2.78732 \mathrm{E} 01$

$-2.78746 \mathrm{E} \quad 01$

$-2.78760 \mathrm{E} 01$

$-2.78774 \mathrm{E} 01$

$-2.78787 \mathrm{E} \quad 01$

-2.78801 E 01

$-2.78814 \mathrm{E} \quad 01$

$-2.78827 \mathrm{E} \quad 01$

$-2.78840 \mathrm{E} \quad 01$

$-2.78853 \mathrm{E} \quad 01$

$-2.78866 \mathrm{E} 01$

$\begin{array}{lll}-2.78879 \mathrm{E} & 01\end{array}$

$-2.78892 \mathrm{E} 01$

$-2.78904 \mathrm{E} \quad 0 \mathrm{l}$

$-2.78917 \mathrm{E} 01$

$-2.78929 \mathrm{E} \quad 01$

$-2.78942 \mathrm{E} 01$ $-2.78954 \mathrm{E} \quad 01$

$-2.78966 \mathrm{E} \quad 01$

$-2.78978 \mathrm{E} 01$

-2.78991 E 01

-4.41527E-02 $-4.47641 \mathrm{E}-02$ $-4.53811 \mathrm{E}-02$ $-4.60036 \mathrm{E}-02$ $-4.66316 \mathrm{E}-02$

$-4.72652 \mathrm{E}-02$ $-4.79044 \mathrm{E}-02$ $-4.85493 \mathrm{E}-02$ $-4.91997 \mathrm{E}-02$ $-4.98559 \mathrm{E}-02$

$-5.05177 \mathrm{E}-02$ $-5.11852 \mathrm{E}-02$ $-5.18585 \mathrm{E}-02$ $-5.25375 \mathrm{E}-02$ $-5.32224 \mathrm{E}-02$

$-5.39130 \mathrm{E}-02$ $-5.46095 \mathrm{E}-02$ $-5.53119 \mathrm{E}-02$ $-5.60201 \mathrm{E}-02$ $-5.67342 \mathrm{E}-02$

$-5.74543 \mathrm{E}-02$ $-5.81803 \mathrm{E}-02$ $-5.89123 \mathrm{E}-02$ $-5.96503 \mathrm{E}-02$ $-6.03944 \mathrm{E}-02$

$-6.11445 \mathrm{E}-02$ $-6.19006 \mathrm{E}-02$ $-6.26629 \mathrm{E}-02$ $-6.34313 \mathrm{E}-02$ $-6.42058 \mathrm{E}-02$

$-6.49865 \mathrm{E}-02$ $-6.57735 \mathrm{E}-02$ $-6.65666 \mathrm{E}-02$ $-6.73660 \mathrm{E}-02$ $-6.81716 \mathrm{E}-02$

$-6.89835 \mathrm{E}-02$ $-6.98018 \mathrm{E}-02$ $-7.06264 \mathrm{E}-02$ $-7.14573 \mathrm{E}-02$ $-7.22947 \mathrm{E}-02$

$-7.31384 \mathrm{E}-02$ $-7.39886 \mathrm{E}-02$ 
ELECTRON RADIAL FUNCTIONS

Positron

$F_{0}$

30.10

30.20

30.30

30.40

30.50

30.60

30.70

30.80

30.90

31.00

31.10

31.20

31.30

31.40

31.50

31.60

31.70

31.80

31.90

32.00

32.10

32.20

32.30

32.40

32.50

32.60

32.70

32.80

32.90

33.00

33.10

33.20

33.30

33.40

33.50

33.60

33.70

33.80

33.90

34.00

34.10

34.20

34.30

34.40

34.50

34.60

34.70

34.80

34.90

35.00

35.10

35.20

35.30

35.40

35.50

35.60

35.70

35.80

35.90

36.00 $f_{1}$

$8.54985 \mathrm{E}-01$ 8.54978E-01 $8.54972 \mathrm{E}-0 \mathrm{I}$ $8.54965 \mathrm{E}-01$ $8.54958 \mathrm{E}-01$

$8.54951 \mathrm{E}-01$ $8.54944 \mathrm{E}-01$ $8.54938 \mathrm{E}-01$ $8.54931 \mathrm{E}-01$ $8.54924 \mathrm{E}-01$

$8.54918 \mathrm{E}-01$ $8.54911 \mathrm{E}-01$ $8.54904 \mathrm{E}-01$ $8.54898 \mathrm{E}-01$ 8.54891 E-01

8.54884E-01 $8.54878 \mathrm{E}-01$ $8.54871 \mathrm{E}-01$ $8.54865 \mathrm{E}-01$ $8.54858 \mathrm{E}-01$

$8.54852 \mathrm{E}-01$ $8.54845 \mathrm{E}-01$ $8.54839 \mathrm{E}-0 \mathrm{l}$ $8.54832 \mathrm{E}-01$ $8.54826 \mathrm{E}-01$

$8.54819 \mathrm{E}-01$ $8.54813 \mathrm{E}-01$ $8.54807 \mathrm{E}-01$ $8.54800 \mathrm{E}-01$ $8.54794 \mathrm{E}-01$

$8.54787 \mathrm{E}-01$ 8.54781 E-01 $8.54775 \mathrm{E}-01$ $8.54769 \mathrm{E}-01$ 8.54762E-01

$8.54756 \mathrm{E}-01$ $8.54750 \mathrm{E}-01$ $8.54744 \mathrm{E}-01$ $8.54737 \mathrm{E}-01$ 8.54731 E-01

$8.54725 \mathrm{E}-01$ $8.54719 \mathrm{E}-01$ $8.54713 \mathrm{E}-01$ $8.54706 \mathrm{E}-01$ $8.54700 \mathrm{E}-01$

$8.54694 \mathrm{E}-01$ $8.54688 \mathrm{E}-0 \mathrm{I}$ $8.54682 \mathrm{E}-01$ $8.54676 \mathrm{E}-01$ $8.54670 \mathrm{E}-01$

$8.54664 \mathrm{E}-0$ $8.54658 \mathrm{E}-01$ $8.54652 \mathrm{E}-01$ $8.54646 \mathrm{E}-01$ $8.54640 \mathrm{E}-01$

$8.54634 \mathrm{E}-01$ $8.54628 \mathrm{E}-01$ $8.54622 \mathrm{E}-01$ $8.54616 \mathrm{E}-01$ $8.54610 \mathrm{E}-01$

$Z=7$

$$
A=14
$$$$
\rho=1.3 A^{1 / 3} F
$$

$g_{1}$

$2.80553 \mathbf{E} 01$

$2.81538 \mathrm{E} \quad 01$ $2.82523 \mathrm{E} \quad 01$ $2.83509 \mathrm{E} \quad 0$ $2.84495 \mathrm{E} \quad 01$

$2.85481 \mathrm{E} \quad 01$ $2.86468 \mathrm{E} \quad 01$ $2.87455 \mathrm{E} \quad 01$ $2.88442 \mathrm{E} 01$ $2.89430 \mathrm{E} \quad 01$

$2.90418 \mathrm{E} \quad 01$ $2.91406 \mathrm{E} 01$ $2.92395 \mathrm{E} \quad 0$ $2.93384 \mathrm{E} \quad 01$ $2.94374 \mathrm{E} \quad 01$

2.95364 E ol $2.96354 \mathrm{E} \quad 01$ $2.97345 \mathrm{E} 01$ $2.98336 \mathrm{E} \quad 0$ $2.99327 \mathrm{E} \quad 0$

$3.00319 \mathrm{E}$ 0l

$3.01311 \mathrm{E} 01$

$3.02304 \mathrm{E} \quad 01$

$3.03297 \mathrm{E} 01$

$3.04290 \mathrm{E} \quad 01$

$3.05284 \mathrm{E} \quad 01$ $3.06278 \mathrm{E} \quad 01$ $3.07273 \mathrm{E} \quad 01$ $3.08268 \mathrm{E} 01$ $3.09263 \mathrm{E} 01$

$3.10258 \mathrm{E} \quad 01$ $3.11254 \mathrm{E} \quad 01$ $3.12251 \mathrm{E} 01$ $3.13248 \mathrm{E}$ 01 $3.14245 \mathrm{E} \quad 01$

$3.15243 \mathrm{E} \quad 01$ $3.16241 \mathrm{E} 01$ $3.17239 \mathrm{E} \quad 01$ $3.18238 \mathrm{E}$ 0

$3.19237 \mathrm{E} \quad 0$

3.20237 E 01 $3.21237 \mathrm{E} \quad 01$ $3.22237 \mathbf{E} 01$ $3.23238 \mathrm{E} 01$ $3.24239 \mathrm{E} \quad 01$

$3.25241 \mathrm{E} \quad 01$ $3.26243 \mathrm{E} \quad 01$ $3.27245 \mathrm{E} \quad 01$ $3.28248 \mathrm{E} \quad 01$

$3.29252 \mathrm{E} 01$

$3.30255 \mathrm{E} \quad 0$ $3.31259 \mathrm{E} 01$ $3.32264 \mathrm{E} \quad 01$ $3.33269 \mathrm{E} 01$ $3.34274 \mathrm{E} \quad 01$

$3.35280 \mathrm{E} \quad 01$ $3.36286 \mathrm{E} 01$ $3.37293 \mathrm{E} 01$ $3.38300 \mathrm{E} 01$ $3.39308 \mathrm{E}$ ol
$1.79174 \mathrm{E} \quad 00$ $1.80569 \mathrm{E} \quad 00$ $1.81970 \mathrm{E} \quad 00$ $1.83376 \mathrm{E} \quad 00$

$1.84788 \mathrm{E} \quad 00$

$1.86206 \mathrm{E} \quad 00$

$1.87629 \mathrm{E} \quad 00$

$1.89059 \mathrm{E} \quad 00$

$1.90493 \mathbf{E} 00$

$1.91934 \mathrm{E} \quad 00$

$1.93380 \mathrm{E} \quad 00$

$1.94832 \mathrm{E} \quad 00$

$1.96289 \mathrm{E} \quad 00$

$1.97752 \mathrm{E} \quad 00$

$1.99221 \mathrm{E} \quad 00$

$2.00696 \mathrm{E} \quad 00$ $2.02176 \mathrm{E} \quad 00$ $2.03662 \mathrm{E} \quad 00$

$2.05153 \mathrm{E} \quad 00$

$2.0665 \mathrm{lE} \quad 00$

$2.08154 \mathrm{E} \quad 00$ $2.09663 \mathrm{E} \quad 00$

$2.11177 \mathrm{E} \quad 00$

$2.12698 \mathrm{E} \quad 00$

$2.14224 \mathrm{E} \quad 00$

$2.15755 \mathrm{E} \quad 00$ $2.17293 \mathrm{E} \quad 00$ $2.18836 \mathrm{E} \quad 00$ $2.20385 \mathrm{E} \quad 00$ $2.21940 \mathrm{E} \quad 00$

$2.23501 \mathrm{E} \quad 00$ $2.25067 \mathrm{E} \quad 00$ $2.26639 \mathrm{E} \quad 00$

$2.28217 \mathrm{E} \quad 00$

$2.29801 \mathrm{E} \quad 00$

$2.31390 \mathrm{E} \quad 00$ $2.32985 \mathrm{E} \quad 00$ $2.34586 \mathrm{E} \quad 00$

$2.36193 \mathrm{E} \quad 00$

$2.37806 \mathrm{E} \quad 00$

$2.39424 \mathrm{E} \quad 00$ $2.41049 \mathrm{E} \quad 00$ $2.42679 \mathrm{E} \quad 00$

$2.44315 \mathrm{E} \quad 00$

$2.45957 \mathrm{E} \quad 00$

$2.47604 \mathrm{E} \quad 00$ $2.49258 \mathrm{E} \quad 00$ $2.50917 \mathrm{E} \quad 00$ $2.52582 \mathrm{E} \quad 00$

$2.54254 \mathrm{E} \quad 00$

$2.55931 \mathrm{E} \quad 00$ $2.57613 \mathrm{E} \quad 00$ $2.59302 \mathrm{E} \quad 00$ $2.60997 \mathrm{E} \quad 00$

$2.62697 \mathrm{E} \quad 00$

$2.64404 \mathrm{E} \quad 00$ $2.66116 \mathrm{E} \quad 00$ $2.67834 \mathrm{E} \quad 00$

$2.69558 \mathrm{E} \quad 00$

$2.71288 \mathrm{E} \quad 00$

$\tan \delta_{1}$
$-8.36660 \mathrm{E}-04$
$-8.37734 \mathrm{E}-04$
$-8.38781 \mathrm{E}-04$
$-8.39795 \mathrm{E}-04$
$-8.40802 \mathrm{E}-04$

$-8.41783 \mathrm{E}-04$

$-8.42734 \mathrm{E}-04$

$-8.43665 \mathrm{E}-04$

$-8.44572 \mathrm{E}-04$

$-8.45460 \mathrm{E}-04$

$-8.46319 \mathrm{E}-04$

$-8.47156 \mathrm{E}-04$

$-8.47969 \mathrm{E}-04$

$-8.48766 \mathrm{E}-04$

$-8.49536 \mathrm{E}-04$

$-8.50284 \mathrm{E}-04$

$-8.51016 \mathrm{E}-04$

$-8.51721 \mathrm{E}-04$

$-8.52408 \mathrm{E}-04$

$-8.53067 \mathrm{E}-04$

$-8.53710 \mathrm{E}-04$

$-8.54330 \mathrm{E}-04$

$-8.54928 \mathrm{E}-04$

$-8.55509 \mathrm{E}-04$

$-8.56062 \mathrm{E}-04$

$-8.56604 \mathrm{E}-04$

$-8.57122 \mathrm{E}-04$

$-8.57621 \mathrm{E}-04$

$-8.58096 \mathrm{E}-04$

$-8.58549 \mathrm{E}-04$

$-8.58984 \mathrm{E}-04$

$-8.59394 \mathrm{E}-04$

$-8.59795 \mathrm{E}-04$

$-8.60170 \mathrm{E}-04$

$-8.60534 \mathrm{E}-04$

$-8.60869 \mathrm{E}-04$

$-8.61192 \mathrm{E}-04$

$-8.61494 \mathrm{E}-04$

$-8.61771 \mathrm{E}-04$

$-8.62029 \mathrm{E}-04$

$-8.62279 \mathrm{E}-04$

$-8.62503 \mathrm{E}-04$

$-8.62711 \mathrm{E}-04$ 


\section{$\bar{F}$}

$1.41479 \mathrm{E}-03$ $1.42420 \mathrm{E}-03$ $1.43364 \mathrm{E}-03$ $1.44311 \mathrm{E}-03$ $1.45262 \mathrm{E}-03$

$1.46215 \mathrm{E}-03$ $1.47172 \mathrm{E}-03$ $1.48132 \mathrm{E}-03$ $1.49095 \mathrm{E}-03$ $1.50061 \mathrm{E}-03$

$1.51030 \mathrm{E}-03$ $1.52002 \mathrm{E}-03$ $1.52977 \mathrm{E}-03$ $1.53956 \mathrm{E}-03$ $1.54938 \mathrm{E}-03$

$1.55922 \mathrm{E}-03$ $1.56910 \mathrm{E}-03$ $1.57901 \mathrm{E}-03$ $1.58895 \mathrm{E}-03$ $1.59892 \mathrm{E}-03$

$1.60893 \mathrm{E}-03$ $1.61896 \mathrm{E}-03$ $1.62903 \mathrm{E}-03$ $1.63912 \mathrm{E}-03$ $1.64925 \mathrm{E}-03$

$1.65941 \mathrm{E}-03$ $1.66960 \mathrm{E}-03$ $1.67982 \mathrm{E}-03$ $1.69007 \mathrm{E}-03$ $1.70036 \mathrm{E}-03$

$1.71067 \mathrm{E}-03$ $1.72102 \mathrm{E}-03$ $1.73140 \mathrm{E}-03$ $1.74180 \mathrm{E}-03$ $1.75224 \mathrm{E}-03$

$1.76271 \mathrm{E}-03$ $1.77322 \mathrm{E}-03$ $1.78375 \mathrm{E}-03$ $1.79431 \mathrm{E}-03$ $1.80491 \mathrm{E}-03$

$1.81553 \mathrm{E}-03$ $1.82619 \mathrm{E}-03$ $1.83688 \mathrm{E}-03$ $1.84760 \mathrm{E}-03$ $1.85835 \mathrm{E}-03$

$1.86914 \mathrm{E}-03$ $1.87995 \mathrm{E}-03$ $1.89079 \mathrm{E}-03$ $1.90167 \mathrm{E}-03$ $1.91258 \mathrm{E}-03$

$1.92351 \mathrm{E}-03$ $1.93448 \mathrm{E}-03$ $1.94548 \mathrm{E}-03$ $1.95652 \mathrm{E}-03$ $1.96758 \mathrm{E}-03$

$1.97867 \mathrm{E}-03$ $1.98980 \mathrm{E}-03$ $2.00095 \mathrm{E}-03$ $2.01214 \mathrm{E}-03$ $2.02336 \mathrm{E}-03$ $f_{2}$

$2.22578 \mathrm{E} \quad 00$ $2.24067 \mathrm{E} \quad 00$ 2.25561 E 00 $2.27060 \mathrm{E} \quad 00$ $2.28564 \mathrm{E} \quad 00$

$2.30073 \mathrm{E} \quad 00$ $2.31587 \mathrm{E} \quad 00$ $2.33105 \mathrm{E} \quad 00$ $2.34629 \mathrm{E} \quad 00$ $2.36157 \mathrm{E} \quad 00$

$2.37691 \mathrm{E} \quad 00$ $2.39229 \mathrm{E} \quad 00$ $2.40772 \mathrm{E} \quad 00$ $2.42320 \mathrm{E} \quad 00$ $2.43873 \mathrm{E} \quad 00$

$2.45431 \mathrm{E} \quad 00$ $2.46994 \mathrm{E} \quad 00$ $2.48561 \mathrm{E} \quad 00$ $2.50134 \mathrm{E} \quad 00$ $2.51711 \mathrm{E} \quad 00$

$2.53293 \mathrm{E} \quad 00$ 2.54881 E $\quad 00$ $2.56473 \mathrm{E} \quad 00$ $2.58070 \mathrm{E} \quad 00$ $2.59671 \mathrm{E} \quad 00$

$2.61278 \mathrm{E} \quad 00$ $2.62890 \mathrm{E} \quad 00$ $2.64506 \mathrm{E} \quad 00$ $2.66128 \mathrm{E} \quad 00$ $2.67754 \mathrm{E} \quad 00$

$2.69385 \mathrm{E} \quad 00$ $2.71021 \mathrm{E} \quad 00$ $2.72662 \mathrm{E} \quad 00$ $2.74308 \mathrm{E} \quad 00$ $2.75958 \mathrm{E} \quad 00$

$2.77614 \mathrm{E} \quad 00$ $2.79274 \mathrm{E} \quad 00$ $2.80939 \mathrm{E} \quad 00$ $2.82609 \mathrm{E} \quad 00$ $2.84284 \mathrm{E} \quad 00$

$2.85964 \mathrm{E} \quad 00$ $2.87649 \mathrm{E} \quad 00$ $2.89339 \mathrm{E} \quad 00$ $2.91033 \mathrm{E} \quad 00$ $2.92732 \mathrm{E} \quad 00$

$2.94437 \mathrm{E} \quad 00$ $2.96146 \mathrm{E} \quad 00$ $2.97860 \mathrm{E} \quad 00$ $2.99578 \mathrm{E} \quad 00$ $3.01302 \mathrm{E} \quad 00$

$3.03031 \mathrm{E} \quad 00$ $3.04764 \mathrm{E} \quad 00$ $3.06502 \mathrm{E} \quad 00$ $3.08245 \mathrm{E} \quad 00$ $3.09993 \mathrm{E} \quad 00$

$3.11746 \mathrm{E} \quad 00$ $3.13504 \mathrm{E} \quad 00$ $3.15266 \mathrm{E} \quad 00$ $3.17034 \mathrm{E} \quad 00$ $3.18806 \mathrm{E} \quad 00$ $g_{2}$

$8.64858 \mathrm{E}-02$ $8.74290 \mathrm{E}-02$ $8.83789 \mathrm{E}-02$ $8.93356 \mathrm{E}-02$ $9.02992 \mathrm{E}-02$

$9.12696 \mathrm{E}-02$ $9.22469 \mathrm{E}-02$

$9.32311 \mathrm{E}-02$ $9.42222 \mathrm{E}-02$ $9.52202 \mathrm{E}-02$

$9.62253 \mathrm{E}-02$ $9.72373 \mathrm{E}-02$ $9.82563 \mathrm{E}-02$ $9.92824 \mathrm{E}-02$ $1.00316 \mathrm{E}-01$

$1.01356 \mathrm{E}-01$ $1.02403 \mathrm{E}-01$ $1.03458 \mathrm{E}-01$ $1.04519 \mathrm{E}-01$ $1.05588 \mathrm{E}-01$

$1.06664 \mathrm{E}-01$

$1.07747 \mathrm{E}-01$

$1.08838 \mathrm{E}-01$

$1.09936 \mathrm{E}-01$

$1.11041 \mathrm{E}-01$

$1.12153 \mathrm{E}-01$

$1.13273 \mathrm{E}-01$

$1.14400 \mathrm{E}-01$

$1.15534 \mathrm{E}-01$

$1.16676 \mathrm{E}-01$

$1.17826 \mathrm{E}-01$

$1.18983 \mathrm{E}-01$

$1.20147 \mathrm{E}-01$

$1.21319 \mathrm{E}-01$

$1.22498 \mathrm{E}-01$

$1.23685 \mathrm{E}-01$

$1.24880 \mathrm{E}-01$

1.26082 E-01

$1.27292 \mathrm{E}-01$

$1.28509 \mathrm{E}-01$

$1.29734 \mathrm{E}-01$

$1.30967 \mathrm{E}-01$

$1.32208 \mathrm{E}-01$

$1.33456 \mathrm{E}-01$

$1.34712 \mathrm{E}-01$

$1.35976 \mathrm{E}-01$

$1.37248 \mathrm{E}-01$

$1.38527 \mathrm{E}-01$

$1.39815 \mathrm{E}-01$

$1.41110 \mathrm{E}-01$

$1.42413 \mathrm{E}-01$

$1.43724 \mathrm{E}-01$

$1.45044 \mathrm{E}-01$

$1.46371 \mathrm{E}-01$

$1.47706 \mathrm{E}-01$

$1.49049 \mathrm{E}-01$

$1.50400 \mathrm{E}-01$

$1.51760 \mathrm{E}-01$

$1.53127 \mathrm{E}-01$

$1.54503 \mathrm{E}-01$ $\tan \delta$

$f_{-2}$

$-2.79003 \mathrm{E} \quad 01 \quad-8.19339 \mathrm{E}-02$ $-2.79014 \mathrm{E}$ 01 $-8.28497 \mathrm{E}-02$ $-2.79026 \mathrm{E} \quad 01 \quad-8.37723 \mathrm{E}-02$ $-2.79038 \mathrm{E}$ 0l $\quad-8.47016 \mathrm{E}-02$ $-2.79050 \mathrm{E} \quad 01 \quad-8.56376 \mathrm{E}-02$

$\begin{array}{ll}-2.79061 \mathrm{E} & 01\end{array}$ $-2.79073 \mathrm{E} \quad 0 \mathrm{~J}$ $-2.79084 \mathrm{E} 01$ $-2.79095 \mathrm{E} 01$ $-2.79107 \mathrm{E} 01$

$-2.79118 \mathbf{E} \quad 01$ $-2.79129 \mathrm{E} \quad 01$ $-2.79140 \mathrm{E} 01$ $-2.79151 \mathrm{E} \quad 01$ $-2.79162 \mathrm{E} 01$

$-2.79173 \mathbf{E} 01$ $-2.79183 \mathrm{E} \quad 01$ $-2.79194 \mathrm{E} \quad 01$ $-2.79205 \mathrm{E} \quad 01$ -2.79215 E 01

$-2.79226 \mathrm{E} \quad 01$ $-2.79236 \mathbf{E} 01$ $-2.79246 \mathrm{E} \quad 01$ $\begin{array}{lll}-2.79257 \mathrm{E} & 01\end{array}$ -2.79267 E 01

$\begin{array}{lll}-2.79277 \mathbf{E} & 01\end{array}$ $\begin{array}{lll}-2.79287 \mathrm{E} & 01\end{array}$ $-2.79297 \mathrm{E} \quad 01$ $-2.79307 \mathrm{E} \quad 01$ $-2.79317 \mathrm{E} \quad 01$

$\begin{array}{lll}-2.79327 \mathrm{E} & 01\end{array}$ $-2.79337 \mathrm{E} \quad 01$ $-2.79346 \mathrm{E} \quad 01$ $-2.79356 \mathrm{E} \quad 01$

$-2.79366 \mathrm{E} \quad 01$

$-2.79375 \mathrm{E} \quad 01$

$-2.79385 \mathrm{E} \quad 01$

$-2.79394 \mathrm{E} 01$

$-2.79403 \mathrm{E}$ 0l

$-2.79413 \mathrm{E} 01$

$-2.79422 \mathrm{E} \quad 01$

$-2.79431 \mathrm{E} \quad 01$

$-2.79440 \mathrm{E} 01$

$-2.79449 \mathrm{E} \quad 01$

$-2.79458 \mathrm{E} \quad 01$

$\begin{array}{lll}-2.79467 \mathrm{E} & 01\end{array}$

$-2.79476 \mathrm{E} \quad 01$

$-2.79485 \mathrm{E} 01$

$-2.79494 \mathrm{E} \quad 01$

$-2.79502 \mathrm{E} 01$

$\begin{array}{lll}-2.79511 \mathrm{E} & 01\end{array}$

$-2.79520 \mathbf{E} 01$

$-2.79528 \mathrm{E} \quad 01$

$-2.79537 \mathrm{E} \quad 01$

$-2.79545 \mathrm{E} 01$

$-2.79554 \mathrm{E} \quad 01$

$-2.79562 \mathrm{E} \quad 01$

-2.79571 E 01

$-2.79579 \mathrm{E} 01$

$-2.79587 \mathrm{E} \quad 01$

$-8.65804 \mathrm{E}-02$

$-8.75300 \mathrm{E}-02$

$-8.84864 \mathrm{E}-02$

$-8.94496 \mathrm{E}-02$

$-9.04198 \mathrm{E}-02$

$-9.13968 \mathrm{E}-02$ $-9.23807 \mathrm{E}-02$

$-9.33716 \mathrm{E}-02$ $-9.4369+\mathrm{E}-02$ $-9.53742 \mathrm{E}-02$

$-9.63860 \mathrm{E}-02$ $-9.74048 \mathrm{E}-02$ $-9.84307 \mathrm{E}-02$ $-9.94637 \mathrm{E}-02$ -1.00504 E-01

$-1.01551 \mathrm{E}-01$ $-1.02605 \mathrm{E}-01$ $-1.03667 \mathrm{E}-01$ $-1.04736 \mathrm{E}-01$ $-1.05811 \mathrm{E}-01$

$-1.06895 \mathrm{E}-01$ $-1.07985 \mathrm{E}-01$ $-1.09083 \mathrm{E}-01$ $-1.10188 \mathrm{E}-01$ $-1.11300 \mathrm{E}-01$

$-1.12420 \mathrm{E}-01$ $-1.13547 \mathrm{E}-01$ $-1.14682 \mathrm{E}-01$ -1.15824 E- 01 $-1.16973 \mathrm{E}-01$

$-1.18130 \mathrm{E}-01$ $-1.19295 \mathrm{E}-0 \mathrm{l}$ $-1.20467 \mathrm{E}-01$ $-1.21646 \mathrm{E}-01$ $-1.22834 \mathrm{E}-01$

$-1.24028 \mathrm{E}-01$ $-1.25231 \mathrm{E}-01$ $-1.26441 \mathrm{E}-01$ $-1.27658 \mathrm{E}-01$ $-1.28884 \mathrm{E}-01$

$-1.30117 \mathrm{E}-01$ 


\begin{tabular}{|c|c|c|c|c|c|c|c|}
\hline$p$ & $F_{0}$ & $f_{1}$ & & $g_{1}$ & & $\tan \delta_{1}$ & $f_{-1}$ \\
\hline 36.10 & $8.54604 \mathrm{E}-01$ & $3.40316 \mathrm{E}$ & 01 & $2.73024 \mathrm{E}$ & 00 & $-8.63380 \mathrm{E}-04$ & $-2.62267 \mathrm{E}$ \\
\hline 36.20 & $8.54598 \mathrm{E}-01$ & $3.41324 \mathrm{E}$ & 01 & $2.74766 \mathrm{E}$ & 00 & $-8.63261 \mathrm{E}-04$ & $-2.63986 \mathrm{E}$ \\
\hline 36.30 & $8.54593 \mathrm{E}-01$ & $3.42333 \mathrm{E}$ & 01 & $2.76514 \mathrm{E}$ & 00 & $-8.63114 \mathrm{E}-04$ & $-2.65712 \mathrm{E}$ \\
\hline 36.40 & $8.54587 \mathrm{E}-01$ & $3.43342 \mathrm{E}$ & 01 & $2.78268 \mathrm{E}$ & 00 & $-8.62961 \mathrm{E}-04$ & $-2.67443 \mathrm{E}$ \\
\hline 36.50 & $8.54581 \mathrm{E}-01$ & $3.44352 \mathrm{E}$ & 01 & $2.80027 \mathrm{E}$ & 00 & $-8.62786 \mathrm{E}-04$ & $-2.69180 \mathrm{E}$ \\
\hline 36.60 & $8.54575 \mathrm{E}-01$ & $3.45362 \mathrm{E}$ & 01 & $2.81793 \mathrm{E}$ & 00 & $-8.62598 \mathrm{E}-04$ & $-2.70923 \mathrm{E}$ \\
\hline 36.70 & $8.54569 \mathrm{E}-01$ & $3.46372 \mathrm{E}$ & 01 & $2.83564 \mathrm{E}$ & 00 & $-8.62380 \mathrm{E}-04$ & $-2.72672 \mathrm{E}$ \\
\hline 36.80 & $8.54564 \mathrm{E}-01$ & $3.47383 \mathrm{E}$ & 01 & $2.85342 \mathrm{E}$ & 00 & $-8.62164 \mathrm{E}-04$ & $-2.74427 \mathrm{E}$ \\
\hline 36.90 & $8.54558 \mathrm{E}-01$ & $3.48395 \mathrm{E}$ & 01 & $2.87126 \mathrm{E}$ & 00 & $-8.61918 E-04$ & $-2.76188 \mathrm{E}$ \\
\hline 37.00 & $8.54552 \mathrm{E}-01$ & $3.49406 \mathrm{E}$ & 01 & $2.88915 \mathrm{E}$ & 00 & $-8.61673 \mathrm{E}-04$ & $-2.77955 \mathrm{E}$ \\
\hline 37.10 & $8.54546 \mathrm{E}-01$ & $3.50419 \mathrm{E}$ & 01 & $2.90710 \mathrm{E}$ & 00 & $-8.61398 \mathrm{E}-04$ & $-2.79728 \mathrm{E}$ \\
\hline 37.20 & $8.54541 \mathrm{E}-01$ & $3.51431 \mathrm{E}$ & 01 & $2.92512 \mathrm{E}$ & 00 & $-8.61109 \mathrm{E}-04$ & $-2.81507 \mathrm{E}$ \\
\hline 37.30 & $8.54534 \mathrm{E}-01$ & $3.52445 \mathrm{E}$ & 01 & $2.94319 \mathrm{E}$ & 00 & $-8.60808 \mathrm{E}-04$ & $-2.83291 \mathrm{E}$ \\
\hline 37.40 & $8.54528 \mathrm{E}-01$ & $3.53458 \mathrm{E}$ & 01 & $2.96133 \mathrm{E}$ & 00 & $-8.60492 \mathrm{E}-04$ & $-2.85082 \mathrm{E}$ \\
\hline 37.50 & $8.54523 \mathrm{E}-01$ & $3.54472 \mathrm{E}$ & 01 & $2.97952 \mathrm{E}$ & 00 & $-8.60158 \mathrm{E}-04$ & $-2.86879 \mathrm{E}$ \\
\hline 7.60 & $8.54517 \mathrm{E}-01$ & $3.55487 \mathrm{E}$ & 01 & $2.99778 \mathrm{E}$ & 00 & $-8.59812 \mathrm{E}-04$ & $-2.88683 \mathrm{E}$ \\
\hline 37.70 & $8.54511 \mathrm{E}-01$ & $3.56502 \mathrm{E}$ & 01 & $3.01609 \mathrm{E}$ & 00 & $-8.59447 \mathrm{E}-04$ & $-2.90492 \mathrm{E}$ \\
\hline 37.80 & $8.54506 \mathrm{E}-01$ & $3.57517 \mathrm{E}$ & 01 & $3.03446 \mathrm{E}$ & 00 & $-8.59068 \mathrm{E}-04$ & $-2.92307 \mathrm{E}$ \\
\hline 37.90 & $8.54500 \mathrm{E}-01$ & $3.58533 \mathrm{E}$ & 01 & $3.05290 \mathrm{E}$ & 00 & $-8.58673 E-04$ & $-2.94128 \mathrm{E}$ \\
\hline 38.00 & $8.54495 \mathrm{E}-01$ & $3.59549 \mathrm{E}$ & 01 & $3.07139 \mathrm{E}$ & 00 & $-8.58261 \mathrm{E}-04$ & $-2.95955 \mathrm{E}$ \\
\hline 38.10 & $8.54489 \mathrm{E}-01$ & $3.60566 \mathrm{E}$ & 01 & $3.08995 \mathrm{E}$ & 00 & $-8.57841 \mathrm{E}-04$ & $-2.97788 \mathrm{E}$ \\
\hline 38.20 & $8.54483 \mathrm{E}-01$ & $3.61583 \mathrm{E}$ & 01 & $3.10857 \mathrm{E}$ & 00 & $-8.57397 \mathrm{E}-$ & $-2.99628 \mathrm{E}$ \\
\hline 38.30 & $8.54478 \mathrm{E}-01$ & $3.62601 \mathrm{E}$ & 01 & $3.12724 \mathrm{E}$ & 00 & $-8.56943 \mathrm{E}-04$ & $-3.01473 \mathrm{E}$ \\
\hline 38.40 & $8.54472 \mathrm{E}-01$ & $3.63619 \mathrm{E}$ & 01 & $3.14598 \mathrm{E}$ & 00 & $-8.56474 \mathrm{E}-04$ & $-3.03324 \mathrm{E}$ \\
\hline 38.50 & $8.54467 \mathrm{E}-01$ & $3.64638 \mathrm{E}$ & 01 & $3.16+78 \mathrm{E}$ & 00 & $-8.55982 \mathrm{E}-04$ & $-3.05182 \mathrm{E}$ \\
\hline 38.60 & $8.54461 \mathrm{E}-01$ & $3.65657 \mathrm{E}$ & 01 & $3.18363 \mathrm{E}$ & 00 & $-8.55494 \mathrm{E}-04$ & $-3.07046 \mathrm{E}$ \\
\hline 38.70 & $8.54456 \mathrm{E}-01$ & $3.66676 \mathrm{E}$ & 01 & $3.20255 \mathrm{E}$ & 00 & $-8.54974 \mathrm{E}$ & $-3.08915 \mathrm{E}$ \\
\hline 38.80 & $8.54450 \mathrm{E}-01$ & $3.67696 \mathrm{E}$ & 01 & $3.22153 \mathrm{E}$ & 00 & $-8.54455 \mathrm{E}-04$ & $10791 \mathrm{E}$ \\
\hline 38.90 & $8.54445 \mathrm{E}-01$ & $3.68717 \mathrm{E}$ & 01 & $3.24057 \mathrm{E}$ & 00 & $-8.53906 \mathrm{E}-04$ & $-3.12673 \mathrm{E}$ \\
\hline 39.00 & $8.54439 \mathrm{E}-01$ & $3.69738 \mathrm{E}$ & 01 & $3.25967 \mathrm{E}$ & 00 & $-8.53349 \mathrm{E}-04$ & $-3.14561 \mathrm{E}$ \\
\hline 39.10 & $8.54434 \mathrm{E}-01$ & $3.70759 \mathrm{E}$ & 01 & $3.27884 \mathrm{E}$ & 00 & $-8.52779 \mathrm{E}-04$ & $-3.16455 \mathrm{E}$ \\
\hline 39.20 & $8.54428 \mathrm{E}-01$ & $3.71781 \mathrm{E}$ & 01 & $3.29806 \mathrm{E}$ & 00 & $-8.52197 \mathrm{E}$ & $8356 \mathrm{E}$ \\
\hline 39.30 & $8.54423 \mathrm{E}-01$ & $3.72803 \mathrm{E}$ & 01 & $3.31734 \mathrm{E}$ & 00 & $-8.51592 \mathrm{E}-04$ & $-3.20262 \mathrm{E}$ \\
\hline 39.40 & $8.54417 \mathrm{E}-01$ & $3.73826 \mathrm{E}$ & 01 & $3.33669 \mathrm{E}$ & 00 & $-8.50975 \mathrm{E}-04$ & $-3.22174 \mathrm{E}$ \\
\hline 39.50 & $8.54412 \mathrm{E}-01$ & $3.74849 \mathrm{E}$ & 01 & $3.35610 \mathrm{E}$ & 00 & $-8.50348 \mathrm{E}-04$ & $-3.24093 \mathrm{E}$ \\
\hline 39.60 & $8.54406 \mathrm{E}-0 \mathrm{l}$ & $3.75873 \mathrm{E}$ & 01 & $3.37556 \mathrm{E}$ & 00 & $-8.49706 \mathrm{E}-04$ & $-3.26018 \mathrm{E}$ \\
\hline 39.70 & $8.54401 \mathrm{E}-01$ & $3.76897 \mathrm{E}$ & 01 & $3.39509 \mathrm{E}$ & 00 & $-8.49053 \mathrm{~F}$ & $.27949 \mathrm{E}$ \\
\hline 39.80 & $8.54396 \mathrm{E}-01$ & $3.77922 \mathrm{E}$ & 01 & $3.41468 \mathrm{E}$ & 00 & $-8.48386 \mathrm{E}-04$ & $-3.29886 \mathrm{E}$ \\
\hline 39.90 & $8.54390 \mathrm{E}-01$ & $3.78947 \mathrm{E}$ & 01 & $3.43434 \mathrm{E}$ & 00 & $-8.47690 \mathrm{E}-04$ & $-3.31829 \mathrm{E}$ \\
\hline 40.00 & $8.54385 \mathrm{E}-01$ & $3.79973 \mathrm{E}$ & 01 & $3.45405 \mathrm{E}$ & 00 & $-8.46998 \mathrm{E}-04$ & $-3.33779 \mathrm{E}$ \\
\hline 40.10 & $8.54380 \mathrm{E}-01$ & $3.80999 \mathrm{E}$ & 01 & $3.47382 \mathrm{E}$ & 00 & $-8.46287 \mathrm{E}-04$ & $-3.35734 \mathrm{E}$ \\
\hline 40.20 & $8.54374 \mathrm{E}-01$ & $3.82026 \mathrm{E}$ & 01 & $3.49366 \mathrm{E}$ & 00 & $-8.45558 \mathrm{E} \rightarrow 04$ & $-3.37696 \mathrm{E}$ \\
\hline 40.30 & $8.54369 \mathrm{E}-01$ & $3.83053 \mathrm{E}$ & 01 & $3.51356 \mathrm{E}$ & 00 & $-8.44818 \mathrm{E}-04$ & $-3.39664 \mathrm{E}$ \\
\hline 40.40 & $8.54364 \mathrm{E}-01$ & $3.84080 \mathrm{E}$ & 01 & $3.53352 \mathrm{E}$ & 00 & $-8.44069 \mathrm{E}-04$ & $-3.41639 \mathrm{E}$ \\
\hline 40.50 & $8.54358 \mathrm{E}-01$ & $3.85108 \mathrm{E}$ & 01 & $3.55354 \mathrm{E}$ & 00 & $-8.43303 \mathrm{E}-04$ & $-3.43619 \mathrm{E}$ \\
\hline 40.60 & $8.54353 \mathrm{E}-01$ & $3.86137 \mathrm{E}$ & 01 & $3.57362 \mathrm{E}$ & 00 & $-8.42527 \mathrm{E}-04$ & $-3.45606 \mathrm{E}$ \\
\hline 40.70 & $8.54348 \mathrm{E}-01$ & $3.87166 \mathrm{E}$ & 01 & $3.59377 \mathrm{E}$ & 00 & $-8.41739 E-04$ & $-3.47598 \mathrm{E}$ \\
\hline 40.80 & $8.54343 \mathrm{E}-01$ & $3.88196 \mathrm{E}$ & 01 & $3.61398 \mathrm{E}$ & 00 & $-8.40927 \mathrm{E}-04$ & $-3.49598 \mathrm{E}$ \\
\hline 40.90 & $8.54337 \mathrm{E}-01$ & $3.89226 \mathrm{E}$ & 01 & $3.63425 \mathrm{E}$ & 00 & $-8.40110 \mathrm{E}-04$ & $-3.51603 \mathrm{E}$ \\
\hline 41.00 & $8.54332 \mathrm{E}-01$ & $3.90256 \mathrm{E}$ & 01 & $3.65458 \mathrm{E}$ & 00 & $-8.39277 \mathrm{E}-04$ & $-3.53614 \mathrm{E}$ \\
\hline 41.10 & $8.54327 \mathrm{E}-01$ & $3.91287 \mathrm{E}$ & 01 & $3.67497 \mathrm{E}$ & 00 & $-8.38427 \mathrm{E}-04$ & $-3.55632 \mathrm{E}$ \\
\hline & $8.54322 \mathrm{E}-01$ & $3.92319 \mathrm{E}$ & 01 & $3.69543 \mathrm{E}$ & 00 & $-8.37568 E-04$ & $-3.57656 \mathrm{E}$ \\
\hline 4.1 .30 & $8.54316 \mathrm{E}-$ & $3.93351 \mathrm{E}$ & 01 & $3.71595 \mathrm{E}$ & 00 & $-8.36697 \mathrm{E}-04$ & $-3.59687 \mathrm{E}$ \\
\hline 41.40 & $8.54311 \mathrm{E}-01$ & $3.94383 \mathrm{E}$ & 01 & $3.73653 \mathrm{E}$ & 00 & $-8.35815 E-04$ & $-3.61723 \mathrm{E}$ \\
\hline 41.50 & $8.54306 \mathrm{E}-01$ & $3.95416 \mathrm{E}$ & 01 & $3.75717 \mathrm{E}$ & 00 & $-8.34914 \mathrm{E}-04$ & $-3.63766 \mathrm{E}$ \\
\hline 41.60 & $8.54301 \mathrm{E}-01$ & $3.96450 \mathrm{E}$ & 01 & $3.77788 \mathrm{E}$ & 00 & $-8.34003 \mathrm{E}-04$ & $-3.65815 \mathrm{E}$ \\
\hline 41.70 & $8.54296 \mathrm{E}-01$ & $3.97484 \mathrm{E}$ & 01 & $3.79865 \mathrm{E}$ & 00 & $-8.33080 \mathrm{E}-04$ & $-3.67870 \mathrm{E}$ \\
\hline 41.80 & $8.54291 \mathrm{E}-$ & $3.98519 \mathrm{E}$ & 01 & $3.81948 \mathrm{E}$ & 00 & $-8.32146 \mathrm{E}-04$ & $-3.69932 \mathrm{E}$ \\
\hline 41.90 & $8.54285 \mathrm{E}-01$ & $3.99554 \mathrm{E}$ & 01 & $3.84037 \mathrm{E}$ & 00 & $-8.31190 \mathrm{E}-04$ & $-3.72000 \mathrm{E}$ \\
\hline 42.00 & $8.54280 \mathrm{E}-01$ & $4.00589 \mathrm{E}$ & 01 & $3.86133 \mathrm{~F}$ & 00 & $-8.30227 \mathrm{E}-04$ & $-3.74074 \mathrm{E}$ \\
\hline
\end{tabular}

$$
g_{-1}
$$

$\tan \delta_{-1}$

$3.50636 \mathrm{E} \quad 01$ $3.51650 \mathrm{E} 01$ $3.52665 \mathrm{E} \quad 01$ $3.53680 \mathrm{E} \quad 01$ $3.54695 \mathrm{E} \quad 01$

$3.55711 \mathrm{E} \quad 01$ $3.56728 \mathrm{E} \quad 01$ $3.57744 \mathrm{E} \quad 01$ $3.58762 \mathrm{E} \quad 01$ $3.59779 \mathrm{E} \quad 01$

$3.60798 \mathrm{E} \quad 0 \mathrm{l}$ $3.61816 \mathrm{E} \quad 01$ $3.62835 \mathrm{E} 01$ $3.63855 \mathrm{E} \quad 01$ $3.64875 \mathrm{E} \quad 01$

$3.65896 \mathrm{E} \quad 01$ $3.66917 \mathrm{E} \quad 01$ $3.67938 \mathrm{E} 01$ $3.68960 \mathrm{E} \quad 01$ $3.69983 \mathrm{E} \quad 01$

$3.71006 \mathrm{E} \quad 01$ $3.72029 \mathrm{E} \quad 01$ $3.73053 \mathrm{E}$ 0l $3.74077 \mathrm{E} \quad 01$ $3.75102 \mathrm{E} 01$

$3.76127 \mathrm{E} \quad 01$ $3.77153 \mathrm{E} 01$ $3.78179 \mathrm{E} 01$ $3.79206 \mathrm{E}$ ol $3.80233 \mathrm{E} 01$

$3.81261 \mathrm{E} \quad 01$ $3.82289 \mathrm{E} 01$ $3.83318 \mathrm{E} 01$

$3.84347 \mathrm{E} \quad 01$

$3.85377 \mathrm{E} 01$

$3.86407 \mathrm{E} \quad 01$ $3.87438 \mathrm{E} \quad 01$ $3.88469 \mathrm{E} \quad 01$ $3.89500 \mathrm{E} \quad 01$ $3.90533 \mathrm{E} \quad 01$

$3.91565 \mathrm{E}$ 0l $3.92598 \mathrm{E} 01$ $3.93632 \mathrm{E} 01$ $3.94666 \mathrm{E} 01$ $3.95701 \mathrm{E} \quad 01$

$3.96736 \mathrm{E} 01$ $3.97772 \mathrm{E} 01$ $3.98808 \mathrm{E} \quad 01$ $3.99845 \mathrm{E}$ 0l $4.00882 \mathrm{E} \quad 01$

$4.01920 \mathrm{E} \quad 01$ $4.02958 \mathrm{E} \quad 01$ $4.03997 \mathrm{E} \quad 01$ $4.05036 \mathrm{E} \quad 01$ $4.06076 \mathrm{E} \quad 01$

$4.07116 \mathrm{E} \quad 01$ $4.08157 \mathrm{E} \quad 01$ $4.09199 \mathrm{E} 01$

$4.10240 \mathrm{E} \quad 01$

$4.11283 \mathrm{E} \quad 01$
$4.42954 \mathrm{E} \quad 02$ $4.43757 \mathrm{E} \quad 02$ $4.44564 \mathrm{E} \quad 02$ $4.45371 \mathrm{E} \quad 02$ $4.46182 \mathrm{E} \quad 02$

$4.46993 \mathrm{E} \quad 02$ $\begin{array}{ll}4.47810 \mathrm{E} & 02\end{array}$ $4.48625 \mathrm{E} \quad 02$ $4.49444 \mathrm{E} \quad 02$ $4.50263 \mathrm{E} \quad 02$

$\begin{array}{lll}4.51086 \mathrm{E} & 02\end{array}$ $4.51911 \mathrm{E} \quad 02$ $4.52738 \mathrm{E} \quad 02$ $4.53567 \mathrm{E} \quad 02$ $4.54398 \mathrm{E} \quad 02$ $4.55231 \mathrm{E} \quad 02$ $4.56067 \mathrm{E} \quad 02$ $4.56905 \mathrm{E} \quad 02$ $4.57745 \mathrm{E} \quad 02$ $\begin{array}{ll}4.58588 \mathrm{E} & 02\end{array}$

$4.59432 \mathrm{E} \quad 02$ $4.60281 \mathrm{E} \quad 02$ $4.61130 \mathrm{E} \quad 02$ $4.61982 \mathrm{E} \quad 02$ $4.62839 \mathrm{E} \quad 02$

$4.63694 \mathrm{E} \quad 02$ $4.64554 \mathrm{E} \quad 02$ $4.65415 \mathrm{E} \quad 02$ $4.66281 \mathrm{E} \quad 02$ $4.67148 \mathrm{E} \quad 02$

$4.68018 \mathrm{E} \quad 02$ $4.68890 \mathrm{E} \quad 02$ $4.69766 \mathrm{E} \quad 02$ $4.70644 \mathrm{E} \quad 02$ $4.71525 \mathrm{E} \quad 02$ $4.72408 \mathrm{E} \quad 02$ $4.73293 \mathrm{E} \quad 02$ $4.74182 \mathrm{E} \quad 02$ $4.75076 \mathrm{E} \quad 02$ $4.75969 \mathrm{E} \quad 02$

$\begin{array}{ll}4.76866 \mathrm{E} & 02\end{array}$ $4.77768 \mathrm{E} \quad 02$ $\begin{array}{lll}4.78671 \mathrm{E} & 02\end{array}$ $4.79576 \mathrm{E} \quad 02$ $4.80486 \mathrm{E} \quad 02$

$4.81397 \mathrm{E} \quad 02$ $4.82311 \mathrm{E} \quad 02$ $4.83231 \mathrm{E} \quad 02$ $4.84152 \mathrm{E} \quad 02$ $4.85076 \mathrm{E} \quad 02$

$4.86005 \mathrm{E} \quad 02$ $4.86936 \mathrm{E} \quad 02$ $4.87869 \mathrm{E} \quad 02$ $4.88806 \mathrm{E} \quad 02$ $4.89747 \mathrm{E} \quad 02$

$4.90691 \mathrm{E} \quad 02$ $4.91638 \mathrm{E} \quad 02$ $\begin{array}{lll}4.92587 \mathrm{E} & 02\end{array}$ $4.93543 \mathrm{E} \quad 02$ $4.94500 \mathrm{E} \quad 02$ 
ELECTRON RADIAL FUNCTIONS

$$
Z=7 \quad A=14 \quad \rho=1.3 A^{1 / 3} F
$$

$\bar{F}$

$2.03461 \mathrm{E}-03$ $2.04589 \mathrm{E}-03$ $2.05720 \mathrm{E}-03$ $2.06855 \mathrm{E}-03$ $2.07992 \mathrm{E}-03$

36.40

36.50

36.60

$2.09133 \mathrm{E}-03$

36.70

36.80

36.90

37.00

37.10

37.20

37.30

37.40

37.50

37.60

37.70

37.80

37.90

38.00

38.10

38.20

38.30

38.40

38.50

38.60

38.70

38.80

38.90

39.00

39.10

39.20

39.30

39.40

39.50

39.60

39.70

39.80

39.90

40.00

$2.10276 \mathrm{E}-03$

$2.11423 \mathrm{E}-03$

$2.12573 \mathrm{E}-03$

$2.13726 \mathrm{E}-03$

$2.14882 \mathrm{E}-03$

$2.16042 \mathrm{E}-03$

$2.1720+\mathrm{E}-03$

$2.18369 \mathrm{E}-03$

$2.19538 \mathrm{E}-03$

$2.20710 \mathrm{E}-03$

$2.21885 \mathrm{E}-03$

$2.23063 \mathrm{E}-03$

$2.24244 \mathrm{E}-03$

$2.25428 \mathrm{E}-03$

$2.26615 \mathrm{E}-03$

$2.27806 \mathrm{E}-03$

$2.28999 \mathrm{E}-03$

$2.30196 \mathrm{E}-03$

$2.31396 \mathrm{E}-03$

$2.32599 \mathrm{E}-03$

$2.33805 E-03$

$2.35014 \mathrm{E}-03$

$2.36226 \mathrm{E}-03$

$2.37441 \mathrm{E}-03$

$2.38660 \mathrm{E}-03$

$2.39881 \mathrm{E}-03$

$2.41106 \mathrm{E}-03$

$2.42334 \mathrm{E}-03$

$2.43565 \mathrm{E}-03$

$2.44799 \mathrm{E}-03$

$2.46036 \mathrm{E}-03$

$2.47276 \mathrm{E}-03$

$2.48520 \mathrm{E}-03$

$2.49766 \mathrm{E}-03$

40.10

40.20

40.30

40.40

40.50

$2.51016 \mathrm{E}-03$ $2.52269 \mathrm{E}-03$ $2.53525 \mathrm{E}-03$ $2.54784 \mathrm{E}-03$ $2.56046 \mathrm{E}-03$

$40.60 \quad 2.57311 \mathrm{E}-03$

40.70

40.80

40.90

41.00

41.10

41.20

41.30

41.40

41.50

$2.58579 \mathrm{E}-03$

$2.59851 \mathrm{E}-03$

$2.61125 \mathrm{E}-03$

$2.624 .03 \mathrm{E}-03$

41.60

41.70

41.80

41.90

42.00

$2.63684 \mathrm{E}-03$

$2.64968 \mathrm{E}-03$

$2.66255 \mathrm{E}-03$

$2.67545 \mathrm{E}-03$

$2.68838 \mathrm{E}-03$

$2.7013+\mathrm{E}-03$

$2.71434 \mathrm{E}-03$

$2.72736 \mathrm{E}-03$

$2.74042 \mathrm{E}-03$

$2.75351 \mathrm{E}-03$
. $f_{2}$

$3.20583 \mathrm{E} \quad 00$ $3.22365 \mathrm{E} \quad 00$ $3.24152 \mathrm{E} \quad 00$ $3.25944 \mathrm{E} \quad 00$ $3.27740 \mathrm{E} \quad 00$

$3.29541 \mathrm{E} \quad 00$ $3.31347 \mathrm{E} \quad 00$ $3.33158 \mathrm{E} \quad 00$ $3.34974 \mathrm{E} \quad 00$ $3.36795 \mathrm{E} \quad 00$

$3.38621 \mathrm{E} \quad 00$ $3.40451 \mathrm{E} \quad 00$ $3.42286 \mathrm{E} \quad 00$ $3.44126 \mathrm{E} \quad 00$ $3.45971 \mathrm{E} \quad 00$

$3.47821 \mathrm{E} \quad 00$ $3.49675 \mathrm{E} \quad 00$ $3.51535 \mathrm{E} \quad 00$ $3.53399 \mathrm{E} \quad 00$ $3.55268 \mathrm{E} \quad 00$

$3.57142 \mathrm{E} \quad 00$ $3.59021 \mathrm{E} \quad 00$ $3.60904 \mathrm{E} \quad 00$ $3.62792 \mathrm{E} \quad 00$ $3.64686 \mathrm{E} \quad 00$

$3.66584 \mathrm{E} \quad 00$ $3.68486 \mathrm{E} \quad 00$ $3.70394 \mathrm{E} \quad 00$ $3.72307 \mathrm{E} \quad 00$ $3.74224 \mathrm{E} \quad 00$

$3.76146 \mathrm{E} \quad 00$ $3.78073 \mathrm{E} \quad 00$ $3.80005 \mathrm{E} \quad 00$

$3.81941 \mathrm{E} \quad 00$

$3.83883 \mathrm{E} \quad 00$

$3.85829 \mathrm{E} \quad 00$

$3.87780 \mathrm{E} \quad 00$

$3.89736 \mathrm{E} \quad 00$

$3.91696 \mathrm{E} \quad 00$

$3.93662 \mathrm{E} \quad 00$

$3.95632 \mathrm{E} \quad 00$ $3.97607 \mathrm{E} \quad 00$ $3.99587 \mathrm{E} \quad 00$ $4.01572 \mathrm{E} \quad 00$ $4.03561 \mathrm{E} \quad 00$

$4.05556 \mathrm{E} \quad 00$ $4.07555 \mathrm{E} \quad 00$ $4.09558 \mathrm{E} \quad 00$ $4.11567 \mathrm{E} \quad 00$ $4.13581 \mathrm{E} \quad 00$

$4.15599 \mathrm{E} \quad 00$

$4.17622 \mathrm{E} \quad 00$ $4.19650 \mathrm{E} \quad 00$ $4.21682 \mathrm{E} \quad 00$ $4.23720 \mathrm{E} \quad 00$

$4.25762 \mathrm{E} \quad 00$ $4.27809 \mathrm{E} \quad 00$ $4.29861 \mathrm{E} \quad 00$ $4.31917 \mathrm{E} \quad 00$ $4.33979 \mathrm{E} \quad 00$ $g_{2}$

$1.55887 \mathrm{E}-01$ $1.57278 \mathrm{E}-01$ $1.58679 \mathrm{E}-01$ $1.60087 \mathrm{E}-01$

$1.61503 \mathrm{E}-01$

$1.62928 \mathrm{E}-01$

$1.64361 \mathrm{E}-01$

$1.65803 \mathrm{E}-01$

$1.67253 \mathrm{E}-01$

$1.68711 \mathrm{E}-01$

$1.70177 \mathrm{E}-01$

$1.71652 \mathrm{E}-01$

$1.73136 \mathrm{E}-01$

$1.74628 \mathrm{E}-01$

$1.76128 \mathrm{E}-01$

$1.77637 \mathrm{E}-01$

$1.79154 \mathrm{E}-01$

$1.80680 \mathrm{E}-01$

$1.82215 \mathrm{E}-01$

$1.83758 \mathrm{E}-01$

$1.85310 \mathrm{E}-01$

$1.86870 \mathrm{E}-01$

$1.88439 \mathrm{E}-01$

$1.90017 \mathrm{E}-01$

$1.91603 \mathrm{E}-01$

$1.93198 \mathrm{E}-01$

$1.94802 \mathrm{E}-01$

$1.96415 \mathrm{E}-01$

$1.98037 \mathrm{E}-01$

$1.99667 \mathrm{E}-01$

$2.01306 \mathrm{E}-01$

$2.02955 \mathrm{E}-01$

$2.04612 \mathrm{E}-01$

$2.06278 \mathrm{E}-01$

$2.07953 \mathrm{E}-01$

$2.09637 \mathrm{E}-01$

$2.11329 \mathrm{E}-01$

$2.13031 \mathrm{E}-01$

$2.14742 \mathrm{E}-01$

$2.16462 \mathrm{E}-01$

2.18192E-01

$2.19930 \mathrm{E}-01$

$2.21677 \mathrm{E}-01$

$2.23434 \mathrm{E}-01$

$2.25200 \mathrm{E}-01$

$2.26974 \mathrm{E}-01$

$2.28759 \mathrm{E}-01$

$2.30552 \mathrm{E}-01$

$2.32355 \mathrm{E}-01$

$2.34167 \mathrm{E}-01$

$2.35988 \mathrm{E}-01$

$2.37819 \mathrm{E}-01$

$2.39659 \mathrm{E}-01$

$2.41508 \mathrm{E}-01$

$2.43367 \mathrm{E}-01$

$2.45236 \mathrm{E}-01$

$2.47113 \mathrm{E}-01$

$2.49001 \mathrm{E}-01$

$2.50898 \mathrm{E}-01$

$2.52804 \mathrm{E}-01$

$\tan \delta_{2}$

$f_{2}$

$-2.79595 \mathrm{E} \quad 01 \quad-1.49556 \mathrm{E}-0 \mathrm{l}$ $-2.7960+\mathrm{E} 01-1.50916 \mathrm{E}-01$ $-2.79612 \mathrm{E} \quad 0 \mathrm{l}-1.52284 \mathrm{E}-0 \mathrm{l}$ $-2.79620 \mathrm{E} \quad 01 \quad-1.53660 \mathrm{E}-01$ $-2.79628 \mathrm{E} \quad 01-1.55044 \mathrm{E}-01$

$-2.79636 \mathrm{E} \quad 01 \quad-1.56437 \mathrm{E}-01$ $-2.79644 \mathrm{E}$ ol $-1.578 .37 \mathrm{E}-0$ $-2.79651 \mathrm{E} 01 \quad-1.59246 \mathrm{E}-01$ $-2.79659 \mathrm{E}$ 01 $-1.60663 \mathrm{E}-01$

$-2.79667 \mathrm{E} \quad 01-1.62089 \mathrm{E}-01$

$-2.79675 \mathrm{E} \quad 01$

$-2.79683 \mathrm{E} \quad 01$

$-2.79690 \mathrm{E} 01$

$-2.79698 \mathrm{E} \quad 01$

$-2.79705 \mathrm{E} 01$

$-2.79713 \mathrm{E} 01$

$-2.79720 \mathrm{E} \quad 01$

$-2.79728 \mathrm{E} 01$

$\begin{array}{lll}-2.79735 \mathrm{E} & 01\end{array}$

$-2.79743 \mathrm{E} 01$

$-1.63523 \mathrm{E}-01$

$-1.64965 \mathrm{E}-01$

$-1.66415 \mathrm{E}-01$

$-1.67874 \mathrm{E}-01$

$-1.69341 \mathrm{E}-01$

$-2.79750 \mathrm{E} \quad 01$

$-2.79757 \mathrm{E} 01$

$-2.79765 \mathrm{E} 01$

$-2.79772 \mathrm{E} \quad 01$

$-2.79779 \mathrm{E} \quad 01$

$-2.79786 \mathrm{E} \quad 01$

$-2.79793 \mathrm{E} \quad 01$

$-2.79800 \mathrm{E} 01$

$-2.79807 \mathrm{E} \quad 01$

-2.79814 E 01

$-2.79821 \mathrm{E} 01$

$-2.79828 \mathrm{E} 01$

$-2.79835 \mathrm{E} \quad 01$

$-2.79842 \mathrm{E} \quad 01$

-2.79848 E 01

$-1.70817 \mathrm{E}-01$

$-1.72301 \mathrm{E}-01$

$-1.73794 \mathrm{E}-01$

$-1.75295 \mathrm{E}-01$

$-1.76805 \mathrm{E}-01$

$-1.78323 \mathrm{E}-01$

$-1.79850 \mathrm{E}-01$ 
$g_{-1}$

$2.44469 \mathrm{E}-01$

$3.86607 \mathrm{E}-01$

$5.26778 \mathrm{E}-01$

$6.65174 \mathrm{E}-01$

$8.01266 \mathrm{E}-01$

$9.34764 \mathrm{E}-01$

$1.06560 \mathrm{E} \quad 00$

$1.19386 \mathrm{E} \quad 00$

$1.31972 \mathrm{E} \quad 00$

$1.44339 \mathrm{E} \quad 00$

$1.56513 \mathrm{E} \quad 00$

$1.68516 \mathrm{E} \quad 00$

$1.80370 \mathrm{E} \quad 00$

$1.92093 \mathrm{E} \quad 00$

$2.03704 \mathrm{E} \quad 00$

$2.15218 \mathrm{E} \quad 00$

$2.26646 \mathrm{E} \quad 00$

$2.38000 \mathrm{E} \quad 00$

$2.49290 \mathrm{E} \quad 00$

$2.60523 \mathrm{E} \quad 00$

$2.71707 \mathrm{E} \quad 00$ $2.82847 \mathrm{E} \quad 00$ $2.93949 \mathrm{E} \quad 00$

$3.05017 \mathrm{E} \quad 00$

$3.16055 \mathrm{E} \quad 00$

$3.27066 \mathrm{E} \quad 00$ $3.38052 \mathrm{E} \quad 00$ $3.49017 \mathrm{E} \quad 00$ $3.59962 \mathrm{E} \quad 00$ $3.70889 \mathrm{E} \quad 00$

$3.81801 \mathrm{E} \quad 00$ $3.92698 \mathrm{E} \quad 00$ $4.03581 \mathrm{E} \quad 00$

$4.14453 \mathrm{E} \quad 00$ $4.25313 \mathrm{E} \quad 00$

$4.36163 \mathrm{E} \quad 00$ $4.47003 \mathrm{E} \quad 00$ $4.57835 \mathrm{E} \quad 00$ $4.68658 \mathrm{E} \quad 00$

$4.79474 \mathrm{E} \quad 00$

$4.90283 \mathrm{E} \quad 00$ $5.01086 \mathrm{E} \quad 00$ $5.11882 \mathrm{E} \quad 00$ $5.22672 \mathrm{E} \quad 00$ $5.33457 \mathrm{E} \quad 00$

$5.44237 \mathrm{E} \quad 00$ $5.55012 \mathrm{E} \quad 00$ $5.65783 \mathrm{E} \quad 00$ $5.76549 \mathrm{E} \quad 00$ $5.87311 \mathrm{E} \quad 00$

$5.98069 \mathrm{E} \quad 00$ $6.08823 \mathrm{E} \quad 00$ $6.19574 \mathrm{E} \quad 00$ $6.30321 \mathrm{E} \quad 00$ $6.41065 \mathrm{E} \quad 00$

$6.51806 \mathrm{E} \quad 00$ $6.62544 \mathrm{E} \quad 00$ $6.73279 \mathrm{E} \quad 00$ $6.84011 \mathrm{E} \quad 00$ $6.94741 \mathrm{E} \quad 00$ $\tan \delta_{-1}$

$-4.39128 \mathrm{E} 00$ $-8.01017 \mathrm{E} \quad 00$ $-1.17252 \mathrm{E}$ 0l $-1.53936 \mathrm{E} 01$ $-1.89838 \mathrm{E} \quad 01$

$-2.24825 \mathrm{E} \quad 01$ $-2.58832 \mathrm{E} \quad 01$ $-2.91829 \mathrm{E} 01$ $-3.23811 \mathrm{E} \quad 01$ $-3.54784 \mathrm{E} 01$ $-3.84768 \mathrm{E} \quad 01$ $-4.13788 \mathrm{E}$ 0l $-4.41874 \mathrm{E} 01$ $-4.69057 \mathrm{E} \quad 01$ $-4.95372 \mathrm{E} 01$ $-5.20851 \mathrm{E} \quad 01$ $-5.45530 \mathrm{E} \quad 01$ $-5.69439 \mathrm{E} \quad 01$ $-5.92612 \mathrm{E} \quad 01$ $-6.15080 \mathrm{E} 01$

$-6.36872 \mathrm{E} 01$ $-6.58015 \mathrm{E} \quad 01$ $-6.78539 \mathrm{E} 01$ $-6.98468 \mathrm{E} 01$ $-7.17828 \mathrm{E} 01$ $-7.36641 \mathrm{E} \quad 01$ $-7.54930 \mathrm{E}$ 0l $-7.72715 \mathrm{E}$ 01 $-7.90019 \mathrm{E}$ 0l $-8.06858 \mathrm{E} 01$

$-8.23253 \mathrm{E} \quad 01$ $-8.39219 \mathrm{E}$ 01 $-8.54773 \mathrm{E} 01$ $-8.69931 \mathrm{E} 01$ $-8.84708 \mathrm{E} 01$

$-8.99120 \mathrm{E} \quad 01$ $-9.13176 \mathrm{E} 01$ $-9.26893 \mathrm{E} 01$ $-9.40281 \mathrm{E} \quad 01$ $-9.53352 \mathrm{E} 01$

$-9.66118 \mathrm{E} 01$ $-9.78590 \mathrm{E} 01$ $-9.90776 \mathrm{E} 01$ $-1.00269 \mathrm{E} 02$ $-1.01433 \mathrm{E} 02$

$-1.02572 \mathrm{E} \quad 02$ $-1.03686 \mathrm{E} 02$ $-1.04777 \mathrm{E} 02$ $-1.05843 \mathrm{E} 02$ $-1.06888 \mathrm{E} 02$

$-1.07910 \mathrm{E} 02$ $-1.08912 \mathrm{E} 02$ $-1.09893 \mathrm{E} 02$ $-1.10854 \mathrm{E} \quad 02$ $-1.11796 \mathrm{E} 02$

$-1.12720 \mathrm{E} \quad 02$ $-1.13625 \mathrm{E} \quad 02$ $-1.14513 \mathrm{E} 02$ $-1.15384 \mathrm{E} \quad 02$ $-1.16238 \mathrm{E} 02$ 
$Z=6 \quad A=12 \quad \rho=1.1 A^{1 / 3} F$

$\bar{F}$

$4.19769 \mathrm{E}-08$ $9.30472 \mathrm{E}-08$ $1.70385 \mathrm{E}-07$ $2.73370 \mathrm{E}-07$ $4.02173 \mathrm{E}-07$

$5.57041 \mathrm{E}-07$ $7.38198 \mathrm{E}-07$

$9.45834 \mathrm{E}-07$

$1.18010 \mathrm{E}-06$

$1.44112 \mathrm{E}-06$

$1.72898 \mathrm{E}-06$ $2.04375 \mathrm{E}-06$ $2.38551 \mathrm{E}-06$ $2.75428 \mathrm{E}-06$ $3.15010 \mathrm{E}-06$

$3.57301 \mathrm{E}-06$ $4.02302 \mathrm{E}-06$ $4.50014 \mathrm{E}-06$ $5.00439 \mathrm{E}-06$ $5.53579 \mathrm{E}-06$

$6.09434 \mathrm{E}-06$ $6.68004 \mathrm{E}-06$ $7.29291 \mathrm{E}-06$ $7.93295 \mathrm{E}-06$ $8.60015 \mathrm{E}-06$

$9.29452 \mathrm{E}-06$ 1.00161 E- 05 $1.07648 \mathrm{E}-05$

$1.15407 \mathrm{E}-05$

$1.23438 \mathrm{E}-05$

$1.31740 \mathrm{E}-05$ $1.40315 \mathrm{E}-05$ $1.49161 \mathrm{E}-05$ $1.58279 \mathrm{E}-05$ $1.67669 \mathrm{E}-05$

$1.77331 \mathrm{E}-05$ $1.87264 \mathrm{E}-05$ $1.97469 \mathrm{E}-05$ $2.07946 \mathrm{E}-05$ $2.18695 \mathrm{E}-05$

$2.29716 \mathrm{E}-05$ $2.41008 \mathrm{E}-05$ 2.52573 E- 05 $2.64409 \mathrm{E}-05$ $2.76517 \mathrm{E}-05$

$2.88896 \mathrm{E}-05$ $3.01547 \mathrm{E}-05$ $3.14471 \mathrm{E}-05$ $3.27666 \mathrm{E}-05$ $3.41132 \mathrm{E}-05$

$3.54871 \mathrm{E}-05$ 3.68881 E-05 $3.83163 \mathrm{E}-05$ $3.97717 \mathrm{E}-05$ $4.12543 \mathrm{E}-05$

$4.27640 \mathrm{E}-05$ $4.43009 \mathrm{E}-05$ $4.58650 \mathrm{E}-05$ $4.74562 \mathrm{E}-05$ $4.90746 \mathrm{E}-05$ $f_{2}$

$-2.92806 \mathrm{E}-06$ $-1.70641 \mathrm{E}-05$ $-5.09374 \mathrm{E}-05$ $-1.11949 \mathrm{E}-04$ $-2.06140 \mathrm{E}-04$

$-3.38173 \mathrm{E}-04$ $-5.11470 \mathrm{E}-04$ $-7.28431 \mathrm{E}-04$ $-9.90679 \mathrm{E}-04$ $-1.29926 \mathrm{E}-03$

$-1.65484 \mathrm{E}-03$ $-2.05778 \mathrm{E}-03$ $-2.50829 \mathrm{E}-03$ $-3.00647 \mathrm{E}-03$ $-3.55231 \mathrm{E}-03$

$-4.14580 \mathrm{E}-03$ $-4.78688 \mathrm{E}-03$ $-5.47549 \mathrm{E}-03$ $-6.21155 \mathrm{E}-03$ $-6.99501 \mathrm{E}-03$

$-7.82577 \mathrm{E}-03$ $-8.70379 E-03$ $-9.62901 \mathrm{E}-03$ $-1.06014 \mathrm{E}-02$ $-1.16208 \mathrm{E}-02$

$-1.26873 \mathrm{E}-02$

$-1.38008 \mathrm{E}-02$

$-1.49612 \mathrm{E}-02$

$-1.61686 \mathrm{E}-02$

$-1.74228 \mathrm{E}-02$

$-1.87239 \mathrm{E}-02$ $-2.00719 \mathrm{E}-02$ $-2.14667 \mathrm{E}-02$ $-2.29083 \mathrm{E}-02$ $-2.43966 \mathrm{E}-02$

$-2.59317 \mathrm{E}-02$ $-2.75135 \mathrm{E}-02$ $-2.91420 \mathrm{E}-02$ $-3.08173 \mathrm{E}-02$ $-3.25392 \mathrm{E}-02$

$-3.43078 \mathrm{E}-02$ $-3.61231 \mathrm{E}-02$ $-3.79850 \mathrm{E}-02$ $-3.98936 \mathrm{E}-02$ $-4.18488 \mathrm{E}-02$

$-4.38506 \mathrm{E}-02$ $-4.58991 \mathrm{E}-02$ $-4.79941 \mathrm{E}-02$ $-5.01358 \mathrm{E}-02$ $-5.23240 \mathrm{E}-02$

$-5.45588 \mathrm{E}-02$ $-5.68402 \mathrm{E}-02$ $-5.91681 \mathrm{E}-02$ $-6.15426 \mathrm{E}-02$ $-6.39637 \mathrm{E}-02$

$-6.64313 \mathrm{E}-02$ $-6.89455 \mathrm{E}-02$ $-7.15061 \mathrm{E}-02$ $-7.41134 \mathrm{E}-02$ $-7.67671 \mathrm{E}-02$ $g_{2}$

$-3.69642 \mathrm{E}-08$ $-2.15750 \mathrm{E}-07$ $-6.45634 \mathrm{E}-07$ $-1.42378 \mathrm{E}-06$ $-2.63274 \mathrm{E}-06$

$-4.34027 \mathrm{E}-06$ $-6.60078 \mathrm{E}-06$ $-9.45777 \mathrm{E}-06$ $-1.29464 \mathrm{E}-05$ $-1.70958 \mathrm{E}-05$

$-2.19307 \mathrm{E}-05$

$-2.74733 \mathrm{E}-05$

$-3.37436 \mathrm{E}-05$

$-4.07606 \mathrm{E}-05$

$-4.85425 \mathrm{E}-05$

$-5.71071 \mathrm{E}-05$

$-6.64718 \mathrm{E}-05$

$-7.66543 \mathrm{E}-05$

$-8.76718 \mathrm{E}-05$

$-9.95418 \mathrm{E}-05$

$-1.12282 \mathrm{E}-04$

$-1.25909 \mathrm{E}-04$

- 1.40441 E-04

$-1.55896 \mathrm{E}-04$

$-1.72291 \mathrm{E}-04$

$-1.89644 \mathrm{E}-04$

$-2.07973 \mathrm{E}-04$

$-2.27295 \mathrm{E}-04$

$-2.47629 \mathrm{E}-04$

-2.68991 E-04

$-2.91401 \mathrm{E}-04$

$-3.14876 \mathrm{E}-04$

$-3.39434 \mathrm{E}-04$

$-3.65092 \mathrm{E}-04$

$-3.91870 \mathrm{E}-04$

$-4.19785 \mathrm{E}-04$

$-4.48854 \mathrm{E}-04$

$-4.79097 \mathrm{E}-04$

$-5.10531 \mathrm{E}-04$

$-5.43174 \mathrm{E}-04$

$-5.77044 \mathrm{E}-04$

$-6.12159 \mathrm{E}-04$

$-6.48538 \mathrm{E}-04$

$-6.86198 \mathrm{E}-04$

$-7.25158 \mathrm{E}-04$

$-7.65435 \mathrm{E}-04$

$-8.07048 \mathrm{E}-04$

$-8.50015 \mathrm{E}-04$

$-8.94354 \mathrm{E}-04$

$-9.40082 \mathrm{E}-04$

$-9.87220 \mathrm{E}-04$

$-1.03578 \mathrm{E}-03$

$-1.08579 \mathrm{E}-03$

$-1.13726 \mathrm{E}-03$

$-1.19021 \mathrm{E}-03$

$-1.24466 \mathrm{E}-03$

$-1.30063 \mathrm{E}-03$

$-1.35813 \mathrm{E}-03$

$-1.41719 \mathrm{E}-03$

$-1.47781 \mathrm{E}-03$ $\tan \delta_{2}$ $f_{-2}$

$2.32085 \mathrm{E} \quad 00 \quad-5.79498 \mathrm{E}-07$ $4.82351 \mathrm{E} \quad 00 \quad-1.72261 \mathrm{E}-06$ $7.18406 \mathrm{E} \quad 00 \quad-3.48736 \mathrm{E}-06$ $9.40344 \mathrm{E} \quad 00 \quad-5.87039 \mathrm{E}-06$ $\begin{array}{lll}1.14638 \mathrm{E} & 01 & -8.86810 \mathrm{E}-06\end{array}$

$1.33544 \mathrm{E} \quad 01$ $\begin{array}{ll}1.50732 \mathrm{E} & 01\end{array}$ $1.66252 \mathrm{E} \quad 01$ $1.80205 \mathrm{E} \quad 01$ $1.92715 \mathrm{E} \quad 01$

$2.03920 \mathrm{E} \quad 01$ $2.13956 \mathrm{E} 01$ $2.22954 \mathrm{E} 01$ $2.31034 \mathrm{E} 01$ $2.38305 \mathrm{E} \quad 01$

$2.44863 \mathrm{E} \quad 01$ $2.50793 \mathrm{E} 01$ $2.56170 \mathrm{E} \quad 01$ $2.61059 \mathrm{E} \quad 01$ $2.65515 \mathrm{E} 01$

$2.69588 \mathrm{E} \quad 01$ $2.73321 \mathrm{E}$ 01 $2.76751 \mathrm{E} 01$ $2.79911 \mathrm{E} \quad 01$

$2.82828 \mathrm{E} \quad 01$

$2.85528 \mathrm{E} \quad 01$

$2.88032 \mathrm{E} \quad 01$

$2.90360 \mathrm{E} 01$

$2.92528 \mathrm{E} \quad 01$

$2.94551 \mathrm{E} 01$

$2.96443 \mathrm{E} \quad 01$ $2.98215 \mathrm{E} 01$ $2.99877 \mathrm{E} \quad 01$ $3.01440 \mathrm{E} \quad 01$

$3.02911 \mathrm{E} 01$

$3.04298 \mathrm{E} \quad 01$ $3.05607 \mathrm{E} \quad 01$

$3.06844 \mathrm{E} \quad 01$

$3.08016 \mathrm{E} \quad 01$

$3.09127 \mathrm{E} \quad 01$

$3.10181 \mathrm{E} \quad 01$

$3.11182 \mathrm{E} 01$

$3.12135 \mathrm{E}$ 01

$3.13042 \mathrm{E} 01$

$3.13907 \mathrm{E} 01$

$3.14732 \mathrm{E} \quad 01$

$3.15520 \mathrm{E}$ 01

$3.16273 \mathrm{E} 01$

$3.16994 \mathrm{E} \quad 01$

$3.17684 \mathrm{E} \quad 01$

$3.18346 \mathrm{E}$ 01 $3.18981 \mathrm{E} 01$ $3.19590 \mathrm{E} 01$

$3.20176 \mathrm{E} \quad 01$

3.20739 E 01

$3.21280 \mathrm{E} \quad 01$

$3.21802 \mathrm{E} \quad 01$

$3.22304 \mathrm{E} \quad 01$

$3.22788 \mathrm{E} \quad 01$

$3.23255 \mathrm{E} 01$

$-1.24781 \mathrm{E}-05$ $-1.67002 \mathrm{E}-05$ $-2.15373 \mathrm{E}-05$ $-2.69950 \mathrm{E}-05$ $-3.30816 \mathrm{E}-05$

$-3.98077 \mathrm{E}-05$ $-4.71856 \mathrm{E}-05$ $-5.52294 \mathrm{E}-05$ $-6.39539 \mathrm{E}-05$ $-7.33749 \mathrm{E}-05$

$-8.35090 \mathrm{E}-05$ $-9.43731 \mathrm{E}-05$ $-1.05984 \mathrm{E}-04$ $-1.18361 \mathrm{E}-04$ $-1.31519 \mathrm{E}-04$

$-1.45478 \mathrm{E}-04$ $-1.60256 \mathrm{E}-04$ $-1.75870 \mathrm{E}-04$ $-1.92339 \mathrm{E}-04$ $-2.09680 \mathrm{E}-04$

$-2.27912 \mathrm{E}-04$ $-2.47054 \mathrm{E}-04$ $-2.67122 \mathrm{E}-04$ $-2.88137 \mathrm{E}-04$ $-3.10115 \mathrm{E}-04$

$-3.33075 \mathrm{E}-04$ $-3.57035 \mathrm{E}-04$ $-3.82014 \mathrm{E}-04$ $-4.08029 \mathrm{E}-04$ $-4.35099 \mathrm{E}-04$

$-4.63243 \mathrm{E}-04$ $-4.92478 \mathrm{E}-04$ $-5.22822 \mathrm{E}-04$ $-5.54295 \mathrm{E}-04$ $-5.86913 \mathrm{E}-04$

$-6.20696 \mathrm{E}-04$ $-6.55661 \mathrm{E}-04$ 
$\tan \delta_{1}$

$f_{-1}$

$1.36608 \mathrm{E}-03$ $1.42229 \mathrm{E}-03$

$1.47673 \mathrm{E}-03$

$1.52946 \mathrm{E}-03$

$1.58059 \mathrm{E}-03$

$1.63018 \mathrm{E}-03$

$1.67828 \mathrm{E}-03$

$1.72499 \mathrm{E}-03$

$1.77033 \mathrm{E}-03$

$1.81439 \mathrm{E}-03$

$1.85720 \mathrm{E}-03$

$1.89882 \mathrm{E}-03$

$1.93931 \mathrm{E}-03$

$1.97870 \mathrm{E}-03$

$2.01704 \mathrm{E}-03$

$2.05436 \mathrm{E}-03$

$2.09072 \mathrm{E}-03$

$2.12615 \mathrm{E}-03$

$2.16066 \mathrm{E}-03$

$2.19432 \mathrm{E}-03$

$2.22714 \mathrm{E}-03$

$2.25915 \mathrm{E}-03$

$2.29039 \mathrm{E}-03$

2.32087 E-03

$2.35064 \mathrm{E}-03$

$2.37971 \mathrm{E}-03$

$2.40810 \mathrm{E}-03$

$2.43584 \mathrm{E}-03$

$2.46295 \mathrm{E}-03$

2.48945 E-03

$2.51537 \mathrm{E}-03$

$2.54071 \mathrm{E}-03$

$2.56550 \mathrm{E}-03$

$2.58975 \mathrm{E}-03$

$2.61348 \mathrm{E}-03$

$2.63672 \mathrm{E}-03$

$2.65946 \mathrm{E}-03$

$2.68172 \mathrm{E}-03$

$2.70354 \mathrm{E}-03$

$2.72491 \mathrm{E}-03$

$2.74584 \mathrm{E}-03$

$2.76637 \mathrm{E}-03$

$2.78647 \mathrm{E}-03$

$2.80619 \mathrm{E}-03$

2.82551 E- 03

$2.84446 \mathrm{E}-03$

$2.86305 \mathrm{E}-03$

$2.88129 \mathrm{E}-03$

$2.89918 \mathrm{E}-03$

$2.91673 \mathrm{E}-03$

$2.93396 \mathrm{E}-03$ $2.95087 \mathrm{E}-03$

$2.96748 \mathrm{E}-03$

$2.98378 \mathrm{E}-03$

$2.99978 \mathrm{E}-03$

$3.01551 \mathrm{E}-03$ $3.03095 \mathrm{E}-03$ $3.04612 \mathrm{E}-03$

$3.06102 \mathrm{E}-03$

$3.07566 \mathrm{E}-03$
$-2.03138 \mathrm{E}-01$ $-2.07765 \mathrm{E}-01$ $-2.12439 \mathrm{E}-01$ $-2.17159 \mathrm{E}-01$ $-2.21924 \mathrm{E}-01$

$-2.26736 \mathrm{E}-0 \mathrm{i}$ $-2.31594 \mathrm{E}-01$ $-2.36498 \mathrm{E}-01$ $-2.41448 \mathrm{E}-01$ $-2.46444 \mathrm{E}-01$

$-2.51486 \mathrm{E}-01$ $-2.56574 \mathrm{E}-01$ $-2.61708 \mathrm{E}-01$ $-2.66889 \mathrm{E}-01$ $-2.72115 \mathrm{E}-01$

$-2.77388 \mathrm{E}-01$ $-2.82706 \mathrm{E}-01$ $-2.88071 \mathrm{E}-01$ $-2.93482 \mathrm{E}-01$ $-2.98939 \mathrm{E}-01$

$-3.04442 \mathrm{E}-01$ $-3.09991 \mathrm{E}-01$ $-3.15586 \mathrm{E}-01$ $-3.21228 \mathrm{E}-01$ $-3.26915 \mathrm{E}-01$

$-3.32648 \mathrm{E}-01$ $-3.38428 \mathrm{E}-01$ $-3.44254 \mathrm{E}-01$ $-3.50125 \mathrm{E}-01$ $-3.56043 \mathrm{E}-01$

$-3.62007 \mathrm{E}-01$ $-3.68016 \mathrm{E}-01$ $-3.74072 \mathrm{E}-01$ $-3.80174 \mathrm{E}-01$ $-3.86322 \mathrm{E}-01$

$-3.92516 \mathrm{E}-01$ $-3.98756 \mathrm{E}-01$ $-4.05042 \mathrm{E}-01$ $-4.11374 \mathrm{E}-01$ $-4.17752 \mathrm{E}-01$

$-4.24176 \mathrm{E}-01$ $-4.30646 \mathrm{E}-01$ $-4.37163 \mathrm{E}-01$ $-4.43725 \mathrm{E}-01$ $-4.50333 \mathrm{E}-01$

$-4.56987 \mathrm{E}-01$ $-4.63687 \mathrm{E}-01$ $-4.70433 \mathrm{E}-01$ $-4.77225 \mathrm{E}-01$ $-4.84063 \mathrm{E}-01$

$-4.90947 \mathrm{E}-01$ $-4.97877 \mathrm{E}-01$ $-5.04853 \mathrm{E}-01$ $-5.11875 \mathrm{E}-01$ $-5.18942 \mathrm{E}-01$

$-5.26056 \mathrm{E}-01$ $-5.33216 \mathrm{E}-01$ $-5.40421 \mathrm{E}-01$ $-5.47673 \mathrm{E}-01$ $-5.54970 \mathrm{E}-01$

$$
g_{-1}
$$
$\tan \delta_{-1}$

$7.05468 \mathrm{E} \quad 00$ $7.16192 \mathrm{E} \quad 00$ $7.26914 \mathrm{E} \quad 00$ $7.37634 \mathrm{E} \quad 00$ $7.48351 \mathrm{E} \quad 00$

$7.59066 \mathrm{E} \quad 00$ $7.69779 \mathrm{E} \quad 00$ $7.80489 \mathrm{E} \quad 00$ $7.91198 \mathrm{E} \quad 00$ 8.01905 E 00

$8.12609 \mathrm{E} \quad 00$ $8.23311 \mathrm{E} \quad 00$ $8.34012 \mathrm{E} \quad 00$ $8.44711 \mathrm{E} \quad 00$ $8.55408 \mathrm{E} \quad 00$

$8.66103 \mathrm{E} \quad 00$ $8.76796 \mathrm{E} \quad 00$ $8.87488 \mathrm{E} \quad 00$ $8.98178 \mathrm{E} \quad 00$ $9.08866 \mathrm{E} \quad 00$

$9.19552 \mathrm{E} \quad 00$ $9.30237 \mathrm{E} \quad 00$ $9.40920 \mathrm{E} \quad 00$ $9.51601 \mathrm{E} \quad 00$ $9.62281 \mathrm{E} \quad 00$

$9.72960 \mathrm{E} \quad 00$ $9.83636 \mathrm{E} \quad 00$ $9.94312 \mathrm{E} \quad 00$ $1.00499 \mathrm{E} 01$

$1.01566 \mathrm{E} \quad 01$

$1.02633 \mathrm{E} \quad 01$ $1.03700 \mathrm{E} \quad 01$ $1.04766 \mathrm{E} \quad 01$ $1.05833 \mathrm{E} \quad 01$ $1.06900 \mathrm{E} \quad 01$

$\begin{array}{ll}1.07966 \mathrm{E} & 01\end{array}$ $1.09032 \mathrm{E} \quad 01$

$1.10098 \mathrm{E} \quad 01$

$1.11164 \mathrm{E} \quad 01$

$1.12230 \mathrm{E} \quad 01$

$1.13295 \mathrm{E} \quad 01$ $1.14361 \mathrm{E} \quad 01$ $1.15426 \mathrm{E} \quad 01$ $1.16491 \mathrm{E} \quad 01$

$1.17556 \mathrm{E}$ 01

$1.18621 \mathrm{E} 01$

$1.19686 \mathrm{E} 01$

$1.20751 \mathrm{E} \quad 01$

$1.21815 \mathrm{E} \quad 01$

$1.22879 \mathrm{E} \quad 01$

$1.23944 \mathrm{E} \quad 01$ $1.25008 \mathrm{E} \quad 01$ $1.26072 \mathrm{E} \quad 01$

$1.27135 \mathrm{E} \quad 01$

$1.28199 \mathrm{E} 01$

$1.29262 \mathrm{E} \quad 01$ $1.30326 \mathrm{E} \quad 01$ $1.31389 \mathrm{E} \quad 01$

$1.32452 \mathrm{E} \quad 01$

$1.33515 \mathrm{E} 01$
$-1.17076 \mathrm{E} \quad 02$ $-1.17899 \mathrm{E} 02$ $-1.18706 \mathrm{E} \quad 02$ -1.19499 E 02 -1.20278 E 02 $\begin{array}{ll}-1.21042 \mathrm{E} & 02\end{array}$ $-1.21793 \mathrm{E} \quad 02$ $-1.22531 \mathrm{E} 02$ $-1.23256 \mathrm{E} 02$ $-1.23969 \mathrm{E} \quad 02$

$-1.24669 \mathrm{E} \quad 02$ $-1.25358 \mathrm{E} \quad 02$ $-1.26035 \mathrm{E} \quad 02$ $-1.26701 \mathrm{E} 02$ $-1.27356 \mathrm{E} \quad 02$

$\begin{array}{ll}-1.28001 \mathrm{E} & 02\end{array}$ $-1.28635 \mathrm{E} 02$ $-1.29259 \mathrm{E} \quad 02$ $-1.29873 \mathrm{E} \quad 02$ -1.30477 E 02

$-1.31072 \mathrm{E} \quad 02$ $-1.31658 \mathrm{E} 02$ $-1.32235 \mathrm{E} \quad 02$ $-1.32803 \mathrm{E} \quad 02$ $-1.33363 \mathrm{E} 02$ $-1.33914 \mathrm{E} \quad 02$ $-1.34457 \mathrm{E} \quad 02$ $-1.34992 \mathrm{E} \quad 02$ $-1.35520 \mathrm{E} \quad 02$ $-1.36040 \mathrm{E} 02$

$\begin{array}{ll}-1.36553 \mathrm{E} & 02\end{array}$ $-1.37058 \mathrm{E} 02$ $-1.37556 \mathrm{E} 02$ $-1.38047 \mathrm{E} \quad 02$ $-1.38532 \mathrm{E} 02$

$-1.39010 \mathrm{E} \quad 02$ $-1.39481 \mathrm{E} 02$ $-1.39947 \mathrm{E} \quad 02$ $-1.40406 \mathrm{E} \quad 02$ -1.40859 E 02

$\begin{array}{lll}-1.41306 \mathrm{E} & 02\end{array}$ $-1.41747 \mathrm{E} \quad 02$ $-1.42182 \mathrm{E} 02$ $-1.42612 \mathrm{E} 02$ $-1.43037 \mathrm{E} \quad 02$

$-1.43456 \mathrm{E} 02$ $-1.43870 \mathrm{E} 02$ $-1.44279 \mathrm{E} \quad 02$ $-1.44683 \mathrm{E} 02$ -1.45082E 02

$-1.45476 \mathrm{E} \quad 02$ $-1.45865 \mathrm{E} 02$ $-1.46250 \mathrm{E} 02$ $-1.46630 \mathrm{E} \quad 02$ $-1.47006 \mathrm{E} 02$ $-1.47377 \mathrm{E} \quad 02$ $-1.47744 \mathrm{E} \quad 02$ $-1.48107 \mathrm{E} \quad 02$ $-1.48466 \mathrm{E} 02$ $-1.48821 \mathrm{E} 02$ 
$\bar{F}$

$5.07203 \mathrm{E}-05$

$5.23930 \mathrm{E}-05$

$5.40930 \mathrm{E}-05$

$5.58201 \mathrm{E}-05$

$5.75743 \mathrm{E}-05$

$5.93558 \mathrm{E}-05$

$6.11644 \mathrm{E}-05$

$6.30002 \mathrm{E}-05$

$6.48632 \mathrm{E}-05$

$6.67534 \mathrm{E}-05$

$6.86706 \mathrm{E}-05$

$7.06151 \mathrm{E}-05$

$7.25868 \mathrm{E}-05$

$7.45856 \mathrm{E}-05$

$7.66116 \mathrm{E}-05$

$7.86648 \mathrm{E}-05$

$8.07451 \mathrm{E}-05$

$8.28525 \mathrm{E}-05$

$8.49872 \mathrm{E}-05$

$8.71490 \mathrm{E}-05$

$8.93380 \mathrm{E}-05$

$9.15541 \mathrm{E}-05$

$9.37975 \mathrm{E}-05$

$9.60679 \mathrm{E}-05$

$9.83655 \mathrm{E}-05$

$1.00690 \mathrm{E}-04$

$1.03042 \mathrm{E}-04$

$1.05421 \mathrm{E}-04$

$1.07828 \mathrm{E}-04$

$1.10261 \mathrm{E}-04$

$1.12722 \mathrm{E}-04$

$1.15210 \mathrm{E}-04$

$1.17724 \mathrm{E}-04$

$1.20267 \mathrm{E}-04$

$1.22836 \mathrm{E}-04$

$1.25432 \mathrm{E}-04$

$1.28056 \mathrm{E}-04$

$1.30707 \mathrm{E}-04$

$1.33385 \mathrm{E}-04$

$1.36090 \mathrm{E}-04$

$1.38822 \mathrm{E}-04$

$1.41581 \mathrm{E}-04$

$1.44368 \mathrm{E}-04$

$1.47182 \mathrm{E}-04$

$1.50023 \mathrm{E}-04$

$1.52891 \mathrm{E}-04$

$1.55786 \mathrm{E}-04$

$1.58708 \mathrm{E}-04$

$1.61658 \mathrm{E}-04$

$1.64634 \mathrm{E}-04$

$1.67638 \mathrm{E}-04$

$1.70669 \mathrm{E}-04$

$1.73727 \mathrm{E}-04$

$1.76813 \mathrm{E}-04$

$1.79925 \mathrm{E}-04$

$1.83065 \mathrm{E}-04$

$1.86231 \mathrm{E}-04$

$1.89425 \mathrm{E}-04$

$1.92646 \mathrm{E}-04$

$1.95895 \mathrm{E}-04$. $f_{2}$

$g_{2}$

$\tan \delta_{2}$

$f_{-2}$

$-7.94674 \mathrm{E}-02$

$-8.22141 \mathrm{E}-02$

$-8.50074 \mathrm{E}-02$

$-8.78472 \mathrm{E}-02$

$-9.07334 \mathrm{E}-02$

$-9.36662 \mathrm{E}-02$

$-9.66454 \mathrm{E}-02$

$-9.96711 \mathrm{E}-02$

$-1.02743 \mathrm{E}-01$

$-1.05862 \mathrm{E}-01$

$-1.09027 \mathrm{E}-01$

$-1.12239 \mathrm{E}-01$

$-1.15497 \mathrm{E}-01$

$-1.18801 \mathrm{E}-01$

$-1.22152 \mathrm{E}-01$

$-1.25550 \mathrm{E}-01$

$-1.28993 \mathrm{E}-01$

$-1.32484 \mathrm{E}-01$

$-1.36020 \mathrm{E}-01$

$-1.39603 \mathrm{E}-01$

$-1.43233 \mathrm{E}-01$

$-1.46909 \mathrm{E}-01$

$-1.50631 \mathrm{E}-01$

$-1.54400 \mathrm{E}-01$

$-1.58215 \mathrm{E}-01$

$-1.62077 \mathrm{E}-01$

$-1.65984 \mathrm{E}-01$

$-1.69939 \mathrm{E}-01$

$-1.73939 \mathrm{E}-01$

$-1.77987 \mathrm{E}-01$

$-1.82080 \mathrm{E}-01$

$-1.86220 \mathrm{E}-01$

$-1.90406 \mathrm{E}-01$

$-1.94638 \mathrm{E}-01$

$-1.98917 \mathrm{E}-01$

$-2.03242 \mathrm{E}-01$

$-2.07614 \mathrm{E}-01$

$-2.12032 \mathrm{E}-01$

$-2.16496 \mathrm{E}-01$

$-2.21007 \mathrm{E}-01$

$-2.25564 \mathrm{E}-01$

$-2.30167 \mathrm{E}-01$

$-2.34816 \mathrm{E}-01$

$-2.39512 \mathrm{E}-01$

$-2.44254 \mathrm{E}-01$

$-2.49043 \mathrm{E}-01$

$-2.53878 \mathrm{E}-01$

$-2.58759 \mathrm{E}-01$

$-2.63686 \mathrm{E}-01$

$-2.68660 \mathrm{E}-01$

$-2.73680 \mathrm{E}-01$

$-2.78746 \mathrm{E}-01$

$-2.83858 \mathrm{E}-01$

$-2.89017 \mathrm{E}-01$

$-2.94222 \mathrm{E}-01$

$-2.99473 \mathrm{E}-01$

$-3.04770 \mathrm{E}-01$

$-3.10114 \mathrm{E}-01$

$-3.15504 \mathrm{E}-01$

$-3.20940 \mathrm{E}-01$
$-1.54003 \mathrm{E}-03$

$-1.60385 \mathrm{E}-03$

$-1.66930 \mathrm{E}-03$

$-1.73639 \mathrm{E}-03$

$-1.80515 \mathrm{E}-03$

$-1.87558 \mathrm{E}-03$

$-1.94771 \mathrm{E}-03$

$-2.02156 \mathrm{E}-03$

$-2.09714 \mathrm{E}-03$

$-2.17448 \mathrm{E}-03$

$-2.25358 \mathrm{E}-03$

$-2.33448 \mathrm{E}-03$

$-2.41718 \mathrm{E}-03$

$-2.50171 \mathrm{E}-03$

$-2.58807 \mathrm{E}-03$

$-2.67630 \mathrm{E}-03$

$-2.76641 \mathrm{E}-03$

$-2.85842 \mathrm{E}-03$

$-2.95234 \mathrm{E}-03$

$-3.04820 \mathrm{E}-03$

$-3.14600 \mathrm{E}-03$

$-3.24578 \mathrm{E}-03$

$-3.34755 \mathrm{E}-03$

$-3.45132 \mathrm{E}-03$

$-3.55711 \mathrm{E}-03$

$-3.66495 \mathrm{E}-03$

$-3.77484 \mathrm{E}-03$

$-3.88682 \mathrm{E}-03$

$-4.00089 \mathrm{E}-0.3$

$-4.11708 \mathrm{E}-03$

$-4.23540 \mathrm{E}-03$

$-4.35586 \mathrm{E}-03$

$-4.47850 \mathrm{E}-03$

$-4.60332 \mathrm{E}-03$

$-4.73035 \mathrm{E}-03$

$-4.85960 \mathrm{E}-03$

$-4.99110 \mathrm{E}-03$

$-5.12485 \mathrm{E}-03$

$-5.26088 \mathrm{E}-03$

$-5.39920 \mathrm{E}-03$

$-5.53984 \mathrm{E}-03$

$-5.68281 \mathrm{E}-03$

$-5.82813 \mathrm{E}-03$

$-5.97581 \mathrm{E}-03$

$-6.12588 \mathrm{E}-03$

$-6.27836 \mathrm{E}-03$

$-6.43325 \mathrm{E}-03$

$-6.59059 \mathrm{E}-03$

$-6.75038 \mathrm{E}-03$

$-6.91265 \mathrm{E}-03$

$-7.07741 \mathrm{E}-03$

$-7.24469 \mathrm{E}-03$

$-7.41449 \mathrm{E}-03$

$-7.58684 \mathrm{E}-03$

$-7.76176 \mathrm{E}-03$

$-7.93926 \mathrm{E}-03$

$-8.11937 \mathrm{E}-03$

$-8.30209 \mathrm{E}-03$

$-8.67547 \mathrm{E}-03$
$-8.48745 \mathrm{E}-03$
$3.23706 \mathrm{E}$ 0l $-1.56886 \mathrm{E}-03$

$3.24141 \mathrm{E}$ 01 $-1.63129 \mathrm{E}-03$

$3.24562 \mathrm{E}$ 0l $-1.69529 \mathrm{E}-03$

$3.24968 \mathrm{E} \quad 01-1.76087 \mathrm{E}-03$

$3.25362 \mathrm{E}$ 0l $-1.82805 \mathrm{E}-03$

$3.25742 \mathrm{E} \quad 01 \quad-1.89685 \mathrm{E}-03$

$3.26111 \mathrm{E} 01-1.96729 \mathrm{E}-03$

$3.26468 \mathrm{E}$ 01 $-2.03938 \mathrm{E}-03$

$3.26814 \mathrm{E} \quad 01 \quad-2.11314 \mathrm{E}-03$

$3.27149 \mathrm{E} 01 \quad-2.18860 \mathrm{E}-03$

$3.27475 \mathrm{E}$ ol $-2.26576 \mathrm{E}-03$

$3.27790 \mathrm{E} \quad 01 \quad-2.34465 \mathrm{E}-03$

$3.28097 \mathrm{E}$ 0l $-2.42529 \mathrm{E}-03$

$3.28395 \mathrm{E} \quad 01 \quad-2.50769 \mathrm{E}-03$

$3.28684 \mathrm{E} \quad 01 \quad-2.59187 \mathrm{E}-03$

$3.28966 \mathrm{E} \quad 01$

$3.29239 \mathrm{E} \quad 01$

$3.29505 \mathrm{E} \quad 01$

$3.29764 \mathrm{E}$ 01

$3.30016 \mathrm{E} \quad 01$

$3.30261 \mathrm{E} \quad 01$

$3.30500 \mathrm{E}$ 01

$3.30733 \mathrm{E} 01$

$3.30961 \mathrm{E} \quad 01$

$3.31182 \mathrm{E} \quad 01$

$3.31398 \mathrm{E} \quad 01$

$3.31609 \mathrm{E} \quad 01$

$3.31814 \mathrm{E} \quad 01$

$3.32015 \mathrm{E} \quad 01$

$3.32211 \mathrm{E} \quad 01$

$3.32402 \mathrm{E} \quad 01$

$3.32589 \mathrm{E} \quad 01$

$3.32772 \mathrm{E}$ 01

$3.32950 \mathrm{E}$ 01

$3.33125 \mathrm{E}$ 0l

$3.33296 \mathrm{E} \quad 01$

$3.33463 \mathrm{E} \quad 01$

$3.33626 \mathrm{E} \quad 01$

$3.33786 \mathrm{E} \quad 01$

$3.33943 \mathrm{E}$ 01

$3.34096 \mathrm{E} \quad 01$

$3.34246 \mathrm{E}$ 01

$3.34393 \mathrm{E}$ 01

$3.34537 \mathrm{E}$ 01

$3.34678 \mathrm{E} 01$

$3.34817 \mathrm{E} \quad 01$

$3.34952 \mathrm{E}$ ol

$3.35085 \mathrm{E} \quad 01$

$3.35215 \mathrm{E}$ 01

$3.35343 \mathrm{E} \quad 01$

$3.35469 \mathrm{E} \quad 01$

$3.35592 \mathrm{E}$ 01

$3.35713 \mathrm{E}$ 01

$3.35831 \mathrm{E}$ 01

$3.35947 \mathrm{E}$ 0l

$3.36062 \mathrm{E} 01$

$3.36174 \mathrm{E} \quad 01$

$3.36284 \mathrm{E}$ 01

$3.36392 \mathrm{E} 01$

$3.36499 \mathrm{E} \quad 01$

$-2.67786 \mathrm{E}-03$

$-2.70566 \mathrm{E}-03$

$-2.85530 \mathrm{E}-03$

$-2.94679 \mathrm{E}-03$

$-3.04015 \mathrm{E}-03$

$-3.13541 \mathrm{E}-03$

$-3.23257 \mathrm{E}-03$

$-3.33166 \mathrm{E}-03$

$-3.43269 \mathrm{E}-03$

$-3.53569 \mathrm{E}-03$

$-3.64067 \mathrm{E}-03$

$-3.74765 \mathrm{E}-03$

$-3.85664 \mathrm{E}-03$

$-3.96768 \mathrm{E}-03$

$-4.08076 \mathrm{E}-03$

$-4.19592 \mathrm{E}-03$

$-4.31316 \mathrm{E}-03$

$-4.43251 \mathrm{E}-03$

$-4.55399 \mathrm{E}-03$

$-4.67762 \mathrm{E}-03$

$-4.80340 E-03$

$-4.93137 \mathrm{E}-03$

$-5.06153 \mathrm{E}-03$

$-5.19391 \mathrm{E}-03$

$-5.32852 \mathrm{E}-03$

$-5.46539 \mathrm{E}-03$

$-5.60453 \mathrm{E}-03$

$-5.74595 \mathrm{E}-03$

$-5.88968 \mathrm{E}-03$

$-6.03574 \mathrm{E}-03$

$-6.18414 \mathrm{E}-03$

$-6.33490 \mathrm{E}-03$

$-6.48804 \mathrm{E}-03$

$-6.64357 \mathrm{E}-03$

$-6.80153 \mathrm{E}-03$

$-6.96191 \mathrm{E}-03$

$-7.12474 \mathrm{E}-03$

$-7.29005 E-03$

$-7.45784 \mathrm{E}-03$

$-7.62814 \mathrm{E}-03$

$-7.80095 \mathrm{E}-03$

-7.97631 E-03 
$$
\text { p }
$$

12.10

12.20

12.30

12.40

12.50

12.60

12.70

12.80

12.90

13.00

13.10

13.20

13.30

13.40

13.50

13.60

13.70

13.80

13.90

14.00

14.10

14.20

14.30

14.40

14.50

14.60

14.70

14.80

14.90

15.00

15.10

15.20

15.30

15.40

15.50

15.60

15.70

15.80

15.90

16.00

16.10

16.20

16.30

16.40

16.50

16.60

16.70

16.80

16.90

17.00

17.10

17.20

17.30

17.40

17.50

17.60

17.70

17.80

17.90

18.00
$F_{0}$

$1.15376 \mathrm{E} \quad 00$ $1.15374 \mathrm{E} \quad 00$

$1.15371 \mathrm{E} \quad 00$

$1.15368 \mathrm{E} \quad 00$

$1.15366 \mathrm{E} \quad 00$

$1.15363 \mathrm{E} \quad 00$

$1.15361 \mathrm{E} \quad 00$

$1.15358 \mathrm{E} \quad 00$

$1.15356 \mathrm{E} \quad 00$

$1.15353 \mathrm{E} \quad 00$

$1.15351 \mathrm{E} \quad 00$

$1.15349 \mathrm{E} \quad 00$

$1.15346 \mathrm{E} \quad 00$

$1.15344 \mathrm{E} \quad 00$

$1.15342 \mathrm{E} 00$

$1.15340 \mathrm{E} \quad 00$ $1.15337 \mathrm{E} \quad 00$

$1.15335 \mathrm{E} \quad 00$

$1.15333 \mathrm{E} \quad 00$

$1.15331 \mathrm{E} \quad 00$

$1.15329 \mathrm{E} \quad 00$

$1.15327 \mathrm{E} \quad 00$

$1.15325 \mathrm{E} \quad 00$

$1.15323 \mathrm{E} \quad 00$

$1.15321 \mathrm{E} \quad 00$

$1.15319 \mathrm{E} \quad 00$

$1.15317 \mathrm{E} \quad 00$

$1.15315 \mathrm{E} \quad 00$

$1.15313 \mathrm{E} \quad 00$

$1.15311 \mathrm{E} \quad 00$

$1.15309 \mathrm{E} \quad 00$

$1.15307 \mathrm{E} \quad 00$

$1.15305 \mathrm{E} \quad 00$

$1.15303 \mathrm{E} \quad 00$

$1.15301 \mathrm{E} \quad 00$

$1.15299 \mathrm{E} \quad 00$

$1.15298 \mathrm{E} \quad 00$

$1.15296 \mathrm{E} \quad 00$

$1.15294 \mathrm{E} \quad 00$

$1.15292 \mathrm{E} \quad 00$

$1.15291 \mathrm{E} \quad 00$

$1.15289 \mathrm{E} \quad 00$

$1.15287 \mathrm{E} \quad 00$

$1.15285 \mathrm{E} \quad 00$

$1.15284 \mathrm{E} \quad 00$

$1.15282 \mathrm{E} \quad 00$

$1.15280 \mathrm{E} \quad 00$

$1.15279 \mathrm{E} \quad 00$

$1.15277 \mathrm{E} \quad 00$

$1.15276 \mathrm{E} \quad 00$

$1.15274 \mathrm{E} \quad 00$

$1.15272 \mathrm{E} \quad 00$

$1.15271 \mathrm{E} \quad 00$

$1.15269 \mathrm{E} \quad 00$

$1.15268 \mathrm{E} \quad 00$

$1.15266 \mathrm{E} \quad 00$

$1.15265 \mathrm{E} \quad 00$

$1.15263 \mathrm{E} \quad 00$

$1.15262 \mathrm{E} \quad 00$

$1.15260 \mathrm{E} \quad 00$

$$
f_{1}
$$

$-1.23943 \mathrm{E} 01$

$-1.25006 \mathrm{E} 01$

$-1.26069 \mathrm{E} 01$

$-1.27132 \mathrm{E} \quad 01$

$-1.28194 \mathrm{E} 01$

$-1.29257 \mathrm{E} \quad 01$

$-1.30319 \mathrm{E} 01$

$-1.31381 \mathrm{E} 01$

$-1.32443 \mathrm{E} 01$

$-1.33505 \mathrm{E} 01$

$-1.34567 \mathrm{E} 01$

$-1.35629 \mathrm{E} 01$

$-1.36691 \mathrm{E} 01$

$-1.37752 \mathrm{E} 01$

$-1.38813 \mathrm{E} 01$

$-1.39875 \mathrm{E} \quad 01$

$-1.40936 \mathrm{E} 01$

$-1.41997 \mathrm{E} 01$

$-1.43057 \mathrm{E} \quad 01$

$-1.44118 \mathrm{E} 01$

$-1.45179 \mathrm{E} \quad 01$

$-1.46239 \mathrm{E} 01$

$-1.47299 \mathrm{E} 01$

$-1.48359 \mathrm{E} 01$

$-1.49419 \mathrm{E} 01$

$-1.50479 \mathrm{E} \quad 01$

$-1.51539 \mathrm{E} 01$

$-1.52599 \mathrm{E} \quad 01$

$-1.53658 \mathrm{E} 01$

$-1.54718 \mathrm{E} 01$

$-1.55777 \mathrm{E} \quad 01$

$-1.56836 \mathrm{E} 01$

$-1.57895 \mathrm{E} 01$

$-1.58954 \mathrm{E} \quad 01$

$-1.60012 \mathrm{E} 01$

$-1.61071 \mathrm{E} \quad 01$

$-1.62129 \mathrm{E}$ ol

$-1.63188 \mathrm{E} \quad 01$

$-1.64246 \mathrm{E} 01$

$-1.65304 \mathrm{E} 01$

$-1.66361 \mathrm{E} 01$

$-1.67419 \mathrm{E} 01$

$-1.68477 \mathrm{E} 01$

$-1.69534 \mathrm{E} \quad 01$

$-1.70591 \mathrm{E} 01$

$-1.71649 \mathrm{E} \quad 01$

$-1.72706 \mathrm{E} \quad 01$

$-1.73763 \mathrm{E} 01$

$-1.74819 \mathrm{E} \quad 01$

$-1.75876 \mathrm{E} 01$

$-1.76932 \mathrm{E} 01$

$-1.77989 \mathrm{E} \quad 01$

$-1.79045 \mathrm{E} 01$

$-1.80101 \mathrm{E} 01$

$-1.81157 \mathrm{E} \quad 01$

$-1.82212 \mathrm{E} \quad 01$

$-1.83268 \mathrm{E} \quad 01$

$-1.84323 \mathrm{E} 01$

$-1.85379 \mathrm{E} \quad 01$

$-1.86434 \mathrm{E} 01$

\section{$g_{1}$ \\ $-5.71824 \mathrm{E}-01$ \\ $-5.79446 \mathrm{E}-0 \mathrm{l}$ \\ $-5.87115 \mathrm{E}-01$ \\ $-5.94829 \mathrm{E}-01$ \\ $-6.02589 \mathrm{E}-01$}

$-6.10395 \mathrm{E}-01$

$-6.18247 \mathrm{E}-01$

$-6.26145 \mathrm{E}-01$

$-6.34089 \mathrm{E}-01$

$-6.42078 \mathrm{E}-01$

$-6.50114 \mathrm{E}-01$

$-6.58195 \mathrm{E}-01$

$-6.66322 \mathrm{E}-01$

$-6.74495 \mathrm{E}-0 \mathrm{I}$

$-6.82713 \mathrm{E}-01$

$-6.90978 \mathrm{E}-01$ $-6.99288 \mathrm{E}-01$

-7.07644 E-01

$-7.16046 \mathrm{E}-01$

$-7.24494 \mathrm{E}-01$

$-7.32987 \mathrm{E}-01$

$-7.41526 \mathrm{E}-01$

$-7.50111 \mathrm{E}-01$

$-7.58742 \mathrm{E}-01$

$-7.67418 \mathrm{E}-01$

$-7.76140 \mathrm{E}-01$

$-7.84908 \mathrm{E}-01$

$-7.93722 \mathrm{E}-01$

$-8.02581 \mathrm{E}-01$

$-8.11486 \mathrm{E}-01$

$-8.20436 \mathrm{E}-01$

$-8.29433 \mathrm{E}-01$

$-8.38475 \mathrm{E}-01$

$-8.47562 \mathrm{E}-01$

$-8.56696 \mathrm{E}-01$

$-8.65875 \mathrm{E}-01$

$-8.75100 \mathrm{E}-01$

$-8.84370 \mathrm{E}-01$

$-8.93686 \mathrm{E}-01$

$-9.03048 \mathrm{E}-01$

$-9.12455 \mathrm{E}-01$

$-9.21908 \mathrm{E}-01$

$-9.31407 \mathrm{E}-01$

$-9.40951 \mathrm{E}-01$

$-9.50541 \mathrm{E}-01$

$-9.60176 \mathrm{E}-01$ $-9.69857 \mathrm{E}-01$

$-9.79583 \mathrm{E}-01$

$-9.89355 \mathrm{E}-01$

$-9.99173 \mathrm{E}-01$

$-1.00904 \mathrm{E} \quad 00$

$-1.01894 \mathrm{E} 00$

$-1.02890 \mathrm{E} 00$

$-1.03890 \mathrm{E} 00$

$-1.04894 \mathrm{E} \quad 00$

$-1.05903 \mathrm{E} \quad 00$

$-1.06917 \mathrm{E} \quad 00$

$-1.07935 \mathrm{E} 00$

$-1.08958 \mathrm{E} \quad 00$

$-1.09985 \mathrm{E} 00$

$\tan \delta_{1}$

$f_{-1}$

$3.09006 \mathrm{E}-03$

$3.10420 \mathrm{E}-03$

$3.11810 \mathrm{E}-03$

$3.13177 \mathrm{E}-03$

$3.14522 \mathrm{E}-03$

$3.15843 \mathrm{E}-03$

$3.17142 \mathrm{E}-03$

$3.18420 \mathrm{E}-03$

$3.19679 \mathrm{E}-03$

3.20914E-03

$3.22131 \mathrm{E}-03$

$3.23328 \mathrm{E}-03$

$3.24507 \mathrm{E}-03$

$3.25666 \mathrm{E}-03$

$3.26807 \mathrm{E}-03$

$3.27930 \mathrm{E}-03$

$3.29036 \mathrm{E}-03$

$3.30125 \mathrm{E}-03$

$3.31195 \mathrm{E}-03$

$3.32251 \mathrm{E}-03$

$3.33290 \mathrm{E}-03$

$3.34313 \mathrm{E}-03$

$3.35322 \mathrm{E}-03$

$3.36314 \mathrm{E}-03$

$3.37292 \mathrm{E}-03$

$3.38255 \mathrm{E}-03$

$3.39204 \mathrm{E}-03$

$3.40139 \mathrm{E}-03$

$3.41060 \mathrm{E}-03$

$3.41968 \mathrm{E}-03$

$3.42862 \mathrm{E}-03$

$3.43744 \mathrm{E}-03$

$3.44613 \mathrm{E}-03$

$3.45469 \mathrm{E}-03$

$3.46313 \mathrm{E}-03$

$3.47146 \mathrm{E}-03$

$3.47966 \mathrm{E}-03$ 
$\bar{F}$

$1.99170 \mathrm{E}-04$ $2.02473 \mathrm{E}-04$ $2.05803 \mathrm{E}-04$ $2.09159 \mathrm{E}-04$ $2.12543 \mathrm{E}-04$

$2.15954 \mathrm{E}-04$ $2.19393 \mathrm{E}-04$ $2.22858 \mathrm{E}-04$ $2.26351 \mathrm{E}-04$ $2.29871 \mathrm{E}-04$

$2.33418 \mathrm{E}-04$ $2.36992 \mathrm{E}-04$ $2.40593 \mathrm{E}-04$ $2.44221 \mathrm{E}-04$

$2.51560 \mathrm{E}-04$ $2.55270 \mathrm{E}-04$ $2.59007 \mathrm{E}-04$ 2.62771 E-04 $2.66562 \mathrm{E}-04$

$2.70381 \mathrm{E}-04$ $2.74226 \mathrm{E}-04$ $2.78099 \mathrm{E}-04$ $2.81999 \mathrm{E}-04$ $2.85926 \mathrm{E}-04$

$2.89880 \mathrm{E}-04$ $2.93862 \mathrm{E}-04$ $2.97870 \mathrm{E}-04$ $3.01906 \mathrm{E}-04$ $3.05969 \mathrm{E}-04$

$3.10058 \mathrm{E}-04$ $3.14176 \mathrm{E}-04$ $3.18320 \mathrm{E}-04$ $3.22491 \mathrm{E}-04$ $3.26690 \mathrm{E}-04$

15.40

15.50

15.60

15.70

15.80

15.90

16.00

16.10

16.20

16.30

16.40

16.50

16.60

16.70

16.80

16.90

17.00

17.10

17.20

17.30

17.40

17.50

17.60

17.70

17.80

17.90

18.00

$3.30916 \mathrm{E}-04$ $3.35169 \mathrm{E}-04$ $3.39449 \mathrm{E}-04$ $3.43756 \mathrm{E}-04$ $3.48090 \mathrm{E}-04$

$3.52451 \mathrm{E}-04$ $3.56840 \mathrm{E}-04$ $3.61256 \mathrm{E}-04$ $3.65699 \mathrm{E}-04$ $3.70169 \mathrm{E}-04$

$3.74666 \mathrm{E}-04$ $3.79190 \mathrm{E}-04$ $3.83742 \mathrm{E}-04$ $3.88320 \mathrm{E}-04$ $3.92926 \mathrm{E}-04$

$3.97559 \mathrm{E}-04$ $4.02219 \mathrm{E}-04$ $4.06906 \mathrm{E}-04$ $4.11621 \mathrm{E}-04$ $4.16362 \mathrm{E}-04$

$4.21131 \mathrm{E}-04$ $4.25927 \mathrm{E}-04$ $4.30750 \mathrm{E}-04$ $4.35600 \mathrm{E}-04$ $4.40477 \mathrm{E}-04$
2.47877 E-04

$f_{2}$

$-3.26423 \mathrm{E}-01$ $-3.31952 \mathrm{E}-01$ $-3.37526 \mathrm{E}-01$ $-3.43148 \mathrm{E}-01$

$-3.48815 \mathrm{E}-01$

$-3.54528 \mathrm{E}-01$

$-3.60288 \mathrm{E}-01$

$-3.66094 \mathrm{E}-01$

$-3.71946 \mathrm{E}-01$

$-3.77844 \mathrm{E}-01$

$-3.83789 E-01$

$-3.89779 \mathrm{E}-01$

$-3.95816 \mathrm{E}-01$

$-4.01899 \mathrm{E}-01$

$-4.08028 \mathrm{E}-01$

$-4.14203 \mathrm{E}-01$

$-4.20425 \mathrm{E}-01$

$-4.26692 \mathrm{E}-01$

$-4.33006 \mathrm{E}-01$

$-4.39366 \mathrm{E}-01$

$-4.45772 \mathrm{E}-01$

$-4.52224 \mathrm{E}-01$

$-4.58722 \mathrm{E}-01$

$-4.65266 \mathrm{E}-01$

$-4.71857 \mathrm{E}-01$

$-4.78493 \mathrm{E}-01$

$-4.85176 \mathrm{E}-01$

$-4.91904 \mathrm{E}-01$

$-4.98679 \mathrm{E}-01$

$-5.05500 \mathrm{E}-01$

$-5.12367 \mathrm{E}-01$

$-5.19280 \mathrm{E}-01$

$-5.26239 \mathrm{E}-01$

$-5.33244 \mathrm{E}-01$

$-5.40295 \mathrm{E}-01$

$-6.47392 \mathrm{E}-01$ $-5.54536 \mathrm{E}-01$ $-5.61725 \mathrm{E}-01$ $-5.68960 \mathrm{E}-01$ $-5.76241 \mathrm{E}-01$

$-5.83569 \mathrm{E}-01$ $-5.90942 \mathrm{E}-01$ $-5.98361 \mathrm{E}-01$ $-6.05827 \mathrm{E}-01$

$-6.13338 \mathrm{E}-01$

$-6.20895 \mathrm{E}-01$

$-6.28499 \mathrm{E}-01$

$-6.36148 \mathrm{E}-01$

$-6.43843 \mathrm{E}-01$

$-6.51584 \mathrm{E}-01$

$-6.59372 \mathrm{E}-01$

$-6.67205 \mathrm{E}-0 \mathrm{l}$

$-6.75084 \mathrm{E}-01$

$-6.83009 \mathrm{E}-01$

$-6.90980 \mathrm{E}-01$

$-6.98996 \mathrm{E}-01$

$-7.07059 \mathrm{E}-01$

$-7.15168 \mathrm{E}-01$

$-7.23323 \mathrm{E}-01$

$-7.31523 \mathrm{E}-01$ $g_{2}$

$-8.86617 \mathrm{E}-03$

$-9.05955 \mathrm{E}-03$

$-9.25565 \mathrm{E}-03$

$-9.45447 \mathrm{E}-03$

$-9.65604 \mathrm{E}-03$

$-9.86037 \mathrm{E}-03$

$-1.00675 \mathrm{E}-02$

$-1.02774 \mathrm{E}-02$

$-1.04901 \mathrm{E}-02$

$-1.07057 \mathrm{E}-02$

$-1.09241 \mathrm{E}-02$

$-1.11454 \mathrm{E}-02$

$-1.13696 \mathrm{E}-02$

$-1.15967 \mathrm{E}-02$

$-1.18267 \mathrm{E}-02$

$-1.20597 \mathrm{E}-02$ $-1.22956 \mathrm{E}-02$

$-1.25345 \mathrm{E}-02$

$-1.27764 \mathrm{E}-02$

$-1.30213 \mathrm{E}-02$

$-1.32693 \mathrm{E}-02$

$-1.35203 E-02$

$-1.37744 \mathrm{E}-02$

$-1.40315 \mathrm{E}-02$

$-1.42918 \mathrm{E}-02$

$-1.45552 \mathrm{E}-02$

$-1.48217 \mathrm{E}-02$

$-1.50914 \mathrm{E}-02$

$-1.53643 \mathrm{E}-02$

$-1.56403 \mathrm{E}-02$

$-1.59196 \mathrm{E}-02$

$-1.62021 \mathrm{E}-02$

$-1.64879 \mathrm{E}-02$

$-1.67769 \mathrm{E}-02$

$-1.70692 \mathrm{E}-02$

$-1.73649 \mathrm{E}-02$

$-1.76638 \mathrm{E}-02$

$-1.79661 \mathrm{E}-02$

$-1.82717 \mathrm{E}-02$

$-1.85807 \mathrm{E}-02$

$-1.88931 \mathrm{E}-02$

$-1.92089 \mathrm{E}-02$

$-1.95282 \mathrm{E}-02$

$-1.98508 \mathrm{E}-02$

$-2.01770 \mathrm{E}-02$

$-2.05066 \mathrm{E}-02$

$-2.08398 \mathrm{E}-02$

$-2.11764 \mathrm{E}-02$

$-2.15166 \mathrm{E}-02$

$-2.18603 \mathrm{E}-02$

$-2.22076 \mathrm{E}-02$

$-2.25586 \mathrm{E}-02$

$-2.29131 \mathrm{E}-02$

$-2.32712 \mathrm{E}-02$

$-2.36330 \mathrm{E}-02$

$-2.39984 \mathrm{E}-02$

$-2.43675 \mathrm{E}-02$

$-2.47404 \mathrm{E}-02$

$-2.51169 \mathrm{E}-02$

$-2.54971 \mathrm{E}-02$ $\tan \delta_{2}$

$f_{-2}$

$3.36603 \mathrm{E} 01$

$3.36706 \mathrm{E} 01$

$3.36807 \mathrm{E}$ 01

$3.36906 \mathrm{E}$ 01

$3.37004 \mathrm{E} 01$

$3.37099 \mathrm{E} \quad 01$

$3.37194 \mathrm{E} \quad 01$

$3.37286 \mathrm{E} \quad 01$

$3.37378 \mathrm{E} \quad 01$

$3.37468 \mathrm{E} \quad 01$

$3.37556 \mathrm{E} \quad 01$

$3.37643 \mathrm{E} \quad 01$

$3.37728 \mathrm{E} \quad 01$

$3.37813 \mathrm{E} \quad 01$

$3.37896 \mathrm{E} \quad 01$

$3.37977 \mathrm{E} \quad 01$

$3.38058 \mathrm{E} 01$

$3.38137 \mathrm{E} 01$

$3.38215 \mathrm{E} \quad 01$

$3.38292 \mathrm{E} 01$

$3.38367 \mathrm{E} \quad 01$

$3.38442 \mathrm{E} 01$

$3.38515 \mathrm{E} 01$

$3.38588 \mathrm{E} 01$

$3.38659 \mathrm{E}$ 01

$3.38729 \mathrm{E} \quad 01$

$3.38799 \mathrm{E} 01$

$3.38867 \mathrm{E} \quad 01$

$3.38934 \mathrm{E} 01$

$3.39001 \mathrm{E} 01$

$3.39066 \mathrm{E} \quad 01$

$3.39131 \mathrm{E} 01$

$3.39195 \mathrm{E} 01$

$3.39257 \mathrm{E}$ 0l

$3.39320 \mathrm{E}$ ol
$3.39381 \mathrm{E} \quad 01$ $3.39441 \mathrm{E} \quad 01$ $3.39501 \mathrm{E} 01$ $3.39559 \mathrm{E}$ 0l $3.39617 \mathrm{E} \quad 01$

$3.39675 \mathrm{E} \quad 01$ $3.39731 \mathrm{E} \quad 01$ $3.39787 \mathrm{E}$ 01

$3.39842 \mathrm{E}$ 01

$3.39897 \mathrm{E}$ 0l

$3.39950 \mathrm{E}$ 0l

$3.40003 \mathrm{E} \quad 01$

$3.40056 \mathrm{E} \quad 01$

$3.40107 \mathrm{E} \quad 01$

$3.40159 \mathrm{E} 01$

$3.40209 \mathrm{E} \quad 01$ $3.40259 \mathrm{E} 01$ $3.40308 \mathrm{E} 01$ $3.40357 \mathrm{E} \quad 01$ $3.40405 \mathrm{E} 01$

$3.40452 \mathrm{E} \quad 01$ $3.40499 \mathrm{E} \quad 01$

$3.40546 \mathrm{E}$ 0l

$3.40592 \mathrm{E} \quad 01$

$3.40637 \mathrm{E} 01$

$-8.70354 \mathrm{E}-03$ $-8.89187 \mathrm{E}-03$ $-9.08286 \mathrm{E}-03$ $-9.27651 \mathrm{E}-03$ $-9.47285 \mathrm{E}-03$

$-9.67189 \mathrm{E}-03$ $-9.87366 \mathrm{E}-03$ $-1.00782 \mathrm{E}-02$ $-1.02854 \mathrm{E}-02$ $-1.04955 \mathrm{E}-02$

$-1.07083 \mathrm{E}-02$ $-1.09239 \mathrm{E}-02$ $-1.11424 \mathrm{E}-02$ 
$p$

18.10

18.20

18.30

18.40

18.50

18.60

18.70

18.80

18.90

19.00

19.10

19.20

19.30

19.40

19.50

19.60

19.70

19.80

19.90

20.00

20.10

20.20

20.30

20.40

20.50

20.60

20.70

20.80

20.90

21.00

21.10

21.20

21.30

21.40

21.50

21.60

21.70

21.80

21.90

22.00

22.10

22.20

22.30

22.40

22.50

22.60

22.70

22.80

22.90

23.00

23.10

23.20

23.30

23.40

23.50

23.60

23.70

23.80

23.90

24.00
$F_{0}$

$f_{1}$

$\begin{array}{llllll}1.15259 \mathrm{E} & 00 & -1.87489 \mathrm{E} & 01 & -1.11017 \mathrm{E} & 00\end{array}$

$\begin{array}{llllll}1.15257 \mathrm{E} & 00 & -1.88544 \mathrm{E} & 01 & -1.12054 \mathrm{E} & 00\end{array}$

$\begin{array}{llllll}1.15256 \mathrm{E} & 00 & -1.89598 \mathrm{E} & 01 & -1.13094 \mathrm{E} & 00\end{array}$

$\begin{array}{llllll}1.15254 \mathrm{E} & 00 & -1.90653 \mathrm{E} & 01 & -1.14140 \mathrm{E} & 00\end{array}$

$1.15253 \mathrm{E} \quad 00$

$1.15251 \mathrm{E} \quad 00$

$1.15250 \mathrm{E} \quad 00$

$1.15249 \mathrm{E} 00$

$1.15247 \mathrm{E} \quad 00$

$1.15246 \mathrm{E} \quad 00$

$1.15244 \mathrm{E} \quad 00$

$1.15243 \mathrm{E} \quad 00$

$1.15242 \mathrm{E} \quad 00$

$1.15240 \mathrm{E} \quad 00$

$1.15239 \mathrm{E} \quad 00$

$1.15238 \mathrm{E} \quad 00$

$1.15236 \mathrm{E} \quad 00$

$1.15235 \mathrm{E} \quad 00$

$1.15234 \mathrm{E} \quad 00$

$1.15232 \mathrm{E} \quad 00$

$1.15231 \mathrm{E} \quad 00$

$1.15230 \mathrm{E} \quad 00$

$1.15229 \mathrm{E} \quad 00$

$1.15227 \mathrm{E} \quad 00$

$1.15226 \mathrm{E} \quad 00$

$1.15225 \mathrm{E} \quad 00$

$1.15224 \mathrm{E} \quad 00$

$1.15222 \mathrm{E} \quad 00$

$1.15221 \mathrm{E} \quad 00$

$1.15220 \mathrm{E} \quad 00$

$1.15219 \mathrm{E} \quad 00$

$1.15217 \mathrm{E} \quad 00$

$1.15216 \mathrm{E} \quad 00$

$1.15215 \mathrm{E} \quad 00$

$1.15214 \mathrm{E} \quad 00$

$1.15213 \mathrm{E} \quad 00$

$1.15212 \mathrm{E} \quad 00$

$1.15210 \mathrm{E} \quad 00$

$1.15209 \mathrm{E} \quad 00$

$1.15208 \mathrm{E} \quad 00$

$1.15207 \mathrm{E} \quad 00$

$1.15206 \mathrm{E} \quad 00$

$1.15205 \mathrm{E} \quad 00$

$1.15204 \mathrm{E} \quad 00$

$1.15202 \mathrm{E} \quad 00$

$1.15201 \mathrm{E} \quad 00$

$1.15200 \mathrm{E} \quad 00$

$1.15199 \mathrm{E} \quad 00$

$1.15198 \mathrm{E} \quad 00$

$1.15197 \mathrm{E} \quad 00$

$1.15196 \mathrm{E} \quad 00$

$1.15195 \mathrm{E} \quad 00$

$1.15194 \mathrm{E} \quad 00$

$1.15193 \mathrm{E} \quad 00$

$1.15192 \mathrm{E} \quad 00$

$1.15191 \mathrm{E} \quad 00$

$1.15189 \mathrm{E} \quad 00$

$1.15188 \mathrm{E} \quad 00$

$\begin{array}{ll}1.15187 \mathrm{E} & 00\end{array}$

$1.15186 \mathrm{E} \quad 00$
$-1.91707 \mathrm{E} 01$

$-1.92762 \mathrm{E} 01$

$-1.93816 \mathrm{E} 01$

$-1.94870 \mathrm{E} 01$

$-1.95924 \mathrm{E} \quad 01$

$-1.96977 \mathrm{E} \quad 01$

$-1.98031 \mathrm{E} \quad 01$

$-1.99084 \mathrm{E} 01$

$-2.00137 \mathrm{E} \quad 01$

$-2.01191 \mathrm{E} 01$

$-2.02244 \mathrm{E} \quad 01$

$-2.03296 \mathrm{E} \quad 01$

$-2.04349 \mathrm{E} 01$

$-2.05401 \mathrm{E} 01$

$-2.06454 \mathrm{E} \quad 01$

$-2.07506 \mathrm{E} 0 \mathrm{l}$

$-2.08558 \mathrm{E} \quad 01$ $-2.09610 \mathrm{E} 01$

$-2.10661 \mathrm{E} 01$

$-2.11713 \mathrm{E} 01$

$-2.12764 \mathrm{E} 01$

$-2.13816 \mathrm{E} \quad 01$

$-2.14867 \mathrm{E} 01$

$-2.15918 \mathrm{E} \quad 01$

$-2.18019 \mathrm{E} \quad 01$

$-2.19069 \mathrm{E} \quad 01$ $-2.20120 \mathrm{E} \quad 01$

$-2.21170 \mathrm{E} 01$

$-2.22220 \mathrm{E} \quad 01$

$-2.23270 \mathrm{E} 01$

$-2.24319 \mathrm{E} 01$ $-2.25369 \mathrm{E} 01$ $-2.26418 \mathrm{E} 01$ $-2.27467 \mathrm{E} \quad 01$

$-2.28516 \mathrm{E} \quad 01$

$-2.29565 \mathrm{E} \quad 01$ $-2.30614 \mathrm{E} \quad 01$ $-2.31663 \mathrm{E} \quad 01$ $-2.32711 \mathrm{E} 01$ $-2.33759 \mathrm{E} \quad 01$

$-2.34807 \mathrm{E} \quad 01$ $-2.35855 \mathrm{E} \quad 01$ $-2.36903 \mathrm{E} \quad 01$ $-2.37950 \mathrm{E} \quad 01$ $-2.38998 \mathrm{E} 01$

$-2.40045 \mathrm{E} \quad 01$ $-2.41092 \mathrm{E} \quad 01$ $-2.42139 \mathrm{E} 01$ $-2.43186 \mathrm{E} \quad 01$ $-2.44232 \mathrm{E} 01$

$-2.45279 \mathrm{E} 01$ $-2.46325 \mathrm{E} \quad 01$ $-2.47371 \mathrm{E} 01$ $-2.48417 \mathrm{E} 01$ $-2.49462 \mathrm{E} \quad 01$
$-2.16968 \mathrm{E} 01$
$-1.15190 \mathrm{E} 00$ $-1.17304 \mathrm{E} \quad 00$ $-1.18367 \mathrm{E} \quad 00$ $-1.19435 \mathrm{E} \quad 00$ $-1.20508 \mathrm{E} 00$

$-1.21585 \mathrm{E} \quad 00$ $-1.22667 \mathrm{E} \quad 00$ $-1.23754 \mathrm{E} 00$ $-1.24844 \mathrm{E} 00$ $-1.25940 \mathrm{E} 00$

$-1.27040 \mathrm{E} \quad 00$ $-1.28144 \mathrm{E} 00$ $-1.29253 \mathrm{E} 00$ $-1.30367 \mathrm{E} 00$ -1.31485 E 00

$-1.32607 \mathrm{E} \quad 00$ $-1.33735 \mathrm{E} 00$ $-1.34866 \mathrm{E} 00$ $-1.36002 \mathrm{E} \quad 00$ $-1.37143 \mathrm{E} 00$

$-1.38288 \mathrm{E} \quad 00$ $-1.39438 \mathrm{E} \quad 00$ $-1.40593 \mathrm{E} \quad 00$ $-1.41751 \mathrm{E} 00$ -1.42915 E 00

$-1.44083 \mathrm{E} 00$ $-1.45255 \mathrm{E} \quad 00$ $-1.46432 \mathrm{E} \quad 00$ $-1.47613 \mathrm{E} \quad 00$ $-1.48799 \mathrm{E} \quad 00$

$-1.49990 \mathrm{E} \quad 00$ $-1.51185 \mathrm{E} \quad 00$ $-1.52384 \mathrm{E} 00$ $-1.53588 \mathrm{E} 00$

$-1.56010 \mathrm{E} \quad 00$ $-1.57227 \mathrm{E} 00$ $-1.59676 \mathrm{E} \quad 00$ $-1.60907 \mathrm{E} 00$

$-1.62143 \mathrm{E} \quad 00$ $-1.63383 \mathrm{E} 00$ $-1.64628 \mathrm{E} \quad 00$ $-1.65877 \mathrm{E} \quad 00$ $-1.67130 \mathrm{E} \quad 00$

$-1.68388 \mathrm{E} \quad 00$ $-1.69651 \mathrm{E} 00$ $-1.70918 \mathrm{E} 00$ $-1.72190 \mathrm{E} \quad 00$ $-1.73466 \mathrm{E} 00$

$-1.74747 \mathrm{E} \quad 00$ $-1.76032 \mathrm{E} 00$ $-1.77322 \mathrm{E} \quad 00$ $-1.78616 \mathrm{E} \quad 00$ $-1.79914 \mathrm{E} \quad 00$
$-1.16245 \mathrm{E} \quad 00$ $-1.54797 \mathrm{E} 00$ $-1.58450 \mathrm{E} \quad 00$

$\tan \delta_{1}$ $f_{-1}$

$3.64609 \mathrm{E}-03$

$3.65193 \mathrm{E}-03$

$3.65769 \mathrm{E}-03$

$3.66337 \mathrm{E}-03$

$3.66899 \mathrm{E}-03$

$3.67453 \mathrm{E}-03$

$3.68000 \mathrm{E}-03$

$3.68540 \mathrm{E}-03$

$3.69072 \mathrm{E}-03$

$3.69599 \mathrm{E}-03$

$3.70119 \mathrm{E}-03$

$3.70631 \mathrm{E}-03$

$3.71137 \mathrm{E}-03$

$3.71637 \mathrm{E}-03$

$3.72131 \mathrm{E}-03$

$3.72619 \mathrm{E}-03$

$3.73099 \mathrm{E}-03$

$3.73574 \mathrm{E}-03$

$3.74043 \mathrm{E}-03$

$3.74506 \mathrm{E}-03$

$3.74963 \mathrm{E}-03$

$3.75415 \mathrm{E}-03$

$3.75861 \mathrm{E}-03$

$3.76302 \mathrm{E}-03$

$3.76736 \mathrm{E}-03$

$3.77164 \mathrm{E}-03$

$3.77589 \mathrm{E}-03$

$3.78007 \mathrm{E}-03$

$3.78421 \mathrm{E}-03$

$3.78829 \mathrm{E}-03$

3.79232E-03 $3.79630 \mathrm{E}-03$ $3.80024 \mathrm{E}-03$

$3.80411 \mathrm{E}-03$

3.80795E-03

$3.81174 \mathrm{E}-03$ $3.81547 \mathrm{E}-03$

$3.81916 \mathrm{E}-03$

$3.82281 \mathrm{E}-03$

$3.82641 \mathrm{E}-03$

$3.82997 \mathrm{E}-03$ $3.83348 \mathrm{E}-03$

$3.83694 \mathrm{E}-03$

$3.84037 \mathrm{E}-03$

$3.84375 \mathrm{E}-03$

$3.84709 \mathrm{E}-03$ $3.85039 \mathrm{E}-03$ $3.85364 \mathrm{E}-03$ 
ELECTRON RADIAL FUNCTIONS

$Z=6 \quad A=12 \quad \rho=1.1 A^{1 / 3} F$

Elecetron

$\bar{F}$

18.10

18.20

18.30

18.40

18.50

18.60

18.70

18.80

18.90

19.00

19.10

19.20

19.30

19.40

19.50

19.60

19.70

19.80

19.90

20.00

20.10

20.20

20.30

20.40

20.50

20.60

20.70

20.80

20.90

21.00

21.10

21.20

21.30

21.40

21.50

21.60

21.70

21.80

21.90

22.00

22.10

22.20

22.30

22.40

22.50

22.60

22.70

22.80

22.90

23.00

23.10

23.20

23.30

23.40

23.50

23.60

23.70

23.80

23.90

24.00 $f_{2}$

$4.45382 \mathrm{E}-04$

$4.50313 \mathrm{E}-04$

$4.55272 \mathrm{E}-04$

$4.60258 \mathrm{E}-04$

$4.65271 \mathrm{E}-04$

4.70311E-04 $4.75378 \mathrm{E}-04$

$4.80473 \mathrm{E}-04$

4.85594 E-04

$4.90743 \mathrm{E}-04$

$4.95919 \mathrm{E}-04$

$5.01122 \mathrm{E}-04$

$5.06352 \mathrm{E}-04$

$5.11609 \mathrm{E}-04$

$5.16894 \mathrm{E}-04$

$5.22205 \mathrm{E}-04$

$5.27544 \mathrm{E}-04$

$5.32910 \mathrm{E}-04$

$5.38303 \mathrm{E}-04$

$5.43723 \mathrm{E}-04$

$5.49170 \mathrm{E}-04$

$5.54644 \mathrm{E}-04$

$5.60146 \mathrm{E}-04$

$5.65675 \mathrm{E}-04$

$5.71231 \mathrm{E}-04$

$5.76814 \mathrm{E}-04$

$5.82424 \mathrm{E}-04$

$5.88061 \mathrm{E}-04$

$5.93725 \mathrm{E}-04$

$5.99417 \mathrm{E}-04$

$6.05136 \mathrm{E}-04$

$6.10881 \mathrm{E}-04$

$6.16654 \mathrm{E}-04$

$6.22455 \mathrm{E}-04$

$6.28282 \mathrm{E}-04$

$6.34136 \mathrm{E}-04$

$6.40018 \mathrm{E}-04$

$6.45926 \mathrm{E}-04$

$6.51862 \mathrm{E}-04$

$6.57825 \mathrm{E}-04$

$6.63815 \mathrm{E}-04$

$6.69833 \mathrm{E}-04$

$6.75877 \mathrm{E}-04$

$6.81948 \mathrm{E}-04$

$6.88047 \mathrm{E}-04$

$6.94173 \mathrm{E}-04$

$7.00326 \mathrm{E}-04$

$7.06506 \mathrm{E}-04$

$7.12713 \mathrm{E}-04$

$7.18947 \mathrm{E}-04$

$7.25209 \mathrm{E}-04$

$7.31497 \mathrm{E}-04$

$7.37813 \mathrm{E}-04$

$7.44156 \mathrm{E}-04$

$7.50526 \mathrm{E}-04$

$7.56923 \mathrm{E}-04$

$7.63348 \mathrm{E}-04$

$7.69799 \mathrm{E}-04$

$7.76278 \mathrm{E}-04$

$7.82783 \mathrm{E}-04$ $g_{2}$

$-7.39770 \mathrm{E}-01 \quad-2.58812 \mathrm{E}-02$

$-7.48062 \mathrm{E}-01-2.62690 \mathrm{E}-02$

$-7.56401 \mathrm{E}-01 \quad-2.66605 \mathrm{E}-02$

$-7.64785 \mathrm{E}-01 \quad-2.70559 \mathrm{E}-02$

$-7.73215 \mathrm{E}-01$

$2.74551 \mathrm{E}-02$

$-7.81690 \mathrm{E}-01$

$-7.90212 \mathrm{E}-01$

$-7.98780 \mathrm{E}-01$

$-8.07393 \mathrm{E}-01$

$-8.16052 \mathrm{E}-01$

$-8.24757 \mathrm{E}-01$

$-8.33508 \mathrm{E}-01$

$-8.42305 \mathrm{E}-01$

$-8.51147 \mathrm{E}-01$

$-8.60036 \mathrm{E}-01$

$-8.68970 \mathrm{E}-01$

$-8.77950 \mathrm{E}-01$

$-8.86975 \mathrm{E}-01$

$-8.96047 \mathrm{E}-01$

$-9.05164 \mathrm{E}-01$

$-9.14327 \mathrm{E}-01$

$-9.23536 \mathrm{E}-01$

$-9.32790 \mathrm{E}-01$

$-9.42091 \mathrm{E}-01$

$-9.51437 \mathrm{E}-01$

$-9.60828 \mathrm{E}-01$

$-9.70266 \mathrm{E}-01$

$-9.79749 \mathrm{E}-01$

$-9.89278 \mathrm{E}-01$

$-9.98853 \mathrm{E}-01$

$-1.00847 \mathrm{E} \quad 00$

$-1.01814 \mathrm{E} \quad 00$

$-1.02785 \mathrm{E} \quad 00$

$-1.03761 \mathrm{E} \quad 00$

$-1.04741 \mathrm{E} \quad 00$

$-1.05726 \mathrm{E} \quad 00$

$-1.06715 \mathrm{E} \quad 00$

$-1.07709 \mathrm{E} \quad 00$

$-1.08708 \mathrm{E} 00$

$-1.09711 \mathrm{E} \quad 00$

$-1.10719 \mathrm{E} \quad 00$ $-1.11731 \mathrm{E} \quad 00$ $-1.12748 \mathrm{E} 00$ $-1.13769 \mathrm{E} \quad 00$ -1.14795 E 00

$-1.15826 \mathrm{E} 00$ $-1.16861 \mathrm{E} \quad 00$ $-1.17900 \mathrm{E} 00$ $-1.18945 \mathrm{E} \quad 00$

$-1.19993 \mathrm{E} 00$

$-1.21047 \mathrm{E} \quad 00$ $-1.22104 \mathrm{E} \quad 00$ $-1.23167 \mathrm{E} \quad 00$ $-1.24234 \mathrm{E} \quad 00$ -1.25305 E 00

$-1.26381 \mathrm{E} \quad 00$ $-1.27462 \mathrm{E} \quad 00$ -1.28547 E 00 $-1.29637 \mathrm{E} \quad 00$ $-1.30731 \mathrm{E} \quad 00$

$-2.78581 \mathrm{E}-02$

$-2.82650 \mathrm{E}-02$

$-2.86758 \mathrm{E}-02$

$-2.90905 \mathrm{E}-02$

$-2.95090 \mathrm{E}-02$

$-2.99315 \mathrm{E}-02$

$-3.03580 \mathrm{E}-02$

$-3.07884 \mathrm{E}-02$

$-3.12228 \mathrm{E}-02$

$-3.16612 \mathrm{E}-02$

$-3.21036 \mathrm{E}-02$

$-3.25500 \mathrm{E}-02$

$-3.30006 \mathrm{E}-02$

$-3.34551 \mathrm{E}-02$

$-3.39138 \mathrm{E}-02$

$-3.43766 \mathrm{E}-02$

$-3.48434 \mathrm{E}-02$

$-3.53145 \mathrm{E}-02$

$-3.57897 \mathrm{E}-02$

$-3.62691 \mathrm{E}-02$

$-3.72404 \mathrm{E}-02$

$-3.77324 \mathrm{E}-02$

$-3.82287 \mathrm{E}-02$

$-3.87292 \mathrm{E}-02$

$-3.92341 \mathrm{E}-02$

$-3.97432 \mathrm{E}-02$

$-4.02566 \mathrm{E}-02$

$-4.07744 \mathrm{E}-02$

$-4.12965 \mathrm{E}-02$

$-4.18230 \mathrm{E}-02$

$-4.28893 \mathrm{E}-02$

$-4.34290 \mathrm{E}-02$

$-4.39732 \mathrm{E}-02$

$-4.45218 \mathrm{E}-02$

$-4.56326 \mathrm{E}-02$

$-4.61947 \mathrm{E}-02$

$-4.67614 \mathrm{E}-02$

$-4.73326 \mathrm{E}-02$ $-4.79084 \mathrm{E}-02$ $-4.84888 \mathrm{E}-02$ $-4.90737 \mathrm{E}-02$

$-4.96633 \mathrm{E}-02$

$-5.02576 \mathrm{E}-02$ $-5.08565 \mathrm{E}-02$

$-5.14601 \mathrm{E}-02$

$-5.20683 \mathrm{E}-02$

$-5.26813 \mathrm{E}-02$

$-5.32990 \mathrm{E}-02$

$-5.39214 \mathrm{E}-02$

$-5.45487 \mathrm{E}-02$

$-5.51806 \mathrm{E}-02$

$-5.58174 \mathrm{E}-02$ $\tan \delta_{2}$

$3.40682 \mathrm{E}$ 01

- $01-2.56876 \mathrm{E}-02$

$3.40770 \mathrm{E}$ 01 $-2.60704 \mathrm{E}-02$

$3.40813 \mathrm{E}$ 01 $-2.64570 \mathrm{E}-02$

$3.40856 \mathrm{E} \quad 01 \quad-2.68474 \mathrm{E}-02$

$-3.67526 \mathrm{E}-02$

$-4.23540 \mathrm{E}-02$

$-4.50749 \mathrm{E}-02$

$3.40898 \mathrm{E}$ ol $-2.72415 \mathrm{E}-02$

$3.40940 \mathrm{E} \quad 01 \quad-2.76394 \mathrm{E}-02$

$3.40982 \mathrm{E}$ 01 $-2.80411 \mathrm{E}-02$

$3.41023 \mathrm{E}$ 01 $-2.84467 \mathrm{E}-02$

$3.41063 \mathrm{E} \quad 01 \quad-2.88561 \mathrm{E}-02$

$3.41103 \mathrm{E} \quad 01 \quad-2.92694 \mathrm{E}-02$

$3.41143 \mathrm{E}$ 01 $-2.96865 \mathrm{E}-02$

$3.41182 \mathrm{E}$ 0l $-3.01076 \mathrm{E}-02$

$3.41221 \mathrm{E} \quad 01 \quad-3.05326 \mathrm{E}-02$

$3.41259 \mathrm{E}$ ol $-3.09615 \mathrm{E}-02$

$3.41297 \mathrm{E}$ 01 $-3.13944 \mathrm{E}-02$

$3.41335 \mathrm{E} \quad 01 \quad-3.18313 \mathrm{E}-02$

$3.41372 \mathrm{E}$ 01 $-3.22721 \mathrm{E}-02$

$3.41409 \mathrm{E}$ 01 $-3.27170 \mathrm{E}-02$

$3.41445 \mathrm{E} \quad 01-3.31659 \mathrm{E}-02$

$3.41481 \mathrm{E} \quad 01$

$3.41517 \mathrm{E} \quad 01$

$3.41552 \mathrm{E}$ 01

$3.41587 \mathrm{E} \quad 01$

$3.41622 \mathrm{E} \quad 01$

$3.41656 \mathrm{E} \quad 01$

$3.41690 \mathrm{E}$ 01

$3.41723 \mathrm{E} \quad 01$

$3.41757 \mathrm{E} \quad 01$

$3.41790 \mathrm{E} \quad 01$

$3.41822 \mathrm{E} \quad 01$

$3.41854 \mathrm{E} \quad 01$

$3.41886 \mathrm{E} \quad 01$

$3.41918 \mathrm{E}$ 01

$3.41949 \mathrm{E} \quad 01$

$3.41980 \mathrm{E} \quad 01$

$3.42011 \mathrm{E} 01$

$3.42041 \mathrm{E} \quad 01$

$3.42072 \mathrm{E} 01$

$3.42101 \mathrm{E} 01$

$3.42131 \mathrm{E} \quad 01$

$3.42160 \mathrm{E} \quad 01$

$3.42189 \mathrm{E} \quad 01$

$3.42218 \mathrm{E}$ 0l

$3.42247 \mathrm{E}$ 01

$3.42275 \mathrm{E} \quad 01$ 
ELECTRON RADIAL FUNCTIONS

Electron

$$
F_{0}
$$

24.10

24.20

24.30

24.40

24.50

24.60

24.70

24.80

24.90

25.00

25.10

25.20

25.30

25.40

25.50

25.60

25.70

25.80

25.90

26.00

26.10

26.20

26.30

26.40

26.50

26.60

26.70

26.80

26.90

27.00

27.10

27.20

27.30

27.40

27.50

27.60

27.70

27.80

27.90

28.00

28.10

28.20

28.30

28.40

28.50

28.60

28.70

28.80

28.90

29.00

29.10

29.20

29.30

29.40

29.50

29.60

29.70

29.80

29.90

30.00
$Z=6$

\begin{abstract}
$f_{\mathrm{t}}$
\end{abstract}
$g_{1}$

$1.15185 \mathrm{E} \quad 00$

$1.15184 \mathrm{E} \quad 00$

$1.15183 \mathrm{E} \quad 00$

$1.15182 \mathrm{E} 00$

$1.15181 \mathrm{E} \quad 00$

$1.15180 \mathrm{E} \quad 00$

$1.15179 \mathrm{E} \quad 00$

$1.15178 \mathrm{E} \quad 00$

$1.15177 \mathrm{E} \quad 00$

$1.15176 \mathrm{E} \quad 00$

$1.15175 \mathrm{E} \quad 00$

$1.15174 \mathrm{E} \quad 00$

$1.15173 \mathrm{E} \quad 00$

$1.15172 \mathrm{E} \quad 00$

$1.15171 \mathrm{E} \quad 00$

$1.15170 \mathrm{E} \quad 00$

$1.15170 \mathrm{E} \quad 00$

$1.15169 \mathrm{E} \quad 00$

$1.15168 \mathrm{E} \quad 00$

$1.15167 \mathrm{E} \quad 00$

$1.15166 \mathrm{E} \quad 00$

$1.15165 \mathrm{E} \quad 00$

$1.15164 \mathrm{E} \quad 00$

$1.15163 \mathrm{E} \quad 00$

$\begin{array}{ll}1.15162 \mathrm{E} & 00\end{array}$

$1.15161 \mathrm{E} \quad 00$

$1.15160 \mathrm{E} \quad 00$

$1.15159 \mathrm{E} \quad 00$

$1.15158 \mathrm{E} \quad 00$

$1.15158 \mathrm{E} \quad 00$

$1.15157 \mathrm{E} \quad 00$

$1.15156 \mathrm{E} \quad 00$

$1.15155 \mathrm{E} \quad 00$

$1.15154 \mathrm{E} \quad 00$

$1.15153 \mathrm{E} \quad 00$

$1.15152 \mathrm{E} \quad 00$

$1.15151 \mathrm{E} \quad 00$

$1.15151 \mathrm{E} \quad 00$

$1.15150 \mathrm{E} \quad 00$

$1.15149 \mathrm{E} \quad 00$

$1.15148 \mathrm{E} \quad 00$ $1.15147 \mathrm{E} \quad 00$

$1.15146 \mathrm{E} \quad 00$

$1.15145 \mathrm{E} \quad 00$

$1.15145 \mathrm{E} \quad 00$

$1.15144 \mathrm{E} \quad 00$

$1.15143 \mathrm{E} \quad 00$

$1.15142 \mathrm{E} \quad 00$

$\begin{array}{ll}1.15141 \mathrm{E} & 00\end{array}$

$1.15140 \mathrm{E} \quad 00$

$1.15140 \mathrm{E} \quad 00$

$1.15139 \mathrm{E} \quad 00$

$1.15138 \mathrm{E} \quad 00$

$1.15137 \mathrm{E} \quad 00$

$1.15136 \mathrm{E} \quad 00$

$1.15136 \mathrm{E} \quad 00$

$1.15135 \mathrm{E} \quad 00$

$1.15134 \mathrm{E} \quad 00$

$1.15133 \mathrm{E} \quad 00$

$1.15132 \mathrm{E} \quad 00$ $\begin{array}{llll}-2.50508 \mathrm{E} & 01 & -1.81218 \mathrm{E} & 00\end{array}$

$\begin{array}{lllll}-2.51553 \mathrm{E} & 01 & -1.82525 \mathrm{E} & 00\end{array}$

$\begin{array}{llll}-2.52599 \mathrm{E} & 01 & -1.83837 \mathrm{E} & 00\end{array}$

$\begin{array}{llll}-2.53644 \mathrm{E} & 01 & -1.85154 \mathrm{E} & 00\end{array}$

$-2.54689 \mathrm{E} \quad 01$

$\begin{array}{ll}-2.55733 \mathrm{E} & 01\end{array}$

$-2.56778 \mathrm{E} \quad 01$

$-2.57822 \mathrm{E} \quad 01$

$-2.58866 \mathrm{E} \quad 01$

$-2.59910 \mathrm{E} \quad 01$

$-2.60954 \mathrm{E} \quad 01$

$-2.61998 \mathrm{E} 01$

$-2.63041 \mathrm{E} \quad 01$

$-2.64085 \mathrm{E} \quad 01$

$-2.65128 \mathrm{E} \quad 01$

$-2.66171 \mathrm{E} \quad 01$

$-2.67213 \mathrm{E} 01$

$-2.68256 \mathrm{E} \quad 01$

$-2.69298 \mathrm{E} 01$

$-2.70341 \mathrm{E} \quad 01$

$-2.71383 \mathrm{E} \quad 01$

$-2.72425 \mathrm{E} \quad 01$

$-2.73466 \mathrm{E} 01$

$-2.74508 \mathrm{E} 01$

$-2.75549 \mathrm{E} 01$

$-2.76590 \mathrm{E} \quad 01$

$-2.77631 \mathrm{E} 01$

$-2.78672 \mathrm{E} \quad 01$

$-2.79713 \mathrm{E} \quad 01$

$-2.80753 \mathrm{E} \quad 01$

$-2.81794 \mathrm{E} \quad 01$

$-2.82834 \mathrm{E} \quad 01$

$-2.83874 \mathrm{E} \quad 01$

$-2.84913 \mathrm{E} \quad 01$

$-2.85953 \mathrm{E} \quad 01$

$-2.86992 \mathrm{E} \quad 01$

$-2.88032 \mathrm{E} \quad 01$

$-2.89071 \mathrm{E} \quad 01$

$-2.90109 \mathrm{E} \quad 01$

$-2.91148 \mathrm{E} 01$.

$-2.92186 \mathrm{E} \quad 01$

$-2.93225 \mathrm{E} 01$

$-2.94263 \mathrm{E} 01$

$-2.95301 \mathrm{E} \quad 01$

$-2.96338 \mathrm{E} \quad 01$

$-2.97376 \mathrm{E} \quad 01$

$-2.98413 \mathrm{E} 01$

$-2.99450 \mathrm{E}$ 0l

$-3.00487 \mathrm{E} 01$

$-3.01524 \mathrm{E} \quad 01$

$-3.02561 \mathrm{E} 01$

$-3.03597 \mathrm{E} \quad 01$

$-3.04633 \mathrm{E} 01$

$-3.05669 \mathrm{E} 01$

$-3.06705 \mathrm{E} 01$

$-3.07740 \mathrm{E} 01$

$-3.08776 \mathrm{E} 01$

$-3.09811 \mathrm{E} 01$

$-3.10846 \mathrm{E} 01$

$-3.11881 \mathrm{E} \quad 01$
$-1.86475 \mathrm{E} 00$

$-1.87801 \mathrm{E} \quad 00$

$-1.89131 \mathrm{E} \quad 00$

$-1.90465 \mathrm{E} 00$

$-1.91804 \mathrm{E} \quad 00$

$-1.93148 \mathrm{E} 00$

$-1.94496 \mathrm{E} \quad 00$

$-1.95849 \mathrm{E} \quad 00$

$-1.97206 \mathrm{E} \quad 00$

$-1.98567 \mathrm{E} \quad 00$

$-1.99933 \mathrm{E} \quad 00$

$-2.01304 \mathrm{E} \quad 00$

$-2.02679 \mathrm{E} 00$

$-2.04058 \mathrm{E} \quad 00$

$-2.06830 \mathrm{E} \quad 00$

$-2.08223 \mathrm{E} \quad 00$

$-2.09621 \mathrm{E} \quad 00$

$-2.11022 \mathrm{E} \quad 00$

$-2.12429 \mathrm{E} \quad 00$

$-2.13839 \mathrm{E} 00$

$-2.15255 \mathrm{E} \quad 00$

$-2.16674 \mathrm{E} \quad 00$

$-2.18099 \mathrm{E} \quad 00$

$-2.19527 \mathrm{E} \quad 00$

$-2.20960 \mathrm{E} \quad 00$

$-2.22398 \mathrm{E} \quad 00$

$-2.23840 \mathrm{E} \quad 00$

$-2.25286 \mathrm{E} \quad 00$

$-2.26738 \mathrm{E} \quad 00$

$-2.28193 \mathrm{E} \quad 00$

$-2.29653 \mathrm{E} \quad 00$

$-2.31117 \mathrm{E} \quad 00$

$-2.32586 \mathrm{E} \quad 00$

$-2.34059 \mathrm{E} \quad 00$

$-2.35537 \mathrm{E} 00$

$-2.37019 \mathrm{E} \quad 00$

$-2.38506 \mathrm{E} 00$

$-2.41493 \mathrm{E} 00$

$-2.42993 \mathbf{E} 00$

$-2.44497 \mathrm{E} \quad 00$

$-2.47519 \mathrm{E} \quad 00$

$-2.49037 \mathrm{E} \quad 00$

$-2.50560 \mathrm{E} \quad 00$

$-2.52086 \mathrm{E} \quad 00$

$-2.53617 \mathrm{E} \quad 00$

$-2.55153 \mathrm{E} \quad 00$

$-2.56693 \mathrm{E} 00$

$-2.58238 \mathrm{E} \quad 00$

$-2.59786 \mathrm{E} \quad 00$

$-2.61340 \mathrm{E} \quad 00$

$-2.62898 \mathrm{E} \quad 00$

$-2.66027 \mathrm{E} \quad 00$
$-2.05442 \mathrm{E} \quad 00$

$-2.39997 \mathrm{E} \quad 00$

$-2.46006 \mathrm{E} \quad 00$

$-2.64460 \mathrm{E} \quad 00$

$\tan \delta_{1}$

$$
\rho=1.1 A^{1 / 3} F
$$

$3.89243 \mathbf{E}-03$

$3.89516 \mathrm{E}-03$

$3.89785 \mathrm{E}-03$

$3.90051 \mathrm{E}-03$

$3.90313 \mathrm{E}-03$

$3.90572 \mathrm{E}-03$

$3.90828 \mathrm{E}-03$

$3.91080 \mathrm{E}-03$

$3.91329 \mathrm{E}-03$

$3.91575 \mathrm{E}-03$

3.91817 E-03

$3.92057 \mathrm{E}-03$

$3.92293 \mathrm{E}-03$

$3.92527 \mathrm{E}-03$

3.92757E-03

3.92984E-03

$3.93209 \mathrm{E}-03$

$3.93430 \mathrm{E}-03$

$3.93648 \mathrm{E}-03$

$3.93863 \mathbf{E}-03$

$3.94075 \mathrm{E}-03$

$3.94285 \mathrm{E}-03$

$3.94491 \mathrm{E}-03$

$3.94696 \mathrm{E}-03$

$3.94896 \mathbf{E}-03$

$3.95095 \mathrm{E}-03$

$3.95291 \mathrm{E}-03$

3.95484 E-03

$3.95674 \mathrm{E}-03$

$3.95862 \mathrm{E}-03$

$3.96047 \mathrm{E}-03$

$3.96229 \mathrm{E}-03$

$3.96410 \mathrm{E}-03$

$3.96587 \mathrm{E}-03$

$3.96762 \mathrm{E}-03$

$3.96934 \mathrm{E}-03$

$3.97103 \mathrm{E}-03$

$3.97271 \mathrm{E}-03$

$3.97436 \mathrm{E}-03$

$3.97599 \mathrm{E}-03$

$3.97758 \mathbf{E}-03$

$3.97917 \mathrm{E}-03$

$3.98072 \mathrm{E}-03$ 
ELECTRON RADIAL FUNCTIONS

$Z=6 \quad A=12 \quad \rho=1.1 A^{1 / 3} F$

$\bar{F}$

$7.89317 \mathrm{E}-04$

$7.95877 \mathrm{E}-04$

8.02464 E-04

$8.09078 \mathrm{E}-04$

$8.15720 \mathrm{E}-04$

$8.22388 \mathrm{E}-04$

$8.29084 \mathrm{E}-04$

$8.35807 \mathrm{E}-04$

$8.42556 \mathrm{E}-04$

$8.49334 \mathrm{E}-04$

$8.56138 \mathrm{E}-04$

$8.62969 \mathrm{E}-04$

$8.69828 \mathrm{E}-04$

$8.76713 \mathrm{E}-04$

$8.83626 \mathrm{E}-04$

$8.90566 \mathrm{E}-04$

$8.97533 \mathrm{E}-04$

$9.04527 \mathrm{E}-04$

$9.11549 \mathrm{E}-04$

$9.18597 \mathrm{E}-04$

$9.25673 \mathrm{E}-04$

$9.32776 \mathrm{E}-04$

$9.39905 \mathrm{E}-04$

$9.47062 \mathrm{E}-04$

$9.54246 \mathrm{E}-04$

$9.61458 \mathrm{E}-04$

$9.68696 \mathrm{E}-04$

$9.75962 \mathrm{E}-04$

$9.83255 \mathrm{E}-04$

$9.90575 \mathrm{E}-04$.

$9.97922 \mathrm{E}-04$

$1.00530 \mathrm{E}-03$

$1.01270 \mathrm{E}-03$

$1.02013 \mathrm{E}-03$

$1.02758 \mathrm{E}-03$

$1.03506 \mathrm{E}-03$

$1.04257 \mathrm{E}-03$

$1.05011 \mathrm{E}-03$

$1.05767 \mathrm{E}-03$

$1.06527 \mathrm{E}-03$

$1.07288 \mathrm{E}-03$

$1.08053 \mathrm{E}-03$

$1.08820 \mathrm{E}-03$

$1.09590 \mathrm{E}-03$

$1.10363 \mathrm{E}-03$

$1.11138 \mathrm{E}-03$

$1.11916 \mathrm{E}-03$

$1.12697 \mathrm{E}-03$

$1.13481 \mathrm{E}-03$

$1.14267 \mathrm{E}-03$

$1.15056 \mathrm{E}-03$

$1.15848 \mathrm{E}-03$

$1.16642 \mathrm{E}-03$

$1.17439 \mathrm{E}-03$

$1.18239 \mathrm{E}-03$

$1.19041 \mathrm{E}-03$

$1.19847 \mathrm{E}-03$

$1.20655 \mathrm{E}-03$

$1.21465 \mathrm{E}-03$

$1.22279 \mathrm{E}-03$ $f_{2}$

$-1.31830 \mathrm{E} \quad 00$

$-1.32933 \mathrm{E} \quad 00$

$-1.34041 \mathrm{E} 00$

$-1.35154 \mathrm{E} 00$

$-1.36271 \mathrm{E} \quad 00$

$-1.37392 \mathrm{E} 00$

$-1.38518 \mathrm{E} \quad 00$

$-1.39649 \mathrm{E} 00$

$-1.40784 \mathrm{E} \quad 00$

$-1.41924 \mathrm{E} \quad 00$

$-1.43068 \mathrm{E} \quad 00$

$-1.44217 \mathrm{E} \quad 00$

$-1.45370 \mathrm{E} 00$

$-1.46528 \mathrm{E} \quad 00$

$-1.47690 \mathrm{E} 00$

- $1.48857 \mathrm{E} \quad 00$

$-1.50029 \mathrm{E} 00$

$-1.51205 \mathrm{E} 00$

$-1.52385 \mathrm{E} \quad 00$

$-1.53570 \mathrm{E} \quad 00$

$-1.54760 \mathrm{E} \quad 00$

$-1.55954 \mathrm{E} \quad 00$

$-1.57153 \mathrm{E} 00$

$-1.58356 \mathrm{E} \quad 00$

$-1.59564 \mathrm{E} \quad 00$

$-1.60776 \mathrm{E} \quad 00$

$-1.61993 \mathrm{E} 00$

$-1.63214 \mathrm{E} \quad 00$

$-1.64440 \mathrm{E} 00$

$-1.65670 \mathrm{E} 00$

$-1.66905 \mathrm{E} \quad 00$

$-1.68145 \mathrm{E} \quad 00$

$-1.69389 \mathrm{E} 00$

$-1.70637 \mathrm{E} 00$

$-1.71890 \mathrm{E} 00$

$-1.73148 \mathrm{E} \quad 00$

$-1.74410 \mathrm{E} 00$

$-1.75676 \mathrm{E} \quad 00$

$-1.76947 \mathrm{E} \quad 00$

$-1.78223 \mathrm{E} 00$

$-1.79503 \mathrm{E} 00$

$-1.80788 \mathrm{E} 00$

$-1.82077 \mathrm{E} 00$

$-1.83370 \mathrm{E} 00$

$-1.84669 \mathrm{E} \quad 00$

$-1.85971 \mathrm{E} \quad 00$ $-1.87279 \mathrm{E} \quad 00$ $-1.88590 \mathrm{E} \quad 00$ $-1.89906 \mathrm{E} \quad 00$ $-1.91227 \mathrm{E} 00$

$-1.92552 \mathrm{E} \quad 00$ $-1.93882 \mathrm{E} \quad 00$ $-1.95216 \mathrm{E} 00$ $-1.96555 \mathrm{E} \quad 00$ $-1.97898 \mathrm{E} 00$

$-1.99246 \mathrm{E} 00$ $-2.00598 \mathrm{E} 00$ $-2.01955 \mathrm{E} \quad 00$ $-2.03316 \mathrm{E} \quad 00$ $-2.04682 \mathrm{E} \quad 00$ $g_{2}$

$-5.64591 \mathrm{E}-02$

$-5.71055 \mathrm{E}-02$

$-5.77568 \mathrm{E}-02$

$-5.84130 \mathrm{E}-02$

$-5.90740 \mathrm{E}-02$

$-5.97400 \mathrm{E}-02$

$-6.04109 \mathrm{E}-02$

$-6.10867 \mathrm{E}-02$

$-6.17675 \mathrm{E}-02$

$-6.24532 \mathrm{E}-02$

$-6.31439 \mathrm{E}-02$

$-6.38397 \mathrm{E}-02$

$-6.45405 \mathrm{E}-02$

$-6.52463 \mathrm{E}-02$

$-6.59572 \mathrm{E}-02$

$-6.66732 \mathrm{E}-02$

$-6.73942 \mathrm{E}-02$

$-6.81204 \mathrm{E}-02$

$-6.88517 \mathrm{E}-02$

$-6.95882 \mathrm{E}-02$

$-7.03298 \mathrm{E}-02$

$-7.10766 \mathrm{E}-02$

$-7.18287 \mathrm{E}-02$

$-7.25859 \mathrm{E}-02$

$-7.33484 \mathrm{E}-02$

$-7.41161 \mathrm{E}-02$

$-7.48891 \mathrm{E}-02$

$-7.56675 \mathrm{E}-02$

$-7.64511 \mathrm{E}-02$

$-7.72400 \mathrm{E}-02$

$-7.80343 \mathrm{E}-02$

$-7.88340 \mathrm{E}-02$

$-7.96390 \mathrm{E}-02$

$-8.04494 \mathrm{E}-02$

$-8.12653 \mathrm{E}-02$

$-8.20865 \mathrm{E}-02$

$-8.29133 \mathrm{E}-02$

$-8.37455 \mathrm{E}-02$

$-8.45831 \mathrm{E}-02$

$-8.54263 \mathrm{E}-02$

$-8.62750 \mathrm{E}-02$

$-8.71292 \mathrm{E}-02$

$-8.79890 \mathrm{E}-02$

$-8.88544 \mathrm{E}-02$

$-8.97253 \mathrm{E}-02$

$-9.06019 \mathrm{E}-02$

$-9.14841 \mathrm{E}-02$

$-9.23719 \mathrm{E}-02$

$-9.32654 \mathrm{E}-02$

$-9.41645 \mathrm{E}-02$

$-9.50694 \mathrm{E}-02$

$-9.59799 \mathrm{E}-02$

$-9.68962 \mathrm{E}-02$

$-9.78183 \mathrm{E}-02$

$-9.87461 \mathrm{E}-02$

$-9.96797 \mathrm{E}-02$

$-1.00619 \mathrm{E}-01$

$-1.01564 \mathrm{E}-01$

$-1.02515 \mathrm{E}-01$

$-1.03472 \mathrm{E}-01$ $\tan \delta_{2}$

$3.42669 \mathrm{E} 01$

.42694 E 0l $-5.58932 \mathrm{E}-02$

$3.42718 \mathrm{E} 01 \quad-5.65322 \mathrm{E}-02$

$3.42742 \mathrm{E}$ 01 $-5.71759 \mathrm{E}-02$

$3.42766 \mathrm{E}$ ol $-5.78245 \mathrm{E}-02$

$3.42790 \mathrm{E} \quad 01$

$3.42813 \mathrm{E}$ 01

$3.42836 \mathrm{E} \quad 01$

$3.42860 \mathrm{E}$ 01

$3.42882 \mathrm{E} \quad 0 \mathrm{l}$

$3.42905 \mathrm{E} \quad 01$

$3.42928 \mathrm{E} \quad 01$

$3.42950 \mathrm{E} \quad 01$

$3.42972 \mathrm{E} \quad 01$

$3.42994 \mathrm{E} \quad 01$

$3.43016 \mathrm{E} \quad 01$

$3.43038 \mathrm{E} \quad 01$

$3.43059 \mathrm{E} \quad 01$

$3.43080 \mathrm{E} \quad 01$

$3.43101 \mathrm{E} \quad 01$

$3.43122 \mathrm{E} \quad 01$

$3.43143 \mathrm{E} \quad 01$

$3.43164 \mathrm{E} \quad 01$

$3.43184 \mathrm{E} \quad 01$

$3.43205 \mathrm{E} \quad 01$

$3.43225 \mathrm{E} \quad 01$

$3.43245 \mathrm{E}$ 01

$3.43265 \mathrm{E} \quad 01$

$3.43284 \mathrm{E}$ 0l

$3.43304 \mathrm{E} \quad 01$

$3.43323 \mathrm{E} \quad 01$

$3.43342 \mathrm{E} \quad 01$

$3.43362 \mathrm{E} \quad 01$

$3.43380 \mathrm{E}$ 0l

$3.43399 \mathrm{E} 01$

$3.43418 \mathrm{E} \quad 01$

$3.43437 \mathrm{E} \quad 01$

$3.43455 \mathrm{E} \quad 01$

$3.43473 \mathrm{E} \quad 01$

$3.43491 \mathrm{E} \quad 01$

$3.43509 \mathrm{E} \quad 01$

$3.43527 \mathrm{E} \quad 01$

$3.43545 \mathrm{E} \quad 01$

$3.43562 \mathrm{E} \quad 01$

$3.43580 \mathrm{E}$ 0l

$3.43597 \mathrm{E} \quad 01$

$3.43614 \mathrm{E} \quad 01$

$3.43631 \mathrm{E} \quad 01$

$3.43648 \mathrm{E} \quad 01$

$3.43665 \mathrm{E} \quad 01$

$3.43682 \mathrm{E} \quad 01$

$3.43699 \mathrm{E} \quad 01$

$3.43715 \mathrm{E} \quad 01$

$3.43732 \mathrm{E} \quad 01$

$3.43748 \mathrm{E} \quad 01$

$3.43764 \mathrm{E} \quad 01$

$3.43780 \mathrm{E} \quad 01$

$3.43796 \mathrm{E} \quad 01$

$3.43812 \mathrm{E} \quad 01$

$3.43827 \mathrm{E} \quad 01$

$-5.84779 \mathrm{E}-02$ $-5.91362 \mathrm{E}-02$

$-5.97994 \mathrm{E}-02$

$-6.04674 \mathrm{E}-02$

$-6.11404 \mathrm{E}-02$

$-6.18183 \mathrm{E}-02$

$-6.25012 \mathrm{E}-02$

$-6.31890 \mathrm{E}-02$

$-6.38818 \mathrm{E}-02$

$-6.45796 \mathrm{E}-02$

$-6.52825 \mathrm{E}-02$ $-6.59904 \mathrm{E}-02$

$-6.67033 \mathrm{E}-02$

$-6.74213 \mathrm{E}-02$

$-6.81444 \mathrm{E}-02$

$-6.88726 \mathrm{E}-02$ $-6.96059 \mathrm{E}-02$

$-7.03444 \mathrm{E}-02$

$-7.10880 \mathrm{E}-02$

$-7.18369 \mathrm{E}-02$

$-7.25909 \mathrm{E}-02$

$-7.33501 \mathrm{E}-02$

$-7.41146 \mathrm{E}-02$

$-7.48843 \mathrm{E}-02$

$-7.56593 \mathrm{E}-02$

$-7.64395 \mathrm{E}-02$

$-7.72251 \mathrm{E}-02$

$-7.80160 \mathrm{E}-02$

$-7.88122 \mathrm{E}-02$

$-7.96138 \mathrm{E}-02$

$-8.04207 \mathrm{E}-02$

$-8.12331 \mathrm{E}-02$

$-8.20508 \mathrm{E}-02$

$-8.28740 \mathrm{E}-02$

$-8.37026 \mathrm{E}-02$

$-8.45366 \mathrm{E}-02$

$-8.53762 \mathrm{E}-02$

$-8.62212 \mathrm{E}-02$

$-8.70718 \mathrm{E}-02$

$-8.79279 \mathrm{E}-02$

$-8.87895 \mathrm{E}-02$

$-8.96567 \mathrm{E}-02$ 
ELECTRON RADIAL FUNCTIONS

Electron

$Z=6 \quad A=12 \quad \rho=1.1 A^{1 / 3} F$

30.10

30.20

30.30

30.40

30.50

30.60

30.70

30.80

30.90

31.00

31.10

31.20

31.30

31.40

31.50

31.60

31.70

31.80

31.90

32.00

32.10

32.20

32.30

32.40

32.50

32.60

32.70

32.80

32.90

33.00

33.10

33.20

33.30

33.40

33.50

33.60

33.70

33.80

33.90

34.00

34.10

34.20

34.30

34.40

34.50

34.60

34.70

34.80

34.90

35.00

35.10

35.20

35.30

35.40

35.50

35.60

35.70

35.80

35.90

36.00
$F_{0}$

$1.15132 \mathrm{E} \quad 00$

$1.15131 \mathrm{E} \quad 00$

$1.15130 \mathrm{E} \quad 00$

$1.15129 \mathrm{E} \quad 00$

$1.15128 \mathrm{E} \quad 00$

$1.15128 \mathrm{E} \quad 00$

$1.15127 \mathrm{E} \quad 00$

$1.15126 \mathrm{E} \quad 00$

$1.15125 \mathrm{E} \quad 00$

$1.15125 \mathrm{E} \quad 00$

$1.15124 \mathrm{E} \quad 00$

$1.15123 \mathrm{E} \quad 00$

$1.15122 \mathrm{E} \quad 00$

$1.15122 \mathrm{E} \quad 00$

$1.15121 \mathrm{E} \quad 00$

$1.15120 \mathrm{E} \quad 00$

$1.15119 \mathrm{E} \quad 00$

$1.15119 \mathrm{E} \quad 00$

$1.15118 \mathrm{E} \quad 00$

$1.15117 \mathrm{E} \quad 00$

$1.15116 \mathrm{E} \quad 00$

$1.15116 \mathrm{E} \quad 00$

$1.15115 \mathrm{E} \quad 00$

$1.15114 \mathrm{E} \quad 00$

$1.15113 \mathrm{E} \quad 00$

$1.15113 \mathrm{E} \quad 00$

$1.15112 \mathrm{E} \quad 00$

$1.15111 \mathrm{E} \quad 00$

$1.15111 \mathrm{E} 00$

$1.15110 \mathrm{E} \quad 00$

$1.15109 \mathrm{E} \quad 00$

$1.15108 \mathrm{E} \quad 00$

$1.15108 \mathrm{E} \quad 00$

$1.15107 \mathrm{E} \quad 00$

$1.15106 \mathrm{E} \quad 00$

$1.15106 \mathrm{E} \quad 00$

$1.15105 \mathrm{E} \quad 00$

$1.15104 \mathrm{E} \quad 00$

$1.15104 \mathrm{E} \quad 00$

$1.15103 \mathrm{E} \quad 00$

$1.15102 \mathrm{E} \quad 00$ $1.15101 \mathrm{E} \quad 00$ $1.15101 \mathrm{E} \quad 00$

$1.15100 \mathrm{E} \quad 00$

$1.15099 \mathrm{E} \quad 00$

$1.15099 \mathrm{E} \quad 00$

$1.15098 \mathrm{E} \quad 00$

$1.15097 \mathrm{E} \quad 00$

$1.15097 \mathrm{E} \quad 00$

$1.15096 \mathrm{E} \quad 00$

$1.15095 \mathrm{E} \quad 00$

$1.15095 \mathrm{E} \quad 00$

$1.15094 \mathrm{E} \quad 00$

$1.15093 \mathrm{E} \quad 00$

$1.15093 \mathrm{E} \quad 00$

$1.15092 \mathrm{E} \quad 00$

$1.15091 \mathrm{E} \quad 00$

$1.15091 \mathrm{E} \quad 00$

$1.15090 \mathrm{E} \quad 00$

$1.15090 \mathrm{E} \quad 00$ $f_{1}$

$\begin{array}{llll}-3.12916 \mathrm{E} & 01 & -2.67598 \mathrm{E} & 00\end{array}$

$\begin{array}{llll}-3.13950 \mathrm{E} & 01 & -2.69173 \mathrm{E} & 00\end{array}$

$-3.14984 \mathrm{E} \quad 01 \quad-2.70753 \mathrm{E} \quad 00$

$-3.16018 \mathrm{E}$ 01 $-2.72338 \mathrm{E} \quad 00$

$-3.17052 \mathrm{E} 01$

$-3.18086 \mathrm{E} \quad 01$

$-3.19119 \mathrm{E} \quad 01$

$-3.20153 \mathrm{E} 01$

$-3.21186 \mathrm{E} 01$

$-3.22219 \mathrm{E} 01$

$-3.23251 \mathrm{E} \quad 01$

$-3.24284 \mathrm{E} 01$

$-3.25316 \mathrm{E} 01$

$-3.26348 \mathrm{E} \quad 01$

$-3.27380 \mathrm{E} \quad 01$

$-3.28412 \mathrm{E} \quad 01$

$-3.29443 \mathrm{E} 01$

$-3.30474 \mathrm{E} 01$

$-3.31505 \mathrm{E} 01$

$-3.32536 \mathrm{E} 01$

$-3.33567 \mathrm{E} \quad 01$

$-3.34597 \mathrm{E} \quad 01$

$-3.35628 \mathrm{E} 01$

$-3.36658 \mathrm{E} 01$

$-3.37688 \mathrm{E} 01$

$-3.38717 \mathrm{E} \quad 01$

$-3.39747 \mathrm{E} 01$

$-3.40776 \mathrm{E} 01$

$-3.41805 \mathrm{E} \quad 01$

$-3.42834 \mathrm{E} \quad 01$

$-3.43862 \mathrm{E} \quad 01$

$-3.44891 \mathrm{E} \quad 01$

$-3.45919 \mathrm{E} 01$

$-3.46947 \mathrm{E} 01$

-3.47975 E 01

$-3.49002 \mathrm{E} \quad 01$

$-3.50030 \mathrm{E} \quad 01$

$-3.51057 \mathrm{E} 01$

$-3.52084 \mathrm{E} \quad 01$

$-3.53111 \mathrm{E} 01$.

$-3.54137 \mathrm{E} \quad 01$

$-3.55163 \mathrm{E} 01$

$-3.56190 \mathrm{E} 01$

$-3.57215 \mathrm{E} 01$

-3.58241 E 01

$-3.59267 \mathrm{E} \quad 01$

$-3.60292 \mathrm{E} \quad 01$

$-3.61317 \mathrm{E} 01$

$-3.62342 \mathrm{E} 01$

$-3.63366 \mathrm{E} 01$

$-3.64391 \mathrm{E} \quad 01$

$-3.65415 \mathrm{E} \quad 01$

$-3.66439 \mathrm{E} 01$

$\begin{array}{lll}-3.67463 \mathrm{E} & 01\end{array}$

-3.68486 E 01

$-3.69510 \mathrm{E} \quad 01$

$-3.70533 \mathrm{E} \quad 01$

$-3.71556 \mathbf{E} 01$

$-3.72578 \mathrm{E} \quad 01$

$-3.73601 \mathrm{E} 01$

$-3.21927 \mathrm{E} \quad 00$

$-3.57188 \mathrm{E} \quad 00$ $\tan \delta_{1}$

$-2.73927 \mathrm{E} \quad 00$

$-2.75520 \mathrm{E} \quad 00$

$-2.77118 \mathrm{E} \quad 00$

$-2.78720 \mathrm{E} 00$

$-2.80326 \mathrm{E} \quad 00$

$-2.81937 \mathrm{E} \quad 00$

$-2.83553 \mathrm{E} 00$

$-2.85173 \mathrm{E} \quad 00$

$-2.86797 \mathrm{E} 00$

$-2.88426 \mathrm{E} \quad 00$

$-2.90059 \mathrm{E} \quad 00$

$-2.91696 \mathrm{E} \quad 00$

$-2.93338 \mathrm{E} \quad 00$

$-2.94985 \mathrm{E} \quad 00$

$-2.96636 \mathrm{E} \quad 00$

$-2.98291 \mathrm{E} 00$

$-2.99950 \mathrm{E} \quad 00$

$-3.01614 \mathrm{E} \quad 00$

$-3.03283 \mathrm{E} \quad 00$

$-3.04956 \mathrm{E} 00$

$-3.06633 \mathrm{E} 00$

$-3.08315 \mathrm{E} \quad 00$

$-3.10001 \mathrm{E} \quad 00$

$-3.11691 \mathrm{E} \quad 00$

$-3.13386 \mathrm{E} \quad 00$

$-3.15086 \mathrm{E} 00$

$-3.16789 \mathrm{E} \quad 00$

$-3.18497 \mathrm{E} \quad 00$

$-3.20210 \mathrm{E} \quad 00$

$-3.23648 \mathrm{E} \quad 00$

$-3.25374 \mathrm{E} \quad 00$

$-3.27104 \mathrm{E} 00$

$-3.28839 \mathrm{E} \quad 00$

$-3.30577 \mathrm{E} \quad 00$

$-3.32321 \mathrm{E} \quad 00$

$-3.34068 \mathrm{E} \quad 00$

$-3.35821 \mathrm{E} \quad 00$

$-3.37577 \mathrm{E} 00$

$-3.39338 \mathrm{E} \quad 00$

$-3.41103 \mathrm{E} 00$

$-3.42873 \mathrm{E} \quad 00$

$-3.44647 \mathrm{E} \quad 00$

$-3.46425 \mathrm{E} \quad 00$

$-3.48208 \mathrm{E} \quad 00$

-3.49995 E 00

$-3.51787 \mathrm{E} \quad 00$

$-3.53583 \mathrm{E} 00$

$-3.55383 \mathrm{E} 00$

$-3.58997 \mathrm{E} \quad 00$

$-3.60810 \mathrm{E} 00$

$-3.62628 \mathrm{E} \quad 00$

$-3.64450 \mathrm{E} \quad 00$

$-3.66277 \mathrm{E} \quad 00$

$-3.68108 \mathrm{E} 00$

$4.00488 \mathrm{E}-03$

$4.00603 \mathrm{E}-03$

$4.00715 \mathrm{E}-03$

$4.00826 \mathrm{E}-03$

$4.00934 \mathrm{E}-03$

$4.01040 \mathrm{E}-03$

$4.01145 \mathrm{E}-03$

$4.01247 \mathrm{E}-03$

$4.01348 \mathrm{E}-03$

$4.01446 \mathrm{E}-03$

4.01544 E-03

$4.01639 \mathrm{E}-03$

$4.01732 \mathrm{E}-03$

$4.01823 \mathrm{E}-03$

$4.01913 \mathrm{E}-03$

$4.02000 \mathrm{E}-03$

$4.02086 \mathrm{E}-03$

$4.02171 \mathrm{E}-03$

$4.02253 \mathrm{E}-03$

$4.02334 \mathrm{E}-03$

$4.02413 \mathrm{E}-03$

$4.02490 \mathrm{E}-03$

$4.02565 \mathrm{E}-03$

$4.02639 \mathrm{E}-03$

$4.02712 \mathrm{E}-03$

$4.02782 \mathrm{E}-03$

$4.02850 \mathrm{E}-03$

$4.02918 \mathrm{E}-03$

$4.02983 \mathrm{E}-03$

$4.03047 \mathrm{E}-03$

$4.03109 \mathrm{E}-03$

$4.03169 \mathrm{E}-03$

$4.03229 \mathrm{E}-03$

$4.03286 \mathrm{E}-03$

$4.03342 \mathrm{E}-03$

$4.03396 \mathrm{E}-03$

$4.03449 \mathrm{E}-0.3$

$4.03500 \mathrm{E}-03$

$4.03549 \mathrm{E}-0.3$

$4.03597 \mathrm{E}-03$

$4.03644 \mathrm{E}-0.3$

$4.03690 \mathrm{E}-03$

$4.03733 \mathrm{E}-03$

$4.03775 \mathrm{E}-03$

$4.03815 \mathrm{E}-03$

$4.03855 \mathrm{E}-03$ 
ELECTRON RADIAL FUNCTIONS

$Z=6 \quad A=12 \quad \rho=1.1 A^{1 / 3} F$

Elec:trisn

$\bar{F}$

$1.23095 \mathrm{E}-03$

$1.23914 \mathrm{E}-03$

$1.24735 \mathrm{E}-03$

$1.25559 \mathrm{E}-03$

$1.26386 \mathrm{E}-03$

$1.27216 \mathrm{E}-03$

$1.28048 \mathrm{E}-03$

$1.28883 \mathrm{E}-03$

$1.29721 \mathrm{E}-03$

$1.30562 \mathrm{E}-03$

$1.31405 \mathrm{E}-03$

$1.32251 \mathrm{E}-03$

$1.33100 \mathrm{E}-03$

$1.33951 \mathrm{E}-03$

$1.34805 \mathrm{E}-03$

$1.35662 \mathrm{E}-03$

$1.36521 \mathrm{E}-03$

$1.37384 \mathrm{E}-03$

$1.38248 \mathrm{E}-03$

$1.39116 \mathrm{E}-03$

$1.39986 \mathrm{E}-03$

$1.40860 \mathrm{E}-03$

$1.41735 \mathrm{E}-03$

$1.42614 \mathrm{E}-03$

$1.43495 \mathrm{E}-03$

$1.44379 \mathrm{E}-03$

$1.45266 \mathrm{E}-03$

$1.46155 \mathrm{E}-03$

$1.47047 \mathrm{E}-03$

$1.47942 \mathrm{E}-03$

$1.48839 \mathrm{E}-03$

$1.49739 \mathrm{E}-03$

$1.50642 \mathrm{E}-03$

$1.51548 \mathrm{E}-03$

$1.524 .56 \mathrm{E}-03$

$1.53367 \mathrm{E}-03$

$1.54281 \mathrm{E}-03$

$1.55198 \mathrm{E}-03$

$1.56117 \mathrm{E}-03$

$1.57039 \mathrm{E}-03$

$1.57963 \mathrm{E}-03$

$1.58891 \mathrm{E}-03$

$1.59821 \mathrm{E}-03$

$1.60753 \mathrm{E}-03$

$1.61689 \mathrm{E}-03$

$1.62627 \mathrm{E}-03$

$1.63568 \mathrm{E}-03$

$1.64512 \mathrm{E}-03$

$1.654 .58 \mathrm{E}-03$

$1.66407 \mathrm{E}-03$

$1.67359 \mathrm{E}-03$

$1.68313 \mathrm{E}-03$

$1.69270 \mathrm{E}-03$

$1.70230 \mathrm{E}-03$

$1.71193 \mathrm{E}-03$

$1.72158 \mathrm{E}-03$

$1.73126 \mathrm{E}-03$

$1.74097 \mathrm{E}-03$

$1.75070 \mathrm{E}-03$

$1.76046 \mathrm{E}-03$ $f_{2}$

$-2.06052 \mathrm{E} \quad 00$

$-2.07427 \mathrm{E} \quad 00$

$-2.08806 \mathrm{E} \quad 00$

$-2.10190 \mathrm{E} \quad 00$

$-2.11578 \mathrm{E} \quad 00$

$-2.12971 \mathrm{E} \quad 00$

$-2.14368 \mathrm{E} \quad 00$

$-2.15770 \mathrm{E} \quad 00$

$-2.17176 \mathrm{E} \quad 00$

$-2.18587 \mathrm{E} \quad 00$

$-2.20002 \mathrm{E} \quad 00$

$-2.21422 \mathrm{E} \quad 00$

$-2.22846 \mathrm{E} 00$

$-2.24275 \mathrm{E} \quad 00$

$-2.25708 \mathrm{E} \quad 00$

$-2.27145 \mathrm{E} \quad 00$

$-2.28588 \mathrm{E} \quad 00$

$-2.30034 \mathrm{E} \quad 00$

$-2.31485 \mathrm{E} \quad 00$

$-2.32941 \mathrm{E} \quad 00$

$-2.34401 \mathrm{E} \quad 00$

$-2.35865 \mathrm{E} \quad 00$

$-2.37334 \mathrm{E} \quad 00$

$-2.38808 \mathrm{E} \quad 00$

$-2.40286 \mathrm{E} \quad 00$

$-2.41768 \mathrm{E} \quad 00$

$-2.43255 \mathrm{E} \quad 00$

$-2.44746 \mathrm{E} \quad 00$

$-2.46242 \mathrm{E} \quad 00$

$-2.47743 \mathrm{E} \quad 00$

$-2.49247 \mathrm{E} \quad 00$ $-2.50757 \mathrm{E} \quad 00$ $-2.52271 \mathrm{E} \quad 00$

$-2.53789 \mathrm{E} \quad 00$

$-2.55311 \mathrm{E} \quad 00$

$-2.56839 \mathrm{E} \quad 00$

$-2.58370 \mathrm{E} \quad 00$

$-2.59906 \mathrm{E} \quad 00$

$-2.61447 \mathrm{E} \quad 00$

$-2.62992 \mathrm{E} \quad 00$

$-2.64541 \mathrm{E} \quad 00$

$-2.66095 \mathrm{E} \quad 00$

$-2.67654 \mathrm{E} \quad 00$

$-2.69216 \mathrm{E} \quad 00$

$-2.70784 \mathrm{E} \quad 00$

$-2.72355 \mathrm{E} \quad 00$

$-2.73932 \mathrm{E} \quad 00$

$-2.75512 \mathrm{E} \quad 00$

$-2.77097 \mathrm{E} \quad 00$

$-2.78687 \mathrm{E} \quad 00$

$-2.80281 \mathrm{E} \quad 00$ $-2.81880 \mathrm{E} \quad 00$ $-2.83482 \mathrm{E} \quad 00$ $-2.85090 \mathrm{E} \quad 00$ $-2.86702 \mathrm{E} 00$

$-2.88318 \mathrm{E} \quad 00$ $-2.89939 \mathrm{E} \quad 00$ $-2.91564 \mathrm{E} \quad 00$ $-2.93193 \mathrm{E} 00$ $-2.94827 \mathrm{E} \quad 00$ $g_{2}$

$-1.04435 \mathrm{E}-01$

$-1.05404 \mathrm{E}-01$

$-1.06378 \mathrm{E}-01$

$-1.07359 \mathrm{E}-01$

$-1.08345 \mathrm{E}-01$

$-1.09337 \mathrm{E}-01$

$-1.10336 \mathrm{E}-01$

$-1.11340 \mathrm{E}-01$

$-1.12350 \mathrm{E}-01$

$-1.13367 \mathrm{E}-01$

$-1.14389 \mathrm{E}-01$

$-1.15418 \mathrm{E}-01$

$-1.16452 \mathrm{E}-01$

$-1.17493 \mathrm{E}-01$

$-1.18539 \mathrm{E}-01$

$-1.19592 \mathrm{E}-01$

$-1.20651 \mathrm{E}-01$

$-1.21717 \mathrm{E}-01$

$-1.22788 \mathrm{E}-01$

$-1.23866 \mathrm{E}-01$

$-1.24949 \mathrm{E}-01$

$-1.26739 \mathrm{E}-01$

$-1.27136 \mathrm{E}-01$

$-1.28238 \mathrm{E}-01$

$-1.2934 .7 \mathrm{E}-01$

$-1.30462 \mathrm{E}-01$

$-1.31583 \mathrm{E}-01$

$-1.32711 \mathrm{E}-01$

- i.33845 E-01

$-1.34986 \mathrm{E}-01$

$-1.36133 \mathrm{E}-01$

$-1.37286 \mathrm{E}-01$

$-1.38446 \mathrm{E}-01$

$-1.39612 \mathrm{E}-01$

$-1.40785 \mathrm{E}-01$

$-1.41964 \mathrm{E}-01$

$-1.43149 \mathrm{E}-01$

$-1.44341 \mathrm{E}-01$

$-1.45540 \mathrm{E}-01$

$-1.46745 \mathrm{E}-01$

$-1.47957 \mathrm{E}-01$

$-1.49175 \mathrm{E}-01$

$-1.50400 \mathrm{E}-01$

$-1.51632 \mathrm{E}-01$

$-1.52870 \mathrm{E}-01$

$-1.54115 \mathrm{E}-01$

$-1.55366 \mathrm{E}-01$

$-1.56624 \mathrm{E}-01$

$-1.57889 \mathrm{E}-01$

$-1.59161 \mathrm{E}-01$

$-1.60439 \mathrm{E}-01$

$-1.61724 \mathrm{E}-01$

$-1.63016 \mathrm{E}-01$

$-1.64315 \mathrm{E}-01$

$-1.65620 \mathrm{E}-01$

$-1.66932 \mathrm{E}-01$

$-1.68251 \mathrm{E}-01$

$-1.69577 \mathrm{E}-01$

$-1.70910 \mathrm{E}-01$

$-1.72250 \mathrm{E}-01$ $\tan \delta_{2}$

$f_{-2}$

3.43843 E 01

3.43874E $01-1.04303 \mathrm{E}-0 \mathrm{I}$

$3.43889 \mathrm{E}$ 01 $-1.05267 \mathrm{E}-01$

$3.43904 \mathrm{E}$ 0l $-1.06238 \mathrm{E}-01$

$3.43919 \mathrm{E}$ 01 $-1.07214 \mathrm{E}-01$

$3.43934 \mathrm{E}$ 01 $-1.08196 \mathrm{E}-01$

$3.43949 \mathrm{E}$ 01 $-1.09184 \mathrm{E}-01$

$3.43964 \mathrm{E}$ 01 $-1.10178 \mathrm{E}-01$

$3.43979 \mathrm{E}$ 01 $-1.11178 \mathrm{E}-01$

$3.43993 \mathrm{E} \quad 01$

$3.44008 \mathrm{E} \quad 01$

$3.44022 \mathrm{E} 01$

$3.44037 \mathrm{E} \quad 01$

$3.44051 \mathrm{E} \quad 01$

$-1.12184 \mathrm{E}-01$

$-1.13196 \mathrm{E}-01$

$-1.14214 \mathrm{E}-01$

$-1.15238 \mathrm{E}-01$

$-1.16268 \mathrm{E}-01$

$3.44065 \mathrm{E} \quad 01$

$3.44079 \mathrm{E} \quad 01$

$3.44093 \mathrm{E} 01$

$3.44107 \mathrm{E} \quad 01$

$3.44120 \mathrm{E} \quad 01$

3.44.134 E 01

$3.44148 \mathrm{E} \quad 01$

$3.44161 \mathrm{E} \quad 01$

$3.44174 \mathrm{E}$ 0I

$3.44188 \mathrm{E} \quad 01$

$-1.17305 \mathrm{E}-01$

$-1.18347 \mathrm{E}-01$

$-1.19395 \mathrm{E}-01$

$-1.20450 \mathrm{E}-01$

$-1.21510 \mathrm{E}-01$

$3.44201 \mathrm{E} 01$

$3.44214 \mathrm{E} \quad 01$

$3.44227 \mathrm{E} \quad 01$

$3.44240 \mathrm{E} 01$

$3.44253 \mathrm{E} \quad 01$

$3.44266 \mathrm{E} \quad 01$

$3.44279 \mathrm{E} \quad 01$

$3.44291 \mathrm{E} 01$

$3.44304 \mathrm{E} \quad 01$

$3.44316 \mathrm{E} \quad 01$

$3.44329 \mathrm{E} \quad 01$

$3.4434 .1 \mathrm{E} \quad 01$

$3.44353 \mathrm{E} 01$

$3.44366 \mathrm{E} \quad 01$

$3.44378 \mathrm{E} \quad 01$

$3.44390 \mathrm{E} \quad 01$

$3.44402 \mathrm{E} \quad 01$

$3.44414 \mathrm{E} \quad 01$

$3.44426 \mathrm{E} \quad 01$

$3.44437 \mathrm{E} \quad 01$

$3.44449 \mathrm{E} \quad 01$

$3.44461 \mathrm{E} \quad 01$

$3.44472 \mathrm{E} \quad 01$

$3.44484 \mathrm{E} \quad 01$

$3.44495 \mathrm{E} \quad 01$

$3.44506 \mathrm{E} \quad 0 \mathrm{l}$

$3.44518 \mathrm{E} 01$

$3.44529 \mathrm{E} \quad 01$

$3.44540 \mathrm{E} \quad 01$

$3.44551 \mathrm{E} \quad 01$

$-1.22577 \mathrm{E}-01$

$-1.23650 \mathrm{E}-01$

$-1.24729 \mathrm{E}-01$

$-1.25814 \mathrm{E}-01$

$-1.26906 \mathrm{E}-01$

$-1.28004 \mathrm{E}-01$

$-1.29108 \mathrm{E}-01$

$-1.30218 \mathrm{E}-01$

$-1.31335 \mathrm{E}-01$

$-1.32458 \mathrm{E}-01$

$-1.33587 \mathrm{E}-01$ $-1.34723 \mathrm{E}-01$ $-1.35865 \mathrm{E}-01$ $-1.37013 \mathrm{E}-01$

$-1.38168 \mathrm{E}-01$

$-1.39329 \mathrm{E}-01$ $-1.40497 \mathrm{E}-01$ $-1.41671 \mathrm{E}-01$ $-1.42851 \mathrm{E}-01$ $-1.44039 \mathrm{E}-01$

$-1.45232 \mathrm{E}-01$ $-1.46432 \mathrm{E}-01$ $-1.47639 \mathrm{E}-01$ $-1.48852 \mathrm{E}-01$ $-1.50072 \mathrm{E}-01$

$-1.51298 \mathrm{E}-01$ $-1.52531 \mathrm{E}-01$ $-1.53770 \mathrm{E}-01$ $-1.55017 \mathrm{E}-01$ $-1.56269 \mathrm{E}-01$

$3.44562 \mathrm{E} \quad 01$ $3.44573 \mathrm{E}$ 01 $3.44 .584 \mathrm{E} \quad 01$ $3.44595 \mathrm{E} 01$

$3.44606 \mathrm{E} \quad 01$

$-1.57529 \mathrm{E}-01$ 


$$
Z=6 \quad A=12 \quad \rho=1.1 A^{1 / 3} F
$$

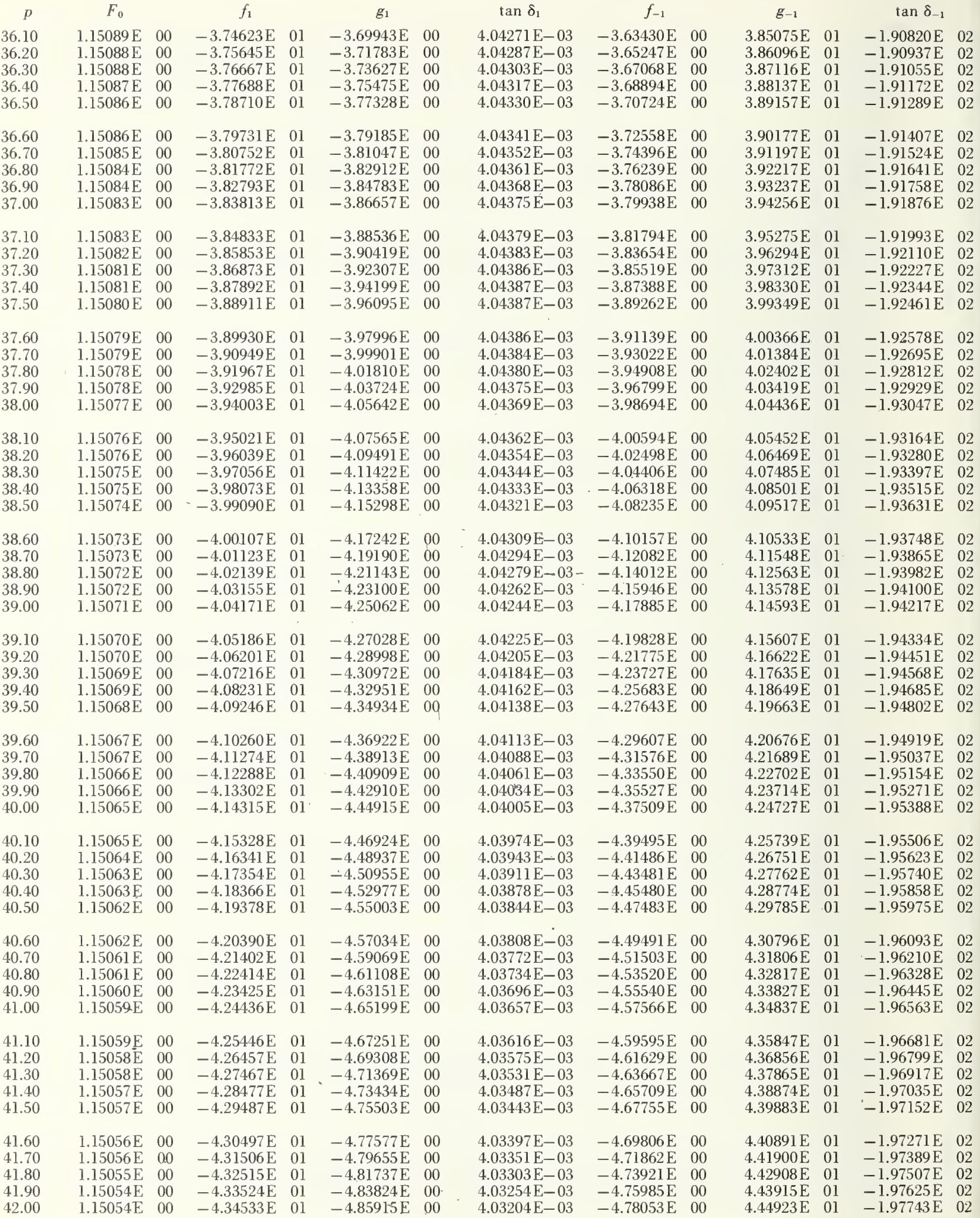


$\bar{F}$

$1.77025 \mathrm{E}-03$ $1.78007 \mathrm{E}-03$ $1.78991 \mathrm{E}-03$ $1.79978 \mathrm{E}-03$ $1.80968 \mathrm{E}-03$

$1.81960 \mathrm{E}-03$ $1.82956 \mathrm{E}-03$ $1.83953 \mathrm{E}-03$ $1.84954 \mathrm{E}-03$ $1.85957 \mathrm{E}-03$

$1.86963 \mathrm{E}-03$ $1.87972 \mathrm{E}-03$ $1.88983 \mathrm{E}-03$ $1.89998 \mathrm{E}-03$ $1.91014 \mathrm{E}-03$

$1.92034 \mathrm{E}-03$ $1.93056 \mathrm{E}-03$ $1.94081 \mathrm{E}-03$ $1.95109 \mathrm{E}-03$ $1.96139 \mathrm{E}-03$

$1.97172 \mathrm{E}-03$ $1.98208 \mathrm{E}-03$ $1.99247 \mathrm{E}-03$ $2.00288 \mathrm{E}-03$ $2.01332 \mathrm{E}-03$

$2.02379 \mathrm{E}-03$ $2.03428 \mathrm{E}-03$ $2.04480 \mathrm{E}-03$ $2.05535 \mathrm{E}-03$ $2.06593 \mathrm{E}-03$

$2.07653 \mathrm{E}-03$ $2.08716 \mathrm{E}-03$ $2.09782 \mathrm{E}-03$ $2.10850 \mathrm{E}-03$ $2.11921 \mathrm{E}-03$

$2.12995 \mathrm{E}-03$ $2.14071 \mathrm{E}-03$ $2.15151 \mathrm{E}-03$ $2.16233 \mathrm{E}-03$ $2.17317 \mathrm{E}-03$

$2.18405 \mathrm{E}-03$ $2.19495 \mathrm{E}-03$ $2.20587 \mathrm{E}-03$ $2.21683 \mathrm{E}-03$ $2.22781 \mathrm{E}-03$

$2.23882 \mathrm{E}-03$ $2.24986 \mathrm{E}-03$ $2.26092 \mathrm{E}-03$ $2.27201 \mathrm{E}-03$ $2.28313 \mathrm{E}-03$

$2.29428 \mathrm{E}-03$ $2.30545 \mathrm{E}-03$ $2.31665 \mathrm{E}-03$ $2.32787 \mathrm{E}-03$ $2.33913 \mathrm{E}-03$

$2.35041 \mathrm{E}-03$ $2.36171 \mathrm{E}-03$ $2.37305 \mathrm{E}-03$ $2.38441 \mathrm{E}-03$ $2.39580 \mathrm{E}-03$

\section{$f_{2}$}

$-2.96466 \mathrm{E} \quad 00$ $-2.98109 \mathrm{E} 00$ $-2.99756 \mathrm{E} \quad 00$ $-3.01408 \mathrm{E} 00$

$-3.03064 \mathrm{E} \quad 00$

$-3.04725 \mathrm{E} 00$ $-3.06390 \mathrm{E} 00$ $-3.08060 \mathrm{E} 00$ $-3.09734 \mathrm{E} \quad 00$

$-3.11412 \mathrm{E} 00$

$-3.13095 \mathrm{E} \quad 00$ $-3.14782 \mathrm{E} \quad 00$ $-3.16474 \mathrm{E} \quad 00$ $-3.18170 \mathrm{E} \quad 00$ $-3.19871 \mathrm{E} 00$

$-3.21576 \mathrm{E} 00$ $-3.23285 \mathrm{E} \quad 00$ $-3.24999 \mathrm{E} \quad 00$ $-3.26717 \mathrm{E} \quad 00$ $-3.28440 \mathrm{E} 00$

$-3.30167 \mathrm{E} \quad 00$ $-3.31898 \mathrm{E} \quad 00$ $-3.33634 \mathrm{E} \quad 00$ $-3.35375 \mathrm{E} 00$ $-3.37120 \mathrm{E} 00$

$-3.38869 \mathrm{E} 00$ $-3.40622 \mathrm{E} \quad 00$ $-3.42380 \mathrm{E} \quad 00$ $-3.44143 \mathrm{E} 00$ $-3.45910 \mathrm{E} \quad 00$

$-3.47681 \mathrm{E} \quad 00$ $-3.49457 \mathrm{E} \quad 00$ $-3.51237 \mathrm{E} \quad 00$ $-3.53021 \mathrm{E} 00$ $-3.54810 \mathrm{E} \quad 00$

$-3.56603 \mathrm{E} \quad 00$ $-3.58401 \mathrm{E} 00$ $-3.60203 \mathrm{E} 00$ $-3.62009 \mathrm{E} \quad 00$ $-3.63820 \mathrm{E} 00$

$-3.65636 \mathrm{E} \quad 00$ $-3.67455 \mathrm{E} \quad 00$ $-3.69280 \mathrm{E} 00$ $-3.71108 \mathrm{E} \quad 00$ $-3.72941 \mathrm{E} 00$

$-3.74778 \mathrm{E} 00$ $-3.76620 \mathrm{E} 00$ $-3.78466 \mathrm{E} 00$ $-3.80316 \mathrm{E} 00$ $-3.82171 \mathrm{E} 00$

$-3.84030 \mathrm{E} \quad 00$ $-3.85894 \mathrm{E} 00$ $-3.87762 \mathrm{E} \quad 00$ $-3.89634 \mathrm{E} 00$ $-3.91511 \mathrm{E} 00$

$-3.93392 \mathrm{E} 00$ $-3.95278 \mathrm{E} 00$ $-3.97168 \mathrm{E} \quad 00$ $-3.99062 \mathrm{E} 00$ $-4.00961 \mathrm{E} 00$ $g_{2}$

$-1.73596 \mathrm{E}-01$ $-1.74950 \mathrm{E}-01$ $-1.76310 \mathrm{E}-01$ $-1.77678 \mathrm{E}-01$ $-1.79052 \mathrm{E}-01$

$-1.80434 \mathrm{E}-01$ $-1.81822 \mathrm{E}-01$ $-1.83217 \mathrm{E}-01$ $-1.84620 \mathrm{E}-01$ $-1.86029 \mathrm{E}-01$

$-1.87446 \mathrm{E}-01$ $-1.88870 \mathrm{E}-01$ $-1.90300 \mathrm{E}-01$ $-1.91738 \mathrm{E}-01$ $-1.93183 \mathrm{E}-01$

$-1.94636 \mathrm{E}-01$ $-1.96095 \mathrm{E}-01$ $-1.97562 \mathrm{E}-01$ $-1.99036 \mathrm{E}-01$ $-2.00517 \mathrm{E}-01$

$-2.02005 \mathrm{E}-01$ $-2.03501 \mathrm{E}-01$ $-2.05003 \mathrm{E}-01$ $-2.06514 \mathrm{E}-01$ $-2.08031 \mathrm{E}-01$

$-2.09556 \mathrm{E}-01$ $-2.11088 \mathrm{E}-01$ $-2.12627 \mathrm{E}-01$ $-2.14174 \mathrm{E}-01$ $-2.15729 \mathrm{E}-01$

$-2.17290 \mathrm{E}-01$ $-2.18860 \mathrm{E}-01$ $-2.20436 \mathrm{E}-01$ $-2.22020 \mathrm{E}-01$ $-2.23612 \mathrm{E}-01$

$-2.25211 \mathrm{E}-01$ $-2.26817 \mathrm{E}-01$ $-2.28432 \mathrm{E}-01$ $-2.30053 \mathrm{E}-01$ $-2.31682 \mathrm{E}-01$

$-2.33319 \mathrm{E}-01$ $-2.34964 \mathrm{E}-01$ $-2.36616 \mathrm{E}-01$ $-2.38276 \mathrm{E}-01$ $-2.39943 \mathrm{E}-01$

$-2.41618 \mathrm{E}-01$ $-2.43301 \mathrm{E}-01$ $-2.44991 \mathrm{E}-01$ $-2.46689 \mathrm{E}-01$ $-2.48395 \mathrm{E}-01$

$-2.50109 \mathrm{E}-01$ $-2.51830 \mathrm{E}-01$ $-2.53559 \mathrm{E}-01$ $-2.55296 \mathrm{E}-01$ $-2.57041 \mathrm{E}-01$

$-2.58794 \mathrm{E}-01$ $-2.60554 \mathrm{E}-0 \mathrm{l}$ $-2.62323 \mathrm{E}-01$ $-2.64099 \mathrm{E}-01$ $-2.65883 \mathrm{E}-0 \mathrm{l}$ $\tan \delta_{2}$

$f_{-2}$

$3.44616 \mathrm{E} 01-1.70495 \mathrm{E}-01$ $3.44627 \mathrm{E}$ 01 $-1.71829 \mathrm{E}-01$ $3.44638 \mathrm{E}$ 01 $-1.73170 \mathrm{E}-01$ $3.44648 \mathrm{E} \quad 01-1.74518 \mathrm{E}-01$ $3.44659 \mathrm{E}$ 0l $-1.75873 \mathrm{E}-01$

$3.44669 \mathrm{E} 01-1.77235 \mathrm{E}-01$ $3.44679 \mathrm{E}$ ol $-1.78603 \mathrm{E}-01$ $3.44690 \mathrm{E}$ 01 $-1.79979 \mathrm{E}-01$ $3.44700 \mathrm{E}$ 01 $-1.81361 \mathrm{E}-01$

$3.44710 \mathrm{E} 01-1.82751 \mathrm{E}-01$

$3.44720 \mathrm{E} 01$ $3.44730 \mathrm{E} \quad 01$ $3.44740 \mathrm{E} 01$ $3.44750 \mathrm{E} \quad 01$ $3.44760 \mathrm{E} \quad 01$

$3.44770 \mathrm{E} \quad 01$ $3.44780 \mathrm{E} \quad 01$ $3.44789 \mathrm{E} \quad 01$ $3.44799 \mathrm{E} \quad 01$ $3.44809 \mathrm{E} \quad 01$

$3.44818 \mathrm{E} \quad 01$ $3.44828 \mathrm{E} 01$

$3.44837 \mathrm{E} \quad 01$

$3.44847 \mathrm{E} \quad 01$

$3.44856 \mathrm{E} \quad 01$

$3.44866 \mathrm{E} \quad 01$ $3.44875 \mathrm{E} \quad 01$ $3.44884 \mathrm{E} \quad 01$ $3.44893 \mathrm{E} 01$ $3.44902 \mathrm{E} 01$

$3.44911 \mathrm{E} \quad 01$ $3.44920 \mathrm{E} \quad 01$ $3.44929 \mathrm{E} 01$ $3.44938 \mathrm{E} 01$ $3.44947 \mathrm{E} \quad 01$

$3.44956 \mathrm{E} \quad 01$

$3.44965 \mathrm{E} \quad 01$

$3.44974 \mathrm{E} \quad 01$

$3.44982 \mathrm{E} \quad 01$

$3.44991 \mathrm{E} 01$

$3.45000 \mathrm{E} \quad 01$

$3.45008 \mathrm{E} \quad 01$

$3.45017 \mathrm{E} \quad 01$

$3.45025 \mathrm{E} \quad 01$

$3.45034 \mathrm{E} \quad 01$

$3.45042 \mathrm{E} \quad 01$ $3.45050 \mathrm{E} \quad 01$ $3.45059 \mathrm{E} \quad 01$ $3.45067 \mathrm{E} \quad 01$ $3.45075 \mathrm{E} \quad 01$

$3.45083 \mathrm{E} \quad 01$ $3.45091 \mathrm{E} \quad 01$ $3.45100 \mathrm{E} 01$ $3.45108 \mathrm{E} \quad 01$ $3.45116 \mathrm{E} \quad 01$

$3.45124 \mathrm{E} \quad 01$ $3.45131 \mathrm{E} 01$ $3.45139 \mathrm{E} 01$ $3.45147 \mathrm{E}$ 0l $3.45155 \mathrm{E}$ 0l

$-1.84148 \mathrm{E}-01$ -1.85551 E-01

$-1.86962 \mathrm{E}-01$ $-1.88380 \mathrm{E}-01$ $-1.89805 \mathrm{E}-01$

$-1.91237 \mathrm{E}-01$ $-1.92676 \mathrm{E}-01$ $-1.94122 \mathrm{E}-01$ $-1.95575 \mathrm{E}-01$ $-1.97036 \mathrm{E}-01$

$-1.98504 \mathrm{E}-01$ $-1.99979 \mathrm{E}-01$ $-2.01461 \mathrm{E}-01$ $-2.02950 \mathrm{E}-01$ $-2.04447 \mathrm{E}-01$

$-2.05951 \mathrm{E}-01$ $-2.07462 \mathrm{E}-01$ $-2.08981 \mathrm{E}-01$ $-2.10506 \mathrm{E}-01$ $-2.12040 \mathrm{E}-01$

$-2.13580 \mathrm{E}-01$ $-2.15128 \mathrm{E}-01$ $-2.16684 \mathrm{E}-01$ $-2.18246 \mathrm{E}-01$ $-2.19816 \mathrm{E}-0 \mathrm{l}$

$-2.21394 \mathrm{E}-01$ $-2.22979 \mathrm{E}-01$ $-2.24572 \mathrm{E}-01$ $-2.26172 \mathrm{E}-01$ $-2.27779 \mathrm{E}-01$

$-2.29394 \mathrm{E}-01$ $-2.31017 \mathrm{E}-01$ $-2.32647 \mathrm{E}-01$ $-2.34285 \mathrm{E}-01$ $-2.35930 \mathrm{E}-01$

$-2.37583 \mathrm{E}-01$ $-2.39244 \mathrm{E}-01$ $-2.40912 \mathrm{E}-01$ $-2.42588 \mathrm{E}-01$ $-2.44272 \mathrm{E}-01$

$-2.45963 \mathrm{E}-01$ 
ELECTRON RADIAL FUNCTIONS

Electron

\begin{tabular}{|c|c|c|c|c|}
\hline$p$ & $F_{0}$ & & $f_{1}$ & $g_{1}$ \\
\hline 0.10 & $3.43664 \mathrm{E}$ & 00 & $-1.46310 \mathrm{E}-02$ & $-3.66201 \mathrm{E}-04$ \\
\hline 20 & $2.07630 \mathrm{E}$ & 00 & $-4.14144 \mathbf{E}-02$ & $-1.03797 \mathrm{E}-03$ \\
\hline 30 & $1.69484 \mathrm{E}$ & 00 & $-8.13111 \mathrm{E}-02$ & $-2.04242 \mathrm{E}-03$ \\
\hline 0.40 & $1.52352 \mathrm{E}$ & 00 & $-1.33043 \mathrm{E}-01$ & $-3.35190 \mathrm{E}-03$ \\
\hline 50 & $1.42868 \mathrm{E}$ & 00 & $-1.95097 \mathrm{E}-01$ & $-4.93362 \mathbf{E}-03$ \\
\hline 0.60 & $1.36967 \mathrm{E}$ & 00 & $-2.65904 \mathrm{E}-01$ & $-6.75354 \mathrm{E}-03$ \\
\hline 70 & $1.33009 \mathrm{E}$ & 00 & $-3.43975 \mathrm{E}-01$ & $-8.77933 \mathrm{E}-03$ \\
\hline 80 & $1.30212 \mathrm{E}$ & 00 & $-4.27973 \mathrm{E}-01$ & $-1.09820 \mathrm{E}-02$ \\
\hline 90 & $1.28158 \mathrm{E}$ & 00 & $-5.16757 \mathrm{E}-01$ & $-1.33369 \mathrm{E}-02$ \\
\hline 00 & $1.26602 \mathrm{E}$ & 00 & $-6.09375 \mathrm{E}-01$ & $-1.58234 \mathrm{E}-02$ \\
\hline 1.10 & $1.25396 \mathrm{E}$ & 00 & $-7.05055 \mathrm{E}-01$ & $-1.84248 \mathrm{E}-02$ \\
\hline 20 & $1.24441 \mathrm{E}$ & 00 & $-8.03178 \mathrm{E}-01$ & $-2.11279 \mathrm{E}-02$ \\
\hline 30 & $1.23672 \mathrm{E}$ & 00 & $-9.03251 \mathrm{E}-01$ & $-2.39219 \mathrm{E}-02$ \\
\hline 40 & $1.23043 \mathrm{E}$ & 00 & $-1.00488 \mathrm{E} \quad 00$ & $-2.67985 \mathrm{E}-02$ \\
\hline 1.50 & $1.22523 \mathrm{E}$ & 00 & $-1.10777 \mathrm{E} \quad 00$ & $-2.97512 \mathrm{E}-02$ \\
\hline 60 & $1.22087 \mathrm{E}$ & 00 & $-1.21166 \mathrm{E} \quad 00$ & $-3.27747 \mathrm{E}-02$ \\
\hline 1.70 & $1.21718 \mathrm{E}$ & 00 & $-1.31637 \mathrm{E}$ & $-3.58648 \mathrm{E}-02$ \\
\hline 1.80 & $1.21403 \mathrm{E}$ & 00 & $-1.42173 \mathrm{E} \quad 00$ & $-3.90184 \mathrm{E}-02$ \\
\hline 1.90 & $1.21131 \mathrm{E}$ & 00 & $-1.52764 \mathrm{E}$ & $-4.22328 \mathrm{E}-02$ \\
\hline 2.00 & $1.20896 \mathrm{E}$ & 00 & $-1.63398 \mathrm{E} \quad 00$ & $-4.55060 \mathrm{E}-02$ \\
\hline 10 & $1.20690 \mathrm{E}$ & 00 & $-1.74069 \mathrm{E}$ & $-4.88362 \mathrm{E}-02$ \\
\hline 20 & $509 \mathrm{E}$ & 00 & $-1.84770 \mathrm{E} \quad 00$ & $2222 \mathrm{E}-02$ \\
\hline 2.30 & $1.20349 \mathrm{E}$ & 00 & $-1.95495 \mathrm{E}$ & $-5.56629 \mathrm{E}-02$ \\
\hline 2.40 & $1.20207 \mathrm{E}$ & 00 & $-2.06240 \mathrm{E}$ & $-5.91573 \mathrm{E}-02$ \\
\hline 2.50 & $1.20079 \mathrm{E}$ & 00 & $-2.17002 \mathrm{E}$ & $-6.27047 \mathrm{E}-02$ \\
\hline 2.60 & $1.19965 \mathrm{E}$ & 00 & $-2.27779 \mathbf{E}$ & $-6.63046 \mathrm{E}-02$ \\
\hline 2.70 & $1.19862 \mathrm{E}$ & 00 & $-2.38567 \mathbf{E}$ & $-6.99564 \mathrm{E}-02$ \\
\hline 2.80 & $1.19769 \mathrm{E}$ & 00 & $-2.49364 \mathrm{E}$ & $-7.36598 \mathrm{E}-02$ \\
\hline 2.90 & $1.19684 \mathrm{E}$ & 00 & $-2.60170 \mathrm{E}$ & $-7.74144 \mathrm{E}-02$ \\
\hline 3.00 & $1.19606 \mathrm{E}$ & 00 & $-2.70983 \mathrm{E} \quad 00$ & $-8.12198 \mathrm{E}-02$ \\
\hline 3.10 & $1.19535 \mathrm{E}$ & 00 & $-2.81801 \mathrm{E}$ & $-8.50760 \mathrm{E}-02$ \\
\hline 20 & $1.19470 \mathrm{E}$ & 00 & $-2.92624 \mathrm{E}$ & $-8.89826 \mathrm{E}-02$ \\
\hline 3.30 & $1.19410 \mathrm{E}$ & 00 & $-3.03450 \mathrm{E}$ & $-9.29396 \mathrm{E}-02$ \\
\hline 3.40 & $1.19355 \mathrm{E}$ & 00 & $-3.14279 \mathrm{E}$ & $-9.69466 \mathrm{E}-02$ \\
\hline 3.50 & $1.19303 \mathrm{E}$ & 00 & $-3.25112 \mathrm{E} \quad 00$ & $-1.01 \mathrm{C} 04 \mathrm{E}-01$ \\
\hline 3.60 & $1.19256 \mathrm{E}$ & 00 & $-3.35945 \mathrm{E}$ & $-1.05111 \mathrm{E}-01$ \\
\hline .70 & $1.19211 \mathrm{E}$ & 00 & $-3.46781 \mathrm{E}$ & $-1.09268 \mathrm{E}$ \\
\hline 80 & $70 \mathrm{E}$ & 00 & $-3.57618 \mathrm{E} \quad 00$ & $474 \mathrm{E}-$ \\
\hline 90 & $31 \mathrm{E}$ & 00 & $-3.68455 \mathrm{E}$ & $-1.17731 \mathrm{E}-01$ \\
\hline 4.00 & $1.19095 \mathrm{E}$ & 00 & $-3.79293 \mathrm{E} \quad 00$ & $-1.22037 \mathrm{E}-01$ \\
\hline 4.10 & $1.19061 \mathrm{E}$ & 00 & $-3.90132 \mathrm{E} \quad 00$ & $-1.26392 \mathrm{E}-01$ \\
\hline & $29 \mathrm{E}$ & 00 & $-4.00970 \mathrm{E}$ & -1 . \\
\hline 0 & $999 \mathrm{E}$ & 00 & $-4.11808 \mathrm{E}$ & $-1.35252 \mathrm{E}-01$ \\
\hline 4.40 & $1.18970 \mathrm{E}$ & 00 & $-4.22647 \mathrm{E}$ & $-1.39756 \mathrm{E}-01$ \\
\hline .50 & $1.18943 \mathrm{E}$ & 00 & $-4.33485 \mathrm{E}$ & $-1.44309 \mathrm{E}-01$ \\
\hline 4.60 & $1.18918 \mathrm{E}$ & 00 & $-4.44322 \mathrm{E} \quad 00$ & $-1.48912 \mathrm{E}-01$ \\
\hline 7 & $1.18893 \mathrm{E}$ & 00 & $-4.55159 \mathrm{E}$ & $-1.53564 \mathrm{E}-01$ \\
\hline & $1.18870 \mathrm{E}$ & 00 & $-4.65995 \mathrm{E}$ & $-1.58266 \mathrm{E}-01$ \\
\hline 4.90 & $1.18848 \mathrm{E}$ & 00 & $-4.76830 \mathrm{E}$ & $-1.63017 \mathrm{E}-01$ \\
\hline & $328 \mathrm{E}$ & 00 & $-4.87665 \mathrm{E}$ & $-1.67817 \mathrm{E}-01$ \\
\hline 5.10 & $1.18808 \mathrm{E}$ & 00 & $-4.98499 \mathrm{E} \quad 00$ & $-1.72666 \mathrm{E}-01$ \\
\hline & $18789 \mathrm{E}$ & 00 & $-5.09331 \mathrm{E}$ & $-1.77565 \mathrm{E}$ \\
\hline & $771 \mathrm{E}$ & 00 & $-5.20163 \mathrm{E}$ & $-1.82513 \mathrm{E}$ \\
\hline & $1.18753 \mathrm{E}$ & 00 & $-5.30994 E$ & $-1.87510 \mathrm{E}-01$ \\
\hline & $1.18737 \mathrm{E}$ & 00 & $-5.41824 \mathrm{E} \quad 00$ & $-1.92557 \mathrm{E}-01$ \\
\hline 5.60 & $1.18721 \mathrm{E}$ & 00 & $-5.52652 \mathrm{E} \quad 00$ & $-1.97653 \mathrm{E}-01$ \\
\hline & $1.18705 \mathrm{E}$ & 00 & $-5.63479 \mathrm{E} \quad 00$ & $-2.02798 \mathrm{E}-01$ \\
\hline & $1.18691 \mathrm{E}$ & 00 & $-5.74306 \mathrm{E} \quad 00$ & $-2.07992 \mathrm{E}-01$ \\
\hline & $1.18676 \mathrm{E}$ & 00 & $-5.85131 \mathrm{E} \quad 00$ & $-2.13235 \mathrm{E}-01$ \\
\hline & & & & \\
\hline
\end{tabular}

$\tan \delta_{1}$

$-2.25885 \mathrm{E}-01$

$-1.06907 \mathrm{E}-01$

$-6.96957 \mathrm{E}-02$

$-5.12808 \mathrm{E}-02$

$-4.02320 \mathrm{E}-02$

$-3.28394 \mathrm{E}-02$

$-2.75314 \mathrm{E}-02$

$-2.35269 \mathrm{E}-02$

$-2.03932 \mathrm{E}-02$

$-1.78709 \mathrm{E}-02$

$-1.57951 \mathrm{E}-02$

$-1.40555 \mathrm{E}-02$

$-1.25758 \mathrm{E}-02$

$-1.13011 \mathrm{E}-02$

$-1.01914 \mathrm{E}-02$

$-9.21619 \mathrm{E}-03$

$-8.35231 \mathrm{E}-03$

$-7.58160 \mathrm{E}-03$

$-6.88965 \mathrm{E}-03$

$-6.26493 \mathrm{E}-03$

$-5.69804 \mathrm{E}-03$

$-5.18127 \mathrm{E}-03$

$-4.70825 \mathrm{E}-03$

$-4.27362 \mathrm{E}-03$

$-3.87287 \mathrm{E}-03$

$-3.50219 \mathrm{E}-03$

$-3.15831 \mathrm{E}-03$

$-2.83841 \mathrm{E}-03$

$-2.54007 \mathrm{E}-03$

$-2.26118 \mathrm{E}-03$

$-1.99989 \mathrm{E}-03$

$-1.75460 \mathrm{E}-03$

$-1.52386 \mathrm{E}-03$

$-1.30644 \mathrm{E}-03$

$-1.10119 \mathrm{E}-03$

$-9.07141 \mathrm{E}-04$

$-7.23390 \mathrm{E}-04$

$-5.49145 \mathrm{E}-04$

$-3.83675 \mathrm{E}-04$

$-2.26351 \mathrm{E}-04$

$-7.65677 \mathrm{E}-05$

$6.61860 \mathrm{E}-05$

$2.02396 \mathrm{E}-04$

$3.32505 \mathrm{E}-04$

$4.56915 \mathrm{E}-04$

$5.75084 \mathrm{E}-04$

$6.90057 \mathrm{E}-04$

$7.99426 \mathrm{E}-04$

$9.04393 \mathrm{E}-04$

$1.00520 \mathrm{E}-03$

$1.10210 \mathrm{E}-03$

$1.19532 \mathrm{E}-03$

$1.28504 \mathrm{E}-03$

$1.37148 \mathrm{E}-03$

$1.45481 \mathrm{E}-03$

$1.53517 \mathrm{E}-03$ $1.61275 \mathrm{E}-03$

$1.68766 \mathrm{E}-03$

$1.76005 \mathrm{E}-03$

$1.83006 \mathrm{E}-03$ $f_{-1}$

$-5.34848 \mathrm{E}-03$

$-8.29807 \mathrm{E}-03$

$-1.12112 \mathrm{E}-02$

$-1.41177 \mathrm{E}-02$

$-1.70145 \mathrm{E}-02$

$-1.99007 \mathrm{E}-02$

$-2.27781 \mathrm{E}-02$

$-2.56507 \mathrm{E}-02$

$-2.85237 \mathrm{E}-02$

$-3.14025 \mathrm{E}-02$

$-3.42926 \mathrm{E}-02$

$-3.71989 \mathrm{E}-02$

$-4.01259 \mathrm{E}-02$

$-4.30777 \mathrm{E}-02$

$-4.60576 \mathrm{E}-02$

$-4.90687 \mathrm{E}-02$

$-5.21134 \mathrm{E}-02$

$-5.51940 \mathrm{E}-02$

$-5.83121 \mathrm{E}-02$

$-6.14694 \mathrm{E}-02$

$-6.46672 \mathrm{E}-02$

$-6.79067 \mathrm{E}-02$

$-7.11886 \mathrm{E}-02$

$-7.45140 \mathrm{E}-02$

$-7.78834 \mathrm{E}-02$

$-8.12975 \mathrm{E}-02$

$-8.47569 \mathrm{E}-02$

$-8.82619 \mathrm{E}-02$

$-9.18129 \mathrm{E}-02$

$-9.54103 \mathrm{E}-02$

$-9.90544 \mathrm{E}-02$

$-1.02746 \mathrm{E}-01$

$-1.06484 \mathrm{E}-01$

$-1.10269 \mathrm{E}-01$

$-1.14103 \mathrm{E}-01$

$-1.17984 \mathrm{E}-01$

$-1.21912 \mathrm{E}-01$

$-1.25889 \mathrm{E}-01$

$-1.29914 \mathrm{E}-01$

$-1.33987 \mathrm{E}-01$

$-1.38108 \mathrm{E}-01$

$-1.42278 \mathrm{E}-01$

$-1.46496 \mathrm{E}-01$

$-1.50762 \mathrm{E}-01$

$-1.55077 \mathrm{E}-01$

$-1.59441 \mathrm{E}-01$

$-1.63853 \mathrm{E}-01$

$-1.68314 \mathrm{E}-01$

$-1.72824 \mathrm{E}-01$

$-1.77382 \mathrm{E}-01$

$-1.81989 \mathrm{E}-01$

$-1.86645 \mathrm{E}-01$

$-1.91350 \mathrm{E}-01$

$-1.96103 \mathrm{E}-01$

$-2.00906 \mathrm{E}-01$

$-2.05757 \mathrm{E}-01$

$-2.10657 \mathrm{E}-01$

$-2.15606 \mathrm{E}-01$

$-2.20604 \mathrm{E}-01$

$-2.25651 \mathrm{E}-01$ $g_{-1}$

$2.61528 \mathrm{E}-01$

$4.05085 \mathrm{E}-01$

$5.45815 \mathrm{E}-01$

$6.84796 \mathrm{E}-01$

$8.21568 \mathrm{E}-01$

$9.55840 \mathrm{E}-01$

$1.08753 \mathrm{E} \quad 00$

$1.21670 \mathrm{E} \quad 00$

$1.34352 \mathrm{E} 00$

$1.46821 \mathrm{E} \quad 00$

$1.59100 \mathrm{E} \quad 00$

$1.71211 \mathrm{E} \quad 00$

$1.83175 \mathrm{E} \quad 00$

$1.95011 \mathrm{E} \quad 00$

$2.06737 \mathrm{E} \quad 00$

$2.18366 \mathrm{E} \quad 00$

$2.29911 \mathrm{E} \quad 00$

$2.41383 \mathrm{E} \quad 00$

$2.52792 \mathrm{E} \quad 00$

$2.64145 \mathrm{E} \quad 00$

$2.75449 \mathrm{E} \quad 00$ $2.86710 \mathrm{E} \quad 00$ $2.97934 \mathrm{E} \quad 00$

$3.09123 \mathrm{E} \quad 00$

$3.20283 \mathrm{E} \quad 00$

$3.31415 \mathrm{E} \quad 00$ $3.42524 \mathrm{E} \quad 00$ $3.53611 \mathrm{E} \quad 00$ $3.64679 \mathrm{E} \quad 00$ $3.75729 \mathrm{E} \quad 00$

$3.86763 \mathrm{E} \quad 00$ $3.97782 \mathrm{E} \quad 00$ $4.08788 \mathrm{E} \quad 00$ $4.19782 \mathrm{E} \quad 00$ $4.30765 \mathrm{E} \quad 00$

$4.41738 \mathrm{E} \quad 00$ $4.52700 \mathrm{E} \quad 00$ $4.63654 \mathrm{E} \quad 00$ $4.74600 \mathrm{E} \quad 00$ $4.85538 \mathrm{E} \quad 00$

$4.96469 \mathrm{E} \quad 00$ $5.07394 \mathrm{E} \quad 00$ $5.18311 \mathrm{E} \quad 00$ $5.29224 \mathrm{E} \quad 00$ $5.40130 \mathrm{E} \cdot 00$

$5.51031 \mathrm{E} \quad 00$ $5.61928 \mathrm{E} \quad 00$ $5.72819 \mathrm{E} \quad 00$ $5.83706 \mathrm{E} \quad 00$ $5.94589 \mathrm{E} \quad 00$

$6.05467 \mathrm{E} \quad 00$ $6.16342 \mathrm{E} \quad 00$ $6.27213 \mathrm{E} \quad 00$ $6.38080 \mathrm{E} \quad 00$ $6.48945 \mathrm{E} \quad 00$

$6.59805 \mathrm{E} 00$ $6.70662 \mathrm{E} \quad 00$ $6.81517 \mathrm{E} \quad 00$ $6.92368 \mathrm{E} \quad 00$ $7.03217 \mathrm{E} \quad 00$ $\tan \delta_{-1}$

$-3.91461 \mathrm{E} \quad 00$ $-6.91789 \mathrm{E} 00$ $-1.00608 \mathrm{E} \quad 01$ $-1.31709 \mathrm{E} 01$

$-1.62135 \mathrm{E} 01$

$-1.91754 \mathrm{E} \quad 01$ $-2.20505 \mathrm{E} \quad 01$ -2.48362 E 01 $-2.75320 \mathrm{E} \quad 01$ $-3.01390 \mathrm{E} 01$

$-3.26590 \mathrm{E} 01$ $-3.50944 \mathrm{E} 01$ $-3.74480 \mathrm{E} 01$ $-3.97229 \mathrm{E} 01$ $-4.19221 \mathrm{E} 01$

$-4.40488 \mathrm{E} \quad 01$ $-4.61059 \mathrm{E} \quad 01$ $-4.80966 \mathrm{E} 01$ $-5.00236 \mathrm{E} 01$ $-5.18898 \mathrm{E} 01$

$-5.36979 \mathrm{E} \quad 01$ $-5.54503 \mathrm{E} 01$ $-5.71495 \mathrm{E} 01$ $-5.87978 \mathrm{E} \quad 01$ $-6.03974 \mathrm{E} 01$

$-6.19503 \mathrm{E} 01$ $-6.34586 \mathrm{E} 01$ $-6.49240 \mathrm{E}$ 0l $-6.63484 \mathrm{E} 01$ $-6.77335 \mathrm{E} 01$

$-6.90808 \mathrm{E} \quad 01$ $-7.03918 \mathrm{E} 01$ $-7.16681 \mathrm{E} 01$ $-7.29109 \mathrm{E} \quad 01$ $-7.41215 \mathrm{E} 01$

$-7.53012 \mathrm{E} 01$ $-7.64512 \mathrm{E} 01$ $-7.75726 \mathrm{E} 01$ $-7.86663 \mathrm{E} 01$ $-7.97336 \mathrm{E} \quad 01$

$-8.07751 \mathrm{E} \quad 01$ $-8.17920 \mathrm{E} 01$ $-8.27851 \mathrm{E} \quad 01$ $-8.37553 \mathrm{E} \quad 01$ $-8.47032 \mathrm{E} \quad 01$

$-8.56297 \mathrm{E} \quad 01$ $-8.65355 \mathrm{E} 01$ $-8.74214 \mathrm{E} \quad 01$ $-8.82879 \mathrm{E} \quad 01$ $-8.91357 \mathrm{E} 01$

$\begin{array}{ll}-8.99654 \mathrm{E} & 01\end{array}$ $-9.07776 \mathrm{E} \quad 01$ $-9.15729 \mathrm{E} 01$ $-9.23517 \mathrm{E} 01$ $-9.31146 \mathrm{E} 01$

$-9.38622 \mathrm{E} 01$ $-9.45948 \mathrm{E} 01$ $-9.53129 \mathrm{E} 01$ $-9.60170 \mathrm{E} 01$ $-9.67074 \mathrm{E} 01$ 
$\bar{F}$

$5.62351 \mathrm{E}-08$

$1.14908 \mathrm{E}-07$

$2.03969 \mathrm{E}-07$

$3.22059 \mathrm{E}-07$

$4.69304 \mathrm{E}-07$

$6.45991 \mathrm{E}-07$

$8.52397 \mathrm{E}-07$

$1.08876 \mathrm{E}-06$

$1.35527 \mathrm{E}-06$

$1.65209 \mathrm{E}-06$

$1.97933 \mathrm{E}-06$

$2.33709 \mathrm{E}-06$

$2.72544 \mathrm{E}-06$

$3.14444 \mathrm{E}-06$

$3.59412 \mathrm{E}-06$

4.07453E-06

$4.58569 \mathrm{E}-06$

$5.12763 \mathrm{E}-06$

$5.70035 \mathrm{E}-06$

$6.30388 \mathrm{E}-06$

$6.93822 \mathrm{E}-06$

$7.60338 \mathrm{E}-06$

$8.29937 \mathrm{E}-06$

$9.02618 \mathrm{E}-06$

$9.78384 \mathrm{E}-06$

$1.05723 \mathrm{E}-0.05$

$1.13917 \mathrm{E}-05$

$1.22419 \mathrm{E}-05$

$1.31229 \mathrm{E}-05$

$1.40318 \mathrm{E}-05$

$1.49775 \mathrm{E}-05$

$1.59511 \mathrm{E}-05$

$1.69555 \mathrm{E}-05$

$1.79908 \mathrm{E}-05$

$1.90569 \mathrm{E}-05$

$2.01539 \mathrm{E}-05$

$2.12817 \mathrm{E}-05$

$2.2+404 \mathrm{E}-05$

$2.36299 \mathrm{E}-05$

$2.48502 \mathrm{E}-05$

$2.61014 \mathrm{E}-05$

$2.73835 \mathrm{E}-05$

$2.86964 \mathrm{E}-05$

$3.00402 \mathrm{E}-05$

$3.14148 \mathrm{E}-05$

$3.28202 \mathrm{E}-05$

$3.42565 \mathrm{E}-0.5$

$3.57236 \mathrm{E}-05$

$3.72215 \mathrm{E}-05$

$3.87504 \mathrm{E}-05$

$4.03100 \mathrm{E}-05$

$4.19005 \mathrm{E}-05$

$4.35218 \mathrm{E}-05$

$4.51740 \mathrm{E}-05$

$4.68571 \mathrm{E}-05$

$4.85709 \mathrm{E}-05$

$5.03156 \mathrm{E}-05$

$5.20911 \mathrm{E}-05$

$5.38974 \mathrm{E}-0.5$

$5.57346 \mathrm{E}-05$ $f_{2}$

$-3.43522 \mathrm{E}-06$ $-1.90295 \mathrm{E}-05$ $-5.58189 \mathrm{E}-05$ $-1.21617 \mathrm{E}-04$

$-2.22806 \mathrm{E}-04$

$-3.64315 \mathrm{E}-04$

$-5.49763 \mathrm{E}-04$

$-7.81696 \mathrm{E}-04$

$-1.06183 \mathrm{E}-03$

$-1.39129 \mathrm{E}-03$

$-1.77077 \mathrm{E}-03$

$-2.20067 \mathrm{E}-03$

$-2.68120 \mathrm{E}-03$

$-3.21248 \mathrm{E}-03$

$-3.79452 \mathrm{E}-03$

$-4.42728 \mathrm{E}-03$

$-5.11071 \mathrm{E}-03$

$-5.84476 \mathrm{E}-03$

$-6.62934 \mathrm{E}-03$

$-7.46438 \mathrm{E}-03$

$-8.34981 \mathrm{E}-03$

$-9.28556 \mathrm{E}-03$

$-1.02716 \mathrm{E}-02$

$-1.13078 \mathrm{E}-02$

$-1.23941 \mathrm{E}-02$

$-1.35306 \mathrm{E}-02$

$-1.47171 \mathrm{E}-02$

$-1.59536 \mathrm{E}-02$

$-1.72401 \mathrm{E}-02$

$-1.85765 \mathrm{E}-02$

$-1.99629 \mathrm{E}-02$

$-2.13991 \mathrm{E}-02$

$-2.28852 \mathrm{E}-02$

$-2.44211 \mathrm{E}-02$

$-2.60068 \mathrm{E}-02$

$-2.76423 \mathrm{E}-02$

$-2.93275 \mathrm{E}-02$

$-3.10625 \mathrm{E}-02$

$-3.28473 \mathrm{E}-02$

$-3.46818 \mathrm{E}-02$

$-3.65659 \mathrm{E}-02$

$-3.84998 \mathrm{E}-02$

$-4.04833 \mathrm{E}-02$

$-4.25166 \mathrm{E}-02$

$-4.45994 \mathrm{E}-02$

$-4.67319 \mathrm{E}-02$

$-4.89140 \mathrm{E}-02$

$-5.11458 \mathrm{E}-02$

$-5.34272 \mathrm{E}-02$

$-5.57581 \mathrm{E}-02$

$-5.81387 \mathrm{E}-02$

$-6.05689 \mathrm{E}-02$

$-6.30486 \mathrm{E}-02$

$-6.55779 \mathrm{E}-02$

$-6.81568 \mathrm{E}-02$

$-7.07852 \mathrm{E}-02$

$-7.34632 \mathrm{E}-02$

$-7.61907 \mathrm{E}-02$

$-7.89677 \mathrm{E}-02$

$-8.17943 \mathrm{E}-02$ $g_{2}$

$-4.95723 \mathrm{E}-08$

$-2.74994 \mathrm{E}-07$

$-8.08494 \mathrm{E}-07$

$-1.76704 \mathrm{E}-06$

$-3.24983 \mathrm{E}-06$

$-5.33796 \mathrm{E}-06$

$-8.09629 \mathrm{E}-06$

$-1.15763 \mathrm{E}-05$

$-1.58194 \mathrm{E}-05$

$-2.08593 \mathrm{E}-05$

$-2.67248 \mathrm{E}-05$

$-3.34410 \mathrm{E}-05$

$-4.10307 \mathrm{E}-05$

$-4.95155 \mathrm{E}-05$

$-5.89157 \mathrm{E}-05$

$-6.92513 \mathrm{E}-05$

$-8.05420 \mathrm{E}-05$

$-9.28072 \mathrm{E}-05$

$-1.06066 \mathrm{E}-04$

$-1.20339 \mathrm{E}-04$

$-1.35645 \mathrm{E}-04$

$-1.52003 \mathrm{E}-04$

$-1.69433 \mathrm{E}-04$

$-1.87955 \mathrm{E}-04$

$-2.07589 \mathrm{E}-04$

$-2.28354 \mathrm{E}-04$

$-2.50270 \mathrm{E}-04$

$-2.73357 \mathrm{E}-04$

$-2.97636 \mathrm{E}-04$

$-3.23125 \mathrm{E}-04$

$-3.49846 \mathrm{E}-04$

$-3.77818 \mathrm{E}-04$

$-4.07061 \mathrm{E}-04$

$-4.37595 \mathrm{E}-04$

$-1.69441 \mathrm{E}-04$

$-5.02619 \mathrm{E}-04$

$-5.37148 \mathrm{E}-04$

$-5.73050 \mathrm{E}-04$

$-6.10344 \mathrm{E}-04$

$-6.49050 \mathrm{E}-04$

$-6.89189 \mathrm{E}-04$

$-7.30782 \mathrm{E}-04$

$-7.73847 \mathrm{E}-04$

$-8.18406 \mathrm{E}-04$

$-8.64479 \mathrm{E}-04$

$-9.12085 \mathrm{E}-04$

$-9.61246 \mathrm{E}-04$.

$-1.01198 \mathrm{E}-03$

$-1.06431 \mathrm{E}-03$

$-1.11826 \mathrm{E}-03$

$-1.17384 \mathrm{E}-03$

$-1.23108 \mathrm{E}-03$

$-1.28999 \mathrm{E}-03$

$-1.35060 \mathrm{E}-03$

$-1.41292 \mathrm{E}-03$

$-1.47698 \mathrm{E}-03$

$-1.54280 \mathrm{E}-03$

$-1.61040 \mathrm{E}-03$

$-1.67980 \mathrm{E}-03$

$-1.75101 \mathrm{E}-03$ $\tan \delta_{2}$

$f_{2}$

$1.94277 \mathrm{E} \quad 00$

$4.11403 \mathrm{E} \quad 00$

$6.14817 \mathrm{E} \quad 00$

$8.05829 \mathrm{E} \quad 00$

$9.83124 \mathrm{E} \quad 00$

$1.14583 \mathrm{E} \quad 01$

$1.29379 \mathrm{E} \quad 01$

$1.42744 \mathrm{E} \quad 01$

$1.54762 \mathrm{E} \quad 01$

$1.65540 \mathrm{E} 01$

$1.75196 \mathrm{E} \quad 01$

$1.83847 \mathrm{E} \quad 01$

$1.91605 \mathrm{E} \quad 01$

$1.98573 \mathrm{E} \quad 01$

$2.04844 \mathrm{E} \quad 01$

$2.10502 \mathrm{E} \quad 01$

$2.15618 \mathrm{E} \quad 01$

$2.20258 \mathrm{E} \quad 01$

$2.24476 \mathrm{E} \quad 01$

$2.28322 \mathrm{E} \quad 0 \mathrm{l}$

$2.31838 \mathrm{E} \quad 01$

$2.35061 \mathrm{E} \quad 01$

$2.38022 \mathrm{E} \quad 01$

$2.40750 \mathrm{E} \quad 01$

$2.43269 \mathrm{E} \quad 01$

$2.45600 \mathrm{E} \quad 01$

$2.47763 \mathrm{E} \quad 01$

$2.49773 \mathrm{E} \quad 0$

$2.51646 \mathrm{E} \quad 01$

$2.53393 \mathrm{E} \quad 01$

$2.55027 \mathrm{E} \quad 01$

$2.56558 \mathrm{E} \quad 01$

$2.57994 \mathrm{E} \quad 01$

$2.59344 \mathrm{E} \quad 01$

$2.60615 \mathrm{E} \quad 01$

$2.61813 \mathrm{E} \quad 01$

$2.62944 \mathrm{E} \quad 01$

$2.64013 \mathrm{E} \quad 01$

$2.65026 \mathrm{E} \quad 01$

$2.65985 \mathrm{E} \quad 01$

$2.66896 \mathrm{E} \quad 01$

$2.67761 \mathrm{E} \quad 01$

$2.68585 \mathrm{E} \quad 01$

$2.69368 \mathrm{E} \quad 01$

$2.70116 \mathrm{E} \quad 01$

$2.70829 \mathrm{E} \quad 01$

$2.71510 \mathrm{E} \quad 01$

2.72161 E 01

$2.72784 \mathrm{E} \quad 01$

$2.73381 \mathrm{E} \quad 01$

$2.73952 \mathrm{E} \quad 01$

$2.74501 \mathrm{E} 01$

$2.75028 \mathrm{E} \quad 01$

$2.75534 \mathrm{E} \quad 01$

$2.76020 \mathrm{E} \quad 01$

$2.76488 \mathrm{E} \quad 01$

$2.76939 \mathrm{E} \quad 01$

$2.77373 \mathrm{E} \quad 01$

$2.77792 \mathrm{E} \quad 01$

2.78195 E 01

$-2.08783 \mathrm{E}-05$

$-2.78335 \mathrm{E}-04$

$-3.25668 \mathrm{E}-04$

$-4.05095 \mathrm{E}-04$

g. $\cdot 2$

$-7.82438 \mathrm{E}-07$

$-2.23268 \mathrm{E}-06$

$-4.4 .4884 \mathrm{~F}-06$

$-7.42615 \mathrm{E}-06$

$-1.11592 \mathrm{E}-0.5$

$-1.56438 \mathrm{E}-05$

$-2.68643 \mathrm{E}-05$

$-3.36071 \mathrm{E}-0.5$

$-4.11150 \mathrm{E}-0.5$

$-4.93990 \mathrm{E}-05$

$-5.84724 \mathrm{E}-0.5$

$-6.83504 \mathrm{E}-05$

$-7.90495 \mathrm{E}-05$

$-9.05869 \mathrm{E}-0.5$

$-1.02981 \mathrm{E}-04$

$-1.16251 \mathrm{E}-04$

$-1.30416 \mathrm{E}-04$

$-1.45495 \mathrm{E}-04$

$-1.61508 \mathrm{E}-04$

$-1.78476 \mathrm{E}-04$

$-1.96418 \mathrm{E}-04$

$-2.15354 \mathrm{E}-04$

$-2.35306 \mathrm{E}-04$

$-2.56293 \mathrm{E}-04$

$-3.01453 \mathrm{E}-04$

$-3.51000 \mathrm{E}-04$

$-3.77468 \mathrm{E}-04$

$-4.33899 \mathrm{E}-04$

$-4.63903 \mathrm{E}-04$

$-4.95125 \mathrm{E}-04$

$-5.27586 \mathrm{E}-04$

$-5.61308 \mathrm{E}-04$

$-5.96309 \mathrm{E}-04$

$-6.32611 \mathrm{E}-04$

$-6.70234 \mathrm{E}-04$

$-7.09199 \mathrm{E}-04$

$-7.49526 \mathrm{E}-04$ 


\section{$p$}

$F_{0}$

$$
f_{1}
$$

$g_{1}$

6.10

6.20

6.30

6.40

6.50

6.60

6.70

6.80

6.90

7.00

7.10

7.20

7.30

7.40

7.50

7.60

7.70

7.80

7.90

8.00

8.10

8.20

8.30

8.40

8.50

8.60

8.70

8.80

8.90

9.00

9.10

9.20

9.30

9.40

9.50

9.60

9.70

9.80

9.90

10.00

10.10

10.20

10.30

10.40

10.50

10.60

10.70

10.80

10.90

11.00

11.10

11.20

11.30

11.40

11.50

11.60

11.70

11.80

11.90

12.00
$1.18650 \mathrm{E} \quad 00$ $1.18637 \mathrm{E} \quad 00$ $1.18625 \mathrm{E} \quad 00$ $1.18613 \mathrm{E} \quad 00$ $1.18601 \mathrm{E} \quad 00$

$1.18590 \mathrm{E} \quad 00$ $1.18580 \mathrm{E} \quad 00$ $1.18569 \mathrm{E} \quad 00$ $1.18559 \mathrm{E} \quad 00$ $1.18550 \mathrm{E} \quad 00$

$1.18540 \mathrm{E} \quad 00$ $1.18531 \mathrm{E} \quad 00$ $1.18522 \mathrm{E} \quad 00$ $1.18513 \mathrm{E} \quad 00$ $1.18505 \mathrm{E} \quad 00$ $1.18488 \mathrm{E} \quad 00$ $1.18481 \mathrm{E} \quad 00$ $1.18473 \mathrm{E} \quad 00$ $1.18466 \mathrm{E} \quad 00$

$1.18458 \mathrm{E} \quad 00$ $1.18451 \mathrm{E} \quad 00$ $1.18444 \mathrm{E} \quad 00$ $1.18437 \mathrm{E} \quad 00$ $1.18431 \mathrm{E} \quad 00$

$1.18424 \mathrm{E} \quad 00$ $1.18418 \mathrm{E} \quad 00$ $1.18412 \mathrm{E} \quad 00$ $1.18406 \mathrm{E} \quad 00$ $1.18400 \mathrm{E} \quad 00$

$1.18394 \mathrm{E} \quad 00$ $1.18388 \mathrm{E} \quad 00$ $1.18377 \mathrm{E} \quad 00$ $1.18372 \mathrm{E} \quad 00$

$1.18367 \mathrm{E} \quad 00$ $1.18361 \mathrm{E} \quad 00$ $1.18356 \mathrm{E} \quad 00$ $1.18351 \mathrm{E} \quad 00$ $1.18346 \mathrm{E} \quad 00$

$1.18341 \mathrm{E} \quad 00$ $1.18337 \mathrm{E} \quad 00$ $1.18332 \mathrm{E} \quad 00$ $1.18327 \mathrm{E} \quad 00$ $1.18323 \mathrm{E} \quad 00$

$1.18318 \mathrm{E} \quad 00$ $1.18314 \mathrm{E} \quad 00$ $1.18310 \mathrm{E} \quad 00$ $1.18305 \mathrm{E} \quad 00$ $1.18301 \mathrm{E} \quad 00$

$1.18297 \mathrm{E} \quad 00$ $1.18293 \mathrm{E} \quad 00$ $1.18289 \mathrm{E} \quad 00$ $1.18285 \mathrm{E} \quad 00$ $1.18281 \mathrm{E} \quad 00$

$1.18277 \mathrm{E} \quad 00$ $1.18270 \mathrm{E} \quad 00$ $1.18266 \mathrm{E} \quad 00$ 1.18263 E 00
$1.18496 \mathrm{E} \quad 00$ $1.18383 \mathrm{E} \quad 00$ $1.18274 \mathrm{E} \quad 00$
$-6.06777 \mathrm{E} \quad 00 \quad-2.23869 \mathrm{E}-01$ $-6.17599 \mathrm{E} \quad 00 \quad-2.29260 \mathrm{E}-01$ $-6.28419 \mathrm{E} \quad 00 \quad-2.34700 \mathrm{E}-01$ $-6.39238 \mathrm{E} \quad 00 \quad-2.40189 \mathrm{E}-01$ $-6.50055 \mathrm{E} \quad 00 \quad-2.45727 \mathrm{E}-01$

$-6.60871 \mathrm{E} \quad 00$ $-6.71686 \mathrm{E} 00$ $-6.82500 \mathrm{E} \quad 00$ $-7.04124 \mathrm{E} \quad 00$

$-7.14933 \mathrm{E} \quad 00$ $-7.25741 \mathrm{E} \quad 00$ $-7.36548 \mathrm{E} \quad 00$ $-7.47354 \mathrm{E} \quad 00$ $-7.58158 \mathrm{E} \quad 00$

$-7.68961 \mathrm{E} \quad 00$ $-7.79763 \mathrm{E} \quad 00$ $-8.01362 \mathrm{E} \quad 00$ $-8.12159 \mathrm{E} \quad 00$

$-8.22955 \mathrm{E} \quad 00$ $-8.33750 \mathrm{E} \quad 00$ $-8.44543 \mathrm{E} \quad 00$ $-8.55335 \mathrm{E} \quad 00$ $-8.66125 \mathrm{E} \quad 00$

$-8.76914 \mathrm{E} \quad 00$ $-8.87702 \mathrm{E} \quad 00$ $-8.98488 \mathrm{E} \quad 00$ $-9.09273 \mathrm{E} \quad 00$ $-9.20057 \mathrm{E} \quad 00$

$-9.30839 \mathrm{E} \quad 00$ $-9.41619 \mathrm{E} \quad 00$ $-9.52399 \mathrm{E} 00$ $-9.63176 \mathrm{E} \quad 00$ $\begin{array}{lll}-9.73953 \mathrm{E} & 00\end{array}$

$-9.84728 \mathrm{E} \quad 00$ $-9.95502 \mathrm{E} \quad 00$ $-1.00627 \mathrm{E} \quad 01$ $-1.01704 \mathrm{E} 01$ $-1.02781 \mathrm{E} \quad 01$

$-1.03858 \mathrm{E} \quad 01$ $-1.04935 \mathrm{E} 01$ $-1.06011 \mathrm{E} 01$ $-1.07088 \mathrm{E} 01$ $-1.08164 \mathrm{E} 01$

$-1.09240 \mathrm{E} \quad 01$ $-1.10316 \mathrm{E} 01$ $-1.11392 \mathrm{E} 01$ $-1.12467 \mathrm{E} 01$ $-1.13543 \mathrm{E} 01$

$-1.14618 \mathrm{E} \quad 01$ $-1.15693 \mathrm{E} 01$ $-1.17843 \mathrm{E} 01$ $-1.18918 \mathrm{E} \quad 01$

$\begin{array}{ll}-1.19993 \mathrm{E} & 01\end{array}$ $-1.21067 \mathrm{E} \quad 01$ $-1.22141 \mathrm{E} 01$ $-1.23215 \mathrm{E} 01$ $-1.24290 \mathrm{E} 01$
$-2.51315 \mathrm{E}-01$

$-2.56951 \mathrm{E}-01$

$-2.62637 \mathrm{E}-01$

$-2.74156 \mathrm{E}-01$

$-2.79989 \mathrm{E}-01$

$-2.91802 \mathrm{E}-01$

$-2.97782 \mathrm{E}-01$

$-3.0381 \mathrm{LE}-01$

$-3.09890 \mathrm{E}-01$

$-3.16017 \mathrm{E}-01$

$-3.28419 \mathrm{E}-01$

$-3.34693 \mathrm{E}-01$

$-3.41017 \mathrm{E}-01$

$-3.47390 \mathrm{E}-0 \mathrm{l}$

$-3.53811 \mathrm{E}-01$

$-3.60282 \mathrm{E}-01$

$-3.66801 \mathrm{E}-01$

$-3.73370 \mathrm{E}-01$ $-3.79987 \mathrm{E}-01$ $-3.86654 \mathrm{E}-01$ $-3.93369 \mathrm{E}-01$

$-4.00134 \mathrm{E}-01$

$-4.06947 \mathrm{E}-01$

$-4.13810 \mathrm{E}-01$

$-4.20721 \mathrm{E}-01$ $-4.27681 \mathrm{E}-01$ $-4.34690 \mathrm{E}-01$

$-4.41748 \mathrm{E}-01$ $-4.48855 \mathrm{E}-01$ $-4.56011 \mathrm{E}-01$ $-4.63216 \mathrm{E}-01$ $-4.70470 \mathrm{E}-01$

$-4.77772 \mathrm{E}-01$ $-4.85124 \mathrm{E}-01$ $-4.92524 \mathrm{E}-01$ $-4.99973 \mathrm{E}-01$ $-5.07471 \mathrm{E}-01$

$-5.15018 \mathrm{E}-01$ $-5.22614 \mathrm{E}-01$ $-5.30258 \mathrm{E}-01$ $-5.37952 \mathrm{E}-01$ $-5.45694 \mathrm{E}-01$

$-5.53485 \mathrm{E}-01$ $-5.61325 \mathrm{E}-0$ $-5.69213 \mathrm{E}-01$ $-5.77151 \mathrm{E}-01$ $-5.85137 \mathrm{E}-01$

$-5.93172 \mathrm{E}-01$ $-6.01256 \mathrm{E}-01$ $-6.09388 \mathrm{E}-01$ $-6.17569 \mathrm{E}-01$ $-6.25799 \mathrm{E}-01$
$-2.68372 \mathrm{E}-01$

$-2.85871 \mathrm{E}-01$

$-3.22193 \mathrm{E}-01$ $-6.93313 \mathrm{E} 00$ $-7.90563 \mathrm{E} 00$ $-1.16768 \mathrm{E} 01$

$\tan \delta_{1}$

$f_{-1}$

$1.89777 \mathrm{E}-03 \quad-2.30747 \mathrm{E}-01$ $1.96331 \mathrm{E}-03 \quad-2.35892 \mathrm{E}-01$

$2.02678 \mathrm{E}-03-2.41086 \mathrm{E}-01$

$2.08827 \mathrm{E}-03-2.46329 \mathrm{E}-01$

$2.14788 \mathrm{E}-03$

$2.20570 \mathrm{E}-03$

$2.26178 \mathrm{E}-03$

$2.31622 \mathrm{E}-03$

$2.36907 \mathrm{E}-03$

$2.42043 \mathrm{E}-03$

$2.47033 \mathrm{E}-03$

$2.51885 \mathrm{E}-03$

$2.56605 \mathrm{E}-03$

$2.61194 \mathrm{E}-03$

$2.65663 \mathrm{E}-03$

$2.70014 \mathrm{E}-03$

$2.74250 \mathrm{E}-03$

$2.78377 \mathrm{E}-03$

$2.82400 \mathrm{E}-03$

$2.86321 \mathrm{E}-03$

$2.90145 \mathrm{E}-03$

$2.93875 \mathrm{E}-03$

$2.97514 \mathrm{E}-03$

$3.01066 \mathrm{E}-03$

$3.04533 \mathrm{E}-03$

$3.07919 \mathrm{E}-03$

$3.11226 \mathrm{E}-03$

$3.14456 \mathrm{E}-03$

$3.17614 \mathrm{E}-03$

$3.20700 \mathrm{E}-03$

$3.23717 \mathrm{E}-03$

$3.26668 \mathrm{E}-03$

$3.29554 \mathrm{E}-03$

$3.32378 \mathrm{E}-03$

$3.35140 \mathrm{E}-03$

$3.37845 \mathrm{E}-03$

$3.40493 \mathrm{E}-03$

$3.43085 \mathrm{E}-03$

$3.45624 \mathrm{E}-03$

$3.48110 \mathrm{E}-03$

$3.50546 \mathrm{E}-03$

$3.52933 \mathrm{E}-03$

$3.55272 \mathrm{E}-03$

$3.57566 \mathrm{E}-03$ 
ELECTRON RADIAL FUNCTIONS

$Z=7 \quad A=14 \quad \rho=1.1 A^{1 / 3} F$

Electron

$\bar{F}$

$f_{2}$

$-8.46705 \mathrm{E}-02$ $-8.75961 \mathrm{E}-02$ $-9.05712 \mathrm{E}-02$ $-9.35958 \mathrm{E}-02$ $-9.66699 \mathrm{E}-02$ $6.33917 \mathrm{E}-05$

$6.53831 \mathrm{E}-05$

$6.74053 \mathrm{E}-05$ $6.94583 \mathrm{E}-05$

$7.15422 \mathrm{E}-05$

$7.36569 \mathrm{E}-05$

$7.58024 \mathrm{E}-05$

$7.79788 \mathrm{E}-05$

$8.01859 \mathrm{E}-05$

$8.24239 \mathrm{E}-05$

$8.46928 \mathrm{E}-05$

$8.69925 \mathrm{E}-05$

$8.93229 \mathrm{E}-05$

$9.16843 \mathrm{E}-05$

$9.40763 \mathrm{E}-05$

$9.64993 \mathrm{E}-05$

$9.89531 \mathrm{E}-05$

$1.01438 \mathrm{E}-04$

$1.03953 \mathrm{E}-04$

$1.06499 \mathrm{E}-04$

$1.09076 \mathrm{E}-04$

$1.11684 \mathrm{E}-04$

$1.14323 \mathrm{E}-04$ $1.16993 \mathrm{E}-04$

$1.19693 \mathrm{E}-04$

$1.22424 \mathrm{E}-04$

$1.25186 \mathrm{E}-04$

$1.27979 \mathrm{E}-04$

$1.30803 \mathrm{E}-04$

$1.33657 \mathrm{E}-04$

$1.36542 \mathrm{E}-04$

$1.39459 \mathrm{E}-04$

$1.42405 \mathrm{E}-04$

$1.45383 \mathrm{E}-04$

$1.48392 \mathrm{E}-04$

$1.51431 \mathrm{E}-04$

$1.54501 \mathrm{E}-04$

$1.57602 \mathrm{E}-04$

$1.60734 \mathrm{E}-04$

$1.63897 \mathrm{E}-04$

$1.67090 \mathrm{E}-04$

$1.70315 \mathrm{E}-04$

$1.73570 \mathrm{E}-04$

$1.76856 \mathrm{E}-04$

$1.80172 \mathrm{E}-04$

$1.83520 \mathrm{E}-04$

$1.86898 \mathrm{E}-04$

$1.90307 \mathrm{E}-04$

$1.93747 \mathrm{E}-04$

$1.97218 \mathrm{E}-04$

$2.00720 \mathrm{E}-04$

$2.04252 \mathrm{E}-04$

$2.07815 \mathrm{E}-04$

$2.11409 \mathrm{E}-04$

$2.15034 \mathrm{E}-04$

$2.18689 \mathrm{E}-04$

$2.22376 \mathrm{E}-04$

$-9.97935 \mathrm{E}-02$

$-1.02967 \mathrm{E}-01$

$-1.06189 \mathrm{E}-01$

$-1.09461 \mathrm{E}-01$

$-1.12783 \mathrm{E}-01$

$-1.16154 \mathrm{E}-01$

$-1.19574 \mathrm{E}-01$

$-1.26563 \mathrm{E}-01$

$-1.30132 \mathrm{E}-01$

$-1.33750 \mathrm{E}-01$

$-1.37418 \mathrm{E}-01$

$-1.41134 \mathrm{E}-01$

$-1.44901 \mathrm{E}-01$

$-1.48717 \mathrm{E}-01$

$-1.52582 \mathrm{E}-01$

$-1.56496 \mathrm{E}-01$

$-1.60460 \mathrm{E}-01$

$-1.64474 \mathrm{E}-01$

$-1.68537 \mathrm{E}-01$

$-1.72649 \mathrm{E}-01$

$-1.76810 \mathrm{E}-01$

$-1.81021 \mathrm{E}-01$

$-1.85281 \mathrm{E}-01$

$-1.89591 \mathrm{E}-01$

$-1.93950 \mathrm{E}-01$

$-1.98358 \mathrm{E}-01$

$-2.02815 \mathrm{E}-01$

$-2.07322 \mathrm{E}-01$

$-2.11879 \mathrm{E}-01$

$-2.16484 \mathrm{E}-01$

$-2.21139 \mathrm{E}-01$

$-2.25843 \mathrm{E}-01$

$-2.30596 \mathrm{E}-01$

$-2.35399 \mathrm{E}-01$

$-2.40251 \mathrm{E}-01$ $-2.45152 \mathrm{E}-01$

$-2.50103 \mathrm{E}-01$

$-2.55103 \mathrm{E}-01$

$-2.60152 \mathrm{E}-01$

$-2.65250 \mathrm{E}-01$

$-2.70398 \mathrm{E}-01$

$-2.75595 \mathrm{E}-01$

$-2.80841 \mathrm{E}-01$

$-2.91481 \mathrm{E}-01$

$-2.96874 \mathrm{E}-01$

$-3.02317 \mathrm{E}-01$

$-3.07810 \mathrm{E}-01$

$-3.13351 \mathrm{E}-01$

$-3.18942 \mathrm{E}-01$

$-3.24581 \mathrm{E}-01$

$-3.30270 \mathrm{E}-01$

$-3.36008 \mathrm{E}-01$

$-3.41796 \mathrm{E}-01$ $g_{2}$

$-1.82406 \mathrm{E}-03$

$-1.89897 \mathrm{E}-03$

$-1.97576 \mathrm{E}-03$

$-2.05445 \mathrm{E}-03$

$-2.13505 \mathrm{E}-03$

$-1.23044 \mathrm{E}-01$

$-2.86136 \mathrm{E}-01$
$-2.21760 \mathrm{E}-03$

$-2.30211 \mathrm{E}-03$

$-2.38860 \mathrm{E}-03$

$-2.47708 \mathrm{E}-03$

$-2.56759 \mathrm{E}-03$

$-2.66014 \mathrm{E}-03$

$-2.75475 \mathrm{E}-03$

$-2.85145 \mathrm{E}-03$

$-2.95024 \mathrm{E}-03$

$-3.05116 \mathrm{E}-03$

$-3.15422 \mathrm{E}-03$

$-3.25945 \mathrm{E}-03$

$-3.36685 \mathrm{E}-03$

$-3.47646 \mathrm{E}-03$

$-3.58829 \mathrm{E}-03$

$-3.70237 \mathrm{E}-03$

$-3.81871 \mathrm{E}-03$

$-3.93734 \mathrm{E}-03$

$-4.05827 \mathrm{E}-03$

$-4.18152 \mathrm{E}-03$

$-4.30712 \mathrm{E}-03$

$-4.43509 \mathrm{E}-03$

$-4.56544 \mathrm{E}-03$

$-4.83338 \mathrm{E}-03$

$-4.97101 \mathrm{E}-03$

$-5.11111 \mathrm{E}-03$

$-5.25369 \mathrm{E}-03$

$-5.39878 \mathrm{E}-03$

$-5.54640 \mathrm{E}-03$

$-5.69657 \mathrm{E}-03$

$-5.84930 \mathrm{E}-03$

$-6.00462 \mathrm{E}-03$

$-6.16255 \mathrm{E}-03$

$-6.32311 \mathrm{E}-03$

$-6.48631 \mathrm{E}-03$ $-6.65219 \mathrm{E}-03$

$-6.82075 \mathrm{E}-03$ $-6.99203 \mathrm{E}-03$

$-7.16603 \mathrm{E}-03$ $-7.52231 \mathrm{E}-03$

$-7.70462 \mathrm{E}-03$

$-7.88974 \mathrm{E}-03$

$-8.07769 \mathrm{E}-03$

$-8.26850 \mathrm{E}-03$

$-8.46217 \mathrm{E}-03$

$-8.65874 \mathrm{E}-03$

$-8.85822 \mathrm{E}-03$

$-9.06063 \mathrm{E}-03$

$-9.26598 \mathrm{E}-03$

$-9.47432 \mathrm{E}-03$

$-9.68563 \mathrm{E}-03$

$-9.89997 \mathrm{E}-03$

$-1.01173 \mathrm{E}-02$
$-4.69820 \mathrm{E}-03$

$-7.34278 \mathrm{E}-03$ $\tan \delta_{2}$

$2.78585 \mathrm{E} \quad 01$

$2.78961 \mathrm{E} \quad 01$

$2.79325 \mathrm{E} \quad 01$

$2.79676 \mathrm{E} \quad 01$

$2.80016 \mathrm{E} \quad 01$

$2.80345 \mathrm{E} \quad 01$

$2.80664 \mathrm{E} \quad 01$

$2.80972 \mathrm{E} \quad 01$

$2.81271 \mathrm{E} \quad 01$

$2.81561 \mathrm{E}$ 0I

$2.81843 \mathrm{E} \quad 01$

$2.82116 \mathrm{E} \quad 01$

$2.82381 \mathrm{E} \quad 01$

$2.82638 \mathrm{E} \quad 01$

$2.82888 \mathrm{E} 01$

$2.83132 \mathrm{E} \quad 01$

$2.83368 \mathrm{E} \quad 01$

$2.83598 \mathrm{E} 01$

$2.83822 \mathrm{E} 01$

$2.84040 \mathrm{E}$ 0I

$2.84252 \mathrm{E} \quad 01$

$2.84459 \mathrm{E} \quad 01$

$2.84660 \mathrm{E} \quad 01$

$2.84856 \mathrm{E}$ ol

$2.85048 \mathrm{E}$ 0l

$2.85234 \mathrm{E} \quad 01$

$2.85416 \mathrm{E} \quad 01$

$2.85594 \mathrm{E} \quad 01$

$2.85768 \mathrm{E} \quad 01$

$2.85937 \mathrm{E} \quad 01$

$2.86102 \mathrm{E} \quad 01$

$2.86264 \mathrm{E} \quad 01$

$2.86422 \mathrm{E} \quad 01$

$2.86577 \mathrm{E} \quad 0$

$2.86727 \mathrm{E} \quad 01$

$2.86875 \mathrm{E} \quad 01$

$2.87020 \mathrm{E} \quad 01$

$2.87161 \mathrm{E} 01$

$2.87299 \mathrm{E} \quad 01$

$2.87434 \mathrm{E}$ ol

$2.87567 \mathrm{E} \quad 01$

$2.87697 \mathrm{E} \quad 01$

$2.87824 \mathrm{E} \quad 01$

$2.87948 \mathrm{E} \quad 01$

$2.88070 \mathrm{E} 01$

$2.88190 \mathrm{E} 01$

$2.88307 \mathrm{E} 01$

$2.88422 \mathrm{E} \quad 01$

$2.88535 \mathrm{E} \quad 01$

$2.88645 \mathrm{E} \quad 01$

$2.88754 \mathrm{E} \quad 01$

$2.88860 \mathrm{E} 01$

$2.88965 \mathrm{E} \quad 01$

$2.89067 \mathrm{E} \quad 01$

$2.89168 \mathrm{E} \quad 01$

$2.89267 \mathrm{E} \quad 01$

$2.89364 \mathrm{E} \quad 01$

$2.89459 \mathrm{E} \quad 01$

$2.89552 \mathrm{E} \quad 01$

$2.89644 \mathrm{E} \quad 01$

$f_{-2}$

$-1.87345 \mathrm{E}-03$

$-1.94709 \mathrm{E}-03$

$-2.02253 \mathrm{E}-03$

$-2.09981 \mathrm{E}-03$

$-2.17893 \mathrm{E}-03$

$-2.25993 \mathrm{E}-03$

$-2.34281 \mathrm{E}-03$

$-2.42761 \mathrm{E}-03$

$-2.51434 \mathrm{E}-03$

$-2.60303 \mathrm{E}-03$

$-2.69368 \mathrm{E}-03$

$-2.78633 \mathrm{E}-03$

$-2.88099 \mathrm{E}-03$

$-2.97768 \mathrm{E}-03$

$-3.07643 \mathrm{E}-03$

$-3.17725 E-03$

$-3.28017 \mathrm{E}-03$

$-3.38520 \mathrm{E}-03$

$-3.49237 \mathrm{E}-03$

$-3.60169 \mathrm{E}-03$

$-3.71318 \mathrm{E}-03$

$-3.82688 \mathrm{E}-03$

$-3.94278 \mathrm{E}-03$

$-4.06093 \mathrm{E}-03$

$-4.18133 \mathrm{E}-03$

$-4.30401 \mathrm{E}-03$

$-4.42898 \mathrm{E}-03$

$-4.55627 \mathrm{E}-03$

$-4.68590 \mathrm{E}-03$

$-4.81789 \mathrm{E}-03$

$-4.95225 \mathrm{E}-03$

$-5.08902 \mathrm{E}-03$

$-5.22820 \mathrm{E}-03$

$-5.36982 \mathrm{E}-03$

$-5.51390 \mathrm{E}-03$

$-5.66046 \mathrm{E}-03$

$-5.80951 \mathrm{E}-03$

$-5.96109 \mathrm{E}-03$

$-6.11521 \mathrm{E}-03$

$-6.27189 \mathrm{E}-03$

$-6.43115 \mathrm{E}-03$

$-6.59301 \mathrm{E}-03$

$-6.75749 \mathrm{E}-03$

$-6.92461 \mathrm{E}-03$ 


$$
Z=7 \quad A=14
$$

$g_{1}$

$\tan \delta_{1}$

$$
\rho=1.1 A^{1 / 3} F
$$

\begin{tabular}{|c|c|c|c|c|c|c|c|}
\hline$p$ & $F_{0}$ & & $f_{1}$ & & $g_{1}$ & $\tan \delta_{1}$ & $f_{-1}$ \\
\hline 12.10 & $1.18259 \mathrm{E}$ & 00 & $-1.25363 \mathrm{E}$ & 01 & $-6.34078 \mathrm{E}-01$ & $3.90561 \mathrm{E}-03$ & $-6.26059 \mathrm{E}-01$ \\
\hline 12.20 & $1.18256 \mathrm{E}$ & 00 & $-1.26437 \mathrm{E}$ & 01 & $-6.42406 \mathrm{E}-$ & $3.92204 \mathrm{E}-$ & $-6.34138 \mathrm{E}-01$ \\
\hline 12.30 & $1.18252 \mathrm{E}$ & 00 & $-1.27511 \mathrm{E}$ & 01 & $-6.50782 \mathrm{E}-01$ & $3.93818 \mathrm{E}-$ & $-6.42265 \mathrm{E}-01$ \\
\hline 12.40 & $1.18249 \mathrm{E}$ & 00 & $-1.28584 \mathrm{E}$ & 01 & $-6.59206 \mathrm{E}-01$ & $3.95405 \mathrm{E}-$ & $-6.50441 \mathrm{E}$ \\
\hline 12.50 & $1.18245 \mathrm{E}$ & 00 & $-1.29657 \mathrm{E}$ & 01 & $-6.67680 \mathrm{E}-01$ & $3.96965 \mathrm{E}-03$ & $-6.58665 \mathrm{E}-01$ \\
\hline 12.60 & $1.18242 \mathrm{E}$ & 00 & $-1.30730 \mathrm{E}$ & 01 & $-6.76202 \mathrm{E}-01$ & $3.98499 \mathrm{E}-03$ & $-6.66939 \mathrm{E}-01$ \\
\hline 2.70 & $1.18238 \mathrm{E}$ & 00 & $-1.31803 \mathrm{E}$ & 01 & $-6.84773 \mathrm{E}-01$ & $4.00006 \mathrm{E}-03$ & $-6.75261 \mathrm{E}-01$ \\
\hline 2.80 & $1.18235 \mathrm{E}$ & 00 & $-1.32876 \mathrm{E}$ & 01 & $-6.93392 E-01$ & $4.01489 \mathrm{E}-$ & $-6.83631 \mathrm{E}$ \\
\hline .90 & $1.18232 \mathrm{E}$ & 00 & $-1.33949 \mathrm{E}$ & 01 & $-7.02061 \mathrm{E}-01$ & $4.02948 \mathrm{E}-$ & $-6.92051 \mathrm{E}-$ \\
\hline 3.00 & $1.18229 \mathrm{E}$ & 00 & $-1.35021 \mathrm{E}$ & 01 & $-7.10778 \mathrm{E}-01$ & $4.04382 \mathrm{E}-03$ & $-7.00519 \mathrm{E}-0$ \\
\hline 3.10 & $1.18226 \mathrm{E}$ & 00 & $-1.36094 \mathrm{E}$ & 01 & $-7.19543 \mathbf{E}-01$ & $94 \mathrm{E}-03$ & $-7.09036 \mathrm{E}-$ \\
\hline .20 & $1.18222 \mathrm{E}$ & 00 & $-1.37166 \mathrm{E}$ & 01 & $-7.28357 \mathrm{E}-01$ & $4.07182 \mathrm{E}-$ & $-7.17601 \mathrm{E}-$ \\
\hline .30 & $1.18219 \mathrm{E}$ & 00 & $-1.38238 \mathrm{E}$ & 01 & $-7.37220 \mathrm{E}-01$ & $4.08548 \mathrm{E}-$ & $-7.26215 \mathrm{E}-$ \\
\hline .40 & $1.18216 \mathrm{E}$ & 00 & $-1.39310 \mathrm{E}$ & 01 & $-7.46131 \mathrm{E}-01$ & $4.09892 \mathrm{E}-$ & $-7.34877 \mathrm{E}-$ \\
\hline 3.50 & $1.18213 \mathrm{E}$ & 00 & $-1.40382 \mathrm{E}$ & 01 & $-7.55092 \mathrm{E}-01$ & $4.11215 \mathrm{E}-03$ & $-7.43589 \mathrm{E}-$ \\
\hline 3.60 & $1.18210 \mathrm{E}$ & 00 & $-1.41453 \mathrm{E}$ & 01 & $-7.64100 \mathrm{E}-01$ & $4.12516 \mathrm{E}-03$ & $-7.52349 \mathrm{E}-$ \\
\hline 3.70 & $1.18207 \mathrm{E}$ & 00 & $-1.42525 \mathrm{E}$ & 01 & $-7.73157 \mathrm{E}-01$ & $4.13797 \mathrm{E}$ & $-7.61157 \mathrm{E}-$ \\
\hline 3.80 & $1.18204 \mathrm{E}$ & 00 & $-1.43596 \mathrm{E}$ & 01 & $-7.82263 \mathrm{E}-01$ & $4.15058 \mathrm{E}-$ & $-7.70014 \mathrm{E}-$ \\
\hline .90 & $1.18201 \mathrm{E}$ & 00 & $44667 \mathrm{E}$ & 01 & $-7.91417 \mathrm{E}-01$ & 4.16 & $-7.78920 \mathrm{E}-$ \\
\hline 4.00 & $1.18199 \mathrm{E}$ & 00 & $45738 \mathrm{E}$ & 01 & $-8.00620 \mathrm{E}-01$ & $21 \mathrm{E}-03$ & $-7.87874 \mathrm{E}-$ \\
\hline 14.10 & $1.18196 \mathrm{E}$ & 00 & $-1.46809 \mathrm{E}$ & 01 & $-8.09872 \mathrm{E}-01$ & $4.18725 \mathrm{E}-03$ & $-7.96877 \mathrm{E}-$ \\
\hline 14.20 & $1.18193 \mathrm{E}$ & 00 & $-1.47880 \mathrm{E}$ & 01 & $-8.19172 \mathrm{E}-01$ & 4.199 & $-8.05928 \mathrm{E}-$ \\
\hline 1.30 & $190 \mathrm{E}$ & 00 & $3950 \mathrm{E}$ & 01 & $-8.28521 \mathrm{E}-01$ & 4.210 & $-8.15028 \mathrm{E}-$ \\
\hline .40 & $187 \mathrm{E}$ & 00 & $0021 \mathrm{E}$ & 01 & -8 & 4.22 & $24177 \mathrm{E}$ \\
\hline 14.50 & $1.18185 \mathrm{E}$ & 00 & $1091 \mathrm{E}$ & 01 & -8 & $4.23356 \mathrm{E}-03$ & $3374 \mathrm{E}-$ \\
\hline 14.60 & $.18182 \mathrm{E}$ & 00 & $-1.52161 \mathrm{E}$ & 01 & $-8.56858 \mathrm{E}-01$ & $4.24470 \mathrm{E}-03$ & $-8.42619 \mathrm{E}-01$ \\
\hline 14.70 & $1.18179 \mathrm{E}$ & 00 & $3231 \mathrm{E}$ & 01 & $-8.66400 \mathrm{E}-$ & 4.25 & $-8.51913 \mathrm{E}-0$ \\
\hline .80 & $77 \mathrm{E}$ & 00 & $300 \mathrm{E}$ & 01 & -8 & 4.26 & $-\varepsilon+2-r+1-1$ \\
\hline 14.90 & $4 \mathrm{E}$ & 00 & $370 \mathrm{E}$ & 01 & -8 & 4.27 & $647 \mathrm{E}-$ \\
\hline 15.00 & $1.18171 \mathrm{E}$ & 00 & $6439 \mathrm{E}$ & 01 & -8.9531 & $64 \mathrm{E}-03$ & $30086 \mathrm{E}-0$ \\
\hline 15.10 & $1.18169 \mathrm{E}$ & 00 & $7509 \mathrm{E}$ & 01 & $056 \mathrm{E}-01$ & $8 \mathrm{E}-03$ & $9574 \mathrm{E}-01$ \\
\hline 5.20 & & 00 & $78 \mathrm{E}$ & 01 & & & \\
\hline 15.30 & $1.18164 \mathrm{E}$ & 00 & $9647 \mathrm{E}$ & 01 & $-9.24675 \mathrm{E}-01$ & 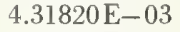 & $-9.08696 \mathrm{E}-$ \\
\hline 15.40 & $1.18161 \mathrm{E}$ & 00 & $-1.60715 \mathrm{E}$ & 01 & $-9.34557 \mathrm{E}-01$ & $4.32810 \mathrm{E}$ & $-9.18329 \mathrm{E}-$ \\
\hline 15.50 & $1.18159 \mathrm{E}$ & 00 & $-1.61784 \mathrm{E}$ & 01 & $-9.44487 \mathrm{E}-01$ & 4.337 & $-9.28011 \mathrm{E}-$ \\
\hline 15.60 & $1.18156 \mathrm{E}$ & 00 & $-1.62852 \mathrm{E}$ & 01 & $-9.54466 \mathrm{E}-01$ & $4.34746 \mathrm{E}-03$ & $7741 \mathrm{E}-$ \\
\hline 15.70 & $54 \mathrm{E}$ & 00 & $921 \mathrm{E}$ & 01 & $E-01$ & 4.35 & $9.47520 \mathrm{E}$ \\
\hline 15.80 & $1.18151 \mathrm{E}$ & 00 & $-1.64989 \mathrm{E}$ & 01 & $-9.74569 \mathrm{E}-01$ & $4.36626 \mathrm{E}$ & $-9.57348 \mathrm{E}-$ \\
\hline 15.90 & $18149 \mathrm{E}$ & 00 & $-1.66057 \mathrm{E}$ & 01 & $-9.84694 \mathrm{E}-01$ & 4.375 & $-9.67223 \mathrm{E}-0$ \\
\hline 16.00 & $47 \mathrm{E}$ & 00 & $7125 \mathrm{E}$ & 01 & $-9.94866 \mathrm{E}-0$ & 4.38 & $-9.77147 \mathrm{E}-0$ \\
\hline 16.10 & $.18144 \mathrm{E}$ & 00 & $-1.68192 \mathrm{E}$ & 01 & $-1.00509 \mathrm{E}$ & $4.39348 \mathrm{E}-03$ & $-9.87120 \mathrm{E}-01$ \\
\hline 16.20 & $3142 \mathrm{E}$ & 00 & $-1.69260 \mathrm{E}$ & 01 & $-1.01536 \mathrm{E}$ & 4.402 & $-9.97141 \mathrm{E}-0$ \\
\hline 16.30 & $8140 \mathrm{E}$ & 00 & $-1.70327 \mathrm{E}$ & 01 & $-1.02567 \mathrm{E}$ & $99 \mathrm{E}$ & $-1.00721 \mathrm{E}$ \\
\hline 16.40 & $37 \mathrm{E}$ & 00 & $394 \mathrm{E}$ & 01 & $-1.03604 \mathrm{E}$ & $56 \mathrm{E}$ & $-1.01733 \mathrm{E}$ \\
\hline 16.50 & $35 \mathrm{E}$ & 00 & $2461 \mathrm{E}$ & 01 & $-1.04645 \mathrm{E} \quad 00$ & $4.42801 \mathrm{E}-03$ & $-1.02749 \mathrm{E}$ \\
\hline 16.60 & $.18133 \mathrm{E}$ & 00 & $-1.73528 \mathrm{E}$ & 01 & $-1.05692 \mathrm{E}$ & 4.43 & $-1.03771 \mathrm{E} \quad 0 \mathrm{C}$ \\
\hline 16.70 & $31 \mathrm{E}$ & 00 & $4595 \mathrm{E}$ & 01 & $-1.06743 \mathrm{E}$ & $6 \mathrm{~F}-2$ & $04797 \mathrm{E}$ \\
\hline 80 & $28 \mathrm{E}$ & 00 & $5661 \mathrm{E}$ & 01 & $-1.07799 \mathrm{E}$ & & $05828 \mathrm{E}$ \\
\hline & & 00 & & 01 & $8860 \mathrm{E}$ & & $.06864 \mathrm{E}$ \\
\hline 17.00 & & 00 & $77793 \mathrm{E}$ & 01 & $-1.09925 \mathrm{E} \quad 00$ & $4.46854 \mathrm{E}-03$ & $-1.07905 \mathrm{E}$ \\
\hline 17.10 & $18122 \mathrm{E}$ & 00 & $-1.78859 \mathrm{E}$ & 01 & $-1.10996 \mathrm{E} \quad 00$ & 4. & $.08950 \mathrm{E} \quad 00$ \\
\hline 17.20 & $3120 \mathrm{E}$ & 00 & & 01 & $-1.12071 \mathrm{E}$ & & $-1.10001 \mathrm{E} \quad 00$ \\
\hline 17.30 & & 00 & $0991 \mathrm{E}$ & 01 & $-1.13151 \mathrm{E}$ & & $-1.11056 \mathrm{E} \quad 00$ \\
\hline 17.40 & $115 \mathrm{E}$ & 00 & $2056 \mathrm{E}$ & 01 & $-1.14236 \mathrm{E}$ & 4.4 & $-1.12116 \mathrm{E} \quad 0 \mathrm{C}$ \\
\hline 17.50 & $1.18113 \mathrm{E}$ & 00 & $-1.83122 \mathrm{E}$ & 01 & $-1.15326 \mathrm{E}$ & $4.50636 \mathrm{E}$ & $-1.13181 \mathrm{E} \quad 0$ \\
\hline 17.60 & $.18111 \mathrm{E}$ & 00 & $-1.84187 \mathrm{E}$ & 01 & $-1.16420 \mathrm{E} \quad 00$ & $4.51362 E-03$ & $-1.14251 \mathrm{E} \quad 00$ \\
\hline & $1.18109 \mathrm{E}$ & 00 & $-1.85252 \mathrm{E}$ & 01 & $-1.17519 \mathrm{E}$ & & $-1.15325 \mathrm{E}$ \\
\hline & $1.18107 \mathrm{E}$ & 00 & $-1.86317 \mathrm{E}$ & 01 & $-1.18624 \mathrm{E}$ & $4.52784 \mathrm{E}$ & $-1.16405 E$ \\
\hline 17.90 & $105 \mathrm{E}$ & 00 & $-1.87381 \mathrm{E}$ & 01 & $-1.19733 \mathrm{E}$ & $4.53481 \mathrm{E}-03$ & $-1.17489 \mathrm{E}$ \\
\hline & & & $88446 \mathrm{E}$ & & $-1.20847 \mathrm{E}$ & $4.54 .169 \mathrm{E}-03$ & \\
\hline
\end{tabular}

$g_{-1}$ $\tan \delta_{-1}$

$1.36111 \mathrm{E} \quad 01$ $1.37185 \mathrm{E} \quad 01$ $1.38258 \mathrm{E}$ 01 $1.39331 \mathrm{E} 01$ $1.40403 \mathrm{E} \quad 01$

$1.41476 \mathrm{E} \quad 01$ $1.42548 \mathrm{E} \quad 01$ $1.43621 \mathrm{E} \quad 01$ $1.44693 \mathrm{E} \quad 01$ $1.45765 \mathrm{E} \quad 01$

$1.46837 \mathrm{E}$ 01 $1.47908 \mathrm{E} \quad 01$ $1.48980 \mathrm{E} \quad 01$

$1.50051 \mathrm{E} \quad 01$

$1.51122 \mathrm{E} \quad 01$

$1.52193 \mathrm{E} \quad 01$ $1.53264 \mathrm{E} \quad 01$ $1.54335 \mathrm{E} \quad 01$ $1.55405 \mathrm{E} \quad 01$

$1.56475 \mathrm{E}$ 01

$\begin{array}{ll}1.57546 \mathrm{E} & 01\end{array}$ $1.58616 \mathrm{E} \quad 01$ $1.59686 \mathrm{E} \quad 01$ $1.60755 \mathrm{E} \quad 01$ $1.61825 \mathrm{E} \quad 01$

$1.62894 \mathrm{E} \quad 01$ $1.63963 \mathrm{E} \quad 01$ $1.65032 \mathrm{E} \quad 01$

$1.66101 \mathrm{E} \quad 01$

$1.67170 \mathrm{E} \quad 01$

$1.68239 \mathrm{E} \quad 01$

$1.69307 \mathrm{E} \quad 01$

$1.70375 \mathrm{E} \quad 01$

$1.71443 \mathrm{E}$ 01

$1.72511 \mathrm{E} 01$

$1.73579 \mathrm{E} \quad 01$ $1.74646 \mathrm{E} \quad 01$ $1.75714 \mathrm{E} \quad 01$ $1.76781 \mathrm{E} 01$ $1.77848 \mathrm{E} \quad 01$

$1.78915 \mathrm{E} \quad 01$ $1.79982 \mathrm{E} \quad 01$ $1.81048 \mathrm{E} \quad 01$ $1.82115 \mathrm{E} \quad 01$ $1.83181 \mathrm{E} \cdot 01$

$1.84247 \mathrm{E} \quad 01$ $1.85313 \mathrm{E} 01$ $1.86379 \mathrm{E} 01$

$1.87444 \mathrm{E} 01$

$1.88509 \mathrm{E} 01$

$\begin{array}{ll}1.89575 \mathrm{E} & 01\end{array}$ $1.90640 \mathrm{E} 01$ $1.91704 \mathrm{E} \quad 01$ $1.92769 \mathrm{E} \quad 01$ $1.93834 \mathrm{E} \quad 01$

$1.94898 \mathrm{E} \quad 01$ $1.95962 \mathrm{E} 01$ $1.97026 \mathrm{E} \quad 01$ $1.98090 \mathrm{E} \quad 01$ $1.99154 \mathrm{E} \quad 01$
$-1.23143 \mathrm{E} \quad 02$ $-1.23420 \mathrm{E} 02$ $\begin{array}{ll}-1.23695 \mathrm{E} & 02\end{array}$ $-1.23966 \mathrm{E} \quad 02$ $-1.24234 \mathrm{E} \quad 02$ $-1.24499 \mathrm{E} \quad 02$ $-1.24762 \mathrm{E} \quad 02$ $-1.25021 \mathrm{E} 02$ $-1.25278 \mathrm{E} \quad 02$ $-1.25533 \mathrm{E} \quad 02$

$-1.25784 \mathrm{E} \quad 02$ $-1.26033 \mathrm{E} \quad 02$ $-1.26280 \mathrm{E} \quad 02$ $-1.26524 \mathrm{E} 02$ $-1.26766 \mathrm{E} \quad 02$

$-1.27005 \mathrm{E} \quad 02$ $-1.27242 \mathrm{E} \quad 02$ $-1.27477 \mathrm{E} \quad 02$ $-1.27709 \mathrm{E} \quad 02$ $-1.27939 \mathrm{E} \quad 02$ $-1.28167 \mathrm{E} \quad 02$ $-1.28393 \mathrm{E} \quad 02$ $-1.28617 \mathrm{E} \quad 02$ $-1.28838 \mathrm{E} 02$ $-1.29058 \mathrm{E} 02$

$-1.29276 \mathrm{E} \quad 02$ $-1.29491 \mathrm{E} \quad 02$ $-1.29705 \mathrm{E} \quad 02$ $-1.29917 \mathrm{E} \quad 02$ $-1.30127 \mathrm{E} \quad 02$

$-1.30335 \mathrm{E} \quad 02$ $-1.30541 \mathrm{E} 02$ $-1.30745 \mathrm{E} 02$ $-1.30948 \mathrm{E} \quad 02$ $-1.31149 \mathrm{E} \quad 02$ $-1.31349 \mathrm{E} \quad 02$ $-1.31546 \mathrm{E} \quad 02$ $-1.31742 \mathrm{E} 02$ $-1.31937 \mathrm{E} \quad 02$ $-1.32130 \mathrm{E} \quad 02$ $-1.32321 \mathrm{E} \quad 02$ $-1.32511 \mathrm{E} \quad 02$ $-1.32699 \mathrm{E} 02$ $-1.32886 \mathrm{E} 02$ -1.33071 E 02

$-1.33255 \mathrm{E} \quad 02$ $-1.33438 \mathrm{E} 02$ $-1.33619 \mathrm{E} 02$ $-1.33799 \mathrm{E} \quad 02$ $-1.33977 \mathrm{E} 02$

$-1.34154 \mathrm{E} \quad 02$ $-1.34330 \mathrm{E} 02$ -1.34505 E 02 $-1.34678 \mathrm{E} 02$ $-1.34850 \mathrm{E} \quad 02$

$-1.35021 \mathrm{E} \quad 02$ $-1.35190 \mathrm{E} 02$ $-1.35359 \mathrm{E} \quad 02$ $-1.35526 \mathrm{E} \quad 02$ $-1.35692 \mathrm{E} 02$ 
ELECTRON RADIAL FUNCTIONS

$Z=7 \quad A=14 \quad \rho=1.1 A^{1 / 3} F$

Electron

$p$

12.10

12.20

12.30

12.40

12.50

12.60

12.70

12.80

12.90

13.00

13.10

13.20

13.30

13.40

13.50

13.60

13.70

13.80

13.90

14.00

14.10

14.20

14.30

14.40

14.50

14.60

14.70

14.80

14.90

15.00

15.10

15.20

15.30

15.40

15.50

15.60

15.70

15.80

15.90

16.00

16.10

16.20

16.30

16.40

16.50

16.60

16.70

16.80

16.90

17.00

17.10

17.20

17.30

17.40

17.50

17.60

17.70

17.80

17.90

18.00
$\bar{F}$

$2.26093 \mathrm{E}-04$

$2.29841 \mathrm{E}-04$

$2.33620 \mathrm{E}-04$

$2.37430 \mathrm{E}-04$

$2.41270 \mathrm{E}-04$

2.45141 E-04

2.49044 E-04

$2.52976 \mathrm{E}-04$

$2.56940 \mathrm{E}-04$

$2.60935 \mathrm{E}-04$

$2.64960 \mathrm{E}-04$

$2.69016 \mathrm{E}-04$

$2.73103 \mathrm{E}-04$

$2.77221 \mathrm{E}-04$

$2.81369 \mathrm{E}-04$

$2.85548 \mathrm{E}-04$

$2.89759 \mathrm{E}-04$

$2.93999 \mathrm{E}-04$

2.98271 E-04

3.02574 E-04

$3.06907 \mathrm{E}-04$

$3.11271 \mathrm{E}-04$

$3.15666 \mathrm{E}-04$

$3.20092 \mathrm{E}-04$

$3.24548 \mathrm{E}-04$

$3.29036 \mathrm{E}-04$

3.33554 E- 04

$3.38103 \mathrm{E}-04$

$3.42683 \mathrm{E}-04$

$3.47293 \mathrm{E}-04$

$3.51934 \mathrm{E}-04$

$3.56607 \mathrm{E}-04$

$3.61310 \mathrm{E}-04$

$3.66043 \mathrm{E}-04$

$3.70808 \mathrm{E}-04$

$3.75603 \mathrm{E}-04$

$3.80429 \mathrm{E}-04$

$3.85286 \mathrm{E}-04$

$3.90174 \mathrm{E}-04$

$3.95093 \mathrm{E}-04$

$4.00042 \mathrm{E}-04$

$4.05022 \mathrm{E}-04$

$4.10033 \mathrm{E}-04$

4.15075 E-04

$4.20147 \mathrm{E}-04$

$4.25250 \mathrm{E}-04$

$4.30384 \mathrm{E}-04$

$4.35549 \mathrm{E}-04$

$4.40745 \mathrm{E}-04$

$4.45972 \mathrm{E}-04$

$4.51229 \mathrm{E}-04$

$4.56517 \mathrm{E}-04$

$4.61836 \mathrm{E}-04$

$4.67185 \mathrm{E}-04$

$4.72566 \mathrm{E}-04$

$4.77977 \mathrm{E}-04$

$4.83419 \mathrm{E}-04$

$4.88892 \mathrm{E}-04$

$4.94395 \mathrm{E}-04$

$4.99930 \mathrm{E}-04$ $f_{2}$

$g_{2}$

$-3.47632 \mathrm{E}-01$

$-3.53518 \mathrm{E}-01$

$-3.59453 \mathrm{E}-01$

$-3.65437 \mathrm{E}-01$

$-3.71470 \mathrm{E}-01$

$-3.77552 \mathrm{E}-01$

$-3.83684 \mathrm{E}-01$

$-3.89864 \mathrm{E}-01$

$-3.96094 \mathrm{E}-01$

$-4.02373 \mathrm{E}-01$

$-4.08700 \mathrm{E}-01$

$-4.15077 \mathrm{E}-01$

$-4.21503 \mathrm{E}-01$

$-4.27978 \mathrm{E}-01$

$-4.34503 \mathrm{E}-01$

$-4.41076 \mathrm{E}-01$

$-4.47698 \mathrm{E}-01$

$-4.54370 \mathrm{E}-01$

$-4.61090 \mathrm{E}-01$

$-4.67859 \mathrm{E}-01$

$-4.74678 \mathrm{E}-01$

$-4.81546 \mathrm{E}-01$

$-4.88462 \mathrm{E}-01$

$-4.95428 \mathrm{E}-01$

$-5.02442 \mathrm{E}-01$

$-5.09506 \mathrm{E}-01$

$-5.16619 \mathrm{E}-01$

$-5.23780 \mathrm{E}-01$

$-5.30991 \mathrm{E}-01$

$-5.38251 \mathrm{E}-01$

$-5.45559 \mathrm{E}-01$

$-5.52917 \mathrm{E}-01$

$-5.60323 \mathrm{E}-01$

$-5.67779 \mathrm{E}-01$

$-5.75283 \mathrm{E}-01$

$-5.82836 \mathrm{E}-01$

$-5.90439 \mathrm{E}-01$

$-5.98090 \mathrm{E}-01$

$-6.05790 \mathrm{E}-01$

$-6.13539 \mathrm{E}-01$

$-6.21337 \mathrm{E}-01$

$-6.29184 \mathrm{E}-01$

$-6.37080 \mathrm{E}-01$

$-6.45024 \mathrm{E}-01$

$-6.53018 \mathrm{E}-01$

$-6.61060 \mathrm{E}-01$

$-6.69151 \mathrm{E}-01$

$-6.77291 \mathrm{E}-01$

$-6.85480 \mathrm{E}-01$

$-6.93718 \mathrm{E}-01$

$-7.02005 \mathrm{E}-01$

$-7.10340 \mathrm{E}-01$

$-7.18724 \mathrm{E}-01$

$-7.27157 \mathrm{E}-01$

$-7.35639 \mathrm{E}-01$

$-7.44170 \mathrm{E}-01$

$-7.52750 \mathrm{E}-01$

$-7.61378 \mathrm{E}-01$

$-7.70055 \mathrm{E}-01$

$-7.78781 \mathrm{E}-01$ $\tan \delta_{2}$

$f_{-2}$

$-1.03378 \mathrm{E}-02$

$-1.05613 \mathrm{E}-02$

$-1.07878 \mathrm{E}-02$

$-1.10175 \mathrm{E}-02$

$-1.12504 \mathrm{E}-02$

$-1.14864 \mathrm{E}-02$

$-1.17255 \mathrm{E}-02$

$-1.19679 \mathrm{E}-02$

$-1.22134 \mathrm{E}-02$

$-1.24622 \mathrm{E}-02$

$-1.27143 \mathrm{E}-02$

$-1.29696 \mathrm{E}-02$

$-1.32282 \mathrm{E}-02$

$-1.34902 \mathrm{E}-02$

$-1.37554 \mathrm{E}-02$

$-1.40240 \mathrm{E}-02$

$-1.42960 \mathrm{E}-02$

$-1.45714 \mathrm{E}-02$

$-1.48502 \mathrm{E}-02$

$-1.51325 \mathrm{E}-02$

$-1.54182 \mathrm{E}-02$

$-1.57073 \mathrm{E}-02$

$-1.60000 \mathrm{E}-02$

$-1.62962 \mathrm{E}-02$

$-1.65959 \mathrm{E}-02$

$-1.68992 \mathrm{E}-02$

$-1.72060 \mathrm{E}-02$

$-1.75164 \mathrm{E}-02$

$-1.78305 \mathrm{E}-02$

$-1.81482 \mathrm{E}-02$

$-1.84695 \mathrm{E}-02$

$-1.87946 \mathrm{E}-02$

$-1.91233 \mathrm{E}-02$

$-1.94557 \mathrm{E}-02$

$-1.97919 \mathrm{E}-02$

$-2.01318 \mathrm{E}-02$

$-2.04755 \mathrm{E}-02$

$-2.08230 \mathrm{E}-02$

$-2.11743 \mathrm{E}-02$

$-2.15295 \mathrm{E}-02$

$-2.18885 \mathrm{E}-02$

$-2.22513 \mathrm{E}-02$

$-2.26181 \mathrm{E}-02$

$-2.29888 \mathrm{E}-02$

$-2.33634 \mathrm{E}-02$

$-2.37420 \mathrm{E}-02$

$-2.41246 \mathrm{E}-02$

$-2.45111 \mathrm{E}-02$

$-2.49017 \mathrm{E}-02$

$-2.52963 \mathrm{E}-02$

$-2.56950 \mathrm{E}-02$

$-2.60977 \mathrm{E}-02$

$-2.65046 \mathrm{E}-02$

$-2.69155 \mathrm{E}-02$

$-2.73306 \mathrm{E}-02$

$-2.77499 \mathrm{E}-02$

$-2.81733 \mathrm{E}-02$

$-2.86009 \mathrm{E}-02$

$-2.90327 \mathrm{E}-02$

$-2.94688 \mathrm{E}-02$

$2.89735 \mathrm{E} \quad 01 \quad-1.01893 \mathrm{E}-02$

$2.89823 \mathrm{E}$ 01 $-1.04074 \mathrm{E}-02$

$2.89911 \mathrm{E} \quad 01-1.06286 \mathrm{E}-02$

$2.89997 \mathrm{E}$ 01 $-1.08528 \mathrm{E}-02$

$2.90081 \mathrm{E} 01 \quad-1.10800 \mathrm{E}-02$

$2.90164 \mathrm{E} \quad 01$

$2.90245 \mathrm{E} 01$

$2.90326 \mathrm{E} \quad 01$

$2.90404 \mathrm{E} \quad 01$

$2.90482 \mathrm{E} \quad 01$

$2.90558 \mathrm{E} \quad 01$

$2.90634 \mathrm{E} \quad 01$

$2.90708 \mathrm{E} \quad 01$

$2.90780 \mathrm{E} \quad 01$

$2.90852 \mathrm{E}$

$2.90923 \mathrm{E} \quad 01$

$2.90992 \mathrm{E} \quad 01$

$2.91061 \mathrm{E} \quad 01$

$2.91128 \mathrm{E} \quad 01$

$2.91195 \mathrm{E} \quad 0$

$2.91260 \mathrm{E} \quad 01$

$2.91325 \mathrm{E} \quad 01$

$2.91388 \mathrm{E} \quad 01$

$2.91451 \mathrm{E} \quad 01$

$2.91512 \mathrm{E} \quad 01$

2.91573 E 01

$2.91633 \mathrm{E} \quad 01$

$2.91692 \mathrm{E} 01$

$2.91750 \mathrm{E} \quad 01$

$2.91808 \mathrm{E} \quad 01$

$2.91864 \mathrm{E} \quad 01$

$2.91920 \mathrm{E} \quad 01$

$2.91975 \mathrm{E} \quad 01$

$2.92030 \mathrm{E} \quad 01$

$2.92083 \mathrm{E} \quad 01$

$2.92136 \mathrm{E} \quad 01$

$-1.13103 \mathrm{E}-02$

$-1.15438 \mathrm{E}-02$

$-1.17804 \mathrm{E}-02$

$-1.20201 \mathrm{E}-02$

$-1.22629 \mathrm{E}-02$ 
ELECTRON RADIAL FUNCTIONS

Electron

$p$

18.10

18.20

18.30

18.40

18.50

18.60

18.70

18.80

18.90

19.00

19.10

19.20

19.30

19.40

19.50

19.60

19.70

19.80

19.90

20.00

20.10

20.20

20.30

20.40

20.50

20.60

20.70

20.80

20.90

21.00

21.10

21.20

21.30

21.40

21.50

21.60

21.70

21.80

21.90

22.00

22.10

22.20

22.30

22.40

22.50

22.60

22.70

22.80

22.90

23.00

23.10

23.20

23.30

23.40

23.50

23.60

23.70

23.80

23.90

24.00
$Z=7$

$\begin{array}{llll}1.18101 \mathrm{E} & 00 & -1.89510 \mathrm{E} & 01\end{array}$

$1.18099 \mathrm{E} 00$

$1.18097 \mathrm{E} \quad 00$

$1.18095 \mathrm{E} \quad 00$

$1.18093 \mathrm{E} 00$

$1.18091 \mathrm{E} \quad 00$

$1.18089 \mathrm{E} \quad 00$

$1.18087 \mathrm{E} \quad 00$

$1.18085 \mathrm{E} \quad 00$

$1.18083 \mathrm{E} 00$

$1.18082 \mathrm{E} \quad 00$

$1.18080 \mathrm{E} \quad 00$

$1.18078 \mathrm{E} \quad 00$

$1.18076 \mathrm{E} \quad 00$

$1.18074 \mathrm{E} \quad 00$

$1.18072 \mathrm{E} \quad 00$

$1.18071 \mathrm{E} 00$

$1.18069 \mathrm{E} \quad 00$

$1.18067 \mathrm{E} \quad 00$

$1.18065 \mathrm{E} \quad 00$

$1.18063 \mathrm{E} \quad 00$

$1.18062 \mathrm{E} \quad 00$

$1.18060 \mathrm{E} \quad 00$

$1.18058 \mathrm{E} \quad 00$

$1.18057 \mathrm{E} \quad 00$

$1.18055 \mathrm{E} \quad 00$

$1.18053 \mathrm{E} \quad 00$

$1.18051 \mathrm{E} \quad 00$

$1.18050 \mathrm{E}-00$

$1.18048 \mathrm{E} \quad 00$

$1.18046 \mathrm{E} \quad 00$

$1.18045 \mathrm{E} \quad 00$

$1.18043 \mathrm{E} \quad 00$

$1.18042 \mathrm{E} \quad 00$

$1.18040 \mathrm{E} \quad 00$

$1.18038 \mathrm{E} \quad 00$

$1.18037 \mathrm{E} \quad 00$

$1.18035 \mathrm{E} \quad 00$

$1.18034 \mathrm{E} \quad 00$

$1.18032 \mathrm{E} \quad 00$

$1.18030 \mathrm{E} \quad 00$

$1.18029 \mathrm{E} \quad 00$

$1.18027 \mathrm{E} \quad 00$

$1.18026 \mathrm{E} \quad 00$

$1.18024 \mathrm{E} \quad 00$

$1.18023 \mathrm{E} \quad 00$

$1.18021 \mathrm{E} \quad 00$

$1.18020 \mathrm{E} \quad 00$

$1.18018 \mathrm{E} 00$

$1.18017 \mathrm{E} \quad 00$

$1.18015 \mathrm{E} \quad 00$

$1.18014 \mathrm{E} \quad 00$

$1.18012 \mathrm{E} \quad 00$

$1.18011 \mathrm{E} \quad 00$

$1.18009 \mathrm{E} \quad 00$

$1.18008 \mathrm{E} \quad 00$

$1.18007 \mathrm{E} \quad 00$

$1.18005 \mathrm{E} \quad 00$

$1.18004 \mathrm{E} \quad 00$

$1.18002 \mathrm{E} \quad 00$

$-1.92702 \mathrm{E} 0$

$-2.06515 \mathrm{E} 01$ $f_{1}$

$g_{1}$

$-1.21965 \mathrm{E} 00$

$-1.23089 \mathrm{E} \quad 00$

$-1.24217 \mathrm{E} \quad 00$

$-1.25350 \mathrm{E} 00$

$-1.26488 \mathrm{E} \quad 00$

$-1.93765 \mathrm{E} 01$

$-1.94829 \mathrm{E} \quad 01$

$-1.95892 \mathrm{E} \quad 01$

$-1.96955 \mathrm{E} \quad 01$

$-1.98018 \mathrm{E} 01$

-1.99081 E 01

$-2.00143 \mathrm{E} \quad 01$

$-2.01206 \mathrm{E} \quad 01$

$-2.02268 \mathrm{E} 01$

$-2.03330 \mathrm{E} 01$

-2.04392 E 01

$-2.05454 \mathrm{E} \quad 01$

$-2.07576 \mathrm{E} \quad 01$

$-2.08637 \mathrm{E} 01$

$-2.09698 \mathrm{E} 01$

$-2.10759 \mathrm{E} \quad 01$

$-2.11820 \mathrm{E} \quad 01$

$-2.12880 \mathrm{E} \quad 01$

$-2.13940 \mathrm{E} \quad 01$

$-2.15000 \mathrm{E} 01$

$-2.16060 \mathrm{E} \quad 01$

$-2.17120 \mathrm{E} 01$

$-2.18180 \mathrm{E} \quad 01$

$-2.19239 \mathrm{E} 01$

-2.20298 E 01

$-2.21357 \mathrm{E} \quad 01$

$-2.22416 \mathrm{E} 01$

$-2.23474 \mathrm{E} 01$

$-2.24533 \mathrm{E} 01$

$-2.25591 \mathrm{E} \quad 01$

$-2.26649 \mathrm{E} 01$

$-2.27707 \mathrm{E} \quad 01$

$-2.28764 \mathrm{E} \quad 01$

$-2.29822 \mathrm{E} 01$

$-2.30879 \mathrm{E} 01$

$-2.31936 \mathrm{E} \quad 01$ $-2.32993 \mathrm{E} 01$

$-2.34050 \mathrm{E} 01$

$-2.35106 \mathrm{E} 01$

$-2.36163 \mathrm{E} 01$

$-2.37219 \mathrm{E} \quad 01$

$-2.38275 \mathrm{E} 01$

$-2.39331 \mathrm{E} 01$

$-2.40386 \mathrm{E} 01$

-2.41442 E 01

$-2.42497 \mathrm{E} \quad 01$

$-2.43552 \mathrm{E} \quad 01$

$-2.44607 \mathrm{E} \quad 01$

$-2.45661 \mathrm{E} \quad 01$

$-2.46716 \mathrm{E} 01$

$-2.47770 \mathrm{E} 01$

$-2.48824 \mathrm{E} \quad 01$

$-2.49878 \mathrm{E} 01$

$-2.50931 \mathrm{E} \quad 01$

-2.51985 E 01
$-1.27631 \mathrm{E} \quad 00$

$-1.28778 \mathrm{E} \quad 00$

$-1.29931 \mathrm{E} 00$

$-1.31088 \mathrm{E} 00$

$-1.32250 \mathrm{E} 00$

$-1.33417 \mathrm{E} \quad 00$ $-1.34588 \mathrm{E} 00$

$-1.35765 \mathrm{E} \quad 00$

$-1.36946 \mathrm{E} \quad 00$

$-1.38132 \mathrm{E} \quad 00$

$-1.39323 \mathrm{E} 00$

$-1.40519 \mathrm{E} 00$

$-1.41719 \mathrm{E} \quad 00$

$-1.44134 \mathrm{E} \quad 00$

$-1.45349 \mathrm{E} \quad 00$

$-1.46569 \mathrm{E} \quad 00$

$-1.47793 \mathrm{E} \quad 00$

$-1.49022 \mathrm{E} \quad 00$

$-1.50256 \mathrm{E} 00$

$-1.51495 \mathrm{E} \quad 00$

$-1.52739 \mathrm{E} \quad 00$

$-1.53987 \mathrm{E} \quad 00$

$-1.55240 \mathrm{E} \quad 00$

$-1.56498 \mathrm{E} \quad 00$

$-1.57761 \mathrm{E} \quad 00$

$-1.59029 \mathrm{E} 00$

$-1.60301 \mathrm{E} 00$

$-1.61578 \mathrm{E} 00$

$-1.62860 \mathrm{E} \quad 00$

$-1.64147 \mathrm{E} \quad 00$

$-1.65438 \mathrm{E} 00$

$-1.66735 \mathrm{E} 00$

$-1.68036 \mathrm{E} 00$

$-1.69342 \mathrm{E} 00$

$-1.70652 \mathrm{E} \quad 00$ $-1.71968 \mathrm{E} \quad 00$

$-1.73288 \mathrm{E} \quad 00$

$-1.74613 \mathrm{E} \quad 00$

$-1.75942 \mathrm{E} \quad 00$

$-1.77277 \mathrm{E} \quad 00$

$-1.78616 \mathrm{E} \quad 00$

$-1.79960 \mathrm{E} \quad 00$

$-1.81309 \mathrm{E} \quad 00$

$-1.82662 \mathrm{E} \quad 00$

$-1.84021 \mathrm{E} \quad 00$

$-1.85384 \mathrm{E} \quad 00$

$-1.86752 \mathrm{E} \quad 00$

$-1.88124 \mathrm{E} \quad 00$

$-1.89502 \mathrm{E} 00$

$-1.90884 \mathrm{E} \quad 00$

$-1.92271 \mathrm{E} \quad 00$

$-1.93662 \mathrm{E} \quad 00$

$-1.95059 \mathrm{E} \quad 00$

$-1.96460 \mathrm{E} 00$
$-1.42924 \mathrm{E} 00$

$\tan \delta_{1}$

$f_{-1}$

$4.54848 \mathrm{E}-03$

$4.55516 \mathrm{E}-03$

$4.56178 \mathrm{E}-03$

$4.56829 \mathrm{E}-03$

$4.57472 \mathrm{E}-03$

$4.58106 \mathrm{E}-03$

$4.58732 \mathrm{E}-03$

$4.59350 \mathrm{E}-03$

$4.59960 \mathrm{E}-03$

$4.60561 \mathrm{E}-03$

$4.61155 \mathrm{E}-03$

$4.61741 \mathrm{E}-03$

$4.62319 \mathrm{E}-03$

$4.62890 \mathrm{E}-03$

$4.63453 \mathrm{E}-03$

$4.64009 \mathrm{E}-03$

$4.64557 \mathrm{E}-03$

$4.65099 \mathrm{E}-03$

$4.65634 \mathrm{E}-03$

$4.66160 \mathrm{E}-03$

$4.66681 \mathrm{E}-03$

$4.67195 \mathrm{E}-03$

$4.67702 \mathrm{E}-03$

$4.68202 \mathrm{E}-03$

$4.68696 \mathrm{E}-03$

$4.69183 \mathrm{E}-03$

$4.69665 \mathrm{E}-03$

$4.70140 \mathrm{E}-03$

$4.70608 \mathrm{E}-03$

$4.71071 \mathrm{E}-03$

$4.71528 \mathrm{E}-03$

$4.71979 \mathrm{E}-03$

$4.72424 \mathrm{E}-03$

$4.72863 \mathrm{E}-03$

$4.73296 \mathrm{E}-03$

4.73724. E-03

$4.74146 \mathrm{E}-03$

$4.74563 \mathrm{E}-03$

$4.74974 \mathrm{E}-03$

$4.75380 \mathrm{E}-03$

$4.75781 \mathrm{E}-03$

$4.76175 \mathrm{E}-03$

$4.76566 \mathrm{E}-03$

$4.76951 \mathrm{E}-03$

4.77331 E-03 
$Z=7 \quad A=14 \quad \rho=1.1 A^{1 / 3} F$

$\bar{F}$

$5.05495 \mathrm{E}-04$

$5.11092 \mathrm{E}-04$

$5.16718 \mathrm{E}-04$

$5.22376 \mathrm{E}-04$

$5.28064 \mathrm{E}-04$

$5.33783 \mathrm{E}-04$

$5.39533 \mathrm{E}-04$

$5.45314 \mathrm{E}-04$

$5.51126 \mathrm{E}-04$

$5.56968 \mathrm{E}-04$

$5.62841 \mathrm{E}-04$

$5.68745 \mathrm{E}-04$

$5.74680 \mathrm{E}-04$

$5.80645 \mathrm{E}-04$

$5.86641 \mathrm{E}-04$

$5.92668 \mathrm{E}-04$

$5.98726 \mathrm{E}-04$

$6.04815 \mathrm{E}-04$

$6.10934 \mathrm{E}-04$

$6.17085 \mathrm{E}-04$

$6.23266 \mathrm{E}-04$ $6.29477 \mathrm{E}-04$ $6.35720 \mathrm{E}-04$

$6.41993 \mathrm{E}-04$

$6.48297 \mathrm{E}-04$

$6.54632 \mathrm{E}-04$ $6.60998 \mathrm{E}-04$ $6.67395 \mathrm{E}-04$ $6.73822 \mathrm{E}-04$ $6.80280 \mathrm{E}-04$

$6.86769 \mathrm{E}-04$

$6.93288 \mathrm{E}-04$

$6.99839 \mathrm{E}-04$

$7.06420 \mathrm{E}-04$

$7.13032 \mathrm{E}-04$

7.19675 E-04

$7.26348 \mathrm{E}-04$

$7.33053 \mathrm{E}-04$

$7.39788 \mathrm{E}-04$

7.46554 E- 04

$7.53350 \mathrm{E}-04$

$7.60178 \mathrm{E}-04$

$7.67037 \mathrm{E}-04$

$7.73926 \mathrm{E}-04$

$7.80846 \mathrm{E}-04$

$7.87796 \mathrm{E}-04$

$7.94778 \mathrm{E}-04$

$8.01790 \mathrm{E}-04$

$8.08833 \mathrm{E}-04$

$8.15907 \mathrm{E}-04$

8.23011 E- 04

$8.30146 \mathrm{E}-04$

$8.37313 \mathrm{E}-04$

$8.44509 \mathrm{E}-04$

$8.51737 \mathrm{E}-04$

$8.58996 \mathrm{E}-04$ $8.66285 \mathrm{E}-04$ $8.73605 \mathrm{E}-04$ $8.80956 \mathrm{E}-04$ $8.88337 \mathrm{E}-04$ $f_{2}$

$-7.87555 \mathrm{E}-01$

$-7.96379 \mathrm{E}-01$

$-8.05251 \mathrm{E}-01$

$-8.23141 \mathrm{E}-01$

$-8.32160 \mathrm{E}-01$

$-8.41226 \mathrm{E}-01$

$-8.50342 \mathrm{E}-01$

$-8.59506 \mathrm{E}-01$

$-8.68719 \mathrm{E}-01$

$-8.77981 \mathrm{E}-01$

$-8.87291 \mathrm{E}-01$

$-9.06058 \mathrm{E}-01$

$-9.15515 \mathrm{E}-01$

$-9.25020 \mathrm{E}-01$

$-9.34573 \mathrm{E}-01$

$-9.44175 \mathrm{E}-01$

$-9.53826 \mathrm{E}-01$

$-9.63526 \mathrm{E}-01$

$-9.73274 \mathrm{E}-01$

$-9.83070 \mathrm{E}-01$

$-9.92916 \mathrm{E}-01$

$-1.00281 \mathrm{E} \quad 00$

$-1.01275 \mathrm{E} \quad 00$

$-1.02274 \mathrm{E} \quad 00$

$-1.04287 \mathrm{E} 00$

$-1.05301 \mathrm{E} \quad 00$

$-1.06319 \mathrm{E} \quad 00$

$-1.07343 \mathrm{E} \quad 00$

$-1.08371 \mathrm{E} \quad 00$

$-1.09404 \mathrm{E} \quad 00$

$-1.10442 \mathrm{E} 00$

-1.11485 E 00

$-1.12532 \mathrm{E} \quad 00$

$-1.13585 \mathrm{E} \quad 00$

$-1.15704 \mathrm{E} \quad 00$

$-1.16771 \mathrm{E} 00$

$-1.18920 \mathrm{E} 00$

$-1.20001 \mathrm{E} 00$

$-1.21088 \mathrm{E} 00$

$-1.22179 \mathrm{E} 00$

$-1.23275 \mathrm{E} \quad 00$

$-1.24376 \mathrm{E} \quad 00$

$-1.25481 \mathrm{E} \quad 00$

$-1.26592 \mathrm{E} 00$

$-1.27707 \mathrm{E} \quad 00$

$-1.28828 \mathrm{E} \quad 00$

$-1.29953 \mathrm{E} \quad 00$

$-1.31083 \mathrm{E} 00$

$-1.32217 \mathrm{E} 00$

$-1.33357 \mathrm{E} \quad 00$

$-1.34501 \mathrm{E} \quad 00$

$-1.35650 \mathrm{E} 00$

$-1.36804 \mathrm{E} \quad 00$

$-1.37963 \mathrm{E} 00$

$-1.39127 \mathrm{E} \quad 00$
$-8.14172 \mathrm{E}-0 \mathrm{I}$

$-8.96650 \mathrm{E}-01$ $-1.03278 \mathrm{E} \quad 00$

$-1.14642 \mathrm{E} \quad 00$

$-1.17843 \mathrm{E} \quad 00$ $g_{2}$

$-2.99091 \mathrm{E}-02$

$-3.03537 \mathrm{E}-02$

$-3.08026 \mathrm{E}-02$

$-3.12558 \mathrm{E}-02$

$-3.17134 \mathrm{E}-02$

$-3.21753 \mathrm{E}-02$

$-3.26416 \mathrm{E}-02$

$-3.31122 \mathrm{E}-02$

$-3.35873 \mathrm{E}-02$

$-3.40668 \mathrm{E}-02$

$-3.45508 \mathrm{E}-02$

$-3.50392 \mathrm{E}-02$

$-3.55322 \mathrm{E}-02$

$-3.60297 \mathrm{E}-02$

$-3.65316 \mathrm{E}-02$

$-3.70382 \mathrm{E}-02$

$-3.75493 \mathrm{E}-02$

$-3.80650 \mathrm{E}-02$

$-3.85854 \mathrm{E}-02$

$-3.91103 \mathrm{E}-02$

$-3.96399 \mathrm{E}-02$

$-4.01742 \mathrm{E}-02$

$-4.07132 \mathrm{E}-02$

$-4.18054 \mathrm{E}-02$

$-4.23585 \mathrm{E}-02$

$-4.29165 \mathrm{E}-02$

$-4.34793 \mathrm{E}-02$

$-4.40468 \mathrm{E}-02$

$-4.46192 \mathrm{E}-02$

$-4.51965 \mathrm{E}-02$

$-4.57786 \mathrm{E}-02$

$-4.63656 \mathrm{E}-02$

$-4.69575 \mathrm{E}-02$

$-4.75544 \mathrm{E}-02$

$-4.81562 \mathrm{E}-02$

$-4.87630 \mathrm{E}-02$

$-4.93747 \mathrm{E}-02$

$-4.99915 \mathrm{E}-02$

$-5.06133 \mathrm{E}-02$

$-5.12402 \mathrm{E}-02$

$-5.18721 \mathrm{E}-02$

$-5.25091 \mathrm{E}-02$

$-5.31513 \mathrm{E}-02$

$-5.37985 \mathrm{E}-02$

$-5.44509 \mathrm{E}-02$

$-5.51085 \mathrm{E}-02$

$-5.57713 \mathrm{E}-02$

$-5.64393 \mathrm{E}-02$

$-5.71124 \mathrm{E}-02$

$-5.77909 \mathrm{E}-02$

$-5.84746 \mathrm{E}-02$

$-5.91636 \mathrm{E}-02$

$-5.98579 \mathrm{E}-02$

$-6.05576 \mathrm{E}-02$

$-6.12626 \mathrm{E}-02$

$-6.19729 \mathrm{E}-02$

$-6.26887 \mathrm{E}-02$

$-6.34098 \mathrm{E}-02$

$-6.41364 \mathrm{E}-02$
$-4.12569 \mathrm{E}-02$ $\tan \delta_{2}$

$2.93262 \mathrm{E} 01$

$2.93300 \mathrm{E} \quad 01$

$2.93338 \mathrm{E} \quad 01$

$2.93375 \mathrm{E}$ 0I

$2.93412 \mathrm{E} 01$

$2.93449 \mathrm{E} \quad 01$

2.93485 E 01

$2.93521 \mathrm{~F} 01$

$2.93556 \mathrm{E} \quad 01$

$2.93591 \mathrm{E} \quad 01$

$2.93626 \mathrm{E} \quad 01$

$2.93660 \mathrm{E} \quad 01$

$2.93694 \mathrm{E} \quad 01$

$2.93728 \mathrm{E} \quad 01$

$2.93794 \mathrm{E}$ 0l

$2.93826 \mathrm{E} \quad 01$

$2.93858 \mathrm{E}$ ol

$2.93890 \mathrm{E} \quad 01$

$2.93922 \mathrm{E} \quad 01$

2.93953 E 01

$2.93984 \mathrm{E} \quad 01$

$2.94014 \mathrm{E} \quad 01$

$2.94044 \mathrm{E} \quad 01$

$2.94074 \mathrm{E} \quad 01$

$2.94104 \mathrm{E} \quad 01$

$2.94133 \mathrm{E} \quad 01$

$2.94162 \mathrm{E} 01$

$2.94191 \mathrm{E} 01$

$2.94219 \mathrm{E} \quad 01$

$2.94247 \mathrm{E} \quad 01$

2.94275 E 01

$2.94303 \mathrm{E} 01$

$2.94330 \mathrm{E} \quad 01$

2.94357 E 01

$2.94384 \mathrm{E} \quad 01$

$2.94411 \mathrm{E} 01$

$2.94437 \mathrm{E} \quad 01$

$2.94463 \mathrm{E} \quad 01$

$2.94489 \mathrm{E} \quad 01$

$2.94515 \mathrm{E} \quad 01$

$2.94540 \mathrm{E} \quad 01$

$2.94565 \mathrm{E} 01$

$2.94590 \mathrm{E} 01$

$2.94614 \mathrm{E} 01$

$2.94639 \mathrm{E} \quad 01$

$2.94663 \mathrm{E}$ 0l

$2.94687 \mathrm{E} \quad 01$

$2.94734 \mathrm{E} \quad 01$

$2.94758 \mathrm{E} \quad 01$

$2.94781 \mathrm{E} 01$

$2.94804 \mathrm{E} \quad 01$

$2.94849 \mathrm{E} \quad 01$

$2.94871 \mathrm{E} \quad 01$ $2.94893 \mathrm{E} \quad 01$

$2.94915 \mathrm{E} \quad 01$

$2.94937 \mathrm{E} \quad 01$

$2.94959 \mathrm{E} 01$
$2.93761 \mathrm{E} \quad 01$

$2.94711 \mathrm{E} 01$

$2.94826 \mathrm{E} 01$

$f_{-2}$

$-2.93178 \mathrm{E}-02$

$-2.97530 \mathrm{E}-02$

$-3.01924 \mathrm{E}-02$

$-3.06360 \mathrm{E}-02$

$-3.10839 \mathrm{E}-02$

$-3.15361 \mathrm{E}-02$

$-3.19925 \mathrm{E}-02$

$-3.24534 \mathrm{E}-02$

$-3.29185 \mathrm{E}-02$

$-3.33881 \mathrm{E}-02$

$-3.38620 \mathrm{E}-02$

$-3.43403 \mathrm{E}-02$

$-3.48231 \mathrm{E}-02$

$-3.53103 \mathrm{E}-02$

$-3.58019 \mathrm{E}-02$

$-3.62981 \mathrm{E}-02$

$-3.67987 \mathrm{E}-02$

$-3.73039 \mathrm{E}-02$

$-3.78137 \mathrm{E}-02$

$-3.83280 \mathrm{E}-02$

$-3.88469 \mathrm{E}-02$

$-3.93703 \mathrm{E}-02$

$-3.98985 \mathrm{E}-02$

$-4.04312 \mathrm{E}-02$

$-4.09687 \mathrm{E}-02$

$-4.15108 \mathrm{E}-02$

$-4.20576 \mathrm{E}-02$

$-4.26092 \mathrm{E}-02$

$-4.31654 \mathrm{E}-02$

$-4.37265 \mathrm{E}-02$

$-4.42923 \mathrm{E}-02$

$-4.48630 \mathrm{E}-02$

$-4.54385 \mathrm{E}-02$

$-4.60188 \mathrm{E}-02$

$-4.66040 \mathrm{E}-02$

$-4.71940 \mathrm{E}-02$

$-4.77890 \mathrm{E}-02$

$-4.83889 \mathrm{E}-02$

$-4.89937 \mathrm{E}-02$

$-4.96035 \mathrm{E}-02$

$-5.02183 \mathrm{E}-02$

$-5.08381 \mathrm{E}-02$

$-5.14629 \mathrm{E}-02$ 


\begin{tabular}{|c|c|c|c|c|c|c|c|c|}
\hline$p$ & $F_{0}$ & & $f_{1}$ & & $g_{1}$ & & $\tan \delta_{1}$ & $f_{-1}$ \\
\hline 24.10 & $1.18001 \mathrm{E}$ & 00 & $-2.53038 \mathrm{E}$ & 01 & $-1.97866 \mathrm{E}$ & 00 & $4.82771 \mathrm{E}-03$ & $-1.94087 \mathrm{E}$ \\
\hline 24.20 & $1.17999 \mathrm{E}$ & 00 & $-2.54091 \mathrm{E}$ & 01 & $-1.99277 \mathbf{E}$ & 00 & $4.83073 \mathbf{E}-$ & $-1.95473 \mathrm{E}$ \\
\hline 24.30 & $1.17998 \mathrm{E}$ & 00 & $-2.55144 \mathrm{E}$ & 01 & $-2.00692 \mathrm{E}$ & 00 & $4.83372 \mathrm{E}$ & $-1.96864 \mathrm{E}$ \\
\hline 24.40 & $1.17997 \mathrm{E}$ & 00 & $-2.56196 \mathrm{E}$ & 01 & $-2.02112 \mathrm{E}$ & 00 & $4.83666 \mathrm{E}-03$ & $-1.98259 \mathrm{E}$ \\
\hline 24.50 & $1.17995 \mathrm{E}$ & 00 & $-2.57249 \mathrm{E}$ & 01 & $-2.03537 \mathrm{E}$ & 00 & $4.83956 \mathbf{E}-03$ & $-1.99659 \mathrm{E}$ \\
\hline 4.60 & $1.17994 \mathrm{E}$ & 00 & $-2.58301 \mathrm{E}$ & 01 & $-2.04967 \mathrm{E}$ & 00 & $4.84242 \mathrm{E}-03$ & $-2.01064 \mathrm{E}$ \\
\hline 4.70 & $1.17993 \mathrm{E}$ & 00 & $-2.59353 E$ & 01 & $-2.06402 \mathrm{E}$ & 00 & $4.84525 \mathrm{E}-03$ & $-2.02474 \mathrm{E}$ \\
\hline .80 & $1.17991 \mathrm{E}$ & 00 & $-2.60405 \mathrm{E}$ & 01 & $-2.07841 \mathrm{E}$ & 00 & $4.84804 \mathrm{E}-$ & $-2.03889 \mathrm{E}$ \\
\hline 4.90 & $1.17990 \mathrm{E}$ & 00 & $-2.61457 \mathrm{E}$ & 01 & $-2.09285 \mathrm{E}$ & 00 & $4.85078 \mathrm{E}-$ & $-2.05308 \mathrm{E}$ \\
\hline 5.00 & $1.17988 \mathrm{E}$ & 00 & $-2.62508 \mathrm{E}$ & 01 & $-2.10733 \mathrm{E}$ & 00 & $4.85349 \mathrm{E}-03$ & $-2.06732 \mathrm{E}$ \\
\hline 5.10 & $1.17987 \mathrm{E}$ & 00 & $-2.63559 \mathrm{E}$ & 01 & $-2.12187 \mathrm{E}$ & 00 & $4.85615 \mathrm{E}-03$ & $-2.08161 \mathrm{E}$ \\
\hline 5.20 & $1.17986 \mathrm{E}$ & 00 & $-2.64610 \mathrm{E}$ & 01 & $-2.13645 \mathrm{E}$ & 00 & $4.85878 \mathrm{E}-03$ & $-2.09594 \mathrm{E}$ \\
\hline 5.30 & $1.17984 \mathrm{E}$ & 00 & $-2.65661 \mathrm{E}$ & 01 & $-2.15108 \mathrm{E}$ & 00 & $4.86139 \mathrm{E}-$ & $-2.11032 \mathrm{E}$ \\
\hline 5.40 & $1.17983 \mathrm{E}$ & 00 & $-2.66712 \mathbf{E}$ & 01 & $-2.16576 \mathrm{E}$ & 00 & $4.86394 \mathrm{E}-$ & $-2.12475 \mathrm{E}$ \\
\hline 5.50 & $1.17982 \mathrm{E}$ & 00 & $-2.67762 \mathbf{E}$ & 01 & $-2.18048 \mathrm{E}$ & 00 & $4.86647 \mathrm{E}-03$ & $-2.13923 \mathrm{E}$ \\
\hline 5.60 & $1.17980 \mathrm{E}$ & 00 & $-2.68812 \mathbf{E}$ & 01 & $-2.19525 \mathrm{E}$ & 00 & $4.86895 \mathbf{E}-03$ & $-2.15376 \mathrm{E}$ \\
\hline .70 & $17979 \mathrm{E}$ & 00 & $-2.69862 \mathbf{E}$ & 01 & $-2.21007 \mathrm{E}$ & 00 & $4.87140 \mathrm{E}$ & $-2.16833 \mathrm{E}$ \\
\hline 25.80 & $17978 \mathrm{E}$ & 00 & $-2.70912 \mathbf{E}$ & 01 & $.22494 \mathrm{E}$ & 00 & $4.87382 \mathrm{E}-$ & $-2.18295 \mathrm{E}$ \\
\hline 25.90 & $1.17977 \mathrm{E}$ & 00 & $-2.71962 \mathbf{E}$ & 01 & $23985 \mathrm{E}$ & 00 & $4.87620 \mathrm{E}-03$ & $-2.19761 \mathrm{E}$ \\
\hline 26.00 & $1.17975 \mathrm{E}$ & 00 & $-2.73011 \mathrm{E}$ & 01 & $-2.25481 \mathrm{E}$ & 00 & $4.87854 \mathrm{E}-03$ & $-2.21233 \mathrm{E}$ \\
\hline 5.10 & $1.17974 \mathrm{E}$ & 00 & $-2.74060 \mathbf{E}$ & 01 & $-2.26982 \mathrm{E}$ & 00 & $4.88085 \mathrm{E}-03$ & $-2.22709 \mathrm{E}$ \\
\hline 5.20 & $7973 \mathrm{E}$ & 00 & $-2.75109 E$ & 01 & $8487 \mathrm{E}$ & 00 & 4.88 & $-2.24190 \mathrm{E}$ \\
\hline 26.30 & $1.17971 \mathrm{E}$ & 00 & $-2.76158 E$ & 01 & $29998 \mathrm{E}$ & 00 & $36 \mathrm{E}-$ & $-2.25676 \mathrm{E}$ \\
\hline 26.40 & $1.17970 \mathrm{E}$ & 00 & $-2.77207 \mathrm{E}$ & 01 & $31513 \mathrm{E}$ & 00 & $57 E-03$ & $-2.27166 \mathrm{E}$ \\
\hline 26.50 & $1.17969 \mathrm{E}$ & 00 & $-2.78255 \mathbf{E}$ & 01 & $-2.33032 \mathrm{E}$ & 00 & $4.88975 \mathrm{E}-03$ & $-2.28661 \mathrm{E}$ \\
\hline 26.60 & $17968 \mathrm{E}$ & 00 & $-2.79303 \mathbf{E}$ & 01 & $-2.34557 \mathrm{E}$ & 00 & $90 E-03$ & $-2.30161 \mathrm{E}$ \\
\hline 26.70 & $17966 \mathrm{E}$ & 00 & $-2.80351 \mathrm{E}$ & 01 & $5086 \mathrm{E}$ & 00 & 4.89 & $1665 \mathrm{E}$ \\
\hline 26.80 & $1.17965 \mathrm{E}$ & 00 & $-2.81399 \mathbf{E}$ & 01 & $-2.37620 \mathrm{E}$ & 00 & $4.89609 \mathrm{E}$ & $-2.33175 \mathrm{E}$ \\
\hline 26.90 & $1.17964 \mathrm{E}$ & 00 & $-2.82446 \mathbf{E}$ & 01 & $-2.39158 \mathrm{E}$ & 00 & $4.89814 \mathrm{E}-$ & $-2.34689 \mathrm{E}$ \\
\hline 27.00 & $1.17963 \mathrm{E}$ & 00 & $-2.83494 \mathbf{E}$ & 01 & $-2.40702 \mathrm{E}$ & 00 & $4.90015 \mathrm{E}-03$ & $-2.36207 \mathrm{E}$ \\
\hline 27.10 & $1.17961 \mathrm{E}$ & 00 & $-2.84541 \mathbf{E}$ & 01 & $-2.42250 \mathrm{E}$ & 00 & $4.90214 \mathrm{E}-03$ & $-2.37731 \mathrm{E}$ \\
\hline 27.20 & $1.17960 \mathrm{E}$ & 00 & $-2.85588 E$ & 01 & $-2.43803 \mathrm{E}$ & 0 & $4.90409 \mathrm{~F}$ & $-2.39259 \mathrm{E}$ \\
\hline 27.30 & $1.17959 \mathrm{E}$ & 00 & $-2.86634 \mathrm{E}$ & 01 & $-2.45360 \mathrm{E}$ & 0 & $4.90602 \mathrm{E}-$ & $-2.40792 \mathrm{E}$ \\
\hline 27.40 & $1.17958 \mathrm{E}$ & 00 & $-2.87681 \mathrm{E}$ & 01 & $46922 \mathrm{E}$ & 00 & $91 \mathrm{E}-$ & $-2.42329 \mathbf{E}$ \\
\hline 27.50 & $1.17957 \mathrm{E}$ & 00 & $-2.88727 \mathrm{E}$ & 01 & $-2.48489 \mathrm{E}$ & 00 & $4.90978 \mathrm{E}-03$ & $-2.43872 \mathrm{E}$ \\
\hline 7.60 & $.17955 \mathrm{E}$ & 00 & $-2.89773 \mathbf{E}$ & 01 & $-2.50061 \mathrm{E}$ & 00 & $4.91161 \mathrm{E}-03$ & $-2.45419 \mathrm{E}$ \\
\hline 27.70 & $7954 \mathrm{E}$ & 00 & $-2.90819 E$ & 01 & $-2.51637 \mathrm{E}$ & 0 & $4.91341 \mathrm{E}$ & $-2.46970 \mathrm{E}$ \\
\hline 27.80 & $53 \mathrm{E}$ & 00 & $1864 \mathrm{E}$ & 01 & $3218 \mathrm{E}$ & 0 & 4.91 & $-2.48527 \mathrm{E}$ \\
\hline 27.90 & & 00 & & 01 & & 00 & 33 & $-2.50088 \mathrm{E}$ \\
\hline 28.00 & $1.17951 \mathrm{E}$ & 00 & $-2.93955 \mathrm{E}$ & 01 & $-2.56395 \mathrm{E}$ & 00 & $4.91865 \mathrm{E}-03$ & $-2.51654 \mathrm{E}$ \\
\hline 28.10 & $17949 \mathrm{E}$ & 00 & $-2.95000 \mathrm{E}$ & 01 & $-2.57990 \mathrm{E}$ & 00 & $4.92034 \mathrm{E}-03$ & $-2.53224 \mathrm{E}$ \\
\hline 28.20 & & 00 & $-2.96045 \mathrm{E}$ & 01 & $59590 \mathrm{E}$ & 00 & 4.9 & $-2.54800 \mathrm{E}$ \\
\hline 28.30 & & 00 & -2 & 01 & $1194 \mathrm{E}$ & 00 & 03 & $-2.56380 \mathrm{E}$ \\
\hline 28.40 & $946 \mathrm{E}$ & 00 & $-2.98134 \mathbf{E}$ & 01 & $2804 \mathrm{E}$ & 00 & 4.92 & $-2.57965 \mathrm{E}$ \\
\hline 28.50 & $1.17945 \mathrm{E}$ & 00 & $-2.99178 \mathbf{E}$ & 01 & $-2.64418 \mathrm{E}$ & 00 & $4.92680 \mathrm{E}-03$ & $-2.59554 \mathrm{E}$ \\
\hline 28.60 & $.17944 \mathrm{E}$ & 00 & $-3.00222 \mathrm{E}$ & 01 & $-2.66037 \mathrm{E}$ & 00 & $4.92836 \mathrm{E}-03$ & $-2.61148 \mathrm{E}$ \\
\hline 28.70 & & 00 & & 01 & & 00 & & $2747 \mathrm{E}$ \\
\hline 28.80 & $941 \mathrm{E}$ & 00 & $-3.02309 \mathbf{E}$ & 01 & $288 \mathrm{E}$ & 00 & 4.93 & $-2.64350 \mathrm{E}$ \\
\hline 28.90 & $1.17940 \mathrm{E}$ & 00 & $-3.03352 \mathbf{E}$ & 01 & $0921 \mathrm{E}$ & 0 & $4.93284 \mathrm{E}-$ & $-2.65959 \mathrm{E}$ \\
\hline 29.00 & $1.17939 \mathrm{E}$ & 00 & $-3.04395 \mathrm{E}$ & 01 & $-2.72558 \mathrm{E}$ & 00 & $4.93428 \mathrm{E}-03$ & $-2.67572 \mathbf{E}$ \\
\hline 29.10 & $1.17938 \mathrm{E}$ & 00 & $-3.05438 \mathbf{E}$ & 01 & $-2.74201 \mathrm{E}$ & 00 & $4.93570 \mathrm{E}-03$ & $-2.69189 \mathrm{E}$ \\
\hline 29.20 & & 00 & $-3.06480 \mathrm{E}$ & 01 & $-2.75848 \mathrm{E}$ & 00 & & $-2.70812 \mathrm{E}$ \\
\hline 29.30 & $1.17936 \mathrm{E}$ & 00 & $-3.07523 \mathbf{E}$ & 01 & $-2.77499 \mathrm{E}$ & 0 & $4.93845 \mathrm{E}-$ & $-2.72439 \mathbf{E}$ \\
\hline 29.40 & $7935 \mathrm{E}$ & 00 & $-3.08565 E$ & 01 & $-2.79155 \mathrm{E}$ & 00 & $4.93979 \mathrm{E}-$ & $-2.74070 \mathrm{E}$ \\
\hline 29.50 & $1.17933 \mathrm{E}$ & 00 & $-3.09607 \mathrm{E}$ & 01 & $-2.80816 \mathrm{E}$ & 00 & $4.94110 \mathrm{E}-03$ & $-2.75707 \mathrm{E}$ \\
\hline 29.60 & $7932 \mathrm{E}$ & 00 & $-3.10648 \mathrm{E}$ & 01 & $-2.82482 \mathrm{E}$ & 0 & $239 \mathrm{E}-03$ & $-2.77348 \mathrm{E}$ \\
\hline & $17931 \mathrm{E}$ & 00 & $-3.11690 \mathrm{E}$ & 01 & $-2.84152 \mathbf{E}$ & 0 & $4.94365 \mathrm{E}-03$ & $-2.78994 \mathrm{E}$ \\
\hline 29.80 & $1.17930 \mathrm{E}$ & 00 & $-3.12731 \mathrm{E}$ & 01 & $-2.85828 \mathrm{E}$ & 00 & $4.94490 \mathrm{E}-03$ & $-2.80644 \mathrm{E}$ \\
\hline 29.90 & $1.17929 \mathrm{E}$ & 00 & $-3.13772 \mathbf{E}$ & 01 & $-2.87507 \mathbf{E}$ & 00 & $4.94611 \mathrm{E}-03$ & $-2.82299 \mathrm{E}$ \\
\hline & $1.17928 \mathrm{E}$ & 00 & $-3.14813 \mathrm{E}$ & 01 & $-2.89192 \mathbf{E}$ & 00 & $4.94729 \mathrm{E}-03$ & $-2.83959 \mathrm{E}$ \\
\hline
\end{tabular}

$2.63691 \mathrm{E} \quad 01$ $2.64743 \mathbf{E} 01$ $2.65795 \mathrm{E} \quad 01$ $2.66847 \mathrm{E}$ 0I $2.67898 \mathrm{E}$ 0I

$2.68950 \mathrm{E} \quad 01$ $2.70001 \mathrm{E} 01$ $2.71051 \mathrm{E} 01$ $2.72102 \mathrm{E}$ 0l 2.73153 E 01

$2.74203 \mathrm{E} \quad 01$ $2.75253 \mathrm{E} 01$ $2.76303 \mathrm{E} \quad 01$ $2.77352 \mathrm{E}$ 01 $2.78402 \mathbf{E} 01$

$2.79451 \mathrm{E} \quad 01$ $2.80500 \mathrm{E} \quad 01$ $2.81549 \mathrm{E}$ 01 $2.82597 \mathrm{E} \quad 01$ $2.83646 \mathrm{E} 01$

$2.84694 \mathrm{E} \quad 01$ $2.85742 \mathrm{E} \quad 01$ $2.86790 \mathrm{E} \quad 01$ $2.87837 \mathrm{E} \quad 01$ $2.88885 \mathrm{E} \quad 01$

$289932 \mathrm{E} \quad 01$ $2.90979 \mathrm{E} \quad 01$ $2.92025 \mathrm{E}$ 01 $2.93072 \mathrm{E} \quad 01$ $2.94118 \mathrm{E} 01$

$2.95164 \mathrm{E} \quad 01$ $2.96210 \mathrm{E}$ 01 $2.97256 \mathrm{E} \quad 01$ $2.98301 \mathrm{E} \quad 01$ $2.99346 \mathrm{E} \quad 01$

$3.00391 \mathrm{E} \quad 01$ $3.01436 \mathrm{E} \quad 01$ $3.02481 \mathrm{E} \quad 01$ $3.03525 \mathrm{E} \quad 01$ $3.04569 \mathrm{E} 01$

$3.05613 \mathrm{E} \quad 01$ $3.06657 \mathrm{E} \quad 01$ $3.07700 \mathrm{E} \quad 01$ $3.08744 \mathrm{E} \quad 01$ $3.09787 \mathrm{E} \quad 01$

$3.10830 \mathrm{E} \quad 01$ $3.11872 \mathrm{E} \quad 01$ $3.12915 \mathrm{E} \quad 01$ $3.13957 \mathrm{E}$ 0l $3.14999 \mathrm{E} \quad 01$

$3.16040 \mathrm{E} \quad 01$ $3.17082 \mathrm{E} 01$ $3.18123 \mathrm{E} \quad 01$ $3.19164 \mathrm{E} \quad 01$ $3.20205 \mathrm{E} \quad 01$

$3.21246 \mathrm{E} \quad 01$ $3.22286 \mathrm{E} \quad 01$ $3.23326 \mathrm{E}$ ol $3.24366 \mathbf{E} 01$ $3.25406 \mathrm{E} 01$ $\tan \delta_{-1}$

$\begin{array}{lll}-1.44218 \mathrm{E} & 02\end{array}$ $-1.44339 \mathrm{E} 02$ $-1.44459 \mathrm{E} 02$ $-1.44578 \mathrm{E} 02$ $-1.44698 \mathrm{E} 02$

$-1.44816 \mathrm{E} \quad 02$ $-1.44935 \mathrm{E} 02$ $-1.45052 \mathrm{E} \quad 02$ $-1.45170 \mathrm{E} 02$ $-1.45287 \mathrm{E} \quad 02$

$-1.45404 \mathrm{E} \quad 02$ $-1.45520 \mathrm{E} \quad 02$ $-1.45636 \mathrm{E} 02$ $-1.45752 \mathrm{E} 02$ $\begin{array}{ll}-1.45867 \mathrm{E} & 02\end{array}$

$\begin{array}{ll}-1.45982 \mathrm{E} & 02\end{array}$ $-1.46096 \mathrm{E} 02$ $-1.46211 \mathrm{E} 02$ $-1.46324 \mathrm{E} 02$ $-1.46438 \mathrm{E} \quad 02$

$-1.46551 \mathrm{E} \quad 02$ $-1.46664 \mathrm{E} \quad 02$ $-1.46777 \mathrm{E} \quad 02$ $-1.46889 \mathrm{E} 02$ $-1.47000 \mathrm{E} \quad 02$

$-1.47112 \mathrm{E} \quad \mathrm{C} 2$ $\begin{array}{ll}-1.47223 \mathrm{E} & 02\end{array}$ $-1.47334 \mathrm{E} \quad 02$ $-1.47445 \mathrm{E} \quad 02$ $-1.47555 \mathrm{E} \quad 02$

$-1.47665 \mathrm{E} \quad 02$ $\begin{array}{ll}-1.47775 \mathrm{E} & 02\end{array}$ $-1.47884 \mathrm{E} \quad 02$ $-1.47994 \mathrm{E} \quad 02$ $-1.48103 \mathrm{E} 02$

$-1.48211 \mathrm{E} \quad 02$ $-1.48320 \mathrm{E} 02$ $-1.48428 \mathrm{E} 02$ $-1.48536 \mathrm{E} 02$ $-1.48644 \mathrm{E} 02$

$\begin{array}{ll}-1.48751 \mathrm{E} & 02\end{array}$ $-1.48858 \mathrm{E} \quad 02$ $-1.48965 \mathrm{E} \quad 02$ $-1.49072 \mathrm{E} 02$ $-1.49179 \mathrm{E} 02$

$-1.49285 \mathrm{E} \quad 02$ $-1.49391 \mathrm{E} 02$ $-1.49497 \mathrm{E} \quad 02$ $-1.49602 \mathrm{E} \quad 02$ $-1.49708 \mathrm{E} 02$

$-1.49813 \mathrm{E} \quad 02$ $-1.49918 \mathrm{E} 02$ $-1.50023 \mathrm{E} 02$ $-1.50128 \mathrm{E} 02$ $-1.50232 \mathrm{E} 02$

$-1.50336 \mathrm{E} 02$ $-1.50440 \mathrm{E} 02$ $-1.50544 \mathrm{E} \quad 02$ $-1.50648 \mathrm{E} 02$ $-1.50752 \mathbf{E} 02$ 
ELECTRON RADIAL FUNCTIONS

$Z=7 \quad A=14 \quad \rho=1.1 A^{1 / 3} \mathrm{~F}$

Ejoretron

$\bar{F}$

$8.95750 \mathrm{E}-04$ $9.03193 \mathrm{E}-04$ $9.10667 \mathrm{E}-04$ $9.18172 \mathrm{E}-04$ $9.25707 \mathrm{E}-04$

$9.33273 \mathrm{E}-04$ $9.40870 \mathrm{E}-04$ $9.48498 \mathrm{E}-04$ $9.56157 \mathrm{E}-04$ $9.63846 \mathrm{E}-04$

$9.71566 \mathrm{E}-04$ $9.79317 \mathrm{E}-04$ $9.87099 \mathrm{E}-04$ $9.94911 \mathrm{E}-04$ $1.00275 \mathrm{E}-03$

$1.01063 \mathrm{E}-03$ $1.01853 \mathrm{E}-03$ $1.02647 \mathrm{E}-03$ $1.03444 \mathrm{E}-03$ $1.04243 \mathrm{E}-03$

$1.05046 \mathrm{E}-03$ $1.05852 \mathrm{E}-03$ $1.06661 \mathrm{E}-03$ $1.07473 \mathrm{E}-03$ $1.08288 \mathrm{E}-03$

$1.09106 \mathrm{E}-03$ $1.09927 \mathrm{E}-03$ $1.10752 \mathrm{E}-03$ $1.11579 \mathrm{E}-03$ 1.124.10E-03

$1.13243 \mathrm{E}-03$ $1.14080 \mathrm{E}-03$ $1.14920 \mathrm{E}-03$ $1.15762 \mathrm{E}-03$ $1.16608 \mathrm{E}-03$

$1.17457 \mathrm{E}-03$ $1.18309 \mathrm{E}-03$ $1.19164 \mathrm{E}-03$ $1.20023 \mathrm{E}-03$ $1.20884 \mathrm{E}-03$

$1.21748 \mathrm{E}-03$ $1.22616 \mathrm{E}-03$ $1.23486 \mathrm{E}-03$ $1.24360 \mathrm{E}-03$ $1.25236 \mathrm{E}-03$

$1.26116 \mathrm{E}-03$ $1.26999 \mathrm{E}-03$ $1.27885 \mathrm{E}-03$ $1.28774 \mathrm{E}-03$ $1.29666 \mathrm{E}-03$

$1.30561 \mathrm{E}-03$ $1.31459 \mathrm{E}-03$ $1.32360 \mathrm{E}-03$ $1.33265 \mathrm{E}-03$ $1.34172 \mathrm{E}-03$

$1.35083 \mathrm{E}-03$ $1.35996 \mathrm{E}-03$ $1.36913 \mathrm{E}-03$ $1.37833 \mathrm{E}-03$ $1.38755 \mathrm{E}-03$ $f_{2}$

$-1.40296 \mathrm{E} \quad 00$ $-1.41469 \mathrm{E} 00$ $-1.42647 \mathrm{E} \quad 00$ $-1.43830 \mathrm{E} 00$ $-1.45018 \mathrm{E} \quad 00$

$-1.46211 \mathrm{E} \quad 00$ $-1.47408 \mathrm{E} \quad 00$ $-1.48610 \mathrm{E} \quad 00$ $-1.49817 \mathrm{E} \quad 00$ $-1.51029 \mathrm{E} \quad 00$

$-1.52246 \mathrm{E} \quad 00$ $-1.53468 \mathrm{E} \quad 00$ $-1.54694 \mathrm{E} \quad 00$ $-1.55925 \mathrm{E} \quad 00$ $-1.57161 \mathrm{E} \quad 00$

$-1.58402 \mathrm{E} \quad 00$ $-1.59648 \mathrm{E} \quad 00$ $-1.60898 \mathrm{E} 00$ $-1.62153 \mathrm{E} \quad 00$ $-1.63413 \mathrm{E} \quad 00$

$-1.64678 \mathrm{E} \quad 00$ $-1.65948 \mathrm{E} \quad 00$ $-1.67222 \mathrm{E} \quad 00$ $-1.68501 \mathrm{E} \quad 00$ $-1.69786 \mathrm{E} \quad 00$

$-1.71074 \mathrm{E} \quad 00$ $-1.72368 \mathrm{E} \quad 00$ $-1.73667 \mathrm{E} \quad 00$ $-1.74970 \mathrm{E} 00$ $-1.76278 \mathrm{E} \quad 00$

$-1.77591 \mathrm{E} \quad 00$ $-1.78908 \mathrm{E} \quad 00$ $-1.80231 \mathrm{E} \quad 00$ $-1.81558 \mathrm{E} \quad 00$ $-1.82890 \mathrm{E} \quad 00$

$-1.84227 \mathrm{E} \quad 00$ $-1.85569 \mathrm{E} 00$ $-1.86915 \mathrm{E} \quad 00$ $-1.88266 \mathrm{E} \quad 00$ $-1.89622 \mathrm{E} \quad 00$

$-1.90983 \mathrm{E} \quad 00$ $-1.92349 \mathrm{E} \quad 00$ $-1.93719 \mathrm{E} 00$ $-1.95094 \mathrm{E} \quad 00$ $-1.96474 \mathrm{E} \quad 00$

$-1.97859 \mathrm{E} \quad 00$ $-1.99248 \mathrm{E} \quad 00$ $-2.00642 \mathrm{E} \quad 00$ $-2.02041 \mathrm{E} \quad 00$ $-2.03445 \mathrm{E} \quad 00$

$-2.04854 \mathrm{E} \quad 00$ $-2.06267 \mathrm{E} \quad 00$ $-2.07685 \mathrm{E} \quad 00$ $-2.09108 \mathrm{E} \quad 00$ $-2.10536 \mathrm{E} \quad 00$

$-2.11968 \mathrm{E} \quad 00$ $-2.13406 \mathrm{E} \quad 00$ $-2.14848 \mathrm{E} \quad 00$ $-2.16294 \mathrm{E} 00$ $-2.17746 \mathrm{E} \quad 00$ $g_{2}$

$-6.48684 \mathrm{E}-02$ $-6.56059 \mathrm{E}-02$ $-6.63489 \mathrm{E}-02$ $-6.70973 \mathrm{E}-02$ $-6.78513 \mathrm{E}-02$

$-6.86109 \mathrm{E}-02$ $-6.93759 \mathrm{E}-02$ $-7.01466 \mathrm{E}-02$ $-7.09229 \mathrm{E}-02$ $-7.17048 \mathrm{E}-02$

$-7.24924 \mathrm{E}-02$ $-7.32856 \mathrm{E}-02$ $-7.40845 \mathrm{E}-02$ $-7.48891 \mathrm{E}-02$ $-7.56994 \mathrm{E}-02$

$-7.65154 \mathrm{E}-02$ $-7.73372 \mathrm{E}-02$ $-7.81648 \mathbf{E}-02$ $-7.89982 \mathrm{E}-02$ $-7.98374 \mathbf{E}-02$

$-8.06825 \mathrm{E}-02$ $-8.15333 \mathrm{E}-02$ $-8.23901 \mathrm{E}-02$ $-8.32528 \mathrm{E}-02$ $-8.41214 \mathrm{E}-02$

$-8.49959 \mathrm{E}-02$ $-8.58764 \mathrm{E}-02$ $-8.67628 \mathrm{E}-02$ $-8.76553 \mathrm{E}-02$ $-8.85538 \mathrm{E}-02$

$-8.94582 \mathrm{E}-02$ $-9.03688 \mathrm{E}-02$ $-9.12854 \mathrm{E}-02$ $-9.22082 \mathrm{E}-02$ $-9.31370 \mathrm{E}-02$

$-9.40719 \mathrm{E}-02$

$-9.50131 \mathrm{E}-02$

$-9.59603 \mathrm{E}-02$

$-9.69138 \mathrm{E}-02$

$-9.78735 \mathrm{E}-02$

$-9.88394 \mathrm{E}-02$ $-9.98116 \mathrm{E}-02$ $-1.00790 \mathrm{E}-01$ $-1.01775 \mathrm{E}-01$ $-1.02766 \mathrm{E}-01$

$-1.03763 \mathrm{E}-01$ $-1.04767 \mathrm{E}-01$ $-1.05777 \mathrm{E}-01$ $-1.06793 \mathrm{E}-01$ $-1.07816 \mathrm{E}-01$

$-1.08845 \mathrm{E}-01$ $-1.09881 \mathrm{E}-01$ $-1.10923 \mathrm{E}-01$ $-1.11972 \mathrm{E}-01$ $-1.13027 \mathrm{E}-01$

$-1.14089 \mathrm{E}-01$ $-1.15157 \mathrm{E}-01$ $-1.16232 \mathrm{E}-01$ $-1.17313 \mathrm{E}-01$ $-1.18401 \mathrm{E}-01$ $\tan \delta_{2}$

$2.94980 \mathrm{E} \quad 01$

$2.95001 \mathrm{E} \quad 01 \quad-6.43148 \mathrm{E}-02$

$2.95022 \mathrm{E}$ 0l $-6.50442 \mathrm{E}-02$

$2.95043 \mathrm{E} \quad 01 \quad-6.57791 \mathrm{E}-02$

$2.95064 \mathrm{E} \quad 01 \quad-6.65194 \mathrm{E}-02$

$2.95084 \mathrm{E} \quad 01$ $2.95104 \mathbf{E} 01$ $2.95125 \mathrm{E} 01$ $2.95144 \mathrm{E} \quad 01$

$2.95164 \mathrm{E} \quad 01$

$2.95184 \mathrm{E} \quad 01$

$2.95203 \mathrm{E} \quad 01$

$2.95223 \mathrm{E} \quad 01$

$2.95242 \mathrm{E} \quad 01$

$2.95261 \mathrm{E} 01$

$2.95280 \mathrm{E} \quad 01$ $2.95298 \mathrm{E} \quad 01$ $2.95317 \mathrm{E} 01$ $2.95335 \mathrm{E} \quad 01$ $2.95354 \mathrm{E} \quad 01$

$2.95372 \mathbf{E} 01$ $2.95390 \mathrm{E} \quad 01$ $2.95408 \mathrm{E} \quad 01$ $2.95425 \mathrm{E} \quad 01$ $2.95443 \mathrm{E} \quad 01$

$2.95460 \mathrm{E} \quad 01$ $2.95478 \mathrm{E} \quad 01$ $2.95495 \mathrm{E} \quad 01$ $2.95512 \mathrm{E} \quad 01$ $2.95529 \mathrm{E} \quad 01$

$2.95545 \mathrm{E} \quad 01$ $2.95562 \mathbf{E} 01$ $2.95579 \mathrm{E}$ ol $2.95595 \mathrm{E}$ 01 $2.95611 \mathrm{E} \quad 01$

$2.95627 \mathrm{E} \quad 01$

$2.95643 \mathrm{E} \quad 01$

$2.95659 \mathrm{E} \quad 01$

$2.95675 \mathrm{E} \quad 01$

$2.95691 \mathrm{E} \quad 01$

$2.95706 \mathrm{E} \quad 01$ $2.95722 \mathrm{E} 01$ $2.95737 \mathrm{E} \quad 01$ $2.95752 \mathrm{E} \quad 01$ $2.95767 \mathrm{E} \quad 01$

$2.95782 \mathrm{E} \quad 01$ $2.95797 \mathrm{E} \quad 01$ $2.95812 \mathrm{E} \quad 01$ $2.95827 \mathrm{E} \quad 01$ $2.95841 \mathrm{E} \quad 01$

$2.95856 \mathrm{E} \quad 01$ $2.95870 \mathrm{E}$ 01 $2.95884 \mathrm{E} \quad 01$ $2.95898 \mathrm{E} \quad 01$ $2.95912 \mathrm{E} \quad 01$

$2.95926 \mathrm{E} \quad 01$ $2.95940 \mathrm{E} \quad 01$ $2.95954 \mathrm{E} \quad 01$ $2.95968 \mathrm{E} 01$ 2.95981 E 01

$-6.72652 \mathrm{E}-02$

$-6.80164 \mathrm{E}-02$ $-6.87732 \mathrm{E}-02$ $-6.95355 \mathrm{E}-02$

$-7.03034 \mathrm{E}-02$

$-7.10769 \mathrm{E}-02$ $-7.18559 \mathrm{E}-02$ $-7.26406 \mathrm{E}-02$ $-7.34309 \mathrm{E}-02$ $-7.42268 \mathrm{E}-02$

$-7.50284 \mathrm{E}-02$ $-7.58358 \mathrm{E}-02$ $-7.66488 \mathrm{E}-02$ $-7.74675 \mathrm{E}-02$ $-7.82920 \mathrm{E}-02$

$-7.91223 \mathrm{E}-02$ $-7.99584 \mathrm{E}-02$ $-8.08003 \mathrm{E}-02$ $-8.16480 \mathrm{E}-02$ $-8.25015 \mathrm{E}-02$

$-8.33609 \mathrm{E}-02$ $-8.42263 \mathrm{E}-02$ $-8.50975 \mathrm{E}-02$ $-8.59746 \mathrm{E}-02$ $-8.68577 \mathrm{E}-02$

$-8.77468 \mathrm{E}-02$ $-8.86419 \mathrm{E}-02$ $-8.95429 \mathrm{E}-02$ $-9.04500 \mathrm{E}-02$

$-9.13631 \mathrm{E}-02$

$-9.22823 \mathrm{E}-02$ $-9.32076 \mathrm{E}-02$ $-9.41390 \mathrm{E}-02$ $-9.50765 \mathrm{E}-02$ $-9.60201 \mathrm{E}-02$

$-9.69699 \mathrm{E}-02$ $-9.79259 \mathrm{E}-02$ $-9.88881 \mathrm{E}-02$ $-9.98565 \mathrm{E}-02$ $-1.00831 \mathrm{E}-01$

$-1.01812 \mathrm{E}-01$ $-1.02799 \mathrm{E}-01$ 
ELECTRON RADIAL FUNCTIONS

Electron

$Z=7 \quad A=14 \quad \rho=1.1 A^{1 / 3} F$

$p$

30.10

30.20

30.30

30.40

30.50

30.60

30.70

30.80

30.90

31.00

31.10

31.20

31.30

31.40

31.50

31.60

31.70

31.80

31.90

32.00

32.10

32.20

32.30

32.40

32.50

32.60

32.70

32.80

32.90

33.00

33.10

33.20

33.30

33.40

33.50

33.60

33.70

33.80

33.90

34.00

34.10

34.20

34.30

34.40

34.50

34.60

34.70

34.80

34.90

35.00

35.10

35.20

35.30

35.40

35.50

35.60

35.70

35.80

35.90

36.00
$F_{0}$

$g_{1}$

$\begin{array}{llllll}1.17927 \mathrm{E} & 00 & -3.15853 \mathrm{E} & 01 & -2.90881 \mathrm{E} & 00\end{array}$

$\begin{array}{llllll}1.17926 \mathrm{E} & 00 & -3.16894 \mathrm{E} & 01 & -2.92575 \mathrm{E} & 00\end{array}$

$\begin{array}{llllll}1.17925 \mathrm{E} & 00 & -3.17934 \mathrm{E} & 01 & -2.94273 \mathrm{E} & 00\end{array}$

$\begin{array}{llllll}1.17924 \mathrm{E} & 00 & -3.18974 \mathrm{E} & 01 & -2.95976 \mathrm{E} & 00\end{array}$

$1.17923 \mathrm{E} \quad 00$

$-3.20013 \mathrm{E} \quad 01$

$-2.97684 \mathrm{E}$ o0

$\begin{array}{llll}1.17921 \mathrm{E} & 00 & -3.21053 \mathrm{E} & 01\end{array}$ $\begin{array}{llll}1.17920 \mathrm{E} & 00 & -3.22092 \mathrm{E} & 01\end{array}$ $\begin{array}{llll}1.17919 \mathrm{E} & 00 & -3.23131 \mathrm{E} & 01\end{array}$ $\begin{array}{llll}1.17918 \mathrm{E} & 00 & -3.24170 \mathrm{E} & 01\end{array}$ $\begin{array}{llll}1.17917 \mathrm{E} & 00 & -3.25208 \mathrm{E} & 01\end{array}$

$1.17916 \mathrm{E} \quad 00$ $1.17915 \mathrm{E} \quad 00$ $1.17914 \mathrm{E} \quad 00$ $\begin{array}{ll}1.17913 \mathrm{E} & 00\end{array}$ $1.17912 \mathrm{E} \quad 00$ $-3.27285 \mathrm{E} 01$ $-3.28322 \mathrm{E} \quad 01$ $-3.29360 \mathrm{E} 01$ $-3.30397 \mathrm{E} \quad 01$

$\begin{array}{llll}1.17911 \mathrm{E} & 00 & -3.31435 \mathrm{E} & 01\end{array}$ $\begin{array}{llll}1.17910 \mathrm{E} & 00 & -3.32471 \mathrm{E} & 01\end{array}$ $\begin{array}{llll}1.17909 \mathrm{E} & 00 & -3.33508 \mathrm{E} & 01\end{array}$ $\begin{array}{llll}1.17908 \mathrm{E} & 00 & -3.34545 \mathrm{E} & 01\end{array}$ $\begin{array}{llll}1.17907 \mathrm{E} & 00 & -3.35581 \mathrm{E} & 01\end{array}$

$-2.99397 \mathrm{E} \quad 00$ $-3.01114 \mathrm{E} \quad 00$ $-3.02836 \mathrm{E} 00$ $-3.04562 \mathrm{E} \quad 00$ $-3.06293 \mathrm{E} 00$

$-3.08029 \mathrm{E} \quad 00$ $-3.09770 \mathrm{E} \quad 00$ $-3.11515 \mathrm{E} \quad 00$ $-3.13265 \mathrm{E} \quad 00$ $-3.15019 \mathrm{E}$ 00

$1.17906 \mathrm{E} \quad 00$ $1.17905 \mathrm{E} \quad 00$ $1.17904 \mathrm{E} \quad 00$ $1.17903 \mathrm{E} \quad 00$ $1.17902 \mathrm{E} \quad 00$

$-3.36617 \mathrm{E} \quad 01$ $-3.37652 \mathrm{E} 01$ $-3.38688 \mathrm{E} \quad 01$ $-3.39723 \mathrm{E} 01$

$1.17901 \mathrm{E} \quad 00$ $1.17900 \mathrm{E} \quad 00$ $1.17899 \mathrm{E} \quad 00$ $1.17898 \mathrm{E} \quad 00$ $1.17897 \mathrm{E} \quad 00$

$1.17896 \mathrm{E} \quad 00$ $1.17895 \mathrm{E} \quad 00$ $1.17894 \mathrm{E} \quad 00$ $1.17893 \mathrm{E} \quad 00$ $1.17892 \mathrm{E} \quad 00$

$1.17891 \mathrm{E} \quad 00$ $1.17890 \mathrm{E} \quad 00$ $1.17889 \mathrm{E} \quad 00$ $1.17888 \mathrm{E} \quad 00$ $1.17887 \mathrm{E} \quad 00$

$1.17886 \mathrm{E} \quad 00$ $1.17885 \mathrm{E} \quad 00$ $1.17884 \mathrm{E} \quad 00$ $1.17883 \mathrm{E} \quad 00$ $1.17883 \mathrm{E} \quad 00$

$1.17882 \mathrm{E} \quad 00$ $1.17881 \mathrm{E} \quad 00$ $1.17880 \mathrm{E} \quad 00$ $1.17879 \mathrm{E} \quad 00$ $1.17878 \mathrm{E} \quad 00$

$1.17877 \mathrm{E} \quad 00$ $1.17876 \mathrm{E} \quad 00$ $1.17875 \mathrm{E} \quad 00$ $1.17874 \mathrm{E} \quad 00$ $1.17873 \mathrm{E} \quad 00$

$\begin{array}{ll}1.17872 \mathrm{E} & 00\end{array}$ $\begin{array}{ll}1.17871 \mathrm{E} & 00\end{array}$ $1.17871 \mathrm{E} \quad 00$ $1.17870 \mathrm{E} \quad 00$ $1.17869 \mathrm{E} \quad 00$

$-3.16779 \mathrm{E} \quad 00$ $-3.18542 \mathrm{E} \quad 00$ $-3.20311 \mathrm{E} \quad 00$ $-3.22084 \mathrm{E} \quad 00$ $-3.23862 \mathrm{E} \quad 00$

$-3.25645 \mathrm{E} \quad 00$ $-3.27432 \mathrm{E} \quad 00$ $-3.29223 \mathrm{E} 00$ $-3.31020 \mathrm{E} 00$ $-3.32821 \mathrm{E} 00$

$-3.41793 \mathrm{E} \quad 01$ $-3.42828 \mathrm{E} 01$ $-3.43862 \mathrm{E} 01$ $-3.44896 \mathrm{E} 01$ $-3.45930 \mathrm{E} 01$

$-3.34627 \mathrm{E} \quad 00$ $-3.36437 \mathrm{E}$ 00 $-3.38252 \mathrm{E} \quad 00$ $-3.40072 \mathrm{E} \quad 00$ $-3.41896 \mathrm{E} \quad 00$

$-3.46964 \mathrm{E} \quad 01$

$-3.47997 \mathrm{E} \quad 01$

$-3.49030 \mathrm{E} 01$

$-3.50063 \mathrm{E} 01$

$-3.51096 \mathrm{E} 01$

$-3.52128 \mathrm{E} 01$ $-3.53160 \mathrm{E} 01$ $-3.54192 \mathrm{E} 01$ $-3.55224 \mathrm{E} \quad 01$ $-3.56255 \mathrm{E} 01$

$-3.57286 \mathrm{E} 01$ $-3.58317 \mathrm{E} 01$ $-3.59348 \mathrm{E} 01$ $-3.60379 \mathrm{E} 01$ $-3.61409 \mathrm{E} 01$

$-3.62439 \mathrm{E} 01$ $-3.63469 \mathrm{E} 01$ $-3.64498 \mathrm{E} 01$ $-3.65527 \mathrm{E} 01$ $-3.66556 \mathrm{E} 01$

$-3.67585 \mathrm{E} \quad 01$ $-3.68614 \mathrm{E} 01$ $-3.69642 \mathrm{E} \quad 01$ $-3.70670 \mathrm{E} 01$ $-3.71698 \mathrm{E} 01$

$-3.72725 \mathrm{E} 01$ $-3.73752 \mathrm{E} 01$ $-3.74779 \mathrm{E} \quad 01$ $-3.75806 \mathrm{E} \quad 01$ $-3.76833 \mathrm{E} 01$
$-3.43725 \mathrm{E} \quad 00$

$-3.45559 \mathrm{E} \quad 00$

$-3.47397 \mathrm{E} \quad 00$

$-3.49240 \mathrm{E} \quad 00$

$-3.51088 \mathrm{E} \quad 00$

$-3.52940 \mathrm{E} \quad 00$

$-3.54797 \mathrm{E} 00$

$-3.56658 \mathrm{E} \quad 00$

$-3.58524 \mathrm{E} \quad 00$

$-3.60395 \mathrm{E} 00$

$-3.62270 \mathrm{E} \quad 00$ $-3.64150 \mathrm{E} \quad 00$ $-3.66035 \mathrm{E} \quad 00$ $-3.67924 \mathrm{E} 00$ $-3.69818 \mathrm{E} \quad 00$

$-3.71716 \mathrm{E} \quad 00$ $-3.73619 \mathrm{E} 00$ $-3.75527 \mathrm{E} 00$ $-3.77439 \mathrm{E} \quad 00$ $-3.79356 \mathrm{E} \quad 00$

$-3.81278 \mathrm{E} \quad 00$ $-3.83204 \mathrm{E} \quad 00$ $-3.85135 \mathrm{E} \quad 00$ $-3.87070 \mathrm{E} 00$ $-3.89010 \mathrm{E} 00$

$-3.90955 \mathrm{E} \quad 00$ $-3.92904 \mathrm{E} \quad 00$ $-3.94858 \mathrm{E} \quad 00$ $-3.96816 \mathrm{E} \quad 00$ $-3.98779 \mathrm{E} \quad 00$
$-3.26247 \mathrm{E} 01$

$\tan \delta_{1}$

$4.94846 \mathrm{E}-03$ $4.94960 \mathrm{E}-03$ $4.95071 \mathrm{E}-03$ $4.95180 \mathrm{E}-03$ $4.95287 \mathrm{E}-03$

$4.95392 \mathrm{E}-03$ $4.95494 \mathrm{E}-03$ $4.95594 \mathrm{E}-03$ $4.95692 \mathrm{E}-03$ $4.95788 \mathrm{E}-03$

$4.95881 \mathrm{E}-03$ $4.95972 \mathrm{E}-03$ $4.96060 \mathrm{E}-03$ $4.96147 \mathrm{E}-03$ $4.96231 \mathrm{E}-03$
$4.96313 \mathrm{E}-03$

$4.96394 \mathrm{E}-03$

$4.96472 \mathrm{E}-03$

$4.96548 \mathrm{E}-03$

$4.96621 \mathrm{E}-03$

$4.96693 \mathrm{E}-03$ $4.96763 \mathrm{E}-03$ $4.96830 \mathrm{E}-03$

$4.96896 \mathrm{E}-03$

$4.96959 \mathrm{E}-03$

$4.97021 \mathrm{E}-03$ $4.97080 \mathrm{E}-03$ $4.97137 \mathrm{E}-03$ $4.97193 \mathrm{E}-03$

$4.97246 \mathrm{E}-03$

$4.97297 \mathrm{E}-03$

$4.97346 \mathrm{E}-03$

$4.97394 \mathrm{E}-03$

$497440 \mathrm{E}-03$

$4.97484 \mathrm{E}-03$

$4.97526 \mathrm{E}-03$

$4.97566 \mathrm{E}-03$

$4.97604 \mathrm{E}-03$

$4.97641 \mathrm{E}-03$

$4.97674 \mathrm{E}-03$

$4.97708 \mathrm{E}-03$ $4.97738 \mathrm{E}-03$ $4.97768 \mathrm{E}-03$ $4.97795 \mathrm{E}-03$ $4.97820 \mathrm{E}-03$ $4.97865 \mathrm{E}-03$ $4.97886 \mathrm{E}-03$ $4.97905 \mathrm{E}-03$ $4.97921 \mathrm{E}-03$

$4.97935 \mathrm{E}-03$ $4.97949 \mathrm{E}-03$ $4.97961 \mathrm{E}-03$ $4.97970 \mathrm{E}-03$ $4.97978 \mathrm{E}-03$

$4.97984 \mathrm{E}-03$ $4.97990 \mathrm{E}-03$ $4.97992 \mathrm{E}-03$ $4.97994 \mathrm{E}-03$ $4.97994 \mathrm{E}-03$
$4.97844 \mathrm{E}-03$ 
ELECTRON RADIAL FUNCTIONS

$Z=7 \quad A=14 \quad \rho=1.1 A^{1 / 3} F$

Electron

$\bar{F}$

30.10

30.20

30.30

30.40

30.50

30.60

30.70

30.80

30.90

31.00

31.10

31.20

31.30

31.40

31.50

31.60

31.70

31.80

31.90

32.00

32.10

32.20

32.30

32.40

32.50

32.60

32.70

32.80

32.90

33.00

33.10

33.20

33.30

33.40

33.50

33.60

33.70

33.80

33.90

34.00

34.10

34.20

34.30

34.40

34.50

34.60

34.70

34.80

34.90

35.00

35.10

35.20

35.30

35.40

35.50

35.60

35.70

35.80

35.90

36.00
$1.39681 \mathrm{E}-03$

$1.40610 \mathrm{E}-03$

$1.41542 \mathrm{E}-03$

$1.42477 \mathrm{E}-03$

$1.43416 \mathrm{E}-03$

$1.44357 \mathrm{E}-03$

$1.45301 \mathrm{E}-03$

$1.46249 \mathrm{E}-03$

$1.47199 \mathrm{E}-03$

$1.48153 \mathrm{E}-03$

$1.49110 \mathrm{E}-03$

$1.50069 \mathrm{E}-03$

$1.51032 \mathrm{E}-03$

$1.51998 \mathrm{E}-03$

$1.52967 \mathrm{E}-03$

$1.53939 \mathrm{E}-03$

$1.54914 \mathrm{E}-03$

$1.55892 \mathrm{E}-03$

$1.56874 \mathrm{E}-03$

$1.57858 \mathrm{E}-03$

$1.58845 \mathrm{E}-03$

$1.59836 \mathrm{E}-03$

$1.60829 \mathrm{E}-03$

$1.61826 \mathrm{E}-03$

$1.62826 \mathrm{E}-03$

$1.63829 \mathrm{E}-03$

$1.64835 \mathrm{E}-03$

$1.65844 \mathrm{E}-03$

$1.66856 \mathrm{E}-03$

$1.67871 \mathrm{E}-03$

$1.68889 \mathrm{E}-03$

$1.69910 \mathrm{E}-03$

$1.70935 \mathrm{E}-03$

$1.71962 \mathrm{E}-03$

$1.72992 \mathrm{E}-03$

$1.74026 \mathrm{E}-03$

$1.75063 \mathrm{E}-03$

$1.76102 \mathrm{E}-03$

$1.77145 \mathrm{E}-03$

$1.78191 \mathrm{E}-03$

$1.79240 \mathrm{E}-03$

$1.80292 \mathrm{E}-03$

$1.81347 \mathrm{E}-03$

$1.82405 \mathrm{E}-03$

$1.83467 \mathrm{E}-03$

$1.84531 \mathrm{E}-03$

$1.85598 \mathrm{E}-03$

$1.86669 \mathrm{E}-03$

$1.87743 \mathrm{E}-03$

$1.88819 \mathrm{E}-03$

$1.89899 \mathrm{E}-03$

$1.90982 \mathrm{E}-03$

$1.92068 \mathrm{E}-03$

$1.93157 \mathrm{E}-03$

$1.94249 \mathrm{E}-03$

$1.95344 \mathrm{E}-03$ $1.96442 \mathrm{E}-03$

$1.97543 \mathrm{E}-03$

$1.98648 \mathrm{E}-03$

$1.99755 \mathrm{E}-03$ $f_{2}$

$g_{2}$

$-2.19202 \mathrm{E} \quad 00 \quad-1.19496 \mathrm{E}-01$

$-2.20663 \mathrm{E} \quad 00 \quad-1.20597 \mathrm{E}-01$

$-2.22129 \mathrm{E} \quad 00 \quad-1.21705 \mathrm{E}-01$

$-2.23600 \mathrm{E} \quad 00-1.22819 \mathrm{E}-01$

$-2.25075 \mathrm{E} \quad 00 \quad-1.23941 \mathrm{E}-01$

$-2.26555 \mathrm{E} \quad 00$

$-2.28040 \mathrm{E} \quad 00$

$-2.29530 \mathrm{E} \quad 00$

$-2.31024 \mathrm{E} \quad 00$

$-2.32523 \mathrm{E} \quad 00$

$-2.34027 \mathrm{E} \quad 00$

$-2.35536 \mathrm{E} \quad 00$

$-2.37049 \mathrm{E} \quad 00$

$-2.38567 \mathrm{E} \quad 00$

$-2.40090 \mathrm{E} \quad 00$

$-2.41618 \mathrm{E} \quad 00$

$-2.43150 \mathrm{E} \quad 00$

$-2.44688 \mathrm{E} \quad 00$

$-2.46230 \mathrm{E} \quad 00$

$-2.47776 \mathrm{E} \quad 00$

$-2.49328 \mathrm{E} \quad 00$

$-2.50884 \mathrm{E} \quad 00$

$-2.52445 \mathrm{E} \quad 00$

$-2.54010 \mathrm{E} \quad 00$

$-2.55581 \mathrm{E} \quad 00$

$-2.57156 \mathrm{E} \quad 00$

$-2.58735 \mathrm{E} \quad 00$

$-2.60320 \mathrm{E} \quad 00$

$-2.61909 \mathrm{E} \quad 00$

$-2.63503 \mathrm{E} 00$

$-2.65102 \mathrm{E} \quad 00$

$-2.66706 \mathrm{E} \quad 00$

$-2.68314 \mathrm{E} \quad 00$

$-2.69927 \mathrm{E} \quad 00$

$-2.71545 \mathrm{E} \quad 00$

$-2.73167 \mathrm{E} \quad 00$

$-2.74794 \mathrm{E} \quad 00$

$-2.76426 \mathrm{E} \quad 00$

$-2.78063 \mathrm{E} \quad 00$

$-2.79704 \mathrm{E} \quad 00$

$-2.81350 \mathrm{E} \quad 00$

$-2.8300 \mathrm{H} \quad 00$

$-2.84656 \mathrm{E} \quad 00$

$-2.86316 \mathrm{E} \quad 00$

$-2.87981 \mathrm{E} \quad 00$

$-2.89651 \mathrm{E} \quad 00$

$-2.91325 \mathrm{E} \quad 00$

$-2.93004 \mathrm{E} \quad 00$

$-2.94688 \mathrm{E} \quad 00$

$-2.96377 \mathrm{E} \quad 00$

$-2.98070 \mathrm{E} \quad 00$

$-2.99768 \mathrm{E} \quad 00$

$-3.01470 \mathrm{E} 00$

$-3.03178 \mathrm{E} 00$

$-3.04890 \mathrm{E} 00$

$-3.06606 \mathrm{E} \quad 00$ $-3.08328 \mathrm{E} \quad 00$ $-3.10054 \mathrm{E} \quad 00$ $-3.11785 \mathrm{E} \quad 00$

$-3.13520 \mathrm{E} \quad 00$

$-1.25068 \mathrm{E}-01$

$-1.26203 \mathrm{E}-01$

$-1.27345 \mathrm{E}-01$

$-1.28493 \mathrm{E}-01$

$-1.29648 \mathrm{E}-01$

$-1.30810 \mathrm{E}-01$

$-1.31978 \mathrm{E}-01$

$-1.33154 \mathrm{E}-01$

$-1.34336 \mathrm{E}-01$

$-1.35525 \mathrm{E}-01$

$-1.36722 \mathrm{E}-01$

$-1.37925 \mathrm{E}-01$

$-1.39135 \mathrm{E}-01$

$-1.40352 \mathrm{E}-01$

$-1.41576 \mathrm{E}-01$

$-1.42807 \mathrm{E}-01$

$-1.44045 \mathrm{E}-01$

$-1.45290 \mathrm{E}-01$

$-1.46542 \mathrm{E}-01$

$-1.47801 \mathrm{E}-01$

$-1.49067 \mathrm{E}-01$

$-1.50341 \mathrm{E}-01$

$-1.51621 \mathrm{E}-01$

$-1.52909 \mathrm{E}-01$

$-1.54204 \mathrm{E}-01$

$-1.55506 \mathrm{E}-01$

$-1.56815 \mathrm{E}-01$

$-1.58132 \mathrm{E}-01$

$-1.59456 \mathrm{E}-01$

$-1.60787 \mathrm{E}-01$

$-1.62125 \mathrm{E}-01$

$-1.63471 \mathrm{E}-01$

$-1.64824 \mathrm{E}-01$

$-1.66184 \mathrm{E}-01$

$-1.67552 \mathrm{E}-01$

$-1.68927 \mathrm{E}-01$

$-1.70310 \mathrm{E}-01$

$-1.71699 \mathrm{E}-01$

$-1.73097 \mathrm{E}-01$

$-1.74502 \mathrm{E}-01$

$-1.75914 \mathrm{E}-01$

$-1.77334 \mathrm{E}-01$

$-1.78761 \mathrm{E}-01$

$-1.80196 \mathrm{E}-01$

$-1.81639 \mathrm{E}-01$

$-1.83089 \mathrm{E}-01$

$-1.84546 \mathrm{E}-01$

$-1.86012 \mathrm{E}-01$

$-1.87485 \mathrm{E}-01$

$-1.88965 \mathrm{E}-01$

$\tan \delta_{2}$

$f_{2}$

$2.95995 \mathrm{E} \quad 01 \quad-1.17290 \mathrm{E}-01$

$2.96008 \mathrm{E} \quad 01 \quad-1.18374 \mathrm{E}-01$

$2.96021 \mathrm{E}$ 01 $-1.19464 \mathrm{E}-01$

$2.96035 \mathrm{E}$ 01 $-1.20561 \mathrm{E}-01$

$2.96048 \mathrm{E} 01-1.21665 \mathrm{E}-01$

$2.96061 \mathrm{E} \quad 01$

$2.96074 \mathrm{E} \quad 01$

$2.96087 \mathrm{E} \quad 01$

$2.96099 \mathrm{E} 01$

$2.96112 \mathrm{E} \quad 01$

$2.96125 \mathrm{E} \quad 01$

$2.96137 \mathrm{E} \quad 01$

$2.96150 \mathrm{E} \quad 01$

$2.96162 \mathrm{E} \quad 01$

$2.96174 \mathrm{E} \quad 01$

$2.96186 \mathrm{E} \quad 01$

$2.96199 \mathrm{E} \quad 01$

$2.96211 \mathrm{E} \quad 01$

$2.96223 \mathrm{E} \quad 01$

$2.96234 \mathrm{E} \quad 01$

$-1.22775 \mathrm{E}-01$

$-1.23892 \mathrm{E}-01$

$-1.25015 \mathrm{E}-01$

$-1.26145 \mathrm{E}-01$

$-1.27282 \mathrm{E}-01$

$2.96246 \mathrm{E} \quad 01$

$2.96258 \mathrm{E} \quad 01$

$2.96270 \mathrm{E} \quad 01$

$2.96281 \mathrm{E} \quad 01$

$2.96293 \mathrm{E} \quad 01$

$-1.28426 \mathrm{E}-01$

$-1.29577 \mathrm{E}-01$

$-1.30734 \mathrm{E}-01$

$-1.31898 \mathrm{E}-01$

$-1.33069 \mathbf{E}-01$

$-1.34246 \mathrm{E}-01$

$-1.35431 \mathrm{E}-01$

$-1.36623 \mathrm{E}-01$

$-1.37821 \mathrm{E}-01$

$-1.39026 \mathrm{E}-01$

$2.96304 \mathrm{E} \quad 01$

$2.96316 \mathrm{E} \quad 01$

$2.96327 \mathrm{E} \quad 01$

$2.96338 \mathrm{E} \quad 01$

$2.96349 \mathrm{E} \quad 01$

$2.96360 \mathrm{E} \quad 01$

$2.96371 \mathrm{E} \quad 01$

$2.96382 \mathrm{E} \quad 01$

$2.96393 \mathrm{E} \quad 01$

$2.96404 \mathrm{E} \quad 01$

$2.96415 \mathrm{E} \quad 01$

$2.96425 \mathrm{E} \quad 01$

$2.96436 \mathrm{E} \quad 01$

$2.96446 \mathrm{E} \quad 01$ 
ELECTRON RADIAL FUNCTIONS

Electron

$p$

36.10

36.20

36.30

36.40

36.50

36.60

36.70

36.80

36.90

37.00

37.10

37.20

37.30

37.40

37.50

37.60

37.70

37.80

37.90

38.00

38.10

38.20

38.30

38.40

38.50

38.60

38.70

38.80

38.90

39.00

39.10

39.20

39.30

39.40

39.50

39.60

39.70

39.80

39.90

40.00

40.10

40.20

40.30

40.40

40.50

40.60

40.70

40.80

40.90

41.00

41.10

41.20

41.30

41.40

41.50

41.60

41.70

41.80

41.90

42.00
$Z=7$

$f_{1}$

$1.17868 \mathrm{E} \quad 00$

$1.17867 \mathrm{E} \quad 00$

$1.17866 \mathrm{E} \quad 00$

$1.17865 \mathrm{E} \quad 00$

$1.17864 \mathrm{E} \quad 00$

$1.17863 \mathrm{E} \quad 00$

$1.17863 \mathrm{E} \quad 00$

$1.17862 \mathrm{E} \quad 00$

$1.17861 \mathrm{E} \quad 00$

$1.17860 \mathrm{E} \quad 00$

$1.17859 \mathrm{E} \quad 00$

$1.17858 \mathrm{E} \quad 00$

$1.17857 \mathrm{E} \quad 00$

$1.17856 \mathrm{E} \quad 00$

$1.17856 \mathrm{E} \quad 00$

$1.17855 \mathrm{E} \quad 00$

$1.17854 \mathrm{E} \quad 00$

$1.17853 \mathrm{E} \quad 00$

$1.17852 \mathrm{E} \quad 00$

$1.17851 \mathrm{E} \quad 00$

$1.17850 \mathrm{E} \quad 00$

$1.17850 \mathrm{E} \quad 00$

$1.17849 \mathrm{E} \quad 00$

$1.17848 \mathrm{E} \quad 00$

$1.17847 \mathrm{E} \quad 00$

$1.17846 \mathrm{E} \quad 00$

$1.17846 \mathrm{E} \quad 00$

$1.17845 \mathrm{E} \quad 00$

$1.17844 \mathrm{E} \quad 00$

$1.17843 \mathrm{E} \quad 00$

$\begin{array}{ll}1.17842 \mathrm{E} & 00\end{array}$

$1.17841 \mathrm{E} \quad 00$

$1.17841 \mathrm{E} \quad 00$

$1.17840 \mathrm{E} \quad 00$

$1.17839 \mathrm{E} \quad 00$

$1.17838 \mathrm{E} \quad 00$ $1.17837 \mathrm{E} \quad 00$

$1.17837 \mathrm{E} \quad 00$

$1.17836 \mathrm{E} \quad 00$

$1.17835 \mathrm{E} \quad 00$

$1.17834 \mathrm{E} \quad 00$ $1.17833 \mathrm{E} \quad 00$

$1.17833 \mathrm{E} \quad 00$

$1.17832 \mathrm{E} \quad 00$

$1.17831 \mathrm{E} \quad 00$

$1.17830 \mathrm{E} \quad 00$

$1.17829 \mathrm{E} \quad 00$

$1.17829 \mathrm{E} \quad 00$

$1.17828 \mathrm{E} \quad 00$

$1.17827 \mathrm{E} \quad 00$

$1.17826 \mathrm{E} \quad 00$ $1.17826 \mathrm{E} \quad 00$ $1.17825 \mathrm{E} \quad 00$

$1.17824 \mathrm{E} \quad 00$

$1.17823 \mathrm{E} \quad 00$

$1.17822 \mathrm{E} \quad 00$ $1.17822 \mathrm{E} \quad 00$ $1.17821 \mathrm{E} \quad 00$

$1.17820 \mathrm{E} \quad 00$

$1.17819 \mathrm{E} \quad 00$
-3.77859 E 01 $\begin{array}{ll}-3.78885 \mathrm{E} & 01\end{array}$ $-3.79911 \mathrm{E} \quad 01$ $-3.80936 \mathrm{E} \quad 01$ $-3.81961 \mathrm{E} \quad 01$

\section{$-3.82986 \mathrm{E} \quad 01$}

$-3.84011 \mathrm{E} 01$

$-3.85036 \mathrm{E} \quad 01$

$-3.86060 \mathrm{E} \quad 0 \mathrm{l}$

$-3.87084 \mathrm{E} \quad 01$

$-3.88107 \mathrm{E} \quad 01$

$-3.89131 \mathrm{E} \quad 01$

$-3.90154 \mathrm{E} 01$

$-3.91177 \mathrm{E} \quad 01$

$-3.92200 \mathrm{E} \quad 01$

$\begin{array}{ll}-3.93222 \mathrm{E} & 01\end{array}$

$-3.94244 \mathrm{E} \quad 01$

$-3.95266 \mathrm{E} \quad 01$

$-3.96288 \mathrm{E} 01$

$-3.97309 \mathrm{E} \quad 01$

$-3.98330 \mathrm{E} \quad 01$

$-3.99351 \mathrm{E} \quad 01$

$-4.00372 \mathrm{E} 01$

$\begin{array}{ll}-4.01392 \mathrm{E} & 01\end{array}$

$-4.02412 \mathrm{E} 01$

$-4.03432 \mathrm{E} \quad 01$ $-4.04451 \mathrm{E} 01$ $-4.05471 \mathrm{E} \quad 01$ $-4.06490 \mathrm{E} \quad 01$ $-4.07508 \mathrm{E} \quad 01$

$-4.08527 \mathrm{E} \quad 01$ $-4.09545 \mathrm{E} \quad 01$ $-4.10563 \mathrm{E} 01$ $-4.11581 \mathrm{E} \quad 01$ $-4.12598 \mathrm{E} 01$

$-4.13615 \mathrm{E} 01$ $-4.14632 \mathrm{E} 01$ $\begin{array}{lll}-4.15649 \mathrm{E} & 01\end{array}$ $-4.16665 \mathrm{E} 01$ -4.17681 E 01 .

$-4.18697 \mathrm{E} \quad 01$ $-4.19712 \mathrm{E} 01$ $-4.20728 \mathrm{E} 01$ $-4.21743 \mathrm{E} 01$ $-4.22757 \mathrm{E} \quad 01$

$-4.23772 \mathrm{E} \quad 01$ $-4.24786 \mathrm{E} 01$ $-4.25800 \mathrm{E} 01$ $-4.26814 \mathrm{E} 01$ $-4.27827 \mathrm{E} \quad 01$

$-4.28840 \mathrm{E} 01$ $-4.30865 \mathrm{E} \quad 01$ $-4.31878 \mathrm{E} 01$ $-4.32890 \mathrm{E} \quad 01$

$-4.33901 \mathrm{E} \quad 01$ $-4.34913 \mathrm{E} \quad 01$ $-4.35924 \mathrm{E} \quad 01$ $-4.36935 \mathrm{E} 01$ $-4.37945 \mathrm{E} \quad 01$ $-4.29853 \mathrm{E} \quad 01$ $g_{1}$

$A=14$

$$
\rho=1.1 A^{1 / 3} F
$$

$-4.00747 \mathrm{E} \quad 00$ $-4.02719 \mathrm{E} \quad 00$ $-4.04696 \mathrm{E} \quad 00$ $-4.06677 \mathrm{E} \quad 00$

$-4.08663 \mathrm{E} \quad 00$

$-4.10654 \mathrm{E} \quad 00$

$-4.12649 \mathrm{E} \quad 00$

$-4.14649 \mathrm{E} 00$

$-4.16653 \mathrm{E} \quad 00$

$-4.18662 \mathrm{E} \quad 00$

$-4.20676 \mathrm{E} \quad 00$

$-4.22694 \mathrm{E} \quad 00$

$-4.24717 \mathrm{E} \quad 00$

$-4.26744 \mathrm{E} \quad 00$

$-4.28776 \mathrm{E} \quad 00$

$-4.30812 \mathrm{E} \quad 00$

$-4.32853 \mathrm{E} \quad 00$

$-4.34899 \mathrm{E} \quad 00$

$-4.36949 \mathrm{E} \quad 00$

$-4.39004 \mathrm{E} \quad 00$

$-4.41063 \mathrm{E} \quad 00$

$-4.43127 \mathrm{E} \quad 00$

$-4.45196 \mathrm{E} \quad 00$

$-4.47269 \mathrm{E} \quad 00$

$-4.49346 \mathrm{E} \quad 00$

$-4.51428 \mathrm{E} \quad 00$

$-4.53515 \mathrm{E} \quad 00$

$-4.55606 \mathrm{E} 00$

$-4.57702 \mathrm{E} \quad 00$

$-4.59803 \mathrm{E} \quad 00$

$-4.61908 \mathrm{E} \quad 00$

$-4.64017 \mathrm{E} \quad 00$

$-4.66131 \mathrm{E} \quad 00$

$-4.68250 \mathrm{E} \quad 00$

$-4.70373 \mathrm{E} \quad 00$

$-4.72501 \mathrm{E} \quad 00$

$-4.74633 \mathrm{E} \quad 00$

$-4.76770 \mathrm{E} \quad 00$

$-4.78911 \mathrm{E} \quad 00$

$-4.81057 \mathrm{E} \quad 00$

$-4.83207 \mathrm{E} \quad 00$

$-4.85362 \mathrm{E} \quad 00$

$-4.87522 \mathrm{E} \quad 00$

$-4.89686 \mathrm{E} \quad 00$

$-4.91855 \mathrm{E} \quad 00$

$-4.94028 \mathrm{E} \quad 00$

$-4.96205 \mathrm{E} \quad 00$

$-4.98388 \mathrm{E} \quad 00$

$-5.00574 \mathrm{E} \quad 00$

$-5.02766 \mathrm{E} 00$

$-5.04961 \mathrm{E} \quad 00$

$-5.07162 \mathrm{E} \quad 00$

$-5.09366 \mathrm{E} \quad 00$

$-5.11576 \mathrm{E} \quad 00$

$-5.13790 \mathrm{E} \quad 00$

$-5.16008 \mathrm{E} \quad 00$

$-5.18231 \mathrm{E} \quad 00$

$-5.20458 \mathrm{E} \quad 00$

$-5.22690 \mathrm{E} \quad 00$

$-5.24927 \mathrm{E} \quad 00$

$\tan \delta_{1}$

$4.97992 \mathrm{E}-03$

$4.97989 \mathrm{E}-03$

$4.97984 \mathrm{E}-03$

$4.97978 \mathrm{E}-03$

$4.97969 \mathrm{E}-03$

$4.97960 \mathrm{E}-03$

$4.97947 \mathrm{E}-03$

$4.97935 \mathrm{E}-03$

$4.97920 \mathrm{E}-03$

$4.97905 \mathrm{E}-03$

$4.97887 \mathrm{E}-03$

$4.97868 \mathrm{E}-03$

$4.97847 \mathrm{E}-03$

$4.97825 \mathrm{E}-03$

$4.97802 \mathrm{E}-03$

$4.97777 \mathrm{E}-03$

$4.97750 \mathrm{E}-03$

$4.97722 \mathrm{E}-03$

$4.97692 \mathrm{E}-03$

$4.97661 \mathrm{E}-03$

$4.97629 \mathrm{E}-03$

$4.97595 \mathrm{E}-03$

$4.97559 \mathrm{E}-03$

$4.97522 \mathrm{E}-03$

$4.97483 \mathrm{E}-03$

$4.97445 \mathrm{E}-03$

$4.97403 \mathrm{E}-03$

$4.97361 \mathrm{E}-03$

$4.97317 \mathrm{E}-03$

$4.97272 \mathrm{E}-03$

$4.97225 \mathrm{E}-03$

$4.97177 \mathrm{E}-03$

$4.97127 \mathrm{E}-03$

$4.97076 \mathrm{E}-03$

$4.97024 \mathrm{E}-03$

$4.96970 \mathrm{E}-03$ $4.96916 \mathrm{E}-03$

$4.96860 \mathrm{E}-03$

$f_{-1}$ 
ELECTRON RADIAL FUNCTIONS

$Z=7 \quad A=14 \quad \rho=1.1 A^{1 / 3} F$

Electron

$p$

36.10

36.20

36.30

36.40

36.50

36.60

36.70

36.80

36.90

37.00

37.10

37.20

37.30

37.40

37.50

37.60

37.70

37.80

37.90

38.00

38.10

38.20

38.30

38.40

38.50

38.60

38.70

38.80

38.90

39.00

39.10

39.20

39.30

39.40

39.50

39.60

39.70

39.80

39.90

40.00

40.10

40.20

40.30

40.40

40.50

40.60

40.70

40.80

40.90

41.00

41.10

41.20

41.30

41.40

41.50

41.60

41.70

41.80

41.90

42.00
$\bar{F}$

$2.00866 \mathrm{E}-03$

$2.01979 \mathrm{E}-03$

$2.03096 \mathrm{E}-03$

$2.04215 \mathrm{E}-03$

$2.05338 \mathrm{E}-03$

$2.06464 \mathrm{E}-03$

$2.07593 \mathrm{E}-03$

$2.08725 \mathrm{E}-03$

$2.09860 \mathrm{E}-03$

$2.10998 \mathrm{E}-03$

$2.12140 \mathrm{E}-03$

$2.13284 \mathrm{E}-03$

$2.14431 \mathrm{E}-03$

$2.15582 \mathrm{E}-03$

$2.16736 \mathrm{E}-03$

$2.17892 \mathrm{E}-03$

$2.19052 \mathrm{E}-03$

$2.20215 \mathrm{E}-03$

$2.21381 \mathrm{E}-03$

$2.22549 \mathrm{E}-03$

2.23721 E- 03

$2.24897 \mathrm{E}-03$

$2.26075 \mathrm{E}-03$

$2.27256 \mathrm{E}-03$

$2.28440 \mathrm{E}-03$

$2.29628 \mathrm{E}-03$

$2.30818 \mathrm{E}-03$

$2.32012 \mathrm{E}-03$

$2.33208 \mathrm{E}-03$

$2.34408 \mathrm{E}-03$

$2.35611 \mathrm{E}-03$

$2.36817 \mathrm{E}-03$

$2.38026 \mathrm{E}-03$

$2.39238 \mathrm{E}-03$

2.40453 E-03

$2.41671 \mathrm{E}-03$

$2.42892 \mathrm{E}-03$

$2.44116 \mathrm{E}-03$

$2.45344 \mathrm{E}-03$

$2.46574 \mathrm{E}-03$

$2.47808 \mathrm{E}-03$

$2.49045 \mathrm{E}-03$

$2.50284 \mathrm{E}-03$

$2.51527 \mathrm{E}-03$

$2.52773 \mathrm{E}-03$

$2.54022 \mathrm{E}-03$

$2.55274 \mathrm{E}-03$

$2.56529 \mathrm{E}-03$

$2.57787 \mathrm{E}-03$

$2.59048 \mathrm{E}-03$

$2.60313 \mathrm{E}-03$

$2.61580 \mathrm{E}-03$

$2.62850 \mathrm{E}-03$

$2.64124 \mathrm{E}-03$

$2.65401 \mathrm{E}-03$

$2.66680 \mathrm{E}-03$

$2.67963 \mathrm{E}-03$

$2.69249 \mathrm{E}-03$

$2.70538 \mathrm{E}-03$

$2.71830 \mathrm{E}-03$

\section{$f_{2}$}

$g_{2}$

$-3.15260 \mathrm{E} \quad 00$

$-3.17005 \mathrm{E} 00$

$-3.18755 \mathbf{E} 00$

$-3.20509 \mathbf{E} 00$

$-3.22268 \mathrm{E} 00$

$-3.24032 \mathrm{E} \quad 00$

$-3.25800 \mathrm{E} \quad 00$

$-3.27573 \mathrm{E} \quad 00$

$-3.29351 \mathrm{E} 00$

$-3.31133 \mathrm{E} \quad 00$

$-3.32921 \mathrm{E} \quad 00$

$-3.34712 \mathrm{E} \quad 00$

$-3.36509 \mathrm{E} 00$

$-3.38310 \mathrm{E} \quad 00$

$-3.40116 \mathrm{E} \quad 00$

$-3.41926 \mathrm{E} 00$

$-3.43742 \mathrm{E} \quad 00$

$-3.45561 \mathrm{E} \quad 00$

$-3.47386 \mathrm{E} \quad 00$

$-3.49215 \mathrm{E} 00$

$-3.51049 \mathrm{E} \quad 00$

$-3.52888 \mathrm{E} 00$

$-3.54731 \mathrm{E} 00$

$-3.56579 \mathrm{E} \quad 00$

$-3.58431 \mathrm{E} \quad 00$

$-3.60288 \mathrm{E} \quad 00$

$-3.62150 \mathrm{E} \quad 00$

$-3.64017 \mathrm{E} \quad 00$

$-3.65888 \mathrm{E} \quad 00$

$-3.67764 \mathrm{E} \quad 00$

$-3.69645 \mathrm{E} \quad 00$

$-3.71530 \mathrm{E} \quad 00$

$-3.73420 \mathrm{E} \quad 00$

$-3.75314 \mathrm{E} 00$

$-3.77213 \mathrm{E} 00$

$-3.79117 \mathrm{E} \quad 00$

$-3.81026 \mathrm{E} \quad 00$

$-3.82939 \mathrm{E} 00$

$-3.84856 \mathrm{E} \quad 00$

$-3.86779 \mathrm{E} \quad 00$

$-3.88706 \mathrm{E} \quad 00$

$-3.90638 \mathrm{E} \quad 00$

$-3.92574 \mathrm{E} \quad 00$

$-3.94515 \mathrm{E} \quad 00$

$-3.96460 \mathrm{E} \quad 00$

$-3.98411 \mathrm{E} \quad 00$

$-4.00366 \mathrm{E} \quad 00$

$-4.02325 \mathrm{E} \quad 00$

$-4.04289 \mathrm{E} \quad 00$

$-4.06258 \mathrm{E} \quad 00$

$-4.08232 \mathrm{E} \quad 00$

$-4.10210 \mathrm{E} \quad 00$

$-4.12192 \mathrm{E} \quad 00$

$-4.14180 \mathrm{E} \quad 00$

$-4.16172 \mathrm{E} \quad 00$

$-4.18168 \mathrm{E} \quad 00$

$-4.20169 \mathrm{E} \quad 00$

$-4.22175 \mathrm{E} \quad 00$

$\begin{array}{lll}-4.24186 \mathrm{E} & 00\end{array}$

$-4.26201 \mathrm{E} 00$ $\tan \delta_{2}$

$f_{-2}$

$-1.98010 \mathrm{E}-01$

$-1.99545 \mathrm{E}-01$

$-2.01088 \mathrm{E}-01$

$-2.02638 \mathrm{E}-01$

$-2.04196 \mathrm{E}-01$

$-2.05762 \mathrm{E}-01$

$-2.07336 \mathrm{E}-01$

$-2.08918 \mathrm{E}-01$

$-2.10508 \mathrm{E}-01$

$-2.12105 \mathrm{E}-01$

$-2.13711 \mathrm{E}-01$

$-2.15325 \mathrm{E}-0 \mathrm{l}$

$-2.16946 \mathrm{E}-01$

$-2.18576 \mathrm{E}-01$

$-2.20214 \mathrm{E}-01$

$-2.21859 \mathrm{E}-0 \mathrm{l}$

$-2.23513 \mathrm{E}-01$

$-2.25175 \mathrm{E}-01$

$-2.26845 \mathrm{E}-01$

$-2.28523 \mathrm{E}-01$

$-2.30210 \mathrm{E}-01$

$-2.31904 \mathrm{E}-01$

$-2.33607 \mathrm{E}-01$

$-2.35318 \mathrm{E}-01$

$-2.37037 \mathrm{E}-01$

$-2.38764 \mathrm{E}-01$

$-2.40500 \mathrm{E}-01$

$-2.42244 \mathrm{E}-01$

$-2.43996 \mathrm{E}-01$

$-2.45756 \mathrm{E}-01$

$-2.47525 \mathrm{E}-01$

$-2.49302 \mathrm{E}-01$

$-2.51088 \mathrm{E}-01$

$-2.52882 \mathrm{E}-01$

$-2.54684 \mathrm{E}-01$

$-2.56495 \mathrm{E}-01$

$-2.58314 \mathrm{E}-01$

$-2.60142 \mathrm{E}-01$

$-2.61978 \mathrm{E}-01$

$-2.63823 \mathrm{E}-01$

$-2.65676 \mathrm{E}-01$

$-2.67538 \mathrm{E}-01$

$-2.69409 \mathrm{E}-01$

$-2.71288 \mathrm{E}-01$

$-2.73175 \mathrm{E}-01$

$-2.75071 \mathrm{E}-01$

$-2.76976 \mathrm{E}-01$

$-2.78890 \mathrm{E}-01$

$-2.80812 \mathrm{E}-01$

$-2.82743 \mathrm{E}-01$

$-2.84682 \mathrm{E}-01$

$-2.86631 \mathrm{E}-01$

$-2.88588 \mathrm{E}-01$

$-2.90554 \mathrm{E}-01$

$-2.92528 \mathrm{E}-01$

$-2.94512 \mathrm{E}-01$

$-2.96504 \mathrm{E}-01$

$-2.98505 \mathrm{E}-01$

$-3.00515 \mathrm{E}-01$

$-3.02534 \mathrm{E}-01$

$2.96663 \mathrm{E} \quad 01 \quad-1.94637 \mathrm{E}-01$

$2.96672 \mathbf{E} 01-1.96150 \mathrm{E}-01$

$2.96681 \mathrm{E} \quad 01 \quad-1.97671 \mathrm{E}-01$

$2.96691 \mathrm{E}$ 01 $-1.99200 \mathrm{E}-01$

$2.96700 \mathrm{E} \quad 01 \quad-2.00737 \mathrm{E}-01$

$\begin{array}{lll}2.96709 \mathrm{E} & 01 & -2.02281 \mathrm{E}-01\end{array}$

$2.96718 \mathrm{E} \quad 01 \quad-2.03833 \mathrm{E}-01$

$2.96726 \mathrm{E} \quad 01 \quad-2.05393 \mathrm{E}-01$

$2.96735 \mathrm{E} \quad 01 \quad-2.06961 \mathrm{E}-01$

$2.96744 \mathrm{E} \quad 01 \quad-2.08536 \mathrm{E}-01$

$2.96753 \mathrm{E} \quad 01$

$2.96761 \mathrm{E} \quad 01$

$2.96770 \mathrm{E} \quad 01$

$2.96779 \mathrm{E} \quad 01$

$2.96787 \mathrm{E} \quad 01$

$2.96796 \mathrm{E} \quad 01$

$2.96804 \mathrm{E} \quad 01$

$2.96813 \mathrm{E} \quad 01$

$2.96821 \mathrm{E} \quad 01$

$2.96829 \mathrm{E} \quad 0$

$2.96838 \mathrm{E} \quad 01$

$2.96846 \mathrm{E} \quad 01$

$2.96854 \mathrm{E} 01$

$2.96862 \mathrm{E} \quad 01$

$2.96870 \mathrm{E} 01$

$2.96878 \mathrm{E} \quad 01$

$2.96886 \mathrm{E} \quad 01$

$2.96894 \mathrm{E} \quad 01$

$2.96902 \mathrm{E} 01$

$2.96910 \mathrm{E} 01$

$2.96918 \mathrm{E} \quad 01$

$2.96926 \mathrm{E} \quad 01$

$2.96933 \mathrm{E} \quad 01$

$2.96941 \mathrm{E} \quad 01$

$2.96949 \mathrm{E} \quad 01$

$-2.10120 \mathrm{E}-01$

$-2.11712 \mathrm{E}-01$

$-2.13311 \mathrm{E}-01$

$-2.14918 \mathrm{E}-01$

$-2.16534 \mathrm{E}-01$ 
Positron

$0.10 \quad 1.89053 \mathrm{E}-01$

$0.20 \quad 4.64816 \mathrm{E}-01$

$0.30 \quad 6.05701 \mathrm{E}-01$

$0.40 \quad 6.84935 \mathrm{E}-01$

$0.50 \quad 7.34170 \mathrm{E}-01$

0.60

0.70

0.80

0.90

1.00

1.10

1.20

1.30

1.40

1.50

1.60

1.70

1.80

1.90

2.00

2.10

2.20

2.30

2.40

2.50

2.60

2.70

2.80

2.90

3.00

3.10

3.20

3.30

3.40

3.50

3.60

3.70

3.80

3.90

4.00

4.10

4.20

4.30

4.40

4.50

4.60

4.70

4.80

4.90

5.00

5.10

5.20

5.30

5.40

5.50

5.60

5.70

5.80

5.90

6.00

$7.66973 \mathrm{E}-01$

$8.06645 \mathrm{E}-01$

$8.19145 \mathrm{E}-01$

$8.28726 \mathrm{E}-01$

$8.36214 \mathrm{E}-01$

$8.42166 \mathrm{E}-01$

$8.46966 \mathrm{E}-01$

$8.50885 \mathrm{E}-01$

8.54121 E-01

$8.56820 \mathrm{E}-01$

$8.59091 \mathrm{E}-01$

$8.61017 \mathrm{E}-01$

$8.62663 \mathrm{E}-01$

8.64078E-01

8.65303E-01

$8.66368 \mathrm{E}-01$

$8.67300 \mathrm{E}-01$

$8.68118 \mathrm{E}-01$

$8.68840 \mathrm{E}-01$

$8.69479 \mathrm{E}-01$

$8.70047 \mathrm{E}-01$

$8.70554 \mathrm{E}-01$

$8.71008 \mathrm{E}-01$

$8.71415 \mathrm{E}-01$

$8.71781 \mathrm{E}-01$

$8.72112 \mathrm{E}-01$

$8.72411 \mathrm{E}-01$

8.72681 E-01

$8.72927 \mathrm{E}-01$

$8.73151 \mathrm{E}-01$

$8.73355 \mathrm{E}-01$

$8.73540 \mathrm{E}-01$

$8.73866 \mathrm{E}-01$

$8.74009 E-01$

$8.74140 \mathrm{E}-01$

$8.74260 \mathrm{E}-01$

$8.74371 \mathrm{E}-01$

8.74472 E-01

$8.74566 \mathrm{E}-01$

$8.74652 \mathrm{E}-01$

$8.74732 \mathrm{E}-01$

$8.74806 \mathrm{E}-01$

$8.74873 \mathrm{E}-01$

$8.74936 \mathrm{E}-01$

$8.74994 \mathrm{E}-01$

$8.75097 \mathrm{E}-01$

8.75143E-01

$8.75186 \mathrm{E}-01$

$8.75225 \mathrm{E}-01$

8.75261 E-01

$8.75294 \mathrm{E}-01$

$8.75325 \mathrm{E}-01$
$Z=6 \quad A=12$

$g_{1}$

$3.32832 \mathrm{E}-03$

$$
-4.37908 \mathrm{E}-05
$$

$1.94304 \mathrm{E}-02 \quad-2.55021 \mathrm{E}-04$

$4.84339 \mathrm{E}-02-6.33135 \mathrm{E}-04$

$8.90432 \mathrm{E}-02-1.15759 \mathrm{E}-03$

$1.39720 \mathrm{E}-01-1.80395 \mathrm{E}-03$

$7.89945 \mathrm{E}-01$

$8.73710 \mathrm{E}-01$

$8.75048 \mathrm{E}-01$
$1.98881 \mathrm{E}-01$

$2.65037 \mathrm{E}-01$

$3.36859 \mathrm{E}-01$

$4.13220 \mathrm{E}-01$

4.93190E-01

$5.76015 \mathrm{E}-01$ $6.61098 \mathrm{E}-01$

$7.47966 \mathrm{E}-01$

$8.36247 \mathrm{E}-01$

$9.25650 \mathrm{E}-01$

$1.01595 \mathrm{E} \quad 00$

$1.10696 \mathrm{E} \quad 00$

$1.19854 \mathrm{E} \quad 00$

$1.29058 \mathrm{E} \quad 00$

$1.38300 \mathrm{E} \quad 00$

$1.47572 \mathrm{E} \quad 00$

$1.56868 \mathrm{E} \quad 00$

$1.66185 \mathrm{E} \quad 00$

$1.75518 \mathrm{E} \quad 00$

$1.84864 \mathrm{E} \quad 00$

$1.94221 \mathrm{E} \quad 00$

$2.03588 \mathrm{E} \quad 00$

$2.12962 \mathrm{E} \quad 00$

$2.22342 \mathrm{E} \quad 00$

$2.31728 \mathrm{E} \quad 00$

$2.41117 \mathrm{E} \quad 00$

$2.50511 \mathrm{E} \quad 00$

$2.59907 \mathrm{E} \quad 00$

$2.69305 \mathrm{E} \quad 00$

$2.78706 \mathrm{E} \quad 00$

$2.88108 \mathrm{E} \quad 00$

$2.97512 \mathrm{E} \quad 00$

$3.06916 \mathrm{E} \quad 00$

$3.16322 \mathrm{E} \quad 00$

$3.25729 \mathrm{E} \quad 00$

$3.35135 \mathrm{E} \quad 00$

$3.44543 \mathrm{E} \quad 00$

$3.53951 \mathrm{E} \quad 00$

$3.63359 \mathrm{E} \quad 00$

$3.72768 \mathrm{E} \quad 00$

$3.82177 \mathrm{E} \quad 00$

$3.91586 \mathrm{E} \quad 00$

$4.00995 \mathrm{E} \quad 00$

$4.10405 \mathrm{E} \quad 00$

$4.19814 \mathrm{E} \quad 00$

$4.29224 \mathrm{E} \quad 00$

$4.38633 \mathrm{E} \quad 00$

$4.48043 \mathrm{E} \quad 00$

$4.57453 \mathrm{E} \quad 00$

$4.66863 \mathrm{E} \quad 00$

$4.76273 \mathrm{E}, 00$

$4.85684 \mathrm{E} \quad 00$

$4.95094 \mathrm{E} \quad 00$

$5.04504 \mathrm{E} \quad 00$

5.13915 E 00
$-2.54697 \mathrm{E}-03$

$-3.36280 \mathrm{E}-03$

$-4.23014 \mathrm{E}-03$

$-5.13088 \mathrm{E}-03$

-6.05001 E-03

$-6.97535 \mathrm{E}-03$

$-7.89722 \mathrm{E}-03$

$-8.80792 \mathrm{E}-03$

$-9.70140 \mathrm{E}-03$

$-1.05729 \mathrm{E}-02$

$-1.14186 \mathrm{E}-02$

$-1.22357 \mathrm{E}-02$

$-1.30217 \mathrm{E}-02$

$-1.37749 \mathrm{E}-02$

$-1.44937 \mathrm{E}-02$

$-1.51770 \mathrm{E}-02$

$-1.58238 \mathrm{E}-02$

$-1.64334 \mathrm{E}-02$

$-1.70052 \mathrm{E}-02$

$-1.75385 \mathrm{E}-02$

$-1.80331 \mathrm{E}-02$

$-1.84886 \mathrm{E}-02$

$-1.92813 \mathrm{E}-02$

$-1.96180 \mathrm{E}-02$

$-1.99148 \mathrm{E}-02$

$-2.01715 \mathrm{E}-02$

$-2.03880 \mathrm{E}-02$

$-2.05641 \mathrm{E}-02$

$-2.07000 \mathrm{E}-02$

$-2.07953 \mathrm{E}-02$

$-2.08502 \mathrm{E}-02$

$-2.08646 \mathrm{E}-02$

$-2.08383 \mathrm{E}-02$

$-2.07715 \mathrm{E}-02$

$-2.06640 \mathrm{E}-02$

$-2.05158 \mathrm{E}-02$

$-2.03269 \mathrm{E}-02$

$-2.00973 \mathrm{E}-02$

$-1.98270 \mathrm{E}-02$

$-1.95159 \mathrm{E}-02$

$-1.91641 \mathrm{E}-02$

$-1.87715 \mathrm{E}-02$

$-1.83381 \mathrm{E}-02$

$-1.78640 \mathrm{E}-02$

$-1.73490 \mathrm{E}-02$

$-1.67932 \mathrm{E}-02$

$-1.55592 \mathrm{E}-02$

$-1.48810 \mathrm{E}-02$

$-1.41620 \mathrm{E}-02$

$-1.34021 \mathrm{E}-02$

$-1.26014 \mathrm{E}-02$

$-1.17598 \mathrm{E}-02$

$-1.08774 \mathrm{E}-02$
$-1.89048 \mathrm{E}-02$

$-1.61967 \mathrm{E}-02$

$\tan \delta_{1}$

$1.94195 \mathrm{E}-01$

$9.46184 \mathrm{E}-02$

$6.29416 \mathrm{E}-02$

$4.72058 \mathrm{E}-02$

$3.77508 \mathrm{E}-02$

$3.14202 \mathrm{E}-02$

2.68731 E- 02

$2.34419 \mathrm{E}-02$

$2.07564 \mathrm{E}-02$

$1.85947 \mathrm{E}-02$

$1.68156 \mathrm{E}-02$

$1.53245 \mathrm{E}-02$

$1.40562 \mathrm{E}-02$

$1.29637 \mathrm{E}-02$

$1.20124 \mathrm{E}-02$

$1.11765 \mathrm{E}-02$

$1.04360 \mathrm{E}-02$

$9.77535 \mathrm{E}-03$

$9.18222 \mathrm{E}-03$

$8.64670 \mathrm{E}-03$

$8.16077 \mathrm{E}-03$

$7.71779 \mathrm{E}-03$

$7.31230 \mathrm{E}-03$

$6.93972 \mathrm{E}-03$

$6.59618 \mathrm{E}-03$

$6.27842 \mathrm{E}-03$

$5.98362 \mathrm{E}-03$

$5.70938 \mathrm{E}-03$

$5.45362 \mathrm{E}-03$

5.21452 E- 03

4.99052 E-03

$4.78022 \mathrm{E}-03$

$4.58240 \mathrm{E}-03$

$4.39599 \mathrm{E}-03$

$4.22001 \mathrm{E}-03$

$4.05364 \mathrm{E}-03$

$3.89608 \mathrm{E}-03$

$3.74667 \mathrm{E}-03$

$3.60479 \mathrm{E}-03$

$3.46988 \mathrm{E}-03$

3.34144 E-03

$3.21903 \mathrm{E}-03$

$3.10221 \mathrm{E}-03$

$2.99063 \mathrm{E}-03$

$2.88393 \mathrm{E}-03$

$2.78180 \mathrm{E}-03$

$2.68396 \mathrm{E}-03$

$2.59014 \mathrm{E}-03$

$2.50011 \mathrm{E}-03$

$2.41363 \mathrm{E}-03$

$2.33049 \mathrm{E}-03$

$2.25053 \mathrm{E}-03$ 
ELECTRON RADIAL FUNCTIONS

$Z=6 \quad A=12 \quad \rho=1.1 A^{1 / 3} F$

Ponitron

$\bar{F}$

$2.64424 \mathrm{E}-09$

$2.28822 \mathrm{E}-08$

$6.54104 \mathrm{E}-08$

$1.30333 \mathrm{E}-07$

$2.17400 \mathrm{E}-07$

$3.26339 \mathrm{E}-07$ $4.56913 \mathrm{E}-07$ $6.08922 \mathrm{E}-07$

$7.82207 \mathrm{E}-07$

$9.76642 \mathrm{E}-07$

$1.19213 \mathrm{E}-06$

$1.42859 \mathrm{E}-06$

$1.68596 \mathrm{E}-06$

$1.96420 \mathrm{E}-06$

$2.26325 \mathrm{E}-06$

$2.58311 \mathrm{E}-06$

$2.92374 \mathrm{E}-06$

$3.28512 \mathrm{E}-06$

$3.66724 \mathrm{E}-06$

$4.07008 \mathrm{E}-06$

$4.49363 \mathrm{E}-06$

$4.93788 \mathrm{E}-06$

$5.40284 \mathrm{E}-06$

$5.88848 \mathrm{E}-06$

$6.39481 \mathrm{E}-06$

$6.92181 \mathrm{E}-06$

$7.46950 \mathrm{E}-06$

$8.03785 \mathrm{E}-06$

$8.62688 \mathrm{E}-06$

$9.23657 \mathrm{E}-06$

9.86692E-06

$1.05179 \mathrm{E}-05$

$1.11896 \mathrm{E}-05$

$1.18820 \mathrm{E}-05$

$1.25950 \mathrm{E}-05$

$1.33286 \mathrm{E}-05$

$1.40829 \mathrm{E}-05$

$1.48579 \mathrm{E}-0.5$

$1.56535 \mathrm{E}-05$

$1.64698 \mathrm{E}-05$

$1.73067 \mathrm{E}-05$

$1.81642 \mathrm{E}-05$

$1.90425 \mathrm{E}-05$

$1.99413 \mathrm{E}-05$

$2.08608 \mathrm{E}-05$

$2.18010 \mathrm{E}-05$

$2.27618 \mathrm{E}-05$

$2.37432 \mathrm{E}-05$

$2.47453 \mathrm{E}-05$

$2.57681 \mathrm{E}-05$

$2.68115 \mathrm{E}-05$

$2.78755 \mathrm{E}-05$

$2.89601 \mathrm{E}-05$

$3.00654 \mathrm{E}-05$

$3.11914 \mathrm{E}-05$

$3.23380 \mathrm{E}-05$

$3.35052 \mathrm{E}-05$

$3.46931 \mathrm{E}-05$

$3.59016 \mathrm{E}-05$

$3.71308 \mathrm{E}-05$ $f_{2}$

$7.30867 \mathrm{E}-07$

$8.45200 \mathrm{E}-06$

$3.15469 \mathrm{E}-05$

$7.72869 \mathrm{E}-05$

$1.51558 \mathrm{E}-04$

$2.58855 \mathrm{E}-04$

$4.02439 \mathrm{E}-04$

$5.84561 \mathrm{E}-04$

$8.06710 \mathrm{E}-04$

$1.06982 \mathrm{E}-03$

$1.37445 \mathrm{E}-03$

$1.72090 \mathrm{E}-03$

$2.10931 \mathrm{E}-03$

$2.53972 \mathrm{E}-03$

$3.01207 \mathrm{E}-03$

$3.52634 \mathrm{E}-03$

$4.08241 \mathrm{E}-03$

$4.68022 \mathrm{E}-03$

$5.31967 \mathrm{E}-03$

$6.00068 \mathrm{E}-03$

$6.72316 \mathrm{E}-03$

$7.48704 \mathrm{E}-03$

$8.29225 \mathrm{E}-03$

$9.13874 \mathrm{E}-03$

$1.00264 \mathrm{E}-02$

$1.09553 \mathrm{E}-02$

$1.19253 \mathrm{E}-02$

$1.29364 \mathrm{E}-02$

$1.39885 \mathrm{E}-02$

$1.50816 \mathrm{E}-02$

$1.62158 \mathrm{E}-02$

$1.73908 \mathrm{E}-02$

$1.86068 \mathrm{E}-02$

$1.98638 \mathrm{E}-02$

$2.11616 \mathrm{E}-02$

$2.25002 \mathrm{E}-02$

$2.38798 \mathrm{E}-02$

$2.53002 \mathrm{E}-02$

$2.67613 \mathrm{E}-02$

$2.82634 \mathrm{E}-02$

$2.98062 \mathrm{E}-02$

$3.13898 \mathrm{E}-02$

$3.30141 \mathrm{E}-02$

$3.46793 \mathrm{E}-02$

$3.63852 \mathrm{E}-02$

$3.81318 \mathrm{E}-02$

$3.99191 \mathrm{E}-02$

$4.17472 \mathrm{E}-02$

$4.36159 \mathrm{E}-02$

4.55254 E-02

$4.74756 \mathrm{E}-02$ $4.94663 \mathrm{E}-02$

$5.14978 \mathrm{E}-02$

$5.35698 \mathrm{E}-02$

$5.56825 \mathrm{E}-02$

$5.78357 \mathrm{E}-02$ $6.00294 \mathrm{E}-02$

$6.22637 \mathrm{E}-02$

$6.45383 \mathrm{E}-02$

$6.68534 \mathrm{E}-02$ $\tan \delta_{2}$

$-5.40333 \mathrm{E}-09 \quad-2.31124 \mathrm{E} \quad 00$

$-6.23226 \mathrm{E}-08 \quad-4.78710 \mathrm{E} \quad 00$

$-2.31621 \mathrm{E}-07$

$-5.64121 \mathrm{E}-07$

$-1.09813 \mathrm{E}-06$

$-1.85929 \mathrm{E}-06$

$-2.86202 \mathrm{E}-06$

$-4.11149 \mathrm{E}-06$

$-5.60584 \mathrm{E}-06$

$-7: 33812 \mathrm{E}-06$

$-9.29784 \mathrm{E}-06$

$-1.14721 \mathrm{E}-05$

$-1.38466 \mathrm{E}-05$

$-1.64058 \mathrm{E}-05$

$-1.91337 \mathrm{E}-05$

$-2.20139 \mathrm{E}-05$

$-2.50300 \mathrm{E}-05$

$-2.81652 \mathrm{E}-05$

$-3.14029 \mathrm{E}-05$

$-3.47265 \mathrm{E}-05$

$-3.81194 \mathrm{E}-05$

$-4.15650 \mathrm{E}-05$

$-4.50468 \mathrm{E}-05$

$-4.85484 \mathrm{E}-05$

$-5.20533 \mathrm{E}-05$

$-5.55453 \mathrm{E}-05$

$-5.90080 \mathrm{E}-05$

$-6.24251 \mathrm{E}-05$

$-6.57804 \mathrm{E}-05$

$-6.90576 \mathrm{E}-05$

$-7.22407 \mathrm{E}-05$

$-7.53136 \mathrm{E}-05$

$-7.82599 \mathrm{E}-05$

$-8.10637 \mathrm{E}-05$

$-8.37089 \mathrm{E}-05$

$-8.61794 \mathrm{E}-05$

$-8.84591 \mathrm{E}-05$

$-9.05320 \mathrm{E}-05$

$-9.23820 \mathrm{E}-05$

$-9.39932 \mathrm{E}-05$

$-9.53495 \mathrm{E}-05$

$-9.64350 \mathrm{E}-05$

$-9.72336 \mathrm{E}-05$

$-9.77293 \mathrm{E}-05$

$-9.79061 \mathrm{E}-05$

$-9.77482 \mathrm{E}-05$

$-9.72394 \mathrm{E}-05$

$-9.63639 \mathrm{E}-05$

$-9.51057 \mathrm{E}-05$

$-9.34488 \mathrm{E}-05$

$-9.13773 \mathrm{E}-05$

$-8.88752 \mathrm{E}-05$

$-8.59265 \mathrm{E}-05$

$-8.25155 \mathrm{E}-05$

$-7.86261 \mathrm{E}-05$

$-7.42423 \mathrm{E}-05$

$-6.93483 \mathrm{E}-05$

$-6.39283 \mathrm{E}-05$

$-5.79661 \mathrm{E}-05$

$-5.14461 \mathrm{E}-05$
$-7.10542 \mathrm{E} 00$

$-9.27020 \mathrm{E} \quad 00$

$-1.12671 \mathrm{E} 01$

$-1.30887 \mathrm{E} \quad 01$

$-1.47360 \mathrm{E} 01$

$-1.62163 \mathrm{E} 01$

$-1.75413 \mathrm{E} 01$

$-1.87247 \mathrm{E} \quad 01$

$-1.97809 \mathrm{E} \quad 01$

$-2.07240 \mathrm{E} 01$

$-2.15673 \mathrm{E} \theta \mathrm{l}$

$-2.23226 \mathrm{E} \quad 01$

$-2.30007 \mathrm{E} 01$

$-2.36111 \mathrm{E} \quad 01$

$-2.41621 \mathrm{E} 01$

$-2.46609 \mathrm{E} 01$

$-2.51136 \mathrm{E} \quad 01$

$-2.55258 \mathrm{E} 01$

$-2.59021 \mathrm{E} \quad 01$

$-2.62465 \mathrm{E} \quad 01$

$-2.65627 \mathrm{E} \quad 01$

$-2.68537 \mathrm{E} \quad 01$

$-2.71221 \mathrm{E} 01$

$-2.73703 \mathrm{E} \quad 01$

$-2.76003 \mathrm{E} \quad 01$

$-2.78140 \mathrm{E} \quad 01$

$-2.80129 \mathrm{E} \quad 01$

$-2.81984 \mathrm{E} 01$

$-2.83718 \mathrm{E} \quad 01$

$-2.85341 \mathrm{E} 01$

$-2.86863 \mathrm{E} \quad 01$

$-2.88292 \mathrm{E} 01$

$-2.89638 \mathrm{E} \quad 01$

$\begin{array}{ll}-2.90905 \mathrm{E} & 01\end{array}$

$-2.92102 \mathrm{E} 01$

$-2.93232 \mathrm{E} \quad 01$

$-2.94302 \mathrm{E} 01$

$-2.95316 \mathrm{E} 01$

$-2.96278 \mathrm{E} \quad 01$

$\begin{array}{lll}-2.97192 \mathrm{E} & 01\end{array}$

$-2.98061 \mathrm{E} \quad 01$

$-2.98888 \mathrm{E} 01$

$-2.99676 \mathrm{E} 01$

$-3.00428 \mathrm{E} 01$

$-3.01146 \mathrm{E} \quad 01$

$-3.01832 \mathrm{E} 01$

$-3.02489 \mathrm{E} \quad 01$

$-3.03118 \mathrm{E} 01$

$-3.03720 \mathrm{E} \quad 01$

$-3.04298 \mathrm{E} \quad 01$

$-3.04852 \mathrm{E} 01$

$-3.05385 \mathrm{E} 01$

$-3.05897 \mathrm{E} 01$

$-3.06390 \mathrm{E} \quad 01$

$-3.06864 \mathrm{E} \quad 01$

$-3.07321 \mathrm{E} \quad 01$

$-3.07761 \mathrm{E} 01$

$-3.08185 \mathrm{E} 01$

$f_{-2}$

$1.45292 \mathrm{E}-07$

$8.50065 \mathrm{E}-07$

$2.13665 \mathrm{E}-06$

$3.97388 \mathrm{E}-06$

$6.32427 \mathrm{E}-06$

$9.14844 \mathrm{E}-06$

$1.24079 \mathrm{E}-05$

$1.60664 \mathrm{E}-05$

$2.00911 \mathrm{E}-05$

$2.44522 \mathrm{E}-05$

$2.91226 \mathrm{E}-05$

$3.40778 \mathrm{E}-05$

$3.92953 \mathrm{E}-05$

$4.47541 \mathrm{E}-05$

$5.04344 \mathrm{E}-05$

$5.63174 \mathrm{E}-05$

$6.23850 \mathrm{E}-05$

$6.86195 \mathrm{E}-05$

$7.50039 \mathrm{E}-05$

$8.15213 \mathrm{E}-05$

$8.81550 \mathrm{E}-05$

$9.48885 \mathrm{E}-05$

$1.01706 \mathrm{E}-04$

$1.08590 \mathrm{E}-04$

$1.15526 \mathrm{E}-04$

$1.22497 \mathrm{E}-04$

$1.29487 \mathrm{E}-04$

$1.36480 \mathrm{E}-04$

$1.43461 \mathrm{E}-04$

$1.50412 \mathrm{E}-04$

$1.57319 \mathrm{E}-04$

$1.64166 \mathrm{E}-04$

$1.70936 \mathrm{E}-04$

$1.77613 \mathrm{E}-04$

$1.84182 \mathrm{E}-04$

$1.90627 \mathrm{E}-04$

$1.96932 \mathrm{E}-04$

$2.03080 \mathrm{E}-04$

$2.09057 \mathrm{E}-04$

$2.14846 \mathrm{E}-04$

$2.20432 \mathrm{E}-04$

$2.25797 \mathrm{E}-04$

$2.30927 \mathrm{E}-04$

$2.35806 \mathrm{E}-04$

$2.40418 \mathrm{E}-04$ 
ELECTRON RADIAL FUNCTIONS

Positron

$\begin{array}{cc}p & F_{0} \\ 6.10 & 8.75353 \mathrm{E}-01 \\ 6.20 & 8.75379 \mathrm{E}-01 \\ 6.30 & 8.75403 \mathrm{E}-01 \\ 6.40 & 8.75426 \mathrm{E}-01 \\ 6.50 & 8.75446 \mathrm{E}-01 \\ & \\ 6.60 & 8.75465 \mathrm{E}-01 \\ 6.70 & 8.75482 \mathrm{E}-01 \\ 6.80 & 8.75498 \mathrm{E}-01 \\ 6.90 & 8.75512 \mathrm{E}-01 \\ 7.00 & 8.75525 \mathrm{E}-01\end{array}$

$7.10 \quad 8.75537 \mathrm{E}-01$

$7.20 \quad 8.75547 \mathrm{E}-01$

$7.30 \quad 8.75557 \mathrm{E}-01$

$7.40 \quad 8.75565 \mathrm{E}-01$

$7.50 \quad 8.75573 \mathrm{E}-01$

$7.60 \quad 8.75580 \mathrm{E}-01$

$7.70 \quad 8.75586 \mathrm{E}-01$

$7.80 \quad 8.75591 \mathrm{E}-01$

$7.90 \quad 8.75596 \mathrm{E}-01$

$8.00 \quad 8.75599 \mathrm{E}-01$

$8.10 \quad 8.75602 \mathrm{E}-01$

$8.20 \quad 8.75605 \mathrm{E}-01$

$8.30 \quad 8.75607 \mathrm{E}-01$

$8.40 \quad 8.75608 \mathrm{E}-01$

$8.50 \quad 8.75609 \mathrm{E}-01$

$8.60 \quad 8.75610 \mathrm{E}-01$

$8.70 \quad 8.75609 \mathrm{E}-01$

$8.80 \quad 8.75609 \mathrm{E}-01$

$8.90 \quad 8.75608 \mathrm{E}-01$

$9.00 \quad 8.75606 \mathrm{E}-01$

$9.10 \quad 8.75605 \mathrm{E}-01$

$9.20 \quad 8.75603 \mathrm{E}-01$

$9.30 \quad 8.75600 \mathrm{E}-01$

$9.40 \quad 8.75598 \mathrm{E}-01$

$9.50 \quad 8.75595 \mathrm{E}-01$

$9.60 \quad 8.75592 \mathrm{E}-01$

$9.70 \quad 8.75588 \mathrm{E}-01$

$9.80 \quad 8.75585 \mathrm{E}-01$

$9.90 \quad 8.75581 \mathrm{E}-01$

$10.00 \quad 8.75577 \mathrm{E}-01$

10.10

10.20

10.30

10.40

10.50

10.60

10.70

10.80

10.90

11.00

11.10

11.20

11.30

11.40

11.50

11.60

11.70

11.80

11.90

12.00

$8.75572 \mathrm{E}-01$ $8.75568 \mathrm{E}-01$ $8.75563 \mathrm{E}-01$ $8.75558 \mathrm{E}-01$ $8.75553 \mathrm{E}-01$

$8.75548 \mathrm{E}-01$ $8.75543 \mathrm{E}-01$ $8.75537 \mathrm{E}-01$ $8.75531 \mathrm{E}-01$ $8.75526 \mathrm{E}-01$

$8.75520 \mathrm{E}-01$ $8.75514 \mathrm{E}-01$ $8.75508 \mathrm{E}-01$ $8.75502 \mathrm{E}-01$ $8.75495 \mathrm{E}-01$

$8.75489 \mathrm{E}-01$ $8.75482 \mathrm{E}-01$ $8.75476 \mathrm{E}-01$ $8.75469 \mathrm{E}-01$ $8.75462 \mathrm{E}-01$
$Z=6 \quad A=12$

$5.79790 \mathrm{E} 00$ $f_{1}$

$5.23325 \mathrm{E} \quad 00$

$5.32736 \mathrm{E} \quad 00$

$5.42147 \mathrm{E} \quad 00$

$5.51557 \mathrm{E} 00$

$5.60968 \mathrm{E} 00$

$5.70379 \mathrm{E} \quad 00$

$5.89201 \mathrm{E} \quad 00$

$5.98612 \mathrm{E} \quad 00$

$6.08024 \mathrm{E} \quad 00$

$6.17435 \mathrm{E} \quad 00$

$6.26846 \mathrm{E} \quad 00$

$6.36257 \mathrm{E} \quad 00$

$6.45667 \mathrm{E} \quad 00$

$6.55077 \mathrm{E} \quad 00$

$6.64487 \mathrm{E} \quad 00$

$6.73895 \mathrm{E} \quad 00$

$6.83303 \mathrm{E} \quad 00$

$6.92710 \mathrm{E} \quad 00$

$7.02114 \mathrm{E} \quad 00$

$7.11516 \mathrm{E} \quad 00$

$7.20915 \mathrm{E} \quad 00$

$7.30306 \mathrm{E} \quad 00$

$7.39689 \mathrm{E} \quad 00$
$7.49057 \mathrm{E}$

$7.49057 \mathrm{E} \quad 00$

$7.58400 \mathrm{E} \quad 00$

$7.67695 \mathrm{E} \quad 00$

$\begin{array}{ll}7.76870 \mathrm{E} & 00\end{array}$

$7.85618 \mathrm{E} \quad 00$

$7.85768 \mathrm{E} \quad 00$
0.00

$8.07942 \mathrm{E} \quad 00$ $8.16278 \mathrm{E} \quad 00$ $8.25380 \mathrm{E} \quad 00$

$8.34652 \mathrm{E} \quad 00$

$8.43989 \mathrm{E} \quad 00$

$8.53358 \mathrm{E} \quad 00$ $8.62748 \mathrm{E} \quad 00$ $8.72146 \mathrm{E} \quad 00$ $8.81554 \mathrm{E} \quad 00$ $8.90967 \mathrm{E} \quad 00$

$9.00385 \mathrm{E} \quad 00$ $9.09804 \mathrm{E} \quad 00$ $9.19228 \mathrm{E} \quad 00$ $9.28653 \mathrm{E} \quad 00$ $9.38082 \mathrm{E} \quad 00$

$g_{1}$
$-9.95411 \mathrm{E}-03$
$-8.98999 \mathrm{E}-03$
$-7.98502 \mathrm{E}-03$
$-6.93919 \mathrm{E}-03$
$-5.85250 \mathrm{E}-03$
$-4.72493 \mathrm{E}-03$
$-3.55650 \mathrm{E}-03$
$-2.34720 \mathrm{E}-03$
$-1.09702 \mathrm{E}-03$
$1.94035 \mathrm{E}-04$
$1.52597 \mathrm{E}-03$
$2.89878 \mathrm{E}-03$
$4.31247 \mathrm{E}-03$
$5.76704 \mathrm{E}-03$
$7.26248 \mathrm{E}-03$

$8.79881 \mathrm{E}-03$
$1.03760 \mathrm{E}-02$
$1.19940 \mathrm{E}-02$
$1.36529 \mathrm{E}-02$
$1.53527 \mathrm{E}-02$
$1.70932 \mathrm{E}-02$
$1.88745 \mathrm{E}-02$
$2.06964 \mathrm{E}-02$
$2.25589 \mathrm{E}-02$
$2.44617 \mathrm{E}-02$

3.41461 E-02

$9.47510 \mathrm{E} \quad 00$ $9.56942 \mathrm{E} \quad 00$ $9.66374 \mathrm{E} \quad 00$ $9.75808 \mathrm{E} \quad 00$ $9.85244 \mathrm{E} \quad 00$

$9.94681 \mathrm{E} \quad 00$ $\begin{array}{lll}1.00412 \mathrm{E} & 01\end{array}$ $1.01356 \mathrm{E} \quad 01$ $\begin{array}{lll}1.02300 \mathrm{E} & 01\end{array}$ $1.03244 \mathrm{E} \quad 01$

$1.04188 \mathrm{E} \quad 01$ $1.05133 \mathrm{E} \quad 01$ $\begin{array}{ll}1.06077 \mathrm{E} & 01\end{array}$ $\begin{array}{lll}1.07022 \mathrm{E} & 01\end{array}$ $\begin{array}{ll}1.07967 \mathrm{E} & 01\end{array}$ $g_{1}$

$2.64043 \mathrm{E}-02$

$2.83858 \mathrm{E}-02$

$3.04029 \mathrm{E}-02$

$3.24423 \mathrm{E}-02$

$3.68554 \mathrm{E}-02$ $3.89997 \mathrm{E}-02$

$4.12185 \mathrm{E}-02$

$4.34857 \mathrm{E}-02$

$4.57967 \mathrm{E}-02$

$4.81502 \mathrm{E}-02$

$5.05455 \mathrm{E}-02$

$5.29822 \mathrm{E}-02$

$5.54604 \mathrm{E}-02$

$5.79798 \mathrm{E}-02$

$6.05404 \mathrm{E}-02$

$6.31421 \mathrm{E}-02$

$6.57850 \mathrm{E}-02$

$6.84691 \mathrm{E}-02$

7.11944 E- 02

$\tan \delta_{1}$

$1.64768 \mathrm{E}-03$

$1.59141 \mathrm{E}-03$

$1.53692 \mathrm{E}-03$

$1.48412 \mathrm{E}-03$

$1.43293 \mathrm{E}-03$

$1.38328 \mathrm{E}-03$

$1.33511 \mathrm{E}-03$

$1.28834 \mathrm{E}-03$

$1.24294 \mathrm{E}-03$

$1.19882 \mathrm{E}-03$

$1.15594 \mathrm{E}-03$

$1.11424 \mathrm{E}-03$

$1.07369 \mathrm{E}-03$

$1.03423 \mathrm{E}-03$

$9.95823 \mathrm{E}-04$

$9.58429 \mathrm{E}-04$

$9.22003 \mathrm{E}-04$

$8.86499 \mathrm{E}-04$

$8.51910 \mathrm{E}-04$

$8.18175 \mathrm{E}-04$

$7.85277 \mathrm{E}-04$

$7.53188 \mathrm{E}-04$

$7.21871 \mathrm{E}-04$

$6.91312 \mathrm{E}-04$

$6.61466 \mathrm{E}-04$

$6.32315 \mathrm{E}-04$

$6.03837 \mathrm{E}-04$

$5.76014 \mathrm{E}-04$

$5.48821 \mathrm{E}-04$

$5.22233 \mathrm{E}-04$

$4.96233 \mathrm{E}-04$

$4.70802 \mathrm{E}-04$

$4.45928 \mathrm{E}-04$

$4.21586 \mathrm{E}-04$

3.97761 E-04

$3.74437 \mathrm{E}-04$

$3.51601 \mathrm{E}-04$

$3.29243 \mathrm{E}-04$

$3.07334 \mathrm{E}-04$

$2.85869 \mathrm{E}-04$

$2.64838 \mathrm{E}-04$

$2.44221 \mathrm{E}-04$

$2.24019 \mathrm{E}-04$

$2.04202 \mathrm{E}-04$

$1.84777 \mathrm{E}-04$

$1.65726 \mathrm{E}-04$

$1.47033 \mathrm{E}-04$

$1.28694 \mathrm{E}-04$

$1.10699 \mathrm{E}-04$

$9.30390 \mathrm{E}-05$

$7.57038 \mathrm{E}-05$ $5.86895 \mathrm{E}-05$

$4.19791 \mathrm{E}-05$

$2.55685 \mathrm{E}-05$

$9.45472 \mathrm{E}-06$

$9.75723 \mathrm{E}-02$

$1.00709 \mathrm{E}-01$

$1.03887 \mathrm{E}-01$

$1.07106 \mathrm{E}-01$

$1.10366 \mathrm{E}-01$

$1.13667 \mathrm{E}-01$

$1.17010 \mathrm{E}-01$
$-6.38558 \mathrm{E}-06$

$-2.19323 \mathrm{E}-05$

$-3.72158 \mathrm{E}-05$

$-5.22321 \mathrm{E}-05$

$-6.69877 \mathrm{E}-05$ $f_{-1}$

$3.85161 \mathrm{E}-02$

$3.77641 \mathrm{E}-02$

$3.69710 \mathrm{E}-02$

$3.61368 \mathrm{E}-02$

$3.52613 \mathrm{E}-02$

$3.43448 \mathrm{E}-02$

$3.33871 \mathrm{E}-02$

$3.23882 \mathrm{E}-02$

$3.13483 \mathrm{E}-02$

$3.02672 \mathrm{E}-02$

$2.91451 \mathrm{E}-02$

$2.79818 \mathrm{E}-02$

$2.67775 \mathrm{E}-02$

$2.55321 \mathrm{E}-02$

$2.42456 \mathrm{E}-02$

$2.29180 \mathrm{E}-02$

$2.15493 \mathrm{E}-02$

$2.01396 \mathrm{E}-02$

$1.86888 \mathrm{E}-02$

$1.71969 \mathrm{E}-02$

$1.56640 \mathrm{E}-02$

$1.40900 \mathrm{E}-02$

$1.24749 \mathrm{E}-02$

$1.08187 \mathrm{E}-02$

$9.12150 \mathrm{E}-03$

$7.38321 \mathrm{E}-03$

$5.60385 \mathrm{E}-03$

$3.78342 \mathrm{E}-03$

$1.92191 \mathrm{E}-03$

$1.93178 \mathrm{E}-05$

$-1.92435 \mathrm{E}-03$

$-3.90910 \mathrm{E}-03$

$-5.93494 \mathrm{E}-03$

$-8.00187 \mathrm{E}-03$

$-1.01099 \mathrm{E}-02$

$-1.22590 \mathrm{E}-02$

$-1.44492 \mathrm{E}-02$

$-1.66806 \mathrm{E}-02$

$-1.89530 \mathrm{E}-02$

$-2.12665 \mathrm{E}-02$

$-2.36212 \mathrm{E}-02$

$-2.60170 \mathrm{E}-02$

$-2.84539 \mathrm{E}-02$

$-3.09319 \mathrm{E}-02$

$-3.34511 \mathrm{E}-02$

$-3.60115 \mathrm{E}-02$

$-3.86129 \mathrm{E}-02$

$-4.12556 \mathrm{E}-02$

$-4.39394 \mathrm{E}-02$

$-4.66644 \mathrm{E}-02$

$-4.94305 \mathrm{E}-02$

$-5.22378 \mathrm{E}-02$

$-5.50864 \mathrm{E}-02$

$-5.79761 \mathrm{E}-02$

$-6.09070 \mathrm{E}-02$

$-6.38791 \mathrm{E}-02$

$-6.68924 \mathrm{E}-02$

$-6.99470 \mathrm{E}-02$

$-7.30428 \mathrm{E}-02$

$-7.61798 \mathrm{E}-02$ 
ELECTRON RADIAL, IUNCTIONS

$Z=6 \quad A=12 \quad \rho=1.1 \mathrm{~A}^{1 / 3} \mathrm{H}$

Pusitron

$\bar{F}$

$6.10 \quad 3.83806 \mathrm{E}-0.5$

$6.20 \quad 3.96510 \mathrm{E}-05$

$6.30 \quad 4.09421 \mathrm{E}-05$

$6.40 \quad 4.22538 \mathrm{E}-05$

$6.50 \quad 4.35861 \mathrm{E}-05$

$6.60 \quad 4.49391 \mathrm{E}-0.5$

$6.70 \quad 4.63127 \mathrm{E}-0.5$

$6.80 \quad 4.77070 \mathrm{E}-0.5$

$6.90 \quad 4.91219 \mathrm{E}-0.5$

$7.00 \quad 5.05574 \mathrm{E}-05$

7.10

7.20

7.30

7.40

7.50

7.60

7.70

7.80

7.90

8.00

8.10

8.20

8.30

8.40

8.50

8.60

8.70

8.80

8.90

9.00

9.10

9.20

9.30

9.40

9.50

9.60

9.70

9.80

9.90

10.00

10.10

10.20

10.30

10.40

10.50

10.60

10.70

10.80

10.90

11.00

11.10

11.20

11.30

11.40

11.50

11.60

11.70

11.80

11.90

12.00

$6.44058 \mathrm{E}-0.5$

$6.60477 \mathrm{E}-0.5$

$6.77102 \mathrm{E}-05$

$6.93933 \mathrm{E}-05$

$7.10971 \mathrm{E}-0.5$

$7.28215 \mathrm{E}-0.5$

$7.45665 \mathrm{E}-0.5$

$7.63322 \mathrm{E}-05$

$7.81185 \mathrm{E}-05$

$7.99254 \mathrm{E}-0.5$

$8.17530 \mathrm{E}-0.5$

$8.36012 \mathrm{E}-05$

$8.54700 \mathrm{E}-05$

$8.73594 \mathrm{E}-0.5$

$8.92695 \mathrm{E}-0.5$

$9.12002 \mathrm{E}-0.5$

$9.31515 \mathrm{E}-05$

$9.51236 \mathrm{E}-0.5$

$9.71161 \mathrm{E}-0.5$

$9.91294 \mathrm{E}-05$

$1.01163 \mathrm{E}-04$

$1.03218 \mathrm{E}-04$

$1.05293 \mathrm{E}-04$

$1.07389 \mathrm{E}-04$

$1.09505 \mathrm{E}-04$

$1.11642 \mathrm{E}-04$

$1.13800 \mathrm{E}-04$

$1.15978 \mathrm{E}-04$

$1.18177 \mathrm{E}-04$

$1.20396 \mathrm{E}-04$

$1.22636 \mathrm{E}-04$

$1.24897 \mathrm{E}-04$

$1.27179 \mathrm{E}-04$

$1.29481 \mathrm{E}-04$

$1.31803 \mathrm{E}-04$

$1.34146 \mathrm{E}-04$

$1.36510 \mathrm{E}-04$

$1.38895 \mathrm{E}-04$

$1.41300 \mathrm{E}-04$

$1.43726 \mathrm{E}-04$

$1.46172 \mathrm{E}-04$

$1.48639 \mathrm{E}-04$ $f_{2}$

$6.92087 \mathrm{E}-02$

$7.16041 \mathrm{E}-02$

$7.40395 \mathrm{E}-02$

$7.65146 \mathrm{E}-02$

$7.90290 \mathrm{E}-02$

$8.15823 \mathrm{E}-02$

$8.41731 \mathrm{E}-02$

$8.68004 \mathrm{E}-02$

$8.94603 \mathrm{E}-02$

$9.21463 \mathrm{E}-02$

$9.48381 \mathrm{E}-02$

$9.74508 \mathrm{E}-02$

$9.85156 \mathrm{E}-02$

$1.04336 \mathrm{E}-01$

$1.06858 \mathrm{E}-01$

$1.09715 \mathrm{E}-01$

$1.12673 \mathrm{E}-01$

$1.15693 \mathrm{E}-01$

$1.18765 \mathrm{E}-01$

$1.21883 \mathrm{E}-01$

$1.25045 \mathrm{E}-01$

$1.28251 \mathrm{E}-01$

$1.31500 \mathrm{E}-01$

$1.34789 \mathrm{E}-01$

$1.38121 \mathrm{E}-01$

$1.41494 \mathrm{E}-01$

$1.44908 \mathrm{E}-01$

$1.48363 \mathrm{E}-0 \mathrm{l}$

$1.51859 \mathrm{E}-01$

$1.55396 \mathrm{E}-01$

$1.58974 \mathrm{E}-0 \mathrm{l}$

$1.66253 \mathrm{E}-01$

$1.69954 \mathrm{E}-0 \mathrm{I}$

$1.73695 \mathrm{E}-01$

$1.77478 \mathrm{E}-0 \mathrm{l}$

$1.81301 \mathrm{E}-01$

$1.89069 \mathrm{E}-01$

$1.93014 \mathrm{E}-01$

$1.97001 \mathrm{E}-01$

$2.01027 \mathrm{E}-01$

$2.05095 \mathrm{E}-01$

$2.09203 \mathrm{E}-0]$

$2.13352 \mathrm{E}-01$

$2.17542 \mathrm{E}-01$

$2.21772 \mathrm{E}-01$

$2.26043 \mathrm{E}-01$

$2.30355 \mathrm{E}-01$

$2.34708 \mathrm{E}-01$

$2.39101 \mathrm{E}-01$

$2.48010 \mathrm{E}-01$

$2.57081 \mathrm{E}-01$

$2.61678 \mathrm{E}-01$

$2.66315 \mathrm{E}-01$

$2.70993 \mathrm{E}-01$

$2.75712 \mathrm{E}-01$

$2.80471 \mathrm{E}-01$ $g_{2}$

$\tan \delta_{2}$

$f^{\prime}: 2$

$-4.43523 \mathrm{E}-0.5$ $-3.66689 \mathrm{E}-0.5$ $-2.83802 \mathrm{E}-05$

$-1.94704 \mathrm{E}-0.5$

$-9.92399 \mathrm{E}-06$

$1.24551 \mathrm{E}-04$

$1.62593 \mathrm{E}-01$

$1.85164 \mathrm{E}-01$

$2.43535 \mathrm{E}-01$

$2.52525 \mathrm{E}-01$
$2.74479 \mathrm{E}-07$

$1.11400 \mathrm{E}-0.5$

$2.26871 \mathrm{E}-0.5$

$3.49284 \mathrm{E}-05$

$4.78735 \mathrm{E}-05$

$6.15193 \mathrm{E}-05$

$7.58023 \mathrm{E}-0.5$

$8.93597 \mathrm{E}-05$

$1.08123 \mathrm{E}-04$

$1.42068 \mathrm{E}-04$

$1.60470 \mathrm{E}-04$

$1.79737 \mathrm{E}-04$.

$1.99876 \mathrm{E}-04$

$2.20897 \mathrm{E}-04$.

$\begin{array}{ll}-3.08595 \mathrm{E} & 01\end{array}$

$-3.08991 \mathrm{E} 01$

$-3.09373 \mathrm{E} \quad 01$

$-3.09742 \mathrm{E} \quad 01$

$-3.10100 \mathrm{E} \quad 01$

$-3.10445 \mathrm{E} \quad 01$

$-3.10780 \mathrm{E} 01$

$-3.11104 \mathrm{E} 01$

$-3.11419 \mathrm{E}$ ol

$-3.11723 \mathrm{E} 01$

$-3.12019 \mathrm{E} \quad 01$

$-3.12305 \mathrm{E}$ 0I

$-3.12584 \mathrm{E} \quad 01$

$-3.12854 \mathrm{E} \quad 01$

$-3.13117 \mathrm{E}$ 0l

$-3.13372 \mathrm{E} \quad 01$

$-3.13620 \mathrm{E}$ 0I

$-3.13862 \mathrm{E}$ ol

$-3.14097 \mathrm{E} \quad 01$

$-3.14325 \mathrm{E} \quad 01$

$2.42814 \mathrm{E}-04$

$2.65643 \mathrm{E}-04$

$2.89398 \mathrm{E}-04$

$3.14093 \mathrm{E}-04$

$3.39748 \mathrm{E}-04$

$3.66374 \mathrm{E}-04$

$3.93990 \mathrm{E}-04$.

$4.22611 \mathrm{E}-04$

$4.52252 \mathrm{E}-04$

$4.82929 \mathrm{E}-04$

$5.14659 \mathrm{E}-04$

$5.47457 \mathrm{E}-04$

$5.81339 \mathrm{E}-04$

$6.16321 \mathrm{E}-04$

$6.52419 \mathrm{E}-04$

$6.89649 \mathrm{E}-04$

$7.28027 \mathrm{E}-04$

$7.67568 \mathrm{E}-04$

$8.08289 \mathrm{E}-04$

$8.50205 \mathrm{E}-04$

$9.37688 \mathrm{E}-04$

$9.83286 \mathrm{E}-04$

$1.03014 \mathrm{E}-03$

$1.07828 \mathrm{E}-03$

$1.12770 \mathrm{E}-03$

$1.17843 \mathrm{E}-03$

$1.23048 \mathrm{E}-03$

$1.28388 \mathrm{E}-03$

$1.33862 \mathrm{E}-03$

$1.39474 \mathrm{E}-03$

$1.45224 \mathrm{E}-03$

$1.5111 .5 \mathrm{E}-03$

$1.57147 \mathrm{E}-03$

$1.63323 \mathrm{E}-03$

$1.69644 \mathrm{E}-03$

$1.76111 \mathrm{E}-03$

$1.82727 \mathrm{E}-03$

$1.89492 \mathrm{E}-03$

$1.96409 \mathrm{E}-03$

$-3.14548 \mathrm{E} \quad 0 \mathrm{l}$

$-3.14765 \mathrm{E} 01$

$-3.14976 \mathrm{E} \quad 01$

$-3.15182 \mathrm{E} \quad 01$

$-3.15383 \mathrm{E} 01$

$-3.15579 \mathrm{E} \quad 0 \mathrm{l}$

$-3.15770 \mathrm{E}$ 0I

$-3.15956 \mathrm{E}$ 0I

$-3.16316 \mathrm{E} \quad 01$

$-3.16489 \mathrm{E} \quad 01$

$-3.16659 \mathrm{E} 0 \mathrm{I}$

$-3.16824 \mathrm{E} \quad 01$

$-3.16986 \mathrm{E} \quad 01$

$-3.17145 \mathrm{E} 01$

$-3.17300 \mathrm{E} 01$

$-3.17451 \mathrm{E} \quad 0 \mathrm{I}$

$-3.17599 \mathrm{E} \quad 01$

$-3.17744 \mathrm{E} \quad 01$

$-3.17886 \mathrm{E} 0 \mathrm{l}$

$-3.18025 \mathrm{E} 01$

$-3.18161 \mathrm{E} \quad 01$

$-3.18294 \mathrm{E} \quad 01$

$-3.18424 \mathrm{E} \quad 01$

$\begin{array}{lll}-3.18678 \mathrm{E} & 01\end{array}$

$-3.18800 \mathrm{E} \quad 01$

$-3.18921 \mathrm{E} \quad 01$

$-3.19039 \mathrm{E} 0 \mathrm{l}$

$\begin{array}{lll}-3.19268 \mathrm{E} & 01\end{array}$

$-3.19380 \mathrm{E} 01$

$-3.19489 \mathrm{E} 0 \mathrm{~J}$

$-3.19597 \mathrm{E} 01$

$-3.19806 \mathrm{E} 0 \mathrm{l}$

$\begin{array}{ll}-3.20007 \mathrm{E} & 01\end{array}$

$-3.20105 \mathrm{E} 01$
$8.93334 \mathrm{E}-04$

$-3.16138 \mathrm{E} 01$

$-3.18552 \mathrm{E} 01$

$-3.19155 \mathrm{E} 01$

$-3.19702 \mathrm{E} \quad 01$

$-3.19907 \mathrm{E} \quad 01$

$-3.20201 \mathrm{E} 01$ 
$p \quad F_{0}$

12.10

12.20

12.30

12.40

12.50

12.60

12.70

12.80

12.90

13.00

13.10

13.20

13.30

13.40

13.50

13.60

13.70

13.80

13.90

14.00

14.10

14.20

14.30

14.40

14.50

14.60

14.70

14.80

14.90

15.00

15.10

15.20

15.30

15.40

15.50

15.60

15.70

15.80

15.90

16.00

16.10

16.20

16.30

16.40

16.50

16.60

16.70

16.80

16.90

17.00

17.10

17.20

17.30

17.40

17.50

17.60

17.70

17.80

17.90

18.00 $f_{1}$

$8.75455 \mathrm{E}-01$

$8.75448 \mathrm{E}-01$

$8.75442 \mathrm{E}-01$

$8.75435 \mathrm{E}-01$

$8.75428 \mathrm{E}-01$

$8.75421 \mathrm{E}-01$

$8.75413 \mathrm{E}-01$

$8.75406 \mathrm{E}-01$

$8.75399 \mathrm{E}-01$

$8.75392 \mathrm{E}-01$

$8.75385 \mathrm{E}-01$

$8.75377 \mathrm{E}-01$

$8.75370 \mathrm{E}-0 \mathrm{I}$

$8.75363 \mathrm{E}-01$

$8.75356 \mathrm{E}-01$

$8.75349 \mathrm{E}-01$

8.75341 E-01

$8.75334 \mathrm{E}-0 \mathrm{I}$

$8.75326 \mathrm{E}-01$

$8.75319 \mathrm{E}-01$

$8.75312 \mathrm{E}-01$

$8.75304 \mathrm{E}-01$

$8.75297 \mathrm{E}-01$

$8.75289 \mathrm{E}-01$

$8.75282 \mathrm{E}-01$

$8.75274 \mathrm{E}-01$

$8.75267 \mathrm{E}-01$

$8.75259 \mathrm{E}-01$

$8.75252 \mathrm{E}-01$

$8.75244 \mathrm{E}-01$

$8.75237 \mathrm{E}-01$

$8.75229 \mathrm{E}-01$

$8.75222 \mathrm{E}-01$

$8.75214 \mathrm{E}-01$

$8.75207 \mathrm{E}-01$

$8.75199 \mathrm{E}-0 \mathrm{r}$

$8.75192 \mathrm{E}-01$

$8.75185 \mathrm{E}-01$

$8.75177 \mathrm{E}-01$

$8.75170 \mathrm{E}-01$

$8.75162 \mathrm{E}-01$

$8.75155 \mathrm{E}-01$

$8.75148 \mathrm{E}-01$

$8.75140 \mathrm{E}-01$

$8.75133 \mathrm{E}-01$

$8.75125 \mathrm{E}-01$

$8.75118 \mathrm{E}-01$

$8.75111 \mathrm{E}-01$

$8.75103 \mathrm{E}-01$

$8.75096 \mathrm{E}-01$

$8.75089 \mathrm{E}-01$

$8.75081 \mathrm{E}-01$

$8.75074 \mathrm{E}-01$

$8.75067 \mathrm{E}-01$

$8.75060 \mathrm{E}-01$

8.75052E-01

$8.75045 \mathrm{E}-01$

$8.75038 \mathrm{E}-01$

$8.75031 \mathrm{E}-01$

$8.75023 \mathrm{E}-01$ $g_{1}$

$1.08911 \mathrm{E} \quad 01$ $1.09856 \mathrm{E} \quad 01$

$1.10801 \mathrm{E} \quad 01$

$1.11747 \mathrm{E} \quad 01$

$1.12692 \mathrm{E} \quad 01$

$1.13637 \mathrm{E} \quad 01$

$1.14583 \mathrm{E} \quad 01$

$1.15528 \mathrm{E} \quad 01$

$1.16474 \mathrm{E} \quad 01$

$1.17420 \mathrm{E} \quad 01$

$1.18366 \mathrm{E} \quad 01$

$1.19312 \mathrm{E} \quad 01$

$1.20258 \mathrm{E} \quad 01$

$1.21204 \mathrm{E} \quad 01$

$1.22150 \mathrm{E} \quad 01$

$1.23097 \mathrm{E} \quad 01$

$1.24043 \mathrm{E} \quad 01$

$1.24990 \mathrm{E} \quad 01$

$1.25937 \mathrm{E}$ 01

$1.26884 \mathrm{E} \quad 0$

$1.27831 \mathrm{E} \quad 01$

$1.28778 \mathrm{E} \quad 01$

$1.29725 \mathrm{E} \quad 01$

$1.30673 \mathrm{E}$ ol

$1.31620 \mathrm{E} \quad 01$

$1.32568 \mathrm{E}$ 01

$1.33516 \mathrm{E} \quad 01$

$1.34463 \mathrm{E} \quad 01$

$1.35411 \mathrm{E} \quad 01$

$1.36359 \mathrm{E} \quad 01$

$1.37308 \mathrm{E} \quad 01$

$1.38256 \mathrm{E} \quad 01$

$1.39205 \mathrm{E} \quad 01$

$1.40153 \mathrm{E} \quad 0$

$1.41102 \mathrm{E} \quad 01$

$1.42051 \mathrm{E} \quad 01$

$1.43000 \mathrm{E} \quad 01$

$1.43949 \mathrm{E} \quad 01$

$1.44898 \mathrm{E} \quad 01$

$1.4584 .7 \mathrm{E} \quad 01$

$1.46797 \mathrm{E} \quad 01$

$1.47746 \mathrm{E} \quad 01$ $1.48696 \mathrm{E} \quad 01$

$1.49646 \mathrm{E} \quad 01$

$1.50596 \mathrm{E} \quad 01$

$\begin{array}{ll}1.51546 \mathrm{E} & 01\end{array}$

$1.52496 \mathrm{E} \quad 01$

$1.53447 \mathrm{E} \quad 01$

$1.54397 \mathrm{E} \quad 01$

$1.55348 \mathrm{E} \quad 0$

$1.56299 \mathrm{E} \quad 01$ $1.57250 \mathrm{E} \quad 01$

$1.58201 \mathrm{E} \quad 01$

$1.59152 \mathrm{E} \quad 01$

$1.60103 \mathrm{E} 0 \mathrm{I}$

$1.61055 \mathrm{E} \quad 01$

$1.62006 \mathrm{E} \quad 01$

I.62958 E 01

$1.63910 \mathrm{E} \quad 01$

$1.64862 \mathrm{E} \quad 01$ $\tan \delta_{1}$

$f_{-1}$

$1.20394 \mathrm{E}-01 \quad-8.15029 \mathrm{E}-05$

$1.23819 \mathrm{E}-01$

$1.27285 \mathrm{E}-01$

$1.30792 \mathrm{E}-01$

$1.34341 \mathrm{E}-01$

$-9.57596 \mathrm{E}-05$

$-1.09779 \mathrm{E}-04$

$-1.23569 \mathrm{E}-04$

$-1.37130 \mathrm{E}-04$

$1.37931 \mathrm{E}-01$

$1.41562 \mathrm{E}-01$

$1.45235 \mathrm{E}-01$

$1.48949 \mathrm{E}-01$

$1.52704 \mathrm{E}-01$

$1.56500 \mathrm{E}-01$

$1.60338 \mathrm{E}-01$

$1.64216 \mathrm{E}-01$

$1.68137 \mathrm{E}-01$

$1.72098 \mathrm{E}-01$

$1.76101 \mathrm{E}-01$

$1.80145 \mathrm{E}-01$

$1.84231 \mathrm{E}-01$

$1.88358 \mathrm{E}-01$

$1.92526 \mathrm{E}-01$

$1.96736 \mathrm{E}-01$

$2.00987 \mathrm{E}-01$

$2.05280 \mathrm{E}-01$

$2.09614 \mathrm{E}-01$

$2.13989 \mathrm{E}-01$

$2.18406 \mathrm{E}-01$

$2.22864 \mathrm{E}-01$

$2.27364 \mathrm{E}-01$

$2.31905 \mathrm{E}-01$

$2.36487 \mathrm{E}-01$

$2.41111 \mathrm{E}-01$

$2.45777 \mathrm{E}-01$

$2.50484 \mathrm{E}-01$

$2.55233 \mathrm{E}-01$

$2.60023 \mathrm{E}-01$

$2.64855 \mathrm{E}-01$

$2.69728 \mathrm{E}-01$

$2.74643 \mathrm{E}-01$

$2.79599 \mathrm{E}-01$

$2.84597 \mathrm{E}-01$

$2.89636 \mathrm{E}-01$

$2.94718 \mathrm{E}-01$

$2.99840 \mathrm{E}-01$

$3.05005 \mathrm{E}-01$

$3.10211 \mathrm{E}-01$

$3.15458 \mathrm{E}-01$

$3.20748 \mathrm{E}-01$

$3.26079 \mathrm{E}-01$

$3.31451 \mathrm{E}-01$

$3.36866 \mathrm{E}-01$

$3.42322 \mathrm{E}-01$

$3.47819 \mathrm{E}-01$

$3.53359 \mathrm{E}-01$

$3.58940 \mathrm{E}-01$

$3.64563 \mathrm{E}-01$

$3.70228 \mathrm{E}-01$

$3.75935 \mathrm{E}-01$

$3.81683 \mathrm{E}-01$

$3.87473 \mathrm{E}-01$

$3.93305 \mathrm{E}-01$

$-1.50466 \mathrm{E}-04$

$-1.63580 \mathrm{E}-04$

$-1.76478 \mathrm{E}-04$

$-1.89185 \mathrm{E}-04$

$-2.01668 \mathrm{E}-04$

$-2.13961 \mathrm{E}-04$

$-2.26062 \mathrm{E}-04$

$-2.37973 \mathrm{E}-04$

$-2.49694 \mathrm{E}-04$

$-2.61229 \mathrm{E}-04$

$-2.72588 \mathrm{E}-04$

$-2.83779 \mathrm{E}-04$

$-2.94800 \mathrm{E}-04$

$-3.05641 \mathrm{E}-04$

$-3.16329 \mathrm{E}-04$

$-3.26855 \mathrm{E}-04$

$-3.37221 \mathrm{E}-04$

$-3.47445 \mathrm{E}-04$

$-3.57504 \mathrm{E}-04$

$-3.67417 \mathrm{E}-04$

$-3.77191 \mathrm{E}-04$

$-3.86823 \mathrm{E}-04$

$-3.96314 \mathrm{E}-04$

$-4.05663 \mathrm{E}-04$

$-4.14889 \mathrm{E}-04$

$-4.23973 \mathrm{E}-04$

$-4.32939 \mathrm{E}-04$

$-4.41775 \mathrm{E}-04$

$-4.50485 \mathrm{E}-04$

$-4.59073 \mathrm{E}-04$

$-4.67549 \mathrm{E}-04$

$-4.75897 \mathrm{E}-04$

$-4.84146 \mathrm{E}-04$

$-4.92270 \mathrm{E}-04$

$-5.00282 \mathrm{E}-04$

$-5.08196 \mathrm{E}-04$

$-5.15990 \mathrm{E}-04$

$-5.23691 \mathrm{E}-04$ 
ELECTRON RADIAL FUNCTIONS

$Z=6 \quad A=12 \quad \rho=1.1 A^{1 / 3} F$

Prositron

p $\quad \bar{F}$

12.10

12.20

12.30

12.40

12.50

12.60

12.70

12.80

12.90

13.00

13.10

13.20

13.30

13.40

13.50

13.60

13.70

13.80

13.90

14.00

14.10

14.20

14.30

14.40

14.50

14.60

14.70

14.80

14.90

15.00

15.10

15.20

15.30

I5. 40

15.50

15.60

15.70

15.80

15.90

16.00

16.10

16.20

16.30

16.40

16.50

16.60

16.70

16.80

16.90

17.00

17.10

17.20

17.30

17.40

17.50

17.60

17.70

17.80

17.90

18.00
$1.51127 \mathrm{E}-04$

$1.53635 \mathrm{E}-04$

$1.56164 \mathrm{E}-04$

$1.58714 \mathrm{E}-04$

$1.6 \mathrm{I} 284 \mathrm{E}-04$

$1.63875 \mathrm{E}-04$

$1.66486 \mathrm{E}-04$

$1.69118 \mathrm{E}-04$

$1.71771 \mathrm{E}-04$

$1.74444 \mathrm{E}-04$

$1.77138 \mathrm{E}-04$

$1.79852 \mathrm{E}-04$

$1.82587 \mathrm{E}-04$

$1.85343 \mathrm{E}-04$

$1.88120 \mathrm{E}-04$

$1.90917 \mathrm{E}-04$

$1.93734 \mathrm{E}-04$

$1.96572 \mathrm{E}-04$

$1.99431 \mathrm{E}-04$

$2.02311 \mathrm{E}-04$

$2.05211 \mathrm{E}-04$

$2.08132 \mathrm{E}-04$

$2.11073 \mathrm{E}-04$

$2.14035 \mathrm{E}-04$

$2.17017 \mathrm{E}-04$

2.20021 E-04

2.23045 E-04

$2.26089 \mathrm{E}-04$

$2.29154 \mathrm{E}-04$

$2.32240 \mathrm{E}-04$

2.35346 E- 04

$2.38473 \mathrm{E}-04$

$2.41620 \mathrm{E}-04$

$2.44789 \mathrm{E}-04$

$2.47977 \mathrm{E}-04$

$2.51187 \mathrm{E}-04$

$2.54417 \mathrm{E}-04$

$2.57668 \mathrm{E}-04$

$2.60939 \mathrm{E}-04$

$2.64231 \mathrm{E}-04$

$2.67543 \mathrm{E}-04$

$2.70876 \mathrm{E}-04$

$2.74230 \mathrm{E}-04$

$2.77604 \mathrm{E}-04$

$2.80999 \mathrm{E}-04$

$2.84415 \mathrm{E}-04$

2.87851 E- 04

$2.91308 \mathrm{E}-04$

$2.94786 \mathrm{E}-04$

$2.98284 \mathrm{E}-04$

$3.01802 \mathrm{E}-04$

$3.05342 \mathrm{E}-04$

$3.08902 \mathrm{E}-04$

$3.12482 \mathrm{E}-04$

$3.16083 \mathrm{E}-04$

$3.19705 \mathrm{E}-04$

$3.23347 \mathrm{E}-04$

$3.27010 \mathrm{E}-04$

$3.30694 \mathrm{E}-04$

$3.34398 \mathrm{E}-04$ $f_{2}$

$g_{2}$

$\tan \delta_{2}$

$f_{-2}$

$2.85271 \mathrm{E}-01$

$2.90112 \mathrm{E}-0$

$2.94994 \mathrm{E}-01$

$2.99916 \mathrm{E}-01$

$3.04878 \mathrm{E}-01$

$3.09882 \mathrm{E}-01$

$3.14926 \mathrm{E}-01$

$3.20011 \mathrm{E}-01$

$3.25136 \mathrm{E}-01$

$3.30302 \mathrm{E}-01$

$3.35509 \mathrm{E}-01$

$3.40756 \mathrm{E}-01$

$3.46044 \mathrm{E}-01$

$3.51373 \mathrm{E}-01$

$3.56742 \mathrm{E}-01$

$3.62153 \mathrm{E}-01$

$3.67603 \mathrm{E}-01$

$3.73094 \mathrm{E}-0$

$3.78626 \mathrm{E}-01$

$3.84199 \mathrm{E}-0 \mathrm{I}$

$3.89812 \mathrm{E}-01$

$3.95466 \mathrm{E}-0$

$4.01160 \mathrm{E}-01$

$4.06896 \mathrm{E}-01$

$4.12672 \mathrm{E}-01$

$4.18488 \mathrm{E}-01$

$4.24345 \mathrm{E}-01$

$4.30243 \mathrm{E}-01$

$4.36181 \mathrm{E}-01$

$4.42160 \mathrm{E}-01$

$4.48179 \mathrm{E}-01$

$4.54240 \mathrm{E}-01$

$4.60340 \mathrm{E}-01$

$4.66482 \mathrm{E}-01$

$4.72664 \mathrm{E}-01$

$4.78887 \mathrm{E}-01$

$4.85150 \mathrm{E}-01$

$4.91454 \mathrm{E}-01$

$4.97798 \mathrm{E}-01$

$5.04183 \mathrm{E}-01$

$5.10609 \mathrm{E}-01$

$5.17075 \mathrm{E}-01$

$5.23582 \mathrm{E}-01$

$5.30130 \mathrm{E}-01$

$5.36718 \mathrm{E}-01$

$5.43346 \mathrm{E}-01$

$5.50016 \mathrm{E}-01$

$5.56726 \mathrm{E}-01$

$5.63476 \mathrm{E}-01$

$5.70267 \mathrm{E}-01$

$5.77099 \mathrm{E}-0 \mathrm{I}$

5.83971 E-01

$5.90884 \mathrm{E}-01$

$5.97838 \mathrm{E}-0 \mathrm{I}$

$6.04832 \mathrm{E}-01$

$6.11866 \mathrm{E}-01$

$6.18941 \mathrm{E}-01$

$6.26057 \mathrm{E}-01$

$6.33213 \mathrm{E}-01$

$6.40410 \mathrm{E}-01$
$2.03479 \mathrm{E}-03$ $2.10704 \mathrm{E}-03$

$2.18084 \mathrm{E}-03$

$2.25622 \mathrm{E}-03$

$2.33320 \mathrm{E}-03$

$2.41178 \mathrm{E}-03$

$2.49199 \mathrm{E}-03$

$2.57385 \mathrm{E}-03$

$2.65736 \mathrm{E}-03$

$2.74254 \mathrm{E}-03$

2.82941 E- 03

$2.91798 \mathrm{E}-03$

$3.00828 \mathrm{E}-03$

$3.10031 \mathrm{E}-03$

$3.19409 \mathrm{E}-03$

$3.28965 \mathrm{E}-03$

$3.38698 \mathrm{E}-03$

$3.48612 \mathrm{E}-03$

$3.58707 \mathrm{E}-03$

$3.68986 \mathrm{E}-03$

$3.79449 \mathrm{E}-03$

$3.90099 \mathrm{E}-03$

$4.00936 \mathrm{E}-03$

$4.11964 \mathrm{E}-03$

$4.23182 \mathrm{E}-03$

$4.34593 \mathrm{E}-03$

$4.46199 \mathrm{E}-03$

$4.58000 \mathrm{E}-03$

$4.69999 \mathrm{E}-03$

$4.82197 \mathrm{E}-03$

$4.94595 \mathrm{E}-03$

$5.07196 \mathrm{E}-03$

$5.20001 \mathrm{E}-03$

$5.33011 \mathrm{E}-03$

$5.46228 \mathrm{E}-03$

$5.59654 \mathrm{E}-03$

$5.7329 \mathrm{IE}-03$

$5.87139 \mathrm{E}-03$

$6.01200 \mathrm{E}-03$

$6.15476 \mathrm{E}-03$

$6.29969 \mathrm{E}-03$

$6.44680 \mathrm{E}-03$

$6.59611 \mathrm{E}-03$

$6.74763 \mathrm{E}-03$

$6.90138 \mathrm{E}-03$

$7.05738 \mathrm{E}-03$

$7.21564 \mathrm{E}-03$

$7.37617 \mathrm{E}-03$

$7.53900 \mathrm{E}-03$

$7.70414 \mathrm{E}-03$

$7.87160 \mathrm{E}-03$ $8.04140 \mathrm{E}-03$

$8.21356 \mathrm{E}-03$

$8.38809 \mathrm{E}-03$

$8.56501 \mathrm{E}-03$

8.74433 E-03

$8.92608 \mathrm{E}-03$

$9.11025 \mathrm{E}-03$

$9.29689 \mathrm{E}-03$

$9.48598 \mathrm{E}-03$
$-3.20296 \mathrm{E} \quad 01$

$-3.20389 \mathrm{E} 01$

$-3.20480 \mathrm{E} 01$

$-3.20570 \mathrm{E} 01$

$-3.20658 \mathrm{E} 01$

$-3.20745 \mathrm{E} 01$

$-3.20831 \mathrm{E} 01$

$-3.20915 \mathrm{E} 01$

$-3.20997 \mathrm{E} 01$

$-3.21079 \mathrm{E} \quad 01$

$-3.21159 \mathrm{E} \quad 01$

$-3.21237 \mathrm{E}$ 01

$-3.21315 \mathrm{E} 01$

$-3.21391 \mathrm{E} 01$

$-3.21466 \mathrm{E} 01$

$-3.21540 \mathrm{E}$ 0I

$-3.21613 \mathrm{E}$ 0I

$-3.21684 \mathrm{E} 01$

$-3.21755 \mathrm{E} 01$

$-3.21824 \mathrm{E} \quad 01$

$-3.21893 \mathrm{E}$ 01

$-3.21960 \mathrm{E} 01$

$-3.22027 \mathrm{E}$ 0I

$-3.22092 \mathrm{E}$ 0I

$-3.22157 \mathrm{E} 01$

$\begin{array}{lll}-3.22221 \mathrm{E} & 01\end{array}$

$-3.22283 \mathrm{E} 01$

$-3.22345 \mathrm{E} 01$

$-3.22406 \mathrm{E} 01$

$-3.22466 \mathrm{E} 01$

$-3.22526 \mathrm{E} 01$

$-3.22584 \mathrm{E} 01$

$-3.22642 \mathrm{E} 01$

$-3.22699 \mathrm{E} 01$

$-3.22755 \mathrm{E} 01$

$-3.22810 \mathrm{E}$ 0I

$-3.22865 \mathrm{E}$ 01

$-3.22919 \mathrm{E} 01$

$-3.22972 \mathrm{E} 01$

$-3.23024 \mathrm{E} 01$

$-3.23076 \mathrm{E} 01$

$-3.23127 \mathrm{E}$ 01 
ELECTRON RADIAL FUNCTIONS

Positron

$p$

18.10

18.20

18.30

18.40

18.50

18.60

18.70

18.80

18.90

19.00

19.10

19.20

19.30

19.40

19.50

19.60

19.70

19.80

19.90

20.00

20.10

20.20

20.30

20.40

20.50

20.60

20.70

20.80

20.90

21.00

21.10

21.20

21.30

21.40

21.50

21.60

21.70

21.80

21.90

22.00

22.10

22.20

22.30

22.40

22.50

22.60

22.70

22.80

22.90

23.00

23.10

23.20

23.30

23.40

23.50

23.60

23.70

23.80

23.90

24.00
$F_{0}$

$8.75016 \mathrm{E}-01$ $8.75009 \mathrm{E}-01$ $8.75002 \mathrm{E}-01$ $8.74995 \mathrm{E}-01$ $8.74988 \mathrm{E}-01$

$8.74981 \mathrm{E}-01$ 8.74974E-01 $8.74967 \mathrm{E}-01$ $8.74960 \mathrm{E}-01$ $8.74953 \mathrm{E}-01$

$8.74945 \mathrm{E}-01$ 8.74939E-01 $8.74932 \mathrm{E}-01$ $8.74925 \mathrm{E}-01$ $8.74918 \mathrm{E}-01$

$8.74911 \mathrm{E}-01$ $8.74904 \mathrm{E}-01$ $8.74898 \mathrm{E}-01$ $8.7489] \mathrm{E}-01$ $8.74884 \mathrm{E}-01$

$8.74877 \mathrm{E}-01$ $8.74870 \mathrm{E}-01$ $8.74863 \mathrm{E}-01$ $8.74857 \mathrm{E}-01$ $8.74850 \mathrm{E}-01$

$8.74843 \mathrm{E}-01$ $8.74837 \mathrm{E}-01$ $8.74830 \mathrm{E}-01$ $8.74823 \mathrm{E}-0$ $8.74817 \mathrm{E}-01$

$8.74810 \mathrm{E}-01$ $8.74803 \mathrm{E}-01$ $8.74797 \mathrm{E}-01$ $8.74790 \mathrm{E}-01$ 8.74784E-01

$8.74777 \mathrm{E}-01$ $8.74771 \mathrm{E}-01$ $8.74764 \mathrm{E}-01$ $8.74758 \mathrm{E}-01$ 8.74751 E-01

$8.74745 \mathrm{E}-01$ $8.74738 \mathrm{E}-01$ 8.74732 E-01 $8.74725 \mathrm{E}-01$ $8.74719 \mathrm{E}-01$

$8.74713 \mathrm{E}-01$ $8.74706 \mathrm{E}-01$ $8.74700 \mathrm{E}-01$ 8.74694 E-01 $8.74688 \mathrm{E}-01$

8.74681 E-01 $8.74675 \mathrm{E}-01$ $8.74669 \mathrm{E}-01$ $8.74663 \mathrm{E}-01$ $8.74656 \mathrm{E}-0 \mathrm{l}$

$8.74650 \mathrm{E}-0$ $8.74644 \mathrm{E}-0$ $8.74638 \mathrm{E}-0$ $8.74632 \mathrm{E}-01$ $8.74626 \mathrm{E}-01$

$Z=6$

$f_{1}$

$1.65814 \mathrm{E} \quad 01$ $1.66767 \mathrm{E} \quad 01$ $1.67719 \mathrm{E} \quad 01$

$1.68672 \mathrm{E} \quad 01$

$1.69625 \mathrm{E} \quad 01$

$1.70578 \mathrm{E} \quad 01$

$1.71531 \mathrm{E} \quad 01$

$1.72484 \mathrm{E} \quad 01$

$1.73437 \mathrm{E} \quad 01$

$1.74391 \mathrm{E} \quad 01$

$1.75344 \mathrm{E} \quad 01$

$1.76298 \mathrm{E} \quad 01$

$\begin{array}{ll}1.77252 \mathrm{E} & 01\end{array}$

$1.78206 \mathrm{E} 01$

$1.79161 \mathrm{E} \quad 01$

$1.80115 \mathrm{E} \quad 01$

$\begin{array}{lll}1.81069 \mathrm{E} & 01\end{array}$

$1.82024 \mathrm{E} \quad 01$

$1.82979 \mathrm{E} \quad 01$

$1.83934 \mathrm{E} \quad 01$

$1.84889 \mathrm{E} \quad 01$

$1.85845 \mathrm{E} \quad 01$

$1.86800 \mathrm{E} \quad 01$

$1.87756 \mathrm{E} 01$

$1.88712 \mathrm{E} \quad 01$

$1.89668 \mathrm{E} \quad 01$

$1.90624 \mathrm{E} \quad 01$

$1.91580 \mathrm{E} \quad 01$

$1.92536 \mathrm{E} \quad 01$

$1.93493 \mathrm{E} \quad 01$

$1.94450 \mathrm{E} \quad 01$

$1.95407 \mathrm{E} \quad 01$

$1.96364 \mathrm{E} \quad 01$

$1.97321 \mathrm{E} \quad 01$

$1.98279 \mathrm{E} \quad 01$

$1.99236 \mathrm{E} \quad 01$ $2.00194 \mathrm{E} \quad 01$

$2.01152 \mathrm{E} \quad 01$ $2.02110 \mathrm{E} \quad 01$ $2.03068 \mathrm{E} \quad 01$

$2.04027 \mathrm{E} \quad 01$ $2.04985 \mathrm{E} 01$ $2.05944 \mathrm{E} \quad 01$ $2.06903 \mathrm{E} \quad 01$

$2.07862 \mathrm{E} \quad 01$

$2.08821 \mathrm{E} \quad 01$ $2.09781 \mathrm{E} \quad 01$ $2.10741 \mathrm{E} 01$

$2.11700 \mathrm{E} \quad 01$

$2.12660 \mathrm{E} 01$

$2.13621 \mathrm{E} \quad 01$ $2.14581 \mathrm{E} 01$

$2.15541 \mathrm{E} 01$

$2.16502 \mathrm{E} \quad 01$

$2.17463 \mathrm{E} \quad 01$

2.18424E 01

$2.19385 \mathrm{E} \quad 01$

$2.20347 \mathrm{E} 01$

$2.21308 \mathrm{E} \quad 01$

$2.22270 \mathrm{E} 01$ $g_{1}$

$3.99179 \mathrm{E}-01$

$4.05095 \mathrm{E}-01$

$4.11052 \mathrm{E}-01$

$4.17052 \mathrm{E}-01$

$4.23093 \mathrm{E}-01$

$4.29176 \mathrm{E}-01$

$4.35301 \mathrm{E}-01$

$4.41468 \mathrm{E}-01$

4.47677 E-01

$4.53928 \mathrm{E}-01$

$4.60221 \mathrm{E}-01$

$4.66555 \mathrm{E}-01$

$4.72932 \mathrm{E}-01$

$4.79351 \mathrm{E}-01$

$4.85812 \mathrm{E}-01$

4.92314 E-01

$4.98859 \mathrm{E}-01$

$5.05446 \mathrm{E}-01$

$5.12075 \mathrm{E}-01$

$5.18746 \mathrm{E}-01$

$5.25460 \mathrm{E}-01$

$5.32215 \mathrm{E}-01$

$5.39012 \mathrm{E}-01$

$5.45852 \mathbf{E}-01$

$5.52733 \mathrm{E}-01$

$5.59657 \mathrm{E}-01$

$5.66623 \mathrm{E}-01$

$5.73631 \mathrm{E}-01$

$5.80682 \mathrm{E}-01$

$5.87775 \mathrm{E}-01$

$5.94909 \mathrm{E}-01$ $6.02086 \mathrm{E}-01$ $6.09306 \mathrm{E}-01$ $6.16568 \mathrm{E}-01$

$6.23872 \mathrm{E}-01$

$6.31218 \mathrm{E}-01$ $6.38607 \mathrm{E}-01$ $6.46038 \mathrm{E}-01$ $6.53511 \mathrm{E}-01$ $6.61027 \mathrm{E}-01$

$6.68585 \mathrm{E}-01$ $6.76185 \mathrm{E}-01$ $6.83828 \mathrm{E}-01$ $6.91514 \mathrm{E}-01$ $6.99242 \mathrm{E}-01$

$7.07012 \mathrm{E}-01$ $7.14825 \mathrm{E}-01$ $7.22680 \mathrm{E}-01$

$7.30578 \mathrm{E}-01$

$7.38518 \mathrm{E}-01$

$7.46501 \mathrm{E}-01$

$7.54527 \mathrm{E}-01$

$7.62595 \mathrm{E}-01$

$7.70706 \mathrm{E}-01$

$7.78859 \mathrm{E}-01$

$7.87055 \mathrm{E}-01$

$7.95294 \mathrm{E}-01$

$8.03575 \mathrm{E}-01$

$8.11899 \mathrm{E}-01$

$8.20265 \mathrm{E}-01$

$\tan \delta_{1}$

$f_{-1}$

$-6.46208 \mathrm{E}-04 \quad-3.45876 \mathrm{E}-01$

$-6.52223 \mathrm{E}-04 \quad-3.51588 \mathrm{E}-01$

$-6.58154 \mathrm{E}-04 \quad-3.57342 \mathrm{E}-01$

$-6.64017 \mathrm{E}-04 \quad-3.63138 \mathrm{E}-01$

$-6.69807 \mathrm{E}-04 \quad-3.68976 \mathrm{E}-01$

$-6.75525 \mathrm{E}-04$

$-6.81169 \mathrm{E}-04$

$-6.86753 \mathrm{E}-04$

$-6.92258 \mathrm{E}-04$

$-6.97702 \mathrm{E}-04$

$-7.03081 \mathrm{E}-04$

$-7.08389 \mathrm{E}-04$

$-7.13635 \mathrm{E}-04$

$-7.18820 \mathrm{E}-04$

$-7.23940 \mathrm{E}-04$

$-7.29003 \mathrm{E}-04$

$-7.33998 \mathrm{E}-04$

$-7.38933 \mathrm{E}-04$

$-7.43816 \mathrm{E}-04$

$-7.48633 \mathrm{E}-04$

$-7.53399 \mathrm{E}-04$

$-7.58105 \mathrm{E}-04$

$-7.62753 \mathrm{E}-04$

$-7.67358 \mathrm{E}-04$

$-7.71892 \mathrm{E}-04$

$-7.76377 \mathrm{E}-04$

$-7.80821 \mathrm{E}-04$

$-7.85199 \mathrm{E}-04$

$-7.89528 \mathrm{E}-04$

$-7.93814 \mathrm{E}-04$

$-7.98047 \mathrm{E}-04$

$-8.02222 \mathrm{E}-04$

$-8.06362 \mathrm{E}-04$

$-8.10441 \mathrm{E}-04$

$-8.14479 \mathrm{E}-04$

-8.18474 E- 04

$-8.22413 \mathrm{E}-04$

$-8.26310 \mathrm{E}-04$

$-8.30167 \mathrm{E}-04$ 
$\bar{F}$

$3.38123 \mathrm{E}-04$

$3.41869 \mathrm{E}-04$

$3.45635 \mathrm{E}-04$

$3.49422 \mathrm{E}-04$

$3.53229 \mathrm{E}-04$

$3.57057 \mathrm{E}-04$

$3.60906 \mathrm{E}-04$

$3.64775 \mathrm{E}-04$

$3.68664 \mathrm{E}-04$

$3.72575 \mathrm{E}-04$

$3.76506 \mathrm{E}-04$

$3.80458 \mathrm{E}-04$

$3.84430 \mathrm{E}-04$

$3.88423 \mathrm{E}-04$

$3.92436 \mathrm{E}-04$

$3.96470 \mathrm{E}-04$

$4.00525 \mathrm{E}-04$

$4.04600 \mathrm{E}-04$

$4.08696 \mathrm{E}-04$

$4.12813 \mathrm{E}-04$

$4.16950 \mathrm{E}-04$

$4.21108 \mathrm{E}-04$

$4.25286 \mathrm{E}-04$

$4.29485 \mathrm{E}-04$

$4.33705 \mathrm{E}-04$

$4.37945 \mathrm{E}-04$

$4.42206 \mathrm{E}-04$

$4.46488 \mathrm{E}-04$

$4.50790 \mathrm{E}-04$

$4.55112 \mathrm{E}-04$

$4.59456 \mathrm{E}-04$

$4.63820 \mathrm{E}-04$

4.68204 E-04

$4.72609 \mathrm{E}-04$

$4.77035 \mathrm{E}-04$

$4.81481 \mathrm{E}-04$

$4.85948 \mathrm{E}-04$

$4.90436 \mathrm{E}-04$

$4.94944 \mathrm{E}-04$

$4.99473 \mathrm{E}-04$

$5.04023 \mathrm{E}-04$

$5.08593 \mathrm{E}-04$

$5.13183 \mathrm{E}-04$

$5.17795 \mathrm{E}-04$

$5.22426 \mathrm{E}-04$

$5.27079 \mathrm{E}-04$

$5.31752 \mathrm{E}-04$

$5.36446 \mathrm{E}-04$

$5.41160 \mathrm{E}-04$

$5.45895 \mathrm{E}-04$

$5.50651 \mathrm{E}-04$ $5.55427 \mathrm{E}-04$

$5.60224 \mathrm{E}-04$

$5.65041 \mathrm{E}-04$

$5.69879 \mathrm{E}-04$

$5.74738 \mathrm{E}-04$

$5.79617 \mathrm{E}-04$

$5.84517 \mathrm{E}-04$

$5.89437 \mathrm{E}-04$

5.94378 E-04 $f_{2}$

$6.47648 \mathrm{E}-01$

$6.54926 \mathrm{E}-01$

$6.62245 \mathrm{E}-01$

$6.69604 \mathrm{E}-01$

$6.77003 \mathrm{E}-01$

$6.84444 \mathrm{E}-01$

$6.91925 \mathrm{E}-01$

$6.99446 \mathrm{E}-01$

$7.07008 \mathrm{E}-01$

$7.14610 \mathrm{E}-01$

7.22254 E-01

$7.29937 \mathrm{E}-0 \mathrm{l}$

$7.37661 \mathrm{E}-01$

$7.45425 \mathrm{E}-01$

$7.53231 \mathrm{E}-01$

7.61076E-01

$7.68963 \mathrm{E}-01$

$7.76890 \mathrm{E}-01$

$7.84857 \mathrm{E}-01$

$7.92864 \mathrm{E}-01$

$8.00913 \mathrm{E}-01$

$8.09002 \mathrm{E}-01$

$8.17131 \mathrm{E}-01$

$8.25301 \mathrm{E}-01$

8.33511 E-01

$8.41762 \mathrm{E}-01$

$8.50053 \mathrm{E}-01$

$8.58385 \mathrm{E}-01$

$8.66757 \mathrm{E}-01$

$8.75170 \mathrm{E}-01$

$8.83623 \mathrm{E}-01$

$8.92117 \mathrm{E}-01$

$9.00652 \mathrm{E}-01$

$9.09226 \mathrm{E}-01$

$9.17842 \mathrm{E}-01$

$9.26497 \mathrm{E}-01$ $9.35193 \mathrm{E}-01$

$9.43930 \mathrm{E}-01$

$9.52707 \mathrm{E}-01$

$9.61525 \mathrm{E}-01$

$9.70383 \mathrm{E}-01$ $9.79282 \mathrm{E}-01$

$9.88221 \mathrm{E}-01$

$9.97200 \mathrm{E}-01$

$1.00622 \mathrm{E} \quad 00$

$1.01528 \mathrm{E} \quad 00$

$1.02438 \mathrm{E} \quad 00$

$1.03352 \mathrm{E} \quad 00$

$1.04270 \mathrm{E} \quad 00$

$1.05193 \mathrm{E} \quad 00$

$1.06119 \mathrm{E} \quad 00$

$1.07049 \mathrm{E} \quad 00$

$1.07983 \mathrm{E} \quad 00$

$\begin{array}{ll}1.08922 \mathrm{E} & 00\end{array}$

$1.09864 \mathrm{E} \quad 00$

$1.10811 \mathrm{E} \quad 00$

$1.11761 \mathrm{E} \quad 00$

$1.12716 \mathrm{E} \quad 00$
0

$1.13674 \mathrm{E} \quad 00$

$1.14637 \mathrm{E} \quad 00$ $g_{2}$

$9.67758 \mathrm{E}-03$

$9.87166 \mathrm{E}-03$

$1.00683 \mathrm{E}-02$

$1.02674 \mathrm{E}-02$

$1.04691 \mathrm{E}-02$

$1.06733 \mathrm{E}-02$

$1.08801 \mathrm{E}-02$

$1.10895 \mathrm{E}-02$

$1.13015 \mathrm{E}-02$

$1.15162 \mathrm{E}-02$

$1.17335 \mathrm{E}-02$

$1.19534 \mathrm{E}-02$

$1.21761 \mathrm{E}-02$

$1.24014 \mathrm{E}-02$

$1.26294 \mathrm{E}-02$

$1.28601 \mathrm{E}-02$

$1.30936 \mathrm{E}-02$

$1.33298 \mathrm{E}-02$

$1.35688 \mathrm{E}-02$

$1.38106 \mathrm{E}-02$

$1.40552 \mathrm{E}-02$

$1.43026 \mathrm{E}-02$

$1.45528 \mathrm{E}-02$

$1.48059 \mathrm{E}-02$

$1.50618 \mathrm{E}-02$

$1.53207 \mathrm{E}-02$

$1.55824 \mathrm{E}-02$

$1.58470 \mathrm{E}-02$

$1.61146 \mathrm{E}-02$

$1.63851 \mathrm{E}-02$

$1.66586 \mathrm{E}-02$

$1.69350 \mathrm{E}-02$

$1.72144 \mathrm{E}-02$

$1.74969 \mathrm{E}-02$

$1.77823 \mathrm{E}-02$

$1.80708 \mathrm{E}-02$

$1.83624 \mathrm{E}-02$

$1.86570 \mathrm{E}-02$

$1.89547 \mathrm{E}-02$

$1.92555 \mathrm{E}-02$

$1.95594 \mathrm{E}-02$

$1.98665 \mathrm{E}-02$

$2.01767 \mathrm{E}-02$

$2.04901 \mathrm{E}-02$

$2.08066 \mathrm{E}-02$

$2.11264 \mathrm{E}-02$

$2.14494 \mathrm{E}-02$

$2.17756 \mathrm{E}-02$

$2.21050 \mathrm{E}-02$

$2.24377 \mathrm{E}-02$

$2.27737 \mathrm{E}-02$

$2.31129 \mathrm{E}-02$

$2.34555 \mathrm{E}-02$

$2.38014 \mathrm{E}-02$

$2.41506 \mathrm{E}-02$

$2.45032 \mathrm{E}-02$

$2.48592 \mathrm{E}-02$

$2.52185 \mathrm{E}-02$

$2.55813 \mathrm{E}-02$

$2.59475 \mathrm{E}-02$ $\tan \delta_{2}$

$f_{2}$

$-3.23987 \mathrm{E}$ 01 $-8.44104 \mathrm{E}-03$

$-3.24027 \mathrm{E} \quad 01$

$-3.24067 \mathrm{E}$ 01

$-3.24106 \mathrm{E} \quad 01$

$-3.24145 \mathrm{E} 01$

$-3.24183 \mathbf{E} 01$

$-3.24221 \mathrm{E} \quad 01$

$-3.24258 \mathrm{E} \quad 01$

$-3.24295 \mathrm{E} 01$

$-3.24332 \mathrm{E} \quad 01$

$-3.24368 \mathrm{E} \quad 01$

$-3.24404 \mathrm{E} 01$

$-3.24440 \mathrm{E} \quad 0 \mathrm{I}$

$-3.24475 \mathrm{E} 01$

$-3.24509 \mathrm{E} 01$

$\begin{array}{lll}-3.24544 \mathrm{E} & 01\end{array}$

$-3.24578 \mathrm{E} \quad 01$

$-3.24611 \mathrm{E} \quad 01$

$-3.24645 \mathrm{E}$ 01

$-3.24678 \mathrm{E} \quad 01$

$-3.24710 \mathrm{E} \quad 01$

$-3.24742 \mathrm{E} 01$

$-3.24774 \mathrm{E} \quad 01$

$-3.24806 \mathrm{E} 01$

$-3.24837 \mathrm{E} \quad 01$

$-3.24868 \mathrm{E} \quad 01$

$-3.24899 \mathrm{E}$ 01

$-3.24929 \mathrm{E} 01$

$-3.24959 \mathrm{E} 01$

$-3.24989 \mathrm{E} 01$

$-3.25018 \mathrm{E} \quad 01$

$-3.25048 \mathrm{E} \quad 01$

$-3.25076 \mathrm{E} 01$

$-3.25105 \mathrm{E} 01$

$-3.25133 \mathrm{E} 01$

$-3.25161 \mathrm{E} \quad 01$ $-3.25189 \mathrm{E} 01$ $-3.25217 \mathrm{E} 01$

$-3.25244 \mathrm{E} 01$

$-3.25271 \mathrm{E} 01$

$-3.25298 \mathrm{E}$ 0I $-3.25324 \mathrm{E} 01$ $-3.25350 \mathrm{E} 01$ $-3.25376 \mathrm{E}$ ol

$-3.25402 \mathrm{E} 01$

$-3.25428 \mathrm{E} 01$ $-3.25453 \mathrm{E} 01$ $-3.25478 \mathrm{E} \quad 01$ $-3.25503 \mathrm{E} 01$ $-3.25527 \mathrm{E} 01$

$-3.25552 \mathrm{E}$ 01 $-3.25576 \mathrm{E} 01$ $-3.25600 \mathrm{E} 01$ $-3.25624 \mathrm{E}$ 01 $-3.25647 \mathrm{E} \quad 01$

$-3.25671 \mathrm{E} 01$

$-3.25694 \mathrm{E} \quad 01$

$-3.25717 \mathrm{E}$ 01

$-3.25739 \mathrm{E} 01$

$-3.25762 \mathrm{E} 01$

$-8.62345 \mathrm{E}-03$

$-8.80833 \mathrm{E}-03$

$-8.99568 \mathrm{E}-03$

$-9.18553 \mathrm{E}-03$

$-9.37789 \mathrm{E}-03$

$-9.57277 \mathrm{E}-03$

$-9.77019 \mathrm{E}-03$

$-9.97017 \mathrm{E}-03$

$-1.01727 \mathrm{E}-02$

$-1.03779 \mathrm{E}-02$

$-1.05856 \mathrm{E}-02$

$-1.07960 \mathrm{E}-02$

$-1.10090 \mathrm{E}-02$

$-1.12247 \mathrm{E}-02$

$-1.14430 \mathrm{E}-02$

$-1.16640 \mathrm{E}-02$

$-1.18877 \mathrm{E}-02$

$-1.21141 \mathrm{E}-02$

$-1.23433 \mathrm{E}-02$

$-1.25752 \mathrm{E}-02$

$-1.28099 \mathrm{E}-02$

$-1.30473 \mathrm{E}-02$

$-1.32876 \mathrm{E}-02$

$-1.35307 \mathrm{E}-02$

$-1.37765 \mathrm{E}-02$

$-1.40253 \mathrm{E}-02$

$-1.42769 \mathrm{E}-02$

$-1.45313 \mathrm{E}-02$

$-1.47887 \mathrm{E}-02$

$-1.50489 \mathrm{E}-02$

$-1.53121 \mathrm{E}-02$

$-1.55782 \mathrm{E}-02$

$-1.58473 \mathrm{E}-02$

$-1.61194 \mathrm{E}-02$

$-1.63944 \mathrm{E}-02$

$-1.66724 \mathrm{E}-02$

$-1.69535 \mathrm{E}-02$

$-1.72376 \mathrm{E}-02$

$-1.75247 \mathrm{E}-02$

$-1.78149 \mathrm{E}-02$

$-1.81082 \mathrm{E}-02$

$-1.84045 \mathrm{E}-02$ 


\begin{tabular}{|c|c|c|c|c|c|c|c|}
\hline$p$ & $F_{0}$ & $f_{1}$ & & $g_{1}$ & $\tan \delta_{1}$ & $f_{-1}$ & $g_{-1}$ \\
\hline 24.10 & $8.74620 \mathrm{E}-01$ & $2.23232 \mathrm{E}$ & 01 & $8.28675 \mathrm{E}-01$ & $-9.04578 \mathrm{E}-04$ & $-7.63241 \mathrm{E}-01$ & $2.32904 \mathrm{E}$ \\
\hline 24.20 & $8.74614 \mathrm{E}-01$ & $2.24194 \mathrm{E}$ & 01 & $8.37127 \mathrm{E}-01$ & $-9.07530 E-04$ & $-7.71492 \mathrm{E}-01$ & $2.33869 \mathrm{E}$ \\
\hline 24.30 & $8.74608 \mathrm{E}-01$ & $2.25157 \mathrm{E}$ & 01 & $8.45622 \mathrm{E}-01$ & $-9.10453 \mathrm{E}-04$ & $-7.79786 \mathrm{E}-01$ & $2.34834 \mathrm{E}$ \\
\hline 24.40 & $8.74602 \mathrm{E}-01$ & $2.26119 \mathrm{E}$ & 01 & $8.54160 \mathrm{E}-01$ & $-9.13341 \mathrm{E}-04$ & $-7.88123 \mathrm{E}-01$ & $2.35799 \mathrm{E}$ \\
\hline 24.50 & $8.74596 \mathrm{E}-01$ & $2.27082 \mathrm{E}$ & 01 & $8.62740 \mathrm{E}-01$ & $-9.16193 \mathrm{E}-04$ & $-7.96503 \mathrm{E}-01$ & $2.36764 \mathrm{E}$ \\
\hline 24.60 & $8.74590 \mathrm{E}-01$ & $2.28045 \mathrm{E}$ & 01 & $8.71364 \mathrm{E}-01$ & $-9.19013 E-04$ & $-8.04926 \mathrm{E}-01$ & $2.37729 \mathrm{E}$ \\
\hline 24.70 & $8.74584 \mathrm{E}-01$ & $2.29008 \mathrm{E}$ & 01 & $8.80030 \mathrm{E}-01$ & $-9.21807 \mathrm{E}-04$ & $-8.13391 \mathrm{E}-01$ & $2.38695 \mathrm{E}$ \\
\hline 80 & $8.74578 \mathrm{E}-01$ & $2.29971 \mathrm{E}$ & 01 & $8.88738 \mathrm{E}-01$ & $-9.24557 \mathrm{E}-04$ & $-8.21900 \mathrm{E}-01$ & $2.39661 \mathrm{E}$ \\
\hline .90 & $8.74572 \mathrm{E}-01$ & $2.30935 \mathrm{E}$ & 01 & $8.97490 \mathrm{E}-01$ & $-9.27287 \mathrm{E}-04$ & $-8.30451 \mathrm{E}-01$ & $2.40627 \mathrm{E}$ \\
\hline .00 & $8.74566 \mathrm{E}-01$ & $2.31899 \mathrm{E}$ & 01 & $9.06285 \mathrm{E}-01$ & $-9.29980 \mathrm{E}-04$ & $-8.39045 \mathrm{E}-01$ & $2.41593 \mathrm{E}$ \\
\hline 25.10 & $8.74560 \mathrm{E}-01$ & $2.32862 \mathrm{E}$ & 01 & $9.15122 \mathrm{E}-01$ & $-9.32636 \mathrm{E}-04$ & $-8.47682 \mathrm{E}-01$ & $2.42560 \mathrm{E}$ \\
\hline .20 & $8.74554 \mathrm{E}-01$ & $2.33826 \mathrm{E}$ & 01 & $9.24003 \mathrm{E}-0]$ & $-9.35275 \mathrm{E}-04$ & $-8.56363 \mathrm{E}-01$ & $2.43526 \mathrm{E}$ \\
\hline 30 & $8.74548 \mathrm{E}-01$ & $2.34791 \mathrm{E}$ & 01 & $9.32926 \mathrm{E}-01$ & $-9.37874 \mathrm{E}-04$ & $-8.65086 \mathrm{E}-01$ & $2.44493 \mathrm{E}$ \\
\hline .40 & $8.74542 \mathrm{E}-01$ & $2.35755 \mathrm{E}$ & 01 & $9.41892 \mathrm{E}-01$ & $-9.40450 \mathrm{E}-04$ & $-8.73852 \mathrm{E}-01$ & $2.45460 \mathrm{E}$ \\
\hline .50 & $8.74537 \mathrm{E}-01$ & $2.36720 \mathrm{E}$ & 01 & $9.50902 \mathrm{E}-01$ & $-9.42991 \mathrm{E}-04$ & $-8.82661 \mathrm{E}-01$ & $2.46427 \mathrm{E}$ \\
\hline 25.60 & $8.74531 \mathrm{E}-01$ & $2.37684 \mathrm{E}$ & 01 & $9.59954 \mathrm{E}-01$ & $-9.45502 \mathrm{E}-04$ & $-8.91513 \mathrm{E}-01$ & $2.47395 \mathrm{E}$ \\
\hline .70 & $8.74525 \mathrm{E}-01$ & $2.38649 \mathrm{E}$ & 01 & $9.69049 \mathrm{E}-01$ & $-9.47986 \mathrm{E}-04$ & $-9.00408 \mathrm{E}-01$ & $2.48362 \mathrm{E}$ \\
\hline .80 & $8.74519 \mathrm{E}-01$ & $2.39615 \mathrm{E}$ & 01 & $9.78187 \mathrm{E}-01$ & $-9.50439 \mathrm{E}-04$ & $-9.09347 \mathrm{E}-01$ & $2.49330 \mathrm{E}$ \\
\hline .90 & $8.74513 \mathrm{E}-01$ & $2.40580 \mathrm{E}$ & 01 & $9.87369 \mathrm{E}-01$ & $-9.52866 \mathrm{E}-04$ & $-9.18328 \mathrm{E}-01$ & $2.50298 \mathrm{E}$ \\
\hline 26.00 & $8.74508 \mathrm{E}-01$ & $2.41546 \mathrm{E}$ & 01 & $9.96593 \mathrm{E}-01$ & $-9.55264 \mathrm{E}-04$ & $-9.27353 \mathrm{E}-01$ & $2.51267 \mathrm{E}$ \\
\hline 6.10 & $8.74502 \mathrm{E}-01$ & $2.42511 \mathrm{E}$ & 01 & $1.00586 \mathrm{E}$ & $-9.57628 E-04$ & $-9.36421 \mathrm{E}-01$ & $2.52235 \mathrm{E}$ \\
\hline .20 & $8.74496 \mathrm{E}-0 \mathrm{l}$ & $2.43477 \mathrm{E}$ & 01 & $1.01517 \mathrm{E}$ & $-9.59973 E-04$ & $-9.45531 \mathrm{E}-01$ & $2.53204 \mathrm{E}$ \\
\hline .30 & $8.74491 \mathrm{E}-01$ & $2.44444 \mathrm{E}$ & 01 & $1.02452 \mathrm{E} \quad 00$ & $-9.62282 \mathrm{E}-04$ & $-9.54685 \mathrm{E}-01$ & $2.54173 \mathrm{E}$ \\
\hline .40 & $8.74485 \mathrm{E}-01$ & $2.45410 \mathrm{E}$ & 01 & $1.03392 \mathrm{E} \quad 00$ & $-9.64573 E-04$ & $-9.63882 \mathrm{E}-01$ & $2.55142 \mathrm{E}$ \\
\hline 26.50 & $8.74479 \mathrm{E}-01$ & $2.46377 \mathrm{E}$ & 01 & $1.04336 \mathrm{E} \quad 00$ & $-9.66829 \mathrm{E}-04$ & $-9.73123 \mathrm{E}-01$ & $2.56111 \mathrm{E}$ \\
\hline 5.60 & $8.74473 \mathrm{E}-01$ & $2.47343 \mathrm{E}$ & 01 & $1.05284 \mathrm{E}$ & $-9.69062 E-04$ & $-9.82406 \mathrm{E}-01$ & $2.57080 \mathrm{E}$ \\
\hline .70 & $8.74468 \mathrm{E}-01$ & $2.48310 \mathrm{E}$ & 01 & $1.06237 \mathrm{E} \quad 00$ & -9 & $-9.91733 \mathrm{E}-0$ & $2.58050 \mathrm{E}$ \\
\hline 26.80 & $8.74462 \mathrm{E}-01$ & $2.49278 \mathrm{E}$ & 01 & $1.07194 \mathrm{E} \quad 00$ & $54 \mathrm{E}-04$ & $-1.00110 \mathrm{E} \quad 00$ & $2.59020 \mathrm{E}$ \\
\hline 26.90 & $8.74456 \mathrm{E}-01$ & $2.50245 \mathrm{E}$ & 01 & $1.08155 \mathrm{E} \quad 00$ & $-9.75606 \mathrm{E}-04$ & $-1.01052 \mathrm{E} \quad 00$ & $2.59990 \mathrm{E}$ \\
\hline 27.00 & $8.74451 \mathrm{E}-01$ & $2.51212 \mathrm{E}$ & 01 & $1.09121 \mathrm{E} \quad 00$ & $-9.77735 \mathrm{E}-04$ & $-1.01997 \mathrm{E} \quad 00$ & $2.60961 \mathrm{E}$ \\
\hline 27.10 & $8.74445 \mathrm{E}-01$ & $2.52180 \mathrm{E}$ & 01 & $1.10091 \mathrm{E} \quad 00$ & $-9.79841 \mathrm{E}-04$ & $-1.02947 \mathrm{E} \quad 00$ & $2.61931 \mathrm{E}$ \\
\hline 27.20 & $40 \mathrm{E}-01$ & $2.53148 \mathrm{E}$ & 01 & $1.11065 \mathrm{E} \quad 00$ & $1 \mathrm{E}-04$ & $-1.03902 \mathrm{E}$ & $62902 \mathrm{E}$ \\
\hline 27.30 & $8.74434 \mathrm{E}-01$ & $2.54116 \mathrm{E}$ & 01 & $1.12044 \mathrm{E}$ & $-9.83978 \mathrm{E}-04$ & $-1.04860 \mathrm{E}$ & $2.63873 \mathrm{E}$ \\
\hline 27.40 & $8.74429 \mathrm{E}-01$ & $2.55085 \mathrm{E}$ & 01 & $1.13027 \mathrm{E}$ & $-9.86004 \mathrm{E}-04$ & $-1.05823 \mathrm{E}$ & $2.64844 \mathrm{E}$ \\
\hline 27.50 & $8.74424 \mathrm{E}-01$ & $2.56054 \mathrm{E}$ & 01 & $1.14014 \mathrm{E} \quad 00$ & $-9.88012 \mathrm{E}-04$ & $-1.06791 \mathrm{E}$ & $2.65816 \mathrm{E}$ \\
\hline 27.60 & $8.74418 \mathrm{E}-01$ & $2.57022 \mathrm{E}$ & 01 & $1.15005 \mathrm{E} \quad 00$ & $-9.89992 \mathrm{E}-04$ & $-1.07762 \mathrm{E} \quad 00$ & $2.66787 \mathrm{E}$ \\
\hline 27.70 & $8.74413 \mathrm{E}-01$ & $2.57991 \mathrm{E}$ & 01 & $1.16001 \mathrm{E} \quad 00$ & $-9.91945 \mathrm{E}-04$ & $-1.08738 \mathrm{E} \quad 00$ & $7759 \mathrm{E}$ \\
\hline 27.80 & $8.74407 \mathrm{E}-01$ & $2.58961 \mathrm{E}$ & 01 & $1.17002 \mathrm{E}$ & $-9.93883 \mathrm{E}-04$ & $-1.09719 \mathrm{E} \quad 00$ & $2.68731 \mathrm{E}$ \\
\hline 27.90 & $8.74402 \mathrm{E}-01$ & $2.59930 \mathrm{E}$ & 01 & $1.18006 \mathrm{E}$ & $-9.95795 \mathrm{E}-04$ & $-1.10704 \mathrm{E}$ & $2.69703 \mathrm{E}$ \\
\hline 28.00 & $8.74396 \mathrm{E}-01$ & $2.60900 \mathrm{E}$ & 01 & $1.19015 \mathrm{E}$ & $-9.97686 \mathrm{E}-04$ & $-1.11693 \mathrm{E}$ & $2.70676 \mathbf{E}$ \\
\hline 28.10 & $8.74391 \mathrm{E}-01$ & $2.61870 \mathrm{E}$ & 01 & $1.20028 \mathrm{E} \quad 00$ & $-9.99541 \mathrm{E}-04$ & $-1.12686 \mathrm{E} \quad 00$ & $2.71649 \mathrm{E}$ \\
\hline 28.20 & $8.74385 \mathrm{E}-01$ & $2.62840 \mathrm{E}$ & 01 & $1.21046 \mathrm{E}$ & $-1.00139 \mathbf{E}-03$ & $-1.13684 \mathrm{E}$ & $2.72622 \mathrm{E}$ \\
\hline 28.30 & $8.74380 \mathrm{E}-01$ & $2.63810 \mathrm{E}$ & 01 & $1.22068 \mathrm{E}$ & $-1.00320 \mathrm{E}-03$ & $-1.14686 \mathrm{E}$ & $2.73595 \mathrm{E}$ \\
\hline 28.40 & $8.74375 \mathrm{E}-01$ & $2.64781 \mathrm{E}$ & 01 & $1.23094 \mathrm{E}$ & $-1.00499 \mathrm{E}-03$ & $-1.15693 \mathrm{E}$ & $2.74568 \mathrm{E}$ \\
\hline 28.50 & $8.74369 \mathrm{E}-01$ & $2.65751 \mathrm{E}$ & 01 & $1.24125 \mathrm{E} \quad 00$ & $-1.00677 \mathrm{E}-03$ & $-1.16703 \mathrm{E}$ & $2.75542 \mathrm{E}$ \\
\hline 8.60 & $8.74364 \mathrm{E}-01$ & $2.66722 \mathrm{E}$ & 01 & $1.25160 \mathrm{E}$ & $-1.00852 \mathrm{E}-03$ & $-1.17719 \mathrm{E}$ & $2.76516 \mathrm{E}$ \\
\hline 28.70 & $8.74359 \mathrm{E}-01$ & $2.67693 \mathrm{E}$ & 01 & $1.26200 \mathrm{E}$ & $-1.01025 \mathrm{E}-03$ & $-1.18738 \mathrm{E}$ & $2.77490 \mathrm{E}$ \\
\hline 28.80 & $8.74353 \mathrm{E}-$ & $2.68665 \mathrm{E}$ & 01 & $1.27243 \mathrm{E}$ & $-1.01196 \mathrm{E}$ & $-1.19762 \mathrm{E}$ & $2.78464 \mathrm{E}$ \\
\hline 28.90 & $8.74348 \mathrm{E}-01$ & $2.69636 \mathrm{E}$ & 01 & $1.28292 \mathrm{E}$ & $55 \mathrm{E}-03$ & $-1.20791 \mathrm{E}$ & $2.79439 \mathrm{E}$ \\
\hline 29.00 & $8.74343 \mathrm{E}-01$ & $2.70608 \mathrm{E}$ & 01 & $1.29344 \mathrm{E} \quad 00$ & $-1.01531 \mathrm{E}-03$ & $-1.21823 \mathrm{E}$ & $2.80413 \mathrm{E}$ \\
\hline 29.10 & $8.74337 \mathrm{E}-01$ & $2.71580 \mathrm{E}$ & 01 & $1.30401 \mathrm{E} \quad 00$ & $6 \mathrm{E}-03$ & $-1.22860 \mathrm{E} \quad 00$ & $2.81388 \mathrm{E}$ \\
\hline 29.20 & $8.74332 \mathrm{E}-$ & $2.72552 \mathrm{E}$ & 01 & $1.31462 \mathrm{E}$ & -1. & $-1.23902 \mathrm{E}$ & $2.82363 \mathrm{E}$ \\
\hline 29.30 & $8.74327 \mathrm{E}-01$ & $2.73525 \mathrm{E}$ & 01 & $1.32528 \mathrm{E} \quad 00$ & -1.0 & $-1.24948 \mathrm{E}$ & $2.83339 \mathrm{E}$ \\
\hline 29.40 & $8.74322 \mathrm{E}-01$ & $2.74498 \mathrm{E}$ & 01 & $1.33598 \mathrm{E} \quad 00$ & $-1.02176 \mathrm{E}-03$ & $-1.25998 \mathrm{E}$ & $2.84315 \mathrm{E}$ \\
\hline 29.50 & $8.74317 \mathrm{E}-01$ & $2.75470 \mathrm{E}$ & 01 & $1.34672 \mathrm{E} \quad 00$ & $-1.02332 \mathrm{E}-03$ & $-1.27052 \mathrm{E}$ & $2.85290 \mathrm{E}$ \\
\hline 29.60 & $8.74311 \mathrm{E}-01$ & $2.76444 \mathrm{E}$ & 01 & $1.35751 \mathrm{E} \quad 00$ & $-1.02487 \mathrm{E}-03$ & $-1.28111 \mathrm{E} \quad 00$ & $2.86267 \mathrm{E}$ \\
\hline 29.70 & $8.74306 \mathrm{E}-01$ & $2.77417 \mathrm{E}$ & 01 & $1.36834 \mathrm{E} \quad 00$ & $-1.02638 \mathrm{E}-03$ & $-1.29175 \mathrm{E} \quad 00$ & $2.87243 \mathrm{E}$ \\
\hline 29.80 & $8.74301 \mathrm{E}-01$ & $2.78390 \mathrm{E}$ & 01 & $1.37921 \mathrm{E} \quad 00$ & $-1.02789 \mathrm{E}-03$ & $-1.30243 \mathrm{E} \quad 00$ & $2.88219 \mathrm{E}$ \\
\hline 29.90 & $8.74296 \mathrm{E}-01$ & $2.79364 \mathrm{E}$ & 01 & $1.39013 \mathrm{E} \quad 00$ & $-1.02937 \mathbf{E}-03$ & $-1.31315 \mathrm{E} \quad 00$ & $2.89196 \mathrm{E}$ \\
\hline & $8.74291 \mathrm{E}-01$ & $2.80338 \mathrm{E}$ & & & & $-1.32391 \mathrm{E} \cdot 00$ & $2.90173 \mathrm{E}$ \\
\hline
\end{tabular}

$\tan \delta_{-1}$ $3.68506 \mathrm{E} \quad 02$ $3.69130 \mathrm{E} \quad 02$ $3.69752 \mathrm{E} \quad 02$ $3.70372 \mathrm{E} \quad 02$ $3.70991 \mathrm{E} \quad 02$

$3.71608 \mathrm{E} \quad 02$ $3.72223 \mathrm{E} \quad 02$ $3.72838 \mathrm{E} \quad 02$ $3.73450 \mathrm{E} \quad 02$ $3.74061 \mathrm{E} \quad 02$

$3.74671 \mathrm{E} \quad 02$ $3.75278 \mathrm{E} \quad 02$ $3.75884 \mathrm{E} \quad 02$ $3.76489 \mathrm{E} \quad 02$ $3.77092 \mathrm{E} \quad 02$ $3.77695 \mathrm{E} \quad 02$ $3.78295 \mathrm{E} \quad 02$ $3.78895 \mathrm{E} \quad 02$ $3.79493 \mathrm{E} \quad 02$ $3.80089 \mathrm{E} \quad 02$

$3.80686 \mathrm{E} \quad 02$ $3.81279 \mathrm{E} \quad 02$ $3.81873 \mathrm{E} \quad 02$ $3.82464 \mathrm{E} \quad 02$ $3.83056 \mathrm{E} \quad 02$

$3.83645 \mathrm{E} \quad 02$ $3.84233 \mathrm{E} \quad 02$ $3.84820 \mathrm{E} \quad 02$ $3.85406 \mathrm{E} \quad 02$ $3.85991 \mathrm{E} \quad 02$

$3.86575 \mathrm{E} \quad 02$ $3.87157 \mathrm{E} \quad 02$ $\begin{array}{ll}3.87739 \mathrm{E} & 02\end{array}$ $3.88320 \mathrm{E} \quad 02$ $3.88899 \mathrm{E} \quad 02$ $3.89478 \mathrm{E} \quad 02$ $3.90056 \mathrm{E} \quad 02$ $3.90632 \mathrm{E} \quad 02$ $3.91208 \mathrm{E} \quad 02$ $3.91782 \mathrm{E} \quad 02$

$3.92357 \mathrm{E} \quad 02$ $3.92929 \mathrm{E} \quad 02$ $3.93502 \mathrm{E} \quad 02$ $3.94074 \mathrm{E} \quad 02$ $3.94645 \mathrm{E} \quad 02$ $3.95214 \mathrm{E} \quad 02$ $3.95783 \mathrm{E} \quad 02$ $3.96351 \mathrm{E} \quad 02$ $3.96918 \mathrm{E} \quad 02$ $3.97485 \mathrm{E} \quad 02$ $3.98051 \mathrm{E} \quad 02$ $3.98616 \mathrm{E} \quad 02$ 3.99181 E . 02 $3.99745 \mathrm{E} \quad 02$ $4.00308 \mathrm{E} \quad 02$

$4.00870 \mathrm{E} \quad 02$ $4.01433 \mathrm{E} \quad 02$ $4.01993 \mathrm{E} \quad 02$ $4.02555 \mathrm{E} \quad 02$ $4.03114 \mathrm{E} \quad 02$ 


\section{$\bar{F}$}

$5.99340 \mathrm{E}-04$ $6.04322 \mathrm{E}-04$ $6.09325 \mathrm{E}-04$ $6.14349 \mathrm{E}-04$ $6.19393 \mathrm{E}-04$

$6.24458 \mathrm{E}-04$ $6.29543 \mathrm{E}-04$ $6.34649 \mathrm{E}-04$ $6.39776 \mathrm{E}-04$ $6.44923 \mathrm{E}-04$

$6.50091 \mathrm{E}-04$ $6.55279 \mathrm{E}-04$ $6.60488 \mathrm{E}-04$ $6.65718 \mathrm{E}-04$ $6.70968 \mathrm{E}-04$

$6.76239 \mathrm{E}-04$ $6.81530 \mathrm{E}-04$ $6.86842 \mathrm{E}-04$ $6.92175 \mathrm{E}-04$ $6.97528 \mathrm{E}-04$

$7.02902 \mathrm{E}-04$ $7.08296 \mathrm{E}-04$ $7.13712 \mathrm{E}-04$ $7.19147 \mathrm{E}-04$ $7.24604 \mathrm{E}-04$

$7.30081 \mathrm{E}-04$

$7.35578 \mathrm{E}-04$

$7.41096 \mathrm{E}-04$

$7.46635 \mathrm{E}-04$

$7.52195 \mathrm{E}-04$

$7.57775 \mathrm{E}-04$

$7.63375 \mathrm{E}-04$

$7.68996 \mathrm{E}-04$

$7.74638 \mathrm{E}-04$

$7.80301 \mathrm{E}-04$

$7.85984 \mathrm{E}-04$

$7.91687 \mathrm{E}-04$

$7.97412 \mathrm{E}-04$

$8.03156 \mathrm{E}-04$

$8.08922 \mathrm{E}-04$

$8.14708 \mathrm{E}-04$ $8.20515 \mathrm{E}-04$

$8.26342 \mathrm{E}-04$

$8.32190 \mathrm{E}-04$

$8.38058 \mathrm{E}-04$

$8.43947 \mathrm{E}-04$ $8.49857 \mathrm{E}-04$ $8.55787 \mathrm{E}-04$

$8.61738 \mathrm{E}-04$

$8.67710 \mathrm{E}-04$

8.73702E-04 $8.79715 \mathrm{E}-04$ $8.85748 \mathrm{E}-04$ $8.91802 \mathrm{E}-04$ $8.97877 \mathrm{E}-04$

$9.03972 \mathrm{E}-04$ $9.10088 \mathrm{E}-04$ $9.16224 \mathrm{E}-04$ 9.22381 E- 04 $9.28558 \mathrm{E}-04$ $f_{2}$

$g_{2}$

$\tan \delta_{2}$

$f_{-2}$

$1.15604 \mathrm{E} \quad 00$ $1.16574 \mathrm{E} \quad 00$ $1.17549 \mathrm{E} \quad 00$ $1.18528 \mathrm{E} \quad 00$ $1.19511 \mathrm{E} \quad 00$

$1.20498 \mathrm{E} \quad 00$

$1.21489 \mathrm{E} \quad 00$ $1.22483 \mathrm{E} \quad 00$

$1.23482 \mathrm{E} \quad 00$

$1.24485 \mathrm{E} \quad 00$

$1.25493 \mathrm{E} \quad 00$

$1.26504 \mathrm{E} \quad 00$

$1.27519 \mathrm{E} \quad 00$

$1.28538 \mathrm{E} \quad 00$

$1.29561 \mathrm{E} \quad 00$

$1.30588 \mathrm{E} \quad 00$

$1.31620 \mathrm{E} \quad 00$

$1.32655 \mathrm{E} \quad 00$

$1.33694 \mathrm{E} \quad 00$

$1.34738 \mathrm{E} \quad 00$

$1.35785 \mathrm{E} \quad 00$

$1.36836 \mathrm{E} \quad 00$

$1.37892 \mathrm{E} \quad 00$

$1.38951 \mathrm{E} \quad 00$

$1.40015 \mathrm{E} \quad 00$

$1.41082 \mathrm{E} \quad 00$

$1.42154 \mathrm{E} \quad 00$

$1.43230 \mathrm{E} \quad 00$

$1.44309 \mathrm{E} \quad 00$

$1.45393 \mathrm{E} \quad 00$

$1.46481 \mathrm{E} \quad 00$

$1.47572 \mathrm{E} \quad 00$

$1.48668 \mathrm{E} \quad 00$

$1.49768 \mathrm{E} \quad 00$

$1.50872 \mathrm{E} \quad 00$

$1.51980 \mathrm{E} \quad 00$

$1.53091 \mathrm{E} \quad 00$

$1.54207 \mathrm{E} \quad 00$

$1.55327 \mathrm{E} \quad 00$

$1.56451 \mathrm{E} \quad 00$

$1.57579 \mathrm{E} \quad 00$

$1.58711 \mathrm{E} \quad 00$

$1.59847 \mathrm{E} \quad 00$

$1.60987 \mathrm{E} \quad 00$

$1.62131 \mathrm{E} \quad 00$

$1.63279 \mathrm{E} \quad 00$

$1.64431 \mathrm{E} \quad 00$

$1.65588 \mathrm{E} \quad 00$

$\begin{array}{ll}1.66748 \mathrm{E} \quad 00 \\ 1.67912 \mathrm{E} & 00\end{array}$

$1.67912 \mathrm{E} \quad 00$

$1.69080 \mathrm{E} \quad 00$

$1.70252 \mathrm{E} \quad 00$

$1.71429 \mathrm{E} \quad 00$

$1.72609 \mathrm{E} \quad 00$

1.73793 E 00

$1.74981 \mathrm{E} \quad 00$

$1.76174 \mathrm{E} \quad 00$

$1.77370 \mathrm{E} \quad 00$

$1.78570 \mathrm{E} \quad 00$

$1.79775 \mathrm{E} \quad 00$
$2.63171 \mathrm{E}-02$ $2.66901 \mathrm{E}-02$

$2.70666 \mathrm{E}-02$

$2.74466 \mathrm{E}-02$

$2.78301 \mathrm{E}-02$

$2.82172 \mathrm{E}-02$

2.86077 E-02

$2.90018 \mathrm{E}-02$

2.93994 E-02

$2.98006 \mathrm{E}-02$

$3.02055 \mathrm{E}-02$

$3.06139 \mathrm{E}-02$

$3.10259 \mathrm{E}-02$

$3.14416 \mathrm{E}-02$

$3.18609 \mathrm{E}-02$

$3.22839 \mathrm{E}-02$

$3.27106 \mathrm{E}-02$

$3.31410 \mathrm{E}-02$

$3.35751 \mathrm{E}-02$

$3.40130 \mathrm{E}-02$

$3.44546 \mathrm{E}-02$ $3.49000 \mathrm{E}-02$

$3.53491 \mathrm{E}-02$

$3.58020 \mathrm{E}-02$

$3.62588 \mathrm{E}-02$

$3.67194 \mathrm{E}-02$

$3.71838 \mathrm{E}-02$

$3.76521 \mathrm{E}-02$

$3.8124 .3 \mathrm{E}-02$

$3.86003 \mathrm{E}-02$

$3.90803 \mathrm{E}-02$ $3.95642 \mathrm{E}-02$ $4.00520 \mathrm{E}-02$ $4.05438 \mathrm{E}-02$

$4.10395 \mathrm{E}-02$

$4.15393 \mathrm{E}-02$

$4.20430 \mathrm{E}-02$

$4.25508 \mathrm{E}-02$

$4.30626 \mathrm{E}-02$

4.35784 E-02

$4.40983 \mathrm{E}-02$

$4.46223 \mathrm{E}-02$

$4.51504 \mathrm{E}-02$

$4.56826 \mathrm{E}-02$

$4.62189 \mathrm{E}-02$

4.67594 E-02

$4.73040 \mathrm{E}-02$

$4.78528 \mathrm{E}-02$

$4.84058 \mathrm{E}-02$

$4.89630 \mathrm{E}-02$

$4.95244 \mathrm{E}-02$

$5.00901 \mathrm{E}-02$

$5.06600 \mathrm{E}-02$

$5.12342 \mathrm{E}-02$

$5.18126 \mathbf{E}-02$

$5.23954 \mathrm{E}-02$

$5.29825 \mathrm{E}-02$

$5.35739 \mathrm{E}-02$

$5.41697 \mathrm{E}-02$

$5.47698 \mathrm{E}-02$
$-3.25784 \mathrm{E} \quad 01$ ol $-2.46450 \mathrm{E}-02$ $-3.25828 \mathrm{E}$ 01 $-2.50066 \mathrm{E}-02$ $-3.25850 \mathrm{E}$ 01 $-2.53717 \mathrm{E}-02$ $-3.25872 \mathrm{E}$ 01 $-2.57402 \mathrm{E}-02$

$-3.25893 \mathrm{E} \quad 01$ $-3.25914 \mathrm{E} \quad 01$ $-3.25935 \mathrm{E} 01$ $-3.25956 \mathrm{E} 01$ $-3.25977 \mathrm{E} \quad 01$

$-3.25998 \mathrm{E} \quad 01$ $-3.26018 \mathrm{E} 01$ $-3.26038 \mathrm{E}$ 0I $-3.26058 \mathrm{E}$ 01 $-3.26078 \mathrm{E} \quad 01$

$-3.26098 \mathrm{E} \quad 01$ $-3.26137 \mathrm{E} 01$ $-3.26156 \mathrm{E} \quad 01$ $-3.26175 \mathrm{E} 01$

$-3.26194 \mathrm{E} 01$ $-3.26213 \mathrm{E} 01$ $-3.26231 \mathrm{E} 01$ $-3.26250 \mathrm{E} 01$ $-3.26268 \mathrm{E} 01$

\section{$-3.26287 \mathrm{E} 01$} $-3.26323 \mathrm{E} 01$ $-3.26340 \mathrm{E} \quad 01$ $-3.26358 \mathrm{E} 01$

$-2.61122 \mathrm{E}-02$ $-2.64876 \mathrm{E}-02$ $-2.68665 \mathrm{E}-02$ $-2.72489 \mathrm{E}-02$ $-2.76349 \mathrm{E}-02$

$-2.80244 \mathrm{E}-02$ $-2.84175 \mathrm{E}-02$ $-2.88141 \mathrm{E}-02$ $-2.92143 \mathrm{E}-02$ $-2.96181 \mathrm{E}-02$

$-3.00255 \mathrm{E}-02$ $-3.04366 \mathrm{E}-02$ $-3.08513 \mathrm{E}-02$

$-3.12697 \mathrm{E}-02$

$-3.16917 \mathrm{E}-02$

$-3.21175 \mathrm{E}-02$

$-3.25470 \mathrm{E}-02$

$-3.29802 \mathrm{E}-02$

$-3.34171 \mathrm{E}-02$

$-3.38578 \mathrm{E}-02$

$-3.43023 \mathrm{E}-02$

$-3.47506 \mathrm{E}-02$

$-3.52027 \mathrm{E}-02$

$-3.56586 \mathrm{E}-02$

$-3.61183 \mathrm{E}-02$

$-3.26376 \mathrm{E} \quad 01$ $-3.26393 \mathrm{E} 01$ $-3.26410 \mathrm{E} 01$ $-3.26427 \mathrm{E} 01$ $-3.26444 \mathrm{E} 01$ $-3.26478 \mathrm{E} 01$ $-3.26495 \mathrm{E} 01$ $-3.26511 \mathrm{E} 01$ $-3.26527 \mathrm{E} 01$

$-3.26544 \mathrm{E} 01$ $-3.26560 \mathrm{E} 01$ $-3.26576 \mathrm{E} 01$ $-3.26592 \mathrm{E} 01$ $-3.26607 \mathrm{E} \quad 01$

$-3.26623 \mathrm{E} 01$ $-3.26654 \mathrm{E} \quad 01$ $-3.26670 \mathrm{E} 01$ $-3.26685 \mathrm{E} \quad 01$

$-3.26700 \mathrm{E} 01$ $-3.26715 \mathrm{E} 01$ $-3.26730 \mathrm{E} 01$ $-3.26745 \mathrm{E} 01$ $-3.26759 \mathrm{E} 01$

\section{$-3.26774 \mathrm{E} \quad 01$}

$-3.26788 \mathrm{E} 0$

$-3.26803 \mathrm{E} 01$

$-3.26817 \mathrm{E} 01$

$\begin{array}{lll}-3.26831 \mathrm{E} & 01\end{array}$

$-3.65819 \mathrm{E}-02$ $-3.70494 \mathrm{E}-02$

$-3.75207 \mathrm{E}-02$

$-3.79960 \mathrm{E}-02$

$-3.84752 \mathrm{E}-02$

$-3.89583 \mathrm{E}-02$

$-3.94453 \mathrm{E}-0$ 2

$-3.99363 \mathrm{E}-02$

$-4.04313 \mathrm{E}-02$

$-4.09303 \mathrm{E}-02$

$-4.14334 \mathrm{E}-02$

$-4.19404 \mathrm{E}-02$

$-4.24515 \mathrm{E}-02$ $-3.26117 \mathrm{E} 01$

$-3.26305 \mathrm{E} \quad 01$

$-3.26461 \mathrm{E} \quad 01$ $-3.26639 \mathrm{E} 01$ 
$p$
30.10

30.20

30.30

30.40

30.50

30.60

30.70

30.80

30.90

31.00

31.10

31.20

31.30

31.40

31.50

31.60

31.70

31.80

31.90

32.00

32.10

32.20

32.30

32.40

32.50

32.60

32.70

32.80

32.90

33.00

33.10

33.20

33.30

33.40

33.50

33.60

33.70

33.80

33.90

34.00

34.10

34.20

34.30

34.40

34.50

34.60

34.70

34.80

34.90

35.00

35.10

35.20

35.30

35.40

35.50

35.60

35.70

35.80

35.90

36.00
$F_{0}$

$8.74286 \mathrm{E}-01$ $8.74281 \mathrm{E}-01$ $8.74276 \mathrm{E}-01$ $8.74270 \mathrm{E}-01$ $8.74265 \mathrm{E}-01$

$8.74260 \mathrm{E}-01$ $8.74255 \mathrm{E}-01$ $8.74250 \mathrm{E}-01$ $8.74245 \mathrm{E}-01$ $8.74240 \mathrm{E}-01$

8.74235 E-01 $8.74230 \mathrm{E}-01$ $8.74225 \mathrm{E}-01$ $8.74220 \mathrm{E}-01$ $8.74215 \mathrm{E}-01$

$8.74211 \mathrm{E}-01$ $8.74206 \mathrm{E}-01$ $8.74201 \mathrm{E}-01$ $8.74196 \mathrm{E}-01$ 8.74191 E-01

$8.74186 \mathrm{E}-01$ $8.74181 \mathrm{E}-0$ $8.74177 \mathrm{E}-01$ $8.74172 \mathrm{E}-01$ $8.74167 \mathrm{E}-01$

$8.74162 \mathrm{E}-01$ $8.74157 \mathrm{E}-01$ $8.74153 \mathrm{E}-01$ $8.74148 \mathrm{E}-01$ $8.74143 \mathrm{E}-01$

$8.74138 \mathrm{E}-01$ 8.74134 E-01 $8.74129 \mathrm{E}-01$ $8.74124 \mathrm{E}-01$ $8.74120 \mathrm{E}-01$

$8.74115 \mathrm{E}-01$ $8.74110 \mathrm{E}-01$ $8.74106 \mathrm{E}-01$ $8.74101 \mathrm{E}-01$ $8.74096 \mathrm{E}-01$

$8.74092 \mathrm{E}-01$ $8.74087 \mathrm{E}-01$ $8.74083 \mathrm{E}-01$ $8.74078 \mathrm{E}-01$ $8.74074 \mathrm{E}-01$

$8.74069 \mathrm{E}-01$ $8.74064 \mathrm{E}-01$ $8.74060 \mathrm{E}-01$ $8.74055 \mathrm{E}-01$ 8.74051 E-01

$8.74046 \mathrm{E}-01$ $8.74042 \mathrm{E}-01$ $8.74037 \mathrm{E}-01$ $8.74033 \mathrm{E}-01$ $8.74029 \mathrm{E}-01$

$8.74024 \mathrm{E}-01$ $8.74020 \mathrm{E}-01$ $8.74015 \mathrm{E}-01$ $8.74011 \mathrm{E}-01$ $8.74006 \mathrm{E}-01$ $f_{1}$

$2.81313 \mathrm{E} \quad 01$ $2.82287 \mathrm{E} \quad 01$ $2.83262 \mathrm{E} 01$ $2.84237 \mathrm{E} \quad 01$ $2.85212 \mathrm{E} \quad 01$

$2.86187 \mathrm{E} \quad 01$ $2.87163 \mathrm{E} \quad 01$ $2.88139 \mathrm{E} 01$

$2.89115 \mathrm{E} \quad 01$

$2.90091 \mathrm{E} \quad 01$

$2.91067 \mathbf{E} 01$ $2.92044 \mathrm{E} \quad 01$ $2.93021 \mathrm{E} \quad 01$ $2.93998 \mathrm{E} \quad 01$ $2.94976 \mathrm{E} \quad 01$

$2.95954 \mathrm{E} \quad 01$ $2.96932 \mathrm{E} \quad 01$ $2.97910 \mathrm{E} 01$ $2.98888 \mathrm{E}$ 01 $2.99867 \mathrm{E} 01$

$3.00846 \mathrm{E} \quad 01$ $3.01825 \mathrm{E} \quad 01$ $3.02804 \mathrm{E} \quad 01$ $3.03784 \mathrm{E} \quad 01$

$3.04764 \mathrm{E} 01$

$3.05744 \mathrm{E} \quad 01$ $3.06724 \mathrm{E} \quad 01$ $3.07705 \mathrm{E} \quad 01$ $3.08686 \mathrm{E} \quad 01$ $3.09667 \mathrm{E} 01$

$3.10648 \mathrm{E} 01$

$3.11629 \mathrm{E} 01$

$3.12611 \mathrm{E} \quad 01$

$3.13593 \mathrm{E} 01$

$3.14576 \mathrm{E} \quad 01$

$3.15558 \mathrm{E}$ 01 $3.16541 \mathrm{E} 01$

$3.17524 \mathrm{E} \quad 01$

$3.18507 \mathrm{E} 01$

$3.19491 \mathrm{E} 01$

$3.20475 \mathrm{E} \quad 01$ $3.21459 \mathrm{E} \quad 01$ $3.22443 \mathrm{E} 01$ $3.23428 \mathrm{E} 01$ $3.24413 \mathrm{E} 01$

$3.25398 \mathrm{E} \quad 01$ $3.26383 \mathrm{E} \quad 01$ $3.27369 \mathrm{E} \quad 01$ $3.28355 \mathrm{E} \quad 01$

$3.29341 \mathrm{E} \quad 01$

$3.30327 \mathrm{E} \quad 01$ $3.31314 \mathrm{E} \quad 01$ $3.32301 \mathrm{E} 01$ $3.33288 \mathrm{E} 01$ $3.34275 \mathrm{E} 01$

$3.35263 \mathrm{E} \quad 01$ $3.36251 \mathrm{E} 01$ $3.37239 \mathrm{E} \quad 01$

$3.38228 \mathrm{E} \quad 01$ $3.39217 \mathrm{E} \quad 01$ $g_{1}$

$1.41210 \mathrm{E} \quad 00$

$\tan \delta_{1}$

$f_{-1}$

$1.42315 \mathrm{E} \quad 00-1.03370 \mathrm{E}-03$

$1.43425 \mathrm{E} \quad 00-1.03510 \mathrm{E}-03$

$\begin{array}{lll}1.44539 \mathrm{E} & 00 & -1.03649 \mathrm{E}-03\end{array}$

$1.45657 \mathrm{E} \quad 00-1.03785 \mathrm{E}-03$

$1.46780 \mathrm{E} \quad 00-1.03920 \mathrm{E}-03$

$1.47907 \mathrm{E} \quad 00-1.04053 \mathrm{E}-03$

$1.49038 \mathrm{E} \quad 00 \quad-1.04184 \mathrm{E}-03$

$1.50174 \mathrm{E} \quad 00-1.04313 \mathrm{E}-03$

$1.51314 \mathrm{E} \quad 00$

$-1.04440 \mathrm{E}-03$

$1.52459 \mathrm{E} \quad 00 \quad-1.04567 \mathrm{E}-03$

$1.53608 \mathrm{E} \quad 00-1.04691 \mathrm{E}-03$

$\begin{array}{lll}1.54761 \mathrm{E} & 00-1.04813 \mathrm{E}-03\end{array}$

$1.55919 \mathrm{E} \quad 00-1.04933 \mathrm{E}-03$

$1.57082 \mathrm{E} \quad 00-1.05052 \mathrm{E}-03$

$1.58248 \mathrm{E} \quad 00$

$1.59419 \mathrm{E} \quad 00$

$1.60595 \mathrm{E} \quad 00$

$1.61775 \mathrm{E} \quad 00$

$1.62959 \mathrm{E} \quad 00$

$1.64148 \mathrm{E} \quad 00$

$1.65342 \mathrm{E} \quad 00$

$1.66539 \mathrm{E} \quad 00$

$1.67742 \mathrm{E} \quad 00$

$1.68948 \mathrm{E} \quad 00$

$1.70159 \mathrm{E} \quad 00$

$1.71375 \mathrm{E} \quad 00$

$1.72595 \mathrm{E} \quad 00$

$1.73819 \mathrm{E} \quad 00$

$1.75048 \mathrm{E} \quad 00$

$\begin{array}{ll}1.76282 \mathrm{E} & 00\end{array}$

$1.77519 \mathrm{E} \quad 00$

$1.78762 \mathrm{E} \quad 00$

$1.80008 \mathrm{E} \quad 00$

$1.81260 \mathrm{E} \quad 00$

$1.82515 \mathrm{E} \quad 00$

$1.83775 \mathrm{E} \quad 00$

$1.85040 \mathrm{E} \quad 00$

$1.86309 \mathrm{E} \quad 00$

$1.87583 \mathrm{E} \quad 00$

$1.88861 \mathrm{E} \quad 00$

$1.90143 \mathrm{E} \quad 00$

$1.91430 \mathrm{E} \quad 00$

$1.92722 \mathrm{E} \quad 00$

$1.94018 \mathrm{E} \quad 00$

$1.95318 \mathrm{E} \quad 00$

$1.96623 \mathrm{E} \quad 00$

$1.97933 \mathrm{E} \quad 00$

$1.99247 \mathrm{E} \quad 00$

$2.00565 \mathrm{E} \quad 00$

$2.01888 \mathrm{E} \quad 00$

$2.03216 \mathrm{E} \quad 00$

$2.04548 \mathrm{E} \quad 00$

$2.05884 \mathrm{E} \quad 00$

$2.07225 \mathrm{E} \quad 00$

$2.08571 \mathrm{E} \quad 00$ $2.09921 \mathrm{E} \quad 00$

$2.11276 \mathrm{E} \quad 00$

$2.12635 \mathrm{E} \quad 00$

$2.13999 \mathrm{E} \quad 00$

$-1.05169 \mathrm{E}-03$

$-1.05284 \mathrm{E}-03$

$-1.05398 \mathrm{E}-03$

$-1.05510 \mathrm{E}-03$

$-1.05620 \mathrm{E}-03$

$-1.05729 \mathrm{E}-03$

$-1.05836 \mathrm{E}-03$

$-1.05941 \mathrm{E}-03$

$-1.06045 \mathrm{E}-03$

$-1.06147 \mathrm{E}-03$

$-1.06247 \mathrm{E}-03$

$-1.06346 \mathrm{E}-03$

$-1.06444 \mathrm{E}-03$

$-1.06539 \mathrm{E}-03$

$-1.06633 \mathrm{E}-03$

$-1.06726 \mathrm{E}-03$

$-1.06817 \mathrm{E}-03$

$-1.06907 \mathrm{E}-03$

$-1.06995 \mathrm{E}-03$

$-1.07082 \mathrm{E}-03$

$-1.07167 \mathrm{E}-03$

$-1.07250 \mathrm{E}-03$

$-1.07333 \mathrm{E}-03$

$-1.07413 \mathrm{E}-03$

- $1.07492 \mathrm{E}-03$

$-1.07571 \mathrm{E}-03$

$-1.07648 \mathrm{E}-03$

$-1.07722 \mathrm{E}-03$

$-1.07796 \mathrm{E}-03$

$-1.07868 \mathrm{E}-03$

$-1.07939 \mathrm{E}-03$

$-1.08008 \mathrm{E}-03$

$-1.08077 \mathrm{E}-03$

$-1.08144 \mathbf{E}-03$

$-1.08209 \mathrm{E}-03$

$-1.08273 \mathrm{E}-03$

$-1.08336 \mathrm{E}-03$

$-1.08397 \mathrm{E}-03$

$-1.08458 \mathrm{E}-03$ 
ELECTRON RADIAL FUNCTIONS

$Z=6 \quad A=12 \quad \rho=1.1 A^{1 / 3} F$

Positron

$\bar{F}$

30.10

30.20

30.30

30.40

30.50

30.60

30.70

30.80

30.90

31.00

31.10

31.20

31.30

31.40

31.50

31.60

$31: 70$

31.80

31.90

32.00

32.10

32.20

32.30

32.40

32.50

32.60

32.70

32.80

32.90

33.00

33.10

33.20

33.30

33.40

33.50

33.60

33.70

33.80

33.90

34.00

34.10

34.20

34.30

34.40

34.50

34.60

34.70

34.80

34.90

35.00

35.10

35.20

35.30

35.40

35.50

35.60

35.70

35.80

35.90

36.00 $f_{2}$

$1.80983 \mathrm{E} \quad 00$

$1.82196 \mathrm{E} \quad 00$

$1.83412 \mathrm{E} \quad 00$

$1.84632 \mathrm{E} \quad 00$

$1.85857 \mathrm{E} \quad 00$

$1.87085 \mathrm{E} \quad 00$

$1.88318 \mathrm{E} \quad 00$

$1.89554 \mathrm{E} \quad 00$

$1.90795 \mathrm{E} \quad 00$

$1.92039 \mathrm{E} \quad 00$

$1.93288 \mathrm{E} \quad 00$

$1.94540 \mathrm{E} \quad 00$

$1.95797 \mathrm{E} \quad 00$

$1.97058 \mathrm{E} \quad 00$

$1.98322 \mathrm{E} \quad 00$

$1.99591 \mathrm{E} \quad 00$

$2.00863 \mathrm{E} \quad 00$

$2.02140 \mathrm{E} \quad 00$

$2.03420 \mathrm{E} \quad 00$

2.04705 E 00

$2.05994 \mathrm{E} \quad 00$

$2.07286 \mathrm{E} \quad 00$

$2.08583 \mathrm{E} \quad 00$

$2.09884 \mathrm{E} \quad 00$

$2.11188 \mathrm{E} \quad 00$

$1.08970 \mathrm{E}-03$

$1.09641 \mathrm{E}-03$

$1.10314 \mathrm{E}-03$

$1.10990 \mathrm{E}-03$

$1.11667 \mathrm{E}-03$

$1.12347 \mathrm{E}-03$

$1.13028 \mathrm{E}-03$

$1.13712 \mathrm{E}-03$

$1.14398 \mathrm{E}-03$

$1.15086 \mathrm{E}-03$

$1.15776 \mathrm{E}-03$

$1.16468 \mathrm{E}-03$

$1.17162 \mathrm{E}-03$

$1.17858 \mathrm{E}-03$

$1.18556 \mathrm{E}-03$

$1.19256 \mathrm{E}-03$

$1.19958 \mathrm{E}-03$ $1.20662 \mathrm{E}-03$ $1.21369 \mathrm{E}-03$ $1.22077 \mathrm{E}-03$ $1.22788 \mathrm{E}-03$

$1.23500 \mathrm{E}-03$ $1.24215 \mathrm{E}-03$ $1.24932 \mathrm{E}-03$ $1.25650 \mathrm{E}-03$ $1.26371 \mathrm{E}-03$

$1.27094 \mathrm{E}-03$ $1.27819 \mathrm{E}-03$ $1.28546 \mathrm{E}-03$ $1.29275 \mathrm{E}-03$ $1.30006 \mathrm{E}-03$

$1.30739 \mathrm{E}-03$ $1.31474 \mathrm{E}-03$ $1.32212 \mathrm{E}-03$ $1.32951 \mathrm{E}-03$ $1.33692 \mathrm{E}-03$
$2.12497 \mathrm{E} \quad 00$ $2.13810 \mathrm{E} \quad 00$ $2.15126 \mathrm{E} \quad 00$ $2.16447 \mathrm{E} \quad 00$ $2.17772 \mathrm{E} \quad 00$

$2.19100 \mathrm{E} \quad 00$ $2.20433 \mathrm{E} \quad 00$ $2.21769 \mathrm{E} \quad 00$ $2.23110 \mathrm{E} \quad 00$ $2.24455 \mathrm{E} \quad 00$

$2.25804 \mathrm{E} \quad 00$ $2.27156 \mathrm{E} \quad 00$ $2.28513 \mathrm{E} \quad 00$ $2.29874 \mathrm{E} \quad 00$ $2.31238 \mathrm{E} \quad 00$

$2.32607 \mathrm{E} \quad 00$ $2.33979 \mathrm{E} \quad 00$ $2.35356 \mathrm{E} \quad 00$ $2.36737 \mathrm{E} \quad 00$ $2.38122 \mathrm{E} \quad 00$

$2.39510 \mathrm{E} \quad 00$ $2.40903 \mathrm{E} \quad 00$ $2.42299 \mathrm{E} \quad 00$ $2.43700 \mathrm{E} \quad 00$ 2.45105 E 00

2.46513E 00 $2.49342 \mathrm{E} \quad 00$ $2.50763 \mathrm{E} \quad 00$ $2.52188 \mathrm{E} \quad 00$

$2.53616 \mathrm{E} \quad 00$ $2.55049 \mathrm{E} \quad 00$ $2.56485 \mathrm{E} \quad 00$ $2.57926 \mathrm{E} \quad 00$ $2.59370 \mathrm{E} \quad 00$ $2.47926 \mathrm{E} \quad 00$ $\tan \delta_{2}$

$f_{-2}$

$5.53743 \mathrm{E}-02$

$5.59832 \mathrm{E}-02$

$5.65965 \mathrm{E}-02$

$5.72143 \mathrm{E}-02$

$5.78365 \mathrm{E}-02$

$5.84631 \mathrm{E}-02$

$5.90942 \mathrm{E}-02$

$5.97298 \mathrm{E}-02$

$6.03699 \mathrm{E}-02$

$6.10145 \mathrm{E}-02$

$6.16637 \mathrm{E}-02$

$6.23174 \mathrm{E}-02$

$6.29757 \mathrm{E}-02$

$6.36386 \mathrm{E}-02$

$6.43060 \mathrm{E}-02$

$6.49781 \mathrm{E}-02$

$6.56548 \mathrm{E}-02$

$6.63362 \mathrm{E}-02$

$6.70222 \mathrm{E}-02$

$6.77129 \mathrm{E}-02$

$6.84083 \mathrm{E}-02$ $6.91084 \mathrm{E}-02$

$6.98132 \mathrm{E}-02$

$7.05228 \mathrm{E}-02$

$7.12371 \mathrm{E}-02$

$7.19562 \mathrm{E}-02$

$7.26801 \mathrm{E}-02$

$7.34087 \mathrm{E}-02$

$7.41423 \mathrm{E}-02$

$7.48806 \mathrm{E}-02$

$7.56238 \mathrm{E}-02$

$7.63718 \mathrm{E}-02$

$7.71247 \mathrm{E}-02$

$7.78825 \mathrm{E}-02$

$7.86453 \mathrm{E}-02$

$7.94130 \mathrm{E}-02$

$8.01856 \mathrm{E}-02$

8.09631 E-02

$8.17457 \mathrm{E}-02$

$8.25332 \mathrm{E}-02$

$8.33257 \mathrm{E}-02$

$8.41233 \mathrm{E}-02$

$8.49259 \mathrm{E}-02$

$8.57335 \mathrm{E}-02$

$8.65463 \mathrm{E}-02$

$8.73641 \mathrm{E}-02$

$8.81870 \mathrm{E}-02$

$8.90150 \mathrm{E}-02$

$8.98482 \mathrm{E}-02$

$9.06864 \mathrm{E}-02$

9.15299E-02

$9.23785 \mathrm{E}-02$

$9.32324 \mathrm{E}-02$

$9.40914 \mathrm{E}-02$

$9.49557 \mathrm{E}-02$

$9.58252 \mathrm{E}-02$ $9.66999 \mathrm{E}-02$

$9.75800 \mathrm{E}-02$

$9.84653 \mathrm{E}-02$

$9.93559 \mathrm{E}-02$
$-3.26845 \mathrm{E} 01$

$-3.26873 \mathrm{E}$ 01 $-5.35466 \mathrm{E}-02$

$-3.26887 \mathrm{E}$ 01 $-5.41462 \mathrm{E}-02$

$-3.26901 \mathrm{E}$ 01 $-5.47503 \mathrm{E}-02$

$-3.26914 \mathrm{E} \quad 01$

$-3.26928 \mathrm{E} 01$

$-3.26941 \mathrm{E} 01$

$-3.26955 \mathrm{E} 01$

$-3.26968 \mathrm{E} 01$

$-3.26981 \mathrm{E} \quad 01$ $-3.26994 \mathrm{E} \quad 01$ $-3.27007 \mathrm{E} 01$ $-3.27020 \mathrm{E} 01$

$-3.27033 \mathrm{E} 01$

$-3.27046 \mathrm{E} 01$ $-3.27059 \mathrm{E} 01$

$-3.27071 \mathrm{E} 01$

$-3.27084 \mathrm{E} \quad 01$

$-3.27096 \mathrm{E} 01$

$-3.27108 \mathrm{E} 01$

$-3.27121 \mathrm{E} 01$

$-3.27133 \mathrm{E} 01$

$-3.27145 \mathrm{E} \quad 01$

$-3.27157 \mathrm{E} 01$

$-3.27169 \mathrm{E} 01$ $-3.27181 \mathrm{E} \quad 01$ $-3.27193 \mathrm{E} 01$ $-3.27204 \mathrm{E} \quad 01$ $-3.27216 \mathrm{E} 01$

$-3.27228 \mathrm{E} \quad 01$

$-3.27239 \mathrm{E} \quad 01$

$-3.27251 \mathrm{E} 01$

$-3.27262 \mathrm{E} 01$

-3.27273 E 01

$-3.27296 \mathrm{E} 01$

$-3.27307 \mathrm{E} 01$

$-3.27329 \mathrm{E} 01$

$-3.27340 \mathrm{E} 01$ $-3.27351 \mathrm{E} 01$ $-3.27361 \mathrm{E} 01$ $-3.27372 \mathrm{E} 01$

$-3.27383 \mathrm{E} 01$

$-3.27393 \mathrm{E} 01$ $-3.27404 \mathrm{E} 01$ $-3.27414 \mathrm{E} 01$ $-3.27425 \mathrm{E}$ 0l

$-3.27435 \mathrm{E} 01$

$-3.27445 \mathrm{E} 01$ $-3.27455 \mathrm{E} 01$ $-3.27466 \mathrm{E} 01$ $-3.27476 \mathrm{E} 01$ $-3.27486 \mathrm{E} 01$

$-3.27496 \mathrm{E} 01$ $-3.27506 \mathrm{E} 0 \mathrm{l}$ $-3.27515 \mathrm{E} 01$ $-3.27525 \mathrm{E} 01$ $-3.27535 \mathrm{E} 01$

$-5.53587 \mathrm{E}-02$ $-5.59716 \mathrm{E}-02$ $-5.65888 \mathrm{E}-02$
$-3.27285 \mathrm{E} \quad 01$

$-3.27318 \mathrm{E} 01$ 


\section{p \\ $F_{0}$}

36.10

36.20

36.30

36.40

36.50

36.60

36.70

36.80

36.90

37.00

37.10

37.20

37.30

37.40

37.50

37.60

37.70

37.80

37.90

38.00

38.10

38.20

38.30

38.40

38.50

38.60

38.70

38.80

38.90

39.00

39.10

39.20

39.30

39.40

39.50

39.60

39.70

39.80

39.90

40.00

40.10

40.20

40.30

40.40

40.50

40.60

40.70

40.80

40.90

41.00

41.10

41.20

41.30

41.40

41.50

41.60

41.70

41.80

41.90

42.00
$8.74002 \mathrm{E}-01$ $8.73998 \mathrm{E}-01$ $8.73993 \mathrm{E}-01$ $8.73989 \mathrm{E}-01$ $8.73985 \mathrm{E}-01$

$8.73980 \mathrm{E}-01$ $8.73976 \mathrm{E}-01$ $8.73972 \mathrm{E}-01$ $8.73967 \mathrm{E}-01$ $8.73963 \mathrm{E}-01$

$8.73959 \mathrm{E}-01$ $8.73955 \mathrm{E}-01$ $8.73950 \mathrm{E}-0 \mathrm{l}$ $8.73946 \mathrm{E}-01$ $8.73942 \mathrm{E}-01$

$8.73938 \mathrm{E}-01$ $8.73933 \mathrm{E}-01$ $8.73929 \mathrm{E}-01$ $8.73925 \mathrm{E}-01$ $8.73921 \mathrm{E}-01$

8.73917E-01 $8.73912 \mathrm{E}-01$ $8.73908 \mathrm{E}-01$ 8.73904 E-01 $8.73900 \mathrm{E}-01$

$8.73896 \mathrm{E}-01$ $8.73892 \mathrm{E}-01$ $8.73888 \mathrm{E}-01$ $8.73884 \mathrm{E}-01$ 8.73879E-01

$8.73875 \mathrm{E}-01$ $8.73871 \mathrm{E}-01$ $8.73867 \mathrm{E}-01$ $8.73863 \mathrm{E}-01$ $8.73859 \mathrm{E}-01$

$8.73855 \mathrm{E}-01$ $8.73851 \mathrm{E}-01$ $8.73847 \mathrm{E}-01$ $8.73843 \mathrm{E}-01$ $8.73839 \mathrm{E}-01$

$8.73835 \mathrm{E}-01$ 8.73831 E-01 $8.73827 \mathrm{E}-01$ $8.73823 \mathrm{E}-01$ 8.73819 E-01

$8.73815 \mathrm{E}-01$ $8.73811 \mathrm{E}-01$ $8.73807 \mathrm{E}-01$ $8.73803 \mathrm{E}-01$ $8.73800 \mathrm{E}-01$

$8.73796 \mathrm{E}-01$ $8.73792 \mathrm{E}-01$ $8.73788 \mathrm{E}-01$ 8.73784 E- 01 $8.73780 \mathrm{E}-01$

$8.73776 \mathrm{E}-01$ $8.73772 \mathrm{E}-01$ $8.73769 \mathrm{E}-01$ $8.73765 \mathrm{E}-01$ 8.73761 E-01 $f_{1}$

$3.40206 \mathrm{E} \quad 01$ $3.41195 \mathrm{E} \quad 01$

$3.42185 \mathrm{E} 01$

$3.43175 \mathrm{E} \quad 01$

$3.44165 \mathrm{E} 01$

$3.45155 \mathrm{E} \quad 01$

$3.46146 \mathrm{E} \quad 01$

$3.47137 \mathrm{E} 01$

$3.48128 \mathrm{E} 01$

$3.49119 \mathrm{E} \quad 01$

$3.50111 \mathrm{E} 01$

$3.51103 \mathrm{E} \quad 01$

$3.52096 \mathrm{E} \quad 01$

$3.53088 \mathrm{E} 01$

$3.54081 \mathrm{E} \quad 01$

$3.55074 \mathrm{E} \quad 01$ $3.56068 \mathrm{E} 01$ $3.57061 \mathrm{E} \quad 01$ $3.58055 \mathrm{E} \quad 01$ $3.59050 \mathrm{E} 01$

$3.60044 \mathrm{E} \quad 01$ $3.61039 \mathrm{E} \quad 01$ $3.62034 \mathrm{E} 01$ $3.63030 \mathrm{E} \quad 01$

$3.64026 \mathrm{E} \quad 01$

$3.65022 \mathrm{E} \quad 01$ $3.66018 \mathrm{E} 01$ $3.67014 \mathrm{E} 01$ $3.68011 \mathrm{E} \quad 01$

$3.69008 \mathrm{E} 01$

$3.70006 \mathrm{E} \quad 01$ $3.71004 \mathrm{E} \quad 01$ $3.72002 \mathrm{E} \quad 01$ $3.73000 \mathrm{E} \quad 01$ $3.73999 \mathrm{E} 01$

$3.74998 \mathrm{E} \quad 01$ $3.75997 \mathrm{E} \quad 01$ $3.76996 \mathrm{E} \quad 01$ $3.77996 \mathrm{E} \quad 01$ $3.78996 \mathrm{E} \quad 01$

$3.79997 \mathrm{E} \quad 01$ $3.80997 \mathrm{E} \quad 01$ $3.81998 \mathrm{E} \quad 01$ $3.83000 \mathrm{E} \quad 01$ $3.84001 \mathrm{E} 01$

$3.85003 \mathrm{E} \quad 01$ $3.86006 \mathrm{E} \quad 01$ $3.87008 \mathrm{E} 01$ $3.88011 \mathrm{E} \quad 01$ $3.89014 \mathrm{E} \quad 01$

$3.90017 \mathrm{E} \quad 01$ $3.91021 \mathrm{E} 01$ $3.92025 \mathrm{E} \quad 01$ $3.93030 \mathrm{E} 01$ $3.94034 \mathrm{E} \quad 01$

$3.95039 \mathrm{E} \quad 01$ $3.96045 \mathrm{E} \quad 01$ $3.97050 \mathrm{E} 01$ $3.98056 \mathrm{E} \quad 01$ $3.99063 \mathrm{E} \quad 01$ $\tan \delta_{1}$ $f_{-1}$

$2.15367 \mathrm{E} \quad 00$ $2.16740 \mathrm{E} \quad 00$

$2.18117 \mathrm{E} \quad 00$

$2.19499 \mathrm{E} \quad 00$

2.20885 E 00

$2.22276 \mathrm{E} \quad 00$

$2.23671 \mathrm{E} \quad 00$

$2.25072 \mathrm{E} \quad 00$

$2.26476 \mathrm{E} \quad 00$

2.27885 E 00

$2.29299 \mathrm{E} \quad 00$

$2.30717 \mathrm{E} \quad 00$

$2.32140 \mathrm{E} \quad 00$

$2.33567 \mathrm{E} \quad 00$

$2.34999 \mathrm{E} \quad 00$

$2.36436 \mathrm{E} \quad 00$

$2.37877 \mathrm{E} \quad 00$

$2.39320 \mathrm{E} \quad 00$

$2.40773 \mathrm{E} \quad 00$

$2.42228 \mathrm{E} \quad 00$

$2.43687 \mathrm{E} \quad 00$

$2.45151 \mathrm{E} \quad 00$

$2.46620 \mathrm{E} \quad 00$

$2.48093 \mathrm{E} \quad 00$

$2.49571 \mathrm{E} \quad 00$

$2.51054 \mathrm{E} \quad 00$

$2.52541 \mathrm{E} \quad 00$

$2.54032 \mathrm{E} \quad 00$

$2.55529 \mathrm{E} \quad 00$

$2.57029 \mathrm{E} \quad 00$

$2.58535 \mathrm{E} \quad 00$

$2.60045 \mathrm{E} \quad 00$

$2.61560 \mathrm{E} \quad 00$

$2.63079 \mathrm{E} \quad 00$

$2.64603 \mathrm{E} \quad 00$

$2.66132 \mathrm{E} \quad 00$

$2.67665 \mathrm{E} \quad 00$

$2.69203 \mathrm{E} \quad 00$

$2.70746 \mathrm{E} \quad 00$

$2.72293 \mathrm{E} \quad 00$

$2.73845 \mathrm{E} \quad 00$ $2.75401 \mathrm{E} 00$ $2.76962 \mathrm{E} \quad 00$

$2.78528 \mathrm{E} \quad 00$

$2.80099 \mathrm{E} \quad 00$

$2.81674 \mathrm{E} \quad 00$

$2.84838 \mathrm{E} \quad 00$

$2.86427 \mathrm{E} \quad 00$

$2.88021 \mathrm{E} \quad 00$

$2.89619 \mathrm{E} \quad 00$

$2.91222 \mathrm{E} \quad 00$

$2.92830 \mathrm{E} \quad 00$

$2.94443 \mathrm{E} \quad 00$

$2.96060 \mathrm{E} \quad 00$

$2.97682 \mathrm{E} \quad 00$ $2.99309 \mathrm{E} \quad 00$ $3.00940 \mathrm{E} \quad 00$

$3.02576 \mathrm{E} \quad 00$

$3.04217 \mathrm{E} \quad 00$

$$
-1.08842 \mathrm{E}-03
$$
$-1.08891 \mathrm{E}-03$ $-1.08940 \mathrm{E}-03$ $-1.08988 \mathrm{E}-03$ $-1.09034 \mathrm{E}-03$

$-1.09078 \mathrm{E}-03$ $-1.09123 \mathrm{E}-03$ $-1.09165 \mathrm{E}-03$ $-1.09206 \mathrm{E}-03$ $-1.09246 \mathrm{E}-03$

$-1.09285 \mathrm{E}-03$ $-1.09322 \mathrm{E}-03$ $-1.09359 \mathrm{E}-03$ $-1.09394 \mathrm{E}-03$

$-1.09429 \mathrm{E}-03$

$-1.09461 \mathrm{E}-03$ $-1.09494 \mathrm{E}-03$ $-1.09524 \mathrm{E}-03$ $-1.09554 \mathrm{E}-03$ $-1.09582 \mathrm{E}-03$

$-1.09610 \mathrm{E}-03$ $-1.09637 \mathrm{E}-03$ $-1.09661 \mathrm{E}-03$ $-1.09685 \mathrm{E}-03$ $-1.09708 \mathrm{E}-03$

$-1.09731 \mathrm{E}-03$ $-1.09751 \mathrm{E}-03$ $-1.09771 \mathrm{E}-03$ $-1.09789 \mathrm{E}-03$ $-1.09807 \mathrm{E}-03$

$-1.09824 \mathrm{E}-03$ $-1.09839 \mathrm{E}-03$ $-1.09853 \mathrm{E}-03$ $-1.09867 \mathrm{E}-03$ $-1.09879 \mathrm{E}-03$

$-1.09890 \mathrm{E}-03$ $-1.09900 \mathrm{E}-03$ $-1.09910 \mathrm{E}-03$ 
ELECTRON RADIAL FUNCTIONS

$Z=6 \quad A=12 \quad \rho=1.1 A^{1 / 3} F$

Ponitron

$\bar{F}$

$1.34436 \mathrm{E}-03$

$1.35181 \mathrm{E}-03$

$1.35929 \mathrm{E}-03$

$1.36678 \mathrm{E}-03$

$1.37430 \mathrm{E}-03$

$1.38184 \mathrm{E}-03$

$1.38940 \mathrm{E}-03$

$1.39698 \mathrm{E}-03$

$1.40457 \mathrm{E}-03$

$1.41219 \mathrm{E}-03$

36.90

37.00

37.10

37.20

37.30

37.40

37.50

37.60

37.70

37.80

37.90

38.00

$1.41984 \mathrm{E}-03$

$1.42750 \mathrm{E}-03$

$1.43518 \mathrm{E}-03$

$1.44288 \mathrm{E}-03$

$1.45060 \mathrm{E}-03$

$1.45835 \mathrm{E}-03$

$1.46611 \mathrm{E}-03$

$1.47390 \mathrm{E}-03$

$1.48170 \mathrm{E}-03$

$1.48953 \mathrm{E}-03$

38.10

38.20

38.30

38.40

38.50

38.60

38.70

38.80

38.90

39.00

39.10

39.20

39.30

39.40

39.50

39.60

39.70

39.80

39.90

40.00

40.10

40.20

40.30

40.40

40.50

40.60

40.70

40.80

40.90

41.00

41.10

41.20

41.30

41.40

41.50

41.60

41.70

41.80

41.90

42.00

$1.49737 \mathrm{E}-03$

$1.50524 \mathrm{E}-03$

$1.51313 \mathrm{E}-03$

$1.52104 \mathrm{E}-03$

$1.52897 \mathrm{E}-03$

$1.53691 \mathrm{E}-03$

$1.54489 \mathrm{E}-03$

$1.55288 \mathrm{E}-03$

$1.56089 \mathrm{E}-03$

$1.56892 \mathrm{E}-03$

$1.57697 \mathrm{E}-03$

$1.58504 \mathrm{E}-03$

$1.59314 \mathrm{E}-03$

$1.60125 \mathrm{E}-03$

$1.60939 \mathrm{E}-03$

$1.61754 \mathrm{E}-03$

$1.62572 \mathrm{E}-03$

$1.63392 \mathrm{E}-03$

$1.64213 \mathrm{E}-03$

$1.65037 \mathrm{E}-03$

$1.65863 \mathrm{E}-03$

$1.66691 \mathrm{E}-03$

$1.67521 \mathrm{E}-03$

$1.68353 \mathrm{E}-03$

$1.69187 \mathrm{E}-03$

$1.70023 \mathrm{E}-03$

$1.70861 \mathrm{E}-03$

$1.71702 \mathrm{E}-03$

$1.72544 \mathrm{E}-03$

$1.73388 \mathrm{E}-03$

$1.74235 \mathrm{E}-03$

$1.75083 \mathrm{E}-03$

$1.75934 \mathrm{E}-03$

$1.76787 \mathrm{E}-03$

$1.77641 \mathrm{E}-03$

$1.78498 \mathrm{E}-03$

$1.79357 \mathrm{E}-03$

$1.80218 \mathrm{E}-03$

$1.81081 \mathrm{E}-03$

$1.81946 \mathrm{E}-03$ $f_{2}$

$2.60819 \mathrm{E} \quad 00$

$2.62271 \mathrm{E} \quad 00$

$2.63728 \mathrm{E} \quad 00$

$2.65188 \mathrm{E} \quad 00$

$2.66653 \mathrm{E} \quad 00$

$2.68121 \mathrm{E} \quad 00$

$2.69593 \mathrm{E} \quad 00$

$2.71070 \mathrm{E} \quad 00$

$2.725 .50 \mathrm{E} \quad 00$

$2.75523 \mathrm{E} \quad 00$

$2.77015 \mathrm{E} \quad 00$

$2.78512 \mathrm{E} \quad 00$

$2.80012 \mathrm{E} \quad 00$

$2.81516 \mathrm{E} \quad 00$

$2.83024 \mathrm{E} \quad 00$

$2.84537 \mathrm{E} \quad 00$

$2.86053 \mathrm{E} \quad 00$

$2.87573 \mathrm{E} \quad 00$

$2.89097 \mathrm{E} \quad 00$

$2.90625 \mathrm{E} \quad 00$

$2.92157 \mathrm{E} \quad 00$

$2.93693 \mathrm{E} \quad 00$

$2.95233 \mathrm{E} \quad 00$

$2.96777 \mathrm{E} \quad 00$

$2.98325 \mathrm{E} \quad 00$

$2.99877 \mathrm{E} \quad 00$

$3.01433 \mathrm{E} \quad 00$

$3.02993 \mathrm{E} \quad 00$

$3.04557 \mathrm{E} \quad 00$

$3.06125 \mathrm{E} \quad 00$

$3.07697 \mathrm{E} \quad 00$

$3.09272 \mathrm{E} \quad 00$

$3.10852 \mathrm{E} \quad 00$

$3.12436 \mathrm{E} \quad 00$

$3.14024 \mathrm{E} \quad 00$

$3.15615 \mathrm{E} \quad 00$

$3.17211 \mathrm{E} \quad 00$

$3.18810 \mathrm{E} \quad 00$

$3.22021 \mathrm{E} \quad 00$

$3.23633 \mathrm{E} \quad 00$

$3.25248 \mathrm{E} \quad 00$

$3.26868 \mathrm{E} \quad 00$

$3.28491 \mathrm{E} \quad 00$

$3.30118 \mathrm{E} \quad 00$

$3.31750 \mathrm{E} \quad 00$

$3.33385 \mathrm{E} \quad 00$

$3.35024 \mathrm{E} \quad 00$

$3.36667 \mathrm{E} \quad 00$

$3.38314 \mathrm{E} \quad 00$

$3.39965 \mathrm{E} \quad 00$

$3.41620 \mathrm{E} \quad 00$

$3.43279 \mathrm{E} \quad 00$

$3.44942 \mathrm{E} \quad 00$

$3.46609 \mathrm{E} \quad 00$

$3.48280 \mathrm{E} \quad 00$

$3.49955 \mathrm{E} \quad 00$

$3.51634 \mathrm{E} \quad 00$

$3.53316 \mathrm{E} \quad 00$
$2.74035 \mathrm{~F}, 00$

$g_{2}$

$\tan \delta_{2}$

$f_{2}$

$1.00252 \mathrm{E}-01 \quad-3.27545 \mathrm{E} \quad 0 \mathrm{l} \quad-9.60655 \mathrm{E}-02$

$1.01153 \mathrm{E}-01 \quad-3.27554 \mathrm{E}$ 01 $-9.69456 \mathrm{E}-02$

$1.02060 \mathrm{E}-0 \mathrm{l}-3.27564 \mathrm{E}$ ol $-9.78310 \mathrm{E}-02$

$1.02972 \mathrm{E}-01-3.27573 \mathrm{E}$ ol $-9.87218 \mathrm{E}-02$

$1.03889 \mathrm{E}-01 \quad-3.27583 \mathrm{E} \quad 01 \quad-9.96178 \mathrm{E}-02$

$1.04812 \mathrm{E}-01$

$1.05740 \mathrm{E}-01$

$1.06674 \mathrm{E}-01$

$1.07613 \mathrm{E}-01$

$1.08557 \mathrm{E}-01$

$1.09507 \mathrm{E}-01$

$1.10463 \mathrm{E}-01$

$1.11424 \mathrm{E}-01$

$1.12390 \mathrm{E}-01$

$1.13362 \mathrm{E}-01$

$1.14340 \mathrm{E}-01$

$1.15323 \mathrm{E}-01$

$1.16312 \mathrm{E}-01$

$1.17306 \mathrm{E}-01$

$1.18306 \mathrm{E}-01$

$3.20414 \mathrm{E} \quad 00$
$1.19311 \mathrm{E}-01$

$1.20323 \mathrm{E}-01$

$1.21339 \mathrm{E}-01$

$1.22362 \mathrm{E}-01$

$1.23390 \mathrm{E}-01$

$1.24424 \mathrm{E}-01$

$1.25464 \mathrm{E}-01$

$1.26509 \mathrm{E}-01$

$1.27560 \mathrm{E}-01$

$1.28617 \mathrm{E}-01$

$1.29680 \mathrm{E}-01$

$1.31823 \mathrm{E}-01$

$1.32903 \mathrm{E}-01$

$1.33989 \mathrm{E}-01$

$1.35081 \mathrm{E}-01$

$1.36179 \mathrm{E}-01$

$1.37282 \mathrm{E}-01$

$1.38392 \mathrm{E}-01$

$1.39507 \mathrm{E}-01$

$1.40629 \mathrm{E}-01$

$1.41756 \mathrm{E}-01$

$1.42889 \mathrm{E}-01$

$1.44029 \mathrm{E}-01$

$1.45174 \mathrm{E}-01$

$1.46325 \mathrm{E}-01$

$1.47483 \mathrm{E}-01$

$1.48646 \mathrm{E}-01$

$1.49816 \mathrm{E}-01$

$1.50991 \mathrm{E}-01$

$1.52173 \mathrm{E}-01$

$1.53361 \mathrm{E}-01$

$1.54554 \mathrm{E}-01$

$1.55754 \mathrm{E}-01$

$1.56961 \mathrm{E}-01$

$1.58173 \mathrm{E}-01$

$1.59392 \mathrm{E}-01$

$1.60616 \mathrm{E}-01$

$1.61847 \mathrm{E}-01$

$1.63084 \mathrm{E}-01$
$1.30749 \mathrm{E}-01$

$\begin{array}{lll}-3.27592 \mathrm{E} & 01 & -1.00519 \mathrm{E}-01 \\ -3.27602 \mathrm{E} & 01 & -1.01426 \mathrm{E}-01 \\ -3.27611 \mathrm{E} & 01 & -1.02338 \mathrm{E}-01 \\ -3.27620 \mathrm{E} & 01 & -1.03256 \mathrm{E}-01 \\ -3.27629 \mathrm{E} & 01 & -1.04179 \mathrm{E}-01\end{array}$

$-3.27639 \mathrm{E} 01$

$-3.27648 \mathrm{E}$ 0I

$-3.27657 \mathrm{E} \quad 01$

$-3.27666 \mathrm{E}$ 0l

$-3.27675 \mathrm{E} 01$

$-3.27684 \mathrm{E} \quad 01$

$-3.27692 \mathrm{E} \quad 01$

$-3.27701 \mathrm{E} \quad 01$

$-3.27710 \mathrm{E}$ 0I

$-3.27719 \mathrm{E} 01$

$-3.27727 \mathrm{E} \quad 01$

$-3.27736 \mathrm{E}$ 0l

$-3.27745 \mathrm{E}$ 0l

$-3.27753 \mathrm{E}$ 0l

$-3.27762 \mathrm{E}$ 0I

$-3.27770 \mathrm{E} \quad 01$

$-3.27778 \mathrm{E}$ 0I

$-3.27787 \mathrm{E} 01$

$-3.27795 \mathrm{E} 01$

-3.27803 E 01

$-3.27811 \mathrm{E} 01$

$-3.27820 \mathrm{E} \quad 01$

$-3.27828 \mathrm{E}$ 0l

$-3.27836 \mathrm{E}$ 0I

$-3.27844 \mathrm{E} 01$

$-3.27852 \mathrm{E}$ 01

$-3.27860 \mathrm{E} \quad 01$

$-3.27868 \mathrm{E} \quad 01$

$-3.27876 \mathrm{E}$ ol

$-3.27883 \mathrm{E} 01$

$-3.27891 \mathrm{E} \quad 01$

$-3.27899 \mathrm{E}$ 0I

$-3.27907 \mathrm{E} \quad 01$

$-3.27914 \mathrm{E} 01$

$-3.27922 \mathrm{E}$ 01

$-3.27930 \mathrm{E} \quad 01$

$-3.27937 \mathrm{E} \quad 01$

$-3.27945 \mathrm{E} \quad 01$

$-3.27952 \mathrm{E} \quad 01$

$-3.27960 \mathrm{E} 01$

$-3.27967 \mathrm{E}$ 0l

$-3.27974 \mathrm{E} 01$

$-3.27982 \mathrm{E} \quad 01$

$-3.27989 \mathrm{E} \quad 01$

$-3.27996 \mathrm{E} 01$

$-1.05107 \mathrm{E}-01$

$-1.06041 \mathrm{E}-01$

$-1.06980 \mathrm{E}-01$

$-1.07925 \mathrm{E}-0 \mathrm{l}$ 


$\begin{array}{cc}p & F_{0} \\ 0.10 & 1.36555 \mathrm{E}-01 \\ 0.20 & 4.04156 \mathrm{E}-01 \\ 0.30 & 5.54684 \mathrm{E}-01 \\ 0.40 & 6.42000 \mathrm{E}-01 \\ 0.50 & 6.97038 \mathrm{E}-01 \\ & \\ 0.60 & 7.33998 \mathrm{E}-01 \\ 0.70 & 7.60007 \mathrm{E}-01 \\ 0.80 & 7.78973 \mathrm{E}-01 \\ 0.90 & 7.93198 \mathrm{E}-01 \\ 1.00 & 8.04115 \mathrm{E}-01 \\ & \\ 1.10 & 8.12656 \mathrm{E}-01 \\ 1.20 & 8.19448 \mathrm{E}-01 \\ 1.30 & 8.24926 \mathrm{E}-01 \\ 1.40 & 8.29399 \mathrm{E}-01 \\ 1.50 & 8.33093 \mathrm{E}-01 \\ & \\ 1.60 & 8.36172 \mathrm{E}-01 \\ 1.70 & 8.38762 \mathrm{E}-01 \\ 1.80 & 8.40958 \mathrm{E}-01 \\ 1.90 & 8.42833 \mathrm{E}-01 \\ 2.00 & 8.44444 \mathrm{E}-01 \\ & \\ 2.10 & 8.45837 \mathrm{E}-01 \\ 2.20 & 8.47048 \mathrm{E}-01 \\ 2.30 & 8.48106 \mathrm{E}-01 \\ 2.40 & 8.49034 \mathrm{E}-01 \\ 2.50 & 8.49852 \mathrm{E}-01 \\ & \\ 2.60 & 8.50575 \mathrm{E}-01 \\ 2.70 & 8.51218 \mathrm{E}-01 \\ 2.80 & 8.51790 \mathrm{E}-01 \\ 2.90 & 8.52301 \mathrm{E}-01 \\ 3.00 & 8.52759 \mathrm{E}-01 \\ & \\ & \\ & \\ & \\ & \\ & \\ & \\ & \\ & \\ & \\ & \\ & \\ & \\ & \end{array}$

3.10

3.20

3.30

3.40

3.50

3.60

3.70

3.80

3.90

4.00

4.10

4.20

4.30

4.40

4.50

4.60

4.70

4.80

4.90

5.00

5.10

5.20

5.30

5.40

5.50

5.60

5.70

5.80

5.90

6.00

$8.53170 \mathrm{E}-01$

$8.53540 \mathrm{E}-01$

$8.53875 \mathrm{E}-01$

$8.54177 \mathrm{E}-01$

$8.54452 \mathrm{E}-01$

$8.54700 \mathrm{E}-01$

$8.54926 \mathrm{E}-01$

$8.55132 \mathrm{E}-01$

$8.55320 \mathrm{E}-01$

$8.55492 \mathrm{E}-01$

$8.55648 \mathrm{E}-01$

$8.55791 \mathrm{E}-01$

$8.55922 \mathrm{E}-01$

$8.56043 \mathrm{E}-01$

$8.56153 \mathrm{E}-01$

$8.56254 \mathrm{E}-01$

$8.56347 \mathrm{E}-01$

$8.56432 \mathrm{E}-01$

$8.56511 \mathrm{E}-01$

$8.56583 \mathrm{E}-01$

$8.56649 \mathrm{E}-01$

$8.56709 \mathrm{E}-01$

$8.56765 \mathrm{E}-01$

$8.56817 \mathrm{E}-01$

$8.56864 \mathrm{E}-01$

$8.56907 \mathrm{E}-01$

$8.56946 \mathrm{E}-01$

$8.56983 \mathrm{E}-01$

$8.57016 \mathrm{E}-01$

$8.57046 \mathrm{E}-01$ $f_{1}$

$2.91537 \mathrm{E}-03$

$1.82769 \mathrm{E}-02$

$4.65353 \mathrm{E}-02$

$8.64046 \mathrm{E}-02$

$1.36343 \mathrm{E}-01$

$1.94762 \mathrm{E}-01$

$2.60167 \mathrm{E}-01$

$3.31230 \mathrm{E}-01$

$4.06820 \mathrm{E}-01$

$4.86006 \mathrm{E}-01$

$5.68036 \mathrm{E}-01$

$7.38362 \mathrm{E}-01$

$8.25814 \mathrm{E}-01$

$9.14378 \mathrm{E}-01$

$1.00383 \mathrm{E} \quad 00$

$1.09398 \mathrm{E} \quad 00$

$1.18470 \mathrm{E} \quad 00$

$1.27588 \mathrm{E} \quad 00$

$1.36742 \mathrm{E} \quad 00$
0

$1.45925 \mathrm{E} \quad 00$
0

$1.55133 \mathrm{E} \quad 00$

$1.64361 \mathrm{E} \quad 00$

$1.73604 \mathrm{E} \quad 00$

$1.82861 \mathrm{E} \quad 00$

$1.92128 \mathrm{E} \quad 00$

$2.01404 \mathrm{E} \quad 00$

$2.10687 \mathrm{E} \quad 00$

$2.29271 \mathrm{E} \quad 00$

$4.71417 \mathrm{E} \quad 00$

$4.80736 \mathrm{E} \quad 00$

$4.90054 \mathrm{E} \quad 00$

$5.08691 \mathrm{E} \quad 00$
$6.52312 \mathrm{E}-01$

$2.19976 \mathrm{E} \quad 00$

$g_{1}$

$-4.62017 \mathrm{E}-05$

$-2.89025 \mathrm{E}-04$

$-7.33312 \mathrm{E}-04$

$-1.35505 \mathrm{E}-03$

$-2.12541 \mathrm{E}-03$

$-3.01462 \mathrm{E}-03$

$-3.99455 \mathrm{E}-03$

$-5.04014 \mathrm{E}-03$

$-6.13005 \mathrm{E}-03$

$-7.24662 \mathrm{E}-03$

$-8.37558 \mathrm{E}-03$

$-9.50554 \mathrm{E}-03$

$-1.06275 \mathrm{E}-02$

$-1.17343 \mathrm{E}-02$

$-1.28204 \mathrm{E}-02$

$-1.38815 \mathrm{E}-02$

$-1.49140 \mathrm{E}-02$

$-1.59153 \mathrm{E}-02$

$-1.68831 \mathrm{E}-02$

$-1.78159 \mathrm{E}-02$

$-1.87122 \mathrm{E}-02$

$-1.95709 \mathrm{E}-02$

$-2.03911 \mathrm{E}-02$

$-2.11722 \mathrm{E}-02$

$-2.19135 \mathrm{E}-02$

$-2.26146 \mathrm{E}-02$

$-2.32751 \mathrm{E}-02$

$-2.38946 \mathrm{E}-02$

$-2.44730 \mathrm{E}-02$

$-2.50099 \mathrm{E}-02$

$2.38569 \mathrm{E} \quad 00 \quad-2.55053 \mathrm{E}-02$

$2.47870 \mathrm{E} \quad 00 \quad-2.59589 \mathrm{E}-02$

$2.57175 \mathrm{E} \quad 00 \quad-2.63707 \mathrm{E}-02$

$2.66481 \mathrm{E} \quad 00 \quad-2.67405 \mathrm{E}-02$

$\begin{array}{lll}2.75790 \mathrm{E} & 00 & -2.70682 \mathrm{E}-02\end{array}$

$2.85100 \mathrm{E} \quad 00 \quad-2.73538 \mathrm{E}-02$

$2.94411 \mathrm{E} \quad 00 \quad-2.75972 \mathrm{E}-02$

$3.03724 \mathrm{E} \quad 00 \quad-2.77984 \mathrm{E}-02$

$3.13037 \mathrm{E} \quad 00 \quad-2.79572 \mathrm{E}-02$

$3.22351 \mathrm{E} \quad 00 \quad-2.80738 \mathrm{E}-02$

$3.31666 \mathrm{E} \quad 00 \quad-2.81479 \mathrm{E}-02$

$3.40981 \mathrm{E} \quad 00 \quad-2.81797 \mathrm{E}-02$

$3.50296 \mathrm{E} \quad 00 \quad-2.81691 \mathrm{E}-02$

$3.59612 \mathrm{E} \quad 00 \quad-2.81160 \mathrm{E}-02$

$3.68928 \mathrm{E} \quad 00 \quad-2.80205 \mathrm{E}-02$

$3.78244 \mathrm{E} \quad 00 \quad-2.78825 \mathrm{E}-02$

$3.87561 \mathrm{E} \quad 00 \quad-2.77021 \mathrm{E}-02$

$3.96878 \mathrm{E} \quad 00-2.74791 \mathrm{E}-02$

$4.06195 \mathrm{E} \quad 00 \quad-2.72136 \mathrm{E}-02$

$4.15512 \mathrm{E} \quad 00 \quad-2.69057 \mathrm{E}-02$

$4.24829 \mathrm{E} \quad 00 \quad-2.65552 \mathrm{E}-02$

$4.34146 \mathrm{E} \quad 00 \quad-2.61621 \mathrm{E}-02$

$4.43464 \mathrm{E} \quad 00 \quad-2.57265 \mathrm{E}-02$

$4.52782 \mathrm{E} \quad 00 \quad-2.52484 \mathrm{E}-02$

$\begin{array}{lll}4.62099 \mathrm{E} \quad 00 & -2.47278 \mathrm{E}-02\end{array}$

$4.99373 \mathrm{E} \quad 00$ $\tan \delta_{1}$

$2.30203 \mathrm{E}-01$

$1.11060 \mathrm{E}-01$

$7.38208 \mathrm{E}-02$

$5.53965 \mathrm{E}-02$

$4.43434 \mathrm{E}-02$

$3.69484 \mathrm{E}-02$

$3.16390 \mathrm{E}-02$

$2.76336 \mathrm{E}-02$

$2.44992 \mathrm{E}-02$

$2.19765 \mathrm{E}-02$

$1.99003 \mathrm{E}-02$

$1.81605 \mathrm{E}-02$

$1.66806 \mathrm{E}-02$

$1.54058 \mathrm{E}-02$

$1.42959 \mathrm{E}-02$

$1.33206 \mathrm{E}-02$

$1.24566 \mathrm{E}-02$

$1.16858 \mathrm{E}-02$

$1.09938 \mathrm{E}-02$

$1.03690 \mathrm{E}-02$

$9.80207 \mathrm{E}-03$

$9.28524 \mathrm{E}-03$

$8.81217 \mathrm{E}-03$

$8.37748 \mathrm{E}-03$

$7.97668 \mathrm{E}-03$

$7.60595 \mathrm{E}-03$

$7.26202 \mathrm{E}-03$

$6.94207 \mathrm{E}-03$

$6.64368 \mathrm{E}-03$

$6.36475 \mathrm{E}-03$

$6.10340 \mathrm{E}-03$

$5.85807 \mathrm{E}-03$

$5.62728 \mathrm{E}-03$

$5.40980 \mathrm{E}-03$

5.20451 E- 03

$5.01040 \mathrm{E}-03$

$4.82660 \mathrm{E}-03$

$4.65230 \mathrm{E}-03$

$4.48677 \mathrm{E}-03$

$4.32939 \mathrm{E}-03$

$4.17955 \mathrm{E}-03$

$4.03674 \mathrm{E}-03$

$3.90047 \mathrm{E}-03$

$3.77030 \mathrm{E}-03$

$3.64582 \mathrm{E}-03$

$3.52669 \mathrm{E}-03$

$3.41255 \mathrm{E}-03$

$3.30311 \mathrm{E}-03$

$3.19808 \mathrm{E}-03$

$3.09720 \mathrm{E}-03$

$3.00023 E-03$

$2.90694 \mathrm{E}-03$

$2.81714 \mathrm{E}-03$

$2.73062 \mathrm{E}-03$

$2.64722 \mathrm{E}-03$

$2.56678 \mathrm{E}-03$

$2.48913 \mathrm{E}-03$

$2.41413 \mathrm{E}-03$

$2.34165 \mathrm{E}-03$

$2.27157 \mathrm{E}-03$ $f_{-1}$

$1.06576 \mathrm{E}-03$

$3.64764 \mathrm{E}-03$

$6.35572 \mathrm{E}-03$

$9.01486 \mathrm{E}-03$

$1.15839 \mathrm{E}-02$

$1.40478 \mathrm{E}-02$

$1.64021 \mathrm{E}-02$

$1.86477 \mathrm{E}-02$

$2.07887 \mathrm{E}-02$

2.28304 E- 02

$2.47788 \mathrm{E}-02$

$2.66399 \mathrm{E}-02$

$2.84192 \mathrm{E}-02$

$3.01217 \mathrm{E}-02$

$3.17520 \mathrm{E}-02$

$3.33138 \mathrm{E}-02$

$3.48106 \mathrm{E}-02$

$3.62452 \mathrm{E}-02$

$3.76203 \mathrm{E}-02$

$3.89378 \mathrm{E}-02$

$4.01996 \mathrm{E}-02$

$4.14074 \mathrm{E}-02$

$4.25625 \mathrm{E}-02$

$4.36661 \mathrm{E}-02$

$4.47191 \mathrm{E}-02$

$4.57225 \mathrm{E}-02$

$4.66770 \mathrm{E}-02$

$4.75833 \mathrm{E}-02$

$4.84420 \mathrm{E}-02$

$4.92535 \mathrm{E}-02$

$5.00184 \mathrm{E}-02$

$5.07371 \mathrm{E}-02$

$5.14098 \mathrm{E}-02$

$5.20369 \mathrm{E}-02$

5.26187 E-02

$5.31554 \mathrm{E}-02$

$5.36473 \mathrm{E}-02$

$5.40944 \mathrm{E}-02$

$5.44971 \mathrm{E}-02$

$5.48555 \mathrm{E}-02$

$5.51697 \mathrm{E}-02$

$5.54398 \mathrm{E}-02$

$5.56660 \mathrm{E}-02$

$5.58483 \mathrm{E}-02$

$5.59869 \mathrm{E}-02$

$5.60818 \mathrm{E}-02$

$5.61332 \mathrm{E}-02$

$5.61410 \mathrm{E}-02$

$5.61054 \mathrm{E}-02$

$5.60264 \mathrm{E}-02$

5.59041 E-02

$5.57385 \mathrm{E}-02$

$5.55297 \mathrm{E}-02$

$5.52776 \mathrm{E}-02$

$5.49825 \mathrm{E}-02$

$5.46442 \mathrm{E}-02$

$5.42628 \mathrm{E}-02$

5.38383 E- 02

$5.33708 \mathrm{E}-02$

$5.28603 \mathrm{E}-02$

$g_{-1}$

$5.21783 \mathrm{E}-02$

$1.78881 \mathrm{E}-01$

$3.12536 \mathrm{E}-01$

$4.44951 \mathrm{E}-01$

$5.74412 \mathrm{E}-01$

$7.00421 \mathrm{E}-01$

$8.22924 \mathrm{E}-01$

$9.42083 \mathrm{E}-01$

$1.05817 \mathrm{E} \quad 00$ 


\begin{tabular}{|c|c|c|c|c|c|c|c|c|}
\hline \multirow[b]{2}{*}{$p$} & \multirow[b]{2}{*}{$\bar{F}$} & \multicolumn{2}{|c|}{$Z=7$} & \multicolumn{3}{|c|}{$\rho=1.1 A^{1 / 3} F$} & \multicolumn{2}{|r|}{ Pusitron } \\
\hline & & $f_{2}$ & $g_{2}$ & $\tan \delta_{2}$ & & $f_{-2}$ & $g=$ & $\tan \delta_{z}$ \\
\hline $\begin{array}{l}0.10 \\
0.20 \\
0.30 \\
0.40 \\
0.50\end{array}$ & $\begin{array}{l}2.23450 \mathrm{E}-09 \\
2.23671 \mathrm{E}-08 \\
6.67545 \mathrm{E}-08 \\
1.35713 \mathrm{E}-07 \\
2.28968 \mathrm{E}-07\end{array}$ & $\begin{array}{l}6.83577 \mathrm{E}-07 \\
8.39413 \mathrm{E}-06 \\
3.19354 \mathrm{E}-05 \\
7.89613 \mathrm{E}-05 \\
1.55664 \mathrm{E}-04\end{array}$ & $\begin{array}{l}-6.09984 \mathrm{E}-09 \\
-7.47335 \mathrm{E}-08 \\
-2.83260 \mathrm{E}-07 \\
-6.96792 \mathrm{E}-07 \\
-1.36489 \mathrm{E}-06\end{array}$ & $\begin{array}{l}-1.93299 \mathrm{E} \\
-4.07750 \mathrm{E} \\
-6.06943 \mathrm{E} \\
-7.92500 \mathrm{E} \\
-9.63454 \mathrm{E}\end{array}$ & $\begin{array}{l}00 \\
00 \\
00 \\
00 \\
00\end{array}$ & $\begin{array}{l}1.55834 \mathrm{E}-07 \\
9.80775 \mathrm{E}-07 \\
2.51969 \mathrm{E}-06 \\
4.73567 \mathrm{E}-06 \\
7.58375 \mathrm{E}-06\end{array}$ & $\begin{array}{l}1.33536 \mathrm{E}-0.5 \\
8.41906 \mathrm{E}-0.5 \\
2.16912 \mathrm{E}-04 \\
4.09276 \mathrm{E}-04 \\
6.58624 \mathrm{E}-04\end{array}$ & $\begin{array}{l}2.31355 \mathrm{E}-01 \\
1.13974 \mathrm{E}-01 \\
7.85227 \mathrm{E}-02 \\
6.18327 \mathrm{E}-02 \\
5.24336 \mathrm{E}-02\end{array}$ \\
\hline $\begin{array}{l}0.60 \\
0.70 \\
0.80 \\
0.90 \\
1.00\end{array}$ & $\begin{array}{l}3.46183 \mathrm{E}-07 \\
4.87056 \mathrm{E}-07 \\
6.51334 \mathrm{E}-07 \\
8.38811 \mathrm{E}-07 \\
1.04933 \mathrm{E}-06\end{array}$ & $\begin{array}{l}2.66766 \mathrm{E}-04 \\
4.15693 \mathrm{E}-04 \\
6.04803 \mathrm{E}-04 \\
8.35654 \mathrm{E}-04 \\
1.10923 \mathrm{E}-03\end{array}$ & $\begin{array}{l}-2.32141 \mathrm{E}-06 \\
-3.58627 \mathrm{E}-06 \\
-5.16796 \mathrm{E}-06 \\
-7.06626 \mathrm{E}-06 \\
-9.27468 \mathrm{E}-06\end{array}$ & $\begin{array}{l}-1.11927 \mathrm{E} \\
-1.26008 \mathrm{E} \\
-1.38656 \mathrm{E} \\
-1.49972 \mathrm{E} \\
-1.60074 \mathrm{E}\end{array}$ & $\begin{array}{l}01 \\
01 \\
01 \\
01 \\
01\end{array}$ & $\begin{array}{l}1.10168 \mathrm{E}-05 \\
1.49892 \mathrm{E}-0.5 \\
1.94582 \mathrm{E}-05 \\
2.43852 \mathrm{E}-05 \\
2.97356 \mathrm{E}-05\end{array}$ & $\begin{array}{l}9.62305 \mathrm{E}-04 \\
1.31789 \mathrm{E}-0.3 \\
1.72330 \mathrm{E}-0.3 \\
2.17680 \mathrm{E}-0.3 \\
2.67701 \mathrm{E}-0.3\end{array}$ & $\begin{array}{l}4.65986 \mathrm{E}-02 \\
4.27492 \mathrm{E}-02 \\
4.01026 \mathrm{E}-02 \\
3.82283 \mathrm{E}-02 \\
3.68714 \mathrm{E}-02\end{array}$ \\
\hline $\begin{array}{l}1.10 \\
1.20 \\
1.30 \\
1.40 \\
1.50\end{array}$ & $\begin{array}{l}1.28275 \mathrm{E}-06 \\
1.53898 \mathrm{E}-06 \\
1.81795 \mathrm{E}-06 \\
2.11958 \mathrm{E}-06 \\
2.44382 \mathrm{E}-06\end{array}$ & $\begin{array}{l}1.42609 \mathrm{E}-03 \\
1.78656 \mathrm{E}-03 \\
2.19078 \mathrm{E}-03 \\
2.63878 \mathrm{E}-03 \\
3.13051 \mathrm{E}-03\end{array}$ & $\begin{array}{l}-1.17823 \mathrm{E}-05 \\
-1.45754 \mathrm{E}-05 \\
-1.76383 \mathrm{E}-05 \\
-2.09540 \mathrm{E}-05 \\
-2.45049 \mathrm{E}-05\end{array}$ & $\begin{array}{l}-1.69088 \mathrm{E} \\
-1.77135 \mathrm{E} \\
-1.84327 \mathrm{E} \\
-1.90768 \mathrm{E} \\
-1.96550 \mathrm{E}\end{array}$ & $\begin{array}{l}01 \\
01 \\
01 \\
01 \\
01\end{array}$ & $\begin{array}{l}3.54781 \mathrm{E}-05 \\
4.15848 \mathrm{E}-05 \\
4.80299 \mathrm{E}-05 \\
5.47898 \mathrm{E}-05 \\
6.18423 \mathrm{E}-05\end{array}$ & $\begin{array}{l}3.22284 \mathrm{E}-0.3 \\
3.81347 \mathrm{E}-03 \\
4.44825 \mathrm{E}-03 \\
5.12670 \mathrm{E}-03 \\
5.84847 \mathrm{E}-03\end{array}$ & $\begin{array}{l}3.58726 \mathrm{E}-02 \\
3.51280 \mathrm{E}-02 \\
3.45678 \mathrm{E}-02 \\
3.41435 \mathrm{E}-02 \\
3.38211 \mathrm{E}-02\end{array}$ \\
\hline $\begin{array}{l}1.60 \\
1.70 \\
1.80 \\
1.90 \\
2.00\end{array}$ & $\begin{array}{l}2.79065 \mathrm{E}-06 \\
3.16002 \mathrm{E}-06 \\
3.55192 \mathrm{E}-06 \\
3.96631 \mathrm{E}-06 \\
4.40319 \mathrm{E}-06\end{array}$ & $\begin{array}{l}3.66592 \mathrm{E}-03 \\
4.24492 \mathrm{E}-03 \\
4.86740 \mathrm{E}-03 \\
5.53327 \mathrm{E}-03 \\
6.24245 \mathrm{E}-03\end{array}$ & $\begin{array}{l}-2.82730 \mathrm{E}-05 \\
-3.22399 \mathrm{E}-05 \\
-3.63872 \mathrm{E}-05 \\
-4.06967 \mathrm{E}-05 \\
-4.51499 \mathrm{E}-05\end{array}$ & $\begin{array}{l}-2.01754 \mathrm{E} \\
-2.06450 \mathrm{E} \\
-2.10700 \mathrm{E} \\
-2.14558 \mathrm{E} \\
-2.18070 \mathrm{E}\end{array}$ & $\begin{array}{l}01 \\
01 \\
01 \\
01 \\
01\end{array}$ & $\begin{array}{l}6.91664 \mathrm{E}-05 \\
7.67418 \mathrm{E}-05 \\
8.45491 \mathrm{E}-05 \\
9.25694 \mathrm{E}-05 \\
1.00784 \mathrm{E}-04\end{array}$ & $\begin{array}{l}6.61328 \mathrm{E}-03 \\
7.42093 \mathrm{E}-03 \\
8.27126 \mathrm{E}-03 \\
9.16417 \mathrm{E}-03 \\
1.00996 \mathrm{E}-02\end{array}$ & $\begin{array}{l}3.35758 \mathrm{E}-02 \\
3.33896 \mathrm{E}-02 \\
3.32490 \mathrm{E}-02 \\
3.31440 \mathrm{E}-02 \\
3.30670 \mathrm{E}-02\end{array}$ \\
\hline $\begin{array}{l}2.10 \\
2.20 \\
2.30 \\
2.40 \\
2.50\end{array}$ & $\begin{array}{l}4.86254 \mathrm{E}-06 \\
5.34435 \mathrm{E}-06 \\
5.84861 \mathrm{E}-06 \\
6.37531 \mathrm{E}-06 \\
6.92444 \mathrm{E}-06\end{array}$ & $\begin{array}{l}6.99485 \mathrm{E}-03 \\
7.79039 \mathrm{E}-03 \\
8.62899 \mathrm{E}-03 \\
9.51059 \mathrm{E}-03 \\
1.04351 \mathrm{E}-02\end{array}$ & $\begin{array}{l}-4.97284 \mathrm{E}-05 \\
-5.44141 \mathrm{E}-05 \\
-5.91887 \mathrm{E}-05 \\
-6.40341 \mathrm{E}-05 \\
-6.89323 \mathrm{E}-05\end{array}$ & $\begin{array}{l}-2.21276 \mathrm{E} \\
-2.24210 \mathrm{E} \\
-2.26903 \mathrm{E} \\
-2.29381 \mathrm{E} \\
-2.31667 \mathrm{E}\end{array}$ & $\begin{array}{l}01 \\
01 \\
01 \\
01 \\
01\end{array}$ & $\begin{array}{l}1.09174 \mathrm{E}-04 \\
1.17723 \mathrm{E}-04 \\
1.26411 \mathrm{E}-04 \\
1.35222 \mathrm{E}-04 \\
1.44136 \mathrm{E}-04\end{array}$ & $\begin{array}{l}1.10774 \mathrm{E}-02 \\
1.20976 \mathrm{E}-02 \\
1.31602 \mathrm{E}-02 \\
1.42650 \mathrm{E}-02 \\
1.54122 \mathrm{E}-02\end{array}$ & $\begin{array}{l}3.30120 \mathrm{E}-02 \\
3.29745 \mathrm{E}-02 \\
3.29508 \mathrm{E}-02 \\
3.29382 \mathrm{E}-02 \\
3.29345 \mathrm{E}-02\end{array}$ \\
\hline $\begin{array}{l}2.60 \\
2.70 \\
2.80 \\
2.90 \\
3.00\end{array}$ & $\begin{array}{l}7.49600 \mathrm{E}-06 \\
8.08998 \mathrm{E}-06 \\
8.70638 \mathrm{E}-06 \\
9.34519 \mathrm{E}-06 \\
1.00064 \mathrm{E}-05\end{array}$ & $\begin{array}{l}1.14026 \mathrm{E}-02 \\
1.24129 \mathrm{E}-02 \\
1.34659 \mathrm{E}-02 \\
1.45618 \mathrm{E}-02 \\
1.57004 \mathrm{E}-02\end{array}$ & $\begin{array}{l}-7.38652 \mathrm{E}-05 \\
-7.88149 \mathrm{E}-05 \\
-8.37635 \mathrm{E}-05 \\
-8.86932 \mathrm{E}-05 \\
-9.35862 \mathrm{E}-05\end{array}$ & $\begin{array}{l}-2.33781 \mathbf{E} \\
-2.35740 \mathbf{E} \\
-2.37559 \mathbf{E} \\
-2.39253 \mathbf{E} \\
-2.40832 \mathbf{E}\end{array}$ & $\begin{array}{l}01 \\
01 \\
01 \\
01 \\
01\end{array}$ & $\begin{array}{l}1.53138 \mathrm{E}-04 \\
1.62209 \mathrm{E}-04 \\
1.71331 \mathrm{E}-04 \\
1.80488 \mathrm{E}-04 \\
1.89661 \mathrm{E}-04\end{array}$ & $\begin{array}{l}1.66017 \mathrm{E}-02 \\
1.78335 \mathrm{E}-02 \\
1.91076 \mathrm{E}-02 \\
2.04239 \mathrm{E}-02 \\
2.17825 \mathrm{E}-02\end{array}$ & $\begin{array}{l}3.29378 \mathbf{E}-02 \\
3.29468 \mathbf{E}-02 \\
3.29603 \mathbf{E}-02 \\
3.29774 \mathbf{E}-02 \\
3.29973 \mathbf{E}-02\end{array}$ \\
\hline $\begin{array}{l}3.10 \\
3.20 \\
3.30 \\
3.40 \\
3.50\end{array}$ & $\begin{array}{l}1.06900 \mathrm{E}-05 \\
1.13961 \mathrm{E}-05 \\
1.21245 \mathrm{E}-05 \\
1.28753 \mathrm{E}-05 \\
1.36486 \mathrm{E}-05\end{array}$ & $\begin{array}{l}1.68816 \mathrm{E}-02 \\
1.81056 \mathrm{E}-02 \\
1.93722 \mathrm{E}-02 \\
2.06814 \mathrm{E}-02 \\
2.20332 \mathrm{E}-02\end{array}$ & $\begin{array}{l}-9.84246 \mathrm{E}-05 \\
-1.03191 \mathrm{E}-04 \\
-1.07867 \mathrm{E}-04 \\
-1.12435 \mathrm{E}-04 \\
-1.16879 \mathrm{E}-04\end{array}$ & $\begin{array}{l}-2.42308 \mathrm{E} \\
-2.43690 \mathrm{E} \\
-2.44985 \mathrm{E} \\
-2.46202 \mathrm{E} \\
-2.47347 \mathrm{E}\end{array}$ & $\begin{array}{l}01 \\
01 \\
01 \\
01 \\
01\end{array}$ & $\begin{array}{l}1.98834 \mathrm{E}-04 \\
2.07988 \mathrm{E}-04 \\
2.17106 \mathrm{E}-04 \\
2.26172 \mathrm{E}-04 \\
2.35166 \mathrm{E}-04\end{array}$ & $\begin{array}{l}2.31834 \mathrm{E}-02 \\
2.46266 \mathrm{E}-02 \\
2.61121 \mathrm{E}-02 \\
2.76398 \mathrm{E}-02 \\
2.92099 \mathrm{E}-02\end{array}$ & $\begin{array}{l}3.30195 \mathrm{E}-02 \\
3.30434 \mathrm{E}-02 \\
3.30686 \mathrm{E}-02 \\
3.30948 \mathrm{E}-02 \\
3.31217 \mathrm{E}-02\end{array}$ \\
\hline $\begin{array}{l}3.60 \\
3.70 \\
3.80 \\
3.90 \\
4.00\end{array}$ & $\begin{array}{l}1.44442 \mathrm{E}-05 \\
1.52622 \mathrm{E}-05 \\
1.61026 \mathrm{E}-05 \\
1.69654 \mathrm{E}-05 \\
1.78506 \mathrm{E}-05\end{array}$ & $\begin{array}{l}2.34276 \mathrm{E}-02 \\
2.48646 \mathrm{E}-02 \\
2.63441 \mathrm{E}-02 \\
2.78662 \mathrm{E}-02 \\
2.94308 \mathrm{E}-02\end{array}$ & $\begin{array}{l}-1.21179 \mathrm{E}-04 \\
-1.25319 \mathrm{E}-04 \\
-1.29280 \mathrm{E}-04 \\
-1.33046 \mathrm{E}-04 \\
-1.36598 \mathrm{E}-04\end{array}$ & $\begin{array}{l}-2.48426 \mathrm{E} \\
-2.49445 \mathrm{E} \\
-2.50407 \mathrm{E} \\
-2.51318 \mathrm{E} \\
-2.52181 \mathrm{E}\end{array}$ & $\begin{array}{l}01 \\
01 \\
01 \\
01 \\
01\end{array}$ & $\begin{array}{l}2.44073 \mathrm{E}-04 \\
2.52874 \mathrm{E}-04 \\
2.61553 \mathrm{E}-04 \\
2.70091 \mathrm{E}-04 \\
2.78472 \mathrm{E}-04\end{array}$ & $\begin{array}{l}3.08222 \mathrm{E}-02 \\
3.24768 \mathrm{E}-02 \\
3.41738 \mathrm{E}-02 \\
3.59130 \mathrm{E}-02 \\
3.76946 \mathrm{E}-02\end{array}$ & $\begin{array}{l}3.31491 \mathrm{E}-02 \\
3.31768 \mathrm{E}-02 \\
3.320+7 \mathrm{E}-02 \\
3.32326 \mathrm{E}-02 \\
3.3260+\mathrm{E}-02\end{array}$ \\
\hline $\begin{array}{l}4.10 \\
4.20 \\
4.30 \\
4.40 \\
4.50\end{array}$ & $\begin{array}{l}1.87582 \mathrm{E}-05 \\
1.96882 \mathrm{E}-05 \\
2.06405 \mathrm{E}-0.5 \\
2.16153 \mathrm{E}-05 \\
2.26124 \mathrm{E}-05\end{array}$ & $\begin{array}{l}3.10379 \mathrm{E}-02 \\
3.26875 \mathrm{E}-02 \\
3.43796 \mathrm{E}-02 \\
3.61142 \mathrm{E}-02 \\
3.78913 \mathrm{E}-02\end{array}$ & $\begin{array}{l}-1.39919 \mathrm{E}-04 \\
-1.42992 \mathrm{E}-04 \\
-1.45799 \mathrm{E}-04 \\
-1.48322 \mathrm{E}-04 \\
-1.50544 \mathrm{E}-04\end{array}$ & $\begin{array}{l}-2.52999 \mathrm{E} \\
-2.53777 \mathrm{E} \\
-2.54516 \mathrm{E} \\
-2.55220 \mathrm{E} \\
-2.55891 \mathrm{E}\end{array}$ & $\begin{array}{l}01 \\
01 \\
01 \\
01 \\
01\end{array}$ & $\begin{array}{l}2.86677 \mathrm{E}-04 \\
2.94690 \mathrm{E}-04 \\
3.02493 \mathrm{E}-04 \\
3.10068 \mathrm{E}-04 \\
3.17399 \mathrm{E}-04\end{array}$ & $\begin{array}{l}3.95184 \mathrm{E}-02 \\
4.13846 \mathrm{E}-02 \\
4.32931 \mathrm{E}-02 \\
4.52439 \mathrm{E}-02 \\
4.72370 \mathrm{E}-02\end{array}$ & $\begin{array}{l}3.32880 \mathrm{E}-02 \\
3.33155 \mathrm{E}-02 \\
3.33426 \mathrm{E}-02 \\
3.33694 \mathrm{E}-02 \\
3.33959 \mathrm{E}-02\end{array}$ \\
\hline $\begin{array}{l}4.60 \\
4.70 \\
4.80 \\
4.90 \\
5.00\end{array}$ & $\begin{array}{l}2.36319 \mathrm{E}-05 \\
2.46737 \mathrm{E}-05 \\
2.57380 \mathrm{E}-05 \\
2.68246 \mathrm{E}-05 \\
2.79337 \mathrm{E}-05\end{array}$ & $\begin{array}{l}3.97108 \mathrm{E}-02 \\
4.15728 \mathrm{E}-02 \\
4.34773 \mathrm{E}-02 \\
4.54242 \mathrm{E}-02 \\
4.74136 \mathrm{E}-02\end{array}$ & $\begin{array}{l}-1.52447 \mathrm{E}-04 \\
-1.54014 \mathrm{E}-04 \\
-1.55228 \mathrm{E}-04 \\
-1.56070 \mathrm{E}-04 \\
-1.56523 \mathrm{E}-04\end{array}$ & $\begin{array}{l}-2.56531 \mathrm{E} \\
-2.57142 \mathrm{E} \\
-2.57726 \mathrm{E} \\
-2.58285 \mathrm{E} \\
-2.58820 \mathrm{E}\end{array}$ & $\begin{array}{l}01 \\
01 \\
01 \\
01 \\
01\end{array}$ & $\begin{array}{l}3.24467 \mathrm{E}-04 \\
3.31256 \mathrm{E}-04 \\
3.37747 \mathrm{E}-04 \\
3.43925 \mathrm{E}-04 \\
3.49770 \mathrm{E}-04\end{array}$ & $\begin{array}{l}4.92725 \mathrm{E}-02 \\
5.13502 \mathrm{E}-02 \\
5.3+703 \mathrm{E}-02 \\
5.56328 \mathrm{E}-02 \\
5.78376 \mathrm{E}-02\end{array}$ & $\begin{array}{l}3.34220 \mathrm{E}-02 \\
3.34476 \mathrm{E}-02 \\
3.34729 \mathrm{E}-02 \\
3.34977 \mathrm{E}-02 \\
3.35221 \mathrm{E}-02\end{array}$ \\
\hline $\begin{array}{l}5.10 \\
5.20 \\
5.30 \\
5.40 \\
5.50\end{array}$ & $\begin{array}{l}2.90651 \mathrm{E}-05 \\
3.02188 \mathrm{E}-05 \\
3.13950 \mathrm{E}-05 \\
3.25935 \mathrm{E}-05 \\
3.38144 \mathrm{E}-05\end{array}$ & $\begin{array}{l}4.94454 \mathrm{E}-02 \\
5.15196 \mathrm{E}-02 \\
5.36362 \mathrm{E}-02 \\
5.57953 \mathrm{E}-02 \\
5.79968 \mathrm{E}-02\end{array}$ & $\begin{array}{l}-1.56570 \mathrm{E}-04 \\
-1.56194 \mathrm{E}-04 \\
-1.55376 \mathrm{E}-04 \\
-1.54099 \mathrm{E}-04 \\
-1.52347 \mathrm{E}-04\end{array}$ & $\begin{array}{l}-2.59332 \mathrm{E} \\
-2.59824 \mathrm{E} \\
-2.60296 \mathrm{E} \\
-2.60749 \mathrm{E} \\
-2.61185 \mathrm{E}\end{array}$ & $\begin{array}{l}01 \\
01 \\
01 \\
01 \\
01\end{array}$ & $\begin{array}{l}3.55266 \mathrm{E}-04 \\
3.60395 \mathrm{E}-04 \\
3.65139 \mathrm{E}-04 \\
3.69482 \mathrm{E}-04 \\
3.73406 \mathrm{E}-04\end{array}$ & $\begin{array}{l}6.00847 \mathrm{E}-02 \\
6.23742 \mathrm{E}-02 \\
6.47059 \mathrm{E}-02 \\
6.70801 \mathrm{E}-02 \\
6.94966 \mathrm{E}-02\end{array}$ & $\begin{array}{l}3.35+60 \mathrm{E}-02 \\
3.35695 \mathrm{E}-02 \\
3.35925 \mathrm{E}-02 \\
3.36151 \mathrm{E}-02 \\
3.36372 \mathrm{E}-02\end{array}$ \\
\hline $\begin{array}{l}5.60 \\
5.70 \\
5.80 \\
5.90 \\
6.00\end{array}$ & $\begin{array}{l}3.50577 \mathrm{E}-05 \\
3.63234 \mathrm{E}-05 \\
3.76114 \mathrm{E}-05 \\
3.89218 \mathrm{E}-05 \\
4.02545 \mathrm{E}-05\end{array}$ & $\begin{array}{l}6.02407 \mathrm{E}-02 \\
6.25270 \mathrm{E}-02 \\
6.48557 \mathrm{E}-02 \\
6.72268 \mathrm{E}-02 \\
6.96403 \mathrm{E}-02\end{array}$ & $\begin{array}{l}-1.50100 \mathrm{E}-04 \\
-1.47342 \mathrm{E}-04 \\
-1.44056 \mathrm{E}-04 \\
-1.40223 \mathrm{E}-04 \\
-1.35827 \mathrm{E}-04\end{array}$ & $\begin{array}{l}-2.61604 \mathrm{E} \\
-2.62007 \mathrm{E} \\
-2.62396 \mathrm{E} \\
-2.62770 \mathrm{E} \\
-2.63132 \mathrm{E}\end{array}$ & $\begin{array}{l}01 \\
01 \\
01 \\
01 \\
01\end{array}$ & $\begin{array}{l}3.76893 \mathrm{E}-04 \\
3.79927 \mathrm{E}-04 \\
3.82489 \mathrm{E}-04 \\
3.84561 \mathrm{E}-04 \\
3.86128 \mathrm{E}-04\end{array}$ & $\begin{array}{l}7.19551 \mathrm{E}-02 \\
7.44565 \mathrm{E}-02 \\
7.70001 \mathrm{E}-02 \\
7.95859 \mathrm{E}-02 \\
8.22142 \mathrm{E}-02\end{array}$ & $\begin{array}{l}3.36589 \mathrm{E}-02 \\
3.36802 \mathrm{E}-02 \\
3.37010 \mathrm{E}-02 \\
3.37214 \mathrm{E}-02 \\
3.37+14 \mathrm{E}-02\end{array}$ \\
\hline
\end{tabular}


ELECTRON RADIAL FUNCTIONS

Positron

$\begin{array}{cc}p & F_{0} \\ 6.10 & 8.57074 \mathrm{E}-01 \\ 6.20 & 8.57099 \mathrm{E}-01 \\ 6.30 & 8.57121 \mathrm{E}-01 \\ 6.40 & 8.57142 \mathrm{E}-01 \\ 6.50 & 8.57161 \mathrm{E}-01 \\ & \\ 6.60 & 8.57178 \mathrm{E}-01 \\ 6.70 & 8.57193 \mathrm{E}-01 \\ 6.80 & 8.57207 \mathrm{E}-01 \\ 6.90 & 8.57219 \mathrm{E}-01 \\ 7.00 & 8.57229 \mathrm{E}-0] \\ & \\ 7.10 & 8.57239 \mathrm{E}-01 \\ 7.20 & 8.57246 \mathrm{E}-01 \\ 7.30 & 8.57254 \mathrm{E}-01 \\ 7.40 & 8.57259 \mathrm{E}-01 \\ 7.50 & 8.57263 \mathrm{E}-01\end{array}$

$7.60 \quad 8.57267 \mathrm{E}-01$

$7.70 \quad 8.57270 \mathrm{E}-01$

$7.80 \quad 8.57272 \mathrm{E}-01$

$7.90 \quad 8.57274 \mathrm{E}-01$

$8.00 \quad 8.57274 \mathrm{E}-01$

$8.10 \quad 8.57274 \mathrm{E}-01$

$8.20 \quad 8.57273 \mathrm{E}-01$

$8.30 \quad 8.57272 \mathrm{E}-01$

$8.40 \quad 8.57269 \mathrm{E}-01$

$8.50 \quad 8.57267 \mathrm{E}-01$

$8.60 \quad 8.57263 \mathrm{E}-01$

$8.70 \quad 8.57260 \mathrm{E}-01$

$8.80 \quad 8.57256 \mathrm{E}-01$

$8.90 \quad 8.57251 \mathrm{E}-01$

$9.00 \quad 8.57247 \mathrm{E}-01$

$9.10 \quad 8.57241 \mathrm{E}-01$

$9.20 \quad 8.57235 \mathrm{E}-01$

$9.30 \quad 8.57229 \mathrm{E}-01$

$9.40 \quad 8.57223 \mathrm{E}-01$

$9.50 \quad 8.57217 \mathrm{E}-01$

$9.60 \quad 8.57210 \mathrm{E}-01$

$9.70 \quad 8.57203 \mathrm{E}-01$

$9.80 \quad 8.57194 \mathrm{E}-01$

$9.90 \quad 8.57187 \mathrm{E}-01$

$10.00 \quad 8.57179 \mathrm{E}-01$

$10.10 \quad 8.57171 \mathrm{E}-01$

$10.20 \quad 8.57163 \mathrm{E}-01$

$10.30 \quad 8.57154 \mathrm{E}-01$

$10.40 \quad 8.57146 \mathrm{E}-01$

$10.50 \quad 8.57137 \mathrm{E}-01$

$10.60 \quad 8.57128 \mathrm{E}-01$

$10.70 \quad 8.57119 \mathrm{E}-01$

$10.80 \quad 8.57110 \mathrm{E}-01$

$10.90 \quad 8.57101 \mathrm{E}-01$

$11.00 \quad 8.57091 \mathrm{E}-01$

$11.10 \quad 8.57082 \mathrm{E}-01$

$11.20 \quad 8.57072 \mathrm{E}-01$

$11.30 \quad 8.57063 \mathrm{E}-01$

$11.40 \quad 8.57053 \mathrm{E}-01$

$11.50 \quad 8.57043 \mathrm{E}-01$

$11.60 \quad 8.57033 \mathrm{E}-01$

$11.70 \quad 8.57023 \mathrm{E}-01$

$11.80 \quad 8.57012 \mathrm{E}-01$

$11.90 \quad 8.57002 \mathrm{E}-01$

$12.00 \quad 8.56992 \mathrm{E}-01$
$Z=7 \quad A=14$

$g_{1}$
$-2.07100 \mathrm{E}-02$
$-1.98913 \mathrm{E}-02$
$-1.90301 \mathrm{E}-02$
$-1.81263 \mathrm{E}-02$
$-1.71799 \mathrm{E}-02$
$-1.61909 \mathrm{E}-02$
$-1.51593 \mathrm{E}-02$
$-1.40851 \mathrm{E}-02$
$-1.29683 \mathrm{E}-02$
$-1.18088 \mathrm{E}-02$

$\tan \delta_{1}$

$\rho=1.1 A^{1 / 3} F$

$f_{1}$

$5.18011 \mathrm{E} \quad 00$

$5.27330 \mathrm{E} \quad 00$

$5.36649 \mathrm{E} \quad 00$

$5.45969 \mathrm{E} \quad 00$

$5.55289 \mathrm{E} \quad 00$

$5.64610 \mathrm{E} \quad 00$

$5.73931 \mathrm{E} \quad 00$

$5.83252 \mathrm{E} \quad 00$

$5.92573 \mathrm{E} \quad 00$

$6.01895 \mathrm{E} \quad 00$

$6.11217 \mathrm{E} \quad 00$

$-1.06068 \mathrm{E}-02$

$6.20540 \mathrm{E} \quad 00 \quad-9.36210 \mathrm{E}-03$

$6.29863 \mathrm{E} \quad 00 \quad-8.07481 \mathrm{E}-03$

$\begin{array}{lll}6.39186 \mathrm{E} & 00 & -6.74489 \mathrm{E}-03\end{array}$

$6.48509 \mathrm{E} \quad 00$

$-5.37233 \mathrm{E}-03$

$6.57833 \mathrm{E} \quad 00$

$-3.95714 \mathrm{E}-03$

$6.67158 \mathrm{E} \quad 00$

$6.76483 \mathrm{E} \quad 00$

$6.85808 \mathrm{E} \quad 00$

$6.95133 \mathrm{E} \quad 00$

$-2.49930 \mathrm{E}-03$

$-9.98821 \mathrm{E}-04$

$5.44309 \mathrm{E}-04$

$2.13009 \mathrm{E}-03$

$7.04460 \mathrm{E} \quad 00$

$7.13786 \mathrm{E} \quad 00$

$7.23112 \mathrm{E} \quad 00$

$7.32439 \mathrm{E} \quad 00$

$7.41766 \mathrm{E} \quad 00$

$7.51094 \mathrm{E} \quad 00$

$7.60420 \mathrm{E} \quad 00$

$7.69748 \mathrm{E} \quad 00$

$7.79074 \mathrm{E} \quad 00$

$7.88400 \mathrm{E} \quad 00$

$3.75853 \mathrm{E}-03$

$5.42963 \mathrm{E}-03$

$7.14339 \mathrm{E}-03$

$8.89982 \mathrm{E}-03$

$1.06989 \mathrm{E}-02$

$1.25407 \mathrm{E}-02$

$1.44251 \mathrm{E}-02$

$1.63522 \mathrm{E}-02$

$1.83220 \mathrm{E}-02$

$2.03344 \mathrm{E}-02$

$7.97726 \mathrm{E} \quad 00$ $8.07049 \mathrm{E} \quad 00$ $8.16370 \mathrm{E} \quad 00$

$8.25688 \mathrm{E} 00$

$8.34999 \mathrm{E} \quad 00$

$8.44301 \mathrm{E} \quad 00$

$8.53583 \mathrm{E} \quad 00$

$8.62833 \mathrm{E} \quad 00$

$8.71995 \mathrm{E} \quad 00$

$8.80837 \mathrm{E} \quad 00$

$8.81205 \mathrm{E} \quad 00$

$9.01939 \mathrm{E} \quad 00$

$9.10557 \mathrm{E} \quad 00$

$9.19691 \mathrm{E} \quad 00$

$9.28932 \mathrm{E} \quad 00$

$9.38217 \mathrm{E} \quad 00$

$9.47527 \mathrm{E} \quad 00$

$9.56848 \mathrm{E} \quad 00$

$9.66176 \mathrm{E} \quad 00$

$9.75511 \mathrm{E} \quad 00$

$9.84852 \mathrm{E} \quad 00$

$9.94195 \mathrm{E} \quad 00$

$1.00354 \mathrm{E} \quad 01$

$1.01289 \mathrm{E} \quad 01$

$1.02224 \mathrm{E} \quad 01$

$1.03159 \mathrm{E} \quad 01$

$1.04095 \mathrm{E} \quad 01$

$1.05030 \mathrm{E} 01$

$1.05966 \mathrm{E} \quad 01$

$1.06902 \mathrm{E} 01$
2.23895 E- 02

$2.44871 \mathrm{E}-02$

$2.66274 \mathrm{E}-02$

$2.88102 \mathrm{E}-02$

$3.10353 \mathrm{E}-02$

$3.33028 \mathrm{E}-02$

$3.56119 \mathrm{E}-02$

$3.79621 \mathrm{E}-02$

$4.03506 \mathrm{E}-02$

$4.27655 \mathrm{E}-02$

$4.47901 \mathrm{E}-02$

$4.78981 \mathrm{E}-02$

$5.04298 \mathrm{E}-02$

$5.30307 \mathrm{E}-02$

$5.56797 \mathrm{E}-02$

$5.83738 \mathrm{E}-02$

$6.11120 \mathrm{E}-02$

$6.38936 \mathrm{E}-02$

$6.67183 \mathrm{E}-02$

6.95862 E-.02

$7.24974 \mathrm{E}-02$

$7.84483 \mathrm{E}-02$

$8.14883 \mathrm{E}-02$

$8.45712 \mathrm{E}-02$

8.76971 E-02

$9.08660 \mathrm{E}-02$

$9.40779 \mathrm{E}-02$

$9.73328 \mathrm{E}-02$

$1.00631 \mathrm{E}-01$
$7.54513 \mathrm{E}-02$
$2.20377 \mathrm{E}-03$

$2.13814 \mathrm{E}-03$

$2.07458 \mathrm{E}-03$

$2.01300 \mathrm{E}-03$

$1.95330 \mathrm{E}-03$

$1.89539 \mathrm{E}-03$

$1.83922 \mathrm{E}-03$

$1.78468 \mathrm{E}-03$

$1.73173 \mathrm{E}-03$

$1.68027 \mathrm{E}-03$

$1.63027 \mathrm{E}-03$

$1.58164 \mathrm{E}-03$

$1.53435 \mathrm{E}-03$

$1.48835 \mathrm{E}-03$

1.44355 E-03

$1.39994 \mathrm{E}-03$

$1.35746 \mathrm{E}-03$

$1.31608 \mathrm{E}-03$

$1.27574 \mathrm{E}-03$

$1.23642 \mathbf{E}-03$

$1.19806 \mathrm{E}-03$

$1.16064 \mathrm{E}-03$

$1.12414 \mathrm{E}-03$

$1.08850 \mathrm{E}-03$

$1.05371 \mathrm{E}-03$

$1.01973 \mathrm{E}-03$

$9.86533 \mathrm{E}-04$

$9.54098 \mathrm{E}-04$

$9.22401 \mathrm{E}-04$

8.91402 E-04

8.61105 E-04

$8.31460 \mathrm{E}-04$

8.02469 E-04

$7.74100 \mathrm{E}-04$

$7.46336 \mathrm{E}-04$

7.19150 E-04

$6.92535 \mathrm{E}-04$

$6.66472 \mathrm{E}-04$

$6.40941 \mathrm{E}-04$

6.15938 E-04

$5.91431 \mathrm{E}-04$

$5.67414 \mathrm{E}-04$

$5.43877 \mathrm{E}-04$

$5.20785 \mathrm{E}-04$

$4.98158 \mathrm{E}-04$

$4.75955 \mathrm{E}-04$

4.54185 E-0 4

$4.32828 \mathrm{E}-04$

$4.11867 \mathrm{E}-04$

3.91295 E-04

$3.71107 \mathrm{E}-04$

$3.51283 \mathrm{E}-04$

$3.31829 \mathrm{E}-04$

$3.12718 \mathrm{E}-04$

$2.93954 \mathrm{E}-04$

$2.75513 \mathrm{E}-04$

$2.57412 \mathrm{E}-04$

$2.39616 \mathrm{E}-04$

$2.22137 \mathrm{E}-04$

2.04949 E-04 $f_{-1}$

$5.23068 \mathrm{E}-02$

$5.17104 \mathrm{E}-02$

$5.10709 \mathrm{E}-02$

$5.03886 \mathrm{E}-02$

$4.96633 \mathrm{E}-02$

$4.88951 \mathrm{E}-02$

$4.80841 \mathrm{E}-02$

$4.72301 \mathrm{E}-02$

4.63333 E-02

4.53937 E-02

$4.44111 \mathrm{E}-02$

$4.33857 \mathrm{E}-02$

$4.23175 \mathrm{E}-02$

$4.12065 \mathrm{E}-02$

$4.00526 \mathrm{E}-02$

$3.88559 \mathrm{E}-02$

$3.76163 \mathrm{E}-02$

$3.63340 \mathrm{E}-02$

$3.50088 \mathrm{E}-02$

$3.36408 \mathrm{E}-02$

$3.22300 \mathrm{E}-02$

$3.07764 \mathrm{E}-02$

$2.92800 \mathrm{E}-02$

$2.77408 \mathrm{E}-02$

$2.61587 \mathrm{E}-02$

$2.45339 \mathrm{E}-02$

$2.28662 \mathrm{E}-02$

$2.11557 \mathrm{E}-02$

$1.94024 \mathrm{E}-02$

$1.76062 \mathrm{E}-02$

$1.57673 \mathrm{E}-02$

$1.38855 \mathrm{E}-02$

$1.19608 \mathrm{E}-02$

$9.99336 \mathrm{E}-03$

$7.98306 \mathrm{E}-03$

$5.92991 \mathrm{E}-03$

$3.83391 \mathrm{E}-03$

$1.69506 \mathrm{E}-03$

$-4.86650 \mathrm{E}-04$

$-2.71122 \mathrm{E}-03$

$-4.97866 \mathrm{E}-03$

$-7.28898 \mathrm{E}-03$

$-9.64218 \mathrm{E}-03$

$-1.20383 \mathrm{E}-02$

$-1.44772 \mathrm{E}-02$

$-1.69591 \mathrm{E}-02$

$-1.94839 \mathrm{E}-02$

$-2.20516 \mathrm{E}-02$

$-2.46622 \mathrm{E}-02$

$-2.73158 \mathrm{E}-02$

$-3.00122 \mathrm{E}-02$

$-3.27517 \mathrm{E}-02$

$-3.55340 \mathrm{E}-02$

$-3.83594 \mathrm{E}-02$

$-4.12276 \mathrm{E}-02$

$-4.41389 \mathrm{E}-02$

$-4.70931 \mathrm{E}-02$

$-5.00904 \mathrm{E}-02$

$-5.31306 \mathrm{E}-02$

$-5.62139 \mathrm{E}-02$

g-1

$\tan \delta_{-1}$

$6.09991 \mathrm{E} \quad 00$ $6.19352 \mathrm{E} \quad 00$ $6.28711 \mathrm{E} \quad 00$

$6.38070 \mathrm{E} \quad 00$ 
ELECTRON RADIAL FUNCTIONS

$Z=7 \quad A=14 \quad \rho=1.1 A^{1 / 3} F$

Pesitron

$\bar{F}$

6.10

6.20

6.30

6.40

6.50

6.60

6.70

6.80

6.90

7.00

7.10

7.20

7.30

7.40

7.50

7.60

7.70

7.80

7.90

8.00

$8.10 \quad 7.34097 \mathrm{E}-05$

$8.20 \quad 7.52346 \mathrm{E}-05$

$8.30 \quad 7.70818 \mathrm{E}-05$

$8.40 \quad 7.89513 \mathrm{E}-05$

$8.50 \quad 8.08433 \mathrm{E}-05$

$8.60 \quad 8.27575 \mathrm{E}-05$

$8.70 \quad 8.46942 \mathrm{E}-05$

$8.80 \quad 8.66532 \mathrm{E}-05$

$8.90 \quad 8.86345 \mathrm{E}-05$

$9.00 \quad 9.06382 \mathrm{E}-05$

$9.10 \quad 9.26643 \mathrm{E}-05$

$9.20 \quad 9.47127 \mathrm{E}-05$

$9.30 \quad 9.67835 \mathrm{E}-05$

$9.40 \quad 9.88767 \mathrm{E}-05$

$9.50 \quad 1.00992 \mathrm{E}-04$

$9.60 \quad 1.03130 \mathrm{E}-04$

$9.70 \quad 1.05290 \mathrm{E}-04$

$9.80 \quad 1.07473 \mathrm{E}-04$

$9.90 \quad 1.09678 \mathrm{E}-04$

$10.00 \quad 1.11905 \mathrm{E}-04$

$10.10 \quad 1.14155 \mathrm{E}-04$

$10.20 \quad 1.16427 \mathrm{E}-04$

$10.30 \quad 1.18721 \mathrm{E}-04$

$10.40 \quad 1.21038 \mathrm{E}-04$

$10.50 \quad 1.23377 \mathrm{E}-04$

$10.60 \quad 1.25738 \mathrm{E}-04$

$10.70 \quad 1.28122 \mathrm{E}-04$

$10.80 \quad 1.30528 \mathrm{E}-04$

$10.90 \quad 1.32957 \mathrm{E}-04$

$11.00 \quad 1.35407 \mathrm{E}-04$

$11.10 \quad 1.37881 \mathrm{E}-04$

$11.20 \quad 1.40376 \mathrm{E}-04$

$11.30 \quad 1.42894 \mathrm{E}-04$

$11.40 \quad 1.45434 \mathrm{E}-04$

$11.50 \quad 1.47997 \mathrm{E}-04$

$11.60 \quad 1.50582 \mathrm{E}-04$

$11.70 \quad 1.53189 \mathrm{E}-04$

$11.80 \quad 1.55819 \mathrm{E}-04$

$11.90 \quad 1.58471 \mathrm{E}-04$

$12.00 \quad 1.61145 \mathrm{E}-04$ $f_{2}$

7.20962 E-02

$7.45944 \mathrm{E}-02$

$7.71349 \mathrm{E}-02$

$7.97178 \mathrm{E}-02$

8.23431 E-02

$8.50106 \mathrm{E}-02$

$8.77204 \mathrm{E}-02$

$9.04724 \mathrm{E}-02$

$9.32668 \mathrm{E}-02$

$9.61033 \mathrm{E}-02$

$9.89818 \mathrm{E}-02$

$1.01902 \mathrm{E}-01$

$1.04865 \mathrm{E}-01$

$1.07869 \mathrm{E}-01$

$1.10915 \mathrm{E}-01$

$1.14002 \mathrm{E}-01$

$1.17128 \mathrm{E}-01$

$1.20294 \mathrm{E}-01$

$1.23494 \mathrm{E}-01$

$1.26723 \mathrm{E}-01$

$1.29942 \mathrm{E}-01$

$1.32787 \mathrm{E}-01$

$1.37349 \mathrm{E}-01$

$1.40490 \mathrm{E}-01$

$1.43897 \mathrm{E}-01$

$1.47382 \mathrm{E}-01$

$1.50924 \mathrm{E}-01$

$1.54515 \mathrm{E}-01$

$1.58150 \mathrm{E}-01$

$1.61831 \mathrm{E}-01$

$1.65556 \mathrm{E}-01$

$1.69323 \mathrm{E}-01$

$1.73133 \mathrm{E}-01$

$1.76987 \mathrm{E}-01$

$1.80883 \mathrm{E}-01$

$1.84821 \mathrm{E}-01$

$1.88803 \mathrm{E}-01$

$1.92827 \mathrm{E}-01$

$1.96894 \mathrm{E}-01$

$2.01003 \mathrm{E}-01$

$2.05154 \mathrm{E}-01$ $2.09348 \mathrm{E}-01$

$2.13584 \mathrm{E}-01$

$2.17863 \mathrm{E}-01$

$2.22185 \mathrm{E}-01$

$2.26548 \mathrm{E}-01$

$2.30954 \mathrm{E}-01$

$2.35403 \mathrm{E}-01$

$2.39894 \mathrm{E}-01$

2.44427 E- 01

$2.49003 \mathrm{E}-01$

$2.53621 \mathrm{E}-01$

$2.58281 \mathrm{E}-01$

$2.62985 \mathrm{E}-01$

$2.67730 \mathrm{E}-01$

$2.72517 \mathrm{E}-01$

$2.77347 \mathrm{E}-01$

$2.82220 \mathrm{E}-01$

$2.87135 \mathrm{E}-01$

$2.92092 \mathrm{E}-01$ $g_{2}$

$-1.30850 \mathrm{E}-04$ $-1.25274 \mathrm{E}-04$

$-1.19082 \mathrm{E}-04$

$-1.12257 \mathrm{E}-04$

$-1.04781 \mathrm{E}-04$

$-9.66358 \mathrm{E}-05$

$-8.78050 \mathrm{E}-05$

$-7.82710 \mathrm{E}-05$

$-6.80163 \mathrm{E}-05$

$-5.70234 \mathrm{E}-05$

$-4.52749 \mathrm{E}-05$

$-3.27534 \mathrm{E}-05$

$-1.94417 \mathrm{E}-05$

$-5.32237 \mathrm{E}-06$

$9.62165 \mathrm{E}-06$

2.54074 E- 05

$4.20514 \mathrm{E}-05$

$5.95698 \mathrm{E}-05$

$7.79754 \mathrm{E}-05$

$9.72781 \mathrm{E}-05$

$1.17456 \mathrm{E}-04$

$1.38125 \mathrm{E}-04$

$1.61592 \mathrm{E}-04$

$1.84441 \mathrm{E}-04$

$2.08535 \mathrm{E}-04$

$2.33686 \mathrm{E}-04$

$2.59889 \mathrm{E}-04$

$2.87151 \mathrm{E}-04$

$3.15485 \mathrm{E}-04$

$3.44911 \mathrm{E}-04$

$3.75444 \mathrm{E}-04$

$4.07099 \mathrm{E}-04$

$4.39895 \mathrm{E}-04$

4.73851 E-04

5.08981 E-04

$5.45304 \mathrm{E}-04$

$5.82838 \mathrm{E}-04$

$6.21599 \mathrm{E}-04$

$6.61605 \mathrm{E}-04$

$7.02873 \mathrm{E}-04$

$7.45422 \mathrm{E}-04$

$7.89267 \mathrm{E}-04$

$8.34428 \mathrm{E}-04$

$8.80921 \mathrm{E}-04$

$9.28764 \mathrm{E}-04$

$9.77974 \mathrm{E}-04$

$1.02857 \mathrm{E}-03$

$1.08056 \mathrm{E}-03$

$1.13398 \mathrm{E}-03$

$1.18883 \mathrm{E}-03$

$1.24514 \mathrm{E}-03$

$1.30292 \mathrm{E}-03$

$1.36219 \mathrm{E}-03$

$1.42297 \mathrm{E}-03$

$1.48526 \mathrm{E}-03$

$1.54911 \mathrm{E}-03$

$1.61451 \mathrm{E}-03$

$1.68148 \mathrm{E}-03$

$1.75005 \mathrm{E}-03$

$1.82024 \mathrm{E}-03$ $\tan \delta_{2}$

$-2.63480 \mathrm{E} 01$ $-2.63817 \mathrm{E} 01$ $-2.64142 \mathrm{E} \quad 01$ $-2.64456 \mathrm{E} 01$

$-2.64760 \mathrm{E}$ 0l

$-2.65055 \mathrm{E}$ 0l

$-2.65339 \mathrm{E} \quad 01$

$-2.65615 \mathrm{E} \quad 01$

$-2.65882 \mathrm{E} \quad 0 \mathrm{l}$

$-2.66142 \mathrm{E} \quad 01$

$-2.66393 \mathrm{E} \quad 01$

$-2.66637 \mathrm{E} \quad 01$

$-2.66874 \mathrm{E} \quad 01$

$-2.67104 \mathrm{E} 01$

$-2.67327 \mathrm{E} \quad 01$

$-2.67544 \mathrm{E} \quad 01$

$-2.67756 \mathrm{E} \quad 01$

$-2.67961 \mathrm{E} \quad 01$

$-2.68161 \mathrm{E} 01$

$-2.68355 \mathrm{E}$ ol

$-2.68545 \mathrm{E} \quad 01$

$-2.68729 \mathrm{E} \quad 01$

$-2.68909 \mathrm{E}$ 0I

$-2.69084 \mathrm{E} \quad 01$

$-2.69255 \mathrm{E}$ 0l

$\begin{array}{lll}-2.69421 \mathrm{E} & 01\end{array}$

$-2.69584 \mathrm{E} \quad 01$

$-2.69743 \mathrm{E} \quad 01$

$-2.69897 \mathrm{E} 01$

$-2.70048 \mathrm{E} \quad 01$

$-2.70196 \mathrm{E} \quad 01$

$-2.70340 \mathrm{E} \quad 01$

-2.70481 E 01

$-2.70619 \mathrm{E} 01$

$-2.70754 \mathrm{E} \quad 01$

$-2.70885 \mathrm{E} \quad 01$

$-2.71014 \mathrm{E} \quad 01$

$-2.71140 \mathrm{E} \quad 01$

$-2.71263 \mathrm{E} \quad 01$

$-2.71384 \mathrm{E} 01$

$-2.71502 \mathrm{E} \quad 01$

$-2.71618 \mathrm{E} \quad 01$

$-2.71731 \mathrm{E} 01$

$-2.71842 \mathrm{E} \quad 01$

$-2.71951 \mathrm{E} 01$

$\begin{array}{lll}-2.72057 \mathrm{E} & 01\end{array}$

$-2.72162 \mathrm{E} \quad 0$

$-2.72264 \mathrm{E} 01$

$-2.72365 \mathrm{E}$ 0l

$-2.72463 \mathrm{E} \quad 01$

$-2.72560 \mathrm{E} \quad 01$

$-2.72655 \mathrm{E} \quad 01$

$-2.72748 \mathrm{E} \quad 01$

$-2.72839 \mathrm{E} \quad 01$

$-2.72929 \mathrm{E} 01$

$-2.73017 \mathrm{E} \quad 01$

$-2.73103 \mathrm{E}$ 01

$-2.73188 \mathrm{E}$ 01

$-2.73271 \mathrm{E} 01$

$-2.73353 \mathrm{E} \quad 01$

$f:$

$3.87171 \mathrm{E}-04$

$3.87673 \mathrm{E}-01$

$3.87616 \mathrm{E}-04$

$3.86982 \mathrm{E}-04$

$3.85756 \mathrm{E}-04$

$3.83918 \mathrm{E}-04$

$3.81452 \mathrm{E}-04$

$3.78340 \mathrm{E}-04$

$3.74564 \mathrm{E}-04$

$3.70108 \mathrm{E}-04$

$3.64954 \mathrm{E}-04$

$3.59084 \mathrm{E}-04$

3.52481 E-04

$3.45127 \mathrm{E}-04$

$3.37005 \mathrm{E}-04$

$3.28098 \mathrm{E}-04$

$3.18387 \mathrm{E}-04$

$3.07857 \mathrm{E}-04$

$2.96488 \mathrm{E}-04$

$2.84264 \mathrm{E}-04$

$2.71168 \mathrm{E}-04$

$2.57181 \mathrm{E}-04$

$2.42287 \mathrm{E}-04$

$2.26467 \mathrm{E}-04$

$2.09705 \mathrm{E}-04$

$1.91983 \mathrm{E}-04$

$1.73283 \mathrm{E}-04$

$1.53589 \mathrm{E}-04$

$1.32882 \mathrm{E}-04$

$1.11145 \mathrm{E}-04$

$8.83612 \mathrm{E}-05$ $6.45125 \mathrm{E}-05$

$3.95816 \mathrm{E}-05$

$1.35510 \mathrm{E}-05$

$-1.35967 \mathrm{E}-05$

$-4.18790 \mathrm{E}-05$

$-7.13132 \mathrm{E}-05$

$-1.01917 \mathrm{E}-04$

$-1.33708 \mathrm{E}-04$

$-1.66703 \mathrm{E}-04$

$-2.00920 \mathrm{E}-04$ 
$p \quad F_{0}$

12.10

12.20

12.30

12.40

12.50

12.60

12.70

12.80

12.90

13.00

13.10

13.20

13.30

13.40

13.50

13.60

13.70

13.80

13.90

14.00

14.10

14.20

14.30

14.40

14.50

14.60

14.70

14.80

14.90

15.00

15.10

15.20

15.30

15.40

15.50

15.60

15.70

15.80

15.90

16.00

16.10

16.20

16.30

16.40

16.50

16.60

16.70

16.80

16.90

17.00

17.10

17.20

17.30

17.40

17.50

17.60

17.70

17.80

17.90

18.00
$8.56982 \mathrm{E}-01$ $8.56972 \mathrm{E}-01$ $8.56961 \mathrm{E}-01$ $8.56950 \mathrm{E}-01$ $8.56940 \mathrm{E}-01$

$8.56929 \mathrm{E}-01$ $8.56919 \mathrm{E}-01$ $8.56908 \mathrm{E}-01$ $8.56897 \mathrm{E}-01$ $8.56887 \mathrm{E}-01$

$8.56877 \mathrm{E}-01$ $8.56866 \mathrm{E}-01$ $8.56855 \mathrm{E}-01$ $8.56844 \mathrm{E}-01$ $8.56834 \mathrm{E}-01$

$8.56823 \mathrm{E}-01$ $8.56812 \mathrm{E}-01$ $8.56802 \mathrm{E}-01$ $8.56791 \mathrm{E}-01$ $8.56780 \mathrm{E}-01$

$8.56769 \mathrm{E}-01$ $8.56759 \mathrm{E}-01$ $8.56748 \mathrm{E}-01$ $8.56738 \mathrm{E}-01$ $8.56727 \mathrm{E}-01$

$8.56716 \mathrm{E}-01$ $8.56705 \mathrm{E}-01$ $8.56694 \mathrm{E}-01$ $8.56684 \mathrm{E}-01$ $8.56673 \mathrm{E}-01$

$8.56662 \mathrm{E}-01$ $8.56652 \mathrm{E}-01$ 8.56641 E-01 $8.56631 \mathrm{E}-01$ $8.56620 \mathrm{E}-01$

$8.56610 \mathrm{E}-01$ $8.56599 \mathrm{E}-01$ $8.56589 \mathrm{E}-01$ $8.56579 \mathrm{E}-01$ $8.56568 \mathrm{E}-01$

$8.56558 \mathrm{E}-01$ $8.56547 \mathrm{E}-01$ $8.56537 \mathrm{E}-01$ $8.56526 \mathrm{E}-01$ $8.56516 \mathrm{E}-01$

$8.56506 \mathrm{E}-01$ $8.56495 \mathrm{E}-01$ $8.56485 \mathrm{E}-01$ $8.56475 \mathrm{E}-01$ $8.56465 \mathrm{E}-01$

$8.56454 \mathrm{E}-01$ $8.56444 \mathrm{E}-01$ $8.56434 \mathrm{E}-01$ $8.56424 \mathrm{E}-01$ $8.56414 \mathrm{E}-01$

$8.56404 \mathrm{E}-01$ $8.56394 \mathrm{E}-01$ $8.56384 \mathrm{E}-01$ $8.56374 \mathrm{E}-01$ $8.56364 \mathrm{E}-01$ $f_{1}$

$1.07838 \mathrm{E} \quad 01$

$1.08774 \mathrm{E} \quad 01$

$1.09711 \mathrm{E} 01$

$1.10647 \mathrm{E} 01$

$1.11584 \mathrm{E} \quad 01$

$1.12521 \mathrm{E} \quad 01$

$1.13457 \mathrm{E} \quad 01$

$1.14394 \mathrm{E} \quad 01$

$1.15332 \mathrm{E} 01$

$1.16269 \mathrm{E} \quad 01$

$1.17206 \mathrm{E} \quad 01$

$1.18144 \mathrm{E} \quad 01$

$1.19081 \mathrm{E} \quad 01$

$1.20019 \mathrm{E} 01$

$1.20957 \mathrm{E} \quad 01$

$1.21895 \mathrm{E} \quad 01$ $1.22833 \mathrm{E} 01$ $1.23772 \mathrm{E} \quad 01$

$1.24710 \mathrm{E} \quad 01$

$1.25649 \mathrm{E} \quad 01$

$1.26587 \mathrm{E} \quad 01$

$1.27526 \mathrm{E} \quad 01$

$1.28465 \mathrm{E} \quad 01$

$1.29404 \mathrm{E} \quad 01$

$1.30344 \mathrm{E} \quad 01$

$1.31283 \mathrm{E} \quad 01$ $1.32222 \mathrm{E} \quad 01$ $1.33162 \mathrm{E} 01$ $1.34102 \mathrm{E} \quad 01$

$1.35042 \mathrm{E} \quad 01$

$\begin{array}{lll}1.35982 \mathrm{E} & 01\end{array}$ $1.36922 \mathrm{E} \quad 01$ $1.37862 \mathrm{E} \quad 01$ $1.38803 \mathrm{E} \quad 01$ $1.39744 \mathrm{E} \quad 01$

$1.40684 \mathrm{E} \quad 01$ $1.41625 \mathrm{E} \quad 01$ $1.42566 \mathrm{E} \quad 01$ $1.43508 \mathrm{E} \quad 01$ $1.44449 \mathrm{E} 01$

$1.45390 \mathrm{E} \quad 01$ $1.46332 \mathrm{E} \quad 01$

$1.47274 \mathrm{E} \quad 01$

$1.48216 \mathrm{E} \quad 01$

$1.49158 \mathrm{E} 01$

$1.50100 \mathrm{E} \quad 01$ $1.51042 \mathrm{E} \quad 01$ $1.51985 \mathrm{E} \quad 01$ $1.52928 \mathrm{E} \quad 01$ $1.53871 \mathrm{E} \quad 01$

$1.54814 \mathrm{E} \quad 01$ $\begin{array}{lll}1.55757 \mathrm{E} & 01\end{array}$ $1.56700 \mathrm{E} \quad 01$ $\begin{array}{ll}1.57644 \mathrm{E} & 01\end{array}$ $1.58587 \mathrm{E} \quad 01$

$1.59531 \mathrm{E} \quad 01$ $1.60475 \mathrm{E} \quad 01$ $1.61419 \mathrm{E} 01$ $1.62363 \mathrm{E} 01$ $1.63308 \mathrm{E} \quad 01$ $g_{1}$

$1.03972 \mathrm{E}-01$

$1.07355 \mathrm{E}-01$

$1.10782 \mathrm{E}-01$

$1.14252 \mathrm{E}-01$

$1.17765 \mathrm{E}-01$

$1.21321 \mathrm{E}-01$

$1.24919 \mathrm{E}-0$

$1.28561 \mathrm{E}-01$

$1.32246 \mathrm{E}-01$

$1.35975 \mathrm{E}-01$

$1.39746 \mathrm{E}-01$

$1.43560 \mathrm{E}-01$

$1.47417 \mathrm{E}-01$

$1.51318 \mathrm{E}-01$

$1.55261 \mathrm{E}-01$

$1.59248 \mathrm{E}-01$

$1.63278 \mathrm{~F}-0$

$1.67350 \mathrm{E}-01$

$1.71467 \mathrm{E}-01$

$1.75626 \mathrm{E}-01$

$1.79828 \mathrm{E}-01$

$1.84074 \mathrm{E}-01$

$1.88363 \mathrm{E}-01$

$1.92695 \mathrm{E}-01$

$1.97070 \mathrm{E}-01$

$2.01489 \mathrm{E}-01$

$2.05950 \mathrm{E}-01$

$2.10455 \mathrm{E}-01$

$2.15004 \mathrm{E}-01$

$2.19595 \mathrm{E}-01$

$2.24230 \mathrm{E}-01$

$2.28908 \mathrm{E}-0$

$2.33630 \mathrm{E}-01$

$2.38395 \mathrm{E}-01$

$2.43203 \mathrm{E}-01$

$2.48054 \mathrm{E}-01$

$2.52949 \mathrm{E}-01$

$2.57888 \mathrm{E}-01$

$2.62869 \mathrm{E}-01$

$2.67895 E-01$

$2.72963 \mathrm{E}-01$

$2.78075 \mathrm{E}-01$

$2.83231 \mathrm{E}-01$

$2.88429 \mathrm{E}-01$

$2.93672 \mathrm{E}-01$

$2.98958 \mathrm{E}-01$ $3.04287 \mathrm{E}-01$ $3.09660 \mathrm{E}-01$

$3.15076 \mathrm{E}-01$

$3.20536 \mathrm{E}-01$

$3.26040 \mathrm{E}-01$ $3.31587 \mathrm{E}-01$ $3.37177 \mathrm{E}-01$ $3.42812 \mathrm{E}-01$ $3.48489 \mathrm{E}-01$

$3.54211 \mathrm{E}-01$ $3.59976 \mathrm{E}-01$ $3.65785 \mathrm{E}-01$ $3.71637 \mathrm{E}-01$ $3.77534 \mathrm{E}-01$

$\tan \delta_{1}$

$f_{-1}$

$1.88067 \mathrm{E}-04-5.93403 \mathrm{E}-02$

$1.71467 \mathrm{E}-04-6.25095 \mathrm{E}-02$

$1.55151 \mathrm{E}-04 \quad-6.57218 \mathrm{E}-02$

$1.39104 \mathrm{E}-04-6.89772 \mathrm{E}-02$

$1.23320 \mathrm{E}-04 \quad-7.22756 \mathrm{E}-02$

$1.07801 \mathrm{E}-04$

$9.25400 \mathrm{E}-05$

$7.75282 \mathrm{E}-05$

$6.27581 \mathrm{E}-05$

$4.82256 \mathrm{E}-05$

$3.39237 \mathrm{E}-05$

$1.98509 \mathrm{E}-05$

$5.99937 \mathrm{E}-06$

$-7.63695 \mathrm{E}-06$

$-2.10592 \mathrm{E}-05$

$-3.42665 \mathrm{E}-05$

$-4.72753 \mathrm{E}-05$

$-6.00774 \mathrm{E}-05$

$-7.27019 \mathrm{E}-05$

$-8.51185 \mathrm{E}-05$

$-9.73614 \mathrm{E}-05$

$-1.09403 \mathrm{E}-04$

$-1.21277 \mathrm{E}-04$

$-1.32975 \mathrm{E}-04$

$-1.44502 \mathrm{E}-04$

$-1.55848 \mathrm{E}-04$

$-1.67039 \mathrm{E}-04$

$-1.78068 \mathrm{E}-04$

$-1.88932 \mathrm{E}-04$

$-1.99644 \mathrm{E}-04$

$-2.10204 \mathrm{E}-04$

$-2.20613 \mathrm{E}-04$

$-2.30867 \mathrm{E}-04$

$-2.40981 \mathrm{E}-04$

$-2.50957 \mathrm{E}-04$

$-2.60787 \mathrm{E}-04$

$-2.70489 \mathrm{E}-04$

$-2.80048 \mathrm{E}-04$

$-2.89484 \mathrm{E}-04$

$-2.98784 \mathrm{E}-04$

$-3.07959 \mathrm{E}-04$ 
ELECTRON RADIAL FUNCTIONS

$Z=7 \quad A=14 \quad \rho=1.1 A^{1 / 3} F$

Positron

$\bar{F}$

$1.63842 \mathrm{E}-04$

$1.66561 \mathrm{E}-04$

$1.69302 \mathrm{E}-04$

$1.72066 \mathrm{E}-04$

$1.74852 \mathrm{E}-04$

$1.77660 \mathrm{E}-04$

$1.80491 \mathrm{E}-04$

$1.83344 \mathrm{E}-04$

$1.86220 \mathrm{E}-04$

$1.89118 \mathrm{E}-04$

$1.92038 \mathrm{E}-04$

$1.94981 \mathrm{E}-04$

$1.97945 \mathrm{E}-04$

$2.00933 \mathrm{E}-04$

2.03942 E-04

$2.06974 \mathrm{E}-04$

$2.10028 \mathrm{E}-04$

$2.13105 \mathrm{E}-04$

$2.16204 \mathrm{E}-04$

$2.19325 \mathrm{E}-04$

13.90

14.00

14.10

14.20

14.30

14.40

14.50

14.60

14.70

14.80

14.90

15.00

15.10

15.20

15.30

15.40

15.50

15.60

15.70

15.80

15.90

16.00

16.10

16.20

16.30

16.40

16.50

$2.22469 \mathrm{E}-04$

$2.25635 \mathrm{E}-04$

$2.28823 \mathrm{E}-04$

$2.32034 \mathrm{E}-04$

$2.35267 \mathrm{E}-04$

$2.38522 \mathrm{E}-04$

$2.41800 \mathrm{E}-04$

$2.45100 \mathrm{E}-04$

$2.48422 \mathrm{E}-04$

$2.51767 \mathrm{E}-04$

$2.55134 \mathrm{E}-04$

$2.58524 \mathrm{E}-04$

$2.61936 \mathrm{E}-04$

$2.65370 \mathrm{E}-04$

$2.68826 \mathrm{E}-04$

$2.72305 \mathrm{E}-04$ $2.75806 \mathrm{E}-04$ $2.79330 \mathrm{E}-04$ $2.82876 \mathrm{E}-04$

$2.86444 \mathrm{E}-04$

$2.90034 \mathrm{E}-04$ $2.93647 \mathrm{E}-04$ $2.97282 \mathrm{E}-04$

$3.00940 \mathrm{E}-04$

$3.04620 \mathrm{E}-04$

16.60

$3.08322 \mathrm{E}-04$

16.70

16.80

16.90

17.00

$3.12047 \mathrm{E}-04$

$3.15793 \mathrm{E}-04$

$3.19563 \mathrm{E}-04$

$3.23354 \mathrm{E}-04$

17.10

17.20

17.30

17.40

17.50

$3.27168 \mathrm{E}-04$

$3.31004 \mathrm{E}-04$

$3.34863 \mathrm{E}-04$

$3.38744 \mathrm{E}-04$

$3.42647 \mathrm{E}-04$

17.60

17.70

17.80

17.90

$3.46573 \mathrm{E}-04$

$3.50521 \mathrm{E}-04$

$3.54491 \mathrm{E}-04$

$3.58484 \mathrm{E}-04$

$3.62499 \mathrm{E}-04$ $f_{2}$

$2.97091 \mathrm{E}-01$

$3.02133 \mathrm{E}-01$

$3.07218 \mathrm{E}-01$

$3.12344 \mathrm{E}-01$

$3.17513 \mathrm{E}-01$

$3.22725 \mathrm{E}-01$

$3.27978 \mathrm{E}-01$

$3.33274 \mathrm{E}-01$

$3.38613 \mathrm{E}-01$

$3.43994 \mathrm{E}-01$

3.494.17E-01

$3.54882 \mathrm{E}-01$

$3.60390 \mathrm{E}-01$

$3.65940 \mathrm{E}-01$

$3.71533 \mathrm{E}-01$

$3.77168 \mathrm{E}-01$

$3.82845 \mathrm{E}-01$

$3.88564 \mathrm{E}-01$

$3.94326 \mathrm{E}-01$

$4.00130 \mathrm{E}-01$

$4.05977 \mathrm{E}-01$

$4.11866 \mathrm{E}-01$

$4.17797 \mathrm{E}-01$

$4.23771 \mathrm{E}-01$

$4.29787 \mathrm{E}-01$

$4.35845 \mathrm{E}-01$

$4.41945 \mathrm{E}-01$

$4.48088 \mathrm{E}-01$

$4.54273 \mathrm{E}-01$

$4.60501 \mathrm{E}-01$

$4.66771 \mathrm{E}-01$

$4.73083 \mathrm{E}-0]$

$4.79437 \mathrm{E}-01$

$4.85833 \mathrm{E}-01$

$4.92273 \mathrm{E}-01$

$4.98754 \mathrm{E}-01$

$5.05278 \mathrm{E}-01$

$5.11844 \mathrm{E}-01$

$5.18452 \mathrm{E}-01$

$5.25103 \mathrm{E}-01$

$5.31796 \mathrm{E}-01$

$5.38531 \mathrm{E}-01$

$5.45308 \mathrm{E}-01$

$5.52128 \mathrm{E}-01$

$5.58990 \mathrm{E}-01$

$5.65894 \mathrm{E}-01$

$5.72841 \mathrm{E}-01$

$5.79830 \mathrm{E}-0 \mathrm{l}$

$5.86861 \mathrm{E}-01$

$5.93935 \mathrm{E}-0 \mathrm{l}$

$6.01050 \mathrm{E}-01$

$6.08208 \mathrm{E}-01$

$6.15409 \mathrm{E}-01$

$6.22651 \mathrm{E}-01$

$6.29936 \mathrm{E}-01$

$6.37263 \mathrm{E}-01$

$6.44633 \mathrm{E}-01$

$6.52044 \mathrm{E}-0]$

$6.59498 \mathrm{E}-0]$

$6.66995 \mathrm{E}-01$ $g_{2}$

$\tan \delta_{2}$

$f_{-2}$

$1.89205 \mathrm{E}-03$

$1.96551 \mathrm{E}-03$

$2.04063 \mathrm{E}-03$

$2.11743 \mathrm{E}-03$

$2.19594 \mathrm{E}-03$

$2.27615 \mathrm{E}-03$

$2.35810 \mathrm{E}-03$

$2.44181 \mathrm{E}-03$

$2.52728 \mathrm{E}-03$

$2.61454 \mathrm{E}-03$

$2.70360 \mathrm{E}-03$

$2.79448 \mathrm{E}-03$

$2.88720 \mathrm{E}-03$

$2.98177 \mathrm{E}-03$

$3.07822 \mathrm{E}-03$

$3.17656 \mathrm{E}-03$

$3.27681 \mathrm{E}-03$

$3.37898 \mathrm{E}-03$

$3.48310 \mathrm{E}-03$

$3.58918 \mathrm{E}-03$

$3.69723 \mathrm{E}-03$

$3.80728 \mathrm{E}-03$

$3.91934 \mathrm{E}-03$

$4.03344 \mathrm{E}-03$

$4.14958 \mathrm{E}-03$

$4.26778 \mathrm{E}-03$

$4.38807 \mathrm{E}-03$

$4.51046 \mathrm{E}-03$

$4.63496 \mathrm{E}-03$

$4.76160 \mathrm{E}-03$

$4.89040 \mathrm{E}-03$

$5.02136 \mathrm{E}-03$

$5.15450 \mathrm{E}-03$

$5.28986 \mathrm{E}-03$

$5.42743 \mathrm{E}-03$

$5.56725 \mathrm{E}-03$

$5.70932 \mathrm{E}-03$

$5.85367 \mathrm{E}-03$

$6.00031 \mathrm{E}-03$

$6.14925 \mathrm{E}-03$

$6.30053 \mathrm{E}-03$

$6.45414 \mathrm{E}-03$

$6.61012 \mathrm{E}-03$

$6.76848 \mathrm{E}-03$

$6.92923 \mathrm{E}-03$

$7.09240 \mathrm{E}-03$

$7.25800 \mathrm{E}-03$

$7.42605 \mathrm{E}-03$

$7.59656 \mathrm{E}-03$

$7.76956 \mathrm{E}-03$

$7.94506 \mathrm{E}-03$

$8.12307 \mathrm{E}-03$

$8.30363 \mathrm{E}-03$

$8.48673 \mathrm{E}-03$

$8.67240 \mathrm{E}-03$

$8.86067 \mathrm{E}-03$

$9.05154 \mathrm{E}-03$

$9.24502 \mathrm{E}-03$

$9.44116 \mathrm{E}-03$

$9.63995 \mathrm{E}-03$
$-2.73434 \mathrm{E}$ 0l $-1.16872 \mathrm{E}-03$

$-2.73513 \mathrm{E}$ 01 $-1.23263 \mathrm{E}-03$

$-2.73591 \mathrm{E}$ 01 $-1.29814 \mathrm{E}-03$

$-2.73667 \mathrm{E}$ 0l $-1.36528 \mathrm{E}-03$

$-2.73742 \mathrm{E}$ 01 $-1.43406 \mathrm{E}-03$

$-2.73816 \mathrm{E} \quad 01$

$-2.73889 \mathrm{E} \quad 01$

$-2.73960 \mathrm{E} \quad 01$

$-2.74030 \mathrm{E} 01$

$-2.74100 \mathrm{E} \quad 01$

$-2.74168 \mathrm{E} \quad 01$

$-2.74235 \mathrm{E} \quad 01$

$-2.74300 \mathrm{E} \quad 01$

$-2.74365 \mathrm{E} \quad 01$

$-2.74429 \mathrm{E} \quad 01$

$-2.74492 \mathrm{E} \quad 01$

$-2.74554 \mathrm{E} \quad 01$

$-2.74615 \mathrm{E} \quad 01$

$-2.74675 \mathrm{E} \quad 01$

$-2.74734 \mathrm{E} \quad 01$

$-2.74792 \mathrm{E} \quad 01$

$-2.74850 \mathrm{E} \quad 01$

$-2.74906 \mathrm{E} \quad 01$

$-2.74962 \mathrm{E} 01$

$-2.75017 \mathrm{E} \quad 01$

$-2.75071 \mathrm{E} \quad 01$

$-2.75124 \mathrm{E}$ 0l

$-2.75177 \mathrm{E} \quad 01$

$-2.75229 \mathrm{E}$ 01

$-2.75280 \mathrm{E} \quad 0 \mathrm{l}$

$-2.75330 \mathrm{E}$ 0l

$-2.75380 \mathrm{E}$ ol

$-2.75429 \mathrm{E} \quad 01$

$-2.75477 \mathrm{E}$ 01

$-2.75525 \mathrm{E} \quad 01$

$-2.75572 \mathrm{E}$ 01

$-2.75619 \mathrm{E}$ 01

$-2.75664 \mathrm{E} \quad 01$

$-2.75710 \mathrm{E} 01$

$-2.75754 \mathrm{E}$ 0l

$-2.75798 \mathrm{E} \quad 01$

$-2.75842 \mathrm{E} \quad 01$

$-2.75885 \mathrm{E} 01$

$-2.75927 \mathrm{E}$ 0l

$-2.75969 \mathrm{E} \quad 01$

$-2.76010 \mathrm{E} \quad 01$

$-2.76051 \mathrm{E} \quad 01$

$-2.76092 \mathrm{E} \quad 01$

$-2.76131 \mathrm{E} 0 \mathrm{l}$

$-2.76171 \mathrm{E}$ 01

$-2.76210 \mathrm{E} \quad 01$

$-2.76248 \mathrm{E} \quad 01$

$-2.76286 \mathrm{E}$ 0l

$-2.76323 \mathrm{E} \quad 01$

$-2.76360 \mathrm{E} 01$

$\begin{array}{lll}-2.76397 \mathrm{E} & 01\end{array}$

$-2.76433 \mathrm{E} 01$

$-2.76469 \mathrm{E} \quad 01$

$-2.76504 \mathrm{E} \quad 01$

$-2.76539 \mathrm{E} 01$

$-1.50449 \mathrm{E}-03$ 


$\begin{array}{cc}p & F_{0} \\ 18.10 & 8.56354 \mathrm{E}-01 \\ 18.20 & 8.56344 \mathrm{E}-01 \\ 18.30 & 8.56334 \mathrm{E}-01 \\ 18.40 & 8.56325 \mathrm{E}-01 \\ 18.50 & 8.56315 \mathrm{E}-01 \\ & \\ 18.60 & 8.56305 \mathrm{E}-01 \\ 18.70 & 8.56295 \mathrm{E}-01 \\ 18.80 & 8.56285 \mathrm{E}-01 \\ 18.90 & 8.56276 \mathrm{E}-01 \\ 19.00 & 8.56266 \mathrm{E}-01 \\ & \\ 19.10 & 8.56256 \mathrm{E}-01 \\ 19.20 & 8.56247 \mathrm{E}-01 \\ 19.30 & 8.56237 \mathrm{E}-01 \\ 19.40 & 8.56228 \mathrm{E}-01 \\ 19.50 & 8.56218 \mathrm{E}-01 \\ & \\ 19.60 & 8.56209 \mathrm{E}-01 \\ 19.70 & 8.56199 \mathrm{E}-01 \\ 19.80 & 8.56190 \mathrm{E}-01 \\ 19.90 & 8.56180 \mathrm{E}-01 \\ 20.00 & 8.56171 \mathrm{E}-01 \\ & \\ 20.10 & 8.56162 \mathrm{E}-01 \\ 20.20 & 8.56152 \mathrm{E}-01 \\ 20.30 & 8.56143 \mathrm{E}-01 \\ 20.40 & 8.56134 \mathrm{E}-01 \\ 20.50 & 8.56124 \mathrm{E}-01\end{array}$

20.60

20.70

20.80

20.90

21.00

21.10

21.20

21.30

21.40

21.50

21.60

21.70

21.80

21.90

22.00

22.10

22.20

22.30

22.40

22.50

22.60

22.70

22.80

22.90

23.00

23.10

23.20

23.30

23.40

23.50

23.60

23.70

23.80

23.90

24.00 $f_{1}$

$1.64252 \mathrm{E} \quad 01$ $\begin{array}{lll}1.65197 \mathrm{E} & 01\end{array}$ $1.66142 \mathrm{E} 01$ $1.67087 \mathrm{E} \quad 01$ $1.68032 \mathrm{E} \quad 01$

$1.68978 \mathrm{E} \quad 01$ $1.69924 \mathrm{E} \quad 01$ $1.70869 \mathrm{E}$ 01 $1.71815 \mathrm{E} \quad 0 \mathrm{l}$ $1.72761 \mathrm{E} \quad 01$

$1.73707 \mathrm{E} \quad 01$ $1.74654 \mathrm{E} \quad 01$ $1.75601 \mathrm{E} 01$ $1.76547 \mathrm{E} \quad 01$ $1.77494 \mathrm{E} \quad 01$

$1.78441 \mathrm{E} \quad 01$ $1.79389 \mathrm{E} 01$ $1.80336 \mathrm{E} \quad 01$ $1.81284 \mathrm{E} \quad 01$ $1.82232 \mathrm{E} \quad 01$

$\begin{array}{ll}1.83180 \mathrm{E} & 01\end{array}$ $1.84128 \mathrm{E} \quad 01$ $1.85076 \mathrm{E} \quad 01$ $1.86025 \mathrm{E} \quad 01$ $1.86973 \mathrm{E}$ 0l

$1.87922 \mathrm{E} \quad 01$ $1.88871 \mathrm{E} \quad 01$ $1.89821 \mathrm{E} \quad 01$ $1.90770 \mathrm{E} \quad 01$ $1.91720 \mathrm{E} \quad 01$

$1.92669 \mathrm{E} 01$ $1.93619 \mathrm{E} \quad 01$ $1.94570 \mathrm{E} \quad 01$ $1.95520 \mathrm{E} \quad 01$ $1.96471 \mathrm{E} \quad 01$

$1.97421 \mathrm{E} \quad 01$ $1.98372 \mathrm{E} \quad 01$ $1.99323 \mathrm{E} \quad 01$ $2.00275 \mathrm{E} \quad 01$ $2.01226 \mathrm{E} \quad 01$

$2.02178 \mathrm{E} \quad 01$ $2.03130 \mathrm{E} \quad 01$ $2.04082 \mathrm{E} \quad 01$ $2.05034 \mathrm{E} \quad 01$ $2.05987 \mathrm{E} \quad 01$

$2.06939 \mathrm{E} \quad 01$ $2.07892 \mathrm{E} \quad 01$ $2.08845 \mathrm{E} \quad 01$ $2.09799 \mathrm{E} \quad 01$ $2.10752 \mathrm{E} \quad 01$

$2.11706 \mathrm{E} \quad 01$ $2.12660 \mathrm{E} \quad 01$ $2.13614 \mathrm{E} \quad 01$ $2.14568 \mathrm{E} \quad 01$ $2.15523 \mathrm{E}$ ol

$2.16477 \mathrm{E} \quad 01$ $2.17432 \mathrm{E}$ 01 $2.18387 \mathrm{E}$ 0l $2.19342 \mathrm{E} 01$ $2.20298 \mathrm{E} \quad 01$

\begin{tabular}{|c|c|c|}
\hline$g_{1}$ & & $f_{-1}$ \\
\hline $\begin{array}{l}.83474 \mathrm{E}-01 \\
.89457 \mathrm{E}-01 \\
.95484 \mathrm{E}-01 \\
.01556 \mathrm{E}-01\end{array}$ & & $\begin{array}{l}-3.26035 \mathrm{E}-01 \\
-3.31806 \mathrm{E}-01 \\
-3.37621 \mathrm{E}-01 \\
-3.43480 \mathrm{E}-01 \\
-3.49383 \mathrm{E}-01\end{array}$ \\
\hline $\begin{array}{l}13829 \mathrm{E}-01 \\
20032 \mathrm{E}-01 \\
26278 \mathrm{E}-01 \\
32568 \mathrm{E}-01 \\
38901 \mathrm{E}-01\end{array}$ & $\begin{array}{l}-5.01 \\
-5.08 \\
-5.14 \\
-5.21 \\
-5.27\end{array}$ & $\begin{array}{l}-3.55329 \mathrm{E}- \\
-3.61319 \mathrm{E}- \\
-3.67353 \mathrm{E}- \\
-3.73431 \mathrm{E}- \\
-3.79553 \mathrm{E}-\end{array}$ \\
\hline $\begin{array}{l}45279 \mathrm{E}-01 \\
51701 \mathrm{E}-01 \\
58166 \mathrm{E}-01 \\
64675 \mathrm{E}-01 \\
71229 \mathrm{E}-01\end{array}$ & $\begin{array}{l}-5.33 \\
-5.39 \\
-5.45 \\
-5.51 \\
-5.57\end{array}$ & $\begin{array}{l}-3.85719 \mathrm{E}-01 \\
-3.91929 \mathrm{E}-01 \\
-3.98182 \mathrm{E}-01 \\
-4.04480 \mathrm{E}-01 \\
-4.10821 \mathrm{E}-01\end{array}$ \\
\hline $\begin{array}{l}77826 \mathrm{E}-01 \\
84467 \mathrm{E}-01 \\
91152 \mathrm{E}-01 \\
97881 \mathrm{E}-01 \\
04654 \mathrm{E}-01\end{array}$ & $\begin{array}{l}-5.63567 \mathrm{E}-04 \\
-5.69334 \mathrm{E}-04 \\
-5.75037 \mathrm{E}-04 \\
-5.80666 \mathrm{E}-04 \\
-5.86217 \mathrm{E}-04\end{array}$ & $\begin{array}{l}-4.17207 \mathrm{E}-01 \\
-4.23636 \mathrm{E}-01 \\
-4.30110 \mathrm{E}-01 \\
-4.36627 \mathrm{E}-01 \\
-4.43189 \mathrm{E}-01\end{array}$ \\
\hline $\begin{array}{l}11471 \mathrm{E}-01 \\
18332 \mathrm{E}-01 \\
25238 \mathrm{E}-01 \\
32187 \mathrm{E}-01 \\
39180 \mathrm{E}-01\end{array}$ & $\begin{array}{l}-5.91716 \mathrm{E}-04 \\
-5.97142 \mathrm{E}-04 \\
-6.02500 \mathrm{E}-04 \\
-6.07800 \mathrm{E}-04 \\
-6.13026 \mathrm{E}-04\end{array}$ & $\begin{array}{l}-4.49794 \mathrm{E}-01 \\
-4.56444 \mathrm{E}-01 \\
-4.63138 \mathrm{E}-01 \\
-4.69875 \mathrm{E}-01 \\
-4.76657 \mathrm{E}-01\end{array}$ \\
\hline $\begin{array}{l}46218 \mathrm{E}-01 \\
53299 \mathrm{E}-01 \\
60425 \mathrm{E}-01 \\
67594 \mathrm{E}-01 \\
74808 \mathrm{E}-01\end{array}$ & $\begin{array}{l}-6.18200 E-04 \\
-6.23311 E-04 \\
-6.28360 E-04 \\
-6.33343 E-04 \\
-6.38269 E-04\end{array}$ & $\begin{array}{l}83484 \mathrm{E}-01 \\
90354 \mathrm{E}-01 \\
97268 \mathrm{E}-01 \\
04227 \mathrm{E}-01 \\
11230 \mathrm{E}-01\end{array}$ \\
\hline $\begin{array}{l}82066 \mathrm{E}-01 \\
89369 \mathrm{E}-01 \\
96715 \mathrm{E}-01 \\
04106 \mathrm{E}-01\end{array}$ & $\begin{array}{l}-6.43142 \mathrm{E}- \\
-6.47949 \mathrm{E}- \\
-6.52705 \mathrm{E}- \\
-6.57401 \mathrm{E}- \\
-6.62040 \mathrm{E}-\end{array}$ & $\begin{array}{l}18277 \mathrm{E}-01 \\
25368 \mathrm{E}-01 \\
32504 \mathrm{E}-01 \\
39684 \mathrm{E}-01 \\
46908 \mathrm{E}-01\end{array}$ \\
\hline $\begin{array}{l}19021 \mathrm{E}-01 \\
26544 \mathrm{E}-01 \\
34112 \mathrm{E}-01 \\
41724 \mathrm{E}-01\end{array}$ & $\begin{array}{l}-6.66632 \mathrm{E}-04 \\
-6.71162 \mathrm{E}-04 \\
-6.75642 \mathrm{E}-04 \\
-6.80061 \mathrm{E}-04 \\
-6.84440 \mathrm{E}-04\end{array}$ & $\begin{array}{l}-5.54177 \mathrm{E}-01 \\
-5.61489 \mathrm{E}-01 \\
-5.68847 \mathrm{E}-01 \\
-5.76248 \mathrm{E}-01 \\
-5.83694 \mathrm{E}-01\end{array}$ \\
\hline $\begin{array}{l}57082 \mathrm{E}-01 \\
64827 \mathrm{E}-01 \\
72617 \mathrm{E}-01 \\
80451 \mathrm{E}-01\end{array}$ & $\begin{array}{l}-6.88758 \mathrm{E}-04 \\
-6.93022 \mathrm{E}-04 \\
-6.97244 \mathrm{E}-04 \\
-7.01417 \mathrm{E}-04 \\
-7.05540 \mathrm{E}-04\end{array}$ & $\begin{array}{l}1185 \mathrm{E}-01 \\
8719 \mathrm{E}-01 \\
6299 \mathrm{E}-01 \\
3922 \mathrm{E}-01 \\
1591 \mathrm{E}-01\end{array}$ \\
\hline $\begin{array}{l}96253 \mathrm{E}-01 \\
04220 \mathrm{E}-01 \\
12232 \mathrm{E}-01 \\
20289 \mathrm{E}-01\end{array}$ & $\begin{array}{l}-7 . \\
-7 . \\
-7 . \\
-7 . \\
-7 .\end{array}$ & $\begin{array}{l}9303 \mathrm{E}-01 \\
7061 \mathrm{E}-01 \\
4863 \mathrm{E}-01 \\
2709 \mathrm{E}-01 \\
0600 \mathrm{E}-01\end{array}$ \\
\hline $\begin{array}{l}36535 \mathrm{E}-01 \\
44725 \mathrm{E}-01 \\
52960 \mathrm{E}-01 \\
61239 \mathrm{E}-01\end{array}$ & $\begin{array}{l}-7.29265 \mathrm{E}-04 \\
-7.33057 \mathrm{E}-04 \\
-7.36810 \mathrm{E}-04 \\
-7.40510 \mathrm{E}-04 \\
-7.44169 \mathrm{E}-04\end{array}$ & $\begin{array}{l}-6.68535 \mathrm{E}-01 \\
-6.76515 \mathrm{E}-01 \\
-6.84540 \mathrm{E}-01 \\
-6.92610 \mathrm{E}-01 \\
-7.00724 \mathrm{E}-01\end{array}$ \\
\hline $\begin{array}{l}77932 \mathrm{E}-01 \\
86345 \mathrm{E}-01 \\
94803 \mathrm{E}-01 \\
03306 \mathrm{E}-01 \\
11853 \mathrm{E}-01\end{array}$ & $\begin{array}{l}-7.47794 \mathrm{E}-04 \\
-7.51367 \mathrm{E}-04 \\
-7.54902 \mathrm{E}-04 \\
-7.58391 \mathrm{E}-04 \\
-7.61837 \mathrm{E}-04\end{array}$ & $\begin{array}{l}-7.08883 \mathrm{E}-01 \\
-7.17086 \mathrm{E}-01 \\
-7.25335 \mathrm{E}-01 \\
-7.33628 \mathrm{E}-01 \\
-7.41965 \mathrm{E}-01\end{array}$ \\
\hline
\end{tabular}

$g_{-1}$ $\tan \delta_{-1}$

$1.73715 \mathrm{E} \quad 01$ $1.74662 \mathrm{E} \quad 01$ $1.75610 \mathrm{E} 01$ $1.76557 \mathrm{E} \quad 0$ $1.77505 \mathrm{E} \quad 01$

$\begin{array}{ll}1.78452 \mathrm{E} & 01\end{array}$ $1.79400 \mathrm{E} \quad 01$ $1.80348 \mathrm{E} 01$ $1.81297 \mathrm{E} \quad 01$ $1.82245 \mathrm{E} \quad 01$

$1.83194 \mathrm{E} \quad 01$ $1.84142 \mathrm{E} 01$ $1.85091 \mathrm{E} \quad 01$ $1.86040 \mathrm{E} \quad 01$ $1.86990 \mathrm{E} \quad 01$

$1.87939 \mathrm{E} \quad 01$ $1.88889 \mathrm{E}$ ol $1.89839 \mathrm{E} \quad 01$ $1.90789 \mathrm{E} 01$ $1.91739 \mathrm{E} \quad 01$

$1.92689 \mathrm{E} \quad 01$ $1.93640 \mathrm{E} \quad 01$ $1.94591 \mathrm{E} \quad 01$ $1.95542 \mathrm{E} \quad 01$ $1.96493 \mathrm{E} \quad 01$

$1.97444 \mathrm{E} \quad 01$ $1.98396 \mathrm{E} \quad 01$ $1.99348 \mathrm{E} 01$ $2.00300 \mathrm{E} \quad 01$ $2.01252 \mathrm{E} 01$

$2.02204 \mathrm{E} \quad 01$ $2.03157 \mathrm{E} \quad 01$ $2.04109 \mathrm{E} \quad 01$ $2.05062 \mathrm{E} 01$ $2.06015 \mathrm{E} \quad 01$

$2.06969 \mathrm{E} \quad 01$ $2.07922 \mathrm{E} \quad 01$ $2.08876 \mathrm{E} \quad 01$ $2.09830 \mathrm{E} \quad 01$

$2.10784 \mathrm{E} \quad 01$

$2.11738 \mathrm{E} \quad 01$ $2.12693 \mathrm{E} \quad 01$ 2.13647 E 01 $2.14602 \mathrm{E} \quad 01$ $2.15557 \mathrm{E} \quad 01$

$2.16513 \mathrm{E} \quad 01$ $2.17468 \mathrm{E} \quad 01$ $2.18424 \mathrm{E}$ 0l $2.19380 \mathrm{E} \quad 01$ $2.20336 \mathrm{E} \quad 01$

$2.21293 \mathrm{E} \quad 0$ $2.22249 \mathrm{E} 01$ $2.23206 \mathrm{E} \quad 01$ $2.24163 \mathrm{E} 01$ $2.25120 \mathrm{E} 01$ $2.26078 \mathrm{E} 01$ $2.27035 \mathrm{E} 01$ $2.27993 \mathrm{E} \quad 01$ $2.28951 \mathrm{E} \quad 01$ $2.29909 \mathrm{E} \quad 01$
$3.04629 \mathrm{E} \quad 02$ $3.05429 \mathrm{E} \quad 02$ $3.06224 \mathrm{E} \quad 02$ $3.07017 \mathrm{E} \quad 02$ $3.07807 \mathrm{E} \quad 02$

$3.08593 \mathrm{E} \quad 02$ $3.09376 \mathrm{E} \quad 02$ $3.10154 \mathrm{E} \quad 02$ $3.10931 \mathrm{E} \quad 02$ $3.11704 \mathrm{E} \quad 02$

$3.12474 \mathrm{E} \quad 02$

$3.13242 \mathrm{E} \quad 02$

$3.14005 \mathrm{E} \quad 02$

$3.14766 \mathrm{E} \quad 02$ $3.15524 \mathrm{E} \quad 02$

$3.16280 \mathrm{E} \quad 02$ $3.17032 \mathrm{E} \quad 02$ 3.17781 E 02 $3.18528 \mathrm{E} \quad 02$ $3.19273 \mathrm{E} \quad 02$

$3.20013 \mathrm{E} \quad 02$ $3.20752 \mathrm{E} \quad 02$ $3.21488 \mathrm{E} \quad 02$ $3.22222 \mathrm{E} \quad 02$ $3.22953 \mathrm{E} \quad 02$

$3.23681 \mathrm{E} \quad 02$ $3.24406 \mathrm{E} \quad 02$ $3.25130 \mathrm{E} \quad 02$ $3.25851 \mathrm{E} \quad 02$ $3.26570 \mathrm{E} \quad 02$

$3.27285 \mathrm{E} \quad 02$ $3.28000 \mathrm{E} \quad 02$ $3.28711 \mathrm{E} \quad 02$ $3.29421 \mathrm{E} \quad 02$ $3.30128 \mathrm{E} \quad 02$

$3.30833 \mathrm{E} \quad 02$ $3.31535 \mathrm{E} \quad 02$ $3.32236 \mathrm{E} \quad 02$ $3.32935 \mathrm{E} \quad 02$ $\begin{array}{lll}3.33631 \mathrm{E} & 02\end{array}$

$3.34326 \mathrm{E} \quad 02$ $3.35019 \mathrm{E} \quad 02$ $3.35710 \mathrm{E} \quad 02$ $3.36398 \mathrm{E} \quad 02$ $3.37084 \mathrm{E} \quad 02$

$3.37769 \mathrm{E} \quad 02$ $3.38451 \mathrm{E} \quad 02$ $3.39133 \mathrm{E} \quad 02$ $3.39813 \mathrm{E} \quad 02$ $3.40490 \mathrm{E} \quad 02$

$3.41166 \mathrm{E} \quad 02$ $3.41840 \mathrm{E} \quad 02$ $3.42512 \mathrm{E} \quad 02$ $3.43183 \mathrm{E} \quad 02$ $3.43853 \mathrm{E} \quad 02$

$3.44519 \mathrm{E} \quad 02$ $3.45185 \mathrm{E} \quad 02$ $3.45849 \mathrm{E} \quad 02$ $3.46513 \mathrm{E} \quad 02$ $3.47174 \mathrm{E} \quad 02$ 
ELECTRON RADIAI, FUNCTIONS

$Z=7 \quad A=14 \quad \rho=1.1 A^{1 / 3} F$

Positron

$\bar{F}$

$3.66536 \mathrm{E}-04$

$3.70596 \mathrm{E}-04$

$3.74678 \mathrm{E}-04$

$3.78782 \mathrm{E}-04$

$3.82909 \mathrm{E}-04$

18.40

$3.87058 \mathrm{E}-04$

$3.91230 \mathrm{E}-04$

$3.95423 \mathrm{E}-04$

$3.99639 \mathrm{E}-04$

$4.03878 \mathrm{E}-04$

18.90

19.00

19.10

19.20

19.30

19.40

19.50

19.60

19.70

19.80

19.90

20.00

20.10

20.20

20.30

20.40

20.50

20.60

20.70

20.80

20.90

21.00

21.10

21.20

21.30

21.40

21.50

21.60

21.70

21.80

21.90

22.00

22.10

22.20

22.30

22.40

22.50

22.60

22.70

22.80

22.90

23.00

23.10

23.20

23.30

23.40

23.50

23.60

23.70

23.80

23.90

24.00

$5.12299 \mathrm{E}-04$

$5.17096 \mathrm{E}-04$

$5.21915 \mathrm{E}-04$

$5.26756 \mathrm{E}-04$

$5.31620 \mathrm{E}-04$

$5.36506 \mathrm{E}-04$

5.41414 E- 04

$5.46345 \mathrm{E}-04$

$5.51298 \mathrm{E}-04$

$5.56274 \mathrm{E}-04$

$5.61271 \mathrm{E}-04$

5.66291 E-04

5.71334 E- 04

$5.76399 \mathrm{E}-04$

$5.81486 \mathrm{E}-04$

$5.86595 \mathrm{E}-04$

$5.91727 \mathrm{E}-04$

5.96881 E-04 $6.02057 \mathrm{E}-04$

$6.07256 \mathrm{E}-04$

$6.12477 \mathrm{E}-04$

$6.17720 \mathrm{E}-04$

$6.22986 \mathrm{E}-04$

$6.28274 \mathrm{E}-04$

$6.33584 \mathrm{E}-04$

$6.38917 \mathrm{E}-04$

$6.44272 \mathrm{E}-04$ $f_{2}$

$8.34171 \mathrm{E}-01$
$6.74533 \mathrm{E}-01$

$6.82114 \mathrm{E}-01$

$6.89737 \mathrm{E}-01$

$6.97402 \mathrm{E}-01$

$7.05109 \mathrm{E}-01$

7.12859 E-01

$7.20651 \mathrm{E}-01$

7.28485 E-01

$7.36361 \mathrm{E}-01$

7.44280 E-01

7.52241 E- 01

$7.60244 \mathrm{E}-01$

$7.68289 \mathrm{E}-01$

$7.76377 \mathrm{E}-01$

$7.84506 \mathrm{E}-01$

$7.92679 \mathrm{E}-01$

$8.00893 \mathrm{E}-01$

$8.09149 \mathrm{E}-01$

$8.17448 \mathrm{E}-01$

$8.25788 \mathrm{E}-01$

$8.42596 \mathrm{E}-01$

$8.51064 \mathrm{E}-01$

$8.59574 \mathrm{E}-01$

$8.68125 \mathrm{E}-01$

$8.76719 \mathrm{E}-01$

$8.85355 \mathrm{E}-01$

$8.94034 \mathrm{E}-01$

$9.02754 \mathrm{E}-01$

$9.11517 \mathrm{E}-01$

9.20322 E-01

$9.29169 \mathrm{E}-01$

$9.38058 \mathrm{E}-01$

$9.46989 \mathrm{E}-01$

$9.55963 \mathrm{E}-01$

$g_{2}$

$9.84142 \mathrm{E}-03$

$1.00456 \mathrm{E}-02$

$1.02524 \mathrm{E}-02$

$1.04620 \mathrm{E}-02$

$1.06744 \mathrm{E}-02$

$1.08895 \mathrm{E}-02$

$1.11074 \mathrm{E}-02$

$1.13280 \mathrm{E}-02$

$1.15515 \mathrm{E}-02$

$1.17778 \mathrm{E}-02$

$1.20070 \mathrm{E}-02$

$1.22390 \mathrm{E}-02$

$1.24740 \mathrm{E}-02$

$1.27118 \mathrm{E}-02$

$1.29525 \mathrm{E}-02$

$1.31962 \mathrm{E}-02$

$1.34428 \mathrm{E}-02$

$1.36924 \mathrm{E}-02$

$1.39450 \mathrm{E}-02$

$1.42006 \mathrm{E}-02$

$1.44592 \mathrm{E}-02$

$1.47209 \mathrm{E}-02$

$1.49856 \mathrm{E}-02$

$1.52534 \mathrm{E}-02$

$1.55243 \mathrm{E}-02$

$1.57983 \mathrm{E}-02$

$1.60754 \mathrm{E}-02$

$1.63557 \mathrm{E}-02$

$1.66391 \mathrm{E}-02$

$1.69257 \mathrm{E}-02$

$1.72155 \mathrm{E}-02$

$1.75085 \mathrm{E}-02$

$1.78047 \mathrm{E}-02$

$1.81042 \mathrm{E}-02$

$1.84070 \mathrm{E}-02$

$9.6+978 \mathrm{E}-01$

$9.74036 \mathrm{E}-01$

$9.83136 \mathrm{E}-01$

$9.92278 \mathrm{E}-01$

$1.00146 \mathrm{E} \quad 00$

$1.01069 \mathrm{E} \quad 00$

$1.01996 \mathrm{E} \quad 00$

$1.02927 \mathrm{E} \quad 00$

$1.03862 \mathrm{E} \quad 00$

$1.04802 \mathrm{E} \quad 00$

$1.05745 \mathrm{E} \quad 00$ $1.06693 \mathrm{E} \quad 00$

$1.07645 \mathrm{E} \quad 00$

$1.08602 \mathrm{E} \quad 00$

$1.09562 \mathrm{E} \quad 00$

$1.10527 \mathrm{E} \quad 00$

$1.11496 \mathrm{E} \quad 00$

$1.12469 \mathrm{E} \quad 00$

$1.13447 \mathrm{E} \quad 00$

$1.14428 \mathrm{E} \quad 00$

$1.15+14 \mathrm{E} \quad 00$

$1.1640 \div \mathrm{E} \quad 00$

$1.17398 \mathrm{E} \quad 00$

$1.18397 \mathrm{E} \quad 00$

$1.19399 \mathrm{E} \quad 00$

$1.87131 \mathrm{E}-02$

$1.90224 \mathrm{E}-02$

$1.93351 \mathrm{E}-02$

$1.96511 \mathrm{E}-02$

$1.9970+\mathrm{E}-02$

$2.02931 \mathrm{E}-02$

$2.06193 \mathrm{E}-02$

$2.09488 \mathrm{E}-02$

$2.12817 \mathrm{E}-02$

2.16181 E-02

$2.19579 \mathrm{E}-02$

$2.23012 \mathrm{E}-02$

$2.26480 \mathrm{E}-02$

$2.29984 \mathrm{E}-02$

$2.33522 \mathrm{E}-02$

$2.37096 \mathrm{E}-02$

$2.40705 \mathrm{E}-02$

$2.44351 \mathrm{E}-02$

$2.48032 \mathrm{E}-02$

$2.51749 \mathrm{E}-02$

$2.55503 \mathrm{E}-02$

$2.59293 \mathrm{E}-02$

$2.63120 \mathrm{E}-02$

$2.66983 \mathrm{E}-02$

$2.7088+\mathrm{E}-02$ $\tan \delta_{2}$

$-2.76573 \mathrm{E} 01$

$\begin{array}{lll}-2.76607 \mathrm{E} & 01\end{array}$

$-2.76641 \mathrm{E} \quad 01$

$-2.76674 \mathrm{E} \quad 01$

$-2.76707 \mathrm{E} 01$

$-2.76740 \mathrm{E} 01$

$-2.76772 \mathrm{E} \quad 01$

$-2.76804 \mathrm{E} \quad 01$

$\begin{array}{lll}-2.76836 \mathrm{E} & 0]\end{array}$

$-2.76867 \mathrm{E} \quad 01$

$-2.76897 \mathrm{E} \quad 01$

$-2.76928 \mathrm{E} 01$

$-2.76958 \mathrm{E}$ ol

$-2.76988 \mathrm{E} \quad 01$

-2.77017 E 01

$-2.77047 \mathrm{E} \quad 01$

$-2.77076 \mathrm{E} \quad 01$

$-2.7710+\mathrm{E} \quad 01$

$-2.77132 \mathrm{E} \quad 01$

$-2.77160 \mathrm{E} 01$

$-2.77188 \mathrm{E} \quad 01$

$-2.77216 \mathrm{E}$ 0I

$-2.77243 \mathrm{E} \quad 01$

$-2.77270 \mathrm{E}$ 0I

$-2.77296 \mathrm{E}$ 0]

$\begin{array}{lll}-2.77323 \mathrm{E} & 01\end{array}$

$-2.77349 \mathrm{E}$ 0l

$-2.77374 \mathrm{E} \quad 0 \mathrm{I}$

$-2.77400 \mathrm{E} 01$

$-2.77425 \mathrm{E}$ 01

$-2.77450 \mathrm{E} \quad 0 \mathrm{l}$

$-2.77475 \mathrm{E} \quad 01$

$-2.77500 \mathrm{E}$ 0I

$-2.77524 \mathrm{E} \quad 01$

$-2.77548 \mathrm{E}$ ol
$-2.77572 \mathrm{E} \quad 01$

$-2.77596 \mathrm{E} \quad 01$

$-2.77619 \mathrm{E}$ ol

$-2.77642 \mathrm{E} \quad 01$

$-2.77665 \mathrm{E}$ 0l

-2.77688 E 01

$-2.77710 \mathrm{E}$ 0I

$-2.77733 \mathrm{E} 01$

$-2.77755 \mathrm{E} \quad 01$

$-2.77777 \mathrm{E}$ 0l

$-2.77798 \mathrm{E} \quad 01$

$-2.77820 \mathrm{E}$ 0l

$-2.77841 \mathrm{E} \quad 01$

$-2.77862 \mathrm{E} \quad 01$

$-2.77883 \mathrm{E} \quad 01$

$\begin{array}{lll}-2.77904 \mathrm{E} & 01\end{array}$

$-2.77925 \mathrm{E}$ 01

$-2.77945 \mathrm{E} \quad 01$

$\begin{array}{lll}-2.77965 \mathrm{E} & 01\end{array}$

$-2.77985 \mathrm{E} \quad 01$

$-2.78005 \mathrm{E} \quad 01$

$-2.78025 \mathrm{E}$ 0l

$-2.780+4 \mathrm{E} 01$

$-2.78063 \mathrm{E} \quad 0 \mathrm{l}$

-2.78083 E 01

$f_{-2}$

$-8.44232 \mathrm{E}-0.3$

$-8.63345 \mathrm{E}-03$

$-8.82722 \mathrm{~F}-03$

$-9.02367 \mathrm{E}-03$

$-9.22280 \mathrm{E}-03$

$-9.42464 \mathrm{E}-03$

$-9.62919 \mathrm{E}-03$

$-9.83649 \mathrm{E}-0.3$

$-1.00465 \mathrm{E}-02$

$-1.02594 \mathrm{E}-02$ 


\begin{tabular}{|c|c|c|c|}
\hline$p$ & $F_{0}$ & $f_{1}$ & \\
\hline 24.10 & $8.55811 \mathrm{E}-01$ & $2.21254 \mathrm{E}$ & 01 \\
\hline 24.20 & $8.55802 \mathrm{E}-01$ & $2.22210 \mathrm{E}$ & 01 \\
\hline 24.30 & $8.55794 \mathrm{E}-01$ & $2.23166 \mathrm{E}$ & 01 \\
\hline 24.40 & $8.55786 \mathrm{E}-01$ & $2.24122 \mathrm{E}$ & 01 \\
\hline 24.50 & $8.55778 \mathrm{E}-01$ & $2.25079 \mathrm{E}$ & 01 \\
\hline 24.60 & $8.55770 \mathrm{E}-01$ & $2.26035 \mathrm{E}$ & 01 \\
\hline 24.70 & $8.55761 \mathrm{E}-01$ & $2.26992 \mathrm{E}$ & 01 \\
\hline 24.80 & $8.55754 \mathrm{E}-01$ & $2.27950 \mathrm{E}$ & 01 \\
\hline 24.90 & $8.55745 \mathrm{E}-01$ & $2.28907 \mathrm{E}$ & 01 \\
\hline 25.00 & $8.55737 \mathrm{E}-01$ & $2.29865 \mathrm{E}$ & 01 \\
\hline 25.10 & $8.55729 \mathrm{E}-01$ & $2.30823 \mathrm{E}$ & 01 \\
\hline 25.20 & $8.55721 \mathrm{E}-01$ & $2.31781 \mathrm{E}$ & 01 \\
\hline 25.30 & $8.55713 \mathrm{E}-01$ & $2.32739 \mathrm{E}$ & 01 \\
\hline 25.40 & $8.55705 \mathrm{E}-01$ & $2.33697 \mathrm{E}$ & 01 \\
\hline 25.50 & $8.55698 \mathrm{E}-01$ & $2.34656 \mathrm{E}$ & 01 \\
\hline 25.60 & $8.55690 \mathrm{E}-01$ & $2.35615 \mathrm{E}$ & 01 \\
\hline 25.70 & $8.55682 \mathrm{E}-01$ & $2.36574 \mathrm{E}$ & 01 \\
\hline 25.80 & $8.55674 \mathrm{E}-01$ & $2.37534 \mathrm{E}$ & 01 \\
\hline 25.90 & $8.55666 \mathrm{E}-01$ & $2.38493 \mathrm{E}$ & 01 \\
\hline 26.00 & $8.55658 \mathrm{E}-01$ & $2.39453 \mathrm{E}$ & 01 \\
\hline 26.10 & $8.55651 \mathrm{E}-01$ & $2.40413 \mathrm{E}$ & 01 \\
\hline 26.20 & $8.55643 \mathrm{E}-01$ & $2.41373 \mathrm{E}$ & 01 \\
\hline 26.30 & $8.55635 \mathrm{E}-01$ & $2.42334 \mathrm{E}$ & 01 \\
\hline 26.40 & $8.55628 \mathrm{E}-01$ & $2.43294 \mathrm{E}$ & 01 \\
\hline 26.50 & $8.55620 \mathrm{E}-01$ & $2.44255 \mathrm{E}$ & 01 \\
\hline 26.60 & $8.55612 \mathrm{E}-01$ & $2.45217 \mathrm{E}$ & 01 \\
\hline 26.70 & $8.55605 \mathrm{E}-01$ & $2.46178 \mathrm{E}$ & 01 \\
\hline 26.80 & $8.55597 \mathrm{E}-01$ & $2.47140 \mathrm{E}$ & 01 \\
\hline 26.90 & $8.55589 \mathrm{E}-01$ & $2.48102 \mathrm{E}$ & 01 \\
\hline 27.00 & $8.55582 \mathrm{E}-01$ & $2.49064 \mathrm{E}$ & 01 \\
\hline 27.10 & $8.55574 \mathrm{E}-01$ & $2.50026 \mathrm{E}$ & 01 \\
\hline 27.20 & $8.55567 \mathrm{E}-01$ & $2.50988 \mathrm{E}$ & 01 \\
\hline 27.30 & $8.55559 \mathrm{E}-01$ & $2.51951 \mathrm{E}$ & 01 \\
\hline 27.40 & $8.55552 \mathrm{E}-01$ & $2.52914 \mathrm{E}$ & 01 \\
\hline 27.50 & $8.55544 \mathrm{E}-01$ & $2.53878 \mathrm{E}$ & 01 \\
\hline 27.60 & $8.55537 \mathrm{E}-01$ & $2.54841 \mathrm{E}$ & 01 \\
\hline 27.70 & $8.55530 \mathrm{E}-01$ & $2.55805 \mathrm{E}$ & 01 \\
\hline 27.80 & $8.55522 \mathrm{E}-01$ & $2.56769 \mathrm{E}$ & 01 \\
\hline 27.90 & $8.55515 \mathrm{E}-01$ & $2.57733 \mathrm{E}$ & 01 \\
\hline 28.00 & $8.55508 \mathrm{E}-01$ & $2.58697 \mathrm{E}$ & 01 \\
\hline 28.10 & $8.55500 \mathrm{E}-01$ & $2.59662 \mathrm{E}$ & 01 \\
\hline 28.20 & $8.55493 \mathrm{E}-01$ & $2.60627 \mathrm{E}$ & 01 \\
\hline 28.30 & $8.55486 \mathrm{E}-01$ & $2.61592 \mathrm{E}$ & 01 \\
\hline 28.40 & $8.55479 \mathrm{E}-01$ & $2.62557 \mathrm{E}$ & 01 \\
\hline 28.50 & $8.55471 \mathrm{E}-01$ & $2.63523 \mathrm{E}$ & 01 \\
\hline 28.60 & $8.55464 \mathrm{E}-01$ & $2.64489 \mathrm{E}$ & 01 \\
\hline 28.70 & $8.55457 \mathrm{E}-01$ & $2.65455 \mathrm{E}$ & 01 \\
\hline 28.80 & $8.55450 \mathrm{E}-01$ & $2.66421 \mathrm{E}$ & 01 \\
\hline 28.90 & $8.55443 \mathrm{E}-01$ & $2.67388 \mathrm{E}$ & 01 \\
\hline 29.00 & $8.55436 \mathrm{E}-01$ & $2.68355 \mathrm{E}$ & 01 \\
\hline 29.10 & $8.55428 \mathrm{E}-01$ & $2.69322 \mathrm{E}$ & 01 \\
\hline 29.20 & $8.55421 \mathrm{E}-01$ & $2.70289 \mathrm{E}$ & 01 \\
\hline 29.30 & $8.55414 \mathrm{E}-01$ & $2.71257 \mathrm{E}$ & 01 \\
\hline 29.40 & $8.55407 \mathrm{E}-01$ & $2.72225 \mathrm{E}$ & 01 \\
\hline 29.50 & $8.55400 \mathrm{E}-01$ & $2.73193 \mathrm{E}$ & 01 \\
\hline 29.60 & $8.55393 \mathrm{E}-01$ & $2.74161 \mathrm{E}$ & 01 \\
\hline 9.70 & $8.55386 \mathrm{E}-01$ & $2.75130 \mathrm{E}$ & 01 \\
\hline 29.80 & $8.55379 \mathrm{E}-01$ & $2.76099 \mathrm{E}$ & 01 \\
\hline 29.90 & $8.55372 \mathrm{E}-01$ & $2.77068 \mathrm{E}$ & 01 \\
\hline 30.00 & $8.55365 \mathrm{E}-01$ & $2.78037 \mathrm{E}$ & 01 \\
\hline
\end{tabular}

\begin{tabular}{|c|c|c|}
\hline$g_{1}$ & $\tan \delta_{1}$ & $f_{-1}$ \\
\hline $\begin{array}{l}.20446 \mathrm{E}-01 \\
.29082 \mathrm{E}-01 \\
.37764 \mathrm{E}-01 \\
.46491 \mathrm{E}-01 \\
.55262 \mathrm{E}-01\end{array}$ & $\begin{array}{r}-7.65246 \mathrm{E}-04 \\
-7.68617 \mathrm{E}-04 \\
-7.71951 \mathrm{E}-04 \\
-7.75237 \mathrm{E}-04 \\
-7.78484 \mathrm{E}-04\end{array}$ & $\begin{array}{l}-7.50349 \mathrm{E}- \\
-7.58776 \mathrm{E}- \\
-7.67248 \mathrm{E}- \\
-7.75765 \mathrm{E}- \\
-7.84327 \mathrm{E}-\end{array}$ \\
\hline $\begin{array}{l}64078 \mathrm{E}-01 \\
72939 \mathrm{E}-01 \\
31845 \mathrm{E}-01 \\
90796 \mathrm{E}-01 \\
99791 \mathrm{E}-01\end{array}$ & $\begin{array}{r}-7.81694 \mathrm{E}-04 \\
-7.84876 \mathrm{E}-04 \\
-7.88014 \mathrm{E}-04 \\
-7.91109 \mathrm{E}-04 \\
-7.94172 \mathrm{E}-04\end{array}$ & $\begin{array}{l}-7.92934 \mathrm{E} \\
-8.01586 \mathrm{E}- \\
-8.10283 \mathrm{E}- \\
-8.19025 \mathrm{E}- \\
-8.27812 \mathrm{E}-\end{array}$ \\
\hline $\begin{array}{l}08832 \mathrm{E}-01 \\
17917 \mathrm{E}-01 \\
27048 \mathrm{E}-01 \\
36224 \mathrm{E}-01 \\
45444 \mathrm{E}-01\end{array}$ & $\begin{array}{l}-7.97187 \mathrm{E}-04 \\
-8.00179 \mathrm{E}-04 \\
-8.03137 \mathrm{E}-04 \\
-8.06054 \mathrm{E}-04 \\
-8.08940 \mathrm{E}-04\end{array}$ & $\begin{array}{l}-8.36643 \mathrm{E}- \\
-8.45520 \mathrm{E}- \\
-8.54442 \mathrm{E}- \\
-8.63409 \mathrm{E}- \\
-8.72421 \mathrm{E}\end{array}$ \\
\hline $\begin{array}{l}54710 \mathrm{E}-01 \\
64021 \mathrm{E}-01 \\
73377 \mathrm{E}-01 \\
82778 \mathrm{E}-01 \\
92224 \mathrm{E}-01\end{array}$ & $\begin{array}{l}-8.11780 \mathrm{E}-04 \\
-8.14600 \mathrm{E}-04 \\
-8.17381 \mathrm{E}-04 \\
-8.20127 \mathrm{E}-04 \\
-8.22835 \mathrm{E}-04\end{array}$ & $\begin{array}{l}-8.81478 \mathrm{E}-01 \\
-8.90580 \mathrm{E}-01 \\
-8.99728 \mathrm{E}-01 \\
-9.08920 \mathrm{E}-01 \\
-9.18158 \mathrm{E}-01\end{array}$ \\
\hline $\begin{array}{ll}00172 \mathrm{E} & 00 \\
01125 \mathrm{E} & 00 \\
02083 \mathrm{E} & 00 \\
03046 \mathrm{E} & 00 \\
04013 \mathrm{E} & 00\end{array}$ & $\begin{array}{l}-8.25513 \mathrm{E}-04 \\
-8.28165 \mathrm{E}-04 \\
-8.30772 \mathrm{E}-04 \\
-8.33355 \mathrm{E}-04 \\
-8.35909 \mathrm{E}-04\end{array}$ & $\begin{array}{l}-9.27441 \mathrm{E}-01 \\
-9.36769 \mathrm{E}-01 \\
-9.46143 \mathrm{E}-01 \\
-9.55562 \mathrm{E}-01 \\
-9.65026 \mathrm{E}-01\end{array}$ \\
\hline $\begin{array}{ll}04985 \mathrm{E} & 00 \\
05961 \mathrm{E} & 00 \\
06942 \mathrm{E} & 00 \\
07927 \mathrm{E} & 00 \\
08917 \mathrm{E} & 00\end{array}$ & $\begin{array}{l}-8.38432 \mathrm{E}-04 \\
-8.40921 \mathrm{E}-04 \\
-8.43371 \mathrm{E}-04 \\
-8.45802 \mathrm{E}-04 \\
-8.48198 \mathrm{E}-04\end{array}$ & $\begin{array}{l}-9.74535 \mathrm{E}-0 \\
-9.84090 \mathrm{E}-0 \\
-9.93690 \mathrm{E}-0 \\
-1.00334 \mathrm{E} \\
-1.01303 \mathrm{E}\end{array}$ \\
\hline $\begin{array}{ll}09912 \mathrm{E} & 00 \\
10911 \mathrm{E} & 00 \\
11914 \mathrm{E} & 00 \\
12922 \mathrm{E} & 00 \\
13935 \mathrm{E} & 00\end{array}$ & $\begin{array}{l}-8.50563 \mathrm{E}-04 \\
-8.52905 \mathrm{E}-04 \\
-8.55211 \mathrm{E}-04 \\
-8.57495 \mathrm{E}-04 \\
-8.59744 \mathrm{E}-04\end{array}$ & $\begin{array}{l}-1.02276 \mathrm{E} \\
-1.03255 \mathrm{E} \\
-1.04237 \mathrm{E} \\
-1.05225 \mathrm{E} \\
-1.06216 \mathrm{E}\end{array}$ \\
\hline $\begin{array}{ll}14952 \mathrm{E} & 00 \\
15973 \mathrm{E} & 00 \\
17000 \mathrm{E} & 00 \\
18031 \mathrm{E} & 00 \\
19066 \mathrm{E} & 00\end{array}$ & $\begin{array}{l}-8.61962 \mathrm{E}-04 \\
-8.64161 \mathrm{E}-04 \\
-8.66323 \mathrm{E}-04 \\
-8.68457 \mathrm{E}-04 \\
-8.70573 \mathrm{E}-04\end{array}$ & $\begin{array}{l}-1.07213 \mathrm{E} \\
-1.08214 \mathrm{E} \\
-1.09219 \mathrm{E} \\
-1.10229 \mathrm{E} \\
-1.11244 \mathrm{E}\end{array}$ \\
\hline $\begin{array}{ll}20106 \mathrm{E} & 00 \\
21150 \mathrm{E} & 00 \\
22199 \mathrm{E} & 00 \\
23253 \mathrm{E} & 00 \\
24311 \mathrm{E} & 00\end{array}$ & $\begin{array}{l}-8.72654 \mathrm{E}-04 \\
-8.74713 \mathrm{E}-04 \\
-8.76748 \mathrm{E}-04 \\
-8.78746 \mathrm{E}-04 \\
-8.80711 \mathrm{E}-04\end{array}$ & $\begin{array}{l}-1.12263 \mathrm{E} \\
-1.13287 \mathrm{E} \\
-1.14315 \mathrm{E} \\
-1.15348 \mathrm{E} \\
-1.16386 \mathrm{E}\end{array}$ \\
\hline $\begin{array}{ll}25374 \mathrm{E} & 00 \\
26441 \mathrm{E} & 00 \\
27513 \mathrm{E} & 00 \\
28590 \mathrm{E} & 00 \\
29671 \mathrm{E} & 00\end{array}$ & $\begin{array}{l}-8.82670 \mathrm{E}-04 \\
-8.84595 \mathrm{E}-04 \\
-8.86494 \mathrm{E}-04 \\
-8.88363 \mathrm{E}-04 \\
-8.90212 \mathrm{E}-04\end{array}$ & $\begin{array}{l}-1.17428 \mathrm{E} \\
-1.18475 \mathrm{E} \\
-1.19526 \mathrm{E} \\
-1.20582 \mathrm{E} \\
-1.21642 \mathrm{E}\end{array}$ \\
\hline $\begin{array}{ll}30756 \mathrm{E} & 00 \\
31847 \mathrm{E} & 00 \\
32941 \mathrm{E} & 00 \\
34041 \mathrm{E} & 00 \\
35145 \mathrm{E} & 00\end{array}$ & $\begin{array}{l}-8.92037 \mathrm{E}-04 \\
-8.93837 \mathrm{E}-04 \\
-8.95609 \mathrm{E}-04 \\
-8.97361 \mathrm{E}-04 \\
-8.99084 \mathrm{E}-04\end{array}$ & $\begin{array}{l}-1.22707 \mathrm{E} \\
-1.23777 \mathrm{E} \\
-1.24851 \mathrm{E} \\
-1.25930 \mathrm{E} \\
-1.27014 \mathrm{E}\end{array}$ \\
\hline $\begin{array}{ll}36254 \mathrm{E} & 00 \\
37367 \mathrm{E} & 00 \\
38485 \mathrm{E} & 00 \\
39607 \mathrm{E} & 00 \\
40734 \mathrm{E} & 00\end{array}$ & $\begin{array}{l}-9.00793 \mathrm{E}-04 \\
-9.02468 \mathrm{E}-04 \\
-9.04130 \mathrm{E}-04 \\
-9.05760 \mathrm{E}-04 \\
-9.07366 \mathrm{E}-04\end{array}$ & $\begin{array}{ll}-1.28102 \mathrm{E} & 00 \\
-1.29194 \mathrm{E} & 00 \\
-1.30292 \mathrm{E} & 00 \\
-1.31393 \mathrm{E} & 00 \\
-1.32500 \mathrm{E} & 00\end{array}$ \\
\hline
\end{tabular}

$g_{-1}$

$2.30868 \mathrm{E} \quad 01$ $2.31827 \mathrm{E} \quad 01$ $2.32786 \mathrm{E} \quad 01$ $2.33745 \mathrm{E} \quad 01$

$2.34704 \mathrm{E} \quad 01$

$2.35664 \mathrm{E} \quad 01$ 2.36624. E 01 $2.37584 \mathrm{E} \quad 01$ 2.38544. E 01 $2.39505 \mathrm{E} \quad 01$

$2.40465 \mathrm{E} \quad 01$ $2.41426 \mathrm{E} \quad 01$ $2.42387 \mathrm{E} \quad 01$ $2.43349 \mathrm{E} \quad 01$ $2.44311 \mathrm{E} 01$

$2.45272 \mathrm{E} \quad 01$ $2.46235 \mathrm{E} \quad 01$ $2.47197 \mathrm{E} \quad 01$ $2.48159 \mathrm{E} \quad 01$ $2.49122 \mathrm{E} 01$

$2.50085 \mathrm{E} \quad 01$ $2.51049 \mathrm{E} \quad 01$ $2.52012 \mathrm{E} \quad 01$ $2.52976 \mathrm{E} \quad 01$ $2.53940 \mathrm{E} 01$

$2.54904 \mathrm{E} \quad 01$ $2.55868 \mathrm{E} \quad 01$ $2.56833 \mathrm{E} \quad 01$ $2.57798 \mathrm{E} \quad 01$ 2.58763 E 01

$2.59729 \mathrm{E} \quad 01$ $2.60694 \mathrm{E} \quad 01$ $2.61660 \mathrm{E} 01$ $2.62626 \mathrm{E} \quad 01$ $2.63593 \mathrm{E} 01$

$2.64559 \mathrm{E} \quad 01$ $2.65526 \mathrm{E} \quad 01$ $2.66493 \mathrm{E} 01$ $2.67461 \mathrm{E} \quad 01$ $2.68428 \mathrm{E} \quad 01$

$2.69396 \mathrm{E} \quad 01$ $2.70364 \mathrm{E} 01$ $2.71333 \mathrm{E} 01$ $2.72302 \mathrm{E} \quad 01$ $2.73270 \mathrm{E} 01$

$2.74240 \mathrm{E} \quad 01$ $2.75209 \mathrm{E} \quad 01$ $2.76179 \mathrm{E} 01$ $2.77148 \mathrm{E} \quad 01$ $2.78119 \mathrm{E} \quad 01$

$2.79089 \mathrm{E} \quad 01$ $2.80060 \mathrm{E} \quad 01$ $2.81031 \mathrm{E} 01$ $2.82002 \mathrm{E} 01$ $2.82973 \mathrm{E} \quad 01$

$2.83945 \mathrm{E} \quad 01$ $2.84917 \mathrm{E} \quad 01$ $2.85889 \mathrm{E} \quad 01$ $2.86862 \mathrm{E} \quad 01$ $2.87835 \mathrm{E} 01$ $\tan \delta_{-1}$

$3.47834 \mathrm{E} \quad 02$ $3.48492 \mathrm{E} \quad 02$ $3.49149 \mathrm{E} \quad 02$ $3.49805 \mathrm{E} \quad 02$ $3.50459 \mathrm{E} \quad 02$

$3.51112 \mathrm{E} \quad 02$ $3.51763 \mathrm{E} \quad 02$ $3.52412 \mathrm{E} \quad 02$ $3.53062 \mathrm{E} \quad 02$ $3.53709 \mathrm{E} \quad 02$

$3.54357 \mathrm{E} \quad 02$ $3.55002 \mathrm{E} \quad 02$ $3.55645 \mathrm{E} \quad 02$ $3.56288 \mathrm{E} \quad 02$ $3.56929 \mathrm{E} \quad 02$

$3.57571 \mathrm{E} \quad 02$ $3.58209 \mathrm{E} \quad 02$ $3.58847 \mathrm{E} \quad 02$ $3.59485 \mathrm{E} \quad 02$ $3.60121 \mathrm{E} \quad 02$

$3.60757 \mathrm{E} \quad 02$ $3.61390 \mathrm{E} \quad 02$ $3.62025 \mathrm{E} \quad 02$ $3.62657 \mathrm{E} \quad 02$ $3.63288 \mathrm{E} \quad 02$

$3.63918 \mathrm{E} \quad 02$ $3.64547 \mathrm{E} \quad 02$ $3.65177 \mathrm{E} \quad 02$ $3.65804 \mathrm{E} \quad 02$ $3.66432 \mathrm{E} \quad 02$

$3.67058 \mathrm{E} \quad 02$ $3.67683 \mathrm{E} \quad 02$ $3.68308 \mathrm{E} \quad 02$ $3.68931 \mathrm{E} \quad 02$ $3.69554 \mathrm{E} \quad 02$

$3.70177 \mathrm{E} \quad 02$ $3.70798 \mathrm{E} \quad 02$ $3.71420 \mathrm{E} \quad 02$ $3.72041 \mathrm{E} \quad 02$ $3.72660 \mathrm{E} \quad 02$

$3.73279 \mathrm{E} \quad 02$ $3.73897 \mathrm{E} \quad 02$ $3.74514 \mathrm{E} \quad 02$ $3.75132 \mathrm{E} \quad 02$ $3.75751 \mathrm{E} \quad 02$

$3.76366 \mathrm{E} \quad 02$ $3.76982 \mathrm{E} \quad 02$ $3.77597 \mathrm{E} \quad 02$ $3.78213 \mathrm{E} \quad 02$ $3.78827 \mathrm{E} \quad 02$

$3.79441 \mathrm{E} \quad 02$ $3.80054 \mathrm{E} \quad 02$ $3.80667 \mathrm{E} \quad 02$ $3.81280 \mathrm{E} \quad 02$ $3.81892 \mathrm{E} \quad 02$

$3.82503 \mathrm{E} \quad 02$ $3.83116 \mathrm{E} \quad 02$ $3.83726 \mathrm{E} \quad 02$ $3.84337 \mathrm{E} \quad 02$ $3.84948 \mathrm{E} \quad 02$ 
ELECTRON RADIAL FUNCTIONS

$Z=7 \quad A=14 \quad \rho=1.1 A^{1 / 3} F$

$\bar{F}$

$6.49650 \mathrm{E}-04$

$6.55049 \mathrm{E}-04$

$6.60471 \mathrm{E}-04$

$6.65916 \mathrm{E}-04$

$6.71382 \mathrm{E}-04$

24.40

24.50

24.60

24.70

24.80

24.90

25.00

25.10

25.20

25.30

25.40

25.50

25.60

25.70

25.80

25.90

26.00

26.10

26.20

26.30

26.40

26.50

26.60

26.70

26.80

26.90

27.00

27.10

27.20

27.30

27.40

27.50

27.60

27.70

27.80

27.90

28.00

28.10

28.20

28.30

28.40

28.50

28.60

28.70

28.80

28.90

29.00

29.10

29.20

29.30

29.40

29.50

29.60

29.70

29.80

29.90

30.00

$9.34020 \mathrm{E}-04$

$9.40491 \mathrm{E}-04$

$9.46985 \mathrm{E}-04$

$9.53501 \mathrm{E}-04$

$9.60039 \mathrm{E}-04$

$9.66600 \mathrm{E}-04$

$9.73183 \mathrm{E}-04$ $f_{2}$

$g_{2}$

$\tan \delta_{2}$

$f_{-2}$

$1.20406 \mathrm{E} \quad 00$ $1.21417 \mathrm{E} \quad 00$

$1.22433 \mathrm{E} \quad 00$

$1.23452 \mathrm{E} \quad 00$

$1.24476 \mathrm{E} \quad 00$

$1.25504 \mathrm{E} \quad 00$

$1.26536 \mathrm{E} \quad 00$

$1.27572 \mathrm{E} \quad 00$

$1.28613 \mathrm{E} \quad 00$

$1.29657 \mathrm{E} \quad 00$

$1.30706 \mathrm{E} \quad 00$

$1.31759 \mathrm{E} \quad 00$

$1.32817 \mathrm{E} \quad 00$

$1.33878 \mathrm{E} \quad 00$

$1.34944 \mathrm{E} \quad 00$

$1.36014 \mathrm{E} \quad 00$

$1.37088 \mathrm{E} \quad 00$

$1.38166 \mathrm{E} \quad 00$

$1.39249 \mathrm{E} \quad 00$

$1.40335 \mathrm{E} \quad 00$

$1.41426 \mathrm{E} \quad 00$

$1.42522 \mathrm{E} \quad 00$

$1.43621 \mathrm{E} 00$

$1.44724 \mathrm{E} \quad 00$

$1.45832 \mathrm{E} \quad 00$

$1.46944 \mathrm{E} \quad 00$

$1.48060 \mathrm{E} \quad 00$

$1.49180 \mathrm{E} \quad 00$

$1.50305 \mathrm{E} \quad 00$

$1.51434 \mathrm{E} \quad 00$

$1.52567 \mathrm{E} \quad 00$

$1.53704 \mathrm{E} \quad 00$

$1.54845 \mathrm{E} \quad 00$

$1.55990 \mathrm{E} \quad 00$

$1.57140 \mathrm{E} \quad 00$

$1.58294 \mathrm{E} \quad 00$

$1.59452 \mathrm{E} \quad 00$

$1.60614 \mathrm{E} \quad 00$

$1.61781 \mathrm{E} 00$

$1.62951 \mathrm{E} \quad 00$

$1.64126 \mathrm{E} \quad 00$

$1.65305 \mathrm{E} \quad 00$

$1.66488 \mathrm{E} \quad 00$

$1.67676 \mathrm{E} \quad 00$

$1.68867 \mathrm{E} \quad 00$

$1.70063 \mathrm{E} \quad 00$

$1.71263 \mathrm{E} \quad 00$

$1.72467 \mathrm{E} \quad 00$

$1.73676 \mathrm{E} \quad 00$

$1.74888 \mathrm{E} \quad 00$

$1.76105 \mathrm{E} \quad 00$

$1.77326 \mathrm{E} \quad 00$

$1.78551 \mathrm{E} \quad 00$

$1.79780 \mathrm{E} \quad 00$

$1.81014 \mathrm{E} \quad 00$

$1.82251 \mathrm{E} \quad 00$

$1.83493 \mathrm{E} \quad 00$

$1.84739 \mathrm{E} \quad 00$

$1.85989 \mathrm{E} \quad 00$

$1.87244 \mathrm{E} \quad 00$
$2.74822 \mathrm{E}-02$ $2.78797 \mathrm{E}-02$

$2.82809 \mathrm{E}-02$

$2.86860 \mathrm{E}-02$

$2.90948 \mathrm{E}-02$

$2.95074 \mathrm{E}-02$

$2.99239 \mathrm{E}-02$

$3.03441 \mathrm{E}-02$

$3.07683 \mathrm{E}-02$

$3.11963 \mathrm{E}-02$

$3.16282 \mathrm{E}-02$

$3.20640 \mathrm{E}-02$

$3.25037 \mathrm{E}-02$

$3.29474 \mathrm{E}-02$

3.33951 E-02

$3.38467 \mathrm{E}-02$

$3.43023 \mathrm{E}-02$

$3.47619 \mathrm{E}-02$

$3.52255 \mathrm{E}-02$

$3.56932 \mathrm{E}-02$

$3.61650 \mathrm{E}-02$

$3.66408 \mathrm{E}-02$

$3.71208 \mathrm{E}-02$

$3.76048 \mathrm{E}-02$

$3.80930 \mathrm{E}-02$

$3.85853 \mathrm{E}-02$

$3.90819 \mathrm{E}-02$

$3.95825 \mathrm{E}-02$

$4.00874 \mathrm{E}-02$

$4.05965 \mathrm{E}-02$

$4.11099 \mathrm{E}-02$

$4.16275 \mathrm{E}-02$

$4.21493 \mathrm{E}-02$

$4.26755 \mathrm{E}-02$

$4.32060 \mathrm{E}-02$

$4.37408 \mathrm{E}-02$

$4.42799 \mathrm{E}-02$

$4.48234 \mathrm{E}-02$

$4.53712 \mathrm{E}-02$

$4.59235 \mathrm{E}-02$

4.64802E-02

$4.70412 \mathrm{E}-02$

$4.76068 \mathrm{E}-02$

$4.87512 \mathrm{E}-02$

$4.93302 \mathrm{E}-02$

$4.99137 \mathrm{E}-02$

$5.05017 \mathrm{E}-02$

$5.10943 \mathrm{E}-02$

$5.16914 \mathrm{E}-02$

$5.22931 \mathrm{E}-02$

$5.28994 \mathrm{E}-02$

$5.35103 \mathrm{E}-02$

$5.41258 \mathrm{E}-02$

$5.47460 \mathrm{E}-02$

$-2.78102 \mathrm{E} \quad 01$

$-2.78139 \mathrm{E}$ 01 $-2.59643 \mathrm{E}-02$

$-2.78158 \mathrm{E}$ 01 $-2.63527 \mathrm{E}-02$

$-2.78176 \mathrm{E}$ ol $-2.67449 \mathrm{E}-02$

$-2.78194 \mathrm{E}$ ol $-2.71408 \mathrm{E}-02$

$-2.78212 \mathrm{E}$ 01 $-2.75404 \mathrm{E}-02$

$-2.78230 \mathrm{E}$ 01 $-2.79439 \mathrm{E}-02$

$-2.78248 \mathrm{E}$ 01 $-2.83511 \mathrm{E}-02$

$-2.78266 \mathrm{E}$ 01 $-2.87621 \mathrm{E}-02$

$-2.78283 \mathrm{E}$ 01 $-2.91770 \mathrm{E}-02$

$-2.78300 \mathrm{E}$ 01 $-2.95957 \mathrm{E}-02$

$-2.78318 \mathrm{E}$ 01 $-3.00183 \mathrm{E}-02$

$-2.78335 \mathrm{E} \quad 01-3.04448 \mathrm{E}-02$

$-2.78351 \mathrm{E} \quad 01 \quad-3.08752 \mathrm{E}-02$

$-2.78368 \mathrm{E} \quad 01 \quad-3.13095 \mathrm{E}-02$

$-2.78385 \mathrm{E}$ 01 $-3.17477 \mathrm{E}-02$

$-2.78401 \mathrm{E} \quad 01 \quad-3.21899 \mathrm{E}-02$

$-2.78418 \mathrm{E}$ 01 $-3.26361 \mathrm{E}-02$

$-2.78434 \mathrm{E}$ 0l $-3.30862 \mathrm{E}-02$

$-2.78450 \mathrm{E}$ 01 $-3.35404 \mathrm{E}-02$

$-2.78466 \mathrm{E}$ 01 $-3.39986 \mathrm{E}-02$

$-2.78482 \mathrm{E}$ 01 $-3.44608 \mathrm{E}-02$

$-2.78498 \mathrm{E}$ 01 $-3.49271 \mathrm{E}-02$

$-2.78513 \mathrm{E}$ 01 $-3.53974 \mathrm{E}-02$

$-2.78529 \mathrm{E}$ 01 $-3.58719 \mathrm{E}-02$

$-2.78544 \mathrm{E}$ 01 $-3.63504 \mathrm{E}-02$

$-2.78559 \mathrm{E}$ 01 $-3.68331 \mathrm{E}-02$

$-2.78574 \mathrm{E}$ 01 $-3.73200 \mathrm{E}-02$

$-2.78589 \mathrm{E}$ 01 $-3.78109 \mathrm{E}-02$

$-2.78604 \mathrm{E} \quad 01$

$-2.78619 \mathrm{E} \quad 01$

$-2.78634 \mathrm{E} \quad 01$

$-2.78648 \mathrm{E} \quad 01$

$-2.78663 \mathrm{E} \quad 01$

$\begin{array}{lll}-2.78677 \mathrm{E} & 01\end{array}$

$\begin{array}{ll}-2.78691 \mathrm{E} & 01\end{array}$

$-2.78706 \mathrm{E} \quad 01$

$-2.78720 \mathrm{E} 01$

$-2.78733 \mathrm{E} 01$

$-3.83061 \mathrm{E}-02$

$-3.88055 \mathrm{E}-02$

$-3.93090 \mathrm{E}-02$

$-3.98169 \mathrm{E}-02$

$-4.03289 \mathrm{E}-02$

$-2.78747 \mathrm{E} \quad 01$

$-2.78761 \mathrm{E} \quad 01$

$-2.78775 \mathrm{E} \quad 01$

$-2.78788 \mathrm{E} \quad 01$

$-2.78802 \mathrm{E} \quad 01$

$-4.08452 \mathrm{E}-02$

$-4.13658 \mathrm{E}-02$

$-4.18907 \mathrm{E}-02$

$-4.24200 \mathrm{E}-02$

$-4.29535 \mathrm{E}-02$

$-2.78815 \mathrm{E} \quad 01$

$-2.78828 \mathrm{E} \quad 01$

$-2.78841 \mathrm{E} \quad 01$

$\begin{array}{lll}-2.78854 \mathrm{E} & 01\end{array}$

$-2.78867 \mathrm{E} \quad 01$

$-4.34914 \mathrm{E}-02$

$-4.40337 \mathrm{E}-02$

$-4.45804 \mathrm{E}-02$

$-4.51314 \mathrm{E}-02$

$-4.56869 \mathrm{E}-02$

$-2.78880 \mathrm{E} \quad 01$

$-2.78893 \mathrm{E} \quad 01$

$-2.78906 \mathrm{E} 01$

$-2.78918 \mathrm{E} 01$

$-2.78931 \mathrm{E} 01$

$-4.62468 \mathrm{E}-02$

$-4.68112 \mathrm{E}-02$

$-4.73801 \mathrm{E}-02$

$-4.79534 \mathrm{E}-02$

$-4.85313 \mathrm{E}-02$

$5.53709 \mathrm{E}-02$

$5.60004 \mathrm{E}-02$

$5.72737 \mathrm{E}-02$

$5.79174 \mathrm{E}-02$ $\begin{array}{lll}-2.78943 \mathrm{E} & 01\end{array}$

$-2.78955 \mathrm{E}$ 0l

$-2.78968 \mathrm{E} \quad 01$

$-2.78980 \mathrm{E} 01$
$-2.78992 \mathrm{E} 01$
$-4.91136 \mathrm{E}-02$ $-4.97005 \mathrm{E}-02$ $-5.02920 \mathrm{E}-02$ $-5.08881 \mathrm{E}-02$ $-5.14887 \mathrm{E}-02$

$-5.20940 \mathrm{E}-02$ $-5.27038 \mathrm{E}-02$ $-5.33184 \mathrm{E}-02$ $-5.39375 \mathrm{E}-02$ $-5.45614 \mathrm{E}-02$
$4.81768 \mathrm{E}-02$

$5.66347 \mathrm{E}-02$ 
ELECTRON RADIAL FUNCTIONS

Positron

\begin{tabular}{|c|c|c|}
\hline$p$ & $F_{0}$ & $f_{1}$ \\
\hline 30.10 & $8.55358 \mathrm{E}-01$ & $2.79007 \mathrm{E}$ \\
\hline 30.20 & $8.55352 \mathrm{E}-01$ & $2.79977 \mathrm{E}$ \\
\hline 30.30 & $8.55345 \mathrm{E}-01$ & $2.80947 \mathrm{E}$ \\
\hline 30.40 & $8.55338 \mathrm{E}-01$ & $2.81918 \mathrm{E}$ \\
\hline 30.50 & $8.55331 \mathrm{E}-01$ & $2.82888 \mathrm{E}$ \\
\hline 30.60 & $8.55324 \mathrm{E}-01$ & $2.83859 \mathrm{E}$ \\
\hline 30.70 & $8.55317 E-01$ & $2.84830 \mathrm{E}$ \\
\hline 30.80 & $8.55311 \mathrm{E}-01$ & $2.85802 \mathrm{E}$ \\
\hline 30.90 & $8.55304 \mathrm{E}-01$ & $2.86773 \mathrm{E}$ \\
\hline 31.00 & $8.55297 \mathrm{E}-01$ & $2.87745 \mathrm{E}$ \\
\hline 31.10 & $8.55291 \mathrm{E}-01$ & $2.88718 \mathrm{E}$ \\
\hline 31.20 & $8.55284 \mathrm{E}-01$ & $2.89690 \mathrm{E}$ \\
\hline 31.30 & $8.55277 \mathrm{E}-01$ & $2.90663 \mathrm{E}$ \\
\hline 31.40 & $8.55271 \mathrm{E}-01$ & $2.91636 \mathrm{E}$ \\
\hline 31.50 & $8.55264 \mathrm{E}-0 \mathrm{l}$ & $2.92609 \mathrm{E}$ \\
\hline 31.60 & $8.55257 \mathrm{E}-01$ & $2.93583 \mathrm{E}$ \\
\hline 31.70 & $8.55251 \mathrm{E}-01$ & $2.94556 \mathrm{E}$ \\
\hline 31.80 & $8.55244 \mathrm{E}-01$ & $2.95531 \mathrm{E}$ \\
\hline 31.90 & $8.55237 \mathrm{E}-01$ & $2.96505 \mathrm{E}$ \\
\hline 32.00 & $8.55231 \mathrm{E}-01$ & $2.97480 \mathrm{E}$ \\
\hline 32.10 & $8.55225 \mathrm{E}-01$ & $2.98454 \mathrm{E}$ \\
\hline 32.20 & $8.55218 \mathrm{E}-01$ & $2.99430 \mathrm{E}$ \\
\hline 32.30 & $8.55212 \mathrm{E}-01$ & $3.00405 \mathrm{E}$ \\
\hline 32.40 & $8.55205 \mathrm{E}-01$ & $3.01381 \mathrm{E}$ \\
\hline 32.50 & $8.55199 \mathrm{E}-01$ & $3.02357 \mathrm{E}$ \\
\hline 32.60 & $8.55192 \mathrm{E}-01$ & $3.03333 \mathrm{E}$ \\
\hline 32.70 & $8.55186 \mathrm{E}-01$ & $3.04310 \mathrm{E}$ \\
\hline 32.80 & $8.55180 \mathrm{E}-01$ & $3.05286 \mathrm{E}$ \\
\hline 32.90 & $8.55173 \mathrm{E}-01$ & $3.06264 \mathrm{E}$ \\
\hline 33.00 & $8.55167 \mathrm{E}-01$ & $3.07241 \mathrm{E}$ \\
\hline 33.10 & $8.55160 \mathrm{E}-01$ & $3.08219 \mathrm{E}$ \\
\hline 33.20 & $8.55154 \mathrm{E}-01$ & $3.09197 \mathrm{E}$ \\
\hline 33.30 & $8.55148 \mathrm{E}-01$ & $3.10175 \mathrm{E}$ \\
\hline 33.40 & $8.55142 \mathrm{E}-01$ & $3.11153 \mathrm{E}$ \\
\hline 33.50 & $8.55135 \mathrm{E}-01$ & $3.12132 \mathrm{E}$ \\
\hline 33.60 & $8.55129 \mathrm{E}-01$ & $3.13111 \mathrm{E}$ \\
\hline 33.70 & $8.55123 \mathrm{E}-01$ & $3.14091 \mathrm{E}$ \\
\hline 33.80 & $8.55116 \mathrm{E}-01$ & $3.15070 \mathrm{E}$ \\
\hline 33.90 & $8.55110 \mathrm{E}-01$ & $3.16050 \mathrm{E}$ \\
\hline 34.00 & $8.55104 \mathrm{E}-01$ & $3.17030 \mathrm{E}$ \\
\hline 34.10 & $8.55098 \mathrm{E}-01$ & $3.18011 \mathrm{E}$ \\
\hline 34.20 & $8.55092 \mathrm{E}-01$ & $3.18992 \mathrm{E}$ \\
\hline 34.30 & $8.55085 \mathrm{E}-01$ & $3.19973 \mathrm{E}$ \\
\hline 34.40 & $8.55079 \mathrm{E}-01$ & $3.20954 \mathrm{E}$ \\
\hline 34.50 & $8.55073 \mathrm{E}-01$ & $3.21936 \mathrm{E}$ \\
\hline 34.60 & $8.55067 \mathrm{E}-01$ & $3.22918 \mathrm{E}$ \\
\hline 34.70 & $8.55061 \mathrm{E}-01$ & $3.23900 \mathrm{E}$ \\
\hline 34.80 & $8.55055 \mathrm{E}-01$ & $3.24882 \mathrm{E}$ \\
\hline 34.90 & $8.55049 \mathrm{E}-01$ & $3.25865 \mathrm{E}$ \\
\hline 35.00 & $8.55043 \mathrm{E}-01$ & $3.26848 \mathrm{E}$ \\
\hline 35.10 & $8.55037 \mathrm{E}-01$ & $3.27832 \mathrm{E}$ \\
\hline 35.20 & $8.55031 \mathrm{E}-01$ & $3.28816 \mathrm{E}$ \\
\hline 35.30 & $8.55025 \mathrm{E}-01$ & $3.29800 \mathrm{E}$ \\
\hline 35.40 & $8.55019 \mathrm{E}-01$ & $3.30784 \mathrm{E}$ \\
\hline 35.50 & $8.55013 \mathrm{E}-01$ & $3.31768 \mathrm{E}$ \\
\hline 35.60 & $8.55007 \mathrm{E}-01$ & $3.32753 \mathrm{E}$ \\
\hline 35.70 & $8.55001 \mathrm{E}-01$ & $3.33739 \mathrm{E}$ \\
\hline & $8.54995 \mathrm{E}-0 \mathrm{l}$ & $3.34724 \mathrm{E}$ \\
\hline 35.90 & $8.54989 \mathrm{E}-01$ & $3.35710 \mathrm{E}$ \\
\hline 36.00 & $8.54983 \mathrm{E}-01$ & $3.36696 \mathrm{E}$ \\
\hline
\end{tabular}

$Z=7 \quad A=14 \quad \rho=1.1 A^{1 / 3} F$

$\begin{array}{lll}g_{1} & \tan \delta_{1} & f_{-1}\end{array}$

$1.41866 \mathrm{E} \quad 00 \quad-9.08949 \mathrm{E}-04$

$\begin{array}{lll}1.43002 \mathrm{E} & 00 & -9.10512 \mathrm{E}-04\end{array}$

$\begin{array}{lll}1.44143 \mathrm{E} & 00 & -9.12051 \mathrm{E}-04\end{array}$

$\begin{array}{lll}1.45289 \mathrm{E} & 00 & -9.13558 \mathrm{E}-04\end{array}$

$\begin{array}{lll}1.46439 \mathrm{E} & 00 & -9.15060 \mathrm{E}-04\end{array}$

$\begin{array}{lll}1.47593 \mathrm{E} & 00 & -9.16537 \mathrm{E}-04\end{array}$

$1.48753 \mathrm{E} \quad 00 \quad-9.17986 \mathrm{E}-04$

$\begin{array}{lll}1.49917 \mathrm{E} & 00 & -9.19417 \mathrm{E}-04\end{array}$

$\begin{array}{lll}1.51085 \mathrm{E} & 00 & -9.20824 \mathrm{E}-04\end{array}$

$\begin{array}{lll}1.52259 \mathrm{E} & 00 & -9.22215 \mathrm{E}-04\end{array}$

$\begin{array}{lll}1.53437 \mathrm{E} & 00 & -9.23579 \mathrm{E}-04\end{array}$

$\begin{array}{lll}1.54619 \mathrm{E} & 00 & -9.24922 \mathrm{E}-04\end{array}$

$\begin{array}{lll}1.55806 \mathrm{E} & 00 & -9.26244 \mathrm{E}-04\end{array}$

$\begin{array}{lll}1.56998 \mathrm{E} & 00 & -9.27550 \mathrm{E}-04\end{array}$

$1.58195 \mathrm{E} \quad 00$

$1.59396 \mathrm{E} \quad 00$

$1.60602 \mathrm{E} \quad 00$

$\begin{array}{lll}1.61812 \mathrm{E} & 00\end{array}$

$1.63027 \mathrm{E} \quad 00$

$1.64247 \mathrm{E} \quad 00$

$1.65471 \mathrm{E} \quad 00$

$1.66700 \mathrm{E} \quad 00$

$1.67934 \mathrm{E} \quad 00$

$1.69173 \mathrm{E} \quad 00$

$1.70416 \mathrm{E} \quad 00$

$1.71663 \mathrm{E} \quad 00$

$1.72916 \mathrm{E} \quad 00$

$1.74173 \mathrm{E} \quad 00$

$1.75435 \mathrm{E} \quad 00$

$1.76701 \mathrm{E} \quad 00$

$1.77973 \mathrm{E} \quad 00$

$1.79249 \mathrm{E} \quad 00$

$1.80529 \mathrm{E} \quad 00$

$1.81814 \mathrm{E} \quad 00$

$1.83104 \mathrm{E} \quad 00$

$1.84399 \mathrm{E} \quad 00$

$1.85699 \mathrm{E} \quad 00$

$1.87003 \mathrm{E} \quad 00$

$1.88312 \mathrm{E} \quad 00$

$1.89625 \mathrm{E} \quad 00$

$1.90943 \mathrm{E} \quad 00$

$1.92266 \mathrm{E} \quad 00$

$1.93594 \mathrm{E} \quad 00$

$1.94927 \mathrm{E} \quad 00$

$1.96264 \mathrm{E} \quad 00$

$1.97606 \mathrm{E} \quad 00$

$1.98952 \mathrm{E} \quad 00$

$2.00304 \mathrm{E} \quad 00$

$2.01660 \mathrm{E} \quad 00$

$2.03021 \mathrm{E} \quad 00$

$2.04387 \mathrm{E} \quad 00$ $2.05757 \mathrm{E} \quad 00$

$2.07132 \mathrm{E} \quad 00$

$2.08512 \mathrm{E} 00$

$2.09897 \mathrm{E} \quad 00$

$2.11286 \mathrm{E} \quad 00$ $2.12680 \mathrm{E} \quad 00$ $2.14079 \mathrm{E} \quad 00$

$2.15483 \mathrm{E} \quad 00$ $2.16892 \mathrm{E} \quad 00$
$-1.33611 \mathrm{E} 00$ $-1.34727 \mathrm{E} 00$ $-1.35847 \mathrm{E} 00$ $-1.36972 \mathrm{E} 00$ $-1.38102 \mathrm{E} \quad 00$

$-1.39236 \mathrm{E} \quad 00$ $-1.40375 \mathrm{E} \quad 00$ $-1.41518 \mathrm{E} 00$ $-1.42667 \mathrm{E} \quad 00$ $-1.43819 \mathrm{E} 00$

$-1.44977 \mathrm{E} \quad 00$ $-1.46139 \mathrm{E} \quad 00$ $-1.47306 \mathrm{E} 00$ $-1.48477 \mathrm{E} \quad 00$ $-1.49653 \mathrm{E} 00$

$-1.50834 \mathrm{E} \quad 00$ $-1.52019 \mathrm{E} \quad 00$ $-1.53209 \mathrm{E} \quad 00$ $-1.54404 \mathrm{E} 00$ $-1.55603 \mathrm{E} 00$

$\begin{array}{ll}-1.56807 \mathrm{E} & 00\end{array}$ $-1.58016 \mathrm{E} \quad 00$ $-1.59230 \mathrm{E} \quad 00$ $-1.60448 \mathrm{E} \quad 00$ $-1.61670 \mathrm{E} 00$

$-] .62898 \mathrm{E} \quad 00$ $-1.64130 \mathrm{E} 00$ $-1.65367 \mathrm{E} 00$ $-1.66608 \mathrm{E} 00$ $-1.67855 \mathrm{E} 00$

$-1.69106 \mathrm{E} \quad 00$ $-1.70361 \mathrm{E} 00$ -1.71621 E 00 $-1.72887 \mathrm{E} 00$ $-1.74156 \mathrm{E} 00$

$-1.75431 \mathrm{E} \quad 00$ $-1.76710 \mathrm{E} 00$ $-1.77994 \mathrm{E} 00$ $-1.79283 \mathrm{E} \quad 00$ $-1.80576 \mathrm{E} \quad 00$

$-1.81874 \mathrm{E} \quad 00$ $-1.83177 \mathrm{E} \quad 00$ $-1.84484 \mathrm{E} 00$ $-1.85797 \mathrm{E} \quad 00$ $-1.87114 \mathrm{E} 00$

$-1.88436 \mathrm{E} 00$ $-1.89762 \mathrm{E} 00$ $-1.91093 \mathrm{E} 00$ $-1.92429 \mathrm{E} 00$ $-1.93770 \mathrm{E} 00$

$-1.95116 \mathrm{E} \quad 00$ $-1.96466 \mathrm{E} 00$ $-1.97821 \mathrm{E} 00$ $-1.99181 \mathrm{E} 00$ $-2.00546 \mathrm{E} 00$

$-2.01915 \mathrm{E} 00$ $-2.03289 \mathrm{E} 00$ $-2.04668 \mathrm{E} \quad 00$ $-2.06052 \mathrm{E} \quad 00$ $-2.07441 \mathrm{E} \quad 00$ $g_{-1}$

$\tan \delta_{-1}$

$2.88808 \mathrm{E} \quad 01$ $2.89781 \mathrm{E} 01$ $2.90755 \mathrm{E} \quad 01$

$2.91728 \mathrm{E} \quad 01$ $2.92703 \mathrm{E} \quad 01$

$2.93677 \mathrm{E} \quad 01$ $2.94652 \mathrm{E} 01$ $2.95626 \mathrm{E} \quad 01$ $2.96602 \mathrm{E} 01$ $2.97577 \mathrm{E} \quad 01$

$2.98553 \mathrm{E} \quad 01$ $2.99529 \mathrm{E} \quad 01$ $3.00505 \mathrm{E} 01$ $3.01482 \mathrm{E} \quad 01$ $3.02459 \mathrm{E} 01$

$3.03436 \mathrm{E} \quad 01$ $3.04413 \mathrm{E} \quad 01$ $3.05391 \mathrm{E} \quad 01$ $3.06369 \mathrm{E}$ ol $3.07347 \mathrm{E} \quad 01$

$3.08326 \mathrm{E} \quad 01$ $3.09304 \mathrm{E} \quad 01$ $3.10284 \mathrm{E} \quad 01$ $3.11263 \mathrm{E} \quad 01$ $3.12243 \mathrm{E} 01$

$3.13223 \mathrm{E} \quad 01$

$3.14203 \mathrm{E} \quad 01$

$3.15183 \mathrm{E} 01$

$3.16164 \mathrm{E} \quad 01$

$3.17145 \mathrm{E} 01$

$3.18127 \mathrm{E} \quad 01$

$3.19108 \mathrm{E} 01$

$3.20090 \mathrm{E} 01$

$3.21073 \mathrm{E} 01$

$3.22055 \mathrm{E} 01$

$3.23038 \mathrm{E} \quad 01$ $3.24021 \mathrm{E} 01$ $3.25005 \mathrm{E} \quad 01$ $3.25989 \mathrm{E} 01$ $3.26973 \mathrm{E} 01$

$3.27957 \mathrm{E} \quad 01$ $3.28942 \mathrm{E} \quad 01$ $3.29927 \mathrm{E} 01$ $3.30912 \mathrm{E} \quad 01$ $3.31897 \mathrm{E} \quad 01$

$3.32883 \mathrm{E} \quad 01$ $3.33869 \mathrm{E} 01$ $3.34856 \mathrm{E} \quad 01$ $3.35843 \mathrm{E} \quad 01$ $3.36830 \mathrm{E} 01$

$3.37817 \mathrm{E} \quad 01$ $3.38805 \mathrm{E} \quad 01$ $3.39793 \mathrm{E} 01$ $3.40781 \mathrm{E} 01$

$3.41770 \mathrm{E}$ ol

$3.42759 \mathrm{E}$ 01 $3.43748 \mathrm{E} 01$ $3.44738 \mathrm{E} \quad 01$

$3.45727 \mathrm{E} 01$ $3.46718 \mathrm{E} 01$
$3.85559 \mathrm{E} \quad 02$ $3.86169 \mathrm{E} \quad 02$ $3.86779 \mathrm{E} \quad 02$ $3.87390 \mathrm{E} \quad 02$ $3.87999 \mathrm{E} \quad 02$

$3.88608 \mathrm{E} \quad 02$ $3.89217 \mathrm{E} \quad 02$ $3.89826 \mathrm{E} \quad 02$ $3.90435 \mathrm{E} \quad 02$ $3.91043 \mathrm{E} \quad 02$

$3.91652 \mathrm{E} \quad 02$ $3.92261 \mathrm{E} \quad 02$ $3.92870 \mathrm{E} \quad 02$ $3.93478 \mathrm{E} \quad 02$ $3.94087 \mathrm{E} \quad 02$

$3.94695 \mathrm{E} \quad 02$ $3.95303 \mathrm{E} \quad 02$ $3.95911 \mathrm{E} \quad 02$ $3.96519 \mathrm{E} \quad 02$ $3.97128 \mathrm{E} \quad 02$

$3.97736 \mathrm{E} \quad 02$ $3.98345 \mathrm{E} \quad 02$ $3.98954 \mathrm{E} \quad 02$ $3.99562 \mathrm{E} \quad 02$ $4.00171 \mathrm{E} \quad 02$

$4.00779 \mathrm{E} \quad 02$ $4.01388 \mathrm{E} \quad 02$ $4.01997 \mathrm{E} \quad 02$ $4.02606 \mathrm{E} \quad 02$ $4.03216 \mathrm{E} \quad 02$

$4.03826 \mathrm{E} \quad 02$ $4.04437 \mathrm{E} \quad 02$ $4.05046 \mathrm{E} \quad 02$ $4.05657 \mathrm{E} \quad 02$ $4.06267 \mathrm{E} \quad 02$

$4.06878 \mathrm{E} \quad 02$ $4.07489 \mathrm{E} \quad 02$ $4.08100 \mathrm{E} \quad 02$ $4.08712 \mathrm{E} \quad 02$ $4.09325 \mathrm{E} \quad 02$

$4.09936 \mathrm{E} \quad 02$ $4.10549 \mathrm{E} \quad 02$ $4.11162 \mathrm{E} \quad 02$ $4.11776 \mathrm{E} \quad 02$ $4.12390 \mathrm{E} \quad 02$

4.13004E 02 $4.13620 \mathrm{E} \quad 02$ $4.14234 \mathrm{E} \quad 02$ $4.14849 \mathrm{E} \quad 02$ $4.15465 \mathrm{E} \quad 02$

4.16083E 02 $4.16699 \mathrm{E} \quad 02$ $4.17317 \mathrm{E} \quad 02$ $4.17935 \mathrm{E} \quad 02$ $4.18554 \mathrm{E} \quad 02$

4.19174E 02 $4.19793 \mathrm{E} \quad 02$ $4.20414 \mathrm{E} \quad 02$ $4.21034 \mathrm{E} \quad 02$ $4.21655 \mathrm{E} \quad 02$ 
ELECTRON RADIAL FUNCTIONS

$Z=7 \quad A=14 \quad \rho=1.1 A^{1 / 3} /$

Pusitrun

\section{$\bar{F}$}

30.10

30.20

30.30

30.40

30.50

30.60

30.70

30.80

30.90

31.00

31.10

31.20

31.30

31.40

31.50

31.60

31.70

31.80

31.90

32.00

32.10

32.20

32.30

32.40

32.50

32.60

32.70

32.80

32.90

33.00

33.10

33.20

33.30

33.40

33.50

33.60

33.70

33.80

33.90

34.00

34.10

34.20

34.30

34.40

34.50

34.60

34.70

34.80

34.90

35.00

35.10

35.20

35.30

35.40

35.50

35.60

35.70

35.80

35.90

36.00 $f_{2}$

$1.01315 \mathrm{E}-03$

$1.01989 \mathrm{E}-03$

$1.02665 \mathrm{E}-03$

$1.03344 \mathrm{E}-03$

$1.04024 \mathrm{E}-03$

$1.04707 \mathrm{E}-03$

$1.05392 \mathrm{E}-03$

$1.06079 \mathrm{E}-03$

$1.06769 \mathrm{E}-03$

$1.07461 \mathrm{E}-03$

$1.08155 \mathrm{E}-03$

$1.08851 \mathrm{E}-03$

$1.09550 \mathrm{E}-03$

$1.10250 \mathrm{E}-03$

$1.10953 \mathrm{E}-03$

$1.11658 \mathrm{E}-03$

$1.12366 \mathrm{E}-03$

$1.13075 \mathrm{E}-03$

$1.13787 \mathrm{E}-03$

$1.14501 \mathrm{E}-03$

$1.15218 \mathrm{E}-03$

$1.15936 \mathrm{E}-03$

$1.16657 \mathrm{E}-03$

$1.17380 \mathrm{E}-03$

$1.18105 \mathrm{E}-03$

$1.18833 \mathrm{E}-03$

$1.19563 \mathrm{E}-03$

$1.20295 \mathrm{E}-03$

$1.21029 \mathrm{E}-03$

$1.21765 \mathrm{E}-03$

$1.22504 \mathrm{E}-03$

$1.23245 \mathrm{E}-03$

$1.23988 \mathrm{E}-03$

$1.2+733 \mathrm{E}-03$

$1.25481 \mathrm{E}-03$

$1.26231 \mathrm{E}-03$

$1.26983 \mathrm{E}-03$

$1.27737 \mathrm{E}-03$

$1.28494 \mathrm{E}-03$

$1.29252 \mathrm{E}-03$

$1.30013 \mathrm{E}-03$

$1.30776 \mathrm{E}-03$

$1.31542 \mathrm{E}-03$

$1.32310 \mathrm{E}-03$

$1.33080 \mathrm{E}-03$

$1.33852 \mathrm{E}-03$

$1.34626 \mathrm{E}-03$

$1.35403 \mathrm{E}-03$

$1.36181 \mathrm{E}-03$

$1.36963 \mathrm{E}-03$

$1.37746 \mathrm{E}-03$

$1.38531 \mathrm{E}-03$

$1.39319 \mathrm{E}-03$

$1.40109 \mathrm{E}-03$

$1.40901 \mathrm{E}-03$

$1.41696 \mathrm{E}-03$

$1.42493 \mathrm{E}-03$

$1 .+3291 \mathrm{E}-03$

$1.44093 \mathrm{E}-03$

$1.44896 \mathrm{E}-03$
$1.88502 \mathrm{E} \quad 00$

$1.89765 \mathrm{E} \quad 00$

$1.91032 \mathrm{E} \quad 00$

$1.92303 \mathrm{E} \quad 00$

$1.93578 \mathrm{E} \quad 00$

$1.94858 \mathrm{E} \quad 00$

$1.96141 \mathrm{E} \quad 00$

$1.97429 \mathrm{E} \quad 00$

$1.98721 \mathrm{E} \quad 00$

$2.00017 \mathrm{E} \quad 00$

$2.01318 \mathrm{E} \quad 00$

$2.02622 \mathrm{E} \quad 00$

$2.03931 \mathrm{E} \quad 00$

$2.05244 \mathrm{E} \quad 00$

$2.06561 \mathrm{E} \quad 00$

$2.07882 \mathrm{E} \quad 00$

$2.09207 \mathrm{E} \quad 00$

$2.10537 \mathrm{E} \quad 00$

$2.11871 \mathrm{E} \quad 00$

$2.13208 \mathrm{E} \quad 00$

$2.14551 \mathrm{E} \quad 00$ $2.15897 \mathrm{E} \quad 00$

$2.17247 \mathrm{E} \quad 00$

$2.18602 \mathrm{E} \quad 00$

$2.19961 \mathrm{E} \quad 00$

$2.21323 \mathrm{E} \quad 00$

$2.22691 \mathrm{E} \quad 00$

$2.24062 \mathrm{E} \quad 00$

$2.25437 \mathrm{E} \quad 00$

$2.26817 \mathrm{E} \quad 00$

$2.28201 \mathrm{E} \quad 00$ $2.29589 \mathrm{E} \quad 00$

$2.30981 \mathrm{E} \quad 00$

$2.32377 \mathrm{~F} \quad 00$

$2.33777 \mathrm{E} \quad 00$

$2.35182 \mathrm{E} \quad 00$

$2.36591 \mathrm{E} \quad 00$

$2.38004 \mathrm{E} \quad 00$

$2.39421 \mathrm{E} \quad 00$

$2.40842 \mathrm{E} \quad 00$

$2.42267 \mathrm{E} \quad 00$

$2.43697 \mathrm{E} \quad 00$

$2.45131 \mathrm{E} \quad 00$

$2.46568 \mathrm{E} \quad 00$

$2.48010 \mathrm{E} \quad 00$

$2.49457 \mathrm{E} \quad 00$

$2.50907 \mathrm{E} \quad 00$

$2.52361 \mathrm{E} \quad 00$

$2.53820 \mathrm{E} \quad 00$

$2.55283 \mathrm{E} \quad 00$

$2.56750 \mathrm{E} \quad 00$

$2.58221 \mathrm{E} \quad 00$

$2.59696 \mathrm{E} \quad 00$

$2.61176 \mathrm{E} \quad 00$

$2.62659 \mathrm{E} \quad 00$

$2.64147 \mathrm{E} \quad 00$

$2.65639 \mathrm{E} \quad 00$

$2.67135 \mathrm{E} \quad 00$

$2.68635 \mathrm{E} \quad 00$

$2.70139 \mathrm{E} \quad 00$ $g_{2}$

$\tan \delta_{2}$

$f-z$

$5.85659 \mathrm{E}-02 \quad-2.79004 \mathrm{E} \quad 01 \quad-5.51900 \mathrm{E}-02$ $5.92191 \mathrm{E}-02$

$5.98771 \mathrm{E}-02$

$6.05400 \mathrm{E}-02$

$6.12076 \mathrm{E}-02$

$6.18801 \mathrm{E}-02$

$6.25575 \mathrm{E}-02$

$6.32397 \mathrm{E}-02$

$6.39268 \mathrm{E}-02$

$6.46188 \mathrm{E}-02$

$6.53158 \mathrm{E}-02$

$6.60177 \mathrm{E}-02$

$6.67245 \mathrm{E}-02$

$6.74364 \mathrm{E}-02$

$6.81532 \mathrm{E}-02$

$6.88750 \mathrm{E}-02$

$6.96019 \mathrm{E}-02$

$7.03338 \mathrm{E}-02$

$7.10708 \mathrm{E}-02$

$7.18128 \mathrm{E}-02$

$7.25600 \mathrm{E}-02$

$7.33123 \mathrm{E}-02$

$7.40697 \mathrm{E}-02$

$7.48322 \mathrm{E}-02$

$7.56000 \mathrm{E}-02$

$7.63728 \mathrm{E}-02$

$7.71510 \mathrm{E}-02$

$7.79343 \mathrm{E}-02$

$7.87228 \mathrm{E}-02$

$7.95166 \mathrm{E}-02$

$8.03157 \mathrm{E}-02$

$8.11201 \mathrm{E}-02$

8.19297 E-02

$8.27447 \mathrm{E}-02$

8.35651 E- 02

$8.43907 \mathrm{E}-02$

$8.52218 \mathrm{E}-02$

$8.60583 \mathrm{E}-02$

$8.69001 \mathrm{E}-02$

$8.77474 \mathrm{E}-02$

$8.86001 \mathrm{E}-02$

$8.94583 \mathrm{E}-02$

$9.03219 \mathrm{E}-02$

$9.11911 \mathrm{E}-02$

$9.20657 \mathrm{E}-02$

$9.29459 \mathrm{E}-02$

$9.38316 \mathrm{E}-02$

$9.47229 \mathrm{E}-02$

$9.56197 \mathrm{E}-02$

$9.65222 \mathrm{E}-02$

$9.74302 \mathrm{E}-02$

$9.83439 \mathrm{E}-02$

$9.92633 \mathrm{E}-02$

$1.00188 \mathrm{E}-01$

$1.01119 \mathrm{E}-01$

$1.02055 \mathrm{E}-01$

$1.02997 \mathrm{E}-01$

$1.03945 \mathrm{E}-01$

$1.04899 \mathrm{E}-01$

$1.05858 \mathrm{E}-01$

$-2.79016 \mathrm{E} 01$

$-2.79028 \mathrm{E} \quad 01$

$-2.79039 \mathrm{E} \quad 01$

$\begin{array}{lll}-2.790 .51 \mathrm{E} & 01\end{array}$

$-2.79063 \mathrm{E} \quad 01$

$-2.79074 \mathrm{E} \quad 01$

$-2.79086 \mathrm{E} \quad 01$

$-2.79097 \mathrm{E} \quad 01$

$-2.79108 \mathrm{E} \quad 01$

$\begin{array}{lll}-2.79119 \mathrm{E} & 01\end{array}$

$-2.79131 \mathrm{E} \quad 01$

$-2.79142 \mathrm{E} \quad 01$

$-2.79153 \mathrm{E} 01$

$-2.79163 \mathrm{E} \quad 01$

$-2.79174 \mathrm{E} \quad 01$

$-2.79185 \mathrm{E}$ 01

$-2.79196 \mathrm{E} \quad 01$

$-2.79206 \mathrm{E} \quad 01$

$-2.79217 \mathrm{E} \quad 01$

$-2.79227 \mathrm{E} \quad 01$

$-2.79238 \mathrm{E} \quad 01$

$-2.79248 \mathrm{E} \quad 01$

$-2.79259 \mathrm{E} \quad 01$

$-2.79269 \mathrm{E} \quad 01$

$-2.79279 \mathrm{E} \quad 01$

$-2.79289 \mathrm{E} \quad 01$

$-2.79299 \mathrm{E} \quad 01$

$-2.79309 \mathrm{E} \quad 01$

$-2.79319 \mathrm{E} 01$

$-2.79329 \mathrm{E} \quad 01$

$-2.79339 \mathrm{E} \quad 01$

$-2.79348 \mathrm{E} \quad 01$

$-2.79358 \mathrm{E} \quad 01$

-2.79368 E 01

$-2.79377 \mathrm{E} \quad 01$

$-2.79387 \mathrm{E} \quad 01$

$-2.79396 \mathrm{E} \quad 01$

$-2.79406 \mathrm{E} 0$

$-2.79415 \mathrm{E} \quad 01$

$-2.79424 \mathrm{E} \quad 01$

$-2.79+33 \mathrm{E} \quad 01$ 
ELECTRON RADIAL FUNCTIONS

Positron

$\begin{array}{cc}p & F_{0} \\ 36.10 & 8.54977 \mathrm{E}-01 \\ 36.20 & 8.54971 \mathrm{E}-01 \\ 36.30 & 8.54965 \mathrm{E}-01 \\ 36.40 & 8.54960 \mathrm{E}-01 \\ 36.50 & 8.54954 \mathrm{E}-01\end{array}$

36.60

36.70

36.80

36.90

37.00

37.10

37.20

37.30

37.40

37.50

37.60

37.70

37.80

37.90

38.00

38.10

38.20

38.30

38.40

38.50

38.60

38.70

38.80

38.90

39.00

39.10

39.20

39.30

39.40

39.50

39.60

39.70

39.80

39.90

40.00

40.10

40.20

40.30

40.40

40.50

40.60

40.70

40.80

40.90

41.00

41.10

41.20

41.30

41.40

41.50

41.60

41.70

41.80

41.90

42.00

$8.54948 \mathrm{E}-01$ $8.54942 \mathrm{E}-01$ $8.54936 \mathrm{E}-01$ $8.54931 \mathrm{E}-01$ $8.54925 \mathrm{E}-01$

8.54919E-01 $8.54913 \mathrm{E}-01$ $8.54907 \mathrm{E}-01$ $8.54901 \mathrm{E}-01$ $8.54896 \mathrm{E}-01$

$8.54890 \mathrm{E}-01$ $8.54884 \mathrm{E}-01$ $8.54879 \mathrm{E}-01$ $8.54873 \mathrm{E}-01$ $8.54867 \mathrm{E}-01$

$8.54862 \mathrm{E}-01$ $8.54856 \mathrm{E}-01$ $8.54851 \mathrm{E}-01$ $8.54845 \mathrm{E}-01$ 8.54840 E-01

$8.54834 \mathrm{E}-01$ $8.54828 \mathrm{E}-01$ $8.54823 \mathrm{E}-01$ $8.54817 \mathrm{E}-01$ 8.54812 E-01

$8.54806 \mathrm{E}-01$ $8.54801 \mathrm{E}-01$ $8.54795 \mathrm{E}-01$ $8.54790 \mathrm{E}-01$ $8.54785 \mathrm{E}-01$

$8.54779 \mathrm{E}-01$ $8.54774 \mathrm{E}-01$ $8.54768 \mathrm{E}-01$ $8.54763 \mathrm{E}-01$ $8.54758 \mathrm{E}-01$

$8.54752 \mathrm{E}-01$ $8.54747 \mathrm{E}-01$ $8.54742 \mathrm{E}-01$ $8.54736 \mathrm{E}-01$ 8.54731 E-01

$8.54726 \mathrm{E}-01$ $8.54720 \mathrm{E}-01$ $8.54715 \mathrm{E}-01$ $8.54710 \mathrm{E}-01$

$8.54700 \mathrm{E}-01$ $8.54694 \mathrm{E}-01$ $8.54689 \mathrm{E}-01$ $8.54684 \mathrm{E}-01$ $8.54679 \mathrm{E}-01$

$8.54674 \mathrm{E}-01$ $8.54668 \mathrm{E}-01$ $8.54663 \mathrm{E}-01$ $8.54658 \mathrm{E}-01$ 8.54653E-01

$Z=7$

$$
A=14
$$

$$
\rho=1.1 A^{1 / 3} F
$$

\section{$f_{1}$}

$3.37683 \mathrm{E} \quad 01$ $3.38669 \mathrm{E} 01$ $3.39656 \mathrm{E} \quad 01$ $3.40644 \mathrm{E} 01$ $3.41631 \mathrm{E} 01$ $8.54705 \mathrm{E}-01$
$3.42619 \mathrm{E} \quad 01$ $3.43608 \mathrm{E} \quad 01$ $3.44596 \mathrm{E} \quad 01$ $3.46574 \mathrm{E} \quad 01$

$3.47564 \mathrm{E} \quad 01$

$3.48554 \mathrm{E} \quad 01$

$3.49544 \mathrm{E} \quad 01$

$3.50534 \mathrm{E} \quad 01$

$3.51525 \mathrm{E} 01$

$3.52516 \mathrm{E} \quad 01$ $3.53507 \mathrm{E} \quad 01$ $3.54499 \mathrm{E} \quad 01$ $3.55491 \mathrm{E} \quad 01$ $3.56484 \mathrm{E} \quad 01$

$3.57476 \mathrm{E} \quad 01$ $3.58469 \mathrm{E} \quad 01$ $3.59463 \mathrm{E} 01$ $3.60456 \mathrm{E} \quad 01$ $3.61450 \mathrm{E} \quad 01$

$3.62445 \mathrm{E} \quad 01$ $3.64434 \mathrm{E} \quad 01$ $3.65430 \mathrm{E} \quad 01$

$3.66425 \mathrm{E} 01$

$3.67421 \mathrm{E} \quad 01$ $3.68418 \mathrm{E} \quad 01$ $3.70411 \mathrm{E} \quad 01$ $3.71409 \mathrm{E} \quad 01$

$3.72406 \mathrm{E} \quad 01$ $3.73404 \mathrm{E} \quad 01$ $3.74403 \mathrm{E} \quad 01$ $3.75401 \mathrm{E} \quad 01$ $3.76400 \mathrm{E} 01$

$3.77399 \mathrm{E} \quad 01$ $3.78399 \mathrm{E} 01$ $3.79399 \mathrm{E} 01$ $3.80400 \mathrm{E} \quad 01$ $3.81400 \mathrm{E} \quad 01$

$3.82401 \mathrm{E} \quad 01$ $3.83403 \mathrm{E} \quad 01$ $3.84404 \mathrm{E} 01$ $3.85407 \mathrm{E} \quad 01$

$3.87412 \mathrm{E} \quad 01$ $3.88415 \mathrm{E} \quad 01$ $3.89418 \mathrm{E} \quad 01$ $3.90422 \mathrm{E} \quad 01$ $3.91427 \mathrm{E} \quad 01$

$3.92431 \mathrm{E} \quad 01$ $3.93436 \mathrm{E} \quad 01$ $3.94441 \mathrm{E} \quad 01$ $3.95447 \mathrm{E}$ 01 $3.96453 \mathrm{E} \quad 01$ $3.45585 \mathrm{E} 01$ $3.63439 \mathrm{E} \quad 01$ $3.69414 \mathrm{E} \quad 01$ $3.86409 \mathrm{E} \quad 01$ $g_{1}$

$\tan \delta_{1}$

$f_{-1}$

$2.18305 \mathrm{E} \quad 00 \quad-9.67972 \mathrm{E}-04$ $2.19723 \mathrm{E} \quad 00 \quad-9.68442 \mathrm{E}-04$

$2.21146 \mathrm{E} \quad 00 \quad-9.68885 \mathrm{E}-04$

$2.22574 \mathrm{E} \quad 00 \quad-9.69324 \mathrm{E}-04$

$2.24006 \mathrm{E} \quad 00 \quad-9.69742 \mathrm{E}-04$

$2.25444 \mathrm{E} \quad 00$

$2.26886 \mathrm{E} \quad 00$

$2.28333 \mathrm{E} \quad 00$

$2.29784 \mathrm{E} \quad 00$

$2.31241 \mathrm{E} \quad 00$

$2.32702 \mathrm{E} \quad 00$

$2.34168 \mathrm{E} \quad 00$

$2.35639 \mathrm{E} \quad 00$

$2.37115 \mathrm{E} \quad 00$

$2.38596 \mathrm{E} \quad 00$

$2.40081 \mathrm{E} \quad 00$

$2.41571 \mathrm{E} \quad 00$

$2.43067 \mathrm{E} \quad 00$

$2.44566 \mathrm{E} \quad 00$

$2.46071 \mathrm{E} \quad 00$

$2.47581 \mathrm{E} \quad 00$

$2.49095 \mathrm{E} \quad 00$

$2.50615 \mathrm{E} \quad 00$

$2.52139 \mathrm{E} \quad 00$

$2.53668 \mathrm{E} \quad 00$

$2.55202 \mathrm{E} \quad 00$

$2.56741 \mathrm{E} \quad 00$

$2.58284 \mathrm{E} \quad 00$

$2.59833 \mathrm{E} \quad 00$

$2.61386 \mathrm{E} \quad 00$

$2.62945 \mathrm{E} \quad 00$

$2.64508 \mathrm{E} \quad 00$

$2.66076 \mathrm{E} \quad 00$

$2.67649 \mathrm{E} \quad 00$

$2.69227 \mathrm{E} \quad 00$

$2.70810 \mathrm{E} \quad 00$

$2.72397 \mathrm{E} \quad 00$

$2.73990 \mathrm{E} \quad 00$

$\begin{array}{lll}2.75587 \mathrm{E} & 00\end{array}$

$2.77190 \mathrm{E} \quad 00$

$2.78797 \mathrm{E} \quad 00$ $2.80409 \mathrm{E} \quad 00$

$2.82026 \mathrm{E} \quad 00$

$2.83648 \mathrm{E} \quad 00$

$2.85276 \mathrm{E} \quad 00$

$2.86907 \mathrm{E} \quad 00$ $2.88544 \mathrm{E} \quad 00$

$2.90186 \mathrm{E} \quad 00$

$2.91833 \mathrm{E} \quad 00$

$2.93485 \mathrm{E} \quad 00$

$2.95141 \mathrm{E} \quad 00$

$2.96803 \mathrm{E}, 00$

$2.98470 \mathrm{E} \quad 00$

$3.00141 \mathrm{E} \quad 00$

$3.01818 \mathrm{E} \quad 00$

$3.03499 \mathrm{E} \quad 00$

$3.05186 \mathrm{E} \quad 00$

$3.06877 \mathrm{E} \quad 00$

$3.08574 \mathrm{E} \quad 00$

$3.10275 \mathrm{E} \quad 00$

$-9.70150 \mathrm{E}-04$

$-9.70529 \mathrm{E}-04$

$-9.70911 \mathrm{E}-04$

$-9.71265 \mathrm{E}-04$

$-9.71622 \mathrm{E}-04$

$-9.71950 \mathrm{E}-04$

$-9.72266 \mathrm{E}-04$

$-9.72572 \mathrm{E}-04$

$-9.72864 \mathrm{E}-04$

$-9.73140 \mathrm{E}-04$

$-9.73406 \mathrm{E}-04$

$-9.73654 \mathrm{E}-04$

$-9.73890 \mathrm{E}-04$

$-9.74112 \mathrm{E}-04$

$-9.74317 \mathrm{E}-04$

$-9.74517 \mathrm{E}-04$

$-9.74694 \mathrm{E}-04$

$-9.74864 \mathrm{E}-04$

$-9.75020 \mathrm{E}-04$

$-9.75154 \mathrm{E}-04$

$-9.75294 \mathrm{E}-04$

$-9.75404 \mathrm{E}-04$

$-9.75516 \mathrm{E}-04$

$-9.75600 \mathrm{E}-04$

$-9.75678 \mathrm{E}-04$

$-9.75744 \mathrm{E}-04$

$-9.75799 \mathrm{E}-04$

$-9.75834 \mathrm{E}-04$

$-9.75858 \mathrm{E}-04$

$-9.75874 \mathrm{E}-04$

$-9.75876 \mathrm{E}-04$

$-9.75869 \mathrm{E}-04$

$-9.75850 \mathrm{E}-04$

$-9.75803 \mathrm{E}-04$

$-9.75762 \mathrm{E}-04$

$-9.75704 \mathrm{E}-04$

$-9.75630 \mathrm{E}-04$

$-9.75545 \mathrm{E}-04$

$-9.75454 \mathrm{E}-04$

$-9.75347 \mathrm{E}-04$

$-9.75231 \mathrm{E}-04$ $-9.75105 \mathrm{E}-04$

$-9.74958 \mathrm{E}-04$

$-9.74806 \mathrm{E}-04$

$-9.74641 \mathrm{E}-04$

$-9.74460 \mathrm{E}-04$ 
$\bar{F}$

$1.45702 \mathrm{E}-03$

$1.46509 \mathrm{E}-03$

$1.47320 \mathrm{E}-03$

$1.48132 \mathrm{E}-03$

$1.48946 \mathrm{E}-03$

36.50

36.60

36.70

36.80

36.90

37.00

$1.49763 \mathrm{E}-03$

$1.50582 \mathrm{E}-03$

$1.51403 \mathrm{E}-03$

$1.52227 \mathrm{E}-03$

$1.53053 \mathrm{E}-03$

37.10

37.20

37.30

37.40

37.50

37.60

37.70

37.80

37.90

38.00

38.10

38.20

38.30

38.40

38.50

38.60

38.70

38.80

38.90

39.00

39.10

39.20

39.30

39.40

39.50

39.60

39.70

39.80

39.90

40.00

40.10

40.20

40.30

40.40

40.50

40.60

40.70

40.80

40.90

41.00

41.10

41.20

41.30

41.40

41.50

41.60

41.70

41.80

41.90

42.00

$1.53881 \mathrm{E}-03$ $1.54711 \mathrm{E}-03$ $1.55543 \mathrm{E}-03$ $1.56378 \mathrm{E}-03$ $1.57215 \mathrm{E}-03$

$1.58054 \mathrm{E}-03$ $1.58895 \mathrm{E}-03$ $1.59739 \mathrm{E}-03$ $1.60584 \mathrm{E}-03$ $1.61432 \mathrm{E}-03$

$1.62283 \mathrm{E}-03$ $1.63135 \mathrm{E}-03$ $1.63990 \mathrm{E}-03$ $1.64847 \mathrm{E}-03$ $1.65706 \mathrm{E}-03$

$1.66567 \mathrm{E}-03$ $1.67431 \mathrm{E}-03$ $1.68297 \mathrm{E}-03$ $1.69165 \mathrm{E}-03$ $1.70035 \mathrm{E}-03$

$1.70908 \mathrm{E}-03$ $1.71783 \mathrm{E}-03$ $1.72660 \mathrm{E}-03$ $1.73539 \mathrm{E}-03$ $1.74421 \mathrm{E}-03$

$1.75304 \mathrm{E}-03$ $1.76190 \mathrm{E}-03$ $1.77078 \mathrm{E}-03$ $1.77969 \mathrm{E}-03$ $1.78862 \mathrm{E}-03$

$1.79756 \mathrm{E}-03$ $1.80653 \mathrm{E}-03$ $1.81553 \mathrm{E}-03$ $1.82454 \mathrm{E}-03$ $1.83358 \mathrm{E}-03$

$1.84264 \mathrm{E}-03$ $1.85173 \mathrm{E}-03$ $1.86083 \mathrm{E}-03$ $1.86996 \mathrm{E}-03$ $1.87911 \mathrm{E}-03$

$1.88828 \mathrm{E}-03$ $1.89747 \mathrm{E}-03$ $1.90669 \mathrm{E}-03$ $1.91593 \mathrm{E}-03$ $1.92519 \mathrm{E}-03$

$1.93447 \mathrm{E}-03$ $1.94378 \mathrm{E}-03$ $1.95311 \mathrm{E}-03$ $1.96246 \mathrm{E}-03$ $1.97183 \mathrm{E}-03$ $f_{2}$ $3.42118 \mathrm{E} \quad 00$ $3.47214 \mathrm{E} \quad 00$
$2.71648 \mathrm{E} \quad 00$ $2.73161 \mathrm{E} \quad 00$ $2.74677 \mathrm{E} \quad 00$ $2.76198 \mathrm{E} \quad 00$ $2.77723 \mathrm{E} \quad 00$

$2.79253 \mathrm{E} \quad 00$ $2.80786 \mathrm{E} \quad 00$ $2.82323 \mathrm{E} \quad 00$ $2.83865 \mathrm{E} \quad 00$ $2.85411 \mathrm{E} \quad 00$

$g_{2}$

$1.06823 \mathrm{E}-01$

$1.07794 \mathrm{E}-01$

$1.08771 \mathrm{E}-01$

$1.09754 \mathrm{E}-01$

1.10742 E-01

$1.11736 \mathrm{E}-01$

$1.12736 \mathrm{E}-01$

$1.13743 \mathrm{E}-01$

$1.14755 \mathrm{E}-01$

$1.15772 \mathrm{E}-01$

$2.86961 \mathrm{E} \quad 00$ $2.88515 \mathrm{E} \quad 00$ $2.90073 \mathrm{E} \quad 00$ $2.91635 \mathrm{E} \quad 00$ $2.93202 \mathrm{E} \quad 00$

$2.94773 \mathrm{E} \quad 00$ $2.96347 \mathrm{E} \quad 00$ $2.97926 \mathrm{E} \quad 00$ $2.99509 \mathrm{E} \quad 00$ $3.01096 \mathrm{E} \quad 00$

$3.02688 \mathrm{E} \quad 00$ $3.04283 \mathrm{E} \quad 00$ $3.05883 \mathrm{E} \quad 00$

$3.07486 \mathrm{E} \quad 00$

$3.09094 \mathrm{E} \quad 00$

$3.10706 \mathrm{E} \quad 00$ $3.12322 \mathrm{E} \quad 00$ $3.13943 \mathrm{E} \quad 00$ $3.15567 \mathrm{E} \quad 00$

$3.17195 \mathrm{E} \quad 00$

$3.18828 \mathrm{E} \quad 00$ $3.20465 \mathrm{E} \quad 00$ $3.22106 \mathrm{E} \quad 00$ $3.23751 \mathrm{E} \quad 00$

$3.25400 \mathrm{E} \quad 00$

$3.27053 \mathrm{E} \quad 00$

$3.28711 \mathrm{E} \quad 00$

$3.30372 \mathrm{E} \quad 00$

$3.32038 \mathrm{E} \quad 00$

$3.33708 \mathrm{E} \quad 00$

$3.35382 \mathrm{E} \quad 00$ $3.37060 \mathrm{E} \quad 00$ $3.38742 \mathrm{E} \quad 00$ $3.40428 \mathrm{E} \quad 00$

$3.43813 \mathrm{E} \quad 00$ $3.45512 \mathrm{E} \quad 00$ $3.48921 \mathrm{E} \quad 00$ $3.50632 \mathrm{E} \quad 00$

$3.52347 \mathrm{E} \quad 00$ $3.54066 \mathrm{E} \quad 00$ $3.55790 \mathrm{E} \quad 00$ $3.57517 \mathrm{E} \quad 00$ $3.59249 \mathrm{E} \quad 00$

$3.60984 \mathrm{E} \quad 00$ $3.62724 \mathrm{E} \quad 00$ $3.64468 \mathrm{E} \quad 00$ $3.66216 \mathrm{E} 00$ $3.67968 \mathrm{E} \quad 00$

$1.16796 \mathrm{E}-01$

$1.17826 \mathrm{E}-01$

$1.18862 \mathrm{E}-01$

$1.19904 \mathrm{E}-01$

1.20952 E-01

$1.22006 \mathrm{E}-01$

$1.23066 \mathrm{E}-01$

$1.24132 \mathrm{E}-01$

$1.25204 \mathrm{E}-01$

$1.26282 \mathrm{E}-01$

$1.27366 \mathrm{E}-01$

$1.28457 \mathrm{E}-01$

$1.2955+\mathrm{E}-01$

$1.30656 \mathrm{E}-01$

$1.31765 \mathrm{E}-01$

$1.32881 \mathrm{E}-01$

$1.34002 \mathrm{E}-01$

$1.35130 \mathrm{E}-01$

$1.36264 \mathrm{E}-01$

$1.37404 \mathrm{E}-01$

$1.38551 \mathrm{E}-01$

$1.39703 \mathrm{E}-01$

$1.40862 \mathrm{E}-01$

$1.42028 \mathrm{E}-01$

$1.43200 \mathrm{E}-01$

$1.44378 \mathrm{E}-01$

$1.45563 \mathrm{E}-01$

$1.47951 \mathrm{E}-01$

$1.49155 \mathrm{E}-01$

$1.50366 \mathrm{E}-01$

$1.51583 \mathrm{E}-01$

$1.52806 \mathrm{E}-01$

$1.54036 \mathrm{E}-01$

$1.55272 \mathrm{E}-01$

$1.56515 \mathrm{E}-01$

$1.57765 \mathrm{E}-01$

$1.59021 \mathrm{E}-01$

$1.60284 \mathrm{E}-01$

$1.61553 \mathrm{E}-01$

$1.62829 \mathrm{E}-01$

$1.64111 \mathrm{E}-01$

$1.65401 \mathrm{E}-01$

$1.66697 \mathrm{E}-01$

$1.67999 \mathrm{E}-01$

$1.69309 \mathrm{E}-01$

$1.70625 \mathrm{E}-01$

$1.71948 \mathrm{E}-01$

$1.73277 \mathrm{E}-01$

$1.74614 \mathrm{E}-01$ $\tan \delta_{2}$

$-2.79598 \mathrm{E} 01$

$-2.79606 \mathrm{E} 01$

$-2.79615 \mathrm{E} 01$

$\begin{array}{lll}-2.79623 \mathrm{E} & 01\end{array}$

$-2.79631 \mathrm{E} 01$

$-2.79639 \mathrm{E} \quad 01$

$-2.79647 \mathrm{E} 01$

$\begin{array}{lll}-2.79655 \mathrm{E} & 01\end{array}$

$-2.79662 \mathrm{E} \quad 01$

$-2.79670 \mathrm{E} 01$

$-2.79678 \mathrm{E} \quad 01$

$-2.79686 \mathrm{E} \quad 01$

$-2.79693 \mathrm{E} 01$

$-2.79701 \mathrm{E} \quad 01$

$-2.79709 \mathrm{E} \quad 01$

$-2.79716 \mathrm{E} \quad 01$

$-2.79724 \mathrm{E} \quad 01$

$-2.79731 \mathrm{E} \quad 01$

$-2.79739 \mathrm{E} 01$

$-2.79746 \mathrm{E} \quad 01$

$-2.79754 \mathrm{E} \quad 01$

$-2.79761 \mathrm{E} \quad 01$

$-2.79768 \mathrm{E} \quad 01$

-2.79775 E 01

-2.79783 E 01

$-2.79790 \mathrm{E} \quad 01$

$-2.79797 \mathrm{E} \quad 01$

$-2.7980+\mathrm{E} \quad$ 0l

$-2.79811 \mathrm{E} 01$

$-2.79818 \mathrm{E} \quad 01$

$1.4675+\mathrm{E}-01$

$\begin{array}{lll}-2.79825 \mathrm{E} & 01\end{array}$

-2.79832 E 01

$-2.79839 \mathrm{E} \quad 01$

$-2.79846 \mathrm{E} 01$

$-2.79853 \mathrm{E} \quad 01$

$-2.79859 \mathrm{E} \quad 01$

$-2.79866 \mathrm{E} \quad 01$

$-2.79873 \mathrm{E} \quad 01$

$-2.79879 \mathrm{E} \quad 01$

$-2.79886 \mathrm{E} 01$

$-2.79893 \mathrm{E} \quad 01$ $-2.79899 \mathrm{E} \quad 01$

$-2.79906 \mathrm{E} \quad 01$

$-2.79912 \mathrm{E} \quad 01$

$-2.79919 \mathrm{E} 01$

$-2.79925 \mathrm{E} \quad 01$

$-2.79932 \mathrm{E} \quad 01$ 
All of the equations and text of this publication were composed on the keyboard of a photocomposition system, eliminating all hand insertion of equation characters. The 392 pages of tables were programmed for the computer and run off on magnetic tape. The magnetic tape was used as input to the photocomposition machine, which produced paper positives to be used as camera copy for offset printing. 



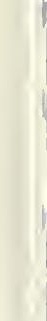




